\title{
Advanced Instrumentation and Control Methods for Small and Medium Reactors with IRIS Demonstration
}

\author{
Final Report \\ Volume 1
}

\section{Executive Summary}

Report No. DE-FG07-07ID14895/UTNE/2011-3

NERI-C Grant Number: DE-FG07-07ID14895

\author{
J. Wesley Hines \\ (Principal Investigator) \\ Belle R. Upadhyaya \\ J. Michael Doster \\ Robert M. Edwards \\ Kenneth D. Lewis \\ (Co-Principal Investigators) \\ Paul Turinsky \\ Jamie Coble
}

The University of Tennessee Nuclear Engineering Department 212 Pasqua Engineering Building

Knoxville, TN 37996-2300

E-mail: jhines2@utk.edu

May 2011

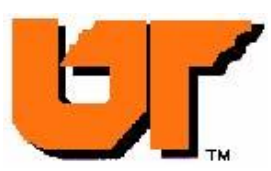




\section{ACKNOWLEDGMENTS}

This research, development, and demonstration (RD\&D) project was performed under a Department of Energy NERI-C grant with the University of Tennessee as the lead University. The collaborating institutions include North Carolina State University, Pennsylvania State University, South Carolina State University, and the Westinghouse Science \& Technology Center as a no-cost participant. We want to acknowledge the support provided by DOE Idaho Operations, and Dr. Dan Ingersoll and Dr. David Holcomb of the Oak Ridge National Laboratory throughout the project period. The technical discussion and the assistance provided by Westinghouse Science \& Technology Center, Pittsburgh, is greatly appreciated.

\section{DISCLAIMER}

Any opinions, findings, and conclusions or recommendations expressed in this report are those of the authors, and do not necessarily reflect the views of the U.S. Department of Energy or Westinghouse Electric Company, LLC. 


\section{TABLE OF CONTENTS}

1 INTRODUCTION AND OVERVIEW

2 PROJECT OBJECTIVES, TASKS, AND SIGNIFICANT RESULTS

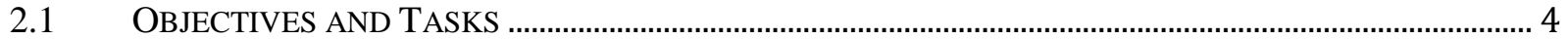

2.2 SUMMARY OF SIGNIFICANT CONTRIBUTIONS OF THE PROJECT R\&D ............................................... 6

2.3 PUBLICATIONS RELATED TO THE PROJECT ……........................................................................................... 8

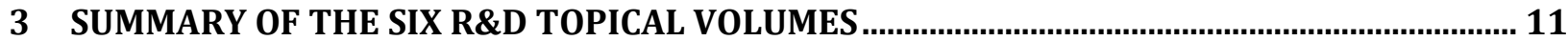

3.1 IRIS SIMULATOR DEVELOPMENT, SENSOR DEPLOYMENT, AND SENSITIVITY ANALYSIS...........11

3.2 DEVELOPMENT OF AUTONOMOUS CONTROL ARCHITECTURE AND MODEL-PREDICTIVE AND

FAULT-TOLERANT CONTROLLERS WITH APPLICATION TO IRIS .................................................................13

3.3 DEVELOPMENT OF MULTI-MODULAR INTEGRAL PRESSURIZED W ATER REACTOR CONTROL

AND OPERATIONAL RECONFIGURATION FOR A FLOW CONTROL LOOP........................................................15

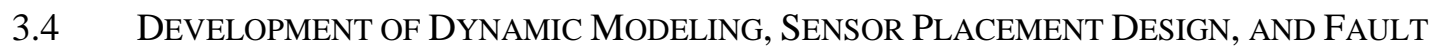

DIAGNOSIS OF NUCLEAR DESALINATION SYSTEMS ..................................................................................18

3.5 ON-LINE MONITORING AND DIAGNOSTICS USING NON-PARAMETRIC METHODS........................19

3.6 DEVELOPMENT OF PROCESS AND EQUIPMENT PROGNOSTICS METHODS .....................................21

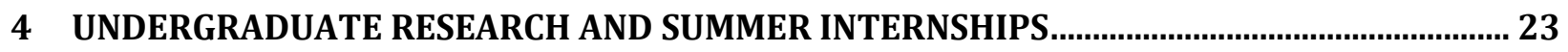

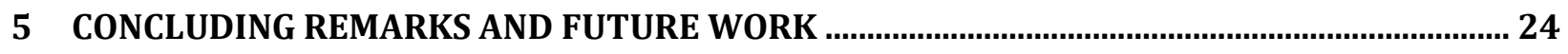

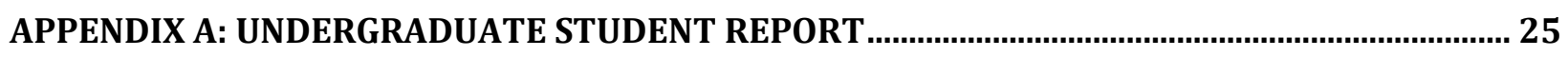

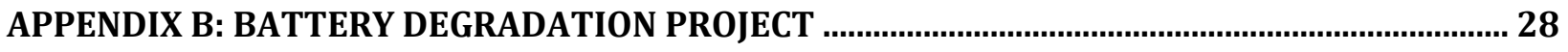

APPENDIX C: UNDERGRADUATE STUDENT RESEARCH REPORT.................................................... 34 


\section{INTRODUCTION AND OVERVIEW}

Development and deployment of small-scale nuclear power reactors and their maintenance, monitoring, and control are part of the mission under the Small Modular Reactor (SMR) program. The objectives of this NERI-consortium research project are to investigate, develop, and validate advanced methods for sensing, controlling, monitoring, diagnosis, and prognosis of these reactors, and to demonstrate the methods with application to one of the proposed integral pressurized water reactors (IPWR). For this project, the IPWR design by Westinghouse, the International Reactor Secure and Innovative (IRIS), has been used to demonstrate the techniques developed under this project. The research focuses on three topical areas with the following objectives.

Objective 1: Develop and apply simulation capabilities and sensitivity/uncertainty analysis methods to address sensor deployment analysis and small grid stability issues.

Objective 2: Develop and test an autonomous and fault-tolerant control architecture and apply to the IRIS system and an experimental flow control loop, with extensions to multiple reactor modules, nuclear desalination, and optimal sensor placement strategy.

Objective 3: Develop and test an integrated monitoring, diagnosis, and prognosis system for SMRs using the IRIS as a test platform, and integrate process and equipment monitoring (PEM) and process and equipment prognostics (PEP) toolboxes.

The research tasks are focused on meeting the unique needs of reactors that may be deployed to remote locations or to developing countries with limited support infrastructure. These applications will require smaller, robust reactor designs with advanced technologies for sensors, instrumentation, and control. An excellent overview of SMRs is described in an article by Ingersoll (2009). The article refers to these as deliberately small reactors. Most of these have modular characteristics, with multiple units deployed at the same plant site. Additionally, the topics focus on meeting two of the eight needs outlined in the recently published "Technology Roadmap on Instrumentation, Control, and Human-Machine Interface (ICHMI) to Support DOE Advanced Nuclear Energy Programs" which was created "to provide a systematic path forward for the integration of new ICHMI technologies in both near-term and future nuclear power plants and the reinvigoration of the U.S. nuclear ICHMI community and capabilities."

The research consortium is led by The University of Tennessee (UT) and is focused on three interrelated topics: 
Topic 1 (simulator development and measurement sensitivity analysis) is led by Dr. Mike Doster with Dr. Paul Turinsky of North Carolina State University (NCSU).

Topic 2 (multivariate autonomous control of modular reactors) is led by Dr. Belle Upadhyaya of the University of Tennessee (UT) and Dr. Robert Edwards of Penn State University (PSU).

Topic 3 (monitoring, diagnostics, and prognostics system development) is led by Dr. Wes Hines of UT.

Additionally, South Carolina State University (SCSU, Dr. Ken Lewis) participated in this research through summer interns, visiting faculty, and on-campus research projects identified throughout the grant period. Lastly, Westinghouse Science and Technology Center (Dr. Mario Carelli) was a no-cost collaborator and provided design information related to the IRIS demonstration platform and defining needs that may be common to other SMR designs. The results of this research are reported in a six-volume Final Report (including the Executive Summary, Volume 1). Volumes 2 through 6 of the report describe in detail the research and development under the topical areas. This volume serves to introduce the overall NERI-C project and to summarize the key results. Section 2 provides a summary of the significant contributions of this project. A list of all the publications under this project is also given in Section 2. Section 3 provides a brief summary of each of the five volumes (2-6) of the report. The contributions of SCSU are described in Section 4, including a summary of undergraduate research experience. The project management organizational chart is provided as Figure 1.

Appendices A, B, and C contain the reports on the summer research performed at the University of Tennessee by undergraduate students from South Carolina State University. 


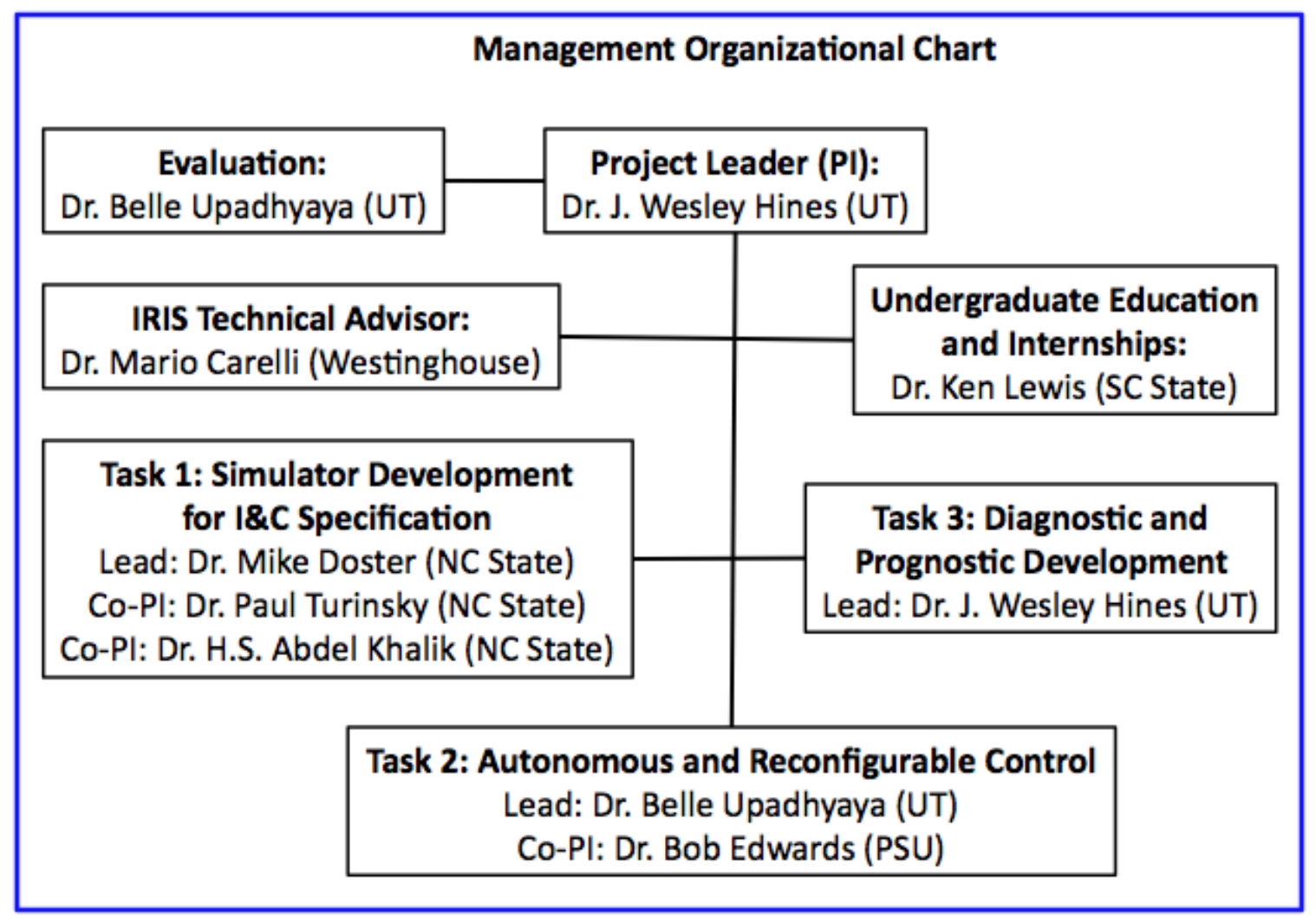

Figure 1: Project Management Organizational Chart 


\section{PROJECT OBJECTIVES, TASKS, AND SIGNIFICANT RESULTS}

\subsection{Objectives and Tasks}

This section summarizes the three main objectives and associated tasks which guided this work. Then, a summary of the significant contributions of the research is given. This is followed by a list of the related publications, including conference publications, journal articles, and theses in completion of degrees.

This research is divided into three separate objectives related to the simulation, control, and monitoring of SMRs. The three objectives and their associated tasks are listed here.

Objective 1: Develop and apply simulation capabilities and sensitivity/uncertainty analysis methodologies to address sensor deployment analysis.

Task 1.1: Define physical model input whose uncertainties will be treated

Task 1.2: Complete simulator model development to address IRIS specific components

Task 1.3: Complete sensitivity study on preliminary system simulation model to determine responses linearity to input data

Task 1.4: Implement uncertainty analysis for system model data inputs using linear or nonlinear approach as appropriate on some input data

Task 1.5: Develop optimum sensor deployment capability for Function (1)

Task 1.6: Develop optimum sensor deployment capability for Function (3)

Task 1.7: Develop optimum sensor deployment capability for Function (2)

Objective 2: Develop and test an autonomous/hierarchical control architecture, with applications to the IRIS design and an experimental flow control loop. Extensions to multi-modular reactors, nuclear desalination and optimal sensor placement.

Task 2.1: Develop and test a nodal model of the IRIS system using the MATLAB-SIMULINK platform, for adaptation to control design.

Task 2.2: Develop a multivariate feedback and feed-forward controller design using linear control algorithms.

Task 2.3: Develop and test a model-predictive controller and a resilient controller for the IRIS system.

Task 2.4: Enhance multivariate controller under model uncertainty.

Task 2.5: Develop a reconfigurable controller design using monitoring and diagnostics information from Research Topic 3 and apply to the experimental flow control loop.

Task 2.6: Demonstrate the digital controller implementation and the fault-tolerant controller in a laboratory multivariate flow control loop.

Task 2.7: Develop an optimal sensor placement strategy for fault detection with IRIS applications.

Task 2.8: Develop a model of a nuclear desalination system and design optimal sensor placement 
to minimize the fault unobservability.

Task 2.9: Develop a multi-modular plant control strategy for the IRIS reactor modules.

Task 2.10: Integrate control and on-line fault monitoring as an autonomous control platform.

Objective 3: Develop and test an integrated monitoring, diagnostic, and prognostic system for SMRs using the IRIS design as a test platform.

Task 3.1: Develop IRIS system hybrid models from Topic 1 models, simulated, tested, and optimized using MATLAB and SIMULINK.

Task 3.2: Develop an automated method for hybrid model adaptation.

Task 3.3: Integrate the adaptive system into a model based FDI system for real time monitoring.

Task 3.4: Develop degradation models for example key systems and components.

Task 3.5: Develop and test the case-based fault identification module.

Task 3.6: Apply the modeling and adaptation methods to a heat exchanger test system and validated in real time.

Task 3.7: Develop a hybrid prognostic framework that combines Type I, II, and III prognostic models.

Task 3.8: Integrate the degradation models from Task 3.4 into the simulation developed in Topic 1 and generate failure data.

Task 3.9: Validate the prognostic algorithm development methodologies using simulated data.

Task 3.10: Integrate monitoring, detection, identification, and prognostic algorithms.

Task 3.11: Test the system using simulated and laboratory data.

Task 3.12: Develop a MATLAB-based system development toolbox and manual.

The research activities performed in completion of these objectives and tasks are divided into six key focus areas. Each of these focus areas is discussed in a separate volume of this final report as summarized below:

- Volume 2: Development of high-fidelity IRIS simulation models, data generation, and sensitivity analysis.

- Volume 3: Development of autonomous control architecture and model-predictive and fault-tolerant controllers with application to IRIS.

- Volume 4: Development of multi-modular integral pressurized water reactor control and operational reconfiguration for a flow control loop.

- Volume 5: Development of dynamic modeling, sensor placement design, and fault diagnosis of nuclear desalination systems.

- Volume 6: Development of on-line monitoring and diagnostics methods with applications to the IRIS system and an experimental flow control loop.

- Volume 7: Development of process and equipment prognostics methods with applications to the IRIS plant components. 


\subsection{Summary of Significant Contributions of the Project R\&D}

All the technical tasks and their reporting have been completed. The NERI-C project has resulted in several original technical contributions. It is envisioned that the results of this project would be implemented in future reactor design, development, and operations. The following is a list of the significant contributions of this 3-year consortium project.

- Reviews of IRIS simulator model, instrumentation and controls, and IRIS preliminary safety report performed to identify input and correlations whose uncertainties would be considered. System attributes such as reactor power, critical heat flux ratio (CHFR), etc. were selected.

- An IRIS specific simulator has been developed capable of simulating a wide range of operational and off normal transients. Detailed systems models allow for simulation of sensor response, degradation, and failure. Versions of the simulator have been distributed to project collaborators. The modeling also includes IRIS reactor to grid model to study the small grid stability issues introduced by sudden loss of generation capacity and reactor islanding.

- Sensitivity analysis at steady state determined that sensor readings would not be useful in data assimilation for thermal-hydraulic input data. Analysis techniques were extended to transient conditions.

- The IRIS simulator has been employed to predict uncertainties in system attributes (e.g. Power, flow, CHFR) as a function of input data uncertainties. Strong nonlinear behavior was noted for certain attributes and input data combinations implying nonlinear analysis techniques must be employed.

- A complete MATLAB/Simulink model of the IRIS system has been developed and used for control demonstration and sensor placement studies.

- Development of a multivariate linear control algorithm with application to the IRIS system and load-following control. Development a model-predictive controller (MPC) with applications to the IRIS system and an experimental control flow loop.

- Development of a fault-tolerant a control system comprised of a Supervisory Controller, a Bank of Robust \& Resilient Controllers, and an Activator \& Filter part.

- Development of a model-based condition monitoring technique for implementation in the autonomous control framework.

- Development of an experimental flow control loop and on-line fault detection and digital control implementation. Demonstration of on-line fault-tolerant control strategy and controller reconfiguration under actuator fault. 
- Development of multi-modular IRIS plant model with steam mixing, and demonstration of load following control and controller robustness.

- Development of an optimal sensor placement method and automated algorithm with application to the IRIS helical coil steam generator.

- Development of a dynamic model of a multi-stage flash (MSF) desalination plant and coupling of this to the IRIS plant for co-generation. Development of an optimal sensor placement for fault isolation of a nuclear desalination plant. Implementation of the sensor placement strategy and demonstration of fault detection and isolation effectiveness.

- Development of IRIS system hybrid models. A PCA-based extended condition monitoring (ECM) method was developed for hybrid model adaptation.

- Integration of the adaptive system into a model-based FDI system and development of degradation models for example key components.

- Development and testing of the case-based fault identification module. Application of the modeling and adaptation methods to a heat exchanger test system and validation in real time.

- Development of a hybrid prognostic framework that combines Type I, II, and III prognostic models, thus leading to the development of a Process and Equipment Prognostics (PEP) toolbox. This is interfaced with the Process and Equipment Monitoring (PEM) toolbox. Demonstration of the PEP toolbox for steam generator tube fouling data generated from the IRIS simulation model.

- Research experience for undergraduate students from South Carolina State University (an HBCU institution). HBCU participation included undergraduate internships during the summer of all the three years at the University of Tennessee and North Carolina State University, and research participation by an SCSU faculty person at the University of Tennessee.

- Information Dissemination: Graduate students from UT, NCSU, and PSU presented papers at the American Nuclear Society (ANS) Meetings, and at the NPIC \& HMIT topical meetings. Three journal papers are published in ANS Nuclear Technology and IEEE Transactions on Nuclear Science. Several manuscripts were completed for journal publications.

- Two of the project investigators, Dr. Hines and Dr. Upadhyaya, served on the Technical Program Committee of NPIC \& HMIT 2009 and 2010. They also participated in the DOE Workshop on On-Line Monitoring, Seattle, June 2010. Dr. Upadhyaya and Dr. Doster participated in the DOE Workshop on Small Modular Reactors (SMR) in Washington, D.C., June 2010. Dr Hines participated in the panel on Instrumentation \& Controls at the DOE-NEET Workshop, Washington, D.C., 2010. Dr. Turinsky presented 
a paper at the International Meeting on Mathematics and Computation, Rio de Janeiro, Brazil. Dr. Hines is on the advisory group for DOE's Light Water Reactor Sustainability (LWRS) Program. A paper, summarizing the recent developments in the project technical task, has been accepted for presentation at the ICI 2011 Conference, Daejeon, South Korea, August 2011.

\subsection{Publications Related to the Project}

The technical information generated by this project has been disseminated at national and international meetings and at workshops and DOE-sponsored meetings. Additionally, several manuscripts have been accepted for publication in ANS Nuclear Technology and IEEE Transactions on Nuclear Science. Several doctoral dissertations and MS theses have been developed based on this research in completion of student graduation requirements which are published and publicly available. A full list of the publications made during the course of this work is given here:

1. B.R. Upadhyaya, F. Li, S.R.P. Perillo, and J.W. Hines, "Control, Co-generation, and Sensor Placement Strategy for Integral Small Modular Reactors," ICI 2011, Daejeon, South Korea, August 2011.

2. F. Li: Dynamic Modeling, Sensor Placement Design, and Fault Diagnosis of Nuclear Desalination Systems, Doctoral Dissertation, The University of Tennessee, May 2011.

3. J. Heo and P. Turinsky, "Data Assimilation and Optimization for the IRIS Reactor System," International Conference on Mathematics and Computational Methods Applied to Nuclear Science and Engineering (M\&C 2011), Rio de Janeiro, Brazil, May 2011.

4. F. Li and B.R. Upadhyaya, "Design of Sensor Placement for an Integral Pressurized Water Reactor Using Fault Diagnostic Observability and Reliability Criteria," Nuclear Technology, Vol. 173, No. 1, pp. 17-25, January 2011.

5. M. Humberstone, B. Wood, J. Henkel, and J.W. Hines, "An Adaptive Model for Expanded Process Monitoring," Nuclear Technology, Vol. 173, No. 1, pp. 35-45, January 2011.

6. S.R.P. Perillo: Multi-Modular Integral Pressurized Water Reactor Control and Operational Reconfiguration for a Flow Control Loop, Doctoral Dissertation, The University of Tennessee, December 2010.

7. B.R. Upadhyaya, Instrumentation, Controls, Monitoring \& Diagnostics of Nuclear Reactor Systems: Overview of Research, Development \& Demonstration, Presented to General Atomics, San Diego, CA, November 2010. 
8. A.S. Rominger and J.M. Doster, "Fast Valving for Small and Medium Sized Nuclear Reactors," ANS International Topical Meeting on Nuclear Plant Instrumentation, Control \& Human Machine Interface Technologies (NPIC\&HMIT 2010), Las Vegas, NV, November 2010.

9. Y. Guo, X. Jin, and R.M. Edwards, "State Observer Design for IRIS Nuclear Reactor Based Dissipation Based High Gain Filter," NPIC \& HMIT 2010, November 2010.

10. Y Guo, X. Jin, R.M. Edwards, and A. Ray, "Data Driven Fault Detection in Nuclear Power Plants under Sensor Degradation,” NPIC\&HMIT 2010, November 2010.

11. S.R.P. Perillo and B.R. Upadhyaya, "Dynamic Modeling and Control Strategy for MultiModular Integrated Pressurized Water Reactors," NPIC\&HMIT 2010, November 2010.

12. F. Li and B.R. Upadhyaya, "Development of an Optimal Sensor Placement Strategy for Nuclear Desalination Plants," NPIC\&HMIT 2010, November 2010.

13. M. Humberstone and J.W. Hines, "Elimination and Similarity Fault Diagnostics," NPIC\&HMIT 2010, November 2010.

14. J. Coble and J. W. Hines, "Application of Failure Prognostics to the IRIS Plant," NPIC\&HMIT 2010, November 2010.

15. J. Coble, M. Humberstone, and J.W. Hines, "Adaptive Monitoring, Fault Detection and Diagnostics, and Prognostics System for the IRIS Nuclear Plant," Prognostics and Health Management Society Conference PHM 2010, Portland, OR, October 2010.

16. X. Jin, A. Ray and R. M. Edwards, "Feature Extraction for Data-driven Fault Detection in Nuclear Power Plants," Transactions of American Nuclear Society, vol. 201, pp. 163-164, June 2010.

17. X. Jin, A. Ray and R. M. Edwards, "Integrated Robust and Resilient Control of Nuclear Power Plants for Operational Safety and High Performance," IEEE Transactions on Nuclear Science, vol. 57, no. 2, part 2, pp. 807-817, Apr. 2010.

18. J. Heo, P. J. Turinsky and J. M. Doster, "Thermal-Hydraulic System Analysis Input Parameters Adjustment for the IRIS Reactor," Transactions of the American Nuclear Society, Vol. 102, pp. 643-644, San Diego, June 2010.

19. J. Coble: Merging Data Source to Predict Remaining Useful Life - An Automated Method to Identify Prognostic Parameters, Doctoral Dissertation, The University of Tennessee: May 2010.

20. M. Humberstone: An Adaptive Nonparametric Modeling Technique for Expanded Condition Monitoring of Processes, Doctoral Dissertation, The University of Tennessee: May 2010.

21. B.R. Upadhyaya and J.W. Hines, "Fault Monitoring in Sensor Networks and Sensor Placement Strategy for Nuclear Desalination Systems," 24th International Forum on Process Analytical Technology, IFPAC-2010, pp. 78-79, Baltimore, MD, January 2010.

22. J.W. Hines, K. Bailey, J. Coble, M. Humberstone, and M. Sharp, "Recent Advances in Empirical Methods for Equipment Condition Monitoring and Prognostics", Proceedings of the 2010 Maintenance and Reliability Conference, Knoxville, TN, March, 2010. 
23. J. Coble and J.W. Hines, "Metrics for Optimal Prognostic Parameter Selection," Presentation to Propulsion Safety and Affordable Readiness Conference P-SAR 2009, Myrtle Beach, SC, March, 2009.

24. M. Humberstone, J. Henkel, J.W. Hines, and B.R. Upadhyaya, "An Adaptive Model for Condition Monitoring Applications," Transactions of the American Nuclear Society, Vol. 101, pp. 299-300, November 2009.

25. S.R.P. Perillo, B.R. Upadhyaya, and J.W. Hines, "On-line Fault Detection in an Experimental Flow Loop and Digital Control Applications," Transactions of the American Nuclear Society, Vol. 101, pp. 305-306, November 2009.

26. X. Xu and B.R. Upadhyaya, "Analysis of Robustness of Model-Predictive Controller for the IRIS Helical Coil Steam Generator," Transactions of the American Nuclear Society, Vol. 101, pp. 317-319, November 2009.

27. M. Humberstone, B. Wood, J. Henkel, and J.W. Hines, "An Adaptive Model for Expanded Process Monitoring,” NPIC\&HMIT 2009, Knoxville, TN, April 2009.

28. J. Coble and J. Hines, "Fusing Data Sources for Optimal Prognostic Parameter Selection", NPIC\&HMIT 2009, Knoxville, Tennessee, April 5-9, 2009.

29. J. Coble and J. W. Hines, "Identifying Optimal Prognostic Parameters from Data: A Genetic Algorithms Approach", International Conference on Prognostics and Health Management 2009, San Diego, September, 2009.

30. M. Humberstone and J.W. Hines, "Differentiating Between Expanded and Fault Conditions Using Principal Component Analysis", Journal of Intelligent Manufacturing special issue on Machinery Health Monitoring, Diagnostics and Prognostics, Springer, Nov., 2009.

31. Xin Jin, R.M. Edwards, and A. Ray, "Integrated Robust and Resilient Control for Nuclear Power Plants," NPIC\&HMIT 2009, April 2009.

32. Fan Li and B.R. Upadhyaya, "Design of Sensor Placement for IRIS Reactor Based on Fault Diagnostics and Reliability Criteria," NPIC\&HMIT 2009, April 2009.

33. S.R.P. Perillo and B.R. Upadhyaya, "Digital Control Implementation in an Experimental Flow Control Loop with Applications to Small and Medium Reactors," NPIC\&HMIT 2009, April 2009.

34. X. Xu and B.R. Upadhyaya, "Modeling and Multivariable Control Design for the IRIS Primary System Using the Model-Predictive Control Approach,” NPIC\&HMIT 2009, April 2009.

35. B.R. Upadhyaya, S.R.P. Perillo, X. Xu, F. Li, "Advanced Control Design, Optimal Sensor Placement, and Technology Demonstration for Small and Medium Nuclear Power Reactors," Proceedings of ICONE 17, Brussels, Belgium, July 2009.

36. F. Li, B.R. Upadhyaya, and J.W. Hines, "Optimal Sensor Placement Strategy for the Steam Generator System in an Integral Pressurized Water Reactor," Transactions of the American Nuclear Society, Vol. 100, pp. 213-214, Atlanta, June 2009. 
37. J.W. Hines, "An Introduction to Prognostics with Application", Society of Maintenance and Reliability Professionals, Austin, Texas, January 2009.

38. D.E. Holcomb and B.R. Upadhyaya, Grid Appropriate Reactors: Instrumentation, Controls, and Human-Machine Interface Technology Development Roadmap and PreConceptual Architecture, Presented at the GNEP/GAR Status and Planning Meeting, Knoxville, TN, August 2008.

39. H.W. Hines and B.R. Upadhyaya, Advanced Instrumentation and Control Methods for Small Export Reactors with IRIS Demonstration, Presented at the GNEP/GAR Status and Planning Meeting, Knoxville, TN, August 2008.

40. B.R. Upadhyaya and X. Xu, "Design of a Model Predictive Controller for the IRIS Helical Coil Steam Generator," Transactions of the American Nuclear Society, Anaheim, CA, Vol. 98, pp. 90-92, June 2008.

41. J. Garvey and J.W., Hines, "Merging Data Sources for Predicting Remaining Useful Life", Maintenance and Reliability Conference, Knoxville, TN, May 2008.

42. J. Garvey and J.W. Hines, "Prognostic Algorithm Categorization with PHM Challenge Application", International Conference on Prognostics and Health Management 2008, Denver, October, 2008.

\section{SUMMARY OF THE SIX R\&D TOPICAL VOLUMES}

This section provides a summary of the six topical volumes $(2-7)$ that describe the project $R \& D$ in detail.

\subsection{IRIS Simulator Development, Sensor Deployment, and Sensitivity Analysis}

The accurate prediction of plant behavior during normal and off-normal conditions requires accurate thermal-hydraulic models, as well as provisions for the simulation of auxiliary systems, mechanical components, and control functions. While these capabilities exist in varying degrees in large reactor safety codes, these codes were developed primarily for the simulation of loss of coolant and other design basis accidents where normal control functions may be assumed to be inactive or to operate in a way that makes the limiting responses of the plant more severe. As such, these codes are not optimized for the development and testing of control systems over the continuous full operating range. Codes of this type also generally require a long learning time and the source code is difficult, if not impossible, to modify. Similar issues are associated with detailed component design codes that have been developed to optimize the performance of a particular system component, such at the reactor core, steam generator, etc. 
This research focuses on the development of a simulation platform and the associated analysis of simulator uncertainty and non-linearity.

This report presents work in the development of a high fidelity simulator of the IRIS reactor design, and in reducing the uncertainty in thermal-hydraulic transient predictions for nuclear power plants (NPP) with a focus on SMRs characterized by the integral PWR design. The objective of a part of the study was to determine the economic benefit of conducting transient experiments on an SMR NPP. To accomplish this, a thermal-hydraulic simulator is used to complete data assimilation for input parameters to the simulator using experimental data generated by the plant. Since no such experimental data exists, it was generated using an altered simulator, referred to as the virtual NPP facilitating the investigation of the benefits of conducting various experiments and sensor deployment. The mathematical approach that is used to complete this analysis depends upon whether the system responses, i.e. sensor signals, and the system attributes, e.g. DNBR, are or are not linearly dependent upon the parameters. A linearity test showed that there exist highly nonlinear as well as mildly nonlinear responses, hence both deterministic and probabilistic methods were used to complete data assimilation and uncertainty quantification. For the mildly nonlinear transient, the Bayesian approach was used to obtain the parameters posteriori distributions assuming Gaussian distributions for the input parameters and responses. For the highly nonlinear transient, the Markov Chain Monte Carlo method was utilized based upon Bayes theorem to estimate the posteriori distributions of parameters. This report also discusses the optimization methodology used to design the plant's experiments so as to reduce a posteriori system attribute uncertainties. The optimization problem decision variables include the selection of sensor types and locations, and experiment type imposing realistic constraints, with the objective of maximizing the savings achieved by utilizing the larger degree of the plant operational freedom created by system attribute uncertainty reduction, offset by the cost of sensors and experiments. Finally this report presents an uncertainty analysis method and result for reactor control problems. For this problem, the goal is to select the optimum algorithm that minimizes the time integrated deviation of the actual state from the desired state accounting for uncertainties introduced during the design phase due to simulator employed and operations phase due to sensor uncertainties. To minimize the deviation from the desired values, multiple control algorithms were created and tested, and the optimum control algorithm was identified. 


\subsection{Development of Autonomous Control Architecture and Model-predictive and Fault-tolerant Controllers with Application to IRIS}

The basic requirement of the modular reactor control system is to achieve stable control at different operation modes and enable a stable transition between these operation modes. The major operation modes include plant start-up, normal operation at different power levels, hot standby mode for system testing, and cold shutdown mode for plant maintenance. One of the important operational modes is the transition from electricity generation to providing process heat. It is anticipated that the primary application of process heat is for water desalination. It may be necessary to partition the total energy output between electricity generation and process heat supply. During the transitional modes, the reactor systems and equipment must be closely monitored and controlled to assure compliance with safety requirements.

In order to meet the remote deployment and proliferation-resistance requirements, as defined by safety, reliability, survivability, and life expectancy, the reactor control system should incorporate advanced features such as fault tolerance, control loop performance monitoring, and safety monitoring. Figure 5 shows the hierarchical control features necessary for the autonomous control of a modular reactor system (Upadhyaya, 2006; Anastaklis et al, 1991).

To realize unattended operation and address remote communication, fault tolerant control with controller reconfiguration, should be an important feature of the control system design. If a fault occurs in a control system, a fault diagnosis system should be able to perform reliable and timely fault detection and isolation before serious consequences may occur. This fault diagnostic information may be further used for both operation planning and controller reconfiguration. The design objective of this fault tolerant control system is to enable a stable control under anticipated conditions with incipient faults in sensors, actuators, and reactor components. This function will be accomplished by the first level tasks in the schematic shown in Figure 3.1. The integration of control performance monitoring and automatic tuning feature provides the reactor control system with adaptive capability. In addition, a computerized emergency operation procedure should be in place to support automatic decision-making and control during accident conditions. Accurate and prompt communication between the reactor site and a centralized command center is a necessary feature of these reactors. This autonomous or semi-autonomous control system can be implemented by a hierarchical paradigm, as shown in Figure 3.1. 
In this hierarchical control paradigm, the bottom level has a direct interface with the reactor systems and is responsible for executing fault detection and isolation, fault tolerant control, and abnormal event control. The middle level acts as a coordination between the plant manager level and the execution level. The coordination includes the determination of what is the performance of current control structure and whether it is necessary to tune the controllers or switch the control modes. Significant uncertainties can be dealt with by designing adaptive control laws for unanticipated situations and producing control sequences based on current operating conditions. The top level is a plant manager that manages plant level performance monitoring, plant condition evaluation, capability assessments, and task planning. In addition, the plant manager can transmit reactor operational data to and receive instructions from a central command, control, and communication $\left(\mathrm{C}^{3}\right)$ system.

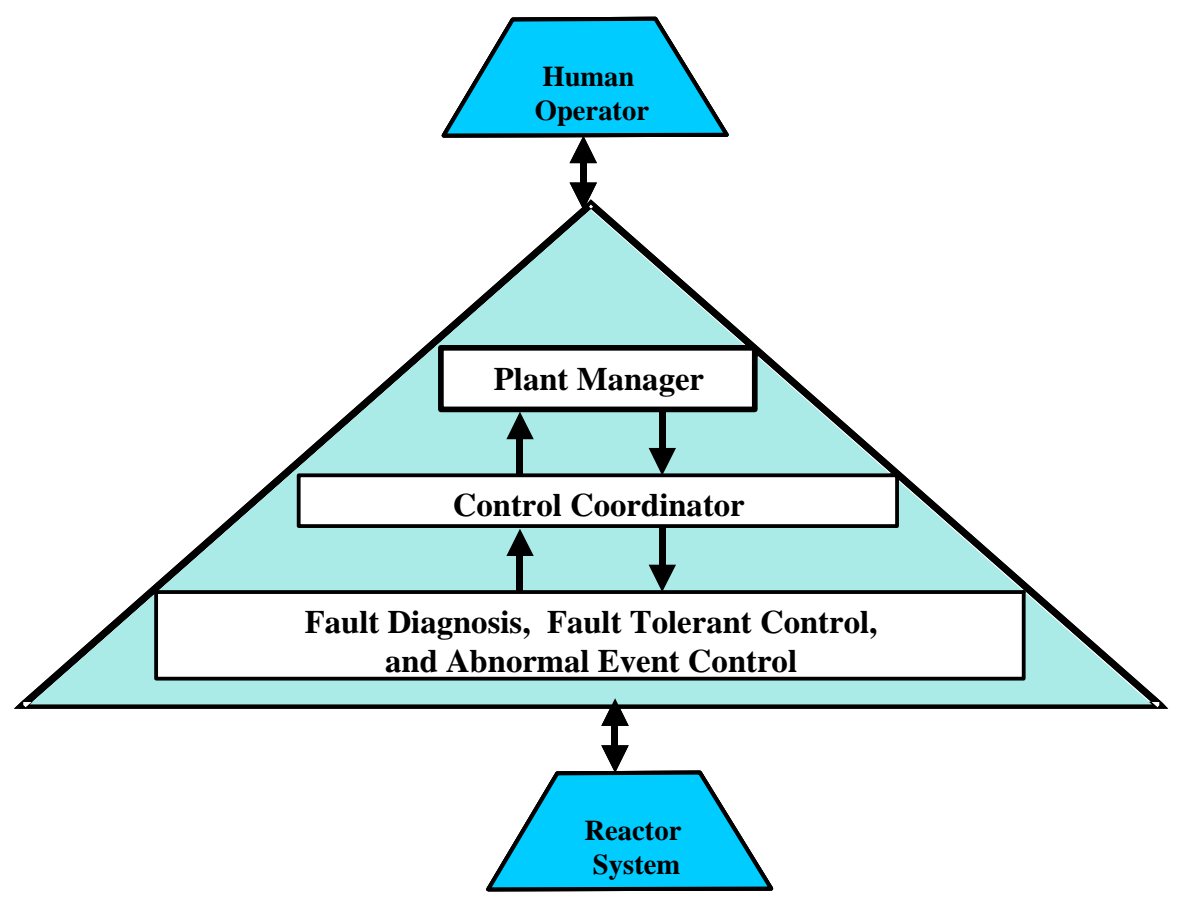

Figure 3.1. Schematic of the autonomous and hierarchical control functional architecture.

The classical proportional-integral (PI) controller, the optimal model-predictive controller (MPC), and a multivariate robust controller are designed and applied to the IRIS dynamic model. This plant model is developed using MATLAB-SIMULINK, and then applied to the 'real plant'. The high-fidelity IRIS simulator (see Section 3.1) is treated as the 'real plant'. 


\subsection{Development of Multi-modular Integral Pressurized Water Reactor Control and Operational Reconfiguration for a Flow Control Loop}

This research focused on the IRIS design since this will likely be one of the designs of choice for future deployment in the U.S and developing countries. With a net 335 MWe output IRIS novel design falls in the "medium" size category and it is a potential candidate for the so called modular reactors, which may be appropriate for base load electricity generation, especially in regions with smaller electricity grids, but especially well suited for more specialized nonelectrical energy applications such as district heating and process steam for desalination.

The first objective of this research is to evaluate and quantify the performance of a Nuclear Power Plant (NPP) comprised of two IRIS reactor modules operating simultaneously with a common steam header, which in turn is connected to a single turbine, resulting in a steammixing control problem with respect to "load-following" scenarios, such as varying load during the day or reduced consumption during the weekend. To solve this problem a single-module IRIS SIMULINK model previously developed by another researcher is modified to include a second module and was used to quantify the responses from both modules. Figure 3.2 shows a block diagram of the dual-module reactor system with a common steam header. A multivariate control strategy was developed to regulate the power levels in the two reactors and at the same maintain the same steam pressure feeding to the mixer.

In order to develop research related to digital instrumentation and control, and equipment and sensor monitoring, the second objective is to build a two-tank multivariate loop in the Nuclear Engineering Department at the University of Tennessee. This loop provides the framework necessary to investigate and test control strategies and fault detection in sensors, equipment and actuators. The third objective is to experimentally develop and demonstrate a fault-tolerant control strategy using this loop. Using six correlated variables in a single-tank configuration, five inferential models and one Auto-Associative Kernel Regression (AAKR) model were developed to detect faults in process sensors. Once detected the faulty measurements were successfully substituted with prediction values, which would provide the necessary flexibility and time to find the source of discrepancy and resolve it, such as in an operating power plant. Finally, using the same empirical models, an actuator failure was 
simulated and once detected the control was automatically transferred and reconfigured from one tank to another, providing survivability to the system. 


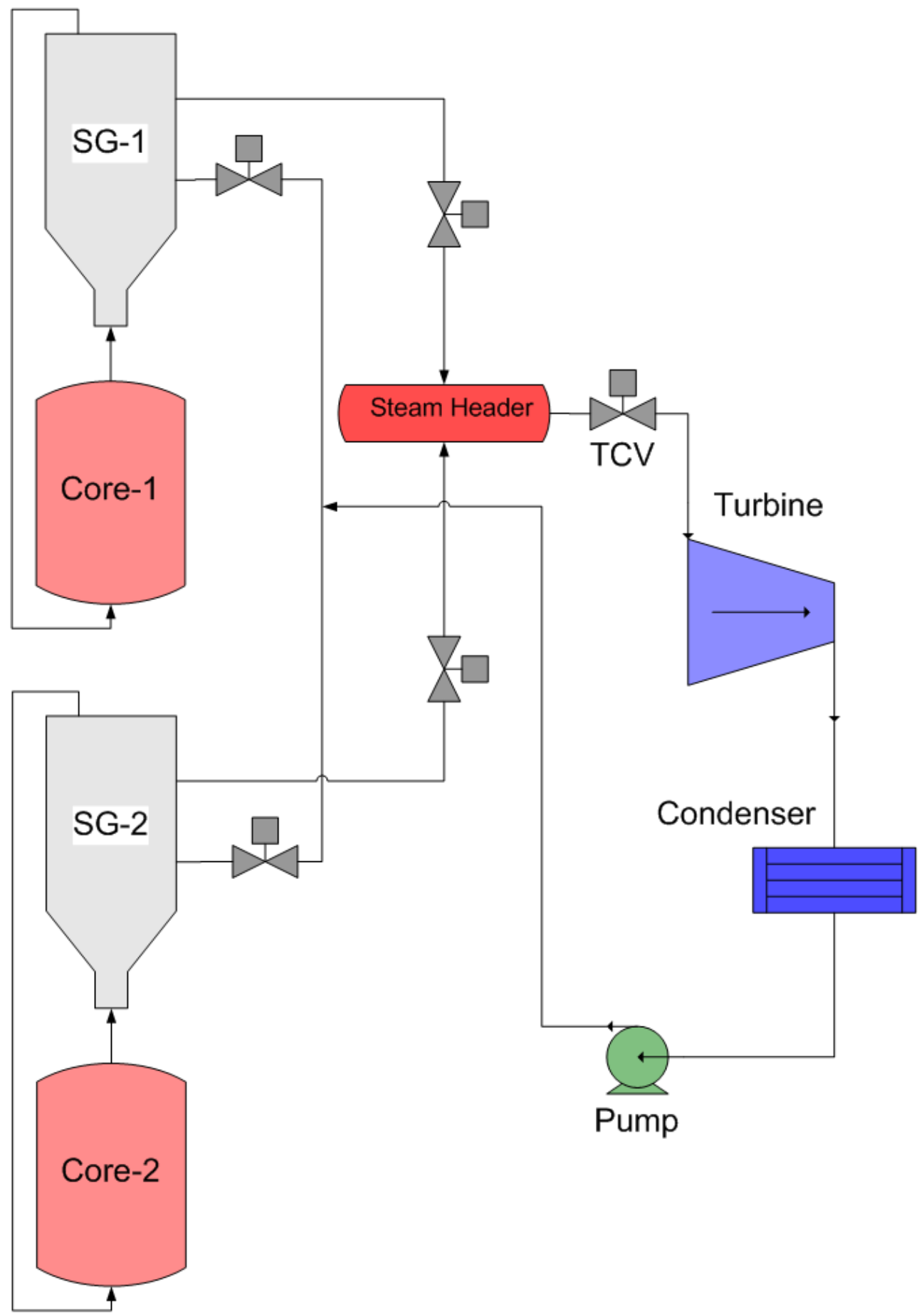

Figure 3.2. Schematic of the dual module reactor system with a common steam header. 


\subsection{Development of Dynamic Modeling, Sensor Placement Design, and Fault Diagnosis of Nuclear Desalination Systems}

Fault diagnosis of sensors, devices, and equipment is an important area in the nuclear industry for effective and continuous operation of nuclear power plants. All the fault diagnostic approaches depend critically on the sensors that measure important process variables. Whenever a process encounters a fault, the effect of the fault is propagated to some or all the process variables. The ability of the sensor network to detect and isolate failure modes and anomalous conditions is crucial for the effectiveness of a fault detection and isolation (FDI) system. With hundreds of process variables available for measurement in either a nuclear power plant or a seawater desalination process, selection of optimum number of sensors and their locations poses a unique challenge. However, the emphasis of most fault diagnostic approaches is primarily on the procedures for performing FDI using a given set of sensors. Little attention has been given to actual sensor allocation for achieving the efficient FDI performance. This report presents a graph-based approach that serves as a solution for the optimization of sensor placement to ensure the observability of faults, as well as the fault resolution to a maximum possible extent. This facilitates an automated approach for sensor placement. Principal component analysis (PCA), a multivariate data-driven technique, is used to capture the relationships in the data, and to fit a hyper-plane to the data. The fault directions for different fault scenarios are obtained from the prediction errors, and fault isolation is then accomplished using new projections on these fault directions. The effectiveness of the use of optimal sensor set versus a reduced set for fault observability and detection is demonstrated using this technique.

Among a variety of desalination technologies, the multi-stage flash (MSF) processes contribute substantially to the desalinating capacity in the world. In this report, both steady-state and dynamic simulation models of a MSF desalination plant are developed. The dynamic MSF model is coupled with a previously developed International Reactor Innovative and Secure (IRIS) model in SIMULINK environment. The proposed methods are illustrated with application

to the coupled nuclear desalination system. The results demonstrate the effectiveness of the proposed integrated approach to performance monitoring and fault diagnosis with optimized sensor network for large industrial systems. 
The contributions of this research task are innovative and significant in that it reports the first comprehensive studies of modeling, simulation, sensor placement design, and fault diagnosis of nuclear desalination processes. The developed methodology would also be applicable to other process industries with the capability to automate the sensor allocation procedure.

Figure 3.3 shows the nuclear desalination system with the MSF plant coupled to the IRIS reactor.

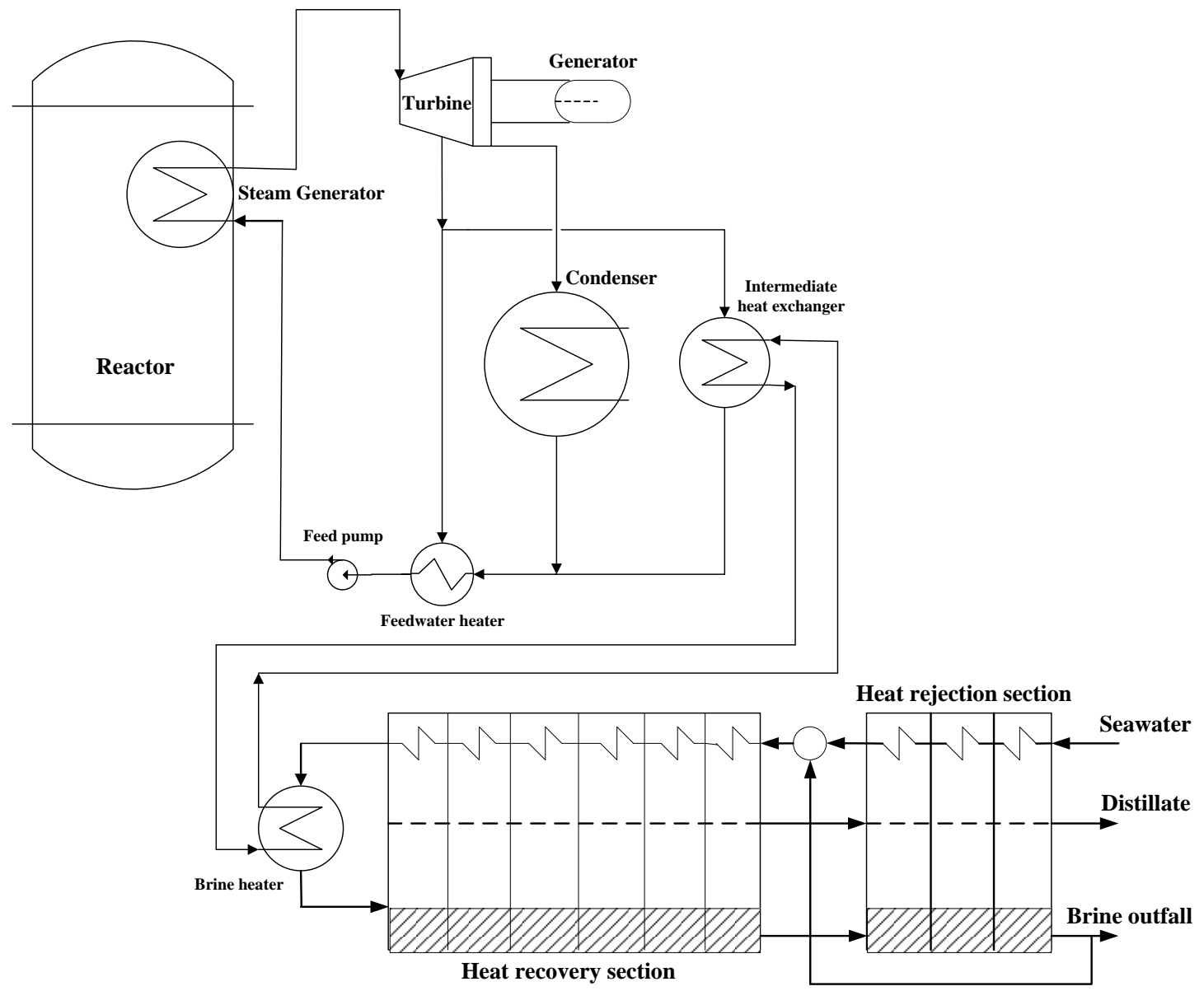

Figure 3.3. Schematic diagram of a MSF plant coupled to the IRIS power conversion system.

\subsection{On-line Monitoring and Diagnostics using Non-Parametric Methods}

Volume 6 of the final report investigates health monitoring methods for small and medium reactors (SMR). Ideally, health monitoring of new, complex engineering systems should begin with the first day of system operation. Health monitoring typically involves a suite 
of modules, including system monitoring, fault detection, fault diagnostics, and system prognostics. However, for systems which have not yet been built, this is challenging. Most available health monitoring modules are empirically based, meaning they are derived from available historic data. For new systems, such data simply does not exist. This volume investigates health monitoring systems for newly designed export reactors. SMRs for remote deployment will have additional condition monitoring, diagnostic, and prognostic needs compared to traditional reactor designs. These needs stem from expectations of availability, safety, proliferation resistance, extended maintenance intervals, reduced staff, and extended operation intervals, all of which are a result of remote operation in countries that may have limited infrastructure and skill sets. Even in domestic reactors, current periodic maintenance practices are increasingly replaced by condition-based strategies, which require methods for monitoring the condition of plant components and systems. The research presented here serves to fulfill objective three of this research and the associated tasks.

A key challenge in automatically adaptive empirical models lies in differentiating between faulted operation and nominal operation which is not well-described by the physicsbased data. Nominal operation may extend beyond the simulated data for many reasons: the system may be operating in un-anticipated environments; the assumptions made in model development may cause inaccuracies in the data; or the relationships modeled may simply be incorrect. Traditional fault detection methods such as those using the sequential probability ratio test are not able to distinguish between unexpected nominal operation and truly faulted operation. However, the main benefit of using adaptive models lies in their ability to accurately learn expanded nominal relationships while detecting and differentiating faulted conditions. For the purposes of accurately adapting a monitoring system, a principal component-based method is proposed to distinguish between these two cases.

The proposed health monitoring system adapts monitoring, diagnostic, and prognostic models as additional, pertinent information (data) becomes available from the plant. The adaptive non-parametric model (ANPM) monitoring system utilizes a hybrid approach which transitions from simulated data to nominal operating data as the plant is running. A principle component analysis (PCA) approach allows the system to automatically determine if a new observation is (a) normal, expected operation, (b) normal operation in an unexpected region, or (c) faulted operation. This approach overcomes the main challenge of online adaptation - 
determining if new observations should be included in the model in an intelligent way. In addition to the new ANPM model and PCA expanded condition monitoring (ECM), several methods for performing fault diagnostics are investigated including a fuzzy inference method, a similarity-based method, and a neural network based method. These techniques were applied to a variety of data sets to highlight the benefits and drawbacks of each. The individual modules are integrated into one complete system to allow for health monitoring from the first day of system operation through end of life. The adaptive nature of the individual components affords the entire system a measure of adaptability when applied to new designs or in new operating regions.

\subsection{Development of Process and Equipment Prognostics Methods}

Unforeseen equipment failure is cost-prohibitive, both in terms of equipment repair costs and lost revenue. Discovery of unanticipated pressure vessel head degradation at the Davis-Besse nuclear plant led to a 25 -month outage and estimated repair costs exceeding $\$ 600$ million. In September, 2008, a turbine generator malfunction at the D.C. Cook nuclear plant resulted in a fire which led to eventual manual plant shutdown. Turbine repairs totaled $\$ 332$ million in addition to lost revenue during the one-year outage. Enterprise server downtime can be even more costly, resulting in a possible loss of \$6.4 million per hour for brokerage operations or \$2.6 million per hour for credit card authorization services. Small- and medium-sized reactors (SMRs) for export will have additional prognostic needs compared to traditional reactor designs, which include increased expectations of availability, safety, proliferation resistance, extended maintenance intervals, reduced staff, and extended operation intervals, all of which are a result of remote operation in countries that may have limited infrastructure and skill sets. Obviously, it is of paramount importance to be aware of impending equipment failures in SMRs so that operations can be adjusted or auxiliary equipment can be employed to avoid unexpected downtime or failure when possible.

The ultimate goal of most prognostic systems is accurate prediction of the remaining useful life (RUL) of individual systems or components based on their specific use and performance. As equipment degrades, measured parameters of the system tend to change; these sensed measurements, or appropriate transformations thereof, may be used to characterize the system degradation. An appropriate degradation measure, also called a prognostic parameter, may be used with a General Path Model (GPM) to make RUL estimates for specific systems or components. A dynamic Bayesian updating methodology is introduced to incorporate prior 
information in the GPM regression parameters, thereby capitalizing on all available information. Incorporating prior knowledge into the regression is particularly useful when only a few observations of the degradation parameter are available or the available observations are contaminated with high noise levels. Degradation measures may include sensed measurements, such as temperature or vibration level, or inferred measurements, such as model residuals or physics-based model predictions. Often, it is beneficial to combine several measures of degradation into a single parameter to provide a more robust prognostic model. Selection of an appropriate parameter is a key for making useful individual-based RUL estimates; typically, identification of a prognostic parameter is left to expert analysis, visual inspection of available data, and knowledge of the degradation mechanisms. This approach is tedious and costly, and scales with the number of available data sources and possible fault modes.

This research introduces a set of metrics which characterize the suitability of a prognostic parameter. Parameter features such as trendability, monotonicity, and prognosability can be used to compare candidate prognostic parameters to determine which is most useful for individual-based prognosis. Trendability indicates the degree to which the parameters of a population of systems have the same underlying shape. Monotonicity characterizes the underlying positive or negative trend of the parameter. Finally, prognosability gives a measure of the variance in the critical failure value of a population of systems. By quantifying these features for a given parameter, the metrics can be used with any traditional optimization technique, such as Genetic Algorithms, to identify the optimal parameter for a given system.

The proposed parameter identification method and prognostic algorithms are illustrated with three applications: first, to simulated failure data of an International Reactor Innovative and Secure (IRIS) system; then, to the simulated turbofan engine data proved in the 2008 Prognostics and Health Management Conference Prognostics Challenge; and, finally, to data collected in a laboratory milling equipment wear experiment. The automated system was shown to identify appropriate parameters in both situations and facilitate Type III prognostic model development. 


\section{UNDERGRADUATE RESEARCH AND SUMMER INTERNSHIPS}

Undergraduate students at South Carolina State University (SCSU) have participated in the NERI-C research through summer internship positions at other university partners. A list of the students and their internship assignments is given in Table 4.1. The bulk of participants studied at the University of Tennessee during the summer, performing research and attending summer classes in relevant topics such as empirical modeling methods and prognostic techniques. The student outcomes, as of July, 2010, are given in

Table 4.2. Four of the seven student participants have graduated from SCSU and one anticipates graduation at the time of this report. The remaining two students have transferred from the department or the school.

Table 4.1: Summer Research Participants

\begin{tabular}{|c|c|c|c|c|}
\hline Year & Participant's Name & Major & Class & Internship Assignment \\
\hline 2008 & Chester Rodrigues & NucE & Junior & University of Tennessee \\
\hline 2008 & Justin Shine & NucE & Soph & University of Tennessee \\
\hline 2008 & Jennifer Conwell & Phys/NE & Soph & North Carolina State \\
\hline 2008 & Bradley Childs & RadChem & Junior & University of Tennessee \\
\hline & & & & \\
\hline 2009 & Chester Rodrigues & NucE & Senior & University of Tennessee \\
\hline 2009 & Justin Shine & NucE & Junior & University of Tennessee \\
\hline 2009 & Rashad Sanders & NucE & Junior & University of Tennessee \\
\hline 2009 & Landis Chambers & NucE & Senior & North Carolina State \\
\hline & & & & \\
\hline 2010 & Chester Rodrigues & NucE & Senior & University of Tennessee \\
\hline 2010 & Katrina Chisolm & NucE & Senior & University of Tennessee \\
\hline 2010 & Dr. Legum Emmanwori & NCSU & Faculty & University of Tennessee \\
\hline
\end{tabular}

Table 4.2: Student Outcomes after Participating in Summer Research Assignments

\begin{tabular}{|c|c|l|}
\hline STUDENT NAME & STATUS & \multicolumn{1}{c|}{ CURRENT ACTIVITY } \\
\hline Chester Rodrigues & $\begin{array}{c}\text { Senior } \\
(5 / 11)\end{array}$ & $\begin{array}{l}\text { Expected to complete BSNE in May 2011. } \\
\text { Participated for three consecutive summers } \\
\text { on the NERI-C project. Plans to apply for } \\
\text { graduate studies in Nuclear Engineering at } \\
\text { the University of Tennessee. }\end{array}$ \\
\hline Katrina Chisolm & $\begin{array}{c}\text { Graduate } \\
(8 / 10)\end{array}$ & $\begin{array}{l}\text { Completing BSNE in August 2010; Senior } \\
\text { project was relevant to reactor control. }\end{array}$ \\
\hline Justin Shine & Changed & Completing Junior year in Mechanical \\
\hline
\end{tabular}




\begin{tabular}{|c|c|l|}
\hline & Major & Engineering Technology \\
\hline Rashad Sanders & Transferred & $\begin{array}{l}\text { Transfer student to Univ. of Tennessee in } \\
\text { materials science }\end{array}$ \\
\hline Landis Chambers & $\begin{array}{c}\text { Graduated in } \\
\text { NucE in 2010 }\end{array}$ & $\begin{array}{l}\text { Accepted a position in Nuclear Engineering } \\
\text { at the Savannah River Site; plans to attend } \\
\text { NC State after some years of work } \\
\text { experience. }\end{array}$ \\
\hline Jennifer Conwell & $\begin{array}{c}\text { Graduated in } \\
2010 \text { in } \\
\text { Physics with } \\
\text { NucE minor }\end{array}$ & $\begin{array}{l}\text { Completed B.S. in Physics, but will attend the } \\
\text { Univ. of Pittsburg in Mechanical Engineering } \\
\text { as a result of NC State experience. }\end{array}$ \\
\hline Bradley Childs & $\begin{array}{c}\text { 2010 in in } \\
\text { RadChem }\end{array}$ & $\begin{array}{l}\text { Completed B.S. in Chemistry and two internships } \\
\text { at Lawrence Berkeley Lab in Radiochemistry. He } \\
\text { will enter Ph.D. studies in RadChem at UNLV. }\end{array}$ \\
\hline
\end{tabular}

During the course of their internships, students worked on a variety of tasks related to the objectives of the NERI-C project. The resulting final reports, which summarize the work completed, are given in the Appendices.

\section{CONCLUDING REMARKS AND FUTURE WORK}

The research presented in this six-volume Final Report describes research completed in the simulation, control, and monitoring of SMRs. This work includes simulation modeling of the IRIS reactor, with associated sensitivity and uncertainty analysis. Methods for optimal sensor placement were developed and investigated, optimizing for such features as fault detectability and observability, sensor reliability, and cost. Several control algorithms were also developed to account for multi-modular design, load following operation, sensor degradation, and faulted operation. Adaptive non-parametric health monitoring techniques and algorithms were developed for system monitoring, fault detection and diagnostics, and equipment prognostics.

Several issues related to SMRs require further work and demonstration. These include but not limited to: economic operation of multi-modular plants, small electric grid stability, automated design of sensor placement, nuclear desalination using SMRs, implementation of online monitoring and equipment prognostics and design phase development of these technologies. 


\title{
Appendix A: Undergraduate Student Report \\ Summary of 2010 Summer Research at the University of Tennessee
}

\author{
Researchers: Dr. Legumchin Emmanwori and Katrina Chisolm \\ Research Topic: PROGNOSTICS APPLICATION FOR THE POWER INDUSTRY
}

This project attempts to examine the applicability of prognostic methodologies to industrial self-priming pump. So the objective was to develop and apply prognostic methodologies to predict the Remaining Useful Life (RUL) of a horizontal industrial selfpriming pump motor. There are several steps to meet this objective, including identifying an optimal degradation parameter pertaining to the state of the pump and developing a suitable prognostic model. We have used four industrial self-priming pumps for this experiment.

Figure A.1 shows a picture of the set up showing the pumps mounted at the top of a six foot structure, instrumentation and collection system.

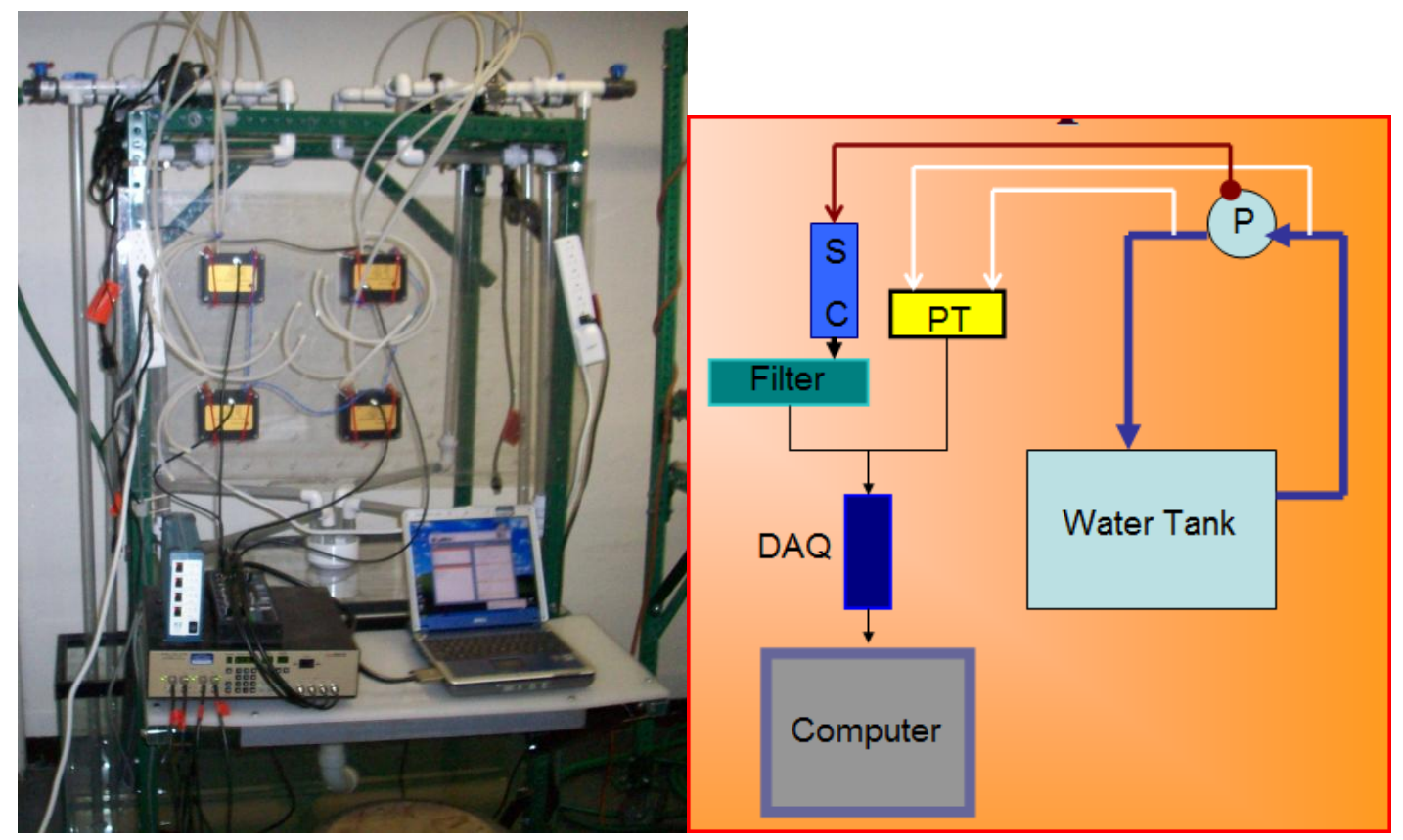

Figure A.1. Experimental Setup. 
Each pump is equipped with a differential pressure transmitter mounted across the pump, and an accelerometer mounted directly onto the metal housing of the pump in the vertical orientation. The system is designed to allow the four pumps to be experimented on at the same time; the data acquisition system collected the vibration and differential pressure of each of the four pumps. A list of the manufacturer and model numbers for the pump units and data collection sensors used is shown in Table A.1.

Table A.1: Component Listing

\begin{tabular}{|c|c|c|}
\hline Name & Model & Manufacturer \\
\hline $\begin{array}{l}\text { Self-Priming Transfer } \\
\text { Pump }\end{array}$ & 12310-Series & $\mathrm{JABSCO}$ \\
\hline ICP Accelerometer & YJM352C18 & $\begin{array}{c}\text { PCB } \\
\text { Piezotronics }\end{array}$ \\
\hline $\begin{array}{c}\text { Modus M Series } \\
\text { Pressure Transmitter }\end{array}$ & M4020ED01R & $\mathrm{GE}$ \\
\hline Amplifier & $\begin{array}{l}\text { Model } \\
482 \mathrm{~A} 16\end{array}$ & $\begin{array}{c}\text { PCB } \\
\text { Piezotronics }\end{array}$ \\
\hline Filter & $\begin{array}{l}\text { Model } \\
3364\end{array}$ & $\begin{array}{l}\text { KH filter } \\
\text { Butterworth }\end{array}$ \\
\hline DAQ & BNC 2120 & $\begin{array}{c}\text { National } \\
\text { Instruments }\end{array}$ \\
\hline
\end{tabular}

For the experimental setup, the four 115 Volt transfer pumps are mounted to a frame drawing its water supply from a common rectangular open top container. The container has water/sand mixture designed to accelerate degradation. Each pump draws the water/sand mixture six feet from the reservoir before returning it via a large mouthed funnel back into the tank to ensure there is minimal outlet end 'suction' to aid the pump in drawing the water.

When the experiment is run, the signals from each accelerometer and differential pressure transducer are amplified, filtered to prevent aliasing and then run through the National Instruments data acquisition system to collect the vibration and differential pressure of each of the four pumps via lab view which stores the data as text files. After this stage, degradation of 
the pump will follow by removing some of the pump's impeller to age failure. After the data is collected, it is cleaned and analyzed to identify a prognostic parameter for further analysis.

Nuclear plants specifically have need for optimizing and regulating maintenance routines. In an industry that is currently looking at lifetime extension and license renewal for many of the plants in operation, prognostics parameters could provide an additional metric to help gauge the suitability of existing equipment for continued use. 


\title{
Appendix B: Battery Degradation Project Summer 2009
}

\begin{abstract}
Through the growth of modern day technology in today's economy, the excessive use of battery-powered devices has grown. However, it is known that the extensive use of a battery over time can cause it to degrade and eventually stop working. This phenomenon is caused by many complex issues contained within the battery and the condition or stress it is operating under within the device. In order to understand this occurrence, multiple batteries will be monitored at high sampling rates and tested closely using a Data Acquisition Board interfaced with LabVIEW Software to provide real-time advanced analysis and data visualization. The NI testing platform was further enhanced to utilize the ability to control the declining capacity and increase internal resistance within each single tested AA battery. Overall, this research encompasses multiple testing techniques and monitoring applications that will ultimately be used to determine the predicted remaining useful life and time to failure (TTF) of an average AA battery used in today's industries.
\end{abstract}

\section{Experimental Procedure}

The first figure below displays the NI data acquisition board that was used as the testing platform to perform multiple life consumption monitoring techniques. The National Instruments USB6229 BNC is a USB high-performance multifunctional data acquisition module optimized for superior accuracy at fast sampling rates. In order to optimize the battery degradation control, the BNC cables used in this experiment were split into a positive and negative node terminal. Once the task was complete, they were then soldered to alligator clips and interfaced along with sixteen separate battery pods. The second figure to the right provides a brief illustration of the completed procedure. The alligator clips provided a strategic approach in changing the internal resistance on each of the four rows of batteries throughout the duration of the testing period. 

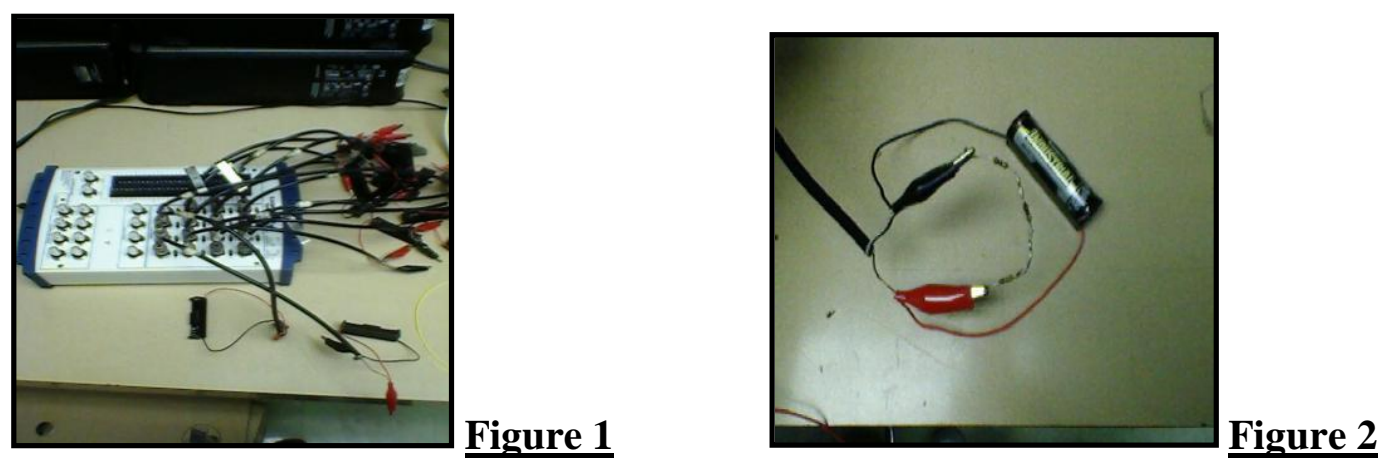

The term internal resistance is technically known as impedance. It determines the performance and lifetime of a battery by measuring the opposition to a sinusoidal electric current. A high internal resistance limits the flow of energy from the battery to the device. A low internal resistance does the exact opposite. The aging of the batteries cell contributes, primarily, to the increase in resistance, not usage. Cell oxidation, which causes high resistance, is non-reversible and is the ultimate cause of battery failure. This causes the cells of the battery to lose its electrons. This means that energy can still be present in the battery, but it can no longer be delivered due to poor conductivity. In order to determine the final energy output, each battery was manually tested using a handheld Voltmeter to be precise that it failed. 


\section{$\underline{\text { Figure } 3}$}
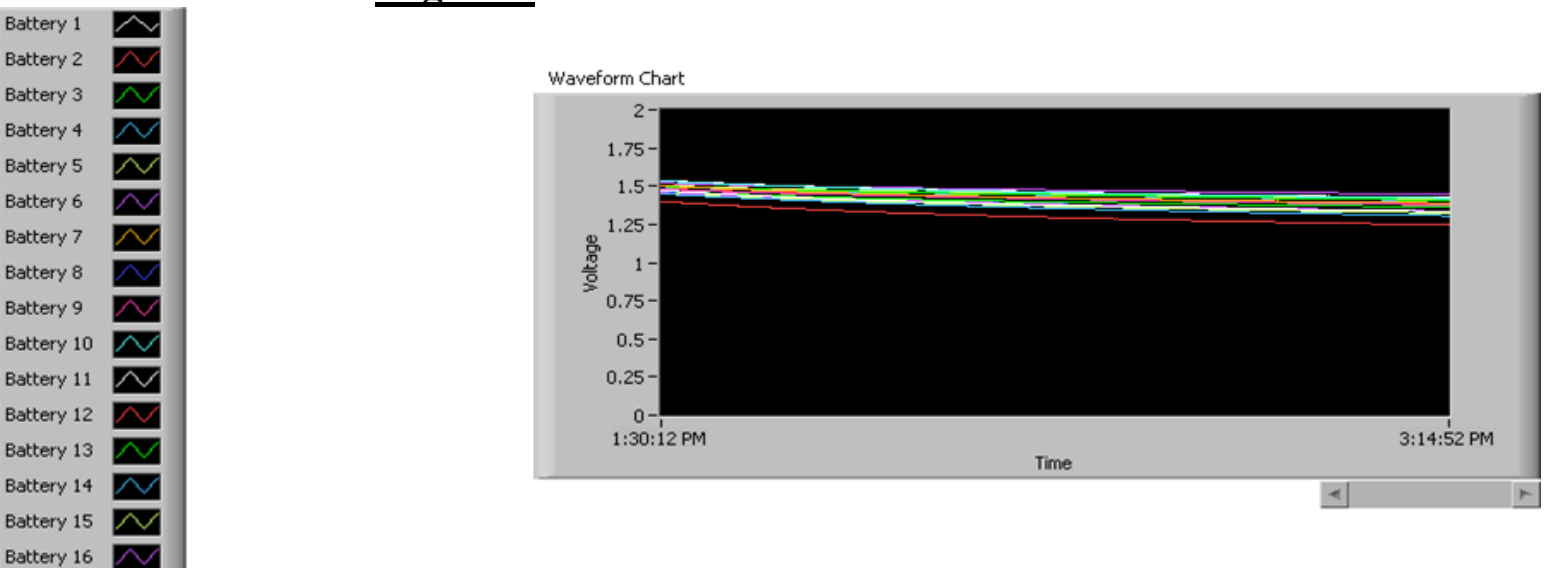

Figure 3 displays the LabVIEW testing panel which is a (NI) computer program that contains a comprehensive set of tools for acquiring, analyzing, displaying, and storing data. The figure displays the user interface or Front panel, with multiple controls and indicators. The controls on the front panel simulate instrument input mechanisms and supply data to the block diagram or back panel (see figure 4) of the VI. Controls are knobs, pushbuttons, or dials. Indicators are graphs, LEDS, or other output displays. Once the front panel was built I added code using VI's and structures to control the back panel. This was done in order to communicate with the NI data acquisition hardware. The input and output data was sampled at a rate of $2500 / \mathrm{s}$ every $10 / \mathrm{sec}$.

The Continuous Operation Mode specified that the task acquires or generates data until stopped 


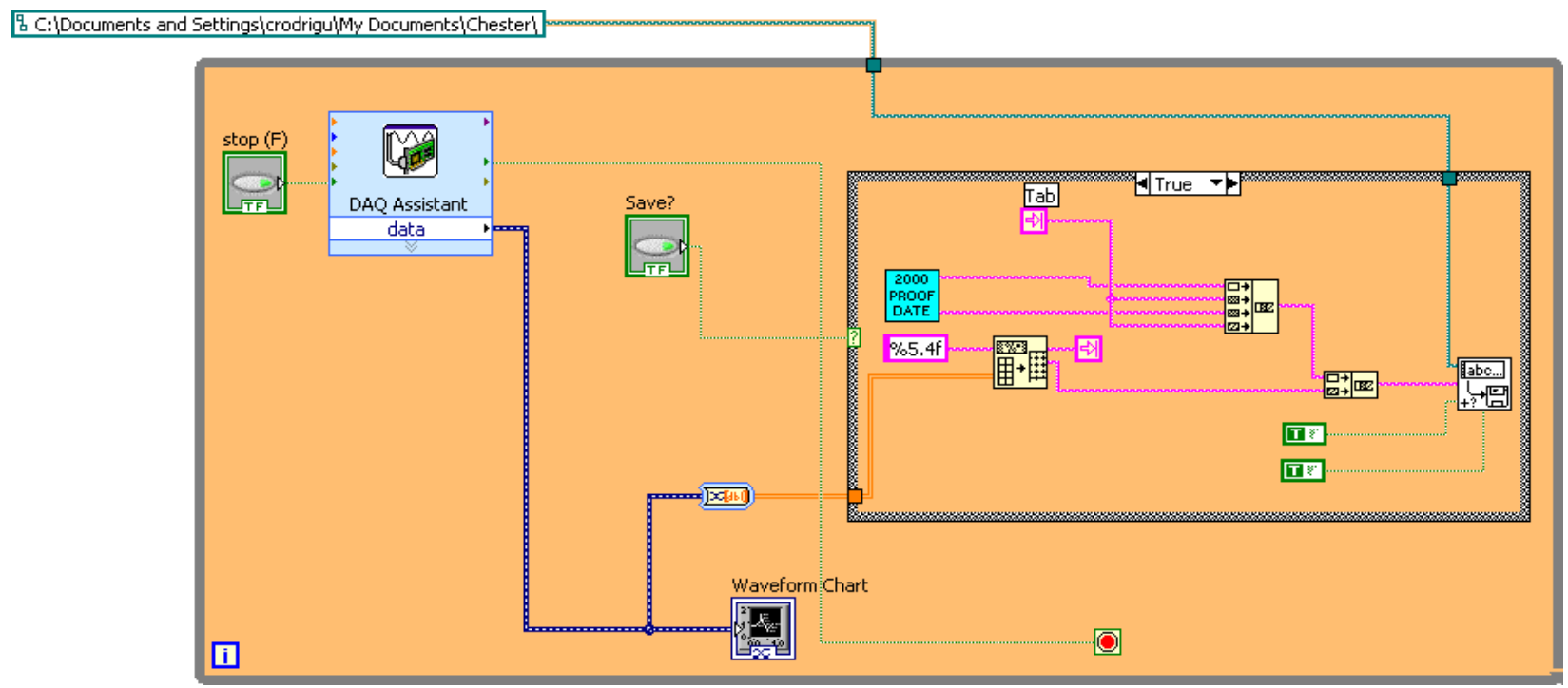

Figure 4 - Back Panel displaying the VI Hierarchy. Sampling rate I/O data at 2500/s every $10 / \mathrm{sec}$.

\section{Methodology}

Below is a brief illustration of the testing methods and Script that are used in the MATLAB editor to run the battery prognostics algorithm. It depicts the necessary procedures that where utilized in this research.

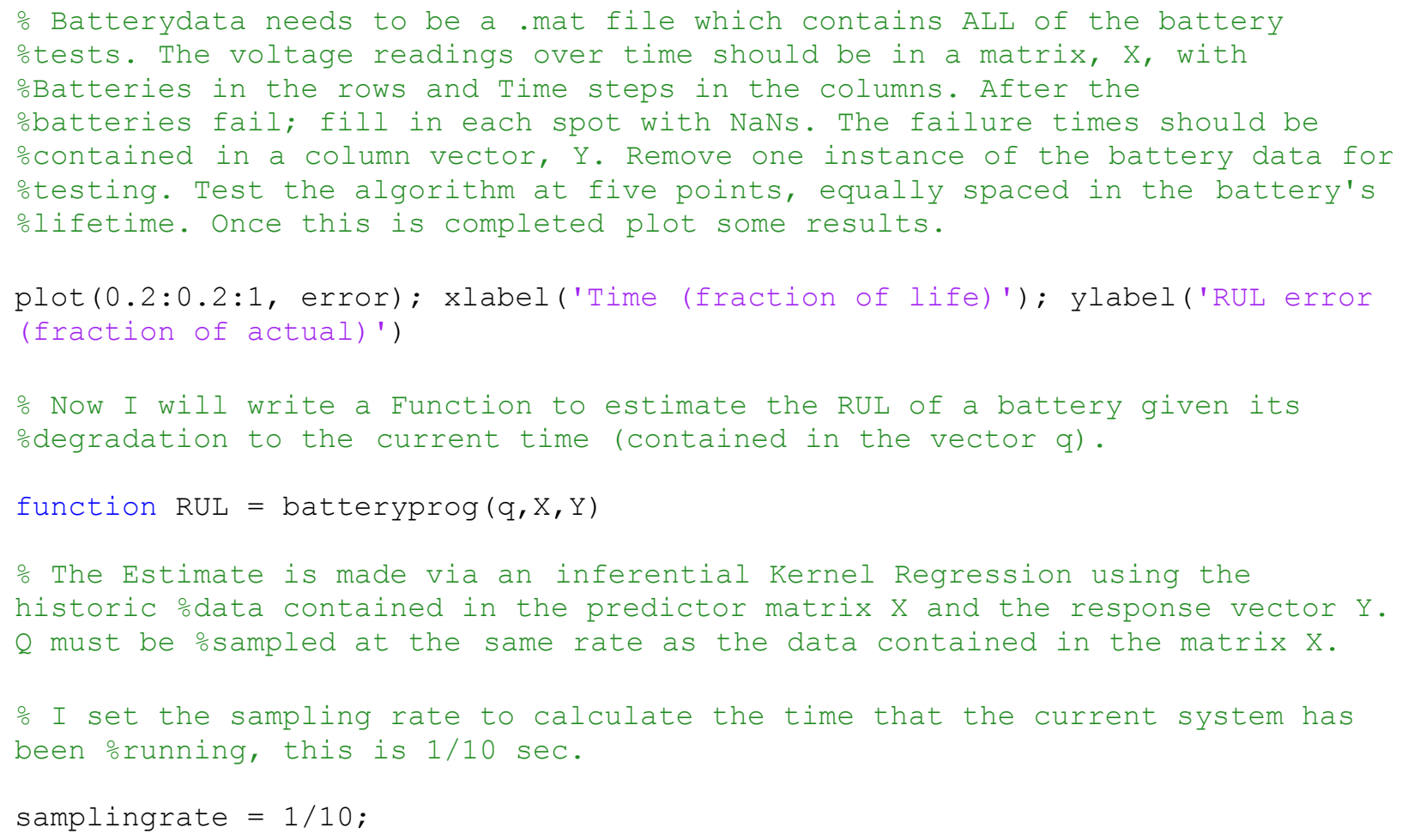




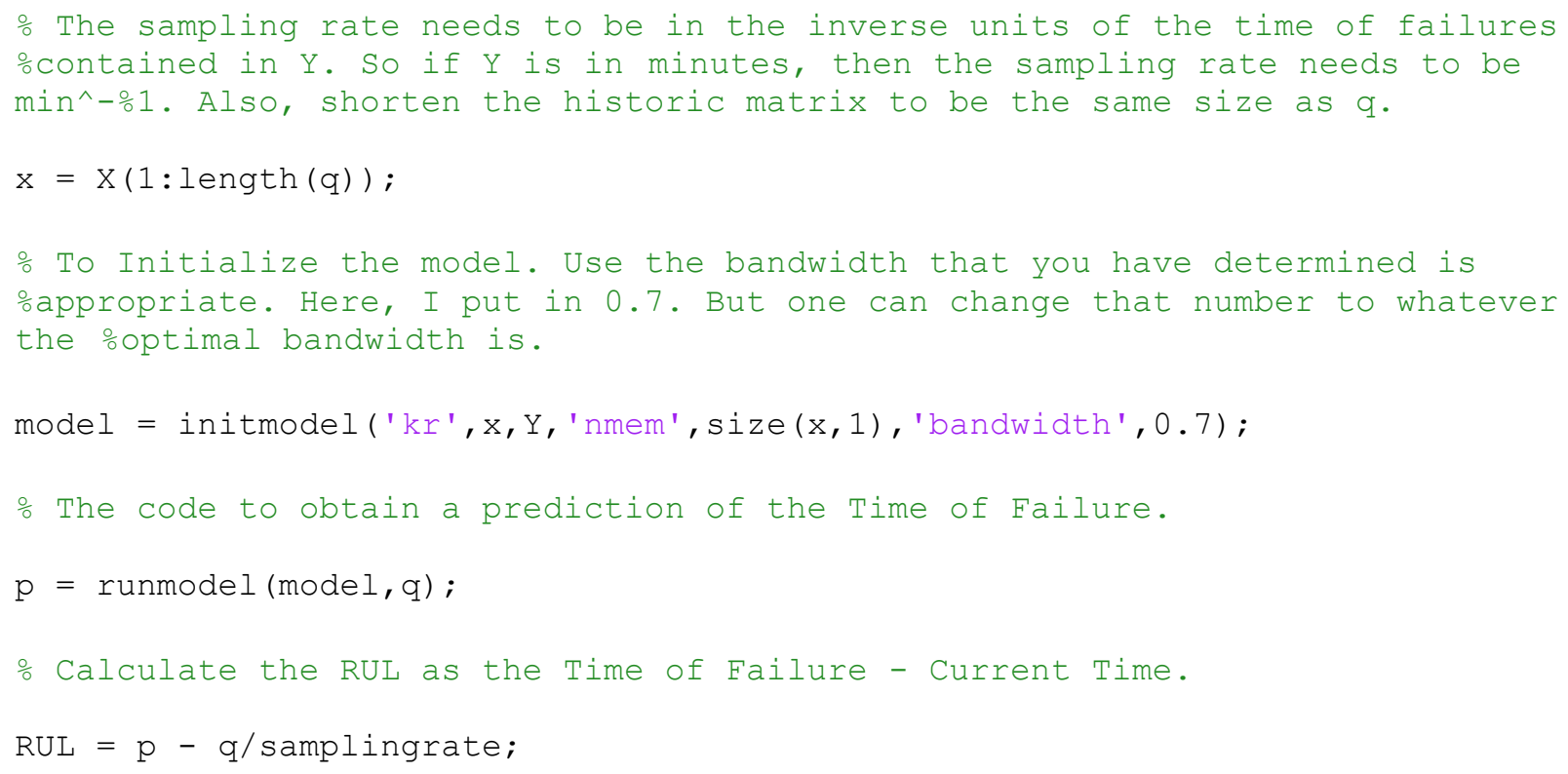

The two figures below display the analyzed voltage degradation of each of the sixteen batteries operating with different resistances overtime. A threshold was determined and used in further analysis

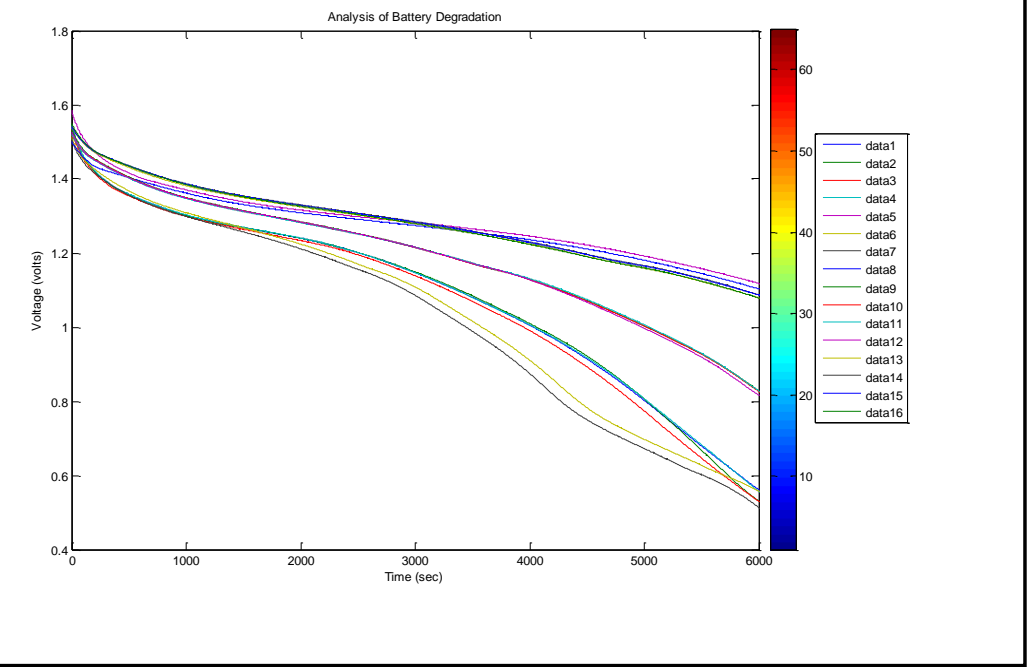

Two common voltage measurements are DC and AC.

- $D C$ voltages are useful for measuring parameters that change slowly with time (Example: Temperature, Pressure, or Strain)

- $A C$ voltages are waveforms that constantly increase, decrease, and reverse polarity. Most power lines deliver AC voltage. 


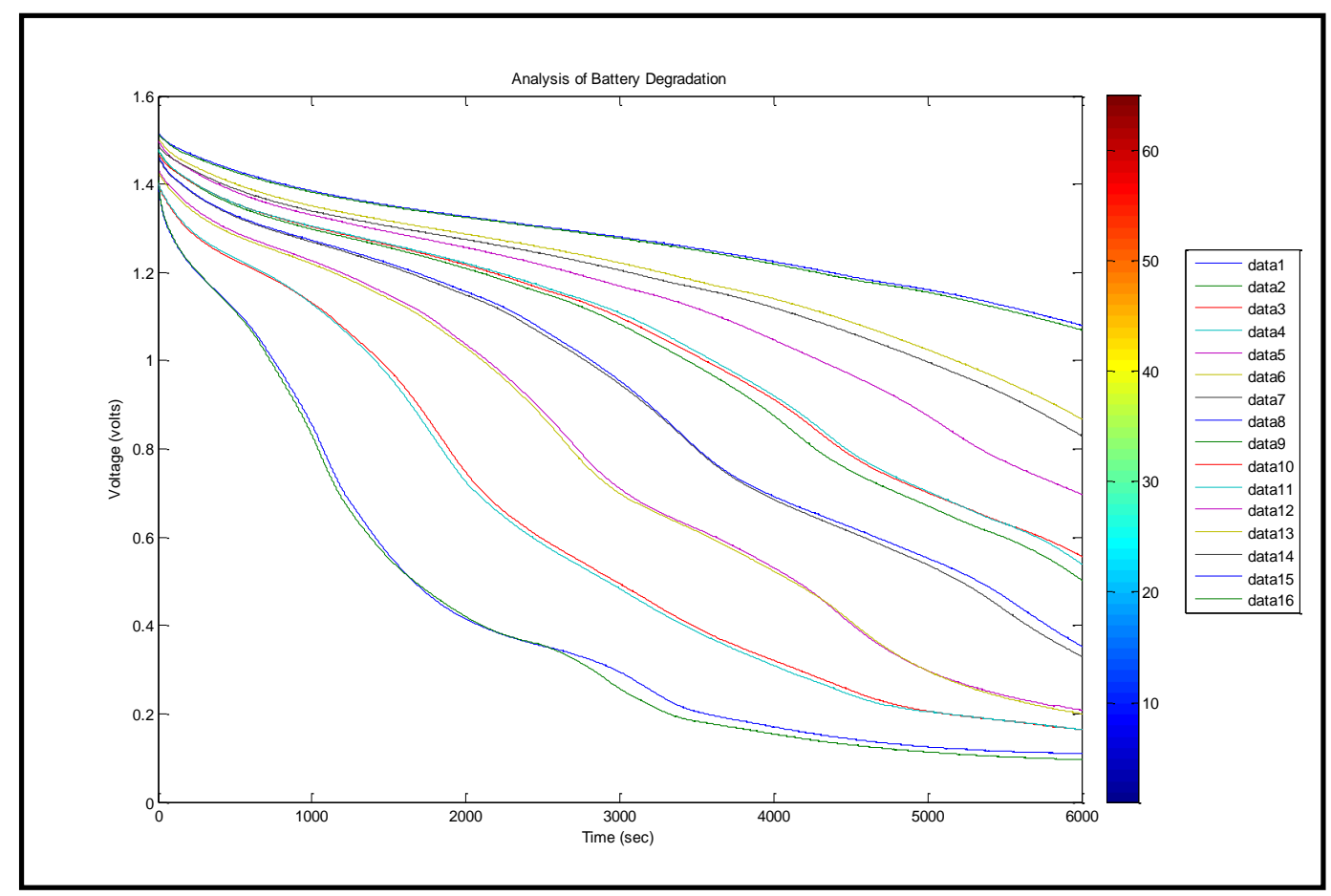

The next step was to perform a data-driven strategy. The two basic data-driven strategies involve (1) modeling cumulative damage (or, equivalently, health) and then extrapolating out to a damage (or health) threshold, or (2) estimating the remaining useful life directly.

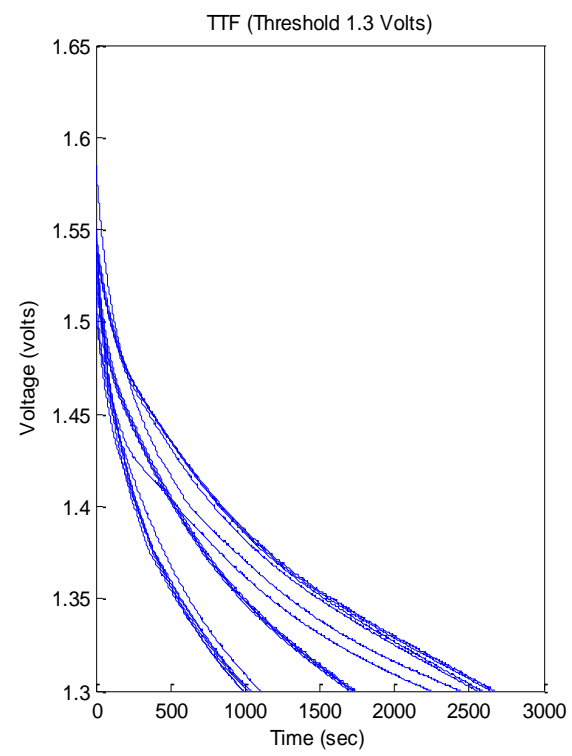




\title{
Appendix C: Undergraduate Student Research Report
}

\section{Control System Fault Detection Using Monitoring and Diagnostic} Techniques

\author{
Prepared by \\ Chester Rodrigues III \\ Bradley Childs \\ Justin Shine \\ South Carolina State University \\ Orangeburg, S.C \\ Undergraduate Internship \\ University Of Tennesee, Knoxville \\ Nuclear Engineering
}

Project Research Period: 6/1/2008 - 8/8/2008 


\section{Acknowledgments}

The research project we conducted at the University of Tennessee was a task performed for the United States Department of Energy (DOE), and sponsored by the Nuclear Energy Research Initiative (NERI-C) program. This proposed research task will use the IRIS reactor as a working platform to demonstrate the implementation of advanced controls, plant monitoring, diagnostics, and prognostics technologies to be developed under the NERI consortium. 


\begin{abstract}
In this research, a SIMULINK model for the two-tank water level control system was developed, and a PID controller was designed to control the water levels in both tanks during operation by adjusting the inlet flow rates. Aside from the theoretical work, our research group fabricated a new piping system and installed new components into the process flow loop laboratory Two-Tank Loop (TTL) at the University of Tennessee (Knoxville). The process flow loop laboratory will be ran by LabVIEW software interfaced with Virtual Instrumentation (VI) and ultimately control the systems such as the (CV, TFM) used to regulate temperature, pressure, flow rate, speed and practically every other variable for which measurement we desire. The operation condition of a plant must be fine tuned in order to make the additional plant design produce to a desired target. These advanced monitoring techniques simulate everyday applications of Nuclear Power Plants, Chemical Plants, Fossil Fuel Processes, and other advanced utilities used in today's industry.

The new installed components consist of four manually operated control valves (Bray Series), and three turbine flow meters. The valves and turbine flow meters are operated electronically by LabVIEW software interfaced with three data acquisition boards (DAQ) in order to produce optimal readout. The four manually operated control valves will control the systems flow rate by inputting different voltage signals. In addition, the upgraded turbine flow meters will measure the flow rates. It is necessary to monitor the functionality of these systems and components, by identifying the measurements that reflect the health of its equipment.

Prognostic methods were developed to predict the remaining useful life (RUL), time to failure (TTF), and probability of failure (POF) in instrumentation and equipment. In this case, one can think of diagnosing conditions of plant components and systems by inputting data that characterizes fault detection. A brief example of fault detection can be as simple as crud layer thickness deposited on a control valves inner tube walls. This can be done by empirical modeling techniques such as auto-associative kernel regression. These models give predictions of the sensor readings and compare them to the actual recorded data. This can be used to discover degradation parameters that can be used for prognostics. Some of the techniques presented here are considered the modified version of the general path model or the Markov Chain model. These techniques are extremely vital in industrial applications involving active components.
\end{abstract}




\section{Introduction}

In order to understand the complexity of various systems used in today's industries, one must understand the importance of "Control" and the role it plays in maintaining an optimal plant performance. Controllers are used in the industry to regulate temperature, pressure, flow rate, chemical composition, speed and practically every other variable for which a measurement exists. The operation condition of a plant must be fine tuned in order to make the additional plant design produce to a desired target. In order to conduct progressive research, we used the MATLAB toolbox software known as SIMULINK for the blue-print loop design, because it provided a graphical user interface that could be connected to form as many complex systems as desired. It essentially produces a live display that lets the user observe variables and output data as the simulation runs and can be manipulated at will.

The proportional-integral-derivative (PID) application is extremely effective and proves to be imperative in this project, because it provides users vital information as the model undergoes operation over time. Because of its wide use, the PID controller is available in nearly all commercial digital control systems, so that efficiently programmed and well-tested implementations are available. The LabVIEW software is used to adjust the input signal controls of each component in the process flow loop. If introduced to failure during operation, it is desired that the system will produce accurate output values, and maintain safety, without having to change any system input parametric values.

In order to fully understand this project, it is helpful to understand how a typical light water reactor (LWR) works because it involves dynamic systems operating continuously under stressful conditions. The IRIS reactor is an innovatively designed LWR, developed by an international team led by Westinghouse Nuclear Division. During operation, these reactors produce heat which is transferred to the primary coolant pumps and later converted into electricity. In this project the coolant is water that is pumped at a high pressure throughout the reactor (loop-system) and monitored closely by orifice and turbine flow meters. There are typically two loops in an LWR called the primary loop and secondary loop. The benefit of this is that radioactivity can mostly be contained within the primary loop, which covers a large portion of the safety control in the reactor. 
Controlling the operation of this process is not only vital to safety, but profit optimization also impacts plant operation on a large scale in the duration of the plant's useful life. In order to maximize long-term operation with minimum downtime, applying monitoring, diagnostics, and prognostic techniques are of top priority. Online readings are measured during operation, and a team of engineers monitor the data to detect faults and make sure values are in proper range of good operating conditions. Aside from the immediate personnel, other engineers study the data from the plant to monitor the long-term trends of operation, thus, determining whether or not a plant is gaining or losing output efficiency. Since no system is perfect, occasional errors may occur due to physical problems. In some instances the signal output from the system may read out normal operation, but actual values may be higher or lower than normal.

The purpose of applying diagnostic and prognostic methodology to the loop system is to eliminate control system masking. Control system fault errors substantially degrade the performance of process control systems. Such model errors result from measurement noise unmeasured disturbances, imperfect input adjustments, and applying simple linear models to truly nonlinear processes.

The following issues are vital in developing innovative autonomous control strategies:

* Safety

* Product Quality

Profit Optimization

Equipment Protection

Environmental Protection

Smooth Plant Operation and Production Rate

\subsection{Objectives and Procedures}

Motivation: Control System Fault Detection Using Monitoring and Diagnostic Techniques

Experimental Procedure: (Details located in section 2.2)

Instrumentation, Control, and Human Machine Interface (ICHMI) 
Develop Blue Print: Two-Tank Loop

Product Design/Construct Model: Simulink Model (P.I.D. controller), LabVIEW Software

Create Dimensions/Measured Variables/Control-Systems: (Pressure, Flow rate, Temperature, Heaters, Condensers, Sensors, and Calibration) using LabVIEW software.

Graduate Level Course: NE-579 Advanced Monitoring and Diagnostic Techniques

This NERI-C project was developed for a Westinghouse IRIS Reactor. The primary part of this research stems toward the use of an actual system that is introduced to errors that can be monitored and controlled. Process and equipment prognostics will be further implemented into the system to understand the degradation mechanism during actual operations over time. The motivation behind this project is to upgrade the process flow loop laboratory and design a process and control system to improve the dynamic operating performance. Thus, minimizing control system fault errors. Once the primary objective is completed, reliability testing (RT) will be further examined while the system is functioning under multiple conditions. The methods introduced in this document are vital in plant operation, reliability testing, and implementation. 


\section{Experimental Design Model}

Figure 1. Demonstration of Process Flow Loop System

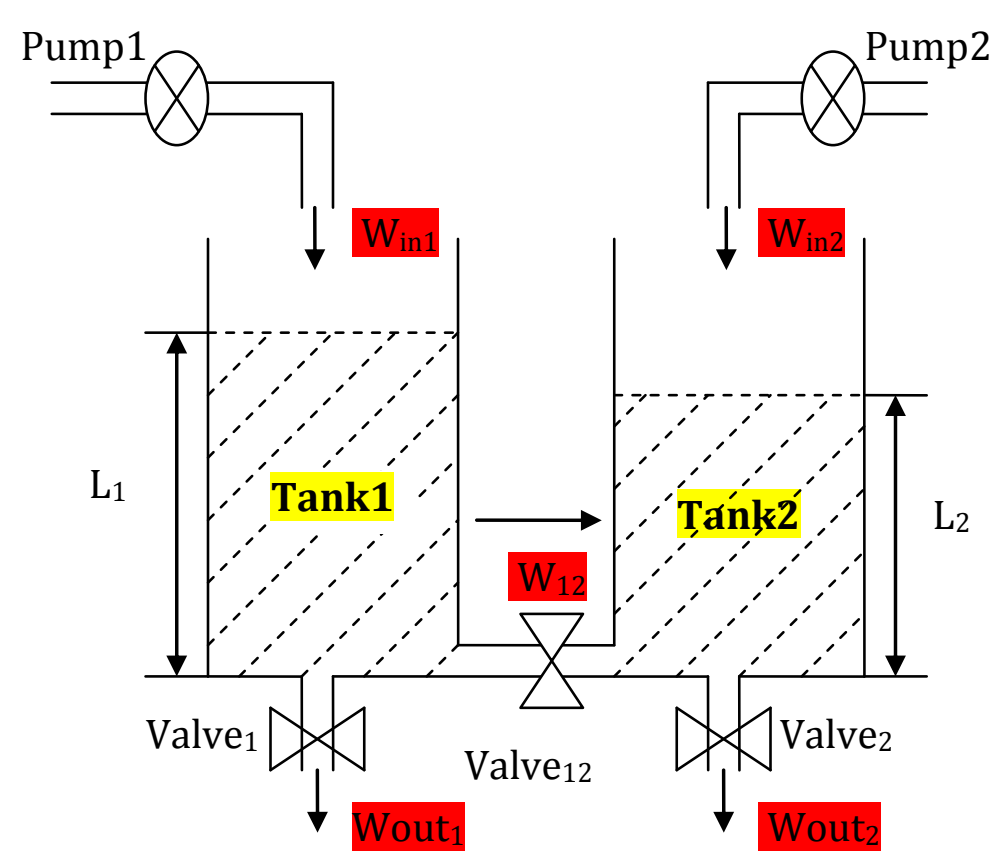

Figure shows a schematic of the two-tank experimental flow control loop used throughout the duration of this project. The physical model above is based on the conservation of mass principle. It will be modified accordingly and compared to general path modeling (GPM) and proportional hazard modeling (PHM) data that was initially used in correlation with the diagnostic and prognostic methodology.

\subsection{Equations of the Physical Model}

$$
\begin{array}{ll} 
& \rho A \frac{d L_{1}}{d t}=W_{i n 1}-W_{\text {out } 1} \pm W_{12} \\
* & \rho A \frac{d L_{2}}{d t}=W_{\text {in } 2}-W_{\text {out } 2} \mp W_{12} \\
* & W_{\text {outi }}=\rho a \sqrt{2 g L_{i}}, \quad \mathbf{i}=\mathbf{1}, 2 \\
* & W_{12}=\rho a \sqrt{2 g\left|L_{1}-L_{2}\right|}
\end{array}
$$

The subscripts 1 and 2 indicate Tank 1 and Tank 2 .

* $\Delta y=L_{\text {set }}-L_{A C T}$ 


$$
\begin{aligned}
& g=9.8 \mathrm{~m} / \mathrm{sec}^{2} \\
& \mathbf{A}=0.01767 \mathrm{~m}^{2} \\
& \mathbf{a}=3.14 \times 10^{-4} \mathrm{~m}^{2} \\
& \rho=1 \times 10^{3} \mathrm{~kg} / \mathrm{m}^{3}
\end{aligned}
$$

\begin{tabular}{|l|l|}
\hline \multicolumn{1}{|c|}{ Variable } & \multicolumn{1}{c|}{ Definition } \\
\hline$L$ & Water Level \\
\hline$W_{\text {in }}$ & Inlet Flow rate \\
\hline$W_{\text {out }}$ & Outlet Flow rate \\
\hline$W_{12}$ & Flow in between Tank 1 and Tank 2 \\
\hline$a$ & Area of the Pipe \\
\hline$A$ & Area of the Tank \\
\hline$\rho$ & Water Density \\
\hline$g$ & Gravitational Acceleration \\
\hline
\end{tabular}

The dynamic state of the system can be described in a physics based model. This twotank water level control loop is multi-input/multi-output system. The two inputs are $W_{i n 1}$ and $W_{i n 2}$ and the two outputs are $L_{1}$ and $L_{2}$. The P-I controllers are designed to control the water levels in both tanks by adjusting the valve position, which leads to the change of inlet flow rate in both tanks.. The outlet flow rate is proportional to the square root of the water level. The equations above relate directly to the system design of the P-I Controller and response variables.

\subsection{Laboratory Control Loop and LabVIEW Interface}

\section{Installation Techniques:}

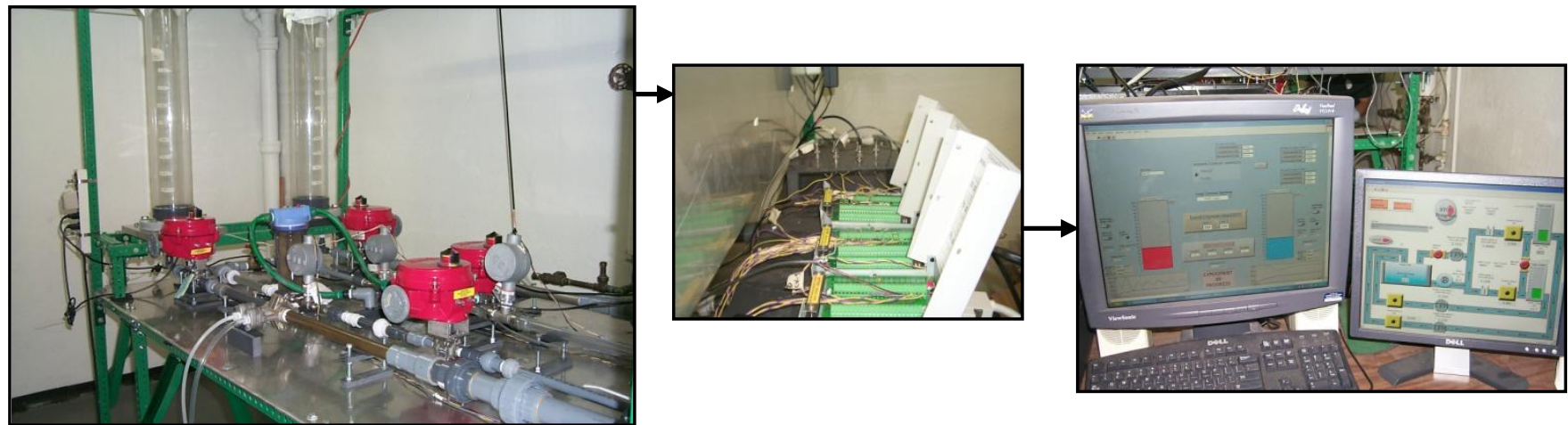

From top left to right are photos illustrating the two-tank loop (TTL), DAQ, and LabVIEW user interface. The first picture shows the deployment of the active components in the 
TTL system. They include four control valves, three turbine flow meters, two orifice meters and four pressure meters. They are used to measure the water levels, inlet and outlet flow rates, and $\mathrm{I} / \mathrm{O}$ voltages distributed to the control valves. All these active components are connected to the DAQ boards with signal wires displayed in the second picture. The DAQ board will change the analog signals to digital signals which is then sent to the PC. A friendly user interface was developed using LabVIEW software which is displayed in the third picture above. The users can adjust parameters and monitor the system's response.

\section{Motor-Operated Control Valve (MOV)}

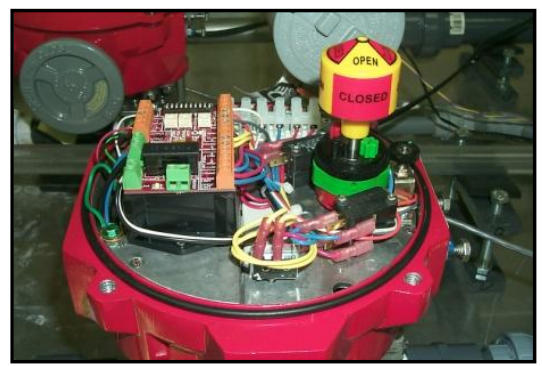

Control Valve (CV): Displaying I/O Signal wiring and Power Supply Wiring. Installation required the fabrication of piping mounts for correct fitting. Once the valve was mounted, a 10 volt power source provided power. After power was established, the valves I/O were connected to data acquisition (DAQ) boards to be recognized by the LabVIEW software. Also, each valve's open/close cam required slight adjustments in order to calibrate the speed of the valves.

\section{Turbine Flow Meter}

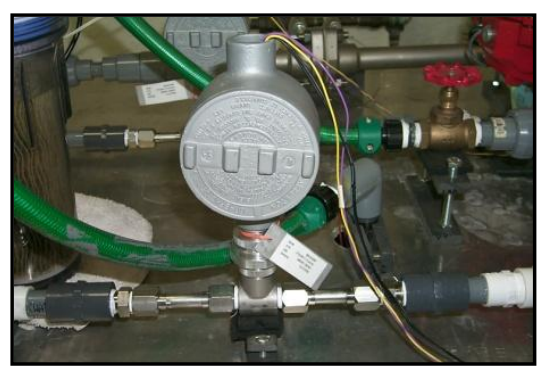

Turbine Flow Meter: Exhibiting the advanced fabricated piping mounts that were handmade by our research group will be used to allow optimal flow rate measurements. They will provide adequate accuracy in determining the control system fault detection occurring in the two tank flow loop.

\section{Theoretical System Response}

The PID control algorithm is a simple, single equation, but it provides good control performance for many different processes in equipment monitoring. This flexibility is achieved through adjusting several parameters, whose values can be selected to modify the behavior of the feedback system. The procedure for selecting the values is termed as tuning, and the adjustable parameters are known as tuning constants.

$u=k_{p} \times \Delta y+k_{i} \int_{t} \Delta y d t+k_{D} \frac{d \Delta y}{d t}$

$$
u_{\mathrm{p}}=k_{p} \times \Delta y: \text { System Response Unit Variable (Reaction) }
$$




$$
\begin{aligned}
& u_{\mathrm{I}}=k_{i} \int \Delta y d t: \text { Integral action eliminates offset } \\
& u_{\mathrm{d}}=k_{d} \frac{d \Delta y}{d t}: \text { Stability Overshoot Control }
\end{aligned}
$$

\subsection{The effect of $\left(K_{p}\right)$ to the (PID) control system}

The first part of the PID controller is the proportional term. The proportional term is the expression that is used so that the output can be made proportional to the value at which the error is currently. In saying this, the proportional term has the ability to determine the reaction according to how the error is put out. The equation that is used to determine the proportional term of the PID is listed below.

$$
P_{\text {out }}=K_{p} e(t)
$$

\subsection{The effect of $\left(K_{i}\right)$ to the (PID) control system}

The second term in the PID controller is the integral term. When the integral term is broken down, it measures the sums of all the errors that have been put together. This value should not only be in proportion with how much error is present, but it also will be in proportion with the length of time that the error occurs. Once this process is complete, the instantaneous error is then added over a certain period of time, and an accumulated error is then produced. An equation defining the integral term is listed below.

$$
I_{\text {out }}=K_{i} \int_{0}^{t} e(t) d t
$$

The third and final term in the PID controller is the derivative term. This value is used to help determine the rate of change of the error that is currently going on in the process. This is a fairly easy calculation by determining the slope of the error and dividing time by that value. In simpler terms, this would be the first derivative with respects to time or the value $t$ and stabilizes the process. The last equation can be found and listed below. 


$$
D_{o u t}=K_{d} \frac{d e}{d t}
$$

\subsection{Stability of the control system}

Each element of the algorithm is defined as a mode, and uses the time-dependent behavior of the feedback information in a different manner, as indicated by the name proportional-integral-derivative. The control calculation is part of the feedback loop, and therefore it should be calculated rapidly and reliably. Excessive time for calculations would introduce an extra slow element in the control loop and degrade the control performance. Iterative calculations, which might occasionally not converge, would result in a loss of control at unpredictable times.

Figure 2. PID Controller

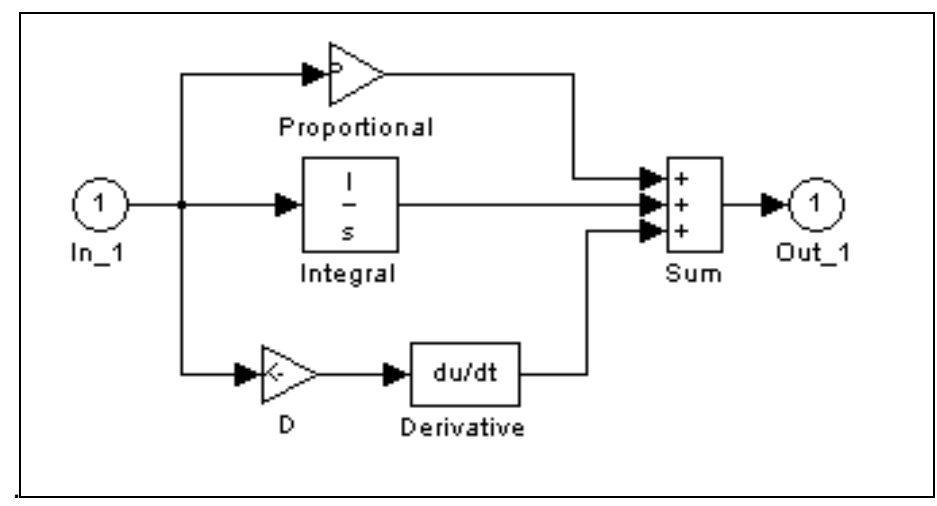

\section{$\underline{\text { Parameters }}$}

- $\mathrm{r}=0.01$; - Meter

- $\mathrm{R}=0.075$; - Meter

- pi=3.14; - Constant

- rho=1e3; - Density

- $\mathrm{g}=9.8$; - Gravity

- $a=\mathrm{pi}^{*} \mathrm{r}^{\wedge} 2$; - Area of Pipe

- $\quad \mathrm{A}=\mathrm{pi}^{*} \mathrm{R}^{\wedge} 2$; - Area of Tank

- $\mathrm{m} \_$in $=5.15$; - Mass flow rate in $(\mathrm{kg} / \mathrm{s})$

\begin{tabular}{|c|c|c|c|c|}
\hline \multicolumn{5}{|c|}{ Effects of Increasing Parameters } \\
\hline Parameter & Rise Time & Overshoot & Settling Time & S.S. Error \\
\hline$K_{\mathrm{p}}$ & Decrease & Increase & Small Change & Decrease \\
\hline$K_{\mathrm{i}}$ & Decrease & Increase & Increase & Eliminate \\
\hline$K_{\mathrm{d}}$ & Small Decrease & Decrease & Decrease & None \\
\hline
\end{tabular}


Since parameters in all control algorithms depend on process models, control algorithms will always be in error despite our best modeling efforts. Therefore, control algorithms should provide good performance when the adjustable parameters have "reasonable" errors. Naturally, all algorithms will give poor performance when the adjustable parameter errors are very large. This event occurs because no single algorithm can address all control requirements. A convenient feature of the PID algorithm is its compatibility with enhancements that provide capabilities not in the basic algorithm. The range of reasonable errors and their effects on accuracy in control performance will be closely studied and thoroughly discussed within this report.

\section{Design of P-I-D Controller}

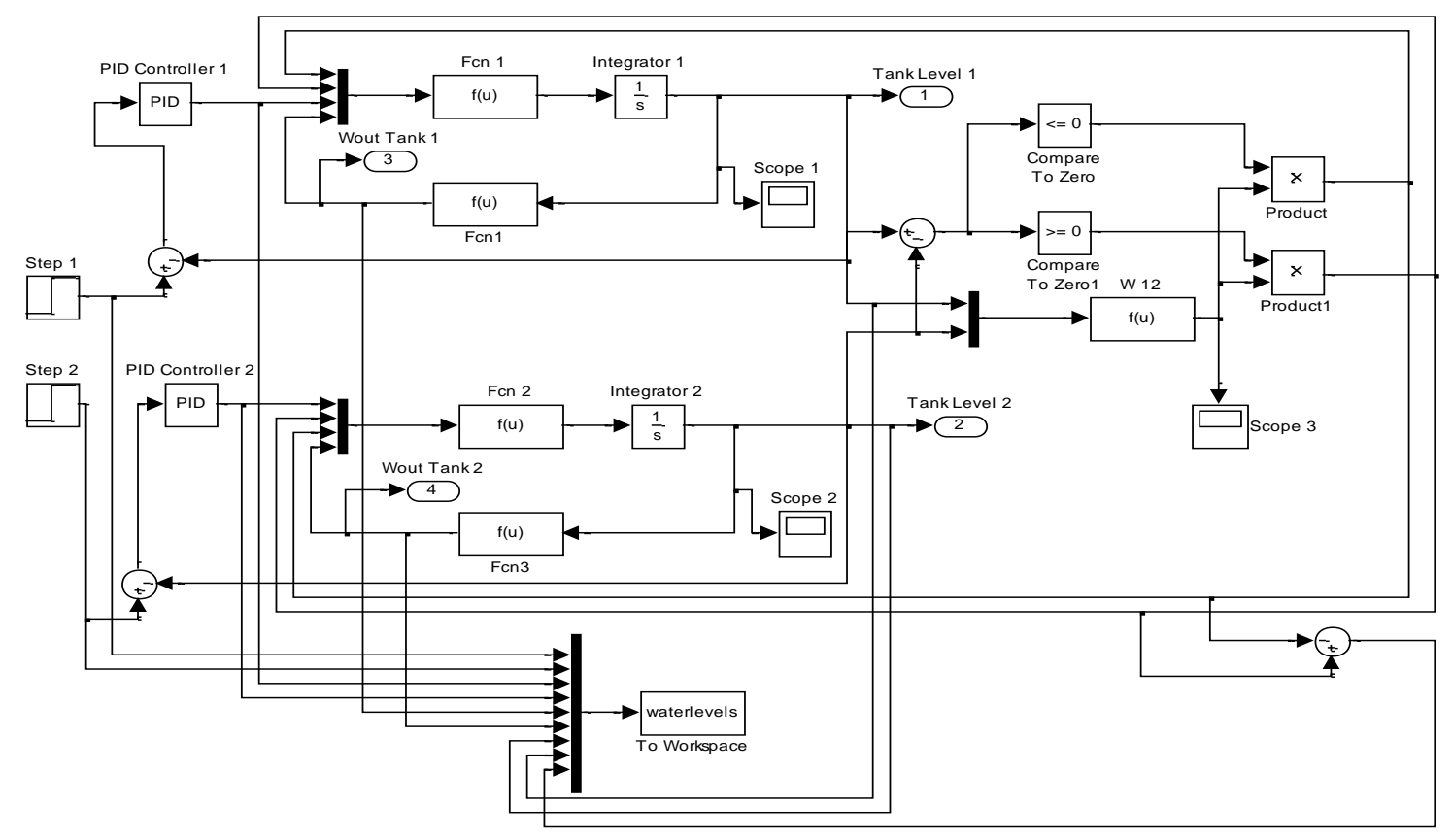

Figure 3. Schematic of Process Flow Loop created using SIMULINK 
The Simulink model in figure 3 displays the use of a proportional-integral-derivative (PID) controller, which attempts to correct the error between the measured process variable and a desired set point within the loop. This is accomplished by calculating and then outputting a corrective action that can adjust the process accordingly. The mux operation, centered on the bottom of the system, combines several input signals into a vector, which measures the water levels in both tanks. The three scopes also supply analytical information.

- Potential anomalies and fault recovery tables must be established to enable the autonomous system to handle different situations.

\subsection{Modeled System - Simulation}

\section{Water Level vs. Time}

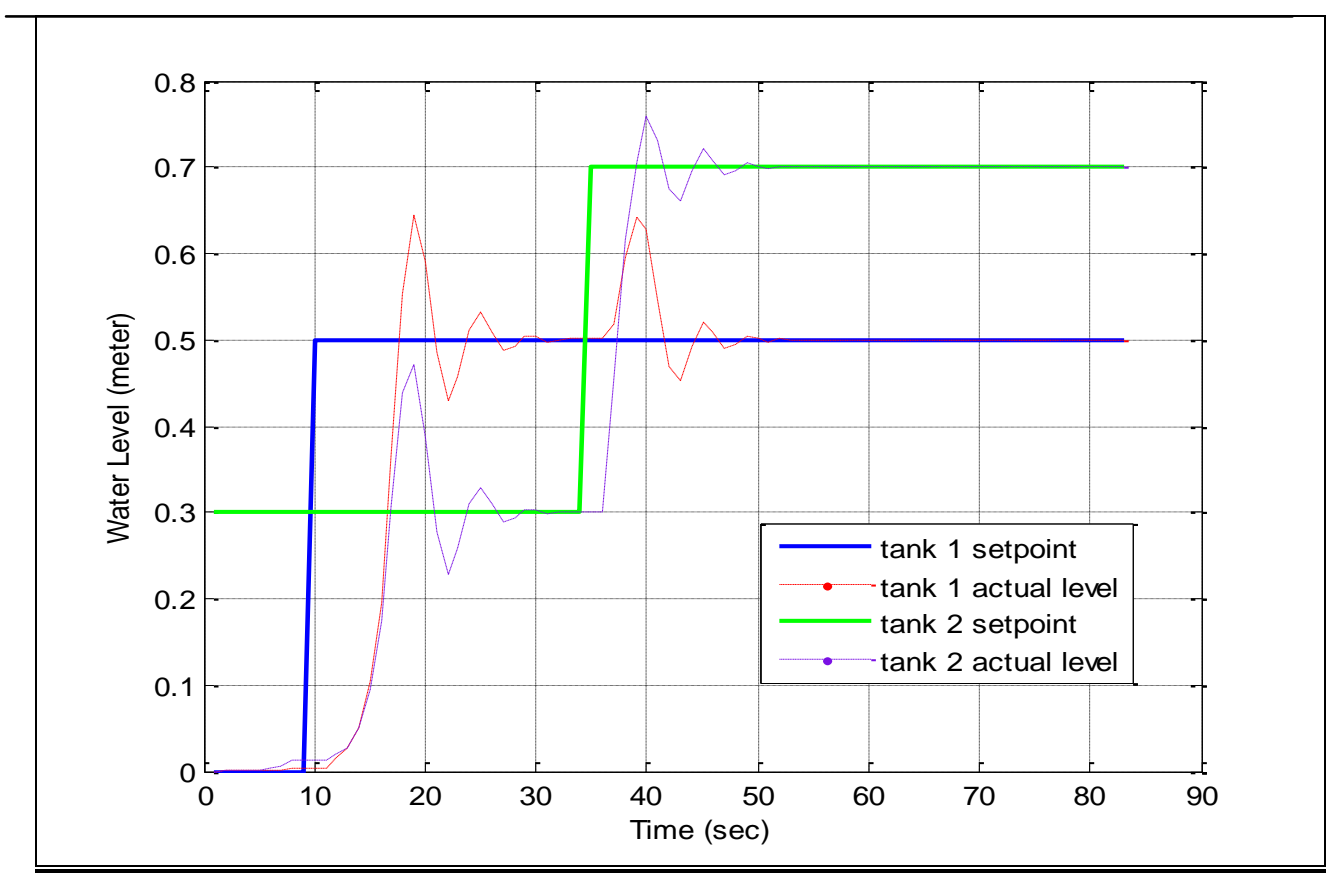

Figure 4. Water Level Change in the Two-Tank System.

Figure 4 displays the change of the water level in each tank over time. The solid blue line illustrates that the set point of water level in tank one goes from 0 to 0.5 meters at ten seconds. The solid green line displays that the set point of water level in tank two goes from 0.3 to 0.7 at 
thirty five seconds. The red-dashed line is the response of the actual water level in tank one and the purple-dashed line is the response of the actual water level in tank two. As you can see, both tanks exhibit oscillations at the beginning of each change. This occurs because the parameters of the P-I controller are not well tuned. Eventually the water levels in both tanks will settle down and adjust to the set points. Figure 5 and Figure 6 show the changes in the inlet and outlet flow rates change in both tanks.

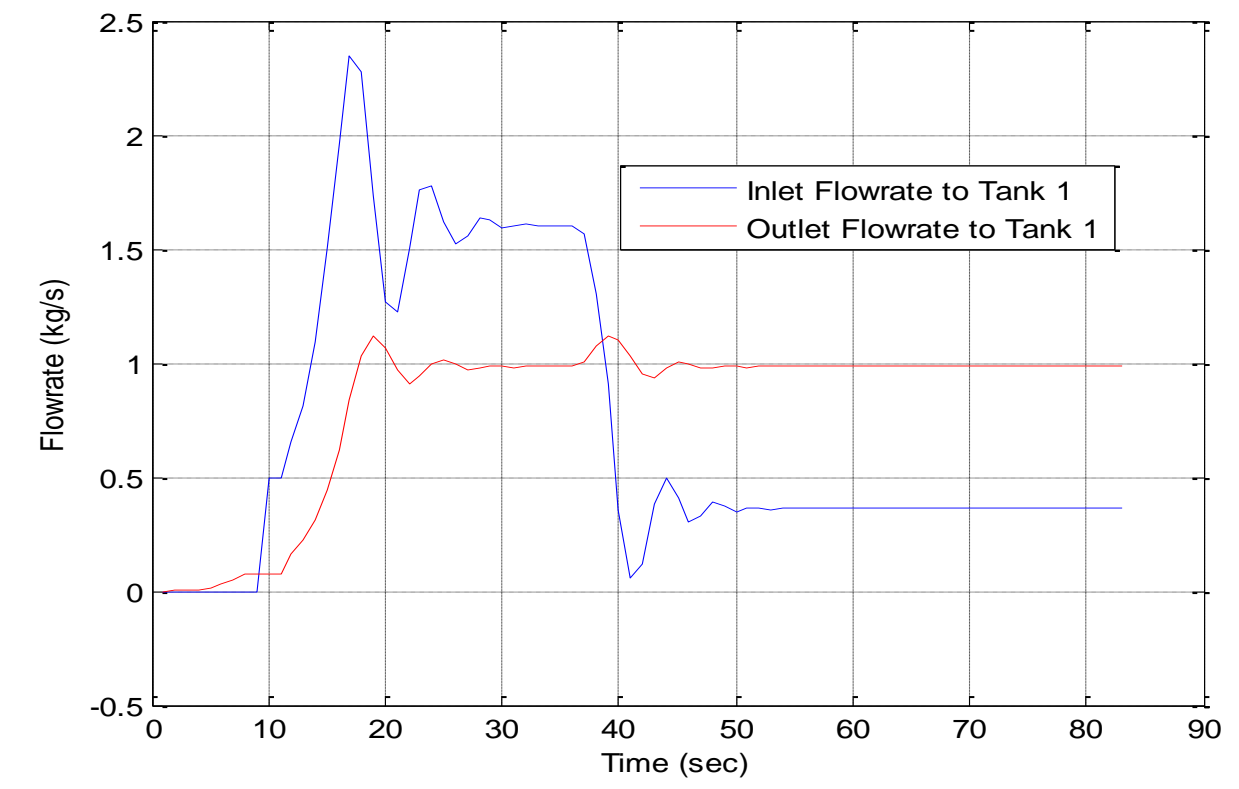

Figure 5. Displays Inlet and Outlet flow rate within Tank 1. 


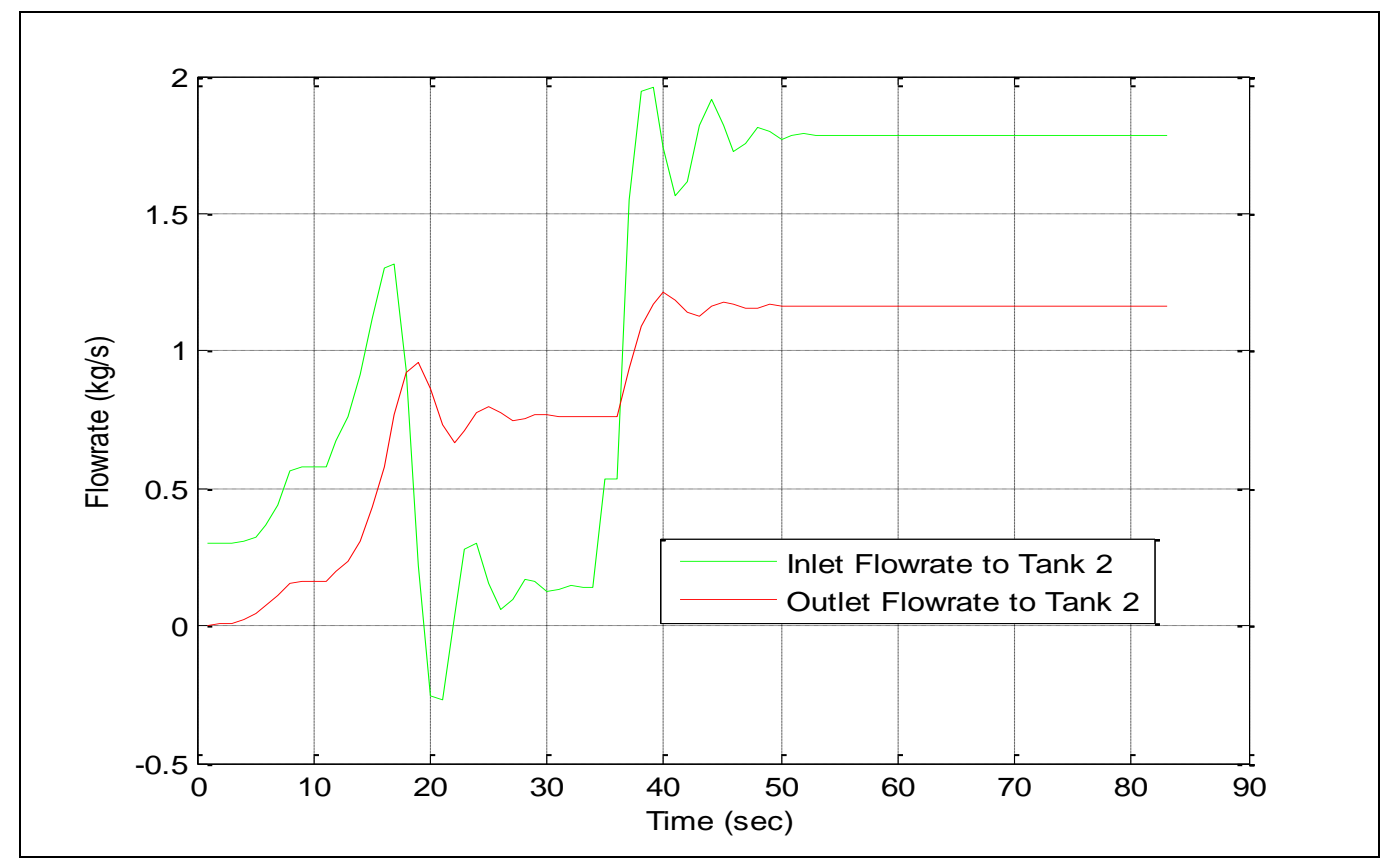

Figure 6. Display of the Inlet and Outlet Flow rates within Tank 2.

The plot in Figure 7 exhibits the flow rate measured in $(\mathrm{L} / \mathrm{sec})$ vs. Valve position at different input voltages. As you can see, the flow rate does not change until CV1 receives an input voltage greater than 4 . This problem is due to calibration and system fault detection error in the control valve. 


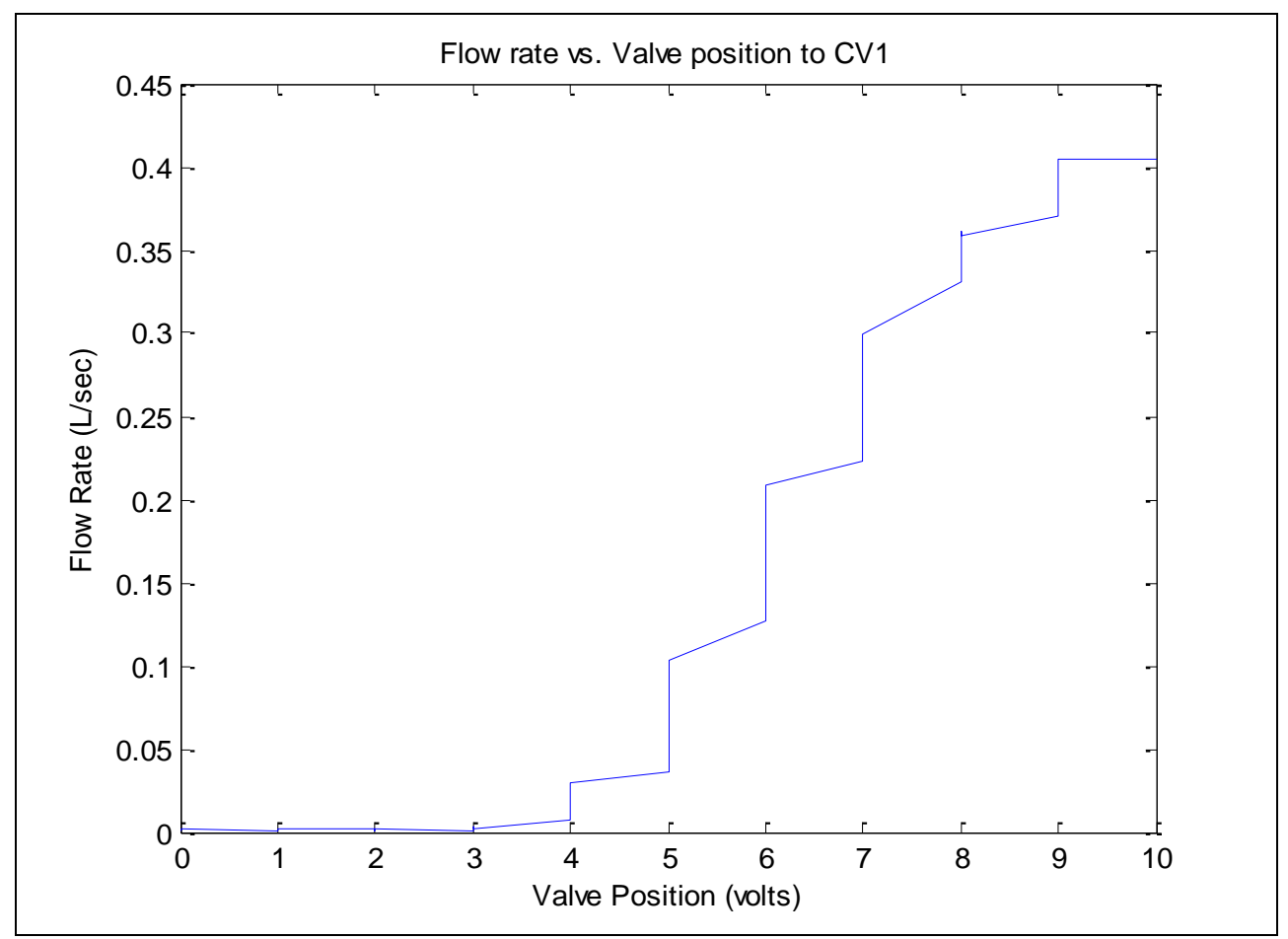

Figure 7

Once the PI algorithm was tuned accordingly, we could quickly detect the fault and maximize the valve positions control by adjusting the input voltage parameters. It has proven to be extremely vital throughout the duration of this research. The tuning technique was implemented to satisfy the systems design and output data of the tank level vs. the valve position, and work autonomously if a control valve where to overshoot or malfunction. The figure below illustrates the tuning and the steady change of the control valve. 


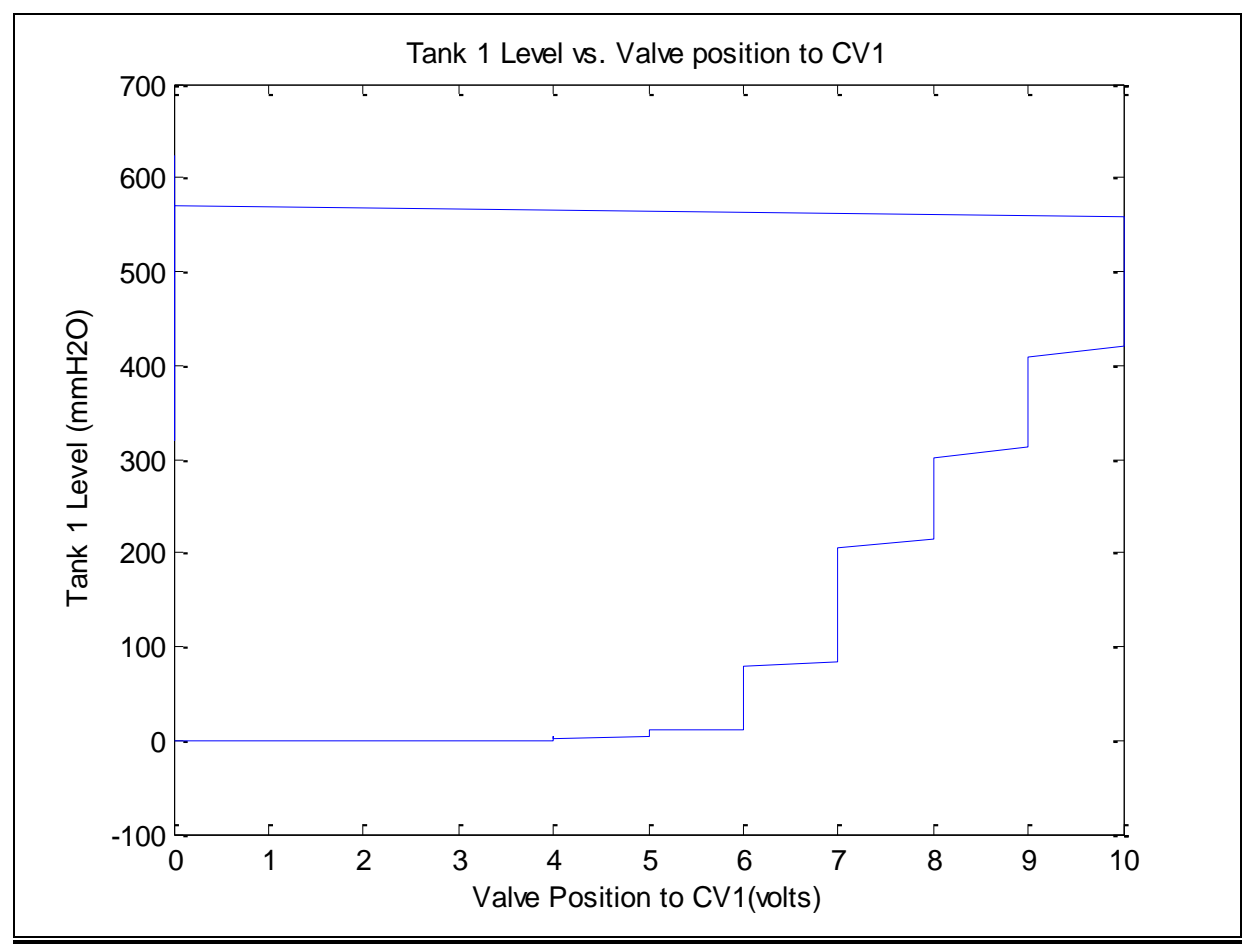

Figure 8

\section{Diagnostic and Prognostic Techniques}

This section discusses the use of empirical methods for process and equipment prognostics. Prognostic methods were developed to predict the remaining useful life (RUL), time-to-failure (TTF), and probability of failure (POF) in instrumentation and equipment. In this case, one can think of diagnosing conditions of plant components and systems by inputting data that characterizes fault detection. A brief example of fault detection can be as simple as crud layer thickness deposited on a control valves inner tube walls.

It is necessary to monitor the functionality of these systems and components, by identifying the measurements that reflect the health of its equipment. This can be done with empirical modeling techniques such as auto-associative kernel regression. These models give predictions of the sensor readings and compare them to the actual recorded data. This can be used to discover degradation parameters that can be used for prognostics.

Some of the prognostic techniques that were investigated in a course on empirical prognostic methods taught at the University of Tennessee, NE-697, can be used to estimate RUL of system components, which can help with maintenance scheduling and failure predictions for 
multiple systems. The prognostic techniques should be chosen based on the historical degradation parameter trends. Some of the prognostic techniques that can be considered are the modified version of the general path model or the Markov Chain model. These techniques are extremely vital in industrial applications involving active components.

\section{Concluding Remarks and Future Work}

* Incorporate advanced reactor monitoring, diagnostics, and prognostics capabilities in the design phase of the IRIS system.

* Test different control algorithms that are being developed under the project. Use the TTL data along with the Simulink model's data to test the adaptive non-parametric model (ANPM).

* Generate data with the operation under various fault conditions in the TTL. These may include sensor faults, actuator faults, controller faults, and etc. Simulate these faults within the TTL to investigate the fault detection capabilities of the ANPM.

* Test the sensitivity of the control algorithms due to uncertainties in physics models.

* Develop strategies for reconfiguring the control action due to faults in the system during operation. 


\section{REFERENCES}

- National Instruments, LabVIEW Measurements Manual, National Instruments, Austin, Texas, July 2000.

- T.E. Marlin, Process Control: Designing Processes and Control Systems for Dynamic Performance, $2^{\text {nd }}$ Edition, McGraw-Hill, New York, 2000.

- B. Mattison, M. Naghedolfeizi, and B.R. Upadhyaya, Development of a Graphical User Interface for a Dynamic Model of a Pressurized Water Reactor Plant, Nuclear Engineering Department, University of Tennessee, Knoxville, TN, September 2007.

- J.W. Hines, J. Garvey, J. Preston, and A. Usynin, Empirical Methods for Process and Equipment Prognostics, Nuclear Engineering Department, University of Tennessee, Knoxville, TN.

- M.R.A. Ali, Lumped Parameter, State Variable Dynamic Models for U-Tube Recirculation Type Nuclear Steam Generators, Doctoral Dissertation, Nuclear Engineering Department, The University of Tennessee, Knoxville, 1976.

- D.E. Holcomb, B.R. Upadhyaya, R.A. Kisner, J. O’Hara, D.D. Dudenhoeffer, E.L. Quinn, D.W. Miller, R.T. Wood, and S.R. Perillo, Instrumentation, Controls, and Human-Machine Interface Technology Development Roadmap for Grid-Appropriate Reactors, final report prepared for the Office of Nuclear Energy, Oak Ridge National Laboratory, Report Number: DE-AC05-00OR22725, June 2008. 


\section{Acronyms}

ANPM - Adaptive Non-Parametric Model

CV - Control Valve

DAQ - Data Acquisition Boards

DOE - United States Department of Energy

ICHMI - Instrumentation, Control, and Human Machine Interface

IRIS. - International Reactor Innovative \& Secure

I/O - Input /Output signal

LWR - Light-Water Reactor

MOV - Motor-Operated Valve

NERI-C - Nuclear Energy Research Initiative - Consortium

RT - Reliability Testing

RUL - Remaining Useful Life

TTL - Two-Tank Loop

TTF - Time to Failure

TFM - Turbine Flow Meter

VI -Virtual Instrumentation

POF - Probability of Failure

PID - Proportional-Integral-Derivative (controller) 


\title{
Advanced Instrumentation and Control Methods for Small and Medium Reactors with IRIS Demonstration
}

\author{
Final Report \\ Volume 2
}

IRIS Simulator Development and Measurement Sensitivity Analysis

Report No. DE-FG07-07ID14895/UTNE/2011-4

NERI-C Grant Number: DE-FG07-07ID14895

\author{
J. Michael Doster \\ (Co-Principal Investigator) \\ Paul J. Turinsky \\ North Carolina State University \\ Department of Nuclear Engineering \\ Raleigh, NC 27695 \\ E-mail: doster@eos.ncsu.edu \\ Lead Institution: \\ The University of Tennessee, Knoxville
}

May 2011 


\begin{abstract}
The accurate prediction of plant behavior during normal and off normal conditions requires accurate thermal-hydraulic models, as well as provisions for the simulation of auxiliary systems, mechanical components, and control functions. While these capabilities exist in varying degrees in large reactor safety codes, these codes were developed primarily for the simulation of loss of coolant and other design basis accidents where normal control functions may be assumed to be inactive or to operate in a way that makes the limiting responses of the plant more severe. As such, these codes are not optimized for the development and testing of control systems over the continuous full operating range. Codes of this type also generally require a long learning time and the source code is difficult, if not impossible, to modify. Similar issues are associated with detailed component design codes that have been developed to optimize the performance of a particular system component, such at the reactor core, steam generator, etc. This research focuses on the development of a simulation platform and the associated analysis of simulator uncertainty and non-linearity.

This report presents work in development of a high-fidelity simulator of the International Reactor Innovative and Secure (IRIS) reactor design, and in reducing the uncertainty in thermalhydraulic transient predictions for nuclear power plants (NPP) with a focus on SMRs characterized by the integral PWR design. The objective of a part of the study was to determine the economic benefit of conducting transient experiments on an SMR NPP. To accomplish this, a thermal-hydraulic simulator is used to complete data assimilation for input parameters to the simulator using experimental data generated by the plant. Since no such experimental data exists, it was generated using an altered simulator, referred to as the virtual NPP facilitating the investigation of the benefits of conducting various experiments and sensor deployment. The mathematical approach that is used to complete this analysis depends upon whether the system responses, i.e. sensor signals, and the system attributes, e.g. DNBR, are or are not linearly dependent upon the parameters. A linearity test showed that there exist highly nonlinear as well as mildly nonlinear responses, hence both deterministic and probabilistic methods were used to complete data assimilation and uncertainty quantification. For the mildly nonlinear transient, the Bayesian approach was used to obtain the parameters posteriori distributions assuming Gaussian distributions for the input parameters and responses. For the highly nonlinear transient, the Markov Chain Monte Carlo method was utilized based upon the Bayes theorem to estimate the
\end{abstract}


posteriori distributions of parameters. This report also discusses the optimization methodology used to design the plant's experiments so as to reduce a posteriori system attribute uncertainties. The optimization problem decision variables include the selection of sensor types and locations, and experiment type imposing realistic constraints, with the objective of maximizing the savings achieved by utilizing the larger degree of the plant operational freedom created by system attribute uncertainty reduction, offset by the cost of sensors and experiments. Finally this report presents an uncertainty analysis method and result for reactor control problems. For this problem, our goal is to select the optimum algorithm that minimizes the time integrated deviation of the actual state from the desired state accounting for uncertainties introduced during the design phase due to simulator employed and operations phase due to sensor uncertainties. To minimize the deviation from the desired values, multiple control algorithms were created and tested, and the optimum control algorithm was identified.

\section{DISCLAIMER}

Any opinions, findings, and conclusions or recommendations expressed in this report are those of the authors, and do not necessarily reflect the views of the U.S. Department of Energy or Westinghouse Electric Company, LLC. 


\section{TABLE OF CONTENTS}

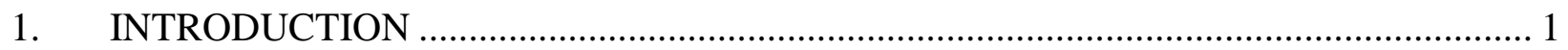

$1.1 \quad$ Significant Contributions ......................................................................................... 1

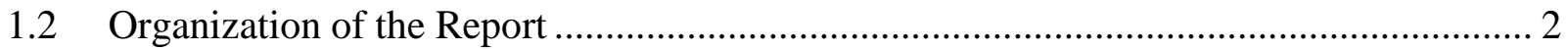

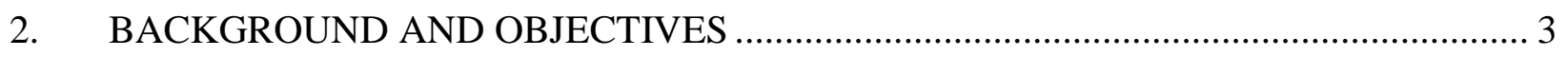

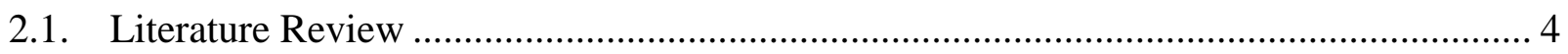

3. IRIS SYSTEM SIMULATOR DEVELOPMENT ……………………................... 5

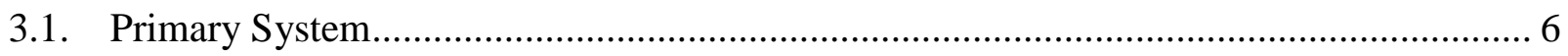

3.2. Secondary System and Balance of Plant …………..................................................... 7

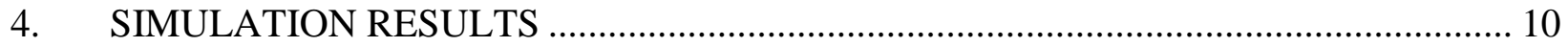

4.1. Normal Operational Transients ............................................................................ 10

4.2. Accident Analysis and Post Trip Behavior..................................................................... 17

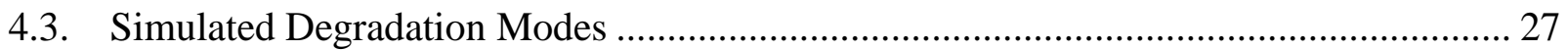

4.3.1. Steam Generator Fouling .................................................................................... 29

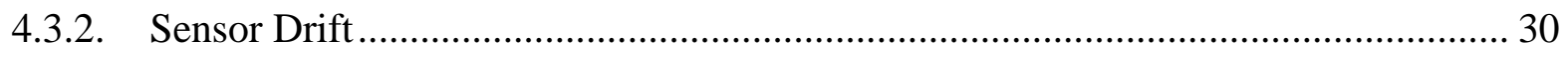

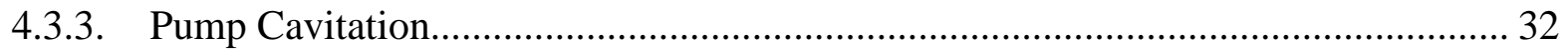

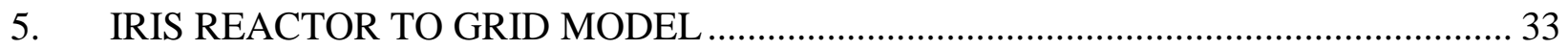

5.1. Fast Valving and Small Nuclear Reactor Applicability …………................................. 33

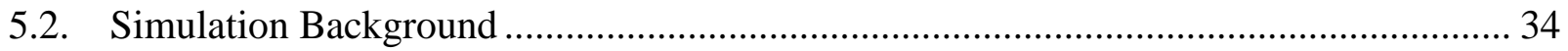

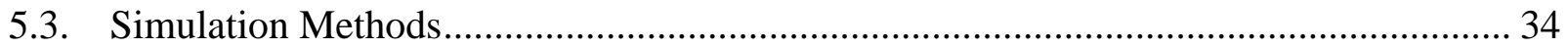

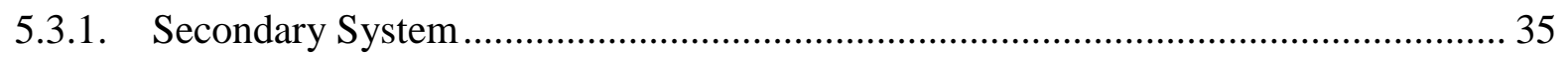

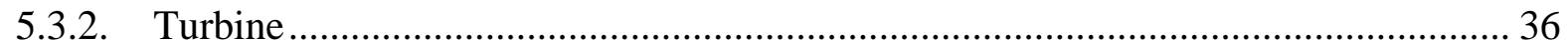

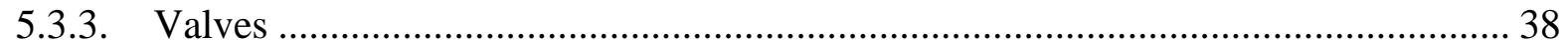

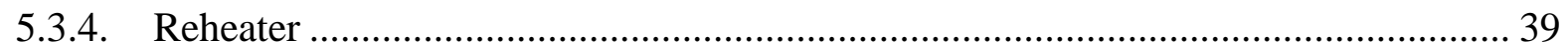

5.4. Generator and Grid Connection.................................................................................. 42

5.5. Low Pressure (LP) Turbine Benchmark ………………............................................... 45

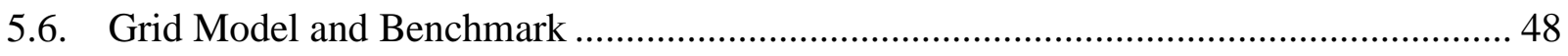

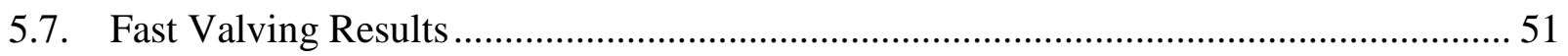

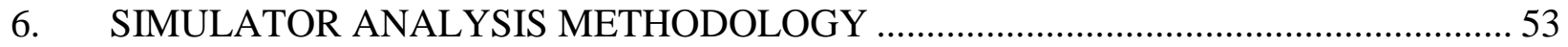

6.1. Linearity Test for the IRIS Reactor System ……………………………….............. 53 


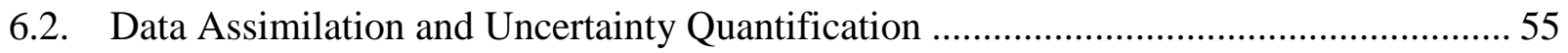

6.2.1. Deterministic Method for the Mildly Nonlinear Problems .................................... 55

6.2.2. MCMC Method for the Nonlinear Problems ....................................................... 63

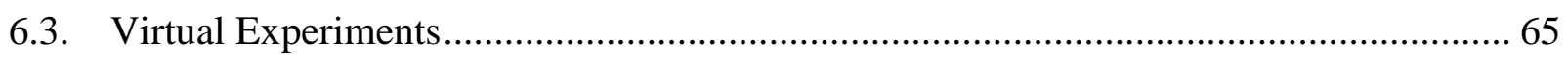

6.3.1. Reactor Coolant Pump (RCP) Trip at $30 \%$ and $80 \%$ Power ............................... 65

6.3.2. Control Bank Withdrawal at 70\% Power............................................................. 65

6.3.3. Feed Control Valve (FCV) Failed Open at 15\% Power ....................................... 66

6.3.4. Turbine Control Valve (TCV) Failed Open at 15\% Power ................................... 66

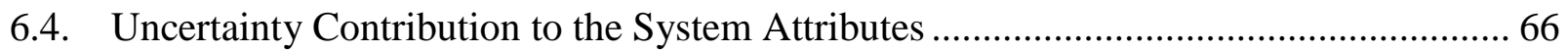

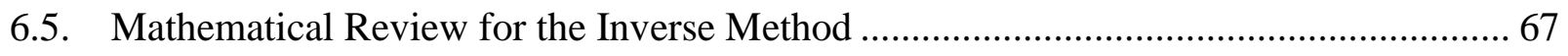

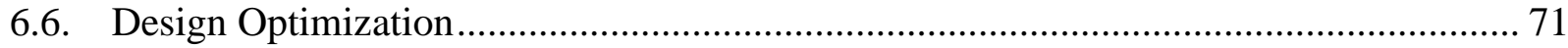

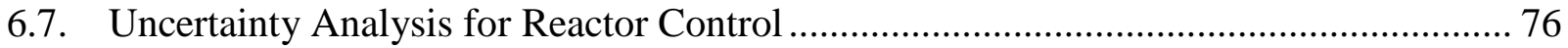

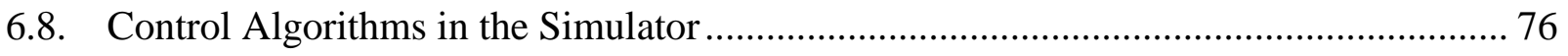

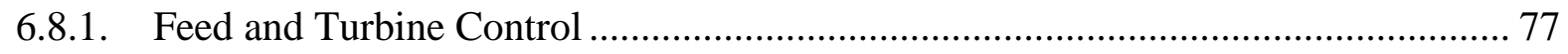

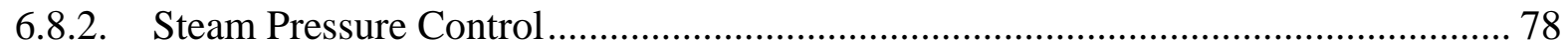

6.8.3. Reactivity (Power) Control ........................................................................ 79

6.8.4. Primary System Pressure Control …................................................................ 80

6.8.5. Case 1: $P_{x} \geq$ Pressure Set point 8 , where $P_{x}$ is the pressurizer pressure ............... 80

6.8.6. Case 2: Pressurizer Set point $8>P_{x} \geq$ Pressurizer Set point 7 ............................. 80

6.8.7. Case 3: Pressurizer Set point 7 $>P_{x}>$ Pressurizer Set point 6 ............................ 81

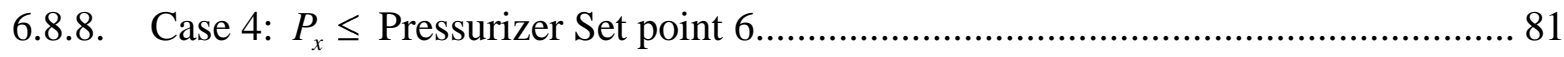

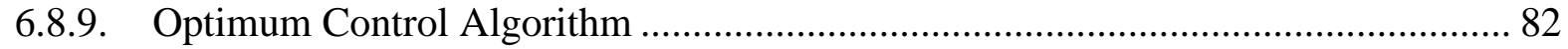

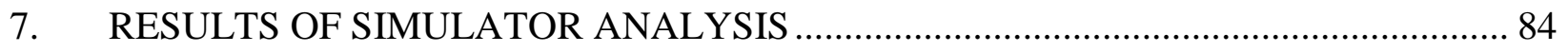

7.1. Mean and Standard Deviation of a Posteriori Input Parameters................................. 84

7.2. Standard Deviation of A Posteriori System Attributes............................................. 86

7.3. Uncertainty Contribution of Each Parameter ......................................................... 90

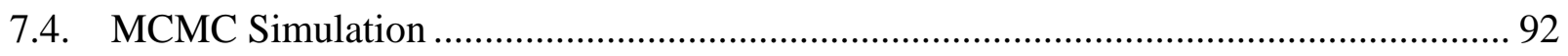

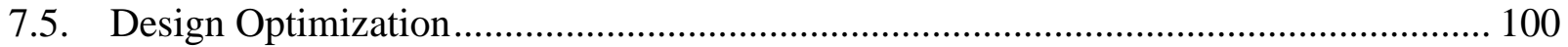

7.6. Uncertainty Analysis for Reactor Control ........................................................ 103

8. CONCLUDING REMARKS AND FUTURE WORK ….......................................... 108 


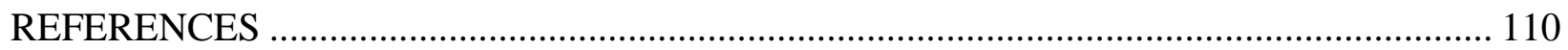

Appendix A. Additional Figures and Results ................................................................. 114

Appendix B. Derivation of the Deterministic Method........................................................ 132 


\section{LIST OF FIGURES}

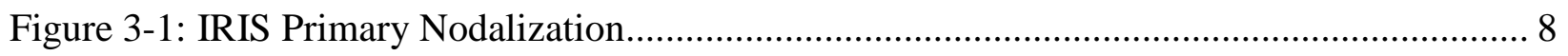

Figure 3-2: Balance of Plant Model................................................................................. 9

Figure 3-3: Feed Train Model ...................................................................................... 9

Figure 4-1: Turbine Output for 10\% Step Reduction in Load .............................................. 10

Figure 4-2: Feed Flow for a 10\% Step Reduction in Load .................................................... 11

Figure 4-3: Reactor Thermal Output and Peak Centerline Temperature for 10\% Step Reduction

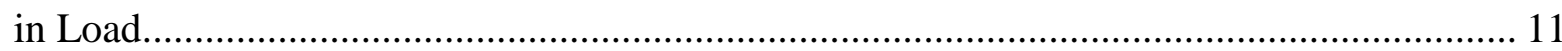

Figure 4-4: Primary System Pressure and Minimum DNB Ratio for 10\% Step Reduction in Load 12

Figure 4-5: Average Primary Coolant Temperature and Pressurizer Level for 10\% Step

Reduction in Load 12

Figure 4-6: Pressurizer Pressure and Heater Output for 10\% Step Reduction in Load ............... 13

Figure 4-7: Indicated and True Steam Pressure, 1\% Bias in Indicated Steam Pressure .............. 14

Figure 4-8: Turbine Control Valve Position, 1\% Bias in Indicated Steam Pressure .................. 14

Figure 4-9: Turbine Output, 1\% Bias in Indicated Steam Pressure ........................................ 15

Figure 4-10: Feed Flow, 1\% Bias in Indicated Steam Pressure............................................ 15

Figure 4-11: Reactor Thermal Output, 1\% Bias in Indicated Steam Pressure........................... 16

Figure 4-12: Feed Flow with Loss of One Hot-well Pump ................................................. 16

Figure 4-13: Feed Pump Speed and Control Valve Position with Loss of One Hot-well Pump.. 17

Figure 4-14: Reactor Power and Heat Transfer Rate............................................................ 18

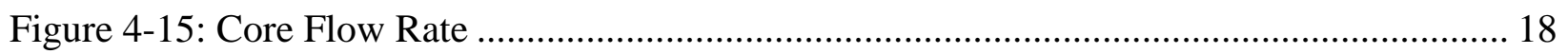

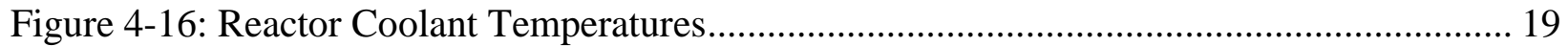

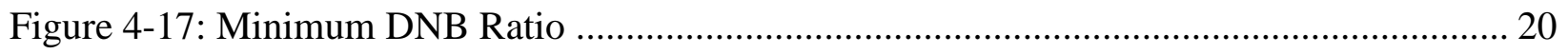

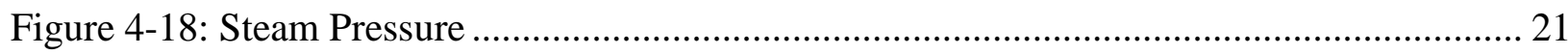

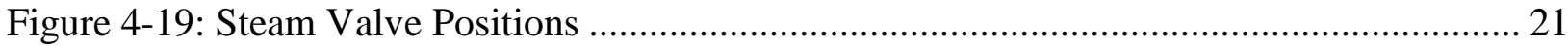

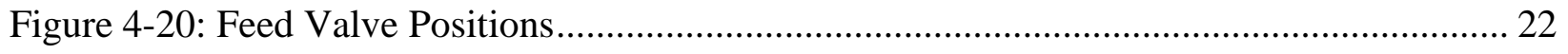

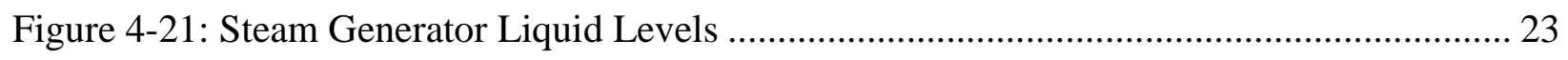

Figure 4-22: Reactor Power and Heat Transfer Rate ........................................................... 24

Figure 4-23: Steam Control Valve Positions .................................................................. 25 


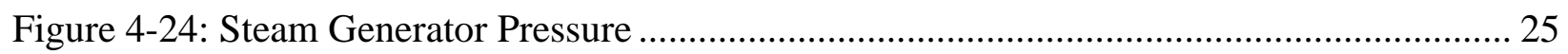

Figure 4-25: Startup Feedwater Valve Position .................................................................. 26

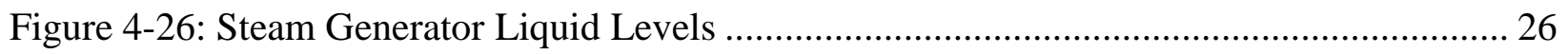

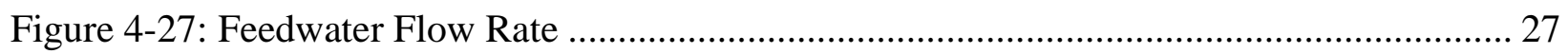

Figure 4-28: Assumed Daily Load Profile ...................................................................... 28

Figure 4-29: Percent Change in Steam Generator Thermal Resistance ......................................... 29

Figure 5-1: Model of the Infinite Bus Connection.................................................................. 35

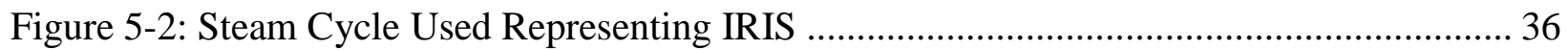

Figure 5-3: Turbine Model Calculation Flow ........................................................................ 37

Figure 5-4: IV Model - Flow Rate and Valve Characteristic ...................................................... 39

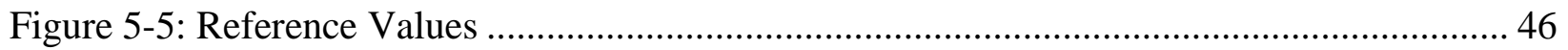

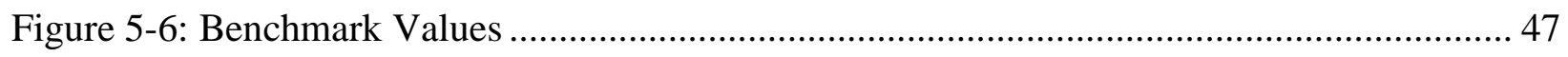

Figure 5-7: 10\% Step Decrease in Programmed Power............................................................ 48

Figure 5-8: Machine Angles through Transient ..................................................................... 49

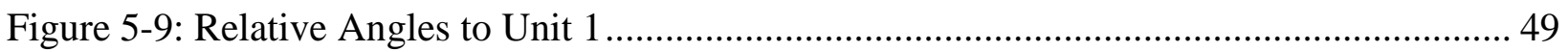

Figure 5-10: Nine Bus Transmission System and Added Load at Bus 8 ................................... 50

Figure 5-11: Rotor Speed through Transient Caused by Extra Electricity Demand...................... 50

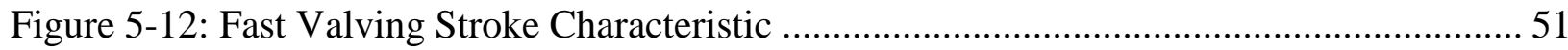

Figure 5-13: (a) Reheater Pressure and Mass Flow Rate and (b) Rotor Angle ............................. 52

Figure 5-14: (a) Hot and Cold Leg Temperature and (b) Dry-out Location.................................. 52

Figure 6-1: Pressurizer Heater Output .................................................................................... 54

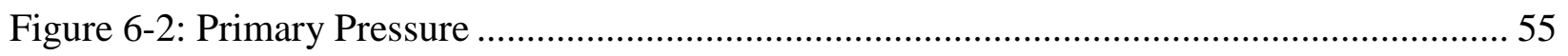

Figure 6-3: A Priori and a Posteriori System Attribute Distributions .......................................... 74

Figure 6-4: Pressurizer Heater Set points .............................................................................. 80

Figure 7-1: Standard Deviations for the Input Parameters for Experiment A at 80\% Power....... 86

Figure 7-2: Standard Deviations for the Input Parameters for Experiments A, B, C, and D........ 86 Figure 7-3: Standard Deviations for Average Fuel Temperature for RCP Trip Using Experiment A at $80 \%$ Power for Data Assimilation 87

Figure 7-4: Standard Deviations for Hot Channel Fuel Centerline Temperature for RCP Trip using Experiment A at 80\% Power for Data Assimilation..................................................... 88 
Figure 7-5: Standard Deviations for Hot Channel MDNBR for RCP Trip using Experiment A at $80 \%$ Power for Data Assimilation. 88

Figure 7-6: Standard Deviations for Average Fuel Temperature for RCP Trip using Experiment

A, B, C and D for Data Assimilation 89

Figure 7-7: Standard Deviations for Hot Channel Fuel Centerline Temperature for RCP Trip

using Experiment A, B, C and D for Data Assimilation 89

Figure 7-8: Standard Deviations for Hot Channel MDNBR for RCP Trip using Experiment A, B, $\mathrm{C}$ and $\mathrm{D}$ for Data Assimilation. 90

Figure 7-9: Each Parameter's Uncertainty Contribution to the System Attributes for RCP Trip 91 Figure 7-10: Each Parameter's Uncertainty Contribution to the System Attributes for RCP Trip as a Result of Multiple Experiments ....................................................................... 92

Figure 7-11: Accepted input parameter vector samples ..................................................... 94

Figure 7-12: A posteriori distribution of the parameters using RCP Trip at 30\% Power........... 95

Figure 7-13: A posteriori distribution of the parameters using RCP Trip at 30\% Power (Cont.) 96 Figure 7-14: A posteriori distribution of the parameters using RCP Trip at 30\% Power (Cont.) 97 Figure 7-15: A posteriori distribution of the parameters using RCP Trip at 80\% Power............ 98 Figure 7-16: A posteriori distribution of the parameters using RCP Trip at 80\% Power (Cont.) 99 Figure 7-17: A posteriori distribution of the parameters using RCP Trip at $80 \%$ Power (Cont.)

Figure 7-18: Design Savings for Each Scenario 102

Figure 7-19: System Responses obtained Simulating 100 Samples for 10\% Step Load Changes at 70\% Power using Control Algorithm F1Q1 for Uncertainty Analysis 106

Figure 7-20: System Responses obtained Simulating 100 Samples for 20\% Ramp Load Changes at 70\% Power using Control Algorithm F1Q1 for Uncertainty Analysis . 107

Figure A-1: Standard Deviations for the Input Parameters for Experiment B ....................... 115

Figure A-2: Standard Deviations for the Input Parameters for Experiment C ....................... 116

Figure A-3: Standard Deviations for the Input Parameters for Experiment D ....................... 116 Figure A-4: Standard Deviations for Average Fuel Temperature for Control Bank Withdrawal using Experiment B for Data Assimilation 117

Figure A-5: Standard Deviations for Hot Channel Fuel Centerline Temperature for Control Bank Withdrawal using Experiment B for Data Assimilation ............................................. 117 
Figure A-6: Standard Deviations for Hot Channel MDNBR for Control Bank Withdrawal using

Experiment B for Data Assimilation 118

Figure A-7: Standard Deviations for Average Fuel Temperature for FCV Failed Open using

Experiment $\mathrm{C}$ for Data Assimilation. 118

Figure A-8: Standard Deviations for Hot Channel Fuel Centerline Temperature for FCV Failed

Open using Experiment $\mathrm{C}$ for Data Assimilation.

Figure A-9: Standard Deviations for Hot Channel MDNBR for FCV Failed Open using

Experiment $\mathrm{C}$ for Data Assimilation.

Figure A-10: Standard Deviations for Average Fuel Temperature for TCV Failed Open using

Experiment D for Data Assimilation.

Figure A-11: Standard Deviations for Hot Channel Fuel Centerline Temperature for TCV Failed

Open using Experiment D for Data Assimilation

Figure A-12: Standard Deviations for Hot Channel MDNBR for TCV Failed Open using

Experiment D for Data Assimilation.

Figure A-13: Standard Deviations for Average Fuel Temperature for Control Bank Withdrawal

using Experiment A, B, C and D for Data Assimilation.

Figure A-14: Standard Deviations for Hot Channel Fuel Centerline Temperature for Control

Bank Withdrawal using Experiment A, B, C and D for Data Assimilation..... 122

Figure A-15: Standard Deviations for Hot Channel MDNBR for Control Bank Withdrawal using

Experiment A, B, C and D for Data Assimilation. 122

Figure A-16: Standard Deviations for Average Fuel Temperature for FCV Failed Open using

Experiment A, B, C and D for Data Assimilation. 123

Figure A-17: Standard Deviations for Hot Channel Fuel Centerline Temperature for FCV Failed

Open using Experiment A, B, C and D for Data Assimilation 123

Figure A-18: Standard Deviations for Hot Channel MDNBR for FCV Failed Open using

Experiment A, B, C and D for Data Assimilation. 124

Figure A-19: Standard Deviations for Average Fuel Temperature for TCV Failed Open using

Experiment A, B, C and D for Data Assimilation. 124

Figure A-20: Standard Deviations for Hot Channel Fuel Centerline Temperature for TCV Failed

Open using Experiment A, B, C and D for Data Assimilation 125 
Figure A-21: Standard Deviations for Hot Channel MDNBR for TCV Failed Open using

Experiment A, B, C and D for Data Assimilation.

Figure A-22: Each Parameter's Uncertainty Contribution to the System Attributes for Control

Bank Withdrawal

Figure A-23: Each Parameter's Uncertainty Contribution to the System Attributes for FCV

Failed Open

Figure A-24: Each Parameter's Uncertainty Contribution to the System Attributes for TCV

Failed Open

Figure A-25: Each Parameter's Uncertainty Contribution to the System Attributes for Control

Bank Withdrawal as a Result of Multiple Experiments

Figure A-26: Each Parameter's Uncertainty Contribution to the System Attributes for FCV

Failed Open as a Result of Multiple Experiments.

Figure A-27: Each Parameter's Uncertainty Contribution to the System Attributes for TCV

Failed Open as a Result of Multiple Experiments. 


\section{LIST OF TABLES}

Table 4-1: Control and Process Sensor Noise...................................................................... 28

Table 4-2: Impact of Steam Generator Fouling at $100 \%$ power ............................................. 29

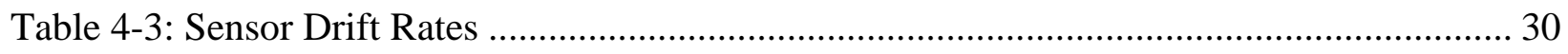

Table 4-4: Impact of Steam Pressure Sensor Drift at $100 \%$ power ......................................... 31

Table 4-5: Impact of Primary Pump Degradation at 100\% Power ........................................... 32

Table 7-1: Virtual Experiment Values and Input Parameters Adapted for Experiment A at 80\%

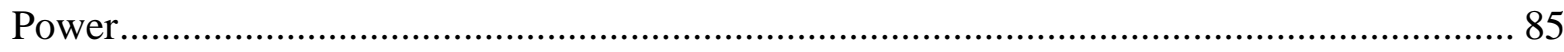

Table 7-2: Virtual Experiment Values and Input Parameters Adapted using Experiments A, B, C,

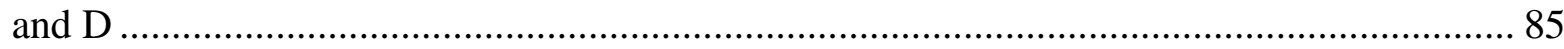

Table 7-3: Deviations for 10\% Step Load Changes at 70\% and 90\% Power using Four Different Control Algorithms 104

Table 7-4: Operational Constraint Probabilities for 10\% Step Load Changes at $70 \%$ and $90 \%$ Power using Four Different Control Algorithms 104

Table 7-5: Deviations for 20\% Ramp Load Changes at 70\% Power using Four Different Control Algorithms..... 105

Table 7-6: Operational Constraint Probabilities for 20\% Ramp Load Changes at 70\% Power

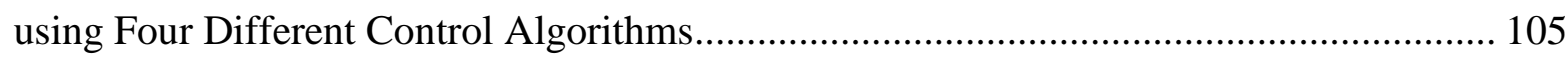

Table A-1: Virtual Experiment Values and Input Parameters Adapted for Experiment B ........ 114

Table A-2: Virtual Experiment Values and Input Parameters Adapted for Experiment C ........ 114 Table A-3: Virtual Experiment Values and Input Parameters Adapted for Experiment D ....... 114 


\section{INTRODUCTION}

The accurate prediction of plant behavior during normal and off normal conditions requires accurate thermal-hydraulic models, as well as provisions for the simulation of auxiliary systems, mechanical components, and control functions. While these capabilities exist in varying degrees in large reactor safety codes, these codes were developed primarily for the simulation of loss of coolant and other design basis accidents where normal control functions may be assumed to be inactive or to operate in a way that makes the limiting responses of the plant more severe. As such, these codes are not optimized for the development and testing of control systems over the continuous full operating range. Codes of this type also generally require a long learning time and the source code is difficult, if not impossible, to modify. Similar issues are associated with detailed component design codes that have been developed to optimize the performance of a particular system component, such at the reactor core, steam generator, etc. This research focuses on the development of a simulation platform with the capability to support:

1) Sensitivity/uncertainty analysis methodologies to address sensor deployment analysis.

2) The development of hierarchical control architectures with model-based, robust controllers.

3) Integrated monitoring, diagnostic and prognostic systems for SMRs.

4) The integration of SMRs into finite grids and the impact of these systems on grid stability.

\subsection{Significant Contributions}

The following is a summary of the achievements of the project during the project period.

- A high-fidelity simulation of the IRIS system was developed to simulate normal and faulted operation.

- Faulted operation was simulated for four fault types: steam generator fouling, control sensor drift, process sensor drift, and pump cavitation.

- The effects of fast valving were investigated and simulated for small reactor applications.

- The impact of SMRs on a modeled grid and its stability were studied.

- Chi-Squared tests on the simulated results were completed for transients assuming inactive reactor controllers to determine the degree of nonlinearity of the system responses and attributes with respect to parameters. 
- Data assimilation was completed on the parameters by solving an inverse problem using regularization and iteration method to address mild nonlinearity. This was done by defining realistic experiments, defining limiting accidents, and determining the posteriori uncertainties of the key system attributes for the limiting accidents.

- The Markov Chain Monte Carlo (MCMC) simulation was completed to determine the posteriori uncertainties of the key system attributes for the Reactor Coolant Pump Trip transient simulation for which one observed relatively large Chi-Squared values.

- Each parameter's uncertainty contribution to the system attributes was determined to identify major sources of uncertainty.

- Mathematical review for the inverse method was completed to identify the major source of disagreement between the a posteriori and the virtual experiment model parameters, assuming data assimilation is performed using one time dependent observable, two parameters and their covariance.

- An optimization problem was solved to determine sensor types and locations, and experiment type with the objective of maximizing the savings achieved by utilizing the larger plant design space achieved by uncertainty reduction.

- The uncertainty analysis accounting for simulator and sensor uncertainties, and used in an optimization, was conducted for reactor control problems to minimize the time integrated deviation of the actual state from the desired state, imposing both safety and operational constraints. The optimum control algorithm was identified by creating and testing multiple control algorithms.

\subsection{Organization of the Report}

This report presents the work performed to complete the tasks and contributions outlined above. The following chapter discusses pertinent background information on simulation and uncertainty analysis. Chapter 3 describes the development of a high-fidelity IRIS simulator, and Chapter 4 summarizes the results of nominal and faulted simulation. Chapter 5 introduces the methods used to model the IRIS reactor attachment to a grid and fast valving for grid stability. The methodologies used in assessing the simulator in terms of uncertainty and non-linearities are outlined in Chapter 6. The results of those analyses are discussed in Chapter 7. Finally, Chapter 8 gives conclusions drawn from this work and suggestions for future work. Additional figures and mathematical derivations are given in the appendices. 


\section{BACKGROUND AND OBJECTIVES}

Estimating nuclear reactor performance during a transient has been the main issue in thermal hydraulic safety research since nuclear energy was first used to produce electricity. For the IRIS reactor that may be built and operated for the first time, simulation models have been developed and tested for the safety research using multiple thermal-hydraulic equations coupled with each other [Shen, 2006; Hines et al., 2008; Hines et al., 2009]. The quality of the plant system simulator predictions will impact the reactor economy through the introduction of margins on the reactor design to ensure an operation, in which the safety and operational limits are satisfied with a high degree of certainty. How tight or relaxed these margins are depends on how accurate the predictions of reactor behavior are. The uncertainties of reactor simulator calculations are thus important to the determination of these margins. The IRIS research has been focused on a design that provides the highest degree of safety and cost effective arrangement. That is, by reducing uncertainties, safety can be maintained while costs are reduced.

The fidelity of the prediction cannot be evaluated directly unless the validation is performed using suitable measurements in nuclear plants. A great deal could be learned from experiment data to enhance simulation fidelity for a nuclear reactor. In particular, input parameters can be refined assuming it is the major source of uncertainties. Using adjusted thermal hydraulic input parameters and their reduced uncertainties, subsequent reactor simulation improves the prediction of key system attributes. The objective of this work is to reduce the uncertainties on limiting system attributes during accident transients. To accomplish this experiments are to be performed, data collected, and the data utilized to complete data assimilation on the thermal-hydraulic input parameters to the system simulation code, in our case the IRIS system simulator. This was done by using the IRISN.Ver08.Mod06 [Shen, 2006] developed at NC State to complete virtual experiments and generate their associated experimental data. Historical research on the thermal hydraulic reactor system indicates that the system responses are usually nonlinear, which adds complexity to data assimilation. To determine whether the nonlinearities would exist for experiments performed at lower power initial conditions, and if so, utilize a data assimilation method capable of addressing nonlinearity, IRIS system simulations can be completed. 


\subsection{Literature Review}

The uncertainty and sensitivity analysis for the thermal hydraulic reactor system has been performed for uncertainty evaluation of best estimate Loss of Coolant Accident (LOCA) analysis. The best estimate plus uncertainty (BEPU) method was introduced and developed in 1980s to support licensing. The code scaling, applicability, and uncertainty (CSAU) evaluation method was developed for large break loss of coolant accident in a pressurized water reactor [Boyack et al., 1990]. The CSAU methodology contains 14 steps organized into three major elements: Requirements and Code Capabilities, Assessment and Ranging of Parameters, and Sensitivity and Uncertainty Analysis. It was developed based on 25 years of experimental data, and included development of the embedded Phenomena Identification \& Ranking Table (PIRT) methodology. The PIRT has become a standard accepted throughout the international nuclear community providing guidance in executing cost effective BEPU applications. In 1996, the USNRC approved a best estimate loss of coolant accident method based on CSAU and the WCOBRA/TRAC thermal hydraulic code. Since the first uncertainty analysis performed with the CSAU method, several different methods have been proposed for uncertainty and sensitivity analysis. One of them is Uncertainty Analysis Methodology based on Accuracy Extrapolation (UMAE) which introduces the model validation [D'Auria et al., 1995]. It is based on the extrapolation of the accuracy resulting from a comparison between the code prediction and the experimental data obtained in small scale facilities.

Westinghouse developed a new BEPU method called Automated Statistical Treatment of Uncertainty Method (ASTRUM) which was approved in 2004 [Frepoli et al., 2004]. The ASTRUM is still based on the WCOBRA/TRAC thermal hydraulic code but it utilizes a nonparametric (distribution free) statistical sampling technique. Since it eliminates the superposition penalty, this technique is expected to reduce the predicted Peak Cladding Temperature (PCT). The BEMUSE (Best Estimate Method - Uncertainty and Sensitivity Evaluation) phase 3 benchmark assesses the reliable application of high quality best estimate and uncertainty and sensitivity evaluation methods [De Crecy et al., 2007]. It shows the method and results of the peak cladding temperature estimation conducted by ten participants from nine organizations and seven countries. The main characteristic of the approach used by participants is the propagation of the input parameters' uncertainties relying on probabilistic analysis associated with Wilks' formula. In Monte Carlo analysis, a probabilistic based sampling is used to develop a mapping 
from input parameters to system responses. Several possible sampling methods exist, including simple random sampling, stratified sampling and Latin Hypercube Sampling (LHS). Due to demanding calculation requirements, the Monte Carlo method is usually not applicable to a large scale thermal hydraulic system simulation code. For the neutronic problem, a more deterministic approach was used for the BEPU analysis. Efficient Subspace Method (ESM) was developed utilizing Singular Value Decomposition (SVD) and used to deal with ill conditioned matrices [Abdel-Khalik, 2004; Jessee, 2008]. ESM is an efficient method for the neutronic system which has multiple parameters. In order to deal with the reactor physics problem that has many more parameters than the thermal hydraulic problem has, adjoint sensitivity analysis was also developed to reduce the calculation effort [Cacuci, 2000]. The adjoint method is known to be very powerful for a system that has multiple input parameters, but sometimes inapplicable for the large scale thermal hydraulic system due to nonlinearity of the responses over their ranges of uncertainties. Nowadays BEPU methods are widely used in the world and the industry is more focusing on development of new licensing BEPU methods. Even though many papers deal with the uncertainty evaluation methods, none of them presents the method which tremendously reduces the computing demand for thermal hydraulic system calculations. In addition, limited research has been conducted to value the benefits of performing additional experiments for the large scale thermal hydraulic system associated with a power reactor, which can possibly reduce a posteriori uncertainties of the system attributes. In the following section, the methods that solve the nonlinear thermal hydraulic system based on the virtual experimental model using both deterministic and probabilistic methods will be presented.

\section{IRIS SYSTEM SIMULATOR DEVELOPMENT}

The accurate prediction of plant behavior during normal and off normal conditions requires accurate thermal-hydraulic models, as well as provisions for the simulation of auxiliary systems, mechanical components, and control functions. While these capabilities exist in varying degrees in large reactor safety codes, these codes were developed primarily for the simulation of loss of coolant and other design basis accidents where normal control functions may be assumed to be inactive or to operate in a way that makes the limiting responses of the plant more severe. As such, these codes are not optimized for the development and testing of control systems over the continuous full operating range. Codes of this type also generally 
require a long learning time and the source code is difficult, if not impossible, to modify. Similar issues are associated with detailed component design codes that have been developed to optimize the performance of a particular system component, such at the reactor core, steam generator, etc. In this work, the emphasis is on the simulation of plant response and control over the full range of operating conditions. Functionally, the plant simulation should execute quickly, require

minimal up-front effort by the user, be easily modified, and allow for ease in the integration and testing of new control strategies and components. The models should allow the simulation of both the physical attributes of the system, as well as the responses of the sensors employed to measure various attributes that are observable, including uncertainties in these measurements.

A high fidelity, full plant simulator of the IRIS reactor [Carelli, 2003] has been developed, and is capable of predicting its dynamic response during normal and off-normal operating conditions. The IRIS simulator includes a model of the IRIS pressurizer, a full kinetics model, a decay heat model and a hot channel/DNB model. In the IRIS design, the steam generators are helical coils, where the secondary fluid flows on the tube side of the heat exchanger. Detailed models have been developed to describe the dynamics of steam generators of this design.

The model includes balance of plant, instrumentation and control systems, and plant protection systems including the normal spectrum of reactor trips and engineered safety features. Options are available to degrade sensor signals for flow, level, pressure, etc. as the result of noise and drift, in addition to failing the sensor all together. Additional sensor models can be easily added. The simulator models also provide ease of access to correlations for heat transfer, pressure loss, void fraction, etc.

\subsection{Primary System}

The IRIS simulator assumes the primary side fluid remains sub-cooled, i.e. two phase flow on the primary side is not considered. Twenty six nodes are considered for computing the primary side fluid temperature distribution and the time dependent flow equations allow for the simulation of both forced (pumped) and natural circulation flows. The reactor coolant pump model allows for a user specified pump performance curve. In the power range, reactor power is determined through a six delayed group, point kinetics model, with rod position controlled through a user specified $\mathrm{T}_{\text {ave }}$ program. An eleven group decay heat model allows for the 
simulation of power history dependent decay heat rates. Reactivity feed backs include fuel and moderator temperature, where fuel temperature is determined by solution of the time dependent heat conduction equation.

Figure 3-1 illustrates the primary side nodalization.

\subsection{Secondary System and Balance of Plant}

Secondary system models are typical of current Light Water Reactor (LWR) designs, and consist of the main steam lines, turbine/generator and the condensate/feed system. Models are also provided for steam dump and turbine by-pass systems, main steam safety valves and steam and feed isolation systems. The balance of plant model is illustrated in Figure 3-2 below. A turbine model had been incorporated to allow electrical demand to be specified as a boundary condition and the feed control model upgraded to reflect that envisioned for the final IRIS design. The turbine control valves operate to meet the steam demand while maintaining a constant pressure in the steam generators. Similarly the turbine bypass valves are currently assumed to operate only in the pressure control mode. To support preliminary investigations of grid stability and grid upset conditions, a turbine-generator/grid model has been added to allow examination of these issues.

Feed control in the power range is based upon electrical demand, similar to control strategies employed in B\&W Once Through Steam Generators. The current feed train model is illustrated in

Figure 3-3. Feed heaters are not modeled explicitly. Instead, feed temperature is assumed to be a function of steam demand (power level). The hot well, condensate and feed pumps are described by user input pump performance curves. The hot well and condensate pumps are fixed speed. The feed pumps are assumed to be variable speed, with the speed controller based on maintaining a constant $\Delta \mathrm{P}$ from the feed manifold to the steam generators. 


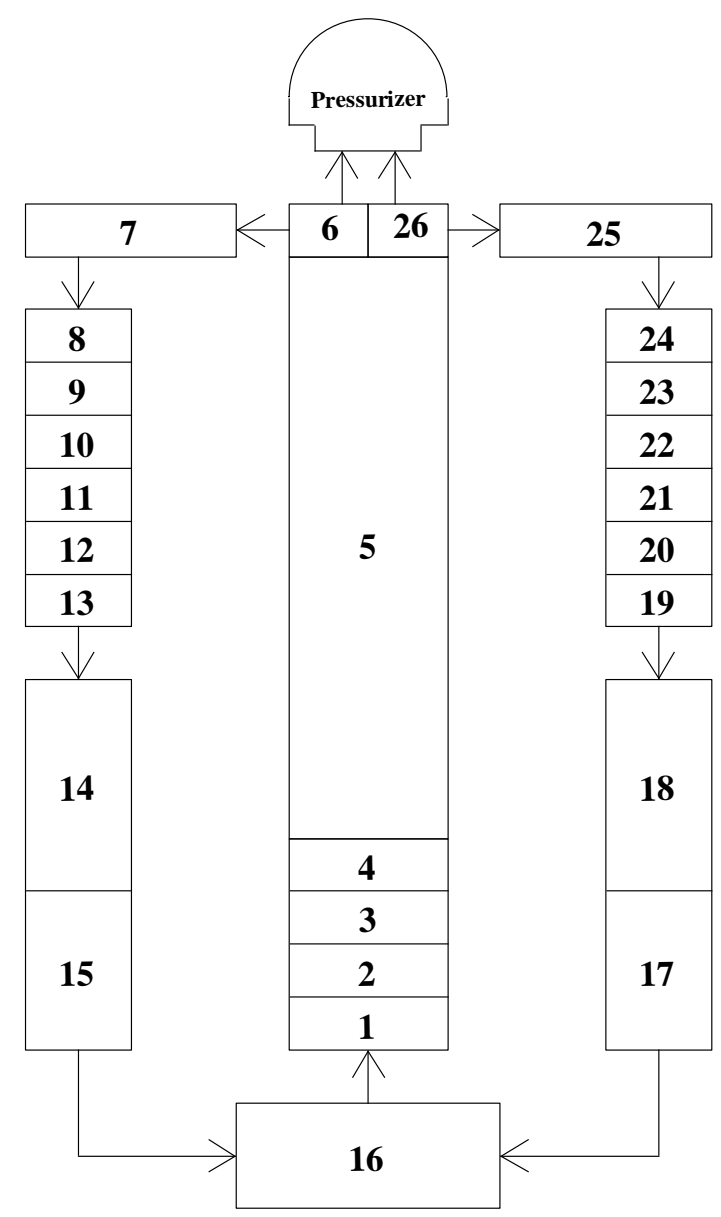

\begin{tabular}{|l|l|}
\hline Nodes & Description \\
\hline $1-4$ & Reactor Core \\
\hline 5 & Riser \\
\hline 6,26 & RCP Inlet Plenum \\
\hline 7,25 & RCP Nodes \\
\hline $8-13,19-24$ & Steam Generator \\
\hline $14-15,17-18$ & Downcomer \\
\hline 16 & Lower Plenum \\
\hline
\end{tabular}

Figure 3-1: IRIS Primary Nodalization 


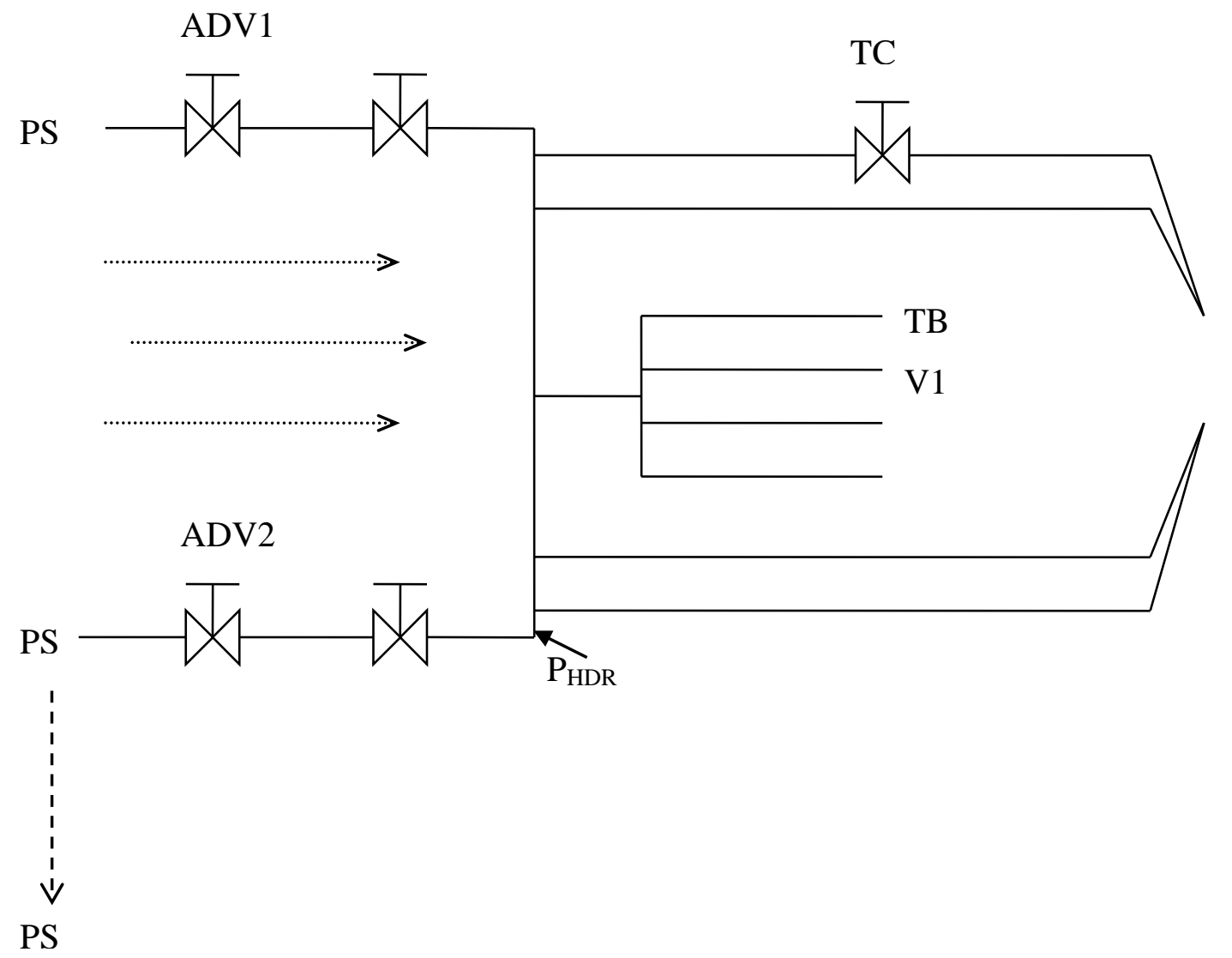

Figure 3-2: Balance of Plant Model

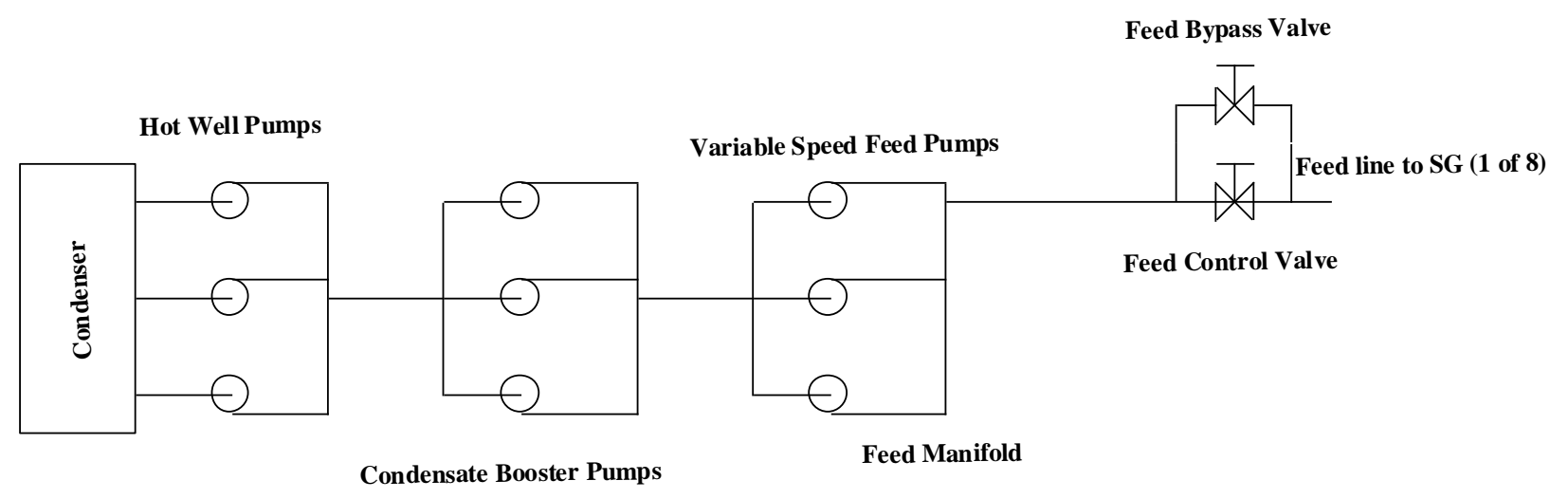

Figure 3-3: Feed Train Model 


\section{SIMULATION RESULTS}

\subsection{Normal Operational Transients}

The simulator is capable of describing normal power maneuvers, as well as a number of upset conditions and sensor failures. Figure 4-1 through Figure 4-6 illustrate a 10\% step reduction in load from $100 \%$ to $90 \%$. Of particular interest is the response of the secondary side to the load reduction. As opposed to Pressurized Water Reactors which operate with recirculating U-Tube steam generators, in the IRIS Once-Through design the turbine control valves modulate to hold steam pressure at its setpoint. Feed demand is effectively proportional to the turbine load signal. As a result, feed demand responds instantly to the step change in load and then is "shimmed" to insure that the turbine output ultimately matches the load. Primary side response is typical of other Pressurized Water Reactors (PWR).

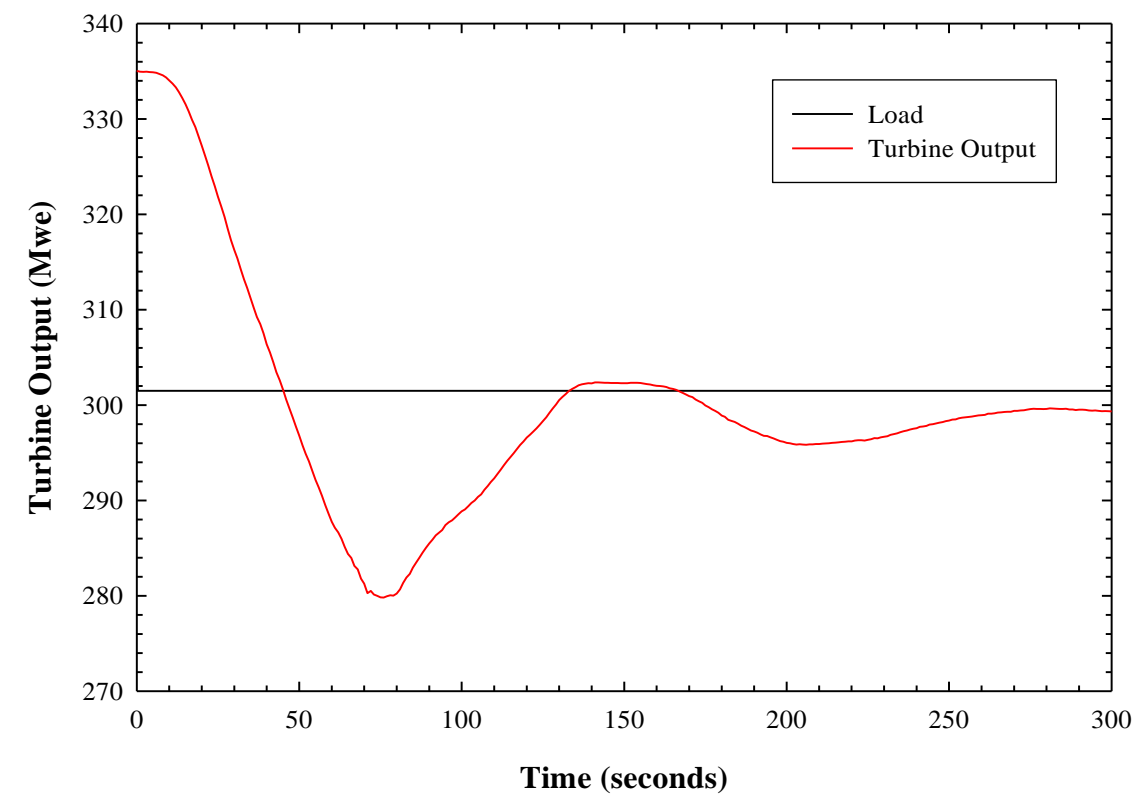

Figure 4-1: Turbine Output for 10\% Step Reduction in Load 


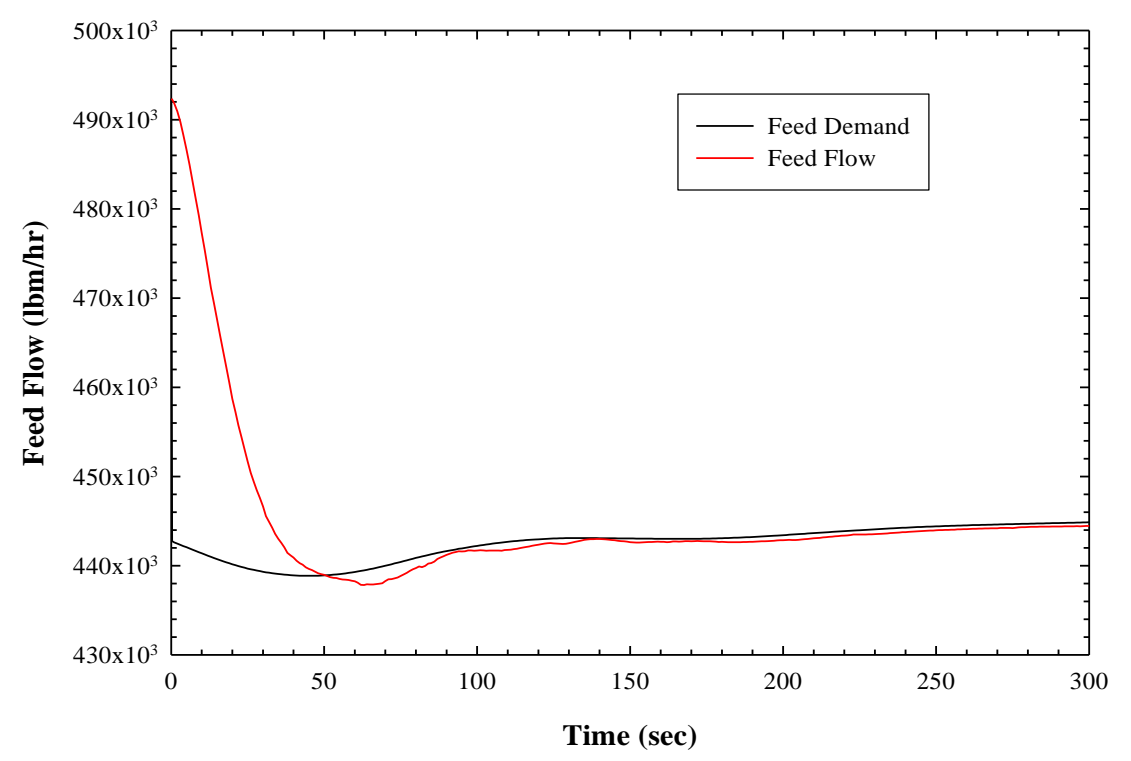

Figure 4-2: Feed Flow for a 10\% Step Reduction in Load

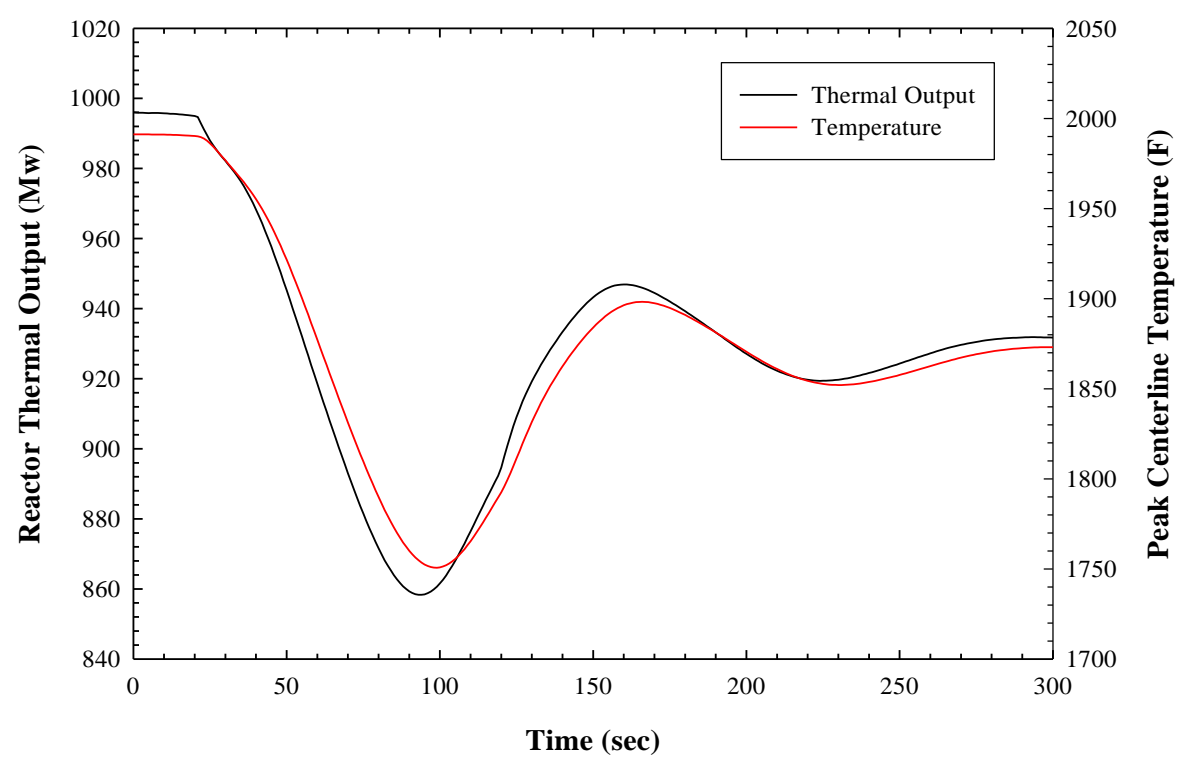

Figure 4-3: Reactor Thermal Output and Peak Centerline Temperature for 10\% Step Reduction in Load 


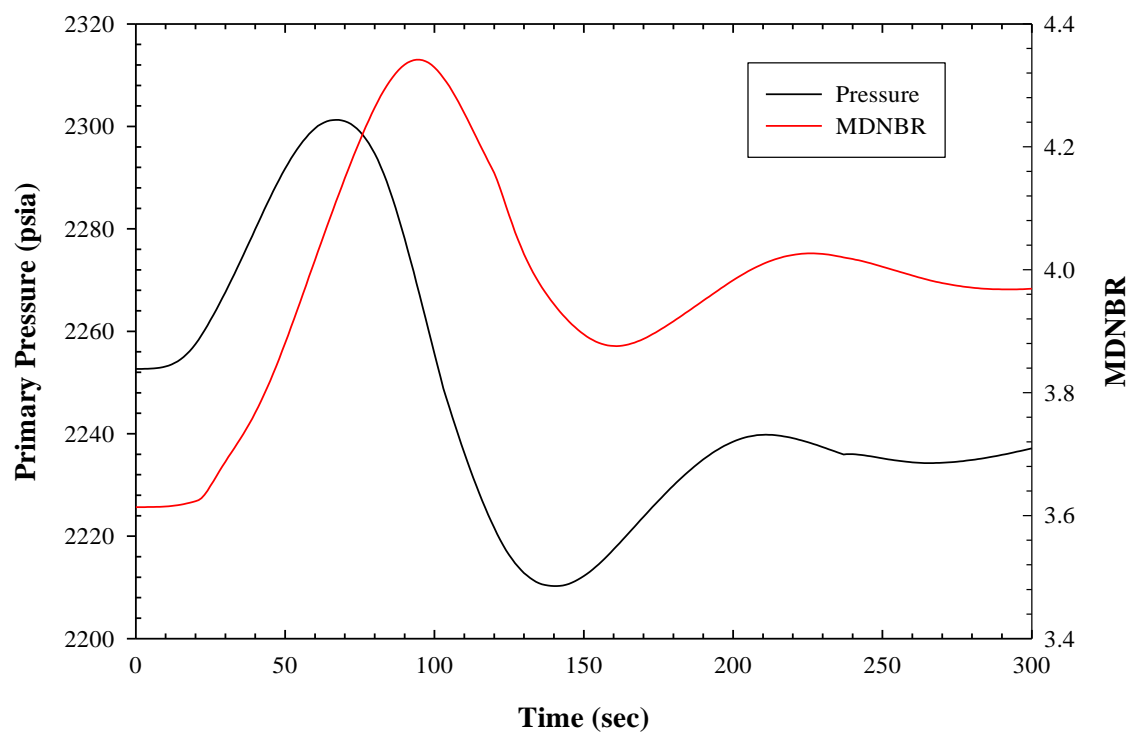

Figure 4-4: Primary System Pressure and Minimum DNB Ratio for 10\% Step Reduction in Load

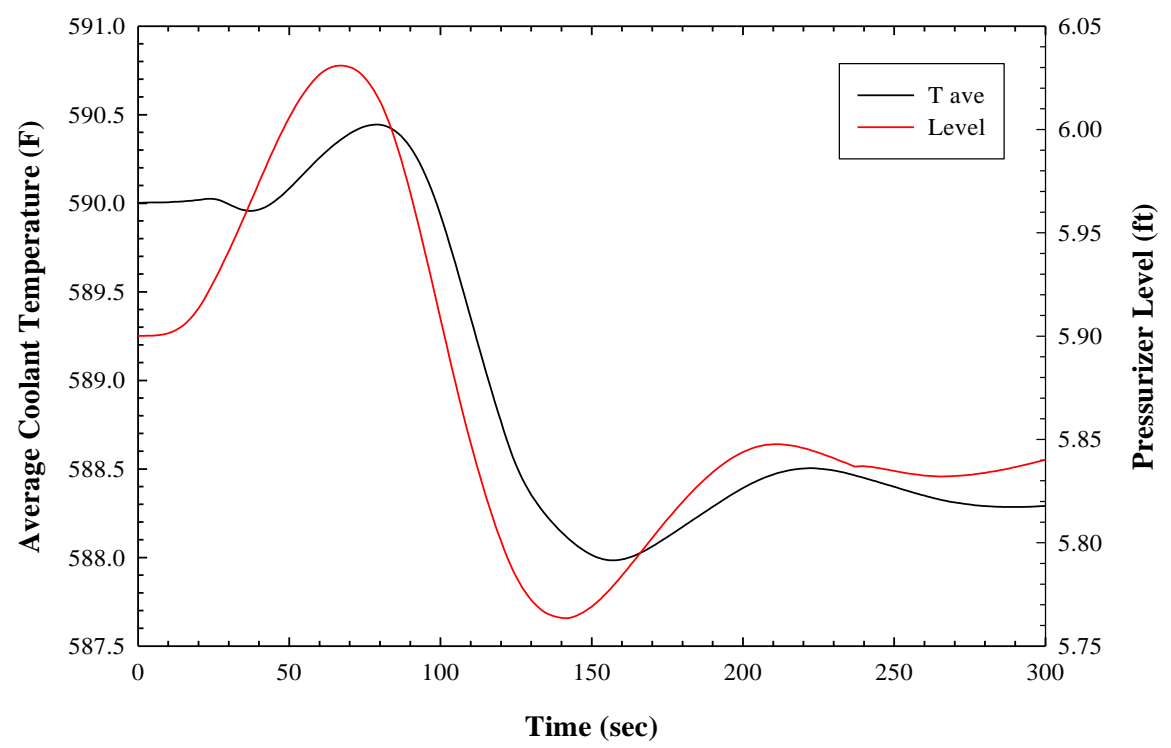

Figure 4-5: Average Primary Coolant Temperature and Pressurizer Level for 10\% Step Reduction in Load 


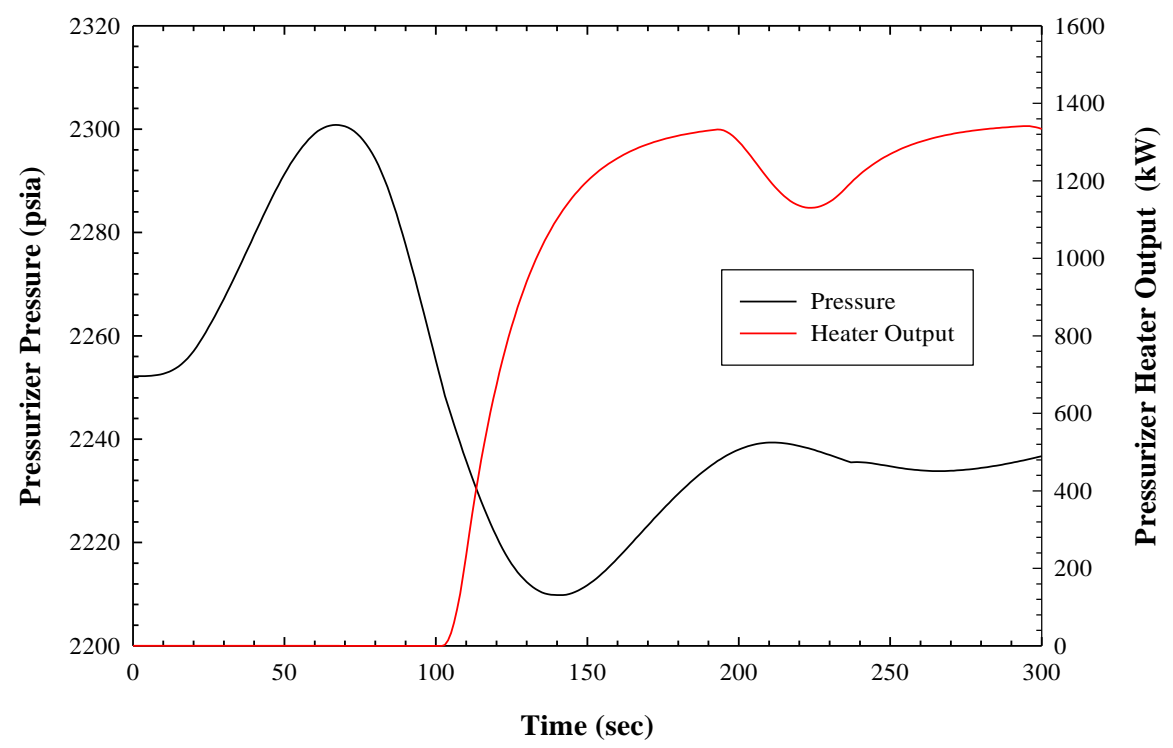

Figure 4-6: Pressurizer Pressure and Heater Output for 10\% Step Reduction in Load

Figure 4-7 - Figure 4-11 illustrate the system response to a $1 \%$ bias being imposed on the steam pressure signal. The increase in indicated steam pressure causes the turbine control valves to open to restore steam pressure to its set point value. The increased steam flow results in an increase in the turbine output and a reduction in the true steam pressure. The mismatch between turbine output and load generates a reduction in the feed demand signal which restores the turbine output to its demand value. The reduction in steam pressure reduces the average primary side temperature causing reactor power to increase. Though not shown, control rods move to bring the average primary side temperature back in line with the $\mathrm{T}_{\mathrm{ave}}$ program. 


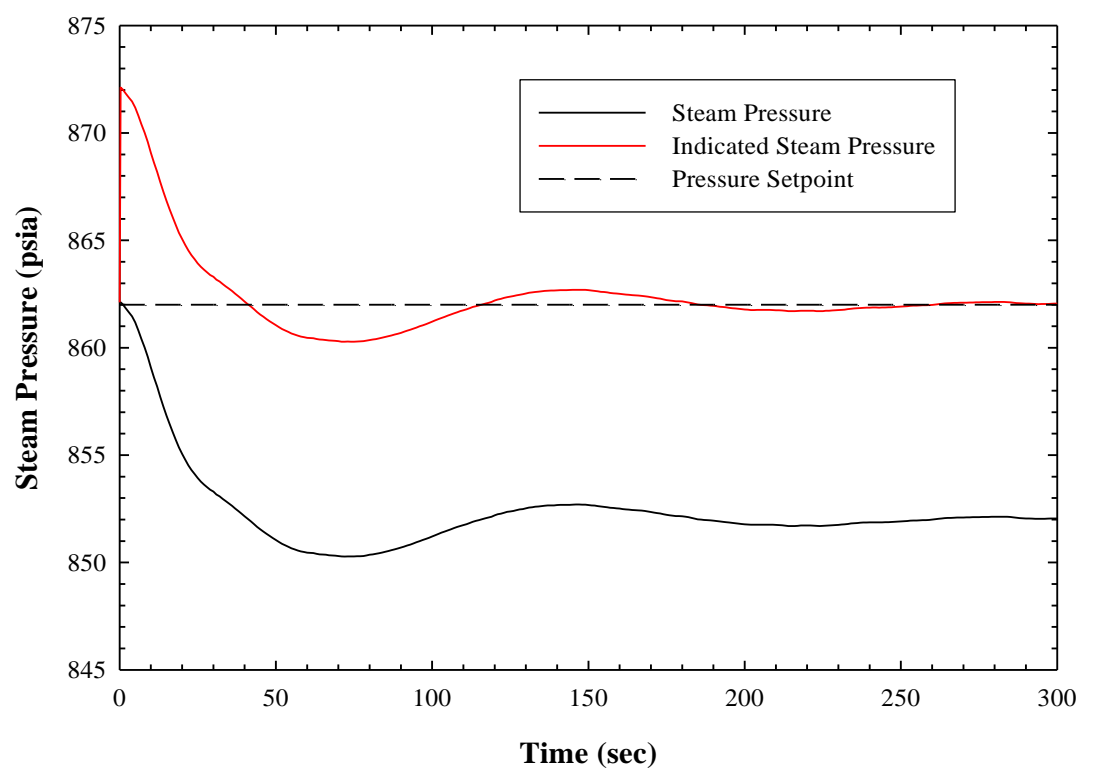

Figure 4-7: Indicated and True Steam Pressure, 1\% Bias in Indicated Steam Pressure

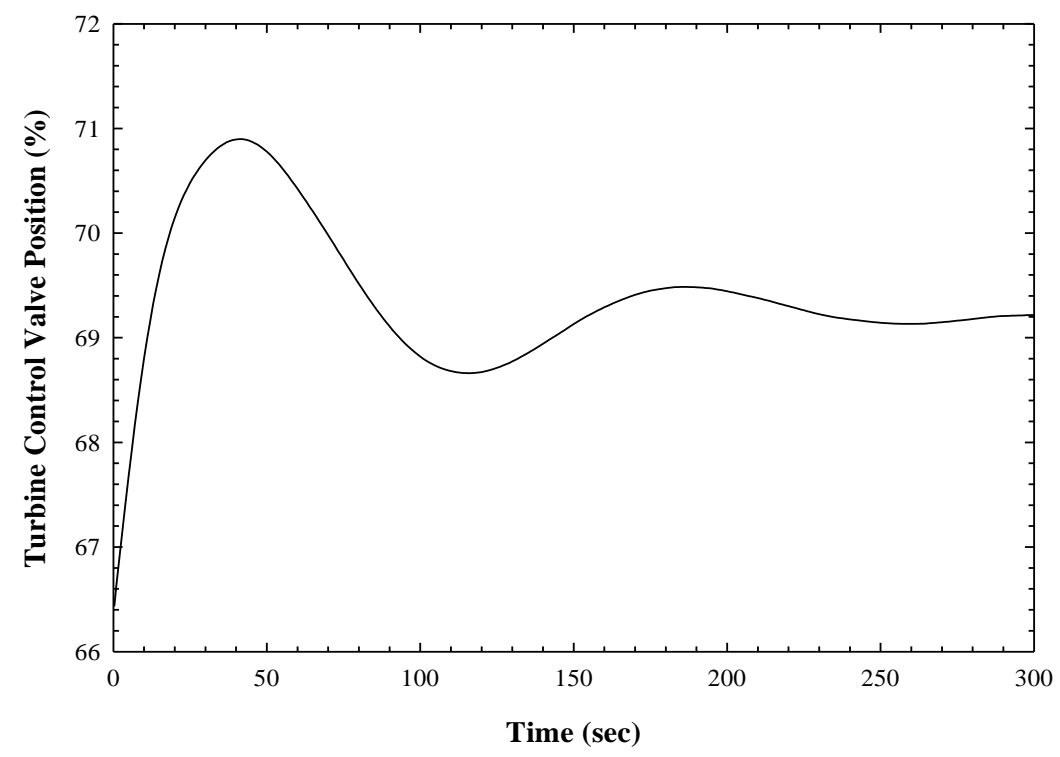

Figure 4-8: Turbine Control Valve Position, 1\% Bias in Indicated Steam Pressure 


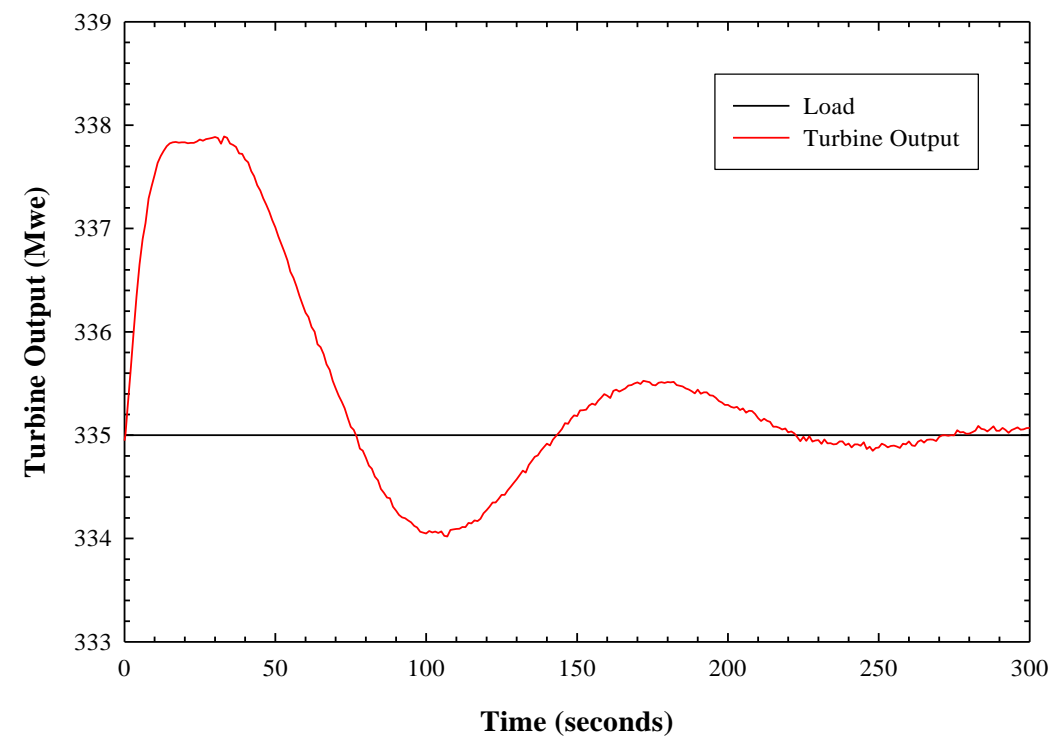

Figure 4-9: Turbine Output, 1\% Bias in Indicated Steam Pressure

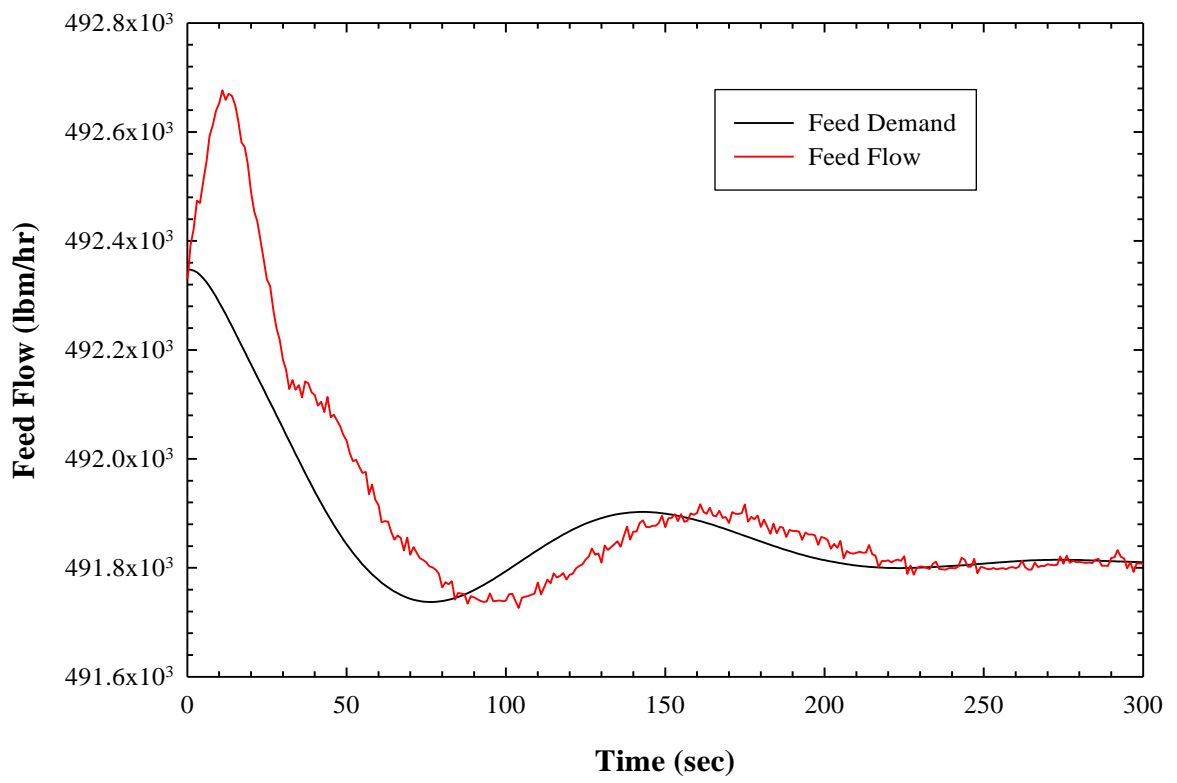

Figure 4-10: Feed Flow, 1\% Bias in Indicated Steam Pressure 


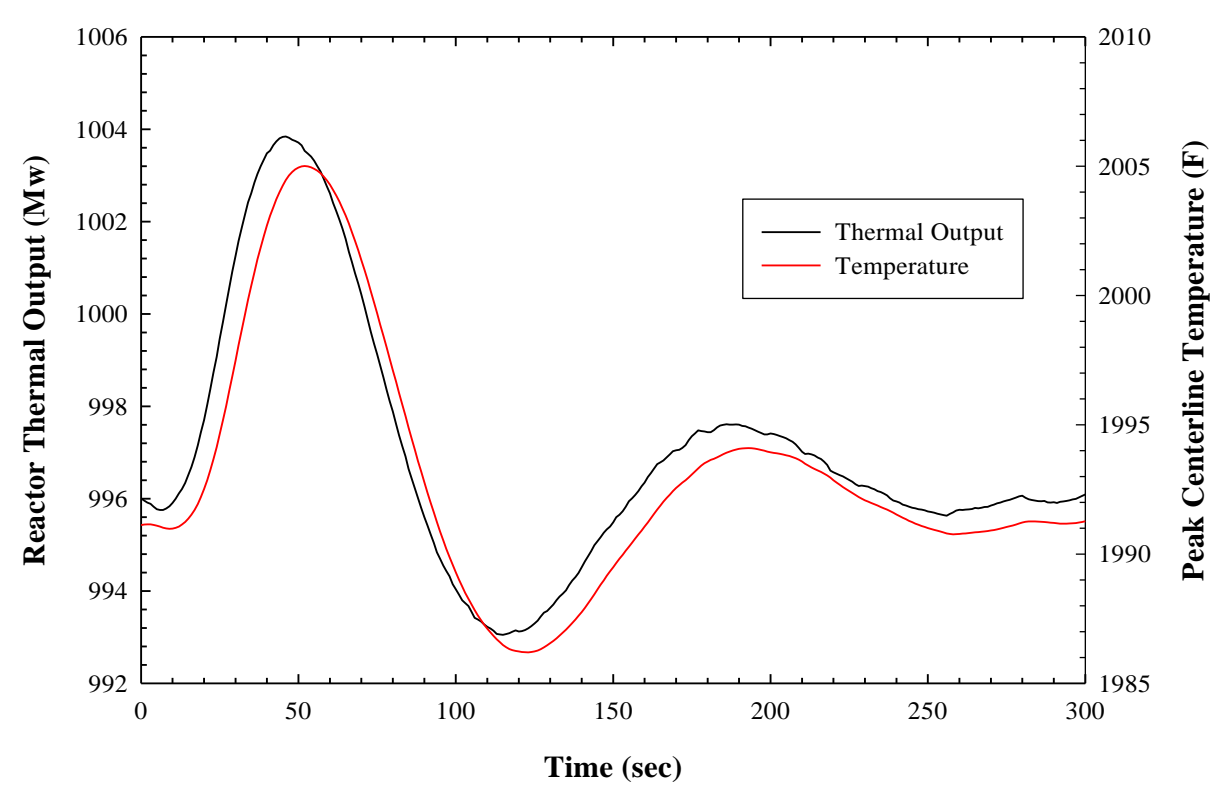

Figure 4-11: Reactor Thermal Output, 1\% Bias in Indicated Steam Pressure

Figure 4-12 and Figure 4-13 illustrate system response to loss of one of the three hot-well pumps. The loss of one hot-well pump results in a drop in the feed flow below its demand value. The feed control valves open and feed pump speed increases to compensate for the loss of flow. Other system parameters are not significantly affected.

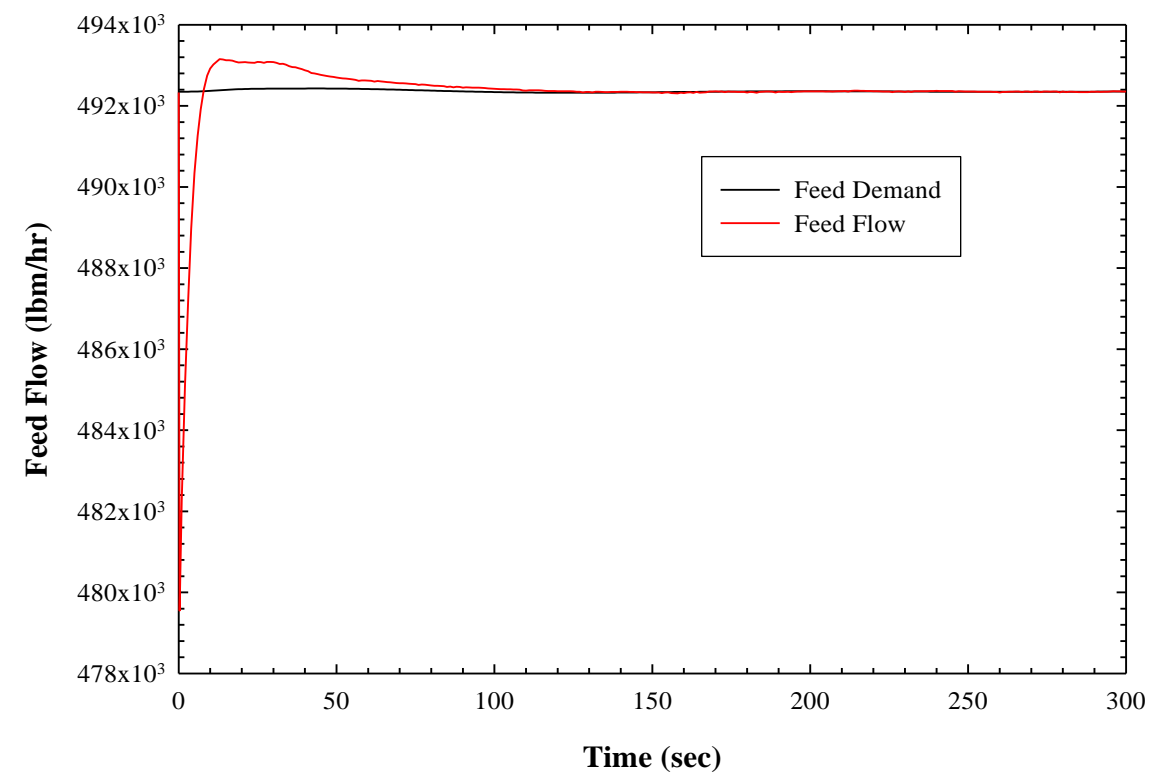

Figure 4-12: Feed Flow with Loss of One Hot-well Pump 


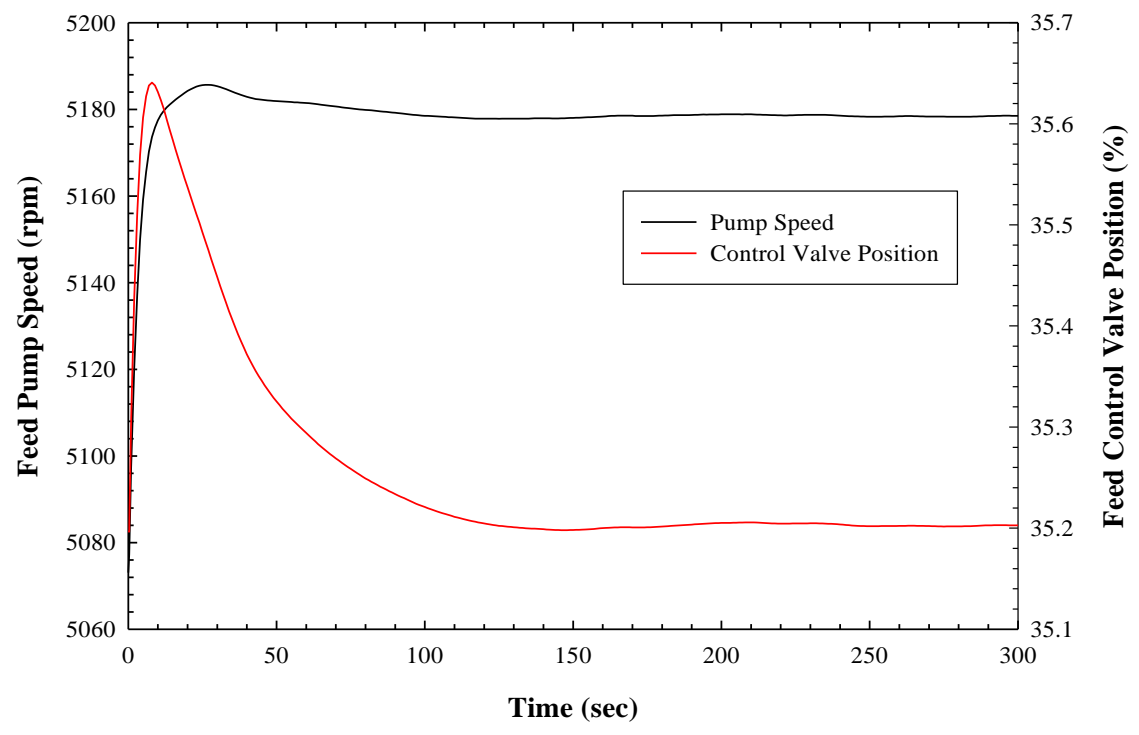

Figure 4-13: Feed Pump Speed and Control Valve Position with Loss of One Hot-well Pump

\subsection{Accident Analysis and Post Trip Behavior}

To support the sensor deployment analysis, a limited suite of accident conditions have been added to the model, including trip logic and actuation of post trip systems, including the turbine bypass system, the feed bypass system and the startup feedwater system. To illustrate this capability, two accidents are considered. The first accident assumes a trip of all reactor coolant pumps from nominal full power conditions. Figure 4-14 and Figure 4-15 show the reactor power, reactor heat transfer rate and the core flow rate for the first 120 seconds following the pump trip. A reactor trip signal is initiated once the core flow rate drops below $87 \%$ of nominal. A user input trip delay of 2 seconds is assumed for all transients. The reduction in core flow results in an increase in moderator temperature as illustrated in Figure 4-16, and an associated reduction in reactor power due to the negative moderator temperature coefficient. Two seconds after the trip signal, the control rods are inserted into the core and the core power level quickly drops to decay heat levels. 


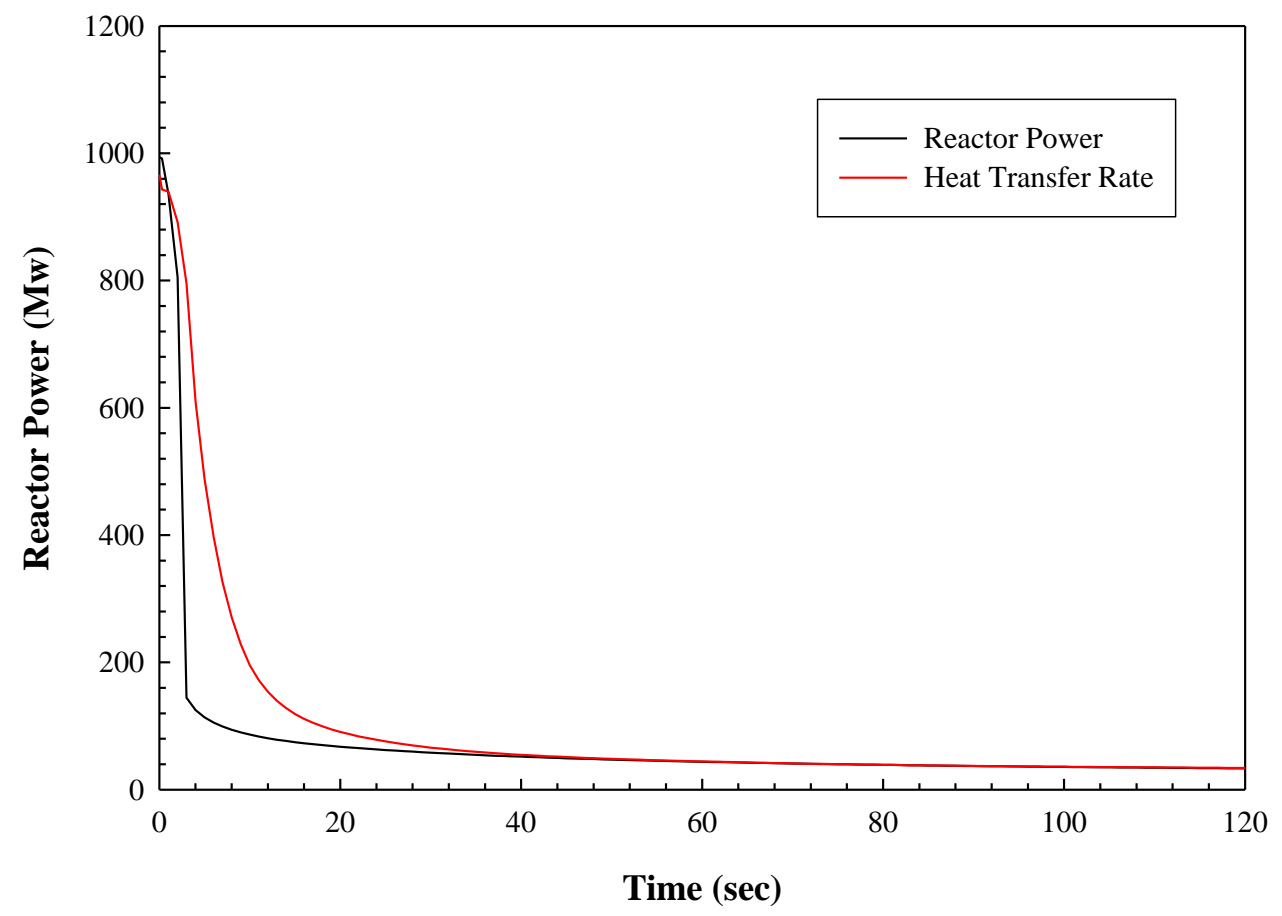

Figure 4-14: Reactor Power and Heat Transfer Rate

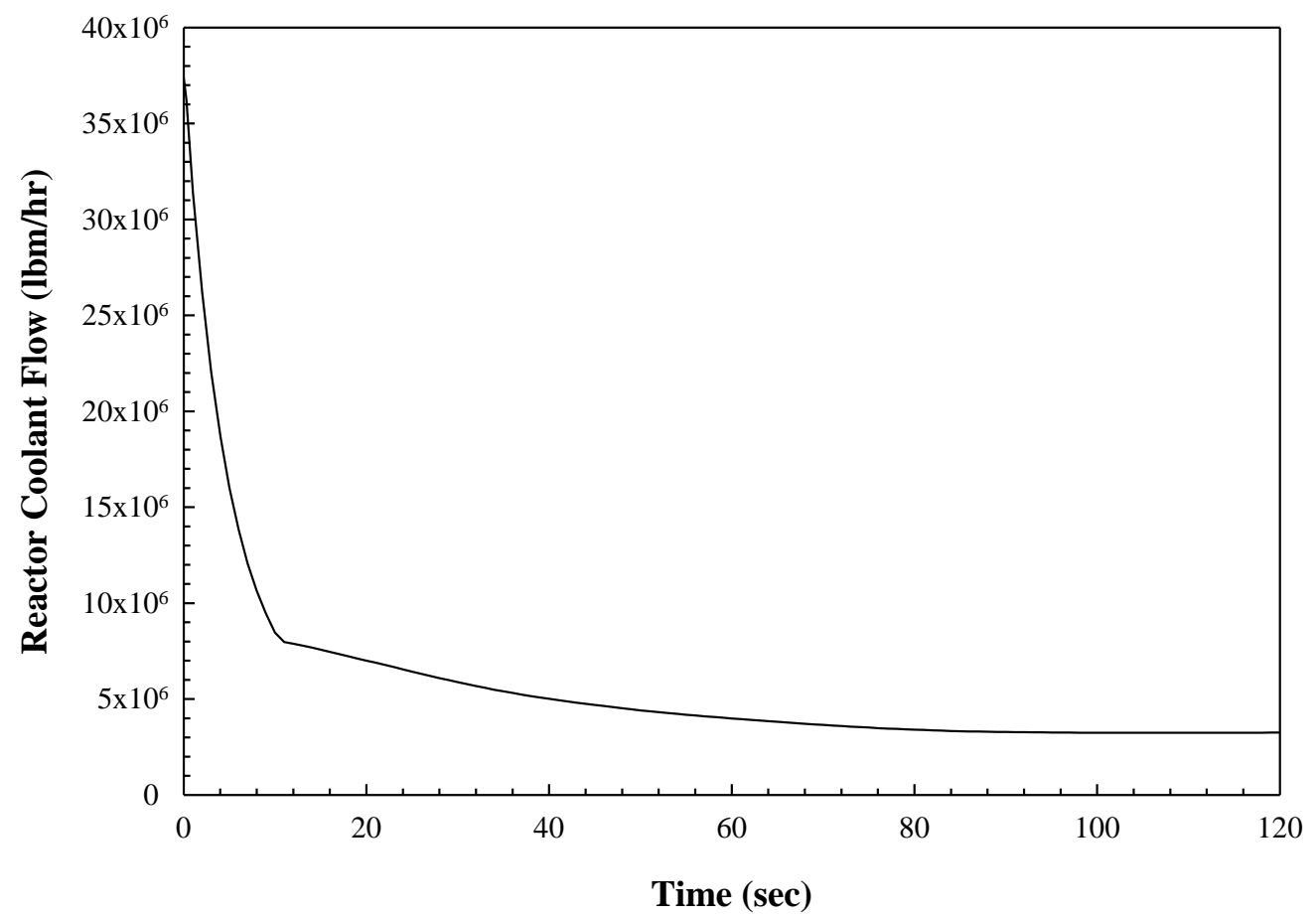

Figure 4-15: Core Flow Rate 


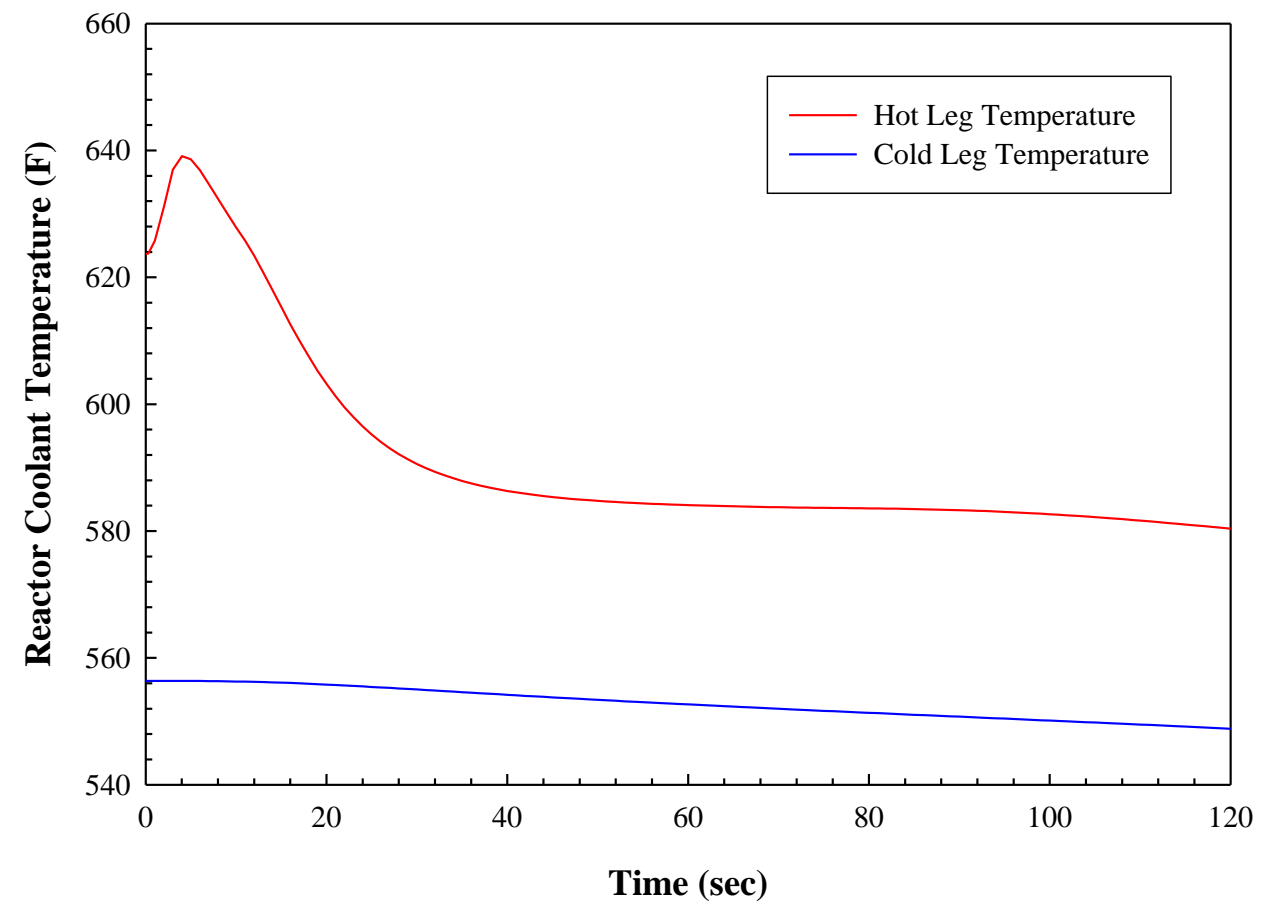

Figure 4-16: Reactor Coolant Temperatures

The principal concern in a transient of this type is the potential for DNB. Figure 4-17 illustrates the Minimum DNB Ratio for this accident, with the MDNBR reaching its lowest value of approximately 1.6 at 88 seconds into the transient. 


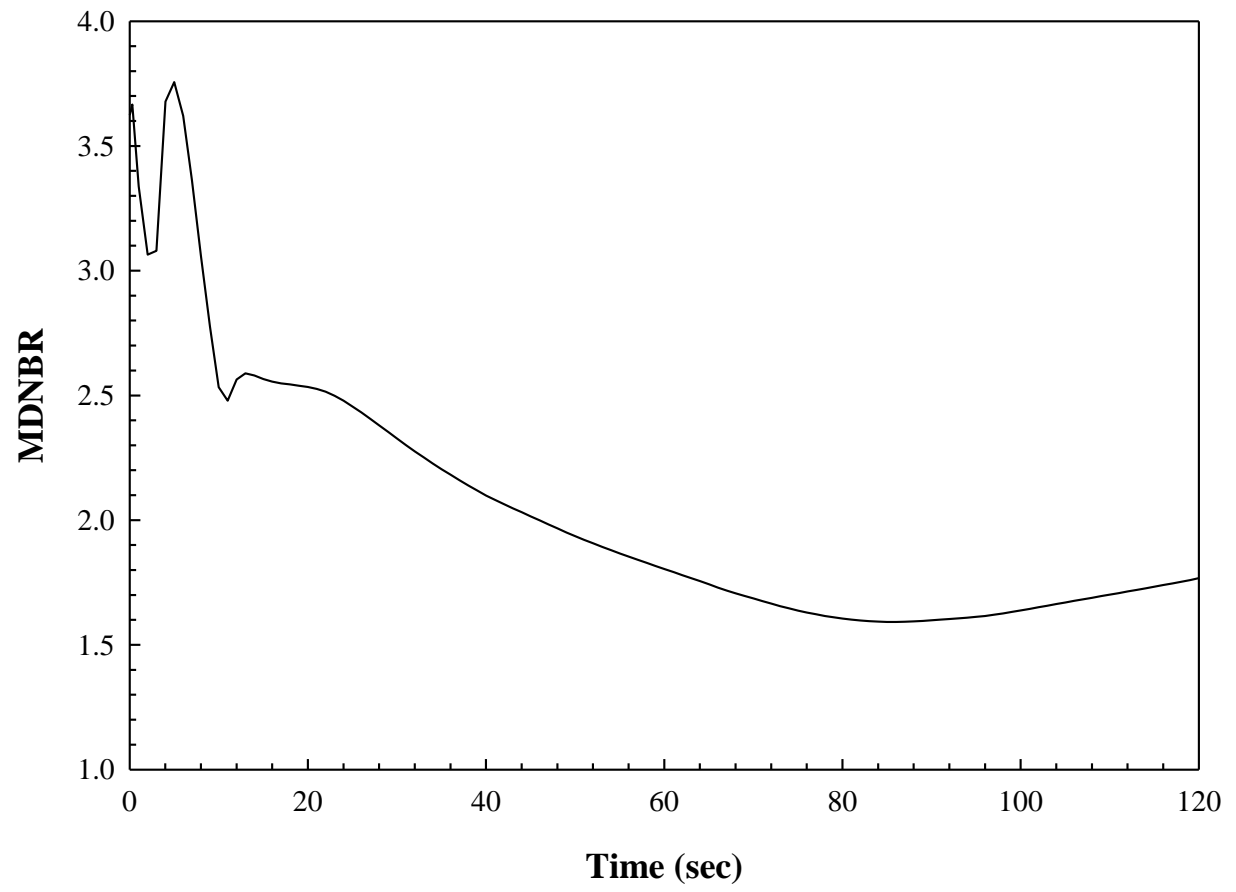

Figure 4-17: Minimum DNB Ratio

The reactor trip signal initiates a turbine trip, with an associated closing of the Turbine Control Valves (TCV) and unlocking of the Turbine Bypass Valves (TBV). Closing of the Turbine Control Valves results in an increase in steam pressure. As the Turbine Bypass Valves operate in pressure control mode, they move to reestablish steam pressure to its programmed set point as illustrated in Figure 4-18 and Figure 4-19. 


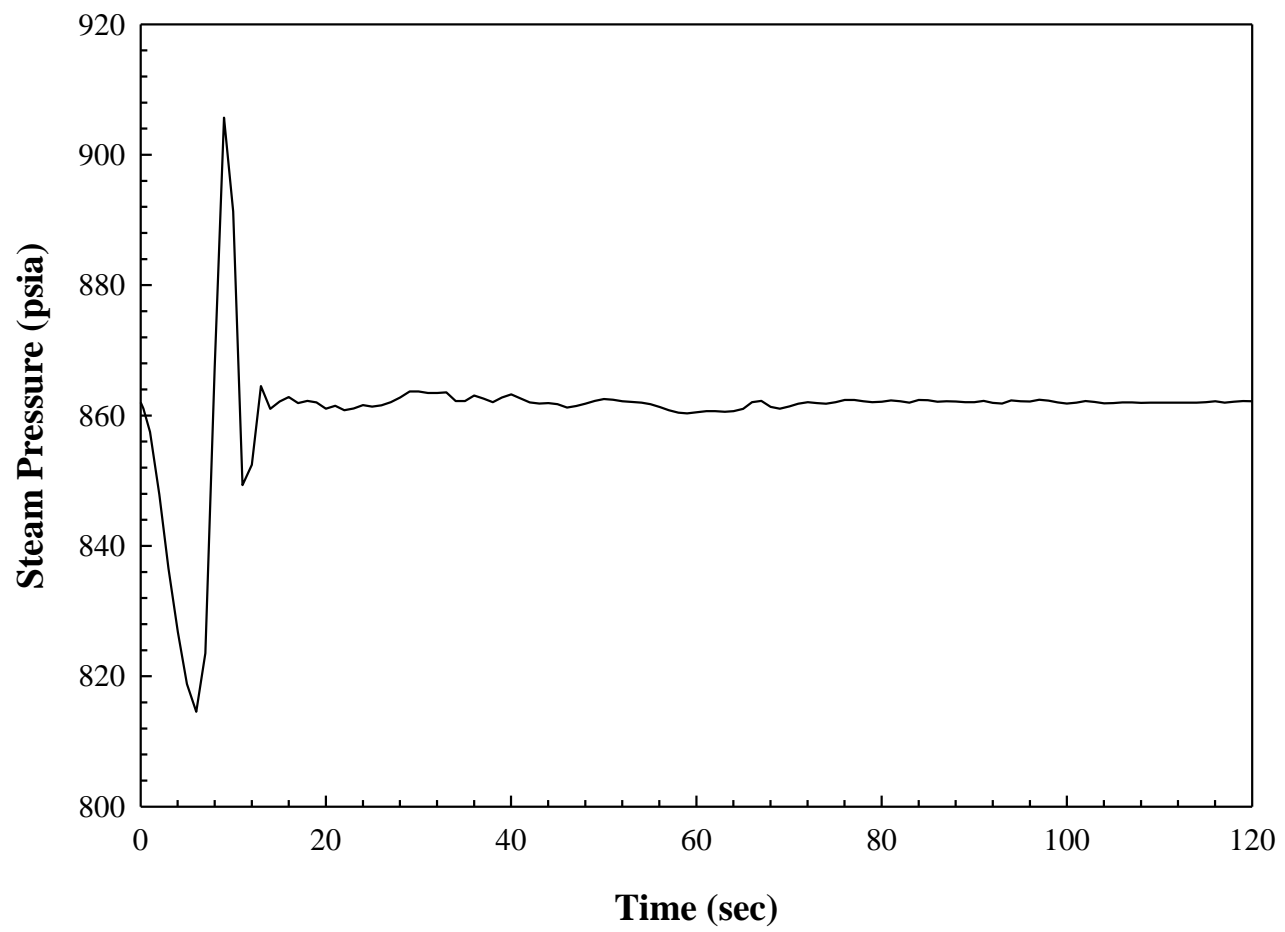

Figure 4-18: Steam Pressure

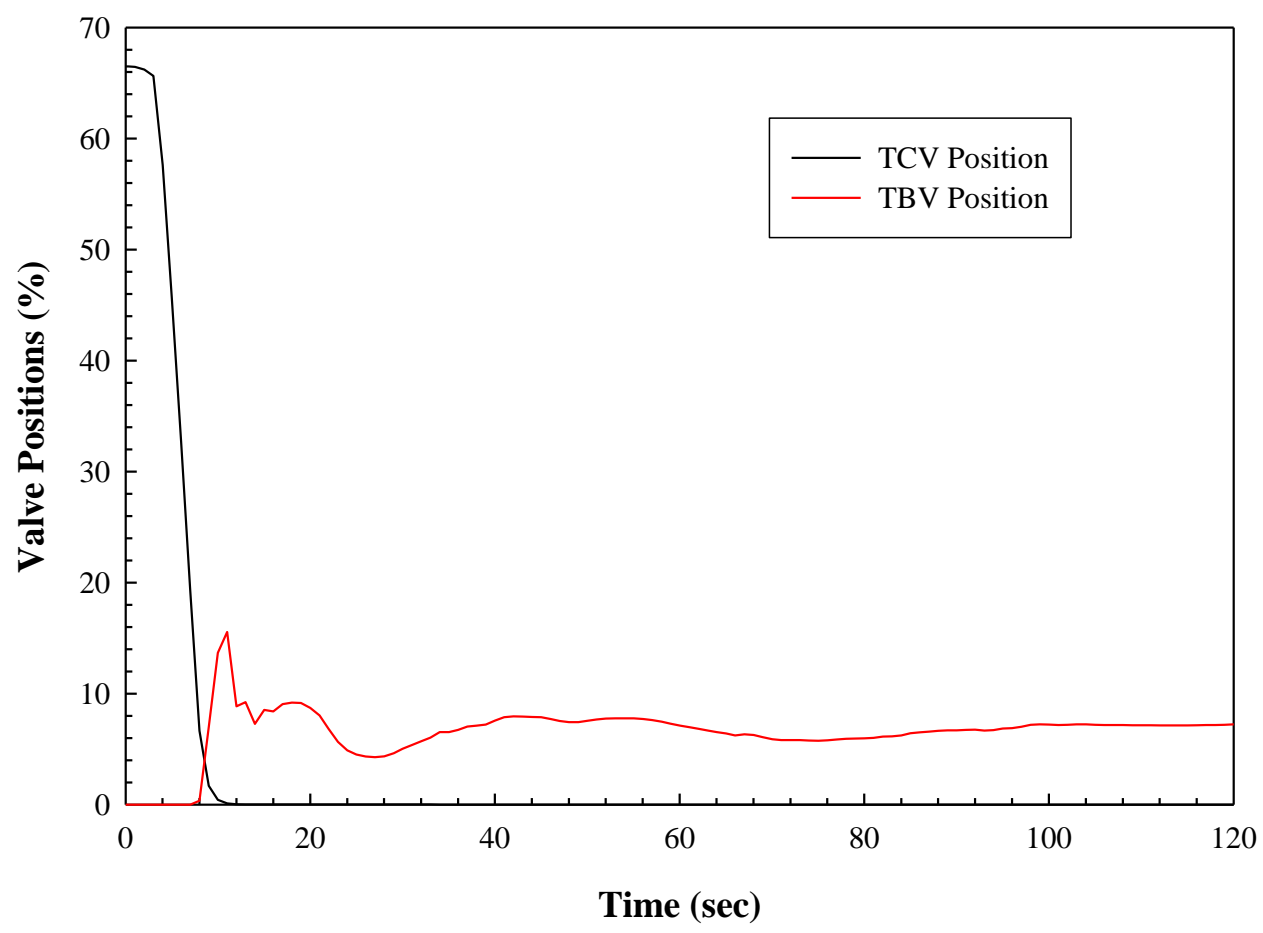

Figure 4-19: Steam Valve Positions 
During normal operation, feed flow is controlled to match power demand. Upon reactor trip, the Feed Control Valves (FCV) close and the Feed Bypass Valves (FBV) open to reestablish feed flow. Feed control at shut down conditions is based on a simple level control algorithm, where level is inferred from the pressure drop across the steam generator. Figure 4-20 and Figure 4-21 show the feed control valve positions, the inferred level and the computed dry-out point within the steam generator. The large indicated level prior to trip demonstrates the problem with using a pressure drop signal to infer level under normal operating conditions in helical coil steam generators. Under these conditions, the pressure drop is dominated by flow losses and is only weakly correlated to the actual liquid inventory in the steam generator. Even at low power, the secondary side flow geometry is primarily horizontal, such that indicated level is relatively insensitive to the dryout point. This behavior is well known.

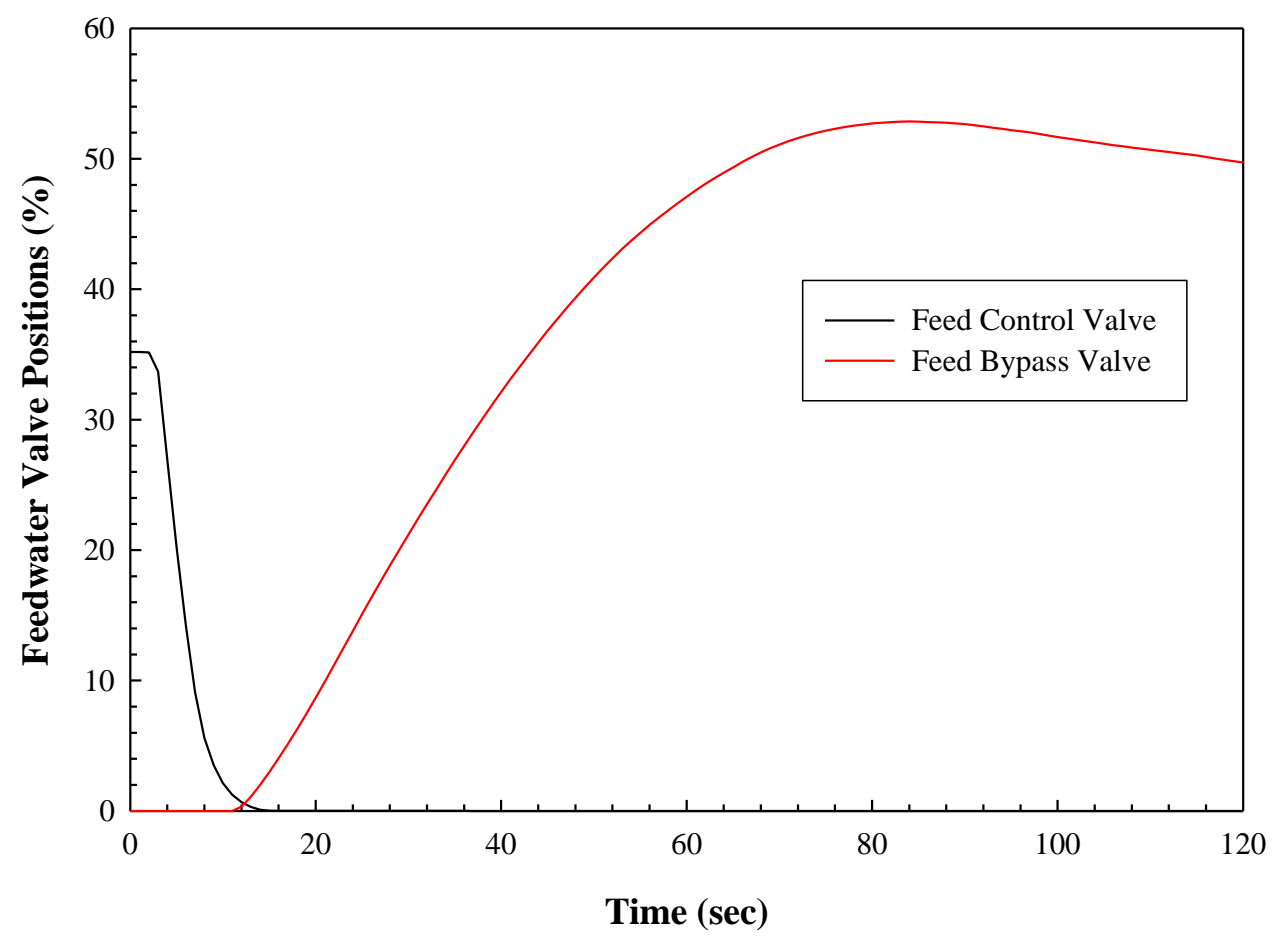

Figure 4-20: Feed Valve Positions 


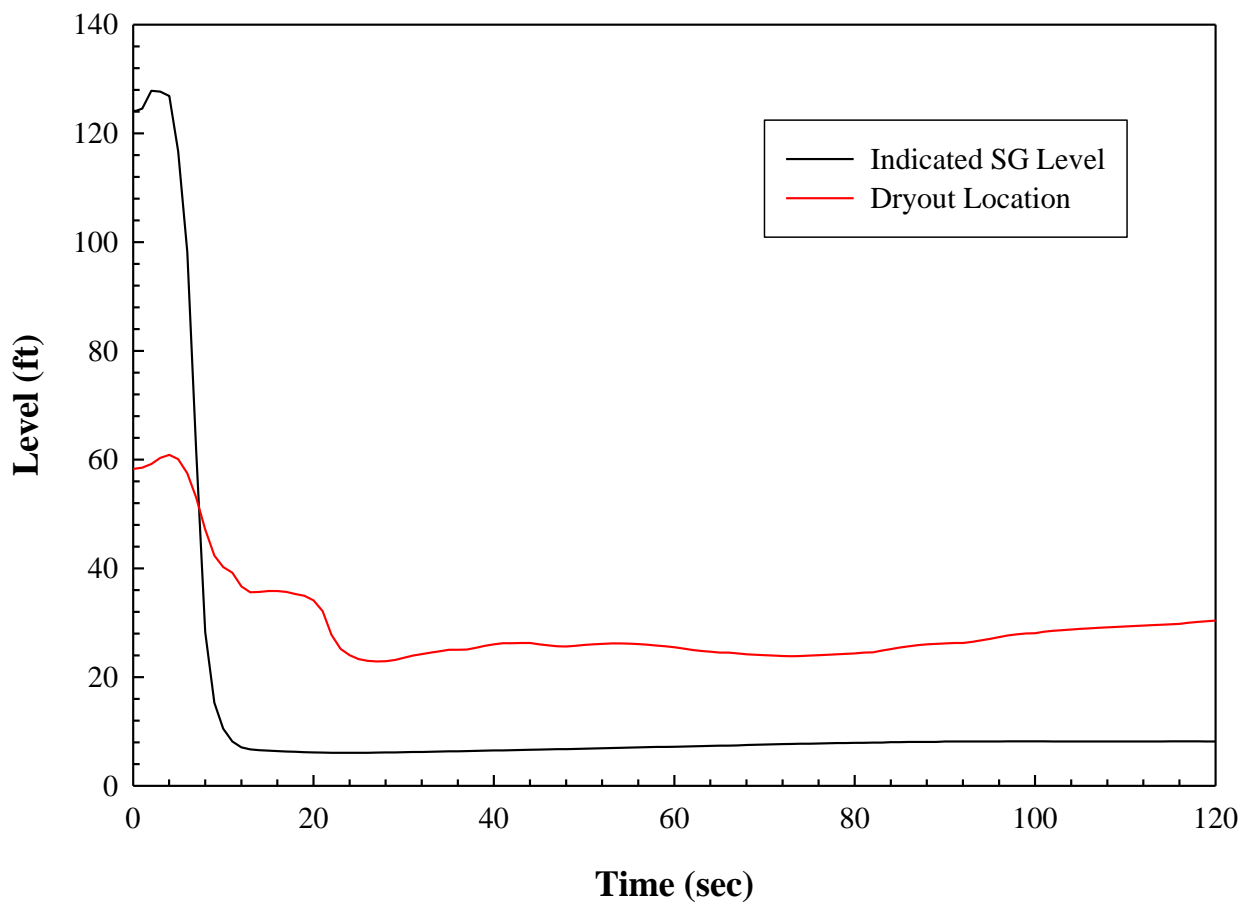

Figure 4-21: Steam Generator Liquid Levels

The second accident considered is the loss of the main feedwater pumps. The reactor trips on a low feedwater signal, again following a two second trip delay. The reactor power and heat transfer rate are given in Figure 4-22. 


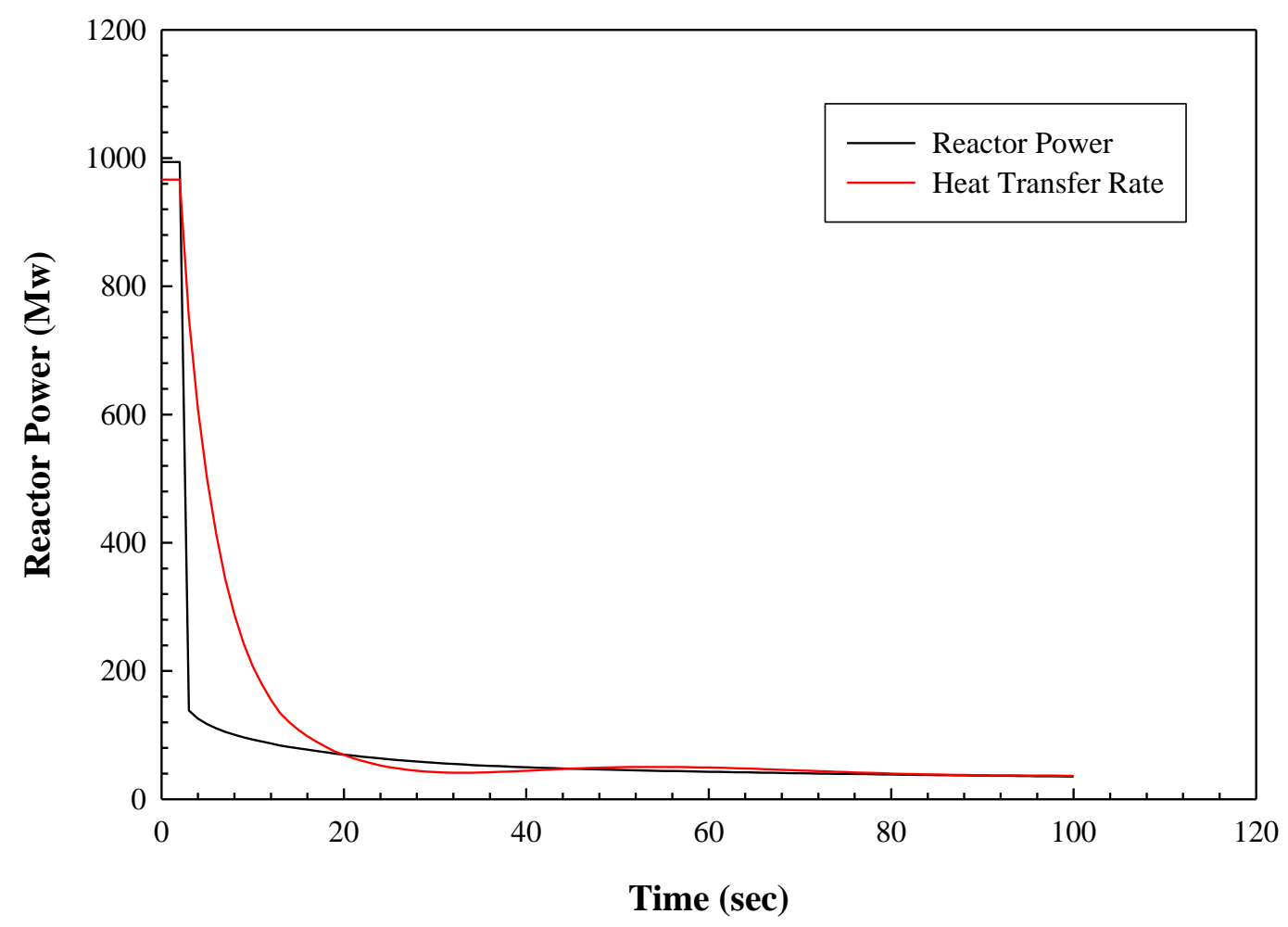

Figure 4-22: Reactor Power and Heat Transfer Rate

As in the previous example, a turbine trip is initiated on reactor trip resulting in closing of the Turbine Control Valves and actuation of the Turbine Bypass System to control steam pressure as illustrated in Figure 4-23 and Figure 4-24. Since the feed pumps are unavailable, feed flow is provided by the Startup Feedwater System (SFWS). Feed control is again based on the indicated level as illustrated in Figure 4-25 - Figure 4-27, with the Startup Feedwater Valves moving to restore indicated level to its reference value. The relatively low secondary side volume associated with the helical coil steam generators results in a rapid reduction in the secondary side mass inventory as the Startup Feedwater System is brought online. 


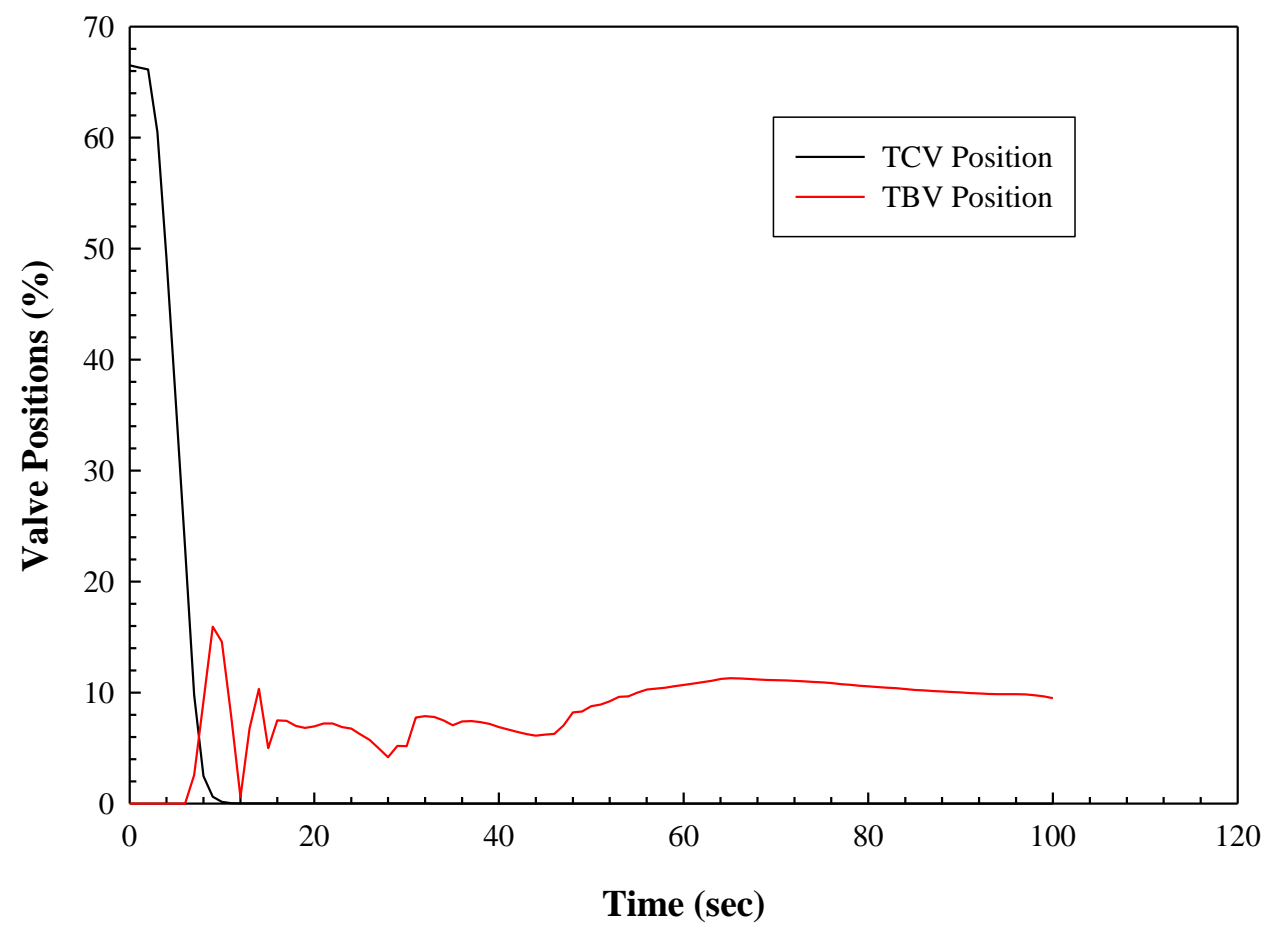

Figure 4-23: Steam Control Valve Positions

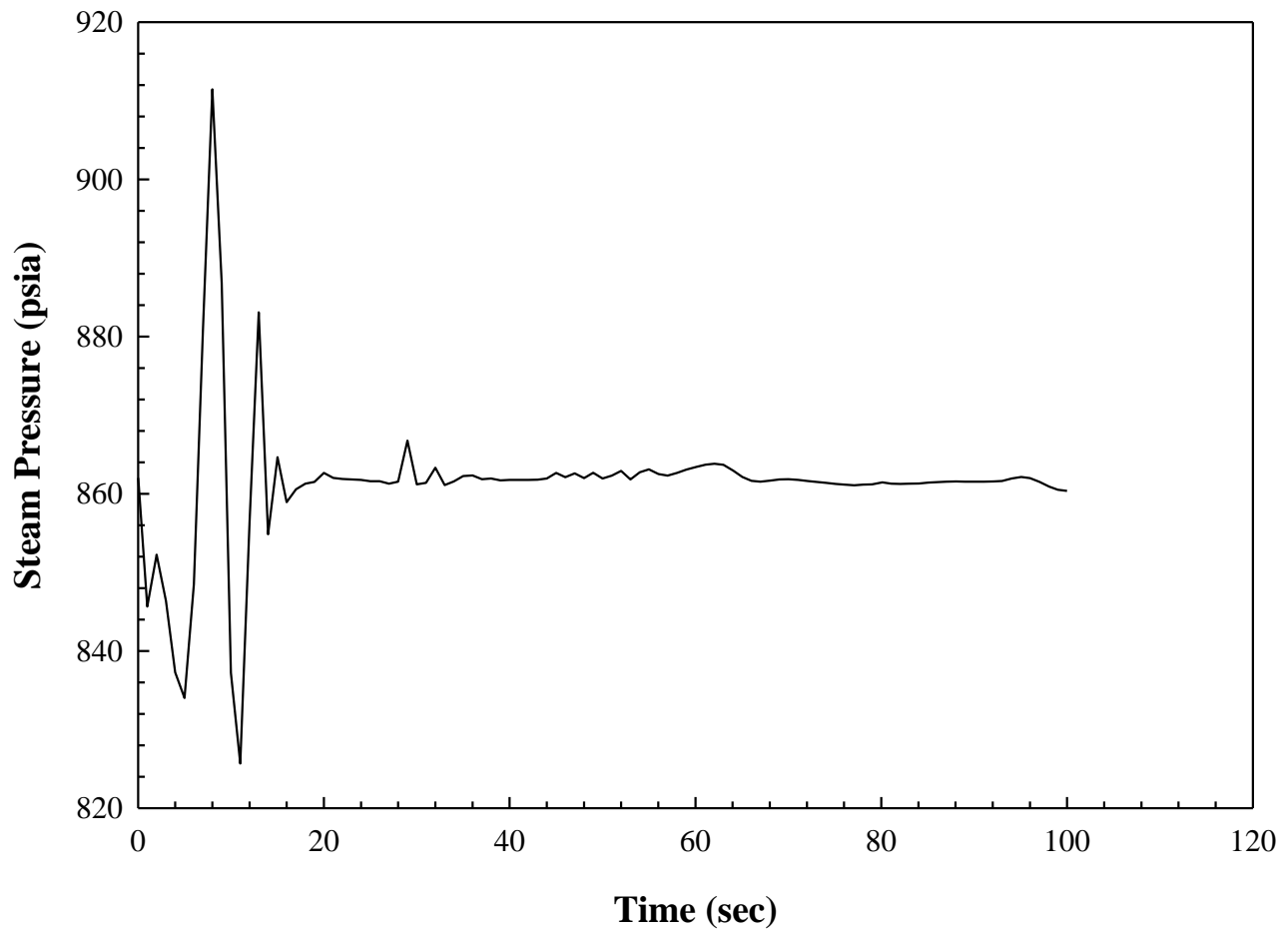

Figure 4-24: Steam Generator Pressure 


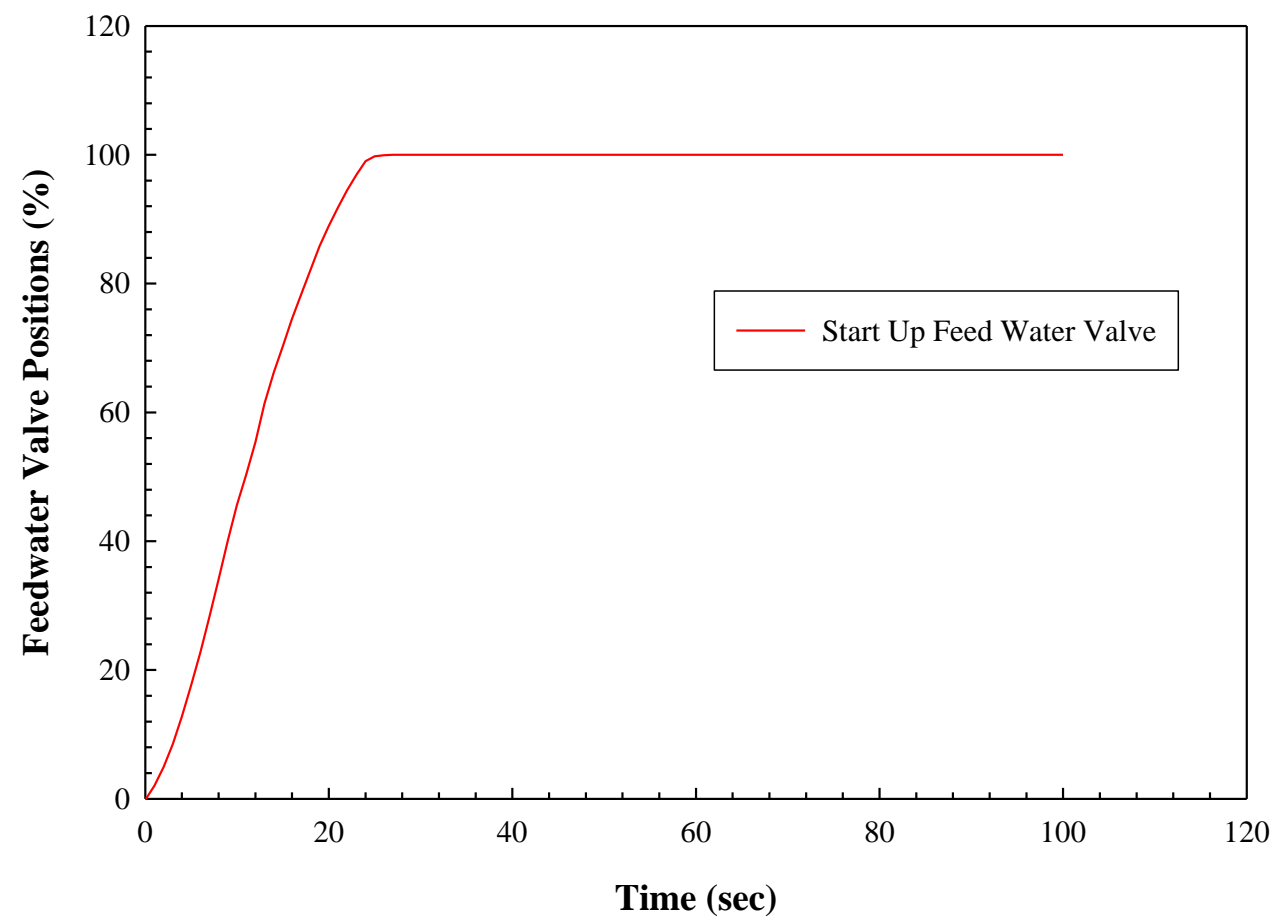

Figure 4-25: Startup Feedwater Valve Position

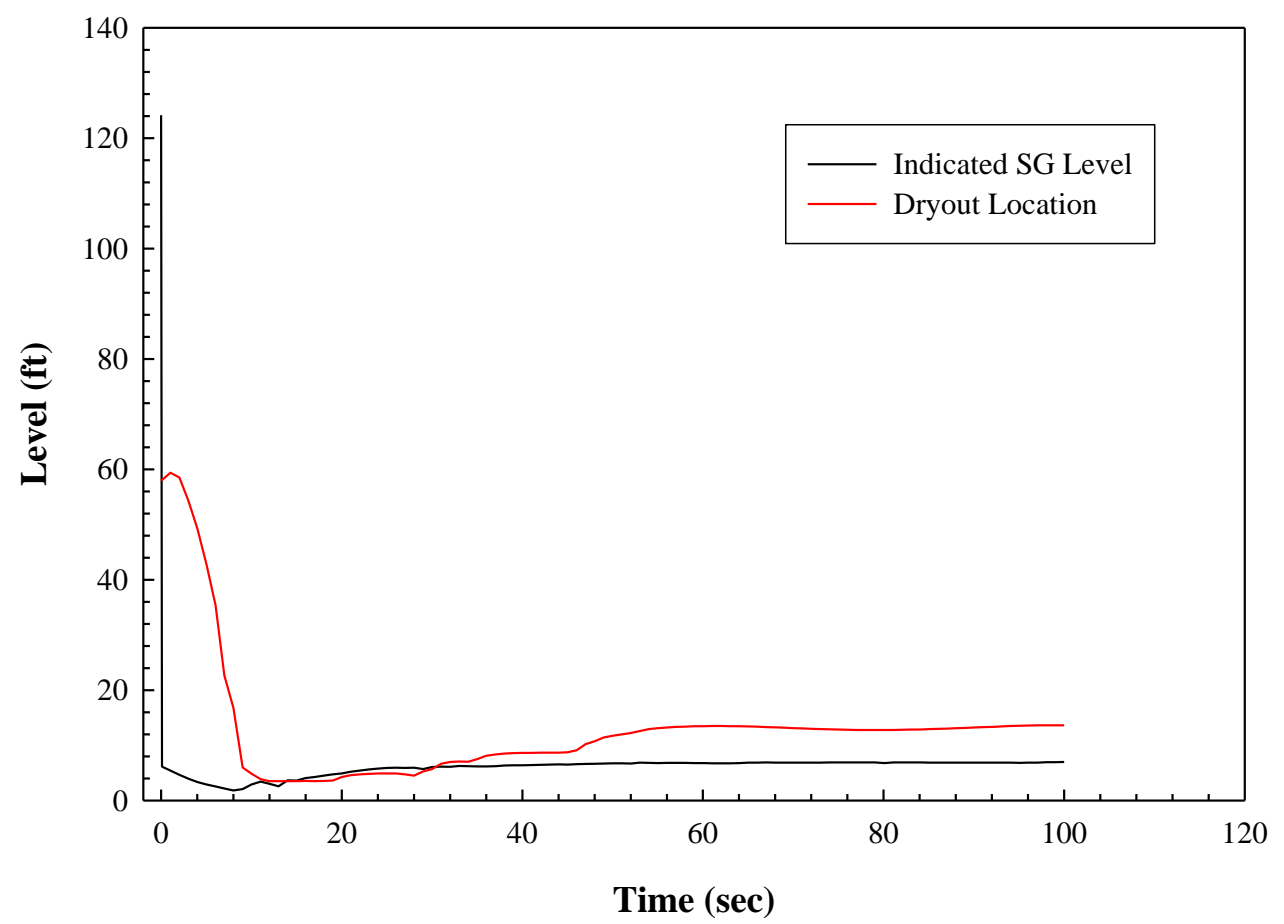

Figure 4-26: Steam Generator Liquid Levels 


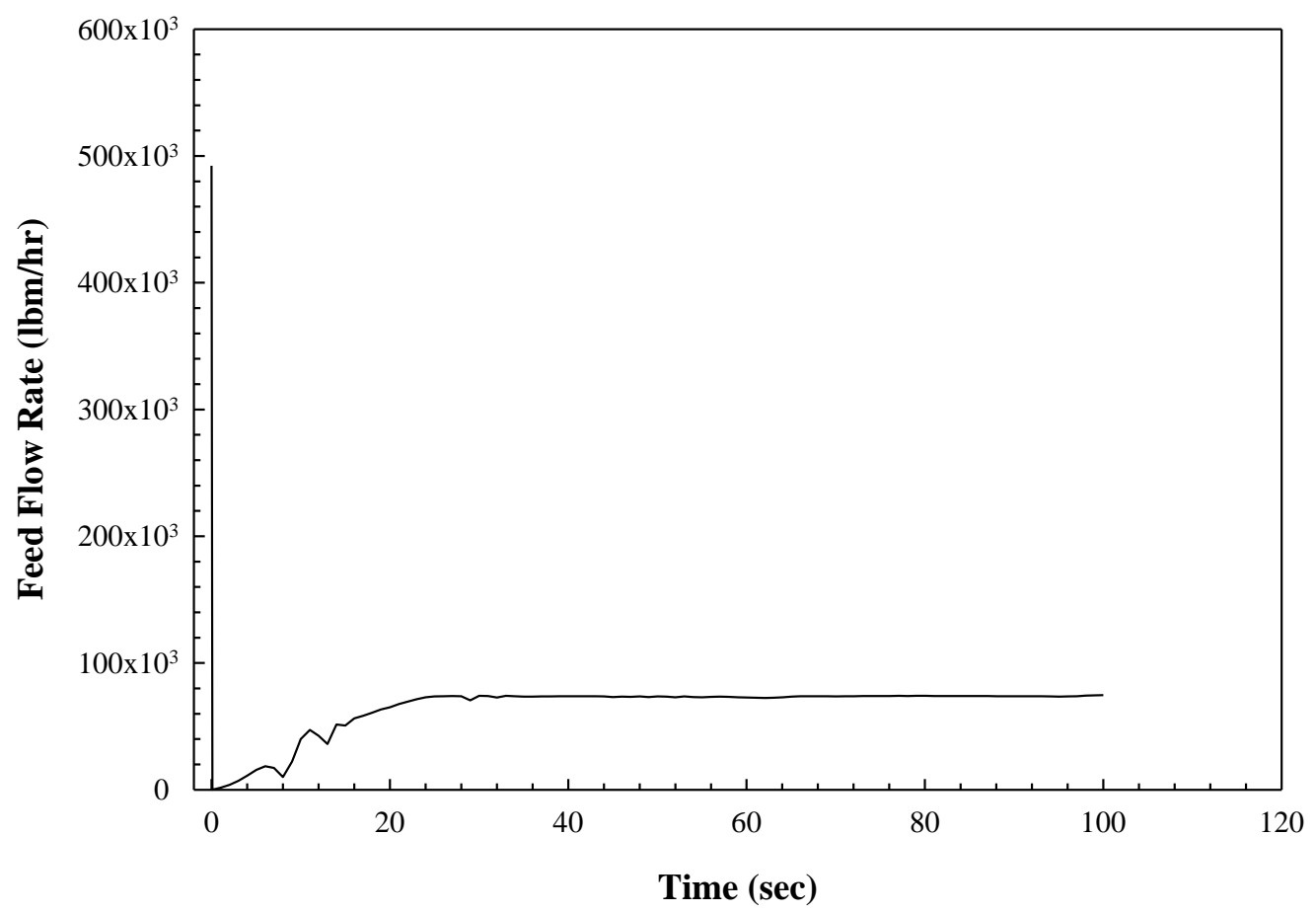

Figure 4-27: Feedwater Flow Rate

\subsection{Simulated Degradation Modes}

In support of the prognostics task, the IRIS simulator was modified to simulate three degradation modes:

1) Steam Generator Fouling

2) Sensor Drift

3) Pump Cavitation

All simulations were run for a representative 24 hour load follow maneuver. The assumed load profile is illustrated in Figure 4-28 below. 


\section{Load Profile}

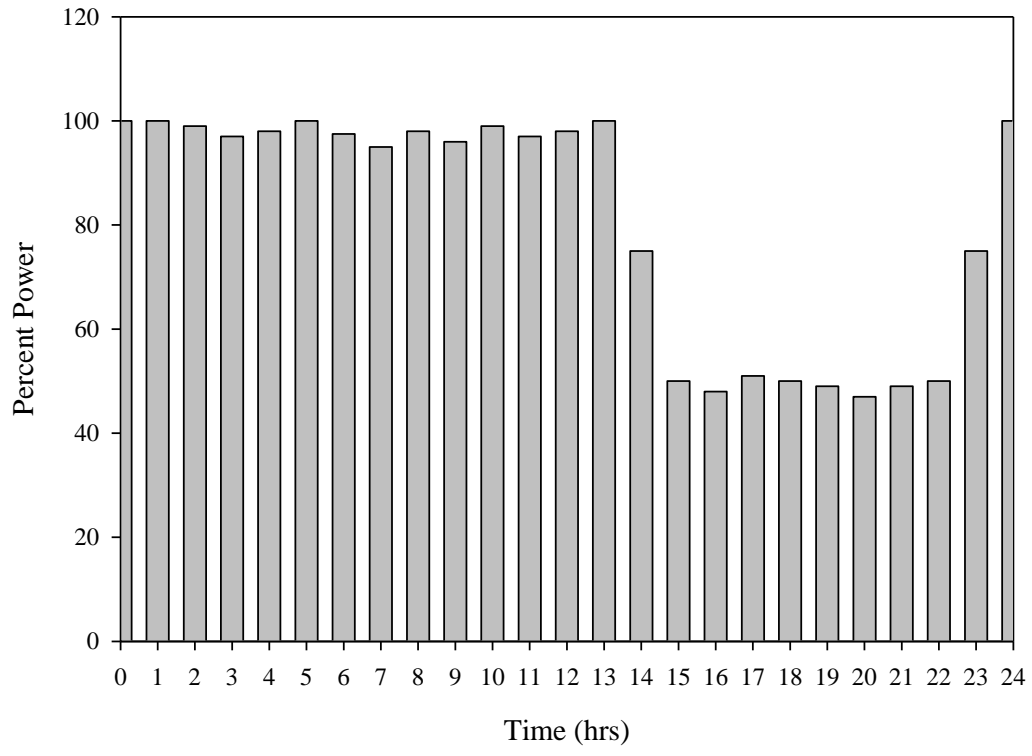

Figure 4-28: Assumed Daily Load Profile

Control and process sensors were assumed to contain noise in the amounts indicated in Table 4-1.

Table 4-1: Control and Process Sensor Noise

\begin{tabular}{|c|c|c|}
\hline Sensor & $\begin{array}{c}\text { Nominal Value }(100 \% \\
\text { Power) }\end{array}$ & $\begin{array}{c}\text { Noise Std. Dev. (\% of } \\
\text { nominal) }\end{array}$ \\
\hline Core Exit Temperature & $624 \mathrm{~F}$ & 0.1 \\
\hline Core Inlet Temperature & $556 \mathrm{~F}$ & 0.1 \\
\hline Steam Pressure & $862 \mathrm{psia}$ & 0.5 \\
\hline Feed Flow & $0.492 \times 10^{6} \mathrm{lbm} / \mathrm{hr}$ & 0.5 \\
\hline Steam Flow & $0.492 \times 10^{6} \mathrm{lbm} / \mathrm{hr}$ & 0.5 \\
\hline Pressurizer Pressure & $2250 \mathrm{psia}$ & 0.5 \\
\hline Pressurizer Level & $5.9 \mathrm{ft}$ & 0.5 \\
\hline Steam Temperature & $619 \mathrm{~F}$ & 0.1 \\
\hline Feed Temperature & $435 \mathrm{~F}$ & 0.1 \\
\hline Steam Generator Delta P & $40 \mathrm{psi}$ & 0.5 \\
\hline
\end{tabular}

Simulation results were output at 10 second intervals for the entire 24 hour period. 


\subsubsection{Steam Generator Fouling}

Steam generator fouling was simulated by increasing the thermal resistance of the heat exchanger tubes over a one year time period according to the fouling schedule illustrated in Figure 4-29 below.

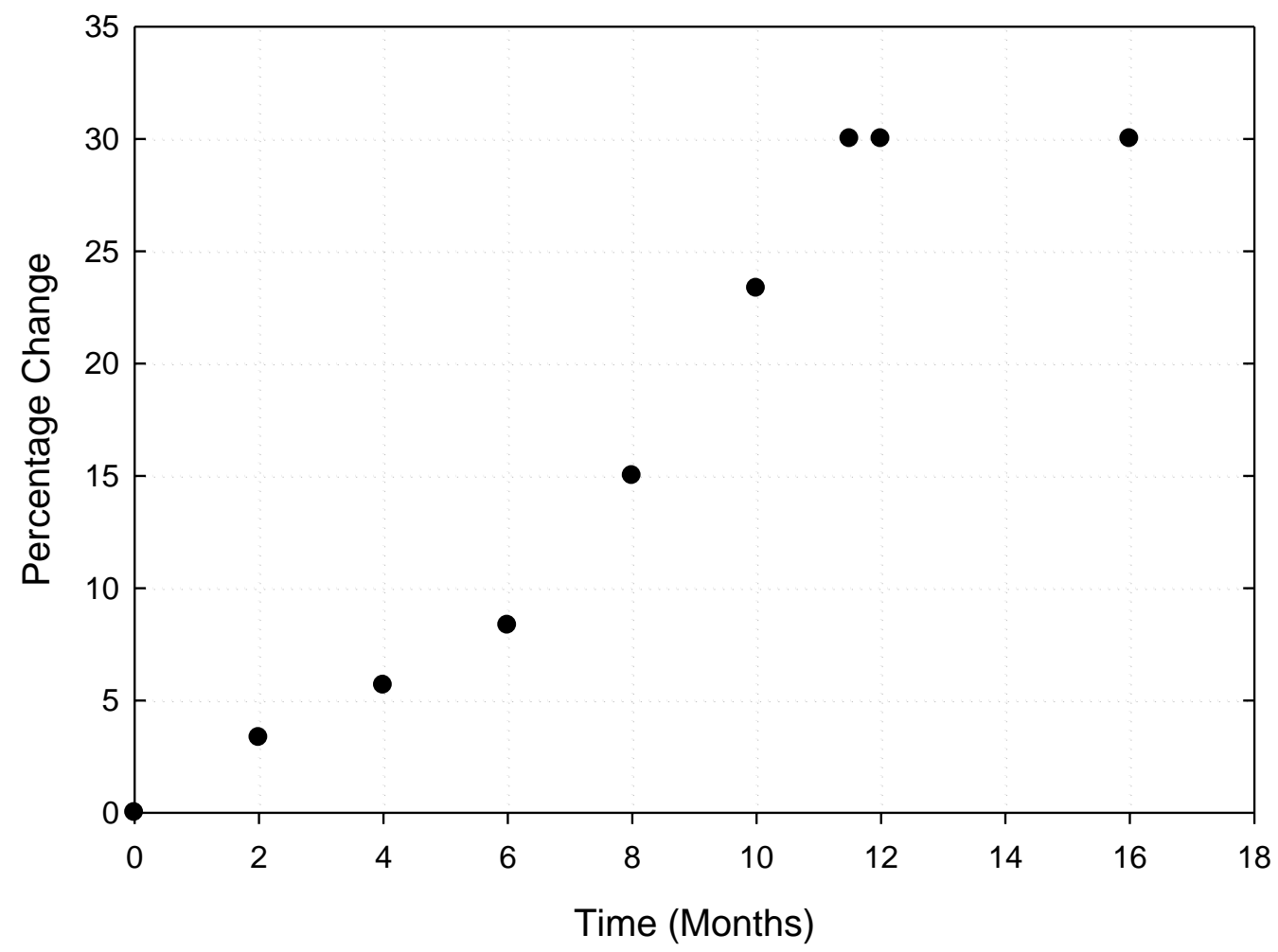

Figure 4-29: Percent Change in Steam Generator Thermal Resistance

The effects of steam generator fouling are to reduce the effectiveness of the heat exchanger and to increase the relative roughness of the tubes. For a fixed power demand, this is seen primarily as a reduction in the steam temperature, an increase in the steam (feed) flow rate and an increase in the pressure drop across the steam generator. This is illustrated in Table 4-2.

Table 4-2: Impact of Steam Generator Fouling at $100 \%$ power

\begin{tabular}{|c|c|c|c|c|c|c|}
\hline Case & $\begin{array}{c}\text { Fouling } \\
(\%)\end{array}$ & $\begin{array}{c}\text { Hot Leg } \\
\text { Temp. } \\
(\mathrm{F})\end{array}$ & $\begin{array}{c}\text { Cold Leg } \\
\text { Temp. }(\mathrm{F})\end{array}$ & $\begin{array}{c}\text { Feed Flow } \\
\left(10^{6}\right. \\
\mathrm{lbm} / \mathrm{hr})\end{array}$ & $\begin{array}{c}\text { Steam } \\
\text { Temp. } \\
(\mathrm{F})\end{array}$ & $\begin{array}{c}\text { SG } \Delta \mathrm{P} \\
(\mathrm{psi})\end{array}$ \\
\hline Reference & 0.00 & 623.7 & 556.4 & 0.4920 & 619.3 & 40.20 \\
\hline Month 1 & 1.36 & 623.7 & 556.4 & 0.4926 & 618.8 & 40.34 \\
\hline
\end{tabular}




\begin{tabular}{|c|c|c|c|c|c|c|}
\hline Month 2 & 3.33 & 623.7 & 556.4 & 0.4929 & 617.9 & 41.30 \\
\hline Month 3 & 4.04 & 623.7 & 556.4 & 0.4931 & 617.5 & 41.31 \\
\hline Month 4 & 5.67 & 623.7 & 556.4 & 0.4935 & 616.6 & 42.15 \\
\hline Month 5 & 7.29 & 623.7 & 556.4 & 0.4940 & 615.4 & 42.09 \\
\hline Month 6 & 8.33 & 623.7 & 556.4 & 0.4943 & 614.6 & 42.88 \\
\hline Month 7 & 11.81 & 623.7 & 556.4 & 0.4956 & 611.3 & 43.39 \\
\hline Month 8 & 15.00 & 623.6 & 556.4 & 0.4974 & 607.3 & 44.61 \\
\hline Month 9 & 18.34 & 623.6 & 556.4 & 0.4998 & 601.4 & 45.64 \\
\hline Month 10 & 23.33 & 623.6 & 556.5 & 0.5054 & 588.5 & 47.06 \\
\hline Month 11 & 27.60 & 623.6 & 556.6 & 0.5133 & 571.4 & 47.69 \\
\hline Month 12 & 30.00 & 623.5 & 556.7 & 0.5191 & 559.1 & 48.87 \\
\hline
\end{tabular}

\subsubsection{Sensor Drift}

Sensor drift was simulated as a linear drift of approximately $1 \%$ occurring over a one year period. Two sensors were considered. The first was a process sensor which only measures a process variable, but is not involved in control actions. The steam flow sensor was chosen for this simulation. The second was a control sensor, which by definition is used to initiate control actions. The steam pressure sensor was chosen for this simulation. The magnitudes of the sensor drift for both simulations are given in Table 4-3. As the drift in the process (steam flow) sensor did not affect control actions, this appeared as a point change in this sensor response alone, with no changes in system response, or other system parameters. In the IRIS design, steam pressure is controlled about a programmed set point. Drift in the steam pressure sensor results in the actual steam pressure being different from the control set point. For the simulations conducted here, the actual steam pressure was lower than the set point value.

Table 4-3: Sensor Drift Rates

\begin{tabular}{|c|c|}
\hline Month & Drift (\% of span) \\
\hline 1 & 0.083 \\
\hline 2 & 0.166 \\
\hline 3 & 0.249 \\
\hline 4 & 0.332 \\
\hline 5 & 0.415 \\
\hline 6 & 0.496 \\
\hline 7 & 0.581 \\
\hline 8 & 0.664 \\
\hline 9 & 0.747 \\
\hline
\end{tabular}




\begin{tabular}{|l|l|}
\hline 10 & 0.830 \\
\hline 11 & 0.913 \\
\hline 12 & 0.996 \\
\hline
\end{tabular}

For a given load, the system will adjust to accommodate the specified load at the reduced pressure. Unless the thermal efficiency of the system changes significantly, the reactor thermal output is then unaffected. Since the reactor operates with fixed flow reactor coolant pumps, the temperature change across the core is unchanged. As the control rods move to maintain a programmed $\mathrm{T}_{\text {ave, }}$ this implies the core inlet and outlet temperatures are unchanged as well. Reduction in steam pressure could potentially impact heat transfer across the steam generators and steam conditions entering the turbine. However, since the turbine load is unchanged, the turbine control valves move to maintain a nearly constant turbine header pressure and steam flow rate. A slight change is seen in the steam exit temperature and steam generator pressure drop, but these are well within the sensor noise.

The most significant change is in feed pump speed, as the pump speed controller adjust to maintain a constant pressure drop across the feed control valves. These results are summarized in Table 4-4.

Table 4-4: Impact of Steam Pressure Sensor Drift at $100 \%$ power

\begin{tabular}{|c|c|c|c|c|c|c|c|c|c|c|}
\hline Case & $\begin{array}{c}\text { Drift } \\
(\%)\end{array}$ & $\begin{array}{c}\text { Hot } \\
\text { Leg } \\
\text { Temp. } \\
(\mathrm{F})\end{array}$ & $\begin{array}{c}\text { Cold } \\
\text { Leg } \\
\text { Temp. } \\
(\mathrm{F})\end{array}$ & $\begin{array}{c}\text { Feed } \\
\text { Flow } \\
\left(10^{6}\right. \\
\text { lbm/hr) }\end{array}$ & $\begin{array}{c}\text { Steam } \\
\text { Temp. } \\
(\mathrm{F})\end{array}$ & $\begin{array}{c}\text { Steam } \\
\text { Pressure } \\
(\mathrm{psia})\end{array}$ & $\begin{array}{c}\text { Turbine } \\
\text { Header } \\
\text { Pressure } \\
(\mathrm{psia})\end{array}$ & $\begin{array}{c}\mathrm{SG} \\
\Delta \mathrm{P} \\
(\mathrm{psi})\end{array}$ & $\begin{array}{c}\text { TCV } \\
(\% \\
\text { Open })\end{array}$ & $\begin{array}{c}\Omega_{f p} \\
(\mathrm{rpm})\end{array}$ \\
\hline Reference & 0.00 & 623.7 & 556.4 & 0.4923 & 619.4 & 862.0 & 726.3 & 40.2 & 66.5 & 5073 \\
\hline Month 1 & 1.36 & 623.7 & 556.4 & 0.4923 & 619.4 & 861.0 & 726.0 & 40.3 & 66.6 & 5071 \\
\hline Month 2 & 3.33 & 623.7 & 556.4 & 0.4923 & 619.5 & 860.7 & 725.8 & 40.4 & 66.8 & 5069 \\
\hline Month 3 & 4.04 & 623.7 & 556.4 & 0.4922 & 619.5 & 860.0 & 726.0 & 40.5 & 67.0 & 5067 \\
\hline Month 4 & 5.67 & 623.7 & 556.4 & 0.4922 & 619.5 & 859.2 & 726.4 & 40.6 & 67.3 & 5065 \\
\hline Month 5 & 7.29 & 623.7 & 556.4 & 0.4921 & 619.6 & 858.6 & 725.7 & 40.7 & 67.4 & 5063 \\
\hline Month 6 & 8.33 & 623.7 & 556.4 & 0.4921 & 619.6 & 857.9 & 725.6 & 40.8 & 67.5 & 5061 \\
\hline Month 7 & 11.81 & 623.7 & 556.4 & 0.4920 & 619.7 & 857.1 & 725.7 & 40.9 & 67.7 & 5059 \\
\hline Month 8 & 15.00 & 623.7 & 556.4 & 0.4920 & 619.7 & 856.4 & 725.6 & 40.1 & 67.9 & 5057 \\
\hline Month 9 & 18.34 & 623.7 & 556.4 & 0.4919 & 619.7 & 855.7 & 725.6 & 40.2 & 68.1 & 5054 \\
\hline Month 10 & 23.33 & 623.7 & 556.4 & 0.4919 & 619.8 & 855.0 & 725.4 & 40.3 & 68.3 & 5052 \\
\hline Month 11 & 27.60 & 623.7 & 556.4 & 0.4919 & 619.8 & 854.3 & 725.3 & 40.4 & 68.5 & 5050 \\
\hline Month 12 & 30.00 & 623.7 & 556.4 & 0.4918 & 619.9 & 853.6 & 725.2 & 40.5 & 68.7 & 5048 \\
\hline
\end{tabular}




\subsubsection{Pump Cavitation}

Pump cavitation is simulated by modifying the pump head curve. Pump head for faulted pumps is computed using a modified volumetric flow rate

$$
\dot{Q}^{*}=\frac{\dot{Q}}{p}
$$

where $\dot{Q}$ is the true volumetric flow rate and $p$ is a number less than 1. For the simulations performed here, $p$ ranged from 0.9 to 0.99 in increments of 0.01 . This transformation has the result of reducing the computed pump head and correspondingly the system flow rate for decreasing values of $p$.

For a given load and reactor thermal output, the impact of reduced primary system flow is to increase the temperature drop across the core. As control rods move to maintain a programmed $\mathrm{T}_{\mathrm{ave}}$, this results in lower core inlet temperature and a higher core exit temperature. The reduced flow rate on the primary side, coupled with the change in the core inlet and outlet temperatures affects heat transfer across the steam generators resulting in a slight increase in the steam exit temperature and reduction in the steam generator pressure drop. These results are still within the noise level of the sensors however. Simulation results are summarized in Table 4-5.

Table 4-5: Impact of Primary Pump Degradation at 100\% Power

\begin{tabular}{|c|c|c|c|c|c|c|}
\hline Case & $\mathrm{P}$ & $\begin{array}{c}\text { Hot Leg } \\
\text { Temp. } \\
(\mathrm{F})\end{array}$ & $\begin{array}{c}\text { Cold Leg } \\
\text { Temp. }(\mathrm{F})\end{array}$ & $\begin{array}{c}\text { Core Flow } \\
\left(10^{8}\right. \\
\mathrm{lbm} / \mathrm{hr})\end{array}$ & $\begin{array}{c}\text { Steam } \\
\text { Temp. } \\
(\mathrm{F})\end{array}$ & $\begin{array}{c}\text { SG } \Delta \mathrm{P} \\
(\mathrm{psi})\end{array}$ \\
\hline Reference & 1.00 & 623.7 & 556.4 & 0.374 & 619.4 & 40.2 \\
\hline Month 1 & 0.99 & 623.8 & 556.3 & 0.373 & 619.4 & 40.1 \\
\hline Month 2 & 0.98 & 623.8 & 556.2 & 0.372 & 619.4 & 40.1 \\
\hline Month 3 & 0.97 & 623.9 & 556.2 & 0.371 & 619.5 & 40.0 \\
\hline Month 4 & 0.96 & 624.0 & 556.1 & 0.370 & 619.5 & 40.0 \\
\hline Month 5 & 0.95 & 624.1 & 556.0 & 0.369 & 619.6 & 39.9 \\
\hline Month 6 & 0.94 & 624.2 & 555.9 & 0.368 & 619.6 & 39.9 \\
\hline Month 7 & 0.93 & 624.3 & 555.8 & 0.367 & 619.6 & 39.8 \\
\hline Month 8 & 0.92 & 624.4 & 555.7 & 0.366 & 619.7 & 39.7 \\
\hline Month 9 & 0.91 & 624.4 & 555.6 & 0.365 & 619.7 & 39.7 \\
\hline Month 10 & 0.90 & 624.5 & 555.5 & 0.364 & 619.8 & 39.6 \\
\hline
\end{tabular}




\section{IRIS REACTOR TO GRID MODEL}

\subsection{Fast Valving and Small Nuclear Reactor Applicability}

Fast valving is a low-cost aid to electric grid stability that responds to large disturbances by rapid valve closure followed by quick opening. The method has been well established in large and modern grid networks. An increasing number of interconnections and increasing scales of individual power plants have increased the stability challenges, increasing the need for such measures [Aanstad and Lokay, 1970]. Additionally, the burden that large nuclear units impose on developed grids by non-participation in voltage support and automatic generation control is expected to become increasingly burdensome and could incur additional costs for new builds [Kirby et al., 2007].

Important past publications addressing grid and nuclear power plant (NPP) interactions have focused on the sufficiency of offsite power supplied to the plant [Kirby et al., 2007; Bickel, 2001], but those reports noted that continued supply of offsite power is dependant on the performance of the nuclear plant itself during transients and thus, controls equipped on the plant to help grid integrity also have a positive effect on nuclear safety by lowering the likelihood of loss of offsite power. Two reports [IAEA, 1983; IAEA, 1987] have addressed the wider range of topics related to the grid-NPP interactions.

There has been continued interest in Small and Medium Sized Reactors (SMR), in part because they can be deployed in a market of smaller and more constrained grids. As such, identification of the contributions that a SMR can make to maintenance of the integrity of the electrical grid could add to the benefits of modularity and contribute to initiatives targeted to improve electrical power grid robustness.

The goal of the present work is to develop comprehensive control strategies that allow the plant to effectively respond to wide swings in load on short to medium time scales, as well as respond to the most severe types of recoverable grid upsets. 


\subsection{Simulation Background}

Defining the set of control variables to be used is of critical importance for development of control systems. For turbine control, the flow area over the turbine control and intercept valves has been taken to be the control variable in literature [Shankar, 1977]. In considering optimal control for load following with a nuclear unit, the control variables have been taken to include differential changes in the rod bank positions, Boron concentrations, steam flow rate, and the primary loop temperature [Yim and Christenson, 1992].

For power system studies, the steam valve positions are often considered by means of the governor equations. Additionally, exciter control has a large role in stability for which many control strategies exist [Anderson and Fouad, 2009]. Inclusion of boiler and feedwater control is considered to be a development area for fast valving studies [Patel et al., 2009].

In terms of dynamic simulation for nuclear plants, the secondary-side of a nuclear plant has comparable time constants to the primary side and warrants consideration for plant control strategies [Shankar, 1977]. For this work, the controls of all but the Boron concentration are considered. Mechanical Shim (MSHIM), is recommended for the IRIS reactor for several reasons including the primary system volume and operational benefits of a low-Boron system.

\subsection{Simulation Methods}

The dynamics of the reactor primary loop are modeled by employing the IRIS simulator described in previous sections. The steam supply system is modeled by appropriately selected parameters for the unit size and steam generator conditions, and the turbine modeled by a detailed simulation with scaled physical parameters from turbomachinery literature [Mynatt et al., 2003].

To address a severe grid fault and fast valving action, the commonly analyzed connection to an infinite bus was considered with a 6-variable generator model and a simple Power System Stabilizer control method for the exciter control [Rominger and Doster, 2009].

The event investigated here is a 3-phase ground fault at the location marked in Figure 5-1. The fault persists for 0.1 seconds, and has a clearing time of 0.5 seconds before the line is restored to its original state. A response is implemented using a fast valving maneuver applied to the butterfly Intercept Valve (IV) between the reheater and the low pressure turbine. Fast 
valving with exclusively the IV has been recommended as the best available option for nuclear units [Kirby et al., 2007].

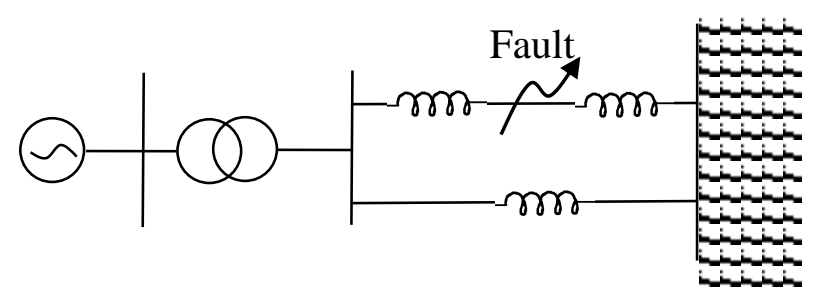

Figure 5-1: Model of the Infinite Bus Connection

A valve characteristic for the IV was constructed using flow-position characteristics found in literature [Aanstad and Lokay, 1970]. Typical values for closure and opening times specific to nuclear units were used for the fast valving maneuver. This is used in conjunction with the reheater, steam supply system, and plant dynamics to perform the simulation.

\subsubsection{Secondary System}

The steam cycle configuration that is assumed for the IRIS reactor in this work is described in previous literature [Kirby et al., 2007]. This cycle was adapted from the AP600 design by Westinghouse, modified slightly for the slightly superheated steam conditions. The schematic is shown in Figure 5-2. 


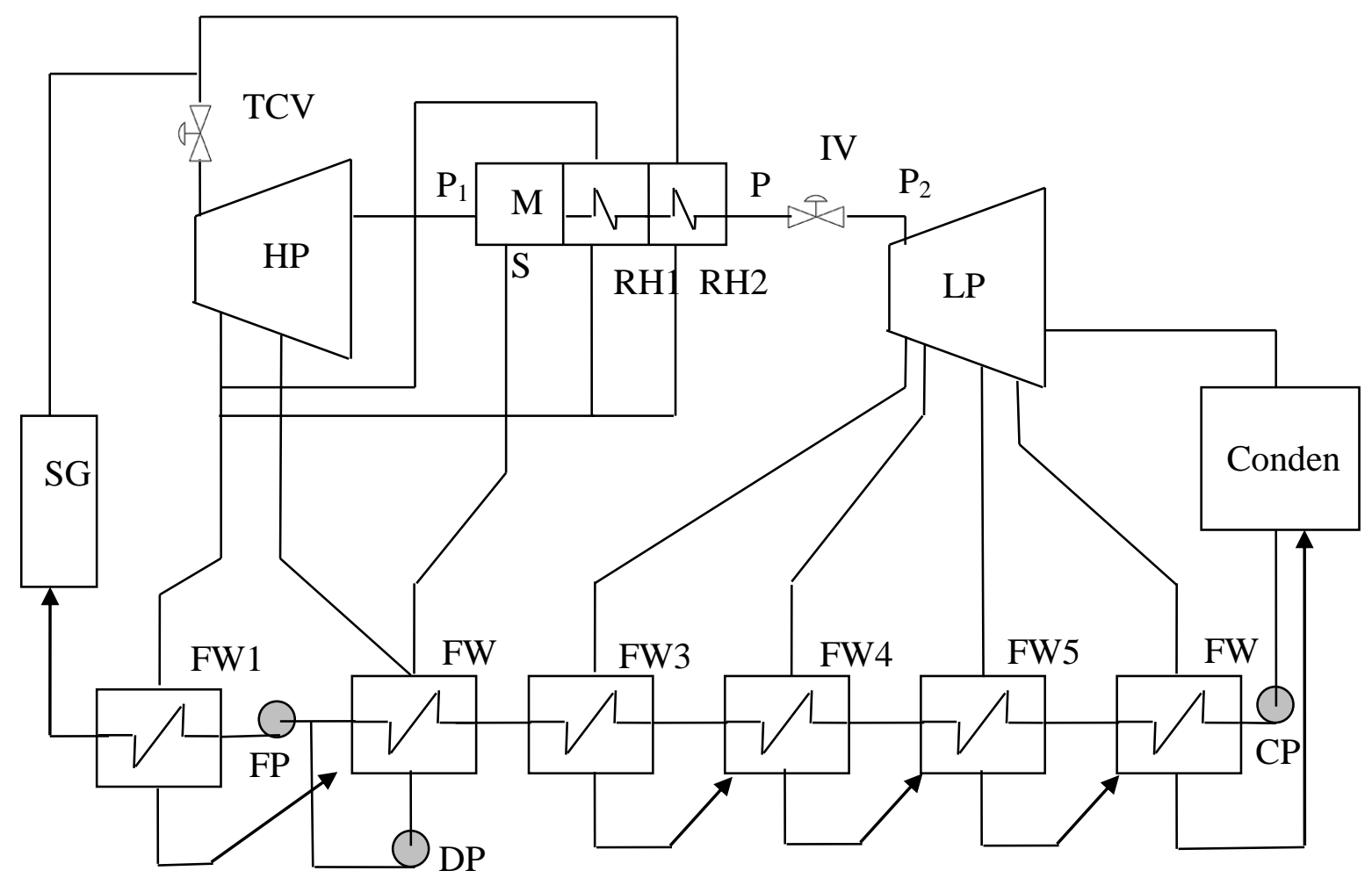

Figure 5-2: Steam Cycle Used Representing IRIS

This represents a system typical of nuclear power plants, being a twice-reheat regenerative reheat cycle with six feedwater heaters and moisture separator. The Turbine Control Valve (TCV) is normally used for the regulation of power, but the IV is the relevant component for the maneuver discussed in this paper.

\subsubsection{Turbine}

The distinguishing feature of the turbine model used in this work is that both the HP and LP turbines in the system are represented by an instance of a row-by-row simulation, connected by the Moisture Separator Reheater (MSR) equations. The row-by-row simulation is performed by an iterative approach based on the row pressures, with the order of calculation shown in Figure 5-3.

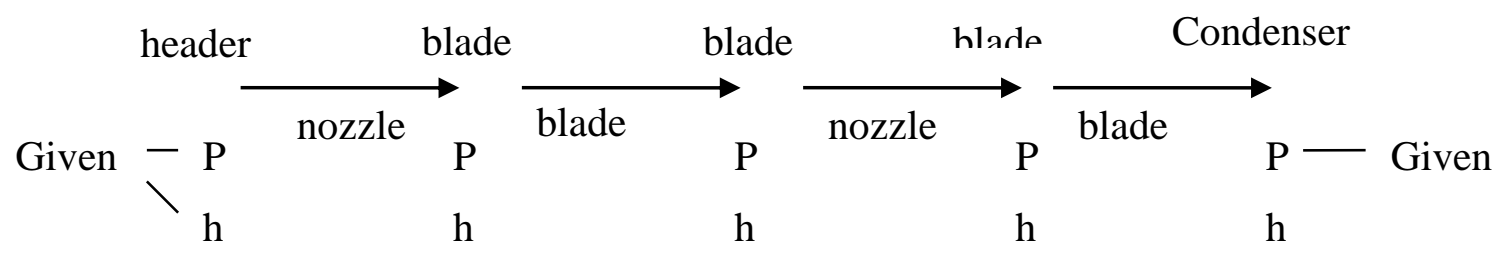


Figure 5-3: Turbine Model Calculation Flow

Equations for the model were built using turbo-machinery literature [Schobeiri, 2005]. A single row is given the index of $i$, and i-1 references the upstream row. The blade speed is related to the rotor speed by

$$
U_{i}=\omega R_{i}
$$

The energy and momentum (efficiency) equations are used to establish all the turbine steam path values for a given set of row pressures in the downstream calculation flow, starting with $i=1$, the first row. The efficiency of the blades and row are comprised of several loss mechanisms, and the functions for these are built with experimentally correlated functions taken from literature.

$$
\begin{aligned}
& \eta_{\mathrm{N} 1}=\text { profile wetness seals }=1-\xi_{p} \quad \alpha_{l}^{\prime}-\alpha_{l} \quad \eta_{\text {wet }, l} \eta_{\text {seals }, l} \\
& \mathrm{~h}_{l+1}=\mathrm{h}_{l}+\frac{1}{2} \mathrm{~V}_{l}^{2}-\eta_{\mathrm{N}, 1}\left(\mathrm{~h}_{l}+\frac{1}{2} \mathrm{~V}_{l}^{2}-\mathrm{h} \_\mathrm{ps} \quad \mathrm{P}_{l}, \mathrm{~s} \_\mathrm{ph}\left(\mathrm{P}_{l}, \mathrm{~h}_{l}\right)\right) \\
& \mathrm{V}_{l+1}=\sqrt{\mathrm{V}_{1}^{2}+2 \mathrm{~h}_{l}-\mathrm{h}_{1+1}} \\
& \mathrm{~V}_{l+1}=\sqrt{\mathrm{V}_{l+1} \sin \left(\alpha_{l+1}\right)-\mathrm{U}^{2}+\mathrm{V}_{l+1}^{2} \cos \left(\alpha_{l+1}\right)^{2}} \\
& \tan \beta_{l+1}^{\prime}=\frac{\mathrm{V}_{l+1} \sin \left(\alpha_{l+1}\right)-\mathrm{U}}{\mathrm{V}_{l+1} \cos \left(\alpha_{l+1}\right)} \\
& \eta_{\mathrm{B}, l+1}=1-\xi_{p} \quad \beta_{l+1}{ }^{\prime}-\beta_{l+1} \quad \eta_{\text {wet }, l+1} \eta_{\text {seals }, l+1} \\
& \mathrm{~h}_{l+2}=\mathrm{h}_{l+2}+\frac{1}{2} \mathrm{~V}_{l+1}{ }^{2}-\eta_{B, l+1}\left(\mathrm{~h}_{l+1}+\frac{1}{2} \mathrm{~V}_{l+1}{ }^{2}-\mathrm{h} \_ \text {ps } \mathrm{P}_{l+2}, \mathrm{~s} \_ \text {ph } \mathrm{P}_{l+1}, \mathrm{~h}_{l+1}\right) \\
& \mathrm{V}_{l+2}{ }^{\prime}=\sqrt{\mathrm{V}_{l+1}^{\prime 2}+2 \mathrm{~h}_{l+1}-\mathrm{h}_{l+2}} \\
& \mathrm{~V}_{l+2}=\sqrt{\mathrm{V}_{l+2}{ }^{\prime} \sin \left(\beta_{l+2}\right)+\mathrm{U}^{2}+\mathrm{V}_{l+2}{ }^{\prime} \cos \left(\beta_{l+2}\right)^{2}}
\end{aligned}
$$




$$
\tan \alpha_{l+2}{ }^{\prime}=\frac{\mathrm{V}_{l+2}{ }^{\prime} \sin \left(\beta_{l+2}\right)+\mathrm{U}}{\mathrm{V}_{l+2}{ }^{\prime} \cos \left(\beta_{l+2}\right)}
$$

Row pressures are obtained iteratively through a Newton-Raphson search. The mass balance forms the nonlinear equation for a single stage. Partial derivatives are found numerically with respect to the inlet velocity and all the row pressures. Then the mass balance for all stages together gives a solvable Newton-Rhapson system that forms a single iteration which is solved every time step

$$
\begin{gathered}
F_{0, l}=\rho_{l} V_{l} A_{l} \cos \alpha_{l}-\rho_{l+1} V_{l+1} A_{l+1} \cos \alpha_{l+1}-\dot{m}_{t a p, l}-V o l \frac{\rho_{1}^{t+\Delta t}-\rho_{1}^{t}}{\Delta t}=0 \\
\vec{U}=V_{1} \quad P_{2} \quad \ldots \quad P_{n}{ }^{T} \\
\overrightarrow{\vec{J}}_{0} \vec{\delta}=-\vec{F}_{0} \\
\vec{U}^{k+1}=\vec{U}^{k}-\vec{\delta}
\end{gathered}
$$

where $\overrightarrow{\vec{J}}_{0}$ represents the Jacobian of the system. Once convergence is reached, the value $\mathrm{V}_{1}$ allows $\dot{m}$ to be returned, and an energy balance allows the mechanical power to be returned.

\subsubsection{Valves}

The valves in the system are modeled utilizing valve data from literature [Aanstad and Lokay, 1970]. The flow-position characteristics are shown in Figure 5-4 as well as the inferred local loss as a function of position. A constant closure rate was assumed, and the valve was taken to move only to a set position before reopening. This final position may be taken as a control system output in an implementation of fast valving logic. 

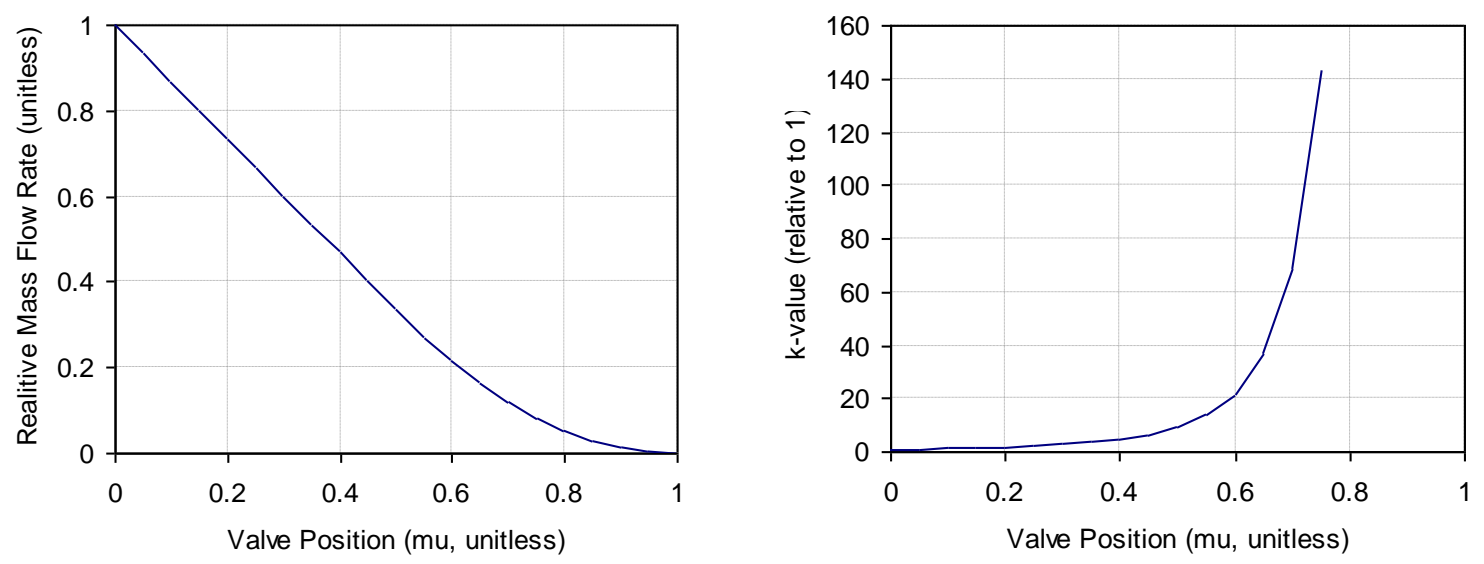

Figure 5-4: IV Model - Flow Rate and Valve Characteristic

The valve nature illustrated in these graphs is characteristic of butterfly valves, where the flow resistance sharply increases as the position nears the fully closed position.

\subsubsection{Reheater}

The reheater model gives a dynamic relationship between the HP outlet, the reheater itself, and the LP inlet conditions. It is modeled as a general steam volume with a heat exchanger imparting heat to the volume. The moisture separator is taken to have a nonnegligible pressure drop, but assumed to achieve perfect steam separation. The energy, mass, and momentum equations are employed with the assumption that the steam reaches stagnation conditions within the reheater, which is reasonable considering the time constant associated with the component.

$$
\begin{gathered}
M=V \rho P, h \\
E=M h \\
\frac{d M}{d t}=\dot{m}_{1}-\dot{m}_{2} \\
\frac{d E}{d t}=\dot{m}_{1} h_{1}-\dot{m}_{2} h+\dot{Q}
\end{gathered}
$$


The interface between the reheater and the LP inlet is defined by forms loss equations over the IV. The calculations are used to give the input parameters for the turbine models at the next time step.

$$
\begin{gathered}
\Delta P=k \frac{V^{2} \rho}{2 g_{c}}=\left(\frac{k}{2 g_{c} A^{2}}\right) \frac{\dot{m}^{2}}{\rho} \square c \frac{\dot{m}^{2}}{\rho} \\
P_{1}-P=\Delta P_{1}=c_{M S} \frac{\dot{m}_{1}^{2}}{\rho_{1}} \\
P-P_{2}=\Delta P_{2}=c_{I V} \mu \frac{\dot{m}_{2}^{2}}{\rho}
\end{gathered}
$$

The high pressure turbine model is called using an exhaust pressure found explicitly from the last time step.

$$
P_{1}^{t+\Delta t}=P^{t}+c_{M S} \frac{\dot{m}_{1}^{t 2}}{\rho_{1}^{t}}
$$

The low pressure turbine model is called using an inlet pressure from the current time step.

$$
P_{2}^{t+\Delta t}=P^{t+\Delta t}-c_{I V} \quad \mu \frac{\dot{m}_{2}^{t 2}}{\rho^{t+\Delta t}}
$$

The reheater values are updated in-between the calculations for the HP turbine and the LP turbine. The HP calculations return $\dot{m}_{1}^{t+\Delta t}$, which is used here, and the final $P_{2}^{t+\Delta t}$ is of relevance to the LP model. A state equation of the form $P=P\left(\frac{E}{M}, \frac{M}{V}\right)$ provides pressure given enthalpy and density.

$$
\begin{gathered}
\frac{M^{t+\Delta t}-M^{t}}{\Delta t}=\dot{m}_{1}^{t+\Delta t}-\dot{m}_{2}^{t} \\
\frac{E^{t+\Delta t}-E^{t}}{\Delta t}=\dot{m}_{1}^{t+\Delta t} h_{1}^{t+\Delta t}-\dot{m}_{2}{ }^{t} h^{t}+\dot{Q}^{t}
\end{gathered}
$$




$$
\begin{gathered}
P=P\left(\frac{E}{M}, \frac{M}{V}\right) \\
P_{2}^{t+\Delta t}=P^{t+\Delta t}-c \frac{\dot{m}_{2}^{t 2}}{\rho^{t+\Delta t}}
\end{gathered}
$$

Finally, the LP model returns $\dot{m}_{2}^{t+\Delta t}$, giving the next time step values. The condensing hot side temperature of the heat exchanger is taken to be spatially uniform in both RH1 and RH2. The log mean temperature difference (LMTD) equation is used for the heat rate calculations.

$$
\Delta T_{m 1}=\frac{T_{\text {sat }} P_{1}-T_{R H 1 \text { out }}}{\ln \left(\frac{T_{h 1}-T_{R H 1 \text { out }}}{T_{h 1}-T_{\text {sat }} P_{1}}\right)}, \Delta T_{m 2}=\frac{T_{R H 1 \text { out }}-T P, h}{\ln \left(\frac{T_{h 2}-T P, h}{T_{h 2}-T_{R H 1 \text { out }}}\right)}
$$

In reality, the hot-side is not a constant temperature if the inlet steam is superheated, which is the case in both of the reheaters of an IRIS steam cycle. However, instead of dealing with the complexity of a region of superheated steam (ss) and region of saturated mixture, the saturation temperature at the hot side pressure is revised upward slightly to reflect the superheating though the effective hot-side temperature defined here.

$$
T_{h}=\frac{\Delta h_{s a t} T_{s a t}+\Delta h_{s s} T_{s s, a v g}}{\Delta h_{s a t}+\Delta h_{s s}}=\frac{h_{f g} T_{s a t}+h-h_{g} \frac{1}{2} T_{s a t}+T}{h-h_{f}}
$$

As one would expect, the latent heat is much greater than the contribution from the superheating, and this makes only a very small change compared to the saturation temperature. For on-design values, RH1 has a difference of $0.1^{\circ} \mathrm{F}$ and $\mathrm{RH} 2$ has a difference of $3.7^{\circ} \mathrm{F}$.

The UA values are easily determined from the known steam cycle values. The $\dot{Q}$ term is found with past time values. The temperature between the two reheaters is calculated using the ratio of the heat rates.

$$
\begin{gathered}
\dot{Q}=U A_{1} \Delta T_{m 1}+U A_{2} \Delta T_{m 2} \\
h_{R H 1 \text { out }}=h_{1}+h_{1}-h \frac{U A_{1} \Delta T_{m 1}}{\dot{Q}}
\end{gathered}
$$




$$
T_{R H 1 \text { out }}=T P, h_{R H 1 \text { out }}
$$

Since the heat rate from the past time value is used, these are all explicit calculations. The MS outlet temperature, the RH1 outlet temperature, and the RH2 outlet temperature are used to calculate a heat rate, then the reheater equations are used to find new outlet and inlet pressures for the HP and LP turbines respectively.

\subsection{Generator and Grid Connection}

The system represented with the generator and grid modeled in Figure 5-1 is a one-line diagram of 3-phase electrical power distribution. A 6-equation model for the generator was used and is described here. A current and voltage phasor represents the state at every bus within the system and for the machine bus-bar, the electrical power comes from the power law which is put into terms of the direct axis and quadrature axis values. Then the kinematics of the rotors of all machines in the system is considered with a momentum equation, which relates the rotor speed to all other values.

$$
\begin{gathered}
P_{e, i}=\bar{V}_{i} * \sum_{j=1}^{N} \bar{Y}_{i j} \bar{V}_{j}=i_{q} \lambda_{d}-i_{d} \lambda_{q} \\
\omega I\left\{\frac{\omega^{t+\Delta t}-\omega^{t}}{\Delta t}\right\}=P_{M} \vec{U}^{t+\Delta t}, \omega^{t+\Delta t}-P_{E} \vec{V}^{t+\Delta t}, \delta^{t+\Delta t}, \omega^{t+\Delta t}
\end{gathered}
$$

Parks transformation is used for converting from the rotor to stator reference frames.

$$
P=\sqrt{2 / 3}\left[\begin{array}{ccc}
\frac{1}{\sqrt{2}} & \frac{1}{\sqrt{2}} & \frac{1}{\sqrt{2}} \\
\cos \theta & \sin \left(\theta-\frac{2 \pi}{3}\right) & \cos \left(\theta+\frac{2 \pi}{3}\right) \\
\sin \theta & \cos \left(\theta-\frac{2 \pi}{3}\right) & \sin \left(\theta+\frac{2 \pi}{3}\right)
\end{array}\right]
$$




$$
\left[\begin{array}{l}
i_{0} \\
i_{d} \\
i_{q}
\end{array}\right]=P\left[\begin{array}{l}
i_{a} \\
i_{b} \\
i_{c}
\end{array}\right],\left[\begin{array}{l}
v_{0} \\
v_{d} \\
v_{q}
\end{array}\right]=P\left[\begin{array}{l}
v_{a} \\
v_{b} \\
v_{c}
\end{array}\right]
$$

Then, by circuit analysis, relationships can be written for generator quantities. Voltage laws are dictated by resistor and inductor properties. The circuits generally have some current, voltage, and flux associated with them. These are all treated as vectors that include quantities for all the rotor (FDQ) and stator quantities (0dq) with the zero sequence left out.

$$
v= \pm \sum R i \pm \sum \frac{d \lambda}{d t}= \pm \sum R i \pm \sum \frac{d L i}{d t}
$$

Vector definitions

$$
\begin{aligned}
& i=\left[\begin{array}{lllll}
i_{d} & i_{F} & i_{D} & i_{q} & i_{Q}
\end{array}\right]^{T} \\
& v=\left[\begin{array}{lllll}
v_{d} & v_{F} & v_{D} & v_{q} & v_{Q}
\end{array}\right]^{T} \\
& \lambda=\left[\begin{array}{lllll}
\lambda_{d} & \lambda_{F} & \lambda_{D} & \lambda_{q} & \lambda_{Q}
\end{array}\right]^{T}
\end{aligned}
$$

The state of the generator is fully determined by flux values, and the time derivatives of the fluxes are integrated for the dynamic simulation. Algebra with Parks Transform is used to obtain a form that only uses 0dq values, thus eliminating troublesome trigonometric identities, the first form of relevance of the voltage laws is as follows.

$$
v=-R+\omega N i-L i
$$

Another approach to reduce the complexity of the equations is to use the mutual flux and inductance for all of the circuits in the $\mathrm{d}$ and $\mathrm{q}$ axis. The flux for AD can be taken as the magnetic flux permeating the entire $d$ axis, which is obtained by proportionally summing the fluxes for all the circuits that lie within that axis. The flux for AQ has similar logic supporting it, but for the $\mathrm{q}$ axis instead. All of the 1 or $\mathrm{L}$ values used here represent inductance and are either given or calculated. 


$$
\begin{gathered}
\lambda_{A D}=\left[\begin{array}{lll}
\frac{L_{m d}}{l_{a}} & \frac{L_{m d}}{l_{f}} & \frac{L_{m d}}{l_{D}}
\end{array}\right]\left[\begin{array}{l}
\lambda_{d} \\
\lambda_{F} \\
\lambda_{D}
\end{array}\right] \\
\lambda_{A Q}=\left[\begin{array}{ll}
\frac{L_{m q}}{l_{a}} & \frac{L_{m q}}{l_{f}}
\end{array}\right]\left[\begin{array}{l}
\lambda_{q} \\
\lambda_{Q}
\end{array}\right]
\end{gathered}
$$

Then the current for each circuit at any given time can be found in a simplified manner using just the flux particular to the circuit and the mutual flux. This is implemented as follows.

$$
\left[\begin{array}{c}
i_{d} \\
i_{F} \\
i_{D} \\
i_{q} \\
i_{Q}
\end{array}\right]=\left[\begin{array}{ccccccc}
1 / l_{a} & & & -1 / l_{a} & & & \\
& 1 / l_{F} & & -1 / l_{F} & & & \\
& & 1 / l_{D} & -1 / l_{D} & & & \\
& & & & 1 / l_{a} & & -1 / l_{a} \\
& & & & & 1 / l_{D} & -1 / l_{D}
\end{array}\right]\left[\begin{array}{c}
\lambda_{d} \\
\lambda_{F} \\
\lambda_{D} \\
\lambda_{A D} \\
\lambda_{q} \\
\lambda_{Q} \\
\lambda_{A Q}
\end{array}\right]
$$

The previous formulations for $\lambda_{D}, \lambda_{A D}$, and $\lambda_{A Q}$ are substituted into this equation. Applying the matrix definitions to the voltage laws, we find the following form.

$$
\begin{gathered}
{\left[\begin{array}{c}
\dot{\lambda}_{d} \\
\dot{\lambda}_{F} \\
\dot{\lambda}_{D} \\
\dot{\lambda}_{q} \\
\dot{\lambda}_{Q}
\end{array}\right]=\left[\begin{array}{cccccc}
-r_{a} & & & & \\
& -r_{F} & & & \\
& & -r_{D} & & \\
& & & -r_{a} & \\
& & & & -r_{Q}
\end{array}\right]\left[\begin{array}{c}
i_{d} \\
i_{F} \\
i_{D} \\
i_{q} \\
i_{Q}
\end{array}\right]+\left[\begin{array}{c}
-\omega \lambda_{d} \\
v_{f} \\
\omega \lambda_{q}
\end{array}\right]+\left[\begin{array}{c}
-v_{t d} \\
\\
-v_{t q}
\end{array}\right]} \\
{\left[\begin{array}{c}
\dot{\dot{\omega}} \\
\dot{\delta}
\end{array}\right]=\left[\begin{array}{c}
T_{m}-\frac{i_{q} \lambda_{d}-i_{d} \lambda_{q}}{3 \tau_{j}} \\
\omega-1
\end{array}\right]}
\end{gathered}
$$

The time derivatives of the fluxes are now functions of the currents, which are functions of the fluxes, giving a non-linear system. 


$$
\begin{gathered}
{\left[\frac{d \bar{\lambda}}{d t}\right]=f \quad \bar{\lambda}, \bar{i}, B C s} \\
{[\bar{i}]=\lambda \text { to i }[\bar{\lambda}]}
\end{gathered}
$$

This gives the basis for establishing a set of non-linear differential equations necessary for simulating the system. The state vector will be defined with the fluxes, angle, and position.

$$
\begin{gathered}
x=\left[\begin{array}{lllllll}
\lambda_{d} & \lambda_{F} & \lambda_{D} & \lambda_{q} & \lambda_{Q} & \omega & \delta
\end{array}\right]^{T} \\
\dot{x}=C x+D
\end{gathered}
$$

Boundary conditions are given by the voltage law applied over the transmission line, which changes throughout the simulation.

\subsection{Low Pressure (LP) Turbine Benchmark}

To verify the accuracy of the turbine model, a 3-stage LP turbine input file was developed that mimicked Petrovic's [Petrovic and Riess] example. There are several differences in how that reference and the present work modeled the steam path, notably, only a 1-D model was used here, whereas a 2-D CFD solution was used in that publication. Simulation results from this research are compared to the literature in Figure 5-5 and Figure 5-6. The 7 main dots represent the different rows of the turbine. These figures shows the steam properties, as well as pressure and quality lines. The temperature of the header was kept constant as the mass flow rate was varied.

A major shortcoming is the behavior in low flow regimes due to the complicated $2 \mathrm{D}$ behavior. This is evident in some of the results, as the exhaust hood and last stage blades are not modeled in a way that is loyal to the reference. The model for this research employed wetness loss, leakage loss, and profile losses in proportions that were consistent with some quoted values in other literature, but can't be expected to match with Petrovic since they are only experimental correlations.

Good correspondence is observed here, but it should be stressed that no great differences should be observed provided that the steam path is given correct treatment. The overall steam 
path values and the total efficiency can be fairly close while the specific row-by-row models for losses are significantly different due to the nature of error propagation for row efficiency. There remains much room for improvement in the way that losses are modeled in the simulation, but these results confirm that it operates correctly as a general turbine model.

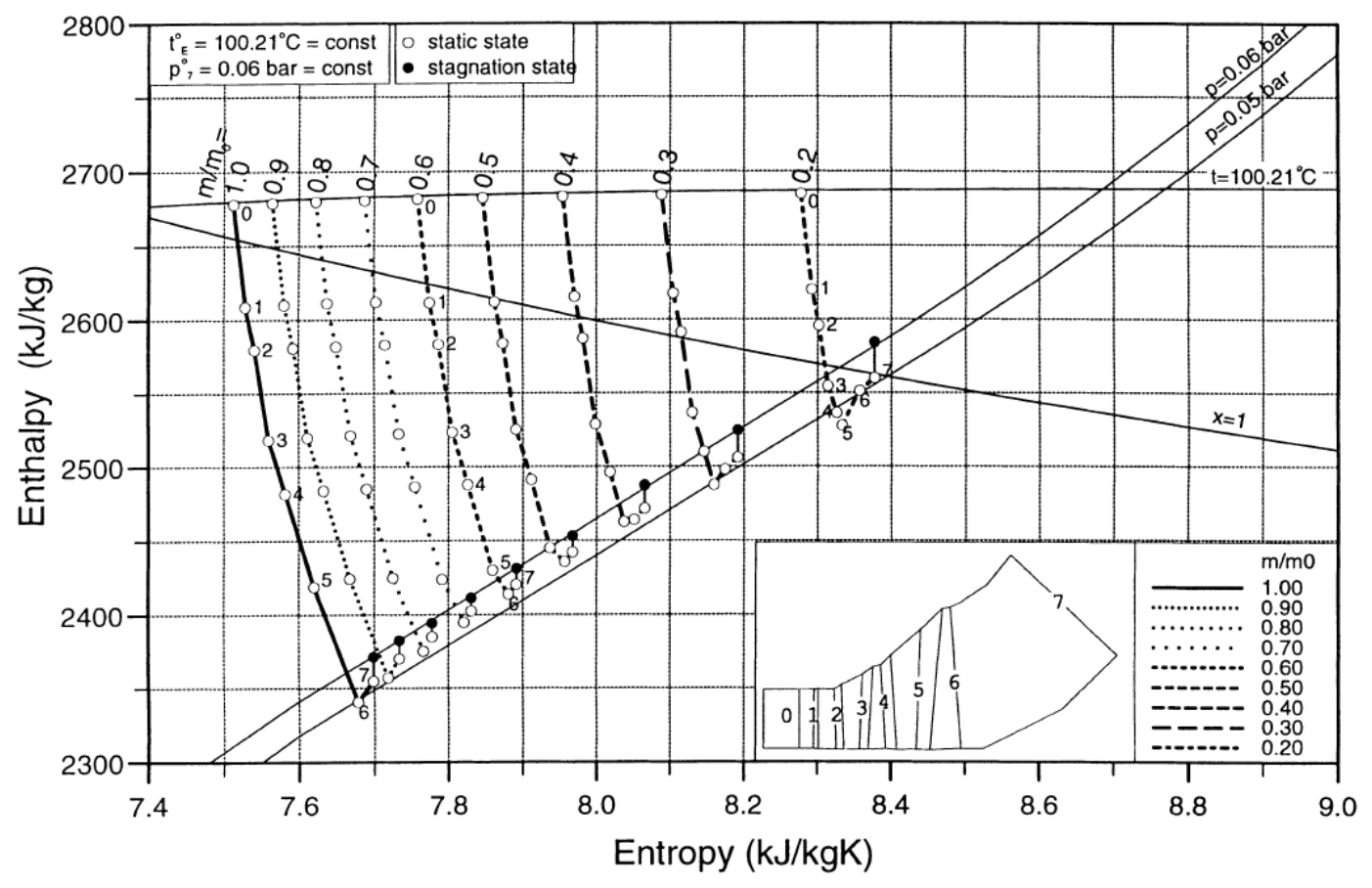

Figure 5-5: Reference Values 
3 Stage LP Benchmark

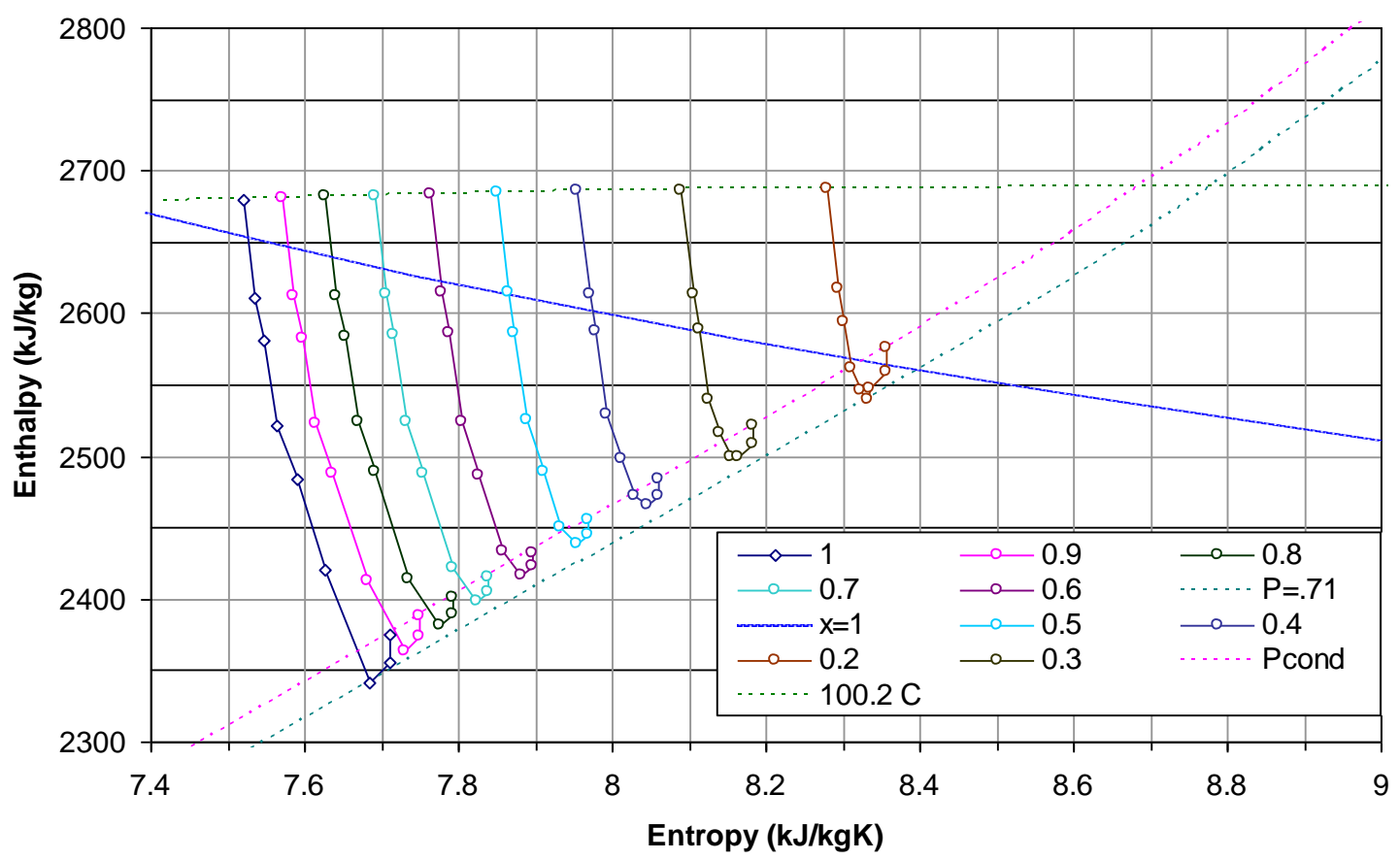

Figure 5-6: Benchmark Values

In addition to this benchmark, data from Mynatt, et. al. [2003] was used to establish the turbine parameters for the rest of the LP and all of the High Pressure (HP) turbine. Ultimately, 11 stages were included, 5 for the HP and 6 for the LP turbine.

This turbine model was coupled to the primary system model with constant tap flows. A program output is set for the plant power and the same control strategy from Shen and Doster [2009] is employed. A step decrease of $10 \%$ in the programmed power, for instance, causes several reactions from the plant, and the behavior of the mechanical power applied to the turbine through such a simulation is shown in Figure 5-7, where the pressure loss through the turbine is modeled with a constant loss coefficient versus the steam path modeling described here with a constant rotor speed. 


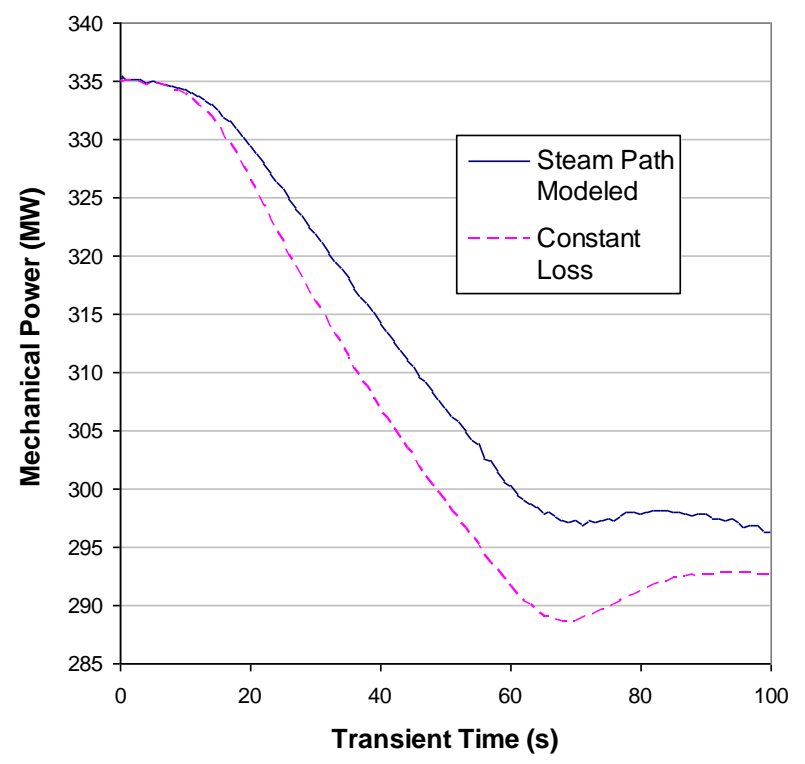

Figure 5-7: 10\% Step Decrease in Programmed Power

A small difference in plant behavior is seen from including the steam path modeling. This can be attributed to the physics of the turbine changing as the flow deviates from the ondesign row velocities. The next step in this simulation was the linking to the grid model.

\subsection{Grid Model and Benchmark}

Example 2.6 and 2.7 from Anderson and Fouad [1970] demonstrate the classic model of rotor angle stability for a 3 machine system. These examples employ a 9 bus system that is reduced to a $3 \times 3$ system for each state of the system (pre-fault, faulted, cleared), then the swing equation forms a dynamic simulation problem that illustrates 3 machine stability. The output for this example was replicated, as shown in Figure 5-8 and Figure 5-9. 


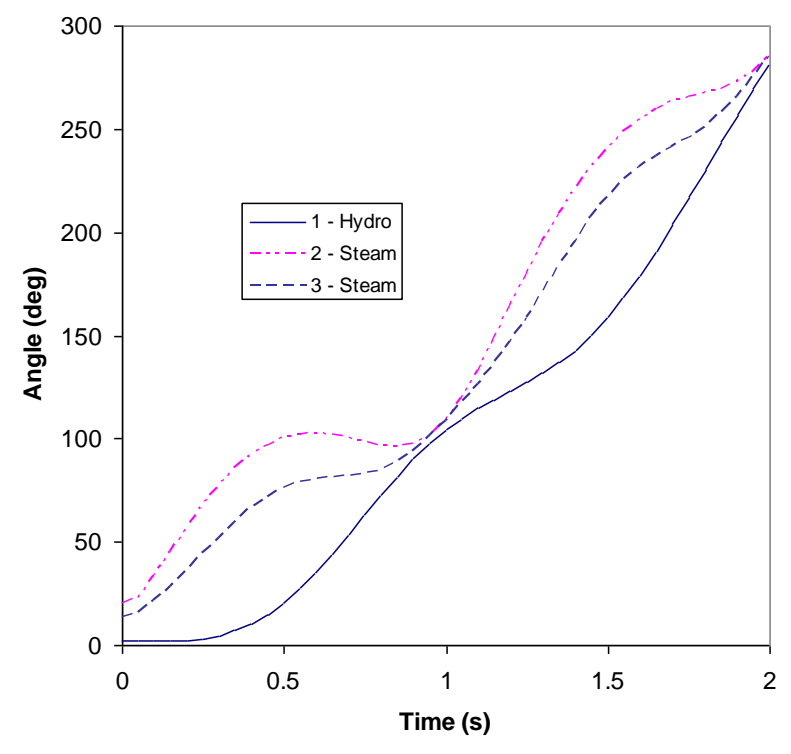

Figure 5-8: Machine Angles through Transient

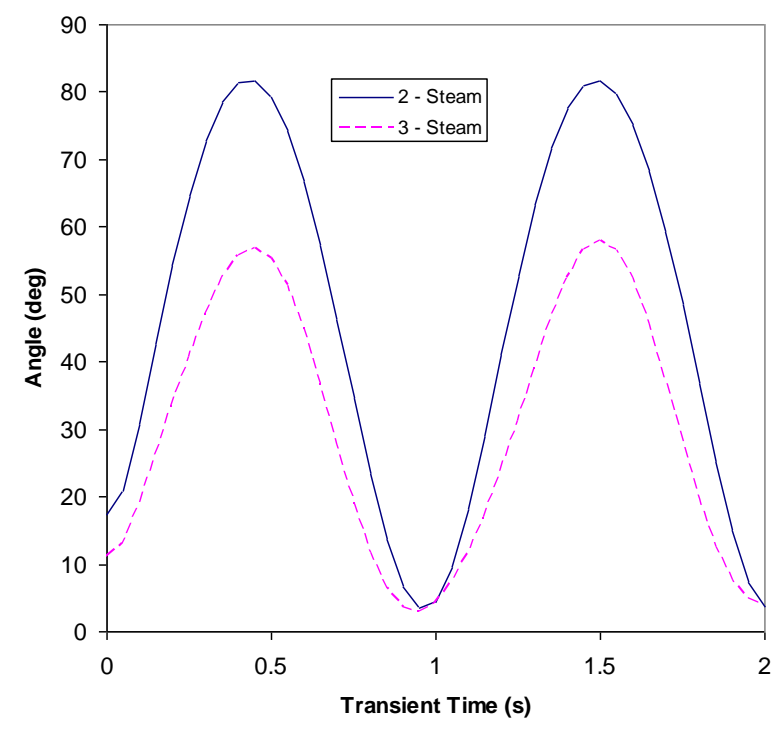

Figure 5-9: Relative Angles to Unit 1

Example 3.4 from the same reference uses the same network with a different fault. This example was coupled to the plant simulation with unit 2 replaced by the IRIS plant rotor. In order to obtain a self-correcting simulation, a $1 \mathrm{MW}$ load at bus 8 was added as shown in Figure 5-10, while at the same time, the programmed plant power was given a 5\% step increase. It was found that for this configuration, the plant behavior arrested deceleration in the underspeed event of adding the electric load (Figure 5-11). 
In developing this coupling, it was found that the time step required by the grid model was significantly smaller than that required for the turbine and primary model. Thus the grid model was decoupled, running several time steps for each that the primary system ran. This was possible to do within the turbine model iteration because the grid model is very computationally light (at this stage) although it has a comparatively higher frequency of oscillation.

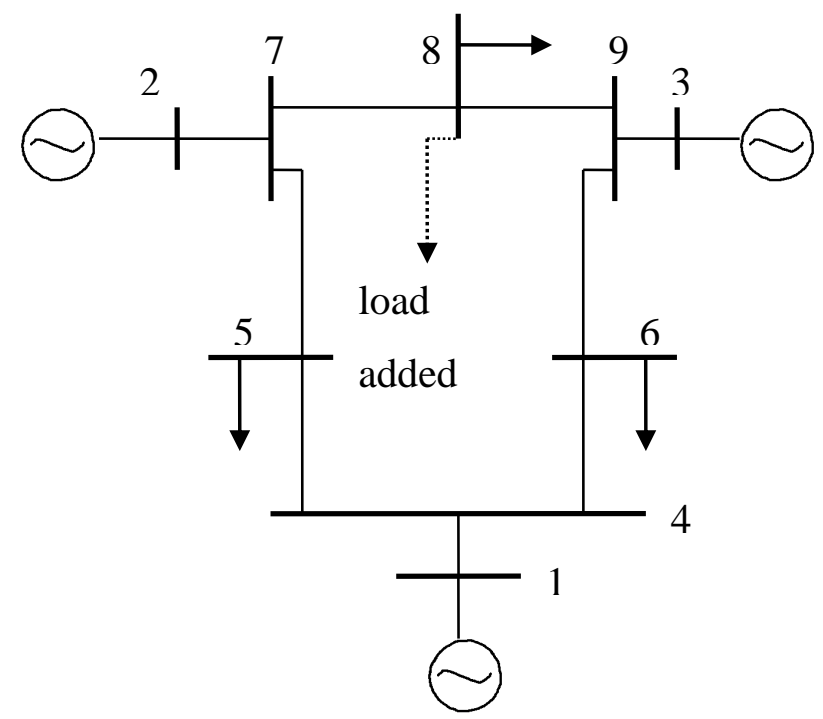

Figure 5-10: Nine Bus Transmission System and Added Load at Bus 8

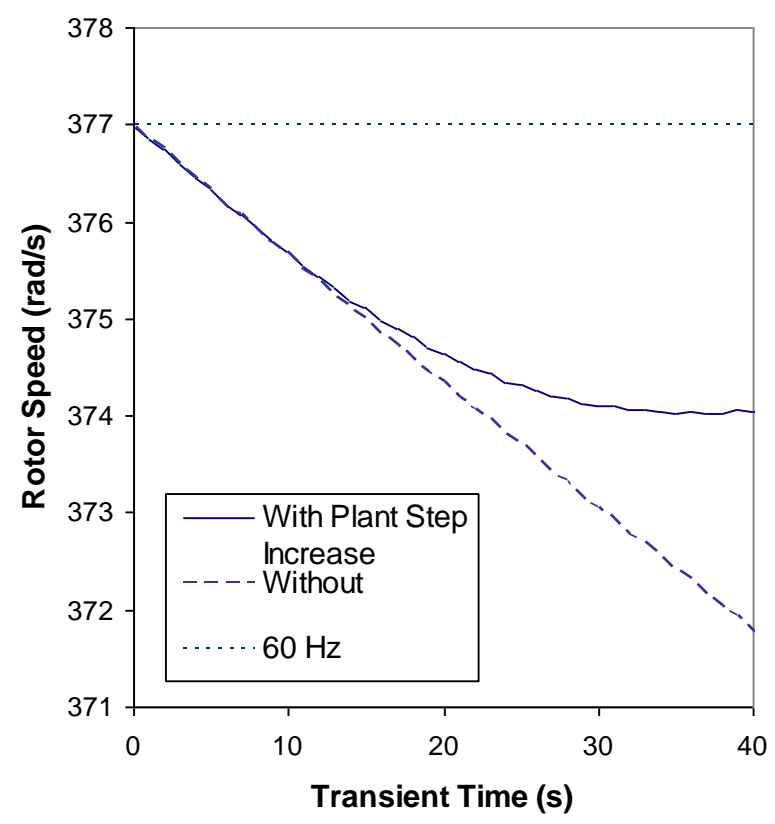

Figure 5-11: Rotor Speed through Transient Caused by Extra Electricity Demand 
The electrical torque quickly increases, causing the initial slowdown (underspeed event). In the run with an increase in programmed power, the mechanical torque slowly catches up and halts the deceleration.

\subsection{Fast Valving Results}

The sample fast valving transient described earlier is presented here, entailing the stroke characteristic shown in Figure 5-12 with a closure, dead, and reopening time of 0.2, 0.5, and 0.8 seconds respectively.

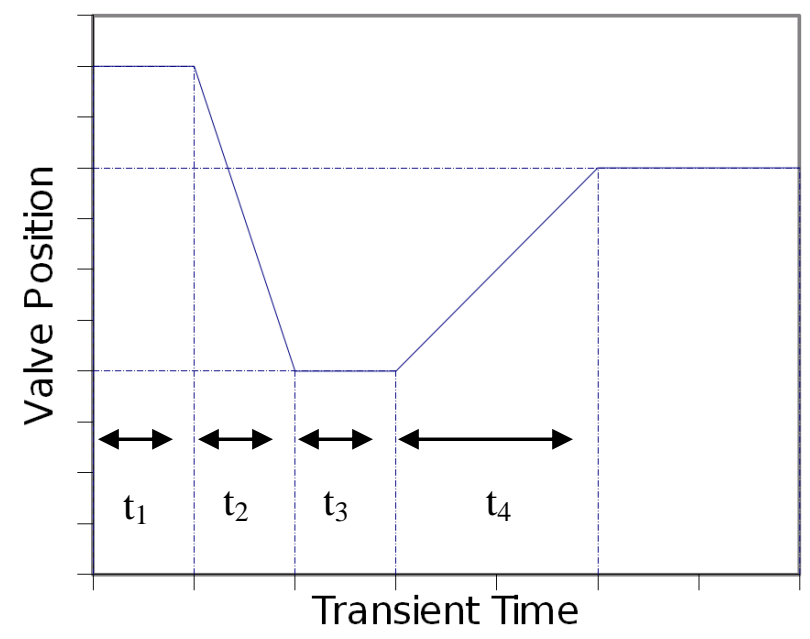

Figure 5-12: Fast Valving Stroke Characteristic

As the valve closes, the reheater volume largely absorbs the energy that is curtailed from being delivered to the grid. This is reflected in increasing pressure, and an initially decreasing flow rate through the low pressure turbine that must be followed by increased flow rate. This is illustrated in Figure 5-13. The rotor angle response is an important metric for response to severe grid upsets such as this. The output from this example is shown in Figure 5-13b, demonstrating the reduction in swing angle as compared to the plant response without fast valving. 

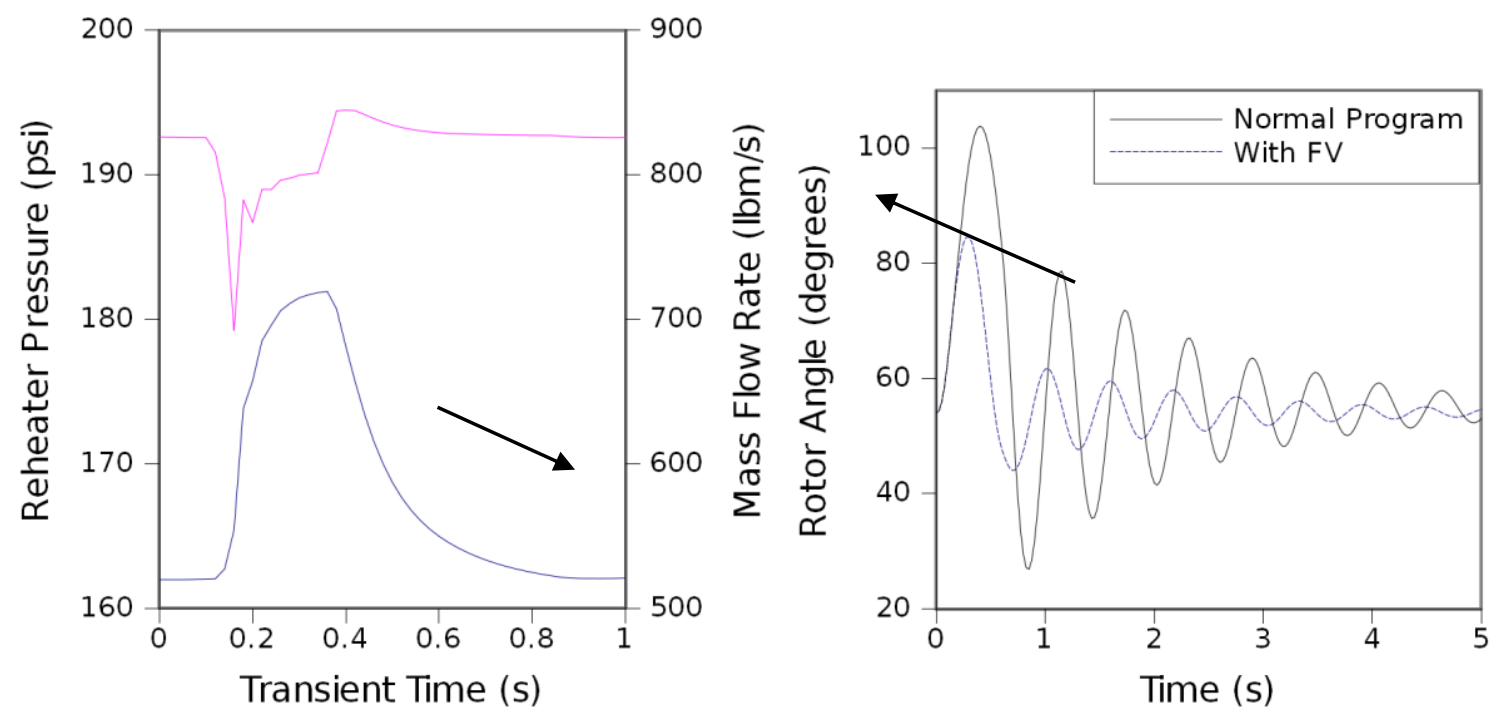

Figure 5-13: (a) Reheater Pressure and Mass Flow Rate and (b) Rotor Angle

The primary system demonstrates more long lived effects, as the pressure propagates through the high pressure turbine before reaching the steam generator. Several of these effects are illustrated in Figure 5-14.
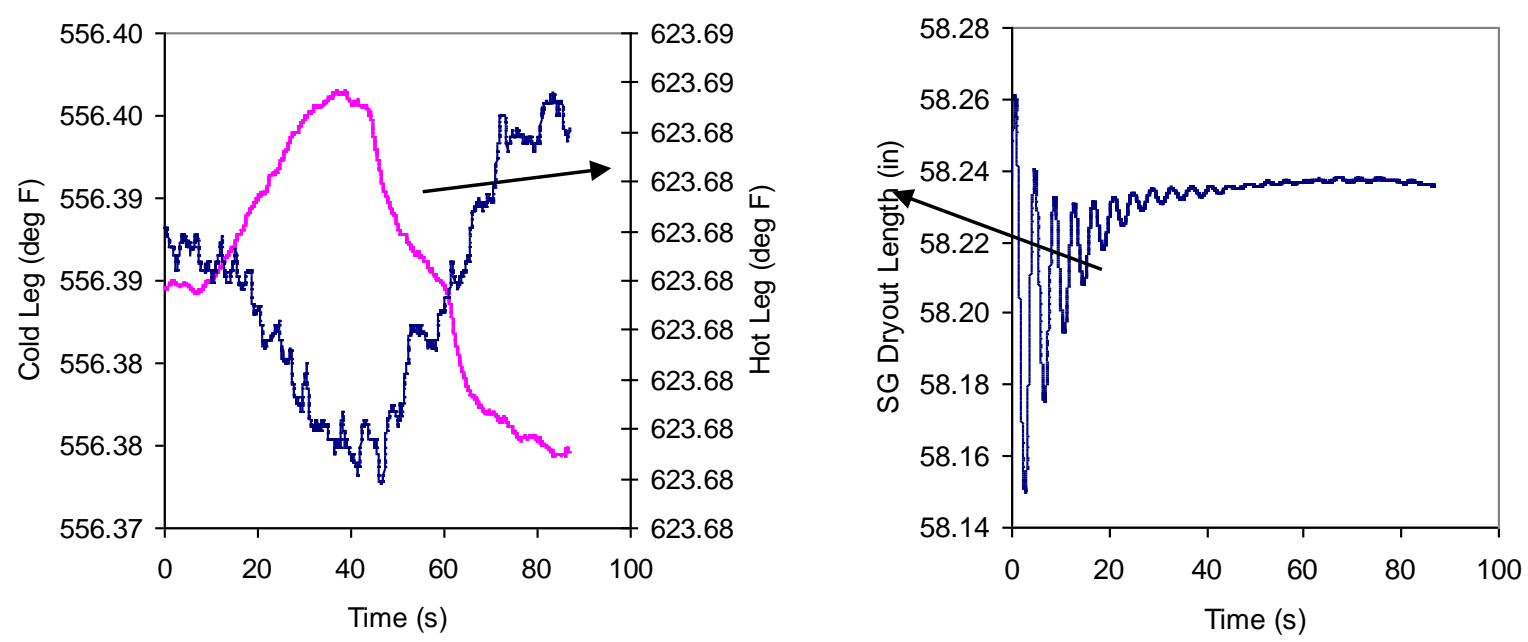

Figure 5-14: (a) Hot and Cold Leg Temperature and (b) Dry-out Location

The effect of the maneuver has a lag associated with it as it has to propagate back through the HP turbine. The steam generator dry out length is an important metric to maintain through the transient, and it will be of interest to identify the severity of faults that can be responded to without having detrimental effects on the steam generator pressure or flow dynamics. 


\section{SIMULATOR ANALYSIS METHODOLOGY}

\subsection{Linearity Test for the IRIS Reactor System}

Since the preferred method for data assimilation and uncertainty quantification is dependent upon whether the observables, i.e. sensor signals, and attributes, e.g. DNBR, sensitivity equations are nearly linear or not, a Chi-Square test is required to confirm the system sensitivity equations linearity. For the Chi-Square goodness of fit, the observables and system attribute data are divided into $\mathrm{K}$ bins and the test statistic is defined as:

$$
\chi^{2}=\sum_{i=1}^{K} \frac{O_{i}-E_{i}{ }^{2}}{E_{i}}
$$

where $O_{i}$ is the observed frequency for bin $i$ and $E_{i}$ is the expected frequency for bin $i$. If the sensitivity equations are linear and the parameters uncertainty distributions are Gaussian, the sensor and attribute distributions will also be Gaussian. Thus using a Gaussian distribution to obtain values for $E_{i}$ and the IRIS simulator to obtain values of $O_{i}$, Chi-Square values can be obtained for the observables and attributes. One can observe that not all observables and/or system attributes are within the desired level of the Chi-Square value, i.e. acceptable range of the linearity, if the reactor controllers are on and/or the transient is rapid. The reactor controllers that are associated with safety systems control certain observables, e.g. primary pressure, causing the reactor system to not behave naturally, which results in nonlinearity. Obviously the rapidness of the transients also causes the nonlinearity, e.g. RCP Trip. Note that the safety controllers, for example, pressurizer heaters and feedwater controllers, could be turned on for certain transients, e.g. feedwater controllers are turned on for a step load change. For further discussion of the ChiSquared test, all of the samples are combined and presented in Figure 6-1 for a 10\% step load change simulation. Figure 6-1 indicates that the pressurizer heater has a limited capacity as constrained by output capacity. This causes nonlinearity in the primary pressure as shown in Figure 6-2. Some of the responses of the observables on the primary side could be affected by this nonlinearity as well. Since the minimum DNBR is a strong function of the system pressure, core flow rate, and coolant temperature, the hot channel minimum DNBR also shows nonlinearity. Similar to the safety control mechanism that produces a nonlinear primary pressure 
response, active feed pumps and feed control valves action during the step load change result in nonlinearity that appears especially on the secondary side of the reactor system.

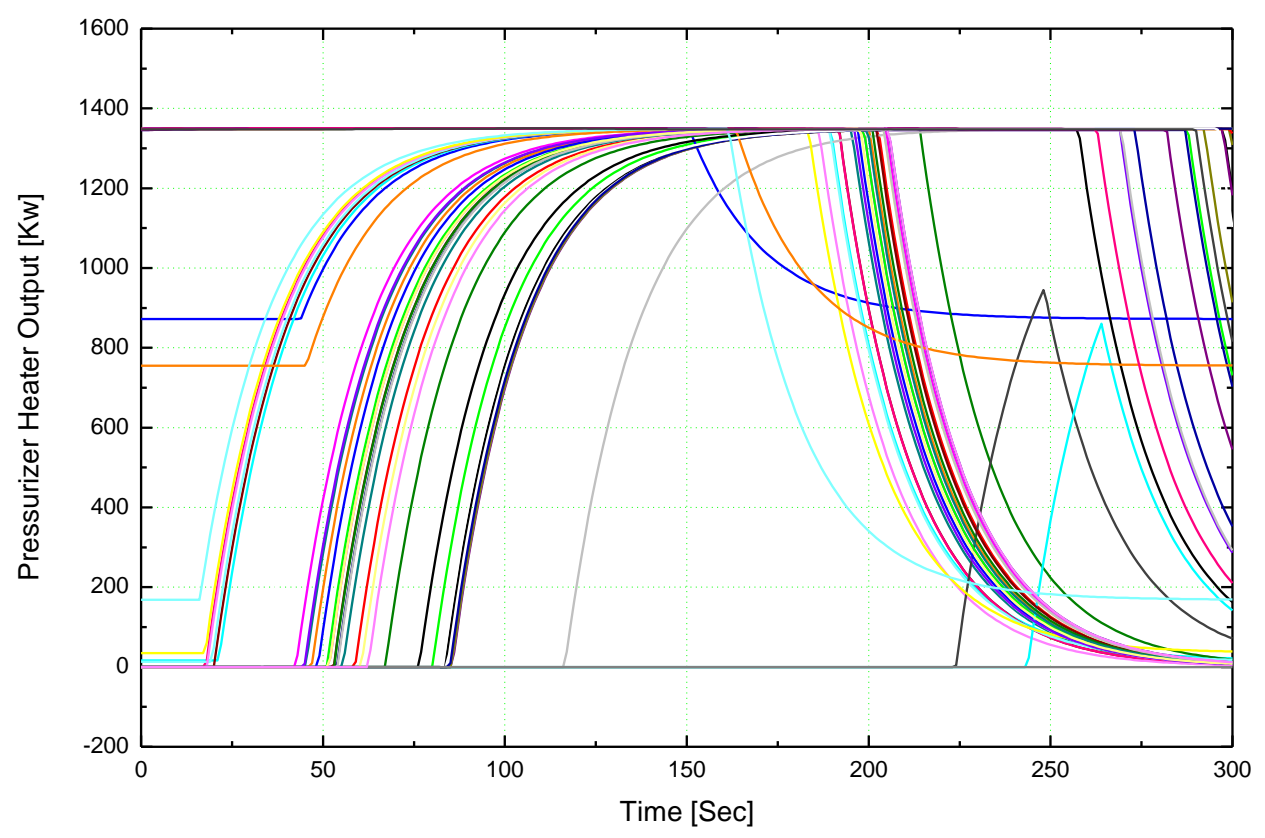

Figure 6-1: Pressurizer Heater Output 


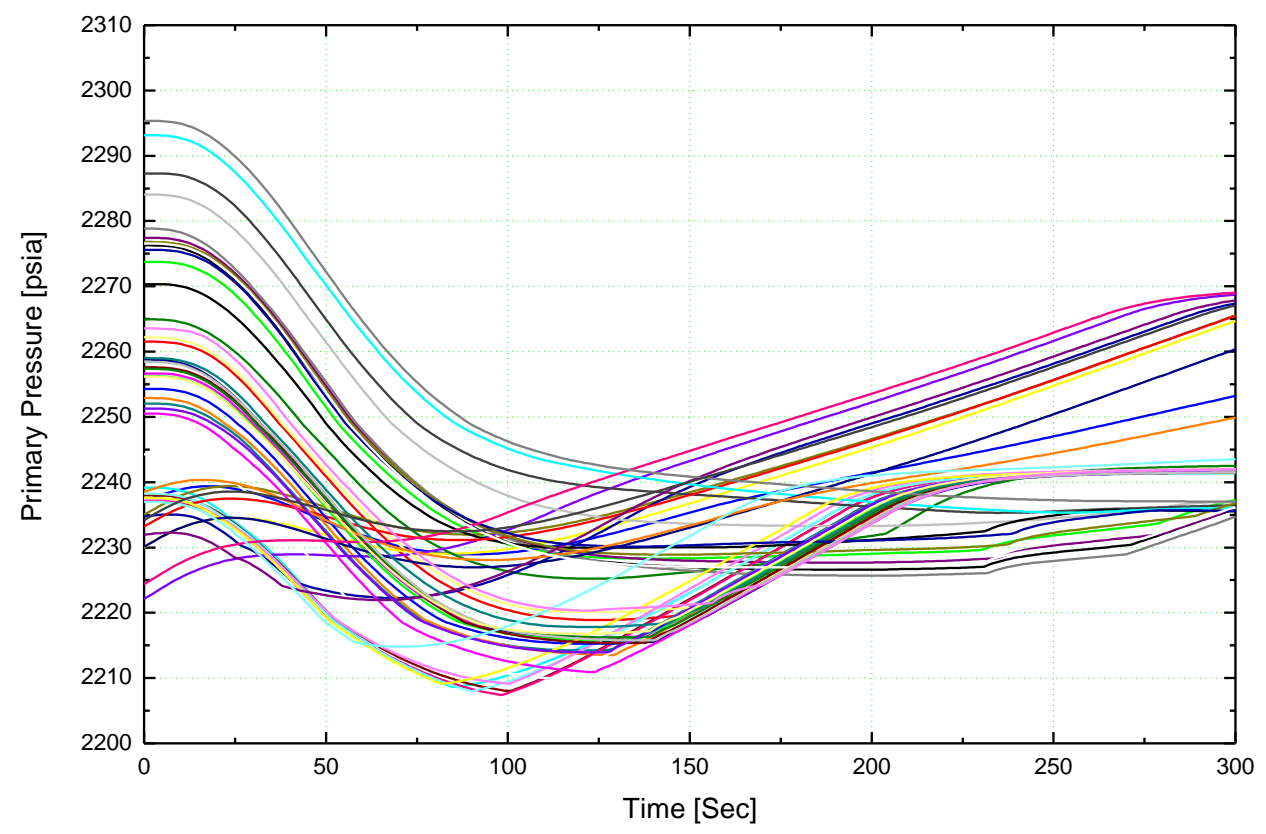

Figure 6-2: Primary Pressure

In order to simulate natural responses of the reactor system, IRIS simulations for several transients assuming inactive reactor controllers were completed for 100 samples of the parameters using Latin Hypercube sampling. With the safety controller, for example, pressurizer heaters, as well as the reactor system controllers assumed to be turned off, the reactor system will behave naturally and the Chi-Square values for most observables and the system attributes decrease versus if these controllers are on.

\subsection{Data Assimilation and Uncertainty Quantification}

\subsubsection{Deterministic Method for the Mildly Nonlinear Problems}

Assuming that parameters have larger uncertainties compared to any other sources of uncertainties, they can be refined to develop a higher fidelity model. Using adjusted thermal hydraulic parameters and their reduced uncertainties, subsequent reactor simulation improves the prediction of key system attributes if closely related to the system observables. In order to accomplish this, given measurements of the observables and a priori distributions of the parameters, one solves an inverse problem adjusting the parameter values to achieve better 
agreement between measured and predicted sensor response values [Tarantola, 2005]. Since IRIS is not operational anywhere, to generate the experimental values of the observables a virtual reactor is employed. By this is implied that a computer simulation is used to predict the experimental values, where perturbed parameter values have been used that are consistent with their uncertainties.

The distribution of the parameters (assumed Gaussian), whose mean is $\overline{\tilde{p}}_{0}$ and whose covariance matrix is $\overline{\bar{C}}_{p}$, is given by:

$$
\rho(\bar{p})=C_{1} \exp \left[-\frac{1}{2}\left\{\frac{\overline{\tilde{p}}-\overline{\tilde{p}}_{0}}{\overline{\tilde{p}}_{0}}\right\}^{\mathrm{T}} \overline{\bar{C}}_{p}^{-1}\left\{\frac{\overline{\tilde{p}}-\overline{\tilde{p}}_{0}}{\overline{\tilde{p}}_{0}}\right\}\right]=C_{1} \exp \left[-\frac{1}{2} \bar{p}-\bar{p}_{0}{ }^{\mathrm{T}} \overline{\bar{C}}_{p}^{-1} \bar{p}-\bar{p}_{0}\right]
$$

where $\bar{p} \square \overline{\tilde{p}} / \overline{\tilde{p}}_{0}$ implying $\bar{p}$ are relative values with respect to the a priori parameters, and $\bar{p}_{0}$ is the unit vector. Note that an over bar indicates a vector and a double over bar denotes a matrix. In general, the expectation of function $f(\overline{\mathrm{x}})$, denoted as $\langle f(\overline{\mathrm{x}})\rangle$, is defined as:

$$
\langle f(\overline{\mathrm{x}})\rangle \equiv \int_{S_{\overline{\mathrm{x}}}} f(\overline{\mathrm{x}}) P(\overline{\mathrm{x}}) d \overline{\mathrm{x}}
$$

where $S_{\overline{\mathrm{x}}}$ represents the space formed by all possible values of $\overline{\mathrm{x}} \cdot P(\overline{\mathrm{x}})$ is recognized as a joint probability density function. The first moment, i.e. $f(\overline{\mathrm{x}})=\overline{\mathrm{x}}$, renders the mean value, and the second moment, i.e. $f(\overline{\mathrm{x}})=[\overline{\mathrm{x}}-\langle\overline{\mathrm{x}}\rangle][\overline{\mathrm{x}}-\langle\overline{\mathrm{x}}\rangle]^{\mathrm{T}}$, renders the covariance matrix. Using these generalized definitions, the a priori parameter covariance matrix is defined as follows:

$$
\overline{\bar{C}}_{p}^{\text {prior }} \equiv\left\langle\left[\bar{p}-\bar{p}_{0}\right]\left[\bar{p}-\bar{p}_{0}\right]^{\mathrm{T}}\right\rangle=\int_{S_{p}}\left[\bar{p}-\bar{p}_{0}\right]\left[\bar{p}-\bar{p}_{0}\right]^{\mathrm{T}} P(\bar{p}) d \bar{p}
$$

where $S_{p}$ is the parameter space.

The sampling model for the observations whose uncertainties can be represented by a Gaussian probability distribution centered at $\bar{r}_{m}$ and with experimental uncertainty covariance matrix $\overline{\bar{C}}_{m}$ is:

$$
\rho\left(\bar{r}_{m} \mid \bar{r}\right)=C_{2} \exp \left[\begin{array}{cccc}
-\frac{1}{2} & \bar{r}-\bar{r}_{m} & { }^{\mathrm{T}} \bar{C}_{m}^{-1} & \bar{r}-\bar{r}_{m}
\end{array}\right]
$$


where, $\bar{r}_{m}$ and $\bar{r}$ denote the virtual experiment sensor signal vector and the design (simulator) model prediction vector, respectively, with the $\bar{p}$ dependence of $\bar{r}$ suppressed. In this work, $\overline{\bar{C}}_{m}$ is assumed diagonal and variance values set to those typical of nuclear power plant sensors. Note that to obtain the virtual reactor sensor signals accounting for observation uncertainty, observation errors are applied to the virtual reactor simulators predicted signal values, $\bar{r}_{m}$. The virtual experiments that were modeled are presented in section 2.3. For $J$ distinct sensors and $T$ discrete times each system response vector $\bar{r}_{j}$ can be represented as follows:

$$
\bar{r}_{j}=r_{j}^{(t)} \mid t=1,2, \ldots, T
$$

Then the vector $\bar{r}$ which contains all of the system response is:

$$
\bar{r}=\left[\begin{array}{cccc}
-\mathrm{T} & -\mathrm{T} & & -\mathrm{T} \\
r_{1} & r_{2} & \ldots & r_{J}
\end{array}\right]^{\mathrm{T}}
$$

If the system is linear or mildly nonlinear, the design model can be linearized by the following first-order Taylor series expansion:

$$
\bar{r} \cong \bar{r}\left(\bar{p}_{0}\right)+\overline{\bar{S}}_{\bar{p}_{0}} \delta \bar{p}=\bar{r}_{0}+\overline{\bar{S}}_{\bar{p}_{0}} \delta \bar{p}
$$

where $\overline{\bar{S}}_{\bar{p}_{0}}$ is the time dependent sensitivity matrix computed about the nominal value of the a priori parameters and $\delta \bar{p} \square \bar{p}-\bar{p}_{0}$. Using the a priori distribution of the parameters, the a priori system attribute covariance can be calculated by the sandwich rule as follows:

$$
\begin{aligned}
& \overline{\bar{C}}_{a}^{\text {prior }} \equiv\left\langle[\bar{a}-\langle\bar{a}\rangle][\bar{a}-\langle\bar{a}\rangle]^{\mathrm{T}}\right\rangle \\
& =\left\langle\left[\bar{a}-\bar{a}_{0}\right]\left[\bar{a}-\bar{a}_{0}\right]^{\mathrm{T}}\right\rangle \\
& =\left\langle\begin{array}{lllll}
\overline{\bar{S}}_{a} & & \delta \bar{p}_{0} \delta \bar{p}_{0}^{-\mathrm{T}} & \overline{\bar{S}}_{a}^{\mathrm{T}} \\
& \bar{p}_{0} & \bar{p}_{0}
\end{array}\right\rangle \\
& =\overline{\bar{S}}_{a}{ }_{\bar{p}_{0}} \overline{\bar{C}}_{p}^{\text {prior }} \overline{\bar{S}}_{a}{ }_{\bar{p}_{0}}^{\mathrm{T}}
\end{aligned}
$$

where $\bar{a} \cong \bar{a}\left(\bar{p}_{0}\right)+\overline{\bar{S}}_{a}{ }_{\bar{p}_{0}} \delta \bar{p}=\bar{a}_{0}+\overline{\bar{S}}_{a_{\bar{p}_{0}}} \delta \bar{p}$

Following the Bayesian approach, a posteriori distribution for the parameter vector $\bar{p}$ is then given by: 


$$
\begin{aligned}
& \rho\left(\bar{p} \mid \bar{r}_{m}\right)=\rho\left(\bar{r}_{m} \mid \bar{r}\right) \times \rho(\bar{p})
\end{aligned}
$$

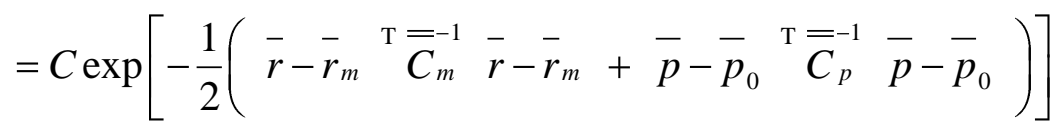

In order to determine the parameter vector that maximizes the Gaussian distribution, i.e. the mean values of the parameters, the parameter vector that minimizes the following equation is sought.

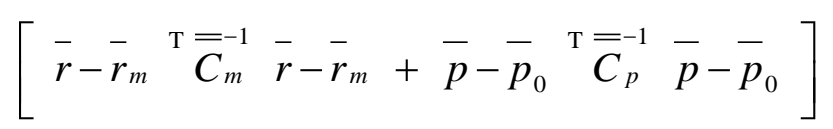

In general, the minimization problem can be formulated with the parameter-response combined vector $\bar{z}$ and the corresponding block covariance matrix $\overline{\bar{C}}$ as follows [Cacuci and Ionescu-Bujor, 2010]:

$$
\bar{z} \equiv\left[\begin{array}{l}
\bar{p}-\bar{p}_{0} \\
\bar{r}-\bar{r}_{m}
\end{array}\right], \overline{\bar{C}}=\left[\begin{array}{ll}
\overline{\bar{C}}_{p} & \overline{\bar{C}}_{p r} \\
\overline{\bar{C}}_{r p} & \overline{\bar{C}}_{m}
\end{array}\right]
$$

where, $\overline{\bar{C}}_{p r}=\overline{\bar{C}}_{r p}^{\mathrm{T}}$ is the parameter-response covariance matrix. This correlation occurs due to the successive linearization iterations one utilizes in solving the weakly nonlinear data assimilation problem, associated with updating the observables sensitivity matrix as updated parameter values become available. If the a priori distribution for the parameters and for the sampling model can be represented by a Gaussian distribution and the system responses are linear to the parameters, then the Gaussian probability density function describes the posteriori uncertainty of $\bar{p}$.

$$
\rho\left(\bar{p} \mid \bar{r}_{m}\right)=\text { const } \cdot \exp \left\{-\frac{1}{2} \bar{z}^{-\mathrm{T}} \overline{\bar{C}}^{-1} \bar{z}\right\}
$$

The mathematical approach for this analysis is presented in 0. Since the thermalhydraulic reactor system model is not highly nonlinear, the a posteriori parameters approach the converged solution quickly and their uncertainties significantly decrease after the first iteration. This makes the parameter adjustments associated with the second linearization iteration small; furthermore, significantly reduced uncertainties of the parameters after the first iteration will cause small parameter-response uncertainties as well. Thus, adding the parameter-response uncertainties does not affect very much the solution to the IRIS thermal-hydraulic inverse 
problem [Hines et al., 2010]. Solution to the minimization problem is then accomplished by differentiating equation $(2.11)$ with respect to $\bar{p}$.

The problem also includes a regularization parameter, $\alpha$, to address any ill-conditioning and to control the amount of parameter adjustments allowed. Thus the minimization problem becomes:

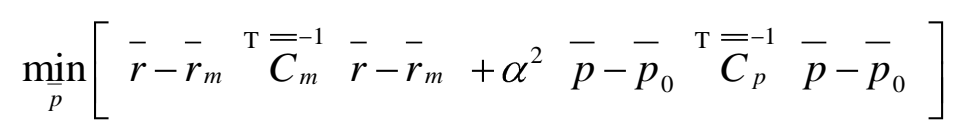

The first term in this equation is the mismatch term between the virtual experiment sensor readings and the design model predictions. The second term in the equation is the regularization term, which shows the change in a priori to a posteriori values of the parameters with respect to the matrix norm of the parameters covariance matrix. Tikhonov regularization [Tikhonov, 1995] was employed, where the weighting sum of the mismatch term and regularization term is minimized by selection of the posteriori input parameter values. The regularization parameter indicates the degree of weighting between the mismatch term and the regularization term. For large $\alpha$ values, a posteriori parameter values will not deviate greatly from their a priori values. This implies the mismatch term will not be reduced very much by data assimilation. For small alpha values, the reverse behavior occurs. The Tikhonov regularization parameter was selected experimentally based on the characteristic L-curve [Engl and Grever, 1994]. For a Bayesian approach, one would set $\alpha=1$ to produce unbiased a posteriori values. The a posteriori parameter vector is obtained without the parameter-response uncertainties as follows [Hines et al., 2009]:

$$
\bar{p}_{0}^{p o s t}=\bar{p}_{0}+\left[\overline{\bar{S}}^{\mathrm{T}} \overline{\bar{C}}_{m}^{-1} \overline{\bar{S}}+\alpha^{2} \overline{\bar{C}}_{p}^{-1}\right]^{-1} \overline{\bar{S}}^{\mathrm{T}} \overline{\bar{C}}_{m}^{-1}\left[\bar{r}_{m}-\bar{r}_{0}\right]
$$

and a posteriori system responses and attributes are approximated as:

$$
\bar{r}^{\text {post }} \cong \bar{r}\left(\bar{p}_{0}^{\text {post }}\right)+\overline{\bar{S}}_{\bar{p}_{0}^{\text {post }}} \delta \overline{\bar{p}}^{\text {post }}=\bar{r}_{0}^{\text {post }}+\overline{\bar{S}}_{\bar{p}_{0}^{\text {post }}} \delta \overline{\bar{p}}^{\text {post }}
$$

and

$$
\bar{a}^{\text {post }} \cong \bar{a}\left(\bar{p}_{0}^{\text {post }}\right)+\overline{\bar{S}}_{a}{ }_{\bar{p}_{0}^{\text {post }}} \delta \bar{p}^{\text {post }}=\bar{a}_{0}^{\text {post }}+\overline{\bar{S}}_{a_{\bar{p}_{0}}^{\text {post }}} \delta \bar{p}^{\text {post }}
$$

The a posteriori parameter covariance matrix can be also computed by: 


$$
\overline{\bar{C}}_{p}^{\text {post }} \equiv\left\langle\left[\bar{p}-\bar{p}_{0}^{\text {post }}\right]\left[\bar{p}-\bar{p}_{0}^{\text {post }}\right]^{\mathrm{T}}\right\rangle
$$

Substituting a posteriori parameters into equation (2.18) produces the following expression for $\overline{\bar{C}}_{p}^{p o s t}$ :

$$
\begin{aligned}
& \overline{\bar{C}}_{p}^{\text {post }}=\left\langle\left[\bar{p}-\bar{p}_{0}-\overline{\bar{S}}^{\mathrm{T}} \overline{\bar{C}}_{m}^{-1} \overline{\bar{S}}+\alpha^{2} \overline{\bar{C}}_{p}^{-1}{ }^{-1} \overline{\bar{S}}^{\mathrm{T}} \overline{\bar{C}}_{m}^{-1} \bar{r}_{m}-\bar{r}_{0}\right]\right. \\
& \left.\times\left[\bar{p}-\bar{p}_{0}-\overline{\bar{S}}^{\mathrm{T}} \overline{\bar{C}}_{m}^{-1} \overline{\bar{S}}+\alpha^{2} \overline{\bar{C}}_{p}^{-1}{ }^{-1} \overline{\bar{S}}^{\mathrm{T}} \overline{\bar{C}}_{m}^{-1} \bar{r}_{m}-\bar{r}_{0}\right]^{\mathrm{T}}\right) \\
& =\overline{\bar{C}}_{p}^{\text {prior }}-\left\langle\left[\bar{p}-\bar{p}_{0}\right]\left[\bar{r}_{m}-\bar{r}+\overline{\bar{S}} \bar{p}-\bar{p}_{0}\right]^{\mathrm{T}}\right\rangle \overline{\bar{C}}_{m}^{-1} \overline{\bar{S}}\left[\overline{\bar{S}^{\mathrm{T}}} \overline{\bar{C}}_{m}^{-1} \overline{\bar{S}}+\alpha^{2} \overline{\bar{C}}_{p}^{-1}\right]^{-\mathrm{T}} \\
& -\left[\overline{\bar{S}}^{\mathrm{T}} \overline{\bar{C}}_{m}^{-1} \overline{\bar{S}}+\alpha^{2} \overline{\bar{C}}_{p}^{-1}\right]^{-1} \overline{\bar{S}}^{\mathrm{T}} \overline{\bar{C}}_{m}^{-1}\left\langle\left[\bar{r}_{m}-\bar{r}+\overline{\bar{S}} \bar{p}-\bar{p}_{0}\right]\left[\bar{p}-\bar{p}_{0}\right]^{\mathrm{T}}\right\rangle \\
& +\left[\overline{\bar{S}}^{\mathrm{T}} \overline{\bar{C}}_{m}^{-1} \overline{{ }^{S}}+\alpha^{2} \overline{\bar{C}}_{p}^{-1}\right]^{-1} \overline{\bar{S}}^{\mathrm{T}} \overline{\bar{C}}_{m}^{-1} \\
& \times\left\langle\left[\bar{r}_{m}-\bar{r}+\overline{\bar{S}} \bar{p}-\bar{p}_{0}\right]\left[\bar{r}_{m}-\bar{r}+\overline{\bar{S}}\left(\bar{c}-\bar{p}_{0}\right]^{\mathrm{T}}\right\rangle\right. \\
& \times \overline{\bar{C}}_{m}^{-1} \bar{S}\left[\overline{\bar{S}^{\mathrm{T}}} \overline{\bar{C}}_{m}^{-1} \overline{\bar{S}}^{2}+\alpha^{2} \overline{\bar{C}}_{p}^{-1}\right]^{-\mathrm{T}}
\end{aligned}
$$

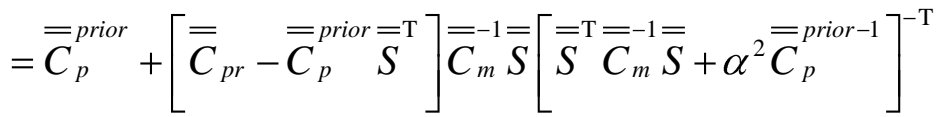

$$
\begin{aligned}
& +\left[\overline{\bar{S}}^{\mathrm{T}} \overline{\bar{C}}_{m}^{-1} \overline{S^{2}}+\alpha^{2} \overline{\bar{C}}_{p}^{\text {prior }-1}\right]^{-1} \overline{\bar{S}}^{\mathrm{T}} \overline{\bar{C}}_{m}^{-1}\left[\overline{\bar{C}}_{r p}-\overline{\bar{S}} \overline{\bar{C}}_{p}^{\text {prior }}\right] \\
& +\left[\overline{\bar{S}}^{\mathrm{T}} \overline{\bar{C}}_{m}^{-1} \overline{\bar{S}}+\alpha^{2} \overline{\bar{C}}_{p}^{\text {prior }-1}\right]^{-1} \\
& \times \overline{\bar{S}}^{\mathrm{T}} \overline{\bar{C}}_{m}^{-1}\left[\overline{\bar{C}}_{m}-\overline{\bar{C}}_{r p} \overline{\bar{S}}^{\mathrm{T}}-\overline{\bar{S}} \overline{\bar{C}}_{p r}+\overline{\bar{S}} \overline{\bar{C}}_{p}^{\text {prior }} \overline{\bar{S}}^{\mathrm{T}}\right] \overline{\bar{C}}_{m}^{-1} \overline{\bar{S}} \\
& \times\left[\overline{\bar{S}}^{\mathrm{T}} \overline{\bar{C}}_{m}^{-1} \overline{\bar{S}}+\alpha^{2} \overline{\bar{C}}_{p}^{\text {prior }-1}\right]^{-\mathrm{T}}
\end{aligned}
$$




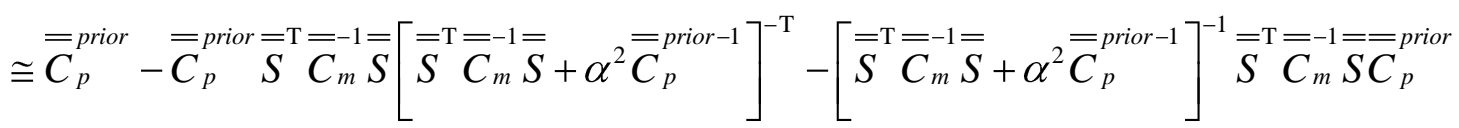

$$
\begin{aligned}
& +\left[\overline{\bar{S}}^{\mathrm{T}} \overline{\bar{C}}_{m}^{-1} \overline{\bar{S}^{2}}+\alpha^{2} \overline{\bar{C}}_{p}^{\text {prior }-1}\right]^{-1} \overline{\bar{S}}^{\mathrm{T}} \overline{\bar{C}}_{m}^{-1}\left[\overline{\bar{C}}_{m}+\overline{\bar{S}}_{\bar{C}}^{\text {prior }} \overline{\bar{S}}^{\mathrm{T}}\right] \overline{\bar{C}}_{m}^{-1} \overline{\bar{S}}\left[\overline{\bar{S}}^{\mathrm{T}} \overline{\bar{C}}_{m}^{-1} \overline{\bar{S}}+\alpha^{2} \overline{\bar{C}}_{p}^{\text {prior }-1}\right]^{-\mathrm{T}}
\end{aligned}
$$

As noted earlier, to address mild nonlinearity, sensitivity coefficient values were redetermined by linearizing about the previous iteration a posteriori parameter values, and inverse theory was once again used to obtain updated a posteriori parameters values. These linearization iterations were continued until a stopping criteria was satisfied.

Best-estimate accident analysis requires not only values of limiting system attributes during accident transients, e.g. minimum departure from nucleate boiling ratio (MDNBR), but also their uncertainties. To obtain those, given a posteriori parameter uncertainties, one can propagate the parameter uncertainties through the simulation model to predict a posteriori uncertainties on core observables and system attributes. If the a posteriori parameter uncertainties are Gaussian and the system responds linearly over the range of the parameter uncertainties, the system attribute uncertainties are Gaussian, which is characterized by the mean values and covariance at the operating power level for the accident " acc" given by:

$$
\left(\overline{\bar{C}}_{a}^{a c c} \text { post }\right)^{o}=\left(\overline{\bar{S}}_{a}^{a c c}\right)_{\bar{p}_{0}^{p o s t}}^{o} \overline{\bar{C}}_{p}^{\text {post }}\left(\overline{\bar{S}}_{a}^{a c c}\right)_{\bar{p}_{0}^{\text {post }}}^{o \mathrm{~T}}
$$

where $\overline{\bar{S}}^{o}$ denotes the sensitivity matrix at operating power. If the system is highly nonlinear, one should propagate parameter uncertainties by Monte Carlo simulation. As done in this work, data assimilation was completed using experiments mostly at lower powers, since experiments actually corresponding to higher power accident conditions would be prohibited. In this case, one should always pay attention to the similarity of physics. If the physics at operating power is significantly different from that at lower power, the calculated a posteriori system attribute uncertainty would in general not represent that associated with the actual transient starting at operating power. Thus the similarity of physics should always be demonstrated if possible when completing an uncertainty analysis.

Literature reviews were completed to identify the uncertainties on parameters and correlations within the model, and which system responses and attributes would need to be 
considered. The following parameters/correlations [Todreas and Kazimi, 1990; Boyack, et al., 1990; RELAP5/MOD3, 1990; Hagrman, 1979] were selected to have their values adjusted via data assimilation:

1. Chen Heat Transfer Correlation for nucleate boiling heat transfer coefficient on the secondary side of steam generator

2. Dittus-Boelter Heat Transfer Correlation for single phase (liquid or gas) heat transfer coefficient on the secondary side of steam generator

3. Fuel Thermal Conductivity

4. Friction Factors

5. Gap Conductance

6. Pellet Diameter

7. Cladding Diameter

8. Cladding Thermal Conductivity

9. Pump Head

10. Moderator Temperature Coefficient The following observables were selected for usage during data assimilation:

1. Neutron Power

2. Reactor Thermal Output

3. Core Flow Rate

4. Primary Pressure

5. Hot Leg Temperature

6. Cold Leg Temperature

7. Average Coolant Temperature

8. Feed Flow Rate per Steam Generator

9. Steam Flow Rate per Steam Generator

10. Turbine Output

11. Steam Generator Exit Temperature

12. Steam Pressure In addition the following system attributes were selected for uncertainty quantification:

1. Average Reactor Fuel Temperature

2. Hot Channel Fuel Center Line Temperature 


\section{Hot Channel Minimum Departure from Nucleate Boiling Ratio}

\subsubsection{MCMC Method for the Nonlinear Problems}

All of the above is based upon the parameters and observables uncertainties being Gaussian and the system sensitivity equations being mildly nonlinear i.e. nearly linear. However according to the linearity test, nonlinear behaviors were observed at the lower power experimental condition, especially on the secondary side of the reactor. The deterministic approach, based upon a first order truncated Taylor series for the responses, to uncertainty analysis is inappropriate to treat this behavior due to the nonlinear relationship between the system responses and the parameters, hence the potential non-Gaussian nature of the a posteriori distribution. This provides motivation that the transients that generate nonlinear system responses be differentiated from those that behave relatively linearly. To address the nonlinear responses in both data assimilation and determining the a posteriori uncertainties of the parameters, the following approach was employed.

Following the Bayesian approach, a posteriori distribution for the parameter vector $\bar{p}$ is then given by equation (6.10). If the system observables are linear with respect to the parameters, then solutions to the inverse problem could be obtained analytically as presented in section 6.2.1. If the system observables are nonlinear with respect to the parameters for certain transients, given a priori parameter uncertainty information, one needs to propagate the parameter uncertainties through the simulation model to predict the a posteriori uncertainties of the parameters using Monte Carlo simulations [Higdon et al., 2004]. This is conducted using the Markov Chain Monte Carlo (MCMC) method which seeks to determine the steady-state Markov distribution by generating Markov chains, which coincides with the target distribution, i.e. the a posteriori distribution of the parameters. A simple MCMC implementation uses the Metropolis algorithm which is presented as follows:

Initialize the parameter vector by guessing it at some value.

Given the current parameter vector is $\bar{p}^{i}$, generate a new parameter vector $\bar{p}^{*}$ in $\left[\bar{p}^{i}-\bar{m}, \bar{p}^{i}+\bar{m}\right]$, where $\bar{m}$ is a random number vector. 
Compute the Metropolis acceptance probability using the following expression: $\alpha=\min \left\{1, \frac{\sigma\left(\bar{p}^{*} \mid \bar{r}_{m}\right)}{\sigma\left(\bar{p}^{i} \mid \bar{r}_{m}\right)}\right\}$

Define $\bar{p}^{i+1}=\left\{\begin{array}{ll}\bar{p}^{*} & \text { with probability } \alpha \\ \bar{p}^{i} & \text { with probability } 1-\alpha\end{array}\right.$ selecting which value to assign via a random number $[0,1]$

\section{Return to step 2.}

At the beginning of the sequence, one needs to run MCMC for awhile to achieve convergence to the target probability density function (pdf). After convergence, one considers a certain number of the first iterations to be discarded as the burn in stage to remove the bias from the initially chosen starting point. The size of the perturbation, i.e. the trial space, can be adapted during the burn in phase to a value that provides a desired acceptance percentage. It has been claimed that, for a wide variety of problems, acceptance probability near 0.5 indicates that the chain has good mixing [Gelman et al., 1996; Muller, 1993; Hanson et al., 1998]. When the percentage is less than 30, i.e. when the trial space is much larger than the target space, the $\bar{p}^{i}$ does not move for long periods, but jumps are large, which implies that one should perform a large number of simulations to obtain a reasonable number of accepted samples that illustrate the a posteriori. When the percentage is more than 70 , i.e. when the trial space is much smaller than the target space, movement across the target pdf is slow, so called unconstrained random walk, and will not efficiently span full range of the target distribution unless the total number of trials is extremely large. In both cases the MCMC simulation is very inefficient since the total number of trials should be sufficiently large to estimate the a posteriori distribution properly. When simulations do not provide the desired acceptance percentage, it is possible to improve mixing by properly adjusting the trial space. Various heuristic rules have been suggested for fixing these problems during a simulation by monitoring the frequency of acceptances in the simulation. While the Metropolis algorithm is running, one can monitor the frequency of acceptances of the Metropolis algorithm; if the acceptance rate is much less or much more than 0.5 , one can alter the size of the perturbation by decreasing or increasing its trial space, respectively. 
MCMC has proven effective for nonlinear response problems with multiple parameters to adjust. However this method is not applicable if there are many parameters and the simulation model requires substantial CPU time to execute due to the computational burden.

\subsection{Virtual Experiments}

Data assimilation and uncertainty quantification were completed by defining the experiments, defining the limiting accidents, and determining the a posteriori uncertainties of the key system attributes for the limiting accidents. In order to identify the limiting accidents, Updated Final Safety Analysis Report (UFSAR) [Duke, 2007] and IRIS Preliminary Safety Assessment Report [Westinghouse, 2003] were reviewed. Simulations were performed to determine virtual sensor signals which are surrogates for unavailable experimental values. A single sample of all the parameter values was used to determine the perturbed parameter values for the virtual reactor (simulator), from which the virtual reactor sensors' readings were obtained. In order to adjust parameters, the following four experiments were simulated using the IRIS system simulation code.

\subsubsection{Reactor Coolant Pump (RCP) Trip at $30 \%$ and $80 \%$ Power}

The simulation is performed for 8 seconds. As mass flow rate decreases below Low Reactor Coolant Flow Trip Set Point, control rods are inserted and the reactor trips. All control systems and safety controllers are assumed to be inactive. Note that RCP trip at $80 \%$ power would not be allowed since it is performed at too high power level, but the simulation was done at relatively high power level to compare the result with that of RCP trip at lower power and to minimize computation induced noise from the simulator.

\subsubsection{Control Bank Withdrawal at $70 \%$ Power}

The simulation is performed for 60 seconds. The control rod worth was assumed to be $0.893 \% \Delta \rho$. The maximum power reached during the transient is slightly below $845 \mathrm{MWt}$ which is approximately $85 \%$ of the operating power and $113 \%$ of the nominal power at $70 \%$ power level. The simulation shows that the reactor does not trip during the experiment since the large volume of the pressurizer compensates for the limited heat sink (The steam volume to 
reactor power ratio is five times larger in IRIS than in current PWRs). All important control systems are assumed to be inactive except the control bank.

\subsubsection{Feed Control Valve (FCV) Failed Open at $15 \%$ Power}

The simulation is performed for 120 seconds. The feed control valve position is assumed to experience a $+3 \%$ step change. All control systems and safety controllers are assumed to be inactive.

\subsubsection{Turbine Control Valve (TCV) Failed Open at $15 \%$ Power}

The simulation is performed for 120 seconds. The turbine control valve position changes from the initial value of $5 \%$ to the final value of $10 \%$. All control systems and safety controllers are assumed to be inactive.

\subsection{Uncertainty Contribution to the System Attributes}

Since identifying major sources of uncertainty, as done in this work, is important in deciding where additional efforts should be given to reduce these uncertainties, each parameter's uncertainty contribution to the system attributes was determined as well. This was evaluated for a specific parameter's uncertainty contribution by determining the system attribute's uncertainty propagated from all parameters and subtracting that obtained by propagating from all but the specific parameter of interest. The associated mathematical derivation is now given.

Assuming no uncertainty for parameter $\alpha_{k}$, the joint distribution function between $\alpha_{k}$ and $\alpha_{j}$ is defined using a Dirac-delta function.

$$
P\left(\alpha_{k}, \alpha_{j}\right)=\delta \alpha_{k}-\left\langle\alpha_{k}\right\rangle P\left(\alpha_{j}\right)
$$

The $(k, j)$ elements of a posteriori parameter covariance matrix are then zeros for all $j \mathrm{~s}$.

$$
\left(\begin{array}{c}
\bar{C}_{p}^{p o s t} \\
{ }^{p, j}
\end{array}\right)_{S_{\alpha_{j}}} \int_{S_{\alpha_{k}}} \alpha_{k}-\left\langle\alpha_{k}\right\rangle \quad \alpha_{j}-\left\langle\alpha_{j}\right\rangle \delta \alpha_{k}-\left\langle\alpha_{k}\right\rangle P\left(\alpha_{j}\right) d \alpha_{k} d \alpha_{j}=0
$$

Likewise $\left(\overline{\bar{C}}_{p}^{\text {post }}\right)_{i, k}$ are zeros for all $i$ s. Thus the matrix $\left(\overline{\bar{C}}_{p}^{\text {post }}\right)_{k}$ which does not account for uncertainty on $\alpha_{k}$ is defined as: 


$$
\left(\overline{\bar{C}}_{p}^{p o s t}\right)_{k} \equiv\left[\begin{array}{cccccccc}
c_{1,1} & c_{1,2} & \cdots & c_{1, k-1} & 0 & c_{1, k+1} & \cdots & c_{1, N p} \\
c_{2,1} & c_{2,2} & \cdots & c_{2, k-1} & 0 & c_{2, k+1} & \cdots & c_{2, N p} \\
\vdots & \vdots & \ddots & \vdots & \vdots & \vdots & & \vdots \\
c_{k-1,1} & c_{k-1,2} & \cdots & c_{k-1, k-1} & 0 & c_{k-1, k+1} & \cdots & c_{k-1, N p} \\
0 & 0 & \cdots & 0 & 0 & 0 & \cdots & 0 \\
c_{k+1,1} & c_{k+1,2} & \cdots & c_{k+1, k-1} & 0 & c_{k+1, k+1} & \cdots & c_{k+1, N p} \\
\vdots & \vdots & & \vdots & \vdots & \vdots & \ddots & \vdots \\
c_{N p, 1} & c_{N p, 2} & \cdots & c_{N p, k-1} & 0 & c_{N p, k+1} & \cdots & c_{N p, N p}
\end{array}\right]
$$

The " $k$ th parameter's uncertainty contribution matrix" is then derived as follows:

$$
\left(\Delta \overline{\bar{C}}_{a}^{\text {post }}\right)_{k} \equiv \overline{\bar{C}}_{a}^{\text {post }}-\left(\overline{\bar{C}}_{a}^{\text {post }}\right)_{k}=\overline{\bar{S}}_{0}\left[\overline{\bar{C}}_{p}^{\text {post }}-\left(\overline{\bar{C}}_{p}^{\text {post }}\right)_{k}\right] \overline{\bar{S}}_{0}^{\mathrm{T}}=\overline{\bar{S}}_{0}\left(\Delta \overline{\bar{C}}_{p}^{\text {post }}\right)_{k} \overline{\bar{S}}_{0}^{\mathrm{T}}
$$

where, $\left(\Delta \bar{C}_{p}^{\text {post }}\right)_{k}=\left[\begin{array}{ccccccc}0 & \cdots & 0 & c_{1, k} & 0 & \cdots & 0 \\ \vdots & \ddots & \vdots & \vdots & \vdots & & \vdots \\ 0 & \cdots & 0 & c_{k-1, k} & 0 & \cdots & 0 \\ c_{k, 1} & \cdots & c_{k, k-1} & c_{k, k} & c_{k, k+1} & \cdots & c_{k, N p} \\ 0 & \cdots & 0 & c_{k+1, k} & 0 & \cdots & 0 \\ \vdots & & \vdots & \vdots & \vdots & \ddots & \vdots \\ 0 & \cdots & 0 & c_{N p, k} & 0 & \cdots & 0\end{array}\right]$

\subsection{Mathematical Review for the Inverse Method}

While the forward problem has a unique solution, the inverse problem could have multiple solutions. In other words, there could be different distributions of input parameters that give the same responses. The regularization term addition addresses this situation making the problem well-posed. Because of this, one needs to make explicit any available a priori uncertainty information on the input parameters. If the information, e.g. input parameter covariances, is not available, the solution of the inverse problem could be misled. The following presentation provides insight into understanding the difficulty that the absence of a priori information on input parameters may cause.

Assuming data assimilation is performed using one time dependent observable, two input parameters and their covariances, the posterior input parameter vector can be calculated as follows: 


$$
\bar{p}_{0}^{p o s t}=\bar{p}_{0}+\left[\begin{array}{cc}
\sum_{t=1}^{T} \frac{s_{1}^{(t) 2}}{c_{r}^{(t)}}+\frac{\alpha^{2} c_{2,2}}{c_{1,1} c_{2,2}-c_{1,2} c_{2,1}} & \sum_{t=1}^{T} \frac{s_{1}^{(t)} s_{2}^{(t)}}{c_{r}^{(t)}}-\frac{\alpha^{2} c_{1,2}}{c_{1,1} c_{2,2}-c_{1,2} c_{2,1}} \\
\sum_{t=1}^{T} \frac{s_{2}^{(t)} s_{1}^{(t)}}{c_{r}^{(t)}}-\frac{\alpha^{2} c_{2,1}}{c_{1,1} c_{2,2}-c_{1,2} c_{2,1}} & \sum_{t=1}^{T} \frac{s_{2}^{(t) 2}}{c_{r}^{(t)}}+\frac{\alpha^{2} c_{1,1}}{c_{1,1} c_{2,2}-c_{1,2} c_{2,1}}
\end{array}\right]^{-1}\left[\begin{array}{l}
\sum_{t=1}^{T} \frac{s_{1}^{(t)}}{c_{r}^{(t)}} \Delta r^{(t)} \\
\sum_{t=1}^{T} \frac{s_{2}^{(t)}}{c_{r}^{(t)}} \Delta r^{(t)}
\end{array}\right]
$$

where, $s_{i}^{(t)}$ is the time $(t)$ dependent sensitivity coefficient with respect to the input parameter $i$, $c_{i, j}$ is the input parameter $(i, j)$ covariance (note $c_{1,2}=c_{2,1}$ ) , $c_{r}^{(t)}$ is the time dependent experimental uncertainty covariance, and $\Delta r^{(t)}$ is the mismatch, $r_{m}^{-(t)}-r_{0}^{-(t)}$. Note that it is assumed that the off diagonal elements of the measurement uncertainty covariance matrix are zeros, which means that the measurement errors do not have correlation. Defining matrix $\overline{\bar{Q}}$ and vector $\bar{\gamma}$ as:

$$
\begin{gathered}
\overline{\bar{Q}} \equiv\left[\begin{array}{c}
\sum_{t=1}^{T} \frac{s_{1}^{(t) 2}}{c_{r}^{(t)}}+\frac{\alpha^{2} c_{2,2}}{c_{1,1} c_{2,2}-c_{1,2} c_{2,1}} \quad \sum_{t=1}^{T} \frac{s_{1}^{(t)} s_{2}^{(t)}}{c_{r}^{(t)}}-\frac{\alpha^{2} c_{1,2}}{c_{1,1} c_{2,2}-c_{1,2} c_{2,1}} \\
\sum_{t=1}^{T} \frac{s_{2}^{(t)} s_{1}^{(t)}}{c_{r}^{(t)}}-\frac{\alpha^{2} c_{2,1}}{c_{1,1} c_{2,2}-c_{1,2} c_{2,1}} \quad \sum_{t=1}^{T} \frac{s_{2}^{(t) 2}}{c_{r}^{(t)}}+\frac{\alpha^{2} c_{1,1}}{c_{1,1} c_{2,2}-c_{1,2} c_{2,1}}
\end{array}\right] \\
\bar{\gamma} \equiv\left[\begin{array}{l}
\sum_{t=1}^{T} \frac{s_{1}^{(t)}}{c_{r}^{(t)}} \Delta r^{(t)} \\
\sum_{t=1}^{T} \frac{s_{2}^{(t)}}{c_{r}^{(t)}} \Delta r^{(t)}
\end{array}\right]
\end{gathered}
$$

and inverting the matrix $\overline{\bar{Q}}$ produces the following expression for the posterior input parameter vector:

$$
\begin{aligned}
\bar{p}_{0}^{\text {post }} & =\bar{p}_{0}+\bar{Q}^{-1} \bar{\gamma} \\
& =\bar{p}_{0}+\frac{1}{\operatorname{det} \overline{\bar{Q}}} \Delta \overline{p^{\prime}}
\end{aligned}
$$

Each component of the vector $\Delta \overline{p^{\prime}}$, which shows the parameter adjustment is then:

$$
\begin{aligned}
\Delta p_{1}^{\prime}= & \sum_{t=1}^{T} \sum_{t^{\prime}=1}^{T} \Delta r^{(t)} \frac{s_{1}^{(t)}}{c_{r}^{(t)}} \frac{s_{2}^{\left(t^{\prime}\right) 2}}{c_{r}^{\left(t^{\prime}\right)}}-\sum_{t=1}^{T} \sum_{t^{\prime}=1}^{T} \Delta r^{(t)} \frac{s_{2}^{(t)}}{c_{r}^{(t)}} \frac{s_{1}^{\left(t^{\prime}\right)} s_{2}^{\left(t^{\prime}\right)}}{c_{r}^{\left(t^{\prime}\right)}} \\
& +\frac{\alpha^{2} c_{1,1}}{c_{1,1} c_{2,2}-c_{1,2} c_{2,1}} \sum_{t=1}^{T} \Delta r^{(t)} \frac{s_{1}^{(t)}}{c_{r}^{(t)}}+\frac{\alpha^{2} c_{1,2}}{c_{1,1} c_{2,2}-c_{1,2} c_{2,1}} \sum_{t=1}^{T} \Delta r^{(t)} \frac{s_{2}^{(t)}}{c_{r}^{(t)}}
\end{aligned}
$$




$$
\begin{aligned}
\Delta p_{2}^{\prime}= & \sum_{t=1}^{T} \sum_{t^{\prime}=1}^{T} \Delta r^{(t)} \frac{s_{2}^{(t)}}{c_{r}^{(t)}} \frac{s_{1}^{\left(t^{\prime}\right) 2}}{c_{r}^{\left(t^{\prime}\right)}}-\sum_{t=1}^{T} \sum_{t^{\prime}=1}^{T} \Delta r^{(t)} \frac{s_{1}^{(t)}}{c_{r}^{(t)}} \frac{s_{2}^{\left(t^{\prime}\right)} s_{1}^{\left(t^{\prime}\right)}}{c_{r}^{\left(t^{\prime}\right)}} \\
& +\frac{\alpha^{2} c_{2,2}}{c_{1,1} c_{2,2}-c_{1,2} c_{2,1}} \sum_{t=1}^{T} \Delta r^{(t)} \frac{s_{2}^{(t)}}{c_{r}^{(t)}}+\frac{\alpha^{2} c_{2,1}}{c_{1,1} c_{2,2}-c_{1,2} c_{2,1}} \sum_{t=1}^{T} \Delta r^{(t)} \frac{s_{1}^{(t)}}{c_{r}^{(t)}}
\end{aligned}
$$

Now assume that the two parameters affect the observable in a similar way, i.e. $s_{2}^{(t)} \cong \Gamma s_{1}^{(t)}$, where $\Gamma$ is a constant. This relation is frequently observed in the thermal hydraulic system, e.g. time dependent sensitivity to the Chen heat transfer coefficient and to the Dittus-Boelter heat transfer coefficient, and will lead to the following:

$$
\begin{aligned}
& \Delta p_{1}^{\prime} \cong \frac{\alpha^{2} c_{1,1}}{c_{1,1} c_{2,2}-c_{1,2} c_{2,1}} \sum_{t=1}^{T} \Delta r^{(t)} \frac{s_{1}^{(t)}}{c_{r}^{(t)}}+\frac{\alpha^{2} c_{1,2}}{c_{1,1} c_{2,2}-c_{1,2} c_{2,1}} \sum_{t=1}^{T} \Delta r^{(t)} \frac{s_{2}^{(t)}}{c_{r}^{(t)}} \\
& \Delta p_{2}^{\prime} \cong \frac{\alpha^{2} c_{2,2}}{c_{1,1} c_{2,2}-c_{1,2} c_{2,1}} \sum_{t=1}^{T} \Delta r^{(t)} \frac{s_{2}^{(t)}}{c_{r}^{(t)}}+\frac{\alpha^{2} c_{2,1}}{c_{1,1} c_{2,2}-c_{1,2} c_{2,1}} \sum_{t=1}^{T} \Delta r^{(t)} \frac{s_{1}^{(t)}}{c_{r}^{(t)}}
\end{aligned}
$$

or

$$
\begin{gathered}
\Delta p_{1}^{\prime} \cong \frac{\alpha^{2}}{1-\Omega_{p}^{2}}\left(\frac{1}{c_{2,2}}+\frac{\Gamma \Omega_{p}}{\sqrt{c_{1,1}} \sqrt{c_{2,2}}}\right) \sum_{t=1}^{T} \Delta r^{(t)} \frac{s_{1}^{(t)}}{c_{r}^{(t)}} \\
\Delta p_{2}^{\prime} \cong \frac{\alpha^{2}}{1-\Omega_{p}^{2}}\left(\Gamma \frac{1}{c_{1,1}}+\frac{\Omega_{p}}{\sqrt{c_{1,1}} \sqrt{c_{2,2}}}\right) \sum_{t=1}^{T} \Delta r^{(t)} \frac{s_{1}^{(t)}}{c_{r}^{(t)}}
\end{gathered}
$$

where the correlation coefficient is given by $\Omega_{p} \square c_{1,2} / \sqrt{c_{1,1}} \sqrt{c_{2,2}}=c_{2,1} / \sqrt{c_{1,1}} \sqrt{c_{2,2}}$. In this case the input parameter adjustment will be determined by the sensitivities, mismatch, variances and covariances. The behavior of the adjustments is as expected. As $\alpha$ increases the magnitude of the adjustments increases. As $c_{r}^{(t)}$ increase the magnitude of the adjustments decrease. Now consider the case of no correlation, i.e. $c_{1,2}=c_{2,1}=0$. This condition would exist if the parameter values were determined by ideal single effect experiments. Under this condition, if $c_{1,1}$ increases relative to $c_{2,2}, p_{1}^{\prime}$ is adjusted more relative to $p_{2}^{\prime}$. For the reverse situation, the reverse adjustment occurs. Again assuming no correlation, if $\Gamma>0$, then the two parameters are adjusted in the same direction; however, if $\Gamma<0$, then the two parameters are adjusted in counter direction. The just noted behaviors are all as expected, but those behaviors could mislead the solution since the a posteriori of each input parameter is obtained using only the sensitivity to the 
parameter itself and a priori uncertainty of the other parameter. For example, consider the case that the virtual experiment input parameters $p_{1}=1+\delta p_{1}$ and $p_{2}=1+\delta p_{2}$, where $\delta p_{1}$ and $\delta p_{2}$ are all positive, and $\delta p_{1} \cong \delta p_{2}$. Also assume that $s_{1}^{(t)}$ is negative, $s_{2}^{(t)}$ is positive and $\Gamma<-1$. $\Delta r^{(t)}$ will be then positive, which results in negative $\Delta p_{1}^{\prime}$ and positive $\Delta p_{2}^{\prime}$. In this case the behavior of one parameter offsets the behavior of the other parameter, implying several combinations of the input parameters can generate the same responses. Now consider the case of positive correlation, i.e. $\Omega_{p}>0$. For $\Gamma>0$, as the correlation becomes stronger the magnitude of the parameter adjustments increase. For $\Gamma<0$, as the correlation becomes stronger the magnitude of the parameter adjustments is decreased from that when $\Gamma>0$. Now consider the case of anti-correlation, i.e. $\Omega_{p}<0$. For $\Gamma>0$, as the anti-correlation becomes stronger the magnitude of the parameter adjustment is decreased from that when there is positive correlation. Finally, for $\Gamma<0$, as the anti-correlation becomes stronger, the $\Delta p_{1}^{\prime}$ and $\Delta p_{2}^{\prime}$ magnitude adjustments both become larger but in opposite direction. Thinking through all these combinations, the parameter adjustments make sense.

Attention is now directed to the a posteriori input parameter covariance matrices. If the regularization parameter is one, a posteriori input parameter covariance matrix is simply:

$$
\overline{\bar{C}}_{p}^{\text {post }}=\left[\overline{\bar{S}}^{\mathrm{T}} \overline{\bar{C}}_{r}^{-1} \overline{\bar{S}}+\overline{\bar{C}}_{p}^{-1}\right]^{-1}
$$

Defining matrix $\overline{\bar{R}}$ as:

$$
\overline{\bar{R}} \equiv\left[\begin{array}{cc}
\sum_{t=1}^{T} \frac{s_{1}^{(t) 2}}{c_{r}^{(t)}}+\frac{c_{2,2}}{c_{1,1} c_{2,2}-c_{1,2} c_{2,1}} & \sum_{t=1}^{T} \frac{s_{1}^{(t)} s_{2}^{(t)}}{c_{r}^{(t)}}-\frac{c_{1,2}}{c_{1,1} c_{2,2}-c_{1,2} c_{2,1}} \\
\sum_{t=1}^{T} \frac{s_{2}^{(t)} s_{1}^{(t)}}{c_{r}^{(t)}}-\frac{c_{2,1}}{c_{1,1} c_{2,2}-c_{1,2} c_{2,1}} & \sum_{t=1}^{T} \frac{s_{2}^{(t) 2}}{c_{r}^{(t)}}+\frac{c_{1,1}}{c_{1,1} c_{2,2}-c_{1,2} c_{2,1}}
\end{array}\right]
$$

a posteriori input parameter covariance matrix can be calculated as follows:

$$
\overline{\bar{C}}_{p}^{\text {post }}=\frac{1}{\operatorname{det} \overline{\bar{R}}}\left[\begin{array}{cc}
\sum_{t=1}^{T} \frac{s_{2}^{(t) 2}}{c_{r}^{(t)}}+\frac{c_{1,1}}{c_{1,1} c_{2,2}-c_{1,2} c_{2,1}} & -\sum_{t=1}^{T} \frac{s_{1}^{(t)} s_{2}^{(t)}}{c_{r}^{(t)}}+\frac{c_{1,2}}{c_{1,1} c_{2,2}-c_{1,2} c_{2,1}} \\
-\sum_{t=1}^{T} \frac{s_{2}^{(t)} s_{1}^{(t)}}{c_{r}^{(t)}}+\frac{c_{2,1}}{c_{1,1} c_{2,2}-c_{1,2} c_{2,1}} & \sum_{t=1}^{T} \frac{s_{1}^{(t) 2}}{c_{r}^{(t)}}+\frac{c_{2,2}}{c_{1,1} c_{2,2}-c_{1,2} c_{2,1}}
\end{array}\right]
$$


Analysis was performed to observe how the two parameters are correlated assuming $s_{2}^{(t)} \cong \Gamma s_{1}^{(t)}$. The a posteriori input parameter correlation is:

$$
\Omega_{p}^{p o s t}=-\frac{\Gamma \sum_{t=1}^{T} \frac{s_{1}^{(t) 2}}{c_{r}^{(t)}}-\frac{\Omega_{p}}{\sqrt{c_{1,1}} \sqrt{c_{2,2}} 1-\Omega_{p}^{2}}}{\sqrt{\left(\Gamma^{2} \sum_{t=1}^{T} \frac{s_{1}^{(t) 2}}{c_{r}^{(t)}}+\frac{1}{c_{2,2} 1-\Omega_{p}^{2}}\right)\left(\sum_{t=1}^{T} \frac{s_{1}^{(t) 2}}{c_{r}^{(t)}}+\frac{1}{c_{1,1} 1-\Omega_{p}^{2}}\right)}}
$$

The denominator is noted to always be positive, so whether posterior is correlated or anticorrelated depends on the sign of the numerator. Assuming no a priori correlation, the posteriori correlation is anti-correlated if $\Gamma>0$ and positively correlated if $\Gamma<0$. When prior correlation exists, i.e. $\Omega_{p} \neq 0$, for $\Gamma>0$ the posteriori is anti-correlated for a small magnitude of $\Omega_{p}$. Positive correlation occurs if now $\Gamma<0$ and $\left|\Omega_{p}\right|$ small. As $\Omega_{p}$ grows positive posteriori can become positively correlated, whereas, if it grows negative the posteriori remains anti-correlated. For $\Omega_{p}$ with a large magnitude versus $\Gamma$ 's magnitude, the posteriori correlation approaches the a priori correlation. An increase in the magnitude of $\Gamma$ will weaken the posteriori correlation with respect to the a priori correlation if both $\Gamma$ and $\Omega_{p}$ have the same signs, and strengthen the posteriori correlation with respect to the a priori correlation if $\Gamma$ and $\Omega_{p}$ have different signs.

What the previous discussion reveals is that although the variance of the posteriori may decrease, the correlation may increase. The implication is that a system attribute of interest posteriori uncertainty may not decrease from a priori depending on the magnitude of parameters'

posteriori correlation and attribute sensitivities to the parameters. Thus just observing the parameters' posteriori variance magnitude could lead to an improper conclusion regarding magnitude of attributes' uncertainty reduction.

\subsection{Design Optimization}

This topic will be focused on a design on the sensor deployment and experiments that provides maximum savings via design modifications that effectively utilize the increased design freedom obtained from the reduced uncertainties achieved, while retaining safety margin. The design feature one is especially interested in is electric power rating, which is limited by not only normal operation but by transient conditions due to safety limits, e.g. CHFR during LOFA. 
Control systems can correct for some changes in NSSS conditions due to accident initiators, but they eventually exceed the capability to control the reactor system, e.g. turbine CV fully open. If it is assumed that the reactor controllers are inactive, then the system will be even more limited. As noted, the degree of design freedom can be increased by performing additional measurements, thereby decreasing the uncertainties on key system attributes via reducing the uncertainties on input parameters to the system simulation code. This gives a posteriori probability distribution of the safety parameter, i.e. $\tilde{p}\left(a^{\text {post }}\right)$. The mean value of a posteriori, $\left\langle a^{\text {post }}\right\rangle$, could be larger or smaller than the mean value of a priori, $\langle a\rangle$, implying it could get better or worse from the safety point of view. The standard deviation of $a^{\text {post }}$ will generally decrease, which is a benefit. Assuming that $\left\langle a^{\text {post }}\right\rangle$ is identical to $\langle a\rangle$, only the standard deviation is relevant. Note that if actual experimental data do not exist, mean values of a posteriori system responses and system attributes determined by simulation are not meaningful.

The basic idea is that even though a new, more economical design of the system could shift the mean value of the system attribute to be closer to the safety limit, e.g. $95 \%$ confidence level of not experiencing DNB, the reduced uncertainty on the system attribute could compensate for it. Quantitatively this can be stated as:

$$
\int_{S_{a}} p(a) d a=\int_{S_{a}} \tilde{p}\left(a^{p o s t}-\delta a\right) d a^{p o s t}
$$

where $S_{a}$ denotes the allowed range of the system attributes, $p(a)$ the a priori probability distribution for the current design, $\tilde{p}\left(a^{\text {post }}-\delta a\right)$ the a posteriori probability distribution for the new design, and $\delta a$ the "margin saving." The margin saving will be discussed later in this section. For safety limits, $\int_{S_{a}} p(a) d a$ is usually $\geq 0.95$.

The optimization problem decision variables include the selection of sensor types and locations, and experiment type imposing realistic constraints, with the objective of maximizing the savings achieved by utilizing the larger degree of the plant operational freedom created by system attribute uncertainty reduction, offset by the cost of sensors and experiments. The above can be implemented via the following algorithm steps: 
1. Obtain $\overline{\bar{C}}_{a}^{\text {post }}(\bar{l}, \overline{\mathrm{x}})$. Vector $\bar{l}$, which has 0 or 1 values, indicates where a certain type of instrument is used at a specific location; and, $\overline{\mathrm{x}}$ is a vector, whose elements are 0 or 1 , indicates whether experiment $n$ is to be completed. A posteriori system attribute uncertainty is then a function of $\bar{l}$ and $\overline{\mathrm{x}}$. Assuming that a Gaussian distribution is applicable for the system attributes, implying the system is linear, $\overline{\bar{C}}_{a}^{\text {post }}(\bar{l}, \overline{\mathrm{x}})$ can be obtained using Equation (2.19) and (2.20), where

$$
\overline{\bar{S}}^{\mathrm{T}} \overline{\bar{C}}_{m}^{-1} \overline{\bar{S}}=\sum_{n=1}^{N n} \sum_{j=1}^{N j} \overline{\mathrm{x}_{n}}{ }_{\bar{l}} \overline{\bar{S}}_{(n, j)}^{\mathrm{T}} \overline{\bar{C}}_{m(n, j)}^{-1} \overline{\bar{S}}_{(n, j)} .
$$

2. Determine the margin saving, which is defined as (see Figure 6-3) for the most limiting system attribute:

$$
\left|\delta a^{(t)}(\bar{l}, \overline{\mathrm{x}})\right| \equiv\left|\left\langle a^{\text {post new }(t)}(\bar{l}, \overline{\mathrm{x}})\right\rangle-\left\langle a^{(t)}\right\rangle\right|=\left|L-\left\langle a^{(t)}\right\rangle\right|-1.96 \sigma_{a}^{\text {post }(t)}(\bar{l}, \overline{\mathrm{x}})
$$

where $\left\langle a^{\text {post new }(t)}(\bar{l}, \overline{\mathrm{x}})\right\rangle$ is the nominal (mean) value of the new design of a posteriori system attribute obtained using vector $\bar{l}$ and $\overline{\mathrm{x}}$ at time $t$, and $\left\langle a^{(t)}\right\rangle$ is the nominal value of a priori system attribute at time $t$ which is assumed identical to $\left\langle a^{\text {post }(t)}(\bar{l}, \overline{\mathrm{x}})\right\rangle$ in this work for lack of real experiments being performed and to isolate the benefits of uncertainty reduction via experiments. The $L$ is the safety limit. If the current system is designed based on a 95/95 safety criteria and all the margin was properly utilized, $\left|L-\left\langle a^{(t)}\right\rangle\right|$ is equal to $1.96 \sigma_{a}^{\text {prior }(t)}$. Note that $\sigma_{a}^{p r i o r(t)}$ and $\sigma_{a}^{\text {post }(t)}(\bar{l}, \overline{\mathrm{x}})$ are the a priori and the a posteriori standard deviations of the limiting system attribute at time $t$, respectively. The $\left\langle a^{\text {post new }(t)}(\bar{l}, \overline{\mathrm{x}})\right\rangle$ is then calculated using Equation (2.40). Do note that it is assumed that the a posteriori uncertainty is unaltered by system design changes, an assumption in practice easily removed but clouds the intuitive understanding. 


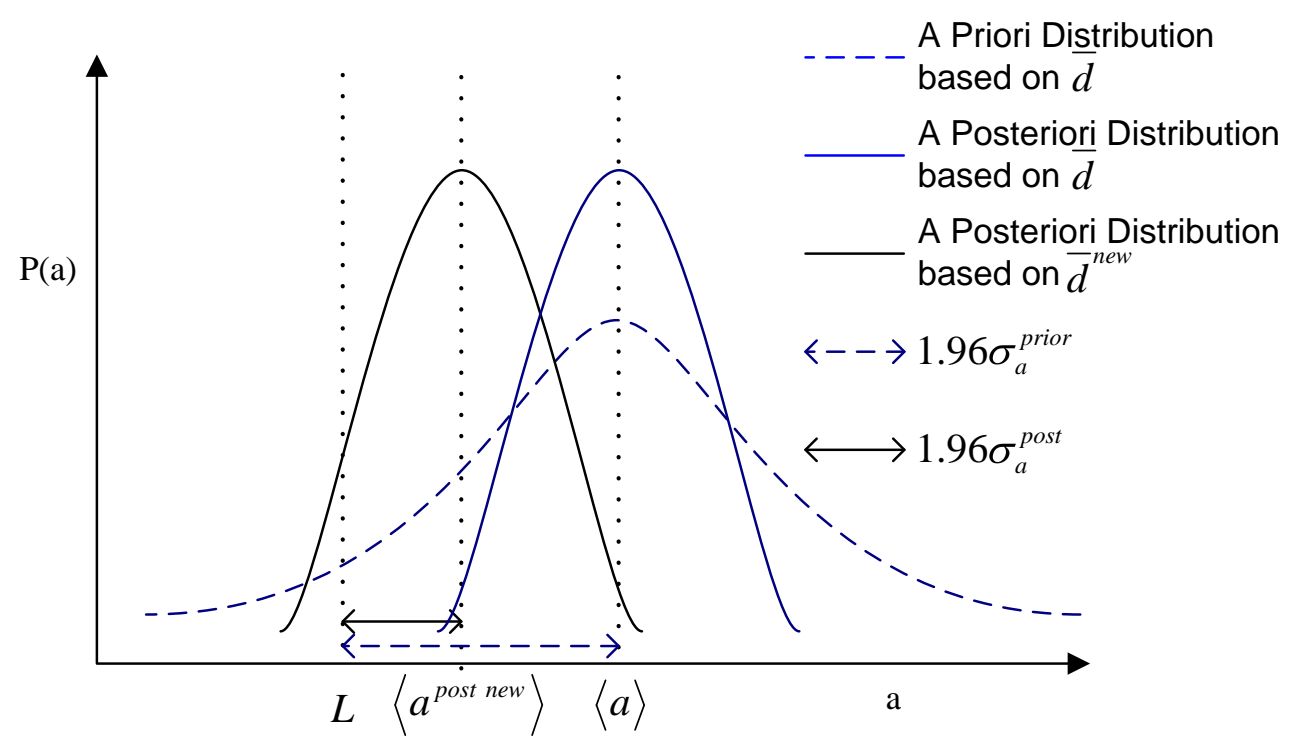

Figure 6-3: A Priori and a Posteriori System Attribute Distributions

3. Determine new design specs which are a function of the system attribute uncertainties. The best altered design specs, which maximize the savings, constrained by the safety criteria will be decided by solving the sub-optimization problem. The allowed difference between the current and new design specs will eventually become larger as more instruments are used and more experiments are conducted, since additional experimental data reduces a posteriori system attribute uncertainties.

Define $\bar{\mu}_{d}$ as the savings associated with each of the design specs. For example one of the elements of design specs would be the pump force, then the corresponding value in $\bar{\mu}_{d}$ would be the saving for the size of the pump. The total savings originating from the a posteriori optimum design specs is then:

$$
\mu_{D}(\bar{l}, \overline{\mathrm{x}})=\bar{\mu}_{d}^{\mathrm{T}} \overline{\delta d}(\bar{l}, \overline{\mathrm{x}})=\bar{\mu}_{d}^{\mathrm{T}}\left[\bar{d}^{\text {new }}(\bar{l}, \overline{\mathrm{x}})-\bar{d}\right]
$$

where $\bar{d}^{\text {new }}$ is the optimum design specs that satisfy the a posteriori safety margins, and $\bar{d}$ is current design specs. Let vector $\bar{C}_{i}$ denotes the cost of each instrument. Then $C_{I}$ is the total instrument cost defined as:

$$
C_{I} \equiv \bar{C}_{i}^{\mathrm{T}} \bar{l}
$$


Defining $\bar{C}_{e}$ as the cost of performing experiment $n$, then $C_{E}$, the total cost of conducting all the experiments, is defined as:

$$
C_{E} \equiv \bar{C}_{e}^{\mathrm{T}} \overline{\mathrm{X}}
$$

The total cost for the instruments and experiments is then:

$$
C_{T}=C_{I}+C_{E}
$$

All of the cost functions can be generalized to have a more complex relationship with the decision variables and design specs than represented here.

The objective is to maximize the savings achieved from the tighter safety margin, i.e. larger degree of the design freedom, offset by the costs of instruments and experiments, which can be expressed as the following objective:

$$
\begin{aligned}
& \max _{\bar{l}, \overline{\mathrm{x}}}\left[\mu_{D}(\bar{l}, \overline{\mathrm{x}})-C_{T}\right] \\
& =\max _{\bar{l}, \overline{\mathrm{x}}}\left[\bar{\mu}_{d}^{\mathrm{T}} \bar{d}^{\text {new }}(\bar{l}, \overline{\mathrm{x}})-\bar{d}-\bar{C}_{i}^{\mathrm{T}} \bar{l}+\bar{C}_{e}^{\mathrm{T}} \overline{\mathrm{x}}\right]
\end{aligned}
$$

constrained to Equation (6.39). This is the optimization problem based on the "design specs saving." In evaluating Equation (6.45), one proceeds as follows. For all the accidents being considered, the posteriori values for each safety attribute must satisfy the safety criteria, e.g. 95/95, at all times during the accident progression for the modified IRIS design. There may be several alternative designs that satisfy the constraints, but we seek the design that maximizes the savings of the modified design. This design must satisfy all the safety criteria for all accidents over the time progression of the accidents. So embedded within the optimization expressed Equation (2.45), whose decision variables are the sensors' selection and locations, and the experiments to conduct, is the sub-optimization to determine the optimum design specs. This presents a significant computational challenge in terms of computational resources required.

The conclusion is in order to maximize the total savings resulting from a new design specs, one should minimize a posteriori system attribute uncertainty by conducting data assimilation and uncertainty quantification. However since the input parameter uncertainties do not go to zero and eventually converges to a certain level due to the measurement error, the rate of the uncertainty reduction deceases as more instruments are deployed and more experiments are performed. At a certain point, due to the technical limitation described above and due to the 
instruments and the experiments cost, it is not economically attractive to add more instruments and to conduct more experiments.

\subsection{Uncertainty Analysis for Reactor Control}

The uncertainty analysis was conducted for reactor control problems. The reactors are controlled by several control algorithms, e.g. $T_{a v e}$ Control, Pressurizer Level Control, Steam Pressure Control and Turbine Control (RPM Control). The purpose of control is to move the system from its current state to the desired state. Controlling some observables, e.g. average temperature, assures future system states, e.g. relative core power, have their desired values. Given a set of alternative control algorithms, our interest is to select the algorithm that minimizes the time integrated deviation of the actual state from the desired state. These deviations originate due to inadequacy of the control system, and uncertainties in the observable values, e.g. average temperature, feed flow rate, steam flow rate, etc. When the system responses change their trajectories following the control algorithm, it could result in the reactor controllers exceeding their capability, for example the pressurizer heater capacity, resulting in lack of control. To ascertain whether this occurs, the capability to complete high fidelity simulation, i.e. small uncertainty, accounting for prediction and measurement uncertainties of control algorithm utilized detector signals is required. In assessing which control algorithm is preferred, those uncertainties in the observable values used as input to the control algorithms must be considered. When simulating the plant, there are also uncertainties in the predicted actual state that could affect selecting the best of the control algorithm to utilize.

\subsection{Control Algorithms in the Simulator}

A Westinghouse report [Petrovic et al., 2005] and the simulation code were reviewed to identify the control algorithms in the IRISN.Ver08.Mod06. The main equipment that the control system controls in the IRIS reactor system are:

1. Control Rods

2. Feed Pumps

3. Feed Control Valves (FCV)

4. Turbine Control Valves (TCV) 


\section{Pressurizer Heaters}

and the main control algorithms for the IRIS reactor are:

1. Feed and Turbine Control

2. Steam Pressure Control

3. Reactor Power Control

4. Primary Pressure Control

The algorithms in the simulator are presented below.

\subsubsection{Feed and Turbine Control}

Generally the feed flow rate follows feed flow demand. The feed flow demand $D_{f}$ is defined as:

$$
D_{f} \equiv \dot{m}_{S G 0} \frac{W_{\text {load }}}{W_{\text {ref }}}+\int_{t_{0}}^{t_{c}} K_{\text {shim }} \dot{m}_{S G 0} \frac{W_{\text {load }}-W_{\text {turb }}}{W_{\text {ref }}} d t
$$

where $t_{0}$ and $t_{c}$ denote initial and current time, respectively, $\dot{m}_{S G 0}$ the nominal steam flow rate ( $\left.4.96 \times 10^{5} \mathrm{lbm} / \mathrm{hr}\right), W_{\text {ref }}$ the reference turbine load $(335 \mathrm{MWe}), W_{\text {load }}$ the turbine load, $W_{\text {turb }}$ the turbine output, and $K_{\text {shim }}$ integral gain of turbine $\left(10 \mathrm{hr}^{-1}\right)$. Note that the values in the parentheses indicate the nominal values for the current IRIS reactor design. The feed shim $S$ is defined as:

$$
S \equiv \int_{t_{0}}^{t_{c}} K_{\text {shim }} \dot{m}_{S G 0} \frac{W_{\text {load }}-W_{\text {turb }}}{W_{\text {ref }}} d t
$$

Thus the feed flow demand follows the turbine load and it is corrected by the feed shim. The feed control valve position change $\Delta V_{F C V}$ at each time step is determined by:

$$
\Delta V_{F C V}=g_{o}^{F C V}+g_{p}^{F C V} \delta \dot{m}+g_{i}^{F C V} \int \delta \dot{m} d t
$$

where $g_{o}^{F C V}$ is controller offset $(0), g_{p}^{F C V}$ is FCV proportional gain (0.05) and $g_{i}^{F C V}$ is FCV integral gain $\left(0.07 \mathrm{hr}^{-1}\right)$. The $\delta \dot{m}$ is the flow error which is defined as:

$$
\delta \dot{m}=\frac{D_{f}-\dot{m}_{F}}{D_{f 0}}=\frac{D_{f}-\dot{m}_{F}}{\dot{m}_{S G 0}}
$$


where $\dot{m}_{F}$ is the feed flow rate per steam generator and $D_{f 0}$ is the nominal flow demand. Note that the nominal flow demand is identical to the nominal steam flow rate.

The feed flow rate per steam generator is controlled by not only the feed control valves but also the feed pumps. The feed pump speed change $\Delta \Omega$ is determined by:

$$
\Delta \Omega=g_{p} \delta P_{D}
$$

where $g_{p}$ is the feed pump speed proportional gain $(0.1)$ and $\delta P_{D}$ is the secondary side pressure drop error which is defined as:

$$
\delta P_{D}=\Delta P_{r e f}-\Delta P=\Delta P_{r e f}+P_{S G}-P_{\text {discharge }}
$$

where $P_{S G}$ is the steam pressure. Feed line discharge pressure $P_{\text {discharge }}$ is defined as:

$$
P_{\text {discharge }}=P_{C D}+\Delta P_{C P}+\Delta P_{H W P}+\Delta P_{F P}-l_{F L}
$$

where $P_{C D}$ indicates condenser pressure, $\Delta P_{C P}$ condensate pump pressure change, $\Delta P_{H W P}$ hotwell pump pressure change, $\Delta P_{F P}$ feed pump pressure change, and $l_{F L}$ feed line loss. $\Delta P_{r e f}$ is the reference (desired) pressure drop across feedwater inlet (122 psia) and $\Delta P$, which is defined as $\Delta P=P_{\text {discharge }}-P_{S G}$, is the actual pressure drop across the feedwater inlet.

\subsubsection{Steam Pressure Control}

The turbine control valve position change, $\Delta \tilde{V}_{T C V}$ at each time step is determined by:

$$
\Delta \tilde{V}_{T C V}=g_{o}^{T C V}+g_{p}^{T C V} \delta P_{S G}+g_{i}^{T C V} \int \delta P_{S G} d t
$$

where $g_{o}^{T C V}$ is controller offset $(0), g_{p}^{T C V}$ is TCV proportional gain $(0.0002)$ and $g_{i}^{T C V}$ is TCV integral gain $\left(0 \mathrm{hr}^{-1}\right)$. The $\delta P_{S G}$ is the steam generator pressure error which is defined as:

$$
\delta P_{S G}=P_{S G}-P_{S G}^{r e f}
$$

where $P_{S G}^{r e f}$ is the reference steam generator pressure (862 psia). If $\Delta \tilde{V}_{T C V} \geq 0$, the actual turbine control valve position change $\Delta V_{T C V}$ is determined by:

$$
\Delta V_{T C V}=\min \Delta \tilde{V}_{T C V}, \Delta \tilde{\tilde{V}}_{T C V}
$$

where $\Delta \tilde{\tilde{V}}_{T C V}=\dot{V}_{\max } \Delta t$, and $\dot{V}_{\max }$ is the maximum speed of the turbine control valve. If $\Delta \tilde{V}_{T C V}<0$ , $\Delta V_{T C V}$ is determined by: 


$$
\Delta V_{T C V}=\max \Delta \tilde{V}_{T C V}, \Delta \tilde{\tilde{V}}_{T C V}
$$

\subsubsection{Reactivity (Power) Control}

The reactor power is controlled based on the average temperature error and the mismatch between the relative electric power and the relative thermal power. The mismatch between the relative electric power and the relative thermal power is:

$$
e_{1}=\frac{W_{t u r b}}{W_{r e f}}-\frac{Q_{t h}}{Q_{t h 0}}
$$

where $Q_{t h}$ is the reactor thermal output and $Q_{t h 0}$ is the nominal full power (1000 MWt). The average temperature mismatch is defined as:

$$
e_{2}=T_{a v e}^{r e f}-T_{a v e}
$$

where $T_{a v e}^{r e f}$ is the reference average temperature defined as:

$$
T_{a v e}^{r e f}=a_{0}+b_{0} \frac{W_{\text {turb }}}{W_{r e f}}
$$

where $a_{0}$ and $b_{0}$ are constants which are designed to be 575.6 and 14.4 , respectively, for the average temperature program. The total error is then:

$$
e=\omega \cdot e_{1}+e_{2}
$$

where $\omega$ is the weight which is selected as 5. The control rod speed $v_{\text {Rod }}$ is then determined by:

$$
v_{\text {Rod }}=g_{\text {Rod }}|| e|-0.5| v_{\text {Rod }}^{\max } \text { if }|e|>0.5
$$

where $g_{\text {Rod }}$ is the rod gain (0.005) and $v_{R o d}^{\max }$ is the maximum rod speed (45 inches/min). The control rod speed is bounded by its minimum and maximum values. In other words if $v_{R o d}>v_{R o d}^{\max }$ at a certain time step, $v_{R o d}=v_{R o d}^{\max }$, or if $v_{R o d}<v_{R o d}^{\min }$, then $v_{R o d}=v_{R o d}^{\min }$, where $v_{R o d}^{\min }$ is the minimum rod speed (3.75 inches/min). The control rod depth $d_{\text {Rod }}$ at time $t$ is determined by:

$$
d_{R o d}^{t}= \begin{cases}d_{R o d}^{t-\Delta t}+v_{R o d}^{t} \Delta t & \text { if } e<0 \\ d_{R o d}^{t-\Delta t}-v_{R o d}^{t} \Delta t & \text { otherwise }\end{cases}
$$

The control rod depth is also constrained by the core geometry, i.e. if $d_{\text {Rod }}^{t}>l_{\text {Rod }}$, then $d_{R o d}^{t}=l_{R o d}$, or if $d_{R o d}<0$, then $d_{R o d}=0$, where $l_{\text {rod }}$ is the control rod length. 


\subsubsection{Primary System Pressure Control}

Figure 6-4 shows the pressurizer heater set points used for the IRIS reactor simulations.

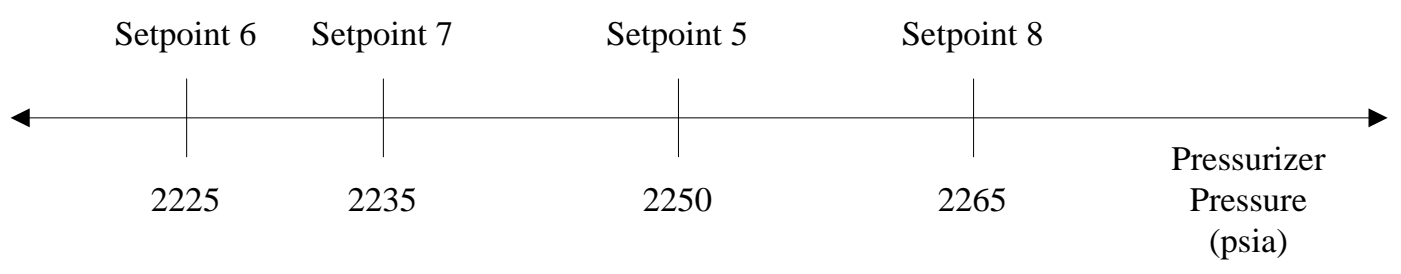

Figure 6-4: Pressurizer Heater Set points

Heater demand is calculated based on the pressurizer pressure ranges shown in the figure. The demands for four different cases are identified and presented as follows:

\subsubsection{Case 1: $P_{x} \geq$ Pressure Set point 8 , where $P_{x}$ is the pressurizer pressure}

In this case, $D_{P}^{P H}=D_{P}^{B H}=0$, where $D_{P}^{P H}$ is proportional heater demand and $D_{P}^{B H}$ is backup heater demand. This means that all heaters are off in this pressure range.

\subsubsection{Case 2: Pressurizer Set point $8>P_{x} \geq$ Pressurizer Set point 7}

Pressurizer pressure error $\delta P$ is defined as:

$$
\delta P=P_{x}^{r e f}-P_{x}
$$

where $P_{x}^{r e f}$ is pressurizer reference pressure $\left(2250\right.$ psia). The pressure demand $D_{P}$ is then determined by:

$$
D_{P}=D_{P 0}+g_{o}^{P}+g_{p}^{P} \delta P+g_{i}^{P} \int \delta P d t
$$

where $g_{o}^{P}$ is controller offset $(0), g_{p}^{P}$ is heater proportional gain $(0.07)$ and $g_{i}^{P}$ is heater integral gain $\left(0.07 \mathrm{hr}^{-1}\right)$. Nominal pressurizer demand $D_{P 0}$ is defined as:

$$
D_{P 0}=\frac{Q_{P H}}{Q_{P H C}}
$$

where $Q_{P H}$ is pressurizer proportional heater output and $Q_{P H C}$ is proportional heater capacity $(1350 \mathrm{KW})$. The proportional heater demand is then:

$$
D_{P}^{P H}=D_{P} Q_{P H C}
$$


and the backup heater demand is zero. Note that if $D_{P}^{P H}>Q_{P H C}$, then $D_{P}^{P H}=Q_{P H C}$, or if $D_{P}^{P H}<0$ , then $D_{P}^{P H}=0$.

\subsubsection{Case 3: Pressurizer Set point 7 $>P_{x}>$ Pressurizer Set point 6}

$$
D_{P}^{P H}=Q_{P H C}
$$

and the backup heater demand is zero.

\subsubsection{Case 4: $P_{x} \leq$ Pressurizer Set point 6}

$$
\begin{aligned}
& D_{P}^{P H}=Q_{P H C} \\
& D_{P}^{B H}=Q_{B H C}
\end{aligned}
$$

where $Q_{B H C}$ is backup heater capacity $(1080 \mathrm{KW})$.

Pressurizer heaters output is then determined using the demand. Pressurizer proportional heater output at time $t$ is:

$$
Q_{P H}^{t}=Q_{P H}^{t-\Delta t} e^{-\frac{\Delta t}{\tau_{h}}}+D_{P}^{P H} t\left(1-e^{-\frac{\Delta t}{\tau_{h}}}\right)
$$

and pressurizer backup heater output at time $t$ is:

$$
Q_{B H}^{t}=Q_{B H}^{t-\Delta t} e^{-\frac{\Delta t}{\tau_{h}}}+D_{P}^{B H t}\left(1-e^{-\frac{\Delta t}{\tau_{h}}}\right)
$$

where $\tau_{h}$ is the heater time constant $(20 \mathrm{sec}$ ). The primary system pressure is also controlled by the pressurizer safety relief valves. Opening set points for pressurizer safety relief valves are all 2500 psia.

Perturbing gains or shim results in different trajectories of the system responses, e.g. feed flow rate. Note that if the gains are all zeros, all reactor controllers are inactive. The idea is one can generate several different control algorithms by perturbing those parameters, which can include the inactive control algorithms. To minimize the deviation from the desired values, multiple control algorithms will be tested and the optimum control algorithm will be identified. The algorithm for determining the controller gains accounting for sensor and simulator introduced uncertainties in the sensor readings used by the control systems is presented in the following section. 


\subsubsection{Optimum Control Algorithm}

1. Define desired values (target values) of the system responses: $\bar{r}^{d}$

2. Identify reactor control algorithm $\tau(\tau=1, \ldots, N \tau)$ by perturbing the gains and the shim etc.

3. Simulate transient whose system responses are controlled by the algorithm $\tau$, and obtain time dependent system responses $\bar{r}$ and its distribution $\rho(\bar{r}, t)$ using the uncertainty propagation method presented below. Note that one should confirm that the system attributes, e.g. MDNBR, are not limited by the reactor control. One should also confirm that the system responses are controlled within their acceptable band of tolerance.

3.1 Sample parameters $\bar{p}^{i}$ using the parameter covariance matrix

3.2 Simulate transient with perturbed parameter $\bar{p}^{i}$ and obtain nominal values of the system responses $\stackrel{-i}{r}$. This is the time dependent system response vector which is calculated assuming the sensors are perfect, i.e. assuming no instrument uncertainties.

3.3 Now generate virtual reactor sensor's reading ${ }^{-i}$. Sensor's reading for the observable $j$ can be obtained by:

$$
\bar{r}_{j}^{i}(t)=\bar{r}_{j 0}^{i}(t)+\delta s_{j}^{i} \cdot \bar{r}_{j 0}^{i}(t)
$$

where $\delta s_{j}^{i}$ is the sensor's error which is randomly generated based on the instrument error standard deviations. The virtual reactor sensor's reading vector ${ }^{-i}$ is then:

$$
\stackrel{-i}{r}^{i}=\left[\begin{array}{llll}
-i \mathrm{~T} & -i \mathrm{~T} & & -i \mathrm{~T} \\
r_{1} & r_{2} & \ldots & r_{J}^{\mathrm{T}}
\end{array}\right]^{\mathrm{T}}
$$

Thus the actual sensor's reading is calculated considering ${ }_{r}^{-i}$ as the nominal value of the measurement, i.e. signal of the perfect instrument, and adding instrument uncertainties.

3.4 Return to step 3.1 until a reasonable numbers of samples are simulated.

3.5 Collect all $\bar{r}^{-i}$ and obtain distributions of the observables, $\rho(\bar{r}, t)$.

4. Calculate the objective:

$$
\frac{1}{t_{f}-t_{0}} \int_{t_{0}}^{t_{f}} \int_{r}\left\langle\bar{\omega},\left(\frac{\bar{r}^{d}(t)-\bar{r}}{\bar{r}^{d}(t)}\right)^{2}\right\rangle \rho(\bar{r}, t) d \bar{r} d t
$$




$$
\cong \frac{1}{t_{f}-t_{0}} \sum_{n=1}^{N t} \sum_{i_{N_{j}}=1}^{N b} \cdots \sum_{i_{2}=1}^{N b} \sum_{i_{1}=1}^{N b}\left[\sum_{j=1}^{N j} \omega_{j}\left(\frac{r_{i_{j}, n}^{d}-r_{i_{j}}}{r_{i_{j}, n}^{d}}\right)^{2}\right] \rho_{i_{1}, i_{2}, \ldots, i_{N j}, t_{n}} \Delta r_{i_{1}} \Delta r_{i_{2}} \cdots \Delta r_{i_{N_{j}}} \Delta t_{n}
$$

together with the safety constraint:

$$
\begin{gathered}
\min _{t} \int_{\min \left(r_{j}\right)}^{\max \left(r_{j}\right)} \rho\left(r_{j}, t\right) d r_{j} \\
\cong \min _{n} \sum_{i_{j}=1}^{N b} \rho_{i_{j}, t_{n}} \Delta r_{i_{j}} \geq \rho_{j}^{s} \text { for all } j \mathrm{~s}, \text { where } r_{j} \in\left[\min \left(r_{j}\right), \max \left(r_{j}\right)\right]
\end{gathered}
$$

and the operational constraint:

$$
\begin{gathered}
\frac{1}{t_{f}-t_{0}} \int_{t_{0}}^{t_{f}} \int_{r_{j}^{d}-\Delta r_{j}}^{r_{j}^{d}+\Delta r_{j}} \rho\left(r_{j}, t\right) d r_{j} d t \\
\cong \frac{1}{t_{f}-t_{0}} \sum_{n=1}^{N t} \sum_{i_{j}=1}^{N b} \rho_{i_{j}, t_{n}} \Delta r_{i_{j}} \Delta t_{n} \geq \rho_{j}^{o} \text { for all } j \mathrm{~s}, \text { where } r_{j} \in\left[r_{j}^{d}-\Delta r_{j}, r_{j}^{d}+\Delta r_{j}\right]
\end{gathered}
$$

where $n$ is a time index and $j$ is an observable index. The $i_{j}$ is a bin index for the $j$-th observable. The $N_{b}$ is the total number of bins. The $\bar{\omega}$ indicates importance of each observables (weight vector). The $\min \left(r_{j}\right)$ and $\max \left(r_{j}\right)$ are safety limits, e.g. $\min \left(r_{j}\right)=1.3$ and $\max \left(r_{j}\right)=\infty$ for the MDNBR. The $\left[r_{j}^{d}-\Delta r_{j}, r_{j}^{d}+\Delta r_{j}\right]$ is the acceptable operational range for observable $j$. The $\rho_{j}^{s}$ and $\rho_{j}^{o}$ are the safety and operation limit probability, respectively, for observable $j$ which should be determined for the licensing regularization. The $\rho(\bar{r}, t)$ and $\rho\left(r_{j}, t\right)$ are the normalized probability distributions. In other words,

$$
\rho(\bar{r}, t)=\hat{\rho}(\bar{r}, t) / \int_{r} \hat{\rho}(\bar{r}, t) d \bar{r}
$$

and

$$
\rho\left(r_{j}, t\right)=\hat{\rho}\left(r_{j}, t\right) / \int_{r_{j}} \hat{\rho}\left(r_{j}, t\right) d r_{j}
$$

where the hatted functions $(\hat{\rho})$ are non-normalized.

5. Go to 2 unless $\tau=N \tau$.

Thus one calculates the objective function: 


$$
\frac{1}{t_{f}-t_{0}} \min _{\tau} \int_{t_{0}}^{t_{f}} \int_{r}\left\langle\bar{\omega},\left(\frac{\bar{r}^{d}(t)-\bar{r}}{\bar{r}^{d}(t)}\right)^{2}\right\rangle \rho\left(\bar{r}^{\tau}, t\right) d \bar{r}^{\tau} d t
$$

constrained to:

$$
\min _{t} \int_{\min \left(r_{j}\right)}^{\max \left(r_{j}\right)} \rho\left(r_{j}^{\tau}, t\right) d r_{j}^{\tau} \geq \rho_{j}^{s} \text { for all } j \mathrm{~s}
$$

and

$$
\frac{1}{t_{f}-t_{0}} \int_{t_{0}}^{t_{f}} \int_{r_{j}^{d}-\Delta r_{j}}^{r_{j}^{d}+\Delta r_{j}} \rho\left(r_{j}^{\tau}, t\right) d r_{j}^{\tau} d t \geq \rho_{j}^{o} \text { for all } j \mathrm{~s}
$$

\section{RESULTS OF SIMULATOR ANALYSIS}

The following sections present the results of the simulator analyses described in Chapter

6. Representative tables and charts are given in the discussion. Additional results can be found in 0 .

\subsection{Mean and Standard Deviation of a Posteriori Input Parameters}

Employing virtual reactor sensor data for the experiments, including the introduction of sensor errors consistent with the sensor signals known uncertainties, the inverse problem using regularization and the iteration method to address mild nonlinearity was solved and a posteriori values were obtained. The model parameters for virtual experiment A, a posteriori parameters and their errors in a posteriori standard deviation units are presented in Table 7-1; results for the remaining experiments are in 0 . Note that the errors show us how realistic the posteriori standard deviation values are. Due to highly correlated observable sensitivities for two or more parameters, lack of covariance information and weak sensitivities with respect to certain parameters, it was shown that adapted parameters are not in good agreement with their values used in the corresponding virtual reactor model. Data assimilation was also completed using the data from all four experiments at the same time with results presented in Table 7-2. Figure 7-1 
and Figure 7-2 show associated a priori and posteriori standard deviations for the parameters. Note that $\sigma_{0}$ indicates a priori standard deviation of the input parameters, and $\sigma_{1}$ and $\sigma_{\text {post }}$ denote a posteriori standard deviations of the parameters calculated after the first and second iteration, respectively. It is shown that a large reduction in uncertainty can be achieved for the parameters if data from multiple experiments are utilized for data assimilation; however, due to the parameters that affect the system in a similar way, it is also shown that the errors increase as more experimental data are added.

Table 7-1: Virtual Experiment Values and Input Parameters Adapted for Experiment A at 80\%

Power

\begin{tabular}{|c|c|c|c|c|}
\hline \multirow{2}{*}{ Index } & Parameter & \multicolumn{2}{|c|}{ Relative Parameter Values } & \multirow{2}{*}{ Error } \\
\cline { 3 - 4 } & & $\begin{array}{c}\text { Virtual } \\
\text { Experiment } \\
\text { Model }\end{array}$ & A Posteriori & \\
\hline 1 & Chen Correlation & 1.129322047 & 1.014566866 & 1.398766966 \\
\hline 2 & Dittus-Boelter Correlation & 1.094106177 & 1.023096863 & 1.110583282 \\
\hline 3 & UO $_{2}$ Thermal Conductivity & 0.985729126 & 0.975832244 & 0.2216251 \\
\hline 4 & Friction Factors & 1.05334116 & 1.021405281 & 4.45995522 \\
\hline 5 & Gap Conductance & 0.806963659 & 1.067984217 & 4.640751644 \\
\hline 6 & Pellet Diameter & 0.996474221 & 1.000511007 & 2.201195638 \\
\hline 7 & Clad Diameter & 1.002919827 & 0.999113131 & 2.147830424 \\
\hline 8 & Clad Thermal Conductivity & 0.984143372 & 0.989605984 & 0.095370202 \\
\hline 9 & Pump Head & 0.987149777 & 0.993102287 & 1.088444156 \\
\hline 10 & MTC & 0.953765678 & 0.933783858 & 0.64321542 \\
\hline
\end{tabular}

Table 7-2: Virtual Experiment Values and Input Parameters Adapted using Experiments A, B, C, and D

\begin{tabular}{|c|c|c|c|c|}
\hline \multirow{2}{*}{ Index } & Parameter & \multicolumn{2}{|c|}{ Relative Parameter Values } & \multirow{2}{*}{ Error } \\
\cline { 3 - 4 } & Chen Correlation & $\begin{array}{c}\text { Virtual } \\
\text { Experiment } \\
\text { Model }\end{array}$ & A Posteriori & \\
\hline 1 & 1.195378183 & 1.119148506 & 1.988067533 \\
\hline 2 & Dittus-Boelter Correlation & 1.011952829 & 1.103939219 & 3.205750513 \\
\hline 3 & $\mathrm{UO}_{2}$ Thermal Conductivity & 0.958773877 & 1.012308383 & 4.642889236 \\
\hline 4 & Friction Factors & 1.027093277 & 1.004192396 & 5.094394314 \\
\hline 5 & Gap Conductance & 1.23124643 & 1.087465374 & 9.299896385 \\
\hline 6 & Pellet Diameter & 0.996822389 & 1.000287263 & 1.756606297 \\
\hline 7 & Clad Diameter & 1.004890791 & 1.005074713 & 0.263385368 \\
\hline
\end{tabular}




\begin{tabular}{|c|c|c|c|c|}
\hline 8 & Clad Thermal Conductivity & 1.074114209 & 1.027732082 & 0.845520076 \\
\hline 9 & Pump Head & 1.013000419 & 1.011671655 & 0.900223765 \\
\hline 10 & MTC & 0.963964368 & 0.966531435 & 0.359255024 \\
\hline
\end{tabular}

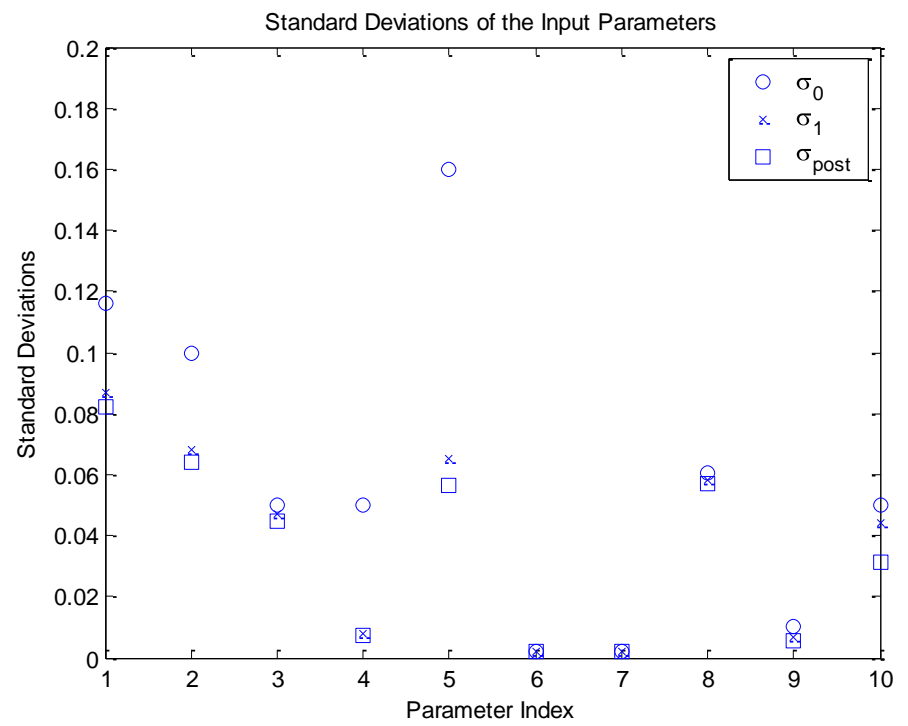

Figure 7-1: Standard Deviations for the Input Parameters for Experiment A at $80 \%$ Power

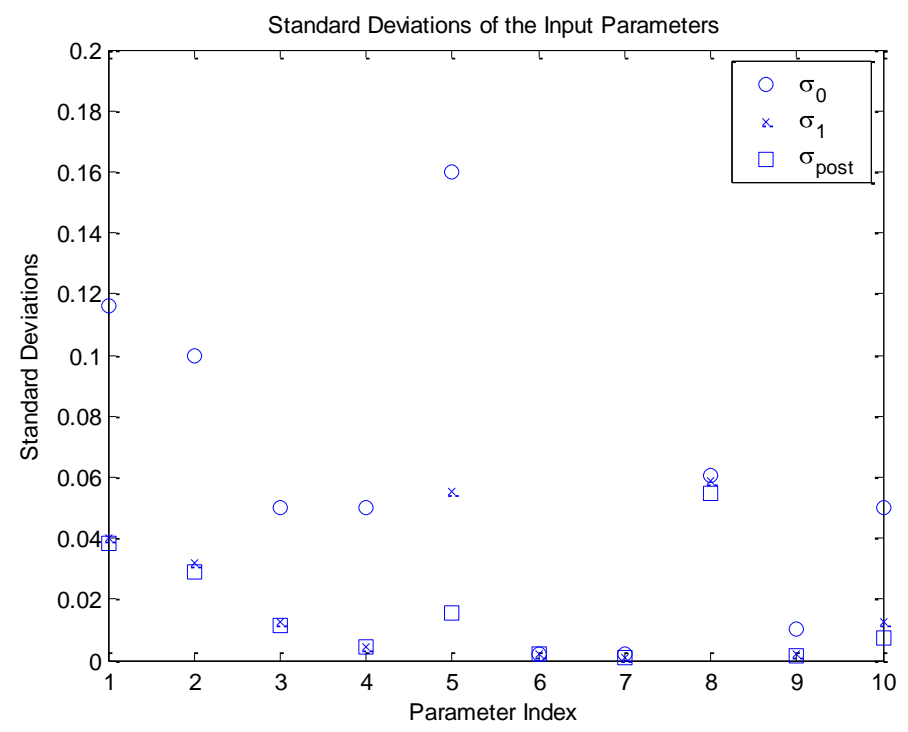

Figure 7-2: Standard Deviations for the Input Parameters for Experiments A, B, C, and D

\subsection{Standard Deviation of A Posteriori System Attributes}

Utilizing the a posteriori covariance of the parameters, the a posteriori distribution of the key system attributes for each of the accidents can be determined by either a linear or nonlinear 
approach. The a priori and posteriori standard deviations for three key system attributes at operating power level were obtained based on the assumption that the system responds linearly; results for experiment A are presented in Figure 7-3 through Figure 7-5. These figures, as well as additional results given in 0 , are obtained based on data assimilation and uncertainty quantification using only a single experiment. Figure 7-6 through Figure 7-8 also show the a priori and posteriori standard deviation for the key system attributes at operating power level experiencing RCP Trip calculated using all four experimental data at the same time. The results of other key accidents based on all four experiments are presented in 0 . These figures indicate that the uncertainties are reduced as more experiments are conducted.

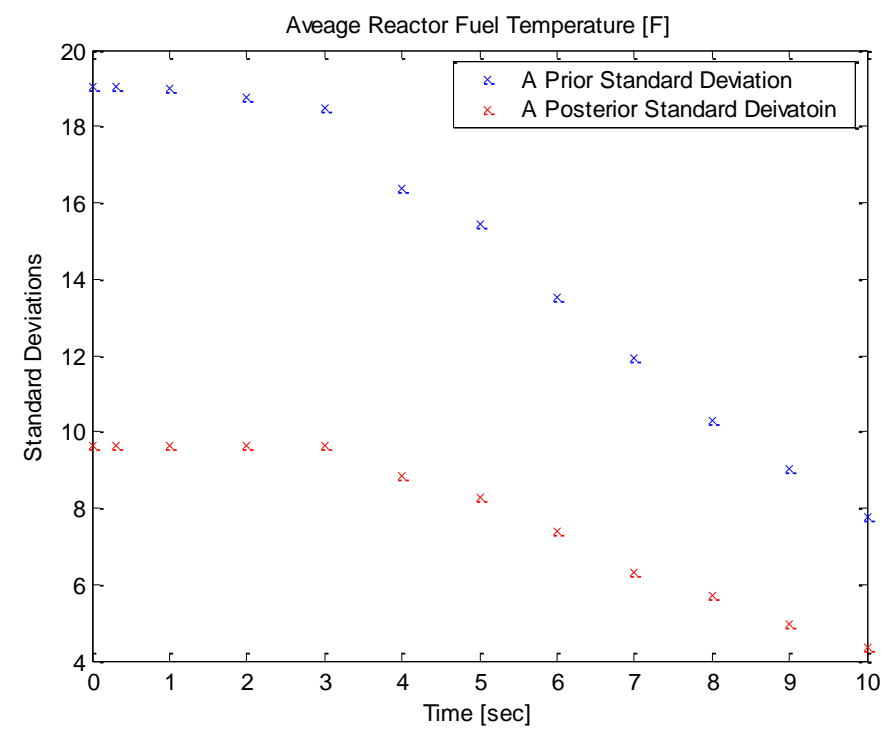

Figure 7-3: Standard Deviations for Average Fuel Temperature for RCP Trip Using Experiment A at $80 \%$ Power for Data Assimilation 


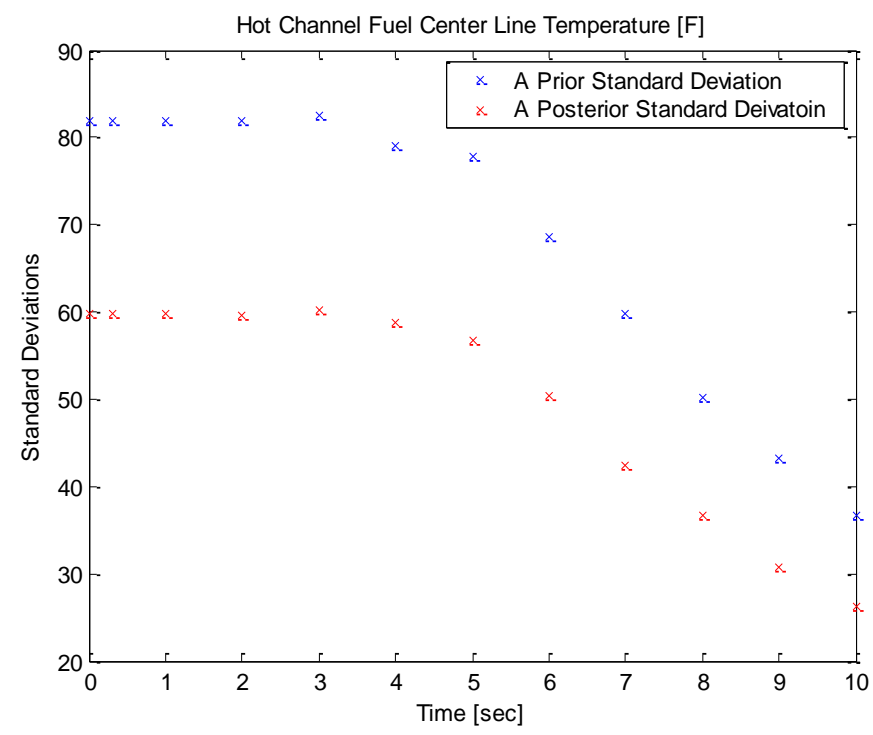

Figure 7-4: Standard Deviations for Hot Channel Fuel Centerline Temperature for RCP Trip using Experiment A at 80\% Power for Data Assimilation

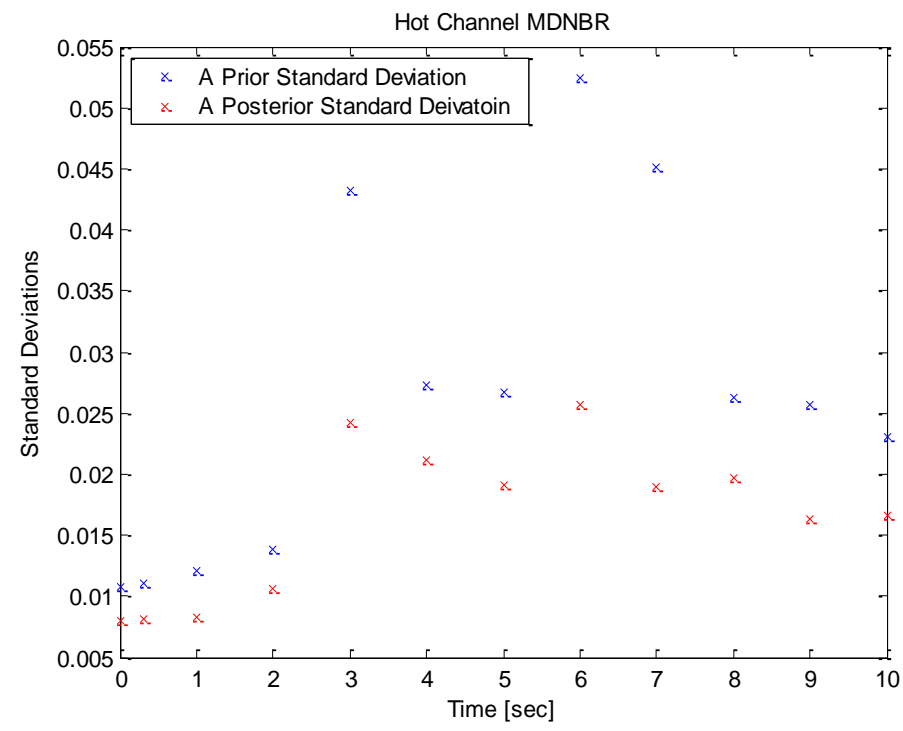

Figure 7-5: Standard Deviations for Hot Channel MDNBR for RCP Trip using Experiment A at 80\% Power for Data Assimilation 


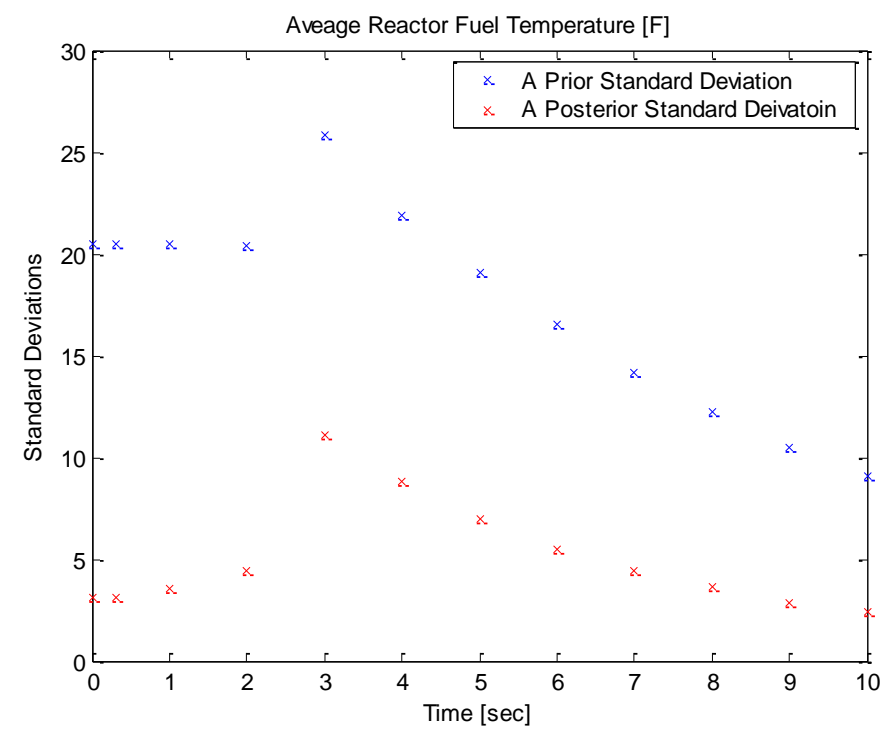

Figure 7-6: Standard Deviations for Average Fuel Temperature for RCP Trip using Experiment A, B, C and D for Data Assimilation

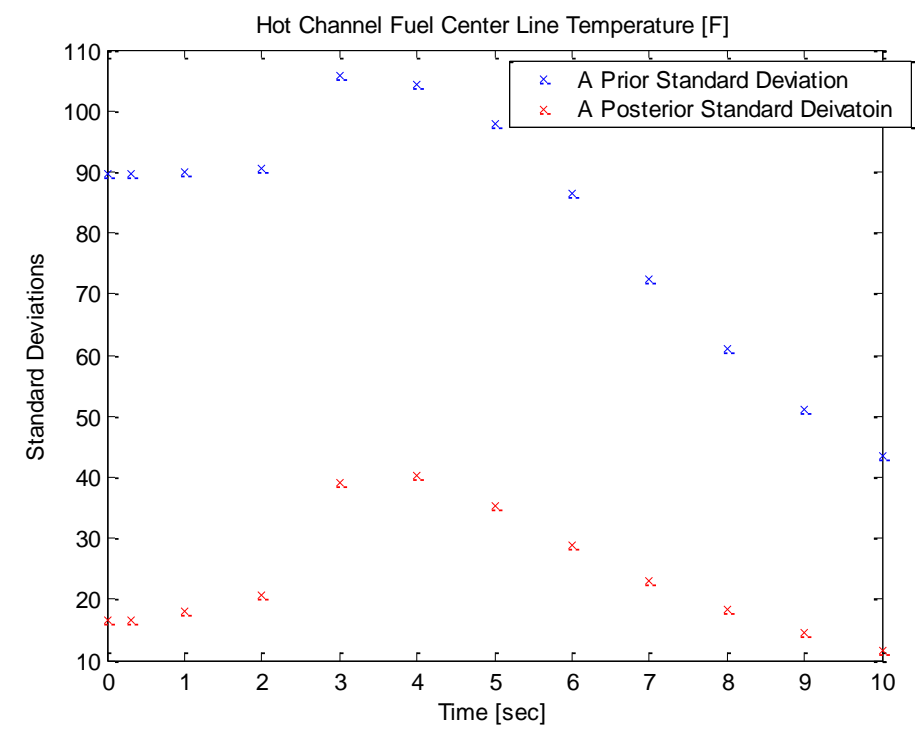

Figure 7-7: Standard Deviations for Hot Channel Fuel Centerline Temperature for RCP Trip using Experiment A, B, C and D for Data Assimilation 


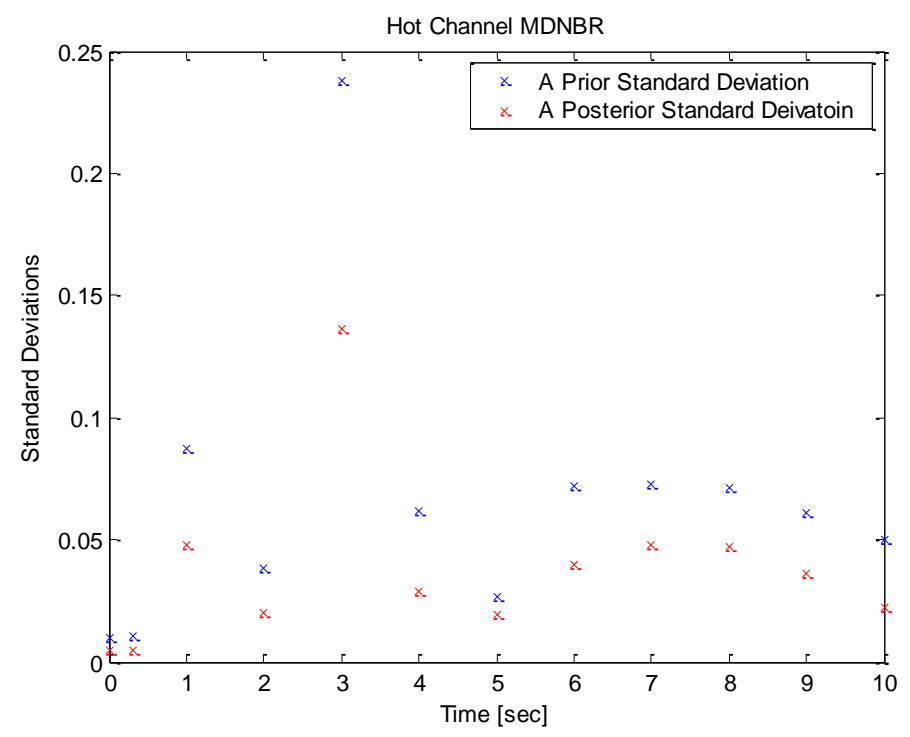

Figure 7-8: Standard Deviations for Hot Channel MDNBR for RCP Trip using Experiment A, B, $\mathrm{C}$ and D for Data Assimilation

\subsection{Uncertainty Contribution of Each Parameter}

Figure 7-9 presents each parameter's uncertainty contribution to the three system attributes during RCP trip, which are obtained using a single experiment's data. Figure 7-10 presents each parameter's uncertainty contribution during RCP trip as a result of using multiple experiments' data at the same time. Results for additional design accidents are given in 0 . As expected, major sources of uncertainty on the average fuel temperature and the hot channel fuel centerline temperature are fuel thermal conductivity and/or gap conductance. For the hot channel MDNBR, the uncertainty contribution depends upon the accident considered, with usually more than four input parameters significantly contributing to this key system attribute. 


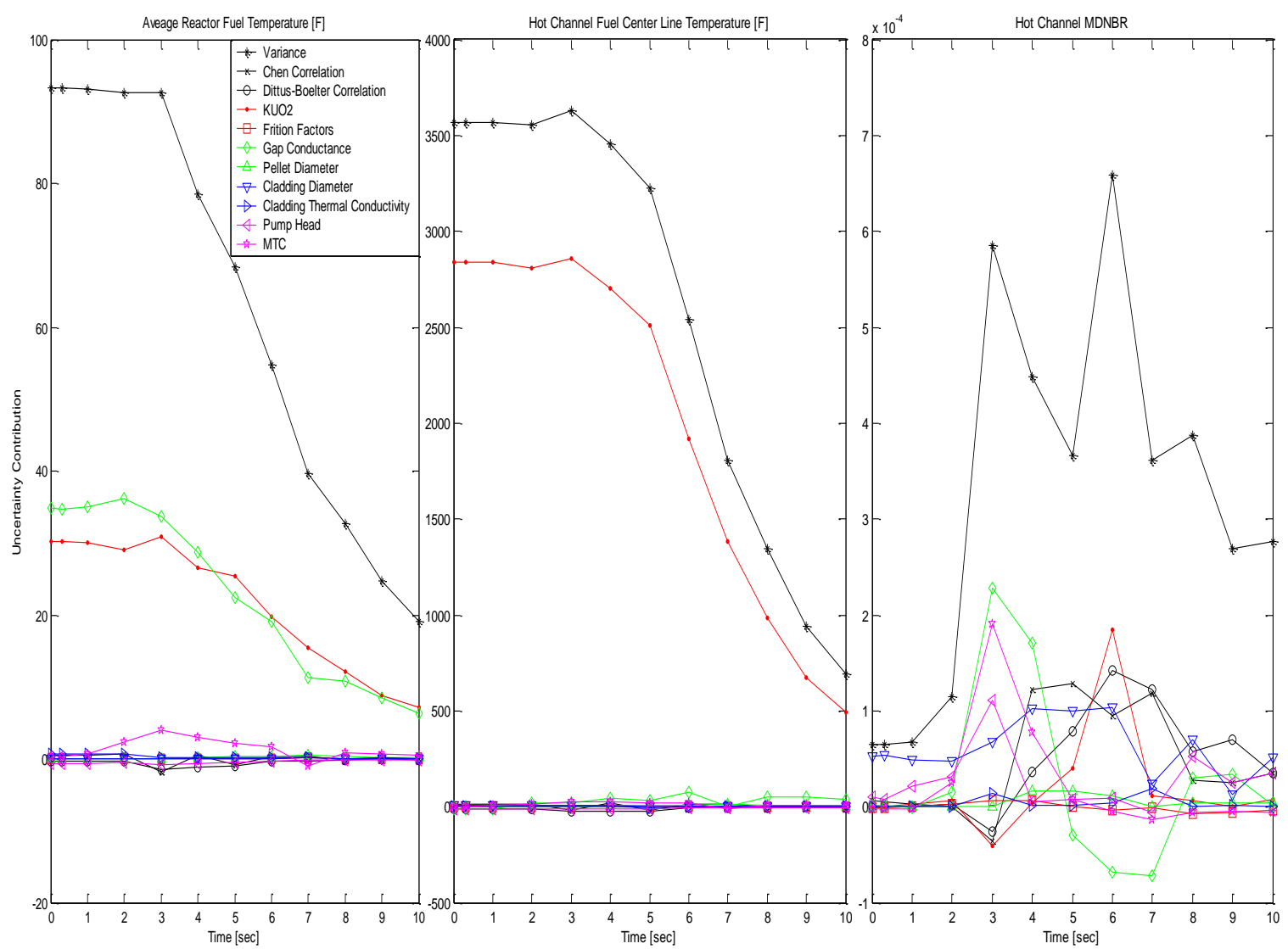

Figure 7-9: Each Parameter's Uncertainty Contribution to the System Attributes for RCP Trip 


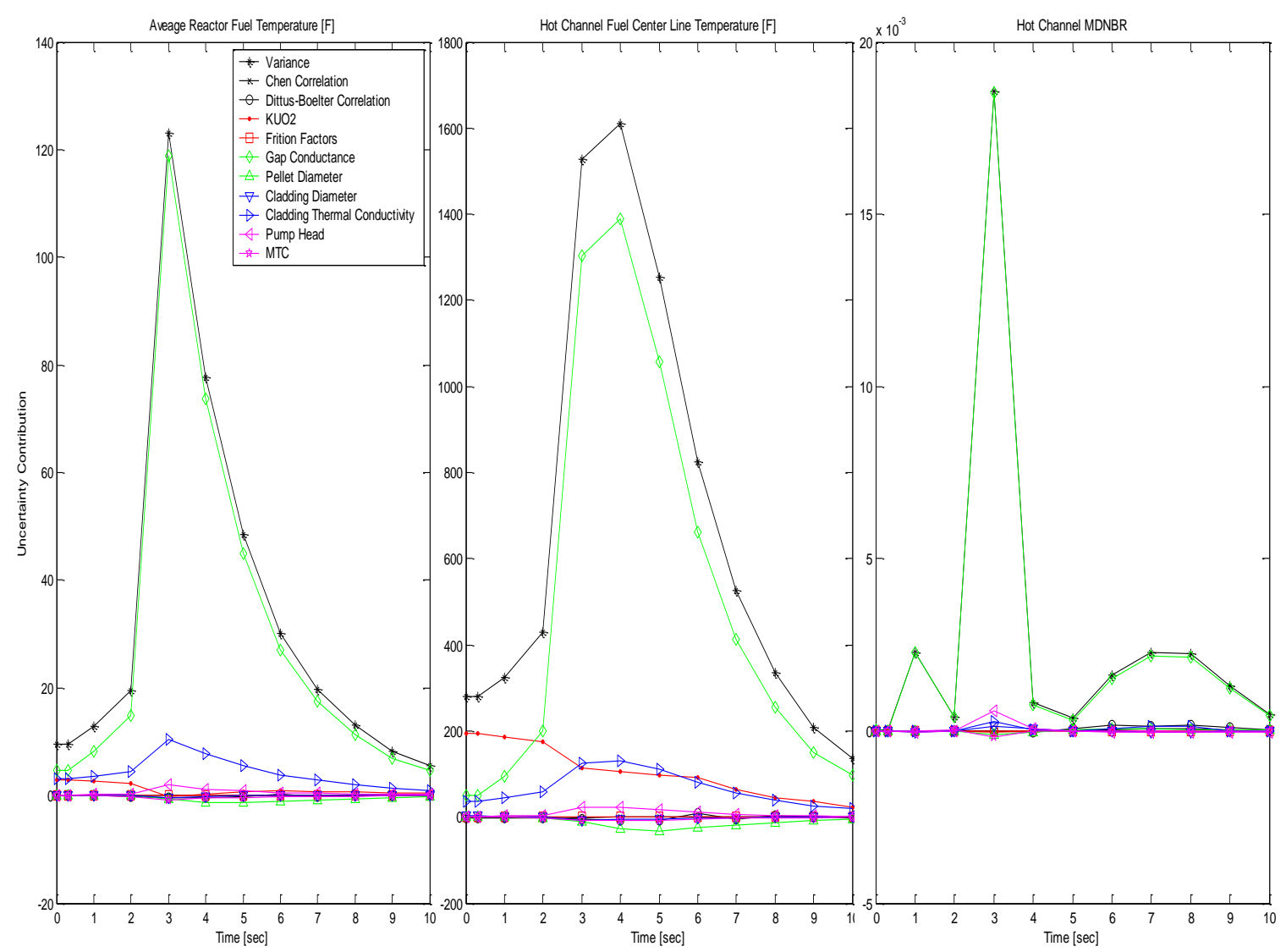

Figure 7-10: Each Parameter's Uncertainty Contribution to the System Attributes for RCP Trip as a Result of Multiple Experiments

\subsection{MCMC Simulation}

The MCMC simulation was completed for the reactor coolant pump trip transient simulation for which one observed relatively large Chi-Squared values. To obtain a posteriori parameter distribution, the true solution vector $\overline{\mathrm{e}}$ was used for the virtual experiment model, which differs from the previous adaptation. Figure 7-11 represents the accepted parameter values during the MCMC iteration for the RCP trip at 30\% power transient. It shows that the chain starts at random numbers and oscillates around the true solution vector. Figure 7-12 through Figure 7-14 present the a posteriori distribution of the parameters using RCP trip at 30\% power, which was computed using about 1200 MCMC samples. As observed, uncertainties are reduced, but nonGaussian distributions are observed as well due to the nonlinearity of the system. The a 
posteriori distributions of the parameters using RCP trip at $80 \%$ power which would be prohibited due to safety concerns was calculated for comparison and are presented in Figure 7-15 though Figure 7-17. Due to the nonlinearity and the different physics at different power levels, the shape of the distribution is not identical, but the standard deviations are not much different, which implies that one can infer the parameter uncertainties at normal operating power from those at lower power level. This also implies that the physics at operating power is not significantly different from that at lower power for this transient. The comparison could be thus one way to demonstrate the similarity of physics when completing an uncertainty analysis. 


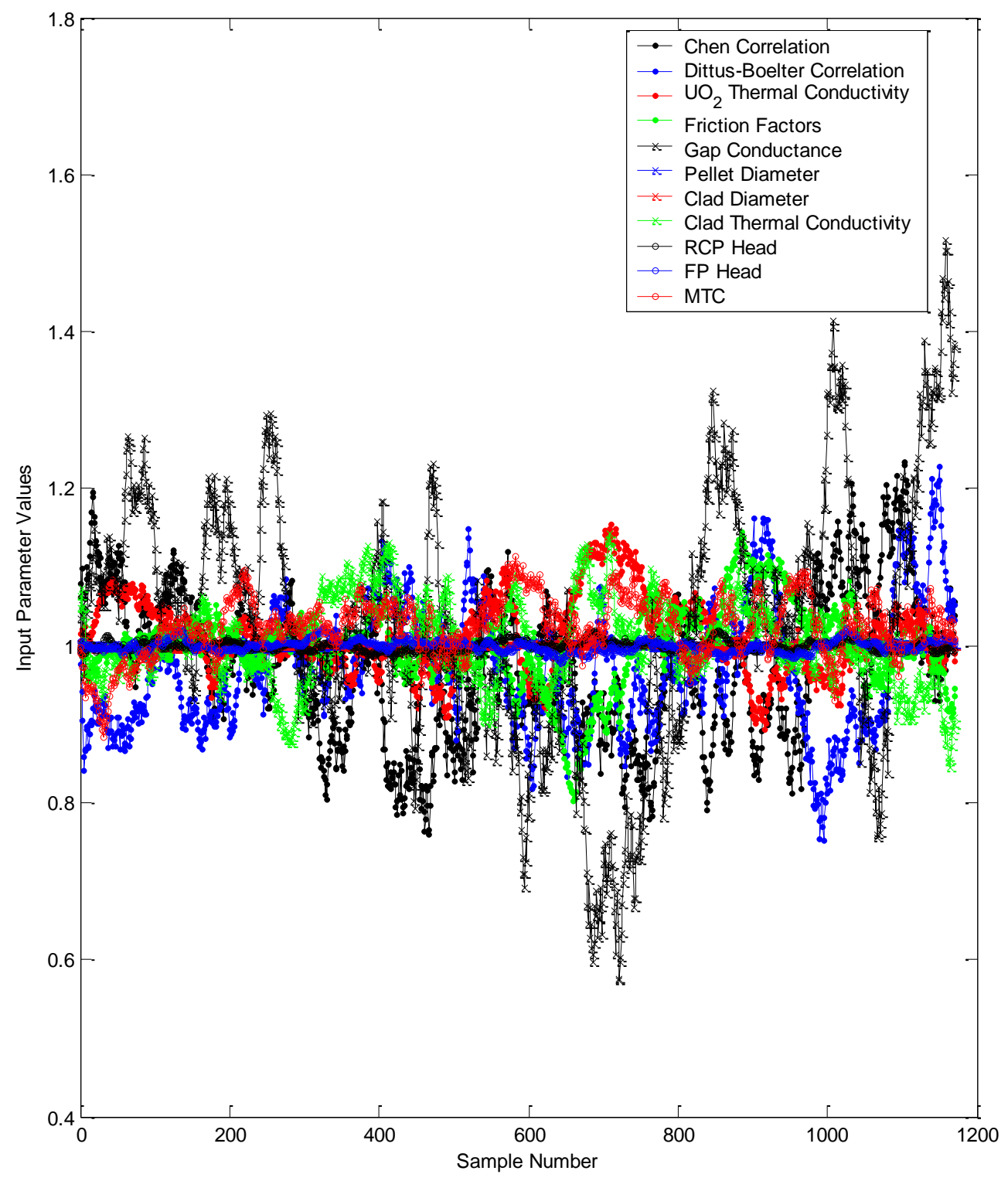

Figure 7-11: Accepted input parameter vector samples 

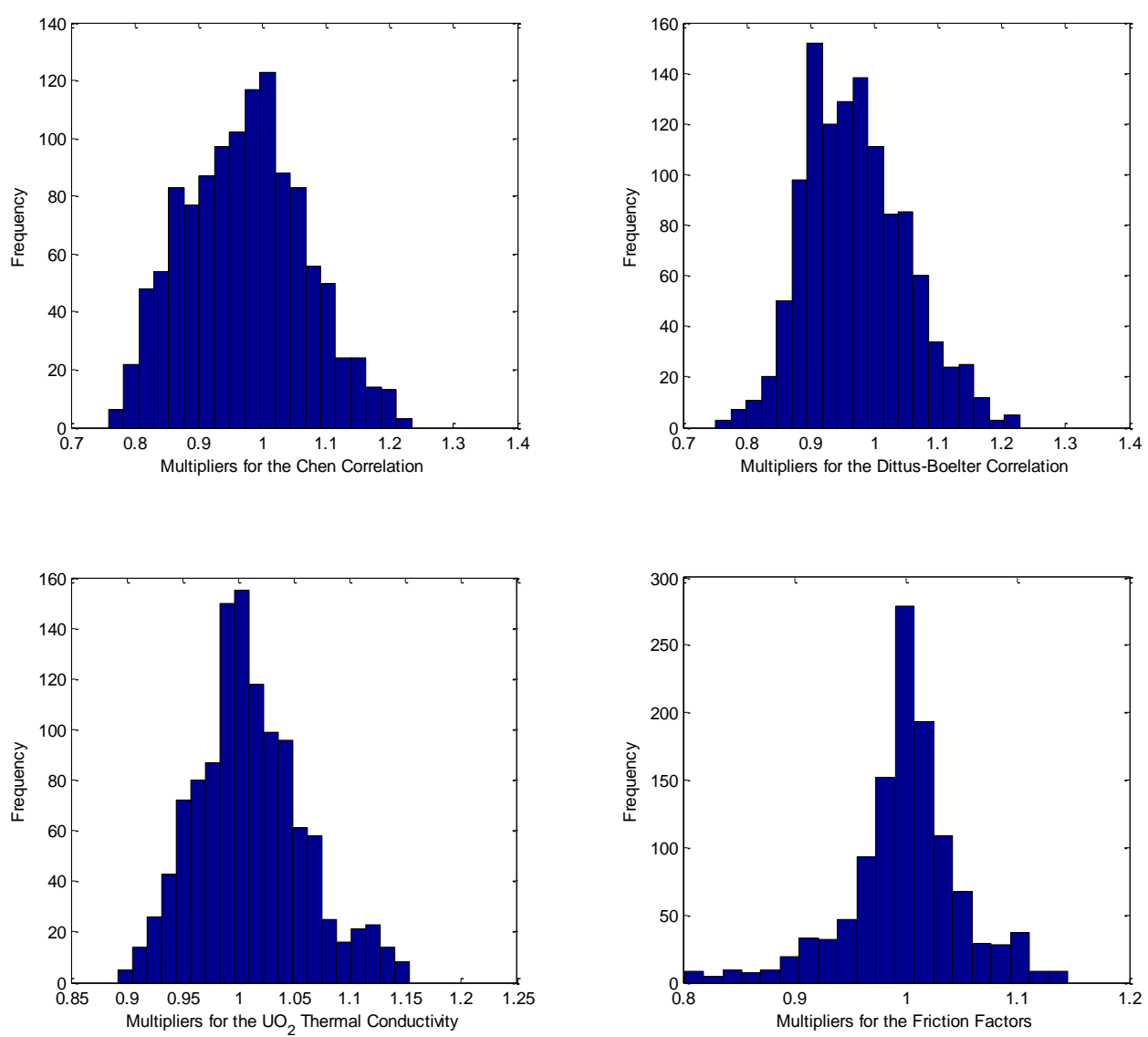

Figure 7-12: A posteriori distribution of the parameters using RCP Trip at 30\% Power 

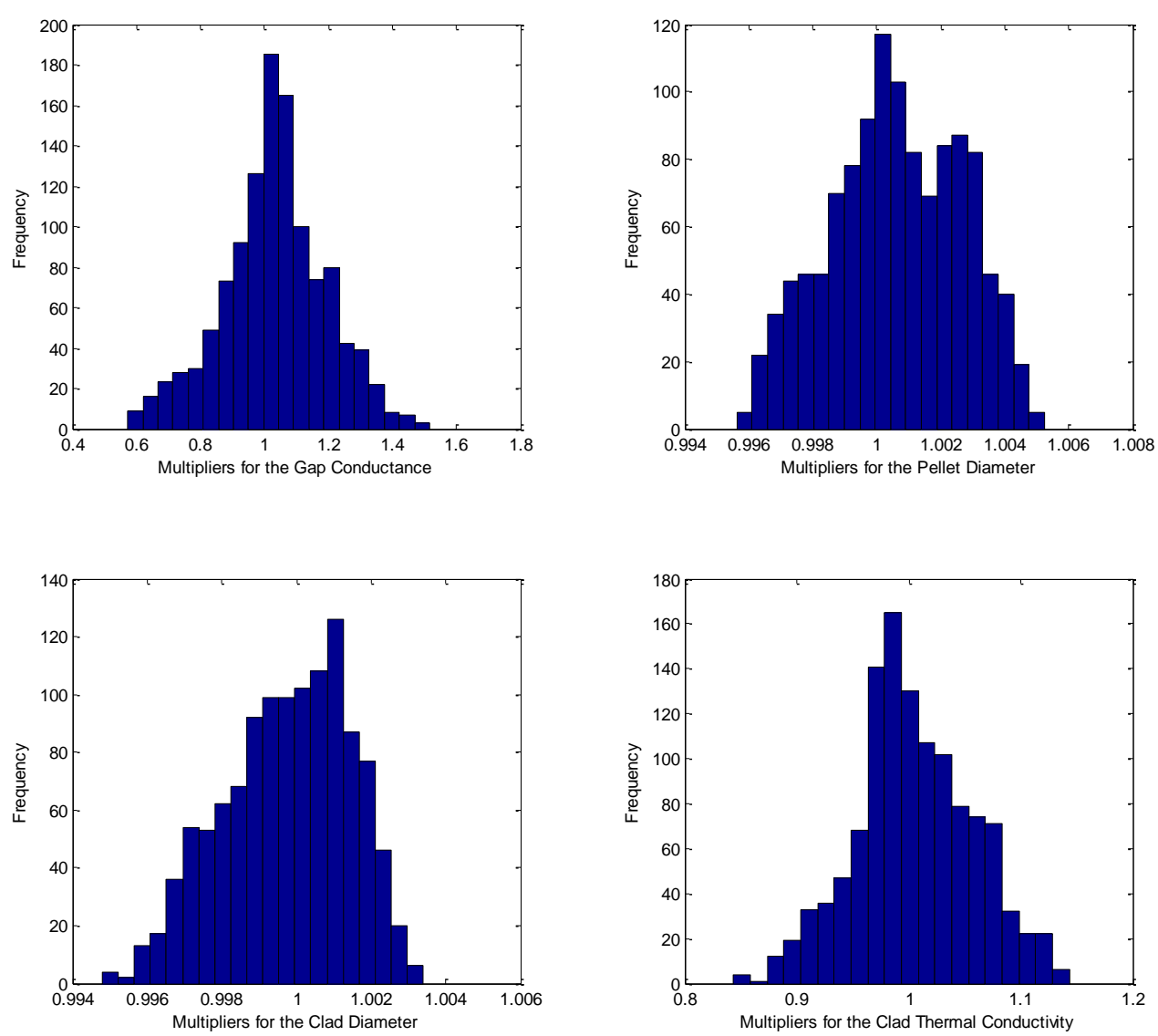

Figure 7-13: A posteriori distribution of the parameters using RCP Trip at 30\% Power (Cont.) 

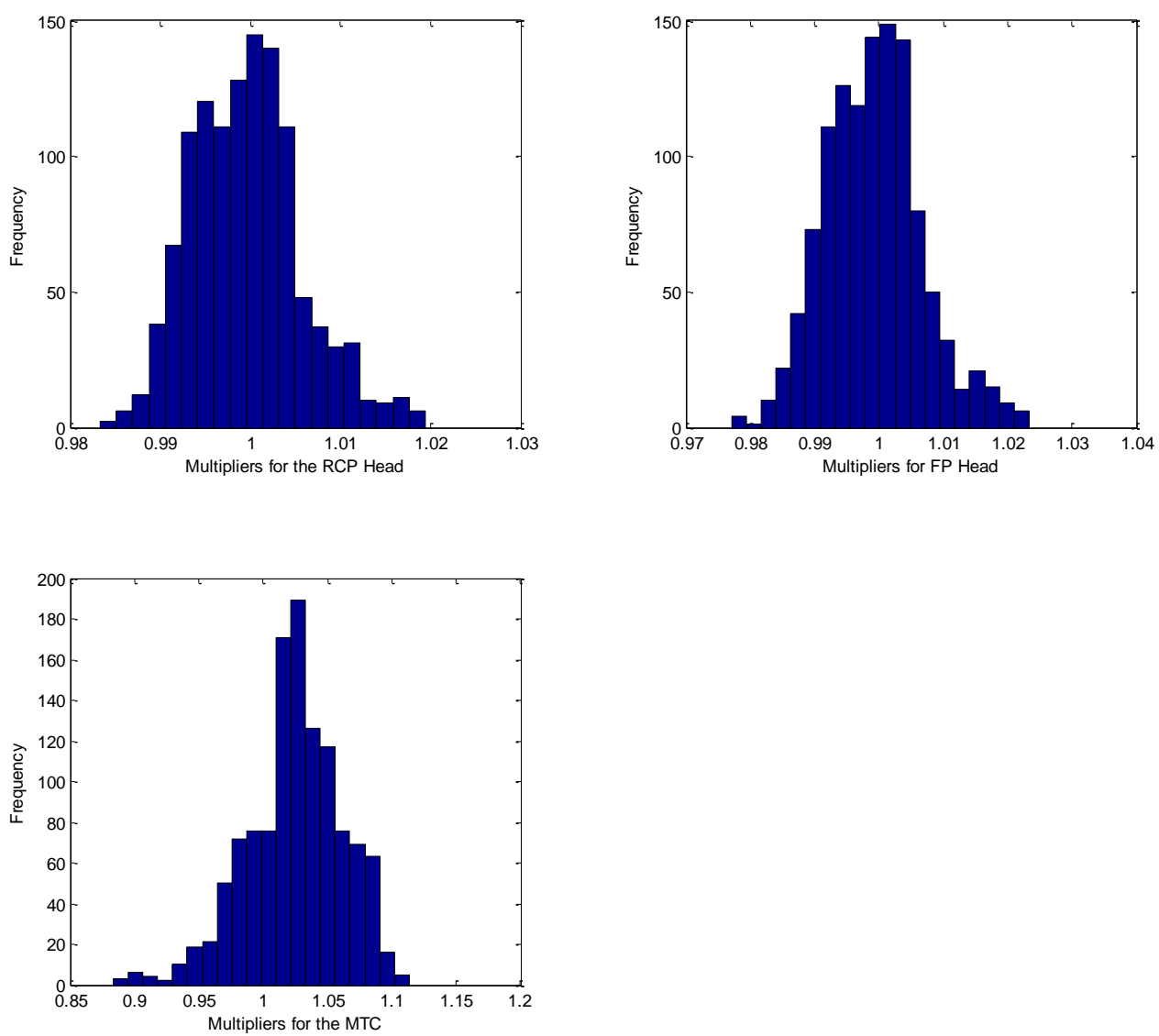

Figure 7-14: A posteriori distribution of the parameters using RCP Trip at 30\% Power (Cont.) 

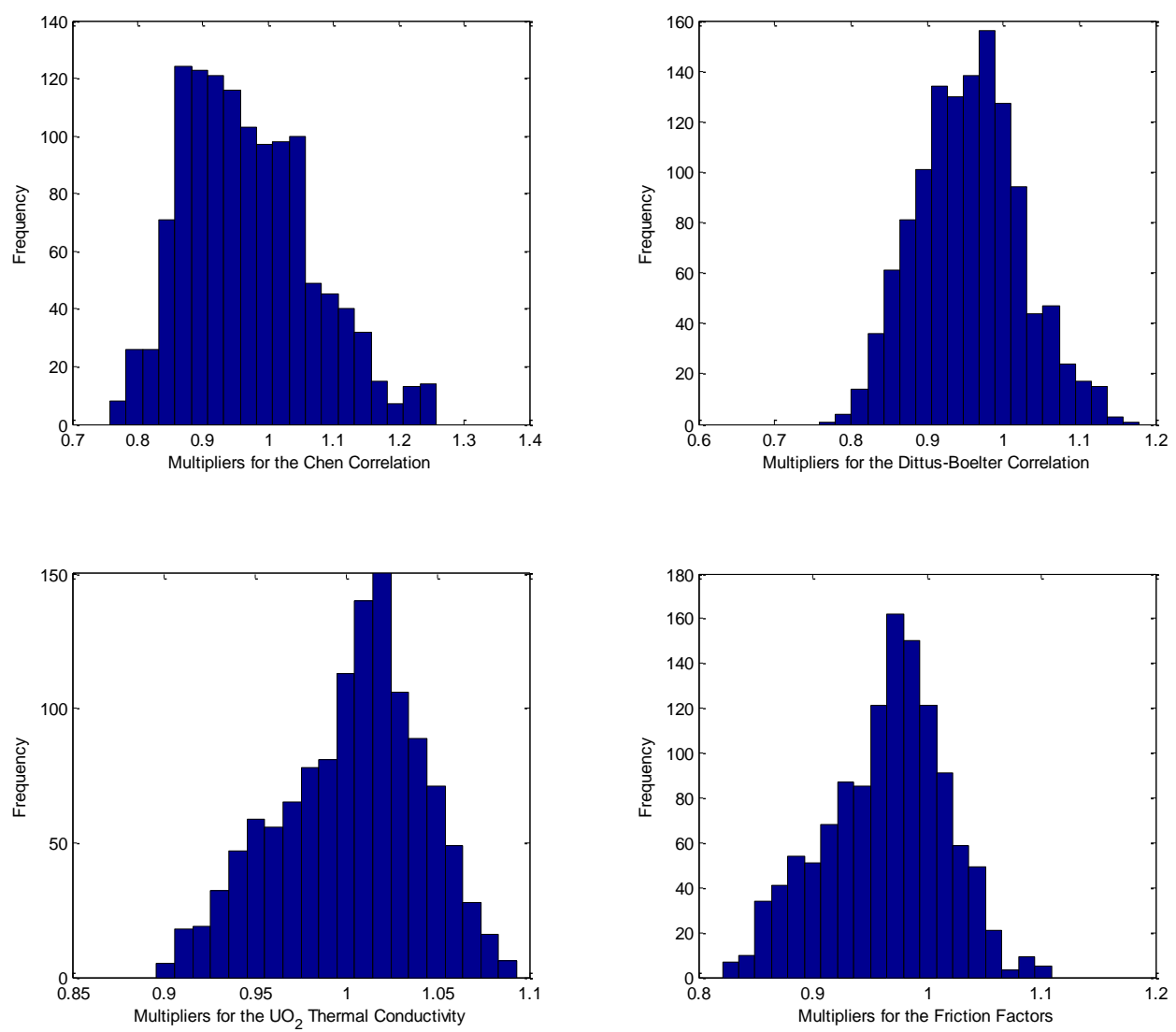

Figure 7-15: A posteriori distribution of the parameters using RCP Trip at $80 \%$ Power 

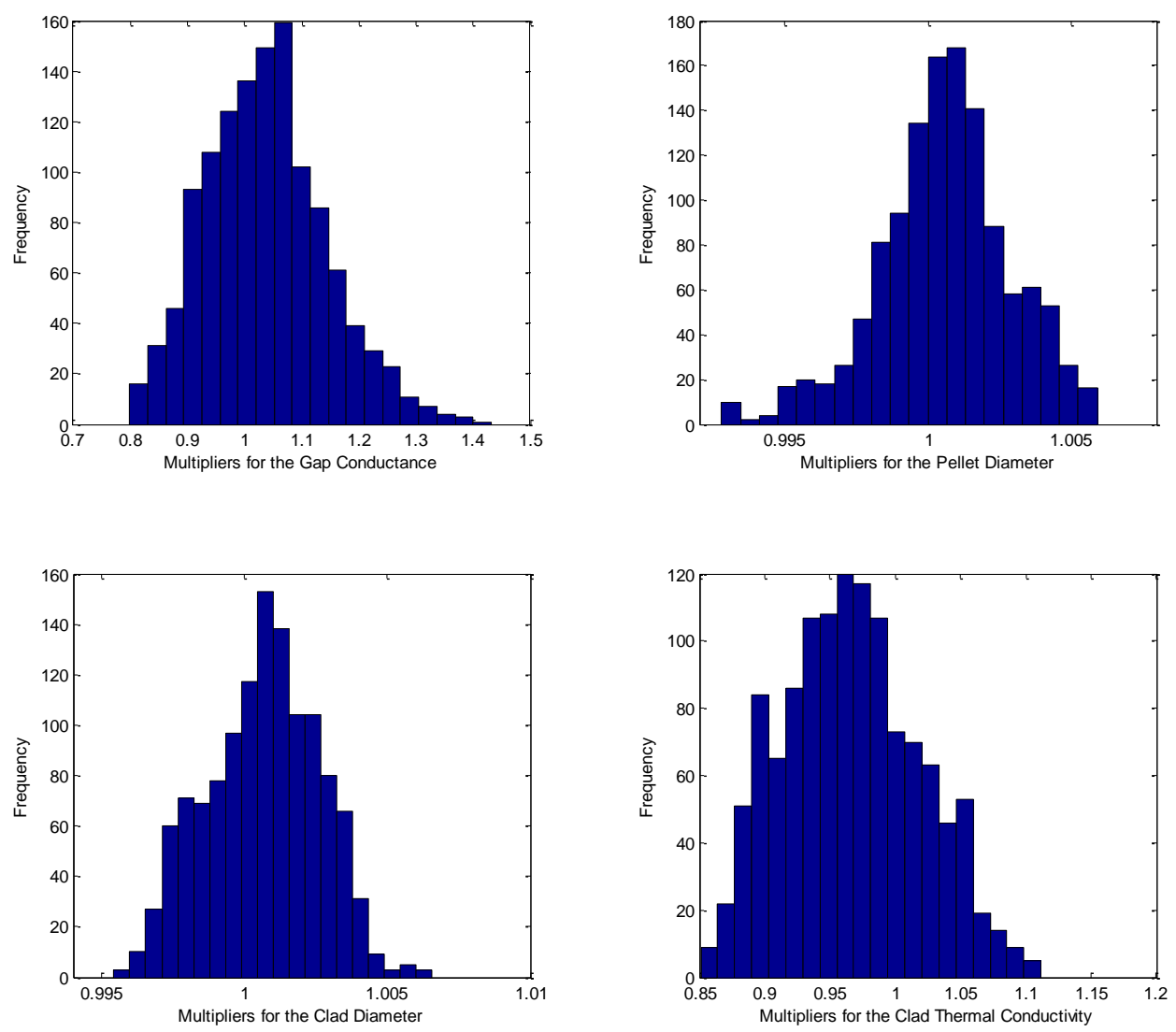

Figure 7-16: A posteriori distribution of the parameters using RCP Trip at $80 \%$ Power (Cont.) 

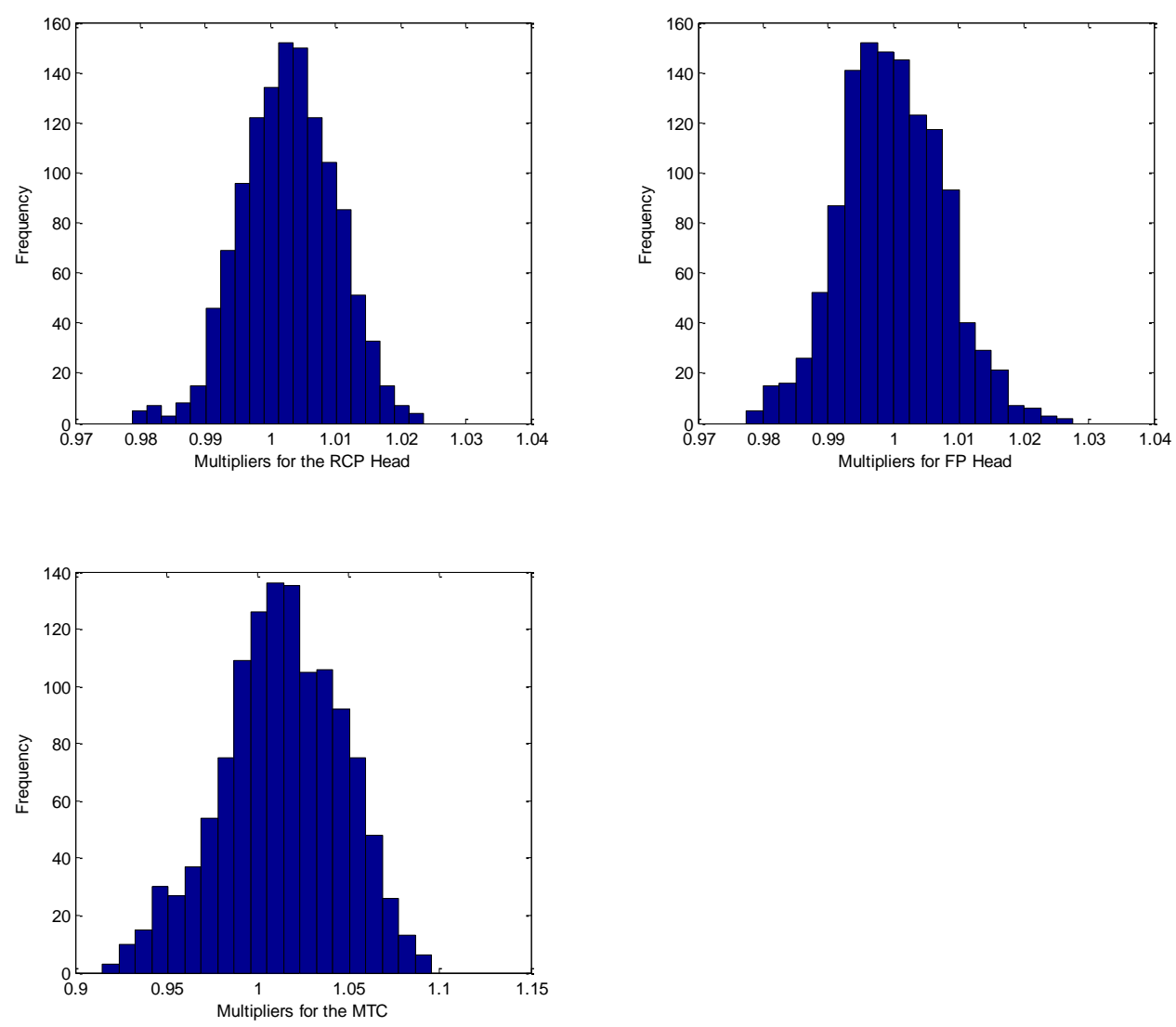

Figure 7-17: A posteriori distribution of the parameters using RCP Trip at $80 \%$ Power (Cont.)

\subsection{Design Optimization}

To solve the optimization problem whose decision variables are binary, one could simply enumerate all of the possible solutions and then choose the best one. This will be possible for very small problems, but it rapidly becomes unworkable for even medium size problems. If the size of the problem is large enough, Equation (2.45) can be solved by possible either a branchand-bound linear programming method or Monte Carlo simulation, e.g. Simulated Annealing, which approach to utilize dependent upon the linearity of the objective function. For the design optimization of the IRIS reactor, a binary $0 / 1$ problem that has 4 experiments and 8 instruments was considered. This will have a maximum of $2^{12}=4096$ solutions to enumerate. The total number of scenarios for performing experiments is $2^{4}-1=15$ since four experiments are 
considered. In addition there are $2^{8}-1=255$ ways to deploy the instruments. This gives $15 \times 255=3825$ solutions to enumerate.

Now consider the computational burden. The number of IRIS simulations is only equal to the total number of experiments, which is 4 . The number of data assimilations is equal to the number of solutions to enumerate, which is 3825. Neither the IRIS simulations or data assimilations presents an undue computational burden. Where the computational burden becomes excessive is in the IRIS simulations required to optimize the IRIS design to take advantage of the increased design freedom achieved by the reduction in uncertainties. Each optimization in general will require a number of IRIS simulations if a stochastic versus deterministic optimization must be employed. Given that the number of IRIS optimizations is equal to the number of solutions to enumerate, the total number of IRIS simulations could approach 250,000 for the case being considered. This may be tractable on a HPC but certainly is beyond the capabilities of current generation PCs unless the IRIS simulation model employed to perform the optimization is very simple.

For the purpose of illustration, a simple problem with one limiting attribute was addressed. It was also assumed that the a posteriori uncertainty of the optimized IRIS design is unchanged from its value for the original design. The IRIS optimization problem was not solved; instead, it was assumed the IRIS cost savings was proportional to the margin reduction. To obtain cost values, the following approach was used for this illustrative example. The algorithm is then simply:

1. Obtain 255 scenarios which determine the instrument deployment.

2. Solve 15 sub-problems for each scenario to determine experiments that will be performed.

One begins by setting the costs equal to the half of the savings.

$$
C_{T}=\bar{C}_{i}^{\mathrm{T}} \bar{l}_{c}+\bar{C}_{e}^{\mathrm{T}} \overline{\mathrm{X}}_{c}=\frac{1}{2} \mu_{a}^{v}\left(\bar{l}_{c}, \overline{\mathrm{x}}_{c}\right) \delta a^{v}\left(\bar{l}_{c}, \overline{\mathrm{x}}_{c}\right)
$$

and substitute an arbitrary number into $\mu_{a}^{v}\left(\bar{l}_{c}, \overline{\mathbf{x}}_{c}\right)$, where $\bar{l}_{c}$ and $\overline{\mathrm{x}}_{c}$ are the complete set of $\bar{l}$ and $\overline{\mathrm{x}}$, i.e. $\bar{l}_{c}=11 \ldots 1^{\mathrm{T}}$ and $\overline{\mathrm{x}}_{c}=1111^{\mathrm{T}}$, respectively. This means that if one uses all the instruments and performs all the experiments, the savings equals to twice the cost. One also sets 
$\bar{C}_{i}=\frac{1}{16} \bar{C}_{i}^{\mathrm{T}} \bar{l}_{c}+\bar{C}_{e}^{\mathrm{T}} \overline{\mathrm{X}}_{c}$ for $j=1, \ldots, 8$ and $\bar{C}_{e}=\frac{1}{8} \bar{C}_{i}^{\mathrm{T}} \bar{l}_{c}+\bar{C}_{e}^{\mathrm{T}} \overline{\mathrm{X}}_{c}$ for $n=1, \ldots, 4$ which is totally arbitrary. Having cost values for this simplified problem, all solutions were enumerated.

The savings were calculated using each scenario set, i.e. \{instrument set, experiment set\}. The first instrument set is $\bar{l}_{1}=00 \ldots 0^{\mathrm{T}}$ and the last instrument set is $\bar{l}_{256}=11 \ldots 1^{\mathrm{T}}$. Likewise the first experiment set is $\overline{\mathrm{x}}_{1}=0000^{\mathrm{T}}$ and the last experiment set is $\overline{\mathrm{x}}_{16}=111^{\mathrm{T}}$. The first scenario set is thus $\left\{\bar{l}_{1}, \overline{\mathrm{x}}_{1}\right\}$ and the second scenario set is $\left\{\bar{l}_{1}, \overline{\mathrm{x}}_{2}\right\}$ and so on. Figure 7-18 indicates the optimum solution which maximizes savings is $\left\{\bar{l}_{162}, \overline{\mathrm{x}}_{4}\right\}$, where $\bar{l}_{162}=$ \{Neutron Power, Core Flow Rate, Steam Pressure $\}$ and $\bar{x}_{4}=\{$ FCV Failed Open, TCV Failed Open\}. As can be seen in Figure 30, there are a number of other \{instrument, experiment combinations nearly with the same savings. Given the simplicity of this illustrative problem, the results should not be assumed to be consistent with what a more sophisticated treatment would render. The work on \{instrument, experiment \} optimum combination is continuing addressing a more realistic problem, with results to be published separate from this report.

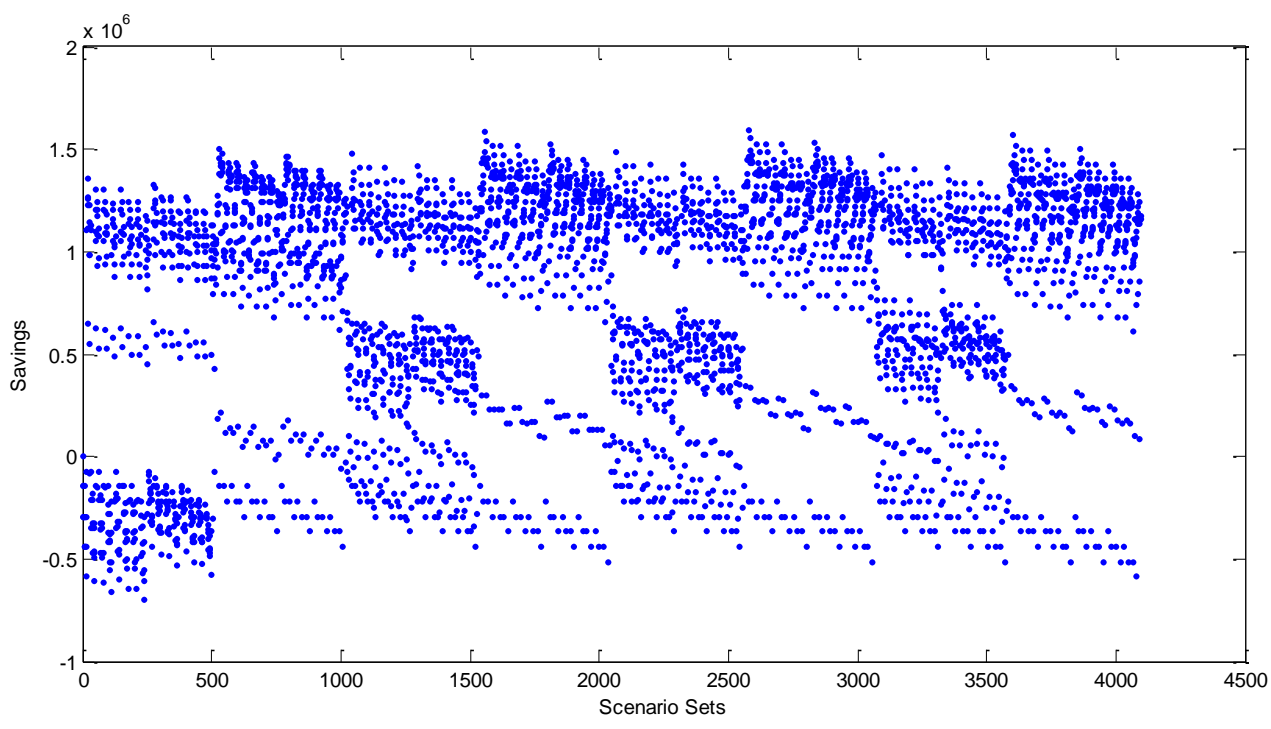

Figure 7-18: Design Savings for Each Scenario 


\subsection{Uncertainty Analysis for Reactor Control}

The $10 \%$ step load changes at $70 \%$ and $90 \%$ power, and $20 \%$ ramp load changes at $70 \%$ power with ramp rate of $15 \% / \mathrm{min}$ [Ouzts, 1972] were simulated with different gains. The following five observables were used to calculate the deviation from the desired state:

1. Neutron Power

2. Reactor Thermal Output

3. Feed Flow Rate per Steam Generator

4. Steam Flow Rate per Steam Generator

5. Turbine Output

Four different algorithms are created by modifying gains and/or reactor control parameters as follows:

1. Algorithm F1Q1:

$\mathrm{FCV}$ gains $=\{0,0.05,0.07\}$

Minimum Rod Speed = 3.75 inches $/ \mathrm{min}$

2. Algorithm F2Q1:

$\mathrm{FCV}$ gains $=\{0,0.01,0.014\}$

Minimum Rod Speed = 3.75 inches $/ \mathrm{min}$

3. Algorithm F3Q1:

$\mathrm{FCV}$ gains $=\{0,0.0025,0.0035\}$

Minimum Rod Speed $=3.75$ inches $/ \mathrm{min}$

4. Algorithm F1Q2:

$\mathrm{FCV}$ gains $=\{0,0.05,0.07\}$

Minimum Rod Speed $=0.75$ inches $/ \mathrm{min}$

Table 7-3 and Table 7-4 present the deviations and the operational constraint probabilities, respectively, for the $10 \%$ step load changes. Table 7-4 shows that as FCV gains increase it generates larger deviations for the primary side observables, but smaller deviations for the secondary side responses. This is because wild feed control which results from the large FCV gains leads the primary side responses to overshoot while the secondary side responses usually follow the feed flow rate (Figure 7-19 and Figure 7-20). In order to reduce the size of overshoot, minimum rod speed was modified, denoted as Algorithm F1Q2, and uncertainty analysis was performed. The results for this analysis are also presented in Table 7-3 and Table 7-4 for 
comparison, which indicates that substantial reductions in the deviations on the responses can be achieved through reducing the minimum rod speed. The deviations and the operational constraint probabilities for the $20 \%$ ramp changes are presented in

Table 7-5 and

Table 7-6. They show a similar behavior to the $10 \%$ step load change. The optimum control algorithm in terms of the deviations is F1Q2 as well. The safety constraint was also calculated and it was confirmed that the system attributes does not exceed the safety limit.

Table 7-3: Deviations for 10\% Step Load Changes at 70\% and 90\% Power using Four Different Control Algorithms

\begin{tabular}{|c|c|c|}
\hline Initial Power & Algorithm Index & Deviations \\
\hline \multirow{3}{*}{$70 \%$} & F1Q1 & 0.008850 \\
\cline { 2 - 3 } & F2Q1 & 0.009770 \\
\cline { 2 - 3 } & F3Q1 & 0.012535 \\
\cline { 2 - 3 } & F1Q2 & 0.007013 \\
\hline \multirow{3}{*}{$90 \%$} & F1Q1 & 0.007210 \\
\cline { 2 - 3 } & F2Q1 & 0.006697 \\
\cline { 2 - 3 } & F3Q1 & 0.015535 \\
\cline { 2 - 3 } & F1Q2 & 0.006327 \\
\hline
\end{tabular}

Table 7-4: Operational Constraint Probabilities for 10\% Step Load Changes at $70 \%$ and $90 \%$ Power using Four Different Control Algorithms

\begin{tabular}{|c|c|c|c|c|c|c|}
\hline \multicolumn{2}{|c|}{ Observable Index } & 1 & 2 & 3 & 4 & 5 \\
\hline \multicolumn{2}{|c|}{$\Delta r_{j}$} & $50 \mathrm{MWt}$ & $50 \mathrm{MWt}$ & $\begin{array}{c}30000 \\
\mathrm{lbm} / \mathrm{hr}\end{array}$ & $\begin{array}{c}30000 \\
\mathrm{lbm} / \mathrm{hr}\end{array}$ & $12 \mathrm{MW}$ \\
\hline $\begin{array}{l}\text { Initial } \\
\text { Power }\end{array}$ & $\begin{array}{l}\text { Algorithm } \\
\text { Index }\end{array}$ & \multicolumn{5}{|c|}{ Operational Constraint Probabilities } \\
\hline \multirow{4}{*}{$70 \%$} & F1Q1 & 0.6972 & 0.6899 & 0.9808 & 0.9419 & 0.8377 \\
\hline & F2Q1 & 0.7234 & 0.7176 & 0.9473 & 0.9085 & 0.7401 \\
\hline & F3Q1 & 0.7617 & 0.7561 & 0.8314 & 0.7929 & 0.6128 \\
\hline & F1Q2 & 0.8198 & 0.8145 & 0.9809 & 0.9431 & 0.8724 \\
\hline \multirow{4}{*}{$90 \%$} & F1Q1 & 0.7164 & 0.7093 & 0.9592 & 0.9079 & 0.7317 \\
\hline & F2Q1 & 0.8198 & 0.8116 & 0.8941 & 0.8562 & 0.6664 \\
\hline & F3Q1 & 0.6430 & 0.5808 & 0.6487 & 0.6287 & 0.2492 \\
\hline & F1Q2 & 0.7883 & 0.7899 & 0.9580 & 0.9141 & 0.7546 \\
\hline
\end{tabular}


Table 7-5: Deviations for 20\% Ramp Load Changes at 70\% Power using Four Different Control Algorithms

\begin{tabular}{|c|c|c|}
\hline Initial Power & Algorithm Index & Deviations \\
\hline \multirow{3}{*}{$70 \%$} & F1Q1 & 0.009739 \\
\cline { 2 - 3 } & F2Q1 & 0.013422 \\
\cline { 2 - 3 } & F3Q1 & 0.021938 \\
\cline { 2 - 3 } & F1Q2 & 0.007763 \\
\hline
\end{tabular}

Table 7-6: Operational Constraint Probabilities for 20\% Ramp Load Changes at 70\% Power using Four Different Control Algorithms

\begin{tabular}{|c|c|c|c|c|c|c|}
\hline \multicolumn{2}{|c|}{ Observable Index } & 1 & 2 & 3 & 4 & 5 \\
\hline \multicolumn{2}{|c|}{$\Delta r_{j}$} & $50 \mathrm{MWt}$ & $50 \mathrm{MWt}$ & $\begin{array}{c}30000 \\
\mathrm{lbm} / \mathrm{hr}\end{array}$ & $\begin{array}{c}30000 \\
\mathrm{lbm} / \mathrm{hr}\end{array}$ & $12 \mathrm{MW}$ \\
\hline $\begin{array}{c}\text { Initial } \\
\text { Power }\end{array}$ & $\begin{array}{c}\text { Algorithm } \\
\text { Index }\end{array}$ & \multicolumn{5}{|c|}{ Operational Constraint Probability } \\
\hline \multirow{4}{*}{$70 \%$} & F1Q1 & 0.6163 & 0.6299 & 0.9906 & 0.9633 & 0.6719 \\
\cline { 2 - 7 } & F2Q1 & 0.5420 & 0.5375 & 0.9310 & 0.8185 & 0.5064 \\
\cline { 2 - 7 } & F3Q1 & 0.6689 & 0.6333 & 0.6231 & 0.6113 & 0.4076 \\
\cline { 2 - 7 } & F1Q2 & 0.7269 & 0.7211 & 0.9899 & 0.9643 & 0.7313 \\
\hline
\end{tabular}



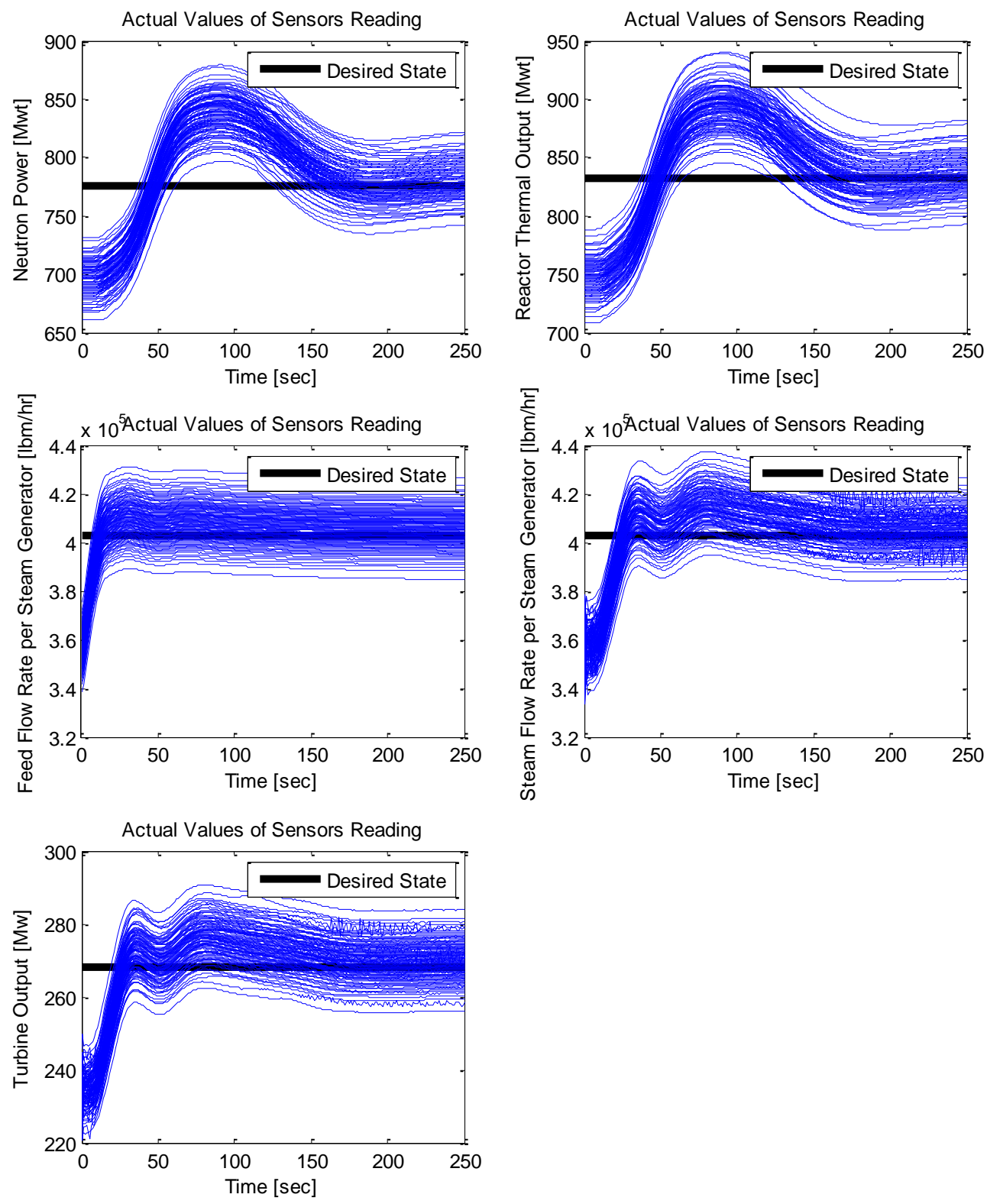

Figure 7-19: System Responses obtained Simulating 100 Samples for 10\% Step Load Changes at 70\% Power using Control Algorithm F1Q1 for Uncertainty Analysis 

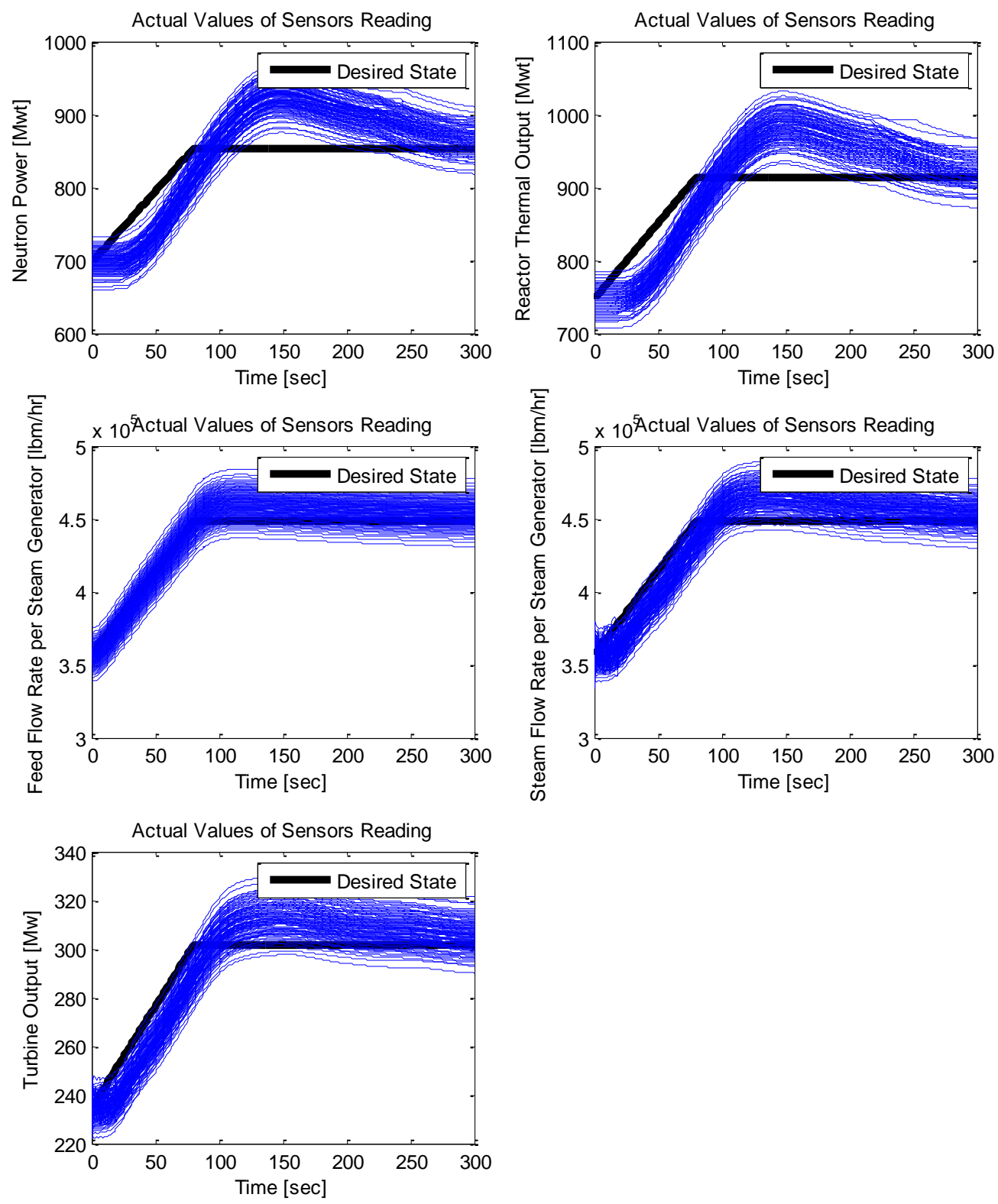

Figure 7-20: System Responses obtained Simulating 100 Samples for 20\% Ramp Load Changes at 70\% Power using Control Algorithm F1Q1 for Uncertainty Analysis 


\section{CONCLUDING REMARKS AND FUTURE WORK}

An effective improvement in the ability to respond to severe grid faults has been demonstrated with the IRIS SMR equipped with fast valving. A great deal of future work remains for this investigation. State of the art non-linear controllers have great applicability to the electrical transmission network and a strong case can be made for application to the steam cycle and the plant control itself.

While exclusive IV closing is usually agreed to be the most efficient method of fast

valving for nuclear units, Turbine bypass valving is another option that offers the advantage of instantly increasing the condensing rate so that later swings are less severe. Using a diverse combination of stability aids may allow a SMR to play a key role in responding to severe load swings and grid events; abilities which are gaining a greater premium through increasing penetration of renewable power and smart grid technology development.

Thermal hydraulic parameters were adjusted for both mildly nonlinear and highly nonlinear transients, and their a posteriori parameter uncertainties were propagated through the simulation model to predict a posteriori uncertainties of the key system attributes. In order to accomplish this, the Bayesian approach modified by regularization is used to incorporate available information in quantifying uncertainties for the IRIS reactor system. The a priori information considered are the parameters and the experimental data together with their uncertainties. To determine the experimental data which do not exist, a virtual experiment model was developed and the following four experiments were simulated using the IRIS system simulation code: RCP Trip at 80\% Power, Control Bank Withdrawal at $70 \%$ Power, Feed Control Valve Failed Open at 15\% Power, and Turbine Control Valve Failed Open at 15\% Power. Each parameter's uncertainty contribution to the system attributes was also calculated to identify major sources of uncertainty. Based on the reduced a posterior system attribute uncertainty, an optimization problem was formulated to maximize design saving, while maintaining safety margin. Finally the uncertainty analysis was conducted for reactor control problems by formulating an objective function together with the safety constraint and the operational constraint. The optimum control algorithm was identified by creating and testing multiple control algorithms. 
The results indicate that substantial reductions in uncertainties on the system attributes can be achieved with experimental data through refining input parameter distributions. Some of the adapted parameters are not in good agreement with their known values, i.e. the virtual experiment model parameters, if those parameters affect the observables in a similar way and/or if they produce small sensitivities. Also, due to the input parameters that have highly correlated response sensitivities, it is shown that the errors can increase as more experimental data are added. However the uncertainties propagated from a posteriori parameter distributions will be correct as long as those parameters generate similar responses for the system attributes during accident transients. 


\section{REFERENCES}

Aanstad, O.J. and Lokay H.E., "Fast Valve Control Can Improve Turbine-Generator Response to Transient Disturbances”, Atompraxis, No. 16, pp. 403-406, 1970.

Abdel-Khalik, H.S., "Adaptive Core Simulation," Doctoral Dissertation, North Carolina State University, 2004

Anderson, P. and A. Fouad, Power Systems Control and Stability, New York: Wiley (2003).

BICKEL, J., "Grid Stability and Safety Issues Associated with Nuclear Power Plants",

Paper prepared for the Workshop on Power Grid Interconnection in Northeast Asia, Beijing, China 2001.

Boyack, B.E., et al., "Quantifying Reactor Safety Margins, Parts 1-6," Nuclear Engineering and Design, 119, 1, 1990

Cacuci, D.G., Sensitivity and Uncertainty Analysis, Volume I, CRC Press, 2000

Cacuci, D.G. and M. Ionescu-Bujor, "Best-Estimate Model Calibration and Prediction Through Experimental Data Assimilation - I: Mathematical Framework," Nuclear Science and Engineering, 165, 18-44, 2010

Carelli, M.D., “IRIS: Final Technical Progress Report,” Westinghouse Electric Co., STDES-03-40 (2003)

Duke Energy Company McGuire Nuclear Station, "Updated Final Safety Analysis Report," Rev 13, May 2007

De Crecy, A., et al., "BEMUSE Phase III Report - Uncertainty and Sensitivity analysis of the LOFT L2-5 Test,” OECD/CSNI Report NEA/CSNI/R(2007)4, 2007

D'Auria, F., et al., "Outline of the Uncertainty Methodology based on Accuracy Extrapolation,” J. Nuclear Technology, 109, 1, 21-38, 1995

Engl, H.W. and W. Grever, "Using the L-Curve for Determining Optimal Regularization Parameters," Numerische Mathematik, 69, 25, 1994

Frepoli, C., et al., "Realistic Large Break LOCA Analysis of AP1000 with ASTRUM," Proc. 6th International Conference on Nuclear Thermal Hydraulics, Operations and Safety (NUTHOS-6), Nara, Japan, Atomic Energy Society of Japan, 2004 
Gelman, A., et al., "Efficient Metropolis jumping rules," Bayesian Statistics, 5, 599-607, Oxford University Press, 1996

Hagrman, D.L., MATPRO - Version 11, A handbook of Materials Properties for Use in the Analysis of Light Water Reactor Fuel Rod Behavior, EG\&G Idaho, Inc., NUREG/CR-0497, February 1979

Hanson, K.M., et al., "Posterior Sampling with Improved Efficiency,” Proc. SPIE 3338, 371-382, 1998

Higdon, D., et al., "Combining Field Data and Computer Simulation for Calibration and Prediction,” SIAM J. Sci. Comput. Vol. 26, No. 2, pp. 448-466, 2004

Hines, J.W., et al., "Advanced Instrumentation and Control Methods for Small and Medium Reactors with IRIS Demonstration,” DE-FG07-07ID14895/UTNE/2008 -4, October 2008.

Hines, J.W., et al., "Advanced Instrumentation and Control Methods for Small and Medium Reactors with IRIS Demonstration,” DE-FG07-07ID14895/UTNE/2009 -4, October 2009.

Hines, J.W., et al., "Advanced Instrumentation and Control Methods for Small and Medium Reactors with IRIS Demonstration,” DE-FG07-07ID14895/UTNE/2010 -1, January 2010.

International Atomic Energy Agency, "Interaction of Grid Characteristics with Design and Performance of Nuclear Power Plants, a Guidebook", Technical Report Series No. 224, Vienna, 1983.

International Atomic Energy Agency, "Introducing Nuclear Power Plants into Electrical Power Systems of Limited Capacity: Problems and Remedial Measures", Technical Report Series No. 271, Vienna, 1987.

Jessee, M.A., "Cross-Section Adjustment Techniques for BWR Adaptive Simulation," Doctoral Dissertation, North Carolina State University, 2008

Kirby, B., et. al., "Nuclear Generating Stations and Transmission Grid Reliability", 39" North American Power Symposium, 2007.

Muller, P., “A Generic Approach to Posterior Integration and Gibbs Sampling,” 1993 
Mynatt, F., et. al., "Design and Layout Concepts for Compact, Factory-Produced Transportable, Generation IV Reactor Systems", NERI Grant Number DE-FG0700SF22168 Final Report, November 12, 2003.

Ouzts, J.E., "Plant Startup Test Report - H. B. Robinson Unit No. 2, Volume I and II," Westinghouse Electric Corporation, WCAP-7844, January 1972

Patel, R., T.S. Bhatti. and D.P. Kothari, "Improvement of Power System Transient Stability Using Fast Valving: A Review", Electric Power Components and Systems, Vol. 29, No. 10, pp. 927-937, 2009.

Petrovic, M. and W. Riess, Off-design flow analysis of low-pressure steam turbines, Proc Institution of Mechanical Engineers Vol. 211 Part A, Pages 215-224.

Petrovic, G., et al., "Instrumentation Needs for Integral Primary System Reactors (IPSRs)," Westinghouse Electric Company LLC, DE-FC07-05ID14690, September 2005

RELAP5/MOD3 Code Manual Volume IV: Models and Correlations (Draft), EG\&G Idaho, Inc., Idaho Falls, Idaho 83415, June 1990

Rominger, A.S. and J.M. Doster, "An IRIS Reactor to Grid Model”, Transactions of the American Nuclear Society, Vol. 101, pp. 595-597, 2009.

Schobeiri, M. Turbomachinery Flow Physics and Dynamic Performance, SpringerVerlag Verlin Heidelberg: Springer (2005).

Shankar, G., "Simulation Model of a Nuclear Reactor Turbine", Nuclear Engineering and Design, Vol. 44, No. 2, 1977.

Shen, H., "Advanced Feedwater Control for Next Generation Nuclear Power Systems," Ph.D. Dissertation, North Carolina State University, 2006.

Shen, H. and J.M. Doster, "Application of a neural network based feedwater controller to helical steam generators", Nuclear Engineering and Design, 239, 1056 (2009).

Tarantola, A., Inverse Problem Theory and Methods for Model Parameter Estimation, Society for Industrial and Applied Mathematics, Philadelphia, 2005

Tikhonov, A.N., Numerical Methods for the Solution of Ill-Posed Problems, Kluwer Academic Publishers, 1995

Todreas, N.E. and M.S. Kazimi, Nuclear Systems, New York: Hemisphere Pub. Corp., 1990. 
Westinghouse Electric Company LLC, "IRIS Preliminary Safety Assessment, Volume I," WCAP-16082-NP, July 2003

Yim, M.S. and Christenson, JM, "Application of Optimal Control Theory to a LoadFollowing Pressurized Water Reactor", Nuclear Technology, Vol. 100, 1992. 


\section{Appendix A. Additional Figures and Results}

Table 0-1: Virtual Experiment Values and Input Parameters Adapted for Experiment B

\begin{tabular}{|c|c|c|c|c|}
\hline \multirow{2}{*}{ Index } & Parameter & \multicolumn{2}{|c|}{ Relative Parameter Values } & \multirow{2}{*}{ Error } \\
\cline { 3 - 4 } & & $\begin{array}{c}\text { Virtual } \\
\text { Experiment } \\
\text { Model }\end{array}$ & A Posteriori & \\
\hline 1 & Chen Correlation & 0.931916448 & 1.053354907 & 1.111678674 \\
\hline 2 & Dittus-Boelter Correlation & 1.157408173 & 0.99905115 & 1.71824379 \\
\hline 3 & $\mathrm{UO}_{2}$ Thermal Conductivity & 0.974167543 & 1.031411167 & 1.294815608 \\
\hline 4 & Friction Factors & 1.061389993 & 1.021863229 & 0.963023336 \\
\hline 5 & Gap Conductance & 1.253420122 & 1.203582807 & 0.653443016 \\
\hline 6 & Pellet Diameter & 0.995822099 & 1.000070991 & 2.125072132 \\
\hline 7 & Clad Diameter & 1.005899091 & 1.00334465 & 1.678090285 \\
\hline 8 & Clad Thermal Conductivity & 1.081842135 & 1.002905555 & 1.314634494 \\
\hline 9 & Pump Head & 1.010500679 & 1.010349603 & 0.048116558 \\
\hline 10 & MTC & 0.961641496 & 0.947026849 & 0.545035714 \\
\hline
\end{tabular}

Table 0-2: Virtual Experiment Values and Input Parameters Adapted for Experiment C

\begin{tabular}{|c|c|c|c|c|}
\hline \multirow{2}{*}{ Index } & Parameter & \multicolumn{2}{|c|}{ Relative Parameter Values } & \multirow{2}{*}{ Error } \\
\cline { 3 - 4 } & & $\begin{array}{c}\text { Virtual } \\
\text { Experiment } \\
\text { Model }\end{array}$ & A Posteriori & \\
\hline 1 & Chen Correlation & 0.931916448 & 1.102411719 & 2.384703664 \\
\hline 2 & Dittus-Boelter Correlation & 1.157408173 & 1.050411901 & 1.458002925 \\
\hline 3 & $\mathrm{UO}_{2}$ Thermal Conductivity & 0.974167543 & 0.980325765 & 0.129547392 \\
\hline 4 & Friction Factors & 1.061389993 & 1.031049652 & 0.787225555 \\
\hline 5 & Gap Conductance & 1.253420122 & 1.073209678 & 1.310304105 \\
\hline 6 & Pellet Diameter & 0.995822099 & 1.00011336 & 2.165726652 \\
\hline 7 & Clad Diameter & 1.005899091 & 1.004639245 & 0.898778351 \\
\hline 8 & Clad Thermal Conductivity & 1.081842135 & 1.002611908 & 1.343794262 \\
\hline 9 & Pump Head & 1.010500679 & 1.011085625 & 0.162505061 \\
\hline 10 & MTC & 0.961641496 & 0.987027606 & 1.789849236 \\
\hline
\end{tabular}

Table 0-3: Virtual Experiment Values and Input Parameters Adapted for Experiment D

\begin{tabular}{|l|l|c|c|c|}
\hline \multirow{2}{*}{ Index } & \multirow{2}{*}{ Parameter } & \multicolumn{2}{|c|}{ Relative Parameter Values } & \multirow{2}{*}{ Error } \\
\cline { 3 - 4 } & $\begin{array}{c}\text { Virtual } \\
\text { Experiment } \\
\text { Model }\end{array}$ & A Posteriori & \\
\hline
\end{tabular}




\begin{tabular}{|c|c|c|c|c|}
\hline 1 & Chen Correlation & 0.931916448 & 1.040895622 & 2.510140746 \\
\hline 2 & Dittus-Boelter Correlation & 1.157408173 & 1.030214805 & 3.532199091 \\
\hline 3 & $\mathrm{UO}_{2}$ Thermal Conductivity & 0.974167543 & 1.017526037 & 1.068096784 \\
\hline 4 & Friction Factors & 1.061389993 & 1.034596433 & 0.76909746 \\
\hline 5 & Gap Conductance & 1.253420122 & 1.190837916 & 0.695937988 \\
\hline 6 & Pellet Diameter & 0.995822099 & 1.000065947 & 2.196746966 \\
\hline 7 & Clad Diameter & 1.005899091 & 1.004139229 & 1.417847299 \\
\hline 8 & Clad Thermal Conductivity & 1.081842135 & 1.004909702 & 1.354266562 \\
\hline 9 & Pump Head & 1.010500679 & 1.010562695 & 0.026657208 \\
\hline 10 & MTC & 0.961641496 & 0.967145026 & 0.491858142 \\
\hline
\end{tabular}

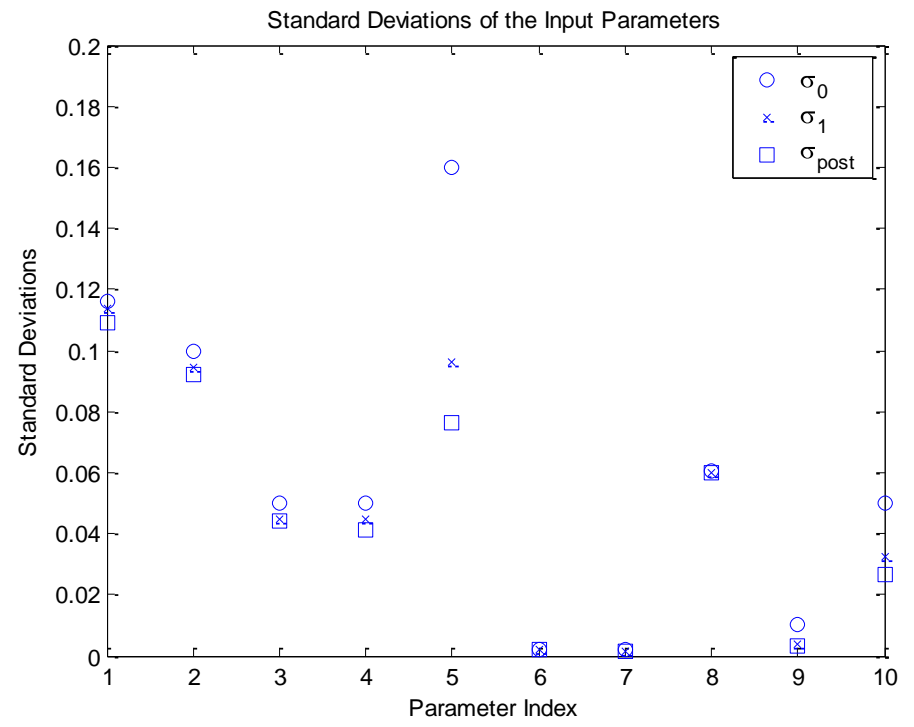

Figure 0-1: Standard Deviations for the Input Parameters for Experiment B 


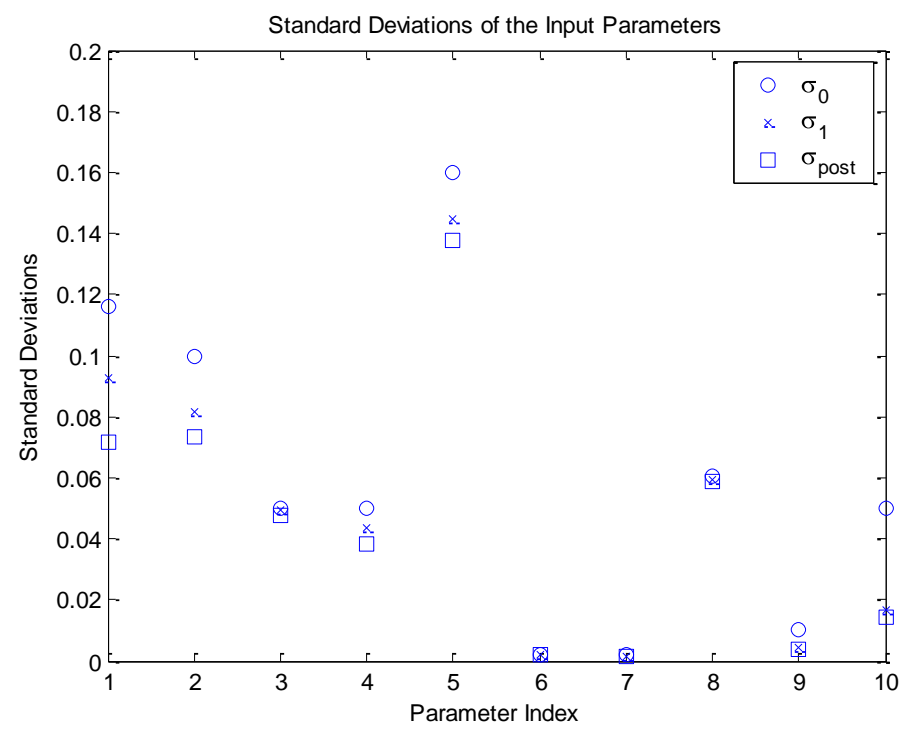

Figure 0-2: Standard Deviations for the Input Parameters for Experiment C

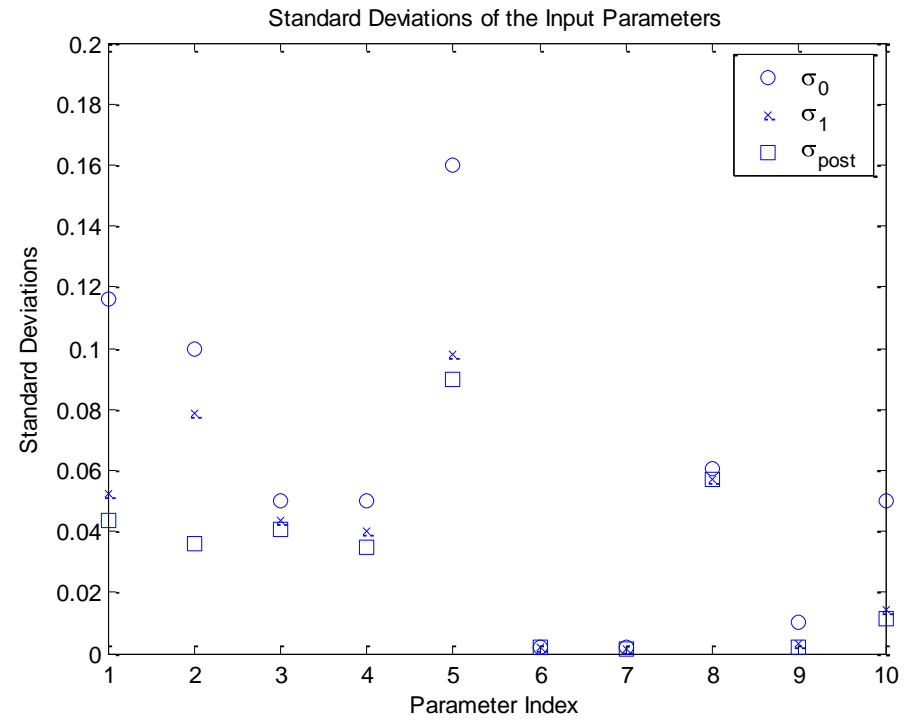

Figure 0-3: Standard Deviations for the Input Parameters for Experiment D 


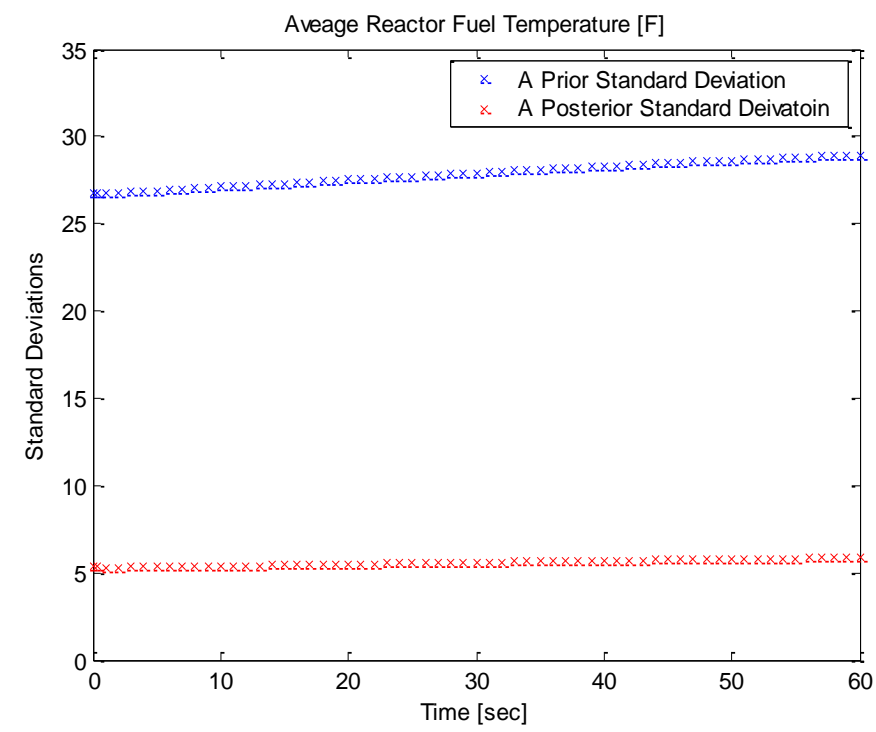

Figure 0-4: Standard Deviations for Average Fuel Temperature for Control Bank Withdrawal using Experiment B for Data Assimilation

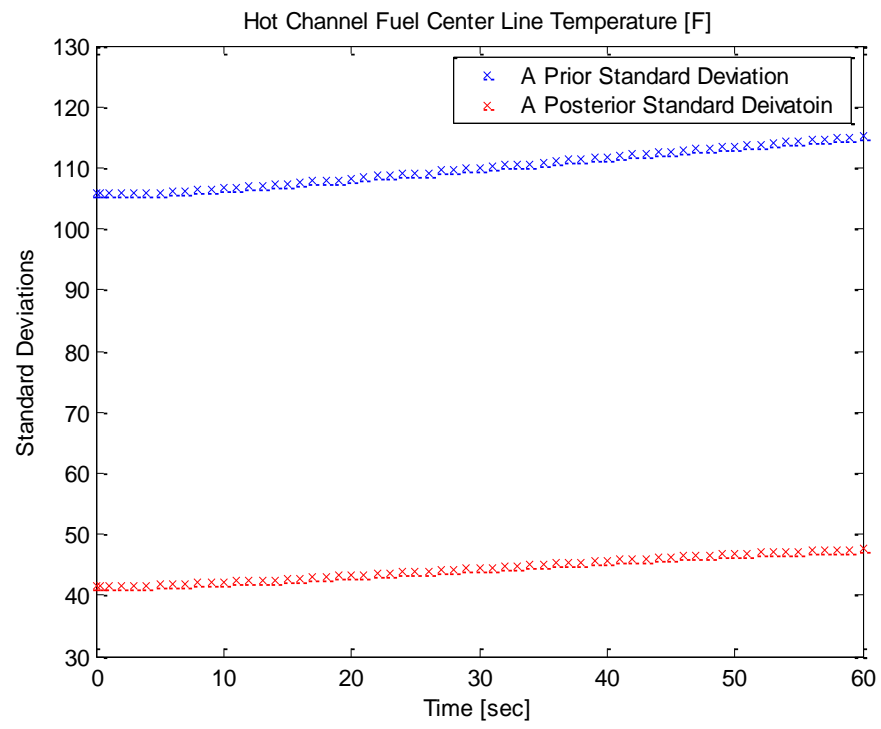

Figure 0-5: Standard Deviations for Hot Channel Fuel Centerline Temperature for Control Bank Withdrawal using Experiment B for Data Assimilation 


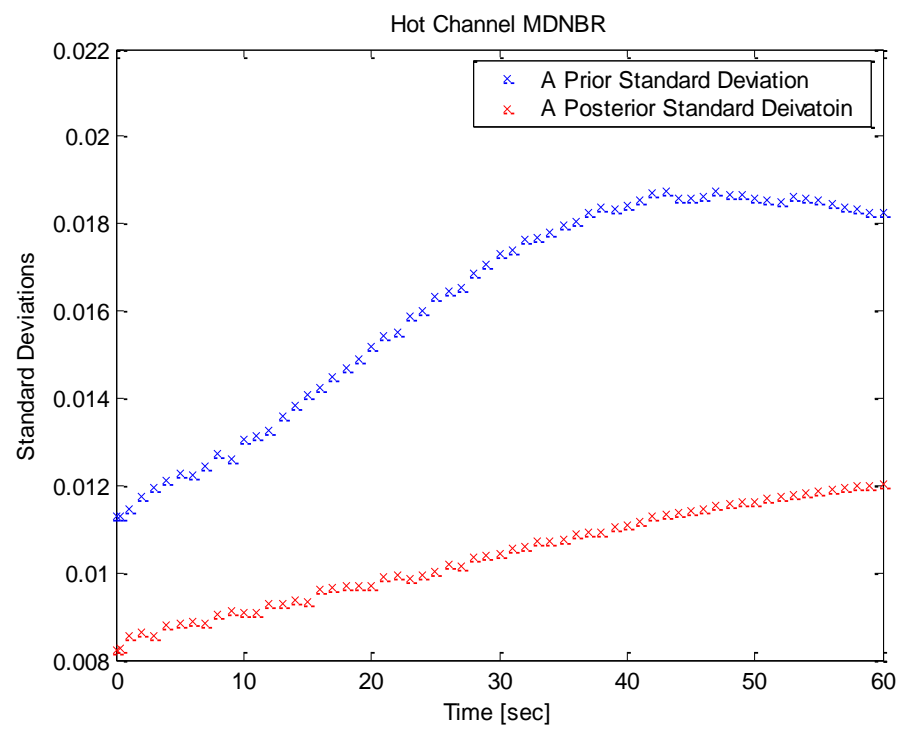

Figure 0-6: Standard Deviations for Hot Channel MDNBR for Control Bank Withdrawal using Experiment B for Data Assimilation

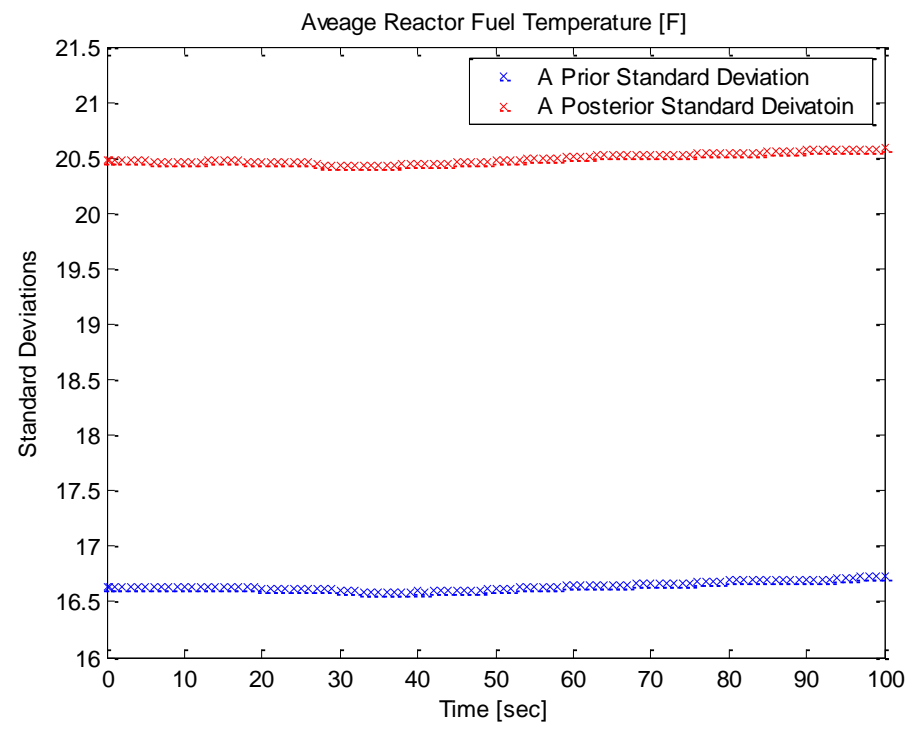

Figure 0-7: Standard Deviations for Average Fuel Temperature for FCV Failed Open using Experiment $\mathrm{C}$ for Data Assimilation 


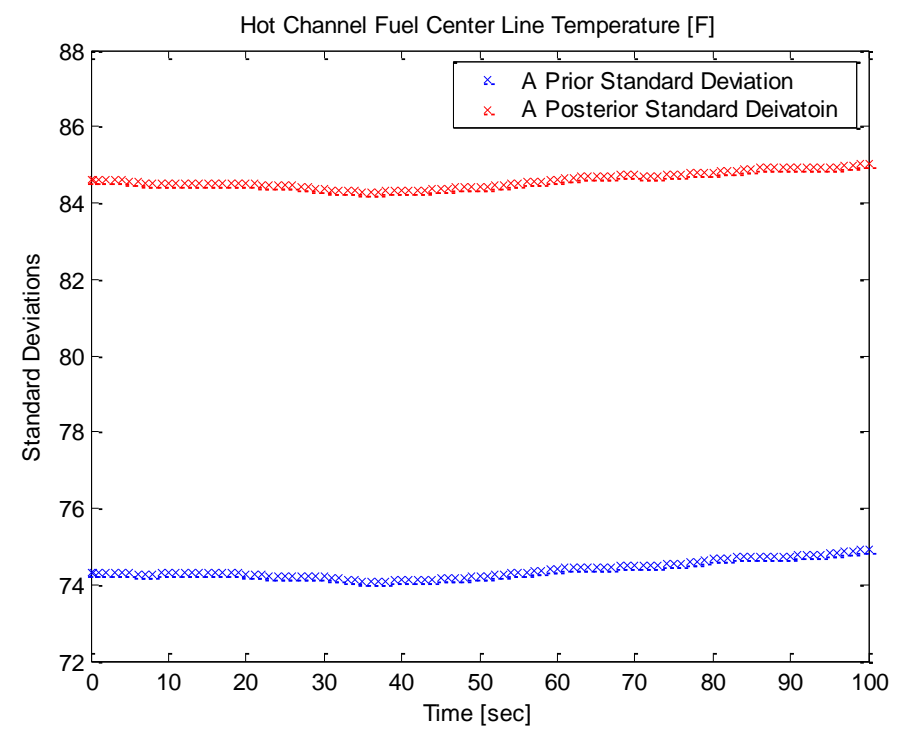

Figure 0-8: Standard Deviations for Hot Channel Fuel Centerline Temperature for FCV Failed Open using Experiment C for Data Assimilation

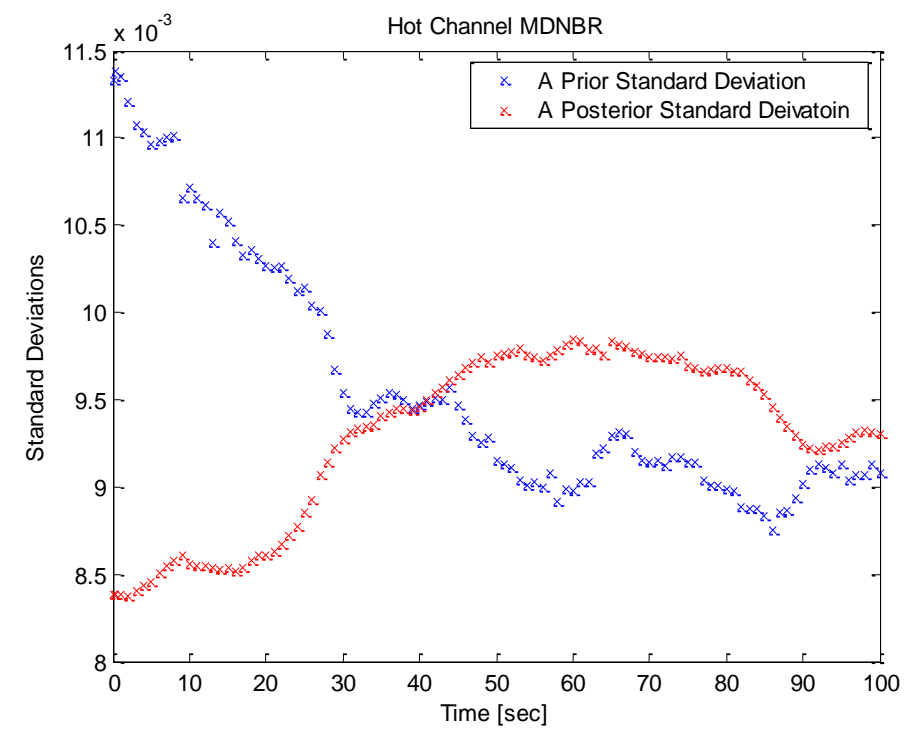

Figure 0-9: Standard Deviations for Hot Channel MDNBR for FCV Failed Open using Experiment $\mathrm{C}$ for Data Assimilation 


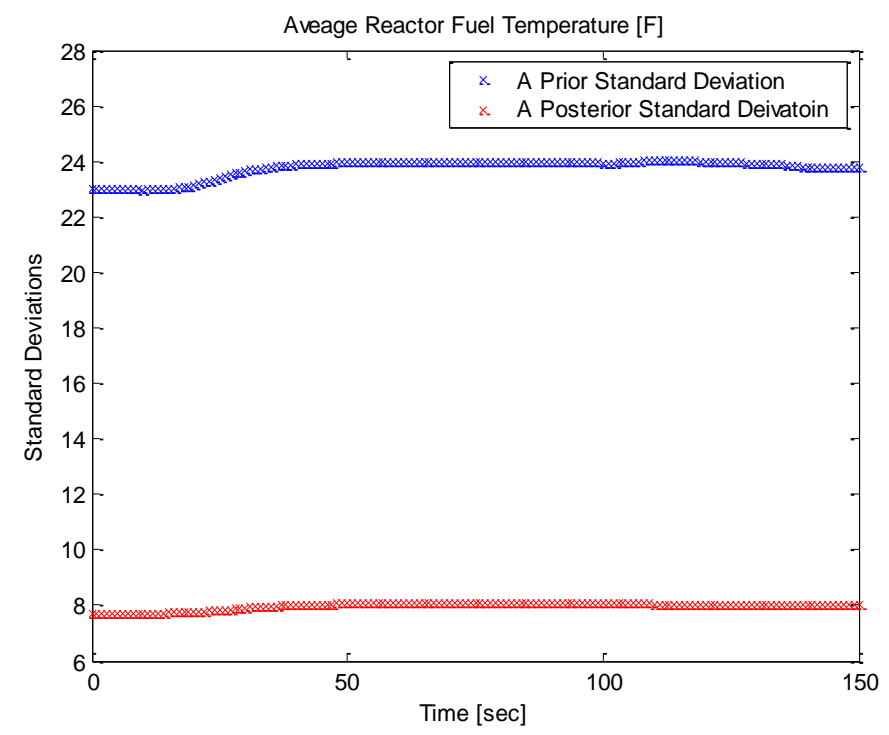

Figure 0-10: Standard Deviations for Average Fuel Temperature for TCV Failed Open using Experiment D for Data Assimilation

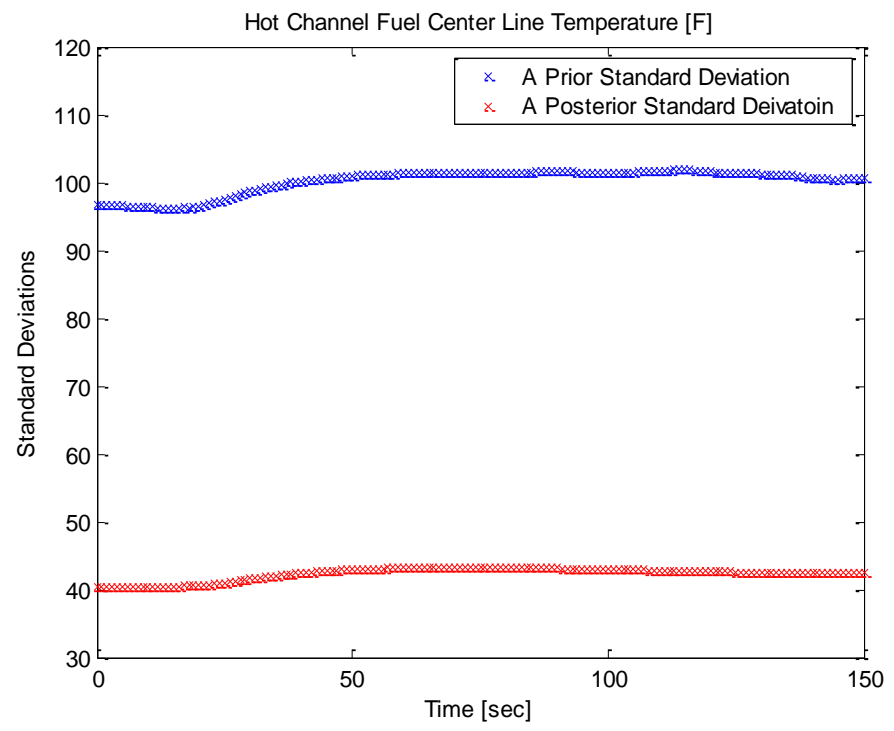

Figure 0-11: Standard Deviations for Hot Channel Fuel Centerline Temperature for TCV Failed Open using Experiment D for Data Assimilation 


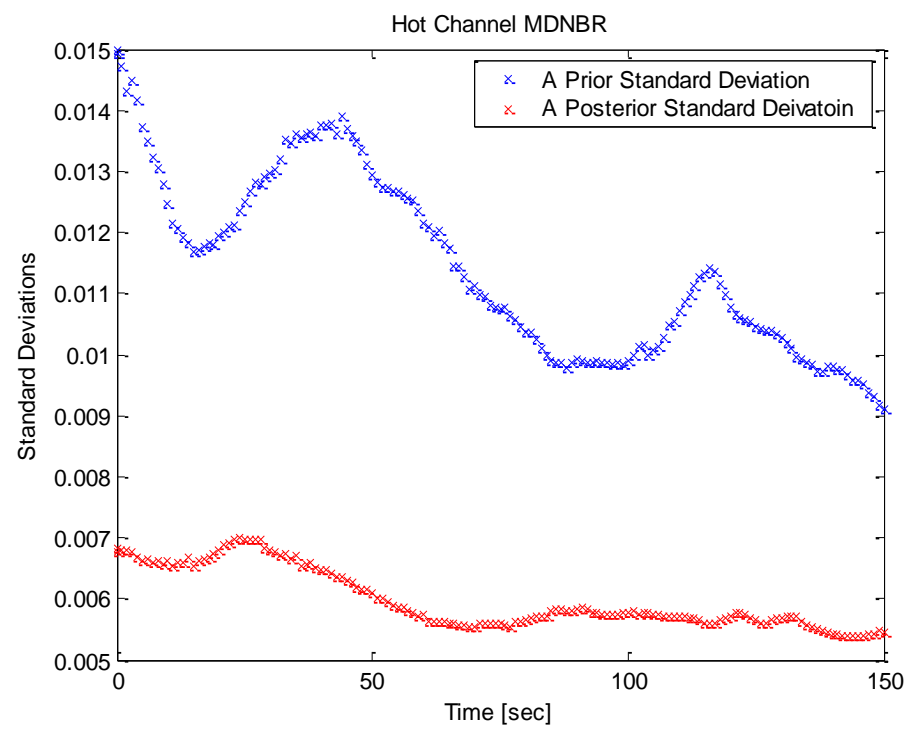

Figure 0-12: Standard Deviations for Hot Channel MDNBR for TCV Failed Open using Experiment D for Data Assimilation

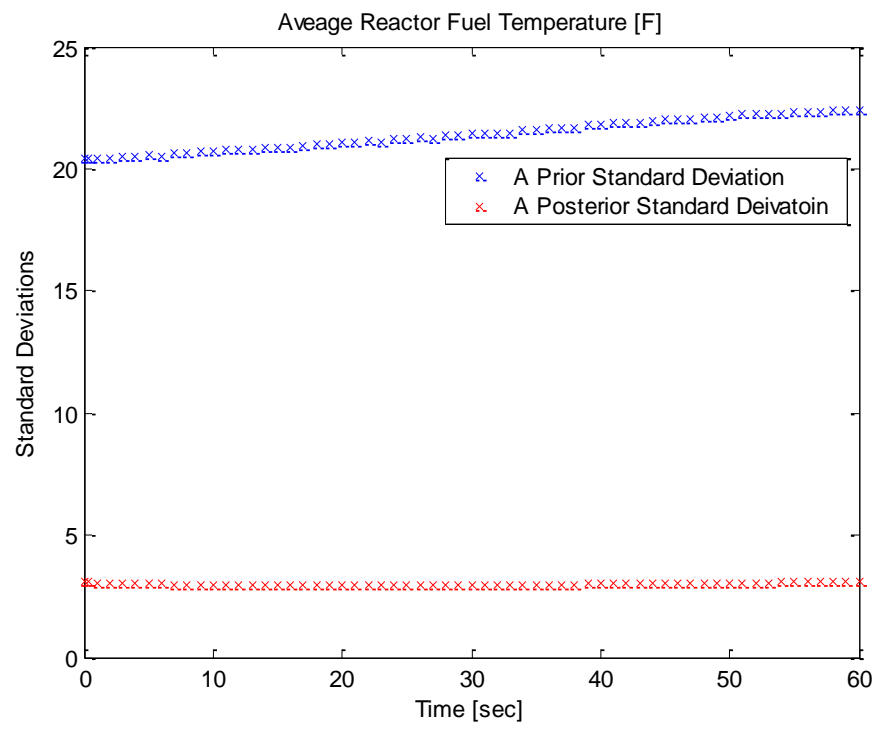

Figure 0-13: Standard Deviations for Average Fuel Temperature for Control Bank

Withdrawal using Experiment A, B, C and D for Data Assimilation 


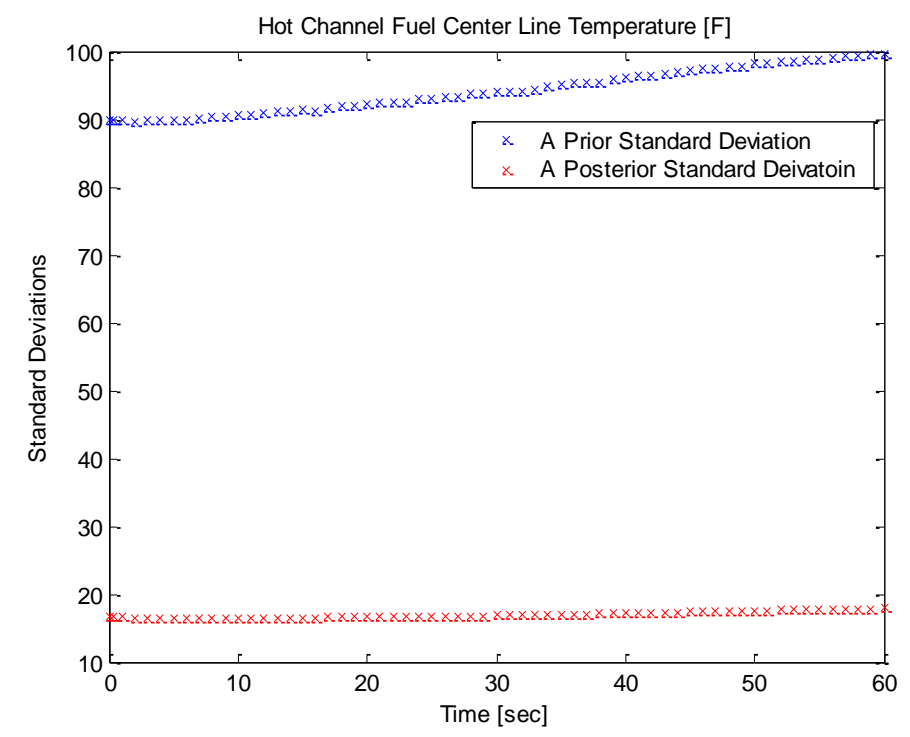

Figure 0-14: Standard Deviations for Hot Channel Fuel Centerline Temperature for Control Bank Withdrawal using Experiment A, B, C and D for Data Assimilation

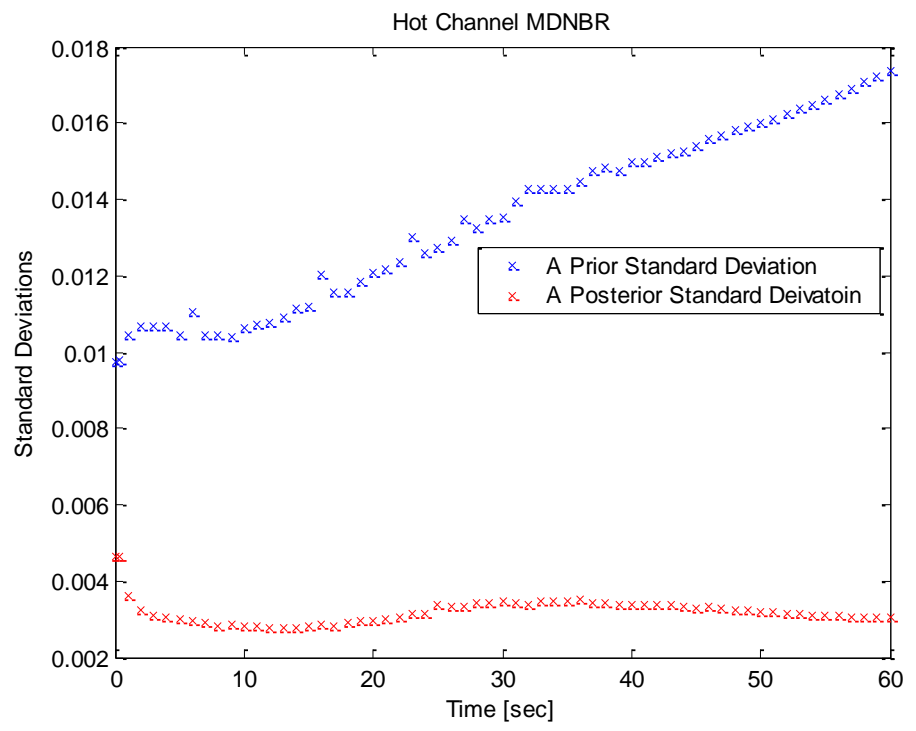

Figure 0-15: Standard Deviations for Hot Channel MDNBR for Control Bank Withdrawal using Experiment A, B, C and D for Data Assimilation 


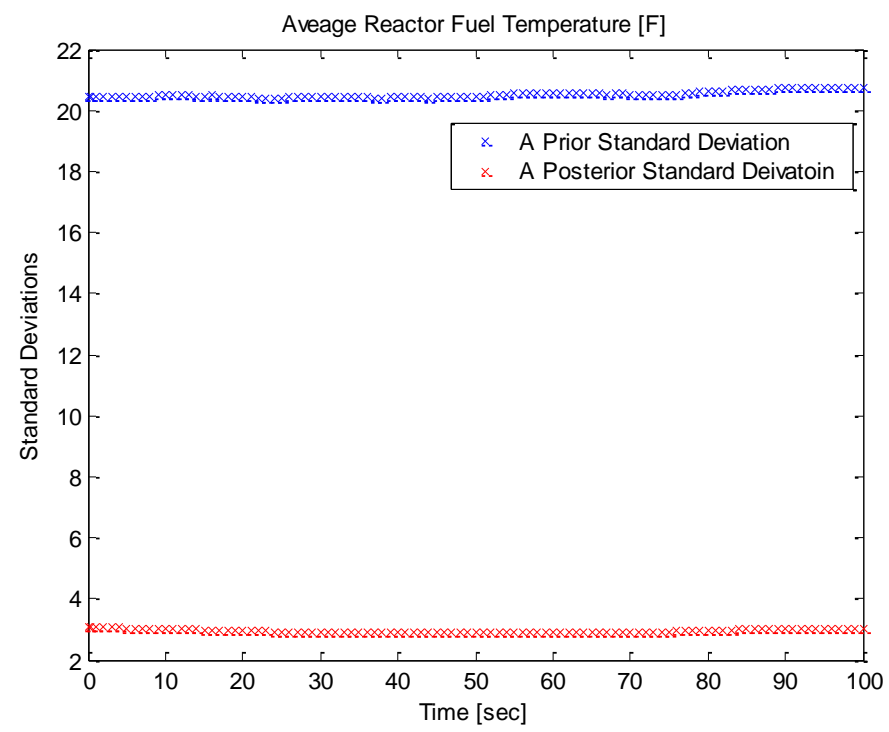

Figure 0-16: Standard Deviations for Average Fuel Temperature for FCV Failed Open using Experiment A, B, C and D for Data Assimilation

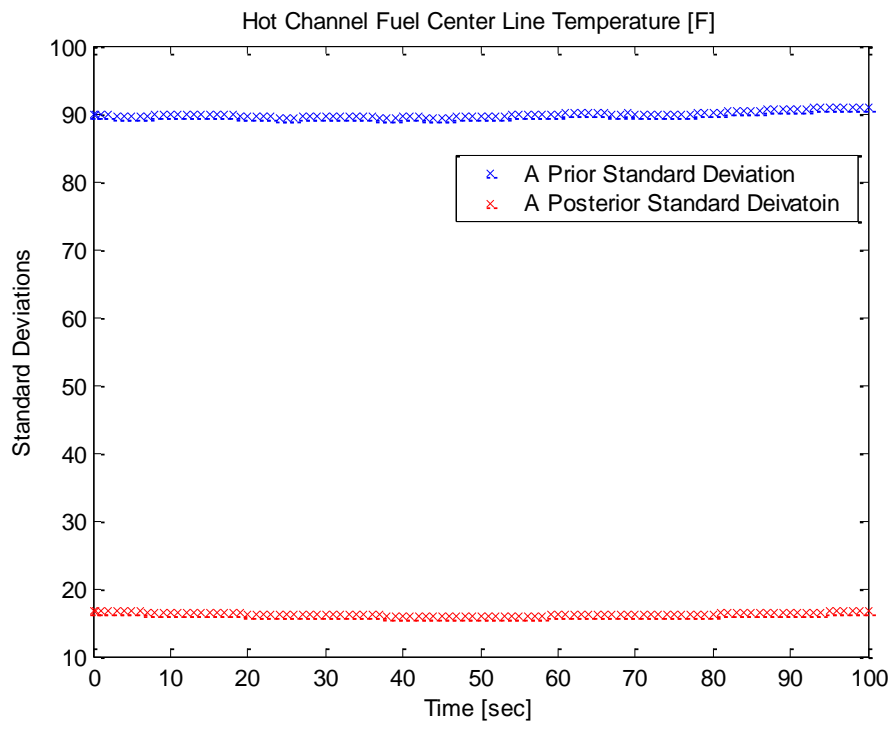

Figure 0-17: Standard Deviations for Hot Channel Fuel Centerline Temperature for FCV Failed Open using Experiment A, B, C and D for Data Assimilation 


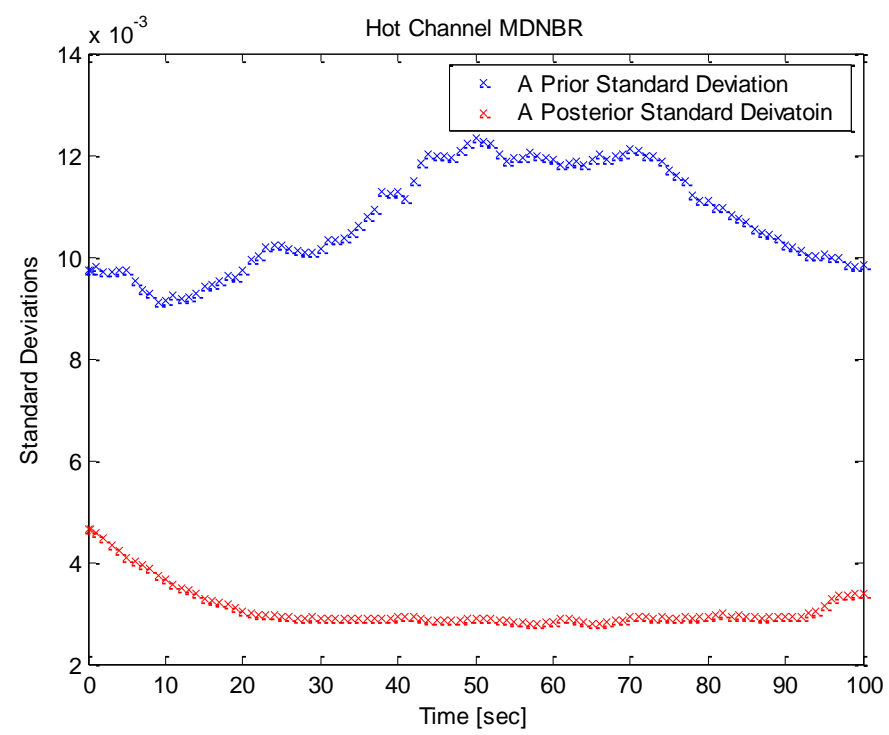

Figure 0-18: Standard Deviations for Hot Channel MDNBR for FCV Failed Open using Experiment A, B, C and D for Data Assimilation

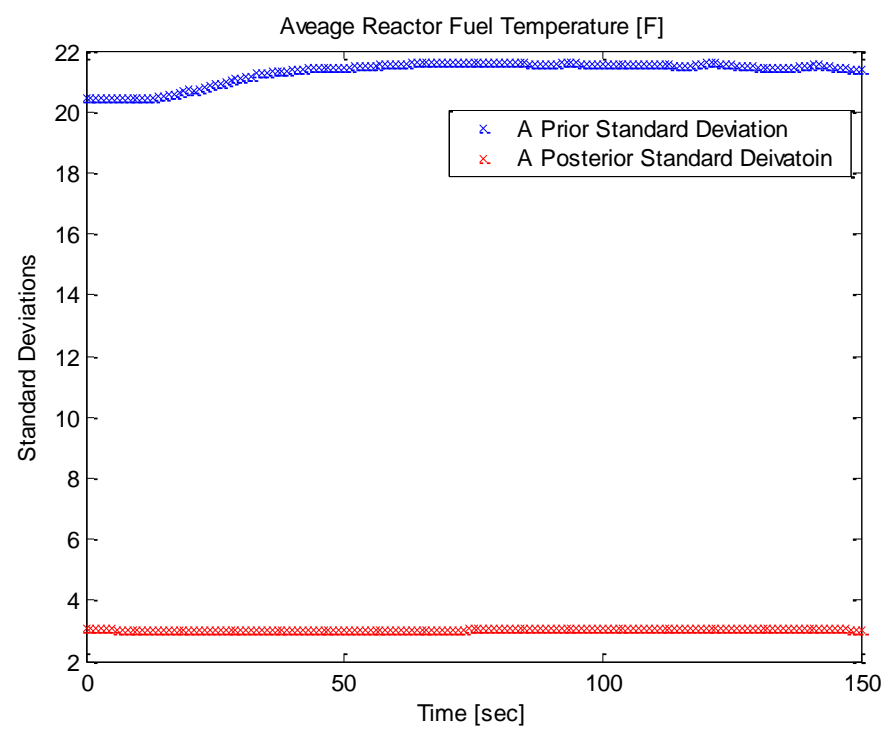

Figure 0-19: Standard Deviations for Average Fuel Temperature for TCV Failed Open using Experiment A, B, C and D for Data Assimilation 


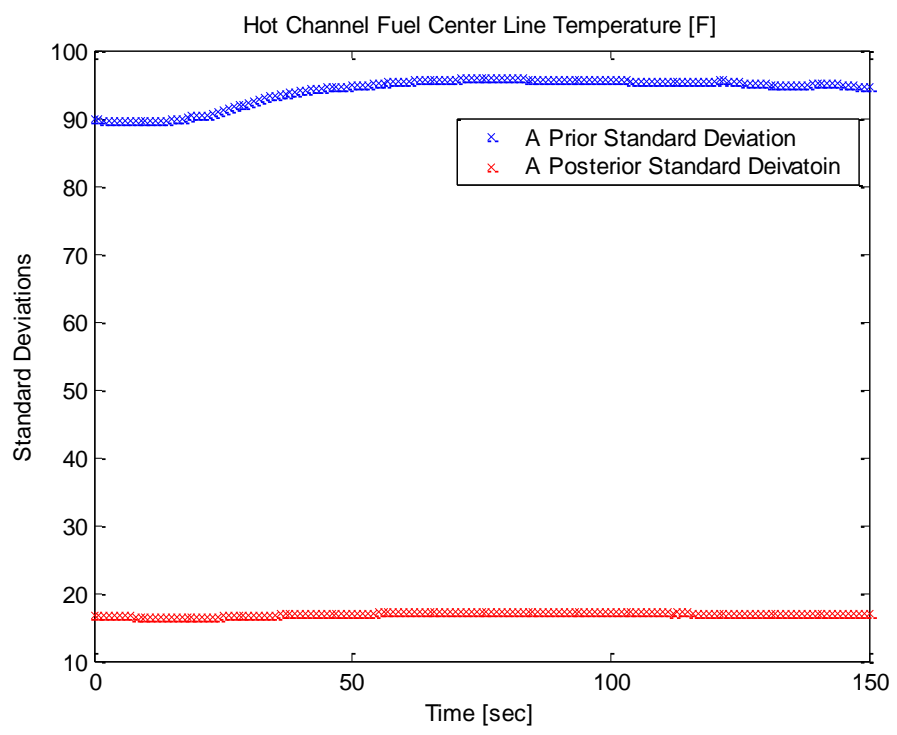

Figure 0-20: Standard Deviations for Hot Channel Fuel Centerline Temperature for TCV

Failed Open using Experiment A, B, C and D for Data Assimilation

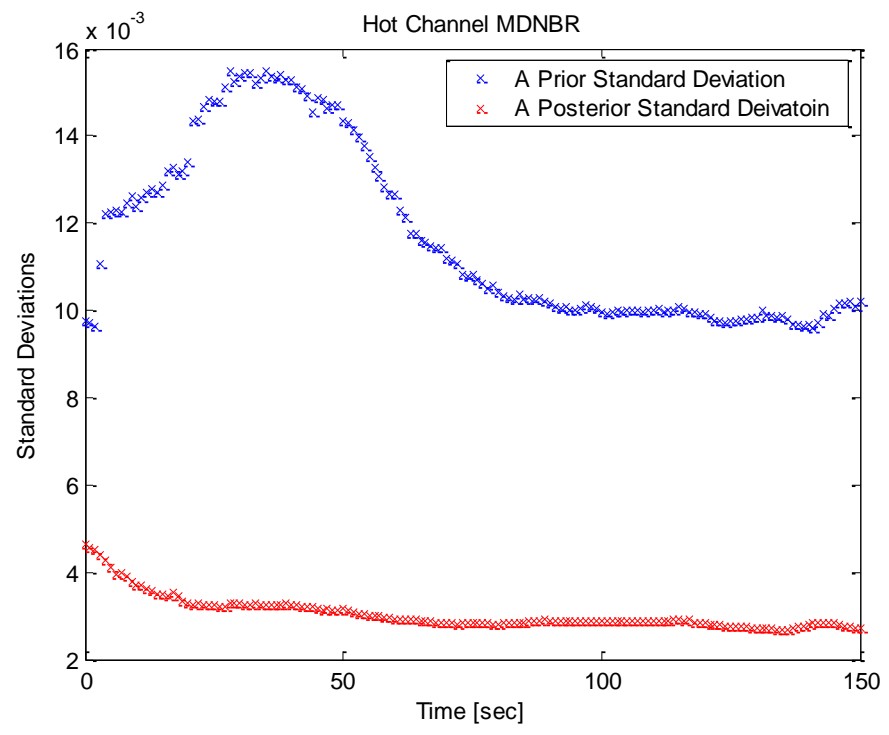

Figure 0-21: Standard Deviations for Hot Channel MDNBR for TCV Failed Open using Experiment A, B, C and D for Data Assimilation 


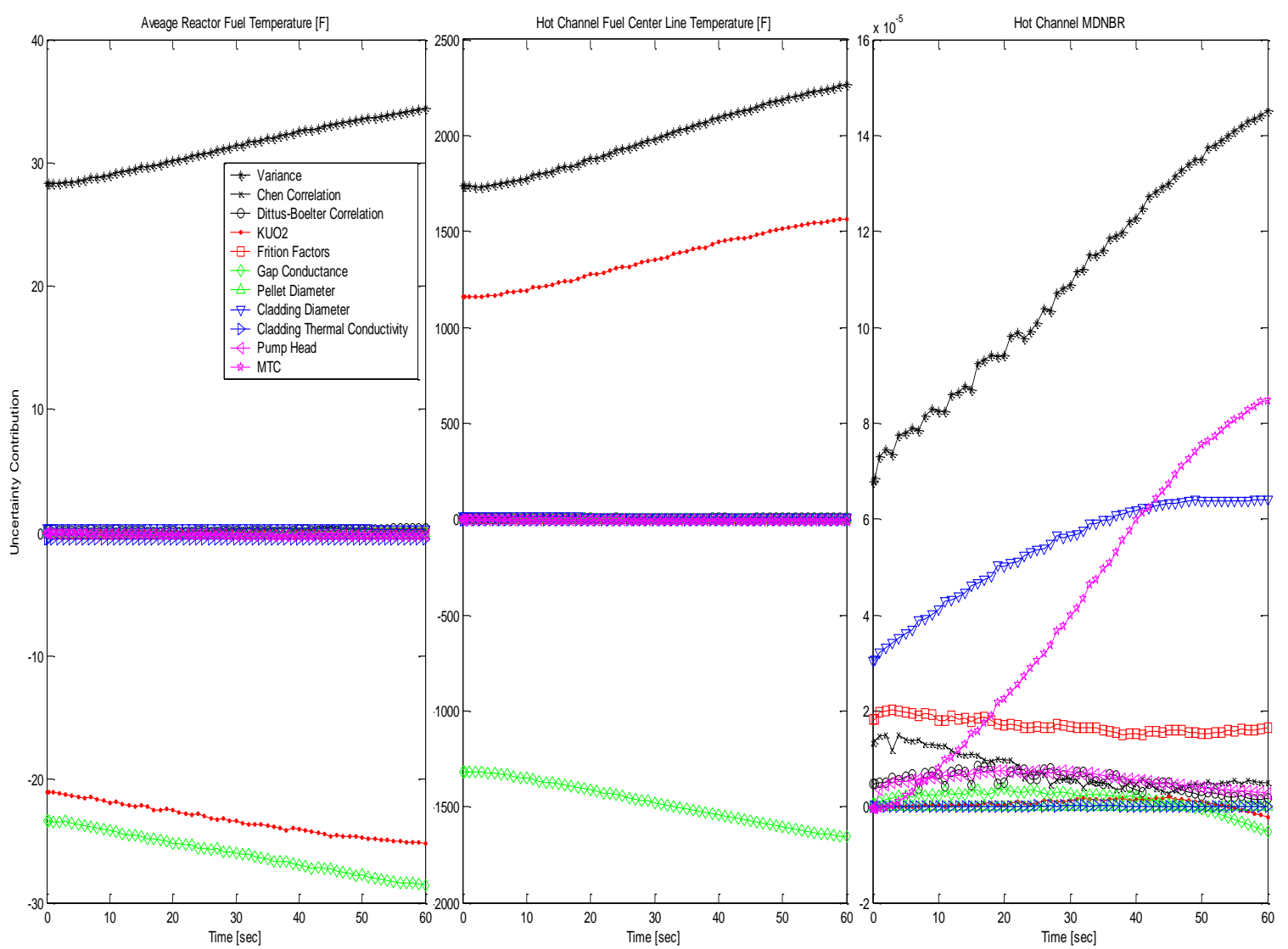

Figure 0-22: Each Parameter's Uncertainty Contribution to the System Attributes for

Control Bank Withdrawal 


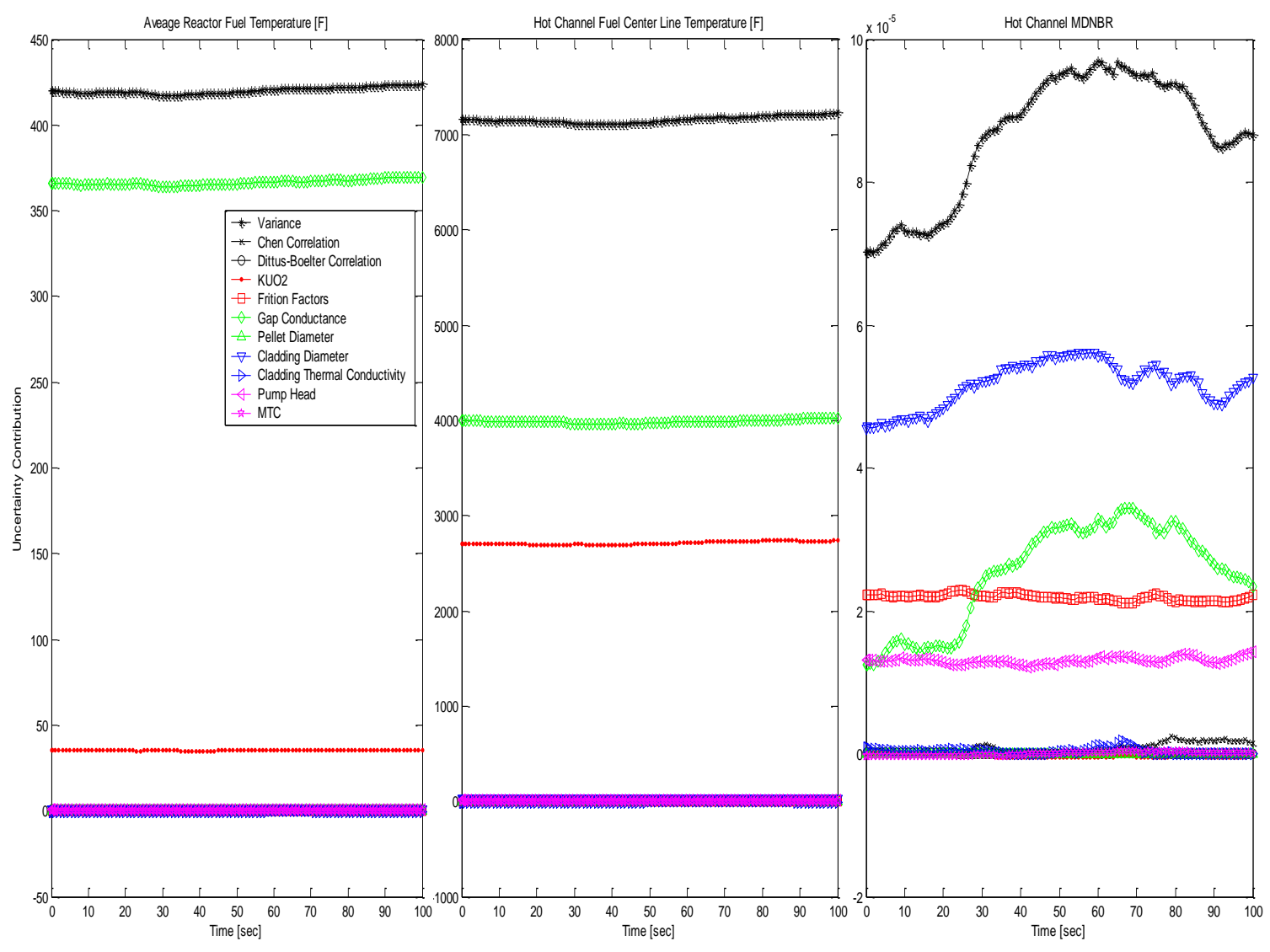

Figure 0-23: Each Parameter's Uncertainty Contribution to the System Attributes for FCV Failed Open 

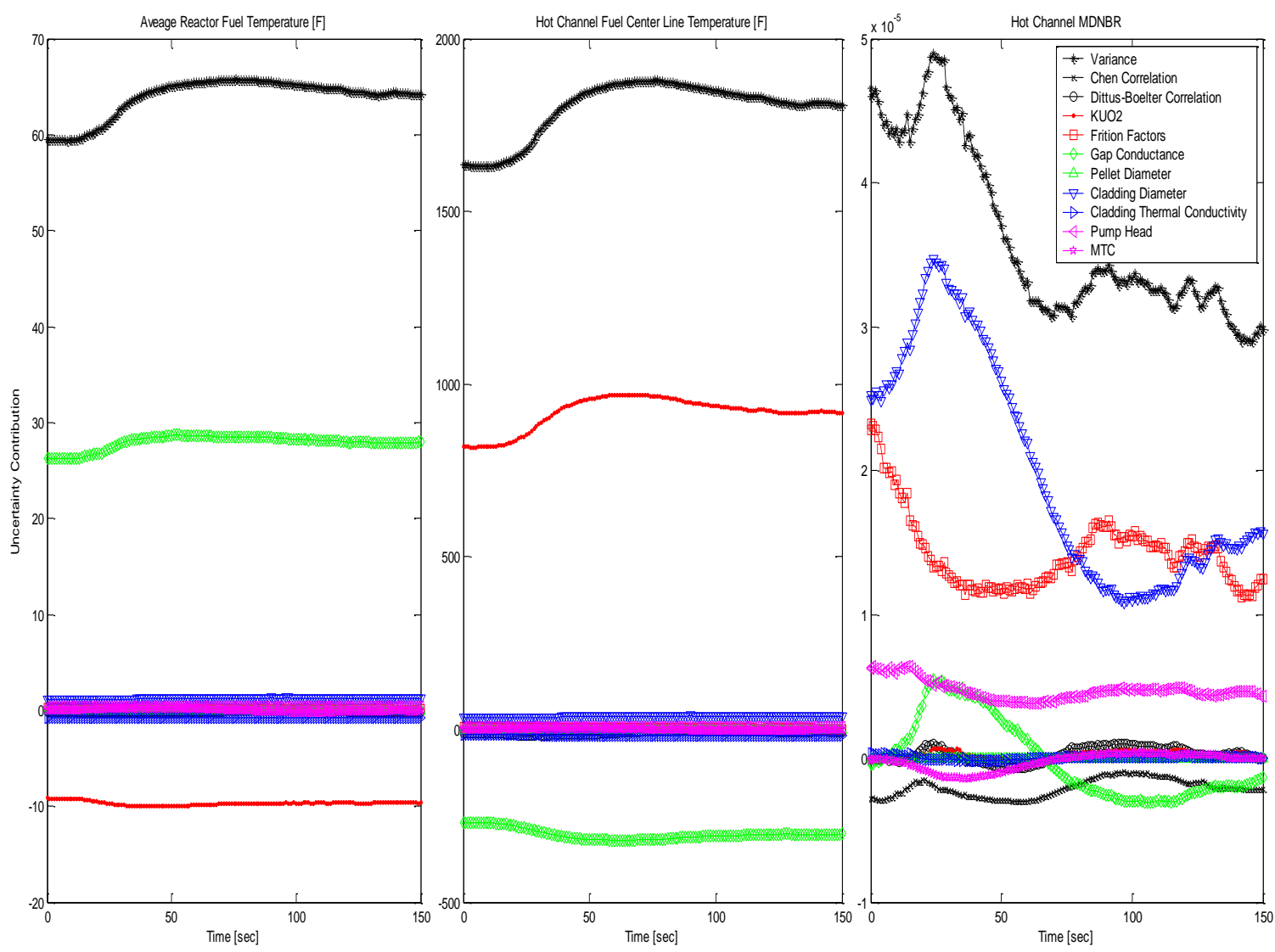

Figure 0-24: Each Parameter's Uncertainty Contribution to the System Attributes for TCV Failed Open 


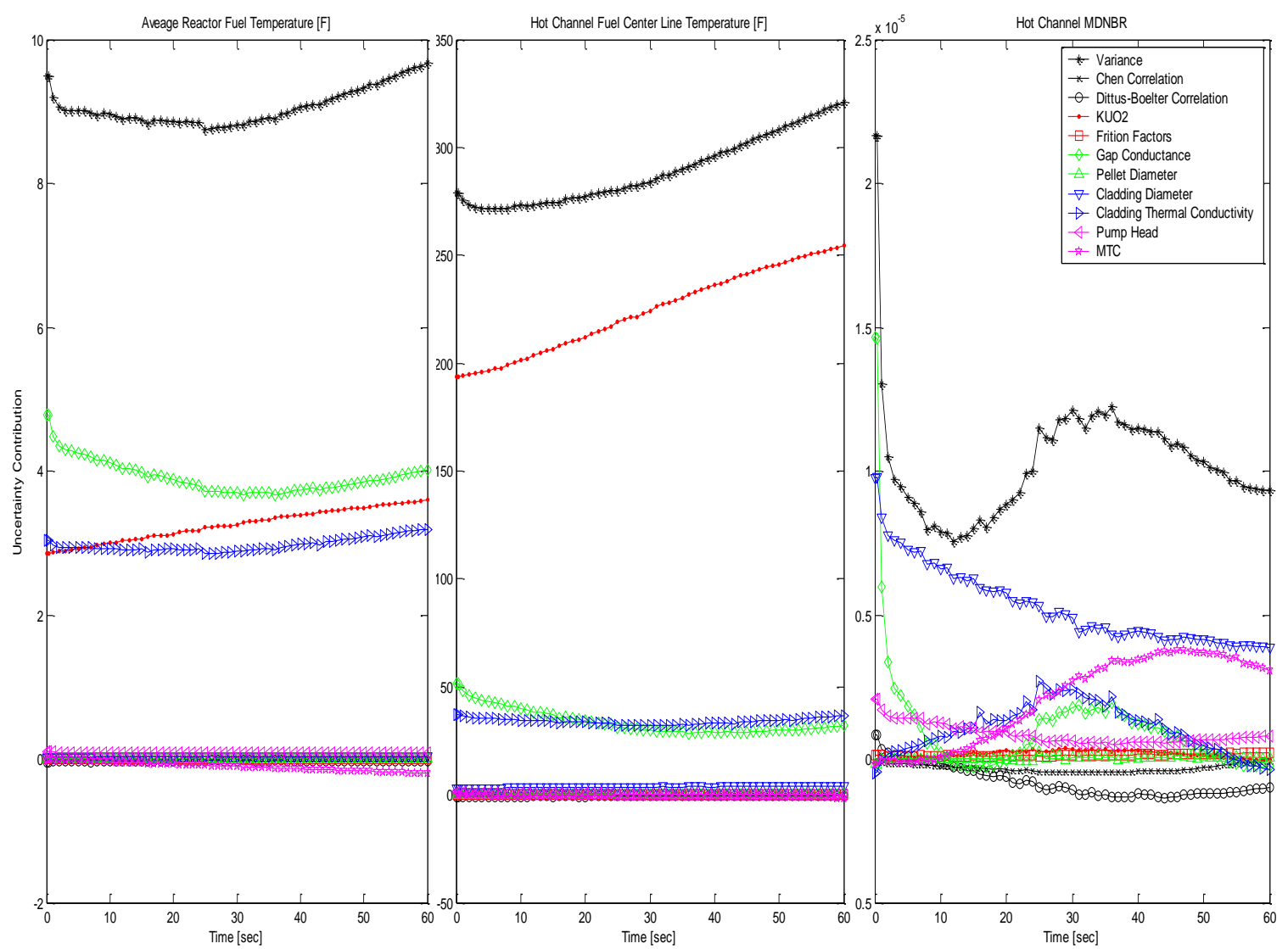

Figure 0-25: Each Parameter's Uncertainty Contribution to the System Attributes for Control Bank Withdrawal as a Result of Multiple Experiments 


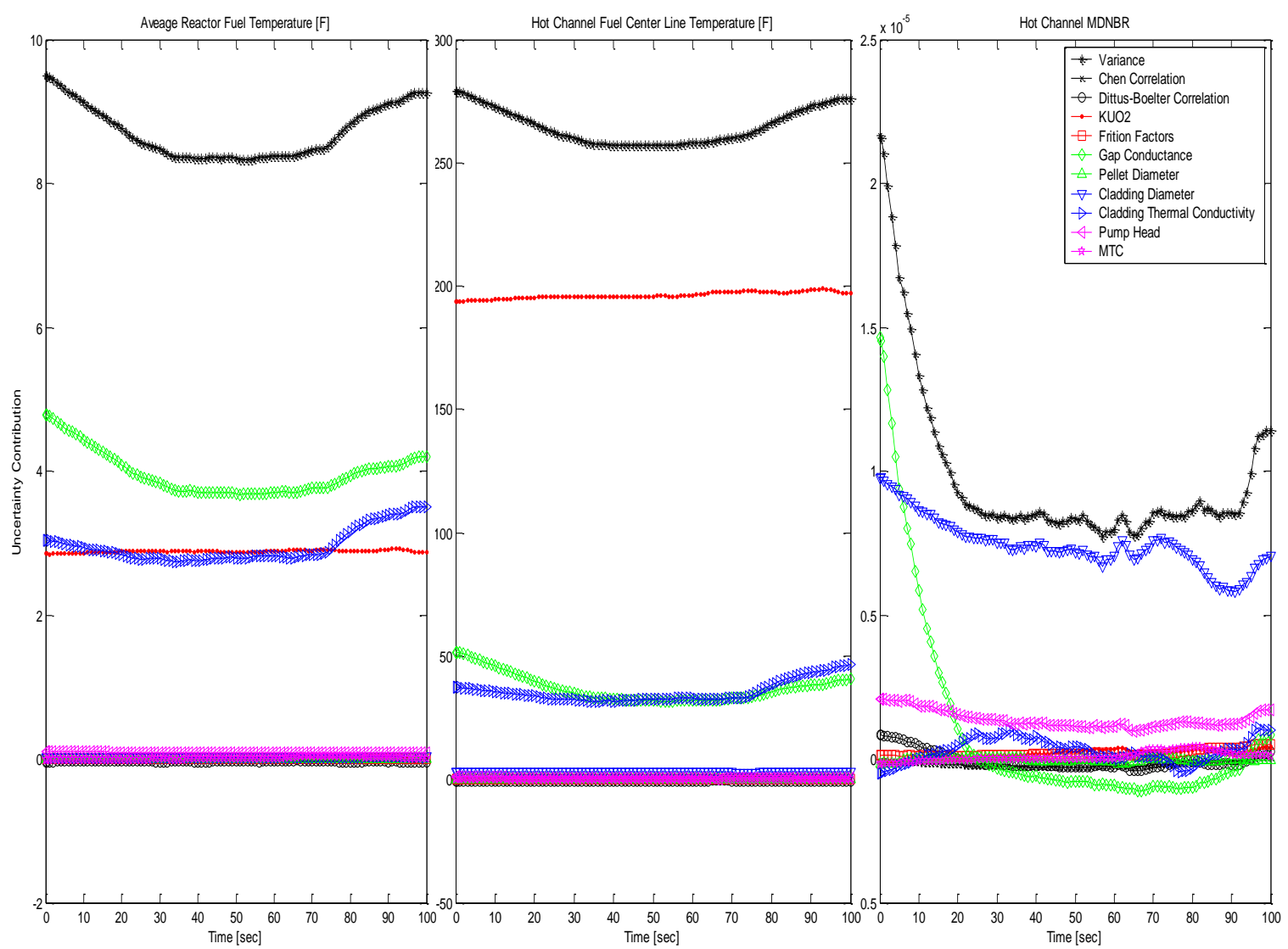

Figure 0-26: Each Parameter's Uncertainty Contribution to the System Attributes for FCV Failed Open as a Result of Multiple Experiments 


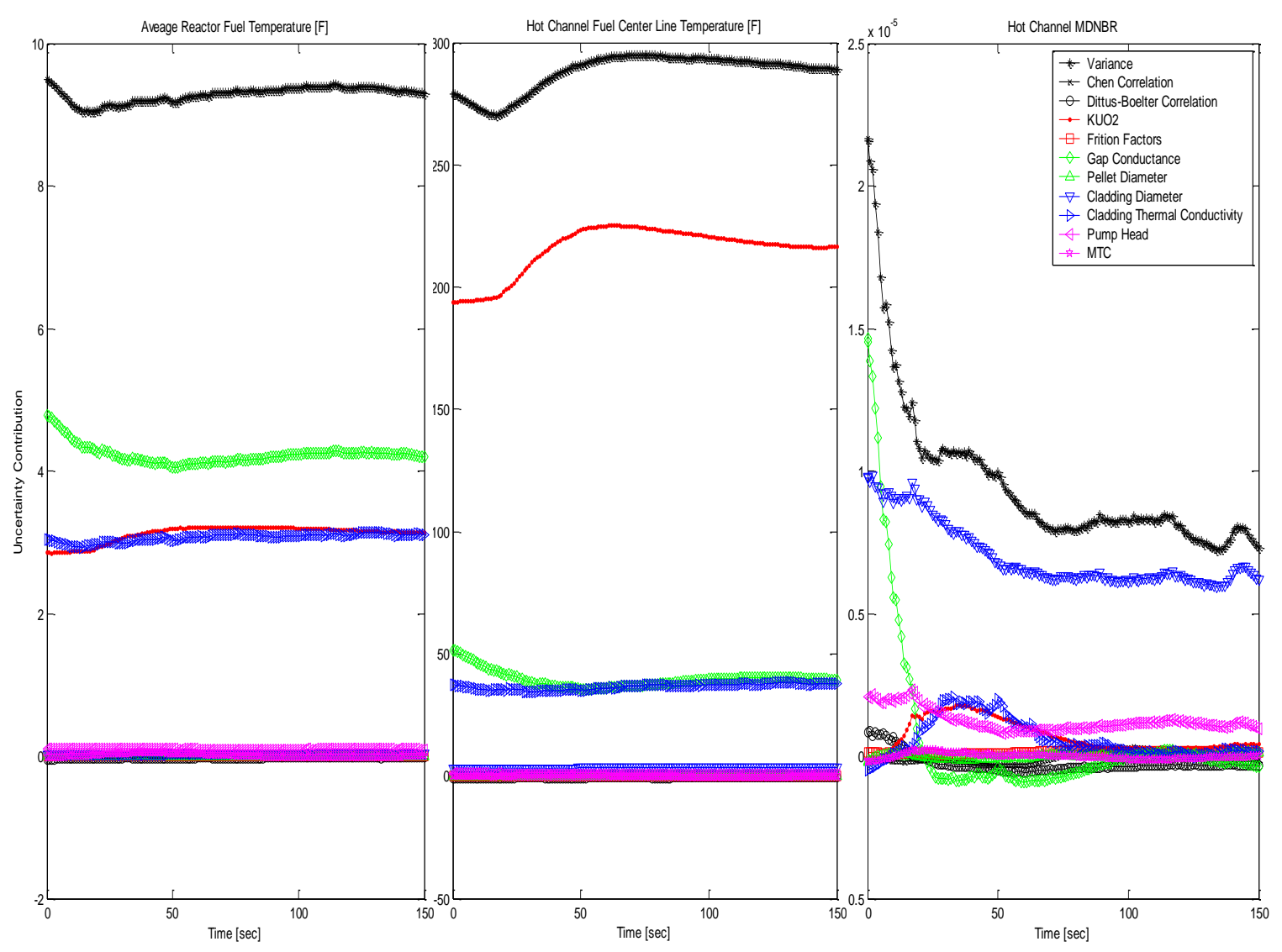

Figure 0-27: Each Parameter's Uncertainty Contribution to the System Attributes for TCV Failed Open as a Result of Multiple Experiments 


\section{Appendix B. Derivation of the Deterministic Method}

For $I$ total input parameters, $J$ distinct responses, and $T$ discrete times, the vector $\bar{p}$ of parameters and the vector $\bar{r}_{j}$ of each system responses can be represented as follows:

$\bar{p}=p_{i}\left|i=1,2, \ldots, I, \bar{r}_{j}=r_{j}^{(t)}\right| t=1,2, \ldots, T$

Then the vector $\bar{r}$ which contains all of the system response is:

$$
\bar{r}=\left[\begin{array}{cccc}
-\mathrm{T} & -\mathrm{T} & & -\mathrm{T} \\
r_{1} & r_{2} & \ldots & r_{J}^{\mathrm{T}}
\end{array}\right]^{\mathrm{T}}
$$

The computed response is linearized around the nominal values of the parameter multipliers.

$$
\bar{r} \cong \bar{r}_{0}+\overline{\bar{S}}_{\bar{p}_{0}} \delta \bar{p}_{0}
$$

The minimization problem can be formulated with the parameter-response combined vector $\bar{z}$ and the corresponding block covariance matrix $\overline{\bar{C}}$ as follows:

$$
\bar{z} \equiv\left[\begin{array}{l}
\bar{p}-\bar{p}_{0} \\
\bar{r}-\bar{r}_{m}
\end{array}\right], \overline{\bar{C}}=\left[\begin{array}{ll}
\overline{\bar{C}}_{p} & \overline{\bar{C}}_{p r} \\
\overline{\bar{C}}_{r p} & \overline{\bar{C}}_{m}
\end{array}\right]
$$

where, $\overline{\bar{C}}_{p r}=\overline{\bar{C}}_{r p}^{\mathrm{T}}$ is the parameter-response covariance matrix. The minimization problem is then given by:

$$
\begin{aligned}
& \min _{\bar{p}} \bar{z} \overline{\bar{C}}^{-1} \bar{z} \\
& =\min _{\bar{p}}\left\{\left[\begin{array}{l}
\bar{p}-\bar{p}_{0} \\
\bar{r}_{m}-\bar{r}
\end{array}\right]^{\mathrm{T}}\left[\begin{array}{ll}
\overline{\bar{C}}_{p} & \overline{\bar{C}}_{p r} \\
\overline{\bar{C}}_{r p} & \overline{\bar{C}}_{m}
\end{array}\right]^{-1}\left[\begin{array}{c}
\bar{p}-\bar{p}_{0} \\
\bar{r}_{m}-\bar{r}
\end{array}\right]\right\} \\
& =\min _{\bar{p}}\left\{\left[\begin{array}{c}
\bar{p}-\bar{p}_{0} \\
\bar{r}_{m}-\overline{r_{0}}-\overline{\bar{S}} \bar{p}-\bar{p}_{0}
\end{array}\right]^{\mathrm{T}}\left[\begin{array}{ll}
\overline{\bar{K}}_{p} & \overline{\bar{K}}_{p r} \\
\overline{\bar{K}}_{r p} & \overline{\bar{K}}_{r}
\end{array}\right]\left[\begin{array}{c}
\bar{p}-\bar{p}_{0} \\
\bar{r}_{m}-\overline{r_{0}}-\overline{\bar{S}} \bar{p}-\bar{p}_{0}
\end{array}\right]\right\}
\end{aligned}
$$




$$
\begin{aligned}
=\min _{p}\{ & {\left[\bar{p}-\bar{p}_{0}\right]^{\mathrm{T}} \overline{\bar{K}}_{p}\left[\bar{p}-\bar{p}_{0}\right]+\left[\bar{r}_{m}-\bar{r}_{0}-\overline{\bar{S}} \bar{p}-\bar{p}_{0}\right]^{\mathrm{T}} \overline{\bar{K}}_{r p}\left[\bar{p}-\bar{p}_{0}\right] } \\
& +\left[\bar{p}-\bar{p}_{0}\right]^{\mathrm{T}} \overline{\bar{K}}_{p r}\left[\bar{r}_{m}-\overline{r_{0}}-\overline{\bar{S}} \bar{p}-\bar{p}_{0}\right] \\
& \left.+\left[\bar{r}_{m}-\bar{r}_{0}-\overline{\bar{S}} \bar{p}-\bar{p}_{0}\right]^{\mathrm{T}} \overline{\bar{K}}_{r}\left[\bar{r}_{m}-\bar{r}_{0}-\overline{\bar{S}} \bar{p}-\bar{p}_{0}\right]\right\}
\end{aligned}
$$

where, $\overline{\bar{K}}=\left[\begin{array}{ll}\overline{\bar{K}}_{p} & \overline{\bar{K}}_{p r} \\ \overline{\bar{K}}_{r p} & \overline{\bar{K}}_{r}\end{array}\right]$ is the pseudo-inverse matrix of $\overline{\bar{C}}$, and matrix dimensions of $\overline{\bar{K}}_{p}, \overline{\bar{K}}_{p r}, \overline{\bar{K}}_{r p}$, and $\overline{\bar{K}}_{r}$ are identical to the dimensions of $\overline{\bar{C}}_{p}, \overline{\bar{C}}_{p r}, \overline{\bar{C}}_{r p}$, and $\overline{\bar{C}}_{m}$, respectively. Solution to the minimization problem is then accomplished by differentiating the above equation with respect to $\bar{p}$ and setting the result equal to zero:

$$
\begin{aligned}
& \alpha^{2} \overline{\bar{K}}_{p}\left[\bar{p}-\bar{p}_{0}\right]-\overline{\bar{S}}^{\mathrm{T}} \overline{\bar{K}}_{r p}\left[\bar{p}-\bar{p}_{0}\right] \\
& +\overline{\bar{K}}_{p r}\left[\bar{r}_{m}-\bar{r}_{0}-\overline{\bar{S}} \bar{p}-\bar{p}_{0}\right]-\overline{\bar{S}}^{\mathrm{T}} \overline{\bar{K}}_{r}\left[\bar{r}_{m}-\bar{r}_{0}-\overline{\bar{S}} \bar{p}-\bar{p}_{0}\right]=0
\end{aligned}
$$

Solving for the a posteriori parameter value produces:

$$
\begin{aligned}
& \bar{p}_{0}^{\text {post }}=\bar{p}_{0}+\left[\overline{\bar{S}} \overline{\bar{K}}_{r} \overline{\bar{S}}-\overline{\bar{S}}^{\mathrm{T}} \overline{\bar{K}}_{r p}-\overline{\bar{K}}_{p r} \overline{\bar{S}}+\alpha^{2} \overline{\bar{K}}_{p}\right]^{-1}\left[\overline{\bar{S}}^{\mathrm{T}} \overline{\bar{K}}_{r}-\overline{\bar{K}}_{p r}\right]\left[\bar{r}_{m}-\bar{r}_{0}\right] \\
& \bar{r}^{\text {post }} \cong \bar{r}\left(\bar{p}_{0}^{\text {post }}\right)+\overline{\bar{S}}_{\bar{p}_{0}^{p o s t}} \delta \bar{p}^{\text {post }}=\bar{r}_{0}^{\text {post }}+\overline{\bar{S}}_{\bar{p}_{0}^{\text {post }}} \delta \bar{p}^{\text {post }}
\end{aligned}
$$

The a posteriori parameter covariance matrix can be computed by:

$$
\overline{\bar{C}}_{p}^{\text {post }} \equiv\left\langle\left[\bar{p}-\bar{p}_{0}^{\text {post }}\right]\left[\bar{p}-\bar{p}_{0}^{\text {post }}\right]^{\mathrm{T}}\right\rangle
$$

Substituting a posteriori parameters into equation (A.8) produces the following expression for $\overline{\bar{C}}_{p}^{p o s t}$ :

$$
\begin{aligned}
\overline{\bar{C}}_{p}^{p o s t} & =\left\langle\left[\bar{p}-\bar{p}_{0}-\left[\overline{\bar{S}}^{\mathrm{T}} \overline{\bar{K}}_{r} \overline{\bar{S}}-\overline{\bar{S}}^{\mathrm{T}} \overline{\bar{K}}_{r p}-\overline{\bar{K}}_{p r} \overline{\bar{S}}+\alpha^{2} \overline{\bar{K}}_{p}\right]^{-1}\left[\overline{\bar{S}}^{\mathrm{T}} \overline{\bar{K}}_{r}-\overline{\bar{K}}_{p r}\right]\left[\bar{r}_{m}-\bar{r}_{0}\right]\right]\right. \\
& \left.\times\left[\bar{p}-\bar{p}_{0}-\left[\overline{\bar{S}} \overline{\bar{K}}_{r} \overline{\bar{S}}-\overline{\bar{S}}^{\mathrm{T}} \overline{\bar{K}}_{r p}-\overline{\bar{K}}_{p r} \overline{\bar{S}}+\alpha^{2} \overline{\bar{K}}_{p}\right]^{-1}\left[\overline{\bar{S}} \overline{\bar{K}}_{r}-\overline{\bar{K}}_{p r}\right]\left[\bar{r}_{m}-\bar{r}_{0}\right]\right]\right\rangle
\end{aligned}
$$




$$
\begin{aligned}
& =\overline{\bar{C}}_{p}^{\text {prior }}-\left\langle\left[\bar{p}-\bar{p}_{0}\right]\left[\bar{r}_{m}-\bar{r}+\overline{\bar{S}} \bar{p}-\bar{p}_{0}\right]^{\mathrm{T}}\right\rangle\left[\overline{\bar{K}}_{r} \overline{\bar{S}}-\overline{\bar{K}}_{r p}\right]\left[\overline{\bar{S}^{\mathrm{T}}} \overline{\bar{K}}_{r} \overline{\bar{S}}-\overline{\bar{S}}^{\mathrm{T}} \overline{\bar{K}}_{r p}-\overline{\bar{K}}_{p r} \overline{\bar{S}}_{+\alpha^{2}} \overline{\bar{K}}_{p}\right]^{-\mathrm{T}}
\end{aligned}
$$

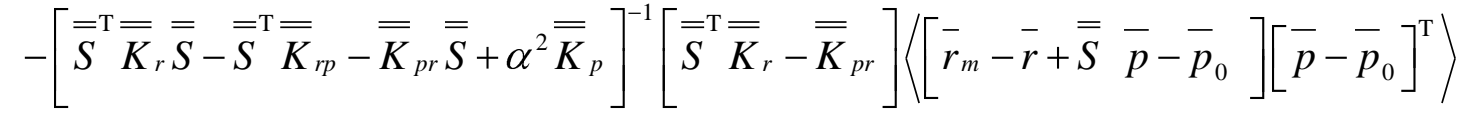

$$
\begin{aligned}
& +\left[\overline{\bar{S}}^{\mathrm{T}} \overline{\bar{K}}_{r} \overline{\bar{S}}-\overline{\bar{S}}^{\mathrm{T}} \overline{\bar{K}}_{r p}-\overline{\bar{K}}_{p r} \overline{\bar{S}}+\alpha^{2} \overline{\bar{K}}_{p}\right]^{-1}\left[\overline{\bar{S}}^{\mathrm{T}} \overline{\bar{K}}_{r}-\overline{\bar{K}}_{p r}\right] \\
& \times\left\langle\left[\bar{r}_{m}-\bar{r}+\overline{\bar{S}}\left(\underline{Q}-\bar{p}_{0}\right) \bar{r}_{m}-\bar{r}+\overline{\bar{S}}\left(p-\bar{p}_{0}\right)\right\rangle\right. \\
& \times\left[\overline{\bar{K}}_{r} \overline{\bar{S}}-\overline{\bar{K}}_{r p}\right]\left[\overline{\bar{S}}^{\mathrm{T}} \overline{\bar{K}}_{r} \overline{\bar{S}}-\overline{\bar{S}}^{\mathrm{T}} \overline{\bar{K}}_{r p}-\overline{\bar{K}}_{p r} \overline{\bar{S}}+\alpha^{2} \overline{\bar{K}}_{p}\right]^{-\mathrm{T}} \\
& =\overline{\bar{C}}_{p}^{\text {prior }}+\left[\overline{\bar{C}}_{p r}-\overline{\bar{C}}_{p}^{p r i o r} \overline{\bar{S}}^{\mathrm{T}}\right]\left[\overline{\bar{K}}_{r} \overline{\bar{S}}-\overline{\bar{K}}_{r p}\right]\left[\overline{\bar{S}}^{\mathrm{T}} \overline{\bar{K}}_{r} \overline{\bar{S}}-\overline{\bar{S}}^{\mathrm{T}} \overline{\bar{K}}_{r p}-\overline{\bar{K}}_{p r} \overline{\bar{S}}+\alpha^{2} \overline{\bar{K}}_{p}\right]^{-\mathrm{T}} \\
& +\left[\overline{\bar{S}}^{\mathrm{T}} \overline{\bar{K}}_{r} \overline{\bar{S}}^{\left.-\overline{\bar{S}}^{\mathrm{T}} \overline{\bar{K}}_{r p}-\overline{\bar{K}}_{p r} \overline{\bar{S}}+\alpha^{2} \overline{\bar{K}}_{p}\right]^{-1}\left[\overline{\bar{S}}^{\mathrm{T}} \overline{\bar{K}}_{r}-\overline{\bar{K}}_{p r}\right]\left[\overline{\bar{C}}_{r p}-\overline{\bar{S}^{p}} \overline{\bar{C}}_{p}\right. \text { prior }}\right] \\
& +\left[\overline{\bar{S}}^{\mathrm{T}} \overline{\bar{K}}_{r} \overline{\bar{S}}-\overline{\bar{S}}^{\mathrm{T}} \overline{\bar{K}}_{r p}-\overline{\bar{K}}_{p r} \overline{\bar{S}}_{+} \alpha^{2} \overline{\bar{K}}_{p}\right]^{-1}\left[\overline{\bar{S}}^{\mathrm{T}} \overline{\bar{K}}_{r}-\overline{\bar{K}}_{p r}\right]\left[\overline{\bar{C}}_{m}-\overline{\bar{C}}_{r p} \overline{\bar{S}}^{\mathrm{T}}-\overline{\bar{S}}_{\bar{S}} \overline{\bar{C}}_{p r}+\overline{\bar{S}} \overline{\bar{C}}_{p}^{p r i o r} \overline{\bar{S}}^{\mathrm{T}}\right]
\end{aligned}
$$

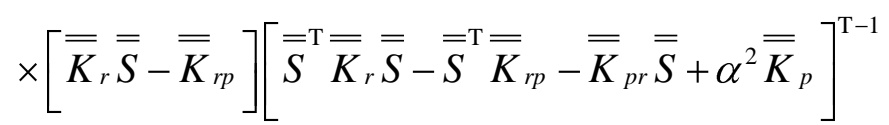

The a posteriori parameter-response covariance matrix can be computed by:

$$
\begin{aligned}
& \overline{\bar{C}}_{p r}^{\text {post }} \equiv \overline{\bar{C}}_{r p}^{\text {post } \mathrm{T}} \equiv\left\langle\left[\bar{p}-\bar{p}_{0}^{\text {post }}\right]\left[\bar{r}^{-}-\bar{r}_{m}\right]^{\mathrm{T}}\right\rangle \\
& \overline{\bar{C}}_{p r}^{p o s t}=\left\langle\left[\bar{p}-\bar{p}_{0}-\left[\overline{\bar{S}}^{\mathrm{T}} \overline{\bar{K}}_{r} \overline{\bar{S}}-\overline{\bar{S}}^{\mathrm{T}} \overline{\bar{K}}_{r p}-\overline{\bar{K}}_{p r} \overline{\bar{S}}+\alpha^{2} \overline{\bar{K}}_{p}\right]^{-1}\left[\overline{\bar{S}}^{\mathrm{T}} \overline{\bar{K}}_{r}-\overline{\bar{K}}_{p r}\right]\left[\bar{r}_{m}-\bar{r}_{0}\right]\right]\left[\bar{r}-\bar{r}_{m}\right]^{\mathrm{T}}\right\rangle \\
& =\left\langle\left[\bar{p}-\bar{p}_{0}\right]\left[\bar{r}-\bar{r}_{m}\right]^{\mathrm{T}}\right\rangle+\left[\overline{\bar{S}}^{\mathrm{T}} \overline{\bar{K}}_{r} \overline{\bar{S}}-\overline{\bar{S}}^{\mathrm{T}} \overline{\bar{K}}_{r p}-\overline{\bar{K}}_{p r} \overline{\bar{S}}+\alpha^{2} \overline{\bar{K}}_{p}\right]^{-1}\left[\overline{\bar{S}}^{\mathrm{T}} \overline{\bar{K}}_{r}-\overline{\bar{K}}_{p r}\right] \\
& \times\left\langle\left[\bar{r}-\bar{r}_{m}-\overline{\bar{S}} \bar{p}-\bar{p}_{0}\right]\left[\bar{r}-\bar{r}_{m}\right]^{\mathrm{T}}\right\rangle \\
& =\overline{\bar{C}}_{p r}-\left[\overline{\bar{S}}^{\mathrm{T}} \overline{\bar{K}}_{r} \overline{\bar{S}}-\overline{\bar{S}}^{\mathrm{T}} \overline{\bar{K}}_{r p}-\overline{\bar{K}}_{p r} \overline{\bar{S}}+\alpha^{2} \overline{\bar{K}}_{p}\right]^{-1}\left[\overline{\bar{S}}^{\mathrm{T}} \overline{\bar{K}}_{r}-\overline{\bar{K}}_{p r}\right]\left[\overline{\bar{C}}_{m}-\overline{\bar{S}_{\bar{C}}} \overline{\bar{C}}_{p r}\right]
\end{aligned}
$$

The subsequent step of the iteration procedure would then be to use the above a posteriori values as a priori information, and compute the new best-estimate quantities by using these formulas again. 


\section{Advanced Instrumentation and Control Methods for Small and Medium Reactors with IRIS Demonstration}

\section{Final Report \\ Volume 3}

\section{Development of an Autonomous and Fault-Tolerant Control Method with Applications to the IRIS System \\ Report No. DE-FG07-07ID14895/UTNE/2011-5 \\ NERI-C Grant Number: DE-FG07-07ID14895}

Belle R. Upadhyaya

(Co-Principal Investigator)

Robert M. Edwards

Pennsylvania State University

(Co-Principal Investigator)

$$
\begin{gathered}
\text { Jenny Xu } \\
\text { Yin Guo } \\
\text { Xin Jin }
\end{gathered}
$$

The University of Tennessee Nuclear Engineering Department 212 Pasqua Engineering Building

Knoxville, TN 37996-2300

E-mail: bupadhya@utk.edu

May 2011

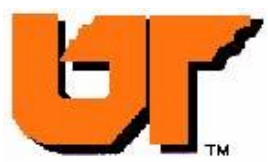




\begin{abstract}
The basic requirement of the modular reactor control system is to achieve stable control at different operation modes and enable a stable transition between these operation modes. The major operation modes include plant start-up, normal operation at different power levels, hot standby mode for system testing, and cold shutdown mode for plant maintenance. One of the important operational modes is the transition from electricity generation to providing process heat. It is anticipated that the primary application of process heat is for water desalination. It may be necessary to partition the total energy output between electricity generation and process heat supply. During the transitional modes, the reactor systems and equipment must be closely monitored and controlled to assure compliance with safety requirements.

In order to meet the remote deployment and proliferation-resistance requirements, as defined by safety, reliability, survivability, and life expectancy, the reactor control system should incorporate advanced features such as fault tolerance, control loop performance monitoring, and safety monitoring. The objective of this task is to develop an autonomous and hierarchical control strategy for the IRIS system and incorporate several control algorithms. These include model-predictive controller (MPC), robust multivariate controller, and resilient controller. Fault-tolerant control performance under model uncertainties, and sensor and actuator fault conditions was evaluated for the IRIS plant. The MPC algorithm was also implemented in an experimental flow control loop.

Several real-time control algorithms were developed and tested. The high-fidelity Fortran model of the IRIS system (developed by NCSU) was used as the 'real plant' and the control actions were generated using the low-order SIMULINK models. The control actions generated using the low-order model was passed to the 'plant' and the resulting response parameters were relayed to the control module. This simulates the on-line implementation of the various controllers.

This volume of the Final Report describes the various control algorithms, their implementation, and the results of application to the complete IRIS system. Hierarchical and fault-tolerant control actions are demonstrated. This report describes the contributions of the research work by Pennsylvania State University and the University of Tennessee. A complete bibliography of publications related to IRIS system and model development, autonomous
\end{abstract}


control, model-predictive and multivariate control algorithms, fault diagnostics, and prognostics are provided at the end of the report.

\section{DISCLAIMER}

Any opinions, findings, and conclusions or recommendations expressed in this report are those of the authors, and do not necessarily reflect the views of the U.S. Department of Energy or Westinghouse Electric Company, LLC. 


\section{TABLE OF CONTENTS}

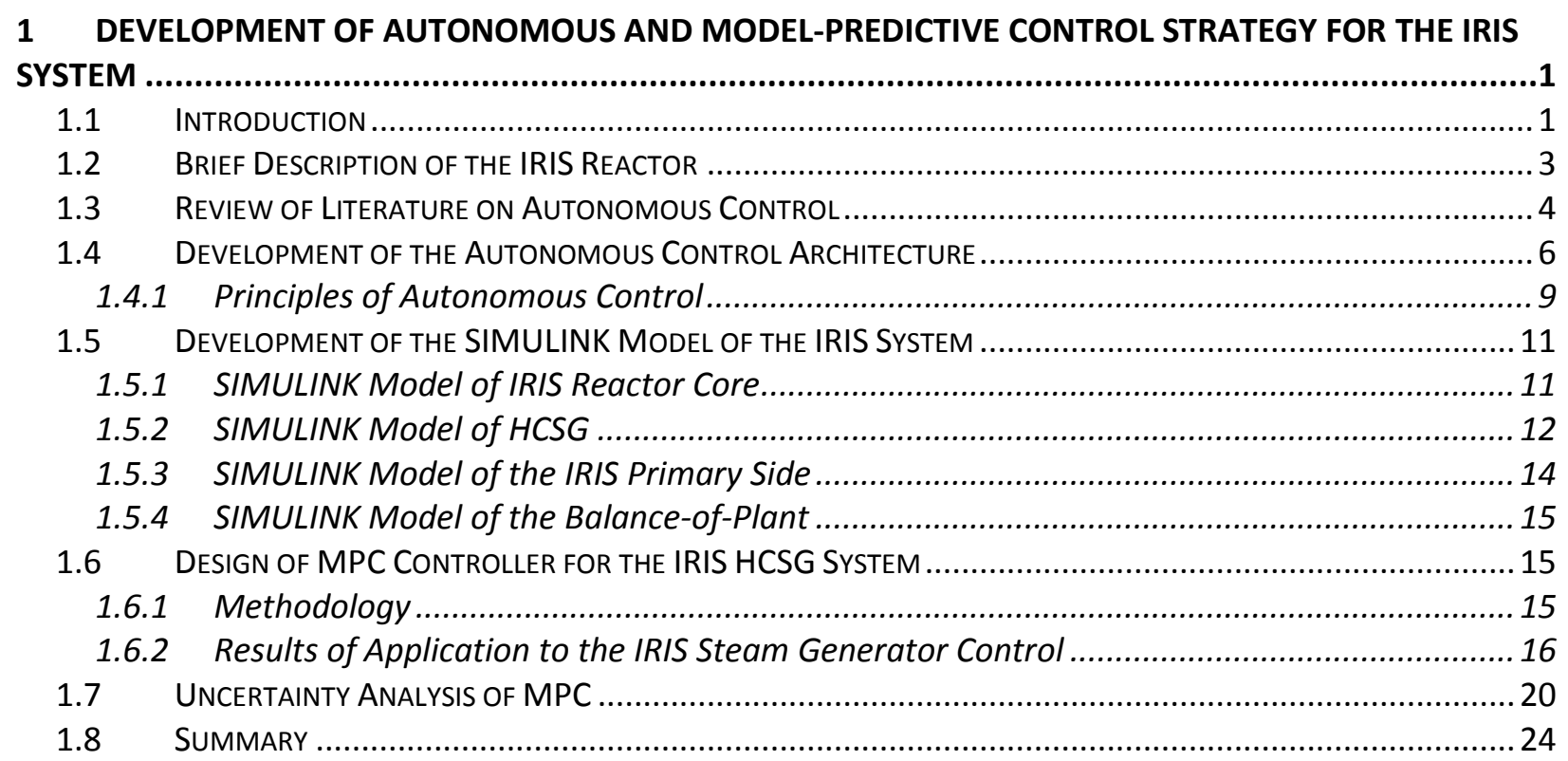

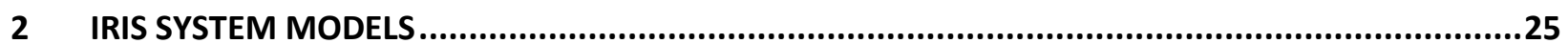

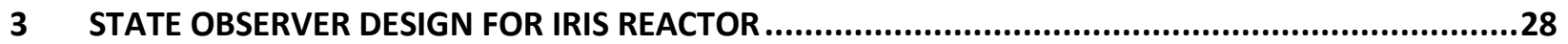

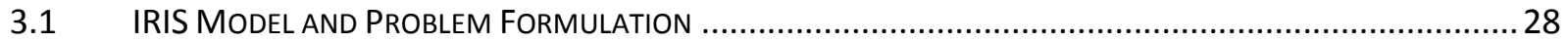

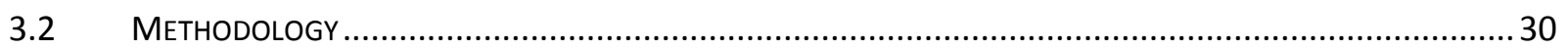

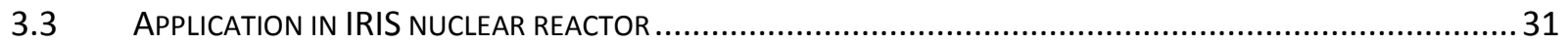

3.3.1 State Space Model of IRIS Core for State-Observer Design .............................................. 31

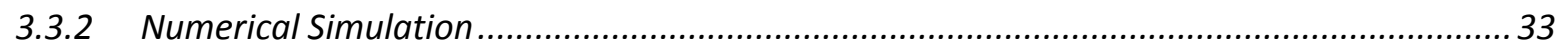

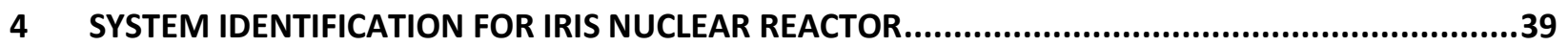

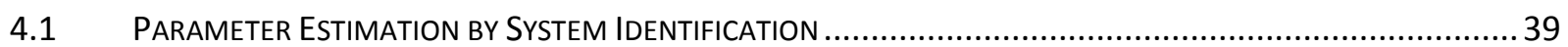

4.2 Prediction ERROR METHOd (PEM) FOR STATE-SPACE Model IDENTIFICATION ..................................... 40

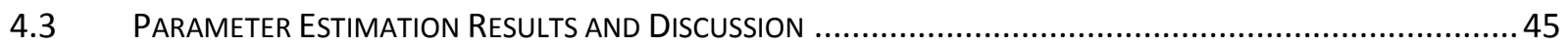

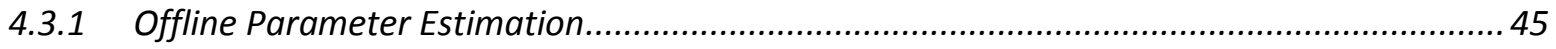

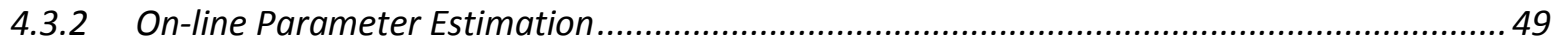

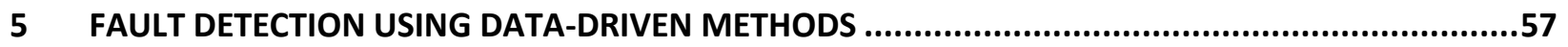

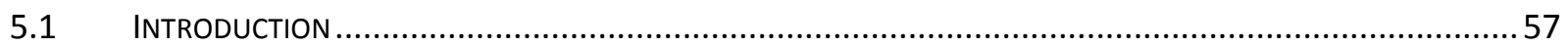

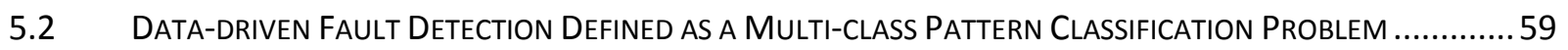

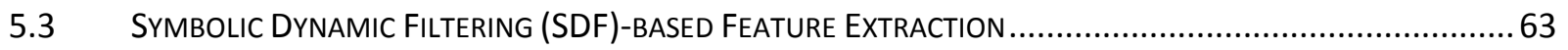

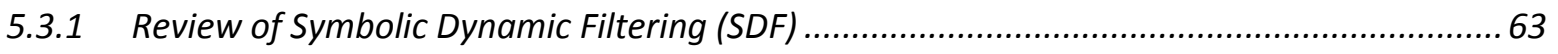

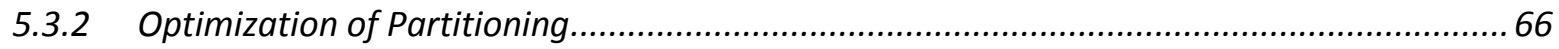

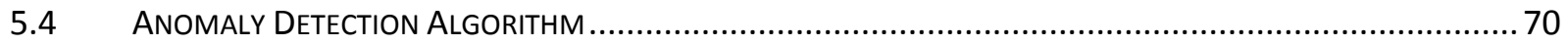

5.4.1 Symbolic Dynamic Filtering for Anomaly Detection ........................................................... 71

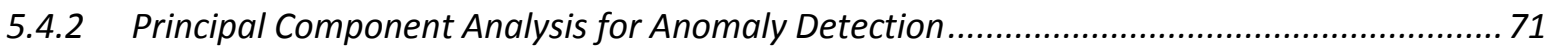

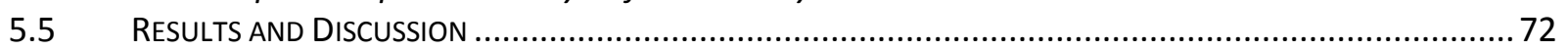

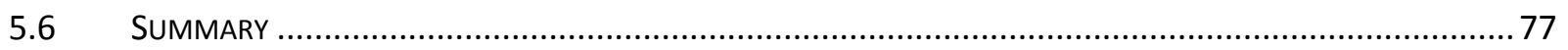

6 ROBUST MULTI-VARIABLE CONTROLLER DESIGN USING THE M-SYNTHESIS APPROACH..............79

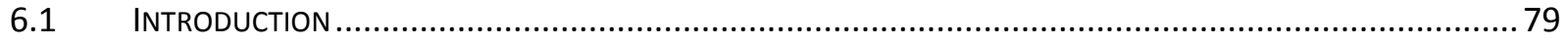




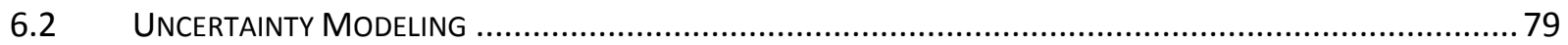

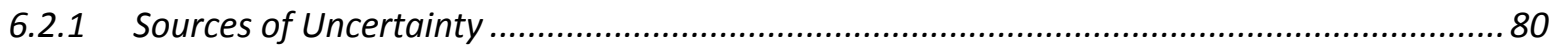

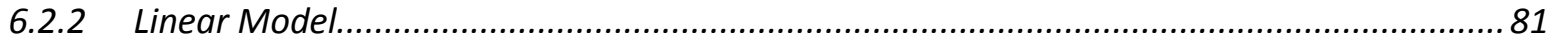

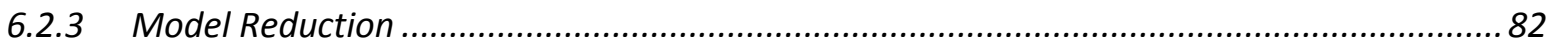

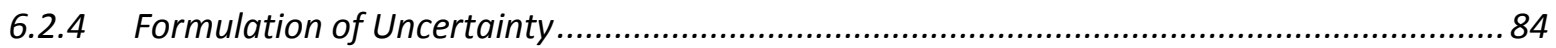

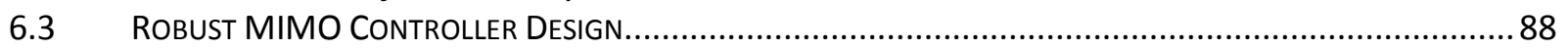

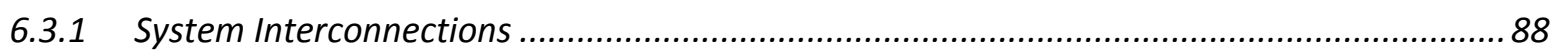

6.3.2 Selection of Performance Weighting Functions ............................................................ 90

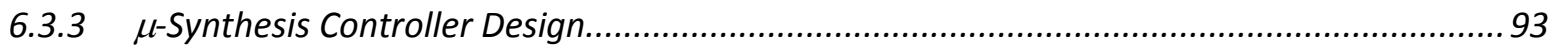

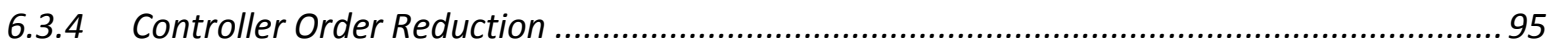

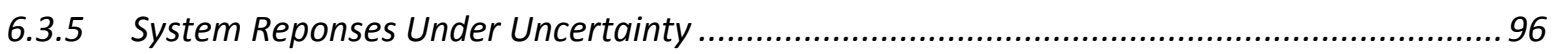

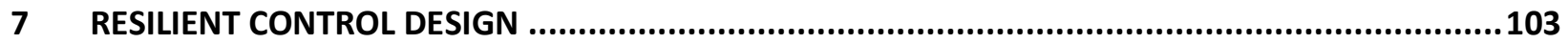

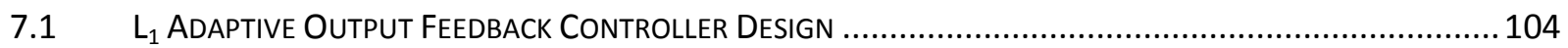

$7.2 \quad L_{1}$ AdaPtive Control LaW for NuClear Power Plant Model ................................................... 107

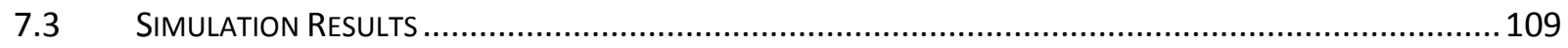

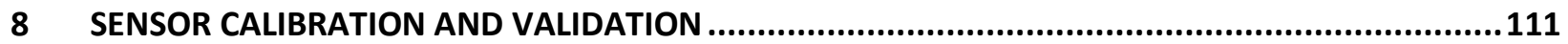

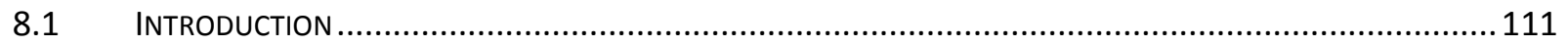

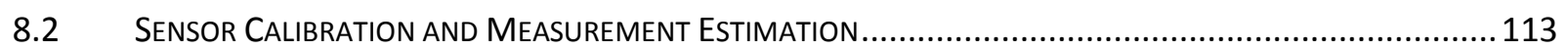

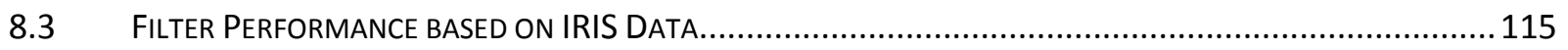

9 RELIABLE LQG CONTROLLER DESIGN WITH SENSOR FAILURE ..............................................119

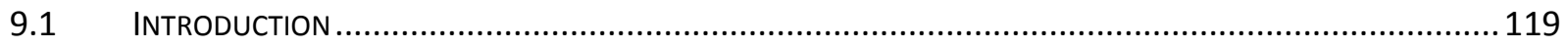

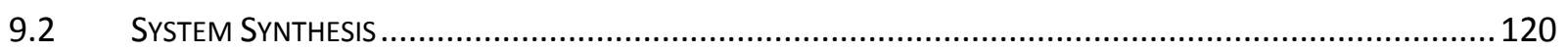

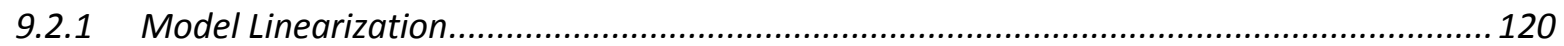

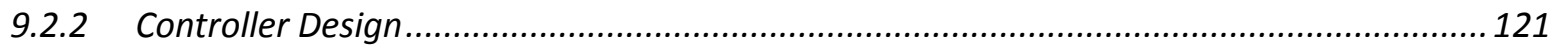

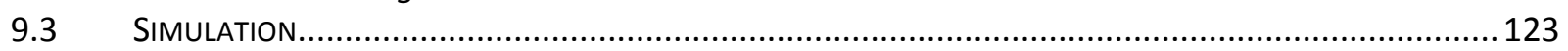

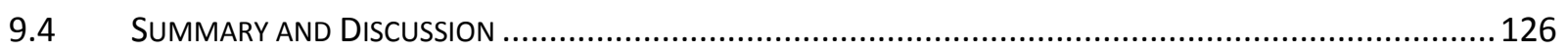

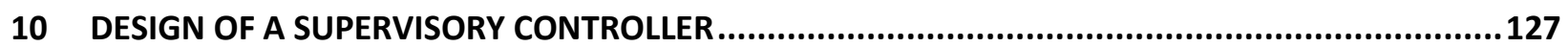

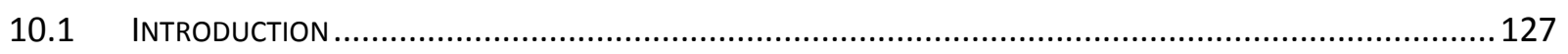

10.2 SUPERVISORY CONTROL ARCHITECTURE FOR DISCRETE-EVENT SYSTEM .......................................... 128

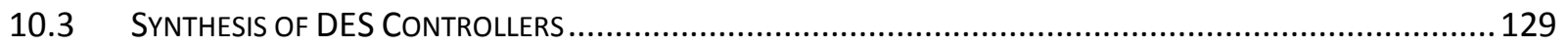

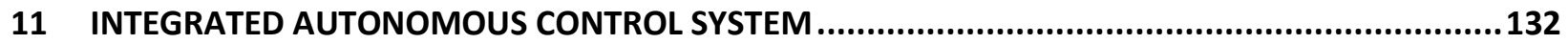

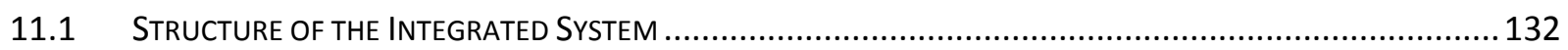

11.2 SYSTEM BEhAVIOR DURING NORMAL OPERATIONS AND FAULTY CONDITIONS .....................................134

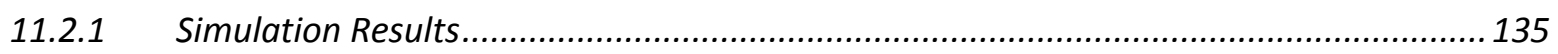

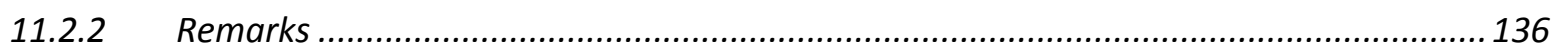

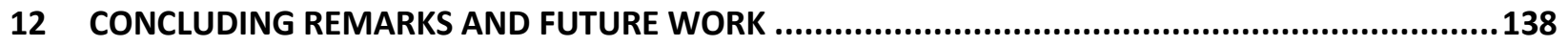

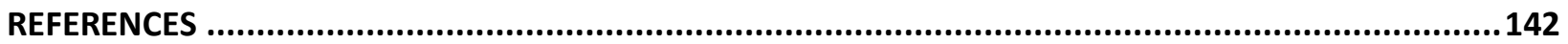

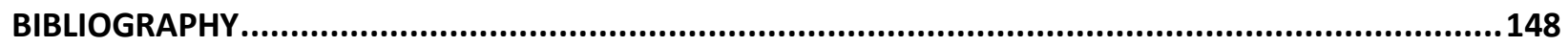




\section{LIST OF FIGURES}

Figure 1.1: Schematic of the autonomous and hierarchical control function architecture ............ 2

Figure 1.2: Layout of the primary side of the IRIS system .............................................. 4

Figure 1.3: Integration of advanced instrumentation and controls modules for small modular

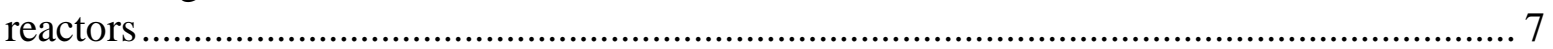

Figure 1.4: Hierarchical Structure of Autonomous Controller for Nuclear Power Plant ............. 9

Figure 1.5: Dynamic responses of the reactor core model to a 10 cent step increase in the external

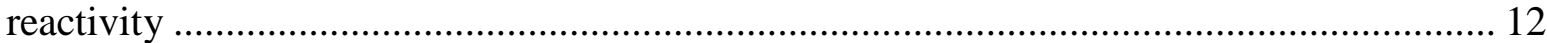

Figure 1.6: HCSG system responses..................................................................... 13

Figure 1.7: Dynamic responses of the reactor primary side model to a 10 cent step increase in the

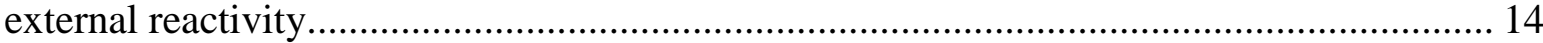

Figure 1.8: Performance of MPC over HCSG model ….................................................... 17

Figure 1.9: MPC performance with 1\% measurement noise ................................................. 18

Figure 1.10: Steam pressure change with actuator failure .................................................. 19

Figure 1.11: Steam flow rate change with actuator failure ................................................. 19

Figure 1.12: The effect of uncertainties in heat transfer coefficient to the MPC performance in

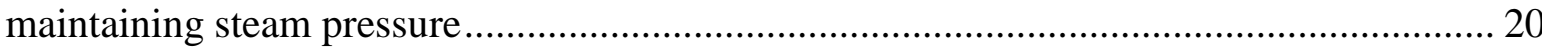

Figure 1.13: The effect of uncertainties in heat transfer coefficient on the MPC performance in

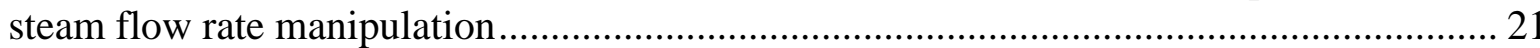

Figure 1.14: The effect of uncertainties in heat transfer coefficient on the MPC performance in

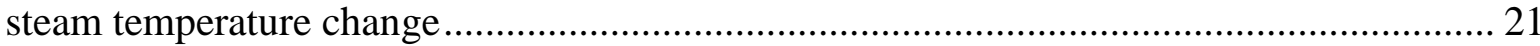

Figure 1.15: MPC performance with system internal parameter change ................................ 23

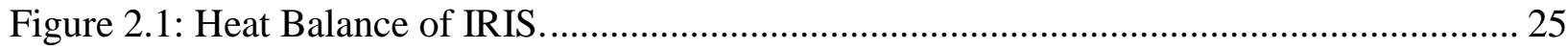

Figure 2.2: Nodalization of IRIS FORTRAN Model [8] ................................................ 26

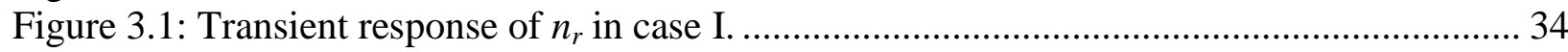

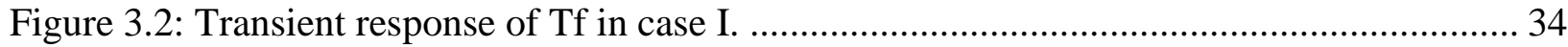

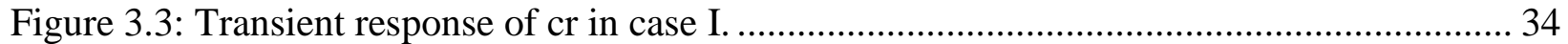

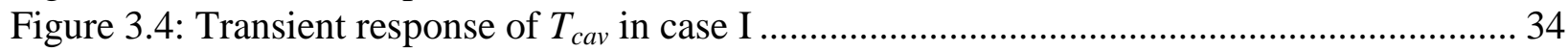

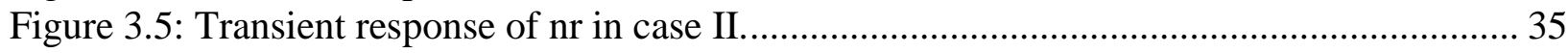

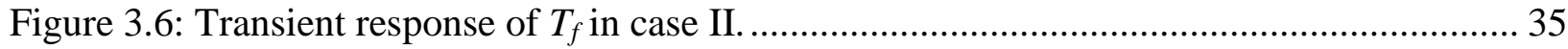

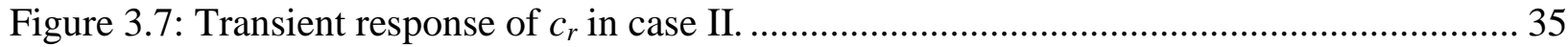

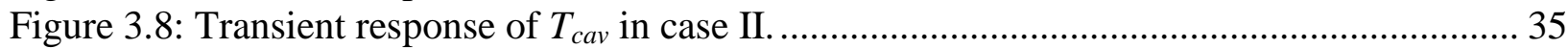

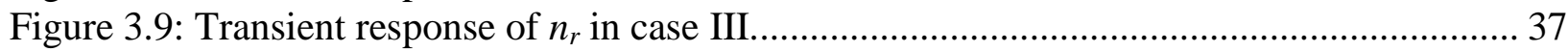

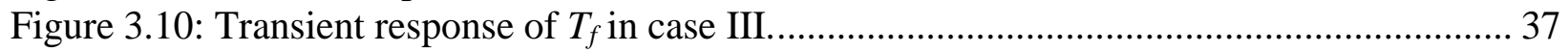

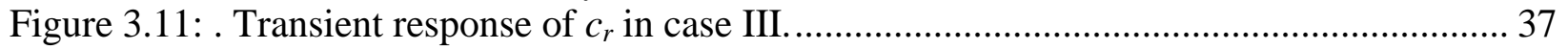

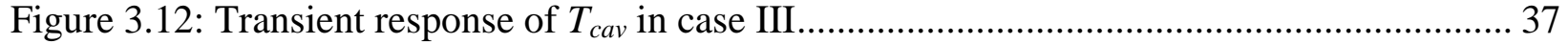

Figure 4.1: Small change reference load signal and reactor relative power response ................. 45

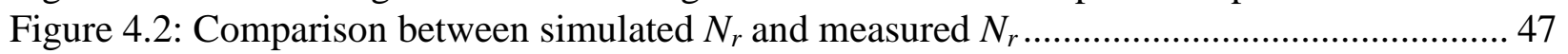

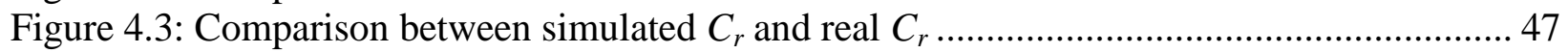

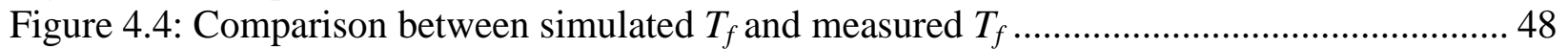

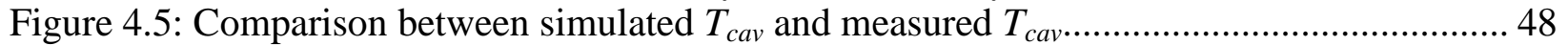

Figure 4.6: Prediction of $N_{r}$ by the offline estimation model ............................................ 49

Figure 4.7: Prediction Error of $N_{r}$ by the offline estimation model....................................... 49

Figure 4.8: Prediction of $C_{r}$ by the offline estimation model .............................................. 50

Figure 4.9: Prediction Error of $C_{r}$ by the offline estimation model......................................... 50 
Figure 4.10: Prediction of $T_{f}$ by the offline estimation model ............................................ 50

Figure 4.11: Prediction Error of $T_{f}$ by the offline estimation model...................................... 51

Figure 4.12: Prediction of $T_{\text {cav }}$ by the offline estimation model ............................................ 51

Figure 4.13: Prediction Error of $T_{c a v}$ by the offline estimation model................................... 51

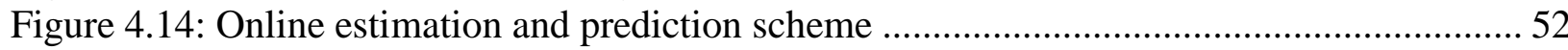

Figure 4.15: Prediction of $N_{r}$ by the online estimation model ............................................ 53

Figure 4.16: Prediction of $C_{r}$ by the online estimation model .......................................... 53

Figure 4.17: Prediction of $T_{f}$ by the online estimation model.............................................. 54

Figure 4.18: Prediction of $T_{\text {cav }}$ by the online estimation model............................................ 54

Figure 4.19: Prediction Error of $N_{r}$ by the offline and online estimation model ....................... 54

Figure 4.20: Prediction Error of $C_{r}$ by the offline and online estimation model ....................... 55

Figure 4.21: Prediction Error of $T_{f}$ by the offline and online estimation model ........................ 55

Figure 4.22: Prediction Error of $T_{\text {cav }}$ by the offline and online estimation model ..................... 55

Figure 5.1: Original class labels for data collection .......................................................... 61

Figure 5.2: Profile of turbine load and turbine output response ............................................ 62

Figure 5.3: Representative time series data for RCP fault and $T_{H L}$ degradation conditions ........ 62

Figure 5.4: Revised class assignment for fault detection............................................... 63

Figure 5.5: Pictorial view of the two time scales: (i) Slow time scale of anomaly evolution and

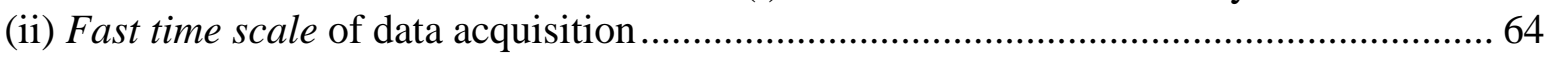

Figure 5.6: Concepts of phase space partitioning for symbolization..................................... 65

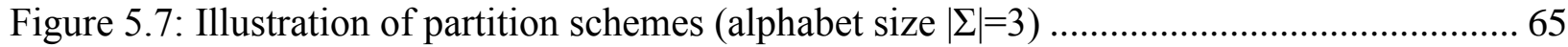

Figure 5.8: General framework for optimization of feature extraction ................................... 69

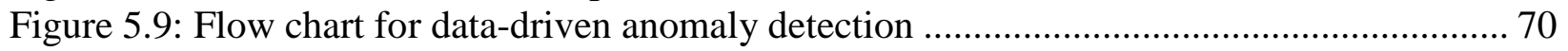

Figure 5.10: Two dimensional objective space for partitioning optimization ........................... 73

Figure 5.11: Feature space of the training set using optimal partitioning ................................ 74

Figure 5.12: Feature space of the training set-uniform partitioning (UP) ............................. 75

Figure 5.13: Feature space of the training set - maximum entropy partitioning......................... 75

Figure 5.14: Classification error vs. neighbor size in $k$-NN classifiers .................................. 76

Figure 6.1: Plant uncertainties between the real plant and reduced model............................... 80

Figure 6.2: Input-Output Representation of the MIMO Plant Model ..................................... 81

Figure 6.3: Frequency responses of original linear model (solid) and minimal model (dotted) .. 85 Figure 6.4: Time domain responses of original linear model (solid) and minimal model (dotted)

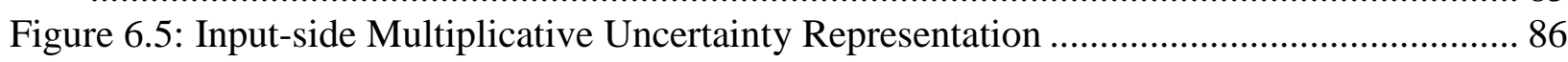

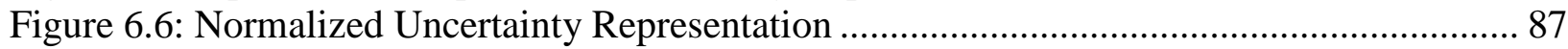

Figure 6.7: Frequency Response of Uncertainty Weighting Function .................................... 88

Figure 6.8: Block diagram of the closed-loop system with performance specifications ............. 89

Figure 6.9: Block diagram of the open-loop interconnection with performance specifications... 89

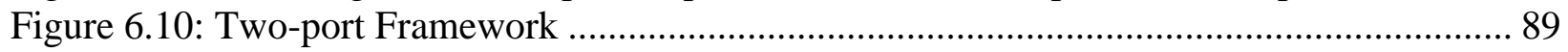

Figure 6.11: Frequency Response of Output Tracking Error Weighting Function .................... 91

Figure 6.12: Frequency Response of Control Action Weighting Function ............................. 92

Figure 6.13: Frequency Response of Sensor Noise Weighting Function ................................ 93

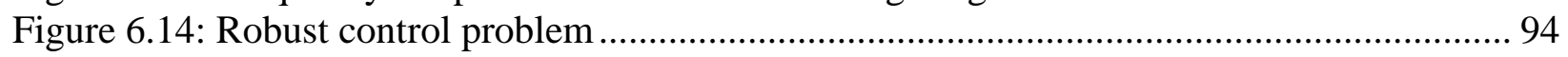

Figure 6.15: Magnitude plots of the full- and reduced-order $\mu$-controllers ............................... 96

Figure 6.16: Phase plots of the full- and reduced-order $\mu$-controllers ..................................... 96

Figure 6.17: Connections between Plant Model and Robust Controller .................................... 97 
Figure 6.18: Comparison of closed-loop system responses at 5\% step decrease of output power load demand, between linear and nonlinear plant models, controlled by reduced-order controller

Figure 6.19: Closed-loop system responses at 5\% step decrease of output power load demand, with linear plant model, controlled by full- and reduced-order controller

Figure 6.20: Linear system response at 5\% step decrease of output power load demand, perturbed by a 0.01 variance zero mean sensor noise

Figure 6.21: Nonlinear system response at 5\% step decrease of output power load demand, perturbed by a 0.01 variance zero mean sensor noise.

Figure 6.22: Closed-loop system responses to disturbance from steam flow ( 0.1 step increase at $\mathrm{t}=50 \mathrm{~s}$, lasting for 10 seconds)

Figure 6.23: Closed-loop system responses to disturbance from rod reactivity ( 0.5 step increase

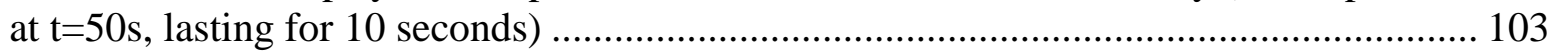

Figure 7.1: Closed loop system with the $\mathrm{L}_{1}$ adaptive controller.......................................... 105

Figure 7.2: Desired system frequency response ............................................................. 108

Figure 7.3: Low-pass filter frequency response............................................................. 109

Figure 7.4: Closed-loop system responses at 5\% step decrease of output power load demand,

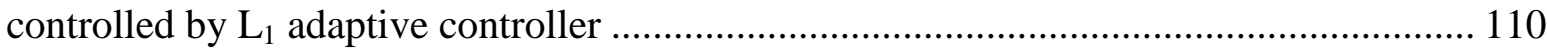

Figure 8.1: Uncalibrated Sensor Data and the Estimate ................................................. 117

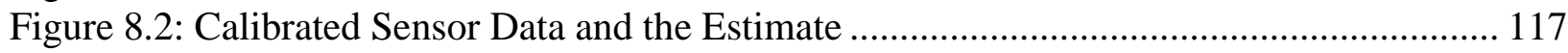

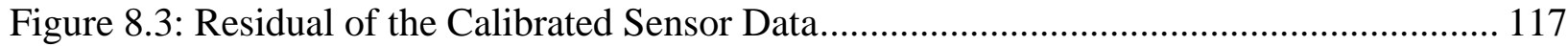

Figure 8.4: Calibrated and Uncalibrated Estimates ........................................................ 118

Figure 8.5: Correction for Sensor Data Calibration........................................................ 118

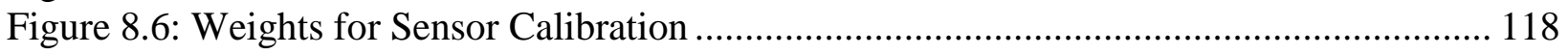

Figure 9.1: Closed loop system sketch. ................................................................... 121

Figure 9.2: Dynamic response of Case I ................................................................... 124

Figure 9.3: Control effects of Case I............................................................................ 124

Figure 9.4: Dynamic response of Case II....................................................................... 125

Figure 9.5: Control effects of Case II .......................................................................... 125

Figure 10.1: Supervisory control architecture of the NPP system ...................................... 129

Figure 10.2: Example of unsupervised plant DFSA model at the plant level......................... 131

Figure 10.3: Example of supervised plant DFSA model at the plant level............................ 131

Figure 11.1: Structure of the integrated autonomous control system ................................... 134

Figure 11.2: Normalized plant outputs ........................................................................ 136

Figure 11.3: Control actions of integrated robust and resilient controller ............................ 137

Figure 11.4: Temperatures of Steam, Primary Inlet and Outlet Flows .................................. 137

Figure 11.5: Finite automaton alphabets and output power set point changes ........................ 137 


\section{LIST OF TABLES}

Table 2.1: Description of Nodes of the IRIS FORTRAN Model ...................................................... 26

Table 3.1: Parameters of DHGF for Simulation of Case III. ......................................................... 37

Table 4.1: Initial value range of coefficients of the point kinetics model.................................... 46

Table 4.2: Estimated coefficients values of IRIS reactor model................................................. 48

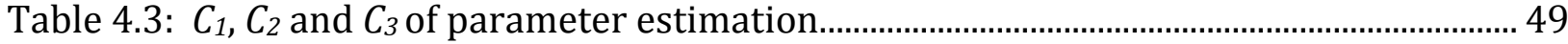

Table 4.4: $C_{1}, C_{2}$ and $C_{3}$ of offline estimation for the four state variables ............................... 52

Table 4.5: $C_{1}, C_{2}$ and $C_{3}$ of online estimation for the four state variables ................................... 56

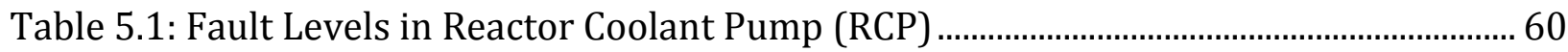

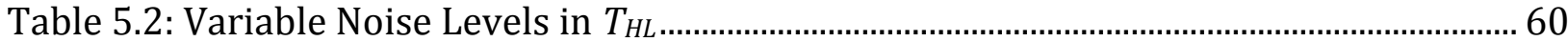

Table 5.3: Comparison of Classification Performance of Different Methods on Test Data Set

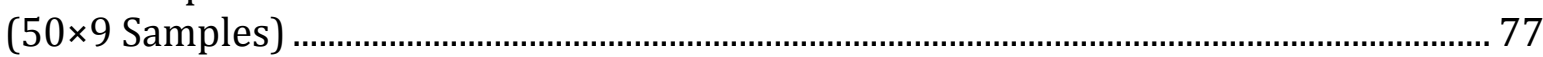

Table 6.1: Comparison of Different Order Reduction Results .................................................... 84

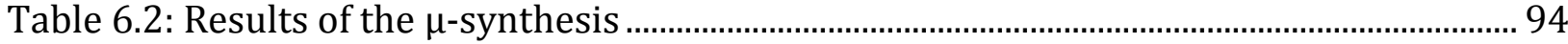

Table 6.3: Summary of different reduced orders of Controller \# 5................................................. 95 


\section{DEVELOPMENT OF AUTONOMOUS AND MODEL-PREDICTIVE CONTROL STRATEGY FOR THE IRIS SYSTEM}

\subsection{Introduction}

The basic requirement of the modular reactor control system is to achieve stable control at different operation modes and enable a stable transition between these operation modes. The major operation modes include plant start-up, normal operation at different power levels, hot standby mode for system testing, and cold shutdown mode for plant maintenance. One of the important operational modes is the transition from electricity generation to providing process heat. It is anticipated that the primary application of process heat is for water desalination. It may be necessary to partition the total energy output between electricity generation and process heat supply. During the transitional modes, the reactor systems and equipment must be closely monitored and controlled to assure compliance with safety requirements.

In order to meet the remote deployment and proliferation-resistance requirements, as defined by safety, reliability, survivability, and life expectancy, the reactor control system should incorporate advanced features such as fault tolerance, control loop performance monitoring, and safety monitoring. Figure $\mathbf{1 . 1}$ shows the hierarchical control features necessary for the autonomous control of a modular reactor system (Upadhyaya, 2006; Anastaklis et al, 1991).

To realize unattended operation and address remote communication, fault tolerant control with controller reconfiguration, should be an important feature of the control system design. If a fault occurs in a control system, a fault diagnosis system should be able to perform reliable and timely fault detection and isolation before serious consequences may occur. This fault diagnostic information may be further used for both operation planning and controller reconfiguration. The design objective of this fault tolerant control system is to enable a stable control under anticipated conditions with incipient faults in sensors, actuators, and reactor components. This function will be accomplished by the first level tasks in the schematic shown in Figure 1.1. The integration of control performance monitoring and automatic tuning feature provides the reactor control system with adaptive capability. In addition, a computerized emergency operation procedure should be in place to support automatic decision-making and control during accident conditions. Accurate and prompt communication between the reactor site and a centralized 
command center is a necessary feature of these reactors. This autonomous or semi-autonomous control system can be implemented by a hierarchical paradigm, as shown in Figure 1.1.

In this hierarchical control paradigm, the bottom level has a direct interface with the reactor systems and is responsible for executing fault detection and isolation, fault tolerant control, and abnormal event control. The middle level acts as a coordination between the plant manager level and the execution level. The coordination includes the determination of what is the performance of current control structure and whether it is necessary to tune the controllers or switch the control modes. Significant uncertainties can be dealt with by designing adaptive control laws for unanticipated situations and producing control sequences based on current operating conditions. The top level is a plant manager that manages plant level performance monitoring, plant condition evaluation, capability assessments, and task planning. In addition, the plant manager can transmit reactor operational data to and receive instructions from a central command, control, and communication $\left(\mathrm{C}^{3}\right)$ system.

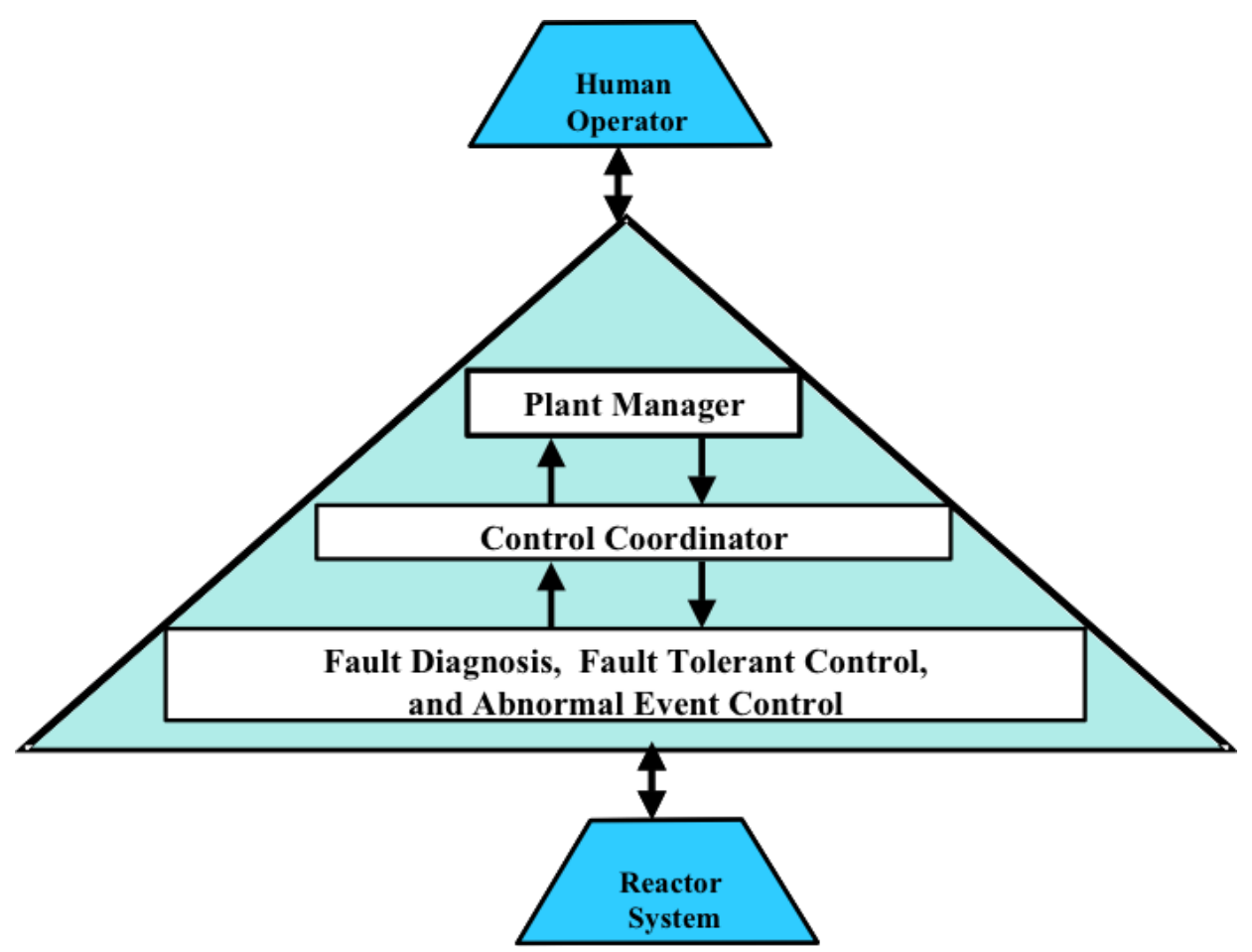

Figure 1.1: Schematic of the autonomous and hierarchical control function architecture

The classical proportional-integral (PI) controller and the optimal model-predictive controller (MPC) designs are evaluated using the IRIS dynamic model. This plant model is developed 
using MATLAB/SIMULINK, and then applied to the 'real plant'. The high-fidelity IRIS simulator is treated as the 'real plant'.

\subsection{Brief Description of the IRIS Reactor}

International Reactor Innovative and Secure (IRIS) is one of the next generation nuclear reactor designs. It is an integral pressurized water reactor (PWR) that houses the steam generators, circulation pumps, and the pressurizer inside the reactor vessel. Figure 1.2 shows the layout of the primary side of the IRIS system. The advantages of this integral design include the following:

$>$ The pressurizer, eight steam generators, and the control rod mechanism are integrated into the pressure vessel with the reactor core. There is no huge pipe used to connect these components. This design avoids the large loss of coolant (LLC) accident.

$>$ The pressure vessel for IRIS reactor is very tall. The whole control rod mechanism is mounted inside the vessel. So that the control rod head penetration failure is avoided.

$>$ The integral design of the primary side also makes the containment vessel much smaller than the traditional PWR pressure vessel.

$>$ IRIS is being designed to fulfill the advantages of the integrated primary system reactor. It improves safety, reduces the site civil works, and improves plant availability for developed and developing countries with large or small electrical grids.

$>$ The development of autonomous and fault-tolerant control strategy is well-suited for remote deployment of small and medium reactors, such as the IRIS. 

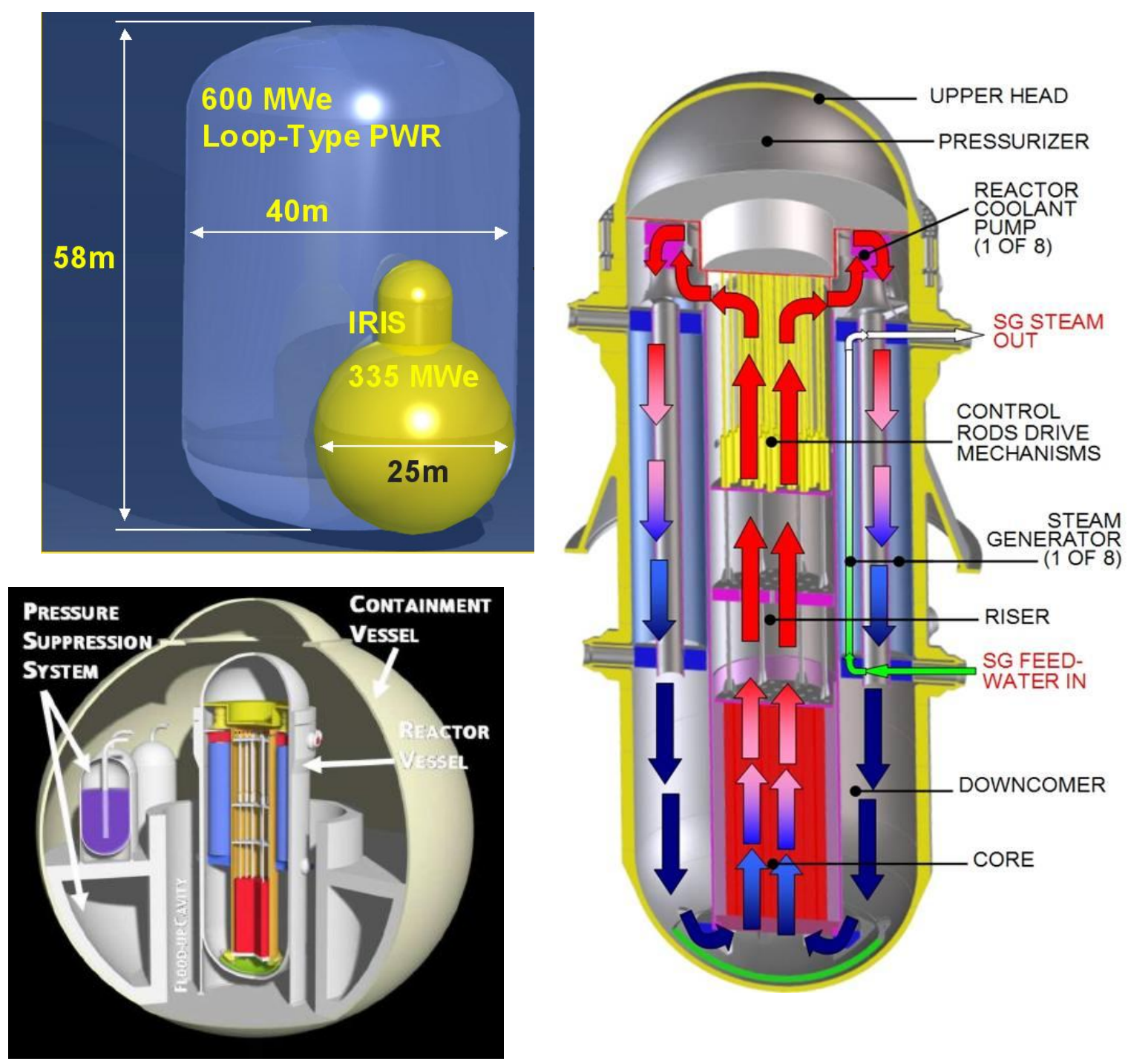

Figure 1.2: Layout of the primary side of the IRIS system

\subsection{Review of Literature on Autonomous Control}

With the fast development of modern industry, autonomous control and fault-tolerant controllers are getting increased attention of researchers and engineers from different areas. From the robotic arms in the auto industry [Rosheim, 1994] to the robot servants [Wilkes, 1997], from the unmanned flight [Mouloua, 2003] to the space vehicles [Wie, 1998], autonomous controllers with different control levels are employed to fulfill different tasks. With a good design of the autonomous control or sophisticated control system, machines can free humans 
from dangerous or unreachable working environments; also it limits the non-safety and nonstability that human factors may bring to the system by incorrect operations.

In this project, we use the IRIS reactor [Carreli, 2000-2003] as the model to develop the autonomous control framework. IRIS reactor is an integral primary system reactor. The reactor core, helical coil steam generators, pressurizer, and the control rod mechanisms are all integrated inside the pressure vessel, and the length of the fuel cycle is 3-3.5 years. If there is a minor anomaly in the system, the operator wants the reactor system to be running under a downgraded condition without violating the safety and stability issues, instead of shutting it down. Therefore, the autonomous control system is needed to fulfill this design concern.

An autonomous control system has the ability to learn, to predict, and to design the routine to reach the goal by itself, when it is running under significant uncertainties in the environment and for an extended period of time. The system must have a high degree of tolerance to failures without external intervention [Antsaklis, 1989,1991,1992]. It must have a high degree of flexibility to reach higher degrees of autonomy [Saridis, 1979]. To design such a sophisticated system, the control designer should have a clear and detailed knowledge base, which includes a complete list of system variables that can be measured, a complete list of system variables that can be controlled, a complete list of potential failures, a table of repair plans to recover the failures, and a well designed learning algorithm to update the system database if the system encounters some unknown situations.

To complete the above list, a well-designed performance monitoring system is needed. Sensor placement is an ongoing research work to find out an effective coverage and surveillance for different sensors to limit the number of sensors being used but to fulfill the control goals [Dhillon, 2003]. Additionally, a detailed fault diagnostic system is needed, which can detect and isolate any type of system potential failure and relay the alarms to the autonomous system [Chen, 1999]. Fault tolerant control and reconfigurable control need to be designed to recover the system from the detected failures [Blanke, 2006; Bodson, 1997]. Reinforcement learning [Sutten et al., 1998] can be employed to update the knowledge base of the autonomous system.

The process of achieving an effective autonomous control design is very complex. There are different areas of knowledge that need to be covered, and requires investigators from different technical areas working together to fulfill the same goal. For our part of the research, 
we are focusing on the framework of autonomous control design. We assume that all necessary measurements are available to us and all potential failures can be successfully detected and identified. The first step of our work is to complete the list of potential failures and design the corresponding recovery plans.

From previous operational experience with traditional PWRs and BWRs, the list of potential failures may be summarized as follows:

- Actuator failure: Feedwater pump trip, reactor coolant pump trip, turbine throttle valve failures, control rod mechanism failure, etc.

- Sensor failure: Temperature sensor failure (primary side coolant inlet temperature, primary side coolant outlet temperature, feed-water temperature, steam temperature), pressure sensor failure (steam pressure, pressurizer pressure, feed-water pressure, coolant pressure), flow-rate sensor failure (coolant flow-rate, feed-water flow-rate, steam flow-rate, bypass flow-rate), etc.

- "Internal" failure: Uncertainty in moderator temperature coefficient of reactivity, uncertainty in fuel temperature coefficient of reactivity, uncertainty coolant heat capacity, uncertainty in feed-water heat capacity, uncertainty in fuel-to-coolant heat transfer coefficient, etc.

\subsection{Development of the Autonomous Control Architecture}

The goal of applying autonomous control to IRIS reactor is to regulate the reactor to generate the desired energy continuously without violating operational safety issues and to provide control actions with minimum operator interference. The fundamental hardware elements of an autonomous system of the IRIS reactor include the components of the system (piping, valves, motors, etc.), sensors (temperature, pressure, flow rate, neutron detectors, etc.), and actuators (control rod, control valves, coolant pumps, etc.).

The architecture of the autonomous control for the IRIS system is given in Figure 1.3 and shows the key functional blocks

1. IRIS reactor: NCSU model, executed in FORTRAN.

2. Simulator: SIMULINK model including reactor core model, HCSG model, and balance of plant model.

3. Controller: Model Predictive Controller (MPC) as the control algorithm. 
4. Perception: Sensor placement and measurements.

5. Diagnoser: Assumes that all the anomalies can be identified correctly.

6. Models/Knowledge base: Updated as a function of current operating condition.

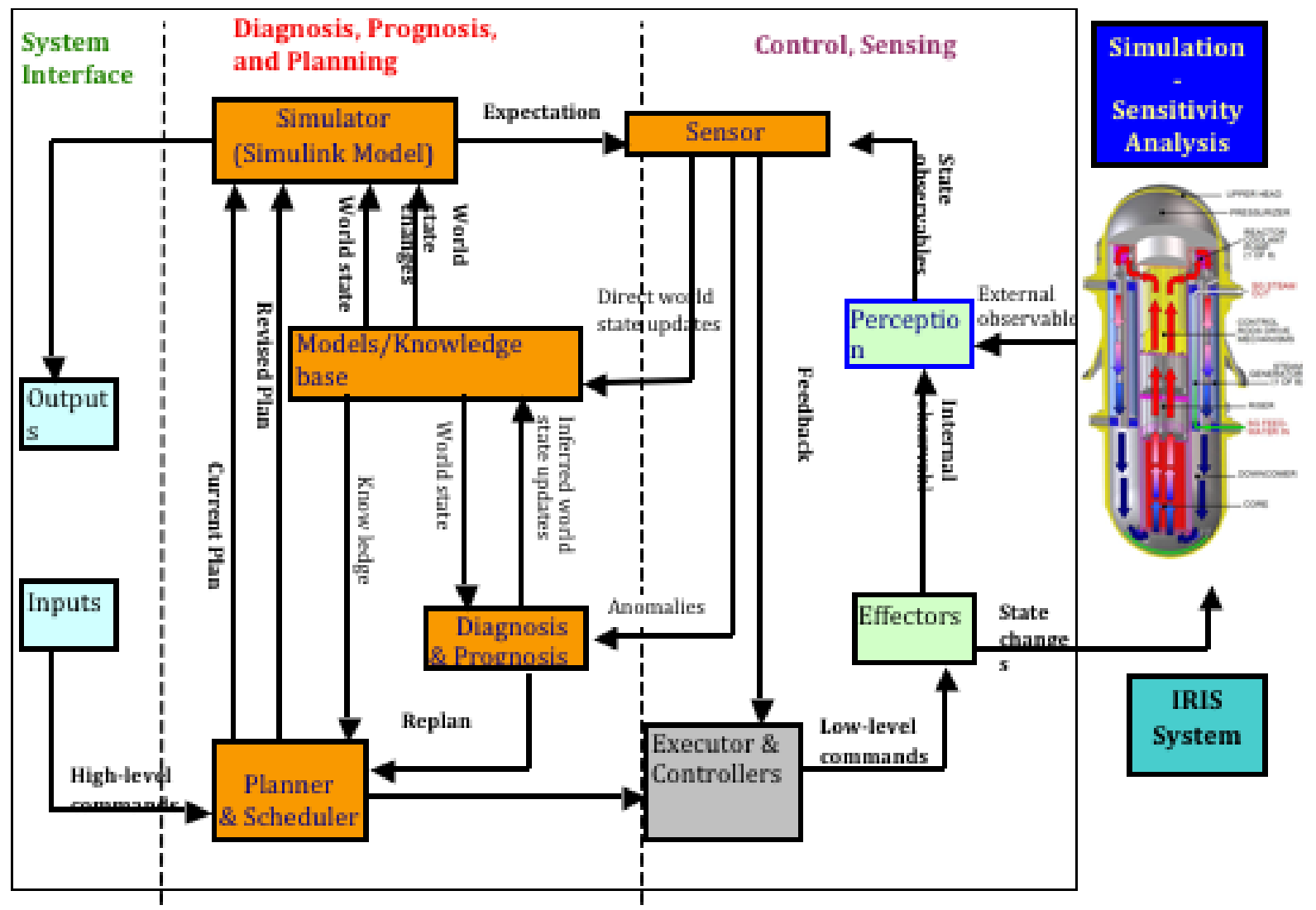

Figure 1.3: Integration of advanced instrumentation and controls modules for small modular reactors

The most important among the above functional blocks is the models/knowledge base, which includes the following models that need to be developed or defined:

- Planning models: Goal table.

- Operations models: State transition table.

Perception models: Sensor placement.

Diagnostic models: Causes-effect table.

Repair models: Fault recovery table. 
The entire autonomous system is the combination of all the hardware components and the software components, such that they work together to achieve a desired goal.

Autonomous control systems have the potential for operating with very high levels of performance and reliability with minimal or no human assistance. To be autonomous, a control system should provide adequate control actions in the presence of significant uncertainties. The key attributes of highly autonomous control systems are that they perform well under all process operating conditions and performance demands and are able to compensate for system failure without external intervention. More complex and sophisticated controllers are necessary to cope with changes in operating conditions relative to the reference model, the presence of large uncertainties in plant parameters and unmeasurable disturbances, the deterioration of components or component failures, etc. The conventional control techniques have evolved substantially over the past several decades from simple proportional-integral-derivative (PID) control to modern control such as optimal, adaptive, and robust control. Recently a novel control algorithm called resilient control has been developed and applied to the area of aerospace [3] and nuclear engineering [4] for operations under emergency situations. With the development of optimal, adaptive, robust, and resilient control the degree of autonomous operation of the controllers has increased. In order to further increase the availability and lower the accident risk and operating costs in nuclear power plants (NPPs), a higher level of automation and fault tolerance will be highly desirable. Recent efforts have been directed toward the development of highly autonomous control systems that can independently perform complex tasks. This trend is gaining momentum as control engineers solve many existing problems and seek to resolve new control problems that involve broader issues and require the full capabilities of available computing technologies. Although it is clear that conventional control will play a major role in the development of such highly automated systems, the main focus in the control community is to integrate the functions of intelligent systems such as fuzzy logic, neural networks, discreteevent supervisory control, genetic algorithms, and knowledge-based systems with the conventional control systems to perform complex tasks more easily. Intelligent control systems are typically able to perform one or more of the following functions to achieve autonomous behavior: planning actions at different levels of detail; emulating human expert behavior; learning from past experiences; integrating sensor information; identifying changes, such as failures, that threaten the system behavior; and reacting appropriately. The inclusion of the 
intelligent control aspect with conventional control techniques will encompass adaptation and learning; planning in the presence of large uncertainties; coping with huge data sets; and accommodation of plant dynamics, especially in complex nonlinear multivariable plants with numerous immeasurable parameters [5].

\subsubsection{Principles of Autonomous Control}

Antsaklis et al. [1] have defined functions, characteristics, and behaviors of an autonomous system that could be used as guidelines in the design of such a system. They have also suggested that a hierarchical control structure is desirable to achieve an increasingly sophisticated autonomous controller. This architecture contains three levels: execution level (lowest), coordination level, and management and organization level (highest). The general idea of the structure is that commands are issued by higher levels to lower levels and response data flows from lower levels to upper levels in the tier structure, as shown in Fig. 1.4. The functions of each of these levels are described below.

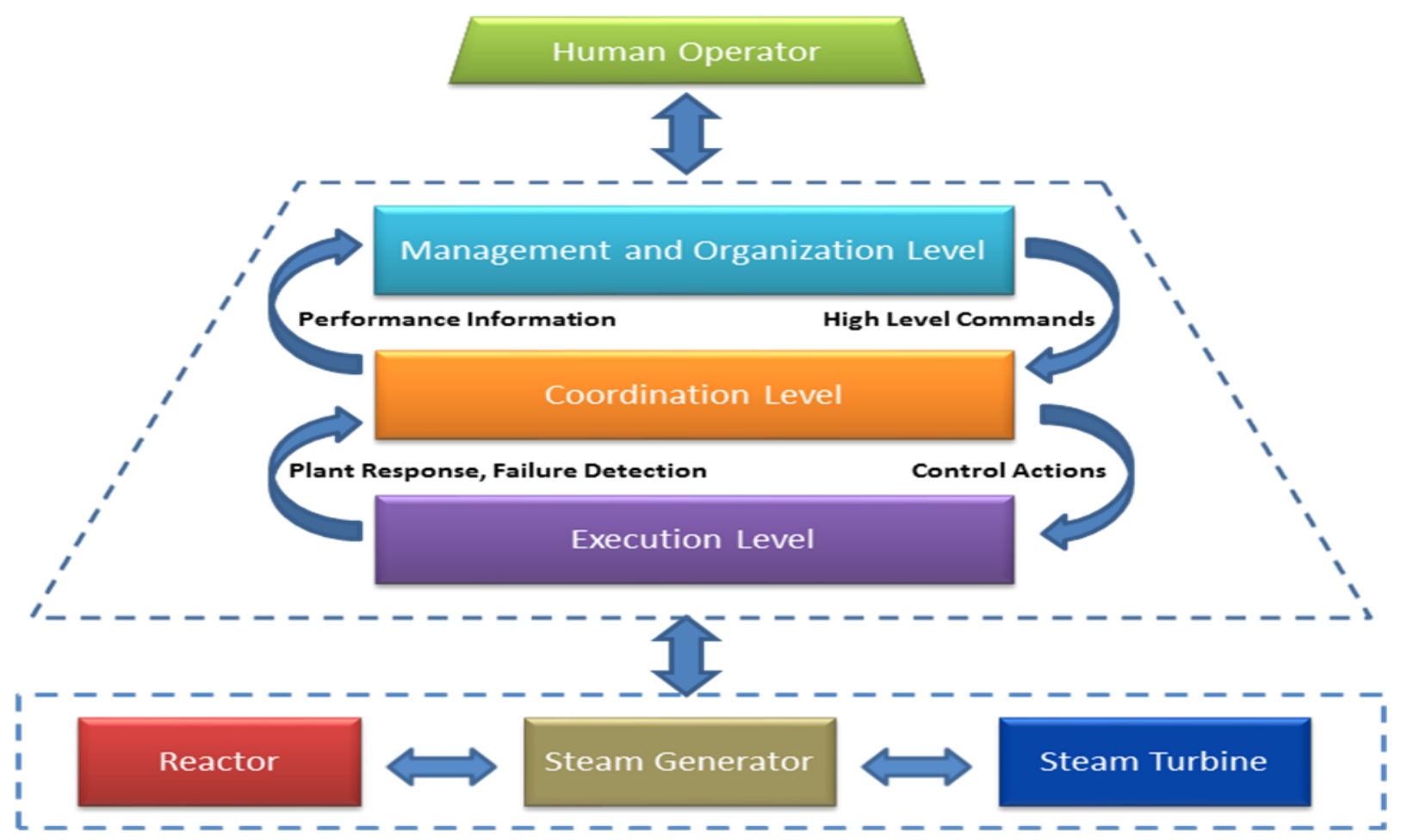

Figure 1.4: Hierarchical Structure of Autonomous Controller for Nuclear Power Plant

1. Execution level: The function of the execution level includes the generation of control actions as dictated by the higher levels and application of this control action to the 
actuators and sensors that drive the plant under control. The execution level also senses the plant responses and environment, processes them to identify parameters, detects any failures, and passes the information to the higher levels. This level possesses conventional adaptive control capabilities and includes failure detection algorithms, state estimators and parameter identifiers.

2. Coordination level: This level translates high-level commands into sequences of action to be passed to the execution level and provides the appropriate sequence of control and identification algorithms. The coordination level can cope with limited pre-determined crisis situations.

3. Management and organization level: This level determines the system goals and supports the human communication interface, and oversees and directs all the activities at both the coordination and execution levels. It is the most intelligent of the three levels.

A similar three-tier structure is also proposed by Williams and Jouse [6]. The proposed controller has been designed for the feedwater heater in an NPP.

It is an established fact that developments of optimal control, adaptive control, robust and resilient control have significantly increased the level of automation by encompassing higher levels of plant characterization uncertainty and parameter changes. However, integration of intelligent components such as fuzzy logic, neural networks, genetic algorithms, support vector machines, and discrete-event supervisory control, with the conventional control methodologies will further enhance the level of automation and permit this automation to be achieved more easily. On the other hand, stability analysis of a system being controlled is needed to verify the performance of the controller. Validation of performance levels through experimental evaluations is needed. Above all, systematic controller design and construction methodologies are needed when intelligent control is used.

The activities in this report deal with different aspects of nuclear operations, but they are all separate components of an autonomous controller for an NPP. The discussions on these components are grouped in the areas such as state observer, system identification, robust control, resilient control, fault detection, identification and accommodation, and supervisory control. In 
the end of this report, a scheme is proposed to integrate those components to implement autonomous control.

\subsection{Development of the SIMULINK Model of the IRIS System}

A modular SIMULINK model of the IRIS plant is developed and includes reactor core, HCSG, pressurizer, and the balance-of-plant (high-pressure turbine, low-pressure turbine, condenser, and feedwater heater). The detailed physical models are listed in Appendix A.

The SIMULINK model is developed based on a previous dynamic model (Naghedolfeizi, 1990) and a SIMULINK model (Mattison, 2007) for a traditional PWR plant. The reactor core is uses the point reactor model. The fuel to coolant heat transfer uses the Mann's model. The pressurizer model and balance-of-the plant are adapted from Mattison's SIMULINK model. The helical coil steam generator (HCSG) is one of the critical components and a major contributor to the cost of IRIS design. The major difference between a traditional PWR and the IRIS reactor is in the steam generator model.

The IRIS reactor has eight HCSGs. The use of helical tubing reduces the size of the steam generator, and results in an efficient heat transfer with a larger heat transfer area per unit volume than straight tube steam generators. The HCSG generates superheated steam, with the secondary water flowing through the tubes and the primary water surrounding the tubes and circulating in a downward direction. The control objective of the HCSG system is to supply adequate amount of steam to meet the turbine demand. As power demand changes, the turbine throttle valve changes its position to follow the set point changes of turbine header pressure. In the meantime, in order to prevent the carryover of water to the turbine system or dry-out of the steam generator tubes, a feed-forward controller is used to minimize the mismatch between steam outlet flow rate and feed water inlet flow rate.

\subsubsection{SIMULINK Model of IRIS Reactor Core}

The IRIS core neutron kinetics and heat transfer model (SIMULINK) has been developed using steps similar to that for a typical PWR. IRIS design parameters are used for simulation. The performance characteristics of core dynamics are shown in Figure 1.5. The state variables are 
reactor power (fraction), fuel temperature change (deg F), and core-exit temperature change (deg F) for a 10-cent increase in the reactivity.
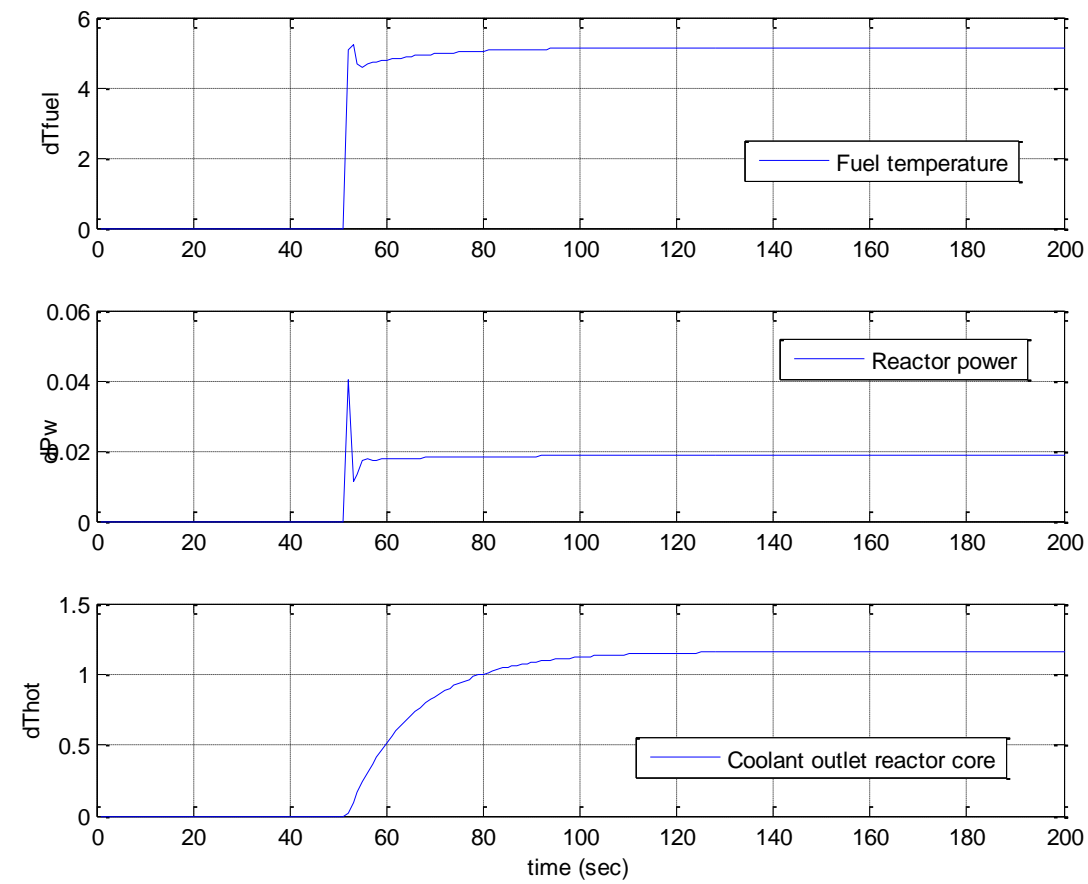

Figure 1.5: Dynamic responses of the reactor core model to a 10 cent step increase in the external reactivity

\subsubsection{SIMULINK Model of HCSG}

The steam generator (HCSG) is the key component that makes the IRIS reactor more efficient and more compact. When we constructed the SIMULINK model of the HCSG, we isolated the HCSG system from the reactor core and the balance-of-plant. There are two parameters we can manipulate to change the pressure of steam that flows out of HCSG. One is the outlet coolant temperature from reactor core, Tpout. The other is the steam flow rate that flows out of HCSG, Wsteam. Figure 1.6 shows HCSG system performance when these two variables are changed. A proportional-integral (PI) controller is integrated to the HCSG model to regulate the steam pressure to the set values by adjusting the steam flow rate. At the $100^{\text {th }}$ second, Tpout is increased by $2 \%$, which is shown in the top plot in Figure 1.6. In the third plot, the steam pressure started to increase corresponding to the increase in Tpout, but it came back to the set value after around 200 seconds. The set-point is indicated by the dashed red line in the $3^{\text {rd }}$ 
plot. Due to the increase of Tpout, the temperature of coolant that flows through the HCSG and back to the reactor core, Tpin, increases by about $10 \mathrm{deg} \mathrm{F}$, which is shown in the last plot. Another perturbation was made to HCSG model and is shown in the $2^{\text {nd }}$ plot. At the $400^{\text {th }}$ second, the steam flow was increased by $3 \%$. The steam pressure was decreased since more steam is removed from the HCSG tubes. The steam flow change has small impact on the coolant temperature, Tpin.
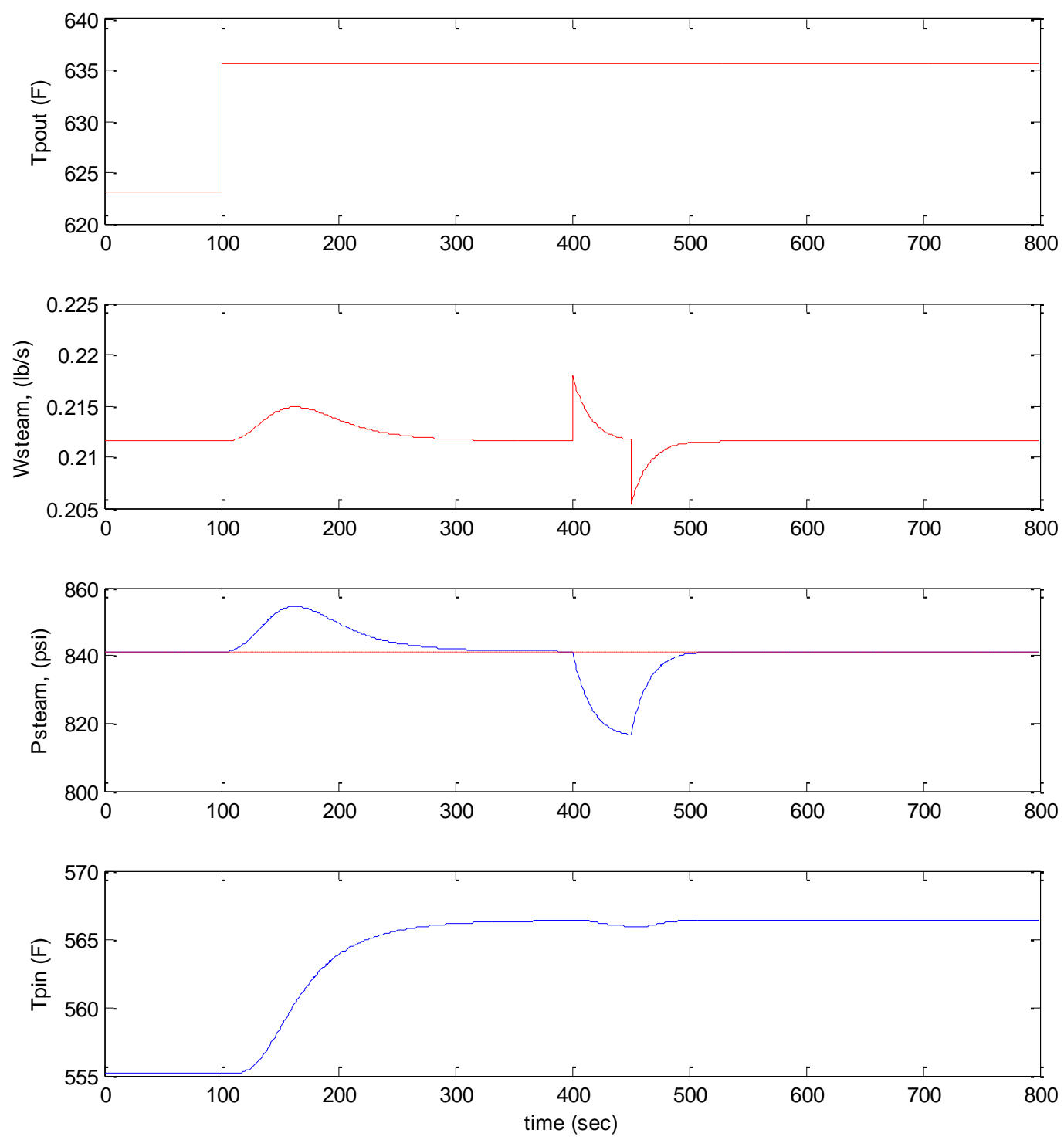

Figure 1.6: HCSG system responses 


\subsubsection{SIMULINK Model of the IRIS Primary Side}

A simple SIMULINK model of the pressurizer was developed, with only a heater input. Then, we integrated the reactor core model, HCSG model, and the pressurizer model to form a simplified primary side of IRIS power system. Figure 1.7 shows the dynamic response of the primary side of the IRIS system for a 10-cent external reactivity insertion. The pressures in both the pressurizer and the steam generators are controlled by traditional PI controllers.
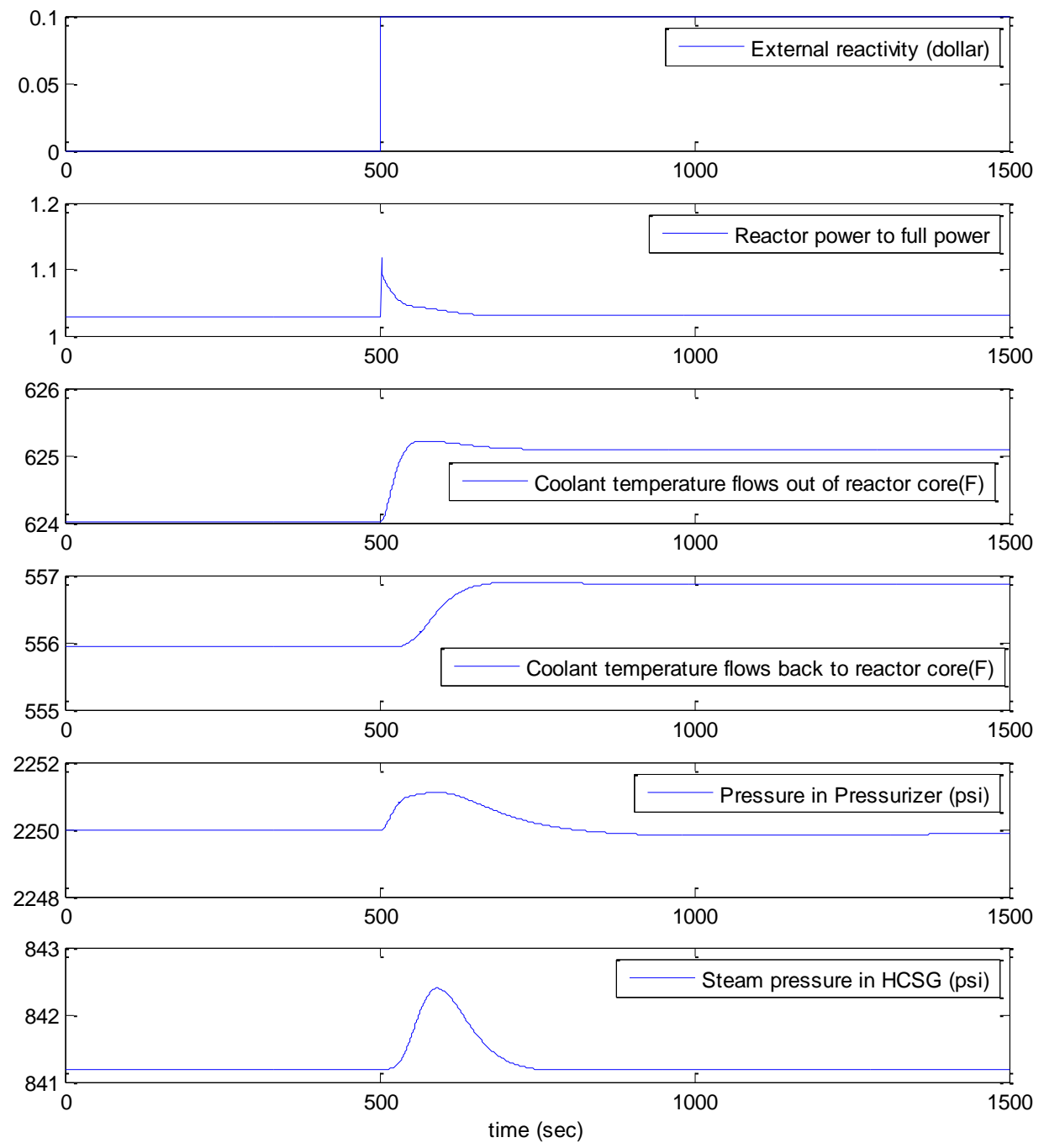

Figure 1.7: Dynamic responses of the reactor primary side model to a 10 cent step increase in the external reactivity 


\subsubsection{SIMULINK Model of the Balance-of-Plant}

The model for balance of plant is the same as that used in a traditional PWR. The electric power generated by the turbines is a function of feedwater flow rate and steam pressure. Some fine tuning is needed to make the output electric power match the desired power.

\subsection{Design of MPC Controller for the IRIS HCSG System}

The design of the Model Predictive Controller (MPC) for IRIS HCSG is one of the major contributions in the development of new control methods and implementation.

The objective of this part of the R\&D is to design a model predictive controller (MPC) for the HCSG. A MATLAB/SIMULINK model of the HCSG is used to design the MPC, in order to regulate the steam pressure. The actuator action is achieved by controlling the steam outlet flow rate. A measurement noise equivalent to $1 \%$ of the steam pressure is added, in order to test the capability of the MPC design to handle noise contamination. In addition, a configurable control strategy is designed to overcome turbine control valve malfunction.

\subsubsection{Methodology}

This research uses the MPC toolbox available in the MATLAB software. The algorithm is based on solving a generalized dynamic control optimization problem with process input and output constraints. The cost function to be minimized is given by

$$
J=\frac{1}{2} \sum_{j=1}^{N} Q[\hat{y}(t+j \mid t)-w(t+j)]^{2}+\frac{1}{2} \sum_{j=1}^{M} R[\Delta u(t+j-1)]^{2}
$$

$J=$ the cost function

$u=$ process input vector

$\hat{y}(t+j / t)=\mathrm{j}$ step predicted output vector based on the time t's value

$w=$ system output vector

$N=$ prediction horizon

$M=$ control horizon

$Q$ and $R=$ weight matrices. 
The control problem requires the minimization of the cost function, $J$, with respect to the control change, $\Delta u$. The advantage of the MPC over the traditional PID controller is that it can integrate different types of constraints on the inputs and outputs, as shown below:

$$
\left\{\begin{array}{l}
\Delta u(t+j-1)=0 \text { for } j>M \\
u_{\min } \leq u(t) \leq u_{\max } \\
|\Delta u(t)| \leq \Delta u_{\max }
\end{array}\right.
$$

Furthermore, the MPC has the feature of control demand anticipation which is derived from the use of prediction horizon.

\subsubsection{Results of Application to the IRIS Steam Generator Control}

Figure 1.8 shows the changes in the major parameters of the HCSG, when the steam

pressure set point and the hot leg temperature change. At the $50^{\text {th }}$ second, the steam pressure set point is decreased by $1 \%$. With the hot leg temperature kept constant, the steam outlet flow rate initially increases, and the steam temperature and pressure decrease. At the 250th second, the hot leg temperature is perturbed by $+2{ }^{\circ} \mathrm{C}$, but the steam pressure set point is unchanged. In order to regulate the steam pressure, the steam flow rate increases, with a resulting increase in the steam temperature. 

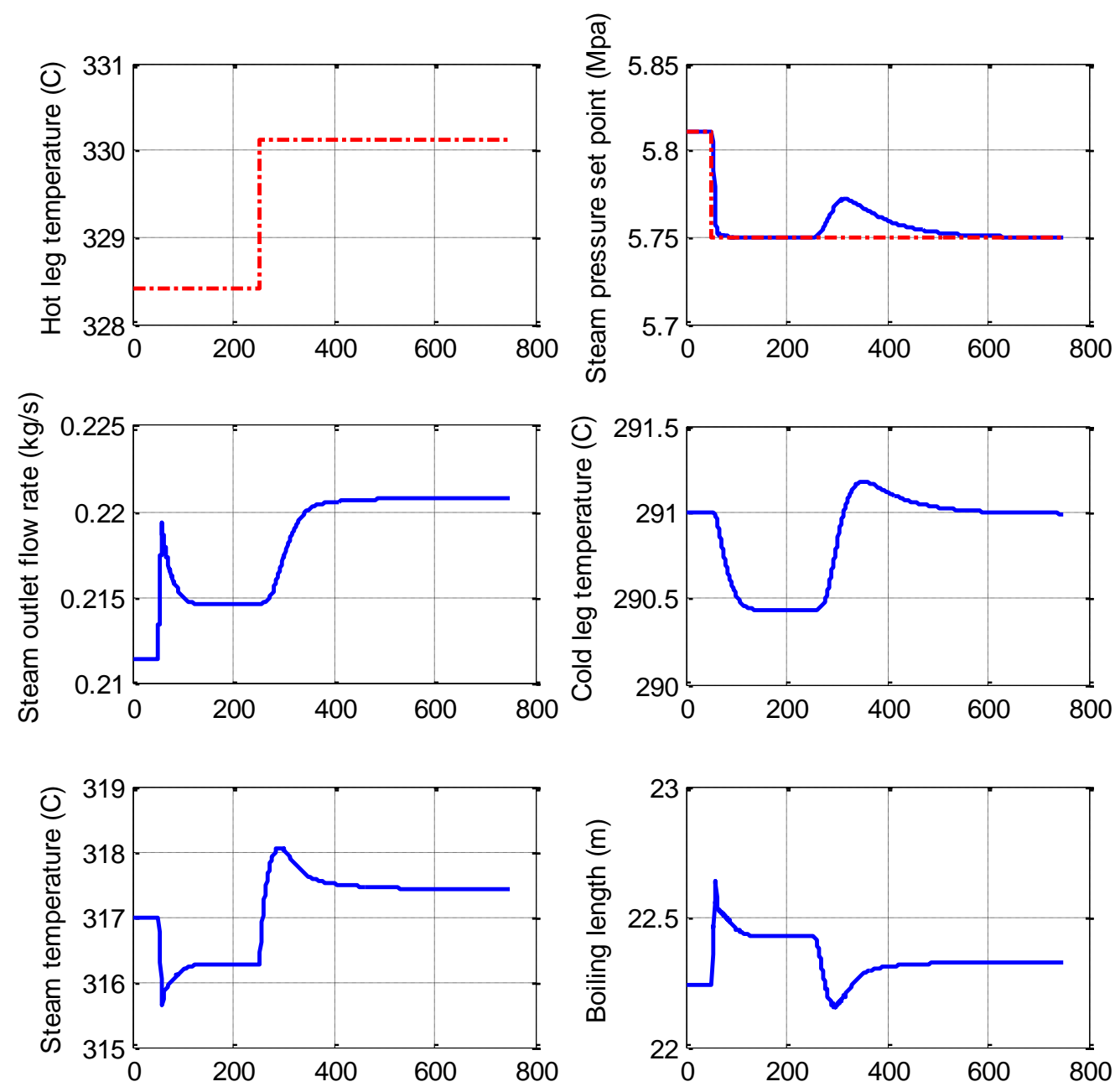

Figure 1.8: Performance of MPC over HCSG model

A more detailed analysis of the MPC using the SIMULINK model of the helical coil steam generator (HCSG) has been completed, and includes the capability of handling measurement noise, actuator failure, and internal failure.

The HCSG system is a single-input and single-output (SISO) system. The input variable is the steam flow rate and the output variable is the steam pressure. When we designed the MPC controller, we were taking the system output as the perturbation variable and the system input as the control variable. So when there is some measurement noise existing in the perturbation variable, it will affect the control performance of the system. Since MPC integrates the input and output constraints to its control algorithm, the difference between the measured value and target 
value will not cause a large jump in the control variable. Therefore the effect of the noise is limited. The MPC can still regulate the actual system output very close to the desired value.

Figure 1.9 shows the performance of the MPC when a $1 \%$ noise is added to the measured value of the steam pressure. The MPC is able to suppress the effect of measurement noise, and maintain the steam pressure very close to the set point value.

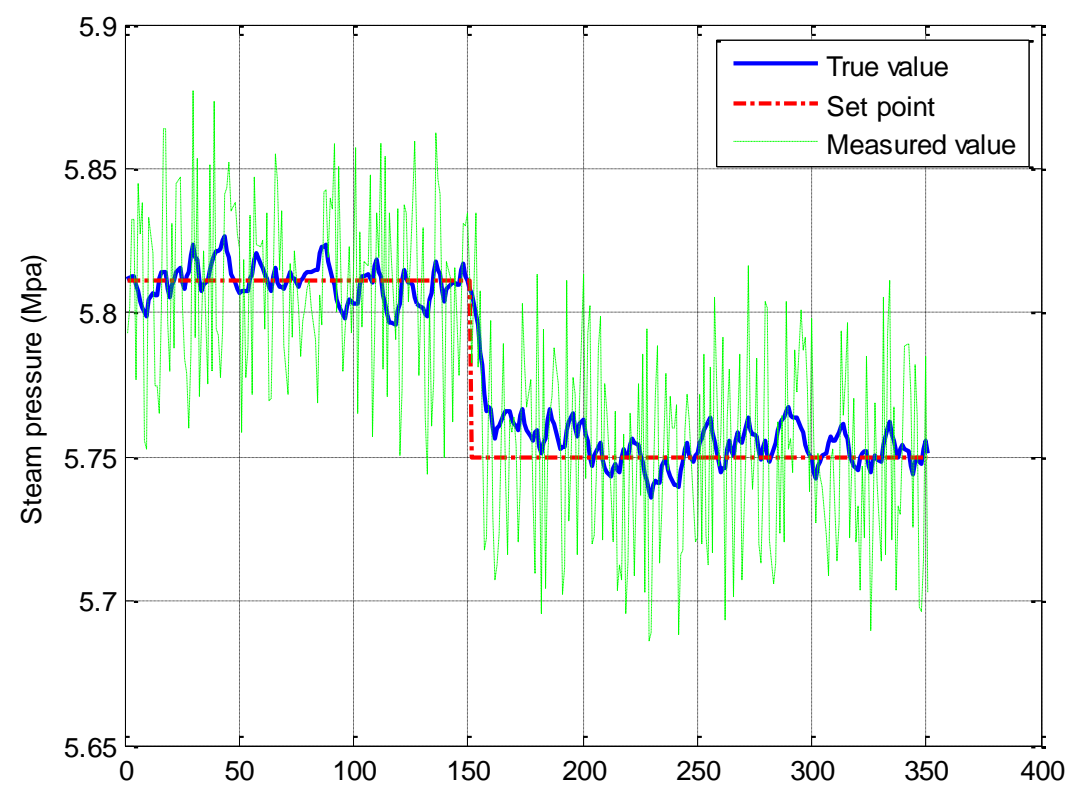

Figure 1.9: MPC performance with 1\% measurement noise

Figures 1.10 and 1.11 show comparison between the control performance of MPC under normal condition and under the $10 \%$ actuator failure. It is assumed that the $10 \%$ of the steam line is blocked. It is similar to saying that if the steam valve is opened $100 \%$ under normal condition, it is just opened $90 \%$ in that actuator failure situation. Figure 1.10 shows the steam pressure change under both normal and abnormal situations. The red graph is the abnormal situation. We can observe that from the plot the steam pressure reaches the desired value but needs more time. Figure 1.11 shows the steam flow-rate change under both normal and abnormal situations. Since the steam line is blocked, the change of the flow-rate under abnormal situation (red line) is not as big as the change of the flow-rate under normal situation (blue line). Those two plots show that the MPC has the capability to handle the actuator failure within a special range. The system performance with $20 \%$ actuator failure was tested, too. However, the system output could not reach the target values. 


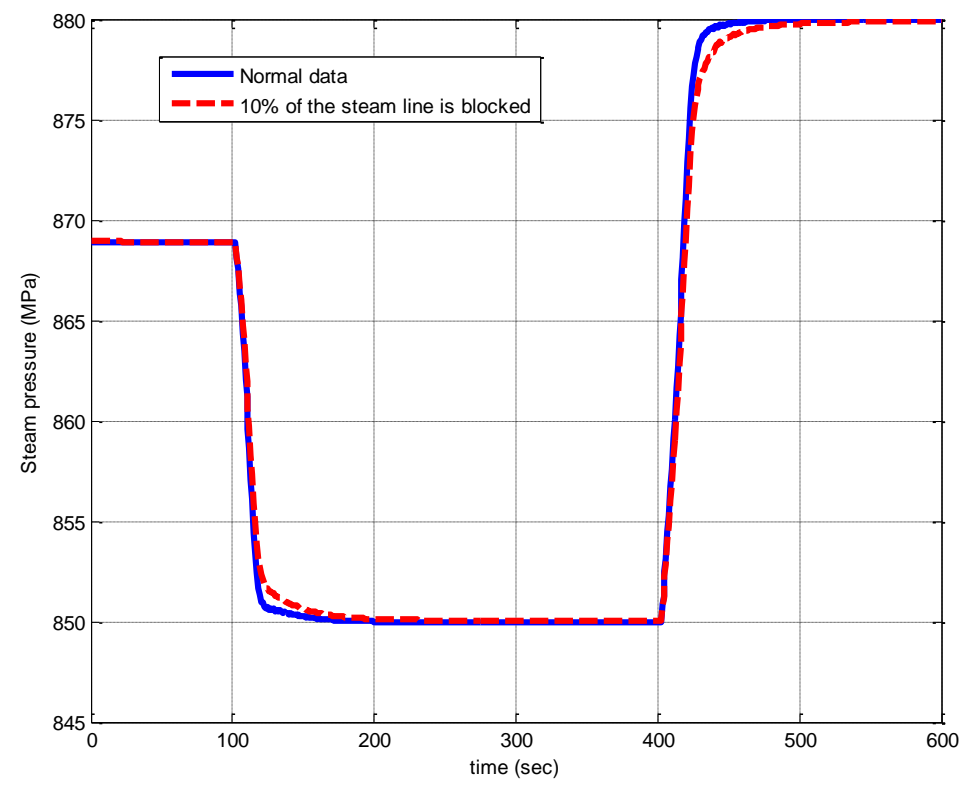

Figure 1.10: Steam pressure change with actuator failure

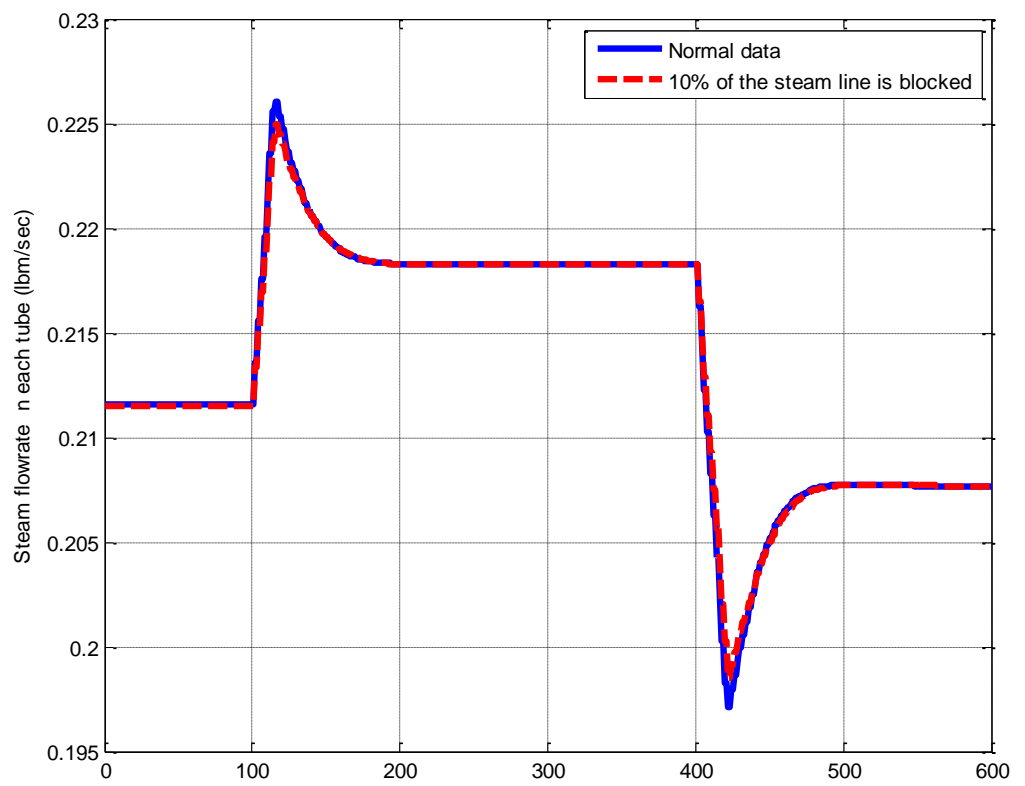

Figure 1.11: Steam flow rate change with actuator failure 


\subsection{Uncertainty Analysis of MPC}

One of the shortcomings of MPC is that the performance of MPC is closely related to the accuracy of the model. The effect of the uncertainty in the heat transfer coefficient between the tube wall and coolant on the primary side is presented in this section. The experiments are designed to change the value of the heat transfer coefficient in the Simulink model, and then apply to the steam pressure control problem.

Figure 1.12 shows the control results for the controller with different model uncertainties. In this experiment, the primary side coolant temperature was increased by $5 \mathrm{deg} F$. The graphs show that the performance of MPC is not sensitive to the heat transfer coefficient when the uncertainty is below $40 \%$. The MPC can still maintain the steam pressure at the set point value of 841.2 psi. When the uncertainty is beyond $40 \%$, there is a considerable deviation in the steam pressure from the set point. This is because the system reaches the maximum steam flow rate, as shown in Figure 1.13. The additional steam brought about by the change in the heat transfer coefficient cannot be removed fast enough; the steam pressure increases until it reaches another equilibrium value (Figure 1.12).

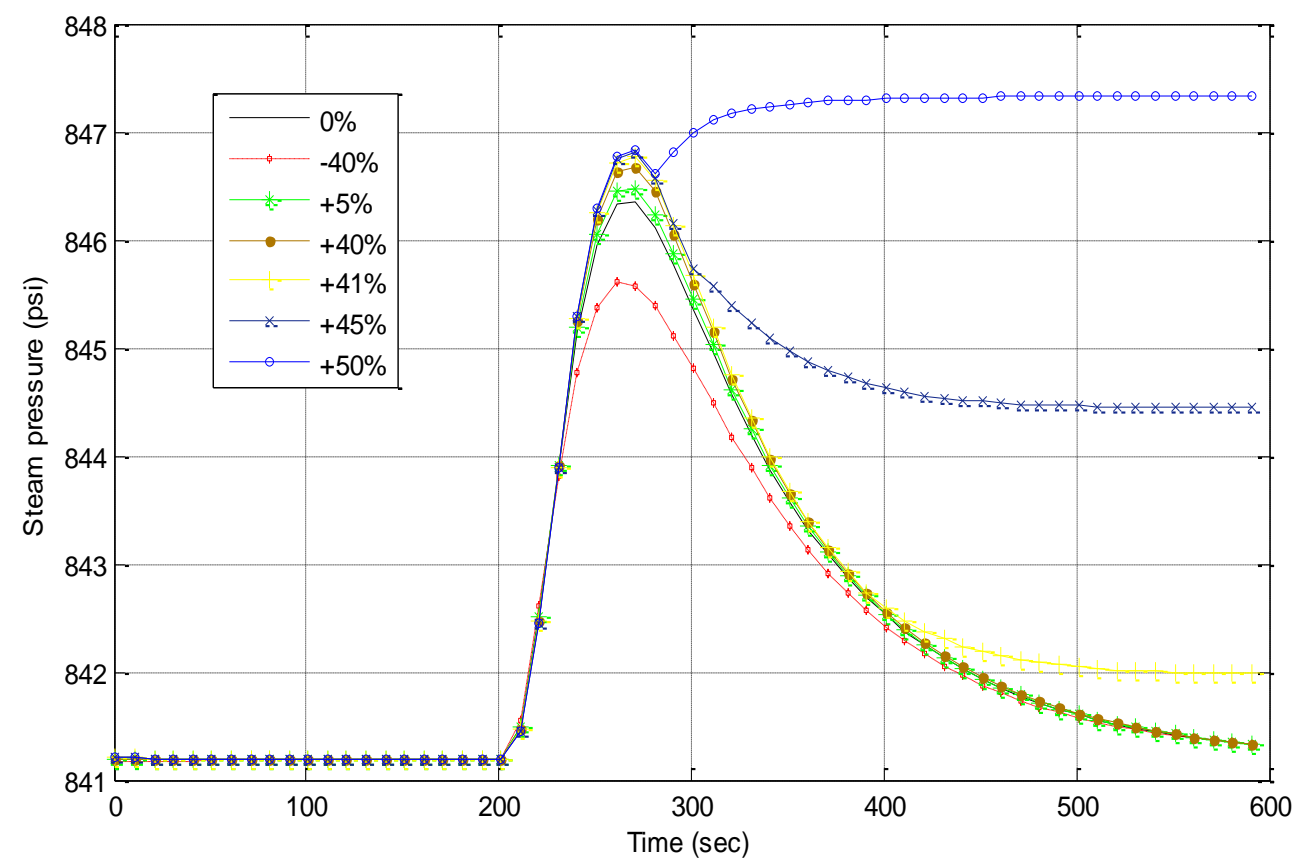

Figure 1.12: The effect of uncertainties in heat transfer coefficient to the MPC performance in maintaining steam pressure 


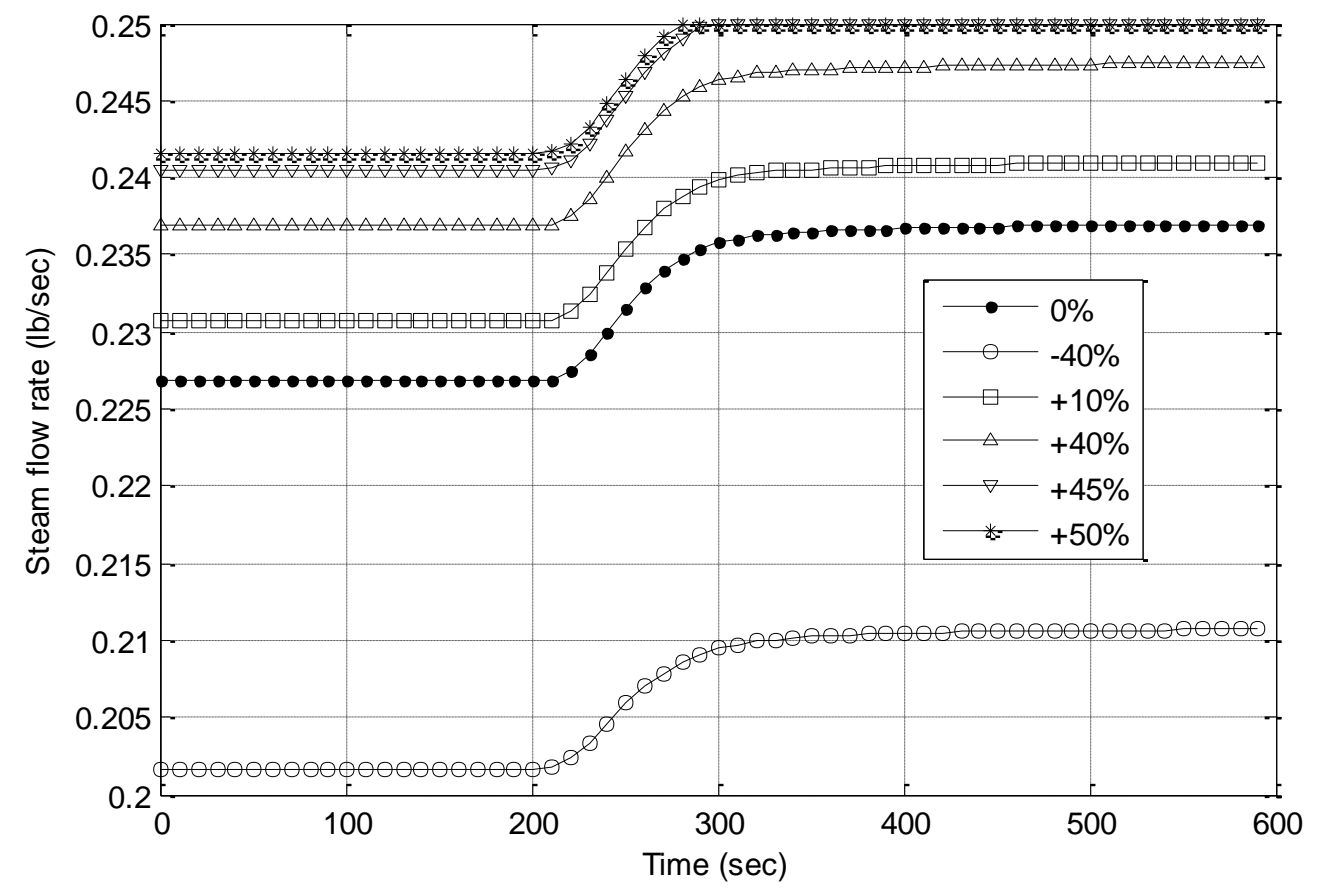

Figure 1.13: The effect of uncertainties in heat transfer coefficient on the MPC performance in steam flow rate manipulation

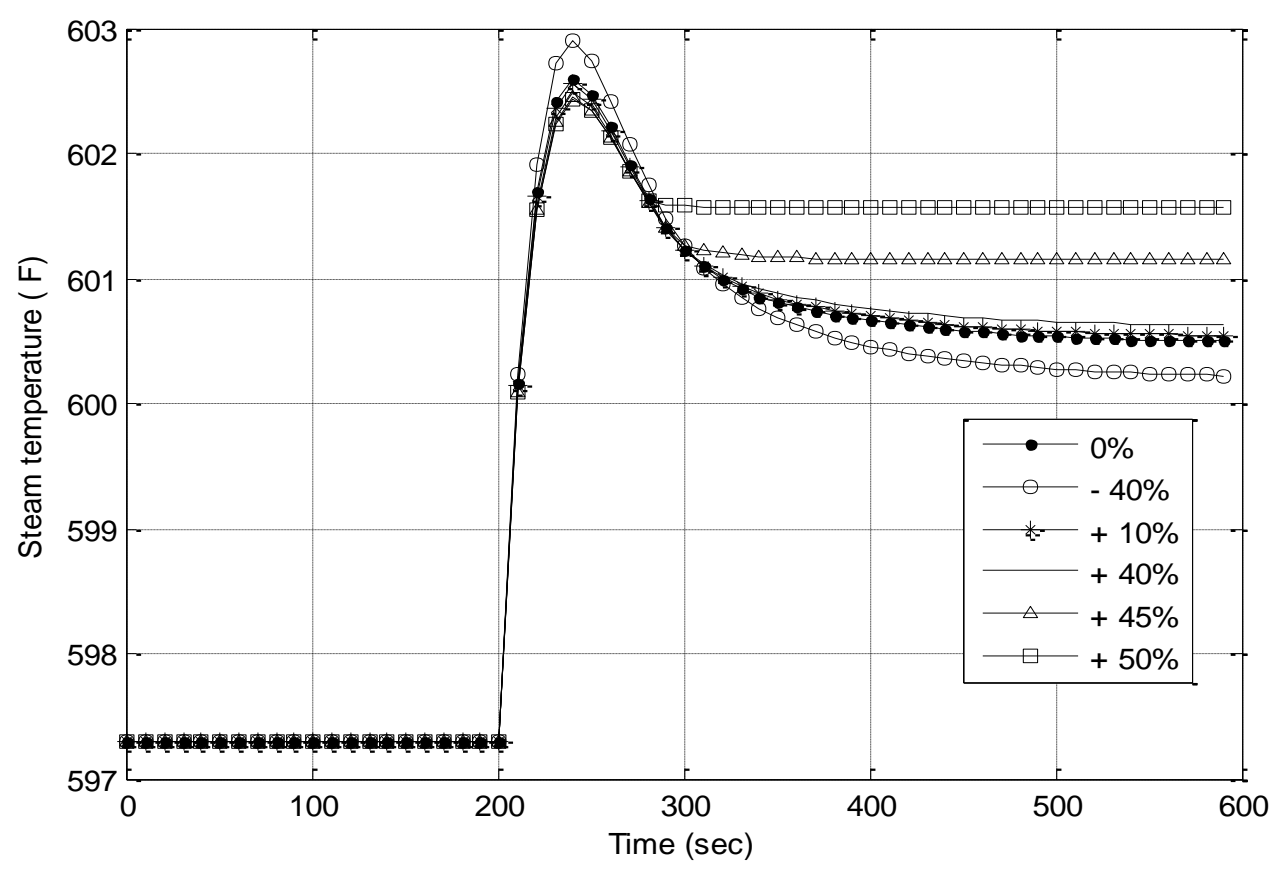

Figure 1.14: The effect of uncertainties in heat transfer coefficient on the MPC performance in steam temperature change 
The corresponding changes in the steam temperature are shown in Figure 1.14. Figure 1.15 shows the control system performance with changes in the system internal parameters. Since we are only focusing on the steam generator, the HCSG model is not very flexible. There are not many parameters we can adjust. In the first case we changed the heat capacity of the coolant in the primary loop, and in the second case we changed the heat capacity of the feedwater in the secondary loop. Figures 1.15(a) and 1.15(c) show the steam pressure change under different conditions. The blue line is the system performance under normal condition. The red line is the system performance when the heat capacity of the coolant degrades by $10 \%$, and the green line is the system performance when the heat capacity of the coolant upgrades by $20 \%$. By comparing Figures 1.15(a) and 1.15(c), we observe that the control system is taking almost the same performance when the heat capacity of the coolant in primary loop and secondary loop are degraded or upgraded. When the capacity is degraded, the heat transfer is getting slower, so it takes longer for the system to reach the steady state. When the heat capacity is increased, the heat transfer becomes faster, and results in an overshoot at each changing point. Comparing Figures 1.15(b) and 1.15(d), we can observe that the changes of the steam flow rate are almost taking the same pattern. However, we can see the change in the heat capacity of the primary coolant has a greater impact on the system. 


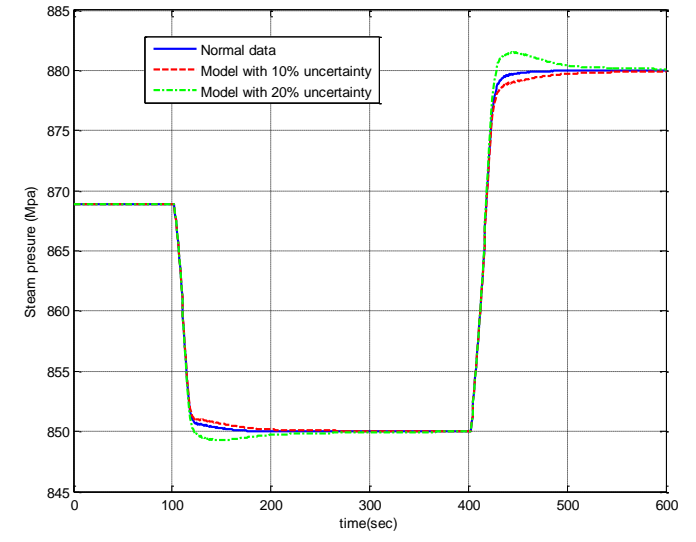

(a) Steam pressure with coolant $\mathrm{Cp}$ change in the primary loop

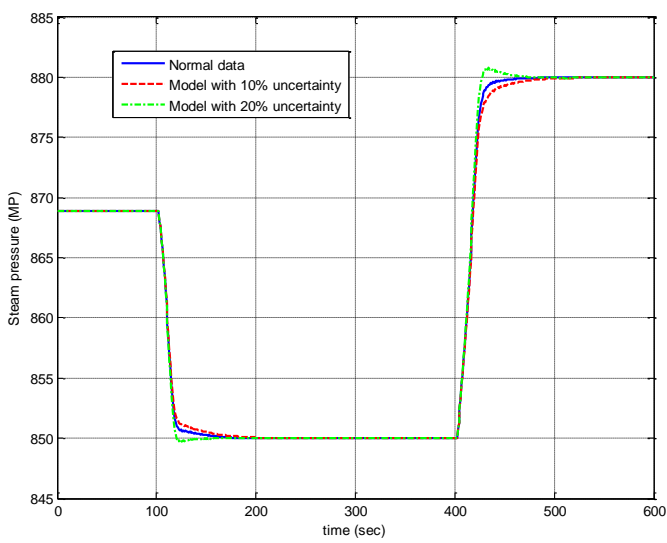

(c) Steam pressure with feed water $\mathrm{Cp}$ change in the secondary loop

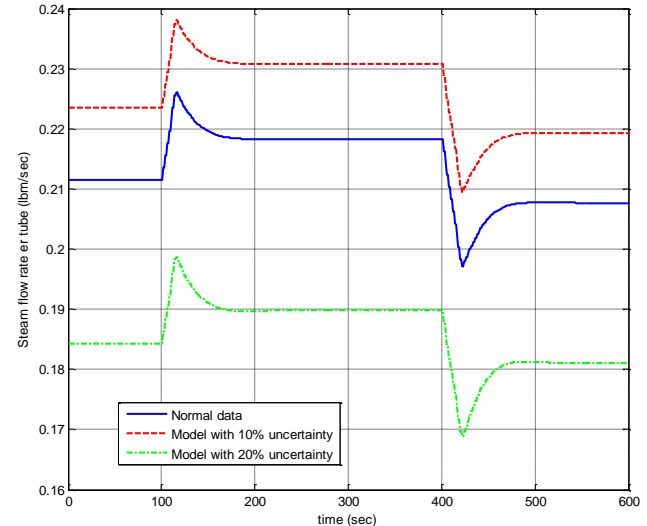

(b) Steam flow rate with coolant $\mathrm{Cp}$ change in the primary loop

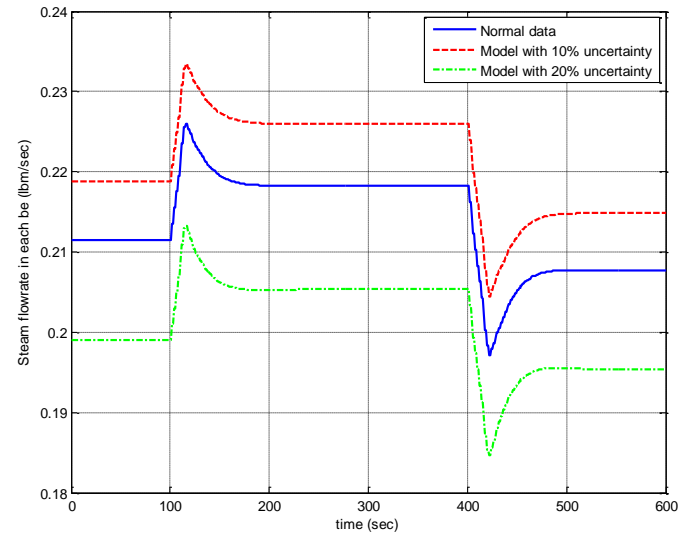

(d) Steam flow rate with feed water $\mathrm{Cp}$ change in the secondary loop

Figure 1.15: MPC performance with system internal parameter change 


\subsection{Summary}

In this research, the MATLAB-SIMULINK model was developed to show the dynamic behavior of the IRIS steam generator (HCSG). The model predictive controller is illustrated successfully using the physics model of the HCSG system to regulate the steam pressure

The following conclusions are drawn from the above analysis:

1. MPC has the capability of suppressing the measurement noise within a limited range.

2. MPC has the capability of overcoming the actuator failure within a limited range.

3. When the system model includes uncertainties, the MPC can regulate the system output to meet the desired values. 


\section{IRIS SYSTEM MODELS}

Two simulators for testing and validation of control algorithms are developed, one by North Carolina State University [8] and the other by the University of Tennessee. The first testbed is built using FORTRAN programming language. This FORTRAN model includes a reactor core model, a helical coil steam generator (HCSG) model and a pressurizer model. Figure 2.1 shows a plant schematic. Figure 2.2 represents the corresponding node structure of the FORTRAN model. Twenty-six nodes are considered for computing the primary side heat generation, heat transportation and coolant temperature distribution. Table 2.1 defines each node.

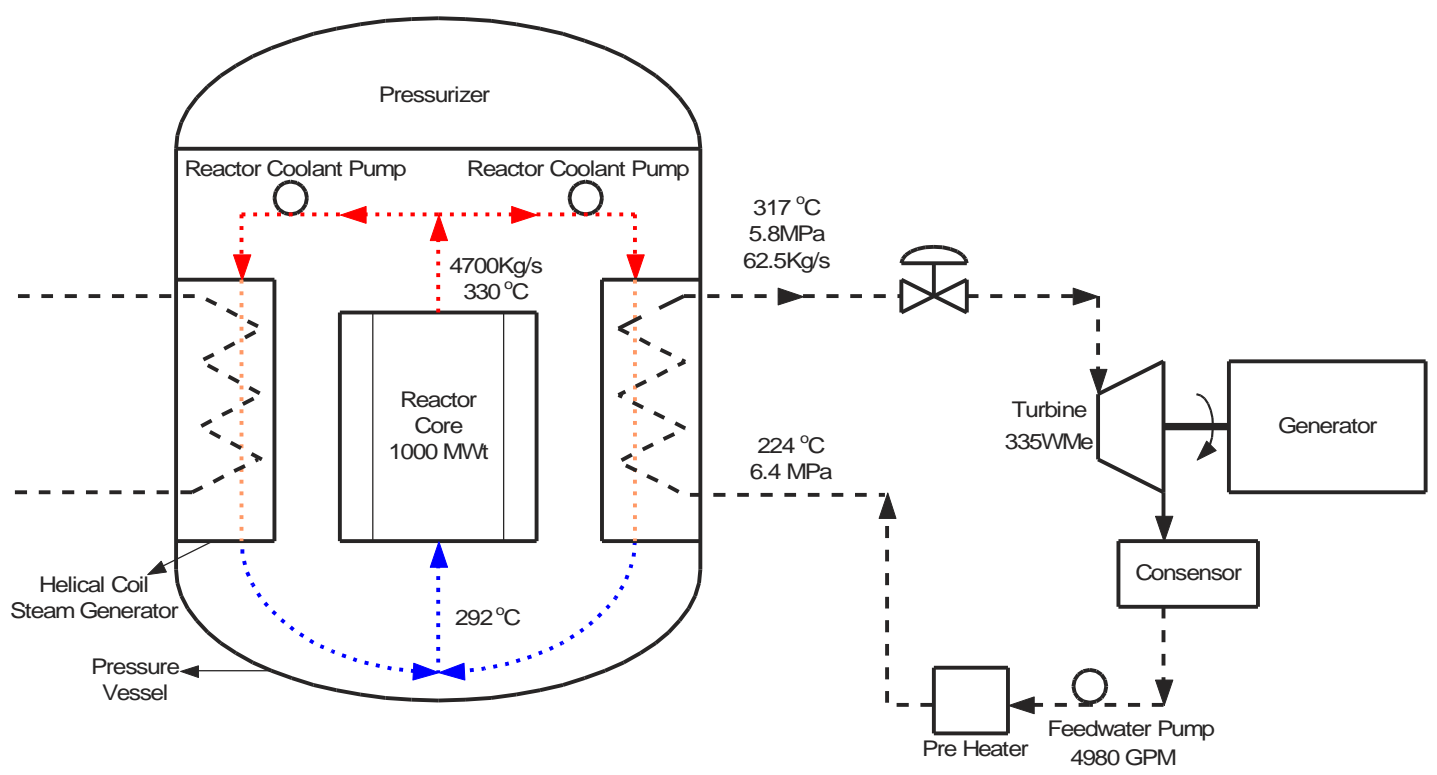

Figure 2.1: Heat Balance of IRIS. 


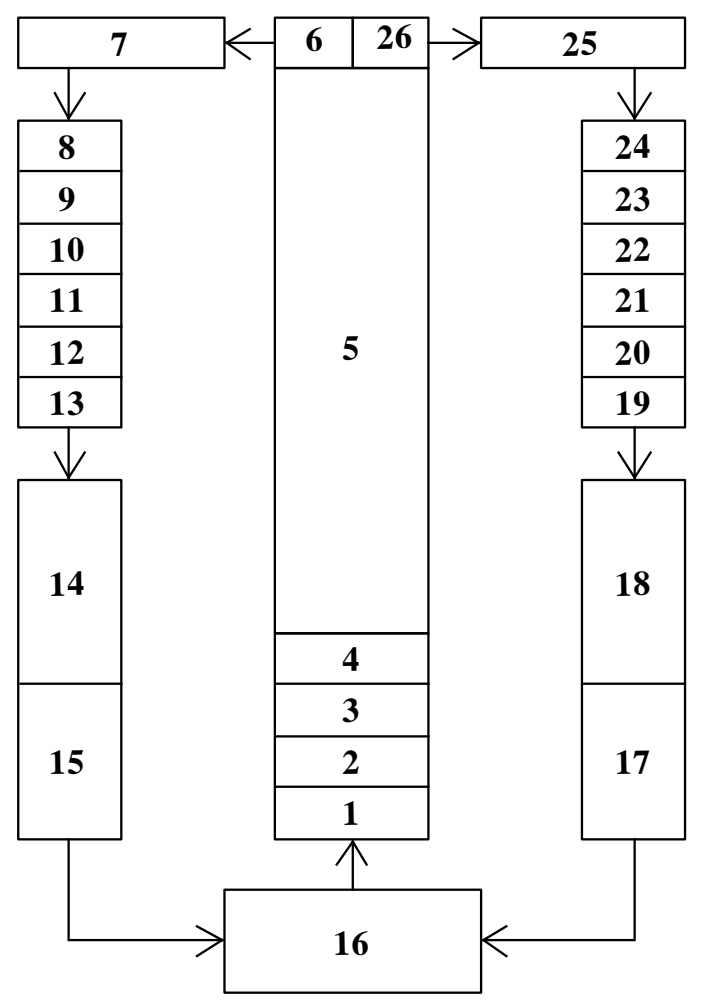

Figure 2.2: Nodalization of IRIS FORTRAN Model [8]

Table 2.1: Description of Nodes of the IRIS FORTRAN Model

\begin{tabular}{|c|c|}
\hline Nodes & Description \\
\hline $1-4$ & Reactor Core \\
\hline 5 & Riser \\
\hline 6,26 & RCP Inlet Plenum \\
\hline 7,25 & RCP Nodes \\
\hline $8-13,19-24$ & Steam Generator \\
\hline $14-15,17-18$ & Downcomer \\
\hline 16 & Lower Plenum \\
\hline
\end{tabular}

The FORTRAN IRIS simulator is operated in the integrated control mode through builtin PID controllers, which regulate all three systems (ithat is, turbine, feedwater flow and control rods) to combine the rapid response of the unregulated system with the stability of the turbinefollowing system. This IRIS FORTRAN Simulator is capable of simulating normal operation conditions at different operational modes (such as normal power maneuvers, reactor start up, turbine loading, etc. [8]), as well as various faulty scenarios such as actuator failures, sensor failures, internal faults etc. 
The second IRIS simulator is built using the MATLAB-SIMULINK platform which makes it more convenient for control system design and validation. However, it is not as detailed as the FORTRAN model. Depending on the applications of different research topics, the two simulators are utilized accordingly. 


\section{STATE OBSERVER DESIGN FOR IRIS REACTOR}

\subsection{IRIS Model and Problem Formulation}

The design of a state observer for a dynamic system, an appropriate mathematical model is needed that describes the process dynamics. This model is usually built based on physical laws such as mass conservation, energy conservation. Generally, to make the estimation more accurate, the corresponding dynamic model should give more information about the real physical process. However, note that if the dynamic model which is used to design the state observer is complex and with high nonlinearity just like models used for simulation, then the observer design will become very difficult and note that some lower order models also give acceptable and reasonable description of the dynamic process, so here, some reasonable simplifications are made. First, the one-delayed neutron group point kinetic is used here. Then, consider the whole fuel rod as a cell, the reactor dynamic model used in this report is the nonlinear point kinetics with one delayed neutron group and temperature feedback from fuel and coolant temperature given in Eq. (3.1) [9]. This model is also used for controller design in many references [10][11].

$$
\left\{\begin{array}{l}
\frac{d n_{r}}{d t}=\frac{\delta \rho_{r}-\beta}{\Lambda} n_{r}+\frac{\beta}{\Lambda} c_{r}+\frac{\alpha_{f} n_{r}}{\Lambda}\left(T_{f}-T_{f 0}\right)+\frac{\alpha_{c} n_{r}}{\Lambda}\left(T_{c a v}-T_{c a v 0}\right) \\
\frac{d c_{r}}{d t}=\lambda\left(n_{r}-c_{r}\right) \\
\frac{d T_{f}}{d t}=-\frac{\Omega}{\mu_{f}} T_{f}+\frac{\Omega}{\mu_{f}} T_{c a v}+\frac{P_{0}}{\mu_{f}} n_{r} \\
\frac{d T_{c a v}}{d t}=-\frac{2 M+\Omega}{\mu_{c}} T_{c a v}+\frac{\Omega}{\mu_{c}} T_{f}+\frac{2 M}{\mu_{c}} T_{c i n}
\end{array}\right.
$$

In Eq. (3.1), $\mathrm{nr}$ is the neutron density relative to density at rated condition. $\mathrm{cr}$ is the precursor density relative to the density of one-group delayed fission neutrons at rated condition. $\beta$ is the fraction of all the delayed fission neutrons. $\Lambda$ is the effective prompt neutron time. $\lambda$ is the effective precursor radioactive decay constant of the delayed fission neutrons. $\alpha f$ is the temperature feedback coefficient of the average fuel temperature. $\alpha$ is the temperature feedback coefficient of the average coolant temperature. Tf is the average reactor fuel temperature. Tf0 is the equilibrium average temperature of fuel. Tcav is the average temperature of the coolant. 
Tcav0 is the equilibrium average temperature of the coolant. $\Omega$ is the heat transfer coefficient between the fuel and coolant. $\mu \mathrm{f}$ is the total heat capacity of the fuel. $\mu \mathrm{c}$ is the total heat capacity of the coolant. M is the value of the coolant flow rate times heat capacity of the coolant. P0 is the rated power distributed in the fuel. Tcin is the temperature of reactor inlet coolant.

Rewrite nonlinear dynamic model Eq. (3.1) in a general form

$$
\left\{\begin{array}{l}
\dot{\boldsymbol{x}}(t)=f(\boldsymbol{x}, \boldsymbol{u}) \\
\boldsymbol{y}=h(\boldsymbol{x}, \boldsymbol{u})
\end{array}\right.
$$

where $x(t) \in R n$ is the state, $u \in R q$ is the system input, $y \in R m$ is the system output and both the functions $\mathrm{f}(\cdot): \mathrm{Rn}+\mathrm{q} \rightarrow \mathrm{Rn}$ and $\mathrm{h}(\bullet): \mathrm{Rn}+\mathrm{q} \rightarrow \mathrm{Rm}$ are smooth. Suppose the system in Eq. (3.2) has the following state observer:

$$
\left\{\begin{array}{l}
\dot{\hat{\boldsymbol{x}}}=f(\hat{\boldsymbol{x}}, \boldsymbol{u})+K(\boldsymbol{y}-\hat{\boldsymbol{y}}) \\
\hat{\boldsymbol{y}}=h(\hat{\boldsymbol{x}}, \boldsymbol{u})
\end{array}\right.
$$

where $\hat{\boldsymbol{x}} \in \mathrm{Rn}$ is the state-observation, $\mathrm{y} \in \mathrm{Rm}$ is the system output, $\mathrm{K} \in \mathrm{Rn} \times \mathrm{m}$ is the observer gain,

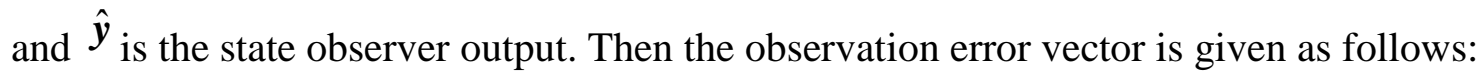

$$
\boldsymbol{e}=\boldsymbol{x}-\hat{\boldsymbol{x}}
$$

Differentiating both sides of Eq. (3.4) and substitute Eq. (3.2) and Eq. (3.3) into Eq. (3.4), the error dynamic is given in Eq. (3.5)

$$
\dot{\boldsymbol{e}}=f(\boldsymbol{x}, \boldsymbol{u})-f(\hat{\boldsymbol{x}}, \boldsymbol{u})-\boldsymbol{K}(\boldsymbol{y}-\hat{\boldsymbol{y}})
$$

Express $f(\boldsymbol{x}, \boldsymbol{u})$ and $h(\boldsymbol{x}, \boldsymbol{u})$ as a Taylor expansion around $\hat{\boldsymbol{x}}$ :

$$
\begin{aligned}
& f(\boldsymbol{x}, \boldsymbol{u})-f(\hat{\boldsymbol{x}}, \boldsymbol{u})=\left.\frac{\partial f(\boldsymbol{x}, \boldsymbol{u})}{\partial \boldsymbol{x}}\right|_{x=\hat{\boldsymbol{x}}}(\boldsymbol{x}-\hat{\boldsymbol{x}})+\ldots=f_{e}(\boldsymbol{e}, \boldsymbol{u}) \\
& h(\boldsymbol{x}, \boldsymbol{u})-h(\hat{\boldsymbol{x}}, \boldsymbol{u})=\left.\frac{\partial h(\boldsymbol{x}, \boldsymbol{u})}{\partial \boldsymbol{x}}\right|_{x=\hat{\boldsymbol{x}}}(\boldsymbol{x}-\hat{\boldsymbol{x}})+\ldots=h_{e}(\boldsymbol{e}, \boldsymbol{u})
\end{aligned}
$$

where $\mathrm{fe}(\cdot): \mathrm{Rn}+\mathrm{q} \rightarrow \mathrm{Rn}$ and $\mathrm{he}(\bullet): \mathrm{Rn}+\mathrm{q} \rightarrow \mathrm{Rm}$ are both smooth functions. Therefore error dynamics Eq. (3.5) can be rewritten as 


$$
\dot{\boldsymbol{e}}=f_{e}(\boldsymbol{e}, \boldsymbol{u})-K h_{e}(\boldsymbol{e}, \boldsymbol{u})
$$

So the objective of the observer design problem for IRIS nuclear reactor can be formulated as: find an appropriate observer gain $\mathrm{K}$ such that the state observer Eq. (3.3) is asymptotically stable. Furthermore, taking into account that the nuclear reactor is highly nonlinear, some coefficients of the nonlinear state space model vary with operation power, coolant temperature, flow rate and the previous operation condition. Meanwhile, many coefficients cannot be measured easily or the measurement involves a large uncertainty. So, the designed state observer should be robust over model uncertainty, model coefficient uncertainties and exterior disturbances.

\subsection{Methodology}

The DHGF is an effective method proposed by Dong [12] to build a nonlinear state observer which can guarantee the asymptotical stability if the dynamic process meets certain requirements. The DHGF theorem is presented here. First, the concept of zero-state detectability is introduced as follows:

Definition 1 [12][13]: Consider the nonlinear systems taking the form as

$$
\left\{\begin{array}{l}
\dot{\xi}=\Phi(\xi) \\
\eta=\Theta(\xi)
\end{array}\right.
$$

Where $\xi \in R n$ is the state-vector, $\eta \in R m$ is the system output, and functions $\Phi(\bullet): R n \rightarrow R n$ and $\Theta(\bullet): R n \rightarrow R m$ are smooth. The system in Eq. (3.9) is called zero state detectable if $\eta=0$ $\forall t \geq 0$ implies $\lim _{t \rightarrow \infty} \xi=o$

Based on the definition of zero-state observability, Theorem 1 is given as follows, which gives the solution of problem formulated before

Theorem 1 [12]: Consider the nonlinear systems described by dynamic Eq. (3.2) with state-observer taking the form as Eq. (3.3). Define

$$
e_{y}=y-\hat{y}
$$


and assume that there exists a smooth function $\mathrm{H}(\bullet): \mathrm{Rm} \rightarrow \mathrm{R}+$ such that $\mathrm{H}($ ey) is only minimal at ey=o and

$$
\boldsymbol{e}_{\boldsymbol{y}}=\boldsymbol{L}^{T}\left[\frac{\partial H\left(\boldsymbol{e}_{\boldsymbol{y}}\right)}{\boldsymbol{e}}\right]^{T}
$$

where $L \in R n \times m$ is a given matrix. Moreover, suppose that the error dynamics Eq. (3.8) with the output defined as ey is zero-state detectable. Therefore the state-observer Eq. (3.3) is asymptotically stable if

$$
\boldsymbol{K}=\frac{1}{\varepsilon} \boldsymbol{L}
$$

where $\varepsilon$ is a small enough positive scalar. The proof of this theorem is given in reference [12].

\subsection{Application in IRIS nuclear reactor}

\subsubsection{State Space Model of IRIS Core for State-Observer Design}

In this section, the DHGF for monitoring the IRIS reactor state will be realized. A nonlinear state space model is given in Eq. (3.1). However, note that the Eq. (3.1) does not meet the requirement of zero-state detectability, to make the dynamic model meet the requirement of zero-state detectability, rewrite the state space model Eq. (3.1) in the incremental form. Choose the state vector as: $\mathrm{x}=[\delta \mathrm{nr} \delta \mathrm{cr} \delta \mathrm{Tf} \delta \mathrm{Tcav}]$ and then dynamic Eq. (3.1) can be written as

$$
\dot{\boldsymbol{x}}=\boldsymbol{f}(\boldsymbol{x}, u)=\boldsymbol{f}\left(\boldsymbol{x}, \delta \rho_{r}\right)=\left[\begin{array}{c}
-\frac{\beta}{\Lambda} \delta n_{r}+\frac{\beta}{\Lambda} \delta c_{r}+\frac{\alpha_{f} n_{r 0}}{\Lambda} \delta T_{f}+\frac{\alpha_{c} n_{r 0}}{\Lambda} \delta T_{c a v}+\frac{n_{r 0}}{\Lambda} \delta \rho_{r} \\
+\frac{\alpha_{f}}{\Lambda} \delta n_{r} \delta T_{f}+\frac{\alpha_{c}}{\Lambda} \delta n_{r} \delta T_{c a v}+\frac{1}{\Lambda} \delta n_{r} \delta \rho_{r} \\
\lambda \delta n_{r}-\lambda \delta c_{r} \\
-\frac{\Omega}{\mu_{f}} \delta T_{f}+\frac{\Omega}{\mu_{f}} \delta T_{c a v}+\frac{P_{0}}{\mu_{f}} \delta n_{r} \\
-\frac{2 M+\Omega}{\mu_{c}} \delta T_{c a v}+\frac{\Omega}{\mu_{c}} \delta T_{f}+\frac{2 M}{\mu_{c}} \delta T_{c i n}
\end{array}\right]
$$

where,

$$
u=\delta \rho_{r}, \delta n_{r}=n_{r}-n_{r 0}, \delta c_{r}=c_{r}-c_{r 0}, \delta T_{f}=T_{f}-T_{f 0}, \delta T_{c a v}=T_{c a v}-T_{c a v 0}
$$


In Eq. (3.13), only $\delta \mathrm{nr}$ and $\delta \mathrm{Tcav}$ are measurable, let $\mathrm{y}=\mathrm{h}(\mathrm{x})=[\delta \mathrm{nr} \delta \mathrm{Tcav}] \mathrm{T}$, so ey= $\left[\delta n_{r}-\delta \hat{n}_{r} \delta T_{c a v}-\delta \hat{T}_{c a v}\right]$

T. In the following, the zero-state observability of observation error dynamics Eq. (3.8) will be determined. Choose the state-observer gain matrix as Eq. (3.12), and then substitute $\mathrm{ey}=\mathrm{o}$ to Eq. (3.8), we have

$$
\left\{\begin{array}{l}
0=\frac{\beta}{\Lambda}\left(\delta c_{r}-\delta \hat{c}_{r}\right)+\frac{\alpha_{f}\left(n_{r 0}+\delta n_{r}\right)}{\Lambda}\left(\delta T_{f}-\delta \hat{T}_{f}\right)+\frac{\alpha_{f}\left(n_{r 0}+\delta n_{r}\right)}{\Lambda}\left(\delta \rho_{r}-\delta \hat{\rho}_{r}\right) \\
\frac{d\left(\delta c_{r}-\delta \hat{c}_{r}\right)}{d t}=-\lambda\left(\delta c_{r}-\delta \hat{c}_{r}\right) \\
\frac{d\left(\delta T_{f}-\delta \hat{T}_{f}\right)}{d t}=-\frac{\Omega}{\mu_{f}}\left(\delta T_{f}-\delta \hat{T}_{f}\right) \\
0=\frac{\Omega}{\mu_{c}}\left(\delta T_{f}-\delta \hat{T}_{f}\right)+\frac{2 M}{\mu_{c}}\left(\delta T_{c i n}-\delta \hat{T}_{c i n}\right)
\end{array}\right.
$$

From the 4th equation of Eq. (3.14), if we want to get $\delta T_{f}-\delta \hat{T}_{f}=0$, then $\delta T_{c i n}-\delta \hat{T}_{c i n}=0$ is required. Since Tcin is the core inlet temperature of the coolant, it is measurable. So, to meet the zero-state detectability requirement, here, let $\delta \hat{T}_{c i n}$ is always equal to $\delta$ Tcin. Then we get:

$$
\delta T_{f}-\delta \hat{T}_{f}=0
$$

Substitute Eq. (3.15) to Eq. (3.14),

$$
\left\{\begin{array}{l}
0=\frac{\beta}{\Lambda}\left(\delta c_{r}-\delta \hat{c}_{r}\right)+\frac{\alpha_{f}\left(n_{r 0}+\delta n_{r}\right)}{\Lambda}\left(\delta \rho_{r}-\delta \hat{\rho}_{r}\right) \\
\frac{d\left(\delta c_{r}-\delta \hat{c}_{r}\right)}{d t}=-\lambda\left(\delta c_{r}-\delta \hat{c}_{r}\right)
\end{array}\right.
$$

Since $\delta \rho r$ is the control rod reactivity to the reactor and $\delta \hat{\rho}_{r}$ is the control rod reactivity to the state observer, so $\delta \rho \mathrm{r}=\delta \hat{\rho}_{r}=\mathrm{u}$ then we get

$$
\delta c_{r}-\delta \hat{c}_{r}=0
$$


From Eq. (3.15) and Eq. (3.17), we can conclude that if ey=o, then $\lim _{t \rightarrow \infty} \boldsymbol{e}=\boldsymbol{o}$. That is, the error dynamic Eq. (3.7) is zero-detectable. Therefore the DHGF can be utilized to observe the state of the IRIS reactor. Choose the Hamiltonian function as

$$
H\left(\boldsymbol{e}_{y}\right)=\frac{1}{2}\left[k_{1}\left(\delta n_{r}-\delta \hat{n}_{r}\right)^{2}+k_{2}\left(\delta T_{c a v}-\delta \hat{T}_{c a v}\right)^{2}\right]
$$

then

$$
\boldsymbol{e}_{y}=\boldsymbol{L}^{T}\left[\frac{\partial H\left(\boldsymbol{e}_{y}\right)}{\partial \boldsymbol{e}}\right]
$$

where

$$
\boldsymbol{L}=\left[\begin{array}{cccc}
k_{1} & 0 & 0 & 0 \\
0 & 0 & 0 & k_{2}
\end{array}\right]
$$

$\mathrm{k}_{1}$ and $\mathrm{k}_{2}$ are two positive numbers. Recall Eq. (3.12), the observer gain $\mathrm{K}=\mathrm{L} / \varepsilon$ is chosen by adjusting the $\varepsilon$. Here, we will directly choose $\mathrm{k}_{1}$ and $\mathrm{k}_{2}$.

\subsubsection{Numerical Simulation}

The DHGF is applied to the IRIS simulation model. The simulation result by choosing two different sets of $(\mathrm{k} 1 \mathrm{k} 2)$ are compared to illustrate the influence of the gain $\mathrm{K}$ in state observer Eq. (3.3).

Three different dynamic processes are simulated to show the performance of the designed DHGF. Here, the values of state variables given by a high order model of IRIS simulator (6delayed group neutron and 4 core cells) will be considered as the real measurement. The calculation values given by a simplified low order model (1-delayed group neutron and 1 core cell) which is usually used for control purpose will be compared with estimation given by the DHGF. 


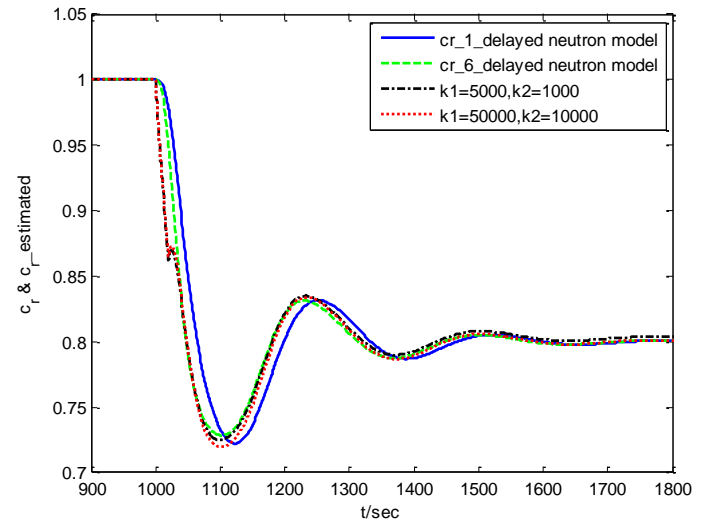

Figure 3.1: Transient response of $n_{r}$ in case I.

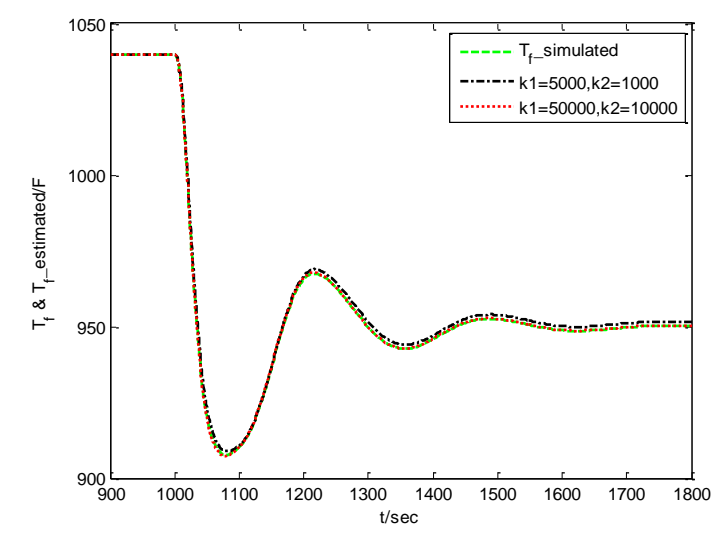

Figure 3.2: Transient response of $\mathrm{Tf}$ in case I.

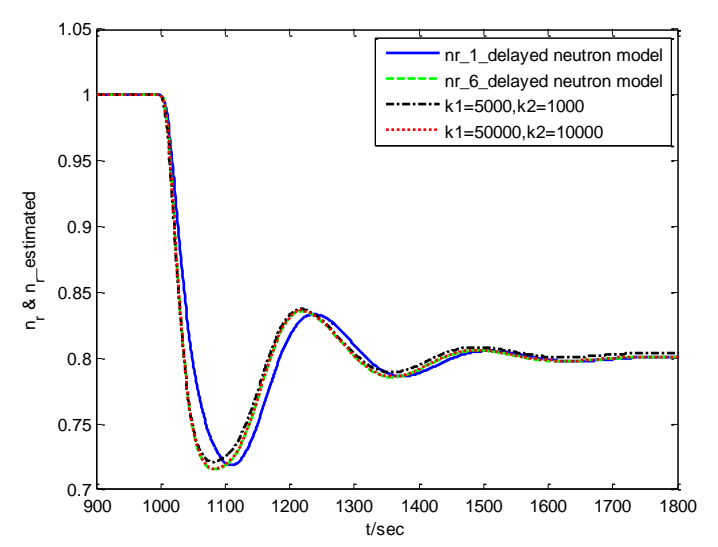

Figure 3.3: Transient response of $\mathrm{cr}$ in case I.

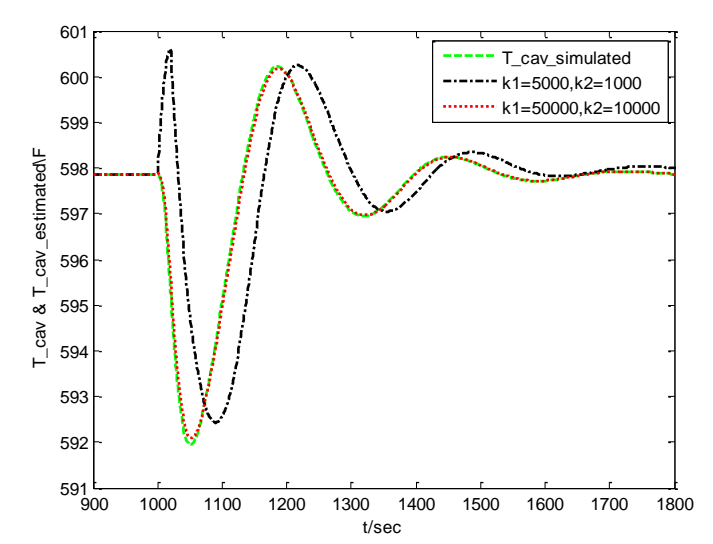

Figure 3.4: Transient response of $T_{\text {cav }}$ in case I

\section{Case I: Reference power step from 1.0 to 0.8 in 20 seconds and the plant parameters used for the estimation is correct.}

Figures $3.1-3.4$ shows the transient response of the four state variables. The relative power nr and average coolant temperature Tcav are measurable while precursor density cr and average fuel temperature $\mathrm{Tf}$ are not measurable. cr and $\mathrm{Tf}$ will be estimated by nr and Tcav. The results simulated by different choice of set (k1 k2) are also shown. From Figure 3.1 and Figure 3.2, there is a difference between the transient value of $\mathrm{nr}$, cr calculated by the 1-delay group point kinetic model and nr, cr calculated by the 6-delay group point kinetic model. The estimated nr, cr calculated by the DHGF follows the real measurement quite well. Figure 3.3 and Figure 
3.4 show that as the gain increases, the tracking performance becomes better. The high gain can effectively reduce the tracking error in the dynamic process.

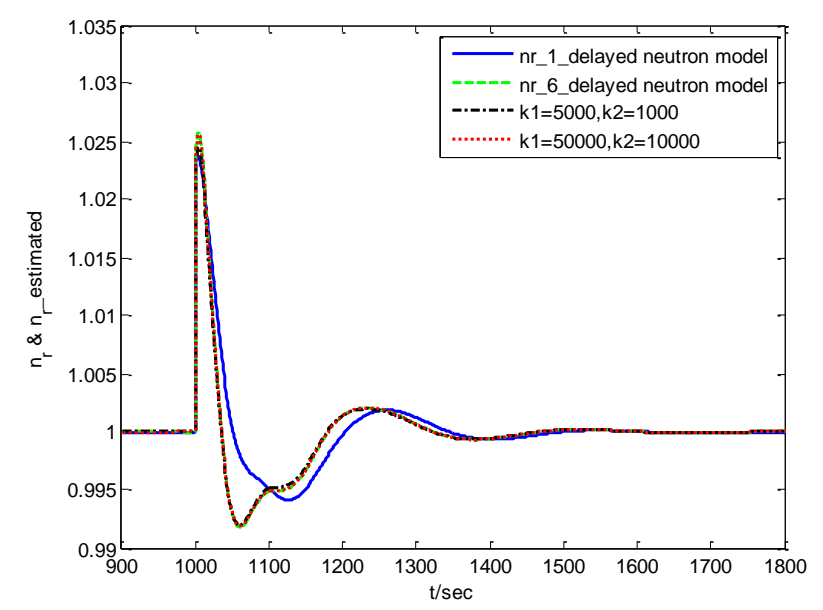

Figure 3.5: Transient response of $\mathrm{nr}$ in case II.

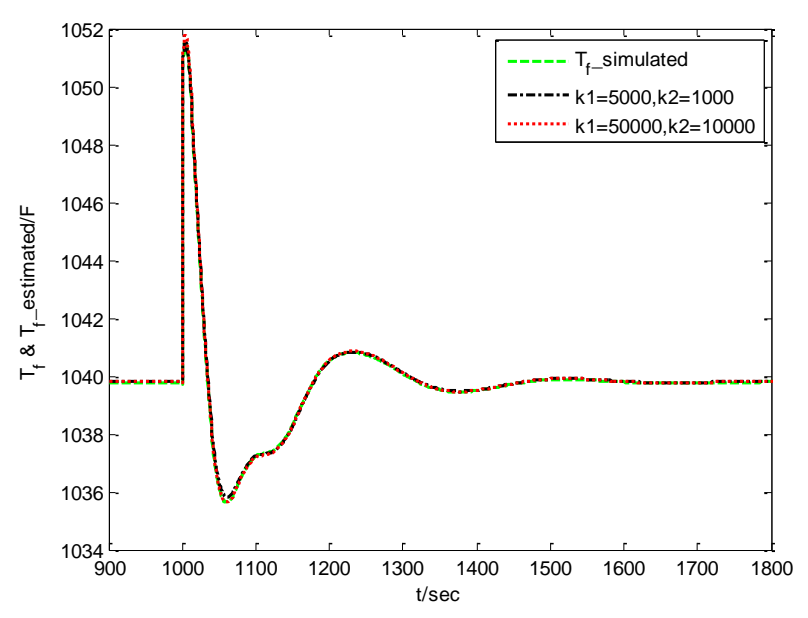

Figure 3.6: Transient response of $T_{f}$ in case II.

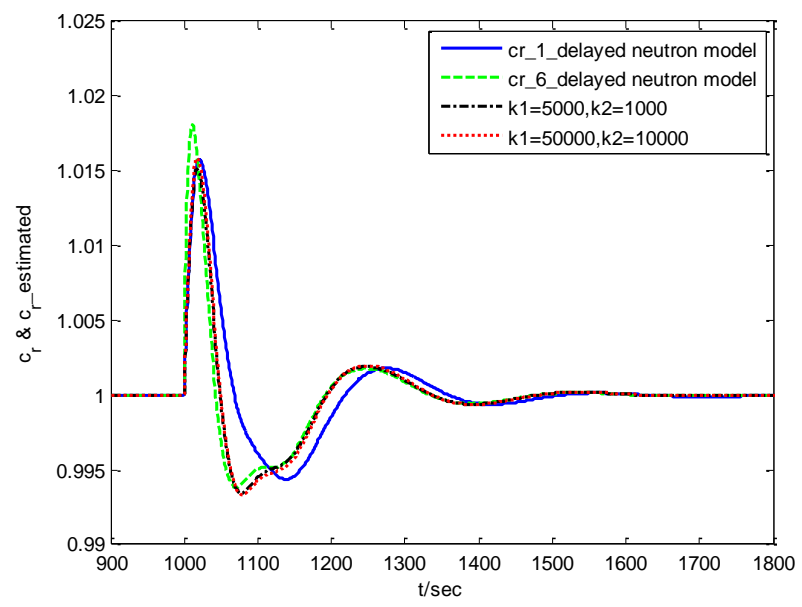

Figure 3.7: Transient response of $c_{r}$ in case II.

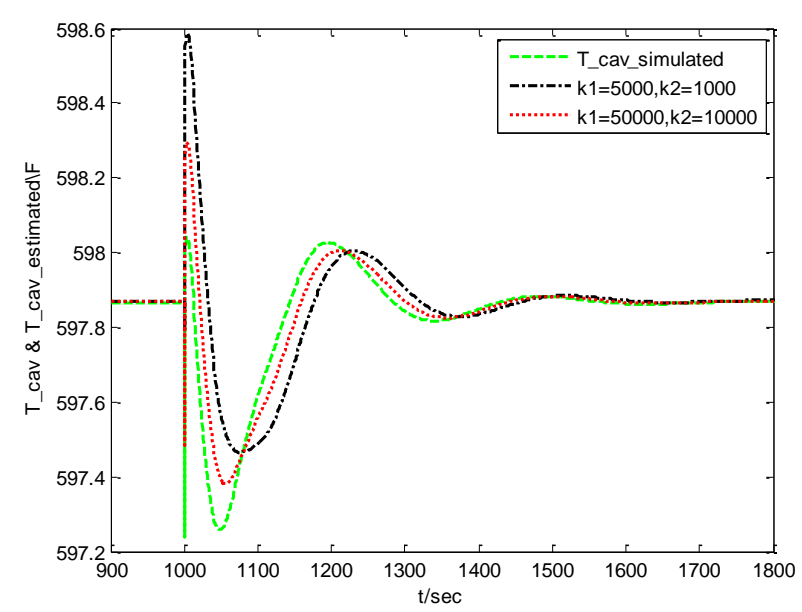

Figure 3.8: Transient response of $T_{\text {cav }}$ in case II.

\section{Case II: Reactor coolant pumps (RCPs) overspeed $2 \%$ with observer with accurate plant parameters.}

Eight RCPs are integrated into the pressure vessel of the IRIS reactor. RCPs are one of the most important components in nuclear power systems because RCPs maintain a forced convective coolant flow in the primary circulation loop which keeps the temperature of reactor core components such as fuel element below the specified values and maintain the heat balance 
of the reactor steam-generator turbine system. No matter that RCPs overspeed or underspeed, the heat balance of a steady state will be destroyed, and the control system will be activated to drive the system to a new steady state. In Case II, it is simulated that RCPs overspeed $2 \%$ of rated value. The transient responses of the four state variables are shown in Figure 3.5, 3.6, 3.7 and 3.8 respectively.

From the simulation result, it can be concluded that there exists error between the estimation of the simplified low order model and the simulation model during the dynamic process. The maximum error of relative nuclear power is about 0.0075. Comparatively, when the state observer gain $\mathrm{K}$ is big enough, the DHGF can estimate the state valuables very well by using a low order model.

\section{Case III: RCPs overspeed $2 \%$ and the state observer with inaccurate model coefficients}

In real application, the following problems are always encountered when modeling the process and designing control systems. 1). many coefficients of the dynamic model cannot be determined directly. 2) the measured values of coefficients involve large uncertainties. 3). model coefficients vary with time, operation condition etc. Specifically, for the reactor model, the average fuel temperature feedback coefficient $\alpha$, the average coolant temperature feedback coefficient $\alpha \mathrm{c}$, the total heat capacity of the fuel $\mu \mathrm{f}$ and the total heat capacity of the coolant $\mu \mathrm{c}$ etc. vary with the reactor power and temperature [9]. In Case III, the performance of DHGF with inaccurate model coefficients is simulated. The inaccurate parameters of DHGF for simulation of Case III are shown in Table I. The transient responses of the four state variables are shown in Figure 3.9, 3.10, 3.11 and 3.12 respectively.

According to Figure 3.11, with inaccurate model coefficients, there exist a large steady state error between Tcav simulated by low order model and that simulated by high order model. Comparatively, with enough high gain, the steady state error of Tcav can be eliminated. The DHGF is robust against coefficients uncertainty. 
Table 3.1: Parameters of DHGF for Simulation of Case III.

\begin{tabular}{|c||c|c|c|c|}
\hline Parameters & $\mu_{f}$ & $\mu_{c}$ & $\alpha_{f}$ & $\alpha_{c}$ \\
\hline \hline Value used in Plant & 0.4519 & 0.7357 & $\begin{array}{c}-1.6500 \mathrm{e}- \\
5\end{array}$ & $-4.000 \mathrm{e}-4$ \\
\hline Value used in Observer & 0.5423 & 0.8828 & $\begin{array}{c}-1.4850 \mathrm{e}- \\
5\end{array}$ & $-3.600 \mathrm{e}-4$ \\
\hline
\end{tabular}

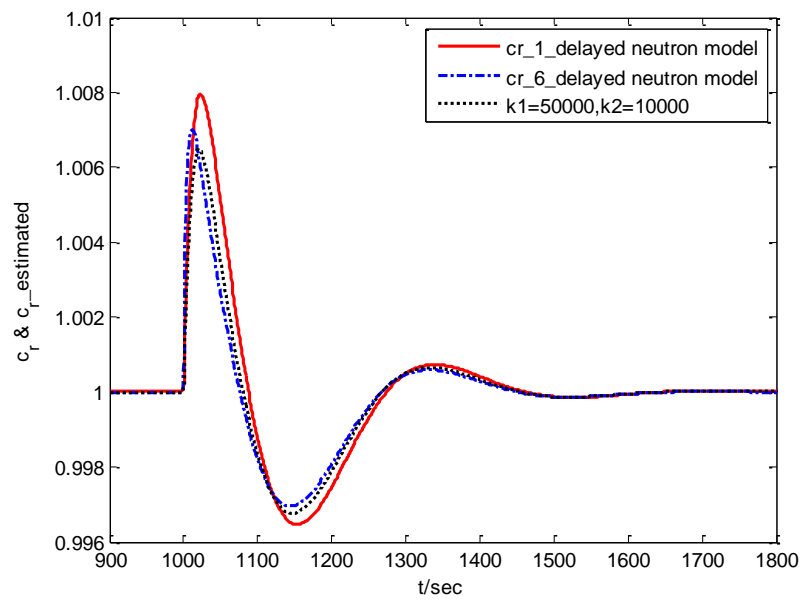

Figure 3.9: Transient response of $n_{r}$ in case III.

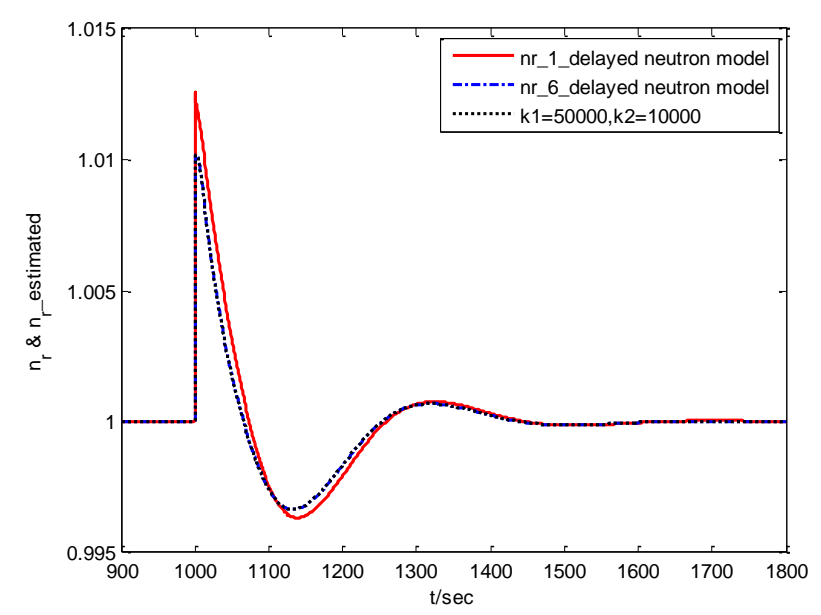

Figure 3.10: Transient response of $T_{f}$ in case III.

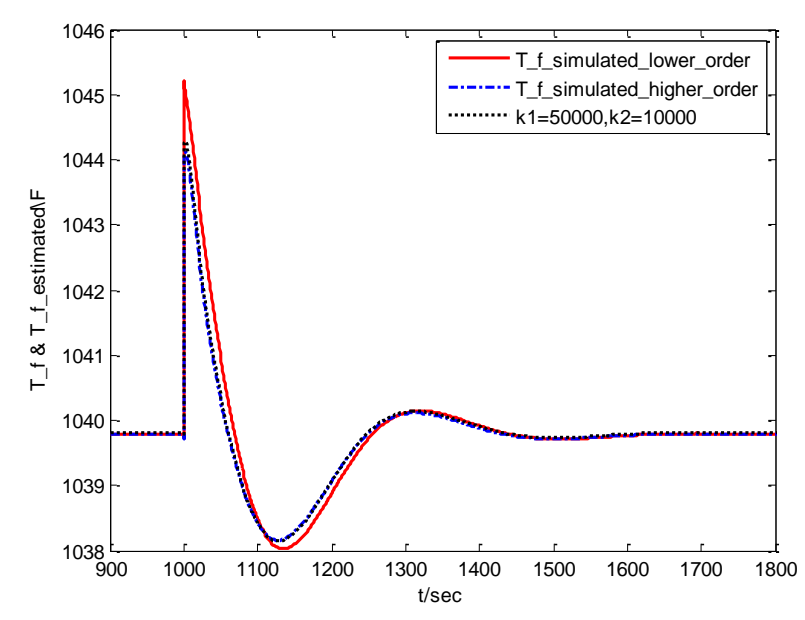

Figure 3.11: . Transient response of $c_{r}$ in case III.

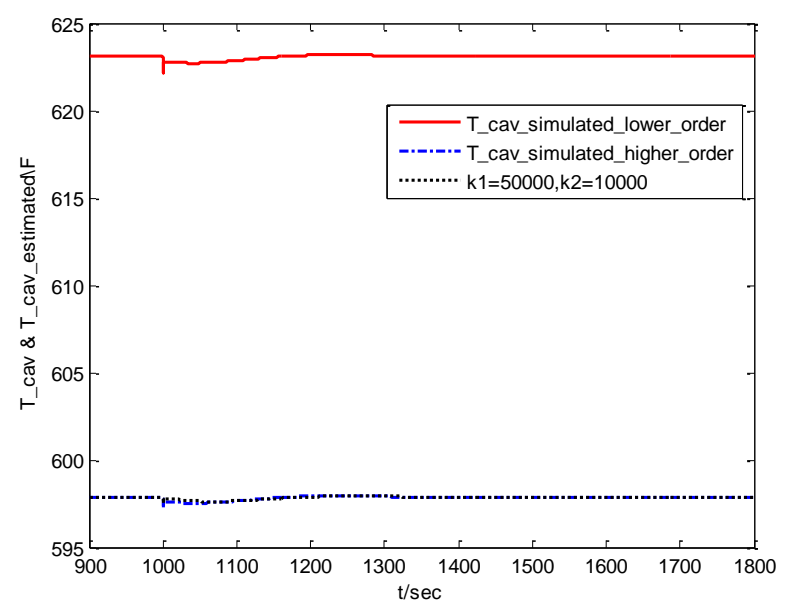

Figure 3.12: Transient response of $T_{c a v}$ in case III

As the simulation results indicate, the DHGF can provide asymptotically stable stateobserver in all three cases. Moreover, the variation of system output leads to the change of output 
error ey $=\mathrm{y}-\hat{\boldsymbol{y}}$, which makes the observed state changes according to the variation of the state vector. According to the Theorem 1 , if $\varepsilon$ is small enough, in other words, $\mathrm{k}_{1}$ and $\mathrm{k}_{2}$ are big enough, then the DHGF is asymptotically stable. This guarantees that the existence of output error ey results in the trend that observation error e becoming smaller, which in turn causes the output difference to be smaller. Based on the concept of zero-state detectability given by Definition 2, it is clear that the state-observation error can be satisfactorily small after a transition period. 


\section{SYSTEM IDENTIFICATION FOR IRIS NUCLEAR REACTOR}

\subsection{Parameter Estimation by System Identification}

In simple terms, system identification is a mathematical problem in which the model or structure of a system is determined from a set of input and output data. Technically, system identification is defined as "the determination on the basis of input and output, of a system (model) within a specified class system (models), to which the system under test is equivalent (in terms of a criterion)" by Zadeh [14]. The system identification procedure can be characterized by the following steps [15].

1) Specify and parameterize a class of mathematical models that represent the system to be identified.

2) Apply an appropriately chosen test signal to the system and record the input-output data.

3) Select the model in the specified class that best fits the statistical data.

4) Estimate the parameters using the given selected model(s)

5) Perform a validation to test to see if the model(s) chosen adequately represent the system with respect to final identification objectives

6) If the validation test passed, the procedure terminates. Otherwise another class of models must be selected and step 2) through 5) repeated.

Generally, there are three approaches to develop model for interested systems. The first one is called "white-box" model which is built based on explicit physical laws that govern the internal behavior of the system, this approach works well for simple systems. For example, an electric circuit including a battery and a resistance follows the Ohm's law. A mass-spring-damp system follows the Newton's kinetic law. But building "white box" model for complicated and nonlinear systems, i.e. nuclear reactor is difficult since either the internal dynamic properties are unknown or it is difficult to express the internal dynamic properties mathematically. On the other hand, the so called "black-box" model is identified from the input/output data totally, rather than 
model the internal dynamic properties based on physical laws. The "grey-box" model is the combination of "white-box" model and "black-box" model. The "grey-box" model is with a model structure derived based on physical laws and the parameters of the model are identified by using the system identification methods.

The "grey-box" modeling approach is utilized to build the IRIS reactor model. The reason is mentioned before. Low-order lumped state space models of reactor are available and these models can describe the internal dynamic characteristic of reactor very well if the coefficients of these models are with acceptable accuracy. And some coefficients of these models cannot be determined directly or easily. Measurements of some coefficients involve large uncertainty; some coefficients vary with operation power level, operation time and so on. So an online adaptive "grey-box" model is a good solution to solve the identification problem of reactor system.

\subsection{Prediction Error Method (PEM) for State-Space Model Identification}

PEM is a widely used model identification technique and studied by many researchers. [16] Generally, PEM consists of the following two procedures: 1). the parameterization of the model to be identified. 2). the determination of the optimal model parameters in such a way that the measurements are "explained" or "fitted" by the parameterized model as accurately as possible. Mathematically, the estimation problem can be formulated as an optimization problem in which the measure of the deviation between the observations and the predictions obtained from the model, as the cost function, is minimized subject to constrains of the model equations, the bounds of the parameters etc. Usually, the least square error is chosen as the cost function. For a single output system, this problem can be expressed as: given a function $J(\theta)$ to be minimized

$$
J(\boldsymbol{\theta})=\sum_{k=1}^{K} \frac{1}{2}(y(k)-\hat{y}(k))^{2}
$$

and an initial state for $\hat{\boldsymbol{\theta}}(\mathbf{0})$, find a minimization direction $\boldsymbol{u ( l )}$ and step size $\eta^{(l)}$, and update the parameters as

$$
\hat{\boldsymbol{\theta}}(l+1)=\hat{\boldsymbol{\theta}}(l)+\eta(l) \boldsymbol{u}(l)
$$


The prediction $\hat{y}(k)=f(\boldsymbol{\theta}, k)$ is a function of the parameter vector $\boldsymbol{\theta}=\left[\begin{array}{ll}\theta_{1}, \cdots, & \theta_{p}\end{array}\right]$, $\boldsymbol{\theta}=\hat{\boldsymbol{\theta}}(l) \quad y(k)$

which is computed using the current parameter vector estimates: ; is the corresponding target observation (measurement); $\mathrm{K}$ is the number of data patterns in the training set.

The task of the minimization is to find optimal values for the direction and the step size, when only local information of the function is available. Repeated application of Eq. (4.2), each time with the optimal direction and step size, will bring $J(\theta)$ to a minimum. As a result of the search, a parameter estimate is obtained which minimized the cost function

$$
\hat{\boldsymbol{\theta}}=\arg \min _{\boldsymbol{\theta}} J(\boldsymbol{\theta})
$$

Note, however, that the globality of the minimum cannot be guaranteed. The optimization techniques are not necessarily restricted to cost functions of the specific form given by Eq. (4.1), as long as the derivatives are computed accordingly.

Let us now focus on finding the minimization direction. Let $\overline{\boldsymbol{\theta}}$ be some fixed parameter vector. The cost function Eq. (4.1) can be written as a Taylor expansion around $\overline{\boldsymbol{\theta}}$ :

$$
J(\boldsymbol{\theta})=J(\overline{\boldsymbol{\theta}})+\sum_{p=1}^{P}\left[\frac{\partial \boldsymbol{J}}{\partial \theta_{p}}\right]_{\boldsymbol{\theta}=\overline{\boldsymbol{\theta}}} \tilde{\theta}_{p}+\sum_{p=1}^{P}\left[\frac{\partial^{2} J}{\partial \theta_{p} \partial \theta_{p^{*}}}\right]_{\boldsymbol{\theta}=\overline{\boldsymbol{\theta}}} \tilde{\theta}_{p} \tilde{\theta}_{p^{*}}+\ldots
$$

where $\tilde{\boldsymbol{\theta}}$ is the deviation from $\overline{\boldsymbol{\theta}}, \boldsymbol{\theta}=\overline{\boldsymbol{\theta}}+\tilde{\boldsymbol{\theta}}$.

If just the first non-constant term of the Taylor expansion is used, this is called the first order methods, expressed by Eq. (4.5).

$$
J(\boldsymbol{\theta}) \approx J(\overline{\boldsymbol{\theta}})+\sum_{p=1}^{P}\left[\frac{\partial J}{\partial \theta_{p}}\right]_{\boldsymbol{\theta}=\overline{\boldsymbol{\theta}}} \tilde{\theta}_{p}
$$

The derivative of $J(\boldsymbol{\theta})$ is given by

$$
\frac{\partial}{\partial \tilde{\theta}_{p}} J(\boldsymbol{\theta})=\left[\frac{\partial J}{\partial \theta_{p}}\right]_{\theta=\overline{\boldsymbol{\theta}}}=\sum_{k=1}^{K}\left([y(k)-\hat{y}(k)]\left[\frac{\partial \hat{y}}{\partial \theta_{p}}(k)\right]_{\boldsymbol{\theta}=\overline{\boldsymbol{\theta}}}\right)
$$


A natural fixed point $\bar{\theta}$ is the current parameter estimate $\theta(l)$. To compute the new estimate, the minimization direction is given by the negative gradient. The learning rule then becomes

$$
\hat{\boldsymbol{\theta}}(l+1)=\hat{\boldsymbol{\theta}}(l)-\eta(l)\left[\frac{\partial \boldsymbol{J}}{\partial \boldsymbol{\theta}}\right]_{\boldsymbol{\theta}=\boldsymbol{\theta}(l)}
$$

The step size $\eta(l)$ is often replaced by a fixed constant, due to lower computational cost. These types of methods are often also referred to as steepest decent, gradient descent, least mean squares, or error back propagation techniques. If the second non-constant term from the Taylor expansion is also considered, the cost function becomes

$$
J(\boldsymbol{\theta}) \approx J(\overline{\boldsymbol{\theta}})+\sum_{p=1}^{P}\left[\frac{\partial \boldsymbol{J}}{\partial \theta_{p}}\right]_{\boldsymbol{\theta}=\overline{\boldsymbol{\theta}}} \tilde{\theta}_{p}+\sum_{p=1}^{P}\left[\frac{\partial^{2} J}{\partial \theta_{p} \partial \theta_{p^{*}}}\right]_{\boldsymbol{\theta}=\overline{\boldsymbol{\theta}}} \tilde{\theta}_{p} \tilde{\theta}_{p^{*}}
$$

and can be written as

$$
J(\boldsymbol{\theta})=J(\overline{\boldsymbol{\theta}})-\boldsymbol{b}^{T} \tilde{\boldsymbol{\theta}}+\frac{1}{2} \tilde{\boldsymbol{\theta}}^{T} \boldsymbol{H}^{T} \tilde{\boldsymbol{\theta}}
$$

where

$$
\boldsymbol{b}=\left[\frac{\partial \boldsymbol{J}}{\partial \boldsymbol{\theta}}\right]_{\boldsymbol{\theta}=\overline{\boldsymbol{\theta}}}
$$

and the elements of the Hessian $\mathrm{H}$ are given as

$$
h_{p, p^{*}}=\left[\frac{\partial^{2} J}{\partial \theta_{p} \partial \theta_{p^{*}}}\right]_{\theta=\bar{\theta}}
$$

Minimum of cost function by Eq. (4.8) is found by setting the derivative to zero, and is located at

$$
\boldsymbol{H} \tilde{\boldsymbol{\theta}}-\boldsymbol{b}=0
$$

from where the optimal $\tilde{\boldsymbol{\theta}}$ can be obtained. It is given by

$$
\tilde{\boldsymbol{\theta}}=\boldsymbol{H}^{-1} \boldsymbol{b}
$$

Thus, the optimization reduces to matrix inversion.

Unfortunately, the calculation of the Hessian $\mathrm{H}$ is computationally prohibitive in practice. Analytical solutions are rare and approximation methods must be applied. Iterative optimization algorithms are utilized to search the minimum of the cost function. The method used in this report is Levenberg-Marquardt method. It is introduced as follows. 
Define a vector $\mathrm{R}$ whose $\mathrm{K}$ components ${ }^{r_{k}}$ are the residuals

$$
r_{k}=\hat{y}(k)-y(k)
$$

$\mathrm{k}=1,2, \ldots \mathrm{K}$, where $\mathrm{K}$ is the number of data samples. The cost function and its derivative can now be expressed as

$$
\begin{gathered}
J(\boldsymbol{\theta})=\frac{1}{2} \boldsymbol{R}(\boldsymbol{\theta})^{T} \boldsymbol{R}(\boldsymbol{\theta}) \\
\frac{\partial J(\boldsymbol{\theta})}{\partial \boldsymbol{\theta}}=\sum_{k=1}^{K} r_{k} \frac{\partial r_{k}}{\partial \boldsymbol{\theta}}=\boldsymbol{G}(\boldsymbol{\theta})^{T} \boldsymbol{R}(\boldsymbol{\theta}) \\
\frac{\partial^{2} J(\boldsymbol{\theta})}{\partial \boldsymbol{\theta}^{2}}=\sum_{k=1}^{K}\left(\frac{\partial r_{k}}{\partial \boldsymbol{\theta}}\left[\frac{\partial r_{k}}{\partial \boldsymbol{\theta}}\right]^{T}+r_{k} \frac{\partial^{2} r_{k}}{\partial \boldsymbol{\theta}^{2}}\right)=\boldsymbol{G}(\boldsymbol{\theta})^{T} \boldsymbol{G}(\boldsymbol{\theta})+\boldsymbol{S}(\boldsymbol{\theta})
\end{gathered}
$$

where $\boldsymbol{G}(\boldsymbol{\theta})$ is the Jacobian matrix, whose elements $g_{k, p}$ are given by

$$
g_{k, p}=\frac{\partial r_{k}}{\partial \theta_{p}}
$$

Note that $\frac{\partial r_{k}}{\partial \theta_{p}}=\frac{\partial \hat{y}}{\partial \theta_{p}}(k)$ and $\boldsymbol{S}(\boldsymbol{\theta})$ is the part of the Hessian matrix that contains the second derivatives of ${ }^{r_{k}}$. The Newton iteration is given by

$$
\hat{\boldsymbol{\theta}}(l+1)=\hat{\boldsymbol{\theta}}(l)-\left[\boldsymbol{G}(\boldsymbol{\theta})^{T} \boldsymbol{G}(\boldsymbol{\theta})+\boldsymbol{S}(\boldsymbol{\theta})\right]^{-1} \boldsymbol{G}(\boldsymbol{\theta})^{T} \boldsymbol{R}(\boldsymbol{\theta})
$$

where the Jacobian $\boldsymbol{G}(\boldsymbol{\theta})$ is easy to calculate, while $\boldsymbol{S}(\boldsymbol{\theta})$ is not. In the Gauss-Newton method, the $\boldsymbol{S}(\boldsymbol{\theta})$ is simply neglected. In the Levenberg-Marquardt method, the step is defined as

$$
\hat{\boldsymbol{\theta}}(l+1)=\hat{\boldsymbol{\theta}}(l)-\left[\boldsymbol{G}(\boldsymbol{\theta})^{T} \boldsymbol{G}(\boldsymbol{\theta})+\mu(l) \boldsymbol{I}\right]^{-1} \boldsymbol{G}(\boldsymbol{\theta})^{T} \boldsymbol{R}(\boldsymbol{\theta})
$$

The following simple procedure was suggested for ${ }^{\mu}$ in the Levenberg-Marquardt method in [17]: whenever a step would result in an increased value $J, \mu(l)$ is increased by multiplying it by some factor larger than 1; when a step reduces the value of $J$, it is divided by some factor larger than 1 . Note, that when ${ }^{\mu}$ is large the algorithm becomes steepest descent with a step size equal to $1 / \mu$, while for small ${ }^{\mu}$ the algorithm becomes Gauss-Newton. Based on the theorem mentioned above, the Levenberg-Marquardt method can be summarized as the following algorithm. 
Given a function $J(\boldsymbol{\theta})$ of the sum of prediction errors

$$
J(\boldsymbol{\theta})=\sum_{k=1}^{K} \frac{1}{2}(y(k)-\hat{y}(k))^{2}
$$

the minimizing parameters

$$
\hat{\boldsymbol{\theta}}=\arg \min _{\boldsymbol{\theta}} J(\boldsymbol{\theta})
$$

can be found by the following algorithm.

1. Initialize:

Set iteration index $l=1$.

Initialize $^{\hat{\boldsymbol{\theta}}(l)}$ and $\mu(l)$ and specify $\eta$.

2. Evaluate the model and the residuals:

Evaluate $\hat{y}(k)$ and $\frac{\partial \hat{y}}{\partial \theta_{p}}(k)$ for all patterns $\mathrm{k}$ and parameters $\mathrm{p}$.

Compose the residual vector $\boldsymbol{R}$

$$
r_{k}=\hat{y}(k)-y(k)
$$

and compute $J(\hat{\theta}(l))$

$$
J(\hat{\boldsymbol{\theta}}(l))=\frac{1}{2} \boldsymbol{R}^{T} \boldsymbol{R}
$$

Compose the Jacobian matrix $\mathrm{G}$

$$
g_{k, p}=\frac{\partial \hat{y}}{\partial \theta_{p}}(k)
$$

3. Solve the parameter update

$$
\Delta \hat{\boldsymbol{\theta}}(l)=-\left[\boldsymbol{G}^{T} \boldsymbol{G}+\mu(l) \boldsymbol{I}\right]^{-1} \boldsymbol{G}^{T} \boldsymbol{R}
$$

4. Repeat step 2 using $\hat{\boldsymbol{\theta}}(l)+\Delta \hat{\boldsymbol{\theta}}(l)$, i.e. compute $J(\hat{\boldsymbol{\theta}}(l)+\Delta \hat{\boldsymbol{\theta}}(l))$.

If $J(\hat{\boldsymbol{\theta}}(l)+\Delta \hat{\boldsymbol{\theta}}(l))<J(\hat{\boldsymbol{\theta}}(l))$ then increase the step size

$$
\mu(l+1)=\mu(l) / \eta
$$

and update the parameters

$$
\hat{\boldsymbol{\theta}}(l+1)=\hat{\boldsymbol{\theta}}(l)+\Delta \hat{\boldsymbol{\theta}}(l)
$$

Otherwise reduce the step size

$$
\mu(l+1)=\eta \mu(l)
$$

5. Set $l=l+1$ and return to Step 2, or quit. 


\subsection{Parameter Estimation Results and Discussion}

\subsubsection{Offline Parameter Estimation}

The grey-box model of IRIS reactor is given in Eq. (3.1). First, offline parameter estimation is implemented to estimated the parameters set $\{\beta \Lambda \alpha$ f $\alpha \mathrm{c} \lambda$ a1 a2 a3 a4 a5 $\}$ of the reactor dynamic model. As stated previously, in order to extract useful information correlating the input and output signals, the input signal must be large enough to perturb the system significantly such that all dynamics are excited, yet small enough not to adversely affect the output. In this study, the dynamics are already given in the ordinary differential equations, we focus on the parameter estimation and future output prediction. The objective of the offline parameter estimation is to estimate the model parameters when the operation power of the reactor is around $100 \%$ rated power. Measurement data from the IRIS FORTRAN simulator were collected for parameter estimation purposes. A small load change periods signal is selected for identification. The reference load signal and reactor relative power response are shown in Figure 4.1.

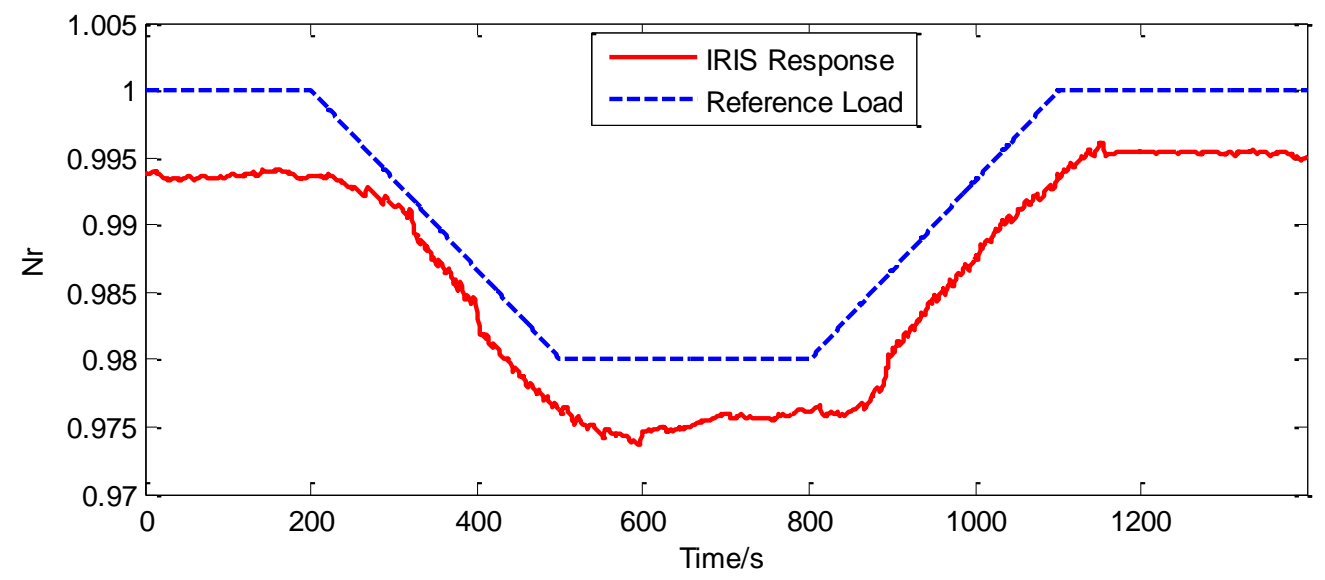

Figure 4.1: Small change reference load signal and reactor relative power response

Before we begin the identification process, three variables are defined to evaluate the prediction performance of estimated model. 


$$
\begin{aligned}
& C_{1}=\max \{|e(1)| \quad|e(2)| \cdots|e(L)|\} \\
& C_{2}=\frac{\sum_{i=1}^{L}(e(i))^{2}}{L} \\
& C_{3}=\frac{\sum_{i=1}^{L} e(i)}{L}
\end{aligned}
$$

where $\mathrm{C}_{1}$ measures the maximum prediction error and $\mathrm{C}_{2}$ measures the variance of prediction error, $\mathrm{C}_{3}$ measures the mean of prediction error .

It is important to note here, since the physically meaningful ranges of the point kinetics model parameters were relatively well known from plant document and literature, the proper selection of initial parameter values was possible. For the IRIS reactor system, the initial value of the coefficients set is chosen as data given in Table 4.1:

Table 4.1: Initial value range of coefficients of the point kinetics model

\begin{tabular}{|c|c|c|c|c|c|}
\hline Coefficients & $\beta$ & $\Lambda$ & $\alpha \mathrm{f}$ & $\alpha \mathrm{c}$ & $\lambda$ \\
\hline Value & $0.002 \sim 0.008$ & $0.00005 \sim 0.002$ & $-4 \mathrm{e}-5 \sim-1 \mathrm{e}-5$ & $-8 \mathrm{e}-4 \sim-1 \mathrm{e}-4$ & $0.04 \sim 0.16$ \\
\hline Coefficients & $a_{1}$ & $\mathrm{a} 2$ & $\mathrm{a} 3$ & $\mathrm{a} 4$ & $\mathrm{a} 5$ \\
\hline Value & $-0.18 \sim-0.14$ & $0.10 \sim 0.22$ & $1.2 \sim 2.9$ & $52 \sim 65$ & $1.0 \sim 2.7$ \\
\hline
\end{tabular}

where $a_{1}=-\frac{\Omega}{\mu_{f}}, a_{2}=\frac{\Omega}{\mu_{c}}, a_{3}=-\frac{2 M+\Omega}{\mu_{c}}, a_{4}=\frac{P_{0}}{\mu_{f}}, a_{5}=\frac{2 M}{\mu_{c}}$

The input data are Tcin and $\rho$ r while the output data is $\mathrm{nr}$ and Tave. The output consists of two components, a weight matrix is applied to calculate the function error, which is shown in Eq. (4.31).

$$
J(\boldsymbol{\theta})=\sum_{k=1}^{K} \frac{1}{2}\left(E(k)^{T} W E(K)\right)^{2}
$$

where

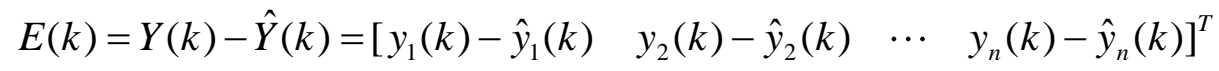

$W=\left[\begin{array}{llll}w_{1} & & & \\ & w_{2} & & \\ & & \ddots & \\ & & & w_{n}\end{array}\right]$ 
The offline estimation result is shown in Figures 4.2-4.5. From Figure 4.2 and 4.5, it can be found that the simulated $\mathrm{Nr}$, Tcav fit the measured $\mathrm{Nr}$, Tcav well. Errors between the simulated $\mathrm{Tf}$ and $\mathrm{Tf}$ given by the IRIS FORTRAN simulator are presented in Figure 4.6. It is interpreted as that there exist certain model uncertainty between the 4th reactor model and the real dynamic in reactor. The algorithm only tries to fit the input/output data. The error of Tf is resulted by the model uncertainty.

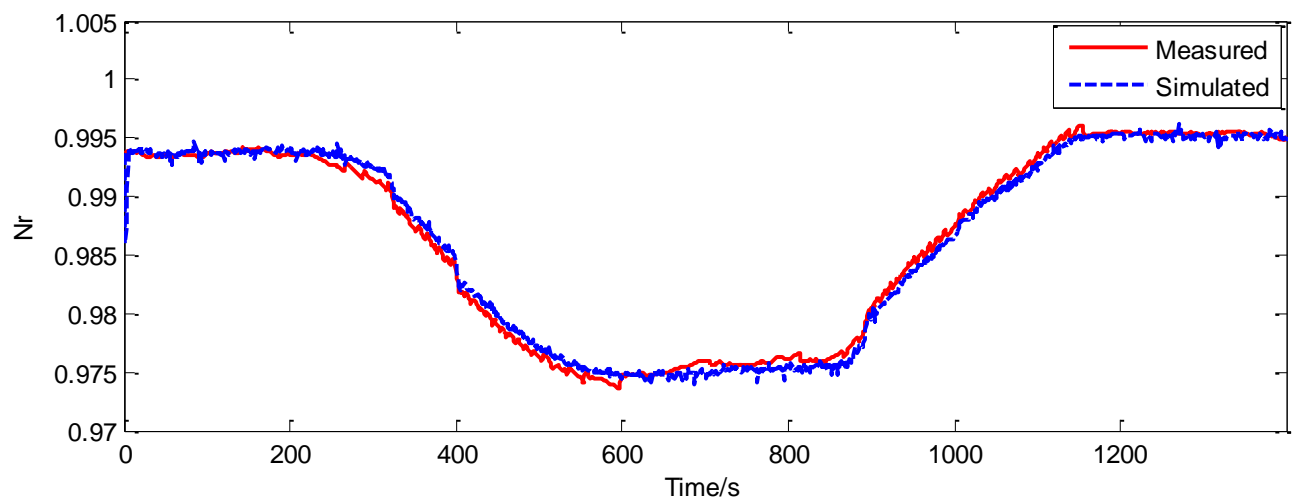

Figure 4.2: Comparison between simulated $N_{r}$ and measured $N_{r}$

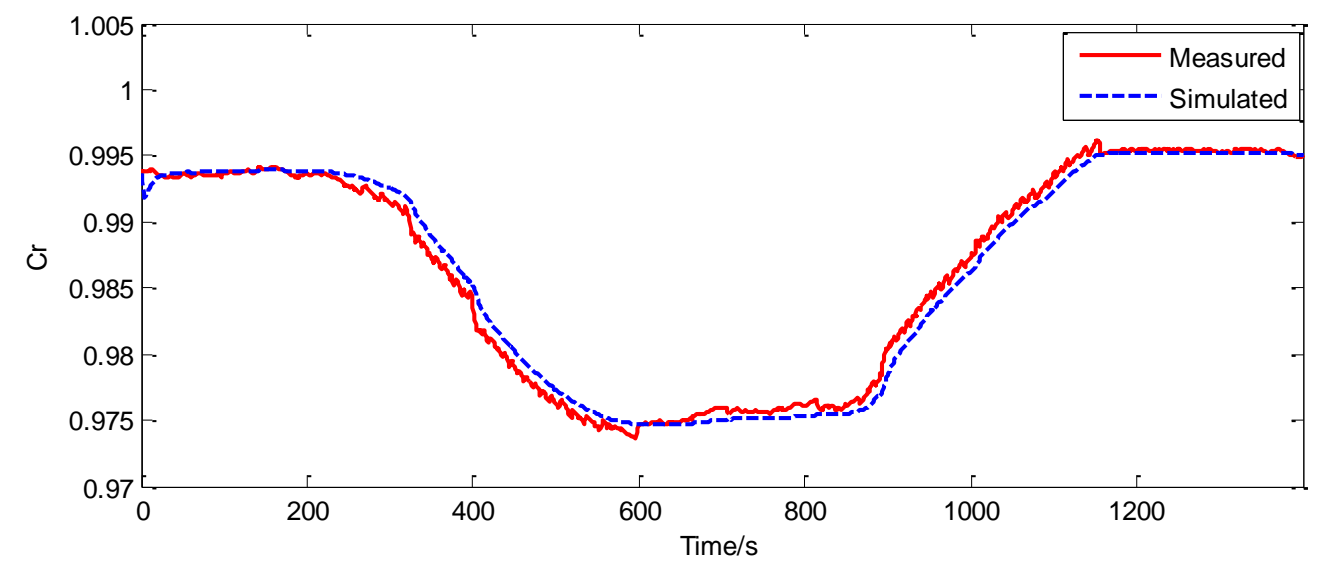

Figure 4.3: Comparison between simulated $C_{r}$ and real $C_{r}$ 


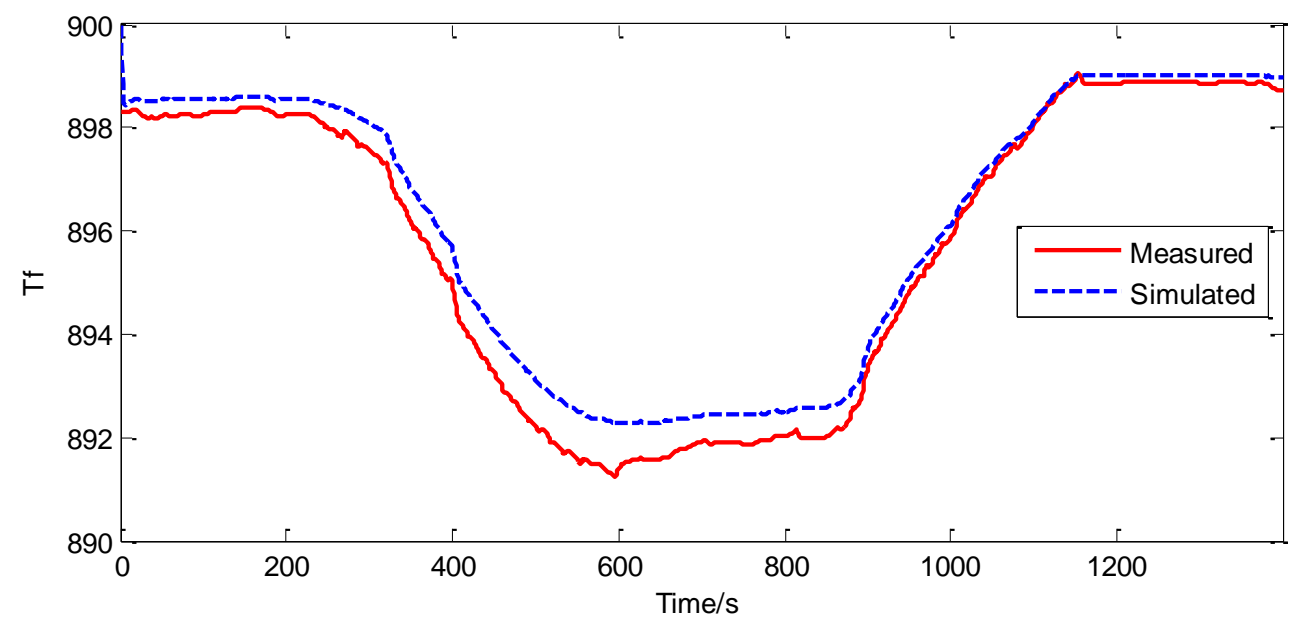

Figure 4.4: Comparison between simulated $T_{f}$ and measured $T_{f}$

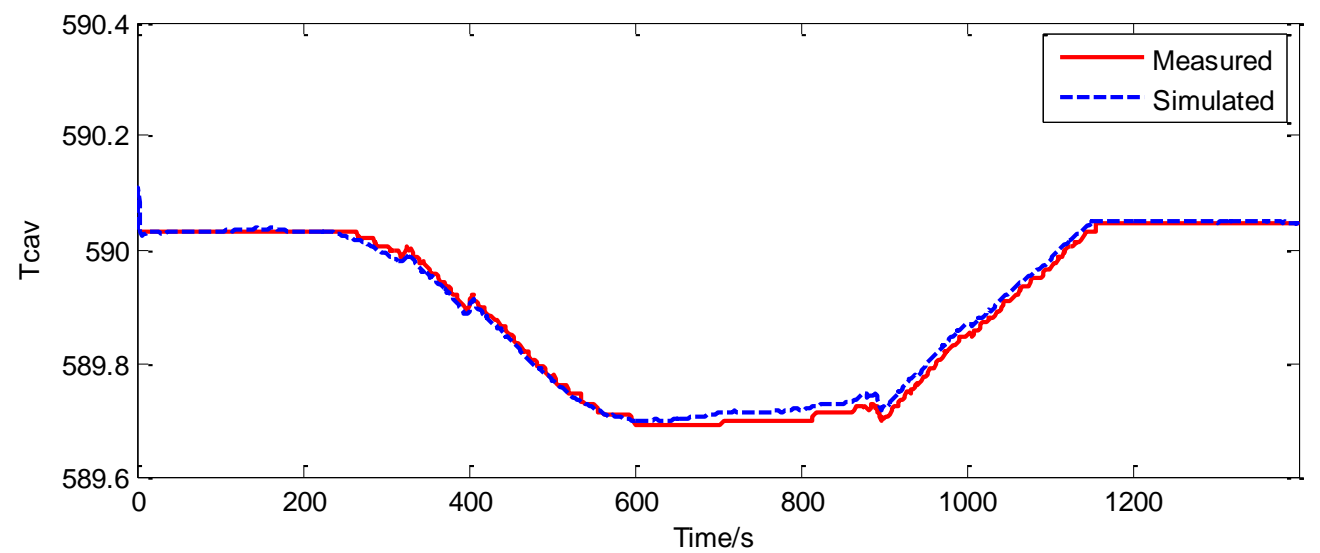

Figure 4.5: Comparison between simulated $T_{\text {cav }}$ and measured $T_{c a v}$

Table 4.2 presents the estimated coefficient values of IRIS reactor model and Table 4.3 shows the fitting error of the four states. It can be concluded that the estimated model can describe the reactor dynamic around $100 \%$ rated power with acceptable accuracy.

Table 4.2: Estimated coefficients values of IRIS reactor model

\begin{tabular}{|c|c|c|c|c|c|}
\hline Coefficients & $\beta$ & $\Lambda$ & $\alpha \mathrm{f}$ & $\alpha \mathrm{c}$ & $\lambda$ \\
\hline Value & 0.00404 & $9.028 \mathrm{e}-5$ & $-1.878-5$ & $-3.8 \mathrm{e}-4$ & 0.1 \\
\hline Coefficients & $a_{1}$ & $\mathrm{a} 2$ & $\mathrm{a} 3$ & $\mathrm{a} 4$ & $\mathrm{a}$ \\
\hline Value & -0.1718 & 0.1287 & -1.4903 & 53.3192 & 1.3724 \\
\hline
\end{tabular}


Table 4.3: $C_{1}, C_{2}$ and $C_{3}$ of parameter estimation

\begin{tabular}{|c|c|c|c|c|}
\hline & $\mathrm{Nr}$ & $\mathrm{Cr}$ & $\mathrm{Tf}$ & Tcav \\
\hline $\mathrm{C} 1$ & 0.0020 & 0.0026 & 1.0469 & 0.0244 \\
\hline $\mathrm{C} 2$ & $4.5437 \mathrm{e}-007$ & $7.5324 \mathrm{e}-007$ & 0.2498 & $1.2208 \mathrm{e}-004$ \\
\hline $\mathrm{C} 3$ & $-6.7318 \mathrm{e}-005$ & $-7.0956 \mathrm{e}-005$ & 0.4234 & 0.0045 \\
\hline
\end{tabular}

\subsubsection{On-line Parameter Estimation}

When the model estimated by input/output data generated around 100\% rated power is used for output prediction at other operation power level, such as $80 \%$ rated power. An appreciable prediction error will be observed, this is because the coefficients vary with the operation power. The prediction and prediction error of the four states are shown in Figure 4.6, $4.7, \ldots, 4.13$.

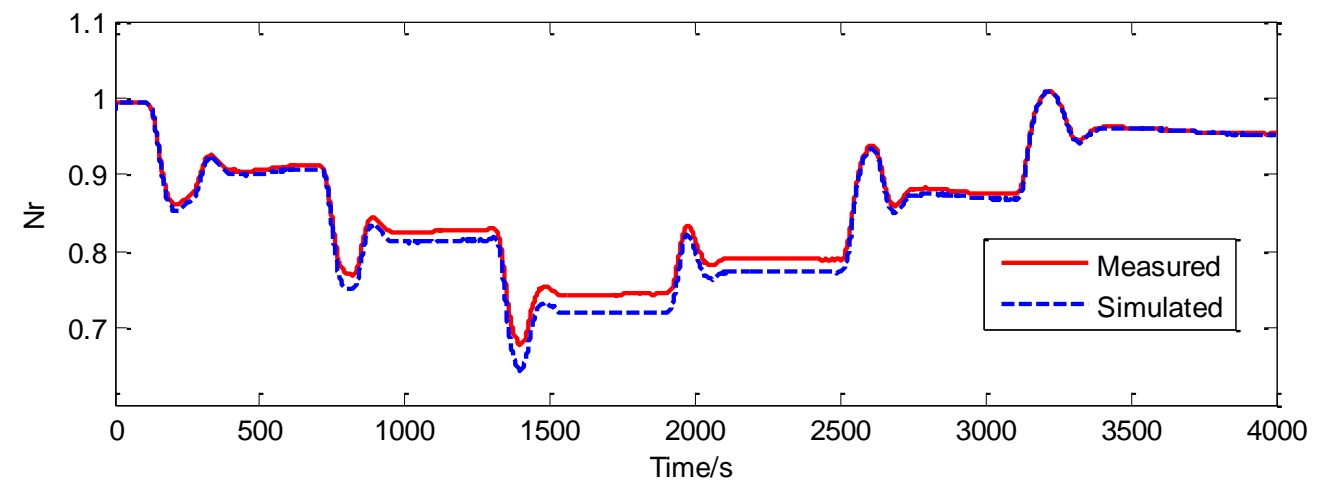

Figure 4.6: Prediction of $N_{r}$ by the offline estimation model

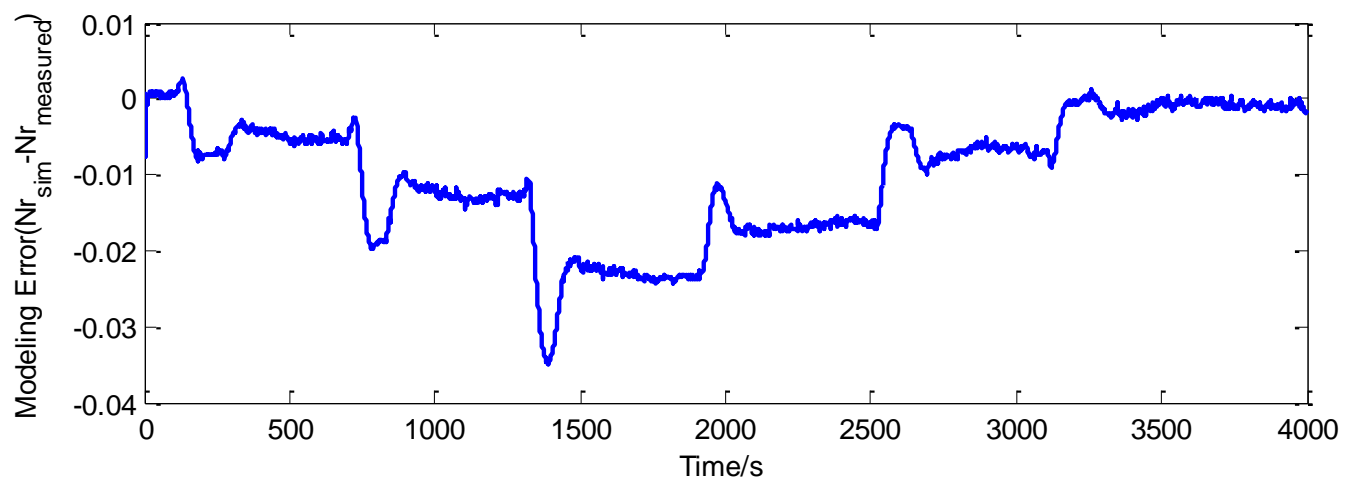

Figure 4.7: Prediction Error of $N_{r}$ by the offline estimation model 


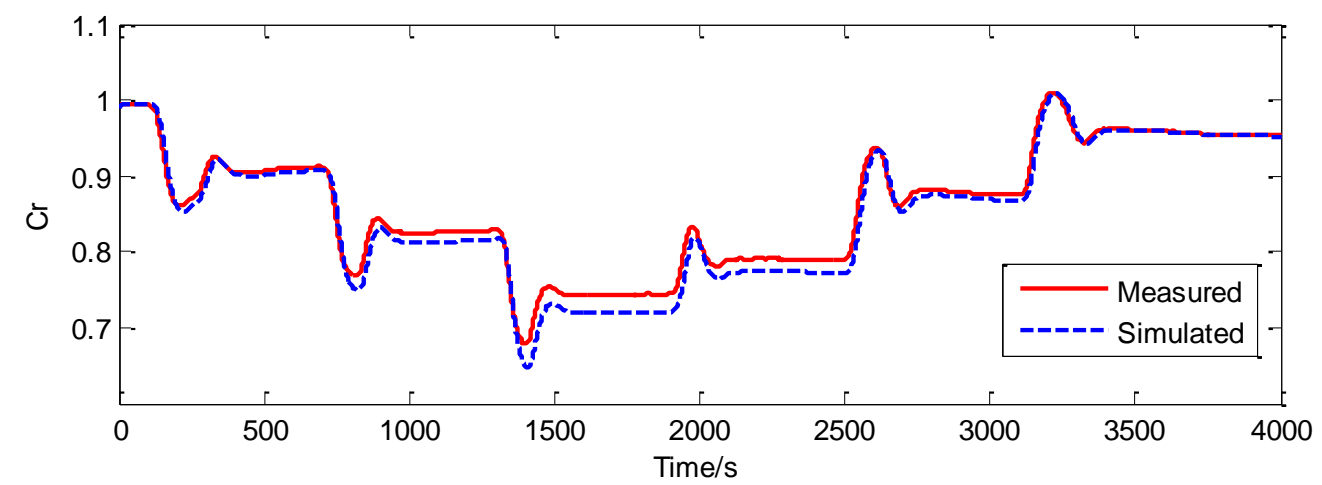

Figure 4.8: Prediction of $C_{r}$ by the offline estimation model

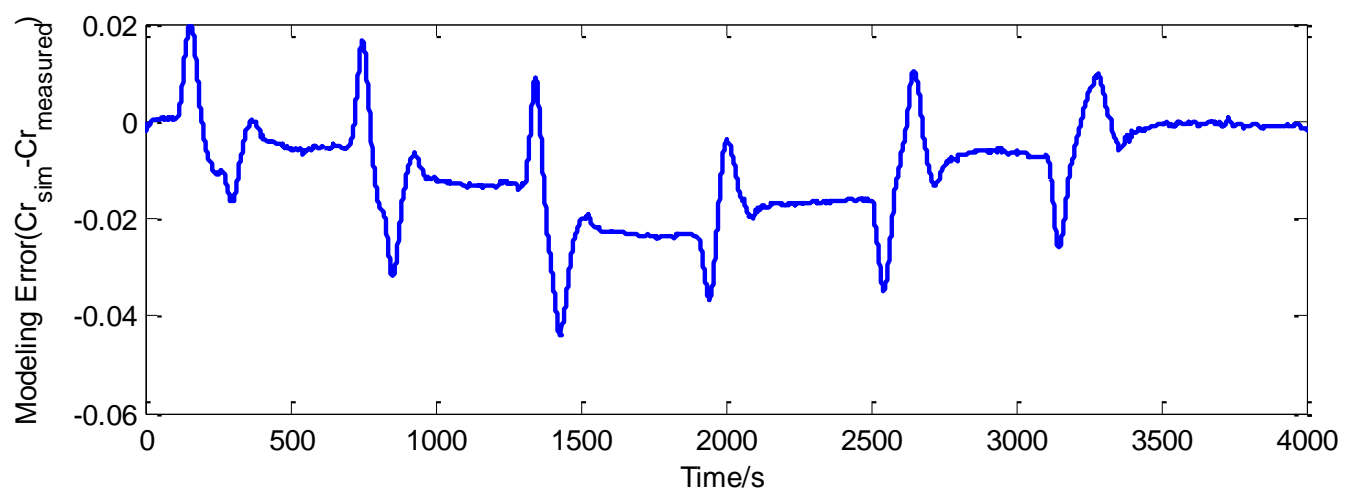

Figure 4.9: Prediction Error of $C_{r}$ by the offline estimation model

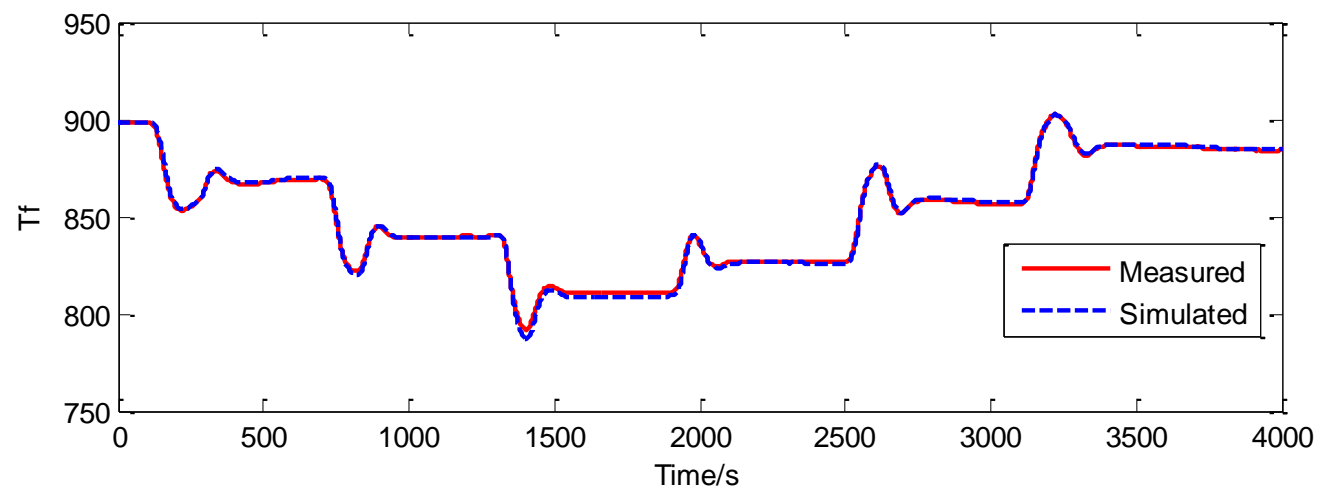

Figure 4.10: Prediction of $T_{f}$ by the offline estimation model 


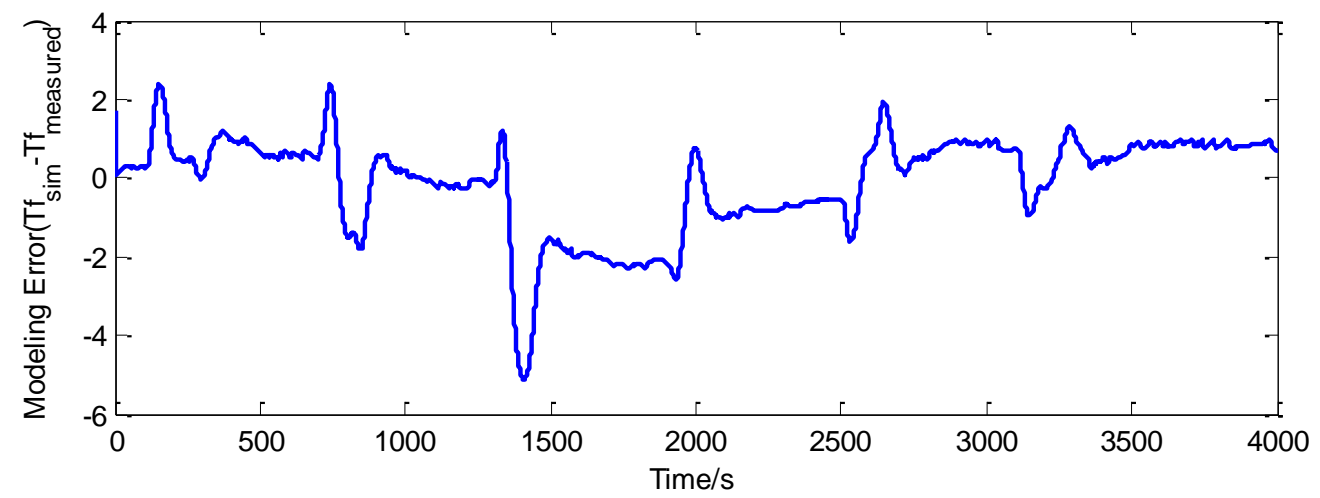

Figure 4.11: Prediction Error of $T_{f}$ by the offline estimation model

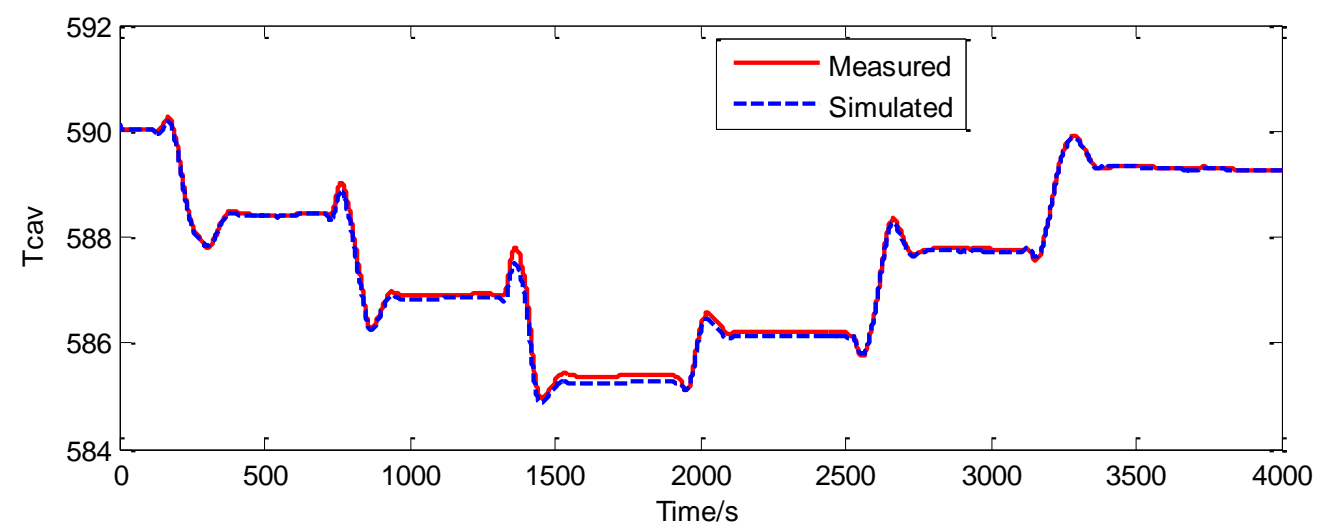

Figure 4.12: Prediction of $T_{\text {cav }}$ by the offline estimation model

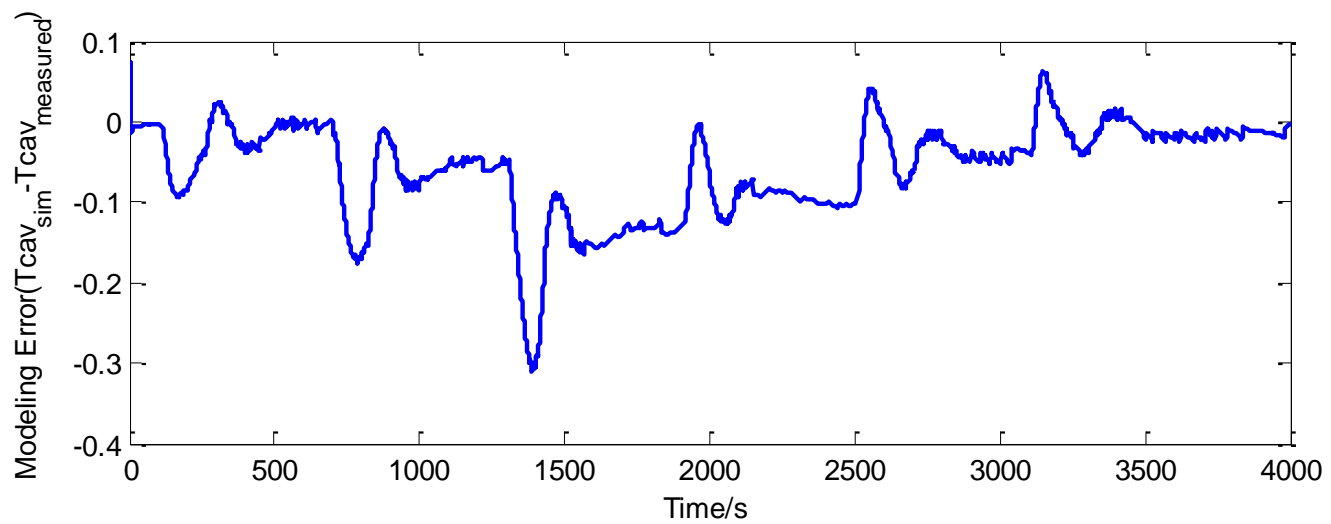

Figure 4.13: Prediction Error of $T_{\text {cav }}$ by the offline estimation model

It can be found that the steady state prediction error and the transient prediction error increase as the difference between the operation power and the rated power increases. Table 4.4 shows the $\mathrm{C} 1, \mathrm{C} 2$ and $\mathrm{C} 3$ for each state variable. 
Table 4.4: $C_{1}, C_{2}$ and $C_{3}$ of offline estimation for the four state variables

\begin{tabular}{|c|c|c|c|c|}
\hline & $\mathrm{Nr}$ & $\mathrm{Cr}$ & $\mathrm{Tf}$ & Tcav \\
\hline $\mathrm{C} 1$ & 0.0350 & 0.0441 & 5.1425 & 0.3106 \\
\hline $\mathrm{C} 2$ & $1.6800 \mathrm{e}-004$ & $2.0802 \mathrm{e}-004$ & 1.5102 & 0.0068 \\
\hline $\mathrm{C} 3$ & -0.0100 & -0.0099 & -0.0915 & -0.0576 \\
\hline
\end{tabular}

The prediction given by the offline estimated model is too large that it cannot be used for modern control algorithms such as model predictive control.

An online parameter adaptation scheme is used in this report to overcome this problem. First it is important to mention here that because the nonlinear minima searching algorithm may fall into local minima, in other words, it is not guaranteed that the global minima were always achieved by the nonlinear iterative optimization algorithm. This will influence the stability of the online parameter estimation. In this study, because prior knowledge about the range and the bound of the change rate of the estimated coefficients are known, after a reasonable initial value was obtained, the optimization algorithm just search the minima over a small subspace of the coefficients space. This online coefficients adaptation scheme has acceptable computation speed and prediction performance. The online system identification scheme is shown in Figure 4.14.

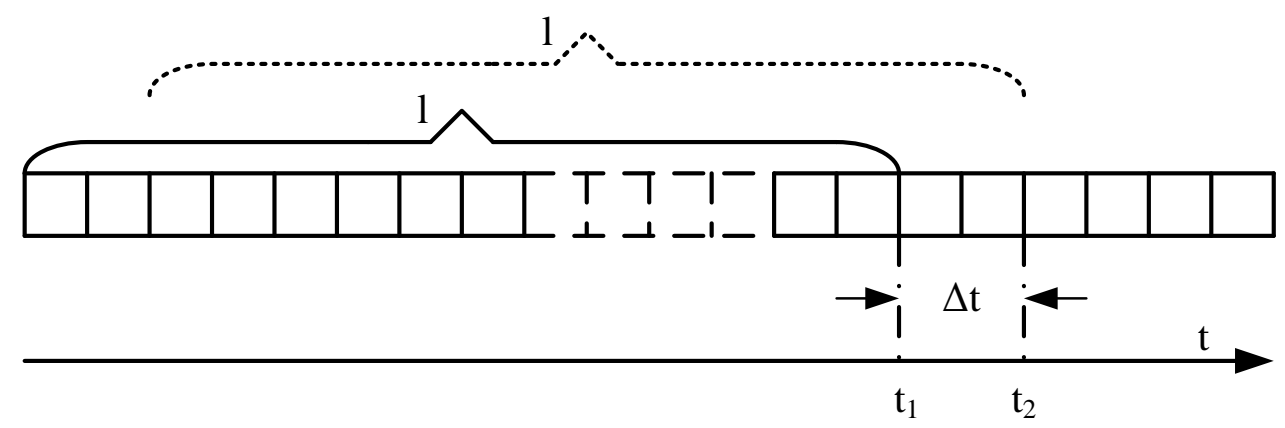

Figure 4.14: Online estimation and prediction scheme

The identification procedure is summarized as follows:

1) At time $t=t 1$, specify the physical meaningful range of the estimated coefficients set $\boldsymbol{\theta}$ based on the current and formal data, such as operation power, coolant temperature etc, this range will serve as the coefficients boundary in the optimization algorithm.

2) Estimated the optimal coefficients set $\boldsymbol{\theta}$ by using the input/output data from t1-1 to t1, here 1 is the width of the moving identification data window. 
3) Predict the output of system during time from $t 1$ to $t 1+\Delta t$ by the estimated model. Then sample new data from $\mathrm{t} 1$ to $\mathrm{t} 2$ where $\mathrm{t} 2=\mathrm{t} 1+\Delta \mathrm{t}$, evaluate the prediction error. Update the time $\mathrm{t}=\mathrm{t} 2$ and repeat from step 1 , here $\Delta \mathrm{t}$ is the time during which the output of the system will be predicted).

In this analysis, the data sampling rate is $1 / \mathrm{sec}$. The width of the moving identification data window 1 is chosen as 20 while $\Delta \mathrm{t}$ is chosen as $5 \mathrm{sec}$, which means the online estimated model will be used to predict the system output response for the future 5 seconds. The online prediction result is presented in Figure 4.15, 4.16, 4.17, 4.18 for the four state variables. The online and offline prediction errors for the four variables were compared in Figure 4.19, 4.20, 4.21, 4.22. The C1, C2 and C3 for online estimation are given in Table 4.5.

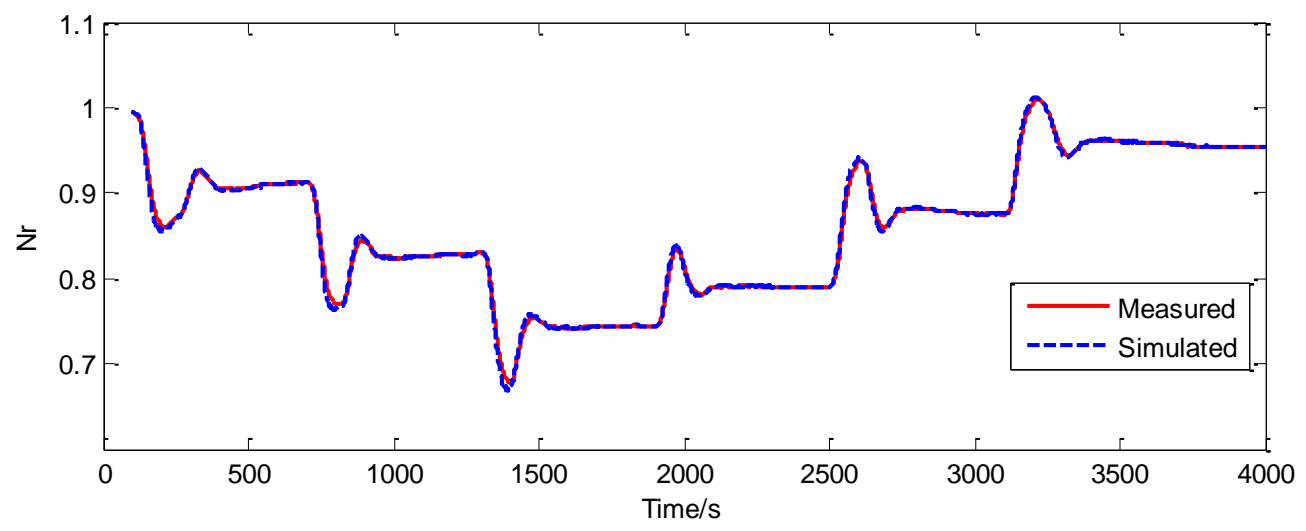

Figure 4.15: Prediction of $N_{r}$ by the online estimation model

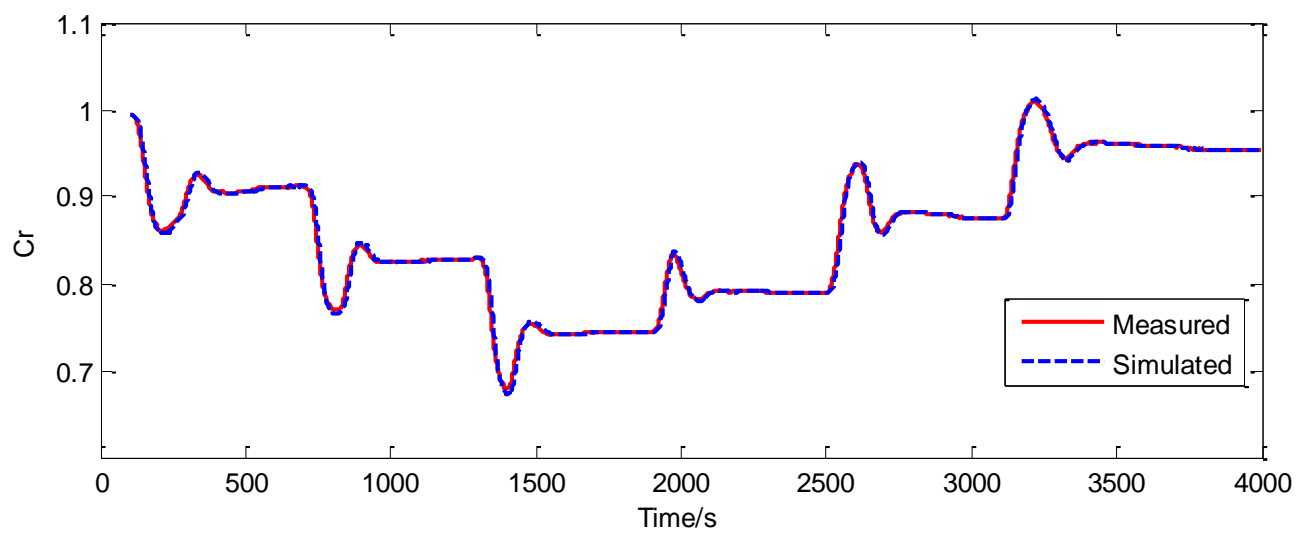

Figure 4.16: Prediction of $C_{r}$ by the online estimation model 


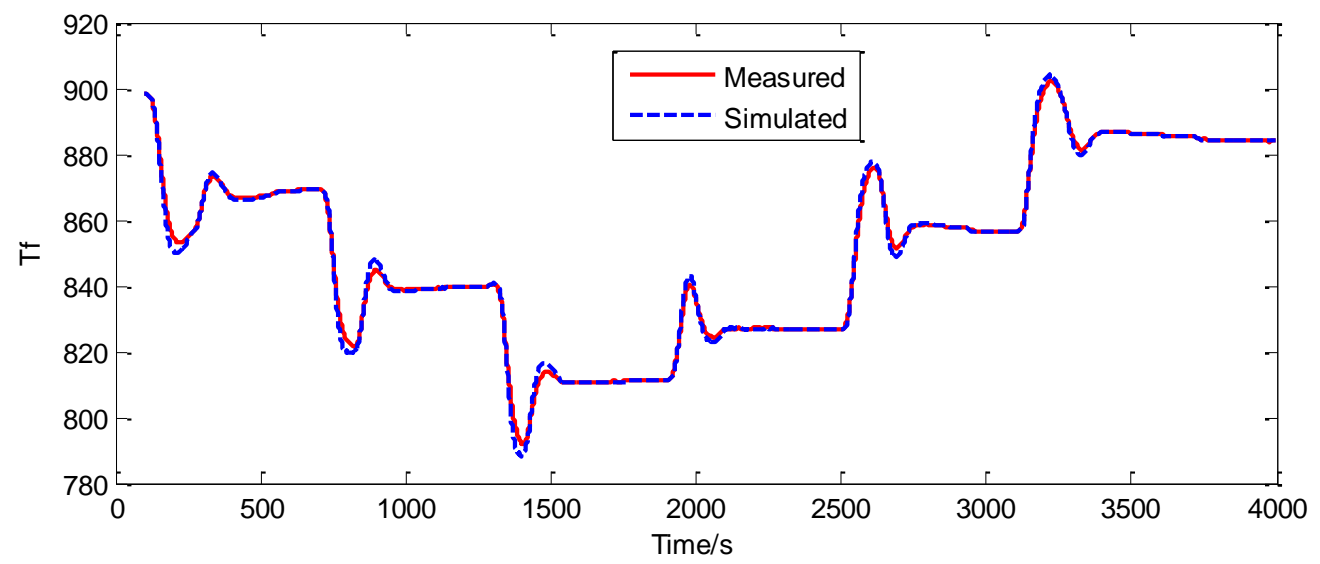

Figure 4.17: Prediction of $T_{f}$ by the online estimation model

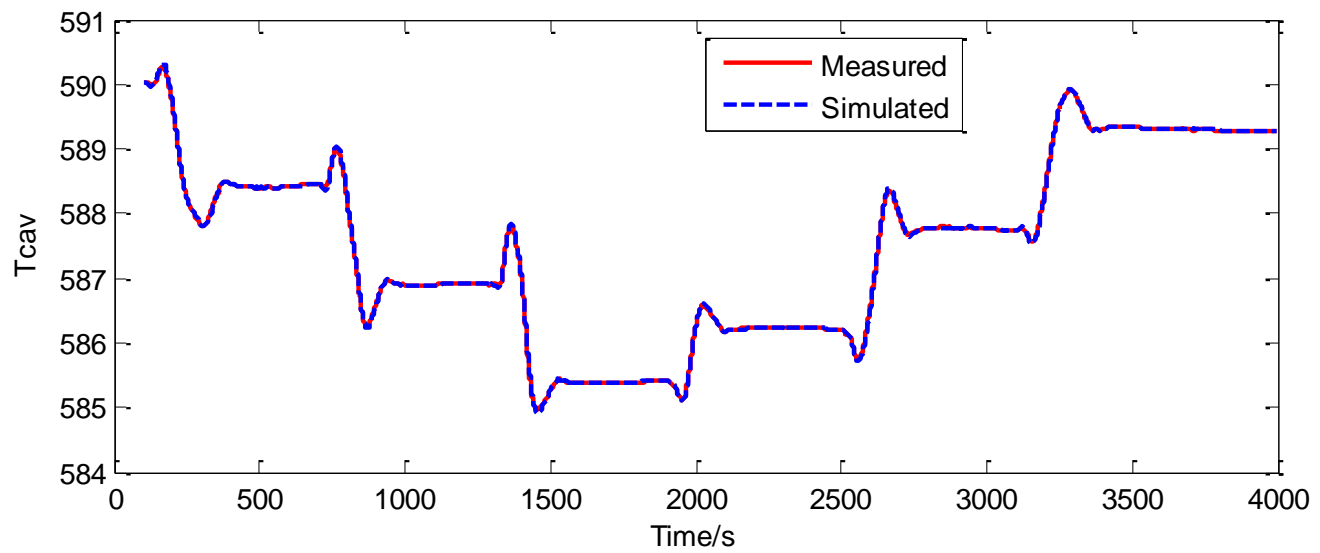

Figure 4.18: Prediction of $T_{\text {cav }}$ by the online estimation model

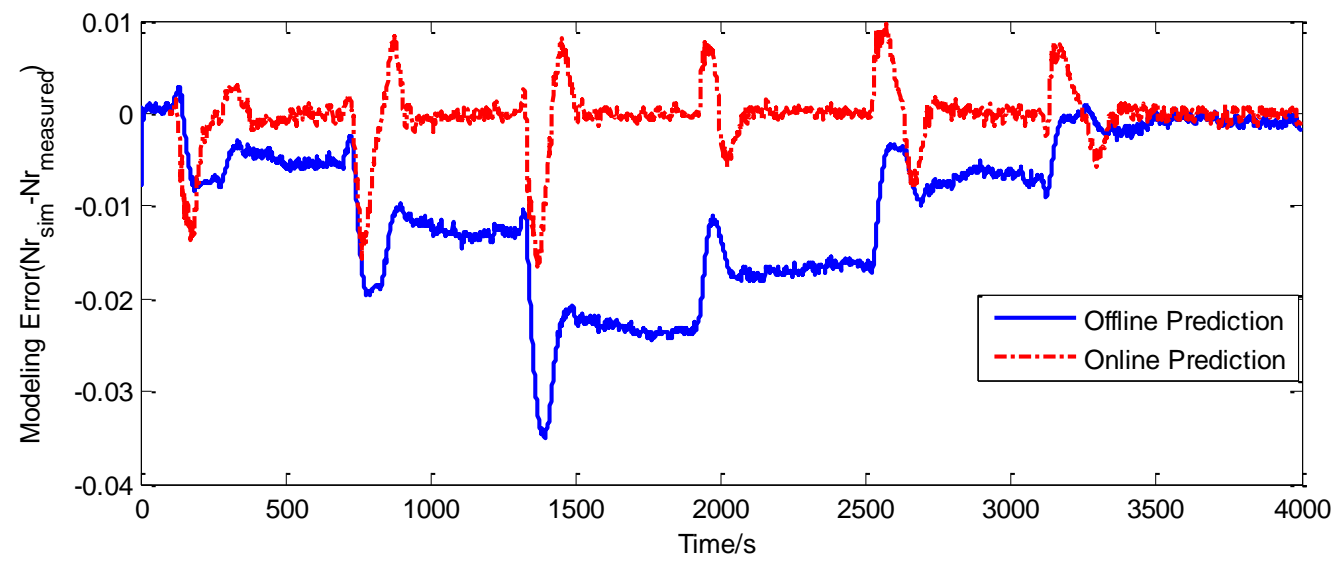

Figure 4.19: Prediction Error of $N_{r}$ by the offline and online estimation model 


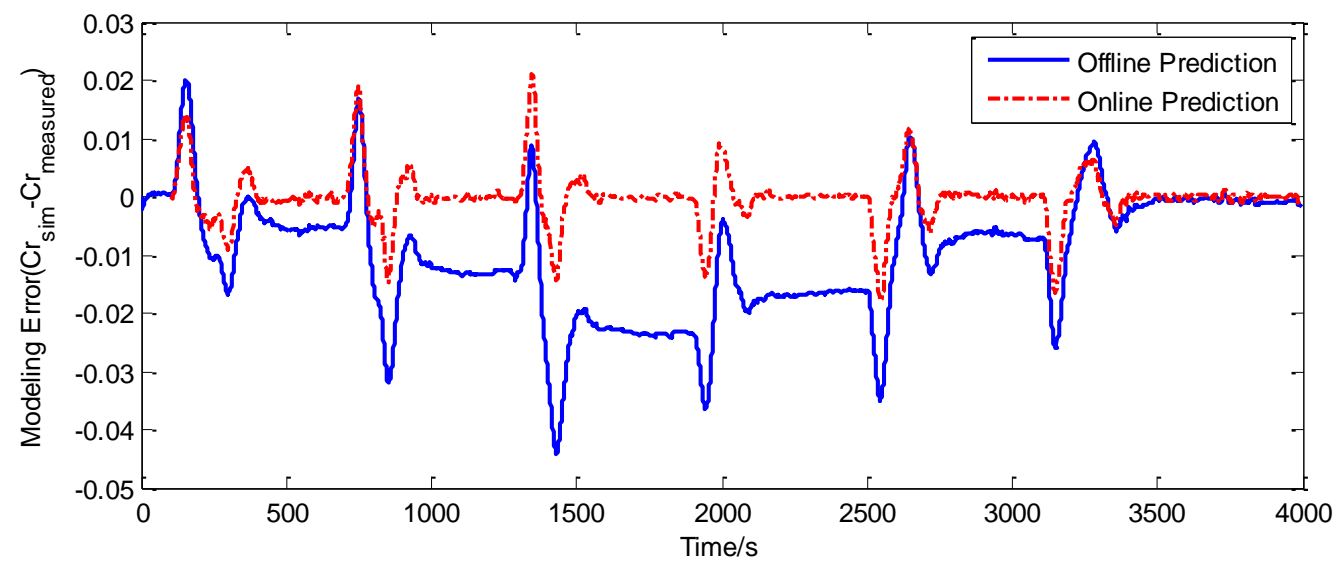

Figure 4.20: Prediction Error of $C_{r}$ by the offline and online estimation model

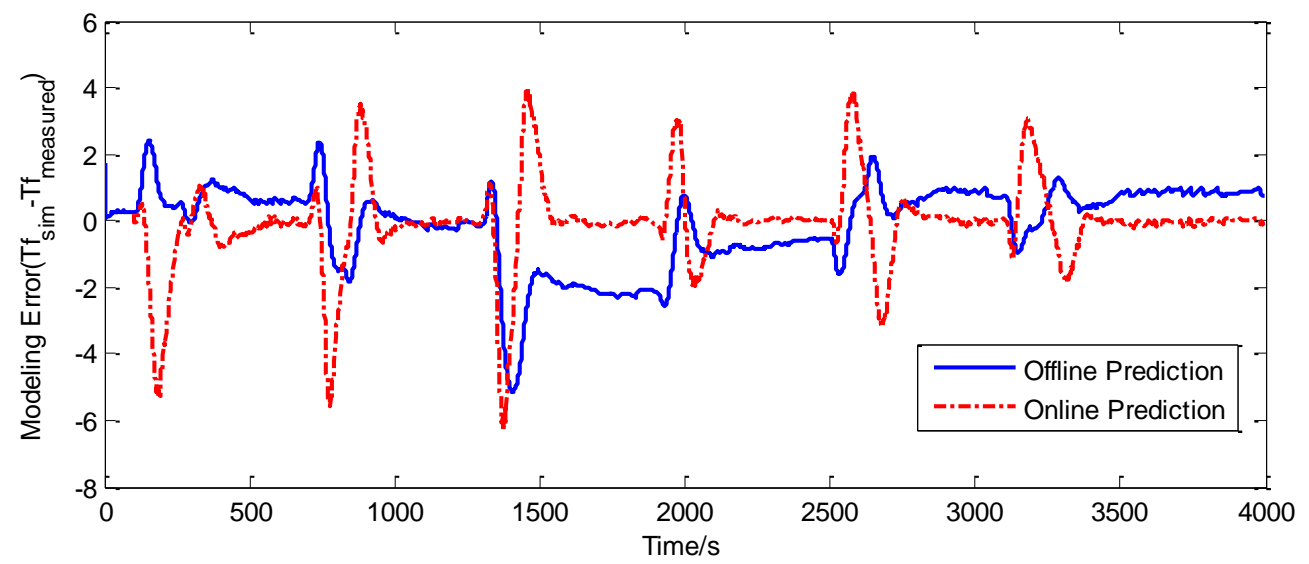

Figure 4.21: Prediction Error of $T_{f}$ by the offline and online estimation model

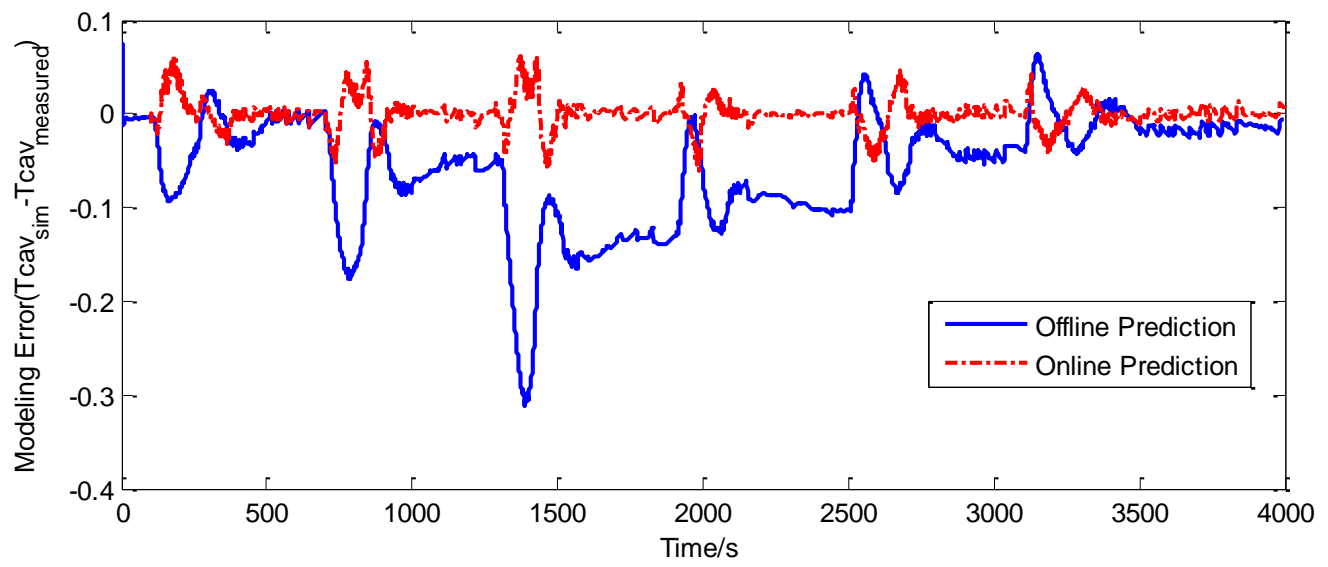

Figure 4.22: Prediction Error of $T_{c a v}$ by the offline and online estimation model 
Table 4.5: $C_{1}, C_{2}$ and $C_{3}$ of online estimation for the four state variables

\begin{tabular}{|c|c|c|c|c|}
\hline & $\mathrm{Nr}$ & $\mathrm{Cr}$ & $\mathrm{Tf}$ & Tcav \\
\hline $\mathrm{C} 1$ & 0.0165 & 0.0212 & 6.2838 & 0.0646 \\
\hline $\mathrm{C} 2$ & $1.1128 \mathrm{e}-005$ & $2.0026 \mathrm{e}-005$ & 1.8015 & $2.3758 \mathrm{e}-004$ \\
\hline $\mathrm{C} 3$ & $-2.9824 \mathrm{e}-004$ & $-1.8449 \mathrm{e}-004$ & -0.0977 & $4.9262 \mathrm{e}-005$ \\
\hline
\end{tabular}

Compared with the offline prediction, the online prediction can effectively reduce the total prediction error of the studied system in both the steady state and dynamic process. 


\section{FAULT DETECTION USING DATA-DRIVEN METHODS}

Tools of data-driven fault detection facilitate performance monitoring of complex dynamical systems if the physics-based models are either inadequate or not available. To this end, many data-driven methods have been developed. An inherent difficulty for a completely data-driven fault detection tool is that the detection performance can deduce drastically in the presence of sensor degradation. Symbolic dynamic filtering (SDF) is recently introduced in the literature as a real-time data-driven pattern identification tool, which is built upon the concepts of Symbolic Dynamics, Information Theory and Statistical Mechanics. This report investigates a SDF-based fault detection algorithm for health monitoring in nuclear power plants under sensor degradation. The proposed fault detection methodology is validated on the International Reactor Innovative \& Secure (IRIS) simulator of nuclear power plants, and its performance is comparatively evaluated with existing fault detection tools.

\subsection{Introduction}

Health monitoring of nuclear power plants (NPPs) are critical for the operational safety. An anomaly in a NPP may be caused by many reasons. It could originate from a fault in a single component or simultaneous faults in multiple components. It may be difficult for the plant operator to detect and locate the faulty component, especially when the fault is small and slowlyevolving. On the other hand, when a fault occurs in a NPP, the operator may be overwhelmed by the shear number of readings from various sensors. It would be beneficial to develop an automatic health monitoring system to assist the plant operator to detect and isolate the faulty component(s).

Health monitoring algorithms are primarily divided into two different categories, namely, model-based and data-driven. Both model-based and data-driven techniques have been reported in the literature for health monitoring of NPPs. Examples of model-based health monitoring can be found in $[18,19]$. Among data-driven approaches, neural networks (NN) and principal component analysis (PCA)-based approaches $[20,21]$ are most popular. A signal pattern matching and verification method [22] was developed for health monitoring in NPPs. 
Although model-based techniques have their advantages in terms of onboard real-time application, their reliability for health monitoring often decreases as the system complexity increases. On the other hand, data-driven techniques may remain reliable and computationally efficient in spite of system complexity if they are required to monitor the input-output information from a limited number of (appropriately calibrated) sensors while considering the entire system as a black-box. However, unless the ensemble of acquired information is systematically handled, data-driven techniques may become computationally intensive and the performance of health monitoring may deteriorate due to sensor degradation. Furthermore, datadriven techniques usually require a high volume of training data (NPP component failure data in the present context).

Tools of real-time data-driven fault detection facilitate performance monitoring of complex dynamical systems if the physics-based models are either inadequate or not available. In this regard, the critical issue is real-time analysis of time series data for information compression into low-dimensional features that capture the relevant information of the underlying dynamics [23]. Time series analysis is a challenging task if the data set is voluminous (e.g., collected at a fast sampling rate), high-dimensional, and noise-contaminated. The problem with handling time series data is its volume and the associated computational complexity; therefore, the available information must be appropriately compressed via transformation of high-dimensional data into a low-dimensional feature space with minimal loss of class separability. In their previous work [24], the authors proposed a nonlinear feature extraction method, namely Symbolic Dynamic Filtering (SDF) for detection of anomalies (i.e., deviations from the nominal condition) in complex systems. This method is shown to be particularly useful for extraction of features from time series data and has been experimentally validated for real-time execution in different applications (e.g., electronic circuits [25] and fatigue damage monitoring in polycrystalline alloys [26]).

A major challenge in any sensor data-driven detection tool is to identify the actual failure in the system in the presence of sensor degradation (e.g., drift and noise) without succumbing to a large number of false alarms or missed detections. The situation becomes even more critical if the control system uses observations from the degraded sensors as feed-back signals and generates the control inputs accordingly. Traditionally, redundant sensors along with methods 
based on analytic redundancy are used for sensor fault identification [27]. This report presents a different approach to this problem, where different class labels are assigned to data sets that are generated from different plant health conditions. The same class labels are assigned to data from plants with similar health conditions and the associated sensor data are subjected to different noise variance at respective sensor degradation levels. To this end, the data-driven fault detection problem is posed as a multi-class pattern classification problem, where the tasks of class assignment and the SDF-based feature extraction are implemented in a supervised manner to mask the sensor degradation signatures while magnifying the system fault signatures. A major step in SDF-based feature extraction is partitioning of time-series data to generate symbol blocks that are subsequently converted to feature vectors by use of the probabilistic finite state automata (PFSA) concept [24].

The major contributions of this section are listed below.

- Formulation of the data-driven fault detection problem at the component level in NPP (in the presence of varying sensor noise condition) as a multi-class classification problem;

- Introduction of the concepts of symbolic dynamics and probabilistic finite state automata (PFSA) for fault detection in nuclear power plants;

- Comparative evaluation of the existing fault detection (such as principal component analysis (PCA)) with the proposed SDF-based fault detection algorithm.

\subsection{Data-driven Fault Detection Defined as a Multi-class Pattern Classification Problem}

Component-level fault diagnosis in nuclear power plants involves identification of the fault type, and location \& quantification of the fault level. Although the present configuration in the IRIS test-bed can be easily extended to simultaneous faults in multiple components, the present report deals with a single component, e.g., reactor coolant pump ( $\mathrm{RCP})$, and the task is to detect a fault and identify its level under varying sensor noise condition.

The RCP overspeed percentage ( $\psi \mathrm{RCP})$ is considered as the health parameter that defines the health status of RCP. Table 5.1 shows the approximate ranges of efficiency health parameters under different fault levels. Here, the low fault level indicate very minimal overspeed in RCP and hence also includes the absolute nominal health condition $(\psi \mathrm{RCP}=0)$. 
Similarly, depending on the noise standard deviation in the hot-leg coolant temperature sensor $T_{H L}$, three sensor noise levels are considered for the study. Table 5.2 shows the approximate ranges of sensor noise standard deviation as a percent of operating point trim values considered under different levels.

Table 5.1: Fault Levels in Reactor Coolant Pump (RCP)

\begin{tabular}{|c|c|}
\hline Fault Level & RCP Overspeed $\psi$ RCP (\%) \\
\hline Low Fault & 0.00 to 0.30 \\
\hline Medium Fault & 0.30 to 0.60 \\
\hline High Fault & 0.60 to 0.90 \\
\hline
\end{tabular}

Table 5.2: Variable Noise Levels in $T_{H L}$

\begin{tabular}{|c|c|}
\hline Noise Level & Standard Deviation Range $\sigma$ THL $\left({ }^{\circ} \mathrm{F}\right)$ \\
\hline Level 1 & 0.005 to 0.025 \\
\hline Level 2 & 0.025 to 0.045 \\
\hline Level 3 & 0.045 to 0.065 \\
\hline
\end{tabular}

Thus, in the above context, there are $3 \times 3=9$ classes of data sets that need to be obtained to define a class by RCP fault level and a noise level of the THL sensor. One hundred simulation runs of the NPP system were performed for each class to generate data sets for analysis, among which 50 samples are chosen as the training sets and the rest of the samples are kept as the testing set. RCP overspeed percentage $\psi \mathrm{RCP}$ and THL standard deviation parameters are chosen randomly from independent uniform distribution such that all of the parameter values are within the prescribed ranges given in Table 5.1 and 5.2. Figure 5.1 plots the samples generated using above logic in the two dimensional parameter space. Different classes of samples are shown in different colors and as well as marked within the class number in the figure. The axis for sensor noise standard deviation $(\sigma \mathrm{THL}$ ) represents actual standard deviation values (not as percent of the operating point trim values of THL reading). 


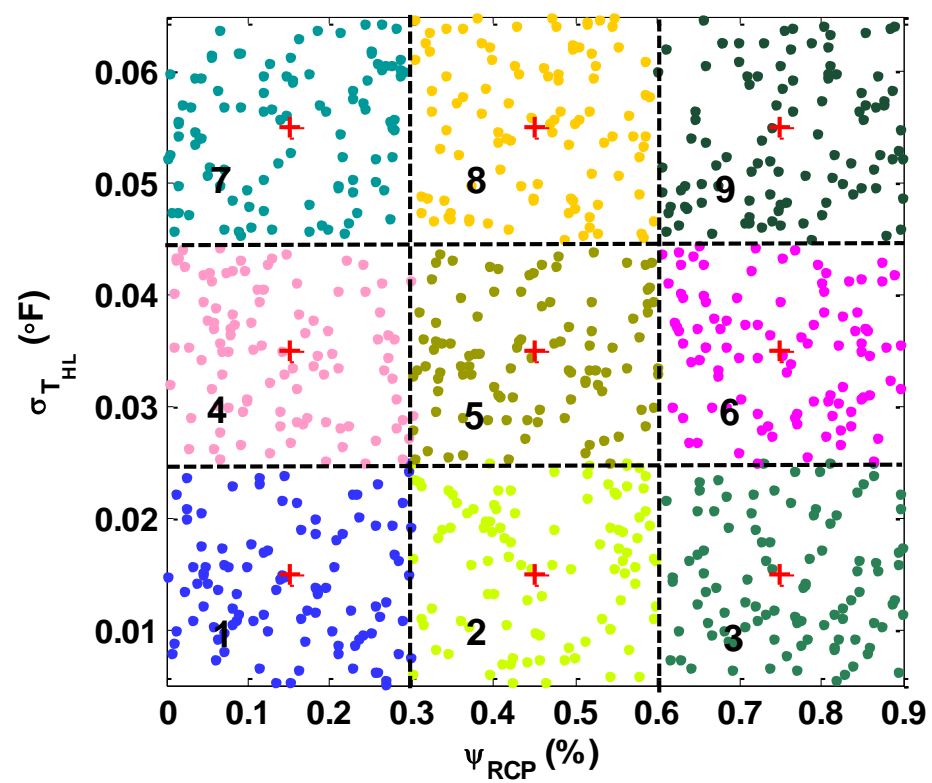

Figure 5.1: Original class labels for data collection

Most degradation in complex systems such as NPPs occurs in a slow-time scale, i.e., the anomaly gradually evolves over a long time span. Sudden failure of components is disastrous, and it usually occurs when the components accumulate sufficient amount of fault and reach the critical point. Therefore, an early detection of the fault is highly desirable for enhancement of operational safety in NPPs. However, component fault is usually very small at early stage and difficult to be detected, especially at steady state. One way to "amplify" the fault signature is perturbing the plant by periodically altering the turbine load such that the plant is in transient state for sufficient long time, which makes it easier to detect the fault.

For each sample point in the parameter space, a time series was collected for the $T_{H L}$ sensor under persistent excitation of turbine load inputs that have truncated triangular profiles with the mean value of $99 \%$ of nominal output power, fluctuations within $\pm 1 \%$ and frequency of $0.00125 \mathrm{~Hz}$ (period $T=800 \mathrm{sec}$ ). In other words, the turbine load is fluctuating between nominal power $(335 \mathrm{MW})$ and $98 \%$ of nominal power $(328.3 \mathrm{MW})$. Figure 5.3 shows the profile of turbine load and a typical example of turbine output power as a result of fluctuation in turbine load. 


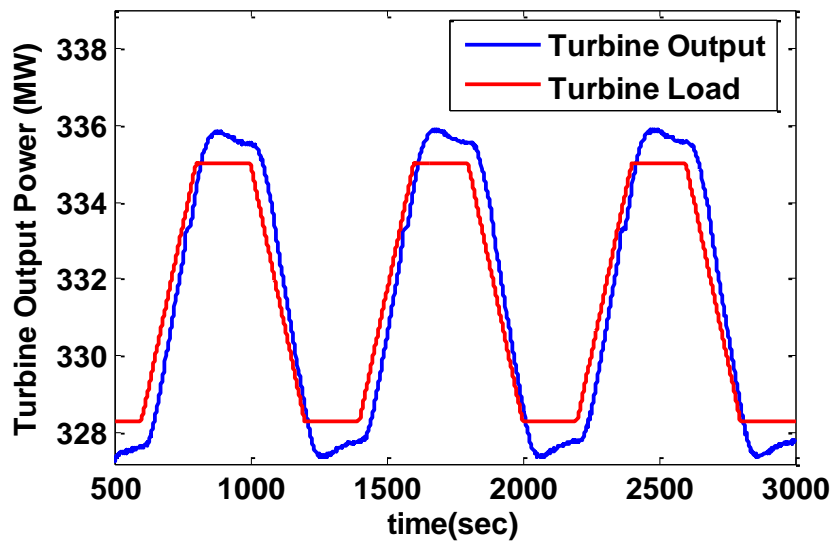

Figure 5.2: Profile of turbine load and turbine output response
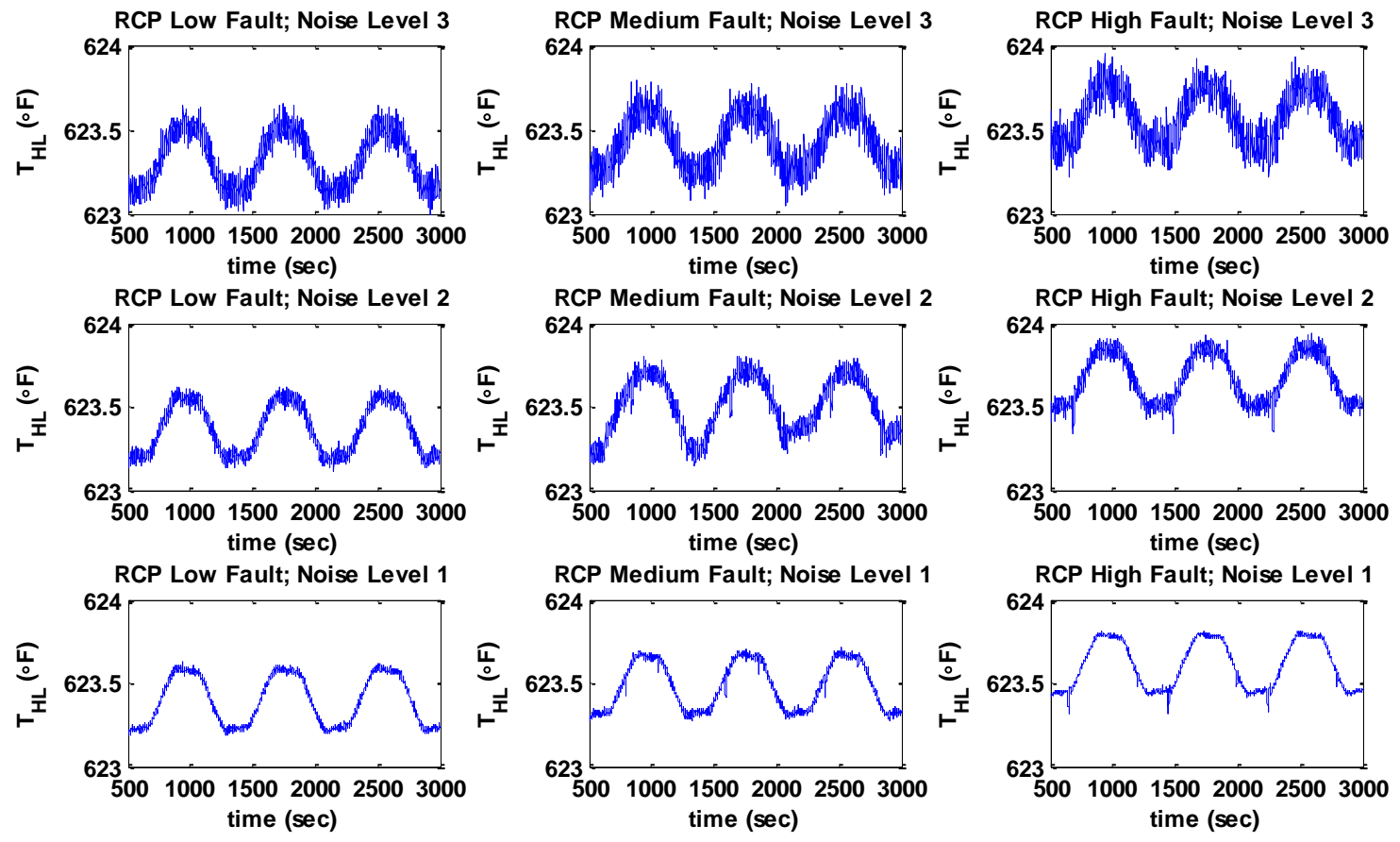

Figure 5.3: Representative time series data for RCP fault and $T_{H L}$ degradation conditions

For each experiment, the sampling frequency for data collection is $1 \mathrm{~Hz}$ (i.e., intersample time of $1 \mathrm{sec}$ ) and the length of the simulation time window is 2,400 seconds, which generate 2,400 data points. The total simulation time for each experiment is 3,000 seconds, the data of the first 600 seconds are not used since the plant is not excited. Figure 5.4 shows representative examples of $T_{H L}$ time series data from each of nine classes. 


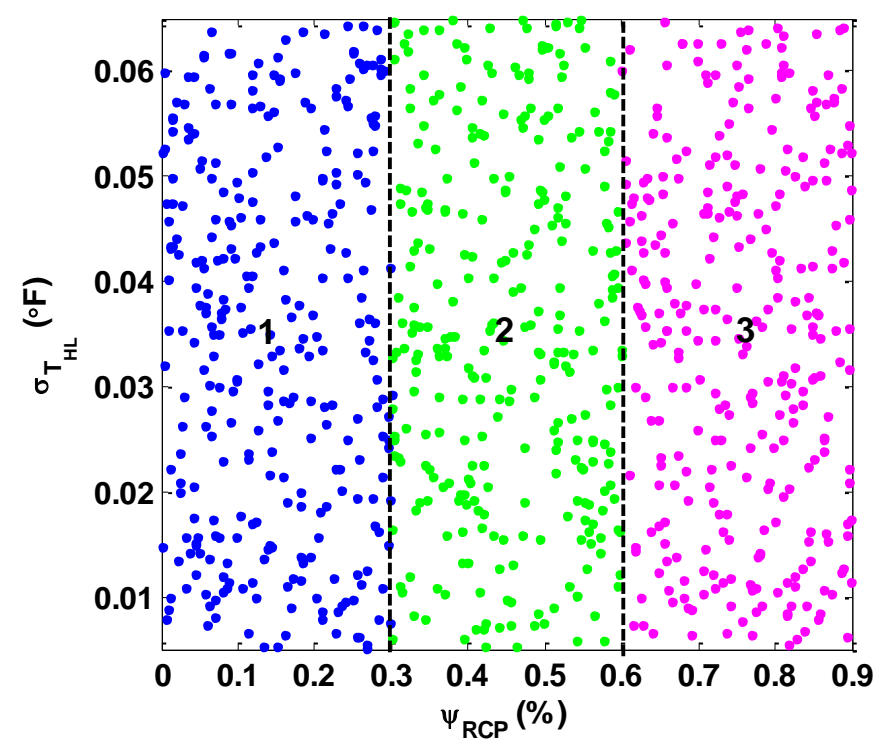

Figure 5.4: Revised class assignment for fault detection

As stated earlier, the objective of the report is to build a data-driven diagnostic algorithm that is robust to varying sensor noise level, provided that the sensor noise is within an allowable range. From this perspective, the definition of a data class is changed and made only dependent on the RCP overspeed parameters. Thus, the 9 original classes are reduced to 3 classes as shown in Fig. 5.4. This is the final class assignment for the data set, where each class has $(50 \times 3)=150$ training samples and $(50 \times 3)=150$ testing samples.

Thus, in the above context, the problem of component level fault detection in presence of varying sensor noise condition in nuclear power plants is formulated as a multi-class classification problem (in the present scenario, number of classes is 3 ).

\subsection{Symbolic Dynamic Filtering (SDF)-based Feature Extraction}

This section briefly reviews the concepts of symbolic dynamic filtering (SDF) [24, 25, 26] for feature extraction, followed by optimization of partitioning.

\subsubsection{Review of Symbolic Dynamic Filtering (SDF)}

Symbolic feature extraction from time series data is posed as a two-time scale problem. Over the span of data acquisition, dynamic behavior of the system is assumed to remain invariant, i.e., the process is quasi-stationary at the fast scale. On the other hand, the slow scale is 
related to the time span over which non-stationary evolution of the system dynamics may occur. It is expected that the features extracted from the fast-scale data will depict statistical nonstationarity between two different slow-scale epochs if the underlying system has undergone a change. The concept of two time scales is illustrated in Fig. 5.5.

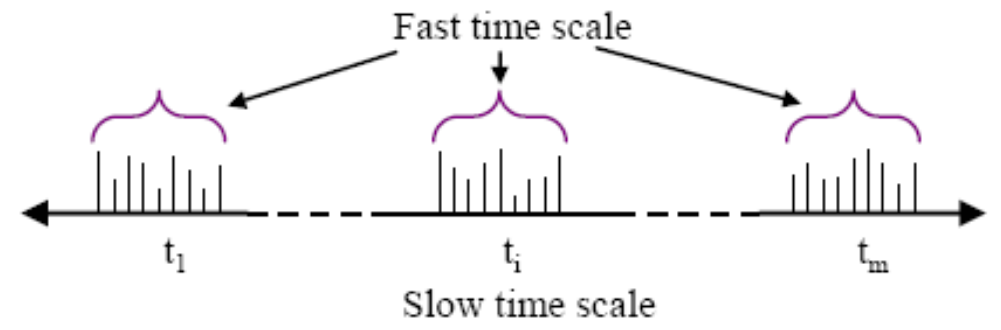

Figure 5.5: Pictorial view of the two time scales: (i) Slow time scale of anomaly evolution and (ii) Fast time scale of data acquisition

The method of extracting features from stationary time series data is comprised of the following steps:

1) Time series data acquisition on the fast scale from sensor and/or analytical measurements (i.e., outputs of a physics-based or an empirical model). Data sets are collected at slow time epochs.

2) Partitioning the time series data. Each segment of a partitioning is assigned a particular symbol from the symbol alphabet set $\Sigma$, as illustrated in Fig. 5.6. This step enables transformation of time series data from the continuous domain to the symbolic domain. The partitioning is generated from the partitioning data and remains fixed for subsequent training and testing stage.

3) Construction of a finite state automaton at each time epoch from alphabet size $|\Sigma|$ and window length $D$.

4) Generation of state probability vectors. The probability vectors are recursively computed as an approximation of the natural invariant density of the dynamical system at the slow time epochs. By calculating the state probability vectors, the dimension of the data sets is decreased from 2,400 (number of data points in each data set) to 4 (assuming the alphabet size $|\Sigma|=4)$. 
5) Identification of the class information. The low-dimensional state probability vectors are input to pattern classifiers to identify the class labels. K-nearest neighbors (k-NN) algorithm is used in this report as the pattern classifier due to its simplicity.

For anomaly detection using SDF, the nominal time-series is partitioned by one of the classical schemes (e.g., uniform partitioning (UP) or maximum entropy partitioning (MEP)), as illustrated in Fig. 5.7. Then, using the steps described earlier, a low-dimensional feature vector $p$ is constructed from each data set.

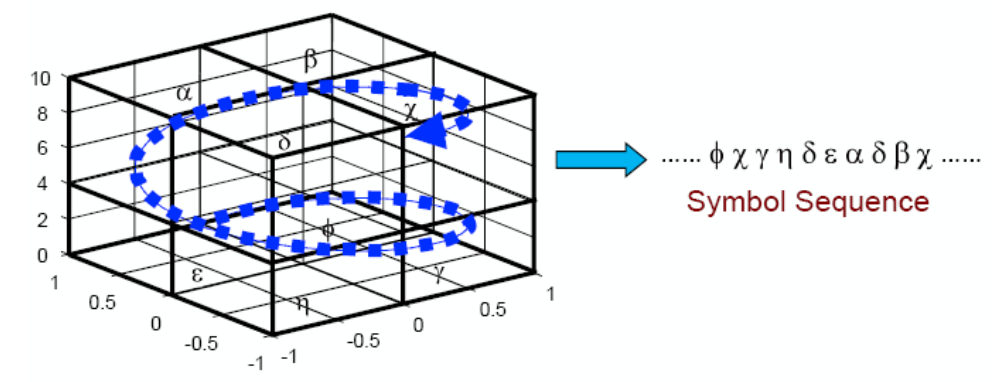

Figure 5.6: Concepts of phase space partitioning for symbolization
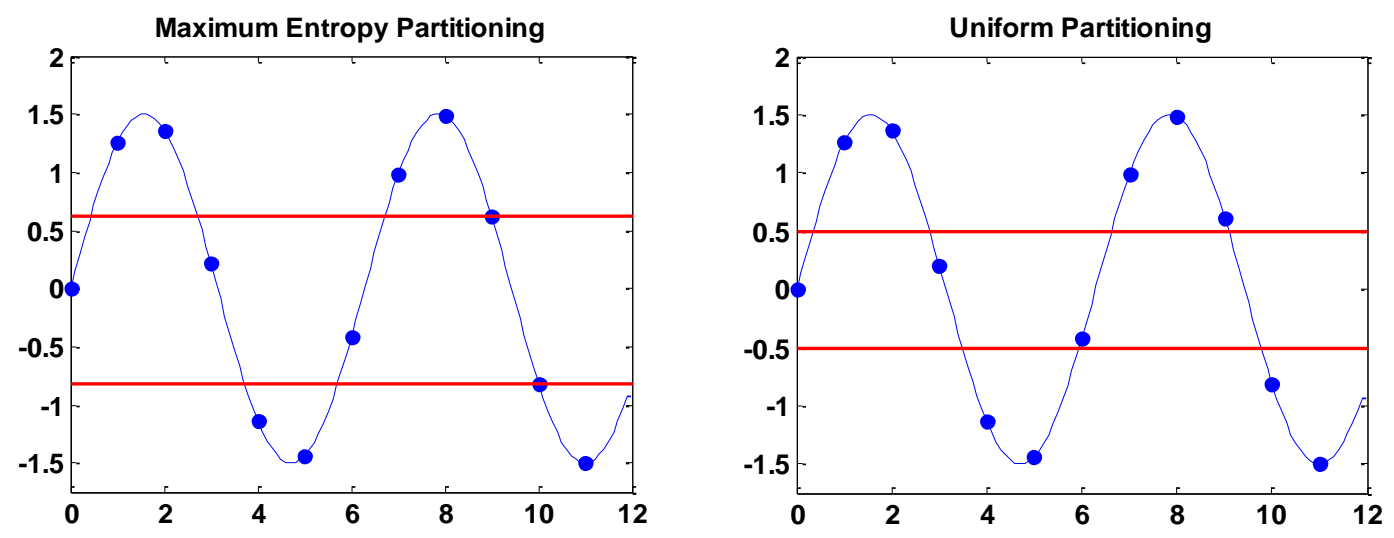

Figure 5.7: Illustration of partition schemes (alphabet size $|\Sigma|=3$ )

Upon completion of feature extraction, a classifier is used to distinguish the set of feature vectors $C l_{i}$, i.e., $\left\{\mathrm{p}^{C l_{i}}\right\}$, from the feature vectors of the other classes, where $i=1,2, \ldots, K$ and $K$ is total number of classes. There are plenty of choices available for design of both parametric and non-parametric classifiers in the literature. Among the parametric type of classifiers, one of the most common techniques is to consider up to two orders of statistics in the feature space. In other words, the mean feature is calculated for every class along with the variance of the feature space 
distribution in the training set. Then, a test feature vector is classified by using the Mahalanobis distance [31] or the Bhattacharrya distance [32] of the test vector from the mean feature vector of each class. However, these methods lack in computational efficiency if the feature space distribution cannot be described by second order statistics (i.e., non-Gaussian in nature). In the present context, Gaussian feature space distribution cannot be ensured due to the nonlinear nature of the partitioning feature extraction technique. Therefore, a non-parametric classifier, such as the k-NN classifier may a better candidate for this study; however, in general, any other suitable classifier, such as the Support Vector Machines (SVM) or the Gaussian Mixture Models (GMM) may also be used.

\subsubsection{Optimization of Partitioning}

Properties and variation of transformation from symbol space to the feature space have been extensively studied in the Mathematics, Computer Science and especially Data Mining literature. Apparently, similar efforts have not been expended to investigate partitioning of time series data to optimally generate symbol blocks for pattern classification and anomaly detection. Symbolic false nearest neighbor partitioning (SFNNP) [33] optimizes a generating partition by avoiding topological degeneracy. However, a major shortcoming of SFNNP is that it may become extremely computationally intensive if the dimension of the phase space of the underlying dynamical system is large. Furthermore, if the time series data become noisecorrupted, the states of SFNNP rapidly grow in number and thus the partitioning may erroneously require a large number of symbols to capture pertinent information on the system dynamics. This shortcoming could be largely alleviated by wavelet space partitioning (WSP) that is particularly effective for noisy data for large-dimensional dynamical systems; maximum entropy partitioning (MEP) was used by Rajagopalan and Ray [25] to generate symbol blocks from time series data by WSP. Although WSP is significantly computationally faster than SFNNP and is suitable for real-time applications, WSP also has several shortcomings such as, requirements of good understanding of signal characteristics for selection of the wavelet basis, identification of appropriate scales, and lossy \& non-unique conversion of the two-dimensional scale-shift domain into a single dimension. Subbu and Ray [30] introduced Hilbert transformbased analytic signal space partitioning (ASSP) as an alternative to WSP. 
The data partitioning techniques, described above, primarily provide a symbolic representation of the underlying dynamical system under a given quasi-stationary condition, rather than capturing the data-evolution characteristics as a consequence of an anomaly. Next we present a partitioning optimization methodology that endeavors to overcome this shortcoming to make SDF a robust data-driven feature extraction tool for pattern classification and anomaly detection.

Optimization criteria that are reported for feature extraction in multi-class classification problems are broadly classified as:

1) Filtering method that makes use of the information content feedback (e.g., Fisher criteria, statistical dependence, and information-theoretic measures) as optimization criteria for feature extraction.

2) Wrapper method that includes the classifier inside the optimization loop to maximize the predictive accuracy (e.g., classification rate using statistical re-sampling or crossvalidation).

The wrapper method is adopted here to minimize the classification error on the training set. The rationale for this choice is the non-binary nature of the problem at hand and the possible non-Gaussian distribution of training samples in the feature space.

In a multi-class problem, ideally one should jointly minimize the off-diagonal elements of the confusion matrix, while maximizing the diagonal elements. However, in that case, the dimension of the objective space blows up with increase in the number of classes which is obviously impractical. Therefore, two costs are defined on the confusion matrix by using another weighting matrix, elements of which denote the relative penalty values for different confusions in the classification process. Formally, let there be $C l 1, \ldots, C l$ n classes of labeled time-series data given as the training set. A partitioning Bis employed to extract features from each sample and a k-NN classifier Kis used to classify them. After the classification process, the confusion matrix $\mathbf{C}$ is obtained, where the value of its element $c_{i j}$ denotes the frequency of data from $\mathrm{class}_{\mathrm{i}} \mathrm{being}$ classified as data from $\mathrm{Cl}_{\mathrm{j}}$. Let $\mathbf{W}$ be the weighting matrix, where the value of its element $\mathrm{w}_{\mathrm{ij}} w_{i j}$ denotes the penalty incurred by the classification process for classifying a data set from $\mathrm{Cl}_{\mathrm{i}} \mathrm{Cl}_{\mathrm{i}}$ as a data set from class $\mathrm{Cl}_{\mathrm{j}}$. With these definitions, two costs that are to be minimized can be defined as follows. The cost due to expected classification error, Cost $\mathrm{E}$ is defined as: 


$$
\operatorname{Cost}_{E}=\frac{1}{N_{S}}{ }_{i j} w_{i j} c_{i j}
$$

where $N_{S}$ is the total number training sample including all classes. The outer sum in the above equation sums the total penalty values for misclassifying each class $C l_{i}$. Thus $\operatorname{Cos} t \mathrm{E}$ is related to the expected classification error. Although, in the current formulation, the total penalty values are equally weighted for all classes, these may be changed based on the prior knowledge about the data and the user requirements.

It is implicitly assumed in many supervised learning algorithms that the training data set is a statistically similar representation of the whole data set. However, this assumption may not be very accurate in practice. A natural solution to this problem is to choose a feature extractor that minimizes the worst-case classification error. In the present setting, that cost due to worstcase classification error, Cost $\mathrm{W}$, can be defined as:

$$
\operatorname{Cost}_{W}=\max _{i} \frac{1}{N_{i}} w_{i} c_{i j}
$$

where $N_{i}$ is the number of training samples in class $C l_{i}$. With such construction, the dimension of the objective space is not a function of the number of classes, which makes it convenient for classification with large number classes. As described earlier, classification needs to be performed on the training data set to calculate the costs during optimization of the feature extractor, i.e., the partitioning.

Figure 5.8 depicts the general outline of the classification process. Labeled time series data from the training set are partitioned and the generated low-dimensional feature vectors (via symbolization and PFSA construction) are fed to the classifier. 


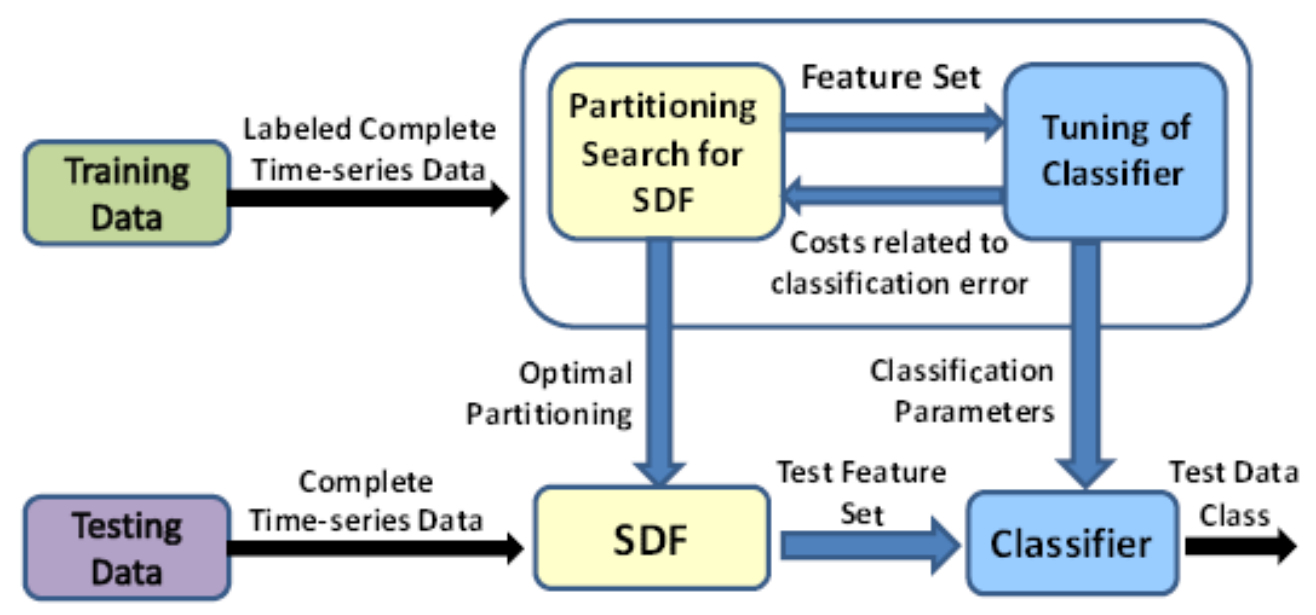

Figure 5.8: General framework for optimization of feature extraction

After classification, the two training error costs defined as above are computed and fed back to the feature extraction block. During classification, the classifier may be tuned to the obtain better classification rates. For example, for k-NN classifiers [34], choice of neighbor size or the distance metric can be tuned. The partitioning is updated to reduce the cost based on the feedback. The iteration is continued until the set of optimal partitioning in a multi-objective scenario and the correspondingly tuned classifier are obtained. Choice of the optimal partitioning is done using the Neyman-Pearson criterion [35] as described later. After the choice is made, the optimal partitioning and the tuned classifier are used to classify the test data set. Although this is the general framework that is being proposed for the optimization methodology, tuning of the classifier has not been performed in this report as the main focus here is to choose the optimal partitioning to minimize the classification error related cost.

Similar to the classical partitioning cases, the Euclidean distance is chosen as the metric. For partitioning optimization, at first, the number of cells $|\Sigma|$ of the partitioning $\mathbb{B}$ is chosen to be 4 in this case. For computation purpose, a suitably fine grid size depending on the data characteristics is then assumed. It should be clear that each of the grid boundaries denote a possible position of a partitioning cell boundary. In this report, the data space region is divided into 40 grid cells, i.e., 39 grid boundaries excluding the boundaries of and the alphabet size is selected to be $|\Sigma|=4$. That is, there are 4 partitioning cells and 3 partitioning boundaries to choose. Hence, the number of elements (i.e., 4-dimensional partitioning vectors) in the space $P$ of all possible partitioning is: $39 C 3=9139$. Since the cardinality of $P$ is computationally 
tractable in this example, a direct search-based Pareto optimization procedure is followed in this report. If the partitioning space happens to be significantly large such that usage of a direct search approach becomes infeasible for evaluation of all possible partitioning, then other searching schemes (e.g., those based on genetic algorithms) should be applied. By searching the partition space, the positions of its elements (i.e., the partitioning vectors) are located in the (twodimensional) $\operatorname{Cost}_{E}-\mathrm{Cost}_{W}$ objective space. The resulting Pareto front is generated by identifying the non-dominated points [35] in the objective space. In the present case, a non-dominated point (i.e., a partitioning vector) is such that no other partitioning has lower values of both $\operatorname{Cost}_{E}$ and Cost $_{W}$. Finally, the Neyman-Pearson criterion [35] is applied to choose the optimal partitioning $\mathbb{B}^{*}$ for minimization of the cost $\operatorname{Cost}_{E}$, while not allowing the cost CostW to exceed the allowable worst-case classification error. In other words, the optimal partitioning $\mathbb{B}^{*}$ is the solution to the following constrained optimization problem:

$\mathbb{B}^{*}=\arg \min _{\mathbb{B}} \operatorname{Cost}_{E}(\mathbb{B})$ such that $\operatorname{Cost}_{W}(\mathbb{B}) \leq \epsilon$

where $\epsilon$ is the allowable worst-case classification error that is denoted by the (possibly scalar) threshold determined by the user.

\subsection{Anomaly Detection Algorithm}

This section describes how the anomaly detection algorithm is formulated based on the concept of symbolic dynamic filtering (SDF). For comparative evaluation, another anomaly detection algorithm is constructed based on the principal component analysis (PCA), which is a linear feature extraction technique and computes the largest eigenvectors of the covariance matrix. A flowchart of the anomaly detection algorithms is shown in Fig. 5.9 and the following steps summarize the procedure.

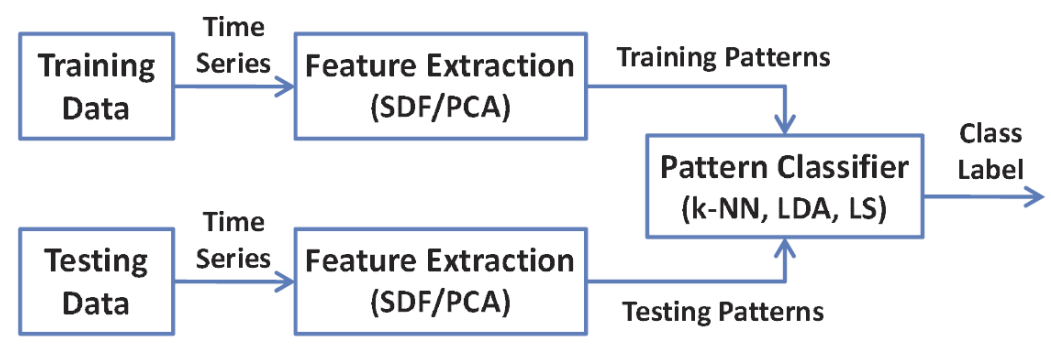

Figure 5.9: Flow chart for data-driven anomaly detection 


\subsubsection{Symbolic Dynamic Filtering for Anomaly Detection}

The following steps summarize the procedure of SDF for anomaly detection.

Time series data acquisition on the fast scale from sensors and/or analytical measurements (i.e., outputs of a physics-based or an empirical model). Data sets of different plant and sensor conditions are collected at slow time epochs, and divided into partitioning, training and testing sets.

Partitioning the time series data: Each segment of the partitioning is assigned a particular symbol from the symbol alphabet set $\Sigma$. This step enables transformation of time series data from the continuous domain to the symbolic domain. The partitioning is generated from the partitioning data and remains fixed for subsequent training and testing stage.

Construction of probabilistic finite state automata from each data set with alphabet size $|\Sigma|$ and window length $D$ : Choices of $|\Sigma|$ and $\mathrm{D}$ depend on the characteristics of the data. In this report, $\mathrm{D}=1$ and $\Sigma=4$ are taken.

Generation of state probability vectors: The state probability vectors $\{\mathrm{p}\}$ are recursively computed as an approximation of the natural invariant density of the dynamical system. For each data set, a state probabilistic vector is generated and serves as the feature vector in the subsequent pattern classification tasks.

Identification of the class information: The low-dimensional state probability vectors are input to pattern classifiers to identify the class labels. The classifier is trained in a supervised manner with the training patterns. The k-nearest neighbors (k-NN) algorithm has been used in this report as the pattern classifier due to its simplicity.

\subsubsection{Principal Component Analysis for Anomaly Detection}

The time series data sets are organized into an $(M \times N)$-dimensional data matrix, where $N$ is the number of data sets, $M$ is the dimension of the time series, i.e., the length of the timeseries data sets. Define $\mathbf{X}$ to be the $(M \times N)$-dimensional data matrix, $\boldsymbol{X}$ to be the centered version of the original data matrix $\mathbf{X}$. Having $N<M$ assures positive definiteness of the $(M \times M)$ covariance matrix $\boldsymbol{S}=\frac{1}{N} \boldsymbol{X} \boldsymbol{X}^{T}$. 
Let $\left\{\boldsymbol{v}_{i}\right\}$ be the eigenvectors of the covariance matrix $\mathbf{S}$ with corresponding eigenvalues $\left\{\lambda_{i}\right\}$ that are arranged in the decreasing order of magnitude. The $m$ largest eigenvalues, where $m \leq M$, and the corresponding eigenvectors are selected such that ${\underset{i=1}{m} \lambda_{i}>\eta}_{i=1}^{M} \lambda_{i}$, where $\eta$ is a real positive fraction close to 1 (e.g., $\eta>0.95$ ). These $m$ eigenvectors can be grouped to form a $(M \times m)$ projection matrix $\boldsymbol{W}_{\boldsymbol{P C A}}$, where $\boldsymbol{W}_{P C A}=\left[\boldsymbol{v}_{1}, \boldsymbol{v}_{2}, \ldots, \boldsymbol{v}_{m}\right]$. Then a new lowdimensional data matrix can be generated by $\boldsymbol{Y}_{\text {train }}=\boldsymbol{W}_{P C A}^{T} \boldsymbol{X}_{\text {train }}$ with the dimensionality of $m \times N$, where each column of $\boldsymbol{Y}_{\text {train }}$ can be considered as a feature vector.

In the training stage, following the above procedure, we can obtain the projection matrix $\boldsymbol{W}_{P C A}$ and the low-dimensional data matrix $\boldsymbol{Y}_{\text {train }}$ from the training data matrix $\boldsymbol{X}_{\text {train }}$. In the testing stage, the low-dimensional data matrix is obtained by $\boldsymbol{Y}_{\text {test }}=\boldsymbol{W}_{P C A}^{T} \boldsymbol{X}_{\text {test }}$.

So far we have obtained a low-dimensional representation of the original data matrix by using PCA as the feature extraction tool, the next step is to use pattern classifier to determine the class labels of the testing data sets. To compare the performance of SDF with PCA, $k$-NN algorithm is also used as the pattern classifier for PCA. Columns of $\boldsymbol{Y}_{\text {train }}$ and $\boldsymbol{Y}_{\text {test }}$ serve as the training patterns and testing patterns, respectively. Other common pattern classifiers (linear discriminant analysis) and least squares algorithm (LS)) are also used for comparative evaluation.

\subsection{Results and Discussion}

This sub-section presents pertinent results for the case study of anomaly detection in the reactor coolant pump (RCP) for comparative evaluation of the optimal partitioning-based SDF tool with those based on the classical methods of partitioning as well as PCA.

At the beginning of the optimization procedure, a weighting matrix $W$ needs to be defined to calculate the cost functionals $\operatorname{Cost}_{E}$ and $\operatorname{Cost}_{W}$ from the confusion matrix for the training data set. In this case study, $\boldsymbol{W}$ is defined according to the adjacency properties of classes in the parameter space, i.e., $w_{i i}=0$ for any $i \in\{1,2,3\}$, i.e. there is no penalty for correct classification. The weights are selected as: $w_{i j}=i-j$, for any $i \in\{1,2,3\}$, i.e., given that a data sample originally from $C l_{i}$ is classified as a member of $C l_{j}$, the penalty incurred by the 
classification process increases with increase in the separation of $C l_{i}$ and $C l_{j}$ in the parameter space. Then, it follows that:

$$
\boldsymbol{W}=\begin{array}{lll}
0 & 1 & 2 \\
1 & 0 & 1 \\
2 & 1 & 0
\end{array}
$$

The data space region $\Omega$ is divided into 40 grid cells, i.e., 39 grid boundaries excluding the boundaries of $\Omega$. Each partitioning in the space $\mathcal{P}$ is evaluated by calculating Cost $_{E}$ and $\operatorname{Cost}_{W}$ to identify the optimal partitioning. Figure 5.10 shows a relevant region of the (twodimensional) Cost $_{E}-$ Cost $_{W}$ objective space, where the elements of the space $\mathcal{P}$ are located. The Pareto front is also generated from this evaluation. The threshold, $\epsilon$, i.e., the maximum allowable $\operatorname{Cost}_{W}$ is taken to be 0.041 in this case and the optimal partitioning (OptP) is chosen by the Neyman-Pearson criterion as discussed earlier. Thus, the user has the option of choosing a classification operating point with selected values of calculating $\operatorname{Cost}_{E}$ and $\operatorname{Cost}_{W}$. The Pareto front is generated after the threshold $\epsilon$ is chosen. Locations of the classical partitioning (i.e., uniform (UP) and maximum entropy (MEP)) are also plotted along with the elements of $\mathcal{P}$ in the figures for comparative evaluation.

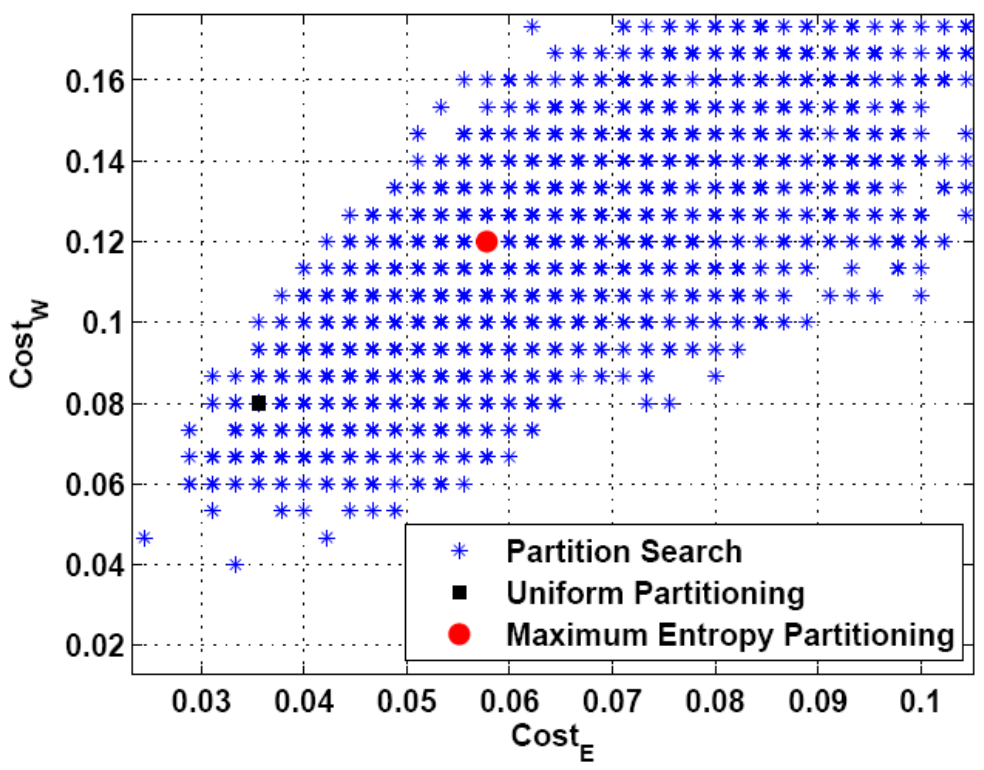

Figure 5.10: Two dimensional objective space for partitioning optimization 


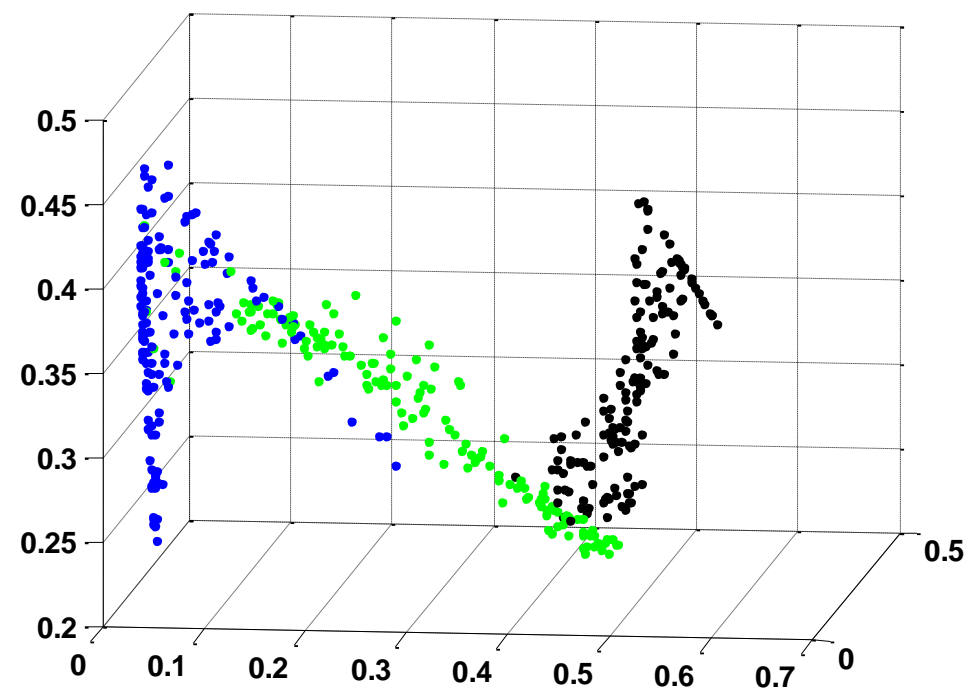

Figure 5.11: Feature space of the training set using optimal partitioning

For SDF analysis, the alphabet size is taken to be $\Sigma=4$ and the depth for constructing PFSA is taken to be $D=1$. Features are classified by a $k-\mathrm{N}$ classifier using the Euclidean distance metric. Figure 5.11 shows locations of the training features in the three-dimensional plot using first three linearly independent elements of the feature vectors obtained by using the chosen optimal partitioning, OptP. Note, only $(\Sigma-1)$ out of its $\Sigma$ elements of a feature vector are linearly independent, because a training feature vector, $\mathbf{p}$ is also a probability vector, i.e., the sum of its elements is constrained to 1 . The class separability is retained by the feature extraction (partitioning) process even after compressing a time series (with 2,400 data points) into 3 numbers.

For comparison purpose, classical partitioning schemes, such as, Uniform Partitioning (UP) and Maximum Entropy Partitioning (MEP) are also used with the same alphabet size, $\Sigma=4$. Figures 5.12 and 5.13 show the location of each training time series in the three dimensional (using first three linearly independent elements of the feature vectors) feature space plot using UP, MEP, respectively.

Finally, the confusion matrices for the SDF-based methods (Opt, UP and MEP) with $k$ $\mathrm{NN}$ on the test data set are given by $\boldsymbol{C}_{\text {test }}^{O p t P+k N N}, \boldsymbol{C}_{\text {test }}^{U P+k N N}, \boldsymbol{C}_{\text {test }}^{M E P+k N N}$, respectively. 


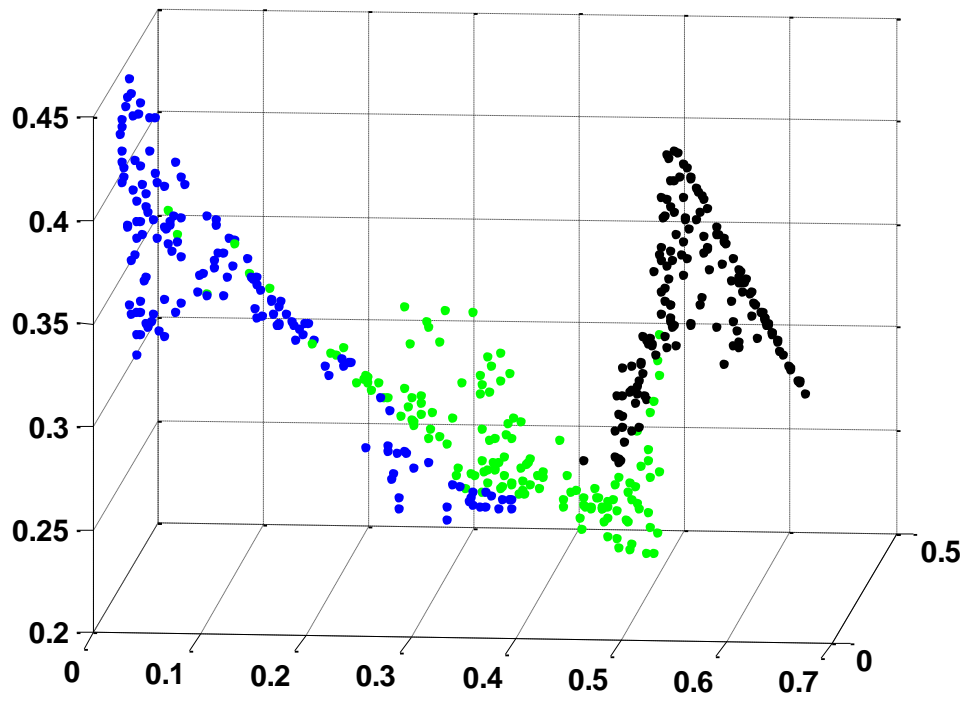

Figure 5.12: Feature space of the training set-uniform partitioning (UP)

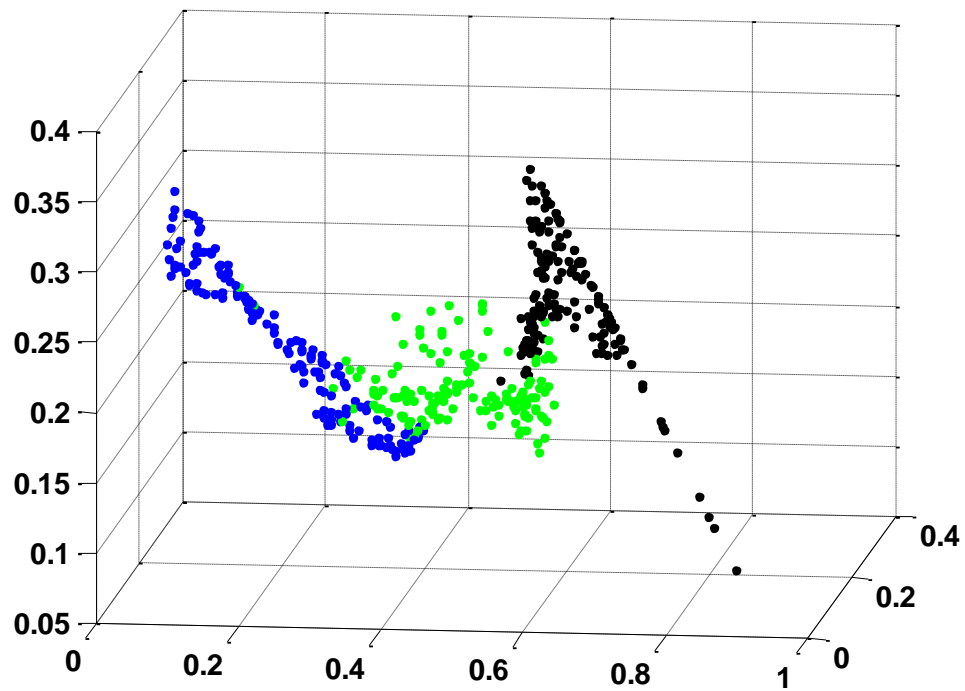

Figure 5.13: Feature space of the training set - maximum entropy partitioning

$$
\begin{array}{lccc}
C_{\text {test }}^{\text {opt }+k N N}= & 142 & 8 & 0 \\
& 14 & 136 & 0 \\
C_{\text {test }}^{U P+k N N} & 0 & 4 & 146 \\
& 145 & 5 & 0 \\
C_{\text {test }}^{M E P+k N N} & 17 & 132 & 1 \\
& 0 & 6 & 144 \\
& 143 & 7 & 0 \\
& 18 & 132 & 0 \\
& 0 & 5 & 145
\end{array}
$$


For comparative evaluation, the data sets are also analyzed using other common pattern recognition tools. In this case study, principal component analysis (PCA) is used as the feature extraction tool, while $k$-NN, linear discriminant analysis (LDA) and least squares (LS) method are used as the pattern classifier. The confusion matrices for PCA with $k$-NN, LDA and LS are given by $\boldsymbol{C}_{\text {test }}^{P C A+k N N}, \boldsymbol{C}_{\text {test }}^{P C A+L D A}, \boldsymbol{C}_{\text {test }}^{P C A+L S}$, respectively.

$$
\begin{array}{rccc}
\boldsymbol{C}_{\text {test }}^{P C A+k N N}= & 149 & 1 & 0 \\
& 40 & 110 & 0 \\
0 & 4 & 146 \\
\boldsymbol{C}_{\text {test }}^{\text {PCA+LDA }} & 136 & 14 & 0 \\
18 & 126 & 6 \\
0 & 3 & 147 \\
\boldsymbol{C}_{\text {test }}^{\text {PCA+LS }}= & 145 & 5 & 0 \\
& 27 & 115 & 8 \\
0 & 0 & 150
\end{array}
$$

Figure 5.14 shows how the neighbor size in the $k$-NN classifiers affect the classification performance, and the performances of OptP, MEP, UP and PCA with different neighbor size $k$ are compared. Only odd values of neighbor size are shown because when using an even value for $k$, it might be necessary to break a tie in the number of nearest neighbors by selecting a random tiebreaker or using the nearest neighbor among the tied groups. It can be seen in Fig. 5.14 the classification performance of SDF-based methods is consistently better than that of PCA-based methods and almost independent of the neighbor size, whereas the classification error of PCA increase with the number of neighbor size. This result shows that the feature vectors extracted by SDF retain better separability among classes than those extracted by PCA.

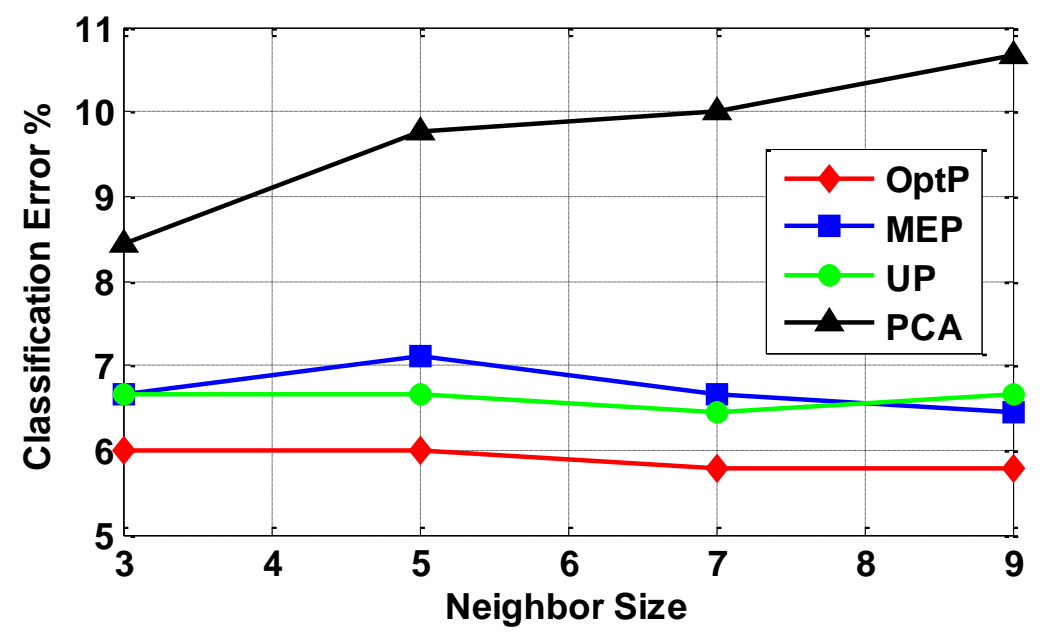

Figure 5.14: Classification error vs. neighbor size in $k$-NN classifiers 
Table 5.3 presents the comparison of the classification error related costs for SDF-based methods and PCA-based methods on the test data sets. The observation made from these results indicates that the classification performance of SDF-based methods are superior to the PCAbased methods. The classification performance of MEP and UP is close to each other, and UP performs slightly better in this case study. The costs due to worst-case classification error and expected classification error are reduced compared to that of uniform partitioning scheme by optimizing the partitioning process over a representative training set.

Table 5.3: Comparison of Classification Performance of Different Methods on Test Data Set (50×9 Samples)

\begin{tabular}{||c||c|c|c||}
\hline \hline Methods & CostE & CostW & Classification Error \% \\
\hline \hline OptP + kNN & 0.0578 & 0.0933 & 5.78 \\
\hline UP + kNN & 0.0644 & 0.1200 & 6.44 \\
\hline MEP + kNN & 0.0667 & 0.1200 & 6.67 \\
\hline PCA + kNN & 0.1000 & 0.2667 & 10.00 \\
\hline PCA + LDA & 0.0911 & 0.1200 & 9.11 \\
\hline PCA + LS & 0.0889 & 0.1800 & 8.89 \\
\hline
\end{tabular}

It should be noted that for some problems, the classical partitioning schemes may perform as well as the optimal one. Therefore, the optimization procedure may also be used to evaluate the capability of any partitioning scheme towards achieving a better classification rate. The evaluation can be performed by using a part of the labeled training data set as the validation set. Finally, although the construction of the cost functions theoretically allow problems with large number of classes, in practice it should be understood that is its upper limit will be constrained by the alphabet size used for partitioning which is also the dimension of the feature space.

\subsection{Summary}

This section presents a data-driven method for detection of component-level faults in nuclear power plants under varying sensor noise condition. The proposed fault detection method is based on Symbolic Dynamic Filtering (SDF) that is recently reported in the literature as a realtime data-driven pattern identification tool. The task of fault detection is viewed as a multi-class 
pattern classification problem, where feature extraction is optimized to enhance the classification rate. The proposed methodology efficiently (i.e., with low memory requirements and fast execution) compresses a high-volume database to execute fault detection for a large number of classes. It has been shown that the SDF-based fault detection algorithm has superior performance compared with the PCA-based fault detection algorithm.

Scalability is a critical issue for data-driven supervised methods of fault detection. A natural way to circumvent this problem is to perform fault isolation before estimating a particular fault level. Furthermore, although this report has shown application of the developed feature extraction method to component fault detection problems, the technique is general enough to be used for detecting sensor and actuator faults under different architectures.

Potential applications of the proposed fault detection method include real-time detection of component-level faults in nuclear power plants (NPPs) with low false alarm and missed detection rates. While the training stage could be accomplished in a NPP simulator (e.g., International Reactor Innovative \& Secure (IRIS) simulator), the testing stage should be ideally performed in real time in an operating nuclear power plant. However, based on the simulation results only, it is found that the plant perturbation needed to execute the proposed fault detection method require small induced changes in the turbine load (e.g., less than $2 \%$ nominal load) over a short period, which does not have any significant adverse effects on stability and performance of the plant operation. 


\section{ROBUST MULTI-VARIABLE CONTROLLER DESIGN USING THE M-SYNTHESIS APPROACH}

\subsection{Introduction}

As the requirement for plant automation has increased as well as benefited from the maturity of reliability and performance of digital equipment in recent years, the control and instrumentation systems of Nuclear Power Plant (NPP) began to adopt digital equipment and network technologies on a plant-wide scale. From the NPP safety point of view, more stability margin is always welcome at all power levels [36]. The controller performances of NPP are affected by uncertainties, such as varying parameters, incomplete plant knowledge, uncertain parameters, and nonlinear plant behavior, etc. The role of a robust controller is to enhance insensitivity of the control system (i.e., plant plus controller) to exogenous disturbances, internal faults, and uncertainties in the plant. Robust decision and control laws are synthesized on an infinite-time time horizon with the objectives of (1) Achieving trade-offs between stability and performance under disturbances and (both structured and unstructured) uncertainties within specified bounds; (2) Mitigation of the detrimental effects of disturbances and uncertainties.

In this section, a methodology for synthesizing a robust multivariable feedback control strategy is proposed for an integrated control of output power, steam pressure in a nuclear power plant. A $\mu$-synthesis robust control approach is used to synthesize the feedback control.

This proposed methodology is demonstrated using the IRIS model which consists of reactor, helical coil steam generators, and a steam turbine. The plant uncertainties including unmodeled dynamics, linearization, and model reduction are characterized and estimated. Based on the specified uncertainty, robust multivariable controllers are designed using D-K iteration according to performance specifications. The order of the obtained controllers is then reduced to an acceptable level by Hankel norm approximation method. Performance of the closed-loop system is evaluated in the linearized and nonlinear NPP model with the reduced order controller.

\subsection{Uncertainty Modeling}

The nonlinear model established using SIMULINK are assumed to represent the dynamics of the actual plant as closely as possible. However, there are always uncertainties due 
to the inability to model all dynamics in an actual plant and the simplification to achieve a mathematical representation. In addition, the linearization process and model reduction processes also introduce uncertainties to the design.

\subsubsection{Sources of Uncertainty}

The plant uncertainty must be specified for the robust control synthesis, and, as a consequence, the robustness of the synthesized controller is subject to the type and size of the uncertainties. An underestimated uncertainty may result in a controller with inadequate robustness for real operation, while an overestimated uncertainty specification may lead to poor performance [36].

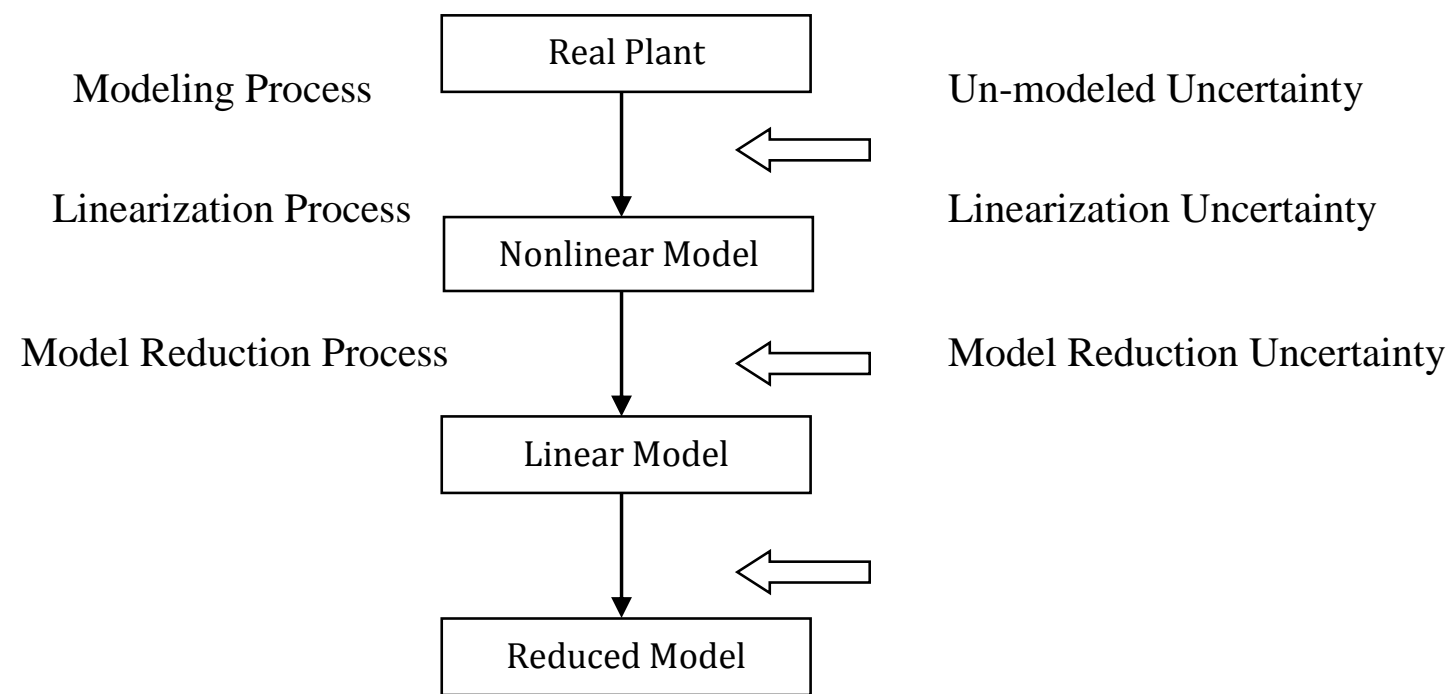

Figure 6.1: Plant uncertainties between the real plant and reduced model

As shown in Figure 6.1, three types of uncertainty are considered in this study. Those uncertainties contribute to the deviation between the real plant and the reduce-order linear model where the robust controller is synthesized. The un-modeled uncertainty is induced from the modeling process where mathematical equations are derived to represent the plant dynamics. The linearization uncertainty is induced from the linearization process, which enables the following robust controller design. The model reduction uncertainty is induced from the model reduction process, which removes unimportant states and thus avoids a large order controller. 


\subsubsection{Linear Model}

A linear model is the basis of modern control system design methods including robust controller synthesis used in this report. In general, there are two ways to derive a linear model from a nonlinear model: symbolic derivation and numerical calculation. The symbolical method is commonly used in obtaining the analytical solution where the state variables of the nonlinear differential equations are split into a steady-state part and a small deviation part around an equilibrium operating point. However, this method is not practical to this plant model discussed in this study in which there contains nonlinear components.

Similar to symbolical method but in a computational way, the numerical method perturbs the states and input variables around the equilibrium operation point to determine the rate of change in the state derivatives and outputs. Then, accordingly, the linear model in state-space form that describes the input-output linearized relationship can be obtained using MATLAB [36]. Owing to the complexity of the model used in this study, the numerical method is used to derive the linear model of the NPP.

To implement the robust multi-input-multi-output (MIMO) control synthesis procedure, the plant model is first configured to a MIMO system with two inputs and two measurable outputs. The control inputs include steam flow and rod reactivity. The measurable plant outputs include output power and steam pressure. In terms of control inputs and system outputs, the MIMO plant model representation is shown in Figure 6.2.

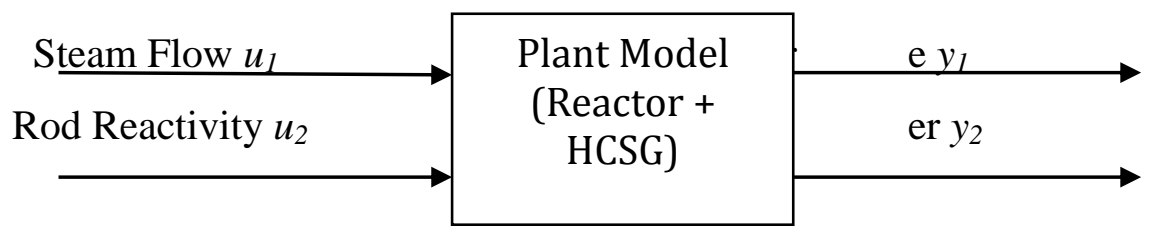

Figure 6.2: Input-Output Representation of the MIMO Plant Model

After the plant nonlinear model is configured as a dual-input-dual-output system, then the linear model can be obtained around a specified operating point using numerical methods. In this study, the "linmod" command from MATLAB is used to generate the linear MIMO model numerically. The resulting linear model has twenty-eight states. 
Of particular note, one of the eigenvalues of the A matrix in the state space representation of the linear model has a small positive value $(5.2563 \times 10-9)$. This eigenvalue can be removed in the following model reduction part, and it will be shown in Section 6.3 that this eigenvalue will not affect the stability of the closed-loop system.

\subsubsection{Model Reduction}

During the linearization process, several states that are neither controllable nor observable are not removed. For robust control synthesis, a low order linear model is desired to avoid a large order controller. So the order of the linearized model must be reduced to remove those uncontrollable and/or unobservable states before the controller design stage. In this study, the balanced realization, Hankel norm approximation and minimal realization methods are used [37].

\section{A) Balanced Realization and Hankel Reduction}

In its simplest form, the balanced realization removes all uncontrollable and/or unobservable modes. And, given a plant $\mathrm{G}(\mathrm{s})$ of full order $\mathrm{n}$, the goal of Hankel norm approximation is to find a reduced order model $\mathrm{Gr}(\mathrm{s})$ of a degree of $\mathrm{r}$ such that the error between $\mathrm{G}(\mathrm{s})$ and $\mathrm{Gr}(\mathrm{s})$ in the form of infinity norm are as close as possible [36].

To obtain the balanced realization, the MATLAB command "sysbal" is used to remove all unobservable and/or uncontrollable modes. "sysbal" also returns a vector of the Hankel singular values of the system, which can be used to further truncate the modes of the input matrix.

However, this command does not work completely for the HCSG model because not all eigenvalues of the matrix A of the plant are negative. One of the eigenvalues is positive, although its value is very small $(5.2563 \times 10-9)$. Due to the small value, the balanced realization may be used followed by Hankel norm approximation to reduce the model to see if this positive eigenvalue can be removed. The resulting system has 26 states. So two unobservable and/or uncontrollable modes have been removed and a balanced realization of 26 states is obtained.

After obtained from the balanced realization, the Hankel norm approximation method can be used to further truncate the modes using MATLAB command "hankmr". The model can be 
reduced to 25 states. Unpack the obtained reduced model and check the eigenvalues of state space, it is found that the positive eigenvalue is still not removed. The H-infinity error between the Hankel reduced order model and the linear model is $3.3231 \times 10-9$. Although the H-infinity error is very small which means the reduced model is very close to the original one, the positive eigenvalue cannot be removed by this method. Some other methods must be used.

\section{B) Minimal Realization}

The Hankel reduction failed due to the existence of the positive eigenvalue of the A matrix of the linearized model. Minimal realization can also eliminate uncontrollable or unobservable states in state-space models, or cancels pole-zero pairs in transfer functions or zero-pole-gain models [38]. The output system model has minimal order and the same response characteristics as the original model.

Implement the algorithm of minimal realization by MATLAB command "minreal" and setting the tolerance to be $1 \times 10-5$, six states are removed and a minimal realization of the linearized model is obtained, as shown in Table 6.1. The positive eigenvalue has been successfully removed and the H-infinity error between the minimal realized model and linear model is $1.1555 \times 10-4$, which is acceptable but not as small as the H-infinity error of Hankel reduced model. This is because more states are removed than the Hankel reduction. The frequency domain responses and time domain responses of the linear model and the minimal realization model are shown in Figure 6.3 and Figure 6.4, respectively.

It can be seen in Figure 6.3 that at the low frequency range, the frequency response of the minimal realized model is lying over the original model, while one pair deviates a little for those frequencies above $200 \mathrm{rad} / \mathrm{s}$. The time responses of the two models, shown in Figure 6.4, are compared in response to a 10 second pulse signal. The same line types are used for the display of the time domain responses. The pulse responses of the two models match with each other perfectly.

The order reduction provides a benefit of handling a lower order plant model during the design process. Throughout this study, the 22nd reduced-order models are used. The loss of the plant dynamics, or the plant uncertainty, will be formulated and determined in the next section. 
Table 6.1: Comparison of Different Order Reduction Results

\begin{tabular}{|c|c|}
\hline Model & Order \\
\hline Linear Model & 28 \\
\hline Balanced Model & 26 \\
\hline Hankel Reduced Model & 25 \\
\hline Minimal Realization & 22 \\
\hline
\end{tabular}

\subsubsection{Formulation of Uncertainty}

The basic idea of formulating a system with uncertainty is to partition the system into nominal part (known) and uncertain part (unknown) in a manner of feedback interconnection, and bind the unknown part to a maximum uncertainty. By this means, a linear dynamical system can be represented as a feedback configuration with a constant matrix containing the nominal plant model and a simple dynamic matrix containing the information of uncertainty [36].

There are two types of uncertainty, structured and unstructured uncertainties. The structured uncertainty represents the uncertainty that has known uncertainty bounds for each element of the uncertainty matrix. It may be caused by variations in parameters associated with the plant properties. The unstructured uncertainty is dynamics related and can be represented by the difference of the magnitude of input-output frequency response at each frequency point. Its bound is defined by the norm of the uncertainty matrix.

In this study, the uncertainties are analyzed in terms of frequency responses although it may be caused from deviations of plant parameters, therefore the unstructured type of uncertainty is used. There are several ways to represent the unstructured uncertainty, and the input-side multiplicative representation is used to represent the uncertainties, the equation is shown as below, and the block diagram form is shown in Figure 6.5.

$$
P_{I M}(s)=P_{0}(s)\left(I+\Delta_{I M}(s)\right)
$$

where $P_{0}(s)$ is the nominal plant model and $P_{I M}(s)$ is the system transfer functions perturbed by input-side multiplicative uncertainty $\Delta_{I M}$. 


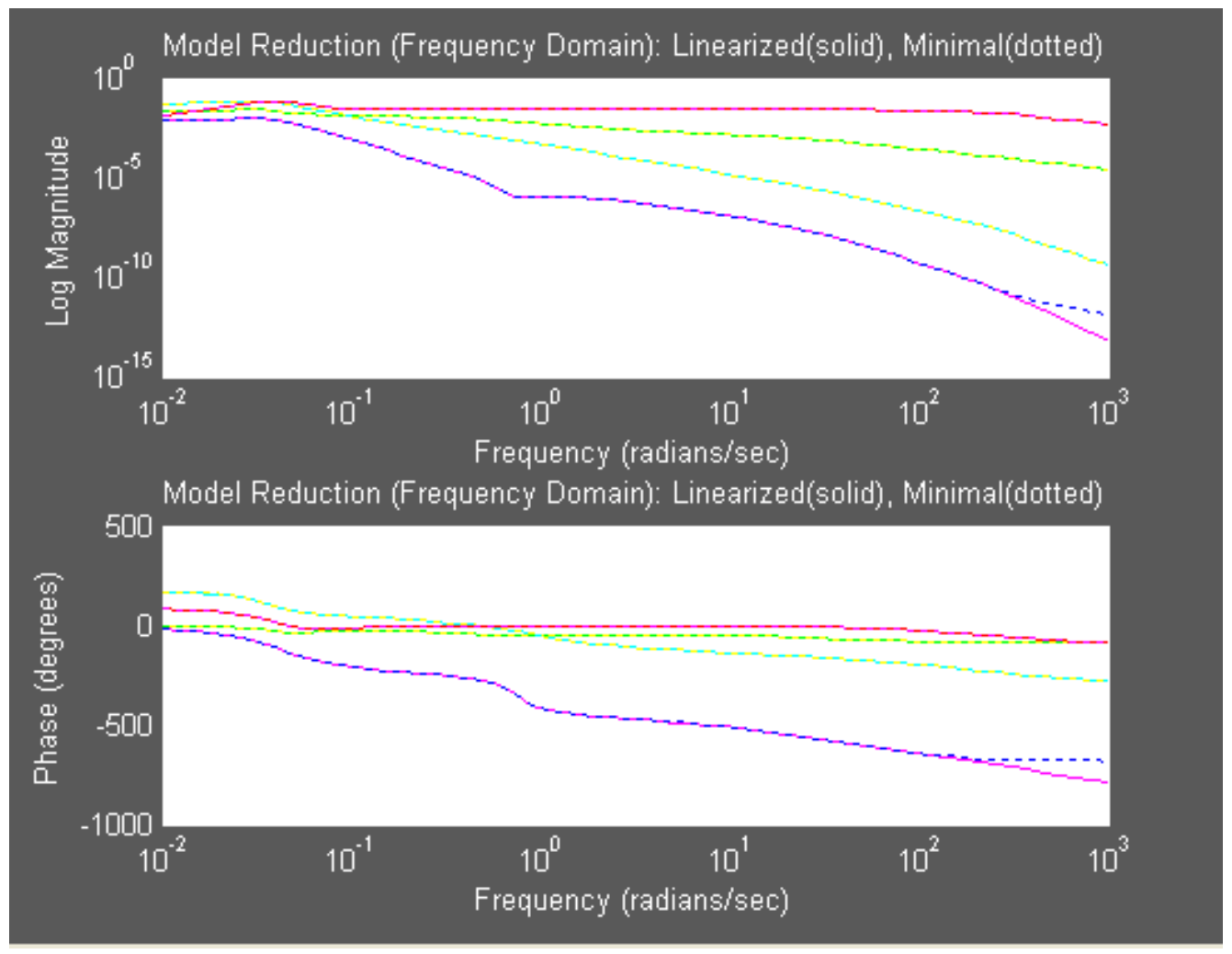

Figure 6.3: Frequency responses of original linear model (solid) and minimal model (dotted)

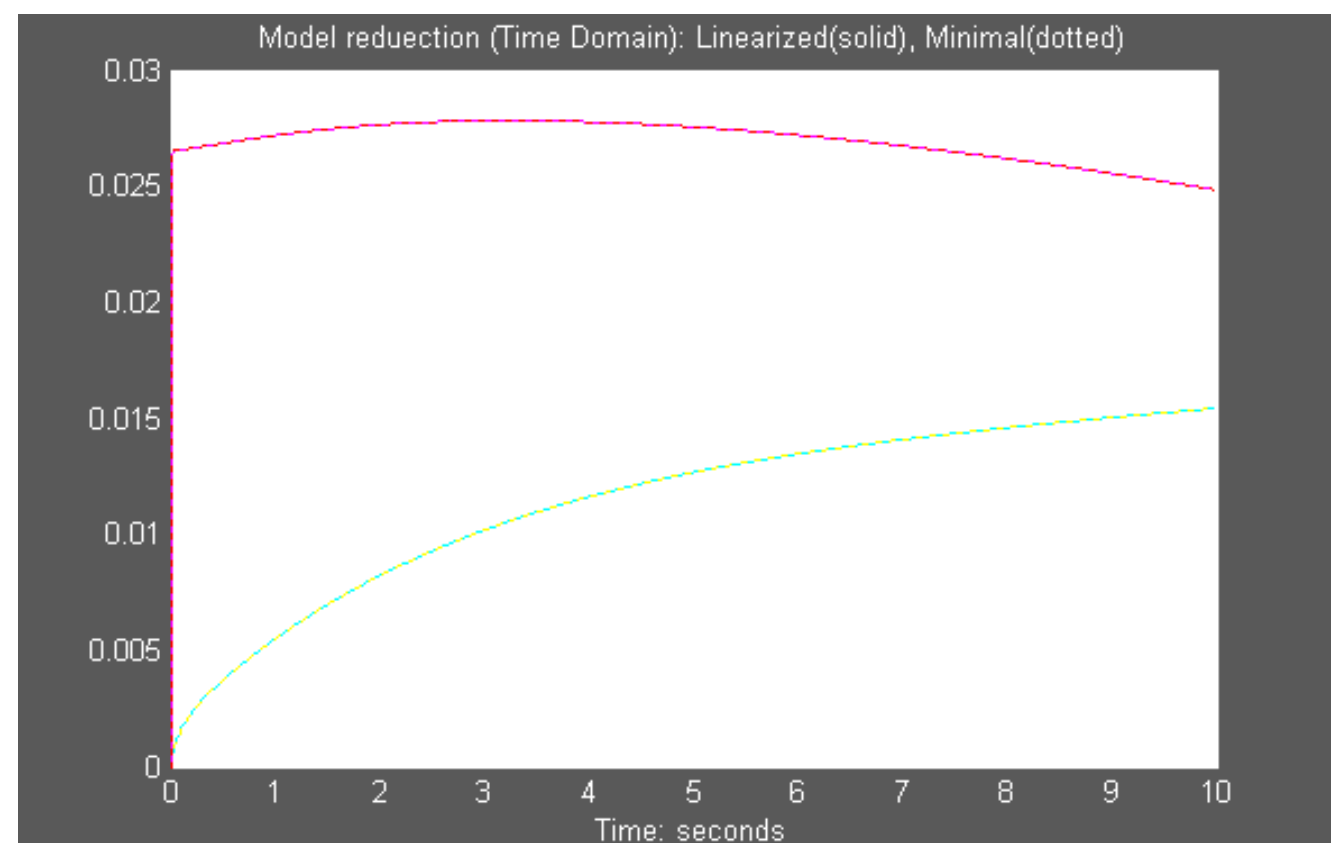

Figure 6.4: Time domain responses of original linear model (solid) and minimal model (dotted) 


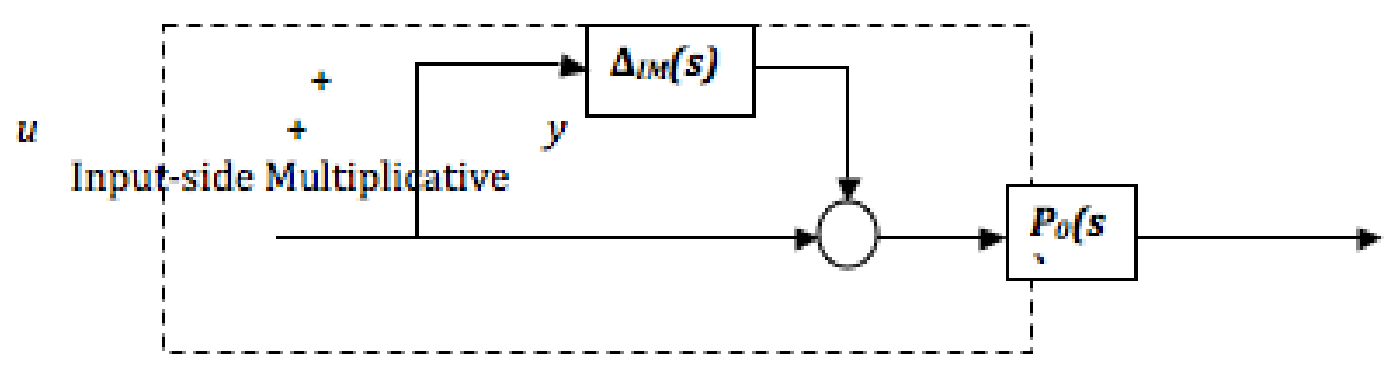

Figure 6.5: Input-side Multiplicative Uncertainty Representation

From Equation 6.1, the input-side multiplicative uncertainty can be calculated from the following equation

$$
\Delta_{I M}(s)=P_{I M}(s) P_{0}^{-1}-I
$$

The unstructured uncertainties are frequency dependent, and the resulting multiplicative uncertainty transfer functions may have very high order. To simplify this problem, a method commonly used is to define a low order uncertainty bound such that it covers the magnitude of the uncertainties at all frequencies. Moreover, for a MIMO uncertain system such as the dualinput-dual-output model in this study, each output is affected by multiple inputs. By adopting the input-side multiplicative uncertainty, the diagonal matrix form that is comprised of two diagonal elements of transfer functions to represent the uncertainties present in the corresponding input, and assume the off-diagonal components to be zero. The unstructured nature distributes these transfer functions across the full MIMO system, so as to describe the overall dynamic uncertainties in the plant model.

The overall uncertainty can be obtained from the summation of the magnitudes of all the uncertainties discussed above, i.e. linearization uncertainty, model reduction uncertainty, and unmodeled uncertainty in input-side multiplicative representation [36].

$$
\left|\Delta_{M}\right|=\left|\Delta_{\text {Linearization }}\right|+\left|\Delta_{\text {Reduction }}\right|+\left|\Delta_{\text {Unmodeled }}\right|
$$

After the overall uncertainties are obtained, the overall uncertainty bound in first-order transfer function form can be determined so as to cover the overall uncertainty.

$$
\left|\Delta_{u}\right| \geq\left|\Delta_{M}\right|
$$


The uncertainty bounds defined as below in diagonal matrix form can cover the overall uncertainty.

$$
\Delta_{u}(s)=\left[\begin{array}{cc}
\frac{0.1(s+60)}{s+600} & 0 \\
0 & \frac{0.1(s+60)}{s+600}
\end{array}\right]
$$

For the purpose of robust control design, it is advantageous to normalize uncertainty with a frequency dependent weighting function. So that as long as the weighting function can cover the overall uncertainty at all frequencies of interest, we can focus the value of the normalized uncertainty to see if it meets the criterion required by robust stability. The uncertainty bound is defined as

$$
\Delta_{u}(s)=\operatorname{diag}\left(\Delta_{u 1}(s), \ldots \Delta_{u n}(s)\right)=W_{u}(s) \delta_{u}
$$

where $W_{u}(s)=\operatorname{diag}\left(W_{u 1}(s), \ldots, W_{u n}(s)\right), \delta_{u}=\operatorname{diag}\left(\delta_{u 1}, \ldots, \delta_{u n}\right)$, as shown in Figure 6.6. The frequency response of uncertainty weighting function $W_{u}(s)$ is shown in Figure 6.7.

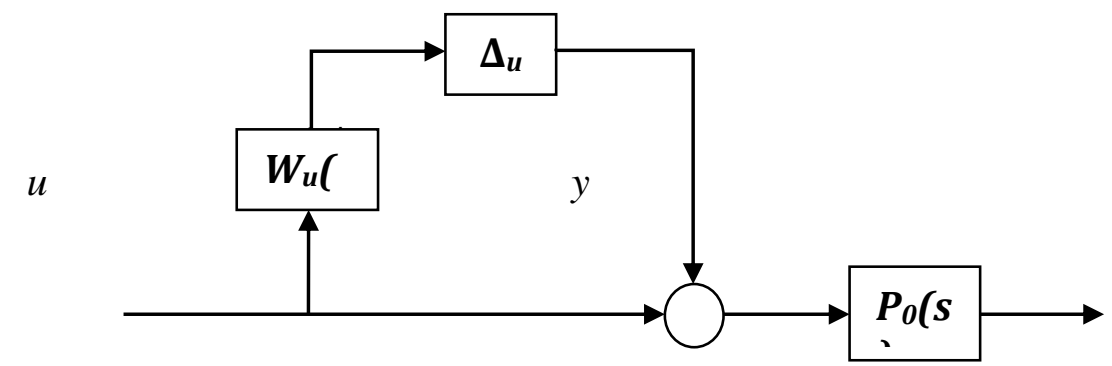

Figure 6.6: Normalized Uncertainty Representation 


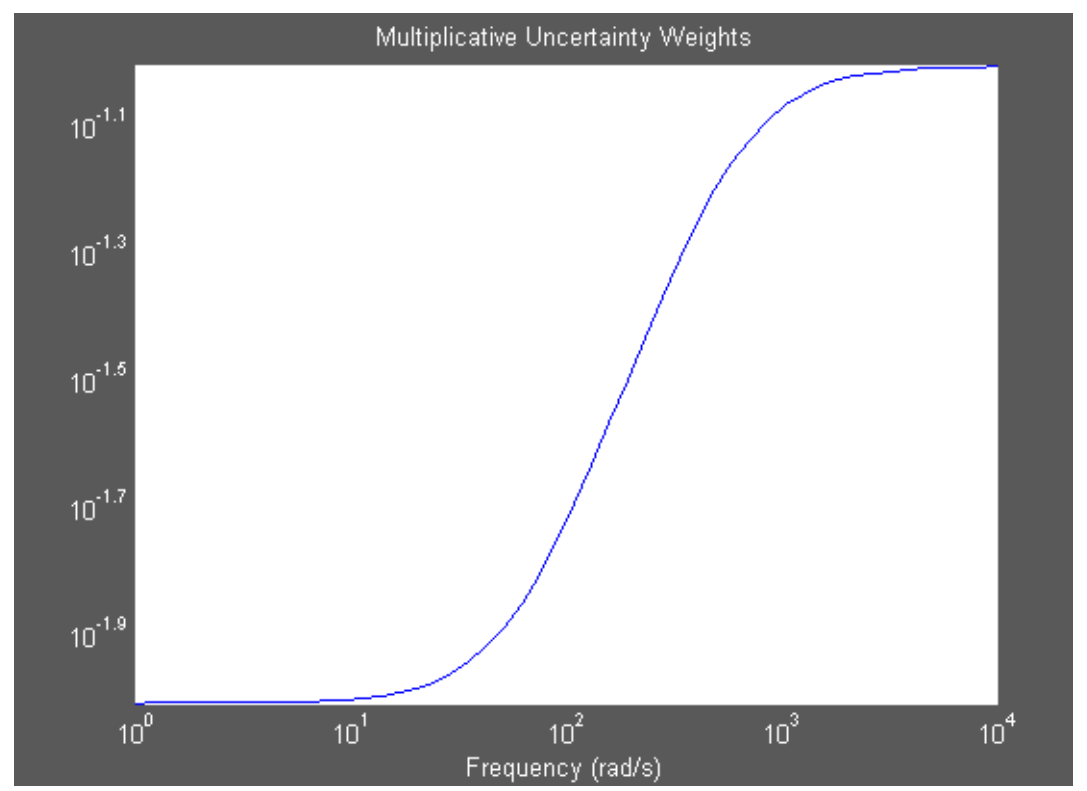

Figure 6.7: Frequency Response of Uncertainty Weighting Function

\subsection{Robust MIMO Controller Design}

The success of a robust controller design heavily depends on the selection of the weighting functions and the interconnected structure of the closed-loop system. The weighting functions that specify the plant uncertainty and performance specification invariably needed to be adjusted iteratively for the success of the design. This part describes the construction of the closed-loop system and the selection of the performance weighting functions. Controller design using $\mu$ synthesis approach and controller order reduction using Hankel norm approximation method are explained in detail in this part. The linear systems controlled responses and the comparison from nonlinear simulation is also presented in the end of this part.

\subsubsection{System Interconnections}

Figure 6.8 shows the closed-loop system interconnection block diagram that specifies the respective weighting functions used in the proposed robust MIMO controller. The interconnection connects the nominal plant model P0(s), the weighting functions of uncertainties and performance specification, as well as the robust controller $\mathrm{K}(\mathrm{s})$ which is to be designed. The weighting functions can be absorbed into the nominal plant model to form an augmented model $\mathrm{P}(\mathrm{s})$ that fits into the two-port frame work from the robust control theory. A diagram of the open- 
loop interconnection is shown in Figure 6.9. A diagram of the two-port framework is shown in Figure 6.10.

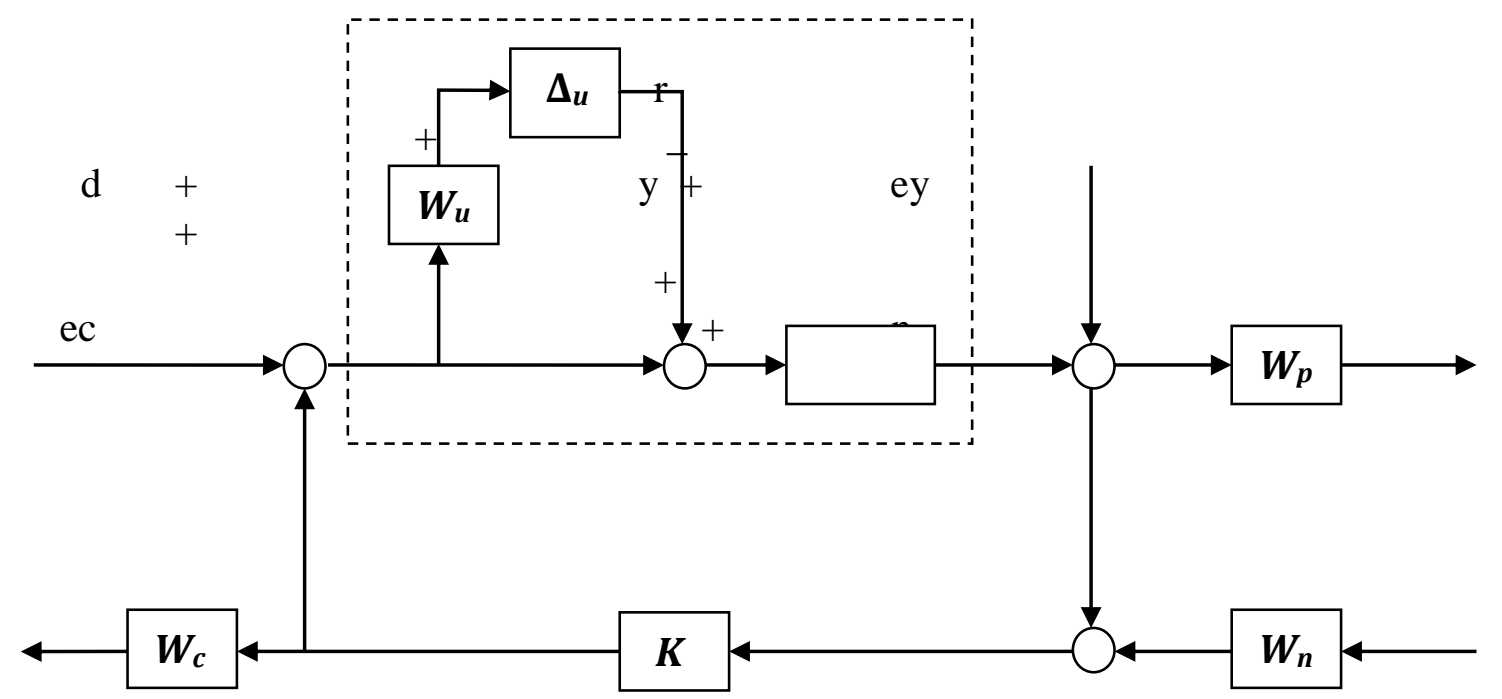

Figure 6.8: Block diagram of the closed-loop system with performance specifications

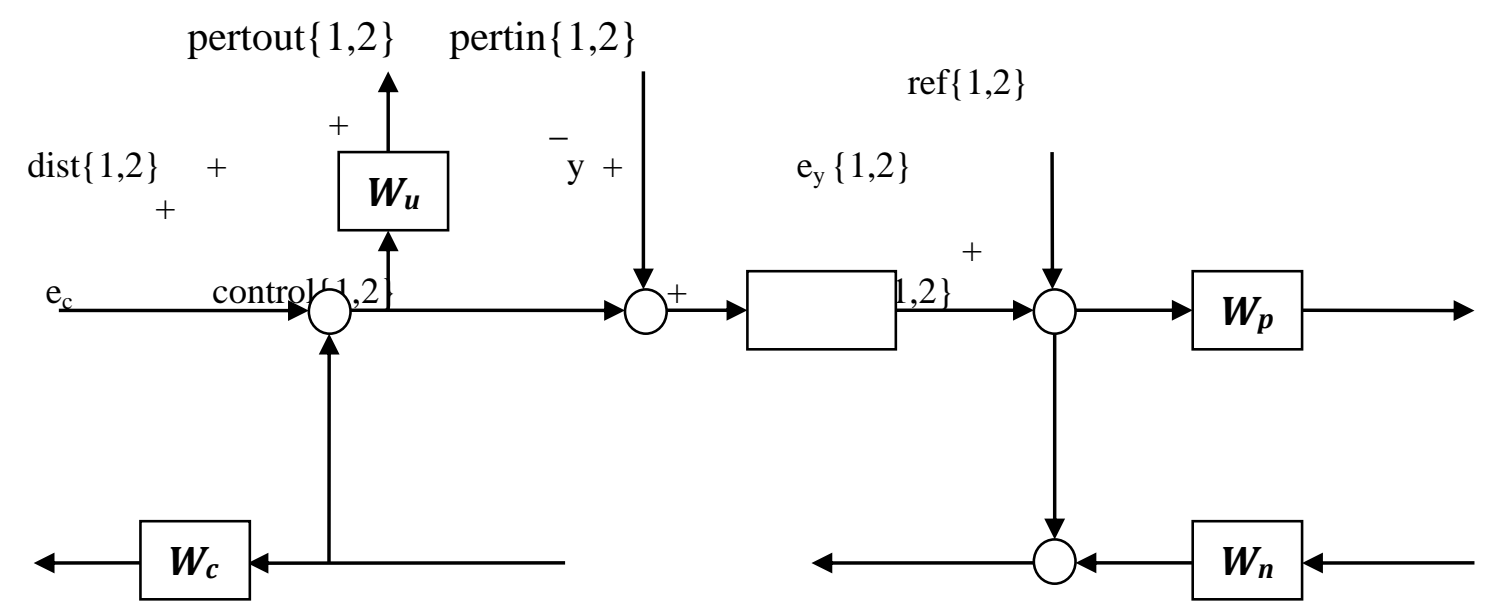

Figure 6.9: Block diagram of the open-loop interconnection with performance specifications

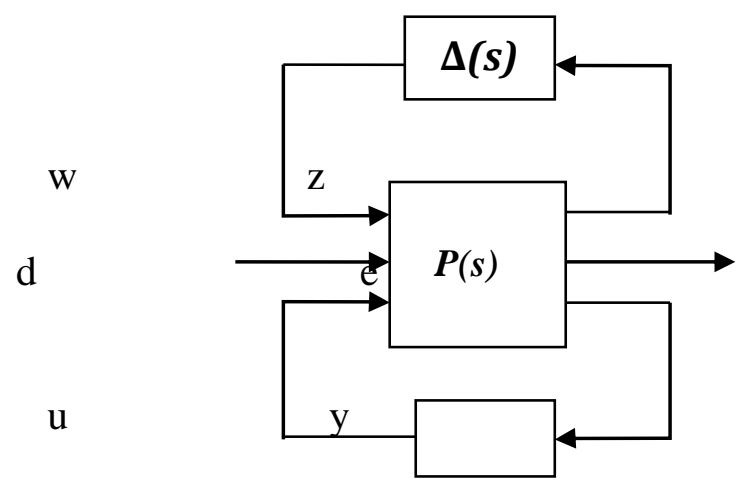

Figure 6.10: Two-port Framework 
As defined in Section 6.2, the linear plant model has two inputs: steam flow and rod reactivity; and two outputs: steam pressure and output power. Therefore, the reference signals $(r)$ includes steam pressure set point and output power load demand. The error signals obtained by subtracting the reference signals from the plant outputs are weighted by $W_{e}(s)$ which specifies the requirement for output tracking. In addition to the signals generated from the plant model, for the purpose of validating the capability of noise rejection of the controller, the error signals are then added with sensor noise signals $n$ to form the measured signals y of the augmented plant, $P(s)$. The control action weighting function $W_{c}(s)$ is used as a means to penalize the control signal. The disturbance $(d)$ is added to the control action to make the resulting controller invulnerable to unexpected disturbance from actuators. The plant uncertainty has been discussed and defined in Section 6.2. The selections of other performance weighting functions are discussed in the following part.

\subsubsection{Selection of Performance Weighting Functions}

The performance weighting functions including tracking error, control action, and sensor noise weighting functions are determined in the following sections.

\section{A) Tracking Error Weighting Function}

To force the plant output, $y$, to track the given reference signal, $r$, the tracking error weighting functions with respect to each plant output, are chosen and put into a diagonal matrix, $\mathrm{Wp}(\mathrm{s})$. The tracking error weighting function is

$$
W_{p}(s)=\left[\begin{array}{cc}
w_{p 1}(s) & 0 \\
0 & w_{p 2}(s)
\end{array}\right]
$$

where $w_{p 1}(s)=\frac{100(s+1)}{1000 s+1}, w_{p 2}(s)=\frac{100(s+1)}{1000 s+1}$

The sensitivity weight indicates that at low frequency, the closed-loop should reject disturbances at the output by a factor of 100-to-1. Expressed differently, steady-state tracking errors in both channels, due to reference step-inputs in either channel should be on the order of 0.01 or smaller. This performance requirement gets less and less stringent at higher and higher frequencies. The closed-loop system should perform better than the open-loop for frequencies up 
to 0.10 radians/second, and for higher frequencies, the closed-loop performance should degrade gracefully. The frequency response of $W_{p}$ is shown in Figure 6.11.

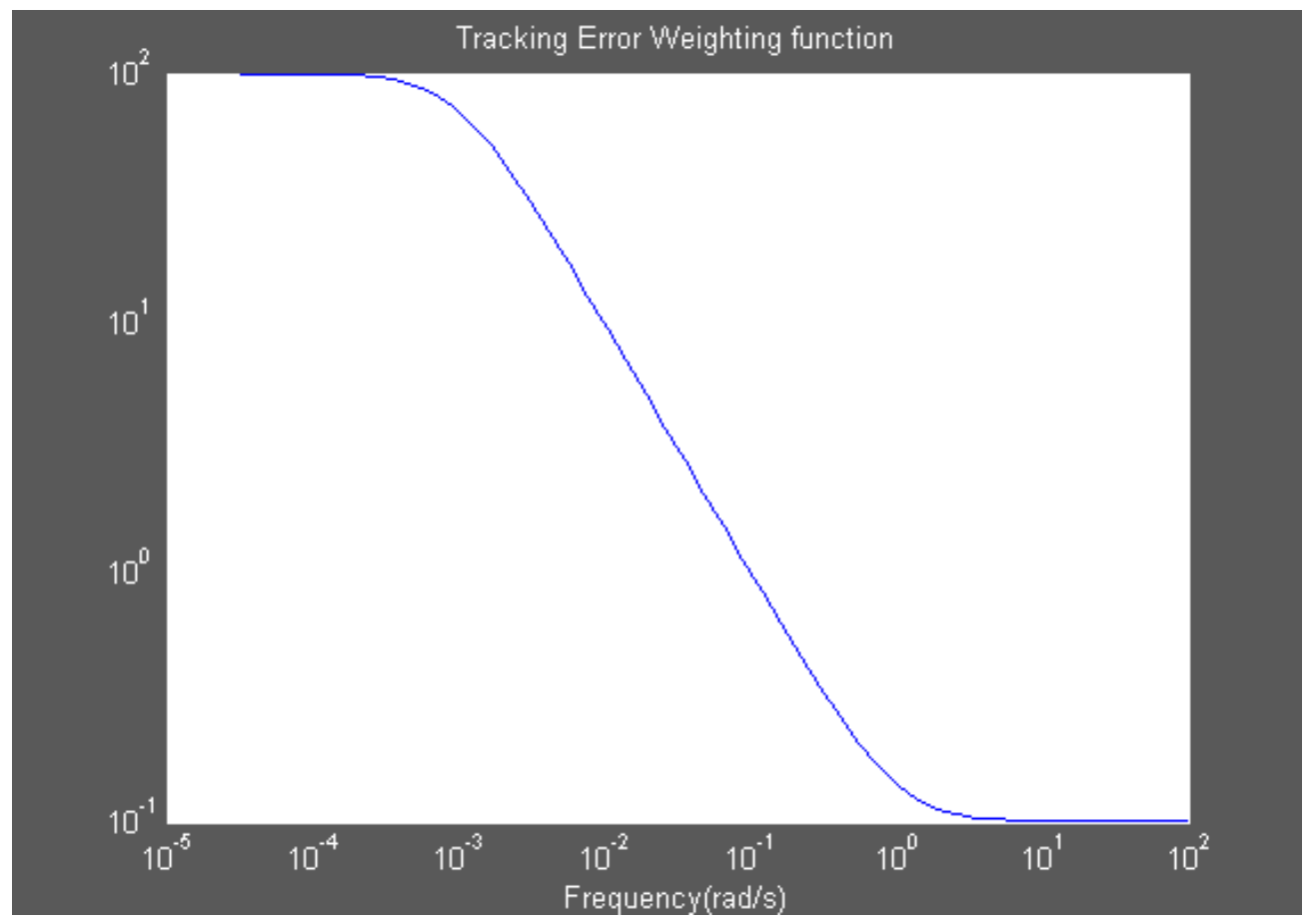

Figure 6.11: Frequency Response of Output Tracking Error Weighting Function

\section{B) Control Action Weighting Function}

The control action diagonal matrix Wc is included in this study to tune the control action effort. For example, when the time response of the controlled system displays excessive control action, the weighting function can be changed to penalize more, whereas when the response is sluggish, the weighting function can be decreased to reduce the penalty [36]. The following diagonal control action matrix $\mathrm{Wc}(\mathrm{s})$ is chosen. The frequency response of $\mathrm{Wc}(\mathrm{s})$ is shown in Figure 6.12.

$$
W_{c}(s)=\left[\begin{array}{cc}
w_{c 1}(s) & 0 \\
0 & w_{c 2}(s)
\end{array}\right]
$$

where $w_{c 1}(s)=\frac{0.0068(10 s+1)}{s+1}, w_{c 2}(s)=\frac{0.0068(10 s+1)}{s+1}$ 


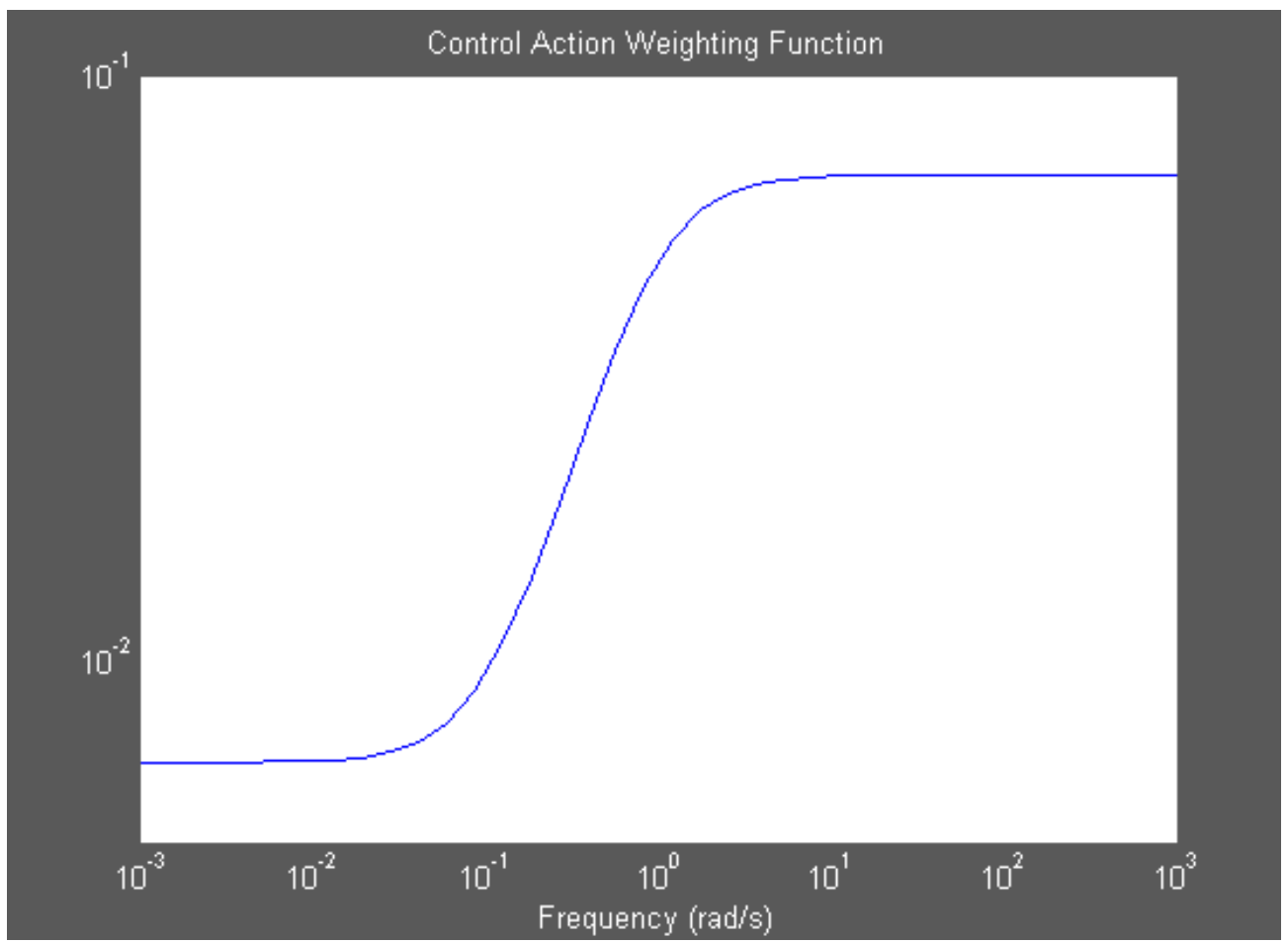

Figure 6.12: Frequency Response of Control Action Weighting Function

\section{C) Sensor Noise Weighting Function}

To represent a more realistic performance specification, a frequency dependent sensor noise weighting function diagonal matrix, $\mathrm{Wn}(\mathrm{s})$, is included in the closed-loop interconnection. It represents the frequency dependent effect that plant sensors could weight the noise. Increasing the sensor noise weight results in a controller which is more insusceptible to sensor noise [36]. The frequency response of the sensor noise weighting function used in this study is shown in Figure 6.13. The diagonal matrix is listed below

$$
W_{n}(s)=\left[\begin{array}{cc}
w_{n 1}(s) & 0 \\
0 & w_{n 2}(s)
\end{array}\right]
$$

where $w_{n 1}(s)=\frac{0.0001(10 s+1)}{s+1}, w_{n 2}(s)=\frac{0.0001(10 s+1)}{s+1}$ 


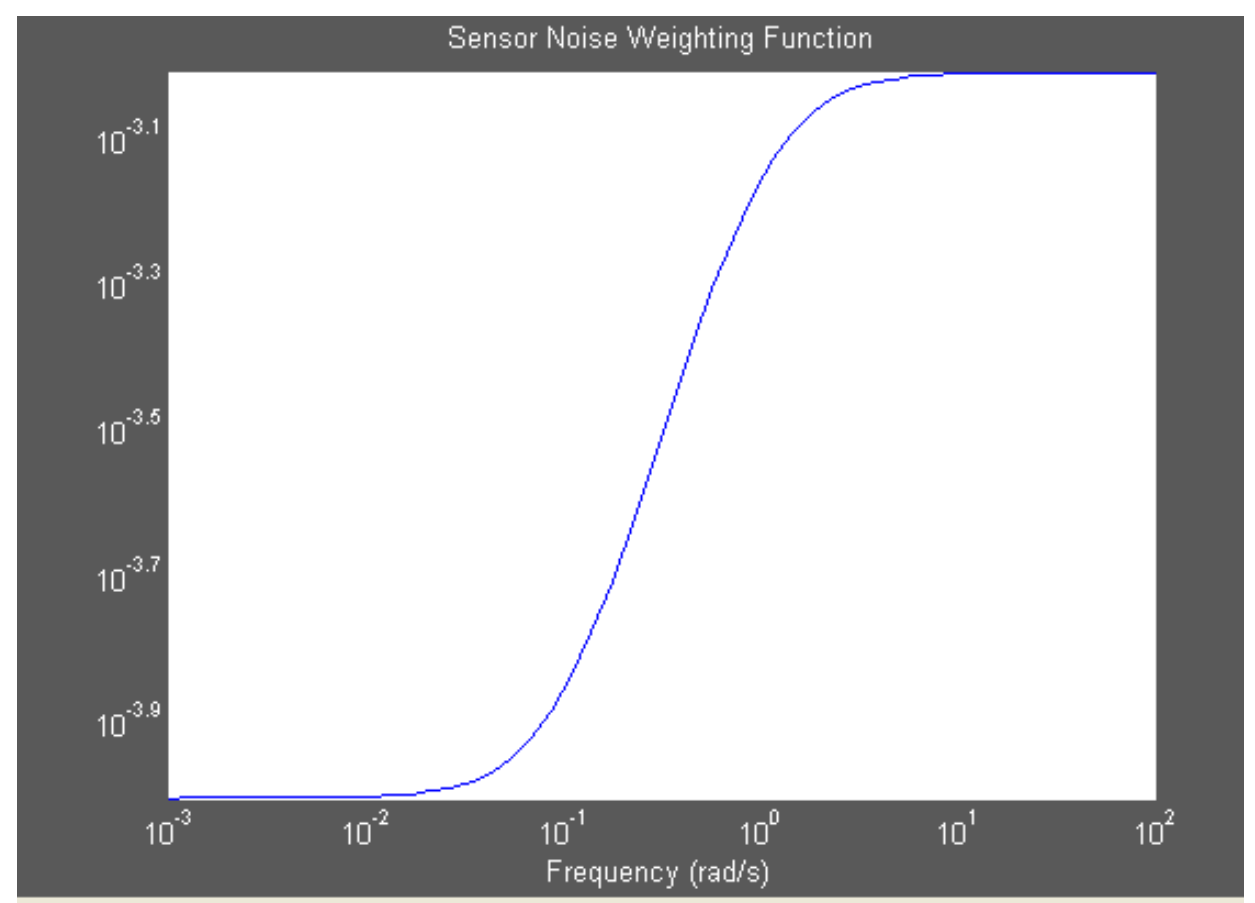

Figure 6.13: Frequency Response of Sensor Noise Weighting Function

\subsection{3 $\mu$-Synthesis Controller Design}

Denote $P(s)$ as the transfer function matrix of the ten-input, eight-output open-loop system consisting of the nuclear power plant model plus the weighting functions and let the block structure $\Delta_{P}$ be defined as

$$
\Delta:=\left\{\left[\begin{array}{cc}
\Delta_{u} & 0 \\
0 & \Delta_{p}
\end{array}\right]: \Delta_{u} \in \square^{2 \times 2}, \Delta_{p} \in \square^{6 \times 4}\right\}
$$

The first block of this matrix corresponds to the uncertainty block $\Delta \mathrm{u}$, used in modeling the uncertainty of the nuclear power plant. The second block $\Delta p$ is a fictitious uncertainty $6 \times 4$ block, introduced to include the performance objectives in the framework of the $\mu$-approach [39]. The inputs to this block are the weighted control action, disturbances and performance signals, and the outputs are the reference and noise signals. Figure 6.14 summaries the complete controller design problem.

To meet the design objectives a stabilizing controller $\mathrm{K}$ is to be found such that, at each frequency $\omega \in 0, \infty$, the structured singular value satisfies the condition

$$
\mu_{\Delta_{p}}\left[F_{L}(P, K)(j \omega)\right]<1
$$


where $F_{L}(P, K)$ represents the Linear Fractional Transformation of $P$ and $K$ [37]. The fulfillment of this condition guarantees robust performance of the closed-loop system.

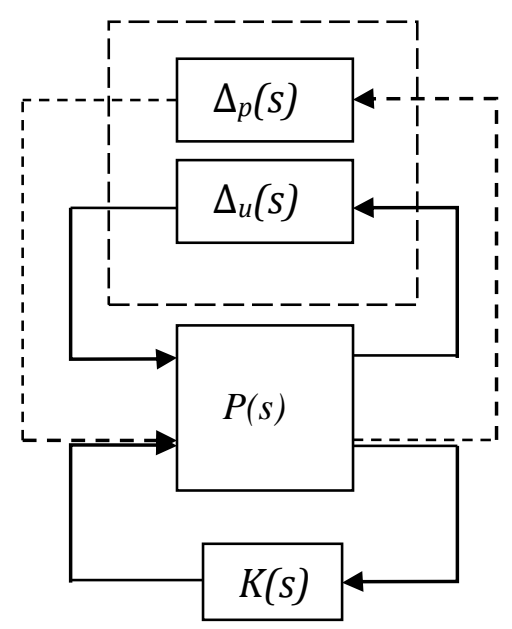

Figure 6.14: Robust control problem

The $\mu$-synthesis is performed by using $\mathrm{D}-\mathrm{K}$ iteration tool in MATLAB. The progress of $\mathrm{D}-\mathrm{K}$ iteration is shown in Table 6.2. The $5^{\text {th }}$ controller is selected since it has lower $\gamma$ and $\mu$ value.

Table 6.2: Results of the $\mu$-synthesis

\begin{tabular}{|c|c|c|c|}
\hline Controller & Order & $\gamma$-Value & $\begin{array}{c}\text { Peak } \mu \text { - } \\
\text { value }\end{array}$ \\
\hline 1 & 30 & 2.441 & 1.515 \\
\hline 2 & 30 & 1.030 & 1.021 \\
\hline 3 & 30 & 0.979 & 0.984 \\
\hline 4 & 30 & 0.986 & 0.988 \\
\hline 5 & 30 & 0.978 & 0.981 \\
\hline 6 & 30 & 0.984 & 0.986 \\
\hline
\end{tabular}

The resulting robust controller with order $n$ in state-space form is represented as

$$
K=\left[\begin{array}{ll}
A_{n \times n} & B_{n \times 2} \\
C_{2 \times n} & D_{2 \times 2}
\end{array}\right]
$$




\subsubsection{Controller Order Reduction}

The estimated controllers have closed-loop $\mu$ value less than 1 . Therefore the robust performance is guaranteed for the prescribed uncertainty and performance. However, the controller obtained from $\mu$-synthesis has a very high order and consists of many insignificant states that can be ignored. In order to simplify the implementation and increase the reality, the balanced realization and Hankel norm approximation method can be used to reduce the order of the controllers [37].

The 5th controller is taken to implement order reduction, and the result is shown below in Table 6.3. The peak $\mu$-values do not increase significantly after order-reduction until the controller order is reduced below 10th order. There is clear gap in $\mu$-values between the 7 th and 6th order controller, and the $\mu$-values of controller with orders less than 6 are greater than 1 . So the controller with 7th reduced-order is selected in the following part of this study.

Table 6.3: Summary of different reduced orders of Controller \# 5

\begin{tabular}{|c|c|c|}
\hline Controller \# & $\begin{array}{c}\text { Reduced } \\
\text { Order }\end{array}$ & $\begin{array}{c}\mu \text {-value after } \\
\text { reduction }\end{array}$ \\
\hline 5 & 30 & 0.9812 \\
\hline 5 & 20 & 0.9812 \\
\hline 5 & 10 & 0.9812 \\
\hline 5 & 8 & 0.9826 \\
\hline 5 & 7 & 0.9828 \\
\hline 5 & 6 & 0.9877 \\
\hline 5 & 5 & 1.0311 \\
\hline
\end{tabular}

In Figures 6.15 and 6.16, the Bode plots of the full-order and reduced-order controllers are compared. The corresponding plots practically coincide with each other, which imply similar performance in the closed-loop systems. There are four pairs of lines in each figure because the controller is two-input-two-output, so four transfer functions exist in each Bode plot. The solid lines represent frequency responses of full-order controller and dashed lines represent frequency responses of reduced-order controller. 


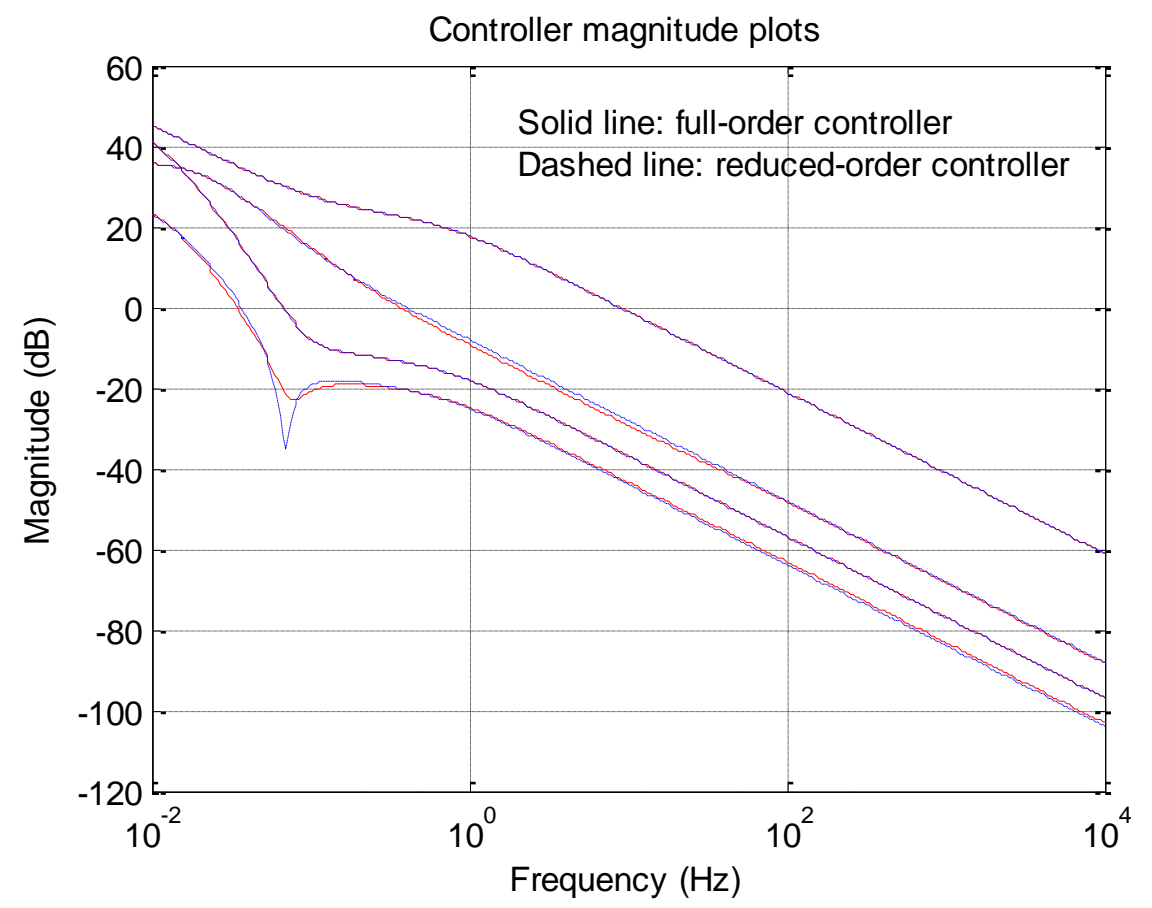

Figure 6.15: Magnitude plots of the full- and reduced-order $\mu$-controllers

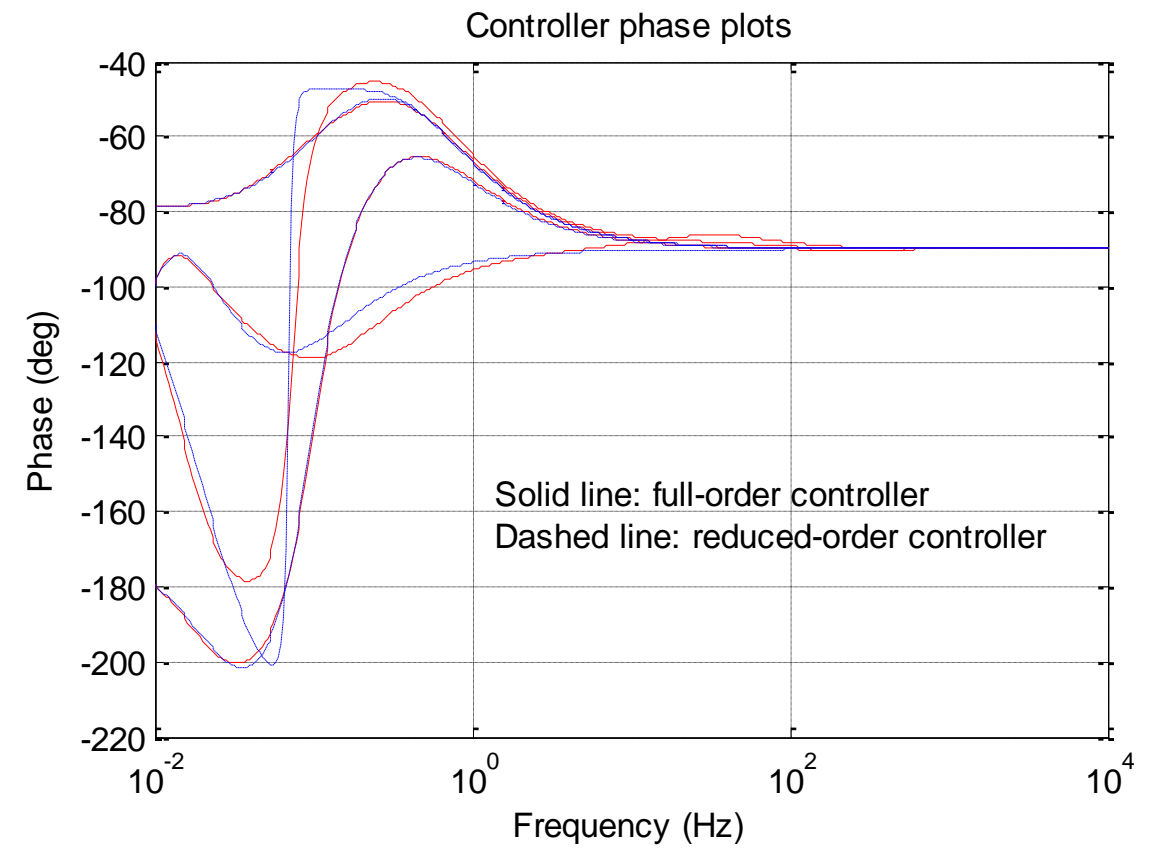

Figure 6.16: Phase plots of the full- and reduced-order $\mu$-controllers

\subsubsection{System Reponses Under Uncertainty}

The performances of the resulting robust controllers are evaluated in this section. The connection between the plant model and the resulting robust controller is shown in 
Figure 6.17 in the form of MATLAB Simulink graphical representation. The outputs of the nonlinear plant model are summed with sensor noise to represent the measured outputs. The load demand reference, and steam pressure setpoint are added to the negative feedback measurements of the output power and steam pressure, respectively. Based on this configuration, the controller performances of both linear and nonlinear models are evaluated with respect to the capability of tracking error, disturbance and sensor noise rejection, in the following sections.

\section{A) Output Tracking}

The output tracking with respect to load demand change achieved by the robust MIMO controller of linear and nonlinear model is evaluated in this section. The controller performance under nominal and perturbed conditions can be observed in the time-domain step responses of the linear and nonlinear system model. The full-order and reduced-order controller obtained in Section 6.3.4 are compared to demonstrate the controller performance.

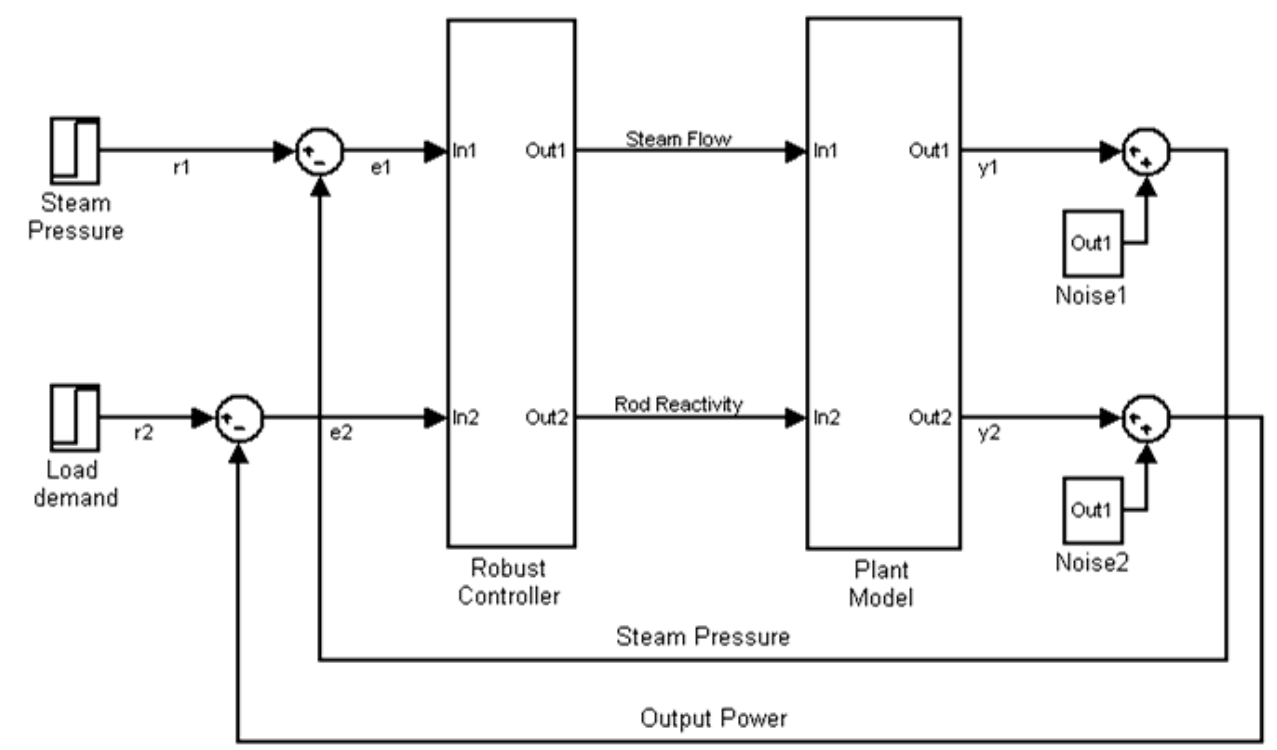

Figure 6.17: Connections between Plant Model and Robust Controller

Figure 6.18 shows the nonlinear and linear closed-loop system at 5\% step decrease of output power load demand, using the reduced-order controller. The steady state value of the output power is $95.03 \%$, which means a $0.03 \%$ steady state error with the desired value. Both models were running for 50 seconds to achieve steady state before the load demand change at $\mathrm{t}=50 \mathrm{~s}$. Since the normal operating point (NOP) of the nonlinear model is 1 whereas the NOP of 
the linear model is 0 , the system responses are represented in relative percentage scale in Figure 3.18 for comparison purpose.

Figure 6.19 shows the behaviors of closed-loop system with linearized plant model using full-order and reduced-order $\mu$ controller. The time domain responses show that the system behaviors controlled by full-order and reduced-order controllers are undistinguishable. So the reduced-order controller is a very good approximation of the full-order controller and satisfies the performance requirements.
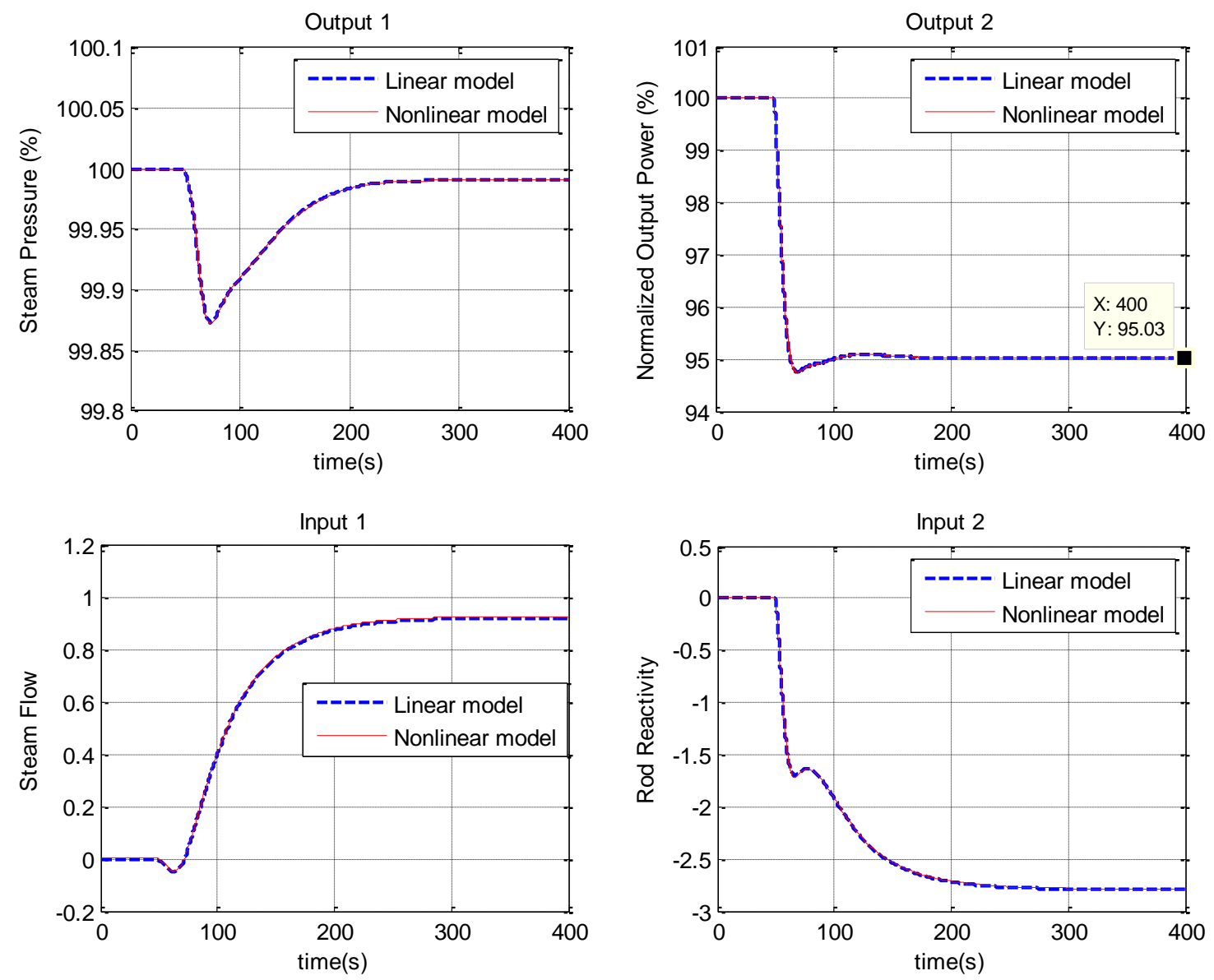

Figure 6.18: Comparison of closed-loop system responses at 5\% step decrease of output power load demand, between linear and nonlinear plant models, controlled by reduced-order controller 

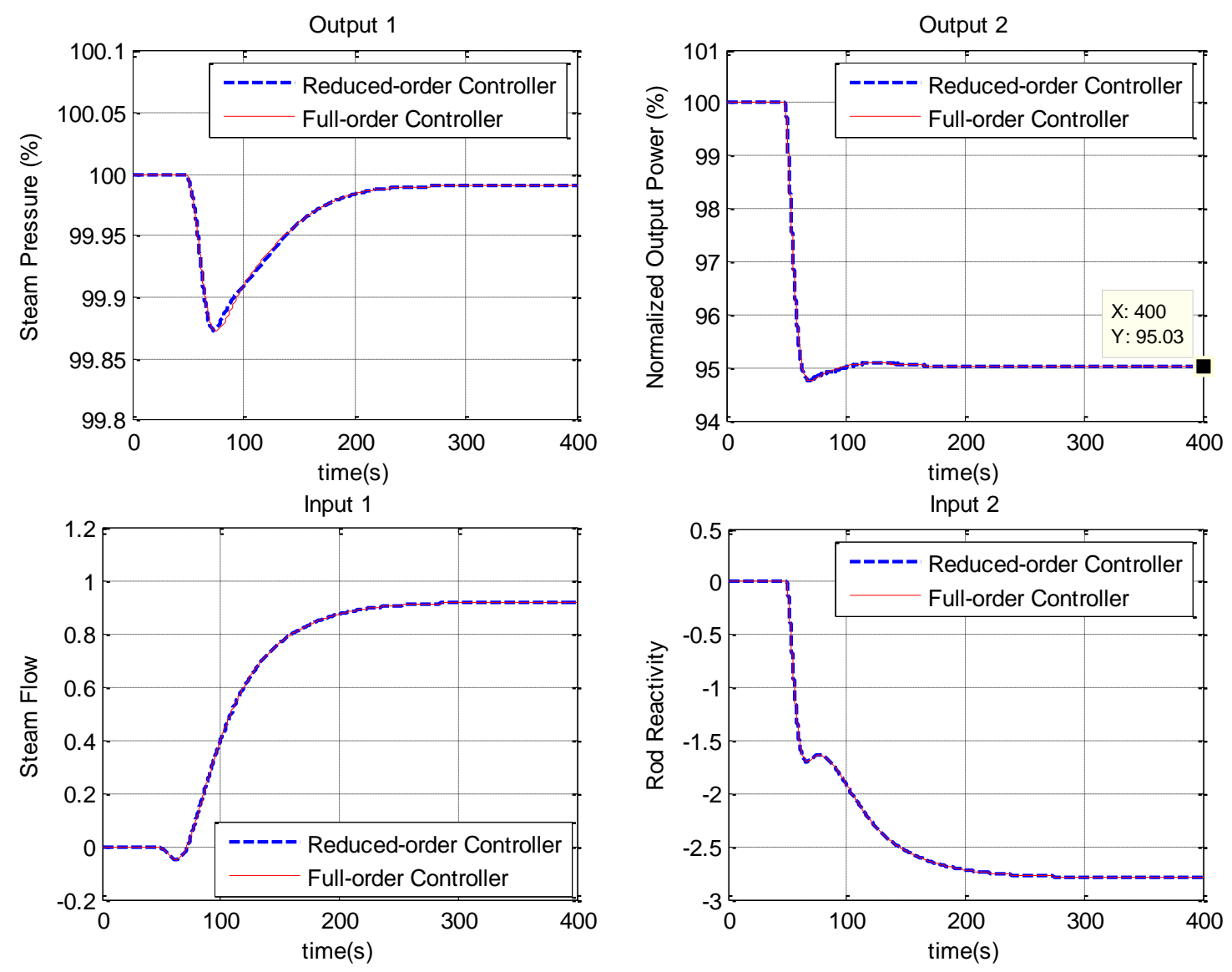

Figure 6.19: Closed-loop system responses at 5\% step decrease of output power load demand, with linear plant model, controlled by full- and reduced-order controller

\section{B) Sensor Noise Rejection}

In order to simulate the robust MIMO controlled responses under the influence of sensor noise, the two outputs of the plant are added with noise signal of zero mean and 0.01 variance. The output responses and control actions are shown in Figure 6.20 and Figure 6.21 for linear model and nonlinear model, respectively, controlled by the reduced-order controller. It can be seen that the plant responses are degraded by the noise. Compared with the output responses and control actions not corrupted by noise in Figure 6.18, it can be seen that the robust controller is not sensitive to noise, and the output power is only varying within a small range although the sensor noise is large. 

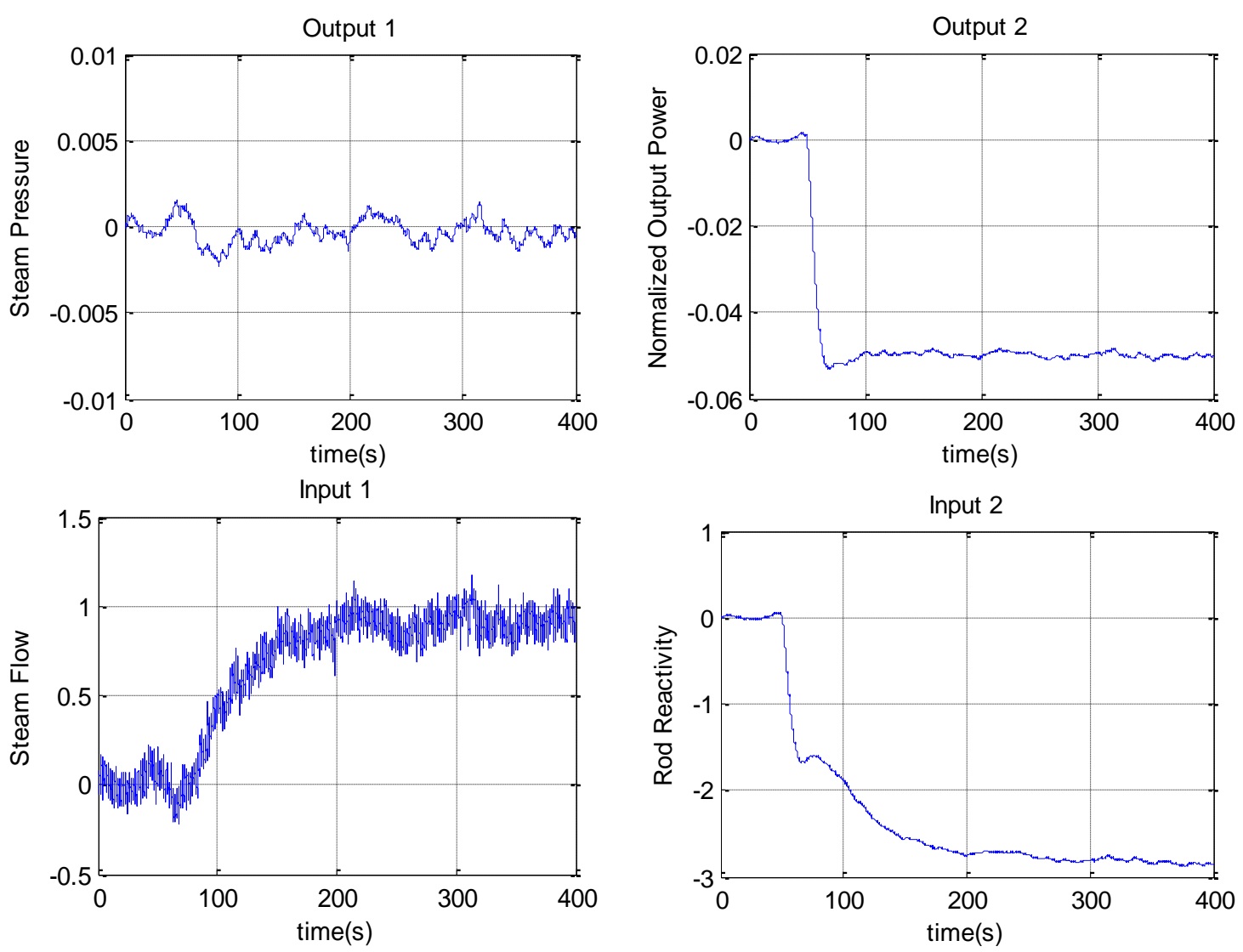

Figure 6.20: Linear system response at 5\% step decrease of output power load demand, perturbed by a 0.01 variance zero mean sensor noise 

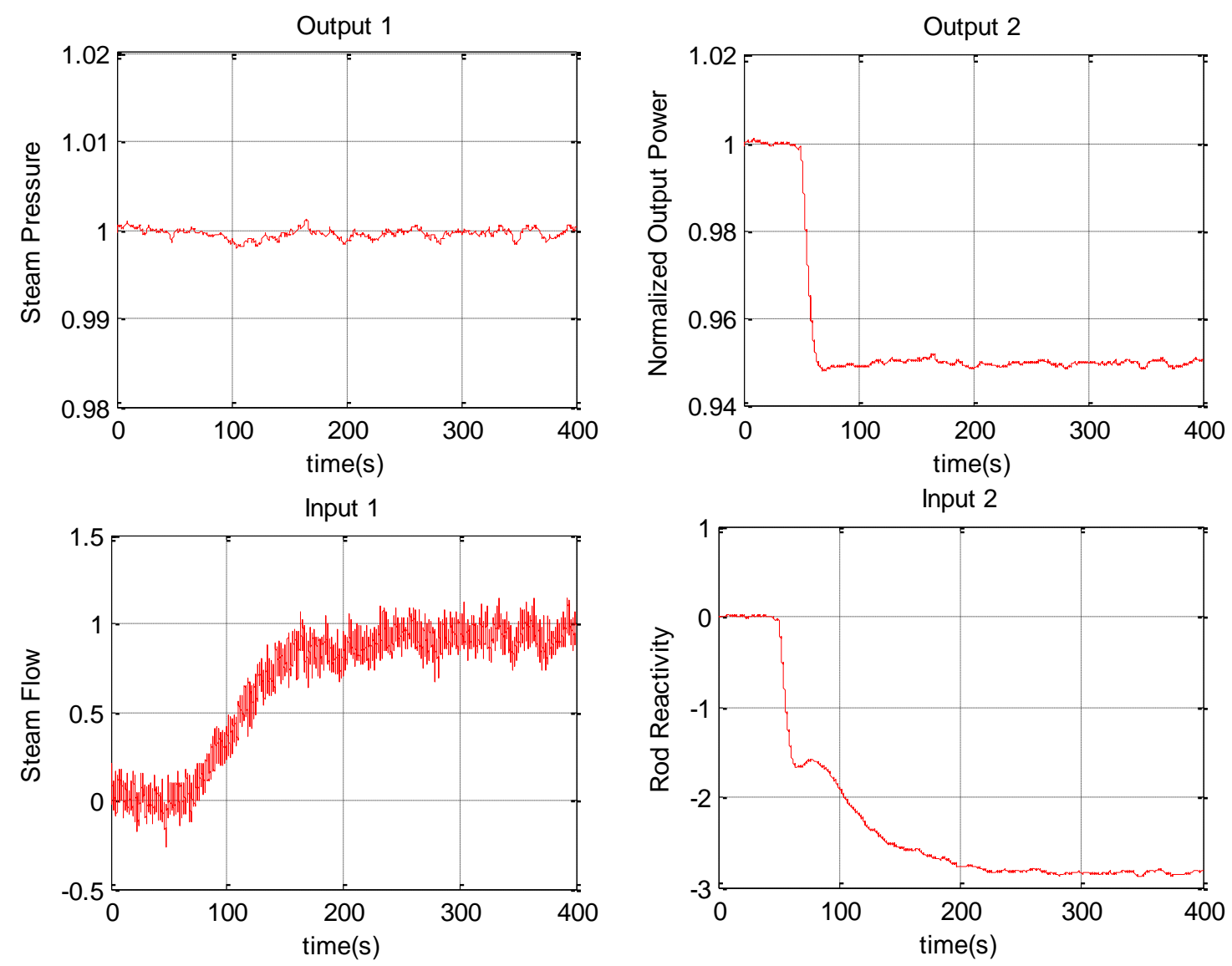

Figure 6.21: Nonlinear system response at 5\% step decrease of output power load demand, perturbed by a 0.01 variance zero mean sensor noise

C) Disturbance Rejection

The capacity of rejecting the disturbance of the robust MIMO controller is evaluated by injecting a steam flow step increase of value 0.1 for 10 seconds at $t=50 \mathrm{~s}$ in the linear model, and injecting a rod reactivity step increase of value 0.5 to the power plant for 10 seconds at $t=50 \mathrm{~s}$ in the linear model. In both cases the nuclear power plant is controlled by the reduce-order controller. Figure 6.22 shows the plant output responses and corresponding control actions to the steam flow disturbance, and Figure 6.23 shows the plant output responses and control actions to the rod reactivity injection.

It can be seen the controller responds to disturbances immediately, and recover to normal operating points quickly after the disturbances are removed. The two outputs (steam pressure and normalized output power) don't change much in both cases. So this controller is very robust against disturbances. 

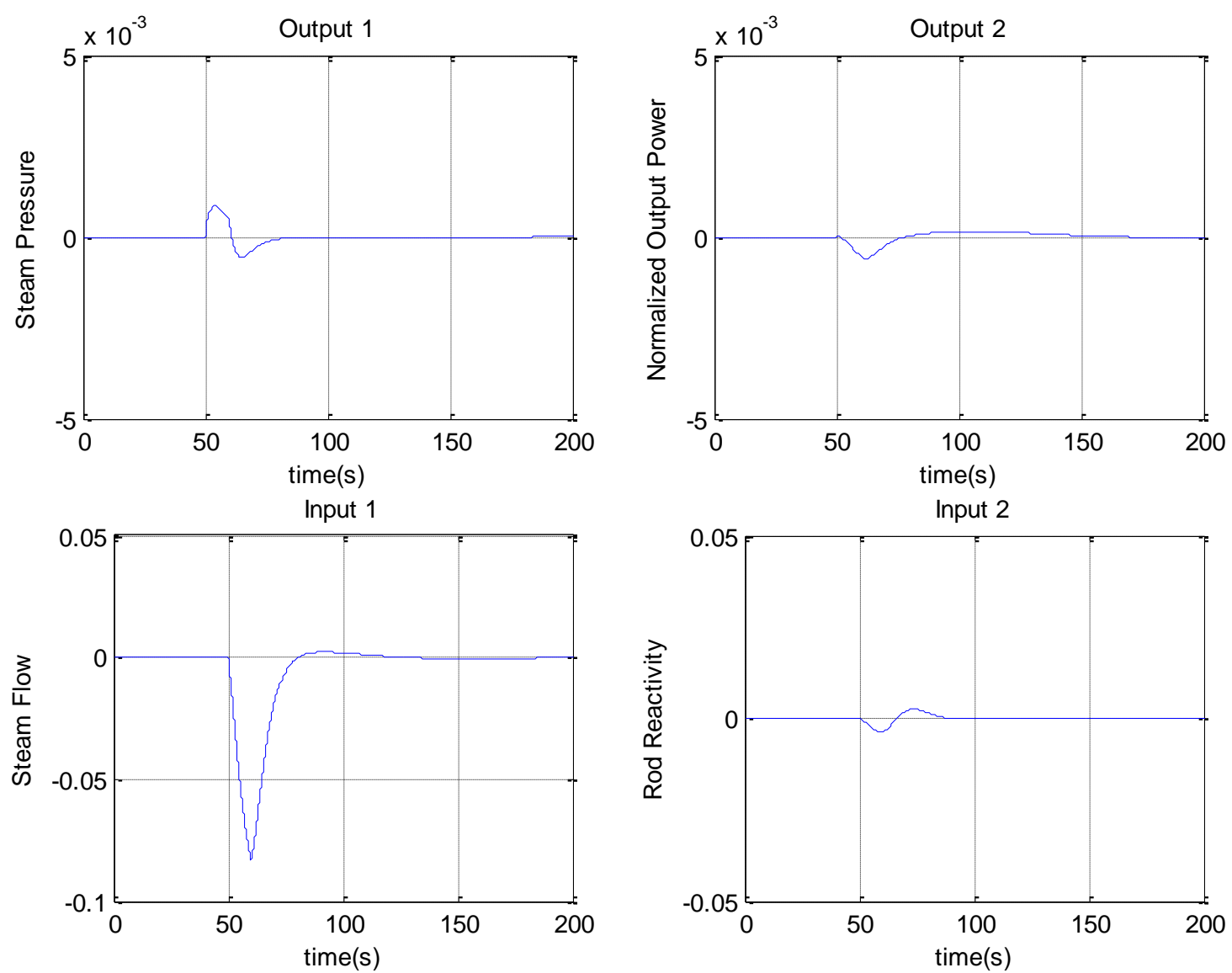

Figure 6.22: Closed-loop system responses to disturbance from steam flow (0.1 step increase at $\mathrm{t}=50 \mathrm{~s}$, lasting for 10 seconds) 

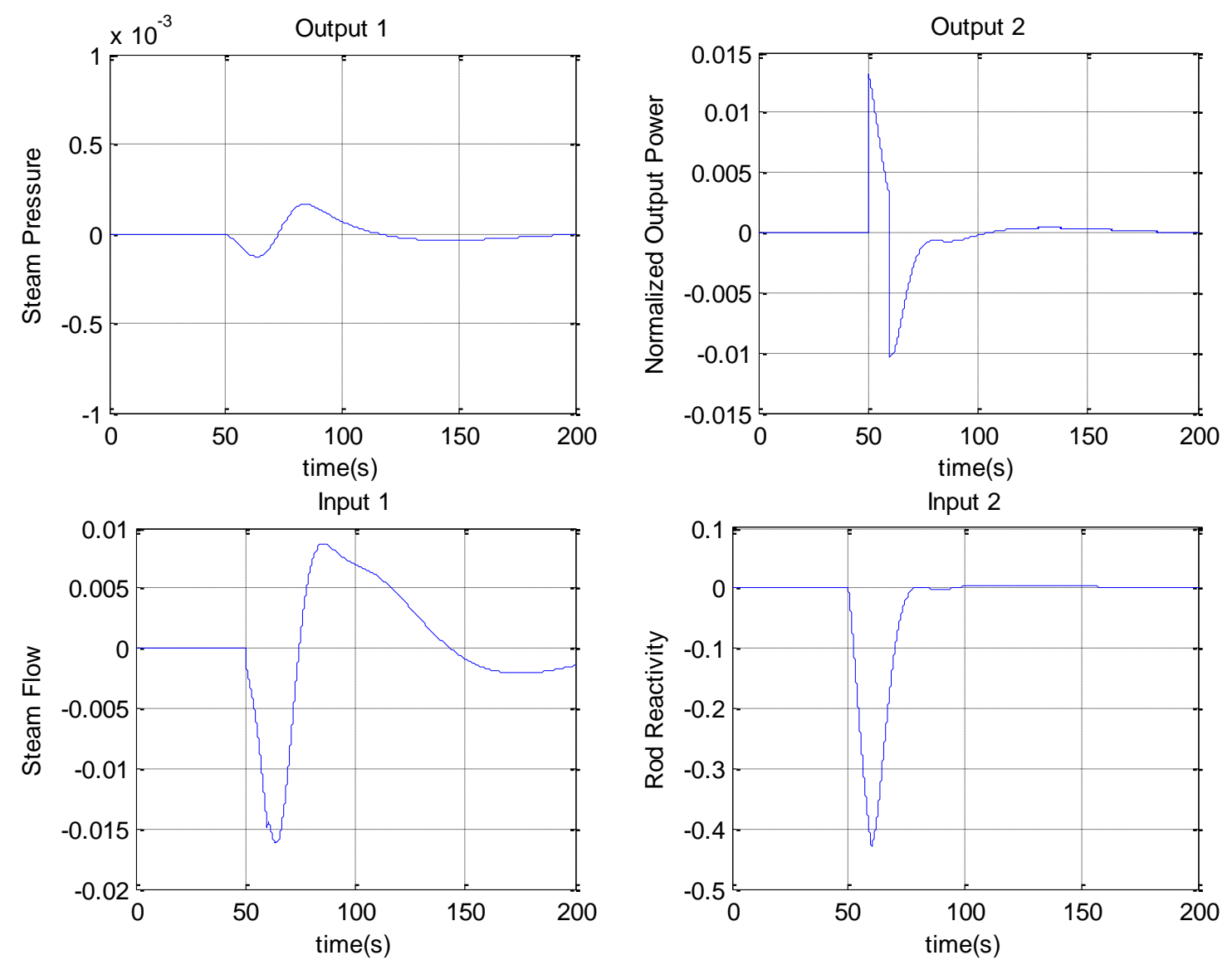

Figure 6.23: Closed-loop system responses to disturbance from rod reactivity ( 0.5 step increase at $\mathrm{t}=50 \mathrm{~s}$, lasting for 10 seconds)

\section{RESILIENT CONTROL DESIGN}

Guo et al. [3] conducted resilient propulsion control research on how engine control systems can improve aircraft safe-landing probabilities under adverse conditions. The idea is in some emergent situations, the conservative design of engine control system may not be the best interest of overall aircraft safety; it may be advantageous to "sacrifice" the engine to "save" the aircraft. The aim of their research is to develop adaptive engine control methodologies to operate the engine beyond the normal domain for emergency operations to maximize the possibility of safely landing the damaged aircraft. Motivated by this idea, resilient controllers can be designed for nuclear power plants to "save" the plant by "sacrificing" performance or some less important parts under adverse conditions. The definition of resilience in this paper is different from [40, 41] in which resilience is defined to be non-fragile or insensitive to some errors in the nominal statespace matrices of controller during implementation. 
The role of a resilient controller is to enhance recovery of the control system from unanticipated adverse conditions and faults as well as from emergency situations by altering its operational envelope in real time. Resilient decision and control laws are synthesized on a finitetime horizon as augmentation of robust decision and control with the objectives of: (1) Reliable and fast recovery from adverse conditions and emergency situations and (2) Restoration of the control configuration upon returning to normalcy or upon graceful degradation within design specifications.

Recently, Hovakimyan and her coworkers developed a novel control algorithm called $\mathrm{L}_{1}$ adaptive control [42]. The advantages of $\mathrm{L}_{1}$ adaptive controller are guaranteed fast adaptation, uniformly bounded transient and asymptotic tracking for system's both signals, input and output, simultaneously [43]. So the $\mathrm{L}_{1}$ adaptive controller would be suitable to implement the function of resilient control.

In this chapter, the algorithm of $\mathrm{L}_{1}$ adaptive output feedback controller is briefly reviewed. Since the dynamics of the nuclear power plant model are coupled in both channels, the SISO framework was extended to the MIMO case. Full nonlinear simulation for the closed loop system was run to evaluate the performance of the $\mathrm{L}_{1}$ adaptive controller. Possible future research is outlined at the end of the report.

\section{1 $\mathrm{L}_{1}$ Adaptive Output Feedback Controller Design}

The $\mathrm{L}_{1}$ adaptive control architecture was first presented by Cao and Hovakimyan [42] for systems constant in unknown parameters using a state feedback approach. The guaranteed timedelay margin of $\mathrm{L}_{1}$ adaptive control architecture was derived in [43]. Later the paradigm was extended to output feedback in [44] for a class of reference systems with strictly positive real (SPR) transfer functions. Extension to nonlinear time-varying systems in the presence of multiplicative and additive un-modeled dynamics is reported in [45 - 47]. In [48], an output feedback extension is presented for a class of uncertain systems that allows for tracking arbitrary reference systems, without imposing an SPR-type requirement on its input-output transfer function. It is this particular architecture that we employ to address the control challenge of the Nuclear Power Plant model. The $\mathrm{L}_{1}$ adaptive output feedback control architecture is presented in Figure 7.1 and a brief overview of it is given below, as reported in [49]. 


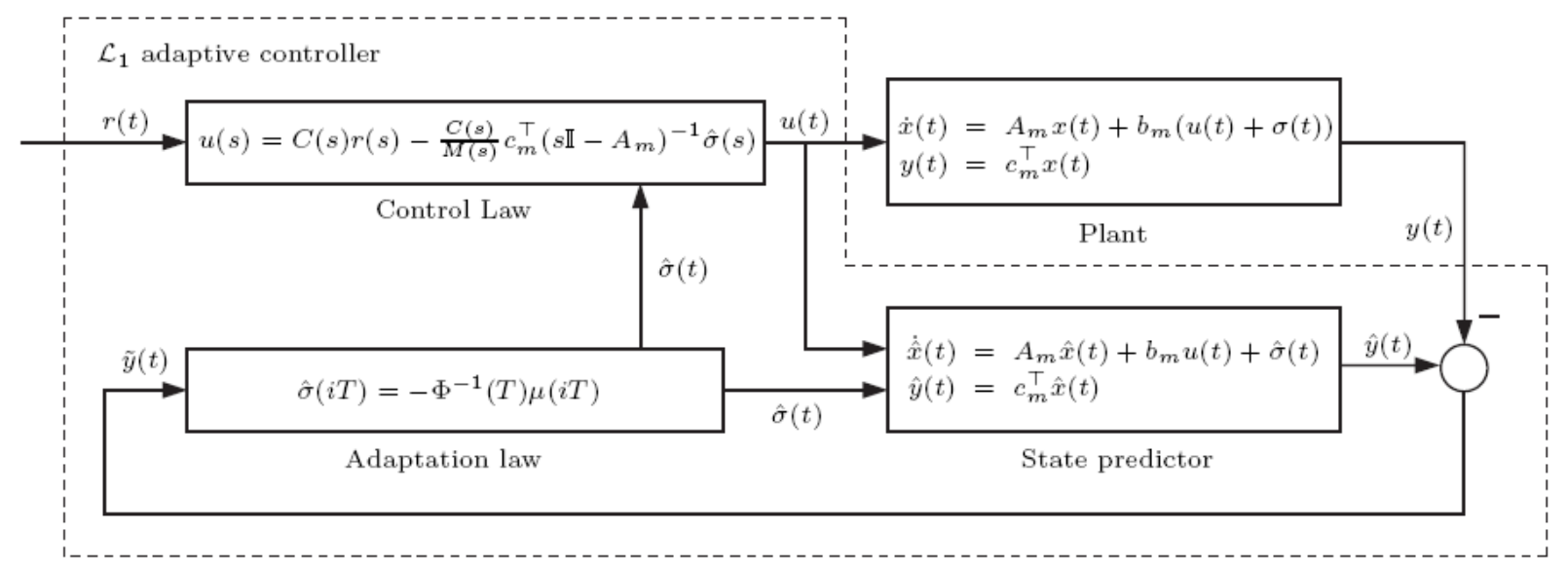

Figure 7.1: Closed loop system with the $\mathcal{L}_{1}$ adaptive controller

Consider the following single-input single-output (SISO) system:

$$
\text { y } s=A(s) u s+d(s)
$$

where $u(t) \in \mathbb{R}$ is the commanded control, $y(t) \in \mathbb{R}$ is the system output, $A(s)$ is a strictly proper unknown transfer function of unknown relative degree $n_{a r}$ for which only a known lower bound $1<n_{r} \leq n_{a r}$ is available, $d(s)$ is the Laplace transform of the time-varying uncertainties and disturbances $d t$. Let $r(t)$ be a given bounded continuous reference input signal. The control objective is to design an adaptive output feedback controller $u(t)$ such that the system output $y(t)$ tracks the reference input $r(t)$ following a desired model

$$
y_{d} s=M s r(s)
$$

where $M S$ is a minimum-phase stable transfer function of relative degree $n_{r}>1$. The system equations in terms of the desired model can be rewritten as:

$$
y s=M s(u s+\sigma(s))
$$

where

$$
\sigma s=(A s-M s u s+A(s) d(s) / M(s)
$$

Next we introduce the closed-loop reference system that defines an achievable control objective for the $\mathrm{L}_{1}$ adaptive controller.

Closed-loop reference system: The reference system is given by

$$
\begin{aligned}
& y_{r e f} s=M s u_{\text {ref }} s+\sigma_{\text {ref }} s \\
& \sigma_{\text {ref }} s=A s-M s u_{\text {ref }} s+A s d_{\text {ref }} s / M s
\end{aligned}
$$




$$
u_{\text {ref }} s=C s\left(r s-\sigma_{\text {ref }} s\right)
$$

where $C s$ is a low pass filter with DC gain $C 0=1$ and $d_{\text {ref }} t=f\left(t, y_{\text {ref }} t\right)$.

According to [23, Lemma 1] the selection of $C(s)$ and $M(s)$ must ensure that

$$
H s=A s M(s) / C s A s+1-C s M(s)
$$

is stable and that the $\mathrm{L}_{1}$-gain of the cascaded system is upper bounded as follows:

$$
H s(1-C(s))_{L_{1}}<1
$$

Then the reference system in (7.2) is stable.

The elements of the $\mathrm{L}_{1}$ adaptive controller are introduced next.

State predictor (passive identifier): Let $\left(A_{m} \in \mathbb{R}^{n \times n}, b_{m} \in \mathbb{R}^{n}, c_{m} \in \mathbb{R}^{n}\right)$ be the minimal realization of $M(s)$. Hence, $\left(A_{m}, b_{m}, c_{m}\right)$ is controllable and observable with $A_{m}$ being Hurwitz . Then the system in (7.1) can be rewritten as

$$
\begin{gathered}
x t=A_{m} x t+b_{m}(u t+\sigma(t)) \\
y t=c_{m}^{T} x(t)
\end{gathered}
$$

The state predictor is given by:

$$
\begin{gathered}
x(t)=A_{m} x t+b_{m}(u t+\sigma(t)) \\
y t=c_{m}^{T} x(t)
\end{gathered}
$$

where $\sigma(t) \in \mathbb{R}^{n}$ is the vector of adaptive parameters. Notice that in the state predictor equations $\sigma(t)$ is not the span of $b_{m}$, whereas in the Equation (7.4) $\sigma(t)$ is in the span of $b_{m}$. Further, let $y t=y t-y(t)$.

Adaptation law: Let $P$ be the solution of the following algebraic Lyapunov equation:

$$
A_{m}^{T} P+P A_{m}=-Q
$$

where $Q>0$. From the properties of $P$ it follows that there always exists a nonsingular $\bar{P}$ such that 


$$
P=\bar{P}^{T} \bar{P}
$$

Given the vector $c_{m}^{T}(\bar{P})^{-1}$, let $D$ be the $(n-1) \times n$-dimensional null space of $c_{m}^{T}(\bar{P})^{-1}$,

$$
D \quad c_{m}^{T} \quad \bar{P}^{-1}{ }^{T}=0
$$

and let

$$
\Lambda={ }_{D}^{c_{m}^{T}} \bar{P} \in \mathbb{R}^{n \times n}
$$

The update law for $\sigma(t)$ is defined via the sampling time $T>0$ (where $T$ defines the sampling rate of the available CPU):

$$
\sigma i T=-\Phi^{-1} T \mu i T, i=1,2, \cdots
$$

where

$$
\Phi \mathrm{T}={ }_{0}^{T} e^{\Lambda A_{m} \Lambda^{-1}(T-\tau)} \Lambda d \tau
$$

and

$$
\mu i T=e^{\Lambda A_{m} \Lambda^{-1} T} \underline{L_{1}} y i T, i=1,2, \cdots
$$

Here $L_{1}$ denotes the basis vector in the space $\mathbb{R}^{n}$ with its first element is equal to 1 and other elements are zero.

Control law: The control law is defined via the output of the low-pass filter:

$$
u s=C s r s-\frac{C(s)}{M(s)} c_{m}^{T} s \mathbb{-}-A_{m}{ }^{-1} \sigma(s)
$$

The complete $\mathrm{L}_{1}$ adaptive controller consists of the state predictor in (7.5), the adaptation law in (7.6), and the control law in (7.7), subject to the $\mathrm{L}_{1}$-gain upper bound in (7.3).

\section{2 $\mathrm{L}_{1}$ Adaptive Control Law for Nuclear Power Plant Model}

The dynamics of the nuclear power plant model are coupled in both channels, thus, requiring an extension of the L1 adaptive output feedback controller to Multi-Input Multi-Output (MIMO) systems. Following the way Kharisov et la did in [49], we extend the SISO $\mathrm{L}_{1}$ framework to MIMO case.

Desired system: The matrix transfer function of the desired system was selected as follows: 


$$
M s=\begin{array}{cc}
M_{1}(s) & 0 \\
0 & M_{2}(s)
\end{array}
$$

where $M_{1}(s)$ and $M_{2}(s)$ are the scalar transfer functions for corresponding channels. It is natural that the desired transfer function has decoupled channels, which implies zeros at non-diagonal elements of the matrix transfer function.

In the current design the following transfer functions were selected:

$$
M_{1} s=M_{2} s=\frac{\omega_{M}{ }^{2}}{s^{2}+2 \xi_{M} \omega_{M} s+\omega_{M}{ }^{2}}
$$

where $\omega_{M}=0.35, \xi_{M}=2$. The bode diagram of $M_{i} s$ is shown in Figure 7.2.

Low-pass filter. The following low-pass filter was selected:

$$
\begin{gathered}
C s=\begin{array}{cc}
C_{1}(s) & 0 \\
0 & C_{2}(s) \\
C_{1} s=C_{2} s= & \frac{\omega_{C}{ }^{2}}{s^{2}+2 \xi_{C} \omega_{C} s+\omega_{C}{ }^{2}}
\end{array}
\end{gathered}
$$

where $\omega_{M}=6, \xi_{M}=1.6$. The bode diagram of $C_{i} s$ is shown in Figure 7.3.

Sample time. The sampling time was set to

$$
T=0.0005
$$

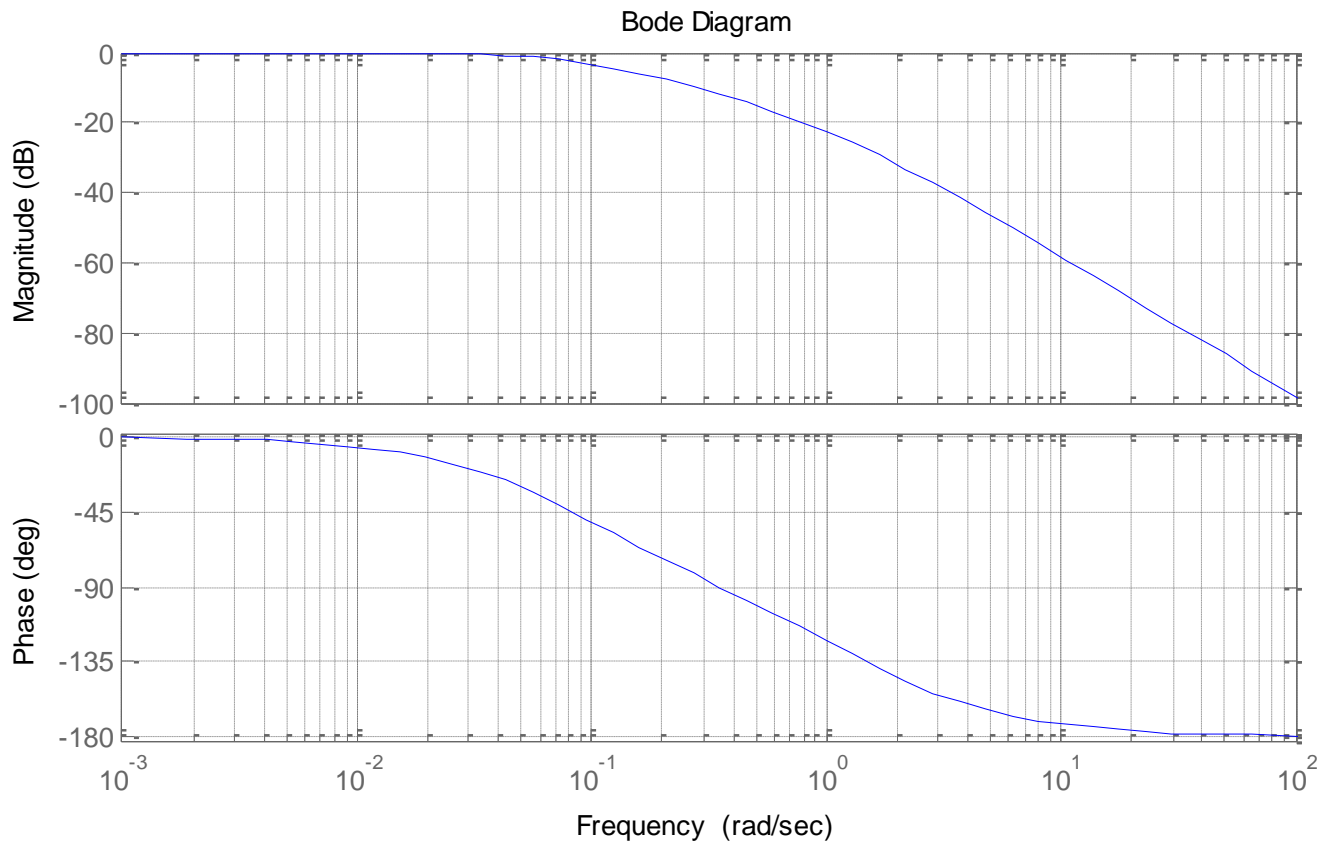

Figure 7.2: Desired system frequency response 


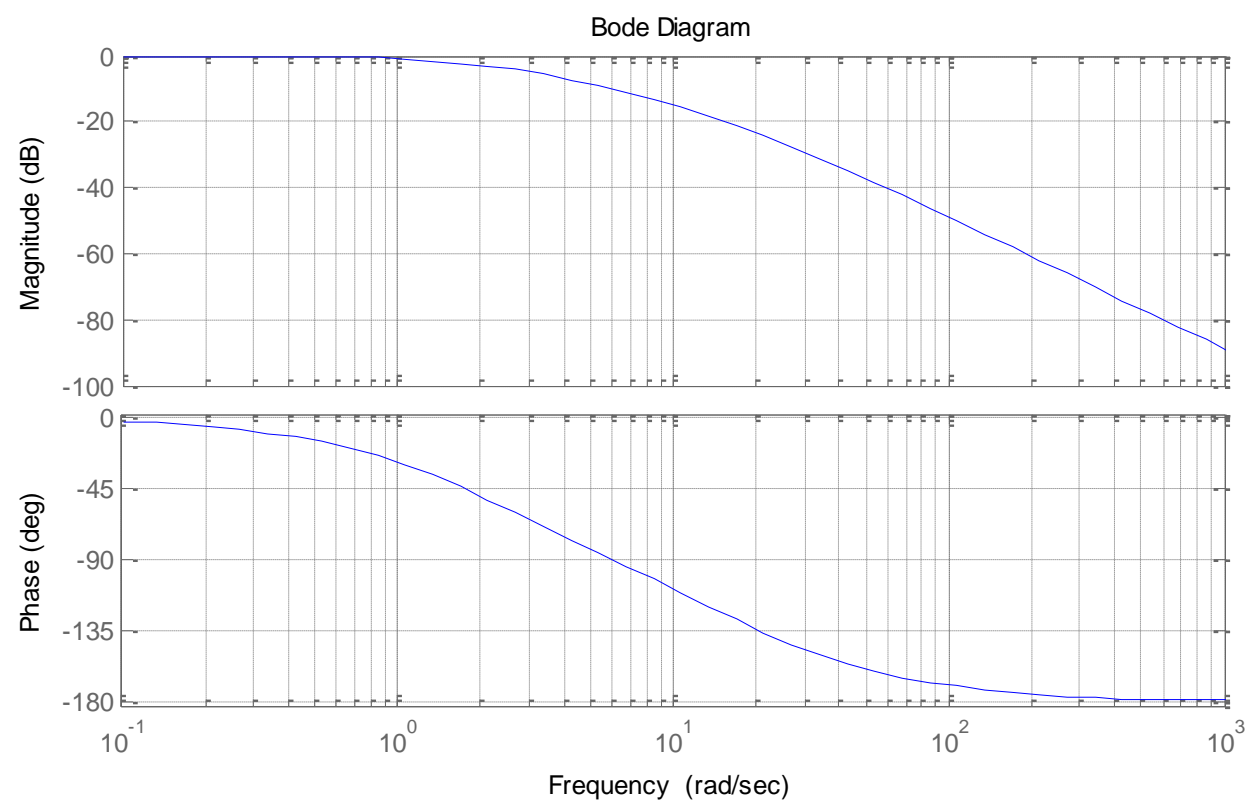

Figure 7.3: Low-pass filter frequency response

\subsection{Simulation Results}

Full nonlinear simulation for the closed loop system was run to evaluate the performance of the L1 adaptive controller defined via (7.5), (7.6), and (7.7). The simulated scenario was similar with what we did before with the robust controller: First run the nuclear power plant model with the L1 adaptive controller for 50 seconds with full loads (both steam pressure load and output power load are 1) to achieve steady state. Then at $\mathrm{t}=50 \mathrm{~s}$, reduce the output power load from 1 to 0.95 .

The simulation results are shown in Figure 7.4 in the next page. Figure 7.4(a) shows the reference commands. Figure 7.4(b) show the plant outputs. It can be seen that the outputs reach steady state in 350 seconds, and the steady state error of the output power is $0.6 \%$, which is very small. Figure 7.4(c) shows the control efforts: steam flow and rod reactivity. Figure 7.4(d) shows the output estimations $y_{1}(t)$ and $y_{2}(t)$. The estimations converge to desired values as time goes on, and finally reach the accurate values: 1 and 0.95 at t=350s. Figure 7.4(d) shows the output errors $y_{1}(t)$ and $y_{2}(t)$. It can be seen that $y_{1}(t)$ converges to 0 , whereas $y_{2}(t)$ converges to $-3.731 \times 10^{-4}$, which in turn results in the steady state error of the actual plant output. Figure 7.4(f) shows the parameter estimation $\sigma(t)$. There are four parameters because the plant is a twoinput two-output system. It can be seen that two parameters converge to non-zero values and the other two parameters converge to 0 asymptotically. 


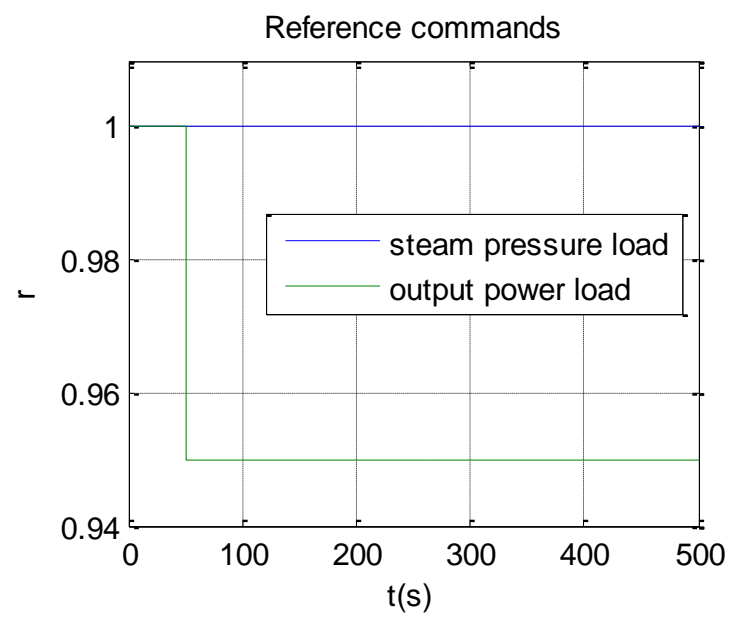

(a) Reference commands

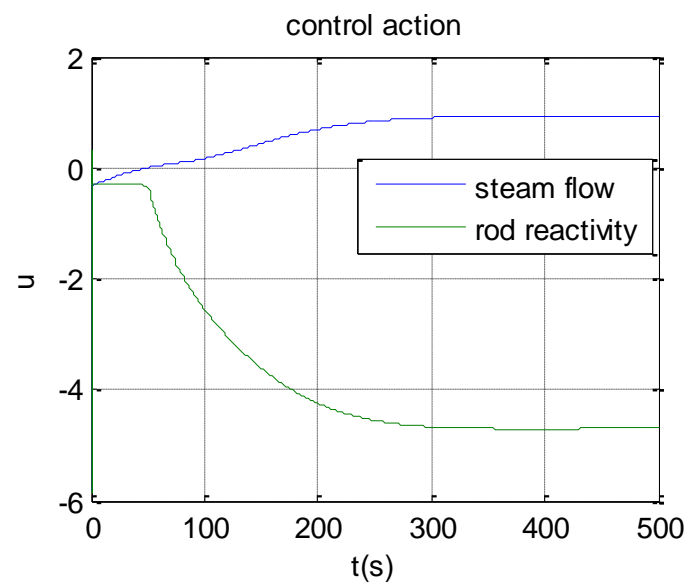

(c) Control efforts

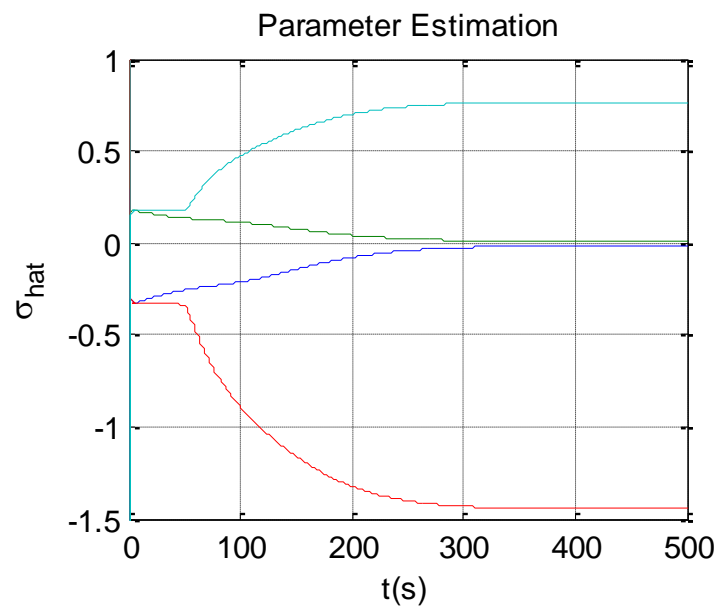

(e) Errors between actual output and estimation

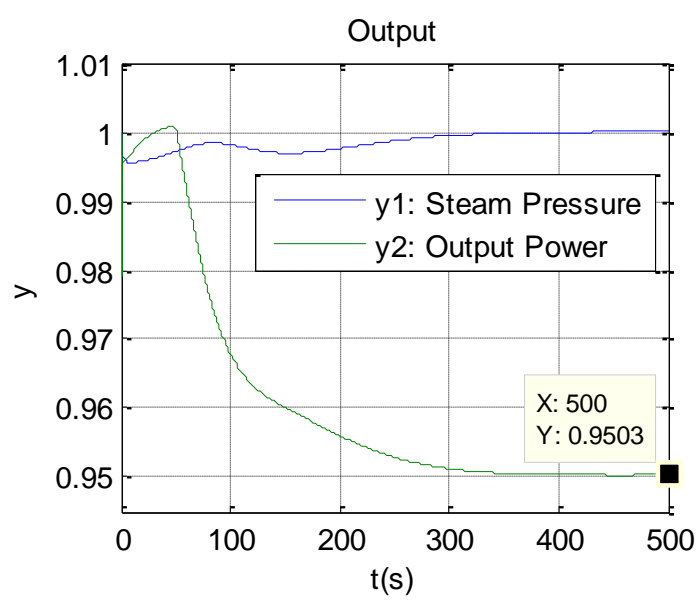

(b) Plant output

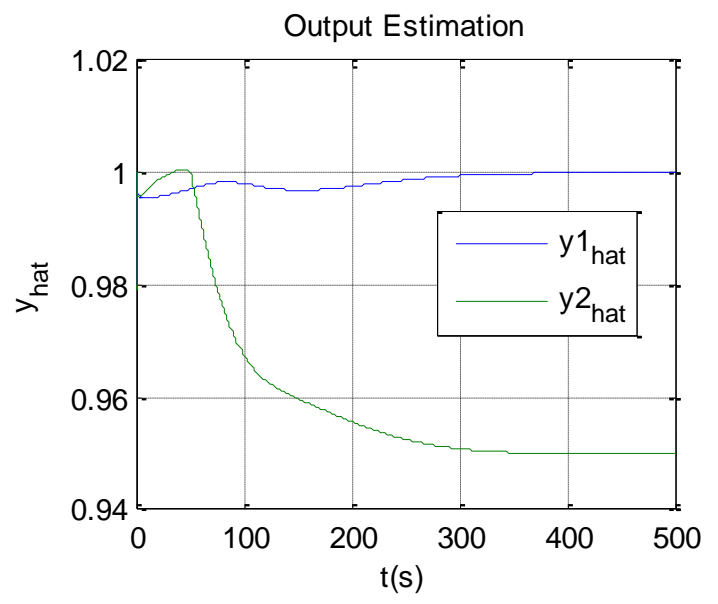

(d) Output Estimation

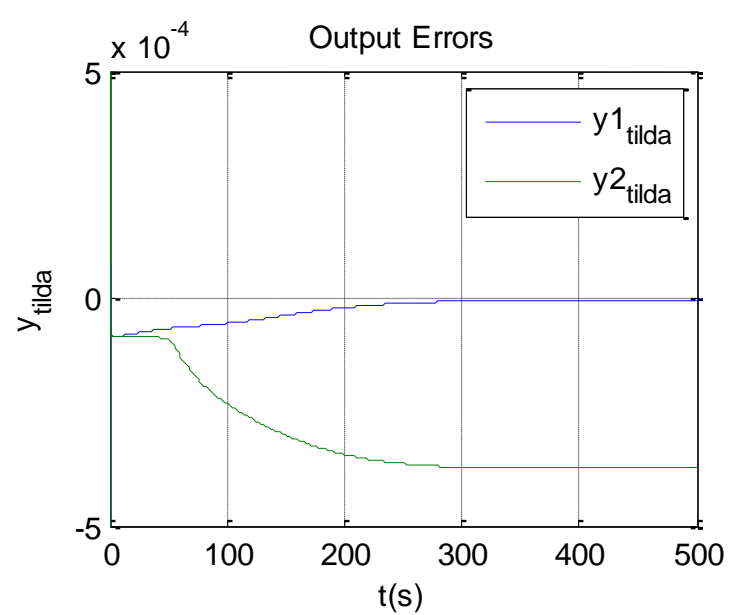

(f) Parameter estimation

Figure 7.4: Closed-loop system responses at 5\% step decrease of output power load demand, controlled by $\mathcal{L}_{1}$ adaptive controller 


\section{SENSOR CALIBRATION AND VALIDATION}

\subsection{Introduction}

Performance, reliability and safety of nuclear power plants depend upon validity and accuracy of sensor signals that measure plant conditions for information display, health monitoring and control [50]. Validity of measurements is important because a sensor failure can have serious consequences. Faulty and conflicting sensor readings may often degrade the performance of the control system, confuse operators, and lead to actions that may compromise the safety of an NPP. Thus, it is essential to regularly ensure correct operation of sensors, in particular for those having great importance for operating safety, to locate and identify any possible degradations and faults. Automation of the sensor validation process will be an important advancement for increased safety and higher autonomy in control functions of an NPP $[5]$.

However, periodic maintenance strategies cause the unnecessary calibration of instruments that are operating correctly which can result in premature aging, damaged equipment, plant downtime, and improper calibration under non-service conditions. Recent studies have shown that less than $5 \%$ of process instrumentation being manually calibrated requires any correction at all. Therefore, plants are interested in monitoring sensor performance during operation and only manually calibrating the sensors that require correction [51].

Redundant sensors are often installed to generate spatially averaged time-dependent estimates of critical variables so that reliable monitoring and control of the plant are assured. For example, temperature, pressure, and flow sensors are installed with redundancy in nuclear power plants. Redundancy can be classified into two groups: direct redundant data and analytical redundant data (using mathematical models of the physical system). In practice, analytical measurements may be the only source of supplemental redundancy for detection of plant component and sensor failures.

This section presents a calibration and estimation filter using adaptive recursive algorithm for redundancy management of sensor data and analytical measurements. The filter is 
validated based on redundant sensor data of primary coolant temperature collected from simulator of IRIS NPP.

The online sensor calibration problem has been investigated by many researchers. An adaptive back-propagation network for signal validation method is introduced in [51]. This work has been extended by Upadhyaya and Eryurek [52] to include additional features for on-line operations. A different approach that utilizes computer-aided techniques to automate the sensor validation process is suggested by Ning and Chou [53] for sensor validation of NPPs. The process empirical modeling (PEM) technique is proposed by Holbert and Upadhyaya [54] for the purpose of signal validation. For signal validation, Fantoni and Mazzola [55] suggest a pattern recognition-ANN-based system that is capable of identifying abnormal condition. The major accomplishment of this work is that the system can validate process signals in case of multiplefailure scenarios, both in steady-state and transient conditions. The signal validation process is accomplished using multilayered supervised ANN with a back-propagation algorithm. A sensor validation system proposed by Heger et al. [56] is based on a fuzzy logic approach and includes the functions such as instantaneous signal validation and measurement of long-term sensor status when two or three redundant sensors are used. A reliable and effective sensor detector proposed by $\mathrm{Na}$ et al. [57] automatically optimizes the number of required input signals and hence reduces the neural network training time. The technique utilizes a fuzzy neural network with an optimal structure constructor. Hines et al. [58] reviewed the on-line monitoring techniques for performance assessment. Dorr et al. [59] explored fault-detection methods using direct and analytical redundancy.

The most critical process temperatures in nuclear power plants are measured using resistant temperature detectors (RTD) and thermocouples. Due to inherent deficiency or aging, temperature sensors can suffer from large calibration shifts, erratic and noisy output, responsetime degradation and saturated output [60].

Individual measurements in a redundant set may often exhibit deviations from each other after a length of time. These differences could be caused by slowly time-varying sensor parameters, plant parameters, transport delays, etc. Consequently, some of the redundant measurements could be deleted by a fault detection and isolation (FDI) algorithm if they are not 
periodically calibrated. On the other hand, failure to isolate a degraded measurement could cause an inaccurate estimate of the measured variable [50]. This problem can be resolved by adaptively filtering the set of redundant measurements as follows: (1) Simultaneously calibrate all measurements online to compensate for their errors; (2) Adaptively update the weights of individual online, based on their respective a posteriori probabilities of failure instead of being fixed a priori.

In the event of an abrupt disruption of a redundant measurement in excess of the allowable bounds, the respective measurement is isolated by the FDI logic, and only the remaining measurements are calibrated to provide an unbiased estimate of the measured variable. On the other hand, if a gradual degradation occurs, the faulty measurement is not immediately isolated by the FDI logic. Instead, the relative weight of the degraded measurement is gradually decreased. Since the weight of a gradually degrading measurement is smoothly reduced, the eventual isolation of the fault would not cause any abrupt change in the estimate [51].

\subsection{Sensor Calibration and Measurement Estimation}

The proposed adaptive recursive algorithm and filter design procedures have been reported in details in a previous paper [50], so only the new design parameters are presented in this chapter.

- The standard deviations of the a priori Gaussian density functions of the four temperature sensors are:

${ }_{1} \sigma=0.6^{\circ} \mathrm{F} \quad 0.33^{\circ} \mathrm{C},{ }_{2} \sigma=0.4^{\circ} \mathrm{F} \quad 0.22^{\circ} \mathrm{C},{ }_{3} \sigma=0.3^{\circ} \mathrm{F} \quad 0.17^{\circ} \mathrm{C},{ }_{4} \sigma=0.4^{\circ} \mathrm{F} \quad 0.22^{\circ} \mathrm{C}$

The initial condition for the measurement noise covariance matrix is set as: $R_{0}=\operatorname{diag}\left[{ }_{j} \sigma\right]$.

The failure threshold parameters are selected as: ${ }_{j} \theta=\frac{{ }_{j} \sigma}{2}$ for $j=1,2,3,4$.

- The probability of intra-sampling failure is assumed to be identical for all four sensors as they are similar in construction and operate under identical environment. The life of the resistance thermometer sensor is assumed to be 2000 days. For a sampling interval of $1 \mathrm{~min}$, this information leads to

$$
{ }_{j} p \approx 3.47 \times 10^{-5} \text { for } \mathrm{j}=1,2,3,4
$$


- The probability of false alarms is selected to have a false alarm after approximately 2000 days of continuous operation. For a sampling interval of $1 \mathrm{~min}$, this information leads to

$$
{ }_{j} \alpha \approx 3.47 \times 10^{-5} \text { for } \mathrm{j}=1,2,3,4
$$

- To allow restoration of a degraded sensor following its recovery, the minimum weight is set as

$$
{ }_{j} w_{\text {min }} \approx 10^{-3} \text { for } \mathrm{j}=1,2,3,4
$$

After conversion of the four temperature sensor data into engineering units, the data into engineering units, the scale factor matrix becomes: $H=\left[\begin{array}{llll}1 & 1 & 1 & 1\end{array}\right]^{T}$. Consequently, the parity space projection matrix becomes:

$$
\begin{aligned}
& \frac{-}{\frac{3}{4}}-\frac{\overline{1}}{12}-\frac{\overline{1}}{2}-\frac{\overline{1}}{12} \\
& V=\begin{array}{lll}
- & \frac{-}{2} & -\frac{1}{3}
\end{array}-\frac{1}{6} \\
& \begin{array}{lll}
0 & 0 & \frac{1}{2}
\end{array}-\frac{\overline{1}}{12}
\end{aligned}
$$

In the event of a sensor being isolated as faulty, sensor redundancy reduces to 3 , for which $H=\left[\begin{array}{lll}1 & 1 & 1\end{array}\right]^{T}$ and

$$
V=\begin{array}{rrr}
\frac{-}{3} & -\frac{1}{6}-\frac{1}{6} \\
0 & \frac{1}{2}-\frac{1}{2}
\end{array}
$$

The ratio, $R_{k}^{-1 / 2} Q R_{k}^{-1 / 2}$, of covariance matrices $Q$ and $R_{k}$ largely determines the characteristics of the minimum variance filter. The filter gain $\Gamma_{k}$ increases with a larger ratio $R_{k}^{-1 / 2} Q R_{k}^{-1 / 2}$ and vice versa. Since the initial steady-state value $R_{0}$ is specified and $R_{k}^{r e a l}$ is recursively generated thereon to calculate $R_{k}$, the choice is left only for selection of $Q$. As a prior information on $Q$ may not be available, its choice relative to $R_{0}$ is a design feature. In this application, we have set $Q=R_{0}$. 


\subsection{Filter Performance based on IRIS Data}

In this section, the results of redundant sensor calibration and estimation for monitoring and control of NPPs are presented. The set of redundant measurements are temperature sensors. The filter simultaneously calibrates the sensors to generate a time-dependent estimate of the temperature difference that is spatially averaged. The simulated scenario is drift error and recovery in a single sensor.

The data were collected from the IRIS simulator over a period of $2.5 \mathrm{~h}$ at the sampling frequency of once every $1 \mathrm{~min}$. The nuclear power plant was under various output power load demand changes within the period. To simulate the sensor degradation, starting at $0.3 \mathrm{~h}$, a drift error was injected into the data stream of Sensor \#1 in the form of additive ramp at the rate of $10^{\circ} \mathrm{F} / \mathrm{h}$. The injected fault was brought to zero at $1.1 \mathrm{~h}$ signifying that the faulty amplifier in the sensor hardware was corrected and reset.

Simulation results in the Figures 8.1 - 8.6 exhibit how the calibration filter responds to a gradual drift in one of the four sensors whereas the remaining three are normally functioning. Figure 8.1 shows the response of the four uncalibrated sensors as well as the estimate generated by simple averaging (i.e., fixed identical weights) of these four sensor readings at each sample. The sensor data profile includes transients lasting from 0.3 to $1.1 \mathrm{~h}$. From time 0 to $0.3 \mathrm{~h}$ when no fault is injected, all sensor readings estimate, shown by a thick solid line, is in close agreement with all four sensors during the period $0 \sim 0.3 \mathrm{~h}$. Sensor \#1, shown by the dotted line, starts drifting at $0.3 \mathrm{~h}$ whereas the remaining sensors stay healthy. Consequently, the uncalibrated estimate starts drifting at one quarter of the drift rate of Sensor \#1 because of equal weighting of all sensors in the absence of the calibration filter. Upon termination of the drift fault at $1.1 \mathrm{~h}$, when Sensor\#1 is brought back to the normal state, the uncalibrated estimate resumes its normal state close to all four sensors for the remaining period from 1.1 to $2.5 \mathrm{~h}$.

Figure 8.2 shows the response of the four calibrated sensors as well as the estimate generated by weighted averaging (i.e., varying non-identical weights) of these four sensor readings at each sample. The calibrated estimate in Fig. 8.2 stays with the remaining three healthy sensors even though Sensor\#1 is gradually drifting. Fig. 8.6 shows that, after the fault injection, Sensor\#1 is weighted less than the remaining sensors. This is due to the fact that the 
residual of Sensor\#1 in Fig. 8.3 increases in magnitude with the drift error. As seen in Fig. 8.6, the weight of Sensor\#1 initially changes very slowly to ensure that it is not sensitive to small fluctuations in sensor data due to spurious noise such as those resulting from thermal-hydraulic turbulence. The significant reduction in weight of Sensor\#1 takes place after about $0.7 \mathrm{~h}$ and eventually reaches the minimum value of $10^{-3}$ when the drift error is sufficiently large. Therefore, the calibrated estimate is practically unaffected by the drifting sensor and stays close to the remaining three healthy sensors. In essence, the calibrated estimate is the average of the three healthy sensors. Upon restoration of Sensor\#1 to the normal state, the calibrated signal temporarily goes down because of the large value of correction at that instant as seen in Fig. 8.5. However, the adaptive filter quickly brings it back to a small value and thereby the residual is reduced and the original weight (i.e., $\sim 1$ ) is regained. Calibrated and uncalibrated estimates are compared in Fig. 8.4 that shows a peak difference of about $1.45^{\circ} \mathrm{F}$ over a prolonged period.

In addition to the accuracy of the calibrated estimate, the filter provides fast and smooth recovery from abnormal conditions under both steady state and transient operations of the power plant. Another important feature of the calibration filter is that it reduces the deviation of the drifting Sensor\#1 from the remaining sensors as seen from a comparison of its responses in Fig. 8.1 and Fig. 8.2. This is very important from the perspectives of fault detection and isolation for the following reason. In an uncalibrated system, Sensor\#1 might have been isolated as faulty due to accumulation of the drift error. In contrast, the calibrated system makes Sensor\#1 temporarily ineffective without eliminating it as faulty. A warning signal can be easily generated when the weight of Sensor\#1 diminishes to a small value. This action will draw the attention of maintenance personnel for possible repair or adjustment. Since the estimate is not poisoned by the degraded sensor, a larger detection delay can be tolerated. Consequently, the allowable threshold for fault detection can be safely increased to reduce the probability of false alarms. 


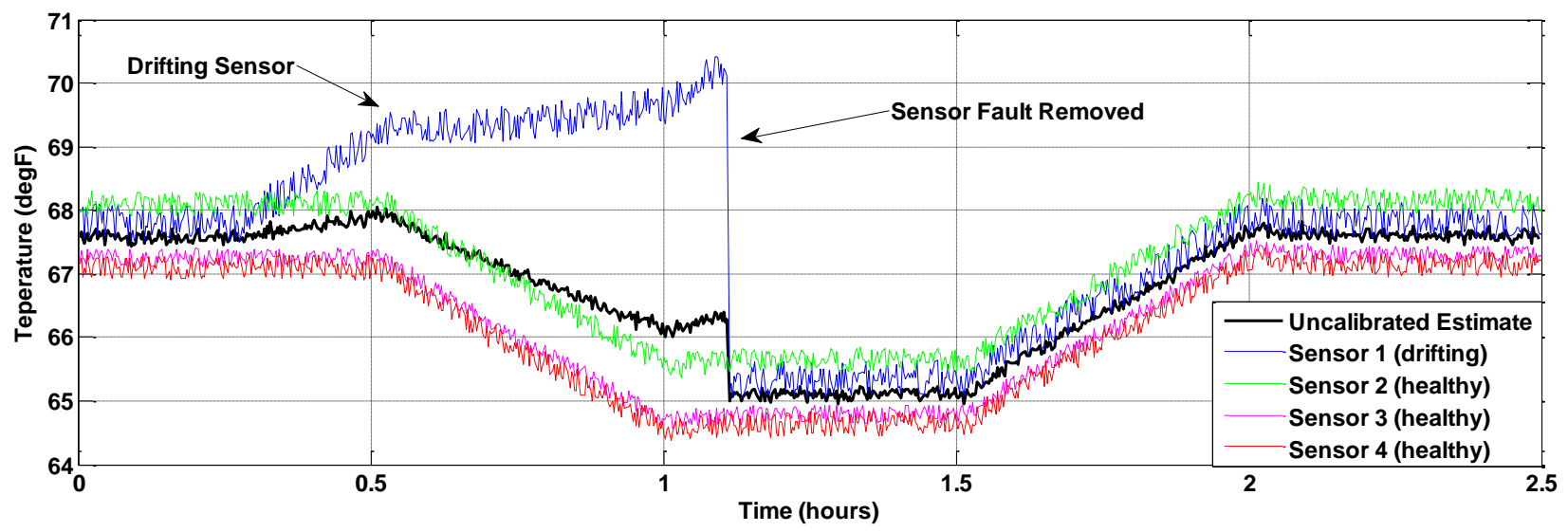

Figure 8.1: Uncalibrated Sensor Data and the Estimate

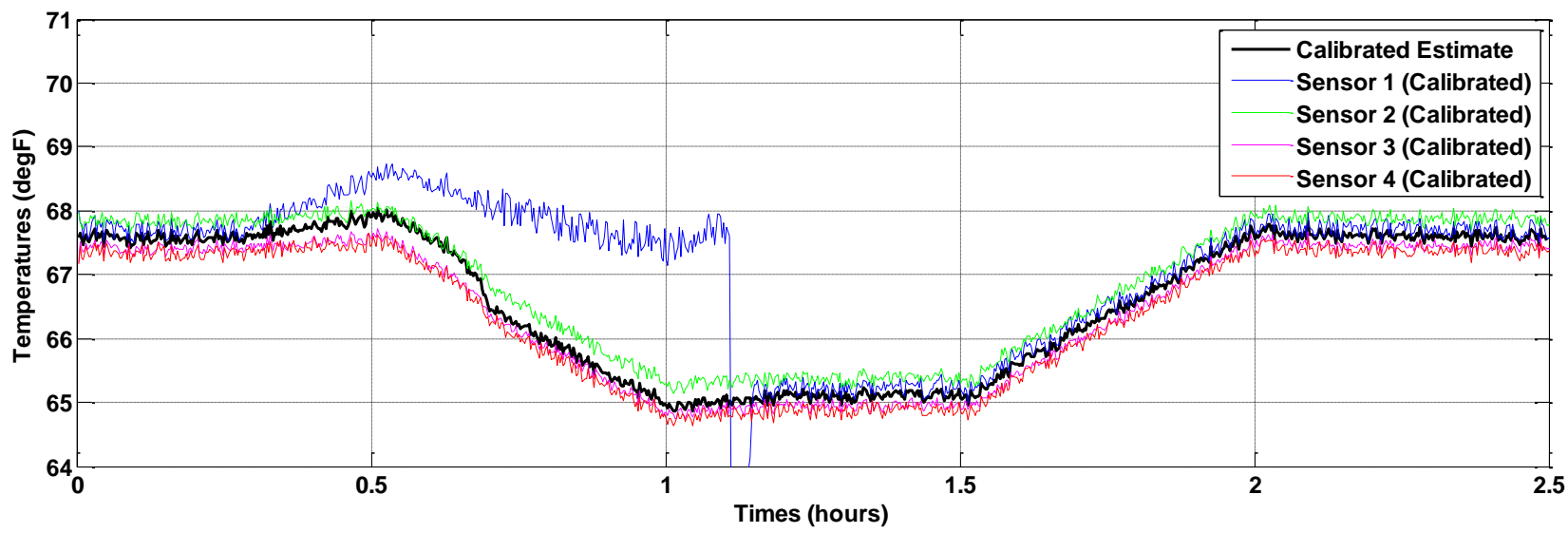

Figure 8.2: Calibrated Sensor Data and the Estimate

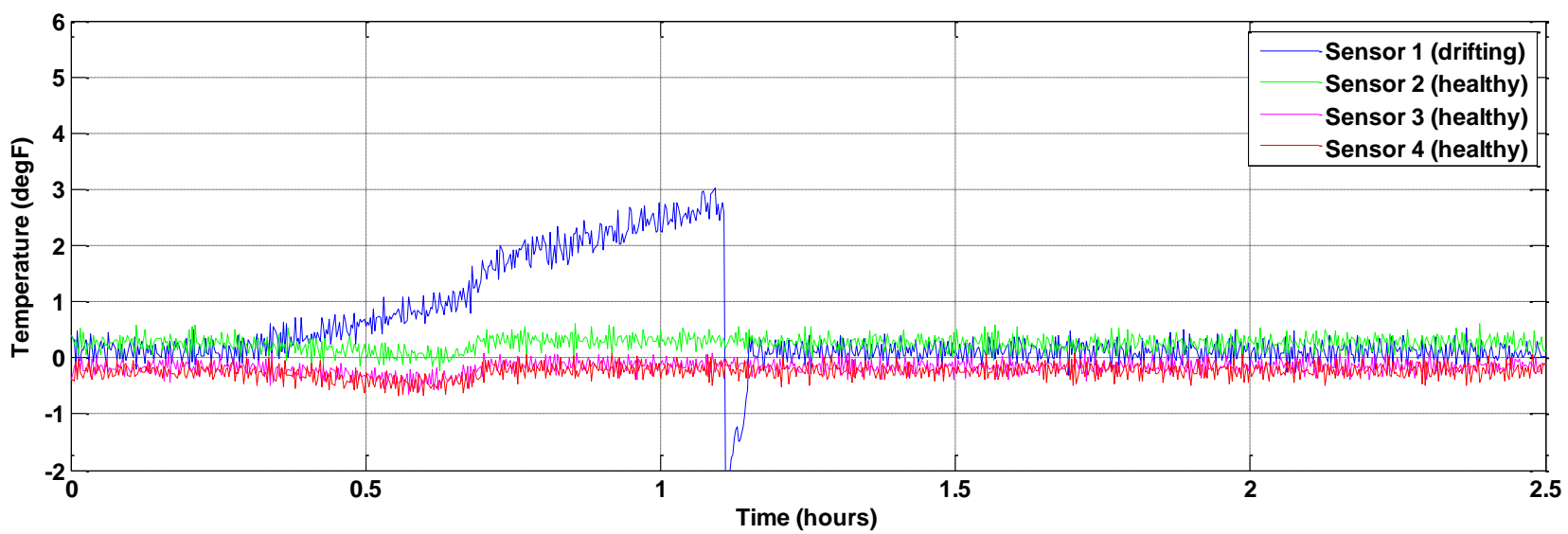

Figure 8.3: Residual of the Calibrated Sensor Data 


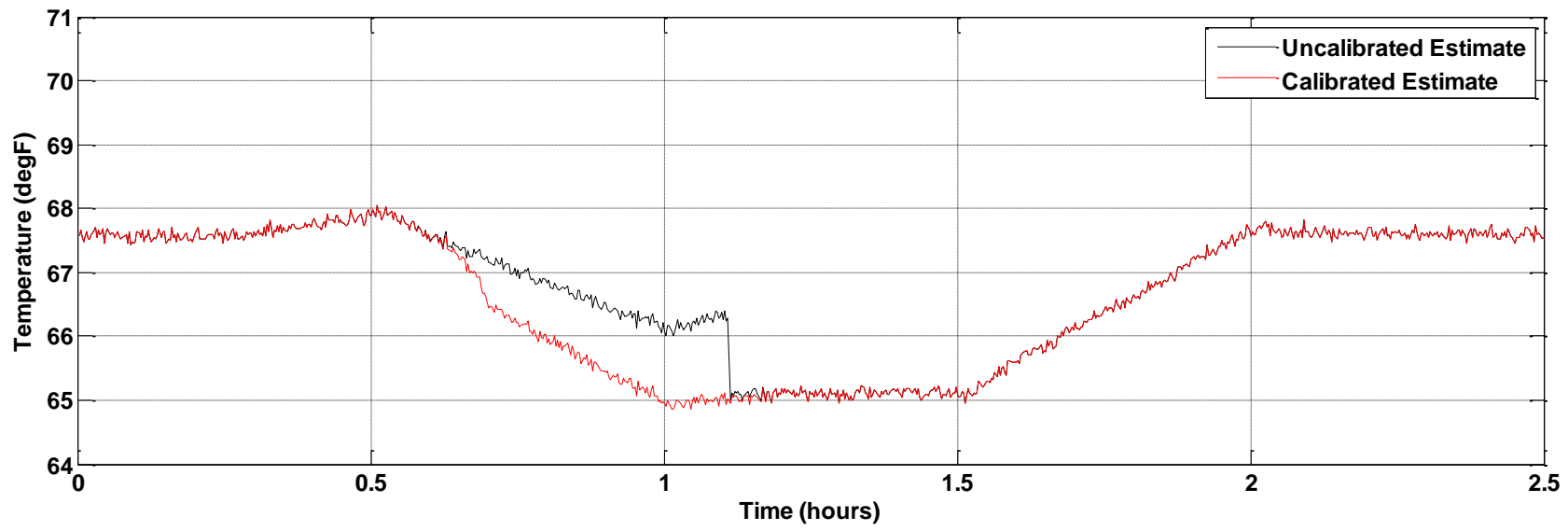

Figure 8.4: Calibrated and Uncalibrated Estimates

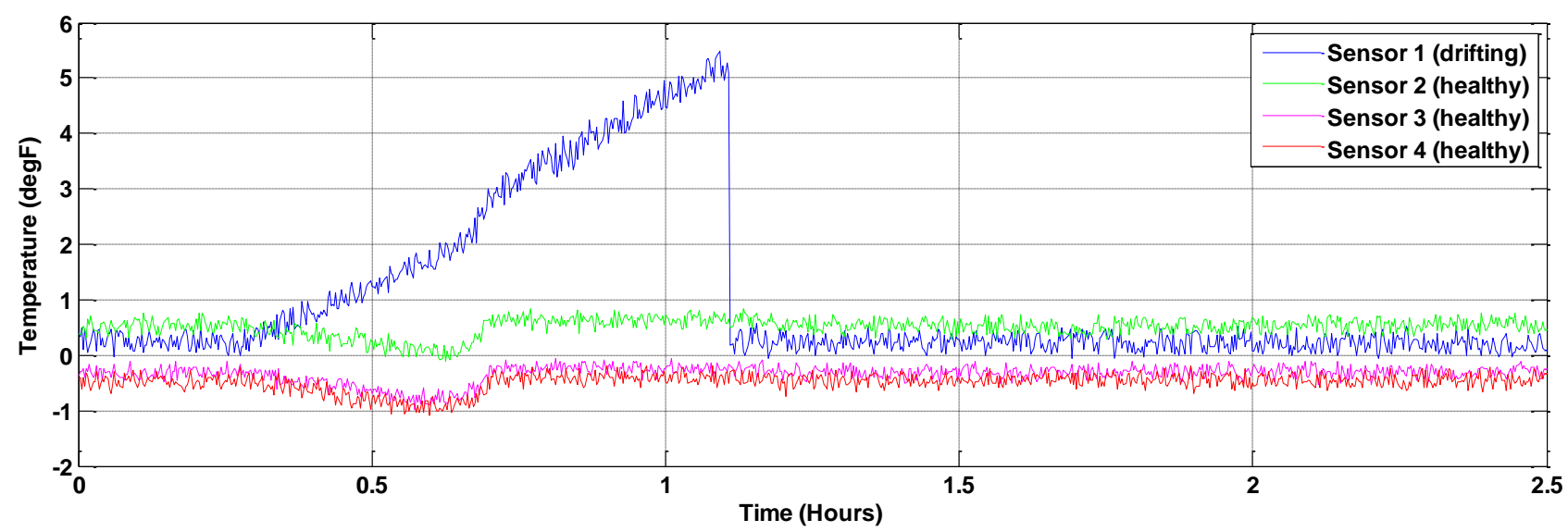

Figure 8.5: Correction for Sensor Data Calibration

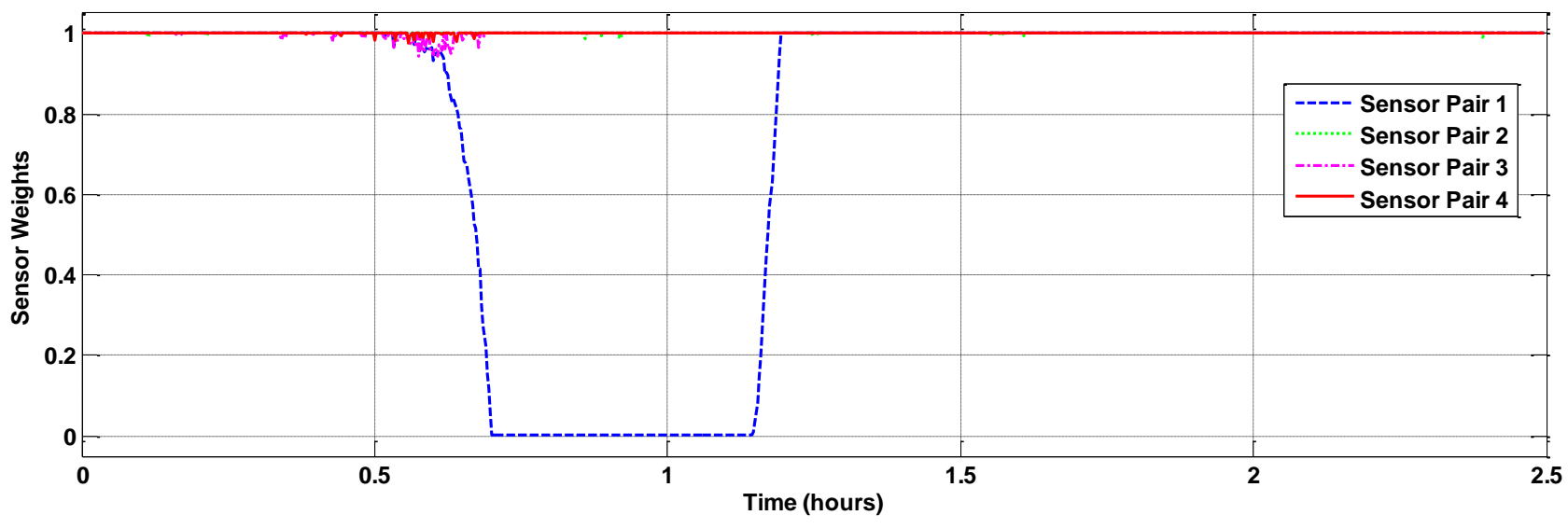

Figure 8.6: Weights for Sensor Calibration 


\section{RELIABLE LQG CONTROLLER DESIGN WITH SENSOR FAILURE}

\subsection{Introduction}

Development and deployment of small and medium-size reactors and their autonomous control and monitoring is part of the objectives for next generation nuclear power plants. Performance, reliability and safety of nuclear power plants depend upon valid and accurate sensor signals that measure plant condition for information display, health monitoring and control [50]. Measurable state variables and output variables such as pressure, power and temperature which are inputs of a controller are measured by different kinds of sensors. Because of rigorous surrounding environment in nuclear reactor core and the inherent deficiency, aging, sensors used in nuclear power plant can suffer from large calibration shifts, erratic and noisy output, response-time degradation and saturated output [60]. Due to the high requirement for safety, redundant sensors are installed in nuclear power plant to make sure reliable monitoring and stability in emergency condition. Spatially averaged time-dependent estimates are used for some parameters and furthermore fault detection and isolation (FDI) mechanism will delete some measurements given by sensors whose measurements are over the allowable bounds. However, if the sensors just drift in the allowable range or the FDI mechanism fails due to some other reasons, the control system should guarantee the stability of system. The reliable control is an effective approach to improve system reliability. A reliable controller is a controller with suitable structure to guarantee stability and satisfactory performance, not only when all control components are in operation, but also in the case when sensors, actuators malfunction [62].

Reliable control for linear and nonlinear systems has been investigated by many researchers during the past two decades and corresponding calculation algorithms have been developed [63-68]. R. J. Veillette [63] presents a methodology for the design of modified linearquadratic (LQ) state feedback controls that can tolerate actuator outages, and presents the sufficient and necessary condition for the existence of such controller. Some LMI approaches are presented to design reliable controller. Li [64] presents an LMI algorithm to design reliable guaranteed cost controller for discrete-time systems with actuator failure. D. Zhang [65] et al. consider a more general condition, investigating the problem of reliable guaranteed cost control with multiple criteria constraints for a class of uncertain discrete-time systems subject to actuator faults. Reliable control for nonlinear system has also been proposed. A Hamilton-Jacobi 
inequality (HJI) approach is employed by [66][67]. Yang et al. [66] present a reliable $H_{\infty}$ controller design for nonlinear system with sensor and/or actuator faults. Liang et al. [67] study the reliable linear quadratic state feedback control problem for nonlinear system with actuator faults which extend Veillette's work on [63]. To avoid solving HJI, Huai-Ning Wu [62] proposes a reliable LQ fuzzy control design which can achieve the LQ performance for continuous-time nonlinear systems with actuator faults.

In this section, a methodology of reliable LQG controller design with direct output feedback of nuclear power plant model using the IRIS SIMULINK model developed by Penn State University is presented. Compared with state feedback LQG controller, the reliable LQG controller proposed by Yang et al. [68] uses output feedback directly. The high order state estimator for complex nonlinear system is not needed. This is an advantage of reliable LQG controller, since for a complex system such as the IRIS, constructing a state estimator may cause estimation error in the closed loop system and thus enhance the complexity of the system.

\subsection{System Synthesis}

\subsubsection{Model Linearization}

For a reliable LQG control synthesis, a linear model with low order is desired to design a low order controller. In this paper, a linear model is first attained by using the control system toolbox of MATLAB, and then the minimal realization of the linear model is attained by removing uncontrollable and/or unobservable states. The minimal realized linear model is used to design the controller. Here, the order of the linear system is reduced from 28 to 22 with allowable $H_{\infty}$ error.

Sensor failure model which covers the cases of partial degradation and outage is considered in reliable LQG controller design. By solving algebraic Riccati equation and linear matrix inequality, reliable LQG control method yields a state space model controller which guarantees the whole system is stable when the drift and noisy output which sensors suffer from are within allowable bound specified by designer. When the sensor errors go beyond the specified bound, corrected or estimated sensor signal which is within the allowable bound is needed for the LQG controller to maintain the closed loop system be stable. Figure 12 shows the structure of the closed loop system. 


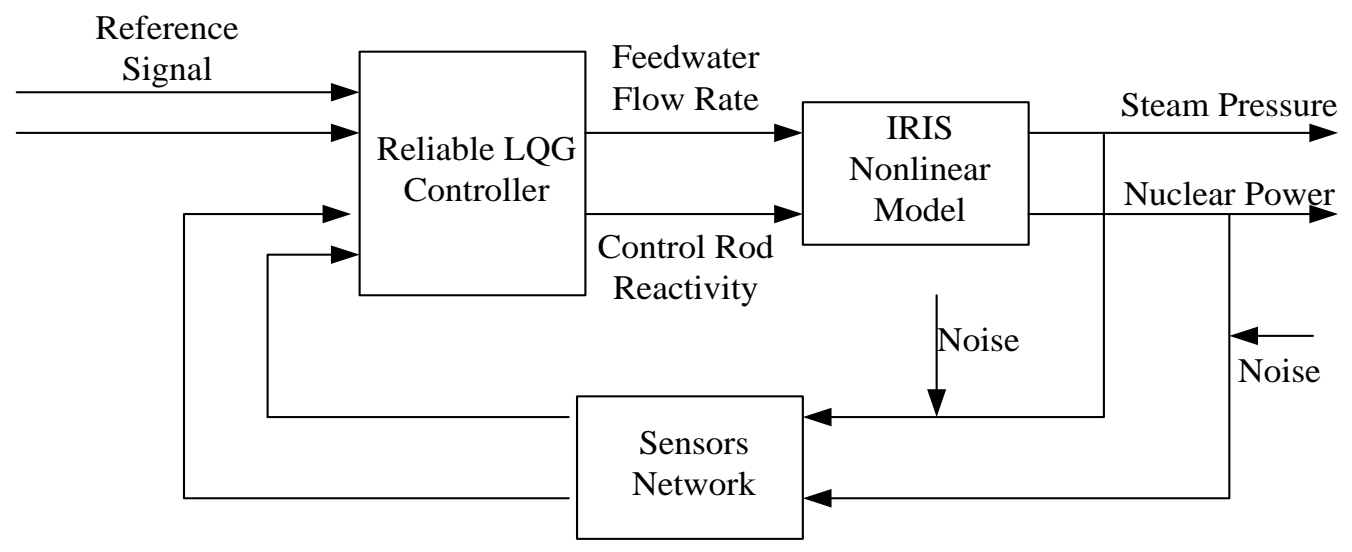

Figure 9.1: Closed loop system sketch.

\subsubsection{Controller Design}

The mathematical description of the IRIS system using linear state space model is presented as follows:

\section{Linear model $\sum$ :}

$$
\begin{aligned}
\Sigma: \dot{x}(t) & =A x(t)+B u(t)+D_{1} \omega(t) \\
y(t) & =C x(t)+D_{2} v(t)
\end{aligned}
$$

Where $x(t) \in R^{n}$ is the state; $u(t) \in R^{m}$ is the control input; $y(t) \in R^{p}$ is the measured output; $\omega(t)$ and $v(t)$ are zero mean white Gaussian noise processes with identical covariance matrix. Furthermore, assume ${ }^{\omega(t)}$ and $v(t)$ is non-correlated, then

$$
\begin{aligned}
& E\left[\omega(t)^{T} \omega(t)\right]=V_{1} \geq 0 \\
& E\left[v(t) v^{T}(t)\right]=V_{2} \geq 0 \\
& E\left[\omega(t) v^{T}(t)\right]=0
\end{aligned}
$$

Associated with this system is the cost function

$$
J=\lim E\left\{\frac{1}{T} \int_{0}^{T}\left[x^{T}(t) R_{1} x(t)+u^{T}(t) R_{2} u(t)\right] d t\right\}
$$

Where $R_{1} \geq 0, R_{2}>0$, and $E\{\}$ denotes the expectation.

\section{Reliable LQG controller $C$ :}




$$
\begin{aligned}
C: \dot{\xi}(t) & =F \xi(t)+G y^{F}(t) \\
u(t) & =K \xi(t)
\end{aligned}
$$

Where $\xi(t) \in R^{n}$ is the controller state

$$
\begin{aligned}
& y^{F}=\operatorname{diag}\left(a_{1}, a_{2} \cdots, a_{m}\right) y=a y \\
& \underline{a}_{i} \leq a_{i} \leq \bar{a}_{i}
\end{aligned}
$$

Equation (9.5) models sensor failures. When $a_{i}=1$, the corresponding sensor work normally, when $a_{i} \neq 1$, the corresponding sensor does not work accurately. An allowable bound $\underline{a}_{i}, \bar{a}_{i}$ should be specified by designer. Applying the controller to system $\sum$ described by Equation 9.1, the closed loop system $C L$ is described by the following equation:

$C L$ :

$$
\left[\begin{array}{c}
\dot{x}(t) \\
\dot{\xi}(t)
\end{array}\right]=\left[\begin{array}{cc}
A & B K \\
G a C & F
\end{array}\right]\left[\begin{array}{l}
x(t) \\
\xi(t)
\end{array}\right]+\left[\begin{array}{c}
D_{1} \\
G a D_{2}
\end{array}\right]\left[\begin{array}{l}
\omega(t) \\
\omega(t)
\end{array}\right]
$$

Yang et al. [4] have proposed the following theorem to solve the reliable LQG controller and the closed loop system is stable when ${ }^{a}$ is in the specified allowable bound.

Theorem: Consider the system $\sum$ described by Equation 9.1. Suppose that

There exists a diagonal matrix $R_{0}>0$ such that the inequality $R_{0} \beta<V_{2}^{-1}$ holds

The following Riccati equation has a stable solution

$$
A Q+Q A^{T}+V_{1}-Q C^{T}\left[\left(I-\beta^{2}\right) V_{2}+R_{0}^{-1} \beta\right]^{-1}\left(I-\beta^{2}\right) C Q=0
$$

There exists symmetric matrices $X_{1}>0, X_{0}>0$ and $W$ such that the following inequalities hold:

$$
\begin{aligned}
& {\left[\begin{array}{cc}
A_{0} X_{1}+X_{1} A_{0}^{T}+B W+W^{T} B^{T}+B_{10} B_{10}^{T} & X_{1} E_{1 \infty}^{T} \\
E_{1 \infty} X_{1} & -I
\end{array}\right]<0}
\end{aligned}
$$

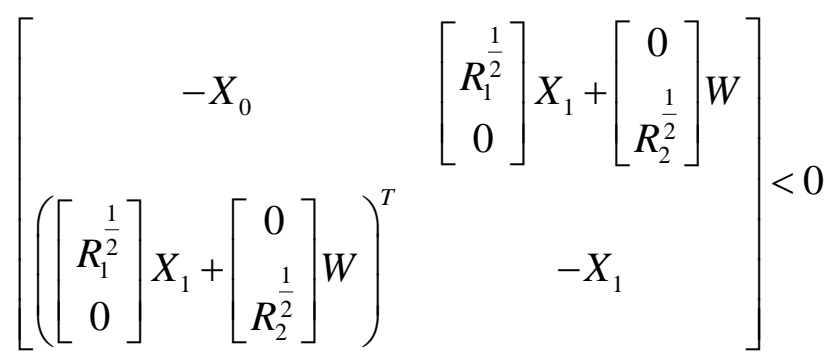

Where 


$$
A_{0}=A+Q E_{1 \infty}^{T} E_{1 \infty}, B_{10}=Q \bar{C}\left(V_{0}+\beta R_{0}^{-1}\right)^{-\frac{1}{2}}
$$

With

$$
\begin{aligned}
& \beta=\operatorname{diag}\left(\beta_{1}, \beta_{2} \cdots, \beta_{p}\right) \\
& \beta_{i}=\frac{\bar{a}_{i}-\underline{a}_{i}}{\bar{a}_{i}+\underline{a}_{i}} \\
& \bar{C}=\left(I+V_{0} \beta R_{0}\right) C \\
& V_{0}=\left(V_{2}^{-1}-\beta R_{0}\right)^{-1} \\
& E_{1 \infty}=\left(I-\beta^{\frac{1}{2}} R_{0}^{\frac{1}{2}} V_{2} \beta^{\frac{1}{2}} R_{0}^{\frac{1}{2}}\right)^{-\frac{1}{2}} \beta^{\frac{1}{2}} R_{0}^{\frac{1}{2}} C
\end{aligned}
$$

Then the corresponding reliable LQG controller is given by:

$$
\begin{aligned}
& F=A_{0}-G a_{0}\left(I-V_{2} \beta R_{0}\right)^{-1} C+B K \\
& K=W X_{1}^{-1} \\
& G=Q C^{T}\left[\left(I-\beta^{2}\right) V_{2}+R_{0}^{-1} \beta\right]^{-1} a_{0}
\end{aligned}
$$

With

$$
a_{0}=\operatorname{diag}\left(a_{01}, a_{02}, \cdots, a_{0 p}\right)
$$

The corresponding algorithm for the design given by this theorem is given as follows:

Step 1: Initialize $R_{0}$ satisfying $R_{0} \beta<V_{2}^{-1}$ and solve Equation (9.)7 for $Q$.

Step 2: Minimize $\operatorname{tr}\left(X_{0}\right)$ subject to LMI constraints.

Step 3: If step 2 is not feasible, then change $R_{0}$ and go to step 1 .

This algorithm can be performed effectively by using MATLAB.

The controller obtained by this algorithm is with the same order with the plant. To make the controller easy to realize, a reduced-order controller is desired. The same method which is used to reduce the plant model is used to reduce the order of controller.

\subsection{Simulation}

According to the theorem given above, a reliable LQG controller which can be tolerant of $\pm 50 \%$ sensors drift is designed. Applying the reliable LQG controller to the nonlinear system, the following running condition is simulated: 
Case I: at $\mathrm{t}=500 \mathrm{~s}$, nuclear power reference signal step from $100 \%$ nominal value to $90 \%$, at $\mathrm{t}=800 \mathrm{~s}$, nuclear power reference signal step from $90 \%$ to $100 \%$, at $\mathrm{t}=1000 \mathrm{~s}$, nuclear power sensor drift $20 \%$ with slope of $1 \% / \mathrm{sec}$, at $\mathrm{t}=1500 \mathrm{~s}$, sensor drift is detected by FDI mechanism and corrected for the drift. Figure 9.2 shows the dynamic response of the closed loop system and Figure 9.3 shows the two control effects: steam generator secondary side feedwater flow rate and control rod reactivity. In the normal case, the controller can make the output of the nonlinear system follow the reference signal with the steady state error less than $0.5 \%$. The response time of nuclear power is $1 \mathrm{sec}$. When nuclear power sensor cannot provide accurate signal, the reliable LQG controller can still maintain output-steam pressure reference signal with steady state error less than $1 \%$ and maintain the system stable.

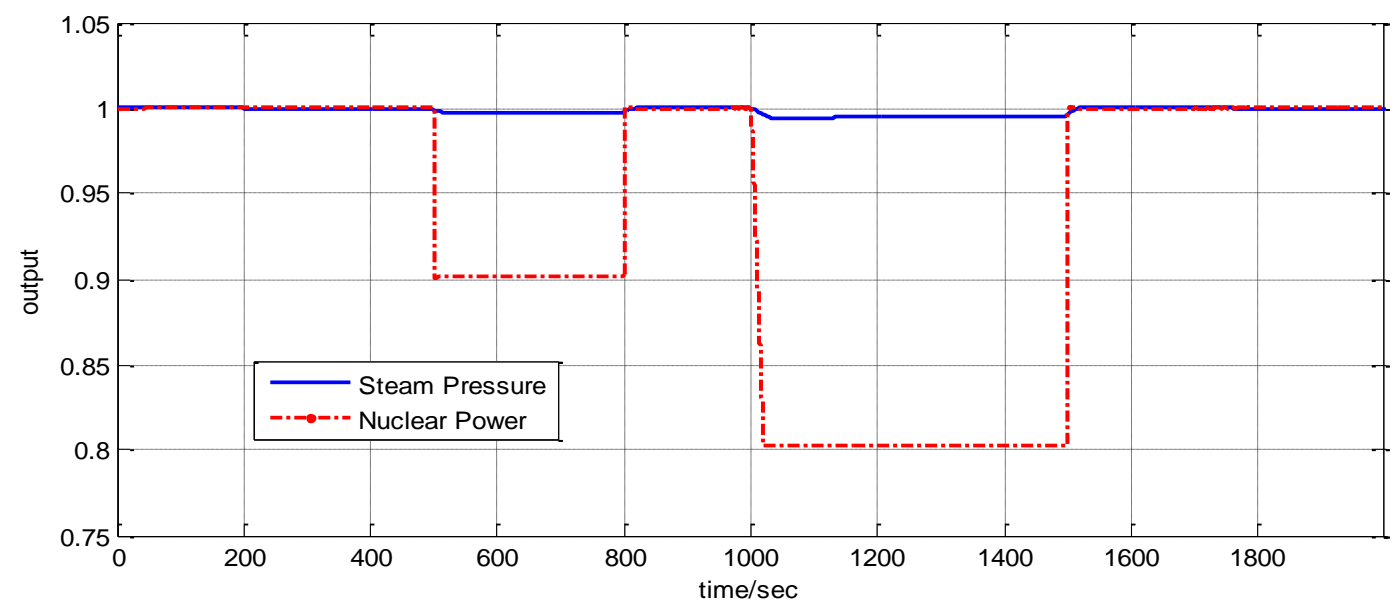

Figure 9.2: Dynamic response of Case I

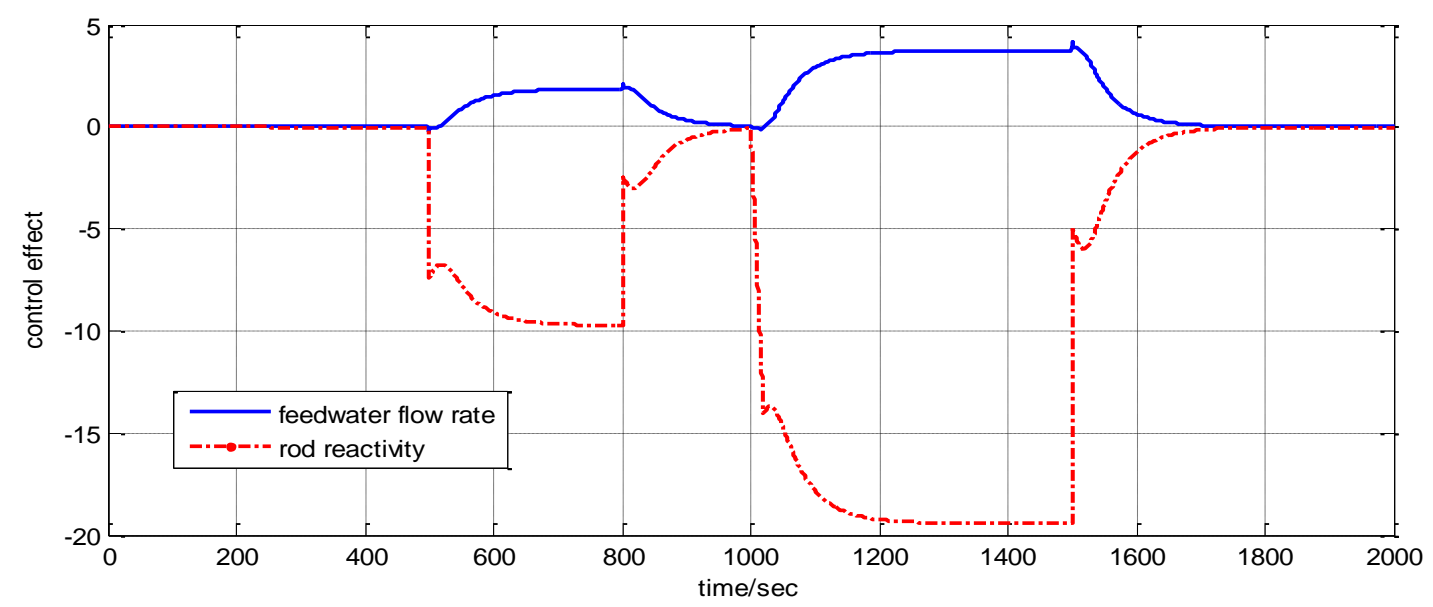

Figure 9.3: Control effects of Case I 
Case II: at $\mathrm{t}=500 \mathrm{~s}$, steam pressure reference signal step from $100 \%$ nominal value to $90 \%$, at $\mathrm{t}=1000 \mathrm{~s}$, steam pressure sensor drift $20 \%$ with slope of $-1 \% / \mathrm{sec}$, at $\mathrm{t}=1500 \mathrm{~s}$, sensor drift is detected by FDI mechanism and corrected for the drift. Figure 9.4 shows the dynamic response of the closed loop system and Figure 9.5 shows the two control effects. Just like case I, in normal operation, the controller can make the output of the nonlinear system follow the reference signal with the steady state error less than $0.5 \%$. The response time of steam pressure is $50 \mathrm{sec}$. When steam pressure sensor cannot provide accurate signal, the reliable LQG controller can still maintain output-nuclear power reference signal with steady state error less than $1 \%$ and maintain the system stable.

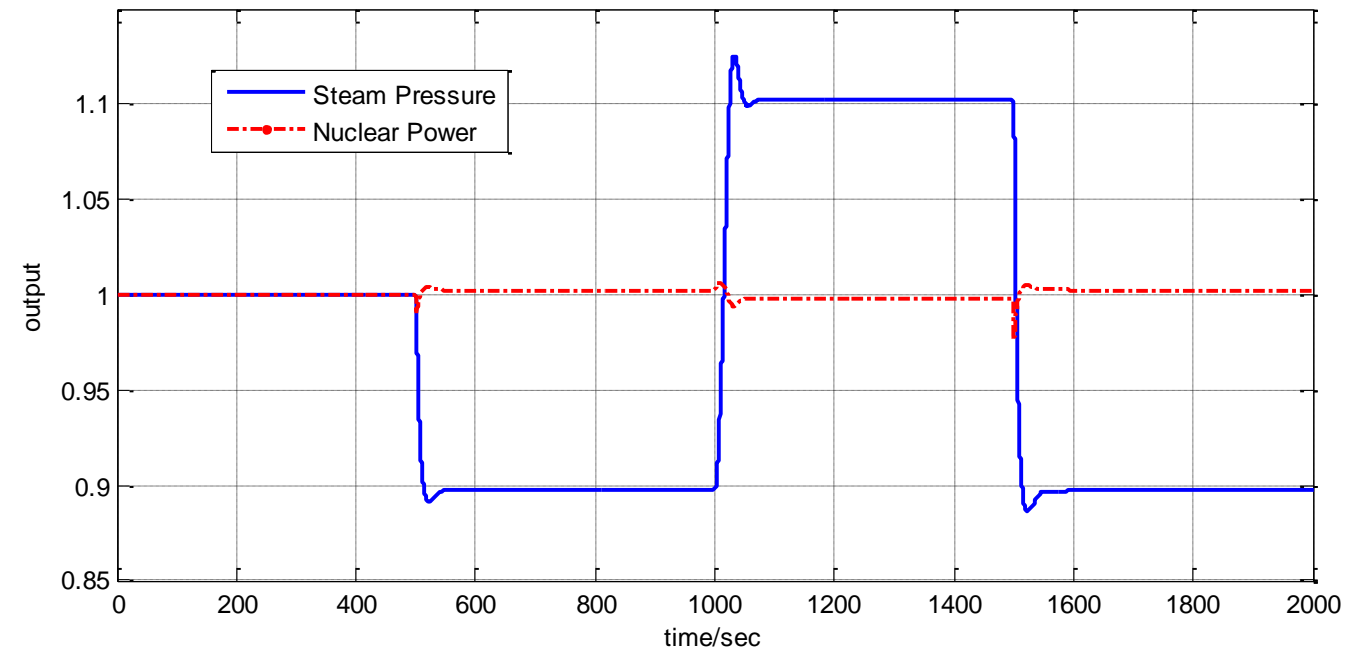

Figure 9.4: Dynamic response of Case II

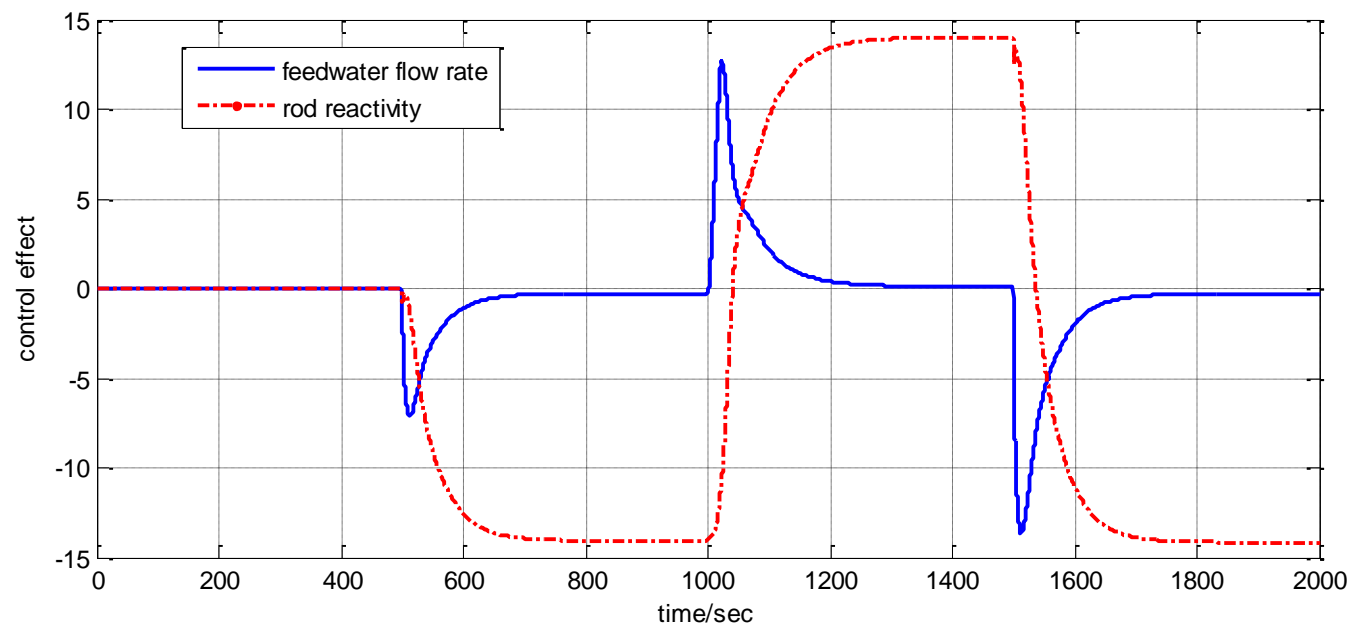

Figure 9.5: Control effects of Case II 


\subsection{Summary and Discussion}

A reliable LQG controller is obtained by solving ARE and LMI. Then, the order of controller is reduced to 9 by using Hankel norm approximation method. From the simulation result, the LQG controller can maintain the system stable when sensors drift in allowable range and perform well in tracking reference signal. However, compared with robust control, for example, $\mathrm{H}_{\infty}$ robust control, the reliable LQG does not perform well in the noise rejection. So, the future work will focus on enhancing the ability to reject noise of reliable LQG control. 


\section{DESIGN OF A SUPERVISORY CONTROLLER}

The supervisory control theory, also known as the Ramadge-Wonham framework, is a method for automatically synthesizing supervisors that restrict the behavior of a plant such that as much as possible of the given specifications are fulfilled. The plant is assumed to spontaneously generate events. The events are in either one of the following two categories: controllable or uncontrollable. The supervisor observes the string of events generated by the plant and might prevent the plant from generating a subset of the controllable events. However, the supervisor has no means of forcing the plant to generate an event [69].

A Discrete Event Supervisory (DES) System is a dynamic system that evolves in accordance with the abrupt occurrence, at possibly unknown irregular intervals, of physical events. For example, an event may correspond to the occurrence of a disturbance or change of set point in a complex control system. DESs arise in the domains of manufacturing, robotics, vehicular traffic, logistics (conveyance and storage of goods, organization and delivery of services), and computer and communication networks. These applications require control and coordination to ensure the orderly flow of events. As controlled (or potentially controllable) dynamic systems, DESs qualify as a proper subject for control theory, a view point that we shall develop in some details for NPPs.

\subsection{Introduction}

Although the theory of DES control has been developed for almost two decades [69], only few applications have been reported in the literature. An apparent reason is that, until recently, no quantitative analytical tool was available for design and evaluation of DES controllers. A quantitative measure of regular languages was reported in [70][71], and a novel application of hierarchical DES control synthesis for the nonlinear complex dynamical system of twin-engine aircraft propulsion was reported in [72].

Discrete-event dynamical behavior of physical plants is often modeled as regular languages that can be realized by finite-state automata. This research focuses on development of intelligent decision and control algorithms based on the theory of discrete event supervisory (DES) control for NPPs. The DES controller design has two important components that serve as 
interfaces between the continuously varying control system and the discrete-event supervisory controller-one is event generator and the other is action generator. Event generator receives continuously varying sensor data from the NPP. The data along with other information like estimated state and external inputs are used to generate events that, in turn, are inputs to the unsupervised deterministic finite state automaton (DFSA) model of NPP operation. The DFSA model is constructed based on the operation scenario. The DFSA model also serves as a state estimator and provides information on plant states and events for the discrete-event supervisor to take appropriate actions. Event behavior in the state-based DFSA model is dependent on the state where the event is generated and not on the history or the path of how the state is reached [72].

The DES control of the NPP aims to enhance operation in the following areas: (i) realtime decision-making, (ii) damage reduction in emergency operation via resilient control, and (iii) enhanced performance and reliability of the operation via robust control.

\subsection{Supervisory Control Architecture for Discrete-Event System}

This section presents a framework for implementation of DES control on a real-time simulation test bed of the IRIS nuclear power plant.

A DES controlled NPP system is proposed to be implemented on a simulation test bed that consists of two networked computers using the client/server concept. One of the two computers hosts the controller for health monitoring of the plant and making intelligent decisions (change of set points, activation of controllers). The other computer runs the IRIS NPP simulation model. The test bed is capable of simulating different scenarios of normal operation and abnormal operation. The NPP simulator integrates the event-driven discrete dynamics modeled by finite state automaton as well as time-driven continuous dynamics modeled by thermal-hydraulic equations through continuous-to-discrete and discrete-to-continuous interfaces. Figure 10.1 shows the supervisory control architecture of the NPP system. This software architecture is flexible to adapt deterministic finite state automaton (DFSA) models and controllers designs for other complex dynamical systems.

The IRIS NPP simulator is based on FORTRAN simulation code which is able to simulate the plant operations from a steady state to transients to (possibly) other steady states, as well as upset conditions. This simulator was originally provided by NCSU with built-in PID 
controllers. The built-in controllers were partially bypassed to import the external control command via an interface with upper level controller. The control command was generated by controllers through MATLAB-SIMULINK. Both the plant and controller works in continuous time. The DES controller interacts with the continuous part of the system through continuous-todiscrete (Event Generator) and discrete-to-continuous (Action Generator) interfaces. The FORTRAN NPP model is implemented on one computer, which communicates with the other computer that hosts the robust \& resilient controller and DES controller through TCP and/or UDP network.

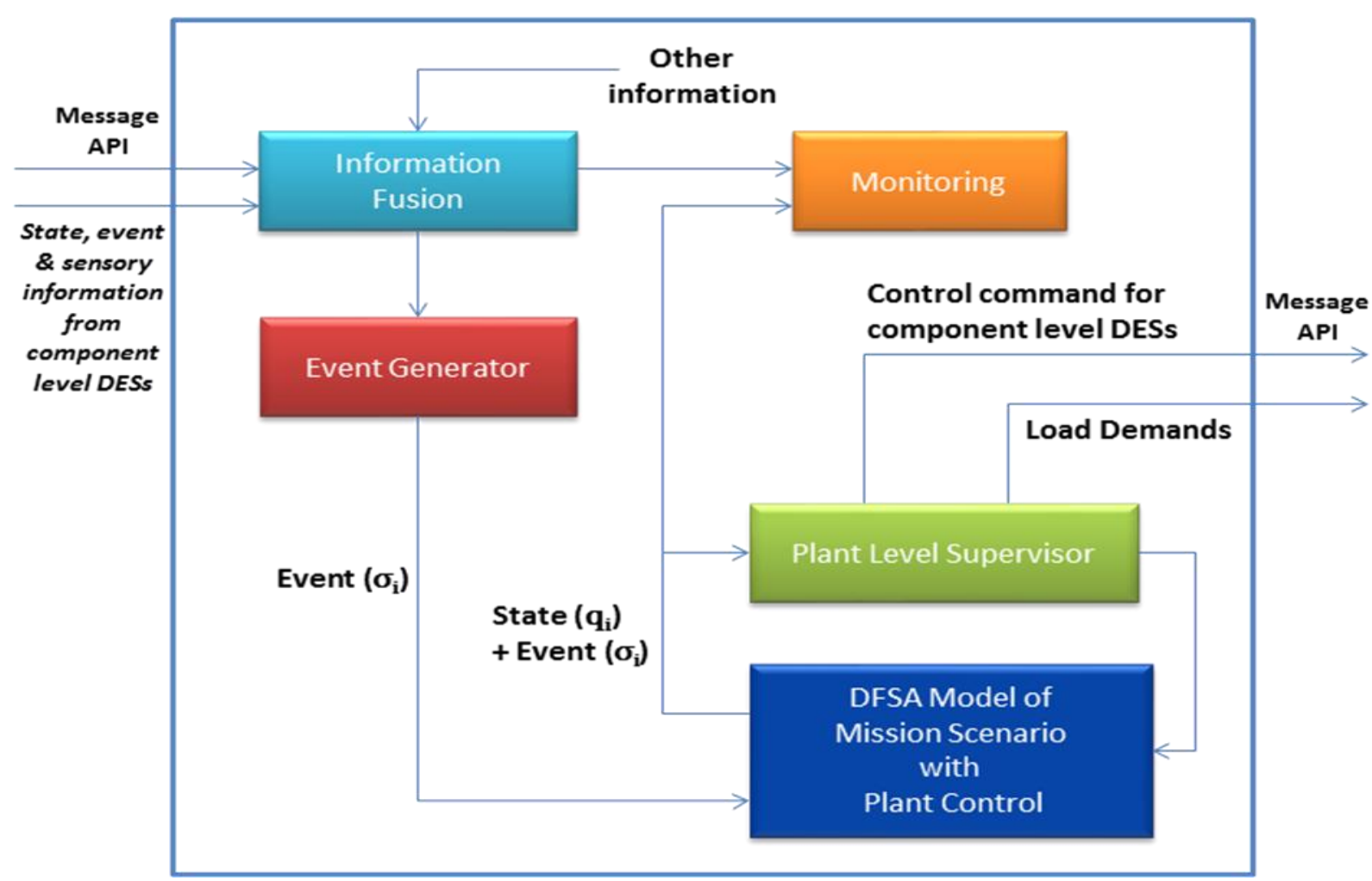

Figure 10.1: Supervisory control architecture of the NPP system

\subsection{Synthesis of DES Controllers}

One of the major tasks of the supervisory decision making is fusion of the (possibly) redundant, conflicting, and incomplete information to make timely decision. Such information can be derived from different types of sensor data [73], as well as operational history and the 
knowledge-base generated from operators' personal experience. Computer-based advanced analytical techniques are necessary for fusion of the time series data available from multiple sensors and other relevant non-sensor-based information to make specific inferences that could not be achieved through the sole usage of the available sensory information. Improved performance may not result simply from an increased volume of sensor data and plant information unless the ensemble of information is systematically processed in the context of the plant operational conditions and mission objectives [74]. In order to achieve the desired performance of a DES controller, it is essential to have an effective event generation algorithm to ensure fusion of the heterogeneous information for: (i) enhanced resolution and reduced ambiguity in decision and control; (ii) advantageous trade-offs between probability of false alarms and missed detection [75].

The unsupervised dynamics of plant operations are modeled as a DFSA, based on postulated scenarios. In the present context, the DFSA model assumes that the NPP is carrying out routine operations. Fig. 10.2 presents an example of the DFSA model of the unsupervised plant operation for DES control. The DFSA model of the supervised plant operations is shown in Fig. 10.3, where the dashed lines indicate those controllable events that are disabled by the optimal control algorithm [72]. The state $\mathbf{q}_{7}$ in Fig. 10.3 becomes unreachable following the disabling action of the supervisor. Therefore, all transitions, originating from the state $\mathbf{q}_{\mathbf{7}}$, are also shown with dashed lines as well as the state itself. 


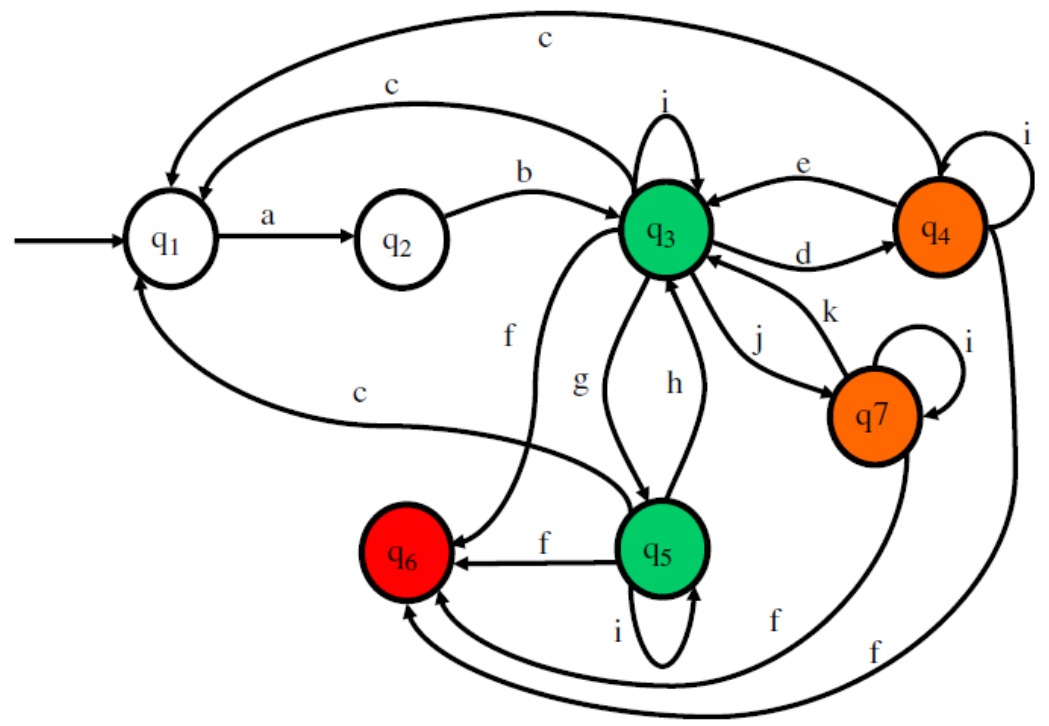

Figure 10.2: Example of unsupervised plant DFSA model at the plant level

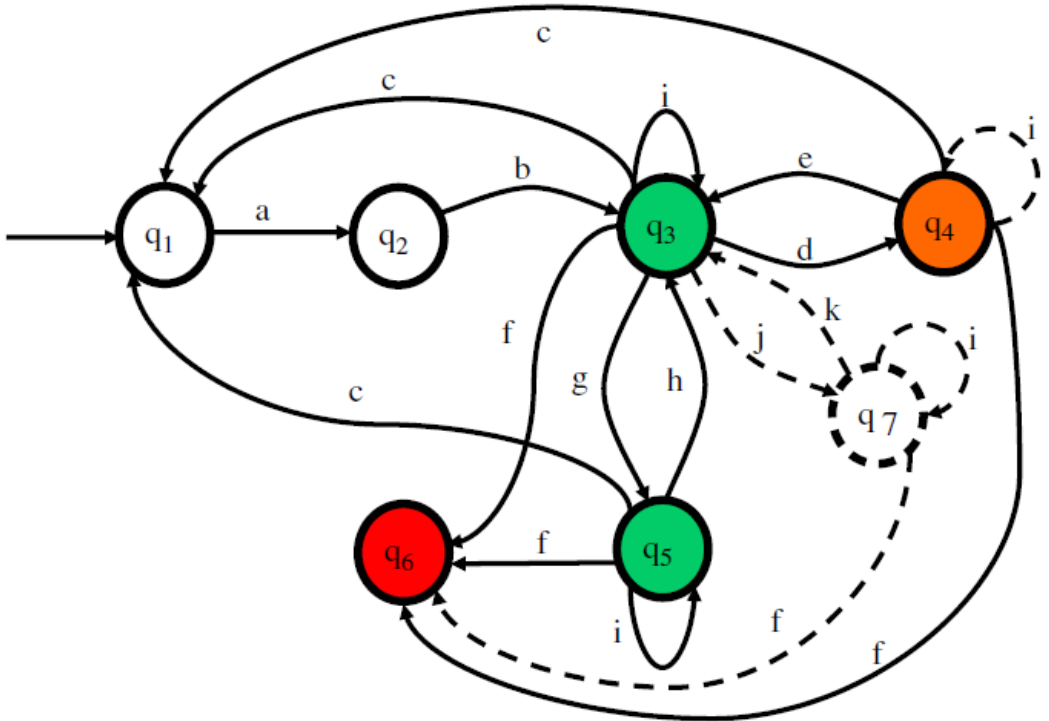

Figure 10.3: Example of supervised plant DFSA model at the plant level 


\section{INTEGRATED AUTONOMOUS CONTROL SYSTEM}

In the previous sections, different fault detection and identification methods and control strategies are introduced and some application results are presented. These control and diagnostics systems not only can work individually, but also can be integrated as a comprehensive autonomous control system, where the individual systems cooperate with each other to perform overall management, decision making, and diagnosis of the NPP.

\subsection{Structure of the Integrated System}

The structure of this integrated system is shown in Figure 11.1. The structure of the system is three-tier: Execution level, Coordination level, and Management level.

The execution level implements the control actions generated by the higher levels through actuators, and senses the plant responses and environment, and passes the information to the higher levels for parameter identification and failure detection. The NPP can be divided into three major components: Reactor, Steam Generator, and Turbine. Information regarding the operation and safety of the plant, such as coolant temperature, steam pressure, turbine output power, is collected by the sensing system and passed to the coordination level for further analysis.

The coordination level translates high-level commands into sequences of action to be passed to the execution level and provides the appropriate sequence of control and identification algorithms. The signals collected from the execution level are calibrated and validated (if redundant sensors are available), and then are classified by the anomaly classification module. The anomaly classification part uses symbolic dynamic filtering (SDF) as feature extraction tool and support vector machine as classifier. The classification results are sent to the higher level DES controller through an interface which generates event and convert the continuous signals to discrete-event signals. On the other hand, the Enabled / Disabled Controllable Events are generated by the DES controller and converts to continuous-time commands through the Action Generator, which generates activation signals for the activator \& filter module, and set point signals for the Bank of Robust \& Resilient Controllers module. The robust controllers are 
designed to cope with limited pre-determined different crisis situations, whereas the resilient controllers are designed to handle unanticipated scenarios.

The management and organization level determines the system goals and supports the human communication interface, and oversees and directs all the activities at both the coordination and execution levels. It is the most intelligent of the three levels. Upon detection of an event, the supervisory selects the new set points and the appropriate controller which is defined in the state behavior of DFSA. The set points information is input to the bank of controllers, whereas the controller output is not switched directly from the previous one to the new one immediately. Instead, to ensure smooth transition of the controller during the transition stage, both outputs from the previous controller and the new controller are both connected online and filtered by time-varying weighted filters. The weight of the previous controller outputs decrease exponentially from 1 to 0 whereas that of the new controller output increases exponentially from 0 to 1 . The sum of the weighted controller outputs is the control action of the NPP. The bumpless control transition is highly desirable in the NPP system because abrupt changes in the control action (control rods, feed water flow, turbine valve opening, and etc.) will result in impact to the entire system which might be disastrous. 


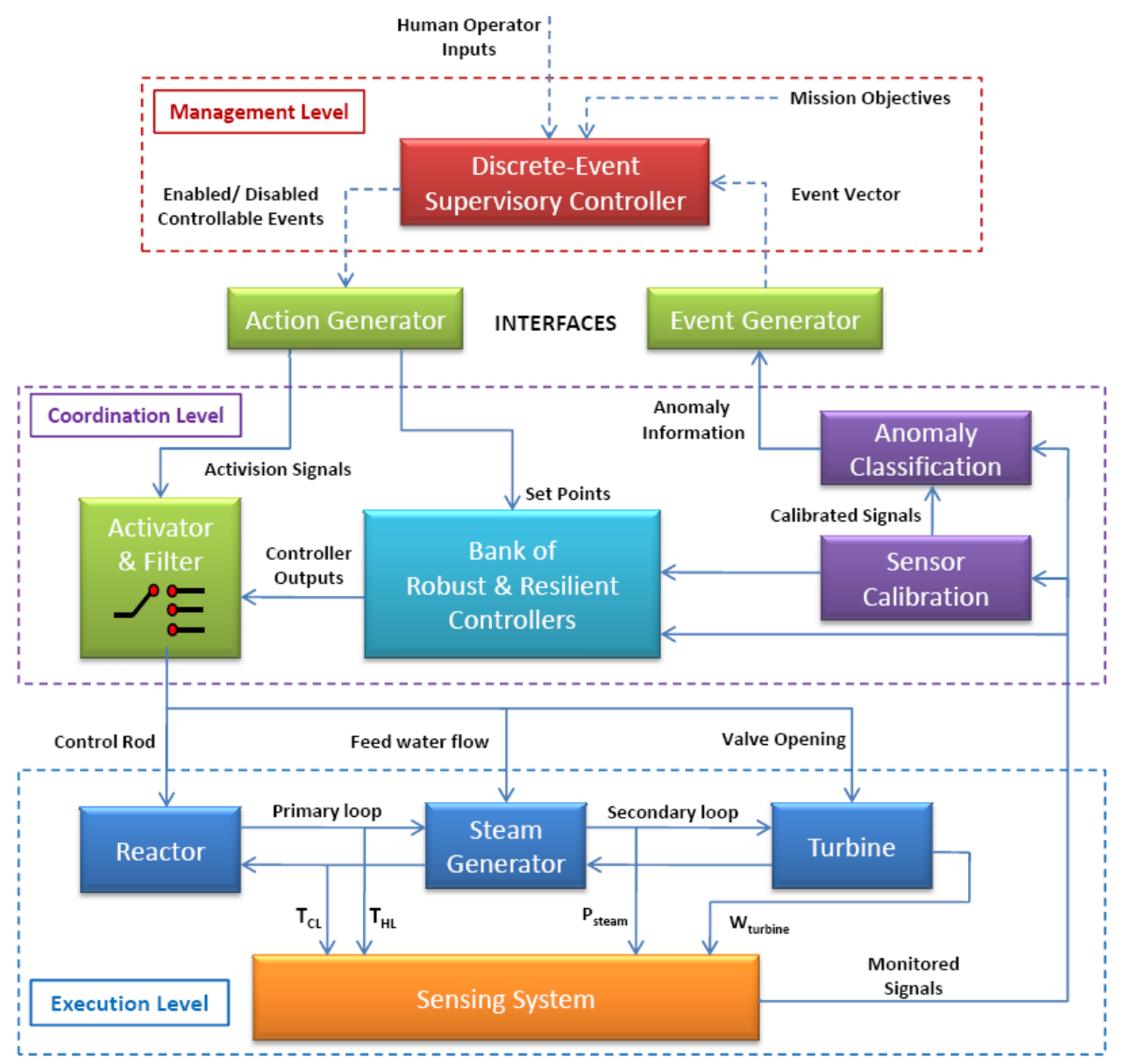

Figure 11.1: Structure of the integrated autonomous control system

\subsection{System Behavior during Normal Operations and Faulty Conditions}

During normal operations (i.e., no fault presents in the system), the plant operates in normal condition controlled by a corresponding robust controller. No anomaly signal will be given to the discrete-event supervisory controller by the anomaly classification module. The Nuclear Power Plant will be operated by the robust controller according to the set points provided by the DES controller.

If sensor fault (drifting, oscillating, and etc.) occurs in the system, the sensor calibration module will calibrate the redundant signals and give calibrated signals to the anomaly classification module. The weight of the faulty sensor will be automatically reduced by the sensor calibration module, so no manual operation is required. After the faulty sensor resumes to 
work, the sensor weight will also automatically resume to normal level (i.e., 1). In this scenario, the DES controller remains unchanged because no plant fault occurs.

On the other hand, if plant fault (for example, reactor coolant pump failure) occurs, the anomaly classification module will immediately recognize the fault class information from the monitored signals and send the fault class information to the DES controller. The DES controller changes the operation envelop of the NPP by choosing appropriate controllers. If the fault is as pre-determined, then the corresponding robust controller will be activated and brought online. If the fault is severe and unanticipated, then resilient controller will be activated to handle the crisis situation, and set points will be changed accordingly as well.

\subsubsection{Simulation Results}

Full nonlinear simulation for the closed loop system, with the robust controller, resilient controller, finite automaton and filters included, was run to evaluate the performance. The simulated scenario is that in the first 100 seconds the nuclear power plant is in normal operating conditions with full output power load and steam pressure load. At $t=100 \mathrm{~s}$, half of the primary flow pumps failed. To save the plant, the output power load and feed water flow are reduced by half immediately by finite automaton, and the control output is shifted quickly from robust controller to resilient controller. The anomaly is removed at $t=900 \mathrm{~s}$, primary flow pumps resume to work. To make the plant return to normalcy, the output power load and feed water flow are reset to nominal value, and control output is shifted smoothly from resilient controller back to robust controller. The smooth transition is ensured by choice of small filter time constant.

Figure 11.2 shows the normalized plant outputs (y1 is steam pressure and y2 is reactor output power). Under normal conditions, both plant outputs are 1 . When half of the primary pumps fail, the plant is not able to generate $100 \%$ power, and thus the output power load is reduced to $50 \%$ by finite automaton. When the anomaly is removed, the output power gradually returns to $100 \%$. Note that there exist spikes in y 2 when anomaly occurs and is removed. Those spikes come from temperature feedback from the reactor due to the delta change in primary flow. An actual anomaly in flow would not occur as a step but as a more gradual degradation. Furthermore, if the output power is turbine output power rather than reactor core output power, 
the spikes will not exist due to the mechanical inertia of the turbine. The turbine model will be integrated into the nuclear power plant model in the future.

Figure 11.3 shows the control efforts ( $u_{1}$ is steam flow and $u_{2}$ is rod reactivity). When the plant is working normally, the normalized control efforts are 0 . After anomaly occurs at $t=100 \mathrm{~s}$, in order to "save" the plant, the steam flow gradually increases to 10 and the rod reactivity reduces to over -80 . Figure 11.4 shows the temperatures (primary inlet, primary outlet, and steam flow temperatures) monitored by sensors inside the plant. $\Delta T$ is the temperature difference between primary inlet and outlet flow, and is used to activate the finite automaton. Figure 11.5 shows alphabet in finite automaton and output power setpoint controlled by the finite automaton. When the plant is working in normal conditions, the alphabet is 0 and output power setpoint is 1 . When anomaly occurs, the alphabet changes to 1 immediately and sets the output power setpoint to 0.5 .

\subsubsection{Remarks}

The simulation results show that the integrated robust and resilient controllers have good performance under both normal and emergency operation conditions. However, the stability of the robust and resilient has not been rigorously proved. In addition, the turbine model has not been integrated into the NPP model in this simulation due to limited time. Those are topics of future research.

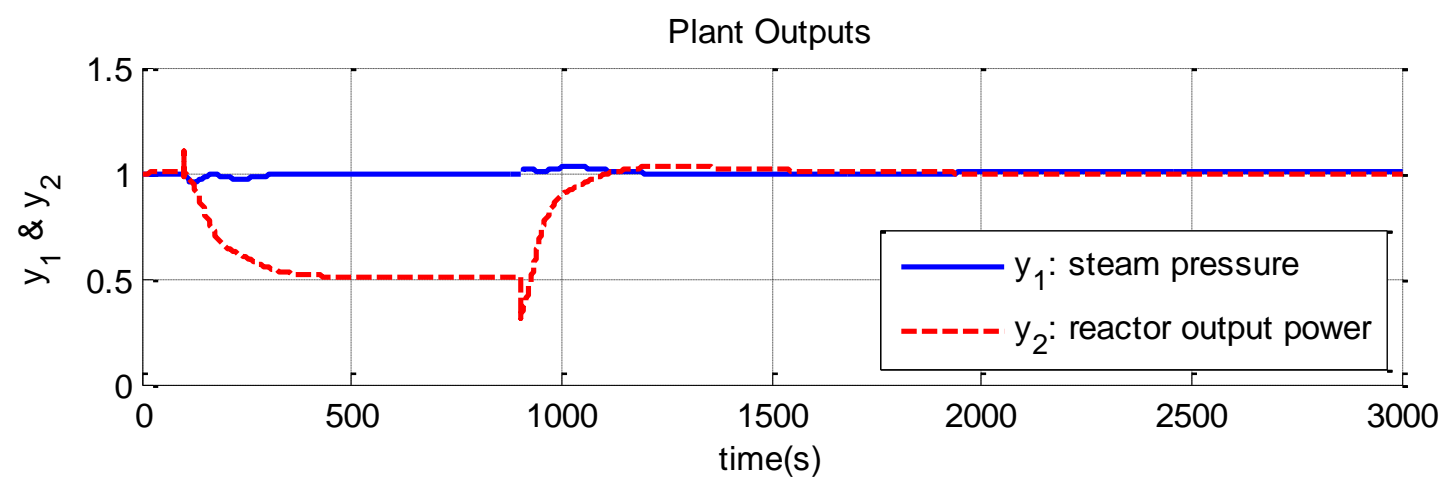

Figure 11.2: Normalized plant outputs 


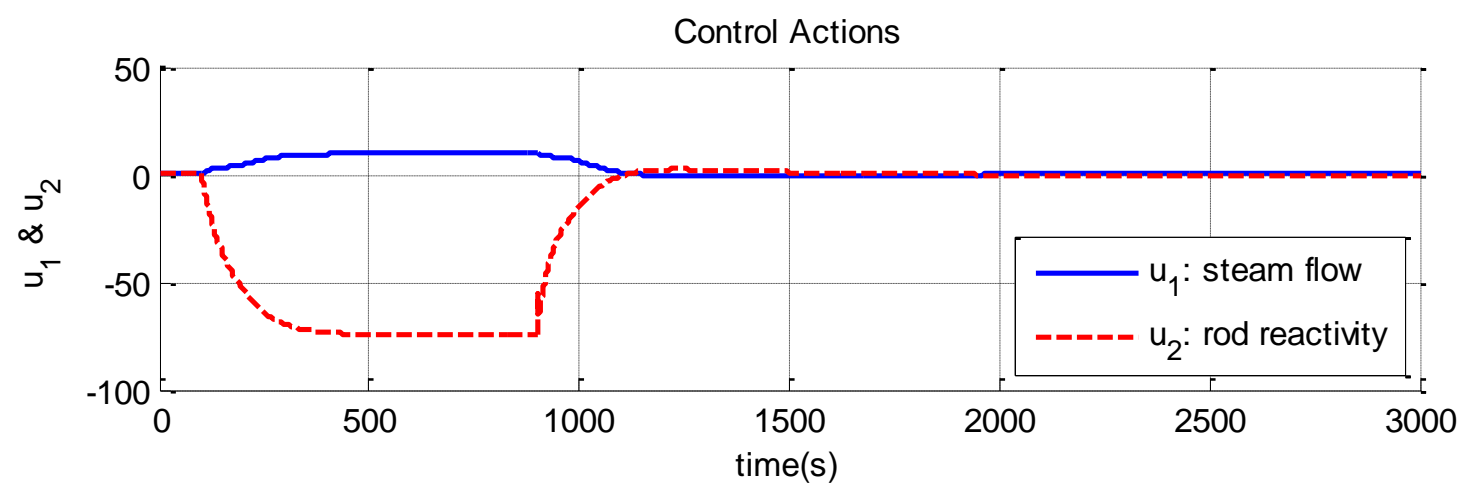

Figure 11.3: Control actions of integrated robust and resilient controller

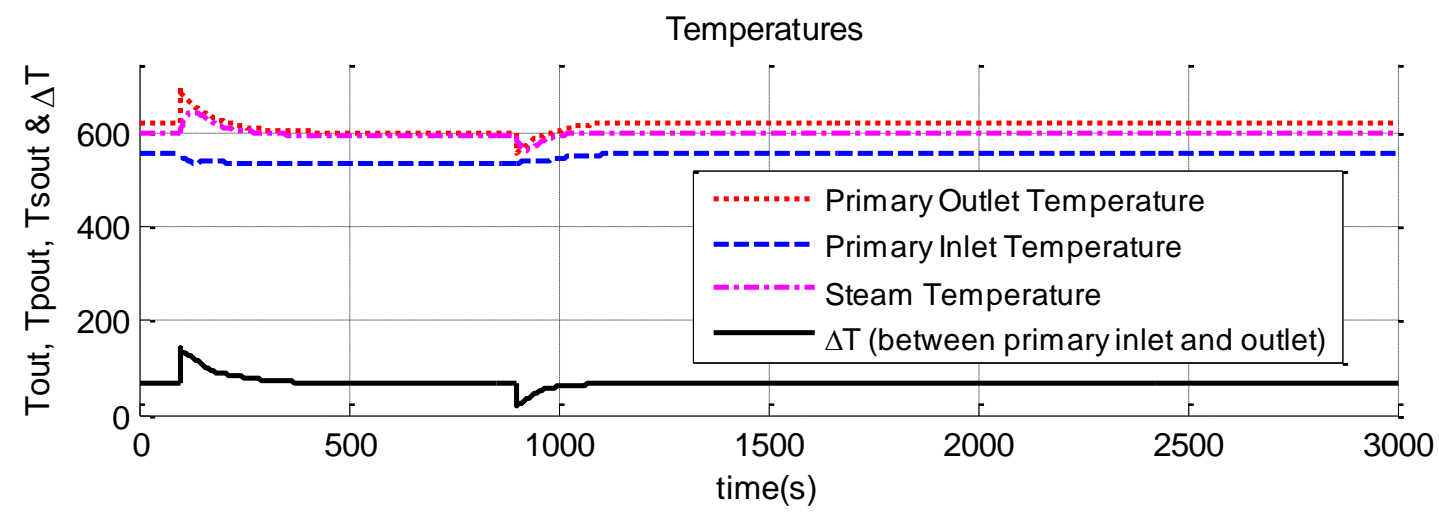

Figure 11.4: Temperatures of Steam, Primary Inlet and Outlet Flows

Finite Automaton Alphabets and Setpoint Change

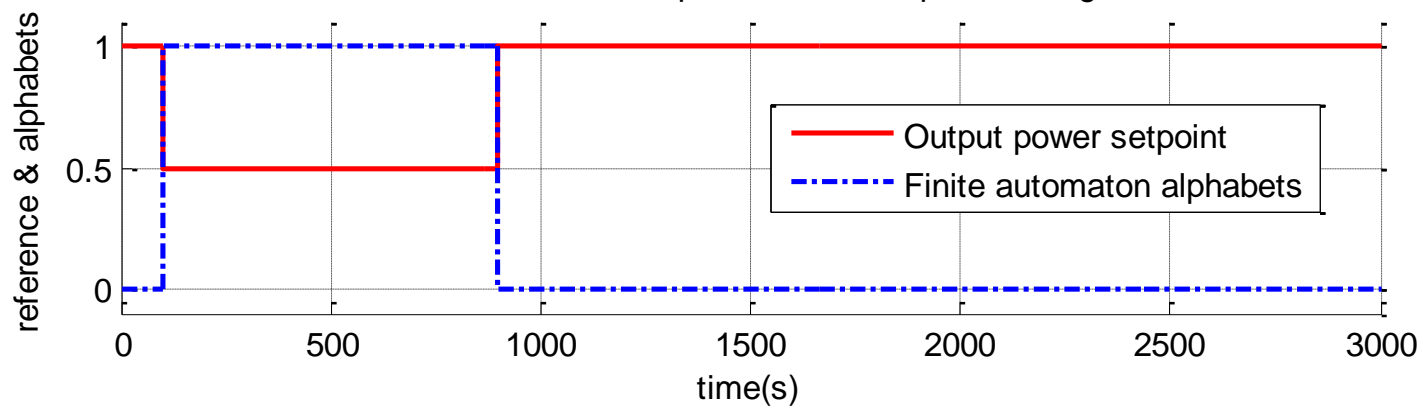

Figure 11.5: Finite automaton alphabets and output power set point changes 


\section{CONCLUDING REMARKS AND FUTURE WORK}

With the ever-increasing demands for quality improvement, reliability, safety, and cost reduction placed on nuclear power plants there is a trend toward autonomous operations in which the tasks of performance monitoring, early fault detection and diagnosis, automated alarm processing, emergency operating procedures, etc., can be integrated. Autonomous control systems must perform well under significant uncertainties in the plant and the environment for extended periods of time, and they must be able to compensate for system failures without external intervention [5].

In order to implement autonomous control of nuclear power plants, several control algorithms and fault detection and identification algorithm have been developed and tested. State observer design, system identification, model-predictive control, robust control, resilient control, and discrete event supervisory control algorithms have been developed and implemented in the IRIS system. Sensor calibration algorithm was introduced to detect sensor fault and isolate the faulty sensors. Fault detection and identification algorithm was introduced to recognize plant fault by using symbolic dynamic filtering (SDF).

A DHGF nonlinear state observer is designed and applied to the IRIS reactor. Three different cases are simulated. Simulation results show that the performance of the DHGF is better than a low order model which is used to estimate state values. The DHGF can be integrated as a component of controller designed by state feedback control law or as a part of online reactor state monitoring system.

The nonlinear model identification of IRIS reactor using time domain data is presented in this report. The identified model is accurate enough such that it can describe the important and major dynamic of the IRIS reactor with acceptable accuracy.

A data-driven method for detection of component-level faults in nuclear power plants under varying sensor noise condition is presented. The proposed fault detection method is based on Symbolic Dynamic Filtering (SDF) that is recently reported in the literature as a real-time data-driven pattern identification tool. The task of fault detection is viewed as a multi-class pattern classification problem, where feature extraction is optimized to enhance the classification 
rate. The proposed methodology efficiently (i.e., with low memory requirements and fast execution) compresses a high-volume database to execute fault detection for a large number of classes. It has been shown that the SDF-based fault detection algorithm has superior performance compared with the PCA-based fault detection algorithm.

The model-predictive controller is applicable to both linear and non-linear systems, and incorporates an on-line control design feature. Robust multi-variable controllers have been designed to enhance insensitivity of the control system to exogenous disturbances, internal faults, and uncertainties in the plant. Simulation results show that the reduced-order robust controllers have good tracking performance, insensitivity to noise and robustness against disturbances.

A novel resilient control algorithm was introduced to enhance recovery of the control system from unanticipated adverse conditions and faults as well as from emergency situations by altering its operational envelope in real time. Resilient decision and control laws were synthesized on a finite-time horizon as augmentation of robust decision and control with the objectives of: (1) Reliable and fast recovery from adverse conditions and emergency situations and (2) Restoration of the control configuration upon returning to normalcy or upon graceful degradation within design specifications.

Individual measurements in a redundant set of sensors may often exhibit deviations from each other after a length of time. These differences could be caused by slowly time-varying sensor parameters, plant parameters, transport delays, etc. Consequently, some of the redundant measurements could be deleted by a fault detection and isolation (FDI) algorithm if they are not periodically calibrated. On the other hand, failure to isolate a degraded measurement could cause an inaccurate estimate of the measured variable. To resolve this problem, an adaptively filtering algorithm for the set of redundant measurements was introduced to (1) simultaneously calibrate all measurements online to compensate for their errors; (2) Adaptively update the weights of individual online, based on their respective a posteriori probabilities of failure instead of being fixed a priori.

NPPs are run and controlled by a team of human operators. Even an experienced operator may be overwhelmed by signals and readings of a large number of sensors and alarms in case of unpredicted and abrupt events. That is why computerized monitoring systems have long been 
used at NPPs with the aim of not only displaying real-time histories of various sensors but to also automatically identify transients and then classify them as specific events. A real-time fault detection and identification algorithm based on time series data collected from multiple sensors in NPP was introduced. The proposed algorithm consists of two inter-related parts: (1) Feature extraction from time-series data using symbolic dynamic filtering; and (2) Pattern classification based on the extracted features using support vector machine.

To implement the functions of the Management and Organization Level of the three-tier hierarchical structure, an intelligent decision and control algorithms based on the theory of discrete event supervisory (DES) control was introduced. A DES controlled NPP system is proposed to be implemented on a simulation test bed that consists of two networked computers using the client/server concept. One of the two computers hosts the controller for health monitoring of the plant and making intelligent decisions. The other computer runs the IRIS NPP simulation model. The NPP simulator integrates the event-driven discrete dynamics modeled by finite state automaton as well as time-driven continuous dynamics modeled by thermal-hydraulic equations through continuous-to-discrete and discrete-to-continuous interfaces.

Finally, a structure which integrates the individual controllers and fault detection \& identification system was presented. This comprehensive autonomous control system has the typical three-tier structure. The execution level is made up of the components of the NPP (reactor, steam generator, steam turbine) and distributed sensing system. The coordination level is comprised of sensor calibration, anomaly identification, bank of robust \& resilient controller, activation and filter modules. The discrete event supervisory controller is the heart of the management level, which communicates with the coordination level through a continuous-todiscrete and discrete-to-continuous interface. The performance of this hierarchical structure has been tested on an accident scenario in which half of the reactor coolant pumps failed. Simulation results show that the integrated structure has fast response during emergency operations, and the feature of the robust controller are retained during normal operating conditions.

The following areas are recommended for future research:

- Evaluate the sensitivity and robustness of this identification model when the measured input/output data are polluted by noises or the measurements involve large uncertainty. 
- Apply system identification methods to identify models of components of IRIS power plant, such as steam generator, pressurizer, and other components.

- Apply system identification to estimate some important internal state variables which are important in nuclear reactor monitoring and operation, such as DNBR, CHF, etc.

- Based on the identified reactor model, design control system for IRIS by using modern control methods, i.e. model predictive control.

- Usage of other classifiers (e.g., Support Vector Machines (SVM) and Gaussian Mixture Models (GMM)) for comparison with the proposed method's performance.

- Inclusion of the step of tuning the classifier inside the optimization loop as described in the general framework shown in Fig. 11.1.

- Extension of the proposed fault detection method to accommodate other types of sensor degradation (e.g., bias and drifting).

- Investigation of anomalous situations with simultaneous faults in multiple plant components, actuators, and sensors.

- Investigate the proposed anomaly identification algorithm using SDF and SVM for multiclass anomaly identification problem

- Generate more accidental scenarios and design the corresponding robust \& resilient controllers

- Implement the proposed DES control algorithm for both normal and emergency operations

- Design the interface between the management level and coordination level

- Develop fault-tolerant control strategies for nuclear plants under actuator malfunction and loss of critical equipment.

- Rigorously establish the stability of the model-predictive, robust, and resilient controllers

- Test the entire integrated autonomous system under different operational scenarios. 


\section{REFERENCES}

[1] P. J. Antsaklis and K. M. Passino, "An Introduction to Intelligent and Autonomous Control", Kluwer Academic Publishers, Boston, 1992

[2] S. Gupta, A. Ray and S. Phoha, "Generalized Ising model for dynamic adaptation in autonomous systems", European Physics Letters, 87 (2009), 10009

[3] T.-H. Guo, J. S. Litt, "Resilient Propulsion Control Research for the NASA Integrated Resilient Aircraft Control (IRAC) Project", AIAA 2007-2802, AIAA 2007 Conference and Exhibit, Rohnert Park, California, May 2007

[4] X. Jin, R. M. Edwards and A. Ray, "Integrated Robust and Resilient Control for Nuclear Power Plants ," Proc. of Sixth ANS International Topical Meeting on NPIC \& HMIT, Knoxville, TN, Apr 2009

[5] H. Basher and J. S. Neal, "Autonomous Control of Nuclear Power Plants," Technical Report ORNL/TM-2003/252, Nuclear Science and Technology Division, Oak Ridge National Laboratory, Oak Ridge, TN, 37831-6238.

[6] J. G. Williams and W. C. Jouse, "Intelligent Control in Safety Systems: Criteria for Acceptance in the Nuclear Power Industry," IEEE Transaction on Nuclear Science, 40(6), 20402044 (Dec. 1993)

[7] M. D. Carelli, “The design and safety features of the IRIS reactor", Nucl. Eng. Design, vol. 230, pp 151-167, 2004.

[8] H. Shen, "Advanced feedwater control for next generation nuclear power systems," Ph.D. dissertation, Department of Nuclear Engineering, North Carolina State University, 2006.

[9] A. Ben Abdennour, R. M. Edwards, and K. Y. Lee, "LQG/LTR robust control of nuclear reactor with improved temperature performance", IEEE Transactions on Nuclear Science. Vol 39, pp. 2286-2294, Dec 1992.

[10] A. Hussein, S. Saeed, "Improved Temperature Control of a PWR Nuclear Reactor Using an LQG/LTR Based Controller”, IEEE Transactions on Nuclear Science. Vol 50, no. 1, Feb 2003.

[11] Z. Dong, J. Feng, and X Huang, "Nonlinear Observer-Based Feedback Dissipation LoadFollowing Control for Nuclear Reactors", IEEE Transactions on Nuclear Science. Vol 56, no. 1, Feb 2009.

[12] Z. Dong, J. Feng, X Huang, and L. Zhang, "Dissipation-based high gain filter for monitoring nuclear reactors", IEEE Transactions on Nuclear Science. Vol 57, no. 1, Feb 2010. 
[13] A. J. Van Der Schaft, L2-Gain and Passivity Techniques in nonlinear control, Springer, Berlin, Germany, 1999.

[14] L. A. Zadeh, “From circuit theory to system theory," Proc. IRE, vol. 50, pp. 856-865, 1962.

[15] J. Schoukens, R. Pintelon, Identification of linear system, Elmsford, NY: Pergamon Press, 1991

[16] E. Ikonen, K. Najim, Advanced process identification and control, Basel, NY: Marcel Dekker, Inc. 2002.

[17] M. Hagan, M. Menhaj, "Training feedforward networks with the marquardt algorithm," IEEE. Trans. on Neural Networks, 5:989-993, 1994.

[18] K. Zhao and B. R. Upadhyaya, "Model based approach for fault detection and isolation of helical coil steam generator systems using principal component analysis," IEEE Transactions on Nuclear Science, 53(4), pp.2343-2352 (2006).

[19] K. C. Gross, R. M. Singer, S. W. Wegerich and J. P. Herzog, "Application of a Model-based Fault Detection System to Nuclear Plant Signals," Proceedings of the 9th International Conference on Intelligent Systems Applications to Power Systems, Seoul, Korea, July 6-10, 1997.

[20] B. Lu and B. R. Upadhyaya, "Monitoring and fault diagnosis of the steam generator system of a nuclear power plant using data-driven modeling and residual space analysis," Annals of Nuclear Energy, 32, pp.897-912 (2005).

[21] K. Hadad, M. Mortazavi, M. Mastali and A. A. Safavi, "Enhanced neural network based fault detection of a VVER nuclear power plant with the aid of principal component analysis," IEEE Transactions on Nuclear Science, 55(6), pp.3611-3619 (2008).

[22] Y.-W. Chang and P.-H. Seong, "A signal pattern matching and verification method using interval means corss correlation and eigenvalues in the nuclear power plant monitoring systems," Annals of Nuclear Energy, 29, pp.1795-1807 (2002).

[23] R. O. Duda, P. E. Hart and D. G. Stork, Pattern Classification, 2nd ed., Wiley Interscience, New York, NY, USA (2001).

[24] A. Ray, "Symbolic dynamic analysis of complex systems for anomaly detection," Signal Processing, 84(7), pp.1115-1130 (2004).

[25] V. Rajagopalan and A. Ray, "Symbolic time series analysis via wavelet-based partitioning," Signal Processing, 86(11), pp.3309-3320 (2006).

[26] S. Gupta, A. Ray and E. Keller, "Symbolic time series analysis of ultrasonic data for early detection of fatigue damage," Mechanical Systems and Signal Processing, 21(2), pp.866-884 (2007). 
[27] E. C. Larson, B. E. Parker Jr., and B. R. Clark, "Model-based sensor and actuator fault detection and isolation," Proceedings of American Control Conference, 2002, pp.5:4215-4219.

[28] S. C. Stultz and J. B. Kitto, Steam: its generation and use, Babcock \& Wilcox Company, (1992).

[29] F. Koushanfar, M. Potkonjak and A. Sangiovanni-Vincentelli , "On-line Fault Detection of Sensor Measurements,” IEEE Sensors, pp.974-980 (2003).

[30] A. Subbu and A. Ray, "Space Partitioning via Hilbert Transform for Symbolic Time Series Analysis," Applied Physics Letters, 92(8), pp.084107(1-3) (2008).

[31] G. J. Mclachlan, Discriminant Analysis \& Statistical Pattern Recognition, WileyInterscience, (2004).

[32] E. Choi and C. Lee, "Feature extraction based on the Bhattacharyya distance," Pattern Recognition, 36(8), pp.1703-1709 (2003).

[33] M. Buhl and M. B. Kennel, "Statistically relaxing to generating partitions for observed timeseries data," Phys. Rev. E, 71(4), p. 046213, Apr. 2005

[34] T. Cover and P. Hart, "Nearest neighbor pattern classification," IEEE Transactions on Information Theory, vol. IT-13, no. 1, pp. 21-27, 1967.

[35] R. Steuer, Multiple Criteria Optimization: Theory, Computations, and Application, John Wiley \& Sons, Inc., New York, USA, 1989.

[36] S.-S. Shyu and R. M. Edwards, "A Robust Multivariable Feedforward / Feedback Controller Design for Integrated Power Control of Boiling Water Reactor Power Plants", Nuclear Technology, 120, 129 (2002)

[37] K. Zhou and J. D. Doyle, Essentials of Robust Control, Prentice Hall, Upper Saddle River, New Jersey (1998)

[38] G. J. Balas, G.C. Doyle, K. Glove, A. Packard, and R. Smith, $\mu$-Analysis and Synthesis Toolbox, Mathworks (1998)

[39] D.-W. Gu, P. Hr. Petkov and M. M. Konstantinov, Robust Control Design with MATLAB, Springer (2005)

[40] J.-S. Yee, J.-L. Wang and G.-H. Yang, "Resilient Ho Control Design for Discrete-Time Uncertain Linear Systems: An Auxiliary System Transformation Approach", Asian Journal of Control, Vol. 4, No. 2, pp. 223-230, June 2002

[41] M. S. Mahmoud, Resilient Control of Uncertain Dynamical Systems, Springer, Berlin, Germany (2004) 
[42] C. Cao and N. Hovakimyan, "Design and Analysis of a Novel L1 Adaptive Control Architecture with Guaranteed Transient Performance", IEEE Transactions on Automatic Control, Vol. 53, No. 3, 2008, pp. 586-591

[43] C. Cao and N. Hovakimyan, "Stability Margins of L1 Adaptive Control Architecture", American Control Conference, 2007, pp. 3931-3936.

[44] C. Cao and N. Hovakimyan, "L1 Adaptive Control Output Feedback Controller for Systems of Unknown Dimension", IEEE Transactions on Automatic Control, Vol. 53, No. 3, pp. 815-821, April 2008

[45] C. Cao and N. Hovakimyan, "L1 Adaptive Controller for Systems in the Presence of Unmodeled Actuator Dynamics", IEEE Conference on Decision and Control, 2007, pp. 891-896

[46] C. Cao and N. Hovakimyan, "Guaranteed Transient Performance with L1 Adaptive Controller for Systems with Unknown Time-Varying Parameters and Bounded Disturbances: Part I," New York, NY, July 2007, pp. 3925-3930

[47] C. Cao and N. Hovakimyan, "Stability Margins of L1 Adaptive Controller: Part II," New York, NY, July 2007, pp. 3931-3936

[48] C. Cao and N. Hovakimyan, "L1 Adaptive Output Feedback Controller for Systems of Unknown Relative Degree", Submitted to American Control Conference, 2009

[49] E. Kharisov, I. M. Gregory, C. Cao, N. Hovakimyan, "L1 Adaptive Control Law for Flexible Space Launch Vehicle and Proposed Plan for Flight Test Validation", AIAA-20087128, AIAA Guidance, Navigation and Control Conference, Honolulu, HI, 2008

[50] A. Ray and S. Phoha, "Calibration and estimation of redundant signals for real-time monitoring and control," Signal Processing, 83, pp. 2593-2605 (2003).

[51] E. Eryurek, and B. R. Upadhyaya, "Sensor Validation for Power Plant using Adaptive Backpropagation Neural Network", IEEE Transaction on Nuclear Science, 37(2), 1040-1047 (Apr. 1990)

[52] B. R. Upadhyaya, and E. Eryurek, "Application of Neural Networks for Sensor Validation and Plant Monitoring", Nuclear Technology, 97, 170-176 (Feb. 1992)

[53] J. N. Ning, and H. P. Chou, "Construction and Evaluation of Fault Detection Network for Signal Validation", IEEE Transaction on Nuclear Science, 39(4), 943-947 (1992).

[54] K. E., Holbert, and B. R. Upadhyaya, "Empirical Process Modeling Techniques for Signal Validation", Annals of Nuclear Energy, 21(7), 387-403 (1994)

[55] P. F. Fantoni, and A. Mazzola, "A Pattern Recognition-Artificial Neural Networks Based Model for Signal Validation in Nuclear Power Plants", Annals of Nuclear Energy, 23(13), 10691076 (1996) 
[56] A. S. Heger, K. H. Holbert, and A. M. Ishaque, "Fuzzy Associative Memories for Instrument Fault Detection", Annals of Nuclear Energy, 23(9), 739-756 (1996)

[57] G. M. Na et al., "Sensor Monitoring Using Fuzzy Neural Network with an Automatic Structure Constructor", IEEE Transaction on Nuclear Science, 50(2), 241-250 (Apr. 2003)

[58] J. W. HINES et. al., "On-Line Monitoring for Calibration Extension: An Overview and Introduction to NUREG/CR-6895," Technical Review of On-Line Monitoring Techniques for Performance Assessment, NUREG-Series Publications, U.S. NRC, May 2009

[59] R. Dorr, F. Kratz, J. Ragot, F. Loisy, and J.-L. Germain, "Detection, Isolation, and Identification of Sensor Faults in Nuclear Power Plants," IEEE Tran. Control System Tech., Vol. 5, No. 1 pp. 42-60 (Jan 1997).

[60] H. M. Hashemian, Maintenance of Process Instrumentation in Nuclear Power Plants, p. 29, Springer, New York (2006).

[61] X. Jin, A. Ray, and R. M. Edwards, "Redundant Sensor Calibration and Estimation for Monitoring and Control of Nuclear Power Plants", Transaction of ANS 2009Winter Meeting, Dec. 2009, Washington, D.C.

[62] H. Wu, "Reliable LQ fuzzy control for continuous-time nonlinear systems with actuator faults," IEEE Transaction on System, Man, and Cybernetics-part B: Cybernetics, Vol. 34, No. 4, August 2004

[63] R. J. Veillette, "Reliable linear-quadratic state-feedback control", Automatic, Vol. 31, No. 1, pp137-143, 1995

[64] L. Yu, "An LMI approach to reliable guaranteed cost control of discrete-time systems with actuator failure", Applied Mathematics and Computation 162(2005) 1325-1331

[65] D. Zhang, H. Su, et. "LMI approach to reliable guaranteed cost control with multiple criteria constraints: The actuator faults case", Int. J. Robust Nonlinear Control 2009; 19: 884-899

[66] G. H. Yang, J. Lam, and J. L. Wang, "Reliable Ho Control for affine nonlinear systems," IEEE Trans. Automat. Contr. Vol, 43, pp. 1112-1117, Aug. 1998

[67] Y. W. Liang, D. C. Liaw, and T. C. Lee, "Reliable control of nonlinear systems," IEEE Trans. Automat. Contr. Vol. 45, No. 4, pp. 706-710, 2000

[68] G.H. Yang, J.L. Wang and Y.C. Soh, "Reliable LQG control with sensor failures", IEEE Proc. Control Theory Application. Vol. 147, No. 4, July 2000

[69] P. J. RAMADGE and W. M. WONHAM, "Supervisory Control of A Class of Discrete Event Processes", SIAM J. Control and Optimization, Vol. 25, No. 1, pp. 206-230 (1987)

[70] A. Ray, "Signed real measure of regular languages for discrete event supervisory control", International Journal of Control, 78(12) 
[71] A. Ray, V. Phoha, and S. Phoha, Quantitative measure for discrete event supervisory control, Springer, New York

[72] M. Yassar, and A. Ray, "Hierarchical control of aircraft propulsion systems: Discrete event supervisory approach," Control Engineering Practice, 15 (2007), 149-162.

[73] A. J. Volponi, T. Brotherton, R. Luppold, and D. L. Simon, "Development of an information fusion system for engine diagnostics and health management", JANNAF 27th airbreathing propulsion subcommittee meeting, Colorado Springs, CO, 2003.

[74] D. K. Tolani, M. Yassar, A. Ray, and V. Yang, "Anomaly detection in aircraft gas turbine engines", Journal of Aerospace Computing, Information, and Communication, 3(2), 44-51.

[75] M. Basseville, and I. V. Nikiforov, Detection of abrupt changes: Theory and application, New Jersey: PTR Prentice-Hall. 


\section{BIBLIOGRAPHY}

\section{IRIS Reactor}

1. Savage , "Session on Generation IV Nuclear Energy Systems: Roadmap and Concepts," ANS Transactions, Milwaukee, WI, June 2001.

2. Computer Models for IRIS Control System Transient Analysis, Westinghouse inner report, 2007

3. D.V. Paramonov, M.D. Carelli, et al, "IRIS Reactor Development," Westinghouse inner report, 2002.

4. G.D. Storrick, B.Patrovic, and L. Oriani, "Computer Models for IRIS Control System Transient Analysis,” Westinghouse Final Report, 2007.

5. "IRIS Preliminary Safety Assessment,” Westinghouse Report, 2003.

6. H.L. Shen, "Advanced Feedwater Control for Next Generation Nuclear Power Systems," Ph.D. Dissertation, North Carolina State University, 2006.

7. H. Nariai, M. Kobayashi, and T. Matsuoka, "Friction Pressure Drop and Heat Transfer Coefficient of Two-Phase Flow in Helically Coiled Tube Once-Through Steam Generator for Integrated Type Marine Water Reactor," Journal of Nuclear Science and Technology, 19(11), pp. 936-947, 1982.

8. http://www.smso.net/International_Reactor_Innovative_and_Secure.

9. J.M. Kujawski, D.M. Kitch, and L.E. Conway, "The IRIS Spool-Type Reactor Coolant Pump,” Proceedings of ICONE10, Arlington, VA, 2002.

10. M.D. Carelli et al., "IRIS Reactor Conceptual Design."

11. M.D. Carelli et al., "IRIS Reactor Development," Proceedings of ICONE-9, Nice, France, April 2001.

12. M.D. Carelli et al., "IRIS, International New Generation Reactor," Proceedings of ICONE-8, Baltimore MD, April 2000.

13. M.D. Carelli, Conway L., Oriani L., Pertovic B., Todreas N. E., et al., "The Design and Safety Features of the IRIS Reactor,” ICONE 11, Tokyo, Japan, April 20-23, 2003.

14. M.D. Carelli, "IRIS: A global approach to nuclear power renaissance," Nuclear News, 2003. 
15. M.E. Ricotti, et al, "Preliminary Safety Analysis of the IRIS Reactor," Proceedings of ICONE10, Arlington, 2002.

16. T. Bajs, et al, "Transient Analysis of the IRIS Reactor," Proceedings of International Conference of Nuclear Energy for New Europe, 2002.

\section{Steam Generator}

17. A. Cioncolini, et al., "Thermal hydraulic Analysis of IRIS Reactor Coiled Tube Steam Generator," Nuclear Mathematical and Computational Sciences: A Century in Review, 2003

18. B.R. Upadhyaya, K. Zhao, and R.T. Wood, "Robust Techniques for Monitoring and Fault Diagnosis of IRIS Helical Coil Steam Generators", GENES4/ANP2003, Kyoto, Japan, September 2003.

19. B.R. Upadhyaya, K. Zhao, R.T. Wood, and D.T. Ingersoll, "Thermal-Hydraulic Analysis of a Helical Coil Steam Generator for Level Monitoring", Transactions of the American Nuclear Society, Vol. 88, pp. 283-284, June 2003.

20. H.Ju, et al, "Research Method and two-Phase Flow Stability of the Steam Generator of HTR-10," Journal of Nuclear Science and Technology, Vol. 38, No. 9, pp. 739-744, 2001.

21. L.Cinotti, et al, "Steam Generator of the International Reactor Innovative and Secure," Proceedings of ICONE10, rlington, VA, 2002.

22. J.I., Choi, et al., Automatic controller for steam generator water level during low power operation. Nucl. Eng. Des. 117, 263-274. 1989.

23. K. Zhao and B.R. Upadhyaya, "Helical Coil Steam Generator (HCSG) Thermal Hydraulic Analysis for Fault Diagnosis," Proceedings of MARCON 2003, Knoxville, TN, May 2003.

24. K. Zhao, and B.R. Upadhyaya, "Model Based Approach for Fault Detection and Isolation of Helical Coil Steam Generator Systems Using Principal Component Analysis," IEEE transactions on nuclear science, vol.53, no. 4, pp. 2343-2352, 2006.

25. K. Zhao, B.R. Upadhyaya, and R.T. Wood, "Modeling and Fault Detection and Isolation of IRIS Helical Coil Steam Generators". Global 2003, ANS Topical Meeting, New Orleans, November 2003.

26. K. Zhao, An Integrated Approach to Performance Monitoring and Fault Diagnosis of Nuclear Power Systems, a Dissertation for Ph.D., the University of Tennessee, 2005.

27. L. Guo, Z. Feng, and X. Chen, "Transient Convective Heat Transfer of Steam-Water Two-Phase Flow in a Helical Tube Under Pressure Drop Type Oscillations," International Journal of Heat and Mass Transfer, Vol. 45, pp. 533-542, 2002. 
28. M.A. Abdalla, "A Nonlinear Dynamic Model of a Once-Through, Helical Coil Steam Generator,” Oak Ridge National Laboratory report, 1993.

29. http://www.nucleartourist.com/systems/sg.htm.

30. R.M. Kuridan and T.D. Beynon, "Analysis of the Steam Generator for the Safe Integral Reactor Concept," Progress in Nuclear Energy, Vol. 31, No. 3, pp. 273-287, 1997.

\section{Autonomous Control}

31. A. Bristow, M. Tharayil, and A. G. Alleyne, "A Survey of Iterative Learning Control, A Learning-Based Method for High-Performance Tracking Control", IEEE Control Systems Magazine, pp. 96-114, 2006.

32. A. Tayebi and C. Chien, "A Unified Adaptive Iterative Learning Control Framework for Uncertain Nonlinear Systems," IEEE Transaction on Automatic Control, Vol. 52, No. 10, pp. 1907-1913, 2007.

33. Alvarex-Ramirex, H. Puebla, and G. Espinosa, "A Cascade Control Strategy for a Space Nuclear Reactor System", Annals of Nuclear Energy, 28 (2), pp. 93-112, 2001.

34. B.Pell et al, "An Autonomous Spacecraft Agent Prototype," Autonomous Robotics, Vol. 5. pp. 1-27, 1998.

35. B. P. Zeigler and S. Chi, Model Based Architecture Concepts for Autonomous Control Systems Design and Simulation, An Introduction to Intelligent and Autonomous Control, Kluwer Academic Publishers, Boston, pp. 57-78, 1992.

36. B. Wie, Space Vehicle Dynamics and Control, Reston, VA, American Institutes of Aeronautics and Astronautics, 1998.

37. B.R. Upadhyaya, M.G. Na, X.Xu, S. R. P. Perillo, “Autonomous Control and Diagnostics of Space Reactor Systems", Proceedings of the International Congress on Advances in Nuclear Power Plants, 2006.

38. Biswas, P. Bonasso, S. Abdelwahed, E. J. Mandersand, J. Wu, D. Kortenkamp, and S. Bell, "Requirements for an Autonomous Control Architecture for Advanced Life Support Systems", SAE International, 2005.

39. M. Boroushaki, M. B. Ghofrani, C. Lucas, and M. J. Yazdanpanah, "An Intelligent Nuclear Reactor Controller for Load Following Operations, Using Recurrent Neural Networks and Fuzzy Systems", Annals of Nuclear Energy, 30 (1), pp. 63-80, 2003.

40. E. Bradley and R. Stolle, "Automatic Construction of Accurate Models of Physical Systems", Annals of Mathematics of Artificial Intelligence, 17 (1), pp. 1-29, 1996.

41. C. Wilhelmsen, R.L. Hurd, and S. Couture, "Tele-Robotic/Autonomous Control Using ControlShell”, American Nuclear Society Seventh Topical Meeting, 1997. 
42. A. Casavola, M. Papini, and G. Franzè, "Supervision of Networked Dynamical Systems Under Coordination Constraints", IEEE Transactions on Automatic Control, 51 (3), pp. 421-437, 2006.

43. S. Chien, B. Smith, G. Rabideau, N. Muscettola, and K. Rajan, "Automated Planning and Scheduling for Goal-Based Autonomous Spacecraft”, IEEE Intelligent Systems, 13 (5), pp. 50-55, 1998.

44. R. Cristi, F. A. Papoulias, and A. J. Healey, "Adaptive Sliding Mode Control of Autonomous Underwater Vehicles in the Dive Plane", IEEE Journal of Oceanic Engineering, 15 (3) pp. 152-160, 1990.

45. D. Wilkes, R. Alford, R. Pack, R. Rogers, R. Peters, and K. Kawamura, "Toward socially intelligent service robots," Applied Artificial Intelligence Journal, vol. 12, pp. 729-766, 1997.

46. E. Rauch, “Autonomous Control Reconfiguration”, IEEE Control Systems Magazine, 15 (6), pp. 37-48, 1995.

47. F. Wang, K. C. Tan, X.D. Jiang, and Y. B. Chen, "A Flexible Automatic Test System for Rotating-Turbine Machinery", IEEE Transactions on Automation Science and Engineering, 2 (1), pp. 1-18, 2005.

48. G. Yasuda, "Distributed Autonomous Control of Modular Robot Systems using Parallel Programming", Journal of Materials Processing Technology, 141 (3), pp. 357-364, 2003.

49. Godbole, T. Samad, and V. Gopal, "Active Multi-Model Control for Dynamic Maneuver Optimization of Unmanned Air Vehicles", IEEE International Conference on Robotics and Automation, 2, pp. 1257-1262, 2000.

50. I. Salsbury, “A Practical Algorithm for Diagnosing Control Loop Problems”, Energy and Buidings, 29 (3), pp. 217-227, 1999.

51. J. Antsaklis and K. M. Passino, "Towards Intelligent Autonomous Control Systems: Architecture and Fundamental Issues", Journal of Intelligent and Robotic Systems, 1 (4), pp. 315-342, 1989.

52. J. Antsaklis, "Defining Intelligent Control, Report of the Task Force on Intelligent Control”, IEEE Control Systems Magazine, pp. 4-5 \& 58-66, 1994.

53. J. Antsaklis, K. M. Passino, and S. J. Wang, "An Introduction to Autonomous Control Systems”, IEEE Control Systems Magazine, pp. 5-13, 1991.

54. K. P. Valavanis, D. Gracanin, M. Matijasevic, R, Kollurur, and G. A. Demetriou, "Control Architectures for Autonomous Underwater Vehicles", IEEE Control Systems Magazine, 17 (6), pp. 48-64, 1997.

55. K.M. Hangos, Intelligent Control Systems: An Introduction with Examples, Kluwer 
Academic Publishers, 2001.

56. A. L. Kuchpil, F. Xavier, J. A. P. da Silva, M. R. Jimenez, "Autonomous Control System for Offshore Oil Explotation Using Digital Acoustic Communication", IEEE Journal of Oceanic Engineering, 2, pp. 849-853, 1997.

57. K.C. Kwon and J. Kim, "Accident Identification in Nuclear Power Plants using Hidden Markov Models”, Engineering Application of Artificial Intelligence, 12 (4), pp. 491-501, 1999.

58. M. Blanke, et al, Diagnosis and Fault-Tolerant Control, Springer, 2006.

59. M.Bodson, et al, "Multivariable adaptive algorithms for reconfigurable flight control," IEEE Transactions on Control Systems Technology, Vol. 5, Issue 2, pp.217-229, 1997.

60. Y. Leu, Industrial Intelligent Control: Fundamentals and Applications, John Wiley \& Sons Ltd., 1996.

61. M. G. Na, "Design of a Genetic Fuzzy Controller for the Nuclear Steam Generator Water Level Control”, IEEE Transactions on Nuclear Science, 45 (4), pp. 2261-2271, 1998.

62. M. L. Moore, V. Gazi, K. M. Passino, W. P Shackleford, and F. M. Proctor, "Complex Control System Design and Implementation", IEEE Control Systems Magazine, 19 (6), pp. 12-27, 1999.

63. M. Mouloua, R. Gilson, and P. Hancock, "Human Centered Design of Unmanned Aerial Vehicles," Ergonomics and Design, Vol. 11, No. 1, pp. 6-11, 2003.

64. M. N. Khajavi, M. B. Menhaj, and A. A. Suratgar, "A Neural Network Controller for Load Following Opeartion of Nuclear Reactors", Annals of Nuclear Energy, 29 (6), pp. 751-760, 2002.

65. M. Surka, "ObjectAgent for Robust Autonomous Control", American Association for Artificial Intelligence, 2001.

66. P. J. Antsaklis, K. M. Passino, Introduction to Intelligent Control Systems with High Degress of Autonomy, Kluwer Academic Publishers, 1993.

67. P. J. Antsaklis, M. Lemmon, and J. A. Stiver, "Learning to be Autonomous: Intelligent Supervisory Control", 1993.

68. P. Piagi and R. H. Lasseter, "Autonomous Control of Microgrids", IEEE PES Meeting, 2006.

69. P. Ridley and P. Corke, "Autonomous Control of an Underground Mining Vehicle", Australian Conference on Robotics and Automation, 2001.

70. R.S. Sutton, and A. G. Barto, Reinforcement learning: An Introduction, MIT Press, 1998. 
71. R. T. Wood, J. S. Neal, C. R. Brittain, J. A. Mullens, “Autonomous Control Capabilities for Space Reactor Power Systems”, Oak Ridge National Laboratory, 2004.

72. S. F. Chew and M. A. Lawley, "Robust Supervisory Control for Production Systems with Multiple Resource Failures", IEEE Transactions on Automation Science and Engineering, 3 (3), pp. 309-323, 2006.

73. S. Ioannidis and N. Tsourveloudis, "Fuzzy Supervisory Control of Manufacturing Systems", IEEE Transactions on Robotics and Automation, 20 (3), pp. 379-389, 2004.

74. S. M. Bragg-Sitton and J. P. Holloway, "Autonomous Reactor Control Using Model Based Predictive Control for Space Propulsion Applications", Annals of Nuclear Energy, 33 (17-18), pp. 1368-1378, 2006.

75. S. S. Choi, S. H. Chung, and D. H. Lee, "Automating Strategies of Emergency Opeartion for Optimal Shutdown in Pressurized Water Reactors", IEEE Transactions on Nuclear Science, 45, pp. 17-29, 1998.

76. S.S. Ge, and F. L. Lewis, Autonomous Mobile Robots: Sensing, Control, Decision Making and Applications, Taylor \& Francis Group, 2006.

77. T. J. Harris, C.T. Seppala, and L. D. Desborough, “A Review of Performance Monitoring and Assessment Techniques for Univariate and Multivariate Control Systems", Journal of Process Control, 9 (1), pp. 1-17, 1999.

78. T. Kourti, and J. F. MacGregor, "Process Analysis, Monitoring and Diagnosis, Using Multivariate Projection Methods", Chemometrics and Intelligent Laboratory Systems, 28 (1), pp. 3-21, 1995.

79. T. L. Johnson, H. A. Sutherland, S. F. Bush, W.Yan, and C. Eaker, "The TRAC Mission Manager Autonomous Control Executive", Aerospace Conference IEEE Proceedings, 2, pp. 639-646, 2001.

80. T. Samad, Active Multimodeling for Autonomous Systems, Automation, Control and Complexity: An Integrated View, John Wiley \& Sons, 2002.

81. T. Wood, "Enabling Autonomous Control For Space Reactor Power Systems", Oak Ridge National Laboratory, 2001.

82. Y. B. Shtessel, "Sliding Mode Control of the Space Nuclear Reactor System", IEEE Transactions on Aerospace and Electronic Systems, 34 (2), pp. 579-589, 1998.

83. Y. Park and P. H. Seong, "Application of a Self-Organizing Fuzzy Logic Controller to Nuclear Steam Generator Level Control”, Nuclear Engineering and Design, 167 (3), pp. 345-356, 1997. 


\section{Robust Control}

1. National Transportation Safety Board, "Aircraft Accident Report, United Airlines Flight 232, McDonnell Douglas DC-1040, Sioux Gateway Airport, Sioux City, Iowa, July 19, 1989," PBSO-910406, NTSB/AAR-SO/06, 1990.

2. Lemaignan, B., "Flying with no Flight Controls: Handling Qualities Analyses of the Baghdad Event," AIAA-2005-5907, AIAA Atmospheric Flight Mechanics Conference and Exhibit, San Francisco, CA, Aug. 15-18, 2005.

3. Burcham, F. W., Maine, T.A., Burken, J. and Pappas, D., "Development and Flight Test of an Augmented Thrust-Only Flight Control System on an MD-11 Transport Airplane," AIAA1996-3742, Guidance,Navigation and Control Conference, San Diego, CA, July 29-31, 1996.

4. Burcham, F.W., Fullerton, C. Gordon, and Trindel A., "Manual Manipulation of Engine Throttles for Emergency Flight Control,” NASA/TM-2004-212045, January 2004.

5. Frankenberger, C.E., III, "Survivable Engine Control Algorithm Development (SECAD)," Proceedings of the 2002 IEEE Aerospace Conference, Vol. 6, 2002, pp. 6-3015-6-3020.

6. Guo, T-H. and Litt, J.S., "Resilient Propulsion Control Research for the NASA Integrated Resilient Aircraft Control (IRAC) Project," AIAA 2007-2802, Infotech Aerospace Conference and Exhibit, 7-10 May 2007, Rohnert Park, CA.

7. Zhou, K., Doyle, J.C., and Glover, K., 1996, Robust and Optimal Control, Prentice-Hall, Upper Saddle River, New Jersey.

8. Chiang, R.Y., Safonov, M.G., Robust Control Toolbox User's Guide, The MathWorks Inc., Natick, MA, 1998.

9. Doyle, J.C., Glove, K, Khargonekar, P.P., and Francis, B.A., "State-space Solutions to Standard $\mathrm{H}_{2}$ and $\mathrm{H}_{\infty}$ Control Problems," IEEE Trans. Automat. Control, AC-34, p831-846, 1987.

10. Balas, G.J, Doyle, G.C., Glove, K., Packard, A., Smith, R., $\underline{\mu-A n a l y s i s ~ a n d ~ S y n t h e s i s ~}$ Toolbox, The MathWorks, Inc., Natick, MA, 1998.

\section{Optimal Sensor Placement}

1. Ali, Y., and S. Narasimhan, "Sensor Network Design for Maximizing Reliability of Linear Processes," American Institute of Chemical Engineering Journal, vol. 39, no. 5, pp. 820- 
$828,1993$.

2. Ali, Y., and S. Narasimhan, "Redundant Sensor Network Design for Linear Processes," American Institute of Chemical Engineering Journal, vol. 41, no. 10, pp. 2237-2249, 1995.

3. Ali, Y., and S. Narasimhan (1996), "Sensor Network Design for Maximizing Reliability of Bilinear Processes, ” American Institute of Chemical Engineering Journal, vol. 42, no. 9, pp. 2563-2575, 1996.

4. Al-Shehabi, A.G. and B. Newman, "Aeroelastic vehicle optimal sensor placement for feedback control applications using mixed gain-phase stability," Proceedings of the American Control Conference, vol. 3, pp. 1848-1852, June 2001.

5. Azam, M., K. Pattipati, and A. Patterson-Hine, "Optimal Sensor Allocation for Fault Detection and Isolation," IEEE International Conference on Systems, Man and Cybernetics, vol. 2, IEEE, pp. 1309-1314, 2004.

6. Bagajewicz, M., “Design and Retrofit of Sensor Networks in Process Plants, " American Institute of Chemical Engineering Journal, vol. 43, no. 9, pp. 2300-2306, 1997.

7. Bagajewicz, M., and M. Sanchez, "Design and Upgrade of Nonredundant and Redundant Linear Sensor Networks," American Institute of Chemical Engineering Journal, vol. 45, no. 9, pp. 1927-1938, 1999.

8. Bagajewicz, M., and M. Sanchez, "Cost-optimal Design of Reliable Sensor Networks," Computers and Chemical Engineering Journal, vol. 23, pp. 1757-1762, 2000.

9. Bagajewicz, M., Process Plant Instrumentation: Design and Upgrade, Technomic Publishing Company, Inc., 2001.

10. Bagajewicz, M., "A Review of Techniques for Instrumentation Design and Upgrade in Process Plants, ” The Canadian Journal of Chemical Engineering, vol. 80, no. 1, pp. 3-16, 2002.

11. Bagajewicz, M., and E. Cabrera, "New MILP Formulation for Instrumentation Network Design and Upgrade, " American Institute of Chemical Engineering Journal, vol. 48, no. 10, pp. 2271-2282, 2002.

12. Bagajewicz, M., A. Fuxman, and A. Uribe, "Instrumentation Network Design and Upgrade for Process Monitoring and Fault Detection," American Institute of Chemical Engineering Journal, vol. 50, no. 8, pp. 1870-1880, 2004.

13. Bhushan, M., and R. Rengaswamy, "Design of Sensor Network Based on the Signed Directed Graph of the Process for Efficient Fault Diagnosis," Industrial and Engineering Chemistry Research, vol. 39, pp. 999-1019, 2000. 
14. Bhushan, M., and R. Rengaswamy, "Design of Sensor Location Based on Various Fault Diagnostic Observability and Reliability Criteria," Computers and Chemical Engineering, vol. 24, pp. 735-741, 2000.

15. Bhushan, M., and R. Rengaswamy, "Comprehensive Design of a Sensor Network for Chemical Plants Based on Various Diagnosability and Reliability Criteria-1. Framework," Industrial and Engineering Chemistry Research, vol. 41, pp. 1826-1839, 2002.

16. Bhushan, M., and R. Rengaswamy, "Comprehensive Design of a Sensor Network for Chemical Plants Based on Various Diagnosability and Reliability Criteria-2. Applications," Industrial and Engineering Chemistry Research, vol. 41, pp. 1840-1860, 2002.

17. Bhushan, M., S. Narasimhan, and R. Rengaswamy, "Robust Sensor Network Design for Fault Diagnosis, " Computers and Chemical Engineering, vol. 32, pp. 1067-1084, 2008.

18. Carelli, M.D., L. Conway, L. Oriani, B. Pertovic, N.E. Todreas, et al., "The Design and Safety Features of the IRIS Reactor," ICONE 11, Tokyo, Japan, April 20-23, 2003.

19. Chen, S.Y. and Y.F. Li, "A method of Automatic Sensor Placement for Robot Vision in Inspection Tasks," Proceedings of the 2002 IEEE International Conference on Robotics \& Automation, vol. 3, pp. 2545-2550, 2002.

20. Chmielewski, D., T. Palmer, and V. Manousiouthakis, "On the Theory of Optimal Sensor Placement," American Institute of Chemical Engineering Journal, vol. 48, no. 5, pp. 10011012, 2002.

21. Dochain, D., N. Tali-Maanar, and J.P. Babary, "On Modeling, Monitoring and Control of Fixed Bed Bioreactors," Computers and Chemical Engineering, vol. 21, pp. 1255-1266, 1997.

22. Eberhart, R. C. and J. Kennedy, "A new optimization using particle swarm theory," Proceedings of the Sixth International Symposium on Micromachine and Human Science, Nagoya, Japan, pp. 39-43, 1995.

23. Faulds, A.L. and B.B. King, "Sensor location in feedback control of partial differential equation systems," Proceedings of the 2000 IEEE International Conference on Control Applications, vol. 1, pp. 536-541, Sept. 2000.

24. Furuya, H. and R.T. Haftka, "Combining Genetic and Deterministic Algorithms for Locating Actuators on Space Structures," Journal of Spacecraft and Rockets, vol. 33, no. 3, pp. 422427, 1996.

25. Giraud, C. and B. Jouvencel, "Sensor Selection: a Geometrical Approach," Proceedings of 1995 IEEE/RSJ International Conference, vol. 2, pp. 555-560, 1995.

26. Glover, F. and M. Laguna, Tabu Search, Kluwer Academic Publishers, Boston, 1997. 
27. Hac, A., and L. Liu, "Sensor and Actuator Location in Motion Control of Flexible Structures, ” Journal of Sound and Vibration, vol. 167, no. 2, pp. 239-261, 1993.

28. Johnson, D.S., C.R. Aragon, L.A. McGeoch, and C. Schevon, "Optimization by Simulated Annealing: An Experimental Evaluation; Part I, Graph Partitioning," Operations Research, vol. 37, pp. 865-893, 1989.

29. Kotecha, P.R., M. Bhushan, R.D. Gudi, and M.K. Keshari, “A Duality Based Framework for Integrating Reliability and Precision for Sensor Network Design," Journal of Process Control, vol. 18, pp. 189-201, 2008.

30. Kretsovalis, A. and R. Mah, "Observability and Redundancy Classification in Multicomponent Process Networks," American Institute of Chemical Engineering Journal, vol. 33, no. 1, pp. 70-82, 1987.

31. Lim, K.B., “A Disturbance Rejection Approach to Actuator and Sensor Placement,” Journal of Guidance, Control and Dynamics, vol. 20, no. 1, pp. 202-204, 1997.

32. Luong, M., D. Maquin, C. Huynh, and J. Ragot, "Observability, Redundancy, Reliability and Integrated Design of Measurement System," Proceedings of 2nd IFAC Symposium on Intelligent Components and Instruments for Control Applications, SICICA'94, 1994.

33. Madron, F., and V. Veverka, "Optimal Selection of Measuring Point in Complex Plants by Linear Models," American Institute of Chemical Engineering Journal, vol. 38, no. 2, pp. 227-236, 1992.

34. Madron, F., J. Papuga, and J. Pliska, "Thermal Performance Monitoring and Assessment in Dukovany Nuclear Power Plant," Proceedings of $5^{\text {th }}$ International Topical Meeting on Nuclear Plant Instrumentation Controls, and Human Machine Interface Technology, NPIC\&HMIT 2006, pp. 814-821, 2006.

35. Maul, W.A., G. Kopasakis, L.M. Santi, T.S. Sowers, and A. Chicatelli, "Sensor Selection and Optimization for Health Assessment of Aerospace Systems, " NASA/TM-2007-214822, 2007.

36. Maurya, M.R., R. Rengaswamy, and V. Venkatasubramanian, "A Signed Directed Graphbased Systematic Framework for Steady-State Malfunction Diagnosis inside Control Loops, " Chemical Engineering Science, vol. 61, pp. 1790-1810, 2006.

37. Mazzour, E.H., D. Hodouin, and S. Makni, "Optimal Sensor Implementation in Metallurgical Plants-An Application to a Generic Mineral Separation Plant,” Int. J. Miner. Processing, vol. 69, pp. 185-203, 2003.

38. Muller, P., and H. Weber, "Analysis and Optimization of Certain Qualities of Controllability and Observability for Linear Dynamical Systems, ” Automatica, vol. 8, pp. 237-246, 1972. 
39. Mushini, R., and D. Simon, "On Optimization of Sensor Selection for Aircraft Gas Turbine Engines," Proceedings of the 18th International Conference on Systems Engineering, ISCEng'05, IEEE, pp. 9-14, 2005.

40. Musulin, E., C. Benqlilou, M. Bagajewicz, and L. Puigjaner, "Instrumentation Design Based on Optimal Kalman Filtering," Journal of Process Control, vol. 15, no. 6, pp. 629-638, 2005.

41. Naimimohasses, R., D.M. Barnett, D.A. Green, and P.R. Smith, "Sensor optimization using neural network sensitivity measures, ” Measurement Science \& Technology, vol. 6, pp.1291$1300,1995$.

42. Narasimhan, S., P. Mosterman, and G. Biswas, "A Systematic Analysis of Measurement Selection Algorithms for Fault Isolation in Dynamic Systems," Proceedings of the 9th International Workshop on Principles of Diagnosis, DX-98, pp. 94-101, 1998.

43. Padula, S.L. and R.K. Kincaid, "Optimization strategies for sensor and actuator placement,” NASA/TM-1999-209126, April 1999.

44. Papadopoulos, M., and E. Garcia, "Sensor Placement Methodologies for Dynamic Testing," AIAA Journal, vol. 36, no. 2, pp. 256-263, 1998.

45. Ponslet, E., R.T. Haftka, and H.H. Cudney, "Optimal Placement of Tuning Masses on Truss Structures by Genetic Algorithm," Collection of Technical Papers - AIAA/ASME Structures, Dynamics and Materials Conference, Part 4, pp. 2448-2457, 1993.

46. Ponzoni, I., M.C. Sanchez, and N.B. Brignole, "A New Hybrid Approach for Instrumentation Design of Chemical Plants."

47. Raghuraj, R., M. Bhushan, and R. Rengaswamy, "Locating sensors in complex chemical plants based on fault diagnostic observability criteria," AIChE Journal, Vol. 45, No.2, pp. 310-322, 1999.

48. Santi, L.M, T.S. Sowers and R.B. Aguilar, "Optimal Sensor Selection for Health Monitoring Systems, ” NASA/TM-2005-213955, 2005.

49. Sen, S., S. Narasimhan, and K. Deb, "Sensor Network Design of Linear Processes Using Genetic Algorithms, ” Computers Chem. Engineering. vol. 22 no.3, pp. 385-390, 1998.

50. Shiozaki, J., B. Shibata, H. Matsuyama, and E. O'Shima, "Fault Diagnosis of Chemical Processes Utilizing Signed Directed Graphs-Improvement by Using Temporal Information," IEEE Transactions on Industrial Electronics, vol. 36, n. 4, pp. 469-474, 1989.

51. Spanache, S., T. Escobet, and L. Travé-Massuyès, "Sensor Placement Optimisation Using Genetic Algorithms," Proceedings of the15th International Workshop on Principles of 
Diagnosis, DX-04, pp. 179-183, 2004.

52. Tylee, J.L., "On-Line Failure Detection in Nuclear Power Plant Instrumentation," IEEE Transactions on Automatic Control, vol. AC-28, n. 3, pp. 406-415, 1983.

53. Udwadia, F.E., "Methodology for Optimum Sensor Locations for Parameter Identification in Dynamic Systems, ”Journal of Engineering Mechanics, vol. 120, n. 2, pp. 368-390, 1994.

54. Van den Berg, F., H. Hoefsloot, H. Boelens, and A. Smilde, "Selection of Optimal Sensor Position in a Tubular Reactor Using Robust Degree of Observability Criteria," Chemical Engineering Science, vol. 55, pp. 827-837, 2000.

55. Wang, H., Z. Song, and H. Wang, "Statistical Process Monitoring Using Improved PCA with Optimized Sensor Locations,” Journal of Process Control, vol. 12, pp. 735-744, 2002.

56. Wang, X., G. Rong, and J. Li, "A New Approach to Design Reliable General Sensor Network on the Basis of Graph Theory,” Ind. Eng. Chem. Res., vol. 46, pp. 2520-2525, 2007.

57. Yan, Y., "Sensor Placement and Diagnosability Analysis at Design Stage," National Research Council of Canada, NRC 47160, 2004.

58. Zhang, G., Optimum Sensor Localization/Selection in a Diagnostic/Prognostic Architecture, $\mathrm{Ph} . \mathrm{D}$. Dissertation, Georgia Institute of Technology, January 2005.

59. Zhang, G. and G. Vachtsevanos, "A Methodology for Optimum Sensor Localization/Selection in Fault Diagnosis," Proceedings of IEEE Aerospace Conference, 2007.

60. Zhang, Y., Product quality modeling \& control based on vision inspection with an application to baking processes, Ph.D. Dissertation, Georgia Institute of Technology, December 2004.

61. Zhao, K., An Integrated Approach to Performance Monitoring and Fault Diagnosis of Nuclear Power Systems, Ph.D. Dissertation, University of Tennessee, Knoxville, May 2005.

\section{Diagnostics and Prognostics}

1. Schmidt, F., R. Henderson, and C. Wolgemuth, Introduction to Thermal Sciences, New York, John Wiley \& Sons, Inc. 1993.

2. Westinghouse Electric Company LLC, STD-AR-05-01, "Instrumentation Needs for Integral Primary System Reactors (IPSRs)", Sept 2005. 
3. Hines, J.W. and D.R. Garvey, "Development and Application of Fault Detectability Performance Metrics for Instrument Calibration Verification and Anomaly Detection", Journal of Pattern Recognition Research, vol. 1, pp2-15, 2006.

4. Hines, J.W. , "Sensor Fault Detectability Measures for Auto-associative Empirical Models", $16^{\text {th }}$ annual Joint ISA POWID/EPRI Control and Instrumentation Conference, $49^{\text {th }}$ Annual ISA POWID Symposium, San Jose CA, June 4-9, 2006.

5. Garvey, D.R. and J.W. Hines An Integrated Fuzzy Inference Based Monitoring, Diagnostic, and Prognostic System, 61th Meeting of the Society for Machinery Failure Prevention Technology, Virginia Beach, Virginia, April, 2007.

6. Hines, J.W., "Empirical Methods for Process and Equipment Condition Monitoring", 52 ${ }^{\text {nd }}$ Annual Reliability and Maintainability Symposium (RAMS), Newport Beach CA, Jan 2326, 2006.

7. Ebeling, C. E. , An Introduction to Reliability and Maintainability Engineering, Waveland Press, Inc., 1997, reissued 2005.

8. Elsayed, E.A., Reliability Engineering, Addison-Wesley, 1996.

9. Meeker, W.O., and L.A. Escobar, Statistical Methods for Reliability Data, John Wiley and Sons, 1998.

10. Abernethy, R. B., The New Weibull Handbook, $2^{\text {nd }}$ edn. ISBN 0965306208 . Abernethy, North Palm Beach, 1996.

11. Cox, DR, D. Oakes, Analysis of survival data Chapman \& Hall, 1984.

12. Lu, C.J, and W.Q. Meeker, "Using Degradation Measures to Estimate a Time-to-Failure Distribution," Technometrics Vol 35 No 2, pp. 161-173, May 1993.

13. Upadhyaya, B.R., M. Naghedolfeizi, and B. Raychaudhuri, "Residual Life Estimation of Plant Components," P/PM Technology, p 22-29, June 1994.

14. Usynin, A, and J.W. Hines, "Use of Linear Growth Models for Remaining Useful Life Prediction," MARCON 2007.

15. Carlin, B.P and T.A. Louis, Bayes and Empirical Bayes Methods for Data Analysis, $2^{\text {nd }}$ edn. Boca Raton: Chapman and Hall/CRC: 2000.

16. Hines, J.W., Usynin, A., "An Introduction to Prognostics with Application", Society of Maintenance and Reliability Professionals, Louisville, Kentucky, 2007.

17. Usynin, A., Hines J.W., and A. Urmanov, "Formulation of Prognostics Requirements", IEEE Aerospace Conference, Big Sky, Montana, 2007. 
18. Coble, J, Merging Data Source to Predict Remaining Useful Life - An Automated Method to Identify Prognostic Parameters, Doctoral Dissertation, The University of Tennessee: May 2010. 


\section{Advanced Instrumentation and Control Methods for Small and Medium Reactors with IRIS Demonstration}

\section{Final Report}

Volume 4

Dynamic Modeling, Sensor Placement Design, and Fault Diagnosis of Nuclear Desalination Systems

Report No. DE-FG07-07ID14895/UTNE/2011-6

NERI-C Grant Number: DE-FG07-07ID14895

Belle R. Upadhyaya

(Co-Principal Investigators)

Fan Li

The University of Tennessee

Nuclear Engineering Department

Knoxville, TN 37996-2300

E-mail: bupadhya@utk.edu

May 2011

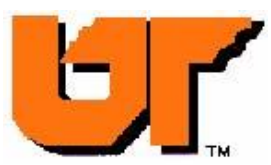




\begin{abstract}
Fault diagnosis of sensors, devices, and equipment is an important topic in the nuclear industry for effective and continuous operation of nuclear power plants. All the fault diagnostic approaches depend critically on the sensors that measure important process variables. Whenever a process encounters a fault, the effect of the fault is propagated to some or all the process variables. The ability of the sensor network to detect and isolate failure modes and anomalous conditions is crucial for the effectiveness of a fault detection and isolation (FDI) system. However, the emphasis of most fault diagnostic approaches found in the literature is primarily on the procedures for performing FDI using a given set of sensors. Little attention has been given to actual sensor allocation for achieving the efficient FDI performance. This research project presents a graph-based approach that serves as a solution for the optimization of sensor placement to ensure the observability of faults, as well as the fault resolution to a maximum possible extent. This would potentially facilitate an automated sensor allocation procedure. Principal component analysis (PCA), a multivariate data-driven technique, is used to capture the relationships in the data, and to fit a hyper-plane to the data. The fault directions for different fault scenarios are obtained from the prediction errors, and fault isolation is then accomplished using new projections on these fault directions. The effectiveness of the use of an optimal sensor set versus a reduced set for fault detection and isolation is demonstrated using this technique.
\end{abstract}

Among a variety of desalination technologies, the multi-stage flash (MSF) processes contribute substantially to the desalinating capacity in the world. In this project, both steady-state and dynamic simulation models of a MSF desalination plant are developed. The dynamic MSF model is coupled with a previously developed International Reactor Innovative and Secure (IRIS) model in the SIMULINK environment. The developed sensor placement design and fault diagnostic methods are illustrated with application to the coupled nuclear desalination system. The results demonstrate the effectiveness of the newly developed integrated approach to performance monitoring and fault diagnosis with optimized sensor placement for large industrial systems. 


\section{TABLE OF CONTENTS}

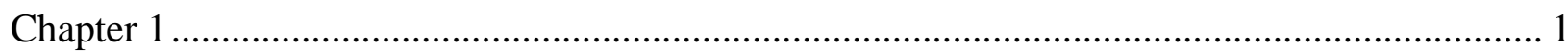

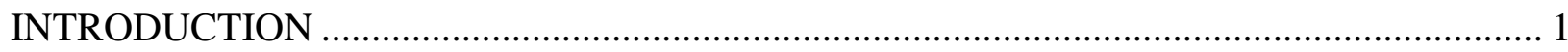

$1.1 \quad$ Background and Motivation ................................................................................ 1

1.2 R\&D Objectives and Significant Contributions …………......................................... 5

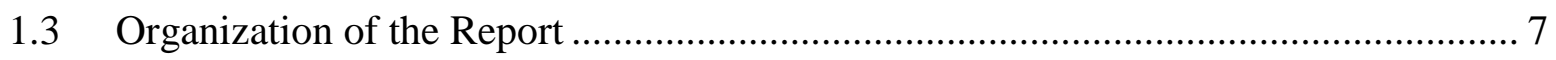

Chapter 2

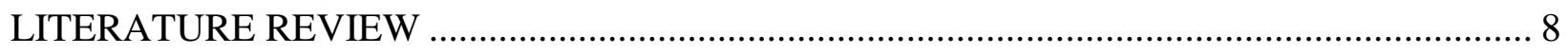

2.1 Optimum Sensor Placement for Process Systems ................................................... 8

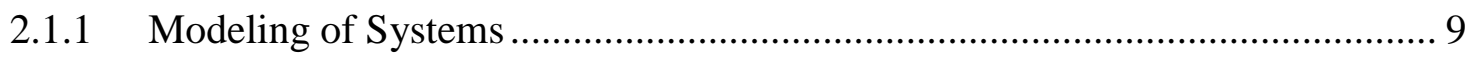

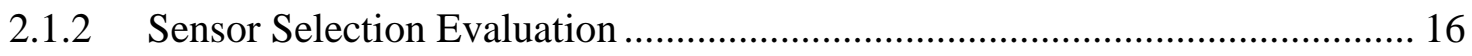

2.1.3 Sensor Optimization Techniques ................................................................ 22

$2.2 \quad$ Sea Water Desalination Methods …………….................................................. 24

2.2.1 Classification of Desalination Processes ..................................................... 24

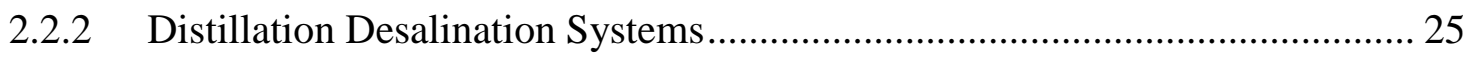

2.2.3 Membrane Desalination Systems ……………………………………...... 31

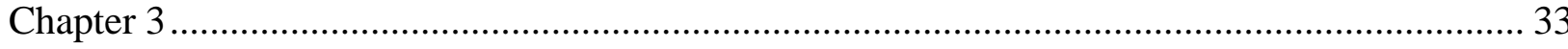

INTEGRATED ARCHITECTURE OF A FAULT DIAGNOSTIC SYSTEM.............................. 33

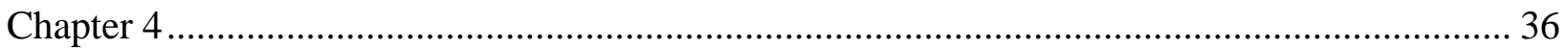

MODELING AND SIMULATION OF A MULTI-STAGE FLASH DESALINATION SYSTEM

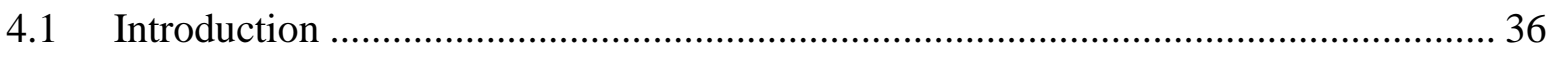

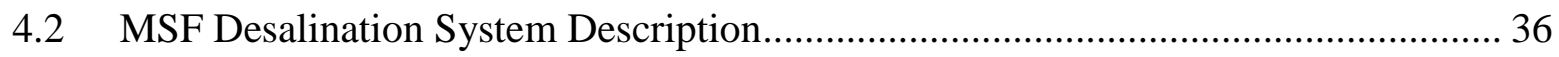

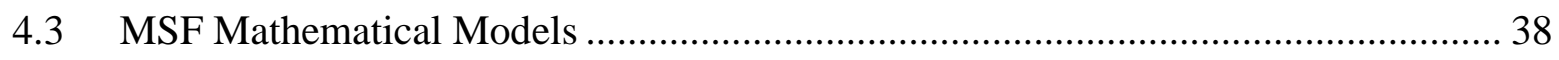

4.3.1 Flashing Stage Dynamic Model ................................................................... 39

4.3.2 Brine Heater Dynamic Model....................................................................... 41

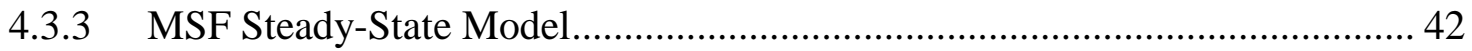

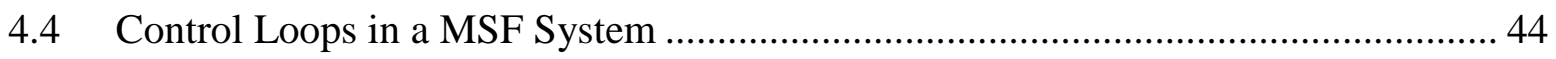

4.5 Measurements and Instrumentation in a MSF System ............................................. 47

4.6 MSF Desalination System Simulation Results ........................................................... 49

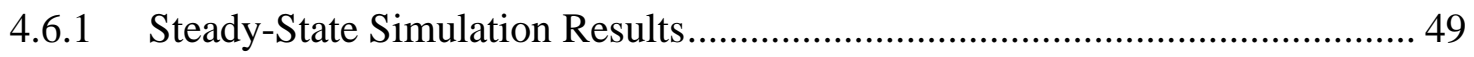

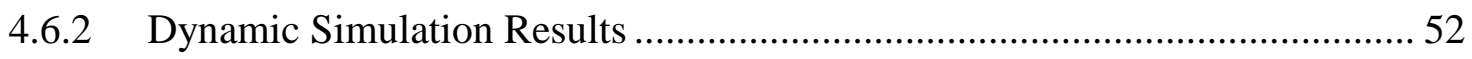

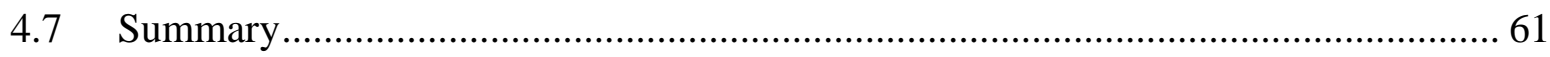

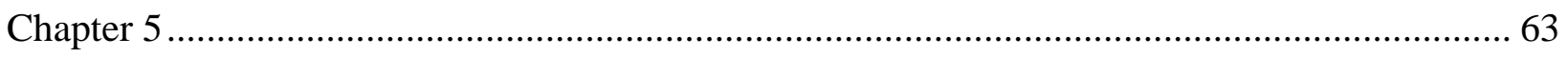

MODELING AND SIMULATION OF A NUCLEAR DESALINATION PLANT …………......... 63

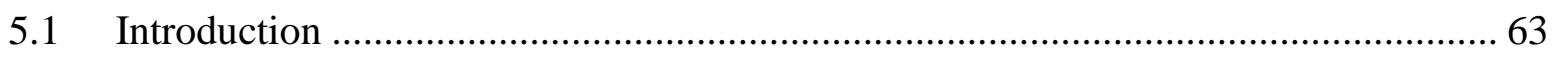

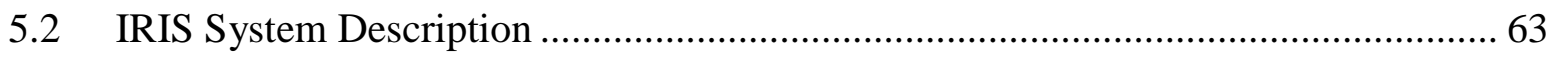

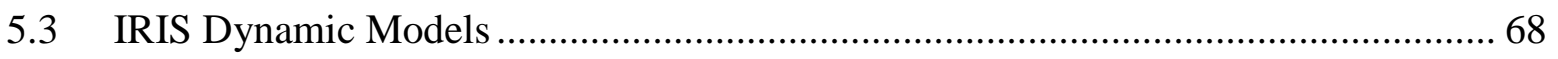

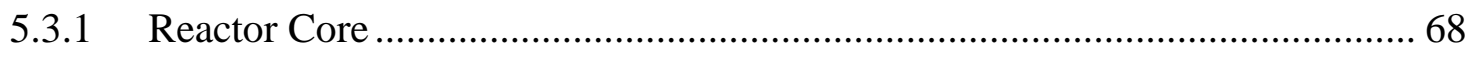




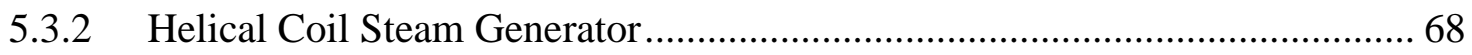

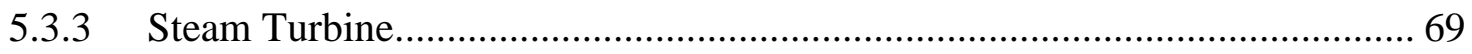

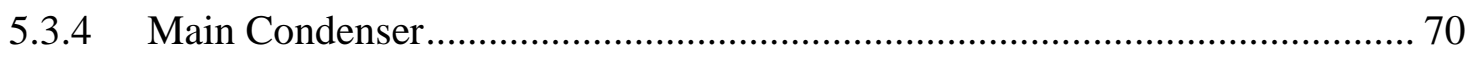

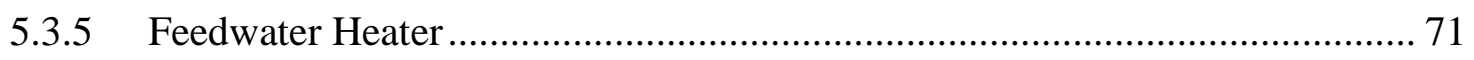

5.4 IRIS-MSF Nuclear Desalination Plant Simulation Results ........................................ 72

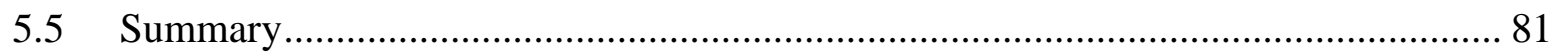

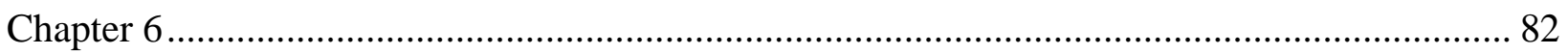

DEVELOPMENT OF SENSOR PLACEMENT DESIGN ALGORITHMS …………................ 82

6.1 Introduction ................................................................................................... 82

6.2 Method of Directed Graph Modeling ……………….......................................... 83

6.3 Sensor Location and Fault Diagnostic Observability Criterion................................... 85

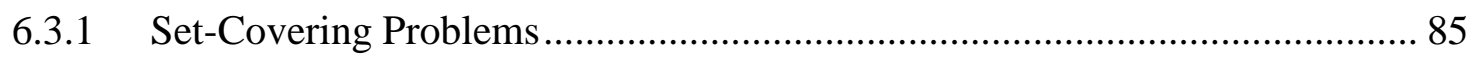

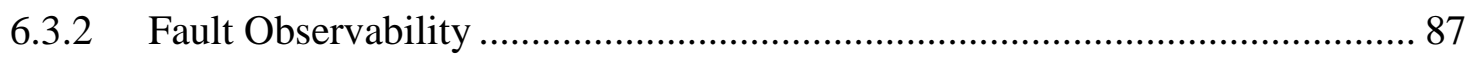

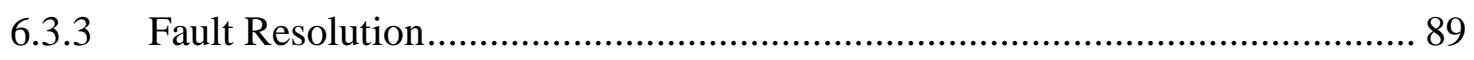

6.4 Sensor Location and System Unobservability Criterion .......................................... 92

6.4.1 Formulation of Unobservability Minimization................................................ 92

6.4.2 One-Step Optimization Formulation …………............................................. 95

6.4.3 Solving Unobservability Minimization Problems …………………………...... 97

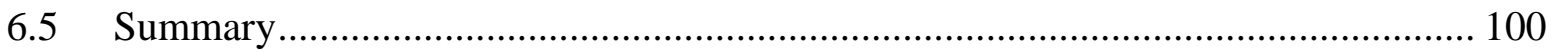

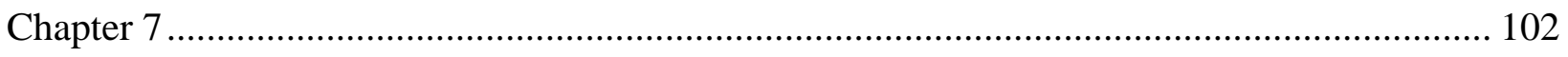

SENSOR PLACEMENT DESIGN AND FAULT DIAGNOSIS APPLICATION FOR A MSF

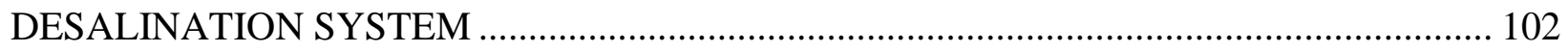

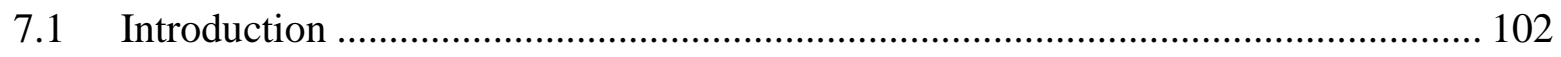

7.2 Principal Component Analysis for Fault Diagnosis ………………........................ 102

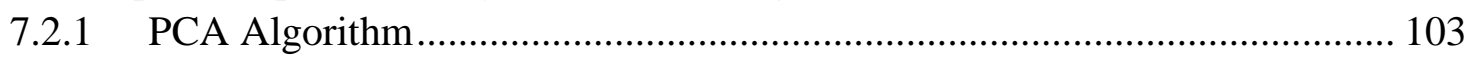

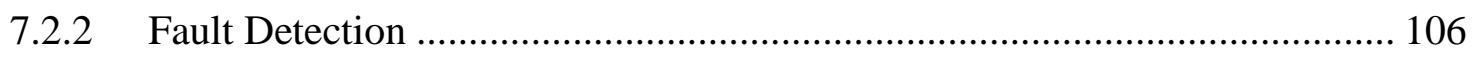

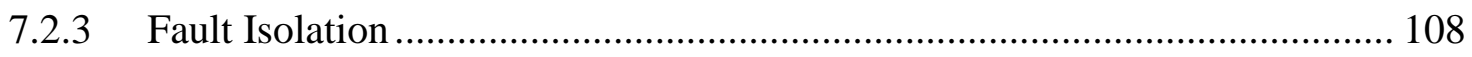

7.3 DG Model Development of a MSF System.......................................................... 110

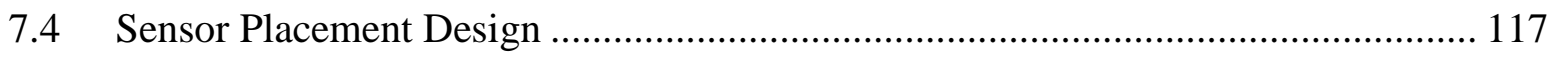

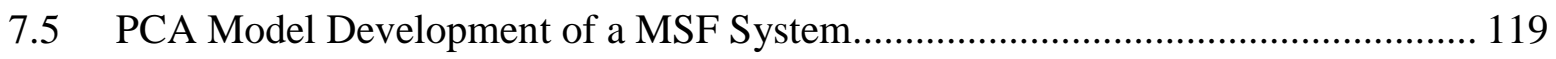

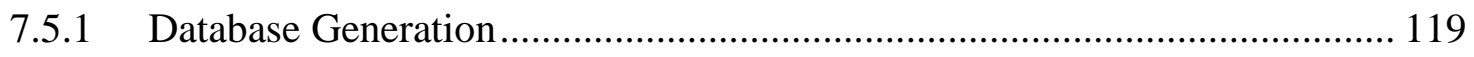

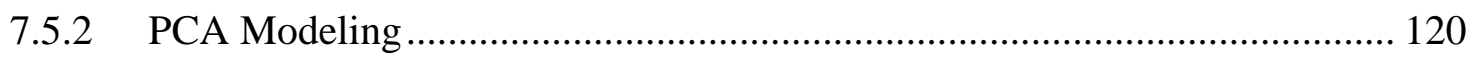

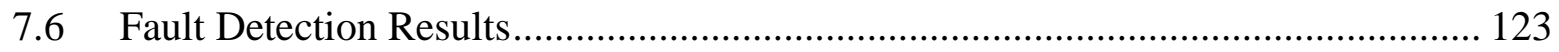

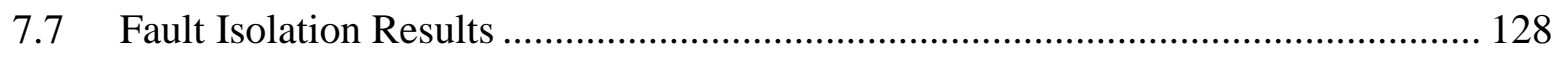

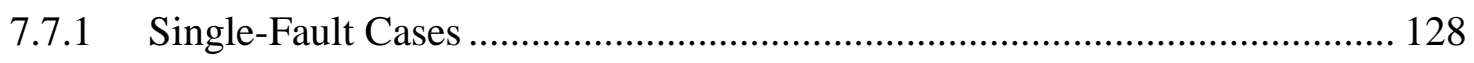

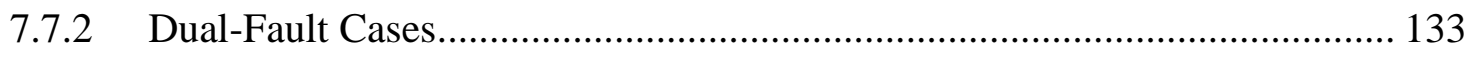

7.8 Summary

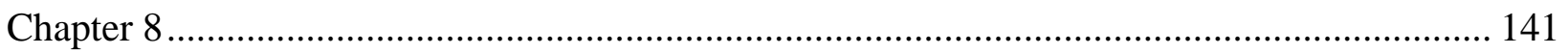

SENSOR PLACEMENT DESIGN AND FAULT DIAGNOSIS APPLICATION FOR HCSG

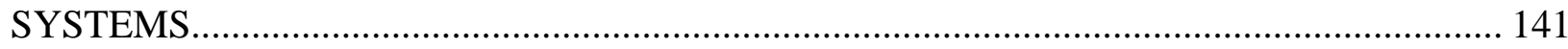

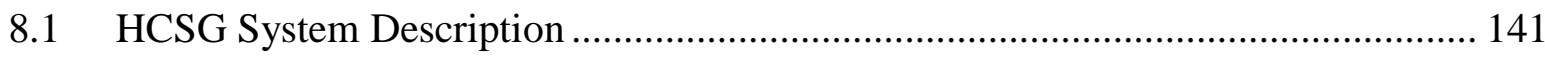




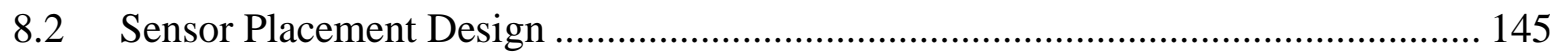

8.2.1 Fault Diagnostic Observability Criterion …………….................................. 145

8.2.2 System Unobservability Criterion ........................................................ 145

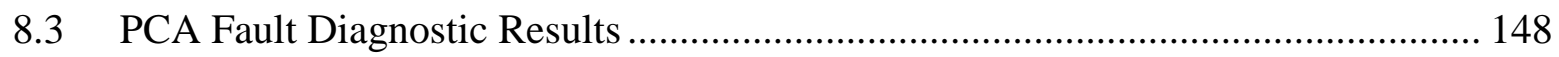

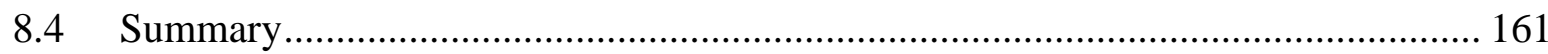

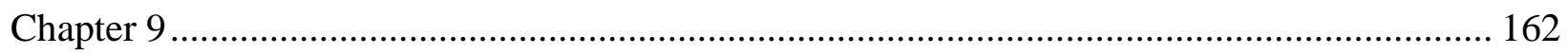

Summary, Conclusions, and Suggestions for Future Research ............................................... 162

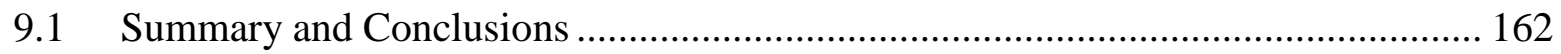

9.2 Suggestions for Future Research ...................................................................... 165

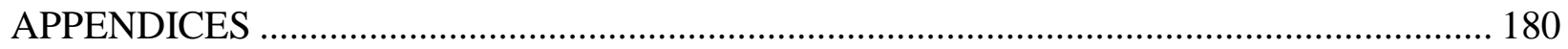

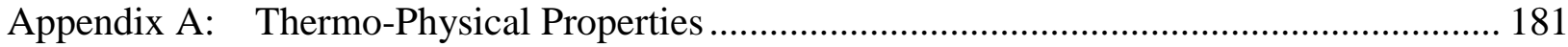

Appendix B: IRIS Balance of Plant Mathematical Models ...................................................... 185

Appendix C: Fault Isolation Index Plots for Dual-Fault Scenarios .......................................... 188

\section{DISCLAIMER}

Any opinions, findings, and conclusions or recommendations expressed in this report are those of the authors, and do not necessarily reflect the views of the U.S. Department of Energy or Westinghouse Electric Company, LLC. 


\section{LIST OF TABLES}

Table 4.1. Measuring instruments used in the desalination industry ................................ 48

Table 4.2. The main characteristics of the plant ........................................................... 50

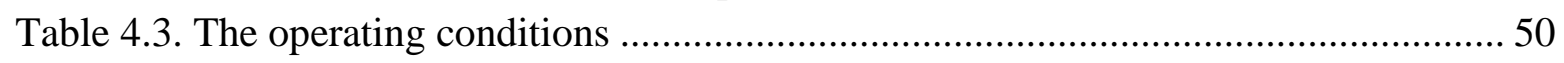

Table 4.4. The condenser tube bundle data..................................................................... 50

Table 4.5. Steady-state model results corresponding to the performance study case.......... 51

Table 4.6. Actual plant operation data [Helal et al., 1986] ............................................. 52

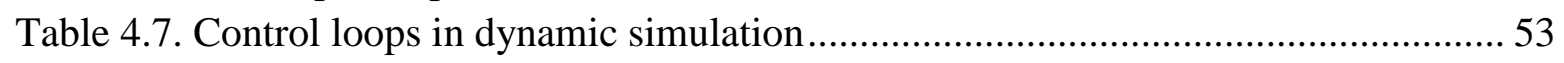

Table 4.8. Dynamic simulation tests ............................................................................... 55

Table 6.1. Set $A$ of sensors for different fault nodes in Figure 6.2 .............................. 91

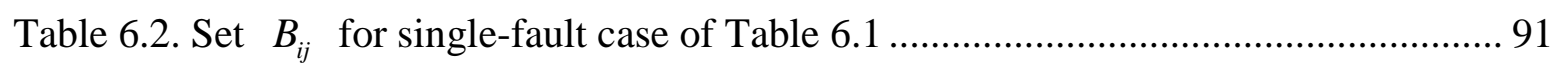

Table 7.1. DG model variables of brine heater .............................................................. 112

Table 7.2. DG model variables of flash stage $i$....................................................... 114

Table 7.3. Two-stage MSF system available variables................................................. 116

Table 7.4. Faults considered for two-stage MSF system .............................................. 117

Table 7.5. MSF variables used to develop PCA models................................................... 120

Table 7.6. PCA fault direction analysis for MSF system under single fault assumption ... 128

Table 8.1. Fault nodes of a pair of HCSGs .................................................................. 143

Table 8.2. Fault data for a pair of HCSGs .................................................................... 146

Table 8.3. Sensor data for a pair of HCSGs................................................................... 146

Table 8.4. Sensor selection results using greedy search heuristic and LINGO 8.0 ........... 147

Table 8.5. HCSG sensor placement results (available cost=1000) ................................ 148

Table 8.6. PCA fault direction analysis for a pair of HCSGs ....................................... 153 


\section{LIST OF FIGURES}

Figure 1.1. Projected regions affected by water shortages [IWMI, 2000]............................. 1

Figure 2.1. Basic components of sensor placement strategy. .............................................. 9

Figure 2.2. Directed graph of a hypothetical system. ………………………………........ 21

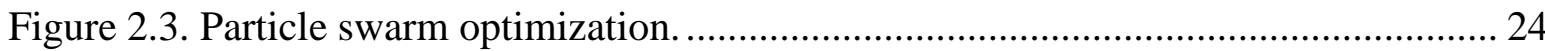

Figure 2.4. Classification of desalination processes [IAEA, 2007] ................................... 25

Figure 2.5. Principle of vapor compression [Khan, 1986]................................................. 27

Figure 2.6. Schematic diagram of a MED system [IAEA, 2007]......................................... 28

Figure 2.7. Operating principle of a single stage MSF [IAEA, 2007] ................................. 29

Figure 2.8. Schematic diagram of an industrial MSF design [IAEA, 2007] ........................ 30

Figure 2.9. Osmosis and reverse osmosis processes [IAEA, 2007] ..................................... 31

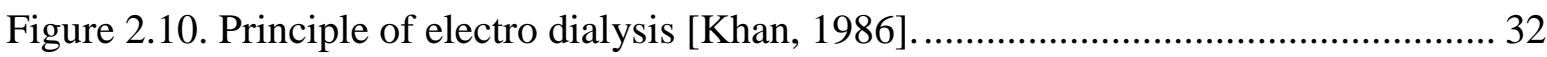

Figure 3.1. An integrated architecture of a fault diagnostic system. ................................... 35

Figure 4.1. Schematic diagram of a MSF process with brine recirculation [Alatiqi et al.,

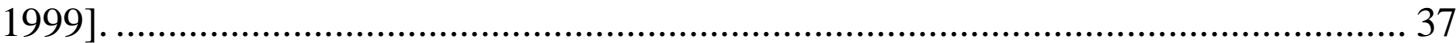

Figure 4.2. Block diagram of a stage of a MSF plant........................................................ 39

Figure 4.3. Single-input single-output control loops of MSF [Alatiqi et al., 1999]............ 44

Figure 4.4. Screenshot of the MSF SIMULINK model...................................................... 54

Figure 4.5. Dynamic responses of process variables to step increase of TBT set point....... 56

Figure 4.6. Dynamic responses of process variables to step increase of incoming cooling

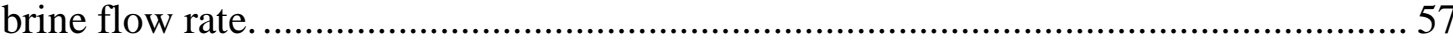

Figure 4.7. Dynamic responses of process variables to step increase of incoming cooling

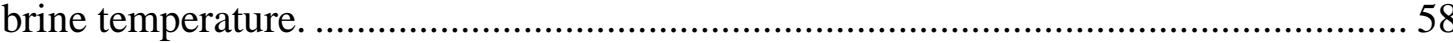

Figure 4.8. Dynamic responses of process variables to step increase of heating steam

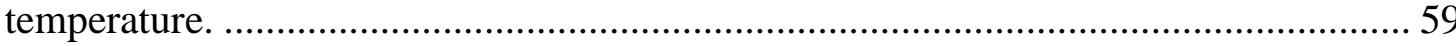

Figure 4.9. Open loop response of TBT to step decrease of heating steam temperature...... 60

Figure 4.10. Open loop response of brine levels to step decrease of heating steam

temperature.

Figure 4.11. Open loop response of last-stage brine level to step decrease of heating steam

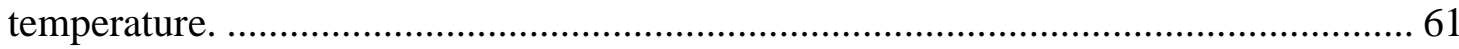

Figure 5.1. IRIS primary system layout [Carelli et al., 2003] ............................................. 66

Figure 5.2. IRIS reactor pressure vessel and compact containment vessel [Carelli et al.,

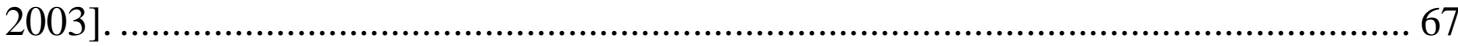

Figure 5.3. Schematic diagram of the turbine condenser model......................................... 71

Figure 5.4. Schematic of a horizontal closed feedwater heater [Thanomsat et al., 1998] ... 72 Figure 5.5. Schematic diagram of a MSF plant coupled to the IRIS power conversion

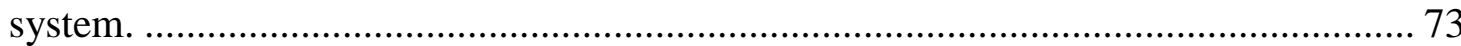

Figure 5.6. Reactor power response to a 10\% power demand step decrease........................ 74 
Figure 5.7. $T_{\text {hot }}$ response to a $10 \%$ power demand step decrease..................................... 75

Figure 5.8. $T_{\text {cold }}$ response to a $10 \%$ power demand step decrease..................................... 75

Figure 5.9. $T_{\text {ave }}$ response to a $10 \%$ power demand step decrease....................................... 76

Figure 5.10. Steam pressure response to a $10 \%$ power demand step decrease..................... 76

Figure 5.11. Steam flow rate response to a 10\% power demand step decrease..................... 77

Figure 5.12. Turbine output response to a 10\% power demand step decrease. .................... 77

Figure 5.13. Feedwater temperature response to a $10 \%$ power demand step decrease. ....... 78

Figure 5.14. Steam temperature response to a $10 \%$ power demand step decrease................ 78

Figure 5.15. Top brine temperature response to a 10\% power demand step decrease. ........ 79

Figure 5.16. Brine heater inlet steam flow rate response to a $10 \%$ power demand step

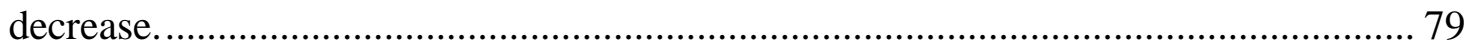

Figure 5.17. Brine heater inlet steam enthalpy response to a $10 \%$ power demand step

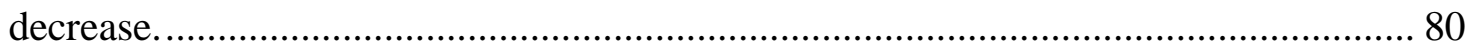

Figure 5.18. Brine level response to a 10\% power demand step decrease. ......................... 80

Figure 6.1. Directed graph of a hypothetical process. ....................................................... 84

Figure 6.2. Fault and sensor node bipartite graph for Figure 6.1 ........................................ 89

Figure 6.3. Flowchart for greedy search algorithm...................................................... 98

Figure 6.4. Bipartite graph for Figure 6.2 with probabilities............................................. 99

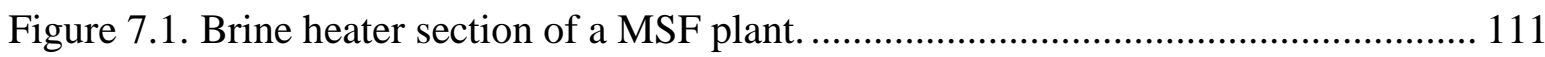

Figure 7.2. Directed graph of brine heater section............................................................ 111

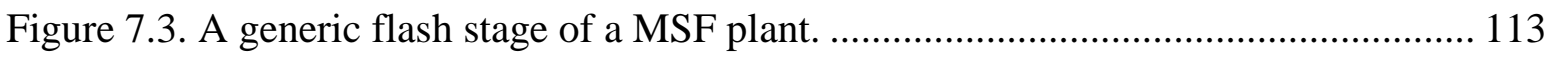

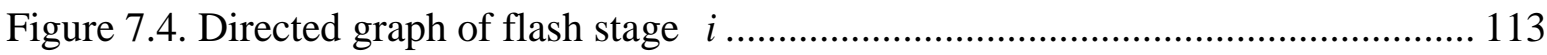

Figure 7.5. Directed graph of a two-stage MSF system. ………...................................... 115

Figure 7.6. Percentage of variance explained by principal components.............................. 121

Figure 7.7. $Q$ statistic for MSF PCA model. ................................................................ 121

Figure 7.8. $T^{2}$ statistic for MSF PCA model. .............................................................. 122

Figure 7.9. Residual pattern for inlet cooling brine temperature sensor drift (Fault \#1).... 124

Figure 7.10. Residual pattern for inlet cooling brine flow sensor drift (Fault \#2).............. 124

Figure 7.11. Residual pattern for inlet cooling brine salinity sensor drift (Fault \#3)......... 125

Figure 7.12. Residual pattern for flashing stage \#1 heat transfer degradation (Fault \#4)... 125

Figure 7.13. Residual pattern for flashing stage \#2 heat transfer degradation (Fault \#5)... 126

Figure 7.14. Residual pattern for top brine temperature sensor drift (Fault \#6)................. 126

Figure 7.15. Residual pattern for top brine temperature controller fault (Fault \#7)........... 127

Figure 7.16. Residual pattern for Stage \#2 brine level controller fault (Fault \#8).............. 127

Figure 7.17. Fault isolation index for inlet cooling brine temperature sensor drift............ 129

Figure 7.18. Fault isolation index for inlet cooling brine flow sensor drift........................ 129

Figure 7.19. Fault isolation index for inlet cooling brine salinity sensor drift. ................. 130 
Figure 7.20. Fault isolation index for flashing stage \#1 heat transfer degradation............. 130

Figure 7.21. Fault isolation index for flashing stage \#2 heat transfer degradation............ 131

Figure 7.22. Fault isolation index for top brine temperature sensor drift........................ 131

Figure 7.23. Fault isolation index for top brine temperature controller fault. .................. 132

Figure 7.24. Fault isolation index for Stage \#2 brine level controller fault...................... 132

Figure 7.25 Fault isolation index for dual-fault case \#1............................................... 137

Figure 7.26 Fault isolation index for dual-fault case \#2............................................ 137

Figure 7.27 Fault isolation index for dual-fault case \#3. ............................................. 138

Figure 7.28 Fault isolation index for dual-fault case \#4. ............................................. 138

Figure 7.29 Fault isolation index for dual-fault case \#5............................................. 139

Figure 7.30 Fault isolation index for dual-fault case \#6............................................... 139

Figure 7.31 Fault isolation index for dual-fault case \#7. ........................................... 140

Figure 8.1. IRIS steam generator layout [Carelli et al., 2003] ....................................... 141

Figure 8.2. A schematic of a pair of HCSGs [Zhao, 2005a] ......................................... 142

Figure 8.3. Directed graph of a pair of HCSGs. ............................................................. 144

Figure 8.4. Decreasing trend of unobservability for system faults of a pair of HCSGs. ... 148

Figure 8.5. Residual pattern for $\mathrm{T}_{\text {hot }}$ sensor fault (Fault \#1)...................................... 150

Figure 8.6. Residual pattern for SG-A thermal degradation (Fault \#2)........................... 151

Figure 8.7. Residual pattern for SG-B thermal degradation (Fault \#3). ........................... 151

Figure 8.8. Residual pattern for secondary flow distribution anomaly (Fault \#4)............. 152

Figure 8.9. Residual pattern for feed temperature sensor fault (Fault \#5)........................ 152

Figure 8.10. Residual pattern for feed flow sensor fault (Fault \#6)................................. 153

Figure 8.11. Fault isolation index for $\mathrm{T}_{\text {hot }}$ sensor fault. ............................................ 154

Figure 8.12. Fault isolation index for SG-A thermal degradation. ................................ 154

Figure 8.13. Fault isolation index for SG-B thermal degradation. ................................ 155

Figure 8.14. Fault isolation index for secondary flow distribution anomaly.................... 155

Figure 8.15. Fault isolation index for feed temperature sensor fault............................... 156

Figure 8.16. Fault isolation index for feed flow sensor fault........................................ 156

Figure 8.17. Fault isolation index for $\mathrm{T}_{\text {hot }}$ sensor fault (reduced sensor set)................... 157

Figure 8.18. Fault isolation index for SG-A thermal degradation (reduced sensor set).... 158

Figure 8.19. Fault isolation index for SG-B thermal degradation (reduced sensor set).... 158

Figure 8.20. Fault isolation index for secondary flow anomaly (reduced sensor set). ....... 159

Figure 8.21. Fault isolation index for feed temperature sensor fault (reduced sensor set). 159

Figure 8.22. Fault isolation index for feed flow sensor fault (reduced sensor set)............ 160

Figure 8.23. Fault isolation index for a pair of HCSGs............................................ 161 


\section{ABBREVIATIONS AND SYMBOLS}

\begin{tabular}{|c|c|}
\hline AIC & Akaike Information Criterion \\
\hline ANN & Artificial Neural Network \\
\hline BOP & Balance-of-Plant \\
\hline BPE & Boiling Point Elevation \\
\hline CBM & Condition-Based Maintenance \\
\hline $\mathrm{CPV}$ & Cumulative Percentage Variance \\
\hline CRDM & Control Rod Drive Mechanism \\
\hline $\mathrm{CV}$ & Control Volume \\
\hline DG & Directed Graph \\
\hline ED & Electro-Dialysis \\
\hline FDI & Fault Detection and Isolation \\
\hline FOM & Figure of Merit \\
\hline GA & Genetic Algorithm \\
\hline GMDH & Group Method of Data Handling \\
\hline GUI & Graphical User Interface \\
\hline HCSG & Helical Coil Steam Generator \\
\hline HTE & Horizontal Tube Evaporator \\
\hline HT-VTE & High Temperature Vertical Tube Evaporation \\
\hline IAEA & International Atomic Energy Agency \\
\hline ILP & Integer Linear Programming \\
\hline INLP & Integer Non-Linear Programming \\
\hline IPSR & Integral Primary System Reactor \\
\hline IRIS & International Reactor Innovative and Secure \\
\hline IWMI & International Water Management Institute \\
\hline LMTD & Log Mean Temperature Difference \\
\hline
\end{tabular}




\begin{tabular}{|c|c|}
\hline LOCA & Loss-of-Coolant Accident \\
\hline LT-HTME & Low Temperature Horizontal Tube Multi-Effect \\
\hline LWR & Light Water Reactor \\
\hline MED & Multi-Effect Distillation \\
\hline MILP & Mixed Integer Linear Programming \\
\hline MINLP & Mixed Integer Non-Linear Programming \\
\hline MSF & Multi-Stage Flash \\
\hline NEA & Non-Equilibrium Allowance \\
\hline PCA & Principal Component Analysis \\
\hline PSO & Particle Swarm Optimization \\
\hline PWR & Pressurized Water Reactor \\
\hline QDG & Quantified Directed Graph \\
\hline $\mathrm{RCP}$ & Reactor Coolant Pump \\
\hline RO & Reverse Osmosis \\
\hline SA & Simulated Annealing \\
\hline SMR & Small Modular Reactor \\
\hline SNR & Signal-to-Noise Ratio \\
\hline SPE & Squared Prediction Error \\
\hline SVD & Singular Value Decomposition \\
\hline TBT & Top Brine Temperature \\
\hline TDM & Tri-Diagonal Matrix \\
\hline $\mathrm{VC}$ & Vapor Compression \\
\hline VRE & Variance Reconstruction Error \\
\hline
\end{tabular}




\section{Chapter 1}

\section{INTRODUCTION}

\subsection{Background and Motivation}

Water is indispensable for the very existence of mankind and for human development. The total quantity of water available on earth is about 1,000 million $\mathrm{km}^{3}$ and covers nearly $70 \%$ of the globe, whereas the total world water consumption does not exceed 2,100 $\mathrm{km}^{3} /$ year. At first thought this would seem rather reassuring. However, $97.5 \%$ of the available water is highly saline or brackish. Of the remaining 2.5\%, nearly $70 \%$ is in the form of ice (Antarctica, Greenland, etc). Yet another large fraction is locked in the soil humidity and in deep underground aquifers. Consequently the effective amount of water, directly accessible to human beings is only $0.007 \%$ (or, about $70,000 \mathrm{~km}^{3}$ ) [IWMI, 2000]. Even this fraction is very unevenly distributed over the planet. Moreover, rapidly increasing populations, rising standards of living, continued development of tourism, progressive industrialization, and expansion of irrigation agriculture have already led to acute water shortages and stresses in many regions of the world as shown in red in Figure 1.1.

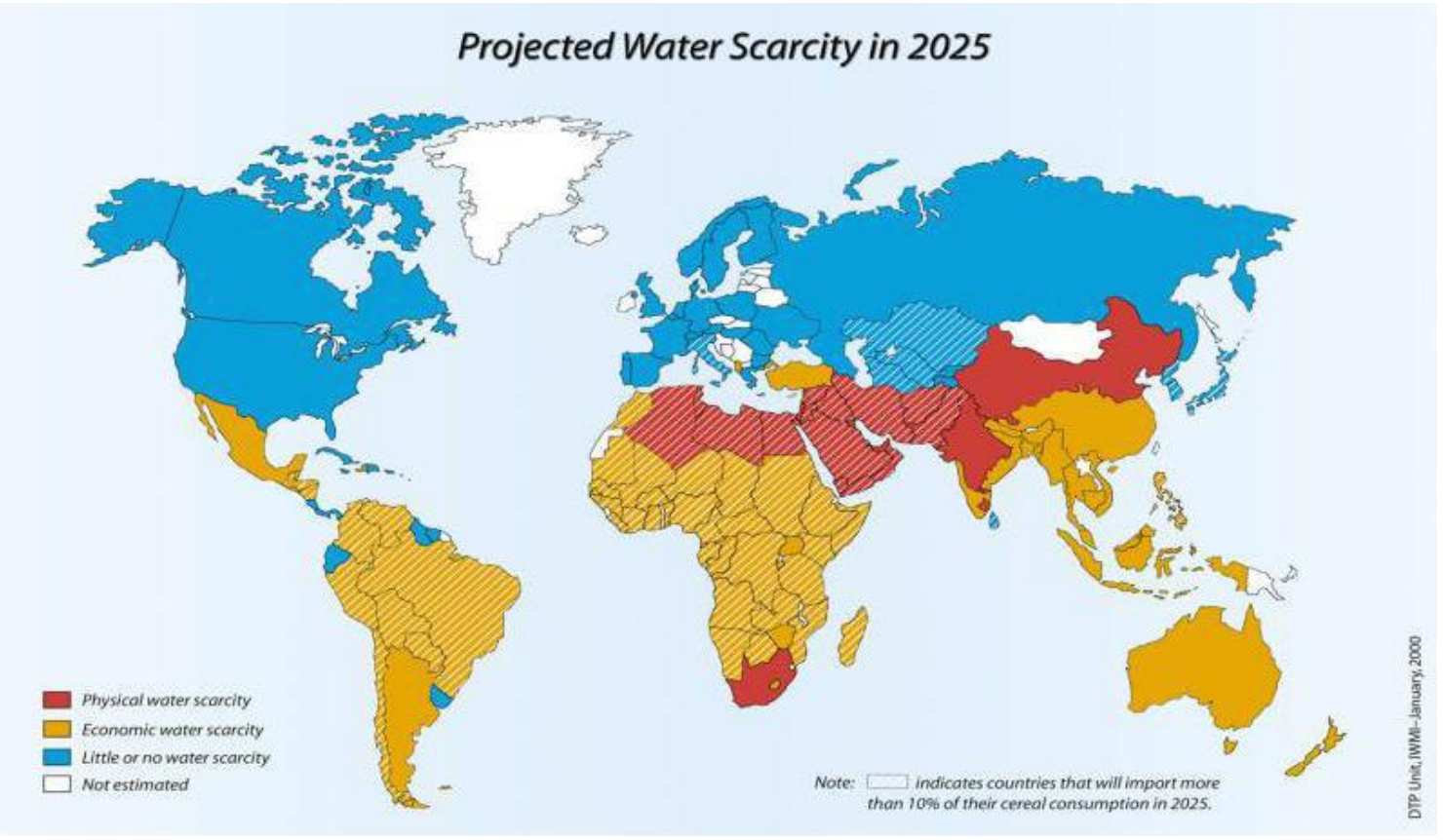

Figure 1.1. Projected regions affected by water shortages [IWMI, 2000]. 
In Figure 1.1, countries which will face "economic water shortages" (i.e. inadequacy of supply and demand) are shown. According to the market survey performed by the World Resources Institute on the future growth of seawater desalination, the worldwide demand for desalination is expected to double approximately every 10 years in the foreseeable future. Most of the demand would arise in the Arabian Gulf and North African regions, but this is likely to expand to other areas.

During the second half of the past century, industrial water desalination became a sustainable source of potable water in several countries across the globe. Out of the more than 12,000 desalination plants currently in operation, only about 10 use heat or electricity provided by nuclear power plants [IAEA, 2002]. Fossil energy sources are the dominant choice. However, due to their constantly depleting quantities and the emitted air pollutants as a result of combustion, fossil fuels should not be considered as a sustainable source of energy. These environmental concerns, coupled with concerns over energy supply security and an anticipated growth in energy demands, are driving a growing interest in the development and expansion of the nuclear energy options. Nuclear energy offers a clean and abundant energy supply. Also, the current generation of nuclear plants has proven that nuclear energy can be safe and economically competitive with alternative options.

In the International Atomic Energy Agency (IAEA) terminology, nuclear desalination is defined as the production of potable water from seawater in a facility in which a nuclear reactor is used as the source of energy for the desalination process. Electrical and/or thermal energy may be used in the desalination process. The facility may be dedicated solely to the production of potable water, or may be used for the generation of electricity and production of potable water, in which case only a portion of the total energy output of the reactor is used for water production [IAEA, 2007]. The prospects of using nuclear energy for seawater desalination on a large scale can be very attractive since desalination is an energy intensive process that can utilize the heat from a nuclear reactor and/or the electricity produced by such plants. The choice of the desalination technology determines the form of energy required: electrical energy for reverse osmosis (RO) systems, and relatively low quality thermal energy for distillation systems, such as multi-stage flash (MSF) distillation. The MSF desalination process has dominated the field of thermal desalination with a market share of over $90 \%$. In addition, market share of MSF 
processes accounts for $70 \%$ of all seawater desalination processes [El-Dessouky et al., 1999]. It is thus logical that the capability of coupling a MSF process to a nuclear power plant as the heat source makes the process increasingly important for future drinking water and electricity generation.

On the other hand, while all nuclear reactor types can provide the energy required by the various desalination processes, it has been shown that small and medium sized reactors (SMR), ranging from $50 \mathrm{MWe}$ to $500 \mathrm{MWe}$ in electricity production, offer the largest potential as the coupling option of nuclear desalination systems in developing countries. The development of innovative reactor concepts and fuel cycles with enhanced safety features as well as their attractive economics are expected to improve the public acceptance and further the prospects of nuclear desalination.

To facilitate the anticipated growth in demand for nuclear energy world-wide, several countries including the United States, have initiated the development of the next generation of nuclear plants that offer even greater safety, reliability, and economics, while also reducing the threat of proliferation of special nuclear materials. The Generation IV nuclear power program was initiated by the U.S. Department of Energy to identify and develop promising next-generation nuclear plant designs, and was expanded to include several other countries. Six advanced reactor designs were selected by the international Generation IV program in 2003 for long-term development and several designs that are viewed as near-term deployable by 2015. One of the reactor types identified for international near-term deployment is integral primary system reactors (IPSR). An example of an IPSR system is the International Reactor Innovative and Secure (IRIS) reactor concept [Carelli et al., 2003]. IRIS is a modular design of a pressurized water reactor (PWR) that has an integral reactor coolant system with enhanced safety and economics. IRIS is especially well suited for deployment in countries with small or medium electricity grids for producing both electricity and fresh water. However, coupling a nuclear plant and a desalination plant involves a number of issues that have to be addressed. Among these issues, performance monitoring and fault diagnosis are of high importance for safe and optimal operation of a coupled nuclear desalination plant. 
The main objective of fault diagnosis is to observe incipient faults and determine the root causes. Nowadays, most processes are extremely well instrumented with a multitude of sensors providing both control and safety-related measurements, especially in a nuclear desalination plant. The faults with control-related sensors can propagate the fault effects through control loops and subsequently disturb process variables throughout the entire process, which will result in a non-optimal performance of the related actuators and plant equipment. The safety-related sensor faults can force a nuclear power plant to de-rate its power and degrade the operational performance. Both types of sensor faults can also mislead operators to take erroneous actions and in turn result in safety problems. The ability of the sensor network to detect and discriminate failure modes and anomalous conditions is critical for the effectiveness of the fault detection and isolation (FDI) system. With hundreds of process variables available for measurement in both nuclear power plants and desalination processes, selection of an optimum number of sensors and their locations poses a unique challenge.

FDI has been considered as an important design feature of the advanced instrumentation and control systems of the IRIS design. However, conventional sensor FDI techniques, such as hardware redundancy, face many challenges in engineering applications. As an integral reactor, IRIS has the entire reactor coolant system housed inside the reactor vessel. This arrangement creates engineering difficulties in placing physically redundant instruments for in-vessel equipment due to the need for extra space and instrument penetrations. In addition, the possible common-cause failures of physically redundant sensors may still constrain the reliability of the instrument system.

Many modern FDI approaches are based on analytical redundancy. Functional relationships among process variables governed by fundamental conservation laws such as mass, momentum, and energy balance, can replace hardware redundancy for plant measurements [Chow et al., 1984; Erbay and Upadhyaya, 1997; Holbert and Upadhyaya, 1990; Upadhyaya et al., 1989; Upadhyaya and Eryurek, 1992]. Residuals, defined as the differences between measured values and estimated values, can be continuously monitored for fault detection. Because different faults cause the violation of different relationships, residual patterns can be used as fault signatures for fault isolation. 
Because of the complexity of either a nuclear power plant or a seawater desalination plant, it is generally very difficult to build physics models with sufficient accuracy for FDI purpose. Thus, the so-called soft computing methods, such as artificial neural networks (ANN) [Kavaklioglu et al., 1994], principal component analysis (PCA) [Wang et al., 2002], fuzzy logic [Zhao et al., 2005], group method of data handling (GMDH) [Farlow, 1984], and other data-based empirical modeling techniques [Hines et al., 1997; Hines et al., 2008], have shown great capabilities of capturing the relationships among the various measurements.

Multivariate statistical process control using empirical techniques, such as PCA for data characterization, has been employed successfully in the process industries. In chemical industry, PCA has been one of the most popular statistical methods for extracting information from process data. Dunia and Qin [1998] proposed a subspace approach for fault detection, reconstruction, isolation, and identification. Due to the difficulty of isolating faults from process parameter changes when the Hotteling's $T^{2}$ test is violated, their method was only based on the $Q$ statistic, or the squared prediction error (SPE), and the $T^{2}$ statistic was not utilized. In fact, any data-based empirical modeling methods, for example, PCA and other multivariate statistical analysis methods, cannot solve the performance monitoring and fault diagnosis problem as a whole if no other information about process is efficiently utilized. Specifically, the emphasis of most PCA-based research has been placed more on monitoring algorithms using a given set of sensors, and less on actual sensor allocation for efficient detection and identification of process malfunctions. Fortunately, many researchers in other fields have resorted to the design of various sensor networks from a fault diagnosis perspective [Bhushan and Rengaswamy, 2002a; Bhushan and Rengaswamy, 2002b; Kramer et al., 1987; Maurya et al., 2006]. If sensors are suitably located based on the knowledge of fault propagation manner within the process, PCA with optimized sensor locations would certainly enable a great improvement on the process FDI performance.

\subsection{R\&D Objectives and Significant Contributions}

The goal of this R\&D project was to develop an integrated approach to address sensor placement design in a fault diagnostic architecture with application to a nuclear desalination system. The contributions are innovative and significant in that, to the best of knowledge, they 
represent the first comprehensive studies of modeling, simulation, sensor placement design, and fault diagnosis of nuclear desalination processes. The original contributions are presented below.

1. Development of steady-state and dynamic models of a MSF desalination plant using MATLAB and SIMULINK: Steady-state models are applicable for design purposes as well as for parametric studies of existing plants. Dynamic models are suitable for simulating transient behavior, studying control strategies, investigating stability problems, and identifying process interactions.

2. Development of a coupled IRIS-MSF nuclear desalination plant model in SIMULINK: This provides a useful platform to facilitate the study of system transients, control behavior, performance monitoring, and fault diagnosis of a nuclear desalination process.

3. Development of an integrated architecture of performance monitoring and fault diagnostics with emphasis on the importance of optimum sensor placement: Causal graph-based algorithms are developed to perform sensor placement design from a fault diagnosis perspective using different design criteria.

4. Development of an optimal and automated sensor allocation procedure for the IRIS system and the MSF desalination plant: An efficient integer linear programming (ILP) embedded greedy search optimization algorithm was developed to solve the formulated sensor allocation optimization problem. This problem of sensor placement design was also solved optimally in this research using a commercial ILP optimization solver, LINGO 8.0, and the optimal solutions are compared with the greedy search algorithm.

5. Application of the sensor placement design strategy to the IRIS-MSF nuclear desalination plant: A PCA-based fault diagnostic approach with optimized sensor selection is able to achieve the satisfactory FDI performance in both single-fault and multi-fault cases. Moreover, fault diagnostic results are examined using reduced sensor sets (non-optimal sensor selection), as opposed to optimal sensor sets. The comparison study indicates that the reduced sensor set is not sufficient for the purpose of fault isolation, thus justifying the use of the developed optimal sensor allocation strategy. 
6. Development of the effective PCA models using the optimal sensor set obtained through sensor allocation design: This provides guidance for choosing measurements necessary for building PCA models, and eliminates the arbitrary decision making in selecting proper measurements that are needed for process performance monitoring and fault diagnosis.

\subsection{Organization of the Report}

This document is divided into eight chapters. Chapter 2 reviews the state-of-the-art optimum sensor placement techniques, which consist of three basic components: a model, an evaluation module, and an optimization algorithm. The details of a variety of desalination technologies are also presented in this chapter. This is followed by the discussion of an integrated architecture of a fault diagnostic system in Chapter 3. Both steady-state and dynamic models for a MSF desalination system are developed in Chapter 4. Simulation results are provided and analyzed in this chapter. Chapter 5 presents simulation results of a coupled IRIS-MSF nuclear desalination plant consisting of the MSF dynamic model developed in this project and an existing IRIS plant model in SIMULINK environment. Next, a complete procedure of implementing optimum sensor placement for fault diagnosis is given in Chapter 6. Different criteria are employed to formulate optimization problems, and a greedy search algorithm is adopted to solve the formulated optimization problems. In Chapter 7 and 8 , the developed sensor placement design algorithms and PCA-based fault diagnostic approaches with optimized sensor placement are demonstrated through the applications to a MSF desalination process and an IRIS helical coil steam generator (HCSG) system, respectively. Finally, the research project is summarized with concluding remarks in Chapter 9, along with suggestions for future research. 


\section{Chapter 2}

\section{LITERATURE REVIEW}

\subsection{Optimum Sensor Placement for Process Systems}

The performance of an industrial process is strongly dependent on available sensor measurements. Inaccurate measurements resulting from insufficient measurements or improper sensor placement can significantly deteriorate fault observability and process control. Therefore, sensor placement has received considerable attention and has been studied in different areas.

Traditionally, sensors are placed mainly to meet control or monitoring objectives. The sensor placement for process control is to determine the controlled variables and the manipulated variables to achieve the designed control objectives. The selection of controlled variables is mainly concerned with the process requirements. The general guideline is that the controlled variables should include: (1) non self-regulating variables; (2) environment and equipment safety critical variables; (3) process performance critical variables; (4) variables that have strong interactions with other control variables; and (5) variables with favorable static and dynamic characteristics [Bagajewicz, 2001].

Faulds and King [2000] introduced the sensor placement problem for feedback control. Al-Shehabi and Newman [2001] employed root locus principles to choose optimum sensor positions for aero-elastic vehicle feedback control applications. Giraud and Jouvencel [1995] addressed the problem of sensor selection in an automatic task, such as the process of data fusion, a sensing task, or the design of a perceptual system for a mobile robot. Chen and $\mathrm{Li}$ [2002] presented an automatic sensor placement technique for robot vision in inspection tasks. One aspect of the NASA Aircraft Morphing program was to determine the optimum number of active control devices (for example, piezoelectric actuators) and their placement in the structure. In this program, Padula and Kincaid [1999] provided a good review of sensor and actuator placement problems. They grouped the sensor and actuator placement research based on

different types of applications (non-aerospace placement problems and aerospace placement problems). 
Some recent works have pertained to other nontraditional activities, such as target tracking, fault detection, and reliability analysis. Sensor placement for fault diagnosis has been studied at two different levels: component level and system level. Some of the sensor placement problems attempted to position sensors in a component's range. Critical systems of interest are characterized as large scale systems consisting of multiple components. For such systems, a fault may propagate through several components when it occurs. Therefore, it is possible that sensors can be placed at any of the components to detect the fault. With hundreds or thousands of possible locations of sensors in a system, the selection of a crucial and optimum sensor location, sensor types, and number of sensors poses an important problem that needs to be solved at the system level before the detailed spatial distribution in a component can be determined.

Although a large body of research work has emerged, the various approaches vary only in their choices of the three basic components: a model of the system of interest, a sensor selection module, and an optimization algorithm, as illustrated in Figure 2.1. The following discussion of sensor placement is based on these three components.

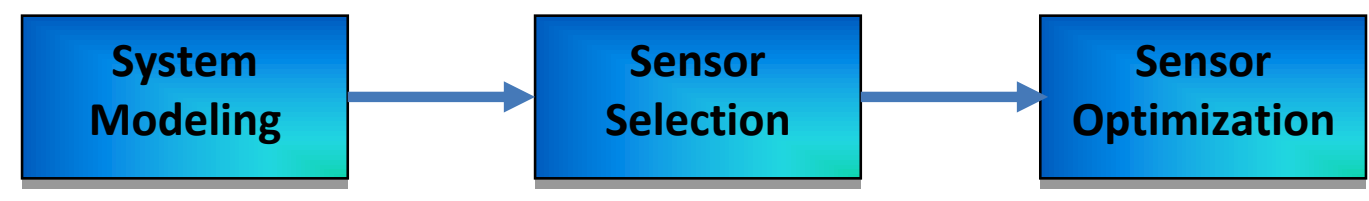

Figure 2.1. Basic components of sensor placement strategy.

\subsubsection{Modeling of Systems}

To date there have been a plethora of different modeling techniques that have been proposed in the literature. These techniques range from the early attempts using fault trees and digraphs, analytical approaches, and knowledge-based systems and neural networks in more recent studies. From a modeling perspective, most of the techniques can be classified as physics-based modeling or empirical modeling. Physics-based models, also known as first principle models, use physics of the system to predict the nominal conditions of the process. Despite its clear benefit of representing the physical relationships among process variables for all the operational conditions, there are a number of drawbacks to using a physics-based model. It is 
not uncommon that the underlying physical processes are not completely understood, thus simplifying assumptions must be made to facilitate model development. Assumptions made in model development may not be fully applicable to real world systems, which limit the applicability of the models. In addition, these models are often computationally expensive for complex system.

Empirical models, on the other hand, use data to fit a model to the relationships seen in real world application. These types of models are built using process parameter measurements that have been collected over the operational range of the system or process of interest. The relationships among these measurements are used within the model architecture to produce accurate predictions. Empirical models are often preferable to physics-based models because they are simple to develop, they capture real world relationships, and they require limited knowledge of the underlying physical phenomena. In practice, there are two fundamentally different empirical modeling approaches. System identification approach identifies empirical models using data obtained from well-designed experiments. Historical empirical modeling approach develops models from routine operation data saved in a historical database. The empirical models developed from system identification techniques are usually causal models because of the careful control of experiments. On the contrary, historical empirical models are usually not causal models because they can only capture the correlations among the variables contained in the historical data.

Due to the large number of modeling methods available in the literature, an exhaustive survey is not practical. The following discussion, however, provides an overview of the basic concepts of the past research in empirical modeling.

\section{Kalman Filter}

Kalman filtering was first introduced by Kalman [1960] as a way of designing a state estimator with minimum estimation error. It is well known that the Kalman filter is a recursive algorithm for state estimation and it has found wide applications in aerospace, chemical, and other industrial processes. A good introduction to the general idea of the Kalman filter can be found in [Maybeck, 1979], while a more complete introductory discussion can be found in [Sorenson, 1970], which also contains some interesting historical narrative. It has been shown 
that a bank of Kalman filters designed on the basis of all the available possible system models under all possible changes can be used for the isolation purpose. Fathi et al. [1993] included adaptive analytical redundancy models in the diagnostic reasoning loop of knowledge based systems. The modified extended Kalman filter is used in designing local detection filters in their work.

The essential Kalman filter theory can be summarized briefly as follows. Describe a linear finite dimensional stochastic system by a discrete-time state-space model:

$$
\begin{gathered}
x(t+1)=A x(t)+B u(t)+w(t) \\
y(t)=C x(t)+v(t), t \geq 0
\end{gathered}
$$

where $x(t)$ is $\mathrm{n}$-dimensional vector, $\mathrm{A}, \mathrm{B}$ and $\mathrm{C}$ are matrices with suitable dimensions, $x_{0}$ has mean $\overline{x_{0}}$ and covariance $\Sigma_{0} ; w(t)$ and $v(t)$ are Gaussian white noise sequences with zero means and the covariance matrix:

$$
E\left\{\left(\begin{array}{c}
w(t) \\
v(t)
\end{array}\right)\left(w^{T}(\tau), v^{T}(\tau)\right)\right\}=\left(\begin{array}{ll}
Q & S \\
S^{\prime} & R
\end{array}\right) \delta_{t-\tau}
$$

where $\delta_{t-\tau}$ is Kronecker's delta, $w(t)$ and $v(t)$ are independent of $\sigma\left(x_{s}: s \leq t\right)$.

In estimating the state $x(t+1)$ based on the observed data $y(t)$ and $u(t)$, the optimal Kalman filter minimizes the function:

$$
J=\lim _{t \rightarrow \infty} E\left(e^{T}(t) e(t)\right)
$$

where $e(t)$ is the estimation error between $x(t)$ and its estimate.

Assume the initial state and noise sequences are jointly Gaussian. Consider the estimator:

$$
\hat{x}(t+1)=E x(t+1) \mid y(t), \ldots, y(0), u(t), \ldots, u(0)
$$


The filtered state $\hat{x}(t+1)$ satisfies:

$$
\begin{gathered}
\hat{x}(t+1)=A \hat{x}(t)+B u(t)+K(t)[y(t)-C \hat{x}(t)] \\
\hat{x}_{0}=\overline{x_{0}}
\end{gathered}
$$

The Kalman filter gain $K(t)$ is given by:

$$
K(t)=\left[A \Sigma(t) C^{T}+S\right]\left[C \Sigma(t) C^{T}+R\right]^{-1}
$$

where $\Sigma(t)$ is a $n \times n$ state error covariance matrix.

\section{Auto-Associative Kernel Regression}

Auto-associative kernel regression (AAKR) is a non-parametric model that uses past normal operational data to correct faulty observations which may be due to system degradation, sensor faults, data acquisition problems, etc [Hines et al., 2008]. The outputs of an AAKR model are the predicted or corrected values of the inputs.

The AAKR model uses a Euclidean distance, which is known as the $\mathrm{L}^{2}$-norm, to compare the input query data to the exemplar vectors which make up the model's memory matrix for $n$ inputs.

$$
d_{i}=\sqrt{\sum_{i=1}^{n}\left(x_{q, i}-m_{i}\right)^{2}}
$$

Next, these distances are transformed to similarity measures used to determine weights by evaluating the Gaussian kernel. This kernel is a function of the Euclidean distance, $d$, and the kernel bandwidth, $h$.

$$
w=K(d, h)=\frac{1}{\sqrt{2 \pi h^{2}}} e^{-d^{2} / h^{2}}
$$

The final prediction is a weighted sum of the exemplar vectors, $m_{i}$. 


$$
\hat{x}_{q}=\frac{\sum_{i=1}^{n_{m}} w_{i} \cdot m_{i}}{\sum_{i=1}^{n_{m}} w_{i}}
$$

The parameters to be optimized in an AAKR model are the memory matrix and the kernel bandwidth, $h$. A researcher must decide how many vectors to include in the memory matrix and how large to make the bandwidth which indirectly controls how many memory vectors shall be weighted heavily during prediction.

\section{Artificial Neural Networks}

Considerable interest has been shown in the literature in the application of artificial neural networks. In general, artificial neural networks can be classified according to the learning strategy such as supervised and unsupervised learning. In supervised learning strategies, by choosing a specific topology for the neural network, the network is parameterized in the sense that the problem at hand is reduced to the estimation of the connection weights. The connection weights are learned by explicitly utilizing the mismatch between the desired and actual values to guide the search. This makes supervised neural networks a good choice for fault classification as the networks are capable of generating, hence classifying, arbitrary regions in space [Cybenko, 1988]. On the other end of the spectrum are neural network architectures which utilize unsupervised estimation techniques. These networks are popularly known as self-organizing neural networks as the structure is adaptively determined based on the input to the network. One such architecture is the ART2 network [Carpenter \& Grossberg, 1988].

The most popular supervised learning strategy in neural networks has been the back-propagation algorithm. Most of the work on improvement of performance of standard back-propagation neural network is based on the idea of explicit feature presentation to the ANN. A number of researchers worked on this issue. Fan et al. [1993] discuss the performance gains through the incorporation of functional inputs in addition to the normal inputs to the neural networks. Incorporation of knowledge into the ANN framework for better diagnosis is discussed by Farell and Roat [1994]. Data processing and filtering is shown to lead to significant performance improvement and reduced training time. Tsai and Chang [1995] propose the integration of feed forward ANN with recurrent ANN for better performance. 
There are also other architectures such as self-organizing maps [Kohonen, 1984]. The objective of these methods is to give credit for patterns that are similar to group together. The similarity measure is usually a distance measure. Whenever a pattern is seen that is not similar (in a distance metric sense) to any of the previously formed classes, a new class is formed and the pattern is retained as the defining pattern for the new class and similarity is measured with respect to this pattern. The crucial elements in these kinds of architectures are the distance metric that one chooses and the threshold for similarity. Clustering is a technique to group samples so as to maximize the separability between these groups. Clustering algorithms specify the number of groups and maximize an objective function that is a measure of separability of these groups. In this manner, clustering becomes a well-defined optimization problem. In the clustering process credit is given to patterns exhibiting similar characteristics. Clustering procedures need two important components. First, they need a measure for estimating similarity between different data points. Without this no credit can be assigned for patterns that are similar. Second, one needs representative patterns against which the similarity of other patterns can be checked.

The most popular clustering algorithm proposed in the literature is the K-means clustering algorithm [Duda and Hart, 1973]. K-means clustering pre-supposes the number of clusters needed and would cluster the data accordingly. It utilizes all the cluster centers so that each of the clusters is guaranteed at least one pattern. Kohonen's self-organizing maps [1984] identify the cluster center closest to the training pattern and update this cluster center and all its topological neighbors. K-means clustering can be shown to be a special case of Kohonen's clustering algorithm. In Kohonen's algorithm, after the neighborhood is decided, the algorithm makes all the clusters in the neighborhood to be the winners of the pattern. This leads to the problem of gravity where all the cluster centers migrate towards dense regions leaving less dense regions unrepresented. Self-organizing neural network structures such as the ART2 network [Carpenter \& Grossberg, 1988] have also been extensively used in fault diagnosis. Whiteley and Davis [1994] demonstrate the use of ART2 network for the interpretation of sensor data. Chen et al. [1999] and Wang et al. [1999] discuss the integration of wavelets with ART networks for the development of diagnostic systems. For a collection of papers on the application of neural networks in solving engineering problems, interested readers are referred to Venkatasubramanian and McAvoy [1992] and Bulsari [1995]. 


\section{Ordinary Least-Squares Regression}

Ordinary least squares regression is a basic algorithm for illustrating inferential techniques. It is also useful for performance bench marketing. Its derivation can be found in [Hastie, 2001] and it is restated here.

Given a linear model:

$$
y=X \beta+\varepsilon
$$

where $y$ is a measurement with $N$ observations; $X$ is p vector of inputs, each vector has $N$ observations; $\varepsilon \square N\left(0, \sigma^{2}\right)$ is the noise.

Estimate the coefficients $\hat{\beta}=\left(\hat{\beta}_{0}, \hat{\beta}_{1}, \ldots, \hat{\beta}_{p}\right)^{T}$ by minimizing the residual sum of squares:

$$
\begin{gathered}
R S S(\hat{\beta})=\sum_{i=1}^{N}\left(y_{i}-\hat{y}_{i}\right)^{2}=\sum_{i=1}^{N}\left(y_{i}-\hat{\beta}_{0}-\sum_{j=1}^{p} x_{i j} \hat{\beta}_{j}\right)^{2} \\
R S S(\hat{\beta})=(y-X \hat{\beta})^{T}(y-X \hat{\beta})
\end{gathered}
$$

The solution is derived by setting derivative of the cost function to zero.

$$
\begin{gathered}
\frac{\partial R S S(\hat{\beta})}{\partial \hat{\beta}}=-2 X^{T}(y-X \hat{\beta}) \\
\frac{\partial^{2} R S S(\hat{\beta})}{\partial \hat{\beta} \partial \hat{\beta}^{T}}=-2 X^{T} X
\end{gathered}
$$

Assuming that $X$ is nonsingular and hence $X^{T} X$ is positive definite, set the first derivative to zero to minimize the RSS.

$$
X^{T}(y-X \hat{\beta})=0
$$

The unique solution can be obtained as:

$$
\hat{\beta}=\left(X^{T} X\right)^{-1} X^{T} y
$$

The predicted values at the training inputs are:

$$
\hat{y}=X \hat{\beta}=X\left(X^{T} X\right)^{-1} X^{T} y
$$

Parameter uncertainties can be estimated using an estimate of the noise $\varepsilon$. 


$$
\begin{gathered}
\operatorname{Var}(\hat{\beta})=\left(X^{T} X\right)^{-1} \sigma^{2} \\
\hat{\sigma}^{2}=\frac{1}{N-p-1} \sum_{i=1}^{N}\left(y_{i}-\hat{y}_{i}\right)^{2}
\end{gathered}
$$

Assume the linear model is correct and the deviations of $\mathrm{Y}$ around its expectation are additive and Gaussian.

$$
\begin{gathered}
Y=\beta_{0}+\sum_{j=1}^{p} X_{j} \beta_{j}+\varepsilon \\
\varepsilon \square N\left(0, \sigma^{2}\right)
\end{gathered}
$$

Then,

$$
\begin{gathered}
\hat{\beta} \square N\left(\beta,\left(X^{T} X\right)^{-1} \sigma^{2}\right) \\
(N-p-1) \hat{\sigma}^{2} \square \sigma^{2} \chi_{N-p-1}^{2}
\end{gathered}
$$

$\hat{\beta}$ and $\hat{\sigma}$ are statistically independent. The mean squared error of an estimator $\hat{y}$ in estimating $y$ is:

$$
\operatorname{MSE}(\hat{y})=E(\hat{y}-y)^{2}=\operatorname{Var}(\hat{y})+(E(\hat{y})-y)^{2}
$$

Least squares estimates have the smallest variance among all linear unbiased estimates. However, there may be a biased estimator with smaller mean squared error. Such an estimator would trade a little bias for a larger reduction in variance. Biased estimators, such as ridge regression, are commonly used. Any method that shrinks or sets to zero some of the least squares coefficients may result in a biased estimate.

\subsubsection{Sensor Selection Evaluation}

The goal of the sensor selection process is to provide a suite of sensors that fulfill specified performance requirements within a set of system constraints. These performance requirements are defined as the figures of merit (FOM) of the system, and considerable research has focused on how to represent these FOM in algorithmic form. The following list of general FOM categories was selected after reviewing the available literature and research. 


\section{Observability}

Of ultimate importance for any sensor suite is the concept of observability. Basically, observability is the capacity of the sensor network to provide information about the state parameters deemed important for performance monitoring, health assessment, and/or control of the system. This information may be provided as direct measurements of the system parameters or, as in data reconciliation [Zhao, 2005b], the reconstruction of unobservable system parameters based upon observable or sensed variables. Approaches vary in the determination of the observability of the sensor network. Some treat observability as a true or false property, while others devised more sophisticated schemes to display the degree of observability.

Some approaches used graph-based analysis for which the structural information of a system is represented by a directed graph (DG), called a digraph. Observability is then based on analysis of cut-sets and cycles generated from the digraph. Kretsovalis [1987] proposed two graphically-based algorithms for classification of observable and redundant variables. Luong et al. [1994] also used graph-based analysis to establish an incidence matrix, relating the process relationships to the state variables qualitatively. Decomposition of this incidence matrix using a Gauss-Jordan elimination process identified whether an unmeasured variable was observable and whether a measured variable was redundant. Using graph theory and cut-sets, Bagajewicz and Sanchez [1999] defined the degree of observability and degree of redundancy for a sensor network. For an unmeasured variable, the degree of observability is the maximum number of sensors that can be eliminated with the variable still observable; for measured variables, the degree of redundancy is the maximum number of sensors that can be eliminated and the measurement remains redundant. These concepts were combined to define a degree to which system variables can be estimated by the sensor network.

Other approaches attempted to define the degree of observability by analyzing the numerical relationships of the system process. By taking advantage of linear state space system theory, observability and controllability can be represented in matrix form. The system is observable or controllable if the observability or controllability matrices are of full rank. These represent binary conditions as to whether the system is observable/controllable or not. Dochain et al. [1997] considered both the rank and condition number of the observability matrix for an 
optimal sensor placement in a bioreactor system. The Gramian of both the observability and controllability matrices have been used by researchers [Muller, 1972; Van den Berg et al., 2000] to develop scalar metrics that indicated the degree of each respective property.

A large amount of research has been devoted to control design and actuator/sensor placement locations, particularly in structural-type problems. Papadopoulos and Garcia [1998] investigated a number of quantitative techniques used to optimize structural sensor placement to select sensor locations that would observe the maximum amount of system information, which is energy in this case. Each technique attempted to characterize the amount of energy the sensors would observe and eliminate potential sensor locations that had minimal observability. Hac and Liu [1993] proposed a methodology that incorporated eigenvalues from both the observability and controllability matrices to select sensor locations for the control of flexible structures.

Several researchers used the predictive error of a given sensor suite relative to a specific reconciliation technique as the performance metric. After performing matrix decomposition on the linear system model coefficients, Madron [1992] proposed a simple local optimization of this baseline set of sensors by computing the prediction precision of unmeasured required state parameters. The baseline sensor suite performance was compared to suite variations where a single sensor was removed and the objective function recomputed. Chmielewski et al. [2002] developed the framework for the justification and incorporation of the error covariance matrices for sensor suite performance properties and demonstrated how that could be optimized with other suite performance constraints using mixed linear integer programming techniques. Musulin et al. [2005] proposed a sensor selection process that maximized a Kalman Filter performance, via the error covariance matrix diagonal terms. He pointed out that although the objective is to maximize the performance of the filter for all variables, maximizing the performance for one particular variable may not be compatible with maximizing it for others, resulting in a conflict. Therefore they proposed two possible approaches; one method would select the variable with the lowest performance value. The second approach would evaluate the performance of each variable relative to an "ideal" sensor suite performance, combining into an overall performance metric. Mushini and Simon [2005] used a similar approach of maximizing a Kalman Filter performance for an aircraft gas turbine engine. The metric was defined as the summation of the error covariance matrix diagonal terms normalized to the reference or ideal system performance. 


\section{Sensor Reliability}

Regardless of the purpose of the sensor network, consideration must be given to the availability of the sensor signal information when it is required. Sensor faults can be common and the potential for interruption of information flow and how that is to be handled is an important consideration in the sensor network design analysis.

Luong et al. [1994] investigated sensor network reliability from a control perspective that the reliability of an instrumentation system is the probability that information required for control are available through measurements or deduction during some time period. Based on that idea, an overall sensor network reliability metric was defined as an expression of the individual sensor reliabilities. Sensor reliabilities were expressed as a function of time; therefore, the integration of the time profile for each sensor network was used as a quantitative comparison metric between competing networks.

Ali and Narasimhan [1993] introduced the concept of reliability of the estimation of variables, which relates the sensor reliability with its availability to provide system state estimations. The focus of the research was for a given sensor network, how many different ways can a variable be estimated and if sensors fail, can a variable still be estimated. The network objective function was defined as the minimum product of the input sensor reliabilities for each estimated parameter. The initial processing routine involved graph theory concepts of chords, cut-sets, and spanning trees to establish sets of estimated variables and their associated sensors used to observe the variable. This objective function was extended to include hardware redundancy (measuring a variable using more than one sensor) and spatial redundancy (more than one way of estimating a variable) [Ali and Narasimhan, 1995]. Within the graph theory algorithm, the sensor network can be optimized with respect to only a single criterion; therefore, this objective function was adapted for processing with genetic algorithms [Sen et al., 1998] and mixed integer nonlinear programming (MINLP) [Bagajewicz and Sanchez, 2000] which enabled it to be evaluated with other performance metrics, such as sensor costs.

Another consideration of the sensor network is the performance in the presence of sensor faults. Sensor networks must be robust to sensor faults, both in being able to perform adequately with a faulty sensor in the network and being able to continue performance adequately if a sensor 
is removed. Bagajewicz [1997] proposed a set of performance metrics for a sensor suite that defined the ability of the sensor network to detect sensor faults (error detectability) and handle sensor faults relative to reconciliation precision performance (availability - precision after failed sensor removed, and resilience - precision in the presence of a sensor fault). Bagajewicz and Cabrera [2002] extended this research from the graphical tree-search-solution procedure to the explicit MINLP formulation and also extended the theory for explicit consideration of hardware redundancy.

\section{Fault Detectability/Fault Discriminability}

For fault diagnosis, the ability of the sensor network to detect and discriminate failure modes and anomalous conditions is of prime importance. This is an extension of the observability analysis where specific state variables or groups of state variables and their response are indicative of health conditions. The performance metric must define the sensor network's ability to distinguish these fault conditions from nominal operation (fault detection) and distinguish these fault conditions from each other (fault discrimination).

Significant research is focused on the qualitative or heuristic representation of the fault progression process. Research conducted by Raghuraj et al. [1999], Bhushan and Rengaswamy [2000a; 2000b], and Bagajewicz et al. [2004] utilized directed or sign directed graphs to generate fault signatures. A directed graph of a hypothetical process with faults $F_{i}$, connecting to sensors $S_{i}$, indicating that the fault will affect the reading of the corresponding sensor, is displayed in Figure 2.2. These signatures provided only an indication that the sensor should respond to the particular fault condition, but the actual signature, magnitude or rate, was not utilized. Spanache et al. [2004] used the physical constraints of the system to develop a set of analytical redundancy relationships between the measured and unmeasured variables and qualitatively established an influence matrix between system components and possible sensor parameters. Fault signatures were assigned to a failed component without regard to specific component failure modes, and hence a fault signature matrix was generated between the component faults and the influenced analytical redundancy relationships. Narasimhan et al. [1998] used temporal causal graphs, and Yan [2004] used a CAD system model to develop qualitative fault signatures. Representing the fault signature response qualitatively avoids the cost of simulating the fault in hardware and/or 
software and often this is the only level of fault information available during the early development of a system.

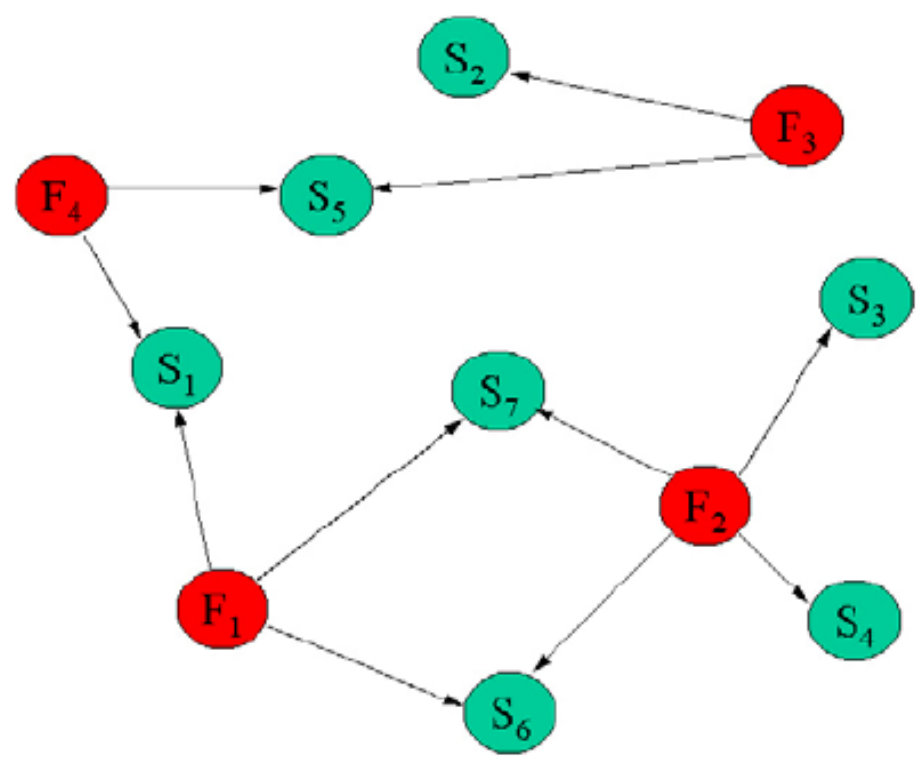

Figure 2.2. Directed graph of a hypothetical system.

In an effort to incorporate more fault information, Zhang [2005] proposed a quantified directed graph (QDG) to model the fault propagation. For the QDG, each node represented a potential sensor with an associated signal-to-noise ratio (SNR), and each arc contained the fault propagation gain and direction between the sensors, along with fault propagation time. A sensor detectability value was computed for each sensor in the suite for a predefined set of faults. Fault detectability was averaged over the available sensor suite and evaluated against an assigned lower bound. Fault resolution was still treated somewhat qualitatively by comparing sets of sensors influenced by each fault.

Azam et al. [2004] proposed a method that used a system model and reliability data to establish cause/effect dependencies between the faults of interest and the effects of faults on observable system parameters. The detection and false alarm probabilities associated with the failure source and observable discrepancy were utilized. A general multiple fault diagnosis algorithm was used to search for the most likely candidate fault that explained the set of observed discrepancies. Three diagnostic performance metrics were established for each potential sensor based on the decision probability error from a number of simulation runs. 
Selection evaluation was performed for candidate sensor networks by summing these performance metrics over the sensor suite and incorporating cost constraints.

Santi et al. [2005] utilized a high-fidelity dynamic model of a rocket propulsion system to generate a simplified linear model. Health parameters were identified in the linear model that represented specific fault conditions. This research focused on mean-shift faults in the system. An inverse diagnostic model was developed which predicted the change in health parameters based on sensor inputs. Fault trajectories were generated and metrics were established for detection timeliness and fault discrimination. Fault detection required sufficient measurement deviation to discriminate a fault from normal operation variance. Detection thresholds were established based on the historical information of measurement variance, anticipated process variance, and defined false alarm constraints of the system. The diagnostic performance metrics were based on the distance measured between fault trajectories for fault discrimination and inferred time to detection threshold areas for detection timeliness. In addition, fault risk reduction measures were incorporated into the evaluation process to allow weighting of individual failure modes based on criticality and occurrence rates.

\subsubsection{Sensor Optimization Techniques}

After a FOM is selected, an algorithm needs to be selected to optimize this FOM. Various optimization algorithms, from random search to heuristic algorithms such as genetic algorithms (GA), have been used for optimizing sensor allocation. Random search is suitable for a small and simple sensor placement problem since it is straightforward and easily implemented. But it is time consuming and inefficient when dealing with a large system. A simulated annealing (SA) method [Johnson et al., 1989] based on random search is used to select a single random subset while seeking to improve the cost function by moving to one of the nearest neighbors of the selected subset. The Tabu search method [Glover and Laguna, 1997] uses probabilistic events for the search of better solutions. Genetic algorithms, based on the Darwinian principle of natural selection, are widely applied in different domains, among the heuristic methods. Sen et al. [1998] designed a sensor network for the linear mass flow process using the GA method. Other examples using the GA method to solve sensor placement problems can be found in [Furuya and Haftka, 1996] and [Ponslet et al., 1993]. 
Other optimization approaches include graph-based methods. A greedy search algorithm based on a bipartite graph was proposed by Raghuraj et al. [1999] to generate a minimal set of sensors to meet observability and maximum resolution requirements. A bipartite graph is one whose vertex set can be partitioned into two sets in such a way that each edge joins a vertex of the first set to a vertex of the second set. Whenever there is a directed path from a root node to a key component, an arc from that root node to the key component is drawn in the bipartite graph.

Particle swarm optimization (PSO) is a population based stochastic optimization technique developed by Eberhart and Kennedy [1995], inspired by social behavior of bird flocking or fish schooling. PSO shares many similarities with evolutionary computation techniques such as genetic algorithms. The system is initialized with a population of random solutions and searches for optima by updating the generations. However, unlike the GA method, PSO has no evolution operators such as crossover and mutation. In PSO, the potential solutions, called particles, fly through the problem space by following the current optimum particles. Each particle keeps track of its coordinates in the problem space which are associated with the best solution (fitness) it has achieved so far. The fitness value is also stored. This value is called "pbest". Another best value that is tracked by the particle swarm optimizer is the best value, obtained so far by any particle in the neighborhood of the particle. This location is called "lbest". When a particle takes all the population members as its topological neighbors, the best value is a global best and is called "gbest" [Zhang, 2004].

The particle swarm optimization concept consists of, at each time step, changing the velocity of (accelerating) each particle toward its "pbest" and "lbest" locations (local version of PSO), as shown in Figure 2.3. Acceleration is weighted by a random term, with separate random numbers being generated for acceleration toward "pbest" and "lbest" locations. In the past several years, PSO has been successfully applied in many research and application areas. It is demonstrated that PSO gets better results in a faster, cheaper manner compared with other methods. 


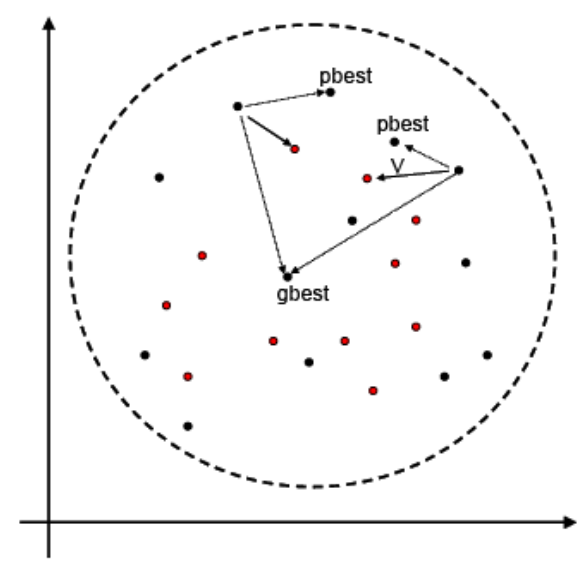

Figure 2.3. Particle swarm optimization.

Another reason that PSO is attractive is that there are few parameters to adjust. One version, with slight variations, works well in a wide variety of applications. Particle swarm optimization has been used for approaches that are employed across a wide range of applications.

\subsection{Sea Water Desalination Methods}

This section introduces the classifications of desalination methods. Also, the principles and operational variables of several widely used desalination technologies are described in this section.

\subsubsection{Classification of Desalination Processes}

Desalination is a process of separating dissolved salts from saline water. Many desalination technologies have been proposed based on different principles of separation. Some of them have been successfully developed and were discussed in detail in [IAEA, 2002], but only a few of them reached commercial operation. Figure 2.4 illustrates the major desalination processes. 


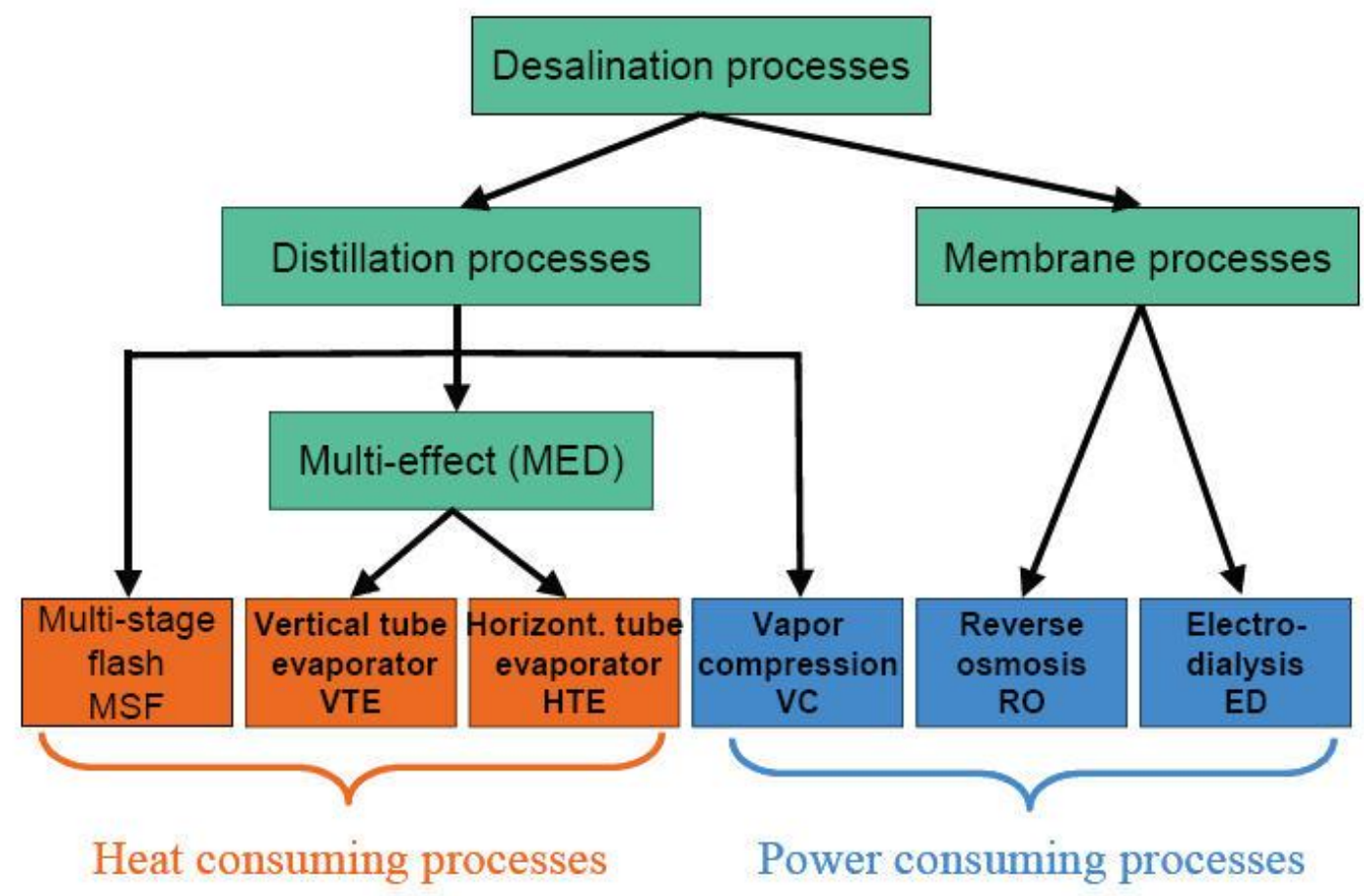

Figure 2.4. Classification of desalination processes [IAEA, 2007].

Desalination processes can be broadly categorized into two main types: processes using heat and process using electricity. The first type of processes is of mainly the distillation processes, multi-stage flash (MSF) or multi-effect distillation (MED). Vapor compression (VC) is a distillation process but it uses electricity, just as the membrane based processes like the reverse osmosis (RO) and the electro-dialysis (ED). Of these, the most commonly used processes are MSF, MED and RO. VC is often combined with MED.

The applicability of any process depends on the salt concentration in the feed water and on its water unit cost. Distillation is the oldest and most commonly-used desalination techniques. In this process, evaporation of the saline water and condensation of the generated vapor occur to obtain fresh water. This process produces fresh water with a better quality as compared with crystallization and membrane processes.

\subsubsection{Distillation Desalination Systems}

In distillation processes (MSF or MED), seawater is heated to evaporate into pure vapor that is subsequently condensed. The heat energy required for distillation is usually supplied as 
low pressure saturated steam, which may be extracted from the exhaust of a low pressure turbine, from a crossover steam duct or from a dedicated, heat only, plant.

\section{Vapor Compression}

Vapor compression distillation uses mechanical energy rather than thermal energy. It is based on a simple principle. Saline water is sprayed over an evaporator tube bundle. The vapor formed at some temperature and pressure is then compressed either thermally in a steam ejector, or mechanically (high and low pressure) in a compressor, causing the condensation temperature and pressure to increase and the volume to decrease. Compressed vapor is passed through the evaporator bundle, where it condenses and forms distilled water. The heat of condensation could be recycled to evaporate more brine. Most vapor-compression plants have single effects, but a multi-effect configuration could be used for a larger product capacity. Figure 2.5 illustrates the principle of vapor compression. The vapor-compression process consumes a small amount of energy and has a low operating cost. However, its capacity is limited, and the quality of water produced and maintenance costs do not match those of other distillation processes. 


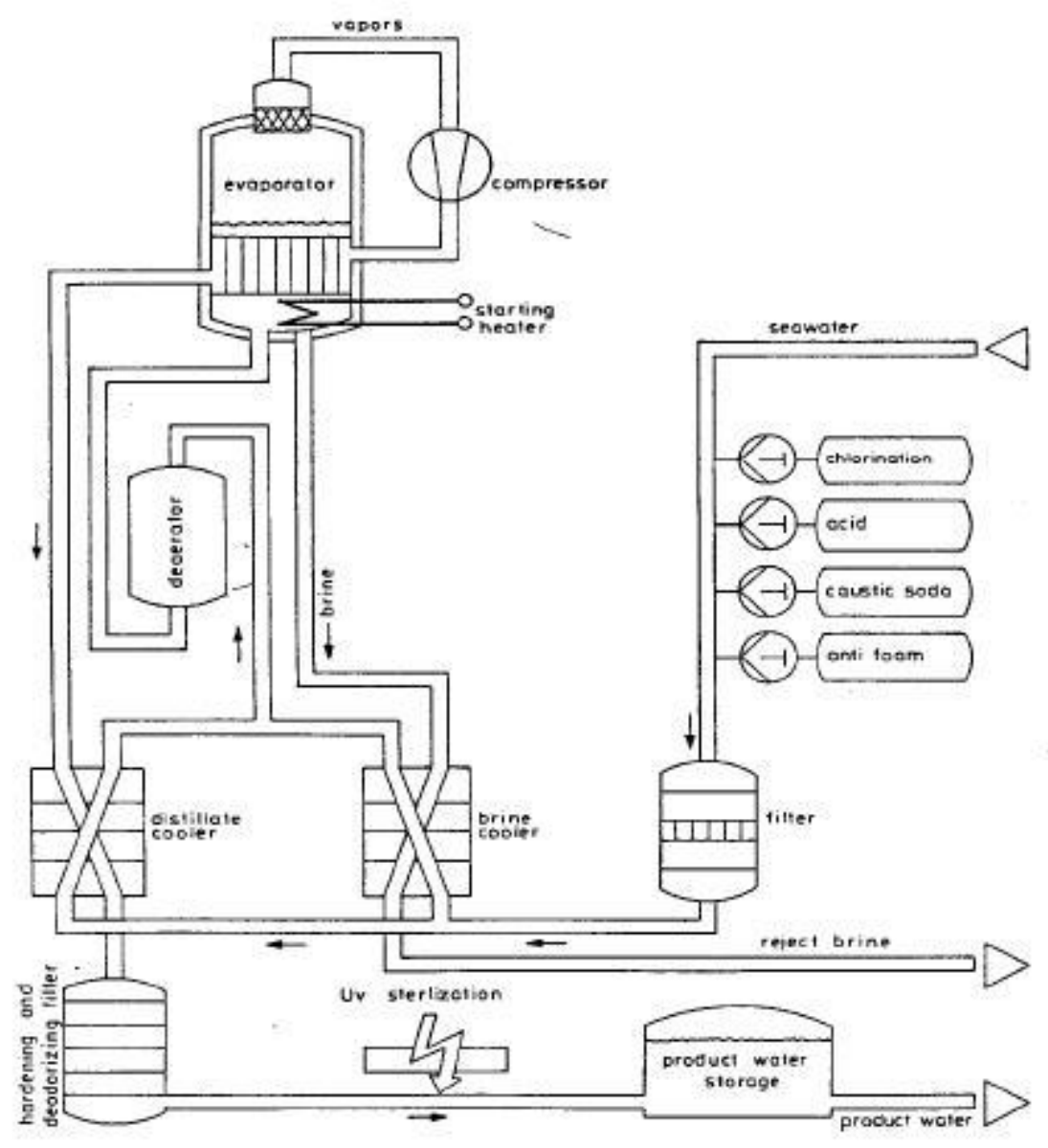

Figure 2.5. Principle of vapor compression [Khan, 1986].

\section{Multi-Effect Distillation}

Figure 2.6 shows the schematic flow diagram of a MED process, using horizontal tube evaporators (HTE). In each effect, heat is transferred from the condensing water vapor on one side of the tube bundles to the evaporating brine on the other side of the tubes.

This process is repeated successively in each of the effects at progressively lower pressure and temperature, driven by the water vapor from the preceding effect. In the last effect, at the lowest pressure and temperature, the water vapor condenses in the heat reject heat exchanger, which is cooled by incoming seawater. The condensate distillate is collected from each effect. 
According to the direction of vapor and brine flow, there are "forward feed" and "backward feed" arrangements. In forward feed MED plants, vapor and brine move through the evaporators as parallel flows from the first high pressure evaporator to the last low pressure one. The pre-heating of feed water occurs in separate heat exchangers. In backward feed MED plants, vapor and brine move through the evaporators in opposite directions, whereby feed water pre-heating is eliminated.

Currently, MED processes with the highest technical and economic potential are the low temperature horizontal tube multi-effect process (LT-HTME) and the high temperature vertical tube evaporation process (HT-VTE). The main differences between LT-HTME plants and HT-VTE plants are in the arrangement of the evaporation tubes, the side of the tube where the evaporation takes place and the evaporation tube materials used. In LT-HTME plants, evaporating tubes are arranged horizontally and evaporation occurs by spraying the brine over the outside of the horizontal tubes creating a thin film from which steam evaporates. In HT-VTE plants, evaporation takes place inside vertical tubes. Furthermore, in LT-HTME plants, the maximum brine temperature is limited to $70{ }^{\circ} \mathrm{C}$, in order to avoid corrosion and scaling problems. Most LT-HTME plants now use low cost materials such as aluminum for heat exchanger and carbon steel as shell material.

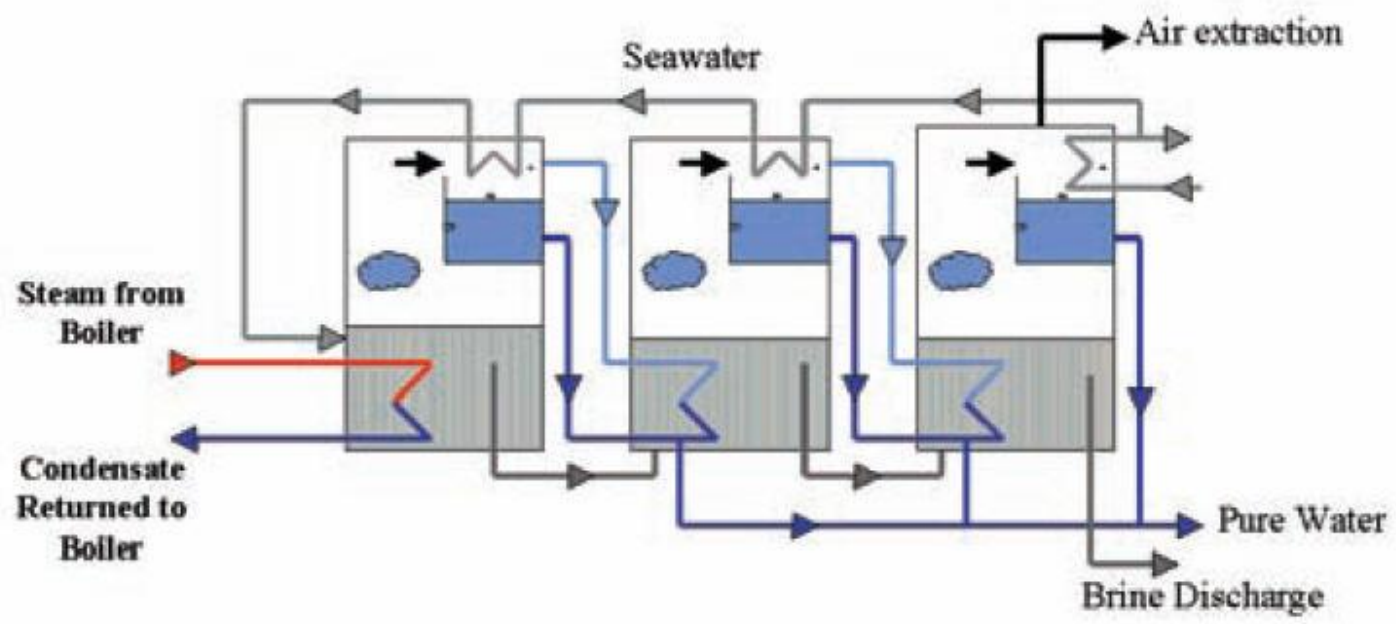

Figure 2.6. Schematic diagram of a MED system [IAEA, 2007]. 


\section{Multi-Stage Flash Distillation}

Figure 2.7 shows the operating principle of a MSF plant. Consider a vessel under vacuum, isolated from its environment and containing only seawater in equilibrium with its vapor at temperature $t$ and pressure $p$. When a heating fluid (generally hot water) at a temperature $t+\Delta t$ is introduced in the vessel, and if the pressure $p$ is less than the saturation pressure, an instantaneous vaporization will be produced by a flash. The latent heat of vapor can then be transferred to cold seawater tubes passing through the vessel and the vapor condensed and recovered in the receptacle.

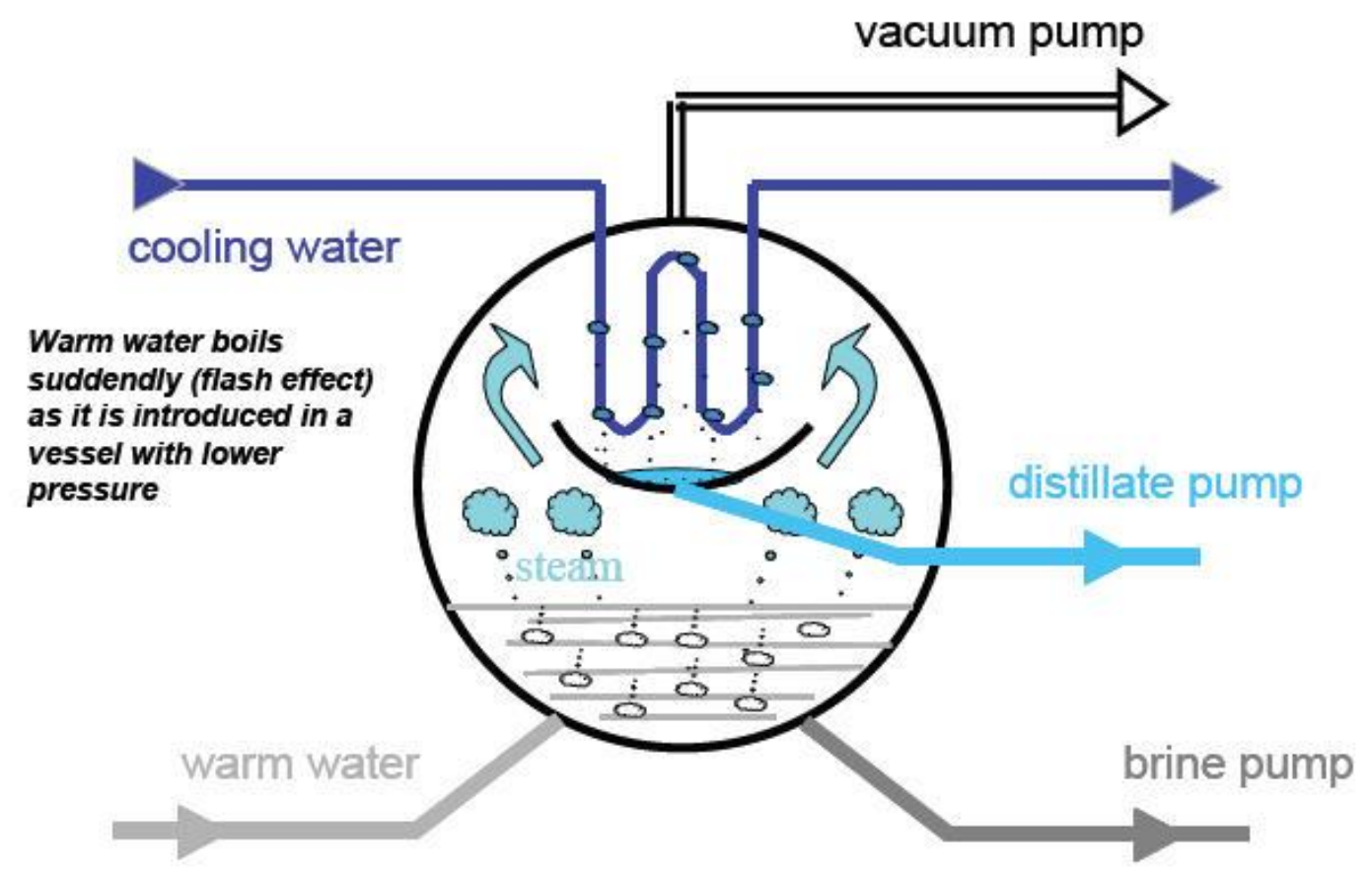

Figure 2.7. Operating principle of a single stage MSF [IAEA, 2007].

Figure 2.8 illustrates the schematic flow diagram of a MSF system. Seawater feed passes through tubes in each evaporation stage where it is progressively heated. Final seawater heating occurs in the brine heater by the heat source. Subsequently, the heated brine flows through nozzles into the first stage, which is maintained at a pressure slightly lower than the saturation pressure of the incoming water. As a result, a small fraction of the brine flashes forming pure steam. 


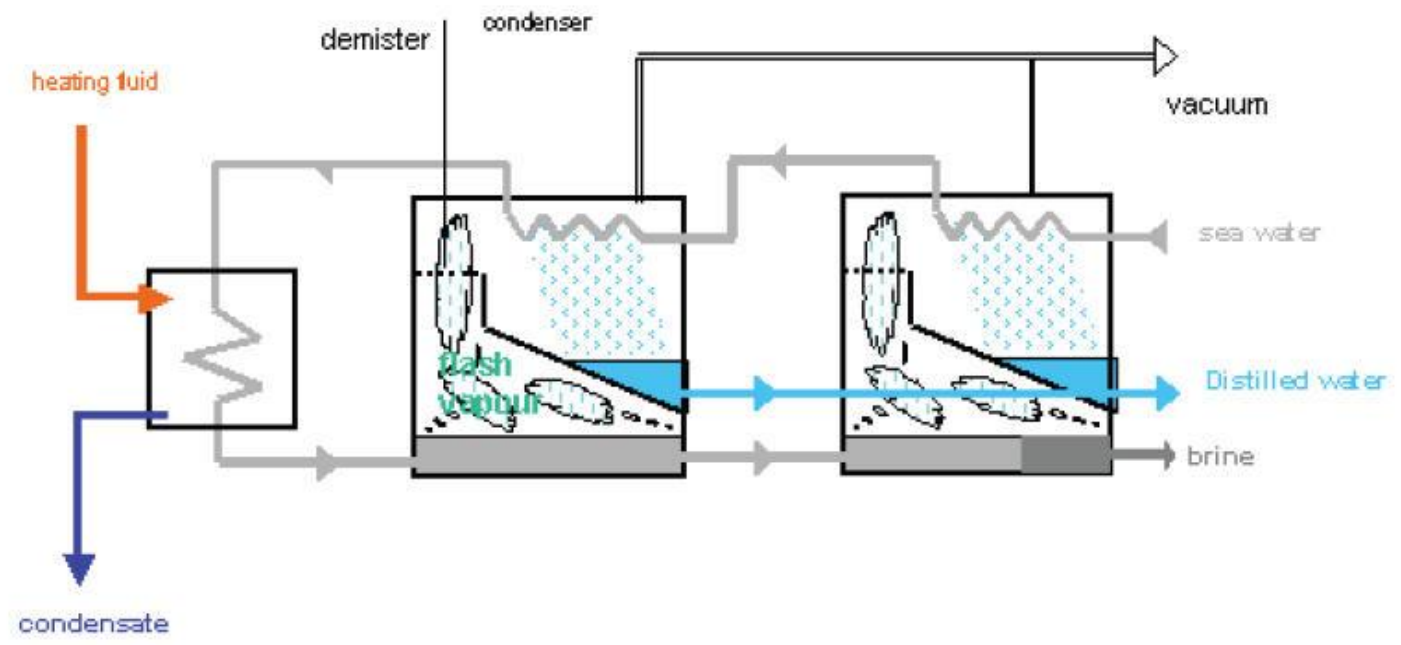

Figure 2.8. Schematic diagram of an industrial MSF design [IAEA, 2007].

The heat needed to flash the vapor comes from cooling of the remaining brine flow, which lowers the brine temperature. Subsequently, the produced vapor passes through a mesh demister in the upper chamber of the evaporation stage where it condenses on the outside of the condensing brine tubes and is collected in a distillate tray. The heat transferred by the condensation warms the incoming seawater feed as it passes through that stage. The remaining brine passes successively through all the stages at progressively lower pressures, where the process is repeated. The hot distillate flows as well from stage to stage and cools itself by flashing a portion into steam which is re-condensed on the outside of the tube bundles.

MSF plants need pre-treatment of the seawater to avoid scaling by adding acid or advanced scale inhibiting chemicals. If low cost materials are used for construction of the evaporators, a separate deaerator is to be installed. The vent gases from the deaeration together with any non-condensable gases released during the flashing process are discharged to the atmosphere. There are two principal arrangements in MSF systems: the brine recycle mode (MSF-BR), and the once-through mode (MSF-OT). The majority of the MSF plants that are built use the brine recycle mode. The brine re-cycle mode was invented in the early years of desalination when seawater corrosion materials and advanced additives were not available or too expensive. Today, corrosion resistant materials are available at reasonable costs as well as high temperature, cost effective anti-scalants. Therefore, MSF-OT plants have already been successfully applied. 


\subsubsection{Membrane Desalination Systems}

Reverse osmosis and electro dialysis are the two most important membrane processes. To affect salt separation, RO uses hydraulic pressure, whereas ED uses electric current.

\section{Reverse Osmosis}

Osmosis is a natural process in which water molecules migrate across a semi-permeable membrane from a solution of low concentration (e.g. pure water) into a solution of higher concentration (e.g. seawater). Reverse osmosis is a separation process in which pure water is "forced" out of a concentrated saline solution by flowing through a membrane at a high static trans-membrane pressure difference (Figure 2.9). This pressure difference must be higher than the osmotic pressure between the solution and the pure water.

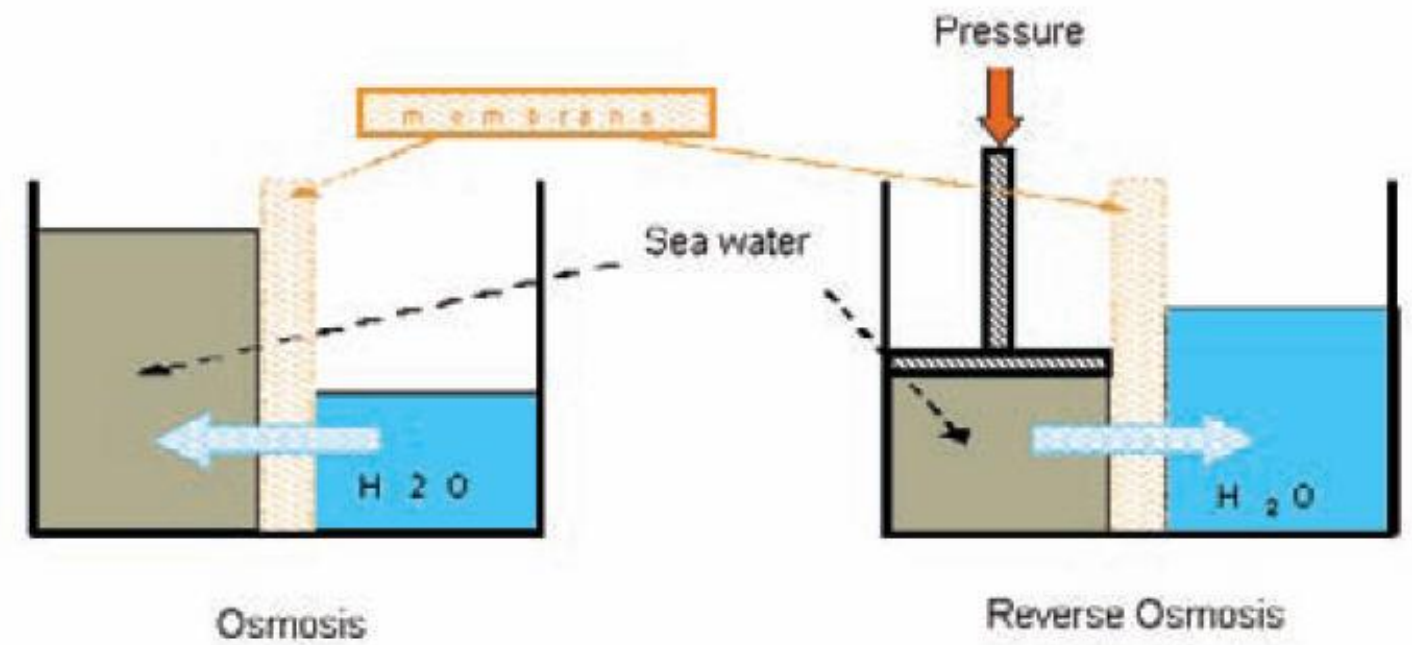

Figure 2.9. Osmosis and reverse osmosis processes [IAEA, 2007].

The saline feed is pumped into a closed vessel where it is pressurized against the membrane. As a portion of water passes through the membrane, the salt content in the remaining brine increases. At the same time, a portion of this brine is discharged without passing through the membrane.

RO membranes are made in a variety of modular configurations: two of the commercially successful configurations are spiral-wound modules and hollow fiber modules. In both 
configurations, module elements are serially connected in pressure vessels, up to 7-8 in the case of spiral wound and up to 2-3 in the case of hollow fiber modules.

\section{Electro-Dialysis}

Figure 2.10 illustrates an ED process, where two types of membranes are used. The cation membrane allows only cations (positive ions) to permeate, and the anion membrane allows only anions (negative ions) to permeate. These exchange membranes are alternately immersed in salty water in parallel, and an electric current is passed through the liquid. The cations will migrate to the cathode, and the anions will migrate to the anode. Therefore, water passing between membranes is split into two streams. One is pure water, and the other is concentrated brine. Because ED uses energy at a rate directly proportional to the quantity of salts to be removed, this process is more useful in desalting brackish water.

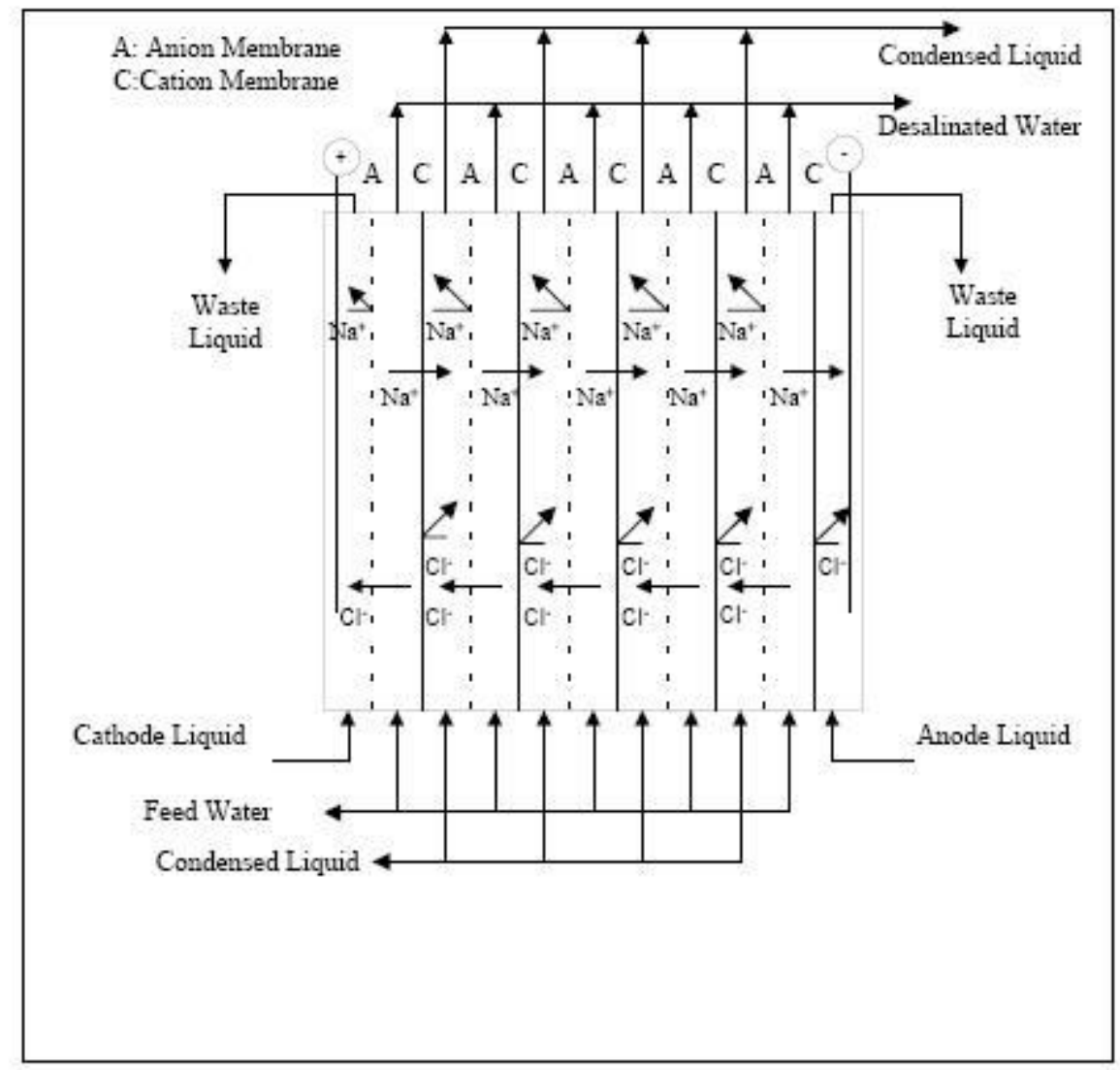

Figure 2.10. Principle of electro dialysis [Khan, 1986]. 


\section{Chapter 3}

\section{INTEGRATED ARCHITECTURE OF A FAULT DIAGNOSTIC SYSTEM}

Large industrial systems, such as power plants, chemical plants, pulp and paper plants, metals and other manufacturing systems, usually employ a multitude of sensors to monitor and control the operating state, which also makes it possible to improve the operational safety and to perform condition-based maintenance (CBM). This results in significant reduction in plant downtime and considerable amount of maintenance cost savings.

Sensors are crucial for automation and process control, both for achieving performance and for guaranteeing plant safety. The technological drive towards automated plants and high energy-intensive processes demands greater intelligence, availability, and reliability of instrumentation. Even when a process is intrinsically safe, loss of a sensor would lead to loss of process control and hence a sustained period of poor quality production. A sensor may be used in control systems, safety systems, or for condition monitoring. For condition monitoring, output of the sensor is normally displayed on a panel or on a computer screen, and not used as an input parameter of any system control. If the faulty sensor is the one used as a monitoring device, then the effect of its fault is one-dimensional, that is, the fault will be observed in its own signature and nowhere else. A multi-dimensional fault shows its effects in multiple measurements. The faults with control-related sensors are multi-dimensional because the fault effects can be propagated through control loops and subsequently disturb many other variables throughout the entire process. For instance, as soon as the sensor drifts, the controller notices the sensor signal variations and actuates in the opposite direction, in an attempt to correctly match the set point.

The detection and isolation of faults in a large industrial system, such as a nuclear power plant or a fossil power plant, is generally complicated due to measurement limitations and uncertainties, various types of processes and equipment, and interactions among the sub-systems. A fault diagnostic system is expected to be able to track incipient equipment and sensor degradation and assist in enhanced operation and maintenance planning. One approach to characterize the measurements is to build empirical models, and to use them in conjunction with a set of rules that defines the cause-and-effect relationships among the process variables. The 
fault diagnostic module proposed in this work is a two-step approach: (1) Development of empirical models for fault residual generation; (2) Detection and isolation of incipient faults based on these residuals. The residuals are computed as the difference between the measured process variables and the empirical model's estimations for those process variables. It is assumed that the residuals are small during normal plant operations. When a fault occurs in a device, equipment, or the process itself, the model residuals deviate from normal allowable values, and indicate a possible abnormal situation.

Sensor placement design is a critical component of a fault diagnostic system, and therefore it is emphasized in the developed integrated approach as illustrated in Figure 3.1. Whenever a process encounters a fault, the effect of the fault is propagated to some or all the process variables. The main objective of fault diagnosis is to observe these fault symptoms and determine the root causes of observed behavior. The ability of the sensor network to detect and discriminate failure modes and anomalous conditions is crucial for the efficiency of the fault diagnostic system. With hundreds of process variables available for measurement in the nuclear plant and the desalination process, selection of optimum number of sensors and their locations poses a unique challenge, and it is indeed an issue that must be addressed in the design phase. 


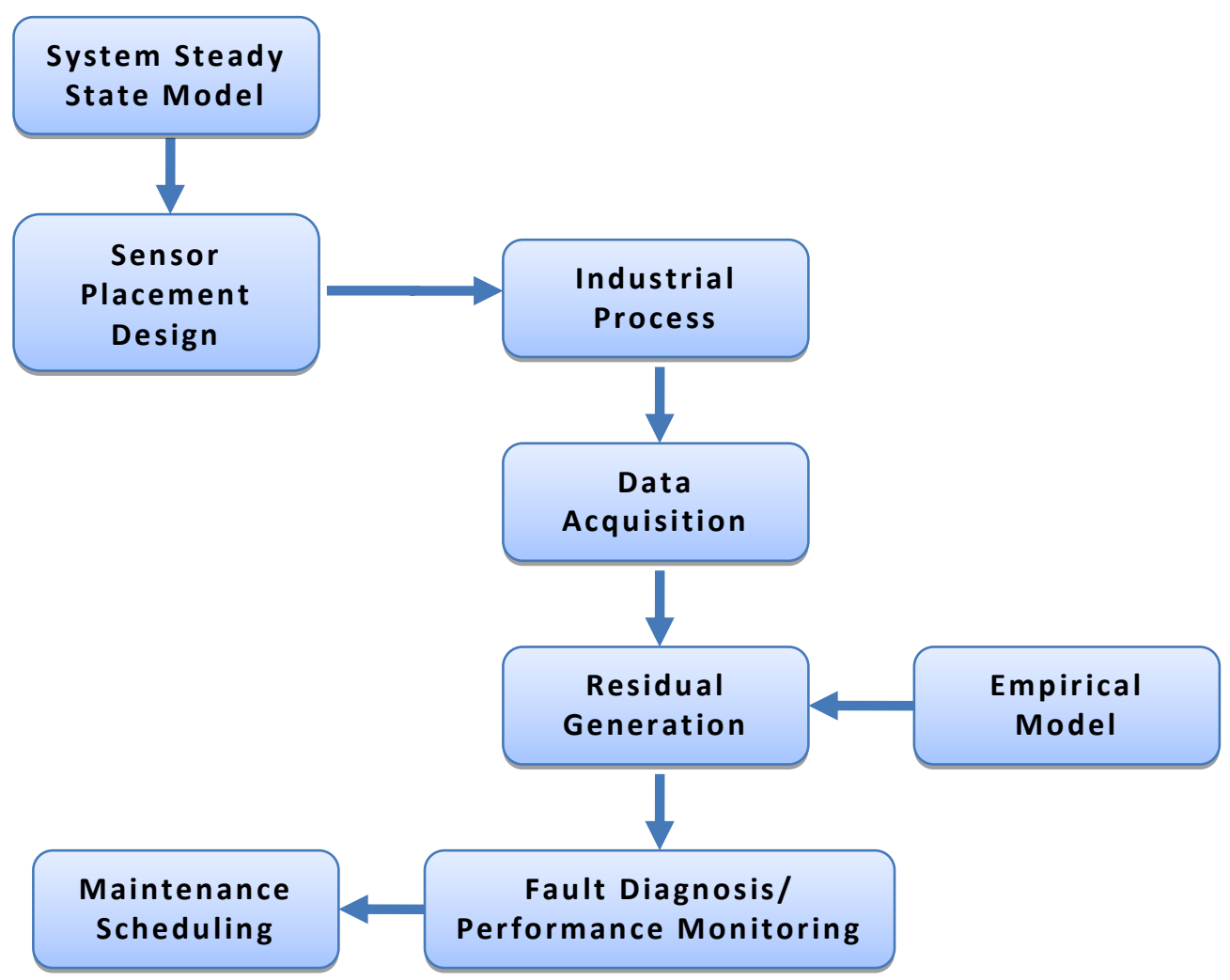

Figure 3.1. An integrated architecture of a fault diagnostic system. 


\section{Chapter 4}

\section{MODELING AND SIMULATION OF A MULTI-STAGE FLASH DESALINATION SYSTEM}

\subsection{Introduction}

Among a variety of desalination technologies, multi-stage flash (MSF) contributes substantially to the desalinating capacity in the world. Market share of the MSF processes accounts for $70 \%$ of all seawater desalination processes [El-Dessouky et al., 1999]. Thus, issues such as optimization of the operation and minimization of the corresponding environmental impact are of the greatest importance. With the objective of addressing all these aspects, mathematical models prove to be a very useful tool. Steady-state models help in plant design and in fixing operating conditions, whereas dynamic models are required for studying control system design, performance optimization, and fault diagnosis and prognosis. The physics model of a MSF desalination plant gives rise to a nonlinear boundary value problem. The stage-by-stage approach to solve the model is characterized by slow convergence and stability problems. Gluek and Bradshaw [1970] were among the earliest to develop a model of MSF plants with a high degree of rigor and few qualifying assumptions. The model takes into account the variation of heat transfer coefficient, vaporization from the product tray, etc. Helal et al. [1986] reported a tri-diagonal matrix (TDM) model for steady-state simulation of MSF plants. The set of equations was solved in a global manner by arranging the stage energy relations in the form of a tri-diagonal matrix. This method is stable and shows fast convergence. Theoretical models which simulate transient behavior of MSF plants under various conditions have also been reported in the literature [Rimawi et al., 1989; Aly and Marwan, 1995]. Generally, the models were based on coupling the dynamic equations of mass and energy for brine and product tray within the flash stages.

\subsection{MSF Desalination System Description}

A brief description of a MSF desalination process is provided in this section, and the process flow sheet is shown in Figure 4.1. The MSF process includes a number of flashing stages 
connected to a brine heater. The flashing stages are composed of two sections - the heat rejection section and the heat recovery section. The feed to the plant, $W_{T}$, is allowed to pass through the heat rejection section, whose function is to reject the surplus thermal energy from the plant and to cool the product and brine to the lowest possible temperature as they emerge from the last (coolest) rejection stage.

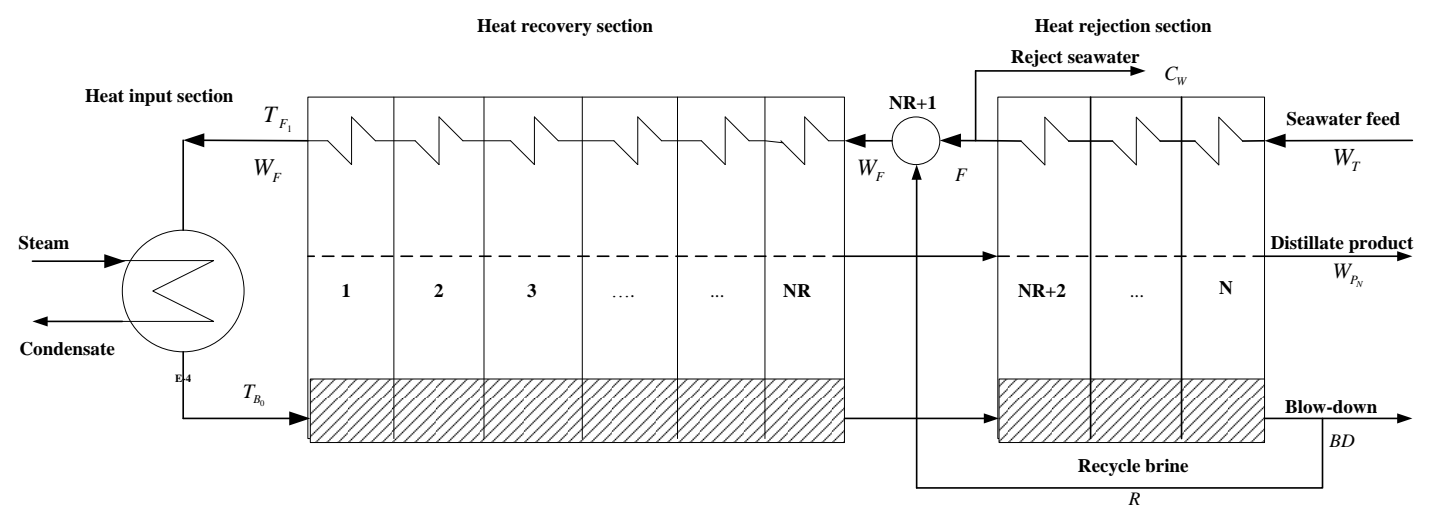

Figure 4.1. Schematic diagram of a MSF process with brine recirculation [Alatiqi et al., 1999].

On leaving the first (warmest) rejection stage the feed stream is split into two parts, reject seawater $C_{W}$, which passes back to the sea and a make-up stream $F$ which is then combined with the recycle stream $R$. The combined stream $W_{F}$ now passes through a series of heat exchangers in the heat recovery section. The temperature of the combined stream rises as it proceeds towards the heat input section of the plant. Passing through the brine heater, the brine temperature is raised from $T_{F_{1}}$ at the inlet of the brine heater to the top brine temperature (TBT) $T_{B_{0}}$ approximately equal to the saturation temperature at the system pressure.

The brine then enters the first heat recovery stage through an orifice thus reducing the pressure. As the brine was already at its saturation temperature for a higher pressure, it will become superheated and flashes to give off water vapor. This vapor passes through a wire mesh (demister) to remove any entrained brine droplets and on to a heat exchanger where the vapor is condensed and drips into a distillate tray. The process is then repeated all the way down the plant as both brine and distillate enter the next stage which is at a lower pressure. The concentrated brine is divided into two parts as it leaves the plant, the blowdown $B D$ which is pumped back to the sea and a recycle stream $R$ which returns to mix with the make-up stream, $F$. 
From the mathematical point of view, the once-through design and the recirculation design can be represented by the same model if the flow rates of the recycle $R$ and the reject seawater $C_{W}$ streams are given zero values. Moreover, there will be no distinction between heat recovery and heat rejection sections in the case of the once-through plant.

\subsection{MSF Mathematical Models}

In order to develop the mathematical models of a multi-stage flash desalination process, the following simplifying assumptions are made [Thomas et al., 1998]:

- The distillate product leaving any stage is salt free

- The heats of mixing for brine solutions are negligible

- No mist or salt is entrained with the vapor

- There is no heat loss from the plant

- The condensate produced in the brine heater is not sub-cooled and flows through the brine heater at a constant temperature

- The accumulation of cooling brine in the tube of the heat exchangers is neglected since its density changes are negligible

- The MSF plant is divided into a brine heater and a number of adiabatic stages while the interaction among them is only through the flow streams connecting them.

- The pumps are not explicitly considered in the model. It is assumed that the change in flow rate in the line due to valve action is transmitted to all parts of the flow line instantaneously.

- The model was developed using lumped parameter analysis, the mass considered to be perfectly mixed and spatial variations were not explicitly considered. 


\subsubsection{Flashing Stage Dynamic Model}

A typical MSF flashing stage can be divided into four control volumes $(\mathrm{CV})$ : the flashing brine tray, the distillate (product) tray, the vapor space, and the condenser tubes [Thomas et al., 1998]. Figure 4.2 shows the block diagram of a single stage. It should be noted that the masses are lumped at the exit of the $\mathrm{CV}$ and hence the exit temperatures $T_{B_{i}}, T_{P_{i}}$, and $T_{F_{i}}$ are well representative of the temperatures of their corresponding CVs.

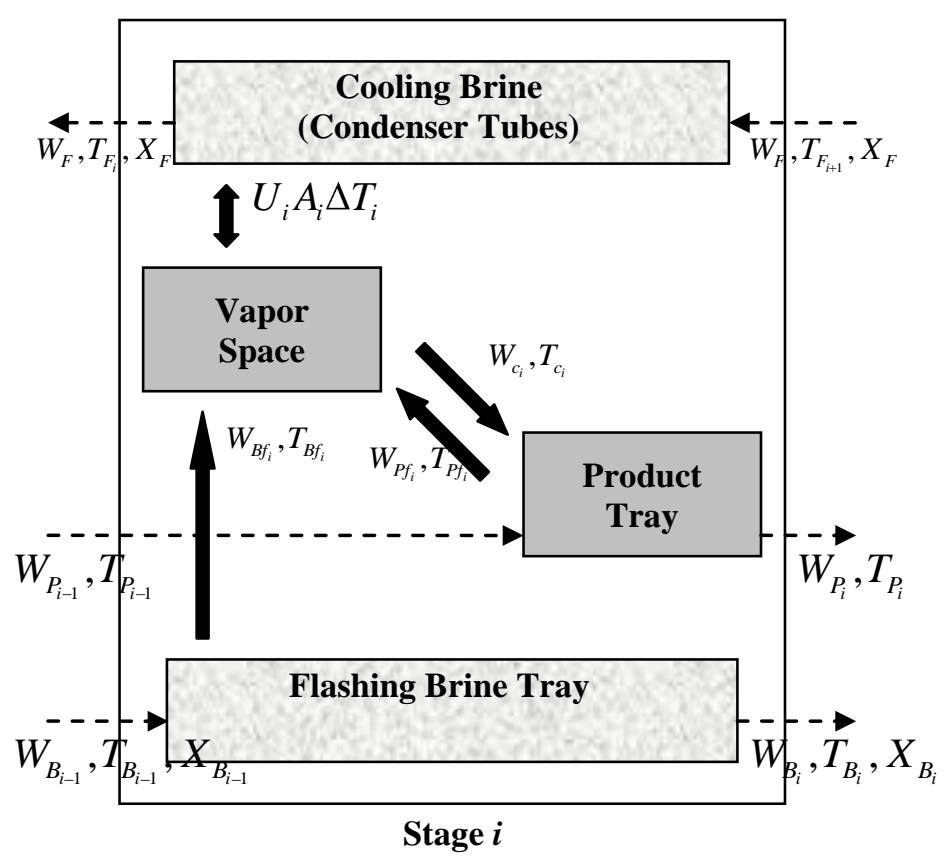

Figure 4.2. Block diagram of a stage of a MSF plant.

The mass balance equation of the flashing brine tray:

$$
\frac{d M_{B_{i}}}{d t}=W_{B_{i-1}}-W_{B_{i}}-W_{B f_{i}}
$$

The balance of salt mass:

$$
\frac{d\left(M_{B_{i}} X_{B_{i}}\right)}{d t}=W_{B_{i-1}} X_{B_{i-1}}-W_{B_{i}} X_{B_{i}}
$$

The energy balance of the flashing brine tray: 


$$
\frac{d\left(M_{B_{i}} h_{B_{i}}\right)}{d t}=W_{B_{i-1}} h_{B_{i-1}}-W_{B_{i}} h_{B_{i}}-W_{B f_{i}} h_{B f_{i}}
$$

The mass balance of the product tray:

$$
\frac{d M_{P_{i}}}{d t}=W_{P_{i-1}}-W_{P_{i}}-W_{P f_{i}}+W_{C_{i}}
$$

The energy balance of the product tray:

$$
\frac{d\left(M_{P_{i}} h_{P_{i}}\right)}{d t}=W_{P_{i-1}} h_{P_{i-1}}-W_{P_{i}} h_{P_{i}}-W_{P f_{i}} h_{P f_{i}}+W_{C_{i}} h_{C_{i}}
$$

The mass balance of the vapor space:

$$
\frac{d M_{V_{i}}}{d t}=W_{B f_{i}}+W_{P f_{i}}-W_{C_{i}}
$$

The energy balance of the vapor space:

$$
\frac{d\left(M_{V_{i}} h_{V_{i}}\right)}{d t}=W_{B f_{i}} h_{B f_{i}}+W_{P f_{i}} h_{P f_{i}}-W_{C_{i}} h_{C_{i}}-U_{i} A_{i} \Delta T_{i}
$$

where $U_{i}$ is the heat transfer coefficient, $A_{i}$ is the heat transfer area, and $\Delta T_{i}$ is the log mean temperature difference (LMTD), given by:

$$
\Delta T_{i}=\frac{T_{F_{i}}-T_{F_{i+1}}}{\ln \left(\frac{T_{P_{i}}-T_{F_{i+1}}}{T_{P_{i}}-T_{F_{i}}}\right)}
$$

The energy balance of the condenser tubes:

$$
M_{F_{i}} \frac{d h_{F_{i}}}{d t}=W_{F}\left(h_{F_{i+1}}-h_{F_{i}}\right)+U_{i} A_{i} \Delta T_{i}
$$


The distillate temperature will be less than the brine temperature by the boiling point elevation (BPE), non-equilibrium allowance (NEA) and loss in saturation temperature due to other pressure losses $(\Delta)$ :

$$
T_{B_{i}}=T_{P_{i}}+B P E_{i}+N E A_{i}+\Delta_{i}
$$

The saturation temperature of the vapor and distillate temperature correlation:

$$
T_{V_{i}}=T_{P_{i}}+\Delta_{i}
$$

The relationship for the evaluation of the stage pressure $f_{1}$ and empirical relationships for the evaluation of the flashing brine and distillate flow rates $f_{2}$ and $f_{3}$ (details in Appendix A):

$$
\begin{gathered}
P_{i}=f_{1}\left(T_{V_{i}}\right) \\
W_{B_{i}}=f_{2}\left(P_{i}, P_{i+1}, M_{B_{i}}, M_{B_{i+1}}\right) \\
W_{P_{i}}=f_{3}\left(P_{i}, P_{i+1}, M_{P_{i}}, M_{P_{i+1}}\right)
\end{gathered}
$$

It is assumed the vapor from the brine and distillate tray are not superheated, the condensate is not sub-cooled, and the vapor space has fully equilibrated with the stage conditions, that is,

$$
T_{C_{i}}=T_{B f_{i}}=T_{P f_{i}}=T_{V_{i}}
$$

\subsubsection{Brine Heater Dynamic Model}

The brine heater $(\mathrm{BH})$ has a steam flow, $W_{\text {STEAM }}$, coming in, which is considered to be saturated. BH is considered as the $0^{\text {th }}$ stage; hence the subscript 0 is used [Thomas et al., 1998].

Enthalpy balance of the cooling brine: 


$$
M_{B_{0}} \frac{d h_{B_{0}}}{d t}=U_{B H} A_{B H} \Delta T_{B H}-W_{F}\left(h_{B_{0}}-h_{F_{1}}\right)
$$

where $U_{B H}$ is the heat transfer coefficient, $A_{B H}$ is the heat transfer area, and $\Delta T_{B H}$ is given by:

$$
\Delta T_{B H}=\frac{T_{B_{0}}-T_{F_{1}}}{\ln \left(\frac{T_{\text {STEAM }}-T_{F_{1}}}{T_{\text {STEAM }}-T_{B_{0}}}\right)}
$$

Enthalpy balance of the condensing steam:

$$
W_{\text {STEAM }} \lambda_{\text {STEAM }}=U_{B H} A_{B H} \Delta T_{B H}
$$

\subsubsection{MSF Steady-State Model}

The mathematical model described in the previous section consists of a system of ordinary differential equations combined with algebraic equations. The differential equations are linear with respect to the time derivatives of the unknown variables of the model. In the

steady-state case, the time derivatives are set to zero. The mass hold-up is no longer a variable in any equation. The enthalpy in the equations is written in terms of the relevant temperature.

Enthalpy balance of the flashing brine tray:

$$
W_{B_{i_{i-1}}} h_{B_{i-1}}=W_{B_{i}} h_{B_{i}}+\left(W_{B_{i-1}}-W_{B_{i}}\right) h_{B f_{i}}
$$

Total material balance:

$$
W_{B_{i-1}}+W_{P_{i-1}}=W_{B_{i}}+W_{P_{i}}
$$

Salt balance of the flashing brine tray:

$$
W_{B_{i-1}} X_{B_{i-1}}=W_{B_{i}} X_{B_{i}}
$$


Overall enthalpy balance of a stage:

$$
\begin{aligned}
W_{F} C p_{A V_{i}}\left(T_{F_{i}}-T_{F_{i+1}}\right)= & W_{P_{i-1}} C p_{P_{i-1}}\left(T_{P_{i-1}}-T^{*}\right)+W_{B_{i-1}} C p_{B_{i-1}}\left(T_{B_{i-1}}-T^{*}\right) \\
& -W_{P_{i}} C p_{P_{i}}\left(T_{P_{i}}-T^{*}\right)-W_{B_{i}} C p_{B_{i}}\left(T_{B_{i}}-T^{*}\right)
\end{aligned}
$$

Heat transfer equation:

$$
W_{F} C p_{A V_{i}}\left(T_{F_{i}}-T_{F_{i+1}}\right)=U_{i} A_{i} \frac{T_{F_{i}}-T_{F_{i+1}}}{\ln \left(\frac{T_{P_{i}}-T_{F_{i+1}}}{T_{P_{i}}-T_{F_{i}}}\right)}
$$

Temperature correction equation:

$$
T_{B_{i}}=T_{P_{i}}+B P E_{i}+N E A_{i}+\Delta_{i}
$$

Brine heater overall enthalpy balance equation:

$$
W_{F} C p_{F_{B H}}\left(T_{B_{0}}-T_{F_{1}}\right)=W_{\text {STEAM }} \lambda_{\text {STEAM }}
$$

Heat transfer equation of brine heater:

$$
W_{F} C p_{F_{B H}}\left(T_{B_{0}}-T_{F_{1}}\right)=U_{B H} A_{B H} \frac{T_{B_{0}}-T_{F_{1}}}{\ln \left(\frac{T_{S T E A M}-T_{F_{1}}}{T_{\text {STEAM }}-T_{B_{0}}}\right)}
$$

For steady-state solution of the MSF plant model, all the input variables (operating conditions), the plant dimensions, and the physical properties of the streams of the plant should be known. The mathematical expressions describing the thermo-physical properties of water, steam, and brine solutions are given in Appendix A. It is noteworthy that the functions representing those properties are highly nonlinear, thus contributing a lot to the overall complexity of the model. A stage-by-stage approach is usually highly iterative and is characterized by slow convergence. Therefore, a global approach presented by Helal et al. [1986] 
is employed in this study. This approach arranges the stage energy equations of all the stages in a TDM form, and is capable of generating stable results with fast convergence.

\subsection{Control Loops in a MSF System}

The main control loops of a MSF process are shown in Figure 4.3. Each of the control loops involves a single-input single-output system. The controlled and manipulated variables include the following:

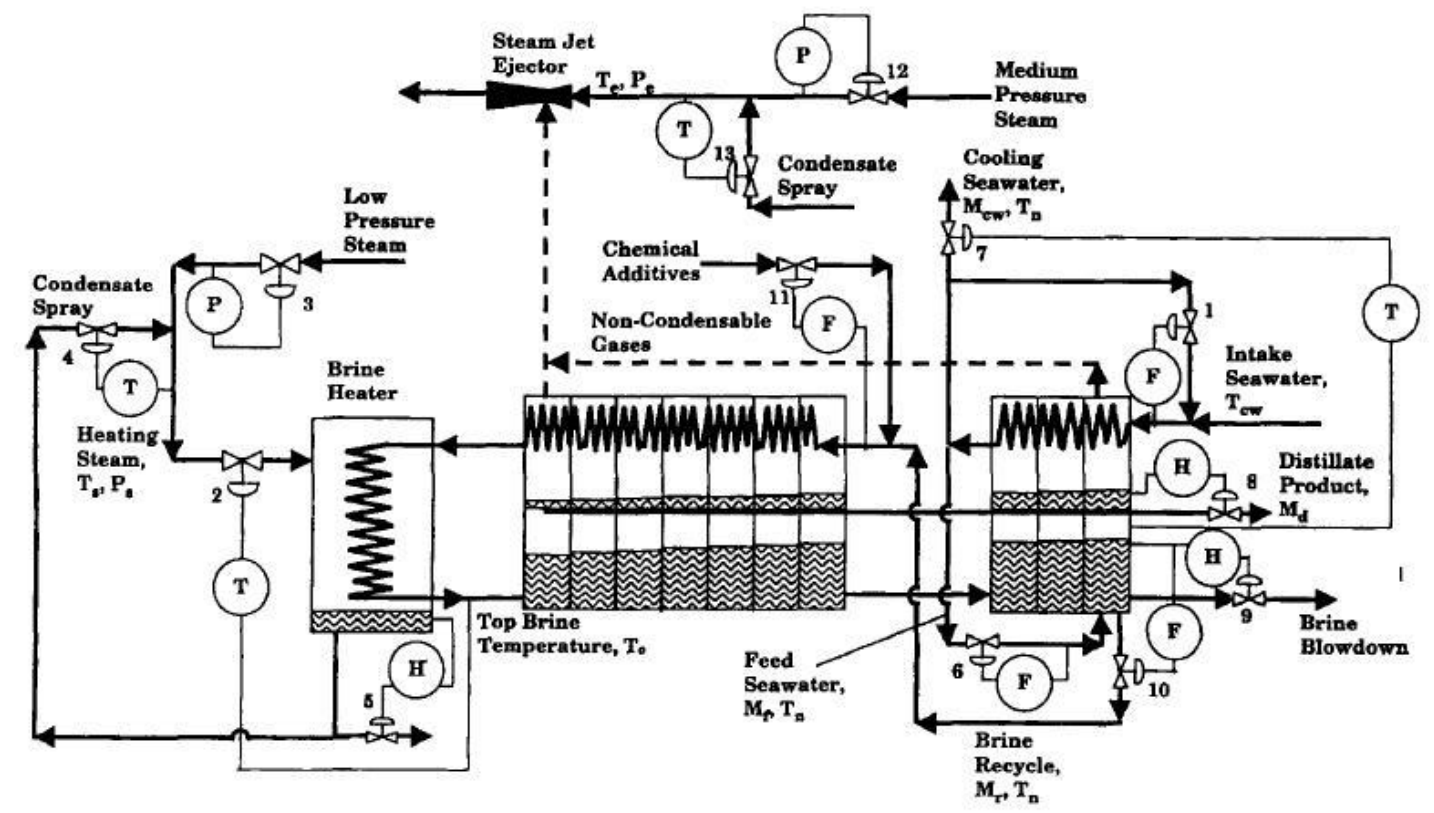

Figure 4.3. Single-input single-output control loops of MSF [Alatiqi et al., 1999].

(1) Temperature of the intake seawater entering the last flashing stage (controlled) and the circulation flow rate of the warm cooling seawater (manipulated). This loop operates only during the winter season when the intake seawater temperature drops to values close to $15^{\circ} \mathrm{C}$. As a result, the control valve and pumping system of the control loop operates to adjust the temperature of the intake seawater. Set-points for the intake seawater temperature vary between $25^{\circ} \mathrm{C}$ for winter operation and $32^{\circ} \mathrm{C}$ for summer conditions. This loop is not found in the once through MSF layout [El-Dessouky et al., 1999].

(2) TBT (controlled) and heating steam flow rate (manipulated). An increase or decrease of the heating steam flow rate is necessary to control the TBT. This might be necessary to take into 
account an increase in the brine circulation rate, fouling and scaling effects, or decrease in the temperature of the brine circulation. The set point for the TBT depends on the type of antiscalant used in the plant where $90^{\circ} \mathrm{C}$ is suitable for a polyphosphate type and $100-110^{\circ} \mathrm{C}$ is used for polymer type additives.

(3) Pressure of the heating steam (controlled) and the opening of the throttling valve (manipulated). Commonly, the low pressure steam has a pressure of 4-7 bars, and it is necessary to reduce its pressure to a value of about 2 bar where it becomes superheated with a temperature close to $170^{\circ} \mathrm{C}$.

(4) Temperature of the heating steam (controlled) and the flow rate of the condensate spray (manipulated). This loop changes the steam quality from superheated to saturated, where its temperature drops from $170^{\circ} \mathrm{C}$ to 100 or $110^{\circ} \mathrm{C}$. The steam temperature is controlled to be higher than the TBT by $5-10^{\circ} \mathrm{C}$. This is necessary to prevent the formation of hot spots and scale formation.

(5) Condensate level in the brine heater (controlled) and the opening of the distillate discharge valve (manipulated). It is necessary to maintain a sufficient static head above the condensate pump to prevent condensate flashing within the discharge line or the pumping unit which would result in violent vibrations, loosening of various connections, and severe erosion. This loop, like all other level loops in the plant, is consistent with the material balance in direction of flow principle.

(6) The flow rate of the feed seawater entering the brine pool of the last stage (controlled) and the valve opening on this line (manipulated). The set point for this control is the flow rate ratio of distillate and brine blow down. This control loop maintains a constant conversion ratio, which is necessary to keep the salinity of the brine blow-down from the last stage at a design value of 70,000 ppm. In the Gulf, the intake seawater salinity varies between 42,000-48,000 ppm and accordingly, the conversion ratio varies between $0.4-0.314$. This sets the controller ratio at 1.5-2.18, with the units of $\mathrm{kg}$ brine blow-down per $1 \mathrm{~kg}$ distillate product.

(7) Temperature of the reject flow rate of the cooling seawater (controlled) and the opening of the discharge valve (manipulated). The set-point of the controller is made in reference to the 
temperature of the last flashing stage. For a constant plant capacity, increase in the stage temperature causes reduction in the flashing range and the system performance ratio with simultaneous increase in the brine recycle flow rate. The last stage temperature is set at $40^{\circ} \mathrm{C}$ for summer operation and $32{ }^{\circ} \mathrm{C}$ for winter operation.

(8) Distillate level in the last stage (controlled) and the distillate product flow rate (manipulated). This control loop has a similar function to the level controller of the heating steam condensate where decrease of the distillate product static head would cause operational problems to the associated pumping unit.

(9) Brine level in the last flashing stage (controlled) and the brine blow-down flow rate (manipulated). This is one of the most important control loops in a MSF plant since the head in the last stage adjusts the head in previous stages. Simultaneously, the flashing efficiency is affected in various stages as well as the amount of distillate product. Increase of the head reduces the flashing rates and the amount of distillate product; consequently, the amount of heat released upon condensation of the distillate vapor becomes lower. Eventually, the temperature of the brine recycle stream entering the brine heater becomes lower, which results in the increase in the amount of heating steam and reduction in the system thermal performance ratio. On the other hand, reduction of the brine head may result in blow-through of the flashed-off vapors across the stages. As a result, the effective number of stages becomes smaller and the plant performance ratio decreases.

(10) Brine level in the last flashing stage (controlled) and the brine recycle flow rate (manipulated). This control loop has a similar function and effects on the system performance as the previous control loop.

(11) Dosing flow rate of the chemical additives (controlled) and the opening of the discharge valve of the chemical additive (manipulated). Variations in the brine circulation flow rate necessitate adjustment of the dosing rate of the chemical additives. Such variations are caused by adjustments in the flow rate of the brine circulation stream which might be necessary to adjust the brine level in the last flashing stage. 
(12) Pressure of the ejector motive steam (controlled) and the opening of the throttling valve (manipulated). Commonly, the medium pressure steam has a pressure of 16 bars and it is necessary to reduce its value to 7 bars.

(13) Temperature of the ejector motive steam (controlled) and the flow rate of the condensate spray (manipulated). This loop reduces the ejector motive steam temperature from $201.4{ }^{\circ} \mathrm{C}$ to $165^{\circ} \mathrm{C}$. Spray of steam condensate results in this temperature reduction.

Selection of the MSF controllers is made subject to the following classification: the controller proportional action works on the set point changes and the controller derivative action does not work on set point changes. This is necessary to obtain a fast response and to limit system disturbances.

\subsection{Measurements and Instrumentation in a MSF System}

Instrumentation and measurements form an essential part in various control loops in desalination plants where measured signals are transmitted to the control system and compared against the desired set points. Subsequently, corrective action in various manipulated parameters takes place to adjust the controlled variable to the desired value. Accordingly, maintaining measuring instrumentation in good working condition requires frequent cleaning, servicing, and calibration. Alatiqi et al. [1999] outlined in detail the main measuring instruments in MSF plants. A summary of these instruments is given in Table 4.1. All of the temperature, pressure, flow rate, level, and $\mathrm{pH}$ measurements are made on-line, while the measured data are taken at equal intervals and logged on a data acquisition system. Other data, such as, detailed water analysis, are made off-line in central analytical laboratories following standard methods. As is shown, some measuring instruments are simple and inexpensive to use; for example, differential manometers. However, a major part of the instrumentation is delicate and requires frequent calibration and replacement of defective parts, such as, $\mathrm{pH}$ and conductivity probes. Also, all of the measuring instrumentation may face problems related to fouling, poor mixing, vapor condensation, and the corrosive nature of the seawater. In various locations around the plant, multiple sensors might be used to obtain an average value or ensure proper measurements of sensitive parameters, i.e., TBT, brine level in the last stage, or pressure and temperature of heating steam. 
All measuring instruments are connected with indicators and alarms commonly located in the central operation and control room. Some instrumentation is tied with a control loop; for example, TBT, brine level in the last stage, and pressure of the heating steam. A small number of the instruments are coupled with an alarm system; this may vary from one plant to another. However, in all plants the TBT is tied with an alarm system. Other alarm units are used for the distillate flow rate, distillate level in the last stage, brine level in the last stage, brine level in the first stage, and temperature and flow rates of intake and feed seawater.

Table 4.1. Measuring instruments used in the desalination industry

\begin{tabular}{|c|c|}
\hline Measured parameter & Typical problems \\
\hline Temperature & $\begin{array}{l}\text { 1. Small temperature differences. Measuring error can be in the same } \\
\text { range as the temperature difference between two stages. } \\
\text { 2. Two phase temperature measurements yield a high error due to } \\
\text { vapor condensation and presence of non-condensable gases. } \\
\text { 3. Temperature non-uniformity, measurement may alter due to } \\
\text { presence of gradients or heat losses. } \\
\text { 4. High corrosion rates of sensors, wires, and cables in presence of } \\
\text { high temperature seawater and brine. }\end{array}$ \\
\hline Pressure & $\begin{array}{l}\text { 1. Vapor condensation in the tubing leading to the sensing sides of } \\
\text { the transducers. } \\
\text { 2. Non-condensable gases may generate large errors. } \\
\text { 3. Pressure variations along the circumference of pipes. } \\
\text { 4. Tap fouling and blockage gives partial readings of pressure and } \\
\text { large errors. }\end{array}$ \\
\hline Flow & $\begin{array}{l}\text { 1. Errors caused by bends, expansion, and contraction. } \\
\text { 2. Flashing effects gives erroneous errors for flow meters with } \\
\text { moving parts. } \\
\text { 3. Fouling and scaling may hinders motion of moving parts and } \\
\text { block small openings. }\end{array}$ \\
\hline Level & $\begin{array}{l}\text { 1. Filling of the vapor/gas leg with condensate may result in large } \\
\text { errors. } \\
\text { 2. Accumulation of dirt in the liquid leg may generate erroneous } \\
\text { errors. } \\
\text { 3. Oscillation effects due to violent flashing effects or turbulence. }\end{array}$ \\
\hline $\mathrm{pH}$ & $\begin{array}{l}\text { 1. Fouling problems around sensors. } \\
\text { 2. Poor reading due the delicate nature of the sensor or bad mixing. }\end{array}$ \\
\hline Gas concentration & $\begin{array}{l}\text { 1. Fouling problems. } \\
\text { 2. Calibration problems. }\end{array}$ \\
\hline Water composition & $\begin{array}{l}\text { 1. Protect sensor from fouling. } \\
\text { 2. Poor mixing and dead zones. } \\
\text { 3. Delicate nature of sensors. }\end{array}$ \\
\hline
\end{tabular}




\subsection{MSF Desalination System Simulation Results}

Steady-state and dynamic behavior of a MSF plant can be predicted from the mathematical model presented in the previous section. However, the model has been derived under certain simplifying assumptions. Thus, it is necessary to validate the given model, obtained from the design data, against the actual plant operation data. Steady-state behavior of the MSF model is presented first.

\subsubsection{Steady-State Simulation Results}

Assuming that stage and brine heater geometries are assigned, together with the values of the fouling factors in all the heat exchangers of the plant, the feed temperature $T_{S E A}$ and concentration $X_{S E A}$, and comparing the number of equations and the number of unknowns of the model allows to determine that the developed model has four degrees of freedom. Below are three different combinations of variables that can be chosen to simulate a MSF plant. Each set comprises four specifications as has been explained before.

(1) The first case is termed as a "performance" calculation. In this case, the following operating variables are specified: $R, C_{W}, F, T_{\text {STEAM }}$.

(2) In the second case the operating parameters $F, C_{W}, T_{B_{0}}$ and the plant capacity $D_{N}$ are specified. This study may be used to investigate the possibility of maintaining a specified plant capacity in the condition of changing feed temperature.

(3) The third case deals with the specified parameters $F, C_{W} / R, W_{\text {STEAM }}$ and $T_{B_{0}}$. This alternative simulates a situation where a specified amount of steam is supplied to the desalination plant by a coupled power plant.

Here we consider performance calculation, where we specify the make-up flow, $F$, the rejected seawater flow rate $C_{W}$, the recycle stream flow rate $R$, and the steam temperature $T_{\text {STEAM }}$. On the basis of the information the model allows to calculate the profiles of temperatures and flow rates in all the stages of the unit. 
The configuration investigated in this work refers to the design data of a real desalination plant located in the Middle East which includes 13 stages in the heat recovery section and 3 heat rejection stages. The plant design data are given in Tables 4.2 - 4.4.

Table 4.2. The main characteristics of the plant

\begin{tabular}{|c|c|}
\hline Type of plant & Brine recycle-cross tube \\
\hline No. of recovery stages & 13 (Stages 1 13) \\
\hline No. of rejection stages & 3 (Stages 14 16) \\
\hline No. of stages & 16 \\
\hline Nominal capacity & 5 MGD \\
\hline
\end{tabular}

Table 4.3. The operating conditions

\begin{tabular}{|c|c|}
\hline Steam temperature to brine heater & $206^{\circ} \mathrm{F}$ \\
\hline Feed Seawater temperature & $95^{\circ} \mathrm{F}$ \\
\hline Feed Seawater concentration & $56,000 \mathrm{ppm}$ \\
\hline Make-up flow rate & $0.125 \times 10^{8} \mathrm{lb} / \mathrm{hr}$ \\
\hline Recycle flow rate & $0.140 \times 10^{8} \mathrm{lb} / \mathrm{hr}$ \\
\hline Reject cooling seawater & $0.124 \times 10^{8} \mathrm{lb} / \mathrm{hr}$ \\
\hline Height of brine level in each stage & $18 \mathrm{in}$. \\
\hline
\end{tabular}

Table 4.4. The condenser tube bundle data

\begin{tabular}{|c|c|c|c|}
\hline Parameter & $\begin{array}{c}\text { Recovery } \\
\text { section }\end{array}$ & $\begin{array}{c}\text { Rejection } \\
\text { section }\end{array}$ & Brine heater \\
\hline No. of tubes per stage & 4300 & 4300 & 4300 \\
\hline Tube length $(f t)$ & 40 & 35 & 40 \\
\hline Inside diameter $($ in. $)$ & 0.866 & 0.9394 & 0.866 \\
\hline Thickness $($ in. $)$ & 0.048 & 0.0303 & 0.048 \\
\hline Stage width $(f t)$ & 40 & 35 & 40 \\
\hline $\begin{array}{c}\text { Thermal conductivity } \\
\left(B T U / h r \cdot f t \cdot{ }^{\circ} F\right)\end{array}$ & 28.9 & 9.4 & 16.8 \\
\hline $\begin{array}{c}\text { Fouling factor } \\
\left(B T U / h r \cdot{ }^{o} F \cdot f t^{2}\right)^{-1}\end{array}$ & $0.681 \times 10^{-3}$ & $0.1136 \times 10^{-3}$ & $0.909 \times 10^{-3}$ \\
\hline $\begin{array}{c}\text { Heat transfer area } \\
\left(f t^{2}\right)\end{array}$ & 43,000 & 38,000 & 38,000 \\
\hline
\end{tabular}


In the performance calculation, the feed seawater temperature and salt concentration are $95^{\circ} \mathrm{F}$ and $5.6 \% \mathrm{wt}$, respectively, while the steam temperature, $T_{\text {STEAM }}$, is $206^{\circ} \mathrm{F}$. The other operating parameters required for the calculation are defined as follows:

$$
F=0.125 \times 10^{8} \mathrm{lb} / \mathrm{hr}, C_{W}=0.124 \times 10^{8} \mathrm{lb} / \mathrm{hr}, \quad R=0.140 \times 10^{8} \mathrm{lb} / \mathrm{hr} .
$$

The results of the performance calculation are reported in Table 4.5. In particular, the values of the flow rates, temperatures and salt concentrations are given. For the sake of comparison, the actual plant values for the principal parameters at operating conditions are given in Table 4.6. It can be observed that the stage temperatures, inter-stage flow rates, and salt concentrations are predicted very well by the steady-state model.

Table 4.5. Steady-state model results corresponding to the performance study case

\begin{tabular}{|c|c|c|c|c|c|c|c|}
\hline Stage \# & $\begin{array}{c}W_{B_{B}} \\
\left.\times 10^{8} l b / h r\right)\end{array}$ & $\begin{array}{c}W_{p_{R}} \\
\left(\times 10^{6} \mathrm{lb} / \mathrm{hr}\right)\end{array}$ & $\begin{array}{l}\mathrm{X}_{B_{B}} \\
(\%)\end{array}$ & $\begin{array}{l}\mathrm{T}_{F_{F}} \\
\left({ }^{\circ} F\right)\end{array}$ & $\begin{array}{l}\mathrm{T}_{R} \\
\left({ }^{\circ} F\right)\end{array}$ & $\begin{array}{l}\mathrm{T}_{B_{i}} \\
\left({ }^{\circ} F\right)\end{array}$ & $\begin{array}{c}\mathrm{U}_{\mathrm{i}} \\
\left(B T U / h r \cdot f t^{2} \cdot{ }^{\circ} F\right)\end{array}$ \\
\hline $\begin{array}{c}0 \\
\text { brine heater }\end{array}$ & 0.2650 & 0 & 6.2412 & 0 & 0 & 200.50 & 696.7 \\
\hline 1 & 0.2636 & 0.1420 & 6.2749 & 190.23 & 192.86 & 194.97 & 676.7 \\
\hline 2 & 0.2621 & 0.2862 & 6.3094 & 184.46 & 187.16 & 189.28 & 674.9 \\
\hline 3 & 0.2607 & 0.4324 & 6.3448 & 178.55 & 181.31 & 183.45 & 673.2 \\
\hline 4 & 0.2592 & 0.5802 & 6.3810 & 172.51 & 175.34 & 177.50 & 671.6 \\
\hline 5 & 0.2577 & 0.7293 & 6.4179 & 166.36 & 169.24 & 171.43 & 670.2 \\
\hline 6 & 0.2562 & 0.8792 & 6.4554 & 160.10 & 163.03 & 165.25 & 668.9 \\
\hline 7 & 0.2547 & 1.0296 & 6.4935 & 153.76 & 156.72 & 158.99 & 667.7 \\
\hline 8 & 0.2532 & 1.1800 & 6.5321 & 147.34 & 150.33 & 152.66 & 666.6 \\
\hline 9 & 0.2517 & 1.3301 & 6.5711 & 140.85 & 143.87 & 146.28 & 665.5 \\
\hline 10 & 0.2502 & 1.4792 & 6.6102 & 134.33 & 137.36 & 139.86 & 664.4 \\
\hline 11 & 0.2487 & 1.6270 & 6.6495 & 127.79 & 130.83 & 133.44 & 663.1 \\
\hline 12 & 0.2473 & 1.7728 & 6.6887 & 121.25 & 124.28 & 127.02 & 661.5 \\
\hline 13 & 0.2458 & 1.9161 & 6.7277 & 114.73 & 117.75 & 120.66 & 659.6 \\
\hline 14 & 0.2448 & 2.0183 & 6.7558 & 110.01 & 112.90 & 116.08 & 614.4 \\
\hline 15 & 0.2438 & 2.1216 & 6.7844 & 105.09 & 108.05 & 111.40 & 611.4 \\
\hline 16 & 0.2427 & 2.2256 & 6.8135 & 100.08 & 103.10 & 106.67 & 607.9 \\
\hline
\end{tabular}


Table 4.6. Actual plant operation data [Helal et al., 1986]

\begin{tabular}{|c|c|c|c|c|c|c|c|}
\hline Stage \# & $\begin{array}{c}W_{B_{B}} \\
\left(\times 10^{8} / b / h r\right)\end{array}$ & $\begin{array}{c}W_{P_{P}} \\
\left(\times 10^{6} l b / h r\right)\end{array}$ & $\begin{array}{c}\mathrm{X}_{B_{i}} \\
(\%)\end{array}$ & $\begin{array}{c}\mathrm{T}_{F_{i}} \\
\left({ }^{\circ} F\right)\end{array}$ & $\begin{array}{c}\mathrm{T}_{p_{i}} \\
\left({ }^{\circ} F\right)\end{array}$ & $\begin{array}{c}\mathrm{T}_{B_{i}} \\
\left({ }^{\circ} F\right)\end{array}$ & $\begin{array}{c}\mathrm{U}_{\mathrm{i}} \\
\left(B T U / h r \cdot . \mathrm{ft}^{2} \cdot{ }^{\circ} F\right)\end{array}$ \\
\hline $\begin{array}{c}0 \\
\text { brine heater }\end{array}$ & 0.2650 & 0 & 6.2412 & 0 & 0 & 199.35 & 696.7 \\
\hline 1 & 0.2633 & 0.1711 & 6.39 & 187.46 & 190.53 & 192.66 & 675.2 \\
\hline 2 & 0.2614 & 0.3440 & 6.44 & 180.74 & 183.66 & 185.80 & 673.3 \\
\hline 3 & 0.2600 & 0.5018 & 6.47 & 174.38 & 177.29 & 179.47 & 671.6 \\
\hline 4 & 0.2584 & 0.6581 & 6.51 & 168.01 & 170.92 & 173.11 & 670.1 \\
\hline 5 & 0.2569 & 0.8116 & 6.55 & 161.70 & 164.57 & 166.81 & 668.8 \\
\hline 6 & 0.2554 & 0.9615 & 6.59 & 155.45 & 158.30 & 160.58 & 667.7 \\
\hline 7 & 0.2539 & 1.1079 & 6.63 & 149.27 & 152.09 & 154.43 & 666.6 \\
\hline 8 & 0.2525 & 1.2509 & 6.67 & 143.16 & 145.95 & 148.36 & 665.7 \\
\hline 9 & 0.2511 & 1.3906 & 6.70 & 137.12 & 139.88 & 142.36 & 664.7 \\
\hline 10 & 0.2497 & 1.5270 & 6.74 & 131.13 & 133.87 & 136.45 & 663.6 \\
\hline 11 & 0.2484 & 1.6602 & 6.78 & 125.22 & 127.93 & 130.62 & 662.4 \\
\hline 12 & 0.2471 & 1.7902 & 6.81 & 119.36 & 122.06 & 124.87 & 660.9 \\
\hline 13 & 0.2458 & 1.9169 & 6.85 & 113.57 & 116.24 & 119.21 & 659.1 \\
\hline 14 & 0.2450 & 1.9980 & 6.87 & 109.73 & 112.36 & 115.57 & 641.8 \\
\hline 15 & 0.2440 & 2.1007 & 6.90 & 104.90 & 107.61 & 110.92 & 639.2 \\
\hline 16 & 0.2429 & 2.2055 & 6.93 & 99.96 & 102.63 & 106.14 & 631.9 \\
\hline
\end{tabular}

\subsubsection{Dynamic Simulation Results}

SIMULINK is a software package for modeling, simulating, and analyzing dynamic systems. It supports linear and nonlinear systems, modeled in continuous time, sampled time, or a hybrid of the two. Systems can also be multi-rate, i.e., have different parts that are sampled or updated at different rates. For modeling, SIMULINK provides a graphical user interface (GUI) for building models as block diagrams, using click-and-drag mouse operations. It includes a comprehensive block library of sinks, sources, linear and nonlinear components, and connectors. Models are hierarchical, so models can be built using both top-down and bottom-up approaches. After the model is defined the simulations can be performed using a choice of integration methods, either from the SIMULINK menus or by entering commands in the MATLAB command window. Model analysis tools include linearization and trimming tools, which can be accessed from the MATLAB command line, plus the many tools in MATLAB and its application toolboxes. 
A SIMULINK model of a MSF plant with three flash stages and the brine heater is developed in this project, as shown in Figure 4.4. Only blocks from the standard SIMULINK library are used, so that the minimal configuration of SIMULINK is necessary to model a MSF process. Proportional-integral controllers are integrated into the MSF model to regulate the top brine temperature and the brine level in the last flashing stage by controlling the heating steam flow rate through the brine heater and the brine blow-down flow rate, respectively. The set points for the controllers are listed in Table 4.7. Regulation of the brine level in the last flashing stage is one of the most important control loops in a MSF plant, since the head in the last stage adjusts the head in previous stages. Simultaneously, the flashing efficiency is affected in various stages as well as the amount of distillate product. An increase in the head reduces the flashing rates and the amount of distillate product; consequently, the amount of heat released upon condensation of the distillate vapor becomes lower. Eventually, the temperature of the brine recycle stream entering the brine heater becomes lower, which results in the increase in the amount of heating steam and reduction in the system thermal performance ratio. On the other hand, reduction of the brine head may result in blow-through of the flashed-off vapors across the stages. As a result, the effective number of stages becomes smaller and the plant performance decreases.

Table 4.7. Control loops in dynamic simulation

\begin{tabular}{|c|c|c|c|c|}
\hline Control loop & Nature & Set point & $\begin{array}{c}\text { Controlled } \\
\text { variable }\end{array}$ & $\begin{array}{c}\text { Manipulated } \\
\text { variable }\end{array}$ \\
\hline 1 & PI & $200.5^{\circ} \mathrm{F}$ & $\begin{array}{c}\text { Top brine } \\
\text { temperature }\end{array}$ & $\begin{array}{c}\text { Steam valve } \\
\text { position }\end{array}$ \\
\hline 2 & PI & $0.57 \mathrm{~m}$ & $\begin{array}{c}\text { Last stage brine } \\
\text { level }\end{array}$ & $\begin{array}{c}\text { Blow-down } \\
\text { valve position }\end{array}$ \\
\hline
\end{tabular}




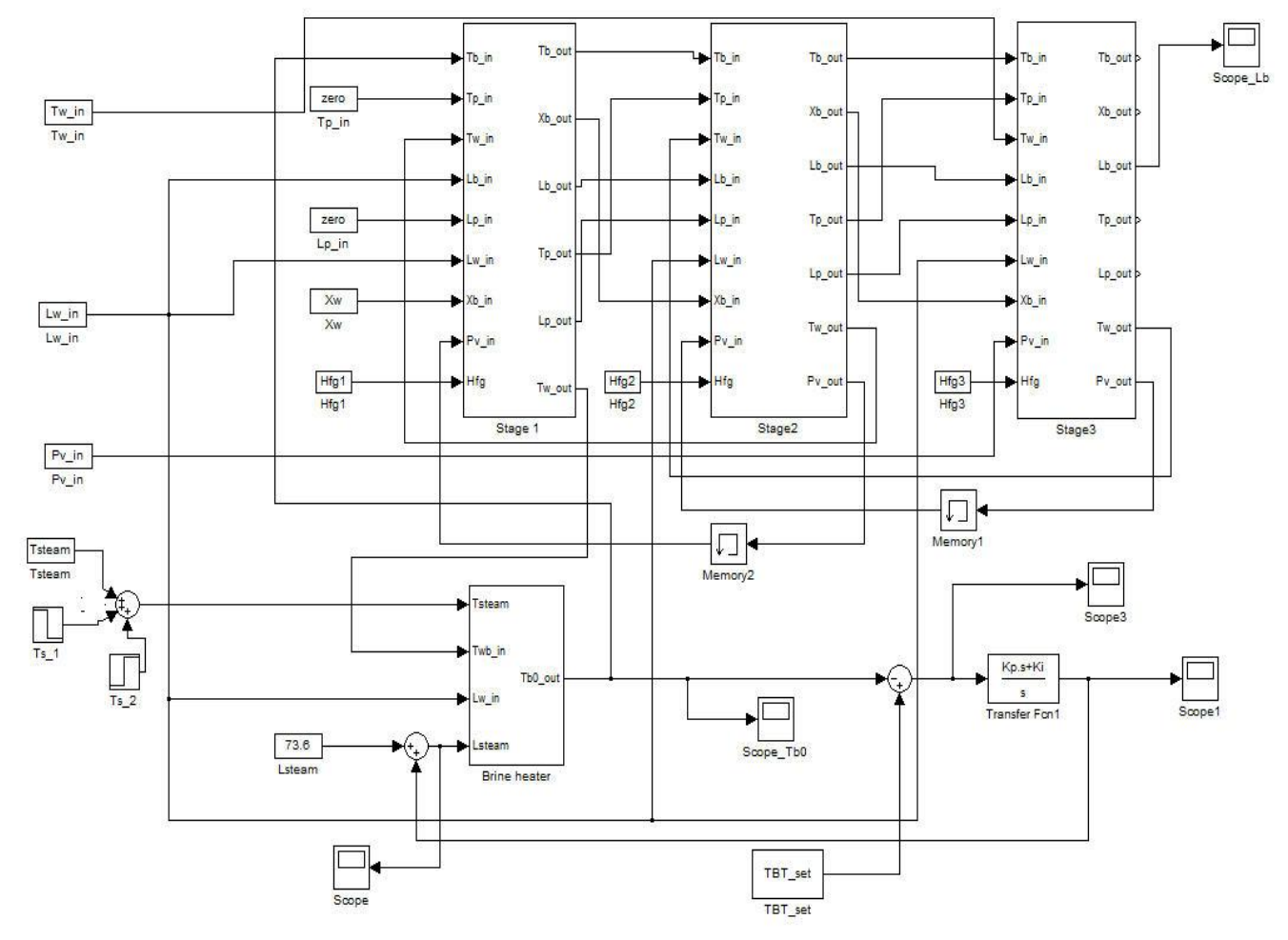

Figure 4.4. Screenshot of the MSF SIMULINK model.

In dynamic simulation, usually, any one or more of the operator controlled variables are perturbed and its effect on the whole plant is studied. Such perturbations, in real conditions, may also be caused by minor failure of some components, i.e., failures which will not lead to immediate shut down of the plant and which gives a possibility of corrective measures. The main operator controlled variables are steam flow rate through the brine heater, brine recirculation flow rate, sea-water reject flow rate, make-up flow rate, sea-water reject flow temperature at entrance to reject section, last stage brine level and last stage distillate level. The other two main input variables, which are location-specific, are sea-water salinity and steam supply pressure. Table 4.8 summarizes the step changes that are introduced in the key variables, such as the heating steam temperature, recirculation brine flow rate, and recirculation brine temperature. It is important to note that the initial conditions of the dynamic model are set to the steady-state values that have been calculated using the steady-state model presented in the previous section. 
Table 4.8. Dynamic simulation tests

\begin{tabular}{|l|}
\hline Step change: \\
$2{ }^{o} \mathrm{~F}$ increase in the set point of top brine temperature \\
$10 \%$ increase in the incoming cooling brine flow rate \\
$2^{\circ} \mathrm{F}$ increase in the incoming cooling brine temperature \\
$5^{\circ} \mathrm{F}$ increase in the heating steam temperature \\
\hline
\end{tabular}

Figures 4.5 to 4.8 present the dynamic responses of the top brine temperature, brine heater inlet steam flow rate, and brine levels in all three stages under different dynamic conditions mentioned in Table 4.8. In all the cases, the steady-state simulation is carried out for 100 seconds before introducing step perturbations. 

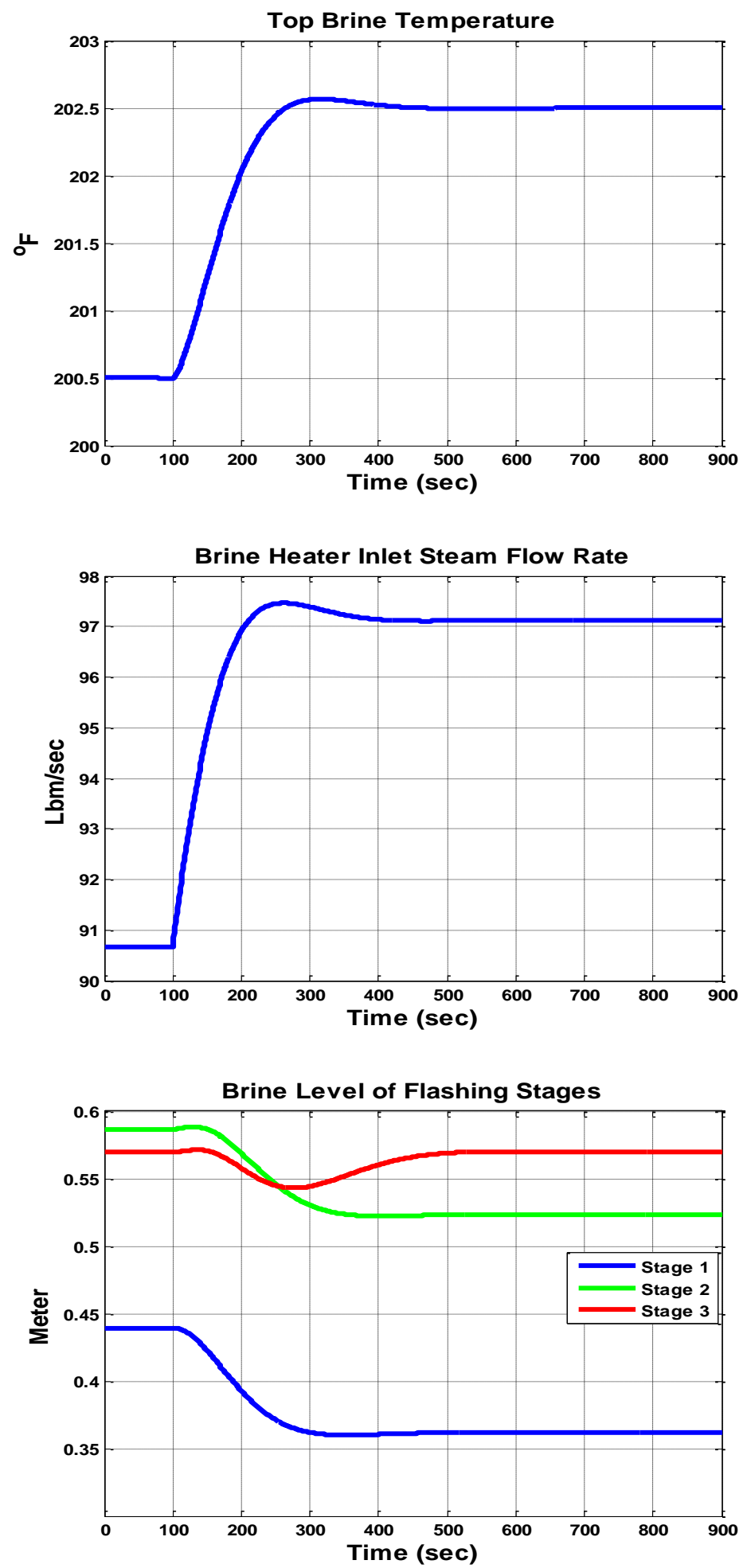

Figure 4.5. Dynamic responses of process variables to step increase of TBT set point. 
(a) Top Brine Temperature

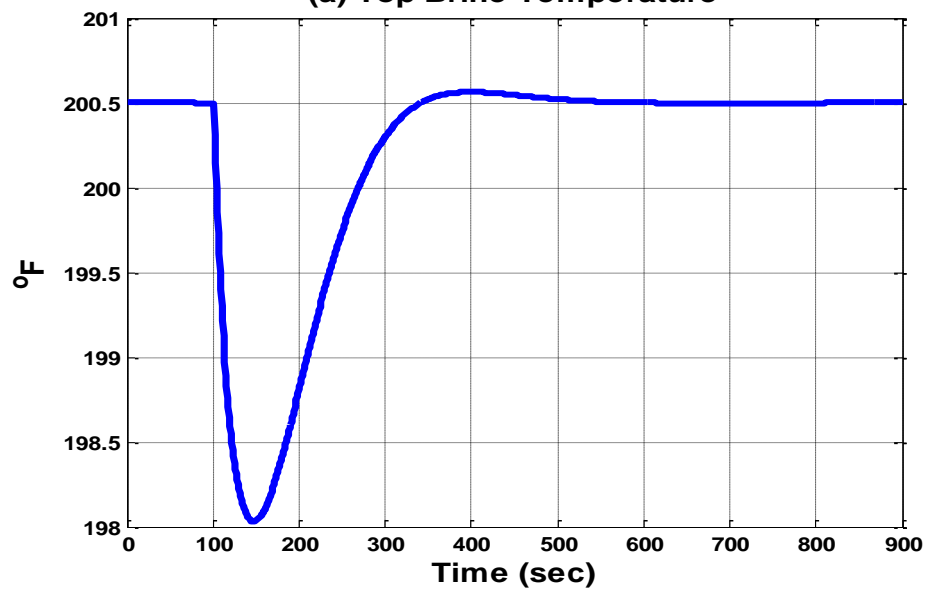

(b) Brine Heater Inlet Steam Flow Rate

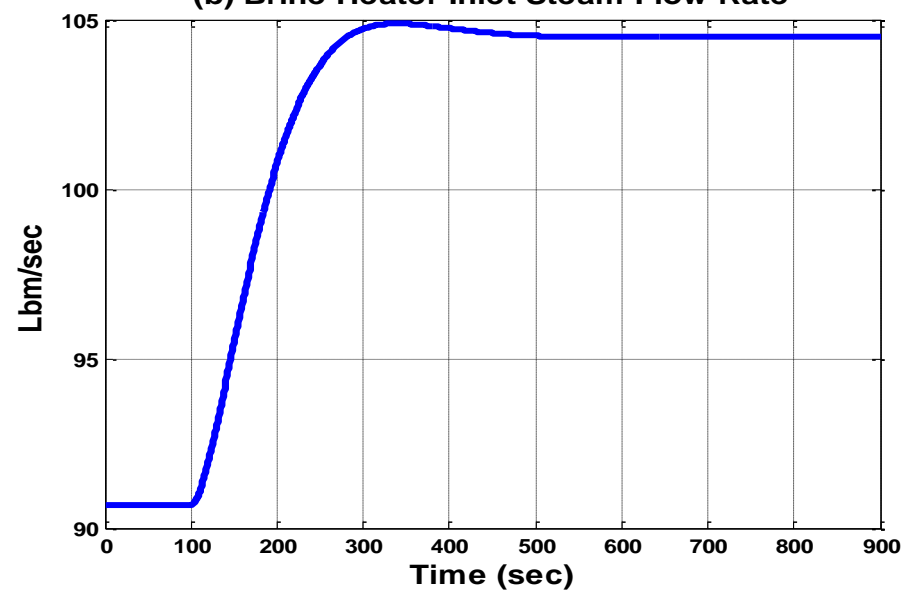

(c) Brine Levels of Flashing Stages

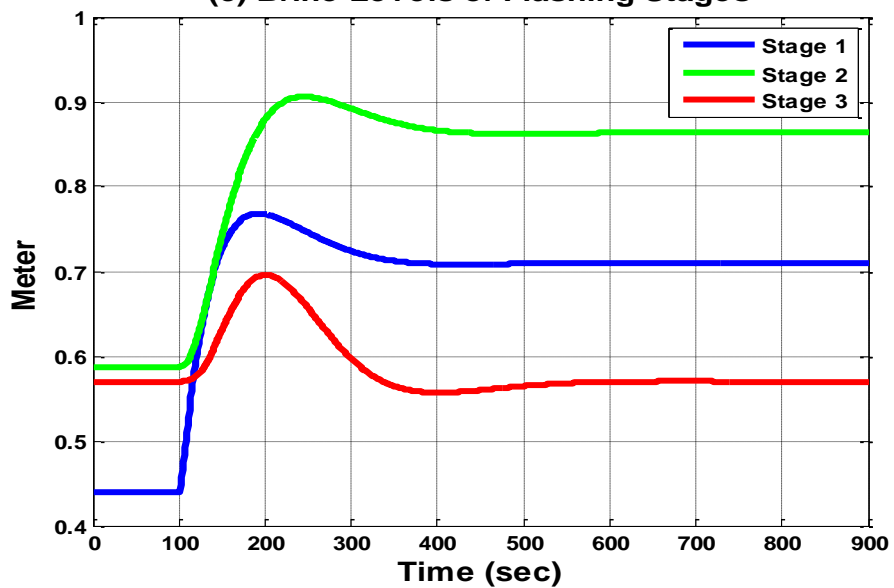

Figure 4.6. Dynamic responses of process variables to step increase of incoming cooling brine flow rate. 
(a) Top Brine Temperature

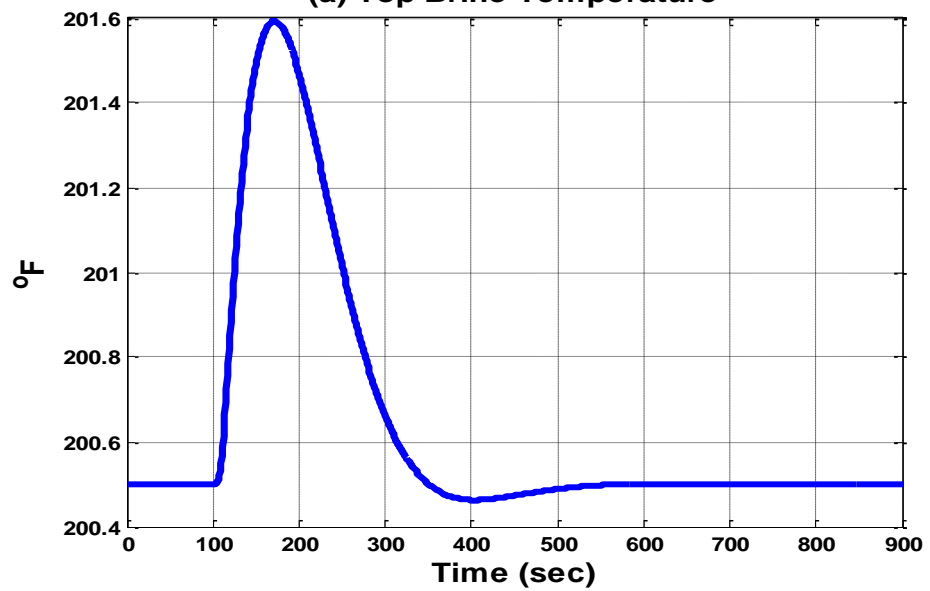

(b) Brine Heater Inlet Steam Flow Rate

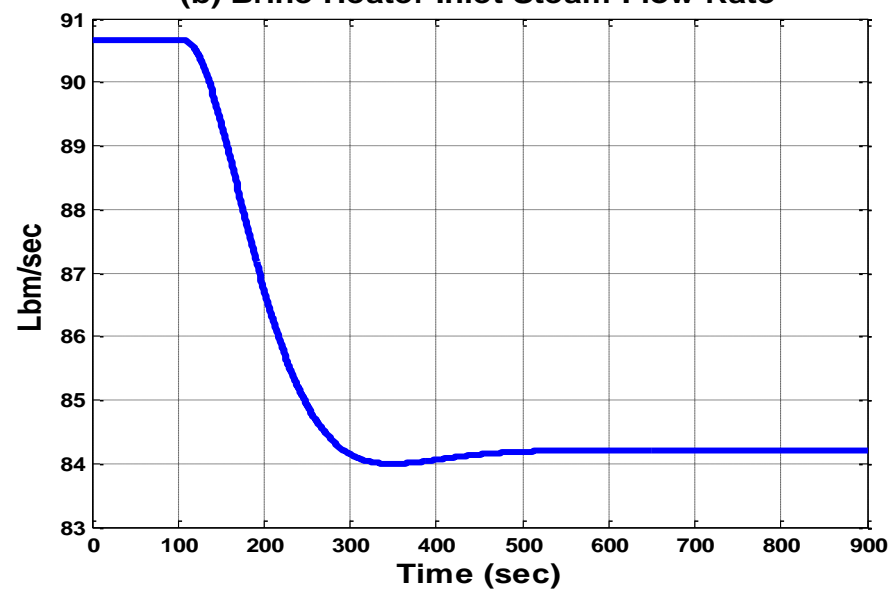

(c) Brine Levels of Flashing Stages

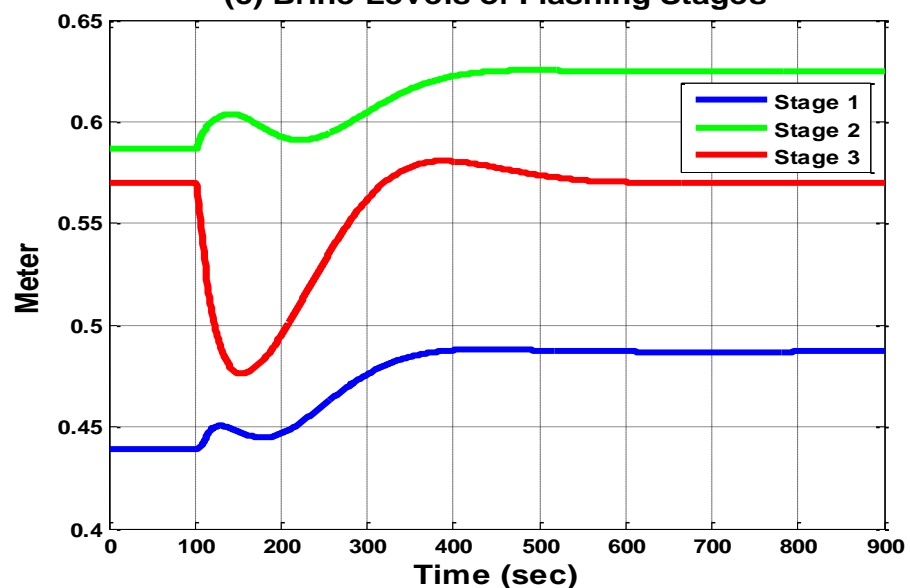

Figure 4.7. Dynamic responses of process variables to step increase of incoming cooling brine temperature. 
(a) Top Brine Temperature

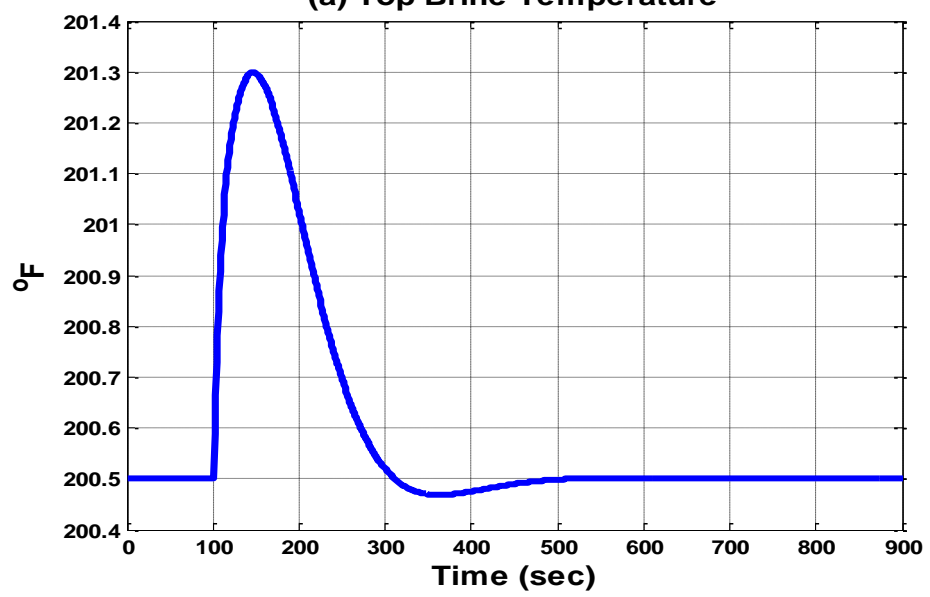

(b) Brine Heater Inlet Steam Flow Rate

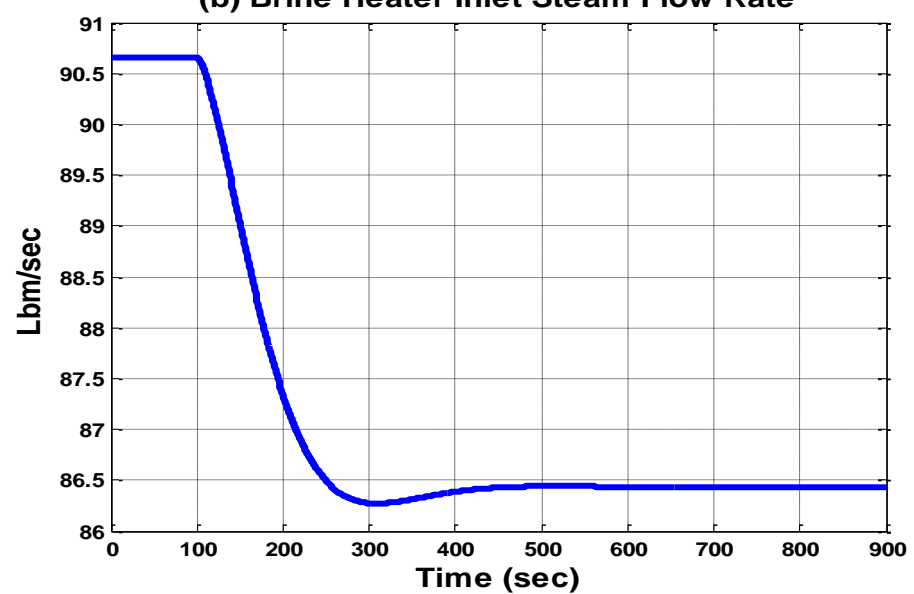

(c) Brine Levels of Flashing Stages

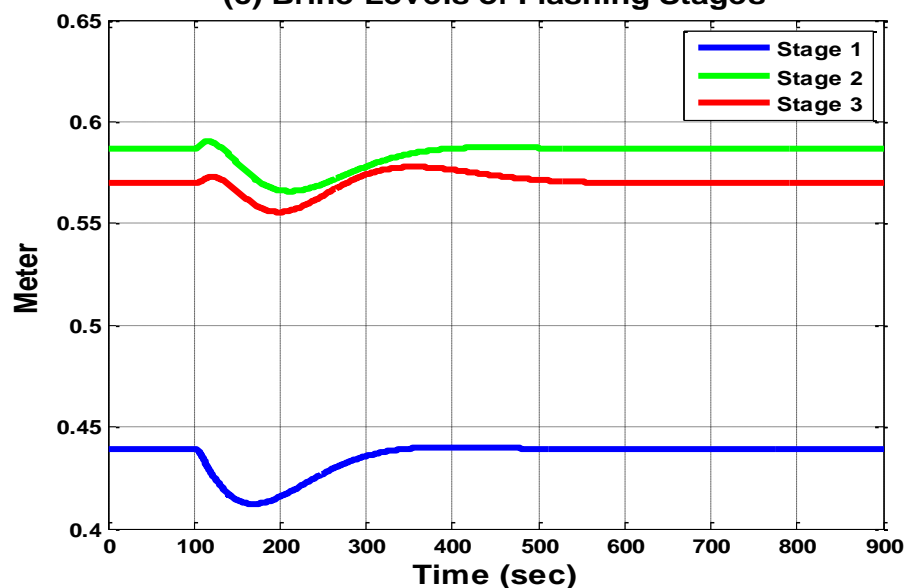

Figure 4.8. Dynamic responses of process variables to step increase of heating steam temperature. 
Figures 4.9-4.10 show the open loop responses of the top brine temperature and the brine levels in all three stages when the TBT control loop is open with a step decrease in the heating steam temperature.

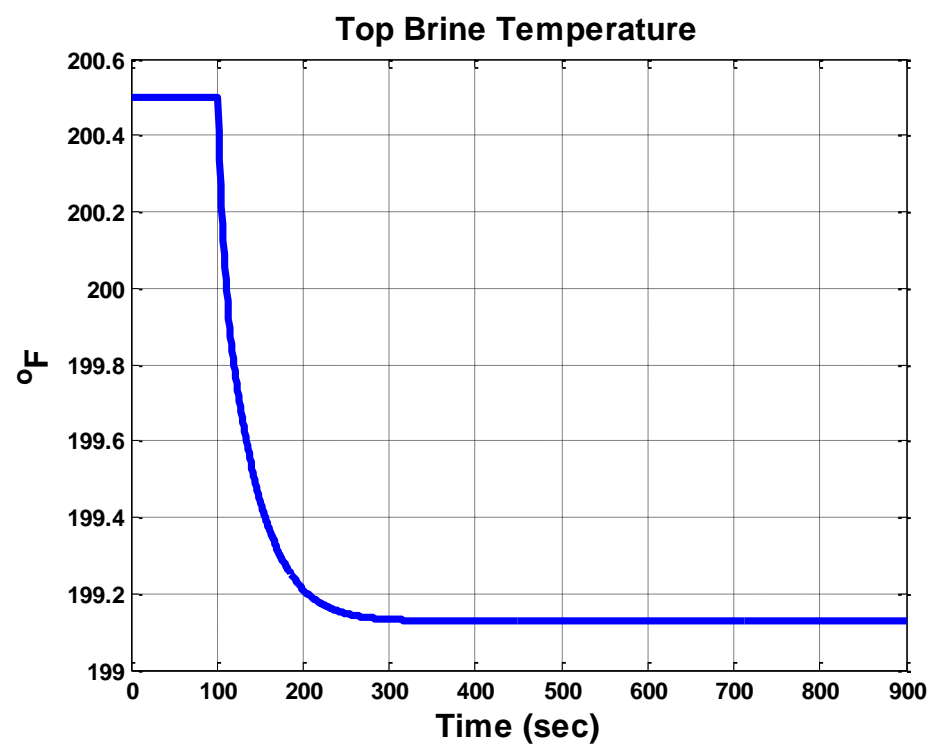

Figure 4.9. Open loop response of TBT to step decrease of heating steam temperature.

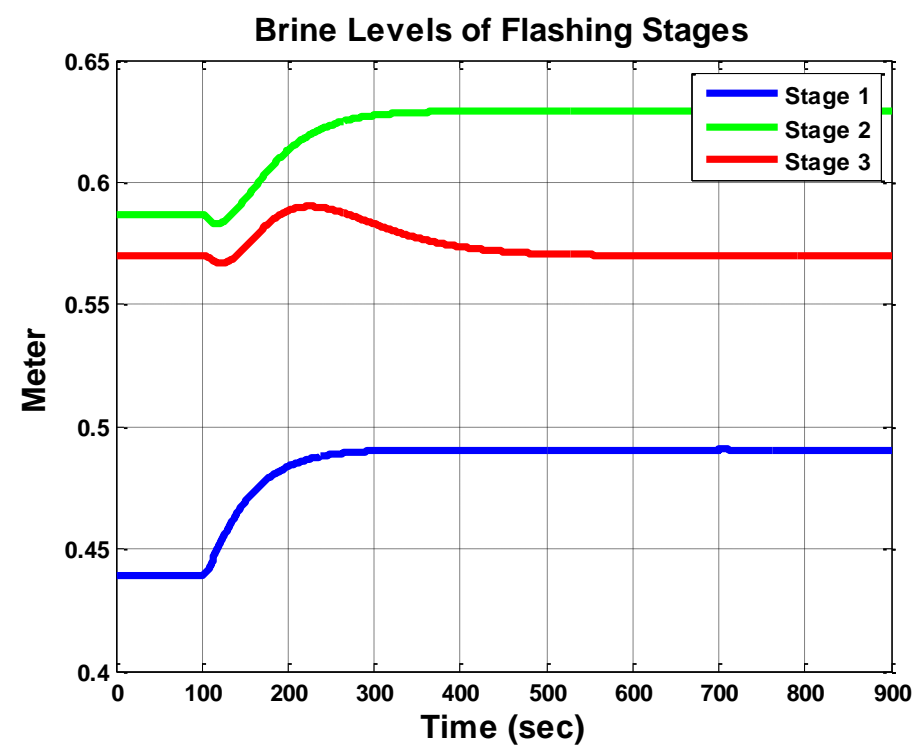

Figure 4.10. Open loop response of brine levels to step decrease of heating steam temperature.

It can be seen from the above figures that the steady state is maintained for the first 100 seconds. When the steam temperature is reduced, the heat transferred to the brine gets reduced and hence TBT also decreases as shown in Figure 4.9. This decrease of TBT reduces the amount 
of vapor flashed from the brine in all stages; hence, the total distillate production also decreases. The decrease of TBT reduces the temperature of the flashing brine and consequently the pressure in the corresponding stages. These changes are transmitted from one stage to the next sequentially. The reduction of pressure in an upstream stage reduces the flow rate through the corresponding orifice. However, as the reduction of pressure has not been transmitted to the stage downstream of the orifice, the outflow of brine from the downstream stage is maintained at the initial value. This will lead to more outflow of brine and hence the decrease in the liquid level in that stage. It can be clearly seen from Figure 4.10 that the decrease in brine level is progressively transmitted from first stage to the third stage. The reduction in brine level is temporary, as the decrease in flashing rates leads to more brine being left behind in the brine tray. Thus a steady increase in the brine levels can be observed. This effect becomes more apparent in Figure 4.11 when the last stage (Stage \#3) brine level is not controlled.

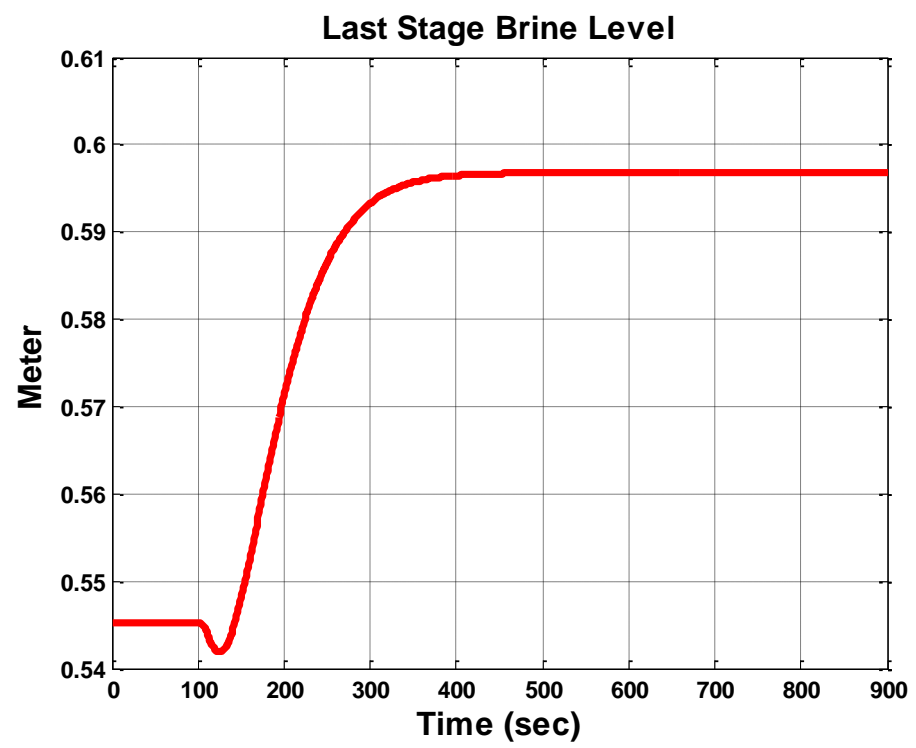

Figure 4.11. Open loop response of last-stage brine level to step decrease of heating steam temperature.

\subsection{Summary}

This chapter presents an overview of modeling and simulation aspects of multi-stage flash desalination plants. Both steady-state and dynamic simulations are discussed. Steady-state models are useful for analyzing the operating conditions of the plant. A mathematical model was obtained by considering the energy and mass conservation equations for the various streams in 
each section of a MSF plant. The steady-state equations were obtained by setting all the time derivative terms to zero. The steady-state model was simulated for a real plant operating conditions and a close agreement has been observed between the predicted results and the actual plant operating parameters.

Dynamic models are suitable for solving problems involving transient behavior, such as studying control strategies, stability problems, process interactions, start-up and shut-down conditions. Two types of dynamic models are possible. The first is an analytical one. The analytical model describes the process through physical relations. Essentially, the lumped parameter dynamic model consists of ordinary differential equations and supporting algebraic equations. The initial conditions are either known by experience or developed by steady-state simulation. The other type of dynamic model is based on a black-box approach. A model with unknown parameters is selected according to previous experience or through experiments. Since the formulation of the analytical model is difficult and complicated, it is common to develop black-box models for complex systems. In this project, the former method was employed for MSF dynamic model development. The developed dynamic model was used to simulate the effect of step changes in several key state variables. The simulation results obtained were physically plausible and similar to those found in the literature. 


\section{Chapter 5}

\section{MODELING AND SIMULATION OF A NUCLEAR DESALINATION PLANT}

\subsection{Introduction}

Nuclear desalination is a viable option as a sustainable source of water and electricity. Nuclear power plants are suitable for large scale desalination application such as MSF, which has been introduced and modeled in Chapter 4. The thermal energy produced in a nuclear plant can provide both electricity and desalted water without the production of greenhouse gases. A particularly attractive option for nuclear desalination is to couple a desalination plant with an advanced, modular, passively safe reactor design, such as the International Reactor Innovative and Secure (IRIS) plant. The design features of the IRIS reactor ensure a safe and reliable source of energy and water even for countries with limited nuclear power experience and infrastructure. In this chapter, the IRIS-MSF nuclear desalination option is explored and analyzed through studying the coupled dynamic SIMULINK models of two plants.

\subsection{IRIS System Description}

IRIS is one of the next generation nuclear reactor designs developed by an international team of industry, national laboratory, and university partners led by Westinghouse Electric Company [Carelli et al., 2006]. The IRIS design is based on proven light water reactor (LWR) technology, but includes several innovative engineering features that enhance its safety and economics relative to other advanced systems. IRIS is a member of the integral primary system reactor class of designs which houses all functions of the primary coolant system inside the reactor pressure vessel. Figure 5.1 shows the layout of the IRIS primary system.

A unique feature of IRIS is the safety-by-design philosophy which means that design choices are made to eliminate the potential for accidents to occur rather than adding systems to respond to the consequences of accidents. By using an integral system, several potentially severe accident scenarios are avoided, such as medium-to-large pipe break loss-of-coolant accidents (LOCA). For those accident scenarios that cannot be precluded, the design is such that the 
consequences of the accident are greatly reduced by the design features. An example is the spherical, high-design-pressure containment vessel that encloses the reactor vessel and safety systems, which is illustrated in Figure 5.2. In the event of a small pipe break in the secondary system, the pressure inside and outside the reactor vessel equalizes very rapidly and prevents the core from being uncovered by coolant.

The novel design features of IRIS are summarized below:

- Scalable in power between $100 \mathrm{MWe}$ and $350 \mathrm{MWe}$. Baseline design is $335 \mathrm{MWe}$ or $1000 \mathrm{MWt}$ in a modular configuration allowing deployment as single units, multiple single units, or multiple twin units.

- All main primary circuit components (core, control rods and drive mechanisms, steam generators, primary coolant pumps, and pressurizer) are integrated into a single reactor vessel.

- The core is comprised of 89 traditional $17 \times 17$-pin PWR fuel bundles containing $4.95 \%$ enriched $\mathrm{UO}_{2}$ fuel and is designed for a 3.5-year cycle with an average burn-up of 50,000 $\mathrm{MWd} / \mathrm{Te}$.

- Reactivity control is accomplished through solid burnable absorbers and control rods, and a limited use of soluble boron in the primary coolant.

- The primary coolant system uses eight helical-coil once-through steam generators and eight spool-type coolant pumps. The steam-regulated pressurizer is located in the upper portion of the 6.2-m-diameter, 22.2-m-high reactor vessel. Normal operating pressure of the primary coolant is $15.5 \mathrm{MPa}$.

- The reactor vessel is placed within a $25-\mathrm{m}$ diameter compact steel containment vessel capable of withstanding 1.4 MPa design pressure.

- All large coolant pipes are eliminated. Only small feed water and steam outlet pipes penetrate the vessel wall (5-cm diameter pipes versus $90-\mathrm{cm}$-diameter pipes for external loop PWRs).

- The total inventory of primary coolant is much larger than for an external loop PWR, 
which increases the heat capacity and thermal inertia of the system and hence yields a much slower response to core heat-up transients.

- The extended riser area provides the possibility for internal placement of the control rod drive mechanisms (CRDM), thus avoiding another potentially serious accident scenario: control rod ejection accident.

Thus, IRIS is designed to fulfill the advantages of the integral primary system reactor. It improves safety, reduces the site civil works, and improves the plant availability for developed and developing countries with large or small electrical grids that can greatly benefit from such design. Thanks to these advantageous design features as well as the specific reasons below, IRIS is well suited for desalination applications.

- The modular sizing of IRIS will allow countries with small-to-medium power requirements to install capacity to their electrical grid in smaller increments and increase additional capacity as their power and water demands warrant and their infrastructure will support.

- The enhanced safety margins will provide additional flexibility in the siting of the reactor to better match the electrical and water use demographics. This will also encourage countries with modest nuclear infrastructure to build and operate nuclear power plants.

- The international nature of the IRIS project team will help to ensure that the design will be licensable for deployment in the global market. 


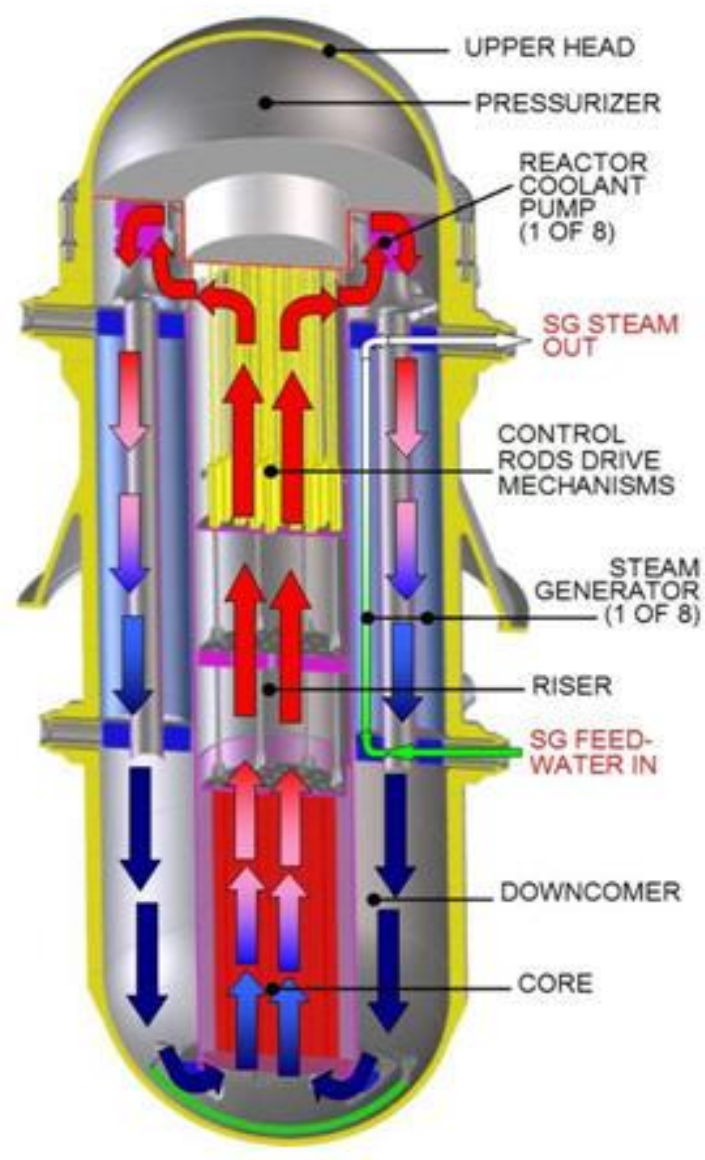

Figure 5.1. IRIS primary system layout [Carelli et al., 2003]. 


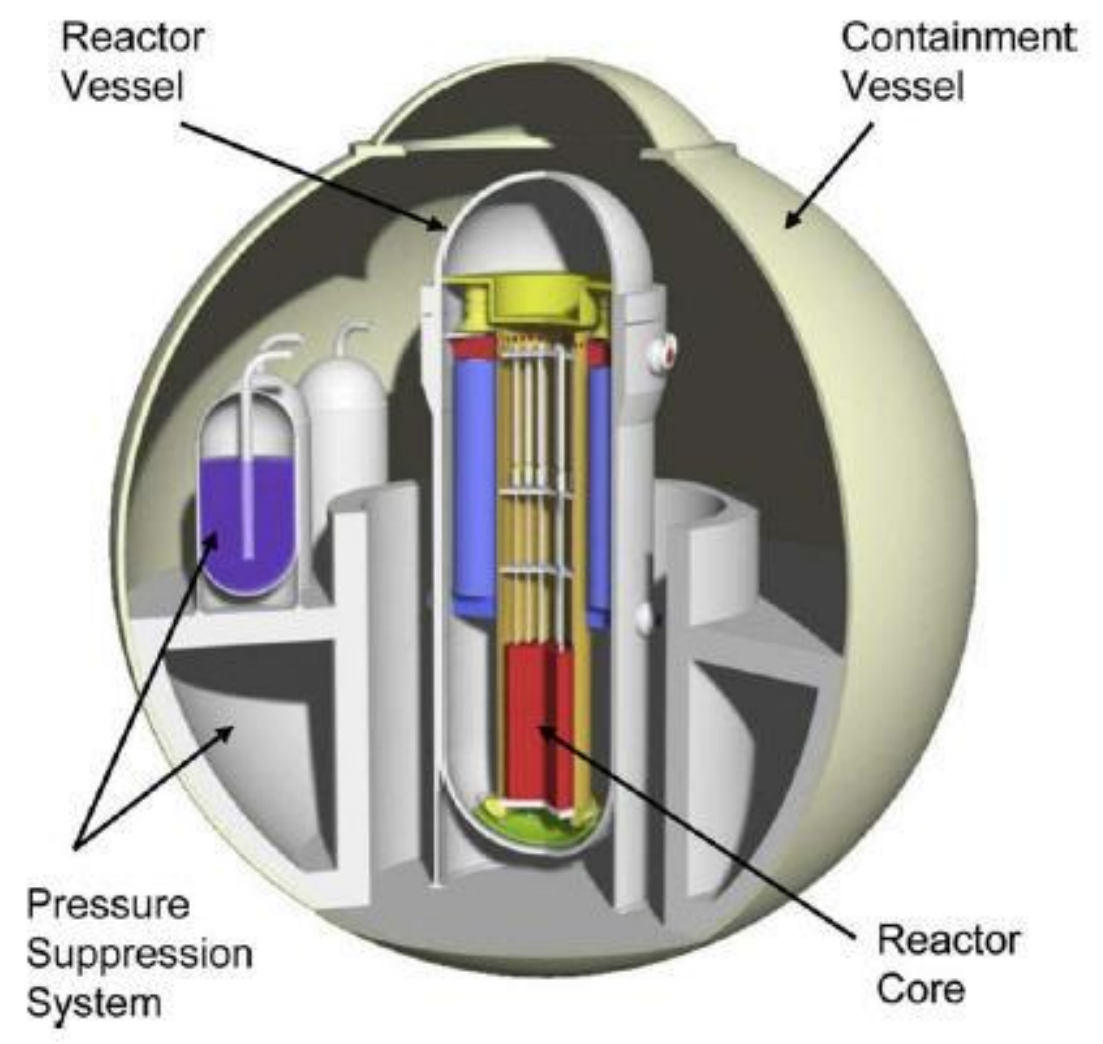

Figure 5.2. IRIS reactor pressure vessel and compact containment vessel [Carelli et al., 2003]. 


\subsection{IRIS Dynamic Models}

An IRIS dynamic model developed previously at the University of Tennessee is used in this project, which includes reactor core, helical coil steam generators (HCSG), and balance-of-plant (BOP) systems [Xu, 2009]. The IRIS dynamic models were developed in SIMULINK, and were based on a conventional PWR dynamic model [Naghedolfeizi, 1990] and a HCSG simulation model [Zhao, 2005a]. Major components of the IRIS plant model are described below, and the mathematics equations of the BOP systems are listed in Appendix B.

\subsubsection{Reactor Core}

The reactor core uses the point reactor kinetics model. The Mann's model is employed to describe the fuel-to-coolant heat transfer in the reactor core. The core model includes the six-group delayed neutron precursor concentration equations, one fuel node temperature, and two coolant node temperature differential equations, along with the fuel and moderator temperature reactivity feedback coefficients. In the reactor core model, the $T_{\text {ave }}$ controller is implemented with the fixed set point of $590^{\circ} \mathrm{F} . T_{a v e}$ is defined as the average moderator temperature between the hot leg temperature $\left(T_{\text {hot }}\right)$ and cold leg temperature $\left(T_{\text {cold }}\right)$.

\subsubsection{Helical Coil Steam Generator}

Helical coil steam generator is one of the critical components as well as being the major difference between traditional PWRs and the IRIS reactor. In the HCSG system, the primary fluid enters at the top of the equipment and flows downward to the bottom on the shell side. The primary side heat transfer is sub-cooled forced convection along the entire steam generator height, while the secondary fluid flows upward inside the coiled tubes from bottom to top. The feed water flows into the sub-cooled region of the steam generator, and in this region the heat transfer is mainly due to single-phase turbulent and molecular momentum transfer and the pressure loss is mainly due to wall friction. The saturated region begins when the bulk temperature becomes saturated. The heat transfer in the saturated boiling region is dominated by nucleate boiling, which is much more efficient than single-phase liquid or steam heat transfer. In the saturated boiling region, the generated bubbles do not disappear in the liquid core and the 
pressure loss is not only due to the wall friction but also due to the interfacial drag between the bubbles and the liquid. The saturated boiling region ends when the critical heat flux is reached and the liquid film disappears. Due to the relatively large mass flow rate, the critical heat flux occurs at relatively high steam quality. When the steam quality becomes one, the liquid evaporation ceases and the steam becomes superheated.

The size of the steam generator can be reduced through the helical coil design. The heat transfer of the coiled configuration is much more efficient than straight tubes because of the larger heat transfer area per unit volume and the secondary flow induced by the coil geometry. The produced superheated steam also avoids the need of a steam-water separator inside the steam generator. The possibility of tube rupture can be reduced because the secondary fluid flows

inside the SG tubes and thus the tubes experience compression force from the outside. The HCSG system control objective is to supply adequate amount of steam to meet the turbine demand, while maintaining the steam pressure at 841 PSI. Therefore, the HCSG model has a proportional-integral steam pressure controller with the fixed set point of 841 PSI, which regulates the steam flow rate through the turbine throttle valve.

The once-through HCSG dynamic model and the steam pressure controller equations can be found in [Zhao, 2005a].

\subsubsection{Steam Turbine}

A steam turbine, found in a fossil power plant, is one in which the thermal energy of the supplied steam is converted to mechanical energy on the turbine shaft. High pressure and temperature steam, produced in the steam generator, is supplied to the steam turbine as a working fluid. The steam passing through the steam turbine is expanded and thus generates mechanical energy on the turbine shaft. The turbine drives an electric generator to produce electricity. It is noted that a portion of the steam is bypassed to a feedwater heater to improve the thermal efficiency of the turbine cycle.

A dynamic model of a turbine cycle has been developed previously by Shankar [1977]. Modifications have been made to reduce the model complexity. In this project, a simple 
one-stage turbine model is used for coupling with the condenser and feedwater heater models, which are described next.

\subsubsection{Main Condenser}

A condenser is a large surface-type heat exchanger, which condenses steam from the exhaust of turbine by transferring steam latent heat to circulating water inside the condenser. The main condenser is equipped with many auxiliary systems such as vacuum and hotwell pumps. The vacuum system maintains a constant pressure in the condenser for transient and steady-state conditions. The hotwell pumps and their control systems discharge the water to a low-pressure heater and control the water level in the hot-wells. The condenser is desired to work under vacuum condition to obtain a maximum mechanical power from the turbine system.

The main condenser is simulated as an equilibrium two-phase tank, as shown in Figure 5.3. The space inside the tank is divided into two independent control volumes, steam and water. They are assumed to be in thermal equilibrium during steady-state condition. Turbine exhaust flow enters the system at the condenser pressure. The water part of the flow falls into the hot-well region and mixes perfectly with the water already present there. The vapor part condenses on the outer surface of metal tubes through which the circulating water flows. The condensation process is associated with a time delay which is due to the dynamics of heat transfer between the vapor and the circulating water. The following assumptions are made to simplify the condenser model.

- Constant pressure exists in the condenser model.

- Mass transfer at steam-liquid interface is assumed to be negligible.

- Wall condensation is considered to be zero. 


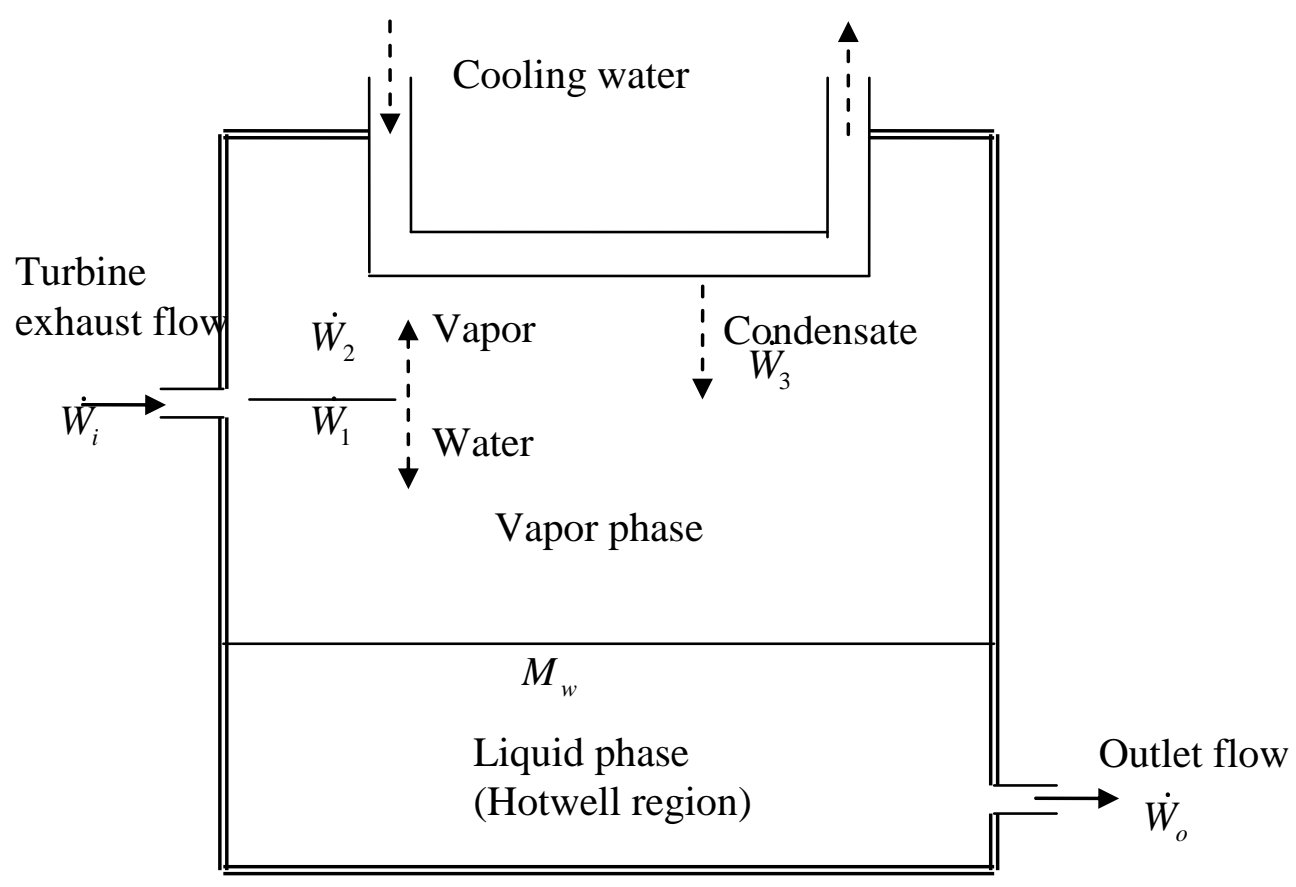

Figure 5.3. Schematic diagram of the turbine condenser model.

\subsubsection{Feedwater Heater}

A closed feedwater heater is basically a shell and tube heat exchanger where the feedwater flows through the tubes and interacts with extracted steam residing along the shell side. As the heat energy from the extraction steam is transferred to the feedwater, the steam condenses and collects at the bottom of the heater. Under normal conditions, the condensate from the heater is passed to the downstream lower pressure heater where it is used to increase the temperature of the feedwater. In emergency conditions, the condensate is directed through the emergency drain valve to the main condenser.

Most feedwater heaters used in power plants are of the shell and U-tube type, horizontal, three-zone configuration. A typical configuration is given in Figure 5.4. Feedwater entering the heater first passes through the drain-cooling region where single-phase convection is the leading heat transfer mechanism from the drains on the shell side to the feedwater on the tube side. The purpose of this section is to cool the drains to a temperature that is close to the shell side saturation temperature of the next heater where it will be mixed with the extraction steam. The feedwater is then passed to the condensing region where the majority of the heat transfer takes 
place. The feedwater temperature can be brought up to within $5^{\circ} \mathrm{C}$ of shell side saturation temperature in this section. Finally, a de-superheating region is used to raise the feedwater temperature even above saturation and cool the steam down to saturation.

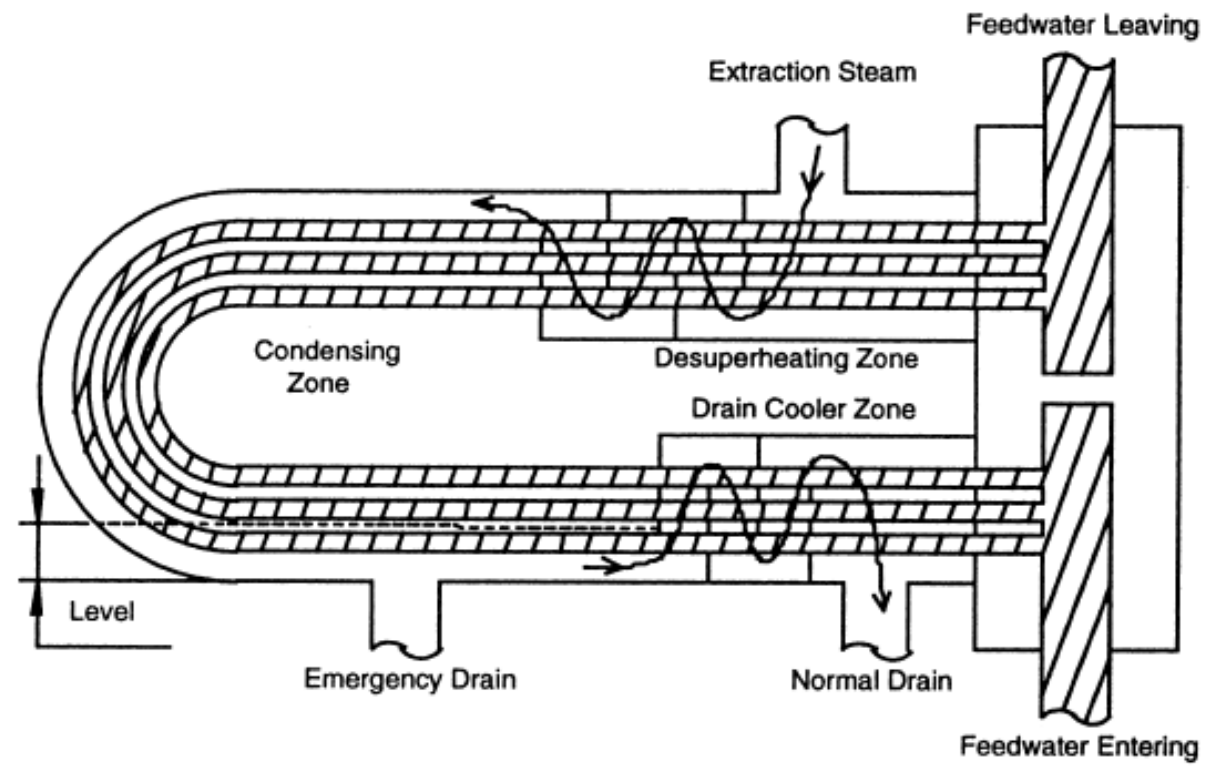

Figure 5.4. Schematic of a horizontal closed feedwater heater [Thanomsat et al., 1998].

\subsection{IRIS-MSF Nuclear Desalination Plant Simulation Results}

Coupling an IRIS system with a MSF desalination plant requires co-location of the two units with a steam supply loop provided from the IRIS secondary system to the MSF plant. Electrical connection is also required to power the MSF pumps and auxiliary equipment. Coupled nuclear desalination plants, in fact, have been in operation for over two decades in countries such as Japan and Kazakhstan. Various concepts of the coupling of nuclear and desalination systems have been presented in the scientific community, some of which are already on their way to being demonstrated. An example of a possible nuclear MSF desalination plant complex is illustrated in Figure 5.5 [Ingersoll et al., 2004]. An intermediate steam loop is proposed for coupling two processes. The pressure of the intermediate loop is maintained above the pressure of the secondary IRIS loop and the pressure of the seawater loop in the brine heater stage of the MSF plant so that a failure of the heat exchanger at either end of the intermediate loop would result in an easily detectable pressure drop in the loop and ensure isolation of the fluids in the two processes. 
In this project, the MSF brine heater steam supply is obtained directly from the exhaust of the low-pressure steam turbine. The heating steam has an initial saturation temperature of $206^{\circ} F$. The brine flows through the tubing in the heat exchanger. The condensate gathered in the sump tank of the brine heater mixes with the flow from the main condenser, and goes to the feedwater heaters.

The overall simulation model of the coupled IRIS-MSF plant is developed by combining the individual SIMULINK models of reactor core, helical coil steam generators, balance-of-plant, as well as the MSF system. The order of the coupled IRIS-MSF SIMULINK model is estimated around more than 100 state variables.

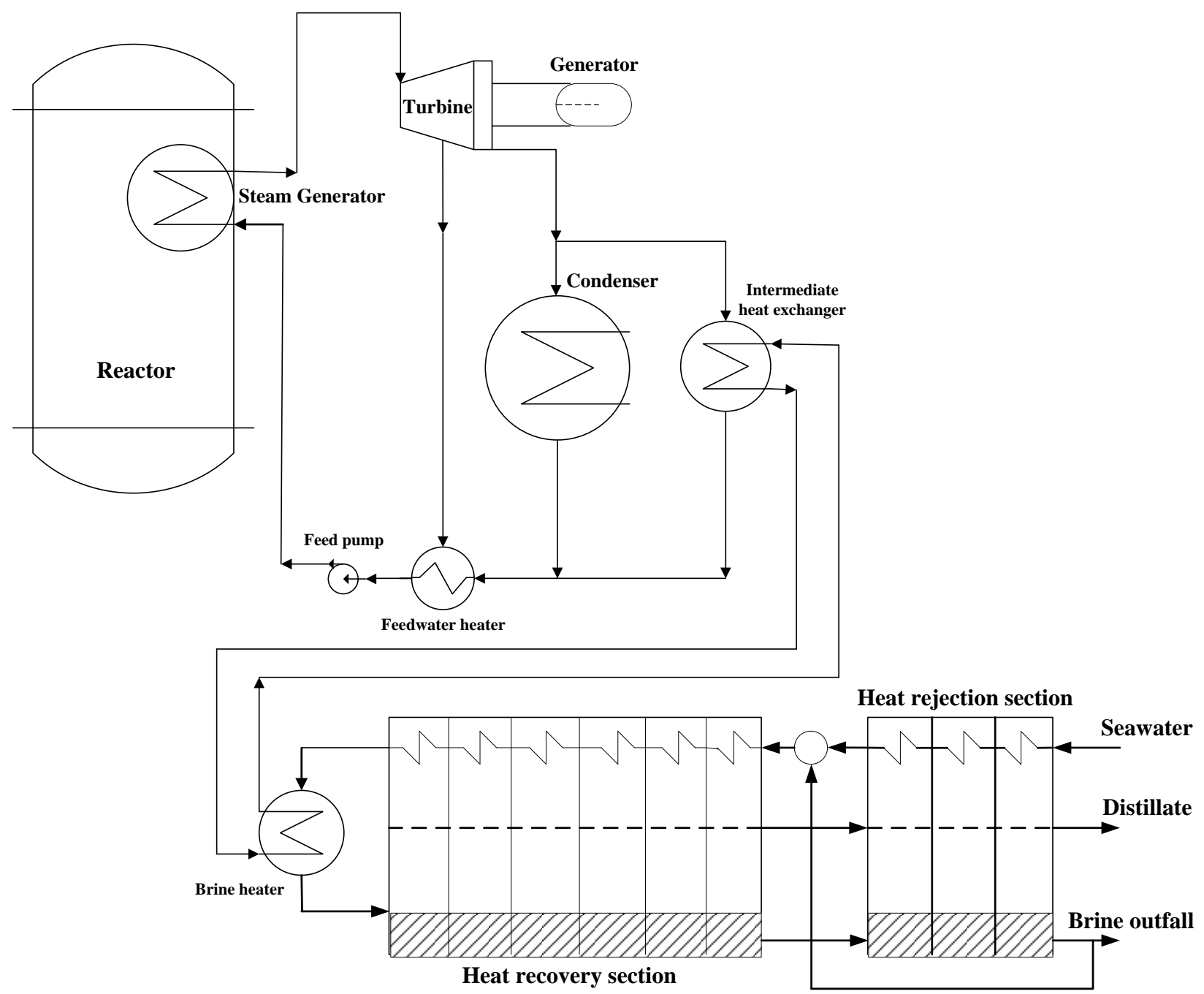

Figure 5.5. Schematic diagram of a MSF plant coupled to the IRIS power conversion system. 
The simulation results of the typical variables in the coupled system due to a $10 \%$ step decrease in the power demand (from 100\% to 90\%) are shown in Figures 5.6 - 5.18.

Figure 5.6 shows the reactor core power change due to the power demand decrease. The core power, which is initially around 100\%, goes to approximately $93.5 \%$ in 400 seconds. Figures 5.7 - 5.9 illustrate the responses of $T_{h o t}, T_{\text {cold }}$, and $T_{\text {ave }}$. As the core power decreases, $T_{\text {hot }}$ goes down as well. The $T_{\text {ave }}$ controller adjusts the external reactivity to maintain a constant average coolant temperature around the set point $\left(590^{\circ} \mathrm{F}\right)$. The steam outlet pressure response shown in Figure 5.10 also varies due to the power change, but the steam pressure controller is able to keep the pressure as close as possible to the set point (841 PSI) by reducing the steam flow rate to the turbine system, shown in Figure 5.11. This, in turn, results in a reduction in the turbine power, as shown in Figure 5.12. It also causes a reduction in the heat flow to the feedwater heater that consequently decreases the feedwater temperature (Figure 5.13). As the power varies, so does the steam generator water level. Hence more heat transfer area is left for the steam to be superheated, thus increasing the steam temperature (Figure 5.14).

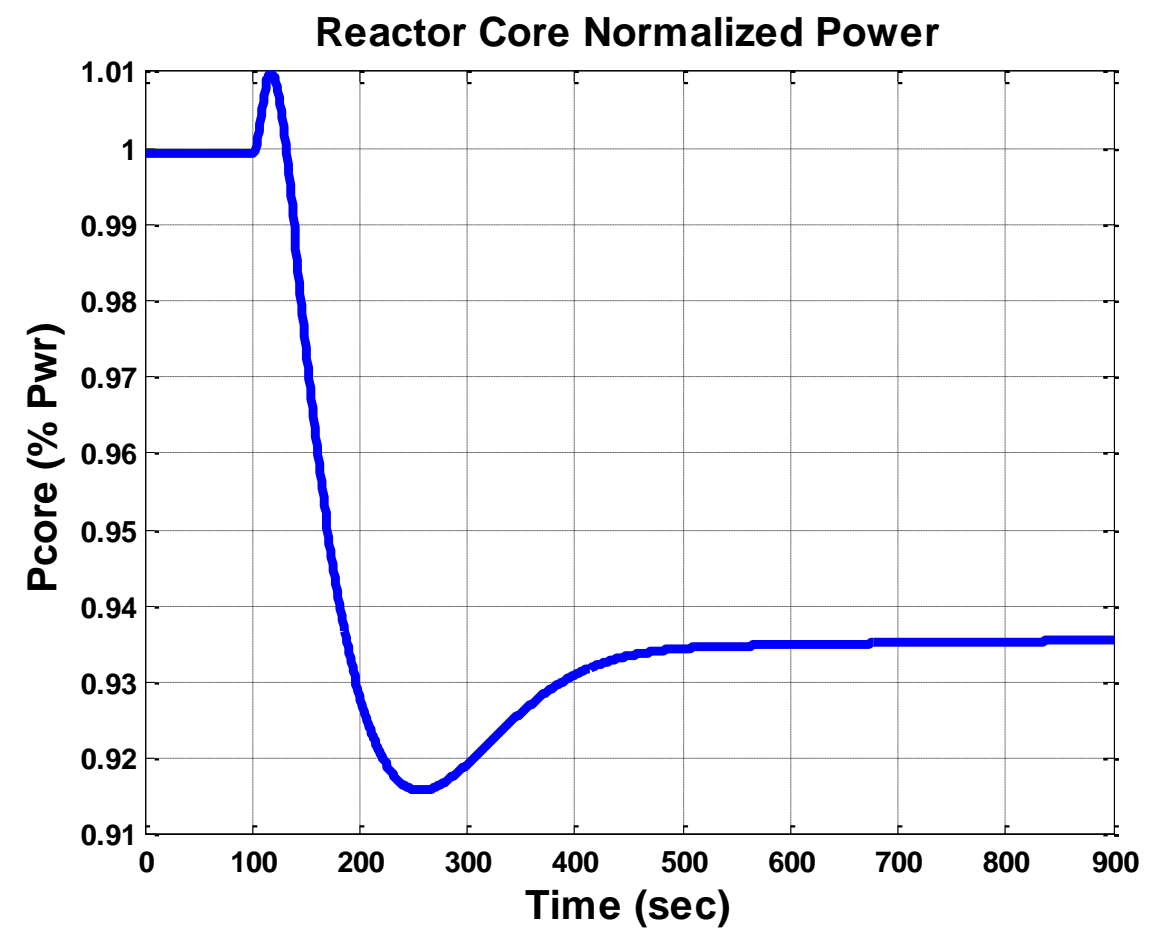

Figure 5.6. Reactor power response to a $10 \%$ power demand step decrease. 


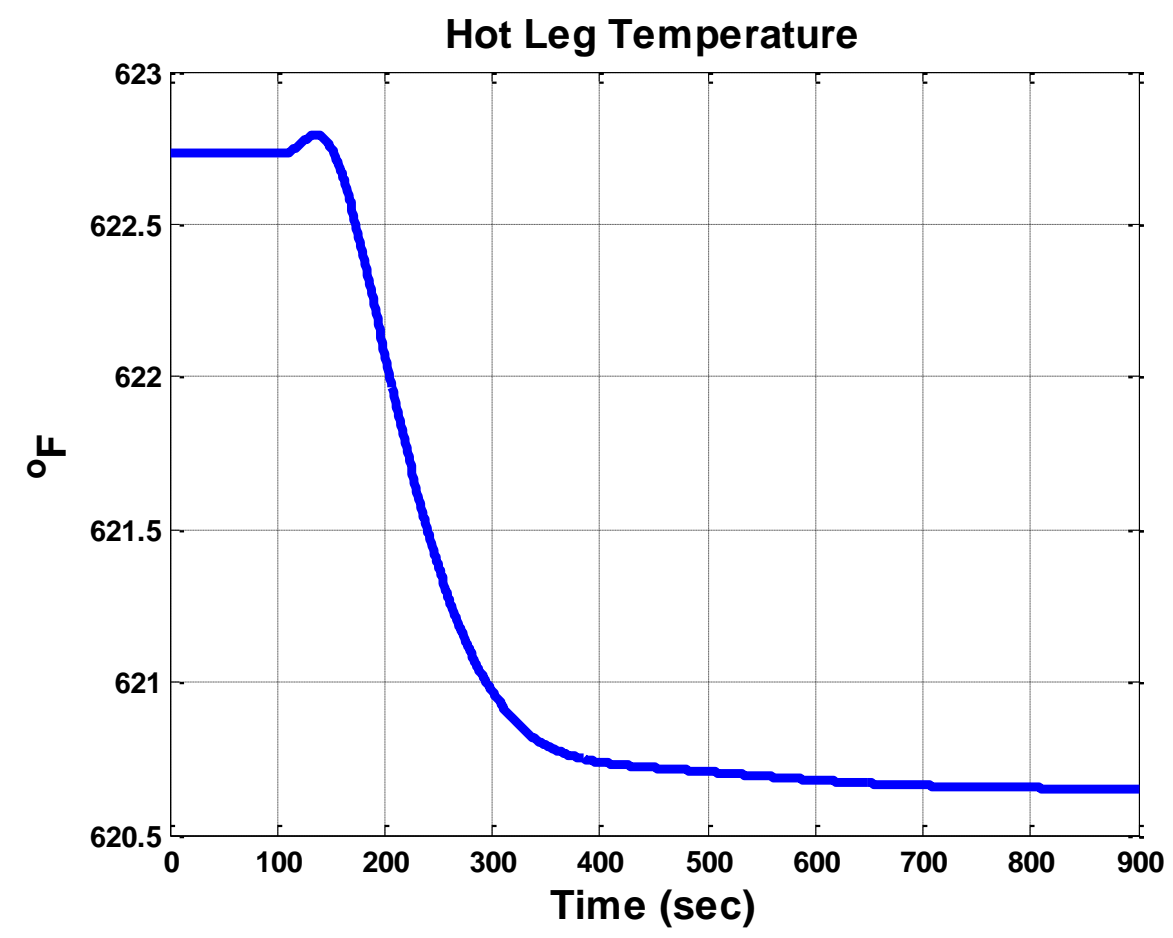

Figure 5.7. $T_{\text {hot }}$ response to a $10 \%$ power demand step decrease.

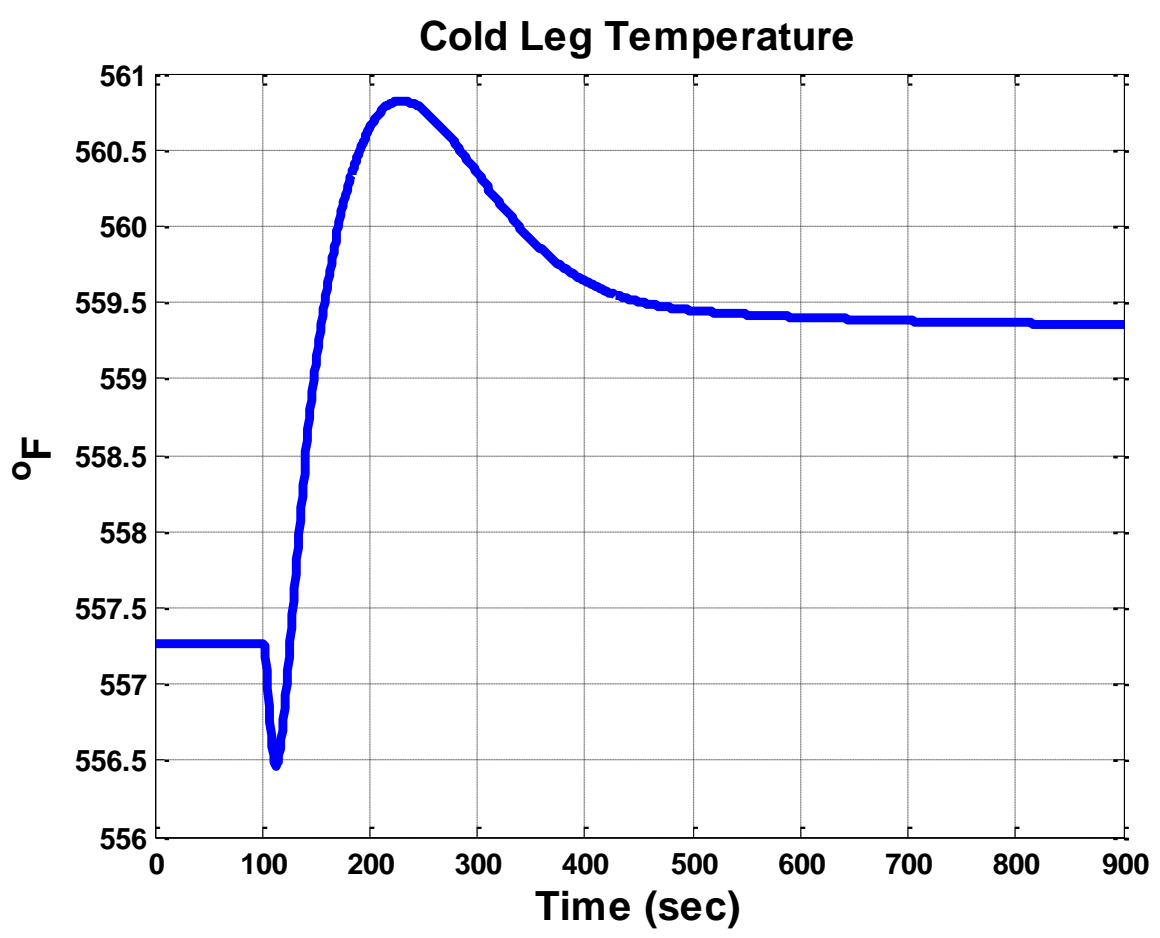

Figure 5.8. $T_{\text {cold }}$ response to a $10 \%$ power demand step decrease. 


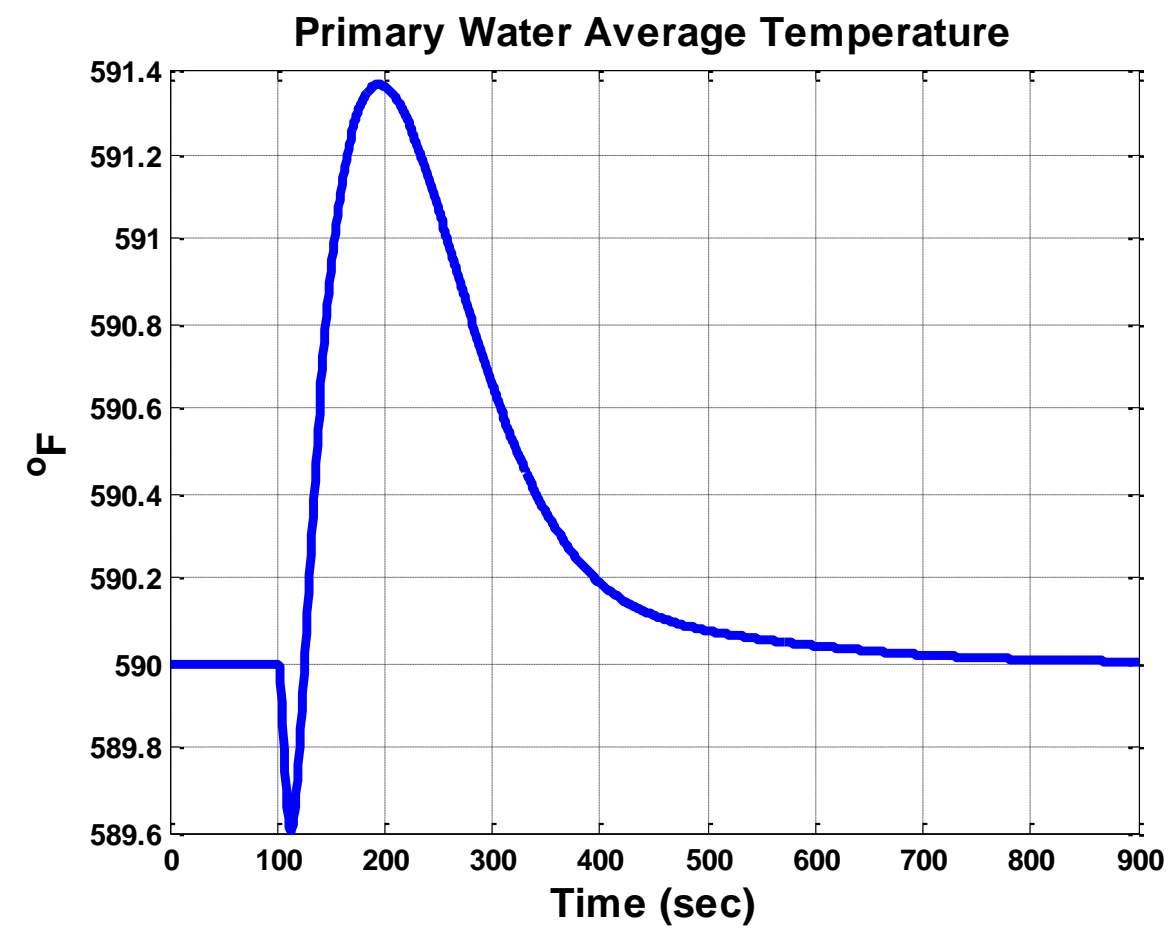

Figure 5.9. $T_{\text {ave }}$ response to a $10 \%$ power demand step decrease.

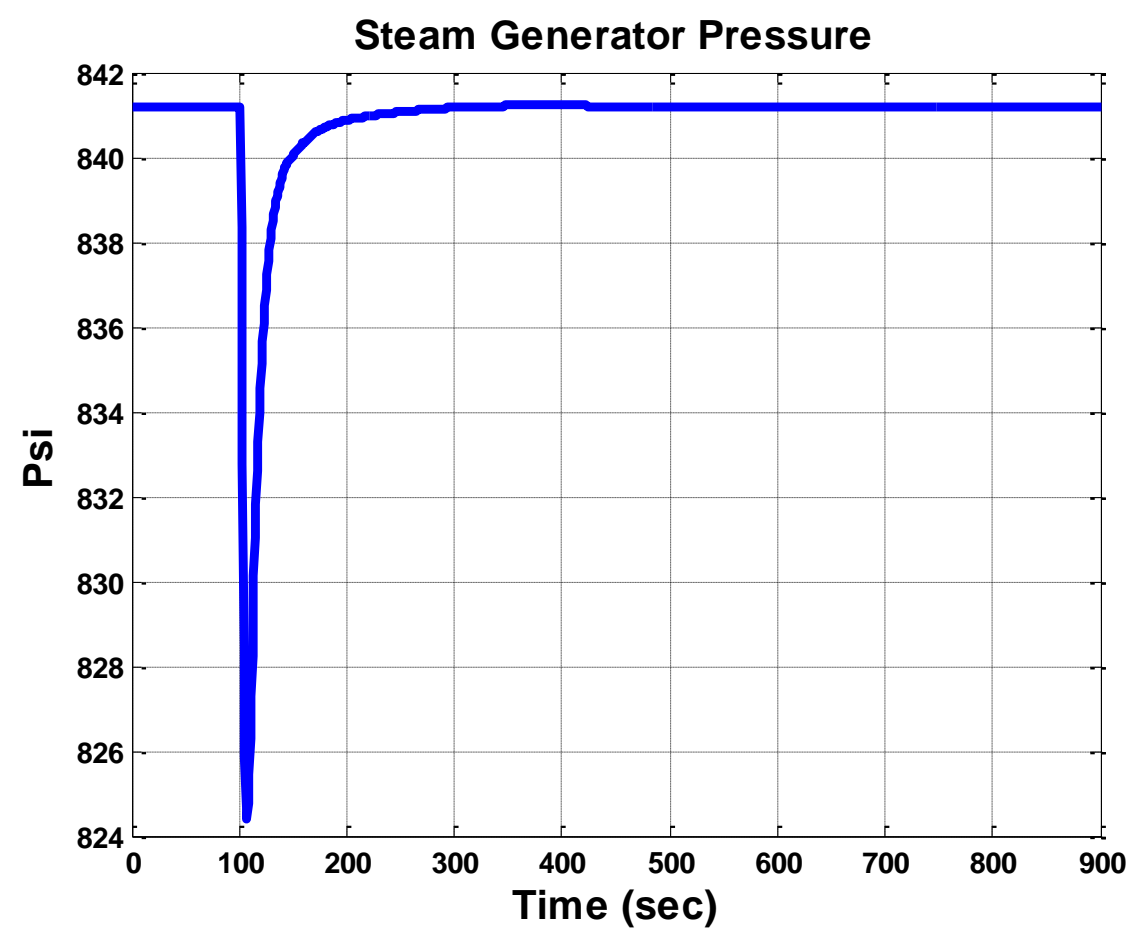

Figure 5.10. Steam pressure response to a $10 \%$ power demand step decrease. 


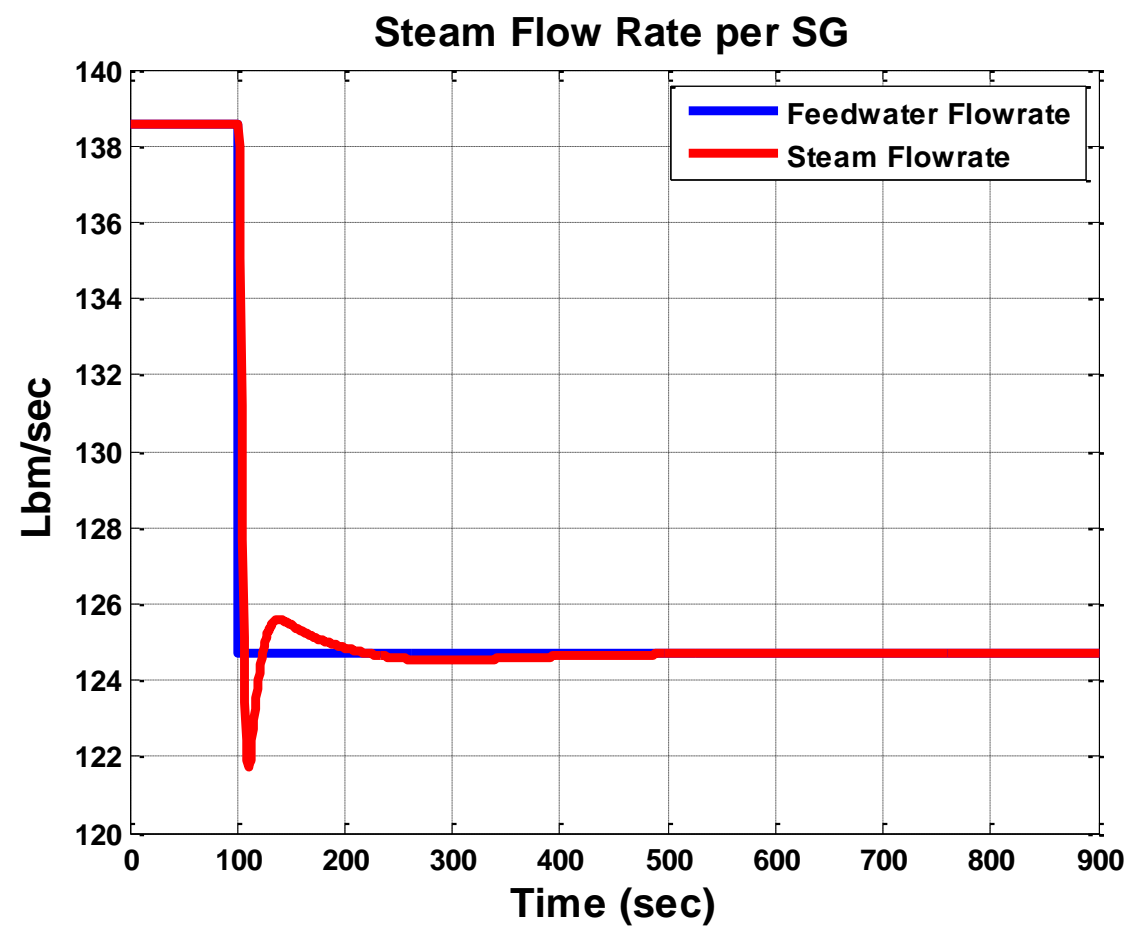

Figure 5.11. Steam flow rate response to a $10 \%$ power demand step decrease.

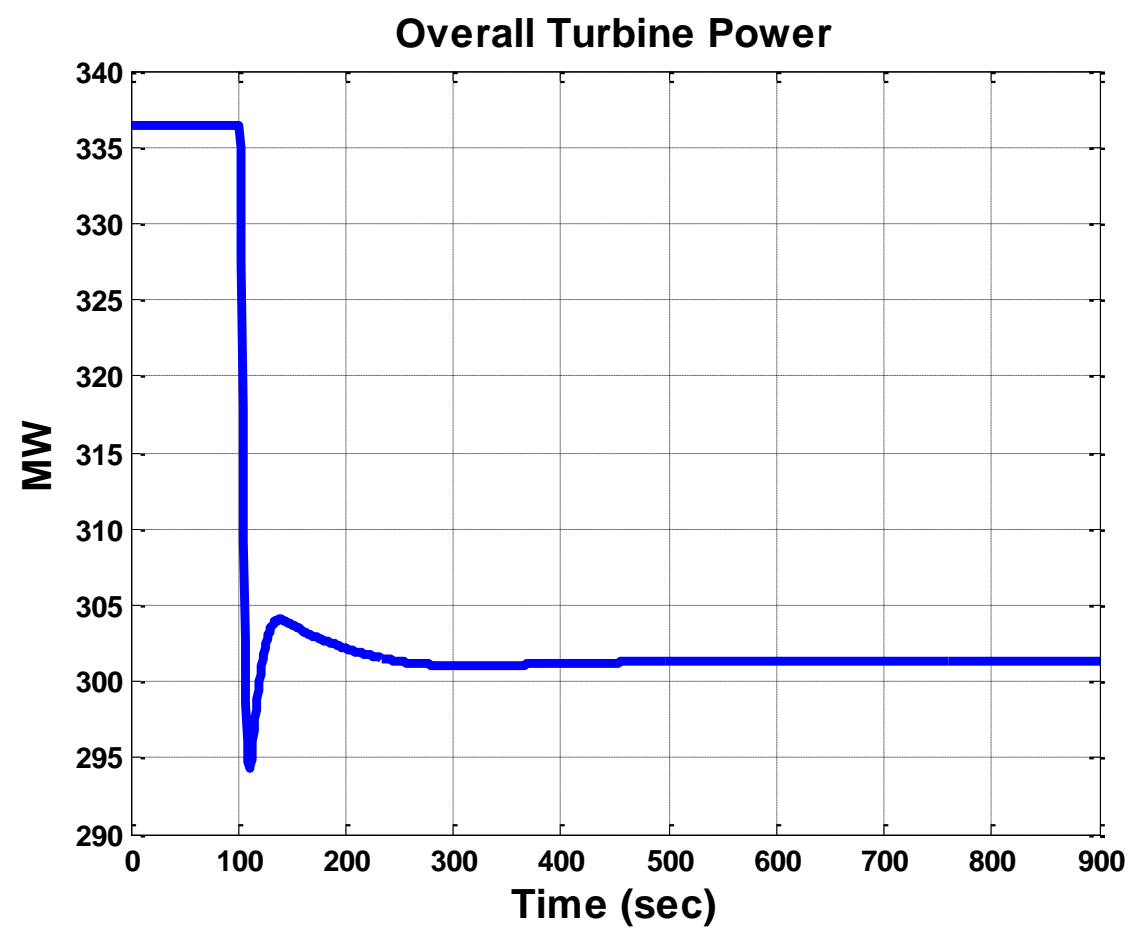

Figure 5.12. Turbine output response to a $10 \%$ power demand step decrease. 


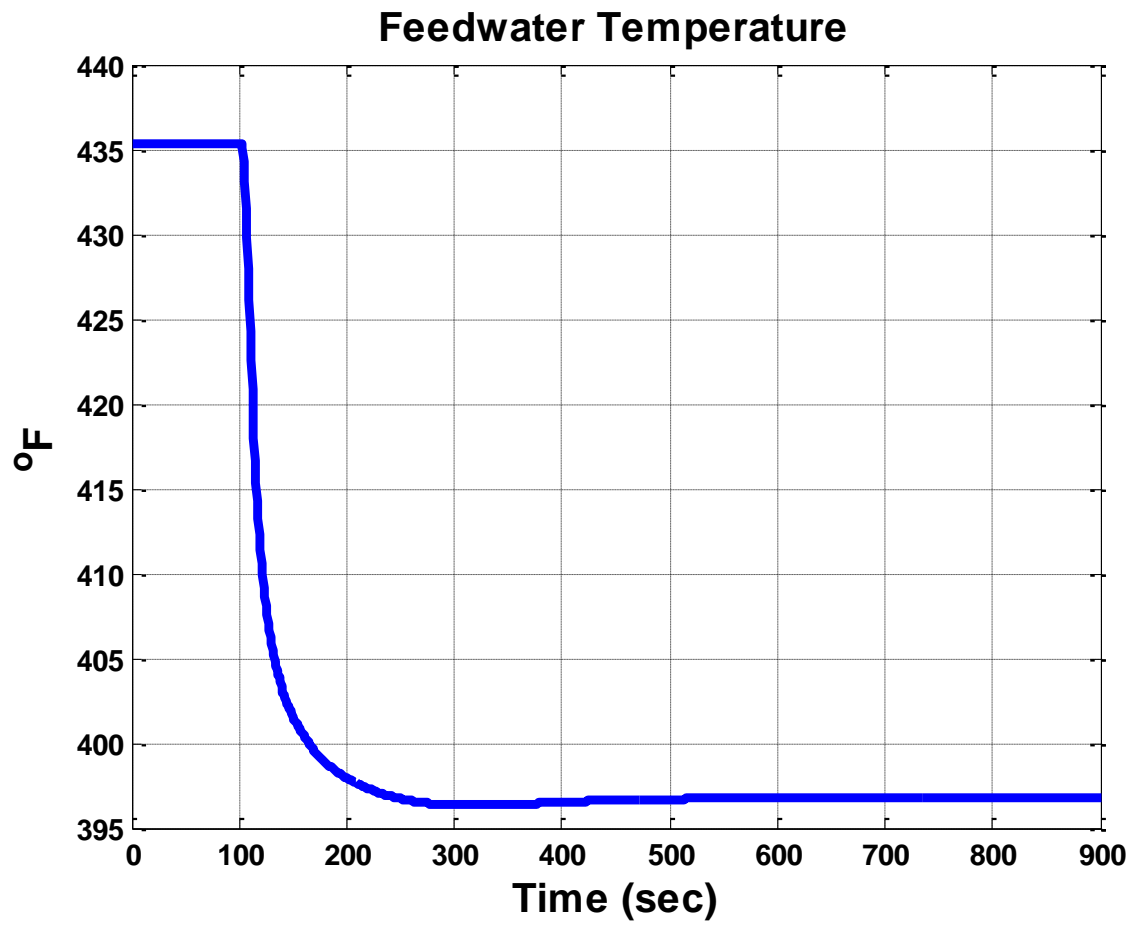

Figure 5.13. Feedwater temperature response to a $10 \%$ power demand step decrease.

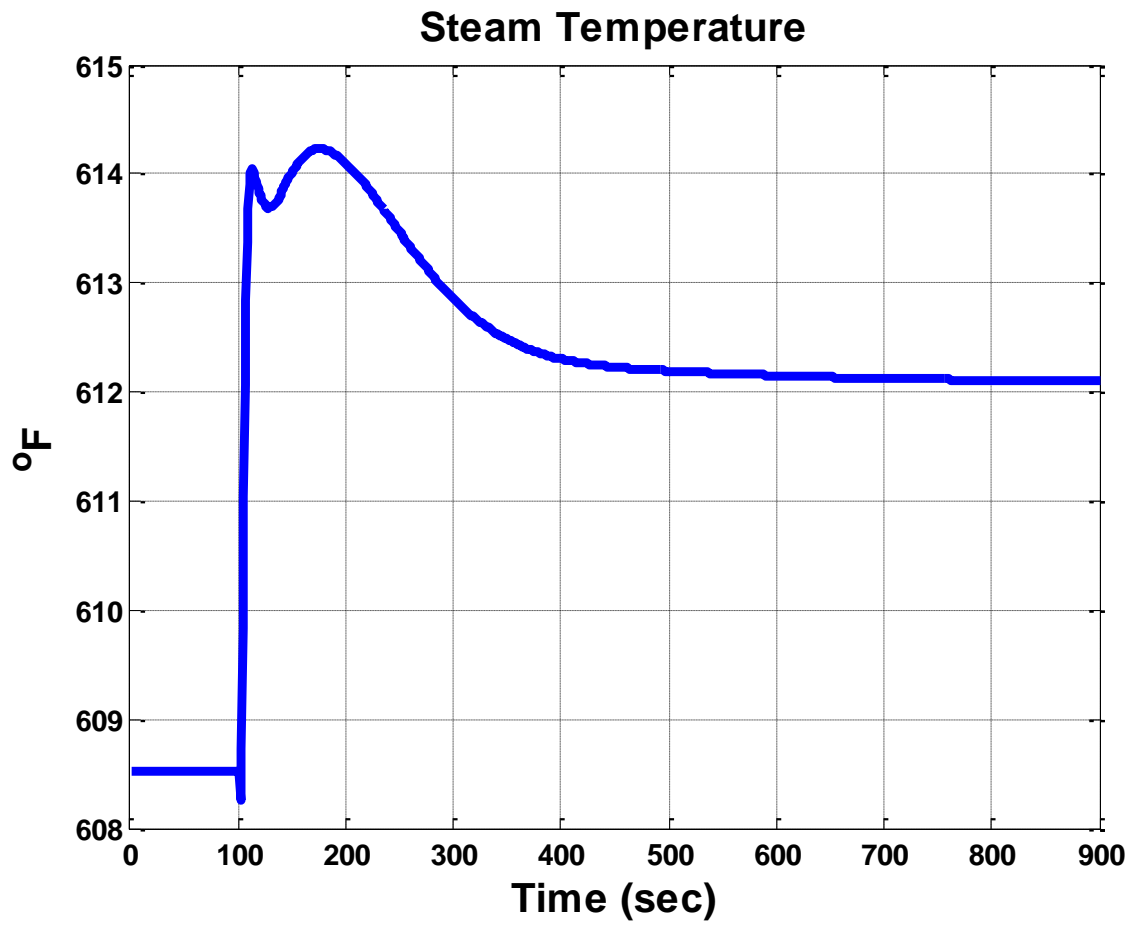

Figure 5.14. Steam temperature response to a $10 \%$ power demand step decrease. 


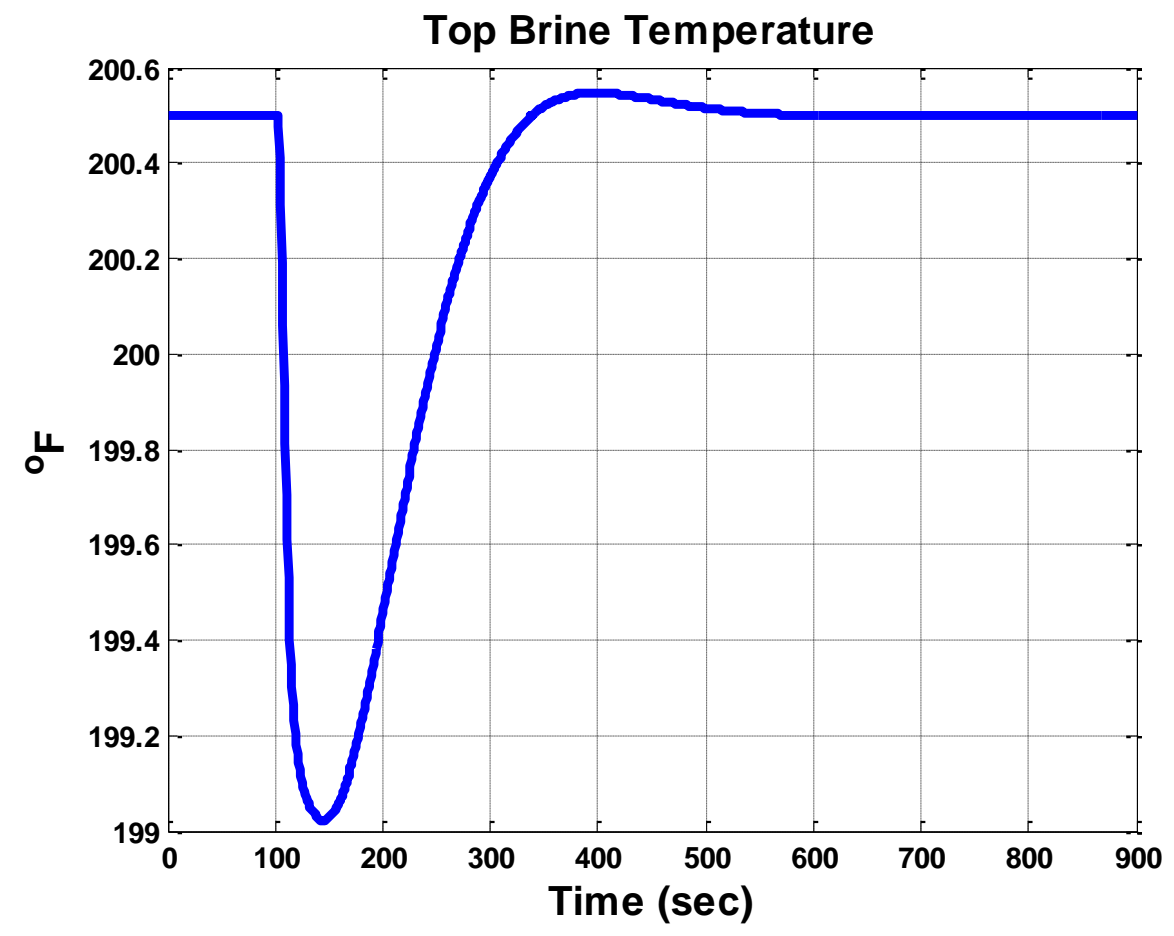

Figure 5.15. Top brine temperature response to a $10 \%$ power demand step decrease.

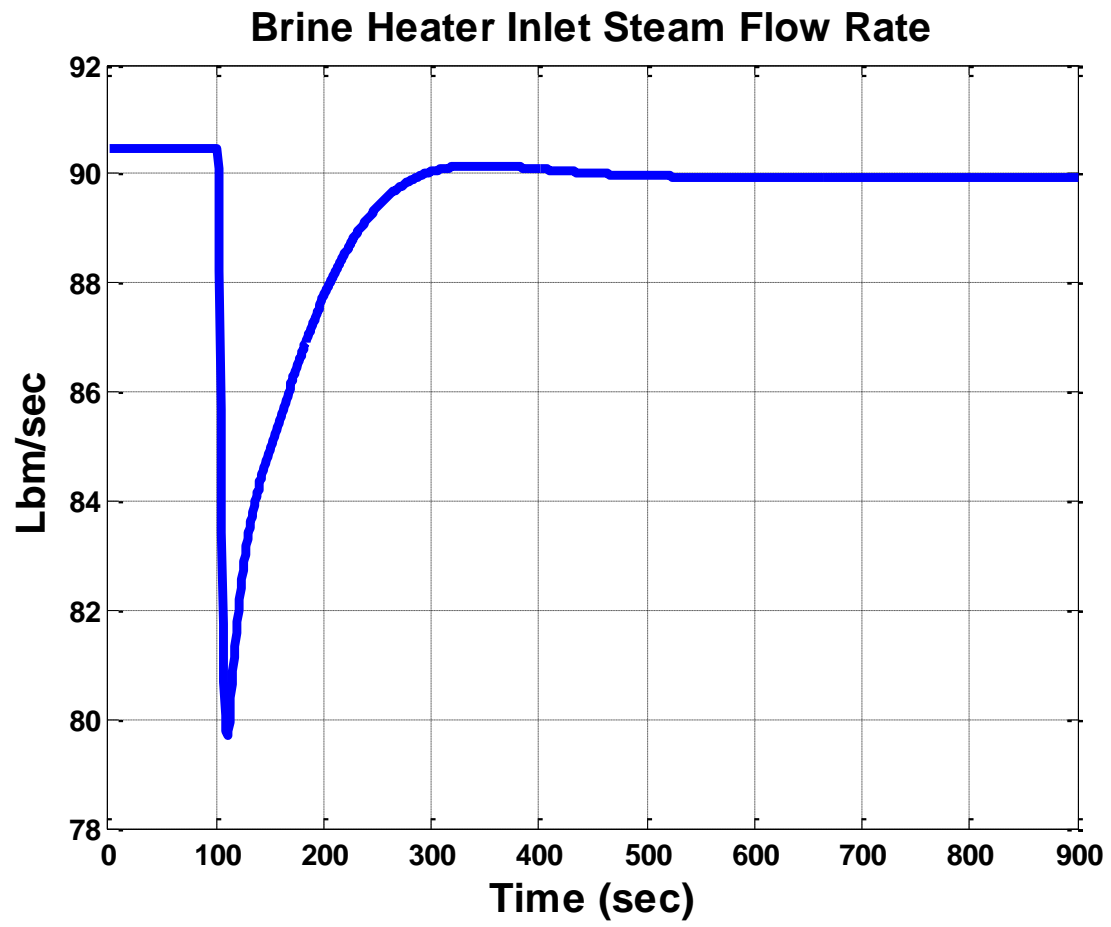

Figure 5.16. Brine heater inlet steam flow rate response to a $10 \%$ power demand step decrease. 


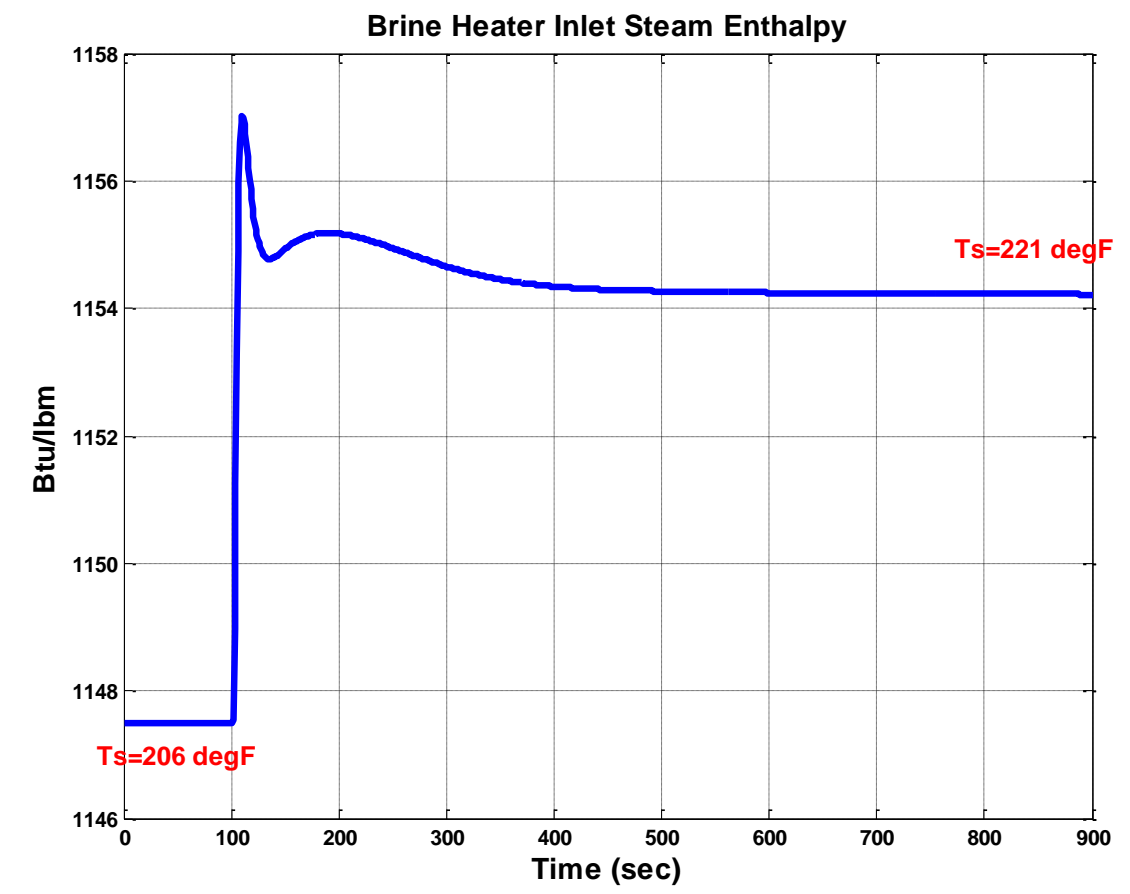

Figure 5.17. Brine heater inlet steam enthalpy response to a $10 \%$ power demand step decrease.

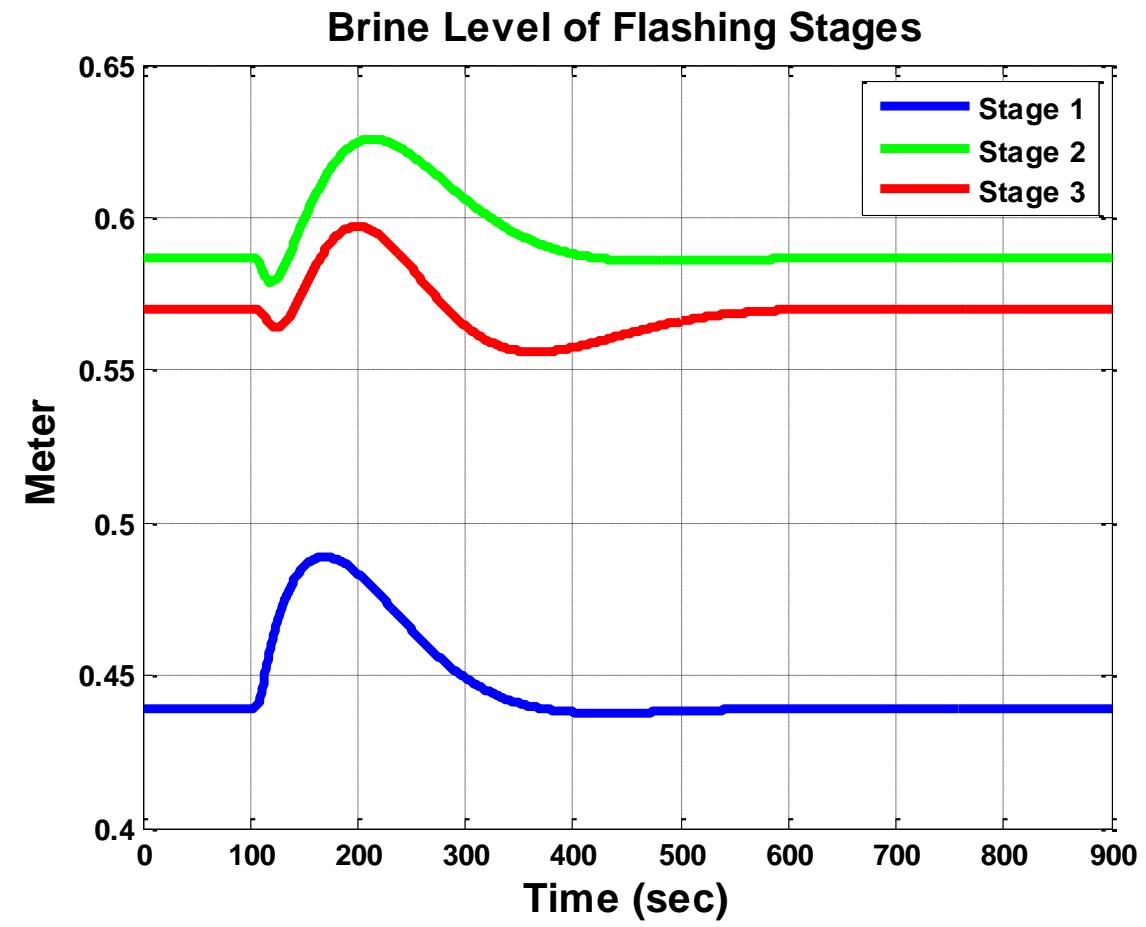

Figure 5.18. Brine level response to a $10 \%$ power demand step decrease. 


\subsection{Summary}

In this chapter, the development of the coupled IRIS-MSF nuclear desalination system model has been described. An existing IRIS plant SIMULINK model was used for coupling with the dynamic MSF model developed and described in Chapter 4. A simple BOP model was also described in this chapter. The BOP model incorporated the subsystem models of the turbine, condenser, and feedwater heater systems. The dynamic model of the coupled plant has been simulated for a $10 \%$ step decrease in the turbine power demand, and the simulation results have been analyzed in this chapter. The results showed that the developed dynamic model was able to characterize the system dynamic behavior with reasonably good accuracy to study the control and fault diagnosis of the IRIS-MSF coupled system. 


\section{Chapter 6}

\section{DEVELOPMENT OF SENSOR PLACEMENT DESIGN ALGORITHMS}

\subsection{Introduction}

Sensor allocation has been treated as an optimization problem in most of the earlier work. The first attempt to present a technique to locate sensors was made by Lambert [1977], where he used probabilistic importance of events in fault trees to decide optimal sensor locations. Vaclavek and Loucka [1976] described the problem of sensor network design and employed graph theory to ensure the observability of a specified set of important variables in a multi-component flow network. Ali and Narasimhan [1993] addressed the concept of reliability of state variable estimation and developed graph-theoretic algorithms for maximizing the reliability. The reliability of the process was defined as the smallest reliability among all of the variables. Unlike the approaches based on graph theory and linear algebra, Bagajewicz [1997] proposed a mixed integer nonlinear programming (MINLP) problem to obtain cost-optimal structures subject to the desired level of precision, residual precision, and error detectability. An alternative mixed integer linear programming (MILP) formulation which was useful for both small- and medium-size problems was presented by Bagajewicz and Cabrera [2002]. Sen et al. [1998] presented a genetic algorithm based approach that can be applied for the design of non-redundant sensor networks using different objective functions.

In this project, the graph-based techniques are used to optimize sensor locations. Issues such as fault detectability and discriminability are discussed to ensure the designed sensor network could observe every defined fault in the process, meanwhile obtain a maximum possible fault resolution. Directed graph (DG) that represents cause-effect behavior of the process is used as a basis for the sensor placement design. DG is employed to describe the propagation of fault effects, and a cause-effect analysis is performed based on the graph theory to design the sensor placement strategy.

The contribution of this research to the field of optimum sensor placement design is innovative in that it develops an optimal and automated sensor allocation algorithm for an 
IRIS-MSF nuclear desalination plant, with considerations of both single-fault and dual-fault assumptions in the application. In addition, this research proposes an efficient integer linear programming (ILP) embedded greedy search algorithm to solve the formulated sensor allocation optimization problem. And the optimal results are compared with those from LINGO 8.0, a commercial ILP optimization solver.

\subsection{Method of Directed Graph Modeling}

The solution to the problem of sensor placement may be broadly broken down into two tasks: (1) fault modeling or prediction of cause-effect behavior of the system, generating a set of variables that are affected whenever a fault occurs, and (2) use of the generated sets to identify sensor locations based on various design criteria, such as fault observability, fault resolution, etc. The fault propagation or cause-effect behavior is derived on the basis of a qualitative model that is used to represent the process.

Directed graph or digraph is such a qualitative cause-effect model that can be used to infer the mechanism of fault propagation in a system. It normally consists of a set of nodes and directed branches. The nodes in a DG model represent states of process variables or malfunctions, and the branches correspond to the causal influences between the nodes. The arrow of a branch reflects the direction of the effect between variables, and the fault propagation pattern can thus be inferred graphically. Based on the DG model of a given process, approaches from the graph theory are then employed to design the sensor networks according to some specified criteria.

The DG modeling is a convenient approach because it clearly illustrates the interactions among the important process variables, and can be easily developed from the empirical relationships or engineering fundamental principles. Figure 6.1 shows a DG diagram of a hypothetical process with the path connections from faults to potential location of sensors, as an illustrating example. In this figure, each node corresponding to a fault $\left(F_{j}\right)$ in the process connects through an arrow to a sensor $\left(S_{i}\right)$, thus indicating that the fault will affect the reading of the corresponding sensor. The method used to solve the problem of sensor location is based on identifying directed paths from root nodes where faults can occur to nodes where effects can be 
measured, called the observability set. Using all these paths, the objective is to choose the minimum subset of sensors from the observability set that would have at least one directed path from every root node.

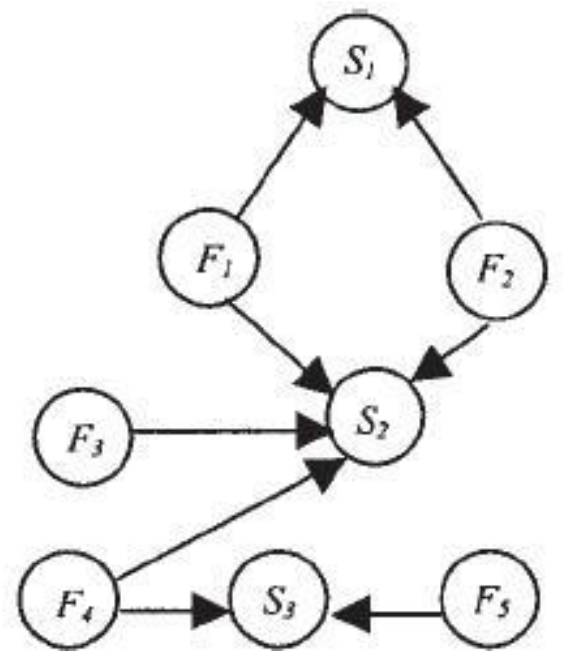

Figure 6.1. Directed graph of a hypothetical process.

The fault-and-sensor maximum connectivity matrix $D$ is also generated for the hypothetical process. The rows of this matrix represent the sensors, and the columns represent the faults. The $(i, j)_{t h}$ entry $D_{i j}$ of this matrix is 1 if fault $F_{j}$ affects the potential sensor location $S_{i}$ and is 0 otherwise $(i=1,2, \ldots, M$ and $j=1,2, \ldots, N)$, where $M$ is the total number of sensors and $N$ is the total number of faults. The generated matrix is given by:

$D=$

\begin{tabular}{c|ccccc} 
& $F_{1}$ & $F_{2}$ & $F_{3}$ & $F_{4}$ & $F_{5}$ \\
\hline$S_{1}$ & 1 & 1 & 0 & 0 & 0 \\
$S_{2}$ & 1 & 1 & 1 & 1 & 0 \\
$S_{3}$ & 0 & 0 & 0 & 1 & 1
\end{tabular}




\subsection{Sensor Location and Fault Diagnostic Observability Criterion}

\subsubsection{Set-Covering Problems}

The proposed sensor placement design that makes use of optimization criteria of fault observability and fault discriminability considers finding the optimal sensor locations as one of choosing the minimum number of sensors that would cover all the faults (root nodes). This is the well-known "minimum set-covering problem". The greedy search algorithm developed by Raghuraj et al.[1999] that was used previously in this research, although one of the fastest solving methods, does not necessarily find all minimal sensor sets [Krysander et al., 2008]. For this purpose, a literature review on solving set-covering problems using heuristic algorithms has been performed.

Set-covering problem is a classical problem in computer science and complexity theory. Set-covering problem is one of the most important discrete optimization problems because it serves as a model for real world problems. Real world problems that can be modeled as set-covering problems include facility location problem, airline crew scheduling, nurse scheduling problem, resource allocation, assembly line balancing, vehicle routing, etc. Set-covering problem is a problem of covering the rows of an $m \times n$ zero-one matrix with a subset of columns at minimal cost [Beasley and Chu, 1996]. Set-covering problem can be formulated as follows:

$$
\operatorname{minimize}\left[\sum_{j=1}^{n} c_{j} x_{j}\right]
$$

subject to

$$
\begin{gathered}
\sum_{j=1}^{n} a_{i j} x_{j} \geq 1, i=1, \ldots, m \\
x_{j} \in\{0,1\}, j=1, \ldots, n
\end{gathered}
$$


Equation (6.1) is the objective function of set-covering problem, where $c_{j}(j=1, \ldots, n)$ is refer to weight or cost of covering column $j$ and $x_{j}$ is decision variable. Equation (6.2) is a constraint to ensure that each row is covered by at least one column where $a_{i j}$ is constraint coefficient matrix of size $m \times n$ whose elements comprise of either " 1 " or " 0 ". A column $j$ $(j=1, \ldots, n)$ is said to cover a row $i(i=1, \ldots, m)$ if $a_{i j}=1$. Set-covering problem calls for a minimum cost subset $\mathrm{S}$, such that each row $i \quad(i=1, \ldots, m)$ is covered by at least one column, $j \in S$. Finally, Equation (6.3) is the integrality constraint in which the value is represented as in Equation (6.4).

$$
x_{j}=\left\{\begin{array}{l}
1 \quad \text { if } j \in S \\
0 \quad \text { otherwise }
\end{array}\right.
$$

Even though it may seem to be a simple problem by judging from the objective functions and constraints of the problem, set-covering problem is a combinational optimization problem.

A number of heuristic algorithms for set-covering problem have been reported in the literature. Beasley, as one of main researcher in set-covering problem had implemented several algorithms in order to solve set-covering problem. Beasley presented an algorithm that combines problem reduction tests with dual ascent, sub-gradient optimization and linear programming. This algorithm had performed well in solving set-covering problem [Beasley, 1987]. It was able to find feasible optimal solutions for all set-covering problem sets. In a different literature, Beasley presented a paper which used Lagrangian relaxation and sub-gradient optimization approach to solve the set-covering problem [Beasley, 1990]. But this method did not perform well compared to his previous method. It was unable to find optimal solutions for several set-covering problems. Haddadi presented a simple Lagrangian heuristic to solve set-covering problem [Haddadi, 1997]. The method is based on Lagrangian duality, greedy heuristic for set-covering problem, sub-gradient optimization and redundant covers. This method had turned out to be efficient for low density set-covering problems with a large number of variables with average deviation of $0.35 \%$. 
Beasley and Chu used genetic algorithm for set-covering problem [Beasley and Chu, 1996]. They presented a new crossover-fusion operator, a variable mutation rate and a heuristic feasibility operator to improve the performance of genetic algorithm. This method performs well in solving most problems. Aickelin proposed an indirect genetic algorithm [Aickelin, 2002]. The indirect genetic algorithm comprises of three phases. In the first phase, the genetic algorithm finds good permutation of the rows to be covered. In the second phase, a decoder builds a solution from the permutations using the parameter provided. And lastly, in the third phase, a hill-climber optimization method is used. The indirect genetic algorithm is able to solve the set-covering problem in a shorter computational time. Monfroglio proposed a linear programming relaxation model and improvement techniques based on simulated neural network [Monfroglio, 1998]. This method is able to find solutions within $0.2 \%$ of optimal solution and increase the overall computational time. Vasko and Wolf adapted heuristic concentration approach to solve the weighted (non-unicost) set-covering problem [Vasko and Wolf, 2001]. Their method is able to solve set-covering problem and find solution with deviation of maximum of $3.27 \%$ from optimum solution.

\subsubsection{Fault Observability}

Fault observability refers to the condition that every fault defined for the process has to be observed by at least one sensor. Given a process DG model, the observability problem becomes one of finding the minimum number of sensors that would cover all the faults in the process (root nodes). This is commonly known as "minimum set-covering problem" [Parker and Rardin, 1988], where the sets to be covered are the sets of sensors affected by each process fault. Although a simple enumeration can be made for a small system, a systematic approach needs to be used for a large system. In this approach, the first step is to build a bipartite graph, which consists of a causal set including all the fault nodes and an observability set including all the sensor nodes with only input arcs in the DG model. A graph is bipartite if a vertex set can be partitioned into two sets in such a way that no two vertices from the same set are adjacent. These two sets constitute a bipartition of the original vertex set [Asratian et al., 1998]. After a bipartite graph is obtained from the DG model, a subset of the key variables can be chosen from the observability set as the minimum sensor requirement for fault detection based on a greedy search algorithm developed by Raghuraj et al.[1999]. 
This search algorithm is summarized as follows:

(1) Initiate $C$ and $G$ as empty sets.

(2) Construct a bipartite graph between the fault nodes and the key variables in the fault observability set.

(3) Select a variable among the unmarked key variables based on the largest number of incident arcs.

(4) Mark the selected key variable in step (2) and store it in $C$.

(5) Determine all the fault nodes covered by $C$.

(6) If there exist uncovered fault nodes, delete all the arcs from the fault nodes covered by the selected variable to all the previously marked key variables; store in a buffer set $G$ all the arcs from the fault nodes covered by the selected variable to the unmarked key variables, then go back to step (3); else, remove the variables from $C$ that do not have arcs incident on them based on the arcs stored in $G$. Output the set $C$ and stop.

\section{(7) End.}

The number of incident arcs used in step (3) is defined as the difference between the actual number of arcs incident on a key variable and the number of arcs incident on the same key variable that have been stored in the buffer set $G$. After the algorithm is completed, the same set of key variables can be obtained in $C$ as is obtained from the greedy search algorithm. However, the redundant key variables will be stored by tracking the arcs stored in the buffer set $G$ that do not have a connection with the marked key variables. To determine the minimum set of sensors, the redundant key variables must be removed from the obtained key variables stored in $C$.

\section{Example:}

Let us use the hypothetical system shown in Figure 6.1 as a simple example to illustrate the greedy search algorithm based on fault observability criteria. In this example, there are five 
fault classes $F_{1}, F_{2}, F_{3}, F_{4}$, and $F_{5}$, and three key variables $S_{1}, S_{2}$, and $S_{3}$ on which sensors can be placed. Figure 6.2 depicts the bipartite graph for the original digraph.

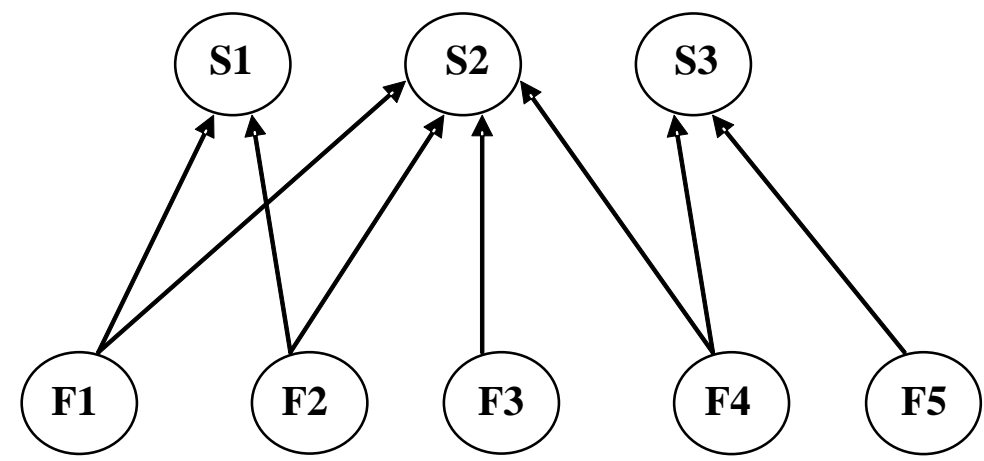

Figure 6.2. Fault and sensor node bipartite graph for Figure 6.1.

Figure 6.2 clearly shows that the set $\left[S_{1}, S_{2}, S_{3}\right]$ form the "observability set", as these are the nodes in the DG model with only input arcs. It is also clear that by placing sensors on these nodes, one could detect all the faults. However, the set $\left[S_{1}, S_{2}, S_{3}\right]$ is not the minimum set. The minimum set is, in fact, $\left[S_{2}, S_{3}\right]$. As a first step in the algorithm, node $S_{2}$ is chosen as the key component, as it has the maximum number of arcs incident on it. In the mean time, the arcs from $F_{1}$ to $S_{1}, F_{2}$ to $S_{1}$, and $F_{4}$ to $S_{3}$ will be stored in set $G$ because $F_{1}, F_{2}$, and $F_{4}$ are covered by the selected variable $S_{2}$, which is marked. In the next step, $S_{3}$ is chosen as the next key component, and with this it is clearly seen that all the fault nodes are now covered. Both $S_{2}$ and $S_{3}$ have the arcs stored in set $G$ incident on it. This is the minimum observability set, as given by the greedy search algorithm.

\subsubsection{Fault Resolution}

Fault resolution refers to the ability to identify the exact fault within a system of interest that has occurred. The maximum resolution that can be attained is restricted by the topology of DG and the position of the fault or root nodes in a DG model. Hence, given the constraints on measurement points, the problem of fault resolution is equivalent to generating sensor locations so that every fault is resolved to the maximum extent possible. This condition is referred to as the "highest fault resolution". As shown by Raghuraj et al. [1999], any fault resolution problem (single-fault resolution, multiple-fault resolution, etc.) can be converted to a suitable fault 
observability problem. Also, the assumption of single-fault or multiple-fault would lead to different fault resolution solutions.

\section{Single-Fault Resolution}

For each pair of faults $i$ and $j$, the fault set $B_{i j}=A_{i} \cup A_{j}-A_{i} \cap A_{j}$ is generated. In this expression, $A_{i}$ and $A_{j}$ are the sets of measurable nodes connected to the $i_{t h}$ fault and $j_{t h}$ fault, respectively. Note that set $B_{i j}$ represents the symmetric difference of sets $A_{i}$ and $A_{j}$, which consists of the set of nodes that can be used for differentiating between a pair of fault

nodes $i$ and $j$. Thus, each $B_{i j}$ is treated as a virtual fault, and a bipartite graph is constructed between these nodes and the sensor nodes. This bipartite graph is then added to the original bipartite graph.

The minimum set of sensors for fault resolution can also be determined using the greedy search algorithm. The steps are summarized as follows:

(1) Determine the set of key variables consisting of the different members of $A_{i}$ and $A_{j}$ that covers the virtual fault $B_{i j}$, which denotes the resolution of the original fault nodes $i$ and $j$.

(2) Construct a bipartite graph between the set of virtual fault nodes and their observability set.

(3) This new bipartite graph is added to the original bipartite graph, which is between root nodes and sensor nodes.

(4) Apply the greedy search algorithm developed for fault observability to the updated bipartite graph to determine the minimum set of sensor nodes that would cover all the root nodes and the virtual fault nodes. The selected sensor set based on this algorithm can ensure that the specified faults can be detected and distinguished.

\section{Example:}


To illustrate the concepts developed, fault diagnosis of the previous example is performed. The greedy search algorithm is used for fault diagnosis under the single-fault assumption. As a first step, the virtual faults $B_{i j}=A_{i} \cup A_{j}-A_{i} \cap A_{j}$ are constructed for all $i$ and $j$. The sensor sets for the different faults are given in Table 6.1. Some faults may affect the identical sensor set. In this case, they have been combined as one fault, and cannot be distinguished from one another. For example, nodes $F_{1}$ and $F_{2}$ share the same sensor set $\left[S_{1}, S_{2}\right]$, and have been combined and denoted by the node $A_{1}$. The set $B_{i j}$ for the faults are shown in Table 6.2. The faults consist of the original faults plus the virtual fault set $B_{i j}$. The greedy search algorithm is applied on this extended fault system and the optimum sensor set is found to be $\left[S_{1}, S_{2}, S_{3}\right]$. All the original faults in Figure 6.2 can be distinguished from each other by the chosen sensor set, except for $F_{1}$ and $F_{2}$.

Table 6.1. Set $A$ of sensors for different fault nodes in Figure 6.2

\begin{tabular}{|c|c|c|}
\hline Fault Nodes & Sensor Nodes & Set A \\
\hline$F_{1}, F_{2}$ & {$\left[S_{1}, S_{2}\right]$} & $A_{1}$ \\
\hline$F_{3}$ & {$\left[S_{2}\right]$} & $A_{2}$ \\
\hline$F_{4}$ & {$\left[S_{2}, S_{3}\right]$} & $A_{3}$ \\
\hline$F_{5}$ & {$\left[S_{3}\right]$} & $A_{4}$ \\
\hline
\end{tabular}

Table 6.2. Set $B_{i j}$ for single-fault case of Table 6.1

\begin{tabular}{|c|c|}
\hline Set B & Sensor Nodes \\
\hline$B_{12}$ & {$\left[S_{1}\right]$} \\
\hline$B_{13}$ & {$\left[S_{1}, S_{3}\right]$} \\
\hline$B_{14}$ & {$\left[S_{1}, S_{2}, S_{3}\right]$} \\
\hline$B_{23}$ & {$\left[S_{3}\right]$} \\
\hline$B_{24}$ & {$\left[S_{2}, S_{3}\right]$} \\
\hline
\end{tabular}




\begin{tabular}{|c|c|}
\hline$B_{34}$ & {$\left[S_{2}\right]$} \\
\hline
\end{tabular}

\section{Multiple-Fault Resolution}

Assuming multiple faults, the sensor placement problem is solved as an extension of the single-fault resolution problem. For the sake of illustration, consider the specific case when a maximum of two faults can occur at a time. For each pair of faults $i$ and $j$, the set $A_{i j}=A_{i} \cup A_{j}$ is formed, where $A_{i j}$ represents the set of nodes which are affected when both of the faults $i$ and $j$ occur simultaneously. The set $A_{i j}$ is treated as a virtual fault, and is therefore added to the original set of faults.

Solving the single-fault resolution problem for this new system gives the sensor locations for resolution under dual-fault assumption. The same methodology can be applied to cases where more than two faults occur simultaneously. Clearly, with a greater number of sets generated, the computational complexity of the approach increases, but as discussed in Raghuraj et al. [1999], one might not be interested in all multiple-fault situations and the above formulation gives the designer a methodology by which different sensor location problems can be posed and solved.

\subsection{Sensor Location and System Unobservability Criterion}

\subsubsection{Formulation of Unobservability Minimization}

The design of a reliable instrumentation system for an industrial process is of the ultimate importance. A sensor network is highly reliable if the probability of any fault occurring without being detected is low. For a given process, the faults in that process have certain occurrence probabilities. The various available sensors also have certain failure probabilities, which depend on the type of sensor and the variable being measured. The only way in which a fault can occur without being detected is that the fault occurs and the sensors monitoring that fault fail simultaneously. The probability of such an event taking place is the product of the fault 
occurrence probability $f_{j}$ and the corresponding sensor failure probability $s_{i}$, which is defined as the unobservability value $U_{j}$ of the $j_{t h}$ fault.

$$
U_{j}=f_{j} \prod_{i=1}^{M} s_{i}^{D_{i j} x_{i}}, j=1,2, \ldots, N .
$$

In Equation (6.5), $x_{i}$ is the number of sensors being placed at a particular location, and the binary number $D_{i j}$ is the $(i, j)_{t h}$ entry of the fault-sensor maximum connectivity matrix. Note that in the proposed algorithm, $x_{i}$ is allowed to be greater than 1, which, in other words, means hardware redundancy is allowed. This feature makes the approach practical because it is reasonable to use more than one sensor to measure a variable if that particular sensor node has a high tendency to fail or if the covered fault has a high probability of occurrence.

Another useful quantity (redundancy value) used in the algorithm is defined in Equation (6.6). This value provides an approximate measure of the total unobservability covered by a sensor.

$$
R_{i}=\sum_{j=1}^{N} D_{i j} U_{j}, i=1,2, \ldots, M
$$

Thus, achieving a minimum unobservability of the system is paramount for the design of a reliable sensor network. The proposed sensor placement formulation is based on minimizing the system overall unobservability, which is defined as the maximum unobservability among all faults $\left(\underset{\forall_{j}}{\operatorname{Max}} U_{j}\right)$. This concept is based on the philosophy that a chain can be no stronger than its weakest link. Therefore, the optimization of sensor placement design is formulated as follows:

$$
\underset{x_{i}}{\operatorname{minimize}}\left[\underset{\forall_{j}}{\operatorname{Max}} U_{j}\right]
$$

subject to 


$$
\begin{gathered}
\sum_{i=1}^{M} c_{i} x_{i} \leq T C \\
\sum_{i=1}^{M} D_{i j} x_{i} \geq 1, j=1,2, \ldots, N . \\
x_{i} \in \square^{+} \cup 0, i=1,2, \ldots, M
\end{gathered}
$$

Equation (6.7) is the objective function of the formulated unobservability optimization problem, where $U_{j}$ is given in Equation (6.5). The constraint given by Equation (6.8) ensures the total cost does not exceed the available resource $T C$, where $c_{i}$ is the cost of placing a sensor at node $i$ and $x_{i}$ is the decision variable. Equation (6.9) is an observability constraint to ensure that each column (fault) is covered by at least one row (sensor location) where $D_{i j}$ is the $(i, j)_{t h}$ entry of the fault-sensor maximum connectivity matrix whose elements comprise of either ' 1 ' or ' 0 '. Finally, Equation (6.10) allows the decision variables $x_{i}$ to take nonnegative integer values which may be greater than 1 . For instance, $x_{i}=4$ indicates that four sensors are placed at the same location.

Even though it may seem to be a simple problem by judging from the objective functions and constraints of the problem, the above problem is an integer nonlinear programming (INLP) problem which is not easy to solve exactly. However, the problem can be converted to a linear integer programming problem through a proper transformation.

The objective function in Equation (6.7) can be replaced by a linear objective function:

$$
\underset{x_{i}}{\operatorname{minimize}}\left[\operatorname{Max}_{\forall_{j}} \ln \left(U_{j}\right)\right]
$$

where 


$$
\ln \left(U_{j}\right)=\ln \left(f_{j}\right)+\sum_{i=1}^{M} D_{i j} x_{i} \ln \left(s_{i}\right), j=1,2, \ldots, N
$$

Thus, $\ln \left(U_{j}\right)$ is linear in the decision variables $x_{i}$ and is obtained by taking the natural logarithm on both sides of Equation (6.5).

The objective function given in Equation (6.11) is still not in the standard ILP form because it involves minimization of the maximum value. Therefore, it is being modified again.

$$
\underset{x_{i}}{\operatorname{minimize}}[U]
$$

subject to

$$
\begin{gathered}
U \geq \ln \left(U_{j}\right), j=1,2, \ldots, N . \\
\sum_{i=1}^{M} c_{i} x_{i} \leq T C \\
\sum_{i=1}^{M} D_{i j} x_{i} \geq 1, j=1,2, \ldots, N . \\
x_{i} \in \square^{+} \cup 0, i=1,2, \ldots, M
\end{gathered}
$$

The objective function given in Equation (6.13) is now in the standard ILP form.

\subsubsection{One-Step Optimization Formulation}

It has been found that the above optimization problem typically has multiple solutions for unobservability of detection with different costs. Lexicographic optimization [Bhushan and Rengaswamy, 2000a] is one way of finding the solution with the least cost among these multiple solutions. This is done by obtaining a solution that optimizes the objectives in an ordered fashion. Minimizing the unobservability is the first objective and minimizing the cost is the next objective. Such an ordering would mean that a higher level objective is more important than a 
lower level objective. We will show below a single objective function that combines system unobservability minimization and cost minimization objectives

$$
\underset{x_{i}}{\operatorname{minimize}}\left[U-\alpha x_{s}\right]
$$

subject to

$$
\begin{gathered}
U \geq \ln \left(U_{j}\right), j=1,2, \ldots, N . \\
\sum_{i=1}^{M} c_{i} x_{i}+x_{s} \leq T C \\
\sum_{i=1}^{M} D_{i j} x_{i} \geq 1, j=1,2, \ldots, N . \\
x_{i} \in \square^{+} \cup 0, i=1,2, \ldots, M \\
x_{s} \in \square^{+} \cup 0
\end{gathered}
$$

In the above problem, the objective function $U$ is the maximum unobservability of detection (on a natural log scale) among all faults, which is ensured by constraints in Equation (6.19). The variable $x_{s}$ is the slack in the cost constraint, which takes nonnegative real values. The higher the value of $x_{s}$, the lower is the cost used for sensor placement. $\alpha$ is a positive constant which has to be selected such that the primary objective (minimizing unobservability) still attains its earlier optimal value. Among all solutions which yield minimum system unobservability, the one which has the highest $x_{s}$ will be chosen. Therefore, if the constant $\alpha$ is appropriately selected, the solution will give a sensor network that has the least cost among all of the networks which yield the minimum system unobservability. 


\subsubsection{Solving Unobservability Minimization Problems}

The problems presented above can be solved by standard solvers for moderate size problems. In this project, the commercial ILP optimization software LINGO 8.0, which employs a branch and bound implicit enumeration algorithm, is used to solve the formulated unobservability minimization problem. Also, a greedy search heuristic is developed to find the optimal number of sensors and their placement. The detailed procedure of the algorithm is described below. At each step the best available sensor is chosen. Also, a flowchart of the algorithm is illustrated in Figure 6.3.

Step 1: Initialize the following variables: current solution $(P S)$, optimal solution $(O S)$, total available cost $(T C)$, cost currently utilized $(C)$, optimal cost utilized $(O C)$.

Step 2: The fault with the highest unobservability value is selected. In the case of more than one fault having the highest unobservability, any one among these faults may be selected.

Step 3: Select a sensor which observes the fault chosen in the previous step, and try to lower the unobservability value of that fault. However, there may be more than one qualified sensor. Among these sensors, only those whose cost is less than the currently available cost can be chosen. Let $S$ be the set of sensors which are connected to the selected fault, as well as cost of each member of $S$ is less than (or equal to) the currently available cost. A sensor of $S$ with the minimum failure probability is to be chosen because this causes a maximum decrease in the unobservability value of the fault under consideration. If more than one element of $S$ satisfies this criteria (that is more than one element of $S$ has the lowest failure probability), then from those, the one with the highest redundancy value is chosen. In case more than one sensor still satisfies this criterion, the one with the lowest cost is chosen. If still there is more than one candidate, any sensor among those may be chosen. A crucial point is that no restriction is placed on the number of sensors that can be placed to observe the same node (variable). Hence, it is possible that at some nodes more than one sensor is placed, while some other nodes are not selected as sensor nodes at all. Then perform the updates on $P S, C$, and $T C-C$. After this sensor selection, recalculate the unobservability values of all faults and the system unobservability (maximum unobservability). If the system unobservability is less than the previous value, then update the 
optimal solution to be the current solution: optimal solution $O S=P S$, optimal cost $O C=C$. Otherwise, go back to step 2.

Step 4: The procedure is continued until the set $S$ becomes empty. This indicates that no more sensors can be selected to decrease the system unobservability. The set of sensors in $P S$ is the selected sensor location. Steps 2 and 3 ensure minimization of the system unobservability. The procedure of updating the optimal solution only if there is a decrease in the system unobservability ensures that the solution is optimal in the lexicographic sense.

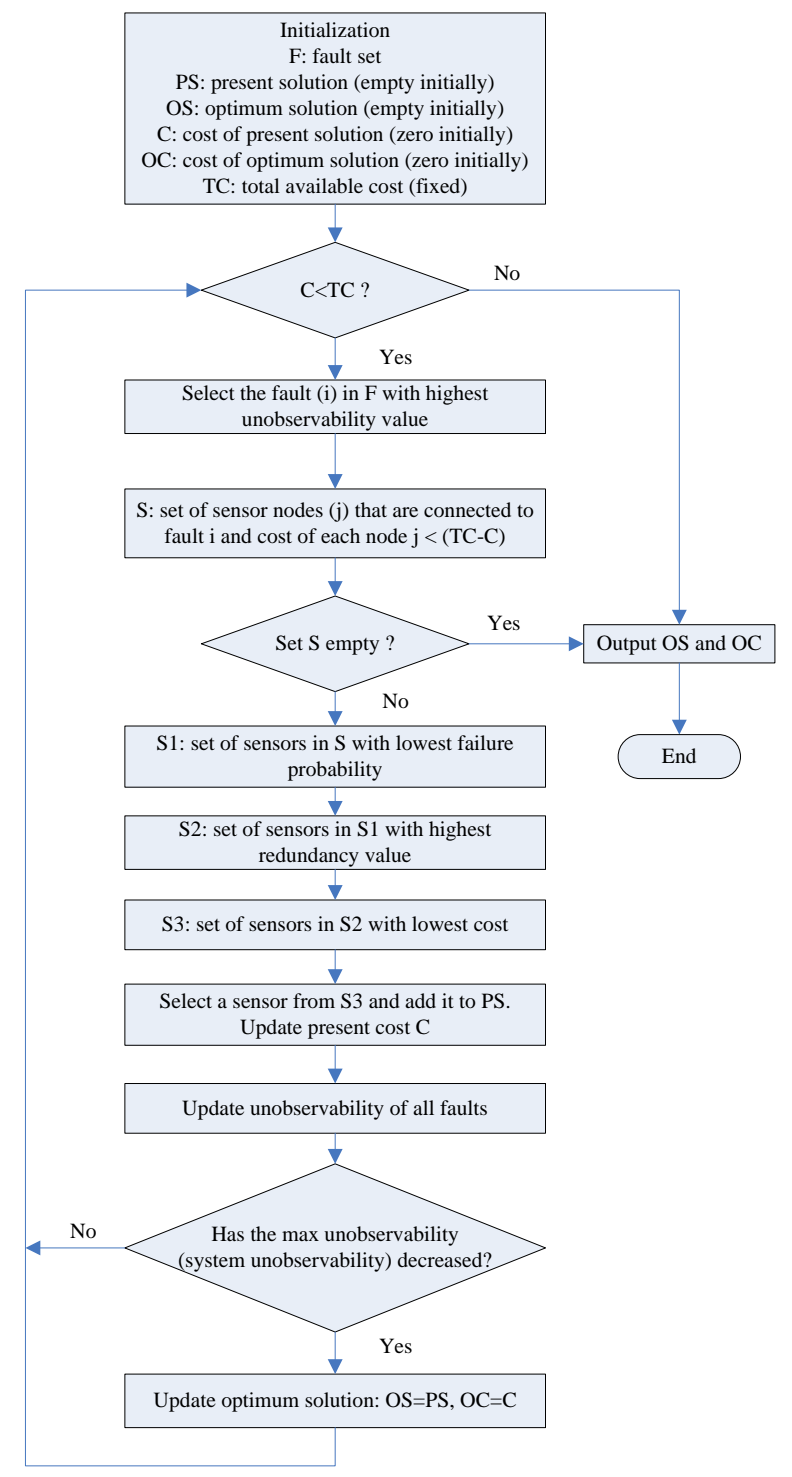

Figure 6.3. Flowchart for greedy search algorithm. 


\section{Example:}

Let us still use the previous example whose bipartite graph is shown in Figure 6.4 to demonstrate the sensor placement algorithm. The probabilities of occurrence of faults are $f_{1}=0.1, f_{2}=0.1, f_{3}=0.01, f_{4}=0.01$, and $f_{5}=0.001$. The probabilities of failures of sensors available to measure the three measurable nodes are $s_{1}=0.1, s_{2}=0.01$, and $s_{3}=0.001$. For the sake of illustration, we shall assume the same cost of 100 for all of the available sensors. The total available cost $T C$ is equal to 400 , which means four available sensors in this specific case. Depending on the scenario considered, different optimum sensor locations may be obtained.

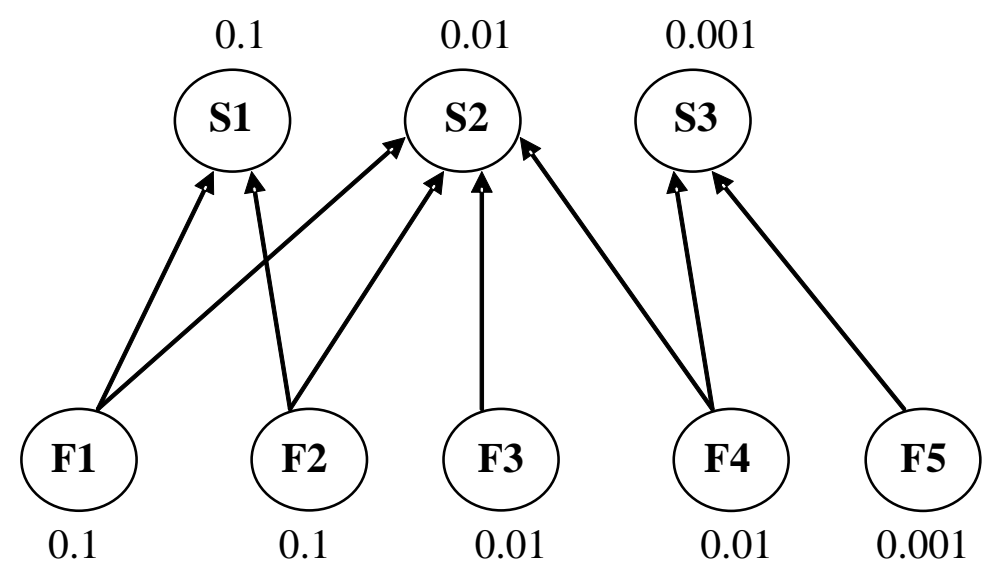

Figure 6.4. Bipartite graph for Figure 6.2 with probabilities.

(a) Only observability of faults is considered: For this case, the optimal sensor network is $\left[S_{2}(3), S_{3}\right]$. Then the unobservability values of faults are as follows:

$U_{1}=1 \times 10^{-7}, U_{2}=1 \times 10^{-7}, U_{3}=1 \times 10^{-8}, U_{4}=1 \times 10^{-11}$, and $U_{5}=1 \times 10^{-6}$

The system unobservability $U$ is the maximum unobservability. Hence,

$U=\max \left(U_{1}, U_{2}, U_{3}, U_{4}, U_{5}\right)=1 \times 10^{-6}$

(b) Resolution for the single-fault case is also considered. Virtual faults are constructed exactly like we did in the previous example.

The probabilities of occurrence of these virtual faults are: 


$$
\begin{aligned}
& f_{12}=\min \left(f_{1}, f_{2}\right)=0.1 \\
& f_{13}=\min \left(f_{1}, f_{3}\right)=0.01 \\
& f_{14}=\min \left(f_{1}, f_{4}\right)=0.01 \\
& f_{15}=\min \left(f_{1}, f_{5}\right)=0.001 \\
& f_{23}=\min \left(f_{2}, f_{3}\right)=0.01 \\
& f_{24}=\min \left(f_{2}, f_{4}\right)=0.01 \\
& f_{25}=\min \left(f_{2}, f_{5}\right)=0.001 \\
& f_{34}=\min \left(f_{3}, f_{4}\right)=0.01 \\
& f_{35}=\min \left(f_{3}, f_{5}\right)=0.001 \\
& f_{45}=\min \left(f_{4}, f_{5}\right)=0.001
\end{aligned}
$$

The virtual faults are then added to the original set of faults. Performing the sensor location algorithm on this extended system gives $\left[S_{1}(2), S_{2}, S_{3}\right]$ as the optimum sensor network. With these sensors, the system unobservability is $U=1 \times 10^{-4}$.

Compared to the previous results based on the fault diagnostic observability and resolution criteria, it is seen that the greedy search-based one-step optimization formulation offers a similar sensor network. The difference lies in the number of sensors that can be put at one location. Because of the additional sensor failure and fault occurrence probability information used in the algorithm, multiple sensors could be placed on any particular nodes in order to minimize the system unobservability, subject to the cost constraints.

\subsection{Summary}

In many cases, it is found that some faults are still indistinguishable using the sensor set obtained from the above sensor placement scheme. Nonetheless, optimal sensor placement design from a fault diagnosis perspective will provide valuable information to a fault diagnostic system based on principal component analysis, as described later in Chapter 7. The overall method as described in the preceding sections consists of the following steps:

(1) Define all faults of interest in a process (including process fault and sensor fault), based on the operation history records and available process knowledge. Then build DG models of the monitored process, which can be implemented by using empirical relationships or fundamental mathematical model of the process. 
(2) Solve the formulated unobservability minimization problem to decide the allocation of sensors. The obtained sensor set would partially guarantee the detection and isolation of all the faults defined in the first step.

(3) Highlight the faults that cannot be isolated by the information provided by the DG models and the sensor network obtained in the steps (1) and (2). Those faults will be left to the PCA-based fault diagnostic system for further detection and isolation. 


\section{Chapter 7}

\section{SENSOR PLACEMENT DESIGN AND FAULT DIAGNOSIS APPLICATION FOR A MSF DESALINATION SYSTEM}

\subsection{Introduction}

Principal component analysis (PCA) is a dimensional reduction method, where original data can be represented by a lower dimensional space without significant loss of the variability. From the modeling point of view, PCA transforms correlated variables into uncorrelated ones and determines the linear combinations with large and low variability [Flury, 1989].

Before original data are transformed into a lower dimensional space, they shall be mean-centered because only the variability of the data is of interest. The data are also standardized with unit variance so that equal weights are given to all the variables as far as the variability is concerned. A complete description of the PCA technique is given in this chapter [Jackson, 1991; Jolliffe, 2002]. Also, the sensor placement algorithms developed in Chapter 6, along with the PCA-based fault diagnostic approaches using the optimized sensor set, are demonstrated with application to a MSF desalination system. Both single-fault and dual-fault scenarios are considered in this application.

\subsection{Principal Component Analysis for Fault Diagnosis}

The basic idea of fault diagnosis using multivariate statistical methods such as PCA is to project the collected data onto a low-dimensional space where the regions of normal operation and abnormal operation can be characterized by fewer state variables.

Because a PCA model represents the variation of normal operation data in a reduced dimensional space, it has better performance of generalization than when the entire measurement space is used. PCA modeling separates the entire measurement space into a model subspace capturing the variation of state variables and a residual subspace containing random variations. The separate characterization of the two subspaces can provide further insights in terms of the changes in operation conditions. In addition, the linear model extracted by PCA enables us to 
determine which variables are most affected by a fault and which variables are most responsible for the fault.

The fault detection and isolation (FDI) methodology consists of building a PCA model to characterize the relationships among various measurements. A breakdown in the relationships is indicated as abnormal prediction residuals or lack of fit with the model and may be used for fault detection. Characteristic patterns in the residuals most probably correspond to faults of a particular type and thus may be used for fault isolation. It shall be emphasized that this is true irrespective of the type of a model that is used. More formally, given a model, the detection and isolation of faults may be achieved using the residuals that signify the mismatch between the model predictions and the actual data. It is worth noting that the isolation procedure developed here requires all possible faults are postulated, thus enabling the generation of fault residual sub-spaces.

\subsubsection{PCA Algorithm}

PCA is a statistical algorithm of dimension reduction by projecting data on to a lower dimensional space such that the major variation of the original data can be preserved. Given a normalized process data matrix $X(m \times n)$ composed of $m$ observations with $n$ measured variables. PCA decomposes $X$ into two components, a predicted value $X$ and an error value $E$, which determine two orthogonal subspaces, i.e., the principal component (PC) subspace and the residual component $(\mathrm{RC})$ subspace, respectively.

$$
\begin{aligned}
& X=X+E \\
& X=T P^{T} \\
& E=T_{E} P_{E}^{T}
\end{aligned}
$$

where $P$ is the orthogonal loading matrix and $T$ is the score matrix. The scores $T$ in the PC subspace explain the dominant variation of the measured variables, and the scores $T_{E}$ in the RC subspace represent the insignificant variation due to model reduction error. The column vectors of principal component loadings $P(n \times l)$ are the eigenvectors corresponding to the $l$ largest 
eigenvalues of the correlation matrix of the data matrix $X$ and the columns of $P_{E}$ are the eigenvectors corresponding to the smallest $n-l$ eigenvalues.

The number of PCs retained in a PCA model, $l$, can be estimated as its corresponding cumulative percentage variance (CPV) greater than a predetermined value $(80 \%$, for example) or using algorithms such as the L-curve method. Valle et al. [1999] proposed a new method of variance reconstruction error (VRE) to select the number of PCs and compared it with ten other methods, including Akaike information criterion (AIC), cross validation (based on the PRESS and $\mathrm{R}$ ratio), and $\mathrm{CPV}$, etc. Here the $\mathrm{CPV}$ is adopted for its simplicity and satisfactory performance. For more complicated applications, other advanced techniques, i.e. VRE, AIC, and cross validation, might be used.

Singular value decomposition (SVD) of the correlation matrix of $X$ can be easily used to obtain the PCs. This technique is briefly described below.

A singular value and corresponding singular vector of a matrix $X \quad(m \times n)$ are a scalar $\delta$ and a pair of vectors $u$ and $v$ that satisfy:

$$
X * v=\delta * u
$$

By comparison, an eigenvalue $\lambda$ and the corresponding eigenvector $e$ of an $n \times n$ square matrix $A=X^{T} X$ is defined by:

$$
A * e=\lambda * e
$$

The corresponding matrices of singular values of $X$ and the eigenvalues of $A$ are written as 


$$
\sum=\left|\begin{array}{cccc}
\delta_{1} & 0 & \ldots & 0 \\
0 & \delta_{2} & \ddots & \vdots \\
\vdots & \ddots & \ddots & 0 \\
\vdots & \ddots & \ddots & \delta_{n} \\
\vdots & \ddots & \ddots & 0 \\
0 & \ldots & \ldots & 0
\end{array}\right|, D=\left|\begin{array}{cccc}
\lambda_{1} & 0 & \cdots & 0 \\
0 & \lambda_{2} & \ddots & \vdots \\
\vdots & \ddots & \ddots & 0 \\
0 & \cdots & 0 & \lambda_{n}
\end{array}\right|
$$

The corresponding singular vectors form the columns of two orthogonal matrices $U$ and $V$, and the eigenvectors of the input matrix $X$ form the matrix $E$ :

$$
\begin{aligned}
& U=\left[u_{1}, u_{2}, \cdots, u_{m}\right] \\
& V=v_{1}, v_{2}, \cdots, v_{n} \\
& E=\left[e_{1}, e_{2}, \cdots, e_{n}\right]
\end{aligned}
$$

The matrices $X$ and $A$ are written as

$$
\begin{aligned}
& X=U^{*} \sum^{*} V^{T} \\
& A=E^{*} D^{*} E^{T}
\end{aligned}
$$

Since $U$ and $V$ are orthogonal matrices, Equation (7.6) becomes the singular value decomposition, and Equation (7.7) is the eigenvalue decomposition.

The singular value decomposition of an $m \times n$ matrix $X$ results in an $m \times m \quad U$ matrix, an $m \times n$ matrix $\sum$, and an $n \times n$ matrix $V$. The column vectors of $V$ are the principal components or eigenvectors of the input matrix $X$, and $V$ is the same as the matrix $E$.

Since

$$
\begin{gathered}
X^{T} X=U \sum V^{T}{ }^{T} U \sum V^{T}=V \sum^{T} U^{T} U \sum V^{T}=V \sum^{T} \sum V^{T} \\
A=X^{T} X=E D E^{T} \text { and } E=V
\end{gathered}
$$


Then

$$
D=\sum^{T} \sum
$$

Therefore, we can always get the same principal components for a matrix using either the SVD technique or through the eigenvalue decomposition of the covariance matrix.

In general, the eigenvalue decomposition is the appropriate tool for analyzing a matrix when it represents a mapping from a vector space onto itself, as it does for an ordinary differential equation. On the other hand, the singular value decomposition is appropriate for analyzing a mapping from one vector space onto another vector space, possibly with a different dimension.

\subsubsection{Fault Detection}

Fault detection can be performed by monitoring the change of the correlation structure of the measured data. Because the variation of data is separated in the PC subspace and RC subspace, two statistics, namely $T^{2}$ and $Q$, are defined to measure the variation in the two spaces, respectively. If a new observation exceeds the effective region in the PC space defined by the normal operation data, a change in operation regime can be detected. If a significant residual is observed in the RC space, a special event, either due to disturbance changes or due to changes in the relationship between variables, can be detected.

The $T^{2}$ statistic measures the variation in the PC subspace, which is defined as:

$$
T_{i}^{2}=t_{i} \lambda^{-1} t_{i}^{T}=x_{i} P \lambda^{-1} P^{T} x_{i}^{T} \leq \delta_{T}^{2}
$$

where $\lambda$ is a diagonal matrix of the first $l$ eigenvalues of the correlation matrix of $X$, which are associated with the PCs retained in the model. $t_{i}=x_{i} P$ is referred to as the principal score vector on the $i_{t h}$ eigenvector of the PCA model. $P$ is the set of PCs kept in the model and $x_{i}$ is the $i_{t h}$ observation. Here $\delta_{T}^{2}$ is the control (or confidence) limit of the $T^{2}$ statistic. 
The $T^{2}$ statistic may be oversensitive when some of the eigenvalues of the correlation matrix are close to zeros. Another limitation of the $T^{2}$ statistic arises from the assumption that the raw data follows multivariate normal distribution. The assumption is true only when the normal operation data are collected at one operating condition. However, the normal operation data are usually collected under different operating conditions for an engineering process where many operation modes are possible. Therefore, one shall be cautious when the $T^{2}$ statistic is used for process monitoring.

The statistic used in the RC subspace is the $Q$ statistic, or the squared prediction error (SPE), which is defined as:

$$
Q_{i}=e_{i} e_{i}^{T}=x_{i}\left(I-P P^{T}\right) x_{i}^{T} \leq \delta_{Q}^{2}
$$

where $\delta_{Q}^{2}$ is the control limit of the $Q$ index.

The $Q$ statistic quantifies the lack of fit between the sample and the model and denotes the distance of the sample from the nominal operation surface. Under the standard assumptions of a multivariate normal distribution for error matrix $E$, control limits at confidence level $\alpha$ may be obtained using the $\chi^{2}$ distribution as [Jackson and Mudholkar, 1979]:

$$
Q_{\alpha}=\Theta_{1}\left[\frac{c_{\alpha} \sqrt{2 \Theta_{2} h_{0}^{2}}}{\Theta_{1}}+1+\frac{\Theta_{2} h_{0}\left(h_{0}-1\right)}{\Theta_{1}^{2}}\right]^{1 / h_{0}},
$$

where

$$
\begin{gathered}
\Theta_{i}=\operatorname{trace}\left(\frac{E E^{T}}{I-1}\right)^{i} \text { for } i=1,2,3 \\
h_{0}=1-\frac{2 \Theta_{1} \Theta_{3}}{3 \Theta_{2}^{2}}
\end{gathered}
$$


And $c_{\alpha}$ is the standard normal deviate $(2.57$ for $\alpha=0.01)$. In the above equations, $i$ is the number of samples in the normal operation set and $\Theta_{i}$ is the sum of the $i_{\text {th }}$ power of the singular values of $E /(I-1)^{0.5}$. For normal operation, the $Q$ statistic would be very small. Detection of faults is accomplished as violations of the control limit, that is, when $Q$ exceeds $Q_{\alpha}$. Because the assumption that the residual vector follows multivariate normal distribution is much more relaxed than multivariate normal distribution of the original measured data, the $Q$ statistic is more appropriate than the $T^{2}$ statistic for process monitoring.

\subsubsection{Fault Isolation}

Building a model to characterize the relationships among the various measurements is an essential part of the FDI methodology. Patterns of the residuals that signify the mismatch between the model and the actual data most likely correspond to faults of a particular type. Specifically, different faults would cause the corresponding residuals to orient toward different directions. These various prediction error directions are referred as the fault directions, and the particular fault may be isolated as the one with maximum projection on the enumerated set of fault directions. The proposed fault isolation scheme is described as follows [Kaistha and Upadhyaya, 2001].

Let $F=f_{1} f_{2} f_{3} \ldots f_{R}$, where $f_{1} f_{2} f_{3} \ldots f_{R}$ are column vectors, denote the fault directions for the various fault scenarios that are observed in an existing database. These fault directions can be extracted from the historical data using clustering techniques. The fault direction $f_{i}$ in the fault matrix $F$ represents the direction in the residual space for the $i_{t h}$ fault such that the samples corresponding to the fault have the maximum projection on $f_{i}$. In other words, if $E_{i}$ denotes the residuals for samples corresponding to the $i_{\text {th }}$ fault, the optimization problem is

$$
J=\max _{f_{i}} f_{i}^{T} E_{i}^{T} E_{i} f_{i}
$$

subject to the constraint 


$$
f_{i}^{T} f_{i}=1
$$

Using the Lagranian multiplier and differentiating $J$ with respect to $f_{i}$ and setting the derivative to zero for maximization,

$$
2 E_{i}^{T} E_{i} f_{i}-2 \sigma f_{i}=0
$$

and

$$
E_{i}^{T} E_{i} f_{i}=\sigma f_{i}
$$

The fault direction $f_{i}$ is thus obtained as the first eigenvector of $E_{i}^{T} E_{i}$. The SVD technique may be used to obtain the eigenvector.

Once the fault matrix $F$ is properly defined, fault isolation is accomplished by calculating the projections onto $F$ and classifying the fault as the one with the maximum projection norm. A fault isolation index for the $i_{t h}$ fault is defined as

$$
F I_{i}=1-Q_{i} / Q
$$

where

$$
Q_{i}=e\left(I-f_{i} f_{i}^{T}\right)\left(I-f_{i} f_{i}^{T}\right) e^{T}
$$

In the above equations, $Q_{i}$ is the distance of the sample from the origin after subtracting the projection of the residuals on the fault direction $f_{i}$. It represents the sum of squares of residuals remaining after removing the contribution from the $i_{t h}$ fault direction. The fault isolation index quantifies the fraction of $Q$ that is due to $f_{i}$. When a particular type of fault scenario, the $j_{t h}$, occurs, the projection of the residuals on $f_{j}$ would be very high. In other 
words, $F I_{i}$ would be the largest and close to 1 for $i=j$, where $i$ varies from 1 to $R$. This results in the isolation of the fault from the various existing scenarios.

In historical databases, the number of fault scenarios and the correspondence of the fault samples to the particular fault scenarios are not known a priori. Classification of the residuals must then be used to establish the number of fault scenarios and also the correspondence of the samples to the particular scenarios. Existing classification algorithms [James, 1985] can be suitably adapted for this purpose.

\subsection{DG Model Development of a MSF System}

A MSF desalination process has been described in Chapter 4. Due to the large size of the MSF plant, it must be appropriately divided into sections before the DG models can be built. The structural decomposition is quite suitable for this case thanks to the similarities among stages. A directed graphical model is built for each stage, which only consists of its process variables. Each DG model is a qualitative model corresponding to a normal stage. It can be obtained either automatically by a computer program using the modular approach or by using the set of equations employed in the quantitative model. The first method builds a DG model by joining the common nodes of the corresponding sub-models (e.g. DGs of valves, pumps, etc). These sub-models are contained in a given model library. In this work, the second method is adopted. That is, DG models are obtained from the equations of the quantitative models, which were described in Chapter 4.

Figure 7.1 illustrates the input and output variables in the brine heater and the first flash stage of the MSF plant; and Table 7.1 lists the definitions of these variables. Figure 7.2 shows the DG model corresponding to the brine heater and the first stage. 


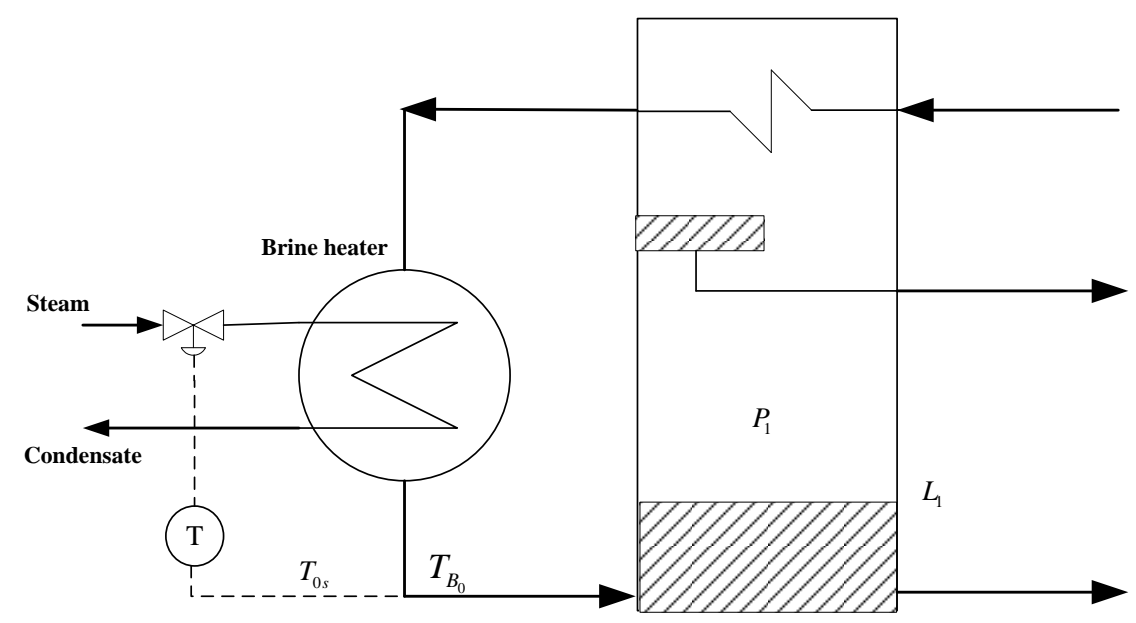

Figure 7.1. Brine heater section of a MSF plant.

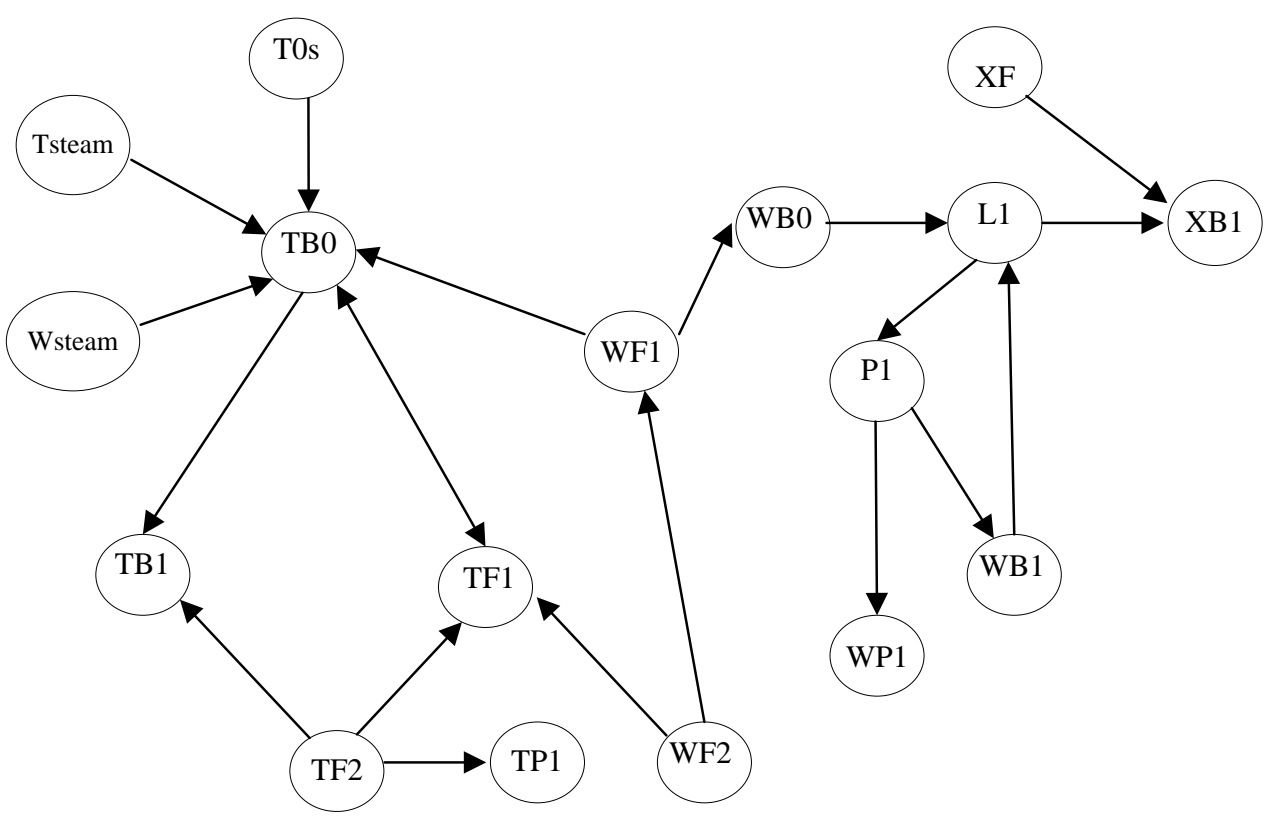

Figure 7.2. Directed graph of brine heater section. 
Table 7.1. DG model variables of brine heater

\begin{tabular}{|c|c|}
\hline Variable & Definition \\
\hline$T_{\text {STEAM }}$ & Inlet steam temperature \\
\hline$W_{\text {STEAM }}$ & Inlet steam flow rate \\
\hline$T_{B_{0}}$ & Top brine temperature \\
\hline$T_{0 s}$ & Top brine temperature set point \\
\hline$T_{B_{1}}$ & Flashing brine temperature exiting stage \#1 \\
\hline$T_{F_{1}}$ & Cooling brine temperature exiting stage \#1 \\
\hline$T_{F_{2}}$ & Cooling brine temperature exiting stage \#2 \\
\hline$T_{P_{1}}$ & Distillate product temperature exiting stage \#1 \\
\hline$W_{F_{1}}$ & Cooling brine flow exiting stage \#1 \\
\hline$W_{F_{2}}$ & Cooling brine flow exiting stage \#2 \\
\hline$W_{B_{0}}$ & Cooling brine flow exiting the brine heater \\
\hline$W_{B_{1}}$ & Flashing brine flow exiting stage \#1 \\
\hline$W_{P_{1}}$ & Distillate product flow exiting stage \#1 \\
\hline$X_{F}$ & Inlet cooling brine salinity \\
\hline$X_{B_{1}}$ & Flashing brine salinity exiting stage \#1 \\
\hline$L_{1}$ & Brine level in stage \#1 \\
\hline$P_{1}$ & Stage \#1 pressure \\
\hline
\end{tabular}


Figure 7.3 shows a flash stage with its input and output variables. The definitions of the variables are listed in Table 7.2. The DG model of a generic flash stage $i$ is illustrated in Figure 7.4 .

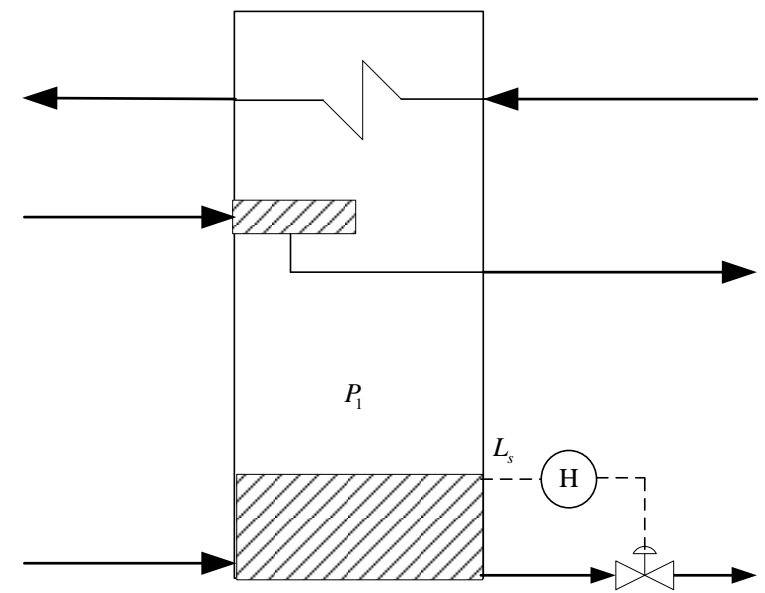

Figure 7.3. A generic flash stage of a MSF plant.

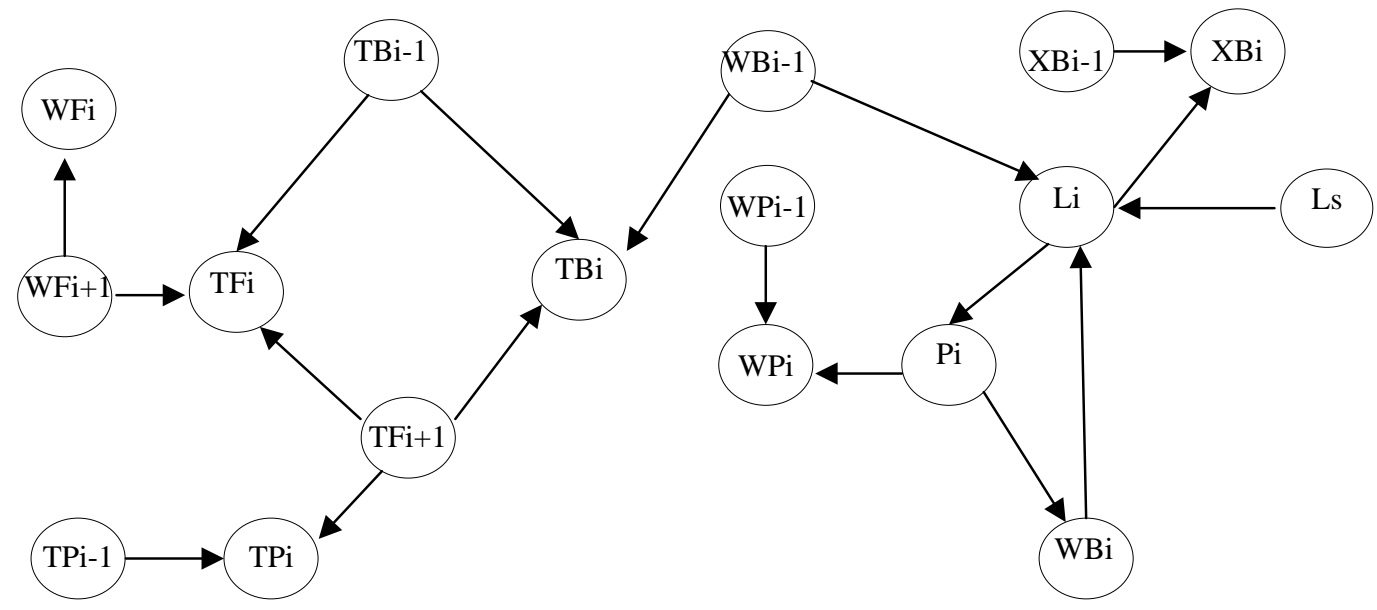

Figure 7.4. Directed graph of flash stage $i$. 
Table 7.2. DG model variables of flash stage $i$

\begin{tabular}{|c|c|}
\hline Variable & Definition \\
\hline$T_{F_{i}}$ & Cooling brine temperature exiting stage $i$ \\
\hline$T_{F_{i+1}}$ & Cooling brine temperature entering stage $i$ \\
\hline$T_{P_{i}}$ & Distillate product temperature exiting stage $i$ \\
\hline$T_{P_{i-1}}$ & Distillate product temperature entering stage $i$ \\
\hline$T_{B_{i}}$ & Flashing brine temperature exiting stage $i$ \\
\hline$T_{B_{i-1}}$ & Flashing brine temperature entering stage $i$ \\
\hline$W_{F_{i}}$ & Cooling brine flow exiting stage $i$ \\
\hline$W_{F_{i+1}}$ & Cooling brine flow entering stage $i$ \\
\hline$W_{P_{i}}$ & Distillate product flow exiting stage $i$ \\
\hline$W_{P_{i-1}}$ & Distillate product flow entering stage $i$ \\
\hline$W_{B_{i}}$ & Flashing brine flow exiting stage $i$ \\
\hline$W_{B_{i-1}}$ & Flashing brine flow entering stage $i$ \\
\hline$X_{B_{i}}$ & Flashing brine salinity exiting stage $i$ \\
\hline$X_{B_{i-1}}$ & Flashing brine salinity entering stage $i$ \\
\hline$L_{i}$ & Brine level in stage $i$ \\
\hline$L_{s}$ & Brine level set point \\
\hline$P_{i}$ & Stage $i$ pressure \\
\hline
\end{tabular}

For the sake of illustration, we shall employ a simplified MSF system with two flash stages and a brine heater to demonstrate the proposed sensor placement and fault diagnostic approaches. The DG model of this system is shown in Figure 7.5. The yellow $F$ nodes represent the root nodes of the system, each of which corresponds to a fault. The 27 measurements that are available are listed in Table 7.3. And Table 7.4 lists eight fault cases that need to be monitored. The fault diagnosis of the simplified MSF system takes into account the 
sensor faults, process faults, and controller faults. The four sensor faults are all sensor drifts; the heat transfer degradation faults of flash stages \#1 and \#2 are considered to be process anomalies in the MSF system. Changes in the set points of the top brine temperature (TBT) controller and the brine level controller of stage \#2 are identified as the two controller faults for the desalination process.

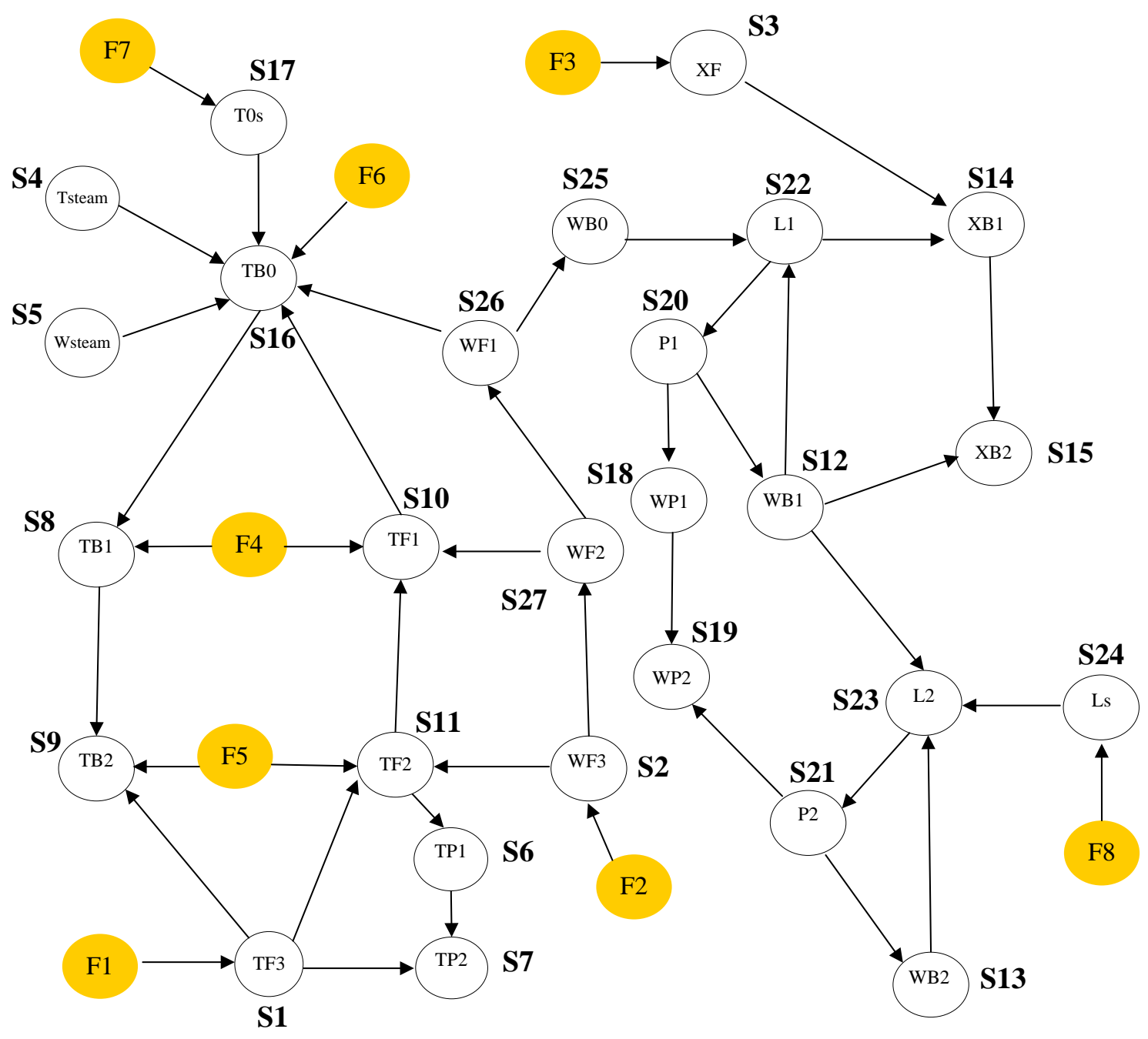

Figure 7.5. Directed graph of a two-stage MSF system. 
Table 7.3. Two-stage MSF system available variables

\begin{tabular}{|c|c|}
\hline Index & Description \\
\hline S1 & Inlet cooling brine temperature \\
\hline S2 & Inlet cooling brine flow \\
\hline S3 & Inlet cooling brine salinity \\
\hline S4 & Inlet steam temperature \\
\hline S5 & Inlet steam flow rate \\
\hline S6 & Distillate product temperature exiting stage \#1 \\
\hline S7 & Distillate product temperature exiting stage \#2 \\
\hline S8 & Flashing brine temperature exiting stage \#1 \\
\hline S9 & Flashing brine temperature exiting stage \#2 \\
\hline S10 & Cooling brine temperature exiting stage \#1 \\
\hline S11 & Cooling brine temperature exiting stage \#2 \\
\hline S12 & Flashing brine flow exiting stage \#1 \\
\hline S13 & Flashing brine flow exiting stage \#2 \\
\hline S14 & Flashing brine salinity exiting stage \#1 \\
\hline S15 & Flashing brine salinity exiting stage \#2 \\
\hline S16 & Top brine temperature \\
\hline S17 & Top brine temperature controller set point \\
\hline S18 & Distillate product flow exiting stage \#1 \\
\hline S19 & Distillate product flow exiting stage \#2 \\
\hline S20 & Stage \#1 pressure \\
\hline S21 & Stage \#2 pressure \\
\hline S22 & Brine level in stage \#1 \\
\hline S23 & Brine level in stage \#2 \\
\hline S24 & Stage \#2 brine level controller set point \\
\hline $\mathrm{S} 25$ & Cooling brine flow exiting the brine heater \\
\hline S26 & Cooling brine flow exiting stage \#1 \\
\hline S27 & Cooling brine flow exiting stage \#2 \\
\hline
\end{tabular}


Table 7.4. Faults considered for two-stage MSF system

\begin{tabular}{|c|c|}
\hline Fault Nodes & System Faults \\
\hline F1 & Inlet cooling brine temperature sensor drift \\
\hline F2 & Inlet cooling brine flow sensor drift \\
\hline F3 & Inlet cooling brine salinity sensor drift \\
\hline F4 & Flashing stage \#1 heat transfer degradation \\
\hline F5 & Flashing stage \#2 heat transfer degradation \\
\hline F6 & Top brine temperature sensor drift \\
\hline F7 & Top brine temperature controller fault \\
\hline F8 & Stage \#2 brine level controller fault \\
\hline
\end{tabular}

\subsection{Sensor Placement Design}

Both the greedy search algorithm and the LINGO solver are applied to find the minimum set of sensors required to observe all the eight faults listed in Table 7.4 for the two-stage MSF system. This is a set-covering problem that has been discussed in Chapter 6. This optimization problem can be formulated as follows:

$$
\operatorname{minimize}\left[\sum_{i=1}^{27} x_{i}\right]
$$

subject to

$$
\begin{aligned}
& \sum_{i=1}^{27} D_{i j} x_{i} \geq 1, j=1, \ldots, 8 \\
& x_{i} \in\{0,1\}, i=1, \ldots, 27
\end{aligned}
$$

Equation (7.17) is the objective function of the set-covering problem, where $x_{i}$ is the decision variable. Equation (7.18) is a constraint to ensure that each column (fault) is covered by at least one row (sensor location) where $D_{i j}$ is the $(i, j)_{t h}$ entry of the fault-sensor maximum connectivity matrix whose elements comprise of either ' 1 ' or ' 0 '. A row $i \quad(i=1, \ldots, m)$ covers a 
column $j(j=1, \ldots, n)$ if $D_{i j}=1$. Finally, Equation (7.19) allows the decision variables $x_{i}$ to only take binary numbers.

Both methods give nodes $\left[S_{9}, S_{15}, S_{16}, S_{23}\right]$ as the sensor set. Although all the faults can be detected, none of them, however, can be distinguished from each other.

To obtain the set of sensors that would give maximum resolution under single-fault assumption, additional virtual faults have to be created as discussed in Chapter 6. Sets $A$ are associated with the original faults. The virtual faults $B_{i j}=A_{i} \cup A_{j}-A_{i} \cap A_{j}$ are also constructed. This involves generation of $C_{8}^{2}=28$ virtual faults, so that the system now has 36 faults. Now each $B_{i j}$ is represented as a fault node, and a bipartite graph is constructed between these nodes and the sensor nodes. The new bipartite graph is added to the original bipartite graph, and the new optimization problem can be modified as follows:

$$
\operatorname{minimize}\left[\sum_{i=1}^{27} x_{i}\right]
$$

subject to

$$
\begin{gathered}
\sum_{i=1}^{27} D_{i j} x_{i} \geq 1, j=1, \ldots, 36 \\
x_{i} \in\{0,1\}, i=1, \ldots, 27
\end{gathered}
$$

The greedy search based heuristic presented for fault diagnostic observability criterion is applied to the new problem. This gives $\left[S_{1}, S_{2}, S_{3}, S_{8}, S_{9}, S_{10}, S_{11}, S_{12}, S_{15}, S_{16}, S_{17}, S_{23}, S_{24}\right]$ as the minimum sensor set for full isolation of the selected faults.

For the dual-fault case, solving the sensor location problem is done by assuming that two simultaneous faults can occur along with the possibility of the occurrence of a single fault. Under this assumption, the sets $A_{i j}=A_{i} \cup A_{j}$ are formed for all faults. Each new virtual fault is treated as a root node along with the original faults. The above greedy algorithm gives 
$\left[S_{1}, S_{2}, S_{3}, S_{8}, S_{9}, S_{10}, S_{11}, S_{12}, S_{15}, S_{16}, S_{17}, S_{23}, S_{24}\right]$ as the set of sensor nodes. An interesting point to note is that the minimum number of sensors for solving single-fault and dual-fault resolution problems turns out to be the same. This would mean that dual-fault resolution cannot be improved with adding more sensors. This is a result that is not obvious from the DG of the process.

\subsection{PCA Model Development of a MSF System}

In the previous section, the design problem of sensor network with maximum fault resolution using fault observability criterion has been presented. The greedy search algorithm

gives $\left[S_{1}, S_{2}, S_{3}, S_{8}, S_{9}, S_{10}, S_{11}, S_{12}, S_{15}, S_{16}, S_{17}, S_{23}, S_{24}\right]$ as the minimum sensor set for fully isolation of the identified faults. The advantage of using this optimized sensor set is that more information about the system is utilized, and some basic properties such as the fault detectability and identifiability are already partially guaranteed before PCA has been employed to monitor system behavior.

\subsubsection{Database Generation}

A normal operation database was generated using the developed two-stage MSF SIMULINK model. The TBT controller set point and the brine level controller set point in the last flashing stage (Stage \#2) were systematically changed one at a time. About 1,728 cases were simulated and the data generated were stored in a database. The list of the measured variables used to develop the PCA model is given in Table 7.5. 
Table 7.5. MSF variables used to develop PCA models

\begin{tabular}{|c|c|}
\hline Variable & Description \\
\hline 1 & Inlet cooling brine temperature (S1) \\
\hline 2 & Inlet cooling brine flow (S2) \\
\hline 3 & Inlet cooling brine salinity (S3) \\
\hline 4 & Flashing brine temperature exiting stage \#1 (S8) \\
\hline 5 & Cooling brine temperature exiting stage \#1 (S10) \\
\hline 6 & Cooling brine temperature exiting stage \#2 (S11) \\
\hline 7 & Flashing brine flow exiting stage \#1 (S12) \\
\hline 8 & Flashing brine salinity exiting stage \#2 (S15) \\
\hline 9 & Top brine temperature (S16) \\
\hline 10 & Top brine temperature controller set point (S17) \\
\hline 11 & Brine level in the stage \#2 (S23) \\
\hline 12 & Stage \#2 brine level controller set point (S24) \\
\hline 13 &
\end{tabular}

\subsubsection{PCA Modeling}

A PCA model is built using the data for the nominal operation case. The nominal operation data matrix is preprocessed by auto-scaling the columns in the data matrix to zero mean and unit variance. This puts all the measurements with their different units on a common unit variance scale. The percentage information explained by each PC is shown in Figure 7.6. Five principal components are retained, and they explain $\sim 99.9 \%$ of the total variation in the data, indicating the high degree of redundancy in the measurements. The $T^{2}$ and $Q$ statistics with 95\% confidence level for the samples in the fault-free database are plotted in Figure 7.7 and Figure 7.8, respectively. If the corresponding statistics exceeds the limit, the confidence to state that the fault free model cannot explain the data is at a level greater than $95 \%$. The two figures illustrate that all the fault free data are well below the limit lines. The probability of false alarms due to process disturbance is low. 


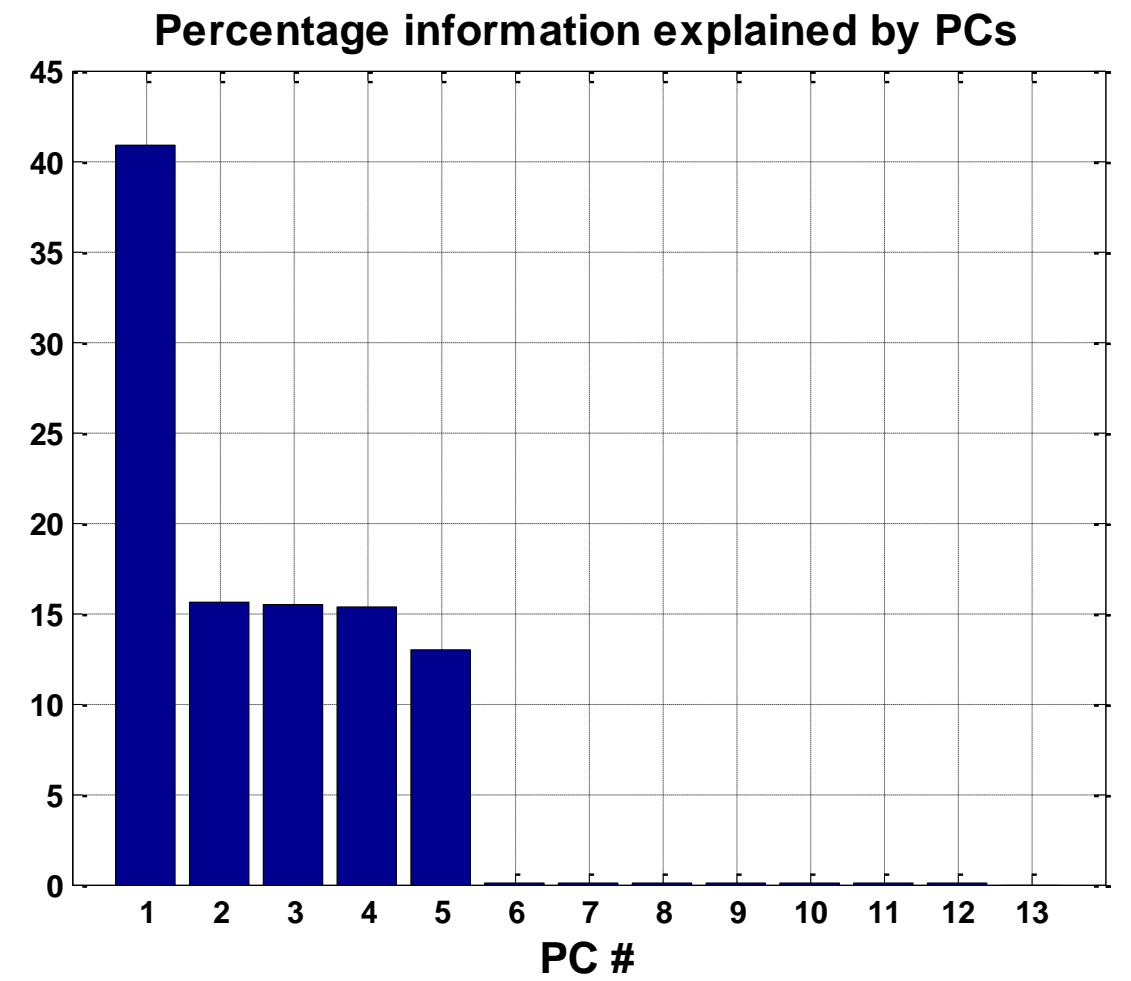

Figure 7.6. Percentage of variance explained by principal components.

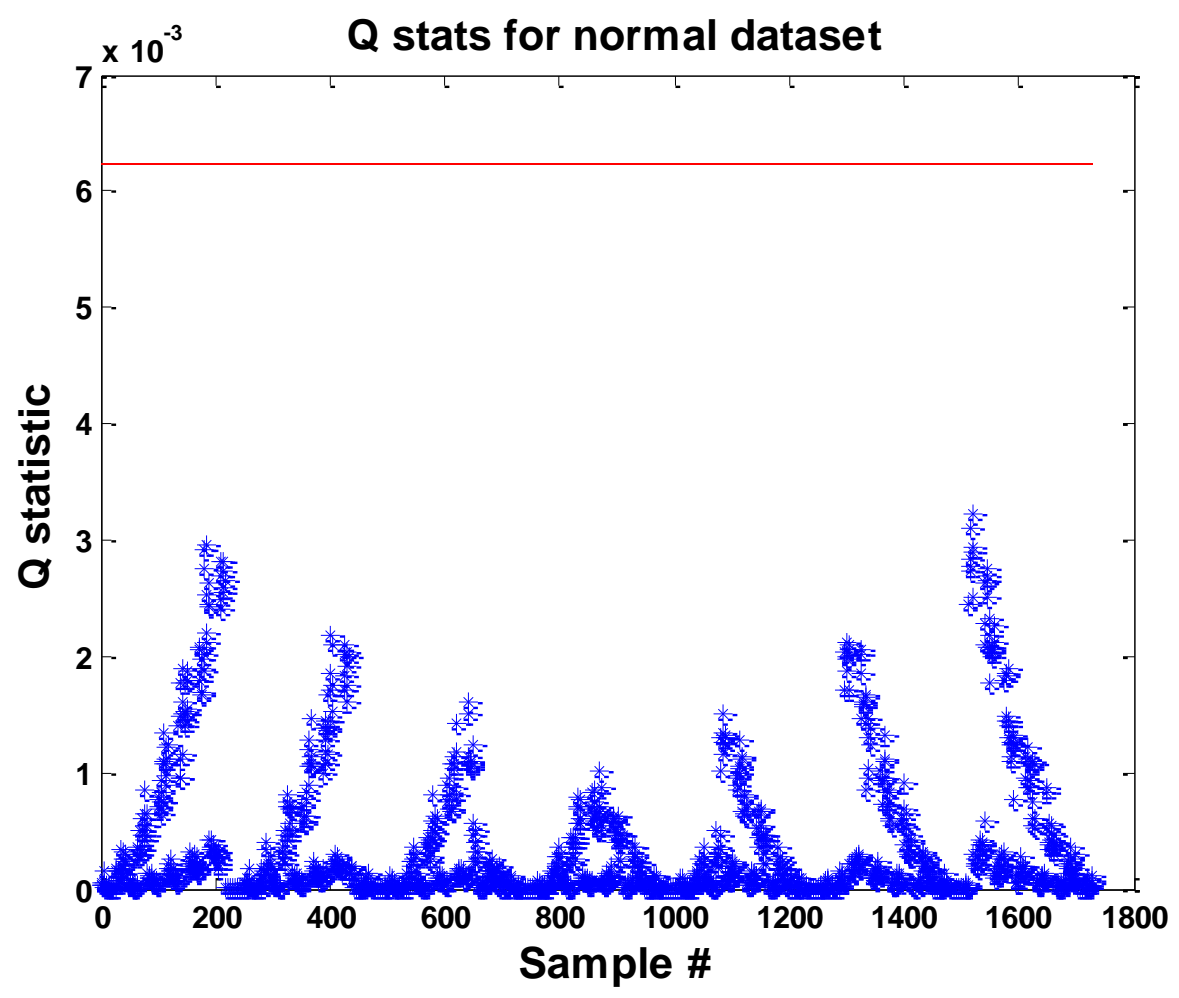

Figure 7.7. $Q$ statistic for MSF PCA model. 


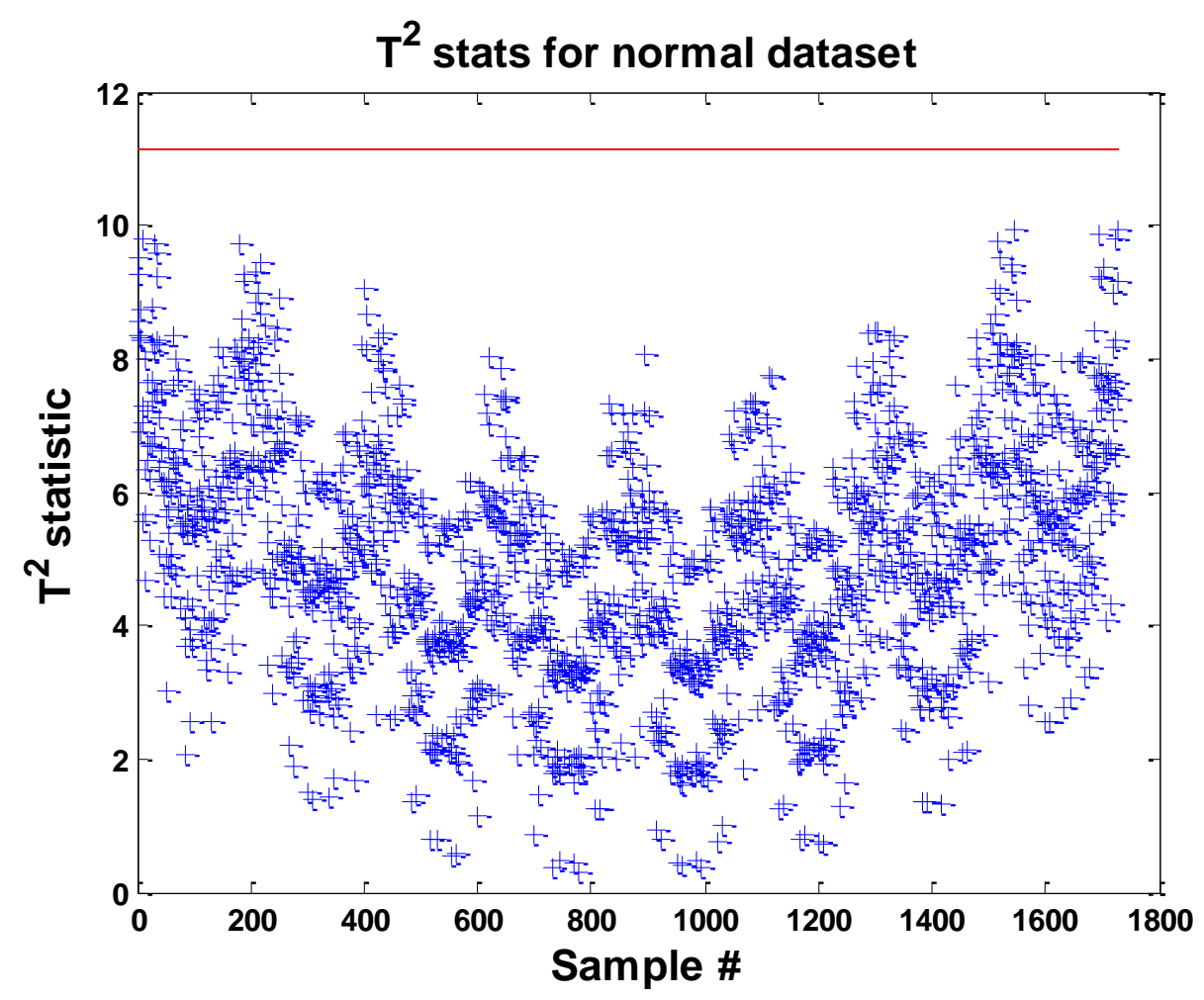

Figure 7.8. $T^{2}$ statistic for MSF PCA model. 


\subsection{Fault Detection Results}

Under normal conditions, small residuals would be generated and limited to a certain range. In the test cases when one or several components in the system are under degradation, the so-called causal relations among these variables will be violated. As a result, the mapping of residuals from residual generators or system models will increase in a specific direction. As an example, Figure 7.9 (a) shows the residual patterns from the PCA models when there is a drift of $0.2 \%$ to $1 \%$ nominal value in the inlet cooling brine temperature sensor (Fault \#1). The residuals are the differences between the measurement values and their PCA model predictions. Figure 7.9 (b) shows a significant increase in the $Q$ statistic of the PCA model exceeding the $95 \%$ confidence level. The $T^{2}$ statistic for the faulty conditions stays within the range, as in Figure 7.9 (c). This would be categorized as the fault scenario where the $Q$ statistic is outside the limits and the $T^{2}$ statistic is within the limits [Humberstone, 2010]. These illustrate the capability of data-driven models in detecting system anomalies. It should be kept in mind that both $T^{2}$ and $Q$ statistics must be used for fault detection. Either statistic being violated will signify that a fault has happened. Violation of the $T^{2}$ statistic represents that the system operates at an abnormal state beyond the model space, while departure of the $Q$ statistic represents that some of the constraint equations defined in the residual space are violated and the system is abnormal.

It is clear that the residuals reflect not only whether there is an abnormal component, but also the severity of the fault, which is very important in helping the operator or the automatic controller to select the correct strategy in order to avoid severe negative effect caused by faulty devices. The residual patterns for the other seven faults are shown in the following figures (Figure 7.10-7.16).

It should be noted that PCA can only deal with steady-state condition or a slow dynamic process. The algorithm to perform PCA based fault detection is only applicable to steady-state condition. The confidence level will affect the false alarm. In a real application, the confidence level needs to be adjusted according to the operation requirements. 

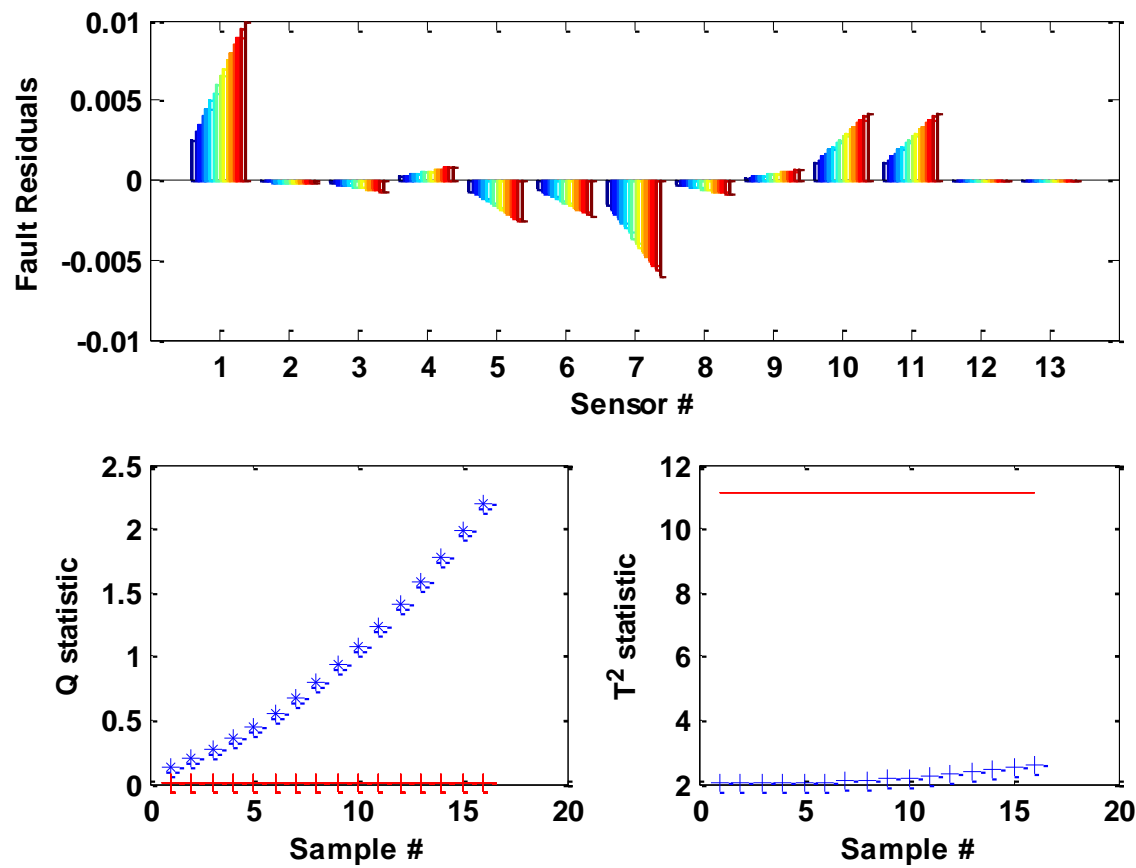

Figure 7.9. Residual pattern for inlet cooling brine temperature sensor drift (Fault \#1).
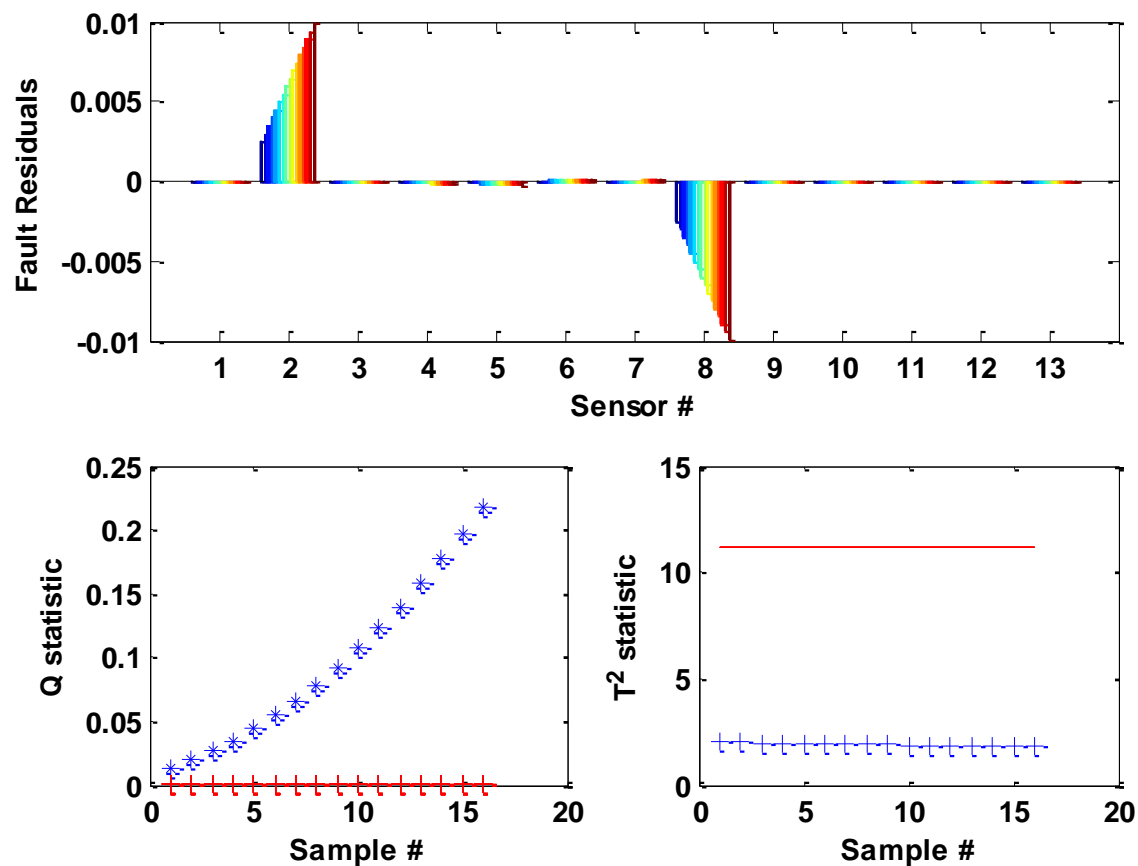

Figure 7.10. Residual pattern for inlet cooling brine flow sensor drift (Fault \#2). 

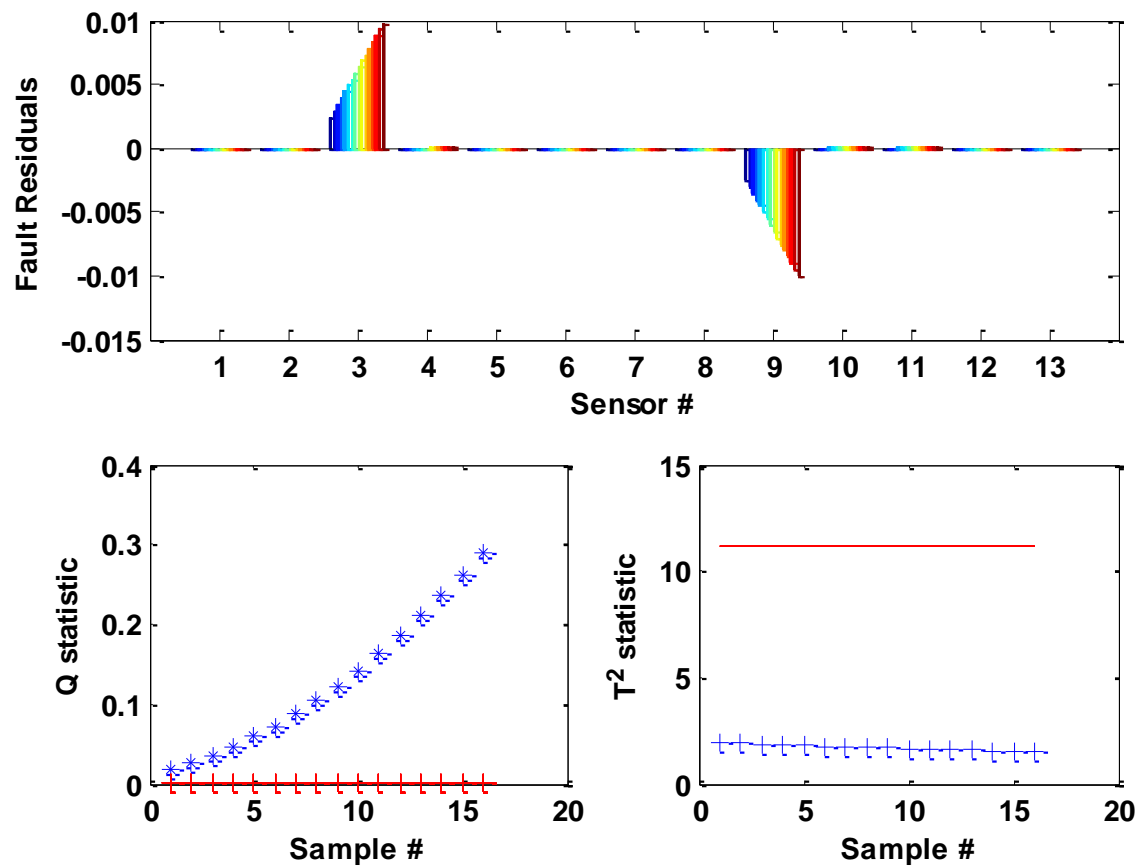

Figure 7.11. Residual pattern for inlet cooling brine salinity sensor drift (Fault \#3).
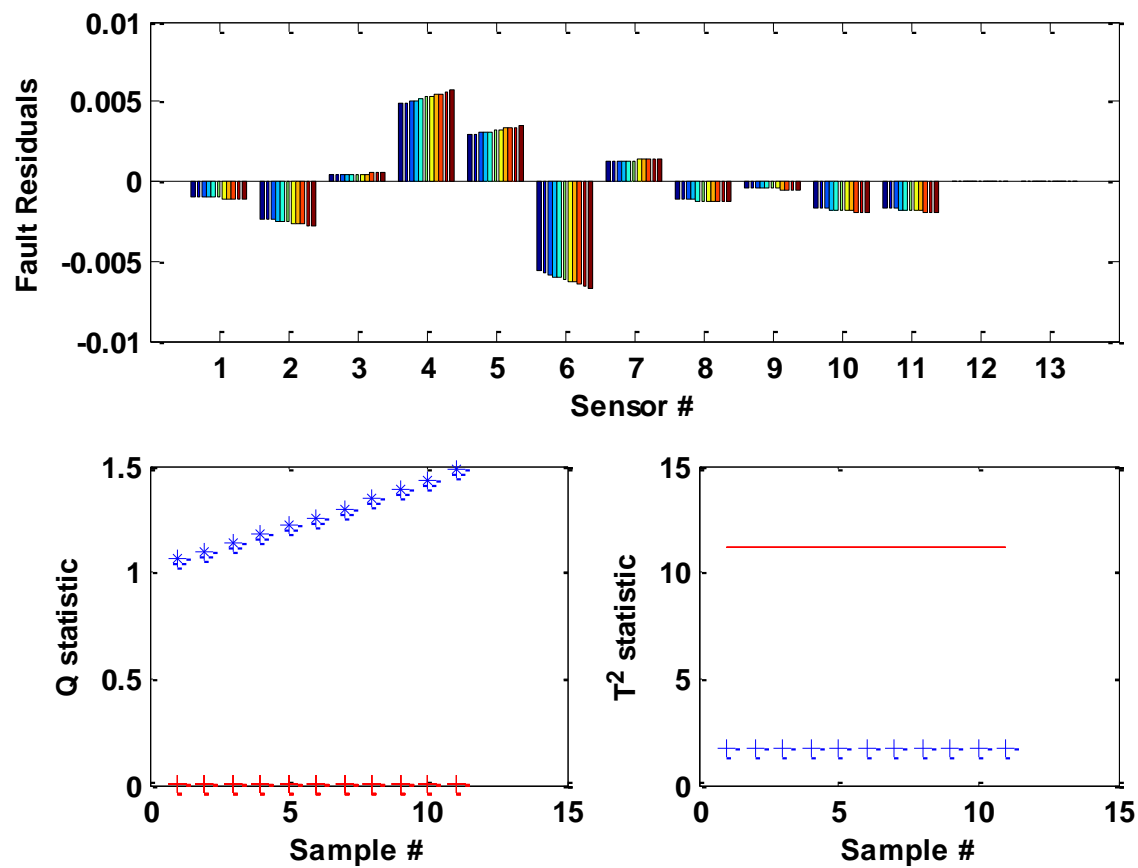

Figure 7.12. Residual pattern for flashing stage \#1 heat transfer degradation (Fault \#4). 

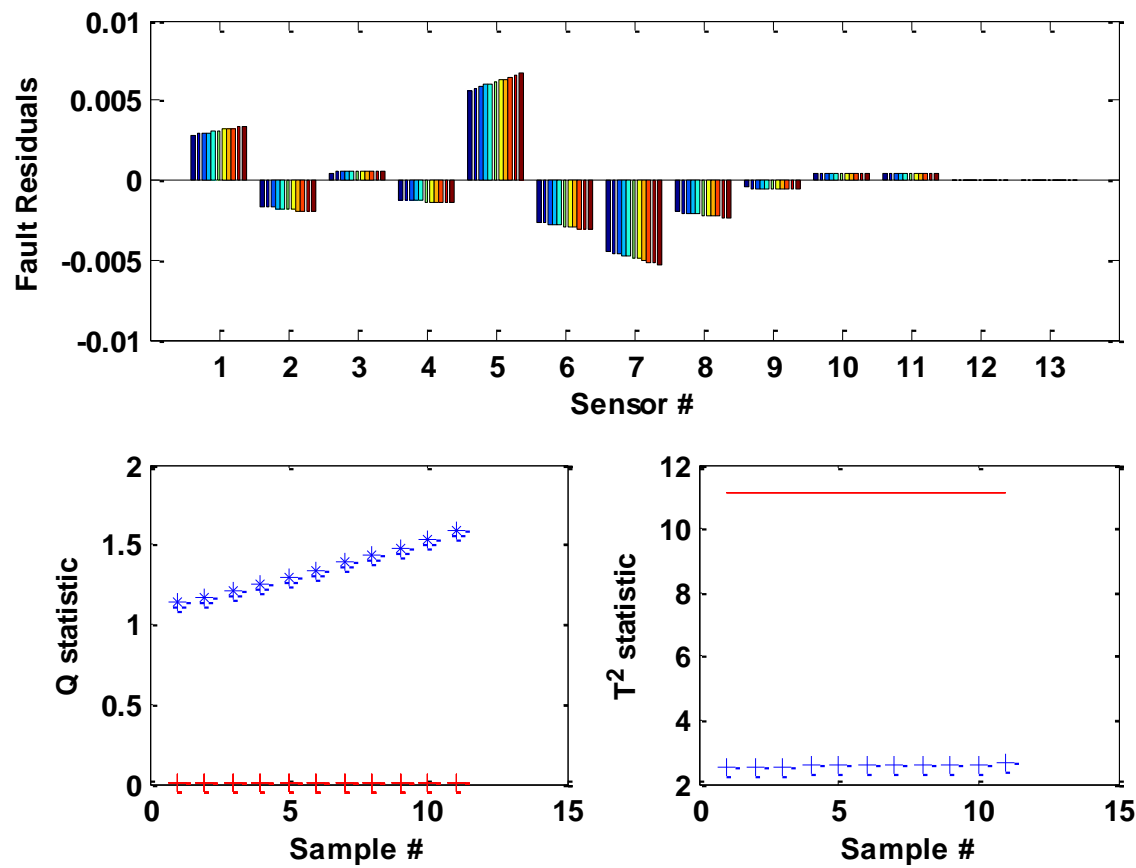

Figure 7.13. Residual pattern for flashing stage \#2 heat transfer degradation (Fault \#5).
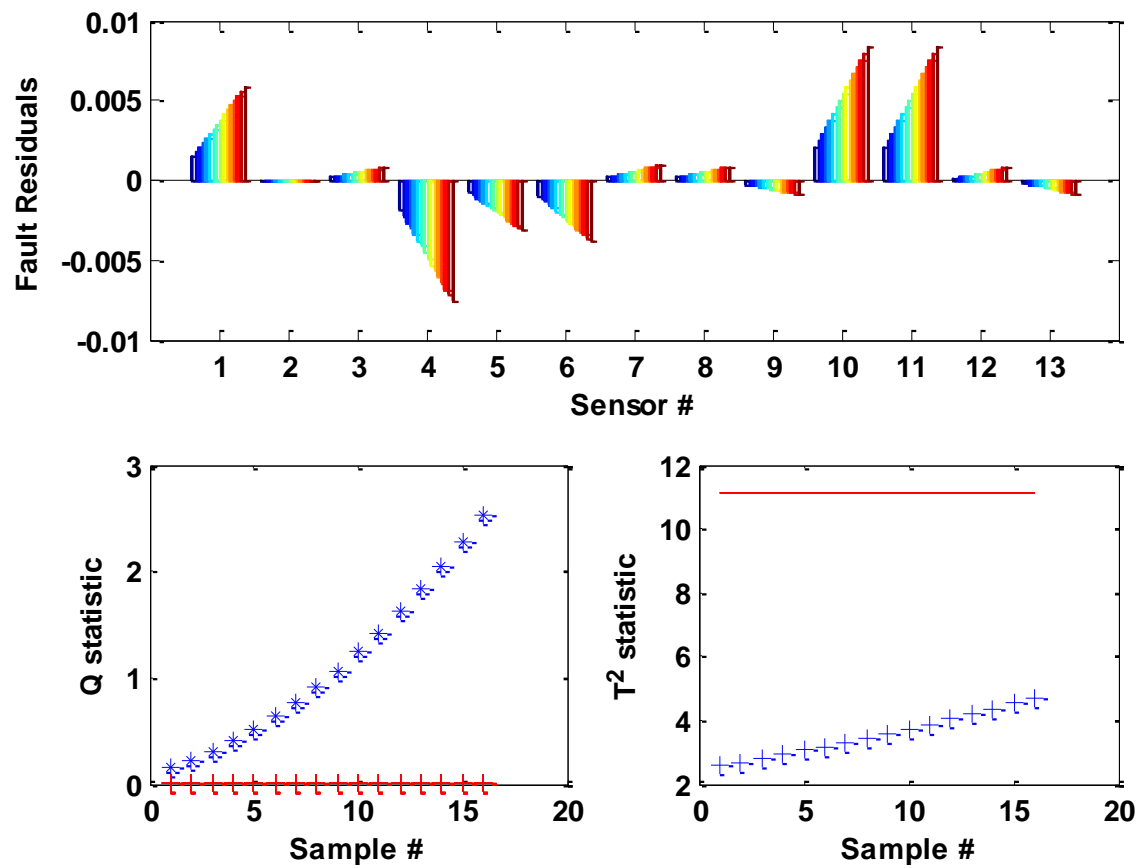

Figure 7.14. Residual pattern for top brine temperature sensor drift (Fault \#6). 

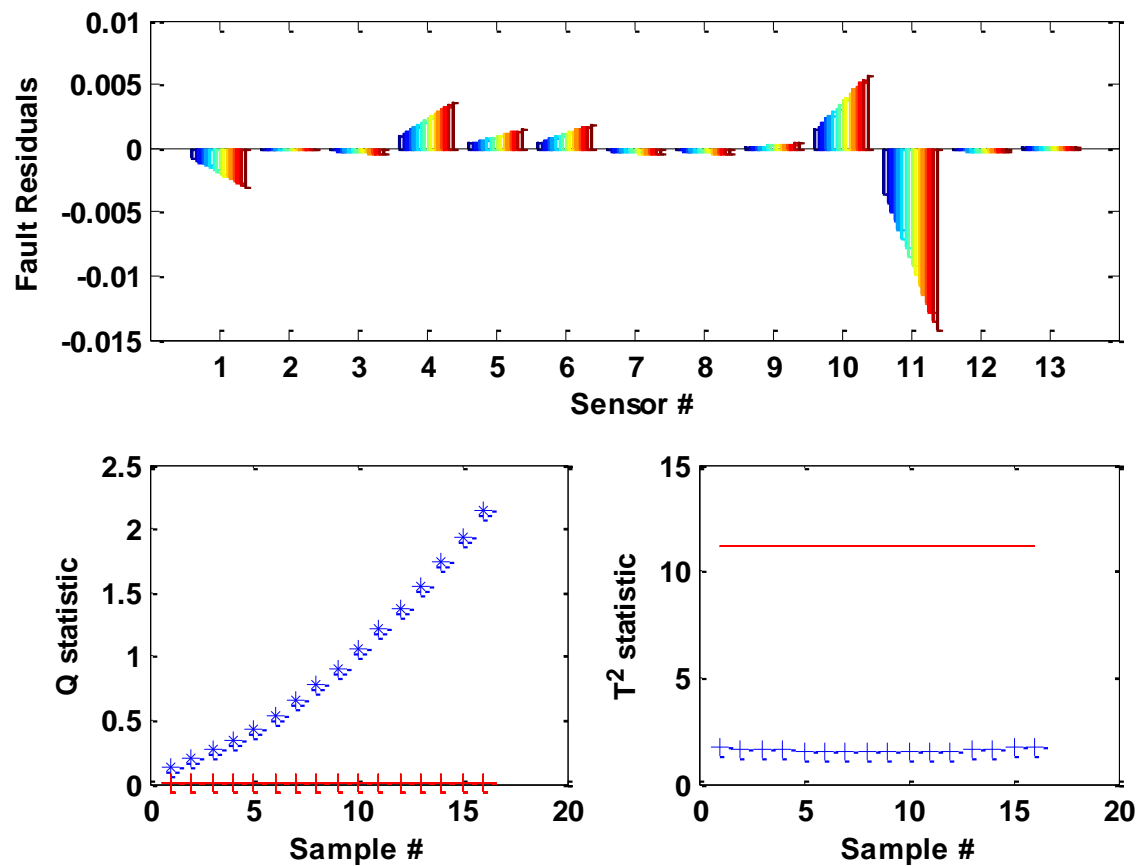

Figure 7.15. Residual pattern for top brine temperature controller fault (Fault \#7).
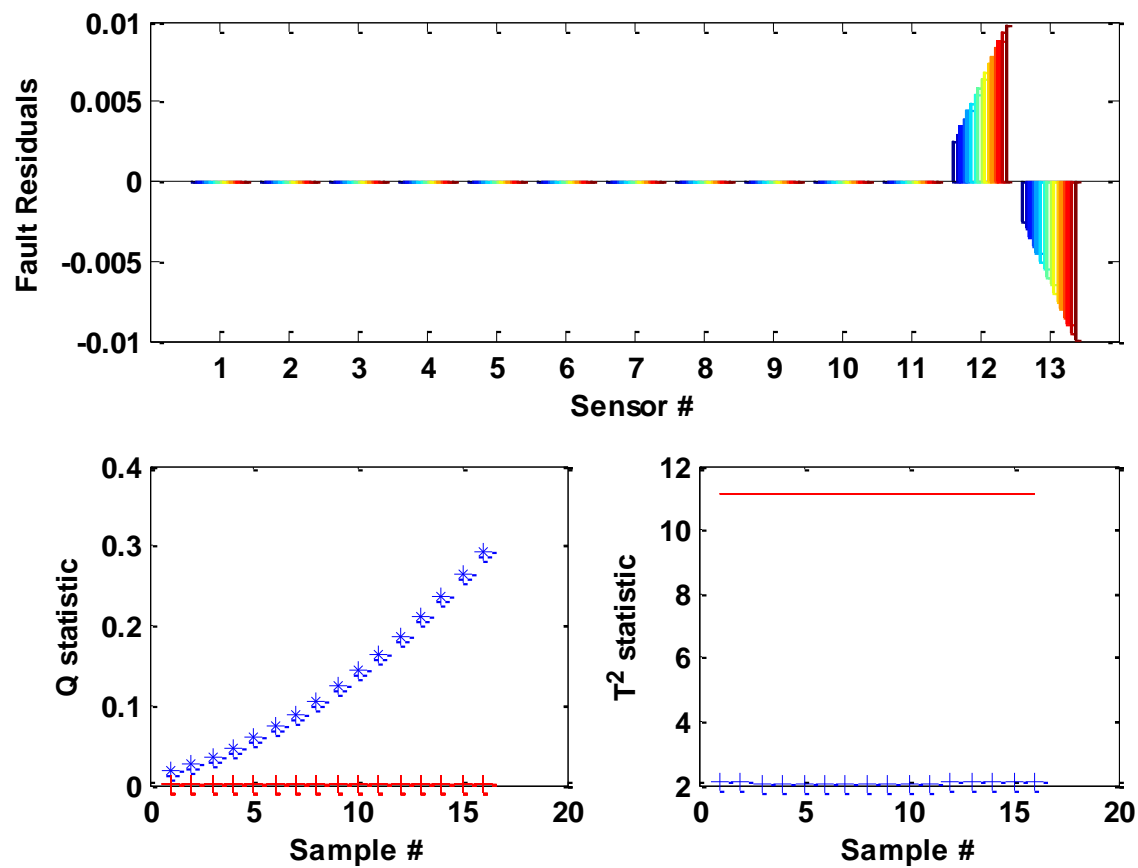

Figure 7.16. Residual pattern for Stage \#2 brine level controller fault (Fault \#8). 


\subsection{Fault Isolation Results}

Under normal conditions, small residuals would be generated and limited to a certain range. In the test cases when one or several components in the system are under degradation, the causal relations among the variables will be violated. As a result, the mapping of residuals from residual generators or system models will increase in a specific direction.

\subsubsection{Single-Fault Cases}

The PCA fault direction analysis is performed through processing the fault residuals. The eight fault directions which correspond to the eight fault scenarios considered for the MSF system are given in Table 7.6.

Table 7.6. PCA fault direction analysis for MSF system under single fault assumption

\begin{tabular}{|c|c|c|}
\hline Fault direction \# & System faults & $\begin{array}{c}\text { o residual variances explained } \\
\text { by the 1st PC }\end{array}$ \\
\hline 1 & Inlet cooling brine temperature sensor drift & $92.56 \%$ \\
\hline 2 & Inlet cooling brine flow sensor drift & $88.29 \%$ \\
\hline 3 & Inlet cooling brine salinity sensor drift & $94.45 \%$ \\
\hline 4 & Flashing stage \#1 heat transfer degradation & $93.68 \%$ \\
\hline 5 & Flashing stage \#2 heat transfer degradation & $89.47 \%$ \\
\hline 6 & Top brine temperature sensor drift & $86.44 \%$ \\
\hline 7 & Top brine temperature controller fault & $87.96 \%$ \\
\hline 8 & Stage \#2 brine level controller fault & \\
\hline
\end{tabular}

The following plots (Figures 7.17 - 7.24) illustrate the fault isolation indices for the eight fault cases. For instance, Figure 7.17 shows the fault isolation index for the inlet cooling brine temperature sensor drift (Fault \#1) scenario in the MSF system. Note that the fault index is close to unity for the particular fault that occurs and is smaller for the others. 


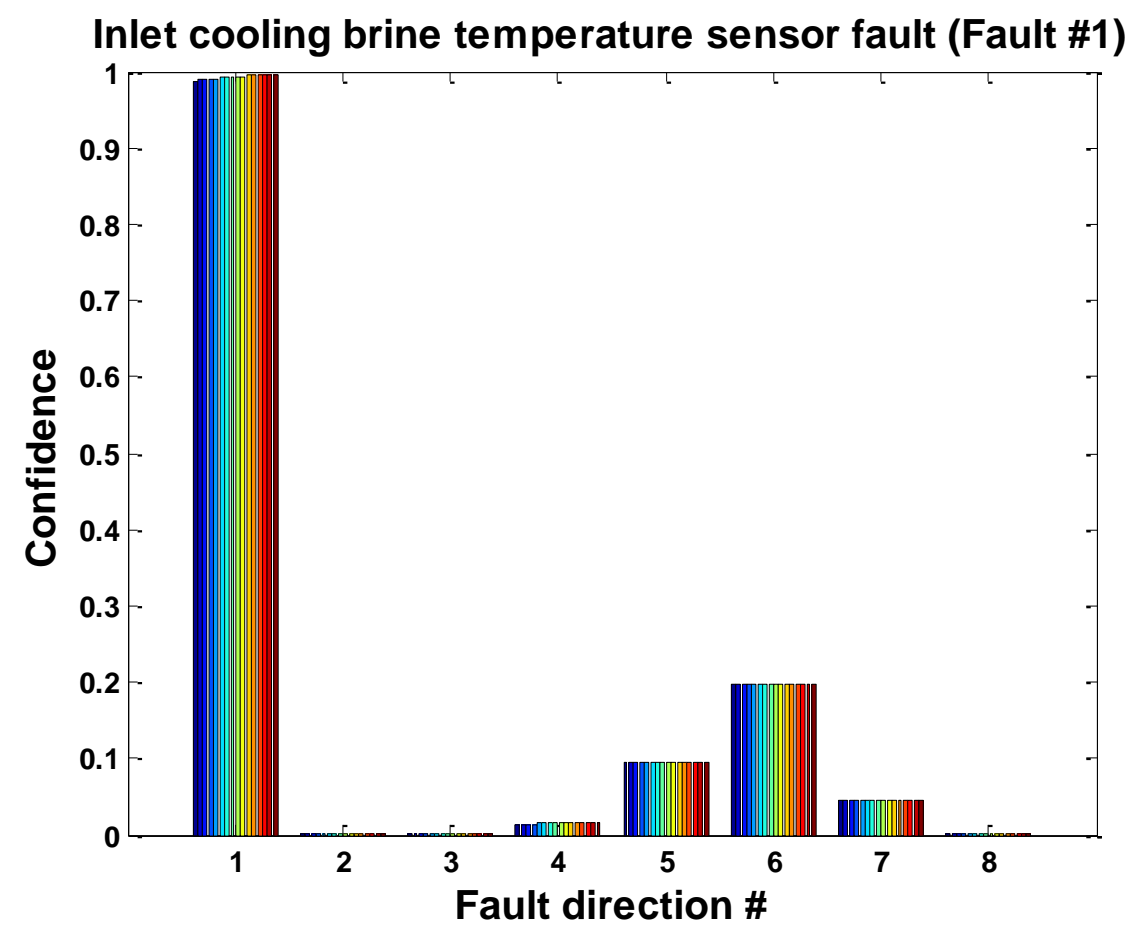

Figure 7.17. Fault isolation index for inlet cooling brine temperature sensor drift.

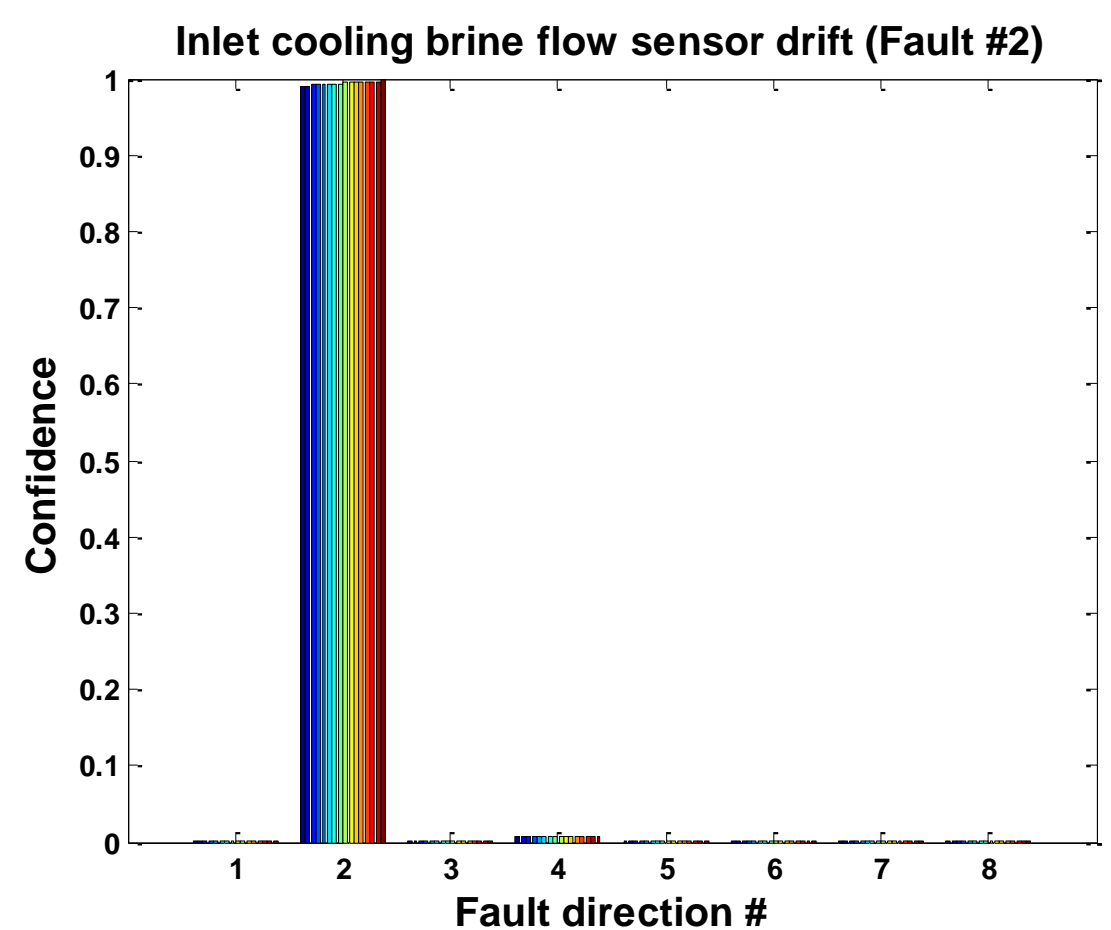

Figure 7.18. Fault isolation index for inlet cooling brine flow sensor drift. 


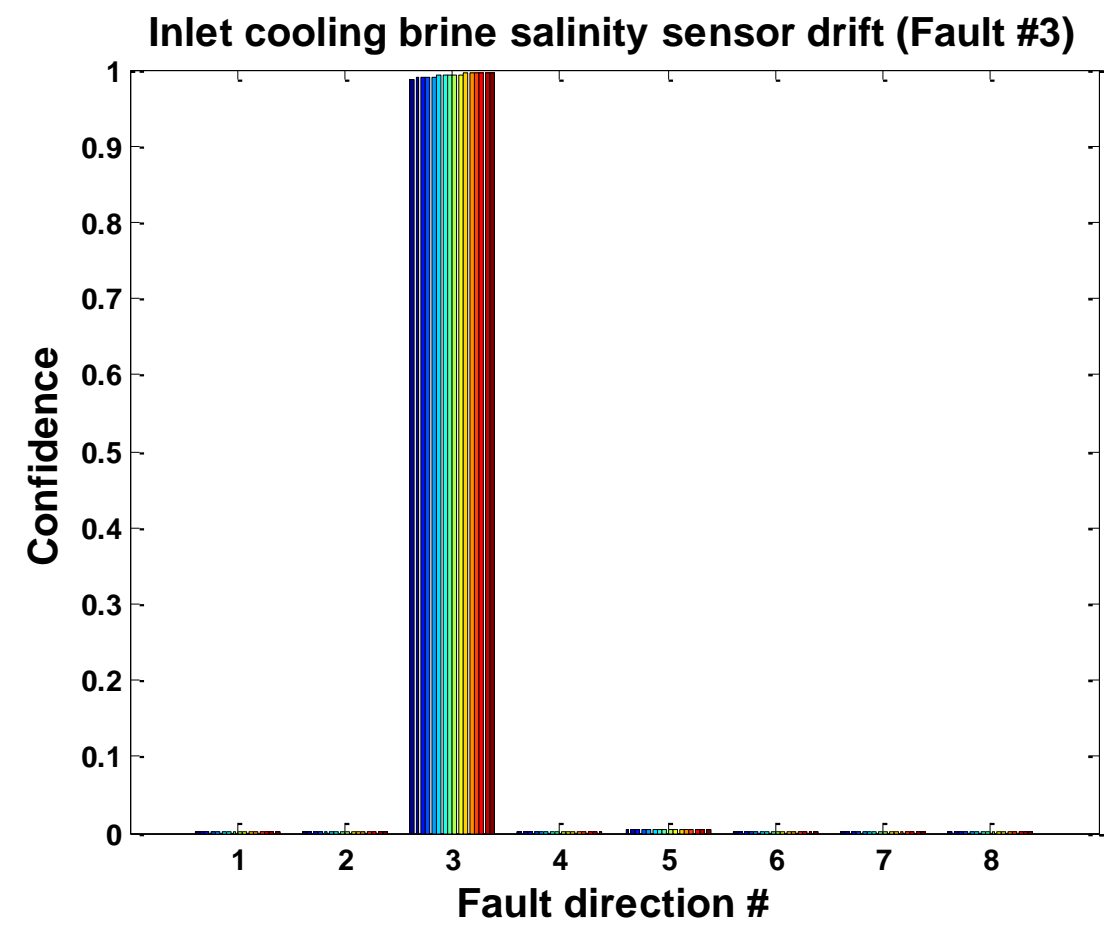

Figure 7.19. Fault isolation index for inlet cooling brine salinity sensor drift.

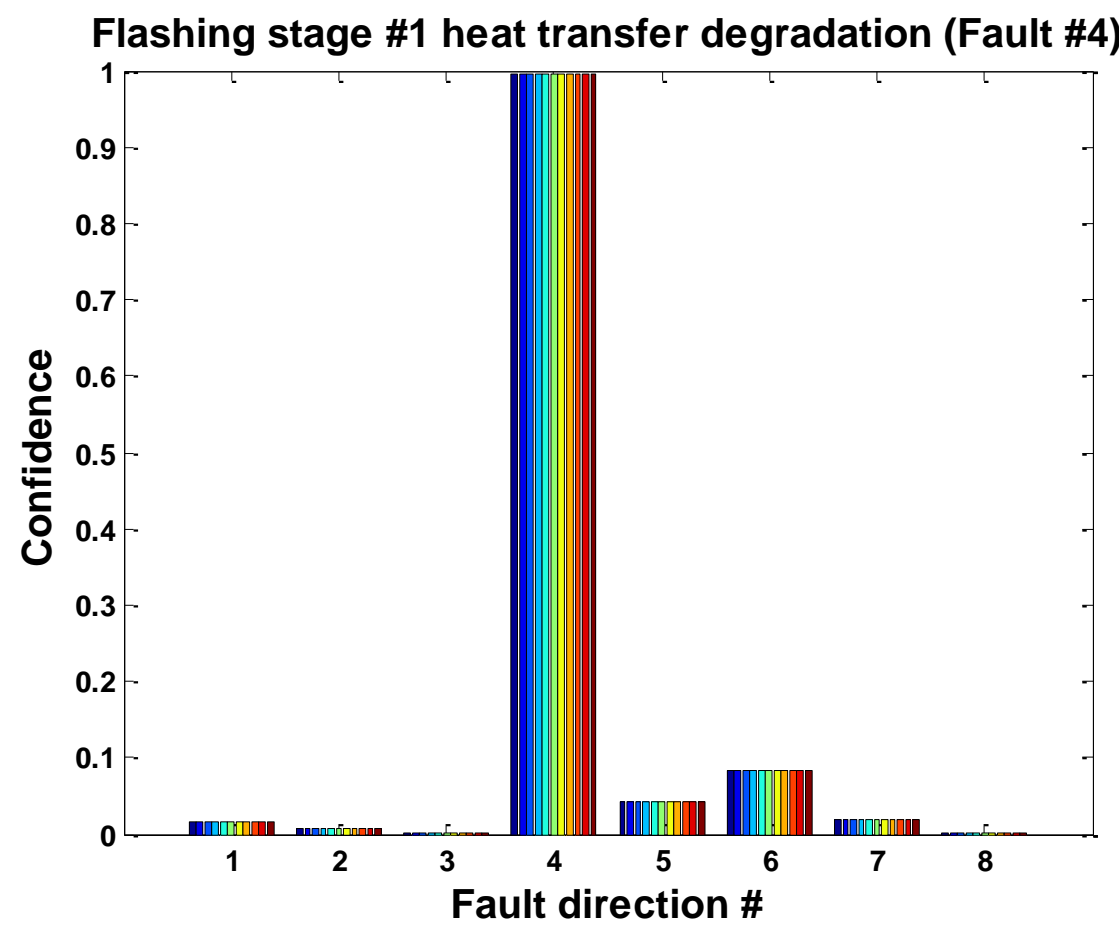

Figure 7.20. Fault isolation index for flashing stage \#1 heat transfer degradation. 


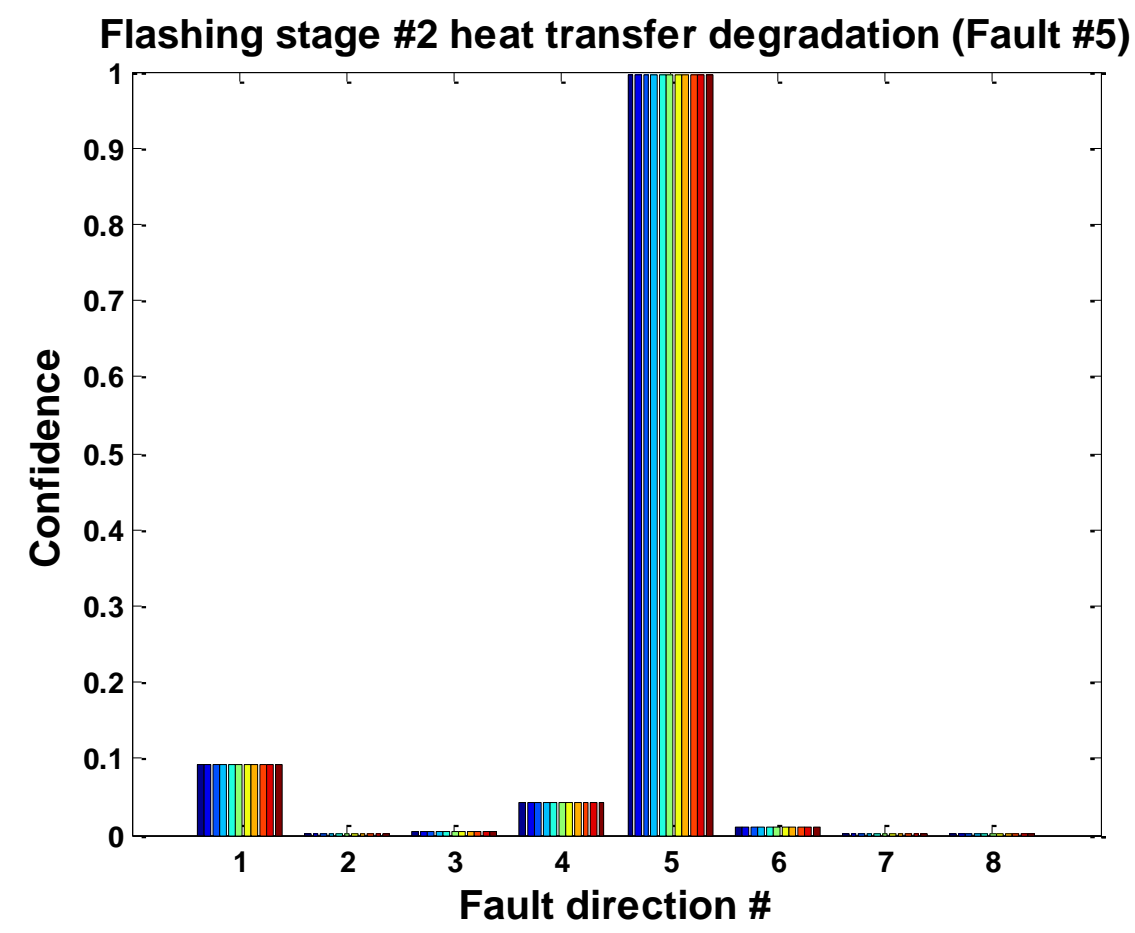

Figure 7.21. Fault isolation index for flashing stage \#2 heat transfer degradation.

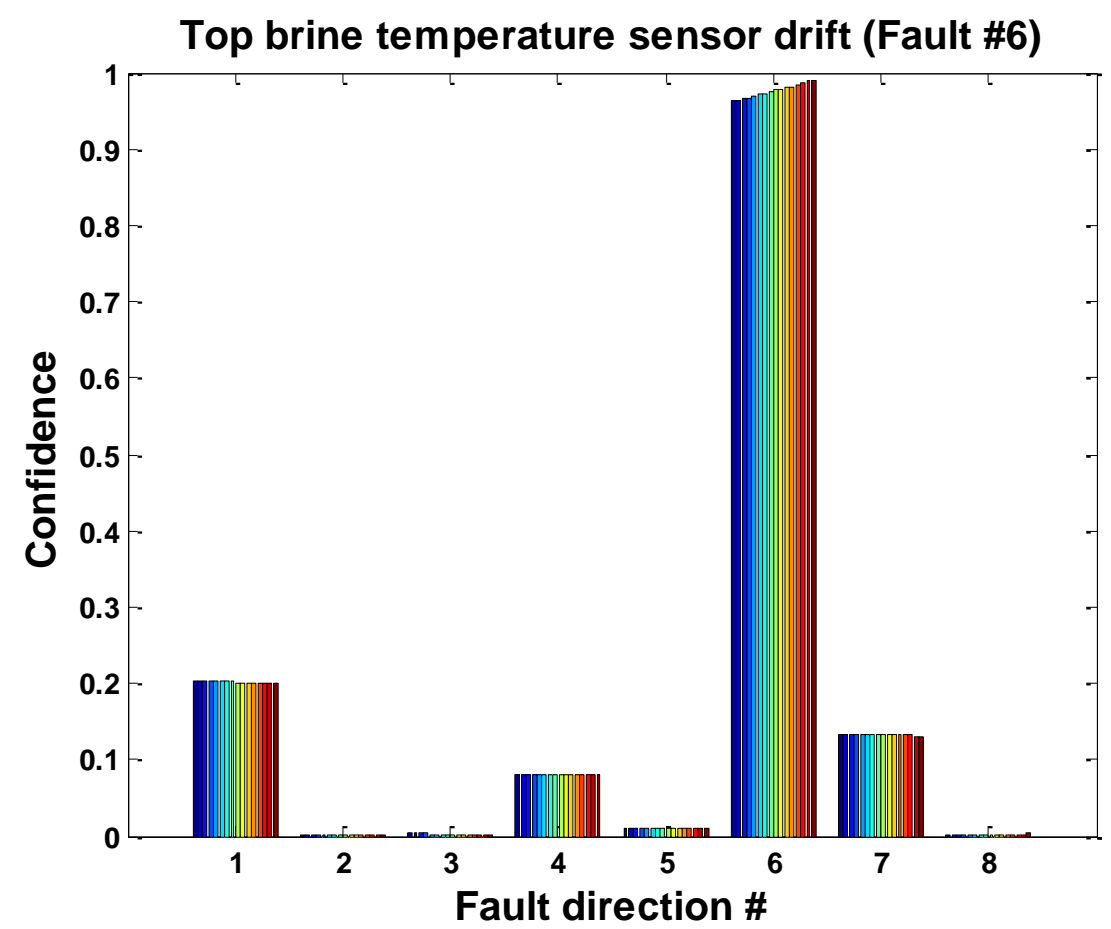

Figure 7.22. Fault isolation index for top brine temperature sensor drift. 


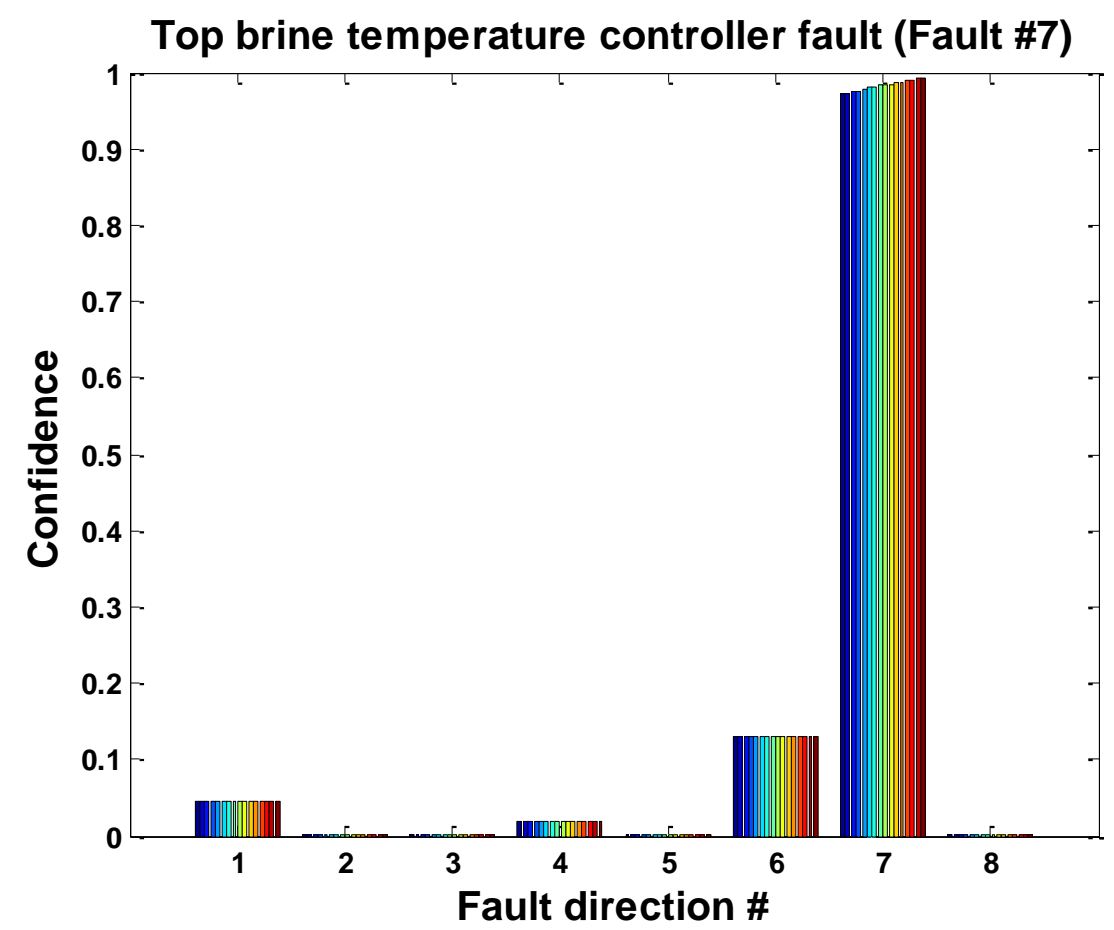

Figure 7.23. Fault isolation index for top brine temperature controller fault.

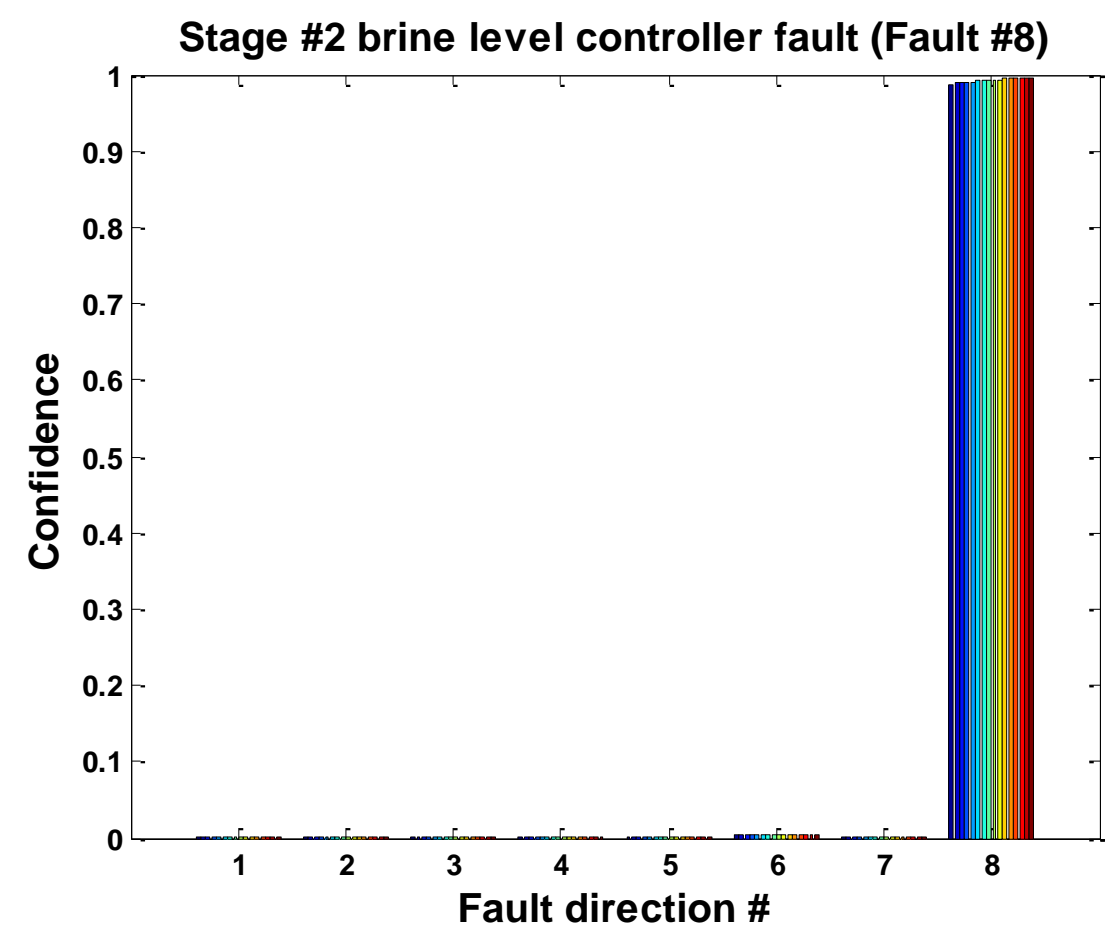

Figure 7.24. Fault isolation index for Stage \#2 brine level controller fault. 
As can be seen from the above figures, all fault cases are diagnosed correctly using the proposed fault isolation approach. Thus, PCA provides a convenient method in dealing with directional residuals. The important assumption that should be emphasized is that the faulty residuals are projected onto the first eigenvector of the residual covariance matrix without losing the direction. In other words, the first PC provides most of the information about the faulty device. However, we shall be cautious about this assumption since it is not always satisfied, especially at the beginning of a transient. Nonetheless, the PCA algorithm demonstrates its effectiveness for fault detection and isolation in complex systems with the optimized sensor allocation, and therefore deserves further study.

\subsubsection{Dual-Fault Cases}

The PCA based directional approach can be extended to detect and isolate multi-dimensional faults such as simultaneous multiple faults. The application to the two-stage MSF system is presented in this section. It is assumed that the possible simultaneous faults are limited to dual faults for all the considered faults in the system. The previous work that is publicly available on simultaneous multiple fault detection is rather limited [Watanabe, 1994]. The research results presented in this section are, therefore, original contributions to the area of fault diagnosis.

The same sensor set is applied to detect and isolate the dual faults. As in the single fault cases, the following eight fault directions which correspond to the eight fault scenarios are defined in the designed FDI scheme:

Single fault direction 1: Inlet cooling brine temperature sensor drifting fault

Single fault direction 2: Inlet cooling brine flow sensor drifting fault

Single fault direction 3: Inlet cooling brine salinity sensor drifting fault

Single fault direction 4: Flashing stage \#1 heat transfer degradation

Single fault direction 5: Flashing stage \#2 heat transfer degradation

Single fault direction 6: Top brine temperature sensor drifting fault

Single fault direction 7: Top brine temperature controller fault

Single fault direction 8: Stage \#2 brine level controller fault

The additional dual-fault directions are considered as well: 
Dual fault direction 1: Inlet cooling brine temperature sensor drifting fault \& Inlet cooling brine flow sensor drifting fault

Dual fault direction 2: Inlet cooling brine temperature sensor drifting fault \& Inlet cooling brine salinity sensor drifting fault

Dual fault direction 3: Inlet cooling brine temperature sensor drifting fault \& Flashing stage \#1 heat transfer degradation

Dual fault direction 4: Inlet cooling brine temperature sensor drifting fault \& Flashing stage \#2 heat transfer degradation

Dual fault direction 5: Inlet cooling brine temperature sensor drifting fault \& Top brine temperature sensor drifting fault

Dual fault direction 6: Inlet cooling brine temperature sensor drifting fault \& Top brine temperature controller fault

Dual fault direction 7: Inlet cooling brine temperature sensor drifting fault \& Stage \#2 brine level controller fault

Dual fault direction 8: Inlet cooling brine flow sensor drifting fault \& Inlet cooling brine salinity sensor drifting fault

Dual fault direction 9: Inlet cooling brine flow sensor drifting fault \& Flashing stage \#1 heat transfer degradation

Dual fault direction 10: Inlet cooling brine flow sensor drifting fault \& Flashing stage \#2 heat transfer degradation

Dual fault direction 11: Inlet cooling brine flow sensor drifting fault \& Top brine temperature sensor drifting fault

Dual fault direction 12: Inlet cooling brine flow sensor drifting fault \& Top brine temperature controller fault

Dual fault direction 13: Inlet cooling brine flow sensor drifting fault \& Stage \#2 brine level controller fault

Dual fault direction 14: Inlet cooling brine salinity sensor drifting fault \& Flashing stage \#1 heat transfer degradation

Dual fault direction 15: Inlet cooling brine salinity sensor drifting fault \& Flashing stage \#2 heat transfer degradation 
Dual fault direction 16: Inlet cooling brine salinity sensor drifting fault \& Top brine temperature sensor drifting fault

Dual fault direction 17: Inlet cooling brine salinity sensor drifting fault \& Top brine temperature controller fault

Dual fault direction 18: Inlet cooling brine salinity sensor drifting fault \& Stage \#2 brine level controller fault

Dual fault direction 19: Flashing stage \#1 heat transfer degradation \& Flashing stage \#2 heat transfer degradation

Dual fault direction 20: Flashing stage \#1 heat transfer degradation \& Top brine temperature sensor drifting fault

Dual fault direction 21: Flashing stage \#1 heat transfer degradation \& Top brine temperature controller fault

Dual fault direction 22: Flashing stage \#1 heat transfer degradation \& Stage \#2 brine level controller fault

Dual fault direction 23: Flashing stage \#2 heat transfer degradation \& Top brine temperature sensor drifting fault

Dual fault direction 24: Flashing stage \#2 heat transfer degradation \& Top brine temperature controller fault

Dual fault direction 25: Flashing stage \#2 heat transfer degradation \& Stage \#2 brine level controller fault

Dual fault direction 26: Top brine temperature sensor drifting fault \& Top brine temperature controller fault

Dual fault direction 27: Top brine temperature sensor drifting fault \& Stage \#2 brine level controller fault

Dual fault direction 28: Top brine temperature controller fault \& Stage \#2 brine level controller fault

Both single-fault directions and dual-fault directions are used as fault signature for dual fault isolation. Vector projection is introduced for isolating different dual faults. In this method, the PCA is used for fault signature collection from the samples corresponding to the different single-fault and dual-fault scenarios. Through the clustering techniques described in the previous sections, the first eigenvector of faulty residuals is obtained as the fault direction in the residual 
space. Finally, the new faulty samples are projected onto each fault direction. If a fault is of a particular type, the projection of the residuals of that fault direction would be higher than those of other directions. In case of a dual-fault scenario occurs, the samples are expected to have large projections on the both single-fault directions in the residual space for the two faults involved. As far as the dual-fault direction is concerned, we expect to see the maximum projection of the faulty sample residuals on the corresponding dual-fault direction.

Figures 7.25 - 7.31 show the plots of the fault directions of the measurements for the first seven dual-fault cases, all of which involve the inlet cooling brine temperature sensor drifting fault. As can be seen, the directional signatures of the studied dual-faults are around 0.2 0.4 when they are projected onto the 8 single-fault directions, as opposed to the much higher values shown in the previous section for the single-fault cases. This is expected due to the difference between single-fault and dual-fault scenarios. Nevertheless, the single-fault directional signatures for the dual-faults are still distinct enough to provide an initial idea of fault isolation. The bottom plots of Figure 7.25-7.31, which are the plots of the residual projections onto the 28 dual-fault directions, clearly illustrate the maximum projection of around one on the corresponding fault direction for each of the seven studied dual-fault cases. All fault cases are diagnosed correctly using the developed fault isolation approach. A caveat with the developed PCA fault diagnostic method is the faults considered for the system of interest must be defined beforehand. In the event of undefined faults occurring in the system, one may be able to observe the faults using the fault directional approach, but the clear fault isolation may not be achievable with the existing fault directions. These faults, if properly identified later, may be included in the fault set.

The fault isolation results for the remaining dual-fault cases are given in Appendix C. 
Fault \#1 \& Fault \#2
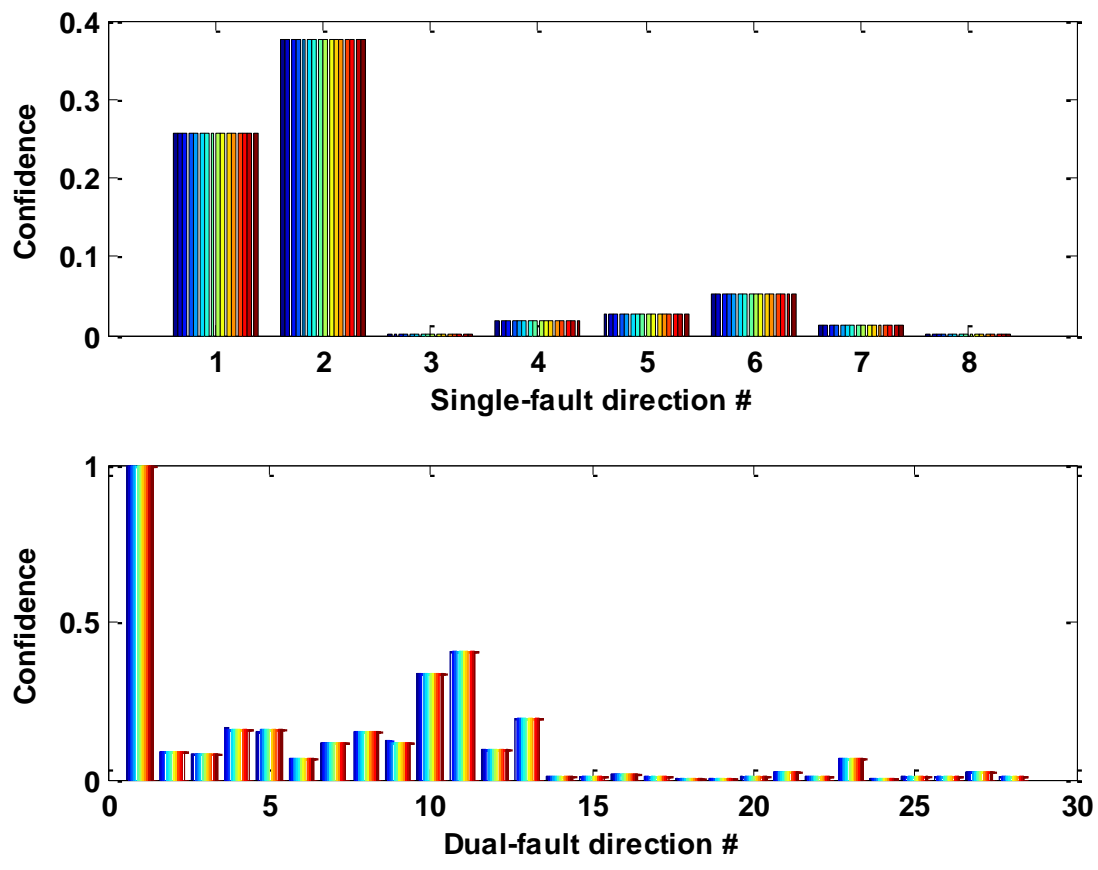

Figure 7.25 Fault isolation index for dual-fault case \#1.

Fault \#1 \& Fault \#3
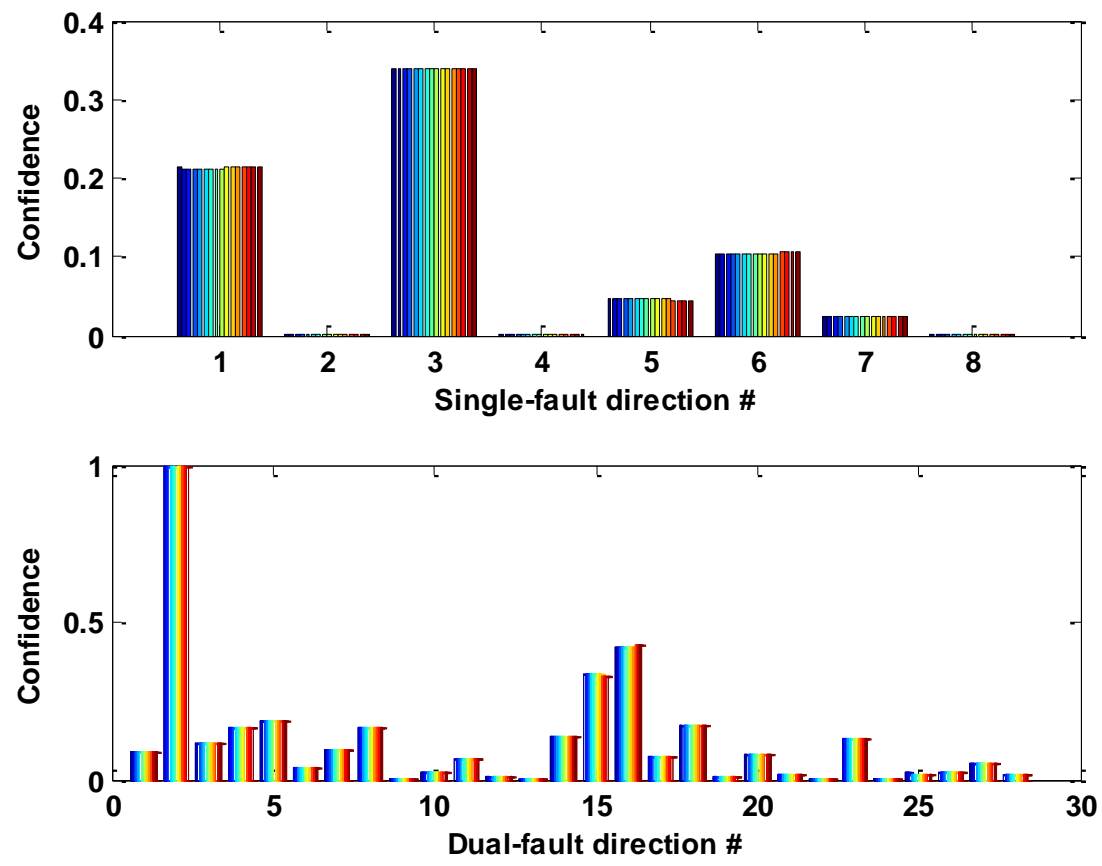

Figure 7.26 Fault isolation index for dual-fault case \#2. 
Fault \#1 \& Fault \#4
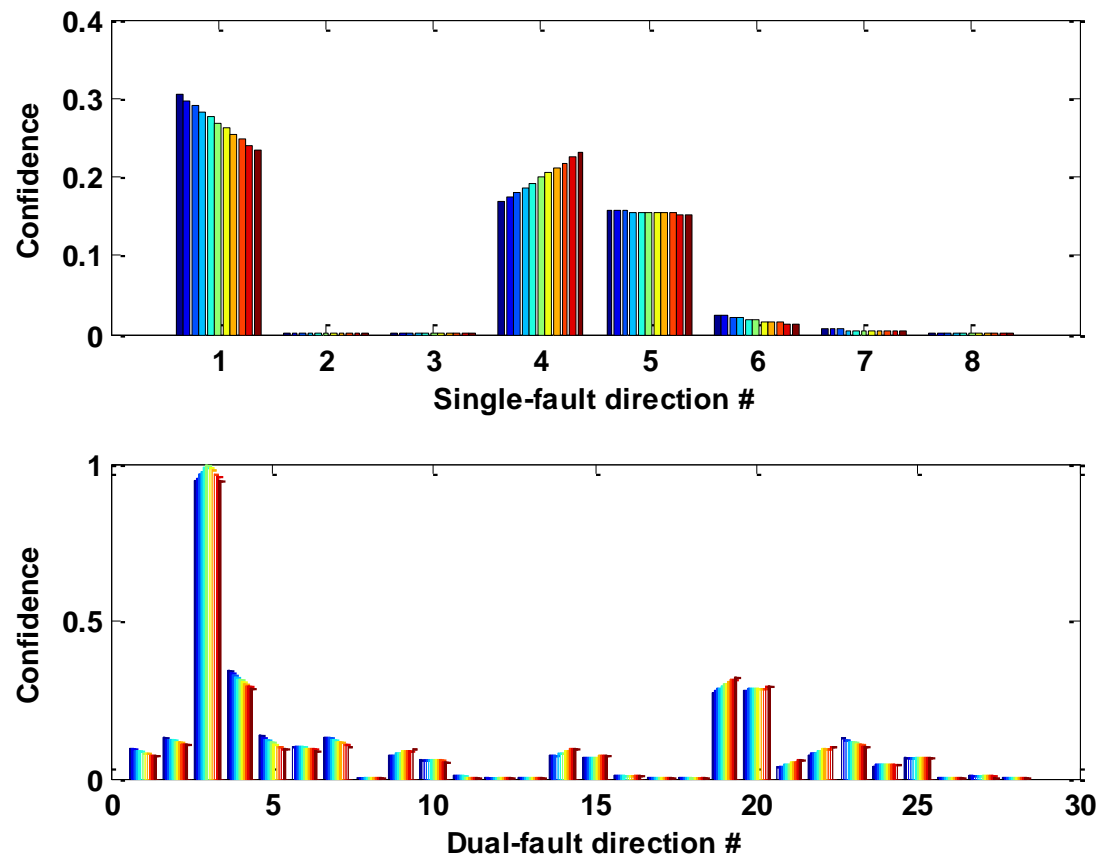

Figure 7.27 Fault isolation index for dual-fault case \#3.

Fault \#1 \& Fault \#5
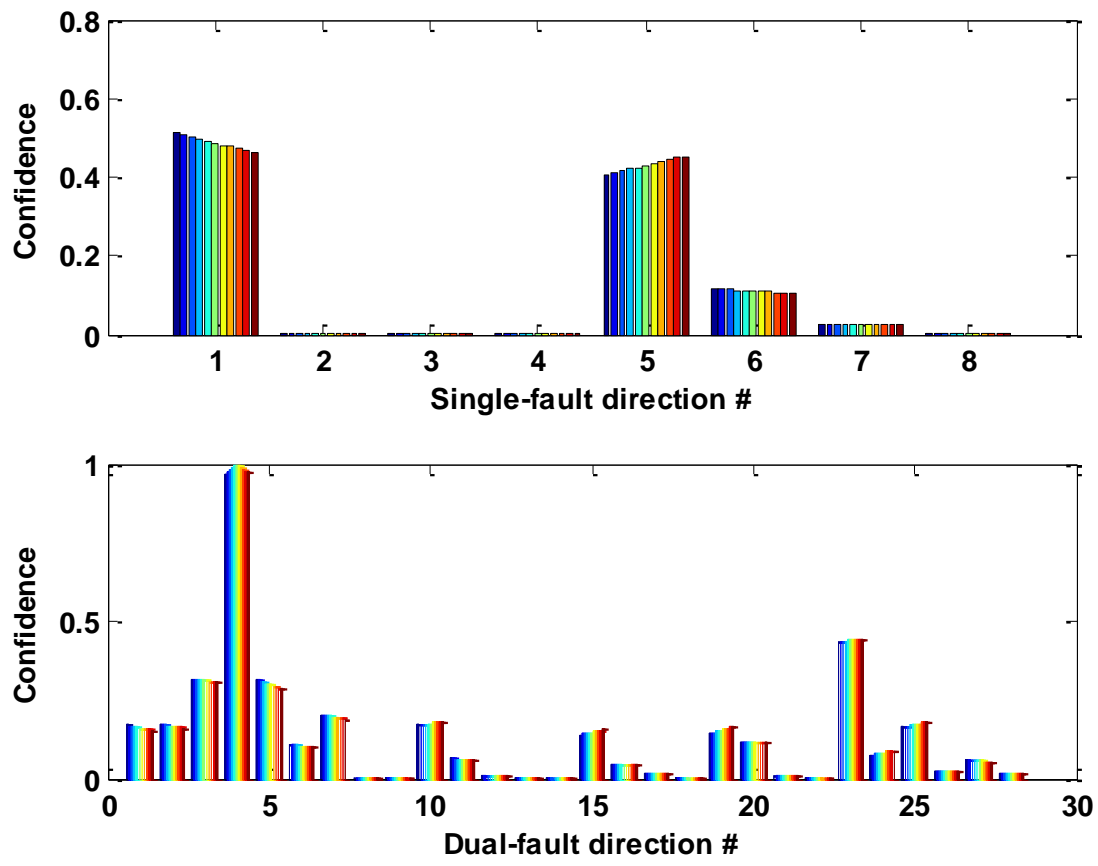

Figure 7.28 Fault isolation index for dual-fault case \#4. 
Fault \#1 \& Fault \#6
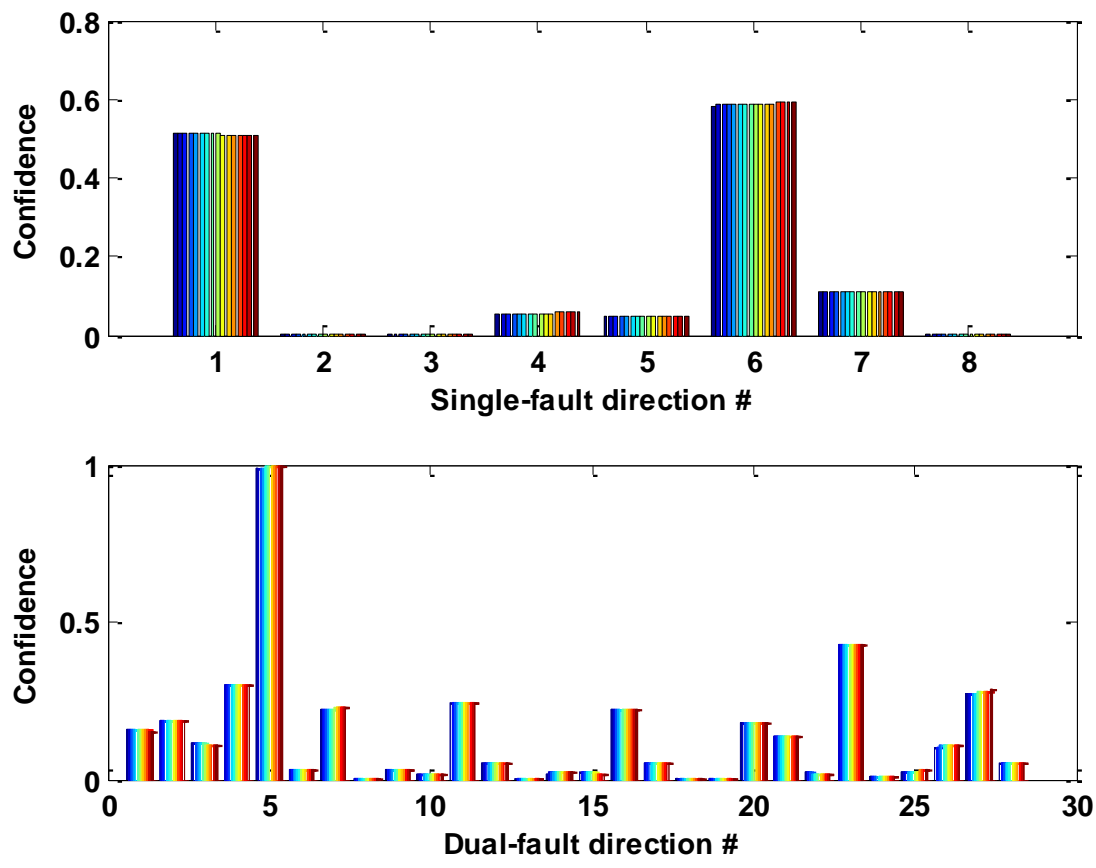

Figure 7.29 Fault isolation index for dual-fault case \#5.

Fault \#1 \& Fault \#7
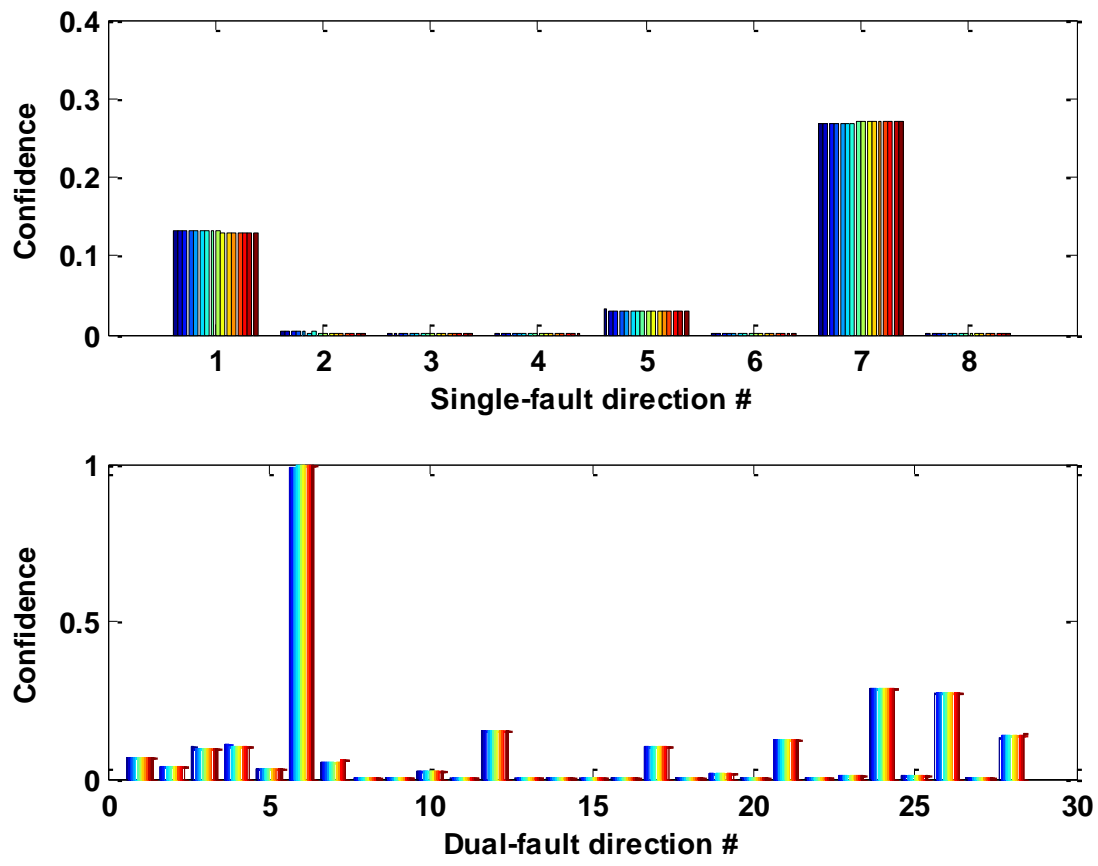

Figure 7.30 Fault isolation index for dual-fault case \#6. 


\section{Fault \#1 \& Fault \#8}
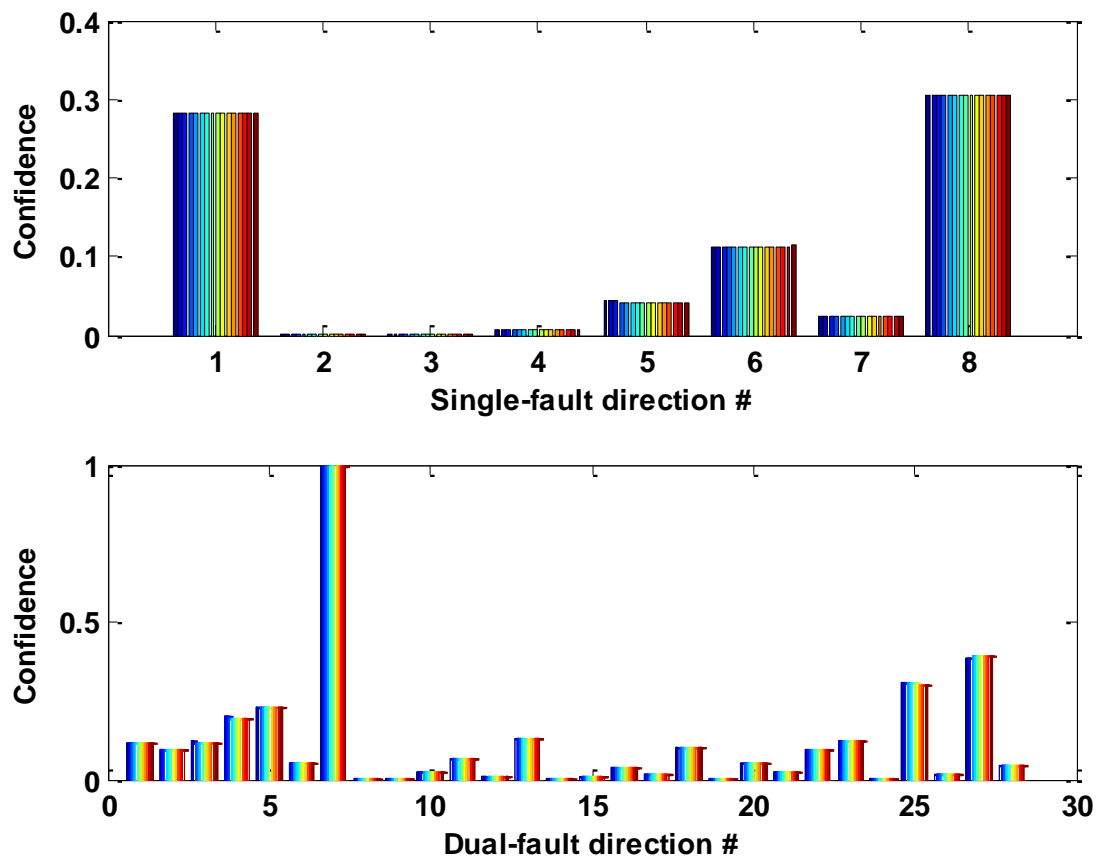

Figure 7.31 Fault isolation index for dual-fault case \#7.

\subsection{Summary}

In this chapter, the previously developed optimum sensor placement design formulations and a PCA-based fault diagnostic method were employed to diagnose the faults defined within a MSF system. When the optimal sensor sets were used, both sensor faults and process faults were correctly detected and isolated using $T^{2}$ and $Q$ statistics, as well as fault isolation index. It was also discovered through the FDI case studies that the PCA fault diagnostic approach could be extended to detect and isolate simultaneous dual-faults using the optimal sensor sets identified for the single-fault cases, in which case both single-fault and dual-fault directions shall be used as fault signatures in order to achieve dual-fault isolation. The fault diagnostic results demonstrated the effectiveness of the proposed FDI methods when employed to complex systems with optimized sensor allocation. 


\section{Chapter 8}

\section{SENSOR PLACEMENT DESIGN AND FAULT DIAGNOSIS APPLICATION FOR HCSG SYSTEMS}

\subsection{HCSG System Description}

International Reactor Innovative and Secure (IRIS) is one of the next generation nuclear reactor designs for near term deployment. This integral LWR type system has eight helical coil steam generators (HCSG) connected to four steam lines and four feed water lines. Eight steam generators are installed in four pairs in the annular space between the core barrel and the reactor vessel wall, which is shown in Figure 8.1 [Carelli et al., 2003]. On the primary side, each reactor coolant pump (RCP) is dedicated to discharging primary coolant into one steam generator. Therefore, each RCP+HCSG module constitutes a separate flow path. On the secondary side, a common feed water supply line splits at the vessel and goes to two steam generators. Similarly, the steam discharge lines from two steam generators join to create a common steam line.

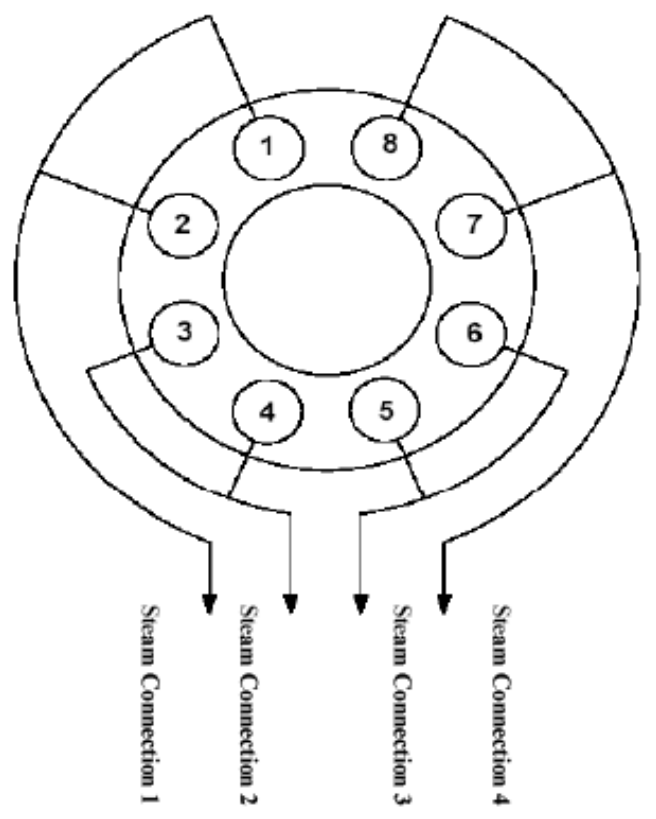

Figure 8.1. IRIS steam generator layout [Carelli et al., 2003]. 
This pairing design of steam generators reduces the number of feed water and steam lines, and the number of penetrations into and out of the containment, but it has an unfavorable consequence on individual steam generator monitoring in terms of its thermal performance and the secondary flow rate. Figure 8.2 shows a schematic of one pair of steam generators.

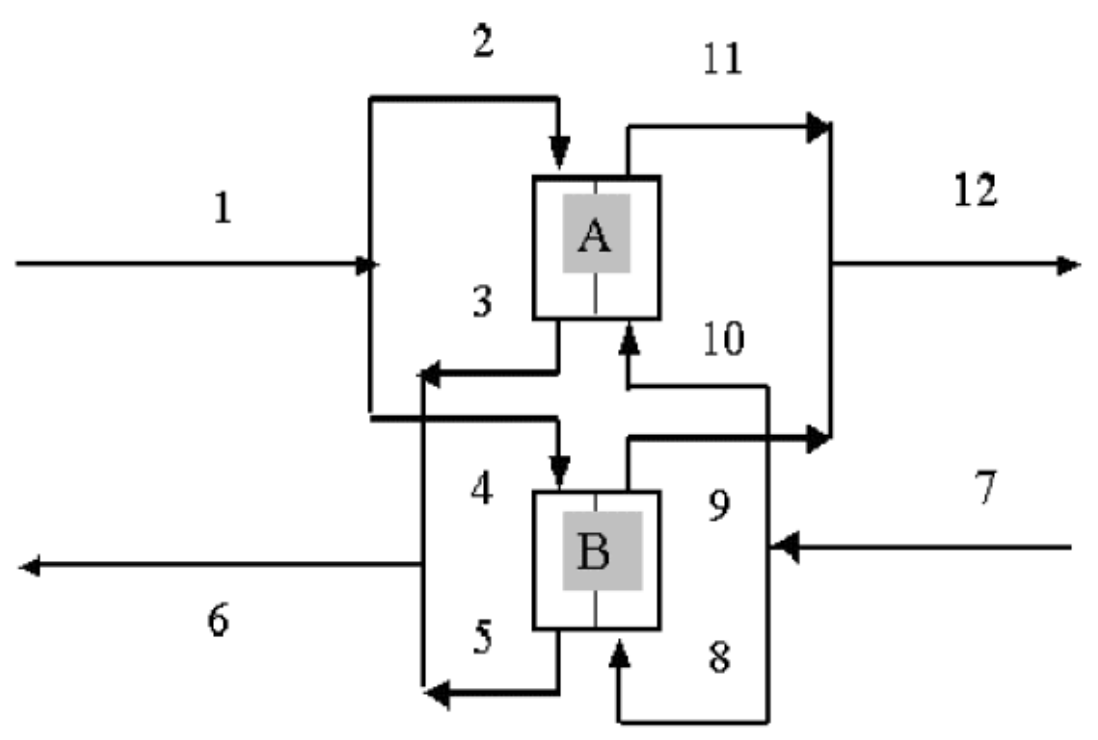

Figure 8.2. A schematic of a pair of HCSGs [Zhao, 2005a].

Twelve potential sensor locations shown in Figure 8.2 are denoted in numerical order as follows:

1. Primary pump outlet;

2. Primary inlet of steam generator 1 ;

3. Primary outlet of steam generator 1 ;

4. Primary inlet of steam generator 2 ;

5. Primary outlet of steam generator 2 ;

6. Cold leg connection line;

7. Feedwater connection line;

8. Secondary inlet of steam generator 2;

9. Secondary outlet of steam generator 2;

10. Secondary inlet of steam generator 1 ;

11. Secondary outlet of steam generator 1 ;

12. Steam connection line. 
For one pair of HCSGs, the steady state models that are based on mass balance and heat balance equations are given as follows.

$$
\begin{aligned}
& W_{1}-W_{2}-W_{4}=0 \\
& W_{2}-W_{3}=0 \\
& W_{4}-W_{5}=0 \\
& W_{6}-W_{3}-W_{5}=0 \\
& W_{12}-W_{9}-W_{11}=0 \\
& W_{10}-W_{11}=0 \\
& W_{8}-W_{9}=0 \\
& W_{7}-W_{8}-W_{10}=0 \\
& T_{1}=T_{2}=T_{4} \\
& T_{7}=T_{8}=T_{10} \\
& W_{6} h_{6}-W_{3} h_{3}-W_{5} h_{5}=0 \\
& W_{12} h_{12}-W_{9} h_{9}-W_{11} h_{11}=0 \\
& W_{2} h_{2}+W_{10} h_{10}-W_{3} h_{3}-W_{11} h_{11}=0 \\
& W_{4} h_{4}+W_{8} h_{8}-W_{9} h_{9}-W_{5} h_{5}=0
\end{aligned}
$$

The symbols $W, h$, and $T$ denote mass flow rate, specific enthalpy, and temperature, respectively. The numeric number appended to the symbols corresponds to a potential sensor location in the HCSG system configuration, as shown in Figure 8.2. It is assumed that the system pressures are constant. Thus, the specific enthalpy can be approximated as a function of local fluid temperature. We shall build a HCSG digraph upon these steady state linear balance equations for one pair of HCSGs due to the symmetry of their configuration.

Table 8.1. Fault nodes of a pair of HCSGs

\begin{tabular}{|c|c|}
\hline Fault Nodes & System Faults \\
\hline F1 & Hot leg temperature sensor fault \\
\hline F2 & SG-A heat transfer degradation \\
\hline F3 & SG-B heat transfer degradation \\
\hline F4 & Secondary flow distribution anomaly \\
\hline F5 & Feedwater temperature sensor fault \\
\hline F6 & Feedwater flow sensor fault \\
\hline
\end{tabular}


The HCSG digraph is shown in Figure 8.3. The yellow nodes represent the root nodes of the system, each of which corresponds to a fault. Table 8.1 lists the six faults that the HCSG diagnostic system needs to monitor. Both process faults and sensor faults are considered in the HCSG fault diagnosis. The three sensor faults considered are sensor drifts; and the thermal degradation of SG-A and SG-B, as well as the secondary flow distribution anomaly, are process faults considered for the HCSG systems. The digraph has clearly illustrated the cause-effect relationships among the involved variables and the propagation pathways from the fault nodes to the other nodes.

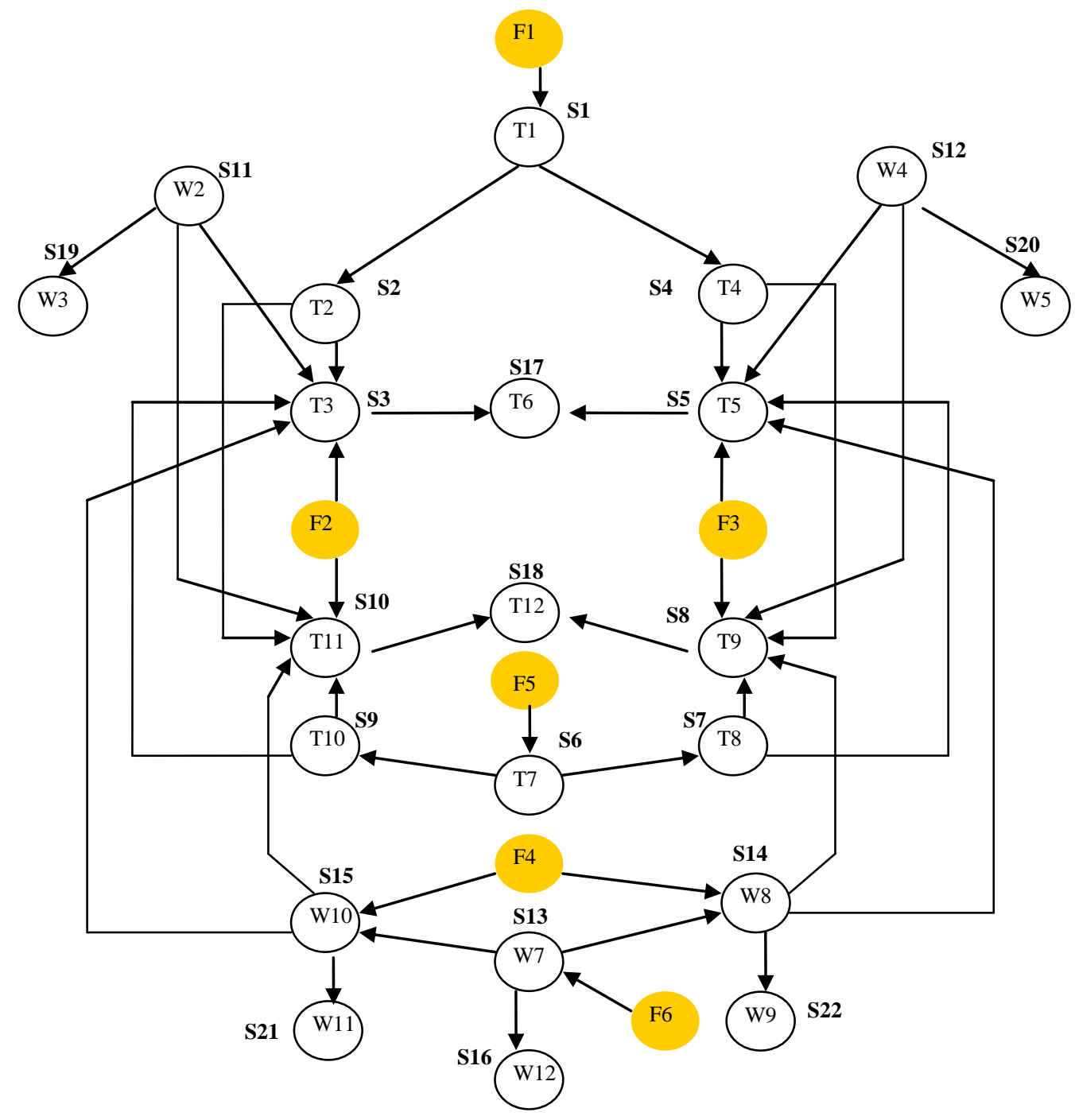

Figure 8.3. Directed graph of a pair of HCSGs. 


\subsection{Sensor Placement Design}

\subsubsection{Fault Diagnostic Observability Criterion}

As a first step, the greedy search algorithm for fault observability is applied to find the minimum set of sensors required to observe all the faults listed in Table 8.1 for one pair of HCSGs. This gives nodes $\left[T_{3}, T_{5}\right]$ as the sensor set. It is noted that in this case, nodes can also be the sensor set, because they are connected to all the faults. Although all the faults can be detected, none of them, however, can be distinguished from each other.

The algorithm is applied to obtain the set of sensors that will give maximum resolution under the single-fault assumption case, which generates $\left[T_{3}, T_{5}, T_{9}, T_{11}, W_{7}, W_{12}\right]$ as the minimum sensor set for fully isolation of the selected faults. Note that none of the fault nodes affect the same sensor set, therefore, they can be distinguished from each other by the selected sensor set.

\subsubsection{System Unobservability Criterion}

In this sub-section, sensor placement design is carried out for the pair of HCSGs by solving the optimization problem that minimizes the system overall unobservability, which is defined as the maximum unobservability across all faults. The objective function of the optimization is given in Equation (6.13). The cost is assumed to have the same value at 100 per sensor for all the sensors. The fault occurrence probabilities are listed in Table 8.2. The sensor failure probabilities are given in Table 8.3. The previously developed greedy search heuristic is employed to solve for optimal selection of the sensors. For the sake of comparison, the one-step optimization problem formulated in Equation (6.18) is solved by the LINGO 8.0 optimization software package under the same cost constraints as greedy search. The one-step approach minimizes the system overall unobservability and, among candidates offering the minimum system unobservability, selects the one with the least cost.

Table 8.4 shows the sensor selection results under a variety of total available cost constraints. The objective function, that is, system overall unobservability, is also reported in this table. It is seen that the greedy search heuristic offers the results similar to those obtained by LINGO 8.0. And the main difference between the two distinct optimization algorithms is that the 
greedy search tends to use all the available resources, while LINGO sometimes achieves the same system unobservability with fewer sensors.

Table 8.2. Fault data for a pair of HCSGs

\begin{tabular}{|c|c|c|c|}
\hline Fault Nodes & $\begin{array}{c}\text { Occurrence } \\
\text { Probability }\left(\log f_{j}\right)\end{array}$ & Fault Nodes & $\begin{array}{c}\text { Occurrence } \\
\text { Probability }\left(\log f_{j}\right)\end{array}$ \\
\hline F1 & -2 & F4 & -1 \\
\hline F2 & -2 & F5 & -1 \\
\hline F3 & -2 & F6 & -1 \\
\hline
\end{tabular}

Table 8.3. Sensor data for a pair of HCSGs

\begin{tabular}{|c|c|c|c|c|c|}
\hline $\begin{array}{c}\text { Sensor } \\
\text { Nodes }\end{array}$ & Variable & $\begin{array}{c}\text { Failure } \\
\text { Probability } \\
\left(\log s_{i}\right)\end{array}$ & $\begin{array}{c}\text { Sensor } \\
\text { Nodes }\end{array}$ & Variable & $\begin{array}{c}\text { Failure } \\
\text { Probability } \\
\left(\log s_{i}\right)\end{array}$ \\
\hline S1 & $T_{1}$ & -2 & $\mathrm{~S} 12$ & $W_{4}$ & -1 \\
\hline S2 & $T_{2}$ & -2 & $\mathrm{~S} 13$ & $W_{7}$ & -1 \\
\hline $\mathrm{S} 3$ & $T_{3}$ & -2 & $\mathrm{~S} 14$ & $W_{8}$ & -1 \\
\hline $\mathrm{S} 4$ & $T_{4}$ & -2 & $\mathrm{~S} 15$ & $W_{10}$ & -1 \\
\hline $\mathrm{S} 5$ & $T_{5}$ & -2 & $\mathrm{~S} 16$ & $W_{12}$ & -1 \\
\hline $\mathrm{S} 6$ & $T_{7}$ & -2 & $\mathrm{~S} 17$ & $T_{6}$ & -2 \\
\hline $\mathrm{S} 7$ & $T_{8}$ & -2 & $\mathrm{~S} 18$ & $T_{12}$ & -1 \\
\hline $\mathrm{S} 8$ & $T_{9}$ & -2 & $\mathrm{~S} 19$ & $W_{3}$ & -1 \\
\hline $\mathrm{S} 9$ & $T_{10}$ & -2 & $\mathrm{~S} 20$ & $W_{5}$ & -1 \\
\hline S10 & $T_{11}$ & -2 & $\mathrm{~S} 21$ & $W_{11}$ & -1 \\
\hline S11 & $W_{2}$ & -1 & $\mathrm{~S} 22$ & $W_{9}$ & \\
\hline
\end{tabular}


Table 8.4. Sensor selection results using greedy search heuristic and LINGO 8.0

\begin{tabular}{|c|c|c|c|}
\hline \multirow{2}{*}{$\begin{array}{c}\text { Total } \\
\text { Cost }\end{array}$} & $\begin{array}{c}\text { System } \\
\text { Unobservability }\end{array}$ & \multicolumn{2}{|c|}{ Sensors Selected } \\
\hline 400 & 0.01 & $\mathrm{~S} 3, \mathrm{~S} 5, \mathrm{~S} 6, \mathrm{~S} 13$ & Greedy Search \\
\hline 500 & 0.01 & $\mathrm{~S} 3, \mathrm{~S} 5, \mathrm{~S} 6, \mathrm{~S} 13$ & $\mathrm{~S} 3, \mathrm{~S} 5, \mathrm{~S} 6, \mathrm{~S} 13$ \\
\hline 800 & 0.001 & $\mathrm{~S} 3, \mathrm{~S} 5, \mathrm{~S} 6, \mathrm{~S} 11_{1}, \mathrm{~S} 12, \mathrm{~S} 13_{(2)}, \mathrm{S} 14$ & $\mathrm{~S} 3, \mathrm{~S} 5, \mathrm{~S} 6, \mathrm{~S} 11_{1}, \mathrm{~S} 12, \mathrm{~S} 13_{(2)}, \mathrm{S} 14$ \\
\hline 1000 & 0.001 & $\mathrm{~S} 3, \mathrm{~S} 5, \mathrm{~S} 6, \mathrm{~S} 11, \mathrm{~S} 12, \mathrm{~S} 13_{(2)}, \mathrm{S} 14$ & $\mathrm{~S} 1, \mathrm{~S} 3, \mathrm{~S} 5_{(2)}, \mathrm{S} 6, \mathrm{~S} 11_{1}, \mathrm{~S} 12, \mathrm{~S} 13_{(3)}$ \\
\hline 1500 & $10^{-4}$ & $\begin{array}{c}\mathrm{S} 1, \mathrm{~S} 3, \mathrm{~S} 5_{(2)}, \mathrm{S} 6, \mathrm{~S} 11_{(2)}, \mathrm{S} 12_{(2)}, \\
\mathrm{S} 13_{(3)}, \mathrm{S} 14\end{array}$ & $\begin{array}{c}\mathrm{S} 1, \mathrm{~S} 3_{(2)}, \mathrm{S} 5_{(2)}, \mathrm{S} 6_{(2)}, \mathrm{S} 11_{(2)}, \\
\mathrm{S} 12_{(2)}, \mathrm{S} 13_{(4)}\end{array}$ \\
\hline 2000 & $10^{-5}$ & $\begin{array}{c}\mathrm{S} 1, \mathrm{~S} 3_{(2)}, \mathrm{S} 5_{(2)}, \mathrm{S} 6_{(2)}, \mathrm{S} 11_{(3)}, \\
\mathrm{S} 12_{(3)}, \mathrm{S} 13_{(4)}, \mathrm{S} 14\end{array}$ & $\begin{array}{c}\mathrm{S} 1_{(2)}, \mathrm{S} 3_{(2)}, \mathrm{S} 5_{(2)}, \mathrm{S} 6_{(2)}, \mathrm{S} 11_{(4)}, \\
\mathrm{S} 12_{(4)}, \mathrm{S} 13_{(4)}\end{array}$ \\
\hline
\end{tabular}

In order to show the decreasing trend of the system unobservability as the iteration progresses, the greedy search algorithm is applied to the HCSG system when the total available cost is 1000 . The results are tabulated in Table 8.5. The first column in the table indicates the number of available sensors. For each extra available sensor, the positions of the already located sensors do not change. Hence only the additional sensor is listed in the second column of Table 8.5. The third column in the table is the unobservability of the fault with the highest value after sensor selection. The sensor location results are shown for ten available sensors.

In order to verify the effectiveness of the greedy search optimization algorithm, the unobservability values of the six faults are calculated. When the total available cost is 1500 , it takes 16 iterations before the algorithm stops. Figure 8.4 shows the trending of unobservability. It can be clearly seen that the greedy search optimization algorithm reduces the unobservability values for all the six faults. 
Table 8.5. HCSG sensor placement results (available cost=1000)

\begin{tabular}{ccc}
\hline $\begin{array}{c}\text { \# of available } \\
\text { sensors }\end{array}$ & Additional sensor & $\begin{array}{c}\text { System unobservability } \\
\text { after sensor selection }\end{array}$ \\
\hline 1 & S5 & 0.1 \\
2 & S3 & 0.1 \\
3 & S6 & 0.1 \\
4 & S13 & 0.1 \\
5 & S13 & 0.01 \\
6 & S1 & 0.01 \\
7 & S11 & 0.01 \\
8 & S12 & 0.01 \\
9 & S13 & 0.001 \\
10 & S5 & 0.001 \\
\hline
\end{tabular}
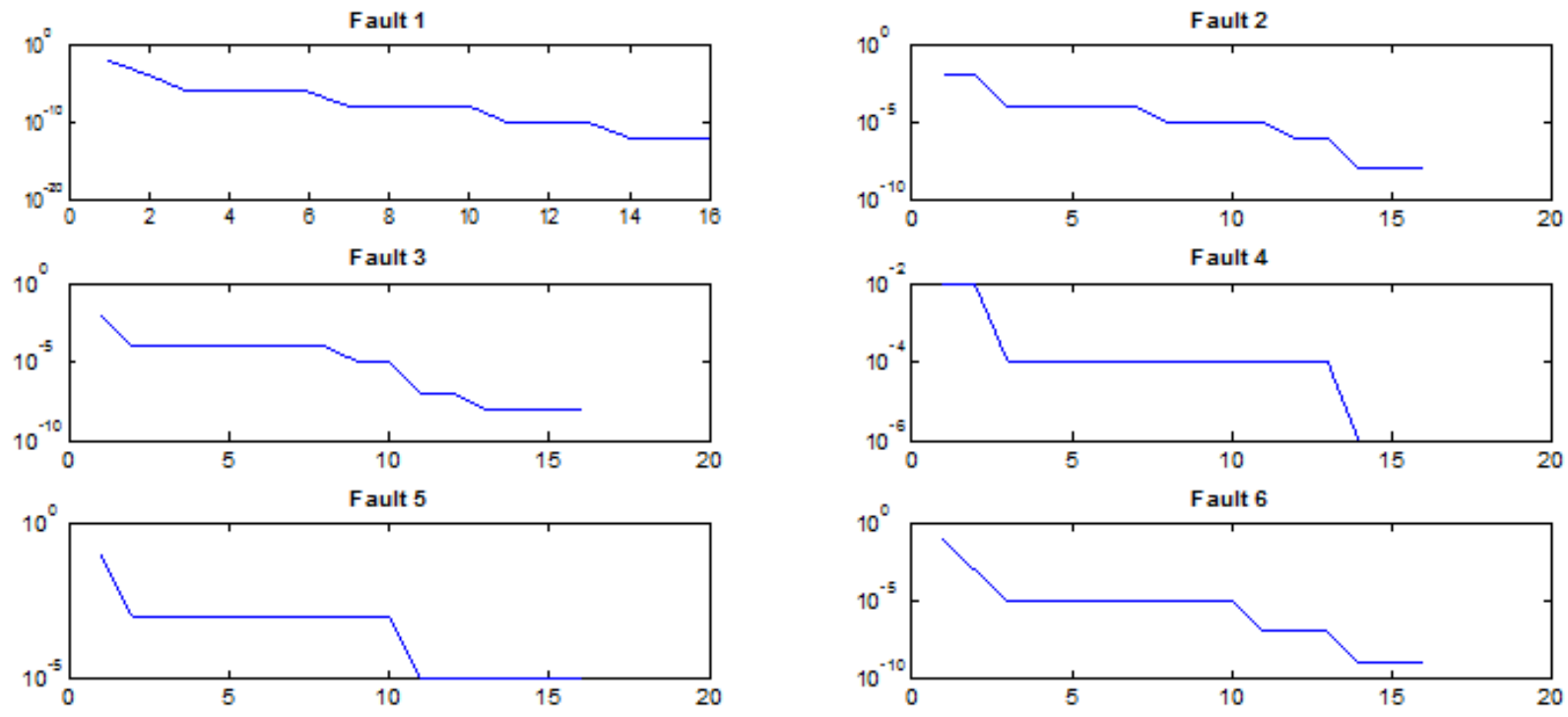

Figure 8.4. Decreasing trend of unobservability for system faults of a pair of HCSGs.

\subsection{PCA Fault Diagnostic Results}

For a pair of HCSGs, the digraph has been constructed based on mass and heat balance equations, as shown in Figure 8.3. In the previous section, the design problem of sensor network with maximum fault resolution using fault observability criterion has been studied. The greedy 
search algorithm gives $\left[T_{3}, T_{5}, T_{9}, T_{11}, W_{7}, W_{12}\right]$ as the minimum sensor set for fully isolation of the selected faults. The advantage of using this optimized sensor set is that more information about the system is utilized, and some basic properties such as the fault detectability and identifiability are already partially guaranteed before PCA has been used to monitor system behavior.

To build the model for nominal operation, a simulation database was created for different power levels ranging from $40 \%$ to $100 \%$ of the plant capacity. The data were generated for one pair of the steam generators, SG-A and SG-B, to simulate the measurements in actual nuclear power plants. For this pair of steam generators, the above six sensors identified as the optimized sensor set are used as the measured variables to provide process information of the HCSG systems. (a) T3: cold leg temperature of SG-A; (b) T5: cold leg temperature of SG-B; (c) T9: steam temperature leaving the secondary side of SG-B; (d) T11: steam temperature leaving the secondary side of SG-A; (e) W7: feed water flow rate into the secondary side of SG-A and SG-B; (f) W12: steam flow rate leaving the secondary side of SG-A and SG-B.

A PCA model was developed using the data for the nominal operation case with the six variables in the minimum sensor set. The nominal operation data matrix is preprocessed by auto-scaling the columns in the data matrix to zero mean and unit variance. This puts all the measurements with their different units on a common unit variance scale. Under normal conditions, small residuals would be generated and limited to a certain range. In the test cases when one or several components in the system are under degradation, the causal relations among the variables will be violated. As a result, the mapping of residuals from residual generators or system models will increase in a specific direction. The residual patterns for different types of faults are shown in Figure 8.5-8.10.

To understand the fault residual patterns, the residual pattern plot for the secondary flow distribution anomaly (Fault \#4), shown in Figure 8.8, is discussed here. The secondary flow distribution anomaly is a process fault in the HCSG systems. When this process fault occurs, the flow rate into the secondary side of each steam generator will be different. However, because the secondary fluid flows inside the helical coil tubes, it is unrealistic to directly measure the flow rate into each steam generator and the fault effects cannot be directly observed based on the flow 
rates. For this reason, the fault needs to be monitored from the other measured variables such as the primary outlet temperatures and the steam outlet temperatures.

The fault data for the secondary flow distribution anomaly (Fault \#4) is generated by linearly reducing the feed flow rate into SG-A from $100 \%$ value to $90 \%$ value, while increasing the feed flow rate into SG-B from $100 \%$ to $110 \%$ value. Thus as shown in Figure 8.8 , the temperatures $\mathrm{T} 3$ and $\mathrm{T} 11$ have positive components, indicating the increase in both measurements when the secondary flow rate into the SG-A decreases; and the temperatures T5 and T9 have negative components, indicating the decrease in them when the secondary flow rate into the SG-B increases.

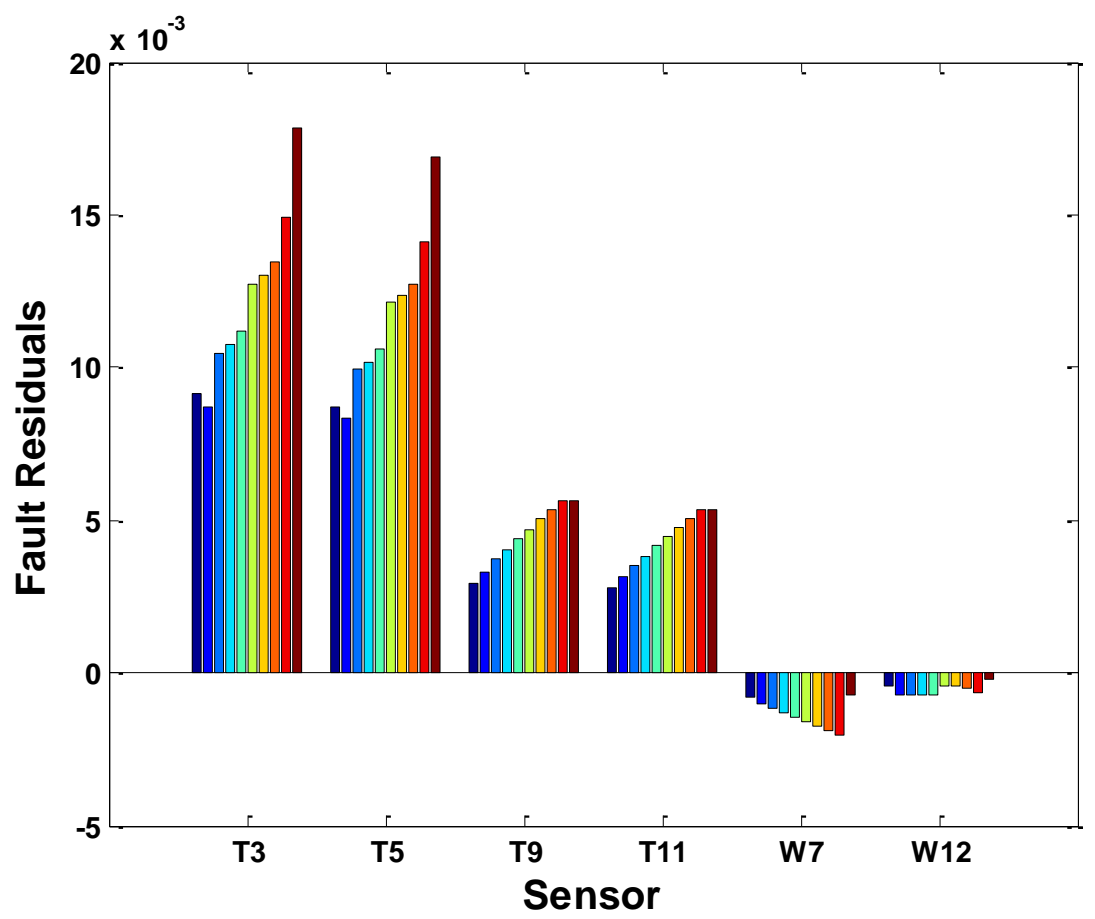

Figure 8.5. Residual pattern for $\mathrm{T}_{\text {hot }}$ sensor fault (Fault \#1). 


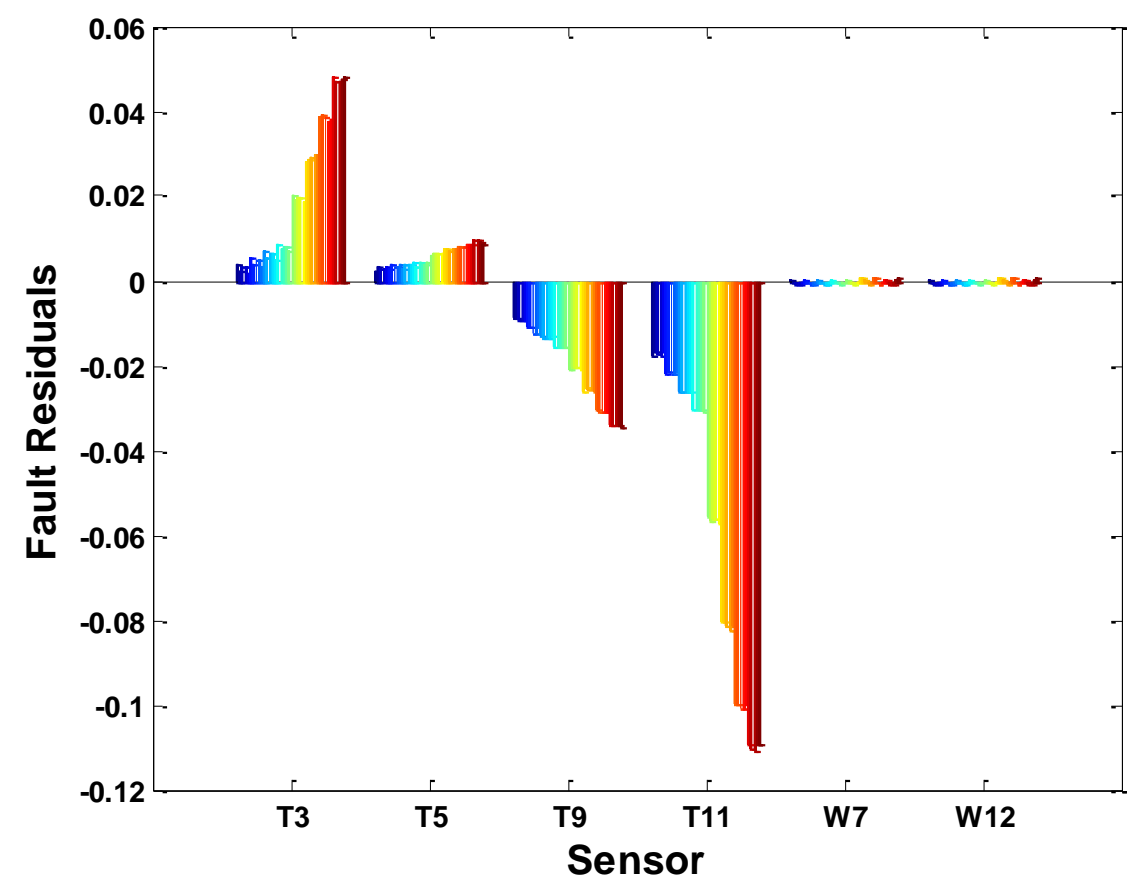

Figure 8.6. Residual pattern for SG-A thermal degradation (Fault \#2).

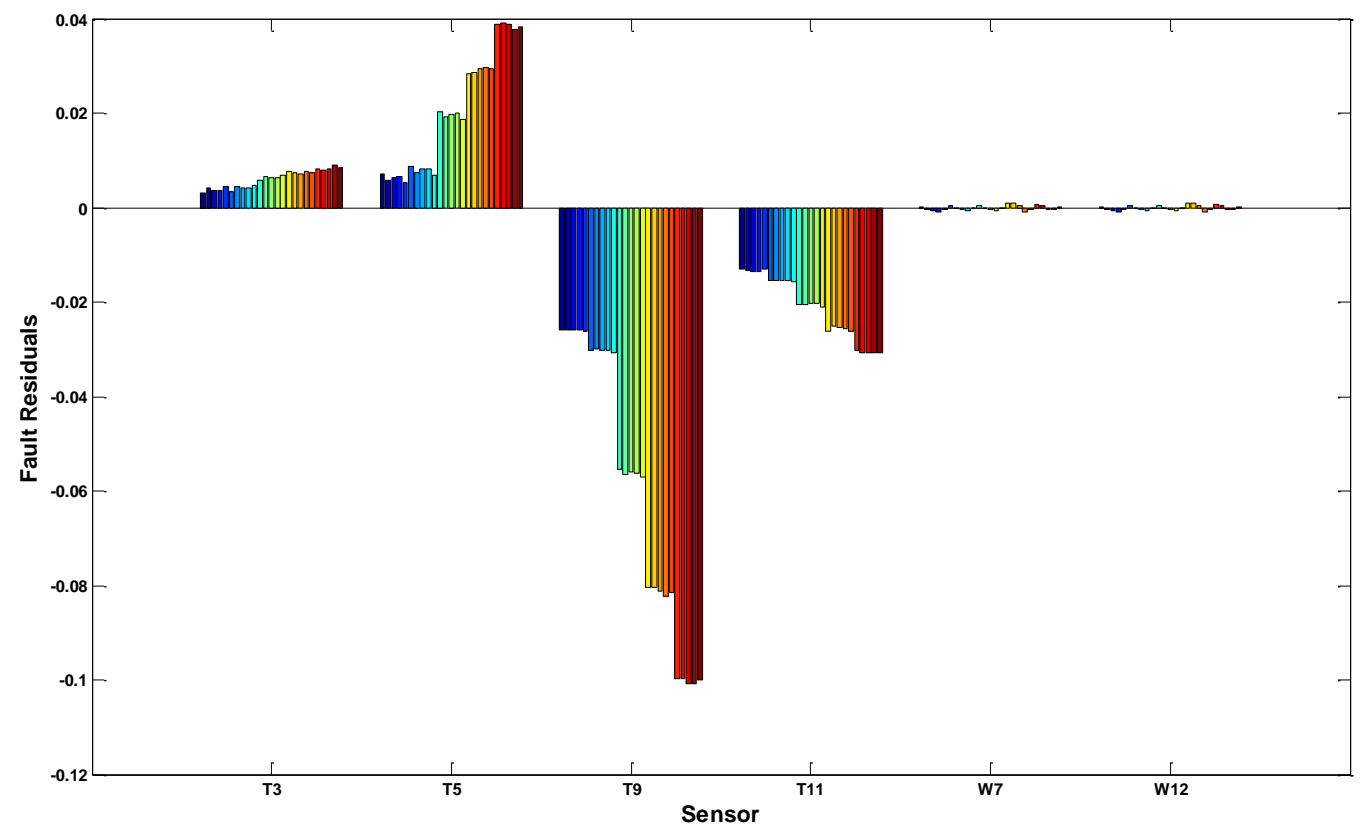

Figure 8.7. Residual pattern for SG-B thermal degradation (Fault \#3). 


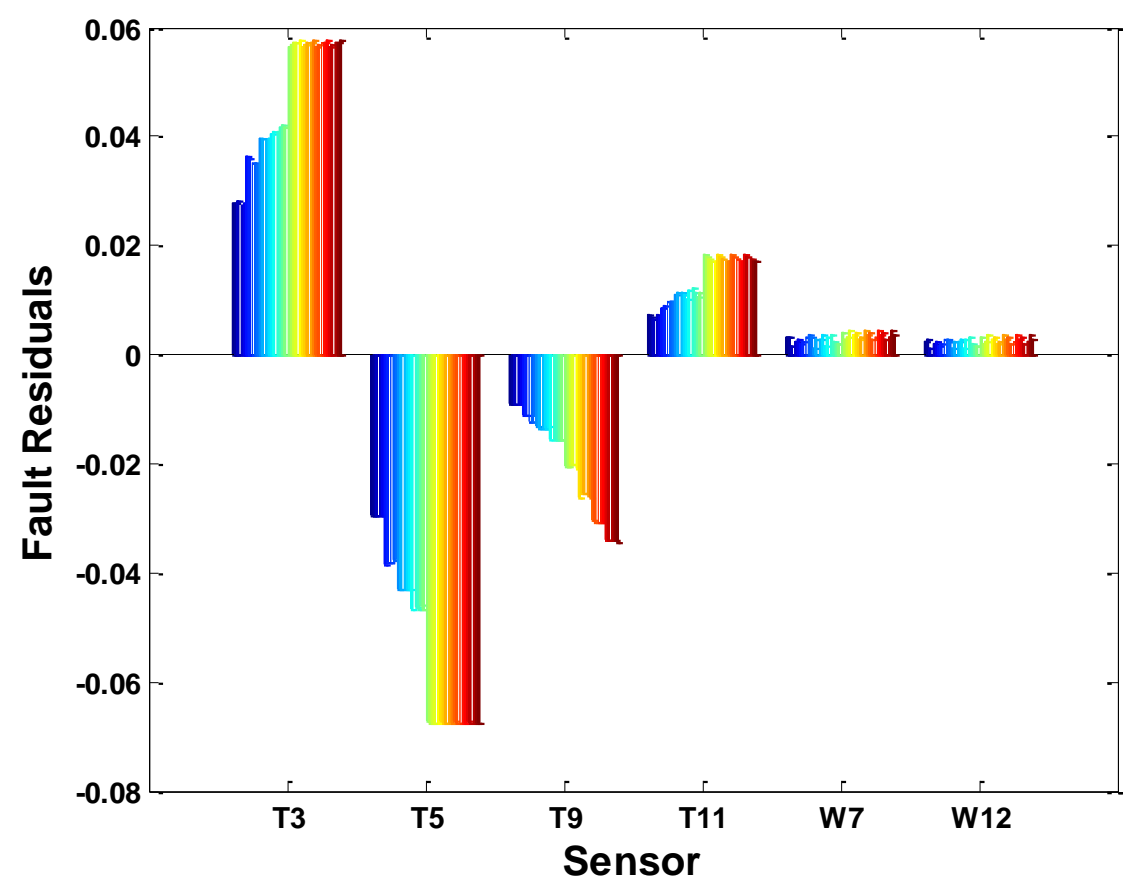

Figure 8.8. Residual pattern for secondary flow distribution anomaly (Fault \#4).

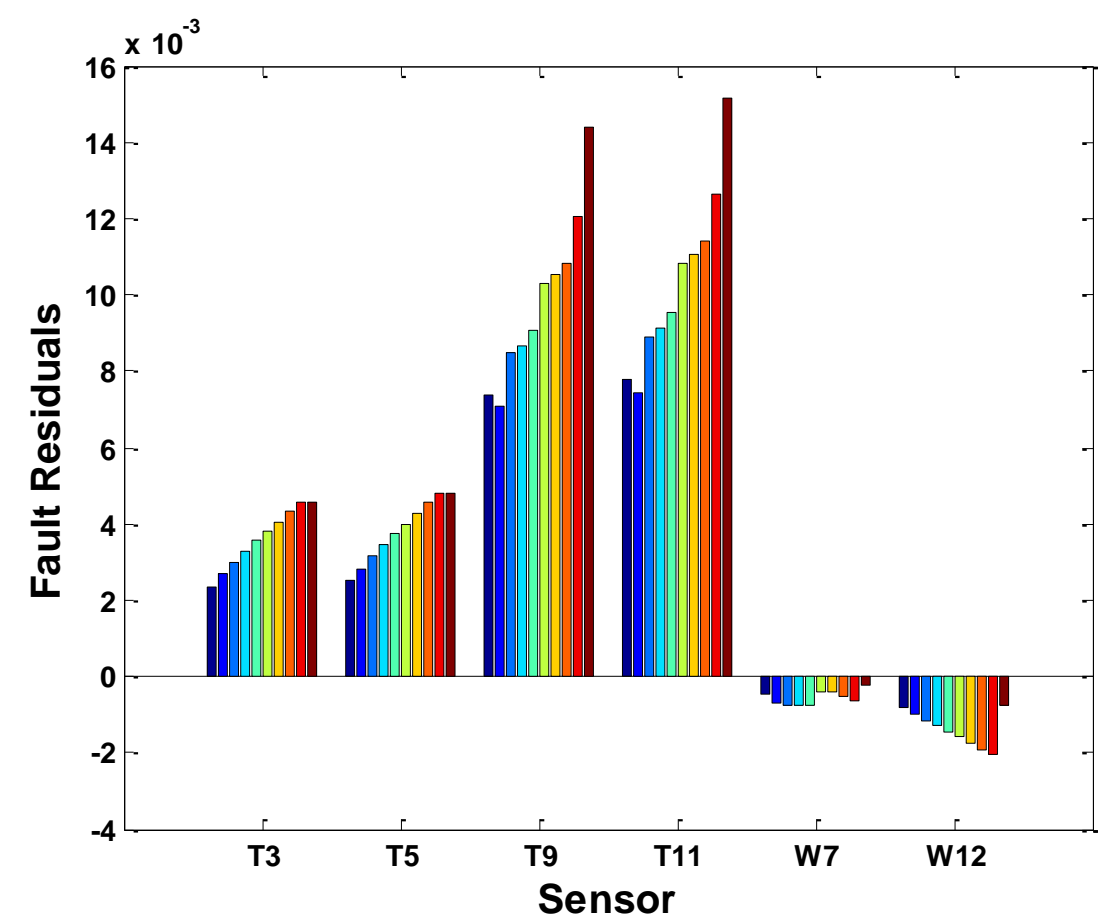

Figure 8.9. Residual pattern for feed temperature sensor fault (Fault \#5). 


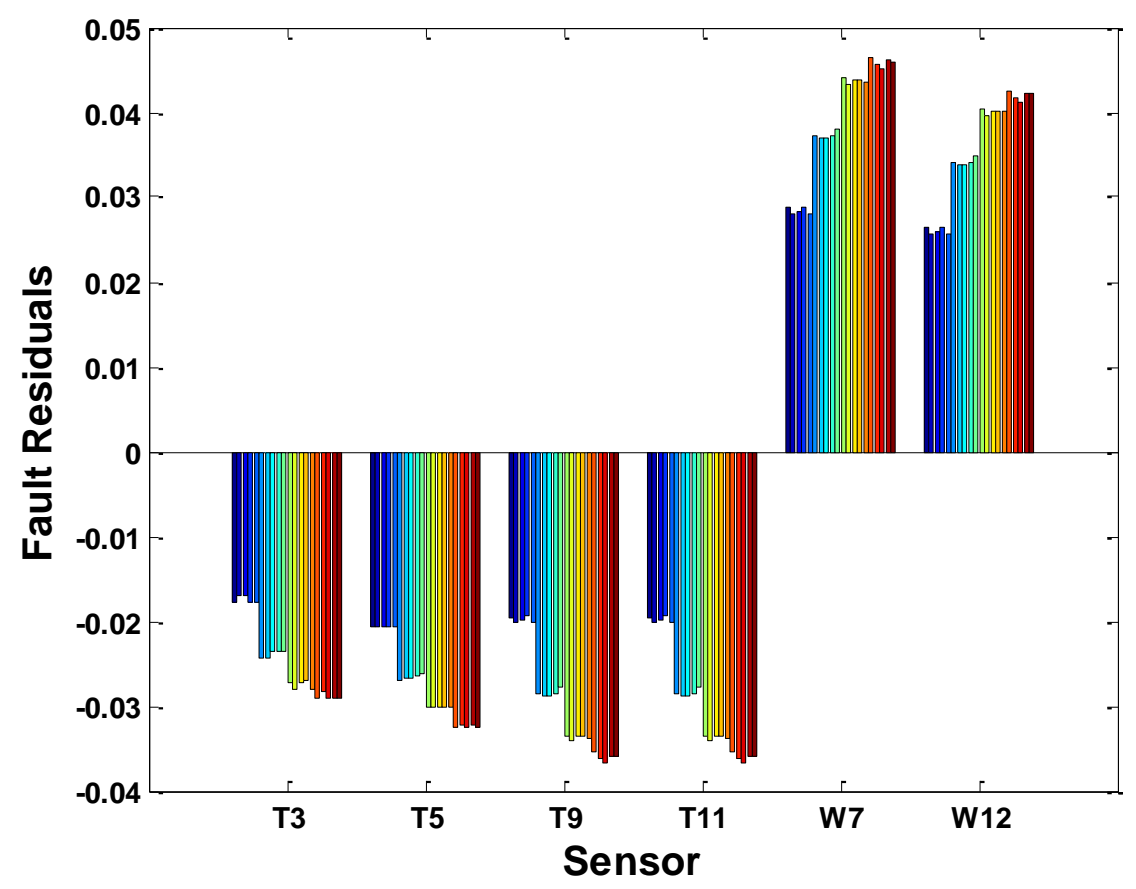

Figure 8.10. Residual pattern for feed flow sensor fault (Fault \#6).

Then, the PCA fault direction analysis is performed for processing the fault residuals. The six fault directions which correspond to the six fault scenarios are listed in Table 8.6.

Table 8.6. PCA fault direction analysis for a pair of HCSGs

\begin{tabular}{|c|c|c|}
\hline Fault direction \# & System faults & $\begin{array}{c}\text { \% of residual variances explained } \\
\text { by the 1st PC }\end{array}$ \\
\hline 1 & Hot leg temperature sensor fault & 98.22 \\
\hline 2 & SG-A thermal degradation & 96.71 \\
\hline 3 & SG-B thermal degradation & 94.52 \\
\hline 4 & Secondary flow distribution anomaly & 92.06 \\
\hline 5 & Feed temperature sensor fault & 95.84 \\
\hline 6 & Feed flow sensor fault & 94.39 \\
\hline
\end{tabular}

The following plots (Figures 8.11-8.16) illustrate the fault isolation indices for the different fault cases. Note that the fault index is close to unity for the particular fault that occurs and is smaller for the others. It is shown that all the fault cases are distinguished correctly using the fault isolation approach when the PCA model includes the six sensors that are chosen by the proposed sensor placement algorithm. 


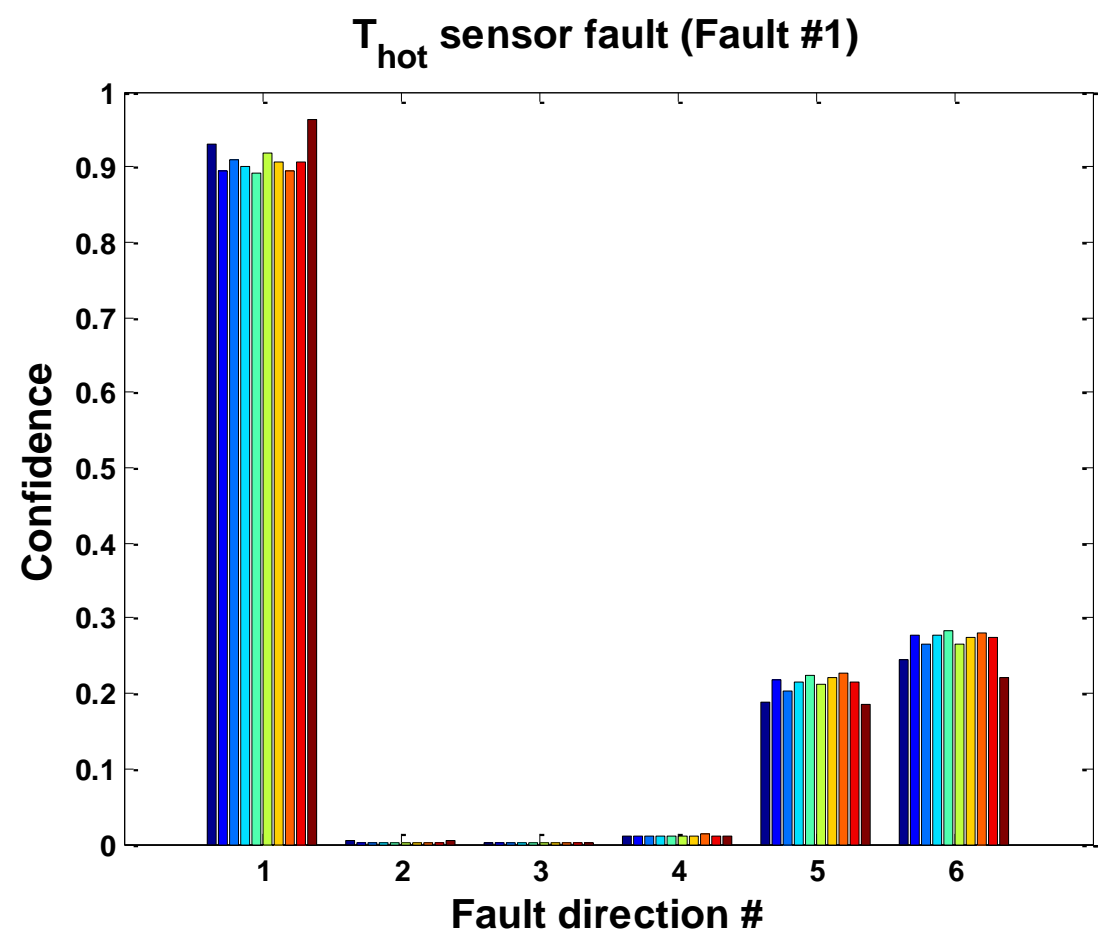

Figure 8.11. Fault isolation index for $\mathrm{T}_{\text {hot }}$ sensor fault.

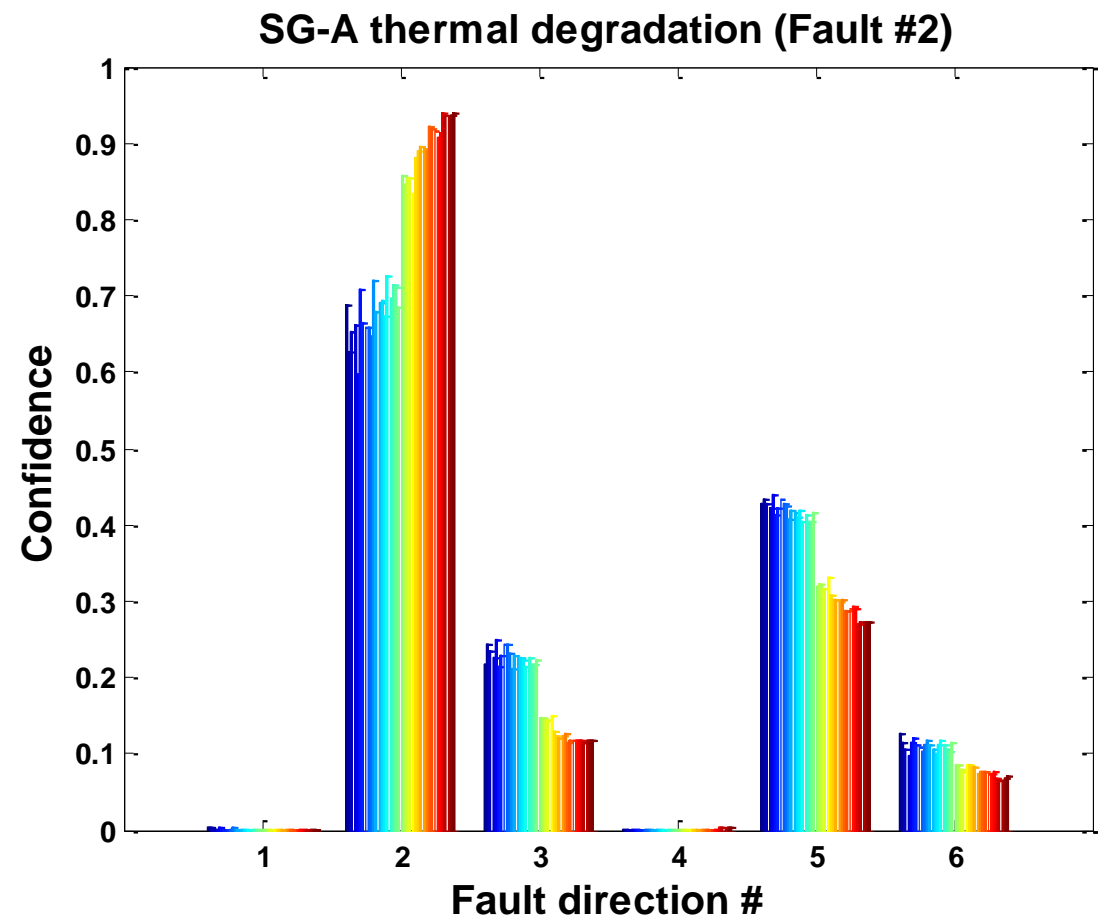

Figure 8.12. Fault isolation index for SG-A thermal degradation. 


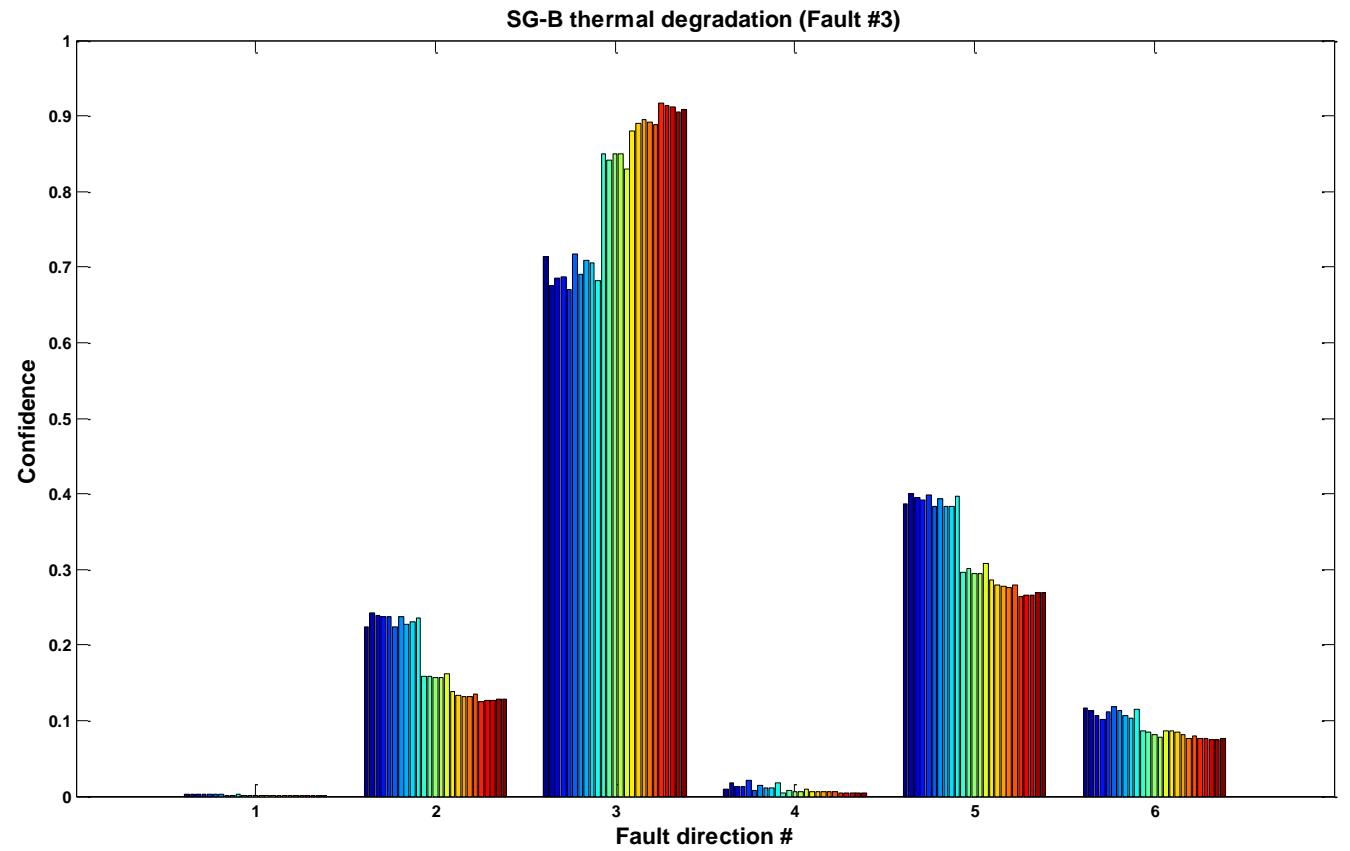

Figure 8.13. Fault isolation index for SG-B thermal degradation.

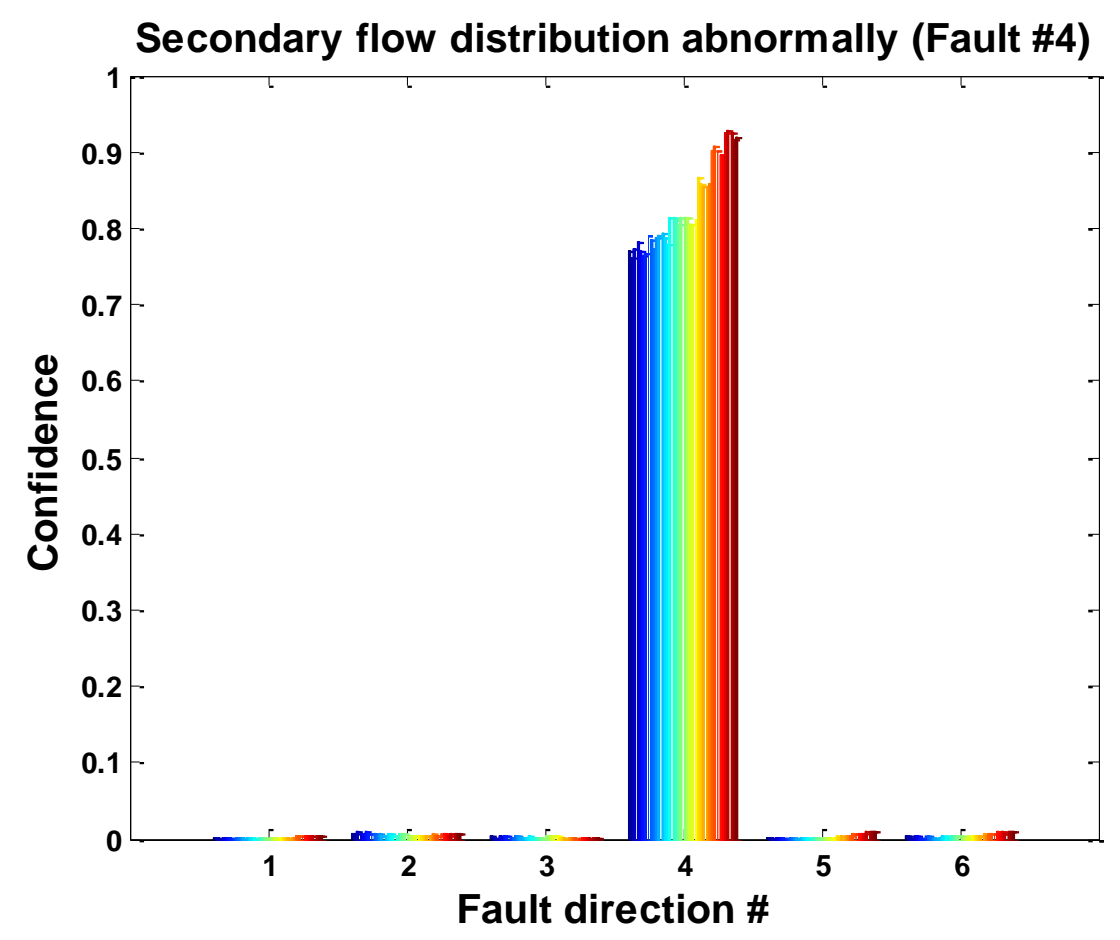

Figure 8.14. Fault isolation index for secondary flow distribution anomaly. 


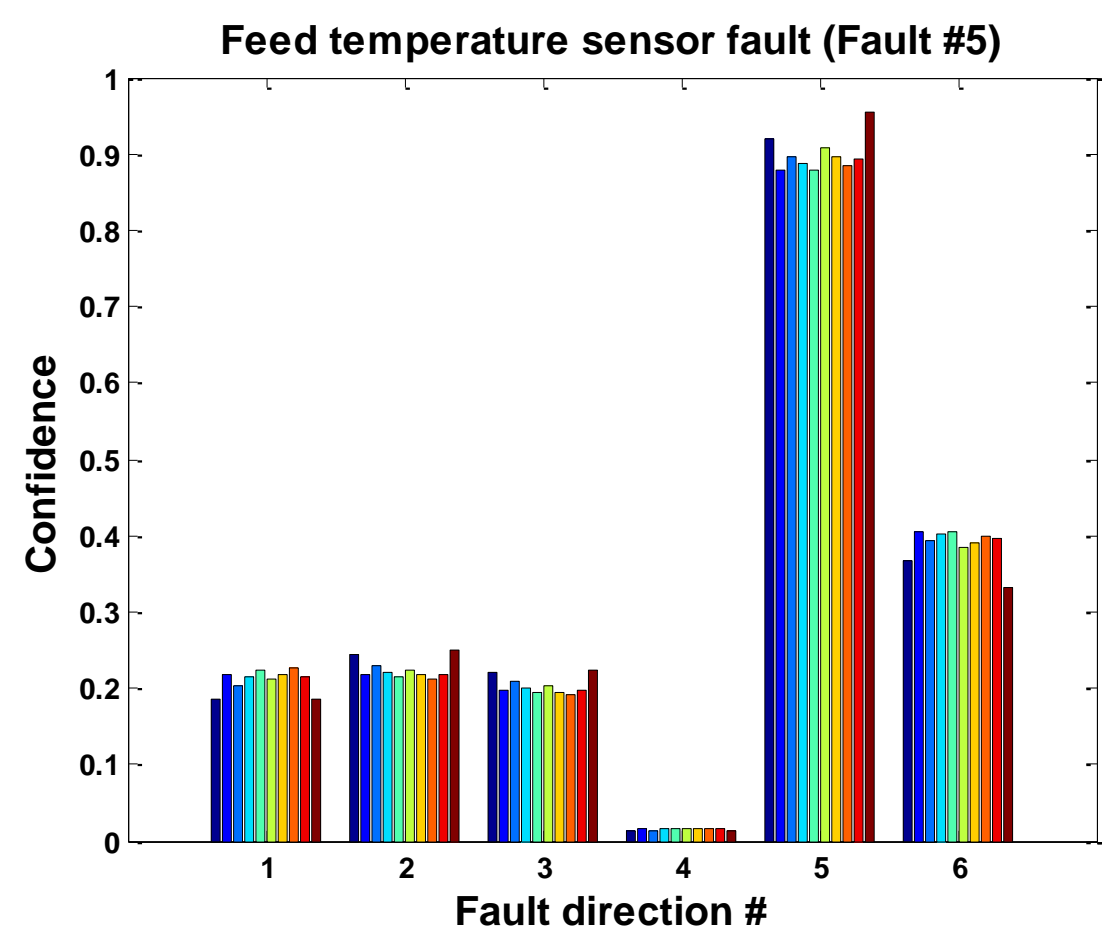

Figure 8.15. Fault isolation index for feed temperature sensor fault.

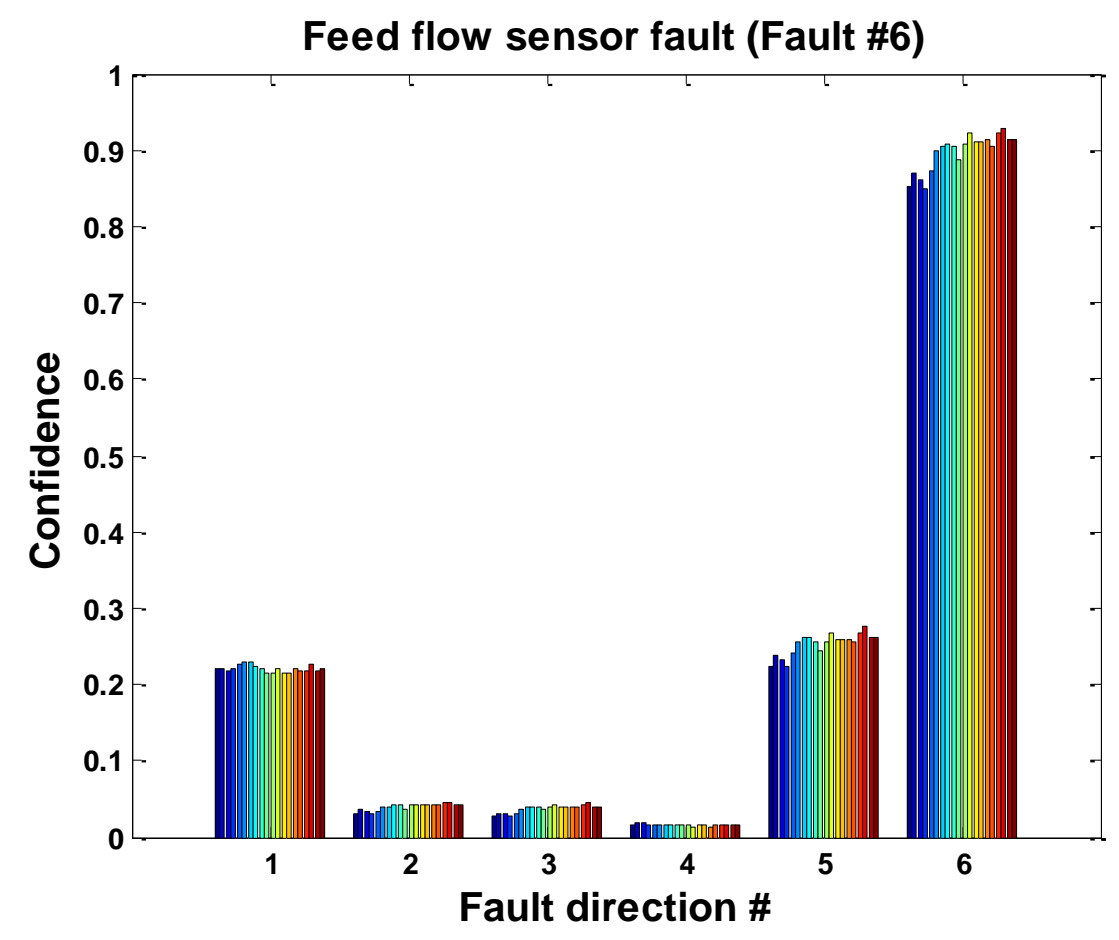

Figure 8.16. Fault isolation index for feed flow sensor fault. 
For the sake of comparison, the following plots (Figures 8.17-8.22) illustrate the fault isolation indices for the different fault cases when the reduced sensor set is used to develop the PCA model for HCSG fault monitoring. For instance, only T3, T11, W7, and W12 are included in this case study.

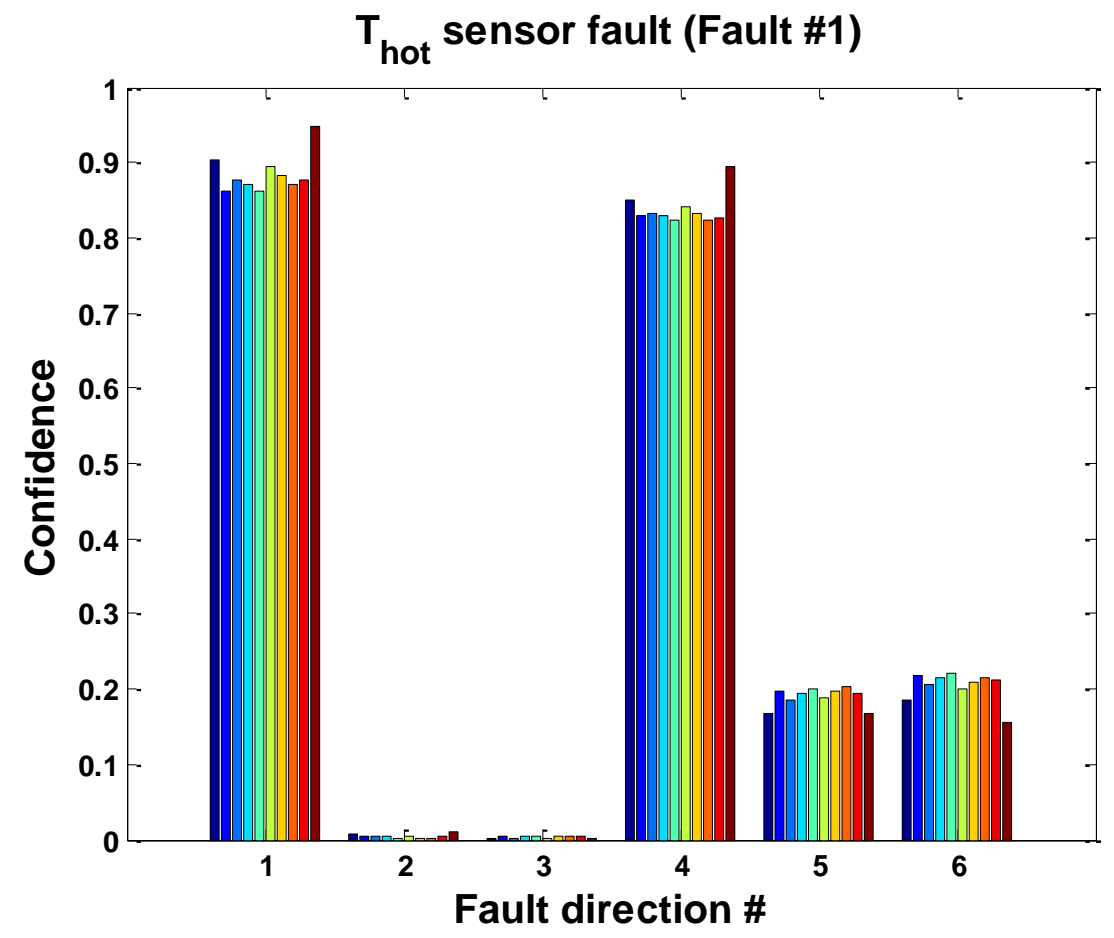

Figure 8.17. Fault isolation index for $\mathrm{T}_{\text {hot }}$ sensor fault (reduced sensor set). 


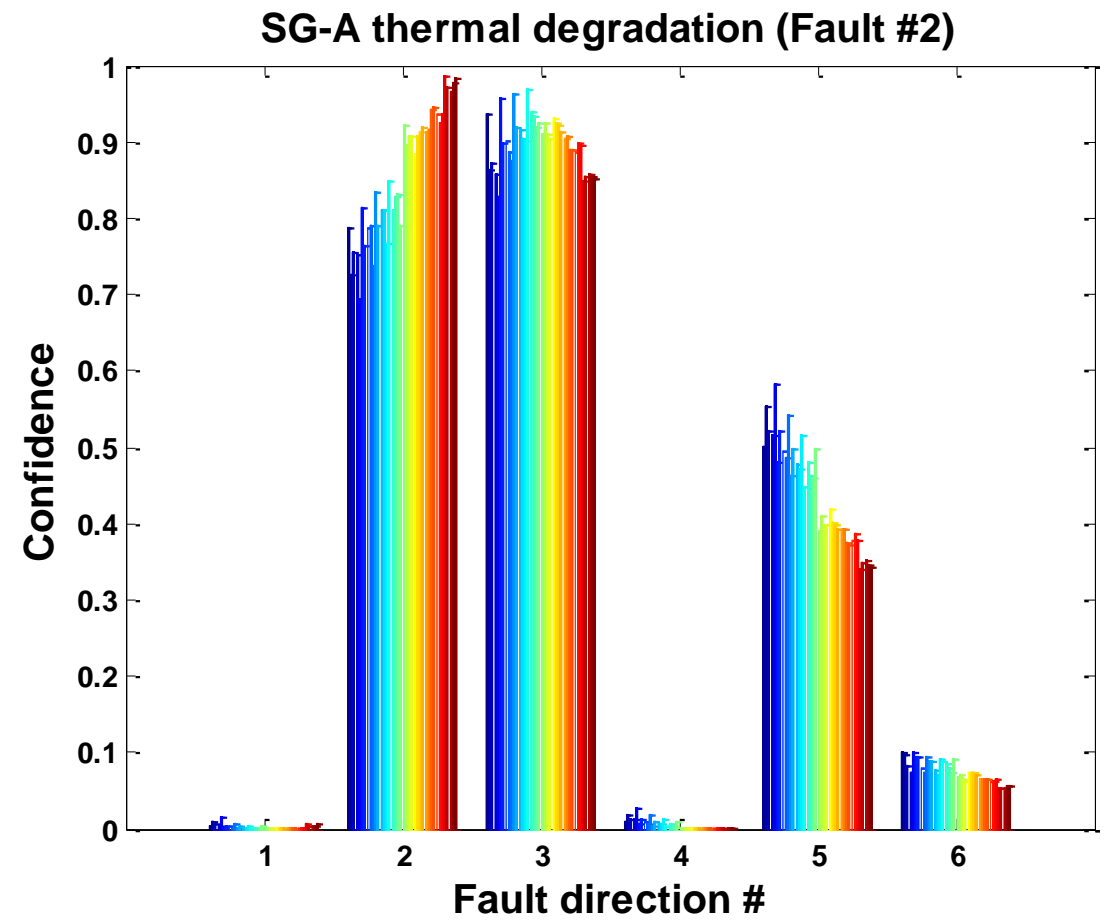

Figure 8.18. Fault isolation index for SG-A thermal degradation (reduced sensor set).

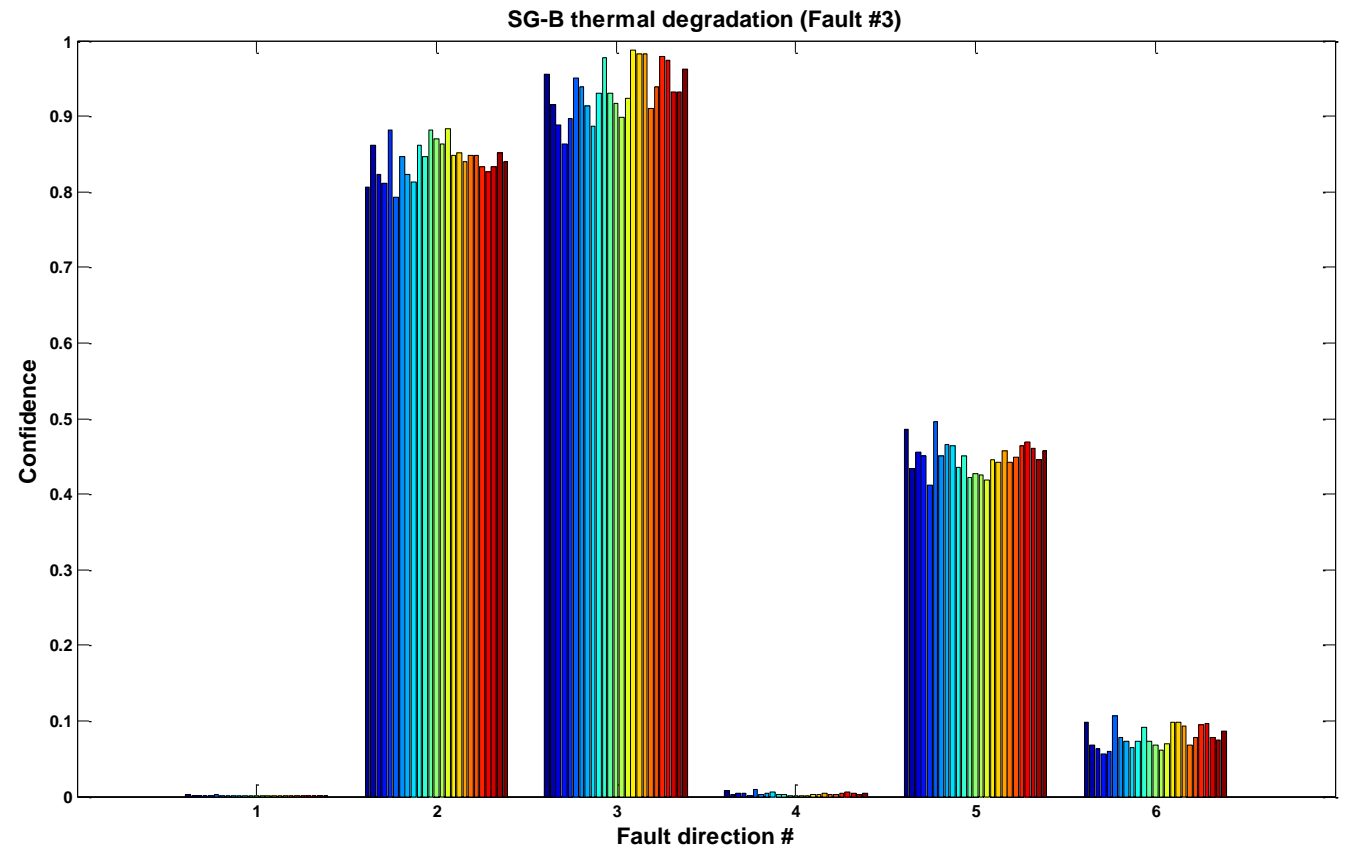

Figure 8.19. Fault isolation index for SG-B thermal degradation (reduced sensor set). 


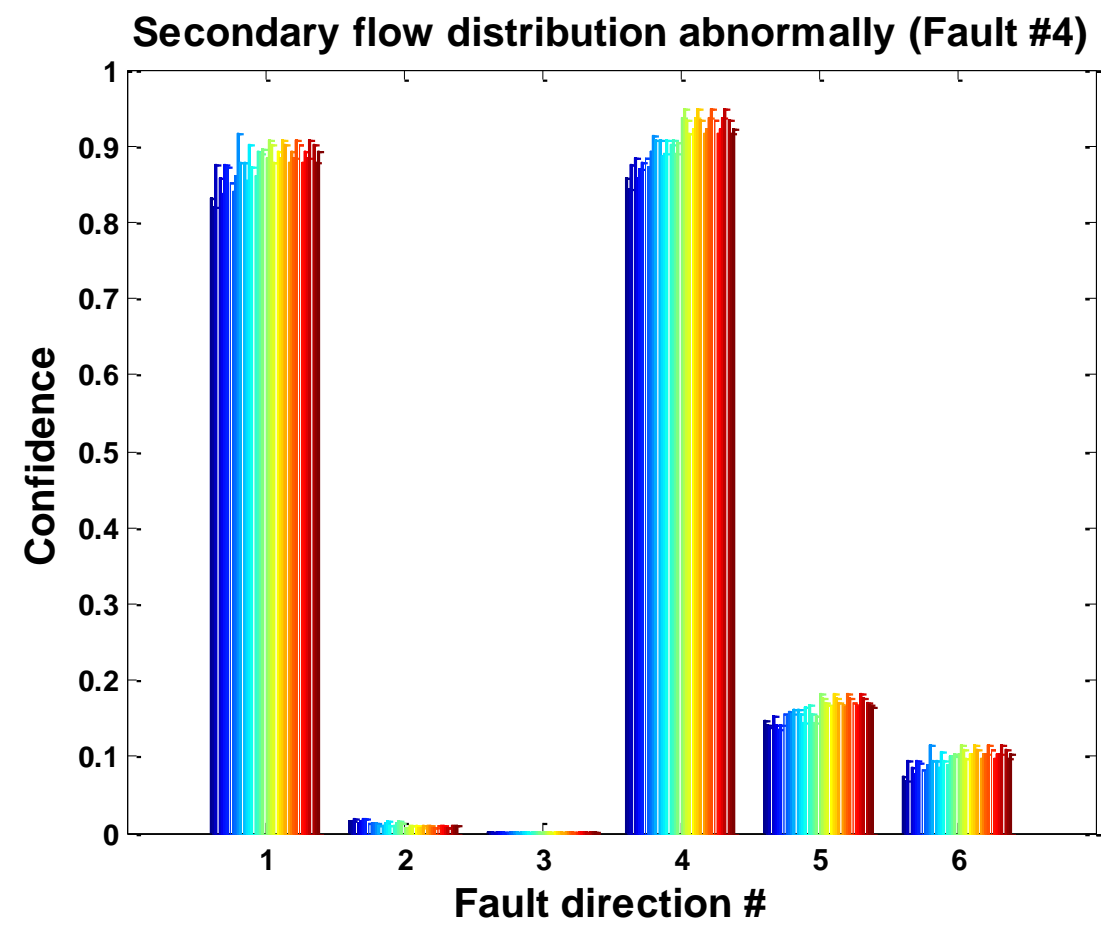

Figure 8.20. Fault isolation index for secondary flow anomaly (reduced sensor set).

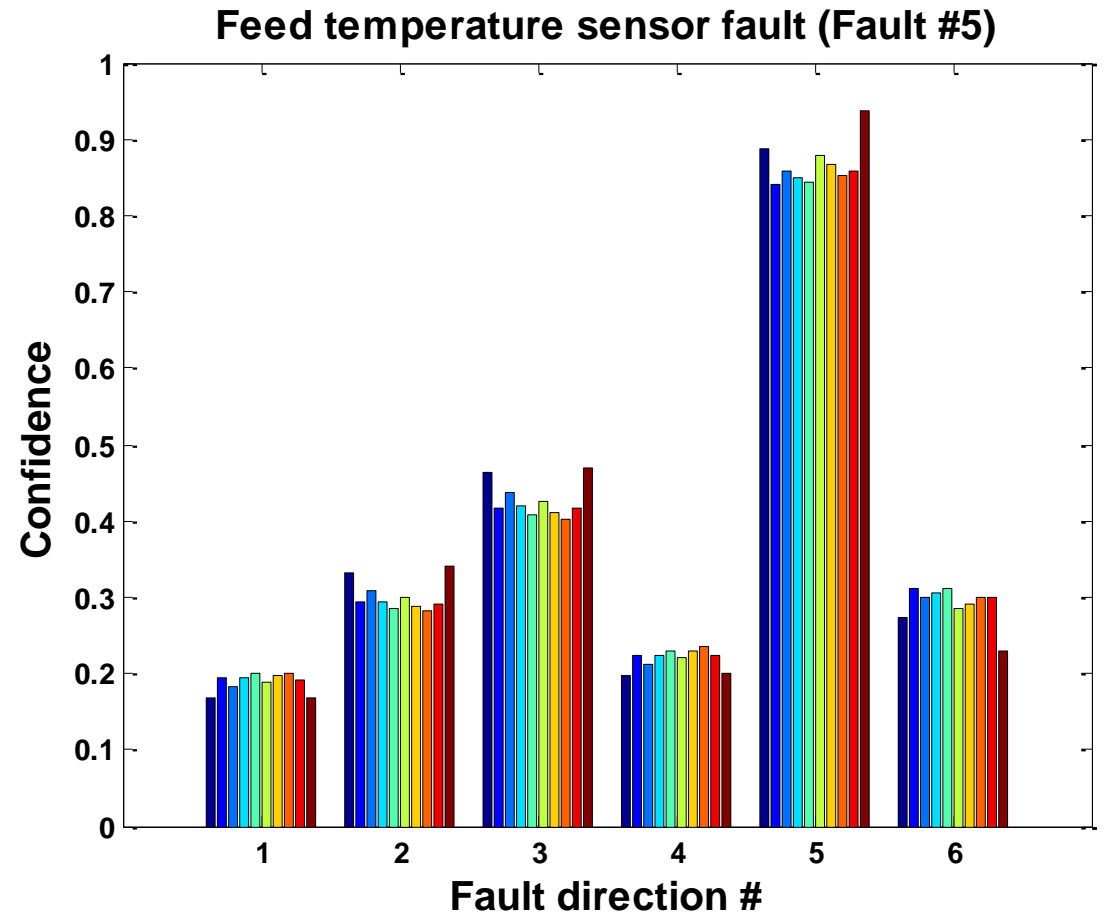

Figure 8.21. Fault isolation index for feed temperature sensor fault (reduced sensor set). 


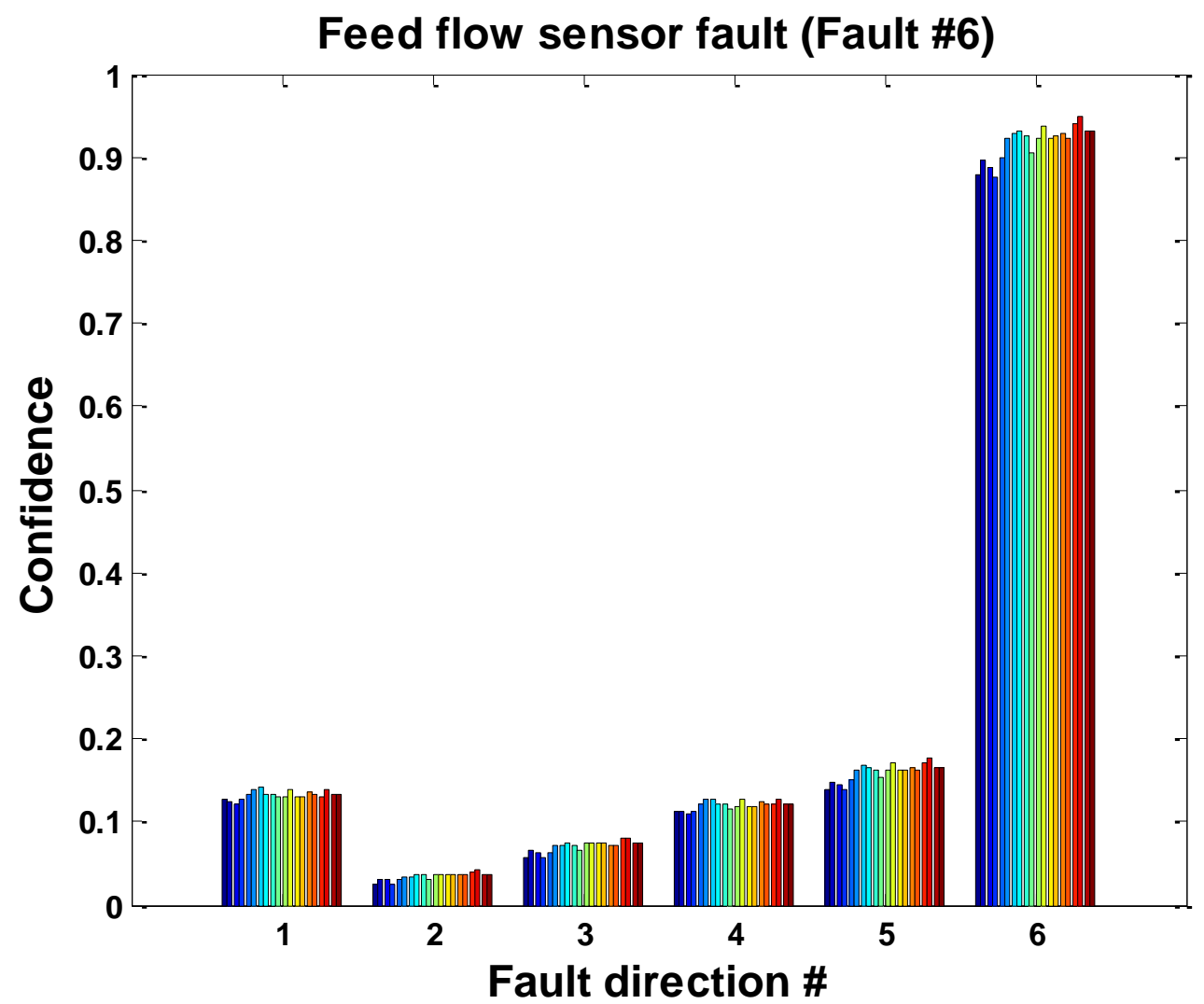

Figure 8.22. Fault isolation index for feed flow sensor fault (reduced sensor set).

In summary, Figure 8.23(a) shows the fault isolation indices for each of the detected fault scenarios in the HCSG system. All the fault cases are detected correctly using the fault isolation approach. In comparison, Figure 8.23(b) illustrates the fault isolation indices when the reduced sensor set (T3, T11, W7, and W12) is used to develop the PCA model for HCSG fault monitoring. It is seen that when the hot leg temperature sensor is under degradation (Fault \#1), the fault isolation indices of both fault direction \#1 and fault direction \#4 have significant values, which are difficult to distinguish. The same problems occur for the fault cases 2, 3, and 4. Therefore, we can draw the conclusion that the reduced sensor set is not sufficient for the purpose of fault isolation in the HCSG systems, thus illustrating the effectiveness of optimal sensor allocation strategy. 
(a) Fault Isolation Index

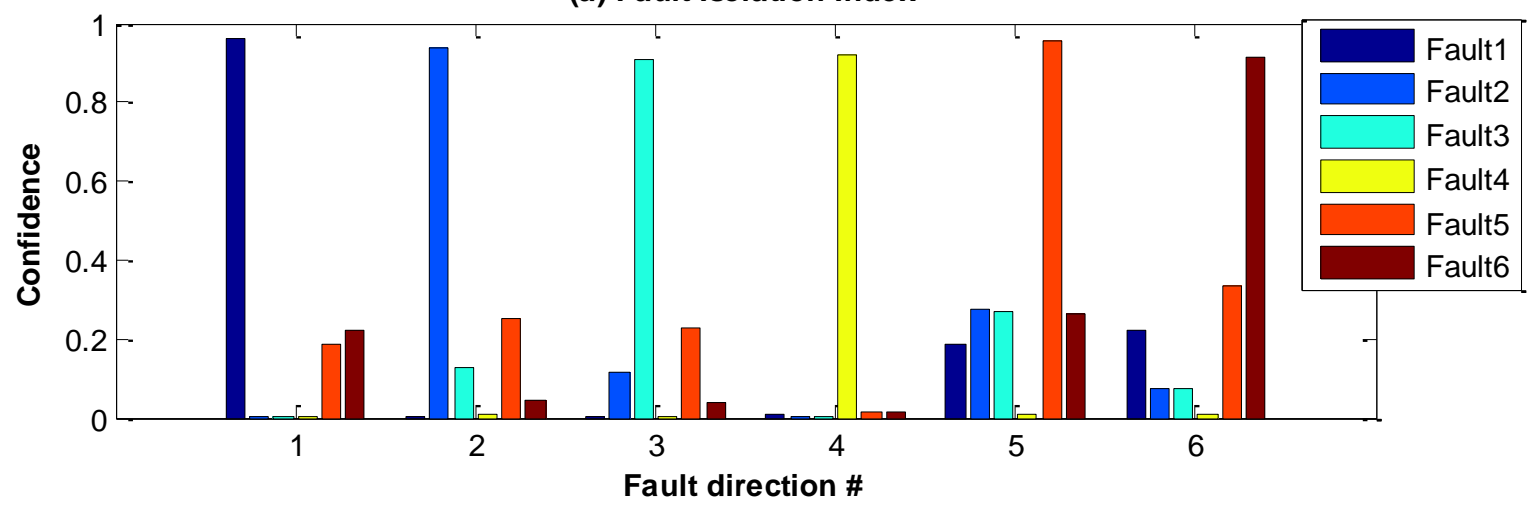

(b) Reduced Sensor Set Fault Isolation Index

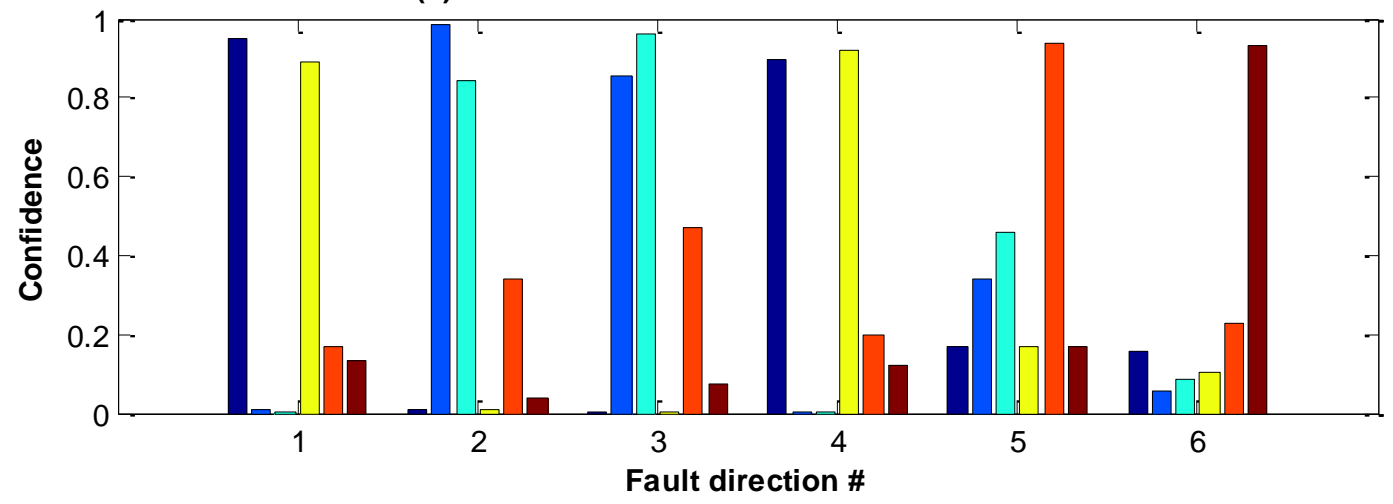

Figure 8.23. Fault isolation index for a pair of HCSGs.

\subsection{Summary}

In this chapter, the application of optimal sensor allocation for the IRIS helical coil steam generator system is demonstrated. A PCA-based fault isolation method is introduced to generate fault signatures of typical faults found in the HCSG systems. The confidence levels on each fault direction were calculated through vector projection. Fault isolation index provides a convenient way to isolate the faults using projections on the fault directions. Fault diagnostic results were also examined using reduced sensor sets (non-optimal sensor selection) for the same fault scenarios, and the results indicated that the reduced sensor sets were not sufficient for the purpose of fault isolation. 


\section{Chapter 9}

\section{Summary, Conclusions, and Suggestions for Future Research}

\subsection{Summary and Conclusions}

Both nuclear and seawater desalination are mature and proven technologies, and are commercially available from a variety of suppliers. Nuclear plants can provide both electrical and thermal energy in an integrated, co-generation fashion to produce a spectrum of energy products including electricity, process heat, district heating, and potentially hydrogen generation. The prospects of using nuclear energy for seawater desalination on a large scale are very attractive since desalination is an energy-intensive process that can utilize the heat and/or the electricity produced by nuclear plants. While all nuclear reactor types can provide the energy required by the various desalination processes, it has been shown that small and medium modular reactors, a burgeoning field in the nuclear community today, offer the largest potential as coupling options of nuclear desalination systems in some developing countries where less stable electrical grids and increasing necessity for potable water sources are often found. IRIS is such a novel SMR with an advanced, modular, passively safe design that is well suited for desalination applications. However, coupling a SMR system and a desalination process involves a number of issues that have to be addressed in the design phase. Among these issues, instrumentation and controls, performance monitoring and fault diagnosis are of high importance for the safe and optimal operation of a coupled nuclear desalination plant.

The preceding chapters have presented an integrated approach to the performance monitoring and fault diagnosis of a large industrial plant, such as nuclear desalination systems using robust data-based empirical methods. Sensor placement design is a critical piece in a fault monitoring system, which typically includes process performance monitoring, fault detection and isolation, and maintenance planning. Thus, it is emphasized in the developed integrated approach. Nowadays, most processes are extremely well instrumented with a multitude of sensors providing both control and safety-related measurements, especially in a nuclear desalination plant. The ability of the sensor network to detect and discriminate failure modes and

anomalous conditions is critical for the effectiveness of any fault monitoring system. With 
hundreds of process variables available for measurement in both nuclear power plants and desalination processes, selection of optimum number of sensors and their locations poses a unique challenge.

Six areas of original contribution are discussed in the project report. These contributions are innovative and significant in that, to the best of the author's knowledge, they represent the first comprehensive studies of modeling, simulation, sensor placement design, and fault diagnosis of a nuclear desalination process. The original contributions are summarized here.

\section{Both steady-state and dynamic models were developed for MSF desalination systems} using MATLAB and SIMULINK. The steady-state model was simulated for a real plant operating conditions and a close agreement was observed between the predicted results and the actual plant operating parameters. Dynamic models were suitable for solving problems involving transient behavior, such as studying control strategies, stability problems, process interactions, start-up and shut-down conditions. In this research, the developed MSF dynamic model is a lumped parameter model that consists of ordinary differential equations and supporting algebraic equations. The initial conditions were known from steady-state simulations. The developed dynamic model was used to simulate the effect of step changes in several key state variables. The simulation results obtained were physically plausible and similar to those found in the literature.

\section{A dynamic SIMULINK model of the coupled IRIS-MSF nuclear desalination system} was also developed in this R\&D project. An existing IRIS SIMULINK model was used for coupling with the developed dynamic MSF model. A simple BOP system was also included in the coupled SIMULINK model. The BOP model incorporated the subsystem models of the turbine, condenser, and feedwater heater systems. The dynamic model of the coupled plant was simulated for a $10 \%$ step decrease in the turbine power demand, and the simulation results were examined and analyzed. The results showed that the developed dynamic model was able to characterize system dynamic behavior with reasonably good accuracy to study the control and fault diagnosis of the IRIS-MSF coupled system.

3. An integrated architecture of performance monitoring and fault diagnostics with emphasis on the importance of optimum sensor placement was developed in this research project. The solution to the problem of optimum sensor placement from a fault diagnosis 
perspective can be broadly broken down into two tasks: (1) fault modeling or prediction of cause-effect behavior of the system, generating a set of variables that are affected whenever a fault occurs, and (2) use of the generated sets to identify sensor locations based on various design criteria, such as fault observability, fault resolution, etc. In this research, the DG causal graph modeling method that represents cause-effect behavior of the process was used as a basis for the sensor placement design. Issues such as fault observability and fault resolution are discussed to ensure the designed sensor network could observe every defined fault in the process, meanwhile obtain a maximum possible fault resolution.

\section{Development of an optimal and automated sensor placement procedure was conducted}

as well. The developed sensor placement design algorithm treats finding the optimal sensor locations as one of choosing the minimum number of sensors that would cover all the faults defined for the system of interest, which is the commonly known "minimum set-covering problem". The fault observability and resolution problems could be solved exactly by enumeration, but with an increasing number of system faults and sensor locations, it may not be computationally feasible to solve the problem in that fashion. In many instances, heuristics often give a quick and reasonably approximate solution. An ILP embedded greedy search heuristic was developed for solving the fault observability and resolution problems in this research. The optimal sensor placement design in terms of fault diagnosis was further formulated as a system unobservability minimization problem which took into account the cost of building a sensor network. Moreover, various information features, such as fault occurrence probability and sensor failure probability, were incorporated in the formulation. The greedy search heuristic was applied to solve the formulated optimization problem, and the optimization performances were evaluated and compared with a commercial optimization solver, LINGO 8.0. Application results showed that the greedy search heuristic and the LINGO solver resulted in optimal sensor sets of comparable performance.

\section{A PCA-based fault diagnostic approach, in conjunction with optimized sensor} selection, was developed in this $R \& D$ project with applications to an IRIS-MSF nuclear desalination plant. Despite using the optimal sensor set obtained from sensor placement design, it was found that some faults of the system were still indistinguishable in many cases. Nonetheless, optimal sensor placement design from a fault diagnosis perspective would provide 
valuable information to a fault monitoring system based on PCA. The PCA algorithm was utilized in this research to describe the relationships among the process variables. The fault directions for various fault scenarios were obtained using the SVD technique on the prediction errors, and fault isolation was then accomplished using new projections on these existing fault directions. The fault isolation index provides a convenient means of isolating the faults as it is close to unity on a particular direction for the corresponding fault whenever it occurs and is smaller for the others. The developed optimum sensor placement design formulations and the PCA-based fault diagnostic methods were demonstrated with application to the coupled IRIS-MSF nuclear desalination plant. When the optimal sensor sets were used, both sensor faults and process faults were correctly detected and isolated based on $T^{2}$ and $Q$ statistics, as well as fault isolation index. Fault diagnostic results were also examined using the reduced sensor sets for the same fault scenarios, and the results indicated that the reduced sensor sets were not enough for the purpose of fault isolation. Another important discovery through the FDI case studies was that the PCA fault diagnostic approach could be extended to detect and isolate simultaneous dual faults using the optimal sensor sets identified for the single-fault cases, in which case both single-fault and dual-fault directions were used as fault signatures for dual-fault isolation. The results demonstrated the effectiveness of the developed methods for fault detection and isolation in complex systems with optimized sensor network.

\section{The results of this $R \& D$ project also showed that it would be sufficient to use the} optimal sensor set to build effective PCA models for FDI. It is not necessary to use all the measurements in the database to develop a PCA representation, but those that are given through optimal sensor selection algorithms. This eliminates the arbitrary decision making in the selection of measurements needed for PCA modeling.

\subsection{Suggestions for Future Research}

The area of FDI holds many opportunities for continuing research beyond the scope of this project report. Although demonstrated through the simulation data generated by a developed SIMULINK model of the IRIS-MSF nuclear desalination plant, the proposed FDI algorithms need to be implemented and tested on a laboratory system and possibly using real nuclear desalination plant data. This would indicate areas where further development is necessary to 
establish a more efficient fault monitoring system. As part of future research, it is suggested that the fault diagnostic procedure be expanded to more fault scenarios and attempts be made to automate the procedure.

Sensor placement design based on directed graph was presented to determine the minimum set of sensors for fault detection and isolation, and was applied to the IRIS-MSF nuclear desalination system. In this project, the DG models were developed manually from engineering models. To facilitate computer aided design, this process should be automated by developing a generic software package that allows an automatic transformation of engineering models into DGs and the determination of minimum requirements of sensor placement from a fault diagnosis perspective.

Future research also should consist of the use of other optimization criteria for sensor placement design, such as process controllability constraint, residual precision, data reconciliation, and gross error robustness, as well as the treatment of the sensor placement design as a multi-objective optimization problem. Development of an integrated sensor network design strategy will make the seamless incorporation of various optimization criteria possible, so that the resulting sensor placement is optimal in a much broader sense, as opposed to the sole consideration of fault diagnostics. 


\section{REFERENCES}

1. Abdel-Jabbar, N.M., H.M. Qiblawey, F.S. Mjalli, and H. Ettouney (2007), "Simulation of large capacity MSF brine circulation plants,” Desalination, vol. 204, pp. 501-514, 2007.

2. Ahn, S.J., C.J. Lee, Y. Jung, C. Han, E.S. Yoon, and G. Lee (2008), "Fault diagnosis of the multi-stage flash desalination process based on signed digraph and dynamic partial least square," Desalination, vol. 228, pp. 68-83, 2008.

3. Aickelin, U. (2002), "An indirect genetic algorithm for set-covering problem," Journal of Operational Research Society, vol. 53, pp. 1118-1126, 2002.

4. Alatiqi, I., H. Ettouney, and H. El-Dessouky (1999), "Process control in water desalination industry: an overview," Desalination, vol. 126, pp. 15-32, 1999.

5. Ali, E., A. Ajbar, and K. Alhumaizi (1997), "Robust control of industrial multi-stage flash desalination plants," Desalination, vol. 114, pp. 289-302, 1997.

6. Ali, E., K. Alhumaizi, and A. Ajbar (1999), "Model reduction and robust control of multi-stage flash (MSF) desalination plants,” Desalination, vol. 121, pp. 65-85, 1999.

7. Ali, E. (2002a), "Understanding the operation of industrial MSF plants Part I: Stability and steady-state analysis," Desalination, vol. 143, pp. 53-72, 2002.

8. Ali, E. (2002b), "Understanding the operation of industrial MSF plants Part II: Optimization and dynamic analysis," Desalination, vol. 143, pp. 73-91, 2002.

9. Ali, Y., and S. Narasimhan (1993), "Sensor Network Design for Maximizing Reliability of Linear Processes," American Institute of Chemical Engineering Journal, vol. 39, no. 5, pp. 820-828, 1993.

10. Ali, Y., and S. Narasimhan (1995), "Redundant Sensor Network Design for Linear Processes," American Institute of Chemical Engineering Journal, vol. 41, no. 10, pp. 2237-2249, 1995.

11. Ali, Y., and S. Narasimhan (1996), "Sensor Network Design for Maximizing Reliability of Bilinear Processes,” American Institute of Chemical Engineering Journal, vol. 42, no. 9, pp. 2563-2575, 1996.

12. Aly, N.H., and M.A. Marwan (1995), "Dynamic behavior of MSF desalination plants," Desalination, vol. 101, pp. 287-293, 1995.

13. Aly, N.H., and M.A. Marwan (1997), "Dynamic response of multi-effect evaporators," Desalination, vol. 114, pp. 189-196, 1997.

14. Al-Shehabi, A.G., and B. Newman (2001), "Aeroelastic vehicle optimal sensor placement for feedback control applications using mixed gain-phase stability," Proceedings of the 
American Control Conference, vol. 3, pp. 1848-1852, June 2001.

15. Asratian, A.S., T.M.J. Denley, and R. Haggkvist (1998), Bipartite Graphs and their Applications, Cambridge University Press, UK, 1998.

16. Azam, M., K. Pattipati, and A. Patterson-Hine (2004), "Optimal Sensor Allocation for Fault Detection and Isolation," IEEE International Conference on Systems, Man and Cybernetics, vol. 2, pp. 1309-1314, 2004.

17. Bagajewicz, M. (1997), "Design and Retrofit of Sensor Networks in Process Plants," American Institute of Chemical Engineering Journal, vol. 43, no. 9, pp. 2300-2306, 1997.

18. Bagajewicz, M., and M. Sanchez (1999), "Design and Upgrade of Nonredundant and Redundant Linear Sensor Networks, ” American Institute of Chemical Engineering Journal, vol. 45, no. 9, pp. 1927-1938, 1999.

19. Bagajewicz, M., and M. Sanchez (2000), "Cost-optimal Design of Reliable Sensor Networks, ” Computers and Chemical Engineering, vol. 23, pp. 1757-1762, 2000.

20. Bagajewicz, M. (2001), Process Plant Instrumentation: Design and Upgrade, Technomic Publishing Company, Inc., 2001.

21. Bagajewicz, M. (2002a), "A Review of Techniques for Instrumentation Design and Upgrade in Process Plants," The Canadian Journal of Chemical Engineering, vol. 80, no. 1, pp. 3-16, 2002.

22. Bagajewicz, M., and E. Cabrera (2002b), "New MILP Formulation for Instrumentation Network Design and Upgrade," American Institute of Chemical Engineering Journal, vol. 48, no. 10, pp. 2271-2282, 2002.

23. Bagajewicz, M., A. Fuxman, and A. Uribe (2004), "Instrumentation Network Design and Upgrade for Process Monitoring and Fault Detection," American Institute of Chemical Engineering Journal, vol. 50, no. 8, pp. 1870-1880, 2004.

24. Beasley, J.E. (1987), "An algorithm for set-covering problem," European Journal of Operational Research, vol. 31, pp 85-93, 1987.

25. Beasley, J.E. (1990), “A Lagrangian heuristic for set-covering problems,” Naval Research Logistics, vol. 37, pp. 151-164, 1990.

26. Beasley, J.E., and P.C. Chu (1996), "A genetic algorithm for the set-covering problem," European Journal of Operational Research, vol. 94, pp. 392-404, 1996.

27. Bennetts, R.G. (1974), "Comment on the evaluation of fault trees," IEEE Transactions on Reliability, vol. 23, no. 1, pp. 54-55, 1974.

28. Bhushan, M., and R. Rengaswamy (2000a), "Design of Sensor Network Based on the Signed Directed Graph of the Process for Efficient Fault Diagnosis," Industrial and 
Engineering Chemistry Research, vol. 39, pp. 999-1019, 2000.

29. Bhushan, M., and R. Rengaswamy (2000b), "Design of Sensor Location Based on Various Fault Diagnostic Observability and Reliability Criteria," Computers and Chemical Engineering, vol. 24, pp. 735-741, 2000.

30. Bhushan, M., and R. Rengaswamy (2002a), "Comprehensive Design of a Sensor Network for Chemical Plants Based on Various Diagnosability and Reliability Criteria-1. Framework," Industrial and Engineering Chemistry Research, vol. 41, pp. 1826-1839, 2002.

31. Bhushan, M., and R. Rengaswamy (2002b), "Comprehensive Design of a Sensor Network for Chemical Plants Based on Various Diagnosability and Reliability Criteria-2. Applications," Industrial and Engineering Chemistry Research, vol. 41, pp. 1840-1860, 2002.

32. Bhushan, M., S. Narasimhan, and R. Rengaswamy (2008), "Robust Sensor Network Design for Fault Diagnosis," Computers and Chemical Engineering, vol. 32, pp. 1067-1084, 2008.

33. Bulsari, A.B. (1995), Neural networks for chemical engineers, Elsevier Science, Amsterdam, 1995.

34. Carelli, M.D., L. Conway, L. Oriani, B. Petrovic, N.E. Todreas (2003), "The Design and Safety Features of the IRIS Reactor, ” ICONE 11, Tokyo, Japan, April 20-23, 2003.

35. Carelli, M.D., and B. Petrovic (2006), "IRIS - progressing toward a worldwide deployment," Proceedings of the International Conference on Advances in Nuclear Power Plants (ICAPP'06), Reno, NV, 2006.

36. Carpenter, G.A., and S. Grossberg (1988), "The art of adaptive pattern recognition by a self-organizing neural network," Computer, vol. 21, no. 3, pp. 77-88, 1988.

37. Chen, B.H., X.Z. Wang, S.H. Yang, and C. Mcgreavy (1999), "Application of wavelets and neural networks to diagnostic system development: I. Feature extraction," Computers and Chemical Engineering, vol. 23, no. 7, pp. 899-906, 1999.

38. Chen, S.Y., and Y.F. Li (2002), “A method of Automatic Sensor Placement for Robot Vision in Inspection Tasks," Proceedings of the IEEE International Conference on Robotics \& Automation, vol. 3, pp. 2545-2550, 2002.

39. Chmielewski, D., T. Palmer, and V. Manousiouthakis (2002), "On the Theory of Optimal Sensor Placement," American Institute of Chemical Engineering Journal, vol. 48, no. 5, pp. 1001-1012, 2002.

40. Chow, E.Y., and A.S. Willsky (1984), "Analytical redundancy and the design of robust detection system," IEEE Transactions on Automatic Control, vol. 29, pp. 603-614, 1984. 
41. Cybenko, G. (1989), "Approximation by superpositions of a sigmoidal function," Mathematics of Control, Signals, and Systems, vol. 2, no. 4, pp. 303-314, 1989.

42. Dardour, S., S. Nisan, and F. Charbit (2005), "Development of a computer-package for MED plant dynamics," Desalination, vol. 182, pp. 229-237, 2005.

43. Delene, J.G., and S.J. Ball (1971), A Digital Computer Code for Simulating Large Multistage Flash Evaporator Desalting Plant Dynamics, Report ORNL-TM-2933, ORNL, Oak Ridge, TN, September 1971.

44. Dochain, D., N. Tali-Maanar, and J.P. Babary (1997), "On Modeling, Monitoring and Control of Fixed Bed Bioreactors," Computers and Chemical Engineering, vol. 21, pp. 1255-1266, 1997.

45. Duda, R.O., and P.E. Hart (1973), Pattern classification and scene classification, Wiley Interscience, New York, 1973.

46. Dunia, R., and S.J. Qin (1998), "Subspace approach to multidimensional fault identification and reconstruction," American Institute of Chemical Engineering Journal, vol. 44, pp. 1813-1831, 1998.

47. Eberhart, R.C., and J. Kennedy (1995), “A new optimization using particle swarm theory,” Proceedings of the 6th International Symposium on Micromachine and Human Science, Nagoya, Japan, pp. 39-43, 1995.

48. El-Dessouky, H.T., H.M. Ettouney, and Y. Al-Roumi (1999), "Multi-stage flash desalination: present and future outlook," Chemical Engineering Journal, vol. 73, no. 2, pp. 173-190, 1999.

49. Erbay, A.S., and B.R. Upadhyaya (1997), "A Personal Computer-Based On-Line Signal Validation System for Nuclear Power Plants," Nuclear Technology, vol. 119, pp. 63-75, 1997.

50. Fan, J.Y., M. Nikolaou, and R.E. White (1993), "An approach to fault diagnosis of chemical processes via neural networks," American Institute of Chemical Engineering Journal, vol. 39, no. 1, pp. 82-88, 1993.

51. Farell, A.E., and S.D. Roat (1994), "Framework for enhancing fault diagnosis capabilities of artificial neural networks," Computers and Chemical Engineering, vol. 18, no. 7, pp. 613-635, 1994.

52. Farlow, S.J. (1984), Self-Organizing Methods in Modeling: GMDH-Type Algorithms, Marcel-Dekker, New York, 1984.

53. Fathi, Z., W.F. Ramirez, and J. Korbiez (1993), "Analytical and knowledge-based redundancy for fault diagnosis in process plants," American Institute of Chemical Engineering Journal, vol. 39, pp. 42-56, 1993. 
54. Faulds, A.L., and B.B. King (2000), "Sensor location in feedback control of partial differential equation systems," Proceedings of the IEEE International Conference on Control Applications, vol. 1, pp. 536-541, Sept. 2000.

55. Flury, B. (1989), Common Principal Components and Related Multivariate Models, John Wiley, New York, 1989.

56. Furuya, H., and R.T. Haftka (1996), "Combining Genetic and Deterministic Algorithms for Locating Actuators on Space Structures," Journal of Spacecraft and Rockets, vol. 33, no. 3, pp. 422-427, 1996.

57. Fussell, J.B., G.J. Powers, and R.G. Bennetts (1974), "Fault trees - state of the art discussion," IEEE Transactions on Reliability, vol. 23, no. 1, pp. 51-55, 1974.

58. Gambier, A., and E. Badreddin (2002), "Application of hybrid modeling and control techniques to desalination plants," Desalination, vol. 152, pp. 175-184, 2002.

59. Gambier, A., and E. Badreddin (2005), "Dynamic Modeling of a Single-stage MSF Plant for Advanced Control Purposes," Proceedings of the IEEE Conference on Control Applications, Toronto, Canada, August 2005.

60. Giraud, C., and B. Jouvencel (1995), "Sensor Selection: a Geometrical Approach," Proceedings of the IEEE/RSJ International Conference, vol. 2, pp. 555-560, 1995.

61. Glover, F., and M. Laguna (1997), Tabu Search, Kluwer Academic Publishers, Boston, 1997.

62. Gluek, A.R., and R.W. Bradshaw (1970), "A mathematical model for a MSF distillation plant," Proceedings of the $3^{\text {rd }}$ International Symposium on Fresh Water from the Sea, vol. 1, pp. 95-108, 1970.

63. Griffith, W.L., and R.M. Keller (1965), Saline: A Fortran Computer Program for the Process Design of Saline Water Conversion Plants using the Multi-Stage, Flash-Evaporation Process, Report ORNL-TM-1299, ORNL, Oak Ridge, TN, November 1965.

64. Hac, A., and L. Liu (1993), "Sensor and Actuator Location in Motion Control of Flexible Structures," Journal of Sound and Vibration, vol. 167, no. 2, pp. 239-261, 1993.

65. Haddadi, S. (1997), "Simple Lagrangian heuristic for the set-covering problem," European Journal of Operational Research, vol. 97, pp 200-204, 1997.

66. Hastie, T., R. Tibshirani, and J. Friedman (2001), The Elements of Statistical Learning: Data Mining, Inference and Prediction, Springer, New York, 2001.

67. Helal, A.M., M.S. Medani, and M.A. Soliman (1986), "A Tridiagonal Matrix Model for Multistage Flash Desalination Plants," Computers and Chemical Engineering, vol. 10, pp. 327-342, 1986. 
68. Hines, J.W., D.J. Wrest, and R.E. Uhrig (1997), "Signal Validation using Adaptive Neural Fuzzy Inference Systems," Nuclear Technology, vol. 119, no. 2, pp. 181-193, 1997.

69. Hines, J.W., and D. Garvey (2007), "Process and equipment monitoring methodologies applied to sensor calibration monitoring," Quality and Reliability Engineering International, vol. 23, no. 1, pp. 123-135, 2007.

70. Hines, J.W. et al. (2008), Technical Review of On-Line Monitoring Techniques for Performance Assessment, vol. 1, 2 \& 3, NUREG/CR-6895, May 2008.

71. Hines, J.W., B.R. Upadhyaya et al. (2008), Advanced Instrumentation and Control Methods for Small and Medium Reactors with IRIS Demonstration, Annual Report prepared for the U.S. Department of Energy, DE-FG07-07ID14895/UTNE/2008-4, October 2008.

72. Hines, J.W., B.R. Upadhyaya et al. (2009), Advanced Instrumentation and Control Methods for Small and Medium Reactors with IRIS Demonstration, Annual Report prepared for the U.S. Department of Energy, DE-FG07-07ID14895/UTNE/2009-4, October 2009.

73. Hines, J.W., B.R. Upadhyaya et al. (2010), Advanced Instrumentation and Control Methods for Small and Medium Reactors with IRIS Demonstration, Annual Report prepared for the U.S. Department of Energy, DE-FG07-07ID14895/UTNE/2010-4, October 2010.

74. Holbert, K.E., and B.R. Upadhyaya (1990), "An Integrated Signal Validation System for Nuclear Power Plants,” Nuclear Technology, vol. 92. no. 3, pp. 411-427, 1990.

75. Holbert, K.E., and B.R. Upadhyaya (1994), "Empirical Process Modeling Technique for Signal Validation," Annals of Nuclear Energy, vol. 21, no. 7, pp. 387-403, 1994.

76. Humberstone, M.J. (2010), An Adaptive Nonparametric Modeling Technique for Expanded Condition Monitoring of Processes, Ph.D. Dissertation, University of Tennessee, Knoxville, May 2010.

77. Husain, A., A. Hassan, D.M.K. Al-Gobaisi, A. Al-Radif, A. Woldai, and C. Sommariva (1993), "Modelling, simulation, optimization and control of multistage flashing (MSF) desalination plants Part I: modelling and simulation," Desalination, vol. 92, pp. 21-41, 1993.

78. Husain, A., A. Woldai, A. Al-Radif, A. Kesou, R. Borsani, H. Sultan, and P.B. Deshpandey (1994), "Modelling and simulation of a multistage flash (MSF) desalination plant," Desalination, vol. 97, pp. 555-586, 1994.

79. International Atomic Energy Agency (2002), Design Concepts of Nuclear Desalination Plants, TECDOC-1326, IAEA, Austria, November 2002.

80. International Atomic Energy Agency (2005), Optimization of the Coupling of Nuclear 
Reactors and Desalination Systems, TECDOC-1444, IAEA, Austria, June 2005.

81. International Atomic Energy Agency (2007), Status of Nuclear Desalination in IAEA Member States, TECDOC-1524, IAEA, Austria, January 2007.

82. Ingersoll, D.T., J.L. Binder, D. Conti, and M.E. Ricotti (2004), "Nuclear Desalination Options for the international Reactor Innovative and Secure (IRIS) Design," Proceedings of the $5^{\text {th }}$ International Conference on Nuclear Option in Countries with Small and Medium Electricity Grids, Dubrovnik, Croatia, May 2004.

83. Ingersoll, D.T. (2009), "Deliberately small reactors and the second nuclear era," Progress in Nuclear Energy, vol. 51, pp. 589-603, 2009.

84. International Water Management Institute (2000), Vision of Water for Food and Rural Development, World Water Forum, Hague, March 2000.

85. Jackson, J.E., and G.S. Mudholkar (1979), "Control procedures for residuals associated with principal component analysis," Technometrics, vol. 21, pp. 341-349, 1979.

86. Jackson, J.E. (1991), A user guide to principal components, John Wiley \& Sons, New York, 1991.

87. James, M. (1985), Classification Algorithms, John Wiley, New York, 1985.

88. Johnson, D.S., C.R. Aragon, L.A. McGeoch, and C. Schevon (1989), “Optimization by Simulated Annealing: An Experimental Evaluation; Part I, Graph Partitioning," Operations Research, vol. 37, pp. 865-893, 1989.

89. Jolliffe, I.T. (2002), Principal Component Analysis, 2nd edition, Springer Series in Statistics, Springer, New York, 2002.

90. Kadu, S.C., M. Bhushan, and R. Gudi (2008), "Optimal sensor network design for multirate systems,” Journal of Process Control, vol. 18, pp. 594-609, 2008.

91. Kaistha, N., and B.R. Upadhyaya (2001), "Incipient fault detection and isolation of field devices in nuclear power systems using principal component analysis," Nuclear Technology, vol. 136, pp. 221-230, 2001.

92. Kalman, R.E. (1960), "A new approach to linear filtering and prediction problems," Journal of Basic Engineering, vol. 1, pp. 35-45, 1960.

93. Kavaklioglu, K., and B.R. Upadhyaya (1994), "Monitoring feedwater flow rate and component thermal performance of pressurized water reactors by means of artificial neural networks," Nuclear Technology, vol. 107, pp.112-123, 1994.

94. Khan, A.H. (1986), Desalination Processes and Multistage Flash Distillation Practice, Elsevier Publishers, New York, 1986. 
95. Kohonen, T. (1984), Self-organization and associative memory, Springer, New York, 1984.

96. Kotecha, P.R., M. Bhushan, R.D. Gudi, and M.K. Keshari (2008a), "A Duality Based Framework for Integrating Reliability and Precision for Sensor Network Design,” Journal of Process Control, vol. 18, pp. 189-201, 2008.

97. Kotecha, P.R., M. Bhushan, and R.D. Gudi (2008b), "Design of robust, reliable sensor networks using constraint programming," Computers and Chemical Engineering, vol. 32, pp. 2030-2049, 2008.

98. Kramer, M.A., and B.L. Palowitch (1987), "A rule-based approach to fault diagnosis using the signed directed graph," American Institute of Chemical Engineering Journal, vol. 33, pp. 1067-1078, 1987.

99. Kretsovalis, A., and R. Mah (1987), "Observability and Redundancy Classification in Multicomponent Process Networks, " American Institute of Chemical Engineering Journal, vol. 33, no. 1, pp. 70-82, 1987.

100. Krysander, M., and E. Frisk (2008), "Sensor placement for fault diagnosis," IEEE Transaction on Systems, Man, and Cybernetics-Part A, vol. 38, no. 6, pp. 1398-1410, 2008.

101. Lambert, H.E. (1977), “Fault Trees for Locating Sensors in Process Systems," Chemical Engineering Progress, vol. 73, no. 8, pp. 81-85, 1977.

102. Lapp, S.A., and G. Powers (1977), "Computer-aided synthesis of fault trees," IEEE Transactions on Reliability, vol. 26, no. 1, pp. 2-13, 1977.

103. Lim, K.B. (1997), “A Disturbance Rejection Approach to Actuator and Sensor Placement," Journal of Guidance, Control, and Dynamics, vol. 20, no. 1, pp. 202-204, 1997.

104. Lior, N., R. Chung, and O. Miyatake (2002), "Correlations (updated) for predicting the flow through MSF plant interstage orifices," Desalination, vol. 151, pp. 209-216, 2002.

105. Luong, M., D. Maquin, C. Huynh, and J. Ragot (1994), “Observability, Redundancy, Reliability and Integrated Design of Measurement System," Proceedings of the 2nd IFAC Symposium on Intelligent Components and Instruments for Control Applications, SICICA'94, 1994.

106. Madron, F., and V. Veverka (1992), "Optimal Selection of Measuring Point in Complex Plants by Linear Models," American Institute of Chemical Engineering Journal, vol. 38, no. 2, pp. 227-236, 1992.

107. Madron, F., J. Papuga, and J. Pliska (2006), "Thermal Performance Monitoring and Assessment in Dukovany Nuclear Power Plant," Proceedings of the $5^{\text {th }}$ International Topical Meeting on Nuclear Plant Instrumentation, Control, and Human Machine Interface 
Technologies (NPIC\&HMIT 2006), Albuquerque, NM, 2006.

108. Maniar, V.M., and P.B. Deshpande (1996), “Advanced controls for multi-stage flash (MSF) desalination plant optimization," Journal of Process Control, vol. 6, no. 1, pp. 49-66, 1996.

109. Mattison, B., M. Naghedolfeizi, and B.R. Upadhyaya (2007), "Development of a Graphical User Interface for a Dynamic Model of a Pressurized Water Reactor Plant," M.S. Project Report, University of Tennessee, Knoxville, 2007.

110. Maul, W.A., G. Kopasakis, L.M. Santi, T.S. Sowers, and A. Chicatelli (2007), Sensor Selection and Optimization for Health Assessment of Aerospace Systems, NASA/TM-2007-214822, 2007.

111. Maurya, M.R., R. Rengaswamy, and V. Venkatasubramanian (2006), “A Signed Directed Graph-based Systematic Framework for Steady-State Malfunction Diagnosis inside Control Loops," Chemical Engineering Science, vol. 61, pp. 1790-1810, 2006.

112. Maybeck, P.S. (1979), Stochastic Models, Estimation, and Control, Volume 1, Academic Press, New York, 1979.

113. Mazzotti, M., M. Rosso, A. Beltramini, and M. Morbidelli (2000), "Dynamic modeling of multistage flash desalination plants," Desalination, vol. 127, pp. 207-218, 2000.

114. Mazzour, E.H., D. Hodouin, and S. Makni (2003), "Optimal Sensor Implementation in Metallurgical Plants-An Application to a Generic Mineral Separation Plant," International Journal of Mineral Processing, vol. 69, pp. 185-203, 2003.

115. Megahed, M.M. (2001), "Nuclear desalination: history and prospects," Desalination, vol. 135, pp. 169-185, 2001.

116. Monfroglio, A. (1998), "Hybrid heuristic algorithm for set-covering," Computers Operational Research, vol. 25, pp. 441-445, 1998.

117. Muller, P., and H. Weber (1972), "Analysis and Optimization of Certain Qualities of Controllability and Observability for Linear Dynamical Systems," Automatica, vol. 8, pp. 237-246, 1972.

118. Mushini, R., and D. Simon (2005), "On Optimization of Sensor Selection for Aircraft Gas Turbine Engines," Proceedings of the 18th International Conference on Systems Engineering, ISCEng'05, IEEE, pp. 9-14, 2005.

119. Musulin, E., C. Benqlilou, M. Bagajewicz, and L. Puigjaner (2005), "Instrumentation Design Based on Optimal Kalman Filtering," Journal of Process Control, vol. 15, no. 6, pp. 629-638, 2005.

120. Naghedolfeizi, M. (1990), Dynamic Modeling of a Pressurized Water Reactor Plant for Diagnostics and Control, M.S. Thesis, University of Tennessee, Knoxville, 1990. 
121. Naimimohasses, R., D.M. Barnett, D.A. Green, and P.R. Smith (1995), "Sensor optimization using neural network sensitivity measures," Measurement Science and Technology, vol. 6, pp.1291-1300, 1995.

122. Narasimhan, S., P. Mosterman, and G. Biswas (1998), “A Systematic Analysis of Measurement Selection Algorithms for Fault Isolation in Dynamic Systems," Proceedings of the 9th International Workshop on Principles of Diagnosis, pp. 94-101, 1998.

123. Nguyen, D.Q., and M.J. Bagajewicz (2008), "Design of Nonlinear Sensor Networks for Process Plants," Industrial and Engineering Chemistry Research, vol. 47, no. 15, pp. 5529-5542, 2008.

124. Padula, S.L., and R.K. Kincaid (1999), Optimization strategies for sensor and actuator placement, NASA/TM-1999-209126, April 1999.

125. Papadopoulos, M., and E. Garcia (1998), "Sensor Placement Methodologies for Dynamic Testing,” AIAA Journal, vol. 36, no. 2, pp. 256-263, 1998.

126. Parker, R.G., and R. L. Rardin (1988), Discrete Optimization, Academic Press, San Diego, 1988.

127. Ponslet, E., R.T. Haftka, and H.H. Cudney (1993), "Optimal Placement of Tuning Masses on Truss Structures by Genetic Algorithm," Collection of Technical Papers - AIAA/ASME Structures, Dynamics and Materials Conference, Part 4, pp. 2448-2457, 1993.

128. Raghuraj, R., M. Bhushan, and R. Rengaswamy (1999), "Locating sensors in complex chemical plants based on fault diagnostic observability criteria," American Institute of Chemical Engineering Journal, vol. 45, no. 2, pp. 310-322, 1999.

129. Rimawi, M.A., H.M. Ettouney, and G.S. Aly (1989), "Transient Model of Multistage Flash Desalination,” Desalination, vol. 74, pp. 327-338, 1989.

130. Rosso, M., A. Beltramini, M. Mazzotti, and M. Morbidelli (1996), "Modeling multistage flash desalination plants," Desalination, vol. 108, pp. 365-374, 1996.

131. Santi, L.M, T.S. Sowers, and R.B. Aguilar (2005), Optimal Sensor Selection for Health Monitoring Systems, NASA/TM-2005-213955, 2005.

132. Sen, S., S. Narasimhan, and K. Deb (1998), "Sensor Network Design of Linear Processes Using Genetic Algorithms," Computers and Chemical Engineering, vol. 22, no. 3, pp. 385-390, 1998.

133. Shankar, G. (1977), "Simulation of a Nuclear Turbine," Journal of Nuclear Engineering and Design, vol. 44, pp. 269-277, 1977.

134. Shiozaki, J., B. Shibata, H. Matsuyama, and E. O'Shima (1989), “Fault Diagnosis of Chemical Processes Utilizing Signed Directed Graphs-Improvement by Using Temporal Information," IEEE Transactions on Industrial Electronics, vol. 36, no. 4, pp. 469-474, 
1989.

135. Sorenson, H.W. (1970), "Least-Squares estimation: from Gauss to Kalman," IEEE Spectrum, vol. 7, no. 7, pp. 63-68, 1970.

136. Spanache, S., T. Escobet, and L. Travé-Massuyès (2004), "Sensor Placement Optimisation Using Genetic Algorithms," Proceedings of the15th International Workshop on Principles of Diagnosis, pp. 179-183, 2004.

137. Tanvir, M.S., and I.M. Mujtaba (2008), "Optimisation of design and operation of MSF desalination process using MINLP technique in gPROMS," Desalination, vol. 222, pp. 419-430, 2008.

138. Tarifa, E.E., and N.J. Scenna (1997), "Fault diagnosis, direct graphs, and fuzzy logic," Computers and Chemical Engineering, vol. 21, pp. 649-654, 1997.

139. Tarifa, E.E., and N.J. Scenna (1998a), "A methodology for fault diagnosis in large chemical processes and an application to a multistage flash desalination process: Part I," Reliability Engineering and System Safety, vol. 60, pp. 29-40, 1998.

140. Tarifa, E.E., and N.J. Scenna (1998b), "A methodology for fault diagnosis in large chemical processes and an application to a multistage flash desalination process: Part II," Reliability Engineering and System Safety, vol. 60, pp. 41-51, 1998.

141. Tarifa, E.E., and N.J. Scenna (2001), “A dynamic simulator for MSF plants,” Desalination, vol. 138, pp. 349-364, 2001.

142. Tarifa, E.E., and N.J. Scenna (2002), "Fault diagnosis for a MSF using a SDG and fuzzy logic,” Desalination, vol. 152, pp. 207-214, 2002.

143. Tarifa, E.E., and N.J. Scenna (2004), "Fault diagnosis for MSF dynamic states using a SDG and fuzzy logic," Desalination, vol. 166, pp. 93-101, 2004.

144. Thanomsat, C., C.W. Taft, and A.M. Annaswamy (1998), "Level Control in Feedwater Heater Systems Using Nonlinear Strategies," ISA Transactions, vol. 37, pp. 299-312, 1998.

145. Thomas, P.J., S. Bhattacharyya, A. Patra, and G.P. Rao (1998), "Steady state and dynamic simulation of multi-stage flash desalination plants: A case study," Computers and Chemical Engineering, vol. 22, pp. 1515-1529, 1998.

146. Tsai, C.S., and C.T. Chang (1995), "Dynamic process diagnosis via integrated neural networks," Computers and Chemical Engineering, vol. 19, pp. 747-752, 1995.

147. Tylee, J.L. (1983), “On-Line Failure Detection in Nuclear Power Plant Instrumentation," IEEE Transactions on Automatic Control, vol. AC-28, no. 3, pp. 406-415, 1983.

148. Udwadia, F.E. (1994), "Methodology for Optimum Sensor Locations for Parameter 
Identification in Dynamic Systems, ” Journal of Engineering Mechanics, vol. 120, no. 2, pp. 368-390, 1994.

149. Ulerich, N.H., and G.A. Powers (1988), "On-line hazard aversion and fault diagnosis in chemical processes: the digraph plus fault tree method," IEEE Transactions on Reliability, vol. 37, no. 2, pp. 171-177, 1988.

150. Upadhyaya, B.R. et al. (1989), Development and Testing of an Integrated Signal Validation System for Nuclear Power Plants, Final Report prepared for the U.S. Department of Energy, DOE/NE/37959-36, September 1989.

151. Upadhyaya, B.R., and E. Eryurek (1992), "Application of Neural Networks for Sensor Validation and Plant Monitoring," Nuclear Technology, vol. 97, no. 2, pp. 170-176, 1992.

152. Vaclavek, V., and M. Loucka (1976), "Selection of measurements necessary to achieve multi-component mass balances in chemical plant," Chemical Engineering Science, vol. 31, no. 12, pp. 1199-1205, 1976.

153. Valle, S., W. Li, and S.J. Qin (1999), "Selection of the number of principal components: the variance of the reconstruction error criterion with a comparison to other methods," Industrial and Engineering Chemistry Research, vol. 38, pp. 4389-4401, 1999.

154. Van den Berg, F., H. Hoefsloot, H. Boelens, and A. Smilde (2000), "Selection of Optimal Sensor Position in a Tubular Reactor Using Robust Degree of Observability Criteria," Chemical Engineering Science, vol. 55, pp. 827-837, 2000.

155. Vasko, J.F., and F.E. Wolf (2001), "A heuristic concentration approach for weighted set-covering problems," Locator: ePublication of Location Analysis, vol. 2, no. 1, pp. 1-14, 2001.

156. Venkatasubramanian, V., and T.J. McAvoy (1992), "Special issue on neural network applications in chemical engineering," Computers and Chemical Engineering, vol. 16, no. 4, 1992.

157. Wang, H., Z. Song, and P. Li (2000), "Improved PCA with Optimized Sensor Locations for Process Monitoring and Fault Diagnosis," Proceedings of the $39^{\text {th }}$ IEEE Conference on Decision and Control, Sydney, Australia, December 2000.

158. Wang, H., Z. Song, and H. Wang (2002a), "Statistical Process Monitoring Using Improved PCA with Optimized Sensor Locations, " Journal of Process Control, vol. 12, pp. 735-744, 2002.

159. Wang, H., Z. Song, and H. Wang (2002b), "Fault detection behavior analysis of PCA-based process monitoring approach," Journal of Chemical Industry and Engineering, vol. 53, no. 3, pp. 297-301, 2002.

160. Wang, H., Z. Song, and P. Li (2002c), "Fault Detection Behavior and Performance Analysis of Principal Component Analysis Based Process Monitoring Methods," Industrial 
and Engineering Chemistry Research, vol. 41, pp. 2455-2464, 2002.

161. Wang, X., G. Rong, and J. Li (2007), “A New Approach to Design Reliable General Sensor Network on the Basis of Graph Theory," Industrial and Engineering Chemistry Research, vol. 46, no. 8, pp. 2520-2525, 2007.

162. Wang, X.Z., B.H. Chen, S.H. Yang, and C. Mcgreavy (1999), “Application of wavelets and neural networks to diagnostic system development: 2. An integrated framework and its application," Computers and Chemical Engineering, vol. 23, no. 7, pp. 945-954, 1999.

163. Watanabe, K., S. Hirota, L. Hou, and D.M. Himmelblau (1994), "Diagnosis of Multiple Simultaneous Faults via Hierarchical Artificial Neural Networks," American Institute of Chemical Engineering Journal, vol. 40, no. 5, pp. 839-848, 1994.

164. Whiteley, J.R., and J.F. Davis (1994), "A similarity-based approach to interpretation of sensor data using adaptive resonance theory," Computers and Chemical Engineering, vol. 18, no. 7, pp. 637-661, 1994.

165. Xu, J., and B.R. Upadhyaya (2009), "Multivariable Control Design for the IRIS Primary System Using the Model-Predictive Control Approach," Proceedings of the $6^{\text {th }}$ International Topical Meeting on Nuclear Plant Instrumentation, Control, and Human Machine Interface Technologies (NPIC\&HMIT 2009), Knoxville, TN, April 5-9, 2009.

166. Yan, Y. (2004), "Sensor Placement and Diagnosability Analysis at Design Stage," National Research Council of Canada, NRC 47160, 2004.

167. Zhang, G. (2005), Optimum Sensor Localization/Selection in a Diagnostic/Prognostic Architecture, Ph.D. Dissertation, Georgia Institute of Technology, January 2005.

168. Zhang, G., and G. Vachtsevanos (2007), "A Methodology for Optimum Sensor Localization/Selection in Fault Diagnosis," Proceedings of the IEEE Aerospace Conference, 2007.

169. Zhang, Y. (2004), Product quality modeling \& control based on vision inspection with an application to baking processes, Ph.D. Dissertation, Georgia Institute of Technology, December 2004.

170. Zhao, K. (2005a), An Integrated Approach to Performance Monitoring and Fault Diagnosis of Nuclear Power Systems, Ph.D. Dissertation, University of Tennessee, Knoxville, May 2005.

171. Zhao, K., and B.R. Upadhyaya (2005b), "Adaptive fuzzy inference causal graph approach to fault detection and isolation of field devices in nuclear power plants," Progress in Nuclear Energy, vol. 46, pp. 226-240, 2005. 


\section{APPENDICES}




\section{Appendix A: Thermo-Physical Properties}

Pressure of saturated steam

$$
\log _{10} \frac{P_{c}}{P}=\frac{X}{T_{S}}\left(\frac{a+b X+c X^{3}}{1+d X}\right)
$$

where

$a=3.2437814$

$b=5.86826 \times 10^{-3}$

$c=1.1702379 \times 10^{-8}$

$d=2.1878462 \times 10^{-3}$

$P=$ pressure $(\mathrm{atm})$

$P_{c}=$ critical pressure $(218.167 \mathrm{~atm})$

$T_{c}=$ critical temperature $(647.27 \mathrm{~K})$

$X=T_{c}-T_{S}$

The above equation is specified for temperatures from 50 to $300^{\circ} \mathrm{F}$

Specific enthalpy of saturated steam

$$
h_{V}=1059.237+0.4833 T-2.558 \times 10^{-4} T^{2}
$$

where

$\mathrm{h}_{\mathrm{V}}=$ specific enthalpy of saturated steam (BTU/lb)

$\mathrm{T}=$ temperature $\left({ }^{\mathrm{o}} \mathrm{F}\right)$

Specific heat capacity of pure water

$$
C p_{w}=1.0011833-6.1666652 \times 10^{-5} T+1.3999989 \times 10^{-7} T^{2}+1.3333336 \times 10^{-9} T^{3}
$$

where

$\mathrm{Cp}_{\mathrm{w}}=$ specific heat capacity of water $\left(\mathrm{BTU} / \mathrm{lb} \cdot{ }^{o} \mathrm{~F}\right)$

$\mathrm{T}=$ boiling temperature of water $\left({ }^{\circ} \mathrm{F}\right)$

The above expression for the heat capacity of saturated water is adequate for computations within the temperature range of $50 \sim 300^{\circ} \mathrm{F}$.

Specific enthalpy of saturated water

$$
h_{W}=-31.92+1.0011833 T-3.0833326 \times 10^{-5} T^{2}+4.6666663 \times 10^{-8} T^{3}+3.333334 \times 10^{-10} T^{4}
$$

where

$\mathrm{h}_{\mathrm{w}}=$ specific enthalpy of saturated water $(\mathrm{BTU} / \mathrm{lb})$

$\mathrm{T}=$ boiling temperature of water $\left({ }^{\circ} \mathrm{F}\right)$

Latent heat of vaporization of water

$$
\lambda_{S}=h_{V}-h_{W}
$$


where

$\lambda_{\mathrm{s}}=$ latent heat of vaporization $(\mathrm{BTU} / \mathrm{lb})$

$\mathrm{h}_{\mathrm{v}}=$ specific enthalpy of saturated steam (BTU/lb)

$h_{w}=$ specific enthalpy of saturated water (BTU/lb)

\section{Specific heat capacity of brine}

$$
C p_{b}=\left[1.0-X_{b}\left(0.011311-0.00001146 T_{b}\right)\right] C p_{w}
$$

where

$\mathrm{Cp}_{\mathrm{b}}=$ specific heat capacity of brine $\left(\mathrm{BTU} / \mathrm{lb} \cdot{ }^{o} \mathrm{~F}\right)$

$\mathrm{Cp}_{\mathrm{w}}=$ specific heat capacity of water $\left(\mathrm{BTU} / \mathrm{lb} \cdot{ }^{o} \mathrm{~F}\right)$

$\mathrm{T}_{\mathrm{b}}=$ brine temperature $\left({ }^{\mathrm{o}} \mathrm{F}\right)$

$\mathrm{X}_{\mathrm{b}}=$ salt concentration $(\mathrm{wt} \%)$

\section{Density of brine}

The expression for the brine density given here is valid for the range of $0 \sim 26 \%$ concentration and $40 \sim 300{ }^{\circ} \mathrm{F}$. Density of pure water is calculated from the given equation putting $X_{b}=0$.

$$
\begin{aligned}
& \rho_{b}=62.707172+49.364088 X_{b}-0.43955304 \times 10^{-2} T_{b}-0.032554667 \mathrm{X}_{\mathrm{b}} T_{b} \\
& -0.46076921 \times 10^{-4} T_{b}^{2}+0.63240299 \times 10^{-4} X_{b} T_{b}^{2}
\end{aligned}
$$

where

$\rho_{b}=$ density $\left(\mathrm{lb} / \mathrm{ft}^{3}\right)$

$\mathrm{T}_{\mathrm{b}}=$ brine temperature $\left({ }^{\circ} \mathrm{F}\right)$

$\mathrm{X}_{\mathrm{b}}=$ salt concentration (mass fraction)

\section{Boiling point elevation}

$$
\begin{aligned}
& B P E=1.8 \times\left[565.757 / \mathrm{T}_{\mathrm{K}}-9.81559+1.54739 \ln \mathrm{T}_{\mathrm{K}}-\left(337.178 / \mathrm{T}_{\mathrm{K}}-6.41981+0.922753 \ln \mathrm{T}_{\mathrm{K}}\right) X\right. \\
& \left.+\left(32.681 / \mathrm{T}_{\mathrm{K}}-0.55368+0.079022 \ln \mathrm{T}_{\mathrm{K}}\right) X^{2}\right] \times\left[X /\left(266919.6 / \mathrm{T}_{\mathrm{K}}^{2}-379.669 / \mathrm{T}_{\mathrm{K}}+0.334169\right)\right]
\end{aligned}
$$

where

$B P E=$ boiling point elevation $\left({ }^{\circ} \mathrm{F}\right)$

$T_{K}=\left(T_{b}+460\right) / 1.8\left({ }^{o} K\right)$

$\mathrm{T}_{\mathrm{b}}=$ brine temperature $\left({ }^{\mathrm{o}} \mathrm{F}\right)$

$X=\left(19.819 X_{b}\right) /\left(1-X_{b}\right)$

$\mathrm{X}_{\mathrm{b}}=$ salt concentration (mass fraction)

\section{Non-equilibrium allowance}

$$
N E A=352 H_{j}^{1.1} \Delta T_{B_{j}}^{-0.25}\left(\omega_{j} \times 10^{-3}\right)^{0.5} T_{S_{j}}^{-2.5}
$$


where

$N E A=$ non - equilibrium allowance $\left({ }^{\circ} \mathrm{F}\right)$

$H_{j}=$ height of brine pool in stage $\mathrm{j}$ (in.)

$\Delta T_{B_{j}}=T_{B_{j-1}}-T_{B_{j}}\left({ }^{o} F\right)$

$\omega_{\mathrm{j}}=\frac{\mathrm{W}}{\mathrm{w}_{\mathrm{j}}}=$ chamber load $\mathrm{lb} / \mathrm{hr}$ per ft width

$\mathrm{w}_{\mathrm{j}}=$ width of stage $\mathrm{j}(\mathrm{ft})$

$\mathrm{W}=$ total mass circulated in the system (recycle $\mathrm{R}+$ make-up $\mathrm{F}, \mathrm{lb} / \mathrm{hr}$ )

Temperature loss across the demister and condenser tubes

$$
\Delta=\exp \left(1.885-0.02063 T_{P_{j}}\right)
$$

where

$\mathrm{T}_{\mathrm{P}_{\mathrm{j}}}=$ temperature of the distillate product $\left({ }^{\mathrm{o}} \mathrm{F}\right)$

\section{Overall heat transfer coefficient}

The data submitted by the Office of Saline Water (OSW), based on the Point Loma Demonstration Plant, have been correlated to calculate the sum of the different resistances included in the classical equation for $U$, except the inside film resistance [Griffith and Keller, 1965]. The correlation ties those resistances with the saturation temperature prevailing in the flash chamber. The polynomial fit is expressed as follows:

$$
\begin{aligned}
z= & 0.1024768 \times 10^{-2}-0.7473939 \times 10^{-5} T_{P_{j}}+0.999077 \times 10^{-7} T_{P_{j}}^{2} \\
& -0.430046 \times 10^{-9} T_{P_{j}}^{3}+0.6206744 \times 10^{-12} T_{P_{j}}^{4}
\end{aligned}
$$

where

$\mathrm{T}_{P_{\mathrm{j}}}=$ temperature of the distillate product $\left({ }^{\mathrm{o}} \mathrm{F}\right)$, the above equation is specified for temperatures ranging from 100 to $250^{\circ} \mathrm{F}$.

$$
y=\frac{\left(v_{j} \times I D_{j}\right)^{0.2}}{\left(160+1.92 T_{F_{j}}\right) v_{j}}
$$

where

$\mathrm{v}_{\mathrm{j}}=$ linear velocity of brine stream $(\mathrm{ft} / \mathrm{sec})$

$\mathrm{ID}_{\mathrm{j}}=$ tube inner diameter (in.)

$\mathrm{T}_{F_{\mathrm{j}}}=$ temperature of the cooling brine at the exit of condenser $\left({ }^{\circ} \mathrm{F}\right)$

From the values of $\mathrm{z}$ and $\mathrm{y}$, the overall heat transfer coefficient $\left(\mathrm{BTU} / \mathrm{hr} \cdot{ }^{o} \mathrm{~F} \cdot \mathrm{ft}^{2}\right)$ is calculated by the equation 


$$
U=\frac{1}{z+y}
$$

\section{Inter-stage flow rate}

$$
\begin{gathered}
Q=\left(K w h \sqrt{\Delta P \rho / 1000} / 1.96 \times 10^{-8}\right) / 0.45359 \\
\Delta P=P_{i-1}-P_{i}+1.0 \times 10^{-5} \rho g(L-C h) \\
C=0.61+0.18 x-0.58 x^{2}+0.7 x^{3} \\
x=\frac{g h \rho / 1000}{100\left(P_{i-1}-P\right)+g L \rho / 1000}
\end{gathered}
$$

where

$\mathrm{Q}=$ inter-stage flow rate $(\mathrm{lb} / \mathrm{hr})$

$\mathrm{w}=$ orifice width $(\mathrm{m})$

$\mathrm{h}=$ orifice height $(\mathrm{m})$

$\mathrm{L}=$ liquid level in the upstream stage (m)

$\rho=$ liquid density $\left(\mathrm{kg} / \mathrm{m}^{3}\right)$

$\mathrm{P}=$ stage pressure (bar)

$\mathrm{K}=$ orifice discharge coefficient

$\mathrm{C}=$ orifice contraction coefficient 


\section{Appendix B： IRIS Balance of Plant Mathematical Models}

\section{Steam Turbine}

The model development of the turbine system incorporates the following assumptions:

- Reversible adiabatic expansion process exists in the nozzle chest and moisture separator.

- Thermodynamic properties of the saturated steam and water are assumed to be linear functions of the steam pressure at each state.

- The bypass flow to the reheater is proportional to the main steam pressure and steam valve coefficient.

- Heat transfer coefficients for the reheater and feedwater heaters are assumed to be constant.

- Steam flow losses in the turbine system are considered to be zero.

To simplify the model complexity, modifications have been made for the evaluation of high pressure and low pressure turbine efficiencies. The efficiencies of the high and low pressure turbines are considered to be constant within a range of \pm 100 PSI deviations in the exhaust pressure of the high pressure turbine from its initial value (at full power of operating condition of the plant).

$$
\begin{gathered}
\eta_{h p}=\frac{h_{c}-h_{2}}{h_{c}-h_{2}^{\prime}} \\
\eta_{l p}=\frac{h_{r}-h_{4}}{h_{r}-h_{4}^{\prime}}
\end{gathered}
$$

\begin{tabular}{|c|c|}
\hline Variable & Definition \\
\hline$\eta_{h p}$ & High pressure turbine efficiency \\
\hline$\eta_{l p}$ & Low pressure turbine efficiency \\
\hline$h_{2}, h_{4}$ & $\begin{array}{c}\text { Steam enthalpy leaving the high and low pressure } \\
\text { turbine at points 2 and 4 respectively }\end{array}$ \\
\hline$h_{2}^{\prime}, h_{4}^{\prime}$ & Isotropic enthalpy at points 2 and 4 \\
\hline$h_{c}$ & Steam enthalpy leaving the nozzle chest \\
\hline
\end{tabular}

\section{Main Condenser}

A condenser is a large surface-type heat exchanger, which condenses steam from the exhaust of turbine by transferring steam latent heat to circulating water inside the condenser. The condenser is desired to work under vacuum condition to obtain a maximum mechanical power from the turbine system.

The main condenser is simulated as an equilibrium tow-phase tank. The space inside the tank is divided into tow independent control volumes, steam and water. They are assumed to be in thermal equilibrium during steady state condition. Turbine exhaust flow enters the system at the condenser pressure. The water part of the flow falls into the hotwell region and mixes perfectly with the water already present there. The vapor part condenses on the outer surface of metal tubes through which the circulating water flows. The condensation process is associated with a 
time delay which is due to the dynamics of heat transfer between the vapor and the circulating water. The following assumptions are made to simplify the mathematical model.

- Constant pressure exists in the condenser model.

- Mass transfer at steam-liquid interface is assumed to be negligible.

- Wall condensation is considered to be zero.

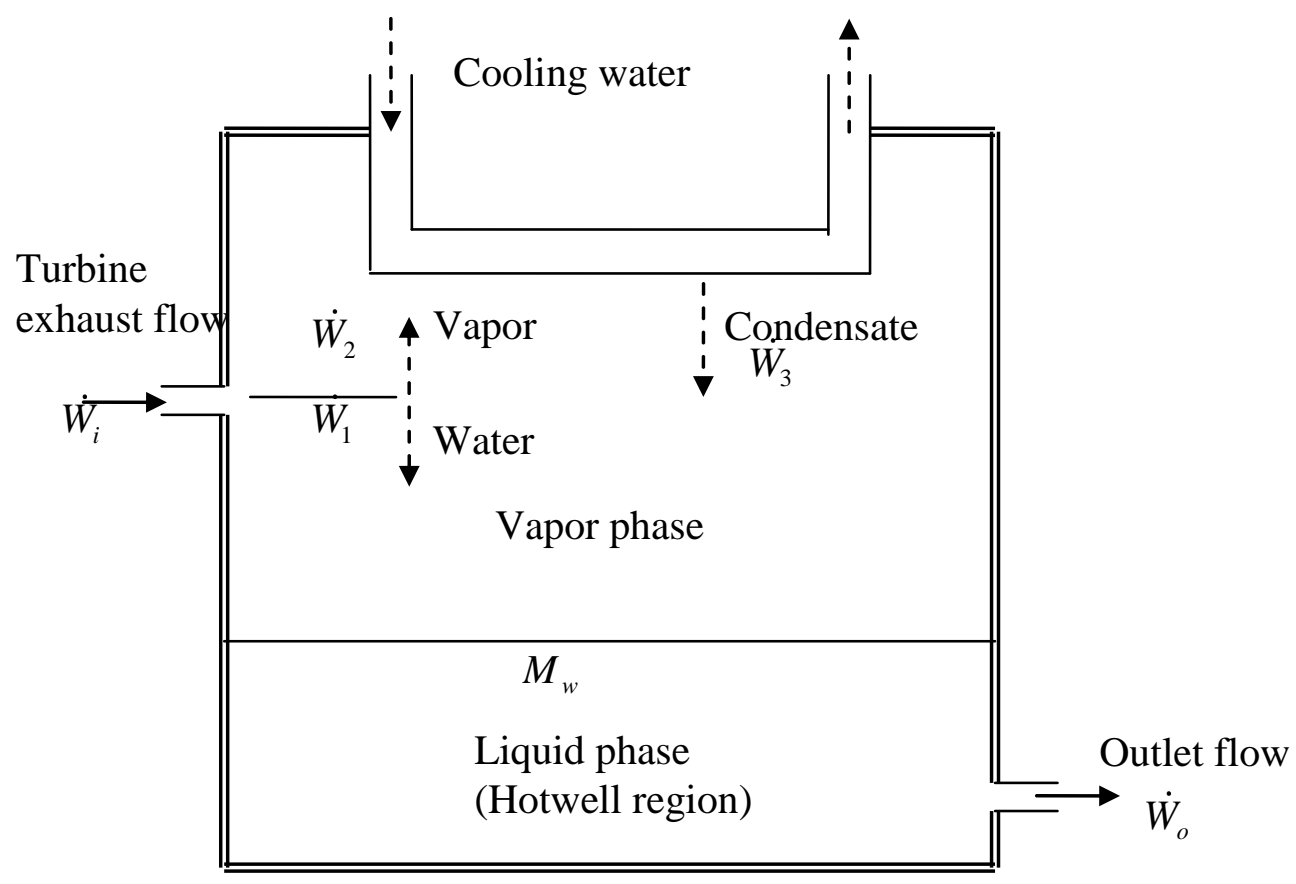

The describing equations of the model are derived based on the conservation of mass and energy equations at each phase. These equations are introduced below.

Mass Balance Equations

Liquid phase:

$$
\frac{d M_{w}}{d t}=\dot{W}_{1}+\dot{W}_{3}-\dot{W}_{o}
$$

Vapor phase:

$$
\frac{d \dot{W}_{3}}{d t}=\frac{\dot{W}_{2}-\dot{W}_{3}}{\tau_{\infty}}
$$

Energy Equations

$$
\begin{gathered}
\frac{d}{d t} M_{w} h_{o}=\dot{W}_{1}+\dot{W}_{3} h_{f}-\dot{W}_{o} h_{o} \\
\frac{d h_{o}}{d t}=\frac{\dot{W}_{1}+\dot{W}_{3} h_{f}-h_{o}}{M_{w}}
\end{gathered}
$$

Constitutive Relations

Mass balance:

$$
\dot{W}_{1}=\dot{W}_{i}-\dot{W}_{2}
$$


where

$$
\dot{W}_{2}=\dot{W}_{i} \frac{\left(h_{i}-h_{f}\right)}{h_{f g}}
$$

\begin{tabular}{|c|c|}
\hline Variable & Definition \\
\hline$M_{w}$ & Water mass inside the condenser \\
\hline$\dot{W}_{1}$ & Water droplet rate into the hotwells \\
\hline$\dot{W}_{3}$ & Water condensation flow rate \\
\hline$\dot{W}_{o}$ & Outlet flow rate to the low pressure feed water heater \\
\hline$\dot{W}_{2}$ & Steam flow rate in the condenser \\
\hline$\tau_{c o}$ & Time constant of the condensation process \\
\hline$h_{f}$ & Enthalpy of the saturated water \\
\hline$h_{o}$ & Enthalpy of the outlet water \\
\hline$\dot{W}_{i}$ & Entering flow rate to the condenser \\
\hline$h_{f g}$ & Latent enthalpy of the water \\
\hline$h_{i}$ & Enthalpy of the entering flow rate \\
\hline
\end{tabular}

\section{Feedwater Heater}

Low Pressure Feedwater Heater Equations

Energy balance:

$$
\frac{d h_{f w 1}}{d t}=\frac{Q_{h 1}}{\tau_{h 1} W_{f w}}+\frac{h_{o}-h_{f w 1}}{\tau_{h 1}}
$$

Heat flow relation:

$$
Q_{h 1}=H_{f w 1}\left(W_{h p 1}+W_{b l p}\right)
$$

High Pressure Feedwater Heater Equations

Mass balance:

$$
\frac{d W_{h p 1}}{d t}=\frac{W_{b h p}+W_{m s}+W_{p r 1}-W_{h p 1}}{\tau_{h 2}}
$$

Energy balance:

$$
\frac{d h_{f w 2}}{d t}=\frac{Q_{h 2}}{\tau_{h 2} W_{f w}}+\frac{h_{f w 1}-h_{f w 2}}{\tau_{h 2}}
$$

Heat flow relation:

$$
Q_{h 2}=H_{f w 2}\left(W_{m s}+W_{p r 1}+W_{b h p}\right)
$$




\section{Appendix C: Fault Isolation Index Plots for Dual-Fault Scenarios}

\section{Dual-fault case \#8:}

Fault \#2 \& Fault \#3
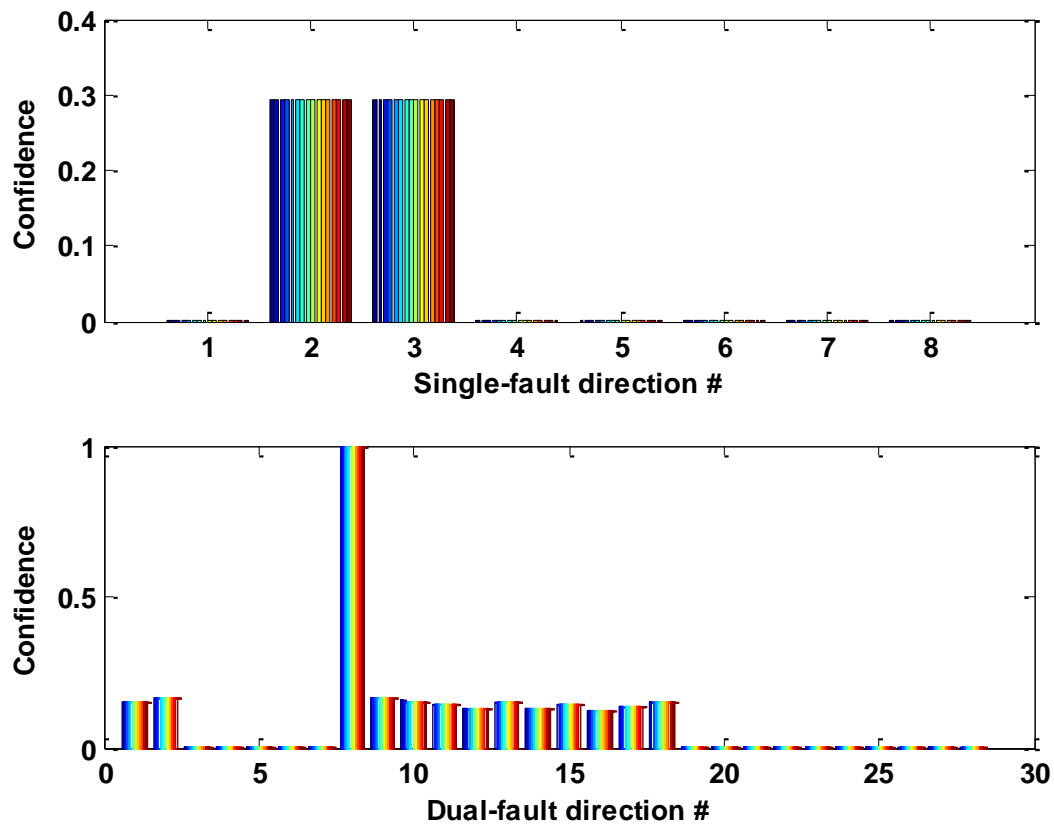

Dual-fault case \#9:

Fault \#2 \& Fault \#4
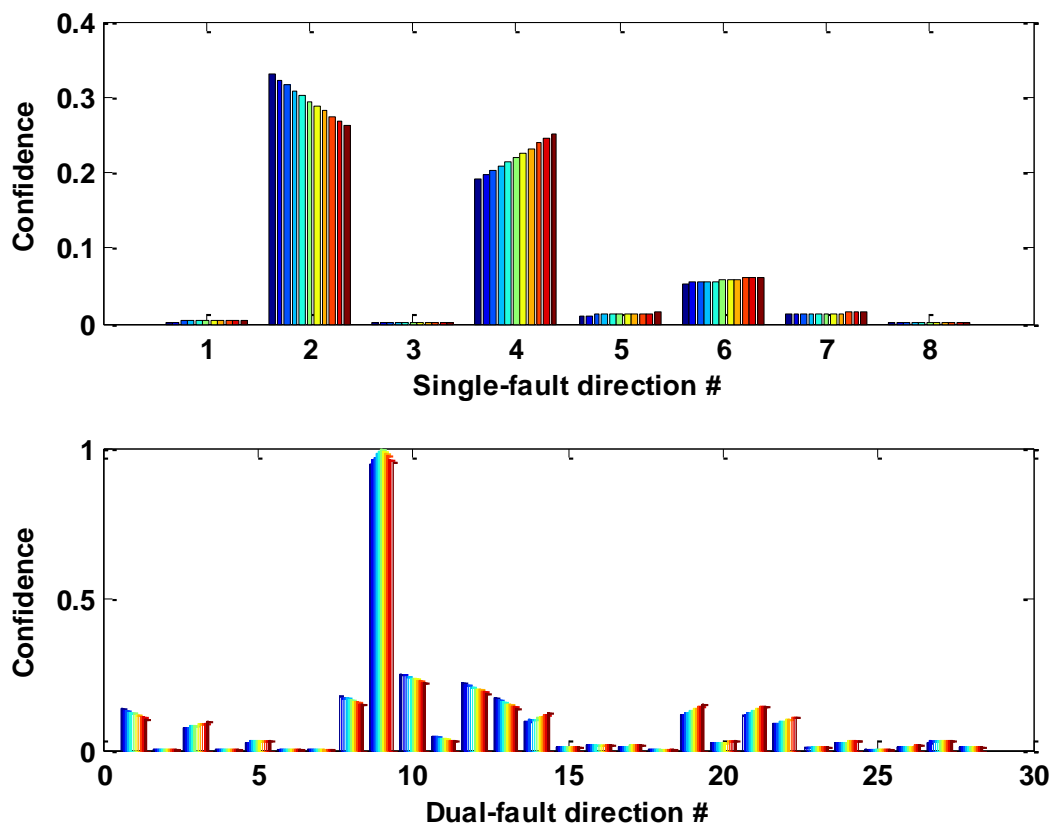
Dual-fault case \#10:

Fault \#2 \& Fault \#5
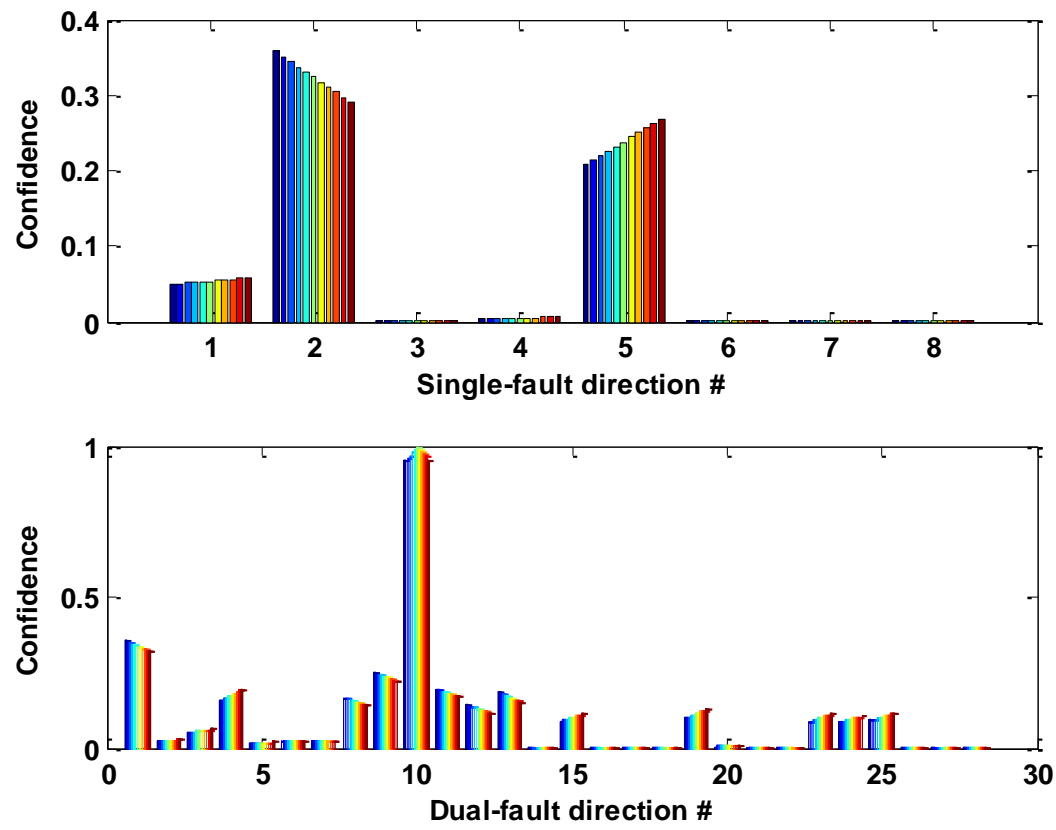

Dual-fault case \#11:

Fault \#2 \& Fault \#6
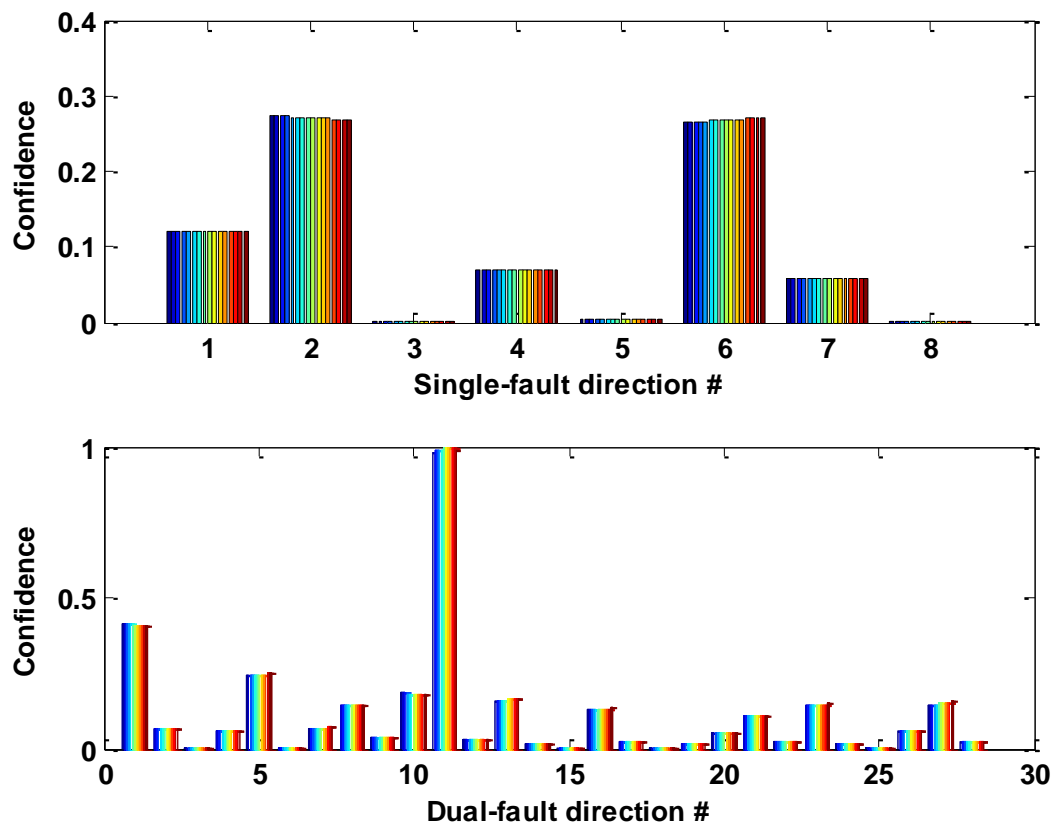
Dual-fault case \#12:

Fault \#2 \& Fault \#7
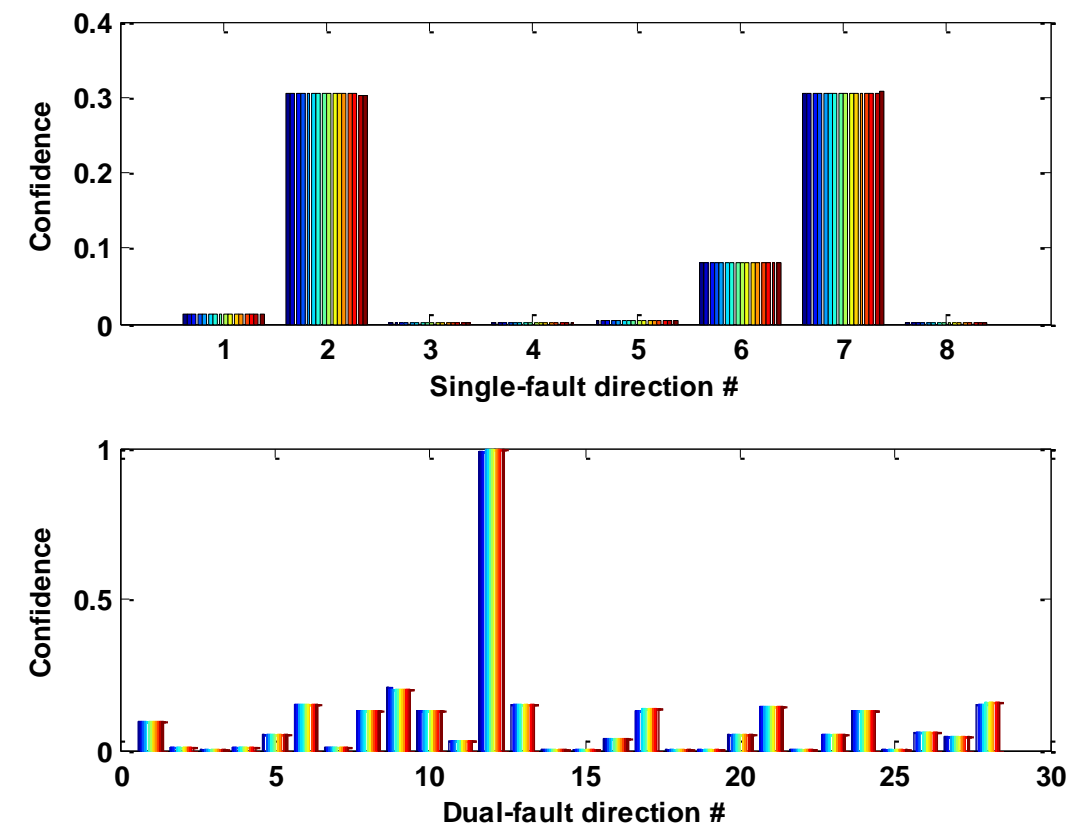

Dual-fault case \#13:

Fault \#2 \& Fault \#8
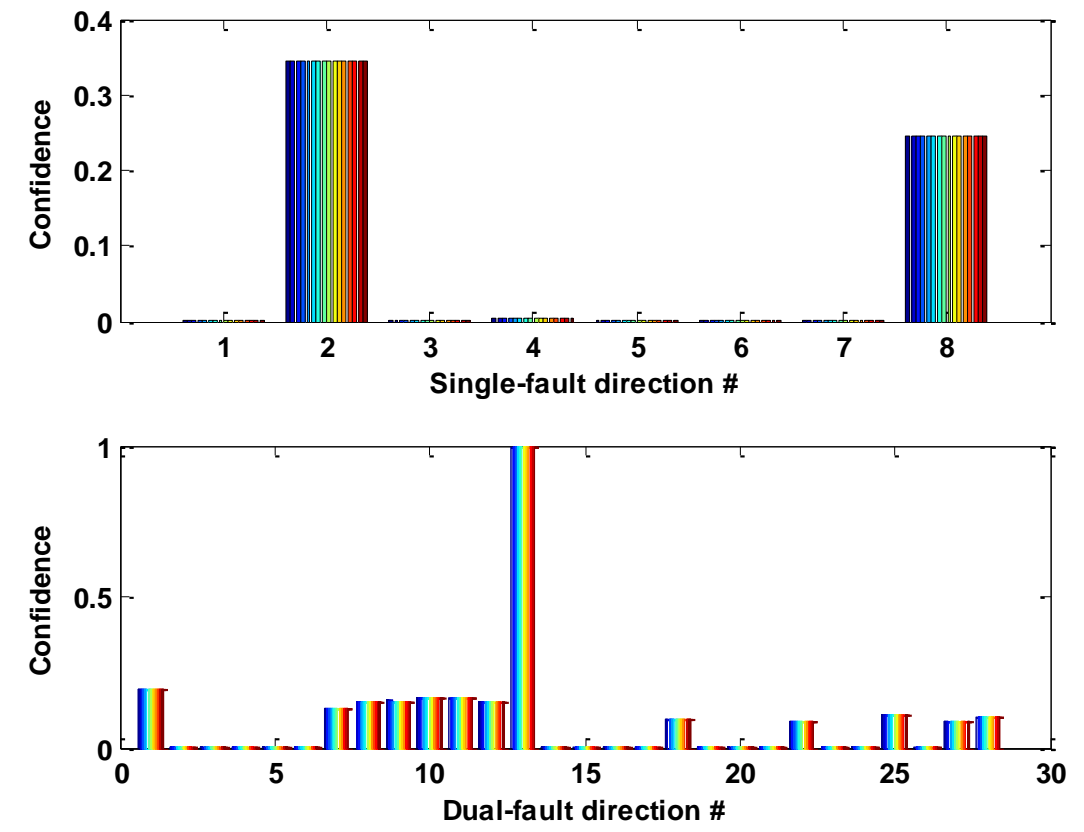
Dual-fault case \#14:

Fault \#3 \& Fault \#4
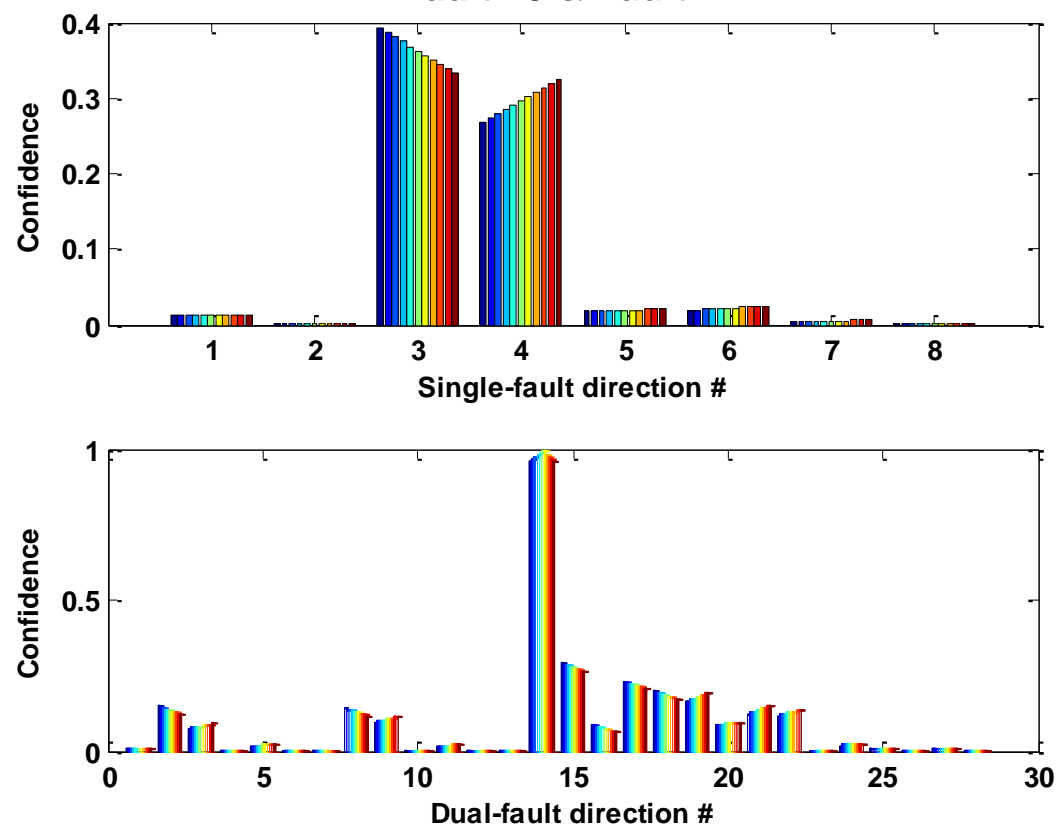

Dual-fault case \#15:

Fault \#3 \& Fault \#5
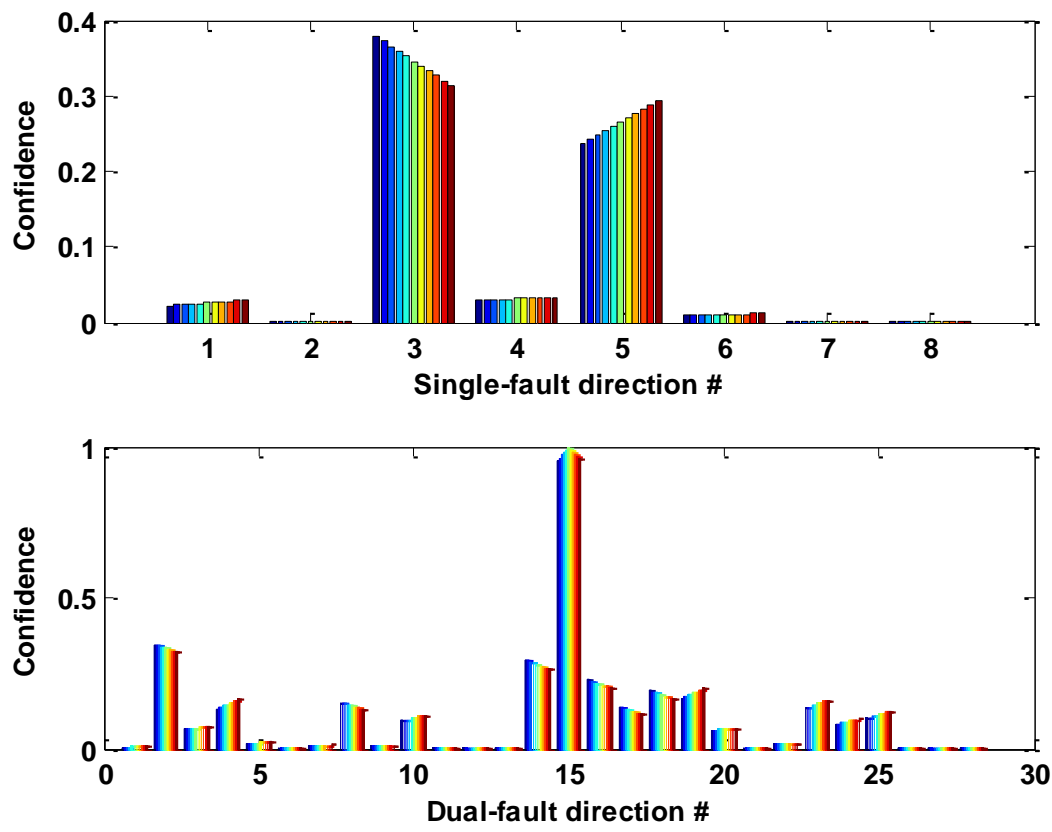
Dual-fault case \#16:

Fault \#3 \& Fault \#6
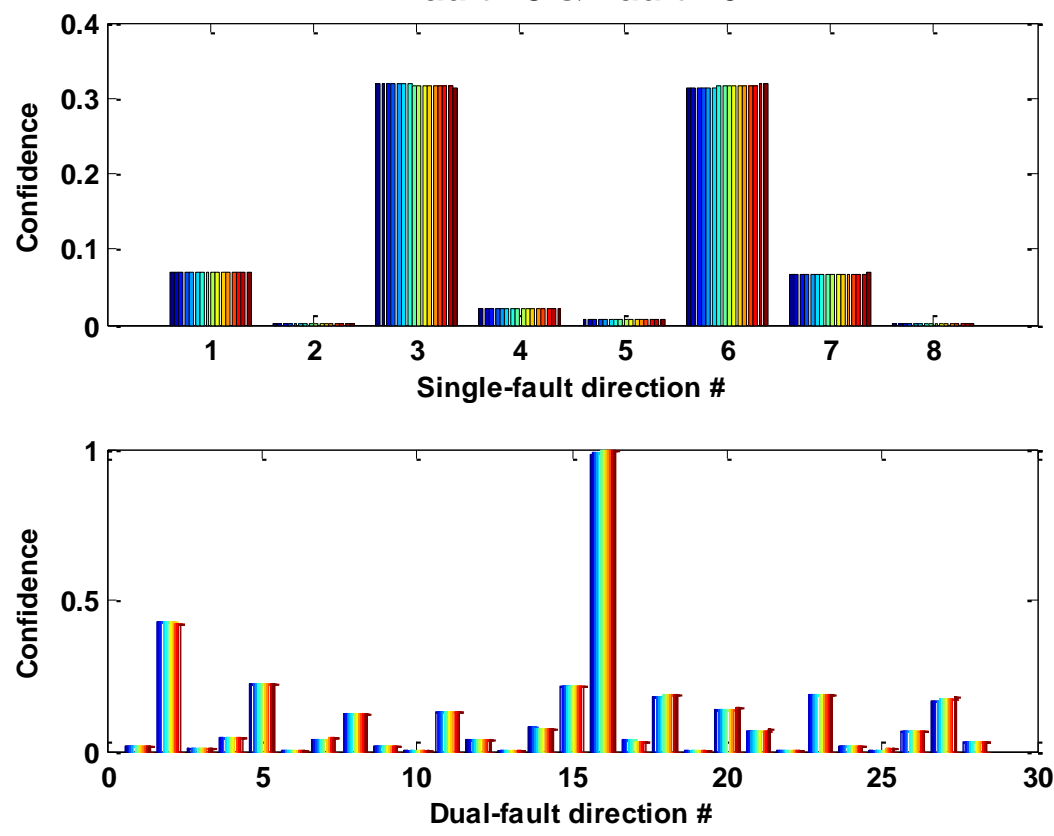

Dual-fault case \#17:

Fault \#3 \& Fault \#7
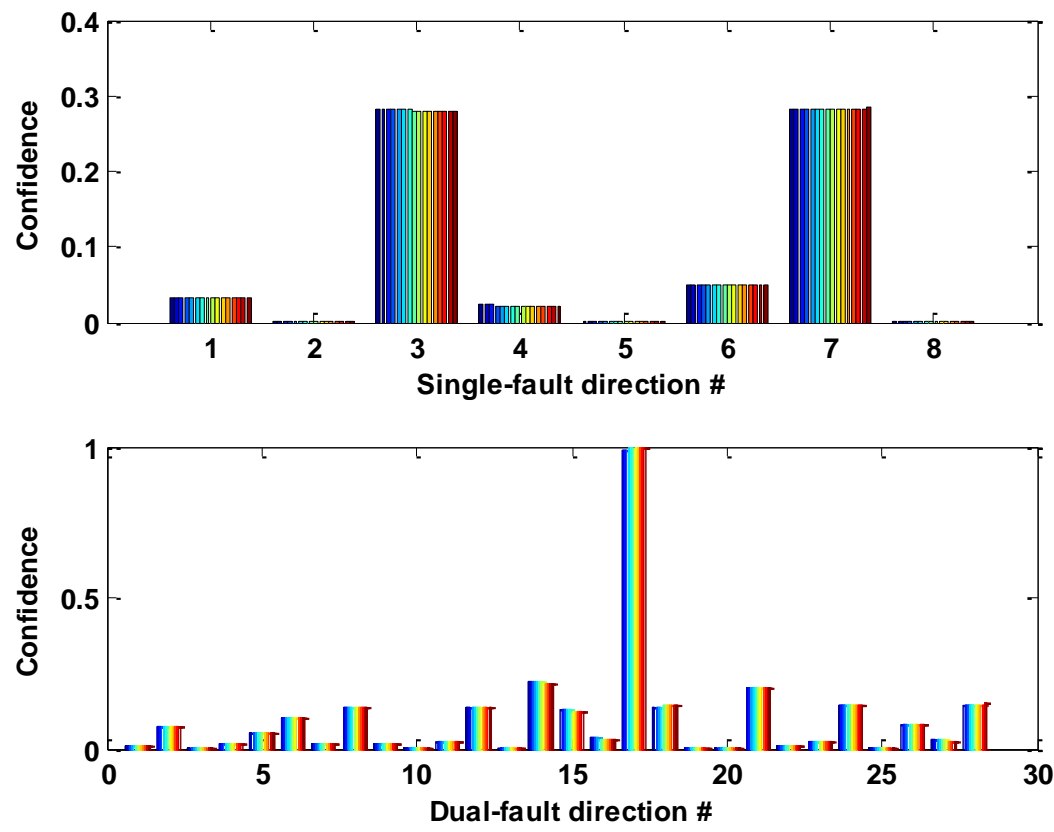
Dual-fault case \#18:

Fault \#3 \& Fault \#8
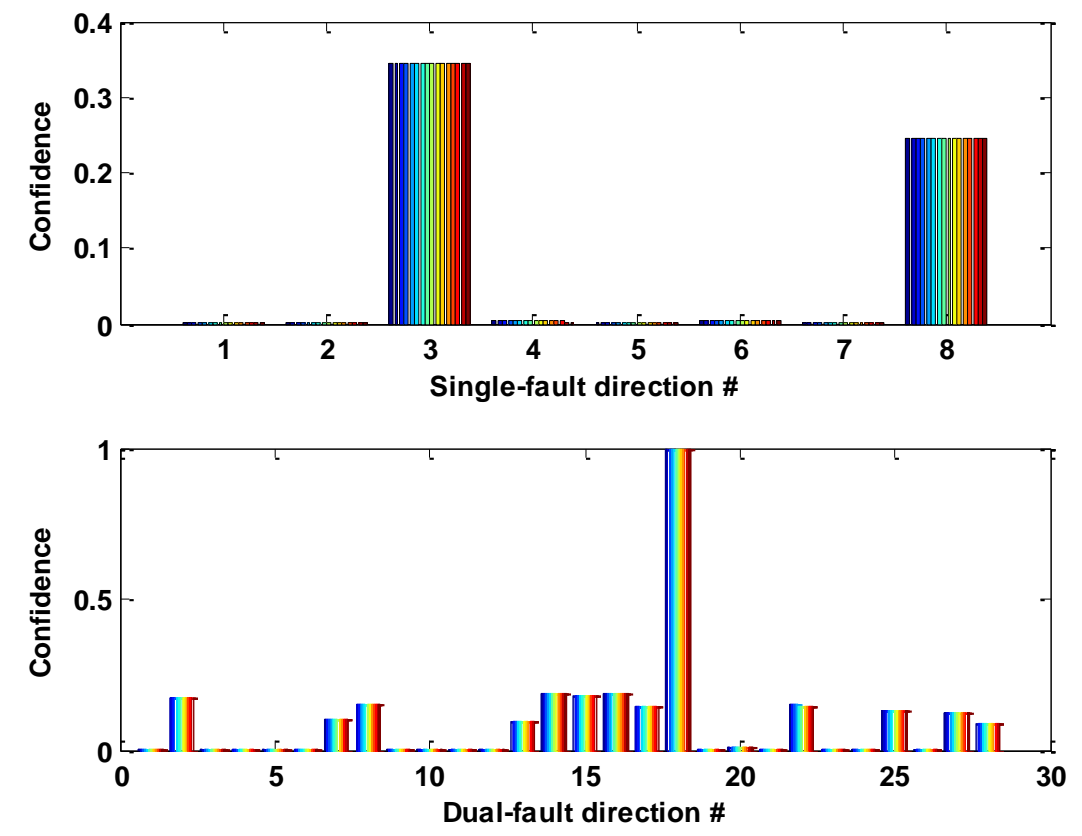

Dual-fault case \#19:
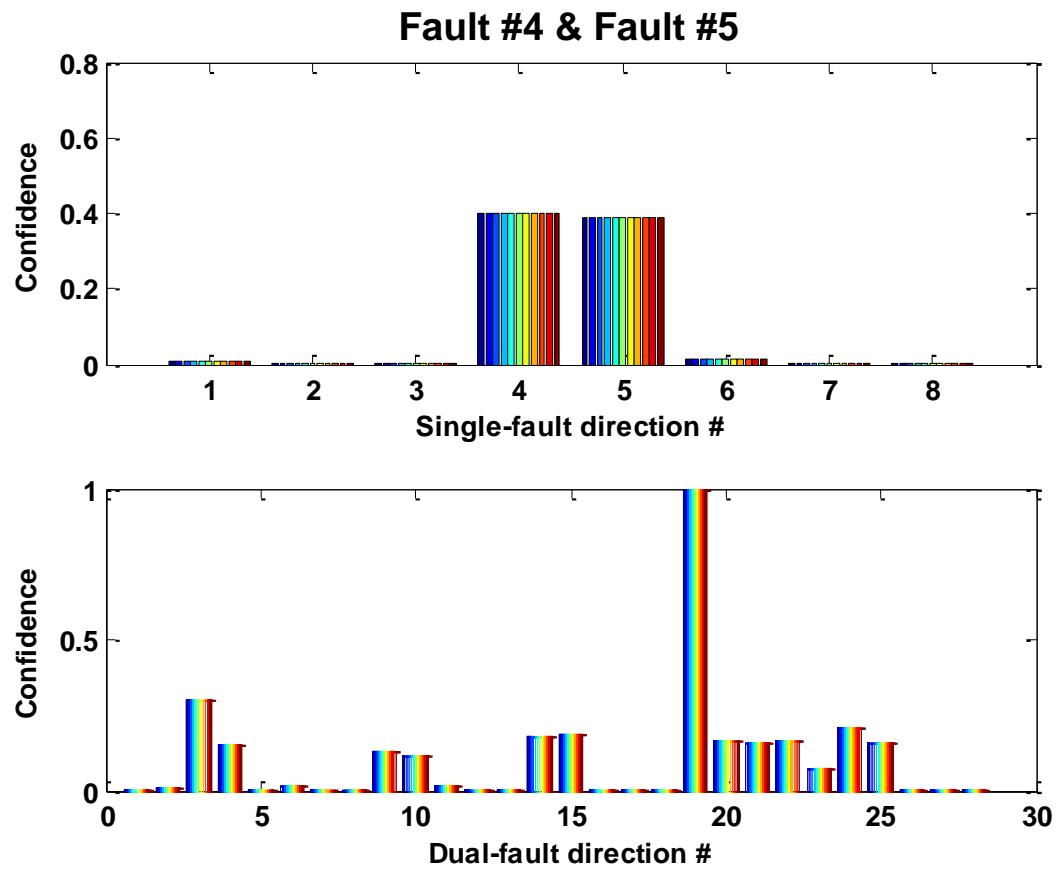
Dual-fault case \#20:

Fault \#4 \& Fault \#6
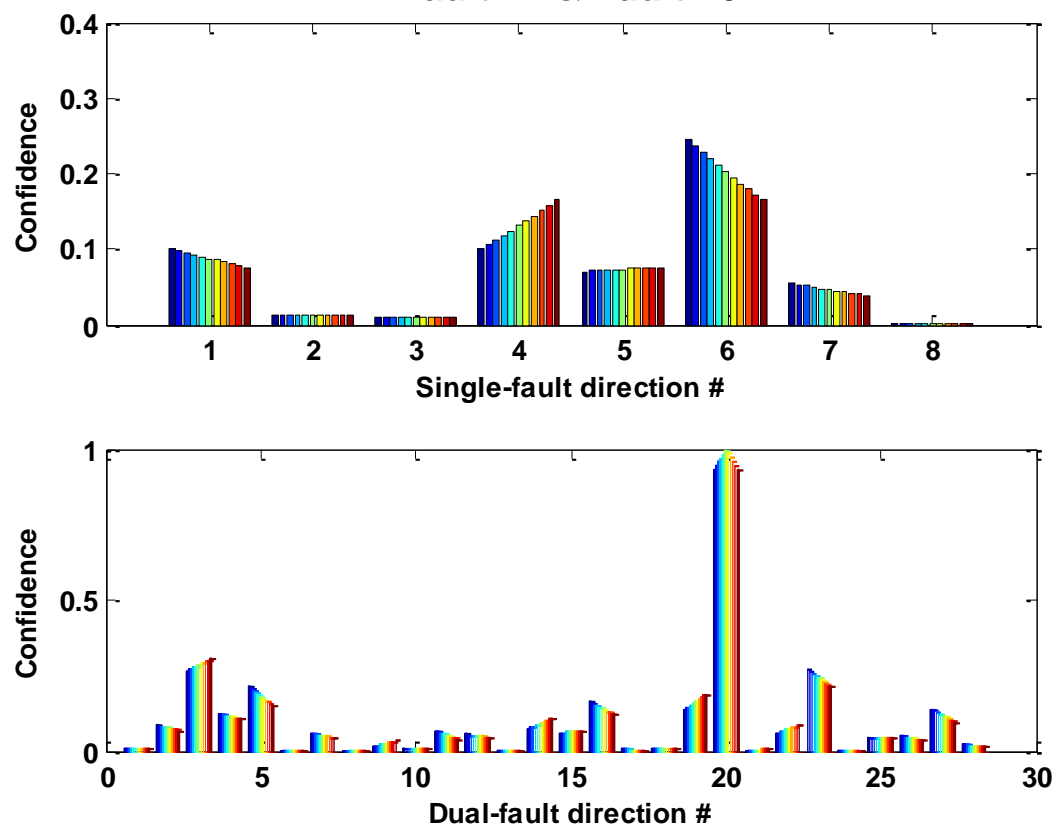

Dual-fault case \#21:
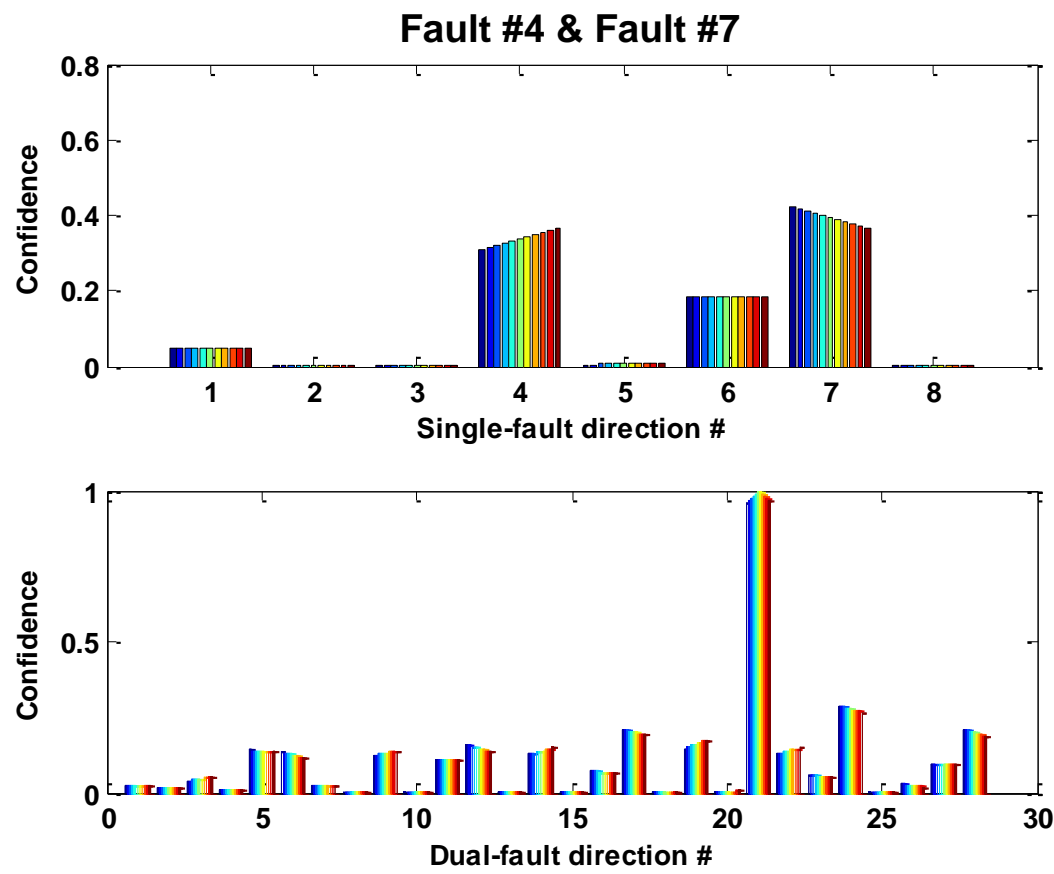
Dual-fault case \#22:

Fault \#4 \& Fault \#8
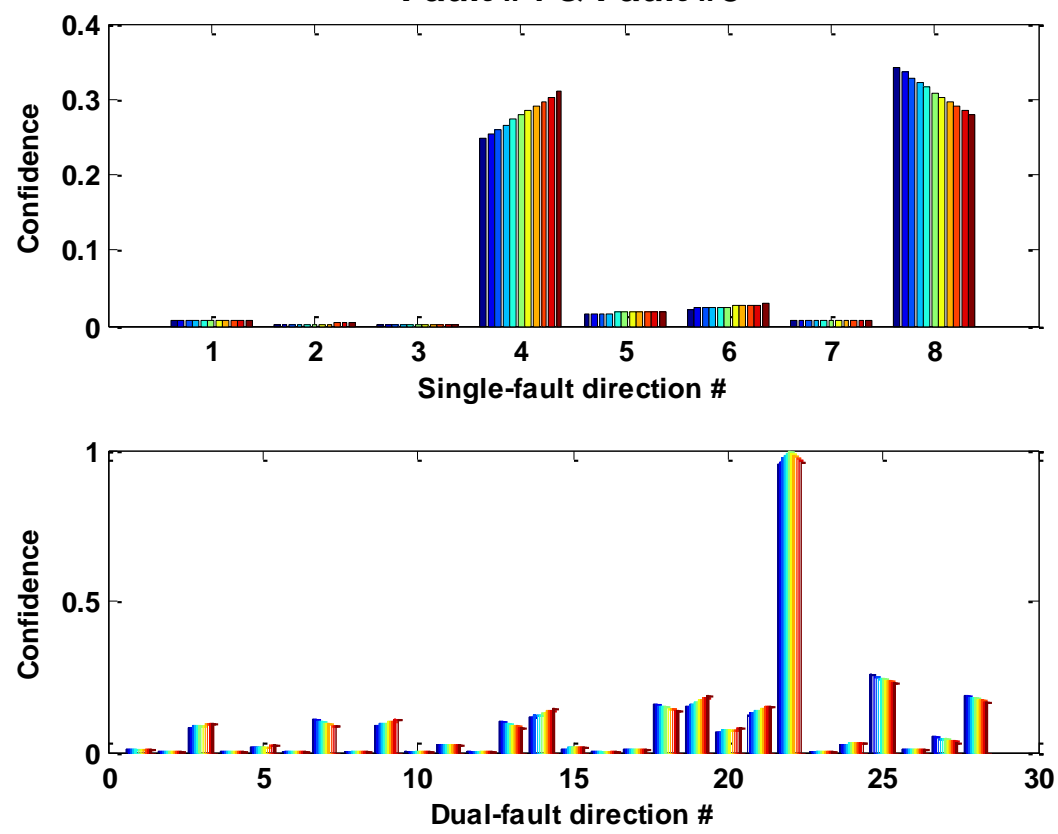

Dual-fault case \#23:

Fault \#5 \& Fault \#6
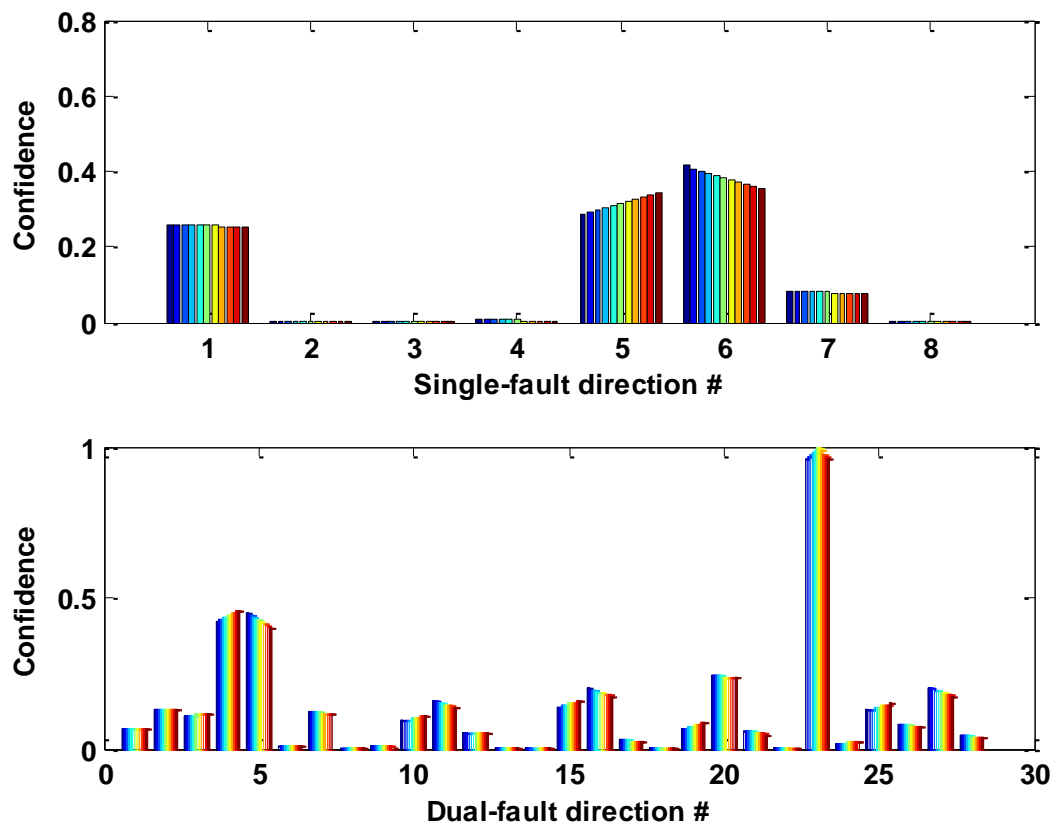
Dual-fault case \#24:

Fault \#5 \& Fault \#7
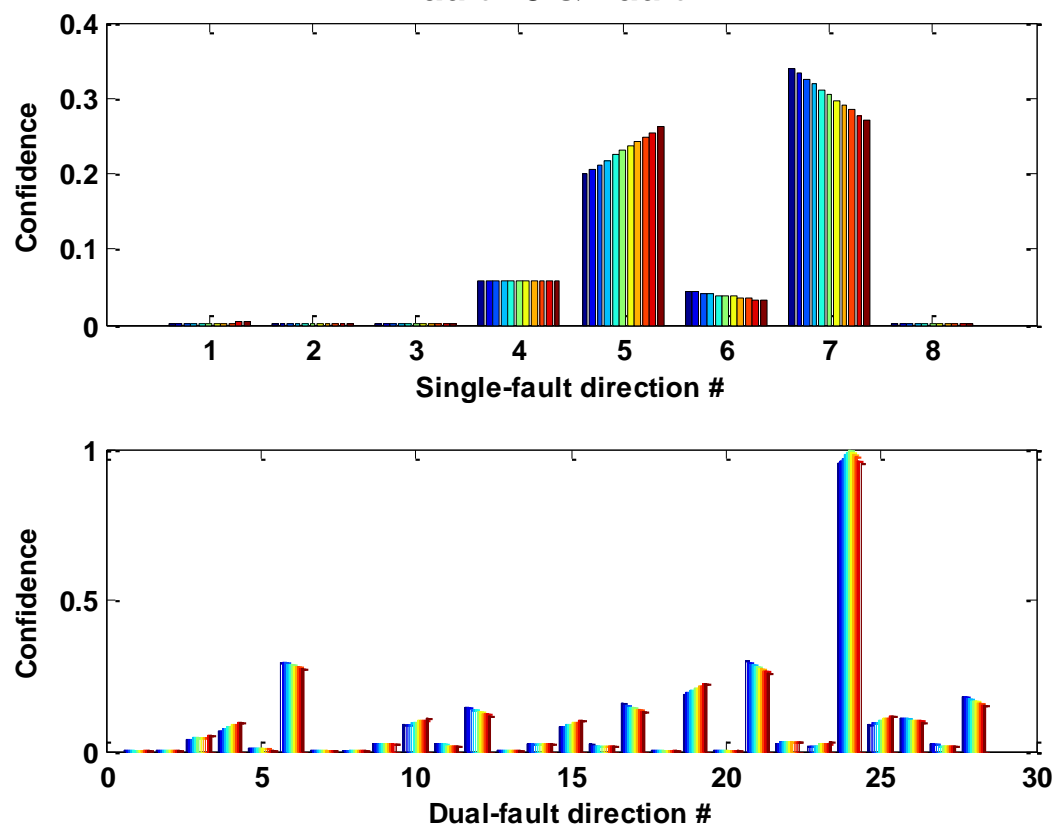

Dual-fault case \#25:

Fault \#5 \& Fault \#8
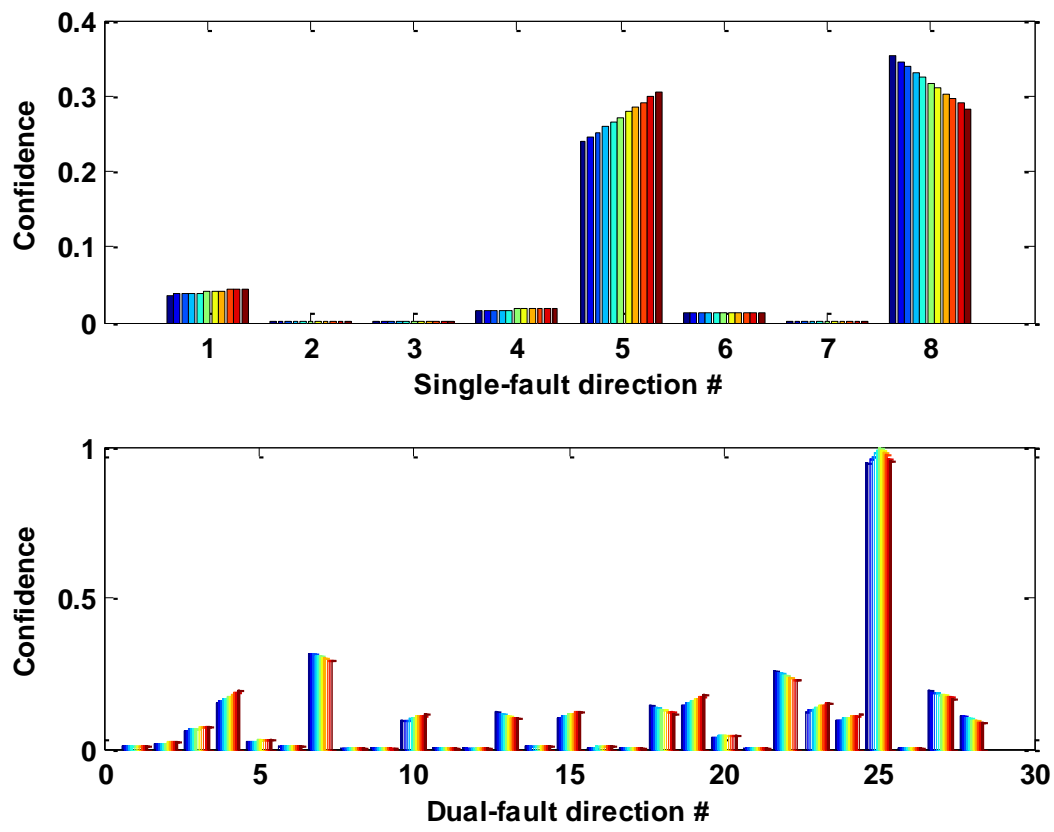
Dual-fault case \#26:

Fault \#6 \& Fault \#7
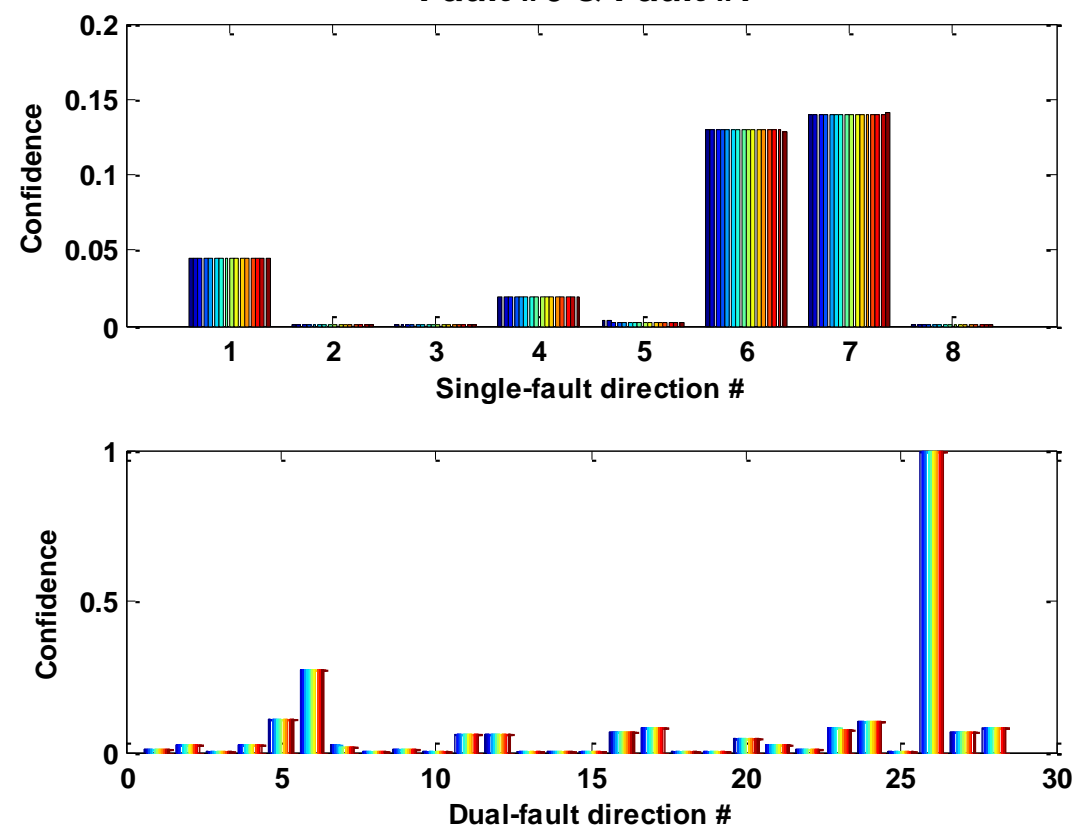

Dual-fault case \#27:

Fault \#6 \& Fault \#8
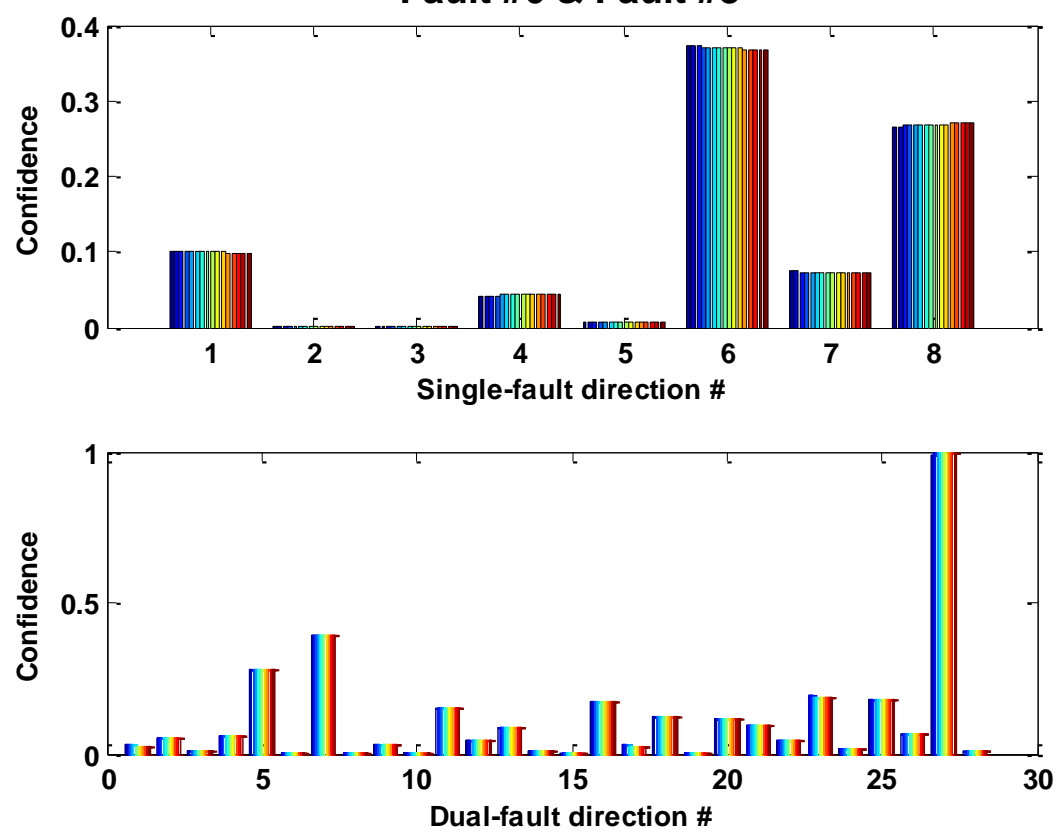
Dual-fault case \#28:

Fault \#7 \& Fault \#8
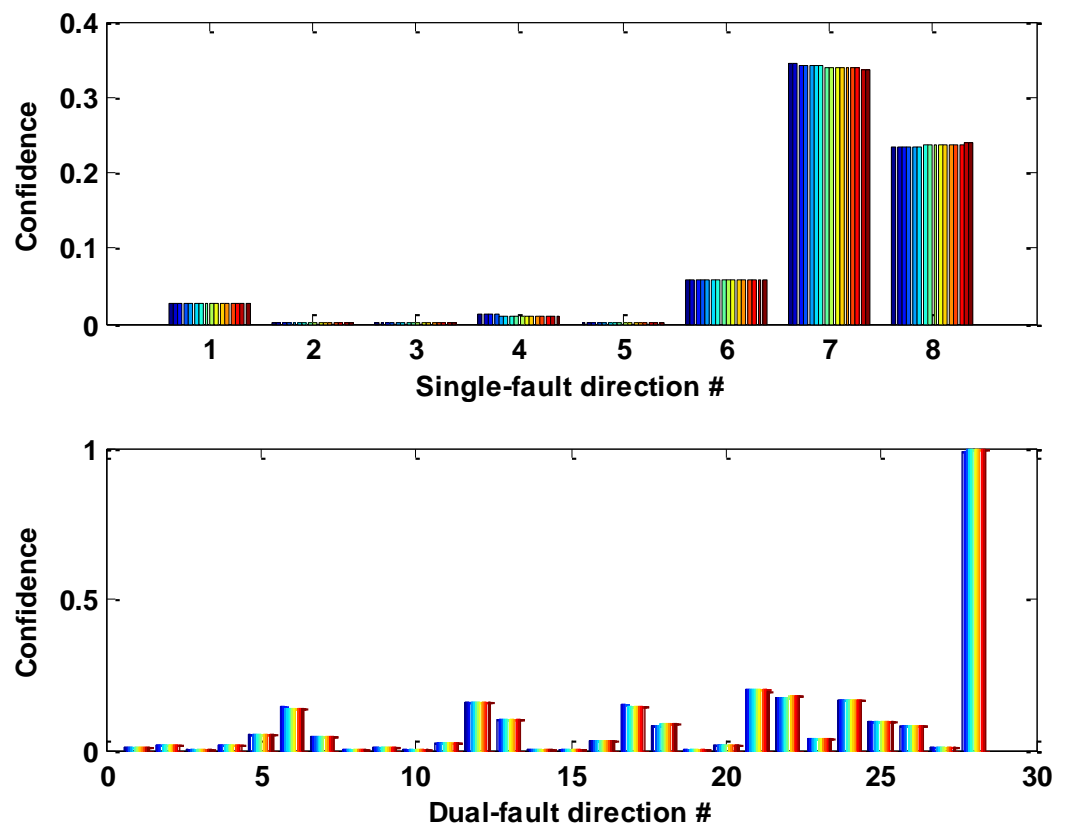


\section{Advanced Instrumentation and Control Methods for Small and Medium Reactors with IRIS Demonstration}

\section{Final Report \\ Volume 5}

\section{Multi-Modular Integral Pressurized Water Reactor Control and Operational Reconfiguration for a Flow Control Loop}

Report No. DE-FG07-07ID14895/UTNE/2011-7
NERI-C Grant Number: DE-FG07-07ID14895

Belle R. Upadhyaya

(Co-Principal Investigators)

Sergio R.P. Perillo

The University of Tennessee

Nuclear Engineering Department

Knoxville, TN 37996-2300

E-mail: bupadhya@utk.edu

May 2011

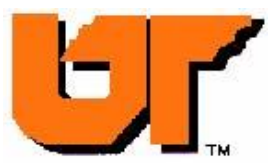




\begin{abstract}
This research focused on the IRIS design since this will likely be one of the designs of choice for future deployment in the U.S and developing countries. With a net $335 \mathrm{MWe}$ output IRIS novel design falls in the "medium" size category and it is a potential candidate for the so called modular reactors, which may be appropriate for base load electricity generation, especially in regions with smaller electricity grids, but especially well suited for more specialized nonelectrical energy applications such as district heating and process steam for desalination.

The first objective of this research is to evaluate and quantify the performance of a Nuclear Power Plant (NPP) comprised of two IRIS reactor modules operating simultaneously with a common steam header, which in turn is connected to a single turbine, resulting in a steammixing control problem with respect to "load-following" scenarios, such as varying load during the day or reduced consumption during the weekend. To solve this problem a single-module IRIS SIMULINK model previously developed by another researcher is modified to include a second module and was used to quantify the responses from both modules.

In order to develop research related to instrumentation and control, and equipment and sensor monitoring, the second objective is to build a two-tank multivariate loop in the Nuclear Engineering Department at the University of Tennessee. This loop provides the framework necessary to investigate and test control strategies and fault detection in sensors, equipment and actuators. The third objective is to experimentally develop and demonstrate a fault-tolerant control strategy using this loop. Using six correlated variables in a single-tank configuration, five inferential models and one Auto-Associative Kernel Regression (AAKR) model were developed to detect faults in process sensors. Once detected the faulty measurements were successfully substituted with prediction values, which would provide the necessary flexibility and time to find the source of discrepancy and resolve it, such as in an operating power plant. Finally, using the same empirical models, an actuator failure was simulated and once detected the control was automatically transferred and reconfigured from one tank to another, providing survivability to the system.
\end{abstract}




\section{TABLE OF CONTENTS}

Chapter

Page

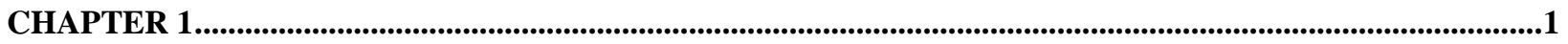

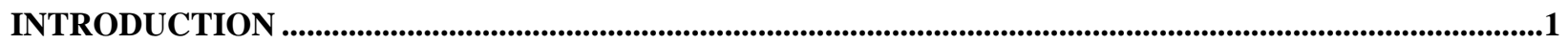

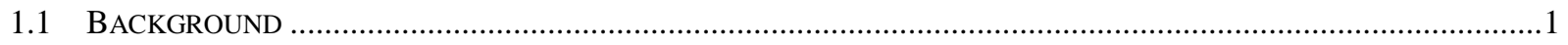

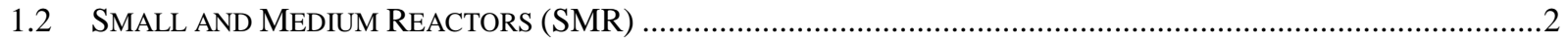

1.3 OVERVIEW of Electrical Grid Stability APPlied to SMall and MEdium Reactors ...........................5

1.4 APPLICATION OF SMRS IN Co-GENERATION AND DESALINATION ……...................................................

1.5 OBJeCtIVES OF THE RESEARCH TASK AND SigNIFICANT CONTRIBUTIONS …….........................................

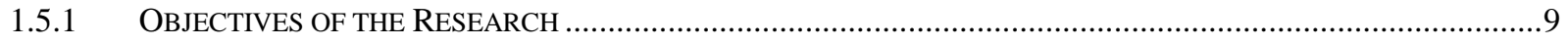

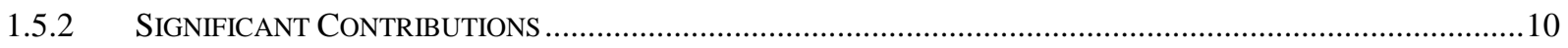

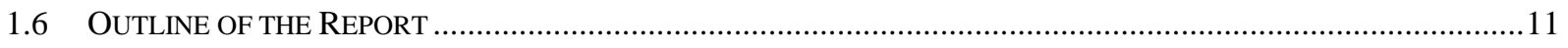

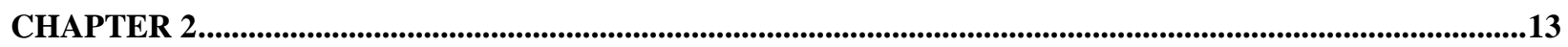

DELIBERATELY SMR DESIGNS AND OVERVIEW OF FAULT TOLERANT AND RECONFIGURABLE

CONTROL .......................................................................................................................................................

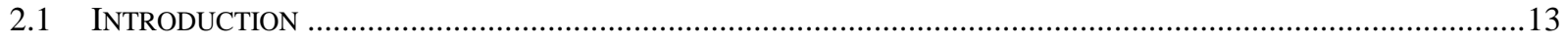

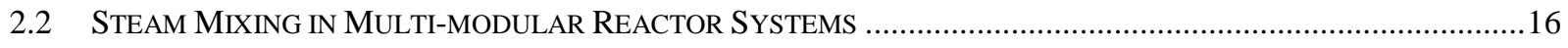

2.2.1 MOdUlar GAS-COOLED REACTOR DeSIGNS ................................................................................18

2.2.2 LARGE PWR MODULAR SYSTEMS.............................................................................................

2.2.2.1 $\mathrm{T}_{\mathrm{ave}}$ Control Program Issue in Multi-modular PWRs ................................................................... 33

2.3 FAUlt TOLERANT AND RECONFIGURABLE CONTROL .......................................................................40

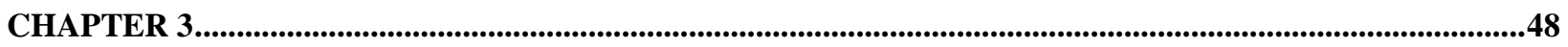

IRIS SYSTEM DESCRIPTION AND THE DEVELOPMENT OF THE REACTOR DYNAMICS MODEL 48

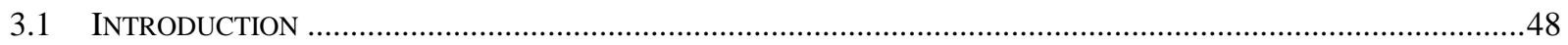

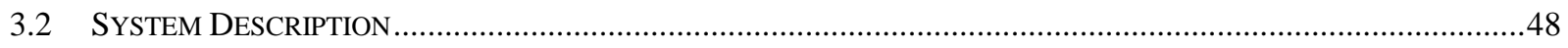

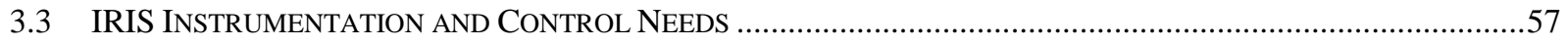


3.4 OnCE-Through Helical COIL Steam Generators (HCSG)

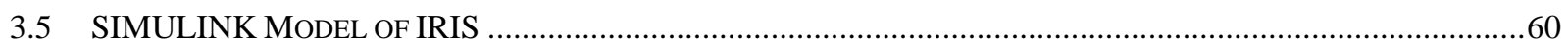

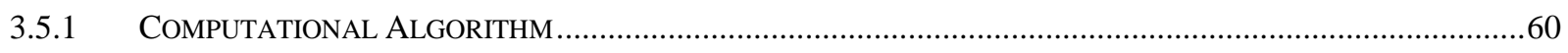

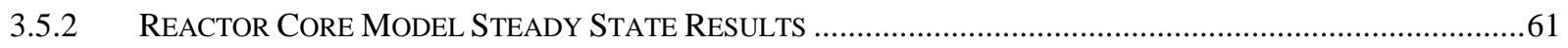

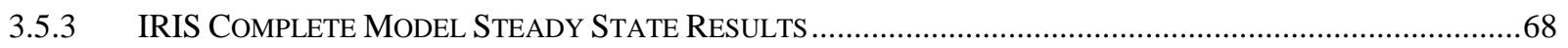

3.6 IRIS SYSTEM GENERAL LOAD FOLLOWING MANEUVER.................................................................

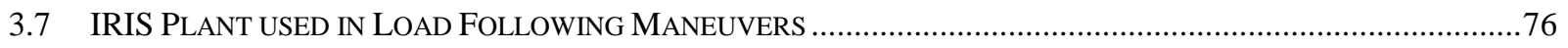

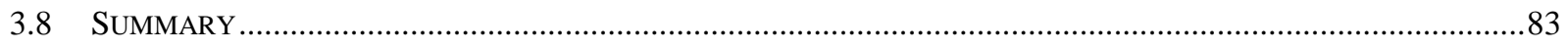

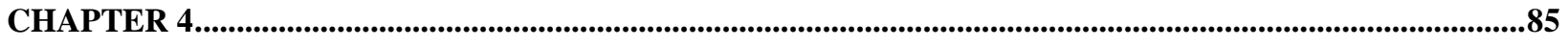

DEVELOPMENT OF A DESIGN APPROACH FOR A MULTI-MODULAR IRIS PLANT .......................85

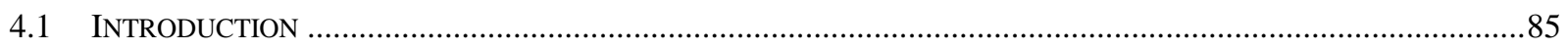

4.2 SYSTEM DESCRIPTION AND MODEL ASSUMPTIONS ......................................................................... 85

4.3 RESULTS OF IRIS DUAL-MODULE USED IN LOAD FOLLOWING MANEUVERS .........................................95

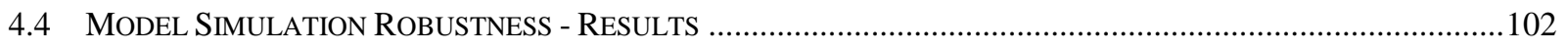

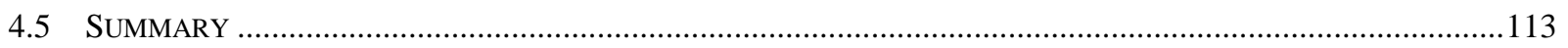

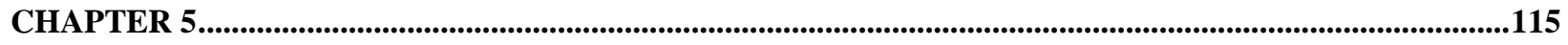

TWO-TANK MULTIVARIATE FLOW CONTROL LOOP .........................................................................115

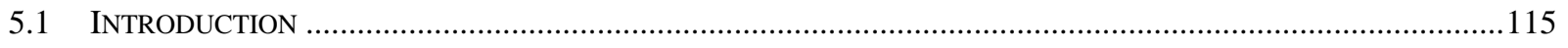

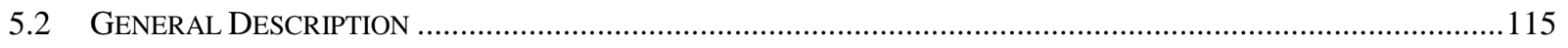

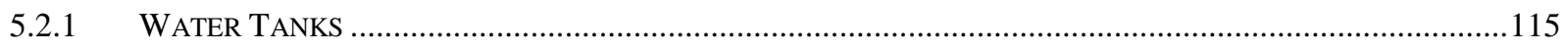

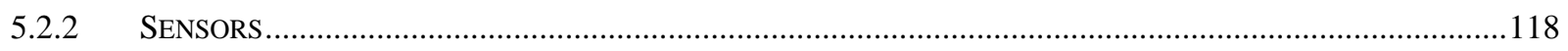

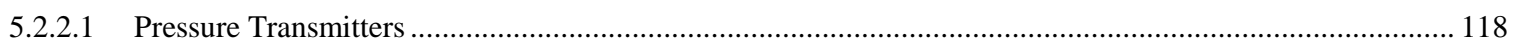

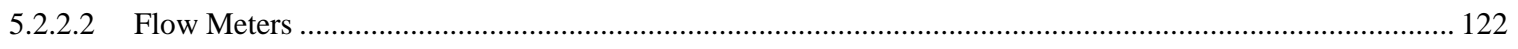

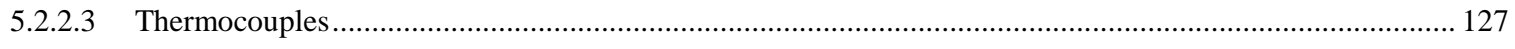

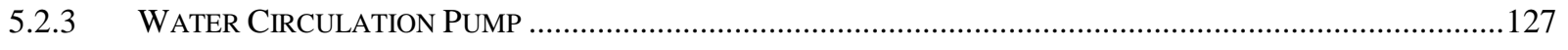

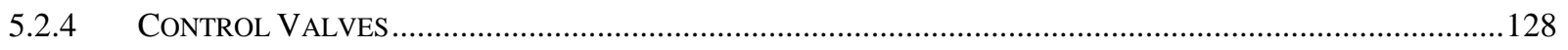

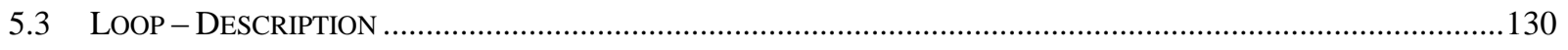

5.3.1 DATA ACQUISITION AND LOOP CONTROL ............................................................................... 134 


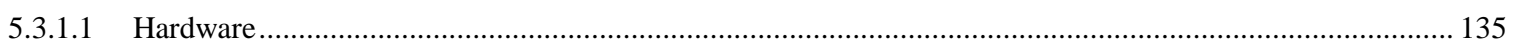

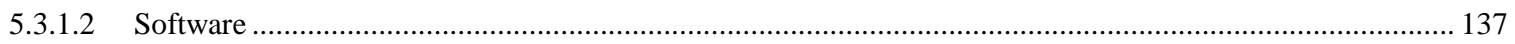

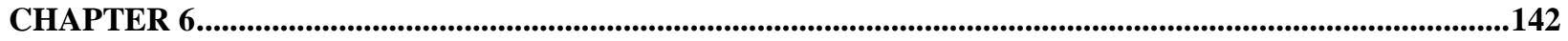

INTEGRATION OF FAULT DETECTION AND CONTROL RECONFIGURATION DURING STEADY

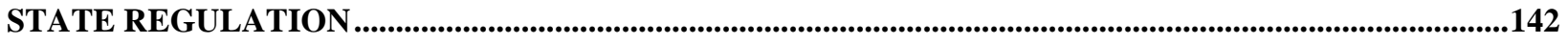

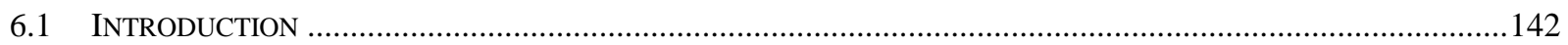

6.2 TOOLS FOR DEVELOPING EMPIRICAL MODELS............................................................................... 142

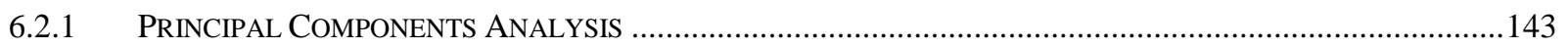

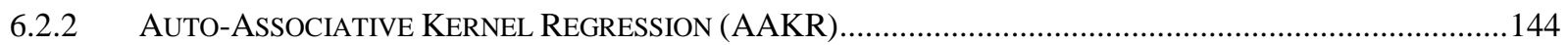

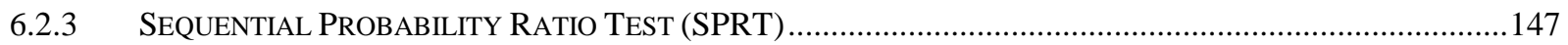

6.2.4 PROCESS AND EQuIPMENT MONITORING (PEM) TOOLBOX......................................................151

6.3 DATA GENERATION AND ModEL DEVELOPMENT …....................................................................... 151

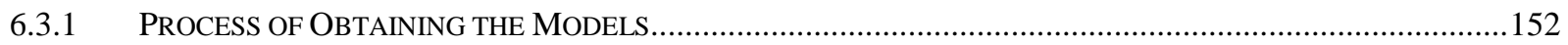

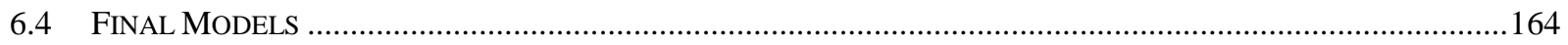

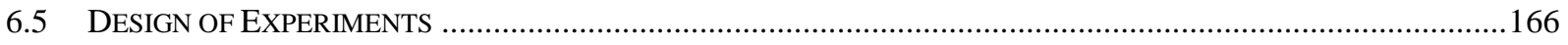

6.6 ApPliCATION OF FAUlty MEASUREMENT IDENTIFICATION AND SUBSTITUTION TO A SingLE-TANK..........167

6.6.1 FAULT DETECTION EXPERIMENTS INVOLVING LEVEL SENSOR..................................................... 167

6.6.2 FAult DETECTION EXPERIMENTS WITH BYPASS, INLET AND OUTLET FlOW RATE SENSORS ................171

6.7 APPliCATION OF RECONFIGURABLE CONTROL TO THE TWO-TANK FLOW CONTROL LOOP .......................174

6.8 PosSIBLE CONTROL RECONFIGURATION APPLICATION TO THE IRIS SYSTEM........................................183

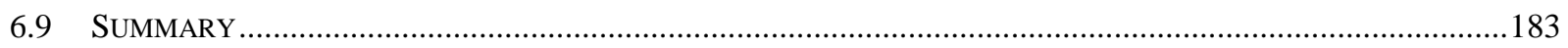

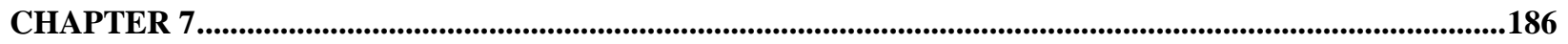

SUMMARY, CONCLUDING REMARKS, AND RECOMMENDATIONS FOR FUTURE WORK ............186

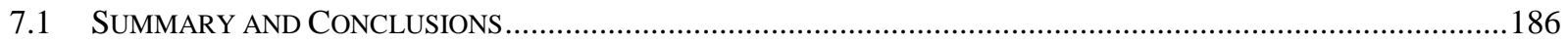

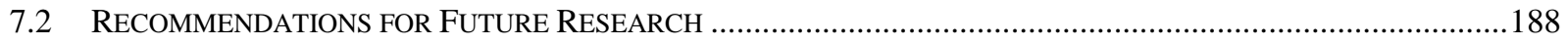

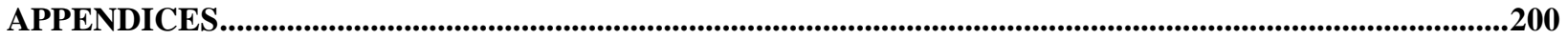

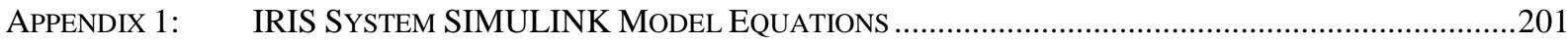

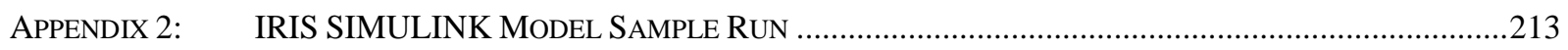

APPENDIX 3: $\quad$ IRIS SIMULINK MODEL SCREENSHOTS ................................................................215 


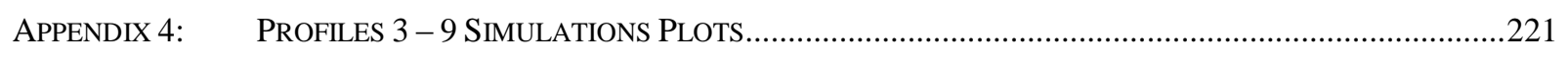

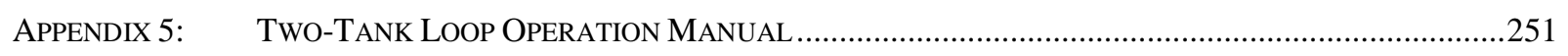

APPENDiX 6: $\quad$ ORIFICE METER CALIBRATION PROCEDURE AND RESUlTS .......................................................256

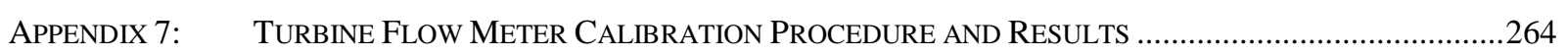

APPENDIX 8: VARIABLES, CHANNELS, AND PATCH PANEL NUMBERS ...........................................................272

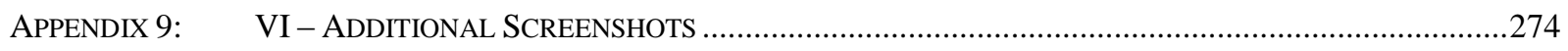

APPENDIX 10: TYPICAL OUtPUt FROM THE CODE USED TO OBTAIN EMPIRICAL MODELS ..............................289

\section{DISCLAIMER}

Any opinions, findings, and conclusions or recommendations expressed in this report are those of the authors, and do not necessarily reflect the views of the U.S. Department of Energy or Westinghouse Electric Company, LLC. 


\section{LIST OF TABLES}

Table

Page

TABLE 1.1. REACTOR TYPES AND DESALINATION PROCESSES [17] ........................................................................

TABLE 2.1. PASSIVE SAFETY RESULTS FROM MATERIAL CHARACTERISTICS AND DESIGN. ..........................................16

TABLE 2.2. ADVANTAGES AND DISADVANTAGES OF DEPLOYMENT OF HTGRS......................................................17

TABLE 2.3. HTR-PM MAIN DESIGN PARAMETERS [28] .......................................................................................23

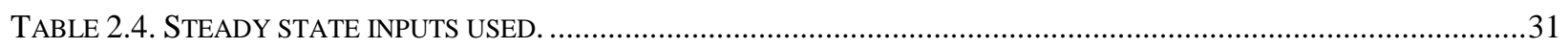

TABLE 2.5. SEQUENCE FOR POWER INCREASE IN SPECIFIED POWER DEMAND MODE [35].........................................38

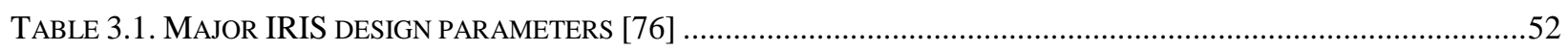

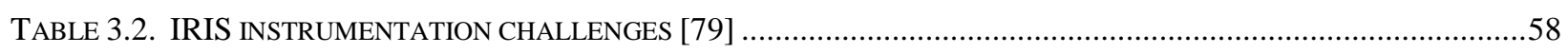

TABLE 3.2. STEADY STATE VALUES FROM SIMULINK AND NCSU IRIS FORTRAN MODELS. ................................74

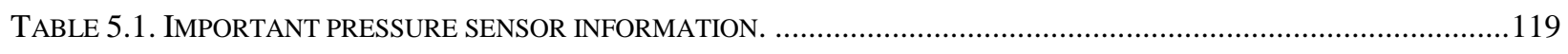

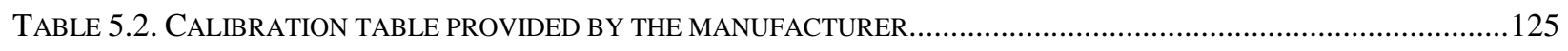

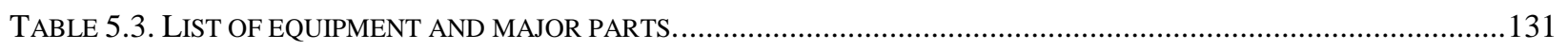

TABLE 6.1. VARIABLES CONSIDERED IN THE EMPIRICAL MODELING PROCESS. ......................................................152

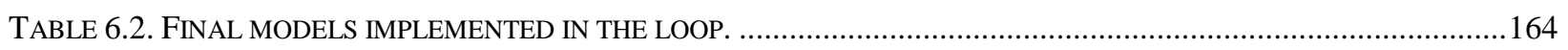




\section{LIST OF FIGURES}

Figure

FIGURE 2.1. B\&W’S 125 MWE MPOWER REACTOR [23] ....................................................................................15

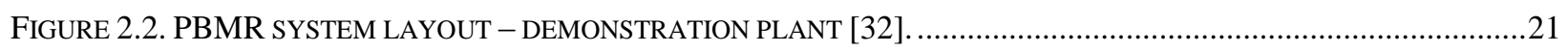

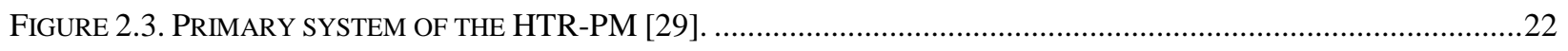

FIGURE 2.4. FLOW DIAGRAM OF THE HTR-PM STEAM TURBINE CYCLE [28] .......................................................23

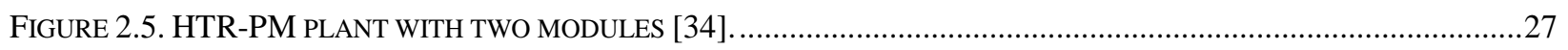

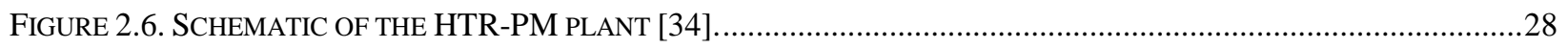

FIGURE 2.7. NODALIZATION OF THE HTR-PM PLANT [34] ................................................................................29

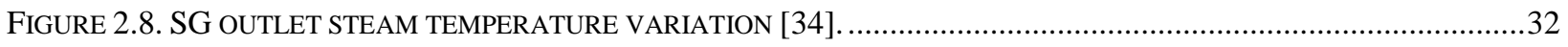

FIGURE 2.9. SCHEMATIC DIAGRAM OF A PWR-TYPE MULTI-MODULAR POWER BLOCK. .............................................35

FIGURE 2.10. OPERATION UNDER SLIDING $\mathrm{T}_{\text {AVE }}$ CONTROL PROGRAM WITH UNBALANCED LOADS [36]..........................36

FIGURE 2.11. A TYPICAL ACTIVE FAULT-TOLERANT CONTROL FOR A STEAM GENERATOR (SG).................................41

FIGURE 2.12. TWO-STAGE STRUCTURE OF AN FDI PROCESS.............................................................................42

FIGURE 2.13. CLASSIFICATION OF DIAGNOSTICS METHODS [53] .....................................................................42

FIGURE 2.14. STEADY-STATE POWER PROFILE UNDER ANALOG CONTROL [65] .....................................................45

FIGURE 2.15. STEADY-STATE POWER PROFILE UNDER DIGITAL CONTROL [68] ......................................................46

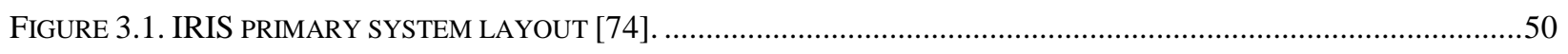

FIGURE 3.2. MODEL OF IRIS PRIMARY SYSTEM AND COMPACT CONTAINMENT VESSEL [76] ......................................51

FIGURE 3.3. IRIS AND LWR CONTAINMENT VESSEL SIZE COMPARISON [77] .........................................................

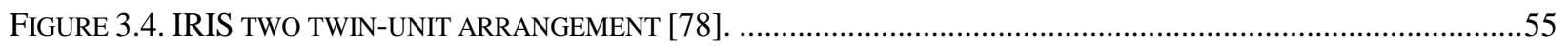

FIGURE 3.5. SCREENSHOT OF THE REACTOR CORE SIMULINK MODEL. ...............................................................63

FIGURE 3.6. POWER PROFILE STARTING FROM POWER $=0$ UNTIL STEADY STATE IS REACHED. ....................................64

FIGURE 3.7. POWER PROFILE SHOWING THE “PROMPT JUMP” PORTION CIRCLED IN RED. ..........................................65 
FIGURE 3.8. A $10^{-3}$ EXTERNAL REACTIVITY INSERTION AT T=700S.

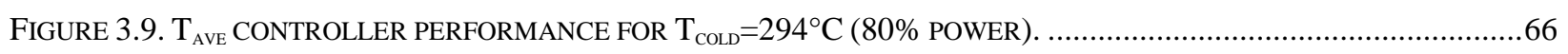

FIGURE 3.10. $\mathrm{T}_{\text {AVE }}$ NOT REACHING SET POINT WITHOUT CONTROLLER ................................................................66

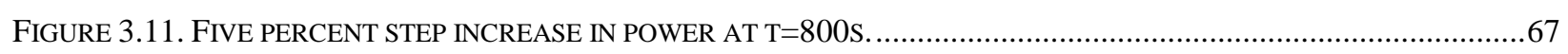

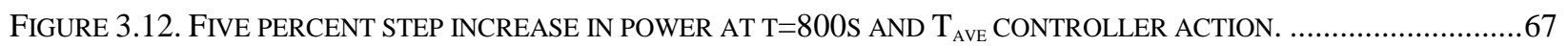

FIGURE 3.13. IRIS COMPLETE SIMULINK MODEL MAIN SCREEN . ...............................................................

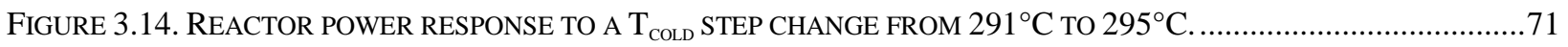

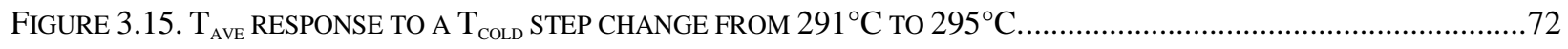

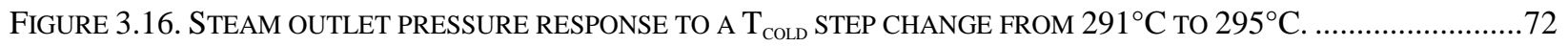

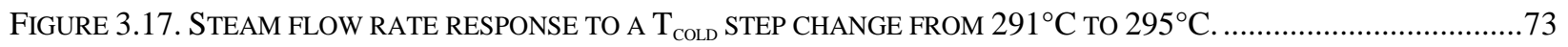

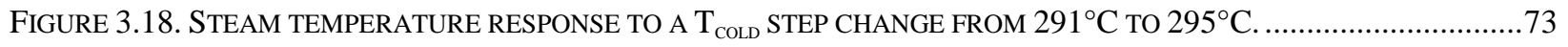

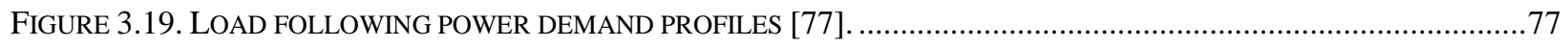

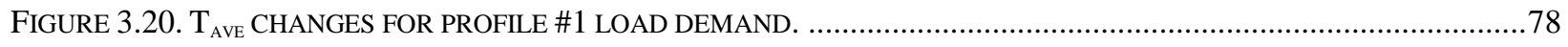

FIGURE 3.21. $\mathrm{T}_{\text {AVE }}$ CHANGES FOR HALF PROFILE \#1 LOAD DEMAND WITHOUT CONTROLLER ..................................78

FIGURE 3.22. POWER CHANGES FOR PROFILE \#1 LOAD DEMAND. .....................................................................

FIGURE 3.23. STEAM FLOW RATE CHANGES FOR PROFILE \#1 LOAD DEMAND.......................................................

FIGURE 3.24. STEAM TEMPERATURE CHANGES FOR PROFILE \#1 LOAD DEMAND......................................................80

FIGURE 3.25. STEAM OUTLET PRESSURE IS CONTROLLED FOR PROFILE \#1 POWER LOAD DEMAND. ...........................80

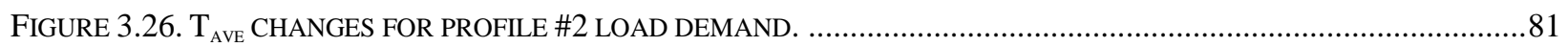

FIGURE 3.27. POWER CHANGES FOR PROFILE \#2 LOAD DEMAND. ................................................................ 82

FIGURE 3.28. STEAM FLOW RATE CHANGES FOR PROFILE \#2 LOAD DEMAND............................................... 82

FIGURE 3.29. STEAM TEMPERATURE CHANGES FOR PROFILE \#2 LOAD DEMAND...............................................83

FIGURE 4.1 SCHEMATIC DIAGRAM OF AN IRIS-TYPE MULTI-MODULAR POWER BLOCK. .......................................86

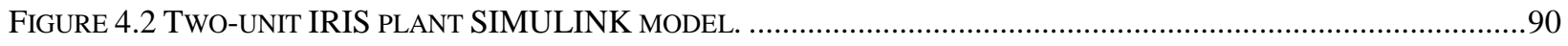

FIGURE 4.3. TWO-UNIT IRIS SHOWING POWER DEMANDS, FEEDWATER FLOW, AND TEMPERATURE BLOCKS...............91

FIGURE 4.4. DETAILS OF THE MIXED STEAM TEMPERATURE CALCULATIONS. ..............................................92 
FIGURE 4.5. DETAILS OF THE HELICAL COIL STEAM GENERATOR MODEL BLOCK.

FIGURE 4.6. SCHEMATIC OF A TWIN-HCSG CONTROL SYSTEM CONNECTED AT THE FEEDWATER FLOW RATE LINE. ....94

FIGURE 4.7. UNIT \#2 POWER PROFILE IN PERCENT FULL POWER. ..................................................................

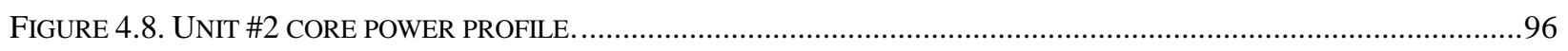

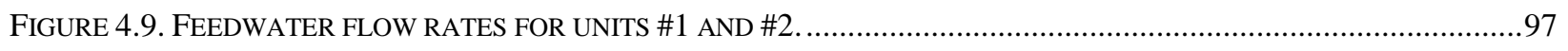

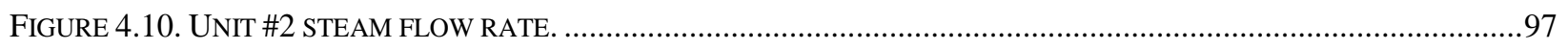

FIGURE 4.11. UNIT \#1 AVERAGE MODERATOR TEMPERATURE. ................................................................98

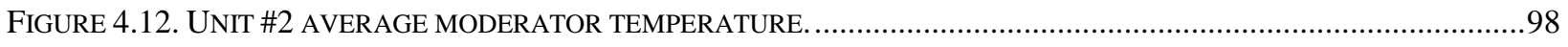

FIGURE 4.13. UNITS \#1 \& \#2 AND MIXED STEAM HEADER TEMPERATURES CHANGES..........................................99

FIGURE 4.14. UNIT \#1 CORE POWER WITH INVERTED PROFILE \#1 LOAD DEMAND.............................................100

FIGURE 4.15. UNIT \#2 CORE POWER WITH INVERTED PROFILE \#1 LOAD DEMAND IN UNIT \#1 .................................100

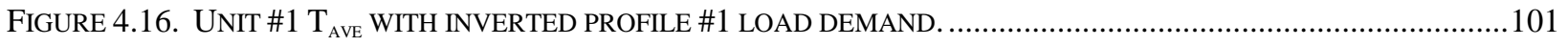

FIGURE 4.17. UNIT \#2 $\mathrm{T}_{\text {AVE }}$ WITH INVERTED PROFILE \#1 LOAD DEMAND IN UNIT \#1 .........................................101

FIGURE 4.18. UNITS \#1 \& \#2 AND COMBINED STEAM HEADER TEMPERATURES CHANGES USING INVERTED PROFILE 1 IN

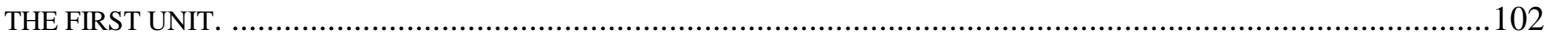

FIGURE 4.19. UNIT 1 CORE POWER PROFILE FOR A $2.8^{\circ} \mathrm{C} \mathrm{T}_{\text {COLD }}$ SENSOR FAULT..................................................104

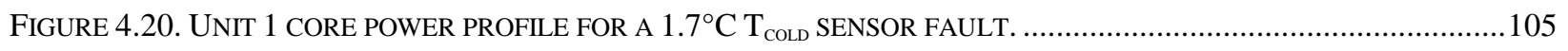

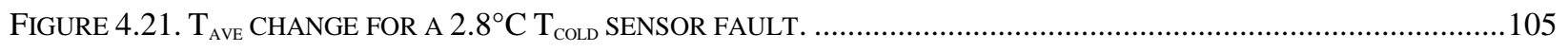

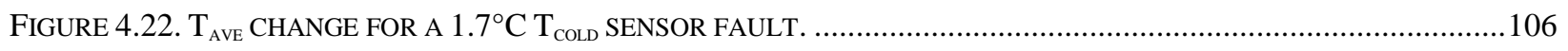

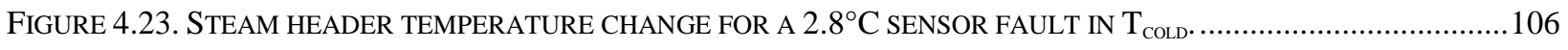

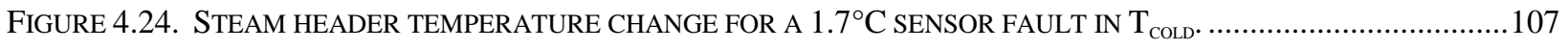

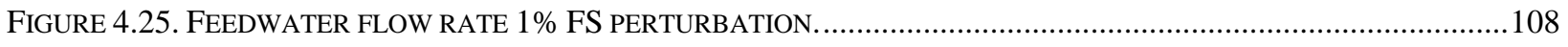

FIGURE 4.26. STEAM AND FEEDWATER FLOW RATE TO 1\% FS PERTURBATION. ................................................108

FIGURE 4.27. STEAM HEADER TEMPERATURE PERTURBATION FOR A NEGATIVE 1\% PERTURBATION IN FEEDWATER

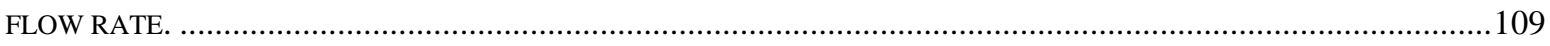

FIGURE 4.28. CORE POWER TRANSIENT FOR A NEGATIVE 1\% PERTURBATION IN FEEDWATER FLOW RATE. ................110

FIGURE 4.29. $\mathrm{T}_{\text {AVE }}$ TRANSIENT FOR A NEGATIVE 1\% PERTURBATION IN FEEDWATER FLOW RATE.............................110 
FIGURE 4.30. STEAM HEADER TEMPERATURE TRANSIENT FOR A NEGATIVE $1.7^{\circ} \mathrm{C}$ PERTURBATION IN FEEDWATER FLOW RATE.

FIGURE 4.31. STEAM PRESSURE TRANSIENT FOR A NEGATIVE $1.7^{\circ} \mathrm{C}$ PERTURBATION IN FEEDWATER FLOW RATE........112

FIGURE 4.32. TAVE TRANSIENT FOR A NEGATIVE $1.7^{\circ} \mathrm{C}$ PERTURBATION IN FEEDWATER FLOW RATE..........................112

FIGURE 4.33. CORE POWER TRANSIENT FOR A NEGATIVE $1.7^{\circ} \mathrm{C}$ PERTURBATION IN FEEDWATER FLOW RATE. .............113

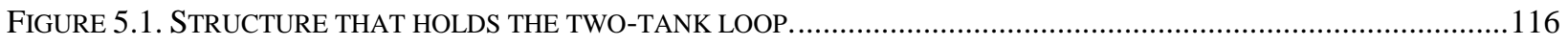

FIGURE 5.2. DRILLING WORK OF A 1/4” THICK ALUMINUM SHEET. .......................................................................116

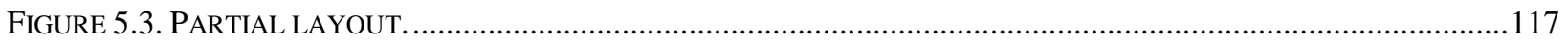

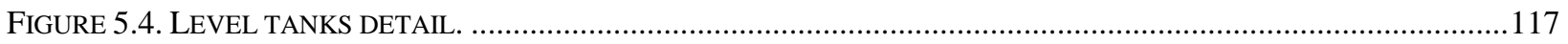

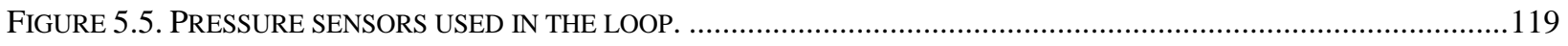

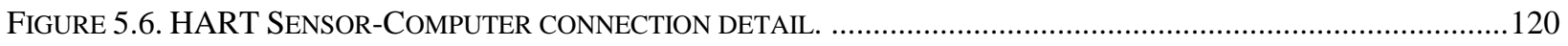

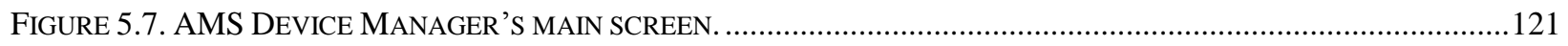

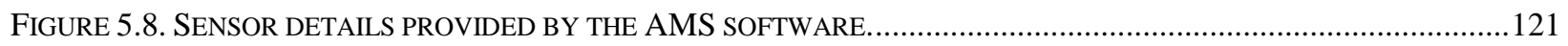

FIGURE 5.9. SENSOR DETAIL AND STATUS PROVIDED BY AMS SOFTWARE ………………………….....................122

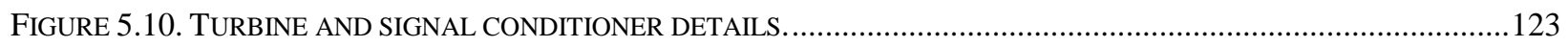

FIGURE 5.11. DETAILS OF THE ORIFICE PLATE AND THE PRESSURE LINES. .............................................................124

FIGURE 5.12. SIGNAL CONDITIONER CALIBRATION CURVE AND PREDICTED VALUES................................................126

FIGURE 5.13. TURBINE FLOWMETER CALIBRATION CURVE AND PREDICTED VALUES...............................................126

FIGURE 5.14. CENTRIFUGAL PUMP AND THERMOCOUPLE DETAIL. .......................................................................129

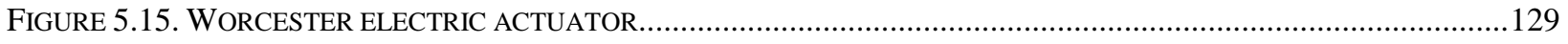

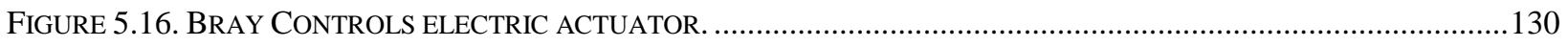

FIGURE 5.17. SCHEMATIC OF THE TWO-TANK EXPERIMENTAL CONTROL LOOP ......................................................133

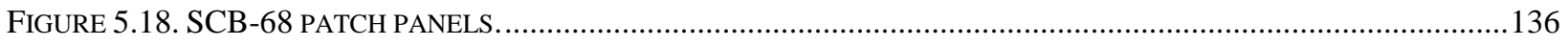

FIGURE 5.19. CONTROL VI FRONT PANEL SHOWING THE MANUAL CONTROL OPTION. ...............................................138

FIGURE 5.20. CONTROL VI FRONT PANEL SHOWING THE AUTOMATIC CONTROL FOR TANK 2..................................139

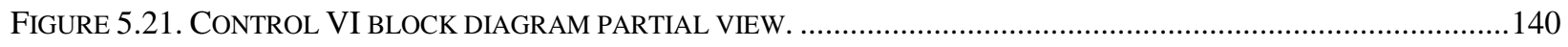

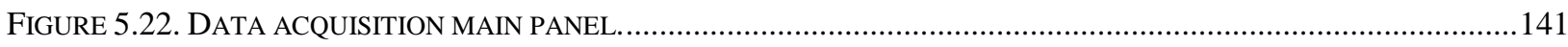


FIGURE 6.1. SPRT IS BASED ON COMPARING STATISTICAL DIFFERENCES.

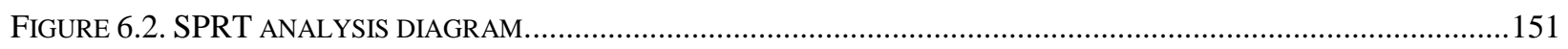

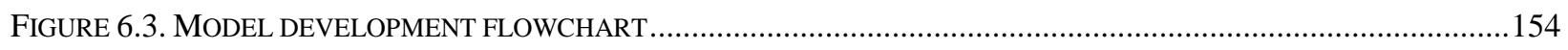

FIGURE 6.5. MAPE FOR LINEAR REGRESSION, KERNEL REGRESSION, AND AAKR MODELS. ...................................157

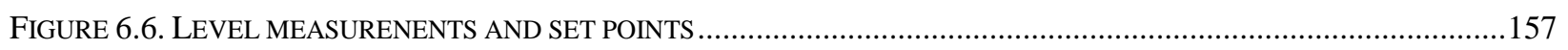

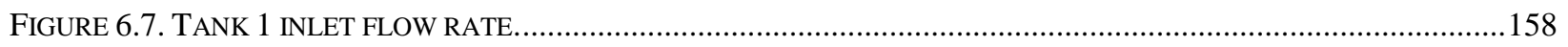

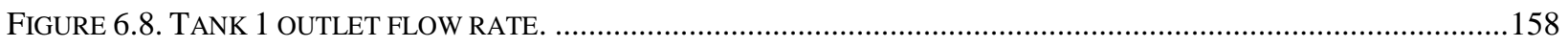

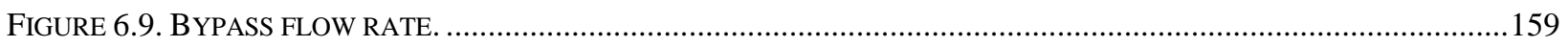

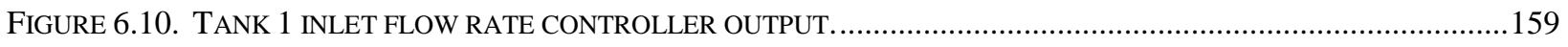

FIGURE 6.11. TANK 1 INLET FLOW RATE CONTROL VALVE FEEDBACK. ......................................................

FIGURE 6.12. MEASURED WATER LEVEL VERSUS PREDICTED VALUES . ......................................................161

FIGURE 6.13. MEASURED OUTLET FLOW RATE VERSUS PREDICTED VALUES ...............................................162

FIGURE 6.14. MEASURED BYPASS FLOW RATE VERSUS PREDICTED VALUES. ..................................................

FIGURE 6.15. MEASURED INLET FLOW RATE VERSUS PREDICTED VALUES. ......................................................163

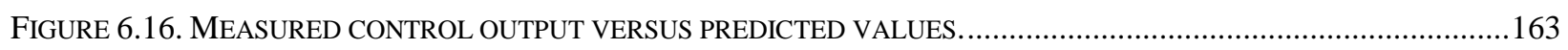

FIGURE 6.17. MEASURED CONTROL VALVE FEEDBACK VERSUS PREDICTED VALUES...........................................164

FIGURE 6.18. FAULTY LEVEL MEASUREMENT SUBSTITUTION FOR SLOW CHANGING DRIFT.....................................169

FIGURE 6.19. WATER LEVEL ABSOLUTE DIFFERENCE FOR SLOW CHANGING DRIFT................................................

FIGURE 6.20. FAULTY LEVEL MEASUREMENT AND SUBSTITUTION FOR FAST CHANGING DRIFT. ............................170

FIGURE 6.21. WATER LEVEL ABSOLUTE DIFFERENCE FOR FAST CHANGING DRIFT. ..........................................170

FIGURE 6.22. FAULTY BYPASS MEASUREMENT DETECTION AND SUBSTITUTION FOR A 2\% DRIFT OVER 3 MINUTES....171

FIGURE 6.23. FAULTY BYPASS LEVEL MEASUREMENT FAULT DETECTED BY LEVEL. ........................................172

FIGURE 6.24. FAULTY INLET FLOW RATE MEASUREMENT DETECTION FOR A 60\% DRIFT OVER 3 MINUTES............... 173

FIGURE 6.25. FAULTY OUTLET FLOW RATE MEASUREMENT DETECTION FOR A 2\% DRIFT OVER 3 MINUTES...............173

FIGURE 6.26. FAULTY OUTLET FLOW RATE MEASUREMENT FAULT DETECTED BY LEVEL. ..................................174

FIGURE 6.27. INLET FLOW RATE CONTROL VALVE POSITION AT 6.5V WITH CONTROL SWITCH OVER TO TANK $2 . . . . . . .176$ 
FIGURE 6.28. CV1 STUCK AT 6.5V FAULT DETECTION USING CV MODELS.......................................................177

FIGURE 6.29. CV1 STUCK AT 6.5V FAULT DETECTION USING TANK 1 LEVEL AND INLET FLOW RATE MODELS. ...........177

FIGURE 6.30. CV1 STUCK AT 6.5V FAULT DETECTION USING OUTLET AND BYPASS FLOW RATE MODELS..................178

FIGURE 6.31. BYPASS FLOW RATE TRANSIENT ORIGINATED BY A CONTROL VALVE STUCK AT 6.5V ........................178

FIGURE 6.32. INLET FLOW RATE CONTROL VALVE POSITION AT 5.5V WITH CONTROL SWITCH OVER TO TANK 2........180

FIGURE 6.33. CV1 STUCK AT 5.5V FAULT DETECTION USING LEVEL AND INLET FLOW RATE MODELS......................180

FIGURE 6.34. INLET FLOW RATE CONTROL VALVE POSITION AT 4.5V WITH CONTROL SWITCH OVER TO TANK 2 ........181

FIGURE 6.35. INLET FLOW RATE CONTROL VALVE POSITION AT 3.5V WITH CONTROL SWITCH OVER TO TANK $2 \ldots . . . . .182$

FIGURE 6.36. CV1 STUCK AT 3.5V FAULT DETECTION USING OUTLET AND BYPASS FLOW RATE MODELS..................182 


\section{Chapter 1}

\section{INTRODUCTION}

\subsection{Background}

Nuclear power plants generate about 20 percent of the electricity produced in the U.S. alone, and more than $15 \%$ of worldwide generation of electricity; however, all recent electricgenerating capacity additions and projected future additions are primarily fueled by natural gas. Despite the excellent performance of current nuclear plants with capacity factors of over $90 \%$ in recent years, and decisions by power plant owners to seek license renewal and power up-rates, no new plant has been built for more than 25 years.

As of May of 2010, there are 104 commercial nuclear generating units in the United States, mostly built between 1967 and 1990, distributed in 31 states, operated by 30 different companies. In 2008 alone, the U.S. generated 4,119 billion kWh net of electricity, with $49 \%$ of it from coal-fired plants, $22 \%$ from gas, $6 \%$ from hydro and 809 billion $\mathrm{kWh}$ from nuclear. Of these 104 reactors, 69 are pressurized water reactors (PWR) totaling 65,100 net megawatts (electric) and 35 units are boiling water reactors (BWR) totaling 32,300 net megawatts (electric).

There have been no new construction starts since 1977, largely because for a number of years gas generation was considered more economically attractive and because construction schedules were frequently extended by opposition, compounded by heightened safety fears following the Three Mile Island accident in 1979. Despite a near halt in new construction for more than 30 years, US reliance on nuclear power has continued to grow. In 1980, nuclear plants produced 251 billion $\mathrm{kWh}$, accounting for $11 \%$ of the country's electricity generation. As stated earlier, in 2008 that output had risen to 809 billion kWh and nearly 20\% of electricity, providing more than $30 \%$ of the electricity generated from nuclear power worldwide. Much of the increase came from the 47 reactors, all approved for construction before 1977, that came on line in the late 1970s and 1980s, more than doubling US nuclear generation capacity. Another PWR unit (Watts Bar 2) is expected to start up by 2012 following Tennessee Valley Authority's decision in 2007 to complete the construction of the unit. The US nuclear industry has also 
achieved remarkable gains in power plant utilization through improved refueling, enhanced maintenance and safety systems at existing plants.

But with annual electricity demand projected to increase to 5,000 billion $\mathrm{kWh}$ in 2030 (annual per capita electricity consumption is currently around $12,400 \mathrm{kWh}$ ) this scenario is rapidly changing. The U.S. Nuclear Regulatory Commission (NRC) is responsible for regulating and licensing all commercial nuclear activities in the United States, and has received, as of March of 2008, expressions of interest for building 32 new reactors and have received four license applications for combined construction and operation, and several utilities have submitted Early Site Permits (ESP), including Southern Nuclear (Georgia Power) and Duke Power. Many countries are building or planning to build new reactors, including Canada, Brazil, England, France, Bulgaria, Ukraine, Finland, Russia, China, India, Japan, South Korea, and others.

The reason behind this resurgence in nuclear energy interest stems from various reasons from public acceptance rates (75\% or better in every aspect) to environmental concerns with global warming, from cost of producing electricity to technological maturity and safety. People have become aware that for several decades, nuclear power has had an incredibly good record of safety, environmental protection and low costs, and the public wants a way to produce electricity that minimizes pollution. But most importantly the US government Research and Development (R\&D) funding for nuclear energy is being revived with the objective of rebuilding US leadership in nuclear technology. In an effort that brings together government research laboratories, industry and academe, the Federal government has significantly stepped up R\&D spending for future plants that improve or go well beyond current designs. There has been particular attention to the Next Generation Nuclear Plant (NGNP) project to develop a Generation IV high-temperature gas-cooled reactor, which would be part of a system that would produce both electricity and hydrogen on a large scale. The US Department of Energy (DOE) has stated that its goal is to have a pilot plant ready at its Idaho National Laboratory (INL) by 2021, with total development cost estimated at $\$ 2$ billion.

\subsection{Small and Medium Reactors (SMR)}

In today's world, small or medium sized reactors can play a significant role in energy

production as the U.S. and other developing countries embark on what is being called the 
"Second Nuclear Era", or most popularly known as "Nuclear Renaissance". The International Atomic Energy Agency (IAEA) has been very active in coordinating the development of smaller sized reactors, especially for their member countries that do not currently use nuclear power. The IAEA defines a "small" reactor as one having electrical output less than $300 \mathrm{MWe}$ and a “medium" reactor as one having output between 300 and 700 MWe. More often, the two sizes are combined into the common term "small and medium-sized reactor" (SMR) representing a reactor with electrical output less than $700 \mathrm{MWe}$. Therefore, a "large" plant is assumed to have a power output greater than $700 \mathrm{MWe}$, although most large plants being planned or constructed today have capacities of less than $1000 \mathrm{MWe}$. The IAEA's definition of an SMR is expressed in terms of the reactor's power output; not surprisingly, most SMRs are also physically smaller than large plants. Both their lower power output and their physical smallness contribute to their associated benefits in areas of plant safety, fabrication, operations, and overall economics.

Out of 442 commercial nuclear power plants (NPP) currently operating worldwide, 139 can be considered SMRs, according to IAEA definition of plant sizes. However, most of these are merely scaled-down versions of large plant designs, and the focus of today's trend is in smaller sized reactor designs that are "deliberately small" 0 , that is novel designs that do not scale to large sizes but rather capitalize on their smaller blueprint to achieve specific performance characteristics. The Integral Primary System Reactor (IPSR), design led by Westinghouse Electric Company [2] [3] [4] is one of the many reactor designs available [5] [6]. It incorporates the reactor core, steam generators, pumps, and pressurizer into a single common pressure vessel that may be appropriate for base load electricity generation, especially in regions with smaller electricity grids. It is also well suited for more specialized non-electrical energy applications such as district heating, process steam partitioning for desalination, and cogeneration. This design is based on very mature Light Water Reactor (LWR) technology, and is currently being developed by a consortium of over 20 different organizations from 10 countries, including the U.S., Italy, Japan, Brazil, U.K., Croatia, Lithuania, Spain, Mexico, and most recently Estonia. The International Reactor Innovative and Secure (IRIS) project was initiated as a DOE-sponsored research project and has evolved into a vendor-led project with international participation of suppliers, national laboratories, and universities, and is estimated for deployment in 2015. 
In June of 2008, an Oak Ridge DOE-supported project published a report that provided a roadmap for the development of what was then called "grid-appropriate reactors" with emphasis on Instrumentation and Control and Human-Machine Interface technology (ICHMI) [7]. In this report, numerous challenges were listed as being absolutely important to be overcome in order to fully realize the benefits of ICHMI for grid-appropriate reactors of the future. The following broad set of projects towards overcoming such problems was recommended:

Sensors and electronics for harsh environments

- Design of control systems for active grid stability support

- Intelligent controls for nearly autonomous operation

- Plant network architecture

- Intelligent aiding technology for operational support

- Human system interaction

- Licensing and regulatory support

- Technologies to support water desalination integration

Also, the instrumentation and control design to avoid and to mitigate the above problems in SMRs requires further research in the design of control and monitoring systems. The necessary R\&D must include a detailed simulation of grid dynamics and the effect of postulated scenarios that cause grid instability. The simulations would identify the power plant response in terms of voltage and frequency, physical limitations on transmission circuits, and the effects of automated load shedding and emergency disconnection of the NPP from the grid. The following section is an excerpt from the document mentioned above. 


\subsection{Overview of Electrical Grid Stability Applied to Small and Medium Reactors}

An electrical grid is said to be stable when "the total mechanical power input from the prime movers to the generators is equal to the sum of all the connected loads, plus all the losses in the system." Grid instability is concerned with the oscillations in the voltages and the mismatch between generator frequency and the grid frequency. If the grid experiences a wide range of operating conditions, then the "coordination among adequate generation, transmission, and operation within the stability limits and load demand becomes a challenging task." In order to maintain the frequency of the turbine generator in synchronous with the grid frequency, it is necessary to regulate the generator frequency using speed governors.

Voltage regulation, power transmission limits across a transmission line, and load disturbances must also be considered as part of the Instrumentation and Control (I\&C) design. In general, frequency stability and voltage stability are affected by load characteristics. In some situations, grid frequency decay (external to the system) or voltage variations affect the performance of induction motors by providing inadequate reactor coolant flow. This form of grid disturbance may require a low-frequency reactor trip. Alternatives to such drives should be considered for SMR equipment.

The engineering considerations of electrical grid interface of NPPs include the following:

- Magnitude and frequency of load rejections, and loss of load to the NPP

- Grid transients involving degraded voltage/frequency

- Complete loss of off-site power to the NPP due to grid disturbances

- NPP unit trip causing a grid disturbance and ultimate grid collapse

The I\&C design to avoid and to mitigate the above problems in SMRs requires further research in the design of control and monitoring systems. The necessary R\&D must include a detailed simulation of grid dynamics and the effect of postulated scenarios that cause grid instability. The simulations would identify the power plant response in terms of voltage and 
frequency, physical limitations on transmission circuits, and the effects of automated load shedding and emergency disconnection of the NPP from the grid. It is suggested that for remote deployed SMRs it would be necessary to install diesel generators and fast start gas turbines (for diversity) in order to provide safety margins for the on-site power system.

About grid stability, the following excerpt is taken from the book authored by Kundur and it explains the stability of power grids [8]:

"Voltage stability is the stability of a power system to maintain steady acceptable voltages at all buses in the system under normal operating conditions and after being subjected to a disturbance. A system enters a state of voltage instability when a disturbance, increase in load demand, or change in system condition causes a progressive and uncontrollable drop in voltage. The main factor causing instability is the inability of the power system to meet the demand for reactive power. The heart of the problem is usually the voltage drop that occurs when active power and reactive power flow through inductive reactances associated with the transmission network."

\subsection{Application of SMRs in Co-Generation and Desalination}

When the first nuclear power reactor at Calder Hall in the United Kingdom came into commercial operation in October 1956, it provided electricity to the grid and heat to a neighboring fuel reprocessing plant. After more than 40 years, the four 50 MWe Calder Hall units are still in operation. In Sweden, the Agesta reactor provided hot water for district heating to a suburb of Stockholm for a decade, starting in 1963 [9].

In recent years, the option of combining nuclear power with brine desalination has been explored to tackle water shortage problem in semi-arid to arid-areas worldwide, especially in developing countries [10] - [17]. The desalination of seawater using nuclear energy is a feasible option to meet the growing demand for potable water. Over 150 reactor-years of operating experience on nuclear desalination has been accumulated worldwide, of which Japan now has over 125 reactor-years. Kazakhstan (Aktau fast reactor BN-350) had accumulated 26 reactoryears of producing $80,000 \mathrm{~m}^{3} /$ day of potable water before shutting down in 1999 . Several demonstration programs of nuclear desalination are also in progress to confirm its technical and 
economical viability under country-specific conditions, with technical coordination or support of IAEA [18]. It is expected that there will be an increased role of nuclear energy in various nonelectrical energy services (desalination, hydrogen production and other industrial process heat applications [19]), which, at the moment, are almost entirely dominated by fossil energy sources. Table 1.1 summarizes past experience as well as current developments and plans for nuclearpowered desalination based on different nuclear reactor types. Most of the technologies in Table 1.1 are land-based, but the table also includes a Russian initiative for barge-mounted floating desalination plants [20] [21]. Floating desalination plants could be especially attractive for responding to temporary demands for potable water. Recent demonstration projects: there are three nuclear demonstration projects presently under construction in India, Pakistan, and Korea. Useful design and operational data are being generated and will be available for these plants. These examples demonstrate the use of nuclear energy that is unfamiliar to many - its capacity to deliver heat for industrial processes and urban needs and to help generate potable water from brine [22]. Some of such applications started at a very early date, practically at the same time when nuclear power reactors were first applied to electricity generation.

Table 1.1. Reactor types and desalination processes [17].

\begin{tabular}{|c|l|l|l|}
\hline Reactor type & \multicolumn{1}{|c|}{ Location } & \multicolumn{1}{|c|}{$\begin{array}{c}\text { Capacities } \\
\left(\mathbf{m}^{\mathbf{3}} / \mathbf{\text { day }}\right)\end{array}$} & \multicolumn{1}{c|}{ Status } \\
\hline \multirow{4}{*}{ LMFR } & Kazakhstan (Aktau) & 80,000 & In service till 1999 \\
\hline \multirow{5}{*}{ PWRs } & $\begin{array}{l}\text { Japan } \\
\text { (Ohi, Takahama, } \\
\text { Ikata, Genkai) }\end{array}$ & $1,000-2,000$ & $\begin{array}{l}\text { In service with operating } \\
\text { experience of over 125 reactor- } \\
\text { years }\end{array}$ \\
\cline { 2 - 5 } & Republic of Korea & 40,000 & Under design \\
\cline { 2 - 5 } & Argentina & 12,000 & Under consideration \\
\hline
\end{tabular}




\begin{tabular}{|c|c|c|c|}
\hline Reactor type & Location & $\begin{array}{c}\text { Capacities } \\
\left(\mathrm{m}^{3} / \text { day }\right)\end{array}$ & Status \\
\hline & Russia & -- & Under design (floating unit) \\
\hline BWR & $\begin{array}{l}\text { Japan } \\
\text { (Kashiwazaki) }\end{array}$ & -- & $\begin{array}{l}\text { Never in service following } \\
\text { testing in } 1980 \text { s, due to } \\
\text { alternative freshwater sources; } \\
\text { dismantled in } 1999\end{array}$ \\
\hline \multirow{2}{*}{ PHWR } & India (Kalpakkam) & 6,300 & Under commissioning \\
\hline & Pakistan (KANUPP) & 4,800 & Under construction \\
\hline NHR & China & 160,000 & Under consideration \\
\hline HTGR & $\begin{array}{l}\text { South Africa, France, } \\
\text { The Netherlands }\end{array}$ & -- & Under consideration \\
\hline
\end{tabular}

\subsection{Objectives of the Research Task and Significant Contributions}

A comprehensive and critical literature review over the most recent progress in small and medium reactors has shown the need for answers to help solve the problems involving the utilization of such novel reactor designs. One of the key features of such design is the employment of Once-Through Steam Generators (OTSGs) instead of the classic recirculation steam generators (SGs), used in most PWRs. Such SGs present a fundamental difference from those used in B\&W PWRs, in that the secondary water flow is inside the tubes. This provides unique challenges such as tube drying and uncertainties in the level of secondary water. The power removed through the SGs depends directly on feedwater flow rate, meaning that, following any large loss of main feedwater, the turbine must be rapidly tripped by closing the Fast Closure Admission Valves (referred to as fast valving). These characteristics of IRIS steam generators make it challenging to implement the classical PWR control logic.

Furthermore, IRIS has a large reactor coolant system inventory of over $16,000 \mathrm{ft}^{3}$, significantly larger than any other PWR, leading to some distinct impacts on the overall system 
behavior such as more time needed during cooldown/heatup, startup and dilution procedures, and most importantly a weak coupling between the reactor core and the steam generators also due to the low flow velocity, which in turn results in a characteristic residence time of about 40 seconds (compared to the 10 seconds in typical PWRs).

In hindsight, having a control system that can help anticipate transients (i.e., a model based control system) can have a significant impact on procedures and lead to better plant design and utilization, specially in view of such large thermal inertia which allows for a relatively large delay between reactor trip and turbine trip to provide adequate initial cooling following a reactor trip.

\subsubsection{Objectives of the Research}

1. The first objective of this research is to evaluate and quantify the performance of a Nuclear Power Plant (NPP) comprised of two IRIS reactor modules operating simultaneously with a common steam header, which in turn is connected to a single turbine, resulting in a steam-mixing control problem with respect to "load-following" scenarios, such as varying load during the day or reduced consumption during the weekend.

2. In order to develop research related to instrumentation and control, and equipment and sensor monitoring, the second objective is to build a two-tank multivariate loop in the Nuclear Engineering Department at the University of Tennessee. This loop provides the framework necessary to investigate and test control strategies and fault detection in sensors, equipment and actuators.

3. The third objective is to experimentally develop and demonstrate a fault-tolerant control strategy using this loop. Using six correlated variables in a single-tank configuration, five inferential models and one Auto-Associative Kernel Regression (AAKR) model were developed to detect faults in process sensors. Once detected the faulty measurements were successfully substituted with prediction values, which would provide the necessary flexibility and time to find the source of discrepancy and resolve it, such as in an operating power plant. Finally, using the same empirical models, an actuator failure 
was simulated and once detected the control was automatically transferred and reconfigured from one tank to another, providing survivability to the system.

As stated earlier, IRIS is to date a conceptual design with several of its features still to be experimentally evaluated and proven. Hence, in addition to the objectives mentioned above, other objectives of this research are:

4. Development and implementation of a multivariate flow control loop with two tanks, a series of control valves and sensors, to provide the necessary framework to develop research related to instrumentation and control strategies, equipment and sensor monitoring.

5. Application of data-based empirical models for on-line characterization of the experimental data generated by the multivariate flow control loop and the use of these models for steady state fault detection.

6. Implementation of on-line, real-time fault detection and isolation, in which the steady-state estimated values were used to replace faulty sensor readings.

7. Steady state actuator fault detection and controller reconfiguration on the multivariate flow control loop.

\subsubsection{Significant Contributions}

1. Existing IRIS single-unit model is extended to a twin-unit IRIS plant.

2. Results show the impact of having steam from two steam generators converging to a common header and subsequently to the turbine (balance-of-plant was not modeled).

3. This solution to the control challenges posed by the operation of a twin-unit IRIS plant with steam mixing is a new contribution. 
4. Evaluation of the control performance of such novel design when subject to different load-following scenarios, resulting in an IRIS model capable of operating as a single-unit plant or as a twin-unit plant.

5. Investigation of the controller robustness in load-following mode under limited fault conditions such as:

Feedwater temperature perturbation.

Feedwater flow rate perturbation.

Hot leg temperature perturbation.

Cold leg temperature perturbation.

6. A digitally-controlled multivariate flow control loop with model-based on-line fault detection and reconfigurable digital controller capacity.

\subsection{Outline of the Report}

Chapter 2 presents a review of the current status of the so called "deliberately small and medium" reactor designs. Deliberately in the sense that such designs are not downscales of large size reactors, but rather these designs capitalize on their smallness to achieve specific performance characteristics, introducing in some cases novel approaches based on years of experience from designing and operating larger reactors. This chapter also provided a short review of fault-tolerant and reconfigurable control as an active approach in utilizing control theory to automatically achieve fault-tolerant control whenever failures occur in sensors or actuators in dynamic systems such as aircraft flight control systems, petro-chemical plants, mineral processing plants, spacecraft maneuvering, robot control systems, ship control, etc., thus providing means of survivability to the systems they control.

Chapter 3 describes the IRIS system highlighting the current status in its development with emphasis on instrumentation and control needs. A description of the current dynamic 
model of the IRIS Helical Coil Steam Generator (HCSG) and reactor core model systems is provided. Load following maneuvers using various power profiles is investigated.

Chapter 4 describes the twin-unit IRIS system approach, providing a description of the model assumptions. A load-following maneuver is used, as well as some controlled faults are introduced in the model to test its robustness and the results are discussed.

Chapter 5 provides a description of the two-tank multivariate experimental flow control loop built to study and develop research related to instrumentation and control, equipment and sensor monitoring, which provides the framework necessary to investigate and test control strategies and fault detection in sensors, equipment, and actuators.

Chapter 6 describes how empirical models were obtained and used to detect faults in six sensors and one actuator using real data obtained from the two-tank flow loop described in the previous chapter. The faulty sensors are isolated and their measurements are substituted with predictions obtained from the models, and in the case of the actuator fault, the control is successfully transferred from tank 1 to tank 2 and reconfigured providing survivability to the system. Results are presented and discussed.

Chapter 7 summarizes the research with concluding remarks, along with recommendations for future work.

Several Appendixes provide additional results of analysis, a user manual for operating the experimental flow control loop, and some typical outputs using the computer codes used in this research. 


\section{Chapter 2}

\section{DELIBERATELY SMR DESIGNS AND OVERVIEW OF FAULT TOLERANT AND RECONFIGURABLE CONTROL}

\subsection{Introduction}

In nuclear power industry, unlike in many industrial undertakings, the long-term viewpoint is predominant. Planning, designing, project preparatory activities, and licensing takes years to be completed for any new nuclear reactor, and is a little easier when renewing and up-rating existing reactors. Reactors are designed and built to last for about 40 years or more, and to achieve the economic benefits expected, they have to be operated with high load factors during their operating lifetime. There are also infrastructure issues, which require time and considerable development efforts, if not already available. These efforts are only justifiable under a long-term perspective directed to a nuclear program.

Siting of nuclear plants has become a major issue, even in those countries that are proceeding with their nuclear programs by initiating new projects because of limited availability of sufficient water for effective rejection of the waste heat from any new nuclear power plant. Such problem is worse for nuclear power plants because of their slightly lower power conversion efficiency compared to fossil plants, dumping two-thirds (typically) of its produced thermal power to the environment, normally an adjacent large body of water. Building additional units at existing nuclear sites has been a standard practice lately, and opening up new sites for nuclear plants are a rare occurrence.

Due to the size effect, nuclear energy economy, in general, is better for larger units. This has led to the development and predominant deployment of large-size reactors in industrialized countries with very large interconnected and stable electrical grid systems. However, today's electricity markets are aspiring for alternatives to large-footprint expensive large reactors, improving the market for small and medium sized power reactors (SMR). As stated earlier, current design SMRs are considered to be not scaled down versions of large commercial reactors, but deliberately small designs and are intended to be economically competitive. A good example is Babcock \& Wilcox’s 125 MWe integral nuclear system design modular pressurized 
water reactor (called the mPower reactor), shown in Figure 2.1, which will be applied for design certification in late 2012, according to an April 1, 2010 letter to NRC, and is expected to be deployed in 2018. This reactor is intended to be manufactured by North American suppliers, and will be built underground. The spent fuel will be stored in a containment-enclosed pool during the 60-year operation. Two to six modules could operate at a typical site, providing power in increments reasonable for mid-sized or small utilities. However, the bottleneck in cases of novel designs like this could be the regulatory body. Budgetary reasons limit NRC's personnel interactions with designers of small power reactors to occasional meetings or other non-resource intensive activities [23]. But just recently the NRC has informed several designers that through the end of fiscal year 2011, the offerings from Babcock \& Wilcox and NuScale Power will be given priority over designs from other manufacturers, clearly showing the ever increasing importance of such designs in the new nuclear industry [24]. Deviating very little from the parameters of PWRs now in service, some of the characteristics of such design are:

- Integral nuclear system design and passive safety systems.

- Underground containment.

- Five-year operating cycle between refueling.

- Scalable, modular design is flexible for local needs.

- Multi-unit (1 to 10+) plant.

- Used fuel stored in spent fuel pool for life of the reactor (60 years).

- North American shop-manufactured.

A South Korea's design called SMART (System-integrated Modular Advanced Reactor) is a $330 \mathrm{MWt}$ pressurized water reactor with integral steam generators and advanced safety features, and is designed by the Korea Atomic Energy Research Institute (KAERI) for generating electricity (up to $100 \mathrm{MWe}$ ) and/or thermal applications such as seawater desalination with a 
design life of 60 years, with a three-year refueling cycle. With a basic design already complete, the absence of any placed orders for an initial reference unit has stalled development. KAERI is now intending to proceed to licensing the design by 2012 .

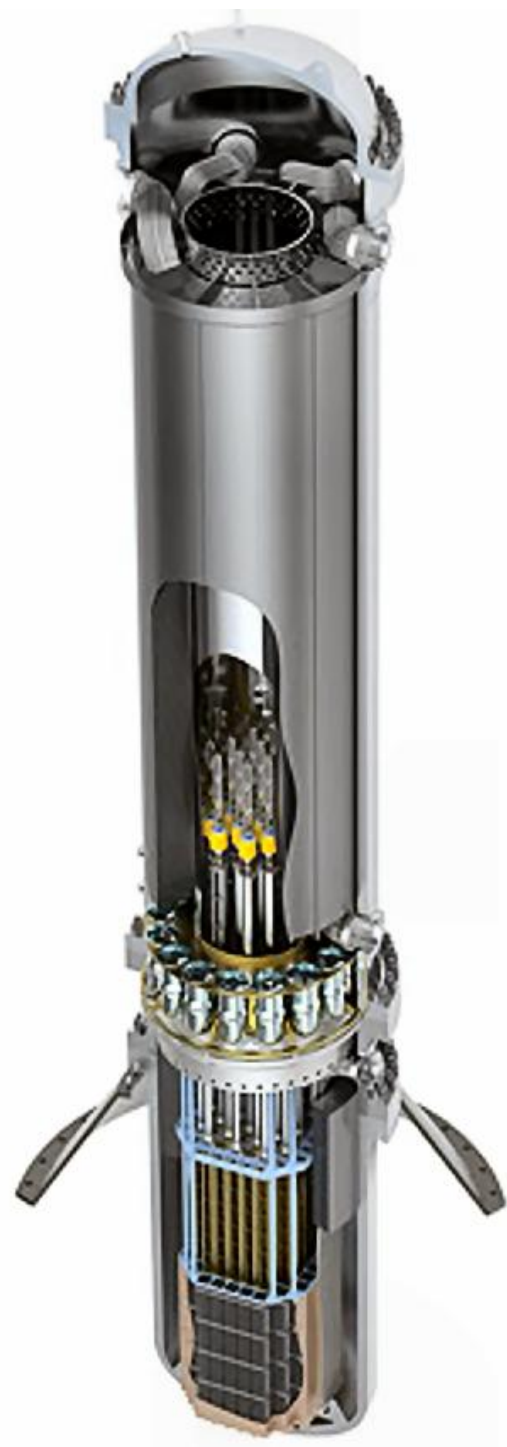

Figure 2.1. B\&W's 125 MWe mPower reactor [23]. 


\subsection{Steam Mixing in Multi-modular Reactor Systems}

This section of the research provides an overview of what has been published to date concerning multi-modular reactor systems.

During the late 1980's Lanning [25] referred to the nuclear status as a "window of opportunity" for innovation in nuclear power technology and regulation. In his paper [25] he introduced the advantages of Modularized High-Temperature Gas-Cooled Reactor (MHTGR) systems, specifically focusing on its capacity of providing passive safety that prevents fuel damage over a wide spectrum of accidents, as well as safety-related cooling components like pump and valves. The concept presented included a system to bridge between the integrated approach to the MHTGR design requirements and the regulatory licensing process. He also discussed designs with the passive safety features, both pebble-bed and prismatic cores, and some incentives and impediments for deployment of the MHTGR. Attractive passive safety features and characteristics resulting from such design are reproduced in Table 2.1, while pros and cons for the deployment of modular MHTGRs are reproduced in Table 2.2.

Table 2.1. Passive safety results from material characteristics and design.

\begin{tabular}{|l|l|}
\hline \multicolumn{1}{|c|}{ Feature } & \multicolumn{1}{c|}{ Result } \\
\hline High temperature stability of reactor and fuel & Fission products retained in coated particles \\
\hline Large negative temperature coefficients & Shutdown without rod motion \\
\hline Small thermal rating & Limiting amount of afterheat \\
\hline Core geometry & Providing for removal of afterheat by \\
\hline Slow heat-up of massive graphite core & passive conduction and radiation \\
\hline
\end{tabular}


Table 2.2. Advantages and disadvantages of deployment of HTGRs.

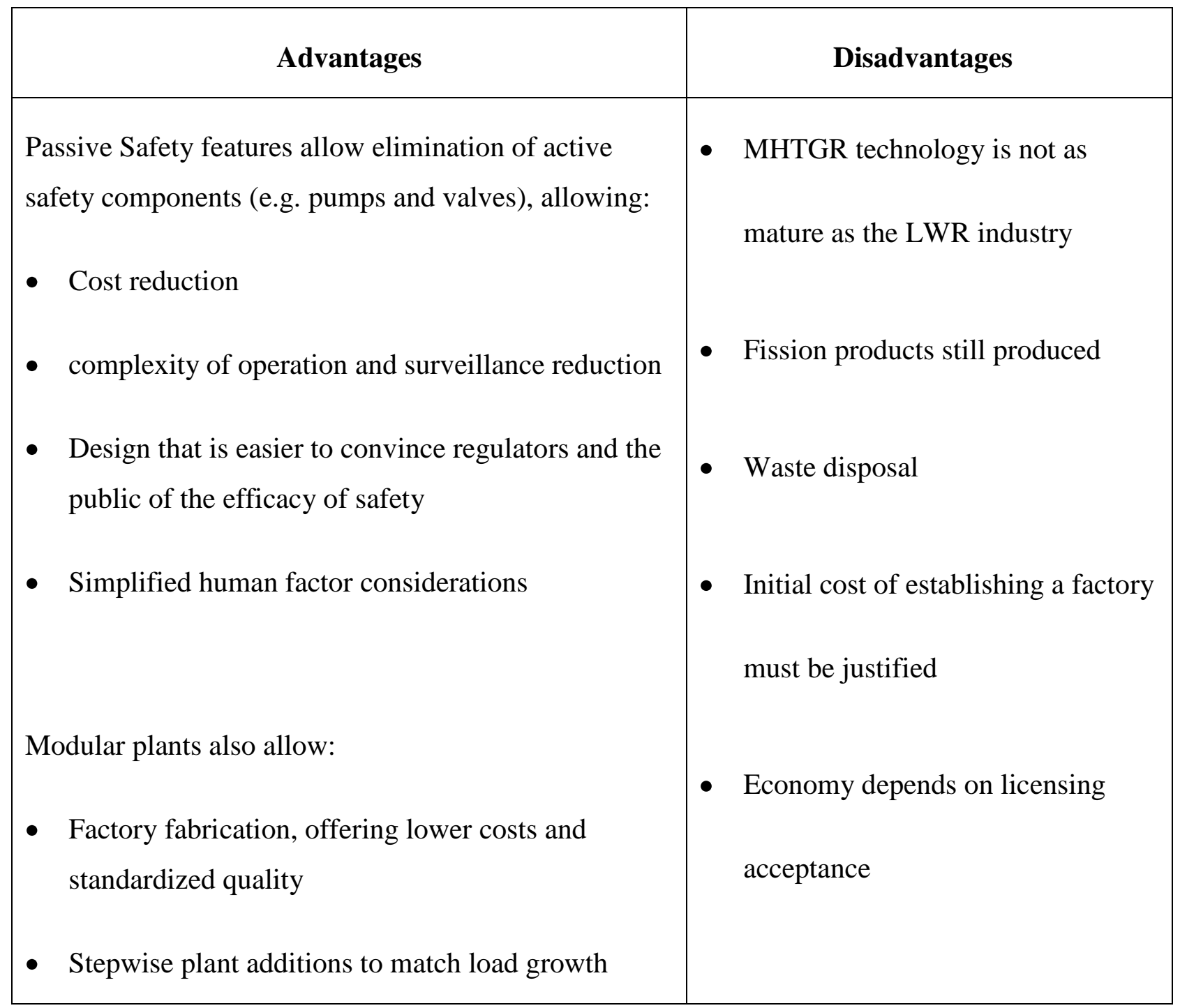

In addition, suggestions for university research related to MHTGR were presented, along with 15 references. Of special interest are modularity issues like multi-modular control, expandable single control room, and optimum module output capabilities.

Concerns with control of multi-modular reactors are not limited to gas-cooled reactors only. Otaduy and Brittain [26] proposed a supervisory, hierarchical control for a Power Reactor 
Innovative, Small Module (PRISM)-like Advanced Liquid-Metal Fast Breeder Reactor (ALMR) multi-modular plant as part of DOE's advanced controls program. Dayal's paper [27] describes a then state-of-the-art multi-modular PRISM 1395 MWe plant comprising of three power blocks, each made up of three identical reactor and steam generator modules connected through a common steam header to one turbine. General Electric's PRISM was a modular liquid metalcooled inherently-safe reactor. Along with national laboratories, GE was developing PRISM during the advanced liquid-metal fast breeder reactor (ALMR) program. No US fast neutron reactor has so far been larger than 66MWe and none has supplied electricity commercially. Today's PRISM is a GE-Hitachi design for compact modular pool-type reactors with passive cooling for decay heat removal. After 30 years of development it represents GE-Hitachi's Generation IV solution to closing the fuel cycle in the USA. Modules are 200 to $360 \mathrm{MWe}$ and operate at high temperature - over $500{ }^{\circ} \mathrm{C}$. The pool-type modules contain the complete primary system with sodium coolant. The Pu and depleted uranium fuel is metal, and obtained from used light water reactor fuel. However, all transuranic elements are removed together in the electrometallurgical reprocessing so that fresh fuel has minor actinides with the plutonium. Fuel stays in the reactor about six years, with one third removed every two years. The commercialscale plant concept uses six reactor modules to provide 1200 to $2200 \mathrm{MWe}$ [28].

\subsubsection{Modular Gas-Cooled Reactor Designs}

Modular reactor designs are not limited to PWR designs. China's experience with MHTGRs is demonstrated in a few important papers, especially with the experience and technology developed based on their 10-MWth research reactor (HTR-10) at Tsinghua University, in Beijing, and their resolve to develop a $200 \mathrm{MWe}$ pebble-bed reactor around year 2013 [29] - [31].

In their paper Zhang et al. [30] delineate the following objectives of such reactors:

- demonstrate the claimed inherent safety features of the system,

- help reveal the potential economic competitiveness, 
- reduce technical risks, employing the rich experiences gained from the HTR-10 and other mature industrial technologies, and

- provide a sound basis for achieving modularized design and construction.

In the 2006 paper by Zhang and Sun [29], provided economical and technical comparisons between a typical PWR plant and a High Temperature Reactor Pebble Bed Module (HTR-PM) plant with comparable power capacity output, that is, 6 to 10 Nuclear Steam Supply Systems (NSSS) with 100 MWe each, are shown, as well as some cost breakdowns, sub-system technical features, and their pros and cons.

Of particular interest is the fact that they are considering only one control room to monitor and control all NSSS modules, the turbine-generator and its auxiliary systems, and that most auxiliary systems should be shared as much as possible among all modules. An exception should be made with the reactor protection system and other relevant nuclear safety systems. In the end, the authors concluded that:

- The costs of the Reactor Pressure Vessel (RPV) and reactor internals of PWRs have very limited impact $(\sim 2 \%)$ in the final cost.

- The costs of an HTR-PM power plant RPVs and reactor internals are significantly higher, but around $50 \%$ of this increase could be compensated by simplification of the systems. The rest is expected to be compensated by the cost reduction of the turbine plant equipments, by benefits of modularization and by a shorter construction schedule as well as by less workload of design and engineering services.

- Estimates show that capital costs of an nth-of-a-kind HTR-PM plant with multiple NSSS modules should be in the range of $90-120 \%$ of the costs of a PWR, with additional reductions being possible.

Ways of attaining economical viability with MHTGRs include:

- Multiple NSSS and only one turbine generator. 
- Reduce RPV and internals by mass production.

- Share auxiliary systems as much as possible.

- MHTGRs modularization and inherently safe characteristics can reduce the workload and engineering management and design, and shorten construction schedules.

- A ready-to-build $2 \times 250$ MWth modular plant cost is only $5 \%$ higher than a $1 \times$ 458 MWth plant, with the first being attractive, given the uncertainties with the latter design.

It includes 4 references, one of which says the resulting construction schedule for PBMR commercial units, after the initial demonstration plant, is planned for 24 months from first concrete to fuel load for a single module or for the first module of a multi-module plant. Of significant informative importance is a paper by Wallace et al. [32], with details on the "field to factory" advantages of pebble bed reactors, to be constructed in South Africa [33]. A demonstration plant layout is shown in Figure 2.2. The HTR-PM reactor primary system is shown in Figure 2.3, with the reactor unit and the steam generator unit arranged in what is called "side-by-side" design, with the main helium circulator sitting on top of the steam generator. Figure 2.4 shows the flow diagram of the HTR-PM steam turbine cycle with all major typical numerical values, and Table 2.3 gives some key parameter designs of HTR-PM. 


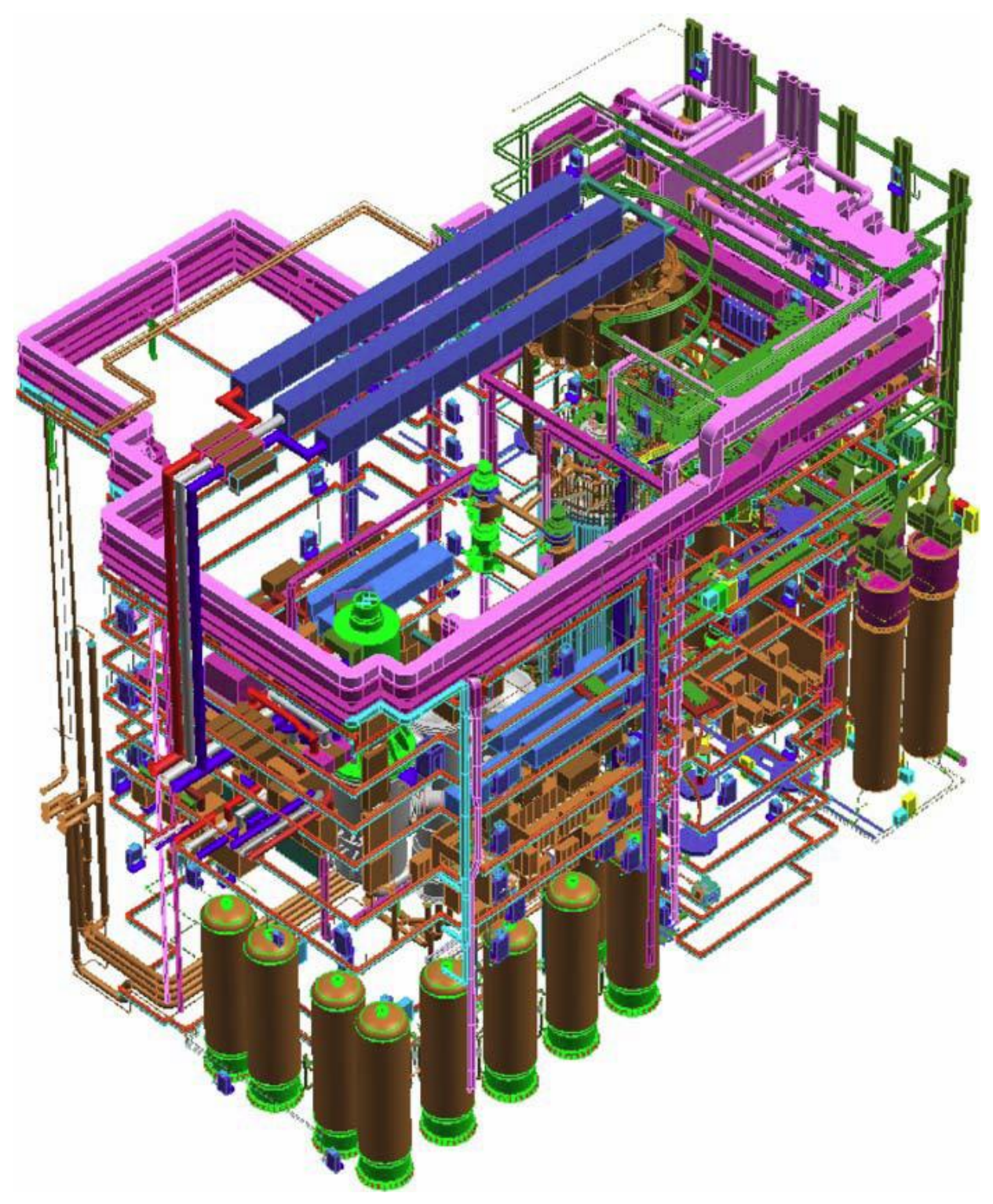

Figure 2.2. PBMR system layout - demonstration plant [32]. 


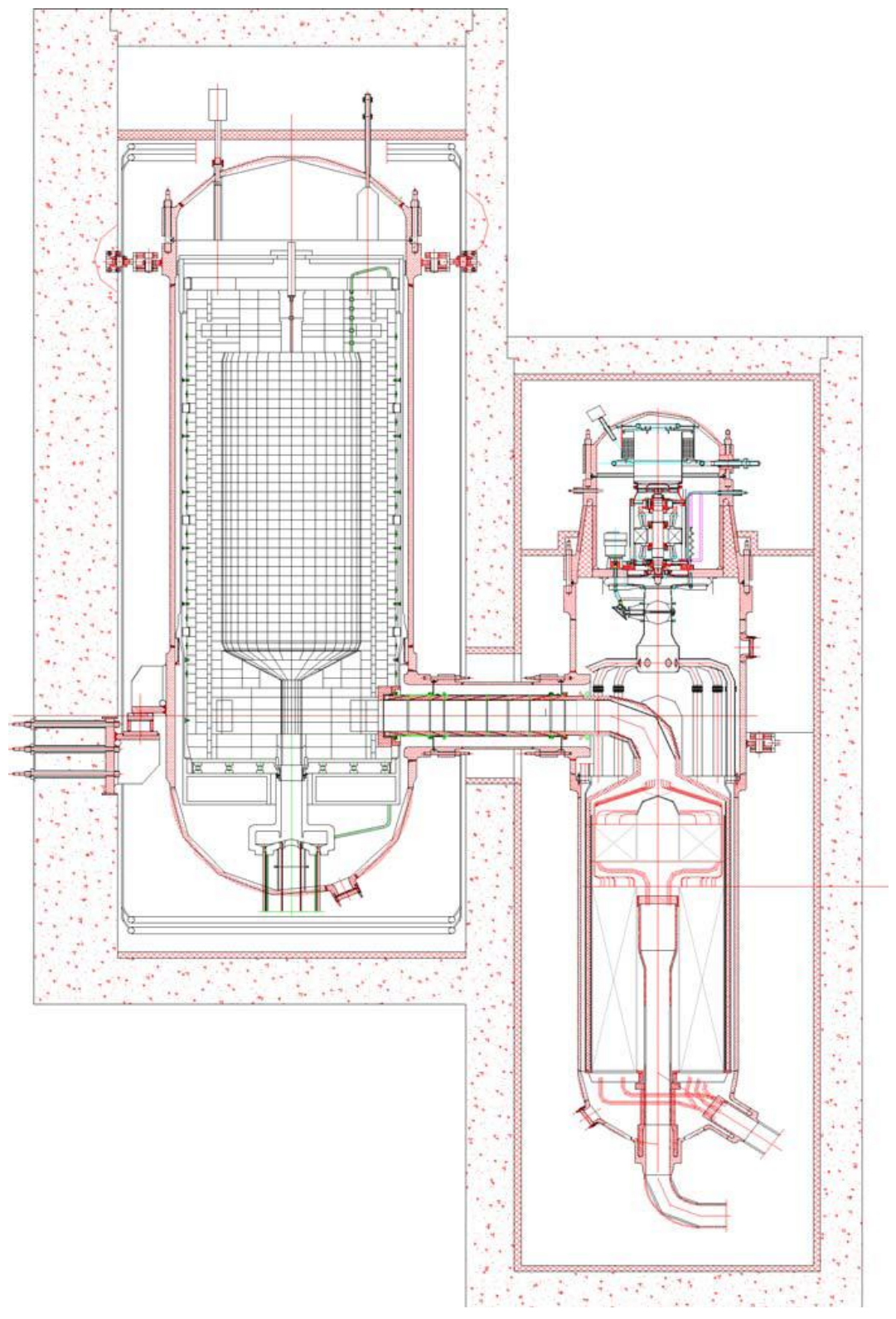

Figure 2.3. Primary system of the HTR-PM [29]. 


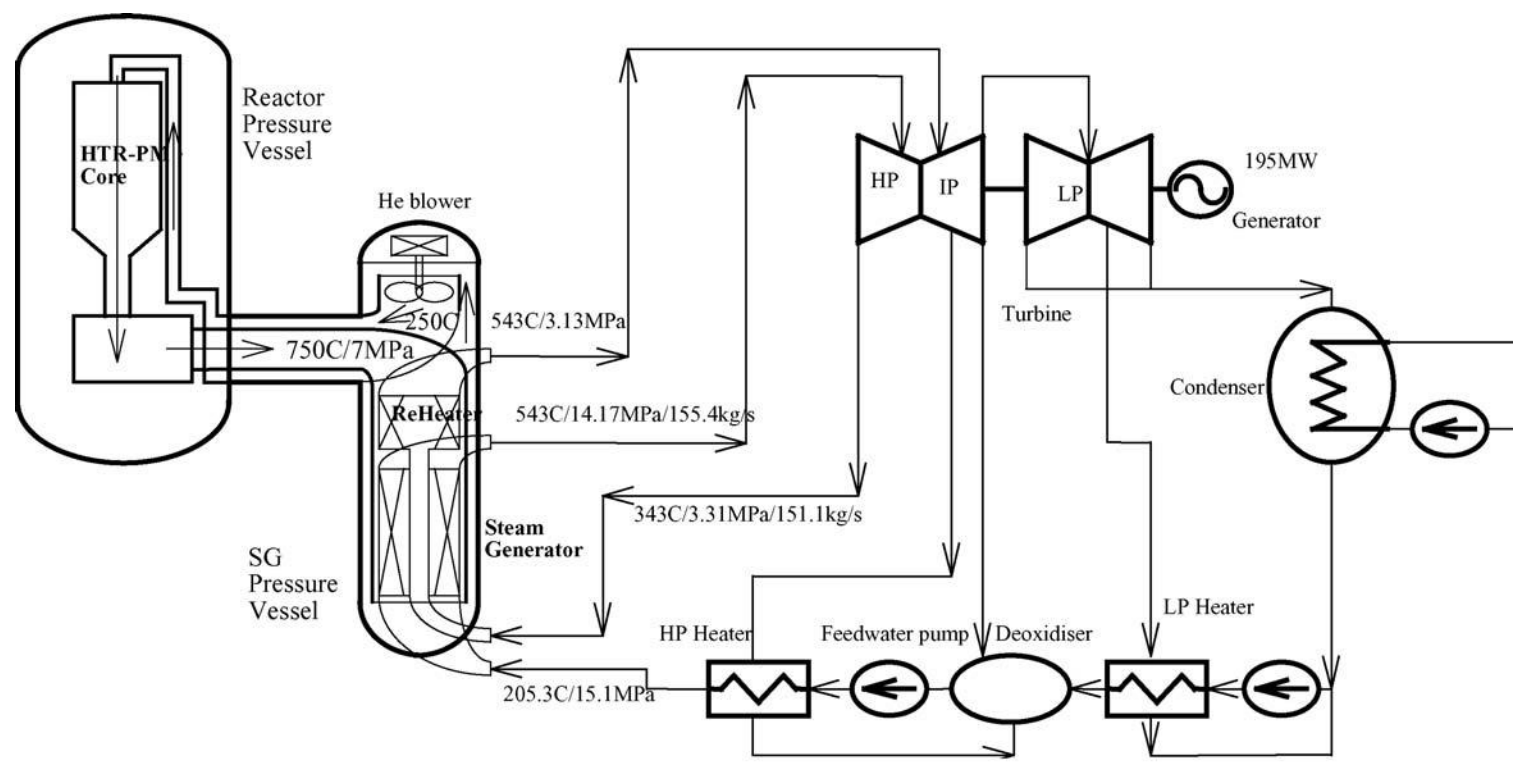

Figure 2.4. Flow Diagram of the HTR-PM steam turbine cycle [28].

Table 2.3. HTR-PM main design parameters [28].

\begin{tabular}{|l|l|}
\hline Reactor thermal power (MW) & 458 \\
\hline Designed operational life time (year) & 60 \\
\hline Expected load factor (\%) & 85 \\
\hline Fuel elements & \\
\hline - Diameter of fuel elements (mm) & 60 \\
\hline$\quad$ Nuclear fuel & $\mathrm{UO} \mathrm{O}_{2}$ \\
\hline - U-235 enrichment of fresh fuel $(\%)$ & 9.08 \\
\hline$\quad$ Heavy metal loading per fuel element (g) & 7 \\
\hline$\quad$ Number of fuel balls & 520,000 \\
\hline
\end{tabular}




\begin{tabular}{|c|c|}
\hline - Number of graphite balls & 225,530 \\
\hline - Average discharge burn-up (MWD/tU) & 80,000 \\
\hline - Fuel loading scheme & Multi-pass (6 times) \\
\hline - Average time of fuel elements in core (EFPD) & 647 \\
\hline - Number of fuel balls discharged each day & 4,821 \\
\hline - Number of fresh fuel balls required each day & 804 \\
\hline - Number of graphite balls discharged each day & 2,091 \\
\hline \multicolumn{2}{|l|}{ Nuclear design parameter } \\
\hline - Diameter of central graphite column $(\mathrm{cm})$ & 220 \\
\hline - Inner/outer diameter of fuel zone $(\mathrm{cm})$ & $220 / 400$ \\
\hline - Average height of active core $(\mathrm{cm})$ & 1,100 \\
\hline - Average power density of fuel zone (MW/m3) & 4.75 \\
\hline - Maximum power density of fuel zone (MW/m3) & 12.85 \\
\hline - Average output power per fuel ball $(\mathrm{kW})$ & 0.881 \\
\hline - Neutron leakage from the core $(\%)$ & 15.08 \\
\hline \multicolumn{2}{|l|}{ Reactivity control } \\
\hline - Number of control rods & 18 \\
\hline - Number of absorber ball units & 18 \\
\hline - Worth of control rods (\%) & 5.25 \\
\hline - Worth of absorb ball units (\%) & 11.32 \\
\hline
\end{tabular}




\begin{tabular}{|c|c|}
\hline - Worth of control rods and absorb ball units (\%) & 14.08 \\
\hline \multicolumn{2}{|l|}{ Coolant } \\
\hline - Primary helium pressure (MPa) & 7.0 \\
\hline - Helium temperature at reactor outlet $\left({ }^{\circ} \mathrm{C}\right)$ & 750 \\
\hline - Helium temperature at reactor inlet $\left({ }^{\circ} \mathrm{C}\right)$ & 250 \\
\hline - Primary helium flow rate $(\mathrm{kg} / \mathrm{s})$ & 176 \\
\hline - Maximum fuel temperature (normal operation) $\left({ }^{\circ} \mathrm{C}\right)$ & 1055 \\
\hline - Maximum fuel temperature under accidents $\left({ }^{\circ} \mathrm{C}\right)$ & 1520 \\
\hline \multicolumn{2}{|l|}{ Reactor pressure vessel } \\
\hline - Inner diameter $(\mathrm{m})$ & 6.7 \\
\hline - Height (m) & 24 \\
\hline - Wall thickness (mm) & $146-250$ \\
\hline \multicolumn{2}{|l|}{ Steam cycle } \\
\hline - Main steam flow rate $(\mathrm{kg} / \mathrm{s})$ & 155.4 \\
\hline - Feed water temperature $\left({ }^{\circ} \mathrm{C}\right)$ & 205.3 \\
\hline - Main steam pressure at turbine inlet (MPa) & 13.5 \\
\hline - Main steam temperature at turbine inlet $\left({ }^{\circ} \mathrm{C}\right)$ & 538 \\
\hline - Generator power (MW) & 195 \\
\hline
\end{tabular}


Haipeng, Huang, and Zhang [34] clearly state in their paper: "Issues in the operation and control of the multi-modular nuclear power plant are complicated." Using fundamental conservation of mass, energy, and momentum equations, a simplified dynamic model was developed and mathematically formulated. The reactor model used in a personal computer simulation was a high temperature gas-cooled reactor, pebble-bed module (HTR-PM) to evaluate the power increase process of the HTR-PM operation. An open-loop operation was first simulated and the results showed that the essential parameter, steam temperature, varied drastically over time, reaching temperatures outside the allowable region for a normal operation. According to the preliminary control strategy of the HTR-PM, a simple steam temperature controller was proposed, using a proportional controller with a time lag, or time delay of 0.1 second. The closed-loop operation with a steam temperature controller was implemented and the simulation results showed that the steam temperature and also other parameters were all within the allowable range. Figure 2.5 shows the proposed design used. It is a two-reactor, two-module design, one module being characterized for having one Nuclear Steam Supply System (NSSS). Each module is basically a 250 MWth reactor, with one helical-coiled once-through, shell-andtube counter-flow steam generator, one blower and appropriate connecting pipes. The steam generated by both modules is connected to a common steam header, which delivers the steam to a 200 MWe turbo-generator. A side-by-side design is used, i.e. the reactor and the steam generator are housed inside two steel pressure vessels and are connected to each other by a third vessel, or hot gas duct. Figure 2.6 shows a HTR-PM schematic with the two vessels and the connecting duct. These three vessels come into contact with the cold helium (about $250{ }^{\circ} \mathrm{C}$ ) as it leaves the blower, which is located on top of the steam generator vessel. The helium enters the main blower and is pressurized before flowing into the outer coaxial pipe of the hot gas duct. It enters the channels in the side reflector, and then flows through these channels from bottom to top. The cold gas directly enters the reactor core and passes through the pebble bed from top to bottom where it is heated to a temperature of about $750{ }^{\circ} \mathrm{C}$. The hot helium leaves the hot gas chamber in the bottom reflector and flows through the hot gas duct to the steam generator. The heat is transferred to water in the secondary circuit and the temperature of the helium falls back down to $250^{\circ} \mathrm{C}$. The secondary feedwater is heated in the tube side to superheated steam. The steam from two modules is then connected to the common steam header, where the steam is then delivered to the turbine for electricity generation. 
A simplified model with lumped parameters was used. As shown in Figure 2.7, the nodalization breaks down the reactor side into: core, reflector, lower plenum, lower header, riser, upper header, downcomer, and outlet header. The steam generator was broken down as economizer, evaporator, and superheater according to the secondary water/steam status, and further in radial direction, the steam generator is divided into primary side, metal tube and secondary side, respectively. The interconnecting sections of the reactor and steam generator are inner duct, outer duct and the blower. The rest of the plant nodal sections are steam header and turbine. Only one of the modules is shown, as they are similar in design.

\section{Module 1}

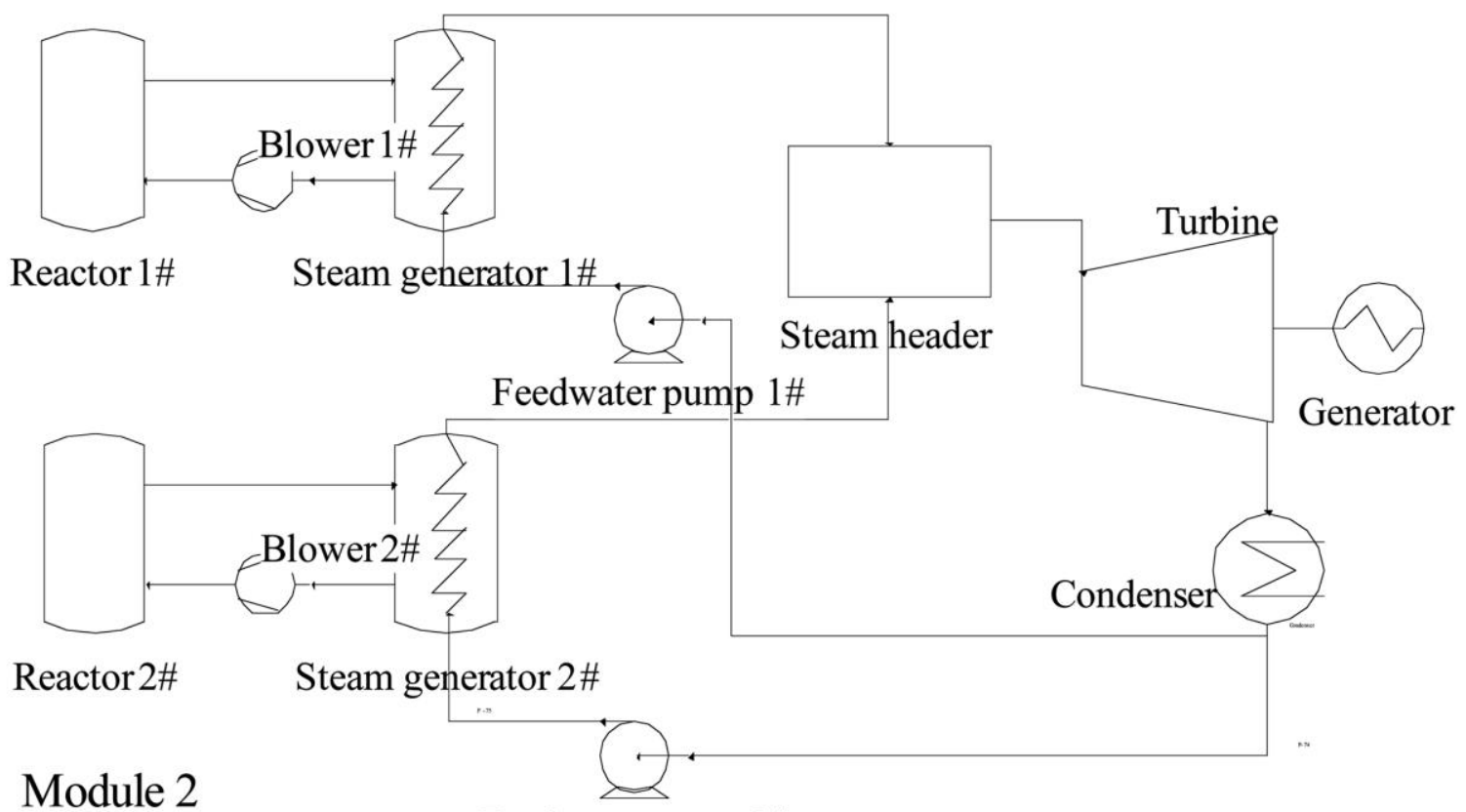

Feedwater pump 2\#

Figure 2.5. HTR-PM plant with two modules [34]. 


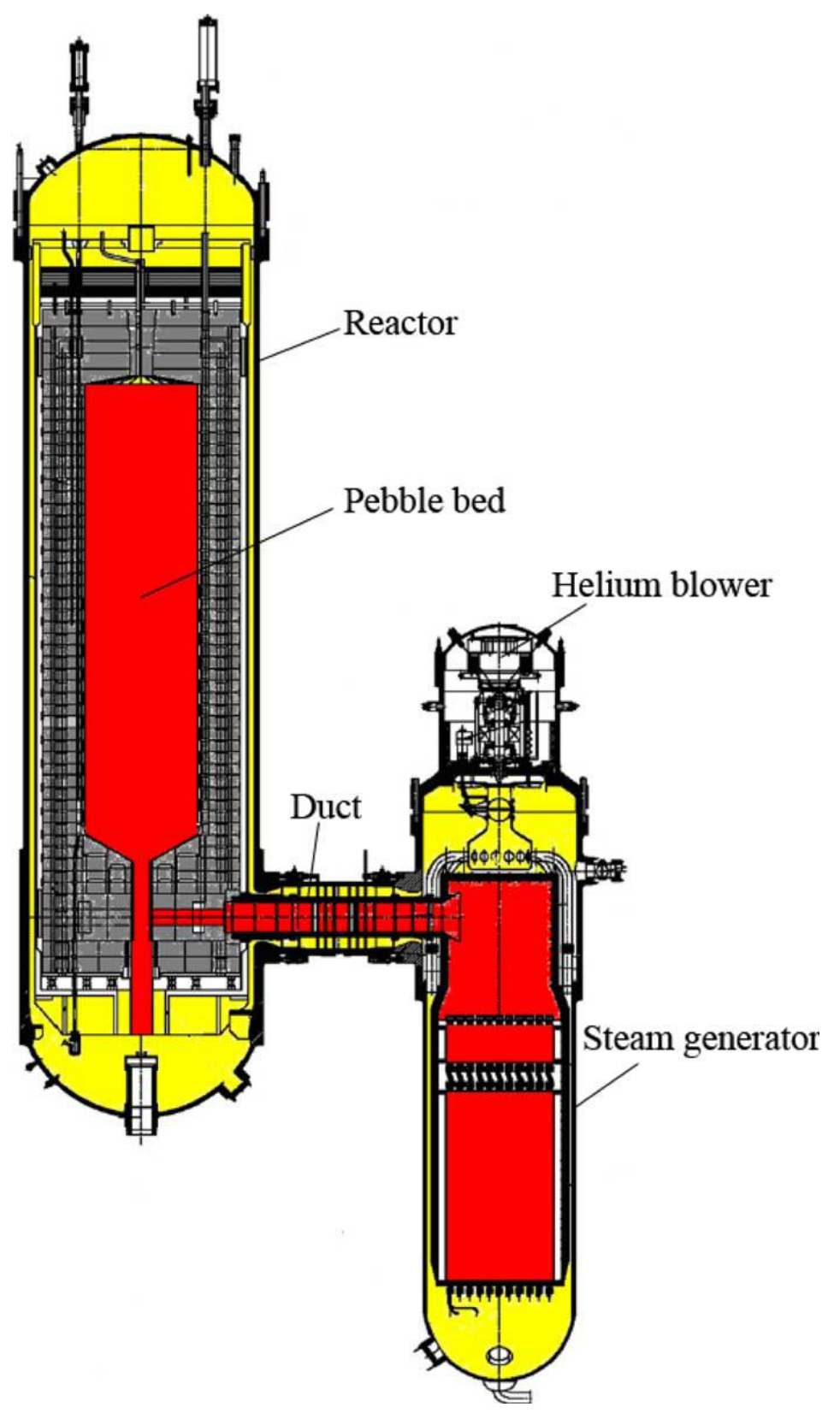

Figure 2.6. Schematic of the HTR-PM plant [34]. 


\section{Module 1}

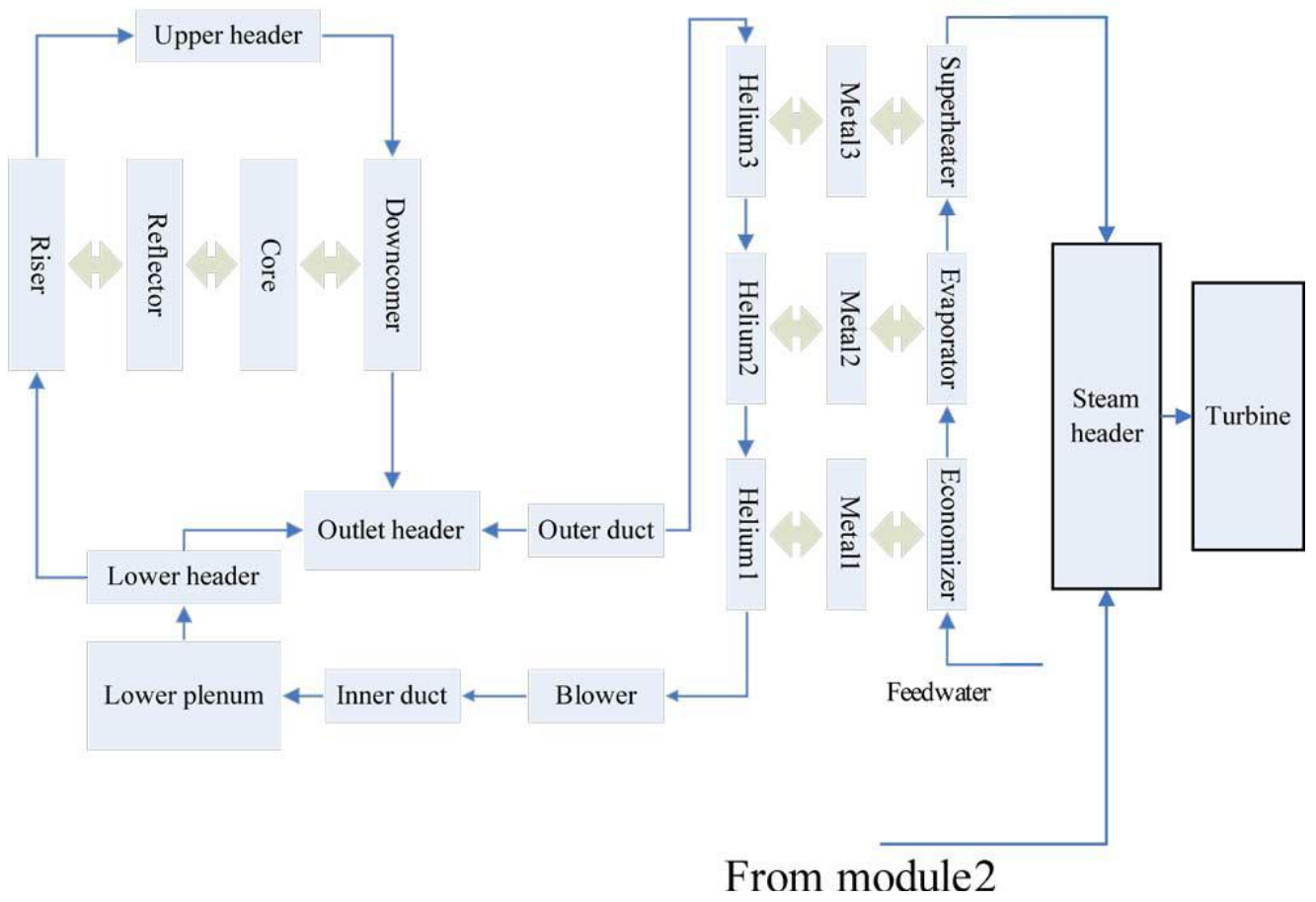

Figure 2.7. Nodalization of the HTR-PM plant [34].

The reactor kinetics model uses a one prompt neutron group point kinetics equations with six delayed neutron precursor groups, and two reactivity feedback mechanisms. Feedback mechanisms in the model are expressed as a function of the mean values of the core and reflector temperatures, resulting in the total reactivity being the control rod reactivity combined with the feedbacks described as: 


$$
\rho=\rho_{r o d}+\boldsymbol{Q}_{f}+\alpha_{m} \boldsymbol{\zeta}_{c}-T_{c 0} \ni \alpha_{r} \boldsymbol{C}_{r}-T_{r 0}-
$$

Where

$$
\begin{aligned}
& \rho \quad: \quad \text { is the reactivity } \\
& \rho_{\text {rod }}: \quad \text { control rod reactivity } \\
& \alpha_{f} \quad: \quad \text { fuel temperature reactivity feedback coefficient } \\
& \alpha_{m}: \quad \text { moderator temperature reactivity feedback coefficient } \\
& \alpha_{r} \quad: \quad \text { reflector reactivity feedback coefficient } \\
& T_{c} \quad: \quad \text { reactor core temperature } \\
& T_{c o} \quad: \quad \text { reactor core temperature at steady state } \\
& T_{r} \quad: \quad \text { reflector temperature } \\
& T_{r 0} \quad: \quad \text { reflector temperature at steady state }
\end{aligned}
$$

The control strategy used was grid load demand, having a power dispatcher to dynamically assign the nominal power that every module should output according to the instant conditions of both modules, leaving the control systems from both modules manipulate to achieve the appropriate outputs. Since the modular nominal power is set by the power dispatcher, the operations of two modules are almost independent of each other. This independence of modules simplifies the operation process, though the modules may operate at different power levels. The controlled variable used was the steam temperature as the input and the output was fed to the primary helium flow rate to close the loop.

Simulation power range span used was from $20 \%$ to $100 \%$ Full Power (FP), with operation ratio limits of $+/-5 \% \mathrm{FP} / \mathrm{min}$, with steady-state inputs to the module shown in Table 2.4. Results indicate the only variable to violate reasonable values during simulation without 
controller was the steam temperature (dashed line), reaching temperatures in excess of $650{ }^{\circ} \mathrm{C}$, as seen in Figure 2.8, where at first the steam temperature hovers at around $540{ }^{\circ} \mathrm{C}$ with constant inputs. With the power increase the steam temperature sharply increases to around $655^{\circ} \mathrm{C}$ and decreases to around $517^{\circ} \mathrm{C}$ after a very short period of time. On the other hand, the effect of the controller on the steam temperature dynamics (solid line) is very clear, with a smooth temperature increase at the beginning of power transient, reaching a maximum value of around $550{ }^{\circ} \mathrm{C}$, slowly decreasing to about $538{ }^{\circ} \mathrm{C}$ and finally stabilizing at around $540{ }^{\circ} \mathrm{C}$, well within what the authors claim to be an allowable range of $540{ }^{\circ} \mathrm{C}+/-10^{\circ} \mathrm{C}$.

Table 2.4. Steady state inputs used.

\begin{tabular}{|c|c|c|c|}
\hline Parameters & Unit & $20 \%$ & $100 \%$ \\
\hline Control Rod Reactivity & & -0.00053 & 0 \\
\hline Primary Helium Pressure & $\mathrm{MPa}$ & 7 & 7 \\
\hline Primary Helium Flow rate & $\mathrm{Kg} / \mathrm{s}$ & 17.87 & 97.53 \\
\hline Secondary Steam Generator Outlet Steam Pressure & $\mathrm{MPa}$ & 13.5 & 13.5 \\
\hline Secondary Steam Generator Inlet Feedwater Flow rate & $\mathrm{Kg} / \mathrm{s}$ & 17.87 & 97.53 \\
\hline Secondary Steam Generator Inlet Feedwater Temperature & ${ }^{\circ} \mathrm{C}$ & 147 & 205 \\
\hline
\end{tabular}




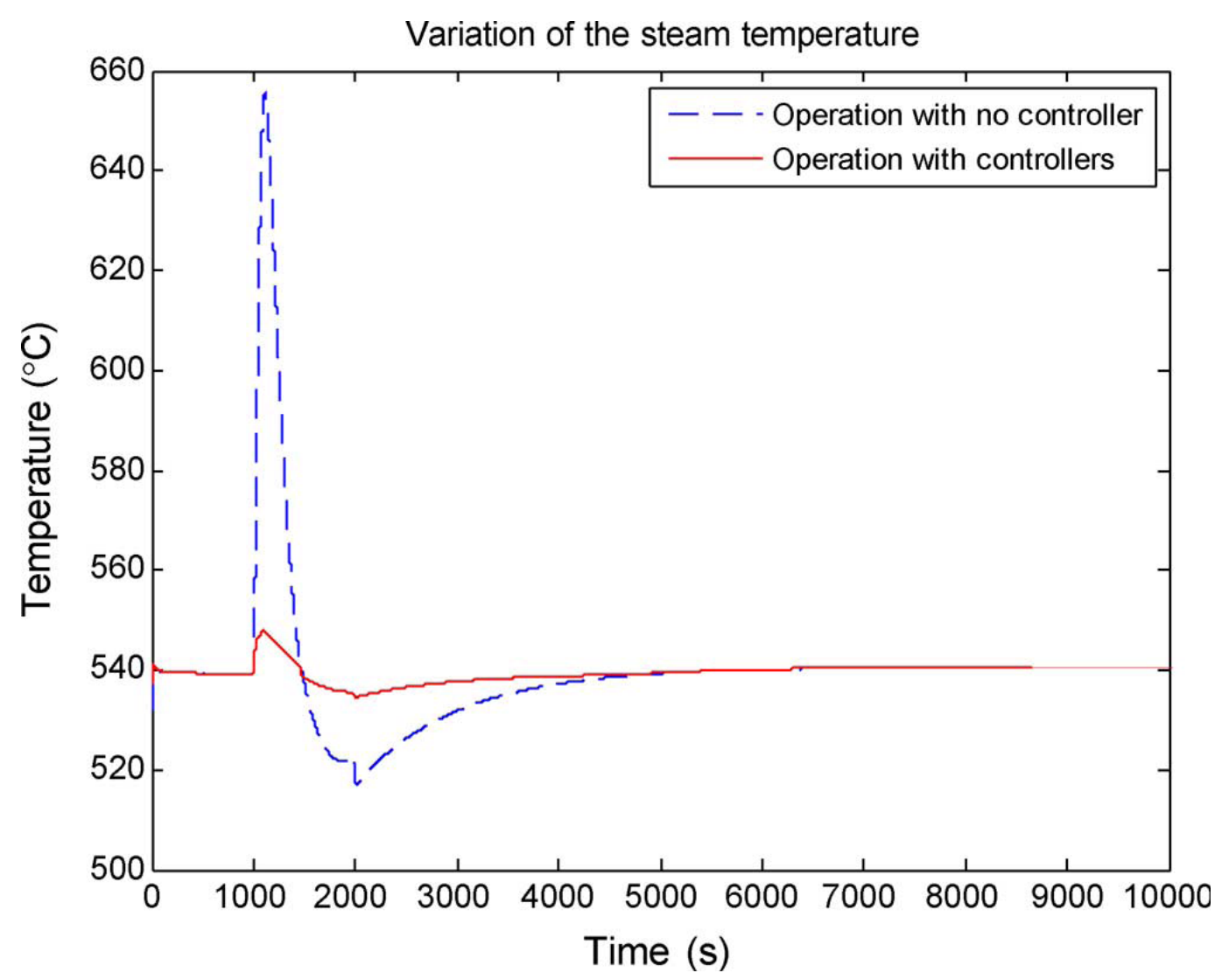

Figure 2.8. SG outlet steam temperature variation [34].

Domestically, another noteworthy novel design is the GT-MHR power plant, developed by General Atomics (GA) which is essentially contained in two interconnected pressure vessels enclosed within a below-ground concrete containment structure. One vessel contains the reactor system and is based on the MHR, which was developed as part of the U.S. Department of Energy's Modular High Temperature Gas-cooled Reactor program. The second vessel contains the power conversion system. The turbo-machine consists of a generator, turbine, and two compressor sections mounted on a single shaft rotating on magnetic bearings. The active magnetic bearings control shaft stability while eliminating the need for lubricants within the primary system. The vessel also contains three compact heat exchangers. The most important of these is a 95\% effective recuperator, which recovers turbine exhaust heat and boosts plant efficiency from $34 \%$ to $48 \%$. 


\subsubsection{Large PWR Modular Systems}

The work by Kim and Bernard [35] [36] in the early 1990's focused on control of PWRtype multi-modular reactor plants, with emphasis on the need for operation under conditions of unbalanced loads, operating strategies for both single- and multi-reactor systems, and the coordinated adjustment of power and temperature. The envisioned multi-modular reactor plant, shown in Figure 2.9, is comprised of several smaller reactors and one common steam header. The paper describes some issues with multi-modular reactor plant control, especially when unbalanced loads are present. The paper also discusses operating strategies for single and multireactor systems and the need for coordinated adjustment of temperature and power. Of special interest is the rationale behind why conventional sliding T-average load maps cannot work in such multi-modular reactors, except for the highest powered unit, and outlines the major differences in operating conditions between single and multi-modular reactor systems.

\subsubsection{1 $\mathrm{T}_{\mathrm{ave}}$ Control Program Issue in Multi-modular PWRs}

Consider a multi-modular PWR plant consisting of two units of identical designs, each being capable of operating between $0 \%$ and $100 \%$ of full power, with any combination of power levels being permitted, Figure 2.10 shows $\mathrm{T}_{\text {ave }}$ and steam generator saturation pressure as a function of module power. At initial state with both modules operating at the same power level, both modules are operated at $Q_{1}$, the pressure in both steam generators being $P_{s g, l}$ and with the main steam line header being $P_{h, l}$. Next, suppose both modules, module \#1 and module \#2, are operating at two different levels, $Q_{1}$, and $Q_{2}$, respectively, with module \#1 operating to follow the original $T_{a v e, 1}$ control program, having its steam generator and main steam line header pressures at $P_{s g, l}$ and $P_{h, 1}$, respectively. If module \#2 were also to be operated according to its original control program, both its steam generator pressure and coolant temperature would be $P_{s g, 2}$ and $T_{a v e, 2}$, respectively, causing instabilities in the system, due to the fact that $P_{s g, 2}$ exceeds

$P_{s g, 1}$. This would cause module \#1 steam flow rate to be cut off, causing module \#2 power to increase in response to the load demand. This instability would eventually die out and module \#1 power would become equal in power. 
In order to avoid such a situation, module \#2's steam generator pressure should be $P_{s g, 2}^{*}$, resulting in module \#2's temperature not being able to be controlled to maintain $T_{\text {ave, } 2 \text {, but }}$ instead, it should be controlled to some lower level value, $T_{a v e, 2}^{*}$ because $P_{\mathrm{sg}, 2}^{*}$ and the corresponding steam generator temperature are less than the values specified by the original $\mathrm{T}_{\text {ave }}$ program for a module operating at power level equal to $Q_{2}$.

Such control issue clearly suggests three different conclusions:

- $\mathrm{T}_{\mathrm{ave}}$ control program applies only to the highest-power reactor in a multi-modular power plant.

- The primary coolant temperatures of the lower-power reactors do not depend solely on their own module's power, but also on that of the highest-power module.

The primary coolant temperature of the highest-power reactor module depends only on its own power. 


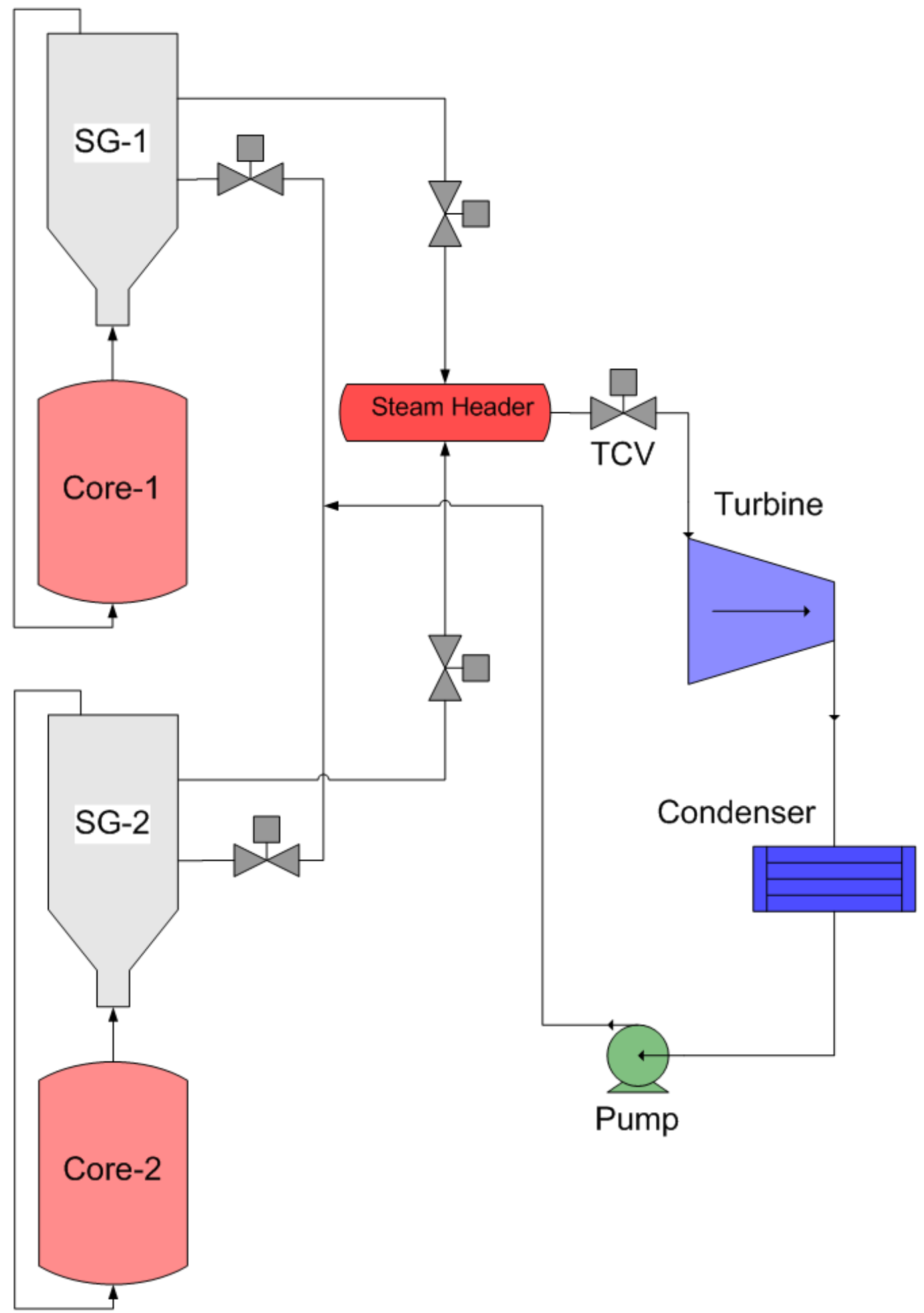

Figure 2.9. Schematic diagram of a PWR-type multi-modular power block. 


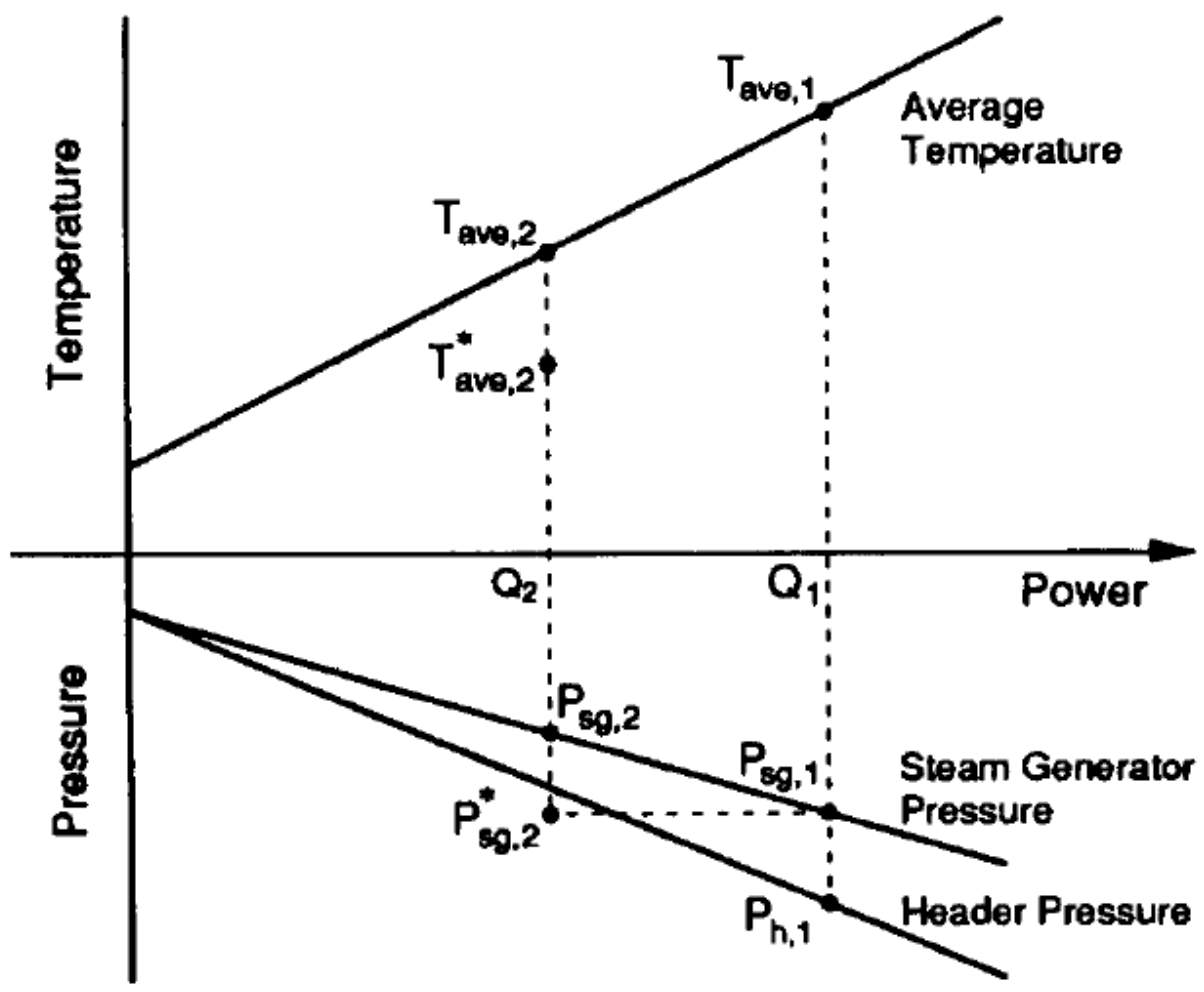

Figure 2.10. Operation under sliding $\mathrm{T}_{\text {ave }}$ control program with unbalanced loads [36]. 
Based on the operating issues posed by a multi-modular reactor plant, the following operating strategies using automatic controllers were proposed, as follows:

- Equal load: where all modules share the load equally, picking up the same fraction of any change in the load.

- Equal Change: operating under unbalanced load conditions, available modules pick up the changes in load, with demand changes being divided by the number of available modules.

- Extreme First: operating under unbalanced load with each available module picking up different fractions of the demand. The lowest power module, in the case of a demand increase, or the highest in the case of a decrease, is changed first, then the next, until desired demand target is met.

- Preset Value: permits load swings in the presence of constant load demand regardless of the initial operating conditions (balanced or unbalanced loads). Changing one module's set point does not change the overall plant output, since the remaining module power levels are automatically shifted (using extreme first or equal change strategy) to compensate the change.

To illustrate the strategies described above, Table 2.5 showcases an example where a multi-modular reactor plant with four different modules, each operating at different power levels, is to have the overall power increased to a base-load equivalent to $100 \%$, using "equal change" strategy as the initial step. 
Table 2.5. Sequence for power increase in specified power demand mode [35].

\begin{tabular}{|c|c|c|c|c|c|c|c|c|}
\hline \multirow{2}{*}{ Phase } & \multicolumn{4}{|c|}{ Module Power } & $\begin{array}{c}\text { Turbine } \\
\text { Power } \\
\mathbf{( \% F P )}\end{array}$ & $\begin{array}{c}\text { Demand } \\
\text { Operating }\end{array}$ & Allocation & $\begin{array}{c}\text { Initiation } \\
\text { Time (s) }\end{array}$ \\
\cline { 2 - 9 } & $\mathbf{\# 1}$ & $\mathbf{\# 2}$ & $\mathbf{\# 3}$ & $\mathbf{\# 4}$ & & & \\
\hline Initial & 95 & 90 & 85 & 80 & 87.5 & Equal Change & Path 1 & 0 \\
\hline $\mathbf{1}$ & 100 & 95 & 90 & 80 & 92.5 & Extreme First & Path 3 & 200 \\
\hline $\mathbf{2}$ & 100 & 95 & 90 & 90 & 93.75 & Extreme First & Path 3 & 400 \\
\hline $\mathbf{3}$ & 100 & 95 & 95 & 95 & 96.25 & Extreme First & Path 3 & 500 \\
\hline $\mathbf{4}$ & 100 & 100 & 100 & 100 & 100 & - & - & - \\
\hline
\end{tabular}

Notes:

1) The demand allocation method depends on the operational mode and operating strategy. The path designations given in the table refer to:

a) Path 1 - Use all modules

b) Path 2 - Use highest-power module and one or more of the lower-power one

c) Path 3 - Use lower-power modules.

2) The "initiation time" denotes the start of each operating strategy.

In the beginning, all modules are operating at different power levels, with the highestpower module being \#1. Performing the operating strategy "equal change" first, all modules are active, and all module powers are increased by $5 \%$ of full power (FP), with a $5.0 \% \mathrm{FP} / \mathrm{min}$ increase ramp rate. Once this module reaches $100 \% \mathrm{FP}$ it no longer can be modified, becoming inactive and ending what it is called phase one. 
Changing the operating strategy to "extreme first", module \#4 becomes active, since it is the lowest-power module. The maximum turbine output power ramp rate is now $1.25 \% \mathrm{FP} / \mathrm{min}$, because only one module is now active. Should a higher power ramp rate be demanded by the load, the plant power controller must either reject it or change the operating strategy, for example, using the "equal change" strategy, with module \#1 being held constant at 100\% and increasing the remaining modules at a maximum turbine power rate equal of $3.75 \% \mathrm{FP} / \mathrm{min}$. Module 4's power continues to increase until it reaches $90 \% \mathrm{FP}$, matching module \#3's power, and ending phase two.

With modules \#3 and \#4 at 90\%FP they both become active, starting phase 3 . Both are now the low-power modules. The transient continues until they attain 95\%FP.

Now that modules \#2, \#3 and \#4 are at the same power level, phase four begins and they are used to steadily increase the power.

In 1992 an MIT report (Bernard et al. [37]) described the theoretical development and the evaluation via both experiment and simulation of digital methods for the closed-loop control of power, temperature, and steam generator level in liquid metal (PRISM), gas-cooled (MHTGR) and PWR-type multi-modular reactors. The major conclusion of the research was that the technology available at the time was able to automate many aspects of the operation of multimodular plants, though an extension to boiling water reactors would require a more realistic model. Another important conclusion was the use of a multiple computer/single task system as an appropriate architecture for the closed-loop digital control of a nuclear reactor as long it is kept isolated from control law software that is updated as plant procedures change, facilitating a fault-tolerant design, software validation and real-time operation at a high numerical throughput.

Signal validation and instrument fault detection was also used in this work by means of a numerical technique called "parity space approach" [38] - [41], which is based on simple algebraic projections and geometry. This method computes a residual vector that is zero when no fault is present and non-zero otherwise. The residual will also be different for different faults. In addition to validating sensor readings, this methodology performs instrument fault checks in which the weighting factor for each sensor is adjusted in proportion to the frequency with which 
its readings are judged to be valid. Thus, reliance on a failing sensor is gradually reduced, thereby assuring a "bumpless" transition when complete failure actually occurs.

\subsection{Fault Tolerant and Reconfigurable Control}

Control reconfiguration, or reconfigurable control, is an active approach utilized in control theory to automatically achieve fault-tolerant control in dynamic systems such as aircraft flight control systems [42] [43], petro-chemical plants [44], brine desalination plants [45], mineral processing plants [46], spacecrafts controls [47], robot control systems [48], ship control [49] [50], etc., and can be used whenever failures occur in actuators or sensors, being capable of maintaining overall system stability and degraded performance [51] in the event of such failures, thus providing survivability to the systems they control.

In many cases, the existing controller can be able to cope even in the presence of such faults long enough to allow corrective procedures to take place. In non-severe cases it can allow the system to function (even at a lower performance rate [52]) until a scheduled maintenance can be performed. However, if the severity of the fault is such that the system behavior changes dramatically and or unexpectedly, the control system may need to undergo modifications by adjusting control parameters so it can work under such different dynamics. Figure 2.11 shows the reconfiguration as being only one of the building modules of a standard active fault-tolerant control system of a steam generator in this example, where the control loop operates to meet the control goals. The other module, depicted in the figure, is the Fault Detection and Isolation (FDI) module. This module monitors and compares measured and predicted values, and triggers an action (fault detection) whenever the difference reaches a preset threshold. The action is dependent on what component has failed (fault isolation) and is passed to the reconfiguration block, which modifies the control loop to reach the control goals in spite of the fault (fault tolerant). Figure 2.12 shows a two-stage fault detection and identification structure for a steam generator controller where faults can occur in the actuator and or sensor, and the decision making are based on such residuals [53].

In general, fault-tolerant control systems fall into two generic categories: passive and active. When controllers are designed to be robust in the presence of uncertainties when a presumed set of pre-determined faults happen, they generally fall in the first category, and no 
fault detection and isolation nor controller reconfiguration is required. If system component failures cause the controller to actively reconfigure by itself and still maintain both stability and reasonable performance, then the fault-tolerant control falls in the active category. Broadly speaking, the fault detection and isolation can also differ not only in the way the process knowledge is used, but also in the way or form such knowledge is required. A classification based on the form of process knowledge was proposed by Dash and Venkatasubramanian [54] and is depicted in Figure 2.13. Examples in the literature range from using Principal Components Analysis (PCA) [55] - [61] to Fuzzy Logic, Genetic Algorithm (GA) and Artificial Neural Networks [55], to data clustering [57] and other residual generation approaches [53]. In many of the papers reviewed more than one approach were used, sometimes combining several of such approaches as tools to obtain residuals and or control algorithms. Two important papers, by Erbay \& Upadhyaya [62], and Holbert \& Upadhyaya [63] show the sctucture of signal validation systems in which they discuss the suitability of this kind of method applied to nuclear power plant data and feasibility of use in real nuclear power plants.

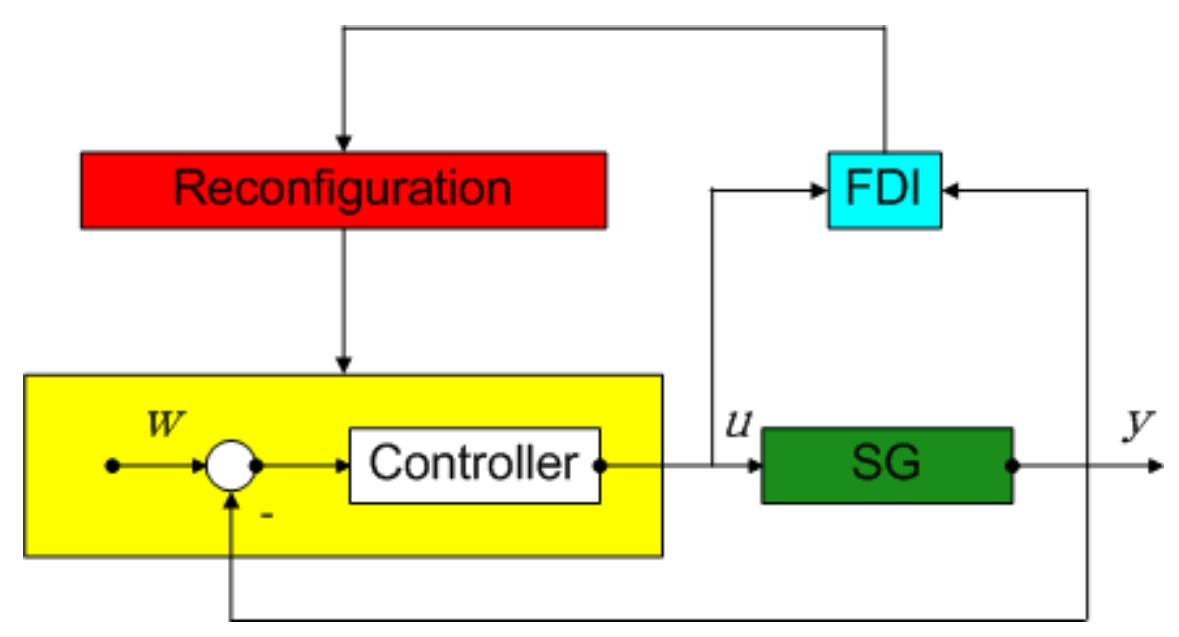

Figure 2.11. A typical active fault-tolerant control for a steam generator (SG). 


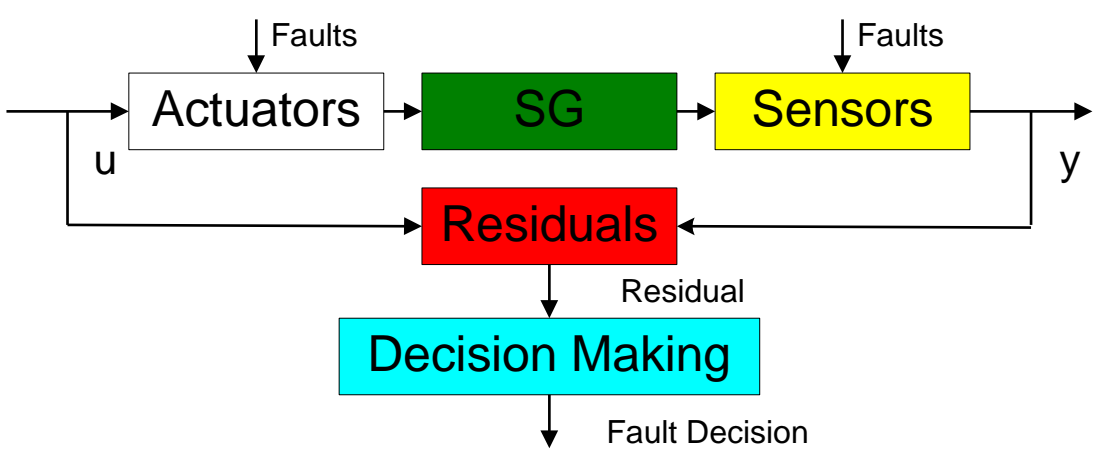

Figure 2.12. Two-stage structure of an FDI process.

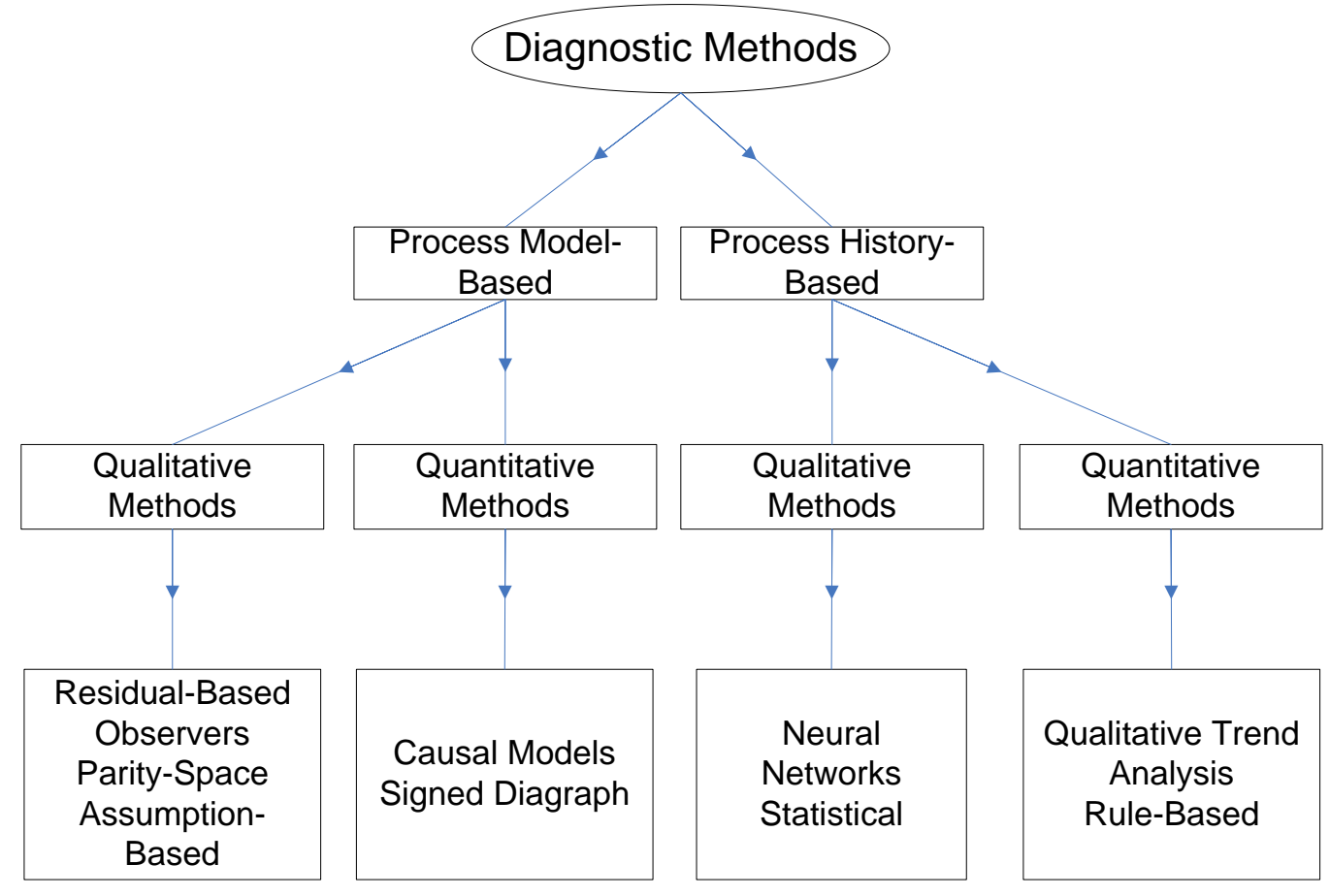

Figure 2.13. Classification of diagnostics methods [53]. 
When considering a reconfigurable controller able to actively reconfigure itself and still maintain stability, a reasonable performance control, and a "bumpless" transition between control strategies, such active fault-tolerant control system consists of four parts:

- A controller that can be reconfigured or restructured.

- A fault detection and isolation system (or module in some cases) with high sensitivity to faults (especially incipient faults), robustness to account for uncertainties, operating condition variations, and external disturbances, to discriminate the fault.

- A controller reconfiguration mechanism (hardware or software) to help reconfigure the controller to achieve pre-fault system performance as much as possible in the presence of uncertainties, time-delays, and account for constraints in the control input and state limits like actuator constraints (output rate, amplitude, stroke time, etc.)

- Command/reference governor validate or verify the control signals (to actuators) and outputs from actuators, and to initiate or terminate the controller reconfiguration action.

Of critical importance is also the amount of time necessary for any fault-tolerant control action to take place in a limited amount of time, such as in safety related nuclear systems, due to fault-detection delays, controller reconfiguration time, actuator speed, etc.

A paper by Zhang and Jiang [67], published in June of 2003, contains a thorough bibliographical review with 250 references on reconfigurable fault-tolerant control systems with papers, books and book chapters, and tutorials dating as early as 1980. The paper also provides a classification of then existing reconfigurable control approaches based on control algorithms such as linear quadratic regulator, pseudo-inverse, model-following, adaptive control, etc., and on application areas such as aircraft, spacecrafts and structures, automotive industry, nuclear, chemical, robots, etc. Of particular importance to the nuclear industry are a few papers, summarized below. 
In 1985 Ray [68] successfully implemented a fault-tolerant, computer-based controller in MIT's 5-MW nuclear research reactor MITR-II, performing digital power control under steadystate and transient operations, via feedback of a number of sensor signals such as neutron flux, primary coolant flow, temperature, and the regulating rod position. The controller used heuristics and learning theory for emulating operator instructions as a monitor displayed in real time the validated data and diagnostics of the relevant instrumentation and equipment. The major functions of the on-line fault-tolerant control system at MITR-II were:

- Regulation of reactor power under steady-state and transient conditions such as xenon oscillations, coolant temperature variations, and rapidly changing load.

- On-line detection, isolation, and reconfiguration of faulty sensors without interrupting the plant operation.

- On-line estimation of both measurable and non-measurable plant variables such as power, coolant flow, temperature, and reactivity using the available sensors and/or analytic measurements that are obtained from real-time models using physical relationships among the plant variables.

- On-line calibration of power sensors to compensate for process disturbances such as changes in the spatial distribution of neutron flux due to xenon transients.

- On-line information display of the critical plant variables and diagnostics of faulty sensors and equipment, assisting the operators to make timely and appropriate decisions.

Experimental results using the fault-tolerant digital controller showed the system was:

- Fault-tolerant to one or two failures in power, flow and $\Delta \mathrm{t}$ sensors.

- Capable of calibrating the power sensors on-line during transients involving xenon variations, fuel depletion, temperature fluctuations, and operator-induced powerdemand changes. 
- The controller maintained the reactor power at the desired level during both steadystate and rapid transient conditions, even though the power sensors themselves could be affected by process disturbances.

- The digital controller was less sensitive to sensor degradation and noise than the original analog controller, as can be seen in Figures 2.14 and 2.15.

- System reliably exhibited on-line information on plant component and sensor failures and estimates of measured variables.

The computer used in the experiment did not have a redundancy and was not faulttolerant. Therefore the experiments were designed such that possible hardware failures in the computer system would trip the controller to the manual mode.

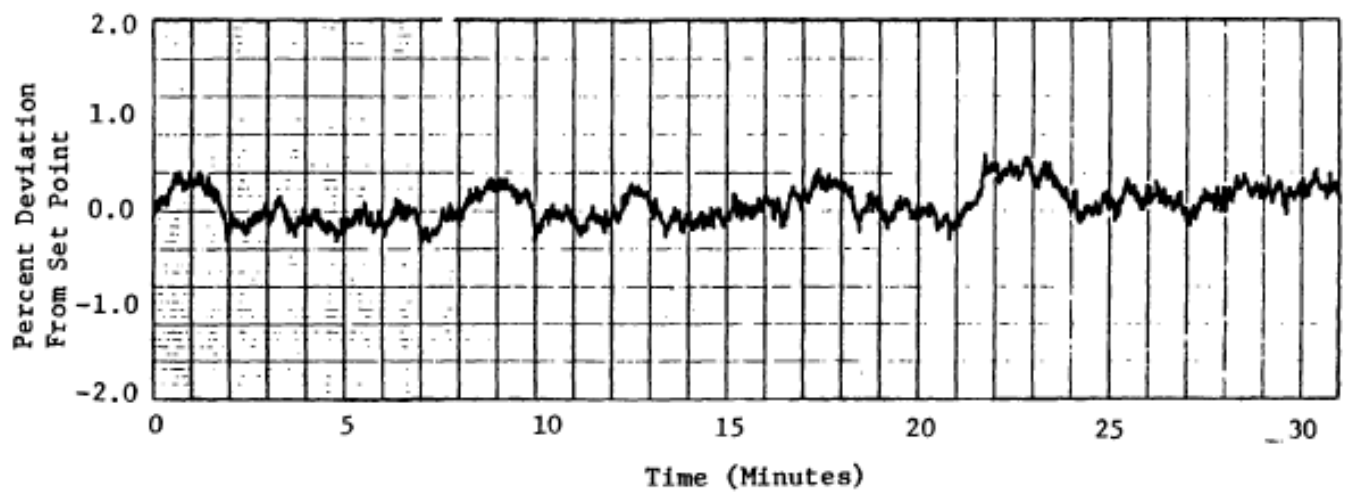

Figure 2.14. Steady-state power profile under analog control [65]. 


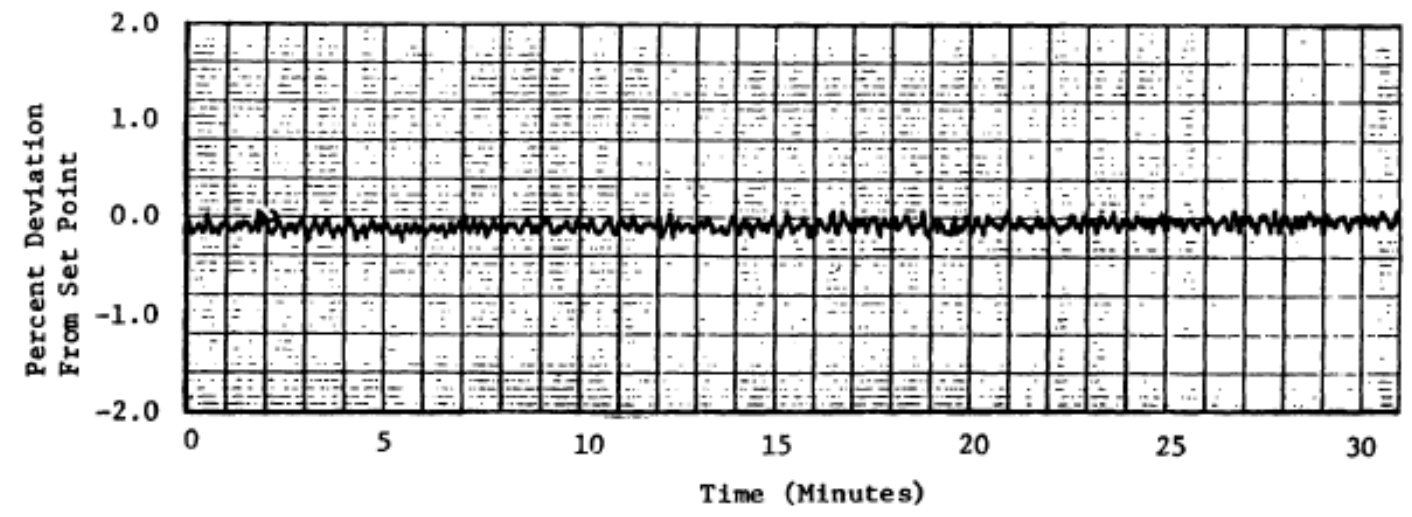

Figure 2.15. Steady-state power profile under digital control [68].

From studies developed by other researchers Garcia et al. [69] used simulations in 1991, on a deaerating feedwater heater based on a model of the Experimental Breeder Reactor (EBRII) at the Argonne National Laboratory site in Idaho, equipped with a water level controller and a pressure controller to investigate the feasibility of using a reconfigurable controller for power plants by incorporating the concept of stochastic learning "automata" ${ }^{1}$. According to the results the controller was able to work well even under unusual events, and propose further research to implement the controller for continuous processes under diverse operating conditions like fossil and nuclear power reactor start-ups and shut-downs. The experiments involving models were subsequently transitioned to the actual reactor feedwater deaerator and the results are presented in a 1995 paper [70]. Argonne's EBR-II stopped operations in 1994 when it lost federal funding. Youn and Lee (2004) [71] used a model-based approach to Younggwang units 3 and 4 deaerator level tank control using operation data to perform fault detection and diagnosis by applying adaptive estimator, a method proposed in 1986 and successful at tracking target maneuvering [72], along with the Kalman filter.

\footnotetext{
${ }^{1}$ An automata is a machine or control mechanism designed to automatically follow a predetermined sequence of operations or respond to encoded instructions. The term stochastic emphasizes the adaptive nature of the automaton.
} 
Other researchers had similar approaches when using fault-tolerant control. In 1995 a paper by Eryurek and Upadhyaya [73] examined the integration of a parallel fault-tolerant control design, signal and command validations, and diagnostics for the main feedwater flow regulation system of a pressurized water reactor (PWR), by combining the steam generator water level controller and the main feed pump speed controller.

The fault-tolerant control and diagnosis utilized three different control algorithms in a parallel manner. The control module incorporated reconstructive inverse dynamics control, which is a model-based adaptive nonlinear control algorithm, fuzzy logic control, which is a nonlinear controller where the qualitative information of the process and approaches of human operators are utilized based on human knowledge about the system, and conventional proportional-integral-derivative (PID) control for feedwater flow regulation. The parallel control included a number of control algorithms, each designed to accomplish the same tasks using different sets of sensor signals, offering a set of possible control solutions that once evaluated could be selected as the final control action based on its suitability for the operation. The signal validation block used two different and independent routines using process empirical modeling, and artificial neural networks with backpropagation. 


\section{Chapter 3}

\section{IRIS SYSTEM DESCRIPTION AND THE DEVELOPMENT OF THE REACTOR DYNAMICS MODEL}

\subsection{Introduction}

International Reactor Innovative and Secure (IRIS) is one of the next generation nuclear reactor designs using mostly established LWR technology (due to its maturity), allowing for an accelerated deployment [74] once the proof of concept is demonstrated and the license is approved. However, an extensive number of tests are still to be performed for new engineering aspects and components that are not yet proven technology in the current PWRs, and present some technological challenges for its deployment since many of its features cannot be proven until a prototype plant is built.

\subsection{System Description}

IRIS is an Integral Primary System Reactor with both the primary and secondary (steam) loops housed inside a large vessel. The upper head acts as the pressurizer to maintain constant primary pressure. Eight spool-type reactor coolant pumps, eight steam generators, and control rod drives are also located within the reactor pressure vessel. Major components of the primary system are shown in Figure 3.1, resulting in a pressure vessel larger than a regular PWR, despite its lower power rating, but largely reducing the size and eliminating dozens of penetrations, virtually eliminating large Loss of Coolant Accidents (LOCAs) and the number of possible small LOCAs [75]. The feedwater flow to a pair of helical coil steam generators has a common feed line, with the primary water being pumped from bottom to top through the core and the riser, the circulation then reverses in a downward direction and the water is forced down by the immersed pumps through the helical tubes. At the steam generator outlet, the flow path goes along the annular downcomer region located outside the core to the plenum and back into the core, closing the path. Figure 3.2 shows details of the primary and secondary systems with the reactor vessel inside the containment vessel. 
IRIS is being designed to fulfill the advantages of the integrated primary system reactor. It improves safety, reduces the site civil works, and improves plant availability for developed as well as developing countries with large or small electrical grids that can greatly benefit from such design. The development of autonomous and fault-tolerant control strategy is well-suited for remote deployment of small and medium reactors, such as the IRIS. Table 3.1 shows the major IRIS parameters currently found in open literature [76]. 


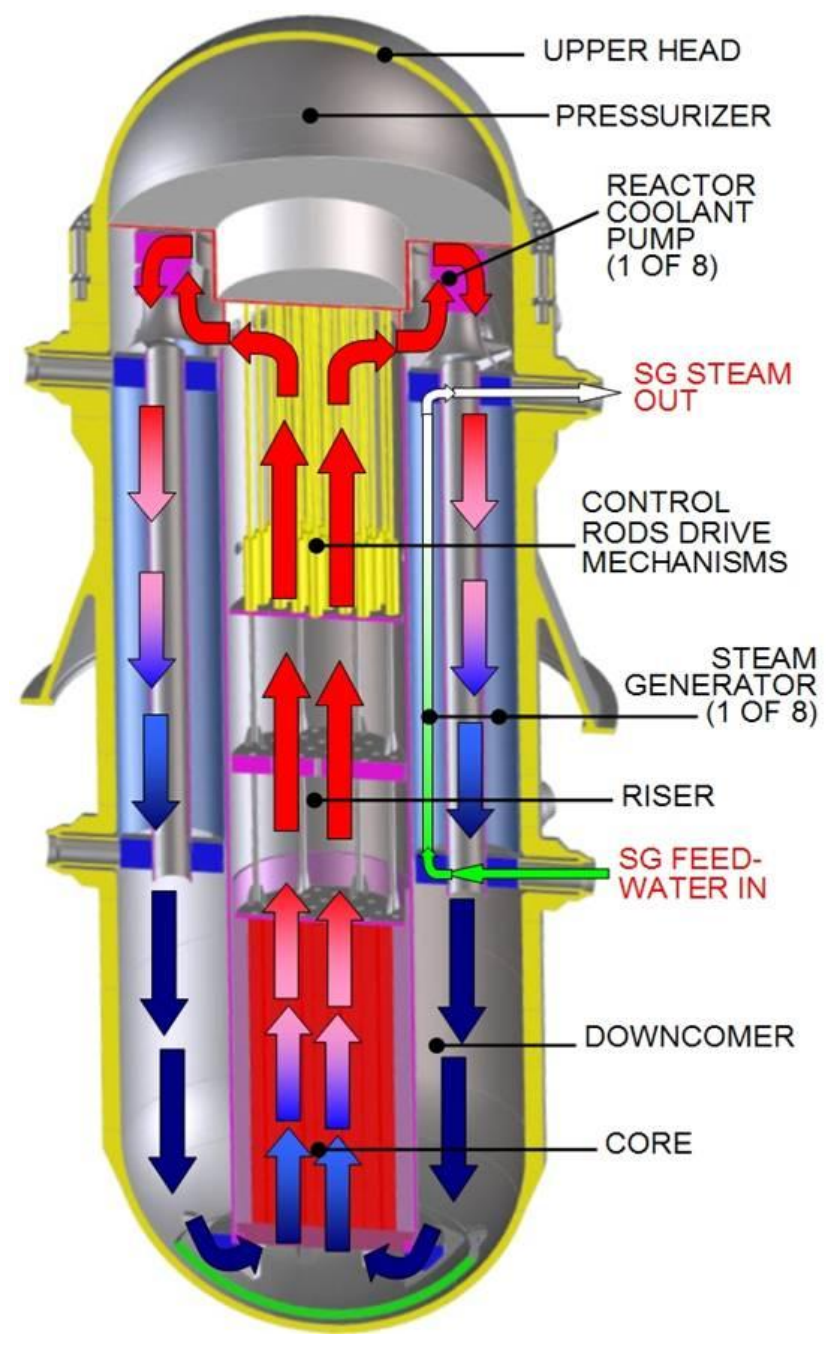

Figure 3.1. IRIS primary system layout [74]. 


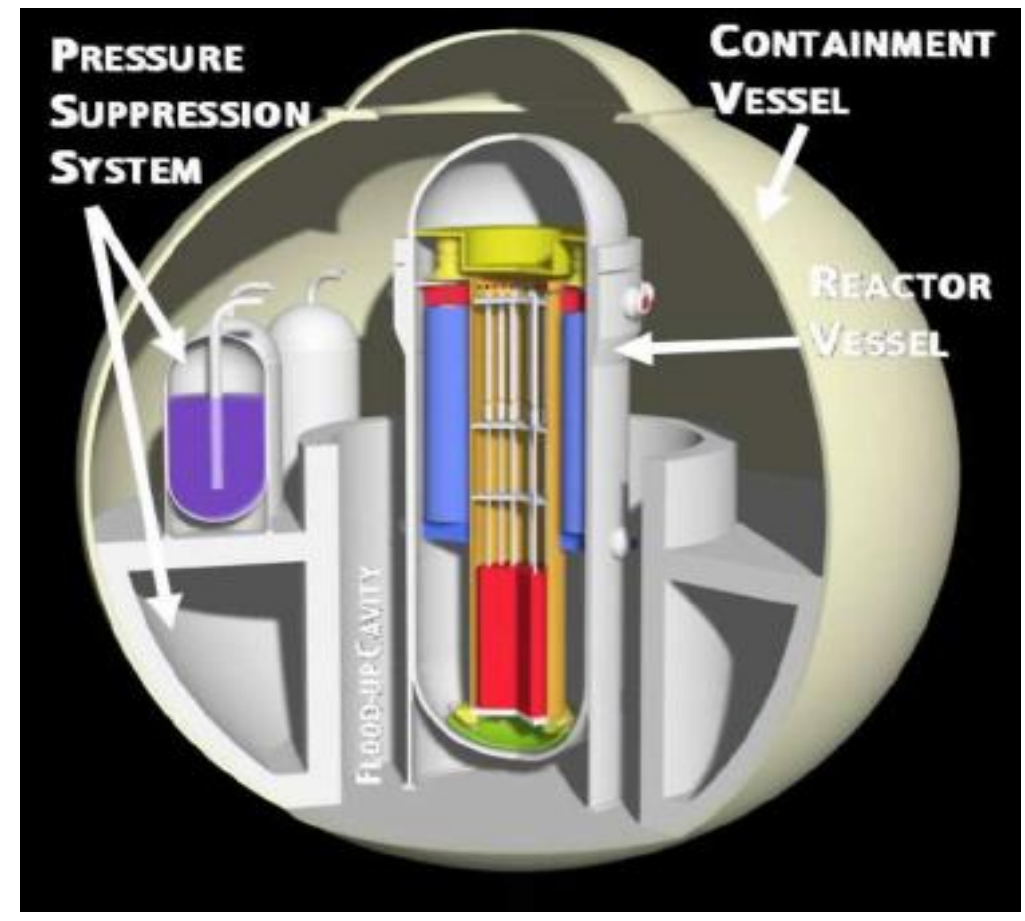

Figure 3.2. Model of IRIS primary system and compact containment vessel [76]. 
Table 3.1. Major IRIS design parameters [76]

\begin{tabular}{|c|c|}
\hline General Plant Data & \\
\hline Power Plant Output (net) & $335 \mathrm{MWe}$ \\
\hline Core Thermal Power & $1000 \mathrm{MWth}$ \\
\hline Nuclear Steam Supply System & \\
\hline Number of Coolant Loops & Integral Reactor Coolant System (RCS) \\
\hline Steam Temperature/Pressure & $317 / 5.8^{\circ} \mathrm{C} / \mathrm{MPa}$ \\
\hline Feedwater Temperature/Pressure & $224 / 6.4^{\circ} \mathrm{C} / \mathrm{MPa}$ \\
\hline Reactor Coolant System & \\
\hline Primary Coolant Flow rate & $4700 \mathrm{Kg} / \mathrm{s}$ \\
\hline Reactor Operating Pressure & $15.5 \mathrm{MPa}$ \\
\hline Core Inlet Temperature & $292{ }^{\circ} \mathrm{C}$ \\
\hline Core (riser) Outlet Temperature & $330{ }^{\circ} \mathrm{C}$ \\
\hline Reactor Core & \\
\hline Fuel Assembly Total Length & $5.207 \mathrm{~m}$ \\
\hline Active Core Height & $4.267 \mathrm{~m}$ \\
\hline Fuel Inventory & $48.5 \mathrm{tU}$ \\
\hline Average Linear Heat Rate & $10.0 \mathrm{~kW} / \mathrm{m}$ \\
\hline Average Core Power Density (volumetric) & $51.26 \mathrm{~kW} / \mathrm{l}$ \\
\hline Fuel Material & Sintered $\mathrm{UO}_{2}$ \\
\hline Rod Array & Square, $17 \times 17$ \\
\hline Number of Fuel Assemblies & 89 \\
\hline Number of Fuel Rods per assembly & 264 \\
\hline
\end{tabular}




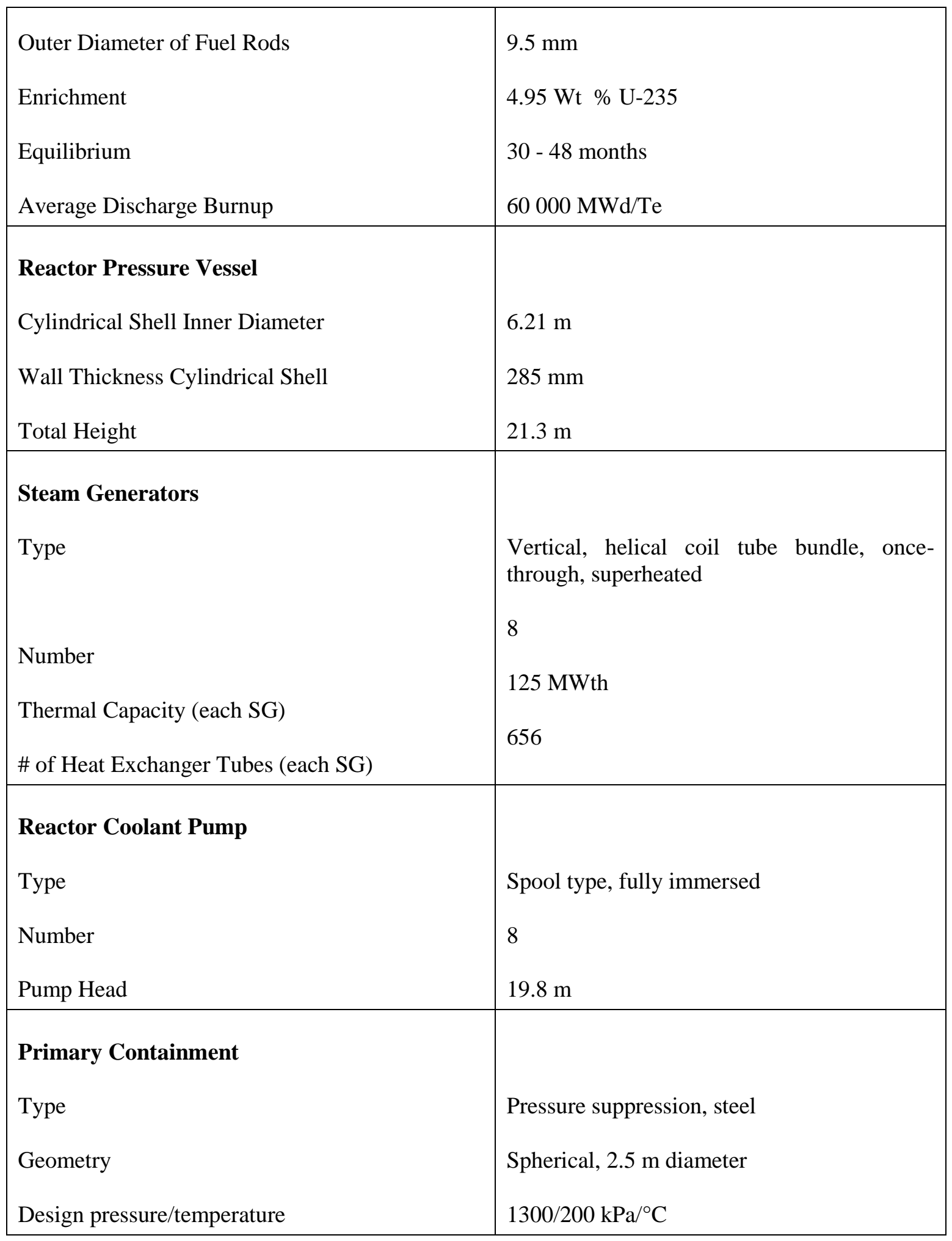


Figure 3.3 shows a size comparison between a typical LWR and an IRIS containment vessel. In Figure 3.4 is shown the preferable plant layout for a two twin-unit $(1,340 \mathrm{MWe})$ configuration, designed to maximize the sharing of auxiliary components and plant economics.

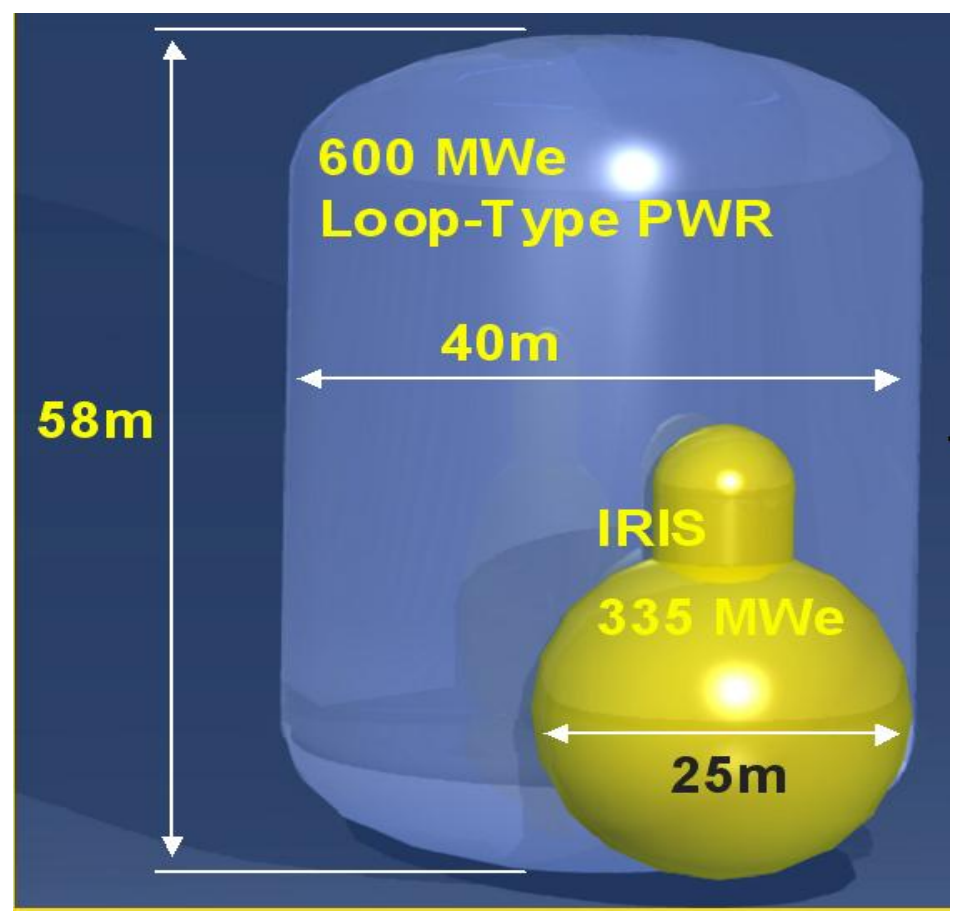

Figure 3.3. IRIS and LWR containment vessel size comparison [77]. 


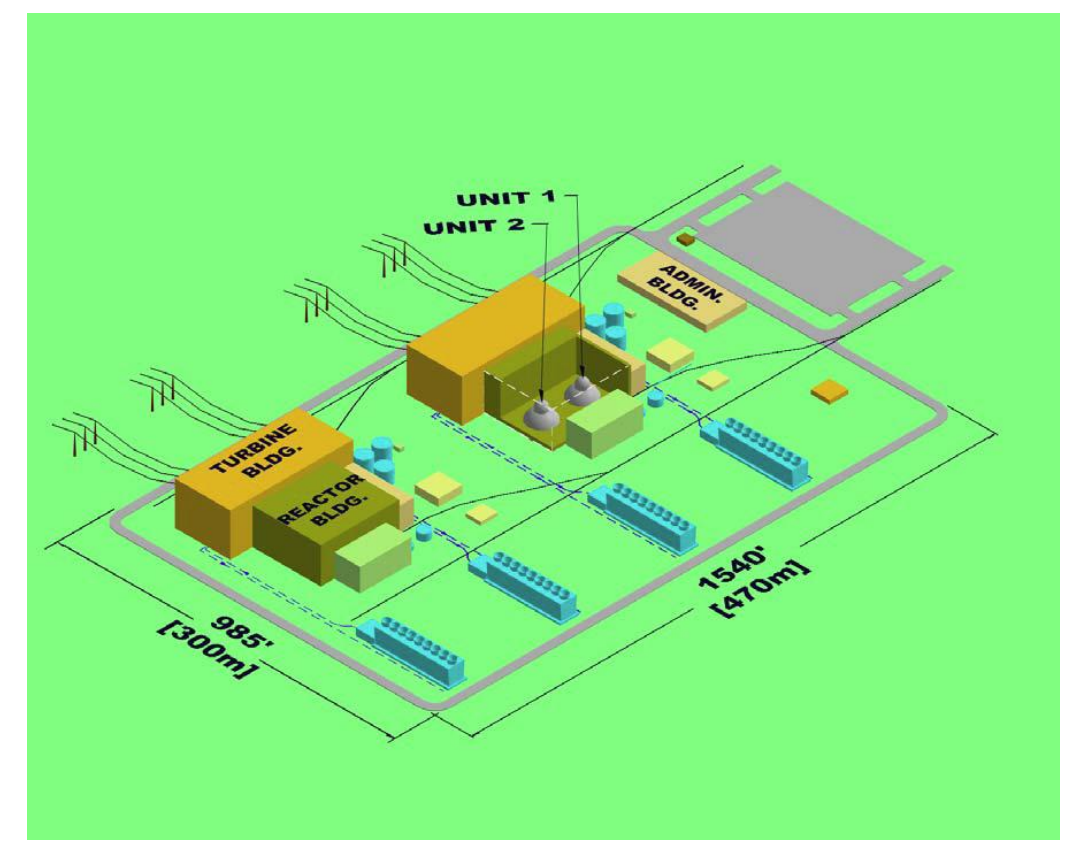

Figure 3.4. IRIS two twin-unit arrangement [78].

The steam generator is a once-through steam generator producing superheated steam. The reactor control requirements specify constant average coolant temperature across the core at constant steam pressure. Such novel, integral design includes the following advantages:

- Scalable in power between 100MWe and 350MWe, the basic design being 335 MWe, or $1000 \mathrm{MWt}$, in modular configuration, allowing scalability as single unit, multiple units, multiple single units and multiple twin units (sharing auxiliary systems and common-header/turbine).

- Reactor core, pressurizer, eight steam generators, coolant pumps and control rod mechanism are all integrated into a single pressure vessel.

- Full natural circulation (safety feature).

- Is designed to be capable of accepting different cores. Fuel will be such that it will not pose any licensing issues.

- The long lifetime core is achieved using 5 percent enriched uranium for the first reactor core and 9 percent enriched uranium for successive reactor cores. 
- The reactor refueling is needed only at the end of the first five years, and afterwards once every eight years.

- Because of the high burn-up, less nuclear waste per unit of reactor power is produced than that in currently operating reactors.

- The reactor core is comprised of 89 LWR 17x17-pin fuel bundles containing $4.95 \%$ enriched UO2 fuel and is designed for a 3.5-year cycle with an average burn-up of 50,000MWd/Te.

- Reactivity control is accomplished through solid burnable absorbers and control rods, and limited boron in the primary coolant [77].

- The primary coolant system uses eight helical-coil once-through steam generators and eight spool-type coolant pumps. The steam-regulated pressurizer is located in the upper portion of the $6.2 \mathrm{~m}$-diameter, $22.2 \mathrm{~m}$-high reactor vessel. Normal operating pressure of the primary coolant is $15.5 \mathrm{MPa}$ (approximately 153 atmospheres or 2,248 PSI).

- All primary piping external to the reactor vessel is eliminated, thus avoiding the Large Loss of Coolant (LLC) accident by design.

- Since the whole control rod mechanism is mounted inside the vessel, control rod ejection accident is also eliminated by design.

- The integral design of the primary side is placed inside a $25 \mathrm{~m}$-diameter compact steel vessel capable of withstanding 1.4 MPa design pressure, making it smaller than the traditional PWR pressure vessel.

- Annular downcomer is larger yielding a separation of the reactor vessel from the core. This way the vessel fluence is decreased by several orders of magnitude (from $1019-1014 \mathrm{n} / \mathrm{cm}^{2}$ ), making the reactor vessel virtually free of radiation damage, eliminating vessel replacement or annealing, no radiation exposure to crew inside the containment, and substantial biological shielding reduction. 


\subsection{IRIS Instrumentation and Control Needs}

In June of 2005, a Westinghouse report [79] with a comprehensive overview of Integral Primary System Reactors (IPSRs) was published using IRIS as the selected representative of such design. It also provided a detailed list of relevant measurement and instrumentation requirements compared to the existing PWR technology and identified areas that were not covered by the existing instrumentation status. Such areas were divided between two distinguished areas: engineering solutions and technological challenges. The first was essentially a matter of modifying or adapting the current technology, while the latter encompassed the true technological challenges for deployment of IRIS as those required new technological developments. Some of the report findings are summarized in Table 3.2, with the reactor system being analyzed listed in the first column and the second column being the current issue(s).

Table 3.2 is not complete, and several other challenges are yet to be resolved by either simulations or small-scaled prototypes like the SPES3 experimental facility for the IRIS reactor simulation [75]. Two good examples of the challenges ahead are IRIS steam generator level and mass inventory that cannot be measured directly, as in current PWRs. In cases like these, other techniques are being applied to try and determine the theoretical values for those parameters, based on simulations. Shen and Doster [81] have proposed an application of Neural Networks (NN) based steam generator feedwater controller to helical steam generators under transient conditions and at low power levels, which is considered the most challenging region for a full range feed water controller, even for a PWR. 
Table 3.2. IRIS instrumentation challenges [79]

\begin{tabular}{|c|c|}
\hline System & Issue \\
\hline Reactor Coolant System & $\begin{array}{l}\text { Flow measurements for IRIS/IPSRs have not yet } \\
\text { been identified. Lack of primary piping } \\
\text { eliminates the possibility of using Venturi tubes. } \\
\Delta \text { p could be used, but this solution would require } \\
\text { a penetration at the bottom of the vessel, spool- } \\
\text { type coolant pumps [80] rotating speed could } \\
\text { also be measured, however this has the } \\
\text { disadvantage of being an indirect measure of } \\
\text { core flow }\end{array}$ \\
\hline Reactor Coolant Temperature & $\begin{array}{l}\text { Not sure where } \mathrm{T}_{\text {hot }} \text { temperature sensors are to be } \\
\text { installed, either thermocouples at the core exit or } \\
\text { RTDs in the riser area. Sensor placement is also } \\
\text { something which is still being addressed }\end{array}$ \\
\hline $\begin{array}{l}\text { Reactor Coolant System Mass } \\
\text { Inventory Measurement }\end{array}$ & Not defined yet. Research ongoing at ORNL \\
\hline Pressurizer Pressure & Sensor placement is still to be decided \\
\hline Pressurizer Mass Inventory & Sensor placement and type is still to be decided \\
\hline $\begin{array}{l}\text { Steam Generator Level and Mass } \\
\text { Inventory }\end{array}$ & Not available \\
\hline
\end{tabular}


Sensor placement is also an important issue that needs to be addressed for both control design and fault diagnosis of IRIS system. Research has been done to help design an efficient monitoring system that will help in quick and accurate identification of faults by optimizing the number of sensors in the system and at the same time maximizing the chances of detecting anomalies. A paper by Li and Upadhyaya [82] addressed this issue by focusing on sensor placement in IRIS reactor with application to the helical coil steam generator using graphical approaches. Another example of a sensor placement research (Wang, [83]) used both graphical tools and an improved Principal Components Analysis to optimize sensor placement.

\subsection{Once-Through Helical Coil Steam Generators (HCSG)}

In the HCSG system the primary fluid enters at the top of the equipment and flows downward to the bottom on the shell side. The primary side heat transfer is sub-cooled, forced convection along the entire steam generator height, while the secondary fluid flows upward inside the 656 coiled tubes from bottom to top. The feed water flows into the sub-cooled region of the steam generator, and in this region the heat transfer is mainly due to single phase turbulent and molecular momentum transfer and the pressure loss is mainly due to wall friction. The saturated region begins when the bulk temperature becomes saturated. The heat transfer in the saturated boiling region is dominated by nucleate boiling, which is much more efficient than single-phase liquid or steam heat transfer. In the saturated boiling region, the generated bubbles do not disappear in the liquid core and the pressure loss is not only due to the wall friction but also due to the interfacial drag between the bubbles and the liquid. The saturated boiling region ends when critical heat flux is reached. After the steam quality becomes greater than 1.0, the liquid evaporation ceases and the steam becomes superheated. The use of helical tubing reduces the size of the steam generator, and results in an efficient heat transfer with a larger heat transfer area per unit volume than straight tube steam generators. The HCSG system objective is to supply adequate amount of steam to meet the turbine demand. A feed-forward controller is used to maintain the outlet steam pressure, while preventing the carryover of water to the turbine system or dry-out of the steam generator tubes, minimizing the mismatch between steam outlet flow rate and feed water inlet flow rate. 


\subsection{SIMULINK Model of IRIS}

SIMULINK is a software package for modeling, simulating, and analyzing dynamic systems. It supports linear and nonlinear systems, modeled in continuous time, sampled time, or a hybrid of the two. Systems can also be multi-rate, i.e., have different parts that are sampled or updated at different rates. For modeling, SIMULINK provides a Graphical User Interface (GUI) for building models as block diagrams, using click-and-drag mouse operations. It includes a comprehensive block library of sinks, sources, linear and nonlinear components, and connectors. Models are hierarchical, so models can be built using both top-down and bottom-up approaches. After the model is defined the simulations can be performed using a choice of integration methods, either from the SIMULINK menus or by entering commands in the MATLAB ${ }^{\circledR}$ command window. Model analysis tools include linearization and trimming tools, which can be accessed from the MATLAB command line, plus the many tools in MATLAB and its application toolboxes.

A SIMULINK modular model of the IRIS plant developed by $\mathrm{Xu}$ [84] is used in this research, and includes reactor core and HCSG models. The helical coil steam generator is one of the critical components and a major contributor to the cost of IRIS design as well as being the major difference between a traditional PWR and IRIS reactor. Typical once-through steam generator equations can be found in [85], and are listed in Appendix 1. The model was developed based on a previous dynamic model [86] and a SIMULINK model [87] for a traditional PWR plant. The reactor core uses the classic point kinetics reactor model (also in Appendix 1) with Mann's model for the fuel to coolant heat transfer.

\subsubsection{Computational Algorithm}

The IRIS model program involves one main m-script file, in which all design constants are properly defined and input matrices setup. One main SIMULINK model and a few userdefined SIMULINK functions or blocks to calculate steam and fluid properties based on either empirical models or steam properties tables are used. The simulation sequence used in this research is the following:

Run the main m-script file to: 
- Define all reactor and steam generator constants.

- Define initial conditions (power demand, core inlet temperature, feedwater temperature, etc.)

- Run both core and HCSG SIMULINK models to use the steady-state results as inputs to the dynamic models.

Run the main SIMULINK model (for one or two reactor units):

- Define the power profile (or transient) to be used.

- Define simulation duration.

- Run core and steam generator dynamic models and store the results.

Run the last part of the m-script file to format and plot the results. Appendix 2 contains an IRIS model sample run to help understand the mechanics of running the code.

\subsubsection{Reactor Core Model Steady State Results}

The initial work with the existing IRIS SIMULINK model (core and HCSG) involved dividing the existing model into separate, independent modules to test and check each one for consistency, with emphasis on the reactor model. Hence, using known input transients and analyzing their responses provided a way to check for consistency of the results. Figure 3.5 shows a screenshot of the reactor core SIMULINK model, with the six delayed neutron precursor concentration differential equations (C1-C6), fuel node temperature (Tf), the two coolant node temperatures (Theta1 and Theta2), fuel and moderator temperature reactivity feedback coefficients, the PID control block, and other variables. Additional model screenshots are shown in Appendix 3. The input to the core model is demand, in percent power, which is sent to a lookup table that translates into moderator inlet temperature, in Fahrenheit. Therefore, power demand can be interchangeably referred to as power demand itself or as $\mathrm{T}_{\text {cold. }}$. The main program outputs are:

- Power profile $\left(\mathrm{P} / \mathrm{P}_{0}\right), \mathrm{P}$ being the power demand, and $\mathrm{P}_{0}$ nominal power. 
- Fuel temperature $\left({ }^{\circ} \mathrm{F}\right)$.

- Moderator core outlet temperature, $\mathrm{T}_{\text {hot }}\left({ }^{\circ} \mathrm{F}\right)$.

- Average moderator temperature, $\mathrm{T}_{\mathrm{ave}}\left({ }^{\circ} \mathrm{F}\right)$.

Core model containing moderator and fuel temperature reactivity feedback coefficients was tested using known perturbations such as external reactivity and pre-set core inlet moderator temperature $\left(\mathrm{T}_{\text {cold }}\right)$ as inputs. The results were consistent with those from PWR core models. Figure 3.6 shows the reactor power simulation with $\mathrm{T}_{\text {cold }}$ equals to $556^{\circ} \mathrm{F}\left(291.1^{\circ} \mathrm{C}\right)$ from $\mathrm{t}=0 \mathrm{~s}$ until steady state was reached. Figure 3.7 is a zoomed-in version of Figure 3.6, showing the "prompt-jump" portion at the beginning of the transient. Starting at steady state, Figure 3.8 shows a 0.001 external reactivity insertion at $\mathrm{t}=700 \mathrm{~s}$, with the resulting core power increasing by about $4 \%$. Such power increase is compatible with a similar transient in a PWR core design for a similar reactivity input change. 


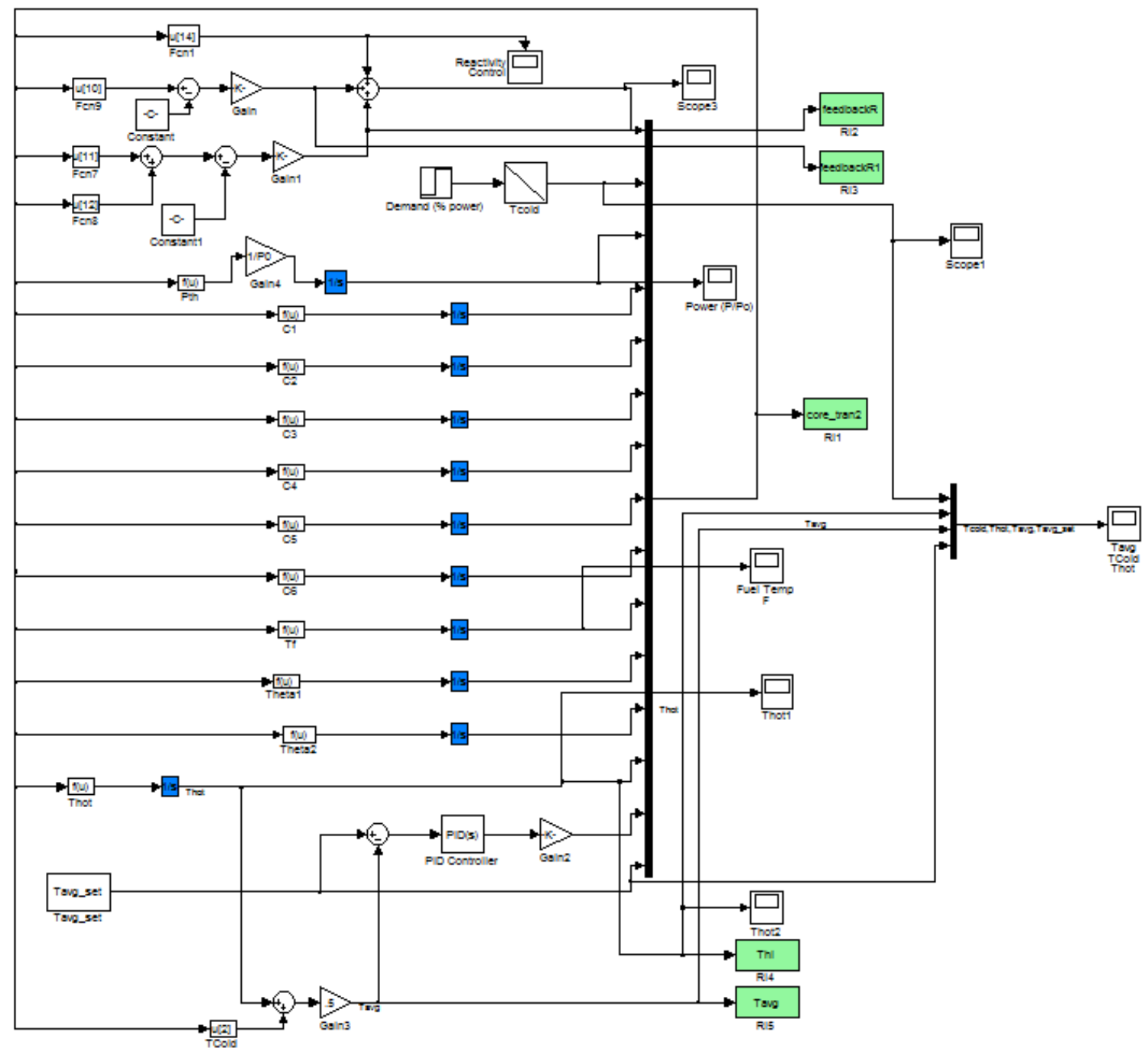

Figure 3.5. Screenshot of the Reactor core SIMULINK model. 
In the available literature, as well as in the complete and comprehensive IRIS FORTRAN code developed by North Carolina State University ([88],[89]), $\mathrm{T}_{\text {ave, }}$ which is the average moderator temperature between core inlet $\left(\mathrm{T}_{\text {hot }}\right)$ temperature and outlet $\left(\mathrm{T}_{\text {cold }}\right)$ temperature is fixed at $590^{\circ} \mathrm{F}$. For this purpose, a $\mathrm{T}_{\text {ave }}$ controller was implemented in the SIMULINK model with its set point fixed at $590^{\circ} \mathrm{F}$. Figure 3.9 shows how outlet temperature adjusts to maintain a constant average coolant temperature around the set point for a $\mathrm{T}_{\text {cold }}$ fixed at $562^{\circ} \mathrm{F}$, contrasting with Figure 3.10 where $T_{\text {ave }}$ does not reach the set point for the same simulation without the controller.

In Figure 3.11 a five percent power step increase at $t=800$ s is shown, where it is possible to check the power increasing from $84 \%$ to about $88 \%$ in 200 seconds, and in Figure 3.12 the $\mathrm{T}_{\mathrm{ave}}$ controller maintains its value at around the set point, for changing $\mathrm{T}_{\text {hot }}$.

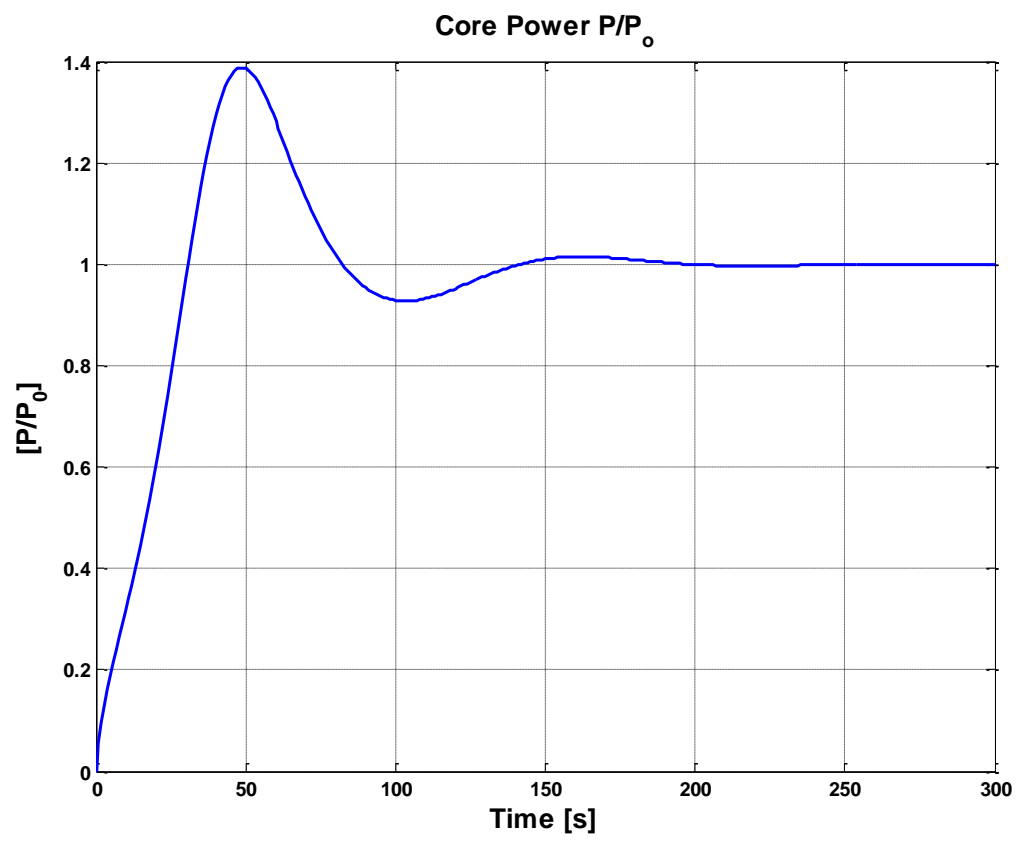

Figure 3.6. Power profile starting from power $=0$ until steady state is reached. 


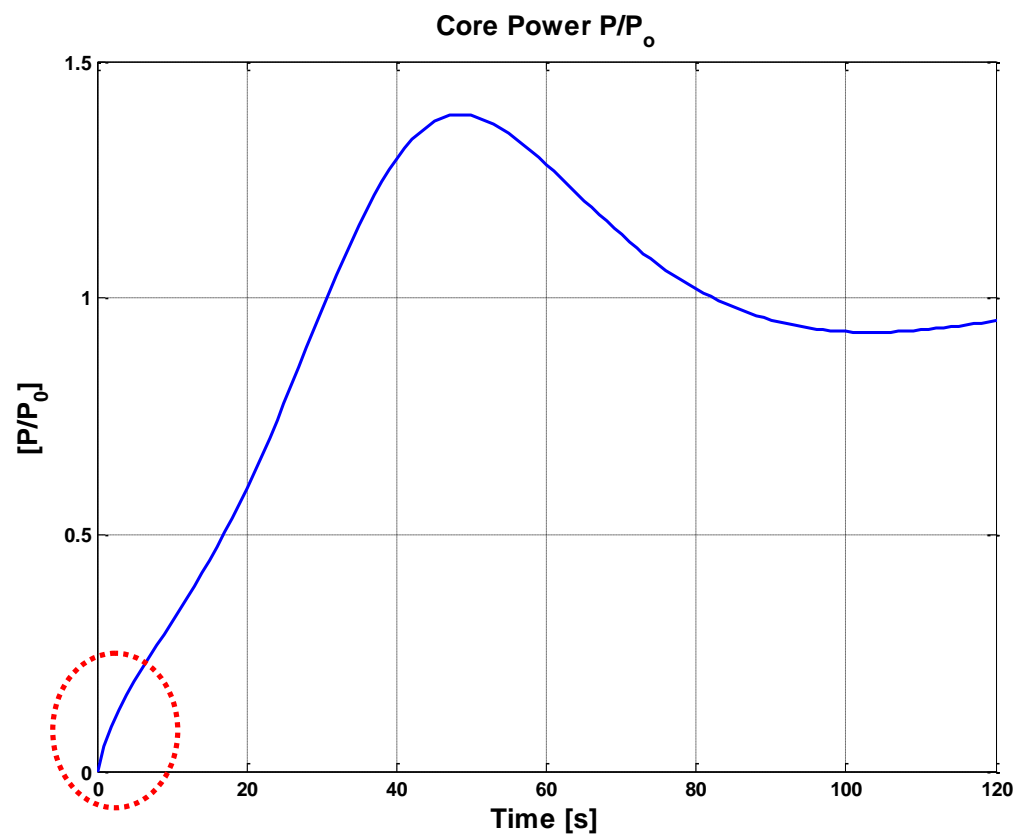

Figure 3.7. Power profile showing the "prompt jump" portion circled in red.

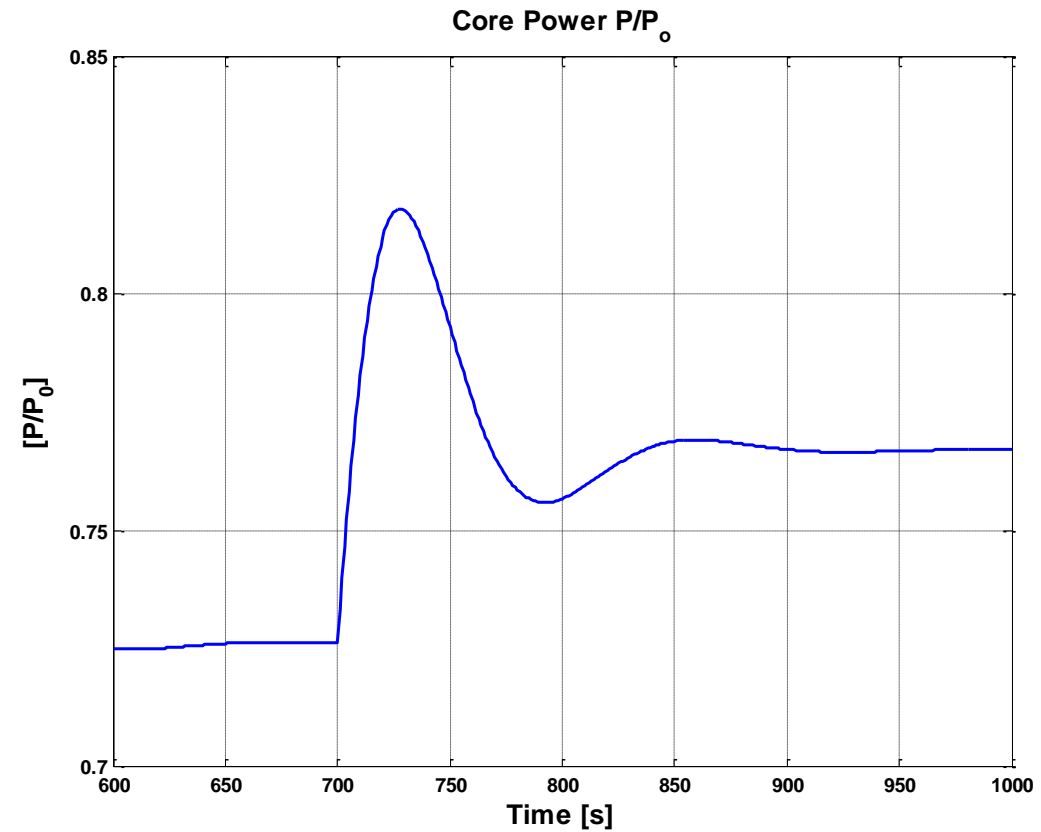

Figure 3.8. A $10^{-3}$ external reactivity insertion at $\mathrm{t}=700 \mathrm{~s}$. 


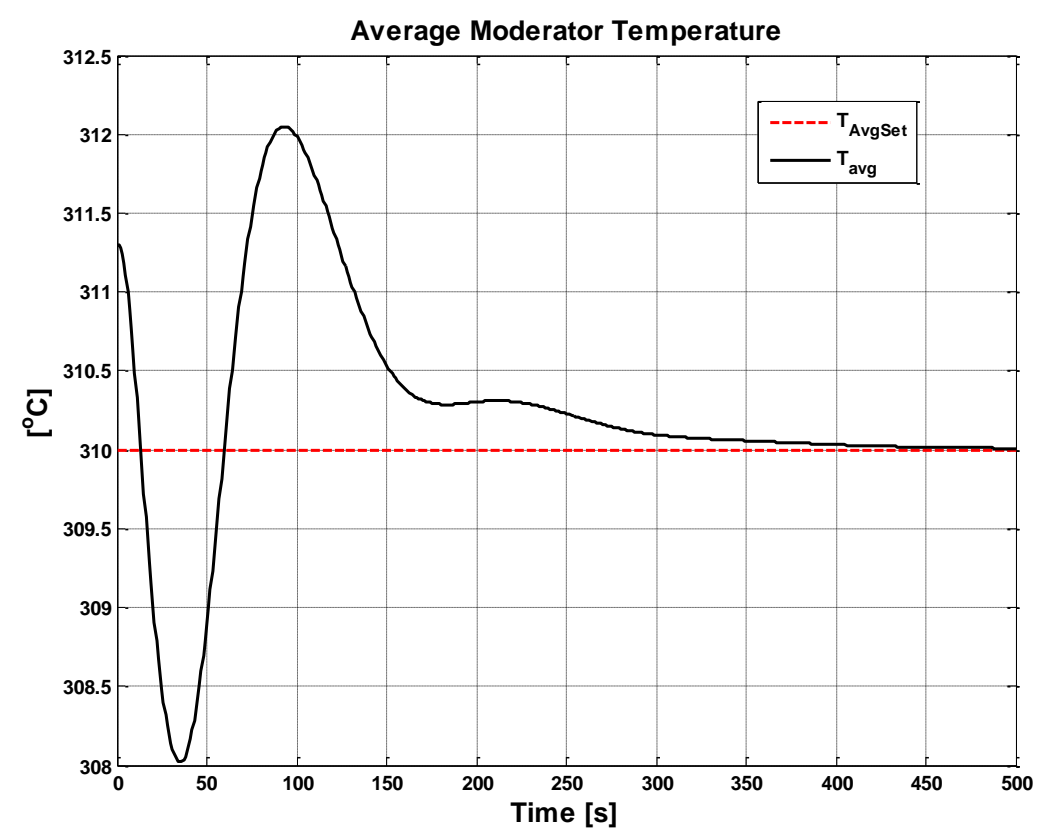

Figure 3.9. $\mathrm{T}_{\text {ave }}$ controller performance for $\mathrm{T}_{\text {cold }}=294^{\circ} \mathrm{C}$ ( $80 \%$ power).

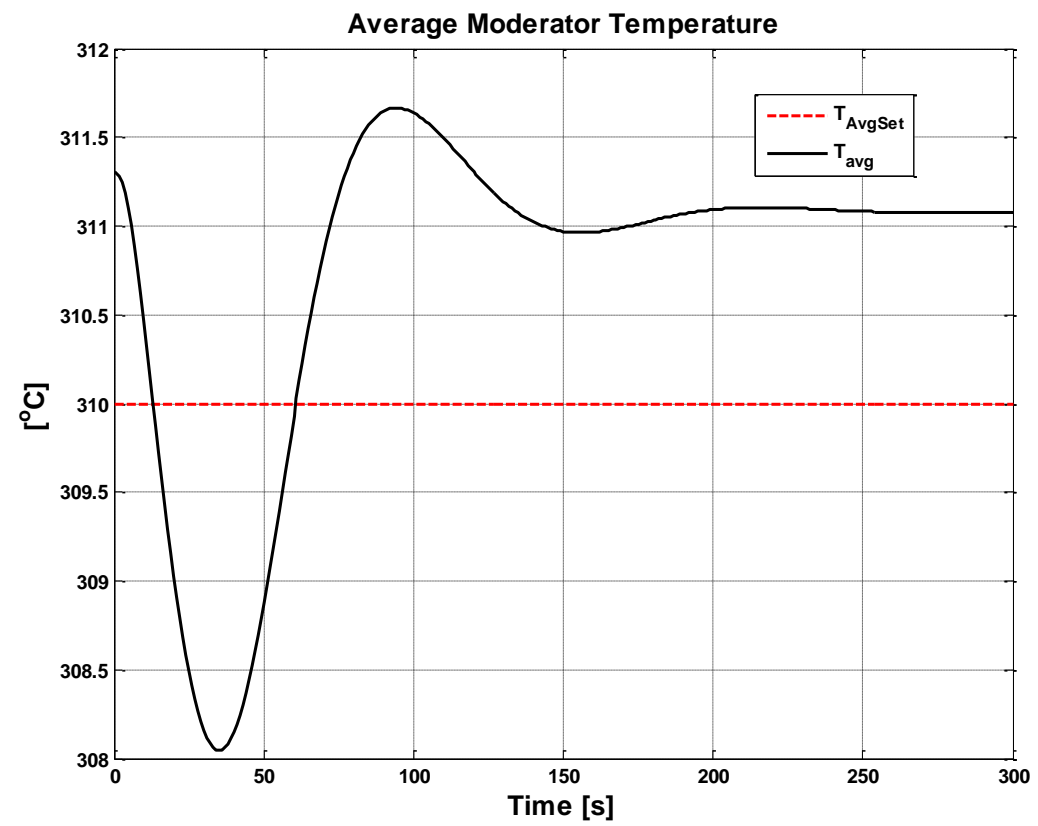

Figure 3.10. $\mathrm{T}_{\mathrm{ave}}$ not reaching set point without controller. 


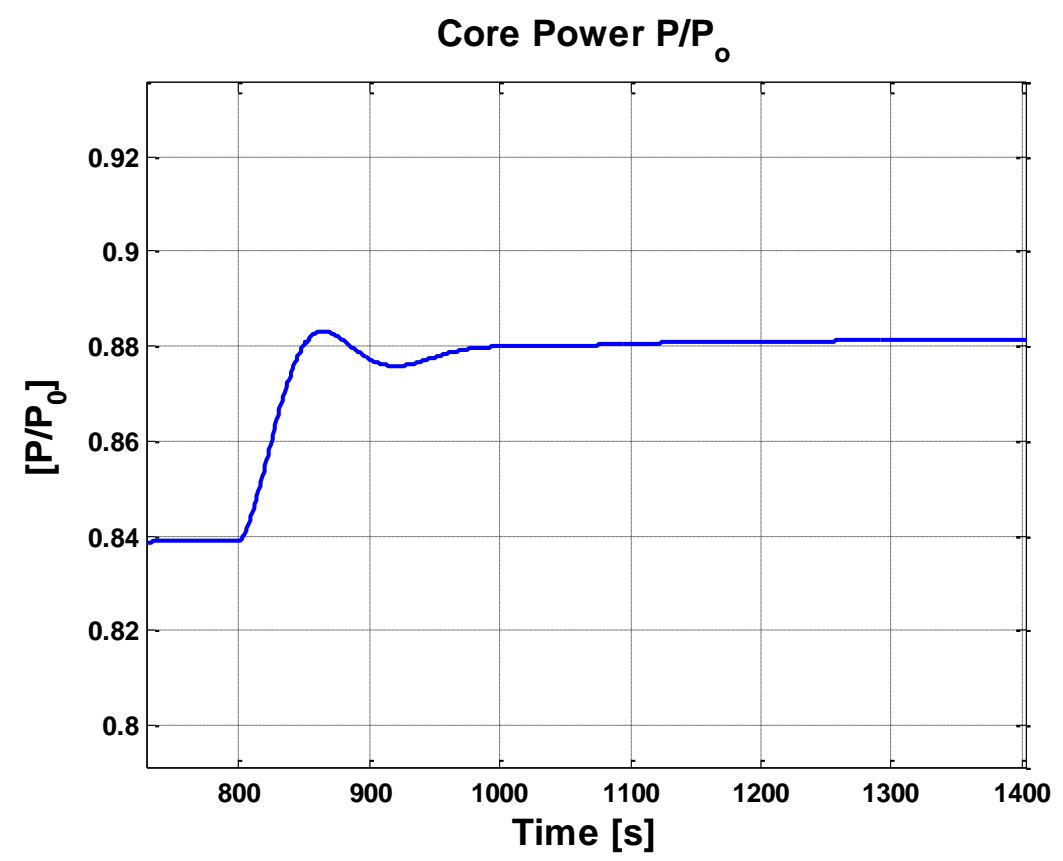

Figure 3.11. Five percent step increase in power at $\mathrm{t}=800 \mathrm{~s}$.

Core Inlet $\left(\mathrm{T}_{\mathrm{CL}}\right)$, Core Exit $\left(\mathrm{T}_{\mathrm{HL}}\right)$, and Average Temperatures

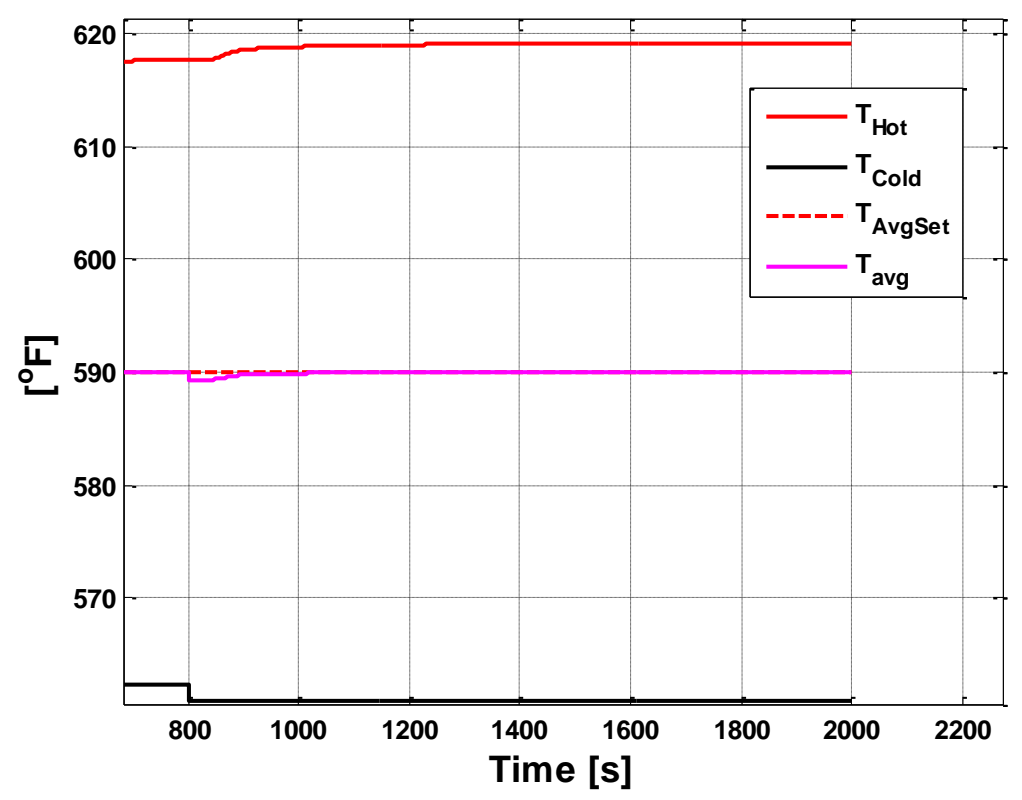

Figure 3.12. Five percent step increase in power at $\mathrm{t}=800 \mathrm{~s}$ and $\mathrm{T}_{\mathrm{ave}}$ controller action. 


\subsubsection{IRIS Complete Model Steady State Results}

After the core model was tested, it was connected back to the HCSG SIMULINK model, with the interface between the two models being both inlet and outlet moderator temperatures. The HCSG has a proportional-integral steam pressure controller to maintain the pressure set point fixed at $5.8 \mathrm{MPa}$ (841 PSI) by varying the steam flow rate. Such controller is based on the following equations [85]:

$$
\dot{W}_{s}=\frac{W_{s 0}\left(1-C_{s t} u\right)-W_{s}}{\tau_{s}}
$$

Where:

$u=$ controller output.

$\tau=$ time constant.

$W_{s 0}=$ initial steam flow rate on the secondary side.

$C_{s t}=$ an adjustable parameter.

If a PI controller is used, the controller output has both the proportional part $u_{1}(t)$ and the integral part $u_{2}(t)$, which is given by:

$$
\begin{aligned}
& u_{1}(t)=k_{1}\left(\frac{P_{t b}}{P_{0}}-\frac{P_{s e t}}{P_{0}}\right) \\
& \frac{d u_{2}(t)}{d t}=k_{2}\left(\frac{P_{t b}}{P_{0}}-\frac{P_{s e t}}{P_{0}}\right)
\end{aligned}
$$

Where:

$k_{1}=$ proportional gain.

$k_{2}=$ integral gain.

$P_{t b}=$ turbine header pressure. 
$P_{\text {set }}=$ turbine header pressure set-point.

$P_{0}=$ turbine nominal pressure set-point.

The feedwater flow rate is determined based on NCSU FORTRAN code, and is set according to the power demand - feed flow program. In this simulation there is no feed-forward controller to quickly move the control rods based on changes on power load demands, and the pressurizer model, as well as the balance-of-plant (BOP) are not included in the simulation, and both are assumed to be functioning well, and temperature of feedwater is assumed to be fixed at $224^{\circ} \mathrm{C}$, which corresponds to $100 \%$ power. The main program window is shown in Figure 3.13 and the main outputs are:

- Moderator core inlet temperature $\left(\mathrm{T}_{\text {cold }}\right)$, referred to in the program as $\mathrm{T}_{\text {pout. }}$.

- Steam outlet pressure, $\mathrm{P}_{\text {sout }}$ in PSI.

- Steam flow rate, $\mathrm{W}_{\text {sout }}$ in $\mathrm{lbm} / \mathrm{s}$ per tube per steam generator.

- Steam outlet temperature, $\mathrm{T}_{\text {sout }}$ in ${ }^{\circ} \mathrm{F}$.

- Steam generator boiling length, $\mathrm{L}_{\mathrm{b}}$ in $\mathrm{ft}$.

- Sub-cooled length, $\mathrm{L}_{\mathrm{sc}}$, in ft.

- Feedwater flow rate, $\mathrm{W}_{\mathrm{fw}}$, in $\mathrm{lbm} / \mathrm{s}$ per tube per steam generator. 


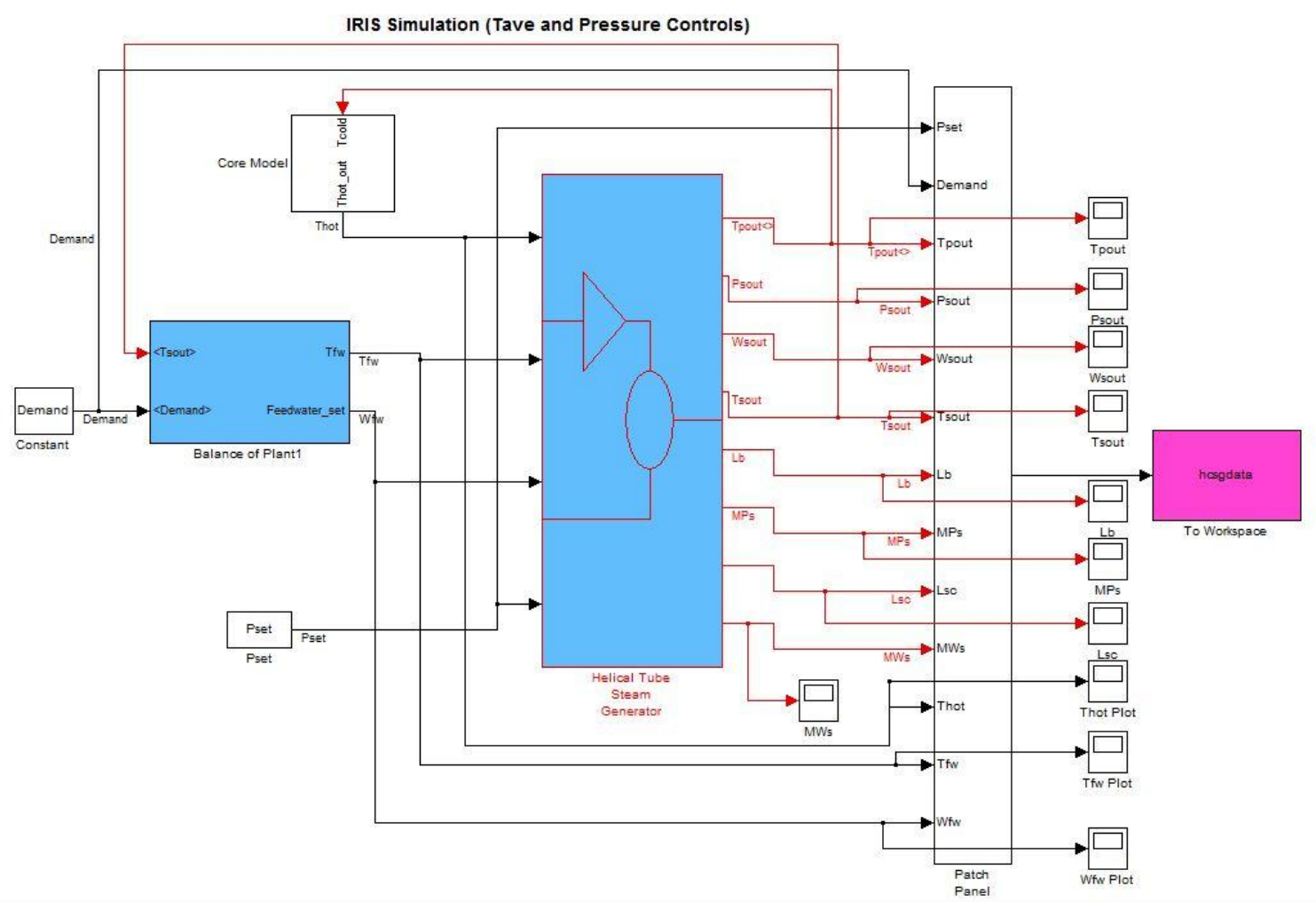

Figure 3.13. IRIS complete SIMULINK model main screen. 
In order to test the entire model, starting at steady state, a step change in $\mathrm{T}_{\text {cold }}$ was performed at $\mathrm{t}=900 \mathrm{~s}$, with initial value of $291^{\circ} \mathrm{C}$ and final value of $295^{\circ} \mathrm{C}$ (or $100 \%$ to $95 \%$ power). Figure 3.14 shows the power change profile due to changes in the inlet moderator temperature propagating through the remaining of the system, with the initial power going from around $99 \%$ to approximately $97 \%$ in 500 seconds. Figure 3.15 shows how the $\mathrm{T}_{\text {ave }}$ value changes around the set point for changes in $\mathrm{T}_{\text {cold }}$. The core outlet temperatures adjust to maintain a constant average coolant temperature around the set point. The steam outlet pressure response shown in Figure 3.16 also varies due to the inlet moderator temperature by changing the steam generator feedwater flow rate, shown in Figure 3.17. And finally, by increasing the moderator inlet temperature the power decreased a few percent points because of the change in moderator density, and so was the steam generator level, leaving more heat transfer area for the steam to be superheated, thus increasing its temperature, as shown in Figure 3.18.

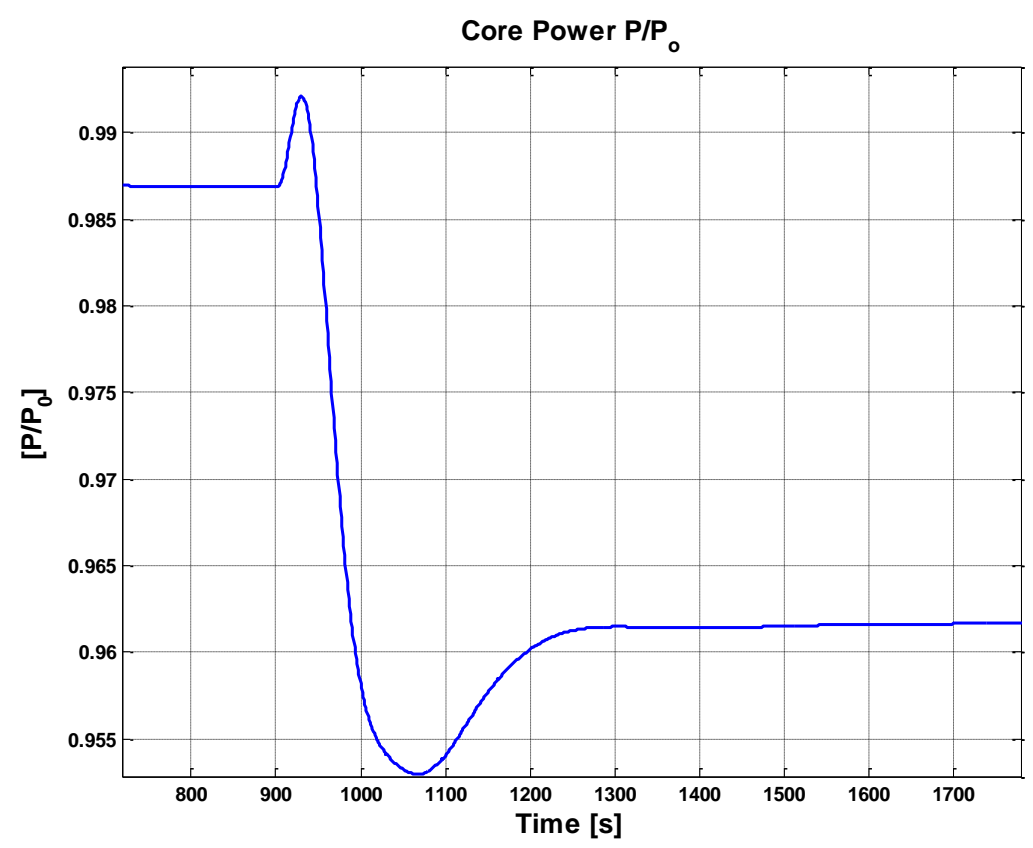

Figure 3.14 . Reactor power response to a $\mathrm{T}_{\text {cold }}$ step change from $291{ }^{\circ} \mathrm{C}$ to $295^{\circ} \mathrm{C}$. 


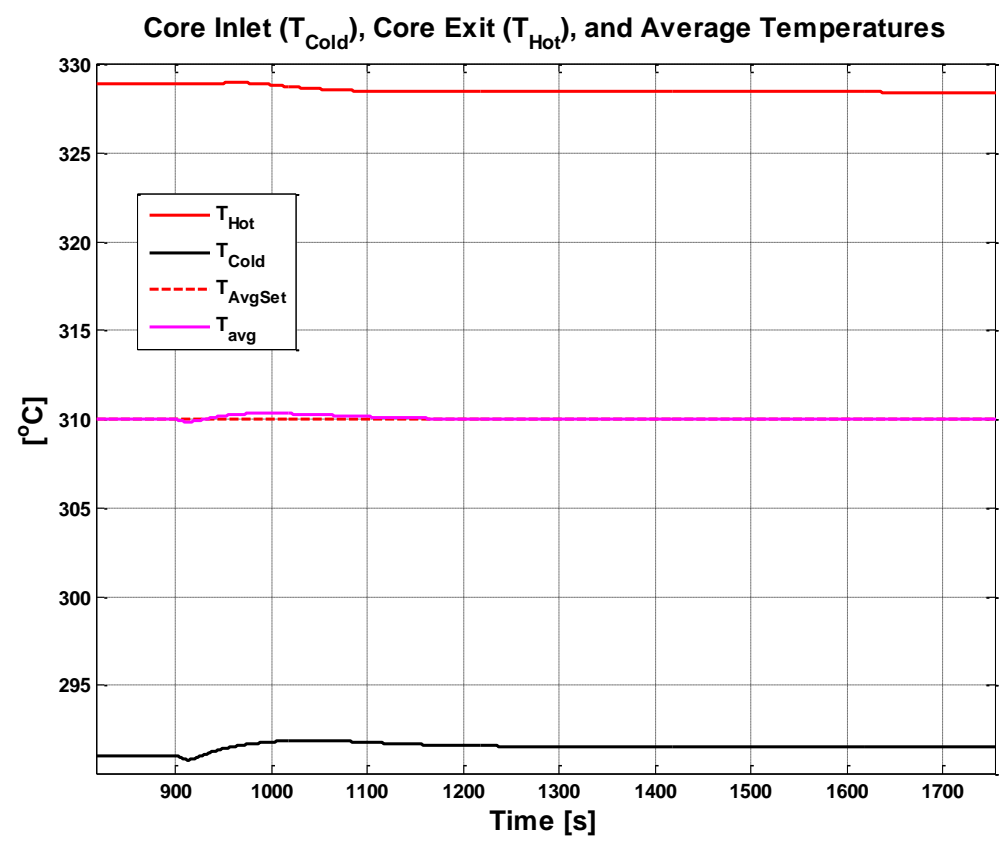

Figure 3.15 . $\mathrm{T}_{\text {ave }}$ response to a $\mathrm{T}_{\text {cold }}$ step change from $291^{\circ} \mathrm{C}$ to $295^{\circ} \mathrm{C}$.

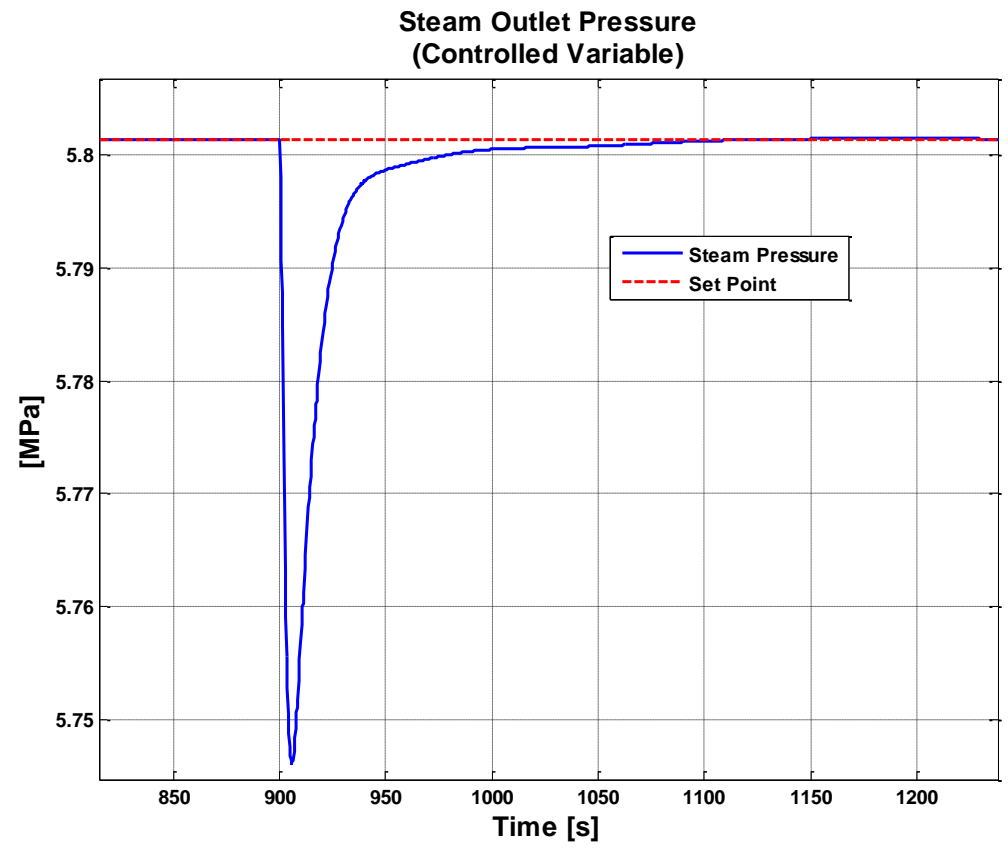

Figure 3.16. Steam outlet pressure response to a $\mathrm{T}_{\text {cold }}$ step change from $291^{\circ} \mathrm{C}$ to $295^{\circ} \mathrm{C}$. 


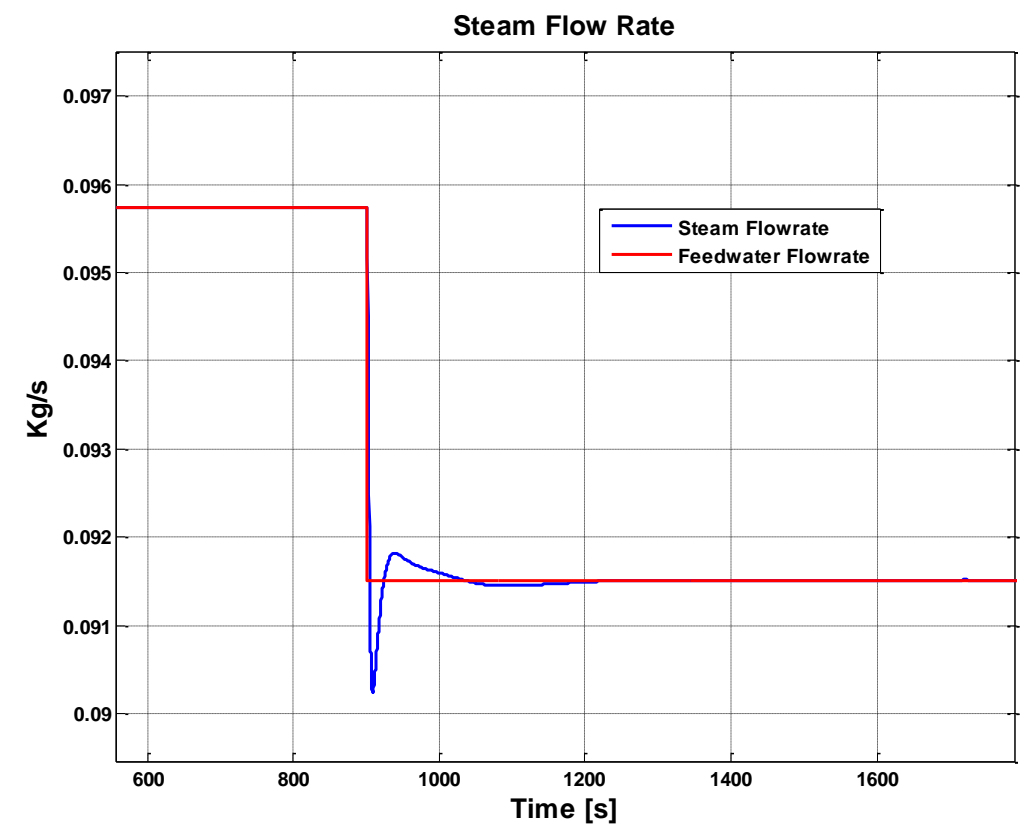

Figure 3.17. Steam flow rate response to a $\mathrm{T}_{\text {cold }}$ step change from $291^{\circ} \mathrm{C}$ to $295^{\circ} \mathrm{C}$.

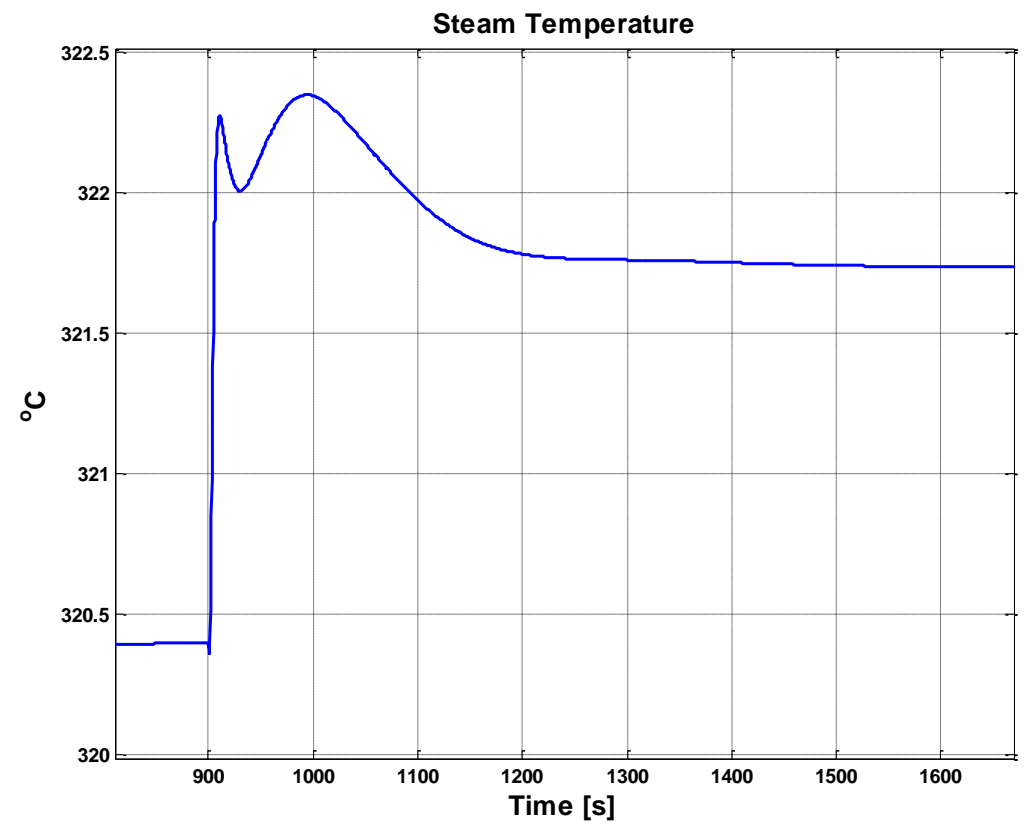

Figure 3.18. Steam temperature response to a $\mathrm{T}_{\text {cold }}$ step change from $291^{\circ} \mathrm{C}$ to $295^{\circ} \mathrm{C}$. 
As stated earlier, NCSU's FORTRAN model is a complete, comprehensive IRIS model, so it is interesting to compare the steady state values with those produced by that code. Table 3.2 summarizes the results obtained from SIMULINK and from NCSU FORTRAN code for a power demand of $100 \%$. The steady state results indicate the SIMULINK model results are very close to those from NCSU.

Table 3.2. Steady state values from SIMULINK and NCSU IRIS FORTRAN models.

\begin{tabular}{|c|c|c|c|}
\hline Reactor Power @ 100\% & $\begin{array}{l}\text { SIMULIN } \\
\mathrm{K}\end{array}$ & NCSU & $\begin{array}{l}\text { Design Parameters }{ }^{[74]} \text { at } 100 \% \\
\text { Power (per SG, per tube) }\end{array}$ \\
\hline Steam outlet Temperature $\left({ }^{\circ} \mathrm{C}\right)$ & 320 & 325 & 317 \\
\hline Steam flow rate $(\mathrm{kg} / \mathrm{s})$ & 0.096 & 0.095 & 0.095 \\
\hline Feedwater flow rate $(\mathrm{kg} / \mathrm{s})$ & 0.096 & 0.096 & 0.095 \\
\hline Feedwater temperature $\left({ }^{\circ} \mathrm{C}\right)$ & 202 & 191 & 224 \\
\hline Steam outlet pressure $(\mathrm{MPa}) *$ & 5.8 & 5.94 & 5.8 \\
\hline $\begin{array}{l}\text { Average moderator temperature* } \\
\left({ }^{\circ} \mathrm{C}\right)\end{array}$ & 310 & 310 & 310 \\
\hline $\mathrm{T}_{\text {cold }}\left({ }^{\circ} \mathrm{C}\right)$ & 291 & 291 & 292 \\
\hline $\mathrm{T}_{\text {hot }}\left({ }^{\circ} \mathrm{C}\right)$ & 329 & 329 & 328 \\
\hline Average fuel temperature $\left({ }^{\circ} \mathrm{C}\right)$ & 449 & 450 & N/A \\
\hline
\end{tabular}

*Controlled variables

\subsection{IRIS System General Load Following Maneuver}

Load following is the capability of a reactor to follow changes in the grid demand, for instance, reduced consumption over the weekend. The following is the sequence of actions 
during a load-following maneuver for the IRIS plant for the case of load change from $80 \%$ to $100 \%$

- Operator sets the desired load program (for example, 5\% ramp change/hour)

- This creates an error signal between the desired load and the actual turbine output. The turbine header pressure (turbine inlet steam pressure) is directly correlated to turbine output.

- There is now a mismatch between turbine output and reactor power, and initiates a signal to move the control rods to increase the reactor power. This may be considered as a feed-forward control action.

- The $\left\{\mathrm{T}_{\mathrm{avg(ref})}-\mathrm{T}_{\mathrm{avg}(\text { actual })}\right\}$ error is generated based on $\mathrm{T}_{\mathrm{avg}(\mathrm{ref})}$ for the actual turbine output. The turbine output is used for calculating the percent power in the $\mathrm{T}_{\mathrm{avg}} \mathrm{vs}$. Power program.

- As the error between the reference $\mathrm{T}_{\mathrm{avg}}$ and measured $\mathrm{T}_{\mathrm{avg}}$ increases, the control rods move to increase the reactor power.

- Turbine control valves move to maintain programmed steam generator pressure.

- Feed flow versus load (desired) program determines the desired feed flow for that load. The feed flow moves to minimize the flow error.

- The error between the desired load and turbine output is used to correct for the feed flow by bumping the feed flow for the given load.

- Increased feed flow increases steam production, and consequently increases the steam pressure. The steam throttle valve is actuated to maintain the pressure.

- As the reactor power increases, the measured $\mathrm{T}_{\text {avg }}$ increases. The control rod motion stops once $\mathrm{T}_{\mathrm{avg} \text { (measured) }}=\mathrm{T}_{\mathrm{avg} \text { (reference) }}$. 
- The pressurizer has no spray control (in IRIS) to reduce the pressure. There are heaters to increase the pressure. No active pressurizer level control, except for an on-off control action.

\subsection{IRIS Plant used in Load Following Maneuvers}

When a power level change is performed, several modifications occur in the core from a neutronic point of view, i.e., the fuel and moderator temperature change, the xenon level is modified, the power distribution skews, etc. These changes need to be adequately counterbalanced to keep both the core critical and the power distribution acceptable. The usual approach is to compensate for the reactivity change due to the power variation by adjusting the soluble boron concentration and moving a limited number of control rod banks. This, however, leads to large volume of liquid effluent, in particular toward the end of cycle, when the soluble boron concentration is low, and it is difficult to dilute. To avoid this, Westinghouse has developed the innovative MSHIM (mechanical shim) strategy in 1988, where the control rods and control banks are designed to allow load follow using only control rods.

According to Franceschini and Petrovic [77], MSHIM capability is a desired feature in AP1000, and it becomes almost a required feature for IRIS. Basically, due to its large inventory of the primary coolant, the dilution/boration strategy becomes not only more expensive, but may additionally be limited by the achievable dilution rate (in particular at the end of cycle). Therefore, MSHIM capability was specified as one of the operational requirements for IRIS. In particular, it is required that IRIS can perform load follow through MSHIM for the Westinghouse design basis load follow maneuvers, plus for additional power changes prescribed by the Electric Power Research Institute for Advanced LWRs. The corresponding nine different power change profiles used in their research are shown in Figure 3.19, and were used as demand input to the IRIS SIMULINK model. The results of the first profile are shown in Figures 3.20 through 3.25. The $\mathrm{T}_{\text {ave }}$ controller in the reactor core module is able to keep the values close to the set point (Figure 3.20) by varying the external reactivity, hence $T_{\text {hot }}$, making the reactor reach the necessary power to match the steam flow rate demanded by the turbine (Figure 3.23). In Figure 3.21 only half of the profile was performed, and it is possible to see that, without $\mathrm{T}_{\text {ave }}$ controller, the average temperature never settles around the set point but there is a bias between 
them. As the power varies (Figure 3.22) so does the steam generator water level, varying the available heat transfer area available for the steam to be heated (Figure 3.24), hence also varying $\mathrm{T}_{\text {cold }}$, while at the same time the pressure controller keeps the pressure as close as possible to the set point (Figure 3.25) by varying the steam flow rate.
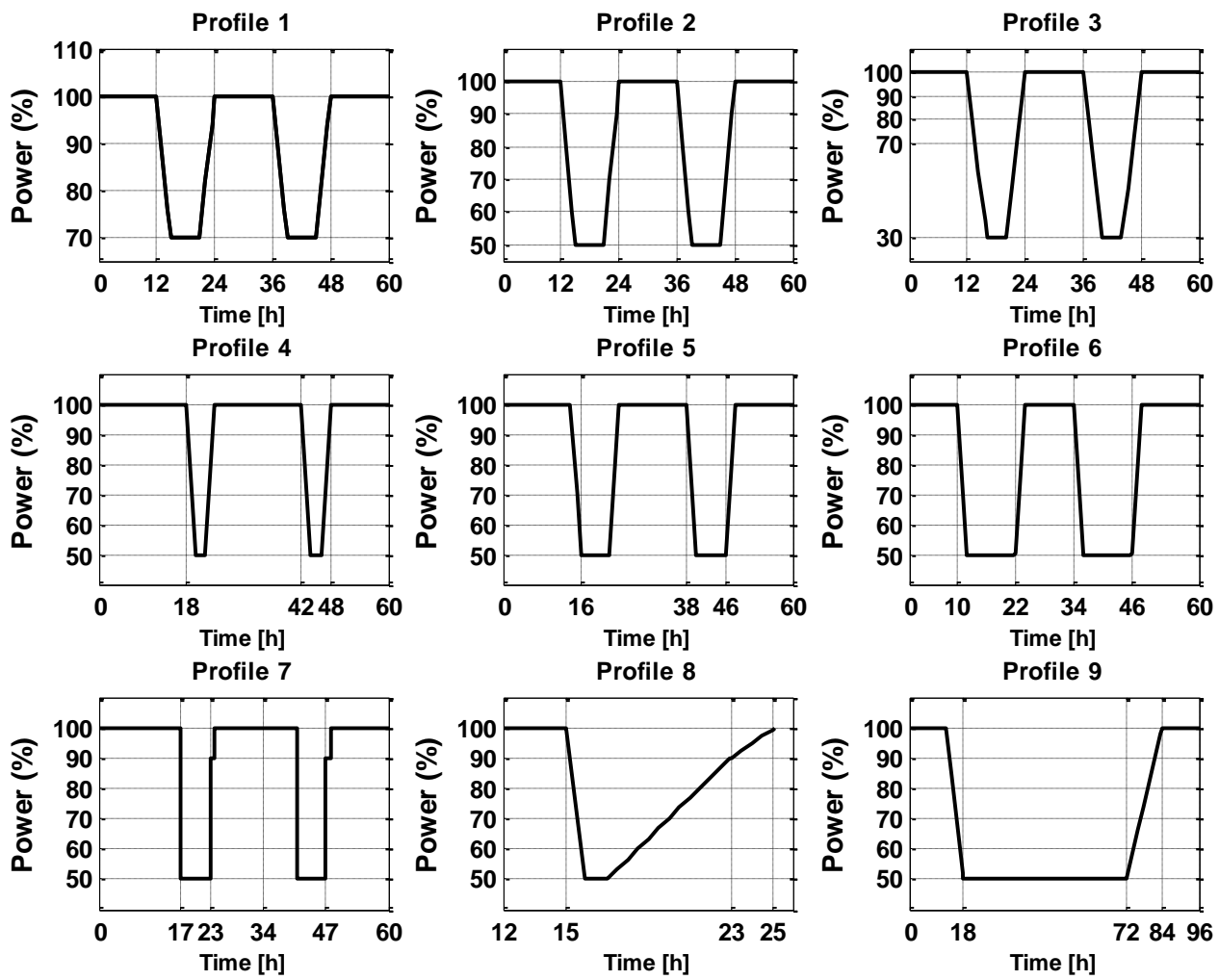

Figure 3.19. Load following power demand profiles [77]. 


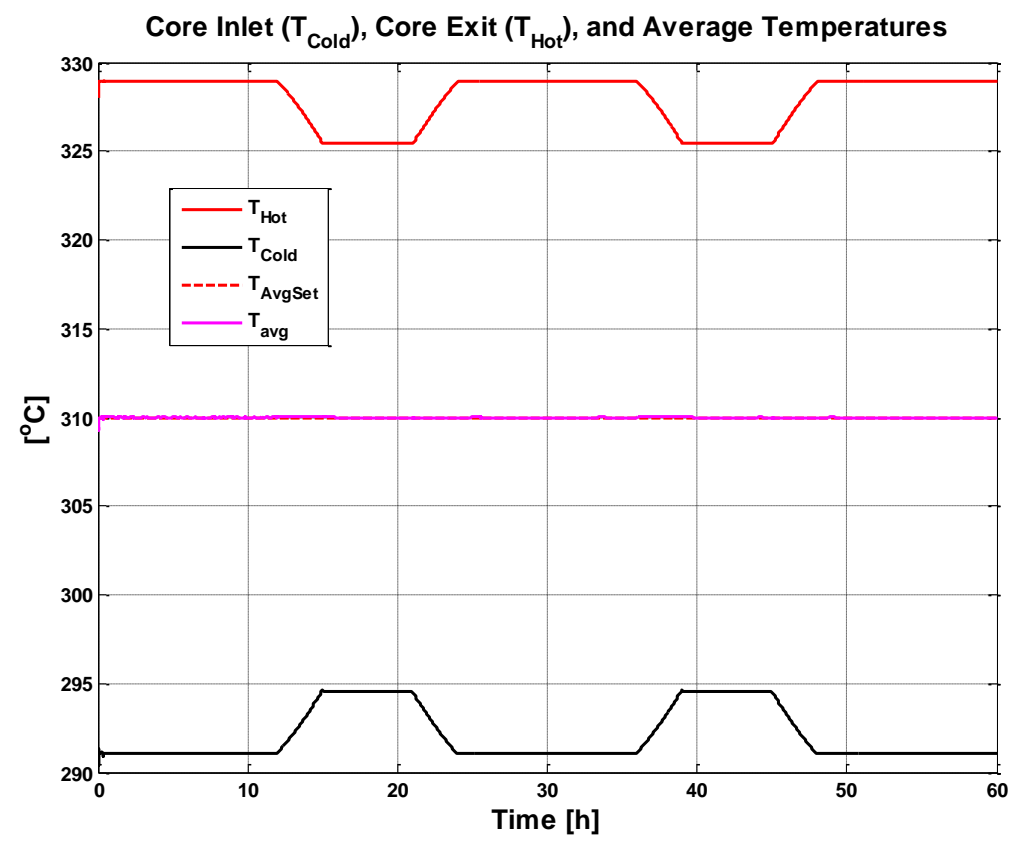

Figure 3.20. $\mathrm{T}_{\mathrm{ave}}$ changes for profile \#1 load demand.

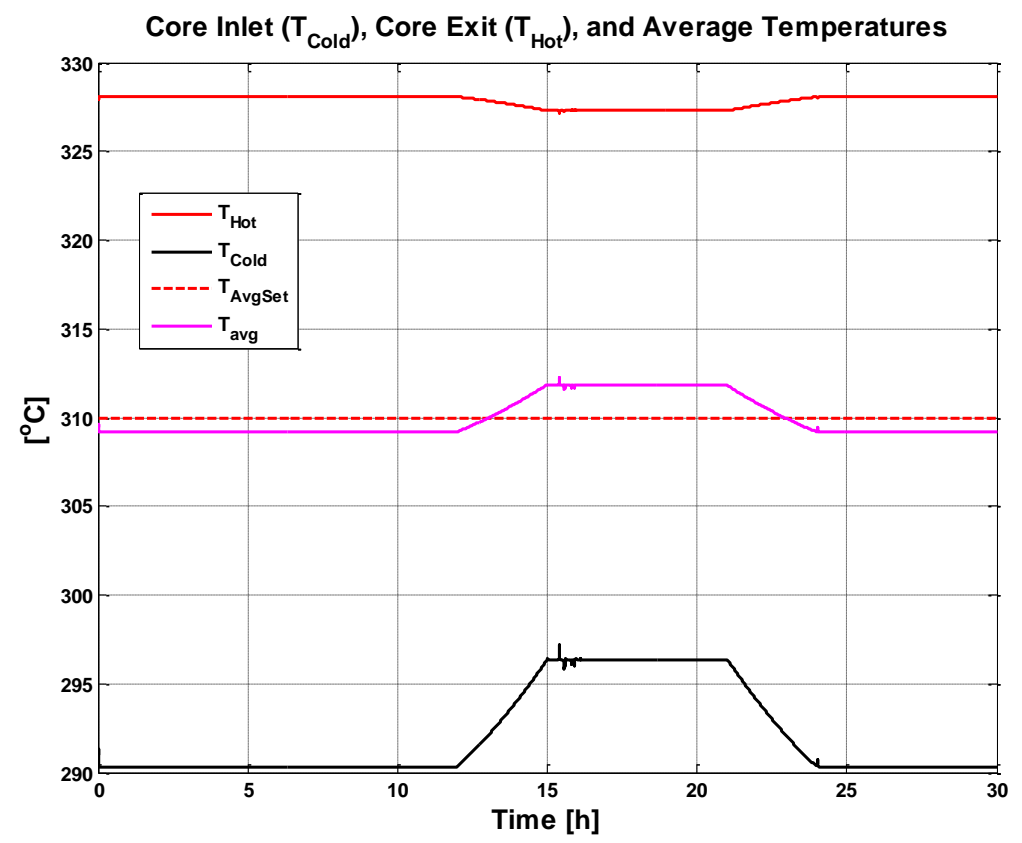

Figure 3.21. $\mathrm{T}_{\mathrm{ave}}$ changes for half profile \#1 load demand without controller. 


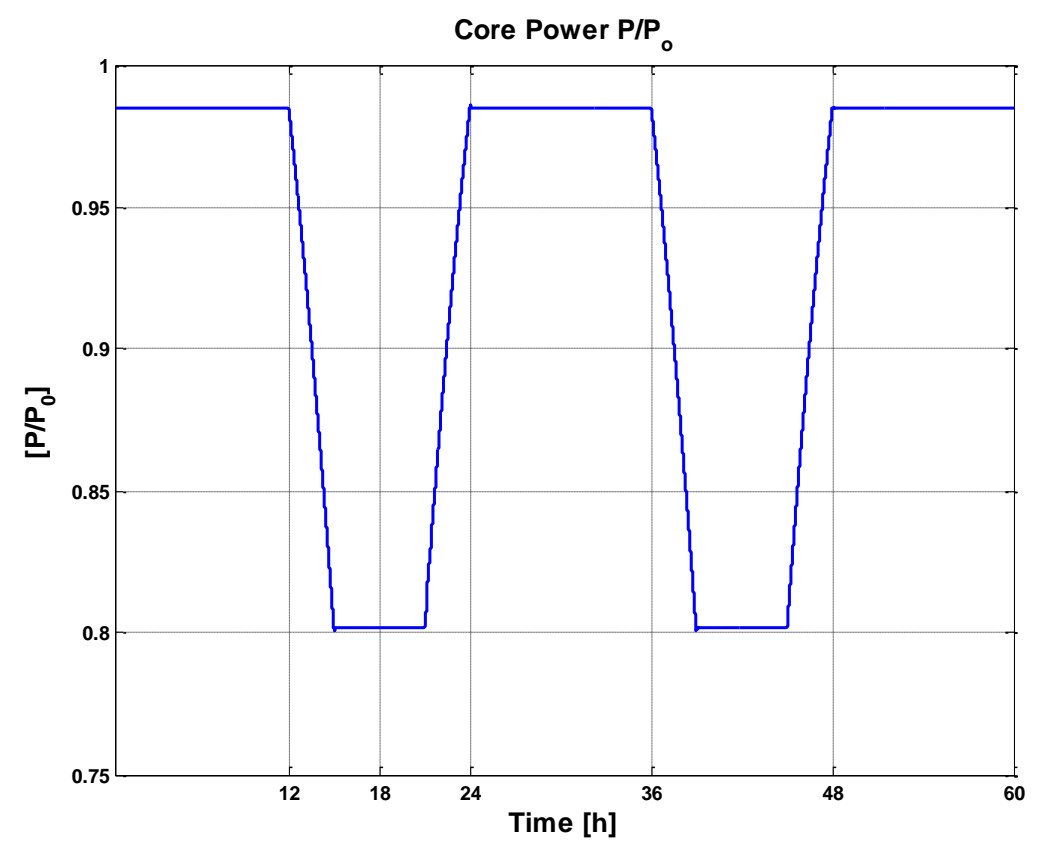

Figure 3.22. Power changes for profile \#1 load demand.

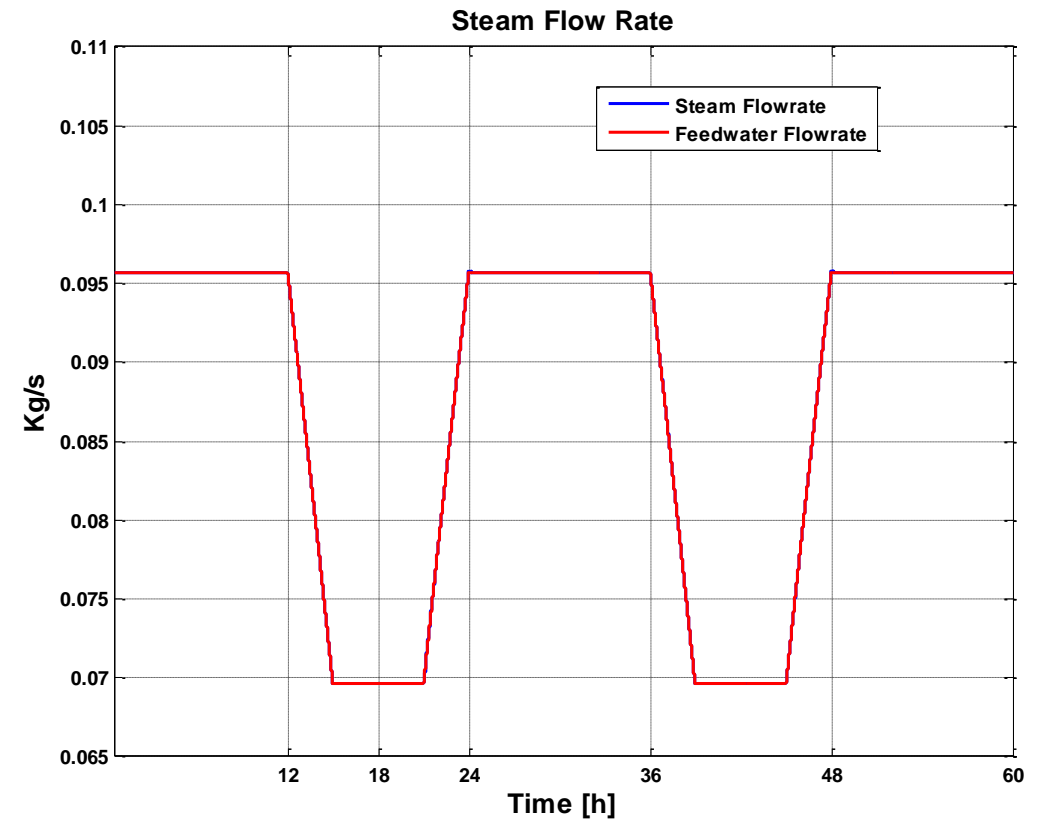

Figure 3.23. Steam flow rate changes for profile \#1 load demand. 


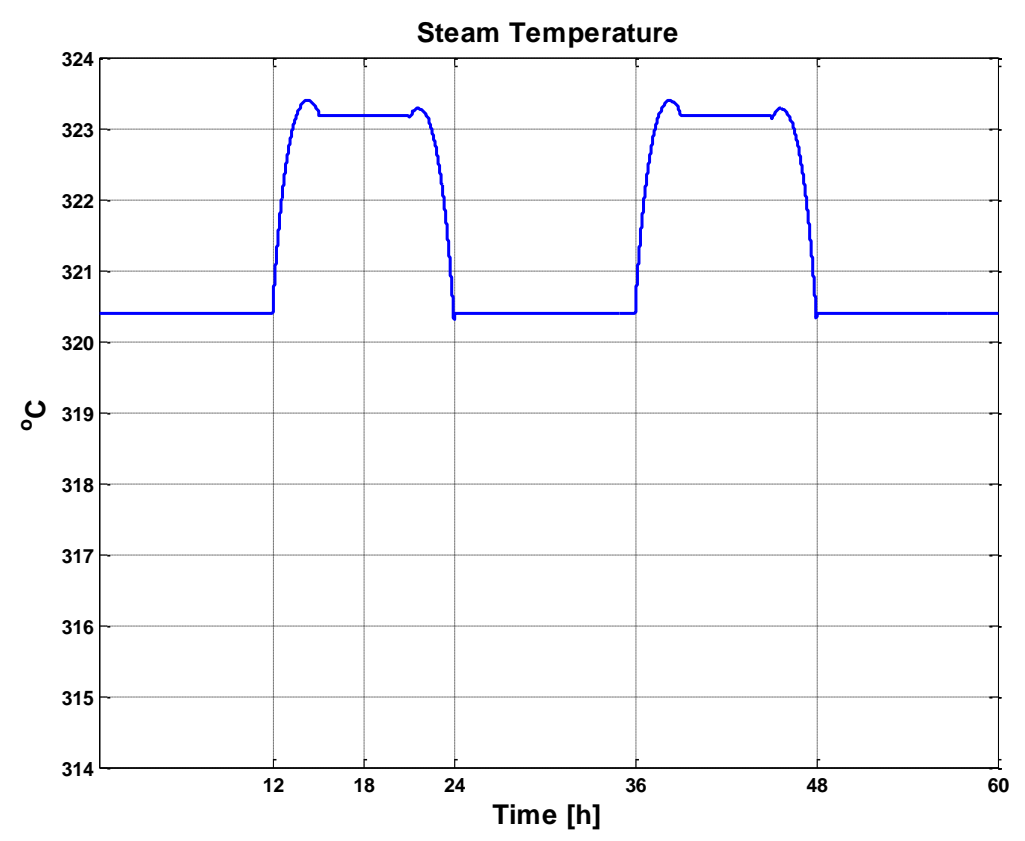

Figure 3.24. Steam temperature changes for profile \#1 load demand.

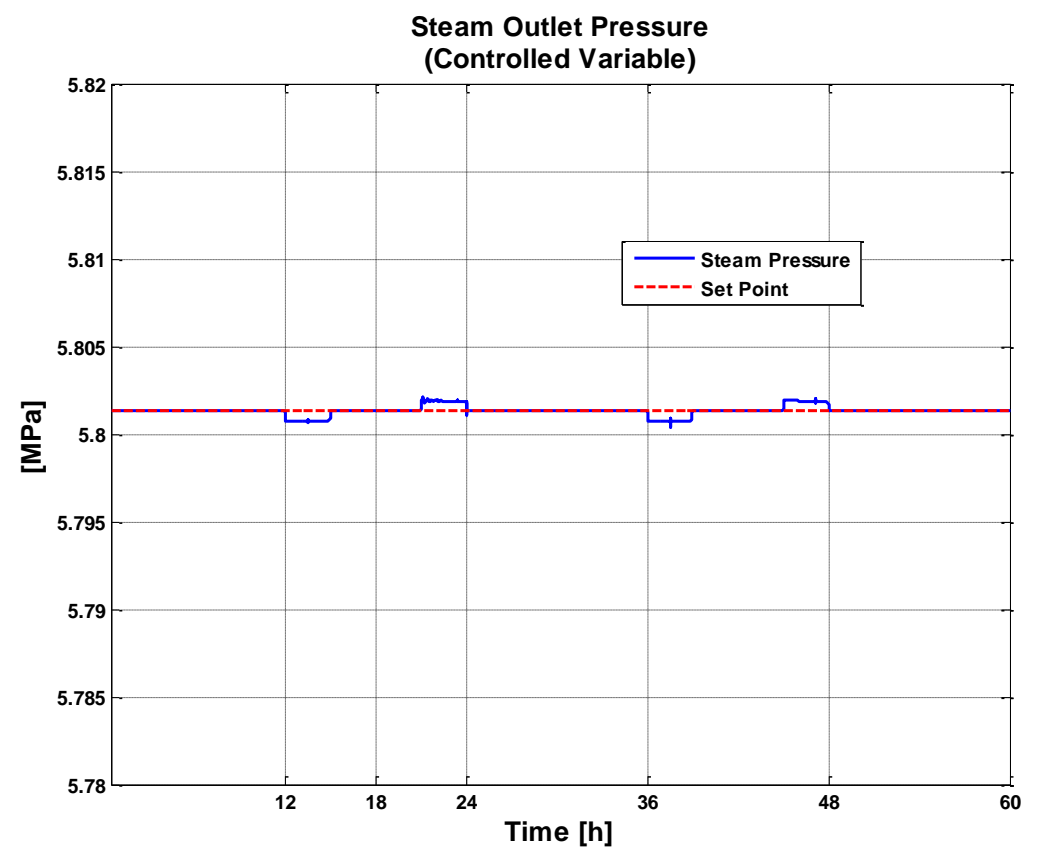

Figure 3.25. Steam outlet pressure is controlled for profile \#1 power load demand. 
As seen from the results using the first profile, the same behavior was observed using the second load following profile, except profile \#2 power change is faster than profile \#1 (Figures 3.26 through 3.29). Again, as power load changes $\mathrm{T}_{\text {ave }}$ controller in the reactor core module is able to keep the values close to the set point by varying $\mathrm{T}_{\text {hot }}$, making the reactor reach the necessary power to match the steam flow rate demanded by the turbine. The spikes in Figures 3.27, 3.28 and 3.29 are localized simulation numerical instabilities. Such instabilities can happen, especially over long simulation times, so it is important to make sure that a stiff variable-step solver is used. There is no exact definition of stiffness for equations. Some numerical methods are unstable when used to solve stiff equations and very small step-sizes are required to obtain a numerically stable solution to a stiff problem. A stiff problem may have a fast changing component and a slow changing component (a large condition number, $\lambda_{\max } / \lambda_{\min }$ ). The results of simulation for the remaining profiles are given in Appendix 4.

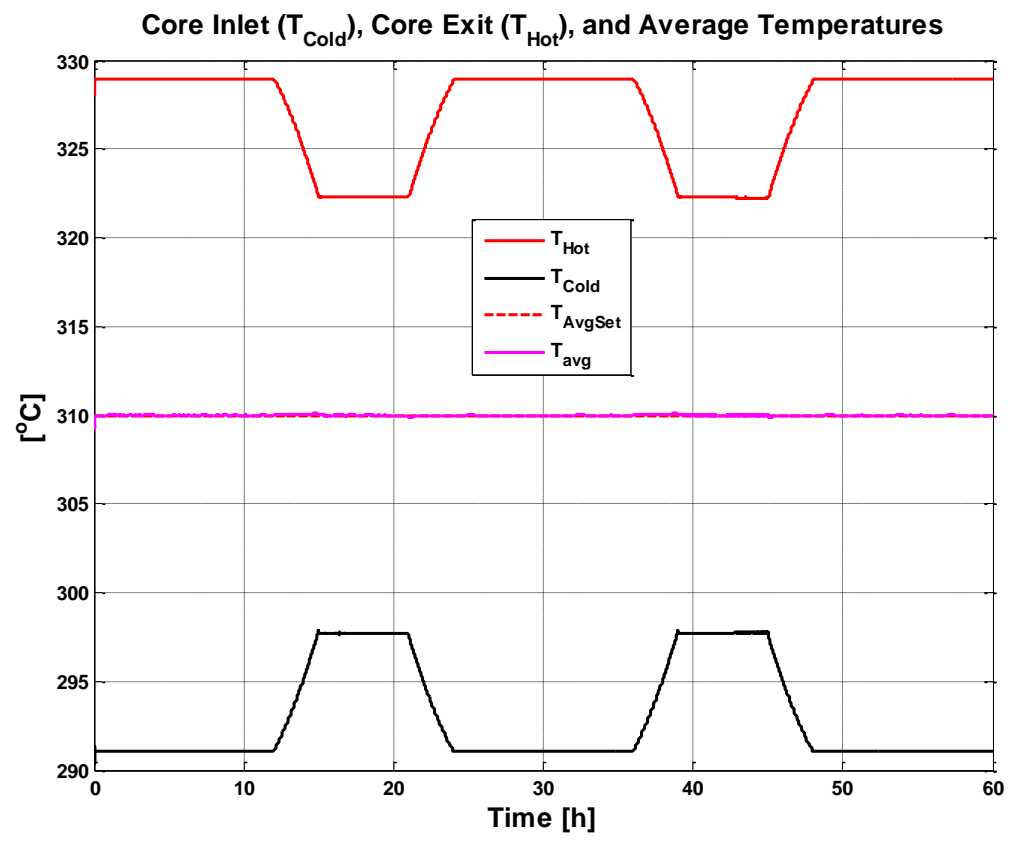

Figure 3.26. $\mathrm{T}_{\mathrm{ave}}$ changes for profile \#2 load demand. 


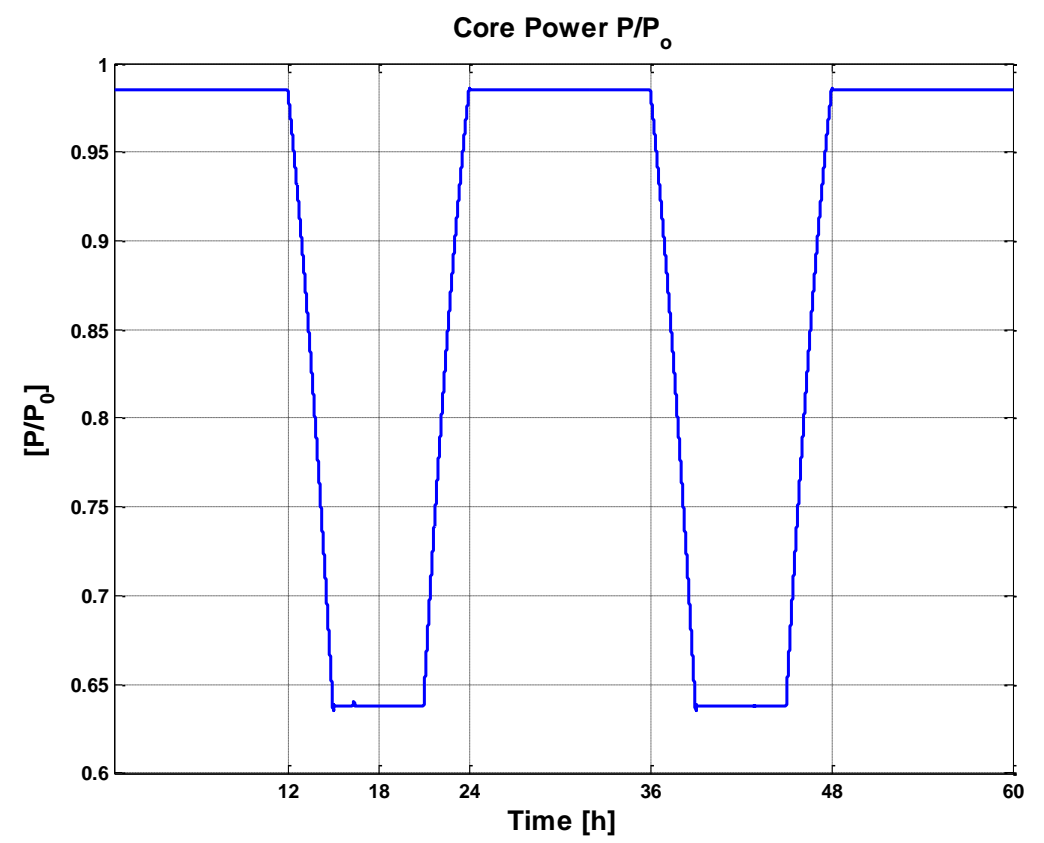

Figure 3.27. Power changes for profile \#2 load demand.

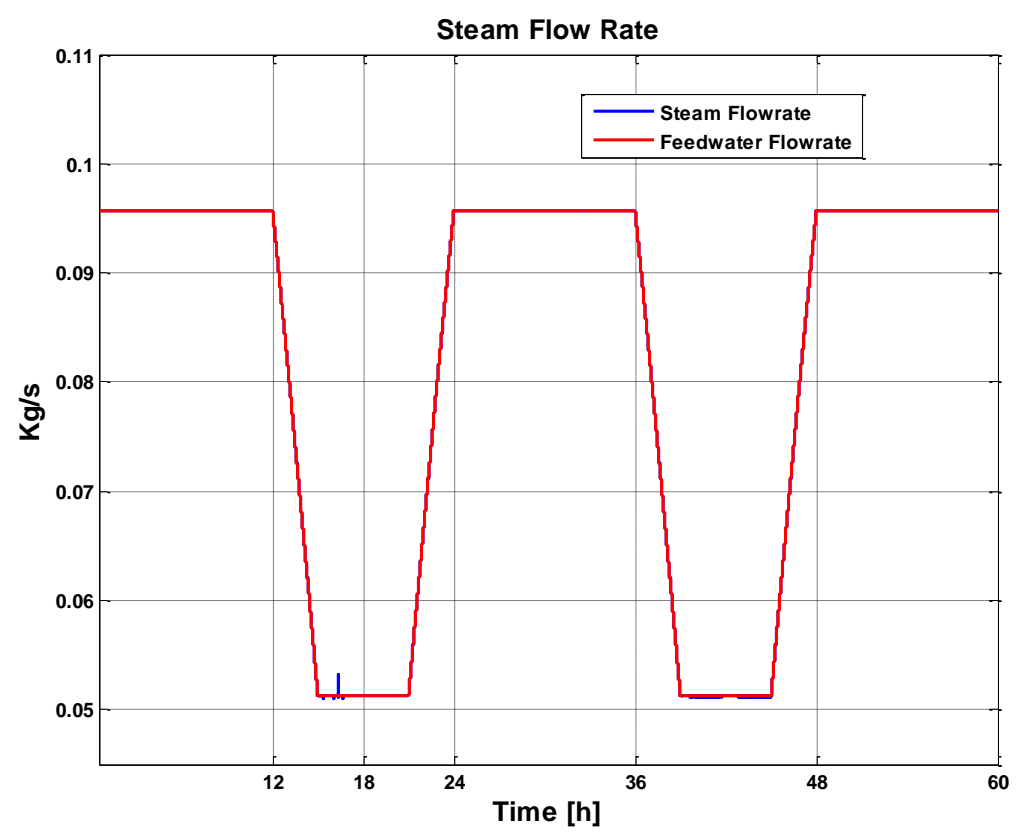

Figure 3.28. Steam flow rate changes for profile \#2 load demand. 


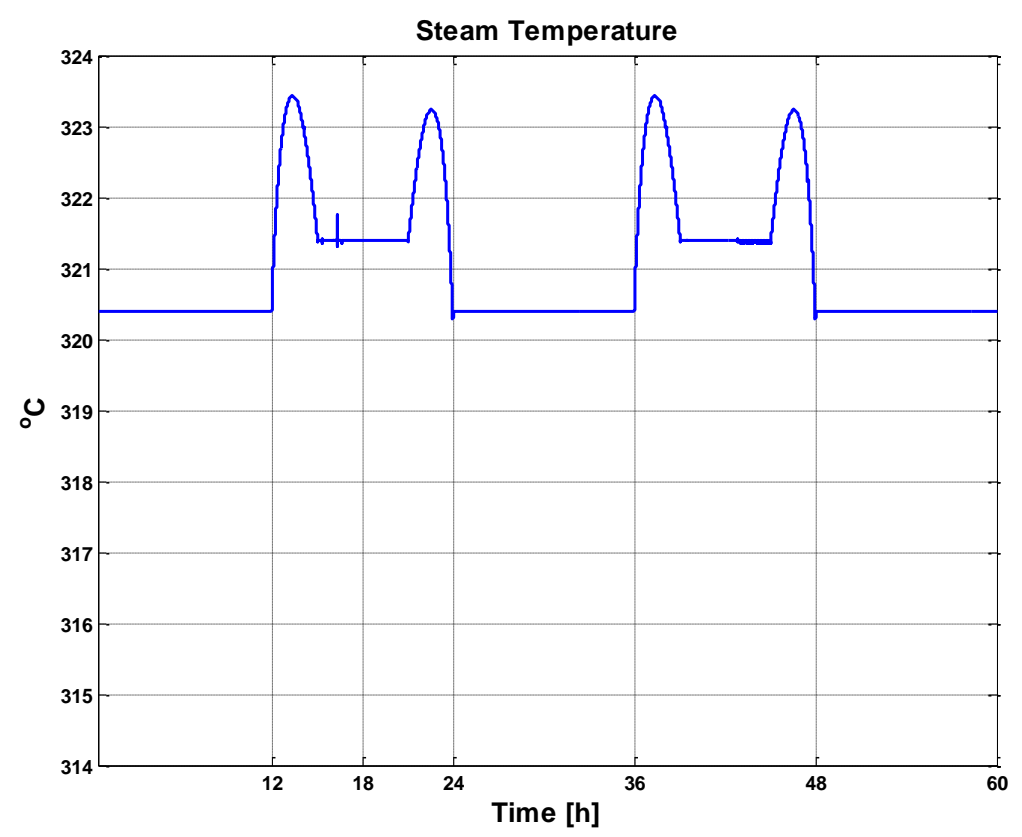

Figure 3.29. Steam temperature changes for profile \#2 load demand.

\subsection{Summary}

The existing IRIS SIMULINK model was studied and tested in this research to be used later on in a steam-mixing scenario (next chapter). The core steady state results are compatible with the literature, and once connected to the helical coil steam generator model, the steady state result values are comparable to those obtained using the more advanced and comprehensive NCSU IRIS FORTRAN code. Both Tave and steam pressure control worked well for different power demand scenarios, with simulations taking around 30 minutes to complete. Special attention was taken using this model to simulate fast changing transients, especially close to inflections points in the profiles, as it became numerically unstable due to the model stiffness and length of simulation. Sometimes, different proportional-integral gains were used in the core model that helped avoid such instabilities, but it involved patience and some guess work but usually, faster changing transients required smaller proportional gains.

Several load demand profiles were investigated, and the simulation results suggest the IRIS design as being suitable for load following applications. But some discretion must be given 
in applying load following profiles, especially profile 7, because it is an unreal situation given no real life power system can be required to go from $100 \%$ power to $50 \%$ instantaneously. Finally, the current SIMULINK model does not include other vital components and systems such as pressurizer, balance-of-plant, secondary feedwater controller. Also, a feed-forward controller to quickly move the control rods based on changes in power load demands, is not included in the model. This makes the response slower than the one from a more complete model. 


\section{Chapter 4}

\section{DEVELOPMENT OF A DESIGN APPROACH FOR A MULTI-MODULAR IRIS PLANT}

\subsection{Introduction}

The use of IRIS as a platform for studying control strategies for multi-modular reactor systems and control issues related to steam mixing with multiple modules that operate in parallel and feed steam to a single turbine was studied and is presented in this chapter. Such a configuration requires mixing steam from two or more modules in a steam header, with steam from all the units maintained at the same condition, with efficient operation of this configuration requiring advanced control strategy. The objective was to evaluate and quantify the performance of a nuclear power plant comprised of two IRIS reactor modules operating simultaneously, with the steam from the two reactors flowing into a common header. This in turn is connected to a single turbine, resulting in a steam-mixing control problem with respect to "load-following" scenarios, such as changing electricity demand. The simulation includes two reactor core models, each with one helical coil steam generator. Each of the units may be operating at different power demands to take full advantage of the multi-modular plant economics.

\subsection{System Description and Model Assumptions}

The multi-modular reactor system used consists of two integral pressurized water reactors of the IRIS design and each unit has a rated power of about 350 MWe that operate in parallel, with the steam from the different units flowing into a common header, as shown in Figure 4.1. 


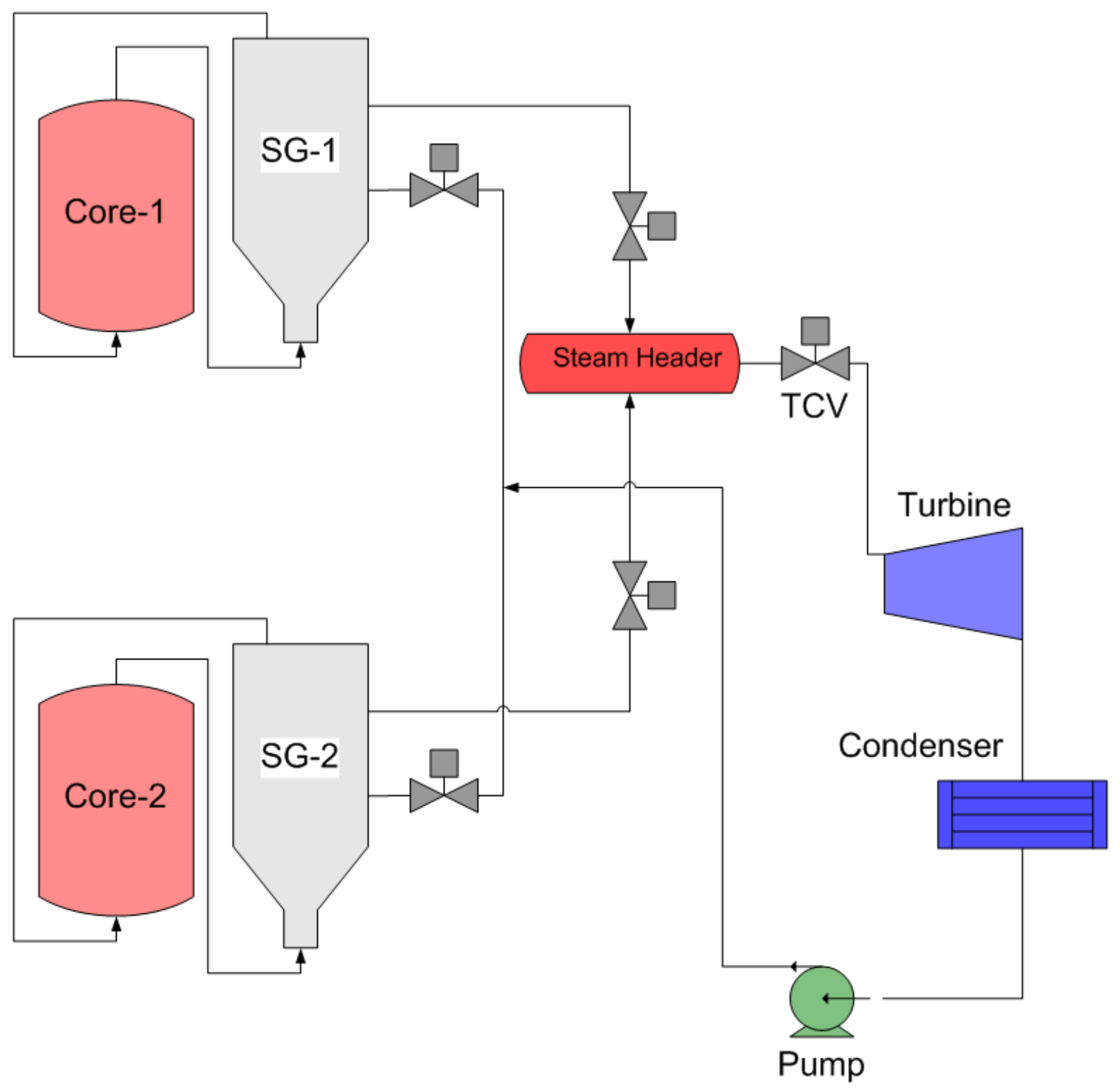

Figure 4.1 Schematic diagram of an IRIS-type multi-modular power block. 
Such power generating stations have the advantages of providing continuous power supply even when one of the units is down for maintenance, and load following features with the modules operating at different power levels. This two-unit model is based on the single model and has the same constants, initial conditions, etc., but there are a few differences between the two versions, most notably a second $\mathrm{T}_{\text {ave }}$ controller and a steam outlet pressure controller set of gains. Steam coming from both units is superheated and any pressure loss between the steam generator exit and the pressure header is neglected. Also, an additional demand input was added to the model to independently set the power in the second unit. But the most important changes are in some of the assumptions (see below), specifically concerning the calculation of the temperature of the mixed steam. Some of the assumptions are the same for the single unit model, and are repeated below for completeness;

- Unlike in PWRs with recirculation-type steam generators, where there is a sliding $\mathrm{T}_{\mathrm{ave}}$ controller, the average core inlet-outlet temperature in IRIS remains constant, with a set point value of $310^{\circ} \mathrm{C}\left(590^{\circ} \mathrm{F}\right)$ over the entire simulation.

- Steam pressure coming out of the HCSGs remains constant at 5.8 MPa ( 841 psi) for the entire range of reactor operation.

- Feedwater temperature is fixed at $223.9^{\circ} \mathrm{C}\left(435.02^{\circ} \mathrm{F}\right)$, corresponding to $100 \%$ power for entire simulations.

- There is no feed-forward controller to quickly move control rods based on changes in power load demands.

- Pressurizer and balance-of-plant models are not included in the simulation. These parameters are assumed to be at fixed values.

- Steam generator feedwater flow rate is set based on FORTRAN code developed by North Carolina State University (NCSU), and is set according to power demand - feed flow program. 
- Steam mixture temperature at the steam header is calculated assuming constant pressure (see bullet above), balance of mass and steam properties, and is calculated as:

$$
\begin{aligned}
& h_{T} \circlearrowleft \frac{h_{1} \bigotimes \dot{x} m_{1}+h_{2} \propto x m_{2}}{m_{T}} \\
& \dot{m}_{T}=\dot{m}_{1}+\dot{m}_{2}
\end{aligned}
$$

Where:

$h_{T}$ is the temperature-dependent total enthalpy.

$h_{1}$ and $h_{2}$ are unit \#1 and unit \#2 temperature-dependent enthalpies, respectively.

$\dot{m}_{T}, \dot{m}_{1}$ and $\dot{m}_{2}$ are total, unit \#1 and unit \#2 mass flow rates, respectively.

The values of $h_{T}$ obtained from the combined steam temperatures are then used to determine the temperature of the mixed steam at the corresponding superheated steam pressure of 5.8 MPa (841 PSI) using a look-up table embedded in the SIMULINK model, assuming steam outlet pressure deviations can be neglected.

In Figure 4.2 a screenshot of the two-unit IRIS reactor plant is shown, with the two reactor cores encircled in red (1), the two helical coil steam generators are encircled in blue (2) and the mixed steam temperature calculation block using the steam flow rates from the two units and the temperatures is encircled in dashed black (3). The outputs of this model are the same as those for a single unit for each unit plus the calculated mixed steam temperature. Because it is a large model to fit in one page, the image shown was zoomed-out, so there is loss of quality and many details are not properly shown. Hence the blocks in the model were rearranged so that partial screenshots showing details could be properly depicted. In Figure 4.2 the power demand to unit 1 is (Demand1) fixed, but the power to unit 2 is allowed to change over time following a power profile which started at specified times with controlled increasing and decreasing slope 
rate values. The saturation blocks limited the input signal to the upper and lower saturation values, preventing the power demand values from going beyond specified limits. As the demand power changed, so did the feedwater flow rate, calculated in the look-up table block. Figure 4.3 shows details of the mixed steam calculation blocks with the values sent to the workspace in MATLAB for analysis and plotting. Finally, Figure 4.5 shows a detailed representation of one of the helical coil steam generators with the inputs and outputs converging to the patch panel. The control schematic of a twin-unit IRIS system, with HCSGs connected by the feedwater line, is shown in Figure 4.6 


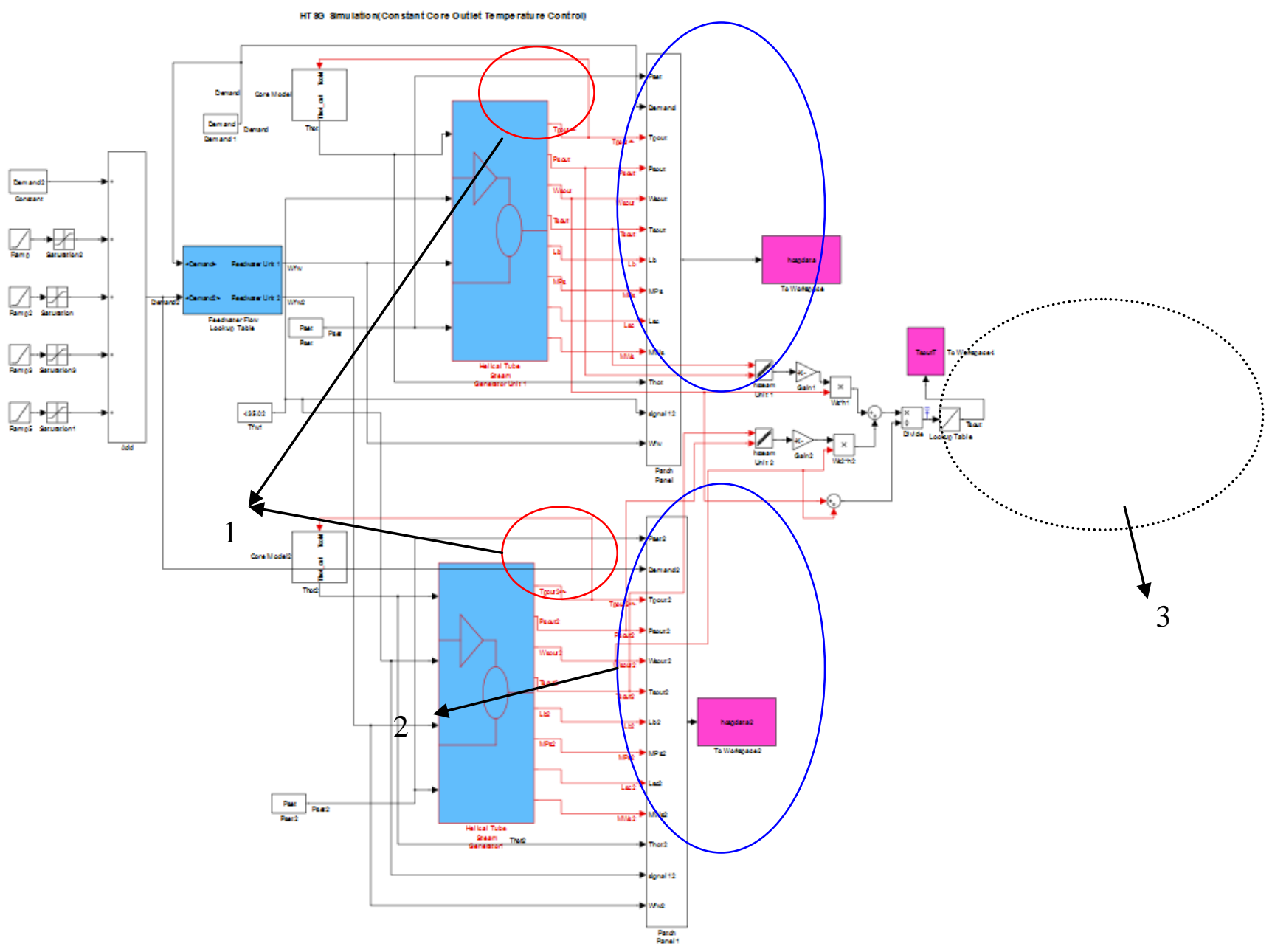

Figure 4.2 Two-unit IRIS plant SIMULINK model. 


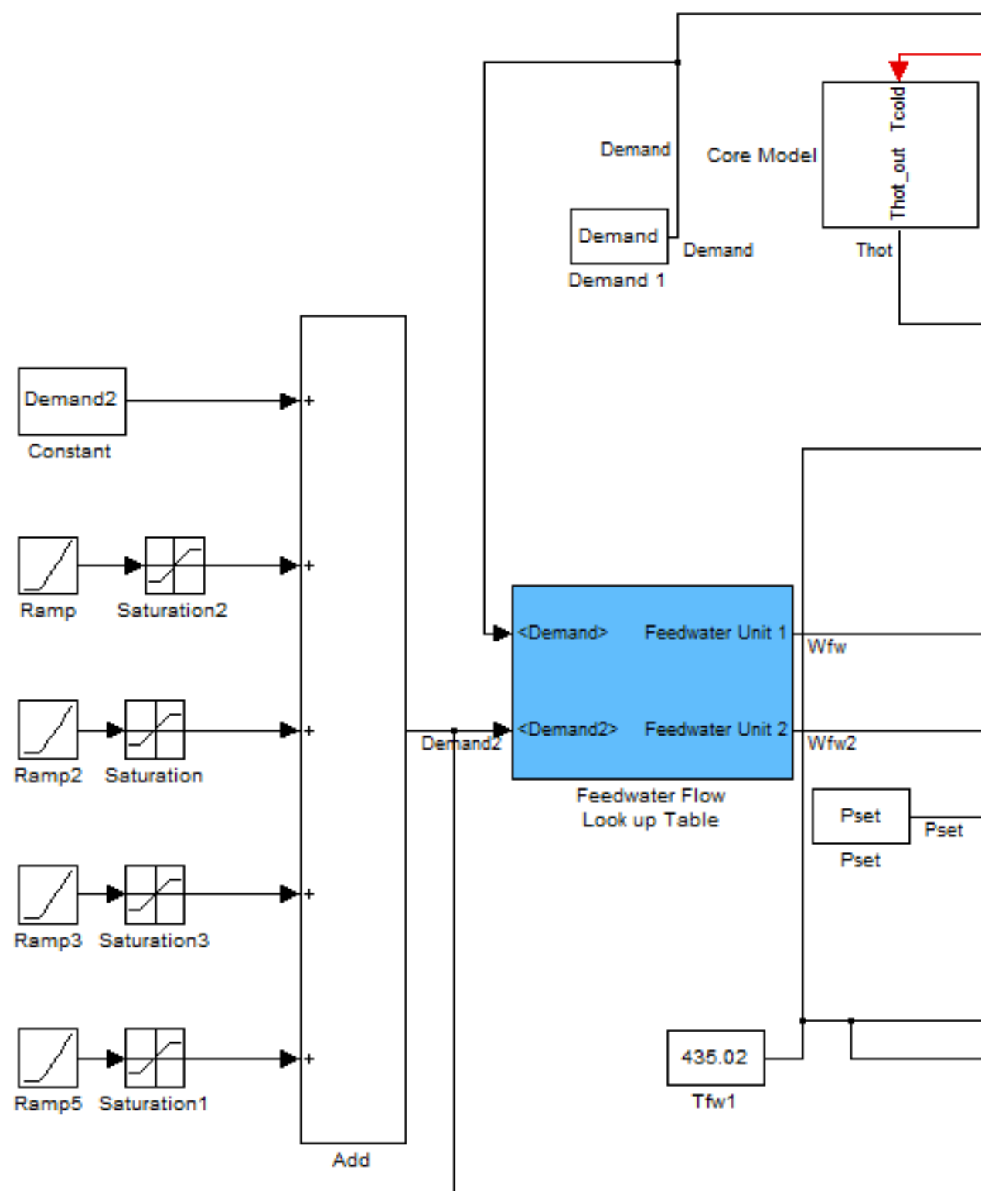

Figure 4.3. Two-unit IRIS showing power demands, feedwater flow, and temperature blocks. 


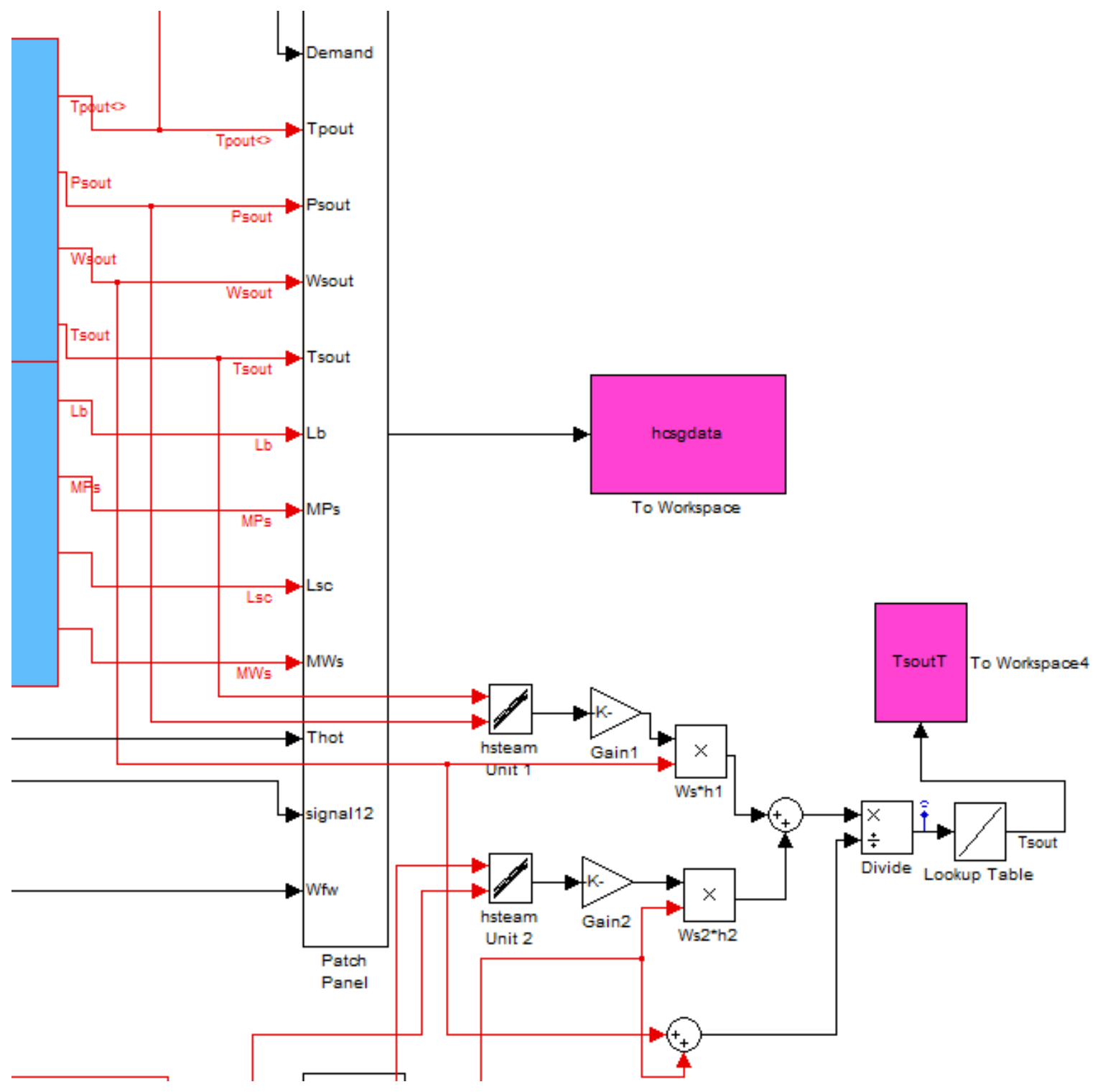

Figure 4.4. Details of the mixed steam temperature calculations. 


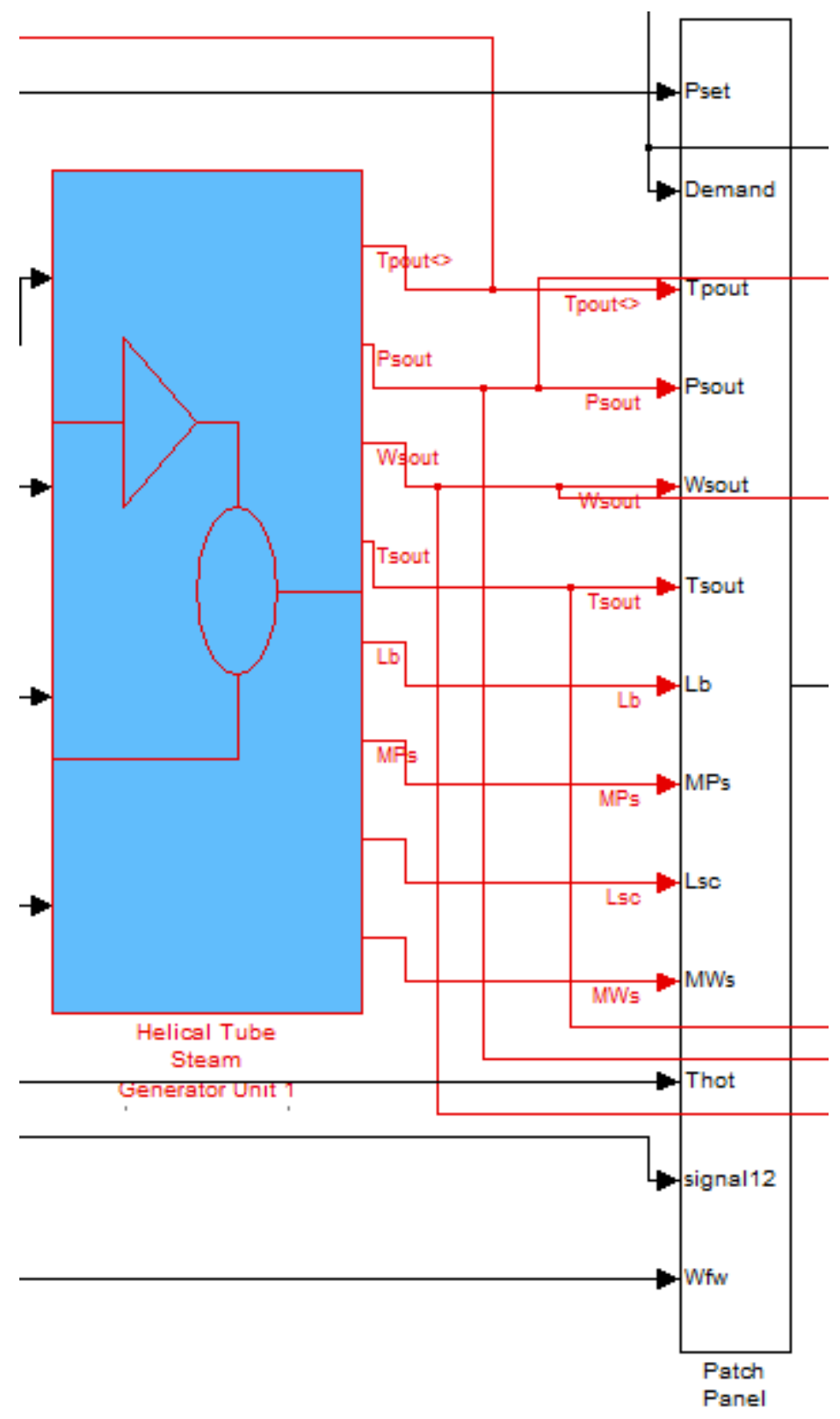

Figure 4.5. Details of the Helical coil steam generator model block. 


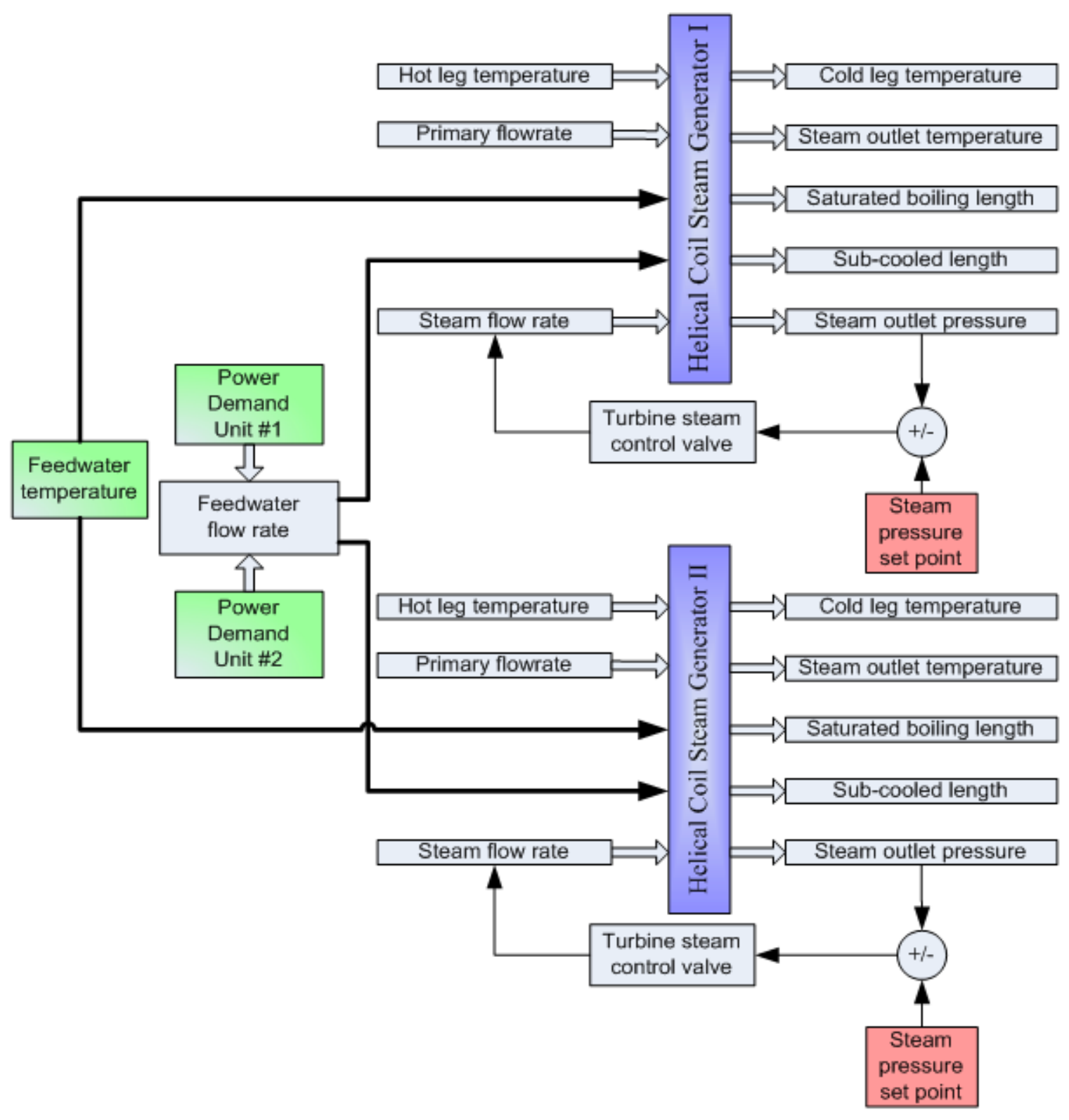

Figure 4.6. Schematic of a Twin-HCSG control system connected at the feedwater flow rate line. 


\subsection{Results of IRIS Dual-Module Used in Load Following Maneuvers}

As stated earlier, load following is the capability of a reactor to follow changes in the grid demand; for example, reduced consumption over the weekend or load changes during the day. Hence, it is desirable from an economical point of view that a multi-modular reactor plant also be able to do just that, although there are currently no regulations in this regard. For this purpose, the two-unit model with steam mixing is subjected to transients similar to profile 1, depicted in Figure 3.19. However, only the first 36 out of the 60 hours of such profile were simulated for three main reasons: running the whole profile is not necessary (and avoid repetition), it is time consuming, and because of numerical stability issues.

The results with the two-unit model using profile 1 (shown in Figure 4.7) load demand as input to the second unit and corresponding core power (Figure 4.8), while maintaining first unit power constant at $100 \%$ are shown in Figures 4.9 to 4.13 . Having both feedwater lines connected, the feedwater flow rates to both units are shown in Figure 4.9. In the figure, unit \#1 flow rate is fixed corresponding to $100 \%$ power, but unit \#2 varied with the power demand, and eventually matched the steam flow rate, shown in Figure 4.10. Figures 4.11 and 4.12 show the $\mathrm{T}_{\mathrm{ave}}$ value changes for units \#1 and \#2 around the set point. In the first unit it started at $309.5^{\circ} \mathrm{C}$ and reached a steady state value $\left(310^{\circ} \mathrm{C}\right)$, while it varied by about $1.5^{\circ} \mathrm{C}$ in unit $\# 2$ because it followed the load demand as the controller varied the external reactivity. Hence, the core inlet and outlet temperatures adjusted to maintain a constant average coolant temperature around the set point. The changes in the steam temperature at each of the reactor modules and the temperature of the mixed steam in the common header are shown in Figure 4.13. At first, both steam temperatures are the same, and remained constant in the first unit while it varied in time in the second unit because of the changes in the power demand. As the power decreased, the area available for heat transfer in the steam generator increased, therefore increasing steam temperature, conversely decreasing following power increase. The control strategy of regulating the average reactor temperature and the steam pressure is robust for this load following operation. The controllers are capable of maintaining both average moderator temperature and steam outlet pressure around their set points in both units. 


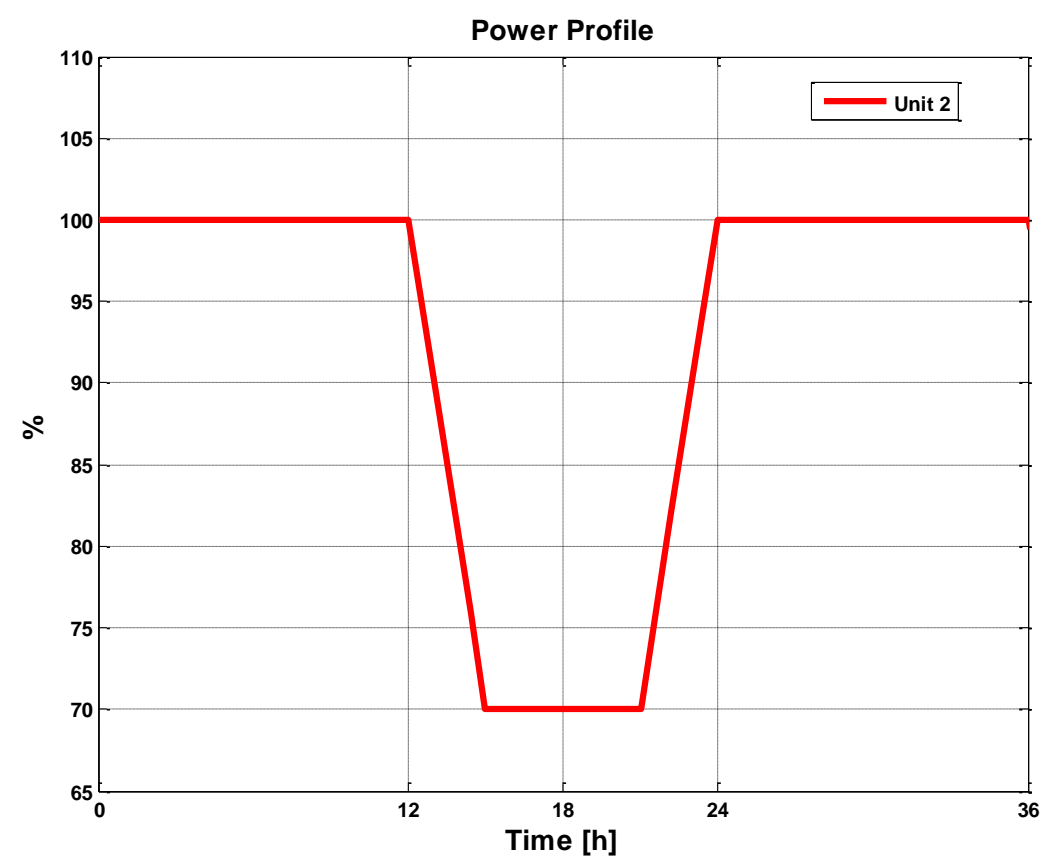

Figure 4.7. Unit \#2 power profile in percent full power.

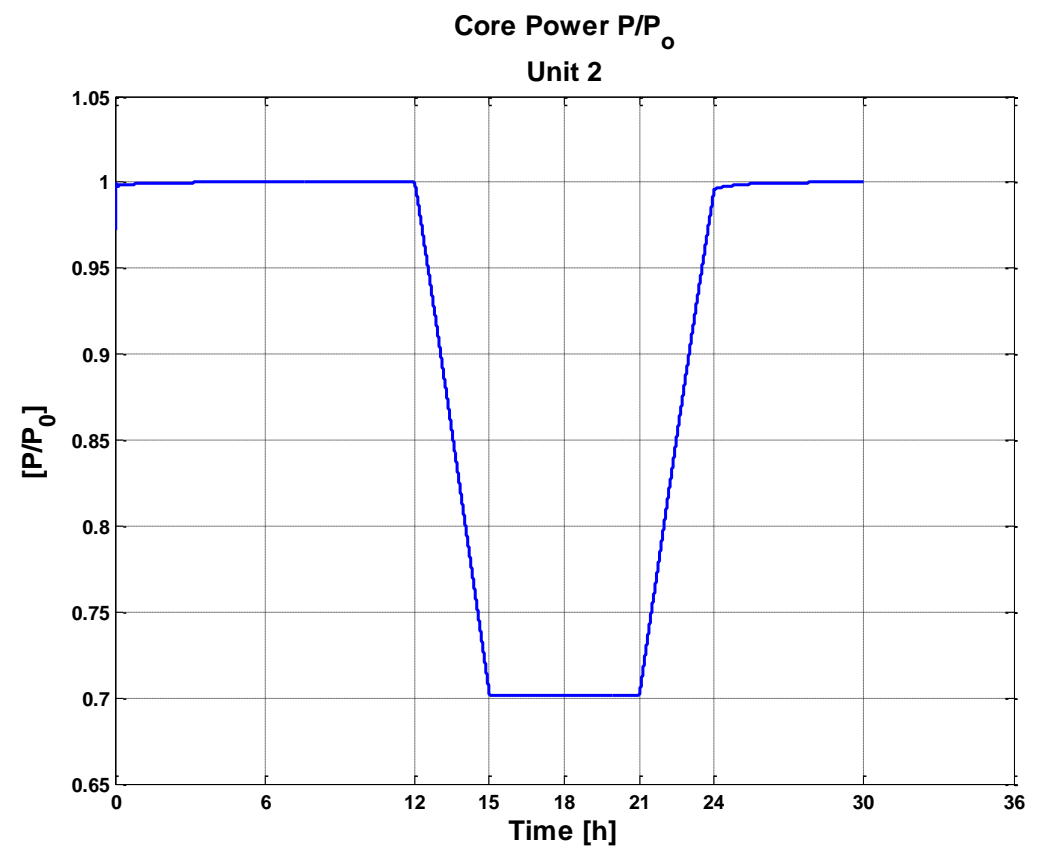

Figure 4.8. Unit \#2 core power profile. 


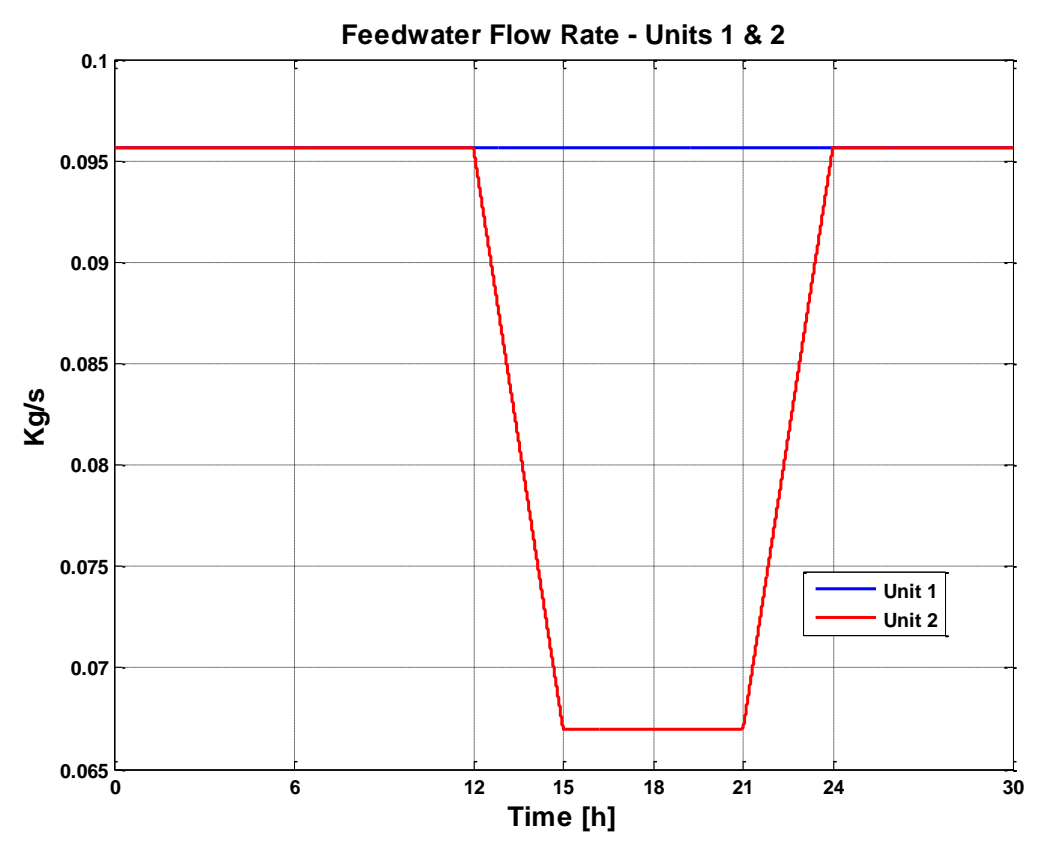

Figure 4.9. Feedwater flow rates for units \#1 and \#2.

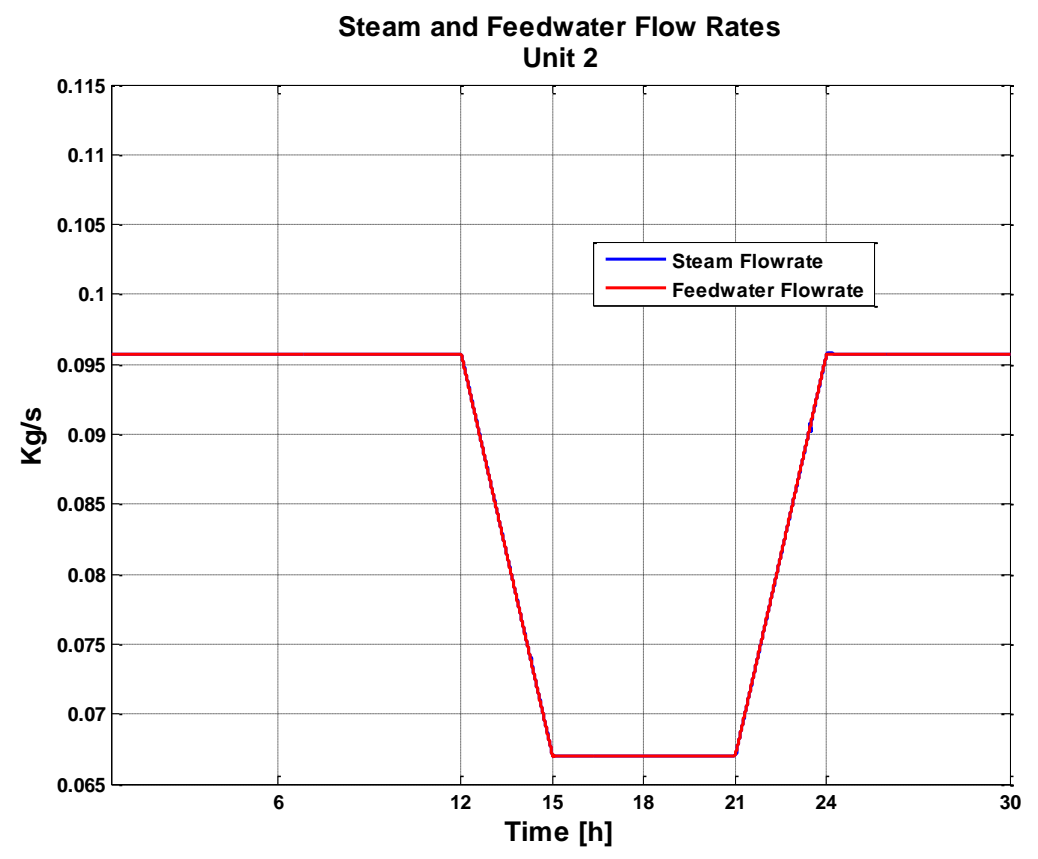

Figure 4.10. Unit \#2 steam flow rate. 


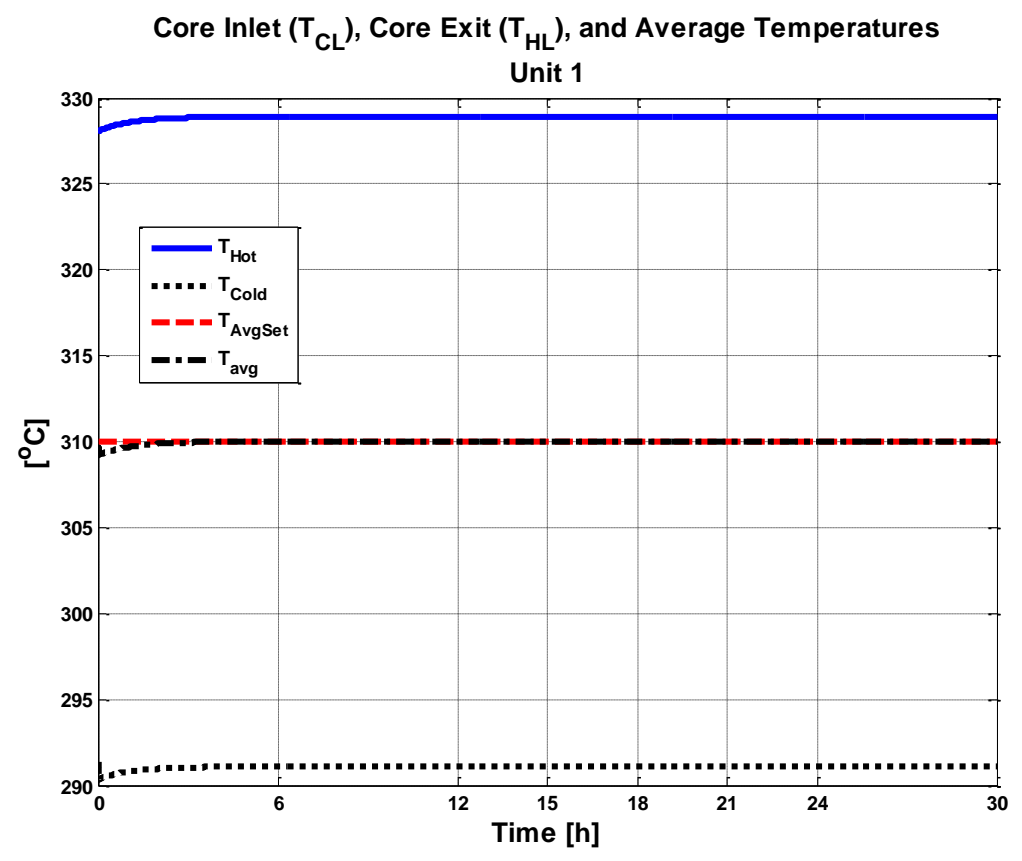

Figure 4.11. Unit \#1 Average moderator temperature.

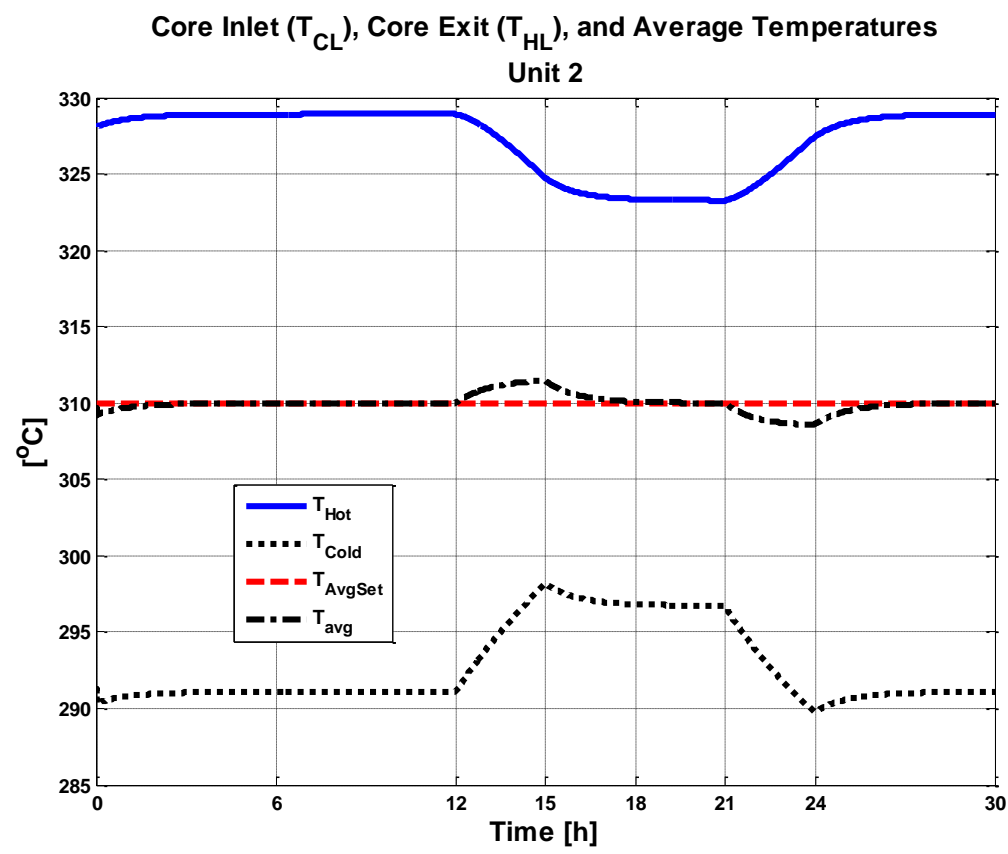

Figure 4.12. Unit \#2 average moderator temperature. 


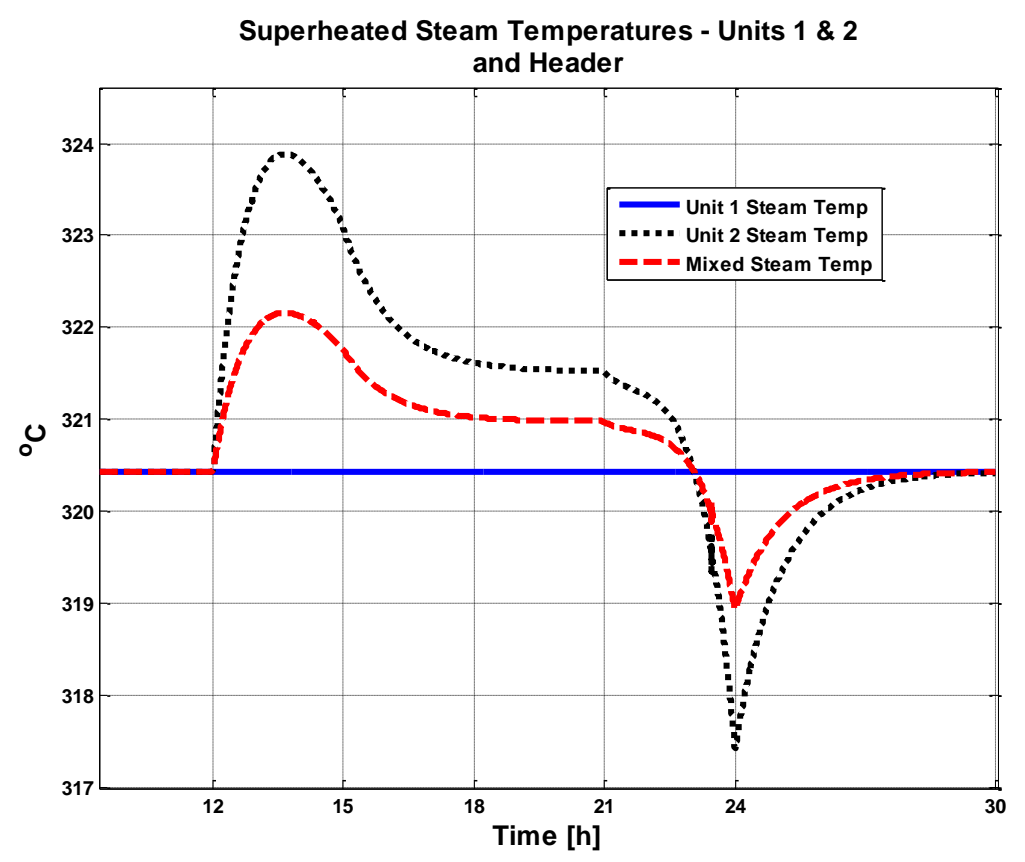

Figure 4.13. Units \#1 \& \#2 and mixed steam header temperatures changes.

Another test was carried out to investigate the behavior of the model when both units are operating at different power levels during transients with both feedwater lines connected, using load demand profile 1 in unit \#2, but an inverted version of it was used in unit \#1, so that while the power demand decreased in one unit it increased in the other at the same time. This profile version is described as 12-hours at 70\%, 3-hour transient from 70\%-100\%, 6 hours at 100\%, 3hour transient down to $70 \%$, and another 12 hours at $70 \%$, and the resulting reactor core power profiles from both units are shown in Figures 4.14 and 4.15. The moderator average temperatures in both units moved in different directions as the power increased in one unit and it decreased in the other at the same time, as well as the steam temperatures coming out of the steam generators. These results show that both units and their controllers are able to operate at different power demands; the results are shown in Figures 4.16, 4.17 and 4.18, respectively. 


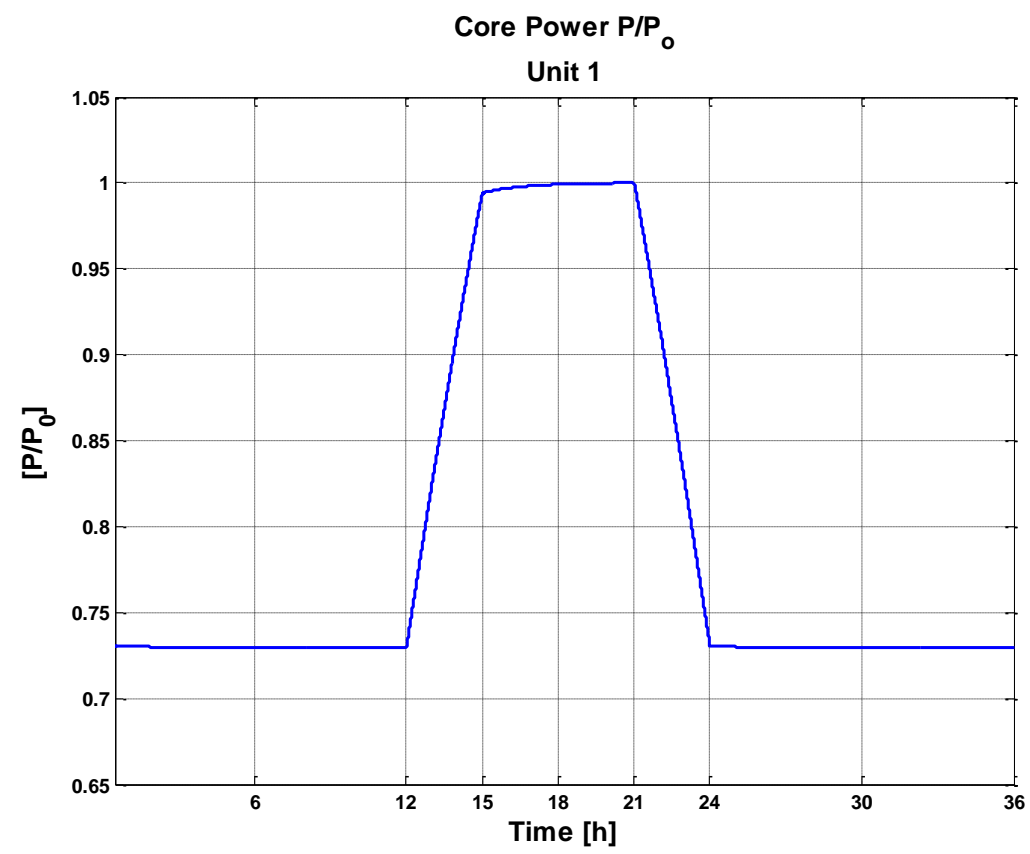

Figure 4.14. Unit \#1 core power with inverted profile \#1 load demand.

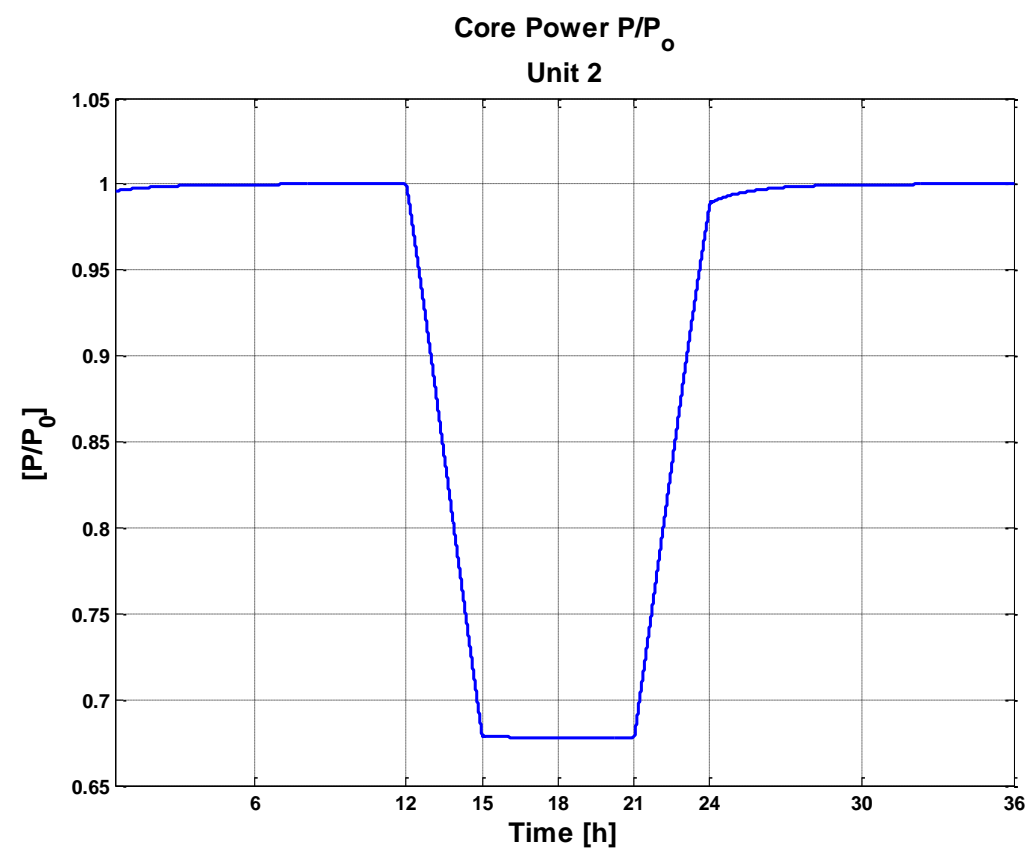

Figure 4.15. Unit \#2 core power with inverted profile \#1 load demand in unit \#1. 


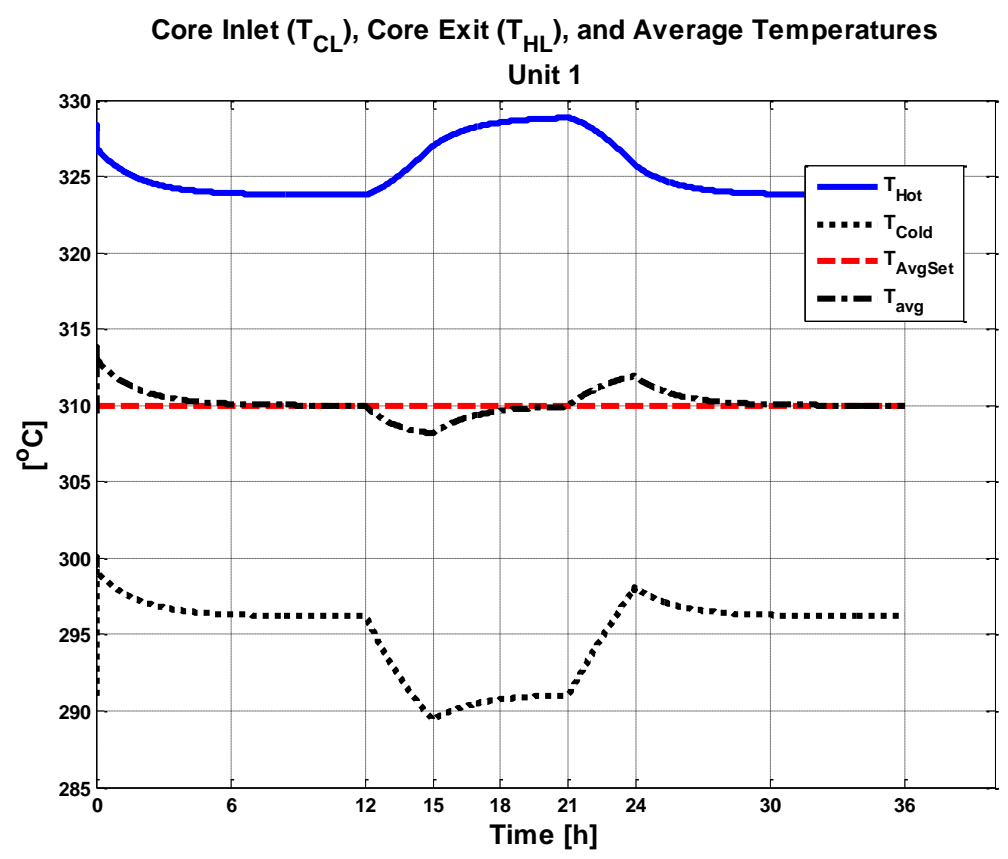

Figure 4.16. Unit \#1 $\mathrm{T}_{\text {ave }}$ with inverted profile \#1 load demand.

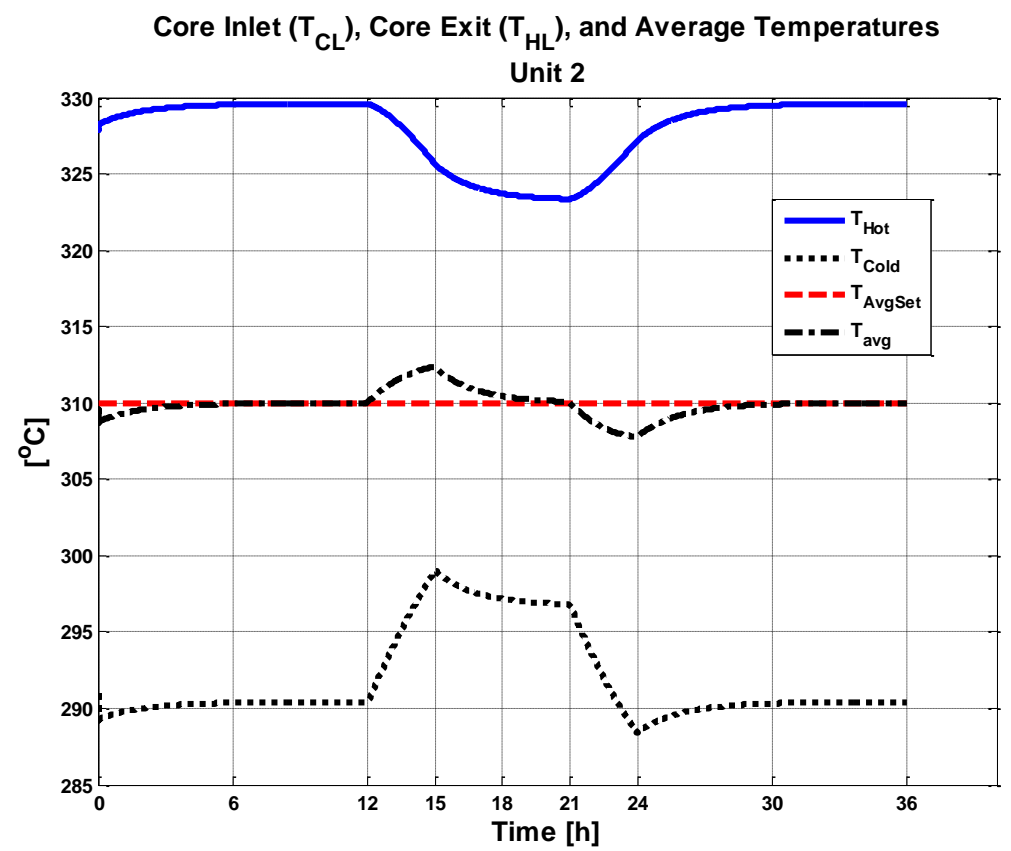

Figure 4.17. Unit \#2 $\mathrm{T}_{\text {ave }}$ with inverted profile \#1 load demand in unit \#1. 


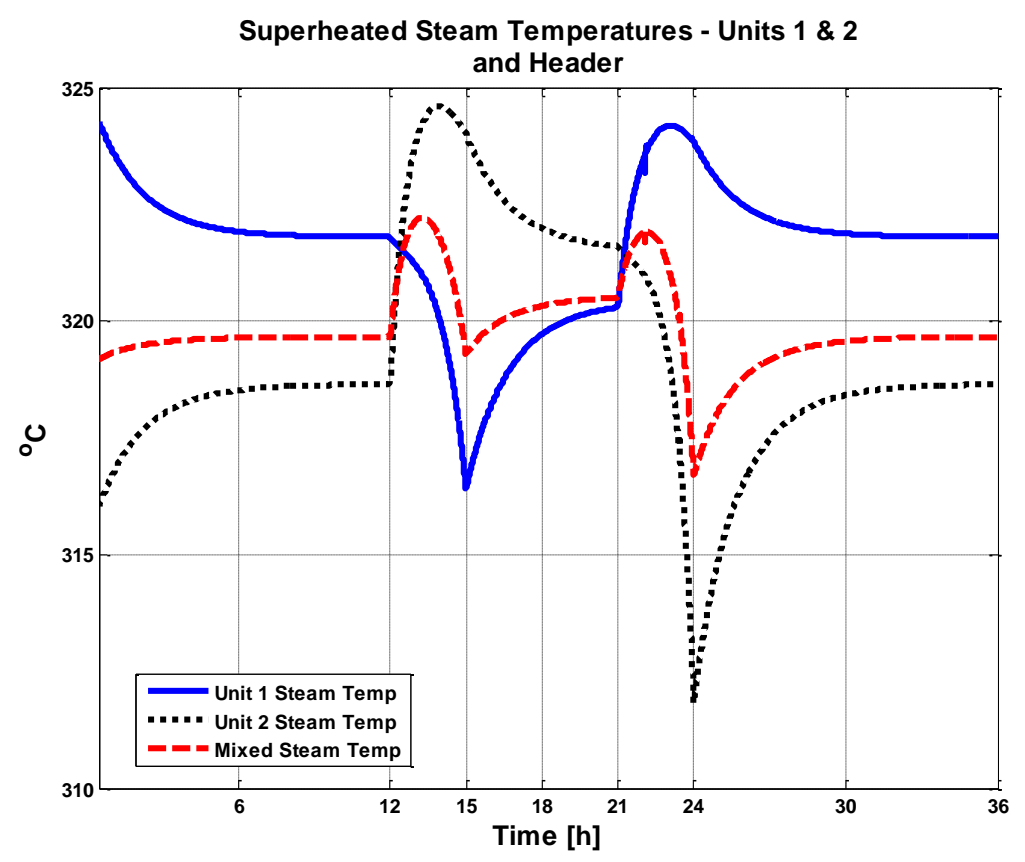

Figure 4.18. Units \#1 \& \#2 and combined steam header temperatures changes using inverted profile 1 in the first unit.

\subsection{Model Simulation Robustness - Results}

Eight different perturbation cases were investigated to analyze the model capability of detecting small perturbations, therefore testing its robustness and sensitivity, and are listed and described below. Cases 1, 2, and 3 involved sensor perturbations, in which the source of perturbation was not in the process itself but in the sensor measurements, but was propagated throughout the system. On the other hand, cases 4, 5, 6, and 7 involved process perturbations due to hypothetical equipment malfunctions, and such perturbations propagated throughout the process.

Case 1:

- $2.8^{\circ} \mathrm{C}\left(5^{\circ} \mathrm{F}\right)$ step perturbation in $\mathrm{T}_{\text {cold }}$ temperature measurements in unit 1 at $\mathrm{t}=6 \mathrm{~h}$, with both units operating steady at $100 \%$ power.

Case 2: 
- $1.7^{\circ} \mathrm{C}\left(3^{\circ} \mathrm{F}\right)$ step perturbation in $\mathrm{T}_{\text {cold }}$ temperature measurements in unit 1 at $\mathrm{t}=6 \mathrm{~h}$, with both units operating steady at $100 \%$ power.

Case 3:

- $2.8^{\circ} \mathrm{C}\left(5^{\circ} \mathrm{F}\right)$ step perturbation in $\mathrm{T}_{\mathrm{hot}}$ temperature measurements in unit 1 at $\mathrm{t}=6 \mathrm{~h}$, with both units operating steady at $100 \%$ power.

Case 4:

- $1 \%$ Full Scale (FS) random perturbation in feedwater flow rate at $\mathrm{t}=6 \mathrm{~h}$ for 5 minutes in both units. Unit 1 operating at 90\% and unit 2 at $95 \%$.

Case 5:

- $1 \%$ FS perturbation in feedwater flow rate in unit 1 at $\mathrm{t}=6 \mathrm{~h}$. Unit 1 operating at $95 \%$ and unit 2 at $100 \%$.

Case 6:

- Negative $1.7^{\circ} \mathrm{C}\left(3^{\circ} \mathrm{F}\right)$ perturbation in feedwater temperature in unit 2 only, at $\mathrm{t}=6 \mathrm{~h}$. Both units operating at $100 \%$ power.

Case 7:

- Negative $1.7^{\circ} \mathrm{C}\left(3^{\circ} \mathrm{F}\right)$ perturbation in feedwater temperature in both units at $\mathrm{t}=6 \mathrm{~h}$. Both units operating at $100 \%$ power.

The first two cases involved a positive step change in the reactor core moderator inlet temperature sensor readings (faulty measurements), which caused the $T_{\text {ave }}$ controller to react by decreasing the reactivity in the reactor, therefore decreasing the reactor core power. In the case involving the $2.8^{\circ} \mathrm{C}\left(5^{\circ} \mathrm{F}\right)$ perturbation, the core power decreased by $0.6 \%$ (Figure 4.19), and in the $1.7^{\circ} \mathrm{C}$ case only about $0.3 \%$ (Figure 4.20 ). 
In the cases involving faulty measurements, the average moderator temperature increased only $1.5^{\circ} \mathrm{C}$ in the first (Figure 4.21 ) case, but the controller brought it back to the set point; as expected, a similar response was seen with the $0.8^{\circ} \mathrm{C}$ jump in the second case (Figure 4.22). It is important to note the transients shown are from the system itself, not from the faulty sensors.

The temperature of steam coming out of unit 1 steam generator was also affected by the perturbation in moderator inlet temperature measurements, as expected, decreasing by $2.9^{\circ} \mathrm{C}$ in the first case and by $1.7^{\circ} \mathrm{C}$ in the second case (Figures 4.23 and 4.24 , respectively). No significant changes were observed in unit 2 dynamics.

The third case, $2.8^{\circ} \mathrm{C}$ step perturbation in $\mathrm{T}_{\text {hot }}$ temperature measurements in unit 1 produced exactly the same results as the first case, that is, a $2.8^{\circ} \mathrm{C}$ increase in $\mathrm{T}_{\text {cold }}$, since both cases produced the very same effect on the measurement error perceived by the controller.

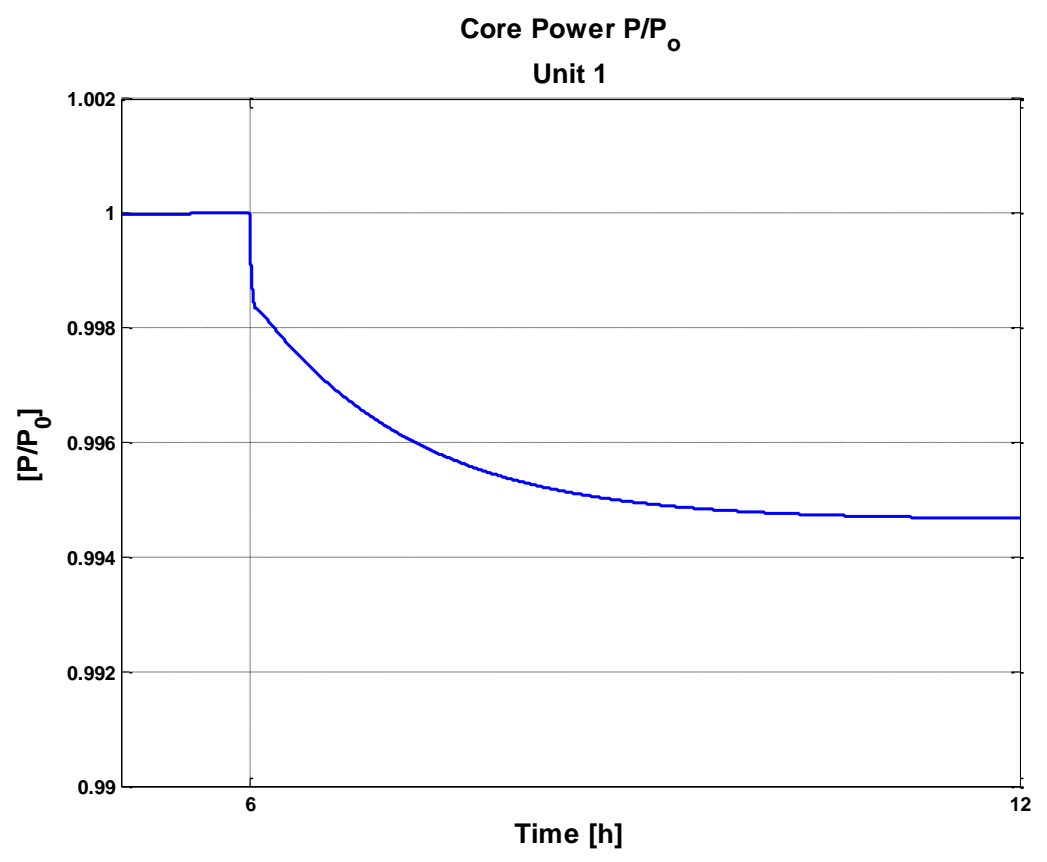

Figure 4.19. Unit 1 core power profile for a $2.8^{\circ} \mathrm{C} \mathrm{T}_{\text {cold }}$ sensor fault. 


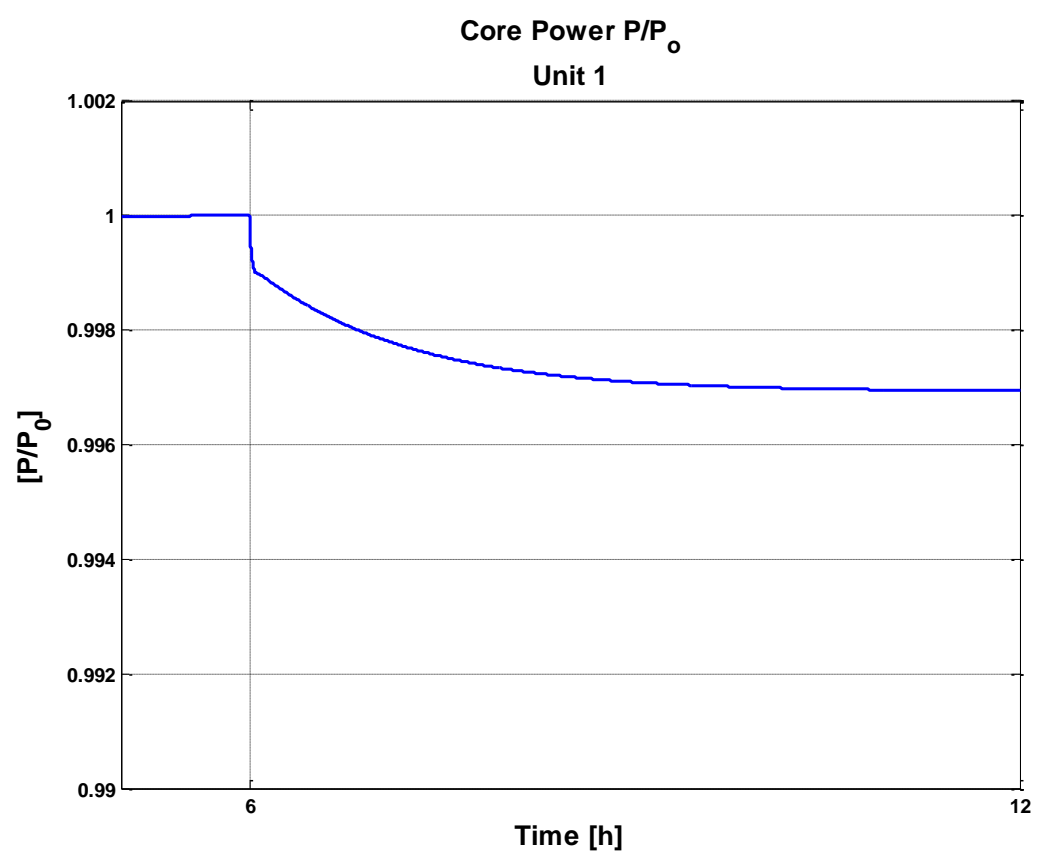

Figure 4.20. Unit 1 core power profile for a $1.7^{\circ} \mathrm{C} \mathrm{T}_{\text {cold }}$ sensor fault.

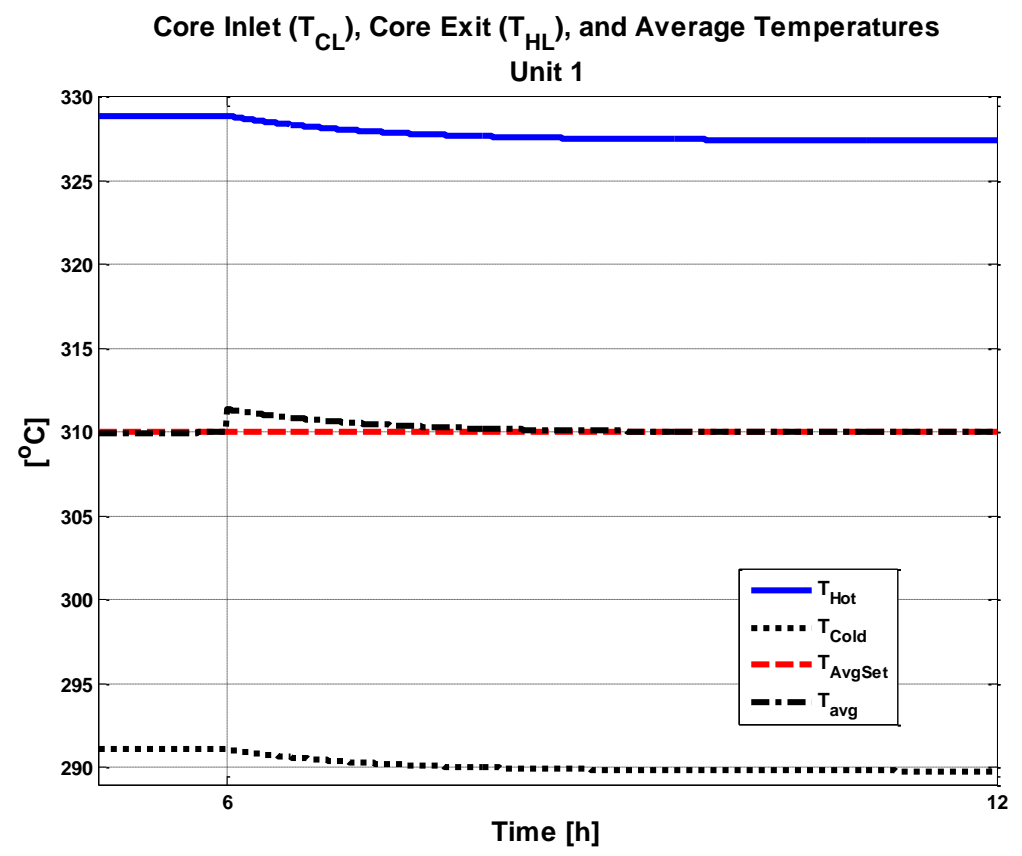

Figure 4.21. $\mathrm{T}_{\mathrm{ave}}$ change for a $2.8^{\circ} \mathrm{C} \mathrm{T}_{\text {cold }}$ sensor fault. 


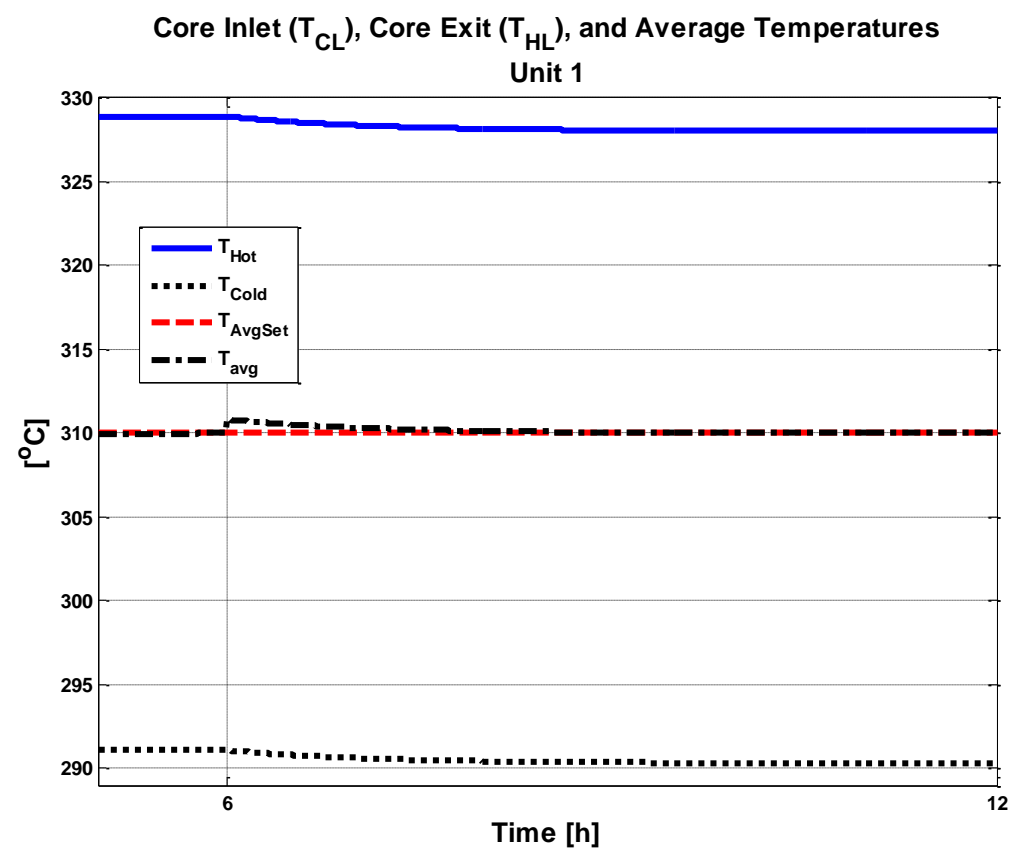

Figure 4.22. $\mathrm{T}_{\mathrm{ave}}$ change for a $1.7^{\circ} \mathrm{C} \mathrm{T}_{\text {cold }}$ sensor fault.

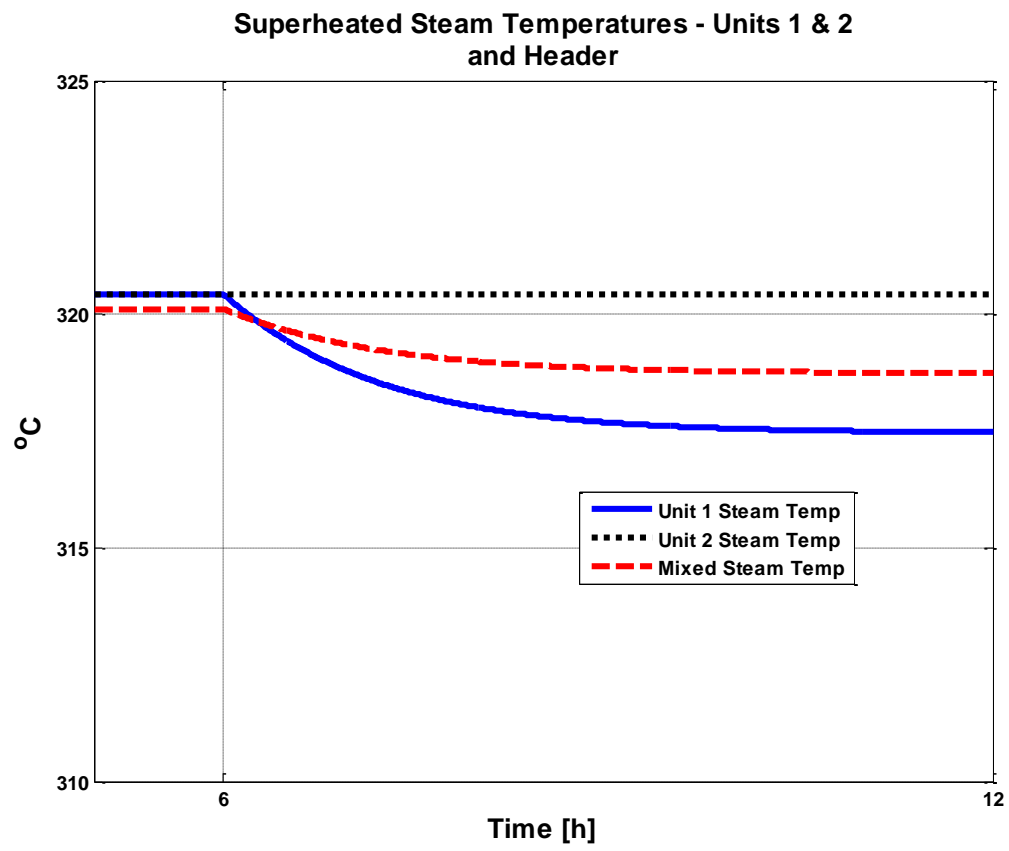

Figure 4.23. Steam header temperature change for a $2.8^{\circ} \mathrm{C}$ sensor fault in $\mathrm{T}_{\text {cold }}$. 


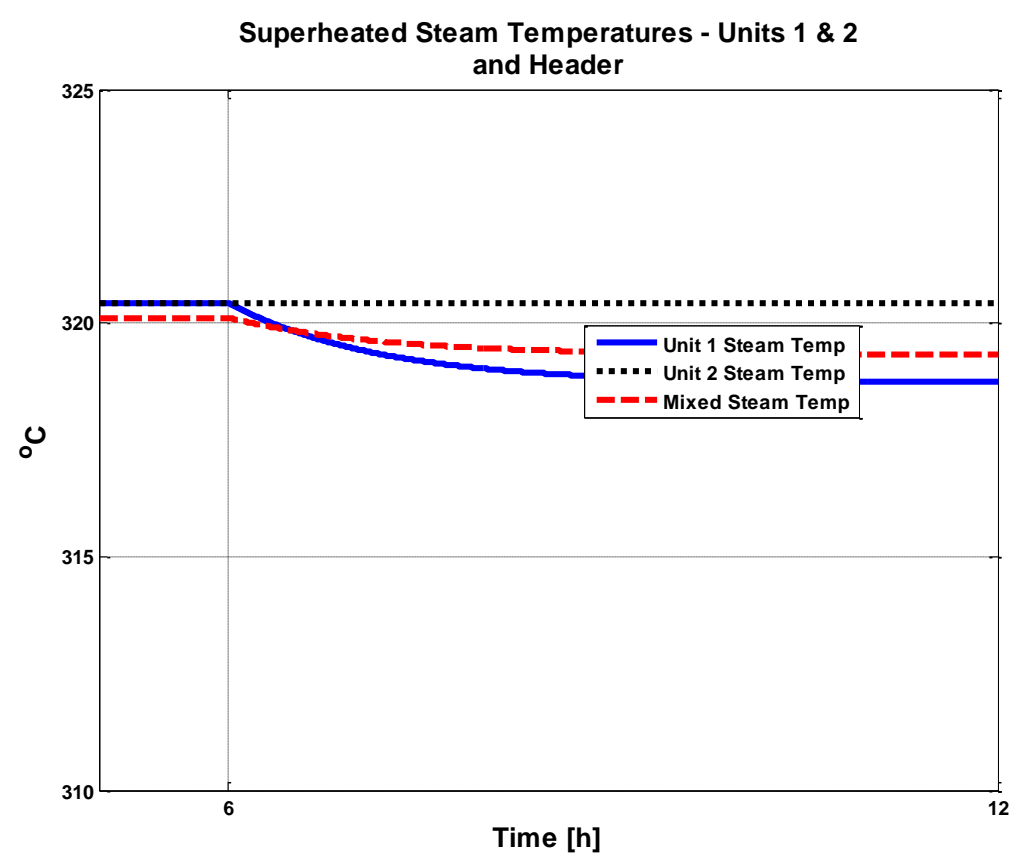

Figure 4.24. Steam header temperature change for a $1.7^{\circ} \mathrm{C}$ sensor fault in $\mathrm{T}_{\text {cold. }}$.

Steam generator feedwater flow rate is set based on a look-up table which relates power and feedwater values. Such table is based on a FORTRAN code developed by North Carolina State University (NCSU), therefore there is no feedwater control in the model used in this research. Multi-modular plants are expected to share components and systems as much as possible to take advantage of their smallness and design features, so a single feedwater line for all modules is not a far fetched assumption, although it is expected each module will have its own feedwater controller. In case 4 a hypothetical $1 \% \mathrm{FS}\left(9.525 \times 10^{-4} \mathrm{Kg} / \mathrm{s}\right.$ amplitude at $100 \%$ power) random temporary perturbation is added to the common feedwater line for five minutes. This signal was generated using SIMULINK block named "Gaussian Noise Generator" with zero mean and enough variance to encompass the $9.525 \times 10^{-4} \mathrm{Kg} / \mathrm{s}$ amplitude and was added to the signal at $\mathrm{t}=6 \mathrm{~h}$. The resulting feedwater input signal is shown in Figure 4.25, Figure 4.26 shows the steam flow rate in unit 2 being manipulated by the controller to keep the steam outlet pressure close to the set point, and how it damps out most of the variation produced by the feedwater flow rate. Changes in the superheated steam in both units were less than $0.7^{\circ} \mathrm{C}$, pressure variations were around $0.03 \mathrm{MPa}$ (4.3 PSI), and changes in core power in both units can 
be neglected, showing that the system was able to absorb the perturbation without significant changes in the dynamics during its duration.

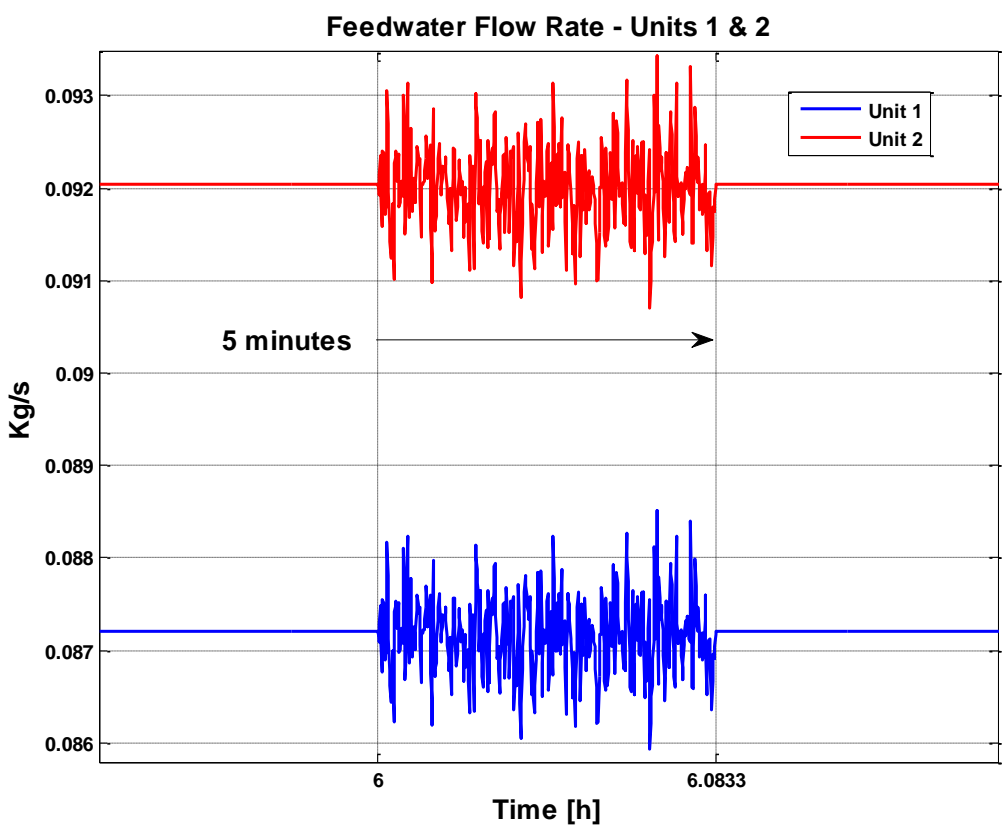

Figure 4.25. Feedwater flow rate 1\% FS perturbation.

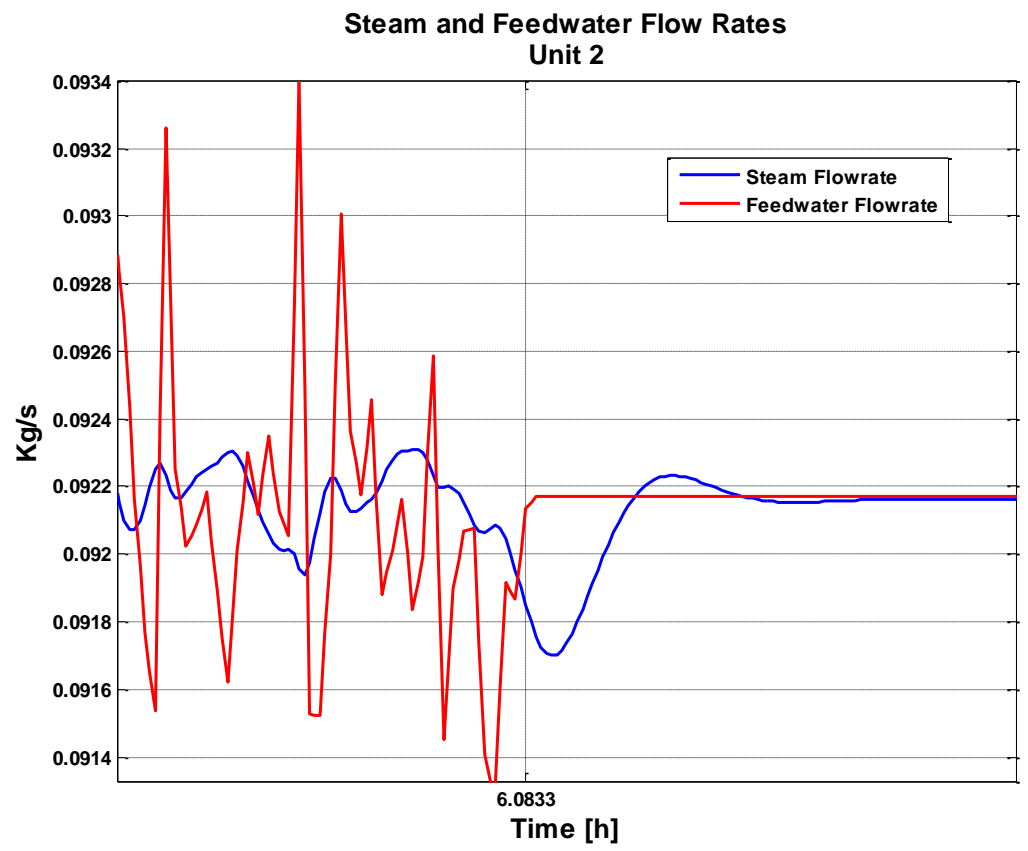

Figure 4.26. Steam and feedwater flow rate to $1 \%$ FS perturbation. 
There is, however, a possibility of future multi-modular plants having independent feedwater lines to each module, or unit, with the only real connection between modules being in the steam header. In case 5 a test involving a negative $1 \%$ step change in feedwater flow rate for only one unit, in this case unit 1 , was carried out. As expected, the superheated steam temperature from unit 1 increased as well (by $0.6^{\circ} \mathrm{C}$ ), subsequently decreasing and stabilizing $0.4^{\circ} \mathrm{C}$ higher. The core power decreased by $0.9 \%$ and the $\mathrm{T}_{\text {ave }}$ temperature in unit one increased by only $0.15^{\circ} \mathrm{C}$. No significant changes in unit 2 dynamics were observed. These results are shown in Figures 4.27, 4.28, and 4.29.

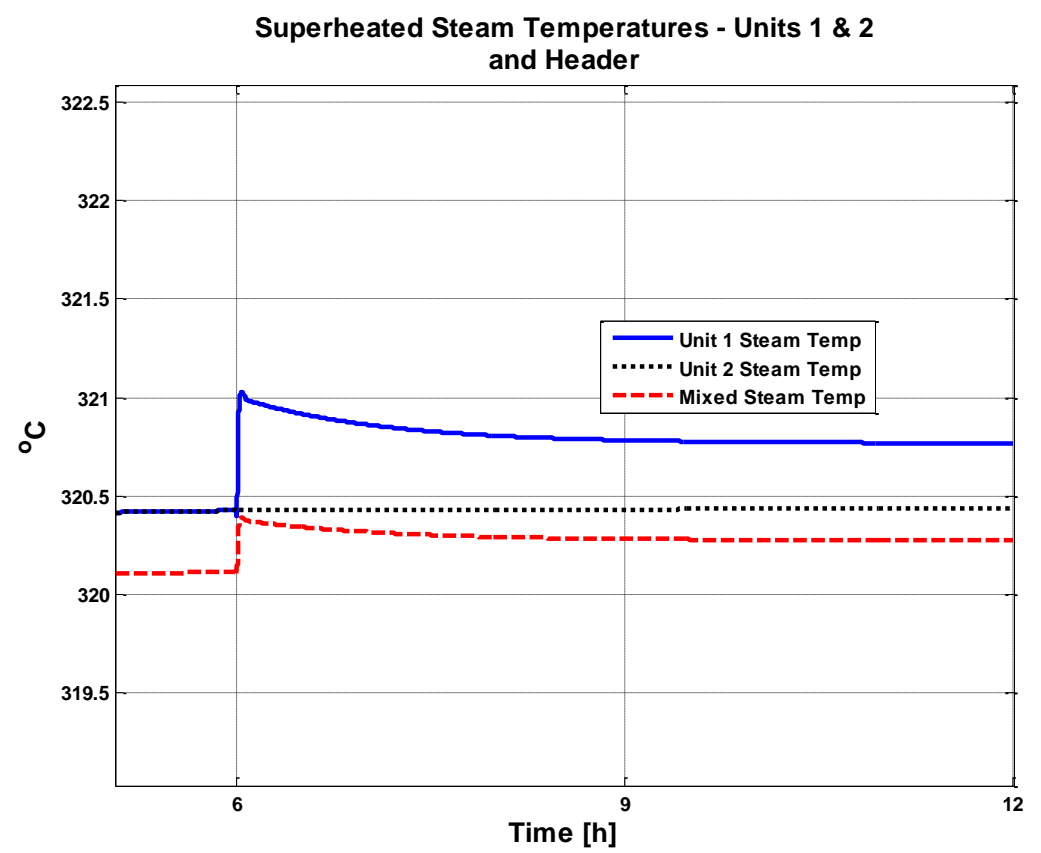

Figure 4.27. Steam header temperature perturbation for a negative $1 \%$ perturbation in feedwater flow rate. 


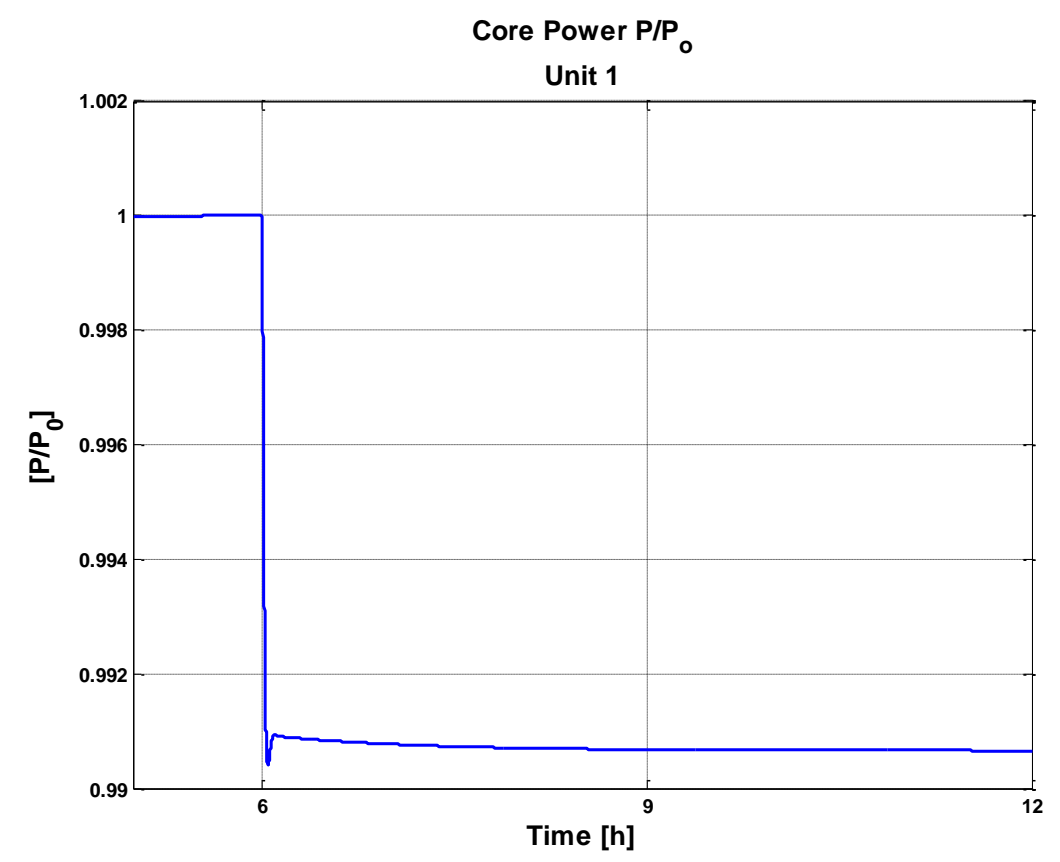

Figure 4.28. Core power transient for a negative $1 \%$ perturbation in feedwater flow rate.

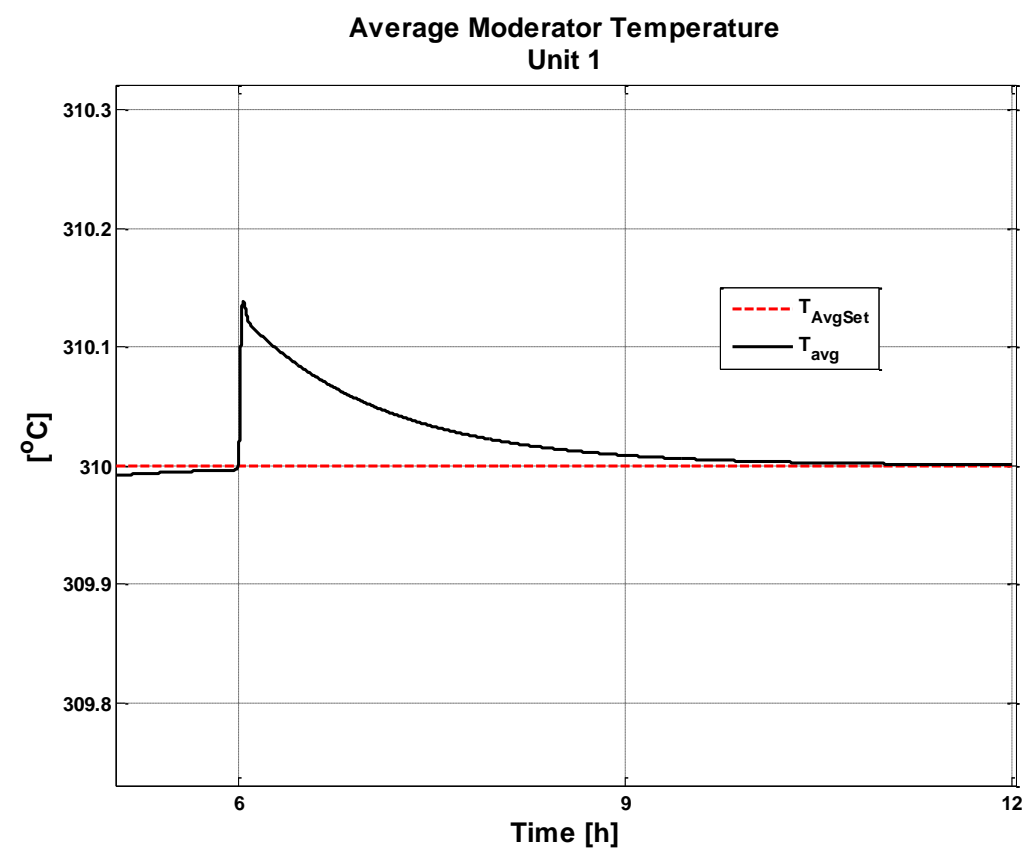

Figure 4.29. $\mathrm{T}_{\text {ave }}$ transient for a negative $1 \%$ perturbation in feedwater flow rate. 
In the last case a small perturbation in the feedwater temperature was introduced in unit 2. A negative $1.7^{\circ} \mathrm{C}\left(3^{\circ} \mathrm{F}\right)$ step perturbation at $\mathrm{t}=6 \mathrm{~h}$ is less than $1 \%$ variation of the nominal value $\left(223.9^{\circ}\right)$, therefore not representing a significant change in temperature. The model, however, was able to pick up the perturbation, which is reflected in the mixed steam temperature in the header, in the steam outlet pressure, in the moderator average temperature and in the core power, shown in Figures 4.30 to 4.33 respectively, but unit 1 was not affected at all. In Figure 4.30 the mixed steam temperature varied only about $0.3^{\circ} \mathrm{C}$. In the steam pressure (Figure 4.31) the perturbation amplitude was only about $0.013 \mathrm{MPa}$ (1.9 PSI) and lasted 46 seconds from start to finish. Such small pressure perturbation amplitude is very small for the steam generator to suffer any significant changes in its dynamics. Same minor effects were seen in both $\mathrm{T}_{\text {ave }}$ (Figure 4.32) and core power in unit 2 (Figure 4.33).

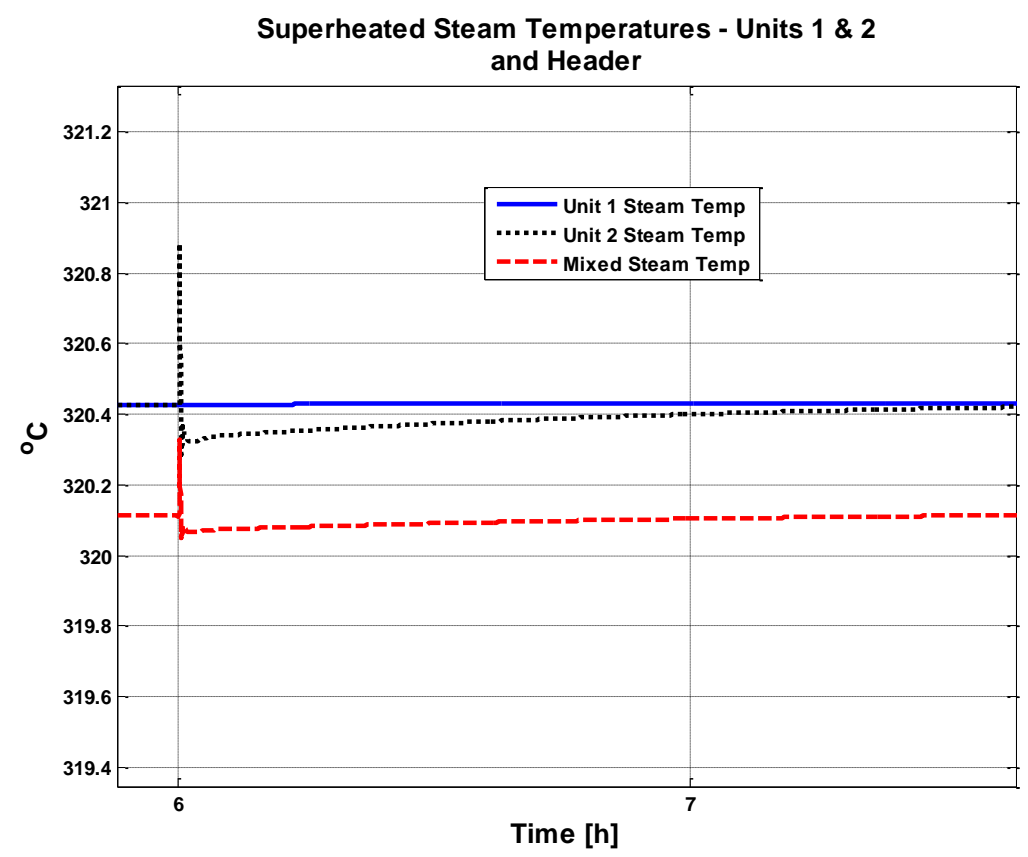

Figure 4.30. Steam header temperature transient for a negative $1.7^{\circ} \mathrm{C}$ perturbation in feedwater flow rate. 


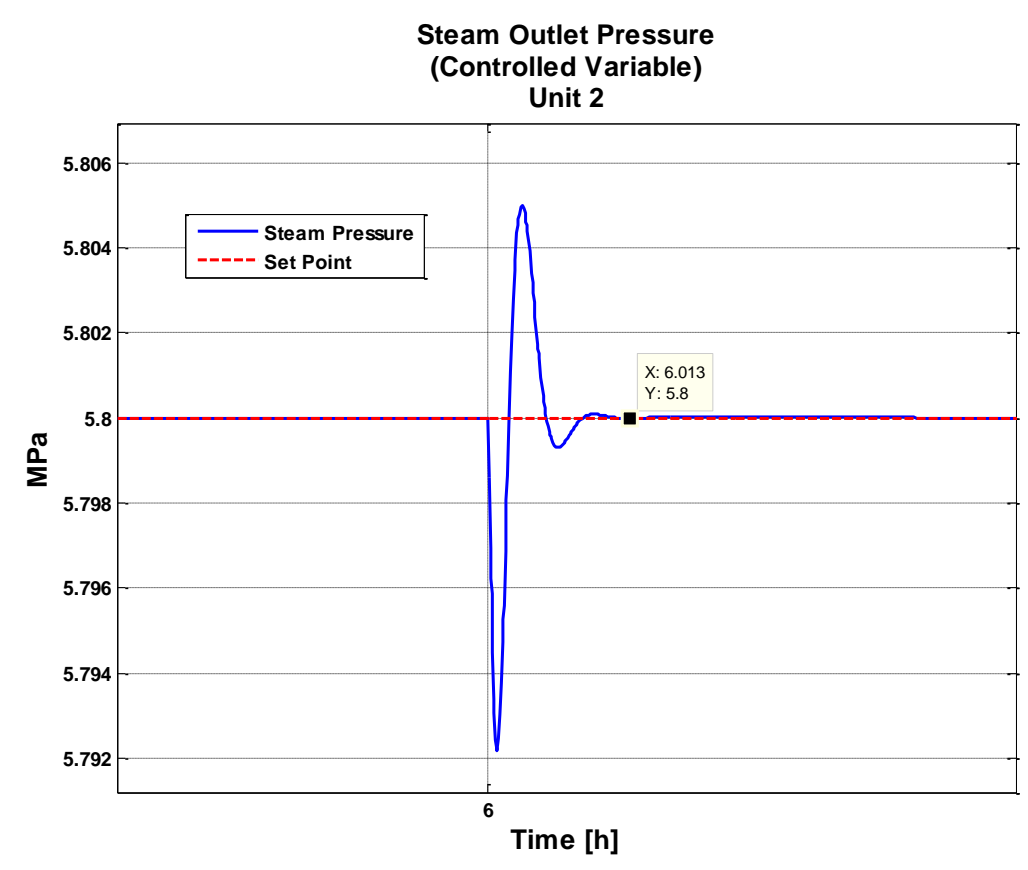

Figure 4.31. Steam pressure transient for a negative $1.7^{\circ} \mathrm{C}$ perturbation in feedwater flow rate.

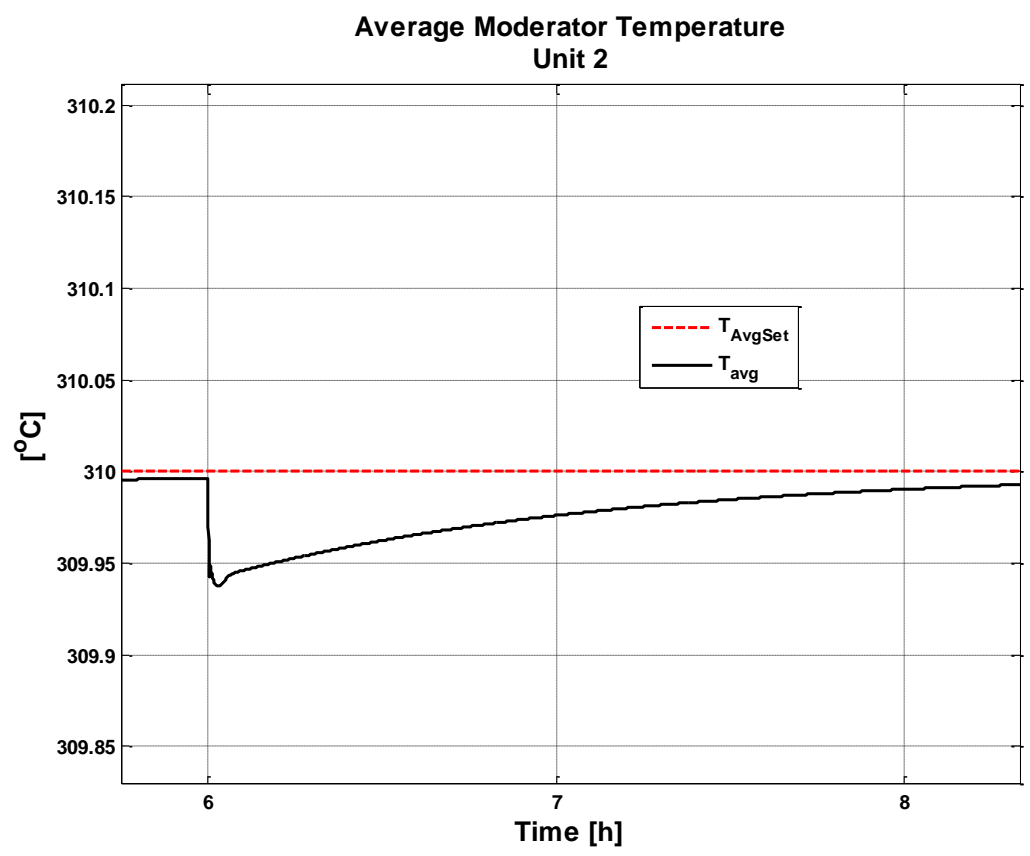

Figure 4.32. Tave transient for a negative $1.7^{\circ} \mathrm{C}$ perturbation in feedwater flow rate. 


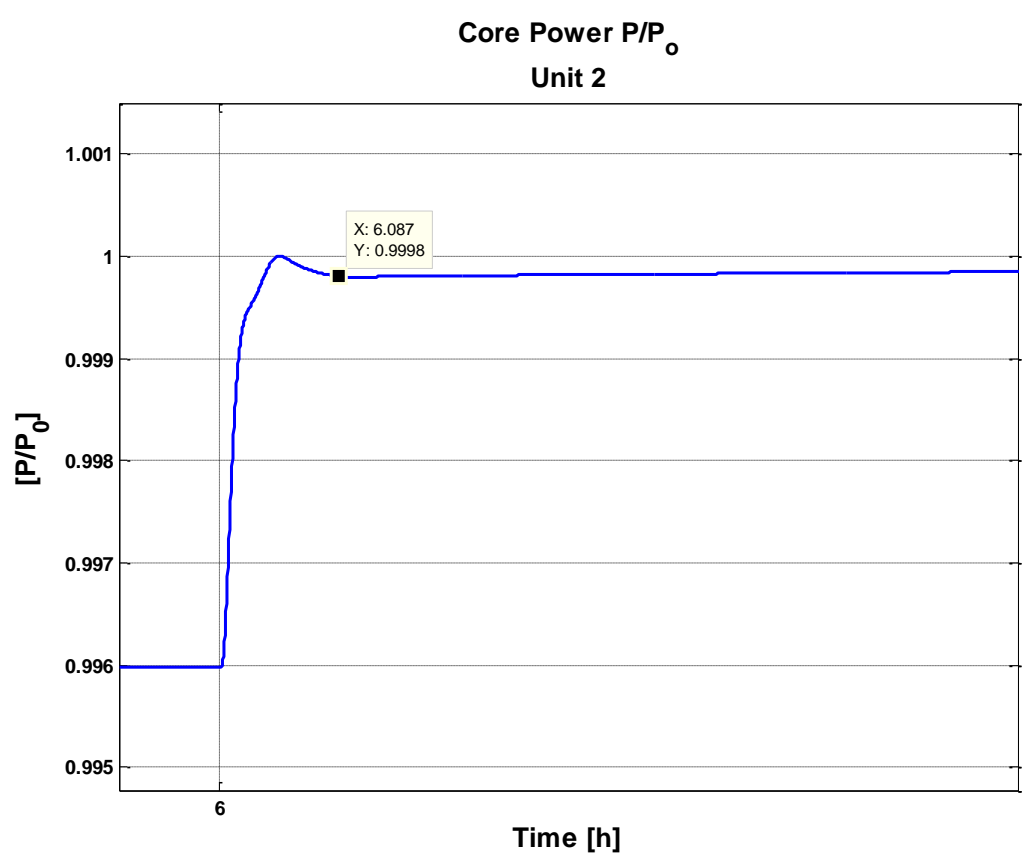

Figure 4.33. Core power transient for a negative $1.7^{\circ} \mathrm{C}$ perturbation in feedwater flow rate.

\subsection{Summary}

The existing IRIS SIMULINK model was extended to include a second unit, or module, with the objective of evaluating and quantifying the performance of a nuclear power plant comprised of two IRIS reactor modules operating simultaneously, with the superheated steam from both units flowing to a common header. This in turn is connected to a single turbine, resulting in a steam-mixing control problem with respect to "load-following" scenarios, such as changing electricity demand. The simulations involved only load following profile number one because the objective was to check if the model would be able to operate in load-following mode and its ability to detect small, controlled faults introduced either in sensor measurements or systems, so either profile could have been used just as well.

A series of perturbation cases were investigated: 3 involving the moderator average temperature controller in the primary system by perturbing $\mathrm{T}_{\text {hot }}$ or $\mathrm{T}_{\text {cold }}$, and 4 involving the 
secondary system ${ }^{2}$ by perturbing the feedwater flow rate or temperature. In all the cases, the unit not being subjected to the fault showed no effect due to this fault. The exception is only for the mixed steam temperature or pressure in the header, even when the modules are connected by a common feedwater flow pipeline. In all cases the steam pressure and $\mathrm{T}_{\text {ave }}$ controllers in both units performed as expected, maintaining the process values well within set points.

Finally, from simulations it is possible to conclude that, for small variations in process variables, both units are somewhat independent of each other even with a common feedwater line connecting both steam generators, and are able to perform well even under such variations, with the real connection between the two units being located in the steam header. The simulation results show the feasibility of having two IRIS modules in a single plant.

\footnotetext{
${ }^{2}$ Such differentiation between primary and secondary systems is not absolutely true in IRIS design since both systems are actually encased inside a common vessel.
} 


\section{Chapter 5}

\section{TWO-TANK MULTIVARIATE FLOW CONTROL LOOP}

\section{$5.1 \quad$ Introduction}

In order to develop research related to instrumentation and control, equipment and sensor monitoring, a two-tank multivariate loop was designed and built in the Nuclear Engineering Department, at the University of Tennessee. Due to its flexibility, this loop provides the framework necessary to investigate and test control strategies and fault detection in sensors, equipment, and actuators.

\subsection{General Description}

The two-tank loop was built on a wheeled table-like steel frame structure seven-foot long, four-foot wide and six-foot high. This structure holds all sensors, piping, pump, sump tank, aircraft aluminum table top, cables, control valves, manual valves, connection boxes, power strips and 2 tanks and can be easily moved around. Since around eighty percent of the piping used to build the loop is made of Chlorinated Polyvinyl Chloride (CPVC) or PVC and union connections were strategically distributed, any maintenance or minor setup modification can easily be carried out. Figure 5.1 shows the main structure at the beginning of the construction. Details of the bypass bore hole drilling operation on the $1 / 4$ " thick aluminum sheet can be seen in Figure 5.2. In Figure 5.3 part of the layout with control valves, flow meters and pressure sensors positioned in place gives an idea of the final layout. A user manual detailing the operation of the loop is provided in Appendix 5.

\subsubsection{Water Tanks}

For the level control there are 2 similar acrylic tanks installed on this loop called Tank 1 and Tank 2, respectively, and their dimensions are: 5-3/4" in diameter and 3-foot long. A 27gallon stainless steel tank is installed underneath the table top to provide the necessary water for the circuit. Figure 5.4 shows the details of the two acrylic tanks used on this loop. 


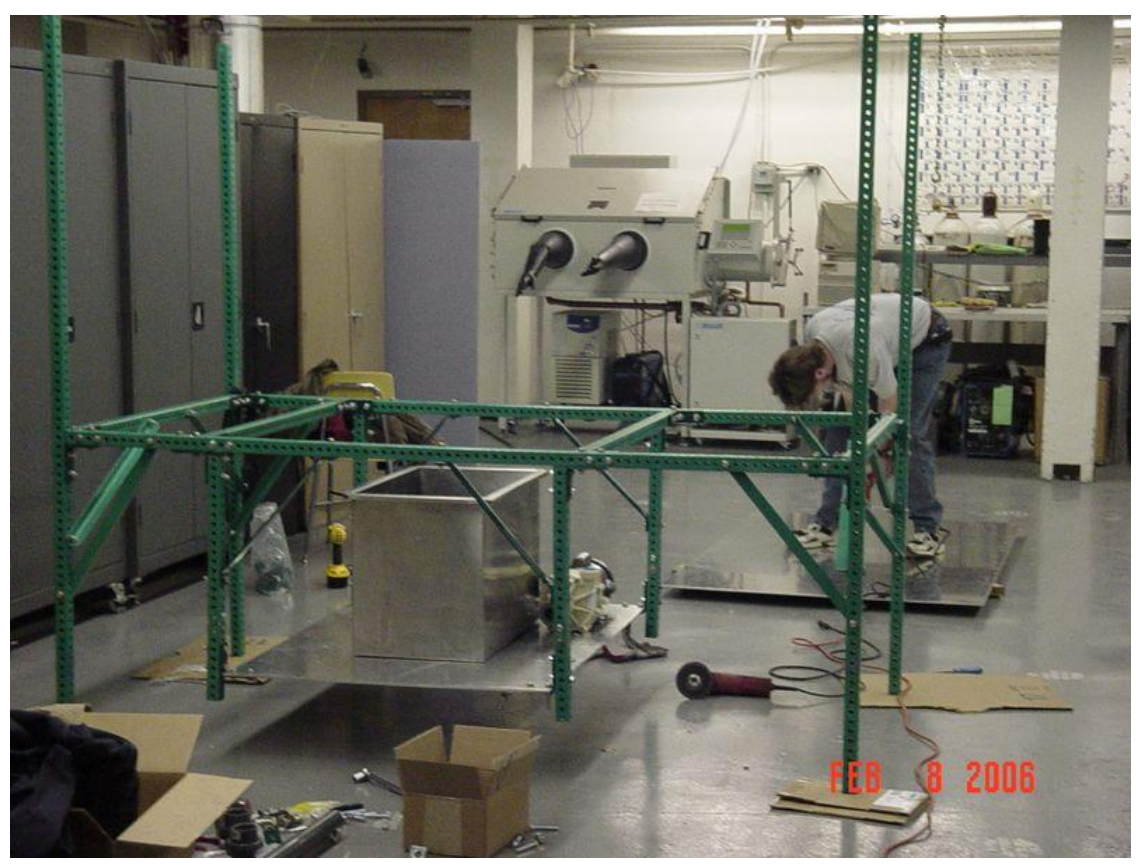

Figure 5.1. Structure that holds the two-tank loop.

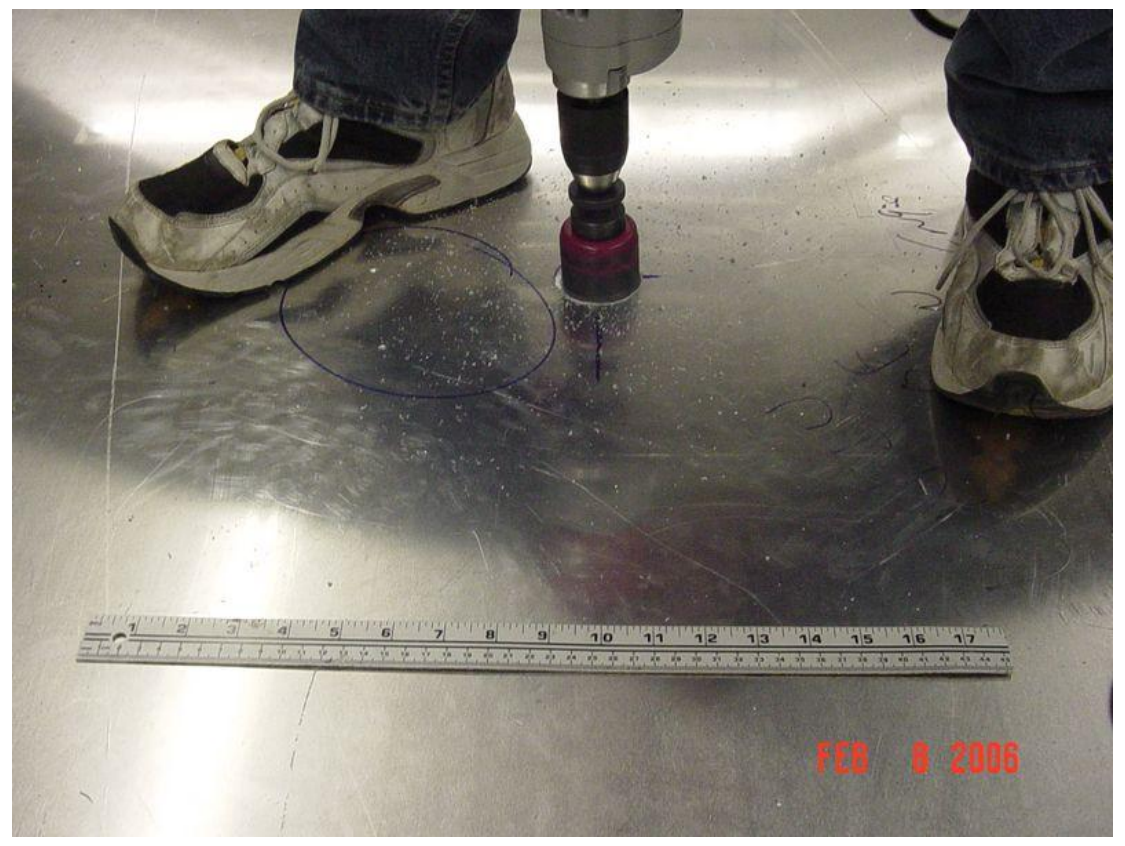

Figure 5.2. Drilling work of a $1 / 4$ " thick aluminum sheet. 


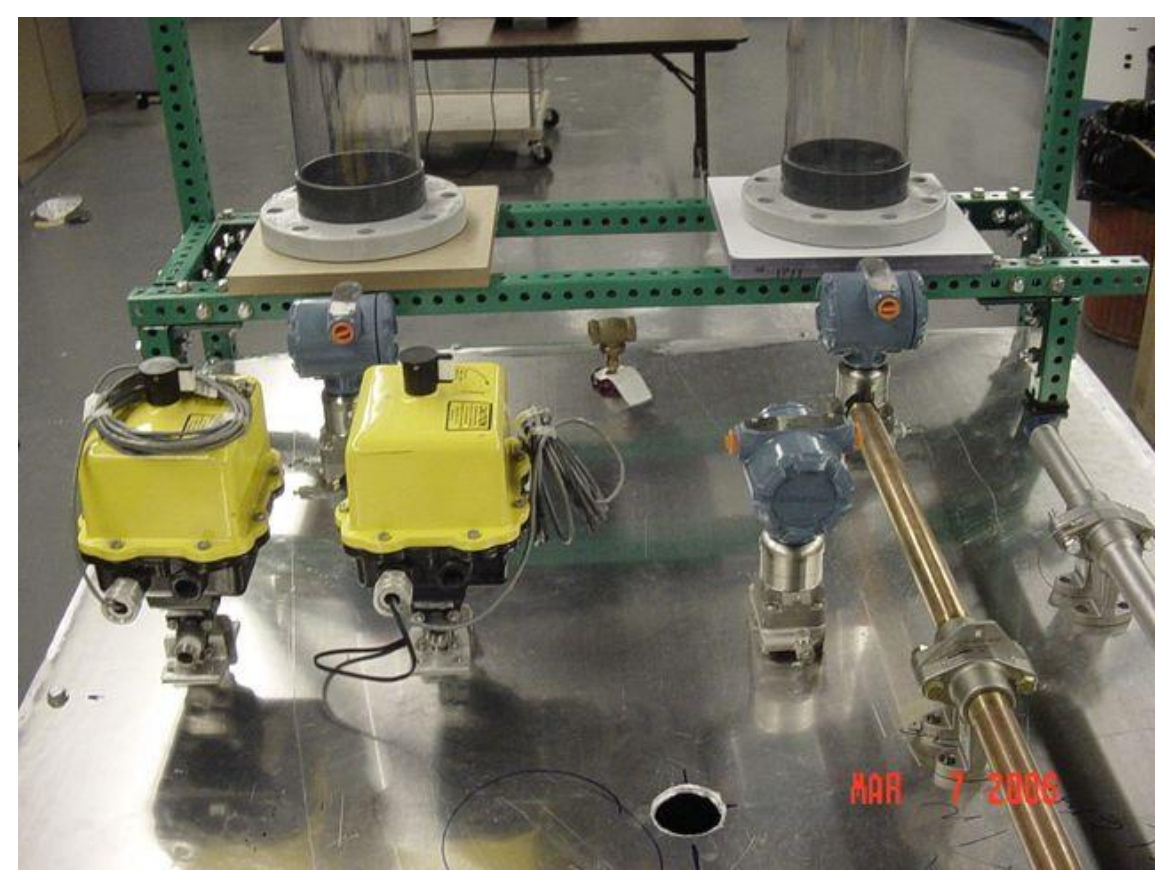

Figure 5.3. Partial layout.

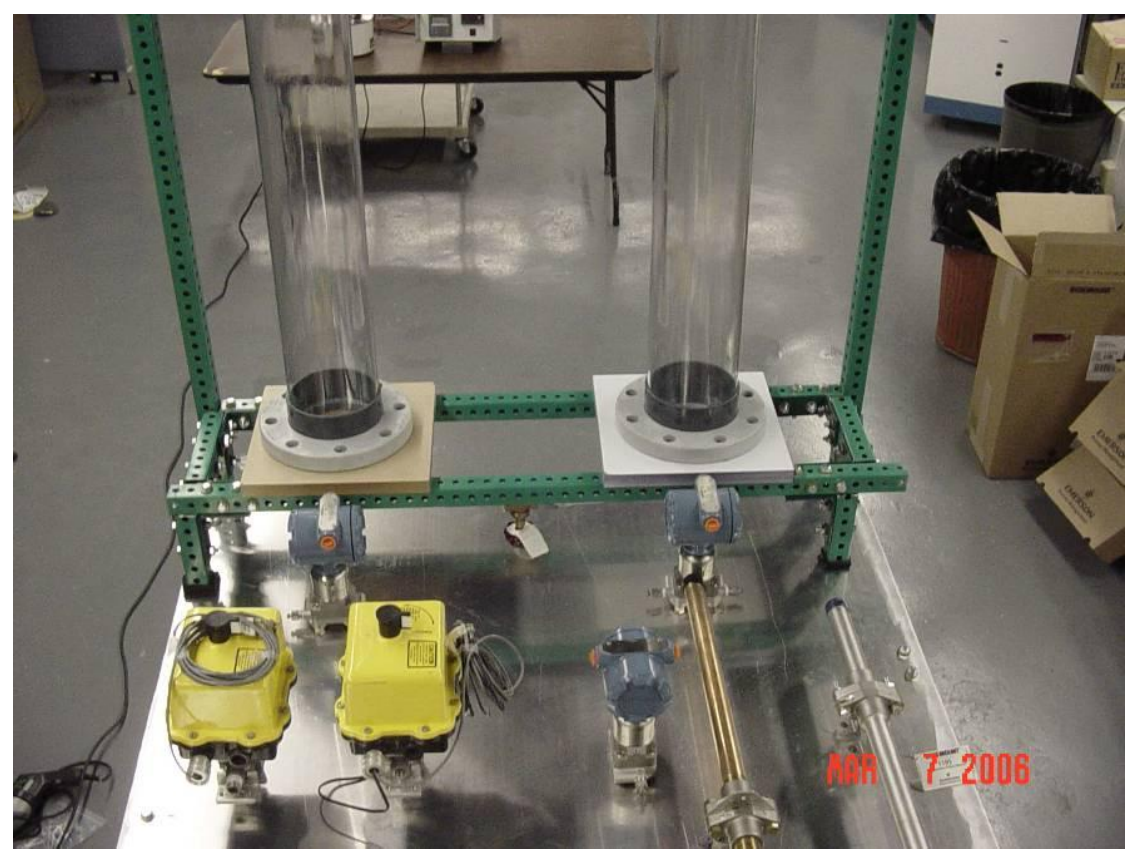

Figure 5.4. Level tanks detail. 


\subsubsection{Sensors}

Several sensors for process measurement are installed in the loop: differential pressure transmitters, thermocouples, turbine flow meters, orifice meters and signal conditioners and transducers. Each sensor type and their role in the loop are discussed below.

\subsubsection{Pressure Transmitters}

Four differential pressure sensors with HART technology, donated by Rosemount ${ }^{\mathrm{TM}}$, are currently installed. The HART (Highway Addressable Remote Transducer) Protocol is the global standard for sending and receiving digital information across analog wires between smart devices and control or monitoring systems. More specifically, HART is a bi-directional communication protocol that provides data access between intelligent field instruments and host systems. A host can be any software application from technician's hand-held device or laptop to a plant's process control, asset management, safety or other system using any control platform.

Two pressure sensors are used to measure the differential pressure between atmosphere and the water column inside each tank, whereas two other pressure sensors are connected to orifice meters and are used to measure the differential pressure caused by the water as it flows through the sensor going into each of the tanks. Figure 5.5 shows the details of the two of the sensors, and in Table 5.1 provides information about the pressure sensors.

To calibrate the range of the pressure sensors and relate their outputs to either level in $\mathrm{mmH}_{2} \mathrm{O}$ or flow rate in liters per second (1/s), a software package called AMS Device Manager

Suite from Emerson ${ }^{\circledR}$ was used (username $=$ admin, no password). This software package provides us with an easy way to calibrate, zero-trim, schedule maintenance and keep record of each and every calibration performed without having to remove the sensor from the loop, attach it to the workbench and physically calibrate it against a known sensor using a pressure source. It comes with a RS-232 modem and cable. On one end it plugs into the computer serial port and on the other end a pair of probes is used to connect to the sensor terminals. 
Table 5.1. Important pressure sensor information.

\begin{tabular}{|c|c|c|c|}
\hline Sensor ID & Function & Output & Calibration Range \\
\hline Tank 1 & Measures Level in Tank 1 & $4-20 \mathrm{~mA}^{*}$ & $0-900 \mathrm{~mm}_{2} \mathrm{O}$ \\
\hline Tank 2 & Measures Level in Tank 2 & $1-5$ volts & $0-900 \mathrm{mmH}_{2} \mathrm{O}$ \\
\hline Flow 1 & Tank 1 Inlet flow & $4-20 \mathrm{~mA}^{*}$ & $0-6303 \mathrm{~mm}_{2} \mathrm{O}$ \\
\hline Flow 2 & Tank 2 Inlet flow & $4-20 \mathrm{~mA}^{*}$ & $0-6303 \mathrm{~mm}_{2} \mathrm{O}$ \\
\hline
\end{tabular}

*A high precision $250 \mathrm{Ohm}$ resistor is used to convert the signal from $\mathrm{mA}$ to VDC.

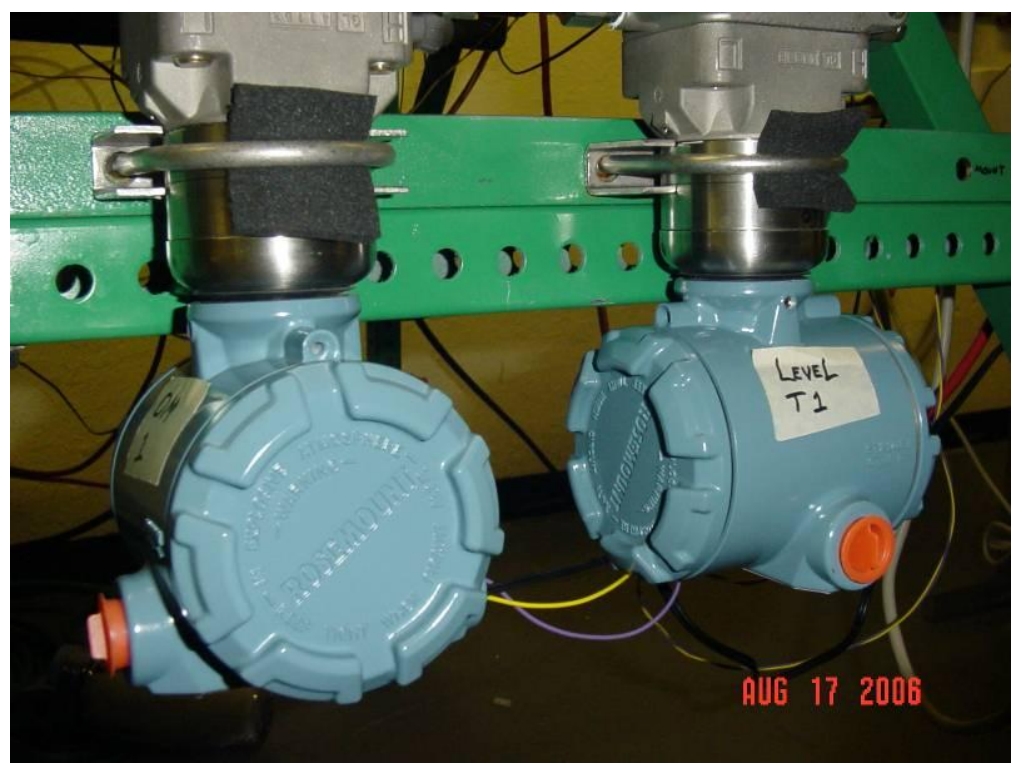

Figure 5.5. Pressure sensors used in the loop.

Details of the connection between the computer and the pressure sensor are shown in Figure 5.6, with the two probes connected to the $250 \Omega$ resistor terminals, and in Figure 5.7 the main screen of the software is shown with the HART modem appearing at the bottom on the left hand side, and the sensor itself on the right hand side. Some important sensor details, for 
example, calibration pressure limits, units, maintenance status, etc., are shown in Figures 5.8 and 5.9 .

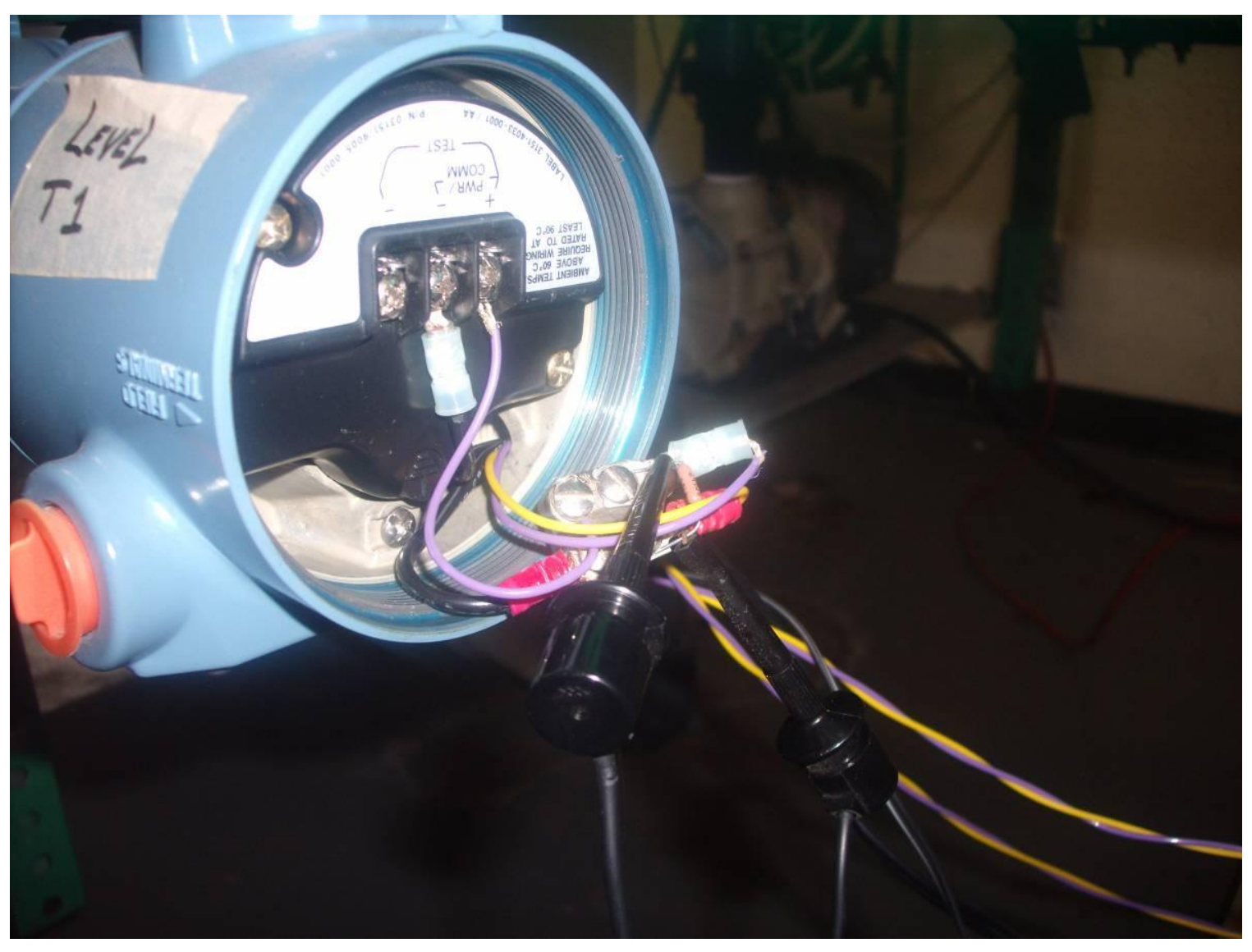

Figure 5.6. HART Sensor-Computer connection detail. 


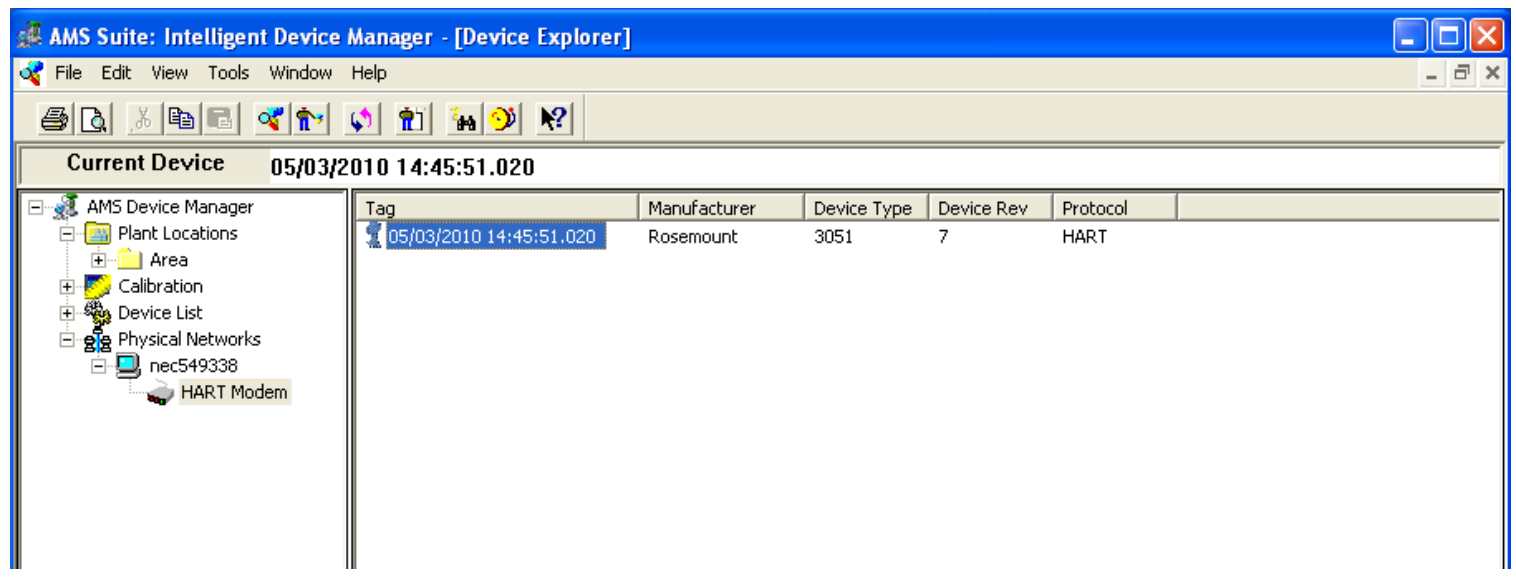

Figure 5.7. AMS Device Manager's main screen.

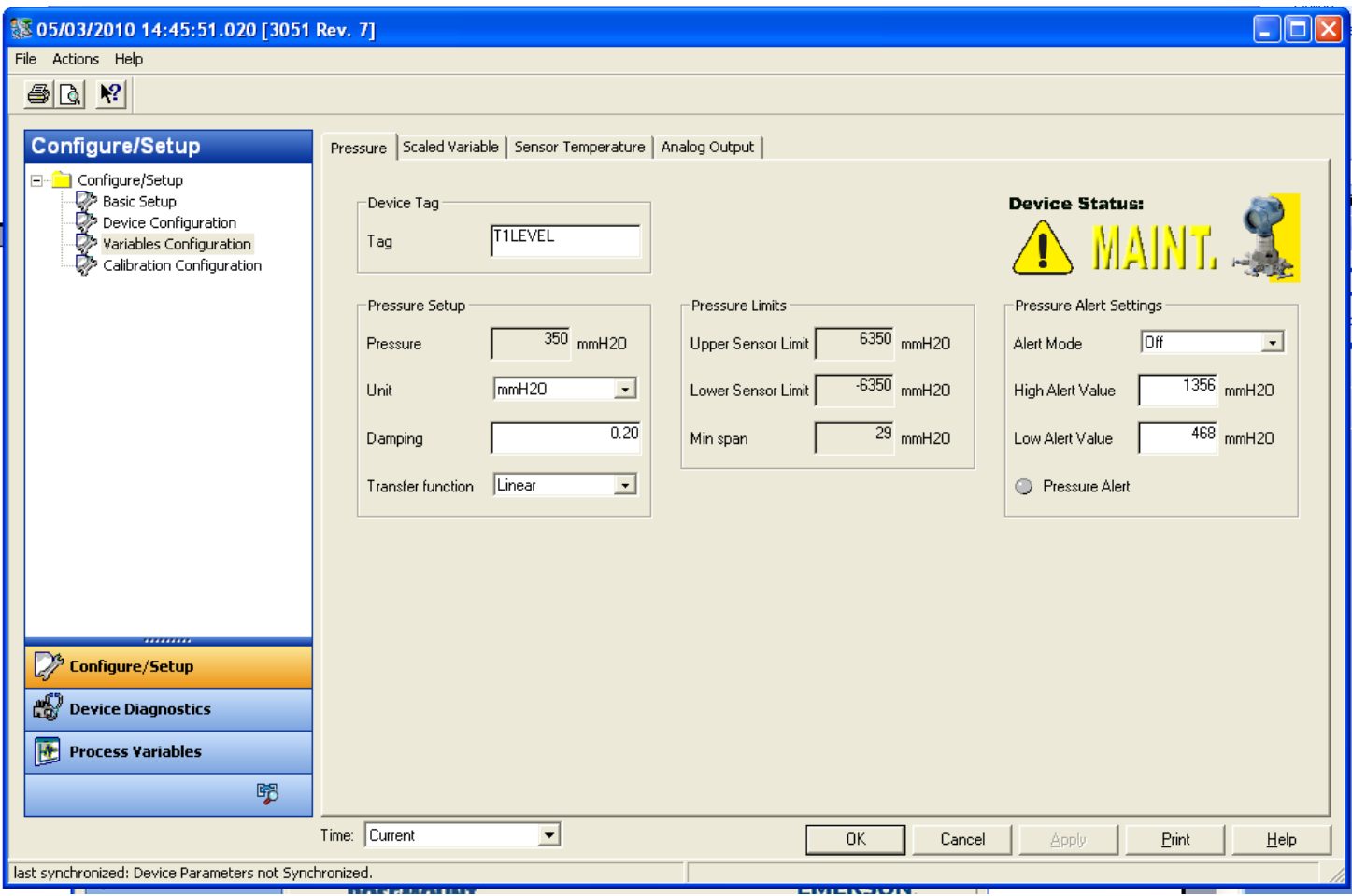

Figure 5.8. Sensor details provided by the AMS software. 


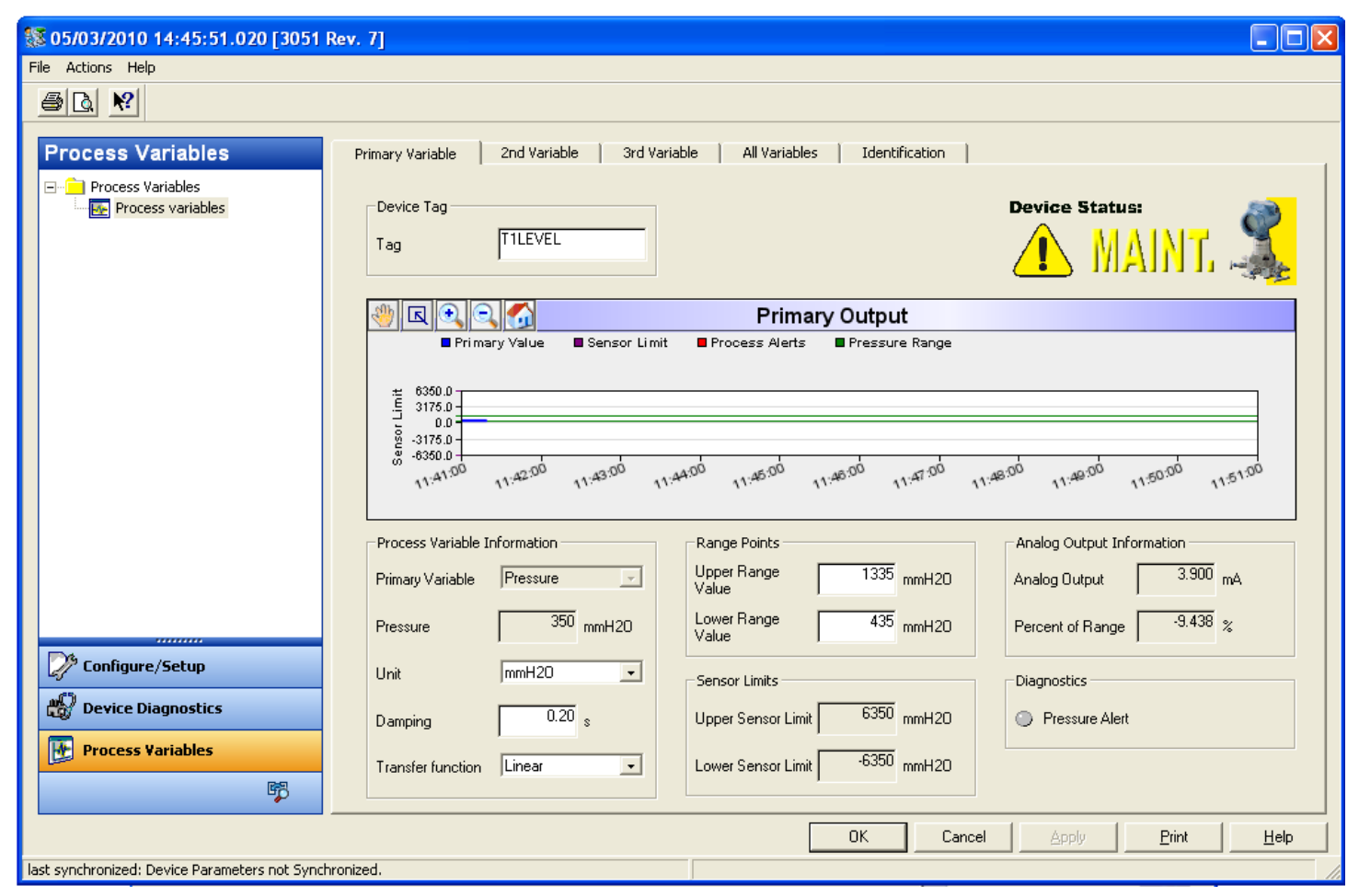

Figure 5.9. Sensor detail and status provided by AMS software

\subsubsection{Flow Meters}

Two different types of flow rate meters are used in the loop: turbine and orifice. Two orifice meters were donated by Rosemount ${ }^{\mathrm{TM}}$ and the turbine flow meters were manufactured by and bought from Omega ${ }^{\mathrm{TM}}$. There are 3 turbines installed in the loop: one at each tank outlet and one in the bypass and all of them are factory calibrated. The electronic circuitry generates a magnetic field inside the turbine case and as a set of metallic blades, similar to those found in domestic fans, spins as the water passes through the turbine a signal is generated with a frequency linearly proportional to the flow rate. A signal conditioner attached to each of the turbines transforms this frequency output $(0-1000 \mathrm{~Hz})$ to a voltage signal $(0-5 \mathrm{~V}$ or $0-10 \mathrm{~V}$, switch selectable) and finally the volumetric flow can be calculated using the calibration tables provided by the manufacturer for both the signal conditioner and the turbine flow meter. In Figure 5.10 a turbine meter and the signal conditioner are shown and Figure 5.11 shows some details of the orifice plate and pressure lines. Table 5.2 is an example of a typical calibration table provided for one of the turbine flowmeters by the manufacturer showing the relationship between 
frequency and voltage, and voltage and volumetric flow rate. Figure 5.12 and Figure 5.13 shows the calibration data points provided by the manufacturer and the predicted values using polynomial fit for both voltage and volumetric flow.

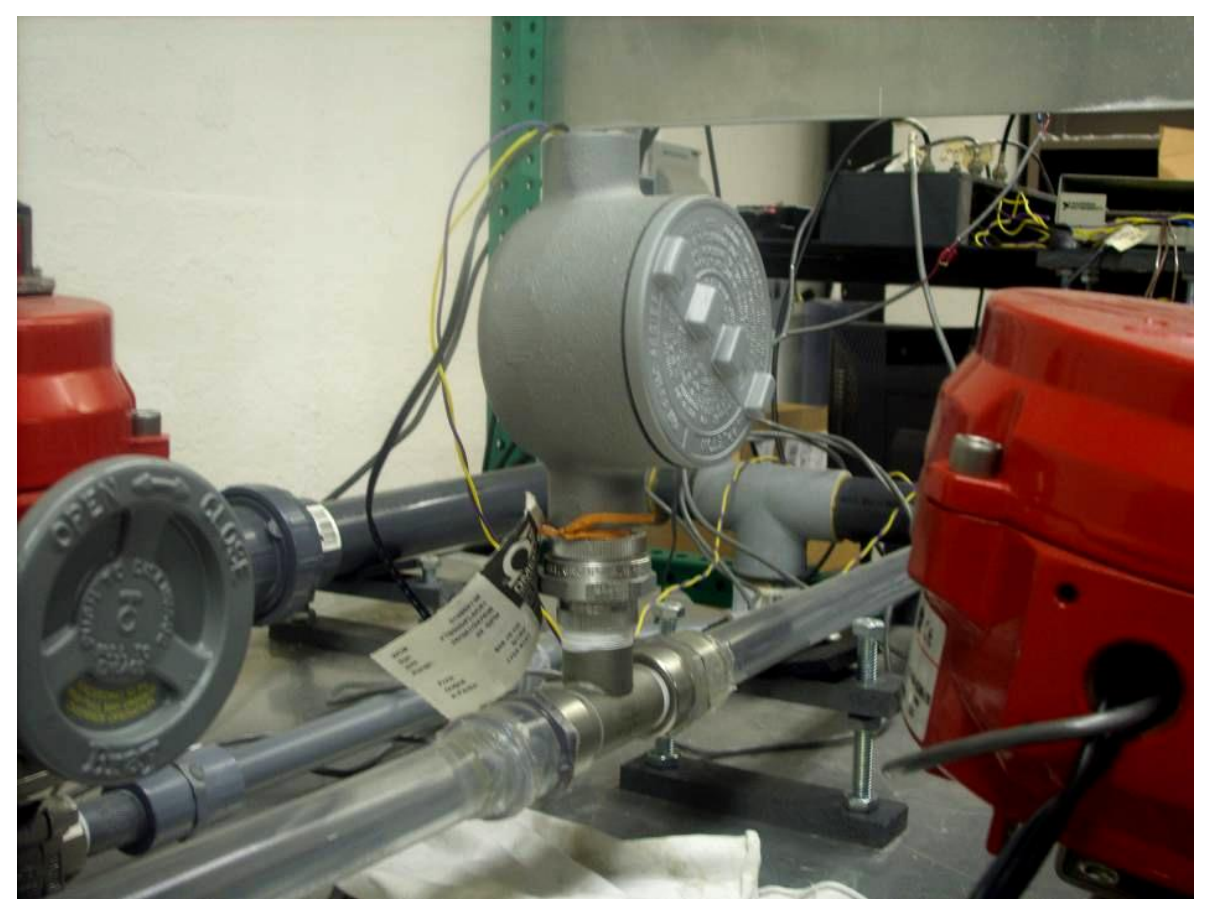

Figure 5.10. Turbine and signal conditioner details. 


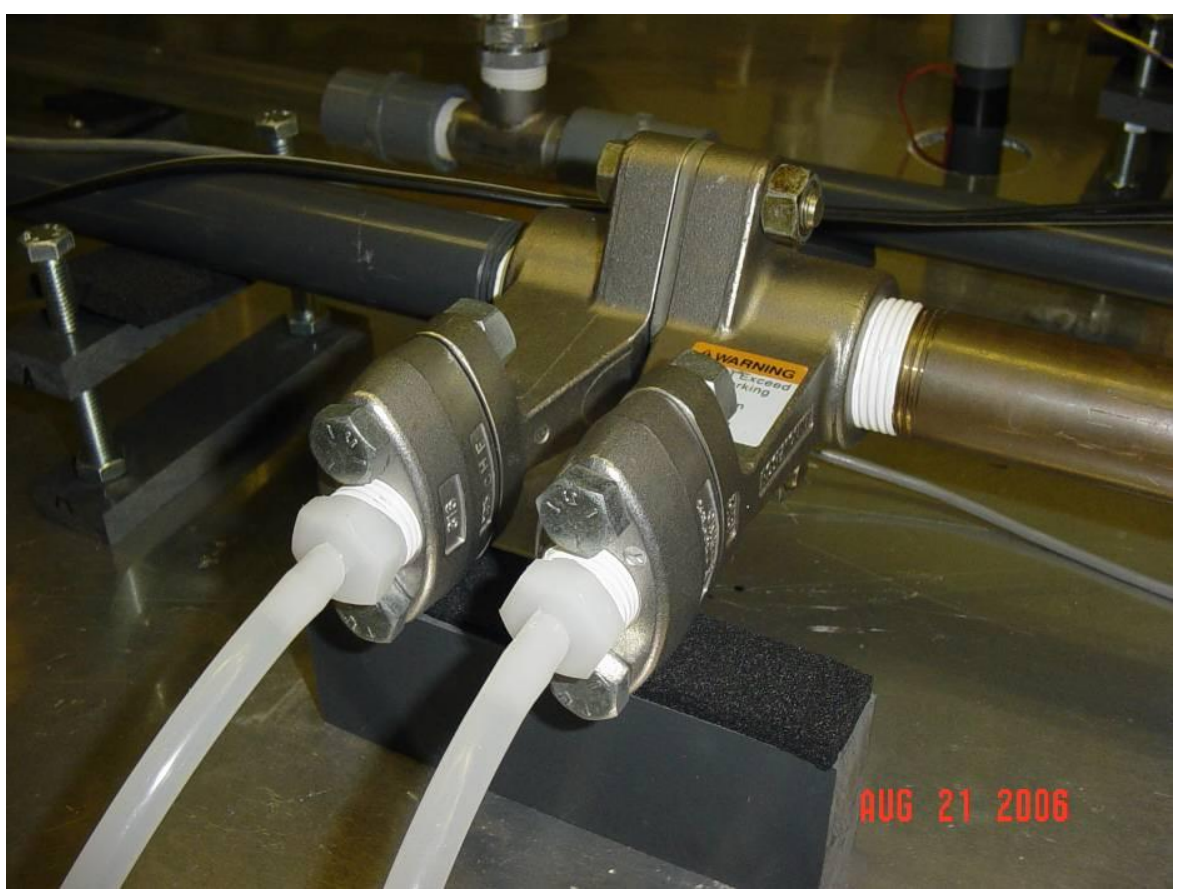

Figure 5.11. Details of the orifice plate and the pressure lines. 
Table 5.2. Calibration table provided by the manufacturer

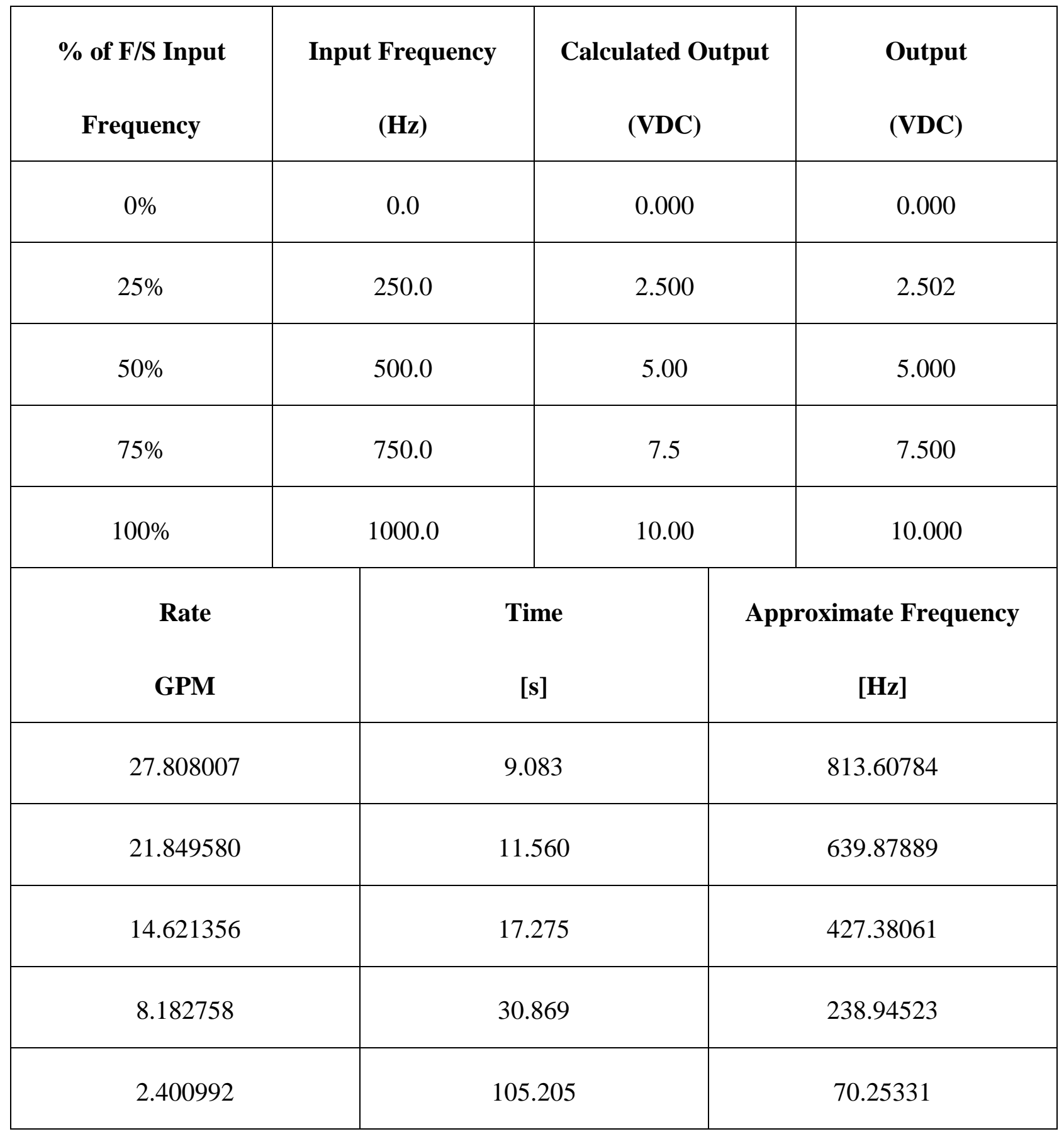




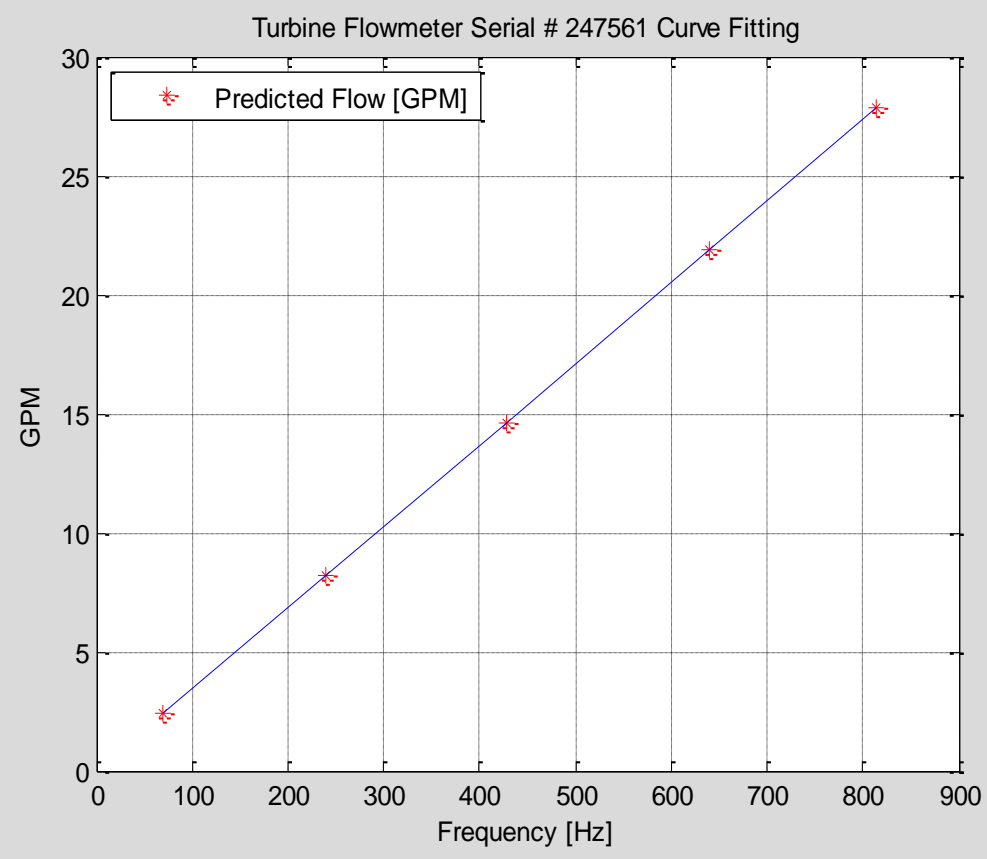

Figure 5.12. Signal conditioner calibration curve and predicted values.

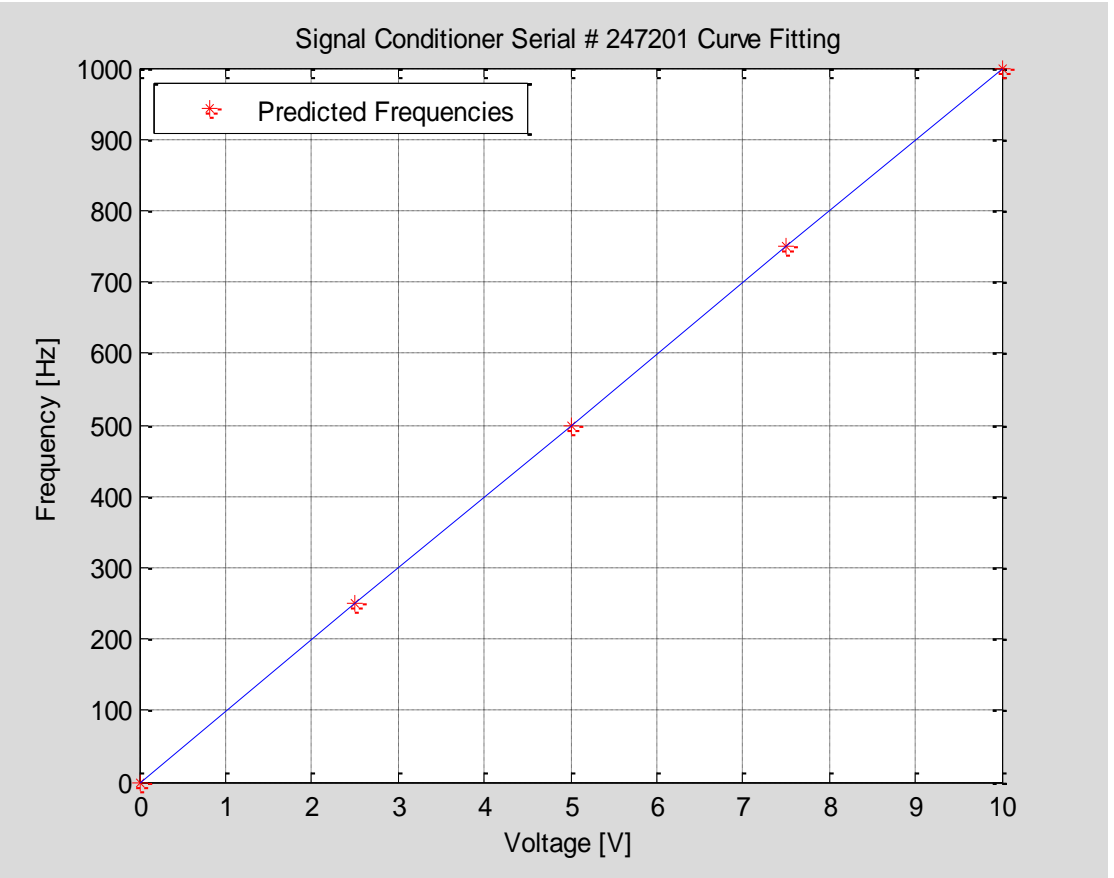

Figure 5.13. Turbine flowmeter calibration curve and predicted values. 
The other flow meters are orifice meters, and their principle of operation is simple: as the fluid approaches the orifice the pressure increases slightly and then drops suddenly as the orifice is passed. It continues to drop until the "vena contracta" is reached and then gradually increases until at approximately 5 to 8 diameters downstream; a maximum pressure point is reached that is lower than the pressure upstream of the orifice. The decrease in pressure as the fluid passes through the orifice is a result of the increased velocity of the fluid passing through the reduced area of the orifice. When the velocity decreases as the fluid leaves the orifice, the pressure increases and tends to return to its original level. All of the pressure loss is not recovered because of friction and turbulence losses in the stream. The pressure drop across the orifice increases when the rate of flow increases. When there is no flow the differential pressure is zero. The differential pressure is proportional to the square of the velocity. It therefore follows that if all other factors remain constant, then the differential pressure is proportional to the square of the rate of flow. The pressure across the orifice plates are measured using differential pressure sensors.

The orifice meter calibration procedures and the results are given in Appendix 6, and a procedure to calibrate the turbine flow meters against the orifice meters is given in Appendix 7.

\subsubsection{Thermocouples}

To monitor the temperature in different points of the loop, four type $\mathrm{J}$ thermocouples are installed: one inside each level tank, one in the reservoir tank and one to monitor the pump temperature. None of these sensors are calibrated and instead a standard calibration curve embedded in the software provided by LabVIEW is used. In the system, the only source of energy is the work generated by the centrifugal pump, which is transferred to the liquid causing the water temperature to rise. During warm days, and running for long periods of time, the water temperature changes from room temperature to about $38^{\circ} \mathrm{C}$, therefore not causing any significant changes in water levels due to density changes.

\subsubsection{Water Circulation Pump}

A constant speed centrifugal Noryl-27 GPM pump is used to drive the water through the loop and is installed underneath the circuit. It has a $0.5 \mathrm{HP}$ motor, 1-1/2" inlet $-1-1 / 2$ " outlet 
piping connection, and 110-230V electric connection. Details of the pump and of a thermocouple used to monitor the pump motor temperature are shown in Figure 5.14.

\subsubsection{Control Valves}

In order to manipulate the water flowing in the loop, five control valves are used: one at each tank inlet, one at each tank exit, and one connecting the piping between the tanks. These control valves have two components: an electric actuator and a $1 / 2$ " ball valve. Four of the electric actuators are manufactured by Bray International ${ }^{\mathrm{TM}}$ and one by Worcester Controls ${ }^{\mathrm{TM}}$, and are shown in Figures 5.16 and 5.15. Although not all five motor-operated valves (MOV) are actually used for control purposes, these actuators are manipulated via software and are responsible for opening and closing the ball valves to regulate the flow according to the experiment being carried out. The actuators are 120 VAC powered, with input and output of 210 VDC and can be locally or remotely operated, with a typical stroke time of 15 seconds. (Stroke time is the time needed to move the valve from the fully closed to fully open position, and conversely).

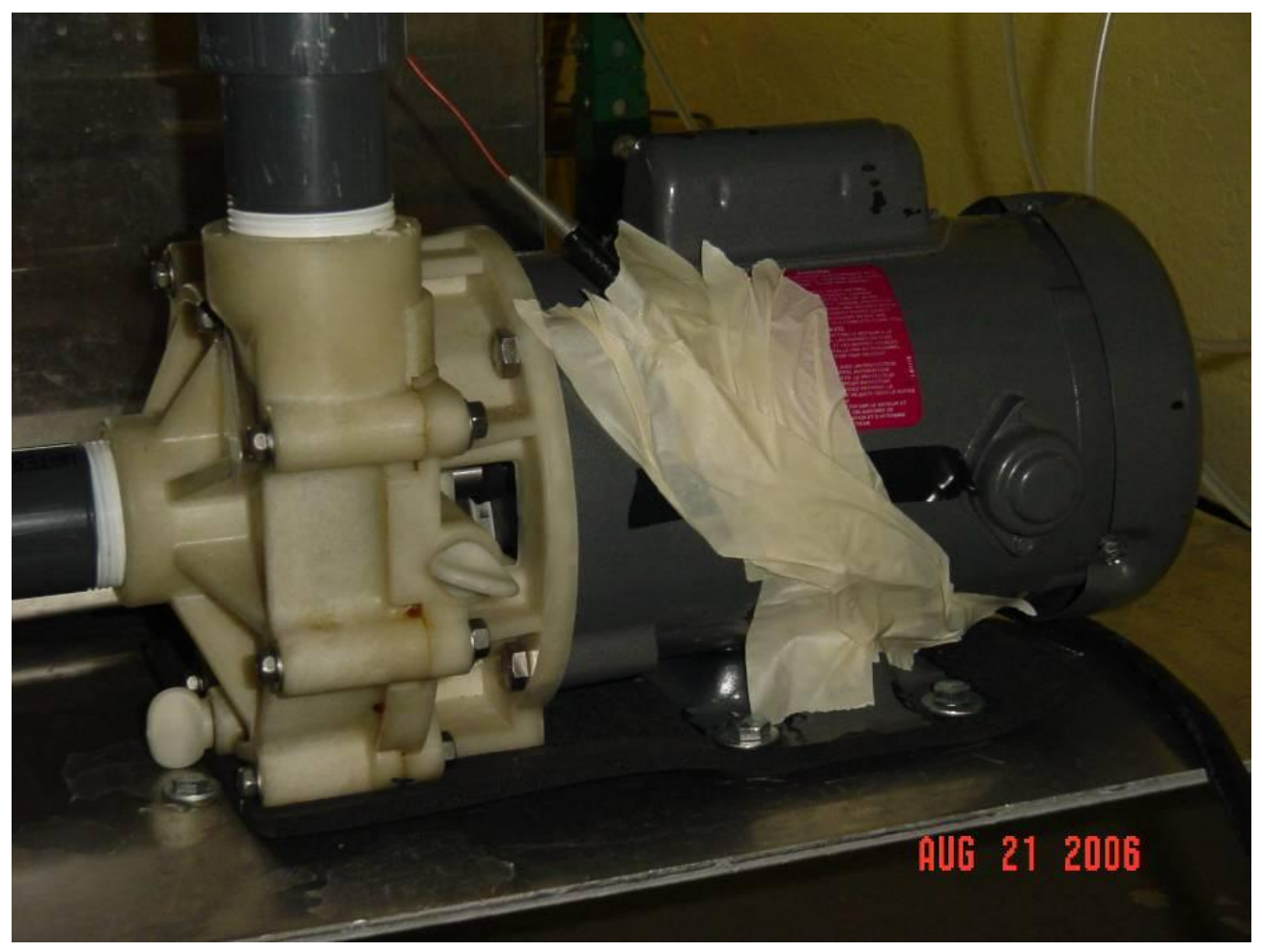


Figure 5.14. Centrifugal pump and thermocouple detail.

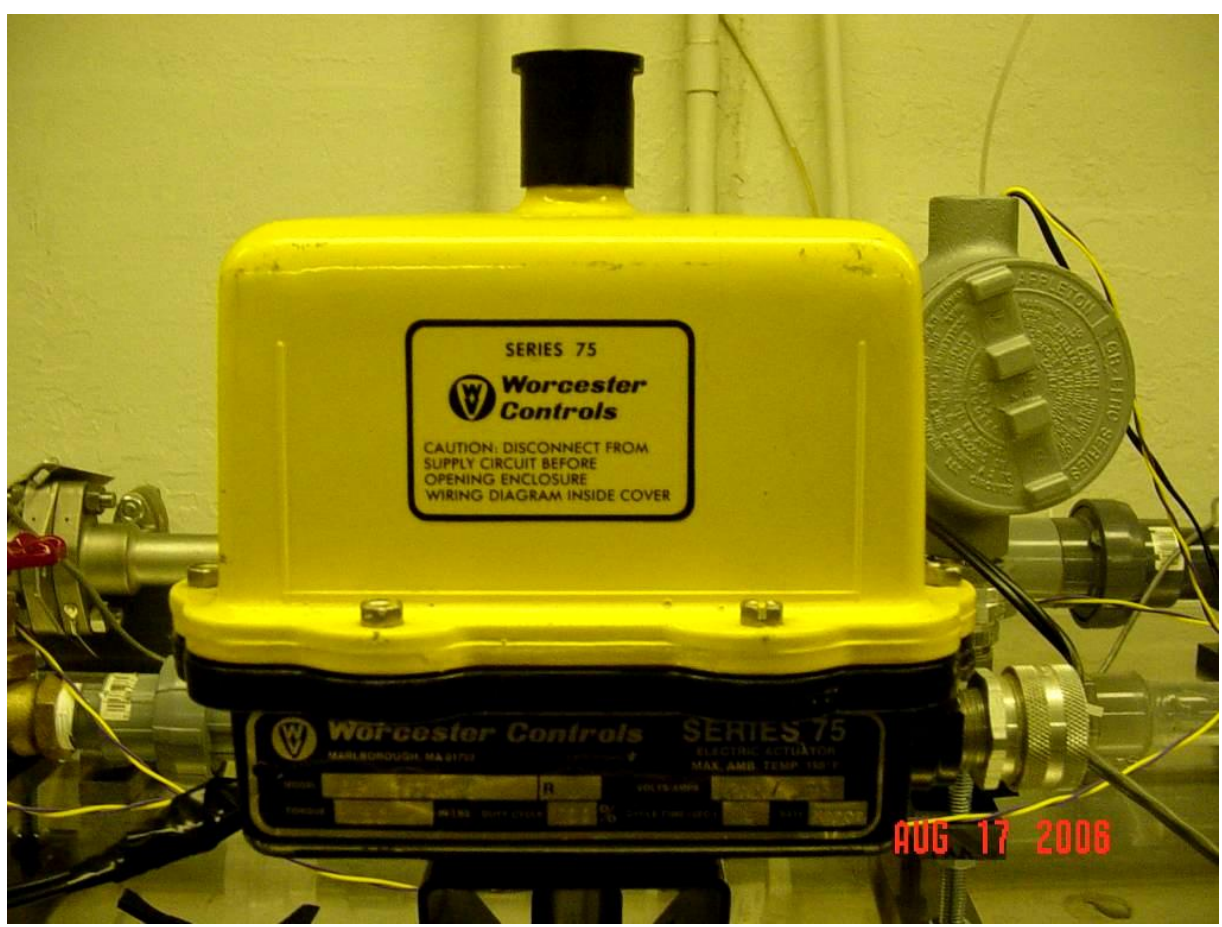

Figure 5.15. Worcester electric actuator. 


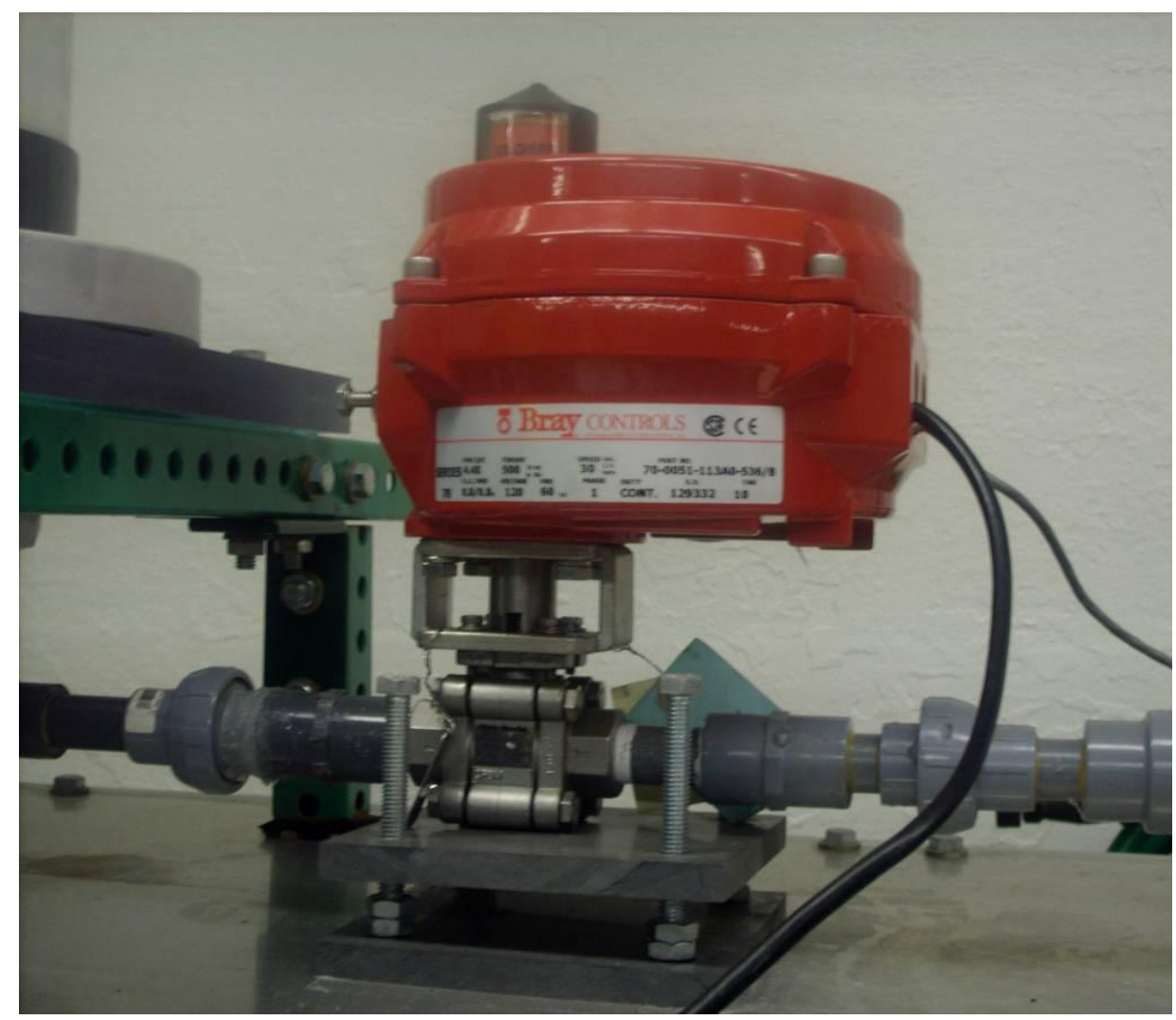

Figure 5.16. Bray Controls electric actuator.

\subsection{Loop - Description}

The two-tank loop was built primarily to provide the necessary framework to develop research related to instrumentation and control strategies, equipment and sensor monitoring, model-predictive control, and the demonstration of fault detection and fault-tolerant control strategy and reconfigurable control. With such objectives in mind, a set of sensors and actuators were placed in key positions throughout the loop to monitor and manipulate the water flow circulating in the loop. The major equipment and parts are listed in Table 5.3. Figure 5.17 shows a schematic of this loop with low-pressure water circulation that is facilitated by a fractional horsepower motor-driven pump. The sensors are identified as follows:

- LT-XX: Level transmitter

- LC-XX: Level control 
- CV-XX: Control valve

- FT-XX: flow rate meter

- V-XX: manual valve

- P-101: Centrifugal pump

Table 5.3. List of equipment and major parts.

\begin{tabular}{|c|c|c|}
\hline ITEM & DESCRIPTION & QUANTITY \\
\hline $\mathbf{1}$ & Rosemount 3051S Differential Pressure Transmitter. & \\
& Part Number: 3051S 1CD 2 A 2 E12 A 1A B4 & 04 \\
\hline $\mathbf{2}$ & Input Range: -250 to 250 inH $\mathrm{O}^{\mathrm{O}}$ & \\
\hline $\mathbf{3}$ & Output: 4 - 20 mA & 02 \\
\hline $\mathbf{4}$ & Rosemount 1195 Integral Orifice Primary Element. & 03 \\
\hline $\mathbf{5}$ & Part Number: 1195 S 010 P1 S 0150 C & 01 \\
\hline $\mathbf{6}$ & Omega Turbine Flow Meters FTB-953 & 04 \\
\hline $\mathbf{7}$ & Worcester Control Valves - Series 75 & 01 \\
\hline $\mathbf{8}$ & Bray Series 70 Control Valve & 02 \\
\hline $\mathbf{9}$ & Ball Valve & 02 \\
\hline
\end{tabular}




\begin{tabular}{|c|c|c|}
\hline ITEM & DESCRIPTION & QUANTITY \\
\hline $\mathbf{1 0}$ & Aluminum Sheets & 02 \\
\hline $\mathbf{1 1}$ & Shertec Centrifugal Pump CMPP12T (36GPM max.) & 01 \\
\hline $\mathbf{1 2}$ & Steel frame, bolts, nuts, brackets, wheels, etc. & - \\
\hline $\mathbf{1 3}$ & Electrical Connections (wires, cables, buttons, etc.) & - \\
\hline $\mathbf{1 4}$ & Computer and Data Acquisition Cards & $1 / 2$ \\
\hline $\mathbf{1 5}$ & LabVIEW, MATLAB & 2 \\
\hline
\end{tabular}




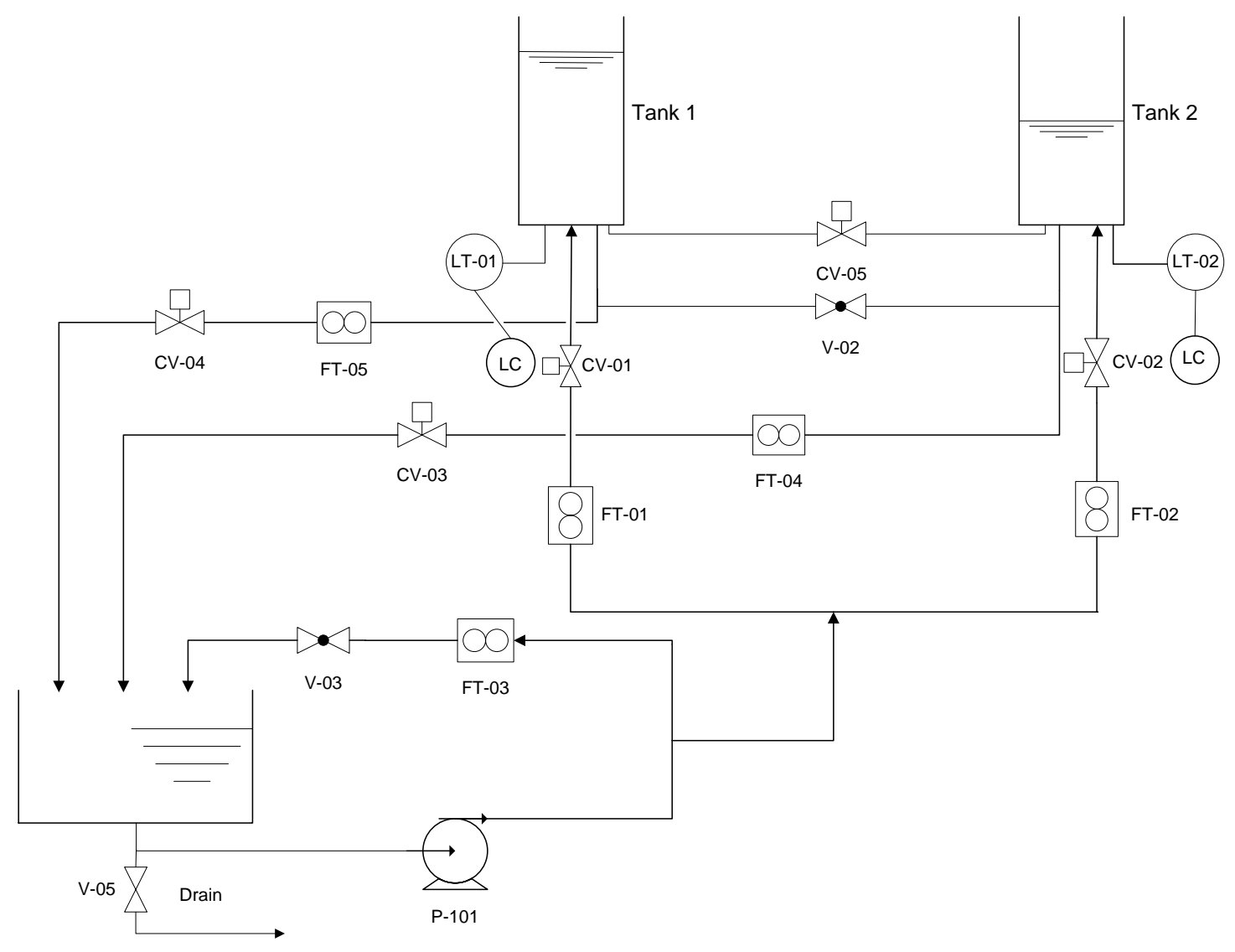

Figure 5.17. Schematic of the two-tank experimental control loop. 
The loop has a centrifugal pump, one stainless steel water reservoir, two acrylic tanks, four flow meters, two level transmitters, five motor-operated control valves (MOV), and three manual valves. The piping is made of either CPVC schedule 80 or PVC, with diameters varying from $1-1 / 2$ " to $1 / 2$ " and with enough flexibility to accommodate minor design changes. A data acquisition and control system developed specially for this loop is used to monitor and control the loop by varying the position of two control valves until a stationary flow throughout the system as well as a pre-set water level in either one or both acrylic tanks is attained. An MOV in between tanks provides the capability to work with both acrylic tanks (connected or not) the same time.

A bypass valve is provided to divert the excess water back to the water reservoir and lower the pressure in the loop. The maximum rated water flow is estimated to be around 32 GPM, provided the necessary pump pressure head is met. Though water temperature is monitored, temperature control is not performed but changes can be implemented for this purpose. The $4 \mathrm{ft}$ wide, $7 \mathrm{ft}$ long and $6 \mathrm{ft}$ high steel frame supports the equipment, up to a maximum load of 800 pounds.

\subsubsection{Data Acquisition and Loop Control}

In order to be able to perform various activities using the loop, a few human-machine software interfaces, called Virtual Instruments (VI), were developed using the National Instruments LabVIEW ${ }^{\circledR}$ - based package and data acquisition (DAQ) hardware. These VIs are capable of controlling the loop in both manual and automatic modes while performing data acquisition, monitoring, and logging the data in computer files for later use. Both software and hardware used in the two-tank loop are discussed in this section. 


\subsubsection{Hardware}

Two different data acquisition (DAQ) cards are installed in the personal computer used to run the loop, and are used for data acquisition/control purposes, and both are manufactured by National Instruments.

The first card is a 16-bit PCIe-6259 with 32 analog inputs and 4 analog outputs channels. This is a fast card capable of acquiring data at a speed of 1MS/s (mega-samples per second) for multi-channels (1.25MS/s for one channel), and output update speed of $2.86 \mathrm{MS} / \mathrm{s}$. The second card is a 12-bit PCI-MIO-16E-4 (discontinued) now known as PCI-6040E with 16 analog inputs and 2 analog outputs, capable of acquiring data at $500 \mathrm{kS} / \mathrm{s}$ (for one channel) or $250 \mathrm{kS} / \mathrm{s}$ for multiple channels, and output update speed of 1MS/s.

Three NI SCB-68 patch panels (shown in Figure 5.18) are used to connect the data acquisition cards to the various sensors and actuators installed in the loop. The NI SCB-68 is a shielded I/O connector block for interfacing I/O signals to plug-in data acquisition (DAQ) devices with 68-pin connectors. Combined with the shielded cables, the SCB-68 provides rugged, very low-noise signal termination, and it has an onboard cold-junction compensation sensor for low-cost thermocouple measurements. The analog outputs from these panels are used to control the MOVs through a CPVC box, also shown in Figure 5.18 on the far right side. Appendix 8 has a table with variables and their corresponding channels and patch panel numbers.

Due to the higher sampling rate speed of the PCIe-6259 card and the greater number of input channels available, it was selected to be used as the hardware interface controller with the loop, whereas the second card, the PCI-MIO-16E-4 is basically used for data acquisition monitoring and data logging, though it is also used to open or close the two control valves installed at the exit of the tanks. 


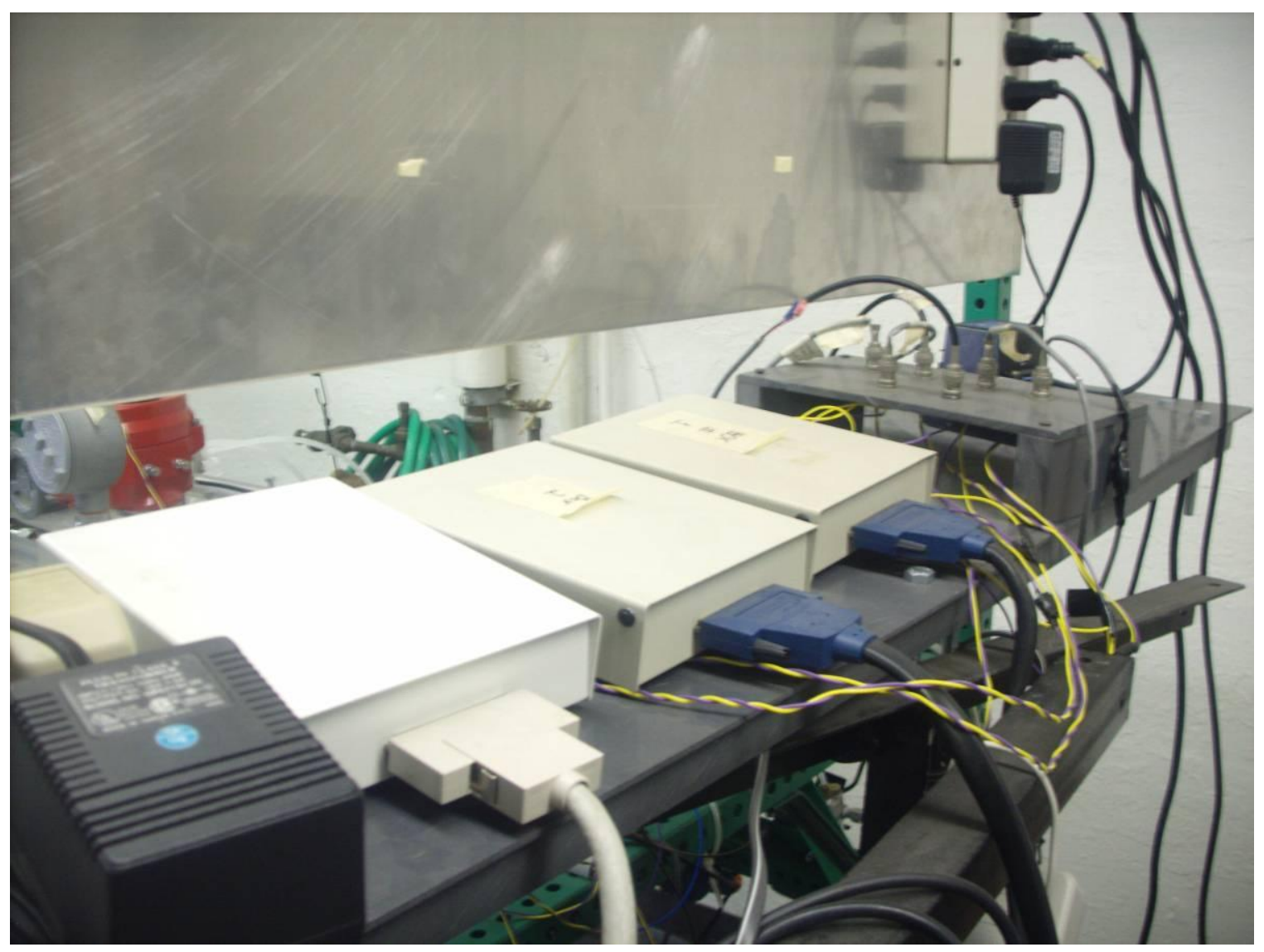

Figure 5.18. SCB-68 patch panels.

The first VI runs on the fastest DAQ and it holds all the control logic, control options (manual or automatic) for either Tank 1, Tank 2, both tanks operating together, tanks connected or disconnected, set point control options (manual or pre-set profile), controlled fault insertion (bias or drift), set point rate control, proportional-integral (PI) control gains for each tank separately and/or connected and gain optimization options using the auto-tune feature provided by LabVIEW, and many other functions. It also contains the MATLAB m-file code that provides the expected values used in the fault detection feature when running in Tank 1-only mode. The front panel automatically changes its appearance based on the configuration of the experiment being performed. Two different front panels are shown in Figures 5.19 and 5.20, respectively. The first panel in Figure 5.19 shows the typical configuration when the loop is running in manual control using both tanks, disconnected or not. A push button located right underneath the MOV manual control input boxes opens or closes the MOV connecting both 
tanks. A second panel is shown in Figure 5.20 with the typical configuration when the loop is running in automatic control using PI and Tank 2. Note that all controls related to Tank 1 are no longer present. Figure 5.21 shows a partial view of the block diagram with connections between blocks and PID filter and dead-band blocks for example. Additional screenshots are found in Appendix 9.

\subsubsection{Software}

The VIs developed to monitor, control and store data acquired from the loop are based on National Instruments LabVIEW, which is a graphical programming environment used to develop sophisticated measurement, test, and control systems using intuitive graphical icons and wires that resemble a flowchart.

Two main VIs were developed for the purpose of monitoring the dynamic condition by showing the current engineering values of all significant variables in SI units (International System of Units), storing the data and controlling the two-tank loop and are described next.

The second VI runs on the second, slowest DAQ, and serves a dual purpose: it is used for monitoring the loop dynamic condition by showing the current engineering values of all significant variables and comparing some of these variables to the expected values (for one single tank) generated in the first VI, and is used to acquire and store the data files on the computer for later use. The current file format used for saving the files is ASCII, which can be imported from and read by a plethora of different software packages. All flow rate equations from turbine and orifice flow meters and unit conversions are located in this VI. The main panel is shown in Figure 5.22, with the fault detection module output circled in red. 


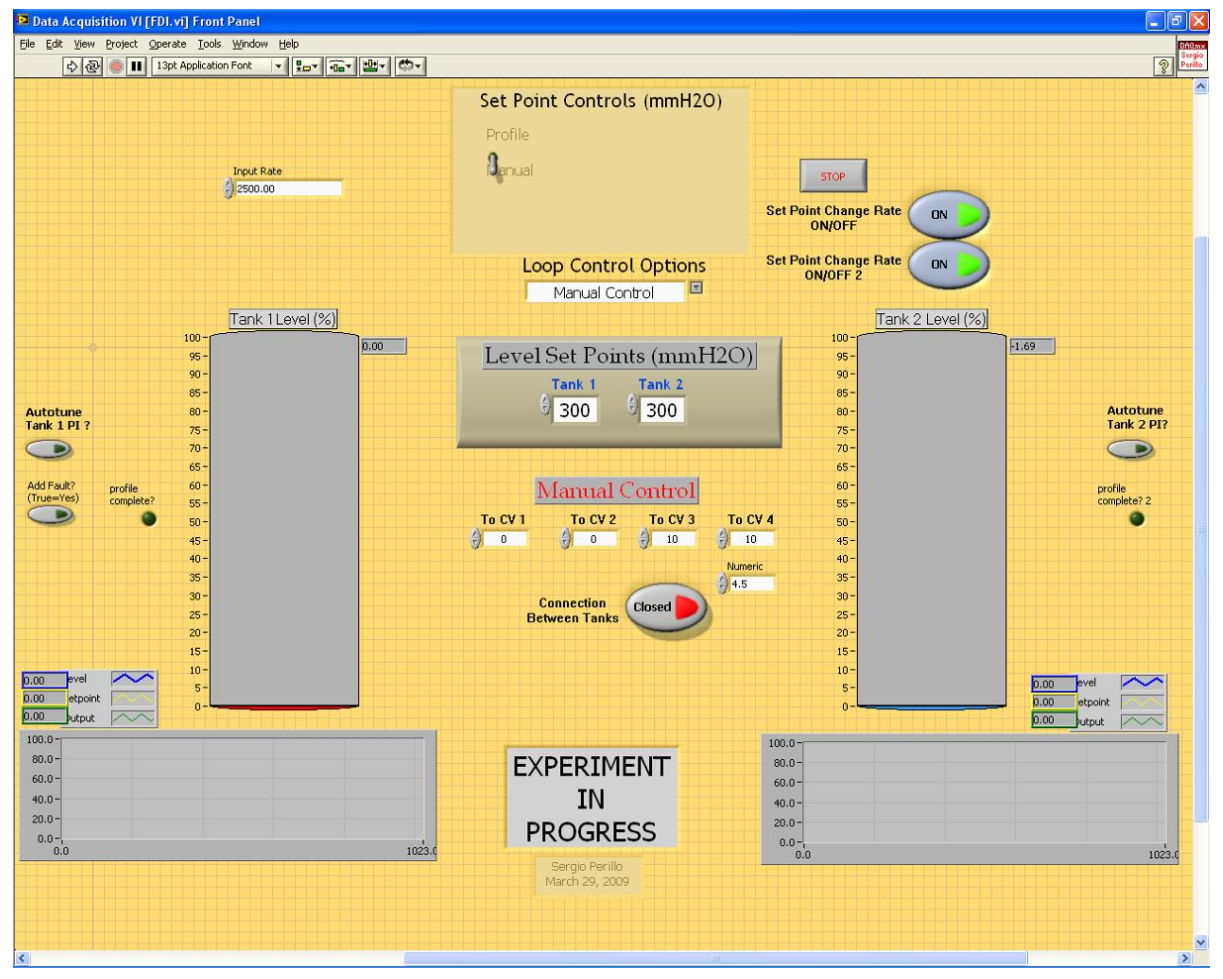

Figure 5.19. Control VI front panel showing the manual control option. 


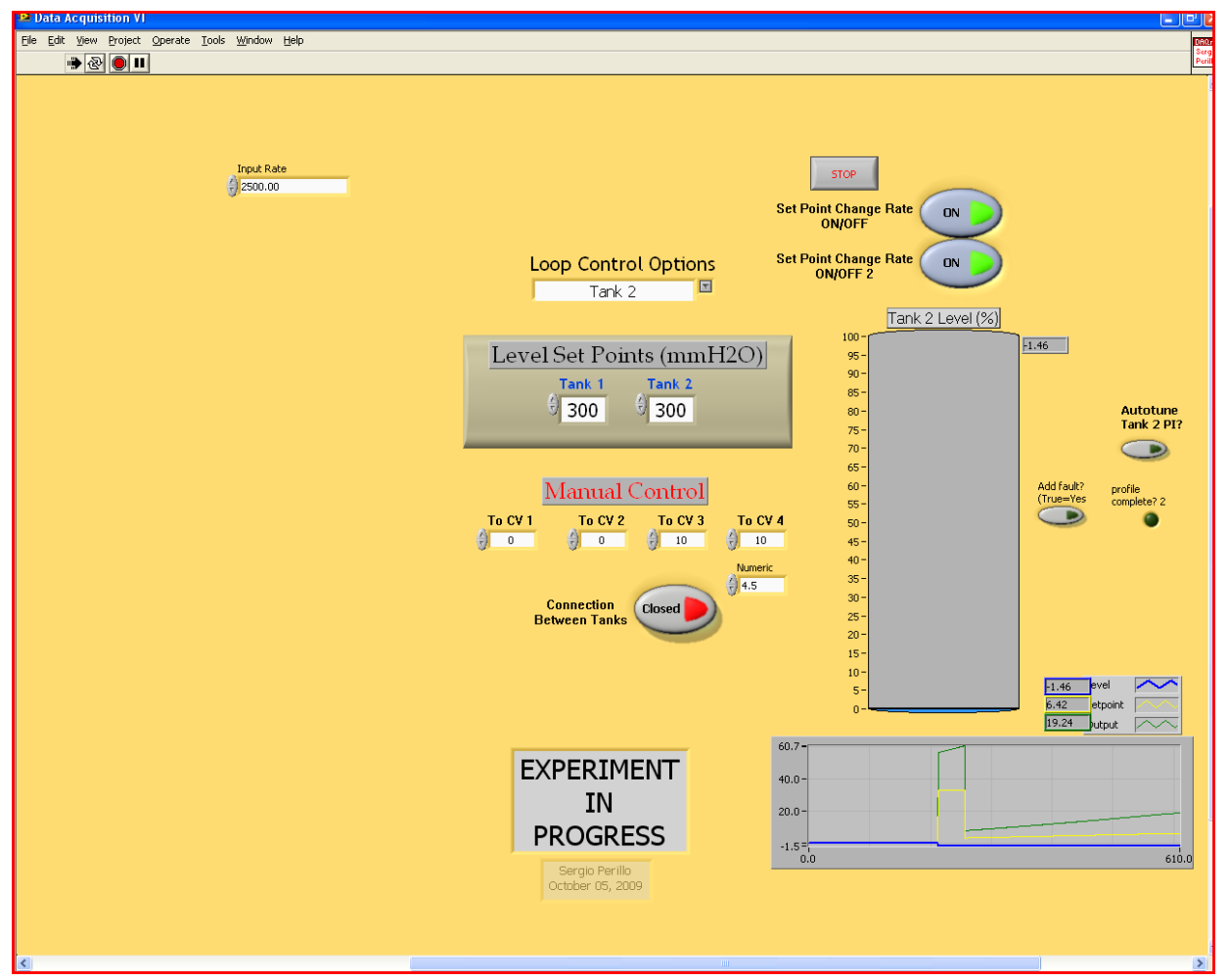

Figure 5.20. Control VI front panel showing the automatic control for Tank 2. 


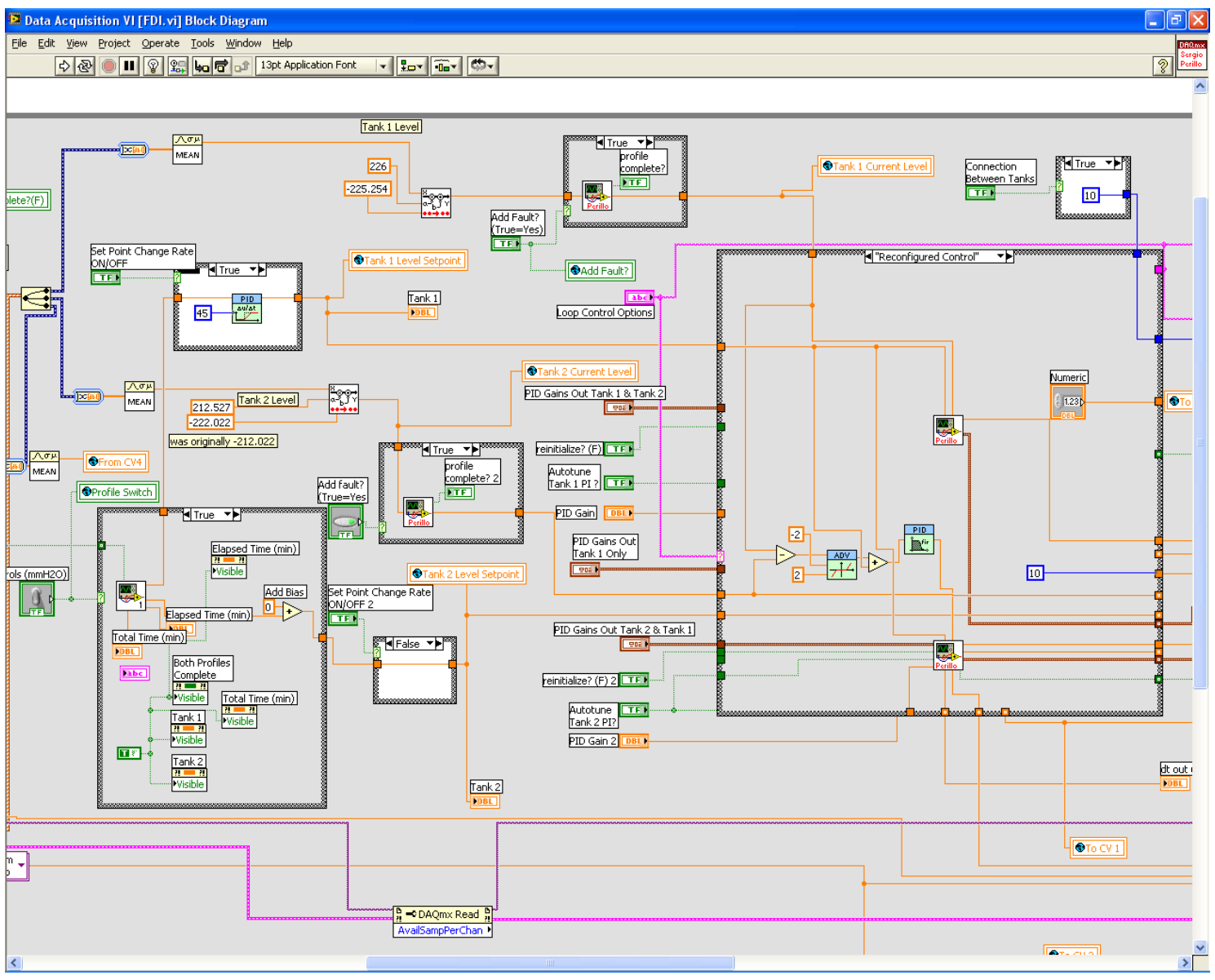

Figure 5.21. Control VI block diagram partial view. 


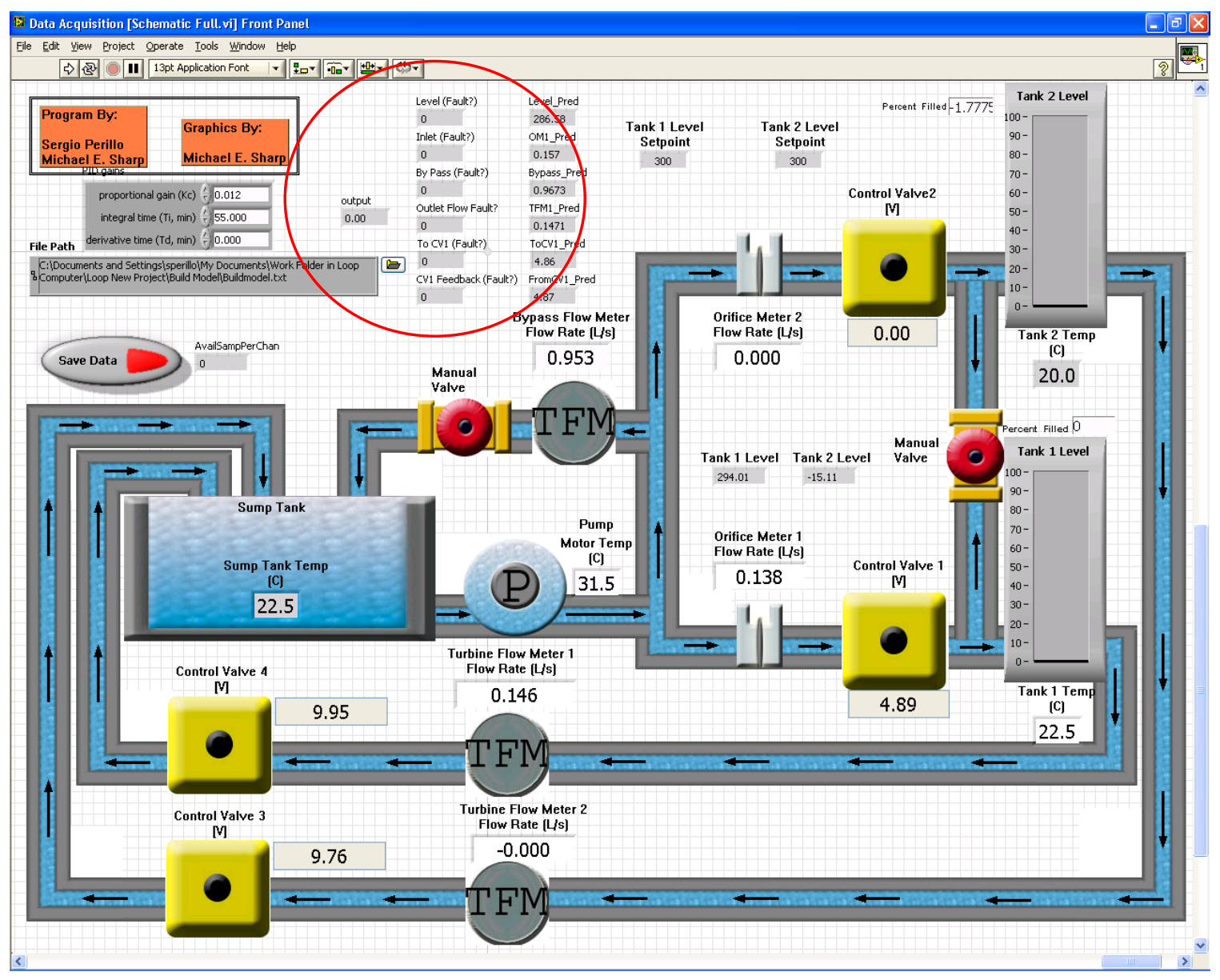

Figure 5.22. Data acquisition main panel. 


\section{Chapter 6}

\section{INTEGRATION OF FAULT DETECTION AND CONTROL RECONFIGURATION DURING STEADY STATE REGULATION}

\subsection{Introduction}

Various techniques are well established for on-line monitoring of equipment and systems in nuclear power plants. Since the early 1970s numerous efforts have been made to detect and identify anomalies and to provide alternative ways to measure critical and non-critical operating parameters in power plants, particularly reactor noise analysis which uses existing sensor signals to detect incipient faults, measure sensor response time, identify blockages in sensor lines, vibration of reactor internals, imbalance in rotating machinery, etc. Such techniques evolved into on-line monitoring to track the vibration of reactor internals, measure reactor stability, verify overall plant thermal performance, leak detection, estimation of remaining useful life of equipment, and others. Early detection of the onset of equipment and instrument channel degradation and failure can prevent loss of operational capability, reduce radiation exposure of plant personnel, enhance plant control, and minimize repair time [88]. The development of online techniques for monitoring and control with application to an experimental flow loop is described in this chapter, corroborating results available in the literature suggesting the applicability of such approach to operating plants with appropriate data acquisition and analytical redundancy. In this chapter the process of obtaining empirical methods based on data acquired from the loop is described. The approach uses data-based methods for characterizing the relationship among a set of measurements and the Sequential Probability Ratio Test (SPRT). Both techniques are part of a MATLAB toolbox software package named Process and Equipment Monitoring, also described in this chapter.

\subsection{Tools for Developing Empirical Models}

A few MATLAB functions were used to obtain each one of the empirical models applied to detect faults in the loop, and they are described in the following sections. Principal Component Analysis (PCA), used to help determine what variables were to be used in each model, AAKR and SPRT are implemented for data analysis and are described in detail. 


\subsubsection{Principal Components Analysis}

Principal Component Analysis (PCA) is a multivariate method used to capture the relationships in the data while reducing the dimensionality of an input space without losing a significant amount of information (variability). The method also makes the transformed vectors orthogonal and uncorrelated and is particularly useful for analysis of ill-conditioned data; hence such transformed vectors can be used by regression techniques without having the problems of collinearity. A lower dimensional input space will also usually reduce the time necessary to train a data-based model and the reduced noise will improve the mapping. The objective of PCA is to reduce the dimensionality and preserve as much of the relevant information as possible. PCA can also be thought of as a method of preprocessing data to extract uncorrelated features from the data.

Consider $m$ samples of $n$ random variables in a matrix $\mathbf{X}$ where the $\mathrm{n}$ columns are the variables and the $\mathrm{m}$ rows are the observations. PCA decomposes $\mathbf{X}$ into a product of scores $\mathbf{T}$ and orthogonal loadings $\mathbf{P}$ as:

$$
\mathbf{X}=\mathbf{T} \mathbf{P}^{\mathbf{T}}+\mathbf{E}
$$

where $\mathbf{E}$ contains the residuals.

The principal components (PCs) in the successive columns of $\mathbf{P}$ are obtained such that maximum variance in $\mathbf{X}$ is explained. Thus, in case the data is highly collinear, the first few PCs explain most of the variability in the data and are retained. The residuals in $\mathbf{E}$ constitute the unexplained variation in the data and contain the higher PCs that are rejected. PCA is thus a very efficient method for data compression. The scores so obtained are uncorrelated, meaning $\mathbf{T}^{\mathbf{T}} \mathbf{T}$ is a diagonal matrix. The PCs can be easily obtained as the right singular vectors of $\mathbf{X}$ using Single Value Decomposition (SVD), described below. 
The Singular Value Decomposition (SVD) algorithm decomposes a matrix $\mathbf{X}$ of dimension ( $n x \quad p$ ) into a diagonal matrix $\mathbf{S}$ of the same dimension as $\mathbf{X}$ containing the singular values, and unitary matrix $\mathbf{U}$ of principle components, and an orthonormal matrix of right singular values $\mathbf{V}$. It is important to use the mean centered data $(\mathbf{X})$ to give all variables the same importance, resulting in:

$$
X=\mathbf{A} \mathbf{L} \mathbf{U}^{\mathbf{T}}
$$

Where:

$\boldsymbol{X}$ is an arbitrary (n x p) matrix.

A is a (n x r) matrix of standardized PC scores with variance $=1 /(n-1)$.

$\mathbf{L}$ is a ( $\mathrm{r} \times \mathrm{r}$ ) diagonal matrix, where $\mathrm{r}$ is the rank of $\mathbf{X}$.

$\mathbf{U}$ is a (p x r) matrix of eigenvectors.

Both A and U have orthonormal columns resulting in:

$$
\mathbf{A}^{\prime} \mathbf{A}=\mathbf{I} \text {, and } \mathbf{U}^{\prime} \mathbf{U}=\mathbf{I}
$$

\subsubsection{Auto-Associative Kernel Regression (AAKR)}

AAKR is a non-parametric, empirical modeling technique that uses historical, fault-free observations and can be used to correct any errors present in current observations. Further details can be found in Hines \& Garvey [89]. The exemplar or memory vectors used to develop 
the empirical model are stored in a matrix $\mathbf{X}$, where $X_{i, j}$ is the $i^{\text {th }}$ observation of the $j^{\text {th }}$ variable. For $\mathrm{n}_{\mathrm{m}}$ observations of $\mathrm{p}$ process variables, this matrix can be written as:

$$
\mathbf{X}=\left[\begin{array}{cccc}
X_{1,1} & X_{1,2} & \cdots & X_{1, p} \\
X_{2,1} & X_{2,2} & \cdots & X_{2, p} \\
\vdots & \vdots & \ddots & \vdots \\
X_{n_{m}, 1} & X_{n_{m}, 2} & \cdots & X_{n_{m}, p}
\end{array}\right]
$$

Using this format, a query vector is represented by a $1 \times p$ vector of process variable measurements: $\mathbf{x}$.

$$
\mathbf{x}=\left[\begin{array}{llll}
x_{1} & x_{2} & \ldots & x_{p}
\end{array}\right]
$$

The corrected version of the input is calculated as a weighted average of historical, errorfree observations termed memory vectors $\left(\mathbf{X}_{\mathrm{i}}\right)$. The mathematical framework of this modeling technique is composed of three basic steps. First, the distance between a query vector and each of the memory vectors is computed. There are several distance functions that may be used, but the most commonly used function is the Euclidean distance, whose equation for the $\mathrm{i}^{\text {th }}$ memory vector is as follows:

$$
d_{i}\left(\mathbf{X}_{\mathrm{i}}, \mathbf{x}\right)=\sqrt{\left(\alpha_{i, 1}-x_{1}{ }^{2}+\alpha_{i, 2}-x_{2}{ }^{2}+\cdots+\alpha_{i, p}-x_{p}{ }^{2}\right.}
$$


For a single query vector, this calculation is repeated for each of the $\mathrm{n}_{\mathrm{m}}$ memory vectors, resulting in an $\mathrm{n}_{\mathrm{m}} \times 1$ matrix of distances: $\mathbf{d}$.

Next, these distances are transformed to similarity measures used to determine weights by evaluating the Gaussian kernel, expressed by:

$$
\mathbf{w}=K_{h}(\mathbf{d})=\frac{1}{\sqrt{2 \pi h^{2}}} e^{-\mathrm{d}^{2} / h^{2}}
$$

Where $h$ is the kernel bandwidth, $\mathbf{w}$ are the weights for the $\mathrm{n}_{\mathrm{m}}$ memory vectors.

Finally, these weigths are combined with the memory vectors to make the predictions according to:

$$
\hat{x}=\frac{\sum_{i=1}^{n_{m}} w_{i} \mathbf{X}_{i}-}{\sum_{i=1}^{n_{m}} w_{i}}
$$

If the scalar $a$ is defined as the sum of the weights, i.e.

$$
a=\sum_{i=1}^{n_{m}} w_{i}
$$

then equation 6.7 can be represented in a more compact matrix notation: 


$$
\hat{\mathbf{x}}=\frac{\mathbf{w}^{\mathrm{T}} \mathbf{X}}{a}
$$

The parameters to be optimized in an AAKR model are the memory matrix $(\mathbf{X})$ and the kernel bandwidth $(h)$. The researcher must decide how many vectors to include in the memory matrix and how large to make the bandwidth which indirectly controls how many memory vectors are weighted heavily during prediction.

\subsubsection{Sequential Probability Ratio Test (SPRT)}

The method chosen to detect faults in sensors and actuators in this research was the Sequential Probability Ratio Test (SPRT), and is based on the assumption that the residuals of your model are normally distributed and uncorrelated. This method, which was originally developed by Wald [90] and applied by many investigators [91], detects changes in signal properties, such as mean and standard deviation of a signal, and is used to identify drifts and changes in noise levels, while minimizing the probability of false alarms.

When performing a hypothesis test between two point hypotheses, the likelihood-ratio test is the most powerful test of size $\alpha$ for a threshold $\eta$. So when

$$
\begin{aligned}
& H_{0}: \theta=\theta_{0} \text {, and } H_{0}: \theta=\theta_{1} \text { the likelihood ratio test rejects the null hypothesis } H_{0} \text { when, }
\end{aligned}
$$

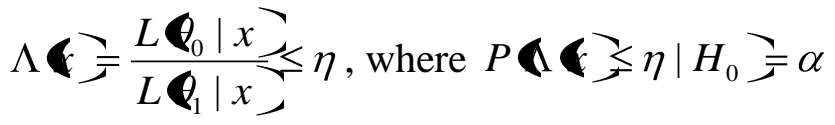

Given the likelihood equation $P$ with residuals $s_{k}$ at time $k$ and mean $m_{i}$ and variance $\sigma_{i}$ for hypothesis I, the likelihood ratio is: 


$$
\lambda_{k}=\frac{P_{1} \boldsymbol{\wp}_{k}, m_{1}, \sigma_{1}}{P_{0} \mathbf{\bigotimes}_{k}, m_{0}, \sigma_{0}}
$$

The log likelihood ratio becomes:

$$
\lambda_{k}=\ln \boldsymbol{\bigotimes}_{k}=\ln \left[\frac{\left.P_{1} \boldsymbol{\zeta}_{k}, m_{1}, \sigma_{1}\right)}{\left.P_{0} \mathbf{(}_{k}, m_{0}, \sigma_{0}\right)}\right]=\sum_{i=1}^{k} \ln \left[\frac{\left.P_{1} \boldsymbol{(}_{i}, m_{1}, \sigma_{1}\right)}{\left.P_{0} \mathbf{(}_{i}, m_{0}, \sigma_{0}\right)}\right]
$$

This can be written in the recurrent form as:

$$
\lambda_{k}=\lambda_{k-1}+\ln \left[\frac{\left.P_{1} \mathbf{C}_{i}, m_{1}, \sigma_{1}\right)}{P_{0}\left(\mathbf{c}_{i}, m_{0}, \sigma_{0}\right)}\right]
$$

According to the Central Limit Theorem (CLT), the distribution of an average tends to be normally distributed, even when the distribution from which the average is computed is decidedly non-normal, so,

$$
\lambda_{k}=\lambda_{k-1}+\ln \left[\frac{\frac{1}{\sqrt{2 \pi \sigma_{1}^{2}}} \exp \left[-\frac{\boldsymbol{\zeta}_{k}-m_{1}^{2}-}{2 \sigma_{1}^{2}}\right]}{\frac{1}{\sqrt{2 \pi \sigma_{0}^{2}}} \exp \left[-\frac{\boldsymbol{\varsigma}_{k}-m_{0}^{2}}{2 \sigma_{0}^{2}}\right]}\right]
$$

Expanding the logarithmic term and simplifying equation (7): 


$$
\lambda_{k}=\lambda_{k-1}+\ln \left(\frac{\sigma_{1}}{\sigma_{0}}\right)+\frac{\mathbf{C}_{k}-m_{0}^{2}}{2 \sigma_{0}^{2}}-\frac{\left.\mathbf{C}_{k}-m_{1}^{2}\right)}{2 \sigma_{1}^{2}}
$$

The residual distributions can be assumed to be normally distributed with zero mean and same variance, so equation (8) becomes:

$$
\lambda_{k}=\lambda_{k-1}+\frac{m_{1}}{\sigma^{2}}\left(s_{k}-\frac{m_{1}}{2}\right)
$$

Using Wald's two-sided test A and B are defined as,

$$
A=\ln \left(\frac{\beta}{1-\alpha}\right) \text { and } B=\ln \left(\frac{1-\beta}{\alpha}\right)
$$

Where:

$\alpha$ is the probability of false alarm, and should be kept small to avoid a Type I error, or false positive.

$\beta$ is the probability of missing an alarm for a Type II error, or false negative.

The status of the equipment being monitored is determined by a comparison of $\mathrm{A}$ and $\mathrm{B}$ with the log likelihood ratio:

For $\lambda_{m}<A$ the sensor can be considered in good condition.

For $\lambda_{m}>B$ the sensor can be considered degraded. 
As depicted in Figure 6.1, rather than computing a new mean and variance at every new sample acquired, the SPRT monitors the equipment's performance by processing the residuals in a sequential fashion. The residual signals, which are the differences between the sensor measurements and the estimates from the model, are used to generate a likelihood ratio (ratio of joint probability density of residuals) based on the statistical properties of the incoming data compared with the statistics in the model. In other words, based on the statistics of the new data coming from the equipment being monitored, the method is capable of detecting differences in such statistical properties and inform if the new data comes from a similar statistical distribution or not. This process of comparing the model predictions with values coming from the equipment is depicted in Figure 6.2, where the likelihood ratio is evaluated by the SPRT threshold for the specified component to make a logical decision concerning its status.

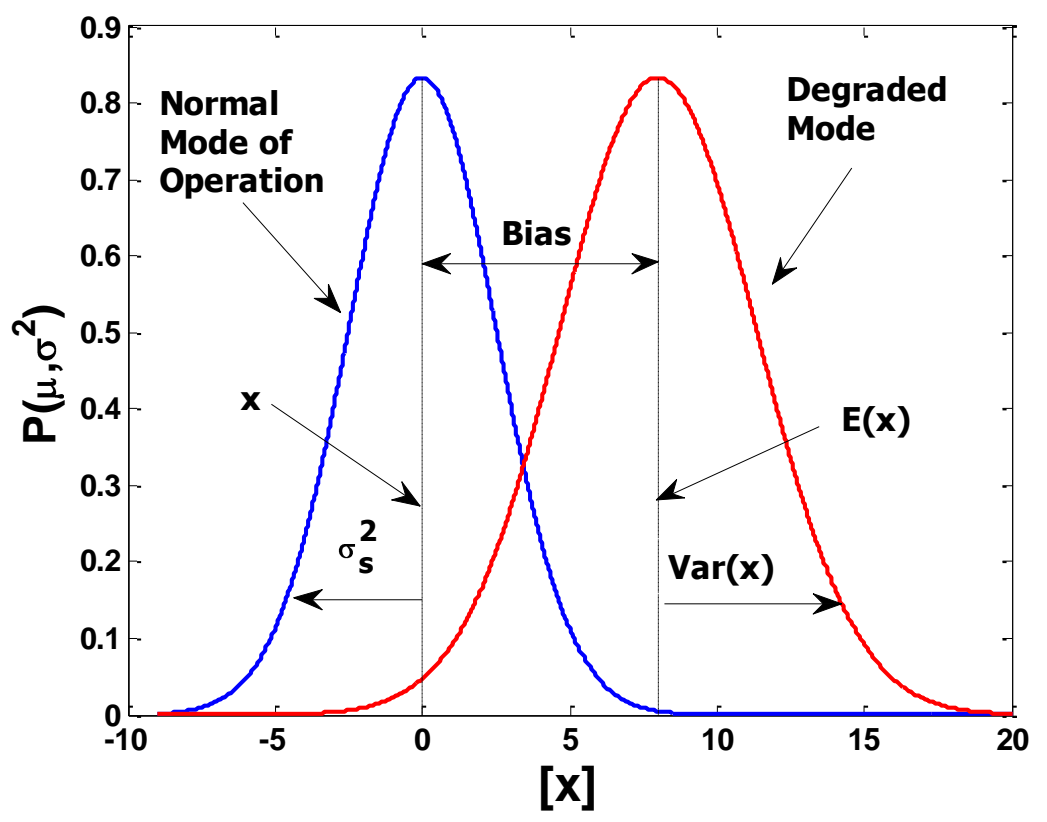

Figure 6.1. SPRT is based on comparing statistical differences. 


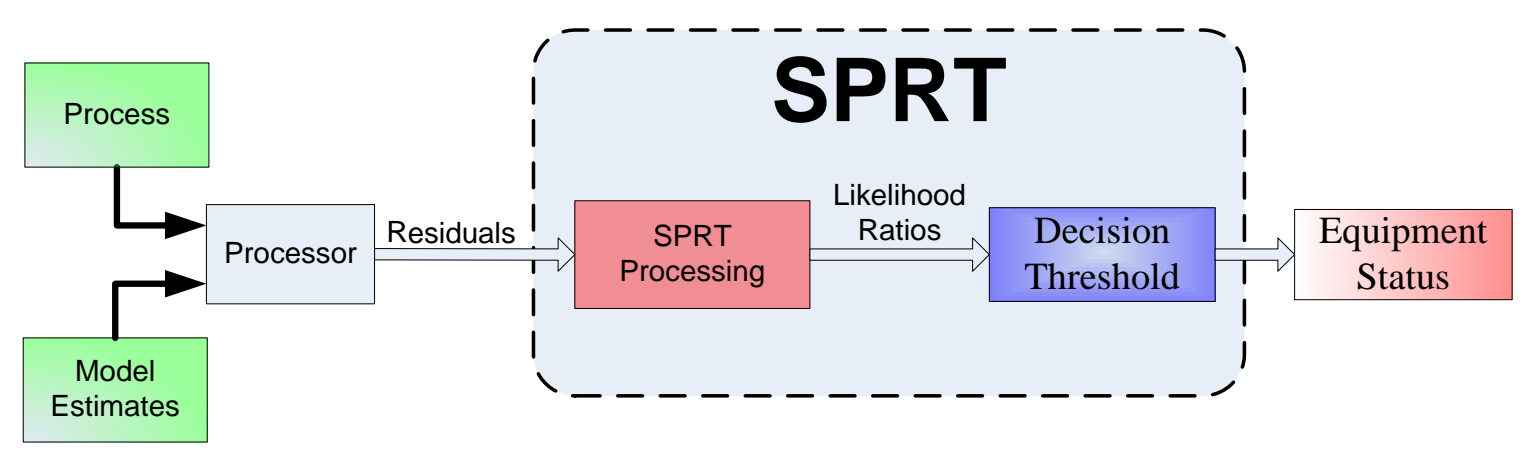

Figure 6.2. SPRT analysis diagram.

\subsubsection{Process and Equipment Monitoring (PEM) Toolbox}

On-line monitoring (OLM) commonly uses an autoassociative empirical modeling architecture to assess equipment performance. An autoassociative architecture predicts a group of correct sensor values when supplied a group of sensor values that is usually corrupted with process and instrument noise, and could also contain faults such as sensor drift or complete failure. The Process and Equipment Monitoring (PEM) Toolbox [92] [93], which was developed at The University of Tennessee, is a set of MATLAB based tools, which have been developed to support the design of process and equipment condition monitoring systems. Its purpose is to provide the necessary tools so that different empirical modeling and uncertainty estimation methods may be easily investigated and compared. In this research several PEM toolbox functions were used to obtain the empirical models necessary to perform fault detection using the sensors and actuators measurements installed in the loop, and as such, some stand-alone, lowlevel PEM-based functions are currently implemented in the data acquisition VI to monitor and compare measurements and predictions.

\subsection{Data Generation and Model Development}

In this research a 2.5 hour long dataset with level set points varying from $300 \mathrm{mmH}_{2} \mathrm{O}$ to $600 \mathrm{mmH}_{2} \mathrm{O}$, containing ten variables for a single-tank configuration was acquired and used to obtain six empirical models: five linear models and one Auto-Associative Kernel Regression (AAKR) model which is a non-parametric technique, commonly called memory based technique, 
that do not compute optimal weights a priori, but instead store all the "training data" and directly use it to compute predictions when a query is made [94], in contrast to parametric techniques, such as neural networks or linear regression, that uses data to "train" a model and determine parameters such as regression coefficients or weights to optimize the performance of the modeled input-output relationships. Of those ten variables, six were considered to be used in the modeling process for being true representatives of the loop dynamics, and these variables are listed in Table 6.1. The process of obtaining the models using the dataset is described below.

Table 6.1. Variables considered in the empirical modeling process.

\begin{tabular}{|c|c|c|}
\hline Tag & Description & Units \\
\hline N/A & Tank 1 water level & $\mathrm{mm}_{2} 0$ \\
\hline OM1 & Tank 1 inlet flow rate & $1 / \mathrm{s}$ \\
\hline TFM1 & Tank 1 outlet flow rate & $1 / \mathrm{s}$ \\
\hline Bypass & Bypass flow rate & $1 / \mathrm{s}$ \\
\hline ToCV1 & Is the control system output to the inlet flow rate control valve actuator & $\mathrm{V}$ \\
\hline FromCV1 & Is the voltage signal from the inlet control valve actuator & $\mathrm{V}$ \\
\hline
\end{tabular}

N/A* - Not applicable

\subsubsection{Process of Obtaining the Models}

As depicted in the flowchart in Figure 6.3, the dataset was acquired in such way to cover water levels ranging from $300 \mathrm{mmH}_{2} \mathrm{O}$ to $600 \mathrm{mmH}_{2} \mathrm{O}$, with one second interval between measurements, and a maximum set point rate of change of $45 \mathrm{mmH}_{2} \mathrm{O} / \mathrm{min}$. The raw data acquired was then visually inspected for outliers and spurious values. Using PEM toolbox functions, the dataset was cleaned up to get rid of stuck values, outliers and spurious values, 
mean-centered, unit variance scaled to give all variables the same importance and a chance to contribute to the models. The scaled dataset was then divided up in to three different blocks using venetian blind method. The training block included 500 of the most significant data points, and both lowest and highest values from each variable to make sure the resulting training set included all the variance present in the dataset. The test set was used to test the models, and the validation set was used as new queries to gauge how well the obtained models performed using unseen or new data. Next, a PCA test was performed using the covariance matrix to obtain the principal component coefficients, also known as loadings. Figure 6.4 shows a graphical representation of how each variable is correlated with each other using the absolute values of the correlation coefficient matrix for all six candidate variables, with dark blue meaning the variables are either weakly or not correlated at all, and dark brown meaning they are highly correlated with each other, and from this representation is possible to conclude:

- Obviously, the water level is highly correlated with the outlet flow rate sensor (TFM1), and somewhat correlated with the inlet flow rate (OM1).

- Inlet flow rate is correlated with the bypass flow rate, somewhat correlated with the outlet flow rate, and highly correlated with the control output (ToCV1) and valve position feedback signal (FromCV1).

- The bypass flow rate is correlated with the inlet flow rate control valve.

- And both control output to the inlet flow rate control valve and the valve feedback signal itself are highly correlated, as expected. 


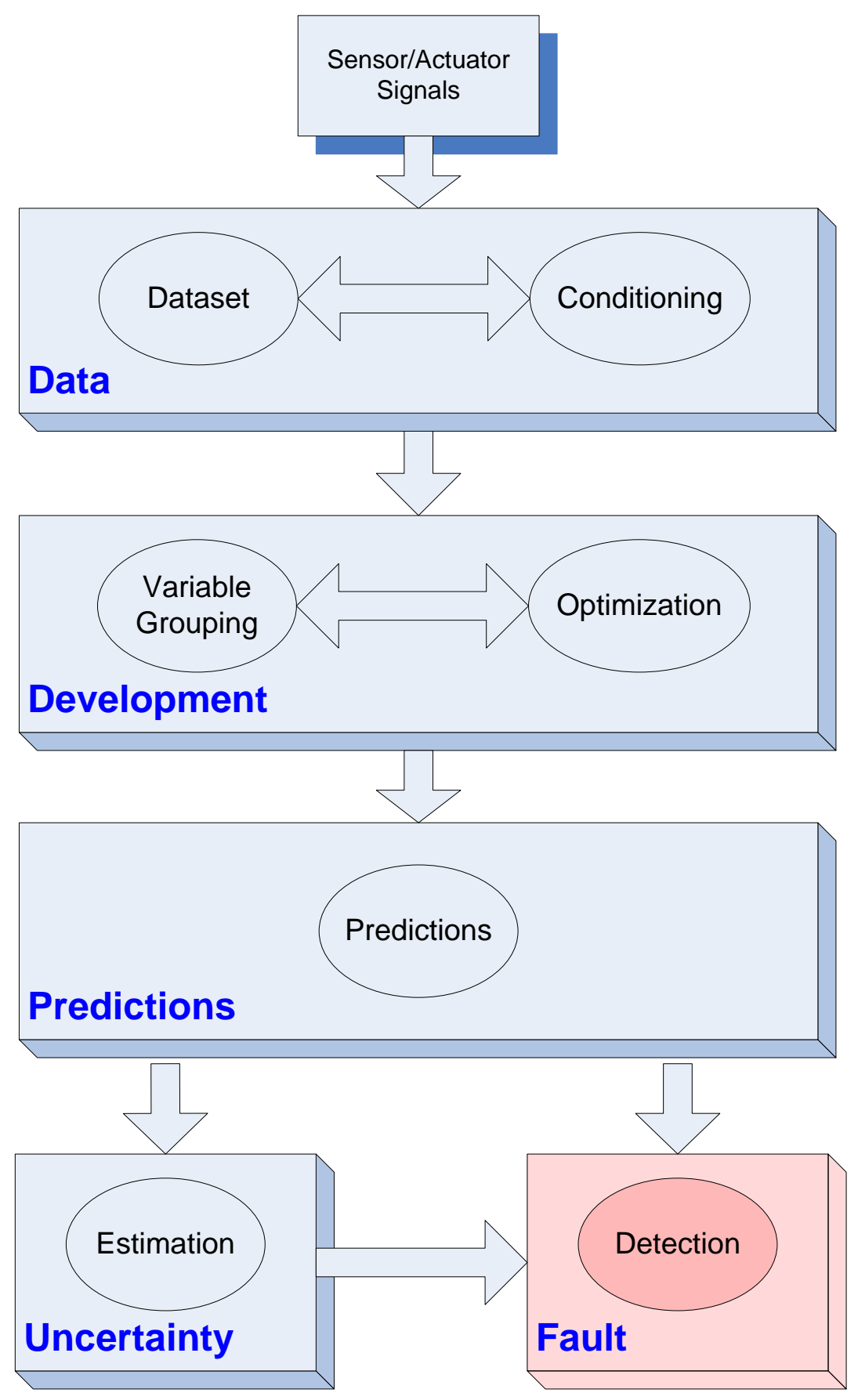

Figure 6.3. Model development flowchart 


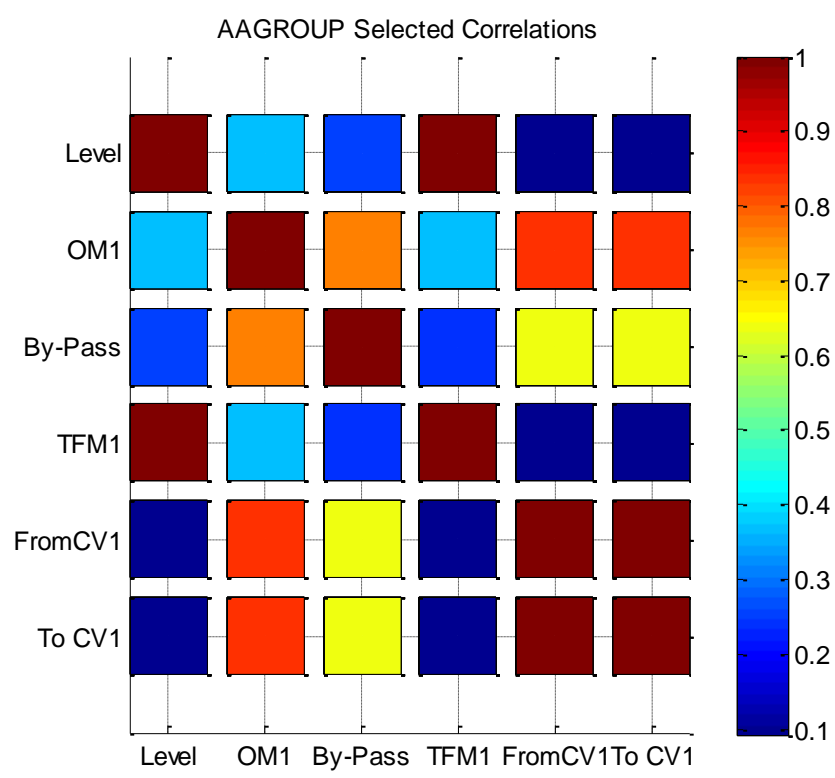

Figure 6.4. Correlation coefficient matrix (absolute values).

It is very important to remember that, since the multivariate loop is an open process, meaning the water inventory in the water reservoir varies over time due to small leaks and evaporation, so the obtained empirical models were obtained under very determined conditions, and are valid only within the range of operation the data was acquired. Any significant deviations from such conditions will cause the predictions to diverge from measurements, causing the fault detection routine developed to misidentify the measurements as coming from a faulty condition.

Based on the PCA findings, each model used variables that are correlated with each other based on their loadings, but making sure at least one not-so-much correlated variable was included in the model to provide the necessary robustness to the fault detection routine currently incorporated in the data acquisition VIs, although risking an increase in model bias. This tradeoff is particularly important in obtaining models that will be used for fault detection. For instance, the outlet flow rate is highly correlated with the water level in the tank, and this variable alone would be enough in the model to predict water level. But if the water level is to be inferred using faulty outlet flow rate sensor readings the predictions would be incorrect, but by including the inlet flow rate readings such one-on-one variable dependency can be diminished, hence adding some robustness to the model. 
Three different models were investigated for each of the six variables: linear regression, kernel regression and $\mathrm{AAKR}$, and the final models implemented in the routine responsible for the fault detection were chosen based upon their Mean Absolute Percent Error (MAPE), defined as:

$$
M A P E=\frac{1}{n} \sum_{i=1}^{n}\left|\frac{\operatorname{Actual}(i)-\operatorname{Predicted}(i)}{\operatorname{Actual}(i)}\right|
$$

Where:

$n$ is the number of fitted points.

$i$ corresponds to the $i$-th value

Figure 6.5 shows MAPE values for all three different modeling methods investigated: linear and kernel regressions, and AAKR. In three of the cases, inlet flow rate control (ToCV1) and control valve feedback signals (FromCV1), the regression models performed just as well as the AAKR models, while linear regression worked well for four variables, outperforming both AAKR and linear. In the case of the inlet flow rate the AAKR model outperformed the other two models, in great part due to non-linearity caused by the control valve hysteresis. All bypass models presented the lowest MAPE of all, with less than $0.5 \%$. In this case the linear model was chosen for being the least complex model of all three. In conclusion, five linear regression and one AAKR models were chosen to be used to perform the predictions and fault detection.

The dataset acquired and used to obtain each of the six models is shown in Figures 6.6 to 6.11. Figure 6.6 shows how close the current water level is to the set point. Both inlet and outlet flow rates are shown in Figures 6.7 and 6.8, respectively, and the flow rate in the bypass is shown in Figure 6.9. The PI controller outputs a voltage signal, which is sent to the control valve which varies the inlet flow rate to regulate the water level inside the tank according to the set point, and is depicted in Figure 6.10. The control valve outputs a voltage signal that can be monitored to assess the control valve position and health, and is shown in Figure 6.11. 


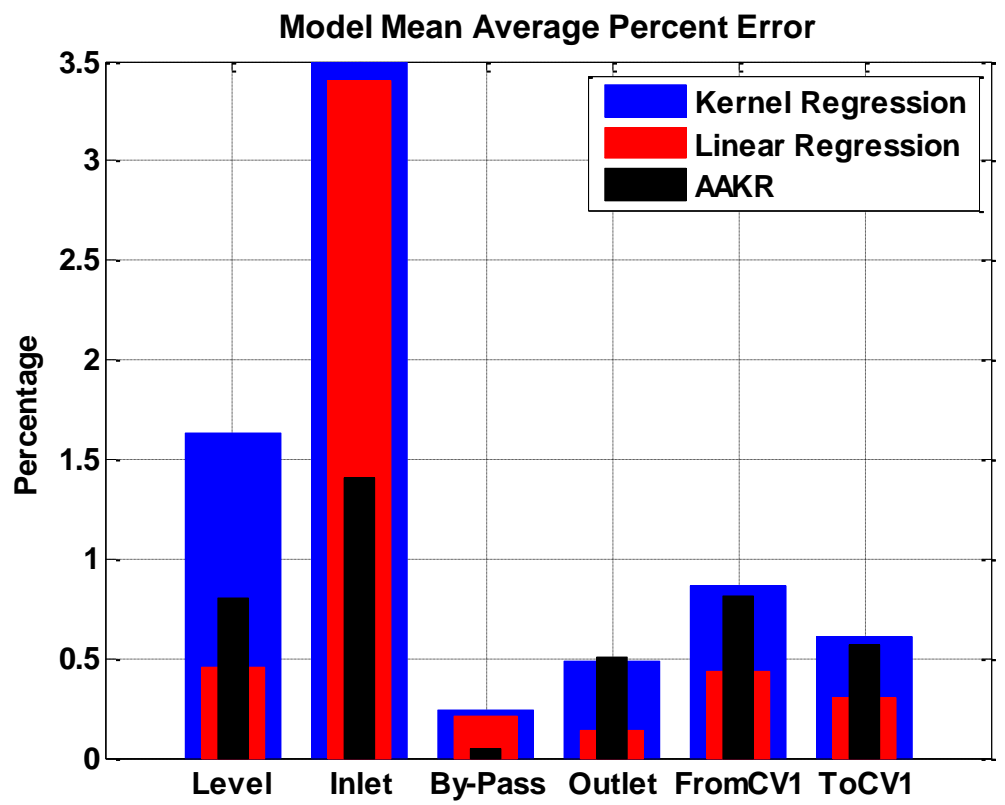

Figure 6.5. MAPE for linear regression, kernel regression, and AAKR models.

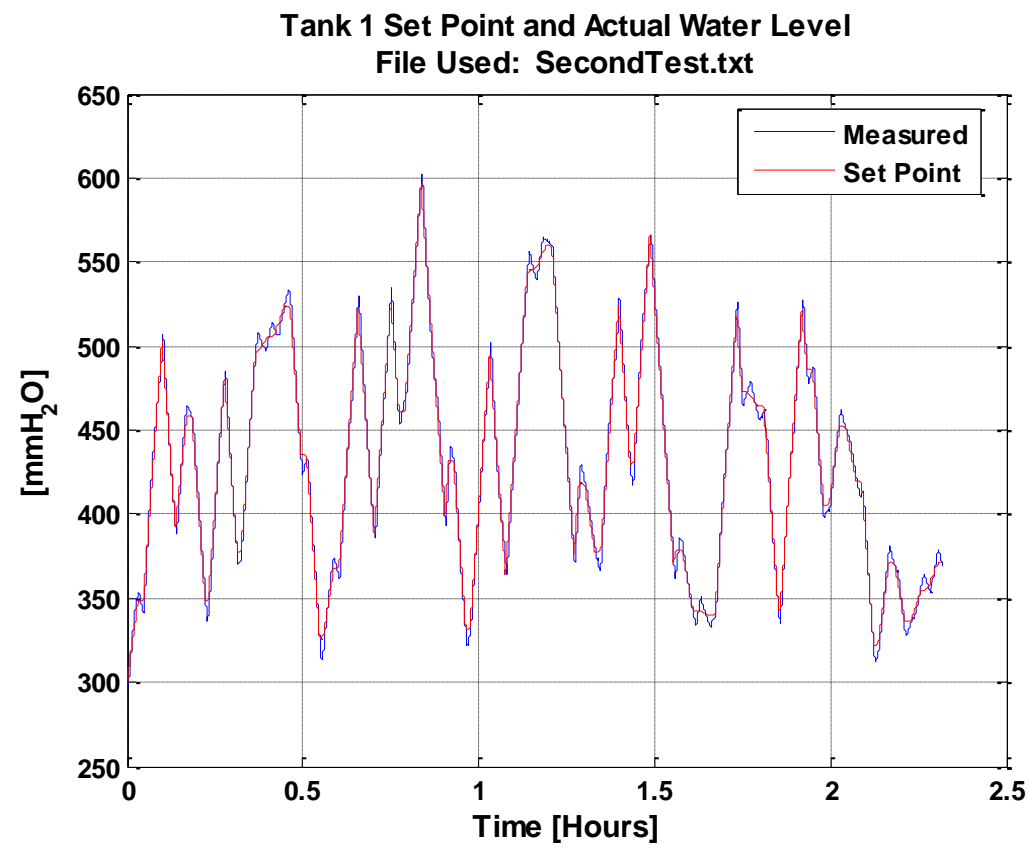

Figure 6.6. Level measurenents and set points 


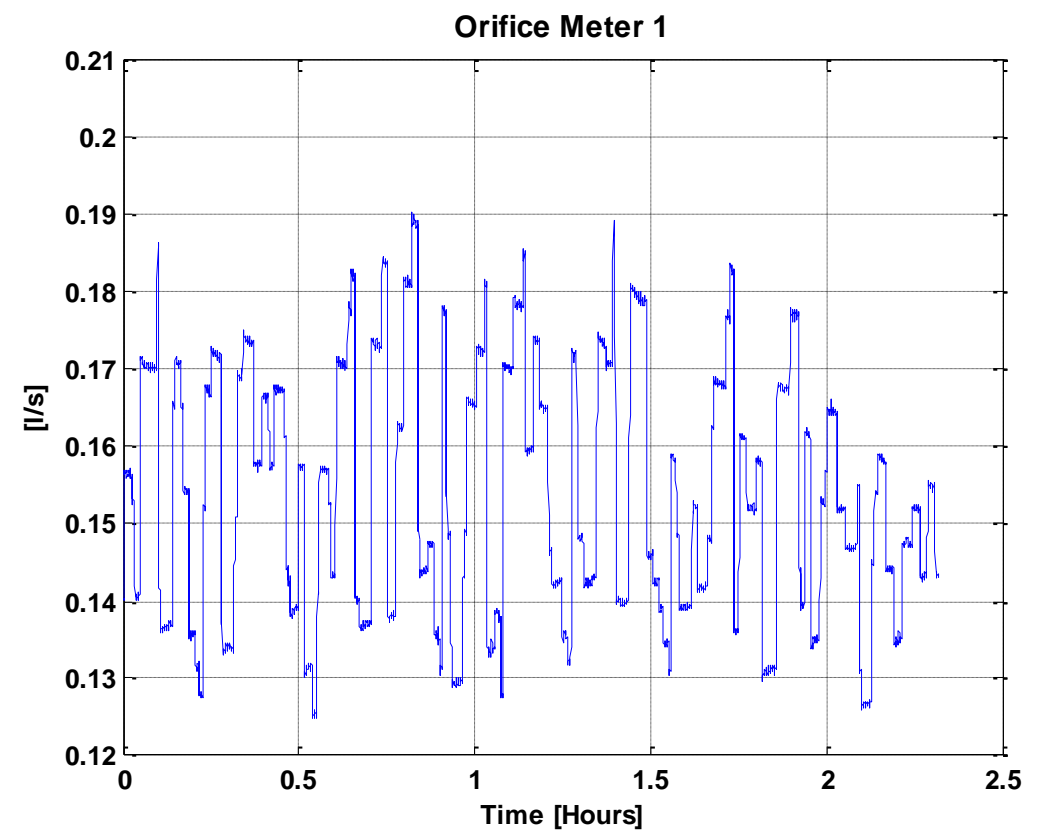

Figure 6.7. Tank 1 inlet flow rate.

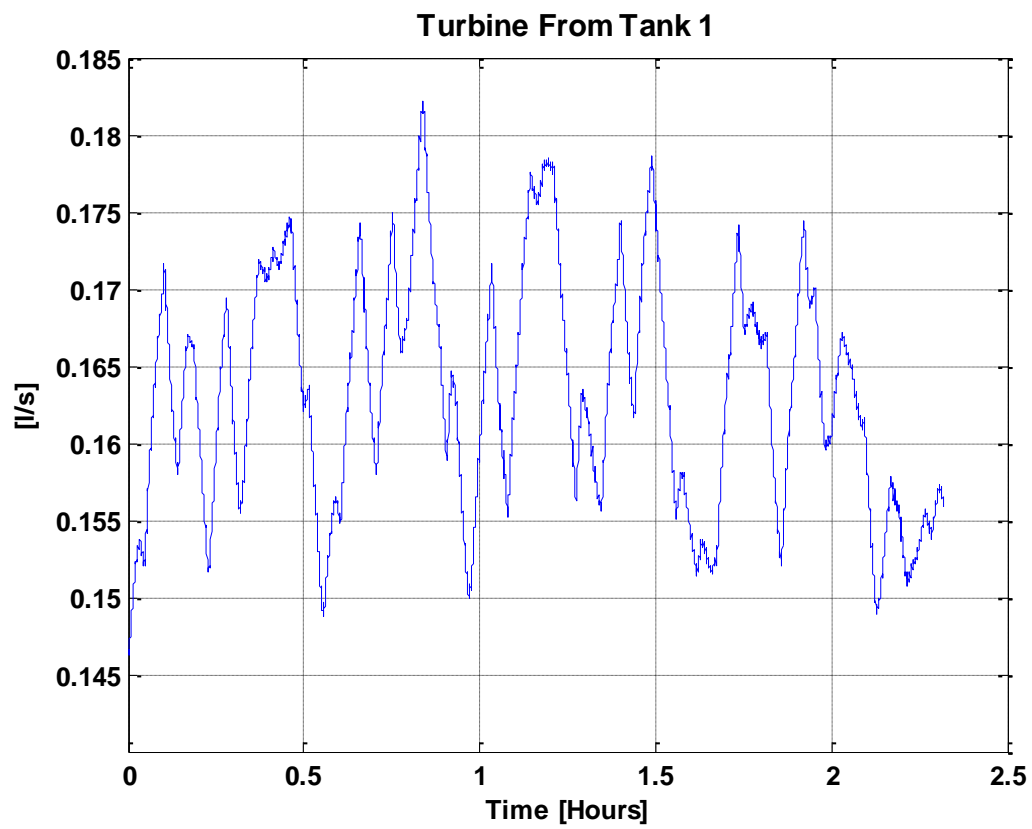

Figure 6.8. Tank 1 outlet flow rate. 


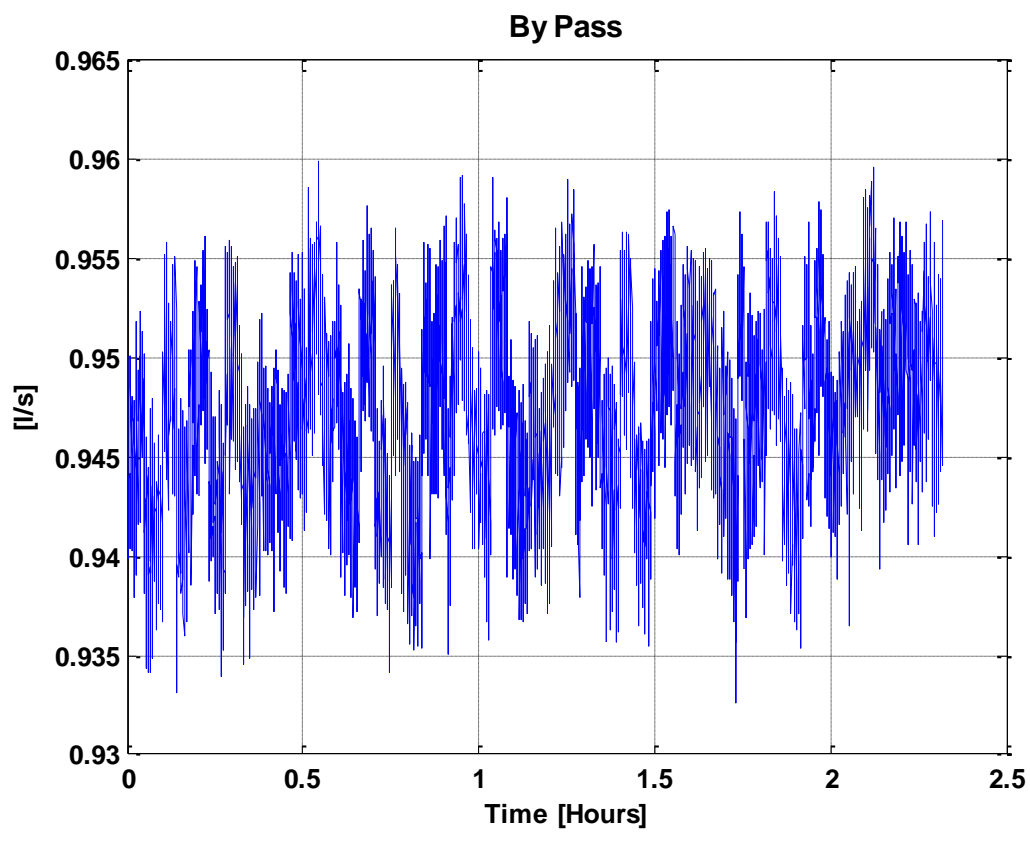

Figure 6.9. Bypass flow rate.

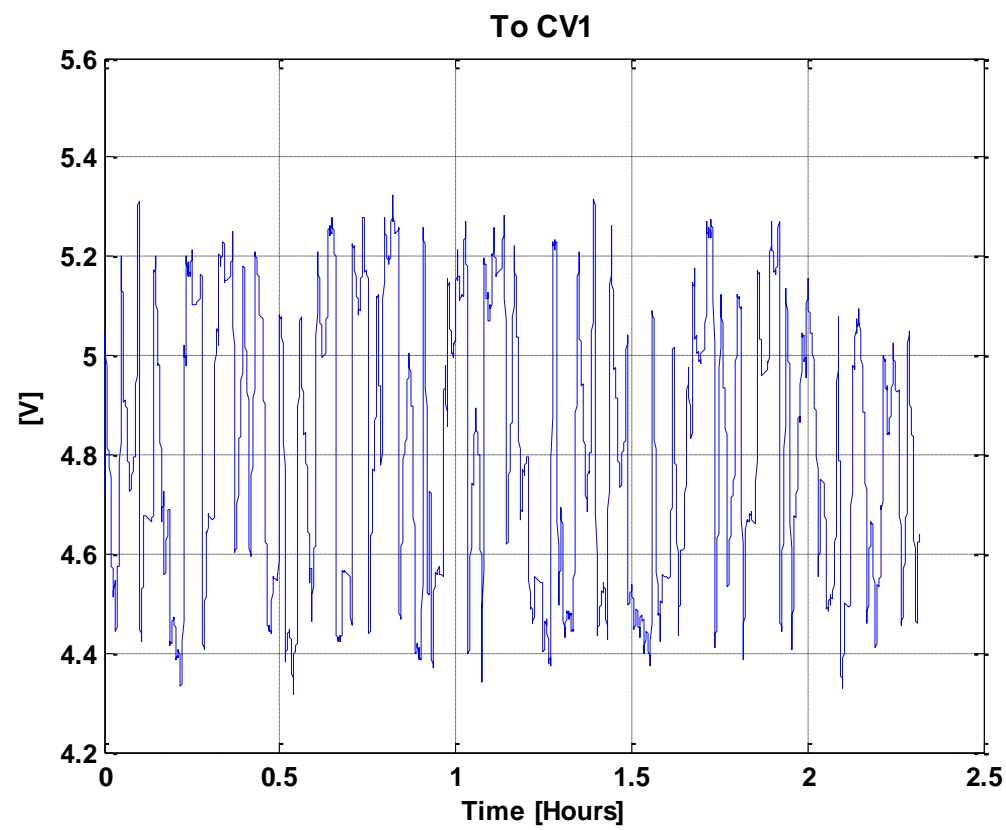

Figure 6.10. Tank 1 inlet flow rate controller output. 


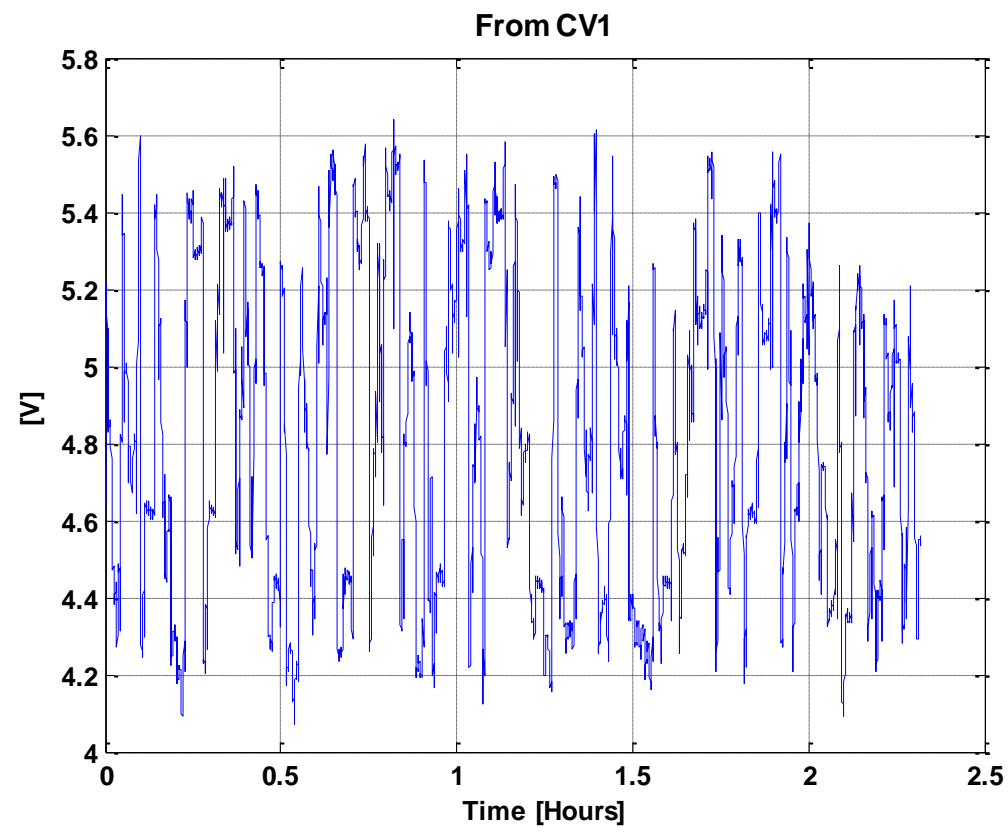

Figure 6.11. Tank 1 inlet flow rate control valve feedback.

After the empirical models were obtained they were implemented in the data acquisition VI and a different dataset was acquired using the same set points as the original dataset used to obtain the models. The new measurements were used as queries to the models, and the results comparing the predicted values with the new query are shown in Figures 6.12 to 6.17. Noteworthy are the level predictions in Figure 6.12 with an average absolute deviation from measured values of about $30 \mathrm{mmH}_{2} \mathrm{O}$, and maximum of $40 \mathrm{mmH}_{2} \mathrm{O}$, and the tank outlet flow rate with an average deviation of about $3 \times 10^{-3} 1 / \mathrm{s}$. Bypass predictions also very low around $2 \%$ of maximum flow rate $(0.92 \mathrm{l} / \mathrm{s})$. The inlet flow rate showed a high absolute difference of about $26 \%$ or $0.04 \mathrm{l} / \mathrm{s}$ with maximum flow rate of around $0.152 \mathrm{l} / \mathrm{s}$, greatly due to the control valve non-linear behavior. 

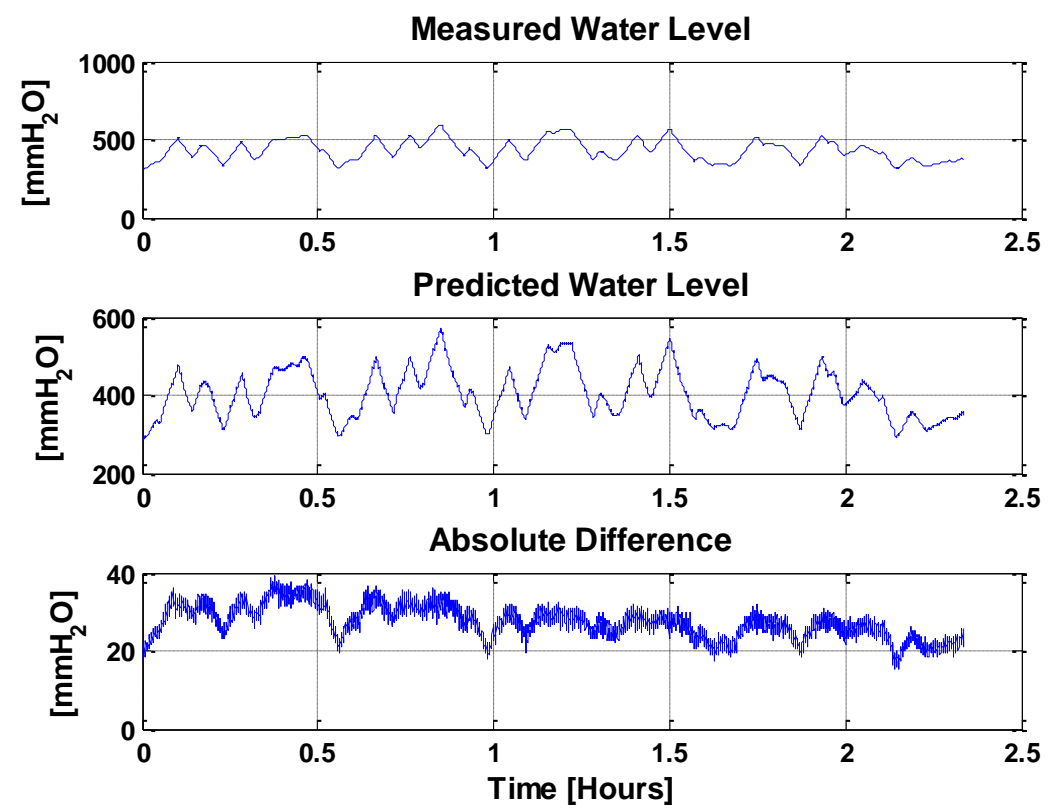

Figure 6.12. Measured water level versus predicted values. 

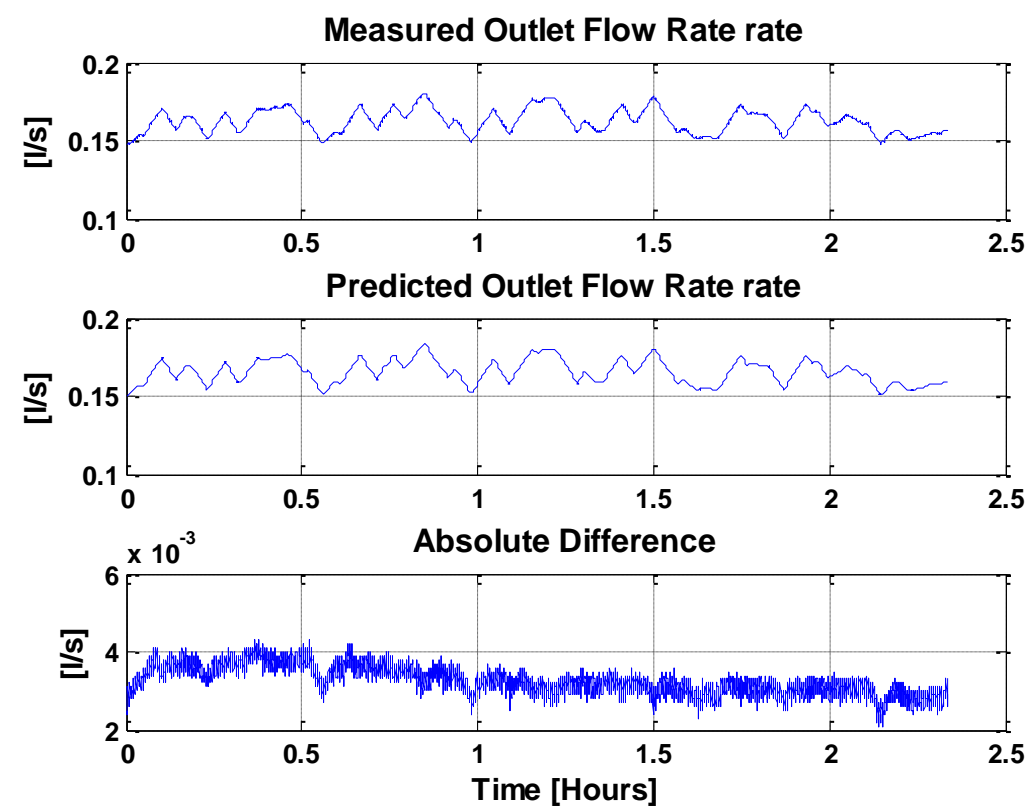

Figure 6.13. Measured outlet flow rate versus predicted values
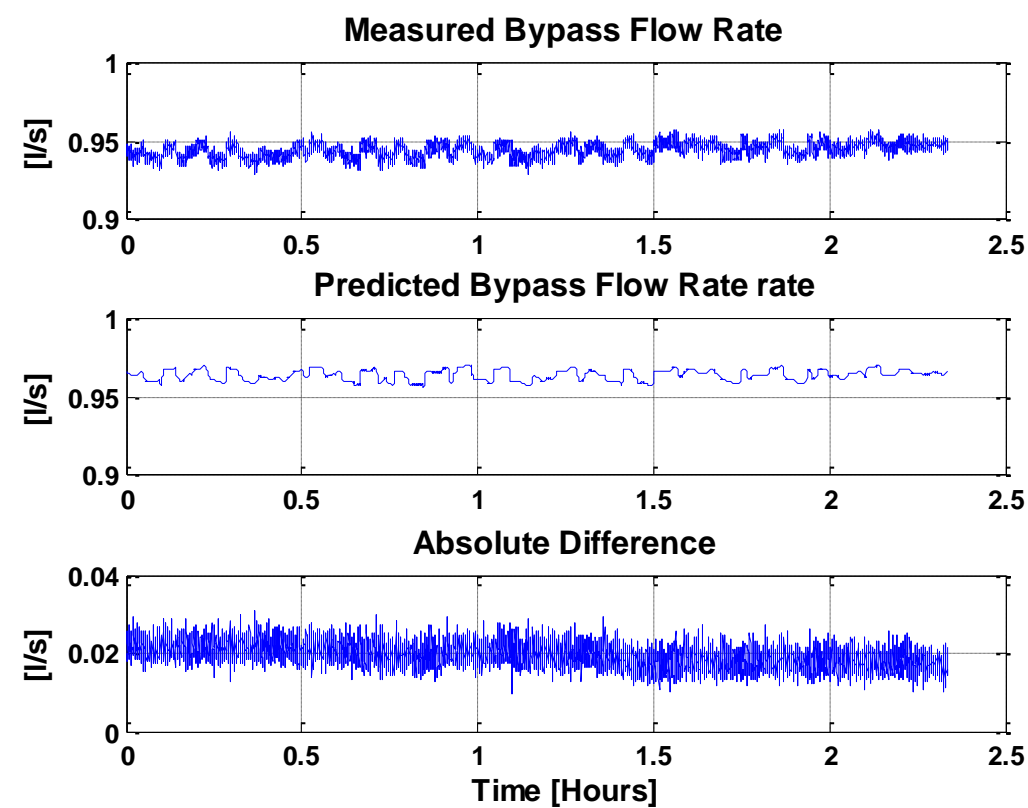

Figure 6.14. Measured bypass flow rate versus predicted values. 

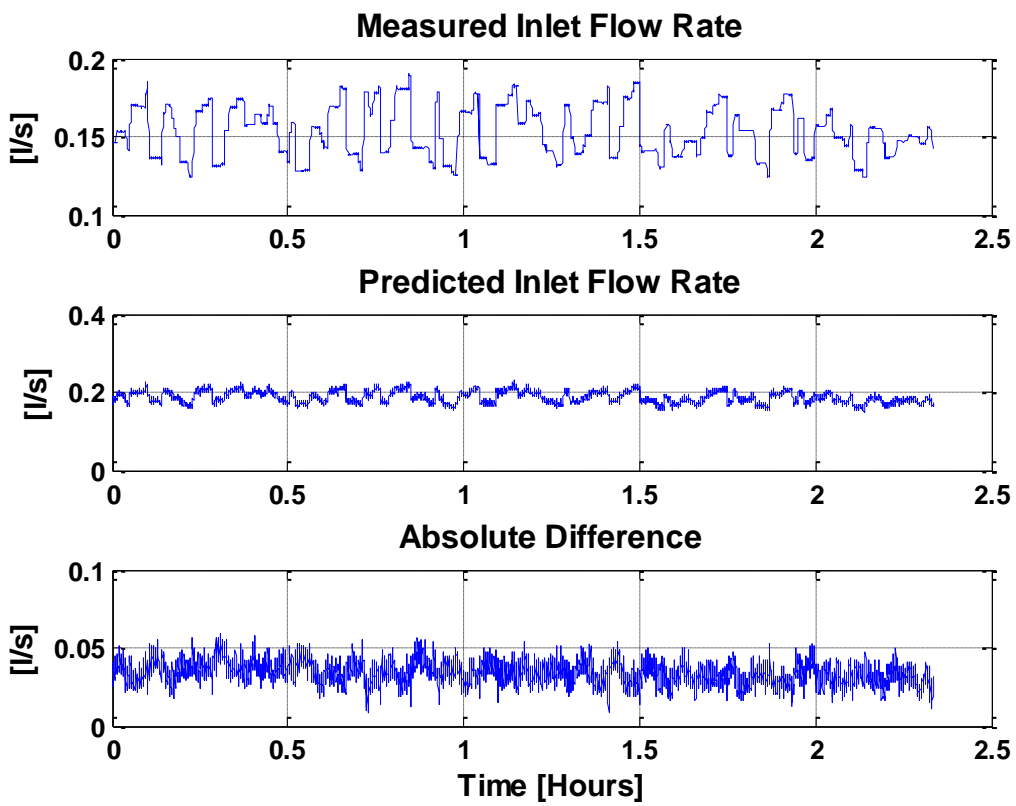

Figure 6.15. Measured inlet flow rate versus predicted values.
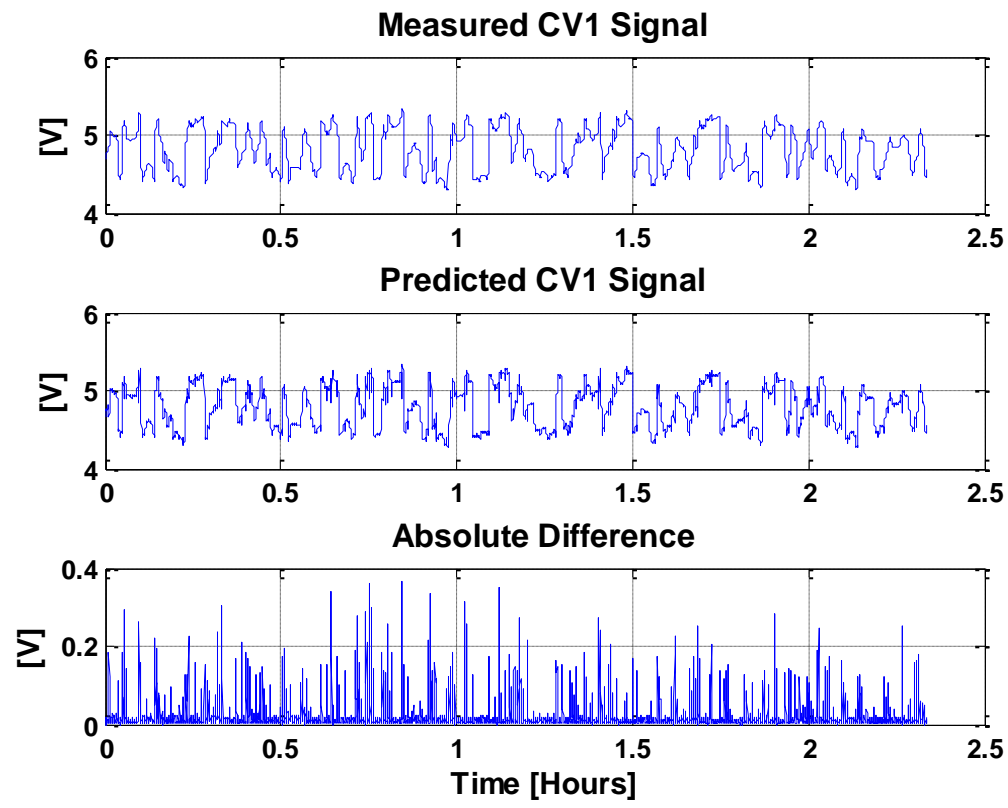

Figure 6.16. Measured control output versus predicted values. 

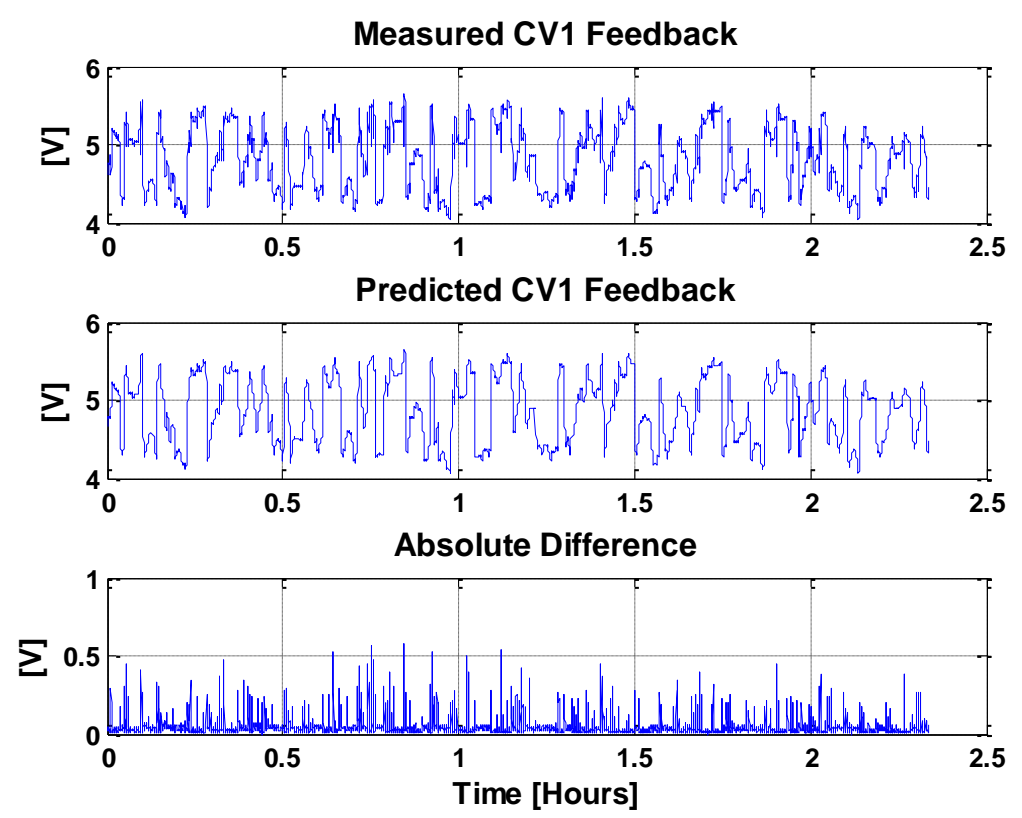

Figure 6.17. Measured control valve feedback versus predicted values.

\subsection{Final Models}

Using the procedure detailed above, the models obtained were implemented in the loop and are listed in Table 6.2. A complete output list using the code developed to obtain the empirical models is found in Appendix 10.

Table 6.2. Final models implemented in the loop.

\begin{tabular}{|c|l|c|c|}
\hline Predicted Variable & \multicolumn{1}{|c|}{ Predictors } & Model Type & Coefficients \\
\hline Level & Inlet flow rate & & -0.0299 \\
& Bypass & Linear & -0.0447 \\
& Outlet flow rate & & 0.9918 \\
\hline Inlet flow Rate & All & AAKR & N/A \\
\hline
\end{tabular}




\begin{tabular}{|c|c|c|c|}
\hline Predicted Variable & Predictors & Model Type & Coefficients \\
\hline By Pass & $\begin{array}{l}\text { Inlet flow rate } \\
\text { Outlet flow rate } \\
\text { FromCV1 } \\
\text { ToCV1 }\end{array}$ & Linear & $\begin{array}{l}-2.2188 \\
-0.8733 \\
2.1610 \\
0.2954 \\
-0.3131\end{array}$ \\
\hline Outlet flow rate & $\begin{array}{l}\text { Level } \\
\text { Inlet flow rate } \\
\text { Bypass }\end{array}$ & Linear & $\begin{array}{l}1.0037 \\
0.0308 \\
0.0436\end{array}$ \\
\hline FromCV1 & $\begin{array}{l}\text { Inlet flow rate } \\
\text { Bypass } \\
\text { ToCV1 }\end{array}$ & Linear & $\begin{array}{l}0.1196 \\
0.0220 \\
0.9160\end{array}$ \\
\hline ToCV1 & $\begin{array}{l}\text { Inlet flow rate } \\
\text { Bypass } \\
\text { FromCV1 }\end{array}$ & Linear & $\begin{array}{r}-0.0990 \\
-0.0184 \\
1.0563\end{array}$ \\
\hline
\end{tabular}




\subsection{Design of Experiments}

A set of controlled faults was implemented in the loop to test the capability of the fault detection routine that was executed in the data acquisition VIs and the suitability of substituting faulty readings with empirical model predictions. Such faults involved controlled drifts in sensor readings to complete fault of the inlet flow control valve. Below is a list of the faults investigated in this research:

- Water level control (single tank):

○ Add $50 \mathrm{mmH}_{2} \mathrm{O}$ drift to the water level sensor over 3 minutes $\left(\sim 17 \mathrm{mmH}_{2} \mathrm{O}\right.$ per minute).

- Manually decrease the level set point to see how well the controller performs using the substituted values.

- Add $50 \mathrm{mmH}_{2} \mathrm{O}$ drift to the water level sensor over 60 seconds, and automatically change the set point at a rate of $45 \mathrm{mmH}_{2} \mathrm{O}$ per minute to see how well the controller acts using substituted values.

- Bypass manual valve:

$\circ$ With the bypass line fully open and indicating around $0.91 \mathrm{l} / \mathrm{s}$, typically, introduce $5 \%$ drift in readings over 3 minutes, and replace faulty measurements with predictions.

- Inlet flow rate sensor readings (orifice meter).

- Introduce a drift in the inlet flow rate readings over 3 minutes, and substitute the faulty measurements. 
- Outlet flow rate sensor readings (turbine flow meter)

- Introduce a $2 \%$ drift in the outlet flow rate readings over 3 minutes, and substitute the faulty measurements.

- Partially closed inlet flow rate control valve:

- This test posed a control challenge because of lack of redundancy in the loop, such as an inlet flow rate bypass line. So, to work around this problem, once the fault was detected, the control logic had to be transferred from tank one to the second tank inlet flow rate control, with significant changes in both tanks control logics.

\subsection{Application of Faulty Measurement Identification and Substitution to a Single-Tank}

With the models and the fault detection module developed implemented, the experiments listed in the previous section were performed and are detailed in this section.

\subsubsection{Fault Detection Experiments Involving Level Sensor}

The first experiment performed to test the fault detection capability and faulty measurement substitution was using the water level measurement sensor. The water level in the tank is measured by a pressure sensor installed at the bottom of the tank and is regulated by varying the volume of water entering and exiting the tank at any given time. By default and under normal operation, the tank exit control valve is left open so that the inlet flow rate control valve is fully responsible for controlling the water level in the tank. Any changes in the level measurement affect the PI controller output signal sent to the control valve.

With empirical models and SPRT continuously running and monitoring each acquired level measurement and comparing its value to predicted values, it was possible to identify each measurement as belonging to either a faulty or a non-faulty condition. When the residuals between prediction and measurement exceeded a pre-determined threshold, based on the 
statistical properties of the dataset used to obtain each of the empirical models, the SPRT flagged that measurement, indicating a faulty condition. Once the faulty condition was detected, the faulty measurements used as inputs to both controller and empirical models were automatically substituted with predicted values. Shortly after the faulty measurements were substituted with predictions, the empirical models misidentified the new situation as being non-faulty and switched back to the faulty measurements coming from the sensor. This situation caused the system to keep switching back and forth, oscillating between faulty and non-faulty modes. To avoid such a problem, a "latch-on" control was added to the fault detection routine, keeping the system from going back to normal condition after identifying and replacing the faulty measurements.

Two different experiments were performed by adding drifts to the level sensor measurements: a slow $+50 \mathrm{mmH}_{2} \mathrm{O}$ over 3 minutes and a fast, $+50 \mathrm{mmH}_{2} \mathrm{O}$ over 60 seconds. The results from the first experiment, or slowest changing drift, are shown in Figure 6.18, with set point values depicted in cyan-dashed line, predictions in solid red at the start of the experiment at steady state, and measurements substituted with predictions shown in solid-black line after a faulty condition was identified. In this experiment, once the difference between measured and predicted values reached around $18 \mathrm{mmH}_{2} \mathrm{O}$, the level SPRT triggered changing from normal to faulty condition (Figure 6.19), causing measured values to be substituted with predicted values, therefore isolating the faulty sensor from the loop. The outlet flow rate model was also able to detect the fault a few seconds later, but not the inlet flow rate until later in the experiment. The controller had difficulties using the prediction values, in large part due to how both VIs were set up and data were transferred between them; but once the PI gains were tuned, the controller was able to successfully change the water level from 600 to $300 \mathrm{mmH}_{2} \mathrm{O}$, although with some performance degradation, showing the applicability of this method for slow-changing processes. Similar results were found with the fastest changing drift, and are shown in Figures 6.20 and 6.21, with level set points changing from 600 to 300 and back to $600 \mathrm{mmH}_{2} \mathrm{O}$, confirming again the controller ability to change the water level using predicted values as inputs and therefore isolating the faulty sensor measurements. 

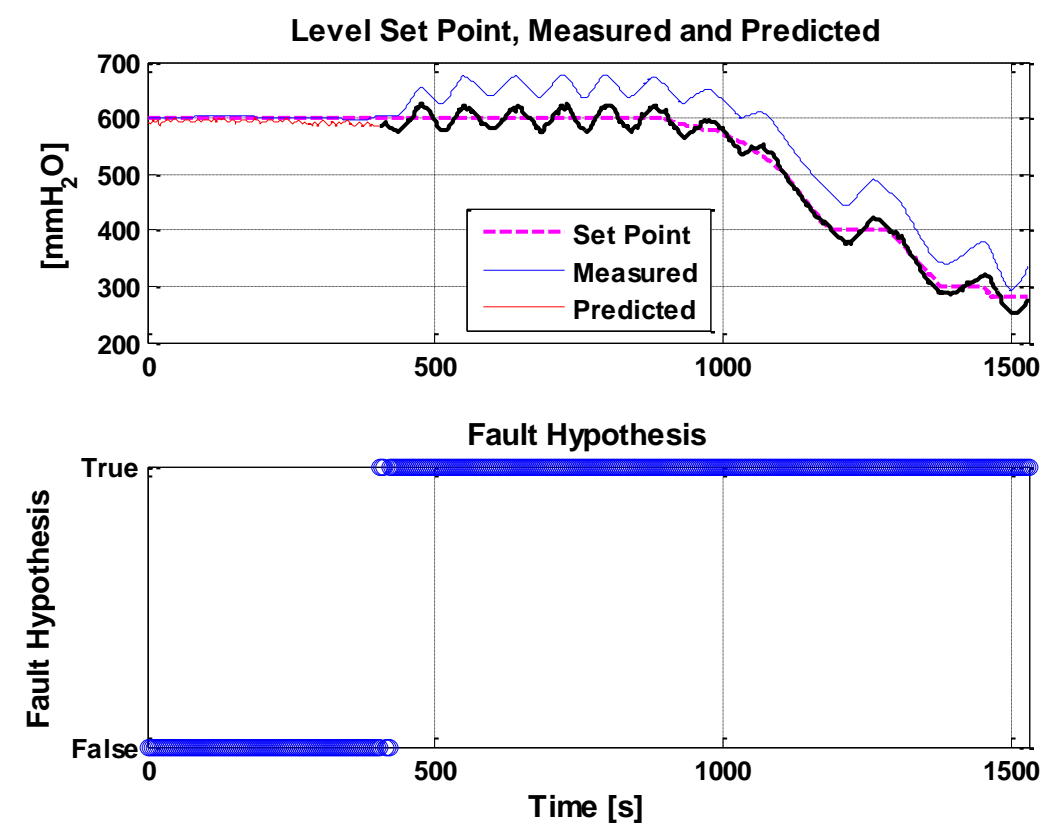

Figure 6.18. Faulty level measurement substitution for slow changing drift.

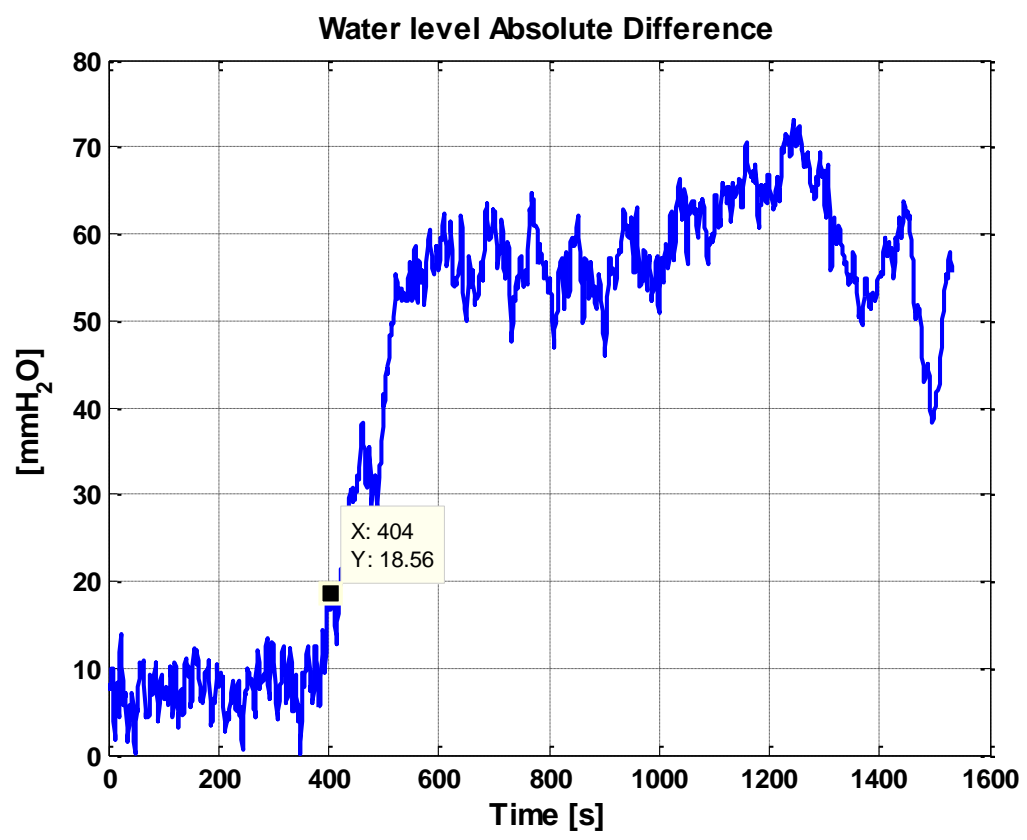

Figure 6.19. Water level absolute difference for slow changing drift. 

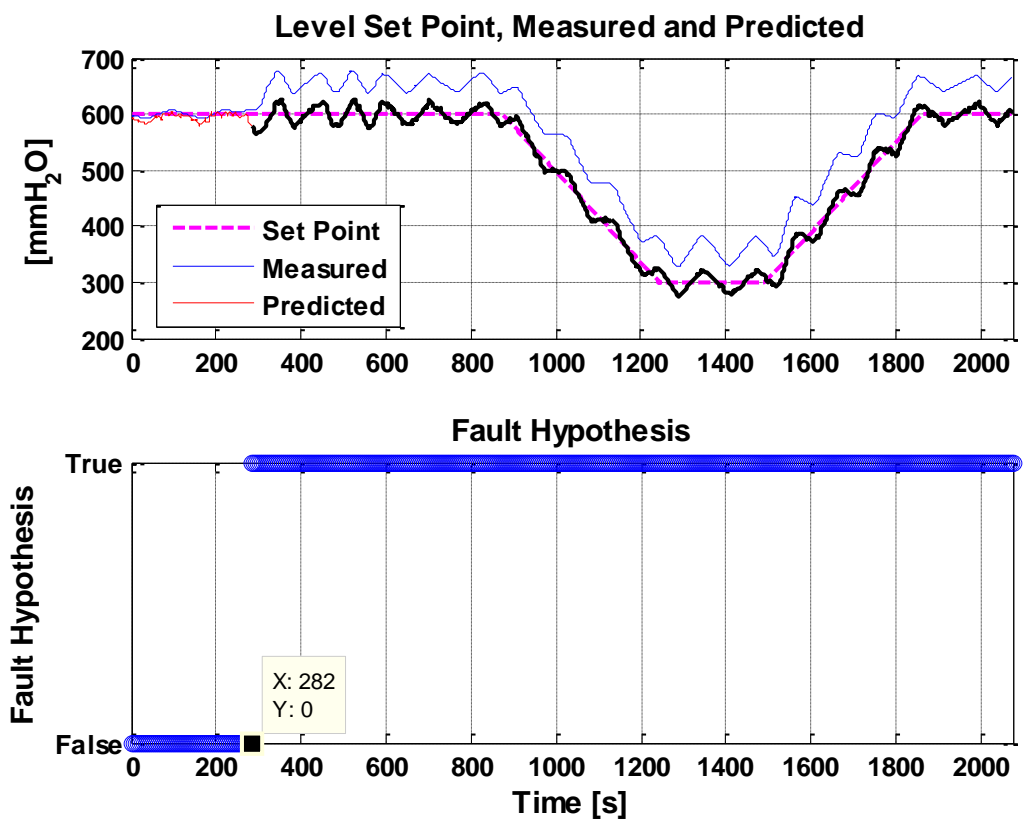

Figure 6.20. Faulty level measurement and substitution for fast changing drift.

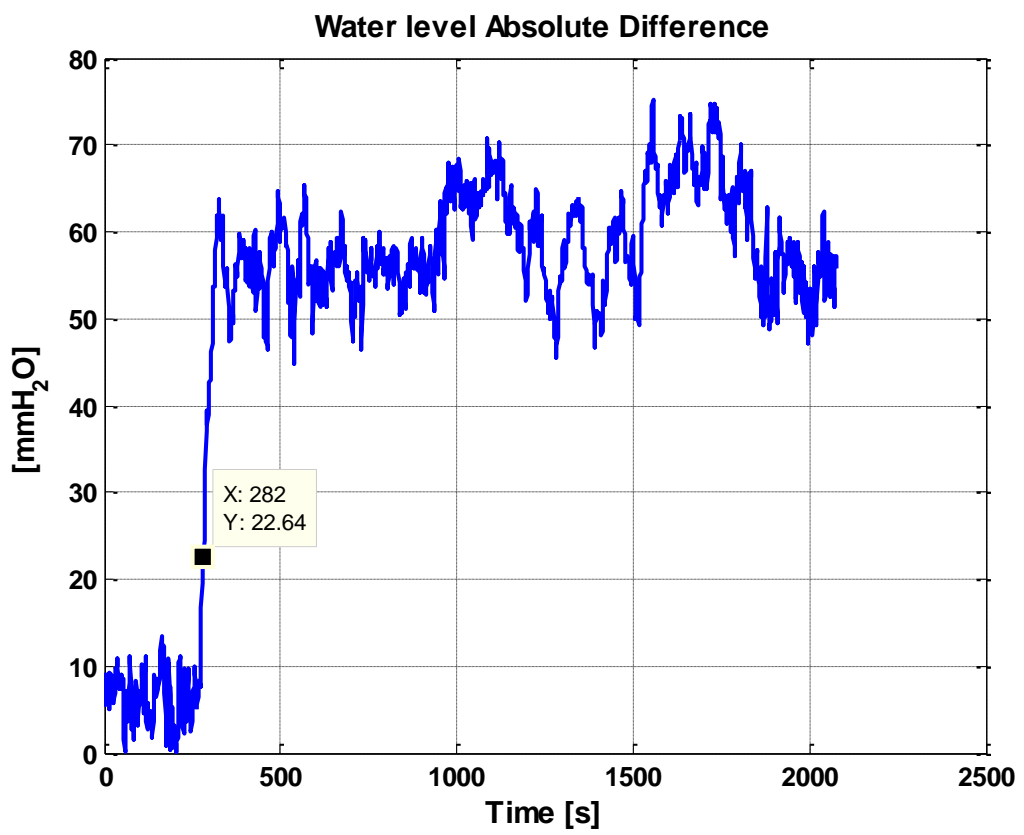

Figure 6.21. Water level absolute difference for fast changing drift. 


\subsubsection{Fault Detection Experiments with Bypass, Inlet and Outlet Flow Rate Sensors}

The following experiments involved detecting and substituting faulty measurements not involved in the water level control, therefore not affecting how the single-tank loop was operated. Following the sequence established in the design of experiments section of this chapter, the bypass measurements were involved in the next experiment. The bypass flow rate was controlled using a manual valve and this valve was kept open by default to divert the excess water back to the tank and lower the pressure in the loop, with typical maximum values of 0.91 $1 / s$, and was kept open when obtaining the empirical models, so any changes to its position would be flagged as a faulty condition. A $5 \%(0.0451 / \mathrm{s})$ drift over 3 minutes was introduced in the bypass measurements, and as expected the SPRT was able to pick up the faulty condition at its

incipient stage when the residuals reached $14 \times 10^{-3} 1 / \mathrm{s}$, as shown in Figure 6.22. The model of the level signal, with which the bypass variable has a correlation of less than 0.3 , was also able to detect the fault a few seconds earlier as shown in Figure 6.23, but not the inlet flow rate model with a correlation of 0.6 , due to the large variance in the inlet flow rate data.

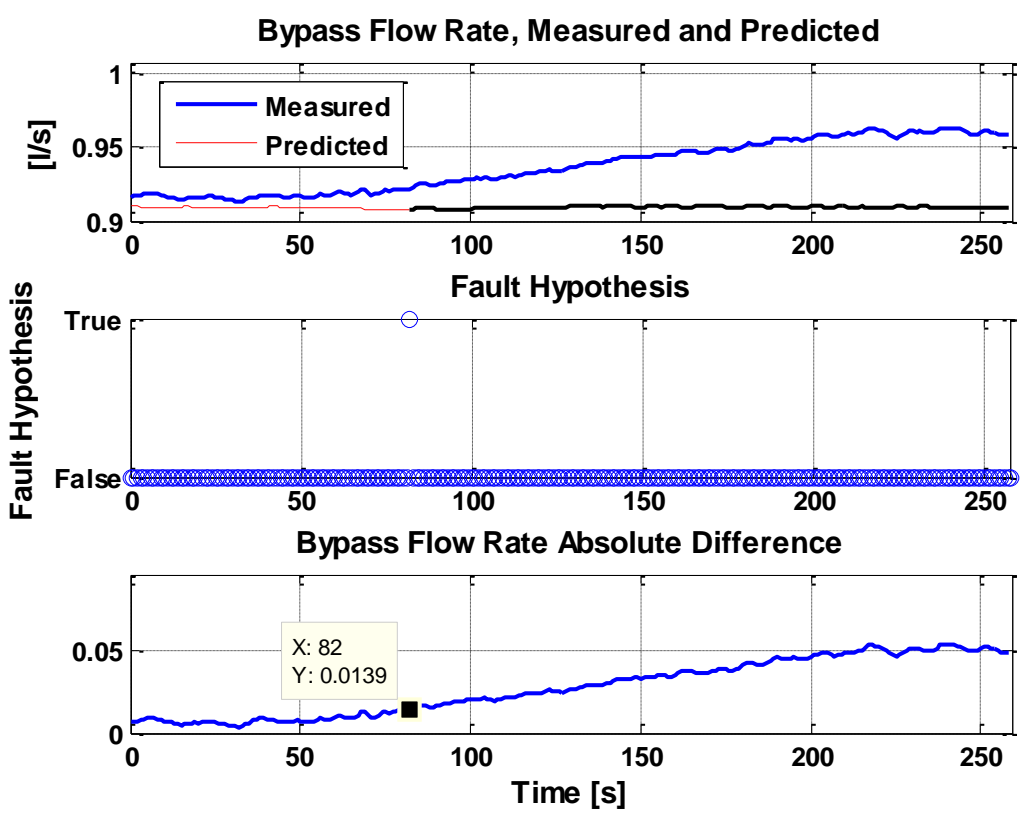

Figure 6.22. Faulty bypass measurement detection and substitution for a $2 \%$ drift over 3 minutes. 


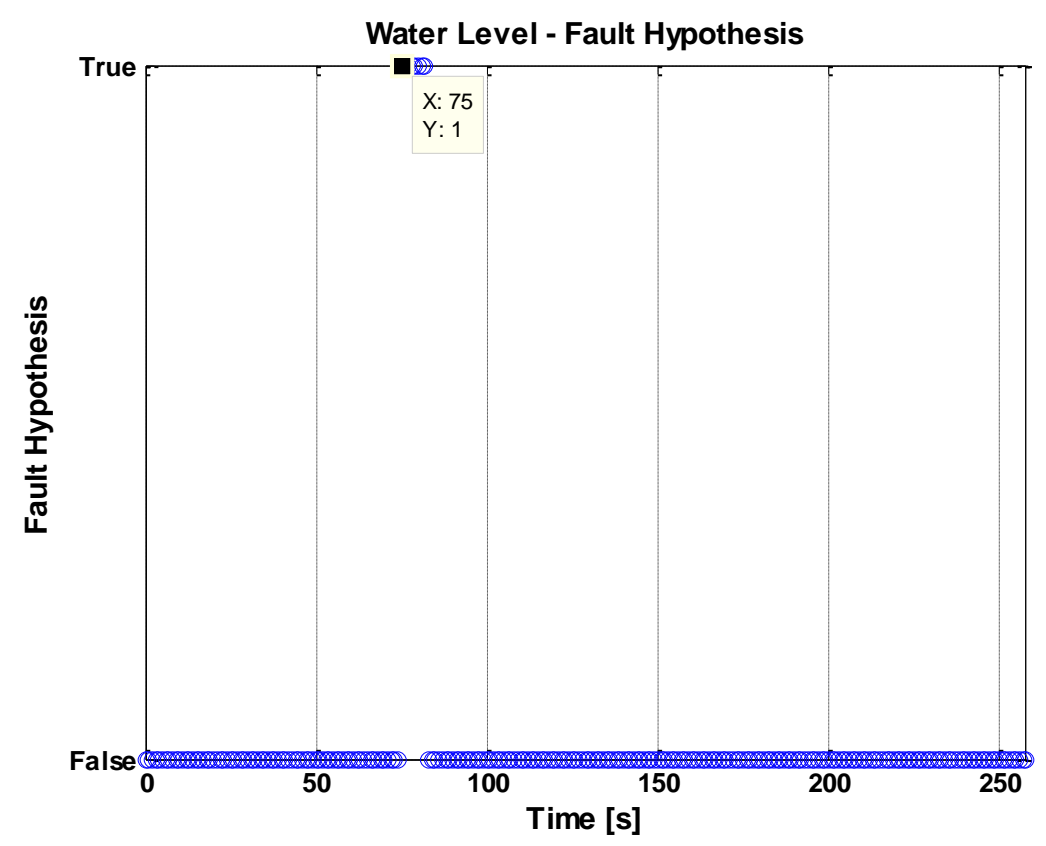

Figure 6.23. Faulty Bypass level measurement fault detected by level.

The inlet flow rate is measured using a differential pressure transducer attached to an orifice meter, and its value is governed by the PI controller output which opens or closes the control valve installed immediately after the orifice meter, closer to the tank inlet. The empirical model showed a difference between measured and predicted values of about $26 \%$, and in this experiment the SPRT flagged the fault at around $0.031 / \mathrm{s}$, or $22 \%$ of the nominal average value $(0.152 \mathrm{l} / \mathrm{s})$, which is in accordance with the inlet flow rate model capability. No other model was able to detect the fault in the inlet flow rate measurements; the results are shown in Figure 6.24.

A turbine flow meter, placed in the tank exit piping, measures the volumetric flow rate of water coming out of the tank. A $2 \%$ drift $(0.003 \mathrm{l} / \mathrm{s})$ over 3 minutes was added to the measurements, and the results are shown in Figure 6.25. The faulty measurements were detected by the outlet flow rate model when the residuals reached $1.4 \times 10^{-3} 1 / \mathrm{s}$ ( $1 \%$ deviation), and the level model detected the fault about 20 seconds earlier, as shown in Figure 6.26. As mentioned earlier, the control valve positioned in this line remained opened throughout the experiment. 


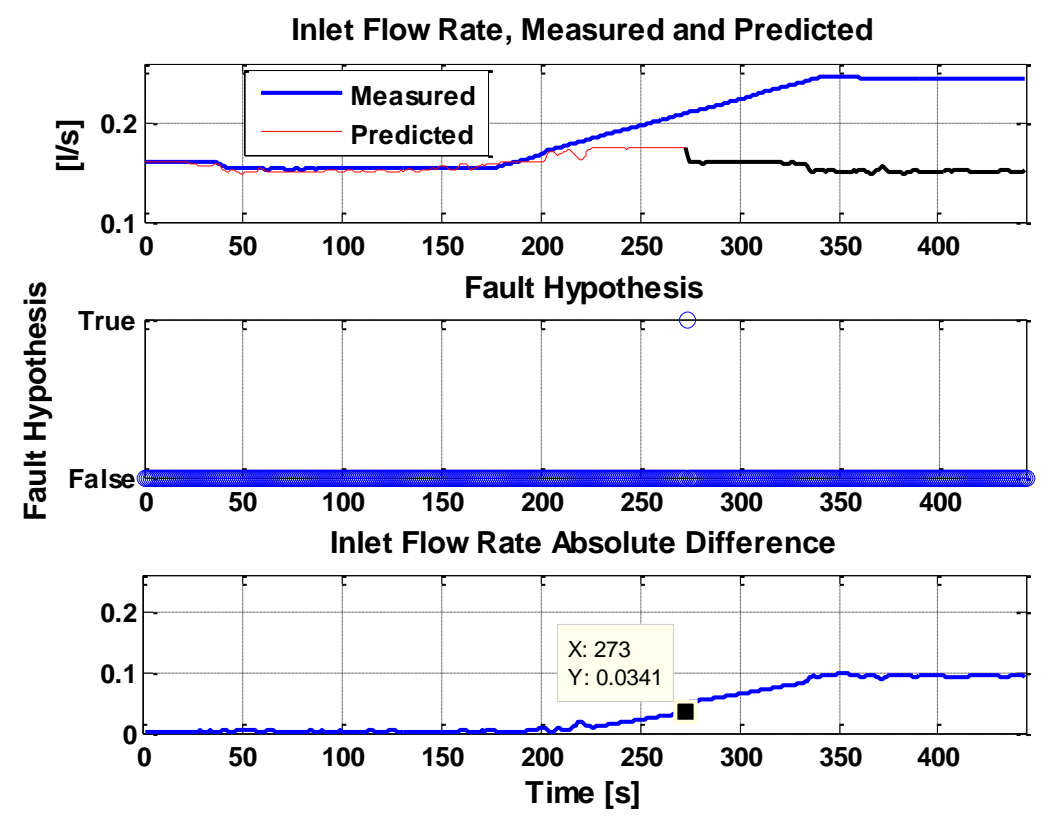

Figure 6.24. Faulty inlet flow rate measurement detection for a $60 \%$ drift over 3 minutes.
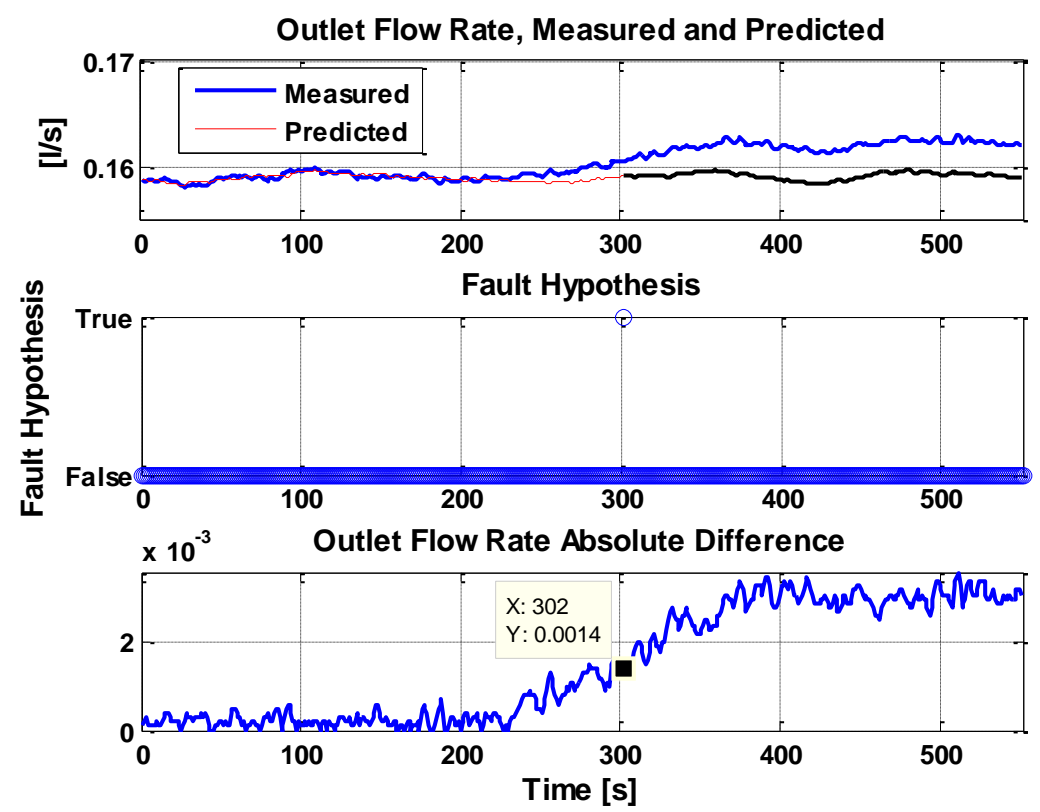

Figure 6.25. Faulty outlet flow rate measurement detection for a $2 \%$ drift over 3 minutes. 


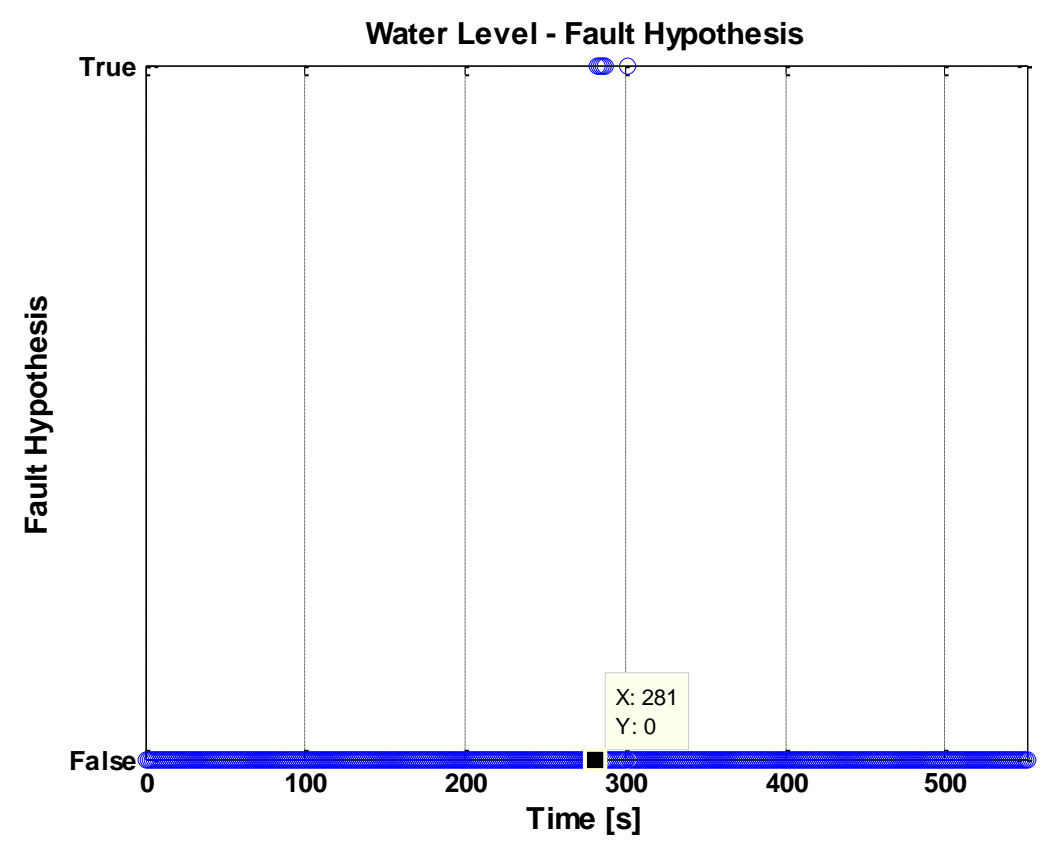

Figure 6.26. Faulty outlet flow rate measurement fault detected by level.

\subsection{Application of Reconfigurable Control to the Two-Tank Flow Control Loop}

The final and most important experiment involved detecting a fault in the inlet flow rate control valve and automatically switching the control action to the second tank inlet control valve, therefore sending the error difference signal between set point and measured water level to the second tank inlet flow rate controller and have it control the water level in the first tank by varying its own level. For this experiment the inlet control valve position was gradually changed at each experiment to see how the controller would work in each case and if it would be able to control the loop under such faulty conditions. In all the cases, the single tank was initially operating at steady state, fault-free, and the water level set point at $500 \mathrm{mmH}_{2} \mathrm{O}$. The signal chosen to flag faulty condition and switching the control over to the second tank in both cases was the signal sent to the control valve, or controller output.

Before performing these experiments it was important to establish the scenario in which such tests were performed prior to the fault was introduced and control switch happened. This was the loop status: 
- Tank 1 was operating at steady state, with water level set point at $500 \mathrm{mmH}_{2} \mathrm{O}$.

- Tank 1 outlet flow rate control valve was at its default position, that is, completely opened.

- Control valve connecting both tanks was closed.

- Tank 2 was completely empty.

- Tank 2 outlet flow rate control valve was half-way opened.

The inlet flow rate control valve positions investigated were, $6.5 \mathrm{~V}, 5.5 \mathrm{~V}, 4.5 \mathrm{~V}$, and $3.5 \mathrm{~V}$, or $92 \%, 78 \%, 64 \%$ and $50 \%$ open, respectively, and substituted the controller output by overriding it in the controller VI. The very second the acquisition VI detected the fault the following changes were automatically performed in the loop status to transfer the control over to the second tank inlet flow rate control valve:

- Control valve connecting both tanks was opened.

- Tank 2 inlet flow rate control valve assumed the control of the first tank, receiving the error signal between tank 1 set point and current water level. The PI controller used the gains that were same as those used when both tanks were running and connected together.

- Tank 2 outlet flow rate control valve completely opened.

Initiating the experiments, the single-tank was running at steady state, and at a certain point the control output was overridden by a $6.5 \mathrm{~V}$ signal sent to the control valve deviating from the normal valve opening for this level of $4.5 \mathrm{~V}$, opening the inlet flow rate to the tank more than necessary, causing the water level to experience a sharp increase. Since this is a slow-changing system, the data acquisition VI was set by default to only flag faulty situations after ten consecutive measurements have been deemed as being faulty by the SPRT (10 out of 10 logic). Ten seconds after the fault was introduced, the SPRT flagged the control output as being faulty and immediately opened the control valve that connects both tanks, while at the same time 
opened both inlet and outlet flow rate control valves to the second tank and transferred the control from the first to the second tank. The results from this first experiment are shown in Figures 6.27 to 6.36. As shown in Figure 6.27 the water level in tank 1 increased sharply while the control was transferred to the second tank. As the control valve between tanks was opened, and the second tank control took over the water in tank 1 reached a temporary steady state, but when the water level in tank 2 started to increase despite the inlet control valve being completely closed the water level in tank 1 resumed increasing again. After a few seconds the experiment was interrupted, before tank 1 overflowed. All empirical models were able to detect the fault at the same time, as shown in Figures 6.28 to 6.30. In Figure 6.31 the bypass flow rate was affected by the sudden increase in the inlet flow rate caused by the opening of the inlet flow rate control valve, then stuck at $6.5 \mathrm{~V}$. With more water being drawn by tank 1 there is less water available for the bypass line, therefore the decrease in bypass flow.

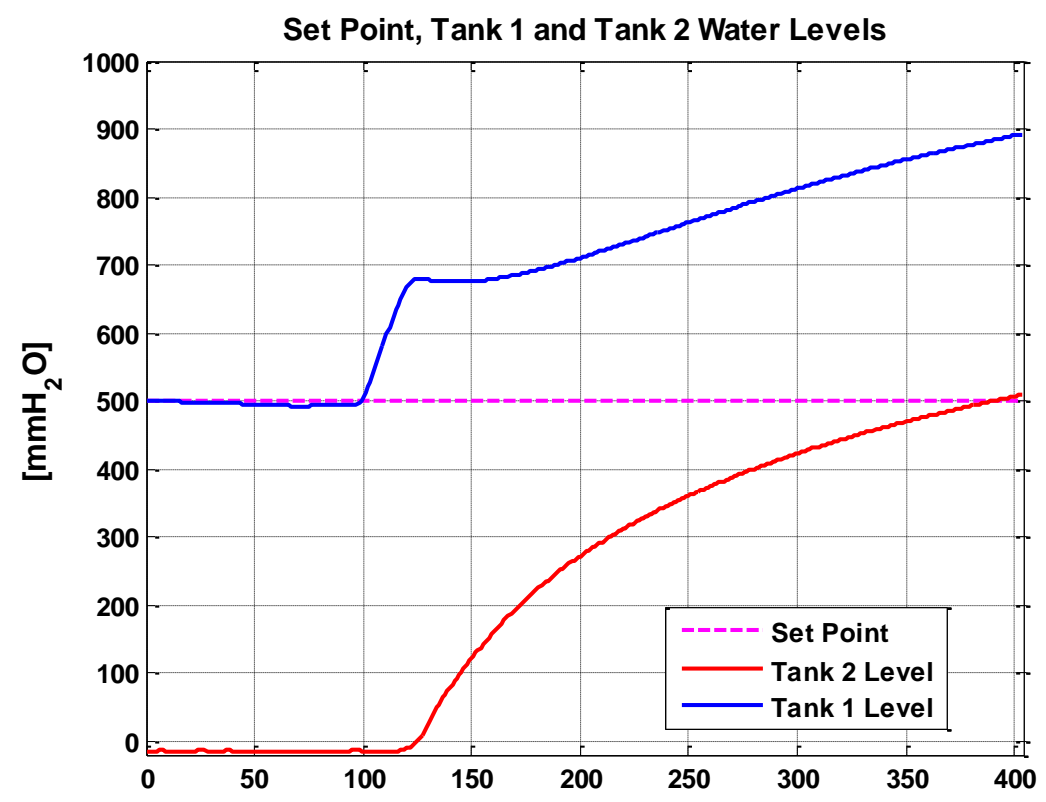

Figure 6.27. Inlet flow rate control valve position at $6.5 \mathrm{~V}$ with control switch over to tank 2 . 
To Control Valve - Fault Hypothesis

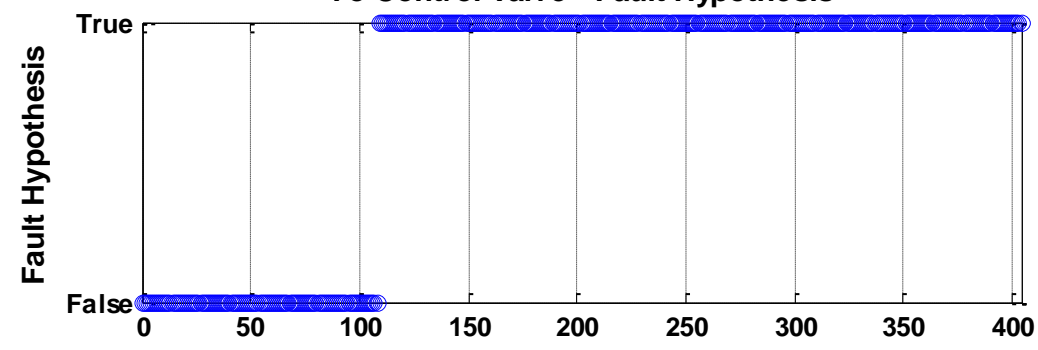

From Control Valve - Fault Hypothesis

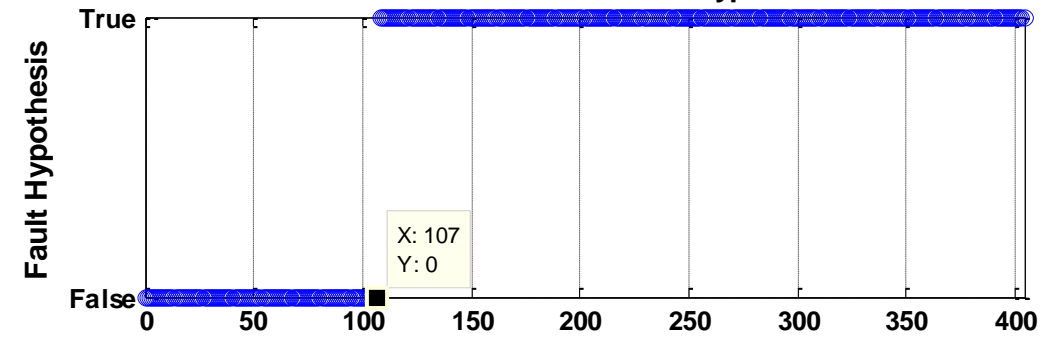

Figure 6.28. CV1 stuck at 6.5V fault detection using CV models.
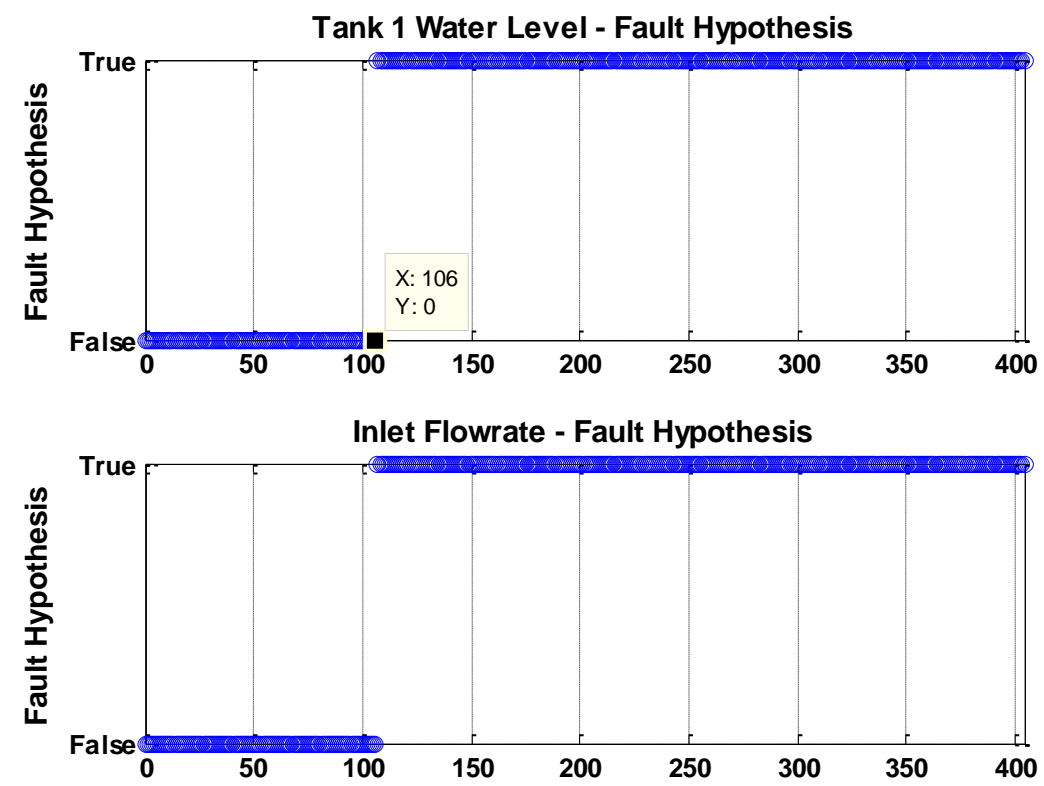

Figure 6.29. CV1 stuck at 6.5V fault detection using tank 1 level and inlet flow rate models. 

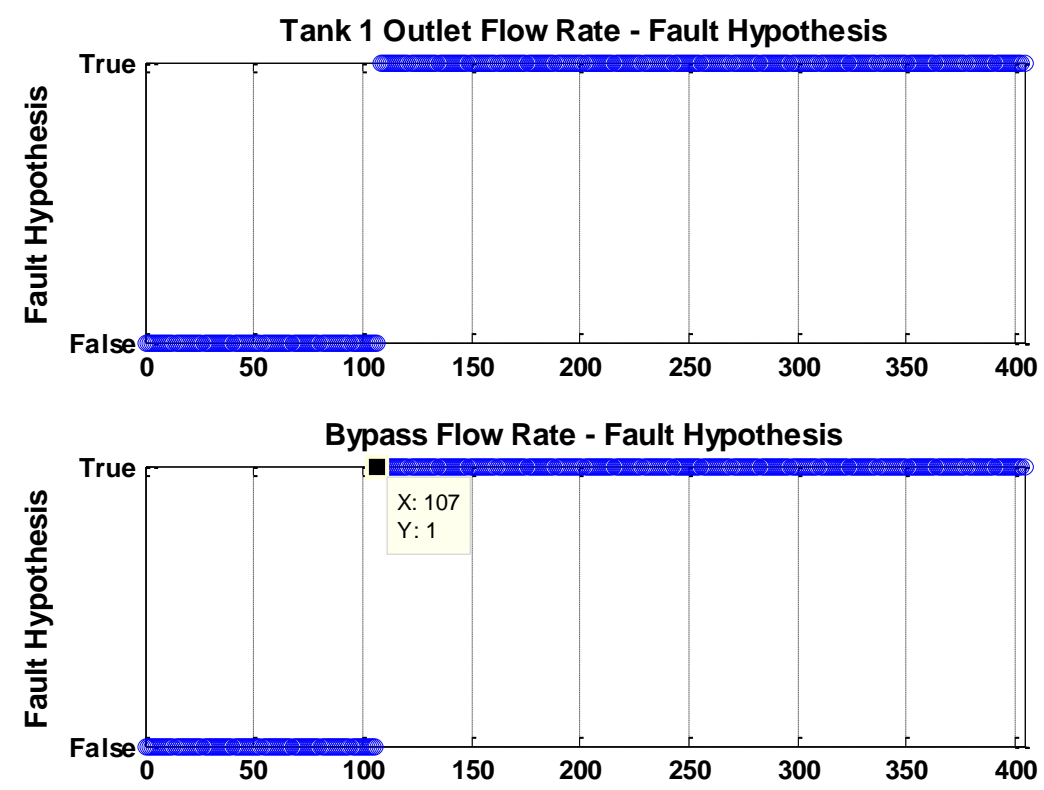

Figure 6.30. CV1 stuck at $6.5 \mathrm{~V}$ fault detection using outlet and bypass flow rate models.

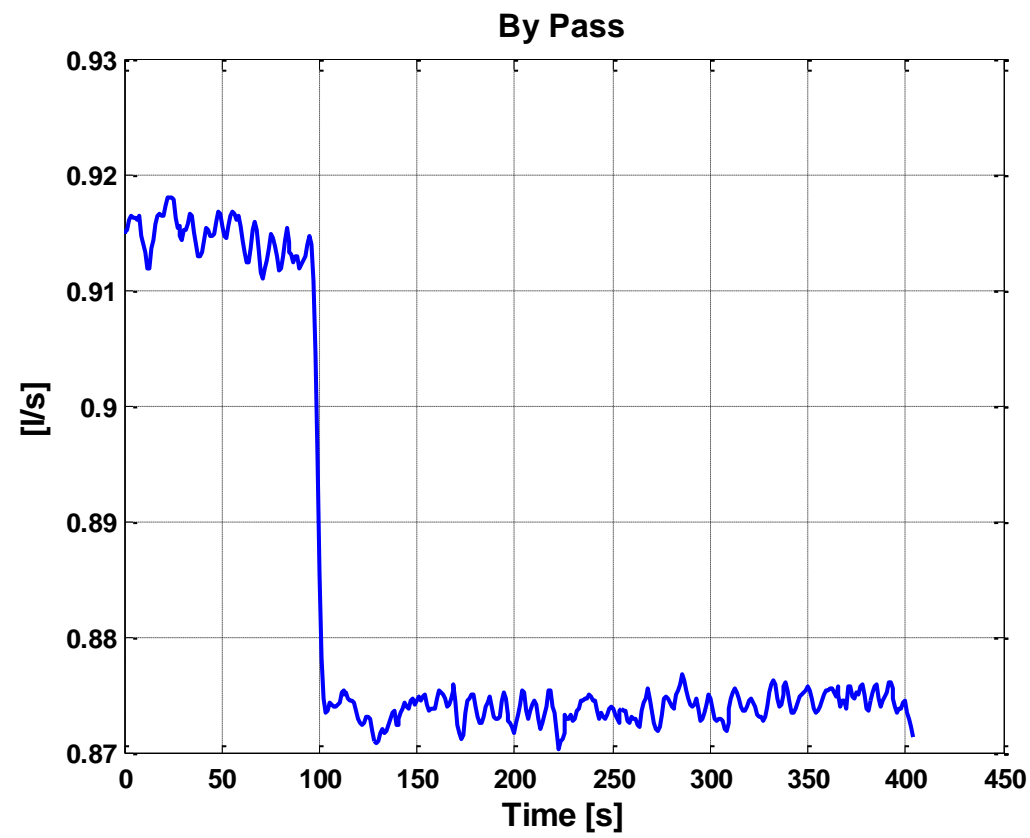

Figure 6.31. Bypass flow rate transient originated by a control valve stuck at $6.5 \mathrm{~V}$. 
In the next experiment a $5.5 \mathrm{~V}$ signal was sent to the valve, and the results are shown in Figures 6.32 and 6.33. As soon as the controller signal was overridden by the $5.5 \mathrm{~V}$ signal there was a sharp increase in the water level in tank 1, followed by a sharp decrease as the control valve between tanks opened and the level in tank 2 increased, but unlike the $6.5 \mathrm{~V}$ experiment the water level in both tanks stabilized at around set point in tank 1 and around $450 \mathrm{mmH}_{2} \mathrm{O}$ in tank 2, with $23 \mathrm{mmH}_{2} \mathrm{O}$ overshoot in tank 1 and cycles of 140 seconds. Being highly correlated with each other, both control valve and inlet flow rate models picked up the fault at the same time. The bypass, water level, and outlet flow rate models picked up 30 seconds later. From Figure 6.33 it is clear how the SPRT kept switching back and forth between faulty and non-faulty modes, but not the inlet flow rate SPRT, which kept signaling the faulty condition with the same frequency as the water level indication. In the next test with the control valve stuck at $4.5 \mathrm{~V}$ there was not an initial increase in the water level as can be seen in Figure 6.34, but the sharp drop due to the connection between tanks being opened. However, there was a significant improvement in the water level measurements with the water level in tank 1 showing a $10 \mathrm{mmH}_{2} \mathrm{O}$ average overshoot with respect to the set point, but still presenting the oscillating condition around set point, with 140 second cycles, the same occurring with the tank 2 level measurements. Since the departure from normal condition started at the same voltage needed to achieve $500 \mathrm{mmH}_{2} \mathrm{O}$ in the tank, the inlet flow rate model took 60 seconds longer and it flagged the faulty condition when the difference between measured and predicted values reached $22 \mathrm{mmH}_{2} \mathrm{O}$, compared to the 20 seconds needed by the level, bypass and outlet flow rate to detect the same fault. The results are shown in Figure 6.34. 


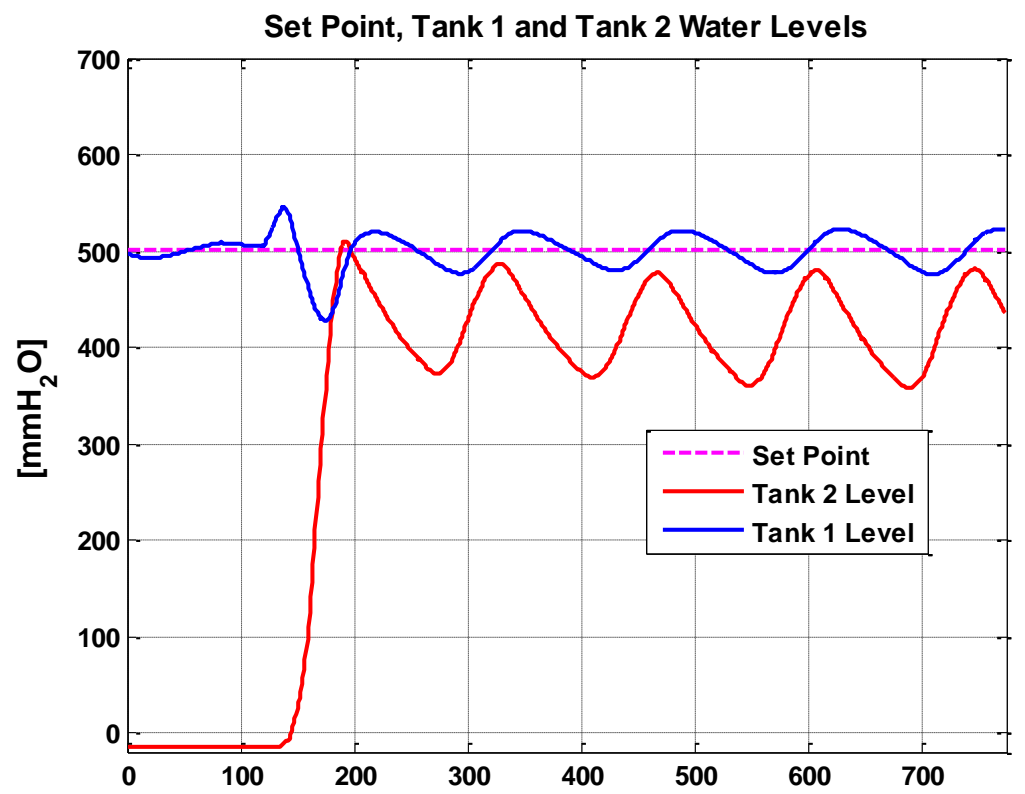

Figure 6.32. Inlet flow rate control valve position at $5.5 \mathrm{~V}$ with control switch over to tank 2 .
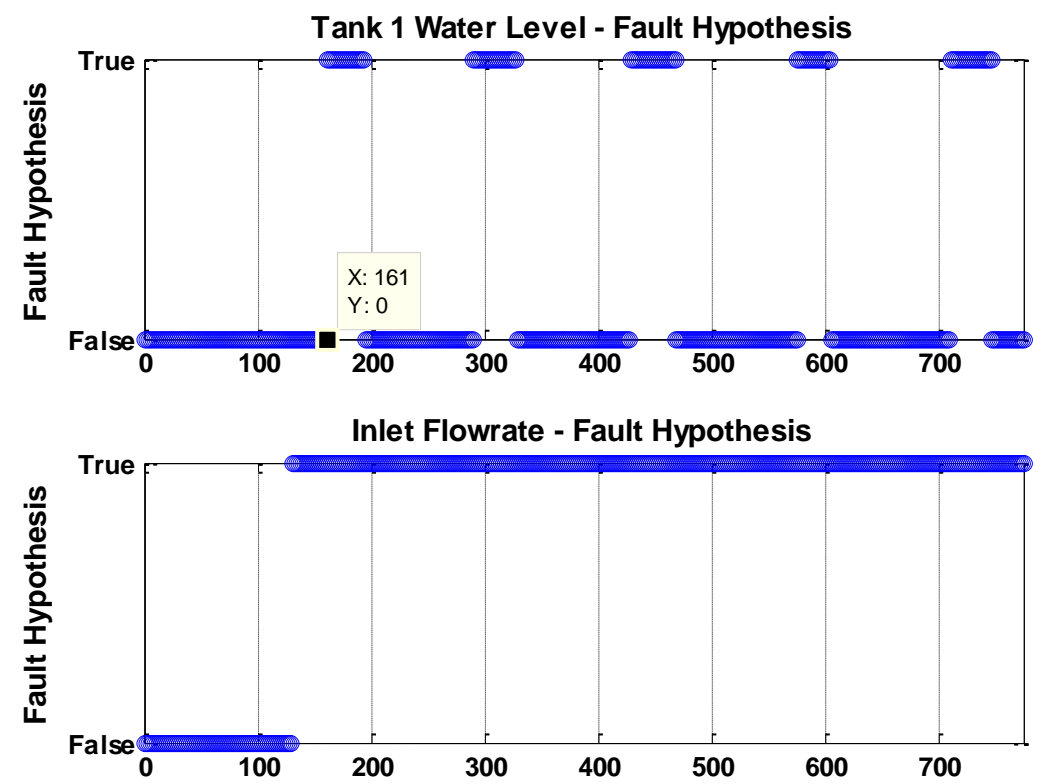

Figure 6.33. CV1 stuck at 5.5V fault detection using level and inlet flow rate models. 


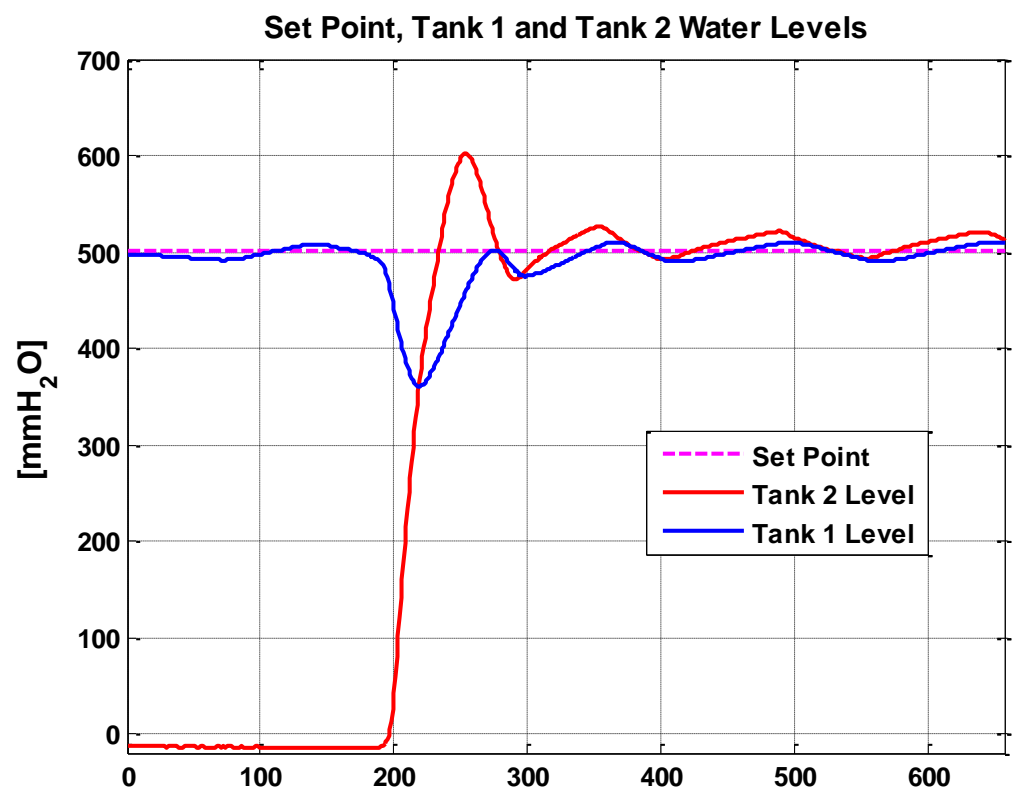

Figure 6.34. Inlet flow rate control valve position at $4.5 \mathrm{~V}$ with control switch over to tank 2 .

The first control switch experiment using the control valve stuck at $6.5 \mathrm{~V}$ proved to be overwhelming to the system with tank 1 almost overflowing at the end of the experiment. The next experiment with $5.5 \mathrm{~V}$ showed an oscillatory behavior around the set point with $20 \mathrm{mmH}_{2} \mathrm{O}$ overshoots. At $4.5 \mathrm{~V}$ the system was still oscillating, but with the water level very close to the set point. The last experiment performed was with the inlet control valve stuck half-open, or $3.5 \mathrm{~V}$, with results shown in Figures 6.35 and 6.36. If the system trend was that of close normalcy as the voltage went down from $6.5 \mathrm{~V}$ to $4.5 \mathrm{~V}$, the valve stuck at $3.5 \mathrm{~V}$ proved to drift away from that trend, with overshoots of $100 \mathrm{mmH}_{2} \mathrm{O}$ in average and the water level in tank 2 reaching its capacity of $850 \mathrm{mmH}_{2} \mathrm{O}$. Being a sharp transient, the inlet flow rate model was able to detect the fault quite rapidly at the same time as the valve signal models, followed 20 seconds later by the other three models. 


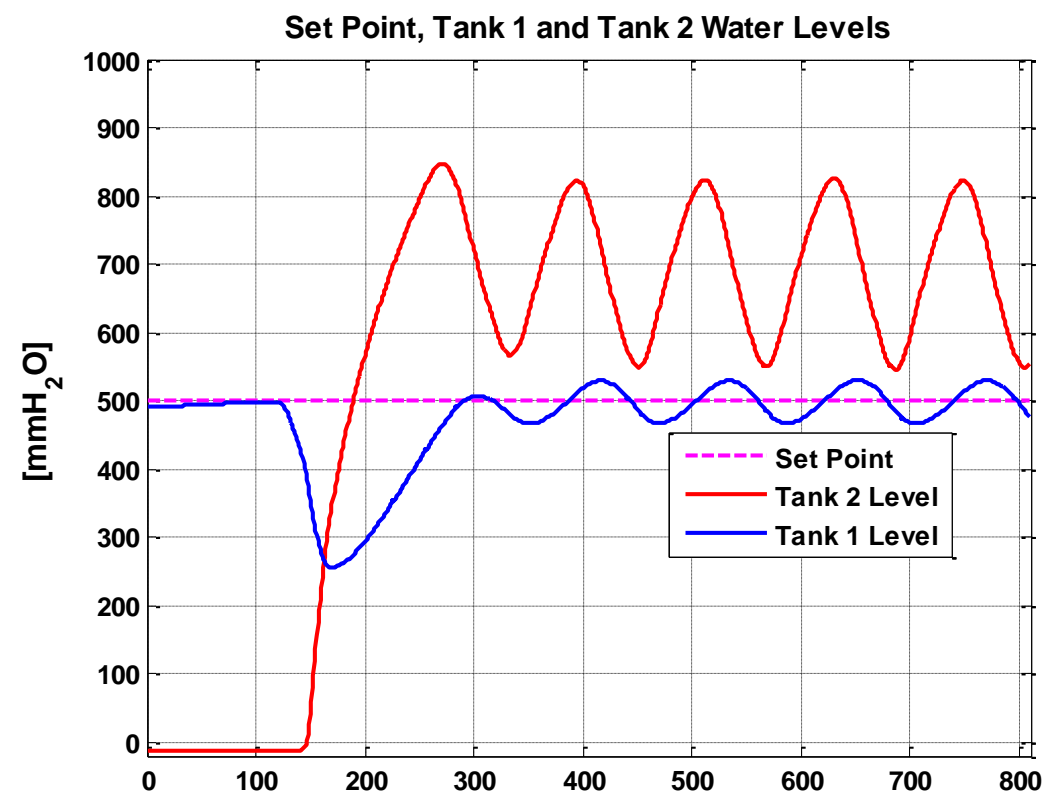

Figure 6.35. Inlet flow rate control valve position at $3.5 \mathrm{~V}$ with control switch over to tank 2 .
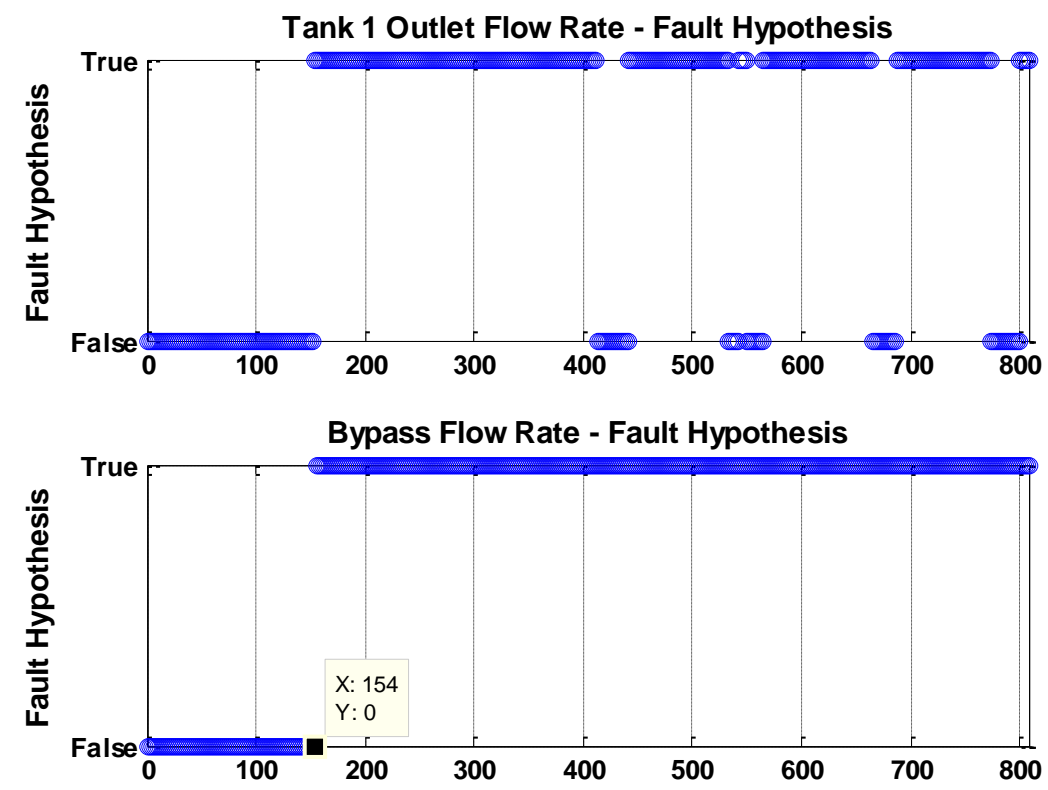

Figure 6.36. CV1 stuck at 3.5V fault detection using outlet and bypass flow rate models. 


\subsection{Possible Control Reconfiguration Application to the IRIS System}

Based on the work developed with the multivariate flow control loop and the results obtained with faulty measurements substitution and control reconfiguration it is possible to devise a scenario in which this kind of approach can be applied. Such scenario involves a small problem where steam control valve position sensor malfunctions indicating a wrong position, or even a malfunction or normal operation wear causing the valve to drift a little bit over a long period of time, hence affecting both power being produced and steam pressure. In both cases an inferential model based on historical fault-free data can easily be used to detect such problems at incipient stages and substitute the faulty measurements with predictions until a scheduled maintenance can be performed. And in the case of very small drifts in the steam control valve, the reconfiguration can be performed to help offset it by performing small changes in the steam generator feedwater flow, similar to reactivity shim control.

\subsection{Summary}

The objective of these experiments was to show the feasibility of using fault detection based on empirical models to substitute faulty measurements with predictions and perform control reconfiguration in the presence of actuator failure in a real system. For this purpose a multivariate loop was used in single-tank configuration where six important variables were subjected to controlled faults. Their measurements were successfully substituted with predictions and provided the system the necessary flexibility to keep on operating even under degraded conditions, thus offering survivability to the system and the time necessary to perform corrective procedures, should that be the necessary. These experiments were particularly important because they offered the opportunity to prove that a system like the multivariate loop can survive degraded circumstances, provided the empirical models used are accurate and representative of the system dynamics.

These demonstrations showed three very distinct scenarios as far as control was concerned. First, simple variable measurement substitution not involving control proved to be a straightforward approach, but special attention was needed to make sure the fault detection system latched on and did not default to normal condition once the faulty measurements exceed 
the thresholds stipulated in the models and were substituted with predictions. However, in the second scenario where the variable measurements were used in the single-tank control logic configuration, the time it took for the fault to be detected depended not only on variable correlation but also on the severity of the fault. In addition, some operational peculiarities, such as having dead-band implemented in the water level error signal, prevented the controller output to adjust the inlet flow rate control valve, SPRT's 10 seconds memory vector, and high-speed rate control loop $(2.5 \mathrm{kHz})$ using model predictions generated at much lower rate $(1 \mathrm{~Hz})$. In this case the faulty level measurement substitution caused some control instabilities, which required controller re-tuning; but in the end the single-tank was successfully controlled, as the results have shown, with the water level closely following the set points changes. The last scenario involved complete actuator fault with the inlet flow rate control valve stuck at various positions, $90 \%, 80 \%, 65 \%$, and $50 \%$, respectively, and total control transfer from tank 1 to tank 2 once such faults were detected. From the results it is clear that the system was perfectly operable with the control valve stuck at $4.5 \mathrm{~V}$, but the system became less stable as the valve position strayed away from this value, a situation that could probably be worked around with new set of controller gains appropriate for each fault level. A simple, and yet important additional test was performed in which the inlet control valve power cord was pulled from the power strip, interrupting the electrical power to the control valve. In this case, since both control valve signals are intrinsically correlated, their empirical models were not able to detect the fault right away. Instead the inlet flow rate measurement as well as the water level models detected it a few seconds later after the cord was pulled, showing that some discretion is needed when relying on empirical models to detect faults. Though this is a slow-transient, non-critical safety system, the important point in this demonstration was to show that a system could have some level of survivability under degraded conditions, provided there is enough analytical and/or physical redundancy. It is important to keep in mind that other critical control systems present in airplanes, spacecrafts, ships, robots, etc., do have the necessary control redundancy to provide them with means to survive system faults.

An important fact to keep in mind when using empirical models in real systems, such as the single-tank experimental loop is that, some of the solutions presented here are system/fault dependent and appropriate fault detection techniques such as expert systems need to be employed to properly identify fault scenarios in order to select the appropriate solution. 


\section{Chapter 7}

\section{SUMMARY, CONCLUDING REMARKS, AND RECOMMENDATIONS FOR FUTURE WORK}

\subsection{Summary and Conclusions}

In the active but still developing realm of small modular reactors, the preceding chapters of this report have presented a comprehensive and critical literature review of the most recent progress in small and medium reactors, with emphasis on integral designs and the application of once-through steam generators instead of the recirculation steam generators, used in most PWRs. The research identified the necessity of utilizing an integrated approach to monitor the condition and perform fault diagnosis of nuclear power systems using robust data driven model based methods for both steady state and dynamic operation conditions which can help anticipate transients (i.e., a model based control system) and have significant impact on procedures and lead to better plant design and utilization. Such novel designs most likely will also be used in applications other than just power generation, but in other important applications such as district heating and most importantly desalination, given the increasing necessity for potable water sources in arid areas of the world. Such increasing interest in integral PWRs has led regulatory agencies, reactor manufacturers and research organizations throughout the world to focus their attention on the development of such smaller designs. The NRC has recently informed several designers that through the end of fiscal year 2011, the offerings from Babcock \& Wilcox and NuScale Power will be given priority over other designs, clearly corroborating the tendency of building plants with smaller footprints that will have proliferation-resistant, fault tolerant and reconfigurable control features. This approach would make them suitable for deployment in developing countries with less stable electrical grids with minimum human interference.

An existing dynamic single-module IRIS Simulink model was studied and tested for consistency and the steady-state values were compared with values provided by North Carolina State University FORTRAN code and those found in the open literature. Both $\mathrm{T}_{\text {ave }}$ and outlet steam pressure controllers in the reactor core and steam generator models, respectively, were successful in keeping process variables close to set points during the simulations. Several load 
demand profiles were investigated, and the simulation results suggest IRIS design as being suitable for load following applications. Numerical instabilities were observed causing the model to crash, especially during long simulation times and close to power inflection points, due in great part to the stiffness of the model. This Simulink model does not include some important components and systems vital to have a complete understanding of the system dynamics such as pressurizer, balance-of-plant, secondary feedwater controller, and feed-forward controller to quickly move the control rods based on changes in power load demands.

IRIS Simulink model was extended in this research to include a second unit, with both modules operating simultaneously, with the superheated steam from both units flowing to a common header. The simulations used one of the load following profiles. The robustness of the new model was tested using small, controlled faults that were introduced either in sensor measurements or in the process. The sensor measurements considered are moderator average temperature controller $\left(\mathrm{T}_{\text {ave }}\right.$ and $\left.\mathrm{T}_{\text {cold }}\right)$ in the primary and the feedwater flow rate and temperature systems in the secondary. From the results, it was possible to conclude that the model was able to detect such faults, and that for small variations the system was able to absorb these effects without significant changes in the dynamics. Also, the results showed that both units were somewhat independent of each other even with a common feedwater line connecting both steam generators. The system was able to perform well even in the presence of small faults in one unit, with the real connection between the two units being located in steam header where most of the transients were observed, and that the prospects of having two IRIS modules in a single plant is feasible from simulation standpoint. It was also possible to notice that with such a large reactor coolant inventory in a single unit $\left(16,000 \mathrm{ft}^{3}\right)$, the coupling between reactor core and the steam generator is very weak, which translates in the reactor core being much less prone to transients due to sudden changes in the steam generator.

A two-tank multivariate control loop was built and used to show the feasibility of using fault detection based on empirical models to substitute faulty measurements with predictions and perform control reconfiguration in the presence of actuator failure in a real system. Six important variables were subject to controlled faults and had their measurements successfully substituted with predictions and provided the system the necessary flexibility to continue operating under degraded conditions, thus offering survivability to the system and the time to perform corrective 
maintenance if necessary. Also, an actuator, responsible for regulating the water level in the tank by varying the inlet flow rate was artificially disabled and the control was successfully transferred to the second tank after the fault had been detected by the fault detection and isolation module. Some loss of control performance was observed, however, but the system was stable and controllable. Residuals between prediction and measurements were monitored on-line. Whenever they exceeded a pre-determined threshold, based on the statistical properties of the dataset used to obtain each of the empirical models, the SPRT flagged that measurement, indicating a faulty condition. Once the faulty condition was identified, or flagged, the faulty measurements used as inputs to both controller and empirical models were automatically substituted with predicted values. In order to prevent the empirical models from misidentifying the new situation as being non-faulty and switch back to the faulty measurements coming from the sensor or actuator a "latch-on" control was added to the fault detection routine, keeping the system from going back to normal after identifying and replacing faulty measurements.

These experiments were particularly important because they offered the opportunity to prove that a real system like the multivariate loop can survive degraded circumstances, provided the empirical models used are accurate and representative of the system dynamics.

\subsection{Recommendations for Future Research}

To the author's knowledge, this research is the only work focused on developing a twinunit IRIS plant to study the impact of having steam from both units converging to a common header, and is the first to offer an original contribution as an initial effort to help solve the control challenges posed by the operation of such a novel design applied to steam mixing when subject to different load-following scenarios, resulting in an IRIS model capable of operating as a single-unit plant or as a twin-unit plant. To further improve the model, a feed-forward controller can be implemented to quickly move the reactor control rods based on power load demands. Also, feedwater controllers (one for each unit) can be developed for implementation at low power operation and at power levels above a certain range.

A multivariate flow control loop with two tanks, a series of control valves and sensors to provide the necessary framework to develop research related to instrumentation and control strategies, equipment and sensor monitoring was built. This provides an excellent tutoring bench 
opportunity for researchers and students to learn about control strategy and equipment monitoring using advanced techniques. Also, through the software application developed to control the loop, and with a few changes, the loop can provide an excellent opportunity for students to perform complete automatic control experiments, either over the departmental local area network or over the internet.

Single-tank empirical models were used in this research to substitute faulty measurements with predictions and perform control reconfiguration in the presence of actuator failure. The experiments carried out can be generalized and extended by including the second tank in the data-based models. 


\section{REFERENCES}

[1] Ingersoll, D.T. "Deliberately small reactors and the second nuclear era." Progress in Nuclear Energy, 51, no. 4-5, 589-603, July 2009.

[2] Carelli, M.D., L. E. Conway, L. Oriani, B. Petrovic, C. V. Lombardi, M. E. Ricotti, A. C. O. Barroso, et al. "The design and safety features of the IRIS reactor." Nuclear Engineering and Design, 230, no. 1-3, 151-167, May 2004.

[3] Storrick, G.D., and M.D. Carelli. "The IRIS (International Reactor Innovative and Secure) and its unique advancement of the state-of the art." In Power and Energy Society General Meeting - Conversion and Delivery of Electrical Energy in the 21 st Century, 2008 IEEE, 1-5, 2008.

[4] Carelli, M.D. "The exciting journey of designing an advanced reactor." Nuclear Engineering and Design, 239, no. 5, 880-887, May 2009.

[5] Lorenzini, P., "Presentation to U.S. Nuclear Regulatory Commission. Resulting Conclusions." Electric Power Research Institute. EPRI RP-1585. Internet:http://www.nuscalepower.com/pdf/NRC_preapp_mtg_072408_INTRODUC TION_1_.pdf, July 24, 2009.

[6] Zhang, Z. et al., "Current status and technical description of Chinese $2 \times 250$ MWth HTR-PM demonstration plant," Nuclear Engineering and Design, 239, no. 7, 12121219, July 2009

[7] Instrumentation, Controls, and Human-Machine Interface Technology Development Roadmap for Grid-Appropriate Reactors, ORNL/GNEP/LTR/2008/041, Oak Ridge National Laboratory, June 2008.

[8] Kundur, P., N.J. Balu, and M.G. Lauby, Power system stability and control (McGrawHill Professional, 1994).

[9] Csik, B.J., Kupitz, J., "Nuclear cogeneration: Supplying heat for homes and industries." Internet: http://www.ecolo.o rg/documents/documents_in_english/cogeneration-nuc-csik-07.html, July 28, 2009.

[10] Sakaba, N., Y. Tachibana, S. Shimakawa, H. Ohashi, H. Sato, Xing Yan, T. Murakami, et al. Examination on Small-Sized Cogeneration HTGR for Developing Countries. Ibaraki, Japan: Japan Atomic Energy Agency, Japan, March 2008. Engineering Village. 
[11] Alonso, G., S. Vargas, and E. del Valle. "Electricity Production and Water Desalination Using IRIS Reactor," presented at the American Nuclear Society Conference, Atlanta, GA, 2009.

[12] Alonso, G., R. Ramirez, C. Gomez, and J. Viais. "IRIS Reactor a Suitable Option to Provide Energy and Water Desalination for the Mexican Northwest." In Americas Nuclear Energy Symposium (ANES 2004), Miami, FL (US), 10/03/2004-10/06/2004.

[13] Ingersom, D.T., J.L. Binder, V.I. Kostin, Y.K Panov, et al, "Cogeneration of Electricity and Potable Water Using The International Reactor Innovative And Secure (IRIS) Design," In Proceedings Conference: Americas Nuclear Energy Symposium (ANES 2004), Miami, FL (US), 10/03/2004-10/06/2004.

[14] Manesh, M.H.K, M. Amidpour, and M.H. Hamedi. "Optimization of the coupling of pressurized water nuclear reactors and multistage flash desalination plant by evolutionary algorithms and thermoeconomic method." International Journal of Energy Research, 33, no. 1, 77-99, 2009.

[15] Darwish, M.A., F.M. Al-Awadhi, and A.M. Darwish. "Energy and water in Kuwait Part I. A sustainability view point." Desalination, 225, no. 1-3, 341-355, May 1, 2008.

[16] Darwish, M.A., and A.M. Darwish. "Energy and water in Kuwait: A sustainability viewpoint, Part II.” Desalination, 230, no. 1-3, 140-152, September 30, 2008.

[17] Misra, B.M. "Seawater desalination using nuclear heat/electricity -- Prospects and challenges." Desalination, 205, no. 1-3, 269-278, February 5, 2007.

[18] Design Concepts of Nuclear Desalination Plants, IAEA-TECDOC-B26, 2002.

[19] Kuhr, Reiner. "HTR's role in process heat applications." Nuclear Engineering and Design 238, no. 11, 3013-3017, November 2008.

[20] Kostin, V.I., Yu.K. Panov, V.I. Polunichev, and I.E. Shamanin. "Floating powergenerating unit with a KLT-40S reactor system for desalinating sea water." Atomic Energy 102, no. 1. At. Energy (USA), 31-5, January 2007.

[21] "KLT-40 floating nuclear heat and power unit with KLT-40S reactor plant," in: Status of Advanced Light Water Reactor Design, IAEA-TECDOC-1391 (2004), pp. 733-754.

[22] Nisan, S., and S. Dardour. "Economic evaluation of nuclear desalination systems." Desalination, 205, no. 1-3, 231-242, February 5, 2009. 
[23] Nuclear News, "B\&W will seek approval for a new modular PWR." Nuclear News, Vol. 52, Number 8, sec. Late News, p. 17, July 2009.

[24] Nuclear News, "NRC to focus attention on integral PWRs." Nuclear News, Vol. 53, Number 8, sec. Power, p. 25, July 2010.

[25] Lanning, D.D. "Modularized high-temperature gas-cooled reactor systems." Nuclear Technology, 88, no. 2: 139-156, 1989

[26] Otaduy, P.J, C.R. Brittain, and L. A. Rovere. "Supervisory, hierarchical control for a multimodular ALMR." In Proc. $7^{\text {th }}$ Power Plant Dynamics, Control, and Testing Symposium, Knoxville, Tennessee, 1989.

[27] Dayal, Y., “Advanced PRISM plant control system,” in Proc. 7th Power Plant Dynamics, Contr. Test. Symp., vol. I, Knoxville, TN, May 15-17, 1989, p. 1.01-13.

[28] "Advanced Nuclear Power Reactors." World Nuclear Association. Internet: http://www.world-nuclear.org/info/inf08.html, July 2009, [Last visited August 5, 2009.

[29] Zhang, Z., Z. Wu, Y. Sun, and F. Li. "Design aspects of the Chinese modular hightemperature gas-cooled reactor HTR-PM.” Nuclear Engineering and Design, 236, no. 5-6, 485-490, March 2006.

[30] Zhang, Z., Z. Wu, D. Wang, Y. Xu, Y. Sun, Fu Li, and Y. Dong. "Current status and technical description of Chinese $2 \times 250$ MWth HTR-PM demonstration plant." Nuclear Engineering and Design, 239, no. 7, 1212-1219, July 2009.

[31] Zhang, Z., and Y. Sun. "Economic potential of modular reactor nuclear power plants based on the Chinese HTR-PM project." Nuclear Engineering and Design, 237, no. 23, 2265-2274, December 2007

[32] Wallace, Edward, R. Matzie, R. Heiderd, and J. Maddalena. "From field to factory-Taking advantage of shop manufacturing for the pebble bed modular reactor." Nuclear Engineering and Design, 236, no. 5-6, 445-453, March 2006. 
[33] Ferreira, T., "PBMR:the future is now," Modern Power Systems, (August, 2001), sec. Nuclear Power Progress, p. 43,44,46.

[34] Li, H., X. Huang, and L. Zhang. "Operation and control simulation of a modular high temperature gas cooled reactor nuclear power plant." IEEE Transactions on Nuclear Science, 55, no. 4. IEEE Trans. Nucl. Sci. (USA), 2357-65, 2008.

[35] Kim, K.K., "Design and Simulation of a Digital Control System for a Multi-Modular Power Plant," Ph.D. dissertation, Dep. Nucl. Eng., Mass. Inst. Technol., Cambridge, MA, Aug. 1992; http://dspace.mit.edu/handle/1721.1/12931.

[36] Kim, K.K., and J.A. Bernard. "Considerations in the control of PWR-type multimodular reactor plants.”, IEEE Transactions on Nuclear Science, 41, no. 6, 2686-2697, 1994

[37] Bernard, J.A., A. F. Henry, D.D. Lanning, and J.E. Meyer. "Studies on the closed-loop digital control of multi-modular reactors," Report No. MITNRL-049, Massachusetts Institute of Technology, November, 1992.

[38] Desai, M., A. Ray, "A fault detection and isolation methodology", Proc. 20th IEEE Conference on Decision and Control including the Symposium on Adaptive Processes, 1981 Volume: 20 Part 1, Dec. 1981, pp 1363-1369

[39] Hagenblad, A., F. Gustafsson, and I. Klein, "A Comparison of Two Methods for Stochastic Fault Detection: The Parity Space Approach and Principal Components Analysis,"13 ${ }^{\text {th }}$ IFAC Symposium on System Identification, 2003.

[40] Upadhyaya, B.R., "Sensor Failure Detection and Estimation," Nuclear Safety, 26 (1), pp. 32-43, 1985.

[41] Zhao, K., B.R. Upadhyaya, and R.T. Wood, "Robust Dynamic Sensor Fault Detection and Isolation of Helical Coil Steam Generator Systems Using a Subspace Identification Technique," Nuclear Technology, 153 (3), pp. 326-340, 2006.

[42] Bodson, M., and J.E. Groszkiewicz, "Multivariable Adaptive Algorithms for Reconfigurable Flight Control," IEEE Transactions on Control Systems Technology, 5 (2), pp. 217-229, 1997. 
[43] Maybeck, P.S., and R.D. Stevens, "Reconfigurable Flight Control Via Multiple Model Adaptive Control Methods," IEEE Transactions on Aerospace and Electronic Systems, 27 (3), pp. 470-480, 1991.

[44] Ruiz, D., J.M. Nougues, and L. Puigjaner, "Fault Diagnosis Support System for Complex Chemical Plants," Computers and Chemical Engineering, 25, pp. 151-160, 2001.

[45] Tarifa, E.E., and N.J. Scenna, “ A Methodology for Fault Diagnosis in Large Chemical Processes and an Application to a Multistage Flash Desalination Process: Part I," Reliability Engineering and System Safety, 60, pp. 29-40, 1998.

[46] Berton, A., and A. Hodouin, "Power of Process and Sensor Fault Detection Methods for Stationary Mineral Processing Plants,"CHEMFAS-4, IFAC, pp. 297-302, 2001.

[47] Williams, B.C., and P.P. Nayak, "A Model-Based Approach to Reactive SelfConfiguring Systems," Proceedings of AAAI-96, 1996.

[48] Braman, J.M., D.A. Wagner, and R.M. Murray, "Fault Tolerance of a Reconfigurable Autonomous Goal-Based Robotic Control System," 2007 International Conference on Robotics and Automation (ICRA), 2007.

[49] Blanke, M., "Diagnosis and Fault-Tolerant Control for Ship Station Keeping," Proceedings of the $13^{\text {th }}$ Mediterranean Conference on Control and Automation, pp. 1379-1384. 2005.

[50] Kerrigan, E.C., and J.M. Maciejowski, "Fault-Tolerant Control of a Ship Propulsion System Using Model Predictive Control," Proceedings of European Control Conference (ECC’99), 1991.

[51] Blanke, M., M. Staroswiecki, and N.E. Wu, "Concepts and Methods in Fault-Tolerant Control," Proceedings of the 2001 American Control Conference, 4, pp. 2606-2620, 2001.

[52] Stengel, R.F. "Intelligent failure-tolerant control." Control Systems Magazine, IEEE 11, no. 4 (1991): 14-23. 
[53] Patton, R.J., and J. Chen, "A Review of Parity Space Approaches to Fault Diagnosis," IFAC SAFEPROCESS Symposium, Baden-Baden, 1991.

[54] Dash, S., and V. Venkatasubramanian, "Challenges in the Industrial Applications of Fault Diagnostic Systems," 24 (2-7), pp. 785-791, 2000.

[55] Na, M.G., et. al., "Failure Detection Using a Fuzzy Neural Network with an Automatic Input Selection Algorithm," Power Plant Surveillance and Diagnostics, 14, pp. 221, 2002.

[56] Kaistha, N., and B.R. Upadhyaya, "Incipient Fault Detection and Isolation of Field Devices in Nuclear Power Systems Using Principal Component Analysis," Nuclear Technology, 136 (2), pp. 221-230, 2001.

[57] He, Q.P., J. Wang, and S.J. Qin, “A New Fault Diagnosis Method Using Fault Directions in Fisher Discriminant Analysis," AIChE Journal, 51 (2), pp. 555-571, 2005.

[58] Dunia, R., and S.J. Qin, "Joint Diagnosis of Process and Sensor Faults Using Principal Component Analysis," Control Engineering Practice, 6 (4), pp. 457-469, 1998.

[59] R. Dunia and S. J. Qin, "Multi-Dimensional Fault Diagnosis Using a Subspace Approach," American Control Conference, 1997.

[60] R. Dunia, and S. J. Qin, "Subspace Approach to Multidimensional Fault Identification and Reconstruction," AIChE Journal, 44 (8), pp. 1813-1831, 2004.

[61] Dunia, R. and S. J. Qin, "A Unified Geometric Approach to Process and Sensor Fault Identification and Reconstruction: The Unidimensional Fault Case," Computers \& Chemical Engineering, 22 (7-8), pp. 927-943, 1998.

[62] A.S. Erbay and B.R. Upadhyaya, "A Personal Computer-Based On-Line Signal Validation System for Nuclear Power Plants," Nuclear Technology, Vol. 119, pp. 6375, July 1997.

[63] K.E. Holbert and B.R. Upadhyaya, "An Integrated Signal Validation System for Nuclear Power Plants," Nuclear Technology, Vol. 92. No. 3, pp. 411-427, December 1990.

[64] Yanh, S.H., B.H. Chen, and X. Z. Wang, "Neural Network Based Fault Diagnosis Using Unmeasurable Inputs," Engineering Applications of Artificial Intelligence, 13 (1), pp. 345-356, 2000. 
[65] Patton, R.J., and J. Chen, "Observer-Based Fault Detection and Isolation: Robustness and Applications,” Control Engineering Practice, 5 (5), pp. 671-682, 1997.

[66] Amand, T., G. Heyen, and B. Kalitventzeff, "Plant Monitoring and Fault Detection Synergy Between Data Reconciliation and Principal Component Analysis," Computers and Chemical Engineering, 25 (4-6), pp. 501-507, 2001.

[67] Zhang, Y., and J. Jiang, "Bibliographical Review on Reconfigurable Fault-tolerant Control Systems",Proceeding of the SAFEPROCESS 2003: 5th Symposium on Detection and Safety for Technical Processes, IFAC, Washington D.C., USA, 265276.

[68] Ray, A., "A Microcomputer-Based Fault-Tolerant Control System for Industrial Applications.” Industry Applications, IEEE Transactions on IA-21, no. 5, 1276-1283, 1985.

[69] Garcia, H.E., A. Ray, and R.M. Edwards. Reconfigurable Control of Power Plants Using Learning Automata. IEEE Control Systems Magazine, 3(2):85-92, 1991.

[70] Garcia, H.E., A. Ray, and R.M. Edwards. "A Reconfigurable Hybrid System and its Application to Power Plant Control." Control Systems Technology, IEEE Transactions on 3, no. 2, 157-170, 1995.

[71] Kim, K.Y., and Y.J. Lee, "Fault Detection and Diagnosis of the Deaerator Level Control System in Nuclear Power Plants," Journal of the Korean Nuclear Society, 36 (1), pp. 73-82, 2004.

[72] Liu, Chi-Min, and Kuo-Guan Wu. "Modified Moose Estimator for Tracking Highly Maneuvering Targets.” Electronics Letters 33, no. 8, 672-674, 1997.

[73] Eryurek, E., and B.R. Upadhyaya. "Fault-tolerant control and diagnostics for large-scale systems.” Control Systems Magazine, IEEE 15, no. 5, 34-42, 1995

[74] Carelli, M.D. IRIS Final Technical Progress Report, 2003. STD-ES-03-40, November 3, 2003,Internet: http://www.osti.gov/energycitations/servlets/purl/816832CfMBvk/native/, [August 11, 2009].

[75] Carelli, Mario, L. Conway, M. Dzodzo, et al., "The SPES3 Experimental Facility Design for the IRIS Reactor Simulation," Science and Technology of Nuclear Installations, vol. 2009, Article ID 579430, 12 pages, 2009. doi:10.1155/2009/579430. 
[76] Nuclear News, “IRIS: A global approach", Nuclear News, Vol. 46, Number 10, page 35, September 2003.

[77] Franceschini, F., and Bojan Petrovic. "Advanced operational strategy for the IRIS reactor: Load Follow Through Mechanical Shim (MSHIM)." Nuclear Engineering and Design 238, no. 12, 3240-3252, December 2008.

[78] "IRIS Official Website - IRIS Home Page.", Internet: http://hulk.cesnef.polimi.it, [September 23, 2009]

[79] Petrovic, B., et al, "Instrumentation Needs for Integral Primary System Reactors (IPSRs), ” Westinghouse Electric Company, STD-AR-05-01, September, 2005.

[80] Kujawski, J.M., D.M., Kitch, and L.E. Conway, “The IRIS Spool-Type Reactor Coolant Pump." Conference Proceedings of 10th International Conference on Nuclear Engineering, 2002, Arlington, VA.

[81] Shen, H., and J.M. Doster. "Application of a neural network based feedwater controller to helical steam generators." Nuclear Engineering and Design 239, no. 6, 1056-1065, 2009

[82] Li, F., and B.R. Upadhyaya, "Design of Sensor Placement for the IRIS Reactor Using Fault Diagnostic Observability and Reliability Criteria," Transactions of the $6^{\text {th }}$ NPIC\&HMIT Meeting, Knoxville, Tennessee, April 2009.

[83] Wang, H., Z. Song, and H. Wang. "Statistical process monitoring using improved PCA with optimized sensor locations*1." Journal of Process Control, 12, no. 6, 735-744, September 2002.

[84] Upadhyaya, B.R., S.R.P. Perillo, X. Xu, and F. Li, "Advanced Control Design, Optimal Sensor Placement, and Technology Demonstration for Small and Medium Nuclear Power Reactor," International Conference on Nuclear Engineering (ICONE17), Brussels, July 2009.

[85] Zhao, K., An Integrated Approach to Performance Monitoring and Fault Diagnosis of Nuclear Power Systems, Doctoral Dissertation, University of Tennessee, 2005. 
[86] Naghedolfeizi, M., Dynamic Modeling of a Pressurized Water Reactor Plant for Diagnostics and Control, Master Thesis, the University of Tennessee, 1990.

[87] Mattison, B., M. Naghedolfeizi, and B.R. Upadhyaya, "Development of a Graphical User Interface for a Dynamic Model of a Pressurized Water Reactor Plant," project report, 2007.

[88] "Advanced Instrumentation and Control Methods for Small and Medium Reactors with IRIS Demonstration," Annual Report No. DE-FG07-07ID14895/UTNE/2008-4, NERI-C Grant Number: DE-FG07-07ID14895.

[89] "Advanced Instrumentation and Control Methods for Small and Medium Reactors with IRIS Demonstration," Annual Report No. DE-FG07-07ID14895/UTNE/2009-4 NERI-C Grant Number: DE-FG07-07ID14895.

[88] "On-line Monitoring for Improving Performance of Nuclear Power Plants Part 1: Instrument Channel Monitoring; Part 2: Process and Component Monitoring and Diagnostics;" " IAEA Technical Report, NP-T-1.1 and NP-T-1.2 (2008).

[89] Hines, J.W., D.R. Garvey, "Development and Application of Fault Detectability Performance Metrics for Instrument Calibration Verification and Anomaly Detection." Journal of Pattern Recognition Research 1, 2006, 2-15.

[90] A Wald, "Sequential Tests of Statistical Hypotheses," Annals of Mathematical Statistics, Vol. 16, pp. 117-186, 1945.

[91] Upadhyaya, B.R., et al., "Development and Testing of an Integrated Signal Validation System for Nuclear Power Plants,” DOE/NE/37959-24, U. S. Department of Energy, (September, 1988).

[92] Hines, J.W. and D. Garvey, "Process and equipment monitoring methodologies applied to sensor calibration monitoring," Quality and Reliability Engineering International 23, no. 1 (2007): 123-135.

[93] Hines, J.W. \& Garvey, D. (2005). The Development of a Process and Equipment Monitoring (PEM) Toolbox and its Application to Sensor Calibration Monitoring. Fourth International Conference on Quality and Reliability (ICQR4). Beijing, China, 
August $9-11$.

[94] Hines, J.W., et al., "Technical Review of On-Line Monitoring Techniques for Performance Assessment," Vol. 1, 2 \& 3, NUREG/CR-6895, (2006-2008).

[95] Athanasopoulou, C. and V. Chatziathanasiou. "Intelligent system for identification and replacement of faulty sensor measurements in Thermal Power Plants (IPPAMAS: Part 1).” Expert Systems with Applications 36, no. 5, 8750-8757, July 2009 


\section{APPENDICES}




\section{Appendix 1: $\quad$ IRIS System SIMULINK Model Equations}

\section{Reactor model: Point Reactor Dynamic Model}

$\frac{d\left(P / P_{0}\right)}{d t}=\frac{\rho-\beta_{t}}{\Lambda} \frac{P}{P_{0}}+\lambda C$

$\frac{d C}{d t}=\frac{\beta}{\Lambda} \frac{P}{P_{0}}-\lambda C$

Reactor Core Heart Transfer Model: Mann’s Model

$$
\begin{aligned}
& \frac{d T_{F}}{d t}= \frac{F_{r} P_{0}}{\left(M C_{p}\right)_{F}} \frac{P}{P_{0}}+\frac{U_{F C} A_{F C}}{\left(M C_{p}\right)_{F}}\left(\theta_{1}-T_{F}\right) \\
& \frac{d \theta_{1}}{d t}= \frac{(1-F)_{r} P_{0}}{2\left(M C_{p}\right)_{c}} \frac{P}{P_{0}}+\frac{U_{F C} A_{F C}}{\left(M C_{p}\right)_{C}}\left(T_{F}-\theta_{1}\right)+\frac{W_{C}\left(T_{c o l d}-\theta_{1}\right)}{2 M_{C}} \\
& \frac{d \theta_{2}}{d t}=\frac{\left(1-F_{r}\right) P_{0}}{2\left(M C_{p}\right)_{C}} \frac{P}{P_{0}}+\frac{U_{F C} A_{F C}}{\left(M C_{p}\right)_{C}}\left(T_{F}-\theta_{1}\right)+\frac{W_{C}\left(\theta_{1}-\theta_{2}\right)}{2 M_{C}} \\
& \frac{d T_{h o t}}{d t}=\frac{W_{C}\left(\theta_{2}-T_{h o t}\right)}{M_{u p}}
\end{aligned}
$$

Table 1: Reactor Model Variables

\begin{tabular}{|l|c|c|}
\hline Variable & Definition & Value(under 100\% power) \\
\hline$A_{F C}$ & Effective heat transfer area \\
between fuel and coolant & $41631.6 \mathrm{ft} 2(3867.7 \mathrm{~m} 2)$ \\
\hline $\mathrm{C}$ & Precursor concentration & \\
\hline$C_{p C}$ & Coolant heat capacity & $1.394 \mathrm{btu} / \mathrm{lbm}-\mathrm{F}(5594.26 \mathrm{~J} / \mathrm{kg}-\mathrm{C})$ \\
\hline
\end{tabular}




\begin{tabular}{|c|c|c|}
\hline Variable & Definition & Value(under $100 \%$ power) \\
\hline$C_{p F}$ & Fuel heat capacity & $0.059 \mathrm{Btu} / \mathrm{lbm}-\mathrm{F} 236.77 \mathrm{~J} / \mathrm{kg}-\mathrm{C}$ \\
\hline$F_{r}$ & $\begin{array}{l}\text { Fraction of the total power } \\
\text { generated in fuel elements }\end{array}$ & 0.97 \\
\hline$U_{F C}$ & $\begin{array}{l}\text { Average overall heat transfer } \\
\text { coefficient }\end{array}$ & 327.4 Btu/hr-ft2-F $1859.03 \mathrm{~J} / \mathrm{sec}-\mathrm{m} 2-\mathrm{C}$ \\
\hline$M_{C}$ & Coolant mass in the core & 1.24 x $104 / 7.45$ x $105 \mathrm{lbm} 5.868 \mathrm{E} 3 \mathrm{~kg}$ \\
\hline$\rho_{C}$ & Coolant density & $43.6 \mathrm{lbm} / \mathrm{ft} 3728.60 \mathrm{~kg} / \mathrm{m} 3$ \\
\hline$M_{F}$ & Fuel mass in the core & $\begin{array}{c}7.66 \mathrm{lbm} / \mathrm{rod} \text { or } 1.8 \times 10^{* * 5} \mathrm{lbm} \text { for the } \\
\text { core } \\
3.6247 \mathrm{~kg} / \mathrm{rod} \text { or } 8.5176 \mathrm{E} 4 \mathrm{~kg}\end{array}$ \\
\hline$M_{u p}$ & Coolant mass in the upriser & \\
\hline $\mathrm{W}$ & Coolant flowrate & $3.7 \times 107 \mathrm{lbm} / \mathrm{hr} 4.8634 \mathrm{E} 3 \mathrm{~kg} / \mathrm{s}$ \\
\hline$P$ & Reactor Core power & $1000 \mathrm{MWt}$ \\
\hline$T_{F}$ & Fuel temperature & $841.8(833.49) \quad(445 。 27 C)$ \\
\hline$T_{\text {cold }}$ & Cold part temperature & $557.6 \mathrm{~F}(292 \mathrm{C})$ \\
\hline$T_{h o t}$ & Hot part temperature & $622.4 \mathrm{~F}(623.12 \mathrm{~F}) 328.2 \mathrm{C}$ \\
\hline$\alpha_{C}$ & Coolant coefficient of reactivity & $-.0004 \mathrm{~F}-1,-0.00072 \mathrm{C}-1$ \\
\hline
\end{tabular}




\begin{tabular}{|l|c|c|}
\hline Variable & Definition & Value(under 100\% power) \\
\hline$\alpha_{F}$ & Fuel coefficient of reactivity & -.0000165 F-1, -0.0000297C-1 \\
\hline$\beta$ & $\begin{array}{c}\text { Delayed neutron group } \\
\text { fraction(One group) }\end{array}$ & 0.0044 \\
\hline$\lambda$ & Average of six group decay & $0.07561 \mathrm{sec}-1$ \\
\hline$\Lambda$ & Nonstant & $0.0001 \mathrm{sec}$ \\
\hline$\rho$ & Total reactivity & \\
\hline$\rho_{e x}$ & External reactivity & 89 \\
\hline$\rho_{f b}$ & Feedback reactivity & 264 \\
\hline & Number of fuel assemblies & \\
\hline & Number of fuel rods per assembly & \\
\hline
\end{tabular}

Note:

$1 \mathrm{lbm}=0.4732 \mathrm{~kg}$

$1 \mathrm{BTU}=1.055 \mathrm{E} 3$ Jour

$1 \mathrm{ft}=0.3048 \mathrm{~m}$

$1 \mathrm{ft} 2=0.0929 \mathrm{~m} 2$

$\mathrm{F}=9 / 5^{*} \mathrm{C}+32$

\section{Dynamic Modeling of HCSG}


A dynamic process is generally modeled as a distributed parameter system characterized by a set of partial differential equations. It is usually rather complicated to solve such a time dependent system with spatial variations. For this reason, a lumped model is used to describe the HCSG dynamic behavior. Each lump has the same averaged properties, so the spatial dependence can be represented simply by the interaction between adjacent lumps. In addition to the assumptions implied in a lumped model, the other major assumptions are as follows:

Only one pressure is used to characterize the superheated region.

The superheated vapor satisfies ideal gas law modified by an expansion coefficient.

The temperature of the second node in the subcooled region is equal to the saturated temperature.

The pressure drop between superheated region and saturated region is constant during any perturbation

The pressure drop between the saturated region and the subcooled region is constant during any perturbation

The steam quality in the boiling region can be assumed as a linear function of the axial coordinate so the density in the boiling region can be approximated as a function of steam pressure.

The steam generation rate assumes to be equal to the boiling rate.

The heat transfer coefficient for the superheated region, the saturated region and the subcooled region is assumed constant. 


\subsection{Nodalization}

Three regions - sub-cooled region, saturated region and superheated region, are used to characterize the significant difference of heat transfer and hydraulic behavior. In each region, two lumps with each volume are used to consider the axial temperature changes.

Correspondingly, six metal nodes are needed to describe the heat transfer from the primary side to the secondary side. For the two lumps of the secondary side in the saturated region, saturated temperature is maintained.

\subsection{Primary Side Equations}

The primary side temperature is given by:

$\frac{d T_{p i}}{d t}=a_{1} \frac{T_{p i-1}-T_{p i}}{L_{i}}-a_{2}\left(T_{p i}-T_{w i}\right)$

where

$L_{i}=\left\{\begin{array}{lll}L_{s} & \text { for } & i=1,2 \\ L_{b} & \text { for } & i=3,4 \\ L_{s c} & \text { for } & i=5,6\end{array}\right.$

$a_{1}=\frac{C_{p p} W_{p}}{\left(A_{x s} C_{p} \rho\right)_{p} / 2}$

$a_{2}=\frac{h_{p w} P_{w p}}{\left(A_{x s} C_{p} \rho\right)_{p} / 2}$

$L_{s}=$ superheated length.

$L_{b}=$ boiling length

$L_{s c}=$ sub-cooled length.

$T$ =primary side temperature. 
$W_{p}=$ coolant flow rate

$C_{p}=$ specific heat.

$\rho=$ density of the primary coolant.

$A_{x s}=$ flow area.

$h=$ heat transfer coefficient.

$P_{w}=$ perimeter for heating.

In the above equations, subscript $p$ and $w$ refer to primary coolant and tube wall respectively.

\subsection{Metal Equations}

The metal temperature is given by:

$$
\begin{aligned}
& \frac{d}{d t} T_{w i}=a_{3}\left(T_{p i}-T_{w i}\right)-a_{4 i}\left(T_{w i}-T_{s i}\right)+\left(T_{w i}-T_{w i-1}\right) \dot{Z}_{i-1} / L_{i}+\left(T_{w i+1}-T_{w i}\right) \dot{Z}_{i} / L_{i} \\
& \text { for } i=1,2,3,4,5,6
\end{aligned}
$$

where

$$
\begin{aligned}
& a_{3}=\frac{h_{p w} P_{w p}}{\left(\rho_{w} A_{w} c_{P w}\right)} \\
& a_{4 i}= \begin{cases}\frac{h_{w s} P_{w s}}{\left(\rho_{w} A_{w} c_{P w}\right)} & i=1,2 \\
\frac{h_{w b} P_{w b}}{\left(\rho_{w} A_{w} c_{P w}\right)} & i=3,4 \\
\frac{h_{w b} P_{w b}}{\left(\rho_{w} A_{w} c_{P w}\right)} & i=5,6\end{cases}
\end{aligned}
$$


$\dot{Z}_{0}=\dot{Z}_{6}=0$

$\dot{Z}_{1}=\dot{L}_{s} / 2$

$\dot{Z}_{2}=\dot{L}_{s}$

$\dot{Z}_{3}=-\left(\dot{L}_{s c}+\dot{L}_{b} / 2\right)$

$\dot{Z}_{4}=-\dot{L}_{s c}$

$\dot{Z}_{5}=-\dot{L}_{s c} / 2$

$h_{w s}, h_{w b}, h_{w s c}=$ heat transfer coefficient on the tube side for superheated steam region, saturated water region, and sub-cooled water region respectively

$P_{w s}, P_{w b}, P_{w s c}=$ heating circumference on the tube side for superheated steam region, saturated water region, and sub-cooled water region respectively

\subsection{Equations for the Superheated Region}

The mass balance of the steam in the superheated steam nodes, node 1 and node 2 , are given by:

$\dot{M}_{s 1}=W_{21}-W_{s}$

$\dot{M}_{s 2}=W_{b}-W_{21}$

where

$M_{s}=$ steam mass in the superheated region.

$W_{s}=$ steam flow rate to turbine, which is an external constraint imposed by the controller.

$W_{b}=$ steam production rate.

The heat balance equations of the two superheated steam nodes, node 1 and node 2, are given by:

$\frac{d}{d t}\left(M_{s 1} H_{s 1}-P_{s} V_{s 1}\right)=Q_{s 1}+W_{21} H_{s 2}-W_{s} H_{s 1}-P_{s 1} \dot{V}_{s 1}$

$\frac{d}{d t}\left(M_{s 2} H_{s 2}-P_{s} V_{s 2}\right)=Q_{s 2}+W_{b} H_{g}-W_{21} H_{s 2}-P_{s 2} \dot{V}_{s 2}$

where 
$M_{s}=$ steam mass in the superheated region.

$P_{s}=$ steam pressure in the superheated region.

$V_{s}=$ steam volume in the superheated region.

$H_{s}=$ specific enthalpy of the steam.

$Q_{s 1}, Q_{s 2}=$ heat transfer rate to the two superheated nodes.

$Q_{s 1}=h_{w s} P_{w s} L_{s}\left(T_{w 1}-T_{s 1}\right) / 2$

$Q_{s 2}=h_{w s} P_{w s} L_{s}\left(T_{w 2}-T_{s 2}\right) / 2$

Assuming the pressure loss in the superheated steam region is small, we have

$P_{s}=P_{s 1}=P_{s 2}$

Since specific enthalpy is a function of temperature and pressure, then we have:

$\dot{H}_{s}=\frac{\partial H_{s}}{\partial T_{s}} \dot{T}_{s}+\frac{\partial H_{s}}{\partial P_{s}} \dot{P}_{s}$

and

$H_{s 2}-H_{s 1}=\frac{\partial H_{s}}{\partial T_{s}}\left(T_{s 2}-T_{s 1}\right)$

Combining with the mass balance equations and the expansion of the specific enthalpy, the energy balance equations can be rewritten as follows:

$$
\begin{aligned}
& M_{s 1} C_{p s} \dot{T}_{s 1}+C_{p s}\left(T_{s 1}-T_{s 2}\right) \dot{M}_{s 1}+\left(M_{s 1} \frac{\partial H_{s}}{\partial P_{s}}-V_{s 1}\right) \dot{P}_{s}=Q_{s 1}-W_{s} C_{p s}\left(T_{s 1}-T_{s 2}\right) \\
& M_{s 2} C_{p s} \dot{T}_{s 2}+\left(M_{s 2} \frac{\partial H_{s}}{\partial P_{s}}-V_{s 2}\right) \dot{P}_{s}=Q_{s 2}-W_{b} C_{p s}\left(T_{s 2}-T_{s a t}\right)
\end{aligned}
$$

The steam pressure in the superheated region can be described by compressibility adjusted ideal gas law, which is given by [18]:

$$
P_{s} V_{s}=Z^{*}{ }_{s} M_{s} R\left(T_{s 1}+T_{s 2}\right) /\left(2 M_{s t m}\right)
$$


The time derivative of the steam pressure can then be determined by the following equation:

$$
\dot{P}_{s}=\left\{\frac{Z^{*}{ }_{s} R}{2 M_{s t m}}\left\{\dot{M}_{s}\left(T_{s 1}+T_{s 2}\right)+M_{s}\left(\dot{T}_{s 1}+\dot{T}_{s 2}\right)\right\}-P_{s} A_{s} \dot{L}_{s}\right\} \frac{1}{A_{s} L_{s}}
$$

where

$M_{s t m}=$ mole mass of steam.

$Z_{s}^{*}=$ steam expansion coefficient.

\subsection{Equations for Boiling Region}

The mass balance equation for the boiling region is given by:

$\frac{d}{d t}\left(\bar{\rho}_{b} A_{s} L_{b}\right)=W_{d b}-W_{b}$

If we notice

$$
\frac{d \bar{\rho}_{b}}{d t}=\frac{d \bar{\rho}_{b}}{d P_{s}} \frac{d P_{s a t}}{d t}
$$

then

$$
A_{s} \bar{\rho}_{b} \dot{L}_{b}+A_{s} L_{b} K_{b} \dot{P}_{s a t}=W_{d b}-W_{b}
$$

where

$$
K_{b}=\frac{\partial \bar{\rho}_{b}}{\partial P}
$$

If we assume that the steam quality is linear function of the axial position along the channel, then

$$
\bar{\rho}_{b}=\frac{\int_{0}^{l} \rho(x) d x}{\int_{0}^{1} d x}=\int_{0}^{1} \frac{d x}{v_{f}+x v_{f g}}
$$

In the operation pressure range, we have [18]:

$$
\bar{\rho}_{b}\left(P_{s}\right)=1.61594+0.00552445 P_{s}
$$


Therefore,

$\dot{L}_{b}=\left\{W_{d b}-W_{b}-A_{s} L_{b} K_{b} \dot{P}_{s a t}\right\} /\left(A_{s} \bar{\rho}_{b}\right)$

$W_{b}=h_{w b} U_{w b}\left(\frac{T_{w 3}+T_{w 4}}{2}-T_{s a t}\right) L_{b} / h_{f g}$

$W_{d b}=$ flow rate leaving sub-cooled region to the saturated region.

$h_{f g}=$ vaporization heat.

2.6. Equations for Sub-cooled Region

In analogy to the boiling region, the mass balance equation for the subcooled region can be given as follows:

$W_{d b}=W_{f w}-\bar{\rho}_{s c} A_{s c} \dot{L}_{s c}-A_{s} L_{s c} K_{s c} \dot{P}_{s c}$

where

$K_{s c}=\frac{\partial \bar{\rho}_{s c}}{\partial P_{s c}}=\frac{\partial}{\partial P_{s c}}\left(\rho_{f w}+\rho_{f}\right) / 2$

$W_{f w}=$ feed water flow rate.

Heat balance equation for the sub-cooled region 1 is given by:

$\frac{d\left(M C_{p} T\right)_{s c 1}}{d t}-V_{s c 1} \dot{P}_{s c}=h_{w s c} P_{w s c} L_{s c}\left(T_{w 5}-T_{s c 1}\right)+C_{p s c}\left(W_{s c} T_{s c 2}-W_{d b} T_{s c 1}\right)$

Since the outlet temperature of the first sub-cooled node can be approximated by the saturated temperature, then

$$
\begin{aligned}
& \frac{A_{s}\left(\rho C_{p}\right)_{s c}}{2}\left[T_{s a t} \dot{L}_{s c}+K_{1} L_{s c} \dot{P}_{s a t}\right]-\frac{A_{s} L_{s c}}{2} \dot{P}_{s c} \\
& =h_{w s c} P_{w s c} L_{s c}\left(T_{w 5}-T_{s a t}\right) / 2+C_{p s c}\left(W_{s c} T_{s c 2}-W_{d b} T_{s a t}\right)
\end{aligned}
$$

where

$$
K_{1}=\frac{\partial T_{s a t}}{\partial P}
$$

Heat balance equation for the sub-cooled region 2: 


$$
\begin{aligned}
& \frac{A_{s}\left(\rho C_{p}\right)_{s c}}{2}\left[T_{s c 2} \dot{L}_{s c}+L_{s c} \dot{T}_{s c 2}\right]-\frac{A_{s} L_{s c}}{2} \dot{P}_{s c} \\
& =h_{w s c} U_{w s c} L_{s c}\left(T_{w 6}-T_{s c 2}\right) / 2+C_{p s c}\left(W_{f w} T_{f w}-W_{s c} T_{s c 2}\right)
\end{aligned}
$$

After simplification, we obtain

$\dot{T}_{s c 2}=\left\{\left\{0.5 * h_{w s c} P_{w s c} L_{s c}\left(T_{w 6}-T_{s c 2}\right)+C_{p s c}\left(W_{f w} T_{f w}-W_{s c} T_{s c 2}\right)\right.\right.$

$\left.\left.+\frac{A_{s} L_{s c}}{2} \dot{P}_{s c}\right\} * 2 /\left(A_{s}\left(\rho C_{p}\right)_{s c}-T_{s c 2} \dot{L}_{s c}\right\}\right\} / L_{s c}$

If we assume that $\dot{M}_{s c 1}=\dot{M}_{s c 2}$, then we have:

$W_{s c}=\left(W_{f w}+W_{d b}\right) / 2$

Substituting the expression of ${ }^{W_{s c}}$ and $W_{d b}$ into the heat balance equation for the subcooled region 1 , we have:

$$
\begin{aligned}
& \dot{L}_{s c}=\frac{1}{0.5\left(A_{s}\left(\rho C_{p}\right)_{s c}\right)\left(T_{s c 2}-T_{s a t}\right)}\left\{\left\{0.5 * h_{w s c} P_{w s c} L_{s c}\left(T_{w 5}-T_{s a t}\right)+C_{p s c}\left(W_{f w} T_{s c 2}-W_{s c} T_{s a t}\right)\right.\right. \\
& \left.\left.-\frac{A_{s} L_{s c}}{2}\left[K_{s c} C_{p s c}\left(T_{s c 2}-2 T_{s a t}\right)-1\right] \dot{P}_{s c}\right\}-0.5 A_{s}\left(\rho C_{p}\right)_{s c} K_{1} L_{s c} \dot{P}_{s a t}\right\}
\end{aligned}
$$

Noticing the pressure relationship between $P_{s c}, P_{s a t}$ and $P_{s}$, we have

$$
\begin{aligned}
& P_{s c}=P_{s a t}+\frac{1}{2}\left(\Delta P_{t p b}+\Delta P_{s p s c}\right) \\
& P_{s a t}=P_{s s}+\frac{1}{2}\left(\Delta P_{t p b}+\Delta P_{s p s s}\right)
\end{aligned}
$$

where

$P_{s c}=$ pressure at the sub-cooled region.

$P_{s a t}=$ pressure at the saturated region.

$\Delta P_{t p b}=$ two-phase pressure loss in the boiling region.

$\Delta P_{s p s c}=$ single phase pressure loss in the sub-cooled region.

$\Delta P_{s p s s}=$ single phase pressure loss in the superheated region. 


\subsection{Equations for the Steam Pressure Controller}

The secondary side pressure is maintained by regulating the steam flow rate. The steam flow rate satisfies the following equation:

$\dot{W}_{s}=\frac{W_{s 0}\left(1-C_{s t} u\right)-W_{s}}{\tau_{s}}$

where

$u=$ controller output.

$\tau=$ time constant.

$W_{s 0}=$ initial steam flow rate on the secondary side.

$C_{s t}=$ an adjustable parameter.

If a PI controller is used, the controller output has both the proportional part $u_{1}(t)$ and the integral part $u_{2}(t)$, which is given by:

$u_{1}(t)=k_{1}\left(\frac{P_{t b}}{P_{0}}-\frac{P_{s e t}}{P_{0}}\right)$

$\frac{d u_{2}(t)}{d t}=k_{2}\left(\frac{P_{t b}}{P_{0}}-\frac{P_{s e t}}{P_{0}}\right)$

where

$k_{1}=$ proportional gain

$k_{2}=$ integral gain

$P_{t b}=$ turbine header pressure.

$P_{s e t}=$ turbine header pressure set-point.

$P_{0}=$ turbine header pressure set-point. 


\section{Appendix 2: $\quad$ IRIS SIMULINK Model Sample Run}

This example applies to the twin-unit SIMULINK model, and the process is similar to the single-unit model.

Step 1: open the SIMULINK profile of your choice (Profile01.mdl through Profile09.mdl).

$>$ Step 2: open IRISMixing.mdl, and make sure all necessary files for both SIMULINK model and M-file are in the same directory or at least in Matlab path.

Step 3: copy and paste the profile of your choice in to the IRIS mixing and connect it to one of units of your choice.

Step 4: adjust the simulation time by opening 'Simulation' -> 'Configuration Parameters.'

$>$ Step 5: open M-file SMixing.m.

Step 6: Adjust power demands (Demand and Demand2) values (lines 144 and 145) and run the file.

Step 7: You will get the following error message:

??? Undefined function or variable 'core_tran'.

Error in ==> SMixing at 408

bb=length(core_tran);

○ This error message appears because it needs outputs from the SIMULINK model that are not yet available, but by doing this all inputs are ready and uploaded in to the computer memory, so you can run the SIMULINK model again and again without having to run the m-file every time you make minor changes in the SIMULINK model. To remove this error and run the whole thing at once, simply uncomment line 390 in the m-file. 
Step 8: After the simulation is complete, skip over to line 392. This section of the mfile plots out all important results. Don't forget to adjust the plotting axes before running it. If the plots don't look good, adjust plotting axes again and run this section again. There's no need to run the whole m-file again, but if you do you'll have to run the SIMULINK model again.

Step 9: If you notice too many spikes in the results, you might want to change the $\mathrm{Kp} 1$ and Kp2 values. Usually, faster transients will need smaller Kp values.

$>$ Step 10: You can pause the SIMULINK model at any time and plot out the results to check if it is going smoothly, and resume the simulation again if you are happy with the results. 


\section{Appendix 3: $\quad$ IRIS SIMULINK Model Screenshots}

MyCore.mdl (created by Xiaojia Xu and with some modifications performed by Sergio Perillo)

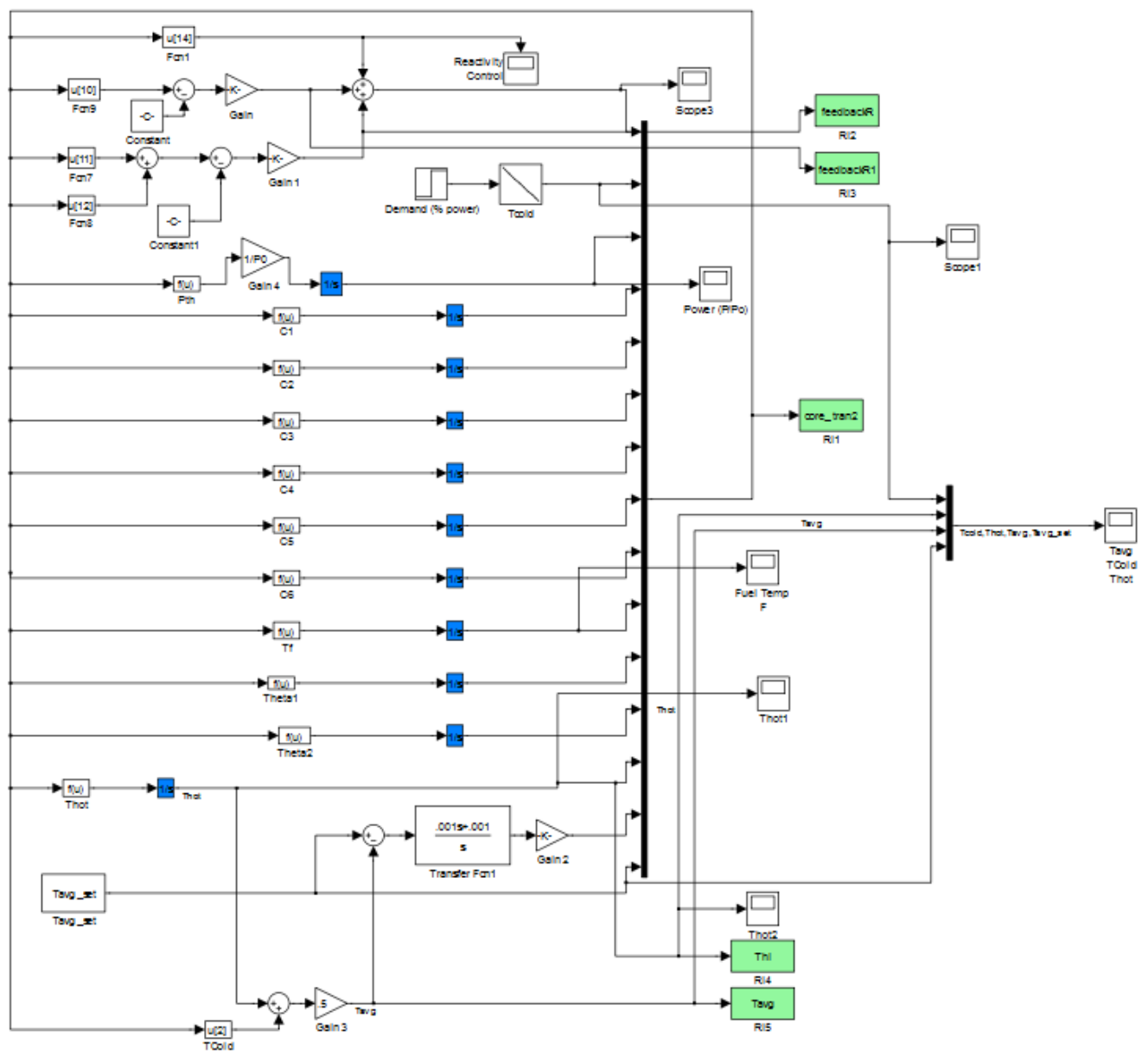


MyHCSG2.mdl (IRIS single unit, created by Xiaojia Xu and modified by Sergio Perillo)

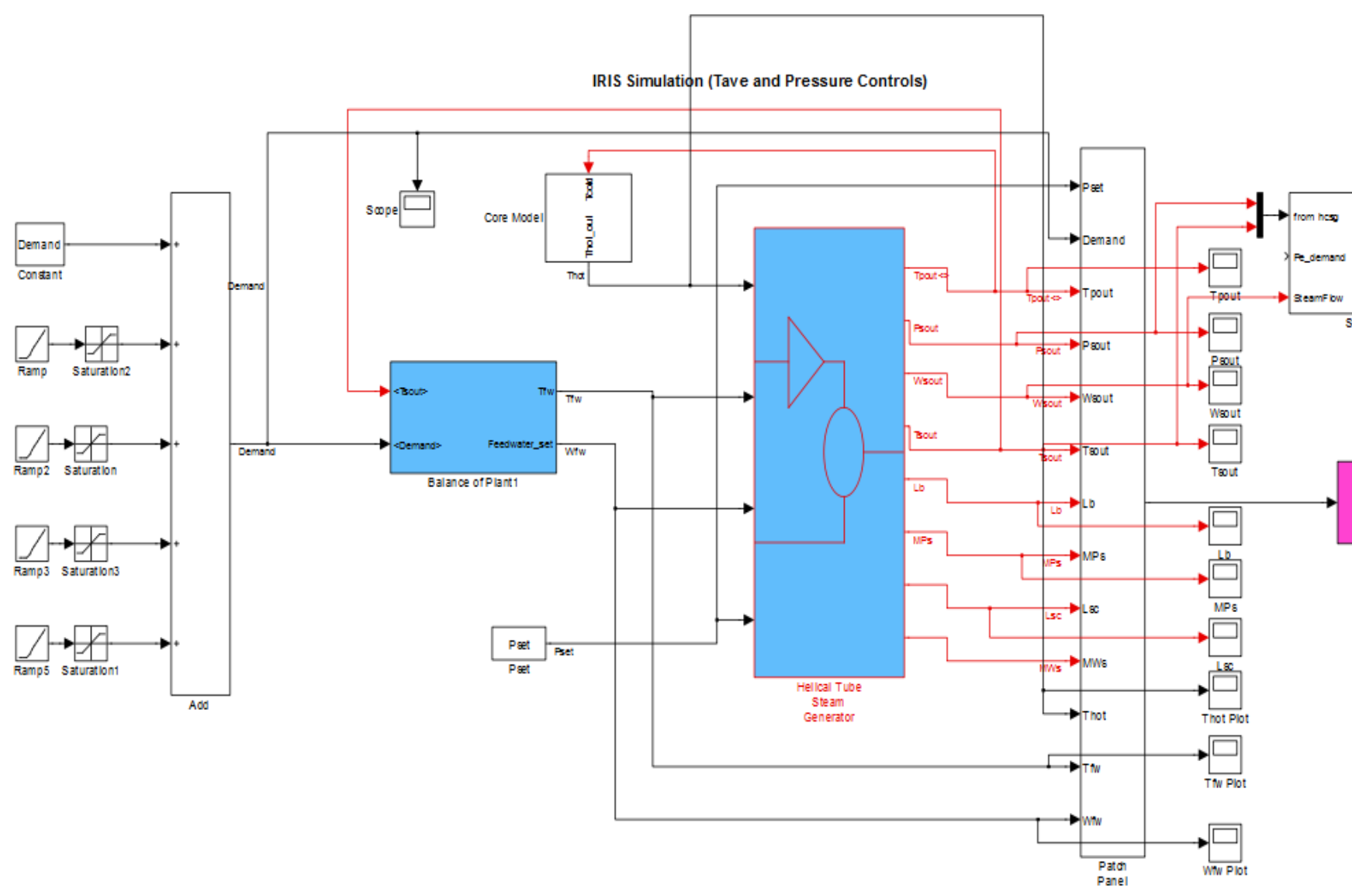


Profile01.mdl (created by Sergio Perillo).

Other profiles are variations from this one.
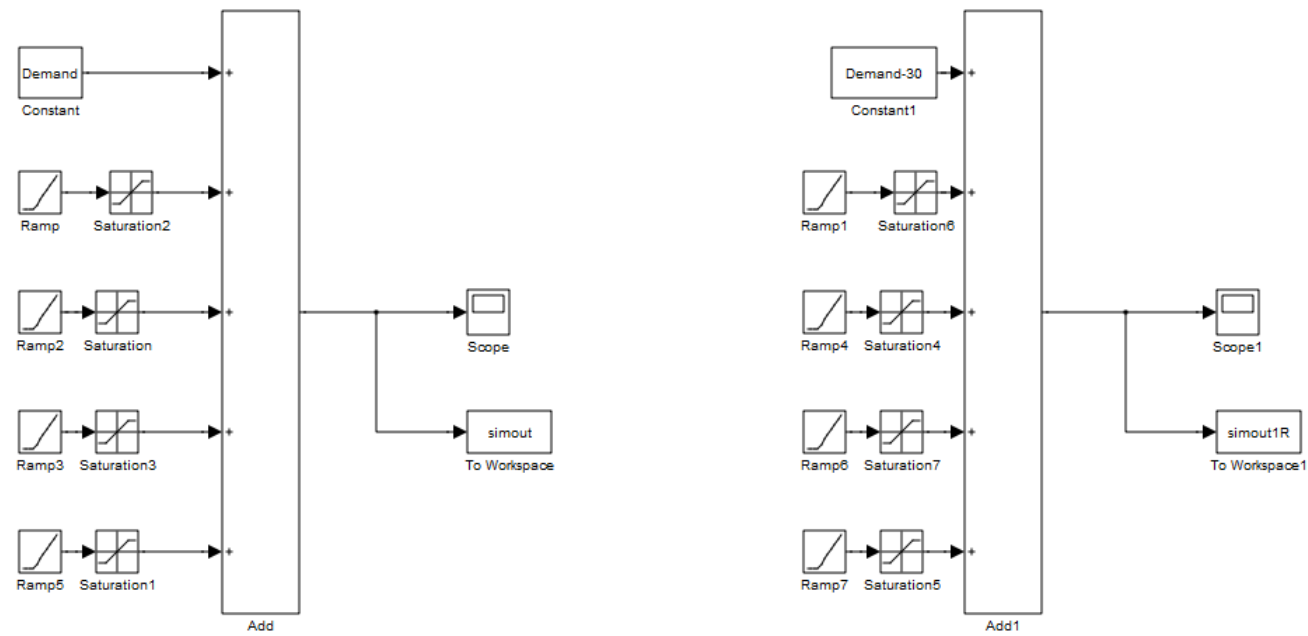

Profile 1

Inverted Profile 1

Profile09.mdl (created by Sergio Perillo). 


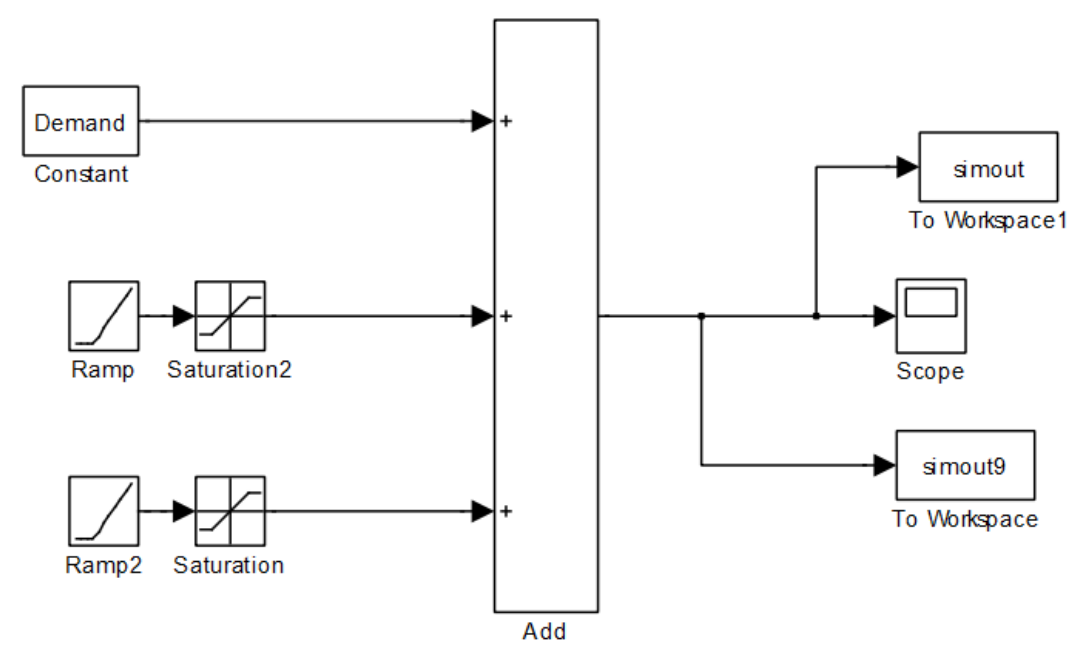


coress.mdl (IRIS reactor core steady-state, without Tave controller, developed by Xiaojia $\mathrm{Xu})$

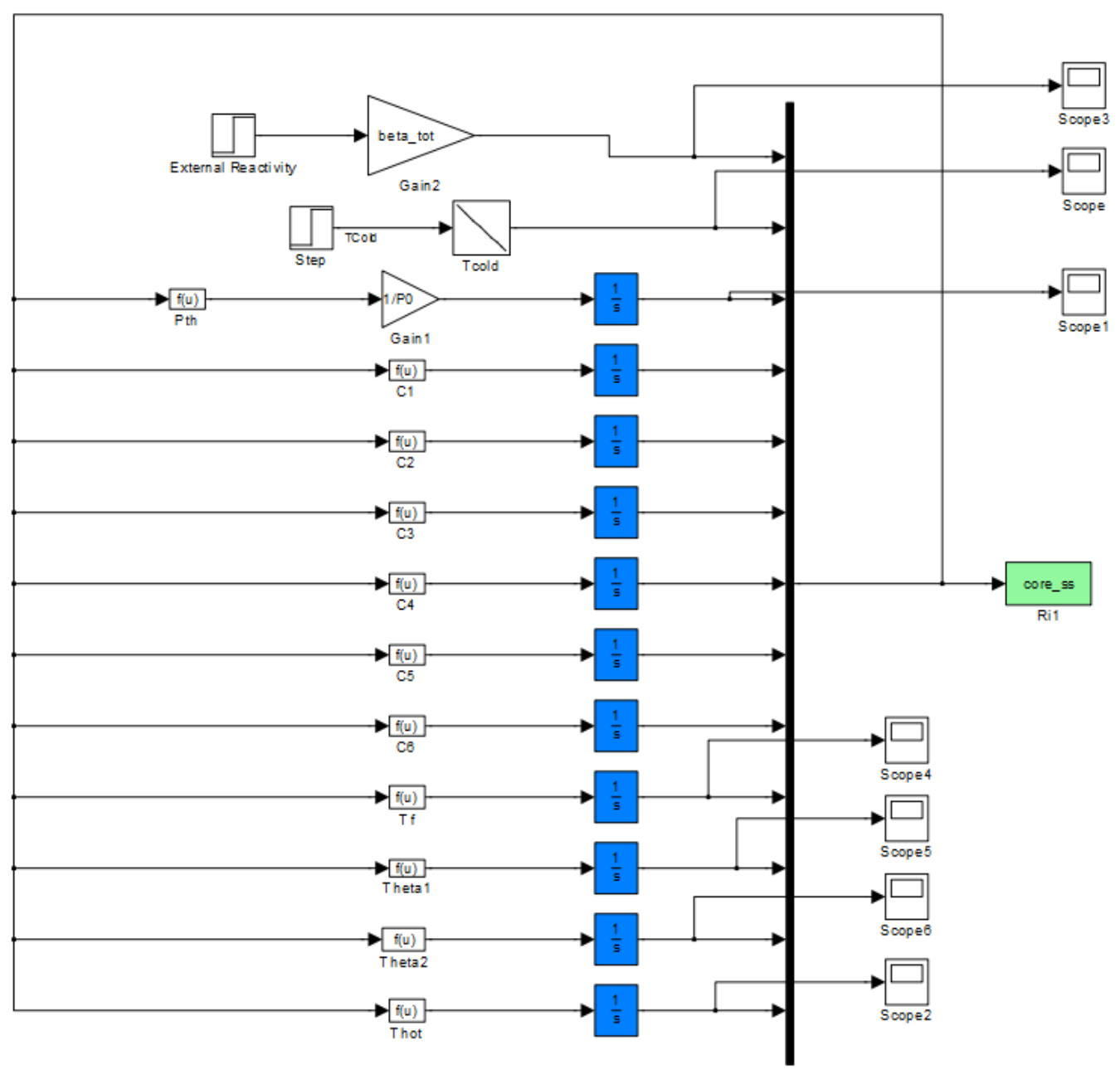


hcsgss.mdl (helical coil steam generator steady-state, without steam pressure controller, developed by Xiaojia Xu)

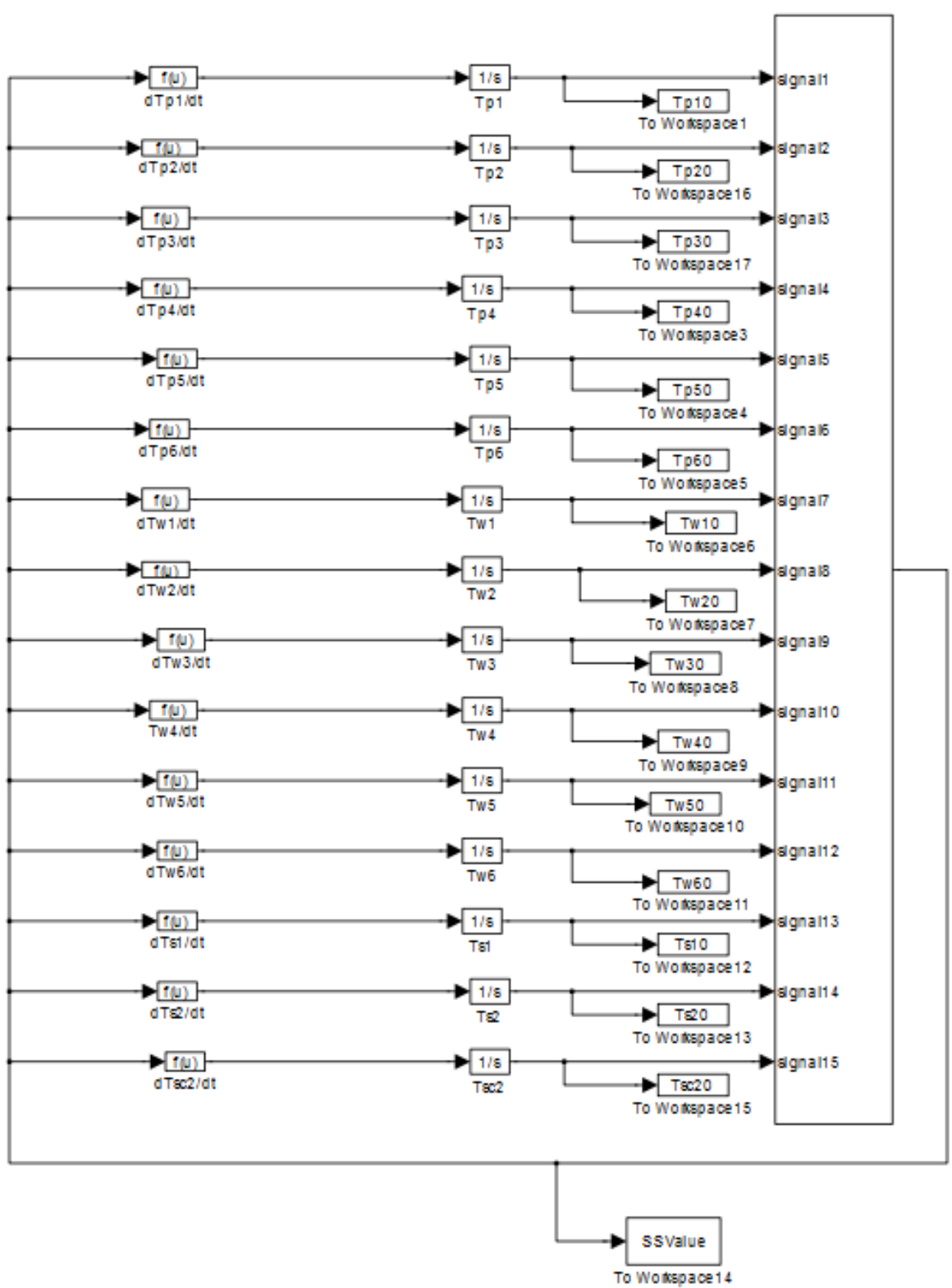


Appendix 4: $\quad$ Profiles 3 - 9 Simulations Plots

Profile \#3:

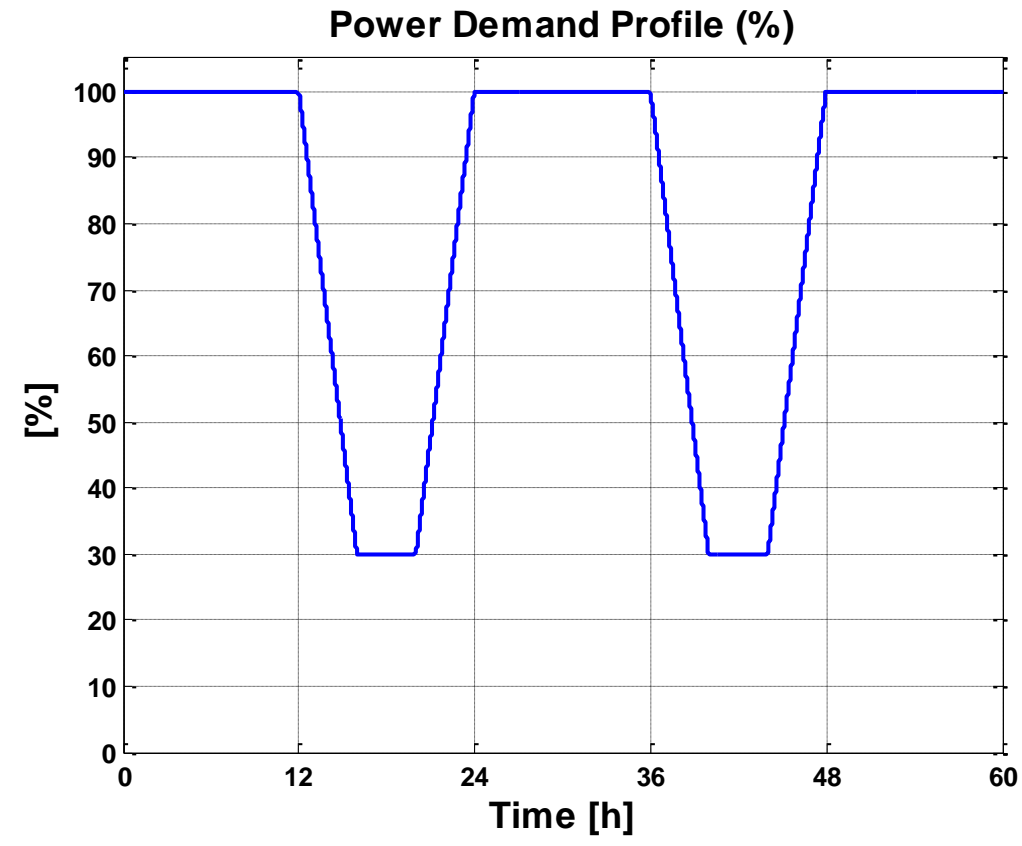




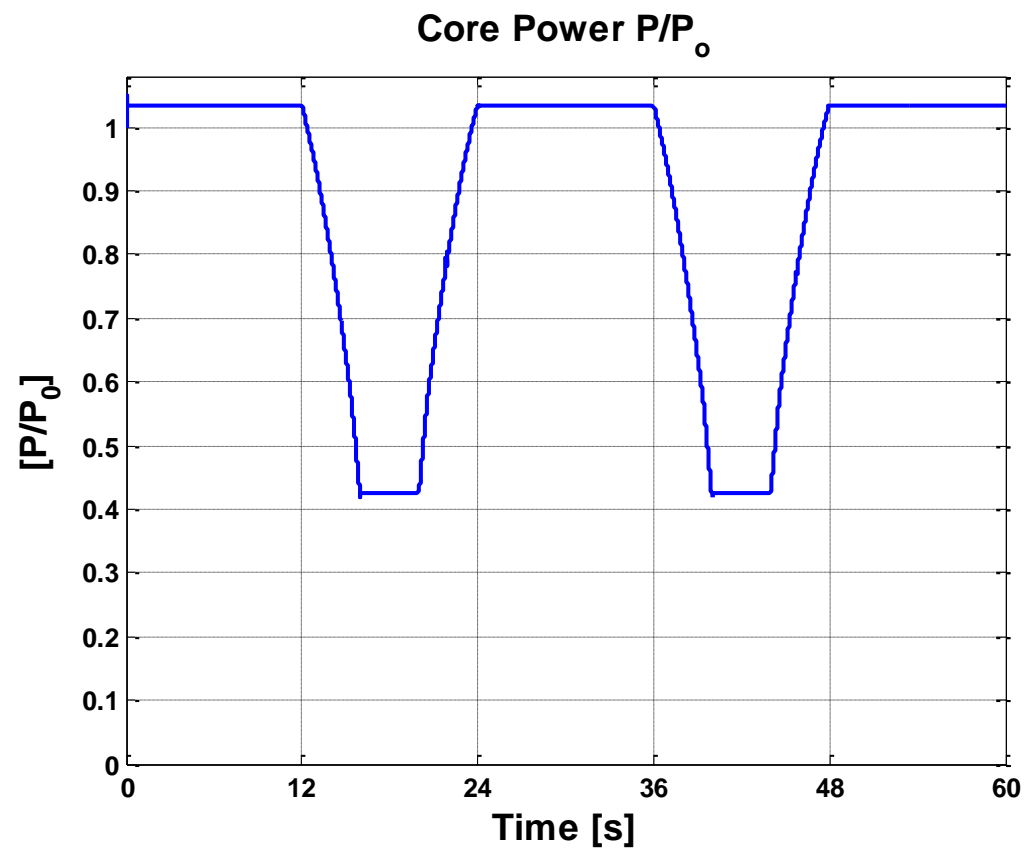


Core Inlet $\left(\mathrm{T}_{\mathrm{CL}}\right)$, Core Exit $\left(\mathrm{T}_{\mathrm{HL}}\right)$, and Average Temperatures
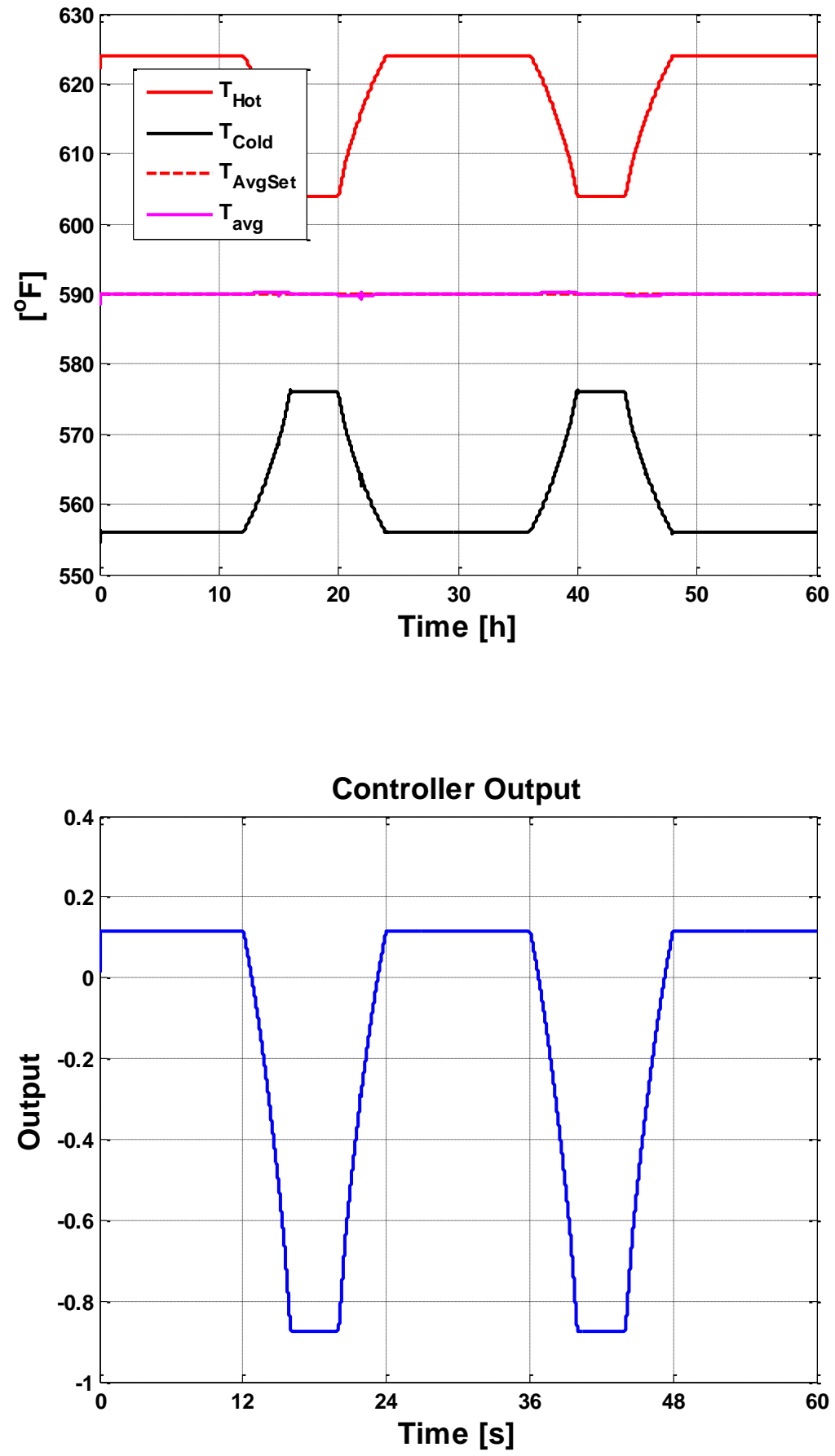

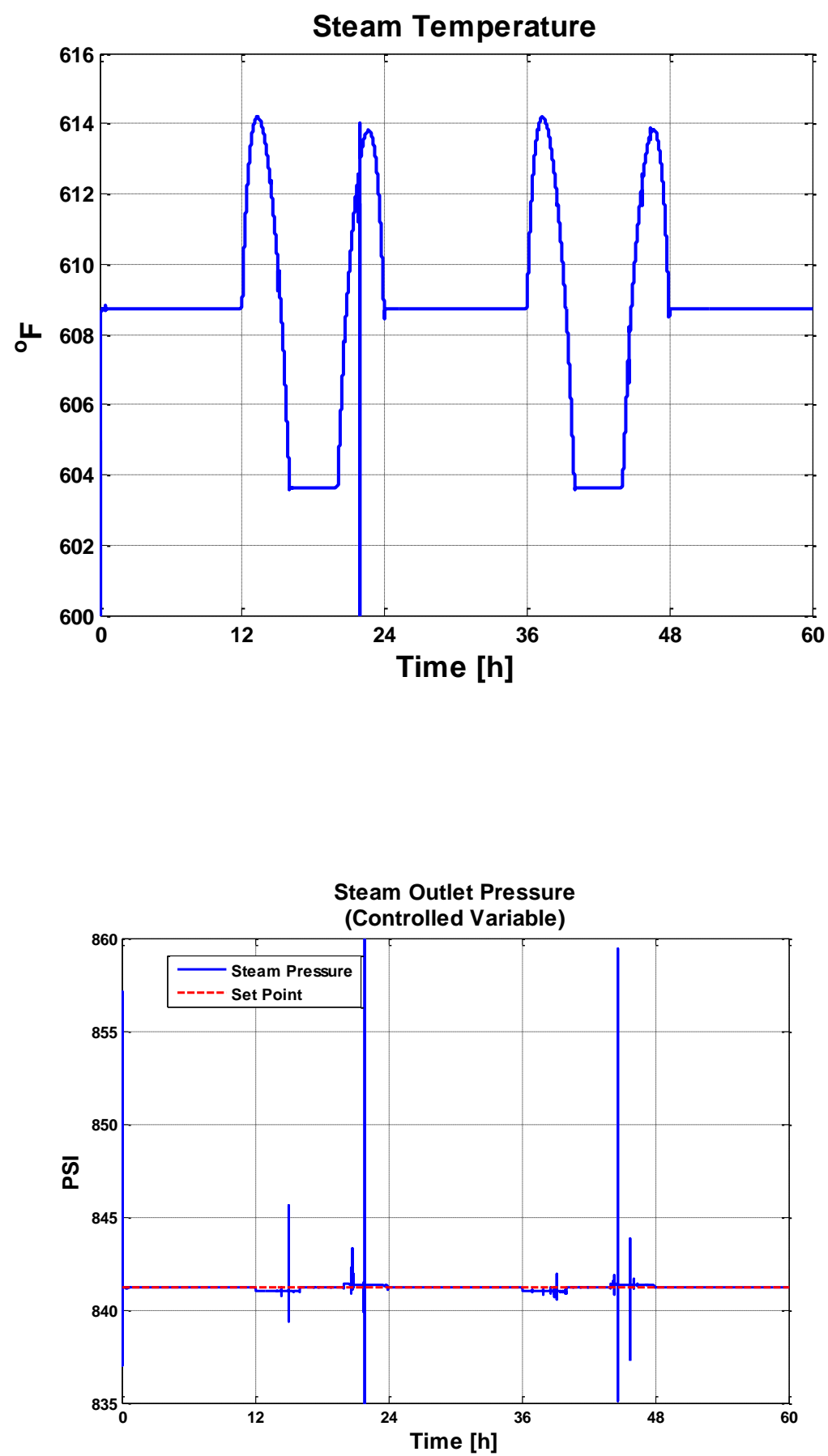


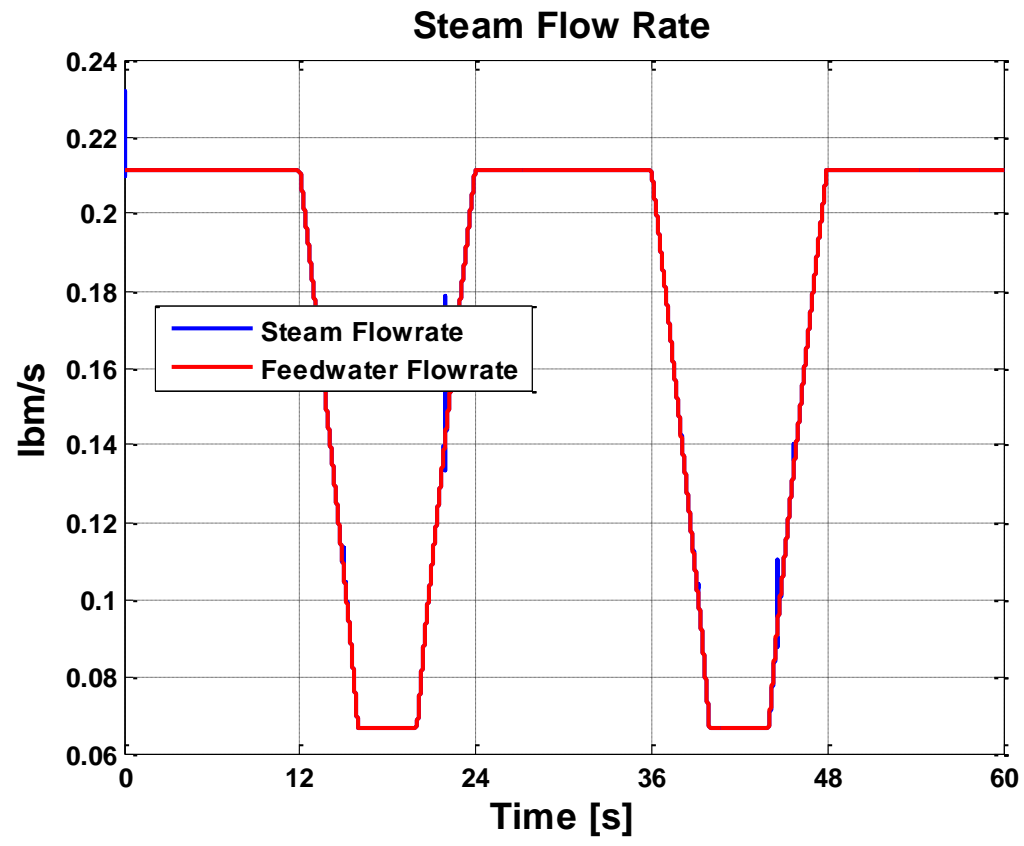

Profile \#4:

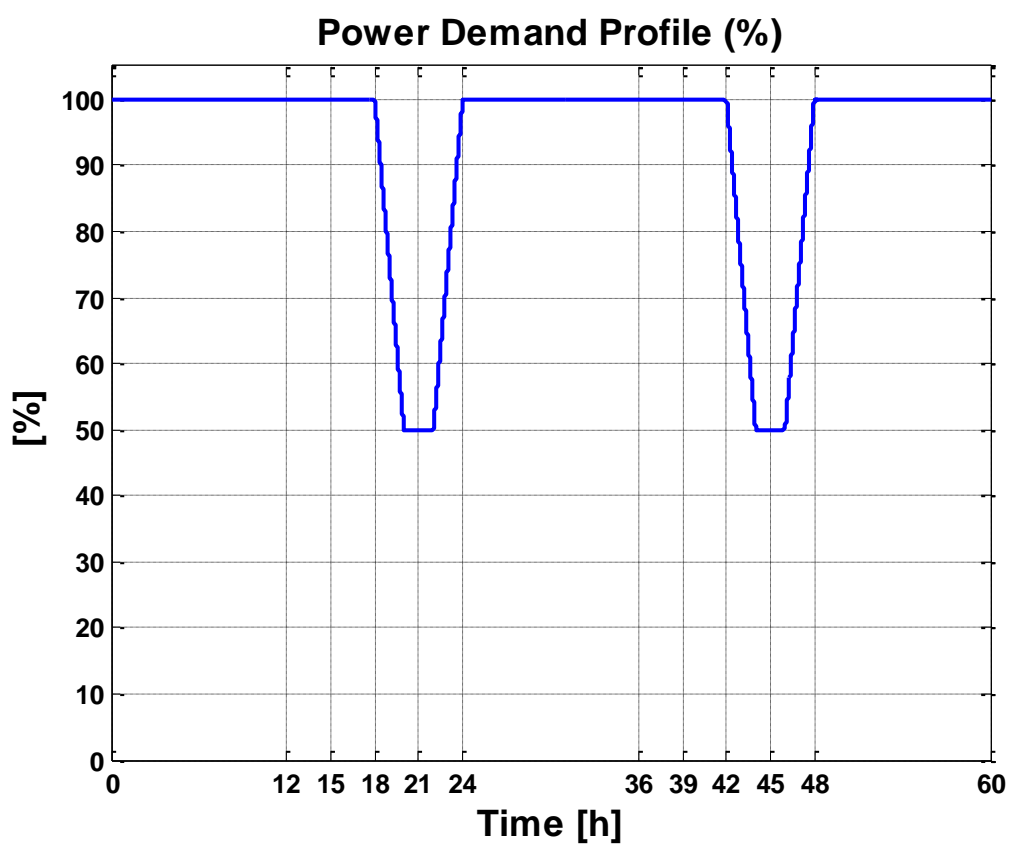




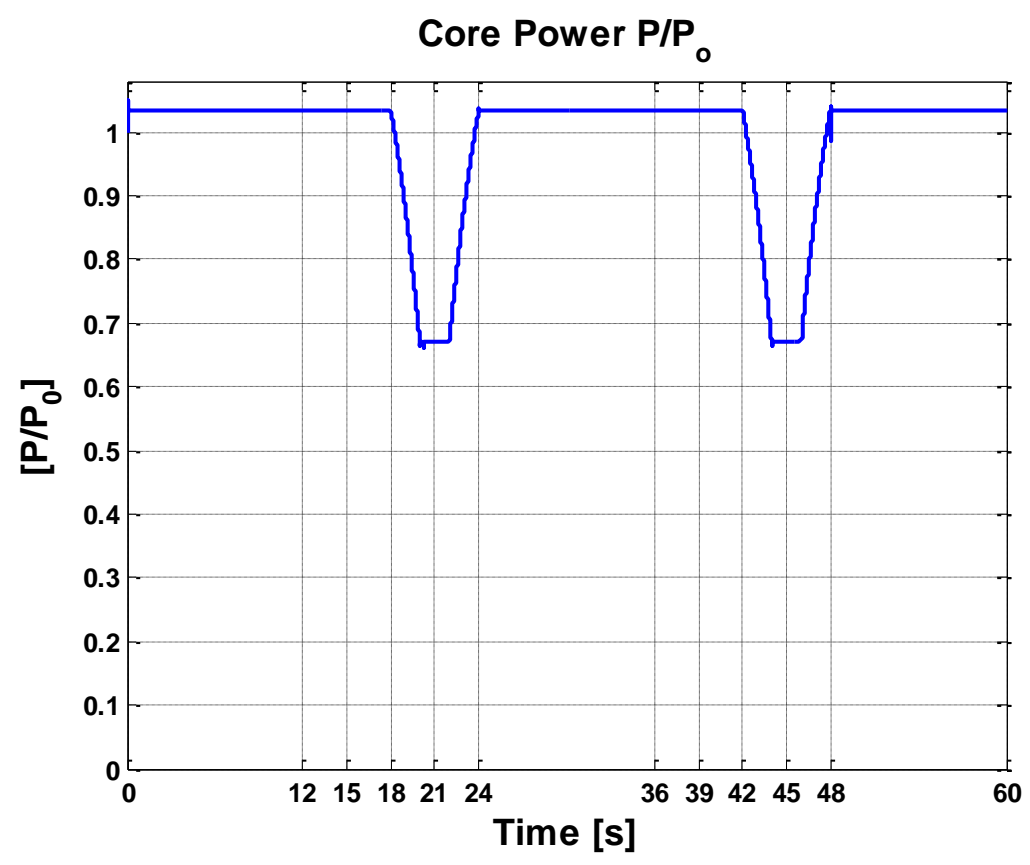

Core Inlet $\left(\mathrm{T}_{\mathrm{CL}}\right)$, Core Exit $\left(\mathrm{T}_{\mathrm{HL}}\right)$, and Average Temperatures

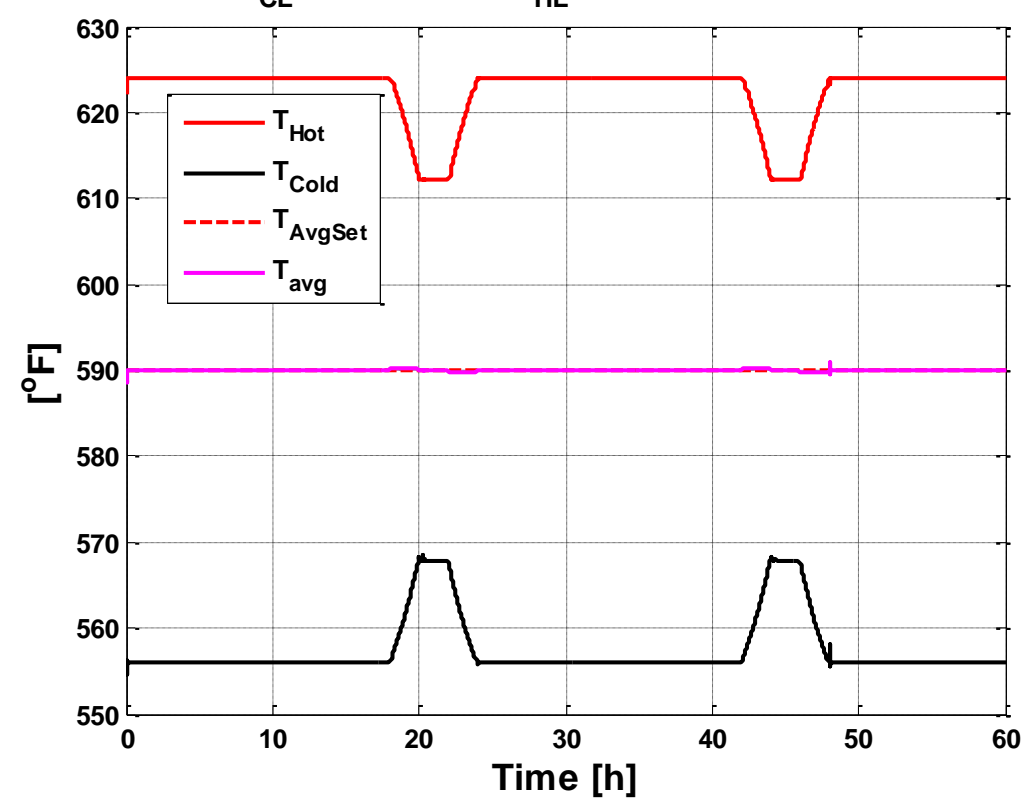



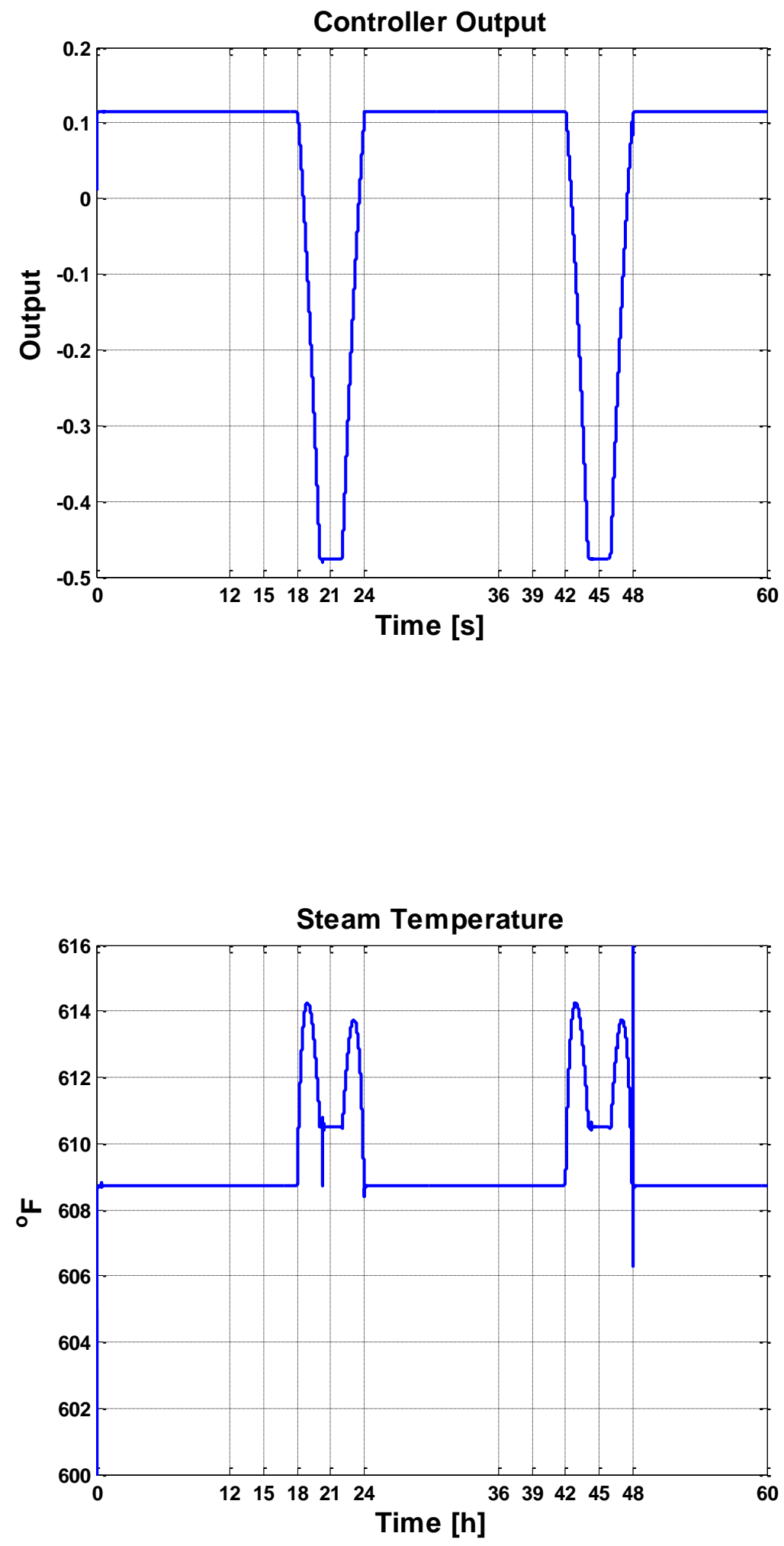

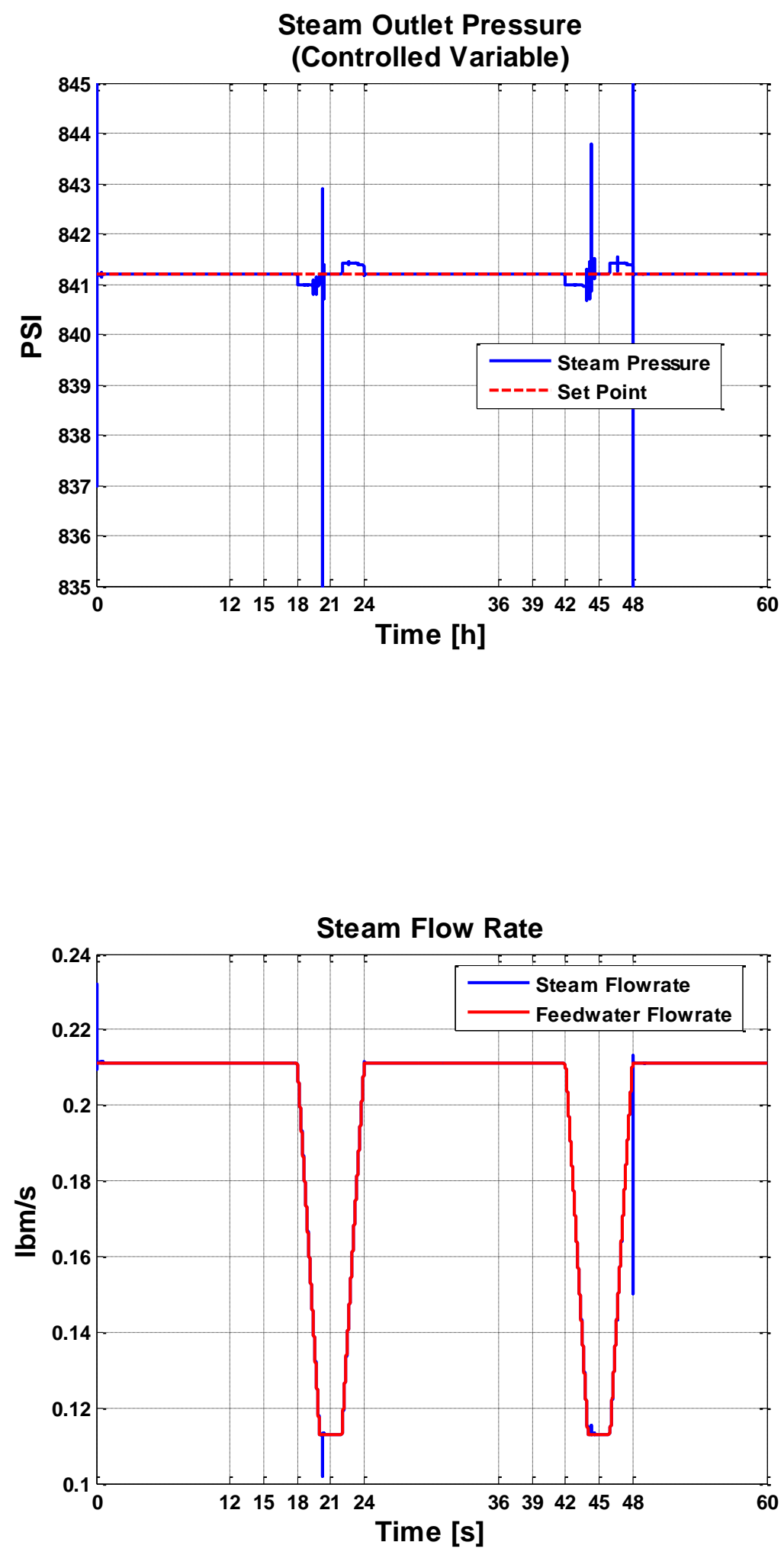


\section{Profile \#5:}
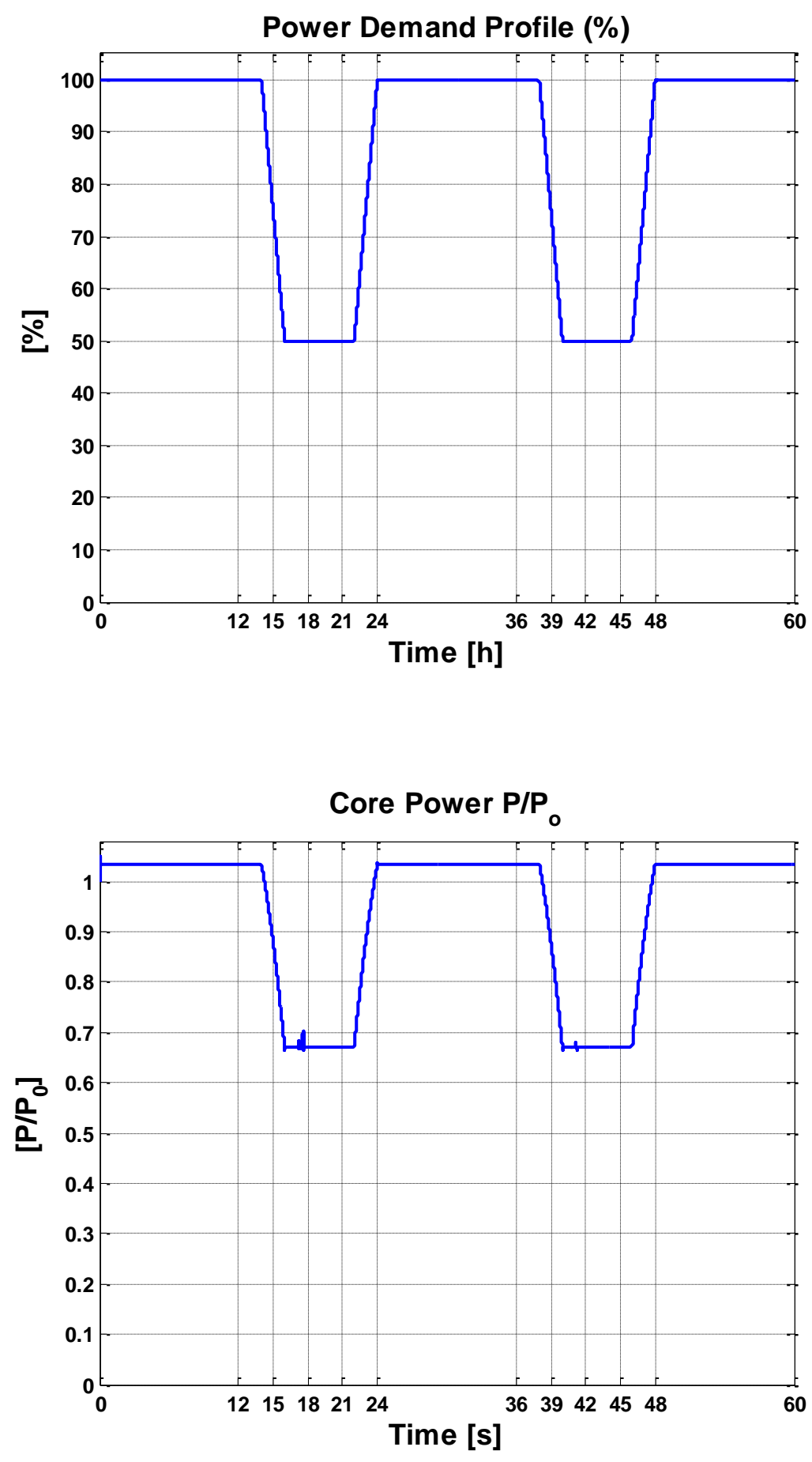
Core Inlet $\left(\mathrm{T}_{\mathrm{CL}}\right)$, Core Exit $\left(\mathrm{T}_{\mathrm{HL}}\right)$, and Average Temperatures
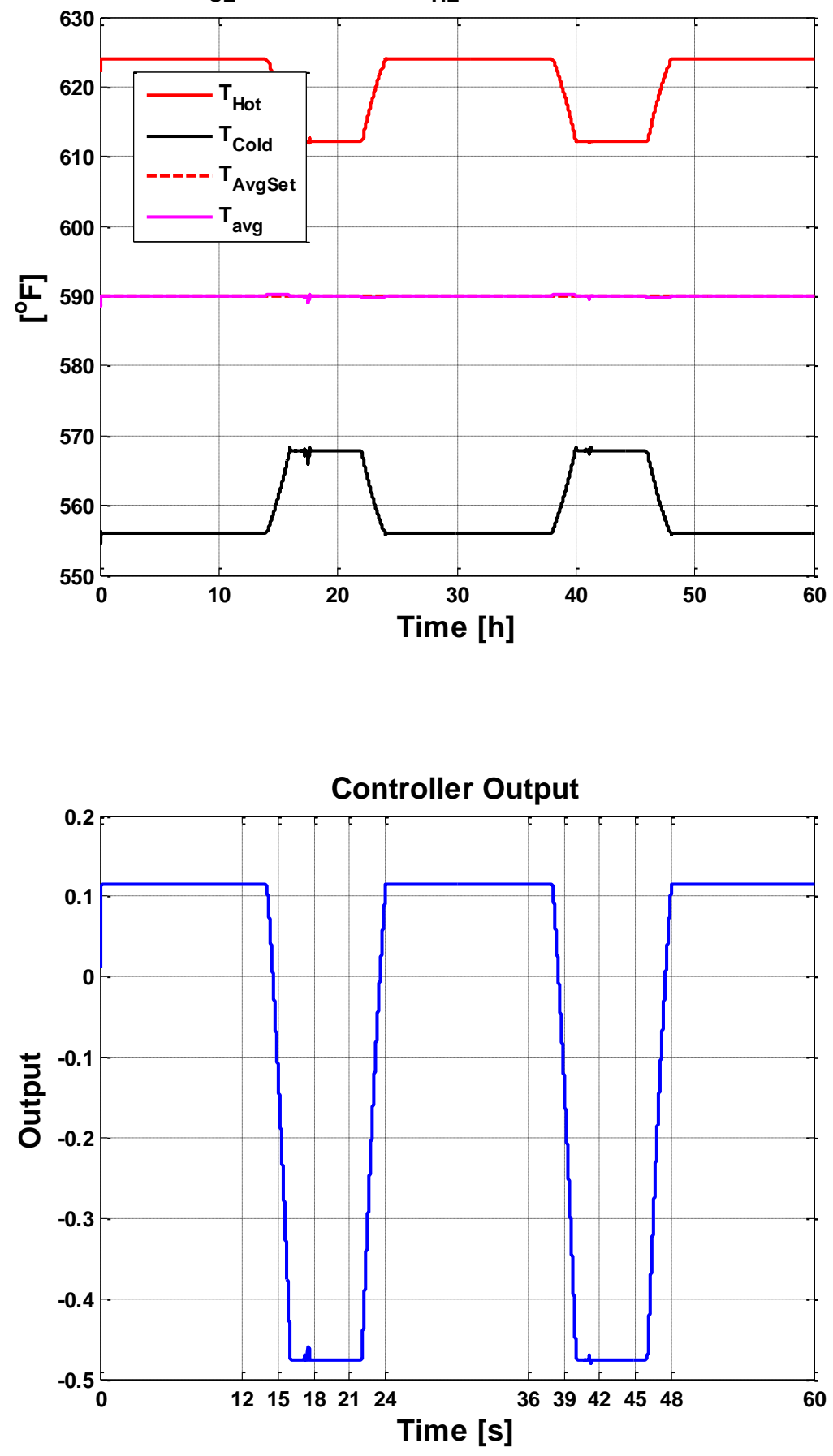

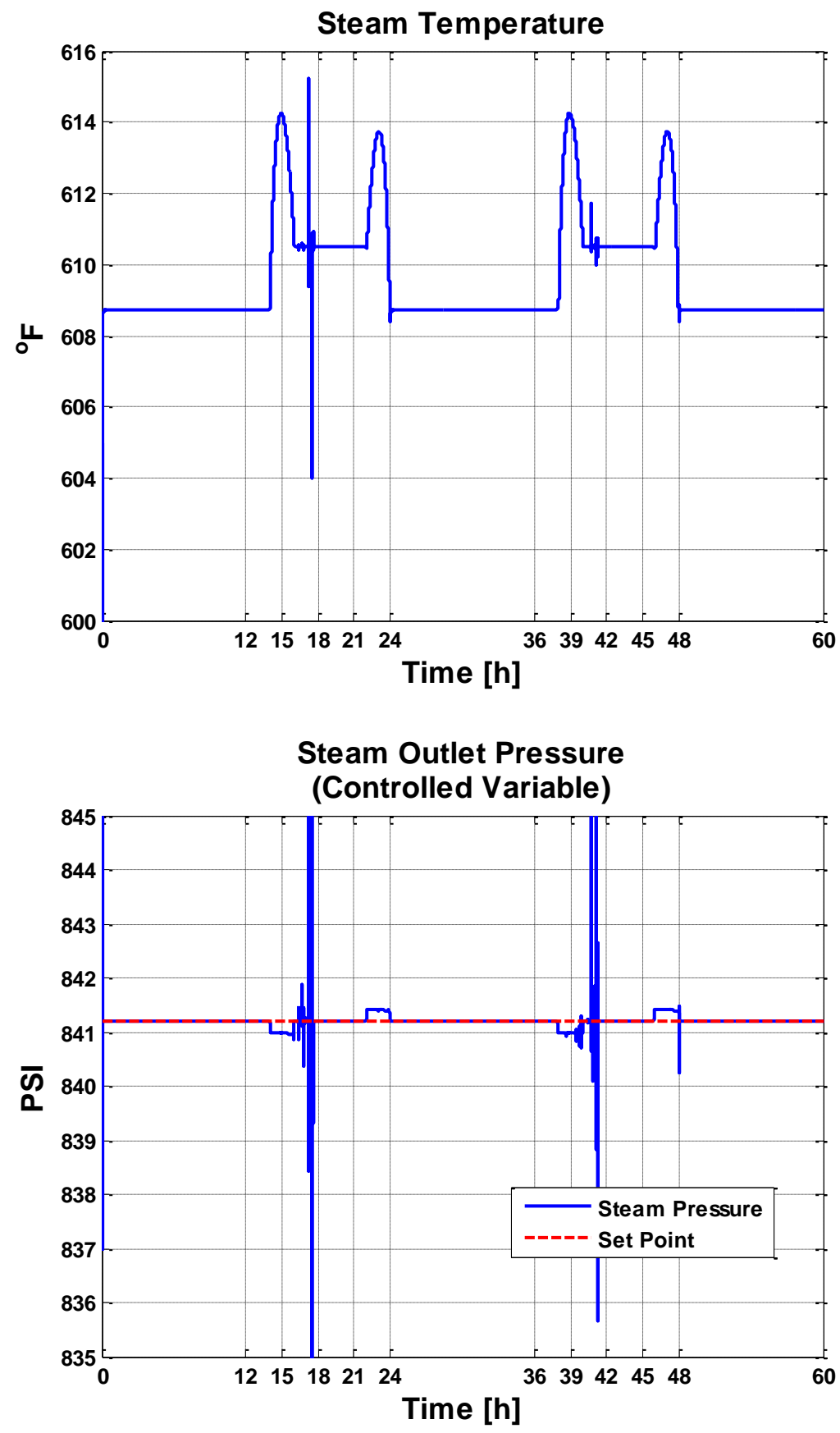

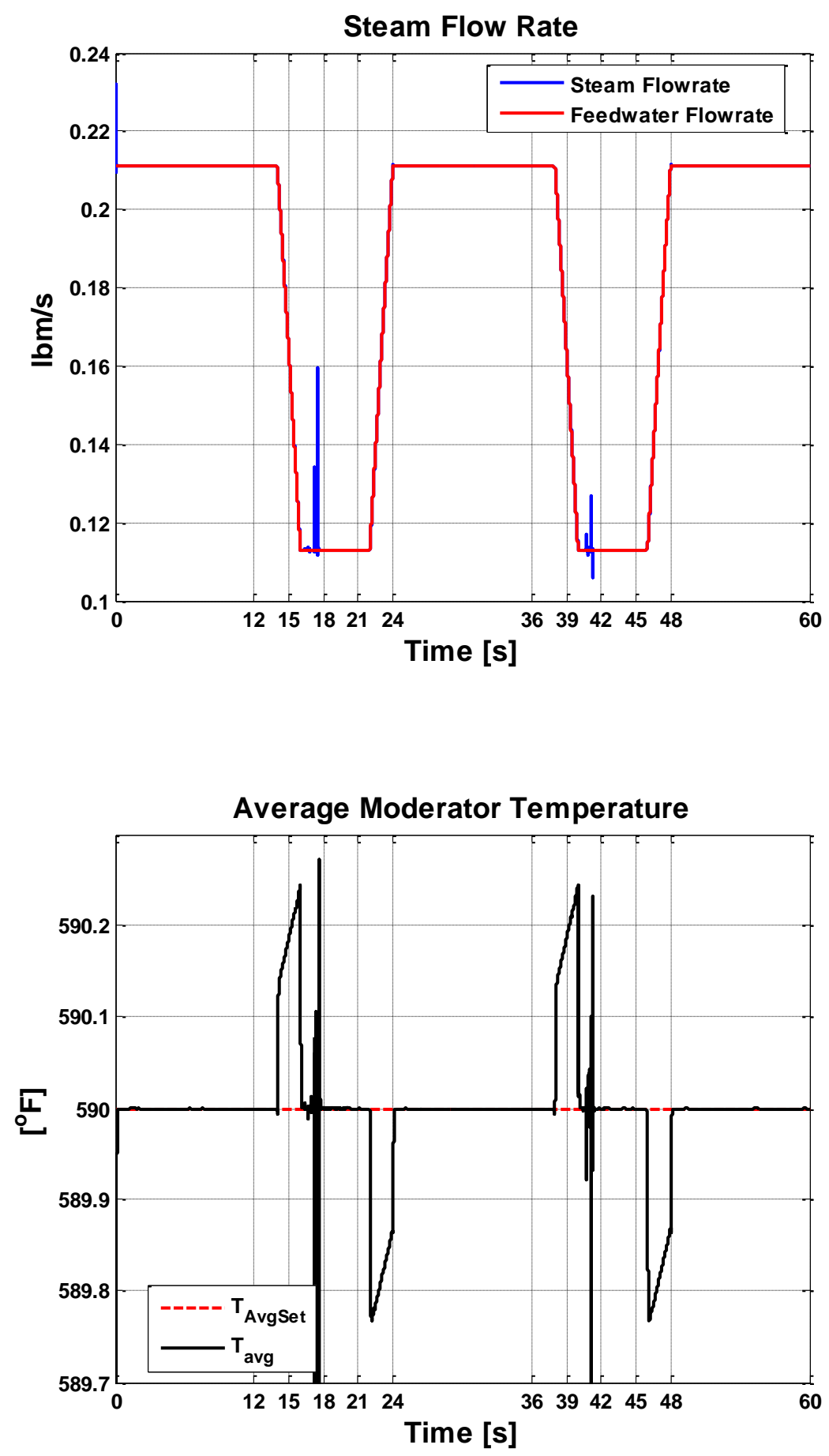


\section{Profile \#6:}
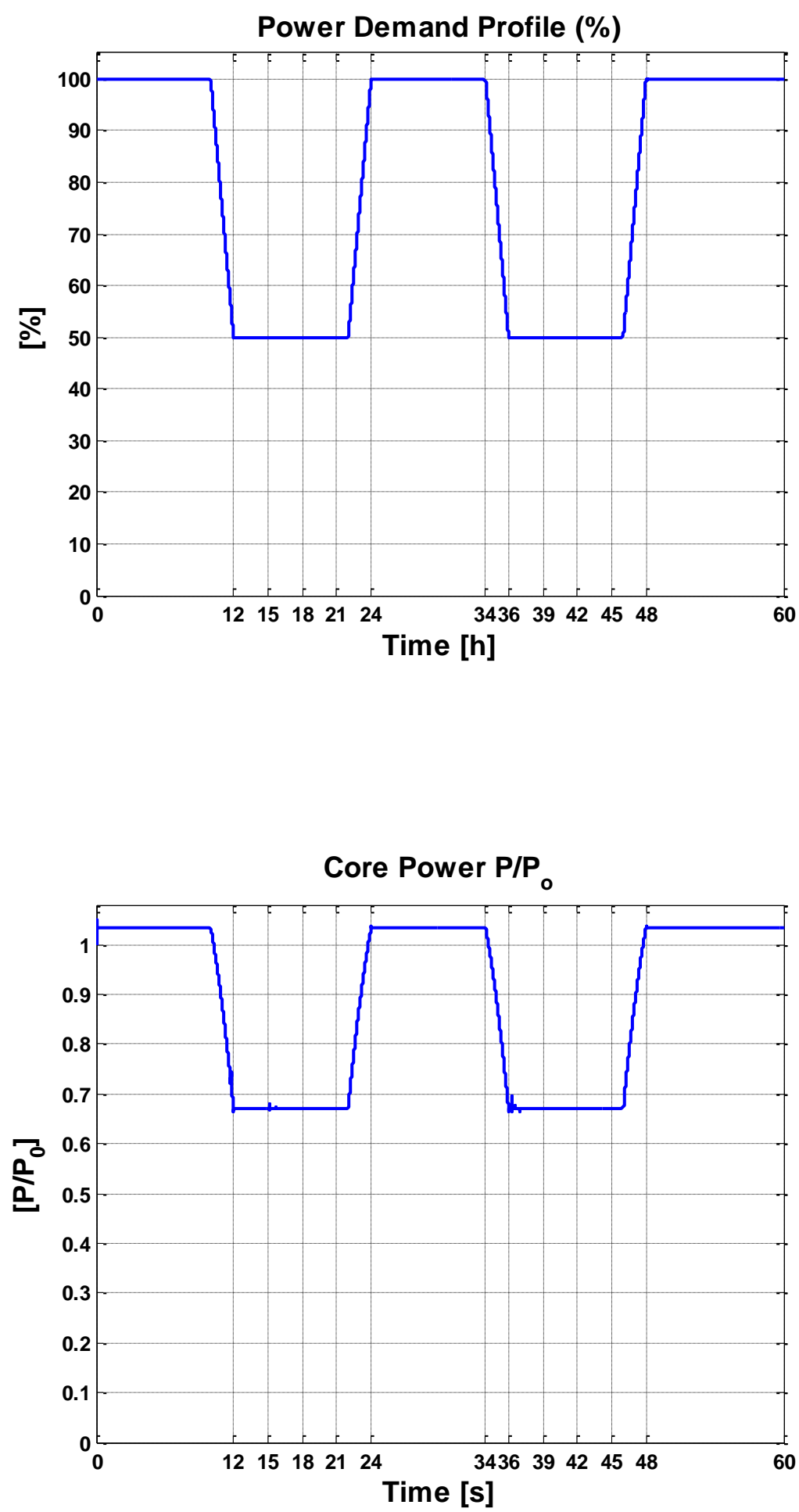
Core Inlet $\left(\mathrm{T}_{\mathrm{CL}}\right)$, Core Exit $\left(\mathrm{T}_{\mathrm{HL}}\right)$, and Average Temperatures
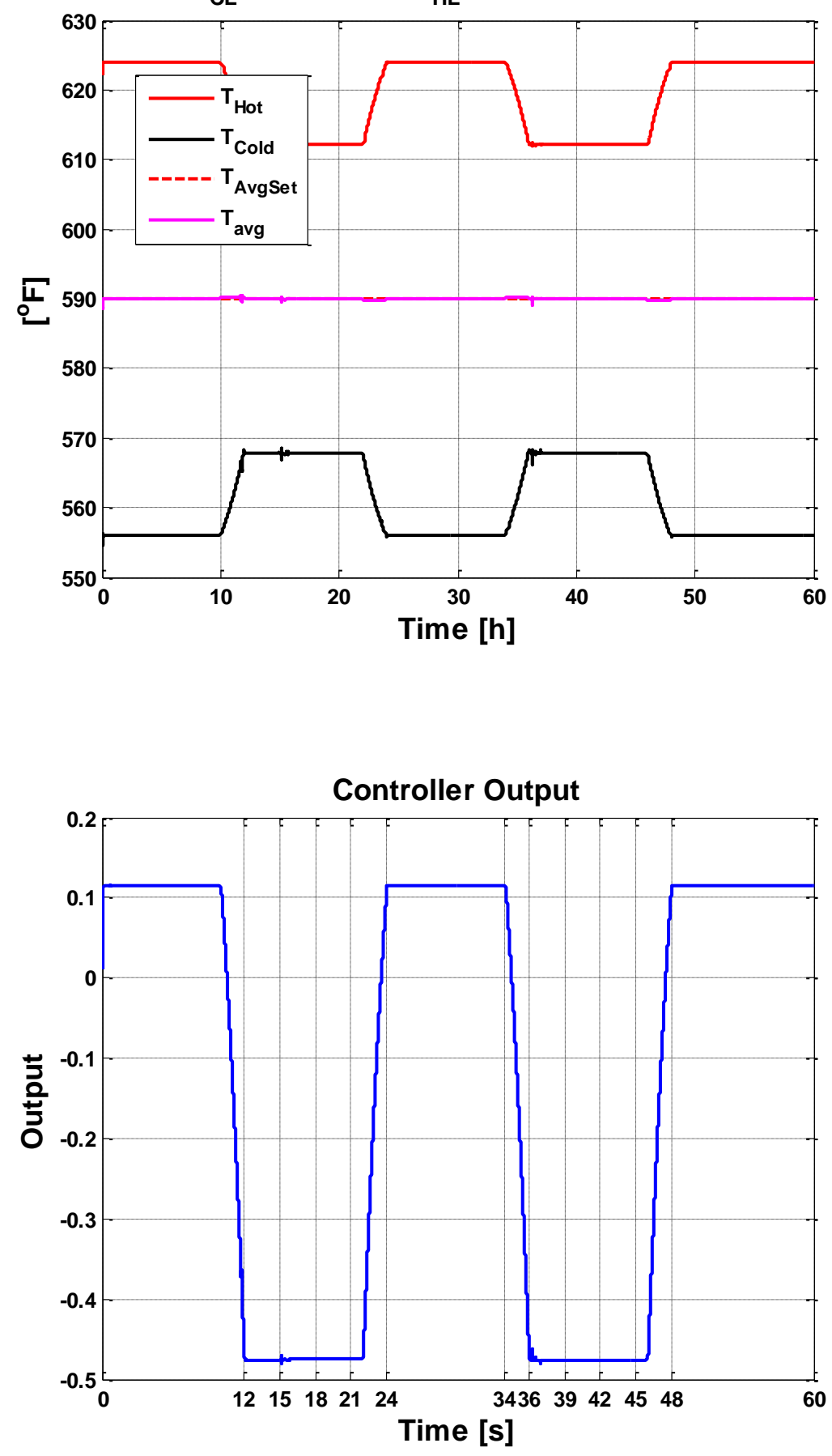

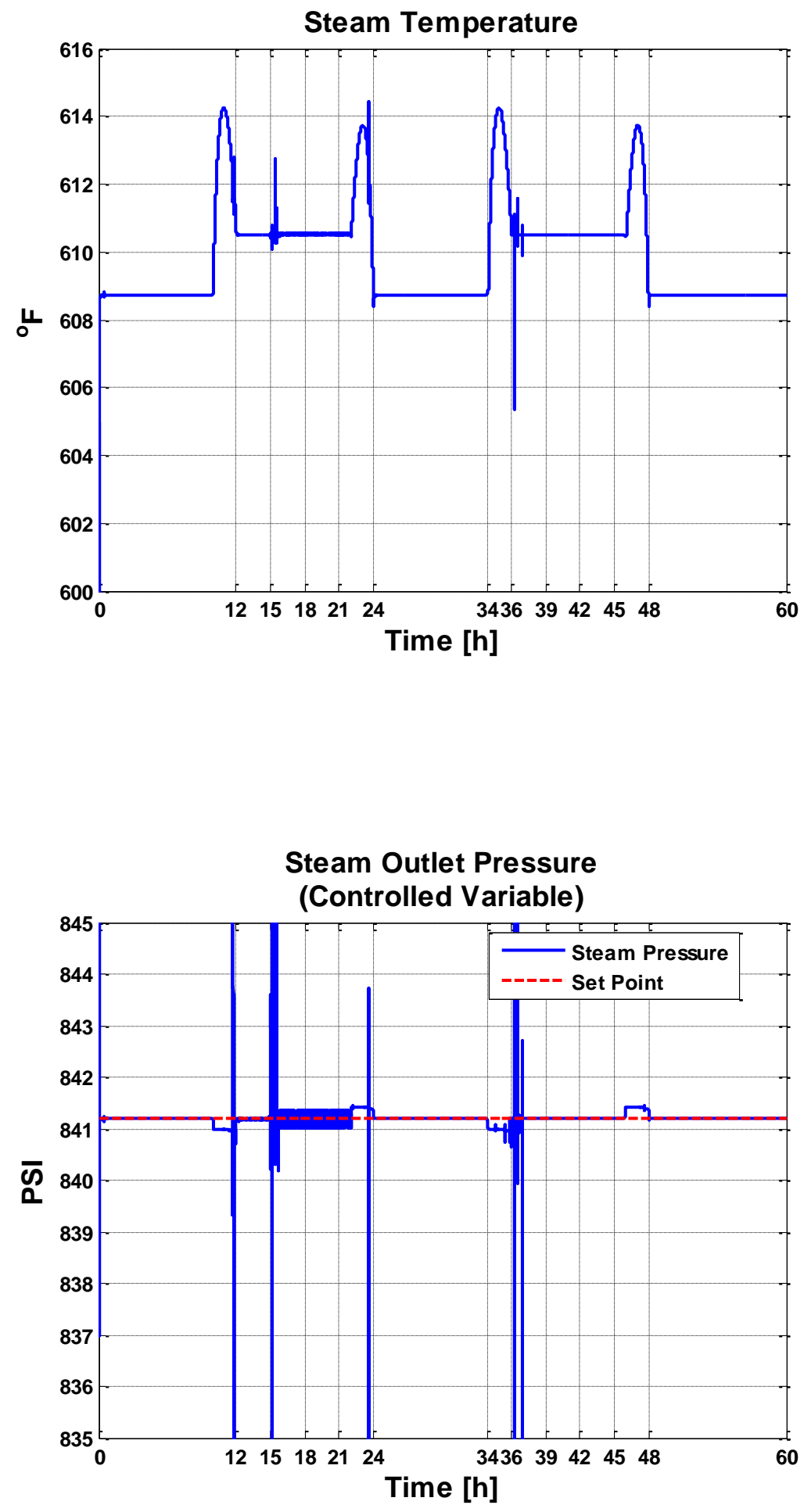

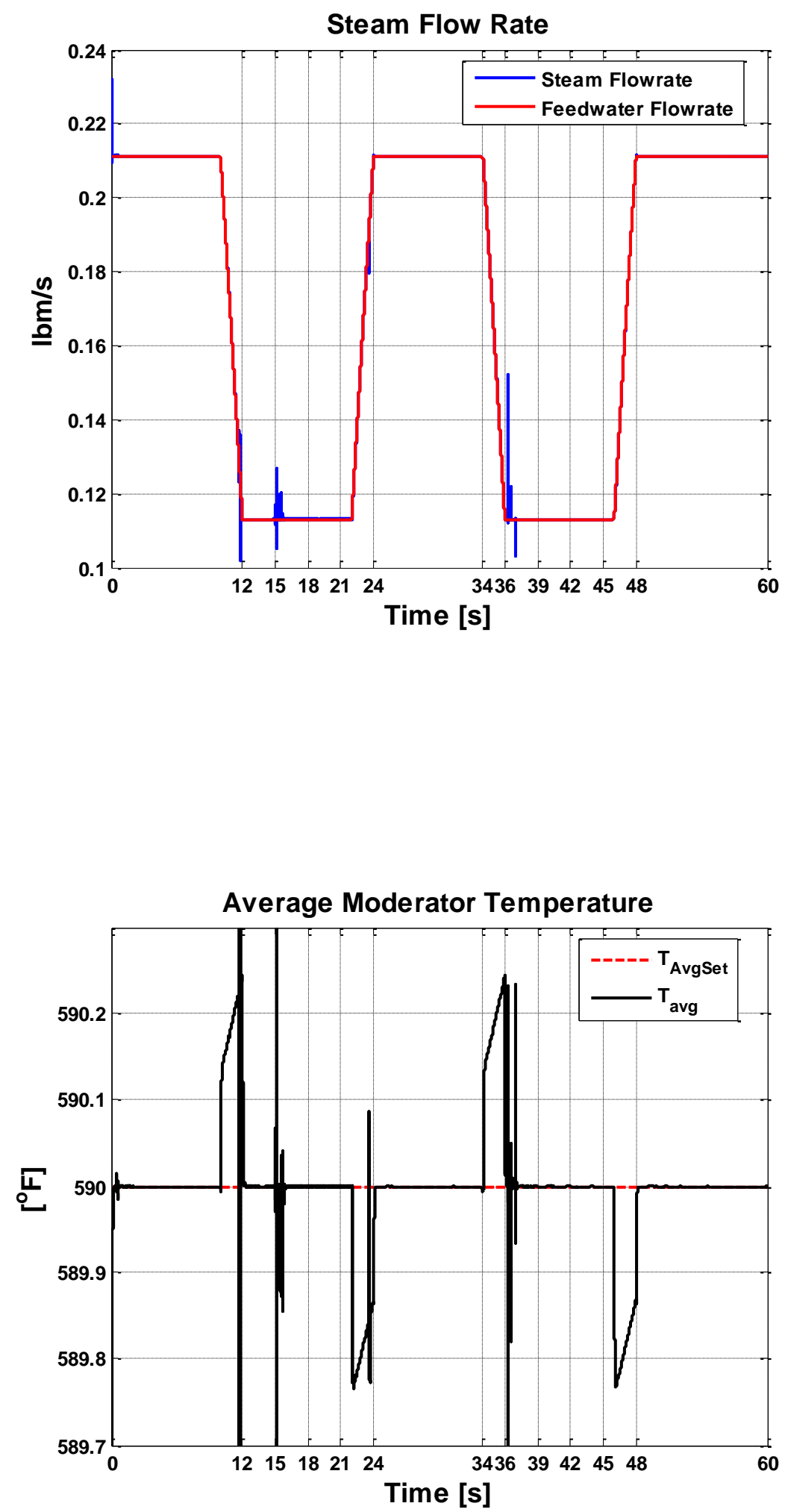


\section{Profile \#7:}
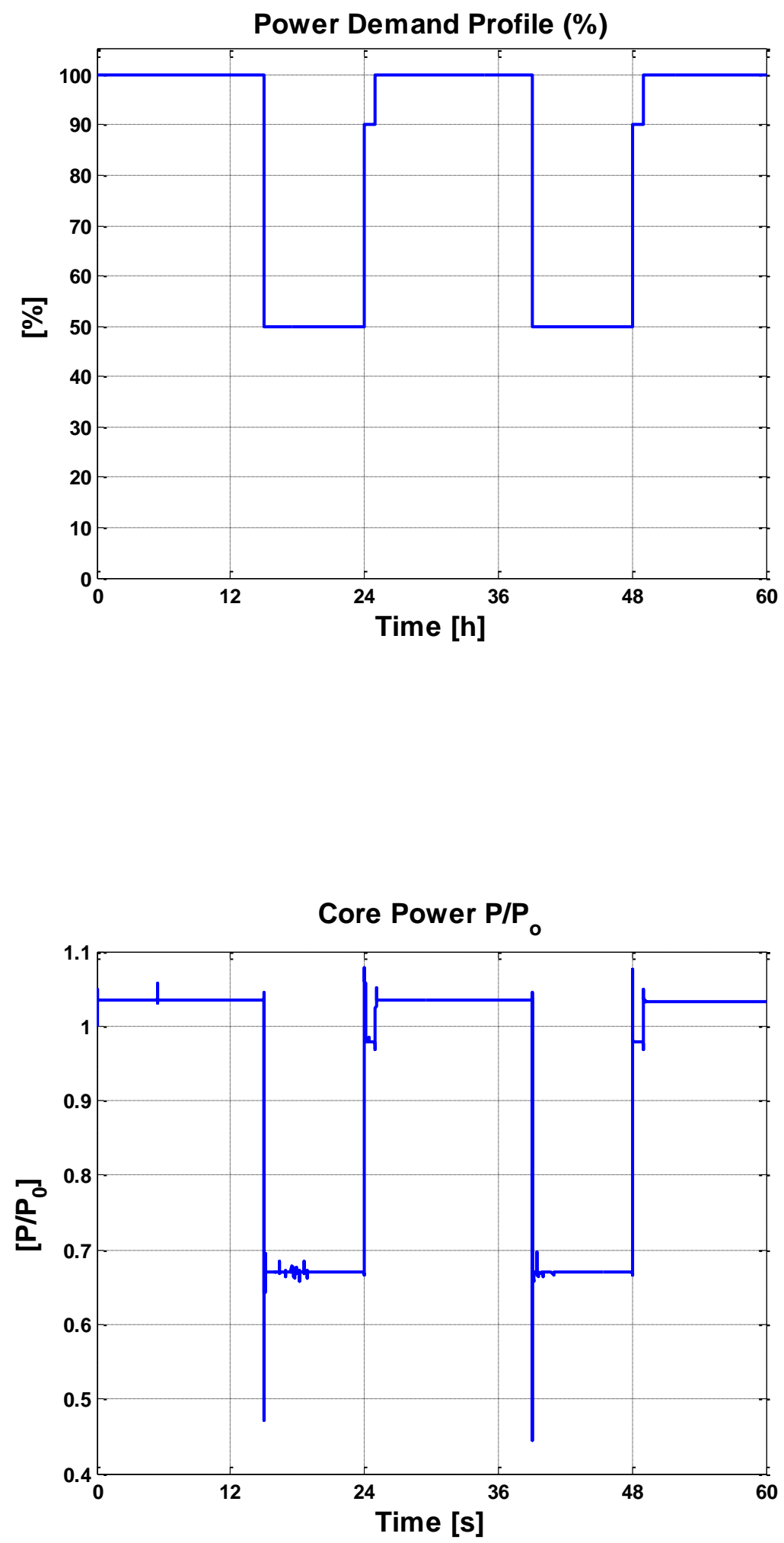
Core Inlet $\left(\mathrm{T}_{\mathrm{CL}}\right)$, Core Exit $\left(\mathrm{T}_{\mathrm{HL}}\right)$, and Average Temperatures
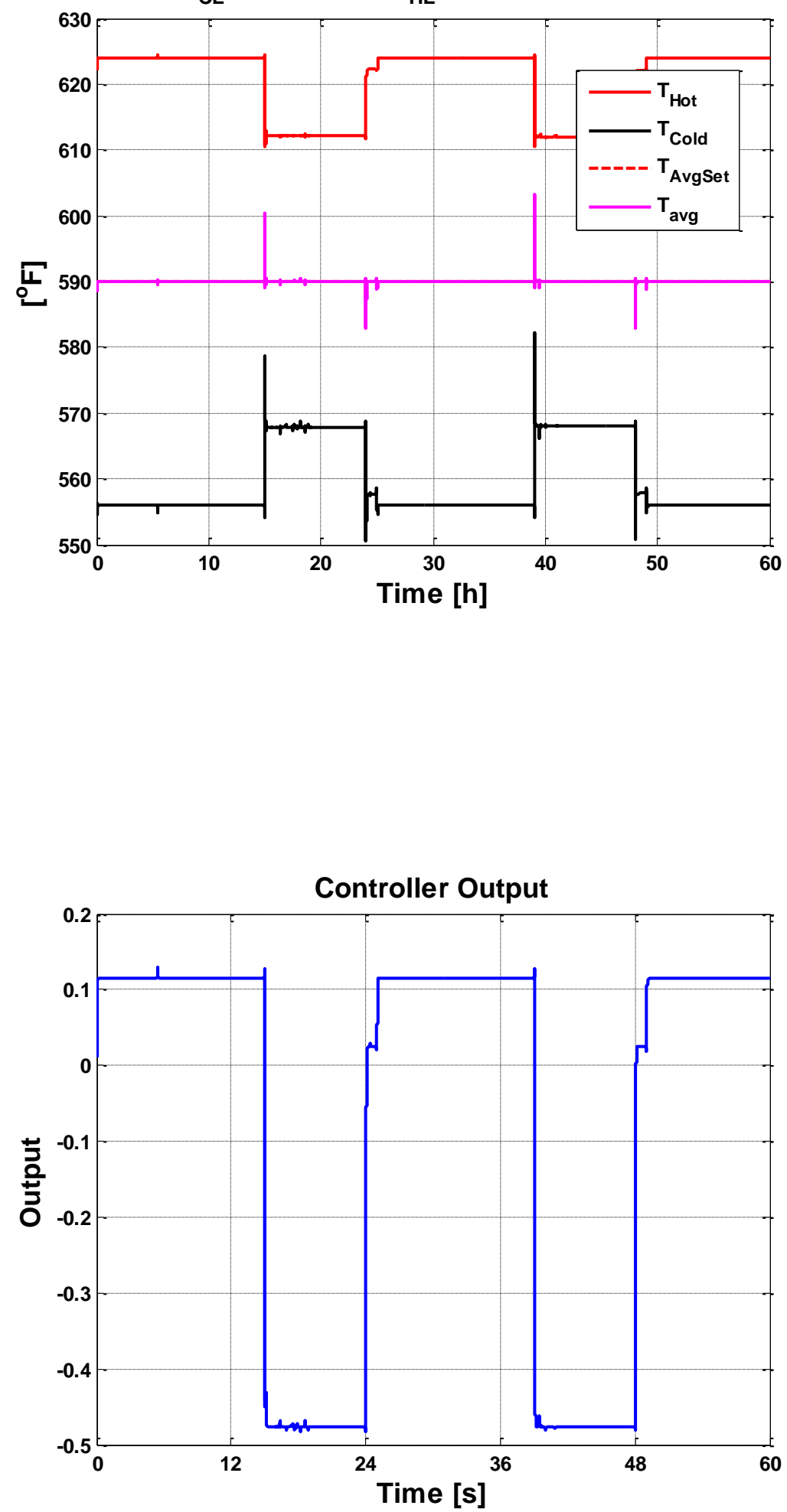

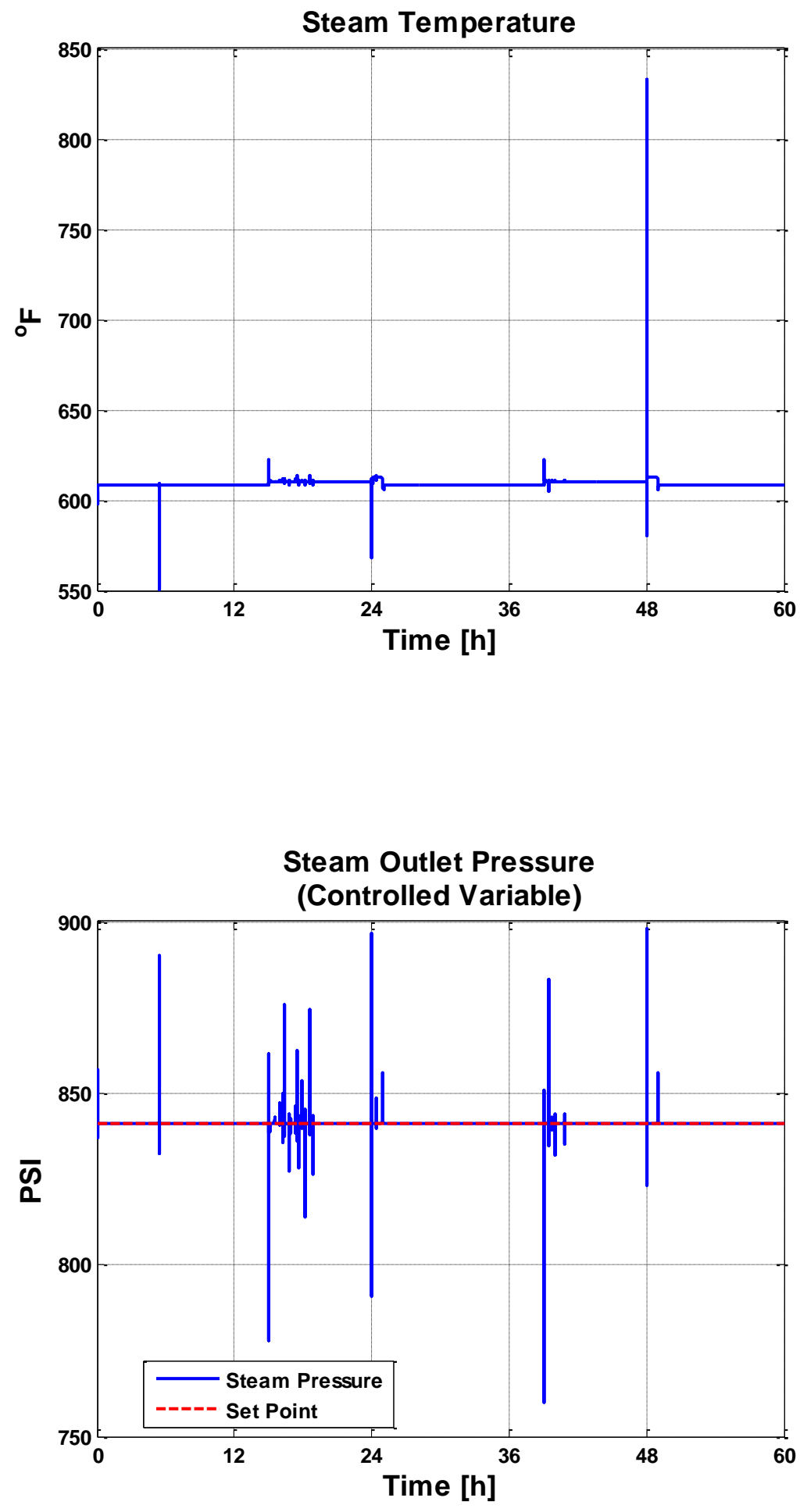

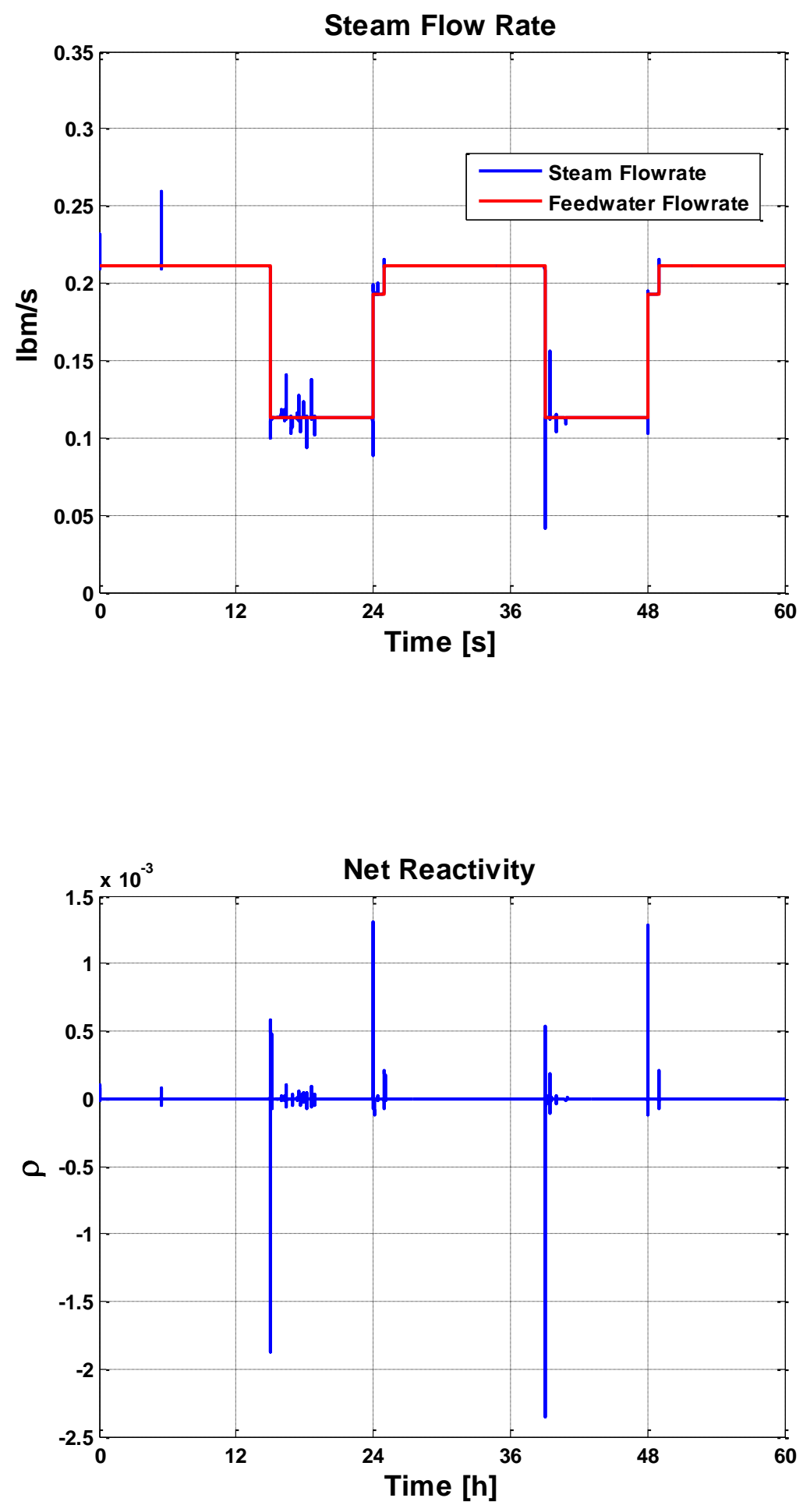


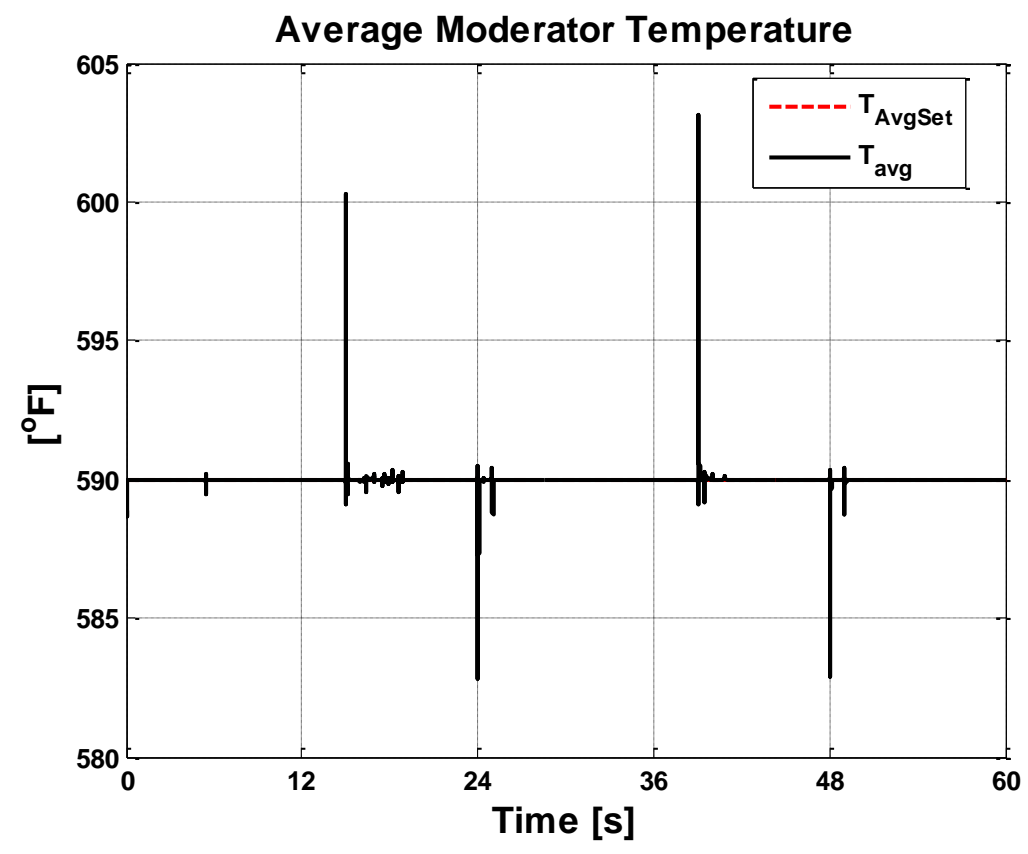

Profile \#8:

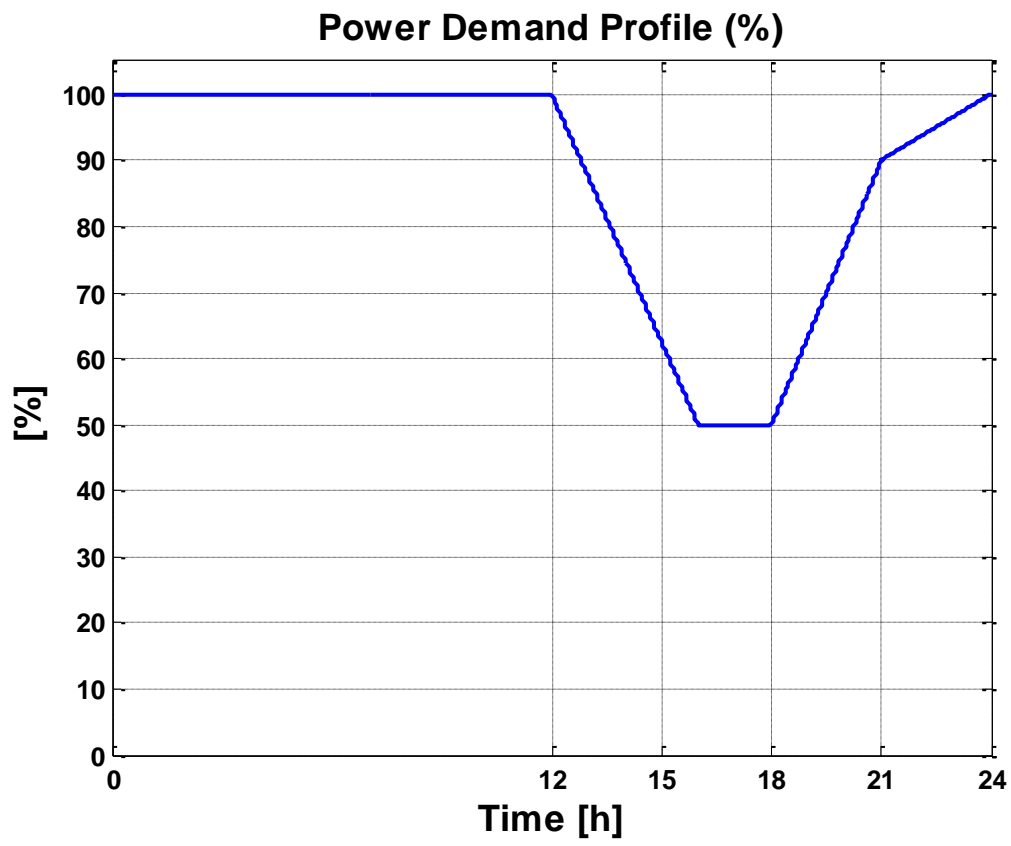




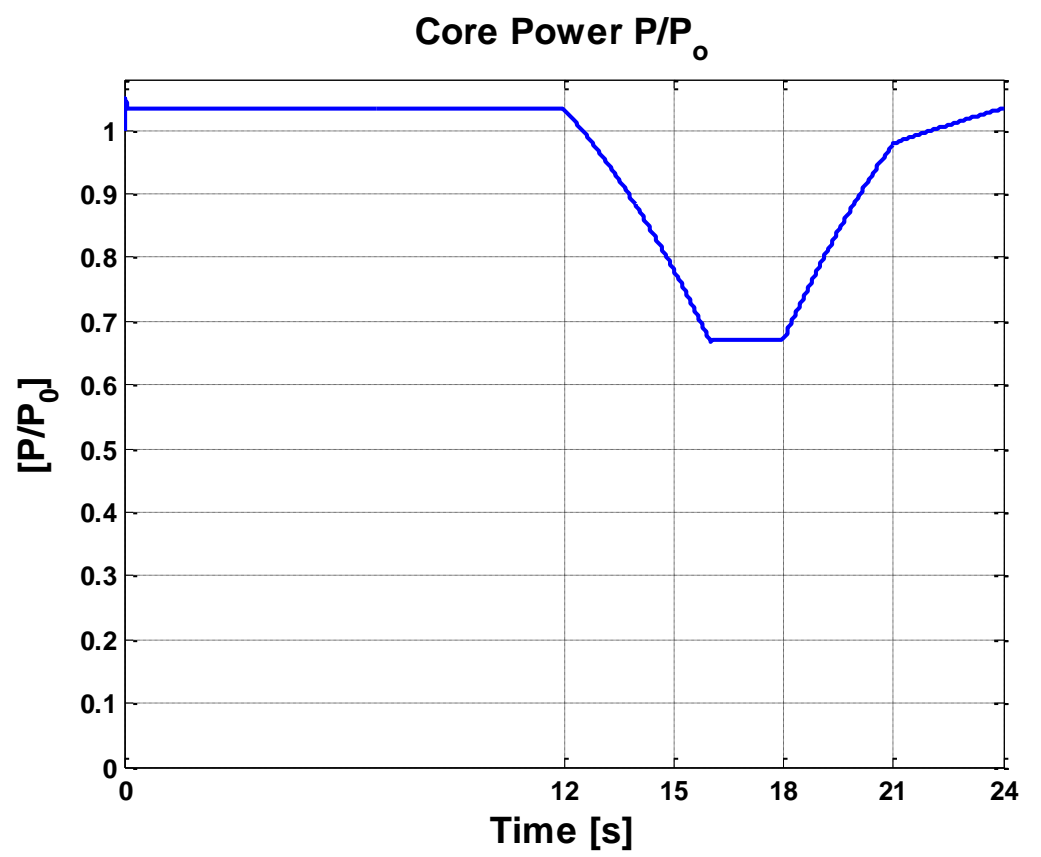

Core Inlet $\left(\mathrm{T}_{\mathrm{CL}}\right)$, Core Exit $\left(\mathrm{T}_{\mathrm{HL}}\right)$, and Average Temperatures

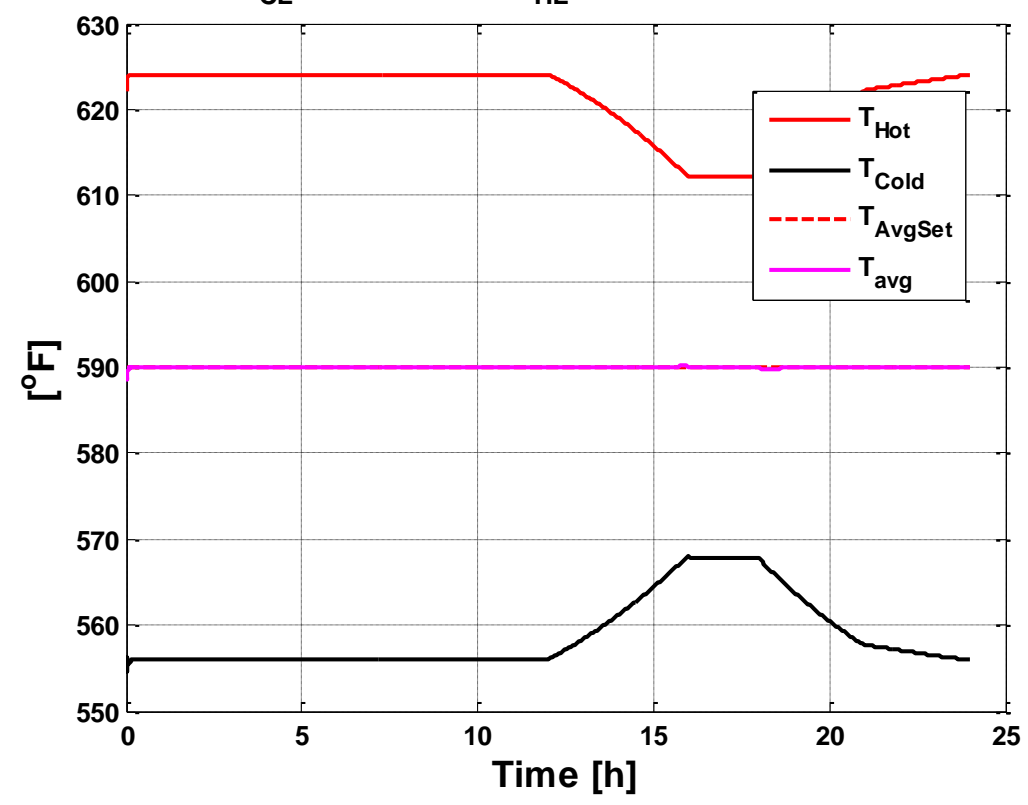



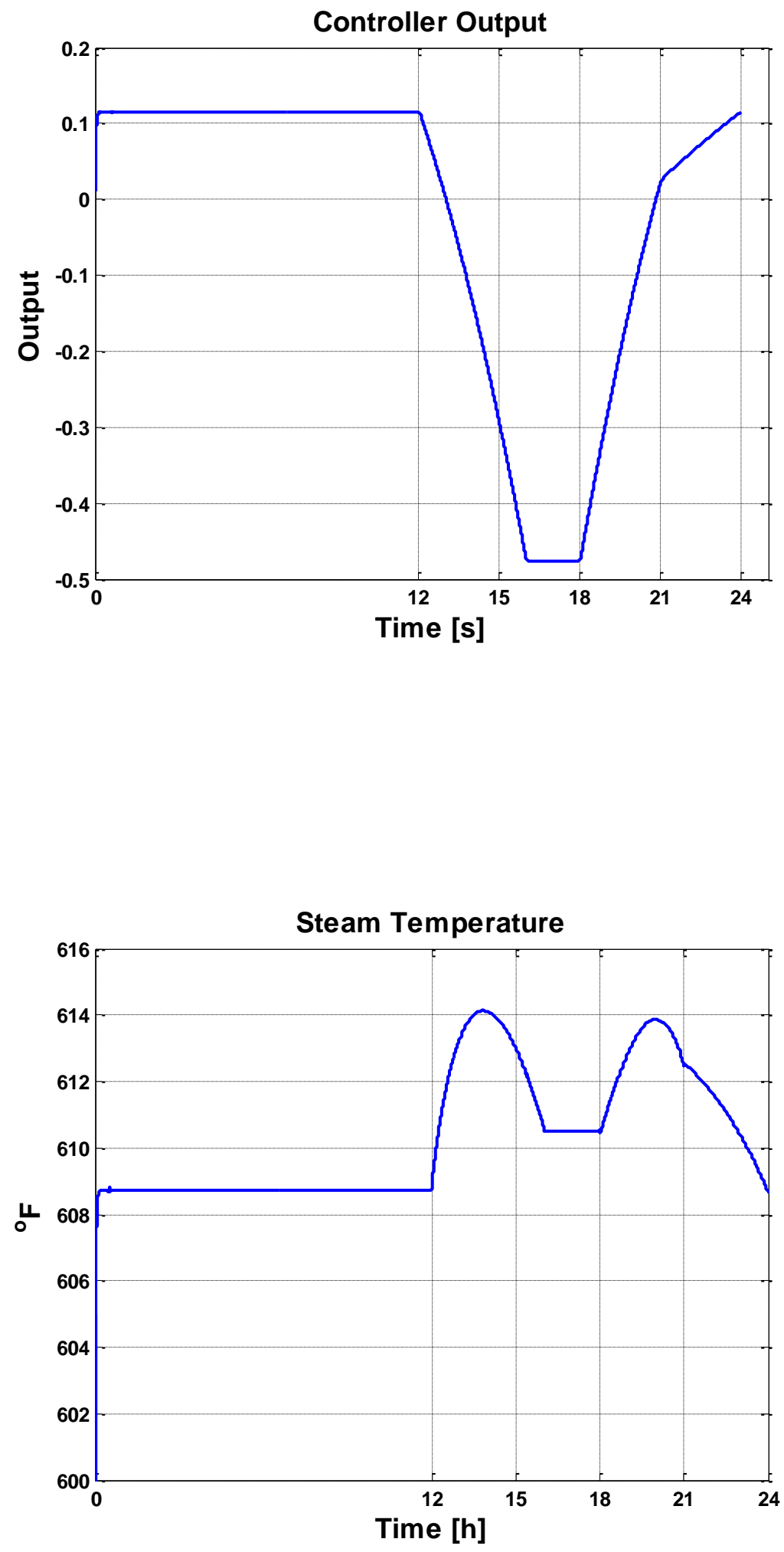

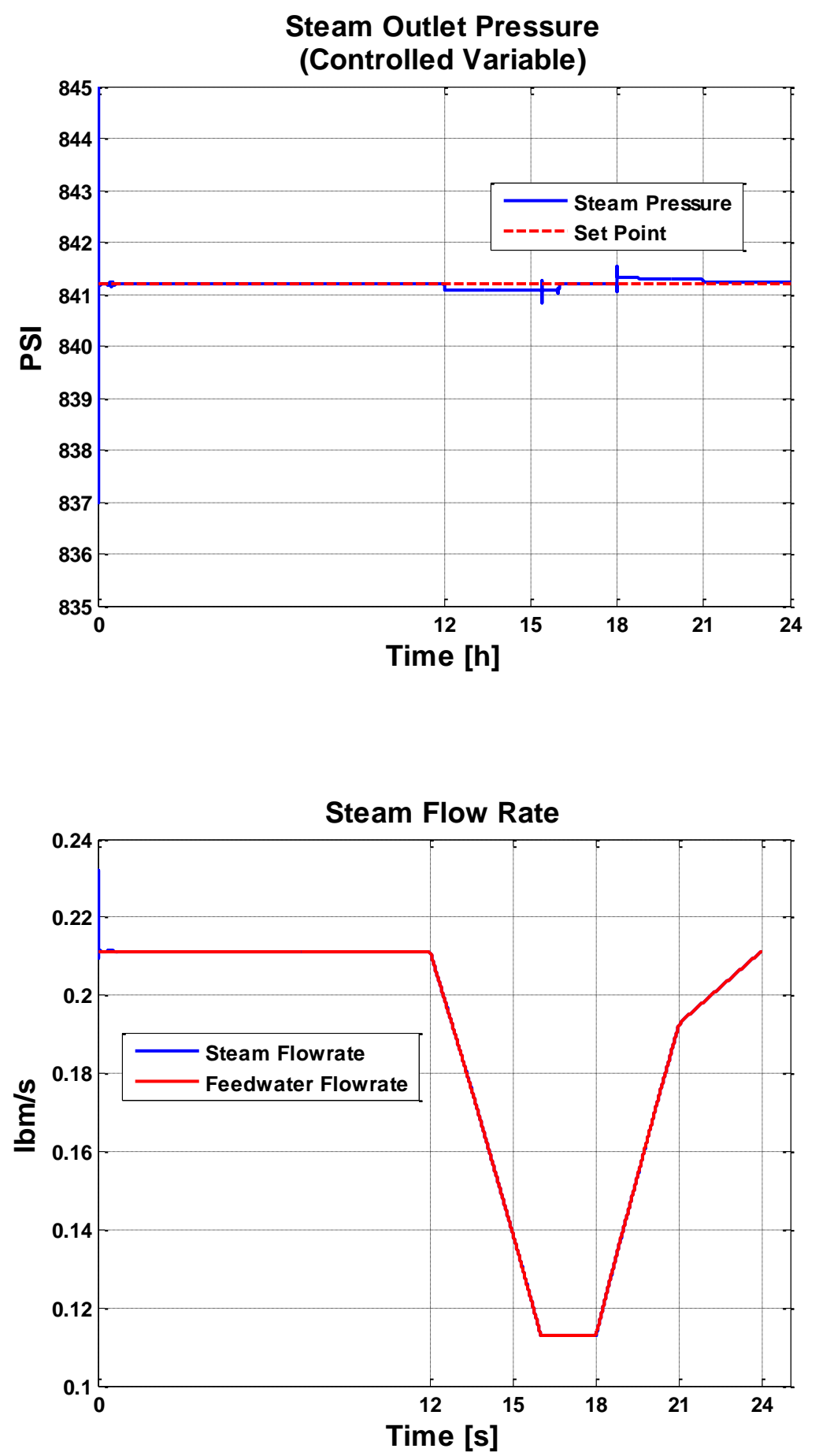

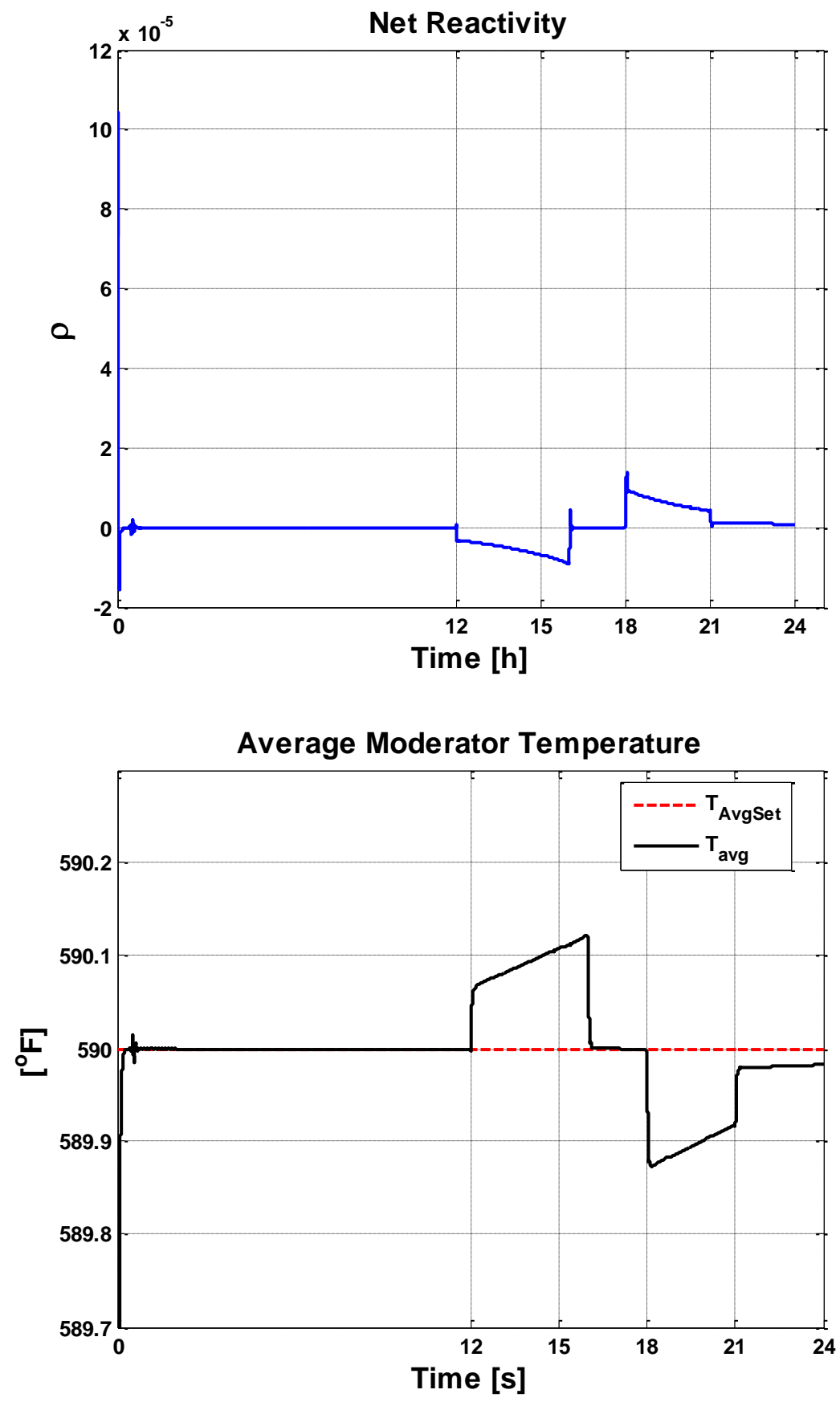


\section{Profile \#9:}
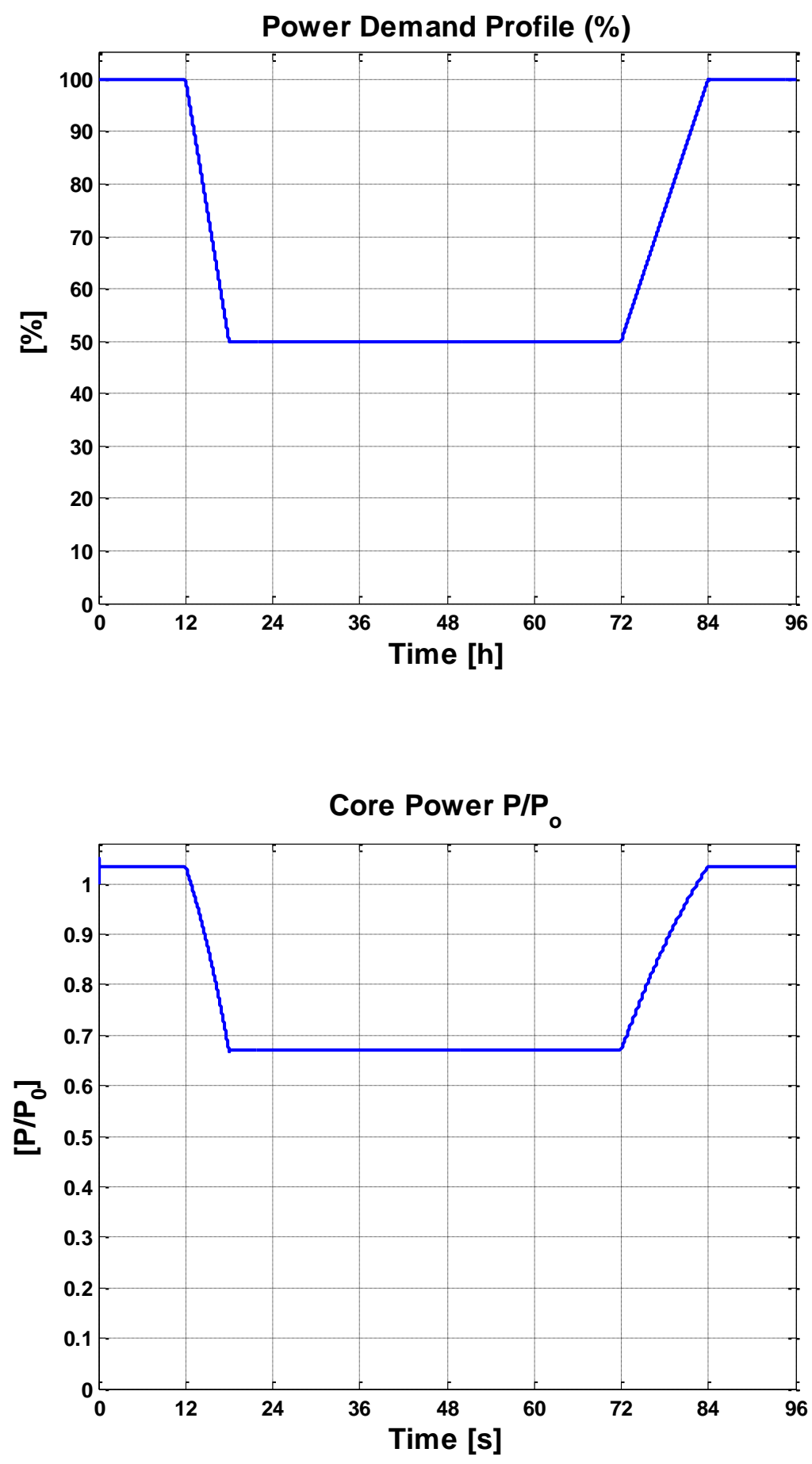

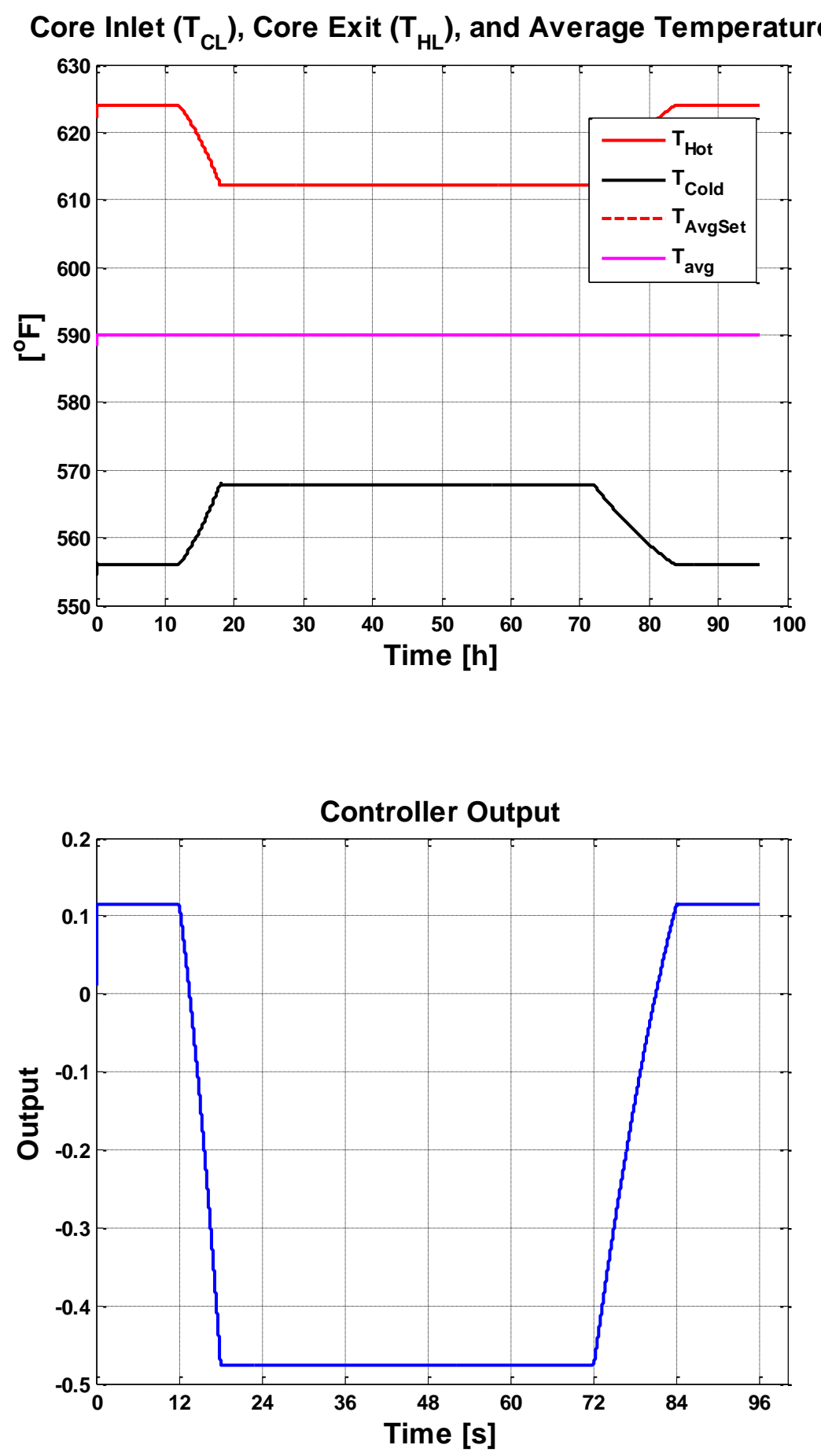

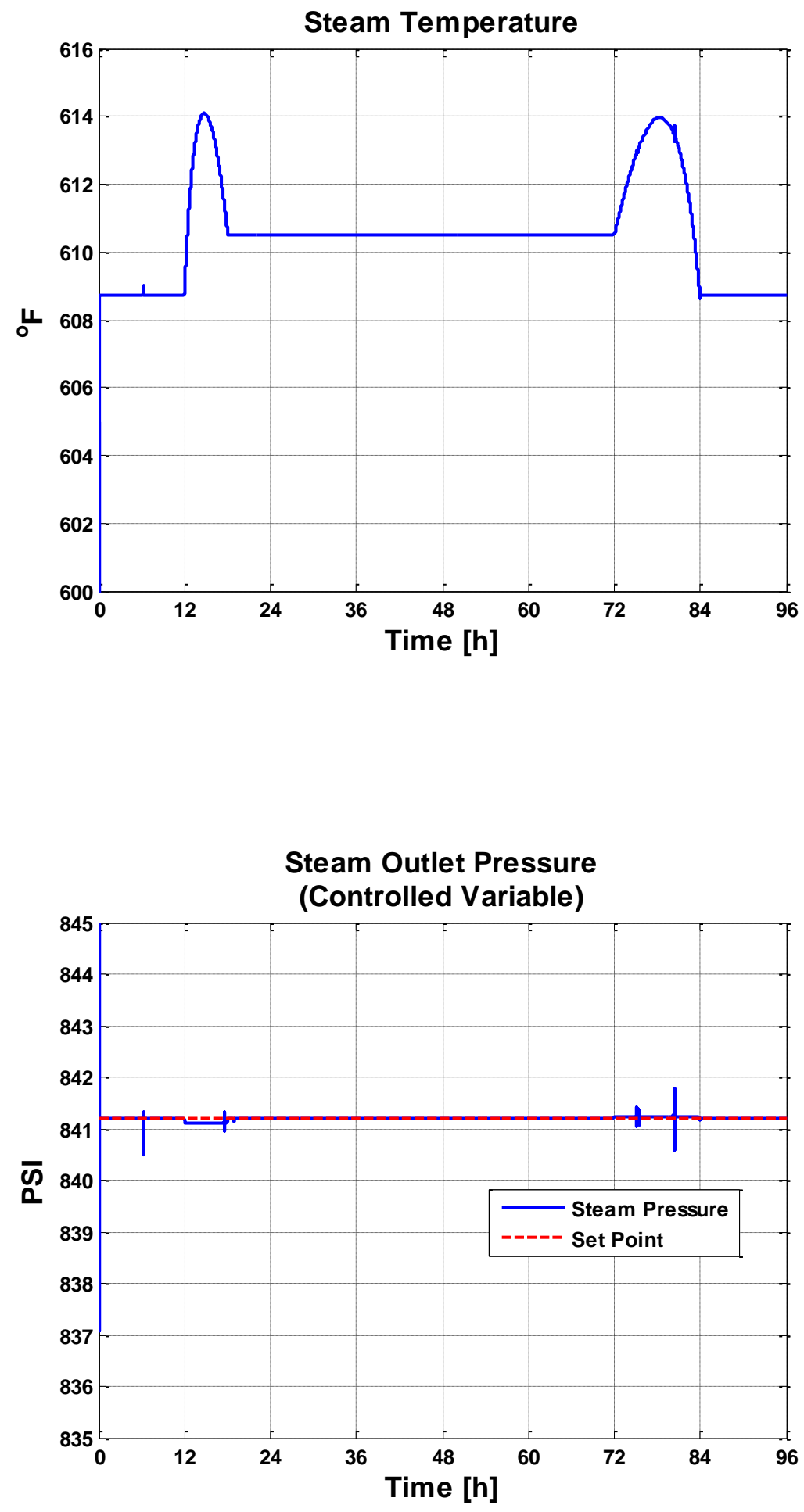

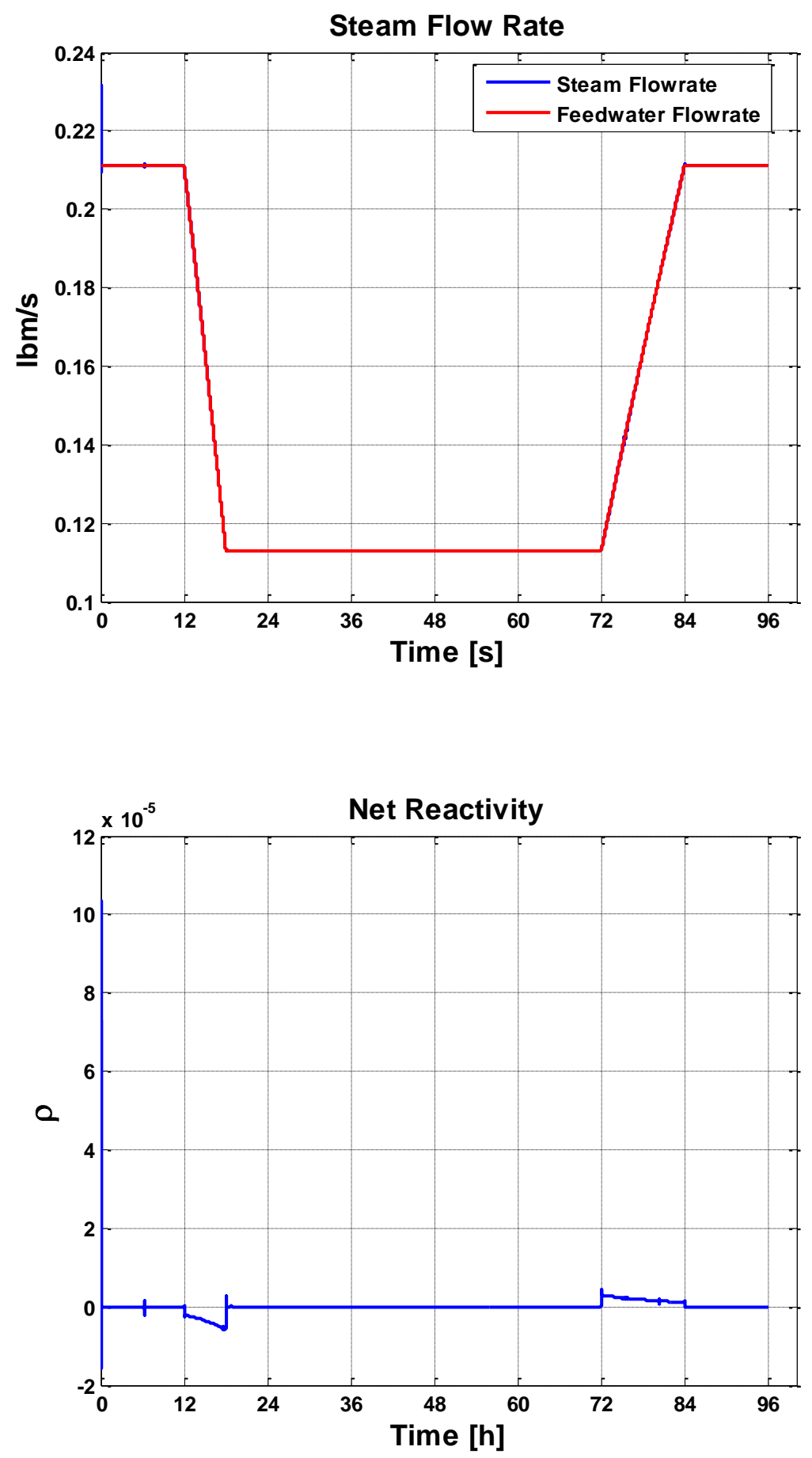


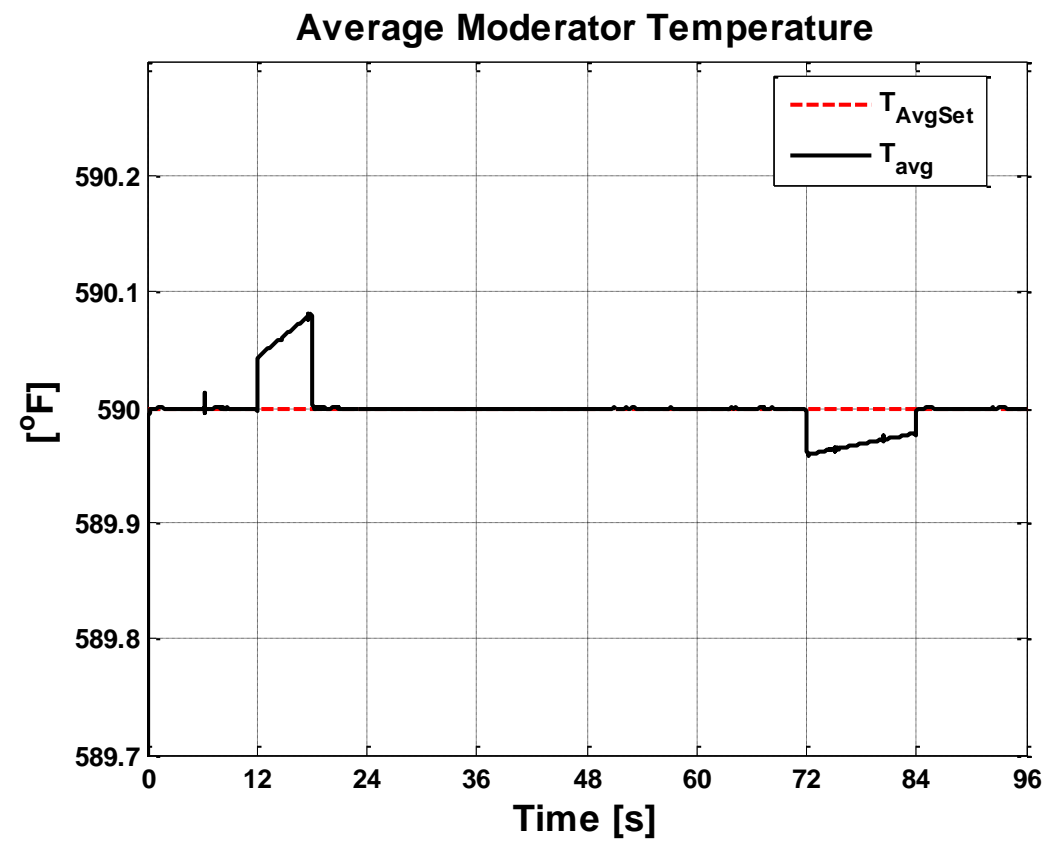




\section{Appendix 5: $\quad$ Two-Tank Loop Operation Manual}

This is a general manual on how to run the loop using the software developed in LabView.

\section{Check list before running the loop:}

Check the water level in the sump tank, located underneath the loop tabletop and refill it if necessary. Remember: the results are heavily dependent on the water level, so always start your runs using the SAME water level every time!

Water is filtered, and filter is located in the kitchen, behind the door.

Open gate valve located at the pump inlet, underneath the sump tank plate support.

$>$ Flowrate inlet control valves should be closed, and outlet flowrate control valves should be open.

Bypass manual valve should be closed. (DO NOT fire up the loop with this valve open!!!!!).

$>$ Power on all power strips, and check if everything else is powered up as well (sensors, control valves, computer screen monitors, etc.).

$>$ Check if computer is on.

\section{Software status check before firing up the loop:}

Open LabView. 
Open file FDI.vi and run it. If LabView don't crash you should see both control and data acquisition Vis pop-up. Matlab workspace window should also pop-up in the background.

Check if the control VI is running on manual mode, if not choose 'manual' from the drop down menu located right in the center of the VI.

Once VIs are running, check if values are being updated, and if they make sense. This check is important because it might indicate a sensor or a control valve not properly powered up.

With the VI in manual, change the values sent to each of the control valves, one at a time, to make sure all control valves are powered up, but leave the inlet control valves closed and the outlet valves open.

Check if the control valve connecting both tanks is closed.

\section{Firing up the loop:}

Once everything has been checked, flip the circuit lever located on the wall. There are 3 boxes on the wall; you are to flip the one in the middle. The third (from left to right) belongs to another experiment, and it might be already on, so don't touch it! If you don't know what you are doing DON'T DO IT! THINK FIRST! Call a professor.

Assuming you were able to fire up the pump, next thing you should do is slowly open the manual bypass valve. There may be air trapped inside the line. That's not a problem, provided you opened the valve slowly. Remember: there is a turbine flowmeter in this line, and it is very, very fragile. And one other thing: don't wait too long to start opening the bypass valve, as it might damage the pump!

With the VI in manual, input 5V to each tank inlet flowrate control valve. 
Check process values after a few seconds to see if everything looks fine.

There may be air trapped in the inlet and outlet lines, so here we go.

Getting rid of air trapped in the piping (this is valid for both tanks, but I'll assume you are interested only in running tank 1)

With the water level below $100 \mathrm{mmH}_{2} \mathrm{O}$ input $10 \mathrm{~V}$ to the inlet control valve. You'll notice some air bubbles coming out of the pipe and into the tank.

Once the water level reaches around $70 \%$ input $0 \mathrm{~V}$ and let the level drop back to around $100 \mathrm{mmH}_{2} \mathrm{O}$.

$>$ Repeat the first and second steps a few times until you are certain there's no more air inside the inlet piping.

Yes I know there is air trapped inside the outlet piping, and getting rid of it will require some ingenuity, skill and body coordination on your part, but here's the kick:

- Bring the water level up to around $700 \mathrm{mmH}_{2} \mathrm{O}$.

- Look underneath the tabletop. Look for the returning pipe, yes, you'll see the water returning back to the tank, that's the returning pipe.

- With your hand, or other tool you can improvise with, temporarily block the water exit. This will increase the backpressure inside the pipe just enough to help expel those air bubbles you should be looking at through the clear section of the pipe right in front of you. Remove your hand and let the backpressure take care of the rest. Repeat this process 3 or 4 times, or until you see the air has left the pipe. But keep an eye on the water tank level! 
Assuming and hoping you successfully completed the previous steps without causing the tank to overflow, the next step is to return to the control table and switch the software from 'manual' to 'tank 1'. This will bring up Tank 1 controller. Select the water set point and the controller will take care of the rest.

$>$ If you decided to run both tanks at the same time, remember you'll have to get rid of the air trapped inside Tank 2 lines (in and out) just the same way you did in Tank 1.

Once the loop is completely free of air, choose 'Tank 1,' 'Tank 2,' or 'Both Tanks'.

The controller VI is wired to hide things you don't need, depending on the choice you made using the drop-down menu.

\section{Turning off the loop:}

Switch the control to manual.

Input $0 \mathrm{~V}$ to control valves 1 and 2 (inlet flowrate control valves, dummy!)

$>$ Input $10 \mathrm{~V}$ to the outlet control valves, if you have changed it to begin with, of course.

Close the control valve between both tanks.

Close the bypass manual valve.

Check to see if the water levels in both tanks are actually coming down.

Remember that switch box lever? Flip it back to "off".

Stop both VIs.

Close the VIs.

Close LabView.

Remember those power strips? Turn them off! But be careful not to turn off the computer. 
Close gate valve located at the pump inlet, underneath the sump tank plate support.

Congratulations! You just managed to successfully operate the loop without an accident, and kept my 3.5-year old Frankenstein-ish offspring, fruit of my hard, back-breaking, mindboggling work from complete disaster! Yes, I know Michael helped me build it, and I'll be forever grateful to him, although it took him a year to figure out what we were doing. 


\section{Appendix 6: $\quad$ Orifice Meter Calibration Procedure and Results}

In order to calibrate the orifice meters, rigorous mechanical timing experiments were performed to find the proportionality of flow to pressure drop across the orifice using the relationship, Flow rate $=\mathrm{K}^{*} \mathrm{SQRT}$ (Pressure difference). Using a timer and pre-calibrated liter marks on the level tanks, average flow rates were determined for different positions of the control valves. Simultaneously, output voltages from the pressure sensors were compared to values set with the calibration software, AMS Device Manager. Using linear interpolation of voltages between the maximum and minimum pressure differences, a $\Delta \mathrm{P}$ was found for each output voltage recorded at each control valve position. Four sets of test positions were performed

for each tank; from these an average proportionality constant was determined. Any anomalous data points were retaken to allow a more accurate calculation to be made.

\section{Procedure:}

1) Find Flow Rate

- Isolate one tank.

- Close all Valves.

- Turn on Pump.

- Open Bypass

- Input test voltage into Control Valve in order to open it.

- As water fills to zero line start timer.

- Stop timer when a whole number a liters are filled.

- Record Time to fill in seconds (T) and Liters filled (L)

- From these Calculate Flow rate for each test point 
2) Find Pressure Difference $(\Delta P)$

- Find Max and Min Voltage and corresponding $\Delta \mathrm{P}$ using calibration software

- Record output voltage from pressure sensor at each test point

- Perform linear interpolation between max and min to determine $\Delta \mathrm{P}$ at each point.

3) Determine Proportionality constant K

- Using flow rate and $\Delta \mathrm{P}$ relationship find $\mathrm{K}$ at each point

- Find Average K

\section{Calculations:}

Flow Rate::

$\mathrm{F}_{\text {Rate }}(\mathrm{L} / \mathrm{s})=\mathrm{L} / \mathrm{T}$

$\Delta \mathrm{P}:$

$\Delta \mathrm{P}\left(\mathrm{mm} \mathrm{H}_{2} 0\right)=\operatorname{SLOPE}\left(\Delta \mathrm{P}_{\min }, \Delta \mathrm{P}_{\max }, \mathrm{V}_{\min }, \mathrm{V}_{\max }\right) * \Delta \mathrm{P}_{\text {test }}+\operatorname{INTERCEPT}\left(\Delta \mathrm{P}_{\min }, \Delta \mathrm{P}_{\max }\right.$, $\left.\mathrm{V}_{\min }, \mathrm{V}_{\max }\right)$

$$
\mathrm{K}=\left(\mathrm{F}_{\text {Rate }} * 0.001\right) / \mathrm{SQRT}(\Delta \mathrm{P} * 9.806652)
$$

\section{Results:}

Orifice Meter 1 


\begin{tabular}{|c|c|c|c|c|c|c|c|}
\hline \multirow[b]{2}{*}{ Voltage to Control Valve 1} & \multicolumn{7}{|l|}{ Average } \\
\hline & $\begin{array}{l}\text { Liters } \\
\text { Filled }\end{array}$ & $\begin{array}{l}\text { Time } \\
\text { To Fill } \\
\text { (sec) }\end{array}$ & $\begin{array}{c}\text { Fill Flow } \\
\text { Rate } \\
\text { (Liter/sec) }\end{array}$ & $\begin{array}{c}\text { Flow Rate } \\
\text { (GPM) }\end{array}$ & $\begin{array}{l}\text { Voltage } \\
\text { Recorded }\end{array}$ & $\begin{array}{c}\Delta \mathrm{P} \text { in } \\
\mathrm{mm} \mathrm{H} 2 \mathrm{O}\end{array}$ & K \\
\hline 0 & $\mathrm{x}$ & $\mathrm{x}$ & $\mathrm{x}$ & $\mathrm{x}$ & $\mathrm{x}$ & $\mathrm{x}$ & $\mathrm{x}$ \\
\hline 1 & $\mathrm{x}$ & $\mathrm{x}$ & $\mathrm{x}$ & $\mathrm{x}$ & $\mathrm{x}$ & $\mathrm{x}$ & $\mathrm{x}$ \\
\hline 2 & $\mathrm{n} / \mathrm{a}$ & $\mathrm{n} / \mathrm{a}$ & $\mathrm{n} / \mathrm{a}$ & $\mathrm{n} / \mathrm{a}$ & $\mathrm{n} / \mathrm{a}$ & $\mathrm{n} / \mathrm{a}$ & $\mathrm{n} / \mathrm{a}$ \\
\hline 3 & 8 & 168.33 & 0.0475257 & 0.7532917 & 0.843152 & 84.36427 & $1.6523 \mathrm{E}-06$ \\
\hline 4 & 8 & 64.54 & 0.1239493 & 1.9646218 & 1.112355 & 585.1418 & $1.63626 \mathrm{E}-06$ \\
\hline 5 & 8 & 41.01 & 0.1950982 & 3.0923448 & 1.582293 & 1459.329 & $1.63086 \mathrm{E}-06$ \\
\hline 6 & 8 & 29.65 & 0.2697918 & 4.2762533 & 2.252593 & 2706.235 & $1.6561 \mathrm{E}-06$ \\
\hline 7 & 8 & 24.38 & 0.3281715 & 5.2015834 & 3.026973 & 4146.753 & $1.62737 \mathrm{E}-06$ \\
\hline 8 & 7 & 18.72 & 0.3740315 & 5.9284745 & 3.712115 & 5421.27 & $1.62217 \mathrm{E}-06$ \\
\hline 9 & 7 & 17.36 & 0.4032839 & 6.3921302 & 4.149825 & 6235.507 & $1.63085 \mathrm{E}-06$ \\
\hline & & & & & & $\operatorname{Avg~K}$ & $1.63656 \mathrm{E}-06$ \\
\hline
\end{tabular}



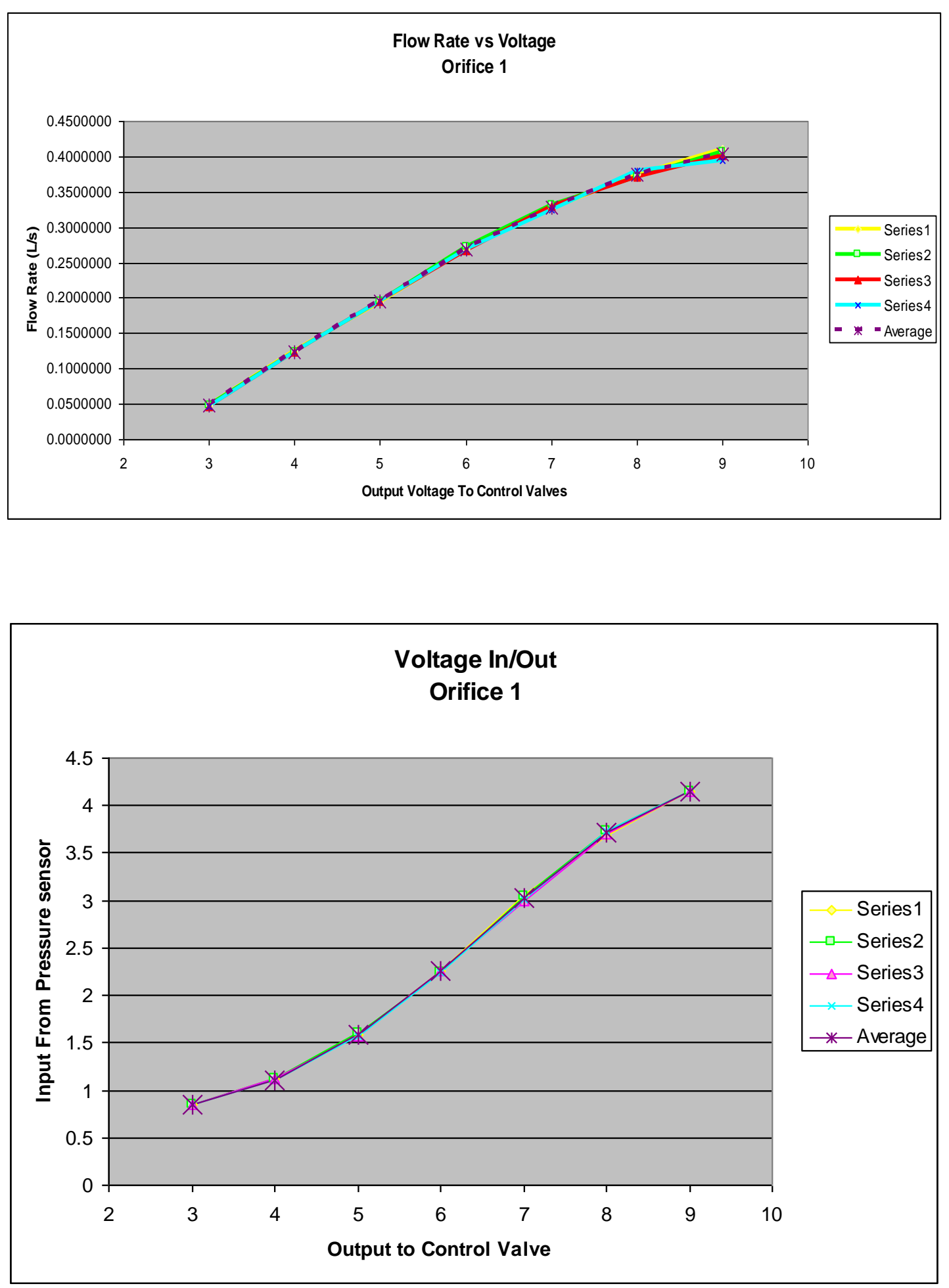


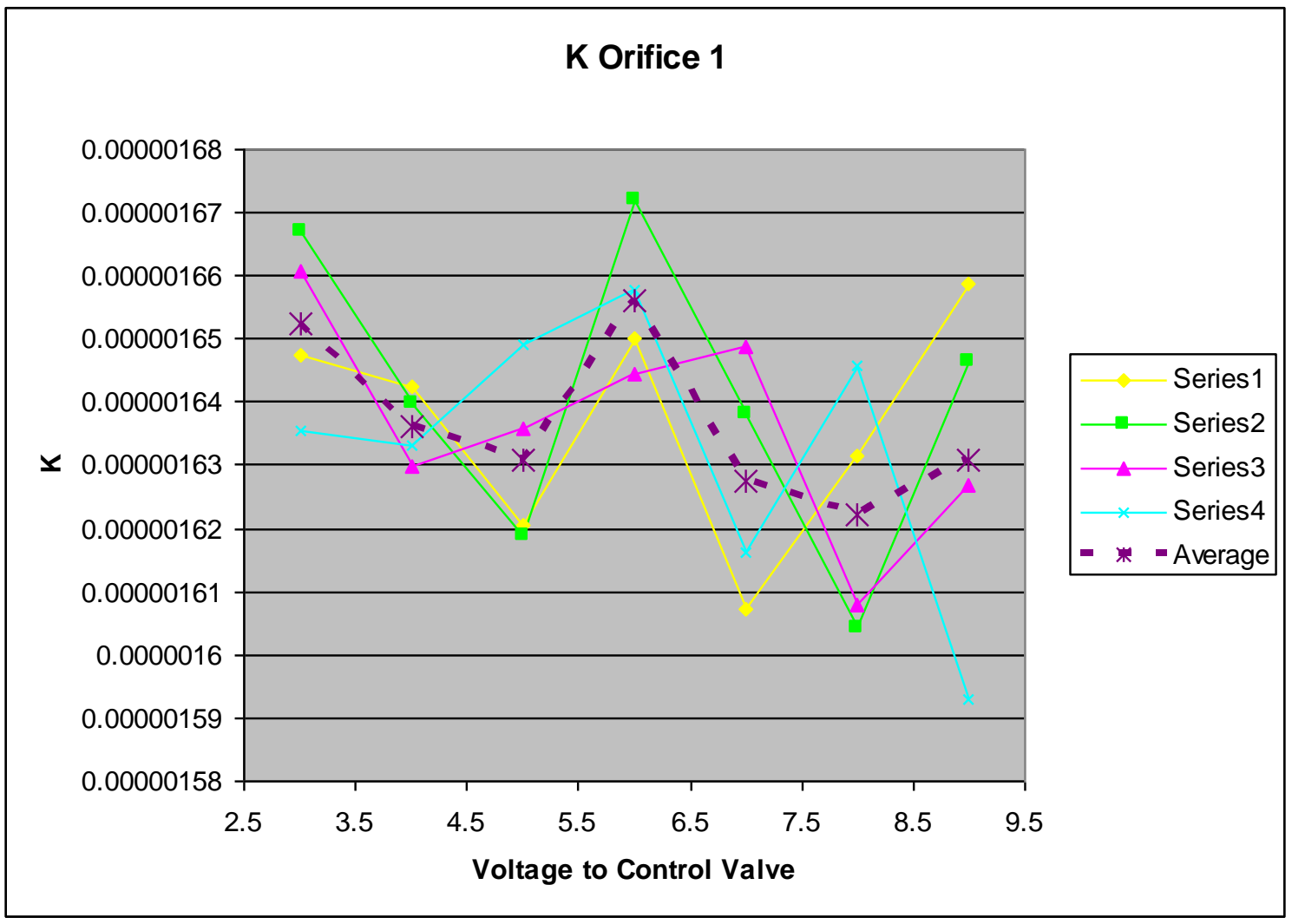

Orifice Meter 2

\begin{tabular}{|c|c|c|c|c|c|c|c|}
\hline \multirow[b]{2}{*}{ Voltage to Control Valve 2} & \multicolumn{7}{|l|}{ Average } \\
\hline & $\begin{array}{l}\text { Liters } \\
\text { Filled }\end{array}$ & $\begin{array}{c}\text { Time } \\
\text { To Fill } \\
(\mathrm{sec})\end{array}$ & $\begin{array}{l}\text { Fill Flow } \\
\text { Rate } \\
\text { (Liter/sec) }\end{array}$ & $\begin{array}{c}\text { Flow Rate } \\
\text { (GPM) }\end{array}$ & $\begin{array}{l}\text { Voltage } \\
\text { Recorded }\end{array}$ & $\begin{array}{c}\Delta \mathrm{P} \text { in } \\
\mathrm{mm} \mathrm{H} 2 \mathrm{O}\end{array}$ & K \\
\hline 0 & $\mathrm{x}$ & $\mathrm{x}$ & $\mathrm{x}$ & $\mathrm{x}$ & $\mathrm{x}$ & $\mathrm{x}$ & $\mathrm{x}$ \\
\hline 1 & $\mathrm{x}$ & $\mathrm{x}$ & $\mathrm{x}$ & $\mathrm{x}$ & $\mathrm{x}$ & $\mathrm{x}$ & $\mathrm{x}$ \\
\hline 2 & $\mathrm{n} / \mathrm{a}$ & $\mathrm{n} / \mathrm{a}$ & $\mathrm{n} / \mathrm{a}$ & $\mathrm{n} / \mathrm{a}$ & $\mathrm{n} / \mathrm{a}$ & $\mathrm{n} / \mathrm{a}$ & $\mathrm{n} / \mathrm{a}$ \\
\hline 3 & 8 & 86.43 & 0.0925578 & 1.4670593 & 0.971 & 318.8873 & $1.65514 \mathrm{E}-06$ \\
\hline 4 & 8 & 46.30 & 0.1727768 & 2.7385476 & 1.403 & 1128.569 & $1.64233 \mathrm{E}-06$ \\
\hline 5 & 8 & 32.54 & 0.2458513 & 3.8967916 & 2.007 & 2260.625 & $1.65119 \mathrm{E}-06$ \\
\hline
\end{tabular}




\begin{tabular}{|c|c|c|c|c|c|c|c|}
\hline 6 & 8 & 25.52 & 0.3134489 & 4.9682280 & 2.7375 & 3629.775 & $1.66137 \mathrm{E}-06$ \\
\hline 7 & 8 & 22.09 & 0.3621548 & 5.7402263 & 3.48 & 5021.416 & $1.632 \mathrm{E}-06$ \\
\hline 8 & 8 & 20.40 & 0.3921569 & 6.2157647 & 4.0175 & 6028.833 & $1.61281 \mathrm{E}-06$ \\
\hline 8.5 & 8 & 19.56 & 0.4089980 & 6.4826994 & 4.16382 & 6303.075 & $1.64507 \mathrm{E}-06$ \\
\hline & & & & & & $\operatorname{Avg} K$ & $1.64284 \mathrm{E}-06$ \\
\hline
\end{tabular}

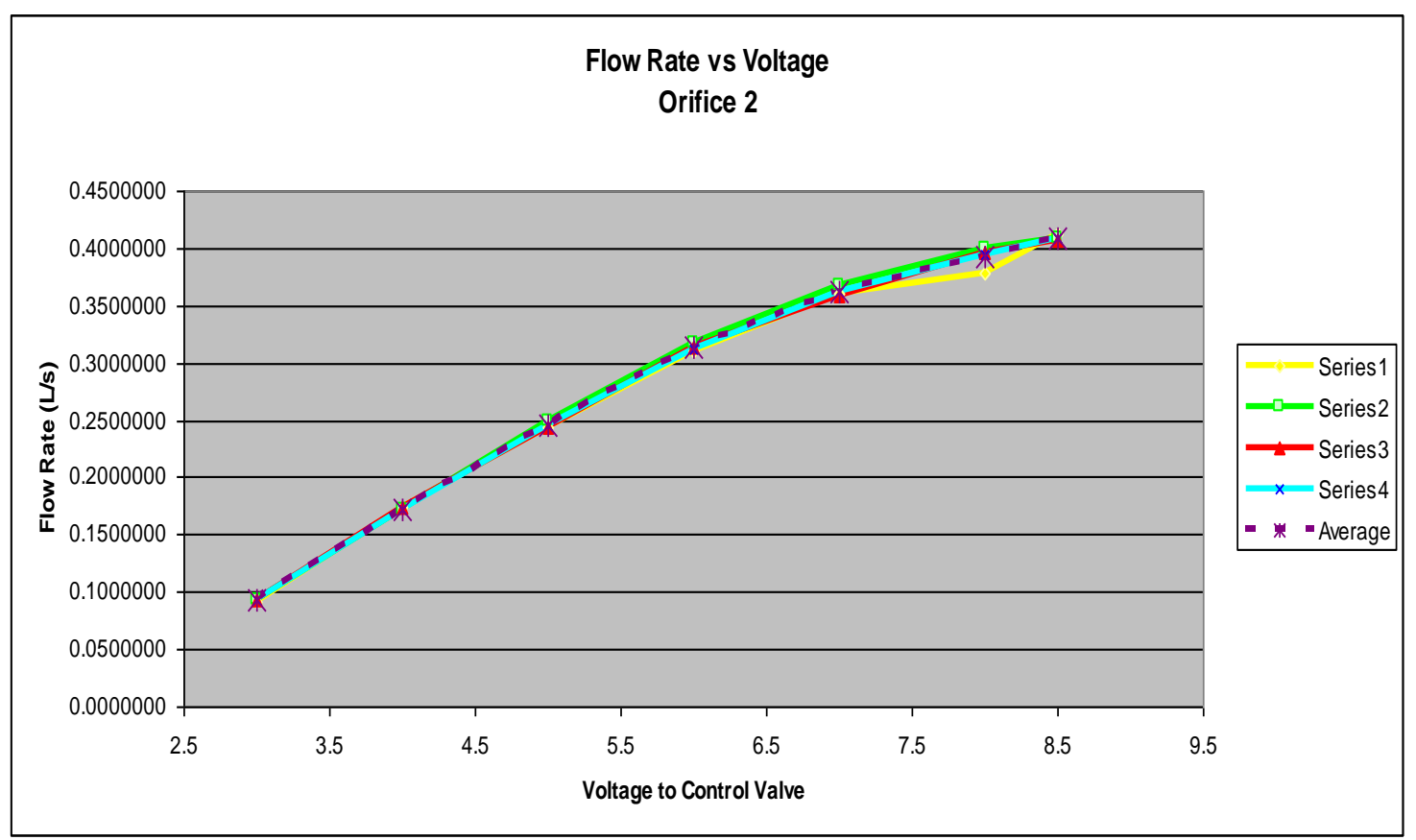




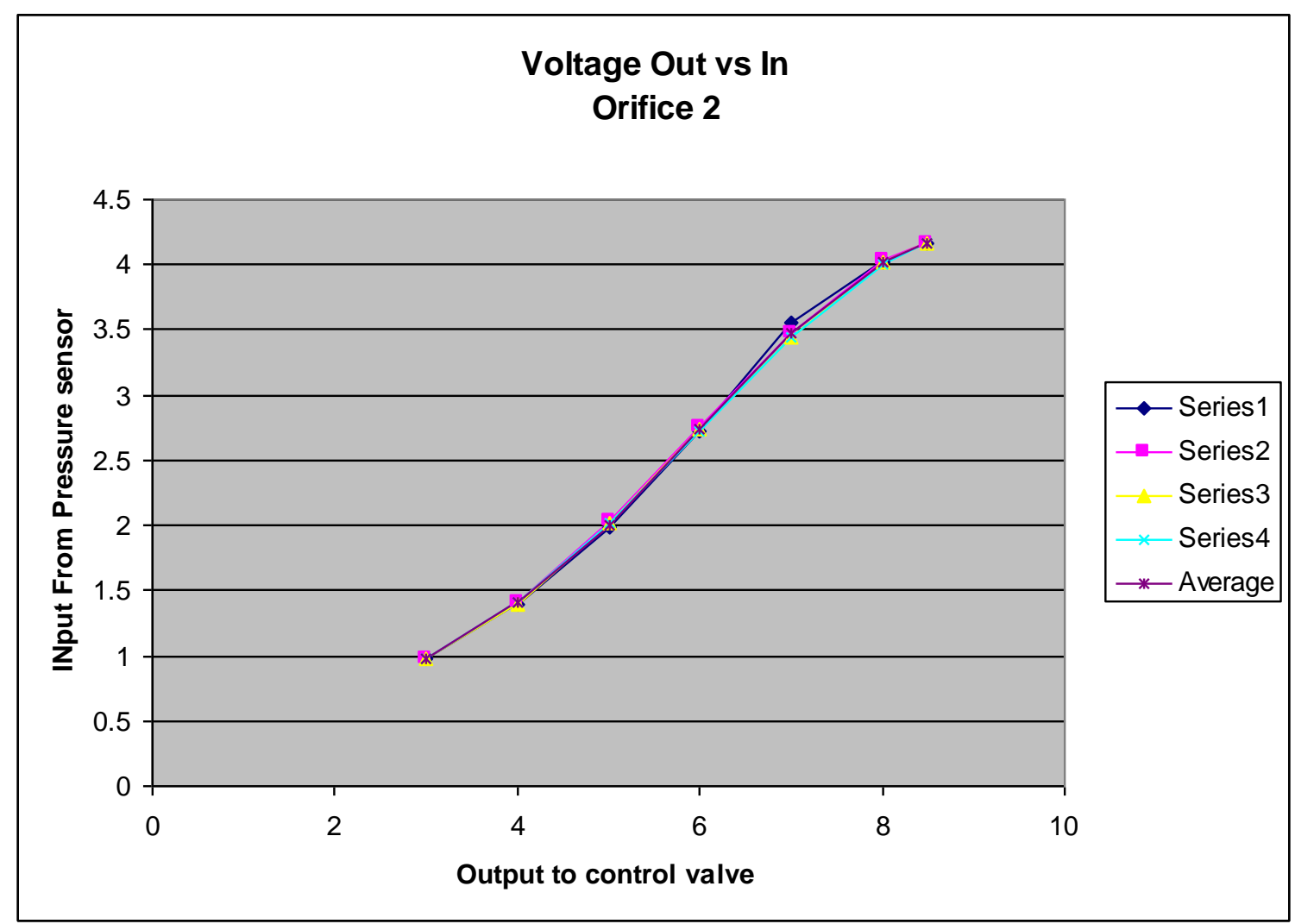




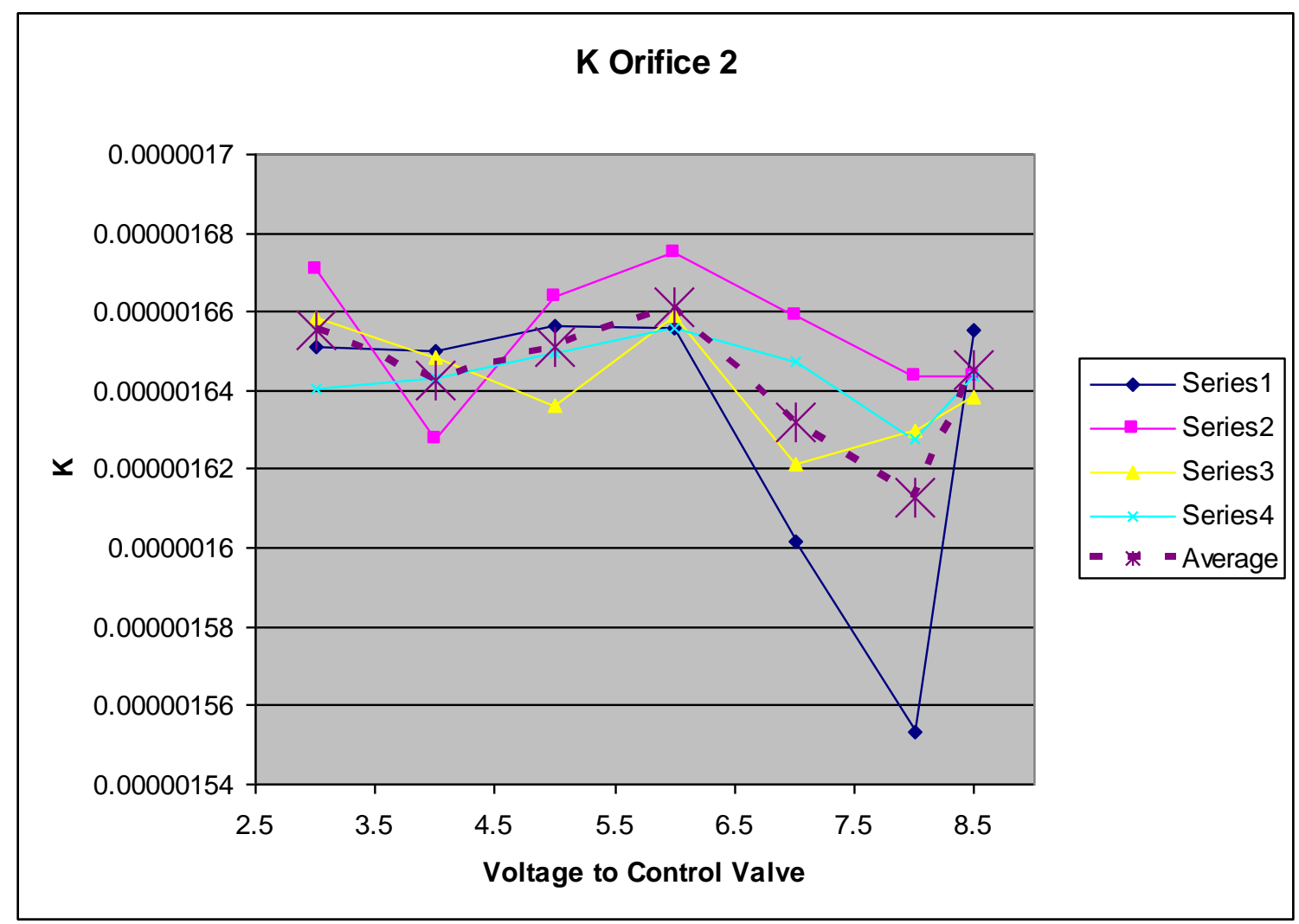

\section{Conclusions:}

After analysis of the data, for orifice meter 1 , the proportionality constant $\mathrm{K}$ is found to be $1.63666 \mathrm{E}-6$, making the calibration curve for Orifice Meter 1, including all conversions:

SQRT $\{(1860.2209 *$ Voltage -1484.084253$) * 9.806652\} * 1.63666 \mathrm{E}-6 * 15850.2=$

Flow Rate (GPM).

For Orifice Meter $2 \mathrm{~K}$ is found to be $1.64296 \mathrm{E}-6$. The corresponding calibration curve is:

SQRT $\{(1874.264032 * x-1501.023093) * 9.806652\} * 1.64296 \mathrm{E}-6 * 15850.2=$

Flow Rate (GPM). 


\section{Appendix 7: $\quad$ Turbine Flow Meter Calibration Procedure and Results}

This is a procedure summary used to calibrate the turbine flow meters installed in the loop against the orifice meters.

- Turn on the loop according to procedure described earlier.

- Isolate both tanks by closing the valve connecting tanks.

- Make sure there is no air trapped inside the piping.

- You can look at the clear parts of the piping to check for entrapped pockets of air.

- If there is, use your hand to plug the water exits located on top of the water tank underneath the loop table top for a few seconds at a time. This simple procedure helps coalesce the trapped air bubbles and the backpressure created inside the pipe helps purge the air out of the pipe as the exit is unplugged.

- Set the first desired water level inside the tank and let the PI controller bring the level to a state as steady as possible (this can take from several minutes to a few hours per data point).

- Monitor the level change inside the tank to make sure it has reached steady state.

- Unplug the corresponding control valve to avoid the controller from making minor changes in the water level while reading the values).

- Place probes in the data acquisition VI diagram to monitor the voltages coming from the orifice meter, and turbine flow meter signal conditioner being calibrated, and build a table similar to the tables below.

- Proceed to the next calibration data point.

- Perform a linear regression using the data points and update equation(s) present in the calibration sub-VI called TFM_Curves.vi, shown in figures below. 


\section{TFM1}

Turbine S/N: 051310332

Signal Conditioner S/N: 16334

Tuesday, May 25, 2010

\begin{tabular}{|l|l|l|l|l|}
\hline \multicolumn{1}{|c|}{ mmH20 } & \multicolumn{1}{|c|}{ Measured mmH2O } & \multicolumn{1}{c|}{ OM1 } & \multicolumn{1}{c|}{ OM1 } \\
{$[1 / \mathrm{s}]$} & & \multicolumn{1}{c|}{ TFM1 } \\
\hline 0 & & & & \\
\hline 400 & 414.14 & .143 & 1.20097 & 2.4526 \\
\hline 500 & 512.4 & .152 & 1.23196 & 2.6298 \\
\hline 600 & 626.2 & .161 & 1.267 & 2.807 \\
\hline 700 & 768.0 & .173 & 1.3114 & 3.017 \\
\hline 800 & 803.7 & .175 & 1.3192 & 3.156 \\
\hline
\end{tabular}

\section{TFM2}

Turbine S/N: 051310300

Signal Conditioner S/N: 16253

Tuesday, May 25, 2010

\begin{tabular}{|c|c|c|c|c|}
\hline $\mathrm{mmH} 20$ & Measured mmH2O & $\begin{array}{c}\text { OM2 } \\
{[1 / \mathrm{s}]}\end{array}$ & $\begin{array}{c}\text { OM2 } \\
\text { [V] }\end{array}$ & $\begin{array}{c}\text { TFM2 } \\
\text { [V] }\end{array}$ \\
\hline 0 & & & & \\
\hline 400 & 400.178 & 0.105 & 1.1740 & 2.30957 \\
\hline
\end{tabular}




\begin{tabular}{|l|l|l|l|l|}
\hline 500 & 470.65 & 0.111 & 1.1968 & 2.441 \\
\hline 600 & 606.73 & 0.120 & 1.2357 & 2.6806 \\
\hline 700 & 760.44 & 0.132 & 1.2801 & 2.927 \\
\hline 800 & 845.4 & .138 & 1.302 & 3.06 \\
\hline
\end{tabular}

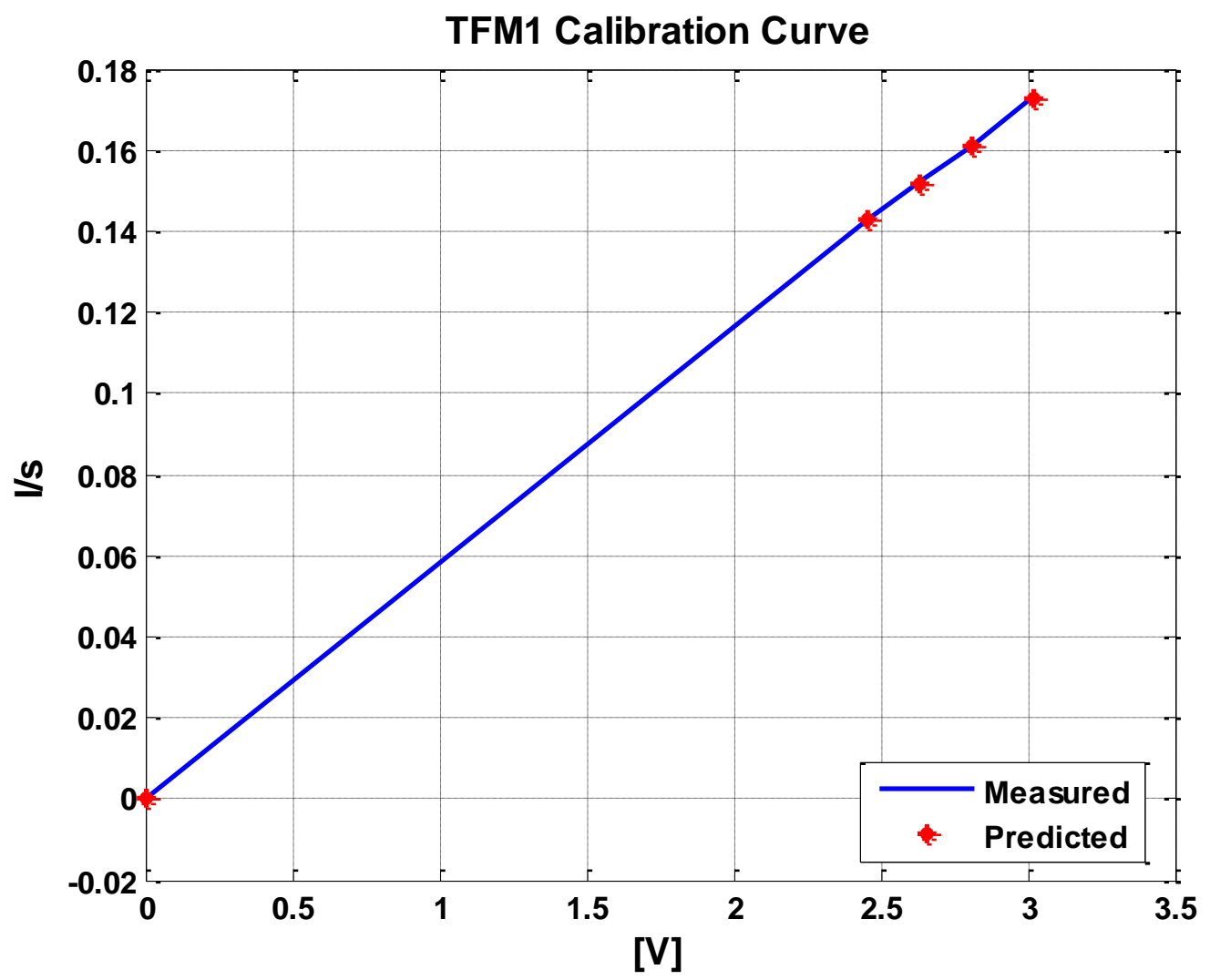




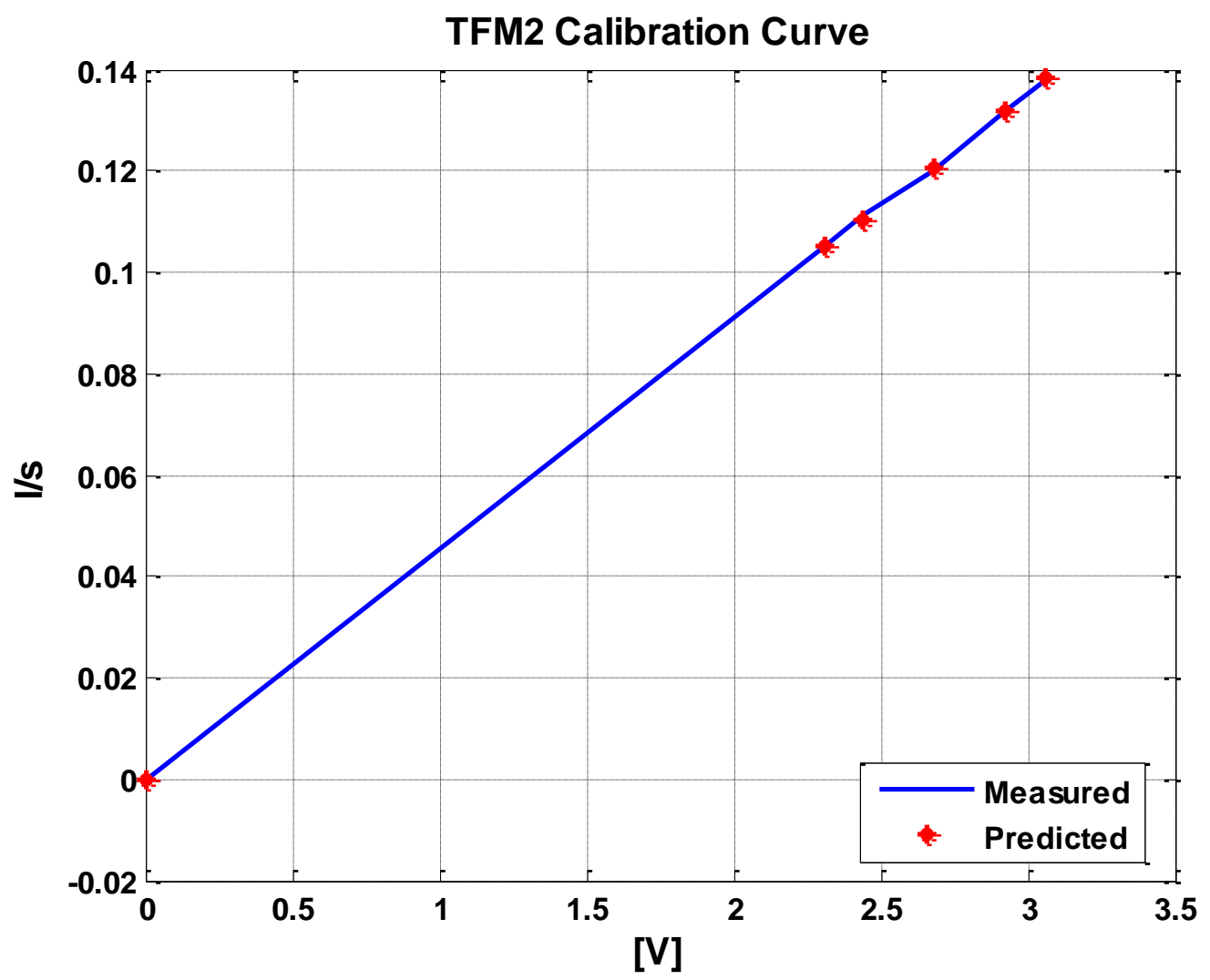




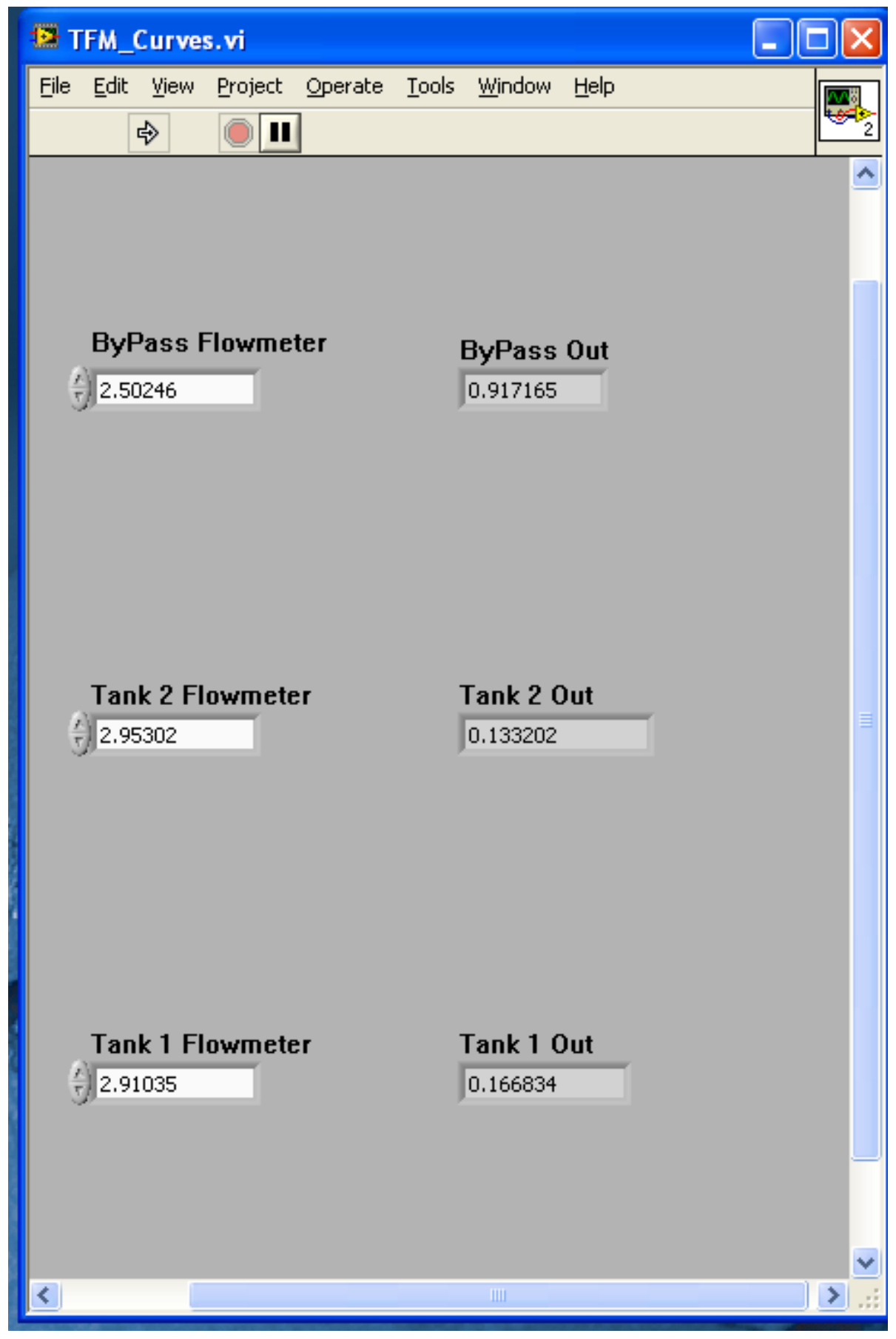




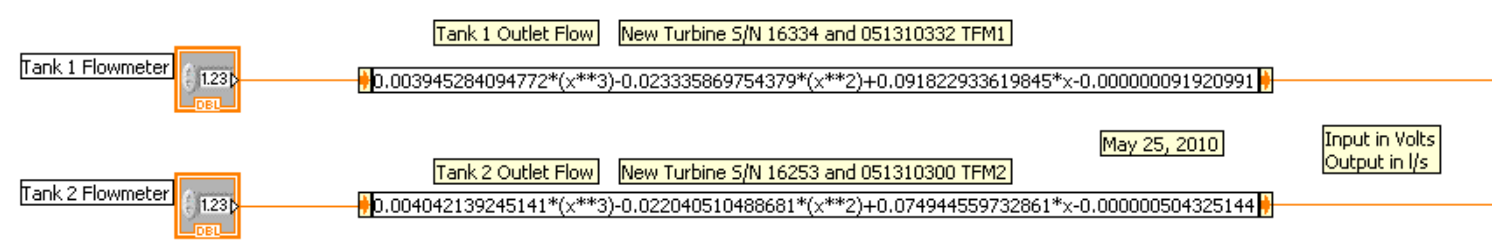

\section{Matlab example code for performing the linear regression:}

$\%$ Calibration of new turbines against OM1\& OM2 respectively

$\%$ S/N: 16334 \& 051310332 (TFM1)

$\% \mathrm{~S} / \mathrm{N}: 16253 \& 051310300(\mathrm{TFM} 1)$

$\%$ May 25th, 2010

$\%$ Sergio Perillo

clear all

clc

close all

$\mathrm{f}=12$;

$\mathrm{tfm} 1=[0 ; 2.453 ; 2.6298 ; 2.807 ; 3.017] ; \% \mathrm{~V}$

om1 $=[0 ; .143 ; .152 ; .161 ; .173] ; \% 1 / \mathrm{s}$

figure(10);

plot(tfm1,om1,'LineWidth',2);grid on;

title('TFM1 Calibration Curve','fontweight','b',...

'fontsize',f);

xlabel('[V]','fontweight','b','fontsize',f);

ylabel('1/s','fontweight','b','fontsize',f);

set(get(gcf,'CurrentAxes'),'fontweight','b'); 
$[\mathrm{p} 1, \mathrm{~s} 1]=$ polyfit $(\mathrm{tfm} 1, \mathrm{om} 1,3)$

[pred1,delta1]=polyval $(\mathrm{p} 1, \mathrm{tfm} 1, \mathrm{~s} 1)$;

hold;

plot(tfm1,pred1,'r*','LineWidth',2)

legend('Measured','Predicted',...

'Location','Best');

set(legend,'fontweight','b')

$\mathrm{tfm} 2=[0 ; 2.3096 ; 2.441 ; 2.6806 ; 2.927 ; 3.06] ; \% \mathrm{~V}$

om2 $=[0 ; .105 ; .111 ; .12 ; .132 ; .138] ; \% 1 / \mathrm{s}$

figure(20);

plot(tfm2,om2,'LineWidth',2);hold;grid on

title('TFM2 Calibration Curve','fontweight','b',...

'fontsize',f);

xlabel('[V]','fontweight','b','fontsize',f);

ylabel('1/s','fontweight','b','fontsize',f);

set(get(gcf,'CurrentAxes'),'fontweight','b');

$[\mathrm{p} 2, \mathrm{~s} 2]=$ polyfit $(\mathrm{tfm} 2, \mathrm{om} 2,4)$

[pred2, delta2]=polyval(p2,tfm2,s2);

plot(tfm2,pred2,'r*','LineWidth',2)

legend('Measured','Predicted',... 
'Location','Best');

set(legend,'fontweight','b') 
Appendix 8: $\quad$ Variables, channels, and Patch Panel Numbers

Box SCB-68 \#1 with PCI-6259

\begin{tabular}{|c|c|c|c|}
\hline Sensor ID & $\begin{array}{c}\text { PCI-6259 Pin } \\
\text { Out }\end{array}$ & SCB Pin Out & Pins \\
\hline Temp Tank 1 & ai01-ai09 & $\mathrm{ACH} 1-\mathrm{ACH} 9$ & $\begin{array}{c}33(+) \\
66(-)\end{array}$ \\
\hline Temp Tank 2 & ai02-ai10 & $\mathrm{ACH} 2-\mathrm{ACH} 10$ & $\begin{array}{l}65(+) \\
31(-)\end{array}$ \\
\hline $\mathrm{CJC}$ & ai00-ai08 & $\mathrm{ACH} 0-\mathrm{ACH} 8$ & $\begin{array}{c}68(+) \\
34(-)\end{array}$ \\
\hline Sump Tank Temp & ai03-ai11 & $\mathrm{ACH} 3-\mathrm{ACH} 11$ & $\begin{array}{l}30(+) \\
63(-)\end{array}$ \\
\hline From CV 1 & ai04-ai12 & $\mathrm{ACH} 4-\mathrm{ACH} 12$ & $\begin{array}{l}28(+) \\
61(-)\end{array}$ \\
\hline From CV 2 & ai05-ai13 & $\mathrm{ACH} 5-\mathrm{ACH} 13$ & $\begin{array}{l}60(+) \\
26(-)\end{array}$ \\
\hline From CV 3 & ai06-ai14 & $\mathrm{ACH} 6-\mathrm{ACH} 14$ & $\begin{array}{c}25(+) \\
58(-)\end{array}$ \\
\hline To CV 1 & Dev5/ao00 & DACO Out & $\begin{array}{c}22(+) \\
55(-)\end{array}$ \\
\hline To CV4 & Dev2/ao3 & DACO Out & \\
\hline
\end{tabular}


Box SCB-68 \#2 with PCI-6259

\begin{tabular}{|c|c|c|c|}
\hline Sensor ID & $\begin{array}{c}\text { PCI-6259 Pin } \\
\text { Out }\end{array}$ & SCB Pin Out & Pins \\
\hline Level 1 & ai23-ai31 & $\mathrm{ACH} 7-\mathrm{ACH} 15$ & $\begin{array}{l}57(+) \\
23(-)\end{array}$ \\
\hline OM 1 & ai17-ai25 & ACH1-ACH9 & $\begin{array}{l}33(+) \\
66(-)\end{array}$ \\
\hline TFM 1 & ai18-ai26 & $\mathrm{ACH} 2-\mathrm{ACH} 10$ & $\begin{array}{l}65(+) \\
31(-)\end{array}$ \\
\hline Bypass FM & ai19-ai27 & $\mathrm{ACH} 3-\mathrm{ACH} 11$ & $\begin{array}{l}30(+) \\
63(-)\end{array}$ \\
\hline Level 2 & ai20-ai28 & $\mathrm{ACH} 4-\mathrm{ACH} 12$ & $\begin{array}{l}28(+) \\
61(-)\end{array}$ \\
\hline OM 2 & ai21-ai29 & ACH5-ACH13 & $\begin{array}{l}60(+) \\
26(-) \\
\end{array}$ \\
\hline TFM 2 & ai22-ai30 & $\mathrm{ACH} 6-\mathrm{ACH} 14$ & $\begin{array}{l}25(+) \\
58(-)\end{array}$ \\
\hline To CV 2 & Dev5/ao1 & DAC1 Out & \\
\hline $\begin{array}{l}\text { To CV } 3 \\
\text { (Bypass) }\end{array}$ & $\mathrm{ao} 2$ & DACO Out & $\begin{array}{l}22(+) \\
55(-)\end{array}$ \\
\hline
\end{tabular}




\section{Appendix 9: $\quad$ VI - Additional Screenshots}

Control VI Screenshots

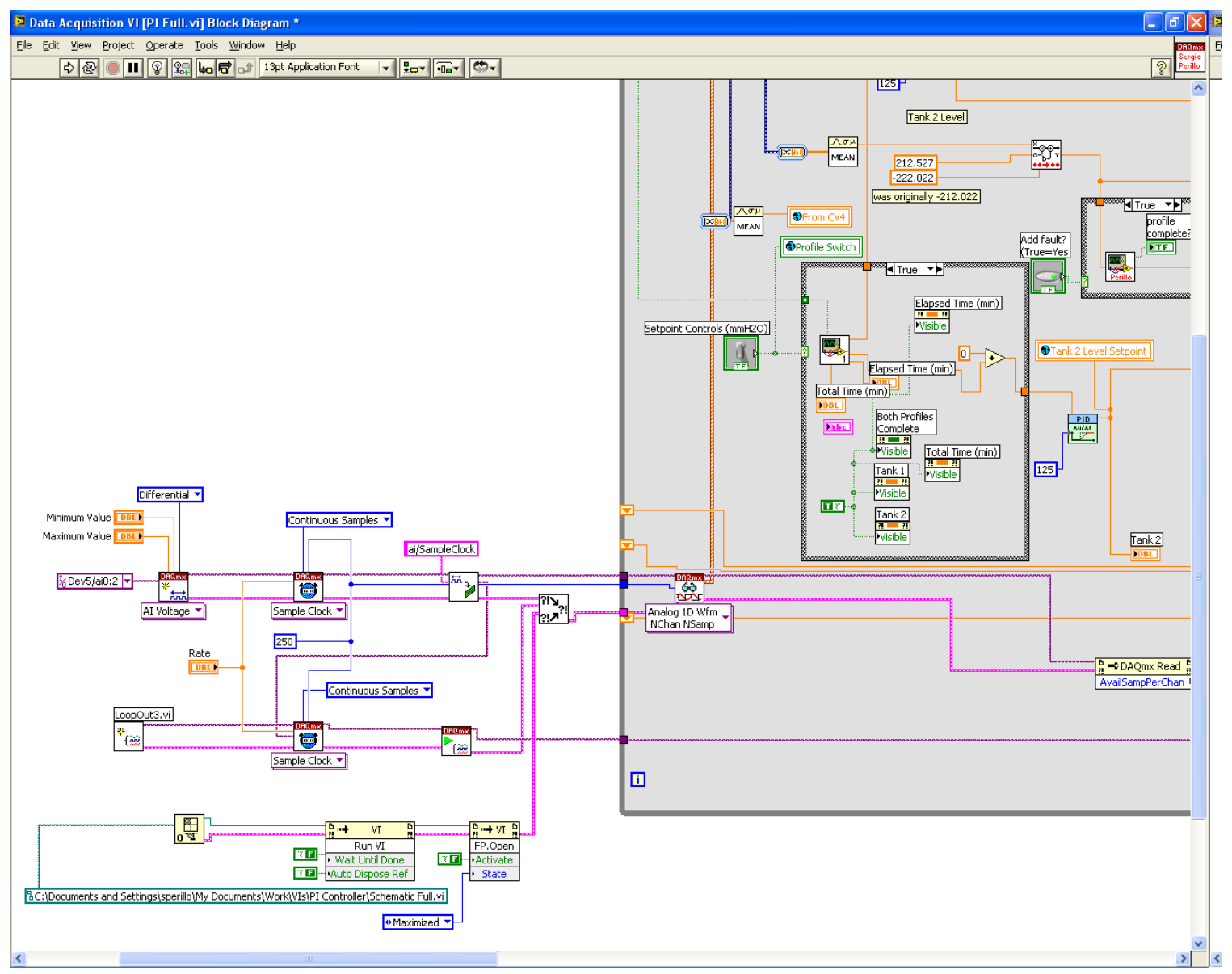




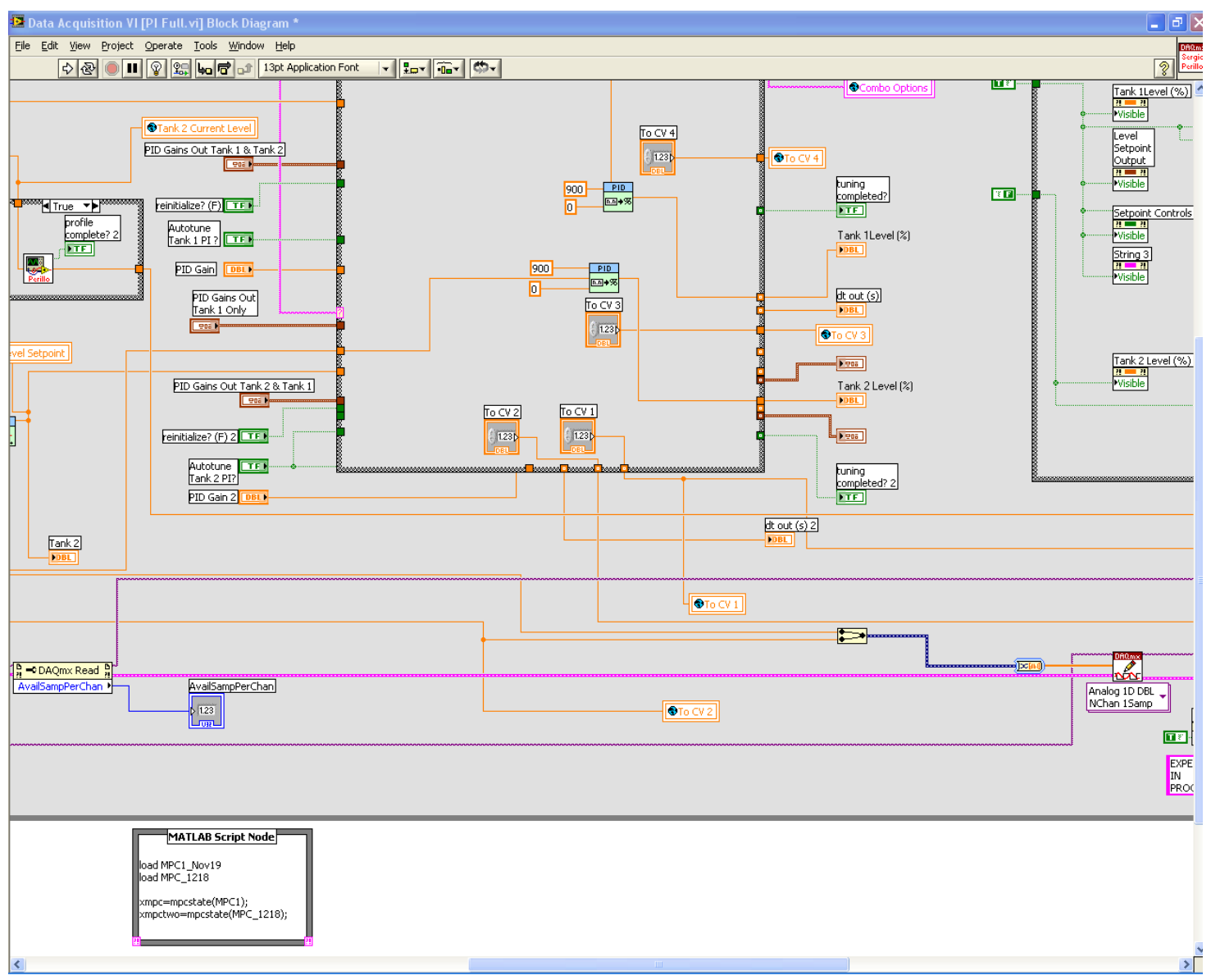




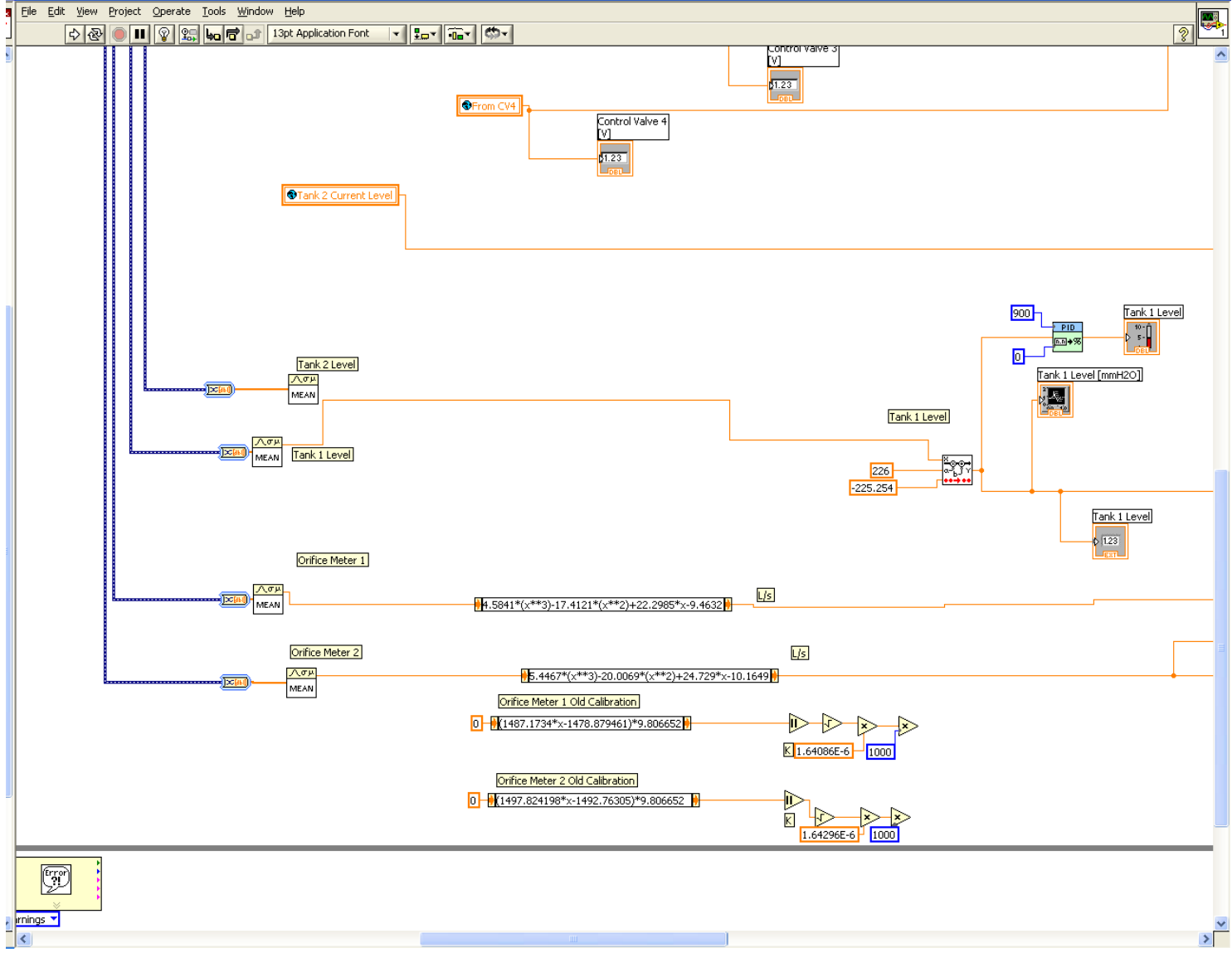




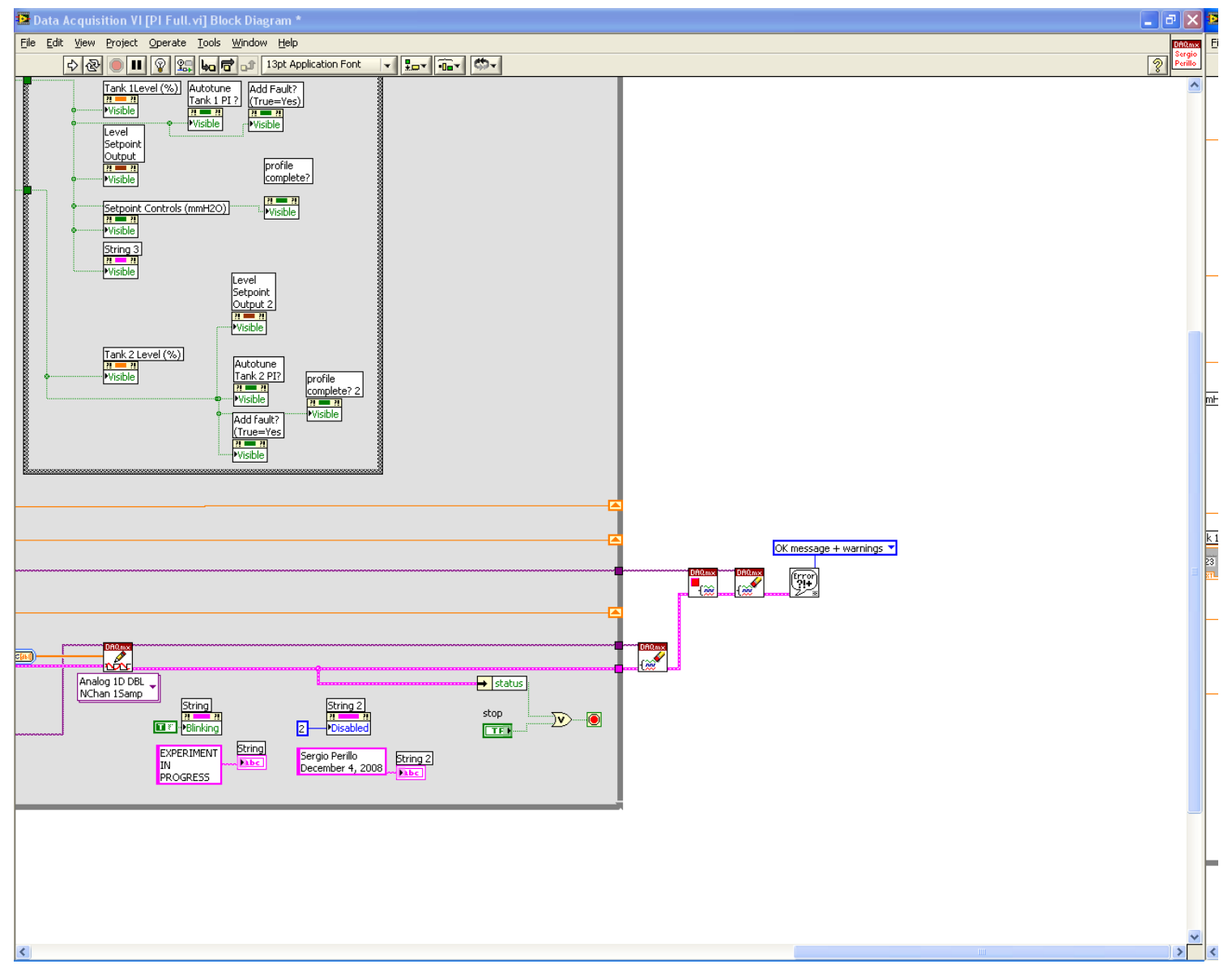




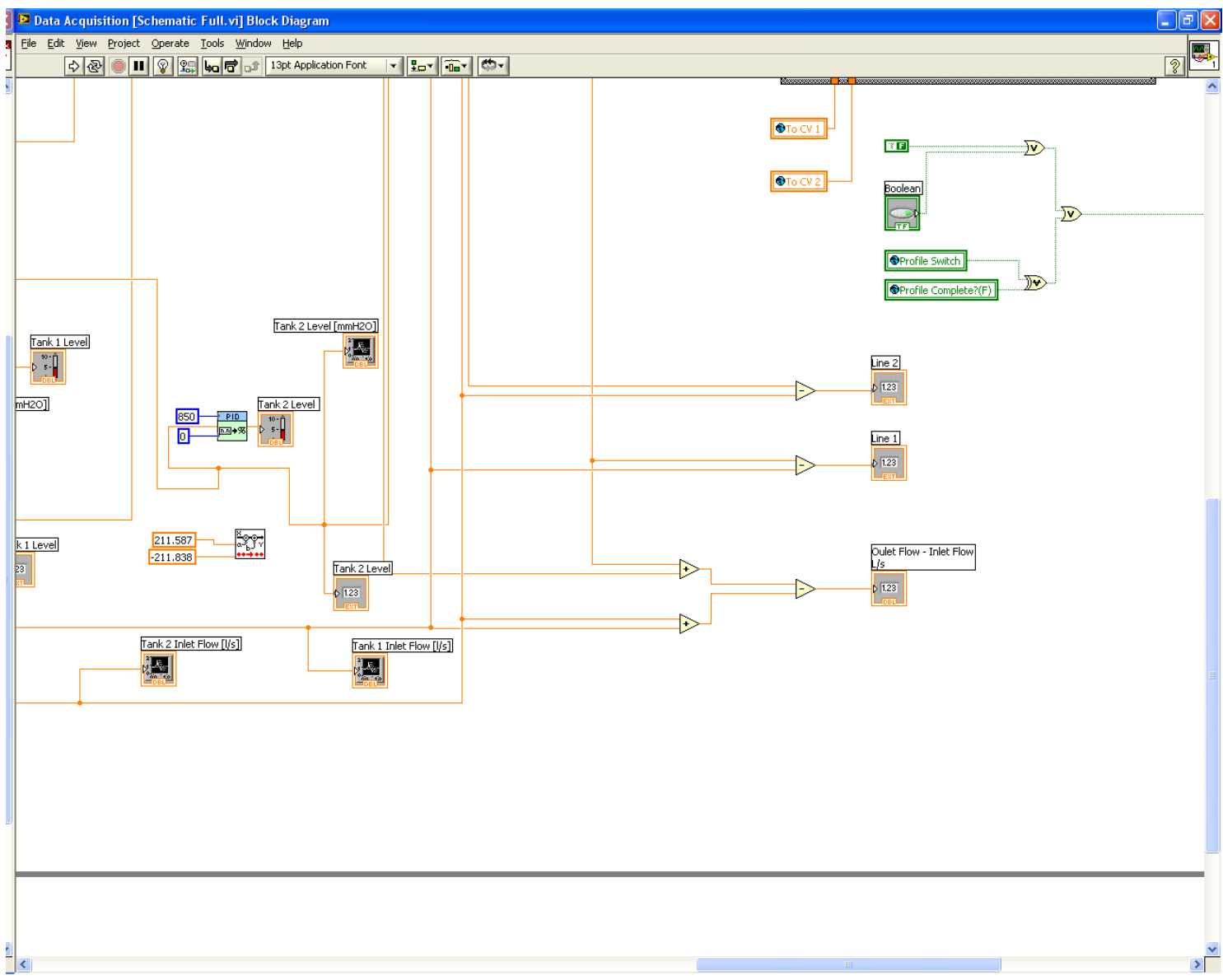




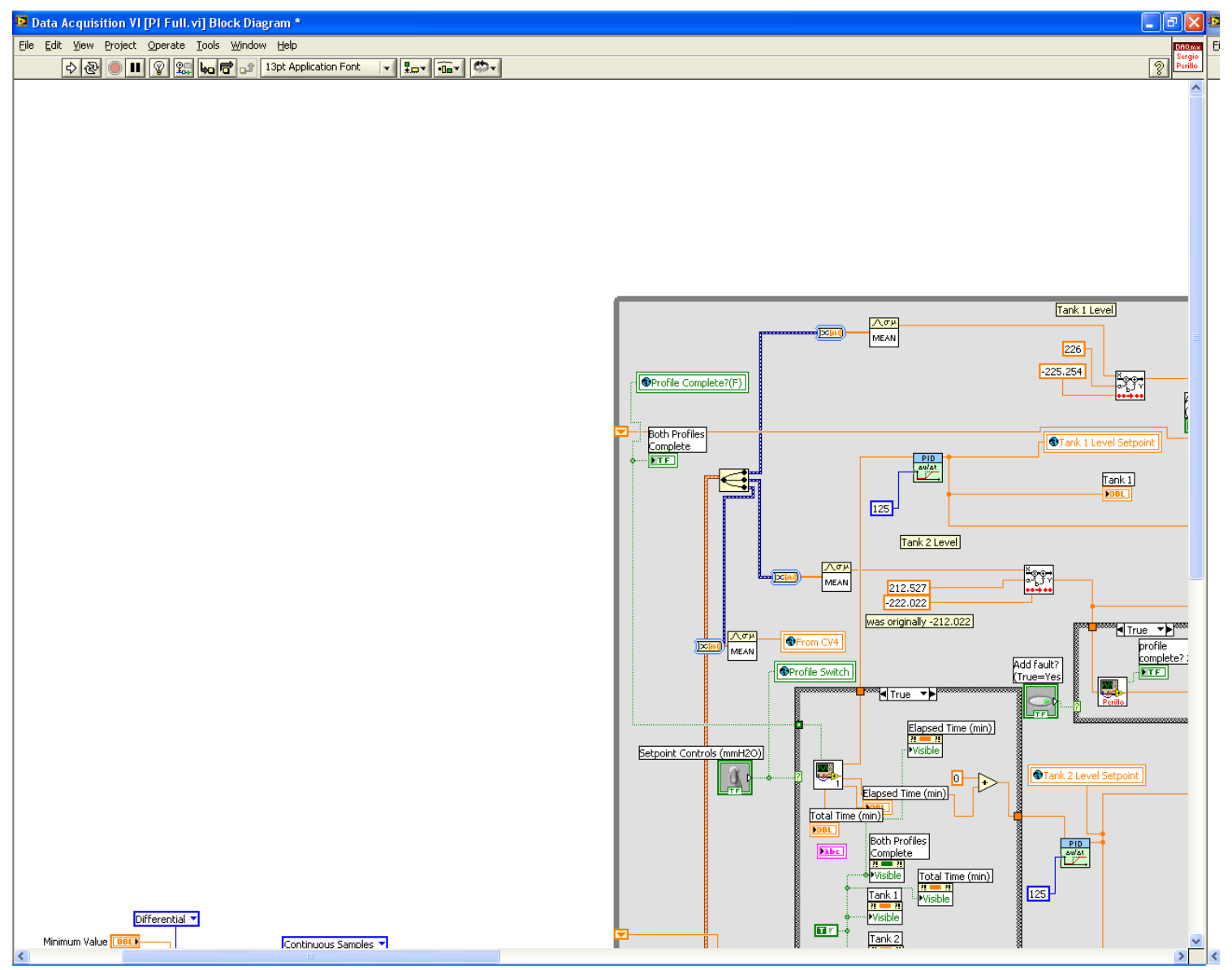




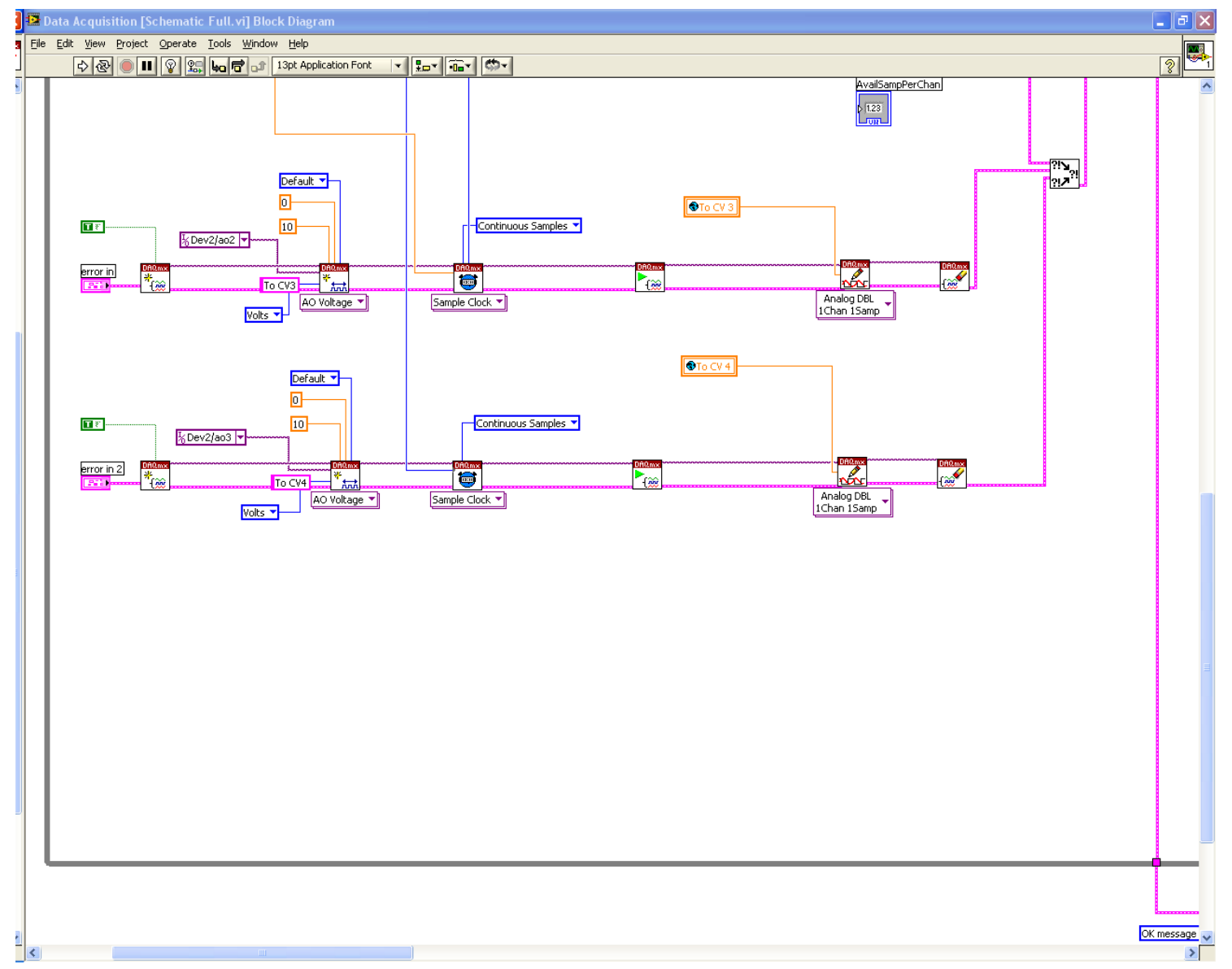



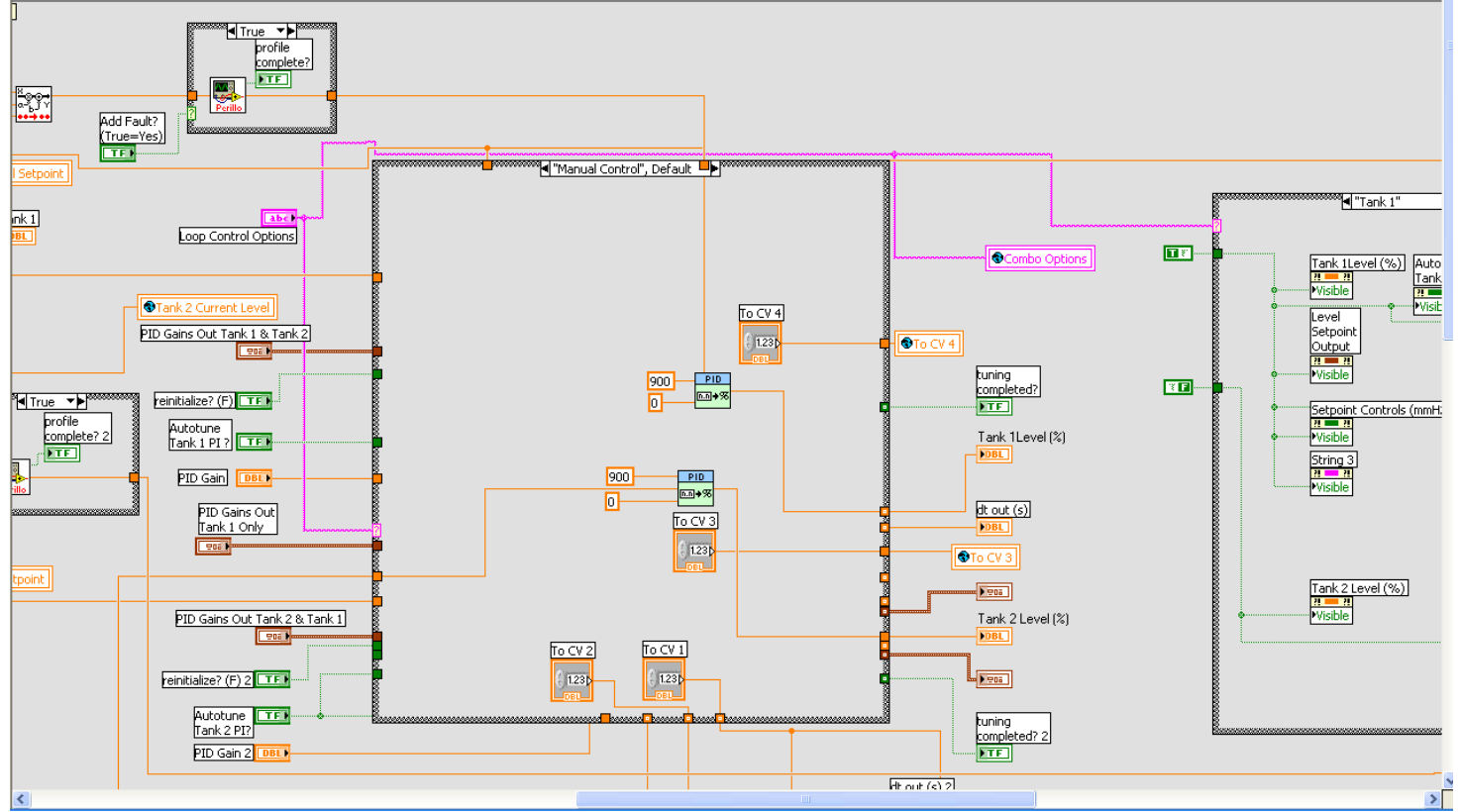


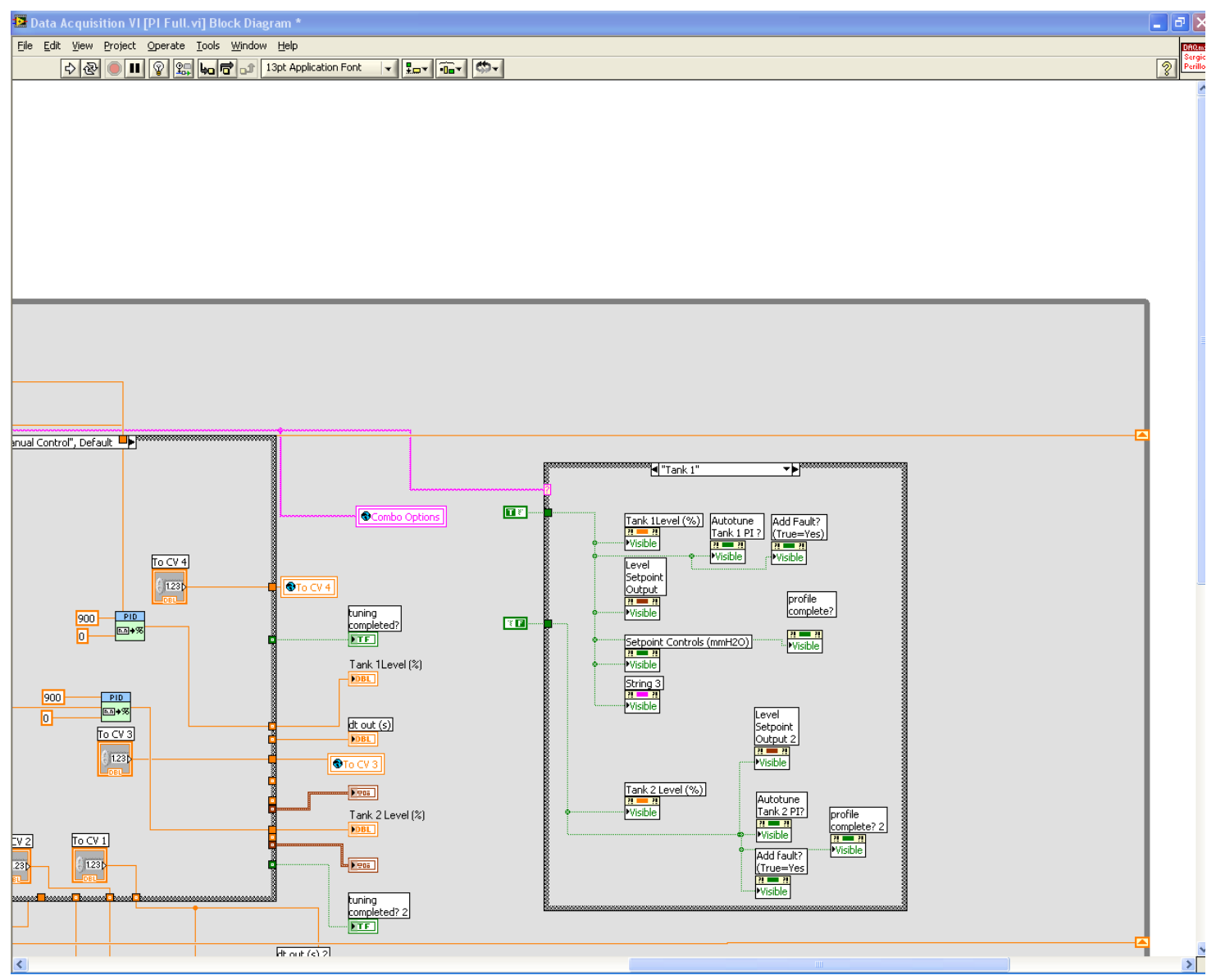




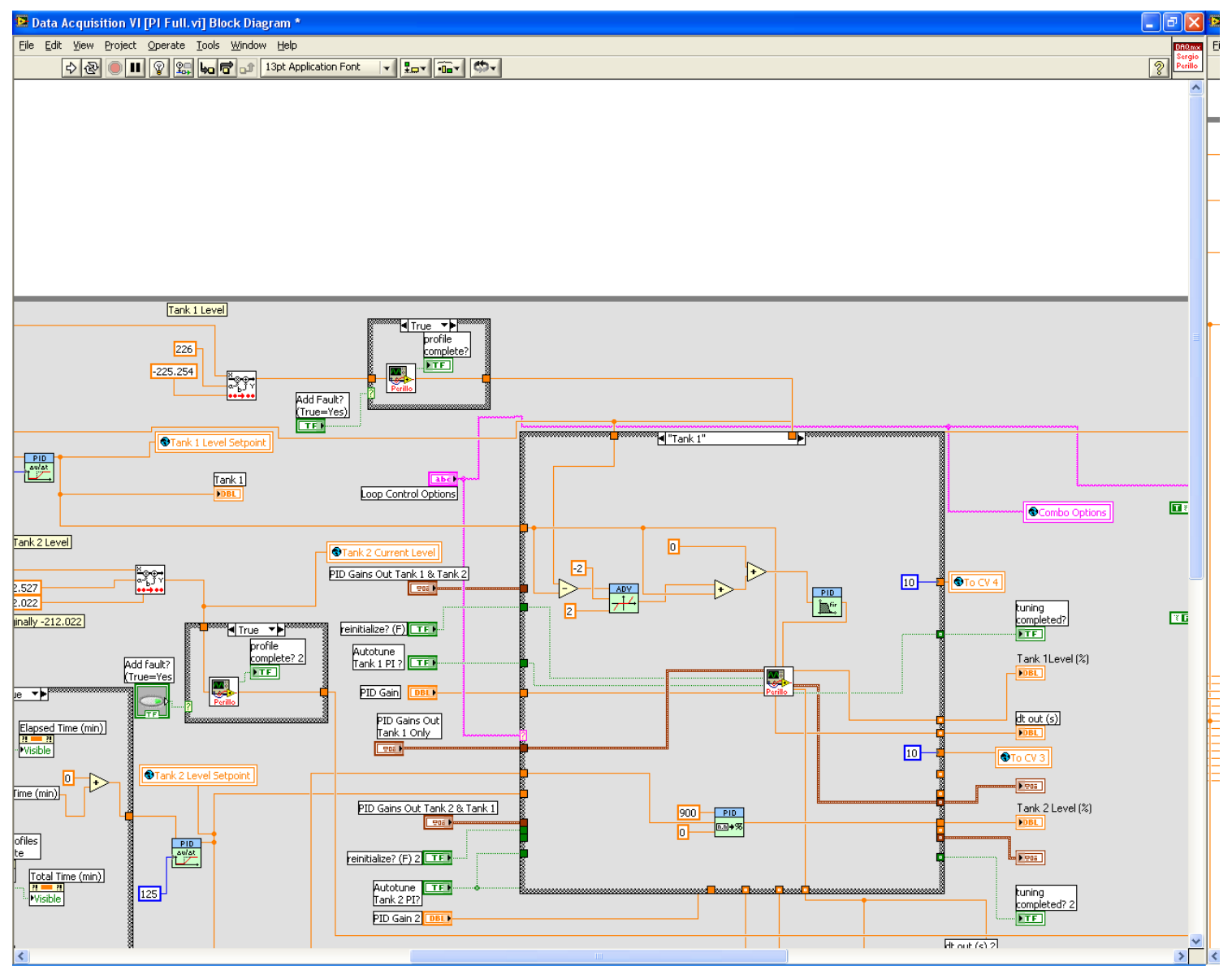




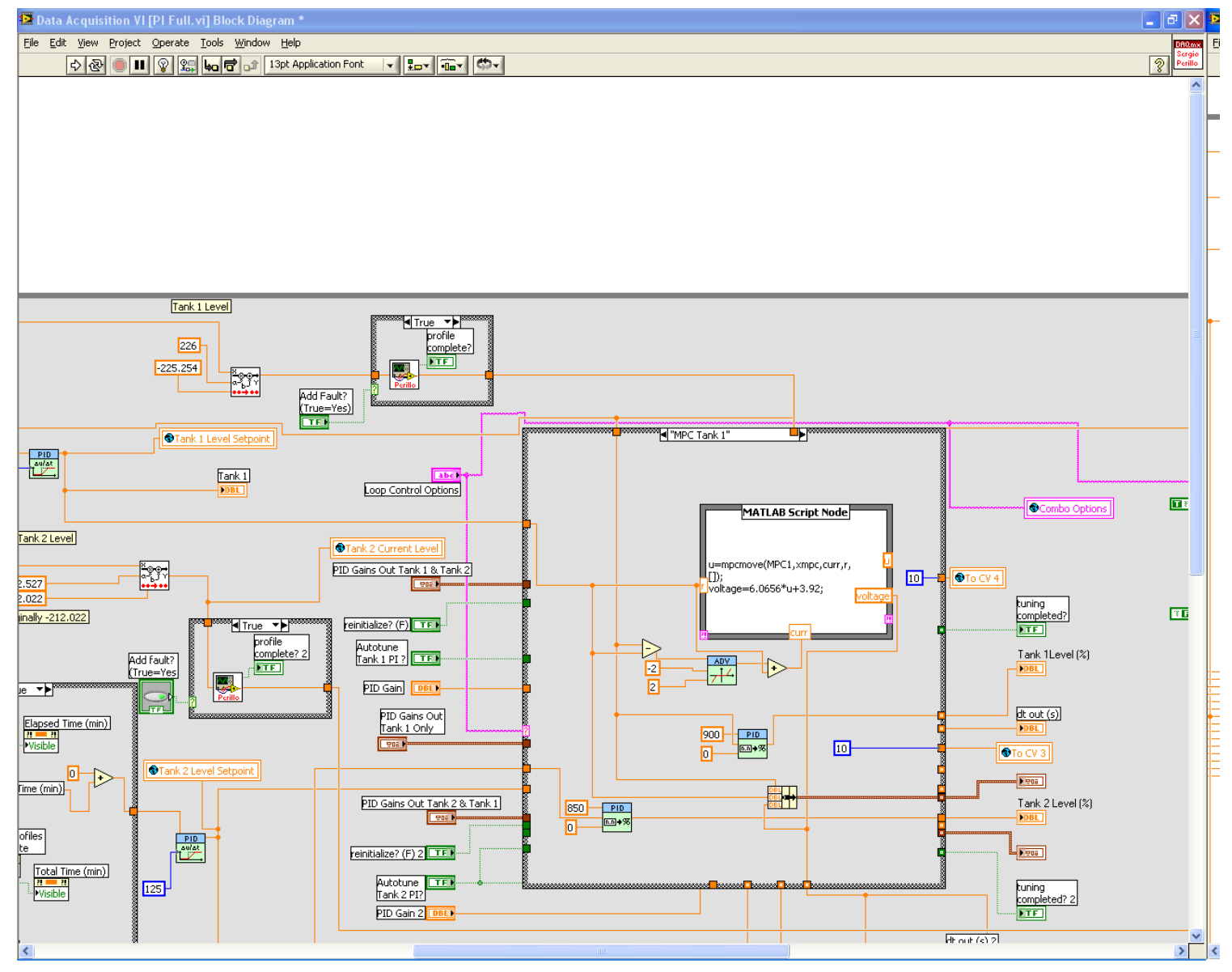


Data Acquisition VI Screenshots

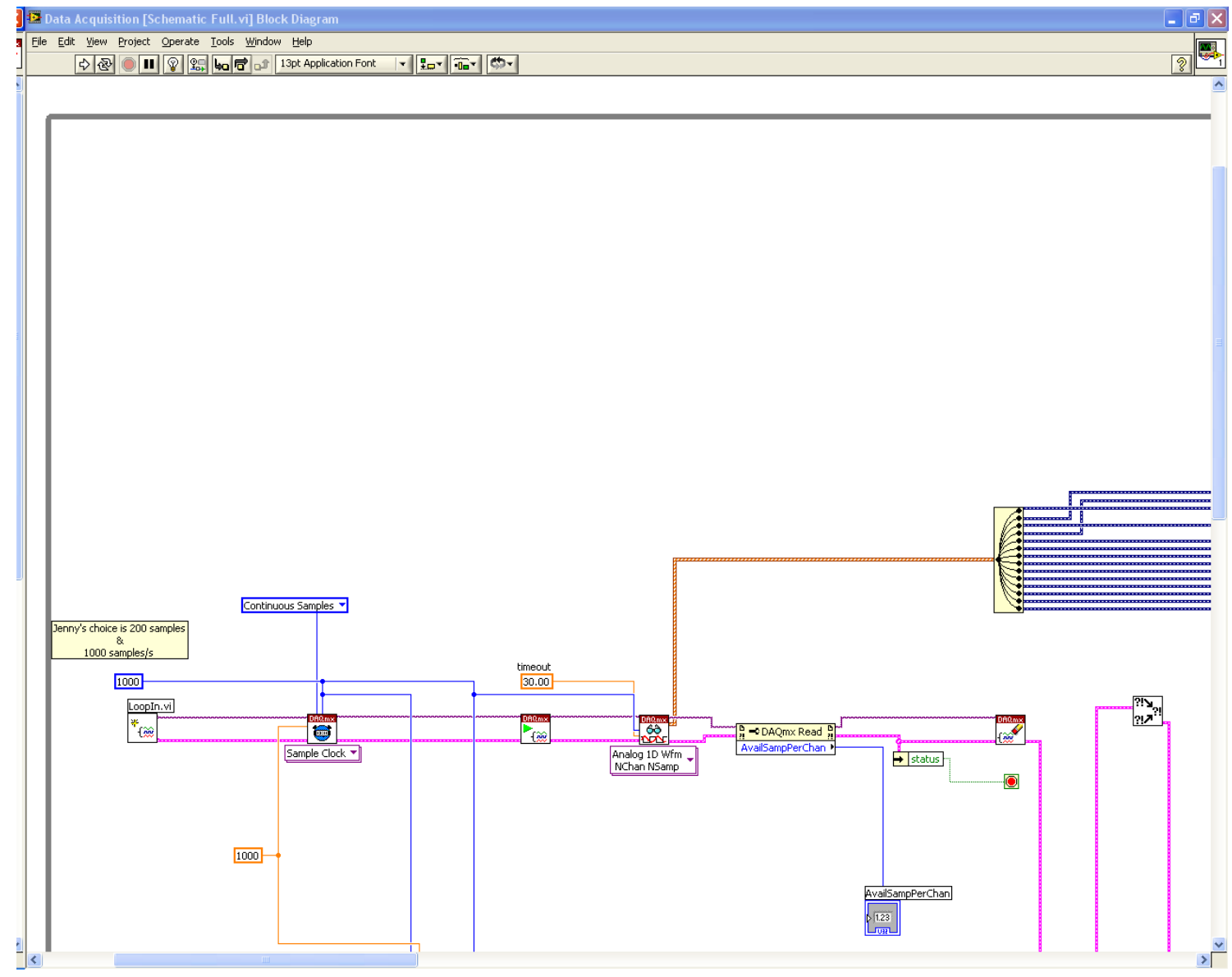




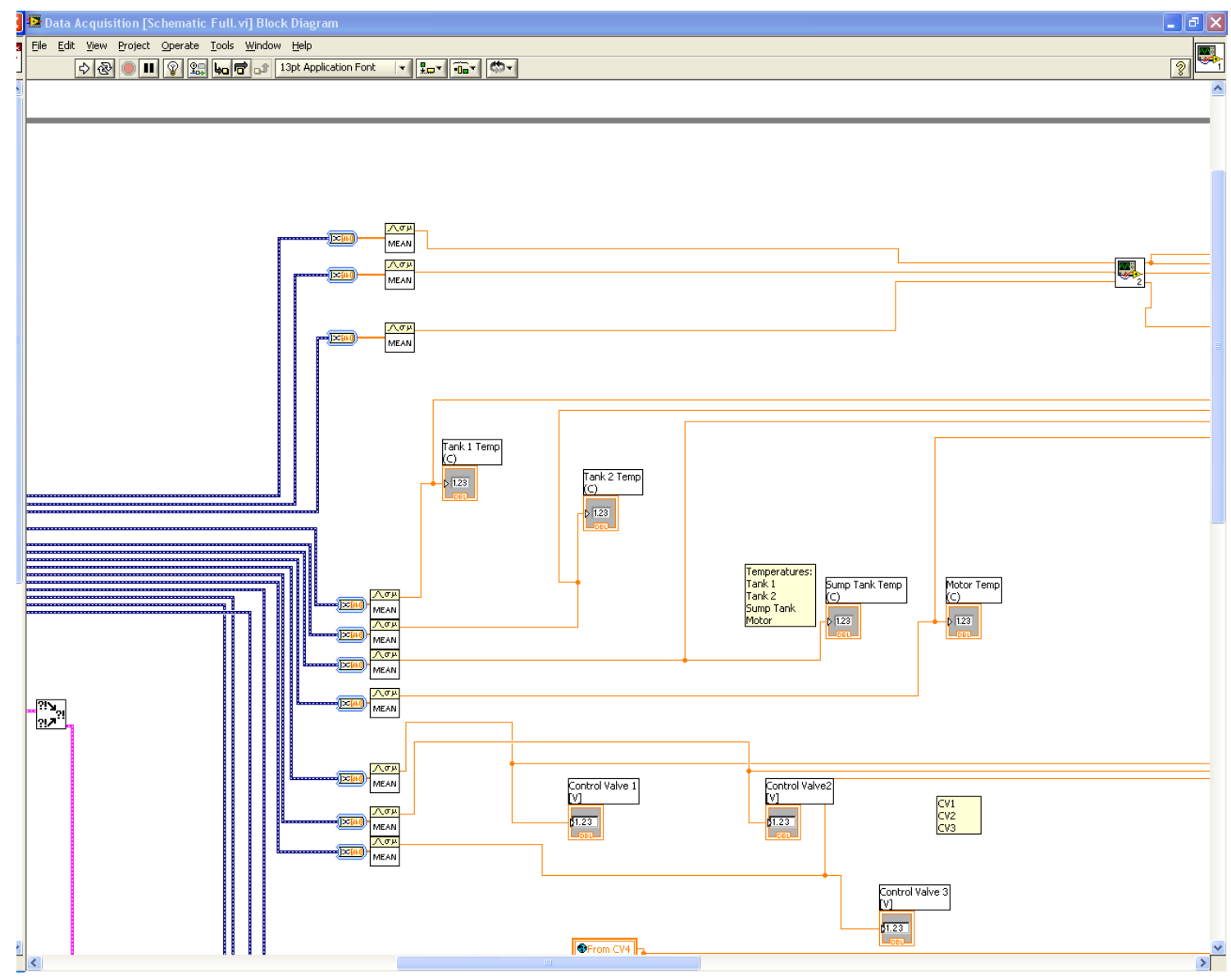




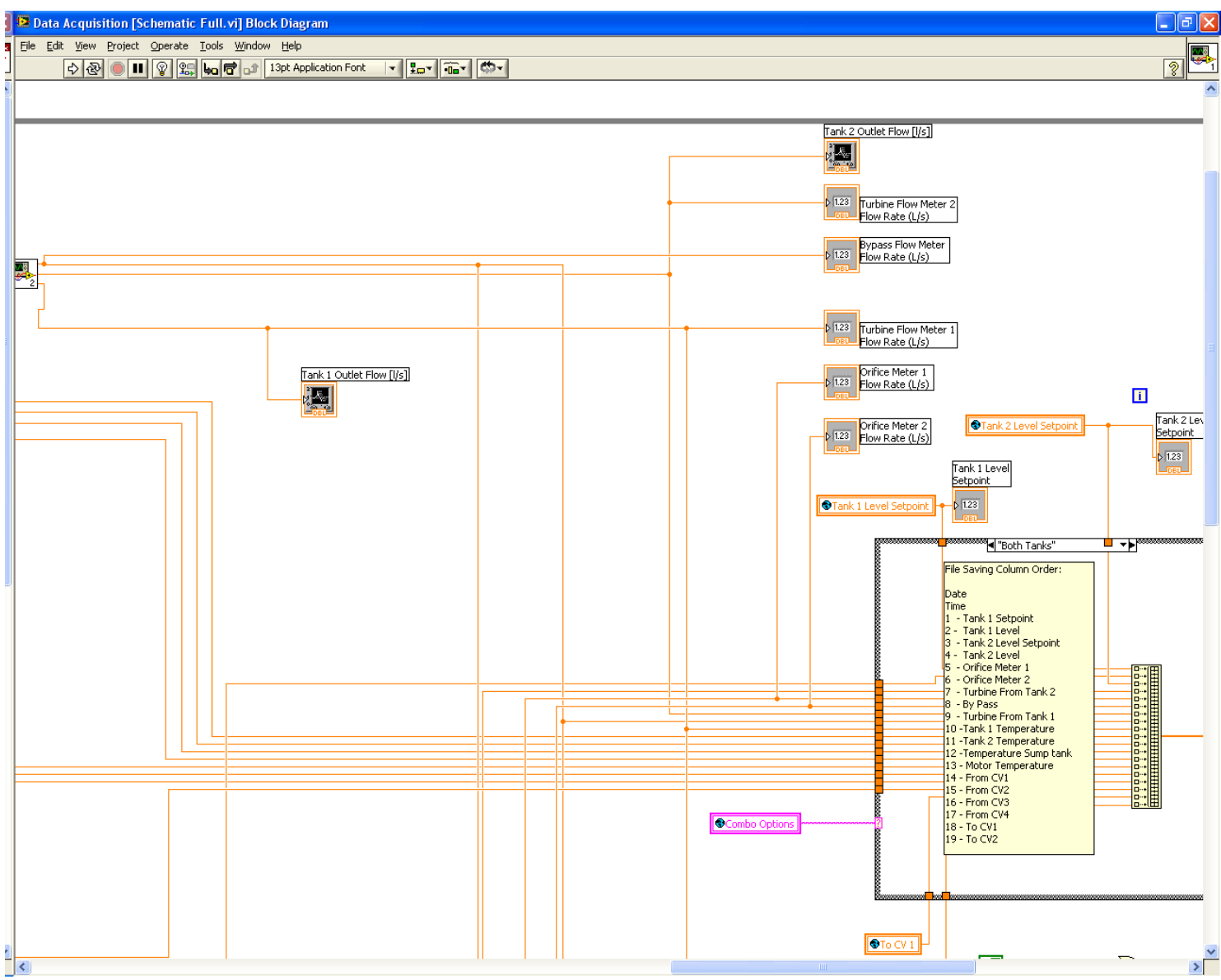




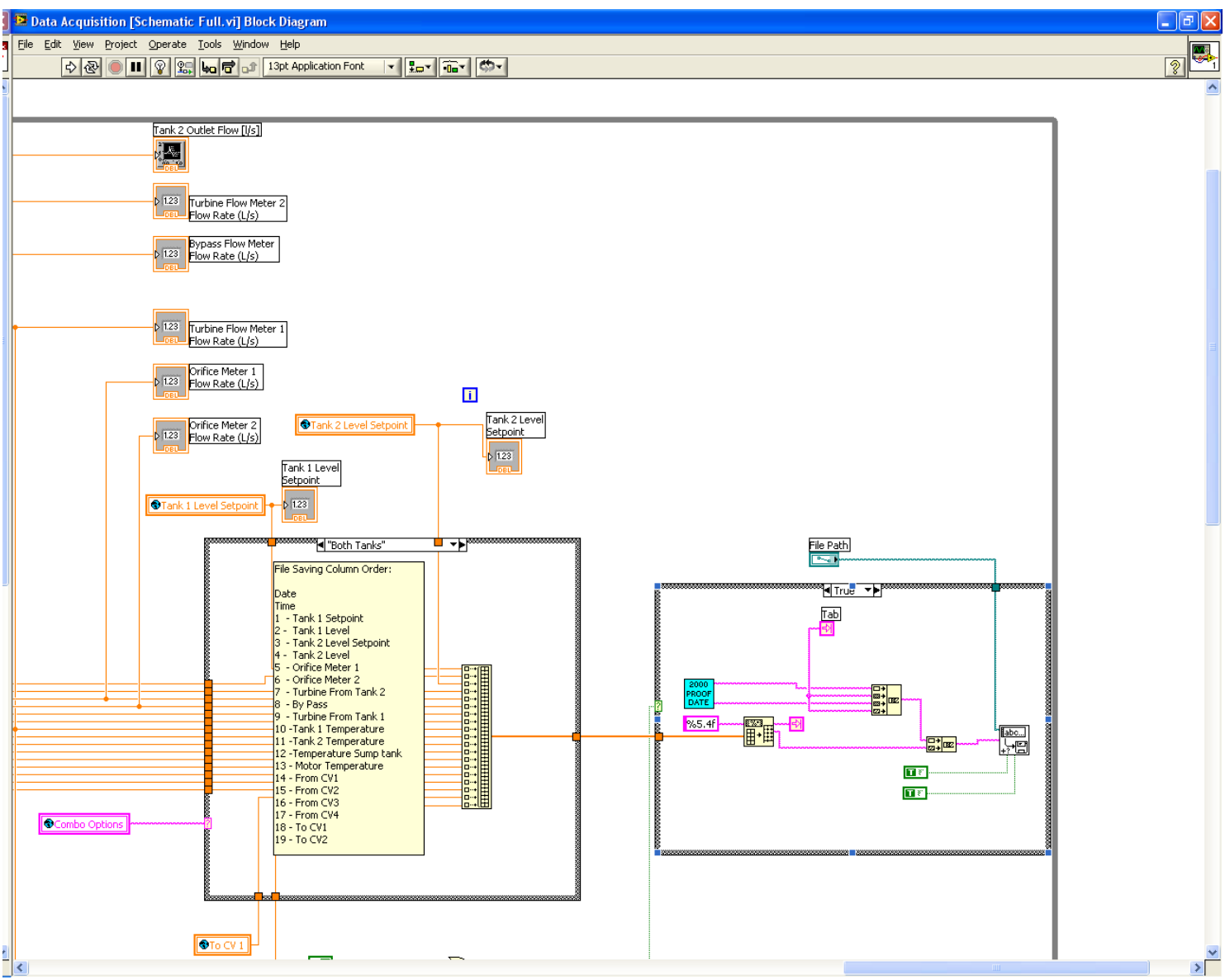


Appendix 10: Typical Output from the Code Used to Obtain Empirical Models

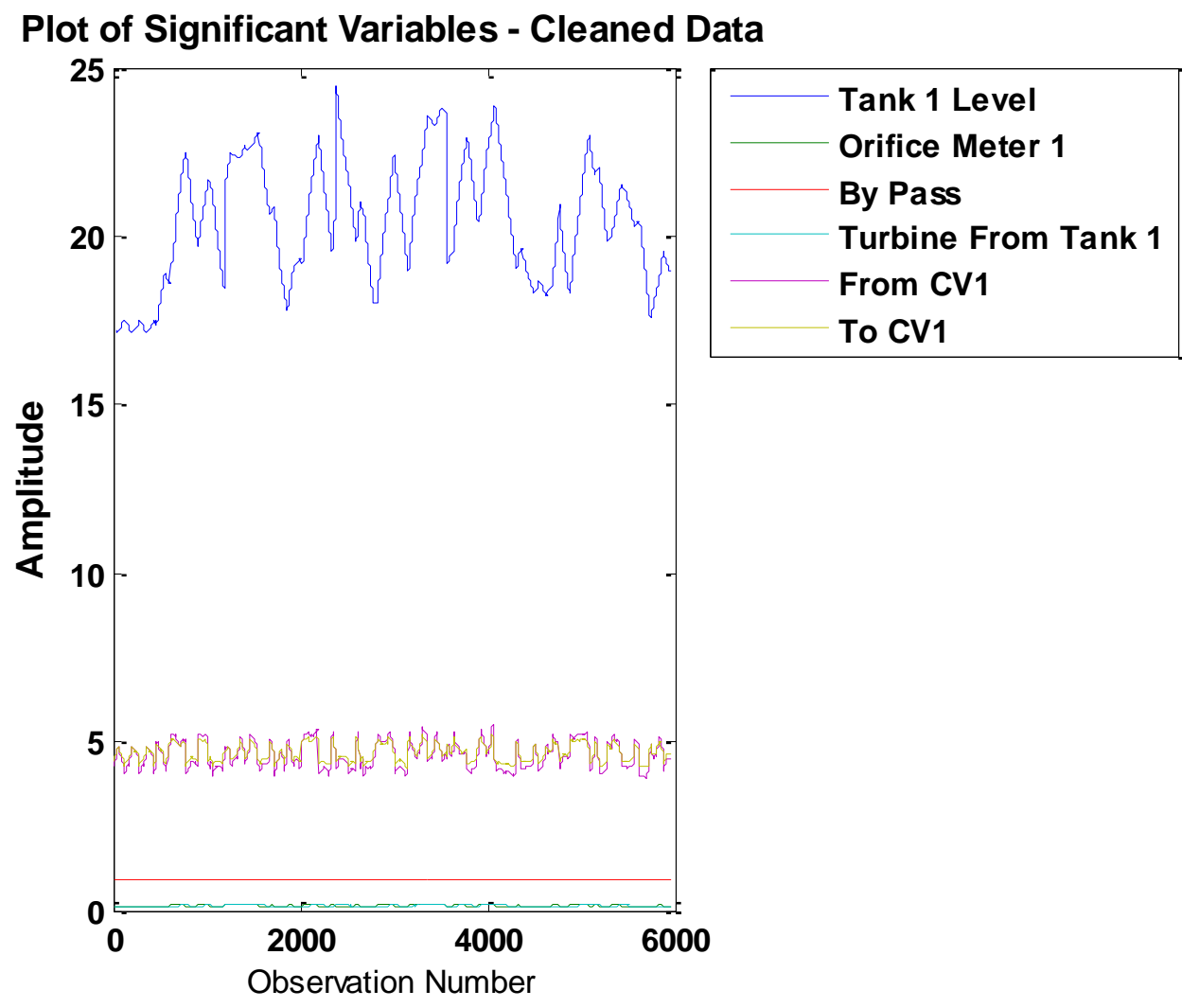



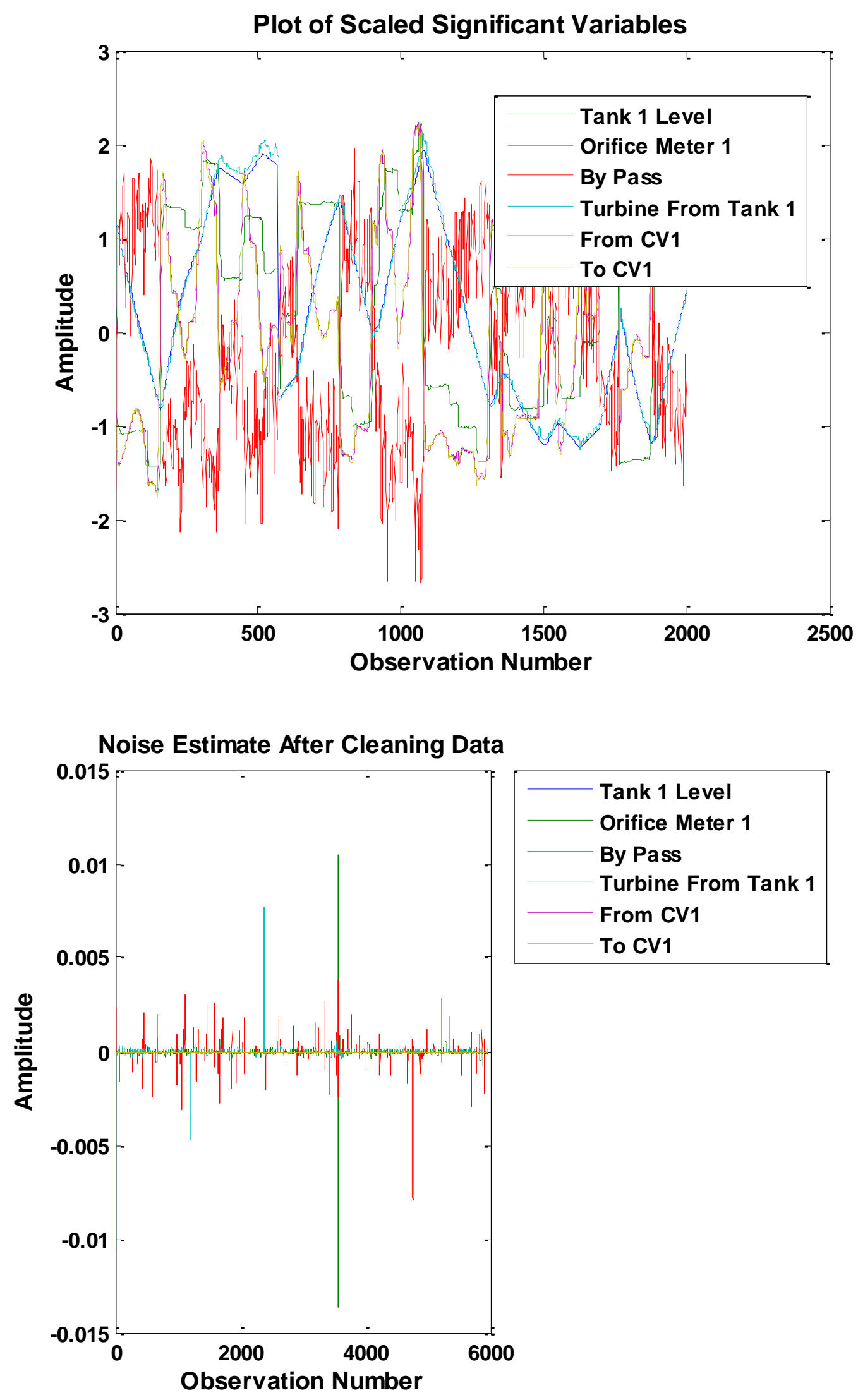
Signal-to-Noise-Ratio

Using Raw Data

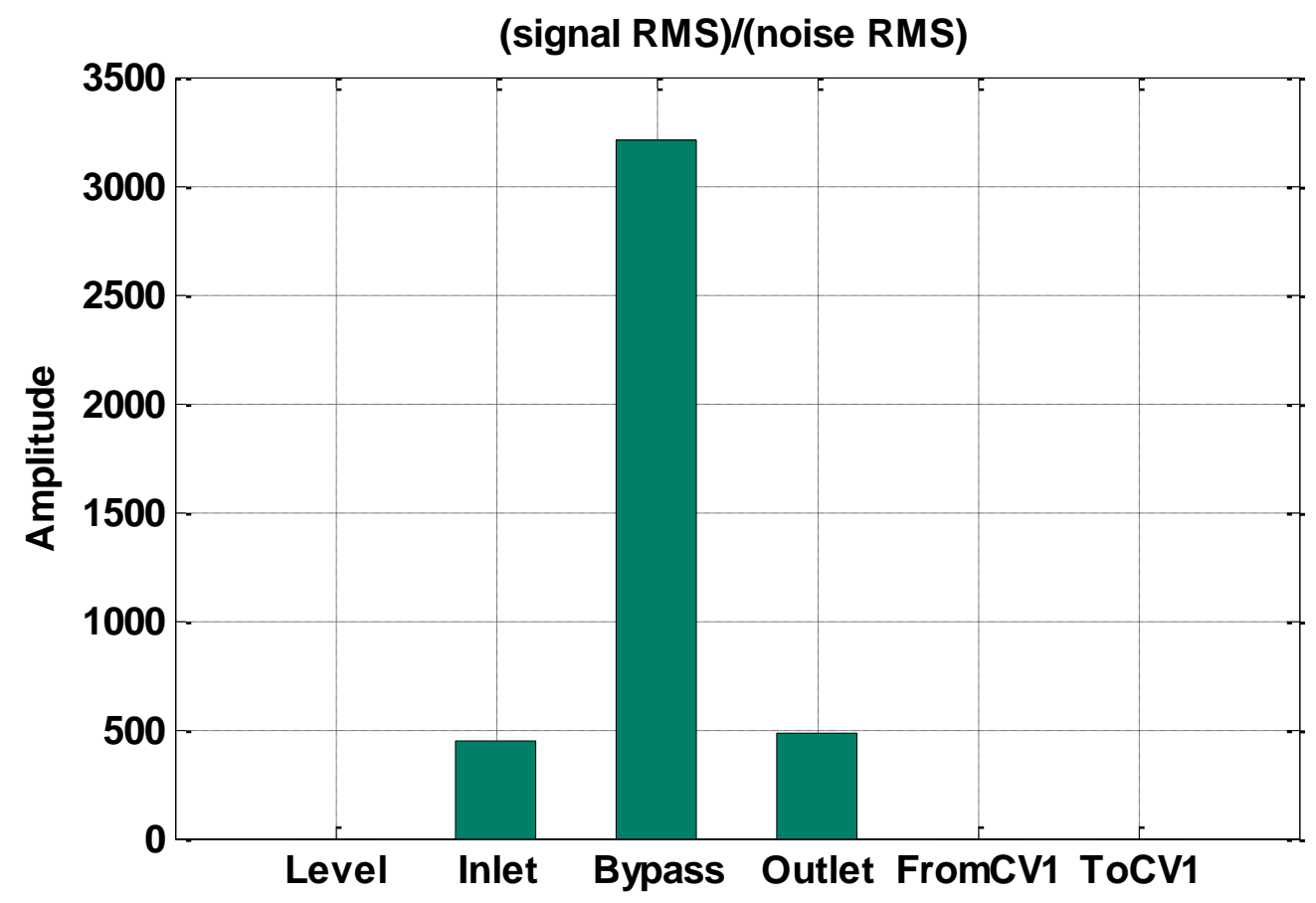




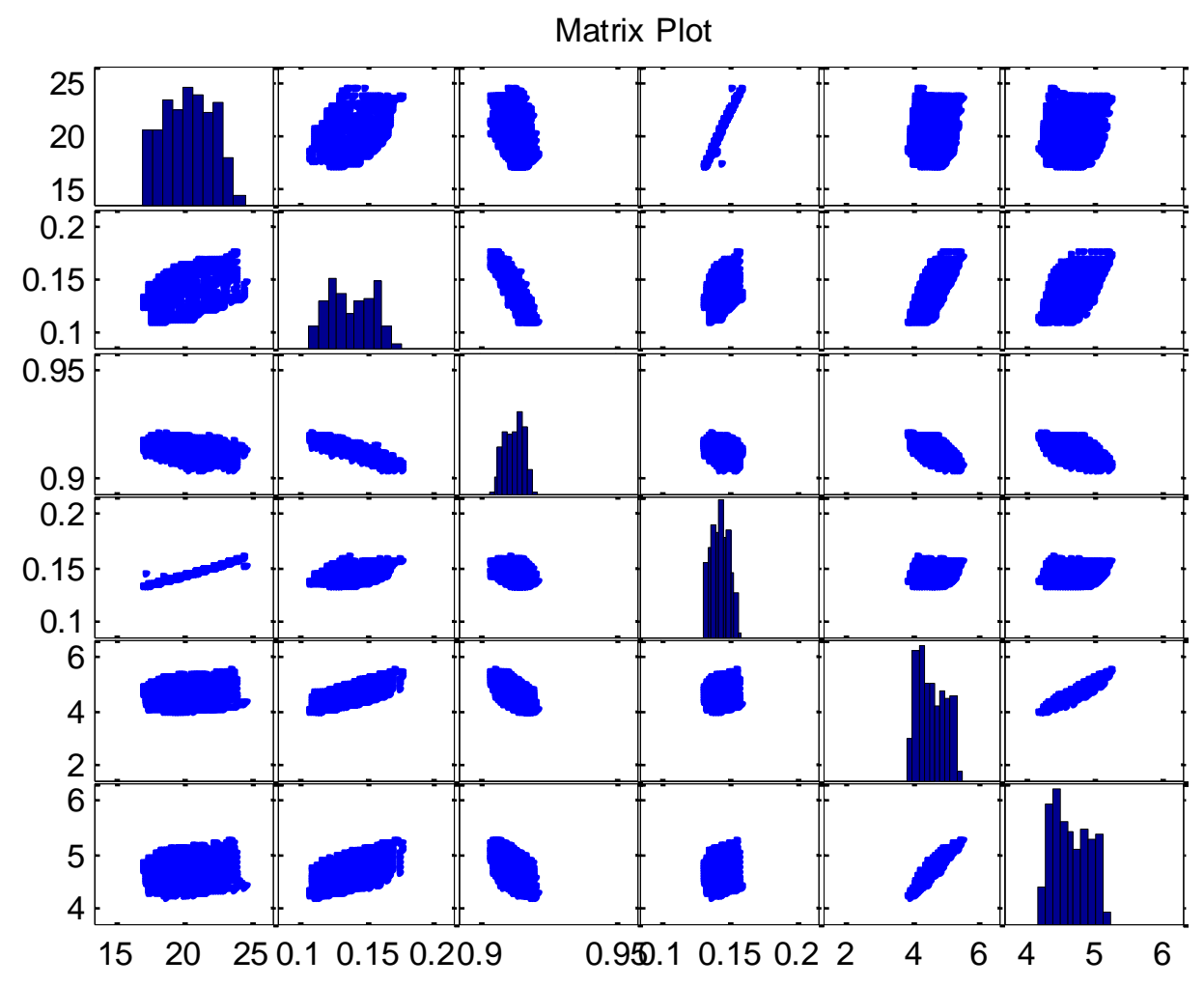


Eigenvalues

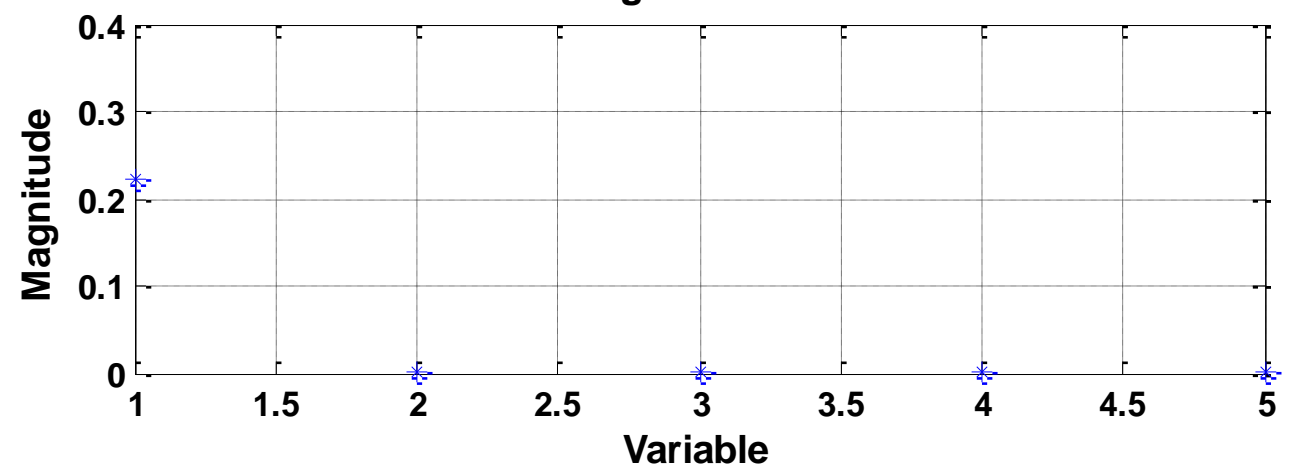

Cumulative \% Variance Explained

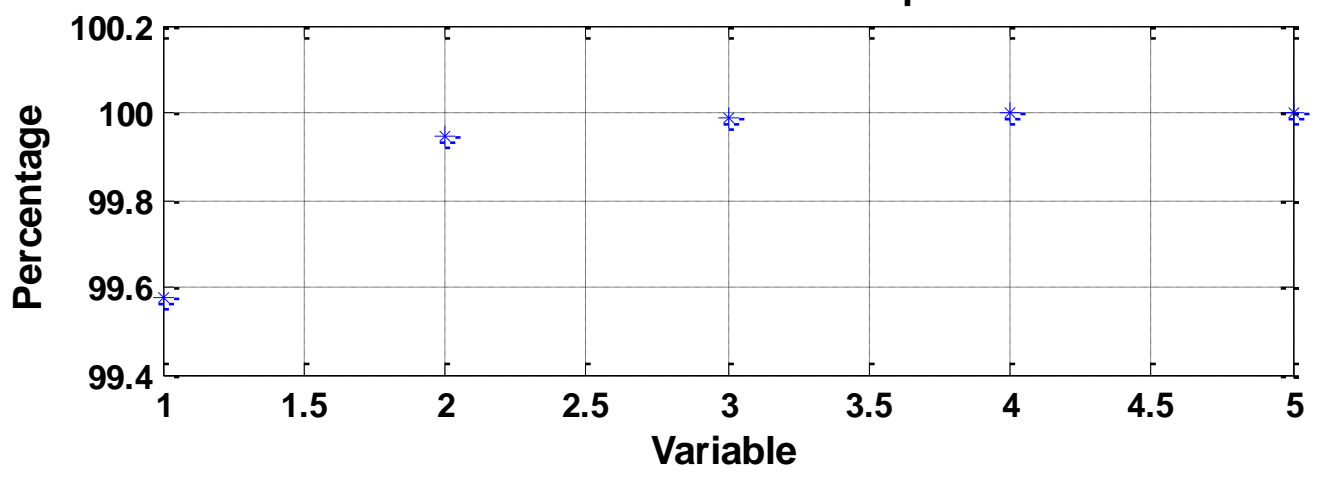

Uncertainties from Optimized and Unoptimized Models

Using Kernel Regression

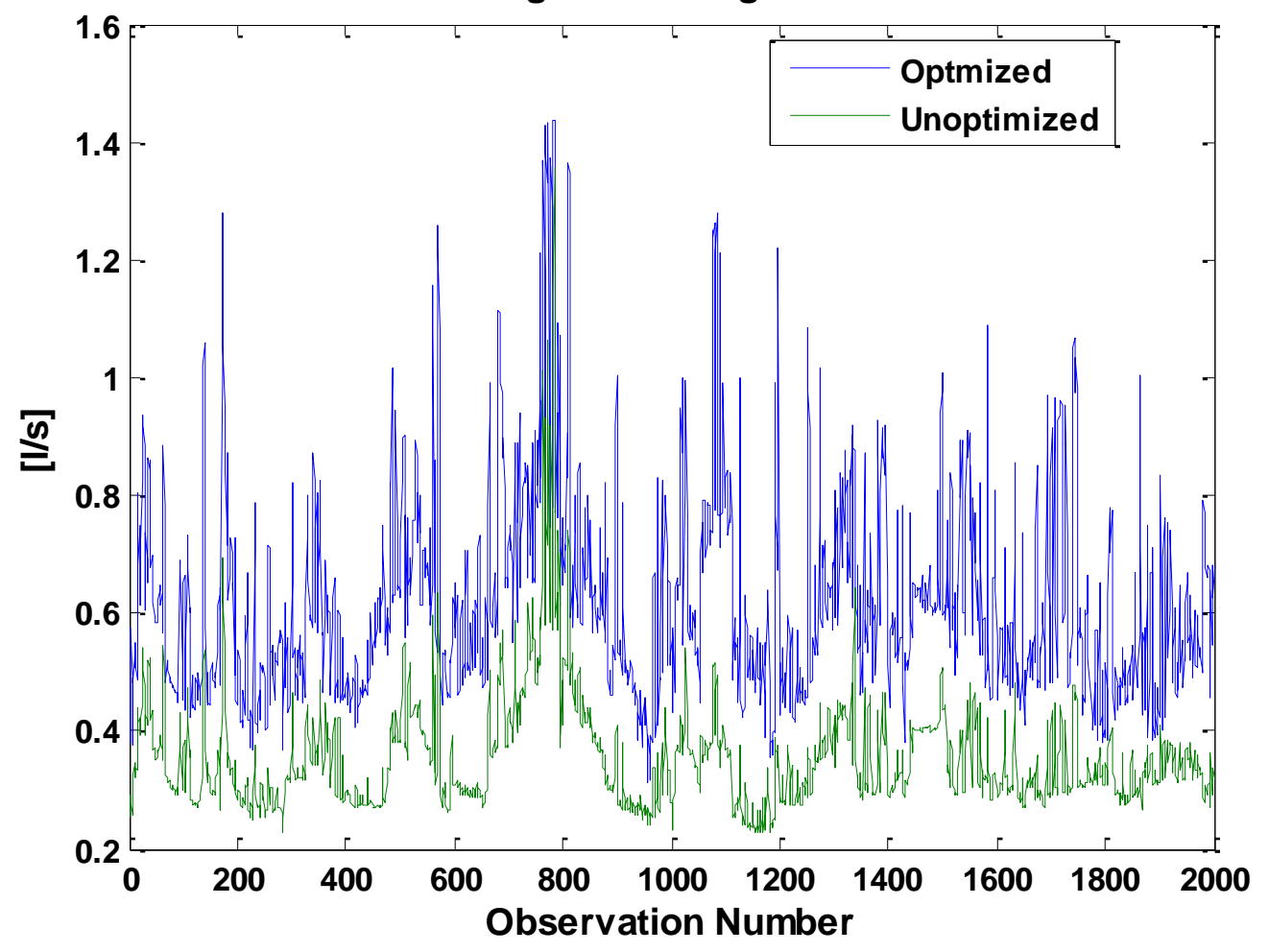




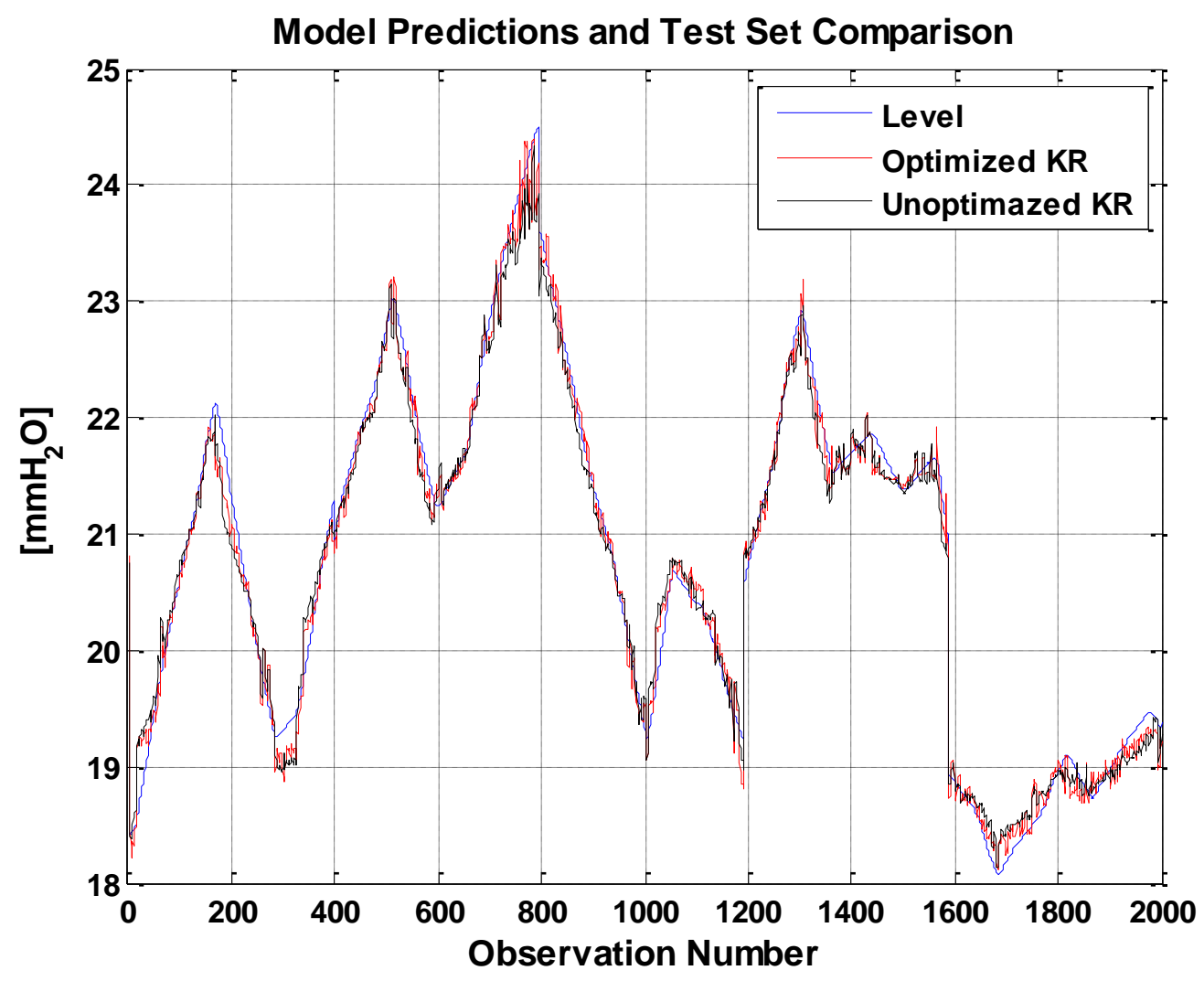



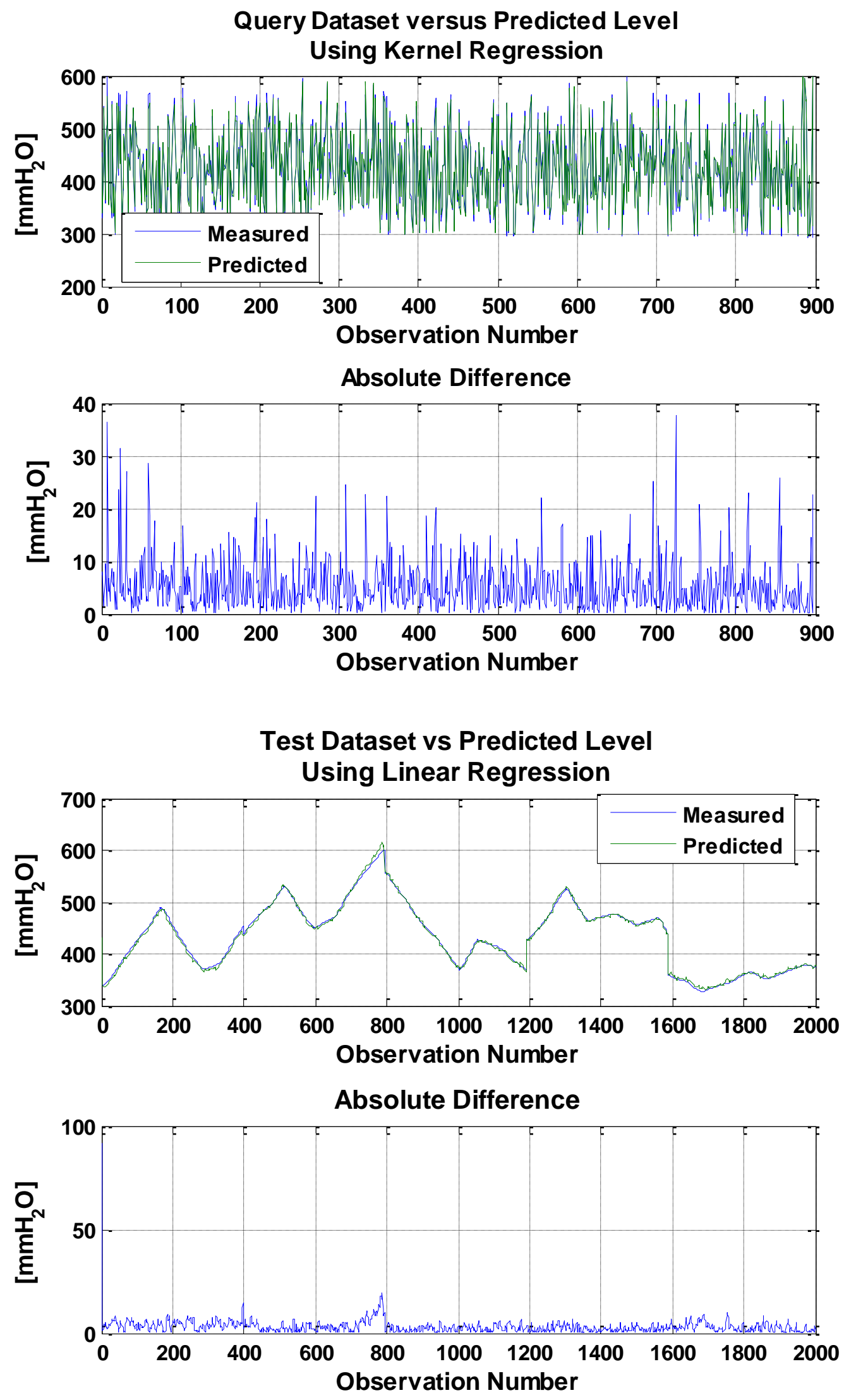
Inlet Flow Rate Uncertainties from Optimized and Unoptimized Models Using Kernel Regression

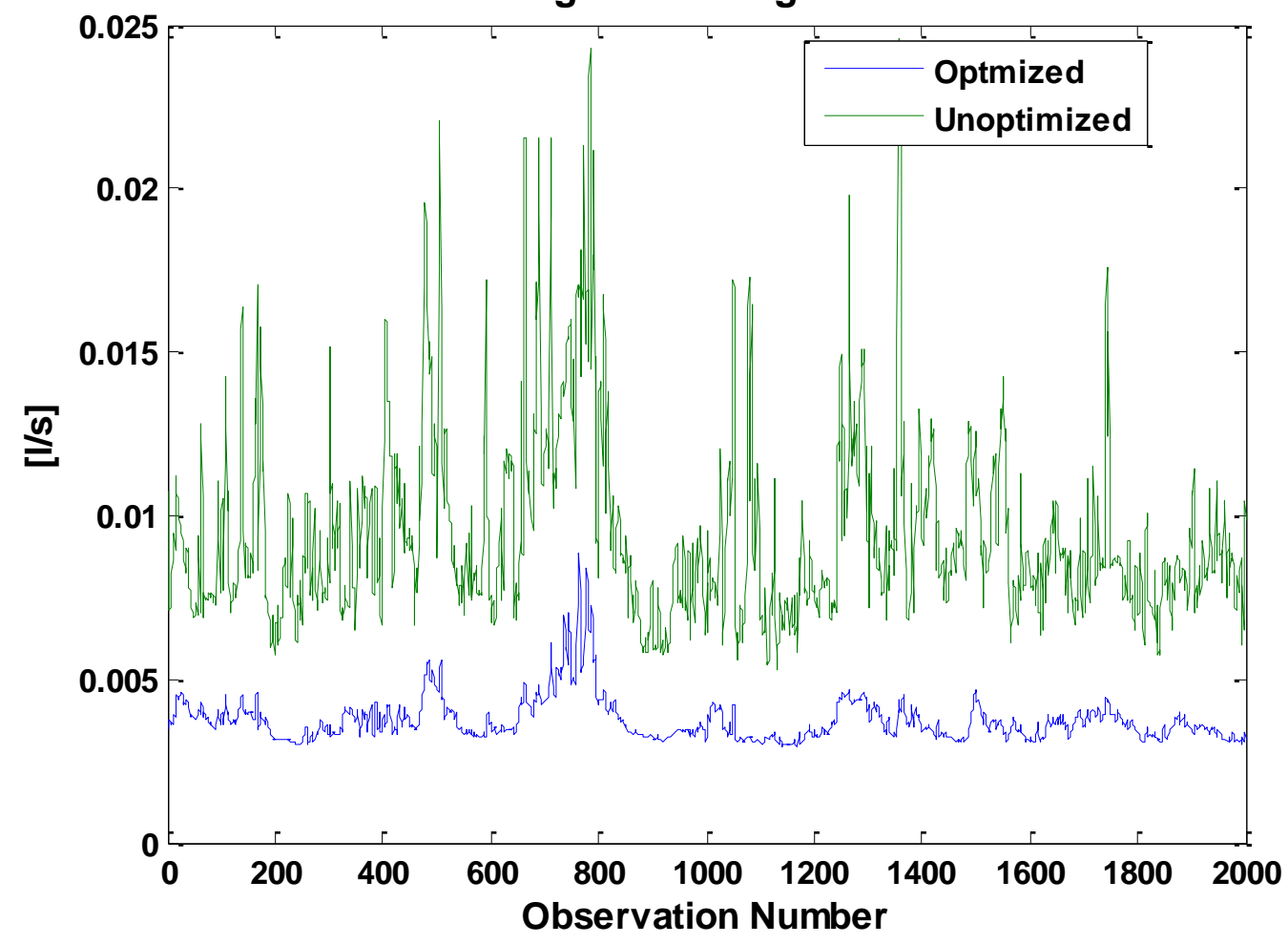


Inlet Flow Rate Model Predictions and Test Set Comparison

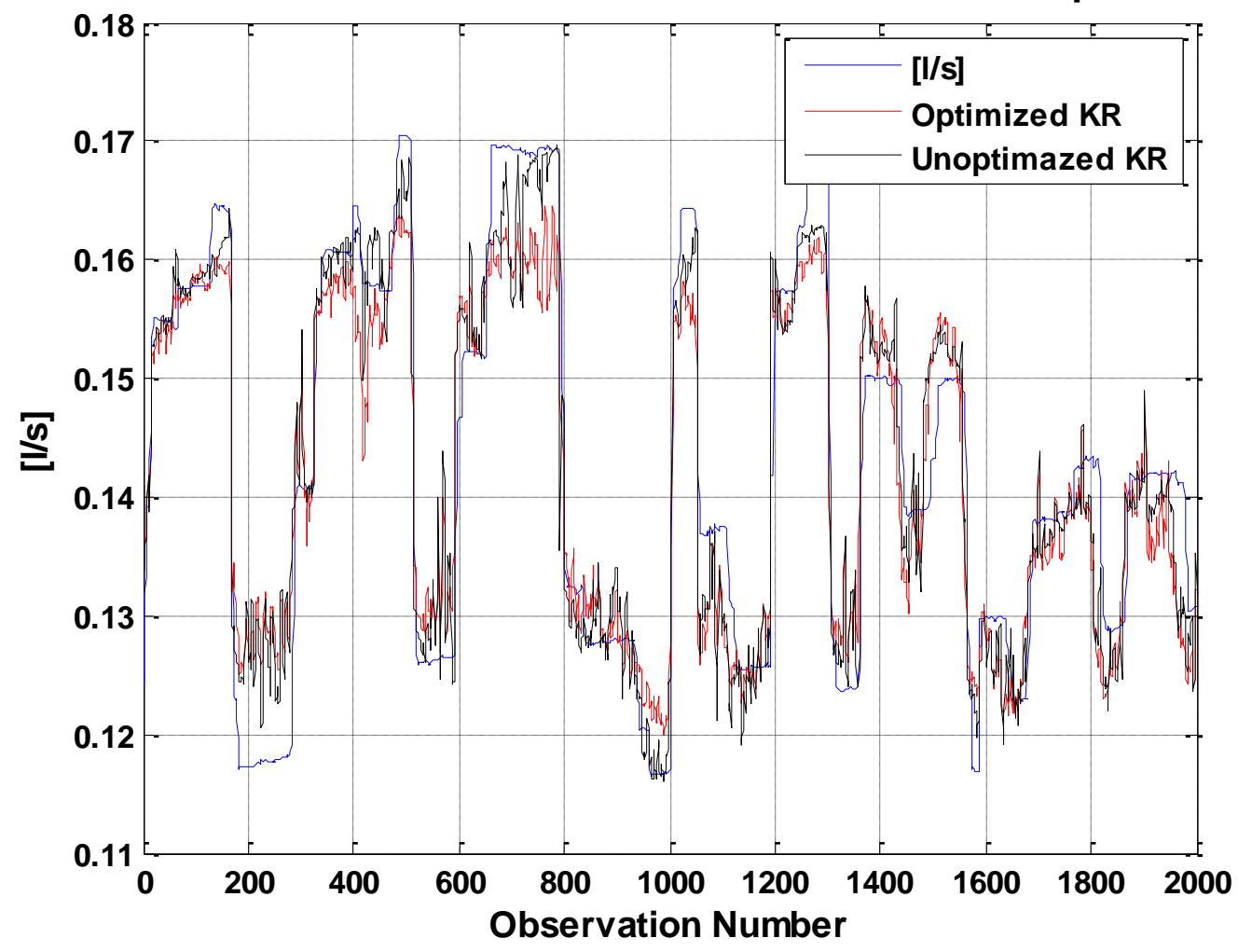



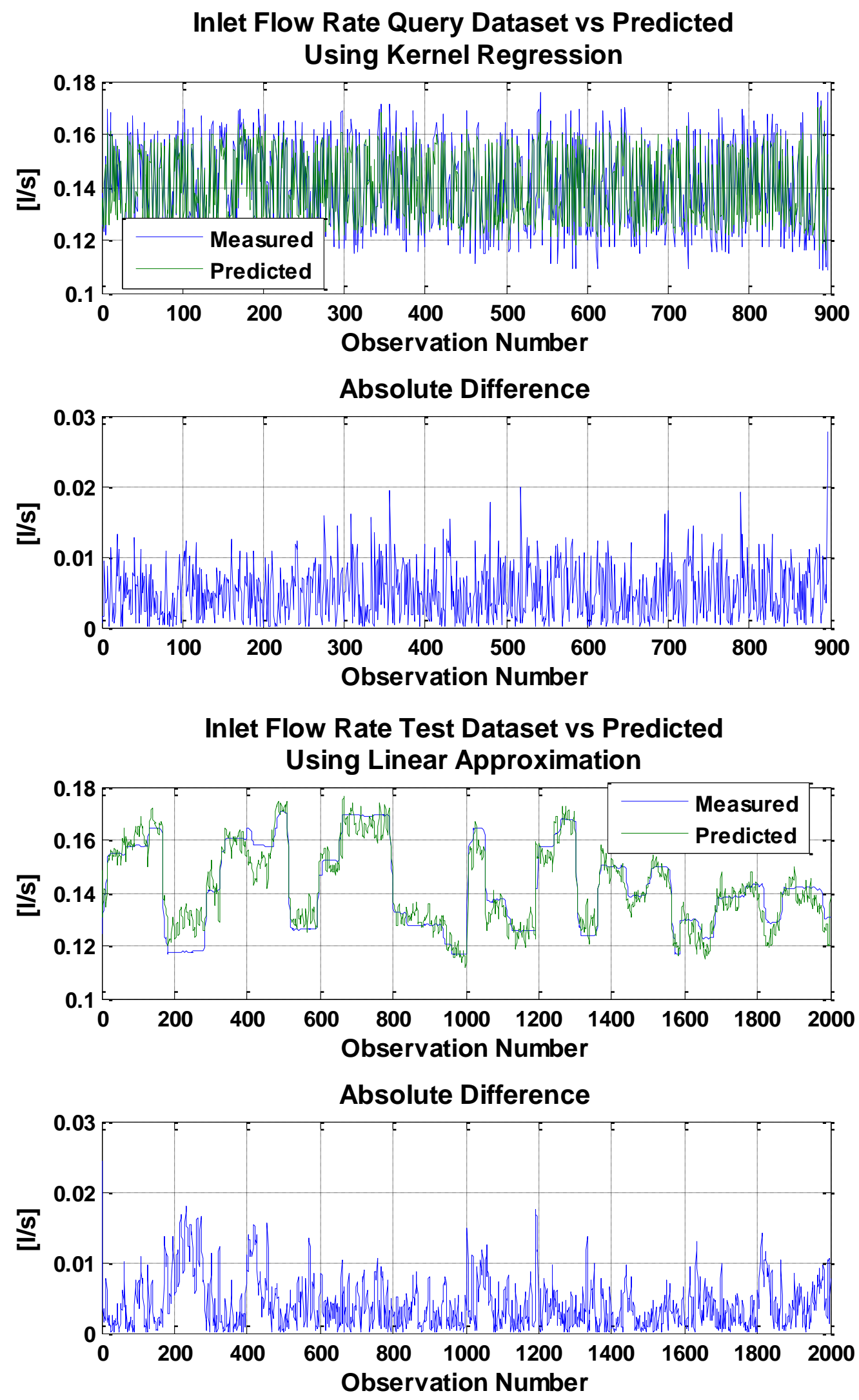
Bypass Flow Rate Uncertainties from Optimized and Unoptimized Models

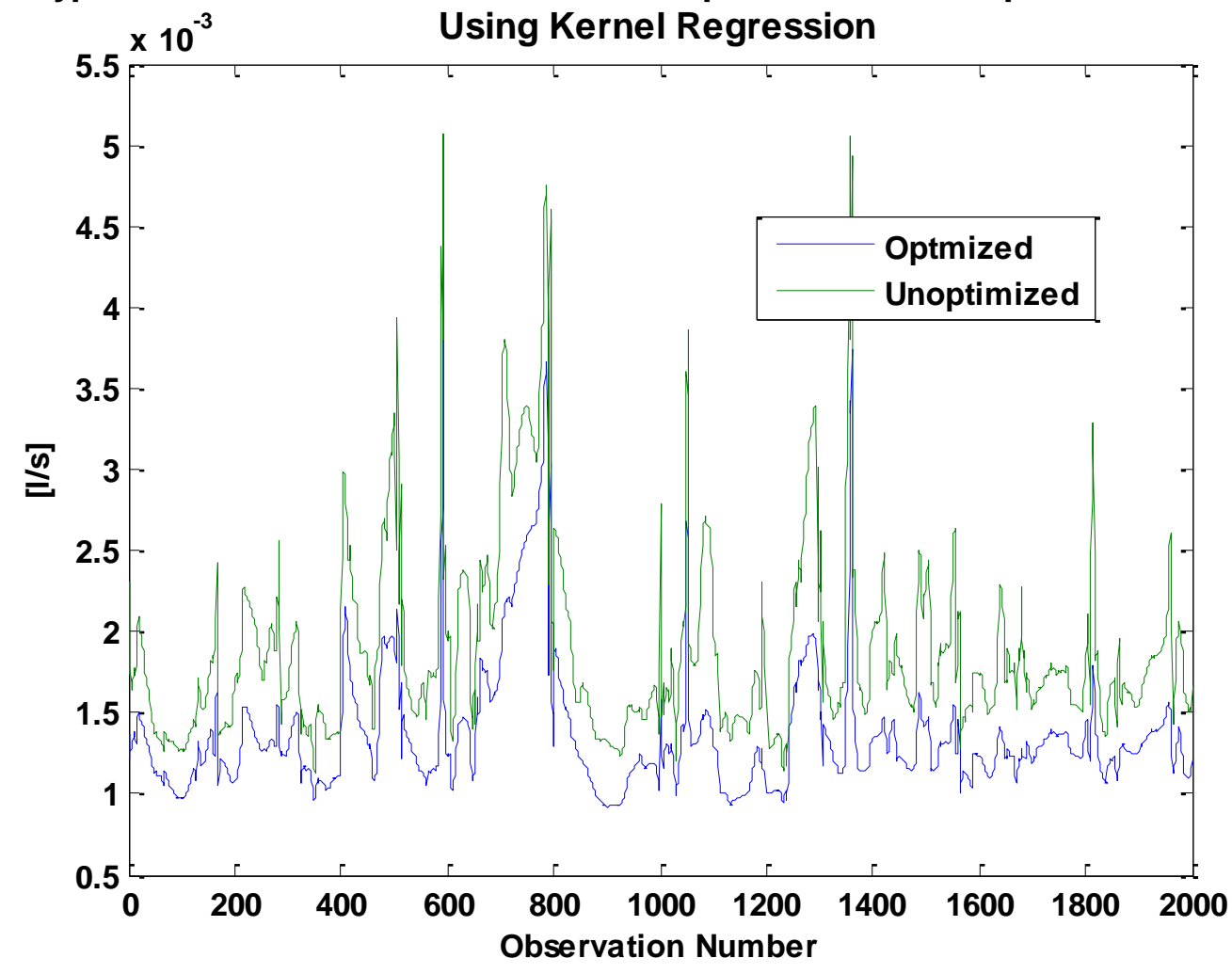

Bypass Flow Rate Model Predictions and Test Set Comparison

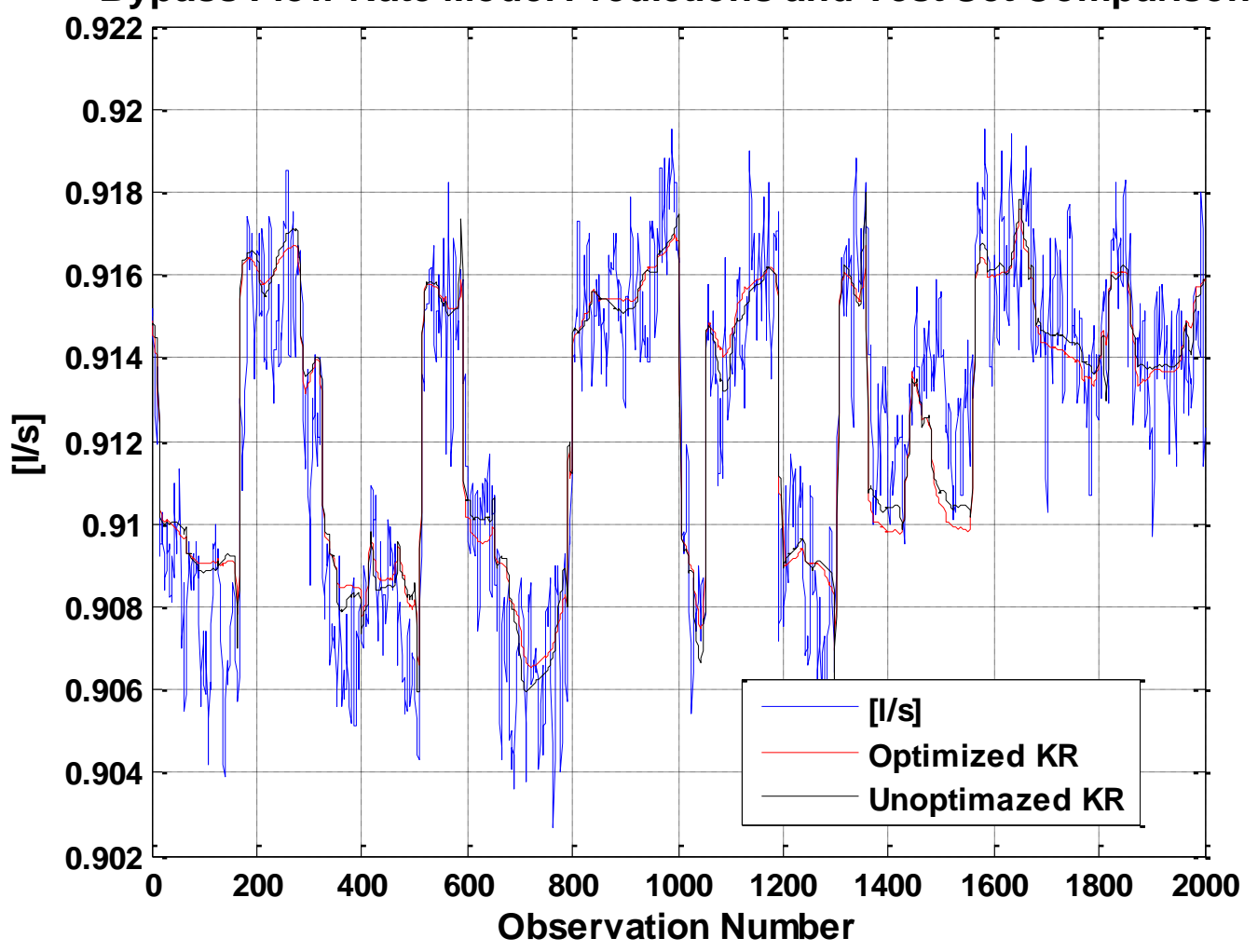



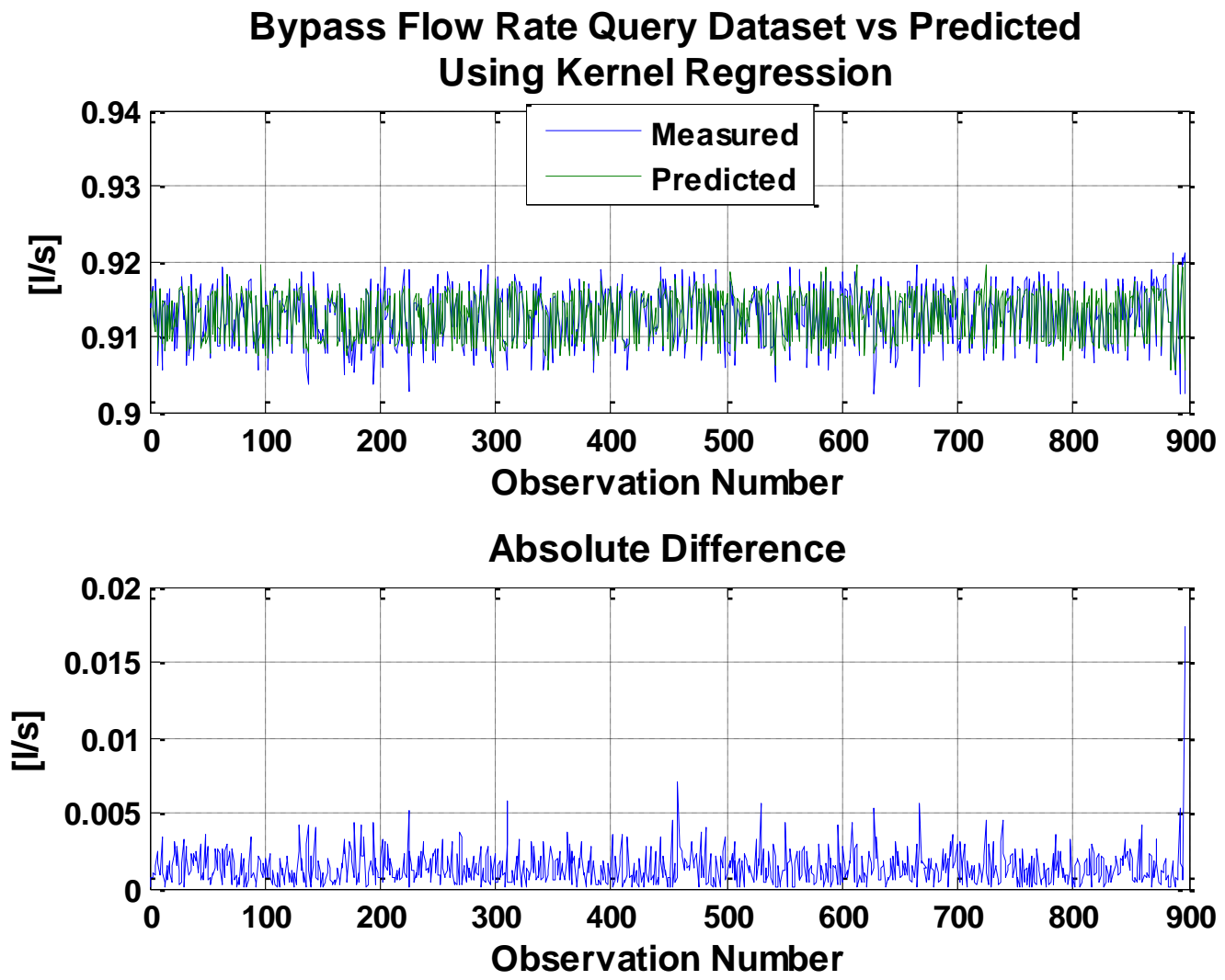

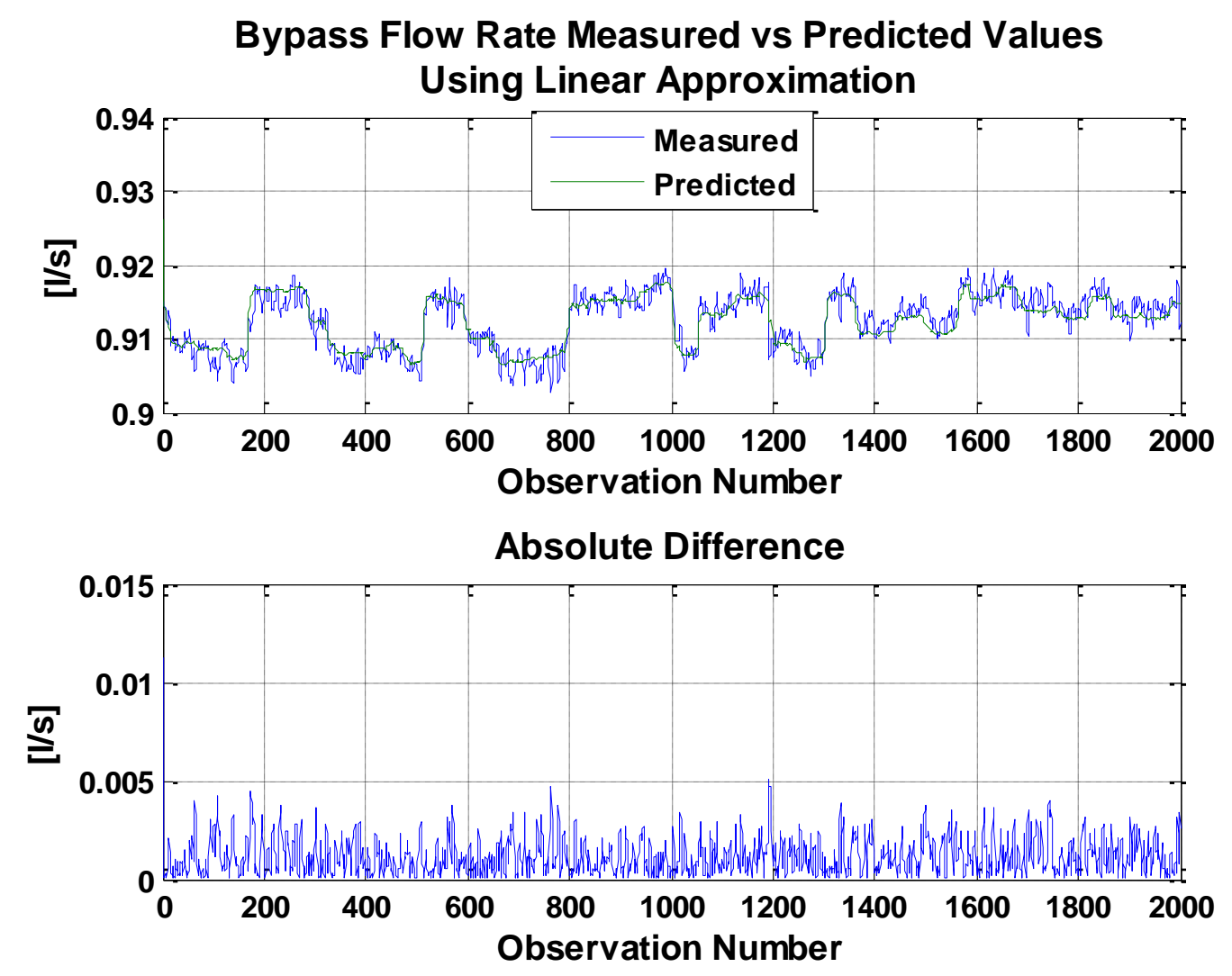
Outlet Flow Rate Uncertainties from Optimized \& Unoptimized Models

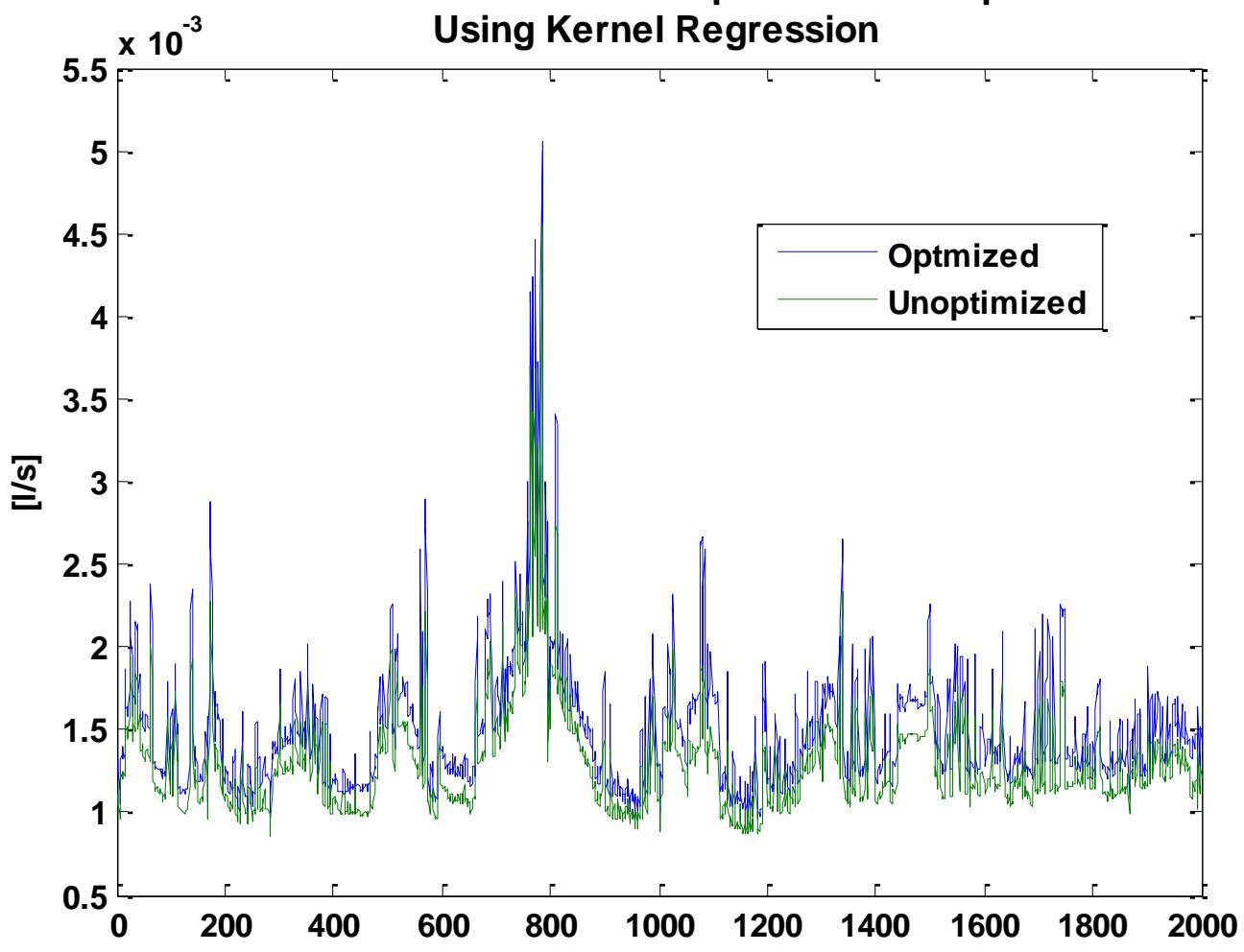

Outlet Flow Rate Model Predictions and Test Set Comparison

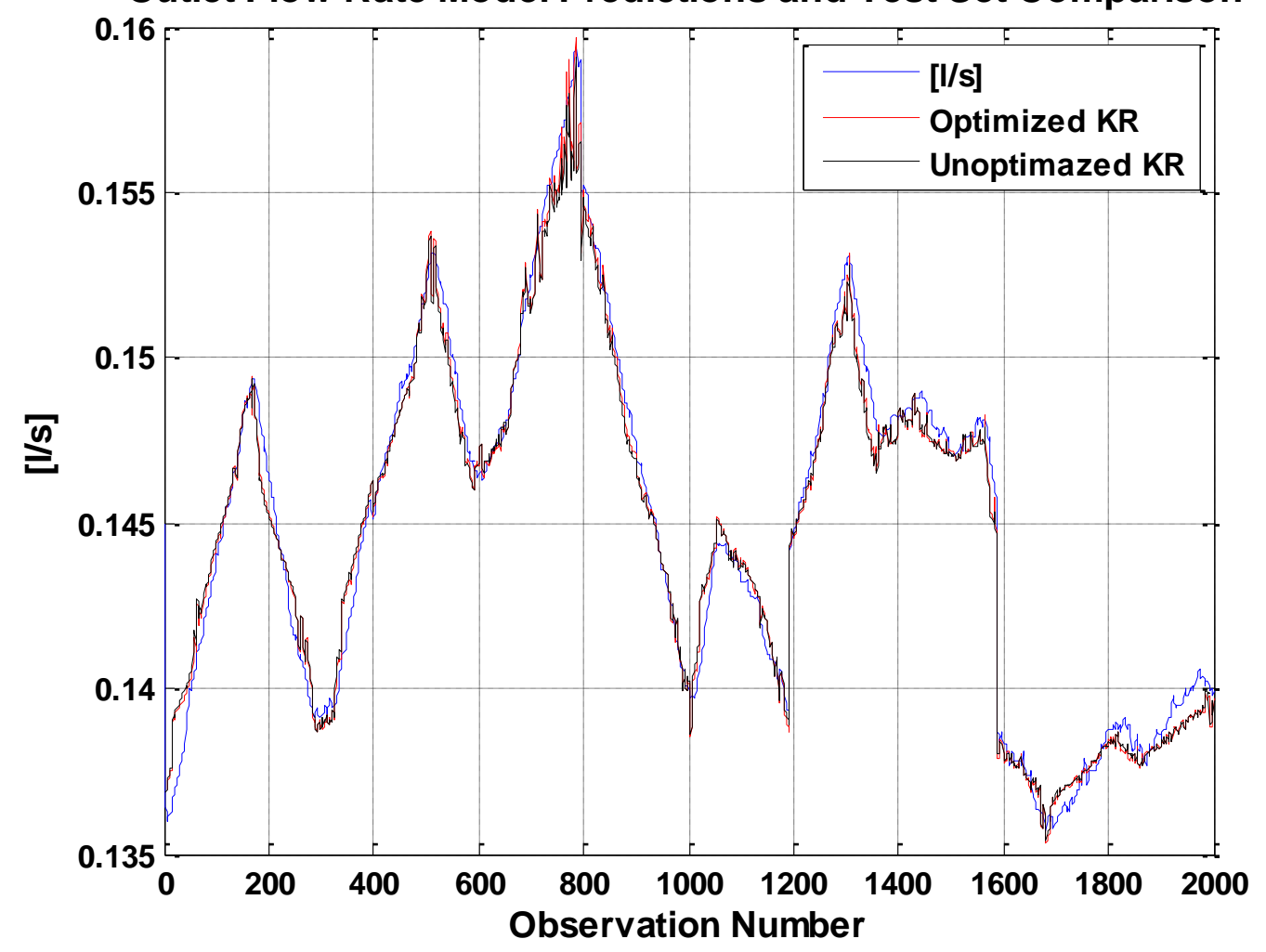



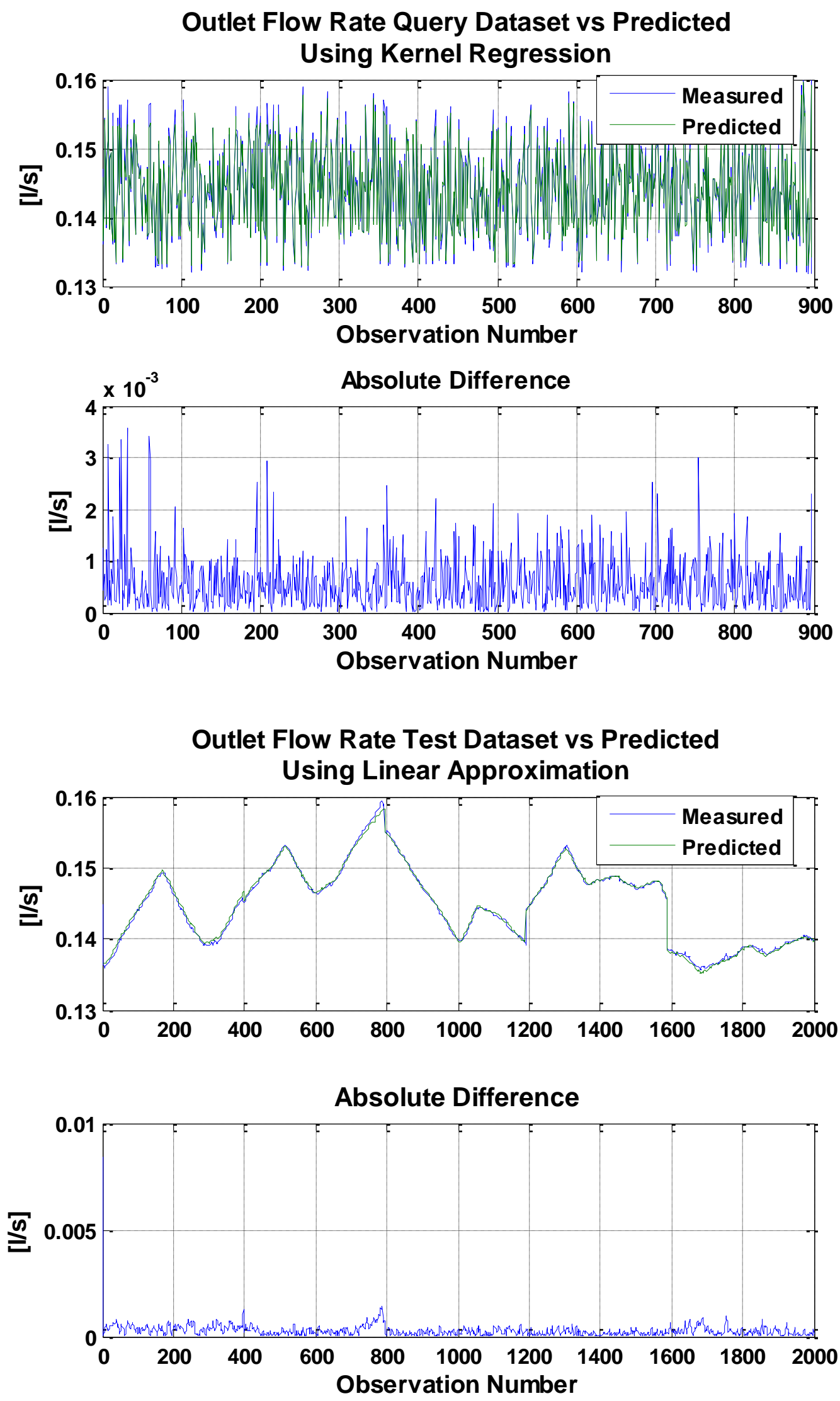
CV1 Feedback Uncertainties with Optimized and Unoptimized Models Using Kernel Regression

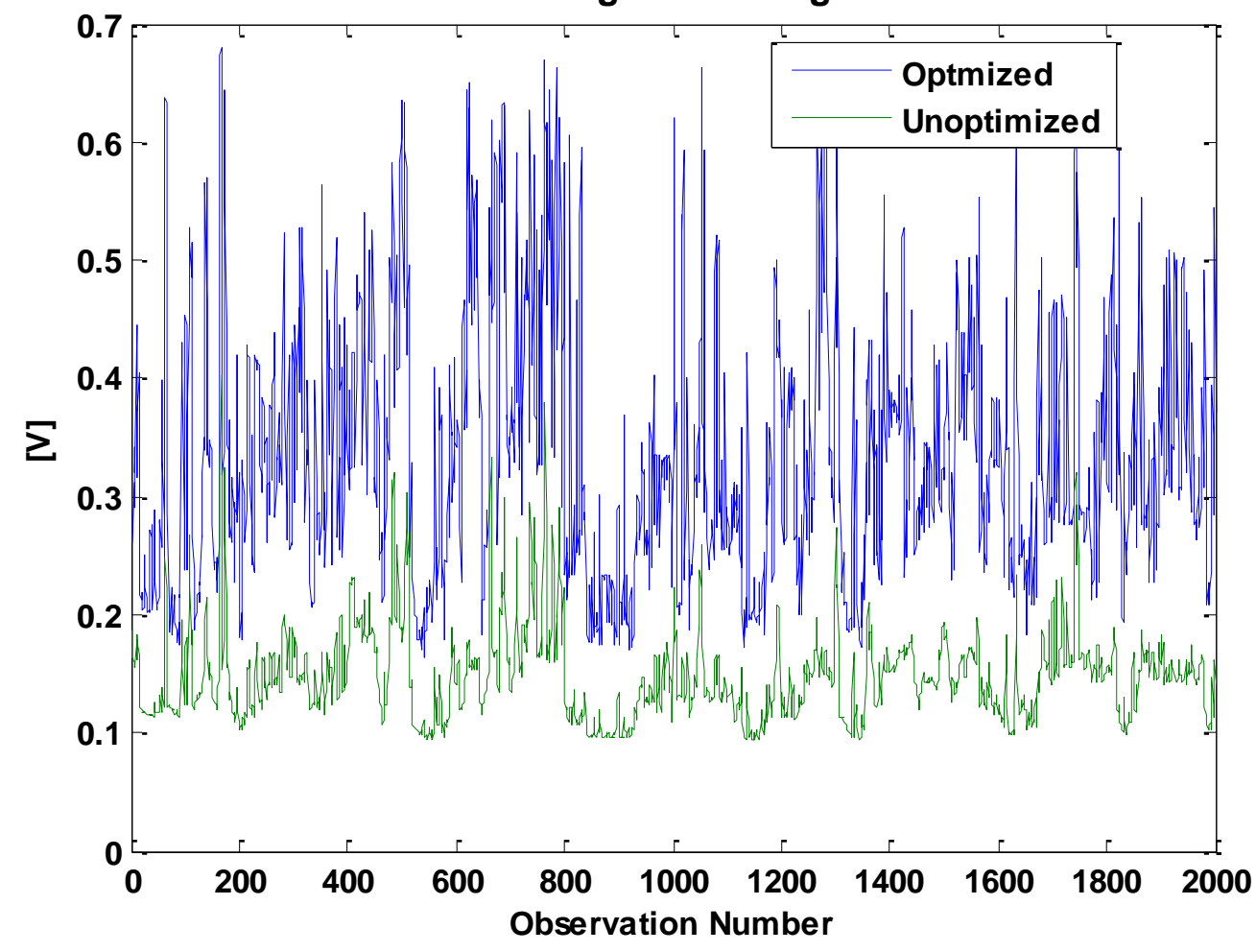


CV1 Feedback Signal Model Predictions and Test Set Comparison Using Kernel Regression

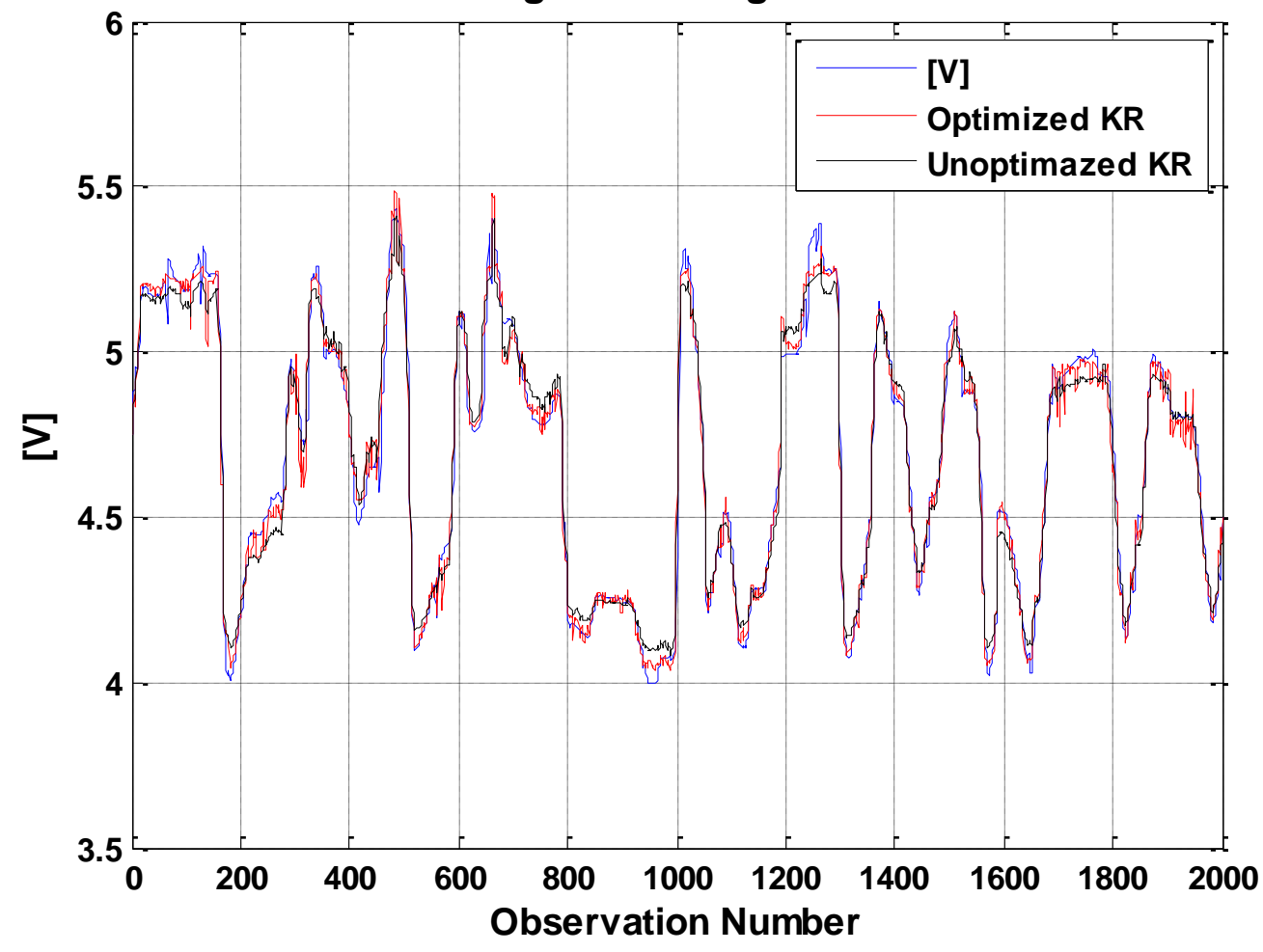

\section{CV1 Feedback Query Dataset vs Predicted Using Kernel Regression}
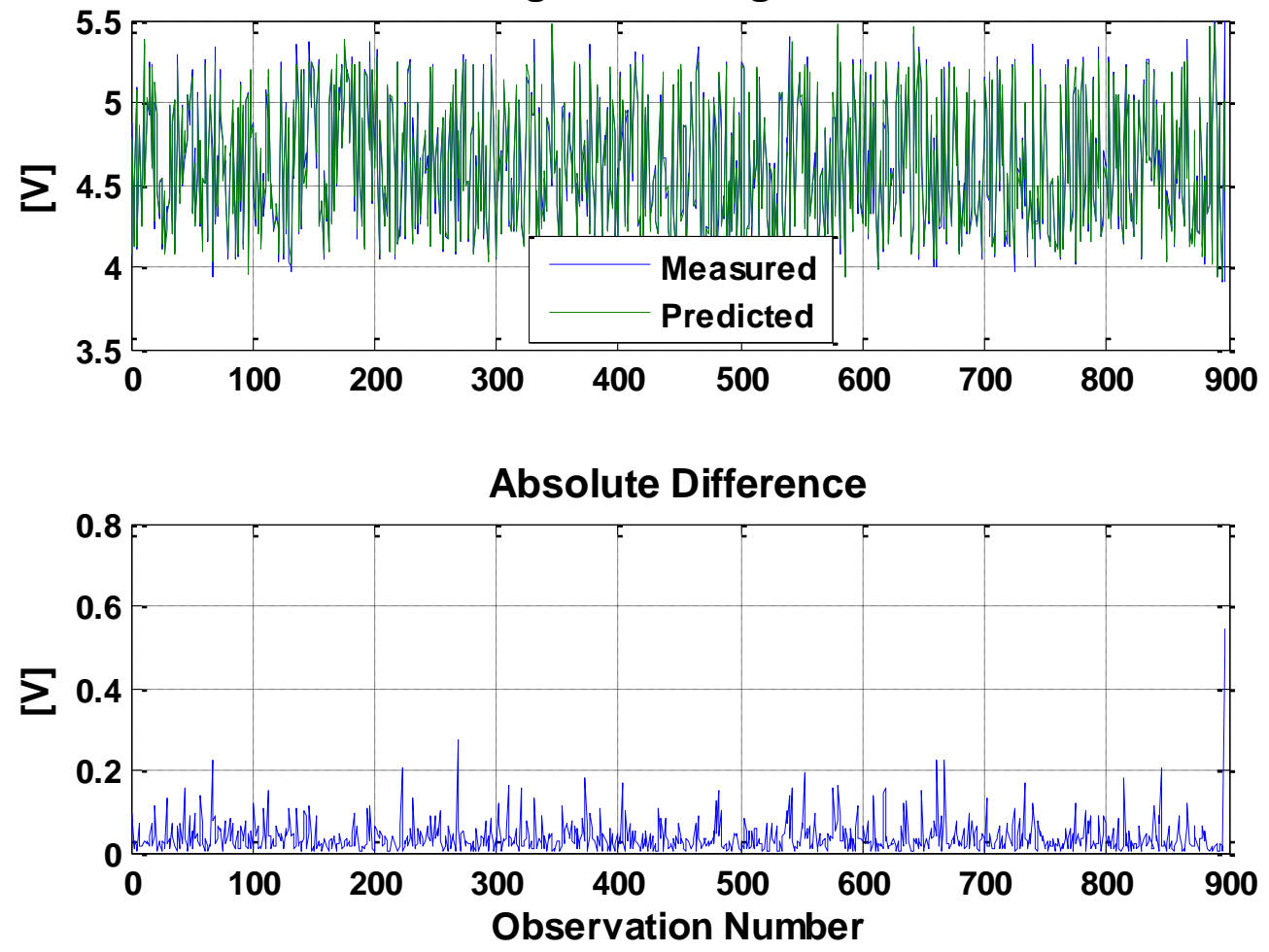

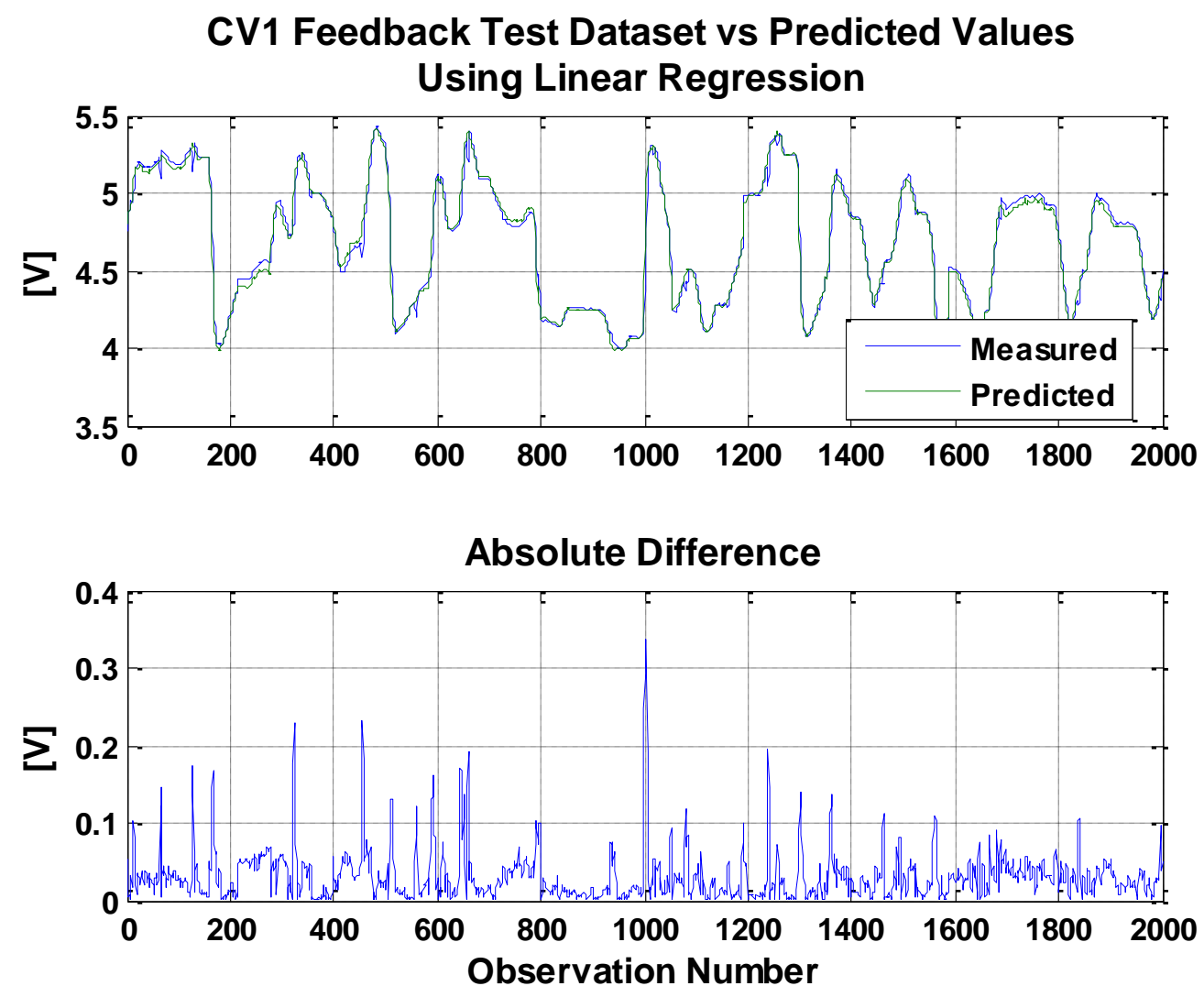

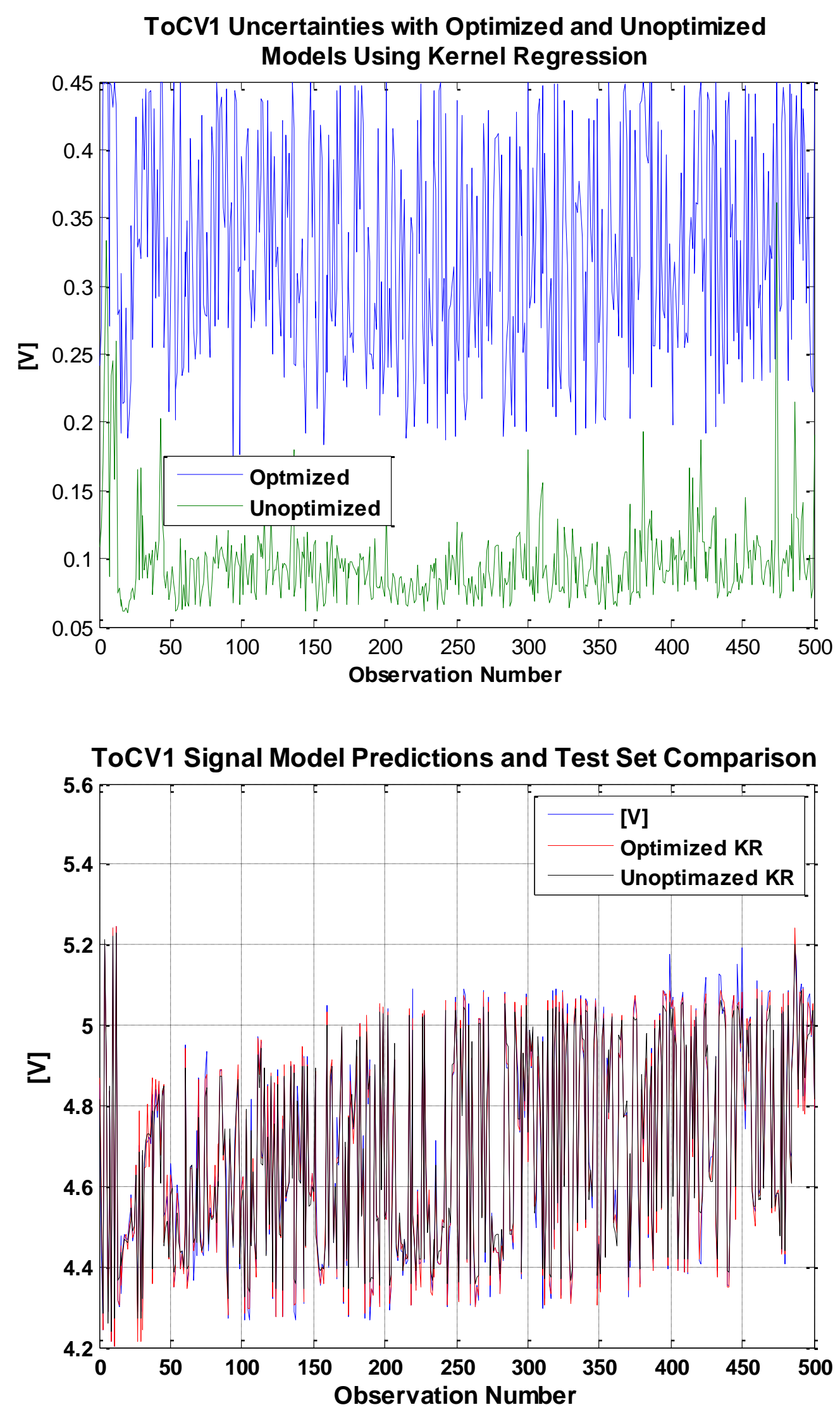
To CV1 Query Dataset vs Predicted Using Kernel Regression
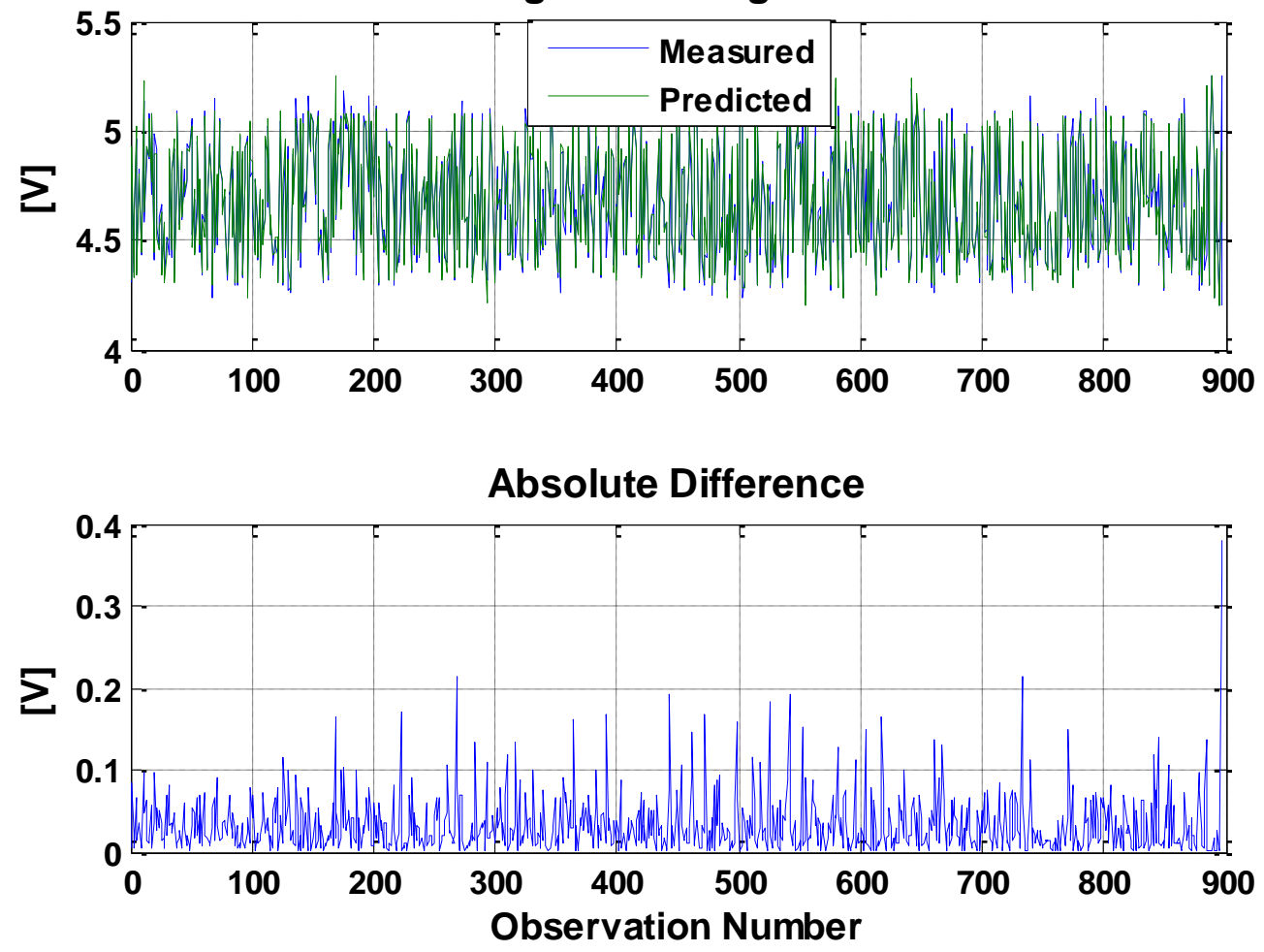

To CV1 Test Dataset vs Predicted

Using Linear Regression

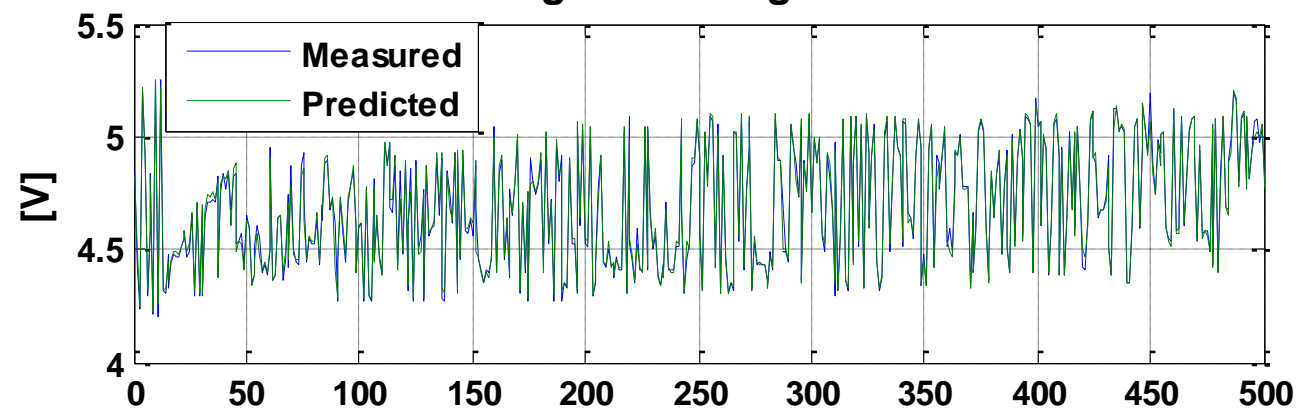

Absolute Difference

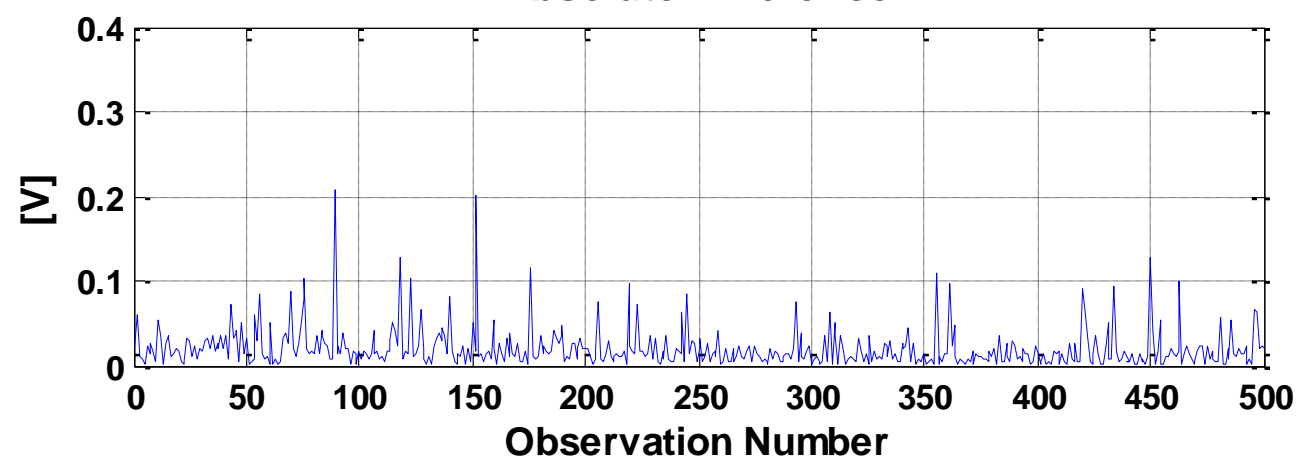



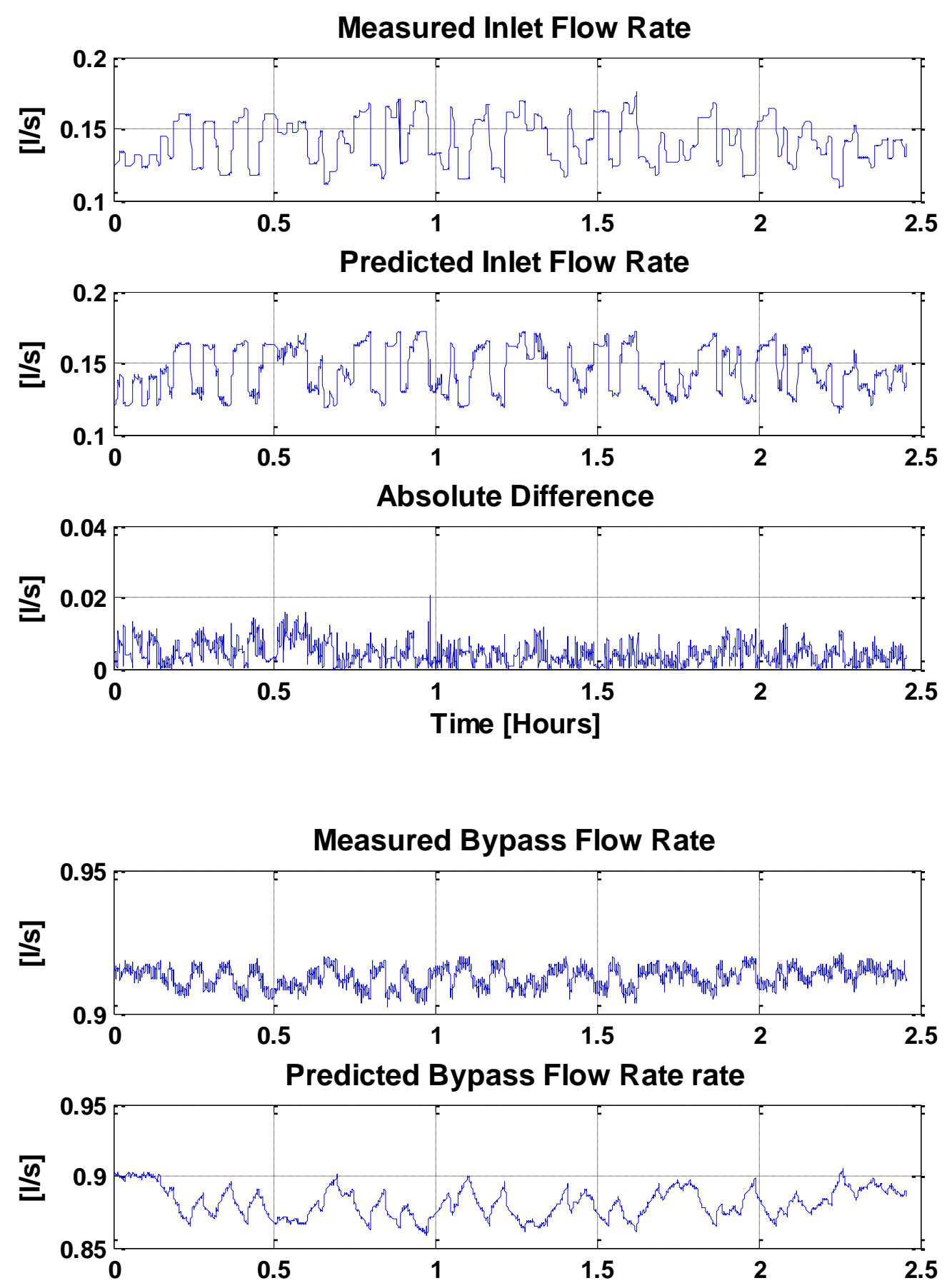

Absolute Difference

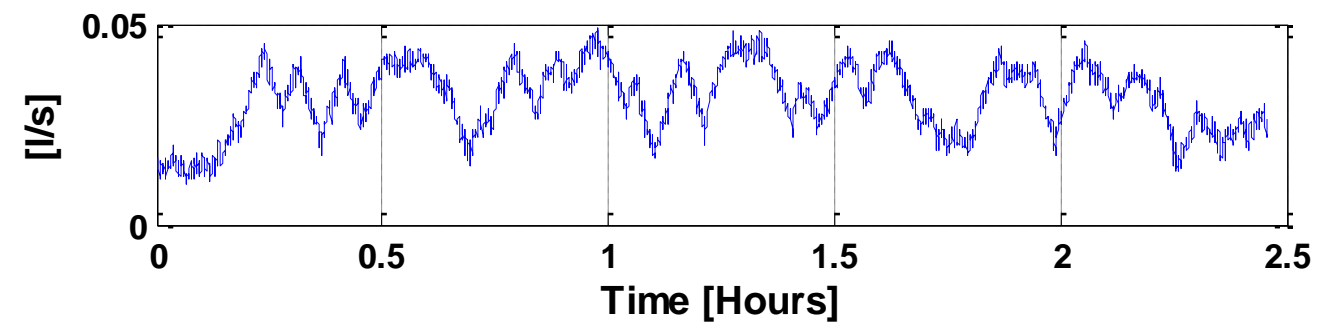



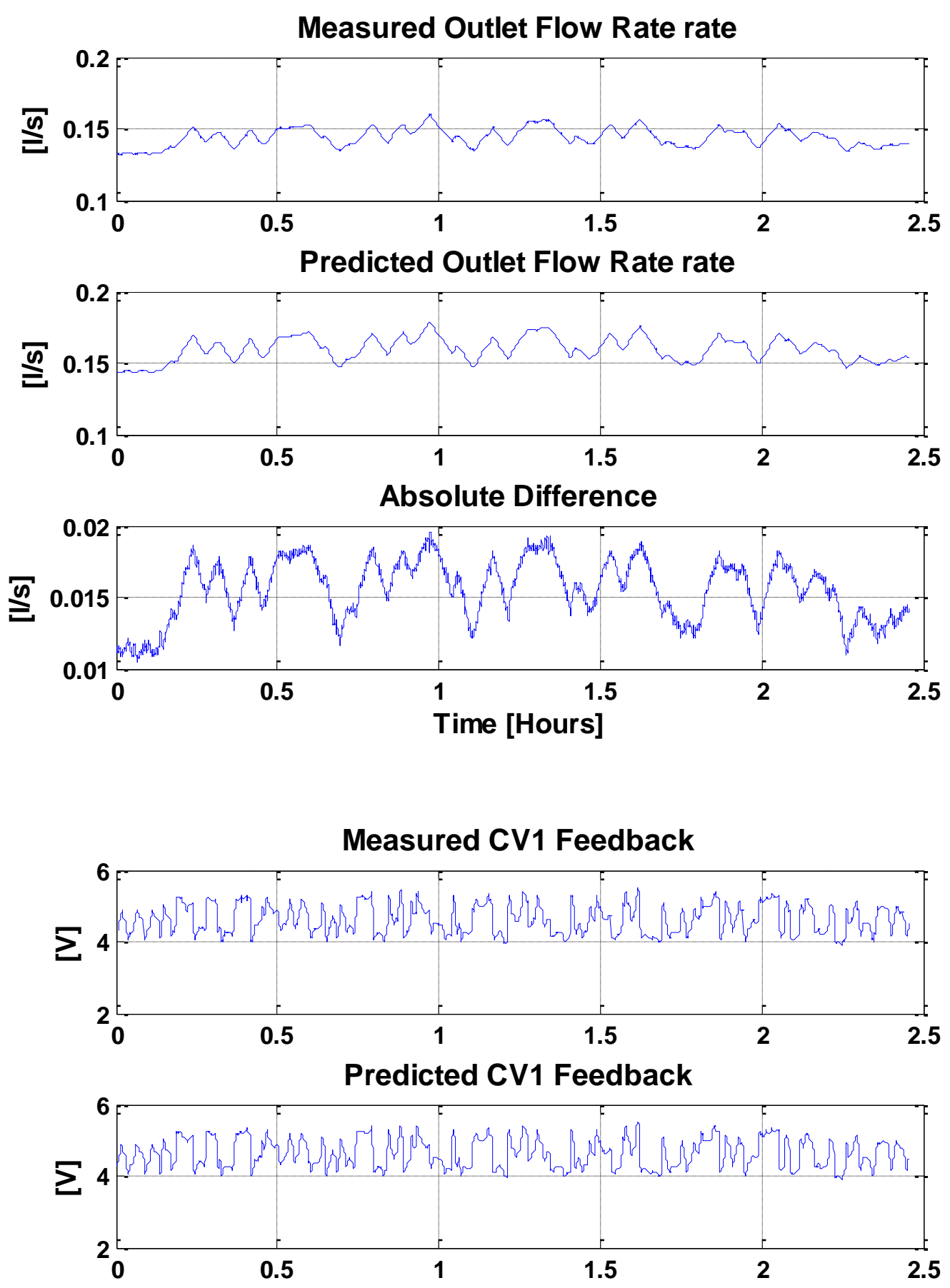

Absolute Difference

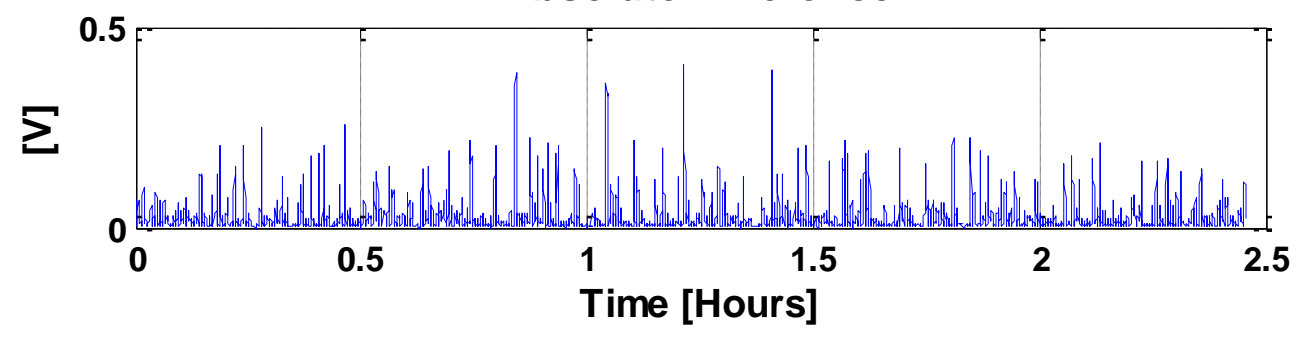



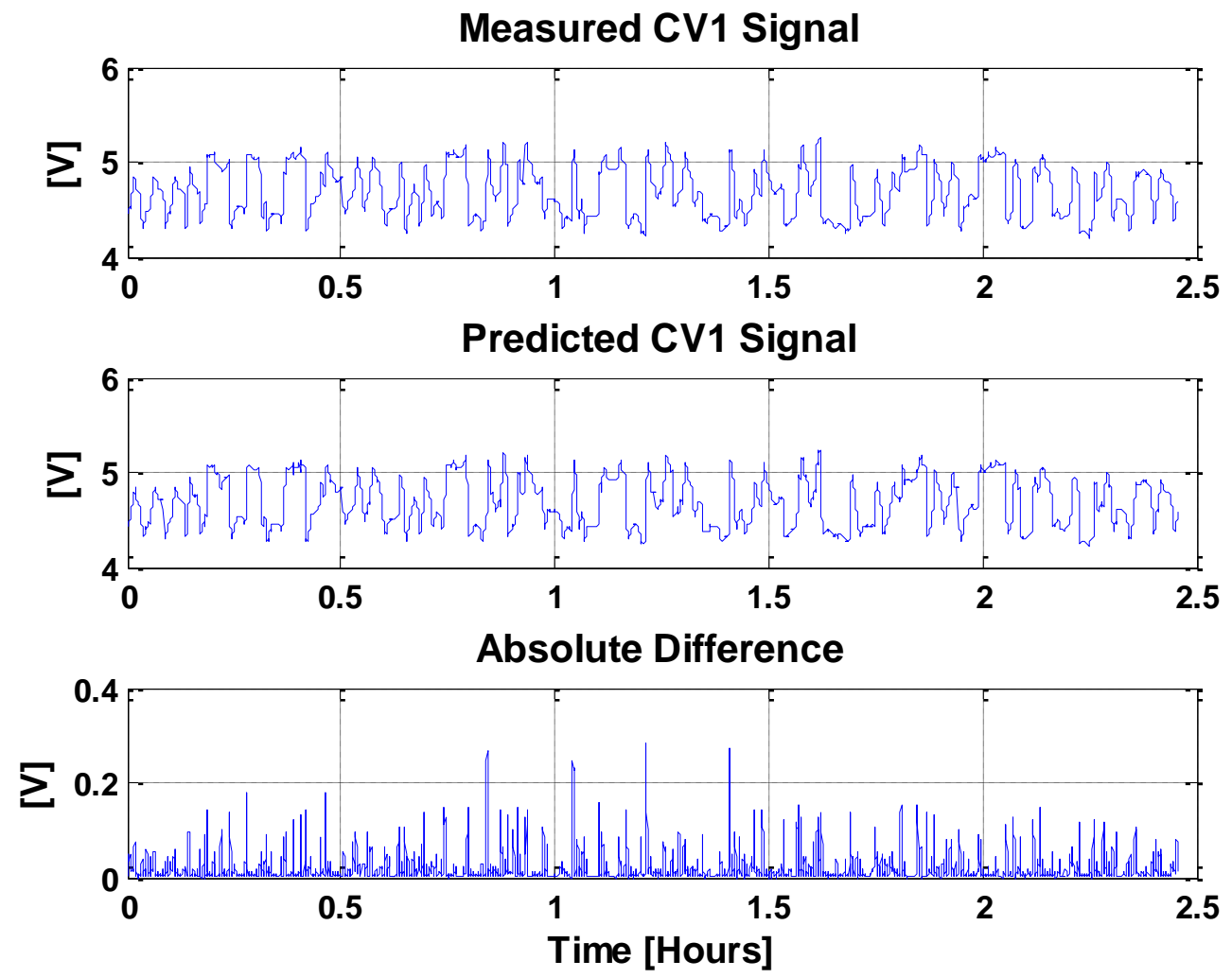


\title{
Advanced Instrumentation and Control Methods for Small and Medium Reactors with IRIS Demonstration
}

\author{
Final Report \\ Volume 6
}

On-Line Monitoring and Diagnostics Methods with Applications to the IRIS System and an Experimental Flow Control Loop

Report No. DE-FG07-07ID14895/UTNE/2011-8

NERI-C Grant Number: DE-FG07-07ID14895

\author{
J. Wesley Hines \\ (Principal Investigator) \\ Jamie Coble \\ Matthew Humberstone \\ The University of Tennessee \\ Nuclear Engineering Department \\ Knoxville, TN 37996-2300 \\ E-mail: jhines2@utk.edu
}

May 2011

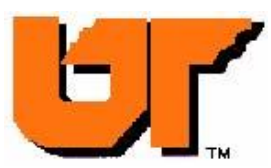




\begin{abstract}
Ideally, health monitoring of new, complex engineering systems should begin with the first day of system operation. Health monitoring typically involves a suite of modules, including system monitoring, fault detection, fault diagnostics, and system prognostics. However, for systems which have not yet been built, this is challenging. Most available health monitoring modules are empirically based, meaning they are derived from available historic data. For new systems, such data simply does not exist. This volume investigates health monitoring systems for newly designed export reactors. SMRs for remote deployment will have additional condition monitoring, diagnostic, and prognostic needs compared to traditional reactor designs. These needs stem from expectations of availability, safety, proliferation resistance, extended maintenance intervals, reduced staff, and extended operation intervals, all of which are a result of remote operation in countries that may have limited infrastructure and skill sets. Even in domestic reactors, current periodic maintenance practices are increasingly replaced by conditionbased strategies, which require methods for monitoring the condition of plant components and systems. The research presented here serves to fulfill objective three of this research and the associated tasks.
\end{abstract}

A key challenge in automatically adaptive empirical models lies in differentiating between faulted operation and nominal operation which is not well-described by the physicsbased data. Nominal operation may extend beyond the simulated data for many reasons: the system may be operating in un-anticipated environments; the assumptions made in model development may cause inaccuracies in the data; or the relationships modeled may simply be incorrect. Traditional fault detection methods such as those using the sequential probability ratio test are not able to distinguish between unexpected nominal operation and truly faulted operation. However, the main benefit of using adaptive models lies in their ability to accurately learn expanded nominal relationships while detecting and differentiating faulted conditions. For the purposes of accurately adapting a monitoring system, a principal component-based method is proposed to distinguish between these two cases.

The proposed health monitoring system adapts monitoring, diagnostic, and prognostic models as additional, pertinent information (data) becomes available from the plant. The adaptive non-parametric model (ANPM) monitoring system utilizes a hybrid approach which transitions from simulated data to nominal operating data as the plant is running. A principle 
component analysis (PCA) approach allows the system to automatically determine if a new observation is (a) normal, expected operation, (b) normal operation in an unexpected region, or (c) faulted operation. This approach overcomes the main challenge of online adaptation determining if new observations should be included in the model in an intelligent way. In addition to the new ANPM model and PCA expanded condition monitoring (ECM), several methods for performing fault diagnostics are investigated including a fuzzy inference method, a similarity-based method, and a neural network based method. These techniques were applied to a variety of data sets to highlight the benefits and drawbacks of each. The individual modules are integrated into one complete system to allow for health monitoring from the first day of system operation through end of life. The adaptive nature of the individual components affords the entire system a measure of adaptability when applied to new designs or in new operating regions.

\section{DISCLAIMER}

Any opinions, findings, and conclusions or recommendations expressed in this report are those of the authors, and do not necessarily reflect the views of the U.S. Department of Energy or Westinghouse Electric Company, LLC. 


\section{TABLE OF CONTENTS}

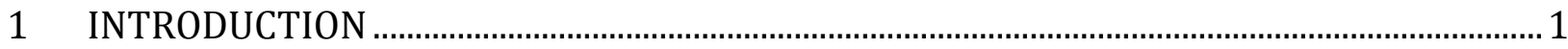

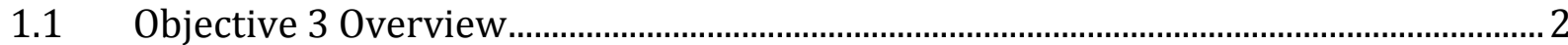

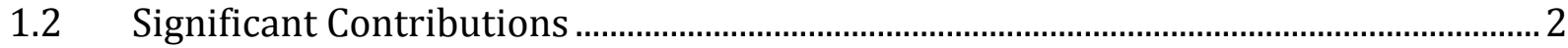

$1.3 \quad$ Organization of this Report................................................................................................. 3

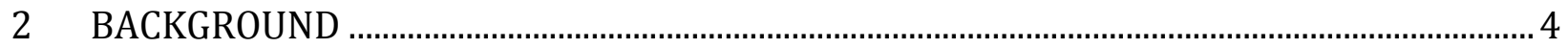

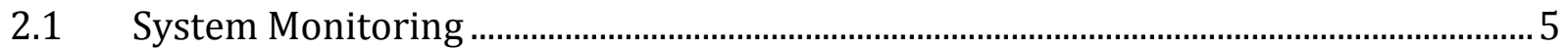

2.1.1 Traditional Monitoring Systems................................................................................. 6

2.1.2 Hybrid Monitoring Systems …………………………………………………......... 8

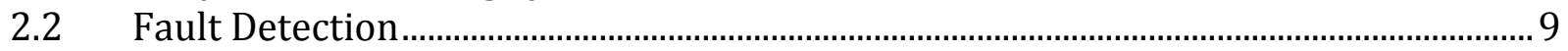

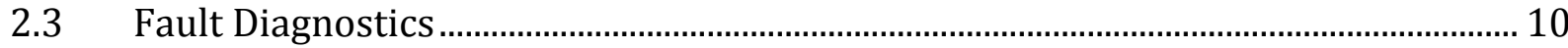

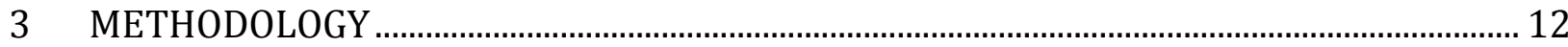

3.1 Adaptive System Monitoring …………………………………................................... 12

3.1.1 Autoassociative Kernel Regression ....……………………………………………. 13

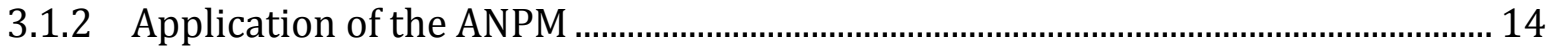

3.2 Fault Detection and Expanded Condition Monitoring ……………………………..... 17

3.3 Fault Diagnostics........................................................................................................... 21

3.3.1 Fault Identification Algorithm............................................................................. 22

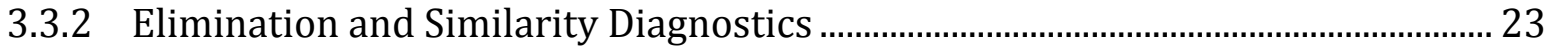

3.3.3 Fault Identification through Neural Networks ......................................................... 26

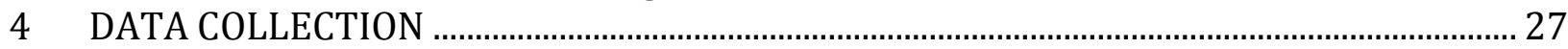

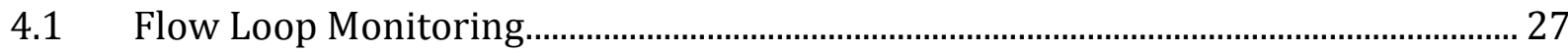

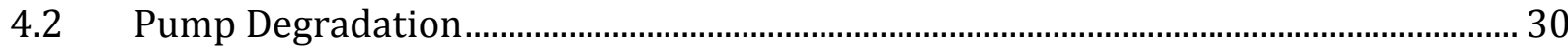

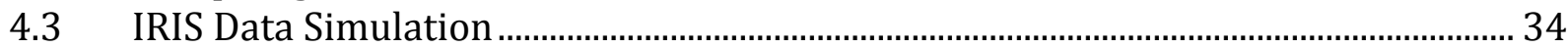

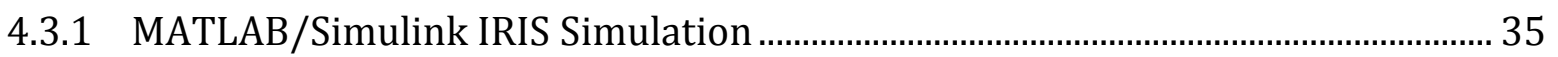

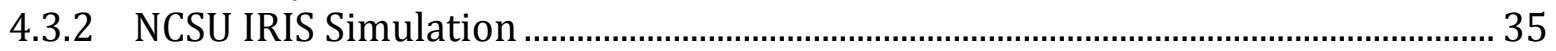

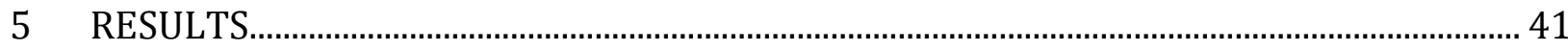

5.1 ANPM Adaptation ....................................................................................................... 41

$5.2 \quad$ PCA-Based Fault Detection............................................................................................ 42

5.2.1 Heat Exchanger Fouling.......................................................................................... 44

5.2.2 Pump Degradation .................................................................................................. 47

5.2.3 Process Sensor Drift...................................................................................................... 47

5.2.4 Control Sensor Drift .................................................................................................. 48

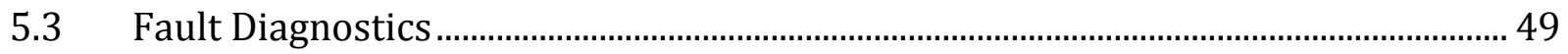

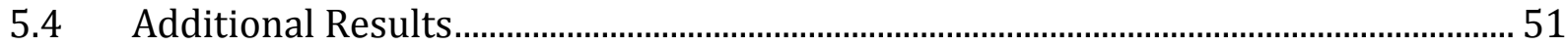

5.4.1 Application of the ECM Technique to Flow Loop Data .............................................51

5.4.2 FIA Diagnosis of Flow Loop Faults …………………………………………………. 56

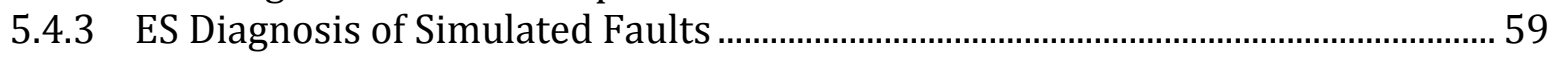

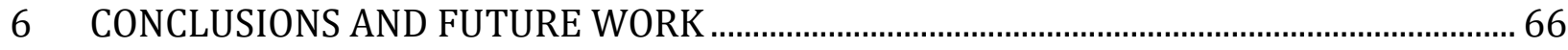

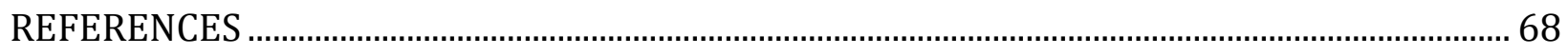

Appendix A: Additional Figures................................................................................................. 75

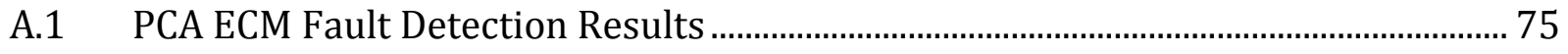

A.1.1 Heat Exchanger Fouling …………………….................................................... 75

A.1.2 Pump Degradation ............................................................................................. 82 


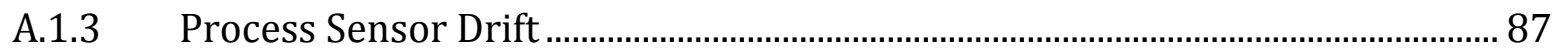

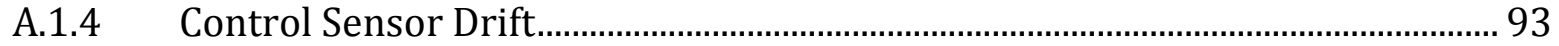

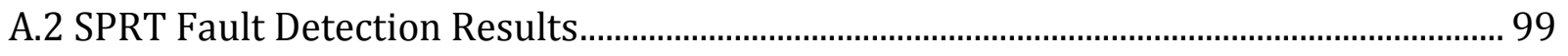

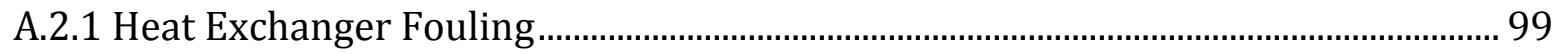

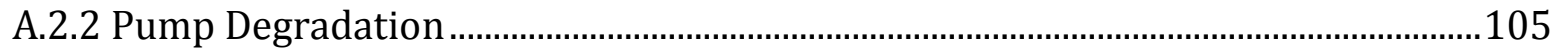

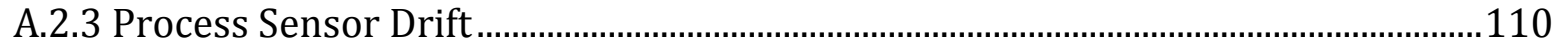

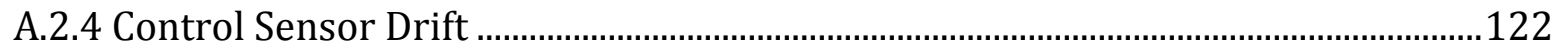

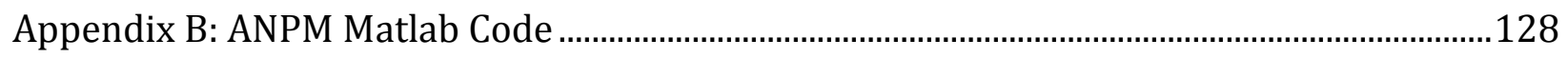

Appendix C: Tutorial for Developing Adaptive Health Monitoring Systems ..........................156 


\section{LIST OF FIGURES}

Figure 2-1: Suite of Modules in a Health Monitoring System ....................................................... 5

Figure 2-2: Adaptive Modeling Fault Procedure........................................................................... 10

Figure 3-1: ANPM Three phase approach ………………………………………………….... 15

Figure 3-2: Phase 1 Overview Diagram........................................................................................ 16

Figure 3-3: Memory matrix decay …………………………………………………………… 17

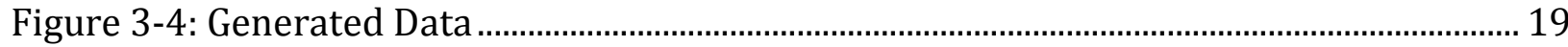

Figure 3-5: Generated Faults ..................................................................................................... 19

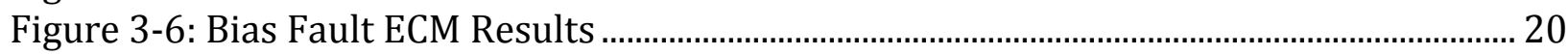

Figure 3-7: Expanded Condition ECM Results ....………………………………………………... 21

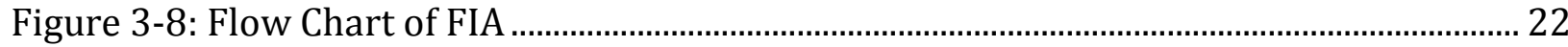

Figure 3-9: Classification of Fault Signatures ……………………………………………….... 25

Figure 3-10: Failure Similarity Process...………………………………………………………. 26

Figure 4-1: Schematic of Two-Tank Flow Loop ......................................................................... 28

Figure 4-2: Pump Degradation Experimental Setup ................................................................. 31

Figure 4-3: Differential Pressure Signal Before and After Adjusting Setup for Air Bubbles. 32

Figure 4-4: First Level of Impeller Degradation .............................................................................. 33

Figure 4-5: Increased Impeller Degradation ................................................................................. 34

Figure 4-6: Load Profile for Simulated IRIS Data ......................................................................... 36

Figure 4-7: Change in Thermal Resistivity due to Fouling [Upadhyaya et al., NEER] .............. 38

Figure 4-8: Identification of the actual duty point (recreated from [Grist 1999]) ................... 39

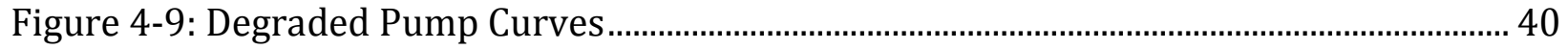

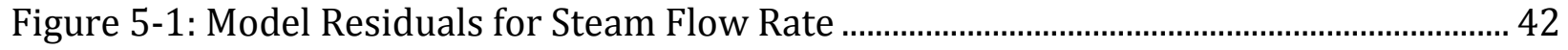

Figure 5-2: Nominal PCA-based Fault Detection Results ................................................................ 44

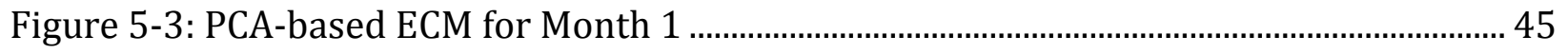

Figure 5-4: PCA-based ECM for Month 4 ................................................................................... 46

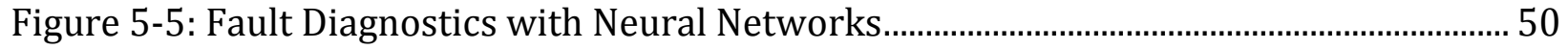

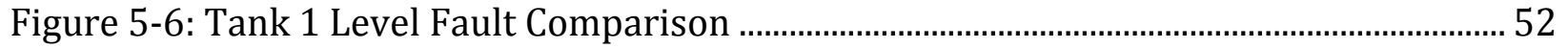

Figure 5-7: PCA ECM Application on Profile 1 ……………………………………………....... 53

Figure 5-8: PCA ECM Application on Profile 2 ……………………………………….............. 53

Figure 5-9: PCA ECM Application on Profile 1, 60 mm Drift ....................................................... 54

Figure 5-10: PCA ECM Application on Profile 2, 60 mm Drift ...................................................... 54

Figure 5-11: PCA ECM Application on Profile 1, 60 mm Drift using 6 Sensors........................... 55

Figure 5-12: PCA ECM Application on Profile 2, 60 mm Drift using 6 Sensors........................... 56

Figure 5-13: Possibility Rank of Actual Fault................................................................................ 57

Figure 5-14: Non-faulty residuals......................................................................................... 59

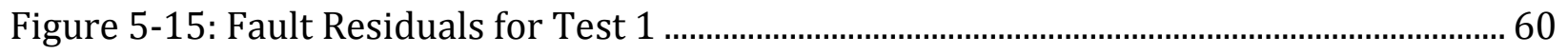

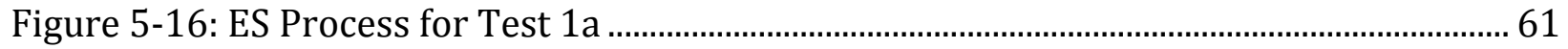

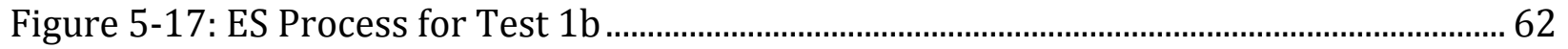

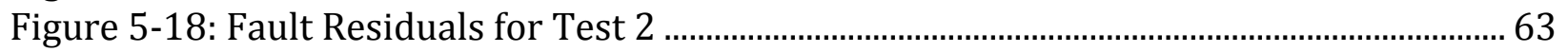

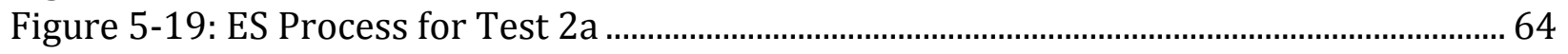

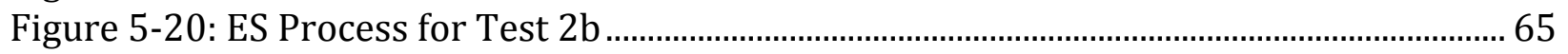

Figure 5-21: ES Process for Test 2c …………………............................................................. 66 


\section{LIST OF TABLES}

Table 1-1: Objective 3 Tasks.........................................................................................................

Table 3-1: Possible Scenarios................................................................................................. 18

Table 4-1: Flow Loop Variables for Fault Identification ............................................................... 28

Table 4-2: Flow Control Loop Fault Types..................................................................................... 29

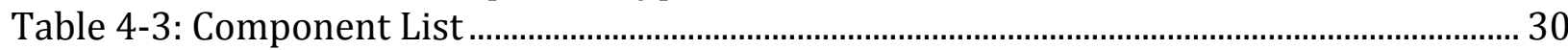

Table 5-1: Mean Squared Error of Predictions...……………………………………………..... 42

Table 5-2: Monitoring System Variables....................................................................................... 43

Table 5-3: Fault Detection Results for Heat Exchanger Fouling .................................................. 46

Table 5-4: Fault Detection Results for Pump Degradation ............................................................ 47

Table 5-5: Fault Detection Results for Process Sensor Drift ......................................................... 48

Table 5-6: Fault Detection Results for Control Sensor Drift........................................................... 48

Table 5-7: Fault Diagnostic Results using PCA Contributions...................................................... 50

Table 5-8: Ordered Faults with Corresponding Possibilities........................................................ 58

Table 5-9: Fault Signature Vector for Test 1 ………………………………………………….. 59

Table 5-10: Fault Signature Vector for Test 2 ……………………………………........................ 62 


\section{ABBREVIATIONS}

AAKR Auto-associative Kernel Regression

AANN Auto-associative Neural Network

ANN Artificial Neural Network

ANPM Adaptive Non-Parametric Model

CBM Condition Based Maintenance

DAQ Data Acquisition System

DNB Departure from Nucleate Boiling

ECM Expanded Condition Monitoring

ES Elimination and Similarity

EULM Error Uncertainty Limit Monitoring

FDI Fault Detection and Identification

FIA Fault Identification Algorithm

FIS Fuzzy Inference System

FPM First Principle-based Model

GLS Generalized Least Squares

GNEP Global Nuclear Energy Partnership

GPM General Path Model

IPSR Integral Primary System Reactor

IRIS International Reactor Innovative and Secure

kNN k-Nearest Neighbor

LVQ Learning Vector Quantization

MLE Maximum Likelihood Estimation

MSET Multivariate State Estimation Technique

MTTF Mean Time to Failure

NCSU North Carolina State University

NI National Instruments

OLM Online Monitoring

OLS Ordinary Least Squares

PACE Path Classification and Estimation

PC Principal Component 


$\begin{array}{ll}\text { PCA } & \text { Principal Component Analysis } \\ \text { PEM } & \text { Process and Equipment Monitoring } \\ \text { PEP } & \text { Process and Equipment Prognostics } \\ \text { PI } & \text { Prediction Interval } \\ \text { PWR } & \text { Pressurized Water Reactor } \\ \text { RCP } & \text { Reactor Coolant Pump } \\ \text { RUL } & \text { Remaining Useful Life } \\ \text { SDG } & \text { Signed Directed Graph } \\ \text { SG } & \text { Steam Generator } \\ \text { SMR } & \text { Small- and Medium-sized Reactor } \\ \text { SPE } & \text { Squared Prediction Error } \\ \text { SPRT } & \text { Sequential Probability Ratio Test } \\ \text { SSE } & \text { Sum of Squared Error } \\ \text { TOF } & \text { Time of Failure } \\ \text { UT } & \text { University of Tennessee } \\ \text { WPP } & \text { Weighted Path Prognostics }\end{array}$




\section{INTRODUCTION}

Small- and medium-sized reactors (SMRs) for remote deployment will have additional condition monitoring, diagnostic, and prognostic needs compared to traditional reactor designs. These needs stem from expectations of availability, safety, proliferation resistance, extended maintenance intervals, reduced staff, and extended operation intervals, all of which are a result of remote operation in countries that may have limited infrastructure and skill sets. Current periodic maintenance practices will be increasingly replaced by condition-based strategies, which will require methods for monitoring the condition of plant components and systems. Ideally, health monitoring of new, complex engineering systems should begin with the first system operation. Health monitoring typically involves a suite of modules, including system monitoring, fault detection, fault diagnostics, and system prognostics. However, for systems which have not yet been built, this is challenging. Most available health monitoring modules are empirically based, meaning they are derived from available historic data. For new systems, such data simply does not exist. This research proposes an adaptive modeling system which builds empirical models from high-fidelity simulated data. This data suffers from the common problems of data simulation caused by complicated physics-of-failure mechanisms and simplifying assumptions made in model development. As actual system data becomes available, the models adapt in an automated and intelligent way to account for real-world, nominal data relationships.

The need for an adaptive empirical modeling technique arises when a system of interest changes from the original training data relationships. Dynamic systems can suffer this problem because a training data set which covers all future ranges may be difficult to develop due to system complexity and uncertainties. This training data problem can also be caused simply by inadequate training data sets that do not cover the system's operating range appropriately. Additionally, adaptive models may be required due to non-availability of data for model training, as is the case for newly designed systems. In this case, an adaptive model must first be built on simulated operational data. First principle-based models (FPMs) are typically developed during the design phase of system development. 
Objective 3 of this research states: Develop and test an integrated monitoring, diagnostic, and prognostic system for SMRs using an experimental flow control loop and the International Reactor Innovative and Secure (IRIS) design as test platforms. This volume will focus on development and application of monitoring and diagnostic systems. Prognostic systems are left to Volume 7.

\subsection{Objective 3 Overview}

Objective 3 includes twelve tasks, spanning three years, as summarized in Table 1-1. These twelve tasks can be separated into four main research topics: monitoring and fault detection, fault diagnostics, system prognostics, and the integration of these three functions into one system. This report summarizes the work performed over the last three years in completion of the monitoring and fault detection and fault diagnostic research tasks. Additionally, integration of the full health monitoring system is discussed.

Table 1-1: Objective 3 Tasks

\begin{tabular}{|c|c|c|}
\hline Year 1 & Year 2 & Year 3 \\
\hline $\begin{array}{c}\text { 3.1 Develop IRIS system } \\
\text { hybrid models }\end{array}$ & $\begin{array}{l}\text { 3.5 Develop and test the } \\
\text { case-based fault } \\
\text { identification module }\end{array}$ & $\begin{array}{c}\text { 3.8 Integrate the } \\
\text { degradation models from } \\
\text { Task } 3.4 \text { into the simulation } \\
\text { developed in Topic } 1 \text { and } \\
\text { generate failure data }\end{array}$ \\
\hline $\begin{array}{c}\text { 3.2 Develop an automated } \\
\text { method for hybrid model } \\
\text { adaptation }\end{array}$ & $\begin{array}{l}\text { 3.6 Apply the modeling and } \\
\text { adaptation methods to a } \\
\text { heat exchanger test system } \\
\text { and validate in real time }\end{array}$ & $\begin{array}{l}\text { 3.9 Validate the prognostic } \\
\text { algorithm development } \\
\text { methodologies using } \\
\text { simulated data. }\end{array}$ \\
\hline $\begin{array}{c}\text { 3.3 Integrate the adaptive } \\
\text { system into a model based } \\
\text { FDI system }\end{array}$ & \multirow{3}{*}{$\begin{array}{c}\text { 3.7 Develop a hybrid } \\
\text { prognostic framework that } \\
\text { combines Type I, II, and III } \\
\text { prognostic models }\end{array}$} & $\begin{array}{l}\text { 3.10 Integrate monitoring, } \\
\text { detection, identification, and } \\
\text { prognostic algorithms }\end{array}$ \\
\hline \multirow{2}{*}{$\begin{array}{l}\text { 3.4 Develop degradation } \\
\text { models for example key } \\
\text { systems and components }\end{array}$} & & $\begin{array}{c}\text { 3.11 Test the system using } \\
\text { simulated and laboratory } \\
\text { data }\end{array}$ \\
\hline & & $\begin{array}{c}\text { 3.12 Develop a Matlab- } \\
\text { based system development } \\
\text { toolbox manual }\end{array}$ \\
\hline
\end{tabular}

\subsection{Significant Contributions}

Research work in completion of Objective 3 of this project has resulted in several significant contributions to the field of health monitoring. 
1. Development of an adaptive system monitoring approach that can be used in a nonparametric modeling environment. The model utilizes a hybrid approach that begins with simulated data and slowly adapts to nominal operating data in an automated way as it becomes available. This increases the predictive capability of the model from initial operation through end of life.

2. Development of a principal component analysis (PCA) based expanded condition monitoring (ECM) technique. When an anomaly is detected in the operating data, this ECM technique differentiates between expanded nominal operating conditions and a fault condition. This technique allows the model to adapt in an automated way without learning faulty system behavior.

3. Development of several possible diagnostic techniques for fault identification. These techniques utilize a variety of data, information, and artificial intelligence frameworks.

4. Development of individual-based prognostic models for simulated common fault types in nuclear power plants. The techniques used to develop these models are straightforward, such that additional models can be developed as new failure modes are identified.

5. Integration of the separate modules into one complete health monitoring system: adaptive monitoring, fault detection, diagnostics, and prognostics. The adaptive nature of the individual components affords the entire system a measure of adaptability when applied to new designs or in new operating regions.

\subsection{Organization of this Report}

This report summarizes the work performed in completion of Objective 3 over approximately three years. The following section discusses pertinent background information about system monitoring, fault detection and diagnostics, and prognostics. Next, the specific methodologies developed for this research, including the adaptive monitoring module, expanded condition monitoring, fault diagnostic methods, and prognostic methods, are described. The Process and Equipment Monitoring (PEM) toolbox is discussed, as it pertains to the current

research. Data collection experiments performed to support this research are described, 
including flow loop monitoring, pump degradation, and first principle IRIS simulation. Then, results of the application of developed techniques to appropriate test bed data are summarized. Finally, conclusions of this research and areas of future work are outlined.

\section{BACKGROUND}

Full health monitoring systems, also called Condition Based Maintenance (CBM) systems, are the focus of much research. Health monitoring systems typically include several modules, such as system monitoring, fault detection, fault diagnostics, system prognostics, and management or operations and maintenance (O\&M) planning. Callan, et al. [2006] outline a five-step CBM system which includes: Data Acquisition, Data Manipulation, Condition Monitoring, Health Assessment, and Prognostics. By applying the entire suite of modules, one can accomplish the goals of most CBM systems: increased productivity; reduced downtime; reduced number and severity of failures, particularly unanticipated failures; optimized operating performance; extended operating periods between maintenance; reduced unnecessary planned maintenance; and reduced life-cycle cost. Figure 2-1 gives a diagram of a typical health monitoring system. Data collected from a system of interest is monitored for deviations from normal behavior. Monitoring can be accomplished through a variety of methods, including first principle models, empirical models, and statistical analysis [Hines et al., 2006]. The monitoring module can be considered an error correction routine; the model gives its best estimate of the true value of the system variables. These estimates are compared to the data collected from the system to generate a time-series of residuals. Residuals characterize system deviations from normal behavior and can be used to determine if the system is operating in an abnormal state. A common test for anomalous behavior is the Sequential Probability Ratio Test (SPRT) [Wald, 1945]. This statistical test considers a sequence of residuals and determines if they are more likely from the distribution that represents normal behavior or a faulted distribution, which may have a shifted mean value or altered standard deviation from the nominal distribution. If a fault is detected, it is often important to identify the type of fault; systems will likely degrade in different ways depending on the type of fault and so different prognostic models will be applicable. Expert systems, such as fuzzy rule-based systems, are common fault diagnosers. Finally, a prognostic model is employed to estimate the Remaining Useful Life (RUL) of the system. This model may include information from the original data, the monitoring system 
residuals, and the results of the fault detection and isolation routines. The following sections discuss three of the modules of a health monitoring system in more detail: system monitoring, fault detection, and fault diagnostics; system prognostics is presented in Volume 7 of this report. This brief review of the relevant literature is then followed by discussion of the specific health monitoring techniques developed and employed in this research.

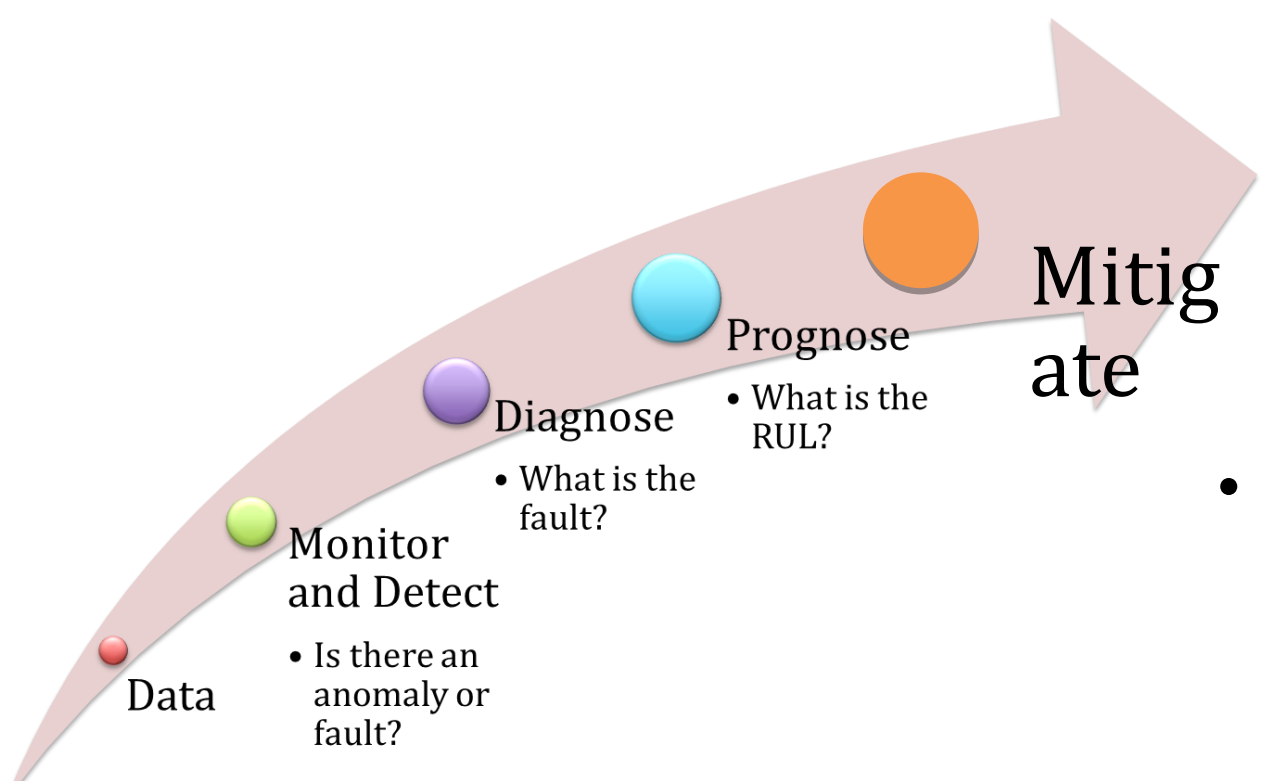

Figure 2-1: Suite of Modules in a Health Monitoring System

\subsection{System Monitoring}

Many recently developed systems for advanced monitoring and diagnostics, incorporate empirical, data-based models, which require operating process data [Uhrig 2005]. These empirical techniques include artificial neural networks [Hines 1997a, Kavaklioglu and Upadhyaya 1994, Upadhyaya and Eryurek 1992], Statistical Models [Hines 2005], fuzzy logic [Hines 1997b, Holbert 1995], Multivariate State Estimation Technique (MSET) or Autoassociative Kernel Regression [D.R. Garvey 2006, J. Garvey 2007a, 2007b] and hybrid combinations [Ikonomopoulos 1992]. These techniques are applicable to currently operating power plants because utilities regularly collect and store nuclear power plant process data. However, the implementation of these advanced monitoring and diagnostic systems on next generation reactor designs may be hindered or made impossible by the lack of available historical operating data.

The absence of historical operating data is a significant impediment to implementation of diagnostic and prognostic systems for these new reactors. When a new power plant is put into 
service, only first principle models will be available to compare the actual performance to that expected. New "hybrid" methods will be needed to resolve this problem. These hybrid methods will combine the reliability of first principle models with the accuracy of data-driven, empirical models. As the reactor operates, data will be available to improve the monitoring system.

The following sections describe traditional monitoring systems and previously developed hybrid monitoring systems. A novel hybrid monitoring system is developed in the current research and is described in Section 3.1.

\subsubsection{Traditional Monitoring Systems}

There are a number of different monitoring techniques that have been proposed within the literature. These techniques range from FPMs that use physics of the system to predict the nominal conditions of the process to empirical models that use actual system data to build prediction models. An example of using a FPM is shown in the research on the Tokamak energy confinement by Kotschenreuther et al. [1995]. These models can be built during the design phase of the system. There are a number of benefits and drawbacks to using a FPM. One of the benefits of a FPM is the physical equation base which gives the model a direct physics based foundation. Experimental data from the system can be used to verify the FPM and test the accuracy of such a model. Despite the benefits of a FPM there are a number of drawbacks. These include the engineering time and understanding that is required to build a good FPM and the lack of sensitivity needed to detect slight changes in the system. Due to the complex nature of building a FPM it is rare to have a FPM that incorporates the complete system; it is common for a FPM to include portions of the system.

Empirical or data based models have a number of benefits and drawbacks as well. These types of models are built using process parameter measurements that have been collected over the range of the system or process of interest. The relationships between these measurements are used within the model architecture to produce accurate predictions. One of the drawbacks of empirical models is the limitation on accuracy of the models. Since the relationships are usually non-linear the models are only accurate when applied to similar operating conditions as the data used to create the model. When the model predictions are extrapolated outside the training range the results are unreliable. This is different from linear models where predictions can be extrapolated outside the training range. 
There are two commonly used types of empirical model architectures, parametric and nonparametric. In a parametric model a set of parameters are used to define the functional relationship of the system. An example of a common parametric model is linear regression with $\mathrm{p}$ predictor variables and $\mathrm{n}$ observations.

$$
y_{i}=\beta_{1} x_{i 1}+\ldots+\beta_{p} x_{i p}+\varepsilon_{i}
$$

This can be described in matrix form as:

$$
Y=X \beta+\varepsilon
$$

where

$$
y=\left(\begin{array}{c}
y_{1} \\
y_{2} \\
\vdots \\
y_{n}
\end{array}\right), X=\left(\begin{array}{c}
x_{1}^{\prime} \\
x_{2}^{\prime} \\
\vdots \\
x_{n}^{\prime}
\end{array}\right)=\left(\begin{array}{cccc}
x_{11} & x_{12} & \cdots & x_{1 p} \\
x_{21} & x_{22} & \cdots & x_{2 p} \\
\vdots & \vdots & \ddots & \vdots \\
x_{n 1} & x_{n 2} & \cdots & x_{n p}
\end{array}\right), \beta=\left(\begin{array}{c}
\beta_{1} \\
\vdots \\
\beta_{p}
\end{array}\right), \varepsilon=\left(\begin{array}{c}
\varepsilon_{1} \\
\varepsilon_{2} \\
\vdots \\
\varepsilon_{n}
\end{array}\right)
$$

Parametric architectures use the training data to determine the parameters of the model that fit a predefined mathematical model. This is normally done by minimizing some objective function, such as the sum of squared error (SSE) or some other error metric. A number of parameter estimation techniques have been developed. One of the most popular methods is OLS; this method minimizes the sum of squared residuals, and leads to a closed form expression.

$$
\hat{\beta}=\left(X^{\prime} X\right)^{-1} X^{\prime} y
$$

Another parameter estimation technique is the generalized least squares (GLS), which is an extension from OLS where a weighted sum of squared residuals is minimized. This is used when heteroscedasticity or correlation is present within the error terms. Maximum likelihood estimation (MLE) is also used as a parameter estimation technique. MLE can be performed when the distribution of the error terms are known, when this is a normal distribution with mean zero the results are the same as OLS.

The parameters for parametric models are defined in finite-dimensional parameter space. There has been a lot of research done on parametric models, too much to include a complete literature review of the topic. However, interested readers are referred to Bickel et al. [2001], Davidson [2003], Freedman [2009], and Seber et al. [2003]. For more specific modeling needs, the reader is referred to Haykin [1994] for neural networks and Draper et al. [1966] for ordinary least squares (OLS) regression. 
Where parametric models specify the structure before the analysis, a non-parametric model does not specify a structure. Non-parametric models may have parameters; however, these parameters have flexibility and are not defined beforehand. Past data exemplars are stored in the memory and used as the actual model. When a query is made the non-parametric model performs a weighted regression of the training exemplars within the vicinity of the query. The proximity of the training exemplars to the query designated the corresponding weights. There are benefits and drawbacks of both empirical modeling techniques. Atkeson et al. [1997b] describes some of the benefits and drawbacks of non-parametric models, Hines et al. [2006b] addresses some of the drawbacks by proposing a robust vector selection method that improves the quality of the locally weighted regression models. For a complete overview of non-parametric techniques the reader is referred to Wasserman [2007] and Gibbons [2003].

\subsubsection{Hybrid Monitoring Systems}

Process or component models are commonly used in monitoring and fault detection systems. Early research focused on the use of first principles models that use mathematical equations of the system; more recent techniques use data-based models such as artificial neural networks (ANN), the Multivariate State Estimation Technique (MSET) or Autoassociative Kernel Regression (AAKR). First-principle models rarely incorporate all of the functionality of the system to be modeled, and may not provide the sensitivity necessary to detect slight changes in plant degradation. Data-based models are applicable to operating plants and systems where normal operating data has been recorded over the expected operating range. These data based systems are only accurate when applied to the same, or similar, operating conditions under which data was collected. When plant conditions or operations change significantly from what was collected, the model must extrapolate outside the training space and the results should not be trusted. Unlike linear models, which extrapolate in a known linear fashion, non-linear models extrapolate in an unknown manner. Since these empirical (data-based) models must be trained on past plant data, they are not applicable to nuclear plants that have not yet been constructed because operational data are not available. First principle models are not specifically limited to a prior operating range and could be used for new plants without an operating history.

The goal of hybrid modeling is to merge the two techniques to take advantage of the strengths of each method, while simultaneously overcoming their weakness. Hybrid models traditionally are designed to use a baseline first principles model and improved with data based 
models as data becomes available. This method provides the robustness of first principle models with the sensitivity of data-based models. This hybrid framework, although more complicated, has a very important advantage. Purely data based systems are not reliable when the system moves into new operating conditions that may result from configuration changes, new operating practices, or external factors such as unusual cold cooling water temperatures in condensers. Through proper application of hybrid systems, the predictions can be forced to revert towards the first-principle model when new operating conditions are encountered and will incorporate the data-based models when in familiar operating conditions. Several useful overview papers on hybrid modeling include Thompson and Kramer [1994], Wilson and Zorsetto [1997], te Braake and van Can [1998]. A novel hybrid modeling system is developed in this research called the Adaptive Non-Parametric Model (ANPM). The details of this model are given in Section 3.1.

\subsection{Fault Detection}

Empirical modeling techniques are used to assess the system performance and can be used for condition based maintenance. Auto-associative architectures produce process variable predictions which are used with the process variable measurements to create residuals. These residuals are used within a fault detection algorithm such as the sequential probability ratio test (SPRT) to evaluate the state of the system. This is traditionally a fault detection technique and does not have the ability to detect expanded conditions. The complete equipment surveillance system consists of the monitoring, diagnostic and prognostic capabilities. The ANPM increases the accuracy of the monitoring portion which affects the whole equipment surveillance system by increasing the performance of the diagnostics and prognostics. The fault detection ability governs the accuracy of the ANPM and is important for the overall equipment surveillance system.

The main difference between an adaptive model and a non-adaptive model, beyond the capability to adapt, is the ability to differentiate between an expanded condition and a fault. Non-adaptive models classify expanded conditions as faults. Adaptive models, however, need the capability to distinguish the two conditions, as shown in Figure 2-2. 


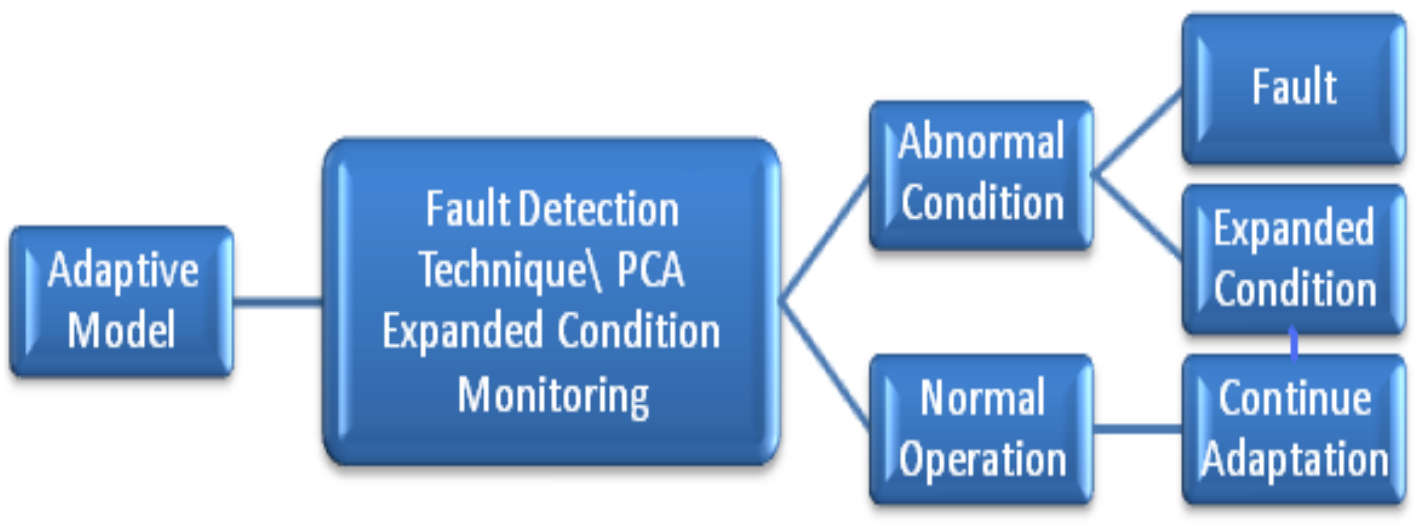

Figure 2-2: Adaptive Modeling Fault Procedure

Adaptive models are dynamic models that rely heavily on the internal fault detection techniques. The fault detection within the model determines the evolution of the model. The correct classification of expanded conditions gives the adaptive model the ability to adjust beyond the initial range of the model. This ability is a desirable attribute that increases the usefulness of the adaptive model. An adaptive model that does not have this ability could be developed without using an expanded condition monitoring technique, but this would affect the potential evolution of the model and limits its true adaptability.

The need for an expanded condition monitoring approach is realized in adaptive modeling environments. Traditional fault detection techniques do not offer the capability to differentiate between faulted conditions and expanded conditions. Both the SPRT [Wald, 1945] and the error uncertainty limit monitoring (EULM) [Garvey et al., 2006] techniques do not have the ability to differentiate between expanded and fault conditions. The proposed Principal Component Analysis (PCA)-based expanded condition monitoring (ECM) technique, however, is shown to have this ability. This newly developed technique is described in Section 3.2.

\subsection{Fault Diagnostics}

This section of the literature review gives a brief overview of the diagnostic methods currently available in the literature. Several diagnostic techniques are described with examples of applications given. Diagnostic is also referred to as identification and isolation; these terms will be used interchangeably throughout this paper. Diagnostics is a key portion of a complete equipment surveillance system, much of the literature focuses on the complete system not 
specifically the diagnostics. Mehra et al. [1971] discusses a general approach using system theory and statistical decision theory for fault detection, diagnosis, and prognosis. A two step diagnostic approach was proposed by Simani et al. [2003]. The first step of this approach was the generation of residuals or symptoms of the system. Where residuals are the discrepancy between the predictions and the actual or expected system values. A number of papers use these residuals as the indicator of incipient faults [Fenu et al., 1998]. The second step is the use of a technique to relate these residuals to the specific fault conditions. This is the basic overview approach that is taken in much of the diagnostic research. There are a number of papers that give a good overview of the challenges and techniques used in diagnostics [Riedesel 1989; Gertler 1988; Frank 1990; Natke 1997; Patton et al. 2000; Milne 1987].

Kreutzer et al. [1987] used predefined tests on the individual systems of a computer network and compared the results to determine which systems were failing. Milne [1987] discusses the evolution of diagnostics from a basic rule-based system to expert systems that can use more complex reasoning techniques. A similar approach was taken by Gertler [1988], where a survey of diagnostic techniques for complex systems was given. He highlights the main components of these techniques as residual generation, signature generation, and signature analysis. Analytic redundancy is used within a number of detection and diagnostic techniques to create the needed residuals [Gertler 1988; Frank 1990]. Frank [1990] discusses model based residual generation using parameter identification. Riedesel [1989] uses a rule-based systems for fault diagnosis and control decisions.

These diagnostic techniques have been developed for a variety of applications. Many of the techniques are focused on the specific systems or situations that are being addressed and not generalized. There are a number of approaches that have been used in diagnostics. One common approach is the mutlivariate statistical method; this is a diagnostic method that uses PCA [Russell et al. 2000; Johnson et al. 1992; Jolliffe 2002]. Another common approach is knowledge-based systems also known as expert systems, these are historically used in fault diagnosis [Germond et al. 1992; Venkatasubramanian et al. 2003]. This technique has commonly been coupled with fuzzy logic or fuzzy inference systems (FIS) for added benefits [Tarifa et al. 1997; Ben-Abdennour et al. 1996; Del Amo et al. 2005; Kavuri et al. 1993]. Tarifa et al. [1997] developed a new model based fault diagnosis approach for chemical processes. They used a signed directed graph (SDG) to determine possible outcomes and then used an expert system and 
fuzzy logic to evaluate the information. Ben-Abdennour et al. [1996] investigates the use of a coordinated intelligent control scheme on power plants.

Another common approach is the nearest neighbor or k-nearest neighbor (kNN) methods. Nearest neighbor classification is a decision rule used to find the nearest set of previously classified points to the observed sample point [Cover et al. 1967]. The kNN classification technique was described as a three step process used to diagnose faults in transformers by Dong et al. [2004]. This technique has been used in various research, such as the geo-location of mobile phones [Wong 2001], and for sign language recognition used for robot guidance [Pook et al. 1994]. Other work has paired the kNN classifier with other techniques such as neural networks [Koutroumbas et al. 1994; Murphy 1990] and learning vector quantization (LVQ) in supervised learning [Geva et al. 1991].

An area where diagnostics has been heavily researched and patented is the automotive industry [Breed 2002; Gayme et al. 2003; Breed 2005; Basir 2004]. There is a wide degree of diagnostic techniques that have been shown to integrate such methods as PCA, fuzzy logic, kNN, expert systems, and many others into diagnostic algorithms. After a fault has been detected and diagnosed, a typical health monitoring system moves to system prognosis to predict the RUL of the system. The next section discusses some of the typical empirical prognostic techniques.

\section{METHODOLOGY}

This chapter gives the details of the specific modeling techniques developed and used in this research, including the Adaptive Non-Parametric Model (ANPM), Expanded Condition Monitoring (ECM), and multiple fault diagnostic methods including a FIS technique and Elimination and Similarity (ES) diagnostics. The final step in health monitoring, prognostics, is left to Volume 7 of this report. A tutorial to guide development of an adaptive health monitoring system from system monitoring through prognosis, is given in Appendix C. This tutorial is meant to serve as a stand-alone guide; much of the information in this chapter is repeated there.

\subsection{Adaptive System Monitoring}

Traditional non-parametric models can only make reliable predictions within the range of the training data. The need for an adaptive modeling technique arises when a monitored system's operation shifts from the original training data relationships. Such a change in data relationships 
can occur for several reasons, including seasonal changes, load changes, and unforeseen operating profiles. Training data sets may be inadequate because the future operating conditions cannot be accurately predicted, development of an inclusive training data set may be complex and uncertain, or no actual operating data is available for new systems and processes. In any of these cases, FPM data can be used in a temporary memory matrix to account for these shortcomings for prediction. However, the actual data will have superior predictive performance to the FPM data. The FPM may include assumptions that don't hold for the process of interest, as well as some modeling error or uncertainty that was not considered in the FPM development. A model with the highest predictive accuracy is desired. As actual operating data is collected, the model should incorporate it in predictions, eventually replacing the FPM data all together. One concern in this process is ensuring data is characteristic of non-fault condition to maintain the fault detection ability of the monitoring system.

The base model used in the ANPM is the AAKR, a non-linear, non-parametric modeling technique. The ease of data addition to the original model is one of the major benefits of this type of model. When appropriate, a new observation can be added to the model by simply appending it to the model's memory matrix. As nominal operation data becomes available, the first principle data can slowly be removed from the model.

The ANPM was developed for the primary use on the IRIS reactor used in the Global Nuclear Energy Partnership (GNEP) program. The IRIS reactor was chosen as the Integral Primary System Reactor (IPSR) for this research [Storrick et al. 2005]. As will be shown through the examples, the ANPM can be applied to a variety of process monitoring systems.

This section presents the development of the ANPM and shows its application to a simple heat exchanger model. The MATLAB code developed to implement the ANPM model is given in Appendix B. Different fault detection techniques have been applied to the ANPM; however, due to the dynamic nature of the model, traditional fault detection is difficult to implement. The traditional methods such as SPRT and EULM do not accurately differentiate faults from expanded operating conditions. A number of fault detection techniques were investigated for implementation with the ANPM.

\subsubsection{Auto-associative Kernel Regression (AAKR)}

The base empirical model uses an AAKR [Hines et al., 2007a] model. AAKR is a nonparametric model that uses past normal operational data to correct faulty observations which may 
be due to system degradation, sensor faults, data acquisition problems, etc. The outputs of an AAKR model are the predicted or corrected values of the inputs.

The AAKR model uses a Euclidean distance, which is known as the $\mathrm{L}^{2}$-norm, to compare the input query data to the exemplar vectors which make up the model's memory matrix. This is for $\mathrm{n}$ inputs.

$$
u_{j}=\sqrt{{ }_{i=1}^{n}\left(\begin{array}{ll}
x_{q}^{i} & m_{j}^{i}
\end{array}\right)^{2}}
$$

The Euclidean distance is then used in a Gaussian kernel similarity function to define weights for the population exemplars. A kernel function should have a large weight for small distances and small weight for large distances. There are advantages and disadvantages specific kernels for each situation; it has been shown that the kernel function does not have a large impact on the performance of the locally weighted models [Cleveland et al. 1994a; Cleveland et al. 1994b]. A common kernel is the Gaussian kernel [Fan et al. 1996]; this kernel is a function of the Euclidean distance and the kernel bandwidth $h$ and is used in the ANPM.

$$
w=K(u, h)=\frac{1}{\sqrt{2 \times h^{2}}} e^{u^{2} / h^{2}}
$$

In general the bandwidth is optimized by minimizing the error for a test data set. This is done initially, before applying the model for system monitoring, to find the optimal bandwidth; this bandwidth is used throughout the adaptation period or phase 1 . The final prediction is a weighted sum of the exemplar vectors, $m_{i}$.

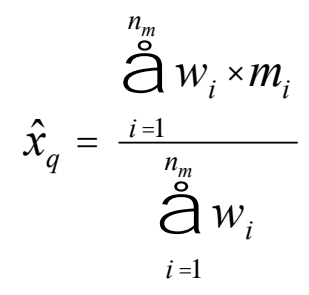

\subsubsection{Application of the ANPM}

A three phase approach is proposed for the application of the ANPM. A diagram of this approach is shown in Fig. 15. This approach starts with phase 1, which uses data from the developed first principle model. This first principle model is based on the underlying physics of 
the system of interest. During phase 1, the ANPM is modified using the actual data collected from the system. The time frame for this phase is subjective, and should be determined on a case by case basis. The time frame needs to allow for data collection sufficient to cover the operating conditions of the system. This is necessary to build a model that will make accurate predictions for the entire span of the operating conditions.

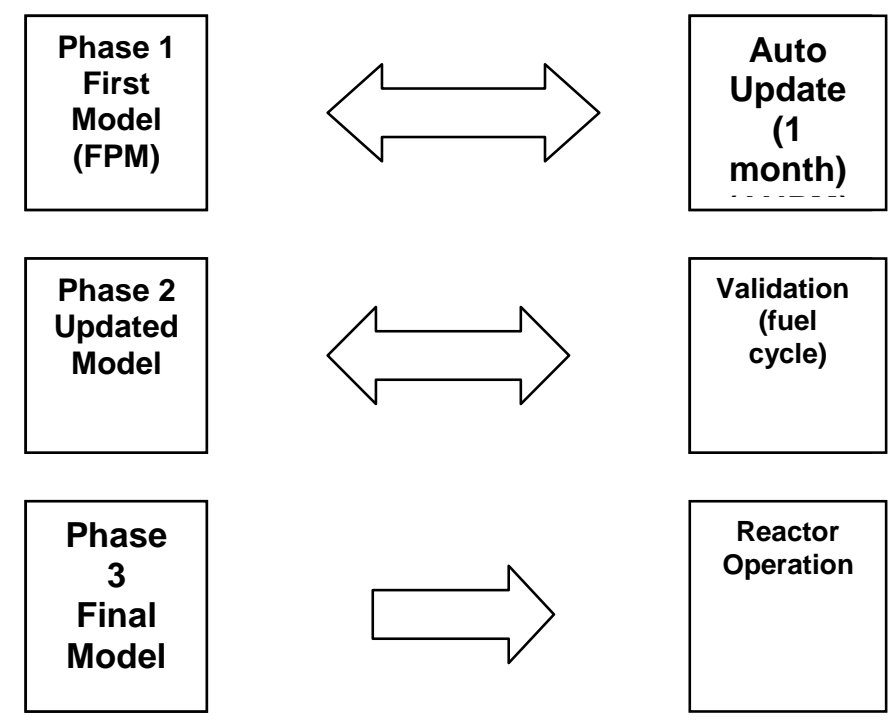

Figure 3-1: ANPM Three phase approach

The second phase of the three phase approach is the validation phase. The updated model is used for monitoring and fault detection without adapting during this phase. In addition, a second model, which is built in the traditional way from the nominal operation data collected in phase 1 , is also used during this phase. This model will be referred to as the non-adaptive model. The new data collected from the system during this phase is used to validate the models, and their performance is compared to determine which model is better suited for monitoring and fault detection. It is believed that the non-adaptive model should be able to perform at least as well as the ANPM developed model; however, further research is needed to validate this claim. This assumption is made because the ANPM may include only a subset of the data used to build the non-adaptive model. During the adaptive phase, the ANPM decides which new observations are important for improving model performance. The ANPM predictive performance depends on how well these decisions are made. 
In the third, and final, phase the final model is chosen from the ANPM and the nonadaptive model and applied to the system. Some model maintenance may be needed over time, but the adaptive phase of model development which is the changing of the data basis is complete. The expanded condition monitoring applications can be applied during the adaptive phase and during the normal operation of the model.

The adaptive portion of ANPM development is completed in phase 1 of the system operation. Figure 3-2 gives a basic overview of phase 1 compared to a traditional hybrid model. This figure shows how the first principle model is used to generate data to populate the original memory matrix in the ANPM. The simulated data is used to make AAKR predictions during initial system operation. These predictions are used to find the residual between the actual data the expected system operation. Analysis is done to determine if the actual system data represents faulted or non-faulted conditions. Then, if the data vector is determined to be from non-faulted data, this new observation can be appended onto the original memory matrix of simulated data. The decision to add the data to the model is based on the fault detection results for the current observation, previous observations, and future observations. In this way, a new model evolves which is based on a combination of the first principle model data and the data collected during system operation.

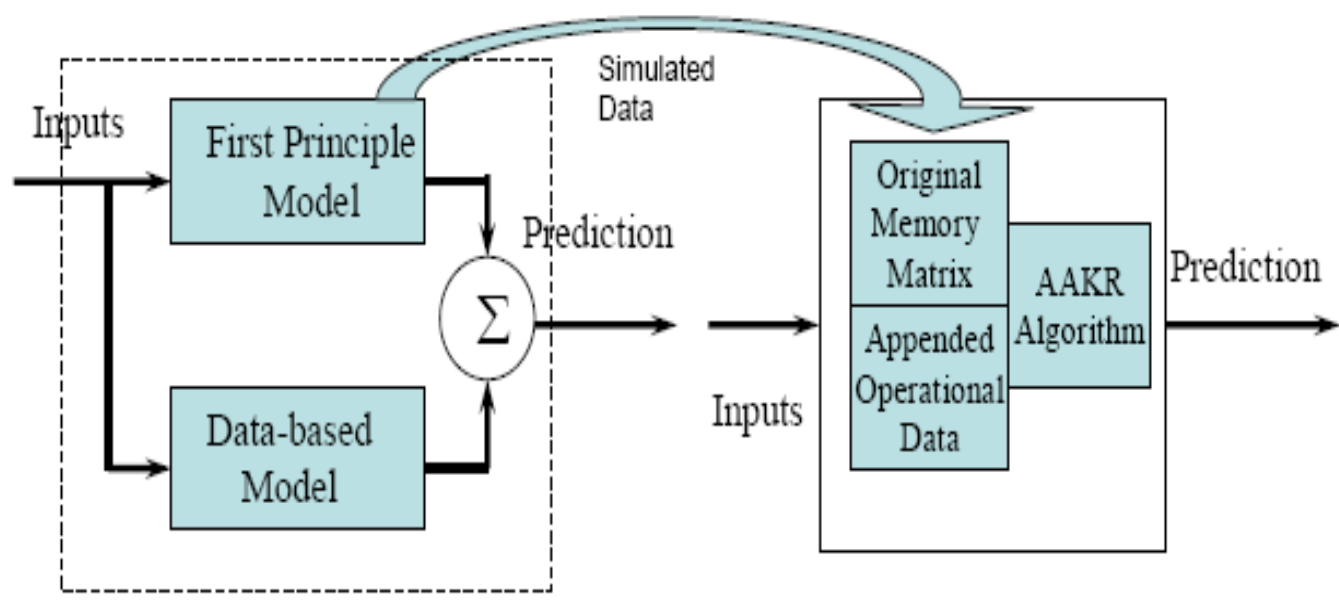

Traditional Hybrid Model

Proposed Adaptive Non-Parametric Model

Figure 3-2: Phase 1 Overview Diagram 
As actual system data is appended to the ANPM memory matrix, the data simulated by the first principle model decays from the model. At the end of phase 1, a model is desired that is based entirely on actual data from the system. This is done by deleting the observations contained in the original memory matrix as actual system observations which characterize the same operating conditions are collected. The method used for vector deletion from the model is a constant rate vector deletion technique. This consists of deleting vectors from the original memory matrix in an evenly spaced time sequence. This is done over the operating period of the ANPM or during phase 1 . The vector with the greatest contributions to past predictions is deleted as is shown in Figure 3-3. This eliminates the vectors that are the most similar to the new vectors that are being appended. This is an important feature of adaptation because it directs the evolution of the model so that the models range is complete. This process is continued, until only the system's data is in the model's memory matrix.

\begin{tabular}{|c|c|}
\hline Memory Matrix & Contribution \\
\hline V1 & 0 \\
\hline V2 & 0 \\
\hline V3 & 0.2 \\
\hline V4 & 0.6 \\
\hline V5 & 0.2 \\
\hline V6 & 0 \\
\hline V7 & 0 \\
\hline \multirow{5}{*}{} \\
\hline \\
\hline \multirow{4}{*}{} \\
\hline
\end{tabular}

Figure 3-3: Memory matrix decay

\subsection{Fault Detection and Expanded Condition Monitoring}

Expanded process monitoring is one of the major challenges within adaptive modeling. Adaptive models have the challenge to balance stability and elasticity. The model should be able to adapt to new operating conditions without losing the capability to differentiate faults from nominal conditions [Humberstone et al. 2009]. Where non-adaptive models may declare any extension beyond the range of the model as a fault, an adaptive model needs the capability to determine if it is a fault or an extended nominal condition. A similar approach to the PCA 
monitoring technique can be applied to the adaptive modeling procedure, which distinguishes the condition of the system.

Two commonly used statistical indices are the Hotteling's $\mathrm{T}^{2}$ statistic and the squared prediction error (SPE), or the Q, statistic. These statistics can be used to indicate where a data point is with respect to the population. The $\mathrm{T}^{2}$ statistic describes the variation within the model, and the Q statistic describes the variation outside the model.

$$
\begin{gathered}
T^{2}=T \Lambda_{k}^{-1} T^{T} \leq \delta_{T}^{2} \\
Q=e^{T}=X\left(I-P_{e} P_{e}^{T}\right) X^{T} \leq \delta_{Q}^{2}
\end{gathered}
$$

This results in one of four possible outcomes which are shown in Table 1 [Wang et al. 2002], where within limits is designated as WL and outside the limits is designated as OL.

Table 3-1: Possible Scenarios

\begin{tabular}{|c|c|c|l|}
\hline Scenarios & Q statistic & $T^{2}$ statistic & Conclusion \\
\hline 1 & WL & WL & Normal Condition \\
\hline 2 & WL & OL & Expanded Condition \\
\hline 3 & OL & WL & Fault \\
\hline 4 & OL & OL & Fault \\
\hline
\end{tabular}

The need for an expanded condition monitoring approach was shown for an adaptive modeling environment. Traditional fault detection techniques do not offer the capability to differentiate between faulted conditions and expanded conditions. However, such capability is crucial for truly adaptive modeling. In the proposed PCA ECM technique, the Q statistic is used as the fault indicator, and the $\mathrm{T}^{2}$ statistic is used as the expanded condition indicator. This is the process that is followed in this research. It is shown that this ECM technique can correctly differentiate between expanded and fault conditions.

The PCA ECM has been tested on a generated data set with linear relationships. Figure 3-4 shows the 5 different generated sensor values over time. The PCA ECM technique was shown to detect all three of the faults that were tested. These included drift faults, bias faults, and stuck sensor faults. Figure 3-5 shows these faults with relationship to the original sensor 2 values. 


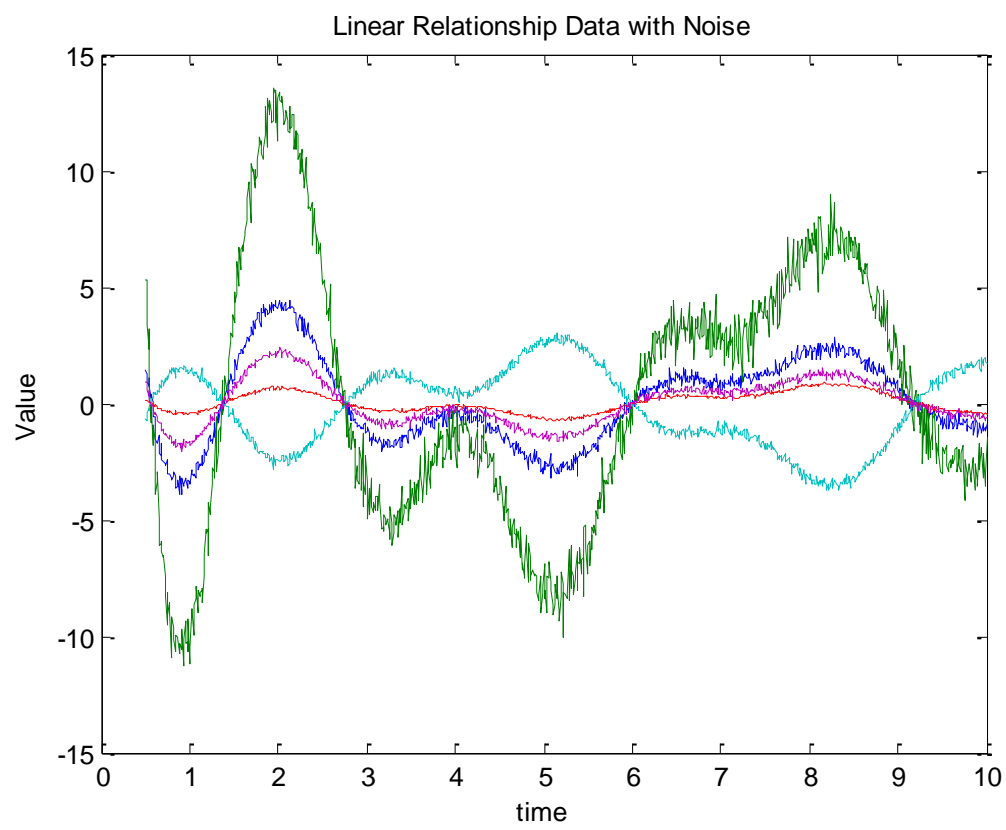

Figure 3-4: Generated Data
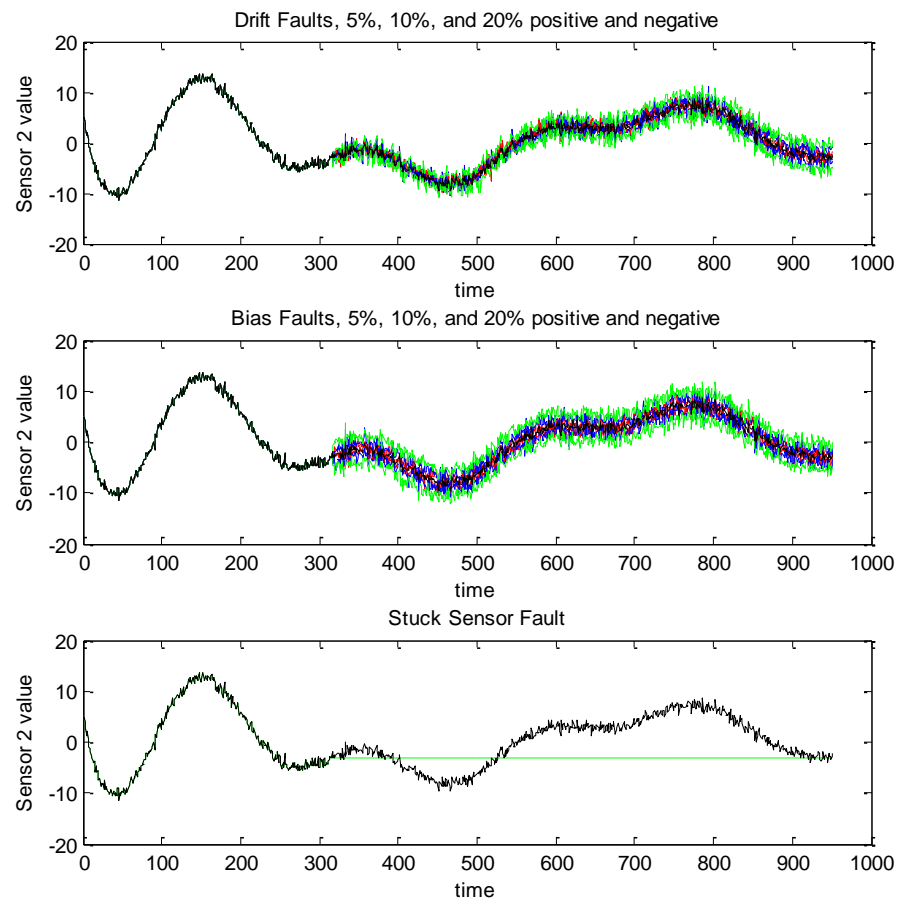

Figure 3-5: Generated Faults

Figure 3-6 shows the positive bias faults that were tested and the corresponding T-squared and Q statistics. The Q-statistic detects the bias faults. The negative bias faults had similar results. 

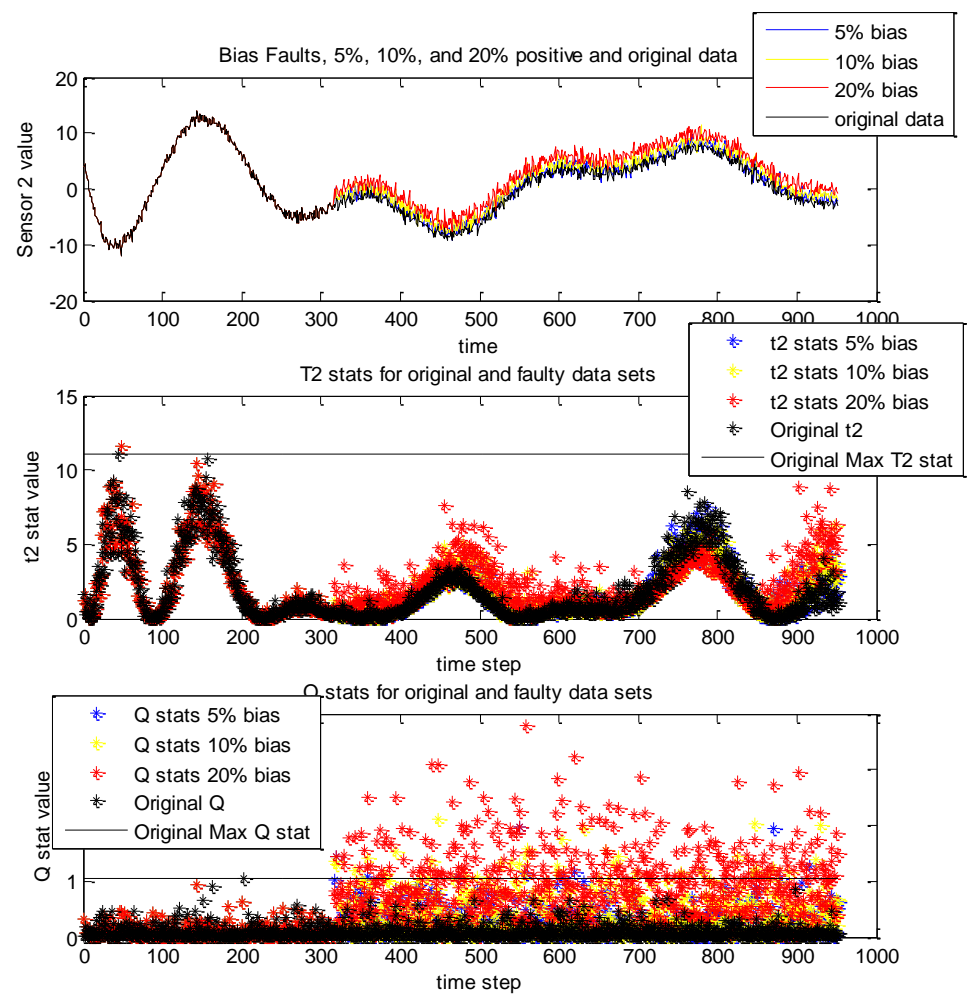

Figure 3-6: Bias Fault ECM Results

The major benefit of this technique was shown in its ability to correctly classify an expanded condition. Figure 3-7 shows the expanded condition and the normal condition. The PCA ECM technique is used, and no faults are detected. The red signal represents the expanded condition and the blue represents the normal operation. Both signals have the same linear relationships with the other signals; the Q statistic range is similar which signifies no faults. 

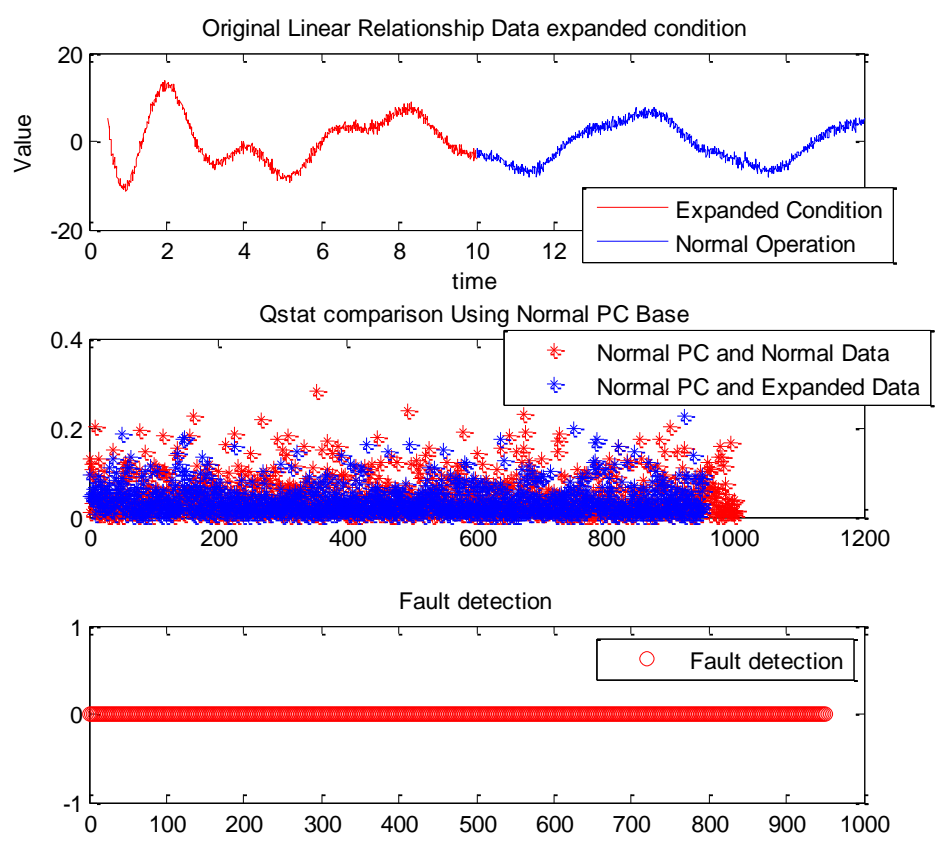

Figure 3-7: Expanded Condition ECM Results

The proposed ECM methodology can be used to differentiation between faults and expanded operating conditions when an anomaly is detected. If an anomaly is determined to be due to faulty conditions, the next step in the health monitoring system is to diagnose the fault.

\subsection{Fault Diagnostics}

For the current research, after a fault has been detected using the proposed PCA method, an expert system or classification algorithm may be used to determine the fault type. Three diagnostic tools have been developed over the course of this research. They utilize different information to make diagnoses and require different data for training. The Fault Identification Algorithm (FIA) uses monitoring system residuals and fuzzy logic to determine the possibility that a detected fault is in each of the historical fault classes. The Elimination and Similarity (ES) method uses two-step process to first eliminate historical faults which are very different from the detected fault and then choose the most likely fault from those classes which remain. The third method utilizes the contributions of each measurement of the faulty observation to the Hotelling's $\mathrm{T}^{2}$ statistic and the Q statistic in the PCA model in a series of ANNs to determine the fault class. Each of the methods is described in detail in the following sections. Similar systems may be built using additional features, such as the results of the PCA fault detection routine, PC 
values, $\mathrm{T}^{2}$ - and Q-statistics, sensor values, expert knowledge, etc. For the current application, this added complexity was unnecessary.

\subsubsection{Fault Identification Algorithm}

The FIA takes the residual vector sets from the AAKR model and compares them to historical exemplar data vector from past faults. The algorithm then uses this comparison to obtain the output. This output is in the form of a vector of possibility values which correspond to the likelihood that each fault occurs. Figure 3-8 outlines this process.

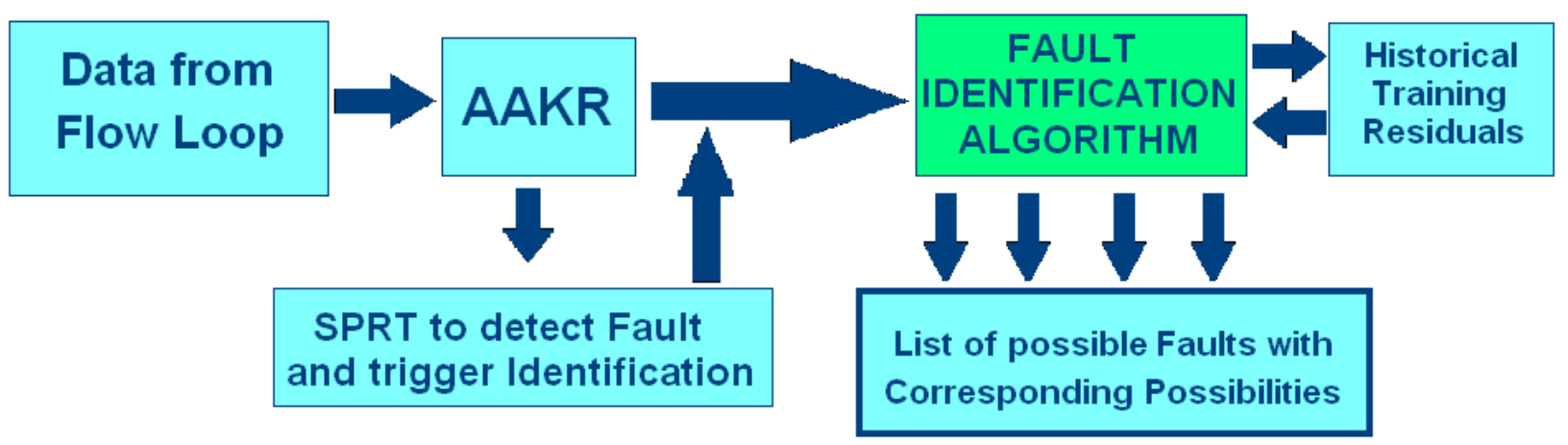

Figure 3-8: Flow Chart of FIA

The input to the FIA is set of query vectors from the AAKR model. This residual query matrix can then be compared to each of the example vectors within the training matrix. First, the similarity of the query residual to the exemplar residuals is calculated according to:

$$
\text { Residual Similarity }=\frac{\sum_{1}^{n}\left(\left[X_{\text {Train }}-X_{\text {Queryy }}\right] /\left[S T D_{\text {profite }}\right]\right)^{2}}{n}
$$

Then, the standard deviation similarity is calculated:

$$
\text { Standard Deviation Similarity }=\sum\left(\left[S T D_{\text {Train }}-S T D_{Q_{\text {wery }}}\right] /\left[S T D_{\text {profile }}\right]\right)^{2}
$$

In these equations $X_{Q u e r y}$ and $S T D_{Q u e r y}$ are the residual vector set and standard deviation for the query matrix. They are compared against the residual vector set, $X_{\text {Train }}$, and standard deviation vector set, $S T D_{\text {Train }}$, for each fault example. The comparisons consider the summed squares of the normalized differences between the sets. The residual similarities are then averaged by dividing by the length of the query data, $n$.

In certain cases, though a sensor may be behaving in a given manner, the residuals may look very different. In these cases, the residual similarities may not compare as well as normal for similar faults. However, in these cases attributes of the residuals, such as standard deviation, 
should still be similar. It is for this reason that both a residual and standard deviation similarity is obtained to make the fault comparison.

These similarity values are converted to fuzzy fault possibilities according to

$$
\begin{gathered}
\text { Weight }_{\text {Fault } X}=C \sqrt{\frac{1}{\operatorname{ResSim}_{1}}+\frac{1}{\text { ResSim }_{2}}+\frac{1}{\text { ResSim }_{2}}+(1-C) \sqrt{\frac{1}{\text { StdSim }_{1}}+\frac{1}{\text { StdSim }_{2}}+\frac{1}{\text { StdSim }_{2}}}} \\
\text { Possibility }_{\text {Fault } X}=\frac{\text { Weight }_{\text {Fault }}}{\sum \text { Weights }}
\end{gathered}
$$

The weight value for each fault is the sum of the inverses of the greatest three similarities from each fault. The weights correspond to the fuzzy likelihood that a particular fault has occurred. If there are fewer than three examples of a particular fault then all available similarities are added to determine a weight. The square root of these sums are then added and multiplied by a residual weighting constant, $C$. This value is a user defined parameter which determines how much emphasis is given to the residual similarity as opposed to the standard deviation similarity. This constant can be any value between zero and one. The, the weights from each fault are normalized by dividing by the sum of the weights to obtain a possibility value between zero and one. This normalization ensures that all the possibilities sum to one. This possibility is a fuzzy measure of how likely it is that the query data was the result of a particular fault. The output of the algorithm is a vector of possibilities for each fault within the training matrix. This fault diagnostic technique was applied to experimental loop control data; the results of this analysis are given in Section 5.4.2.

\subsubsection{Elimination and Similarity Diagnostics}

A number of fault diagnostic techniques have been studied in depth and applied to varying systems of interest. A fault diagnostic module has been created that can be implemented into a complete equipment surveillance system, which will be capable of determining the most likely fault given the faulty residuals. A two step process which consists of fault signature elimination and a weighted path similarity are used to give the best identification. This technique is referred to as the elimination and similarity (ES) diagnostic technique. A detailed description of both steps is provided. Starting with faulty residuals and ending with a most likely fault.

Several fault detection and identification (FDI) techniques have been developed for application with the ANPM. Diagnostics is also referred to as identification and isolation; these terms will be used interchangeably throughout this paper. The approach used in this FDI system 
can be applied to numerous equipment surveillance systems, an adaptive modeling technique is not required. An overview of the basic implementation of this ES diagnostic technique into a complete equipment surveillance system is discussed.

One of the main requirements of the monitoring and fault detection techniques are, the prediction ability of the model which results in residuals, and the fault detection ability that produces a set of faulty residuals corresponding to known sensors, respectively. The fault detection portion of an equipment surveillance system can be a number of different techniques such as the SPRT, EULM, PCA ECM, or many other techniques, and still be compatible with the ES diagnostic module presented. Depending on the system of interest's monitoring needs, different detection methods have different strengths. The SPRT is a sequential hypothesis testing techniques that has good detection results for a number of systems; however, both the SPRT [Wald 1945] and the EULM [Garvey et al. 2006] techniques do not have the ability to differentiate between expanded and fault conditions. The PCA ECM technique [Humberstone et al. 2009], however, is shown to have this ability to detect faults and differentiate expanded conditions in an adaptive environment.

The residuals are the inputs into the ES diagnostic module. These faulty residuals are classified as either a positive drift or bias which is given the signature designation +1 , a negative drift or bias which has a signature designation -1 , or a noise increase which has a signature designation of 3 . The non-fault residuals have a fault signature designation of 0 . This process of classification of fault signatures can be seen in Figure 3-9. The end result is a complete fault signature vector. 


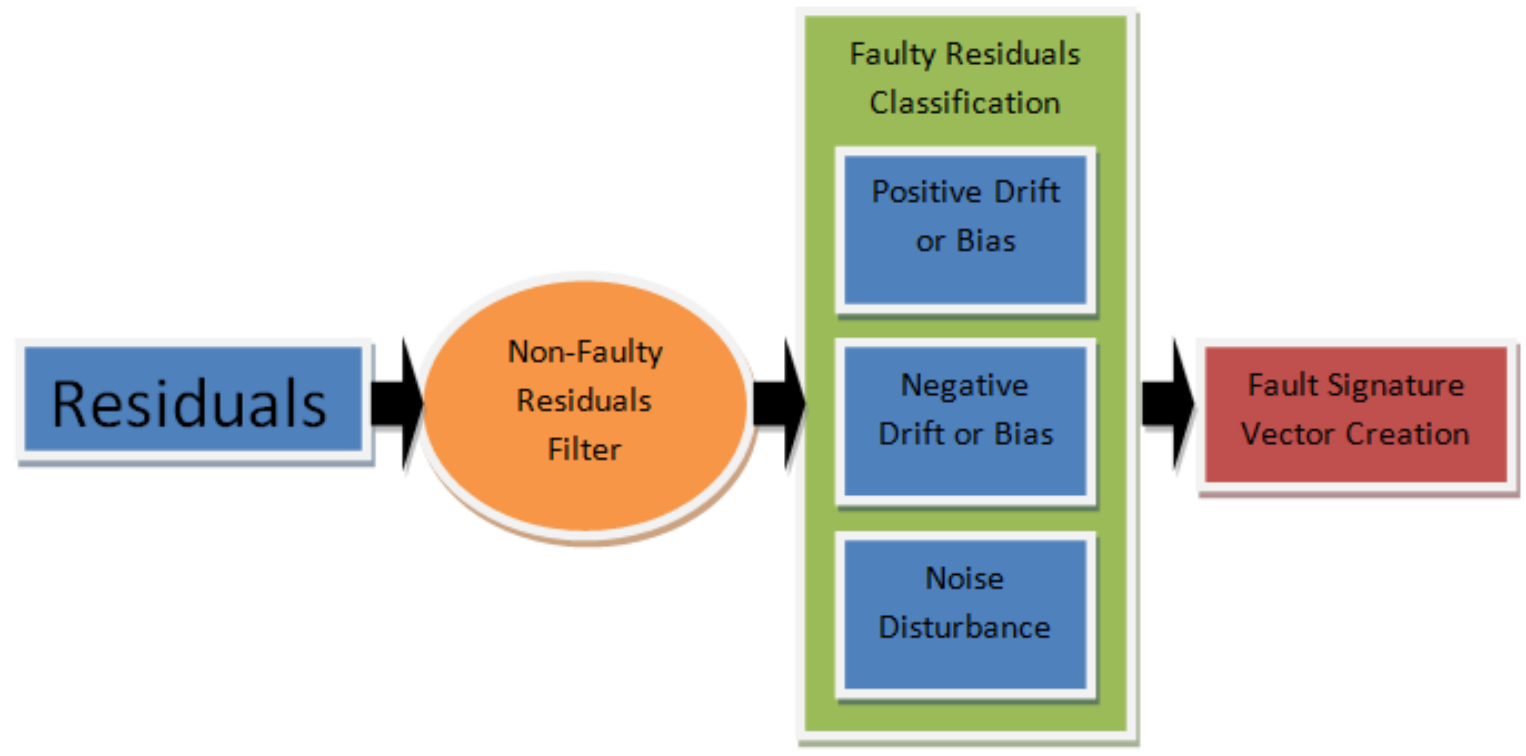

Figure 3-9: Classification of Fault Signatures

The complete fault signature vector is compared to a failure bank of fault signature vectors that correspond to all possible failures. After the possible failures are determined there may be more than one possible fault corresponding to the given fault signature vector. This elimination of possible faults to only include failures with similar fault signature vectors is the first step in the ES diagnostics. A historical residual memory matrix of failures is used to designate which fault is most similar to the current failure. The similarity method uses the Euclidean distance from the past failure residuals and the current faulted residuals.

$$
S=\sum_{i=1}^{n}\left[\sum_{j=1}^{m}\left(P F R_{i j}-C F R_{i j}\right)^{2}\right]
$$

where $\mathrm{n}$ is the number of faulty residuals and $\mathrm{m}$ is the number of observations from the time a fault was initially detected.

First the historical residual memory matrix of failures is trimmed to include only the faults that match the given fault signature vector. And then this matrix is trimmed to include only the residuals that have failures. Figure 3-10 shows the process of comparing the current failure to the past failures that have the same fault signature vector. This example shows that system failures 1, 4, and 6 have the same fault signature vectors. The Euclidean distances between the actual failure residuals and the historic residuals of the designated failures measure the similarity of the failures. 


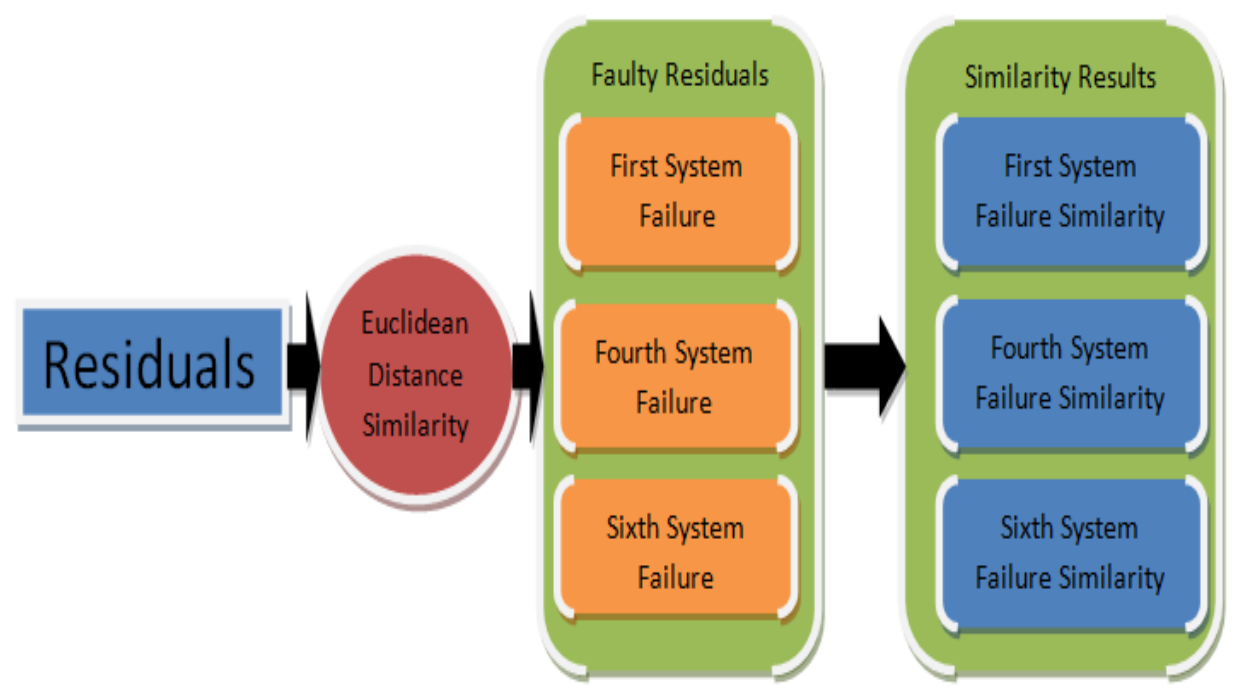

Figure 3-10: Failure Similarity Process

\subsubsection{Fault Identification through Neural Networks}

The final fault diagnostic routine developed utilizes the results of the PCA fault detection algorithm, specifically the contributions of each variable of the faulty observation to the $\mathrm{T}^{2}$ - and Q-statistics. The previously described diagnostic techniques are well suited to systems with a large number of faults to differentiate, but may be overly complicated for systems with only a few faults. To utilize this method, a separate ANN for each fault type is trained. These networks are evaluated in a set order, from the easiest-to-diagnose faults through to more difficult faults. When an observation is determined to be faulty, the appropriate data is cascaded through the networks until one identifies the fault. In this way, the network system has a higher chance of correctly identifying the fault. Because this method requires a separate network for each possible fault, it is not suggested for systems with many faults to be differentiated. However, for the simulated IRIS data described in Section 4.3, only four faults are considered. It is important to note that this method is not well suited to adaptation. If an unexpected fault is identified, the neural networks for each previous fault must be tested on the new fault, and perhaps retrained. A new network must also be built for each new fault type. For a complete discussion of ANNs, including network architecture, training algorithms, and performance optimization, the interested reader is referred to Tsoukalas and Uhrig [1997]. 


\section{DATA COLLECTION}

To support methodology development and model validation, data were collected from multiple sources, including a flow control loop and a small transfer pump degradation experiment located in the Nuclear Engineering Department, University of Tennessee (UT), and two IRIS simulators. Each of these data sources is described here.

\subsection{Flow Loop Monitoring}

An experiment was performed using a flow loop system to emulate various modes of degradation. Sixteen datasets were compiled, each consisting of a 9000-second (2.5-hour) run in which two vertical tanks' water levels were cycled through various set points. The lowest water level used was $300 \mathrm{~mm}$ and the highest was $598 \mathrm{~mm}$, for an average level of about $450 \mathrm{~mm}$. The following 19 variables were measured and recorded in an output deck: tank 1 setpoint, tank 1 level, tank 2 setpoint, tank 2 level, flowrate into tank 1, flowrate into tank 2, flowrate return from tank 1, bypass flowrate, flowrate return from tank 2, tank 1 temperature, tank 2 temperature, sump tank temperature, pump temperature, voltage from valve 1, voltage from valve 2 , voltage from valve 3 , voltage from valve 4 , voltage to valve 1 , and voltage to valve 2 . Figure 4-1 gives a schematic of the experimental flow control loop. 


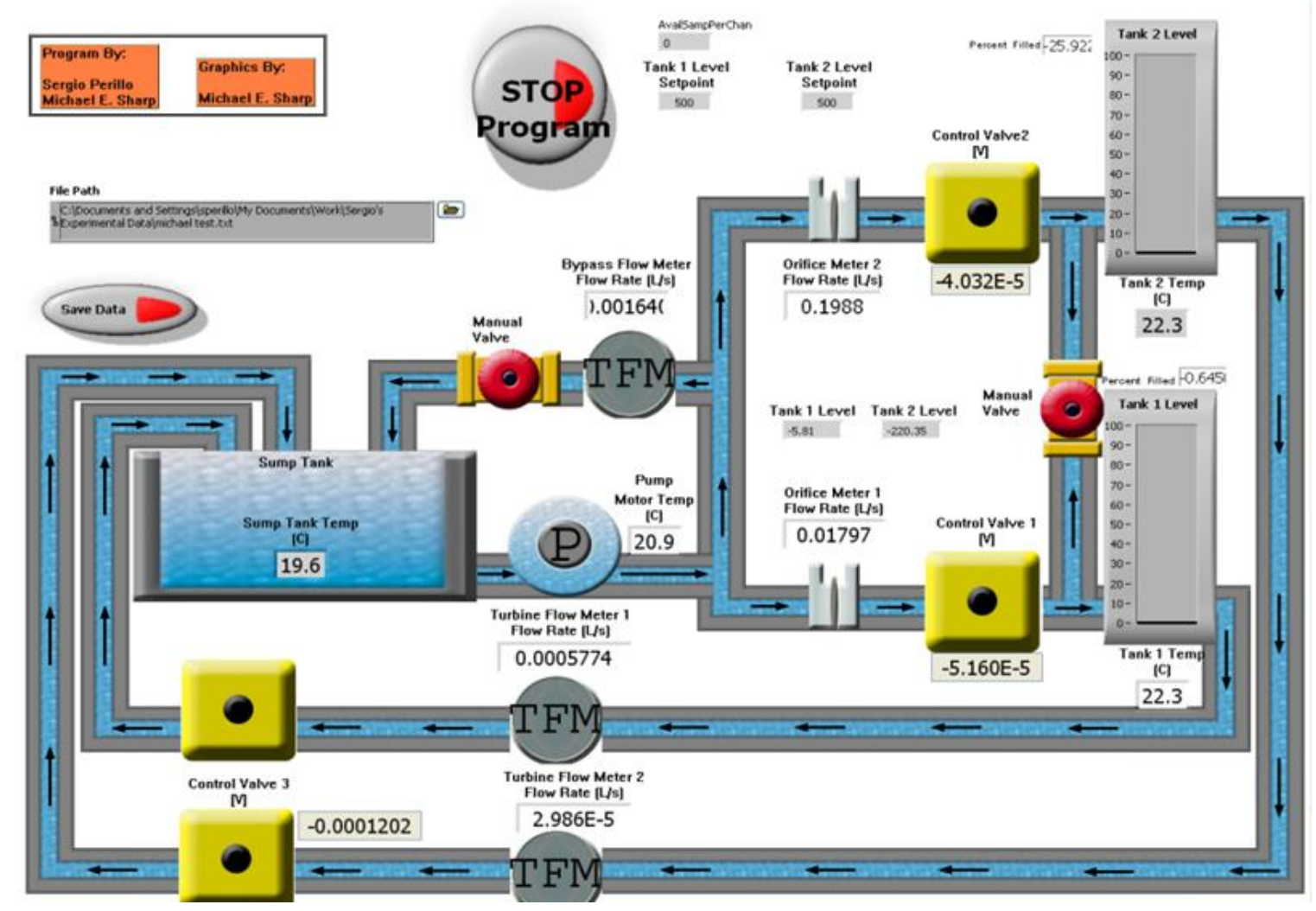

Figure 4-1: Schematic of Two-Tank Flow Loop

The list of preset water level values form a "profile," and when a fault is inserted, the system attempts to mask it by increasing or decreasing the input flowrates into the tanks to maintain this profile. Because of the self-correcting behavior of the system, degradation was not evident in the tank levels themselves, but it was noticeable in the input flowrates for both tanks 1 and 2. The variables used for fault detection are given in Table 4-1.

Table 4-1: Flow Loop Variables for Fault Identification

\begin{tabular}{|l|l|}
\hline Variable \# & Sensor Description \\
\hline Variable 1 & Tank 1 Setpoint \\
\hline Variable 2 & Tank 1 Level \\
\hline Variable 3 & Tank 2 Setpoint \\
\hline Variable 4 & Tank 2 Level \\
\hline Variable 5 & Tank 1 Inlet Flow Rate \\
\hline Variable 6 & Tank 2 Inlet Flow Rate \\
\hline Variable 7 & Tank 2 Outlet Flow Rate \\
\hline Variable 8 & Tank 1 Outlet Flow Rate \\
\hline Variable 9 & Voltage to Control Valve 1 \\
\hline
\end{tabular}


Variable $10 \quad$ Voltage to Control Valve 2

The data collected includes four normal runs in which the tank water levels fluctuate uninterruptedly under normal operating conditions. These data can be considered nominal operating data and were used to make create AAKR monitoring models.

The first degradation concerned the return valves. Both tanks' return valve openings were decreased successively by a factor of 1 from an initial value of 10 -which corresponds to a completely open valve-down to 0 , which is a completely closed valve. Three variations were tested, starting with a reduction every 15 minutes beginning 15 minutes into the run; then a reduction every 10 minutes beginning 30 minutes into the run; and finally every 5 minutes beginning 45 minutes into the run. Each of these tests was performed twice, resulting in a total of 6 return valve degradation sets.

The second mode of failure tested was a level sensor degradation. This fault was programmed into the user interface and involved a $0.02 \%$ per minute drift, a $0.07 \%$ per minute drift, and a $0.15 \%$ per minute drift. By the end of each run, the level sensors were thus degraded by $2.97 \%, 10.38 \%$, and $22.25 \%$, respectively. These percentages are based on the average set point value of $450 \mathrm{~mm}$.

Ten faults were tested or simulated for use with the proposed FIS and ES diagnostic techniques. These faults are summarized in Table 4-2. The results of these analyses are given in Section 5.4.

Table 4-2: Flow Control Loop Fault Types

\begin{tabular}{|l|l|l|l|}
\hline Fault 1 & Return Valve Blocked & Fault 6 & Sensor 7 drift up \\
\hline Fault 2 & Tank Level Drift (60 mm) & Fault 7 & Sensor 6 jump down \\
\hline Fault 3 & Variable 5 jump up & Fault 8 & Sensor 6 drift down \\
\hline Fault 4 & Variable 7 jump down & Fault 9 & Sensor 8 stuck sensor \\
\hline Fault 5 & Variable 9 stuck sensor & Fault 10 & Sensor 8 variance increase \\
\hline
\end{tabular}

The intent of the variations in both the return valve and sensor level degradations was to produce comprehensive datasets to model real-world situations in which degradation typically begins after a certain grace period. In addition, the flow loop uses distilled water to more closely 
mimic the water used in a nuclear power plant. The data collected from this set up was used to develop and test the PCA ECM technique; the results of that analysis are given in Section 5.4.1.

\subsection{Pump Degradation}

The pump setup consists of four transfer pumps atop a six-foot platform pulling and pumping water from and into a single reservoir at floor level. The pumps have to pull the water about five feet from the level of the reservoir. Accelerated aging is achieved by damaging the flexible impellers in the pumps. The damage is inflicted using an x-acto knife. This process continues until failure, which is defined as the point at which the pumps can no longer pull the water up the five feet.

The current experiment is a continuation and improvement of a previous experiment investigating pump degradation [EPRI, 2009]. Each AC motor, Jabsco pumps (Model No. 12310 Series), operates using phenolic plastic flexible impellers. The individual flow loops are each outfitted with a differential pressure gauge across the pump, as well as vibration sensors and current clamps attached to the pump motors themselves. The components used in this experiment are listed in Table 4-3.

Table 4-3: Component List

\begin{tabular}{|c|c|c|c|}
\hline Use & Manufacturer & Name & Model Number \\
\hline Pump & Jabsco & Self-Priming Pump & 12310-0001 \\
\hline Pressure Sensor & $\begin{array}{c}\text { General Electric } \\
\text { Sensing }\end{array}$ & Pressure Transmitter & W30-34E-1-R \\
\hline DC Power Supply & Autonics & Power Supply & SP-0324 \\
\hline Accelerometer & PCB Piezotronics & ICP Accelerometer & YJM352C18 \\
\hline Current Sensor & Fluke & AC Current Clamp & i200s \\
\hline $\begin{array}{c}\text { Signal Receiver } \\
\text { Chassis }\end{array}$ & $\begin{array}{c}\text { National } \\
\text { Instruments }\end{array}$ & NI CompactDAQ & NI cDAQ-9178 \\
\hline $\begin{array}{c}\text { Signal Receiving } \\
\text { Module 1 }\end{array}$ & $\begin{array}{c}\text { National } \\
\text { Instruments }\end{array}$ & $\begin{array}{c}\text { 4-Channel } \\
\text { Selectable IEPE }\end{array}$ & NI 9205 \\
\hline $\begin{array}{c}\text { Signal Receiving } \\
\text { Module 2 }\end{array}$ & $\begin{array}{c}\text { National } \\
\text { Instruments }\end{array}$ & $\begin{array}{c}\text { 32-Channel Analog } \\
\text { Input Module }\end{array}$ & NI 9234 \\
\hline
\end{tabular}

The Data Acquisition System (DAQ) is a National Instruments (NI) based hardware setup connected to a LabView software interface inside the computer. This NI Compact DAQ (NI cDAQ-9178) is the housing for three input modules, a NI 9205 connecting the differential pressure data, and two NI 9234's, which connect the current and vibration data and also provide 
anti-aliasing filters for that data. The data is collected at a sampling rate of $1 \mathrm{kHz}$ for slightly over two minutes after the startup of the pumps.

Once an output was achieved, it was found that the signal was very noisy. This was attributed to the lack of a solid water column in the plastic lines going to the piping. To rectify this, new piping was installed with the taps for the pressure lines entering the bottom of the piping instead of the top as in the previous setup. The outlet piping on each pump was also given an upward bend so that any bubbles in the piping at that point would move towards the top of the bend and not enter the pressure lines. The final setup can be seen in Figure 4-2. Between these two changes, the amount of bubbles in the pressure lines was greatly reduced and the pressure signal was made less noisy (Figure 4-3). A manifold was setup between the high pressure and low pressure lines right against the pressure sensors in order to allow the high pressure line to prime the low pressure line and clear any bubbles.

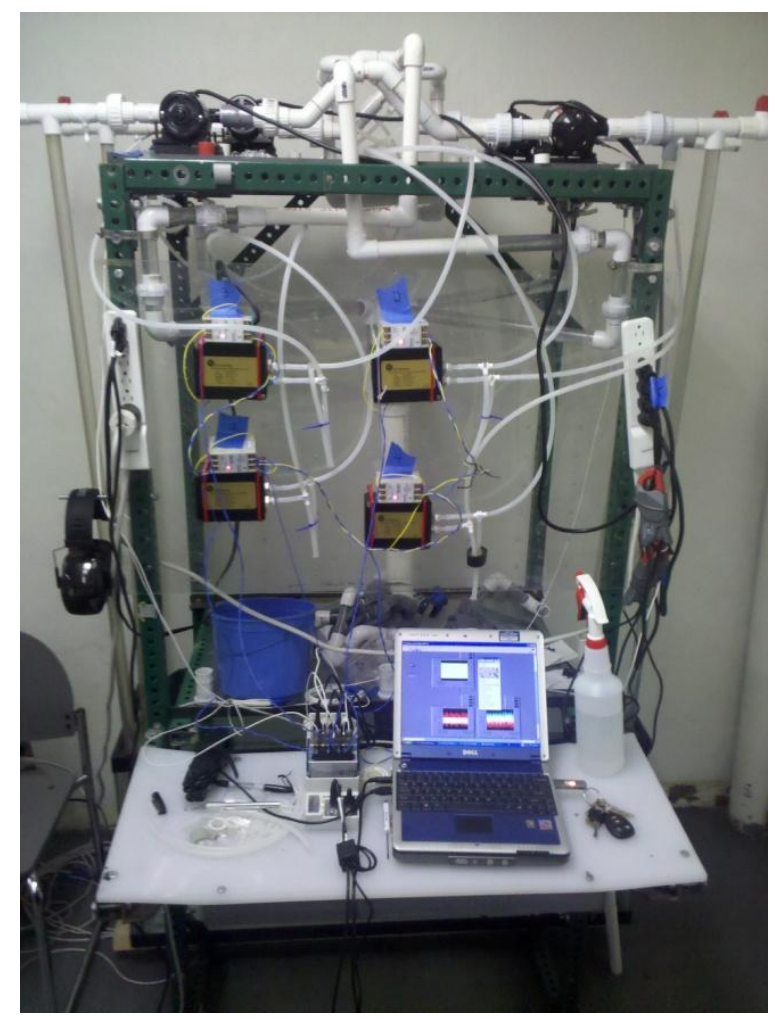

Figure 4-2: Pump Degradation Experimental Setup 


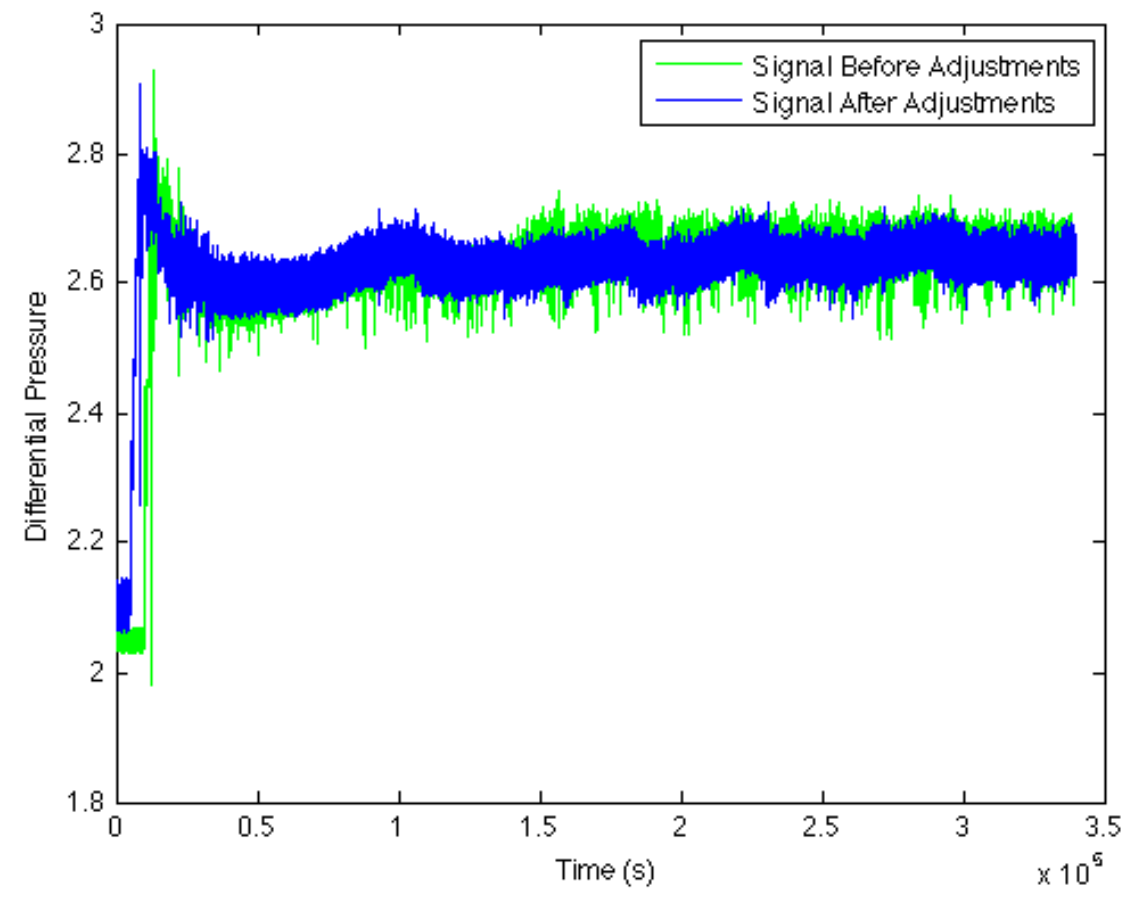

Figure 4-3: Differential Pressure Signal Before and After Adjusting Setup for Air Bubbles

Finally, the data acquisition program was built in LabVIEW. The system was set to collect pressure, vibration, and current data for each pump for 210 seconds. The program writes all of this data to a file and graphically outputs the data for each pump on three charts, one for each data type. The program was actuated and the pumps activated about 4-5 seconds after the program started recording data. The delay is to assure that all start-up data is recorded, as this is the target data for analysis.

The focus of this experiment is to capture the startup transient of these pumps while also collecting a short amount of steady-state data for comparison. In order to accelerate the degradation process, and to precipitate impeller failures in a timely manner, increasingly large notches are cut into the center of the impeller vanes between each test run. Failure of the pumps is defined as the time when the pumps are no longer able to self-prime.

An initial run was performed with a set of impellers with no degradation. These were then set aside and used intermittently for baseline measurements. A new set of impellers was the put in the pumps and data was collected on them with no degradation. A small cut was made into one vane of each of the four impellers to imitate impeller degradation. Figure 4-4 shows an example of the small cuts that were made in one of the vanes of each impeller. 


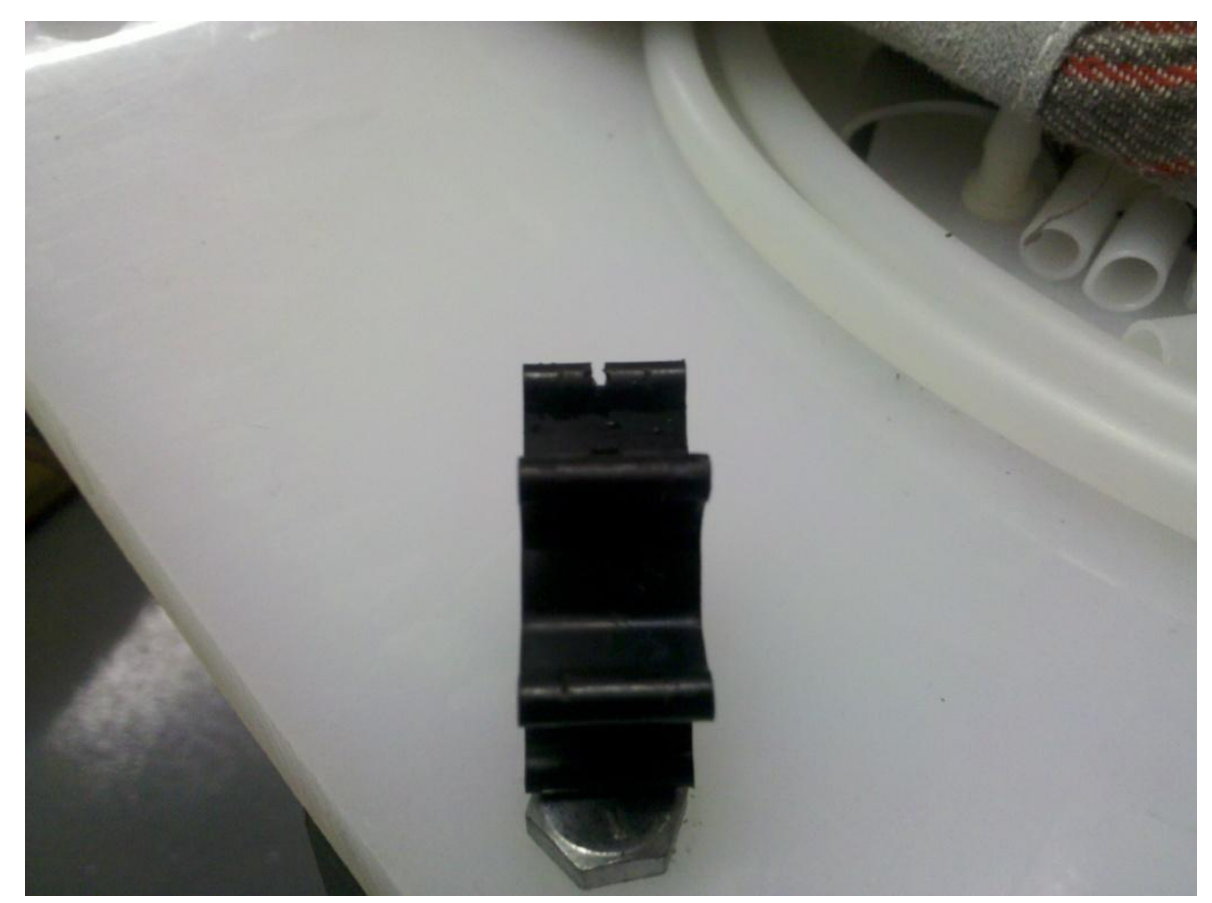

Figure 4-4: First Level of Impeller Degradation

The impellers were the installed into the pumps again. Similar incisions were then made in each other vane, one-by-one, and data was collected for each. Once each vane had a cut, a larger cut was made on one of the vanes, to simulate the initial cut's growth with time, as shown in Figure 4-5. Next, a similar cut was performed on a second vane. As failure had still not occurred, the process was accelerated and the second sized cut was made on all of the remaining vanes of each impeller. This finally caused failure and provided a rough idea of the level of degradation that leads to failure. 


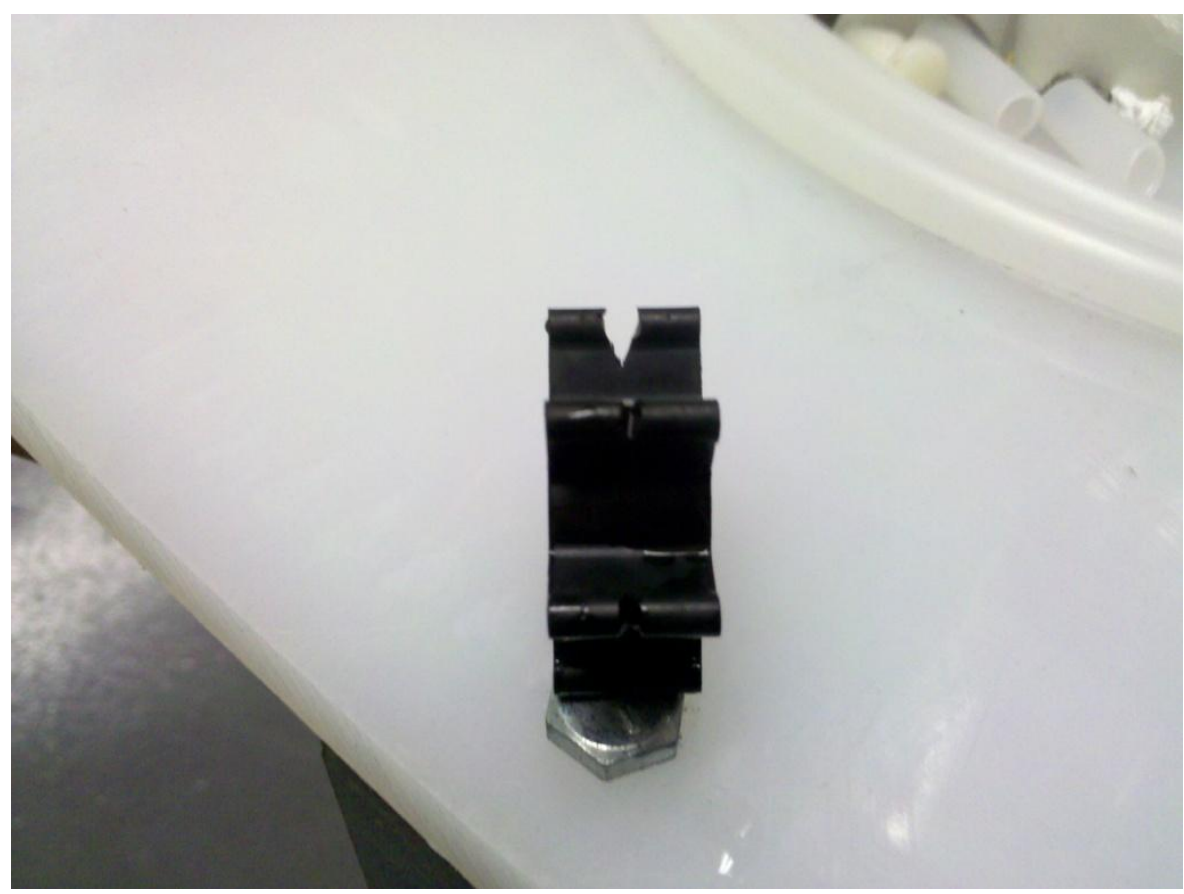

Figure 4-5: Increased Impeller Degradation

After a run with the original baseline impellers, new impellers were again installed in the pumps. A run was performed with the impellers undamaged. A triangular cut was made on the edge of each vane of the impellers as the first degree of degradation. This cut is approximately $2 \mathrm{~mm}$ wide and $2 \mathrm{~mm}$ deep. This caused the pumps to noticeably struggle to pull the water up the required $5 \mathrm{ft}$. The second level of degradation involved cutting another $0.5 \mathrm{~mm}$ from one side of the wedge shaped cut on each vane. This caused pump failure on all four pumps. With this, a much greater understanding of exactly the level of degradation at which failure occurs was gained.

With a fine degree of understanding of the amount of degradation necessary for failure, it was then possible to degrade the impellers at regular intervals knowing the failure point. Thus, the third set was degraded such that it would take four degrees of regular degradation to cause failure. This was done to gain sufficient data points to search for trends indicating approach to failure. Fifteen data collection runs were performed for each degradation level. This resulted in the failure of the last set of impellers.

\subsection{IRIS Data Simulation}

The proposed adaptive monitoring and health management architecture was applied to the IRIS nuclear plant design. The IRIS design is a generation IV small- to medium-size modular 
reactor design that has a proposed 335MW output. The IRIS reactor is an integral Pressurized Water Reactor with eight helical coil steam generators. There are a number of advantages that the IRIS reactor has over traditional pressurized water reactors; most of these are safety related benefits necessary for remote operation in developing nations. Because the IRIS reactor is still in the design stage, no actual operation data is available. However, two simulators have been developed. Data obtained from those simulations is used in this research. Because the two simulators do not track the same set of sensors, model development and testing was performed in two phases. First, the nominal condition adaptation of the ANPM was tested using a reduced number of sensors to utilize both simulators. Then, the PCA-based expanded operation monitoring system was testing using the full data set from the high-fidelity simulation. The two simulators and the data obtained from each are briefly described below. A more detailed discussion of the IRIS simulators is given in the report on Objective 1.

\subsubsection{MATLAB/Simulink IRIS Simulation}

The first simulator, considered to be a low-fidelity simulator, was built by researchers at the University of Tennessee using MATLAB Simulink® (Li et al., 2009). The Simulink model is a modular model which includes the reactor core, the helical coil steam generators, and the balance of plant. In testing the adaptation phase of the ANPM model, the results of this simulator are considered the first-principle model results and are used to seed the original memory matrix.

\subsubsection{NCSU IRIS Simulation}

The second simulator used was developed by Michael Doster at North Carolina State University (NCSU). NCSU has demonstrated experience in developing high fidelity, full plant simulators for predicting the dynamic response of pressurized water reactors during normal and off-normal operational conditions. An IRIS specific simulator has been developed which includes a model of the IRIS Pressurizer, a six delayed neutron group kinetics model, a decay heat model and a hot channel/departure from nucleate boiling (DNB) model. In the IRIS design, the steam generators are helical coils, where the secondary fluid flows on the tube side of the heat exchanger. Detailed models have been developed to describe the dynamics of steam generators of this design. For testing model adaptation, the results of this simulator are used as the high fidelity, "operational" data. 
In support of the Year 3 prognostics tasks, North Carolina State University used their IRIS simulator to generate degradation data for the IRIS system. Three degradation modes were identified for testing:

1. Heat exchanger fouling

2. Pump cavitation

3. Sensor drift

Each of these planned degradation modes are described in some detail in following sections. In addition to nominal operating data, simulations of each fault type are used to develop fault detection, diagnostic and prognostic models for the faults. For each condition, a common load profile was used, shown in Figure 4-6. This profile exhibits the load-following characteristics expected for the IRIS reactor, with full demand during the day and lower demand at night. To alleviate the computational time, each fault was simulated for one 24-hour period each month with increasing fault severity at each simulation. Only one simulation of each fault was available for each fault type. Additionally, we are only concerned with fault modes that present one at a time; that is, we are not considering multiple fault modes occurring at the same time.

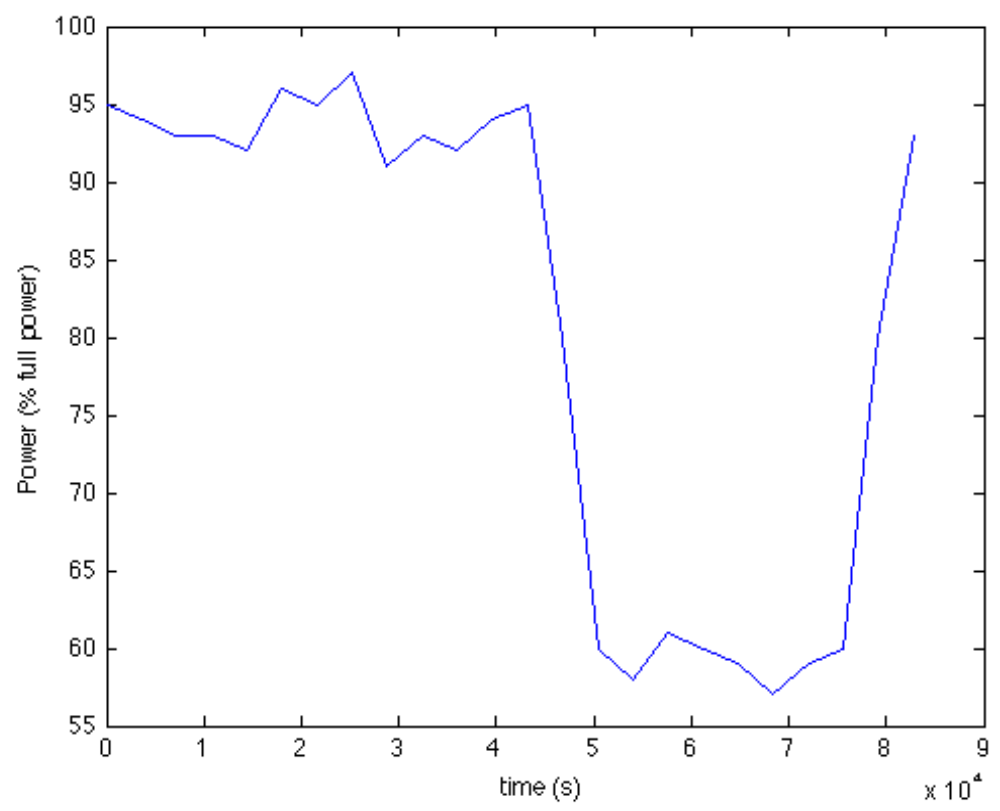

Figure 4-6: Load Profile for Simulated IRIS Data

\subsubsection{Heat Exchanger Fouling}

In general, fouling is the accumulation of unwanted material on the surface of process equipment. Heat exchanger fouling is of particular import for two reasons: first, fouling causes 
an increase in thermal resistivity which reduces heat exchanger effectiveness; second, it reduces the cross sectional area for flow which increases the pressure drop across the flow path. This work will focus on the effect of fouling on the thermal resistivity of the steam generator (SG) heat exchanger. The thermal resistance due to fouling, $\mathrm{R}_{\mathrm{f}}$, is included in the equation for the overall heat transfer coefficient as follows [Piezo Systems]:

$$
\frac{1}{U_{o} A_{o}}=\frac{1}{{ }_{\mathrm{o}} A_{o}}+\frac{\mathrm{R}_{\mathrm{f}, \mathrm{i}}}{\mathrm{A}_{\mathrm{i}}}+\frac{\mathrm{R}_{\mathrm{w}}}{\mathrm{A}_{\mathrm{w}}}+\frac{\mathrm{R}_{\mathrm{f}, \mathrm{o}}}{\mathrm{A}_{\mathrm{o}}}+\frac{1}{{ }_{\mathrm{i}} A_{i}}
$$

$\mathrm{U}_{\mathrm{o}}=$ overall heat transfer coefficient based on outside area of tube wall

$\mathrm{A}=$ tube wall area

$\mathrm{A}_{\mathrm{w}}=$ mean wall area

$\mathrm{R}_{\mathrm{f}}=$ thermal resistance due to fouling

$\mathrm{R}_{\mathrm{w}}=$ thermal resistance of wall

$\alpha=$ convective heat transfer coefficient.

$\mathrm{U}_{\mathrm{o}}$ decreases as the deposit thickness or $\mathrm{R}_{\mathrm{f}}$ increases with time. The thermal fouling resistance, $\mathrm{R}_{\mathrm{f}}$, is defined as:

$$
R_{f}=\frac{1}{\mathrm{U}_{\mathrm{o}, \text { dirty }}}-\frac{1}{\mathrm{U}_{\mathrm{o}, \text { clean }}}
$$

The overall heat transfer coefficient determines how much heat can be transferred between the hot and the cold fluid in a given heat exchanger. When it falls below a tolerable value, the heat exchanger has to be serviced.

To simulate SG heat exchanger fouling, we need only to define the overall heat transfer coefficient of the dirty tubes. Experimental results obtained in [Upadhyaya et al., NEER] are shown below. Figure 1 gives the percent change in overall thermal resistance as a function of runtime. This curve can be used to generate fouled data for the SG heat exchanger with some modifications. Though the results shown below are given for a matter of approximately 170 hours, we would like to extend this degradation mode to occur over the course of approximately one year. In addition, the total change in thermal resistance should be limited to approximately $30 \%$ of the nominal value; this degradation may be unusually high for legacy systems, but it is accepted here because the system is new and untested. In addition to nominal operation data, we would like to have 12 ten-minute "snap-shots" of simulated operation at 12 equally spaced intervals along the curve, to simulate the year long degradation process. 


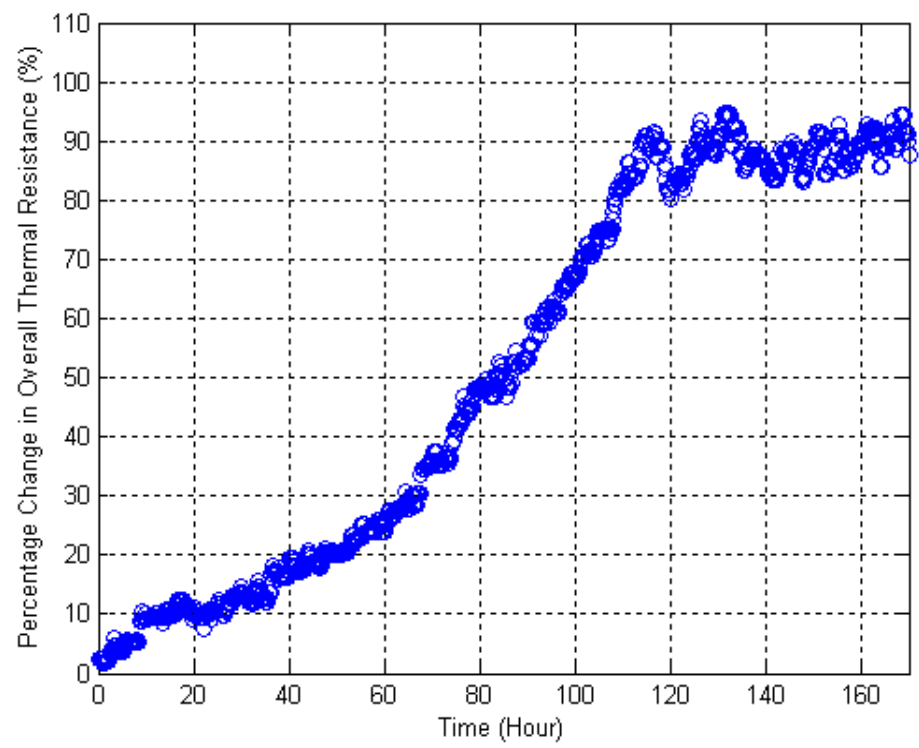

Figure 4-7: Change in Thermal Resistivity due to Fouling [Upadhyaya et al., NEER]

\subsubsection{Pump Cavitation}

Cavitation of centrifugal pumps can limit performance and compromise safe operation of the plant. Grist [1999] identifies four categories of cavitation-induced pump failure: hydraulic performance loss, hydrodynamically induced cavitation surging, thermodynamically induced cavitation surging, and cavitation erosion. This research will focus on hydraulic performance loss due to cavitation in the reactor coolant pump (RCP). This mode of pump failure leads to a continuing failure to provide the required coolant flow rate, which reduces volumetric performance. Such a failure mode appears as a head-drop below the non-cavitating performance characteristics as shown in Figure 4-8. In order to model this type of failure, pump curves corresponding to the cavitated pump performance should be generated and used to give the appropriate flow rate/head-loss relationship. It may be possible to modify the existing pump curves by a simple transformation, which accounts for the loss in flow rate at a given time. 


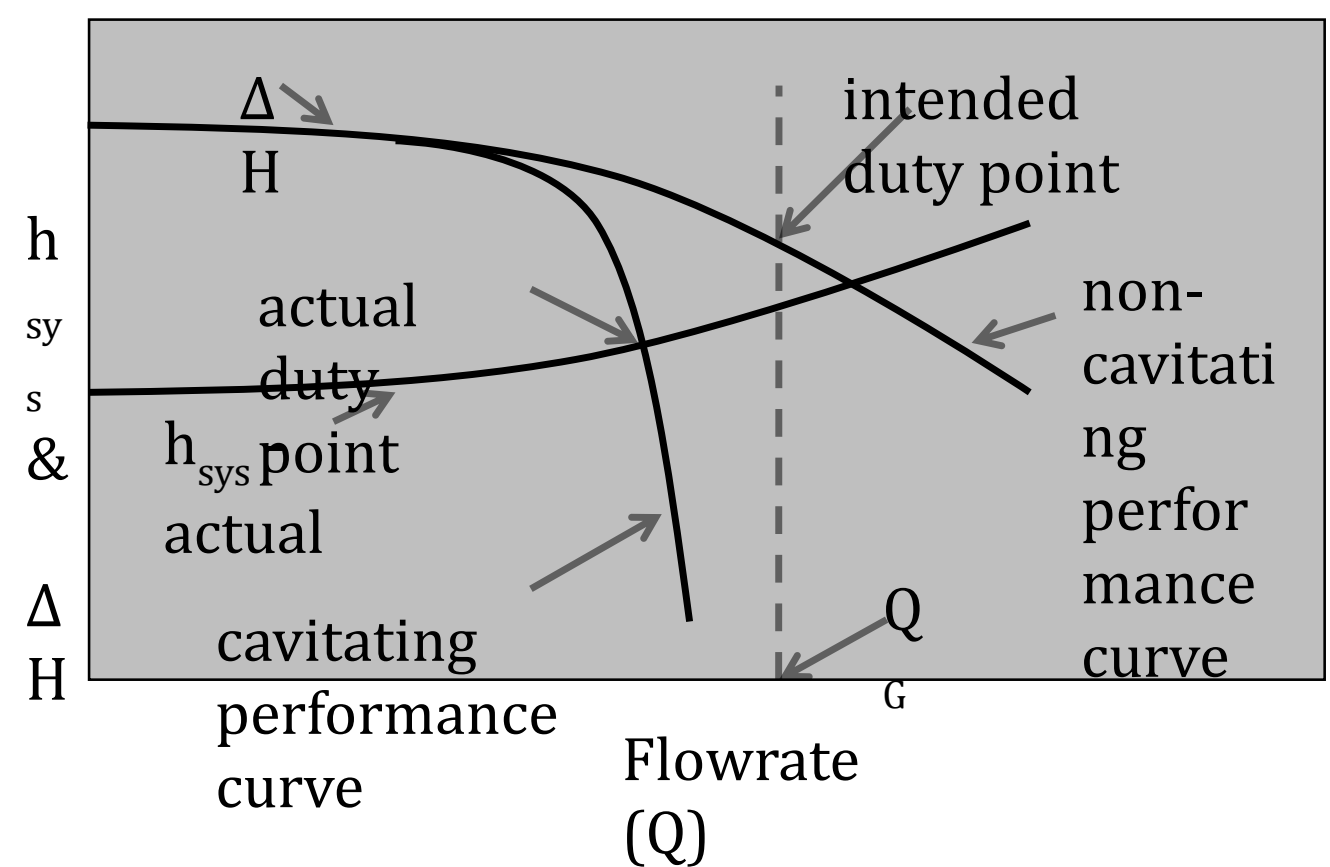

Figure 4-8: Identification of the actual duty point (recreated from [Grist 1999])

Once an appropriate transform to move between nominal and degraded pump performance curves has been identified, then data can be simulated to give "snapshots" of the degraded pump performance over the course of a year. Ideally, we would like to have ten minutes of un-degraded data representing nominal operation. In addition, ten-minute blocks of degraded operation giving a flow rate degradation of $1 \%$ with each block should be simulated resulting in a total degradation of $10 \%$ of flow rate. This can be achieved by regenerating the pump curves as shown in Figure 4-9, where the pump curve is regenerated for each degradation level by making the following transform of the flow rate:

$$
Q_{p}^{*}=\frac{Q}{p}
$$

where $p$ is the fraction of flow rate remaining, i.e. for the first degraded condition, where $99 \%$ of the flow is still available, $p=0.99$. This effectively shifts the pump curves in along the flow variable, adjusting for the lost flow rate. We will consider flow loss of $10 \%$ to be failure. Two types of degradation simulation are desired: first a degradation in only one of the RCPs, and then a degradation in all the parallel RCPs. As in the other fault modes, several histories of these progressions are needed, wherein the fraction of flowrate available may vary randomly by $\sim 0.1 \%$ about the expected loss value. 


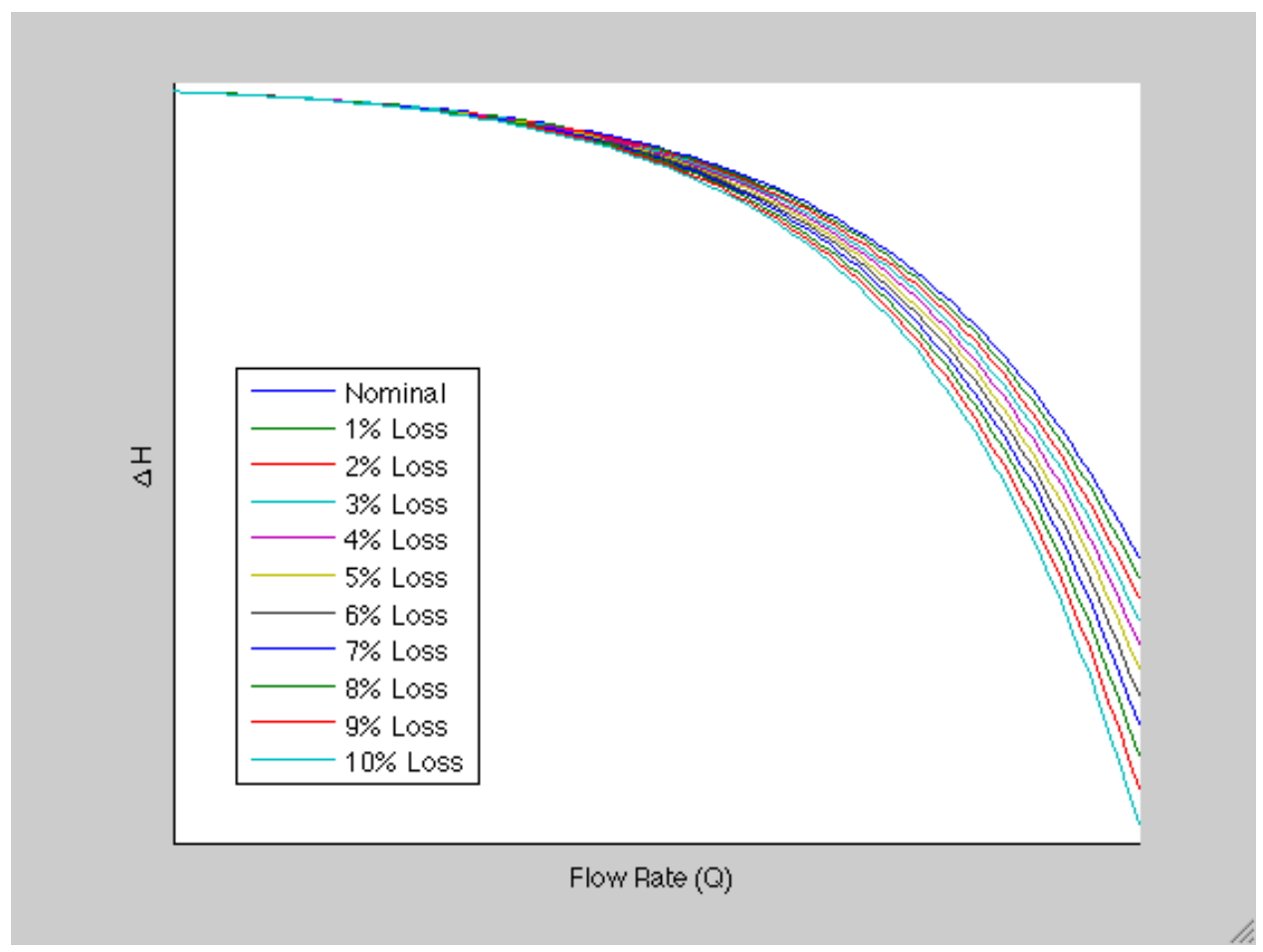

Figure 4-9: Degraded Pump Curves

\subsubsection{Sensor Drift}

Sensor drift is possibly the most straightforward fault. This involves a slow, linear drift in one sensor. This particular fault should be simulated for two different sensors: one process sensor and one control sensor. A process sensor refers to any sensor which measures a variable of the process but is not used by the control algorithms, whereas a control sensor is used for system control. Feedwater flow is a process sensor of particular interest because it is known to have problems in current pressurized water reactor (PWR) plant designs. The drift in the process sensor should not affect the values of any other sensors and will show up simply as a linear drift. The drift in the control sensor, however, will affect the other variables in the control scheme, leading to sensor fault masking. Sensor drift should occur linearly, eventually adding up to a drift of $1 \%$ of the actual sensor value. Ideally, this should occur over a simulated time of approximately one year. Multiple runs of several drift rates would be ideal, where the drift rate is not exact but includes some random component. Simulated drift rates may include $0.083 \%$ per month, $0.091 \%$ per month, and $0.10 \%$ per month, giving approximate degradation times of 13 months, 12 months, and 11 months, respectively including the first month of unfaulted operation.

This chapter has discussed efforts to collect or simulate nominal and degraded operational data to test the developed methodologies for adaptive system monitoring, fault 
detection and diagnostics, and prognostics. The next chapter presents the results of these analyses.

\section{RESULTS}

During the course of this research, model testing has utilized each of the data sources discussed above; however, the major focus has been on the simulated data of the IRIS system. The results of this work are discussed here. As mentioned previously, due to the limitations of the two available IRIS simulators, the experimental results are completed in two phases. In the first phase, the adaptation of the ANPM method is tested with the common sensor outputs of the two simulators, using the MATLAB/Simulink simulator as low fidelity (first principle model) data and the NCSU simulator as high fidelity (actual operation) data. The second phase uses only the data from the NCSU simulator to test for fault detection, differentiation between expanded operations and faults, diagnostics, and prognostics. For systems with more complete data available, it is straightforward to connect the two phases of results.

\subsection{ANPM Adaptation}

The two simulators share five common sensor measurements: hot leg temperature, cold leg temperature, feed flow rate and steam flow rate per steam generator, and feedwater temperature. The adaptation phase of the ANPM utilized these five, highly correlated sensors to illustrate adaptation to nominal operating data. Both simulators generated data for a loadfollowing power profile, which is more likely in a GAR than steady-state operation. The load profile used is shown in Figure 4-6. Figure 5-1 shows the Steam Flow Rate residuals for three models. The worst performer is the model based solely on first-principle generated data. The figure shows that this model was not able to predict the behavior during periods of lower power demand. The ANPM residual remains around zero, indicating that it was able to adapt from the low-fidelity first principle model to a more accurate model. Finally, the "final" model is the result of the adaptation phase, a model built solely on the collected data. This model has the lowest residuals, slightly better even than the adapting ANPM model. The mean squared errors of the predictions for all models and each of the five sensors are shown in Table 5-1. 


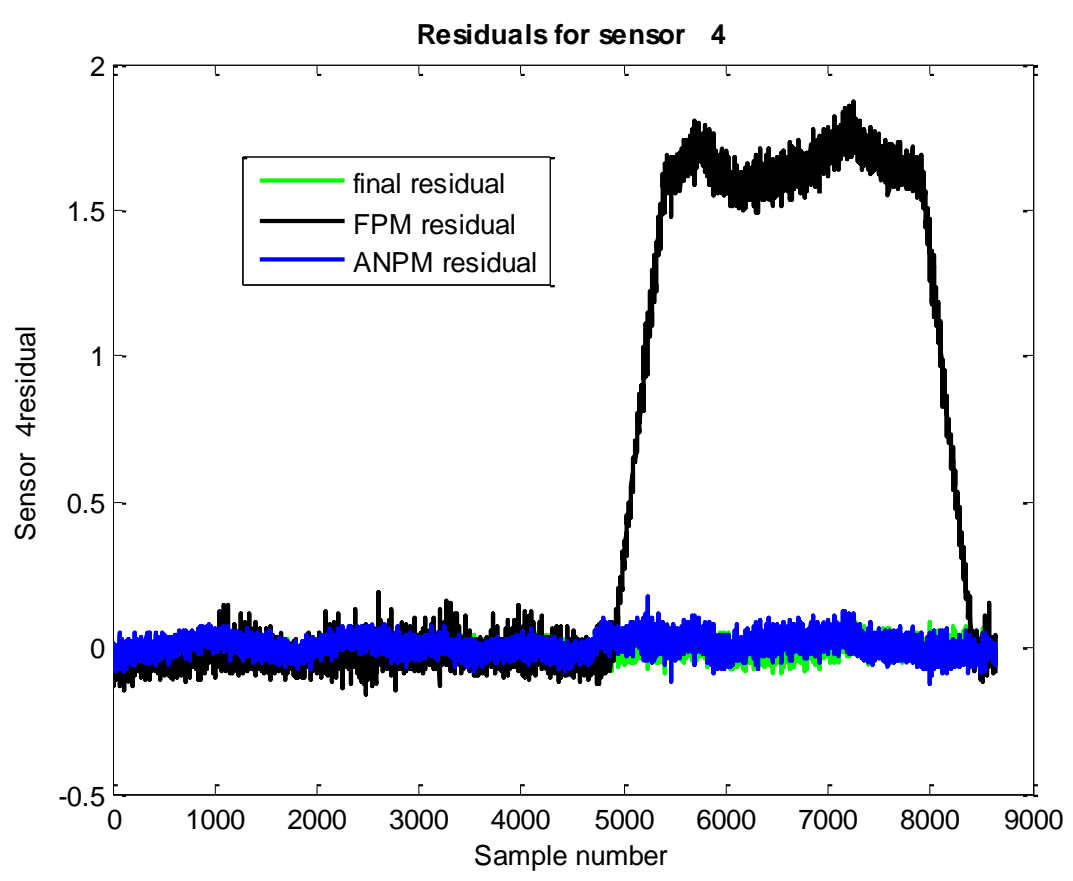

Figure 5-1: Model Residuals for Steam Flow Rate

Table 5-1: Mean Squared Error of Predictions

\begin{tabular}{|c|c|c|c|c|c|}
\cline { 2 - 6 } \multicolumn{1}{c|}{} & \multicolumn{5}{c|}{ Sensor Number } \\
\cline { 2 - 6 } \multicolumn{1}{c|}{} & 1 & 2 & 3 & 4 & 5 \\
\hline FPM & 4.27 & 0.178 & 0.899 & 0.898 & 0.066 \\
\hline ANPM & 0.0011 & 0.0011 & 0.001 & 0.001 & 0.0002 \\
\hline Final & 0.00037 & 0.00027 & 0.00066 & 0.00069 & 0.00016 \\
\hline
\end{tabular}

These results indicate that the ANPM adapts correctly from low-fidelity simulated data to high-fidelity operating condition data. However, the ANPM must be able to identify observations which result from faulted behavior in order to exclude these observations from the memory matrix. To test this feature, a larger model was built using thirteen highly correlated sensors from the NCSU simulator. This model was used with the proposed PCA-based ECM methodology to identify known faulty data. The results of this analysis are given next.

\subsection{PCA-Based Fault Detection}

The NCSU simulator was used to generate data with the four faults described in Section 4.3.2. The generated data includes one day per month of operation under the nominal load profile for ten or twelve months with increasing fault levels at each month. Seven of the 
resulting principle components were used to build a model. This number of PCs was used because it offered the highest overall level of sensitivity for all of the four investigated fault types. Detection limits for the $\mathrm{T}^{2}$ - and Q-statistics were set to the $99^{\text {th }}$ percentile of the values for the nominal data, which corresponds to the commonly accepted false alarm rate of $1 \%$. Resulting fault and expanded condition alarms were then consolidated with a 3 out of 6 logic to eliminate spurious alarms; that is, at least three observations of the preceding minute must be identified as faulty for a specific observation to alarm. This fault alarm logic was chosen to eliminate spurious alarms in the available fault-free data. Of the available sensor readings, thirteen are included in a monitoring model, as listed in Table 5-2. The PCA ECM results for one day of nominal operation using this model are shown in Figure 5-2. The top plot shows the $\mathrm{T}^{2}$ statistic for each observation, with the training data in blue and the test data in red. The fault detection threshold is indicated as the $99 \%$ fault threshold of the training $\mathrm{T}^{2}$ statistics. The second plot shows the contributions to the Q-statistic in a similar fashion. Next, the fault and expanded condition test results are shown, with the fault hypotheses in blue and the expanded condition hypotheses in red. Finally, the consolidated fault results are given in the bottom plot, which indicates that no observations in the nominal data will be flagged as faults or expanded conditions.

Table 5-2: Monitoring System Variables

\begin{tabular}{|c|c|}
\hline Variable & Unit \\
\hline Neutron Power & Mwt \\
\hline Pressurizer Two Phase Mixture Level & $\mathrm{ft}$ \\
\hline Hot Leg Temperature & $* \mathrm{~F}$ \\
\hline Cold Leg Temperature & $* \mathrm{~F}$ \\
\hline Average Temperature & $* \mathrm{~F}$ \\
\hline Feed Flow Rate per Steam Generator & $\mathrm{Ibm} / \mathrm{hr}$ \\
\hline Steam Flow Rate per Steam Generator & $\mathrm{Ibm} / \mathrm{hr}$ \\
\hline Feed Pump Speed & $\mathrm{rpm}$ \\
\hline Steam Generator Exit Temperature & $* \mathrm{~F}$ \\
\hline Feed Temperature & $* \mathrm{~F}$ \\
\hline Steam Pressure & $\mathrm{psia}$ \\
\hline Total Pressure Drop Across the Secondary Side & $\mathrm{psi}$ \\
\hline Rod Depth & $\%$ \\
\hline
\end{tabular}



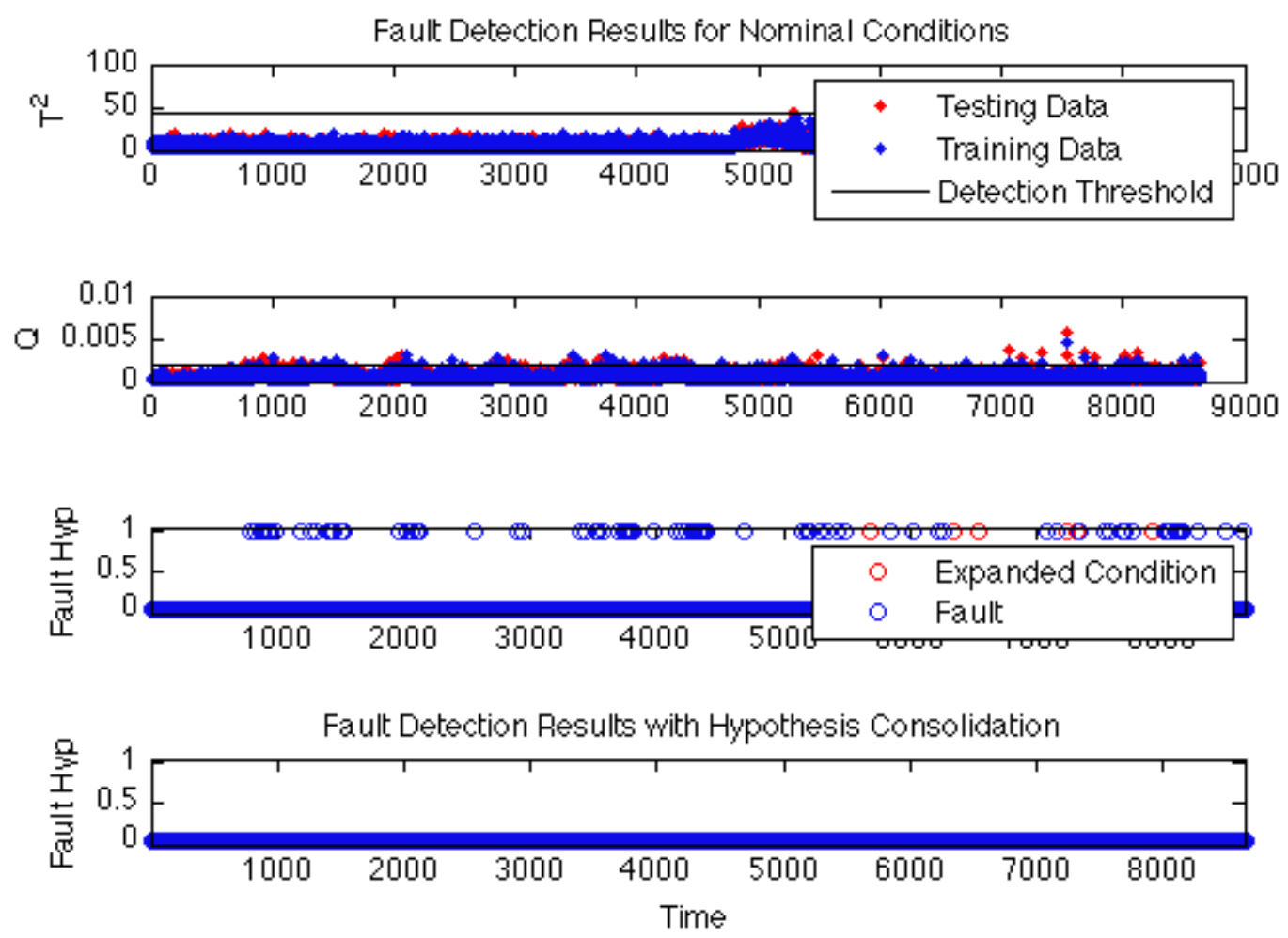

Figure 5-2: Nominal PCA-based Fault Detection Results

The following sections describe the results of the PCA ECM fault detection method for each fault mode during select months of the modeled degradation. Additionally, fault detection results using the SPRT with each model are given. After model adaptation has completed, the PHM system will likely fall back to traditional fault detection methods for continued monitoring. The following sections include select figures for each fault type. A table summarizing the results of both PCA-based and SPRT fault detection is given for each fault. These tables list the fault size at each month and the percentage of observations flagged as faulted for each detection method after fault consolidation is performed. All figures for each fault type and detection method can be found in Appendix A, including the plots shown here and additional results for ease of investigation.

\subsubsection{Heat Exchanger Fouling}

The simulated faulted conditions for heat exchanger fouling involve increasing fouling levels at each month, ranging from $1.4 \%$ fouling in month one to $30 \%$ fouling in month 12 . The 
results of the PCA-based ECM for the first month are shown in Figure 5-3. This included only $1.4 \%$ heat exchanger fouling but was still detected as a fault for $78 \%$ of the observations. By the fourth month of this fault, which included $5.7 \%$ fouling, every observation of the month was identified as faulty (Figure 5-4). For each month following, the PCA-based ECM was able to correctly and completely identify the faulted data. The full results of fault detection are shown in Table 5-3. The SPRT method was able to detect this fault, but not as quickly or reliably as the PCA-based method.

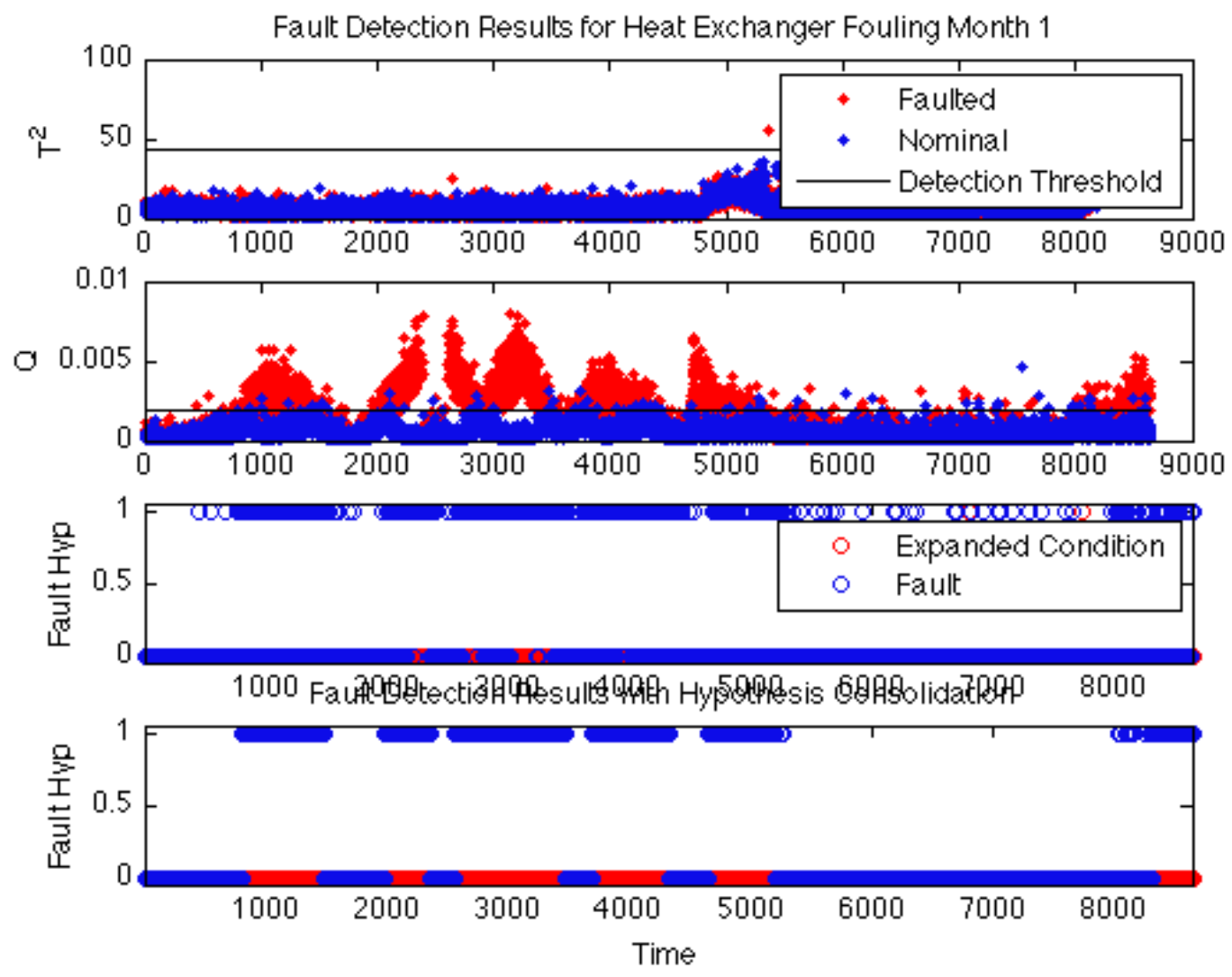

Figure 5-3: PCA-based ECM for Month 1 

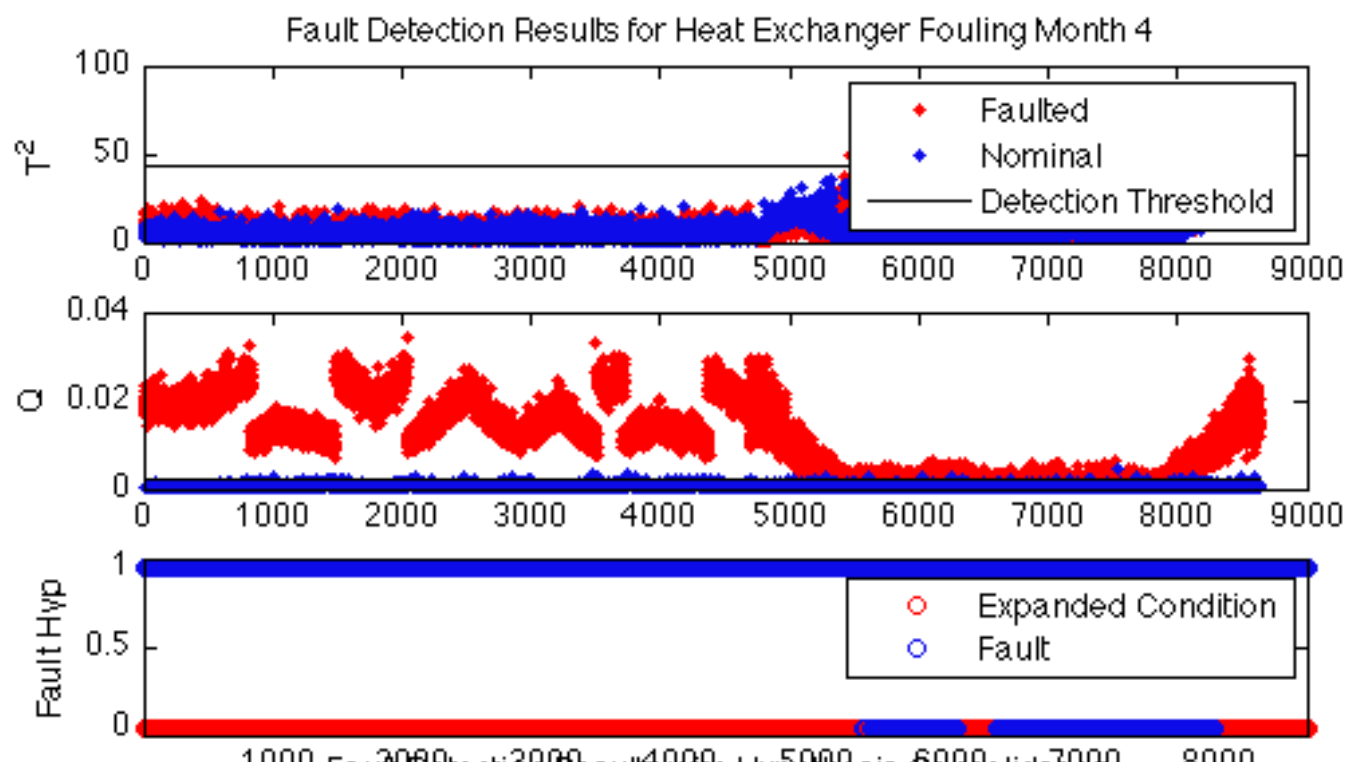

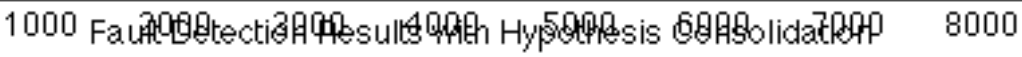

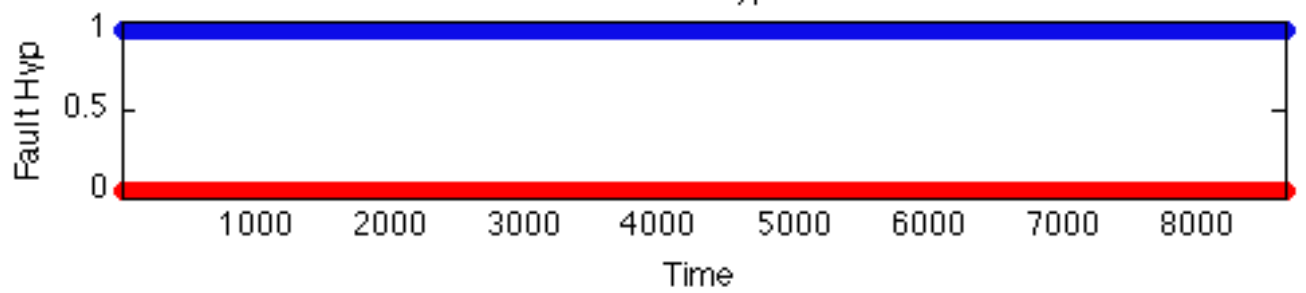

Figure 5-4: PCA-based ECM for Month 4

Table 5-3: Fault Detection Results for Heat Exchanger Fouling

\begin{tabular}{|c|c|c|c|}
\hline \multicolumn{4}{|c|}{ Heat Exchanger Fouling } \\
\hline Month & Fault Size (\%) & PCA (\%) & SPRT (\%) \\
\hline 1 & 1.36 & 45.83 & 0.13 \\
\hline 2 & 3.33 & 78.34 & 0.19 \\
\hline 3 & 4.04 & 88.23 & 0.19 \\
\hline 4 & 5.67 & 100.00 & 0.20 \\
\hline 5 & 7.29 & 100.00 & 0.29 \\
\hline 6 & 8.33 & 100.00 & 0.24 \\
\hline 7 & 11.81 & 100.00 & 1.30 \\
\hline 8 & 15.00 & 100.00 & 6.53 \\
\hline 9 & 18.34 & 100.00 & 34.86 \\
\hline 10 & 23.33 & 100.00 & 60.85 \\
\hline 11 & 27.60 & 100.00 & 63.13 \\
\hline 12 & 30.00 & 100.00 & 64.24 \\
\hline
\end{tabular}




\subsubsection{Pump Degradation}

The pump degradation fault is simulated by reducing the effective flow rate through the pump by one percent during each of ten months. This fault has a small effect on the model simulation and is quite difficult to detect. Neither the PCA nor the SPRT fault detection routines are able to accurately or consistently detect the pump degradation, as shown in Table 5-4. This fault may be more easily detected with additional measureands, but it is not consistently detectable with the available data at such low levels of fault.

Table 5-4: Fault Detection Results for Pump Degradation

\begin{tabular}{|c|c|c|c|}
\hline \multicolumn{4}{|c|}{ Pump Degradation } \\
\hline Month & Fault Size (\%) & PCA $(\%)$ & SPRT $(\%)$ \\
\hline 1 & 1.00 & 0.00 & 0.05 \\
\hline 2 & 2.00 & 0.00 & 0.16 \\
\hline 3 & 3.00 & 0.42 & 0.08 \\
\hline 4 & 4.00 & 0.00 & 0.19 \\
\hline 5 & 5.00 & 1.10 & 0.32 \\
\hline 6 & 6.00 & 0.69 & 0.14 \\
\hline 7 & 7.00 & 0.88 & 0.17 \\
\hline 8 & 8.00 & 3.09 & 0.36 \\
\hline 9 & 9.00 & 9.97 & 0.67 \\
\hline 10 & 10.00 & 10.17 & 0.56 \\
\hline
\end{tabular}

\subsubsection{Process Sensor Drift}

The process sensor drift fault involves a slow drift of a sensor not used in the control system. Here, it is detected using the 13-sensor model discussed previously. This fault will be more easily detected using a set of redundant sensors. A set of seven correlated sensors was used to build a model more typical of those currently available in nuclear plants: two redundant feedwater flow sensors, two redundant steam flow sensors, and three redundant steam generator pressure sensors. It was assumed that these sensors do not experience common-cause failures; that is, only one of the redundant sensors will experience a drift. The results for SPRT fault detection using both models are shown in Table 5-5. 
Table 5-5: Fault Detection Results for Process Sensor Drift

\begin{tabular}{|c|c|c|c|c|}
\hline \multicolumn{5}{|c|}{ Process Sensor Drift } \\
\hline Month & Fault Size (\%) & PCA (\%) & SPRT (\%) & $\begin{array}{c}\text { SPRT (\%) } \\
\text { Redundant Model }\end{array}$ \\
\hline 1 & 0.08 & 0.00 & 0.14 & 12.32 \\
\hline 2 & 0.17 & 0.07 & 0.05 & 7.66 \\
\hline 3 & 0.25 & 1.09 & 0.07 & 10.01 \\
\hline 4 & 0.33 & 1.91 & 0.12 & 15.03 \\
\hline 5 & 0.42 & 3.93 & 0.22 & 15.18 \\
\hline 6 & 0.50 & 10.77 & 0.06 & 21.13 \\
\hline 7 & 0.58 & 14.93 & 0.34 & 24.06 \\
\hline 8 & 0.66 & 24.21 & 0.32 & 28.91 \\
\hline 9 & 0.75 & 39.72 & 0.59 & 29.74 \\
\hline 10 & 0.83 & 59.26 & 0.80 & 34.44 \\
\hline 11 & 0.91 & 83.14 & 1.06 & 42.40 \\
\hline 12 & 1.00 & 97.27 & 1.59 & 48.40 \\
\hline
\end{tabular}

\subsubsection{Control Sensor Drift}

Control sensor drift introduces additional fault detection difficulties because of sensor masking. Because the sensor is controlled to meet a set point, the sensor itself, even in redundant sets, cannot be used to directly detect the fault. However, the PCA fault detection method can be used to effectively detect the control sensor drift because it changes the way that the system variables interact with each other. Although the relationships between variables change due to the control sensor drift, the value of the measured variables does not change significantly, which means that the residuals from a traditional monitoring system are not large. As a result, the SPRT fault detection routine is not as effective at detecting the fault, as can be seen in Table 5-6.

Table 5-6: Fault Detection Results for Control Sensor Drift

\begin{tabular}{|c|c|c|c|}
\hline \multicolumn{4}{|c|}{ Control Sensor Drift } \\
\hline Month & Fault Size (\%) & PCA (\%) & SPRT (\%) \\
\hline 1 & 0.08 & 1.97 & 0.20 \\
\hline 2 & 0.17 & 8.11 & 0.15 \\
\hline 3 & 0.25 & 13.32 & 0.13 \\
\hline 4 & 0.33 & 23.54 & 0.17 \\
\hline 5 & 0.42 & 29.35 & 0.16 \\
\hline 6 & 0.50 & 35.45 & 0.43 \\
\hline 7 & 0.58 & 48.47 & 0.66 \\
\hline
\end{tabular}




\begin{tabular}{|c|c|c|c|}
8 & 0.66 & 60.94 & 1.50 \\
\hline 9 & 0.75 & 76.15 & 2.68 \\
\hline 10 & 0.83 & 92.91 & 5.66 \\
\hline 11 & 0.91 & 98.36 & 8.98 \\
\hline 12 & 1.00 & 99.79 & 15.91 \\
\hline
\end{tabular}

The proposed PCA-based fault detection method outperformed the traditional SPRT method for each of the simulated faults. The SPRT results may be improved with models which incorporate additional inputs and redundant sensor sets, but these are the best results obtained with the data available. After determining through the proposed ECM method or traditional fault detection methods that a fault is present, the health monitoring system next turns to a fault diagnostic routine to identify the type of fault experienced.

\subsection{Fault Diagnostics}

Two fault diagnostic methods have been developed during the course of this research. These methods are attractive because it is relatively easy to update the fault diagnoser as new faults and new examples of known faults become available. However, both methods require multiple runs of each anticipated fault for initial model development. Because of the computational burden of the NCSU IRIS simulator, only one exemplar run of each fault type was available. For this reason, a cascade of neural networks was used for fault diagnostics of the simulated IRIS data. Here, each neural network diagnoses only one fault type; the output is either false (the fault is not the current type) or true (the fault is the current type). The networks are evaluated in a set order, where the easiest-to-diagnose faults are evaluated first moving to more difficult faults. In this way, the networks have a higher probability of correctly diagnosing each fault. The flow of networks in the fault diagnoser is shown in Figure 5-5. The results of this fault diagnoser with the observations identified as faulty through the PCA ECM fault detection routine are shown in Table 5-7. The diagonal elements indicate correctly identified faults. Heat Exchanger Fouling and Control Sensor Drift were correctly identified through this method with 98\% accuracy. Pump Degradation and Process Sensor Drift were more difficult to differentiate with $60 \%$ and $75 \%$ accuracy, respectively. In both cases, the majority of misdiagnosed observations were diagnosed as either Process Sensor Drift or Pump Degradation. 


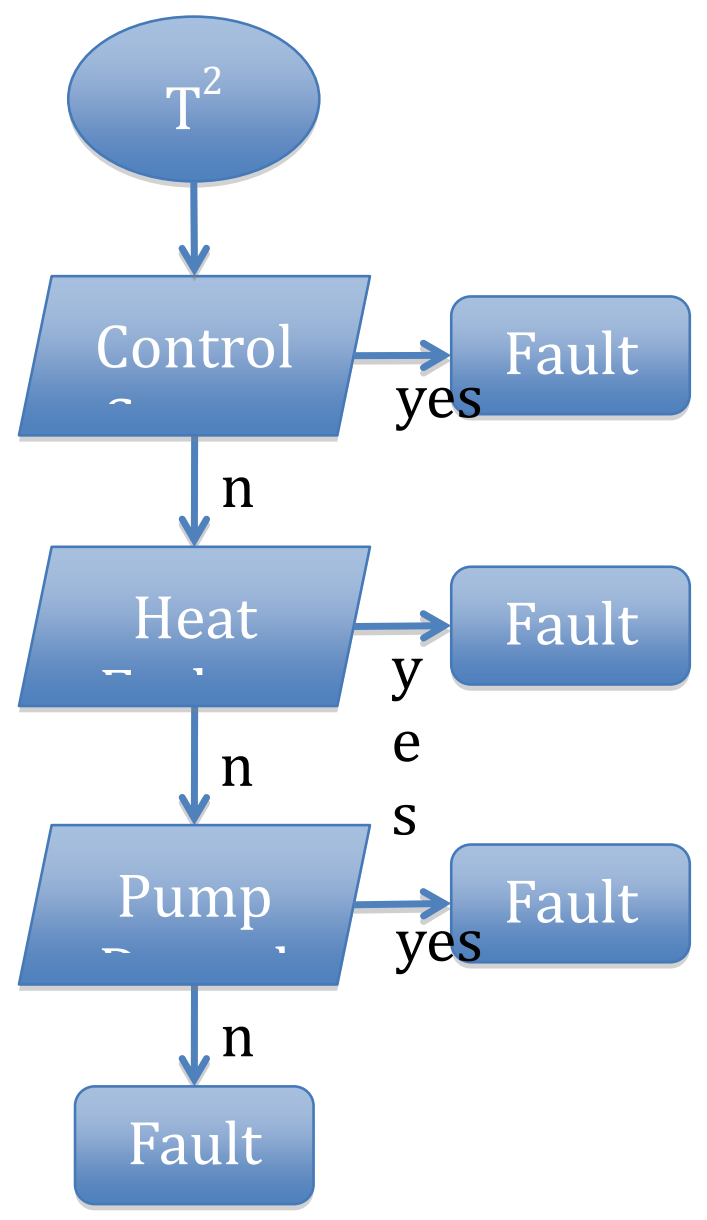

Figure 5-5: Fault Diagnostics with Neural Networks

Table 5-7: Fault Diagnostic Results using PCA Contributions

\begin{tabular}{|c|c|c|c|c|c|}
\hline & \multicolumn{4}{|c|}{ Diagnosed Fault } \\
\hline & & HF & PD & CSD & PSD \\
\hline \multirow{8}{*}{ 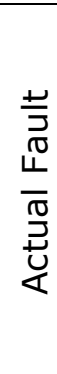 } & \multirow{2}{*}{$\mathrm{HF}$} & 36106 & 118 & 86 & 198 \\
\hline & & 98.90 & 0.32 & 0.24 & 0.54 \\
\hline & \multirow{2}{*}{ PD } & 30 & 2882 & 3 & 1888 \\
\hline & & 0.62 & 60.00 & 0.06 & 39.31 \\
\hline & \multirow{2}{*}{ CSD } & 214 & 17 & 14406 & 24 \\
\hline & & 1.46 & 0.12 & 98.26 & 0.16 \\
\hline & \multirow{2}{*}{ PSD } & 24 & 1266 & 8 & 3954 \\
\hline & & 0.46 & 24.11 & 0.15 & 75.29 \\
\hline
\end{tabular}

This fault diagnostic method is not readily amenable to adaptation. For each possible fault type, a neural network must be trained. If the diagnostic system is built and a new fault type emerges, a new network must be trained to differentiate that fault type, and every other network will need to be retrained to correctly deal with the data for the new fault. This can be a 
long and tedious process. In application, it is suggested that one of the more adaptable and flexible fault diagnostic methods described is used. Because the available data were not well suited to these methods, they couldn't be applied here. However, both methods were applied to other faulted data. The results of these analyses are given in Section 5.4.

\subsection{Additional Results}

In addition to the simulated IRIS data sources, specific modules in the health monitoring system were tested on other data sets. The results of these analyses are given here. First, the application of the ECM technique for differentiating faults and expanded operating conditions is applied to the experimental flow control loop.

\subsubsection{Application of the ECM Technique to Flow Loop Data}

Applying the PCA ECM technique to the flow loop data similar results are shown when signals with linear relationships are used. To be able to compare this application to the ANPM use, the original PCA statistics need to be based on the FPM data. The actual flow loop data is monitored for faults or expanded conditions. Three actual faults were added to the flow loop operation. These faults included a return valve blockage, $60 \mathrm{~mm}$ level drift, and a pump failure simulation. These faults can be detected by analyzing different sensors. Figure 5-6 shows the results on the level of tank 1 during normal operation compared to the different faults under the same profile operation. 

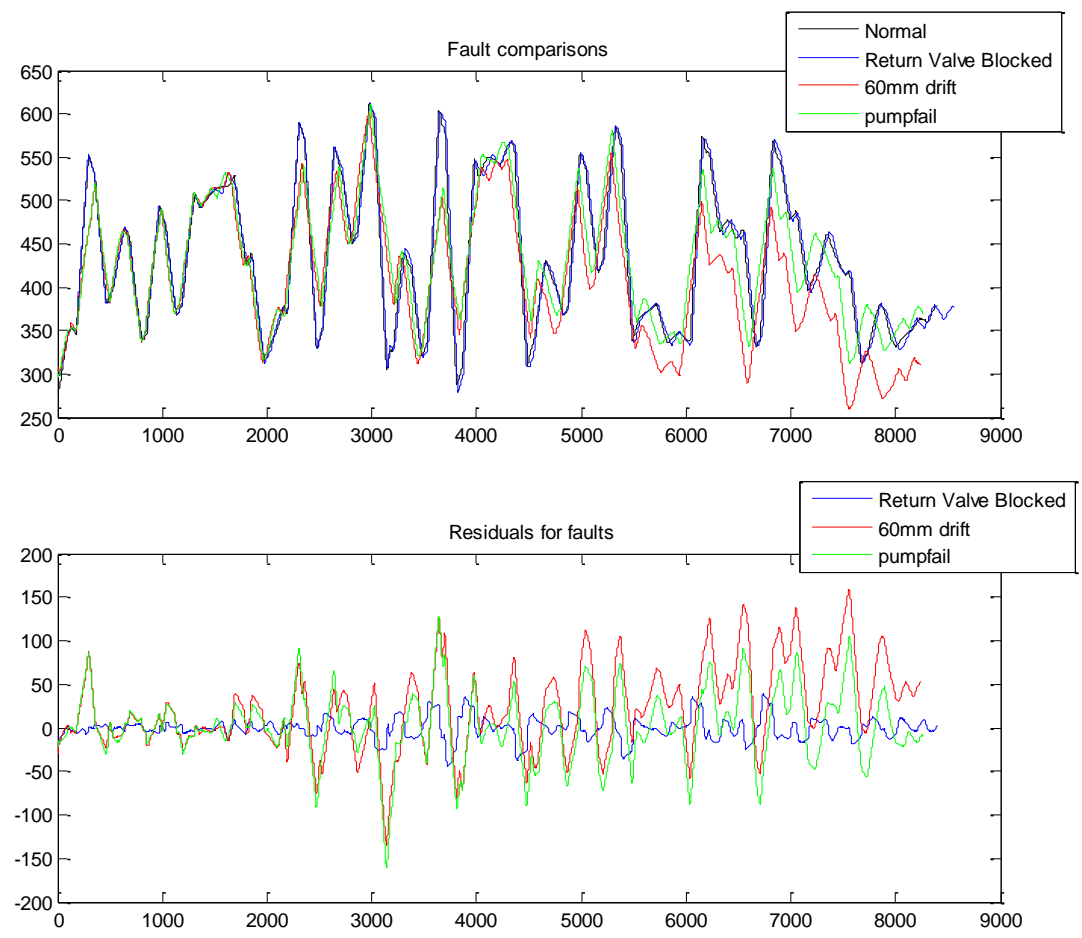

Figure 5-6: Tank 1 Level Fault Comparison

The first four sensors which are the two tank levels and the two setpoints have linear relationships. The PCA ECM technique was applied to the first four sensors using the FPM data as the basis. The first principal component (PC) describes over 99\% of the variance within the data, so only one PC is used. Applying this technique to the normal operation of the flow loop no faults are detected in profile 1 data or profile 2 data. Figure 5-7 shows the profile 1 results, and Figure 5-8 shows the profile 2 results. 

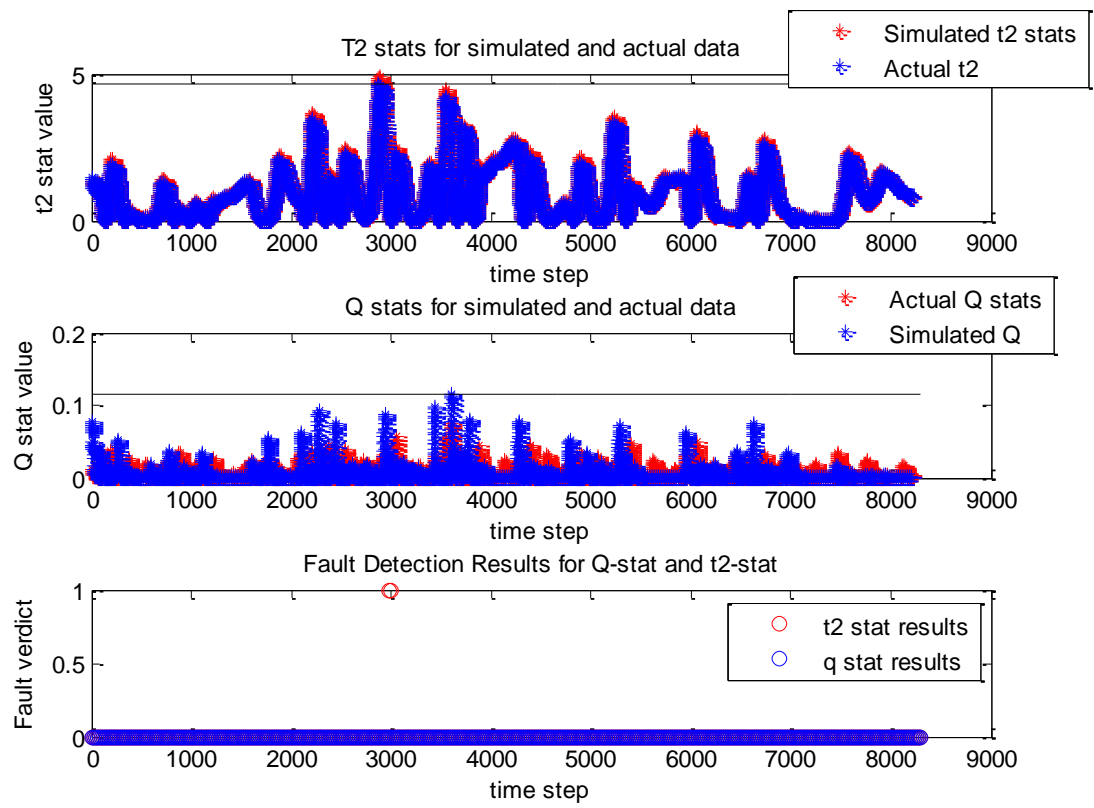

Figure 5-7: PCA ECM Application on Profile 1
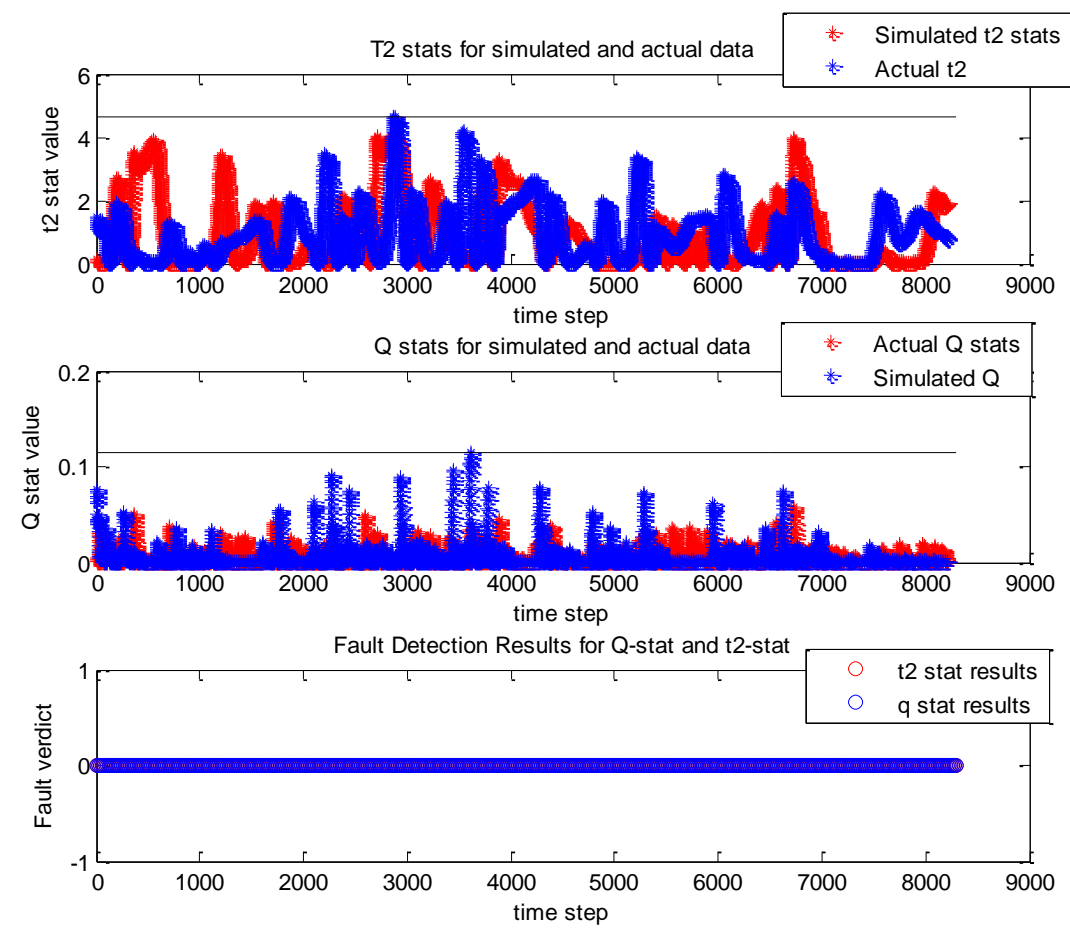

Figure 5-8: PCA ECM Application on Profile 2 
Applying this technique to the $60 \mathrm{~mm}$ level drift fault condition of the flow loop the faults were detected in profile 1 faulty data and profile 2 faulty data. Figure 5-9 shows the profile 1 results, and Figure 5-10 shows the profile 2 results.
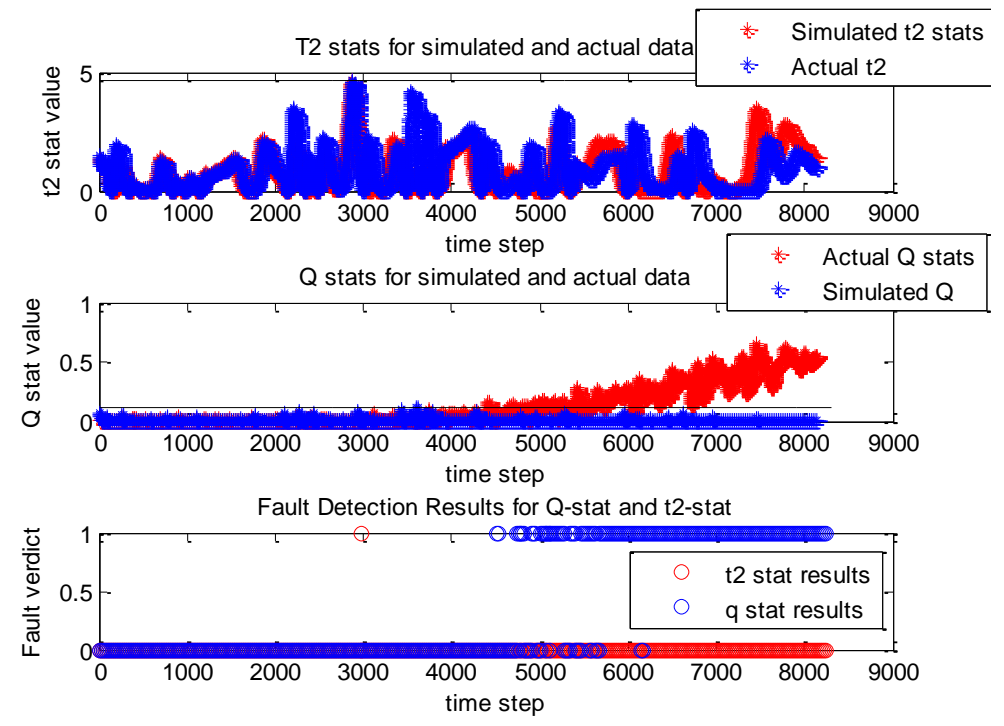

Figure 5-9: PCA ECM Application on Profile 1, $60 \mathrm{~mm}$ Drift
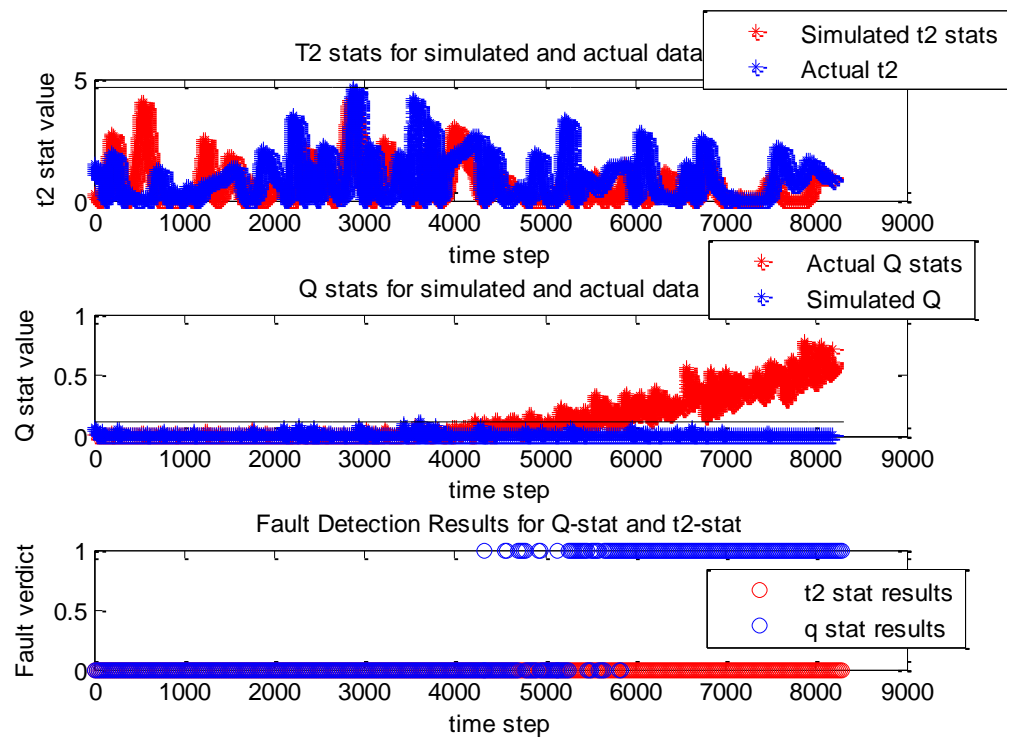

Figure 5-10: PCA ECM Application on Profile 2, $60 \mathrm{~mm}$ Drift

The addition of the two outlet flow sensors creates problems for the PCA ECM technique. This problem is created by the non-linear relationships between the outlet flow and the tank levels. Where above the four sensors had linear relationships, the faults were 
detected and normal operation data was shown to have no faults. The addition of the two flow sensors breaks down the basis for the PCA ECM technique. Figure 5-11 shows the application of the PCA ECM technique on the profile 1, 60mm drift fault. Figure 5-12 shows the application of the PCA ECM technique on the profile 2, 60mm drift fault.
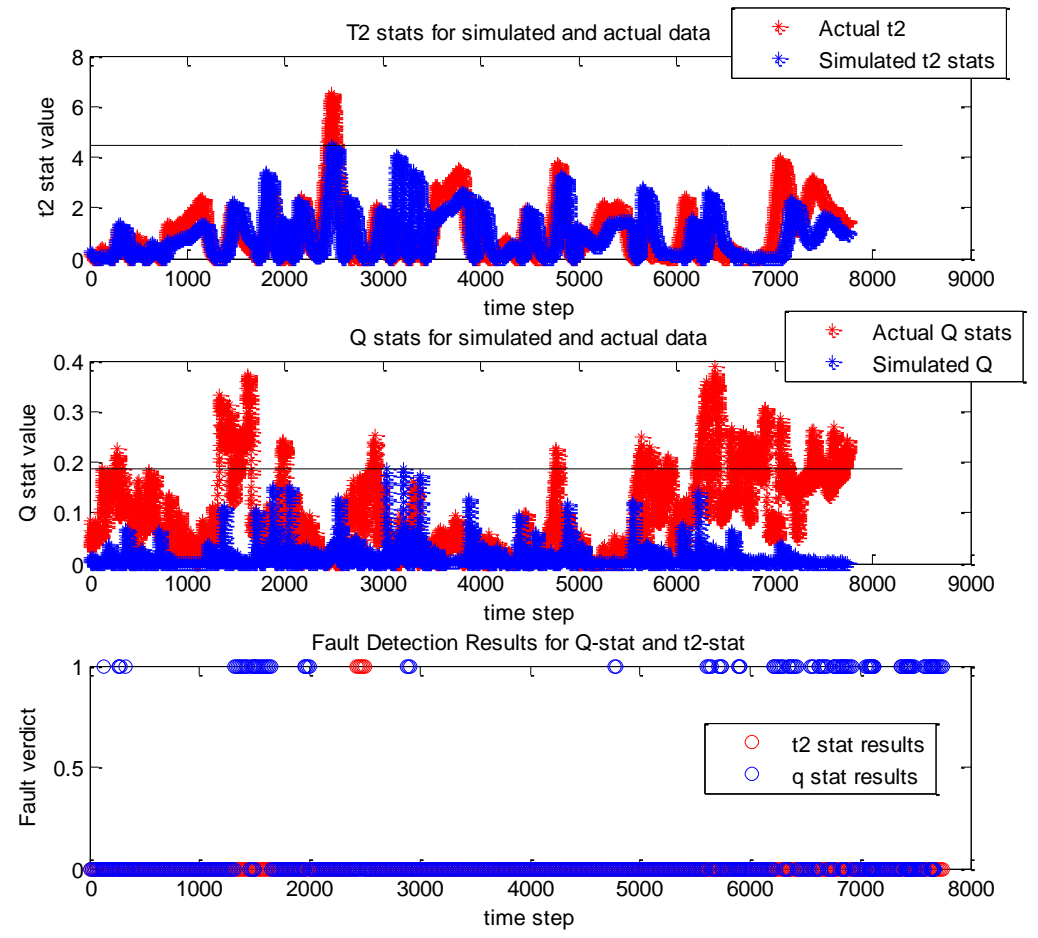

Figure 5-11: PCA ECM Application on Profile 1, 60mm Drift using 6 Sensors 

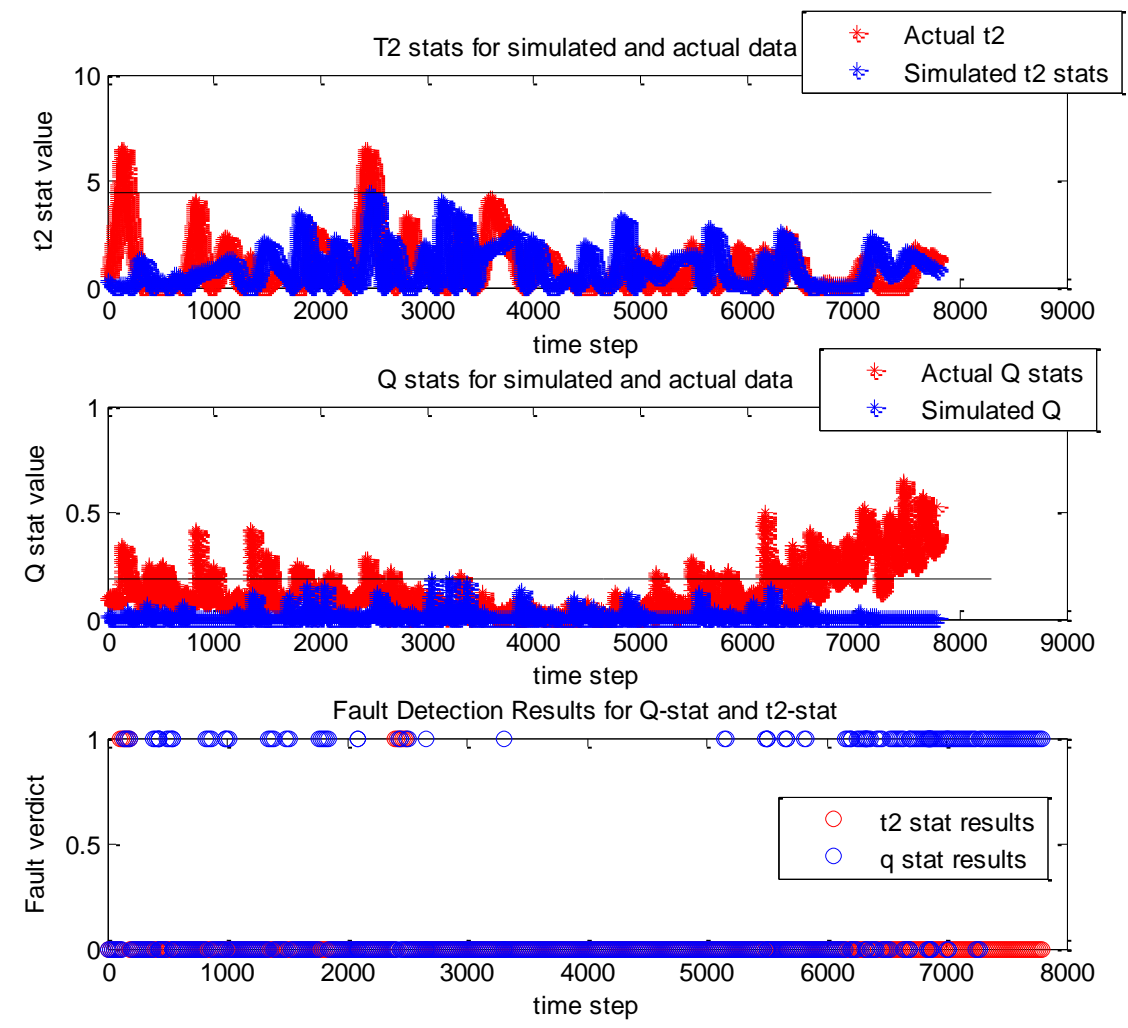

Figure 5-12: PCA ECM Application on Profile 2, 60 mm Drift using 6 Sensors

This demonstration highlights the importance of having linear relationships within the data when using the PCA ECM technique. It was shown on actual data from the flow loop the ability of this technique to detect faults. The detection of faults within the ANPM is very important. This fault detection ability will govern the adaptive ability of the model.

\subsubsection{FIA Diagnosis of Flow Loop Faults}

To assess the performance of the algorithm a test set of each synthetically produced fault was created. They were created in a process similar to how the synthetic training vectors were produced. Appropriate drifts, jumps, variance increases and stuck sensors were added to data obtained from the Flow Loop. Residual sets were created from each of these faults and run through the fault identification program at different lengths of time after the fault occurred. Figure 5-13 shows the results of this analysis. This figure depicts what rank of Possibility the correct fault was given by the algorithm. The algorithm was run using data from one to six minutes after the fault was detected, at one minute intervals. The rank was determined by the Possibility that each fault received. The figure illustrates that in most cases the correct fault 
immediately recognized as the most possible fault. However, in cases where the fault was a drift of some kind (Faults 6 and 8), it took slightly longer for the algorithm to correctly predicted. This trait can be attributed to the nature of drift faults, and that they diverge further with time. Yet even with these two faults the correct fault was identified as the most possible after five or six minutes. It is therefore recommended that the operator wait six minutes or so to react to the algorithms prediction, to verify the identification. This recommendation is especially encouraged if a drift fault is predicted to be most possible.

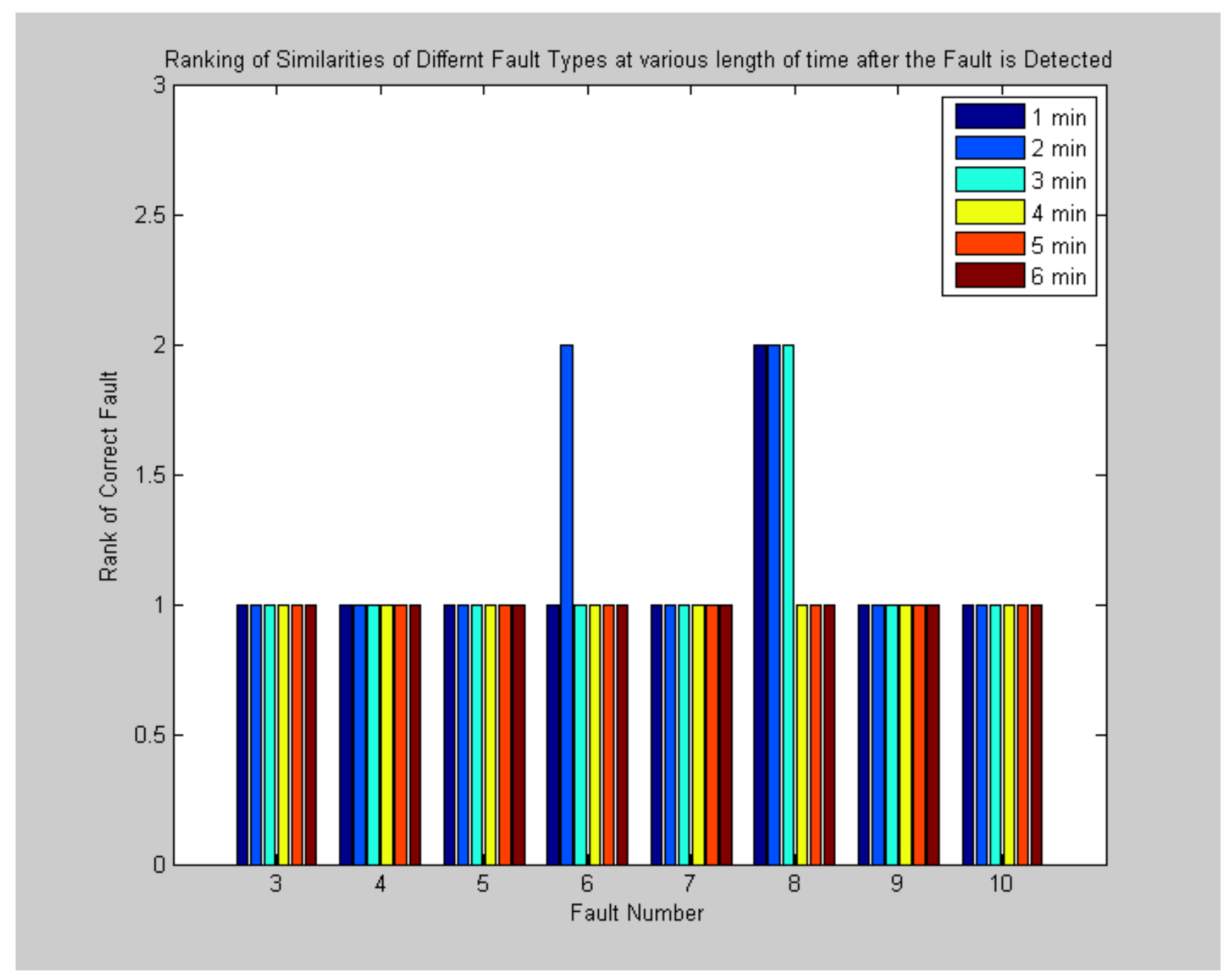

Figure 5-13: Possibility Rank of Actual Fault

The outputs from the identification program are an ordered list of faults with the corresponding Possibility. The results from each fault are displayed in Table 5-8. These results were calculated five minutes after the fault was detected. For each test fault, this table gives an ordered list of the likelihood that it is each particular training fault. The training faults are listed in order of most possible. Notice that the after five minutes the algorithm identifies the correct 
fault as the most Possible fault. The outcomes in both Figure 5-13 and Table 5-8 were calculated with the default residual weighting coefficient of 0.8 .

Table 5-8: Ordered Faults with Corresponding Possibilities

\begin{tabular}{|c|c|c|c|c|c|c|c|c|c|c|c|c|c|c|c|}
\hline \multicolumn{2}{|c|}{ Fault 3} & \multicolumn{2}{|c|}{ Fault 4} & \multicolumn{2}{|c|}{ Fault 5} & \multicolumn{2}{|c|}{ Fault 6} & \multicolumn{2}{|c|}{ Fault 7} & \multicolumn{2}{|c|}{ Fault 8} & \multicolumn{2}{|c|}{ Fault 9} & \multicolumn{2}{|c|}{\begin{tabular}{|l|} 
Fault 10 \\
\end{tabular}} \\
\hline & 0.21 & & 0.13 & & 0.26 & & 0.34 & & 0.13 & & 0.32 & & 0.37 & 1 & 0.21 \\
\hline 3 & 5 & 4 & 3 & 5 & 7 & 6 & 1 & 7 & 2 & 8 & 9 & 9 & 5 & 0 & 3 \\
\hline & 0.13 & & 0.11 & & 0.10 & & 0.29 & & 0.11 & & 0.29 & & 0.08 & & 0.10 \\
\hline 8 & 6 & 2 & 9 & 2 & 2 & 8 & 8 & 3 & 6 & 6 & 9 & 6 & 7 & 9 & 3 \\
\hline & 0.13 & & 0.10 & & 0.10 & & 0.06 & & 0.10 & & 0.06 & & 0.08 & & 0.09 \\
\hline 6 & 3 & 8 & 6 & 3 & 1 & 3 & 5 & 8 & 9 & 3 & 7 & 8 & 7 & 2 & 2 \\
\hline & 0.12 & & 0.10 & & 0.10 & & 0.05 & & 0.10 & & 0.05 & & 0.08 & & 0.09 \\
\hline 7 & 2 & 9 & 6 & 8 & 1 & 4 & 7 & 6 & 8 & 4 & 8 & 4 & 1 & 8 & 1 \\
\hline & 0.11 & & 0.10 & & 0.10 & & 0.05 & & 0.10 & & 0.05 & & 0.08 & & 0.09 \\
\hline 2 & 7 & 6 & 5 & 6 & 0 & 7 & 5 & 5 & 7 & 7 & 7 & 2 & 0 & 6 & 0 \\
\hline & 0.09 & & 0.10 & & 0.10 & & 0.05 & & 0.10 & & 0.05 & & 0.07 & & 0.08 \\
\hline 4 & 8 & 3 & 2 & 7 & 0 & 5 & 4 & 2 & 3 & 5 & 6 & 3 & 5 & 5 & 9 \\
\hline & 0.07 & & 0.10 & & 0.08 & & 0.05 & & 0.10 & & 0.05 & & 0.07 & & 0.08 \\
\hline 5 & 4 & 7 & 2 & 4 & 2 & 2 & 4 & 9 & 1 & 2 & 5 & 7 & 5 & 7 & 8 \\
\hline & 0.05 & & 0.10 & & 0.07 & & 0.04 & & 0.09 & & 0.04 & & 0.07 & & 0.08 \\
\hline 9 & 7 & 5 & 1 & 9 & 4 & 9 & 7 & 4 & 7 & 9 & 8 & 5 & 5 & 3 & 8 \\
\hline & 0.02 & & 0.06 & & 0.04 & & 0.01 & & 0.06 & & 0.01 & 1 & 0.03 & & 0.08 \\
\hline 1 & 8 & 1 & 5 & 1 & 5 & 1 & 5 & 1 & 8 & 1 & 6 & 0 & 4 & 4 & 7 \\
\hline 1 & 0.02 & 1 & 0.06 & 1 & 0.02 & 1 & 0.01 & 1 & 0.05 & 1 & 0.01 & & 0.03 & & 0.05 \\
\hline 0 & 0 & 0 & 1 & 0 & 8 & 0 & 4 & 0 & 9 & 0 & 5 & 1 & 1 & 1 & 9 \\
\hline
\end{tabular}

The results show that the algorithm was able to accurately determine the likelihood that a fault occurred within approximately five minutes of the fault being detected. The fuzzy possibilities provided the user a good idea of the odds that a particular fault has occurred. The results show that the algorithm was able to correctly identify the actual fault as the most possible for all eight synthetic faults. Further work still focus on optimization of the residual weighting constant for best results. 


\subsubsection{ES Diagnosis of Simulated Faults}

A data set was created to test the ES diagnostic technique. There are 10 residuals with a mean of zero and different variances which represent a nominal condition. Below in Figure 5-14 are the non-faulty residuals, which is normally distributed noise with a mean of zero. This is expected when a good monitoring model is used for prediction. The deviation from nominal conditions of the variance or mean of the residual shows some abnormality in the system.

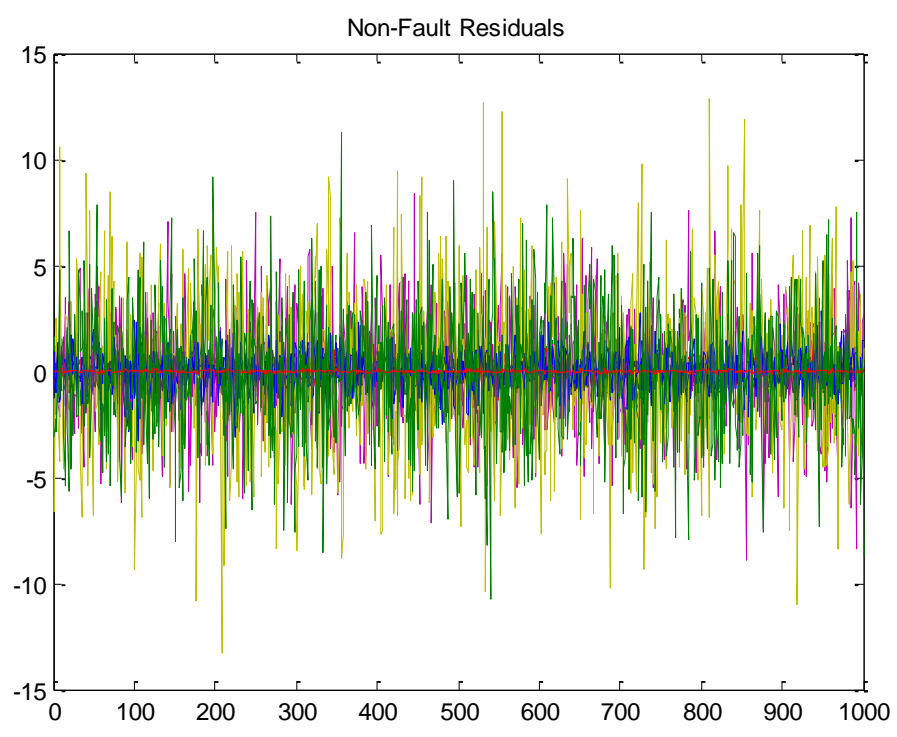

Figure 5-14: Non-faulty residuals

A historical residual memory matrix of failures was created with fifteen possible failures. These failures map to a corresponding fault signature vector. The historical residual memory matrix consists of ten observations for each failure.

To test the complete capability of the ES diagnostic technique a number of different failures need to have the same fault signature vector. The first two tested failures have the same fault signature vectors which can be seen in Table 5-9.

Table 5-9: Fault Signature Vector for Test 1

\begin{tabular}{|c|c|c|c|c|c|c|c|c|c|}
\hline \multicolumn{10}{|c|}{ Sensors and corresponding fault signatures } \\
\hline 1 & 2 & 3 & 4 & 5 & 6 & 7 & 8 & 9 & 10 \\
\hline 1 & 0 & 0 & 1 & 0 & 0 & 0 & 0 & 0 & 0 \\
\hline
\end{tabular}

This fault signature vector shows that a positive drift or bias was found in sensors 1 and 4. All the other sensors had no detected deviations from nominal conditions. However, for this 
example, two failures were created that have this fault signature vector, failure a and failure $\mathbf{b}$. These residuals for sensors 1 and 4 can be seen in Figure 5-15.
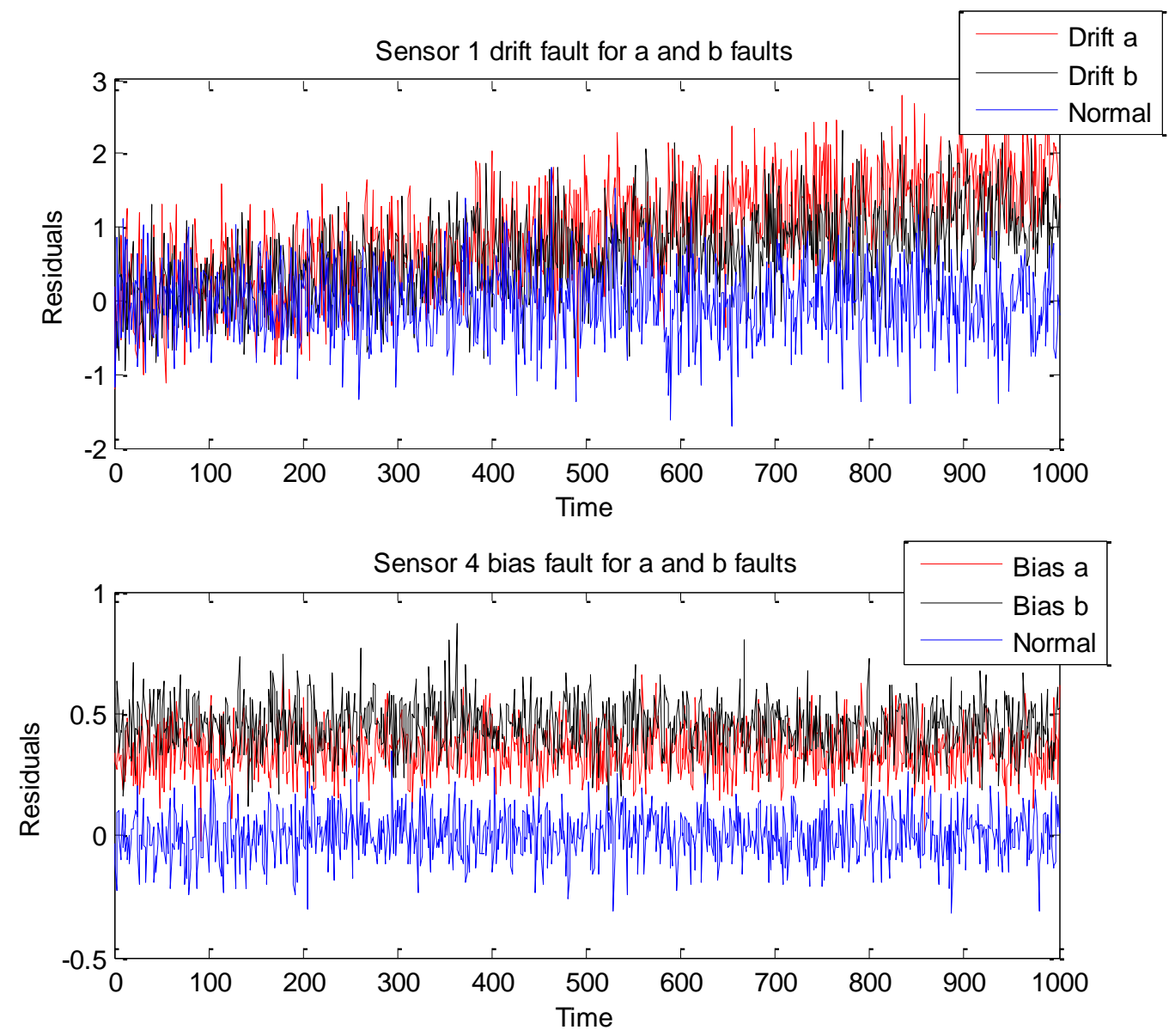

Figure 5-15: Fault Residuals for Test 1

The ES diagnostic technique outputs the fault signature vector followed by the failures that have that fault signature vector. Then the final output is the similarity parameters which has lower values for failures that are more similar to the observed failure. Testing the ES diagnostic technique on the actual failure a gives the following results:

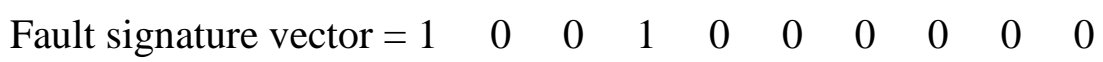

Possible Failures $=2 \quad 13$

Failure Similarities $=115.5691132 .1960$

For this specific fault signature there are two possible failures, failure 2 and 13 . The other 13 possible failures have been eliminated from the possible failure list. A similarity comparison is 
conducted and failure 2 has the lowest similarity parameter of 115.57 which shows this failure is most similar to the observed failure. Where actual failure a is failure 2 so this failure was correctly classified. The classification process for this example can be seen in Figure 5-16.

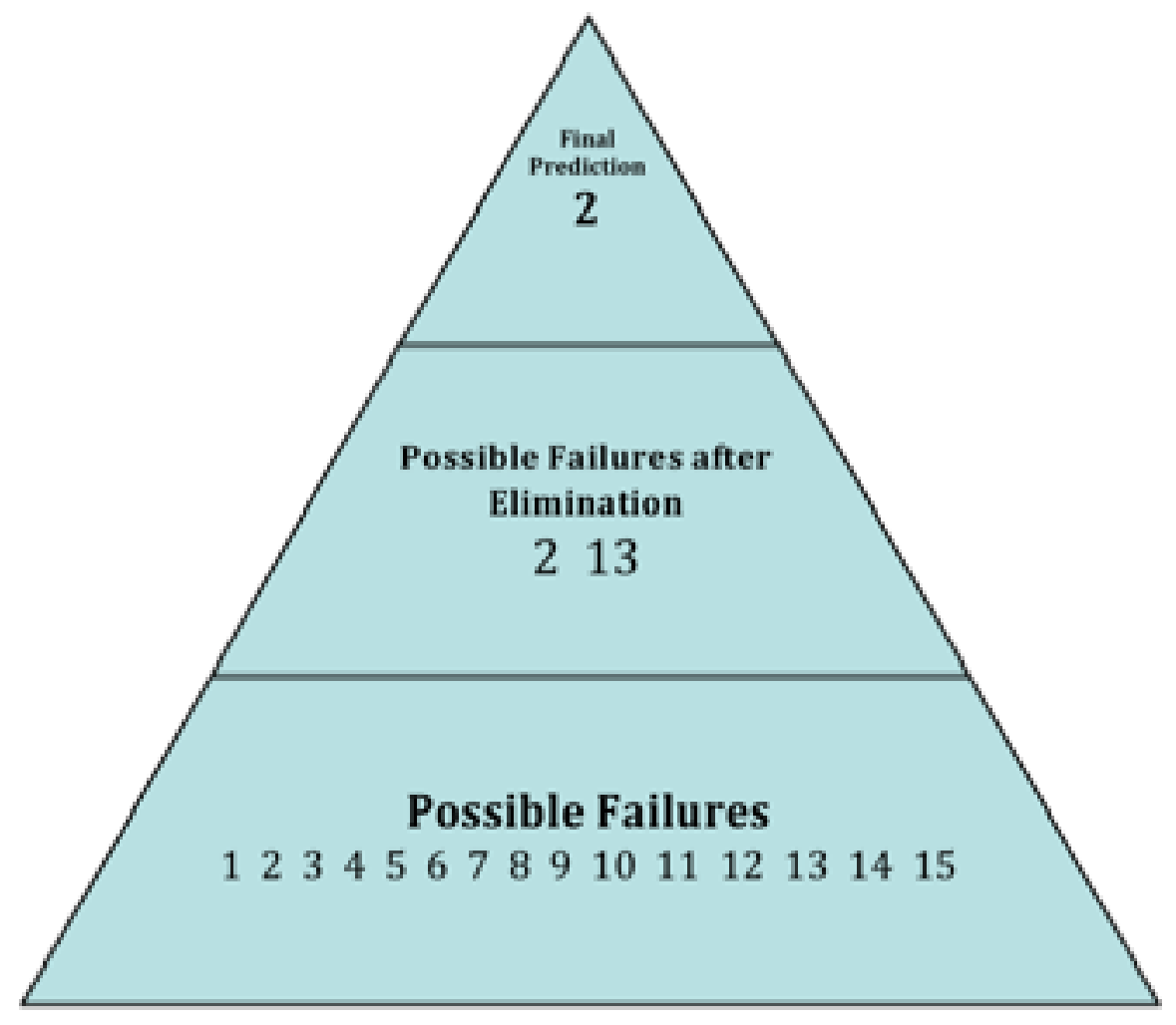

Figure 5-16: ES Process for Test 1a

Testing the ES diagnostic technique on the actual failure $\mathbf{b}$ gives the following results:

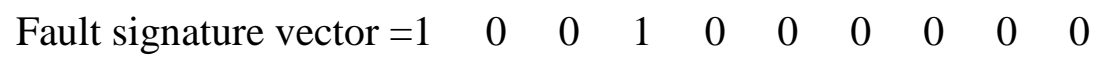

Possible Failures $=2 \quad 13$

Failure Similarities $=140.4501 \quad 122.4915$

For this specific fault signature there are the same two possible failures, failure 2 and 13. The other 13 possible failures have been eliminated from the possible failure list. A similarity comparison is conducted and failure 13 has the lowest similarity parameter of 122.49 which shows this failure is most similar to the observed failure. Where actual failure $\mathbf{b}$ is failure 13 so this failure was correctly classified. The classification process for this example can be seen in Figure 5-17. 


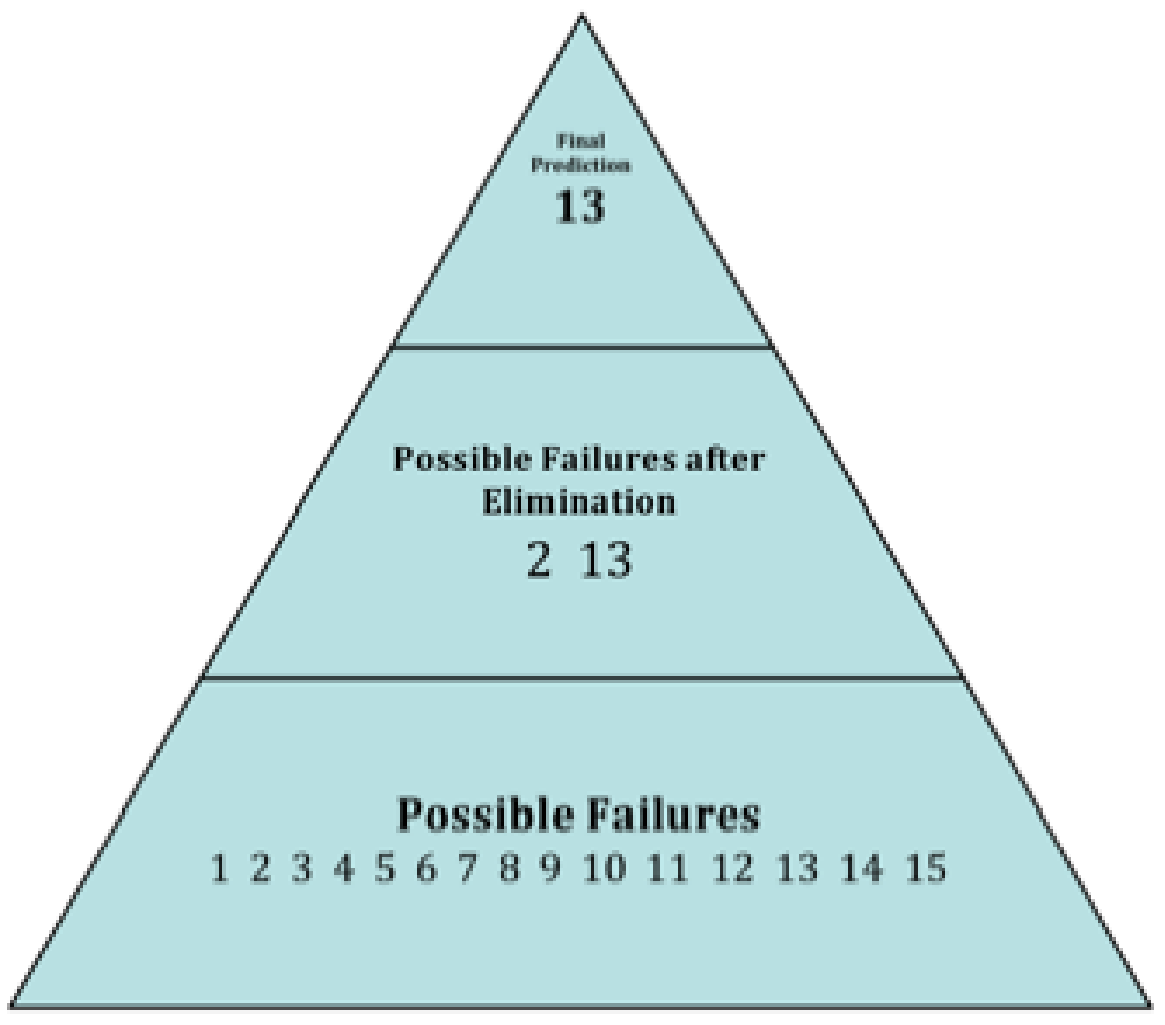

Figure 5-17: ES Process for Test 1b

Three more system failures with the same fault signatures were created to test the ES diagnostic technique, the fault signature vector that these faults can be seen below in Table 5-10.

Table 5-10: Fault Signature Vector for Test 2

\begin{tabular}{|c|c|c|c|c|c|c|c|c|c|}
\hline \multicolumn{1}{|c|}{ Sensors and corresponding faults } \\
\hline 1 & 2 & 3 & 4 & 5 & 6 & 7 & 8 & 9 & 10 \\
\hline 0 & 0 & 1 & 0 & 1 & 0 & 3 & 0 & 0 & 0 \\
\hline
\end{tabular}

This fault signature vector has noise disturbance in sensor 7 and a positive drift or bias in sensors 3 and 5. All the other sensors had no detected deviations from nominal conditions. However, for this example, three failures were created that have this fault signature vector, failure a, failure $\mathbf{b}$ and failure c. The residuals for sensors 3, 5 and 7 can be seen in Figure 5-18. 


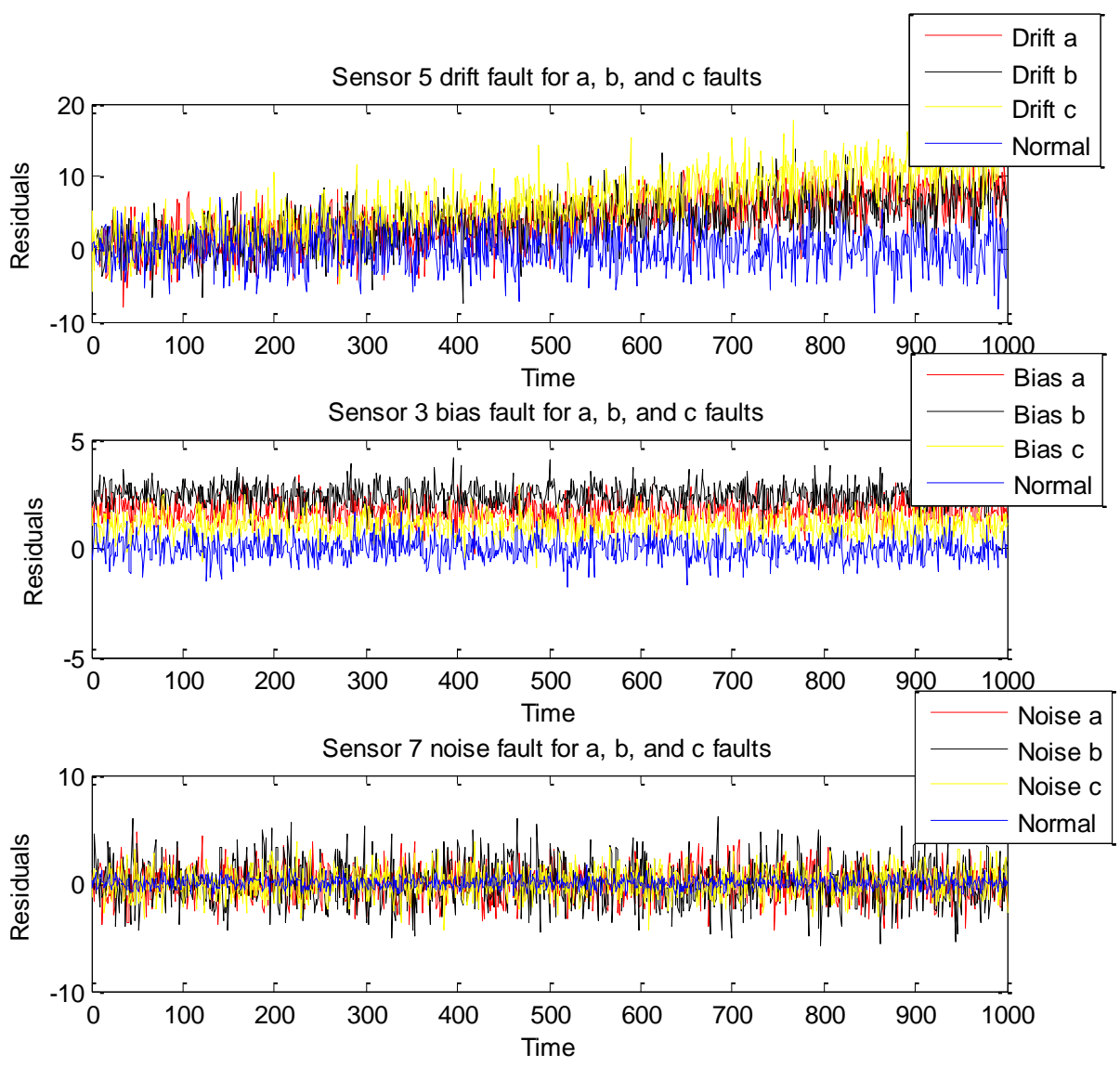

Figure 5-18: Fault Residuals for Test 2

Applying the ES diagnostic technique to the actual failure a gives the following results:

Fault signature vector $=\begin{array}{llllllllll}0 & 0 & 1 & 0 & 1 & 0 & 3 & 0 & 0 & 0\end{array}$

Possible Failures = $5 \quad 11 \quad 15$

Failure Similarities $=119.9847171 .9808137 .0913$

For this specific fault signature there are the three possible failures, failure 5, failure 11 and failure 13. The other 12 possible failures have been eliminated from the possible failure list. A similarity comparison is conducted and failure 5 has the lowest similarity parameter of 119.98 which shows this failure is most similar to the observed failure. The actual failure a is failure 5 so this failure was correctly classified. The classification process for this example can be seen in Figure 5-19. 


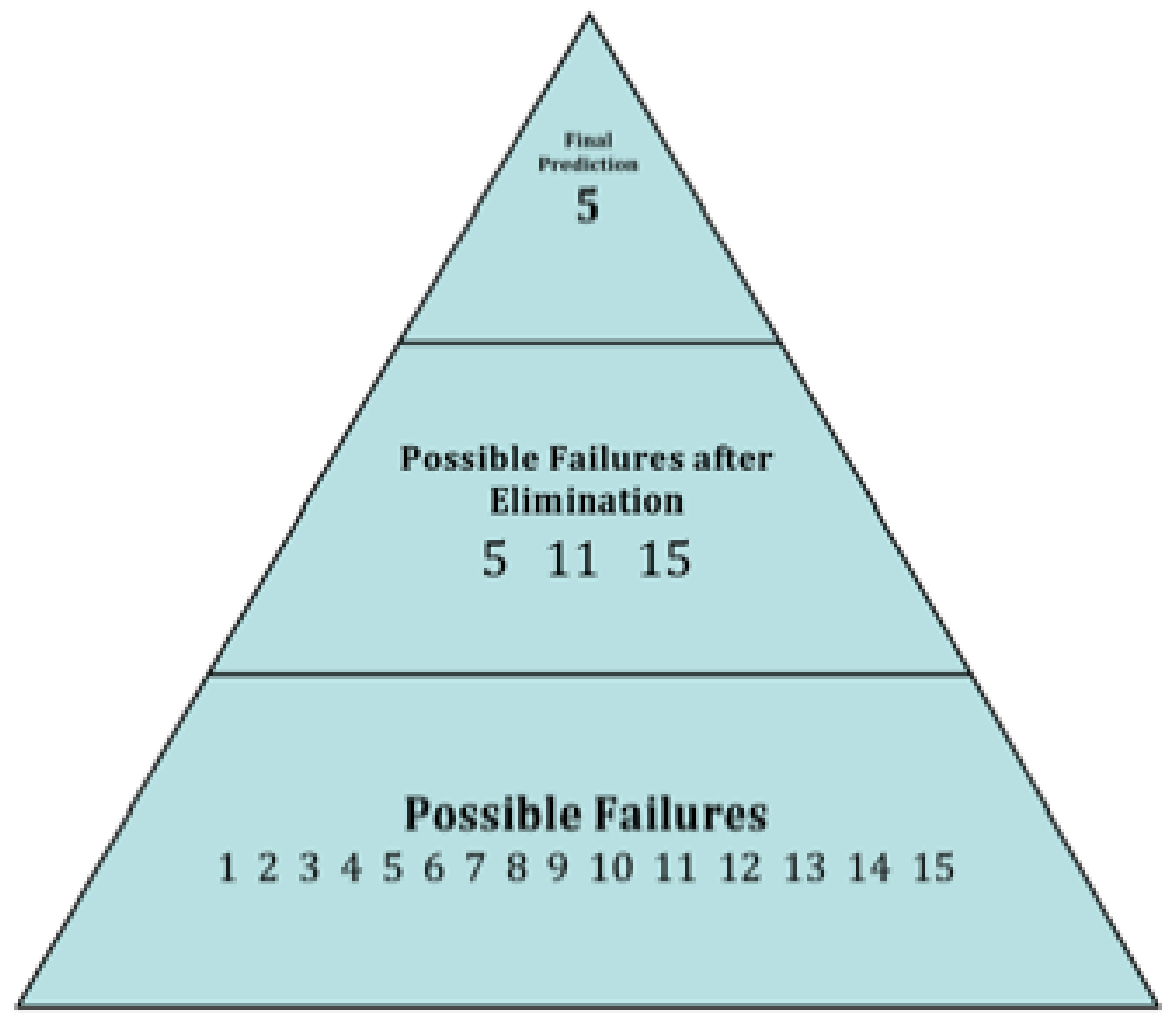

Figure 5-19: ES Process for Test 2a

The ES diagnostic technique results for failure $\mathbf{b}$ are:

Fault signature vector $=\begin{array}{llllllllll}0 & 0 & 1 & 0 & 1 & 0 & 3 & 0 & 0 & 0\end{array}$

Possible Failures $=5 \quad 11 \quad 15$

Failure Similarities $=197.7658158 .9452168 .6357$

There are three possible failures, failure 5, failure 11 and failure 13. A similarity comparison is conducted and failure 11 has the lowest similarity parameter of 158.95 which shows this failure is most similar to the observed failure. The actual failure $\mathbf{b}$ is failure 11 so this failure was correctly classified. The classification process for this example can be seen in Figure 5-20. 


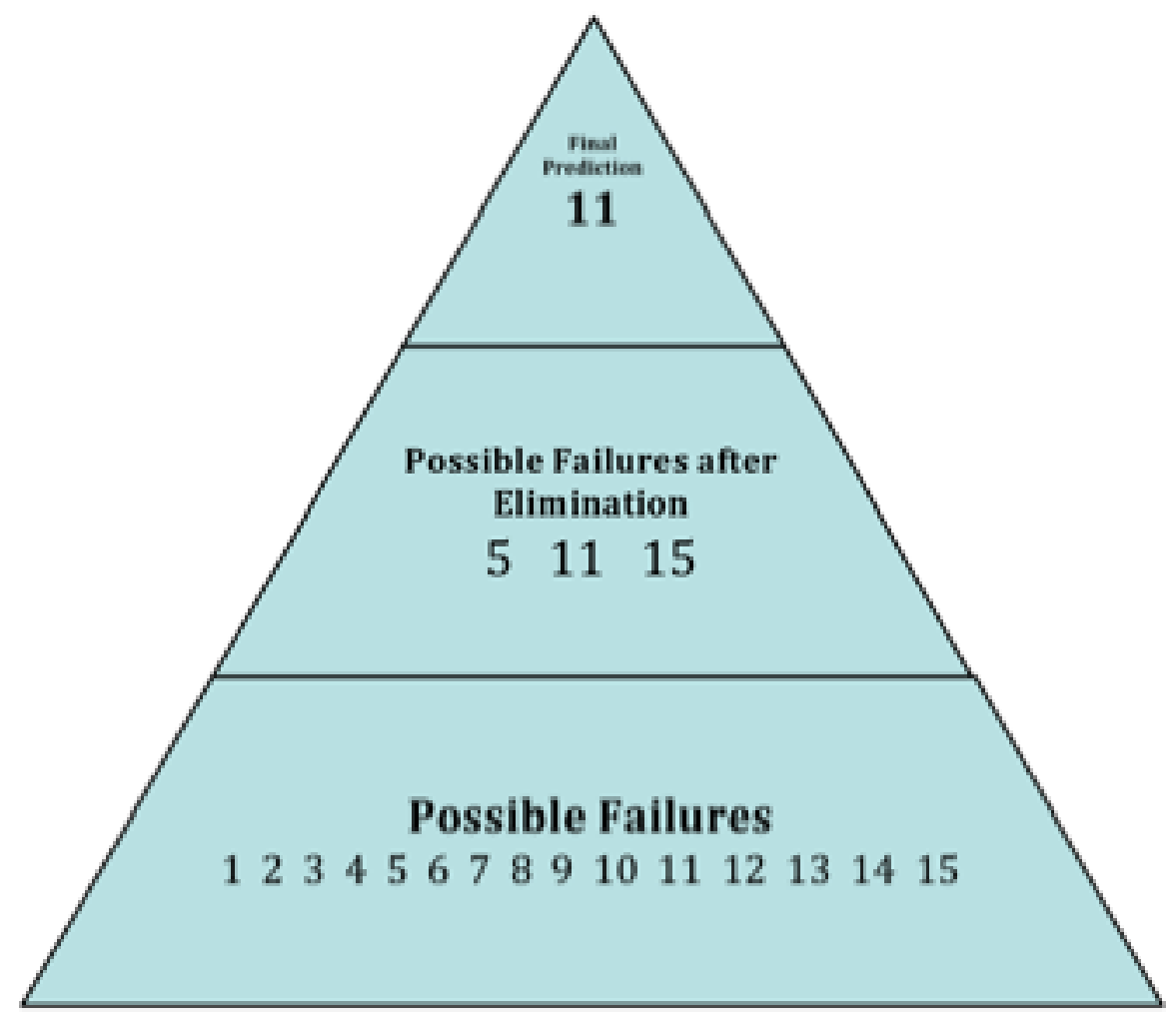

Figure 5-20: ES Process for Test 2b

The ES diagnostic technique results for failure $\mathbf{c}$ are:

Fault signature vector $=\begin{array}{llllllllll}0 & 0 & 1 & 0 & 1 & 0 & 3 & 0 & 0 & 0\end{array}$

Included $=5 \quad 11 \quad 15$

Failure Similarities $=135.1732152 .8051121 .5484$

There are three possible failures, failure 5, failure 11 and failure 13. A similarity comparison is conducted and failure 15 has the lowest similarity parameter of 121.55 which shows this failure is most similar to the observed failure. The actual failure $\mathbf{c}$ is failure 15 so this failure was correctly classified. The classification process for this example can be seen in Figure 5-21. 


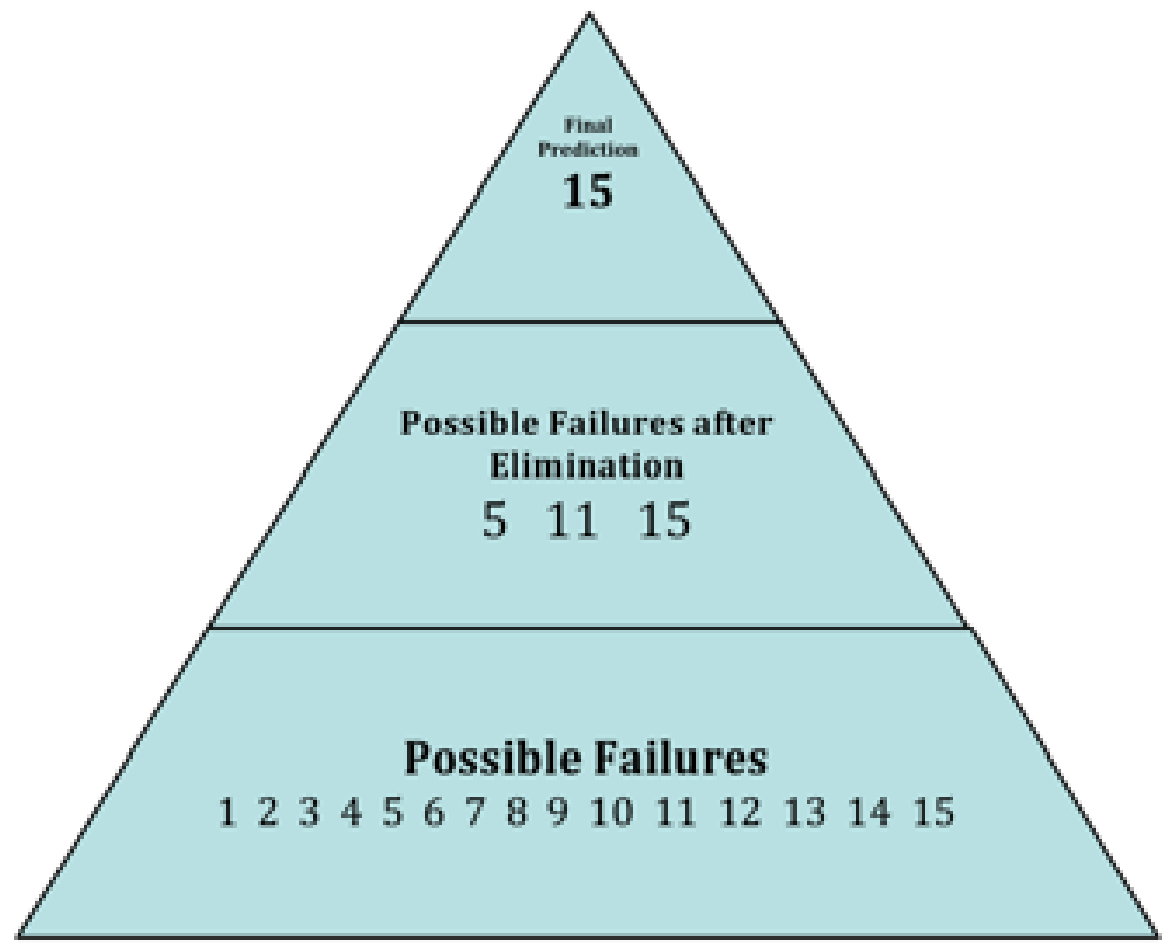

Figure 5-21: ES Process for Test 2c

\section{CONCLUSIONS AND FUTURE WORK}

This report has presented the work pursued in completion of the monitoring and diagnostics tasks of Objective 3 of the NERI-C project. The full health monitoring system, including adaptive system monitoring, fault detection and expanded condition monitoring, fault diagnostics, and system prognostics has been demonstrated on simulated data for the IRIS system; the prognostics portion of this research is presented in Volume 7. The Adaptive NonParametric Model (ANPM) monitoring system is designed for hybrid model adaptation beginning with data simulated from first principle models and moving to actual operational data as it becomes available. Because the monitoring module is adaptive in nature, traditional fault detection techniques are not well suited for this monitoring method. An alternative technique was developed to detect anomalies in the system and differentiate between nominal expanded operating conditions and system faults using principal component analysis. After an anomaly has been positively identified as a fault, the next step in an equipment surveillance system is to diagnose the fault type. Three methods for fault diagnostics have been studied and applied to 
various test cases. The three methods utilize different data sources, different modeling and logic architectures, and the features of different systems.

The proposed health monitoring modules were applied to simulated nominal and degradation data for the IRIS design. These modules are linked together so that the outputs of one module may be used to inform the inputs to the following modules.

Several areas of future work are proposed to augment the research completed here. Application of the developed techniques and methodologies to an actual, complex system with an associated high-fidelity simulation model can be used to further verify the ANPM and PCA ECM techniques. 


\section{REFERENCES}

Atkeson, Christopher G., Andrew W. Moore, and Stefan Schaal (1997b). Locally Weighted Learning for Control, Artificial Intelligence Review, Vol. 11, pp. 75113: 1997.

Basir, O. A. (2004). Vibro-Acoustic Engine Diagnostic System, U.S. Patent Application 20040260454, Carlson, Gaskey \& Olds, P.C., Birmingham, MI: June 14, 2004.

Ben-Abdennour, A. and K.Y. Lee (1996). An Autonomous Control System For BoilerTurbine Units, IEEE Transactions on Energy Conversion, Vol. 11, No. 2, pp.401406: June 1996.

Bickel, Peter, J., Doksum, K. A. (2001). Mathematical Statistics: Basic and Selected Topics, Volume 1, Pearson Prentice-Hall, 2001

Breed, D. S. (2002). Telematics System for Vehicle Diagnostics, U.S. Patent 6,738,697, Automotive Technologies International, Inc., Denville, NJ: July 3, 2002.

Breed, D. S. (2005). Vehicular Information and Monitoring System and Methods, U.S. Patent Application 20050125117, Brian Roffe, ESQ, Valley Stream, NY: January $19,2005$.

Brown, D.W., P.W. Kalgren, C.S. Byington, and M.J. Roemer, "Electronic Prognostics A Case Study using Global Positioning System (GPS)," Microelectronics Reliability 47 2007: 1874 - 1881.

Byington, C.S., M. Watson, D. Edwards, P. Stoelting, “A Model-Based Approach to Prognostics and Health Management for Flight Control Actuators," Proceedings of the IEEE Aerospace Conference, 2004: 3551 - 3562.

Callan, R., B. Larder, and J. Sandiford, “An Integrated Approach to the Development of an Intelligent Prognostic Health Management System," Proceedings of the IEEE Aerospace Conference, 2006: 12.

Carlin, B.P and T.A. Louis, Bayes and Empirical Bayes Methods for Data Analysis, 2nd ed. Boca Raton: Chapman and Hall/CRC: 2000.

Chinnam, R.B., "On-line Reliability Estimation of Individual Components, Using Degradation Signals," IEEE Transactions on Reliability 48 (4) 1999: 403 - 412.

Cleveland, W. S., Loader, C. (1994a).Computational methods for local regression, Technical Report 11, AT\&T Bell Laboratories, Statistics Department, Murray Hill, NJ: 1994.

Cleveland, W. S., Loader, C. (1994b). Smoothing by local regression: Principles and methods, Technical Report 95.3, AT\&T Bell Laboratories, Statistics Department, Murray Hill, NJ: 1994. 
Coble, J. and J.W. Hines (2009), "Development of a Prognostics Toolbox with Application to GPS Degradation Data," 2009 Conference of the Society for Machinery Failure Prevention Technology MFPT 2009, Dayton, Ohio, Apr, 2009.

Coble, J. (2010), Merging Data Source to Predict Remaining Useful Life - An Automated Method to Identify Prognostic Parameters, PhD Thesis, The University of Tennessee.

Cover, T. M. and P. E. Hart (1967). Nearest Neighbor Pattern Classification, IEEE Transactions on Information Theory, Vol. 13, No. 1: January 1967.

Davidson, A.C. (2003). Statistical Models, Cambridge University Press, 2003

Del Amo, A., K. Keller, and K. Swearingen (2005). General Reasoning System for Health Management, Proceedings of the Annual Meeting of the North American Fuzzy Information Processing Society, pp.19-24: June 26-28, 2005.

Dong, M., D. K. Xu, M. H. Li, and X. Yan (2004). Fault Diagnosis Model for Power Transformer Based on Statistical Learning Theory and Dissolved Gas Analysis, Proceedings of the IEEE International Symposium on Electrical Insulation, pp.85-88, Indianapolis, IN: September 19-22, 2004.

Draper, N. R., and H. Smith (1966). Applied Regression Analysis, John Wiley \& Sons, New York: 1966.

Engel, S., B. Gilmartin, K. Bongort, and A. Hess, "Prognostics, the Real Issues Involved with Predicting Life Remaining," Proceedings of the IEEE Aerospace Conference, pp. 457-469, 2000.

Fan, J., Gijbels, I. (1996a). Local Polynomial Modeling and Its Applications, Chapman \& Hall/CRC, New York, NY: 1996.

Fan, J., Gijbels, I. , Hu, T-C., Huang, L.(1996b). A study of variable bandwidth selection for local polynomial regression. Statistica Sinica. 6, 113-127.

Fenu, G. and F. Parisini (1998). "Model-Free Fault Diagnosis for Nonlinear Systems: A Combined Kernel-Regression and Neural Networks Approach", Proceedings of the American Control Conference, Philadelphia, PA: June 1998.

Frank, P.M. (1990). Fault diagnosis in dynamic systems using analytical and knowledge base redundancy-A survey and some new results, Automatica 26 (1990), pp. 459474.

Freedman, D.A. (2009). Statistical Models: Theory and Practice, Second Edition, Cambridge University Press, 2009

Garvey, D.R. and J.W. Hines (2006), "The Development of a Process and Equipment Monitoring (PEM) Toolbox and its Application to Sensor Calibration Monitoring", Quality and Reliability Engineering International, 22, 1-13, 2006.

Garvey D., and J. Hines (2006). Development and Application of Fault Detectability Performance Metrics for Instrument Calibration Verification and Anomaly Detection, Journal of Pattern Recognition Research, vol. 1, pp2-15, 2006. 
Garvey, D.R. (2007), “An Integrated Fuzzy Inference Based Monitoring, Diagnostic, and Prognostic System," Ph.D. Thesis, University of Tennessee, Knoxville: May, 2007.

Garvey, D. and J.W. Hines, "Dynamic Prognoser Architecture via the Path Classification and Estimation (PACE) Model," AAAI Fall Symposium on Artificial Intelligence for Prognostics, Nov 9-11, 2007, Arlington, VA.

Garvey, J., D. Garvey, R. Seibert and J.W. Hines (2007a), "Nuclear Power Plant On-Line Sensor Calibration Monitoring Implementation Issues", International Journal of Nuclear Knowledge Management, Vol. 2, No.3.

Garvey, J., R. Seibert, D. Garvey, and J.W. Hines (2007b), "Application of On-Line Monitoring Techniques to Nuclear Plant Data" in press Nuclear Engineering and Technology, 2007.

Gayme, D. F., S. K. Menon, E. O. Nwadiogbu, D. W. Mukavetz, and C. M. Ball (2003). Fault Detection System and Method Using Augmented Data and Fuzzy Logic, U.S. Patent Application 20050021212, Honeywell International Inc., Morristown, NJ: July 24, 2003.

Gelman, A., J. Carlin, H. Stern, and D. Rubin, Bayesian Data Analysis 2nd ed. Boca Raton: Chapman and Hall/CRC: 2004.

Germond, A. J. and D. Niebur (1992). Survey of Knowledge-Based Systems in Power Systems: Europe, Proceedings of the IEEE, Vol. 80, No. 5: May 1992.

Gertler, J. (1988). Survey of model-based failure detection and isolation in complex plants, IEEE Control Syst. Mag. 8 (1988), pp. 3-11.

Geva, Shlomo and J. Sitte (1991). Adaptive Nearest Neighbor Pattern Classification, IEEE Transactions on Neural Networks, Vol. 2, No. 2, pp.318-322: March 1991.

Gibbons, J. D., Chakraborti, S. (2003). Nonparametric Statistical Inference, $4^{\text {th }}$ Edition, CRC, 2003.

Girish, T., S.W. Lam, J.S.R. Jayaram, "Reliability Prediction Using Degradation Data A Preliminary Study Using Neural Network-based Approach," Proceedings of the European Safety and Reliability Conference (ESREL 2003), Jun. 15-18, 2003: Maastricht, The Netherlands.

Grist, Edward, Cavitation and the Centrifugal Pump: A Guide for Pump Users, Taylor \& Francis: Philadelphia, PA. (1999).

Heo, G.Y., "Condition Monitoring Using Empirical Models: Technical Review and Prospects for Nuclear Applications," Nuclear Engineering and Technology 40 (1) 2008: $49-68$.

Haykin, Simon (1994). Neural Networks: A Comprehensive Foundation, 2nd Edition, Prentice Hall: 1998.

Hess, A., G. Calvello, and P. Frith, "Challenges, Issues, and Lessons Learned Chasing the 'Big P': Real Predictive Prognostics Part 1,' Proceedings of the IEEE Aerospace Conference, pp. 3610 - 3619, March 2005. 
Hines, J.W. and R.E. Uhrig (1997a), "Use of Autoassociative Neural Networks for Signal Validation", Journal of Intelligent and Robotic Systems, Kluwer Academic Press.

Hines, J.W. and D.J. Wrest (1997b), "Signal Validation Using an Adaptive Neural Fuzzy Inference System" Nuclear Technology, August, pp. 181-193.

Hines, J.W. and D. Garvey (2005). "The Development of a Process and Equipment Monitoring (PEM) Toolbox and its Application to Sensor Calibration Monitoring." Fourth International Conference on Quality and Reliability (ICQR4), August 9 - 11, 2005: Beijing, China.

Hines, J.W. and B. Rasmussen (2005), "On-Line Sensor Calibration Monitoring Uncertainty Estimation", Nuclear Technology, Sept, 151(3).

Hines, J.W., R. Seibert, S.A. Arndt, "Technical Review of On-Line Monitoring Techniques for Performance Assessment (NUREG/CR-6895) Vol. 1, State-of-theArt." Published January, 2006.

Hines, J. Wesley and Dustin Garvey (2006b), "Traditional and Robust Vector Selection Methods for Use with Similarity Based Models", 5th International Topical Meeting on Nuclear Plant Instrumentation, Control and Human-Machine Interface Technologies, Albuquerque, NM: November 12-14, 2006.

Hines, J.W., A. Usynin, and A. Urmanov, "Prognosis of Remaining Useful Life for Complex Engineering Systems," American Nuclear Society International Topical Meeting on Nuclear Plant Instrumentation, Control, and Human-Machine Interface Technologies (NPIC\&HMIT), 2006: Albuquerue, NM.

Hines, J.W., D. Garvey, R. Seibert, A. Usynin, (2007a). Technical Review of On-Line Monitoring Techniques for Performance Assessment, NUREG/CR-6895 ORNL/TM-188, Vol 2

Hines, J.W. and A. Usynin, "Current Computational Trends in Equipment Prognostics," International Journal of Computational Intelligence Systems 1 (1) 2008: 1 - 9.

Ho, Y.-C., and D. L. Pepyne, "Simple Explanation of the No Free Lunch Theorem of Optimization”, Cybernetics and Systems Analysis, 38 (2), 2002, pp 292 - 298.

Holbert, K. E., Heger, S. A. and A. M. Ishaque, (1995) " Fuzzy Logic For Power Plant Signal Validation", Proceedings of the 9th Power Plant Control \& Testing Symposium, Knoxville, Tennessee, May 24-26, 20.01 - 20.15.

Humberstone, M., Wood, B., Henkel, J., Hines, J.W. (2009). Differentiating between expanded and fault conditions using principal component analysis, Journal of Intelligent Manufacturing, 2009, 10-1007/s 10845-009-0343-1

Ikonomopoulos, A., R. E. Uhrig, L. H. Tsoukalas (1992), "A Methodology for Performing Virtual Measurements in a Nuclear Reactor System", Transactions of the 1992 American Nuclear Society International Conference on Fifty Years of Controlled Nuclear Chain Reaction: Past, Present, and Future, Chicago, Illinois, 106-107.

Johnson, R. A. and D. W. Wichern (1992). Applied Multivariate Statistical Analysis, 3rd Edition, Prentice Hall, New Jersey: 1992. 
Jolliffe, I. T. (2002). Principal Component Analysis, Second Edition, Springer Series in Statistics, New York, Springer

Kavaklioglu, K., and B.R. Upadhyaya (1994), "Monitoring Feedwater Flow Rate and Component Thermal Performance of Pressurized Water Reactors By Means of Artificial Neural Networks", Nuclear Technology Vol. 107.

Kavuri, S. N. and V. Venkatasubramanian (1993). Using Fuzzy Clustering with Ellipsoidal Units in Neural Networks for Robust Fault Classification, Computers \& Chemical Engineering, Vol. 17, No. 8, pp.765-784: August 1993.

Keller, K., K. Swearingen, J. Sheahan, M. Baily, J. Dunsdon, B. Cleeve, K.W. Przytula, and B. Jordan, "Aircraft Electrical Power Systems Prognostics and Health Management," IEEE Aerospace Conference, 2006: Big Sky, MT.

Koppen, M., "No-Free-Lunch Theorems and the Diversity of Algorithms", Congress on Evolutionary Computation, 1, 2004, pp 235 - 241.

Kothamasu, R., S.H. Huang, and W.H. VerDuin, "System Health Monitoring and Prognostics - A Review of Current Paradigms and Practices," International Journal of Advanced Manufacturing Technology 28 2006: 1012 - 1024.

Kotschenreuther, M., Dorland, W., Beer, M. A., Hammett, G.W. (1995). Quantitative Predictions of Tokamak Energy Confinement from First-Principles Simulations with Kinetic Effects. Phys. Plasmas, Vol 2, 2381: 1995.

Koutroumbas, K. and N. Kalouptsidis (1994). Nearest Neighbor Pattern Classification Neural Networks, Proceedings of the IEEE World Congress on Computational Intelligence and Neural Networks, Vol. 5, pp.2911-2915, Orlando, FL: June 27July 2, 1994.

Kreutzer, S. E. and Hakimi, S. L. (1987). "System-Level Fault Diagnosis: A Survey", Microprocessing and Microprogramming, Vol. 20, No. 4-5, pp.323-330: May 1987.

Li, F., B.R. Upadhyaya and J. Wesley Hines (2009), "Optimal Sensor Placement Strategy for the Steam Generator System in an Integral Pressurized Water Reactor", Invited paper for the Transaction of the American Nuclear Society, Atlanta Georgia.

Lindely, D.V. and A.F. Smith, "Bayes Estimates for Linear Models," Journal of the Royal Statistical Society (B) 34 (1) 1972: 1 - 41.

$\mathrm{Lu}, \mathrm{C} . J$. , and W. Meeker, "Using Degradation Measures to Estimate a Time-to-Failure Distribution," Technometrics 35 (2) 1993: 161 - 174.

Luo, J., M. Namburu, K. Pattipati, L. Qiao, M. Kawamoto, S. Chigusa, "Model-based Prognostic Techniques," IEEE Systems Readiness Technology Conference (AUTOTESTCON), Sep 22-25 2003: Anaheim, CA

Meeker, W.Q., L.A. Escobar, and C.J. Lu, "Accelerated degradation tests: modeling and analysis," Technometrics, vol. 40, no. 2, pp. 89-99, 1998.

Mehra, R.K. and Peshon, I. (1971). An innovations approach to fault detection and diagnosis in dynamic systems, Autmatica 7 (1971), pp. 637-640. 
Milne, R. (1987). Strategies for diagnosis, IEEE Trans. Syst., Man Cybern. SMC-17 (1987), pp. 333-339.

Murphy, O. J. (1990). Nearest Neighbor Pattern Classification Perceptrons, Proceedings of the IEEE, Vol. 78, No. 10, pp.1595-1598: (1990).

Natke, H. Gunther (1997). "Symptom-Based Fault Diagnosis of Vibrating Structures", Proceedings of the 51st Meeting of the MFTP Society, pp. 3-7, Virginia Beach, VA: April 14-18, 1997.

Patton, R. J., F. J. Uppal, and C. J. Lopez-Toribio (2000). "Soft Computing Approaches to Fault Diagnosis for Dynamic Systems: A Survey", Proceedings of the IFAC Symposium SAFEPROCESS 2000, pp.298-311, Budapest: June 14-16, 2000.

Piezo Systems, Inc., Cambridge, Massachusetts, Introduction to Piezo Transducers, (2001). www.piezo.com

Pipe, K., "Practical Prognostics for Condition Based Maintenance," 2008 International Conference on Prognostics and Health Management, Oct 6 - 9, 2008: Denver, $\mathrm{CO}$.

Prognostic Application for the Power Industry: Final Report. EPRI, Palo Alto, CA: 2009. PID: 065702.

Pook, P. K. and D. H. Ballard (1994). Deictic Teleassistance, Proceedings of the IEEE/RSJ/GI International Conference on Intelligent Robots and Systems, Vol. 1, pp.245-252, Munich, Germany: September 12-16, 1994.

Riedesel, J. (1989). "A Survey of Fault Diagnosis Technology", Proceedings of the 24th Intersociety Energy Conversion Engineering Conference, Vol. 1, pp. 183-188, Washington, D.C.: August 6-11, 1989.

Robinson, M.E. and M.T. Crowder, "Bayesian Methods for a Growth-Curve Degradation Model with Repeated Measures," Lifetime Data Analysis 6, 2000: 357 - 374.

Russell, E., Chiang, L. H., and Braatz, R.D. (2000). Data-Driven Techniques for Fault Detection and Diagnosis in Chemical Processes, A Volume in the Advances in Industrial Control Series, Springer, New York, NY: 2000.

Seber, G. A. F., Lee, A. J. (2003). Linear Regression Analysis, $2^{\text {nd }}$ Edition, WileyInterscience, 2003.

Simani, S., Fantuzzi, C., and Patton, R. J. (2003). Model-based Fault Diagnosis in Dynamic Systems Using Identification Techniques, Springer-Verlag London Limited, London: 2003.

Storrick, G.D.,Petrovic, B., Oriani, L., Conway, L.E., and Conti, D., Westinghouse Electric Company LLC, STD-AR-05-01 (2005). Instrumentation Needs for Integral Primary System Reactors (IPSRs), USDOE. Sept 2005

te Braake, H.A.B., H.J.L.V. van Can, and H.B. Verbruggen (1998), "Semi-mechanistic Modeling of Chemical Processes with Neural Networks", Engineering Applications of Artificial Intelligence, 11(4), 507-515. 
Tarifa, E. E. and Nicolas J. S. (1997). Fault Diagnosis, Direct Graphs, and Fuzzy Logic, Computers \& Chemical Engineering, Vol. 21, Supplement 1, pp.S649-S654: May 20, 1997.

Thompson, M.L. and M.A. Kramer (1994), "Modeling Chemical Processes Using Prior Knowledge and Neural Networks", AIChE Journal, 4 (8), 1328-1340.

Tsoukalas, L.H. and R.E. Uhrig, Fuzzy and Neural Approaches in Engineering. John Wiley \& Sons, New York: 1997.

Uhrig, R.E., and J.W. Hines (2005), "Computational Intelligence in Nuclear Engineering", Nuclear Engineering and Technology, Vol. 37, No. 2, pp 127-138.

Upadhyaya, B. R. and E. Eryurek (1992), "Application of Neural Networks for Sensor Validation and Plant Monitoring", Nuclear Technology, Vol. 97, pp. 170-176.

Upadhyaya, B.R., M. Naghedolfeizi, and B. Raychaudhuri, "Residual Life Estimation of Plant Components," P/PM Technology June, 1994: 22 - 29.

Upadhyaya, B, J.W. Hines, B. Lu, and X. Huang, "On-Line Monitoring and Diagnostics of the Integrity of Nuclear Plant Steam Generators and Heat Exchangers," Phase III NEER Annual Report, Report No. DE-FG07-01ID14114/UTNE-06, NEER Grant Number: DE-FG07-01ID14114.

Venkatasubramanian, V., R. Rengaswamy, S.N. Kavuri, and K. Yin (2003). A Review of Process Fault Detection and Diagnosis Part III: Process 324 History Based Methods, Computers \& Chemical Engineering, Vol. 27, No. 3, pp.327-346: March 15, 2003.

Wang, H., Song, Z., and Wang, H., (2002). Fault detection behavior analysis of PCAbased process monitoring approach, Journal of Chemical Industry and Engineering, Vol 53, No 3, pp. 297-301, Mar. 2002

Wang, H., Li, P., and Yuan, Z., (2002). Understanding PCA fault detection results by using expectation analysis method, Proceedings of the $41^{\text {st }}$ IEEE Conference on Decision and Control., Las Vegas, NV, Dec. 2002

Wang, P. and D. Coit, "Reliability and Degradation Modeling with Random or Uncertain Failure Threshold," Proceedings of the IEEE Reliability and Maintainability Symposium (RAMS), 2007: 392 - 397.

Wilson, J.A. and L.F.M. Zorsetto (1997), "A Generalized approach to Process State Estimation Using Hybrid Artificial Neural Network Mechanistic Models", Computers \& Chemical Engineering, 21(9), 951-963.

Wald A. (1945). Sequential Tests of Statistical Hypotheses. Annals of Mathematical Statistics, Vol 16 (2): 117 - 186, June 1945.

Wasserman, L. (2007). All of the Nonparametric Statistics, Springer, 2007.

Wong, D. K. (2001). Geo-Location in Urban Areas Using Signal Strength Repeatability, IEEE Communications Letters, Vol. 5, No. 10, pp.411-413: October 2001. 


\section{Appendix A: Additional Figures}

\section{A.1 PCA ECM Fault Detection Results}

\section{A.1.1 Heat Exchanger Fouling}
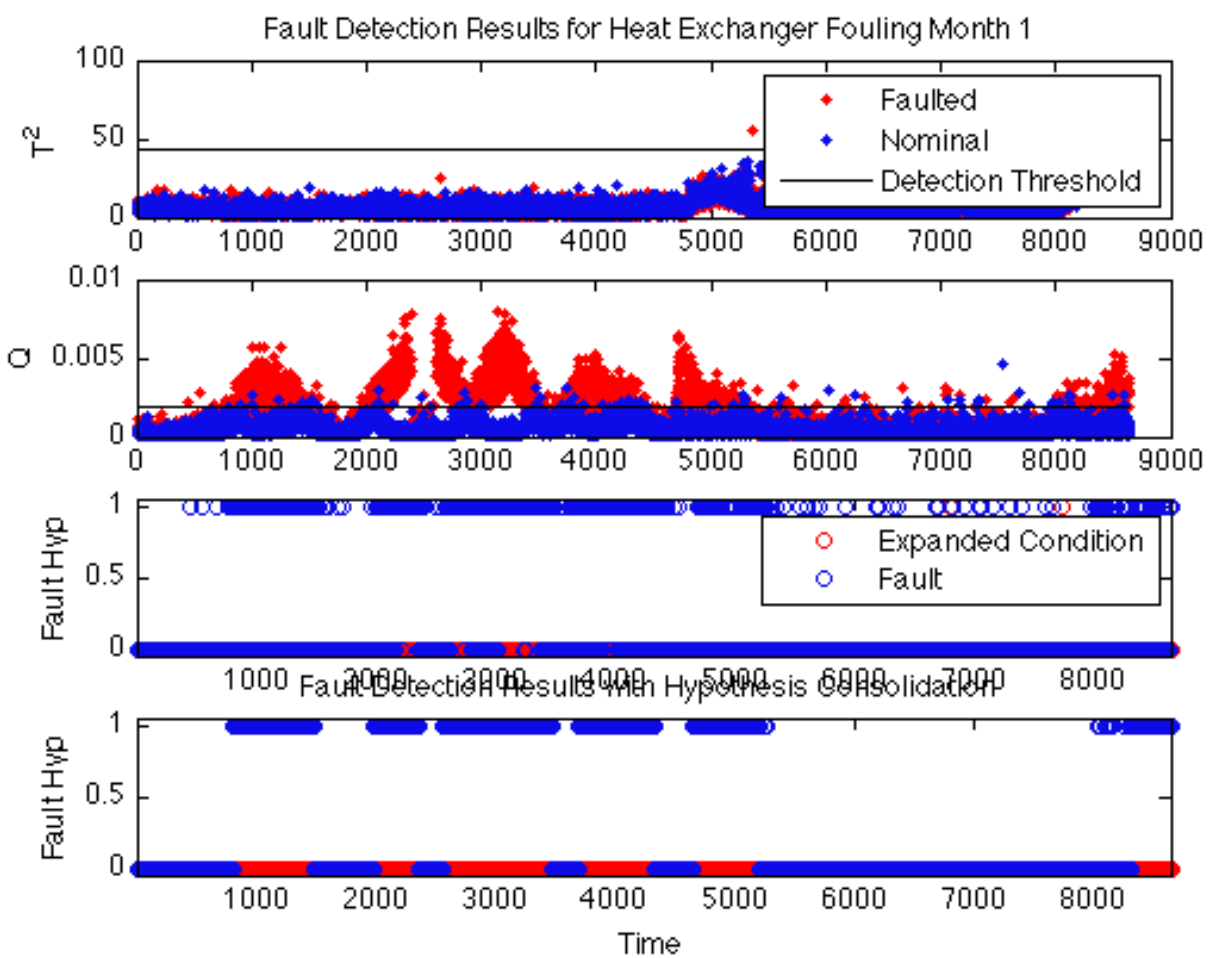
Fault Detection Results for Heat Exchanger Fouling Month 2
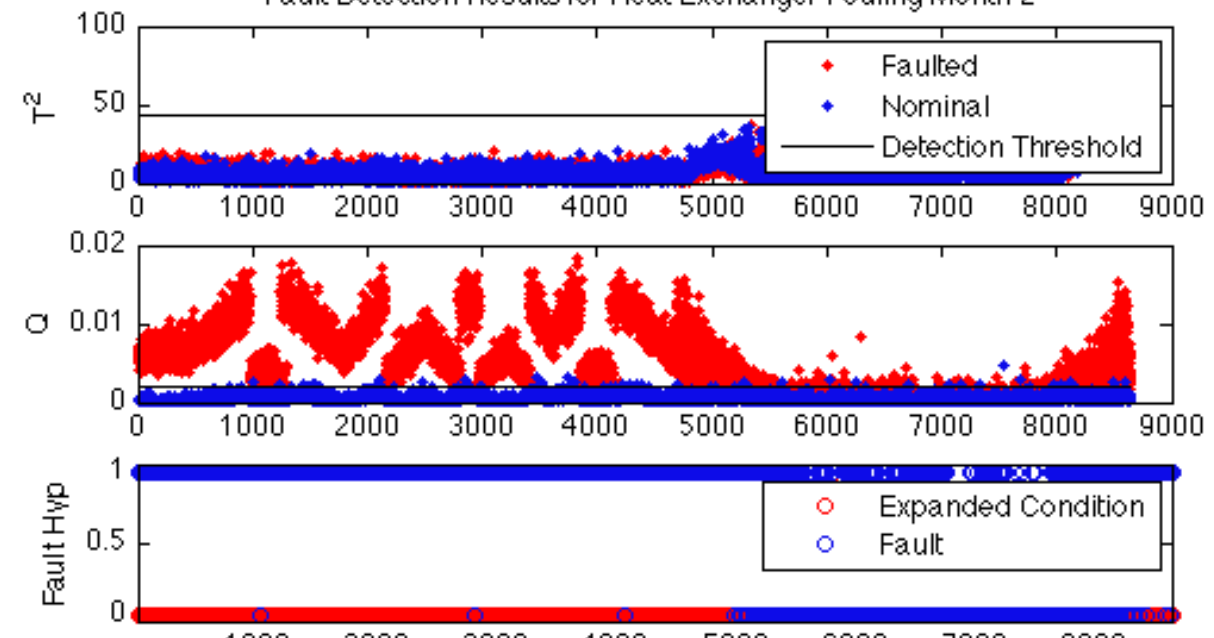

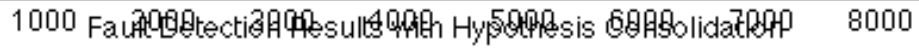

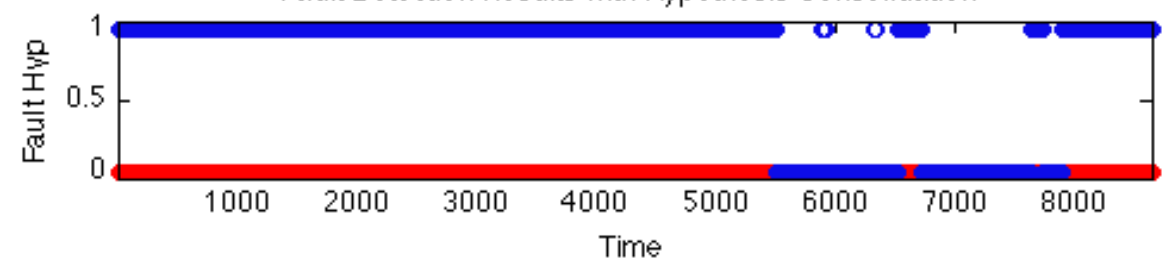


Fault Detection Results for Heat Exchanger Fouling Month 3
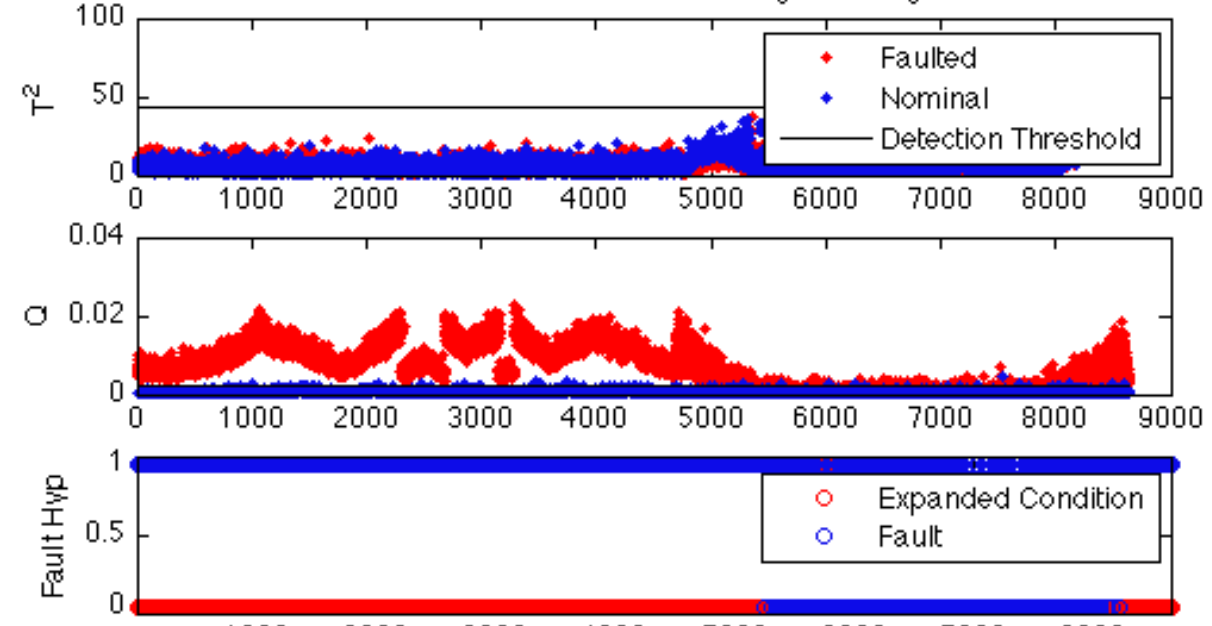

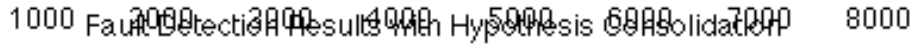

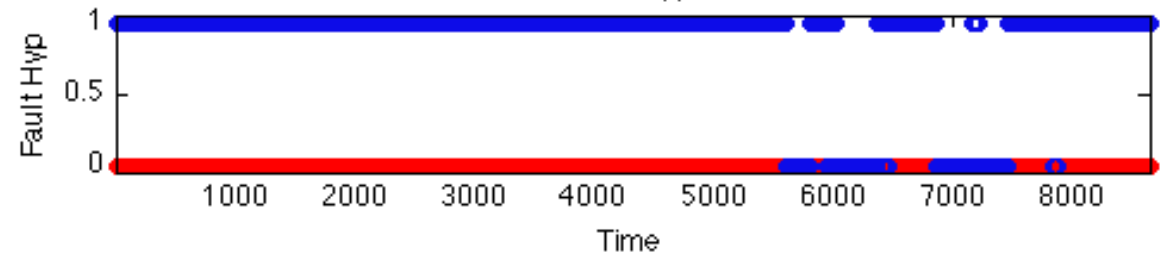

Fault Detection Results for Heat Exchanger Fouling Month 4
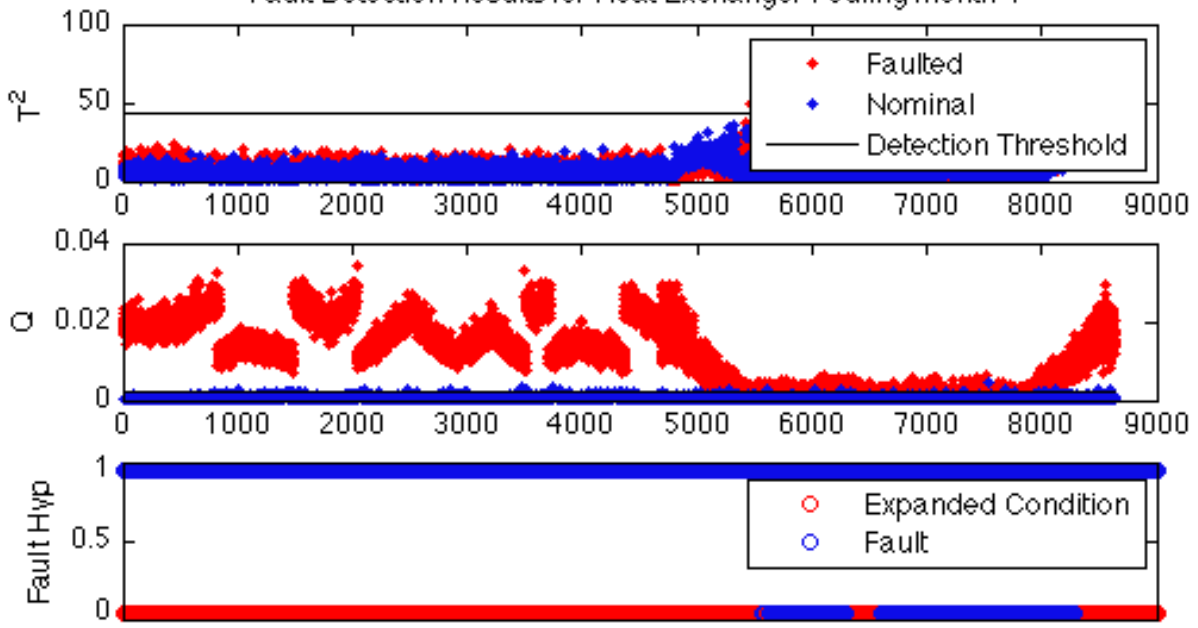

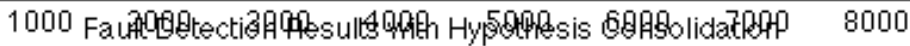

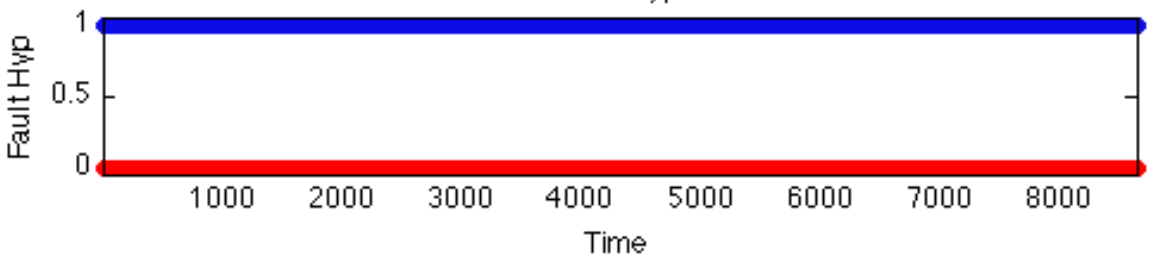


Fault Detection Results for Heat Exchanger Fouling Month 5
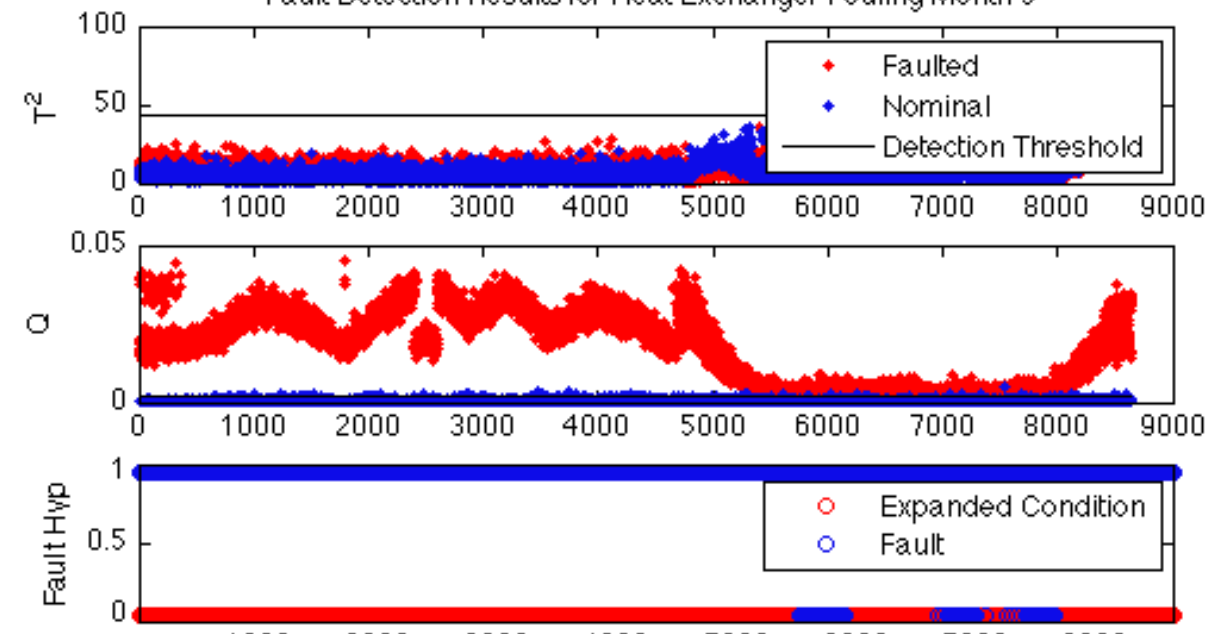

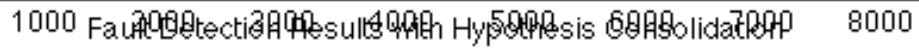

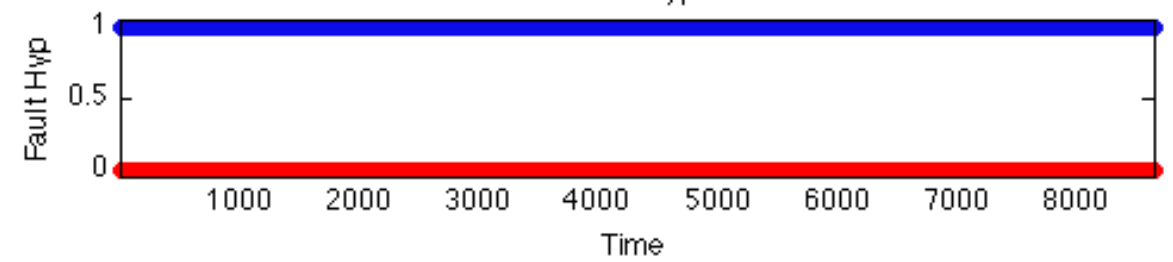

Fault Detection Results for Heat Exchanger Fouling Month 6
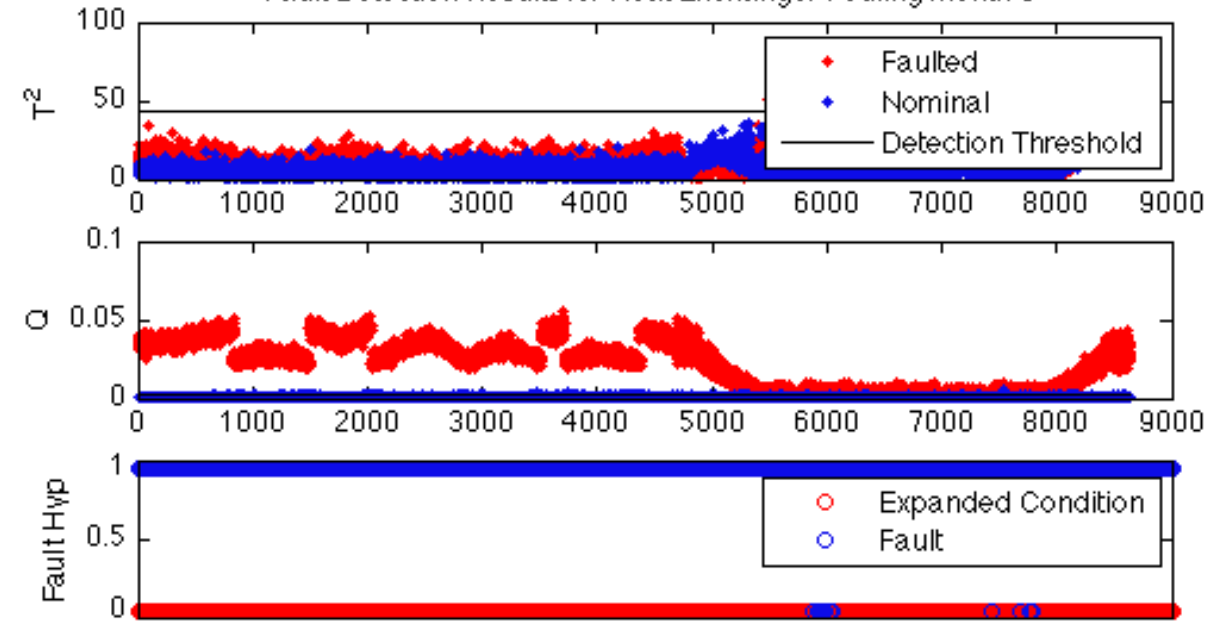

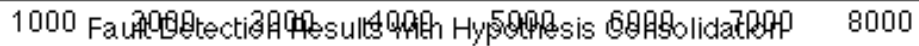

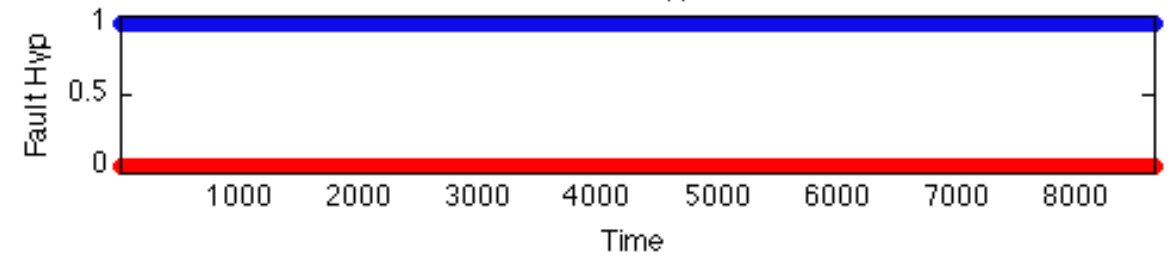


Fault Detection Results for Heat Exchanger Fouling Month 7
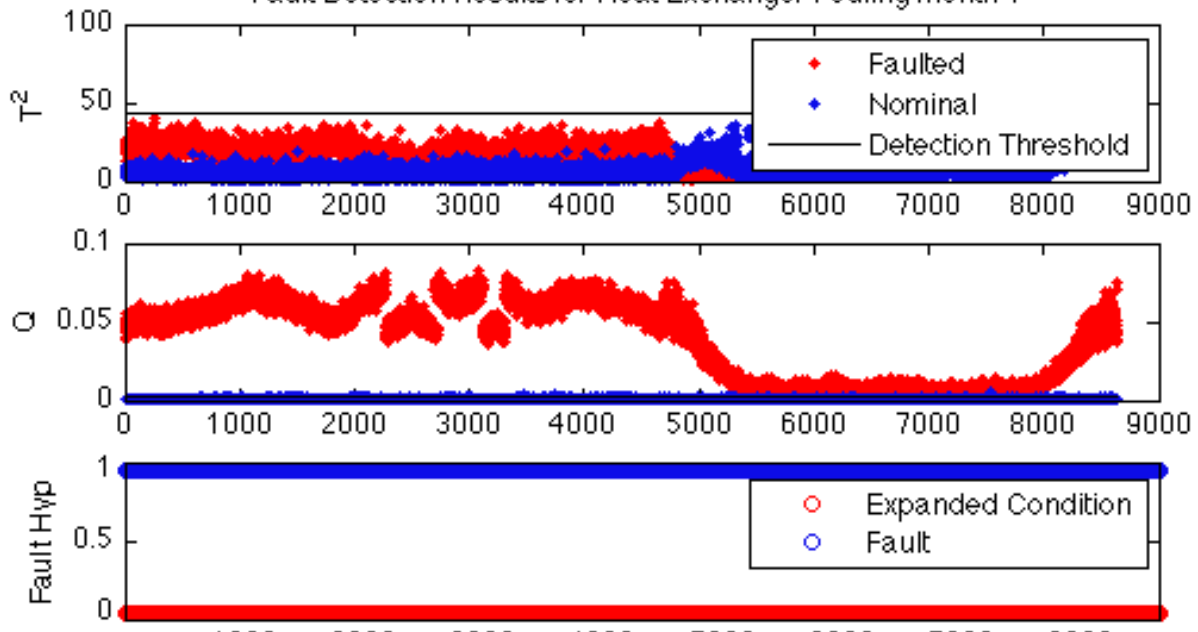

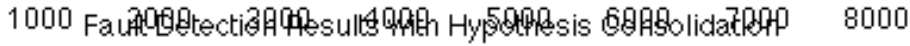

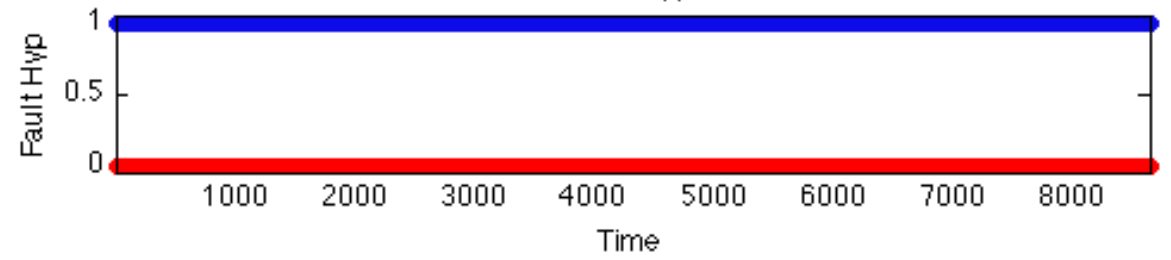

Fault Detection Results for Heat Exchanger Fouling Month 8
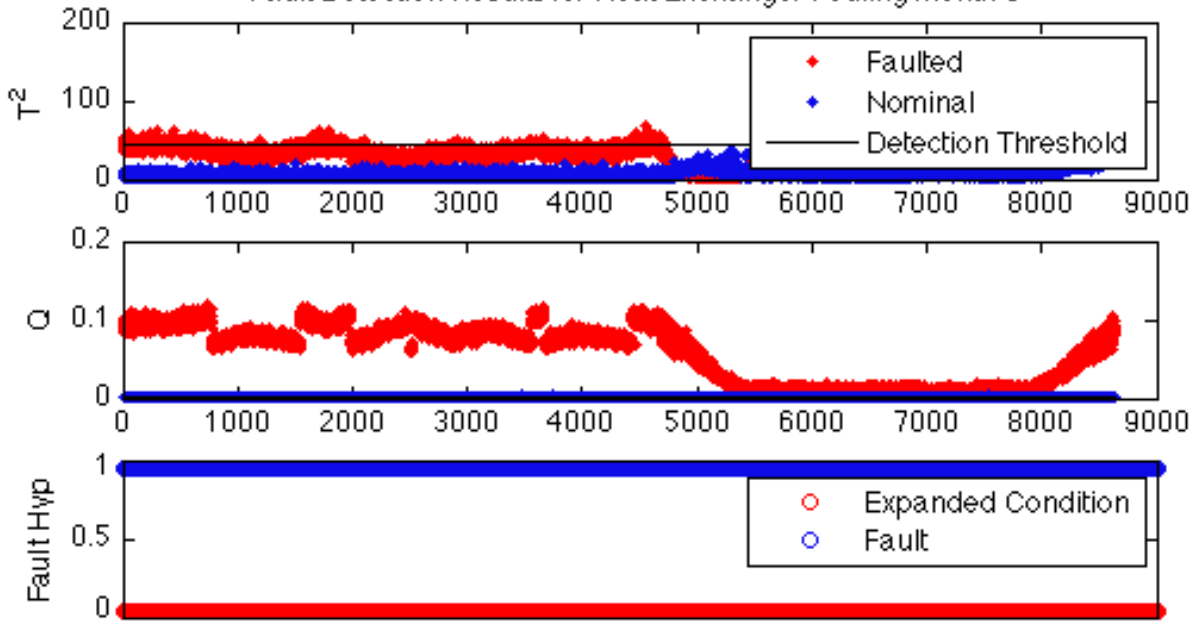

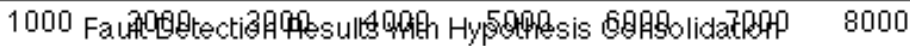

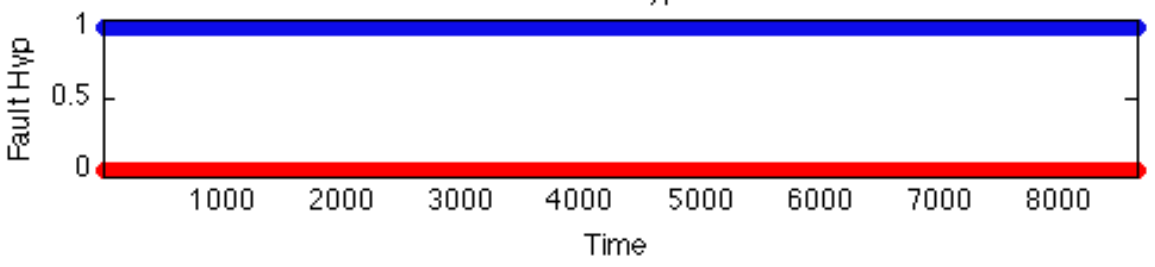


Fault Detection Results for Heat Exchanger Fouling Month 9
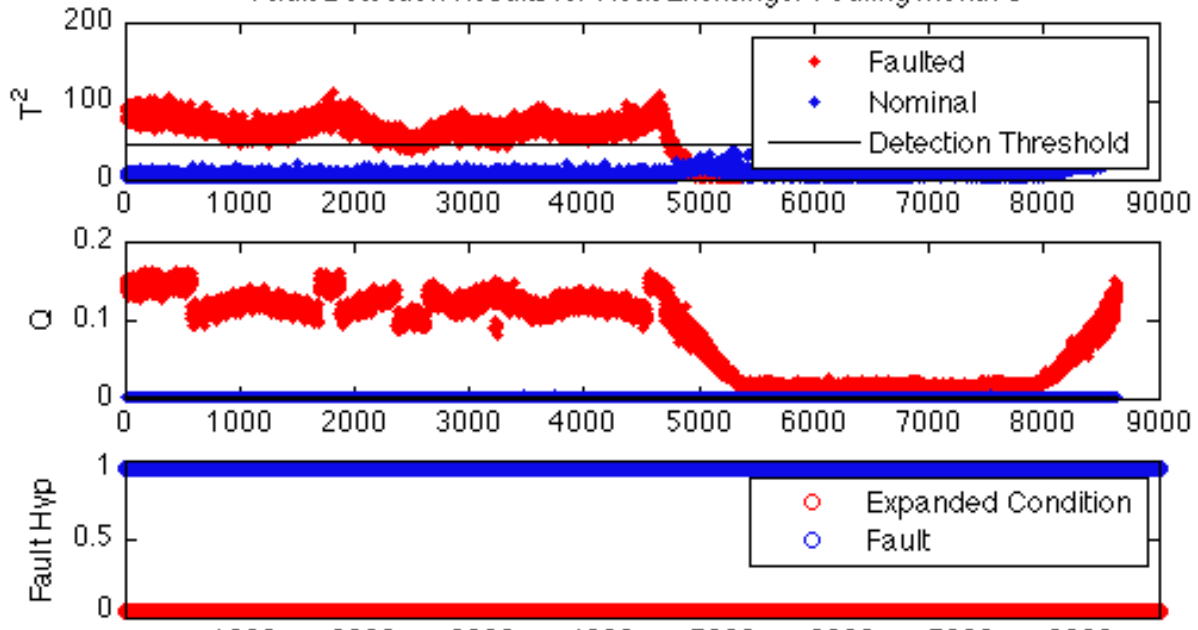

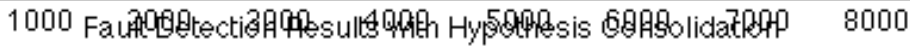

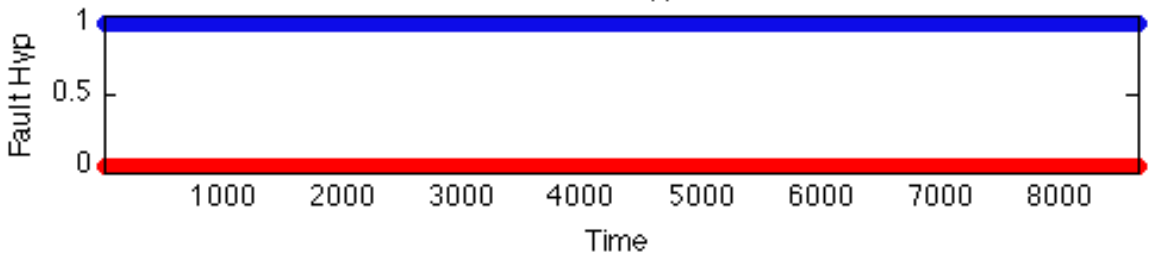

Fault Detection Results for Heat Exchanger Fouling Month 10
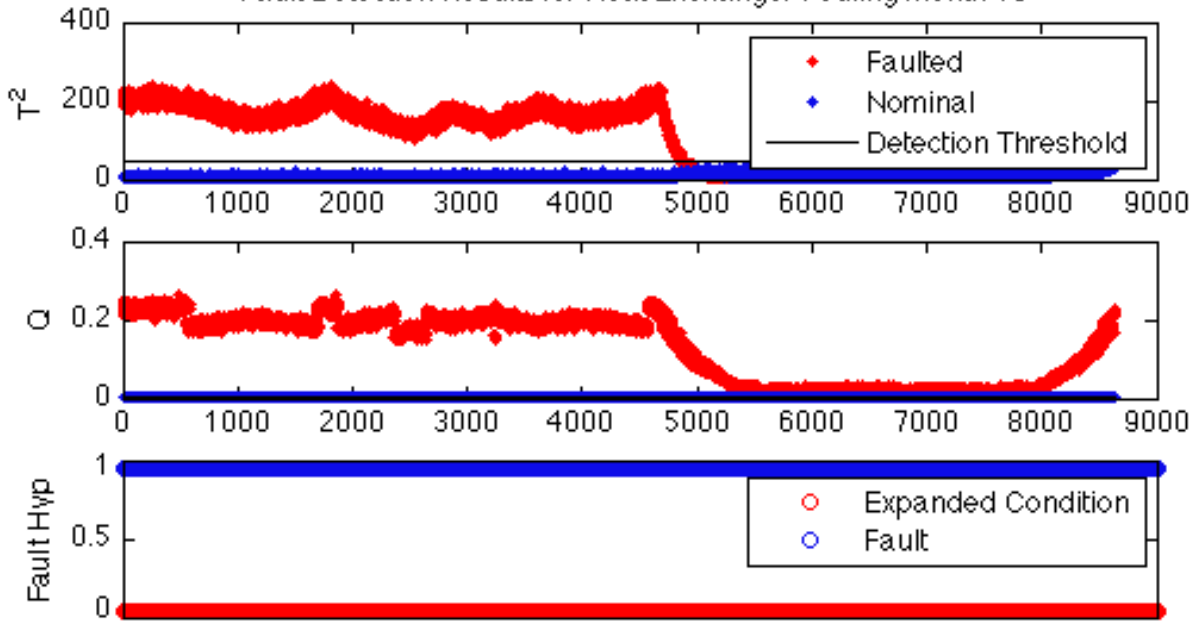

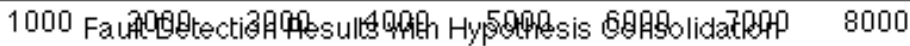

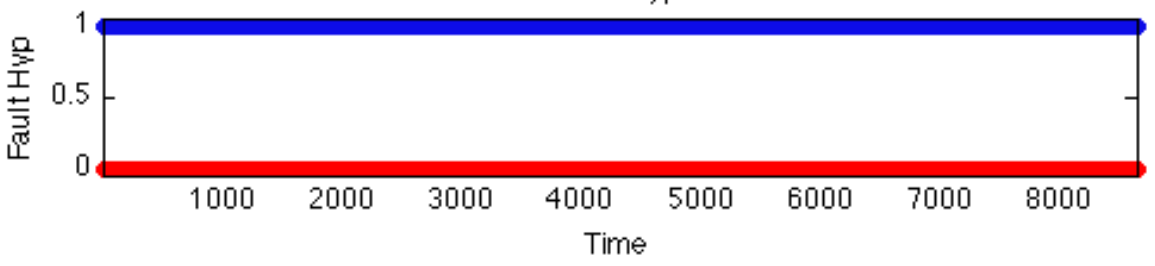


Fault Detection Results for Heat Exchanger Fouling Month 11
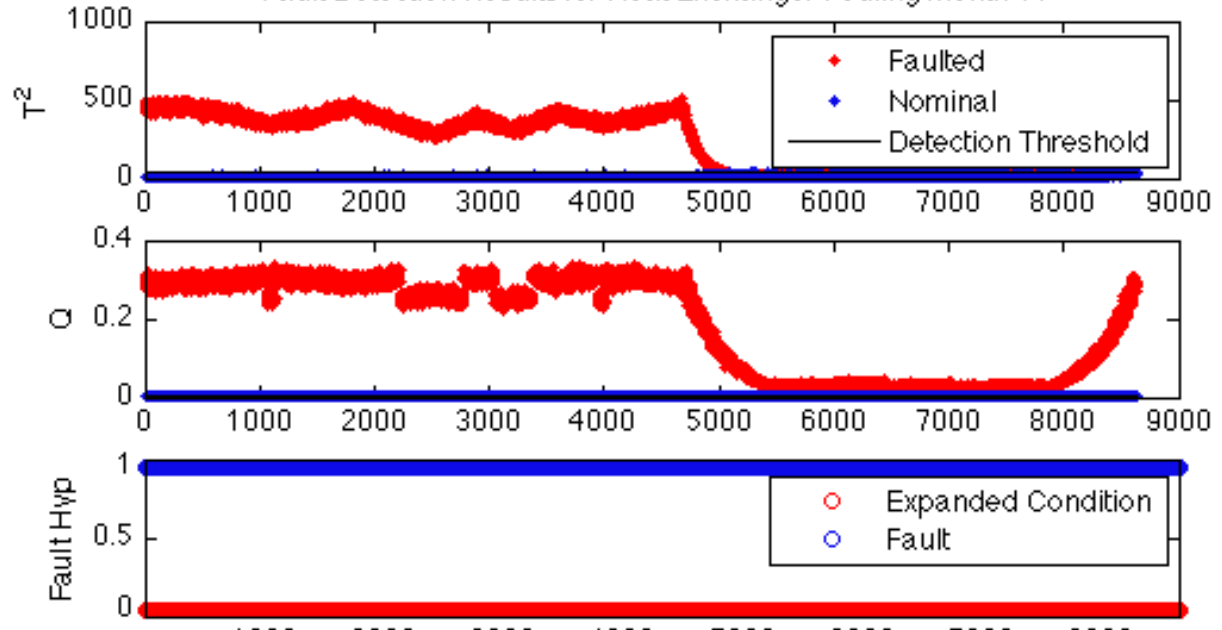

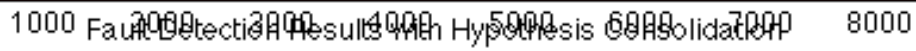

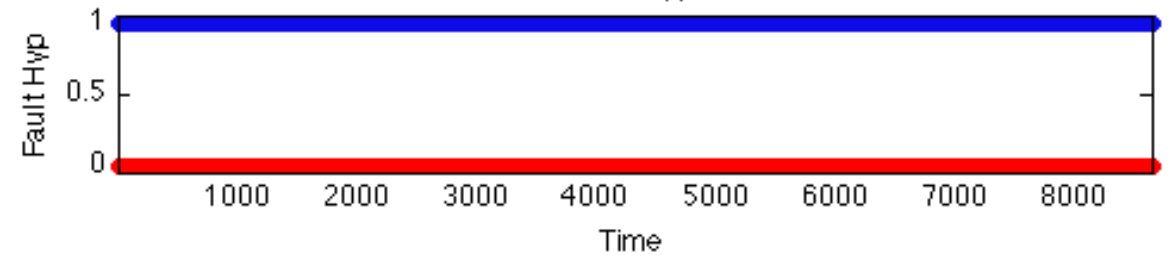

Fault Detection Results for Heat Exchanger Fouling Month 12
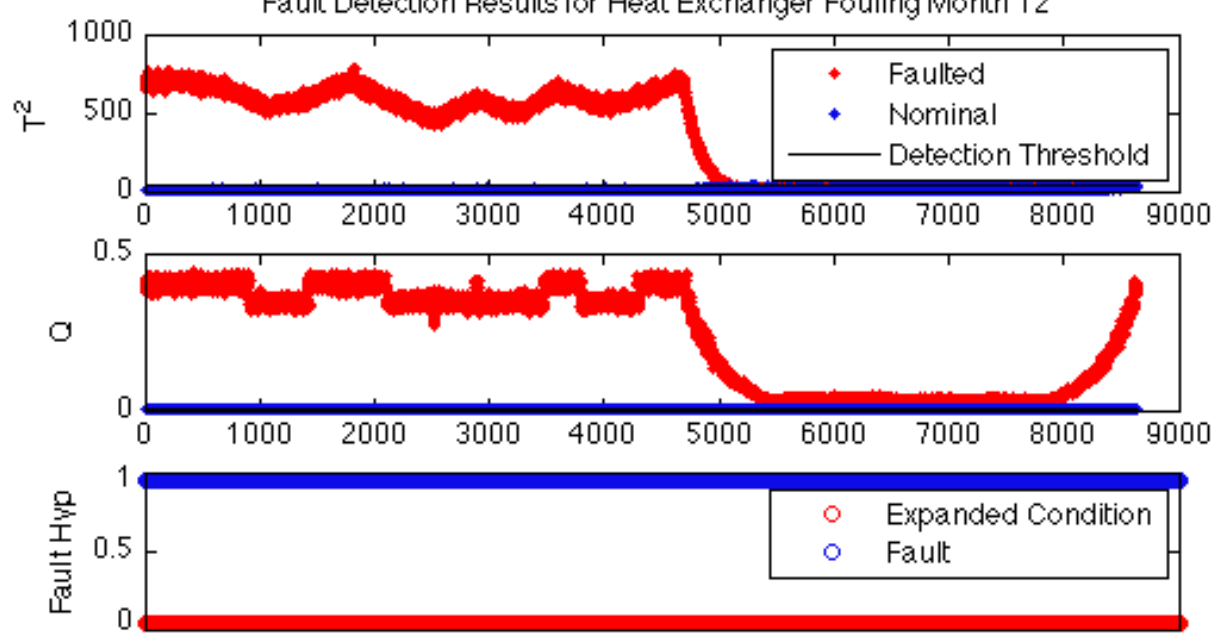

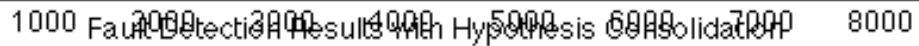

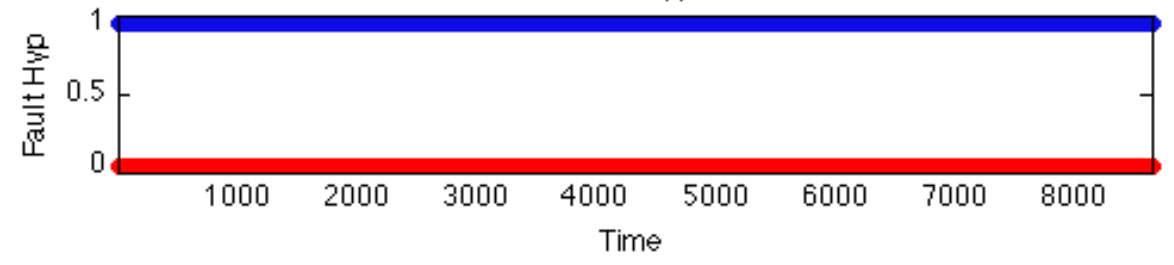




\section{A.1.2 Pump Degradation}

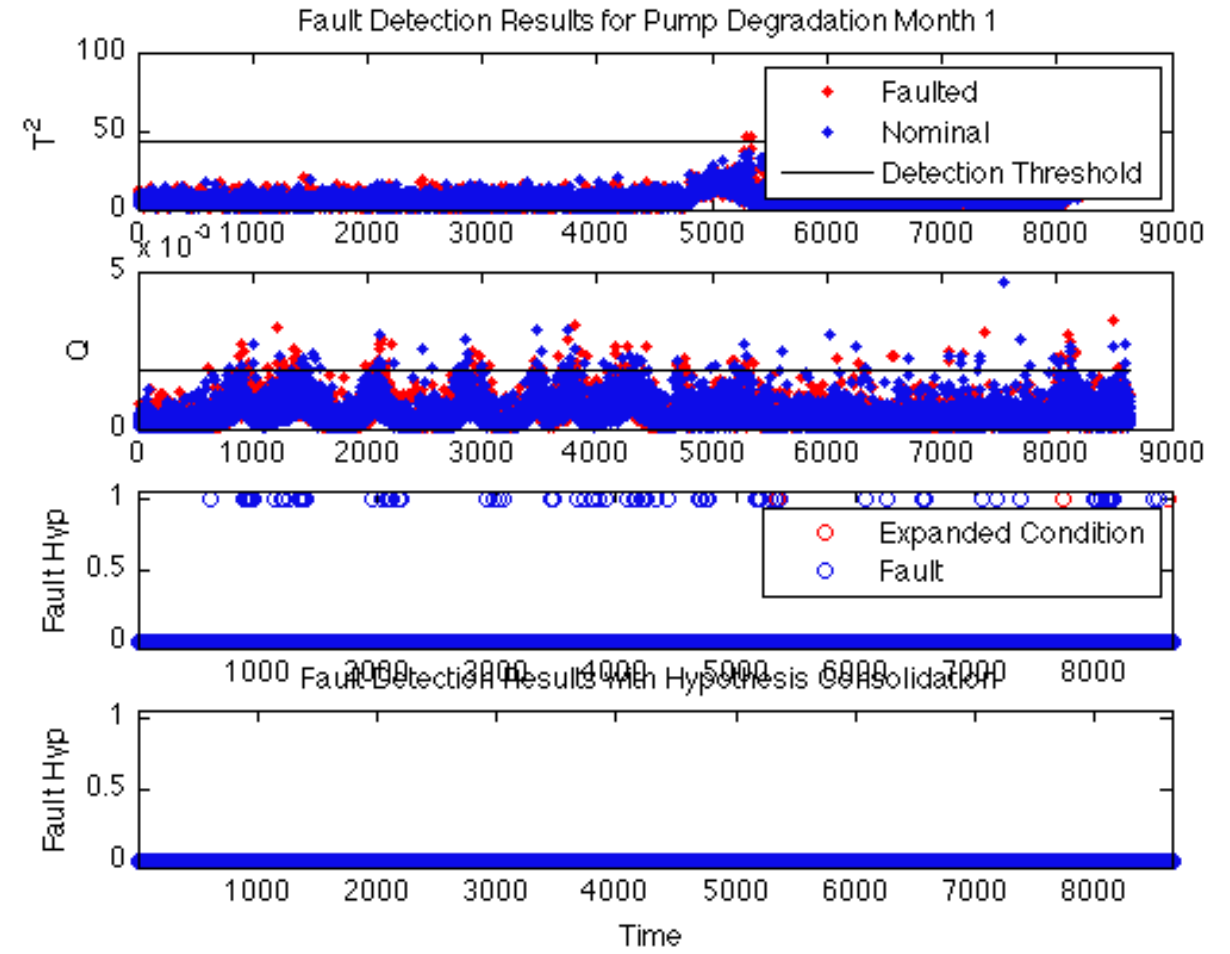

Fault Detection Results for Pump Degradation Month 2

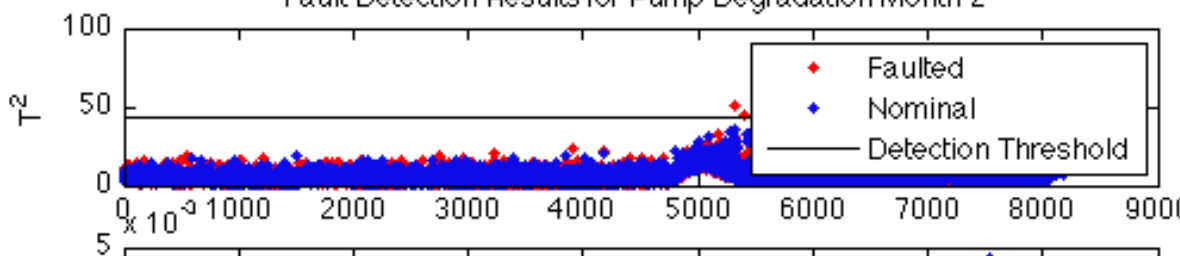

○
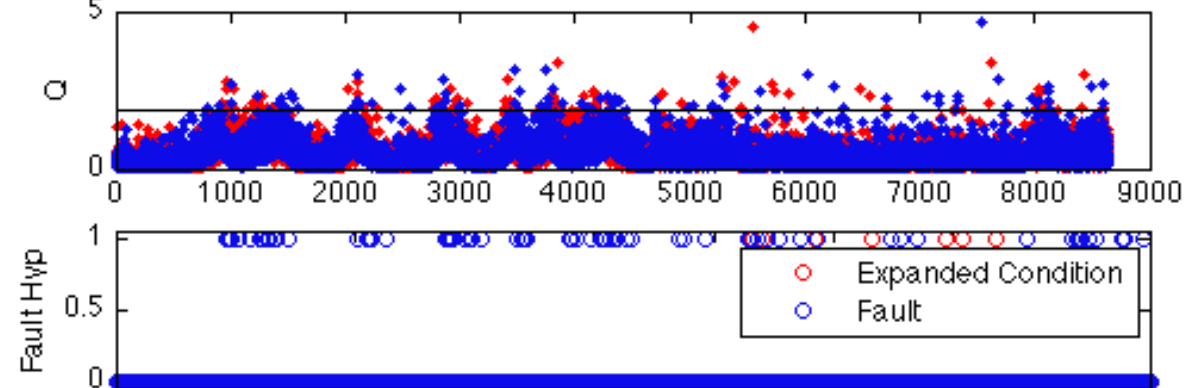

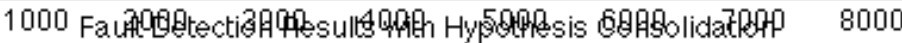

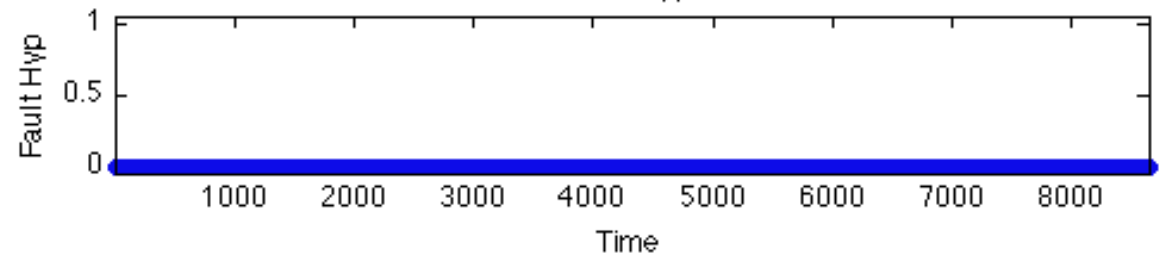


Fault Detection Results for Pump Degradation Month 3
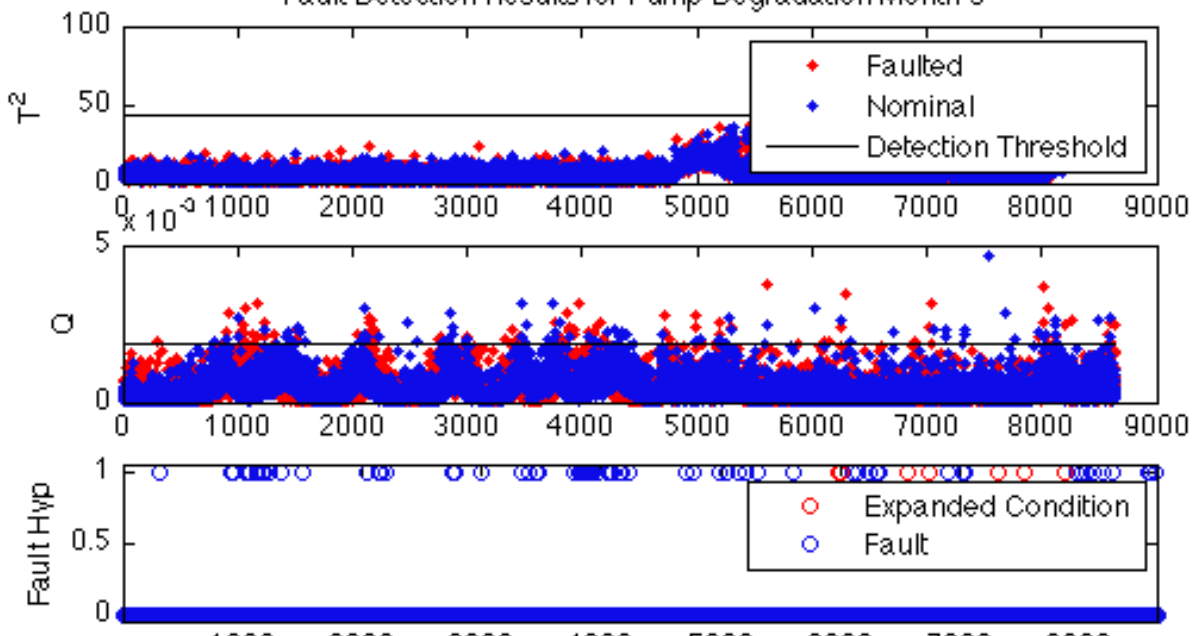

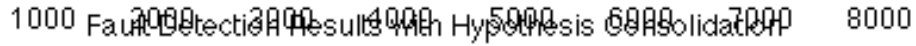

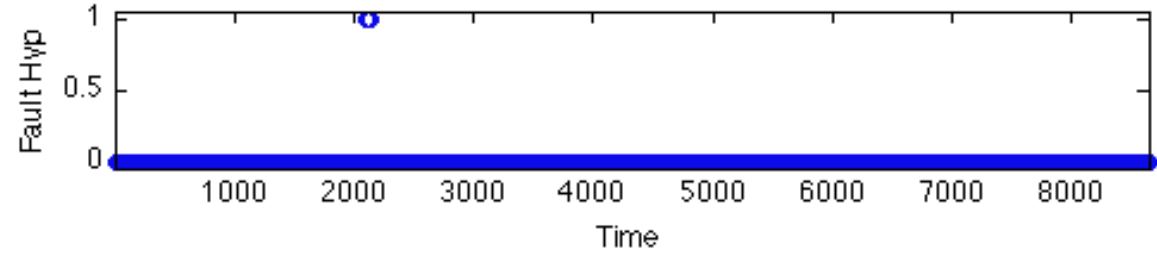

Fault Detection Results for Pump Degradation Month 4
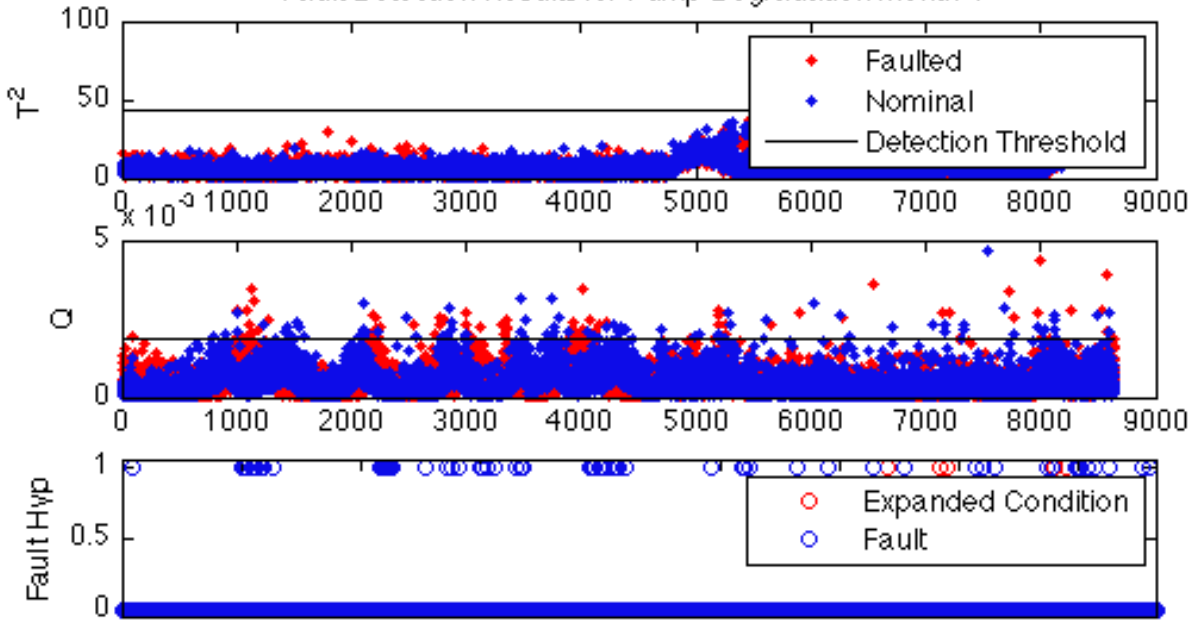

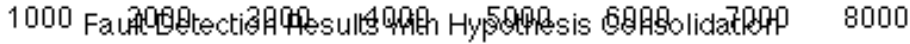

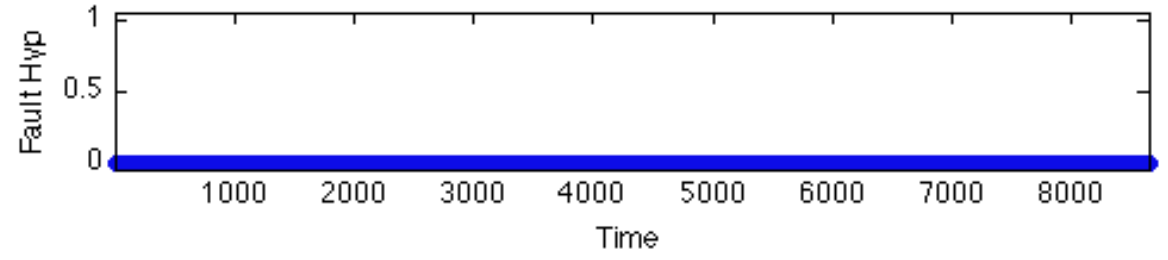


Fault Detection Results for Pump Degradation Month 5
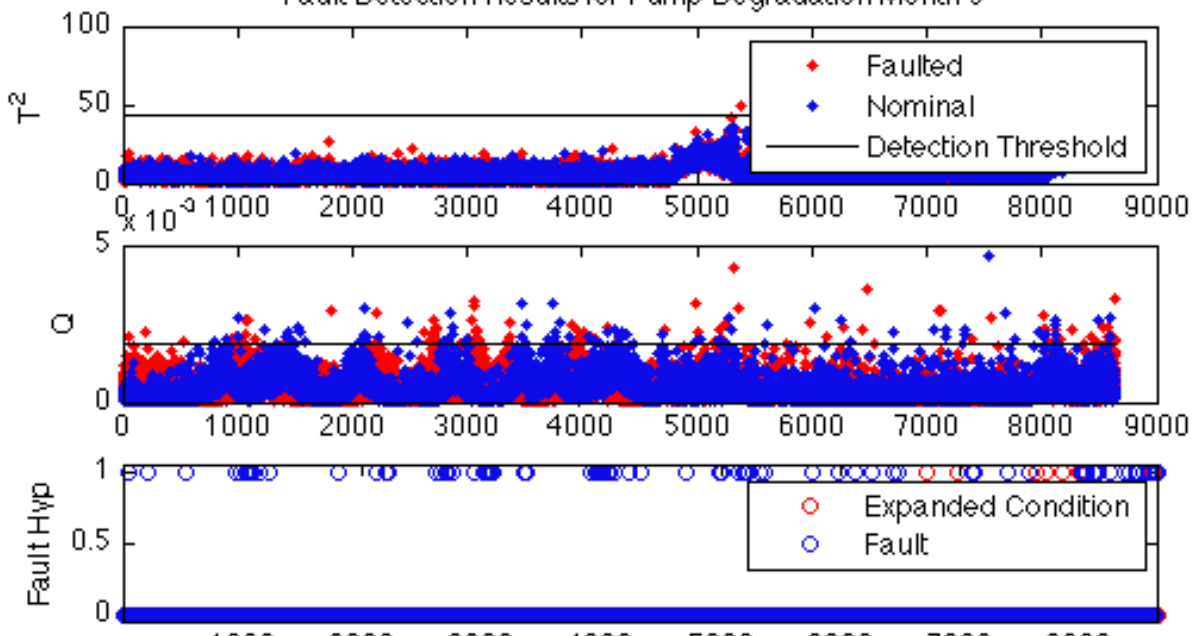

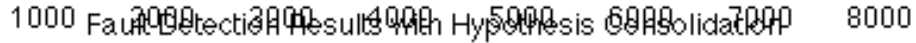

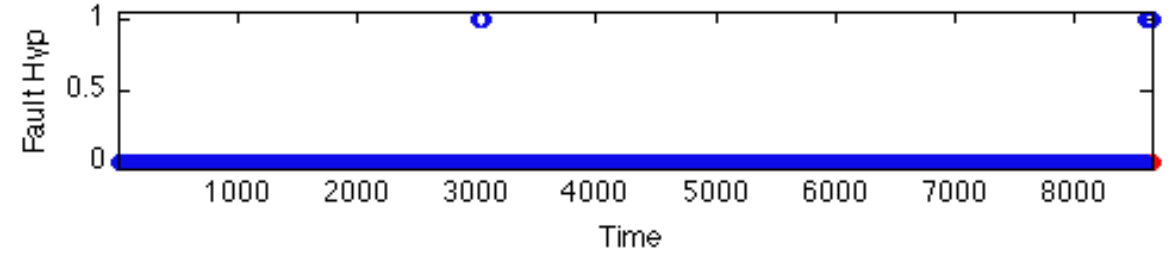

Fault Detection Results for Pump Degradation Month 6
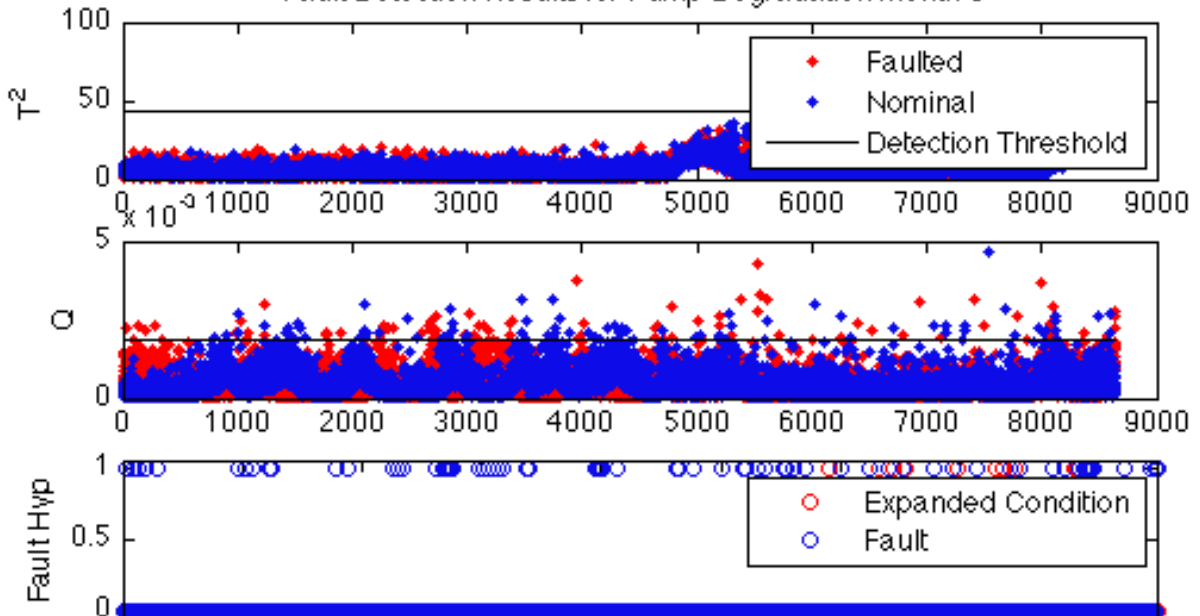

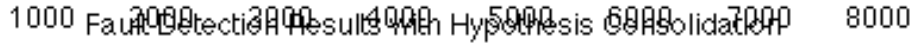

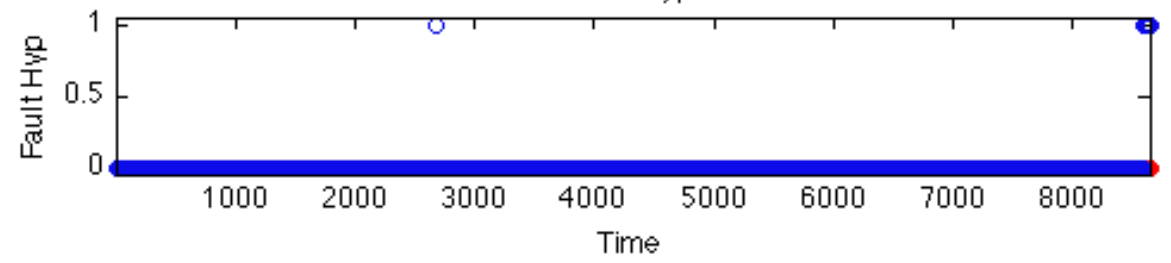


Fault Detection Results for Pump Degradation Month 7
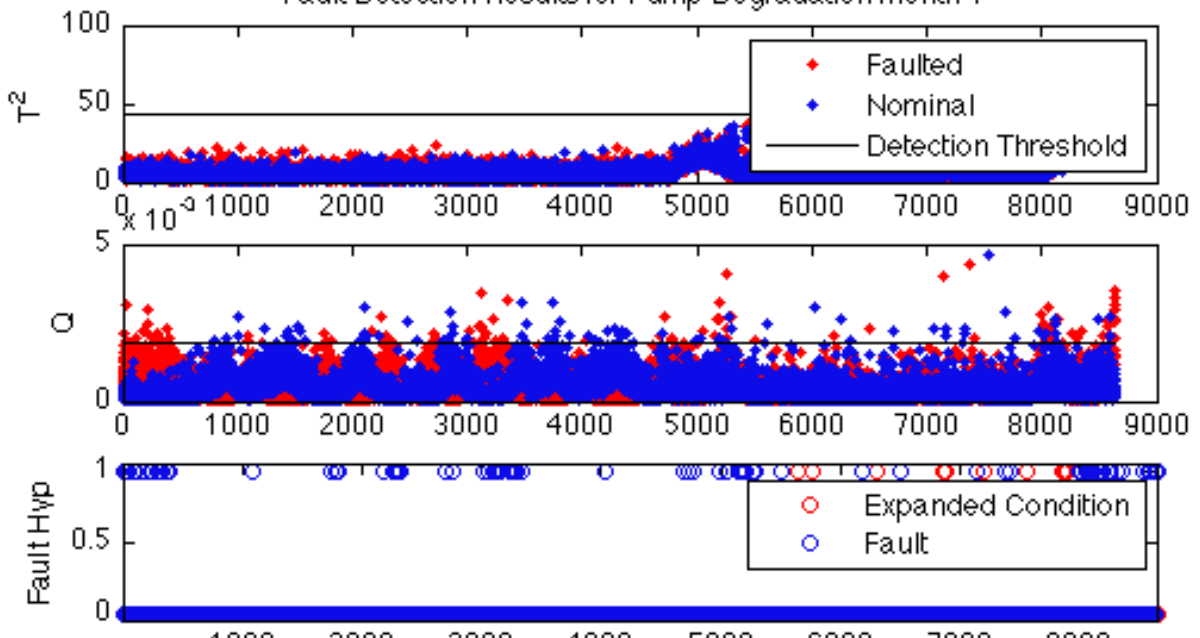

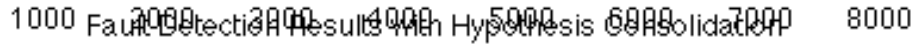

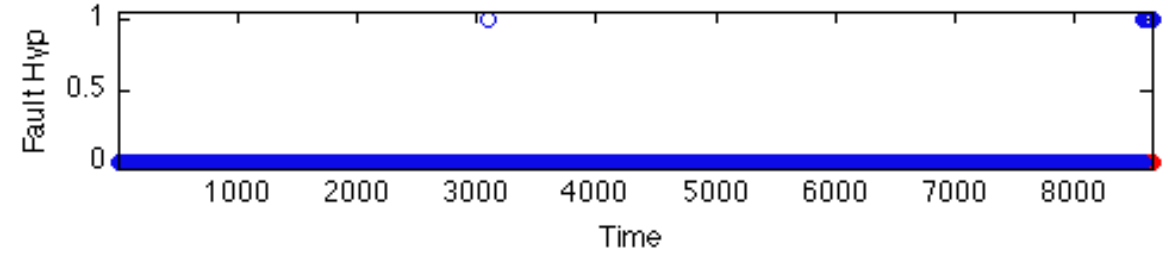

Fault Detection Results for Pump Degradation Month 8
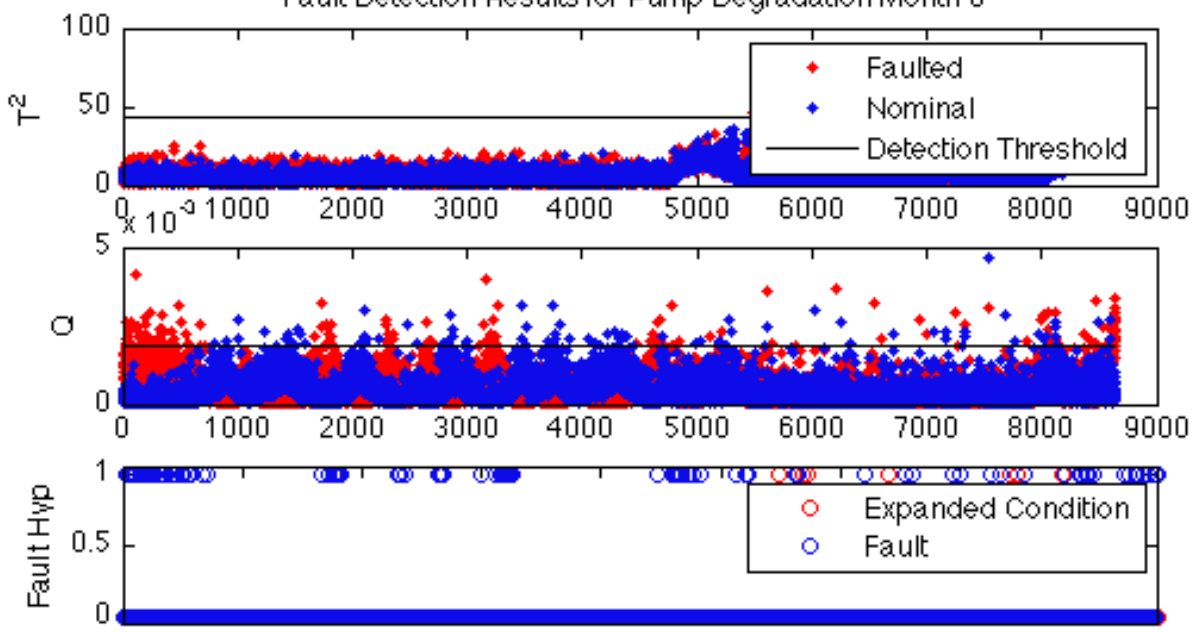

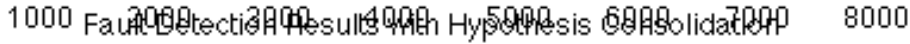

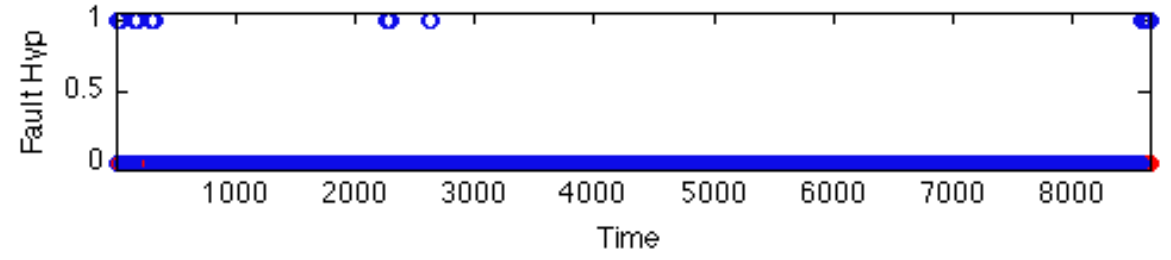


Fault Detection Results for Pump Degradation Month 9

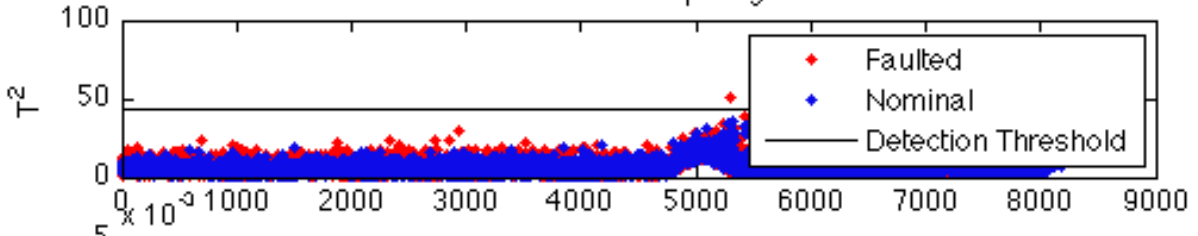

○
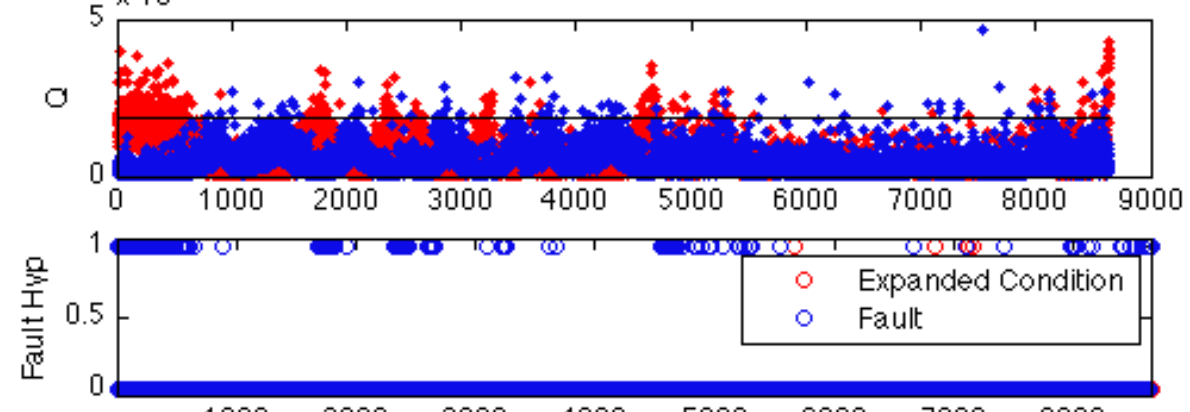

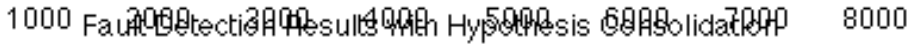

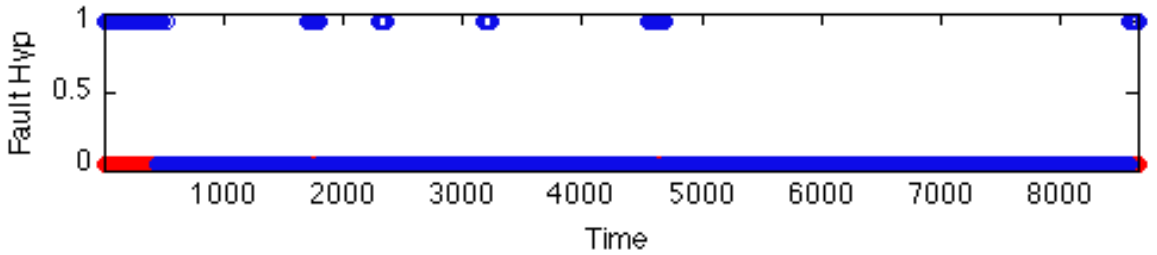

Fault Detection Results for Pump Degradation Month 10
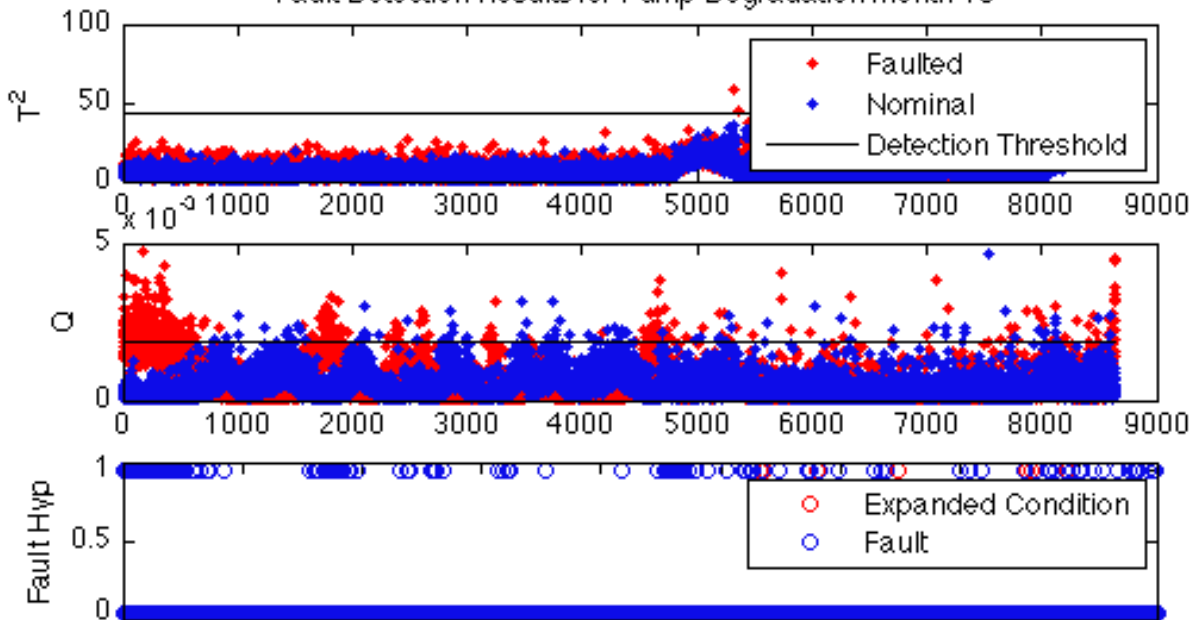

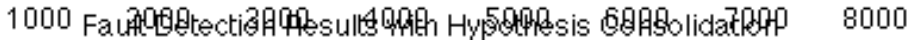

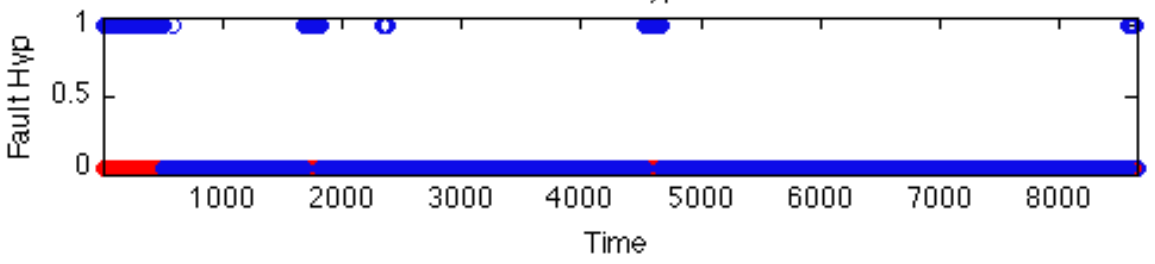




\section{A.1.3 Process Sensor Drift}

Fault Detection Results for Process Sensor Drit Month 1

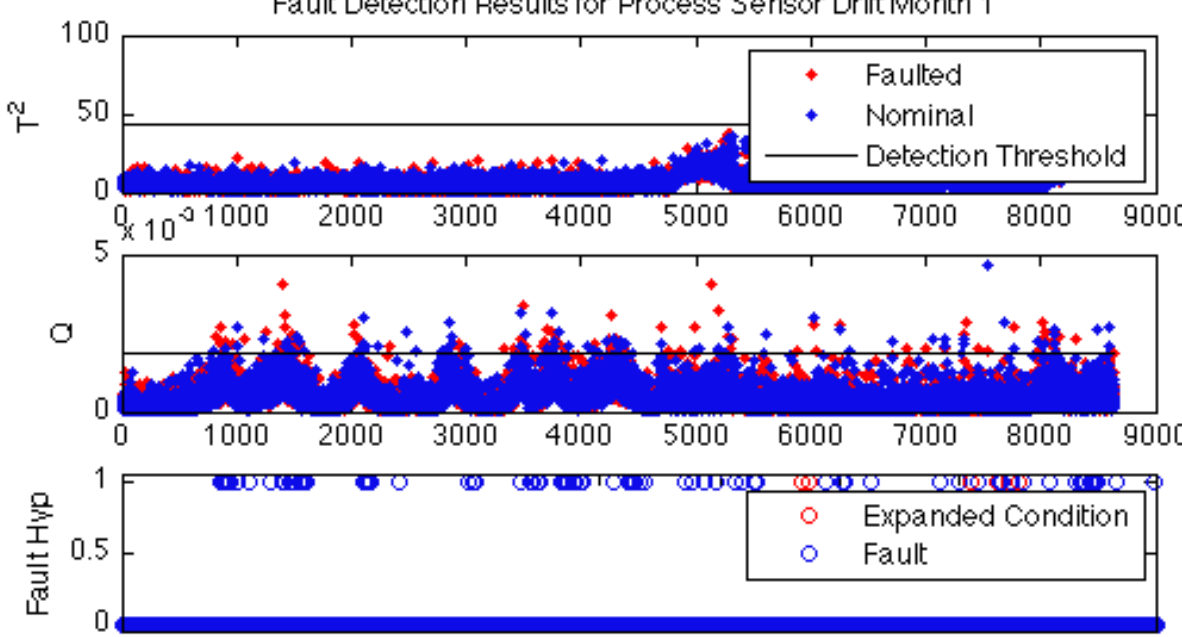

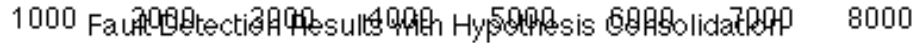

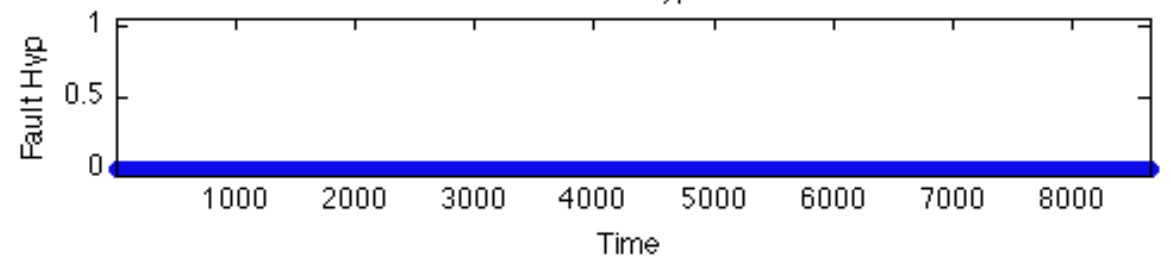

Fault Detection Results for Process Sensor Drit Month 2
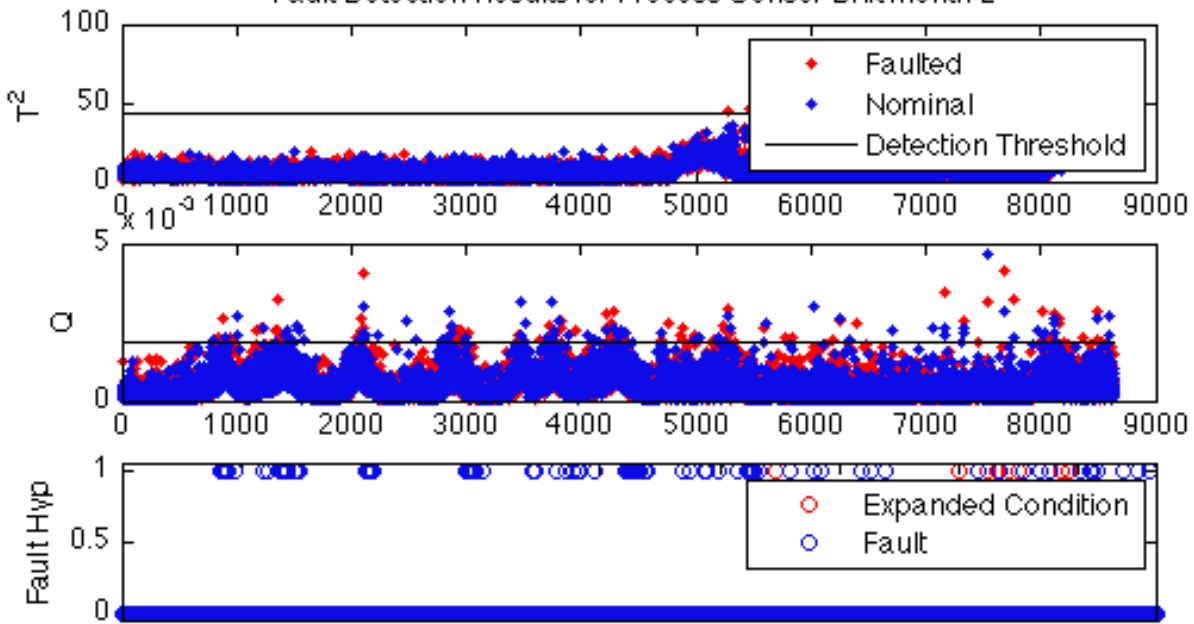

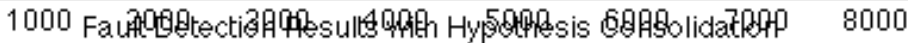

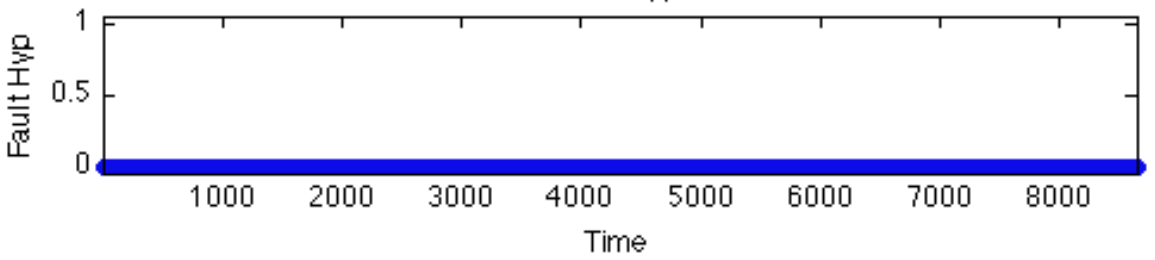


Fault Detection Results for Process Sensor Dritt Month 3

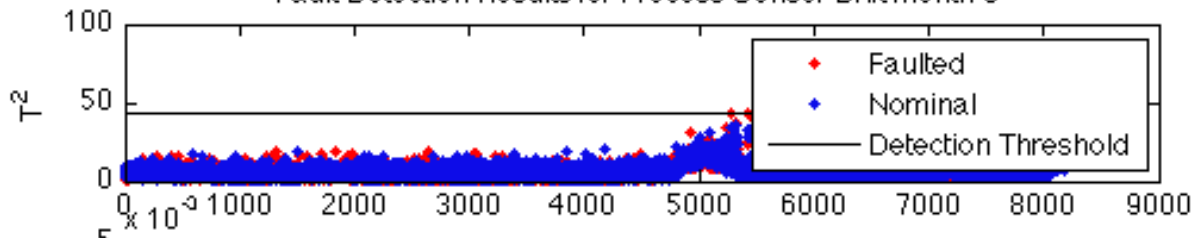

○
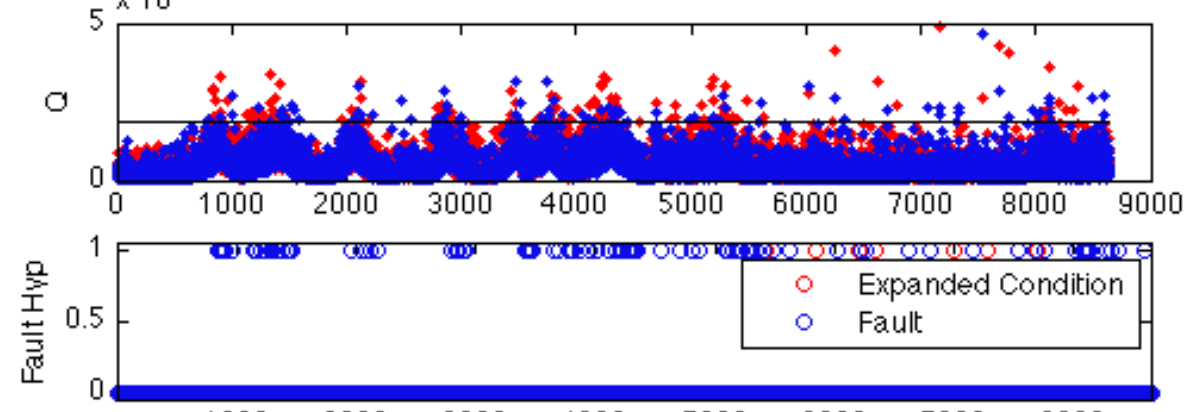

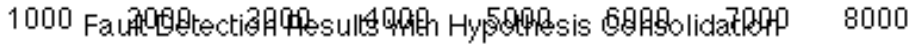

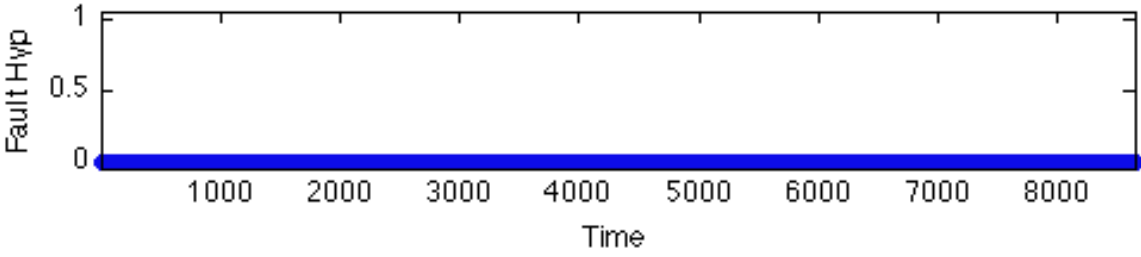

Fault Detection Results for Process Sensor Drit Month 4
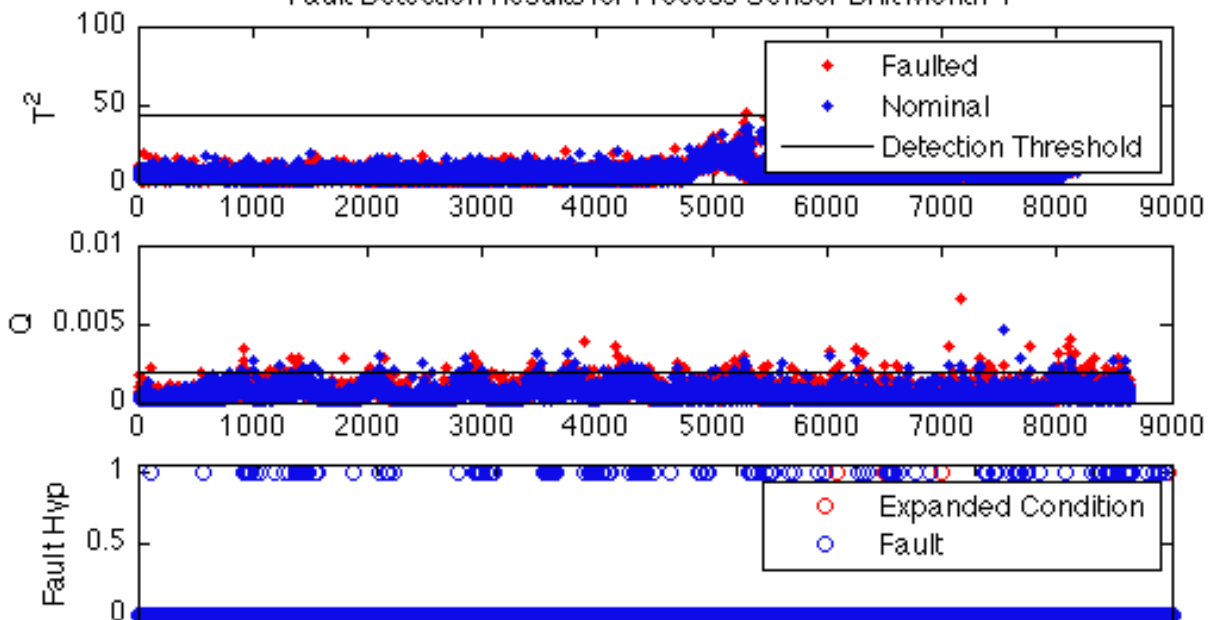

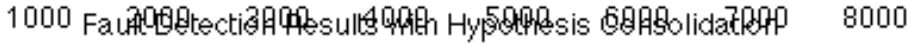

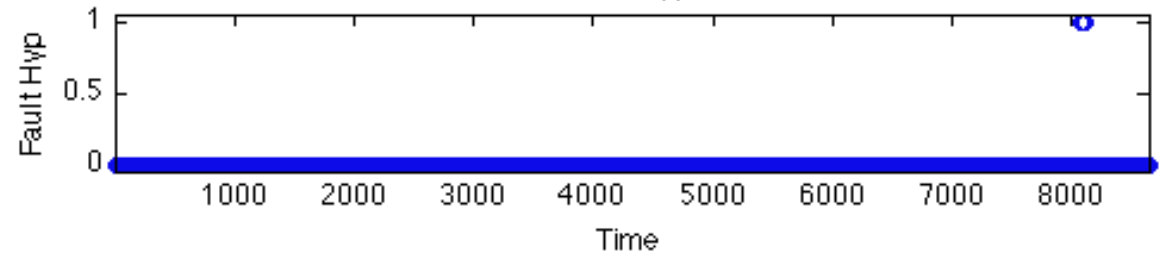


Fault Detection Results for Process Sensor Dritt Month 5
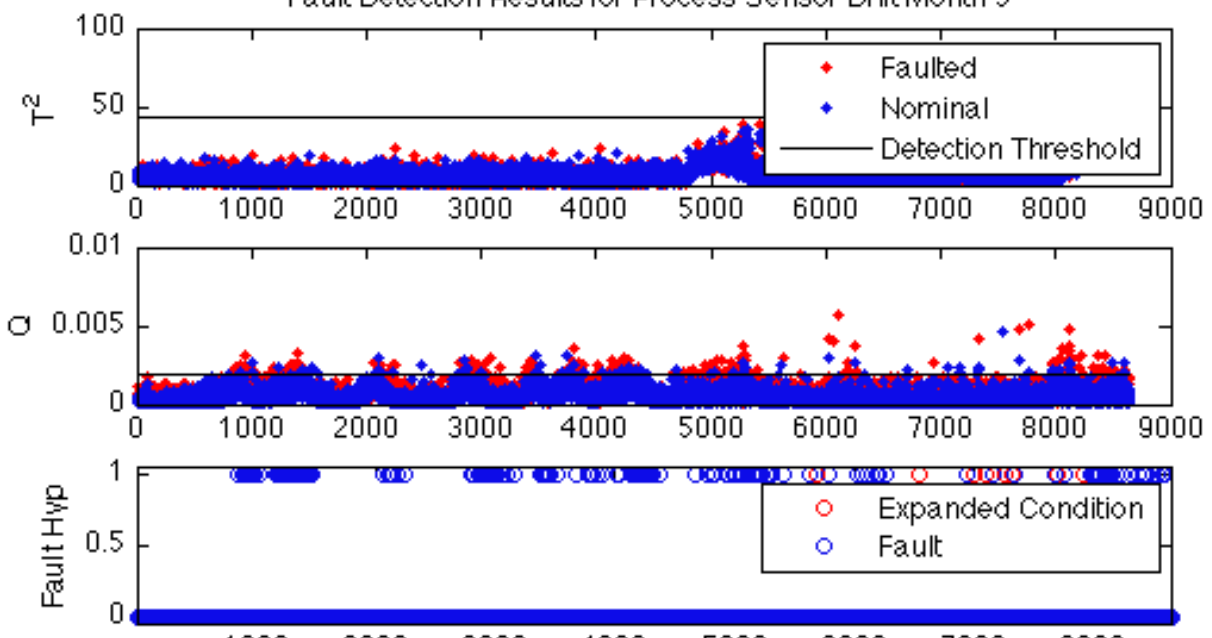

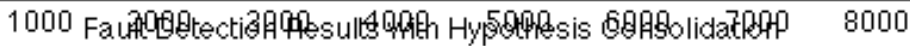

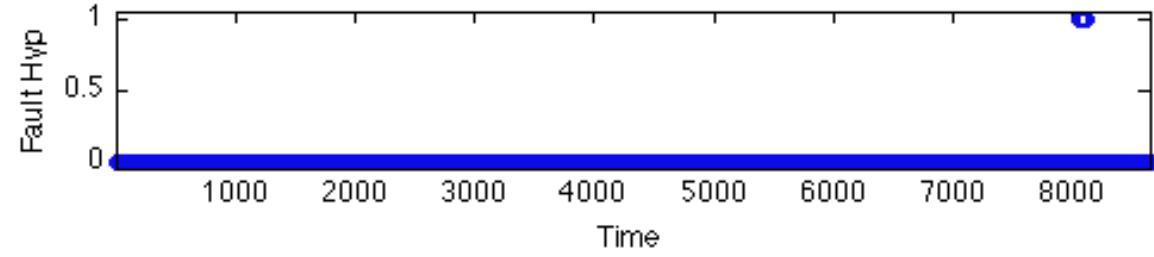

Fault Detection Results for Process Sensor Drift Month 6
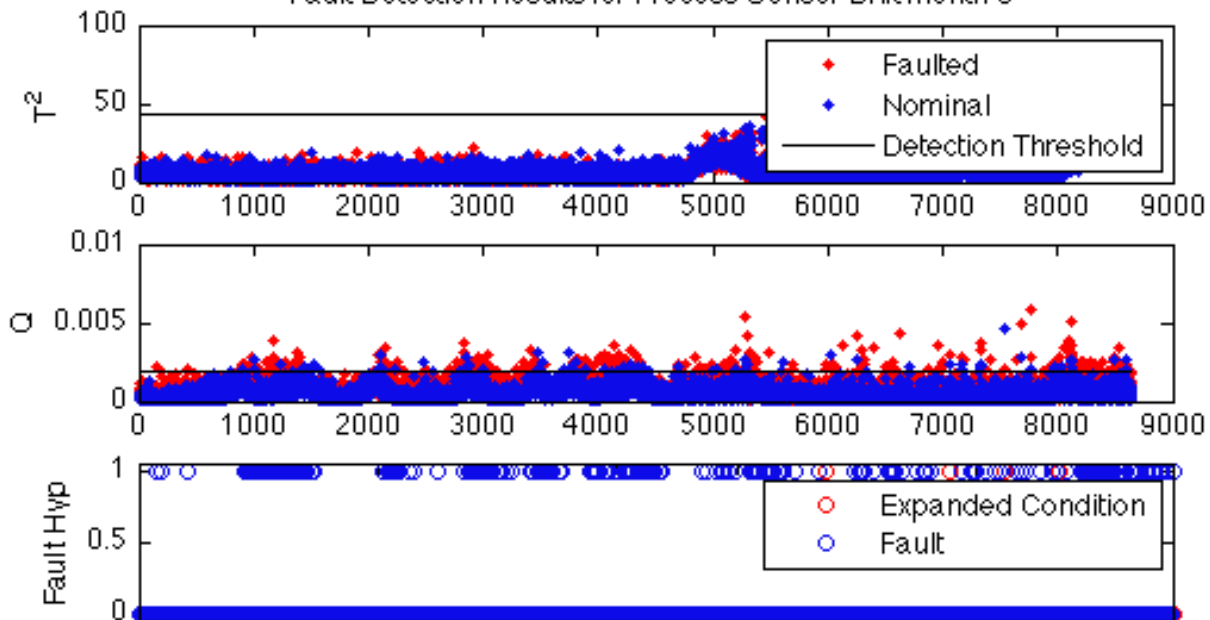

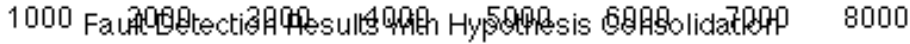

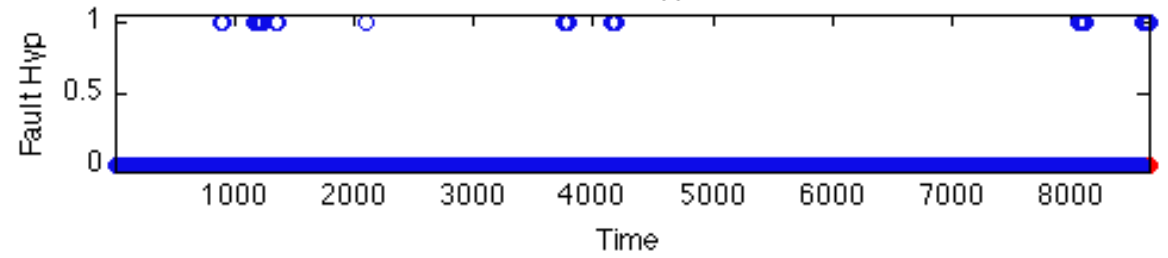


Fault Detection Results for Process Sensor Dritt Month 7
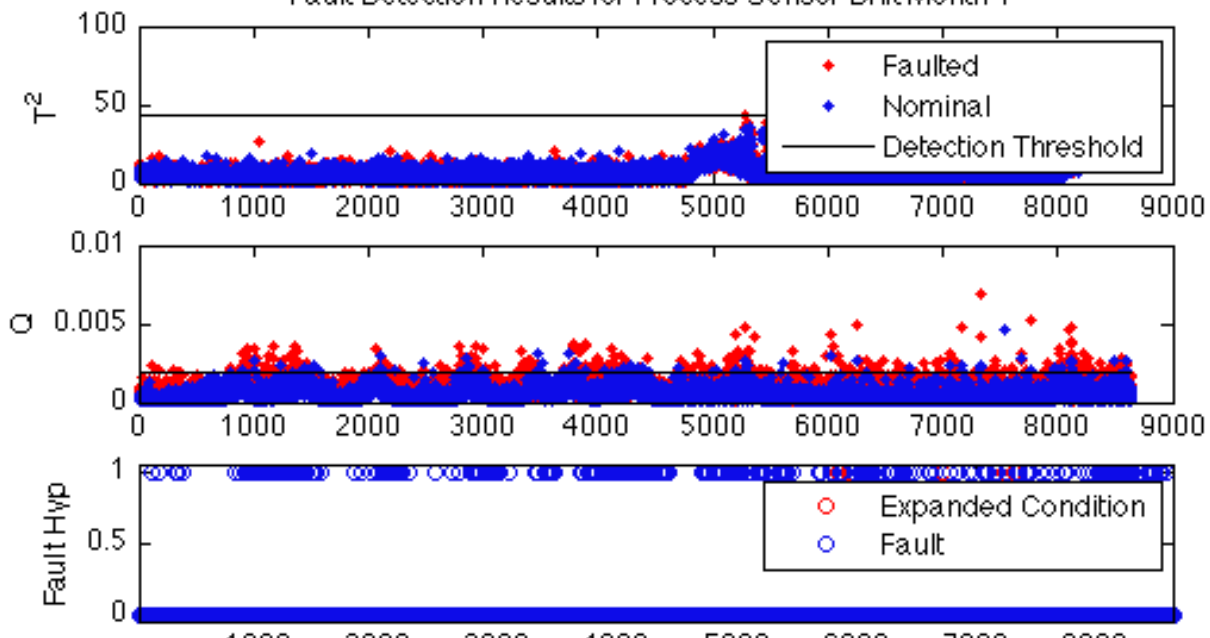

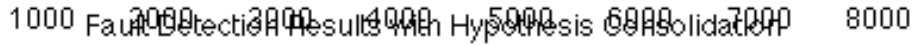

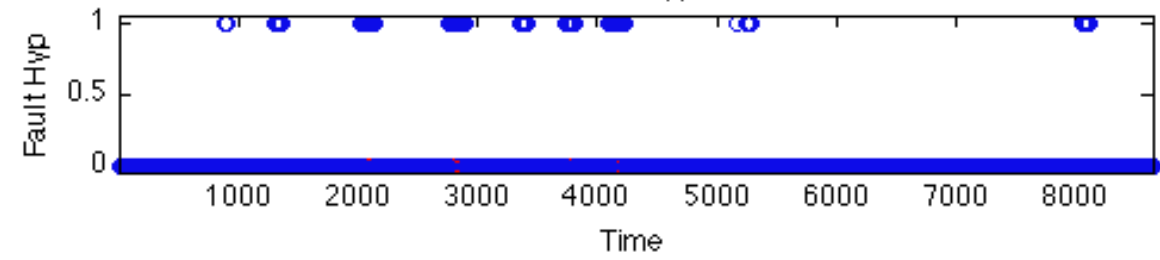

Fault Detection Results for Process Sensor Drift Month 8
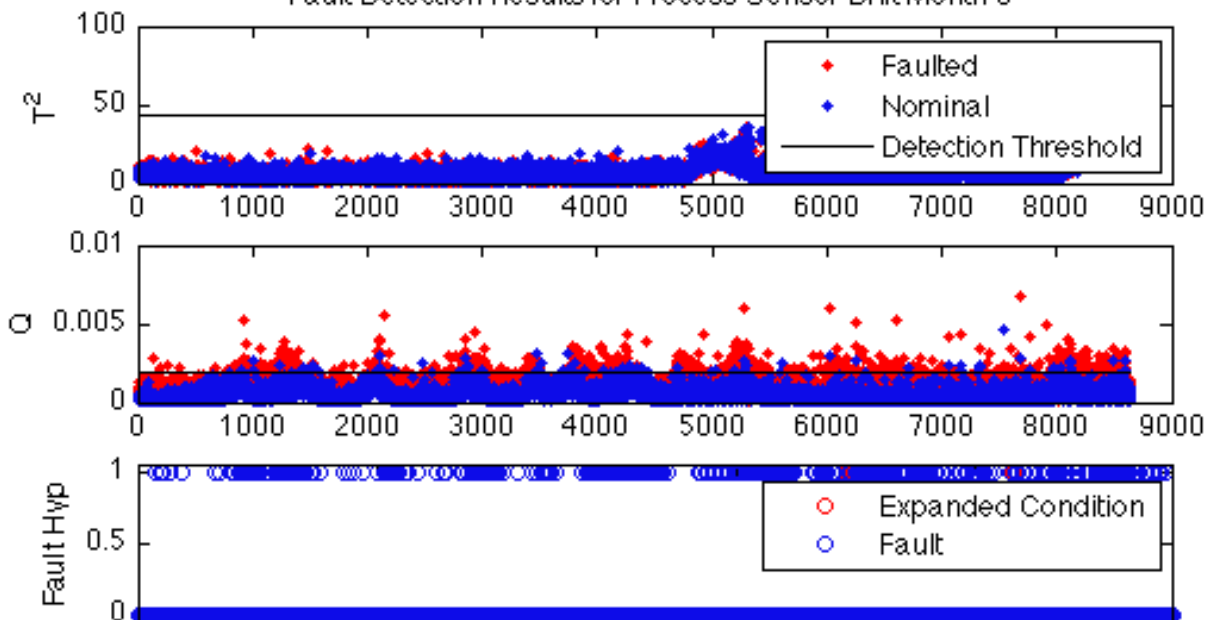

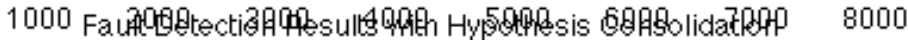

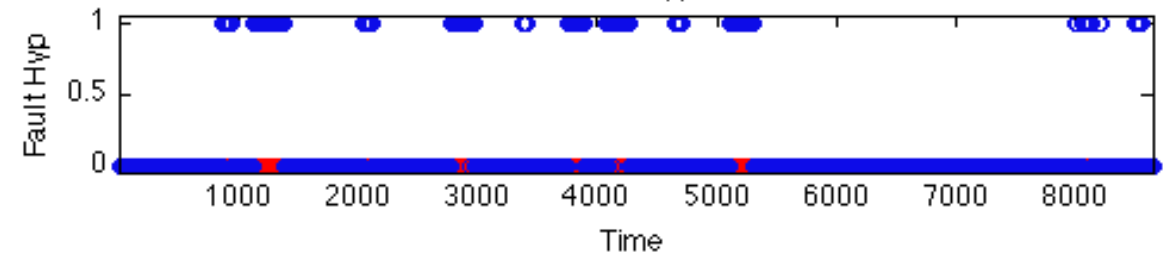


Fault Detection Results for Process Sensor Dritt Month 9
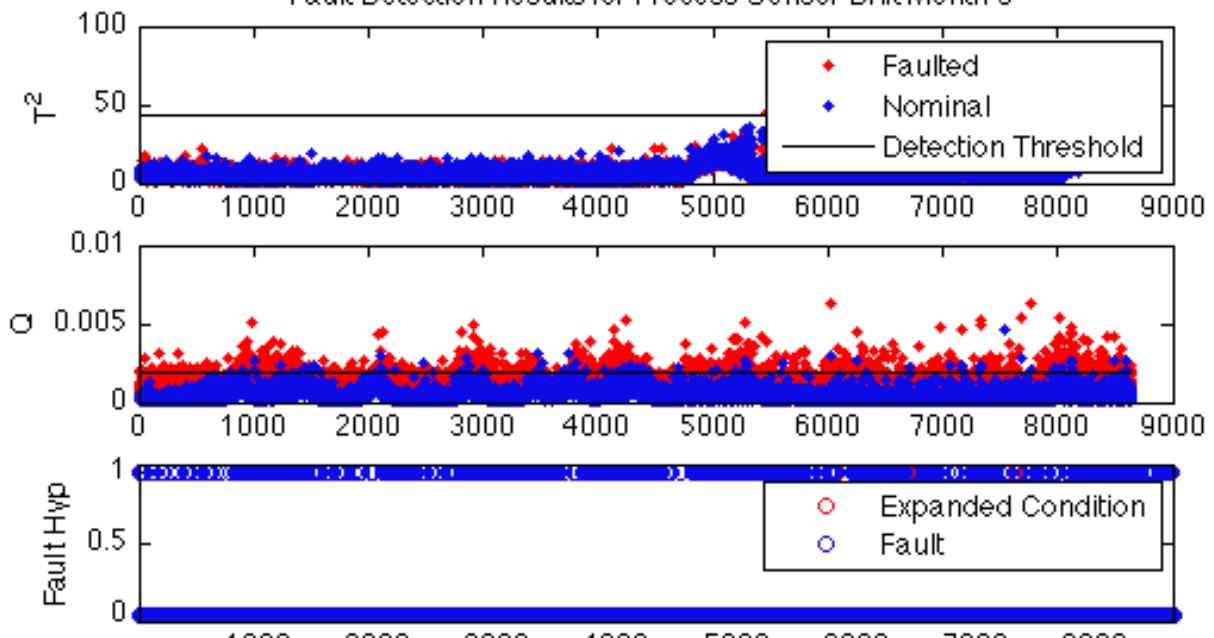

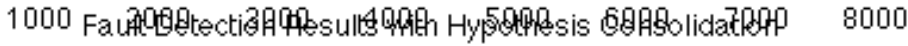

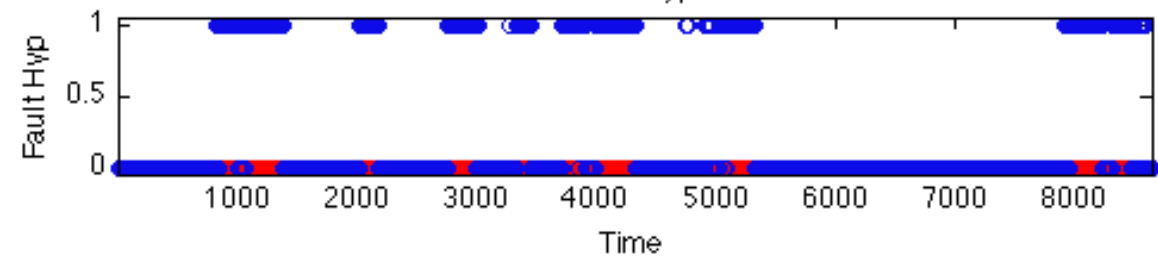

Fault Detection Results for Process Sensor Dritt Month 10
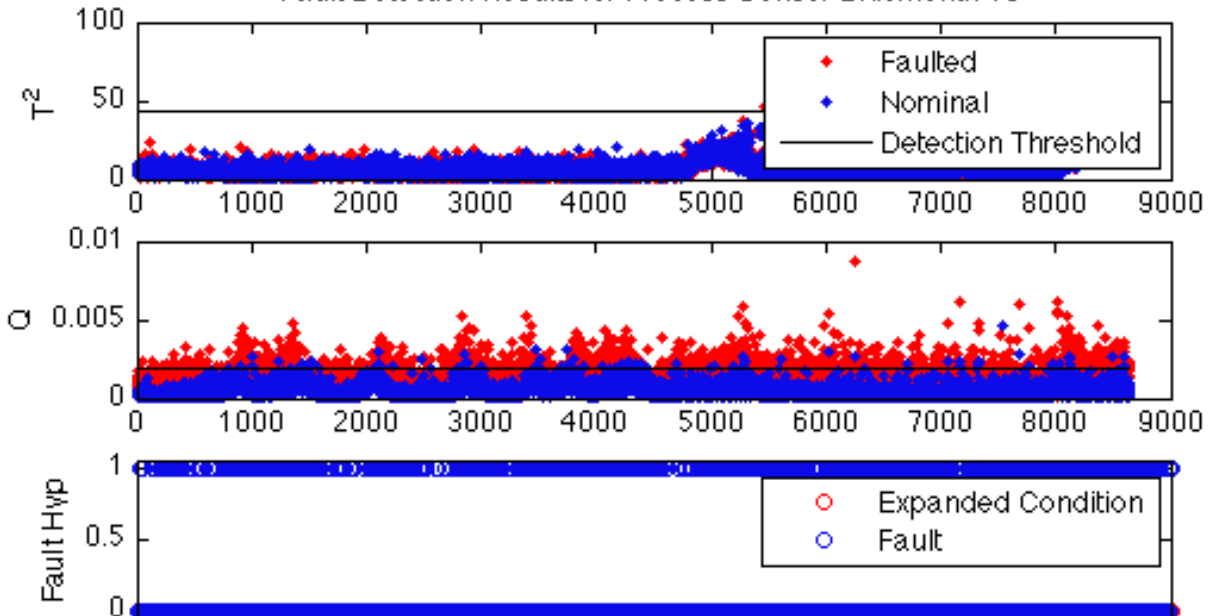

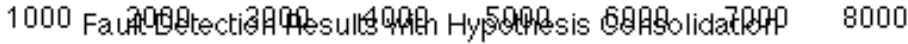

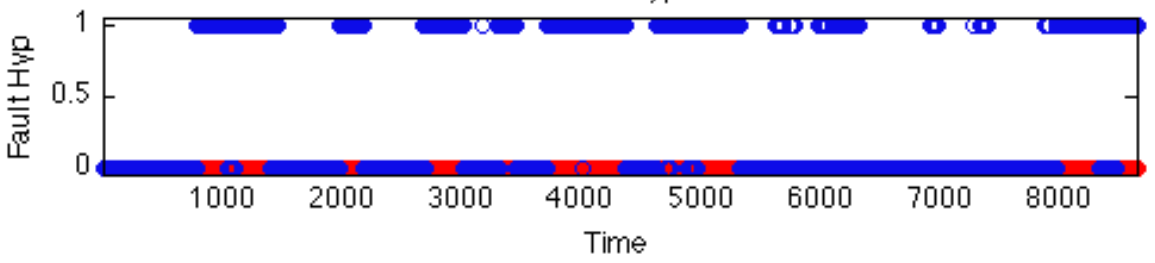


Fault Detection Results for Process Sensor Dritt Month 11
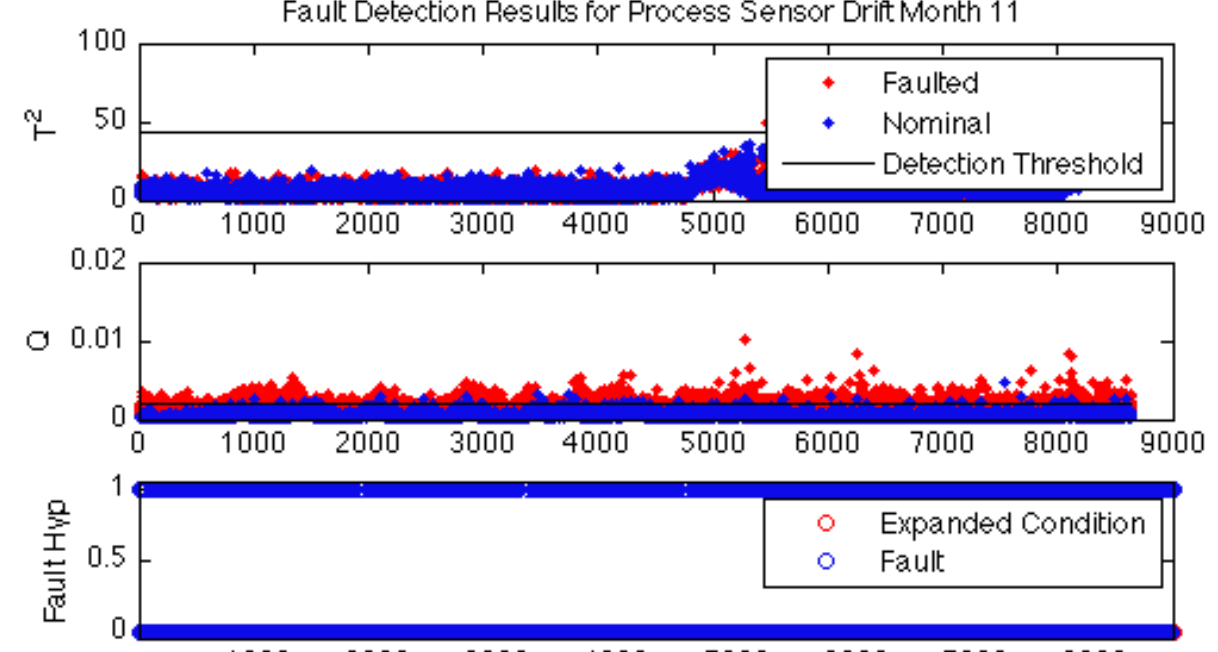

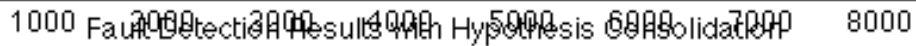

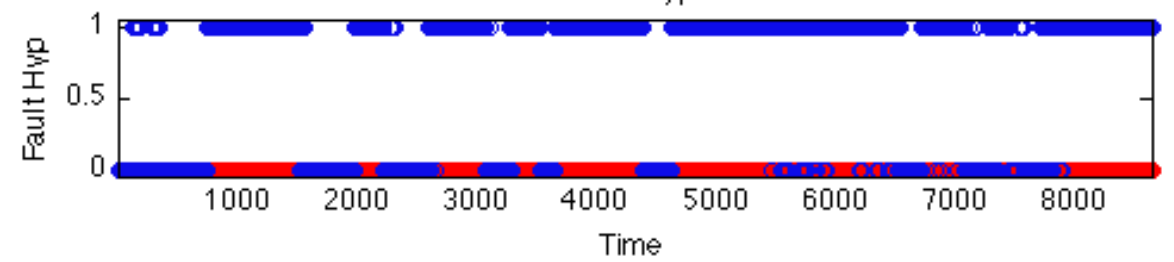

Fault Detection Results for Process Sensor Drift Month 12
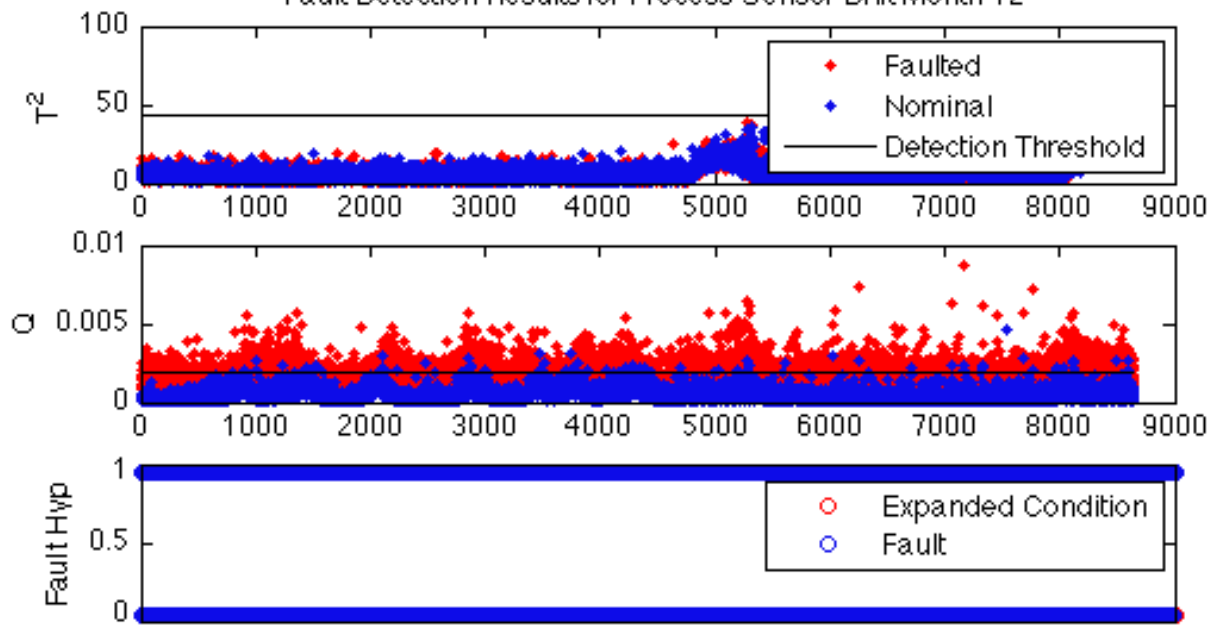

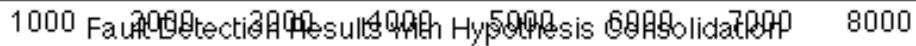

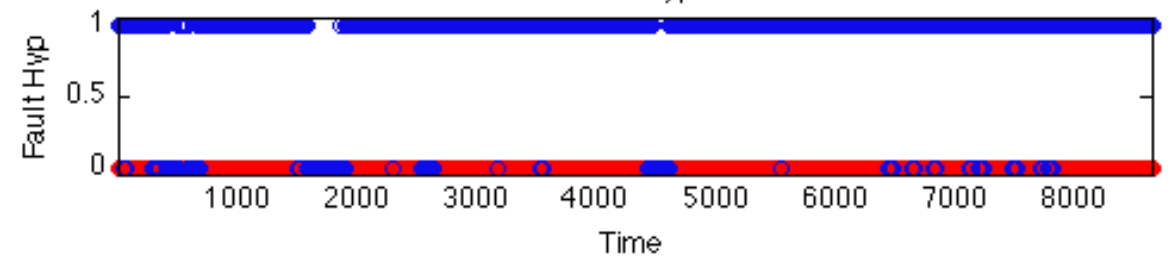




\section{A.1.4 Control Sensor Drift}

Fault Detection Results for Control Sensor Dritt Month 1
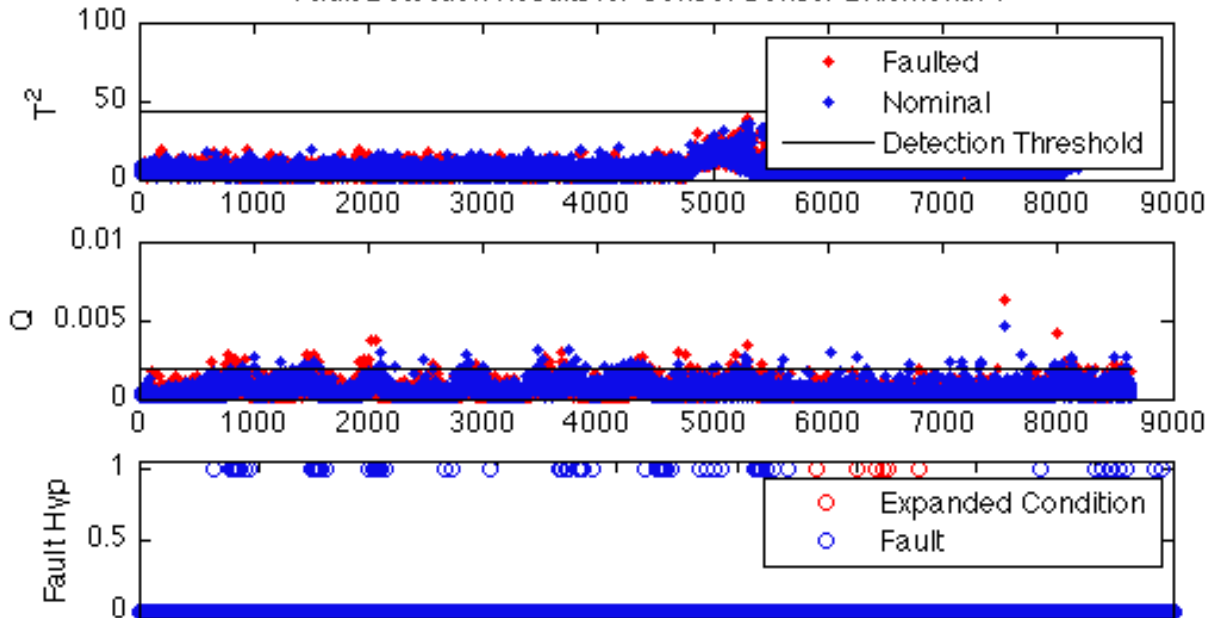

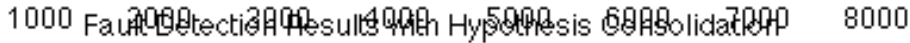

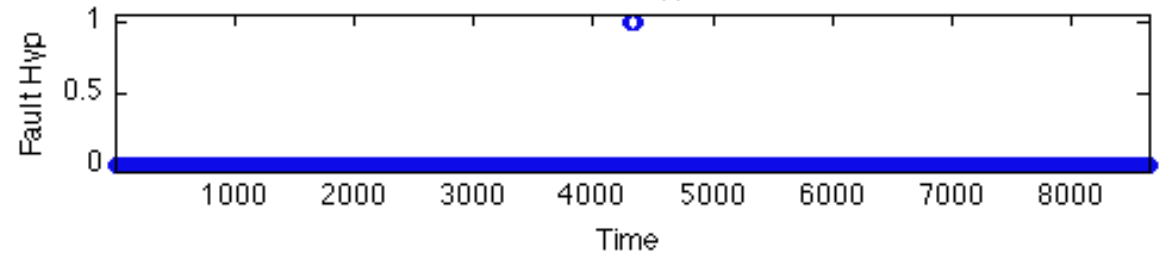

Fault Detection Results for Control Sensor Drit Month 2

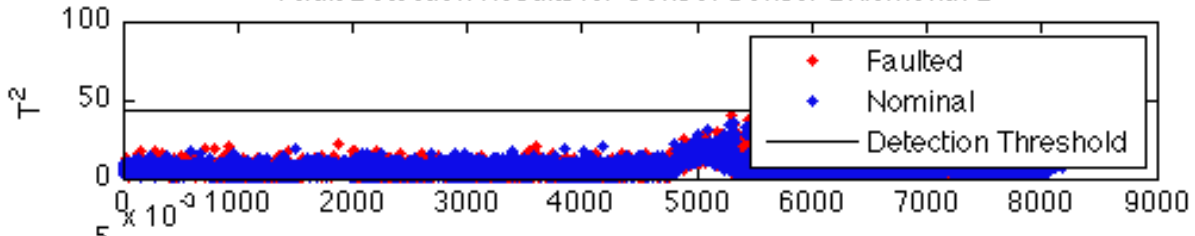

$\bigcirc$
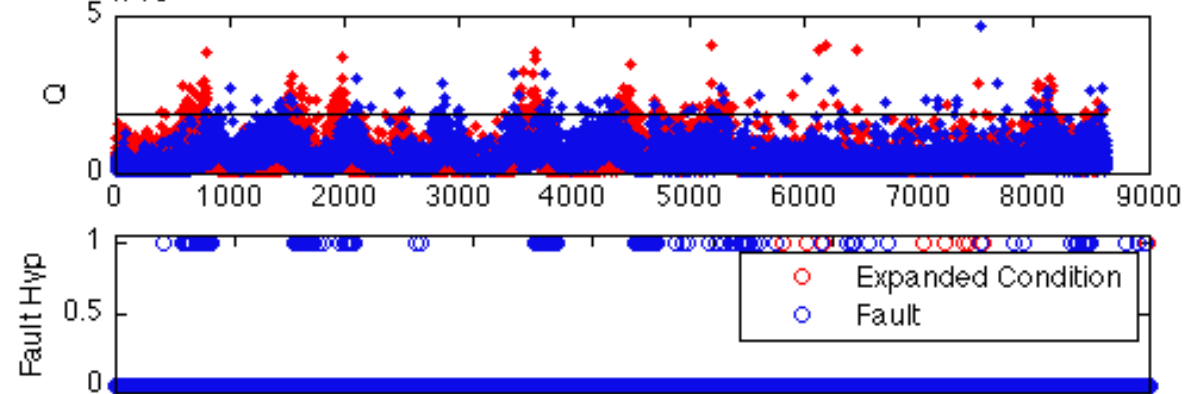

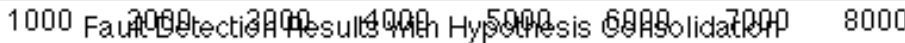

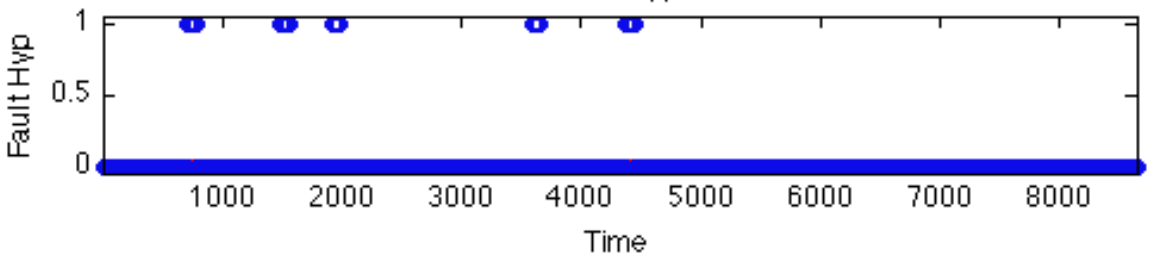


Fault Detection Results for Control Sensor DrittMonth 3
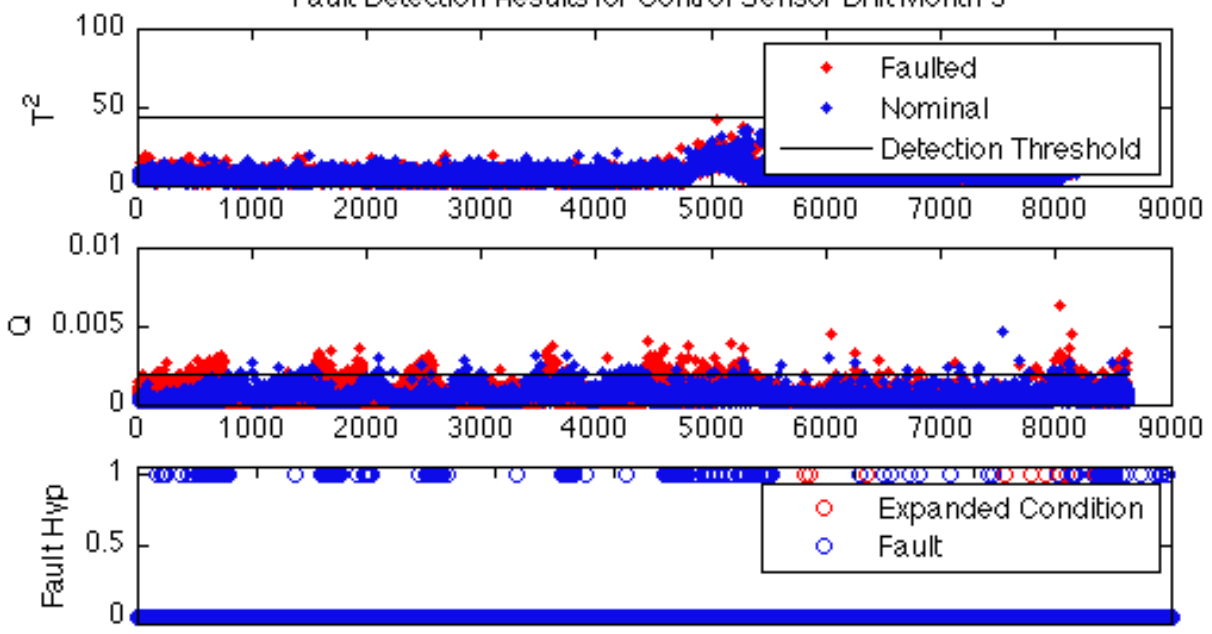

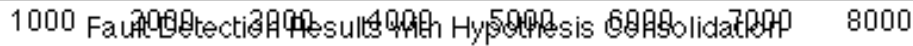

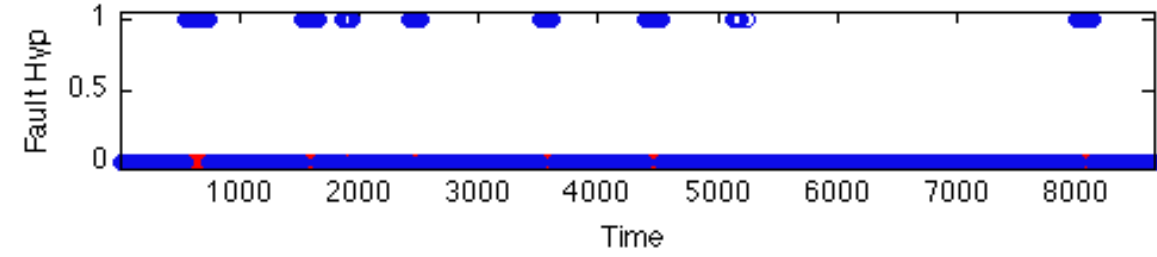

Fault Detection Results for Control Sensor Dritt Month 4
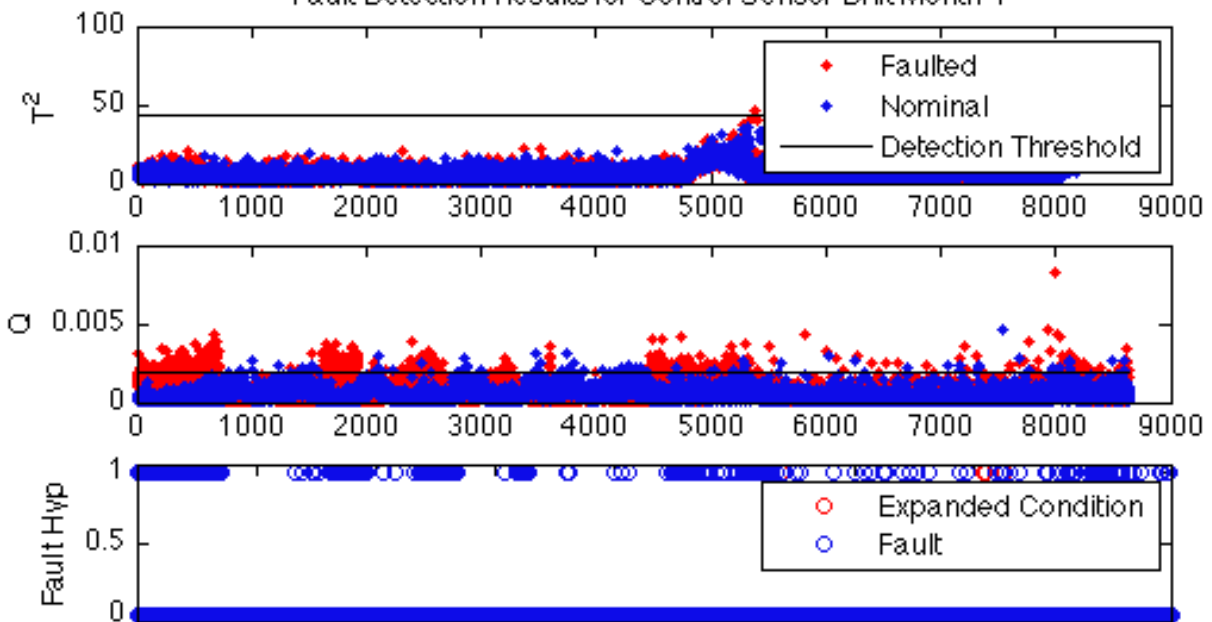

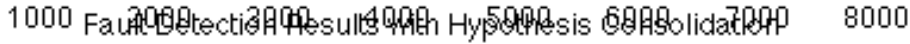

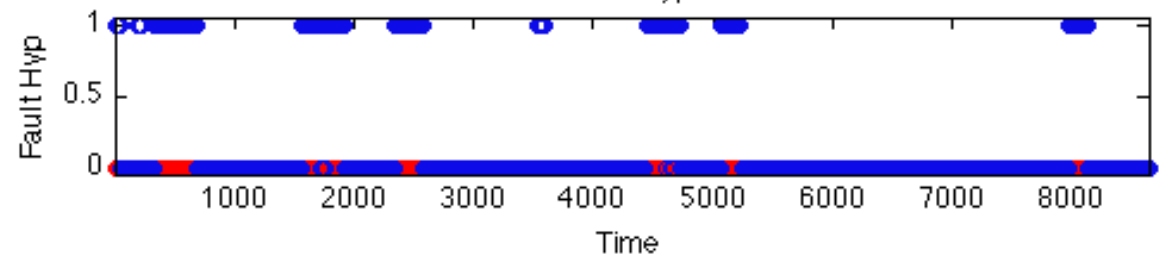


Fault Detection Results for Control Sensor Drift Month 5

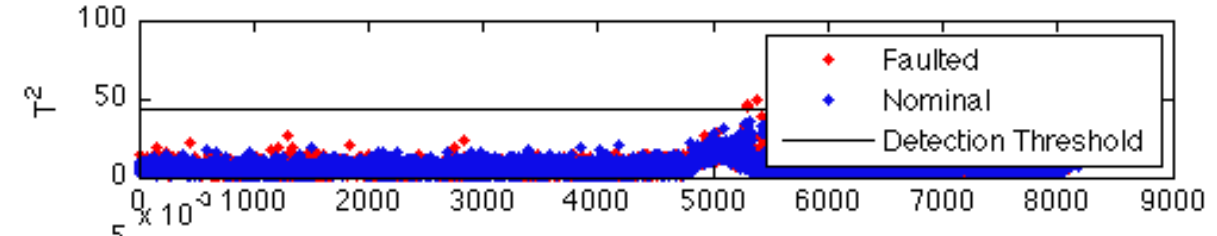

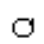
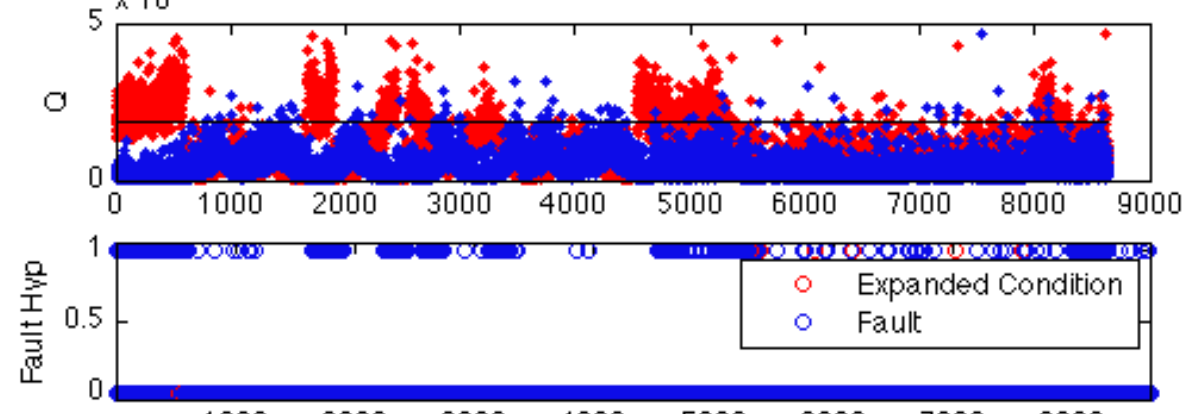

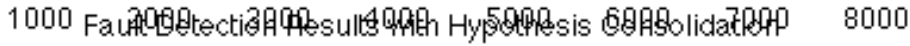

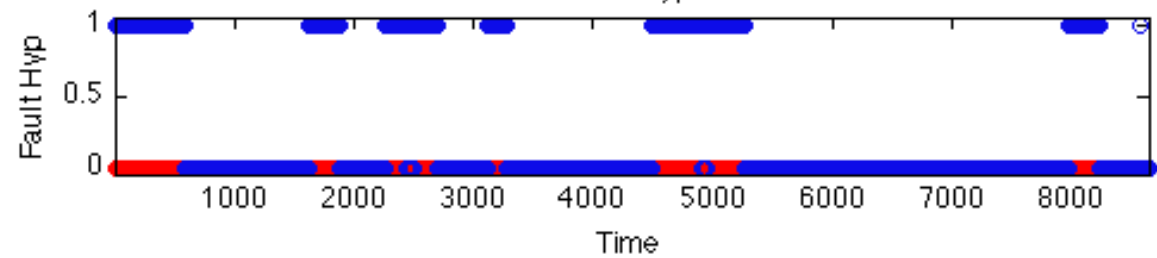

Fault Detection Results for Control Sensor Drift Month 6
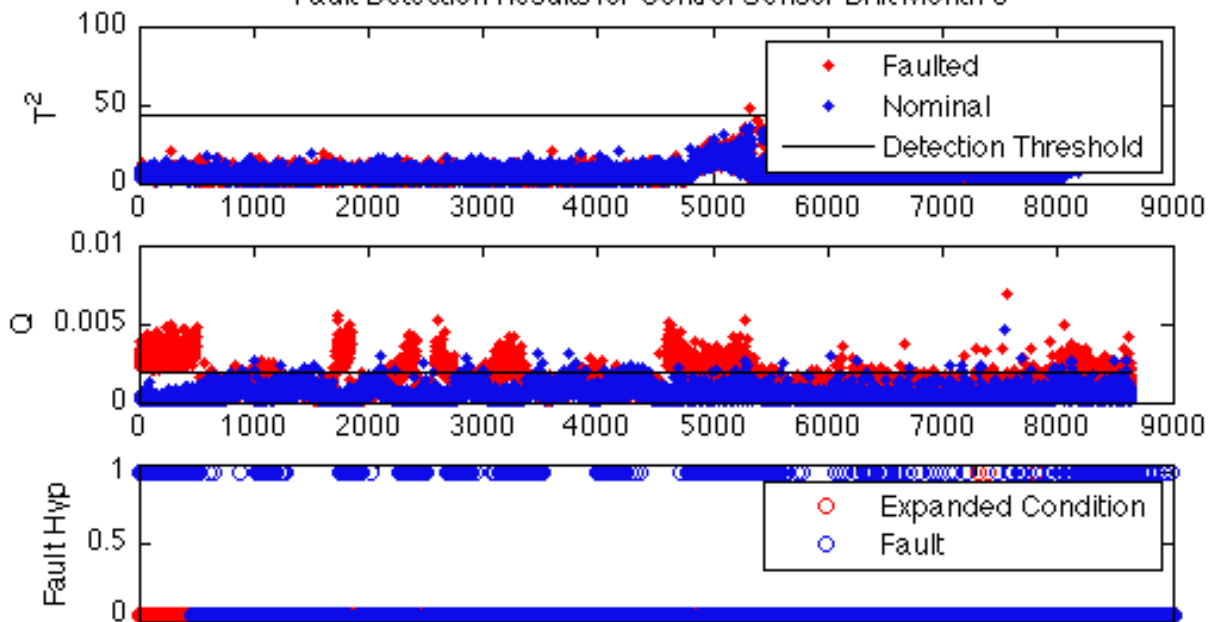

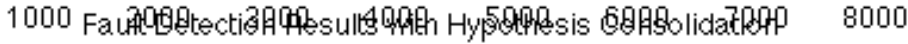

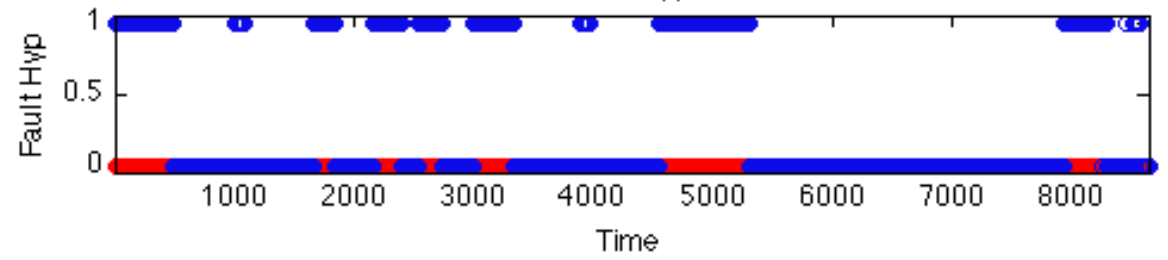


Fault Detection Results for Control Sensor Drift Month 7
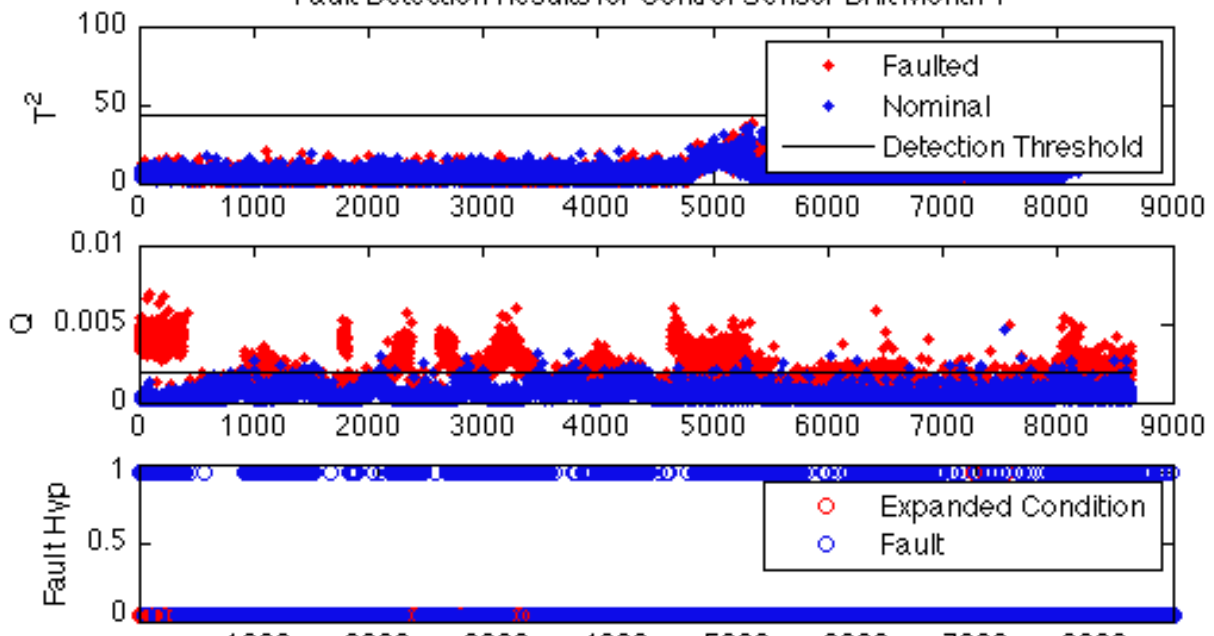

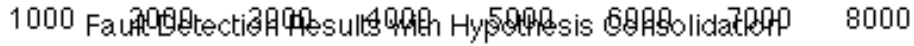

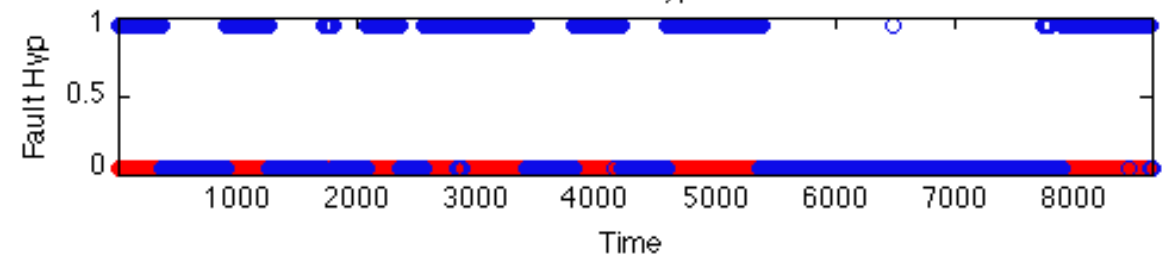

Fault Detection Results for Control Sensor Dritt Month 8
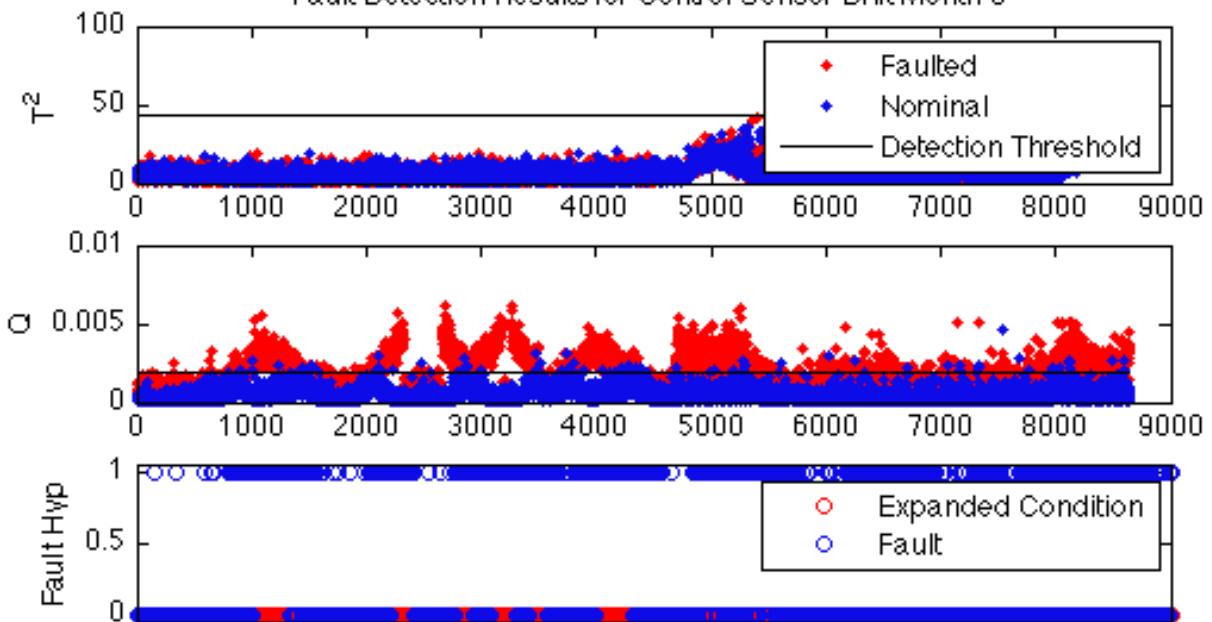

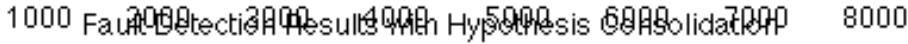

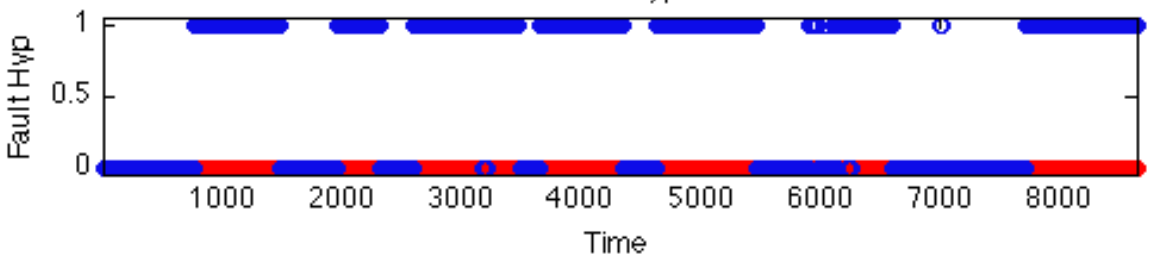


Fault Detection Results for Control Sensor Drift Month 9
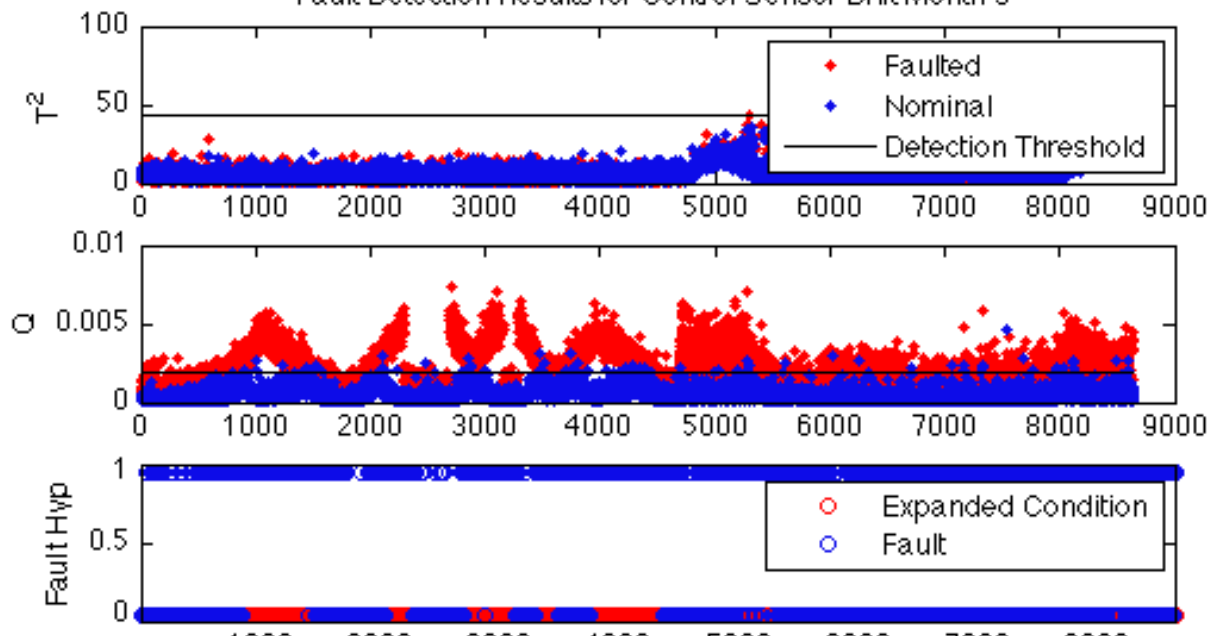

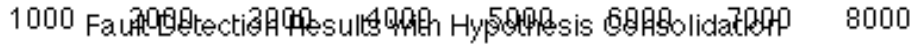

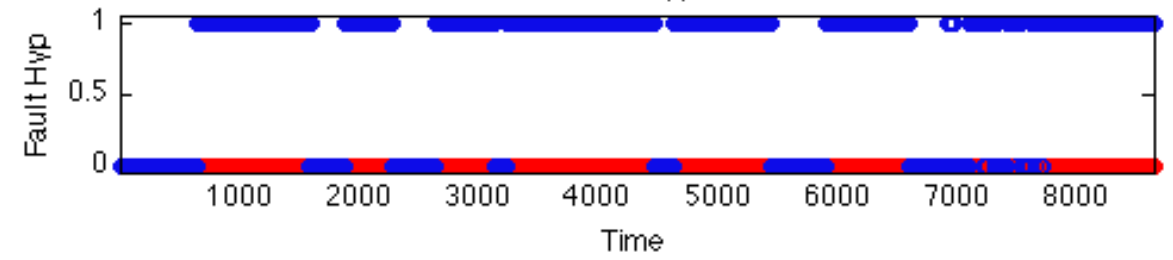

Fault Detection Results for Control Sensor Dritt Month 10
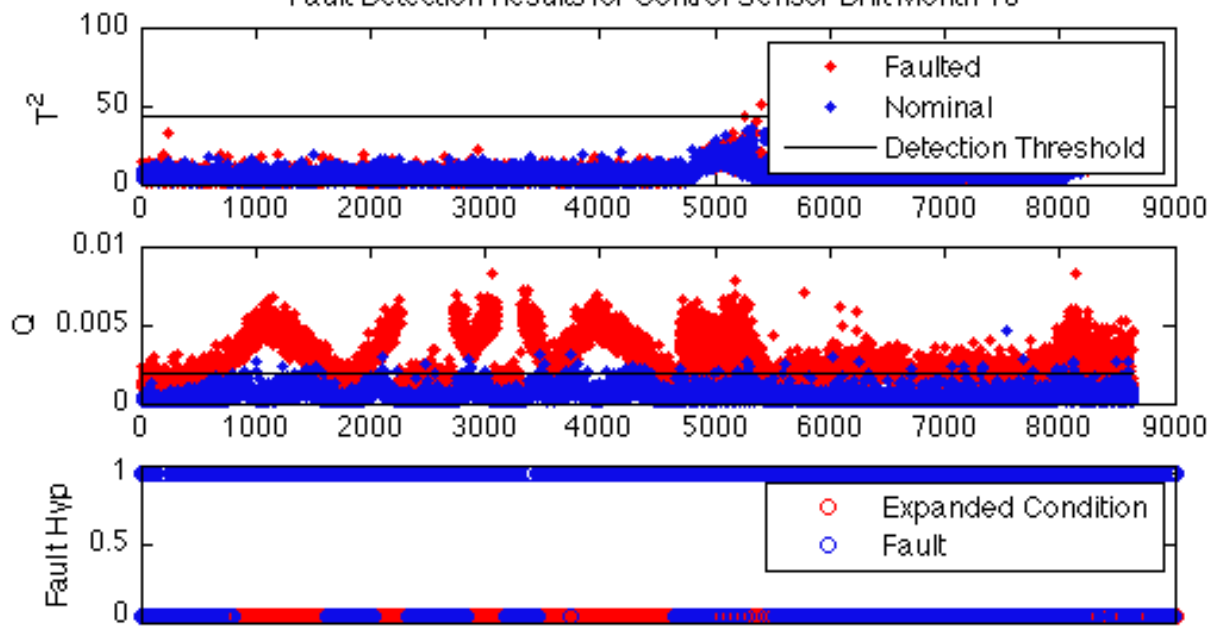

1000 Fau

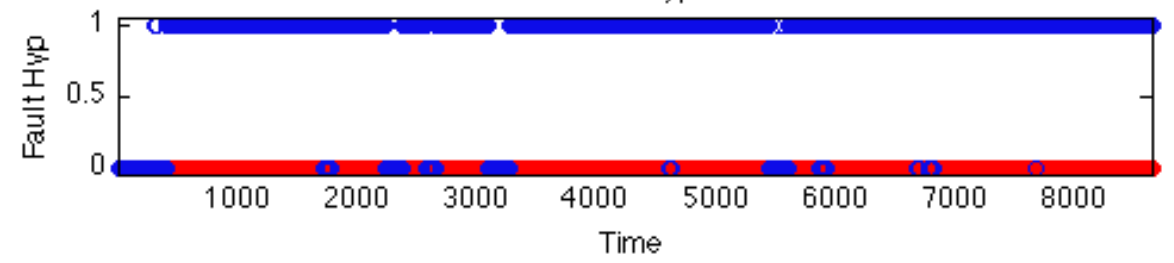


Fault Detection Results for Control Sensor Dritt Month 11
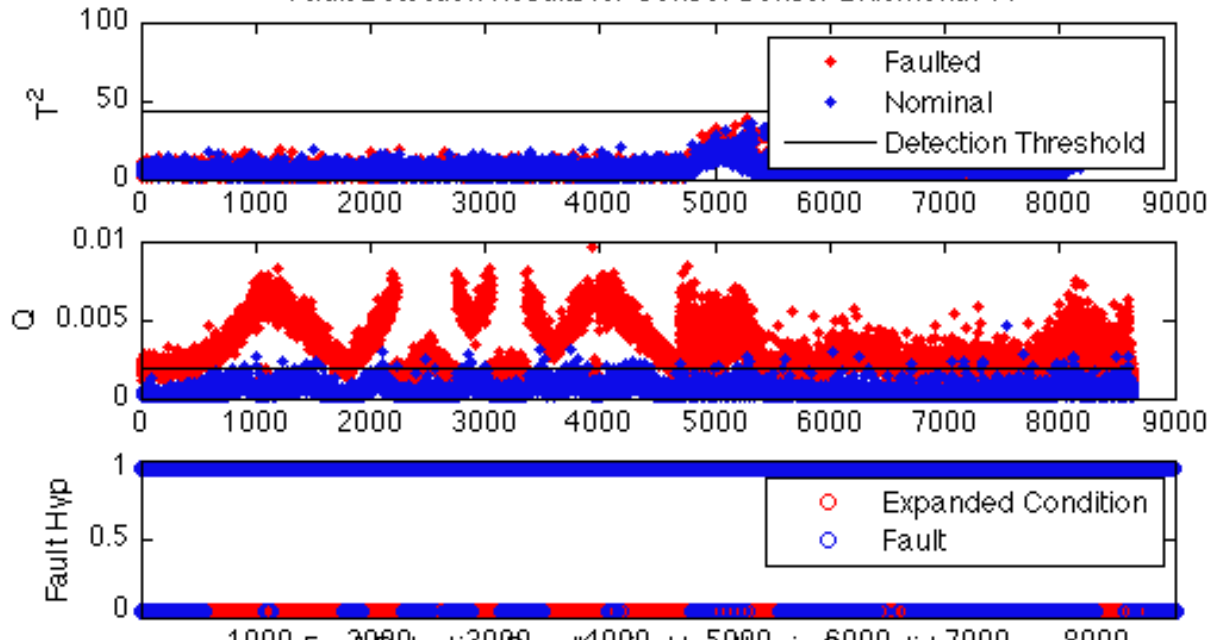

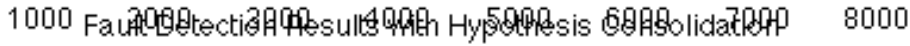

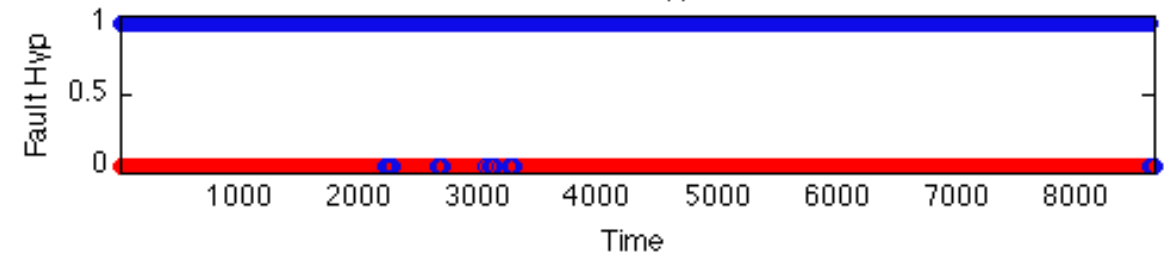

Fault Detection Results for Control Sensor Dritt Month 12
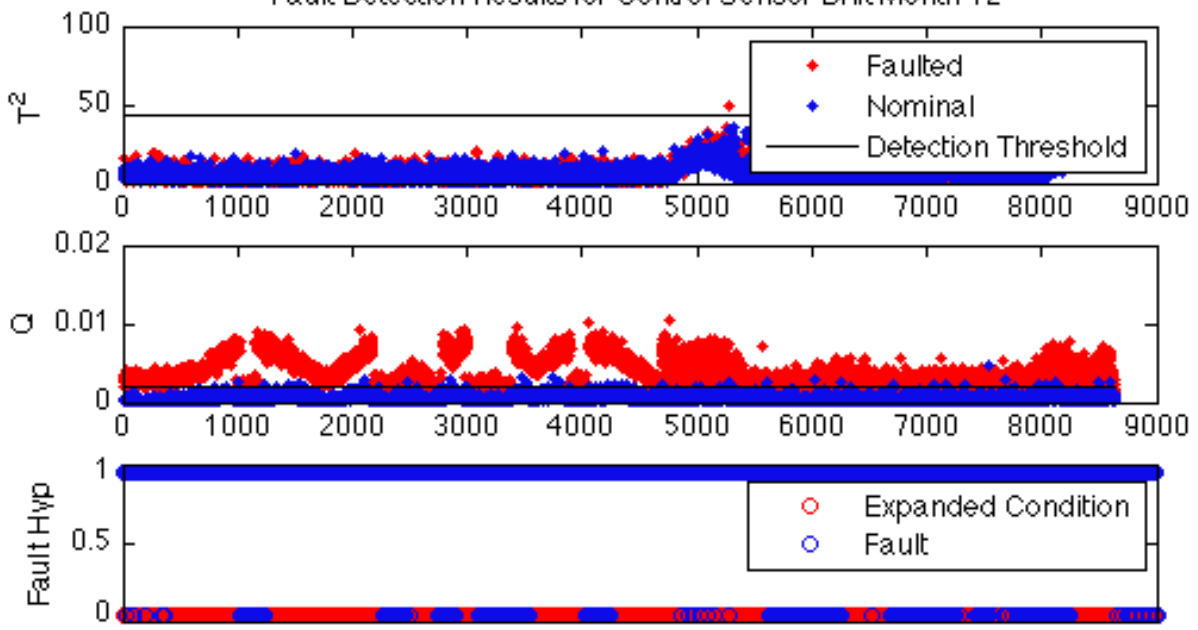

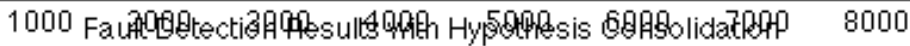

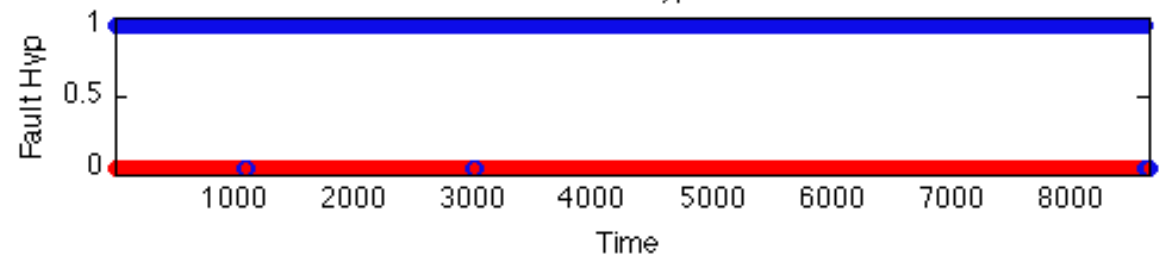




\section{A.2 SPRT Fault Detection Results}

\section{A.2.1 Heat Exchanger Fouling}

Fault Detection Results for Heat Exchanger Fouling Month 1
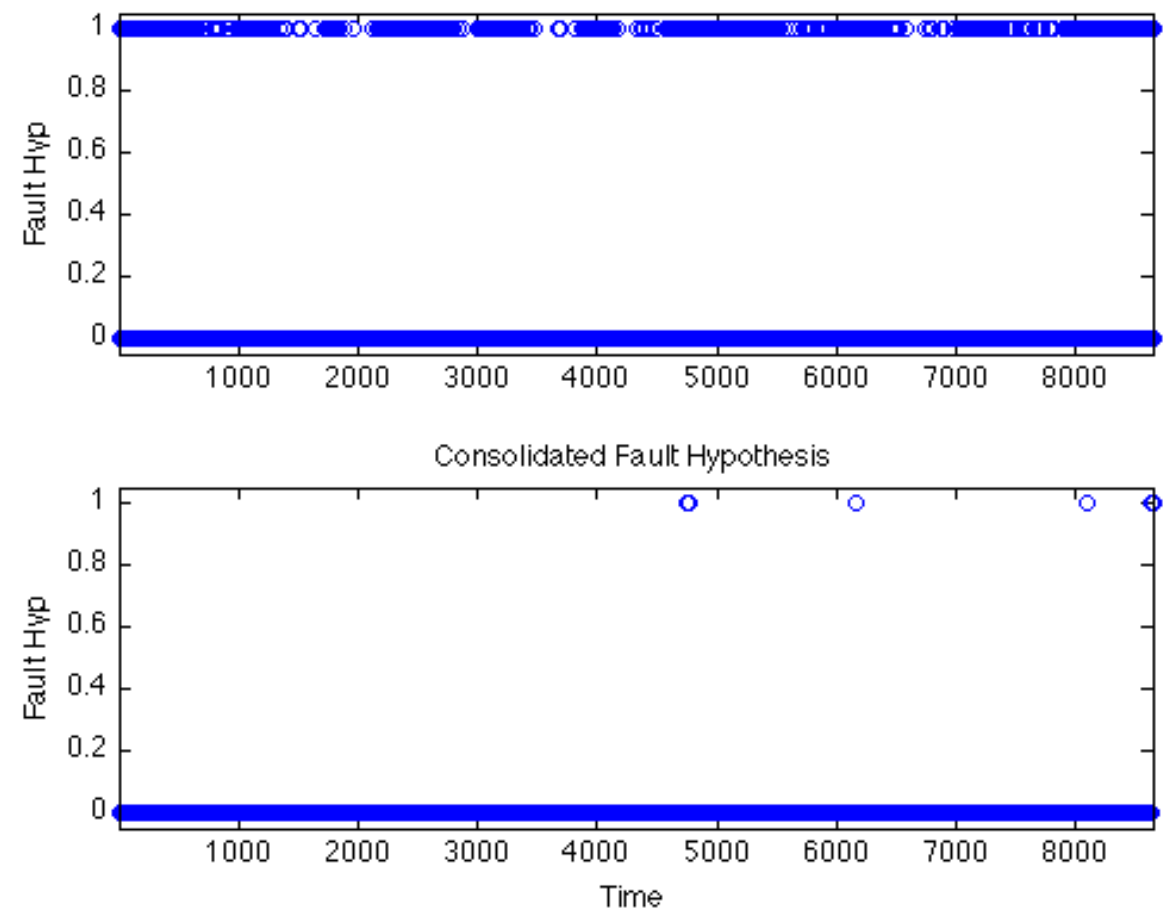

Fault Detection Results for Heat Exchanger Fouling Month 2
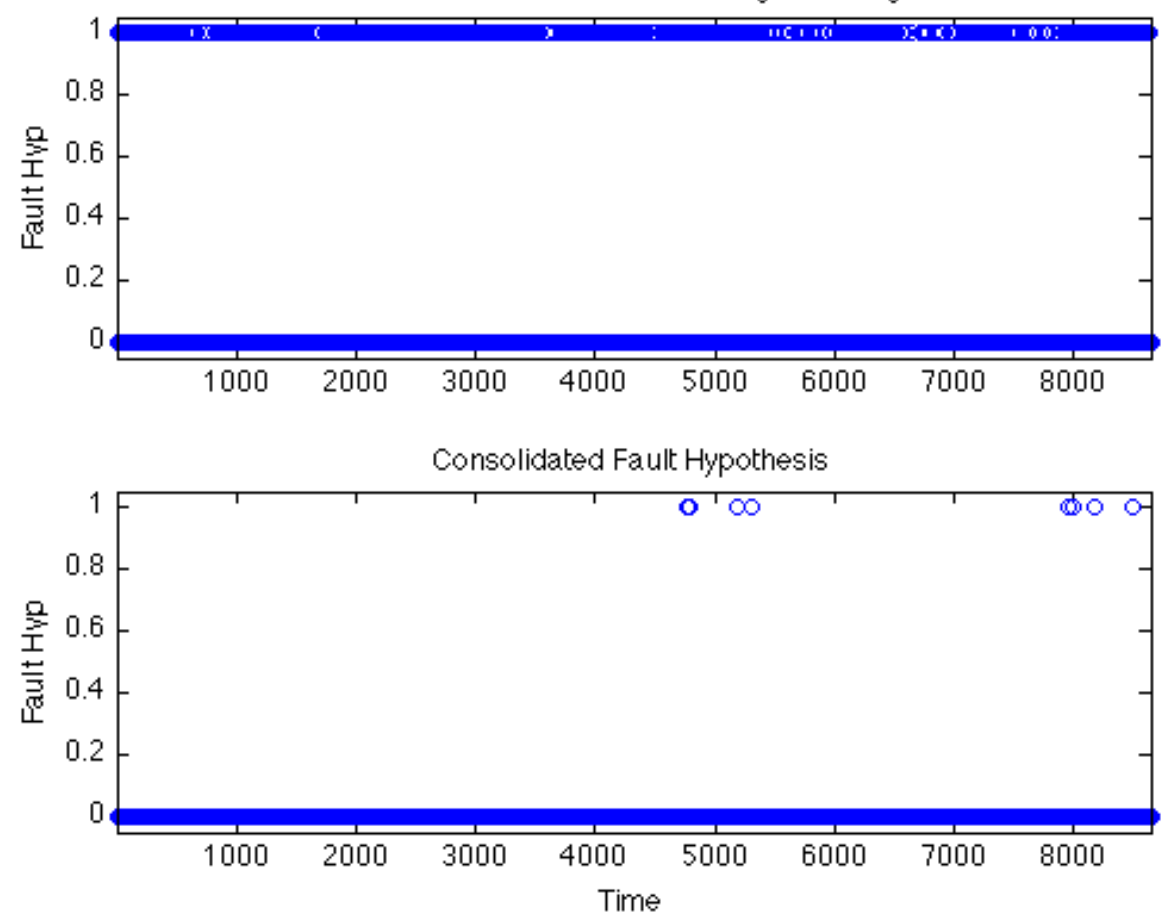
Fault Detection Results for Heat Exchanger Fouling Month 3
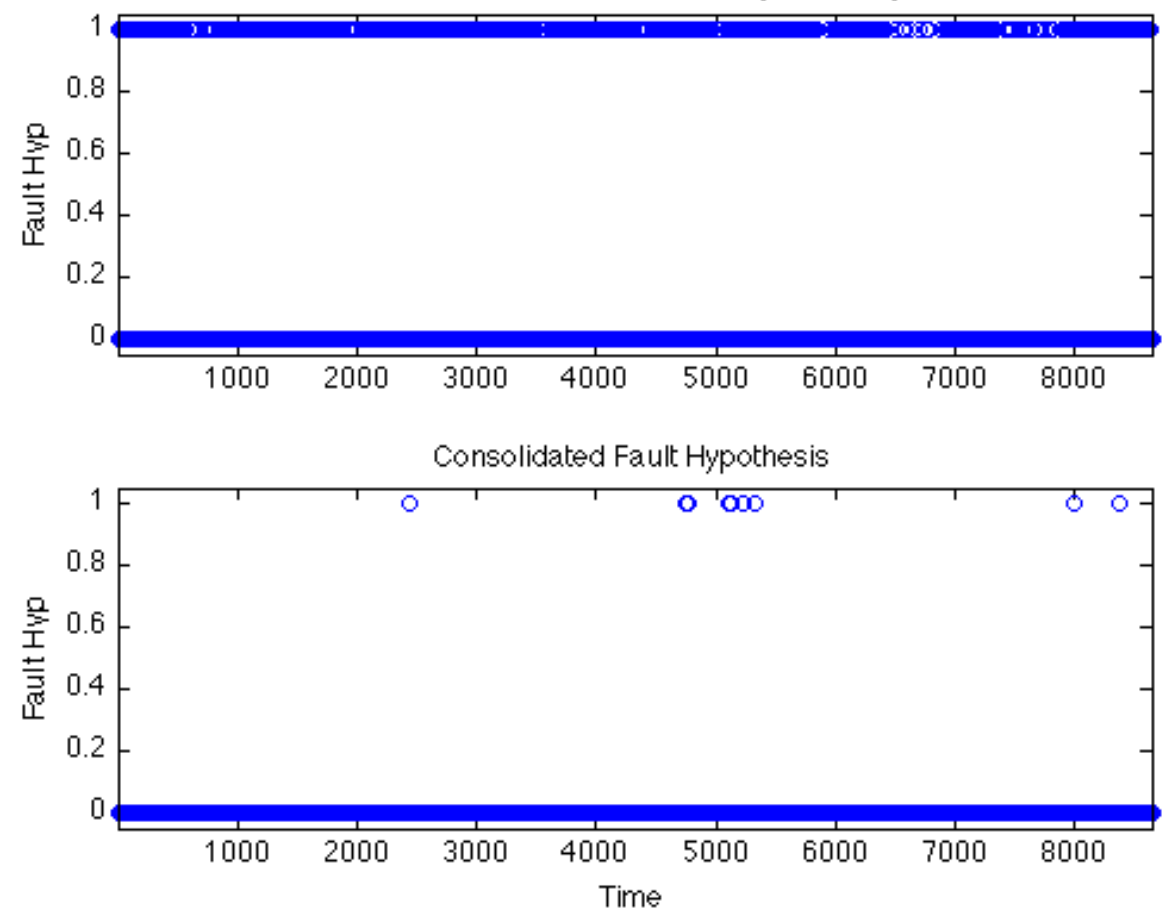

Fault Detection Results for Heat Exchanger Fouling Month 4
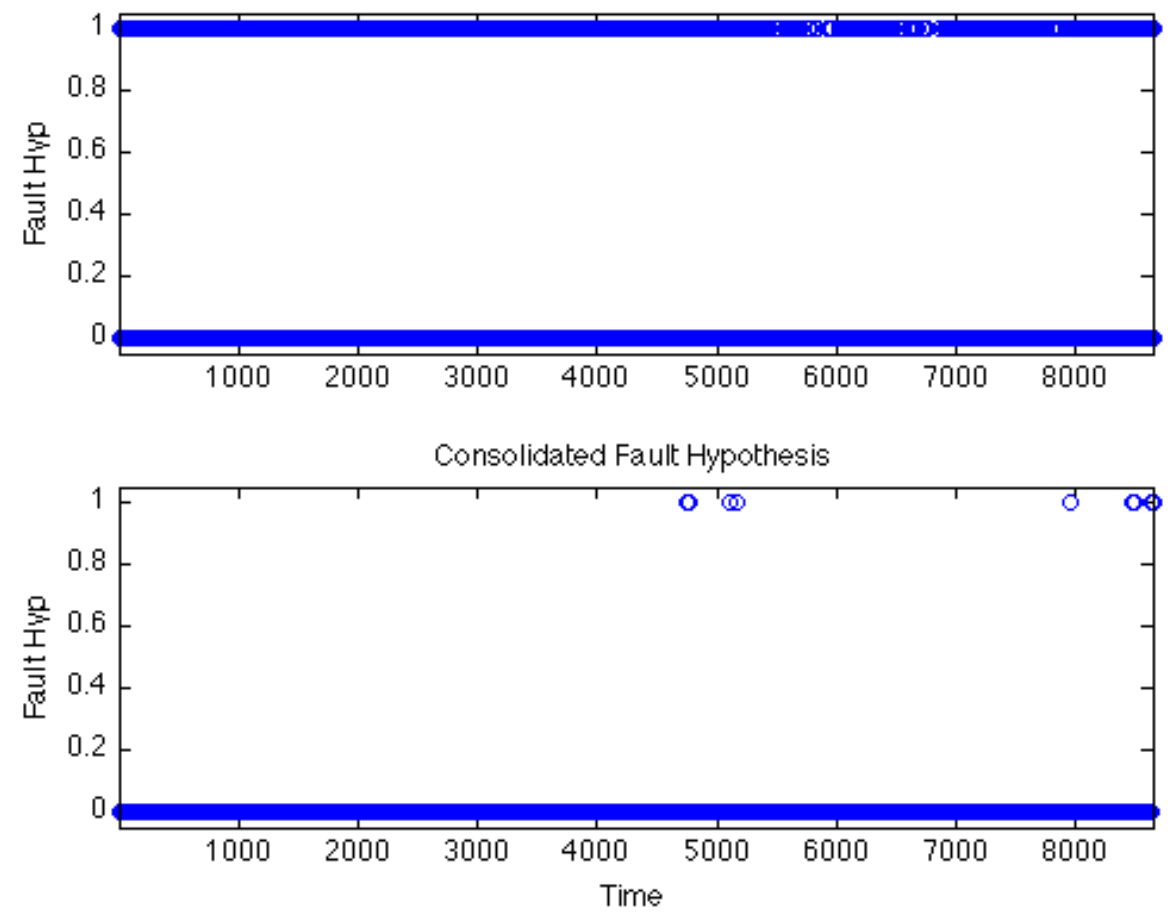
Fault Detection Results for Heat Exchanger Fouling Month 5

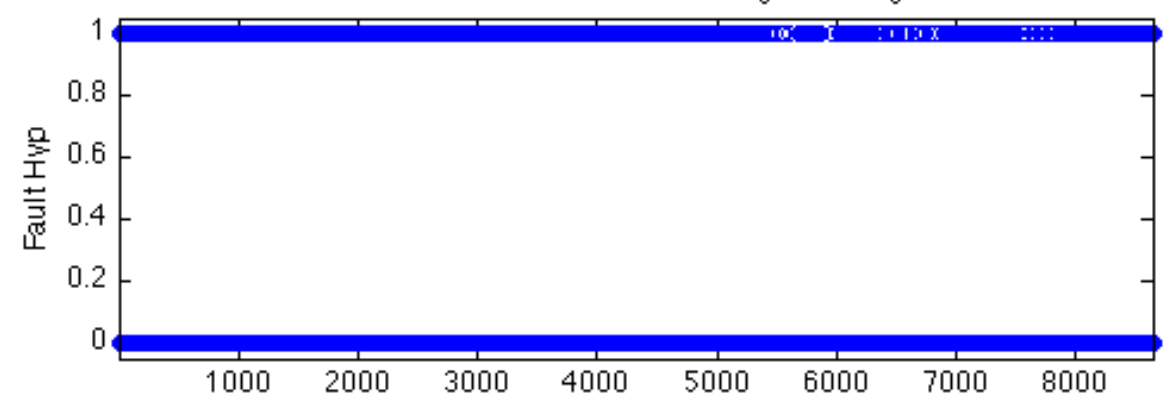

Consolidated Fault Hypothesis

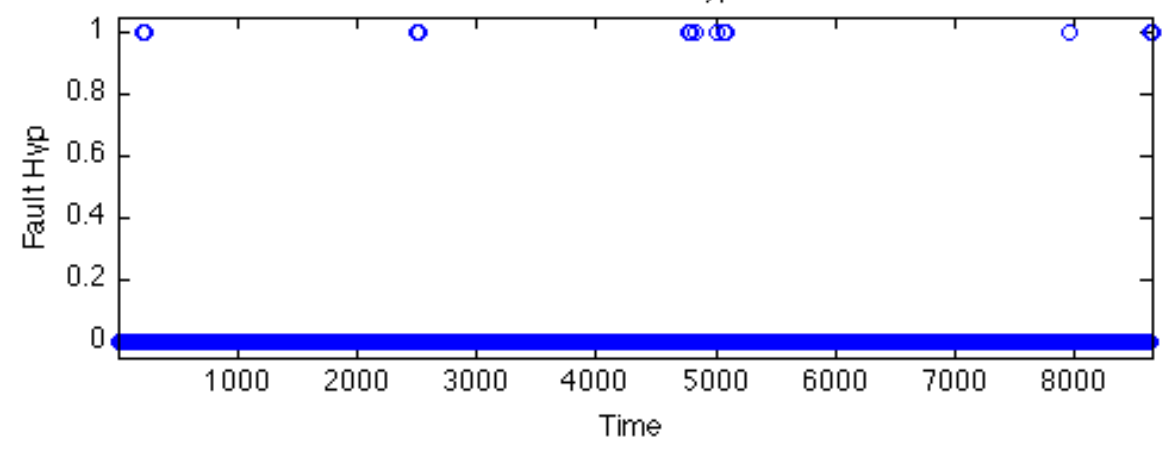

Fault Detection Results for Heat Exchanger Fouling Month 6
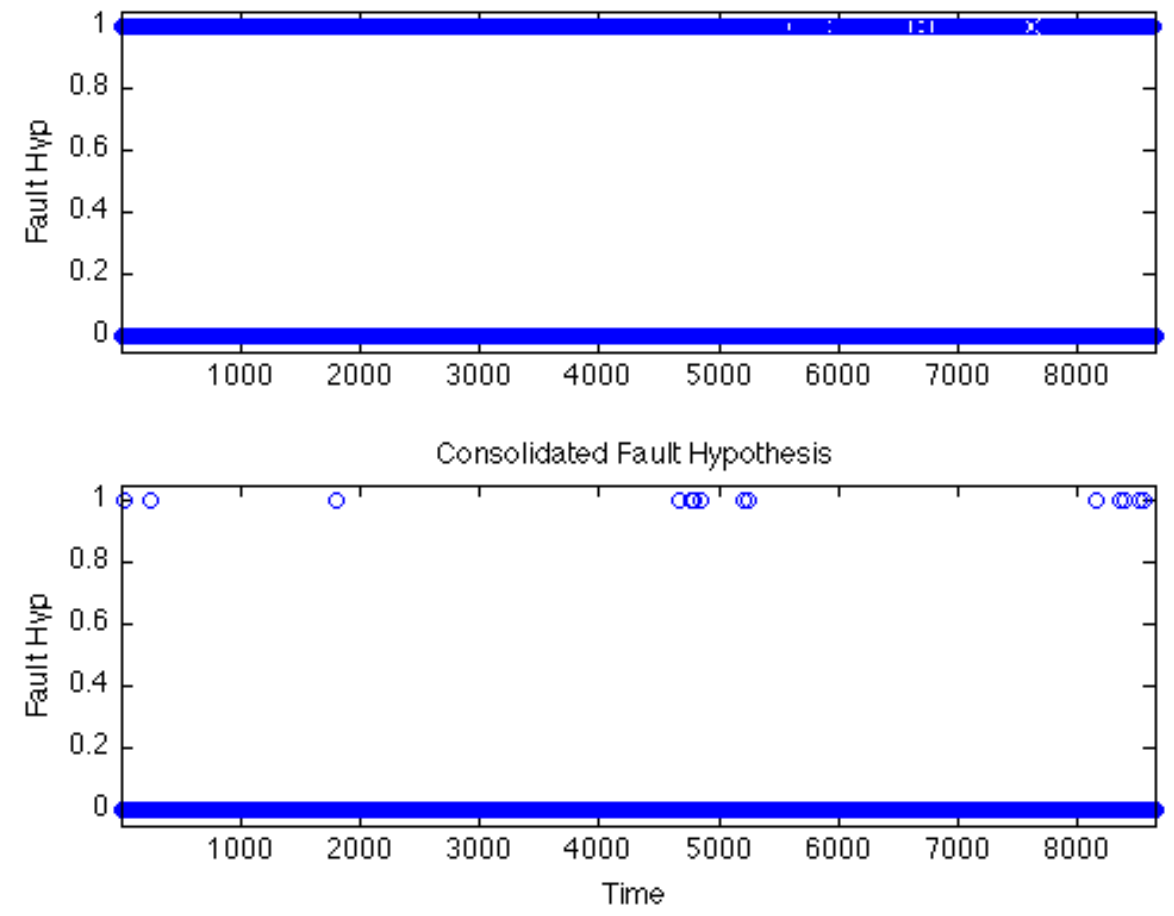
Fault Detection Results for Heat Exchanger Fouling Month 7

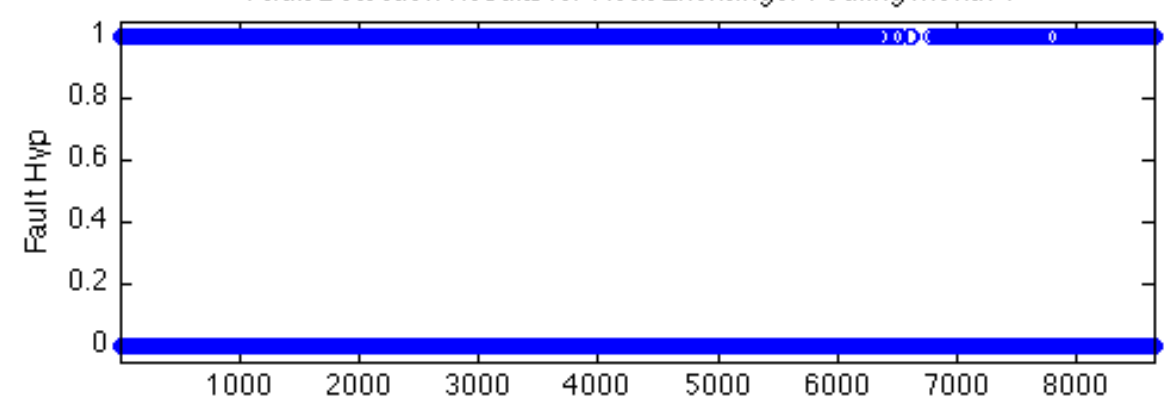

Consolidated Fault Hypothesis

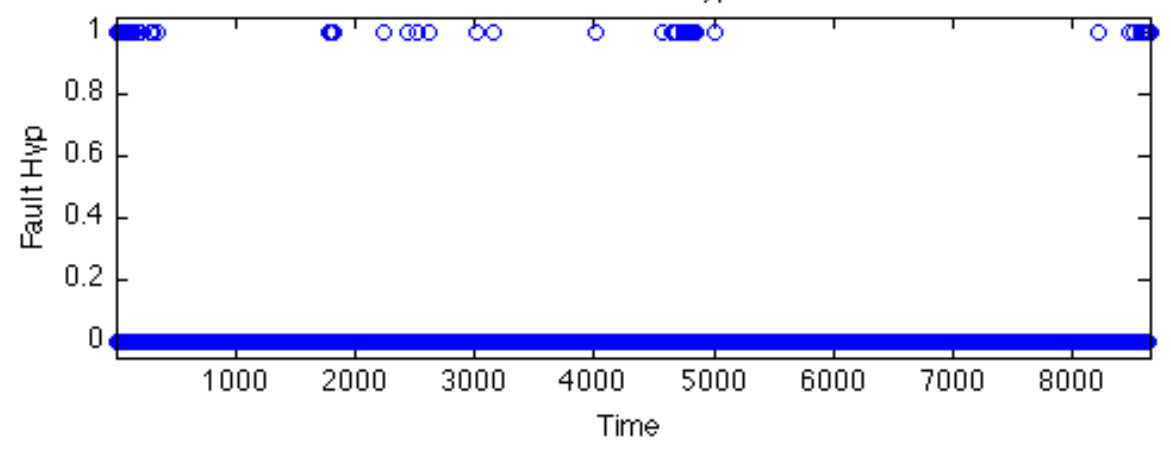

Fault Detection Results for Heat Exchanger Fouling Month 8

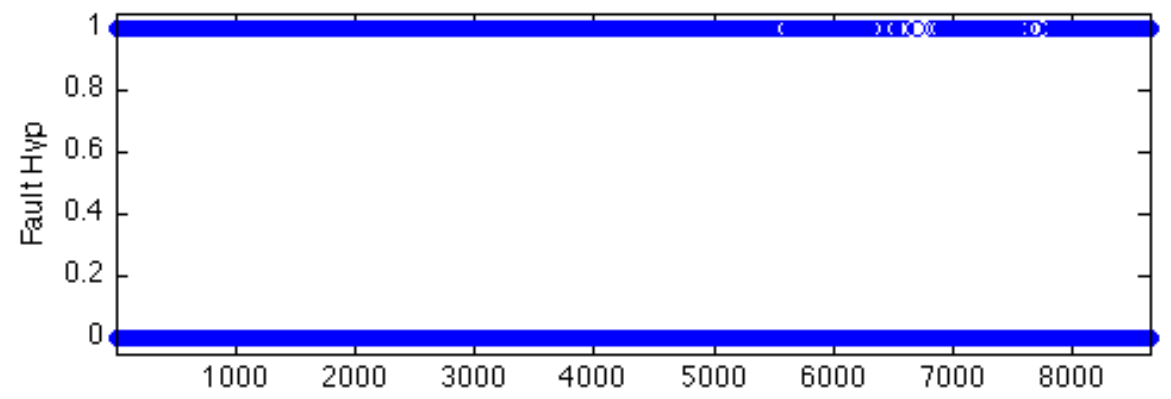

Consolidated Fault Hypothesis

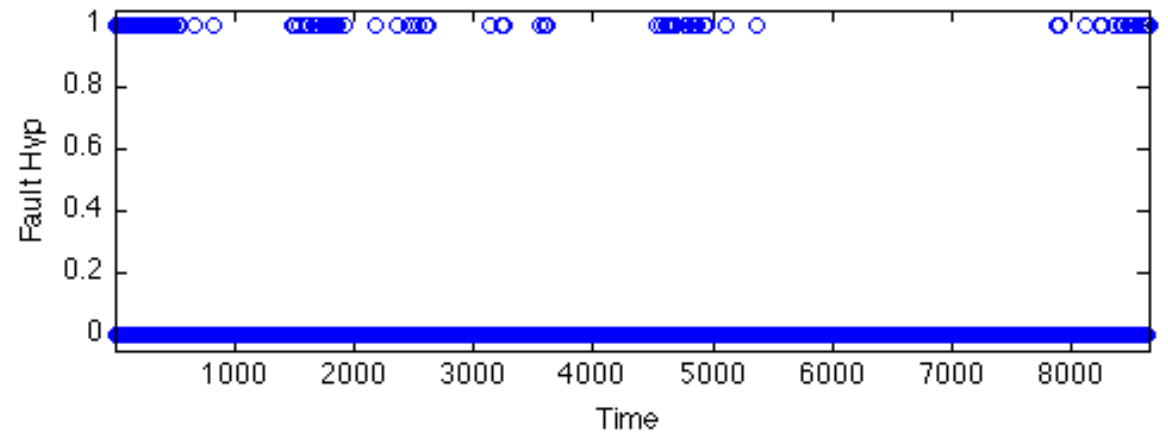


Fault Detection Results for Heat Exchanger Fouling Month 9

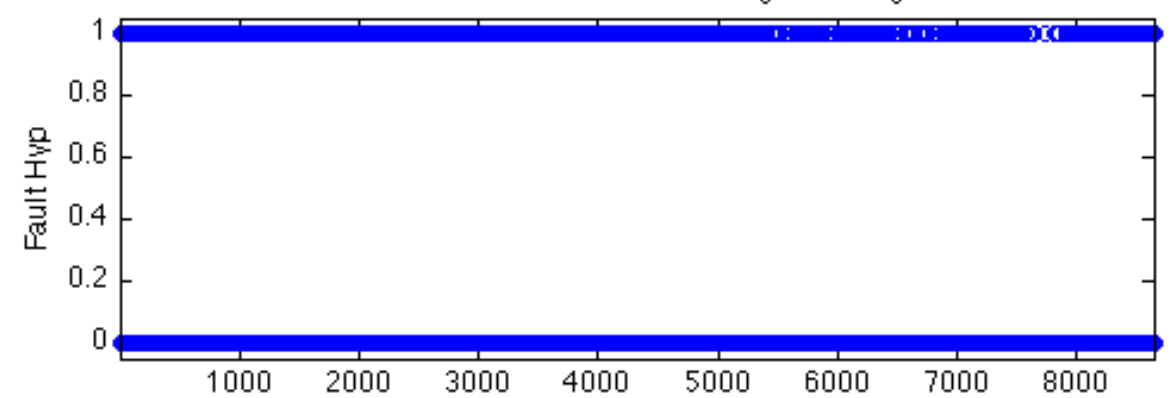

Consolidated Fault Hypothesis

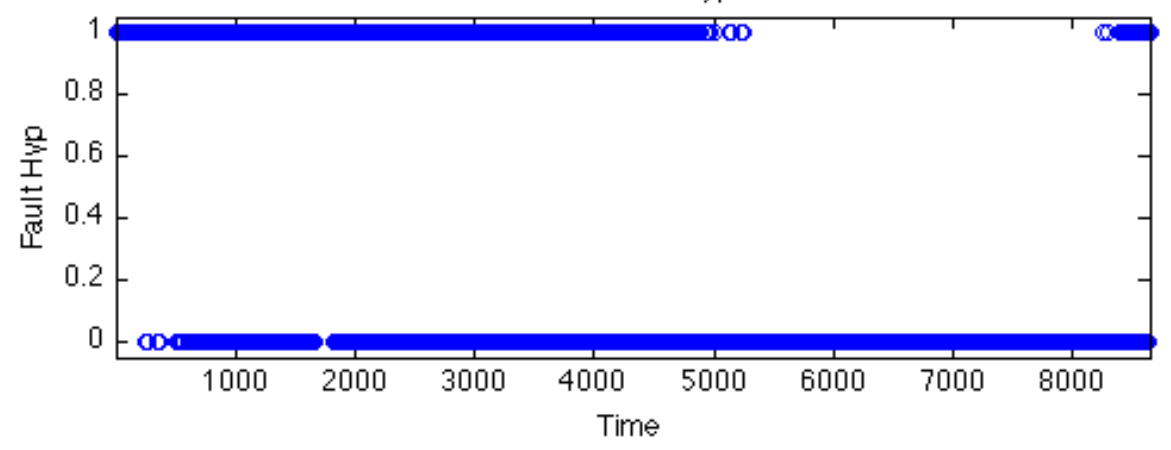

Fault Detection Results for Heat Exchanger Fouling Month 10
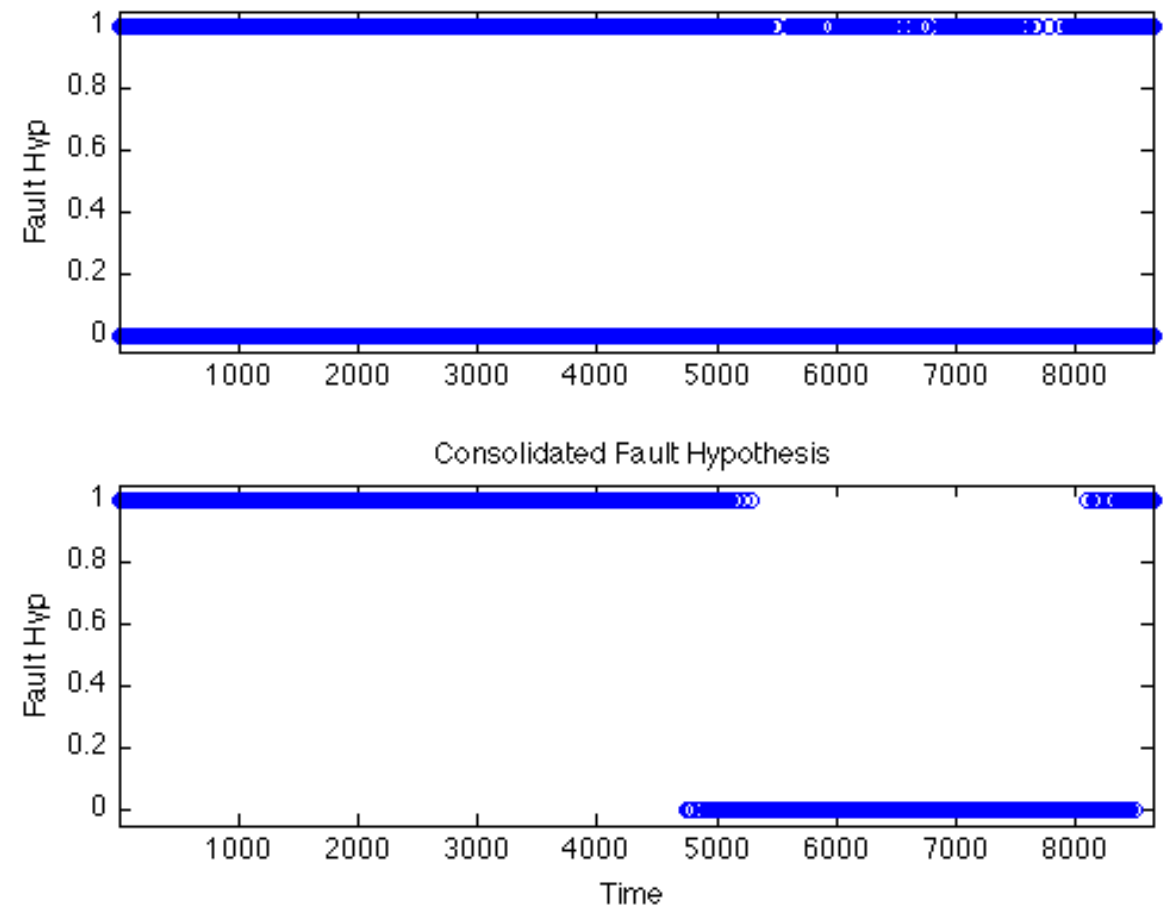
Fault Detection Results for Heat Exchanger Fouling Month 11

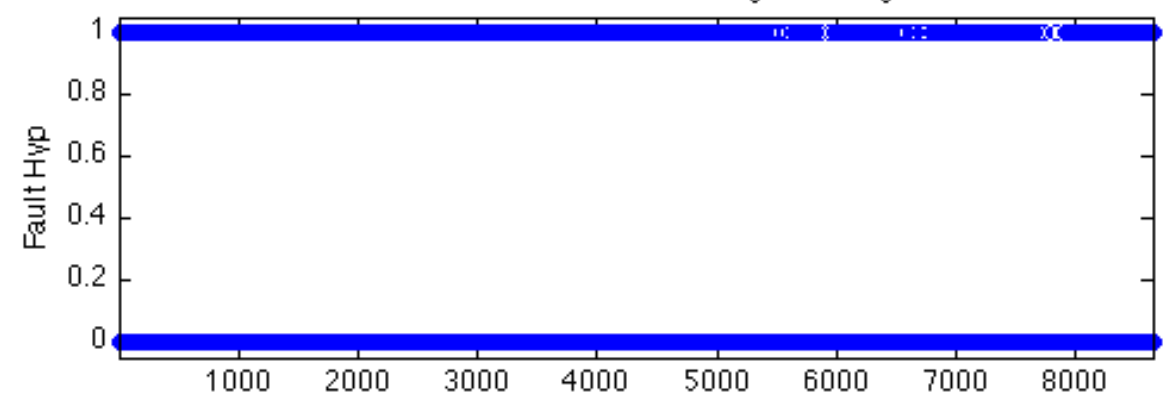

Consolidated Fault Hypothesis

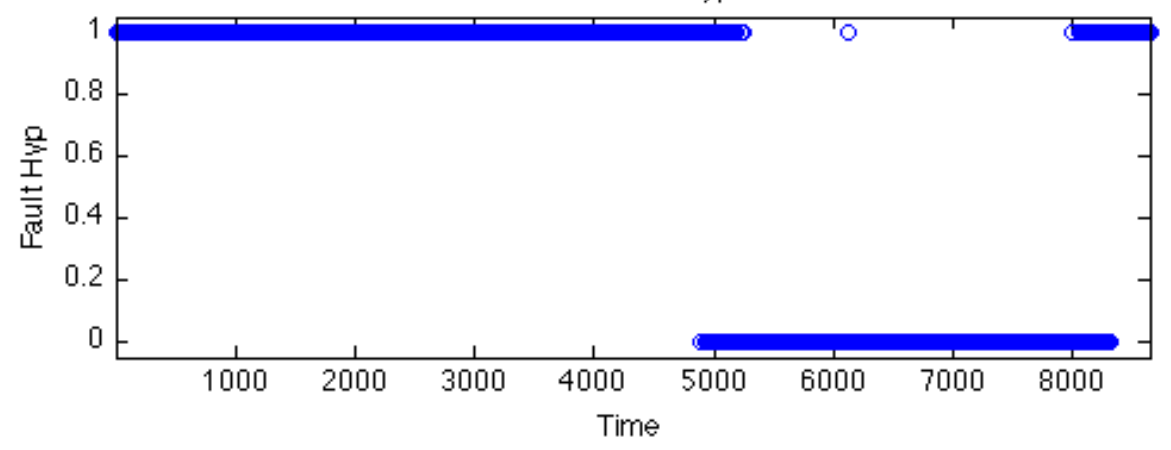

Fault Detection Results for Heat Exchanger Fouling Month 12
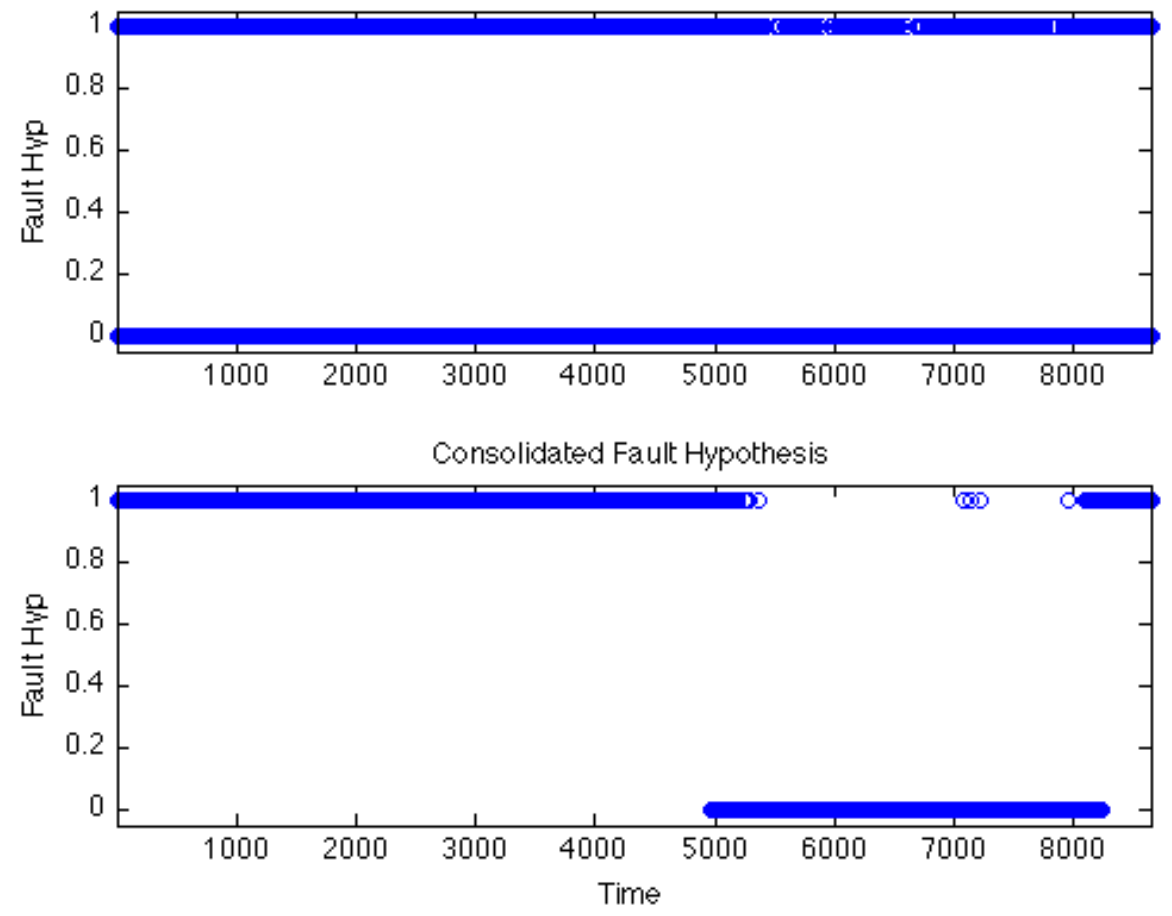


\section{A.2.2 Pump Degradation}

Fault Detection Results for Pump Degradation Month 1

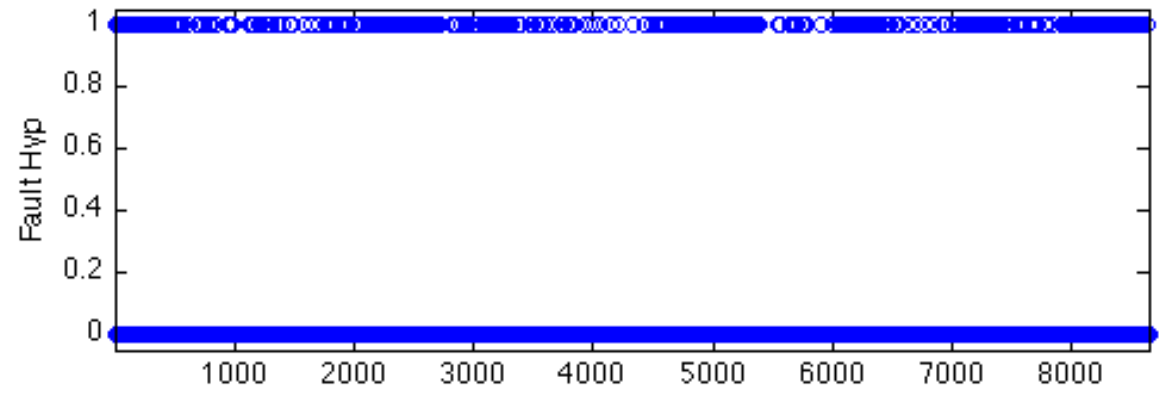

Consolidated Fault Hypothesis

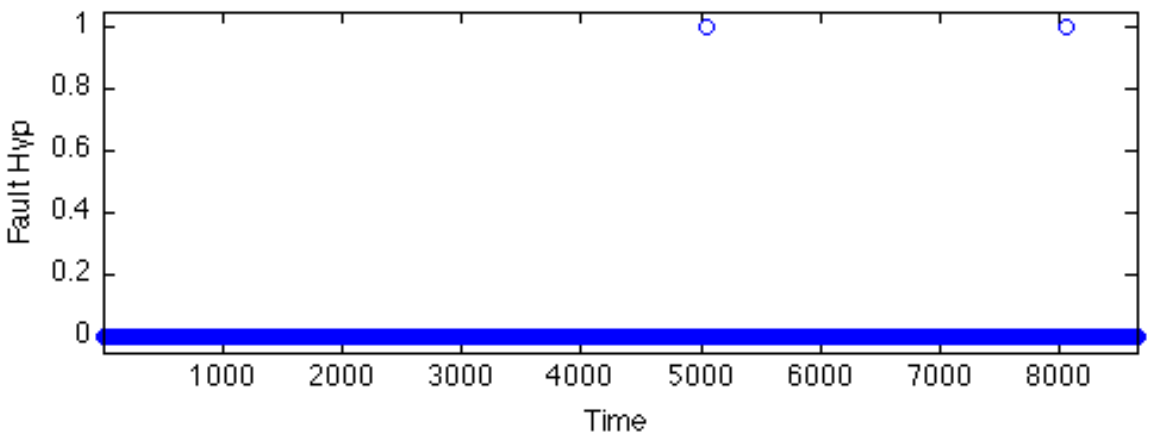

Fault Detection Results for Pump Degradation Month 2

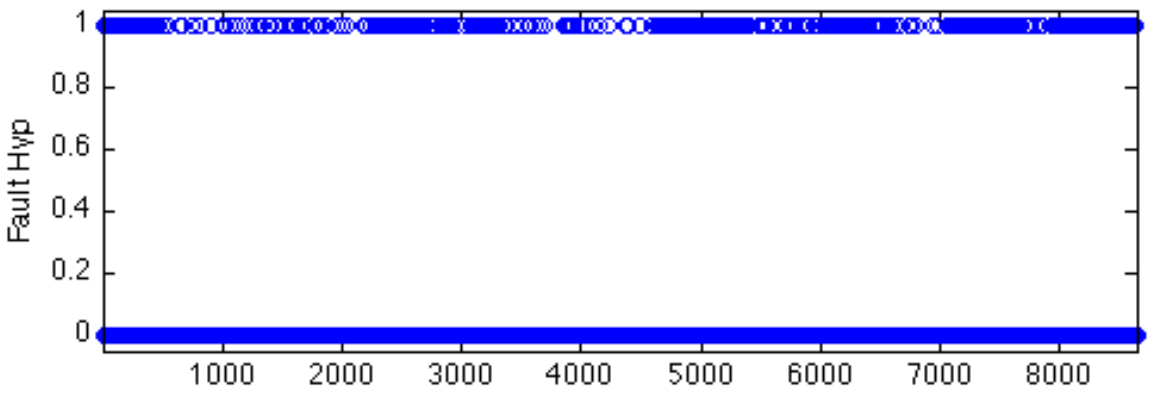

Consolidated Fault Hypothesis

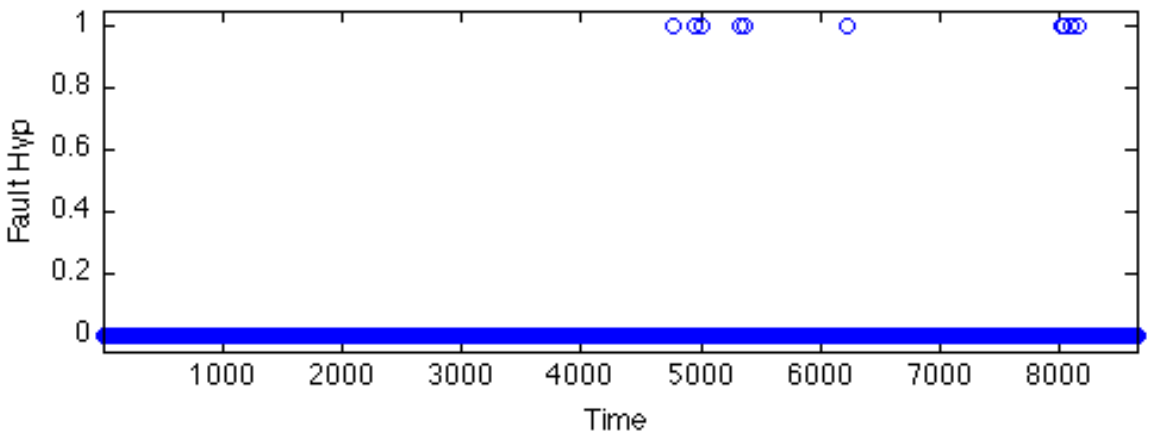


Fault Detection Results for Pump Degradation Month 3

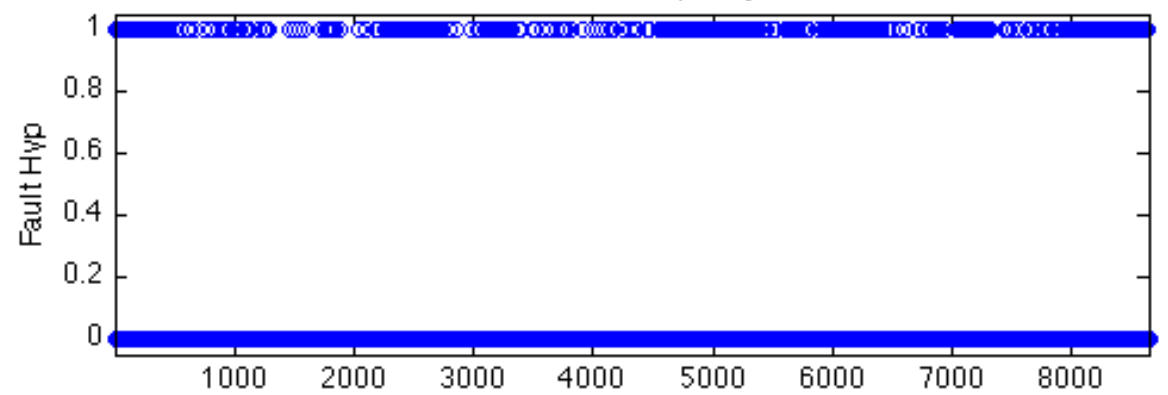

Consolidated Fault Hypothesis

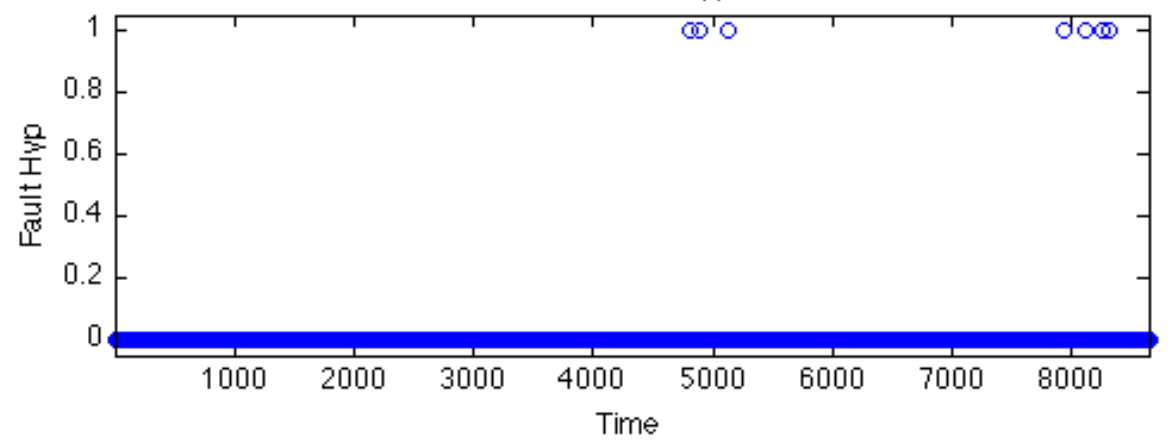

Fault Detection Results for Pump Degradation Month 4
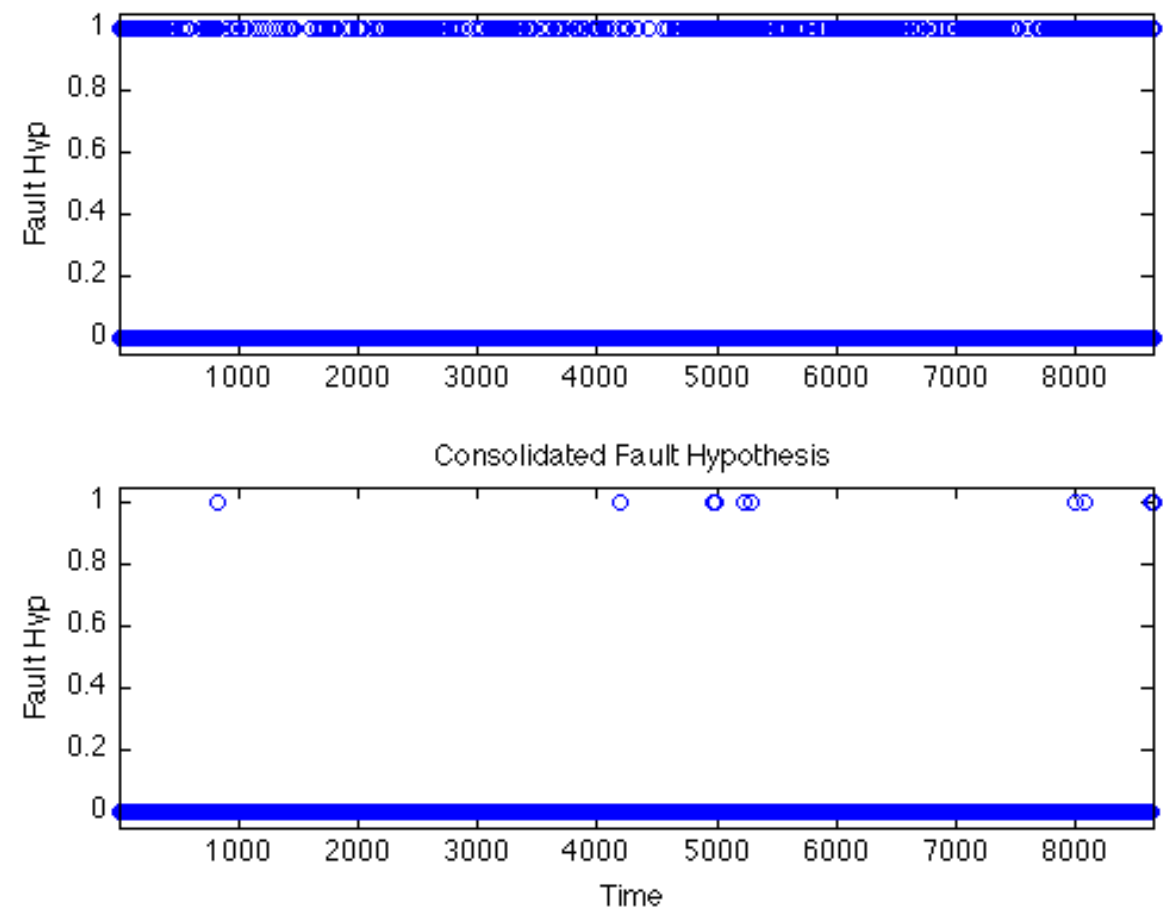
Fault Detection Results for Pump Degradation Month 5

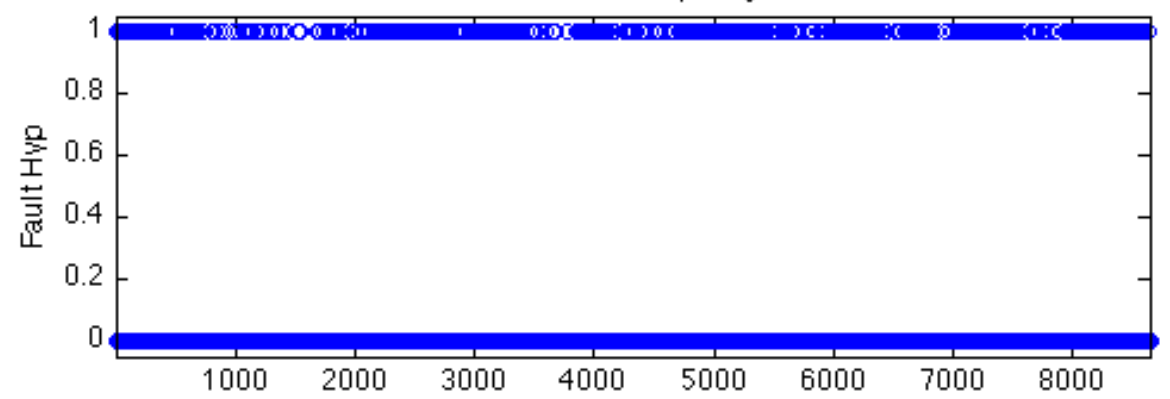

Consolidated Fault Hypothesis

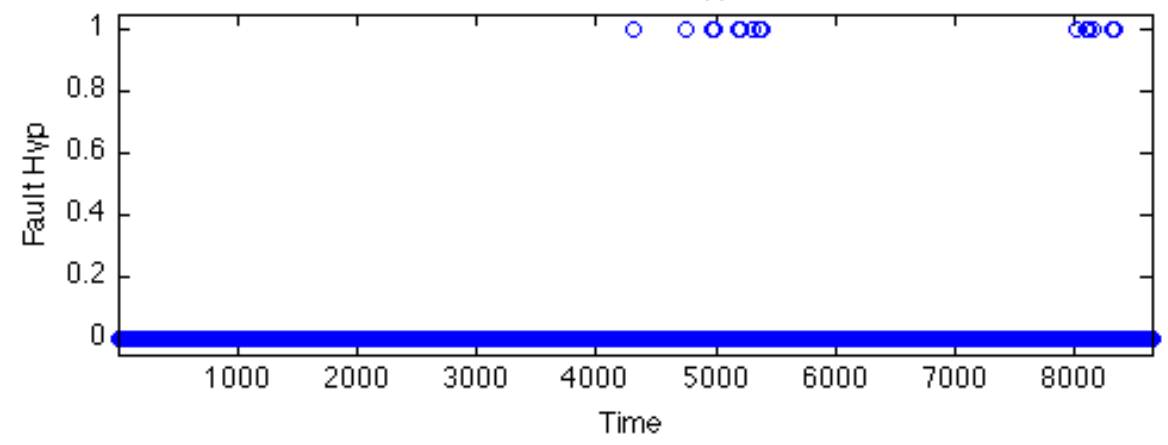

Fault Detection Results for Pump Degradation Month 6
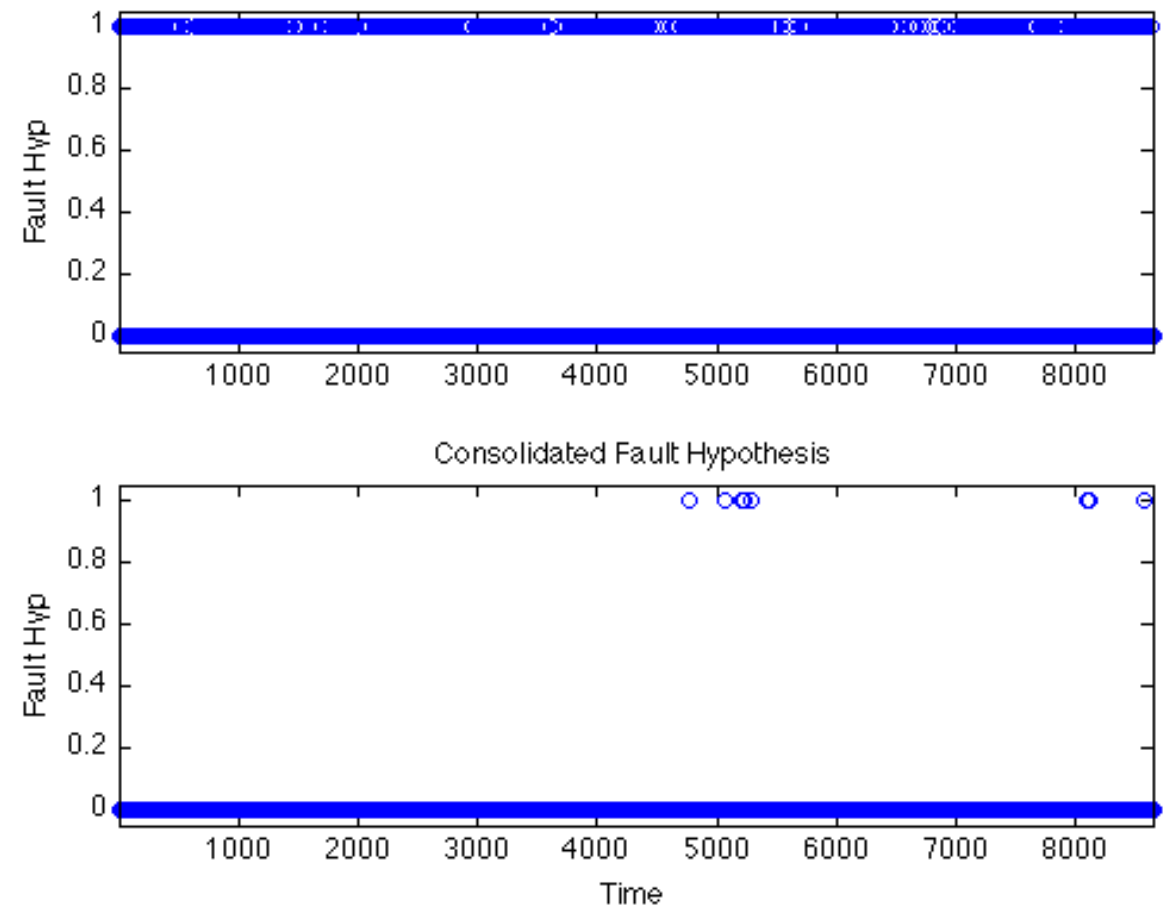
Fault Detection Results for Pump Degradation Month 7

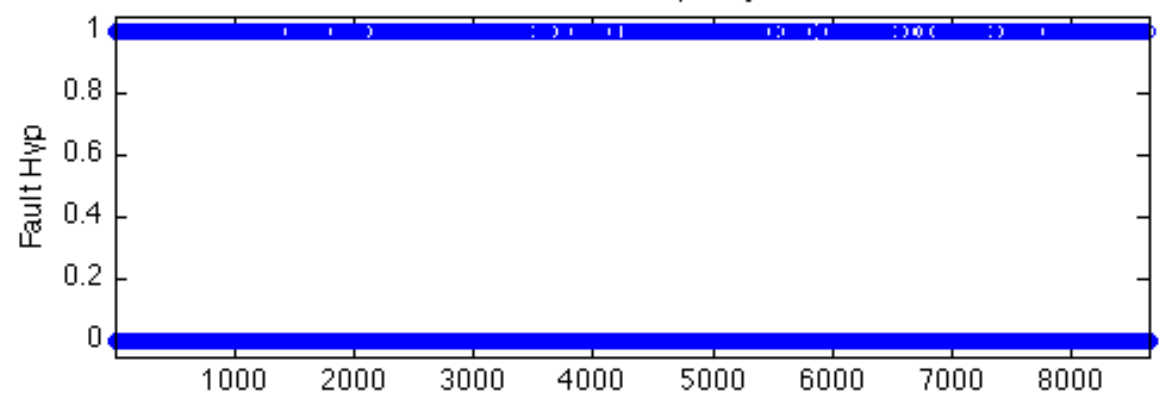

Consolidated Fault Hypothesis

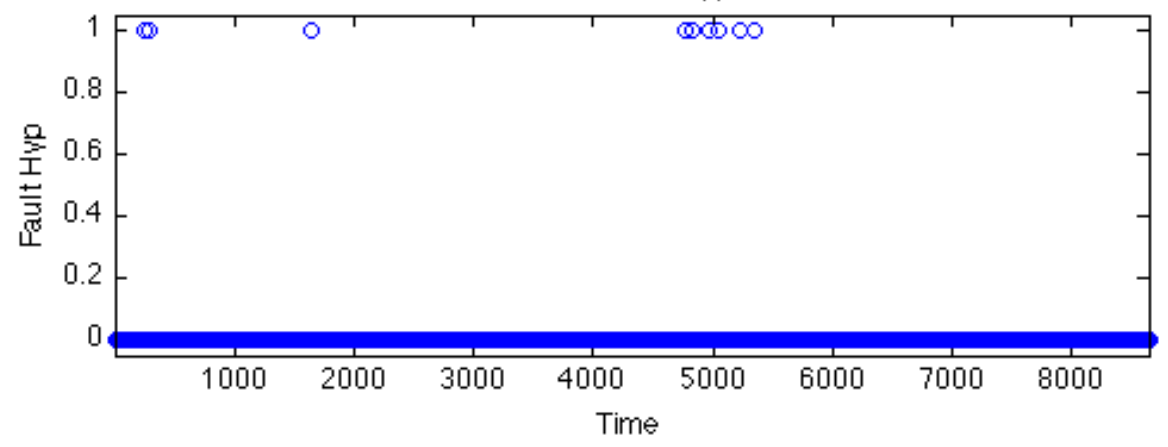

Fault Detection Results for Pump Degradation Month 8
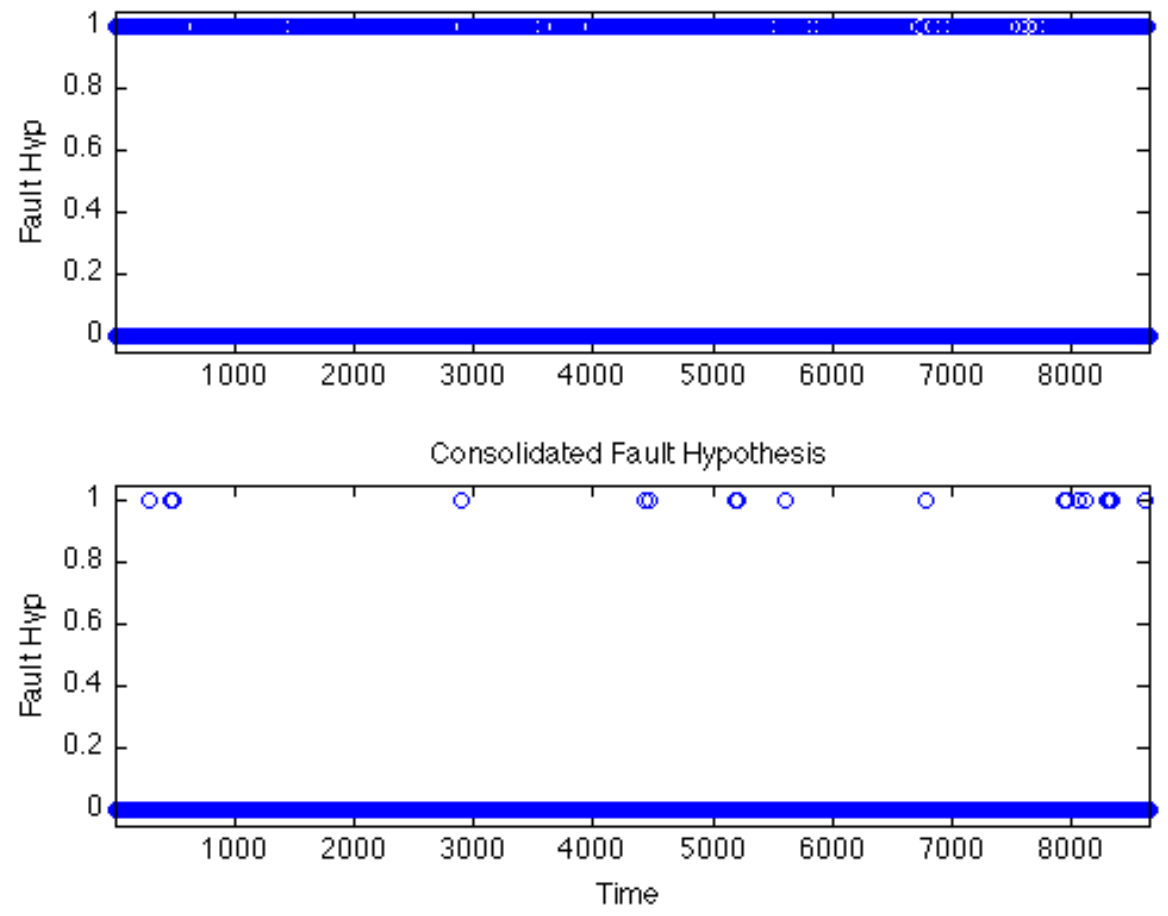
Fault Detection Results for Pump Degradation Month 9

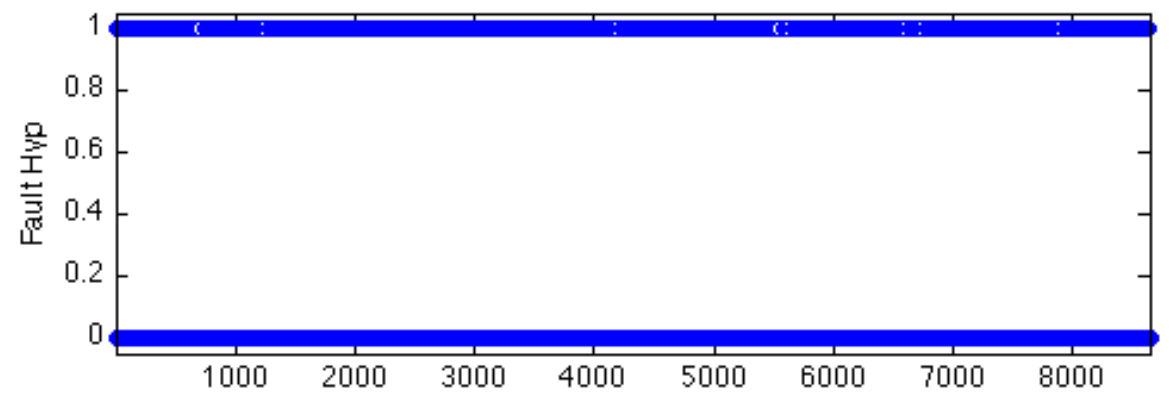

Consolidated Fault Hypothesis

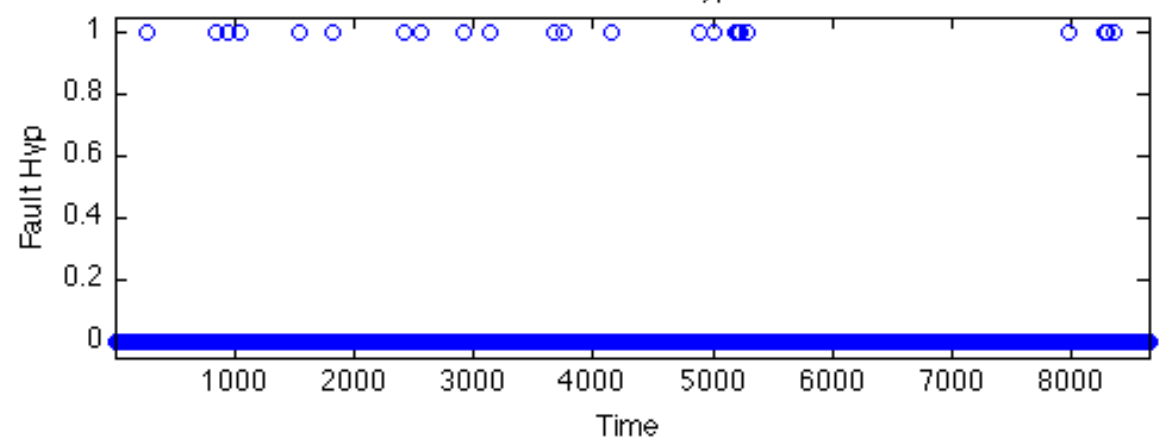

Fault Detection Results for Pump Degradation Month 10

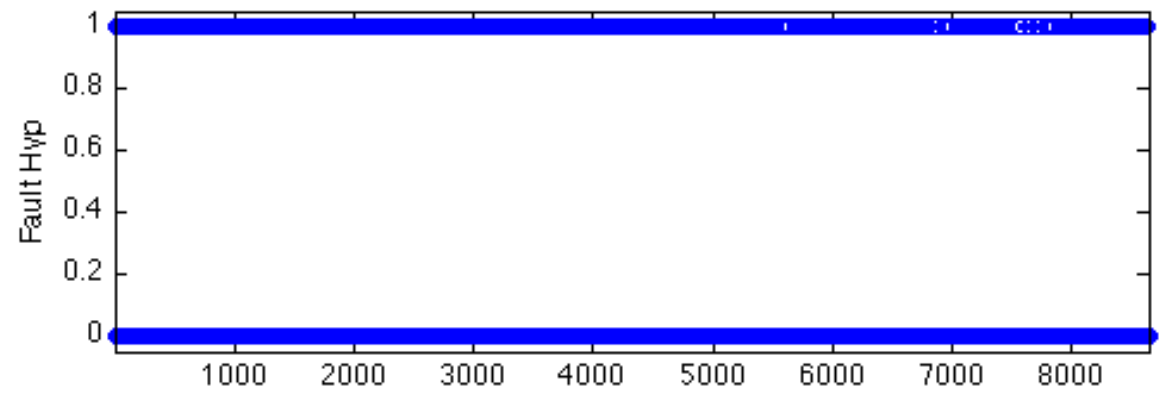

Consolidated Fault Hypothesis

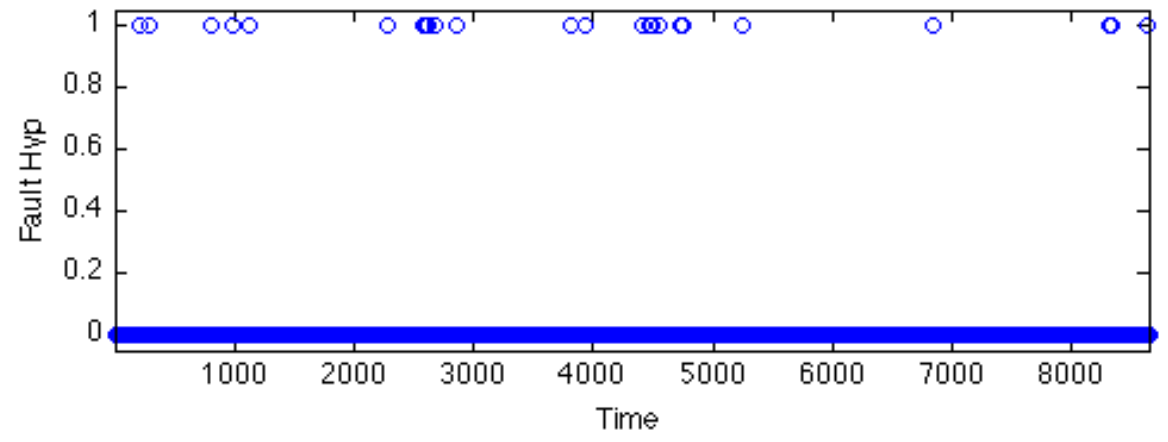




\section{A.2.3 Process Sensor Drift}

Fault Detection Results for Process Sensor Drit Month 1
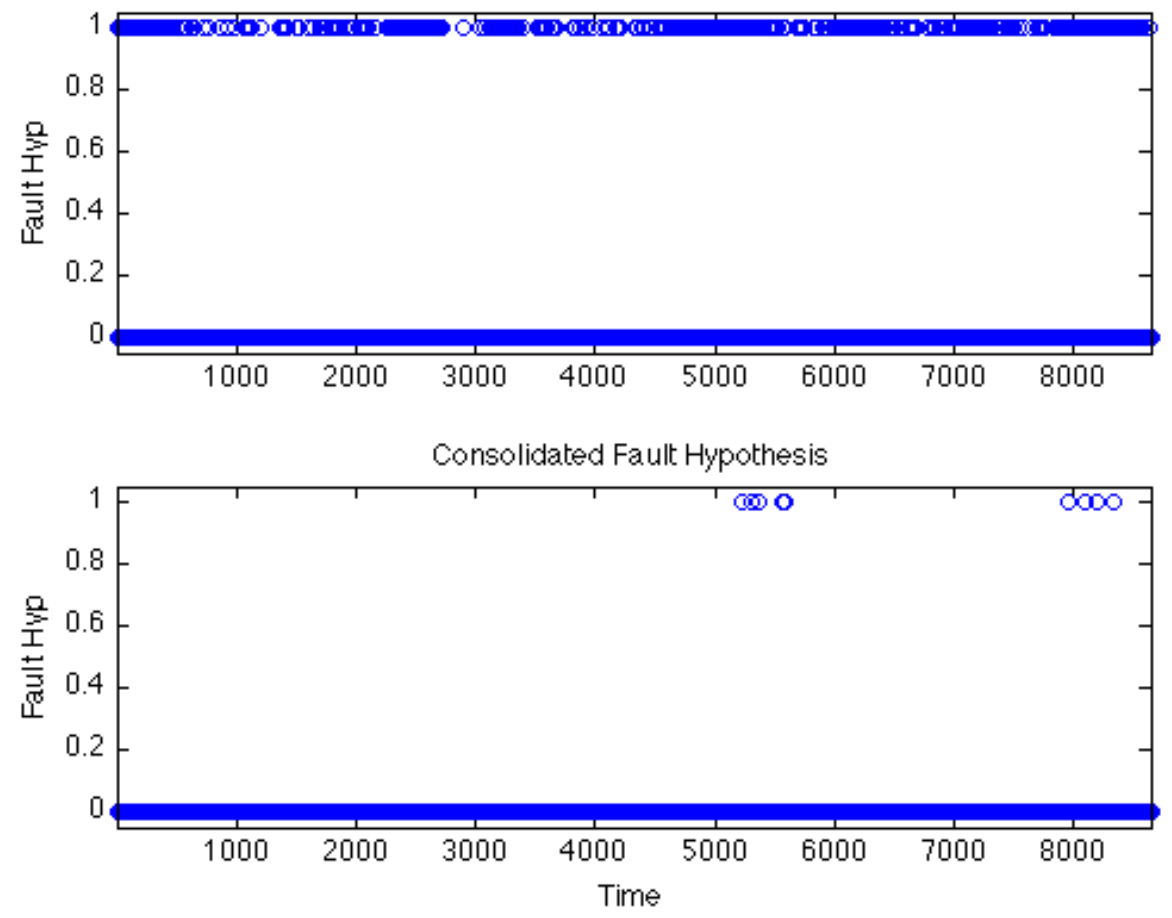

Fault Detection Results for Process Sensor Dritt Month 2
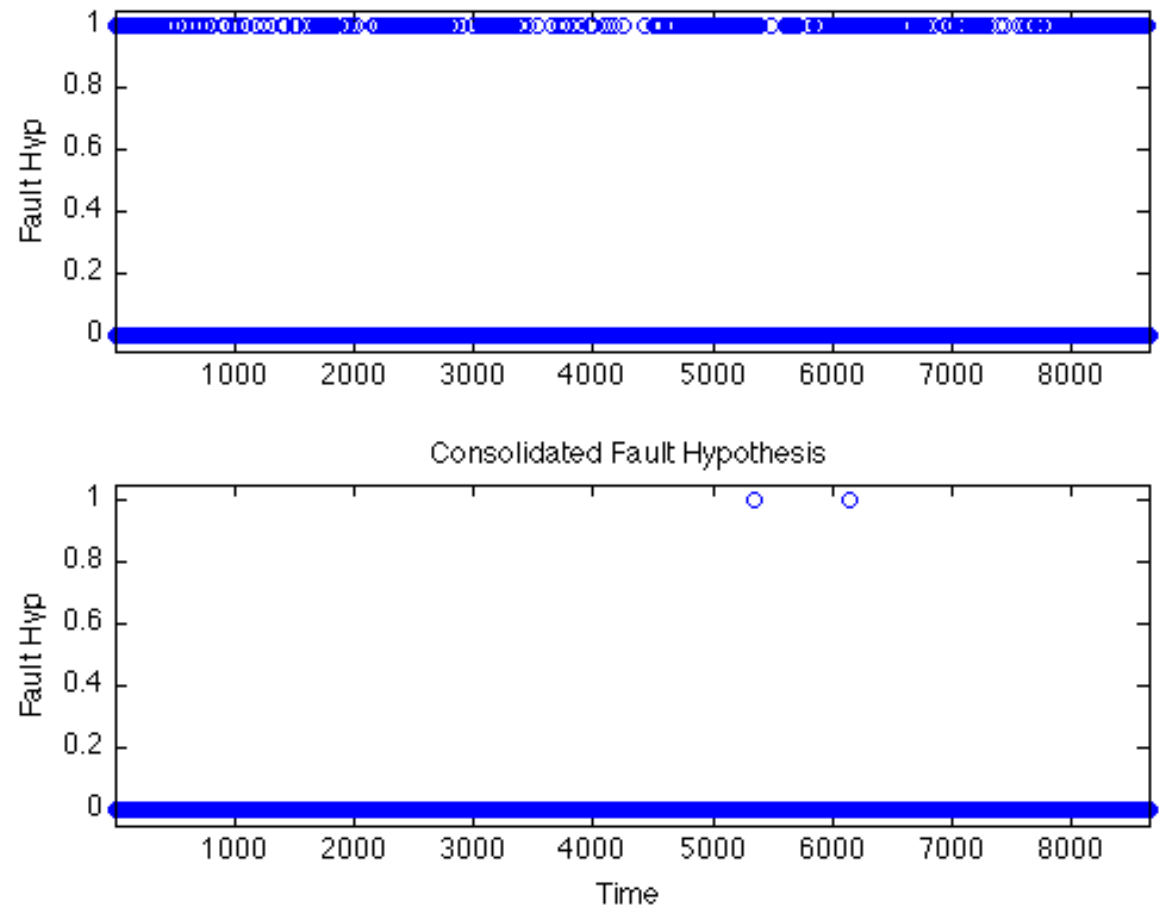
Fault Detection Results for Process Sensor Drift Month 3

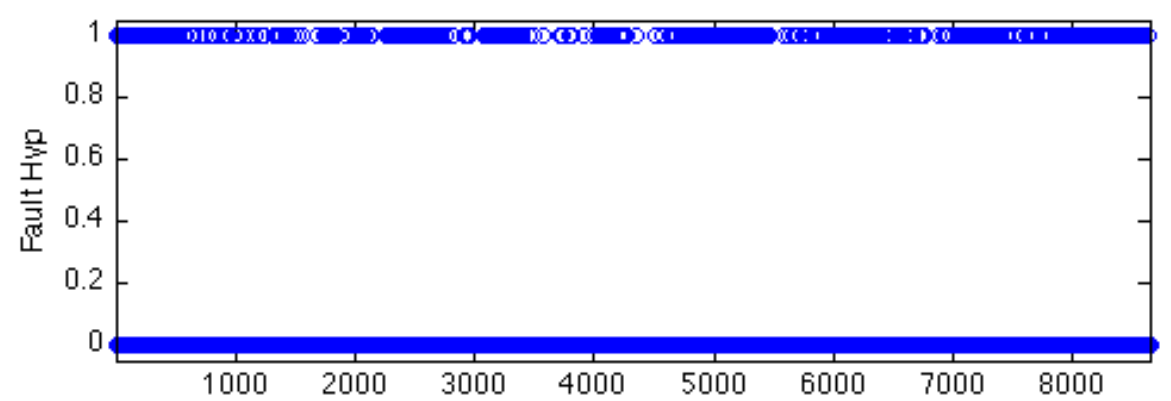

Consolidated Fault Hypothesis

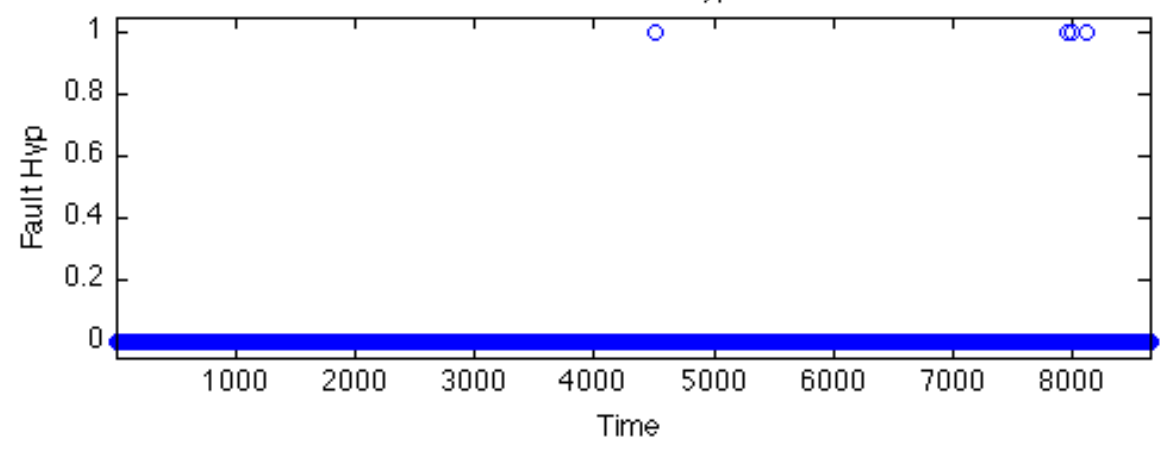

Fault Detection Results for Process Sensor Dritt Month 4
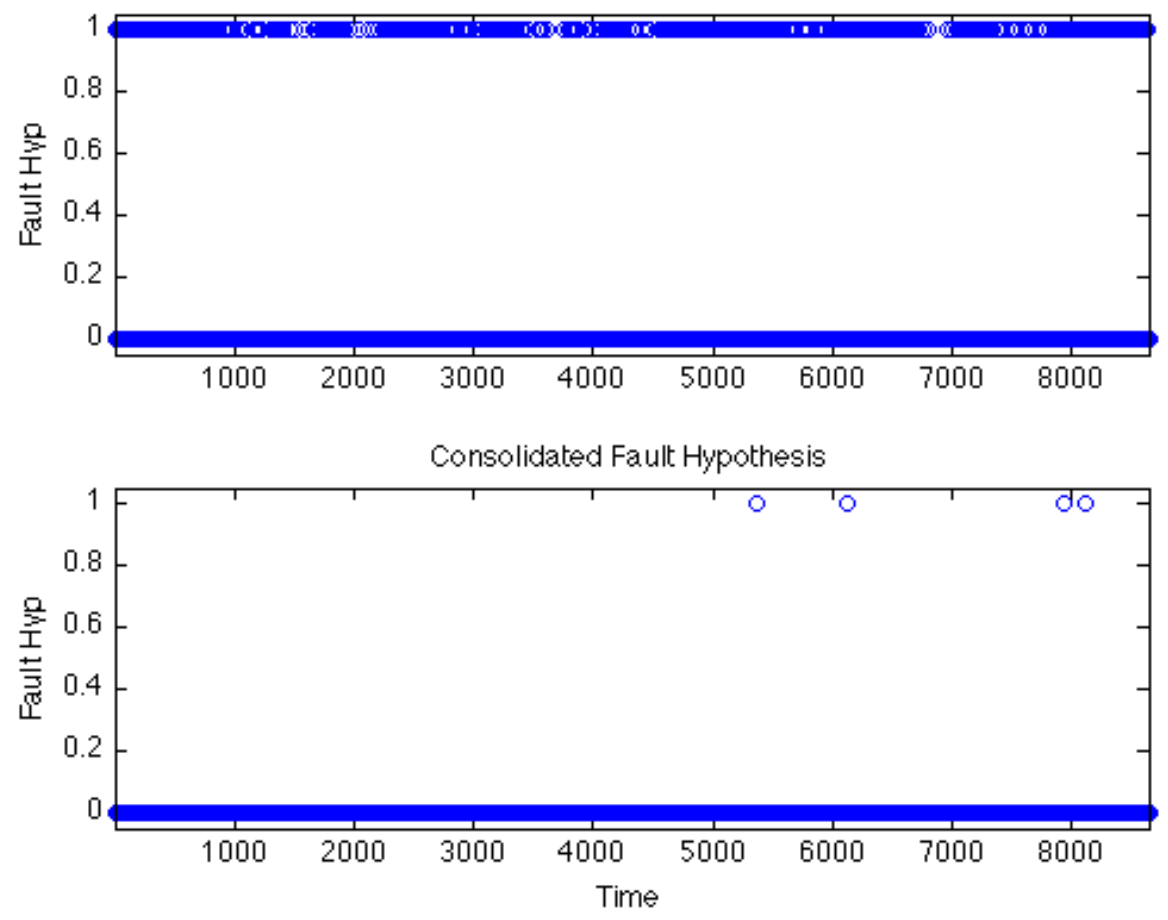
Fault Detection Results for Process Sensor Drift Month 5

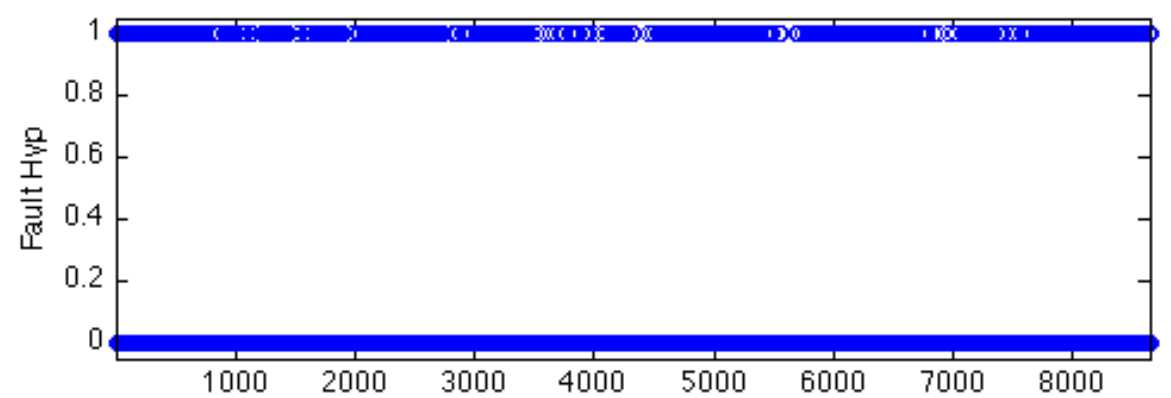

Consolidated Fault Hypothesis

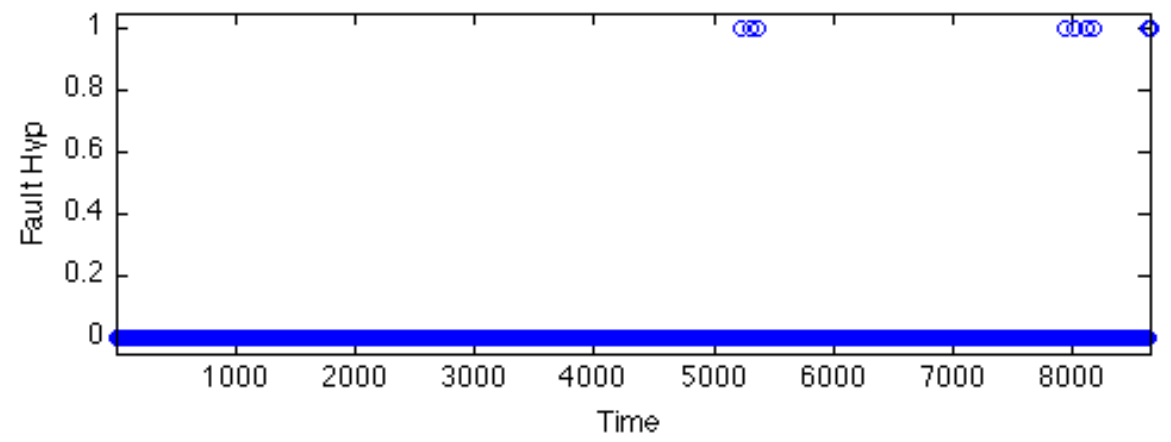

Fault Detection Results for Process Sensor Dritt Month 6
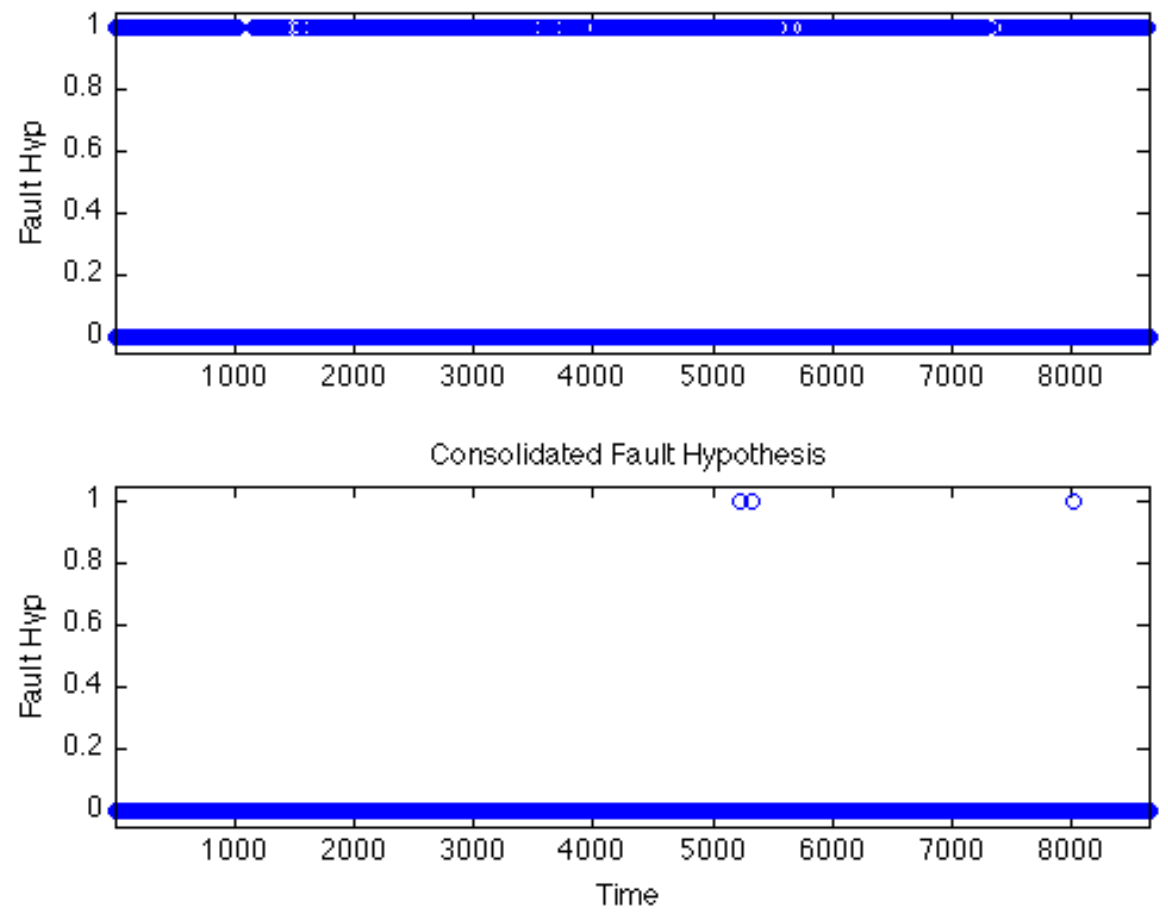
Fault Detection Results for Process Sensor Dritt Month 7

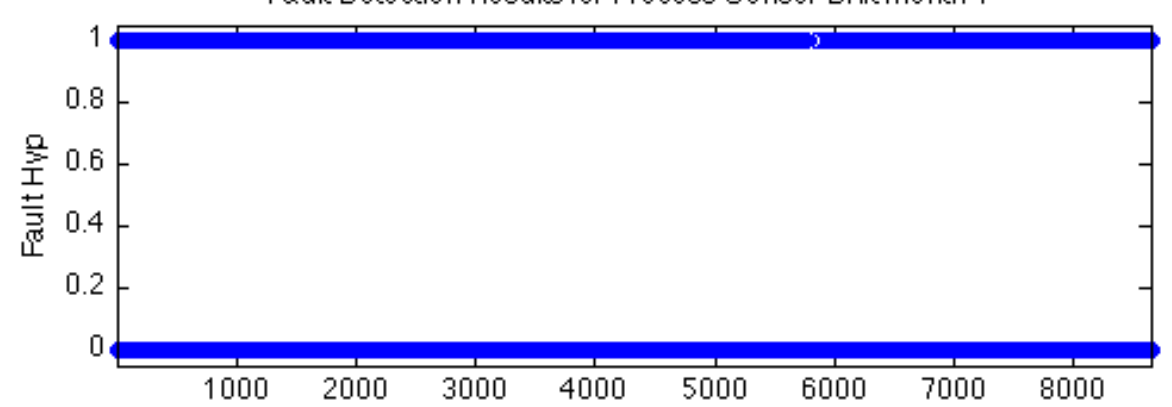

Consolidated Fault Hypothesis

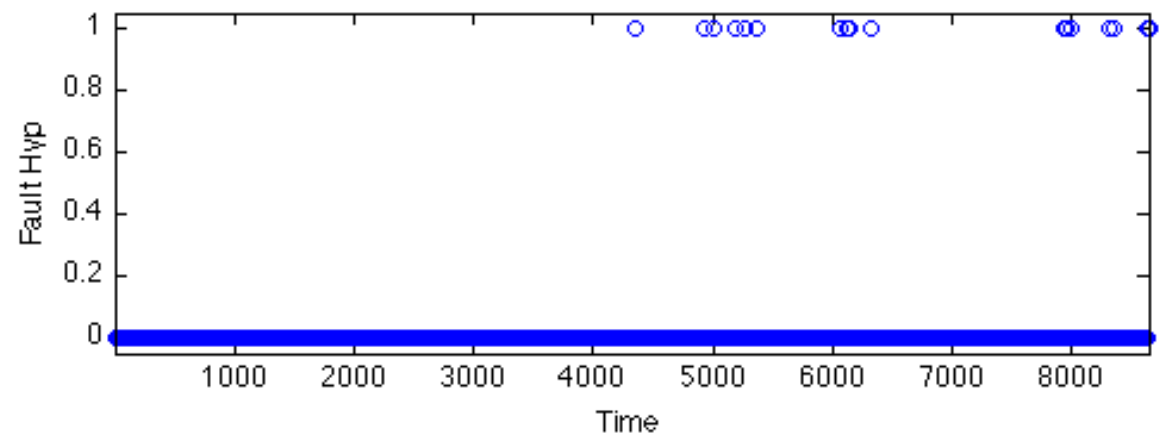

Fault Detection Results for Process Sensor Dritt Month 8
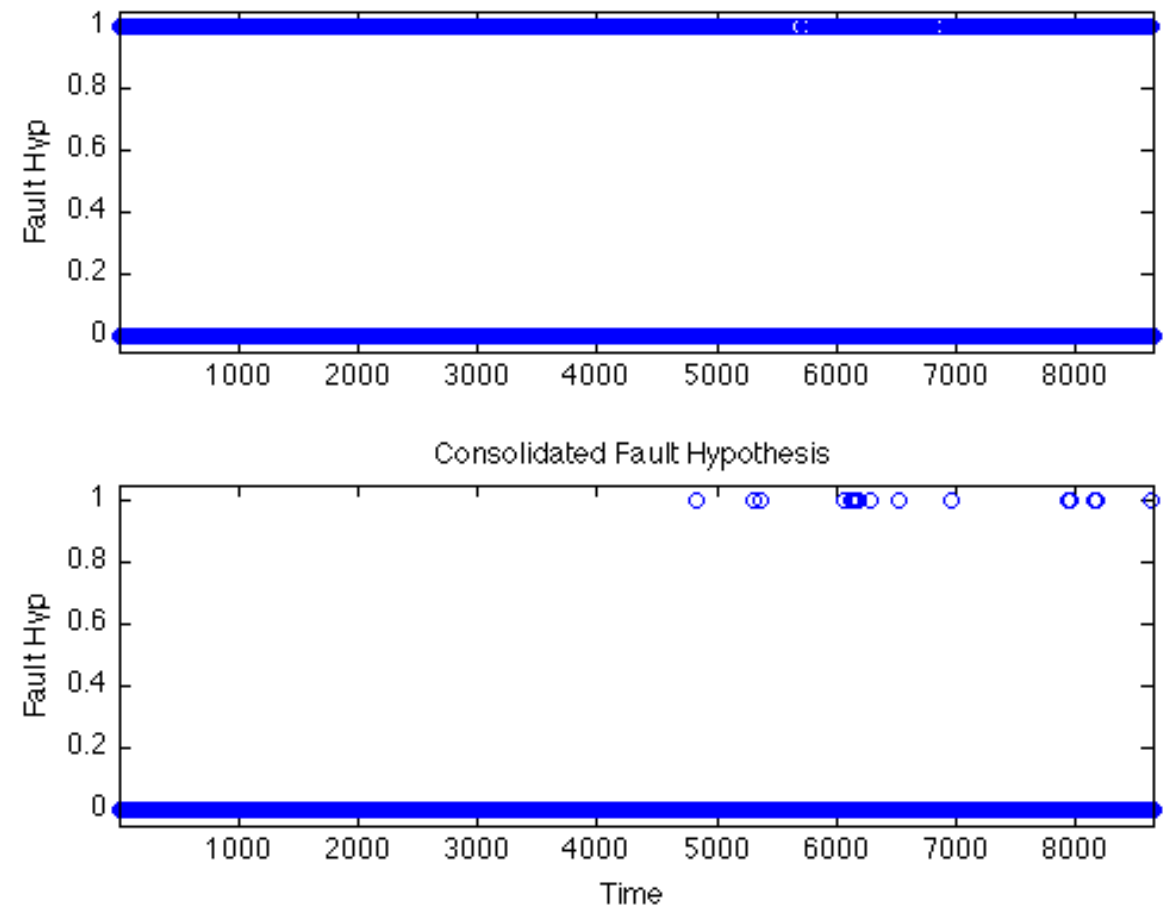
Fault Detection Results for Process Sensor Dritt Month 9

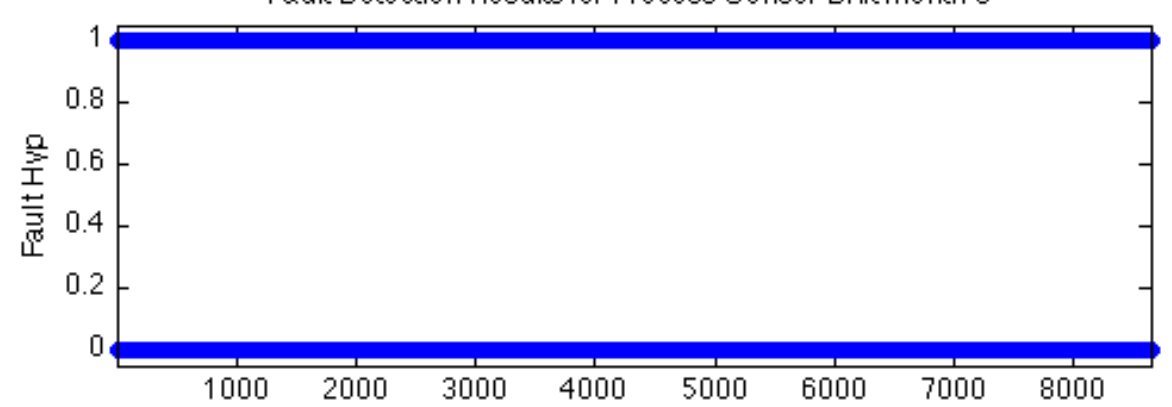

Consolidated Fault Hypothesis

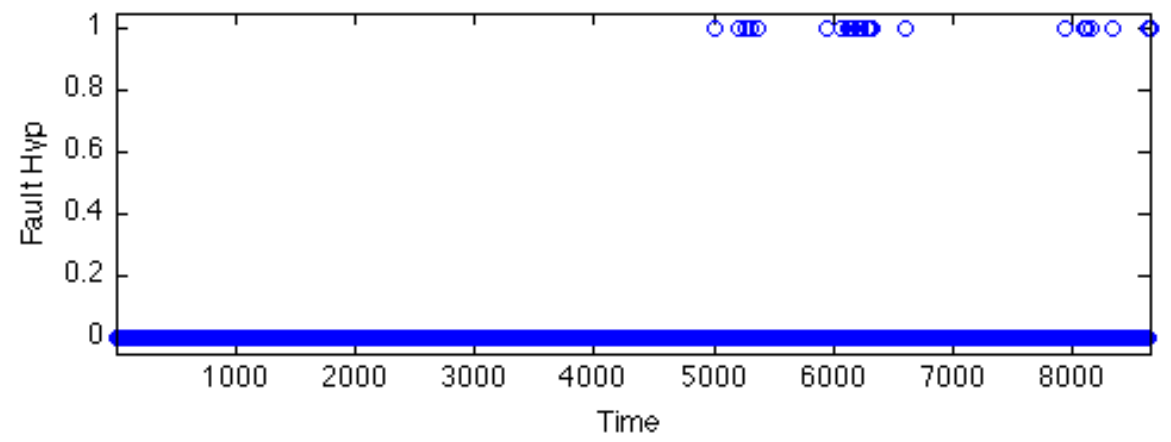

Fault Detection Results for Process Sensor Dritt Month 10
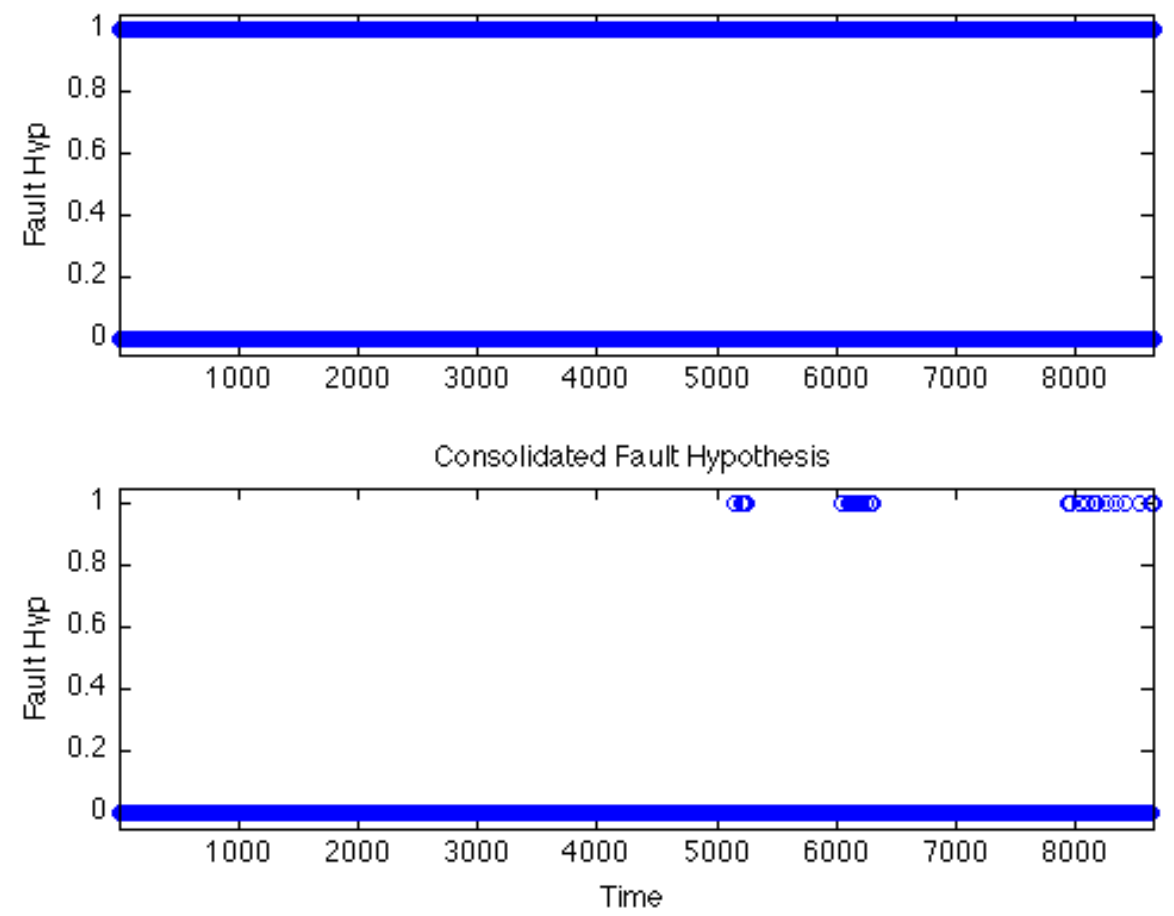
Fault Detection Results for Process Sensor Dritt Month 11

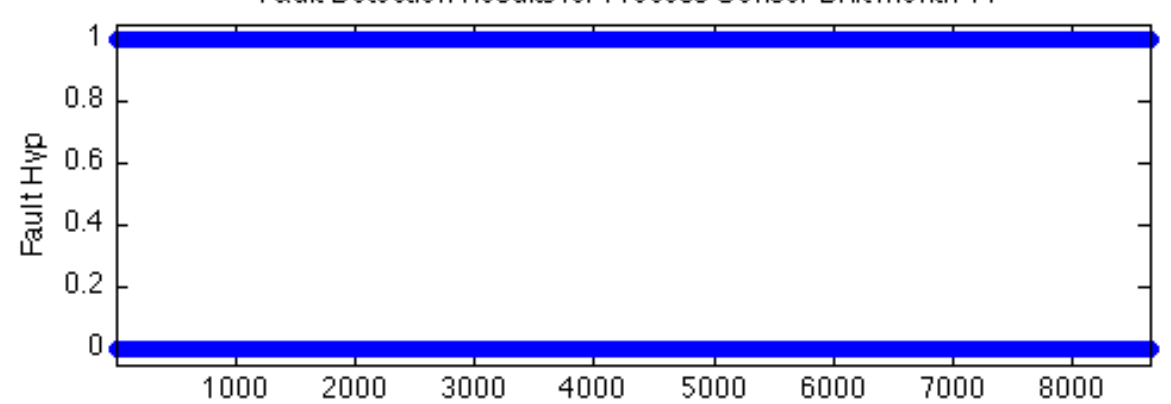

Consolidated Fault Hypothesis

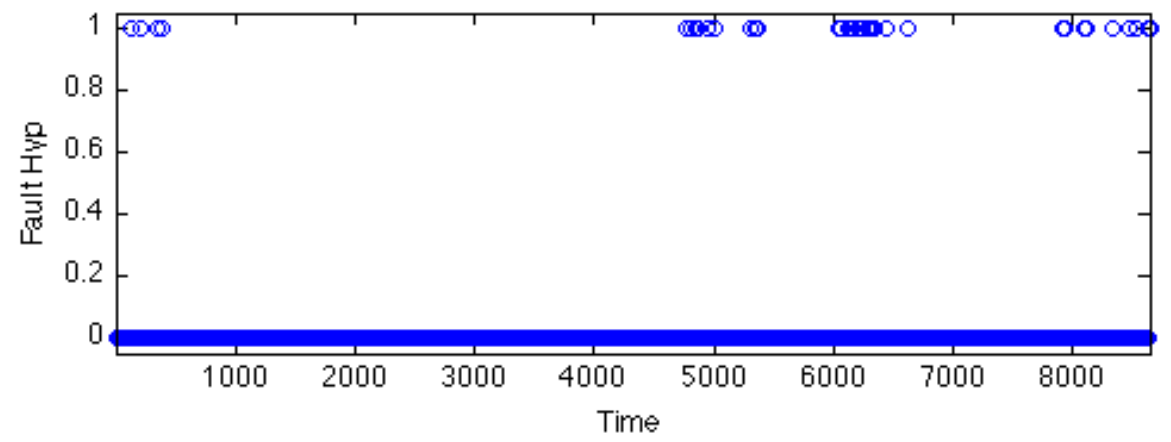

Fault Detection Results for Process Sensor Dritt Month 12

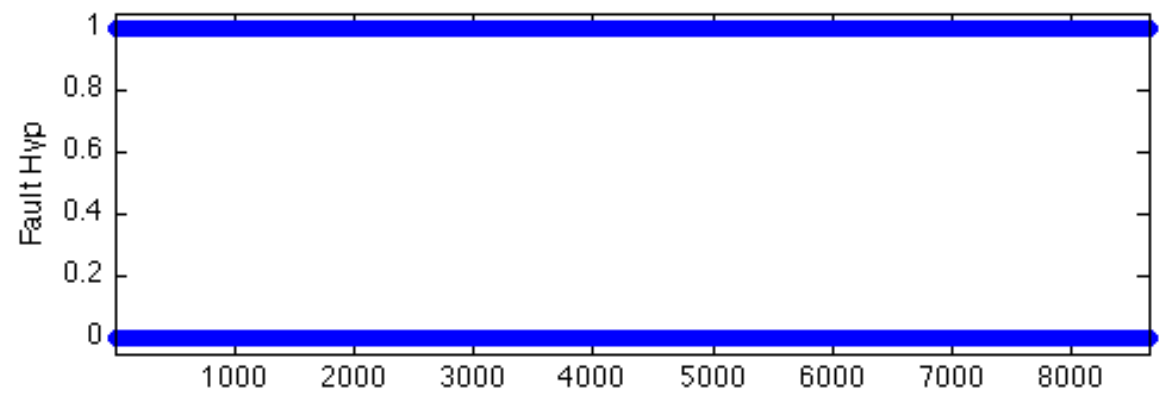

Consolidated Fault Hypothesis

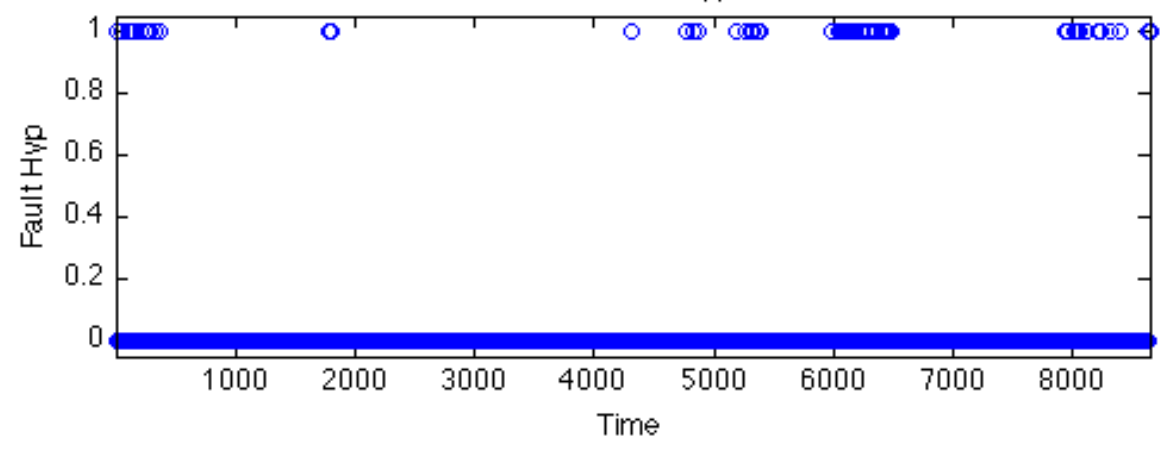


Fault Detection Results for Process Sensor Drift Month 1 Redundant Model

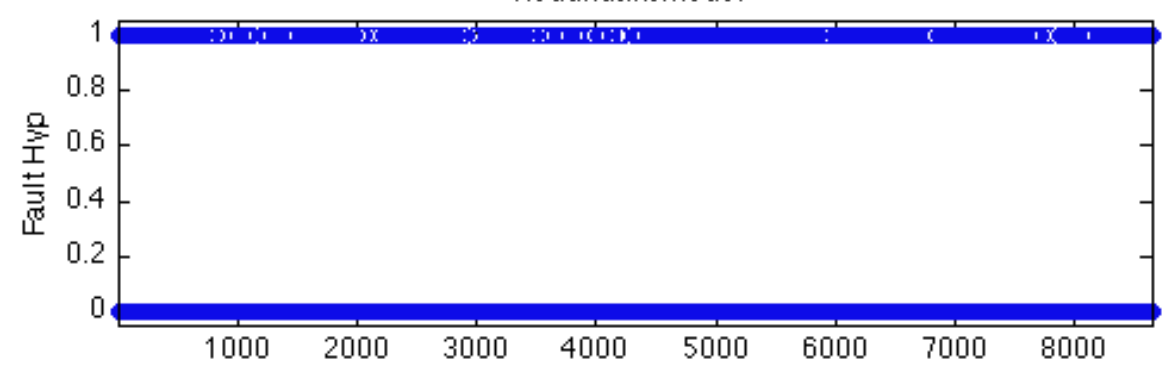

Consolidated Fault Hypothesis

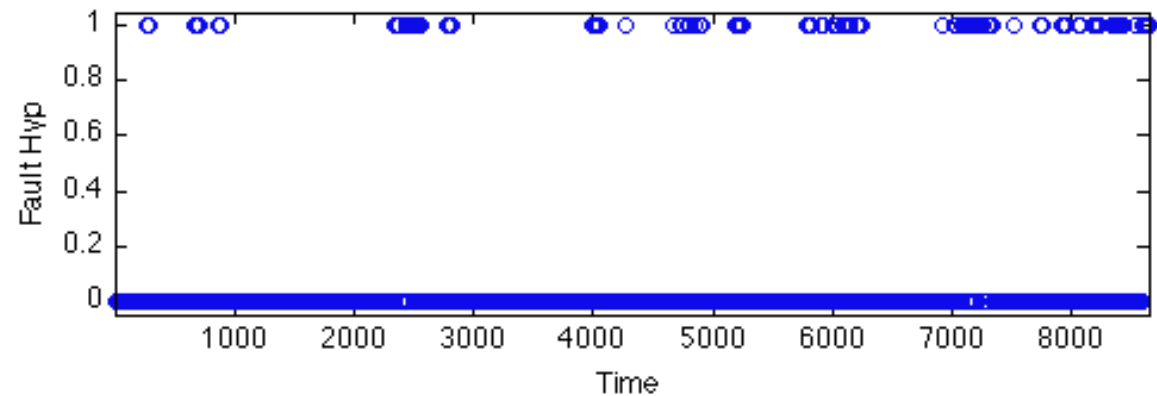

Fault Detection Results for Process Sensor Drift Month 2 Redundant Model

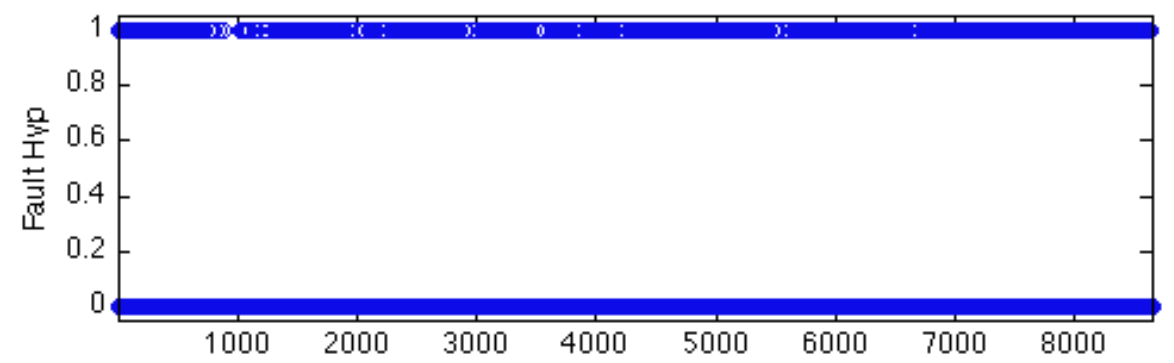

Consolidated Fault Hypothesis

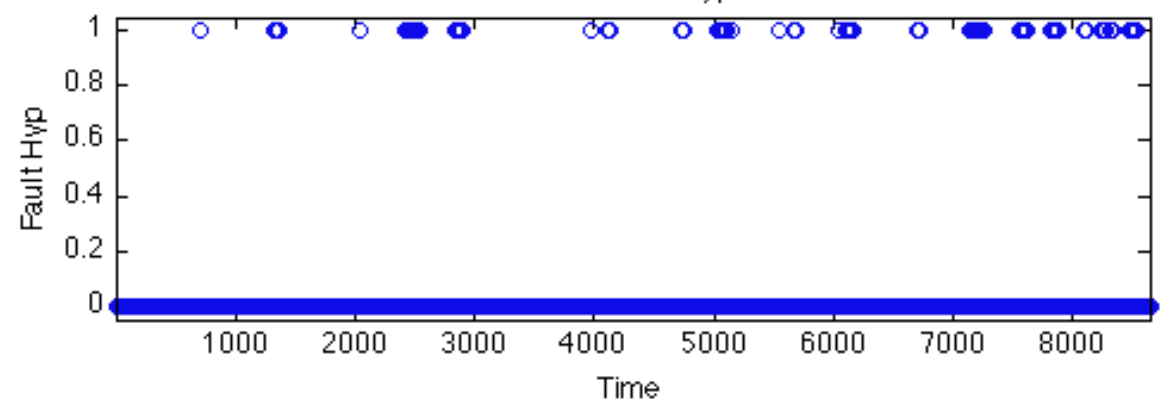


Fault Detection Results for Process Sensor Drift Month 3 Redundant Model

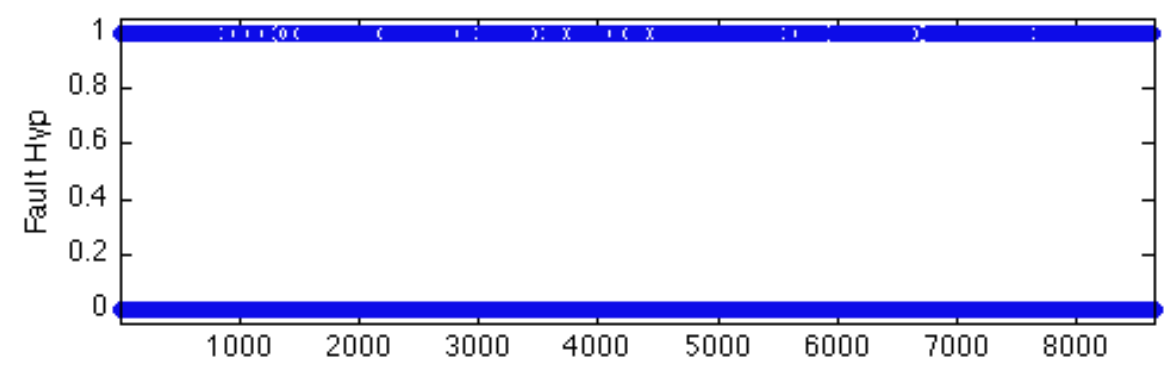

Consolidated Fault Hypothesis

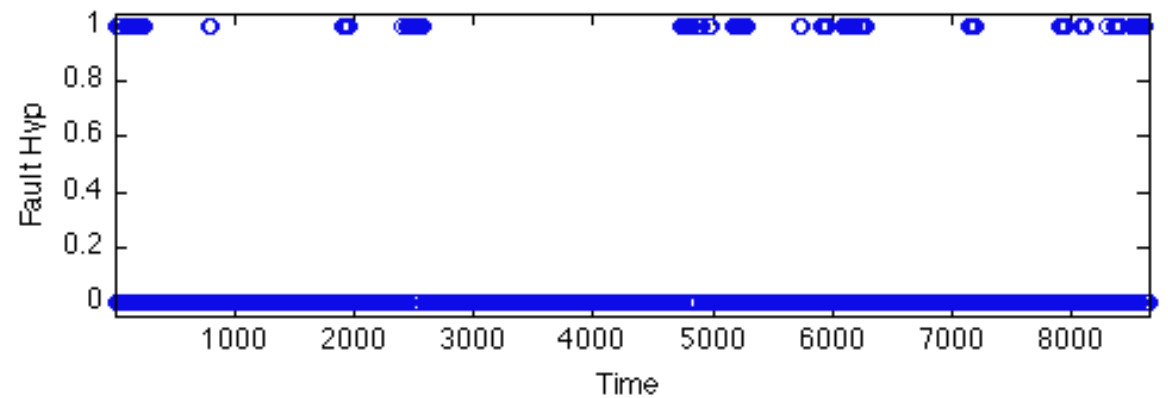

Fault Detection Results for Process Sensor Dritt Month 4 Redundant Model

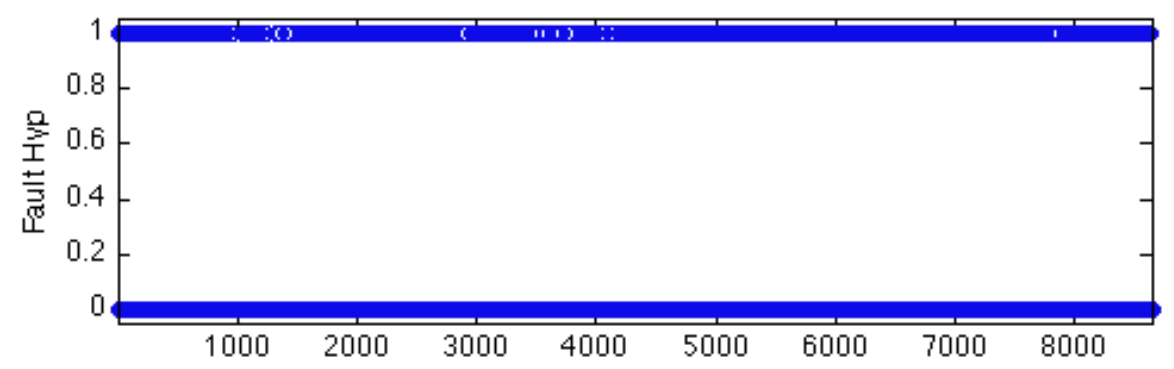

Consolidated Fault Hypothesis

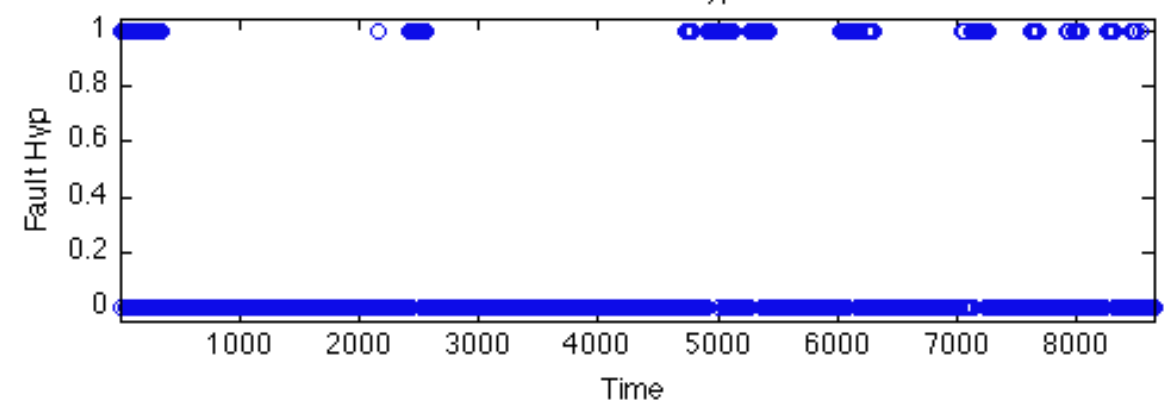


Fault Detection Results for Process Sensor Dritt Month 5 Redundant Model

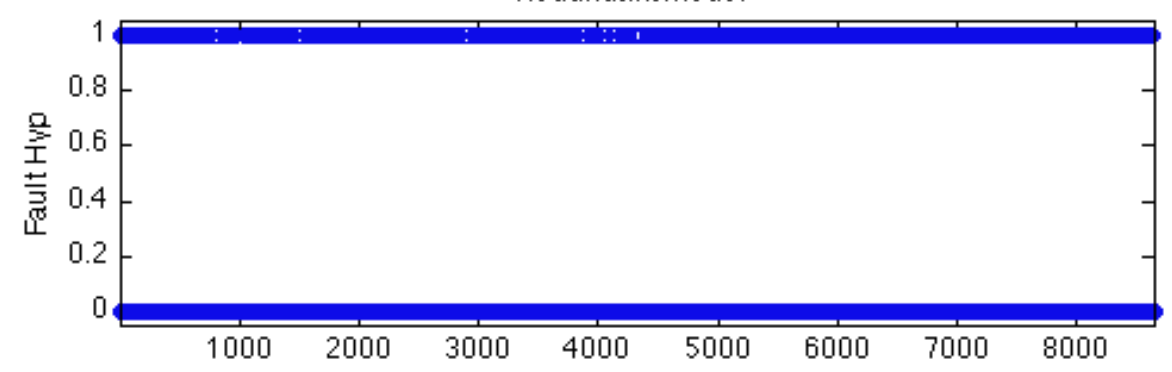

Consolidated Fault Hypothesis

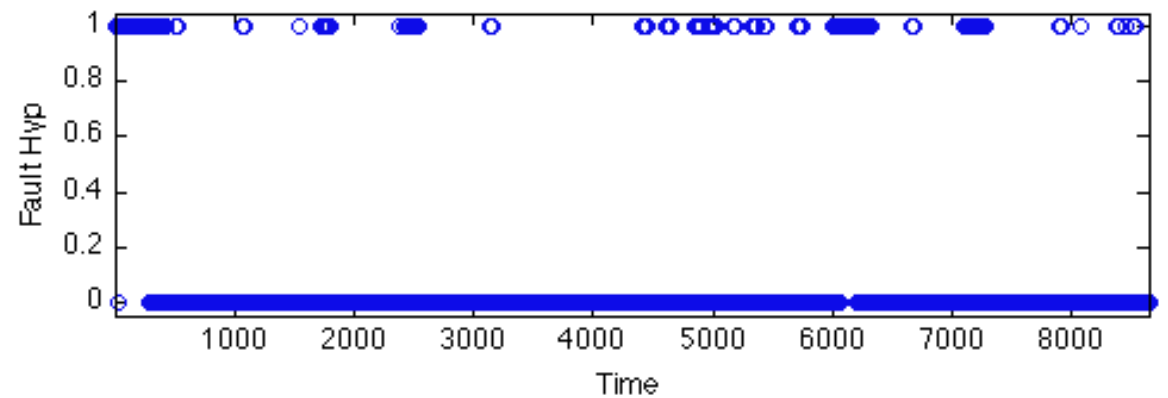

Fault Detection Results for Process Sensor DriftMonth 6 Redundant Model

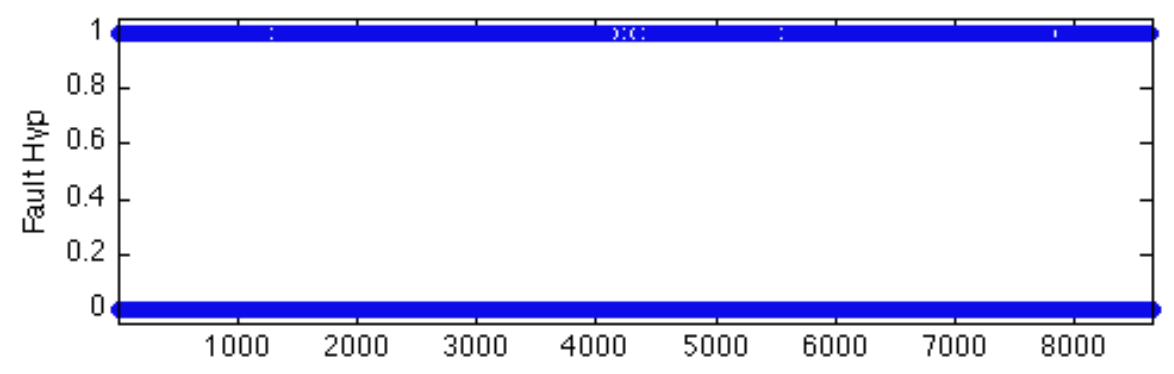

Consolidated Fault Hypothesis

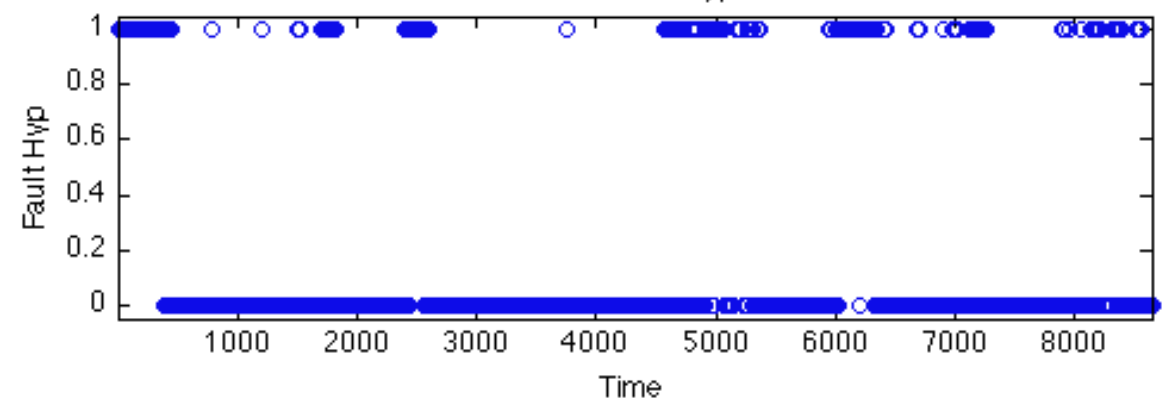


Fault Detection Results for Process Sensor Dritt Month 7

Redundant Model

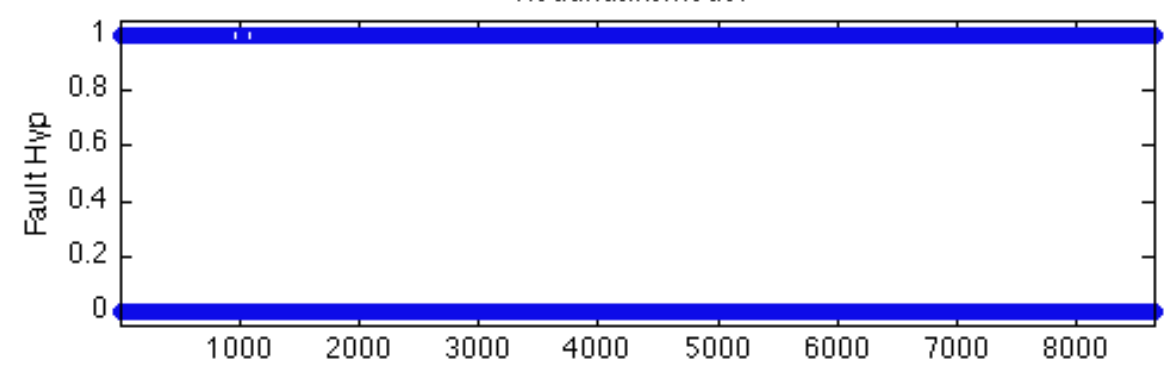

Consolidated Fault Hypothesis

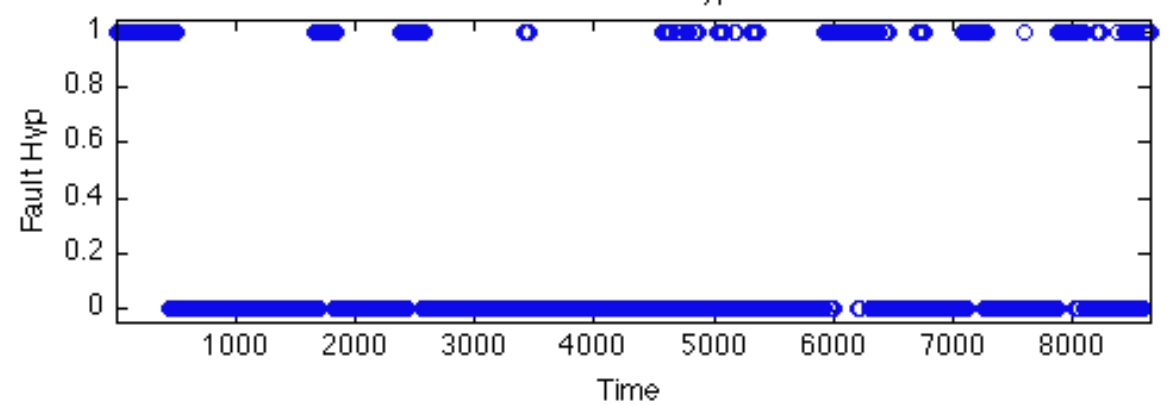

Fault Detection Results for Process Sensor DriftMonth 8 Redundant Model

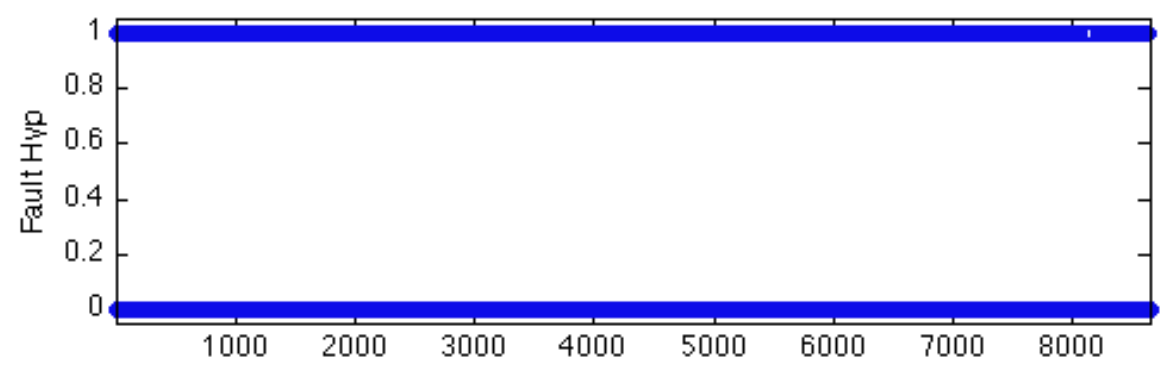

Consolidated Fault Hypothesis

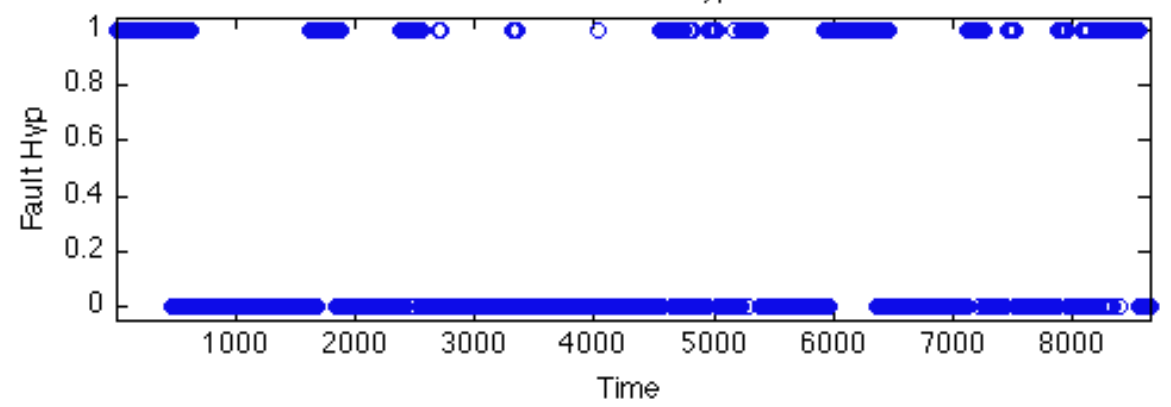


Fault Detection Results for Process Sensor Drift Month 9

Redundant Model

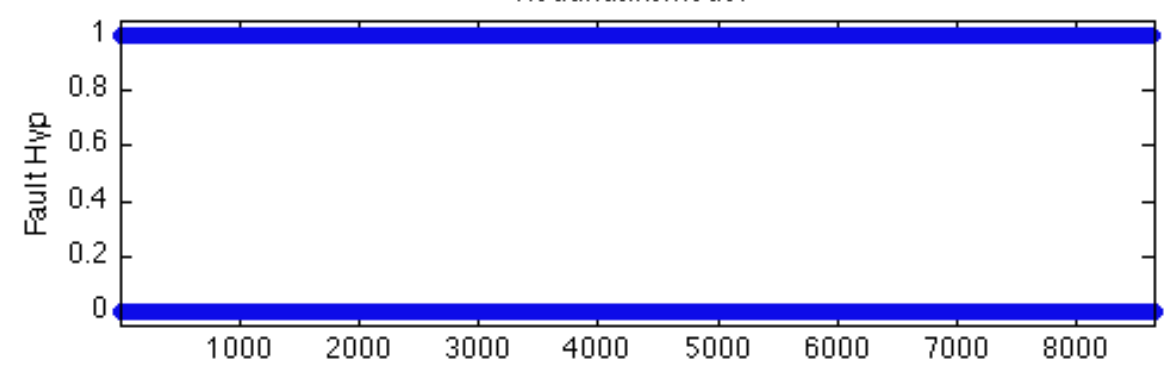

Consolidated Fault Hypothesis

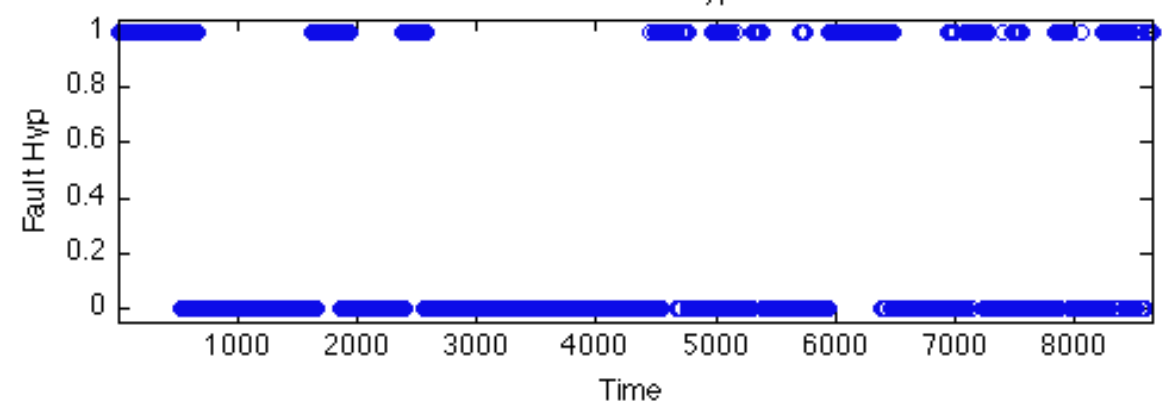

Fault Detection Results for Process Sensor Dritt Month 10 Redundant Model

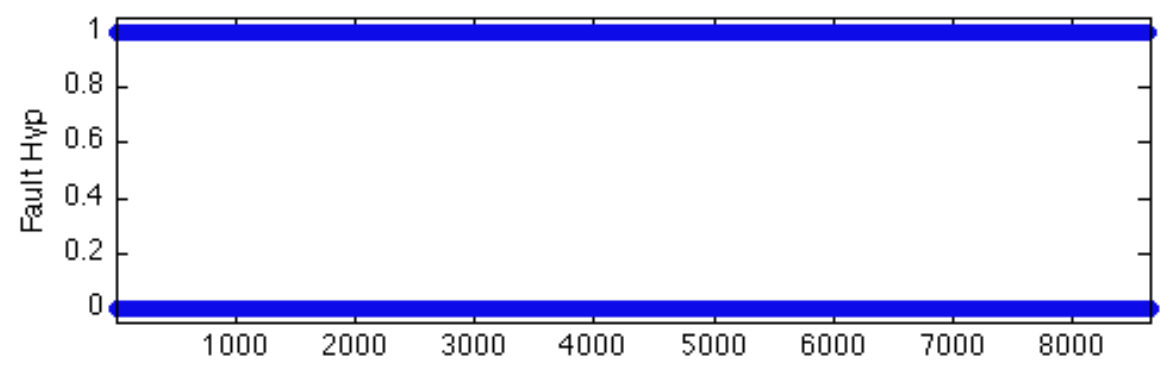

Consolidated Fault Hypothesis

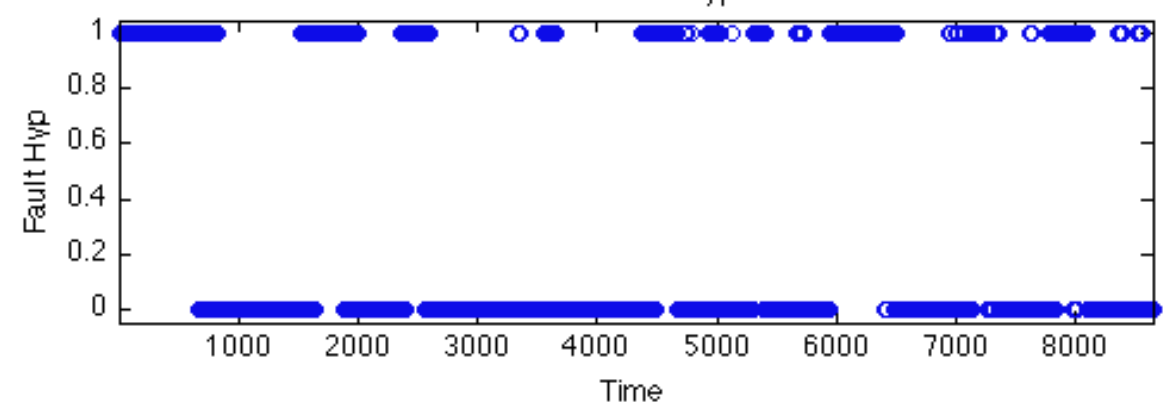


Fault Detection Results for Process Sensor Dritt Month 11 Redundant Model

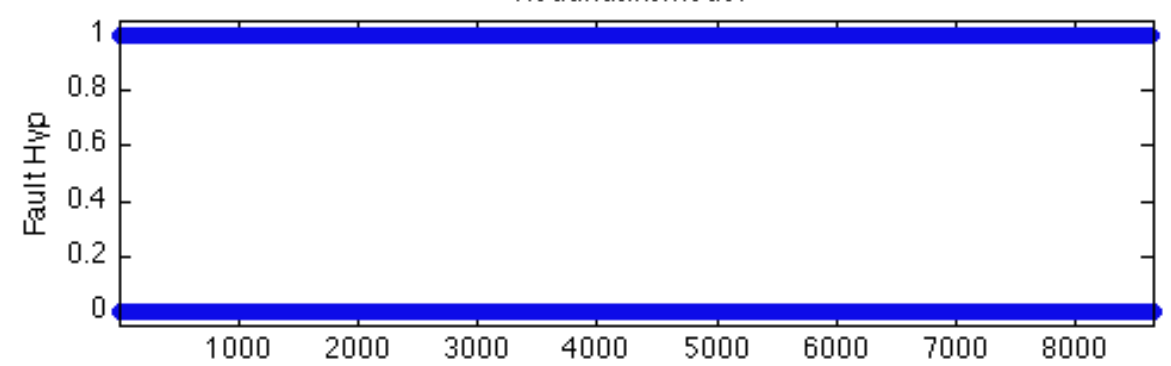

Consolidated Fault Hypothesis

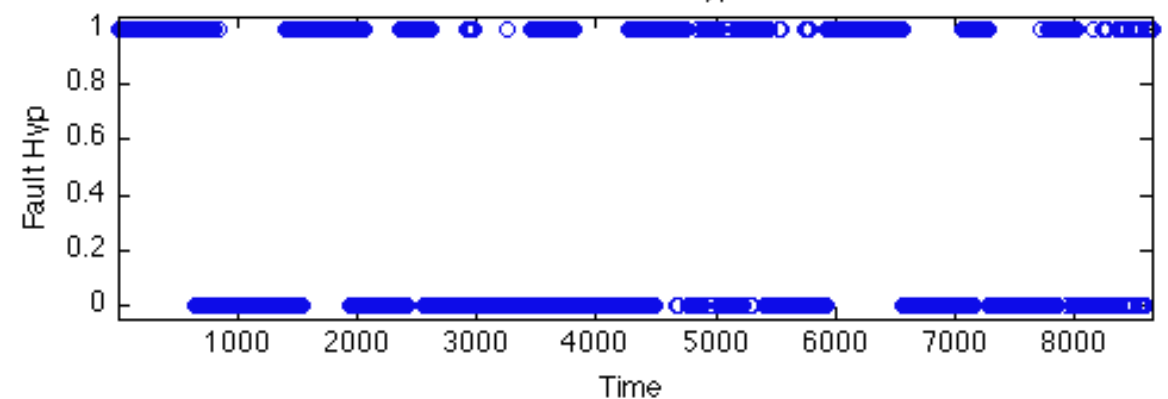

Fault Detection Results for Process Sensor Dritt Month 12 Redundant Model

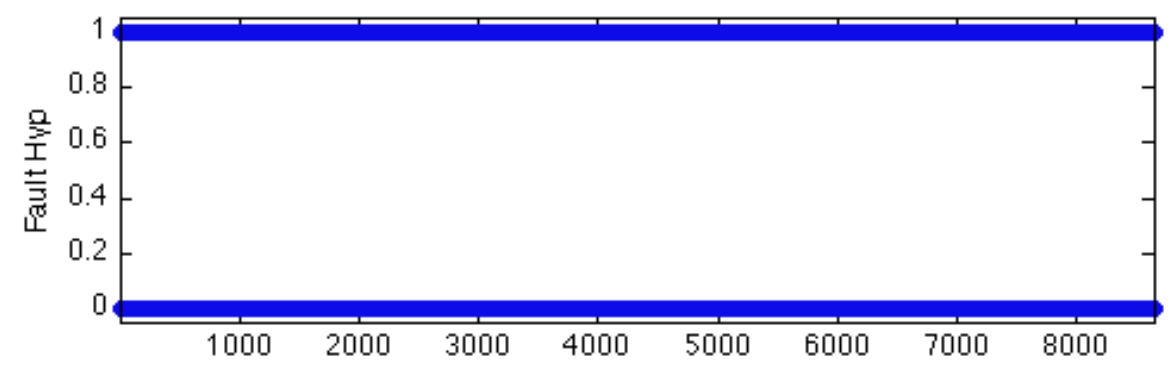

Consolidated Fault Hypothesis

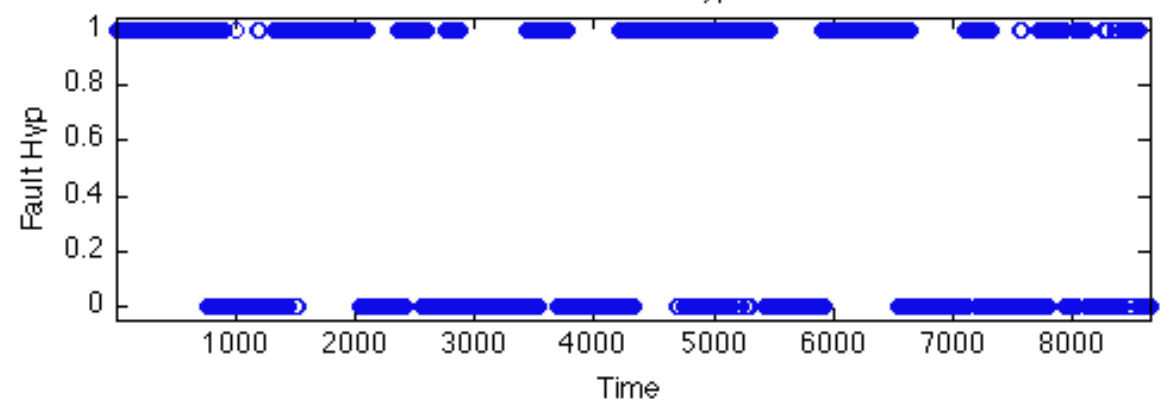




\section{A.2.4 Control Sensor Drift}

Fault Detection Results for Control Sensor Dritt Month 1
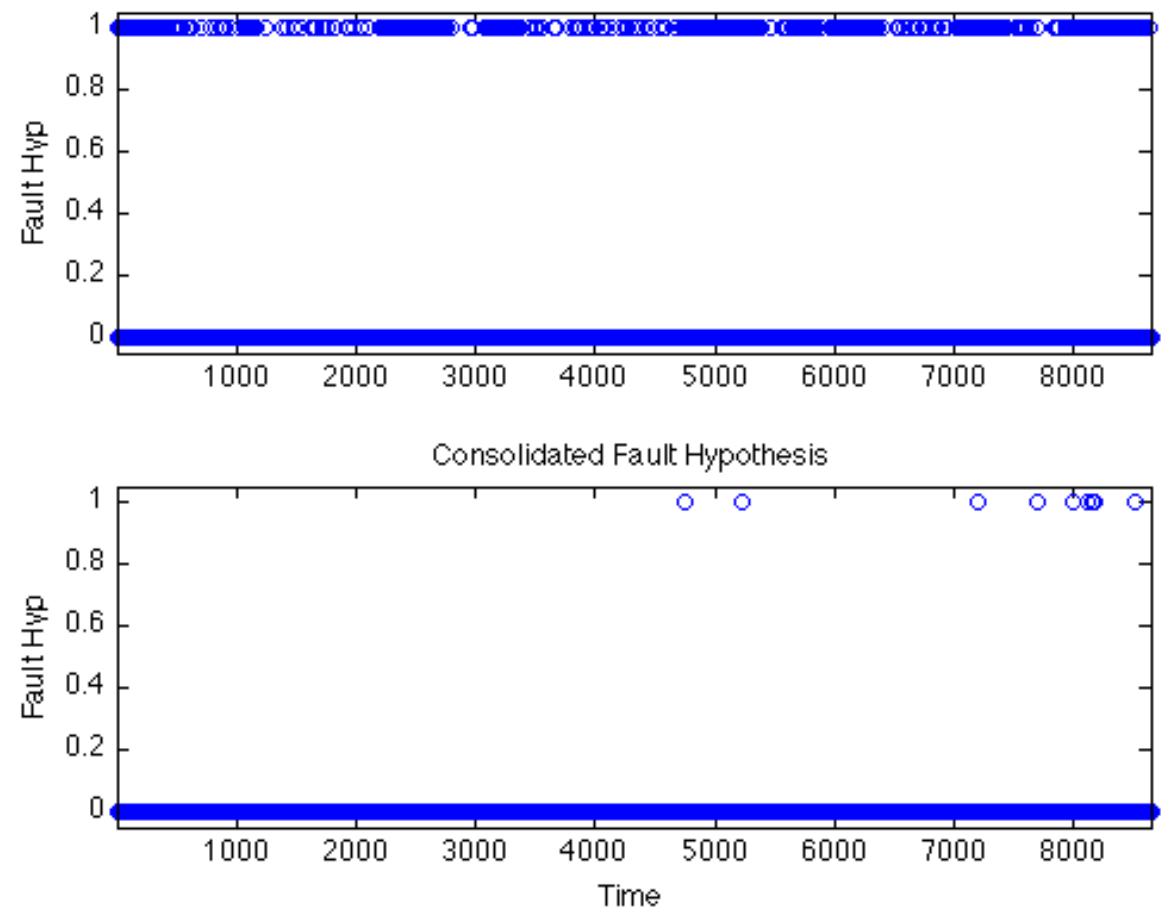

Fault Detection Results for Control Sensor Dritt Month 2
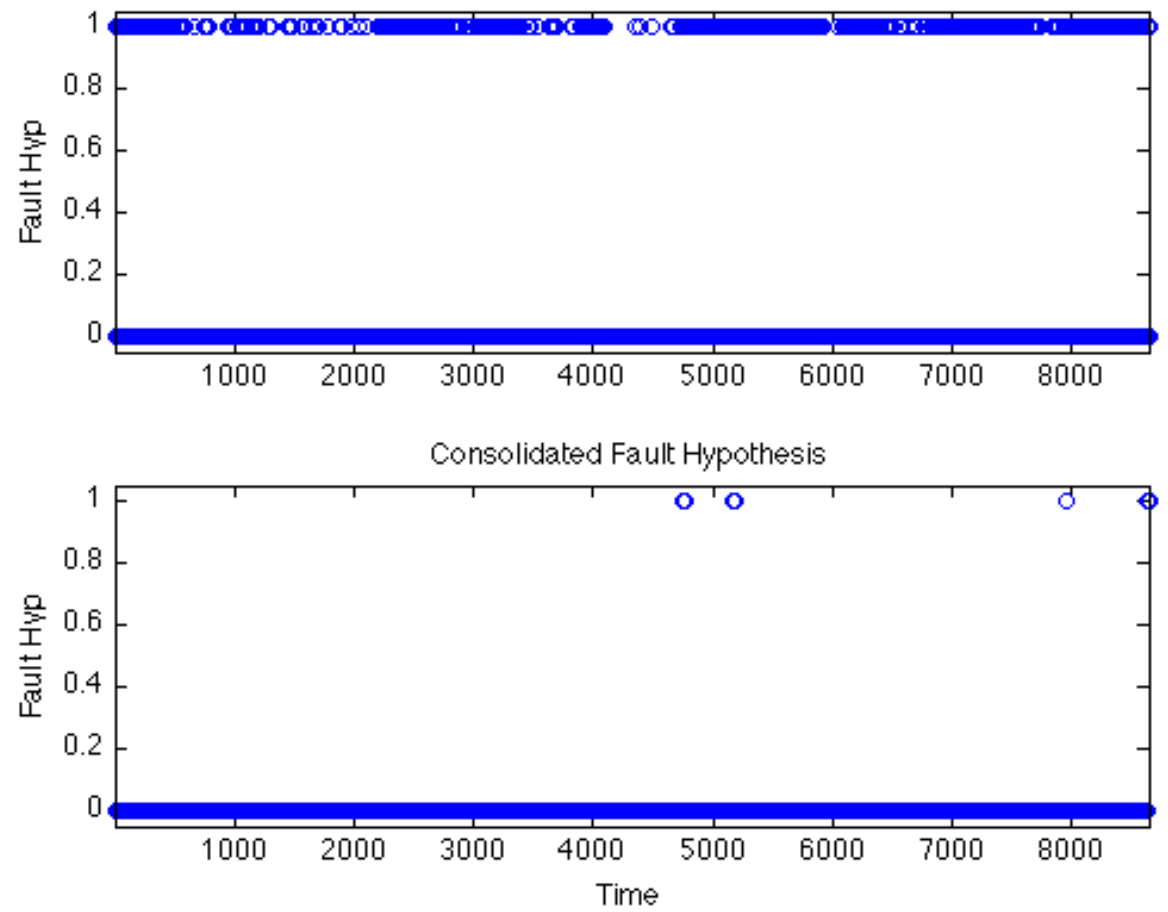
Fault Detection Results for Control Sensor Drift Month 3

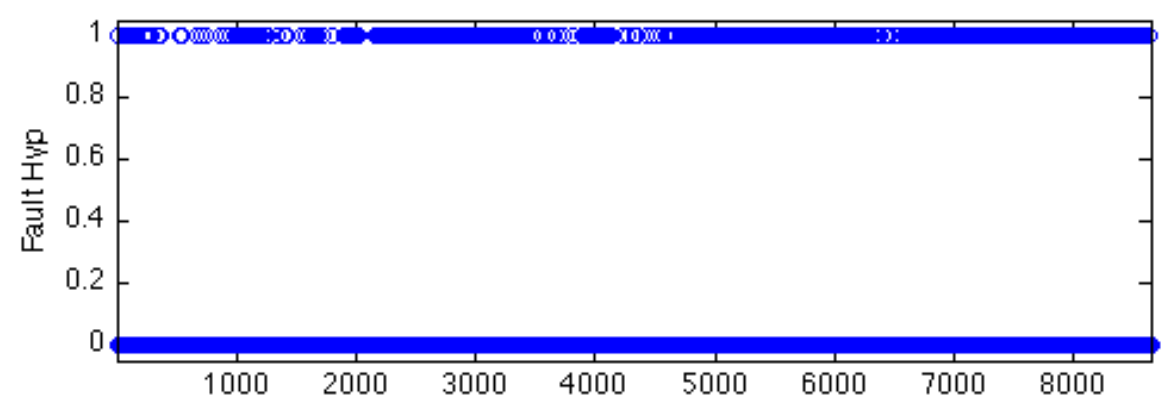

Consolidated Fault Hypothesis

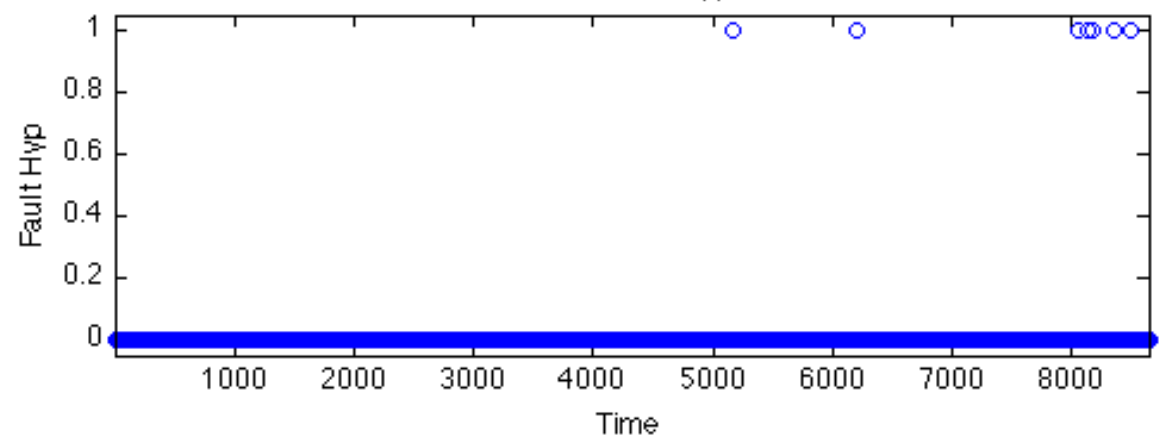

Fault Detection Results for Control Sensor Dritt Month 4
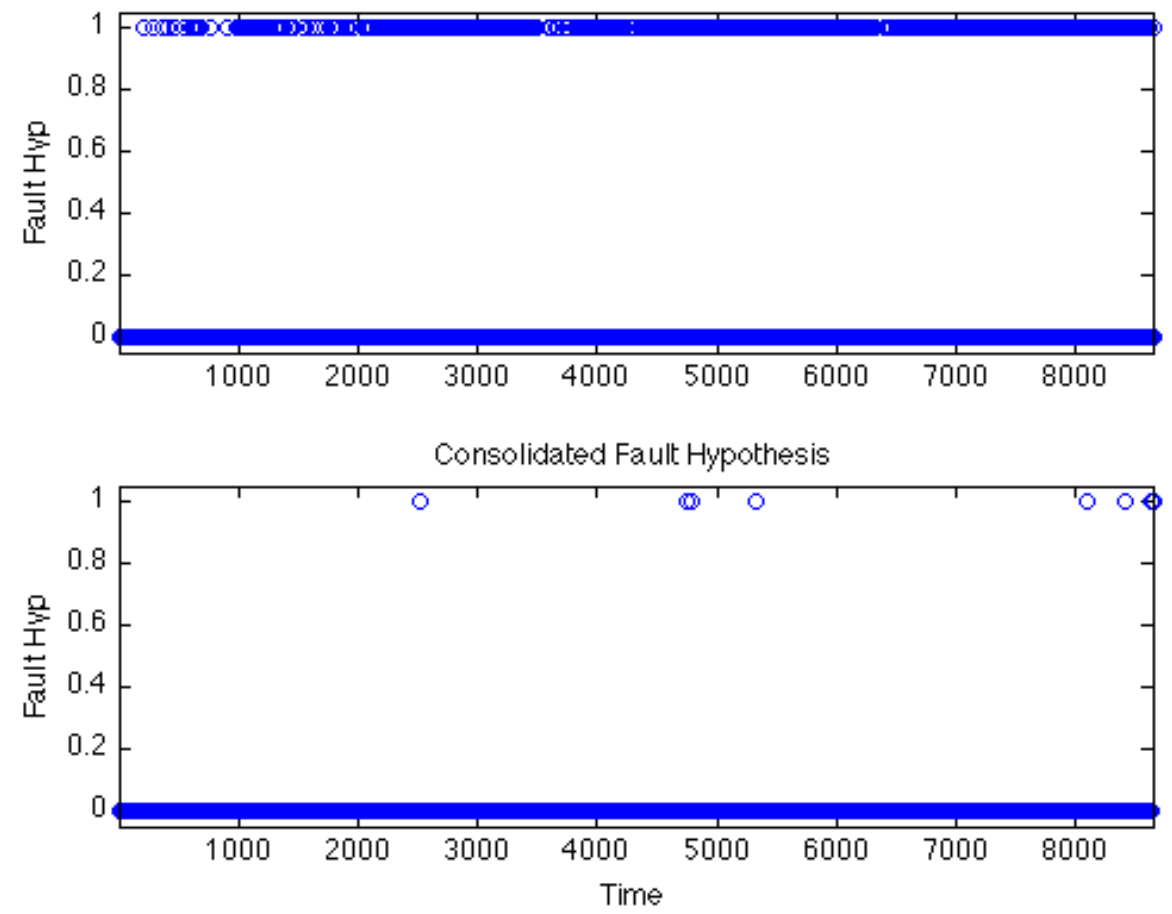
Fault Detection Results for Control Sensor Drit Month 5
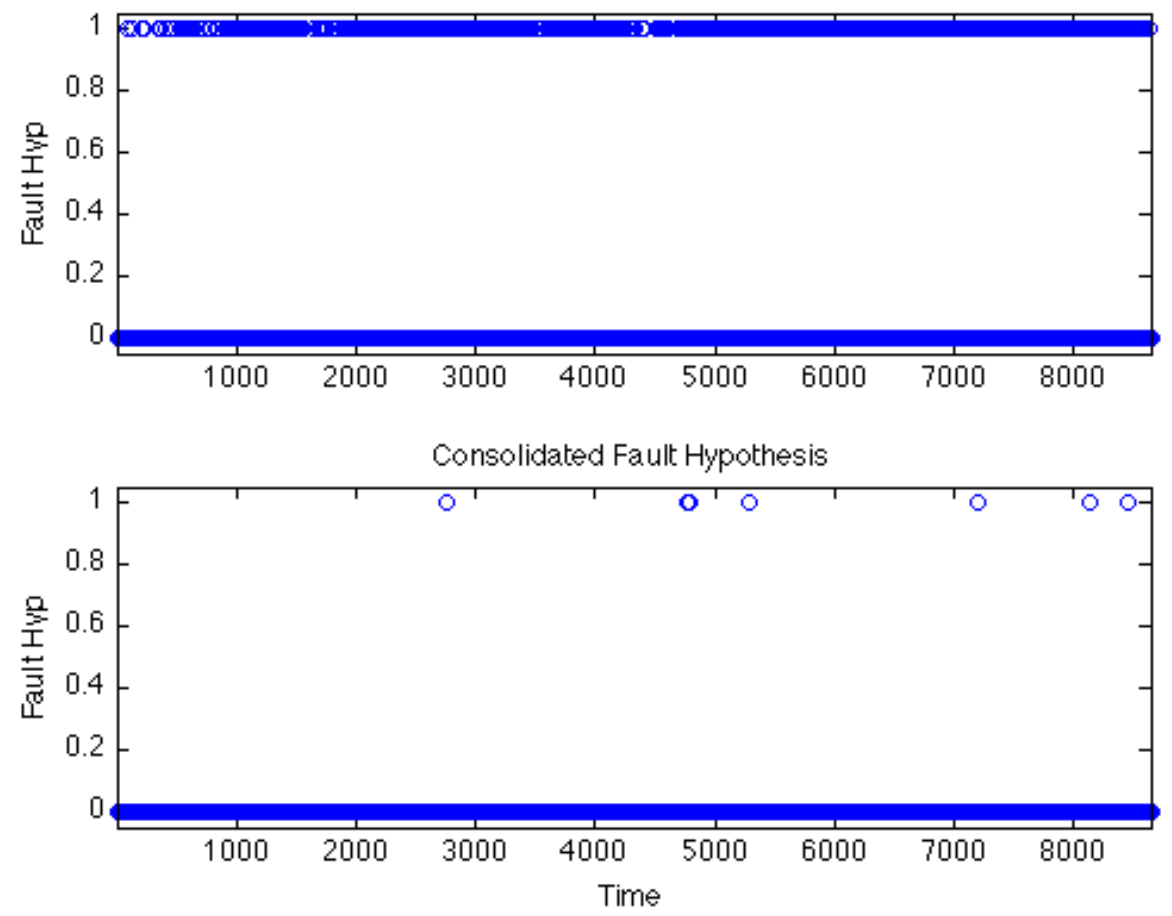

Fault Detection Results for Control Sensor Drit Month 6

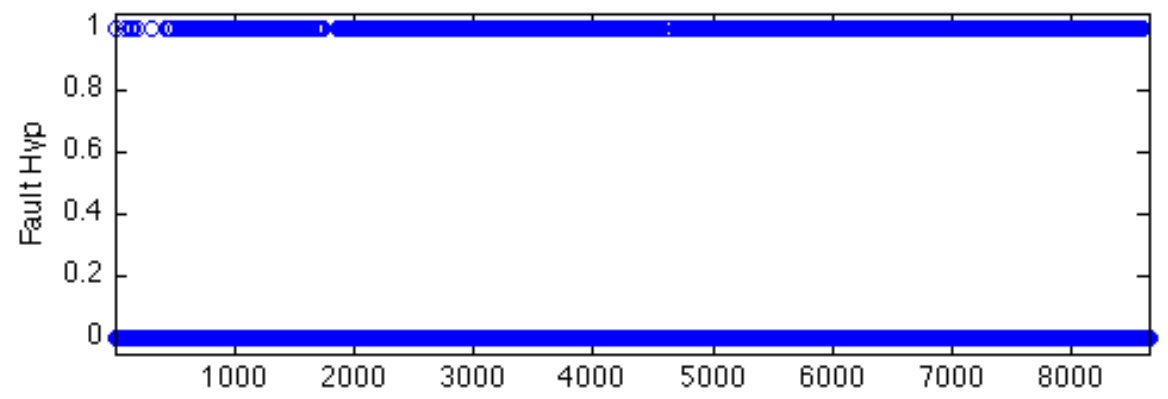

Consolidated Fault Hypothesis

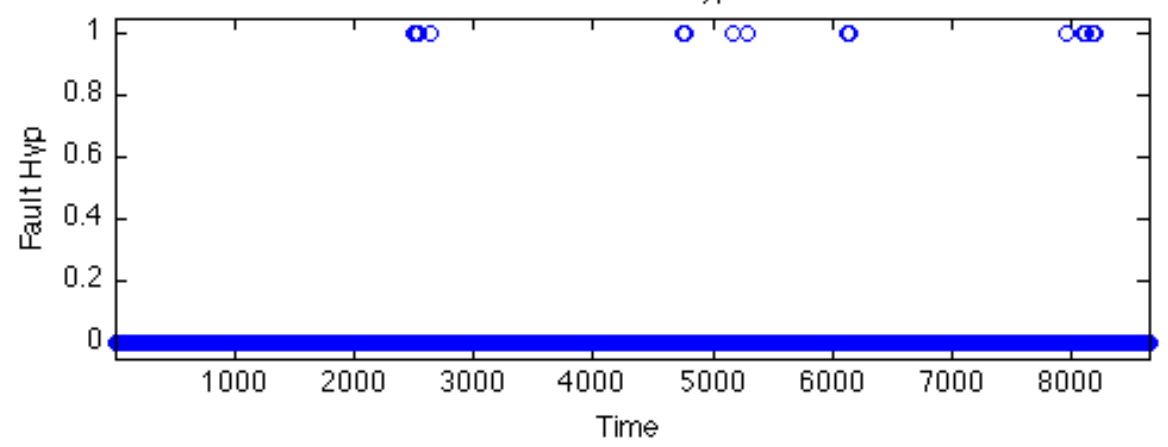


Fault Detection Results for Control Sensor Drift Month 7

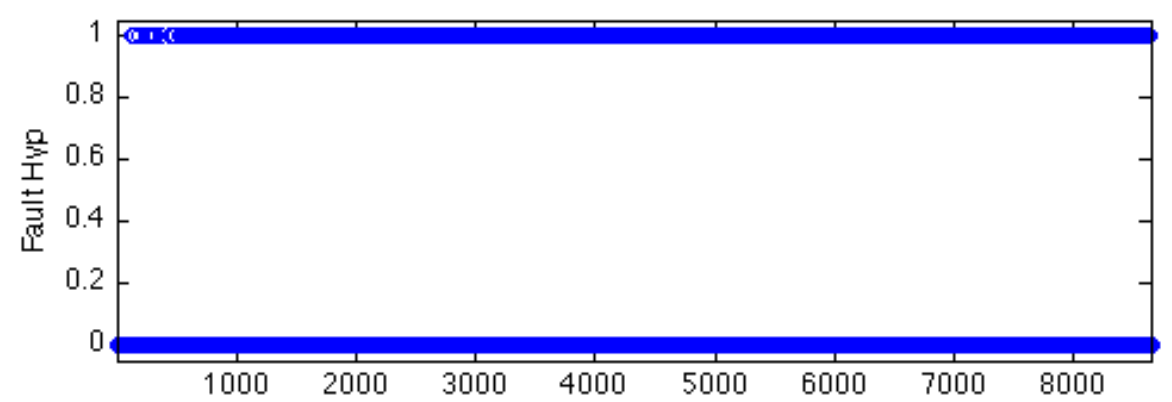

Consolidated Fault Hypothesis

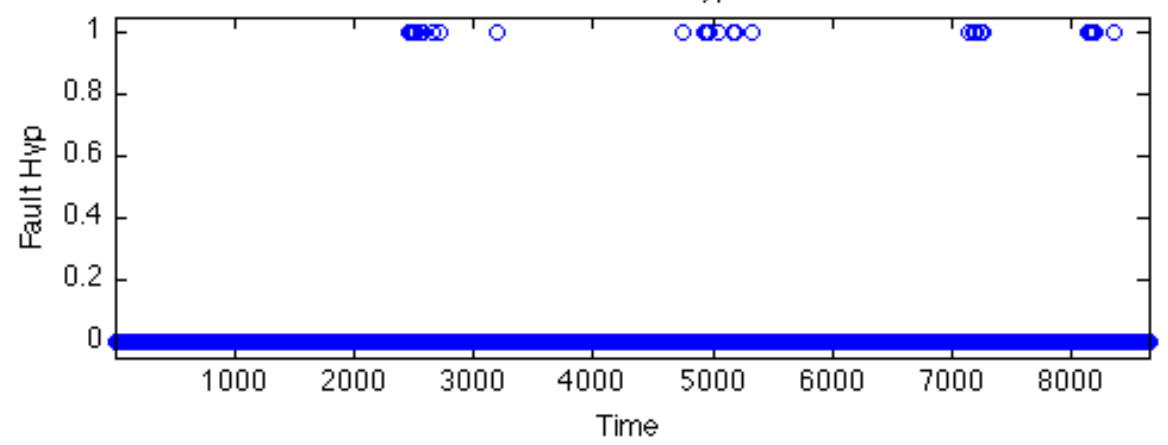

Fault Detection Results for Control Sensor Dritt Month 8

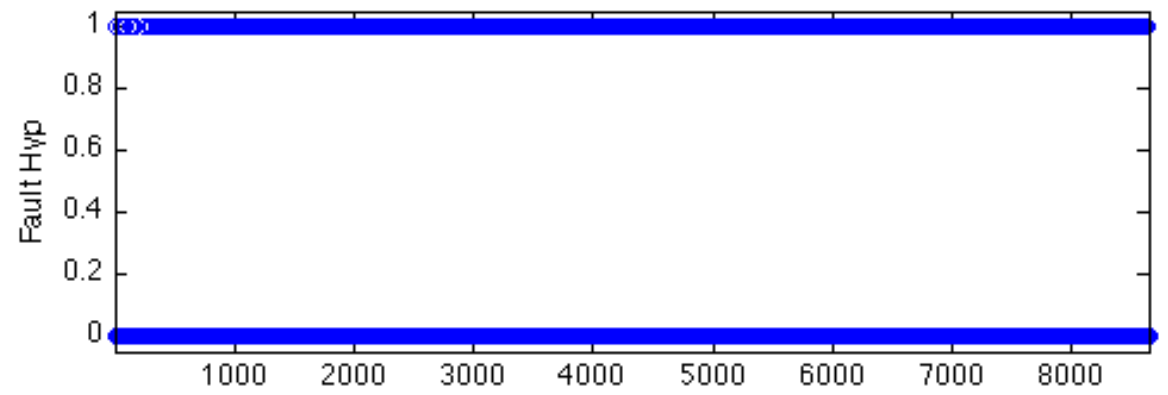

Consolidated Fault Hypothesis

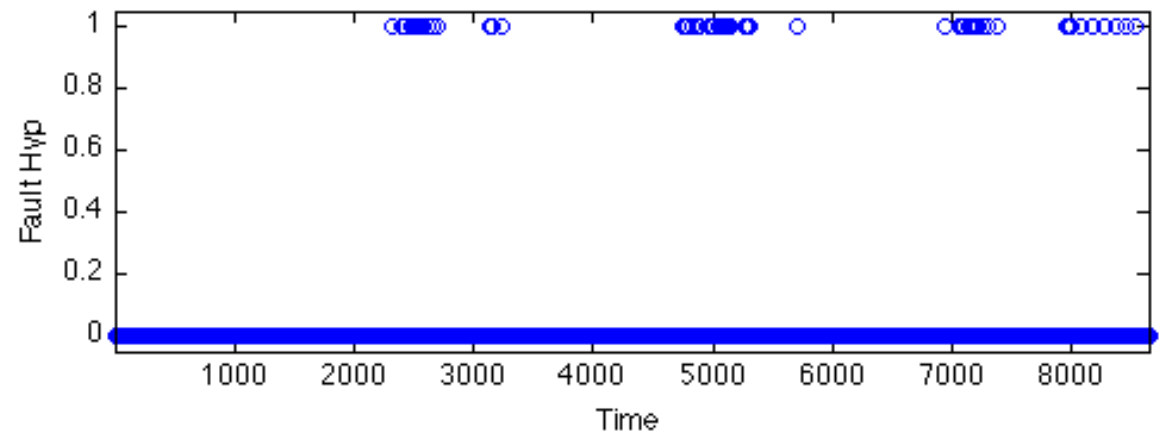


Fault Detection Results for Control Sensor Drift Month 9

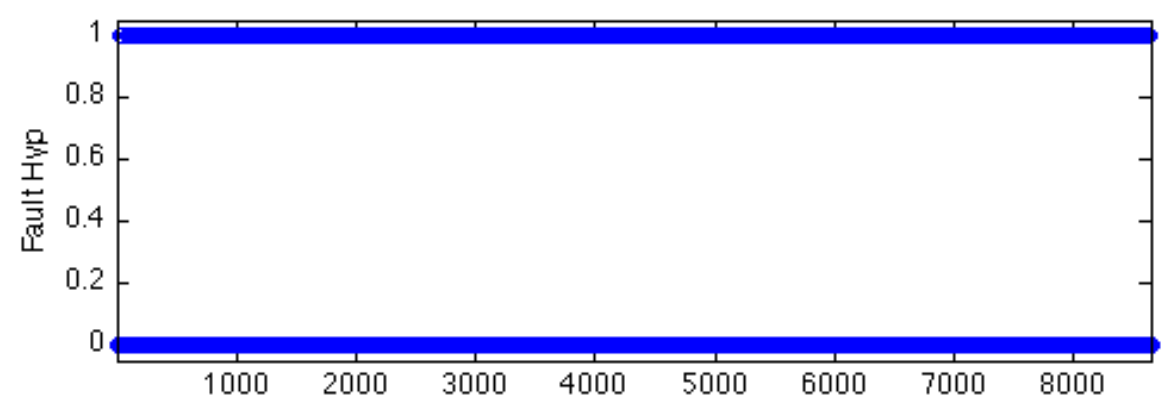

Consolidated Fault Hypothesis

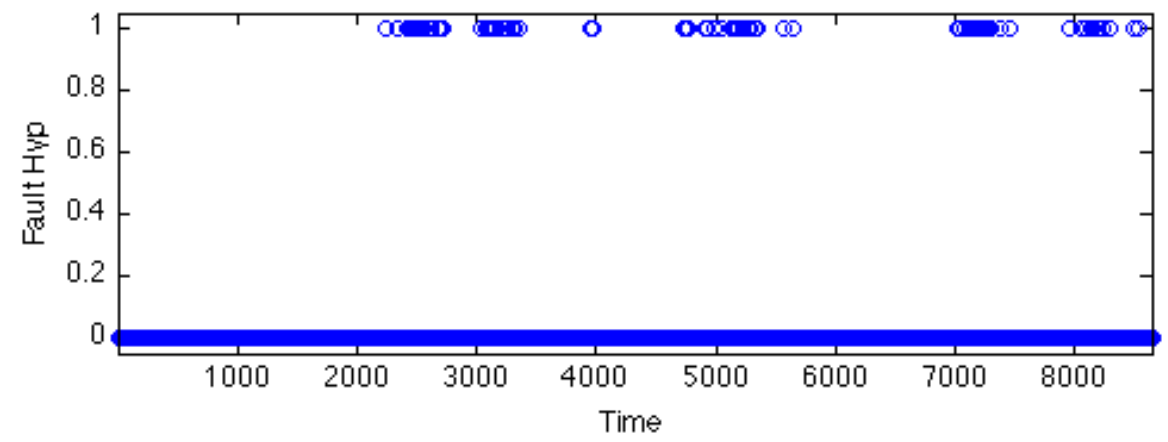

Fault Detection Results for Control Sensor Drit Month 10

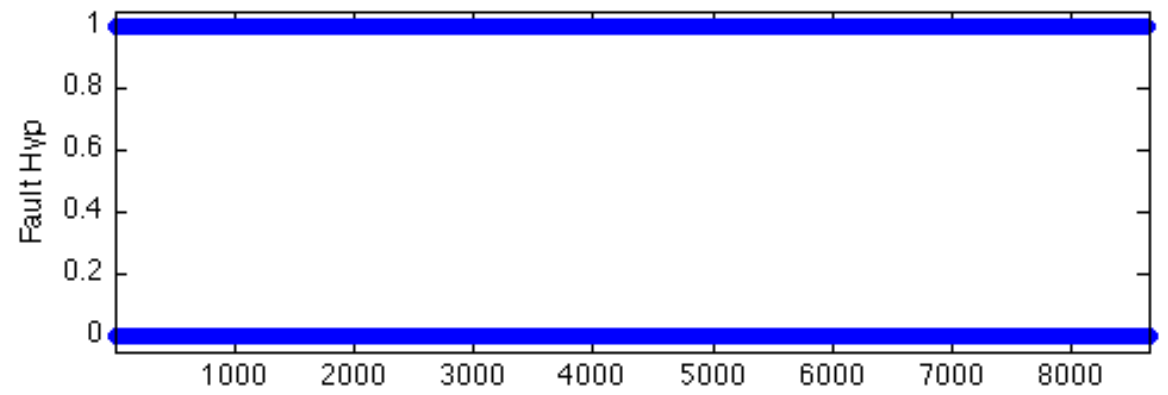

Consolidated Fault Hypothesis

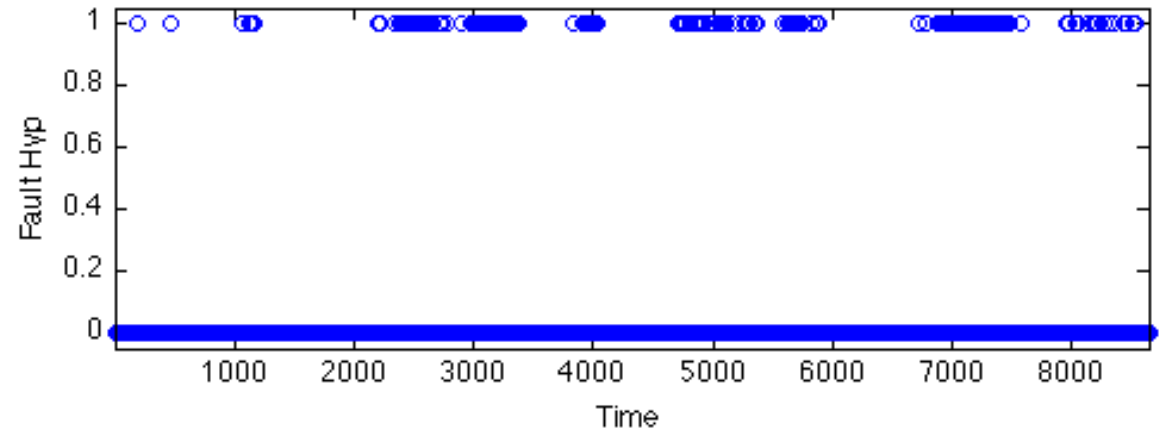


Fault Detection Results for Control Sensor Drift Month 11

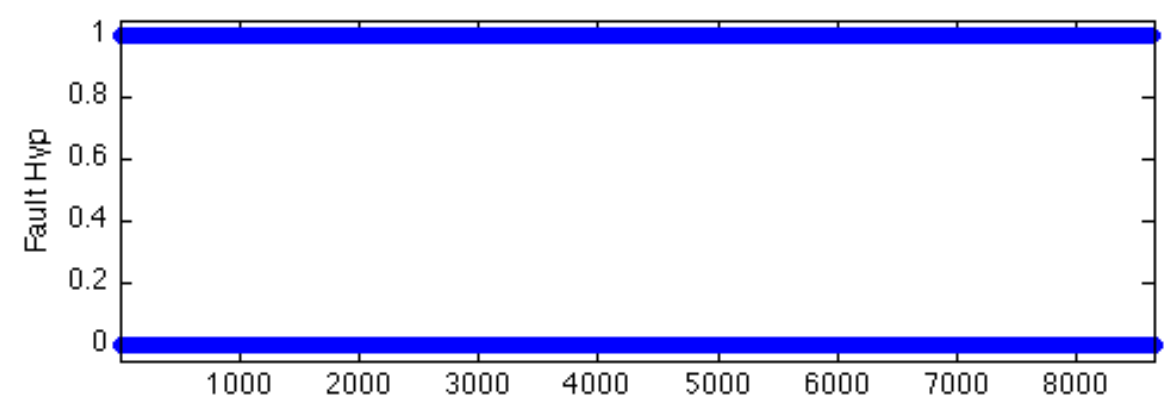

Consolidated Fault Hypothesis

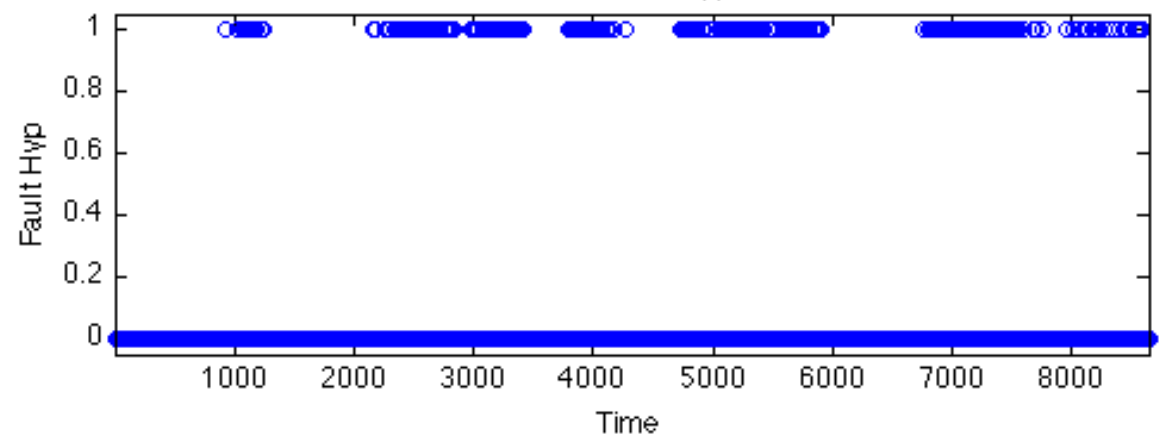

Fault Detection Results for Control Sensor Drift Month 12
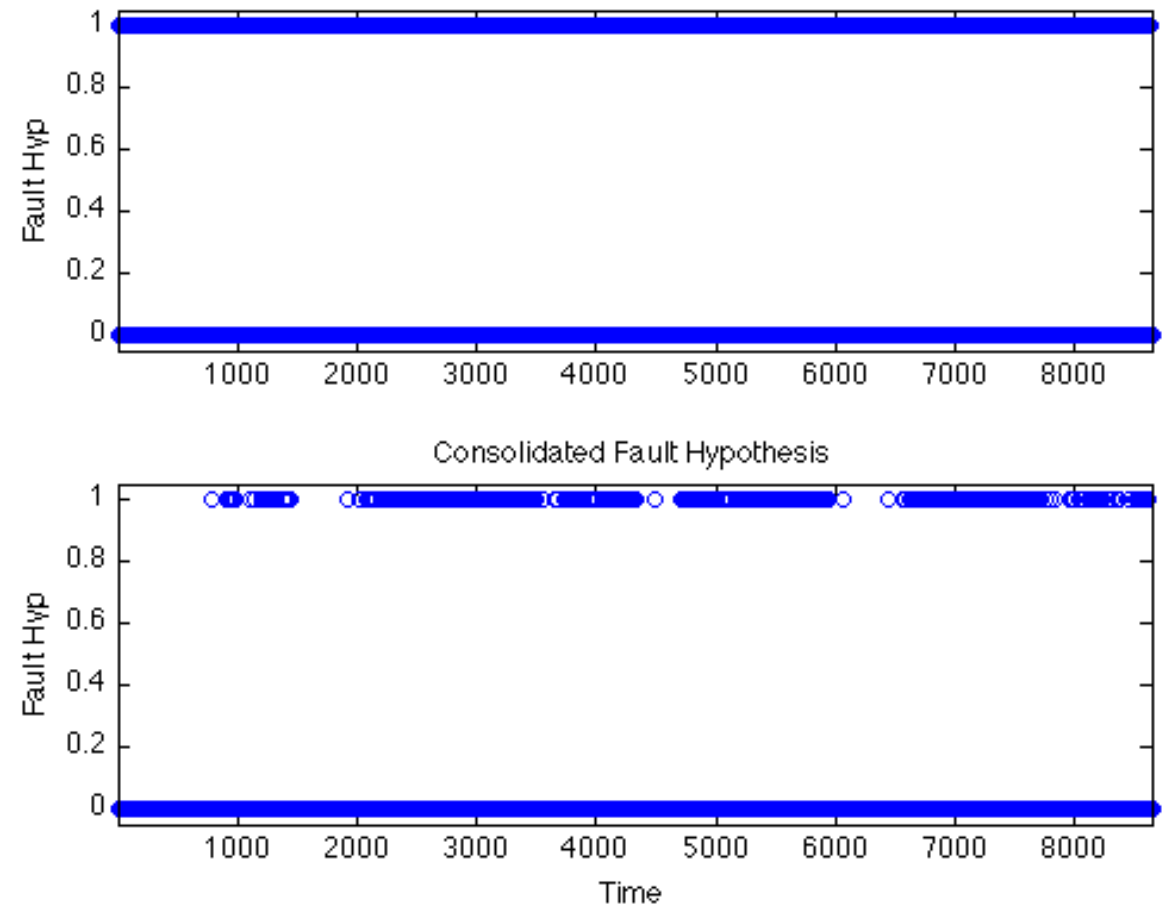


\section{Appendix B: ANPM Matlab Code}

There are a number of versions of the ANPM that have different capabilities.

The ANPM_PCA_T function provides the ANPM capability with the integrated PCA ECM technique is a total combined format.

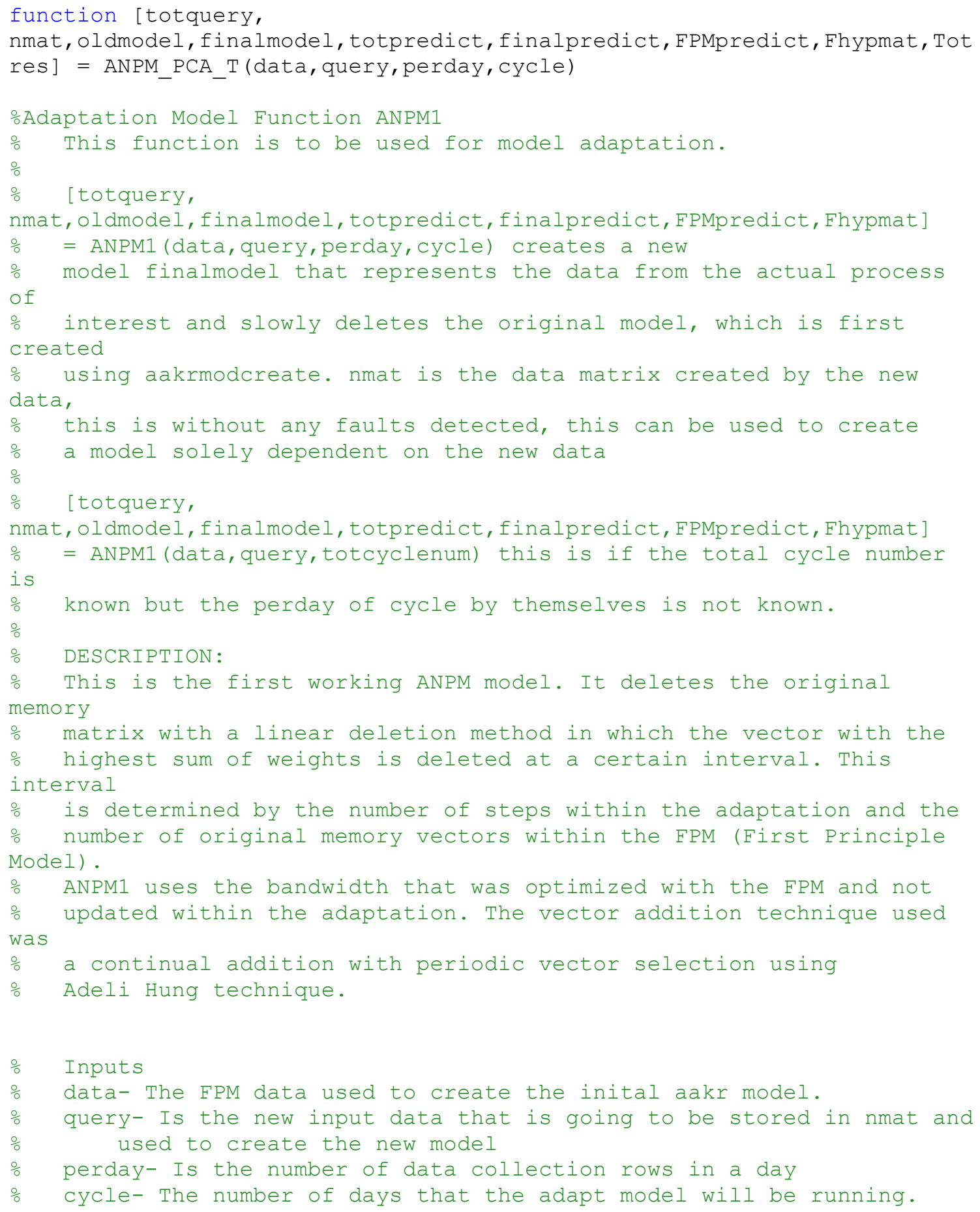




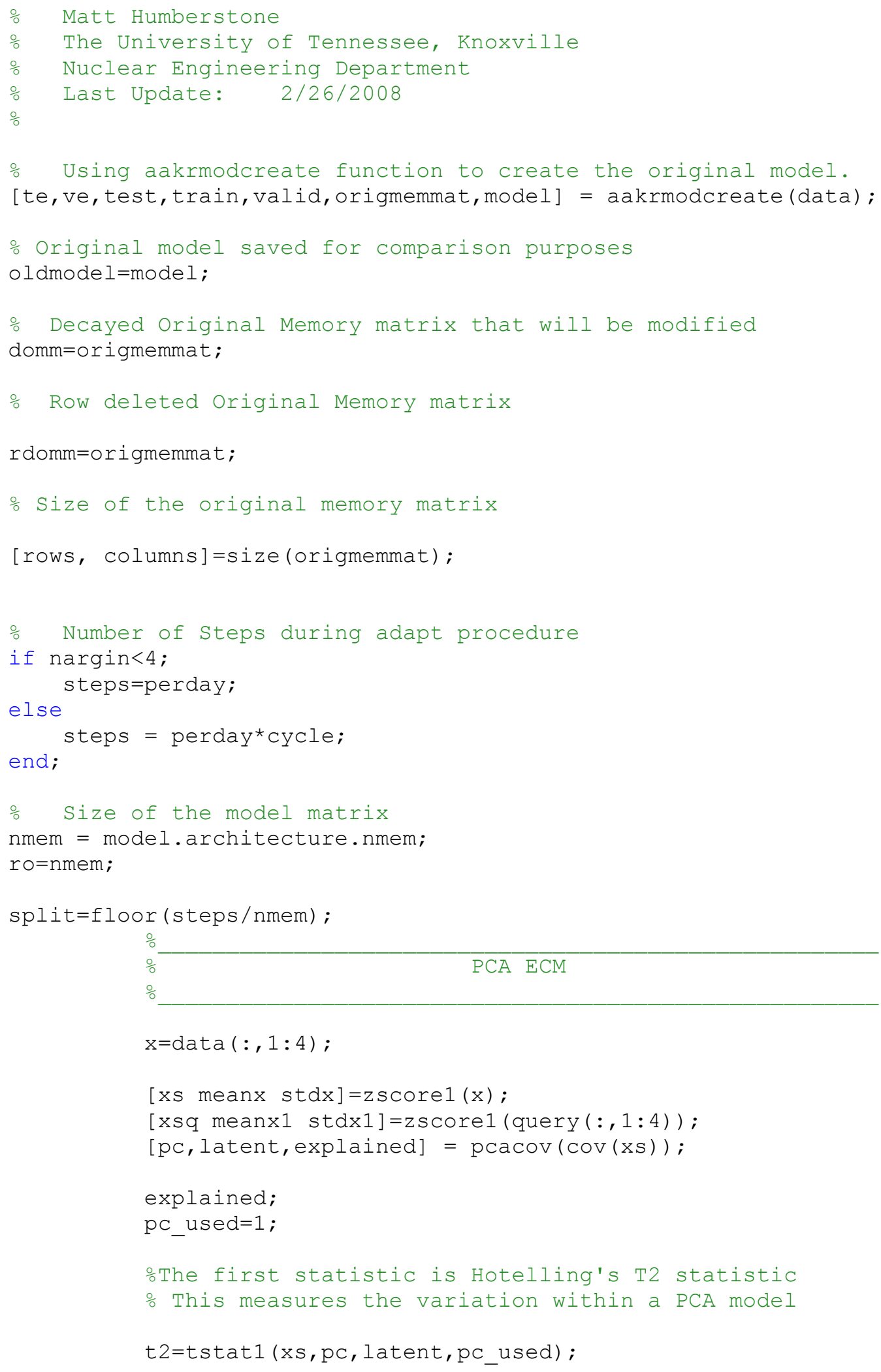


oThe Q statistic measure the variation outside of the PCA

model.

$\mathrm{q}=\mathrm{qs}$ tat $(\mathrm{xs}, \mathrm{pc}, \mathrm{pc}$ _used) ;

$\mathrm{QL}=\max (\mathrm{q})$;

$\mathrm{TL}=\max (\mathrm{t} 2)$;

QLmax_Vec $(1:$ steps $)=Q L$

TLmax_Vec $(1:$ steps $)=T L$;

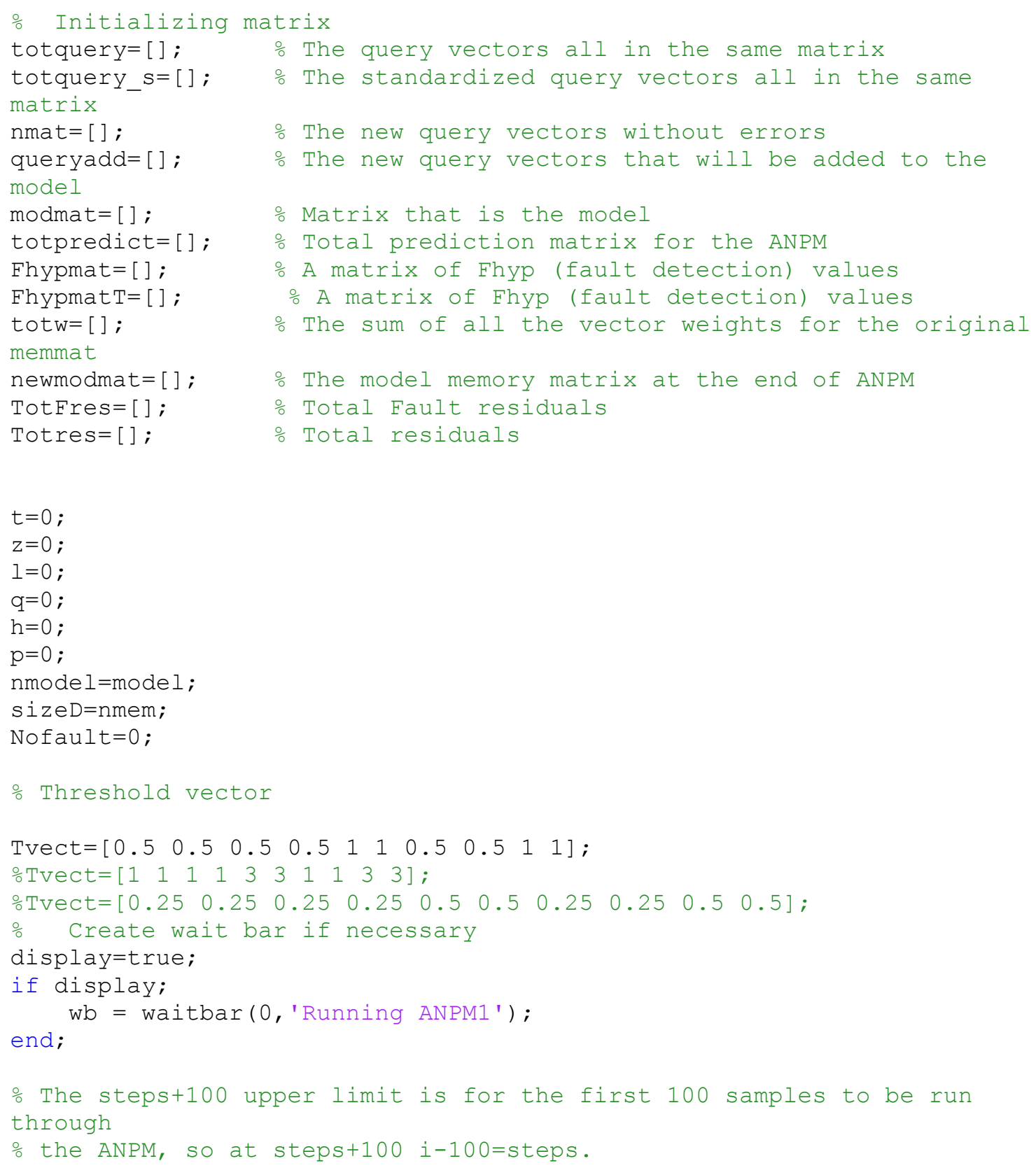




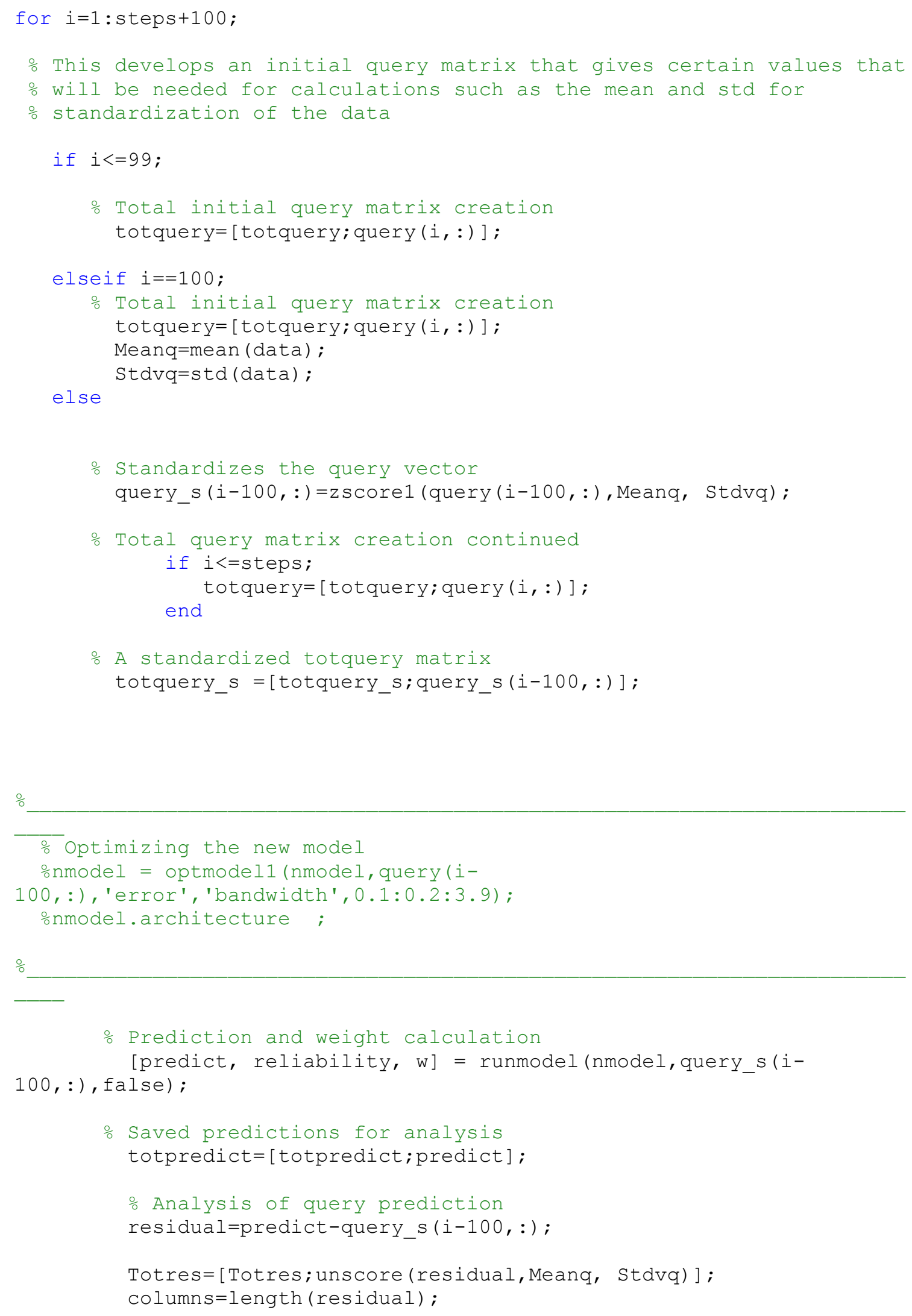


$\frac{\circ}{\circ}$

\% HR based on max weight not each individual weight

․ Find the weight and index

$\div \quad[\mathrm{M}$ ind $]=\max (\mathrm{w})$;

\% Find the hit rate contribution to the decay vector

$\circ \operatorname{HR}($ ind $)=H R($ ind $) * 0.9$

$\frac{0}{\circ}$

$\frac{0}{2}$

PCA ECM

$\div$

sdata $=$ zscore1 (query $(i-100,1: 4)$, meanx, stdx1) ;

t2v(i)=tstat1 (sdata, pc, latent, pc used);

qv $(i)=$ qstat (sdata, pc,pc_used);

if $q v(i)>Q L$;

Fhyp $=1$;

else

FhypP $=0$;

end;

if $\mathrm{FhypP}==1$;

Fhyp $(1: \operatorname{columns})=1 ;$ Nofault=Nofault +1 ;

else

Fhyp $(1:$ columns $)=0$;

end;

\% Saving a matrix of Fhyp values

Fhypmat $=[$ Fhypmat $;$ Fhyp $]$;

\%

: Threshold Fault Detection

for $\mathrm{h}=1$ : columns;

if residual $(\mathrm{h})>=$ Tvect $(\mathrm{h})$;

Fhypt $(1: h)=1 ;$ Nofault=Nofault +1 ;

else

end

Fhypt $(1: \mathrm{h})=0$;

end

FhypmatT $=[$ FhypmatT; Fhypt $]$;

\% Size of Row Deleted Original Memory Matrix

[Rrdomm Cdomm]=size (rdomm); 
- Initializing the totw vector

$\mathrm{p}=\mathrm{p}+1$;

if $\mathrm{p}==1$;

for $j=1:$ Rrdomm;

$\operatorname{totw}(j)=0$;

end;

end;

\% Addition of the weights for only the original memory matrix

portion

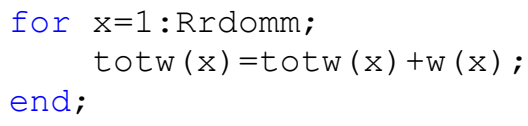

o Decay of initial matrix

$\mathrm{h}=\mathrm{h}+1$;

if $h==s p l i t$;

$\mathrm{h}=0$;

\% max weigth and index found

$[\mathrm{M}$ ind $]=\max ($ totw $)$;

\% Max weight vector deleted from rdomm

rdomm (ind, : ) $=[]$;

totw $($ ind $)=[]$;

end;

\%

- For Dynamic SPRT calculations

o Initial Tolerances for SPRT, set as constants but could be

made

응 dependent on $i$

\%False Alarm Probability

\% alpha $=0.0001 ;$ \%0.01\%

\%Missed Alarm Probability

․ beta $=0.10 ; \quad \frac{\circ}{\circ} 10 \%$

Fault Detection

o SPRT (Sequential Probability Ratio Test)

o Training the SPRT for dynamic alpha and beta

\% [ErrM ErrV Tol] = trainsprt (te, ve, alpha, beta);

응

\%

\% Apply a logic screen to limit the number of alarms

\% 3 out of 5 before a fault is declared

CFhyp $=\operatorname{confh}($ Fhyp, [1 2 $]$ ); \%possibility 
\% Sum of the Fhyp for each sensor

for $k=1$ : columns; sumFhyp=sum $($ Fhyp $(k))$;

end sumFhypT=sum (FhypT (k));

o Add query if no fault detected within vector if sumFhyp $==0$ \&\& sumFhypt $==0$;

if Nofault $<=2$;

$z=z+1$;

\% Query matrix with no faults detected

nmat $=[$ nmat; query_s $(i-100,:)]$;

\% The non fault query vector is added to the queryadd vector queryadd=[queryadd; query_s $(i-100,:)]$;

ㅇ A 1500 vector memory matrix limit is set, then the Adeli-Hung

\% clusstering vector selection is used to set the queryadd

matrix

\% back to 500 memory vector

if $z==1500$;

$\mathrm{z}=0$;

- New queryadd matrix

queryadd=vectsel (nmat, ' h', 500 );

- Model matrix is first defined as the deleted original

o memory matrix

modmat $=r d o m m$;

\% Then the queryadd is added onto the model matrix modmat $=[$ modmat; queryadd $]$;

else

\% Then the queryadd matrix is added to the decayed

original

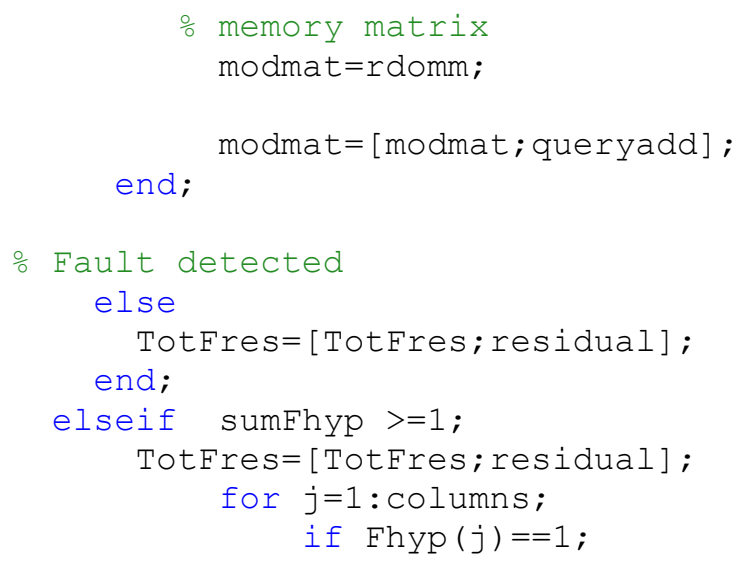




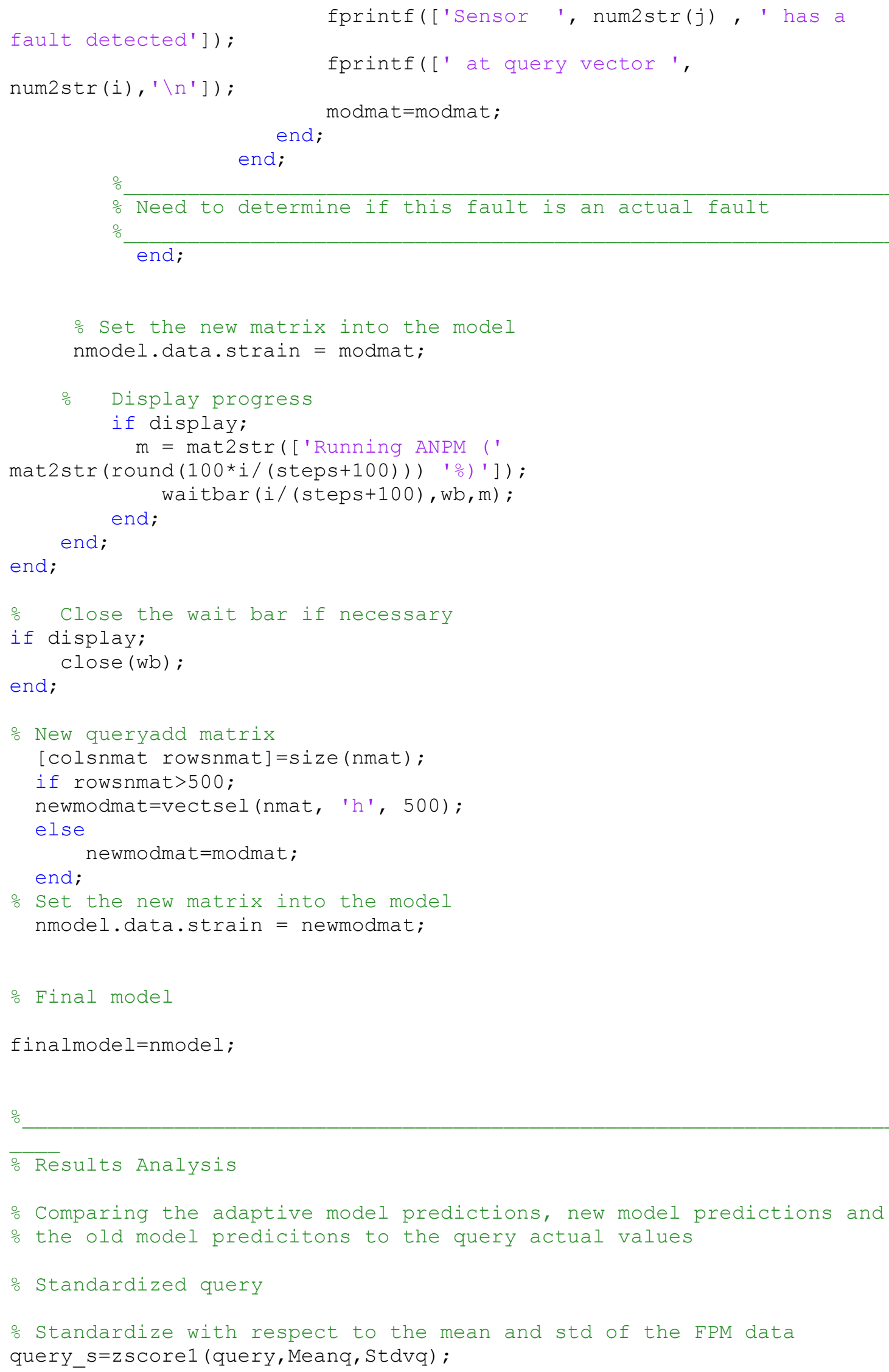




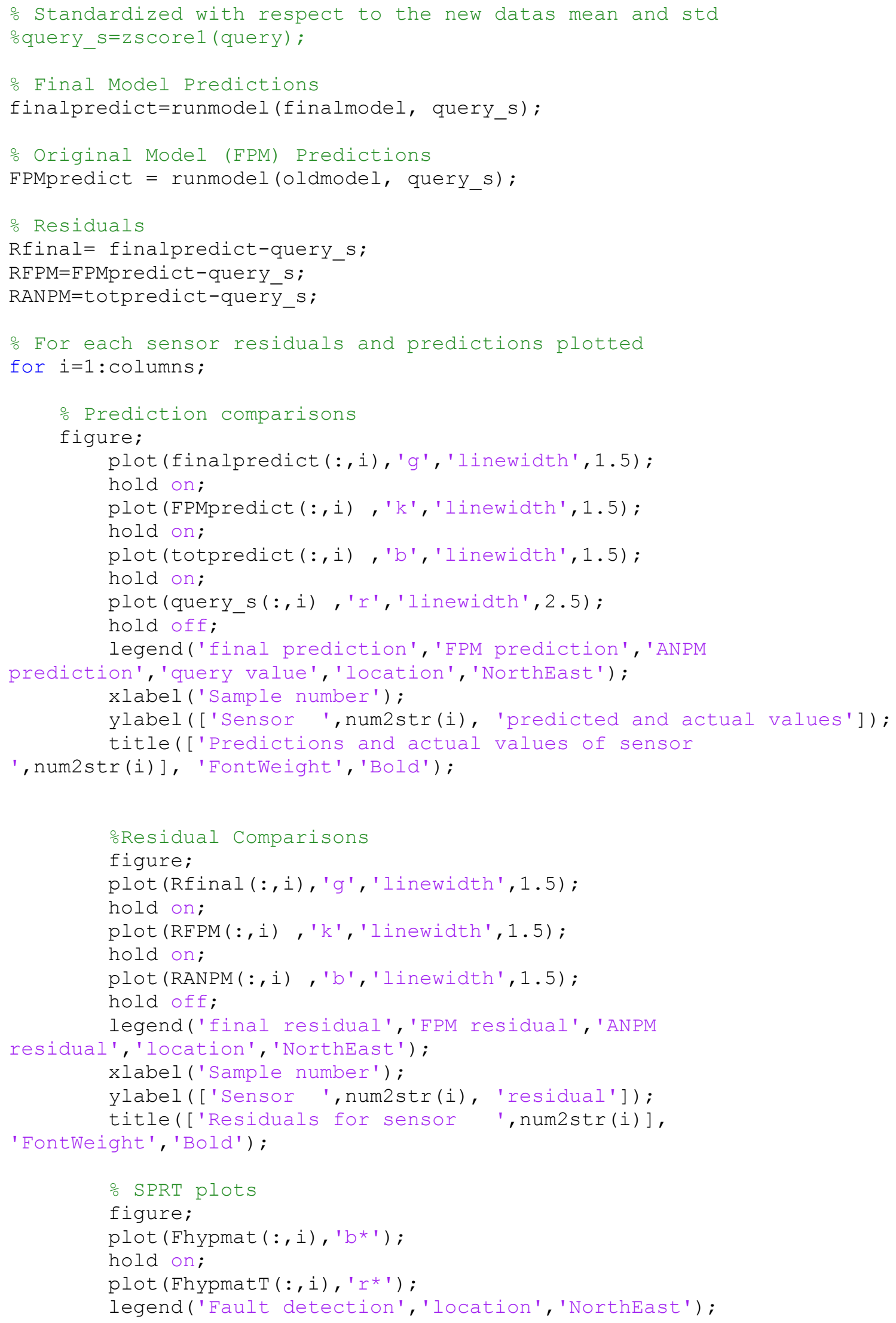




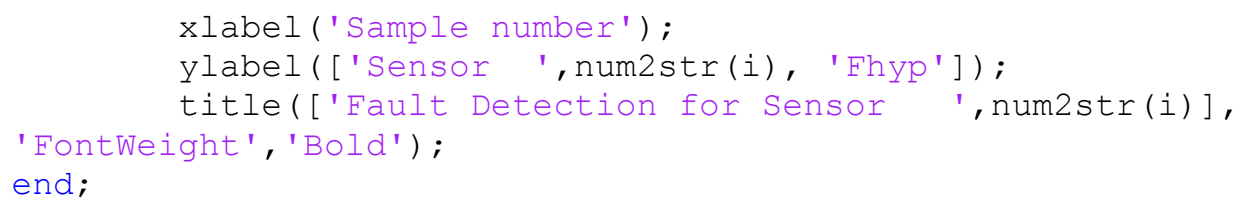




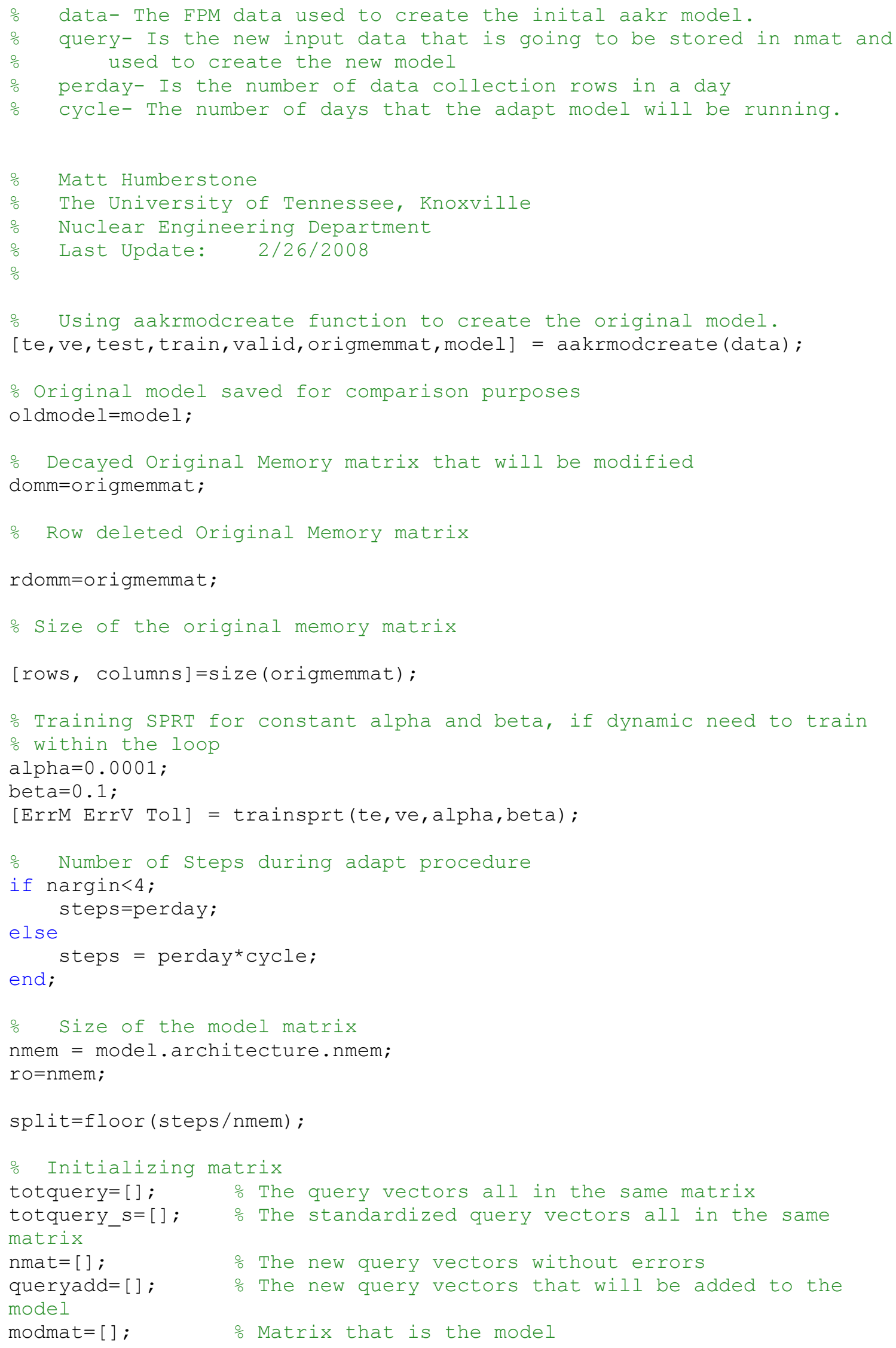




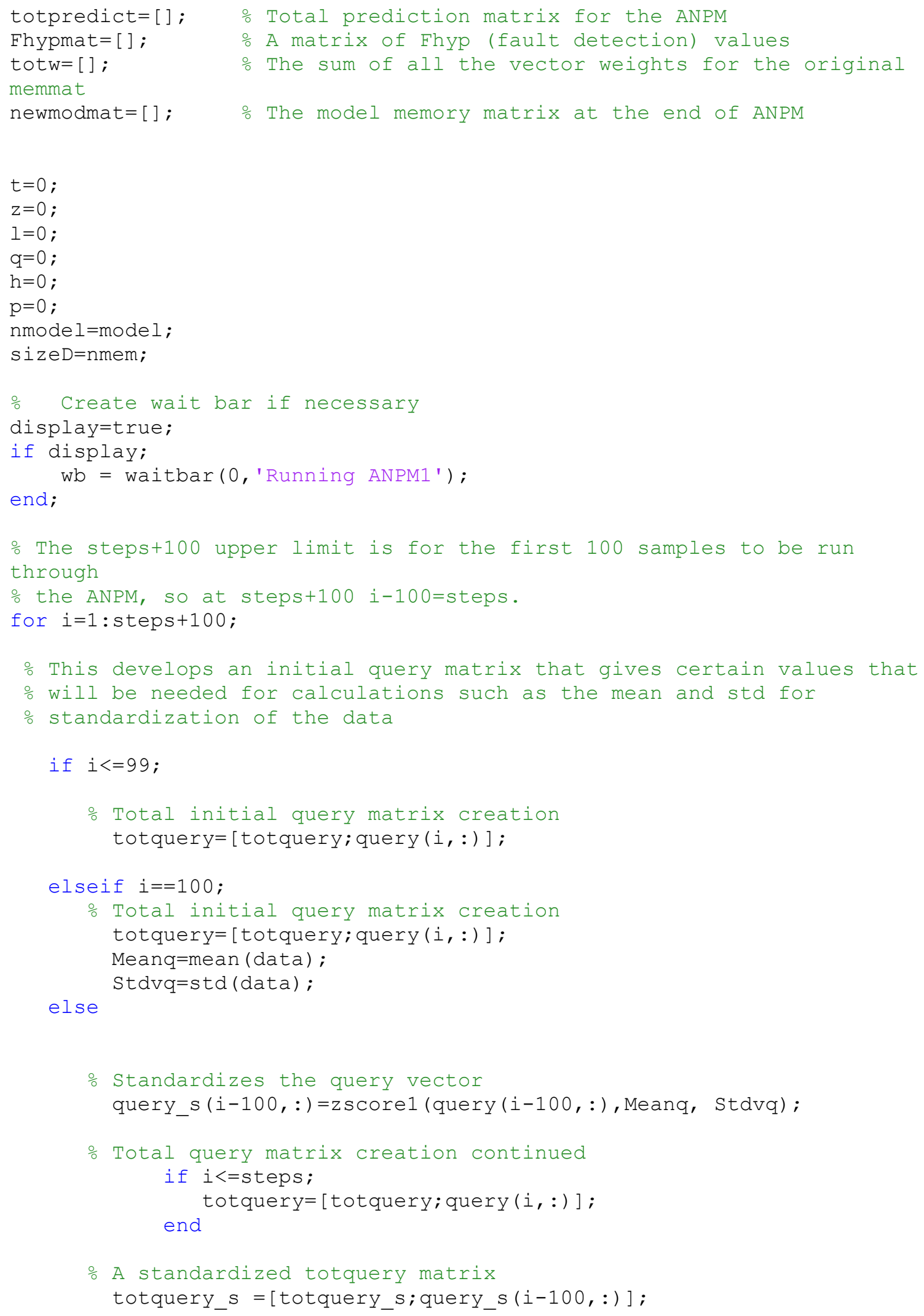


응

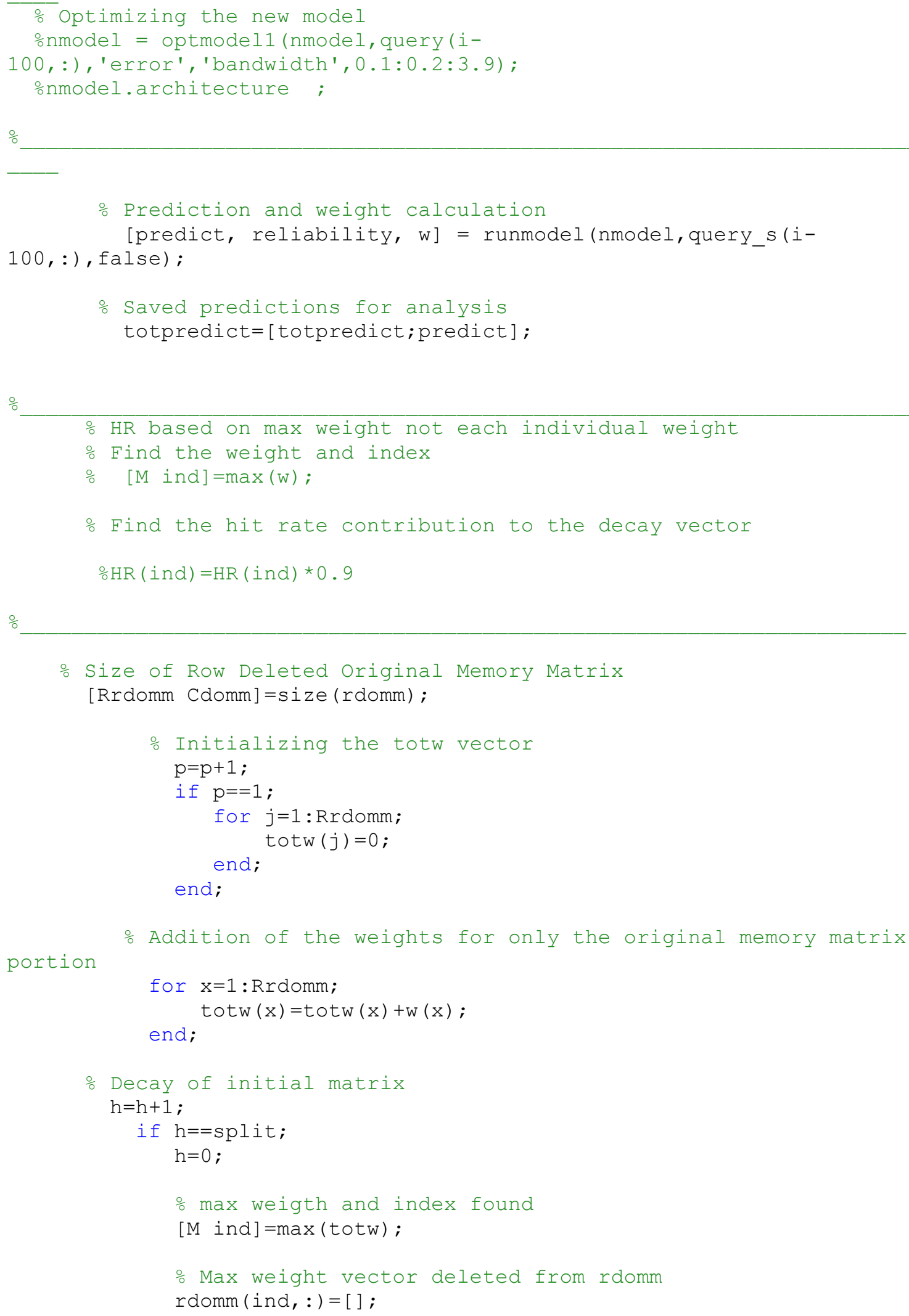




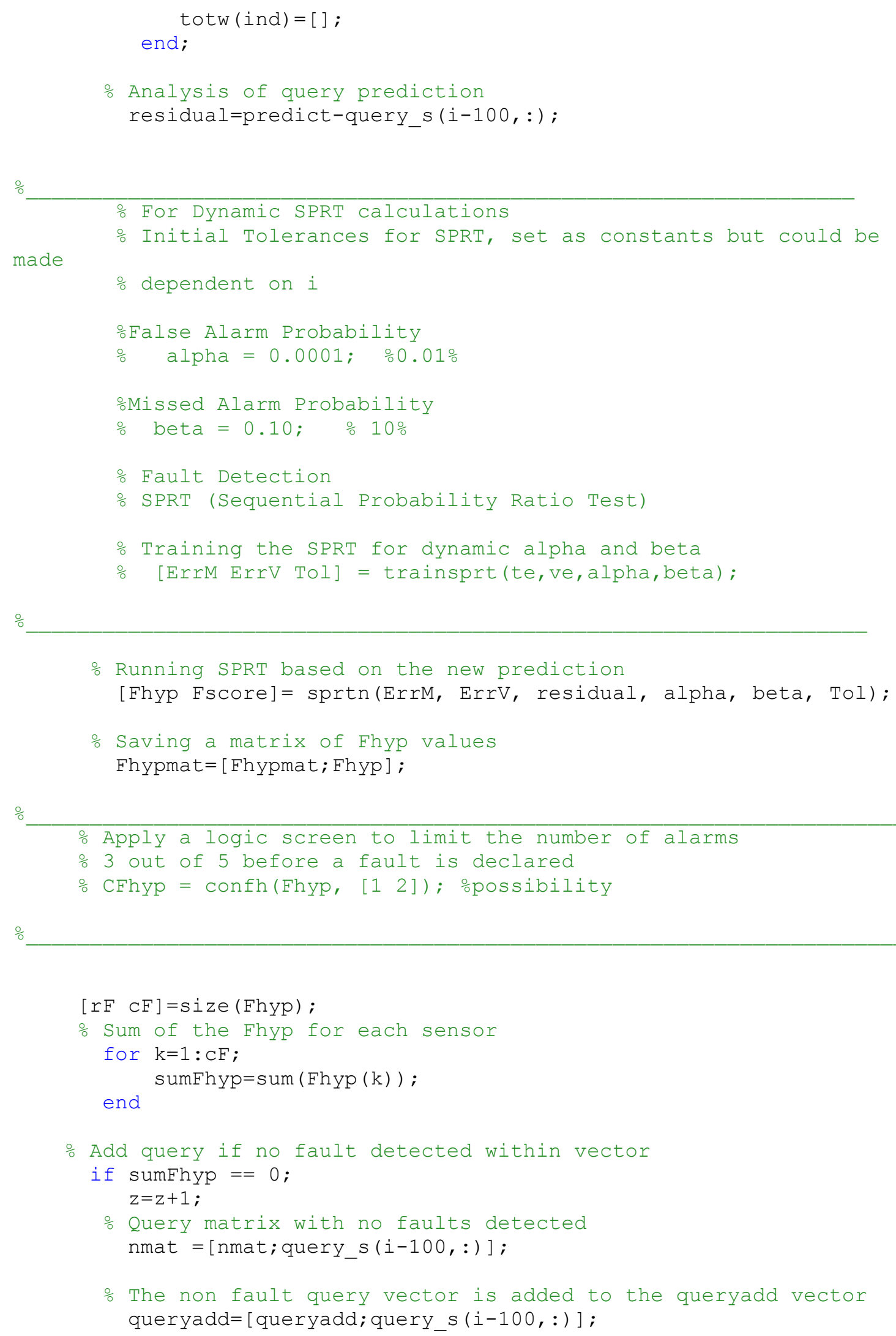

\% The non fault query vector is added to the queryadd vector queryadd=[queryadd; query_s (i-100, : ) ] ; 


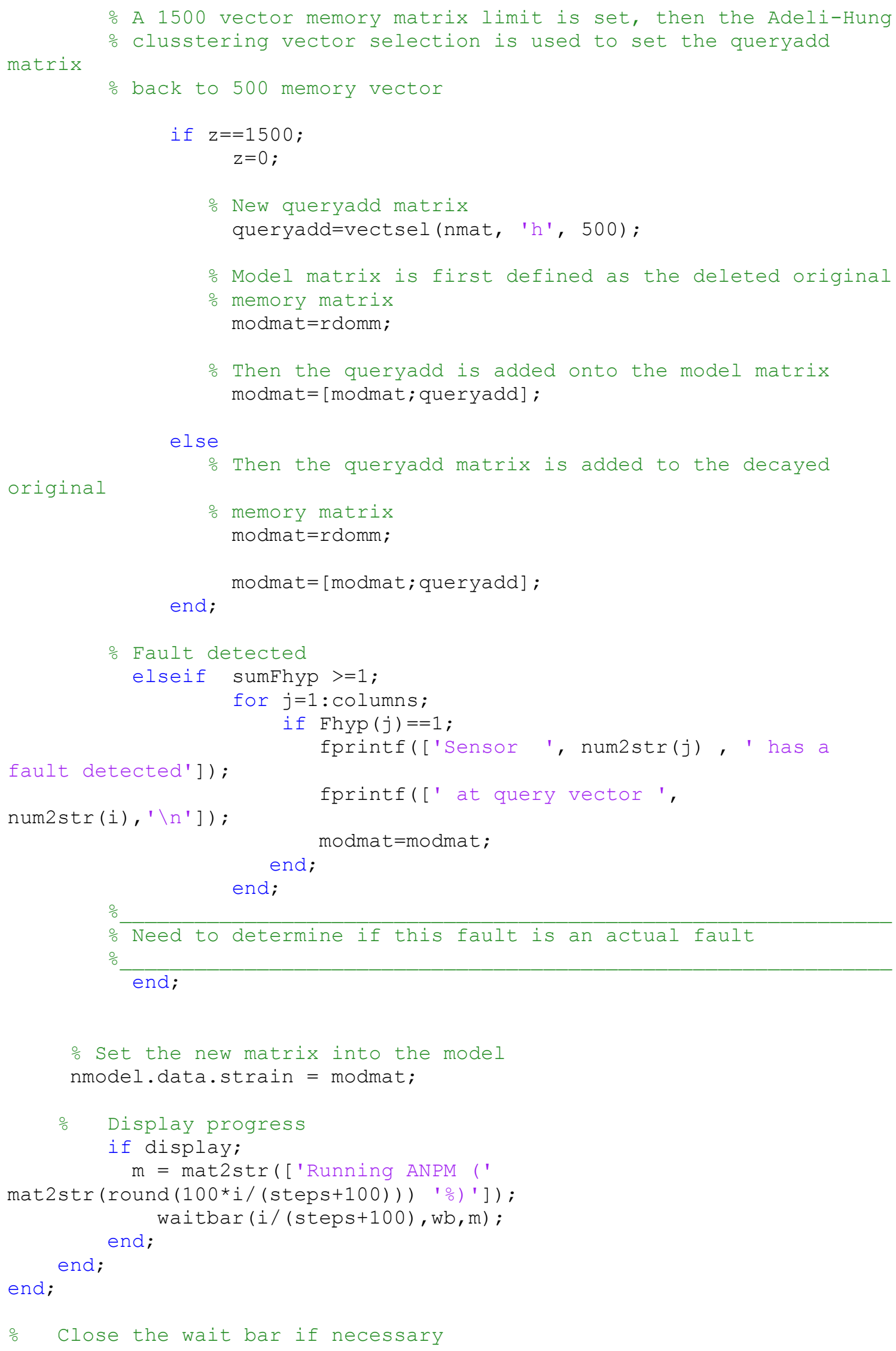




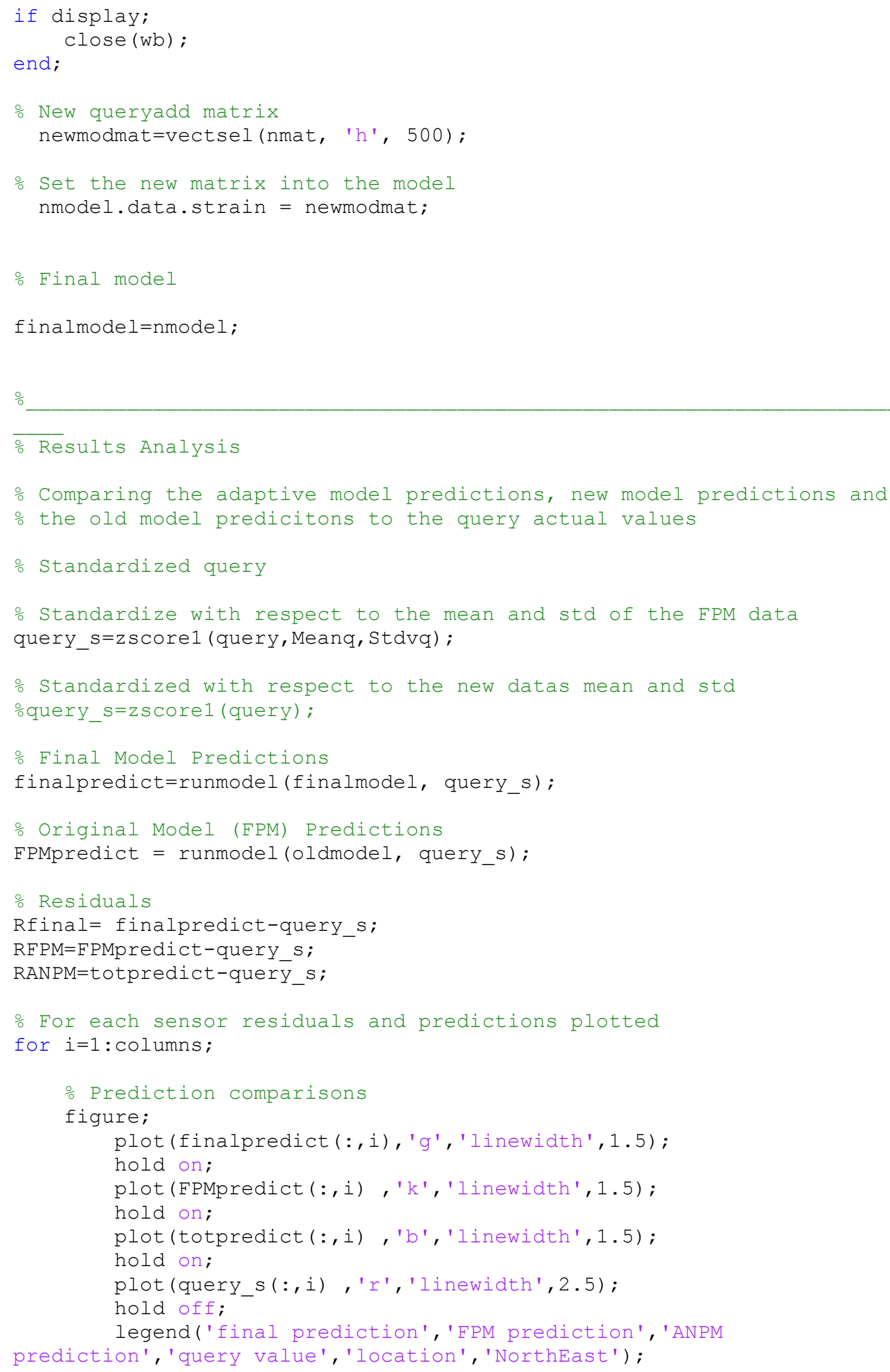




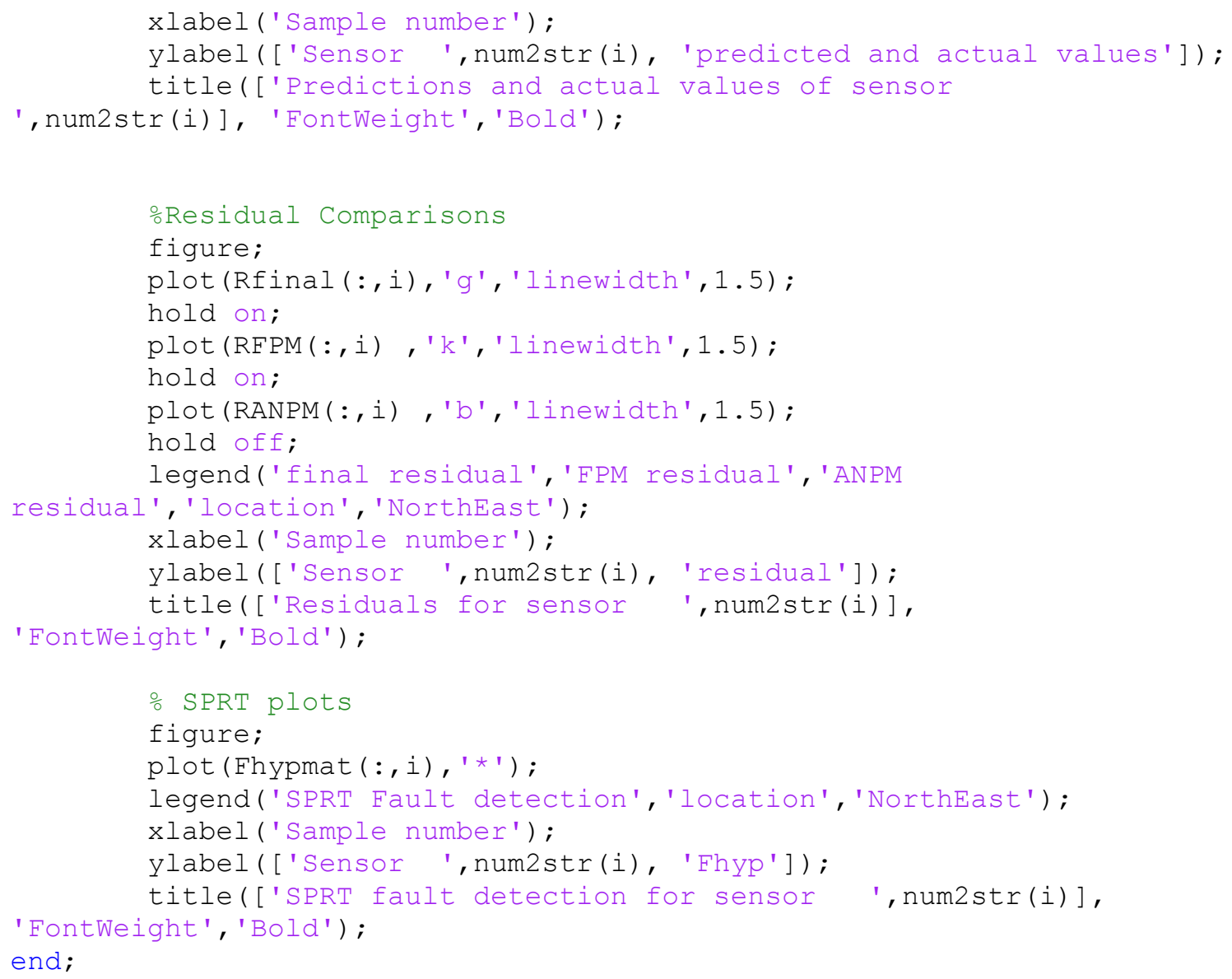

ANPM1_5 function is a basic ANPM function with a step vector addition technique. This function is similar to ANPM1 function as in it was used for testing the adaptation of the model not the fault detection capability.

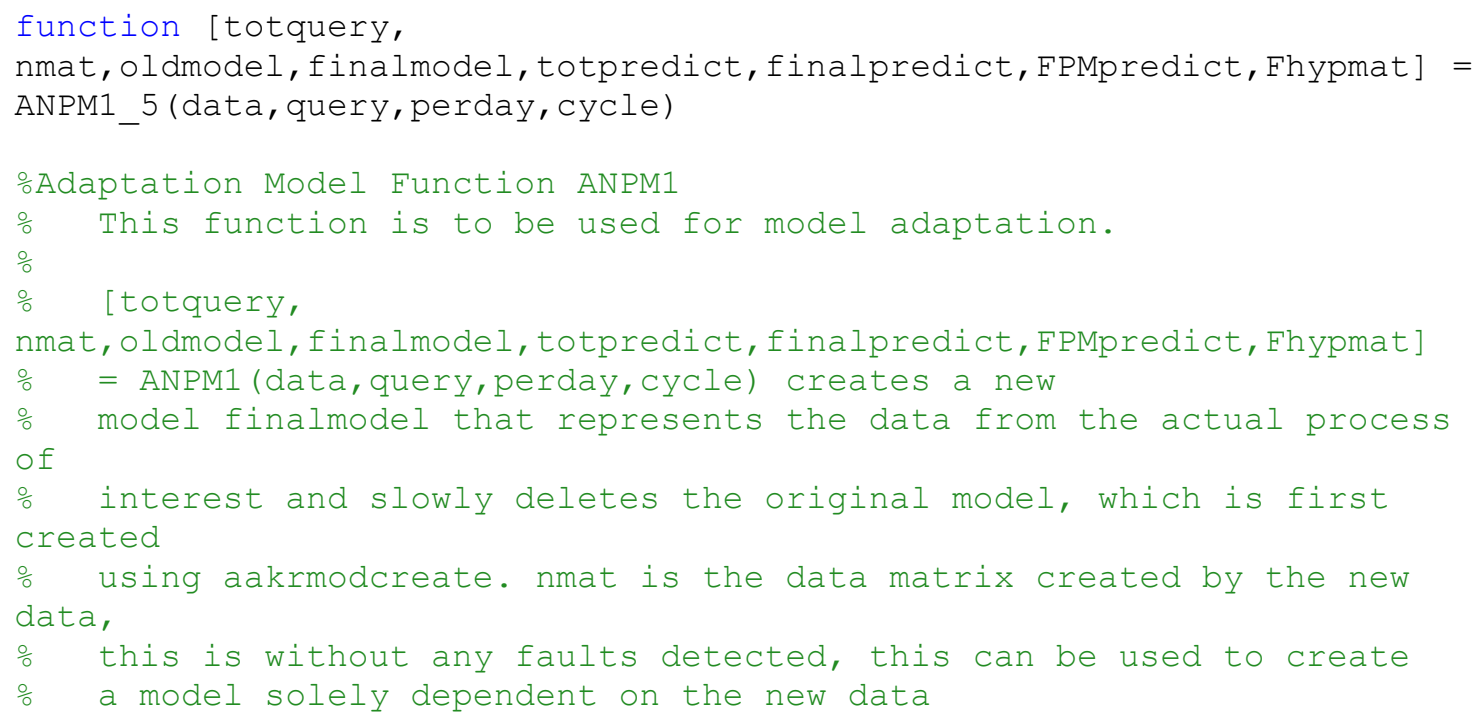




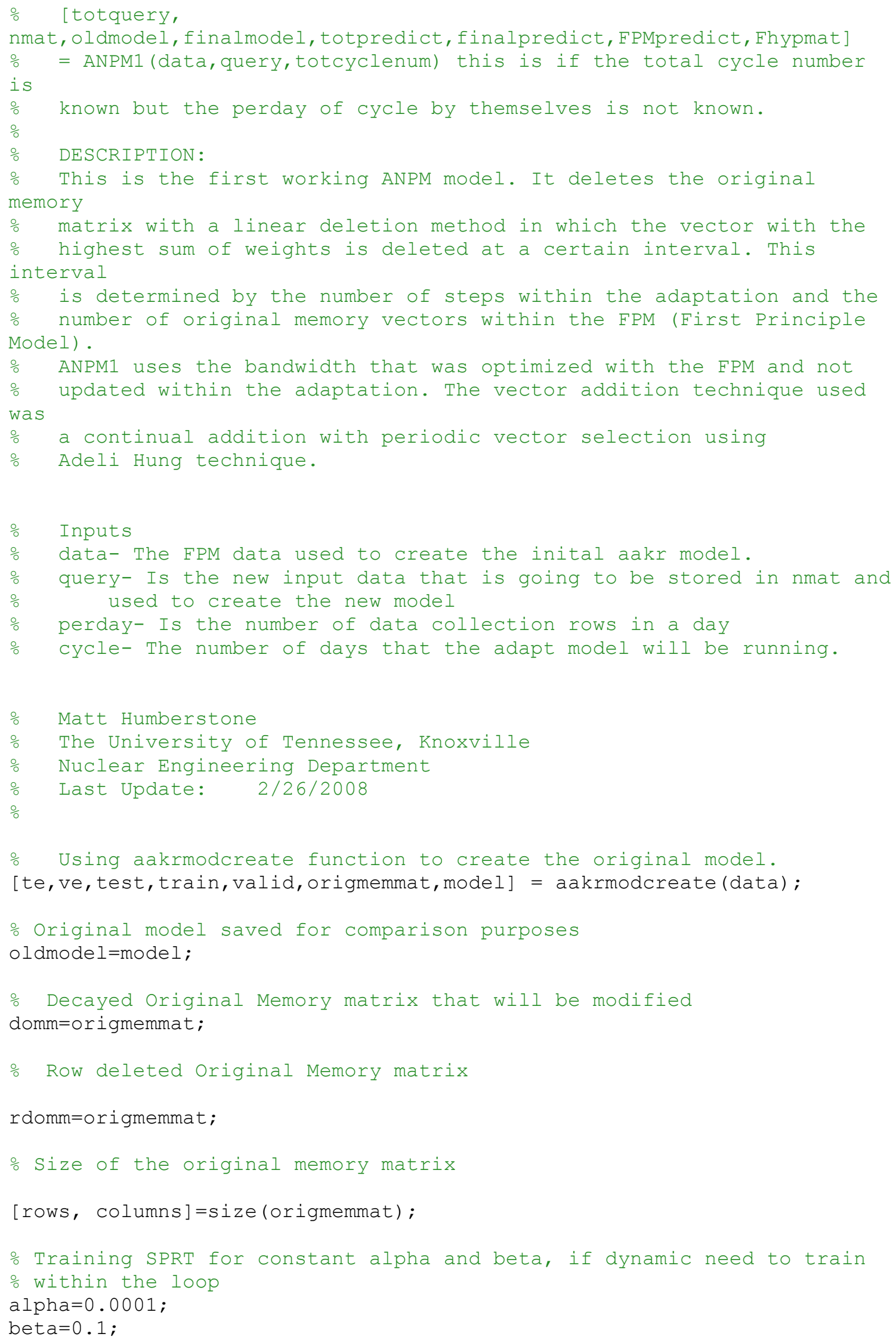




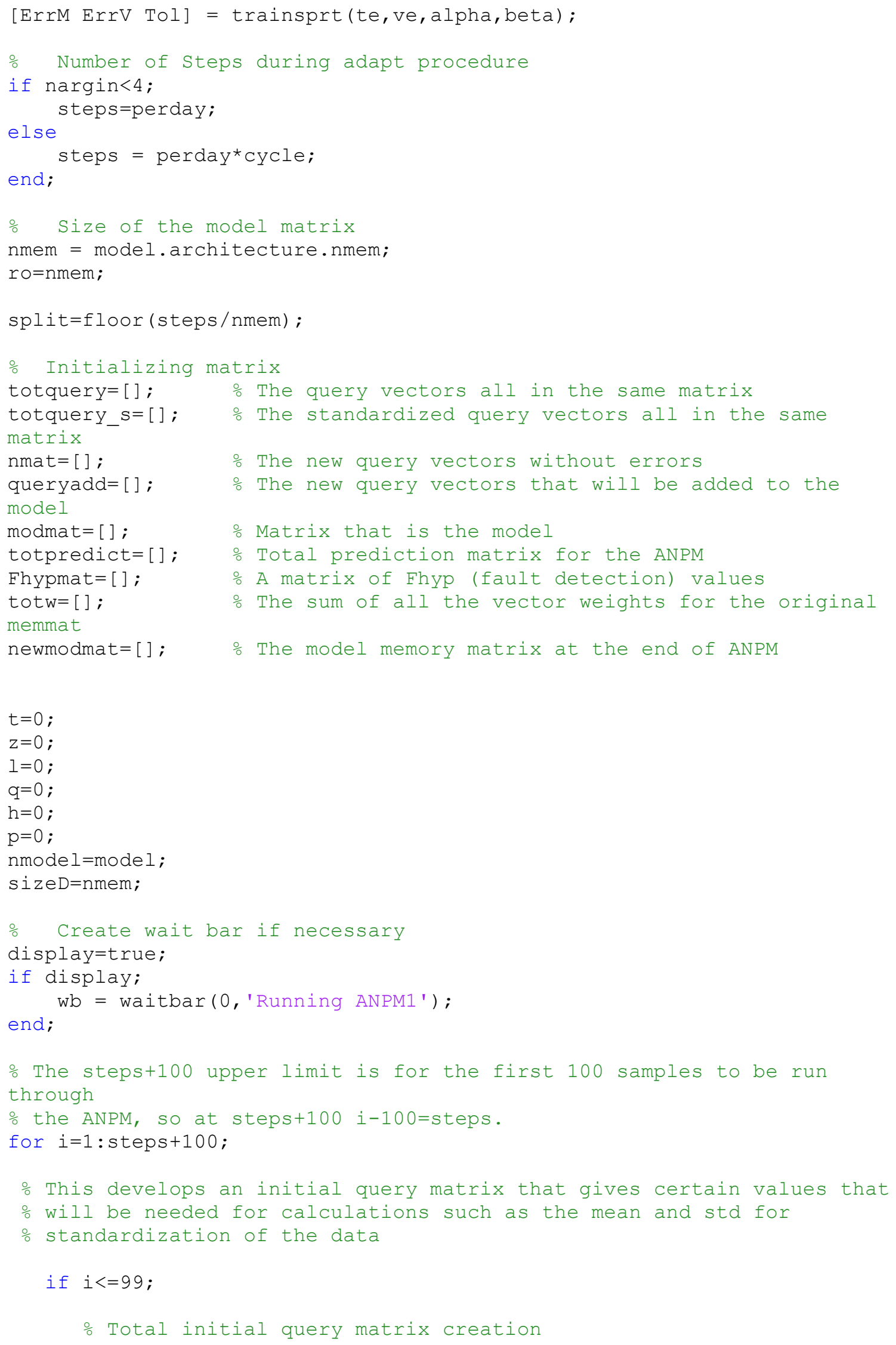




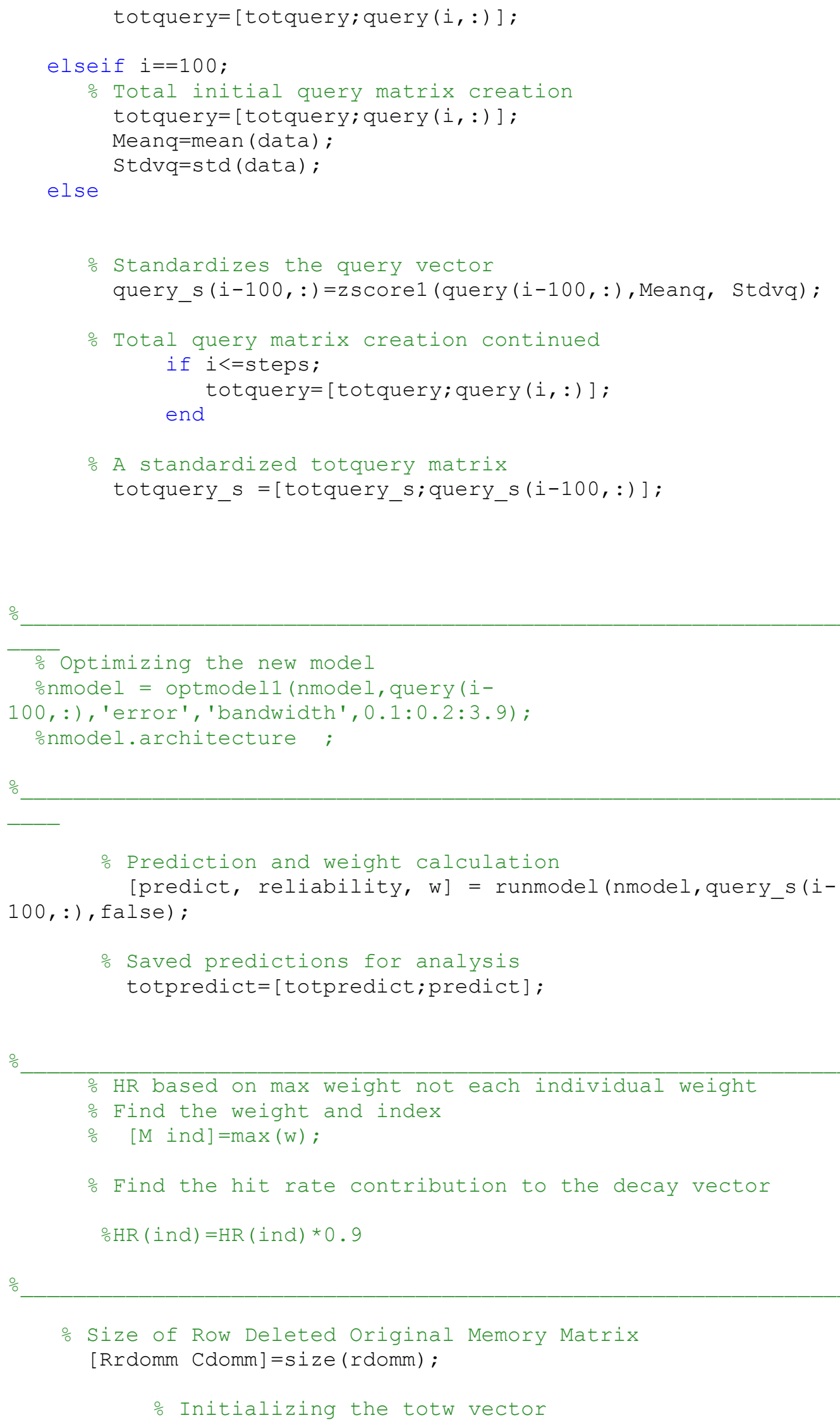




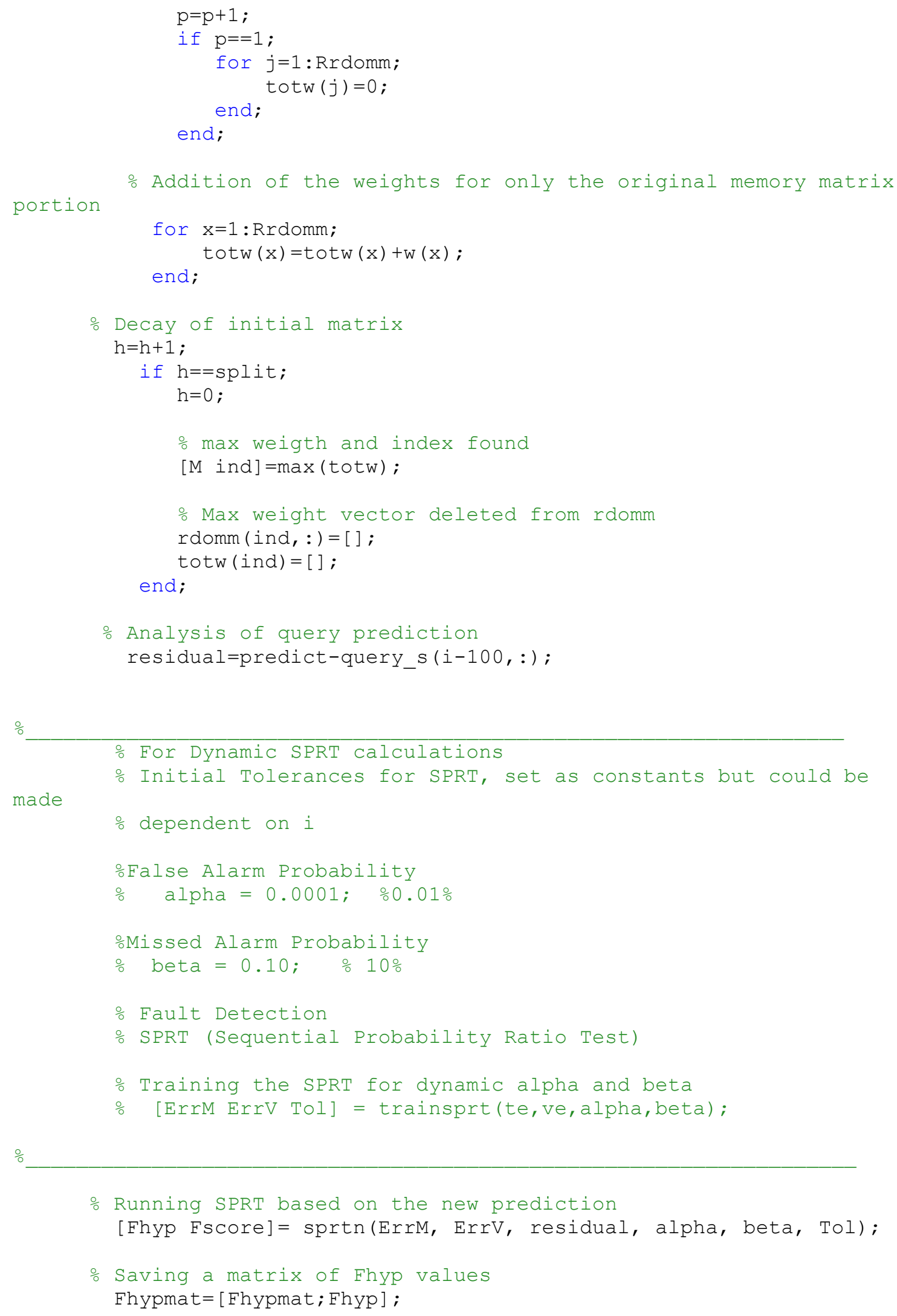




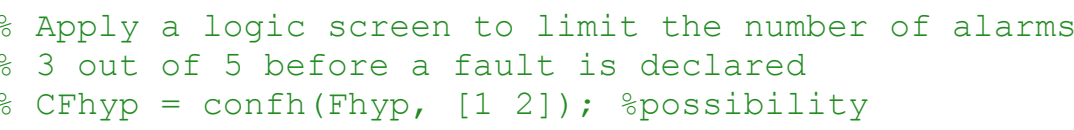

응

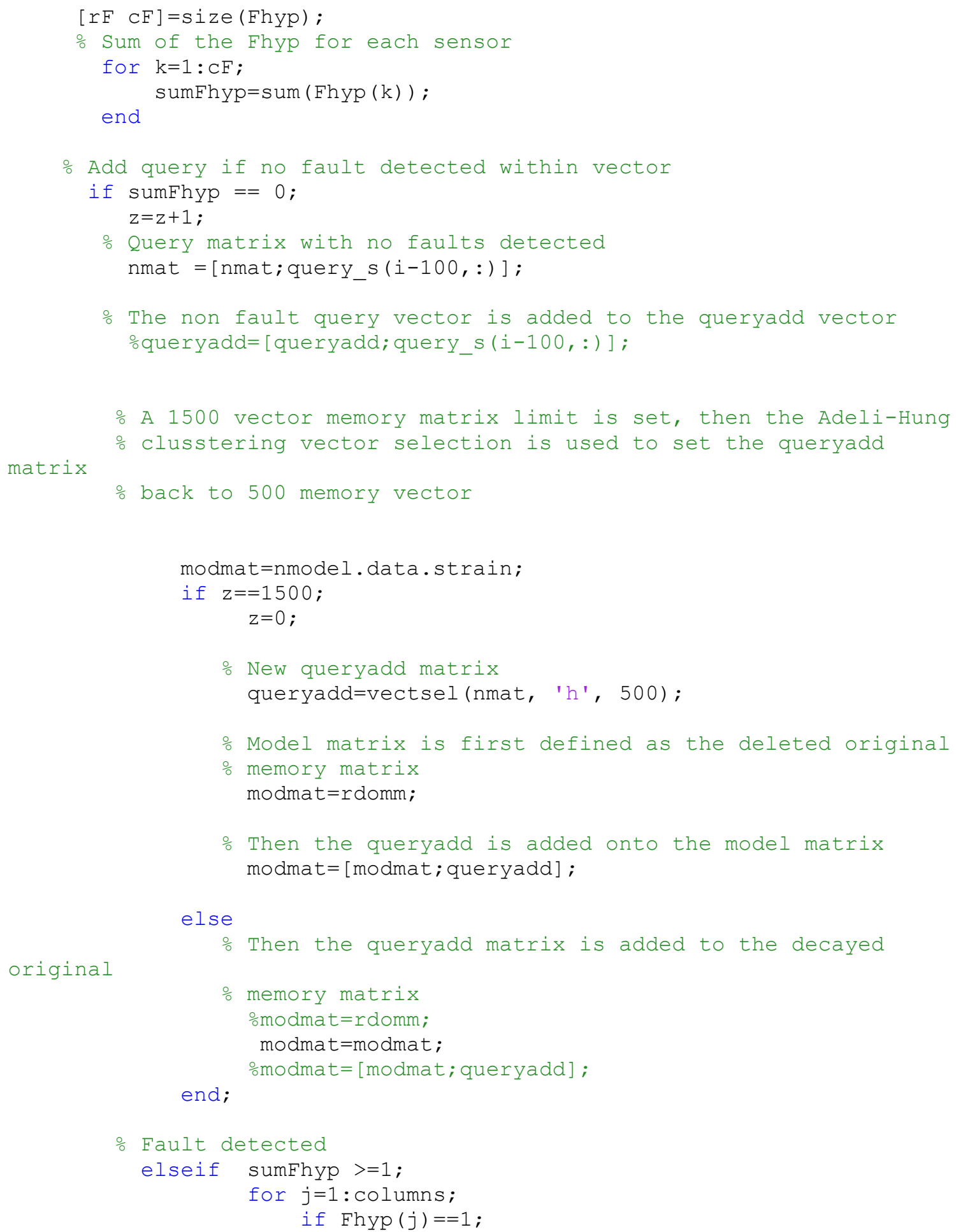




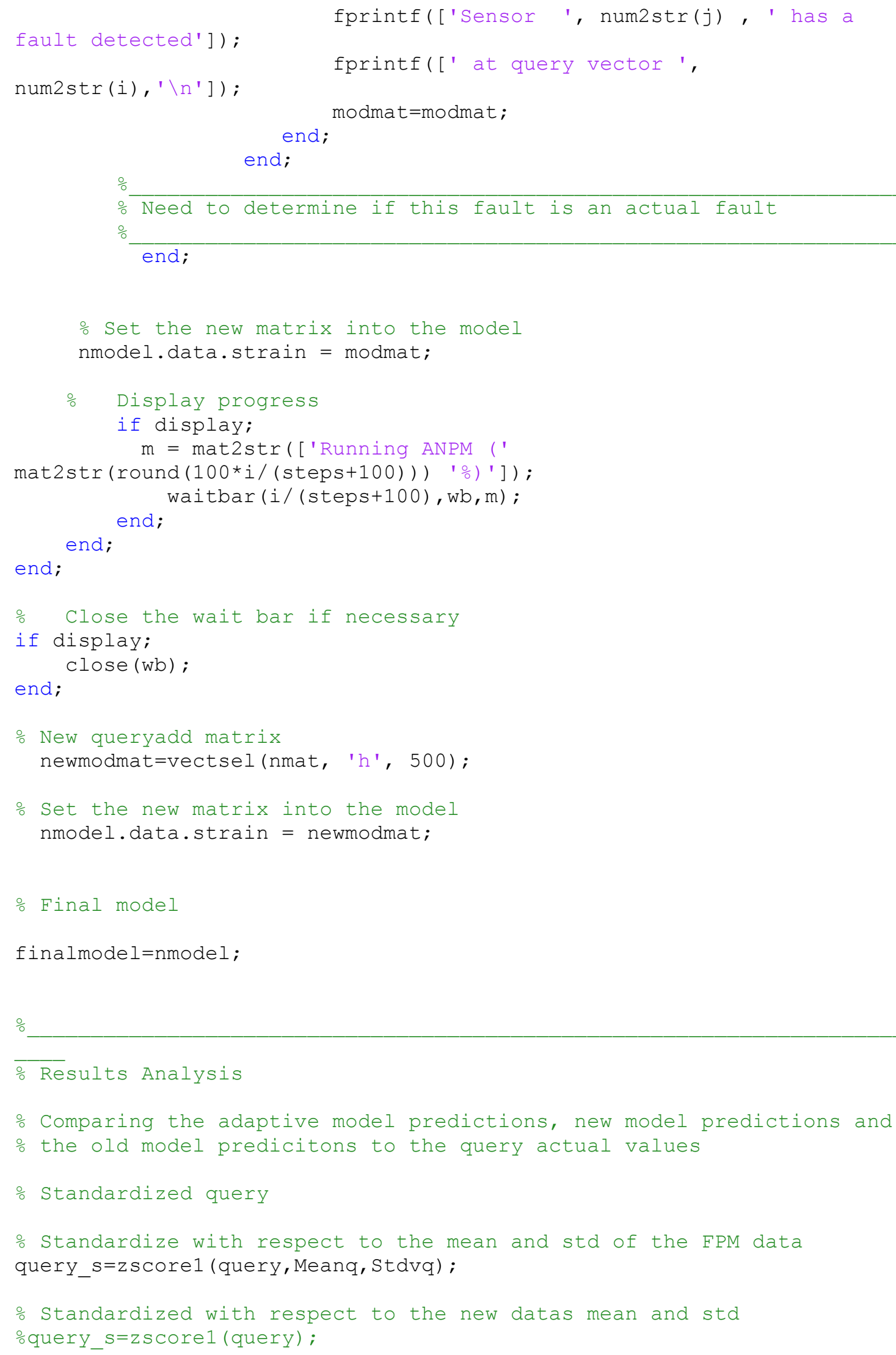




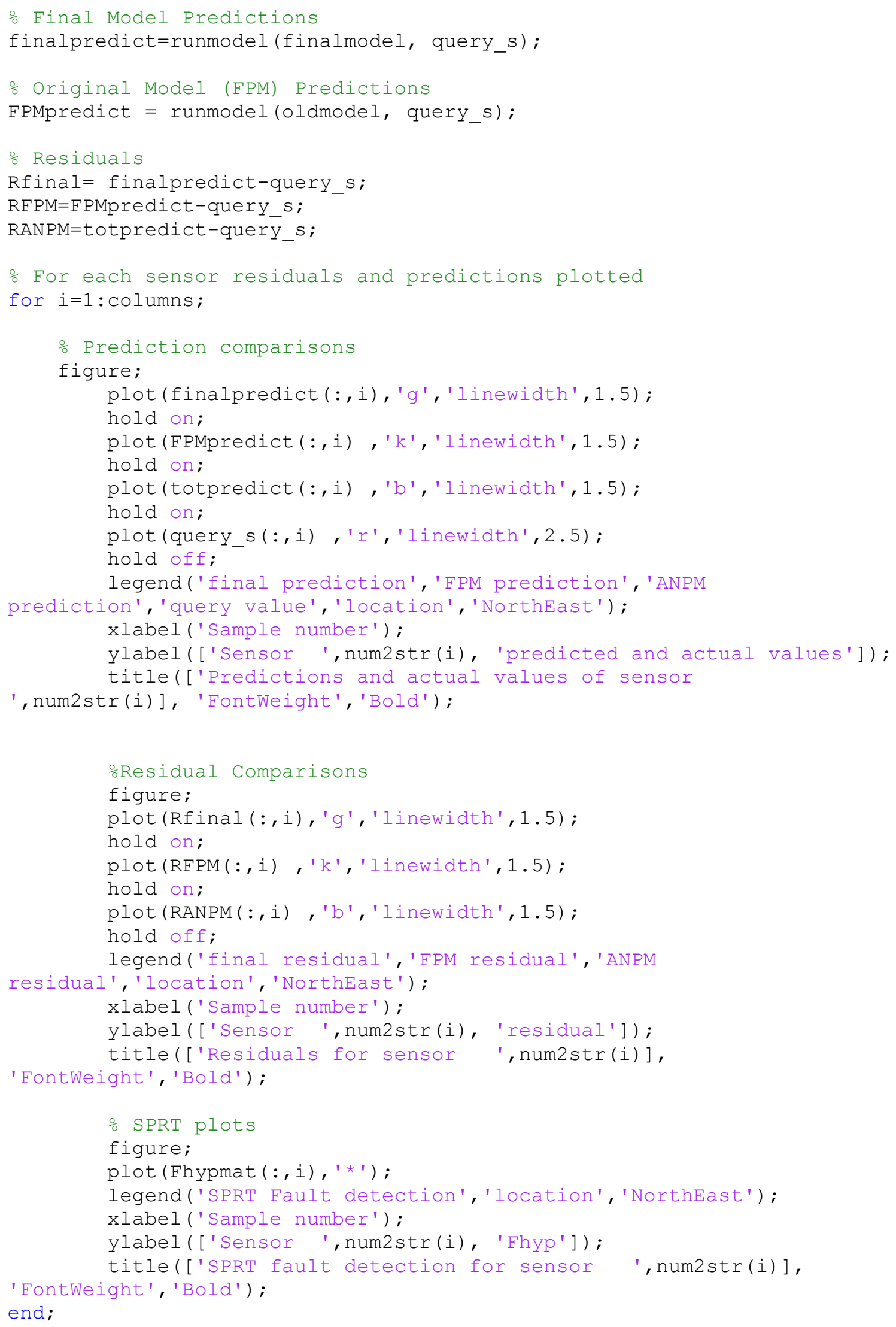


The function aakrmodcreate inputs the original data from the system of interest and separates the data into a train, test, and validation data set. It then optimizes the bandwidth and outputs the model the original memory matrix, the train, test, and validation data sets, and the train and validation error for use in an SPRT algorithm.

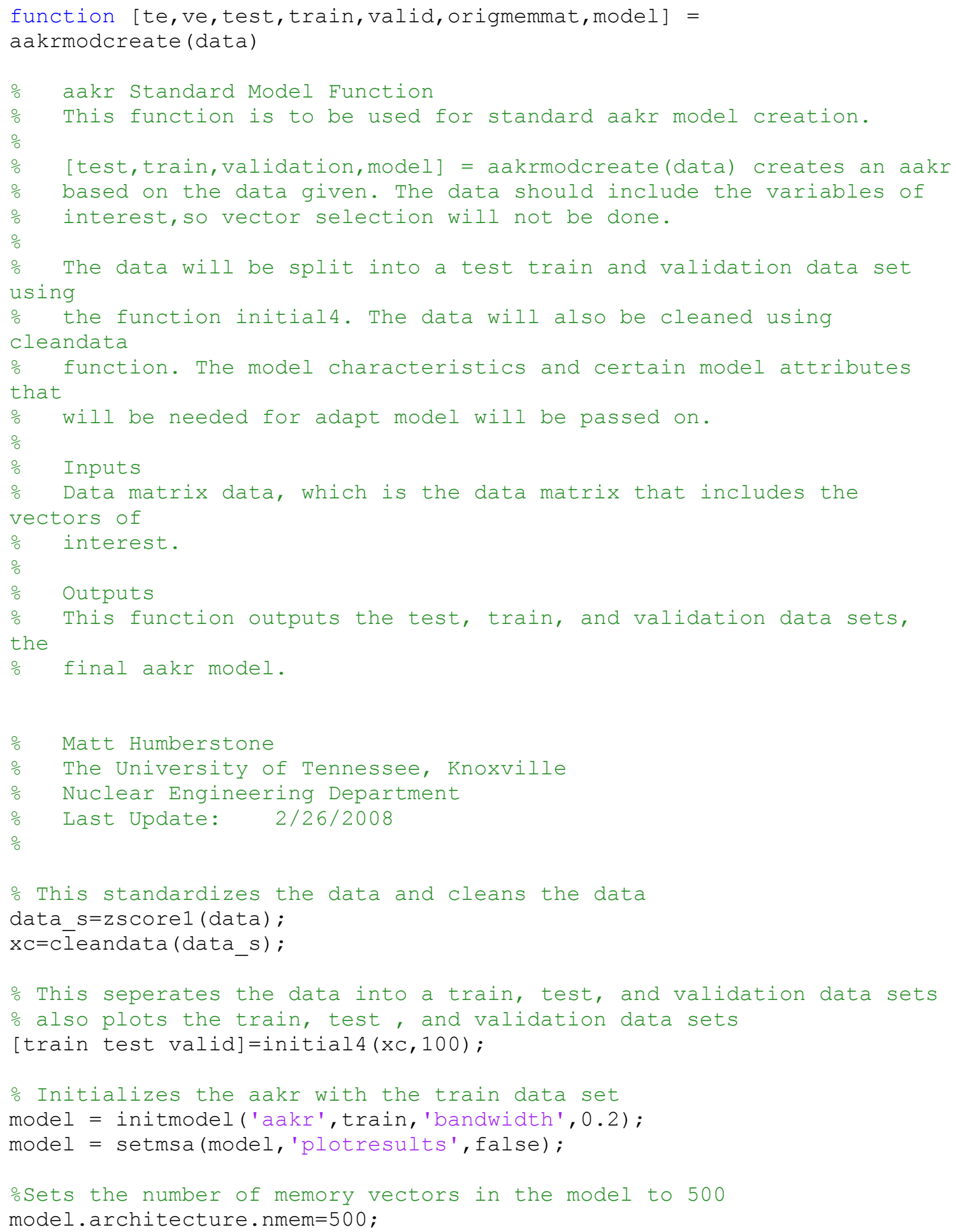




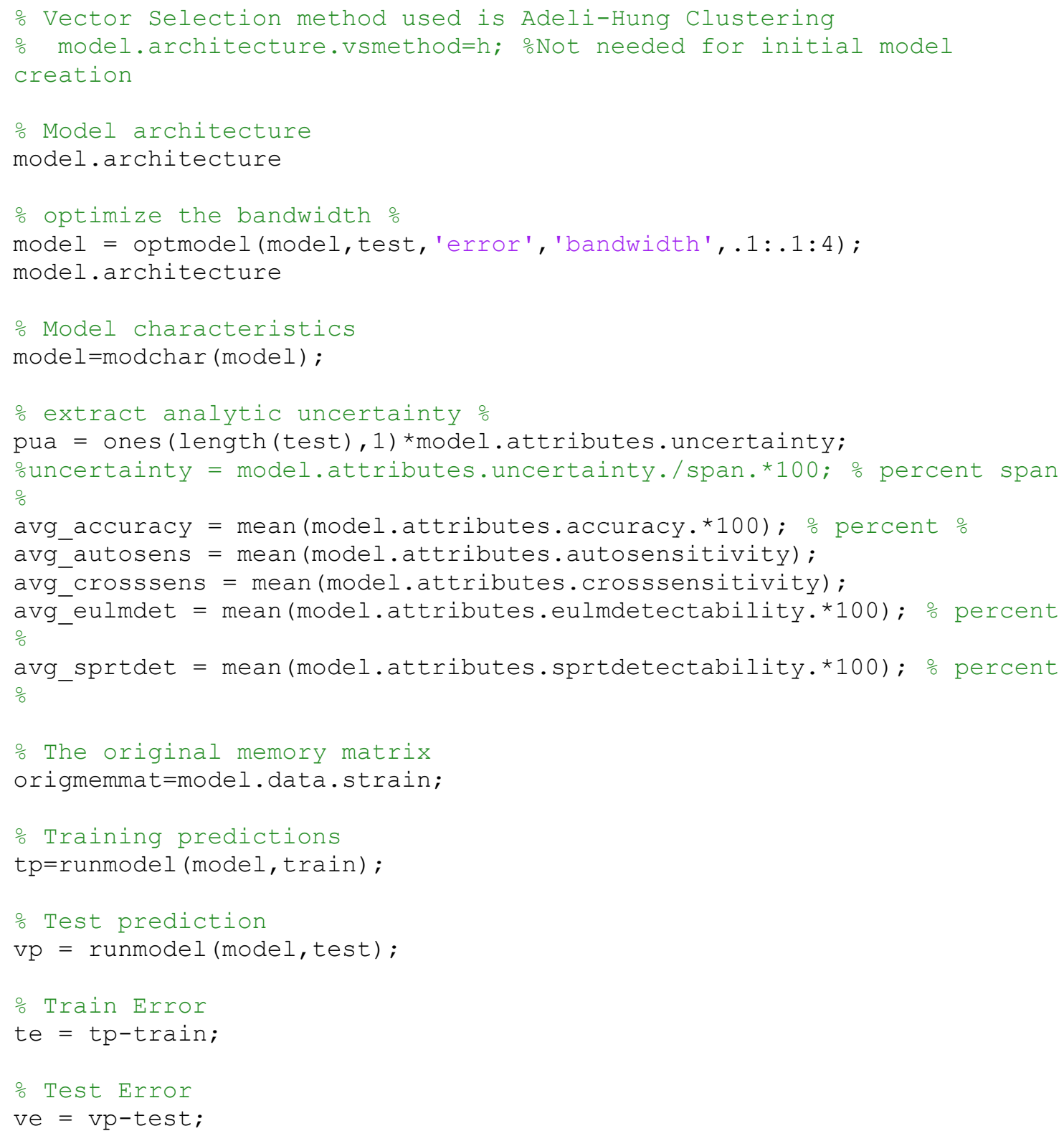




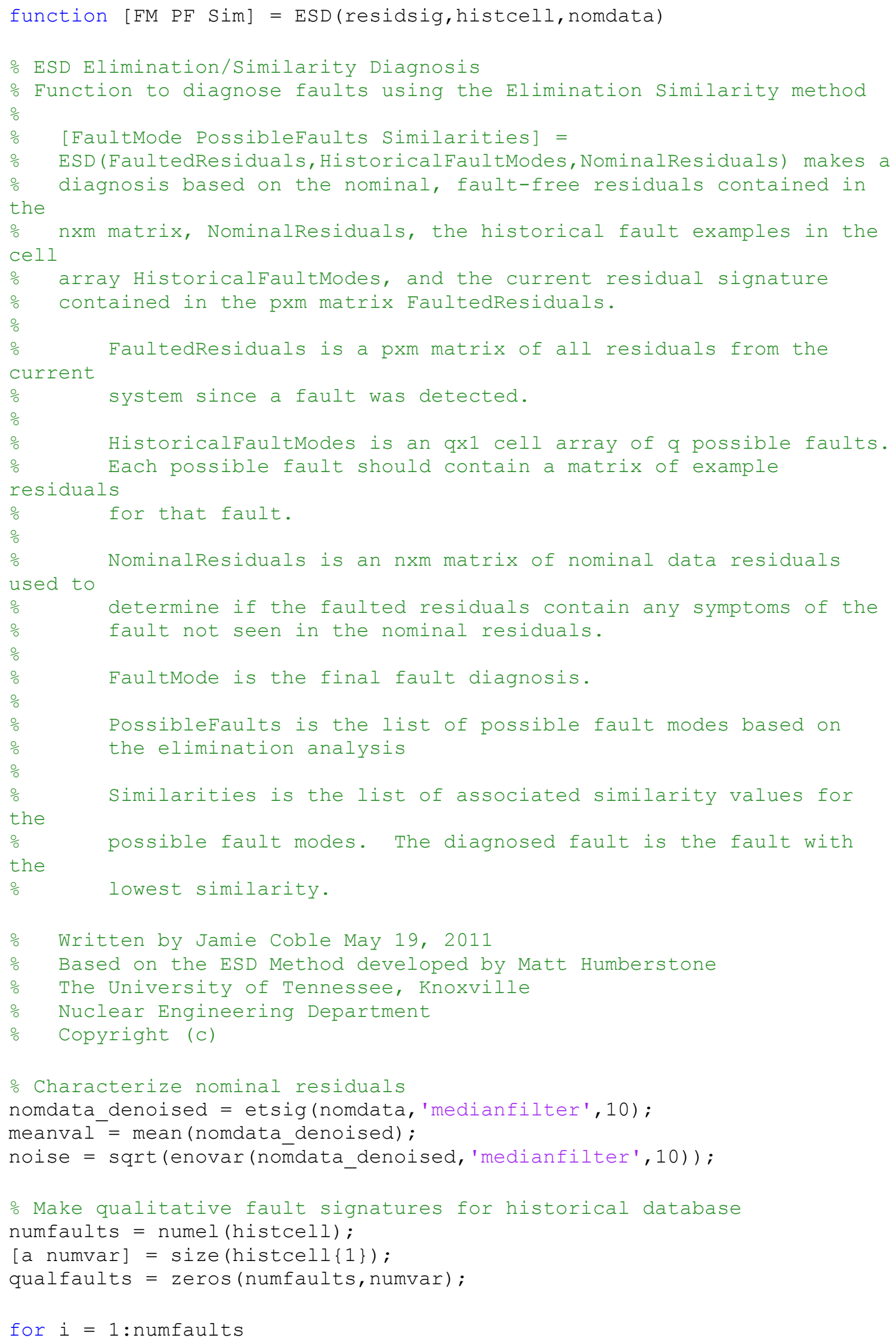




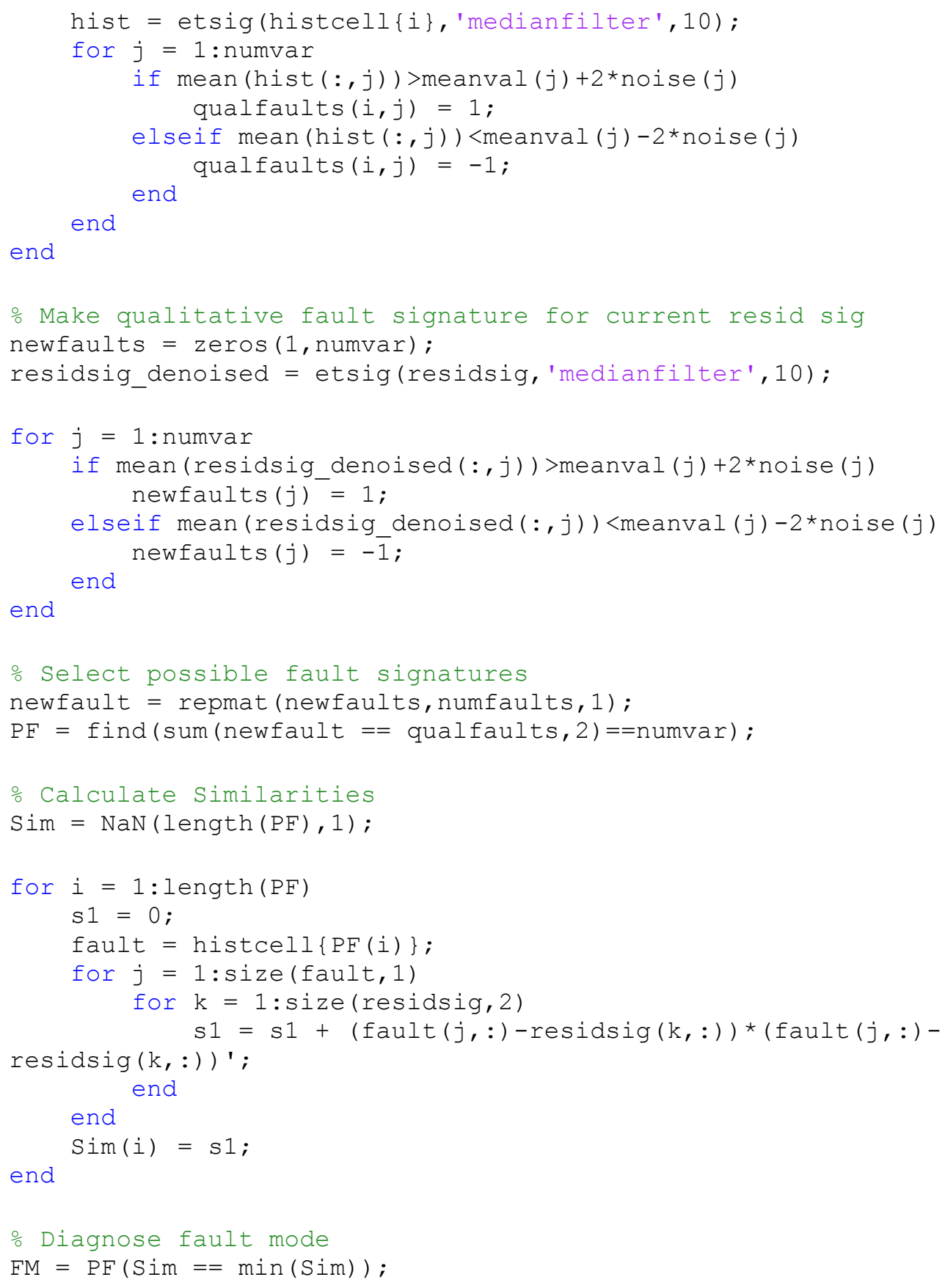


Appendix C: Tutorial for Developing Adaptive Health Monitoring Systems 


\section{A Tutorial for Developing an Adaptive Health Monitoring System}

Dr. Jamie Coble, jcoble1@ utk.edu

Dr. J. Wesley Hines, jhines2@utk.edu

Dr. Matthew Humberstone, mhumbers@utk.edu 


\section{Table of Contents}

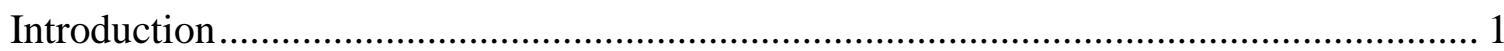

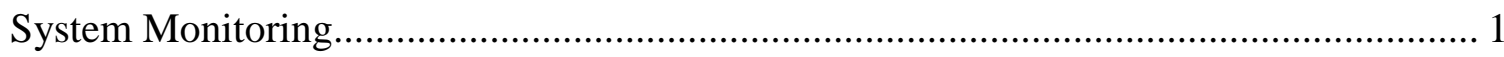

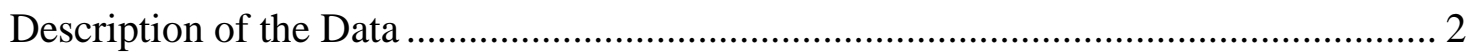

Auto-Associative Kernel Regression ........................................................................ 2

Application of Auto-Associative Kernel Regression.................................................. 4

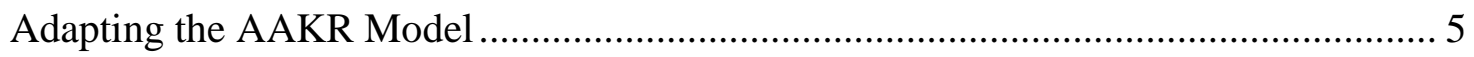

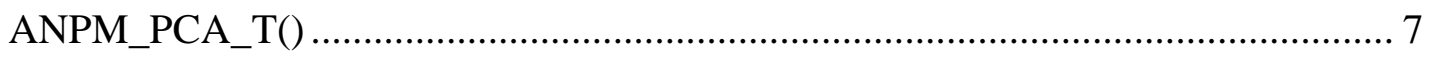

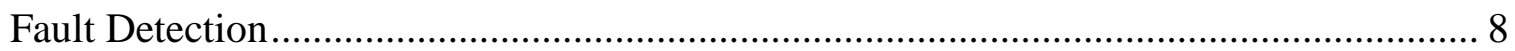

Differentiating Faults and Expanded Conditions - PCA-based Detection .................... 10

Principle Component Analysis Based Expanded Condition Monitoring ................. 10

Application - Expanded Operation..................................................................... 11

Application - Faulted Operation...................................................................... 12

Static Fault Detection - SPRT-based Detection ………………….............................. 13

Application of SPRT Fault Detection .................................................................. 14

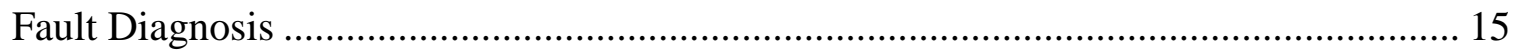

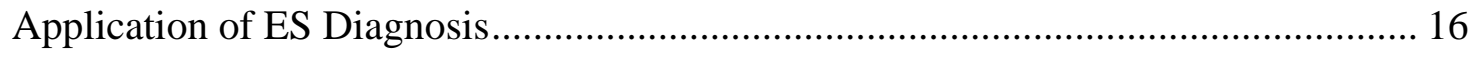

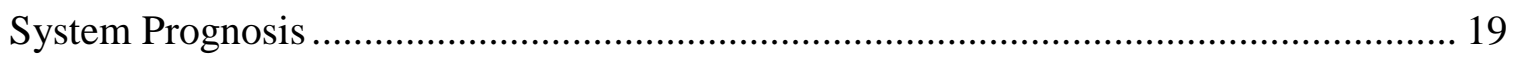

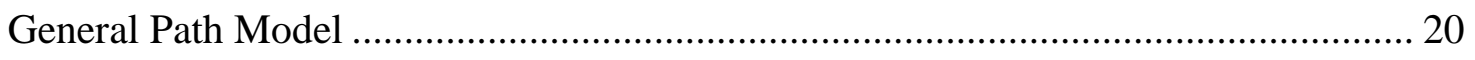

Module Integration.............................................................................................. 22 


\section{List of Figures}

Figure 1: Full Health Monitoring System ............................................................................. 1

Figure 2: Auto-Associative Model Architecture................................................................... 3

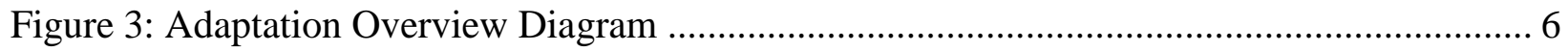

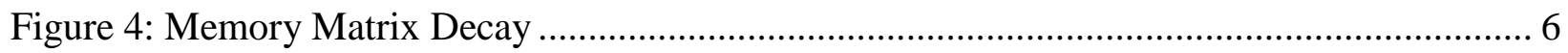

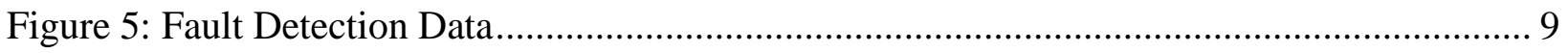

Figure 6: Training and Expanded Nominal Condition Data ....................................................... 9

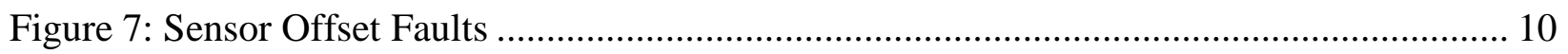

Figure 8: Adaptive Modeling Fault Procedure …………………………………………........ 11

Figure 9: Expanded Nominal Condition PCA-ECM Results ........................................................ 12

Figure 10: Faulted Condition PCA-ECM Results ..................................................................... 13

Figure 11: Illustration of (a) $+\mathrm{M}$ and (b) -M degraded modes .................................................. 14

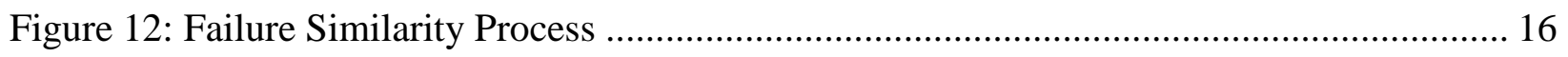

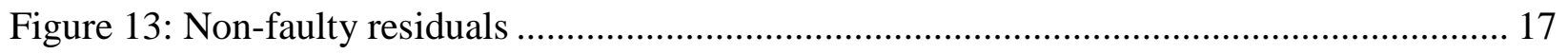

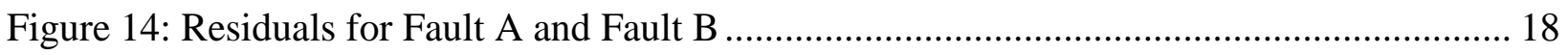

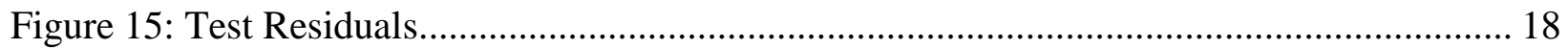

Figure 16: Integration of Health Monitoring Modules ................................................................ 22 


\section{List of Tables}

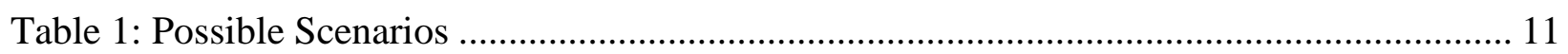

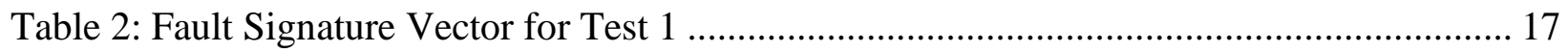




\section{Introduction}

Full health monitoring systems include several modules: system monitoring, fault detection, fault diagnosis, and system prognosis, as shown in Figure 1. Typically, these modules are built for static data sets where the operating conditions and possible faults are well understood. However, for systems that do not meet these assumptions, monitoring systems can be developed which allow a certain amount of flexibility. Similar, but adaptive, modeling techniques and algorithms can be used for these systems. This tutorial outlines the development of an adaptive health monitoring system using the previously developed Process and Equipment Monitoring (PEM) and Process and Equipment Prognostics (PEP) toolboxes, the developed Adaptive Non-Parametric Model (ANPM), and expert diagnostic systems.

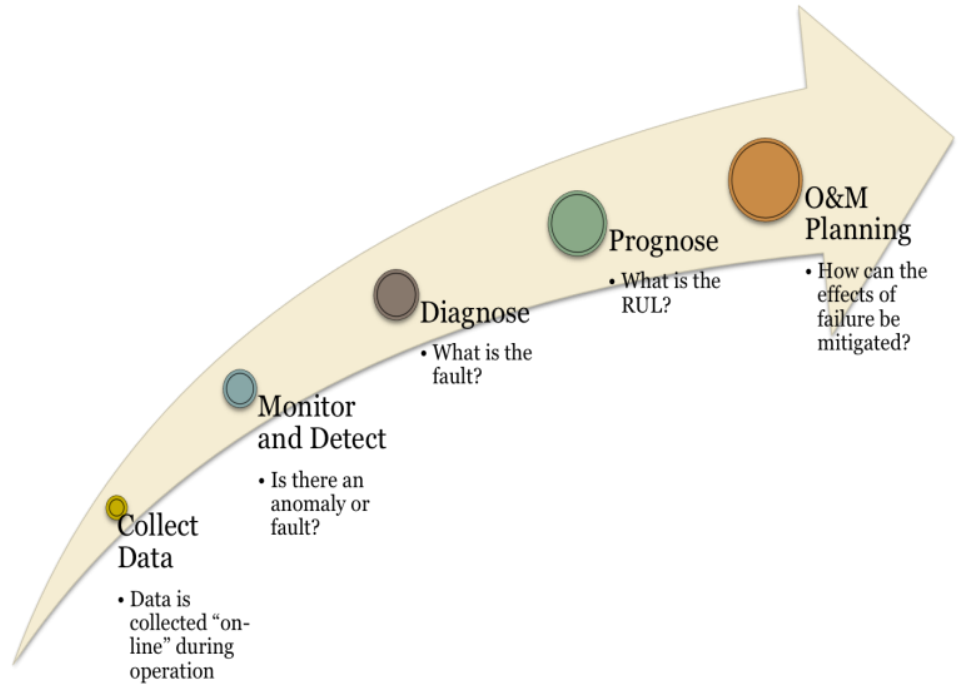

Figure 1: Full Health Monitoring System

This tutorial outlines algorithms and methods that are useful for building an adaptive monitoring system. Each of the modules includes discussion of how to include new information as it becomes available. The following chapters discuss each of the monitoring system modules in turn, provide some insight into the ability for specific algorithms to adapt, and present example applications of the algorithms. This tutorial does not suppose that the algorithms presented here are the only algorithms which allow for model adaptation, but merely a subset that will provide a basic framework for adaptive health monitoring.

\section{System Monitoring}

System monitoring is traditionally accomplished by comparing the system measurements collected on-line to the results of a high-fidelity model of the system. This model can be either a first-principle based model or an empirically derived model. First-principle models have the advantage of making predictions for a wide range of operating conditions. However, these models are difficult and costly to develop, they necessarily include simplifying assumptions, and they may be intractable to run in real- or near-real-time. Conversely, empirically based models 
are simple to develop, they naturally account for any noise sources or idiosyncrasies in the system, and they are typically very fast to run. Unfortunately, empirically based models cannot accurately extrapolate for operating conditions outside of their training regions. Predictions made by empirical models can only be trusted when they are similar to the data used to train the model. This is a major disadvantage when developing adaptive models, as the model necessarily needs to recognize and predict nominal operation in new regimes. The Adaptive Non-Parametric Model (ANPM) capitalizes on the advantages of first-principle and empirical models while minimizing the deficits of each. ANPM is built on the auto-associative kernel regression (AAKR) algorithm. The following sections demonstrate development of a static and a dynamic AAKR model, respectively. Development of a static model utilizes the PEM toolbox, while a dynamic model is built using independently developed code specifically designed for dynamic modeling.

\section{Description of the Data}

The monitoring algorithms described here are illustrated using simulated data from two first principle based simulations of the International Reactor Innovative and Secure (IRIS). Because the two simulators do not track the same set of sensors, model development and testing was performed in two phases. First, the nominal condition adaptation of the ANPM was tested using a reduced number of sensors to utilize both simulators. Then, the PCA-based expanded operation monitoring system was testing using the full data set from the high-fidelity simulation.

The first simulator, considered to be a low-fidelity simulator, was built by researchers at the University of Tennessee using MATLAB Simulink® (Li et al., 2009). The Simulink model is a modular model which includes the reactor core, the helical coil steam generators, and the balance of plant. In testing the adaptation phase of the ANPM model, the results of this simulator are considered to be low-fidelty results and are used to seed the original memory matrix.

The second simulator was developed at North Carolina State University (NCSU). NCSU has demonstrated experience in developing high fidelity, full plant simulators for predicting the dynamic response of pressurized water reactors during normal and off-normal operational conditions. An IRIS specific simulator has been developed which includes a model of the IRIS Pressurizer, a six delayed neutron group kinetics model, a decay heat model and a hot channel/departure from nucleate boiling (DNB) model. In the IRIS design, the steam generators are helical coils, where the secondary fluid flows on the tube side of the heat exchanger. Detailed models have been developed to describe the dynamics of steam generators of this design. For testing model adaptation, the results of this simulator are used as the high fidelity, "operational" data.

\section{Auto-Associative Kernel Regression}

An auto-associative model is a model whose outputs are trained to emulate its inputs over an appropriate dynamic range, as in Figure 2. Because data is often corrupted by noise, faults, and/or biases, an auto-associative model may be considered an error-correction routine, wherein the model predictions are the "correct" measurement values. In other words, an auto-associative model will act to correct its input based on those values that are in agreement with the 
relationships embedded in the model during training. Auto-associative kernel regression (AAKR) is a similarity-based error correction technique.

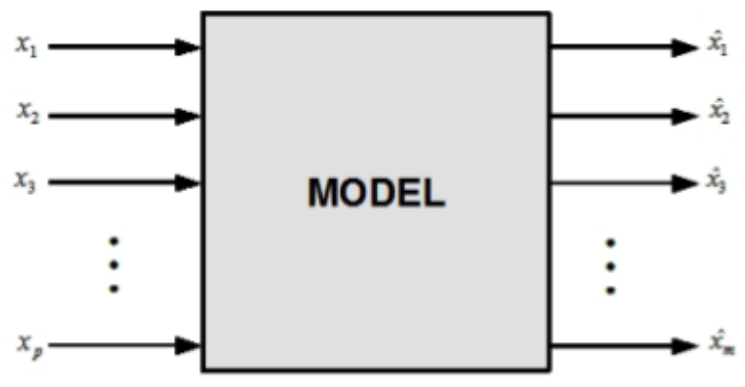

Figure 2: Auto-Associative Model Architecture

AAKR is a nonparametric, empirical modeling technique that uses historical, fault-free observations to correct any errors present in current observations. The exemplar or memory vectors used to develop the empirical model are stored in a matrix $\mathbf{X}$, where $X_{i, j}$ is the $i^{\text {th }}$ observation of the $j^{\text {th }}$ variable. For $n_{m}$ observations of $p$ process variables, this matrix can be written as:

$$
\mathbf{X}=\left[\begin{array}{cccc}
X_{1,1} & X_{1,2} & \cdots & X_{1, p} \\
X_{2,1} & X_{2,2} & \cdots & X_{2, p} \\
\vdots & \vdots & \ddots & \vdots \\
X_{n_{m}, 1} & X_{n_{m}, 2} & \cdots & X_{n_{m}, p}
\end{array}\right]
$$

Using this format, a query vector is represented by a $1 \times p$ vector of process variable measurements. This vector, $\mathbf{x}$, can be written as:

$$
x=\left[x_{1} x_{2} \ldots x_{p}\right] .
$$

The corrected version of the input is calculated as a weighted average of historical, errorfree observations termed memory vectors $\left(\mathbf{X}_{i}\right)$. The mathematical framework of this modeling technique is composed of three basic steps. First, the Euclidean distance between a query vector and each of the memory vectors is computed. The Euclidean distance equation for the $i^{\text {th }}$ memory vector is:

$$
d_{i} \boldsymbol{\alpha}_{i}, \mathbf{x} \bar{\equiv} \sqrt{\mathbf{Q}_{i, 1}-x_{1}{ }^{2}+\boldsymbol{\alpha}_{i, 2}-x_{2}{ }^{2}+\cdots+\mathbf{Q}_{i, p}-x_{p}{ }^{2}} .
$$

For a single query vector, this calculation is repeated for each of the $n_{m}$ memory vectors, resulting in an $n_{m} \times 1$ matrix of distances: $\mathbf{d}$.

Next, these distances are converted into similarity measures or weights by evaluating the Gaussian kernel, expressed by:

$$
\mathbf{w}=K \mathbf{T}=\exp \left(-\frac{\mathbf{d}^{2}}{h^{2}}\right)
$$

Here, $h$ is the kernel bandwidth, and $\mathbf{w}$ are the weights for the $n_{m}$ memory vectors. 
Finally, these weights are used to form a weighted average of the memory vectors according to:

$$
\hat{\mathbf{x}}=\frac{\sum_{i=1}^{n_{m}} \boldsymbol{w}_{i} \mathbf{X}_{i}^{-}}{\sum_{i=1}^{n_{m}} w_{i}}
$$

If the scalar $a$ is defined as the sum of the weights, i.e.

$$
a=\sum_{i=1}^{n_{m}} w_{i},
$$

then the model prediction can be represented in a more compact matrix notation as:

$$
\hat{\mathbf{x}}=\frac{\mathbf{w}^{\mathrm{T}} \mathbf{X}}{a}
$$

The parameters to be chosen in an AAKR model are the memory matrix $(\mathbf{X})$, the kernel bandwidth $(h)$, and the distance function. The model developer must decide how many and which vectors to include in the memory matrix and how large to make the bandwidth, which indirectly controls how many memory vectors are weighted heavily during prediction.

Traditionally, the memory matrix, $\mathbf{X}$, is populated with data collected during previous, fault-free operation. This matrix remains static after initial training, precluding any model adaptation. The following section describes the methods and techniques used to move static AAKR models into an adaptive paradigm.

\section{Application of Auto-Associative Kernel Regression}

To begin, a static AAKR model is initialized with the PEM toolbox.

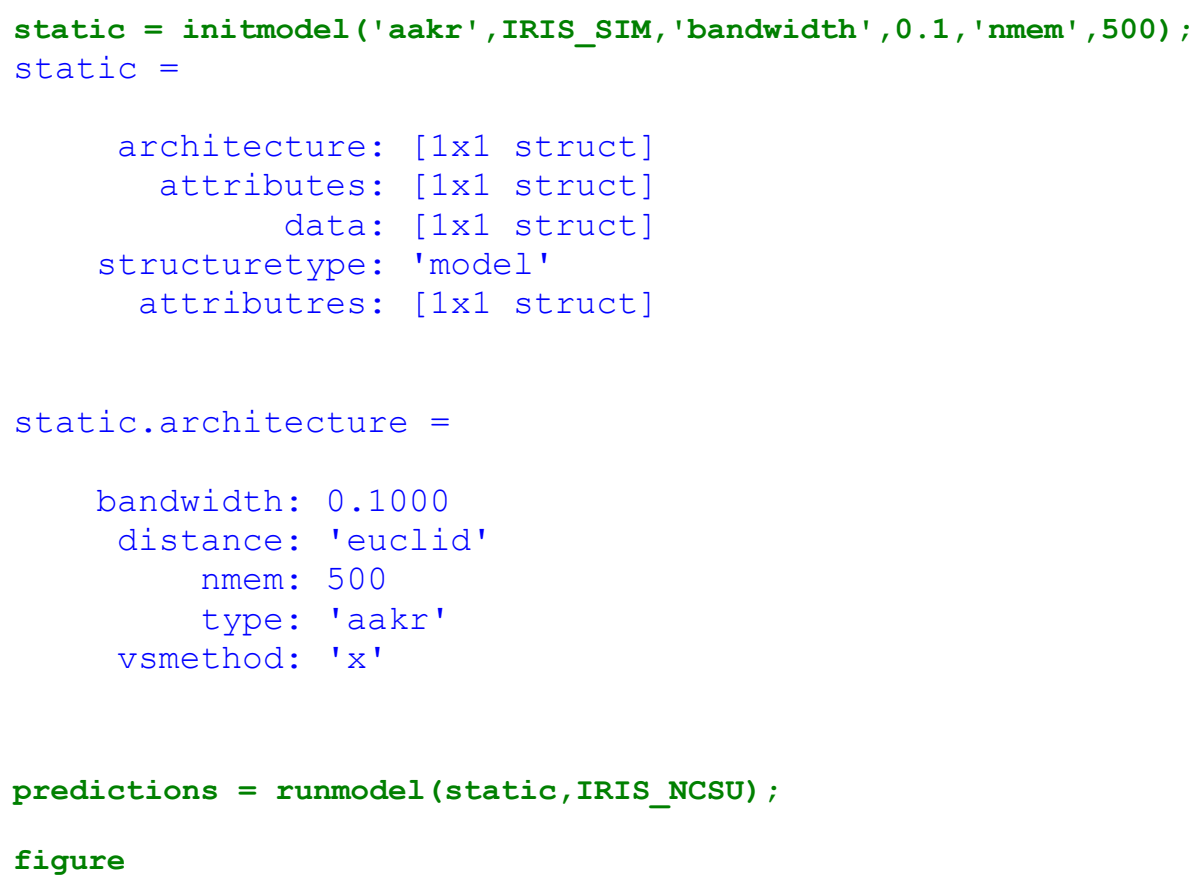


hold on

plot (IRIS_NCSU $(:, 1), '$ ' ')

plot (predictions $(:, 1), ' k^{\prime}$ )

xlabel ('Time (obs)')

ylabel ('Sensor 1')

legend('Actual', 'Predictions')

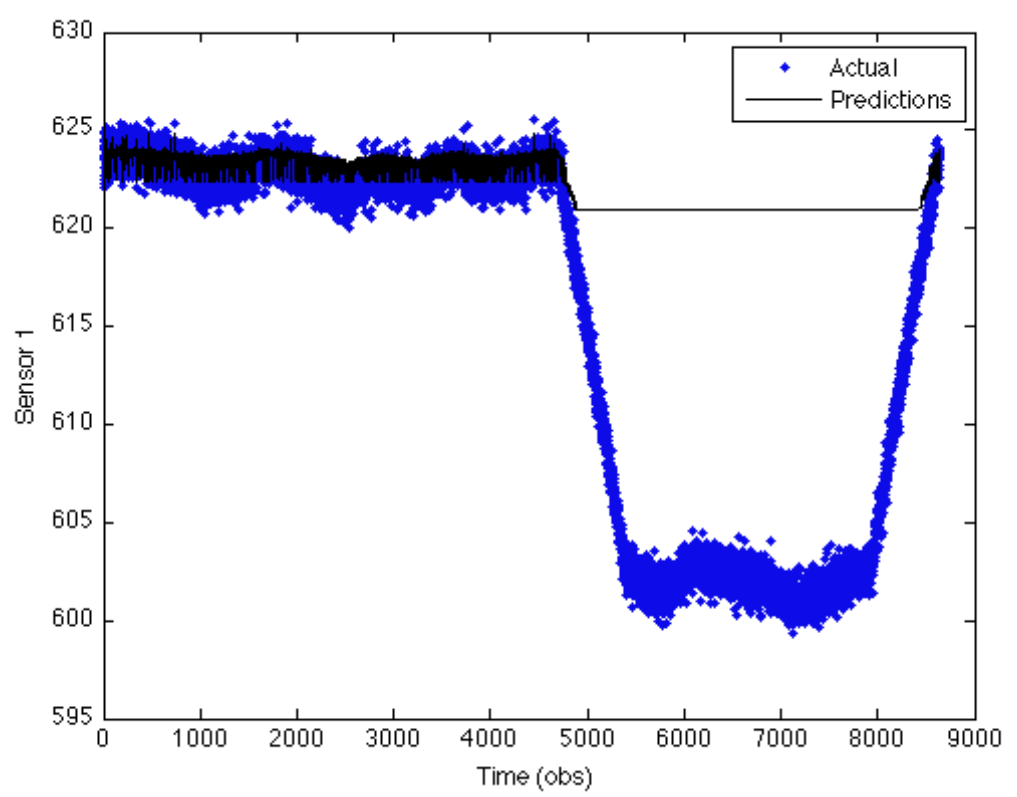

As we can see from the above plot, the static model built on low-fidelity data does not predict all operating regions; the model cannot predict behavior during low power operation (observations $\sim 5000$ to $\sim 8500$ ). It also shows significant clipping in the high power region. The ANPM model, shown next, can account for operation in new conditions.

\section{Adapting the AAKR Model}

Code has been developed specifically for training and adapting ANPM models. The MATLAB function ANPM1 utilizes the PEM toolbox to initialize a model based on low-fidelity data. The model is then updated as new data become available. The results of this model are shown below. These results are obtained using the PCA-based Expanded Condition Monitoring algorithm described in the next section. The ANPM1() function is described and illustrated here.

Figure 3 gives a basic overview of the adaptation phase of the ANPM compared to a traditional hybrid model. This figure shows how the first principle model is used to generate data to populate the original memory matrix in the ANPM. The simulated data is used to make AAKR predictions during initial system operation. These predictions are used to find the residual between the actual data the expected system operation. An expanded condition monitoring system is employed to determine if actual system data represents faulted or non-faulted conditions; this method is described in the next chapter. Then, if the data vector is determined to be from non-faulted data, this new observation can be appended to the original memory matrix of simulated data. The decision to add the data to the model is based on the fault detection results for the current observation, previous observations, and future observations. In this way, a new 
model evolves which is based on a combination of the first principle model data and the data collected during system operation.

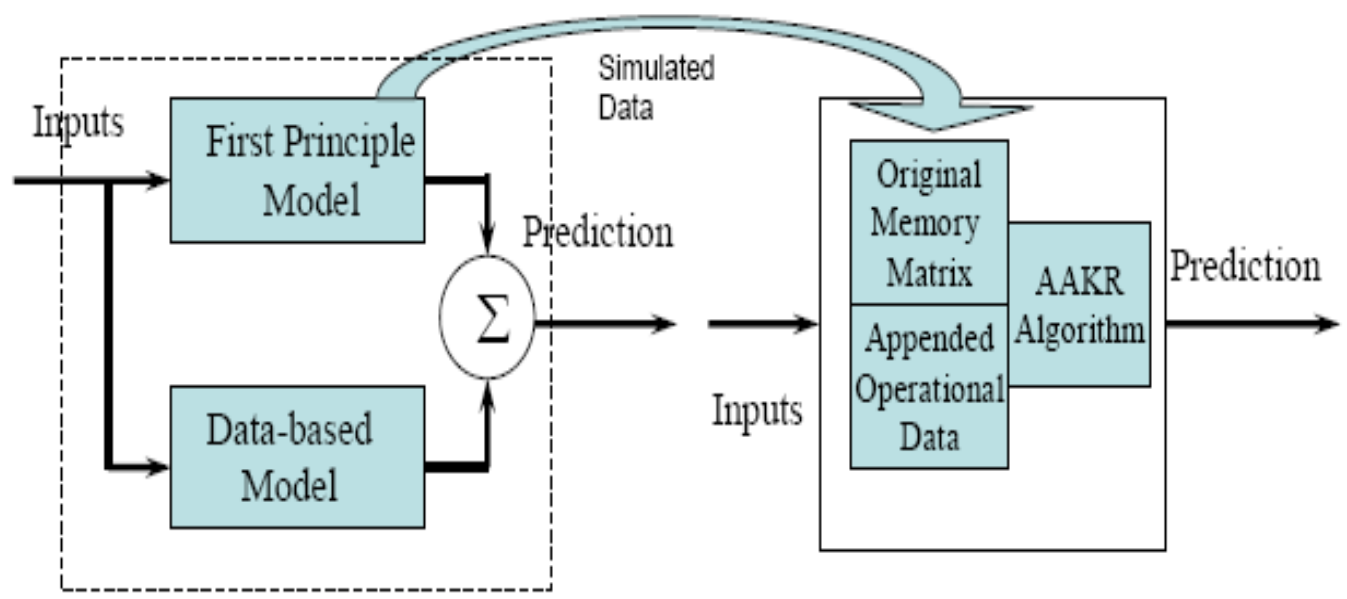

Traditional Hybrid Model Proposed Adaptive Non-Parametric Model

Figure 3: Adaptation Overview Diagram

As actual nominal system data is appended to the ANPM memory matrix, the data simulated by the first principle model decays from the model. At the end of model adaptation, a model is desired that is based entirely on actual data from the system. This is accomplished by deleting the observations contained in the original memory matrix as actual system observations which characterize the same or similar operating conditions are collected. The method used for vector deletion from the model is a constant rate vector deletion technique. This consists of deleting vectors from the original memory matrix in an evenly spaced time sequence. This is done over the operating period of the ANPM or during phase 1 . The vector with the greatest contributions to past predictions is deleted as is shown in Figure 4. This eliminates the vectors that are the most similar to the new vectors that are being appended. This is an important feature of adaptation because it directs the evolution of the model so that the models range is complete. This process is continued until only the system's data is in the model's memory matrix.

\begin{tabular}{|c|c|c|}
\hline Memory Matrix & Contribution & New Memory Matrix \\
\hline V1 & 0 & V1 \\
\hline V2 & 0 & V2 \\
\hline V3 & 0.2 & V3 \\
\hline V4 & 0.6 & New Vector \\
\hline V5 & 0.2 & V5 \\
\hline V6 & 0 & V6 \\
\hline V7 & 0 & V7 \\
\hline
\end{tabular}

Figure 4: Memory Matrix Decay 


\section{ANPM_PCA_T() \\ The ANPM_PCA_T() function utilizes the functionality of the PEM Toolbox in addition to other functions specifically developed for adaptive monitoring. This toolbox and the additional functions must be available for the adaptive model to train, adapt, and make predictions.}

Adaptation Model Function ANPM PCA T

This function is to be used for model adaptation.

[totquery, nmat, oldmodel, finalmodel, totpredict, finalpredict, FPMpredict, Fhypmat

] = ANPM_PCA_T(data, query, perday, cycle) creates a new model finalmodel that represents the data from the actual process of interest and slowly deletes the original model, which is first created using aakrmodcreate. nmat is the data matrix created by the new data, this is without any faults detected, this can be used to create a model solely dependent on the new data

[totquery, nmat, oldmodel, finalmodel, totpredict, finalpredict, FPMpredict, Fhypmat ] = ANPM PCA T (data, query, totcyclenum) this is if the total cycle number isknown but the perday of cycle by themselves is not known.

\section{DESCRIPTION :}

This is the first working ANPM model. It deletes the original memory matrix with a linear deletion method in which the vector with the highest sum of weights is deleted at a certain interval. This interval is determined by the number of steps within the adaptation and the number of original memory vectors within the FPM (First Principle Model).

ANPM_PCA_T uses the bandwidth that was optimized with the FPM and not updated within the adaptation. The vector addition technique used was a continual addition with periodic vector selection using Adeli Hung technique.

\section{Inputs :}

data- The FPM data used to create the inital aakr model. query- Is the new input data that is going to be stored in nmat and used to create the new model perday- Is the number of data collection rows in a day cycle- The number of days that the adapt model will be running.

\section{Outputs :}

totquery- query vectors

nmat- new query vectors without errors

oldmodel- model built on low-fidelity data

finalmodel- adapted model

totpredict- matrix of predictions during adaptation

finalpredict-predictions of final model

FPMpredict- predictions of original model

Fhypmat- matrix of fault hypotheses from SPRT analysis

It is important to note that the predictions and models which are returned by ANPM1 are standardized. Inputs to the models must be standardized according to the mean and standard deviation of the original data before applying any model. Similarly, the predictions need to be unscaled to compare them to actual sensor values. The PEM toolbox functions zscorel and unscale can be used for this purpose. 

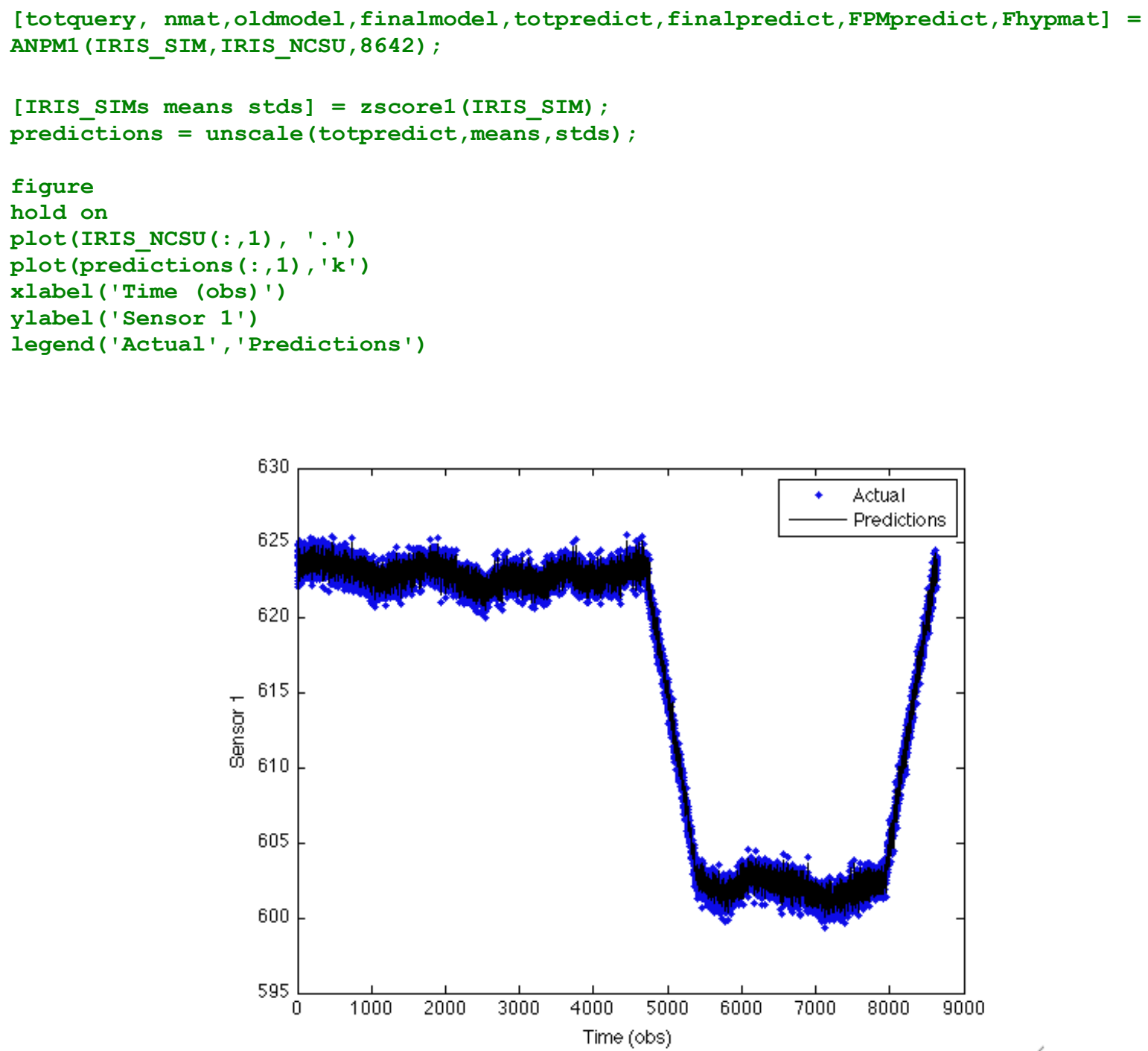

As the plot shows, the ANPM model is able to accurately adapt to the new low-power operating condition. This gives the model flexibility to make predictions for a wide range of nominal operation, provided that the relationships between sensors remain the same.

\section{Fault Detection}

Traditional statistically-based fault detection methods are not suitable for an adaptive environment because there is a sometimes significant lag time between fault inception and fault detection. During this time, the adaptive model would have the opportunity to learn the faulty behavior, instead of remaining robust to it. Identification of faulted operation is paramount to model adaptation. To facilitate this, a principle component-based fault detection routine is introduced for the adaptation phase. After adaptation is complete, it may be desirable to revert to the traditional methods, such as the sequential probability ratio test. This chapter introduces both methods and gives results for fault detection. 
For the purpose of illustrating the two fault detection methods, this section employs a different set of data exhibiting expanded nominal operation and faults. The data investigated here is simulated with linear relationships between sensors. The nominal operation data is shown in Figure 5. Figure 6 shows the training data in blue and the expanded nominal condition data in red for one sensor. The other sensors are divided the same way. Finally, Figure 7 shows the faulted condition investigated: sensor bias.

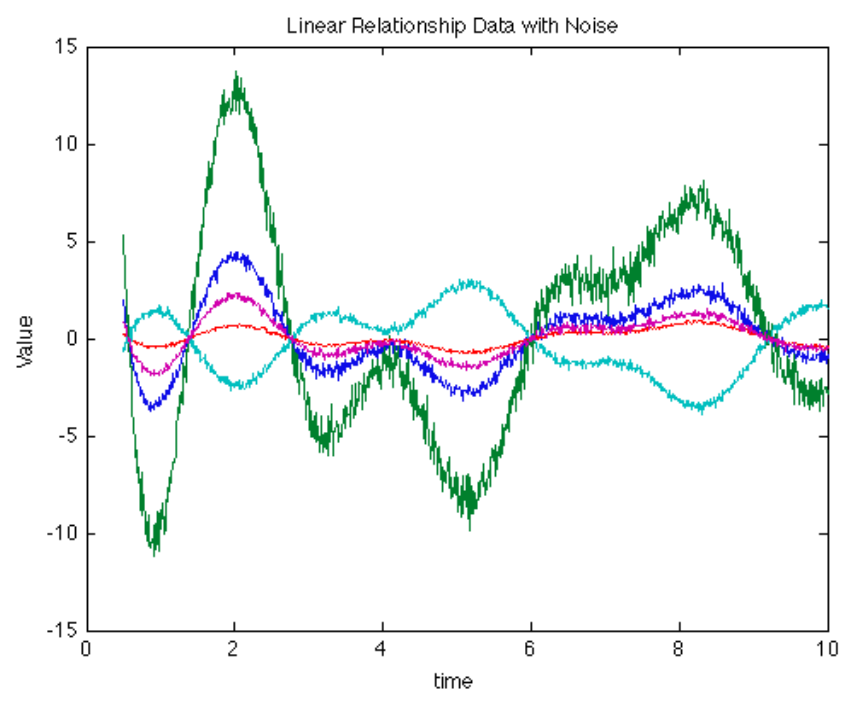

Figure 5: Fault Detection Data

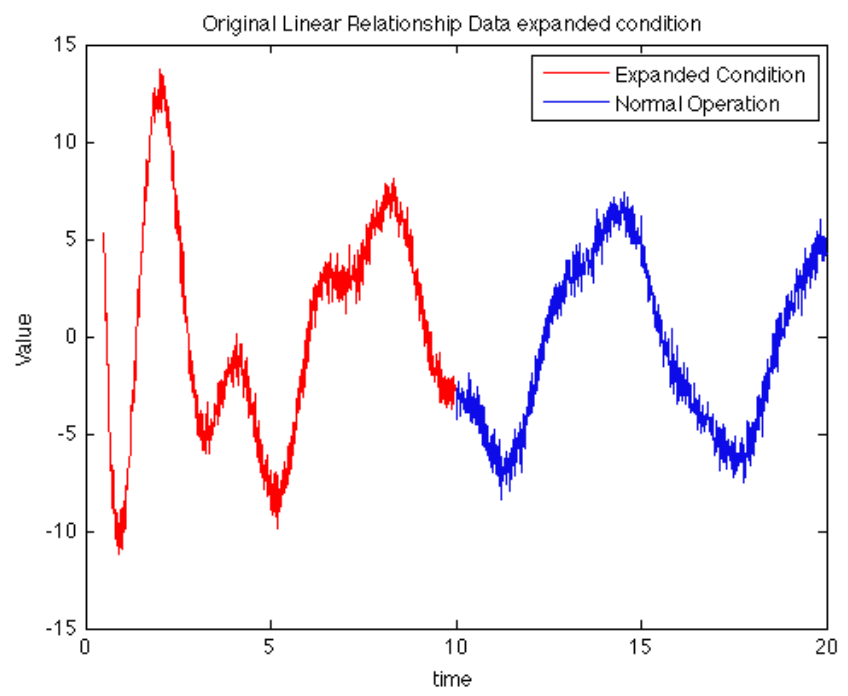

Figure 6: Training and Expanded Nominal Condition Data 


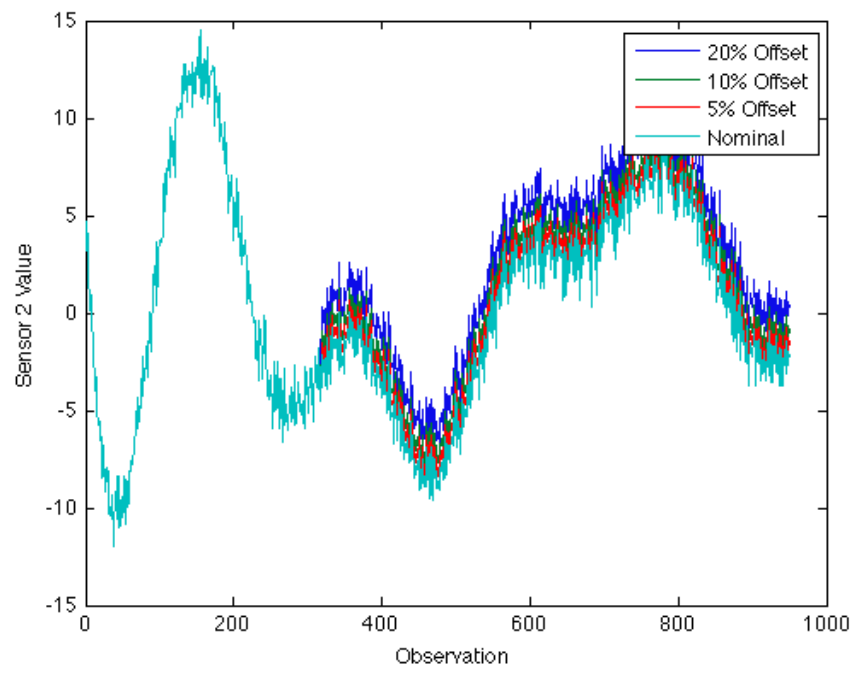

Figure 7: Sensor Offset Faults

\section{Differentiating Faults and Expanded Conditions - PCA-based Detection}

The ANPM adaptation relies on the ability to differentiate between faulted operation and nominal operation in new conditions. This is achieved through a Principle Component Analysis (PCA) method which transforms the data to the PCA space to determine if a new observation is due to (1) expected, nominal operation, (2) nominal operation in a new condition, or (3) a system fault. The following sections look at how the PCA method differentiates between expanded conditions and faulty conditions.

\section{Principle Component Analysis Based Expanded Condition Monitoring}

Principle component analysis (PCA)-based expanded condition monitoring (ECM) is the cornerstone of the automatic adaptation in the ANPM. Adaptive models must strike a balance between being elastic enough to learn new operating conditions and changing nominal relationships in the system, but plastic enough to not learn faulty behavior. Identifying faulty inputs and differentiating them from unexpected nominal conditions is a key challenge in automated model adaptation. Where static models may declare any observation beyond the range of the model training as a fault, an adaptive model needs the capability to determine if this new observation is, in fact, a fault or an extended nominal condition. PCA ECM gives one viable approach to this problem for sensed data with linear or nearly-linear relationships.

PCA ECM begins by performing PCA on the original data, that is, projecting the nominal low-fidelity data onto a principle component (PC) space. As new observations are collected, these observations are projected to the same PC space. These new PC observations are then analyzed with the Hotteling's $\mathrm{T}^{2}$ statistic and the squared prediction error, or $\mathrm{Q}$ statistic. These statistics are used to indicate where a data point is with respect to the PC model of nominal behavior. The $\mathrm{T}^{2}$ statistic describes the variation within the model and the $\mathrm{Q}$ statistic describes the variation outside the model according to the equations below:

$$
T^{2}=T \Lambda_{k}^{-1} T^{T} \leq \delta_{T}^{2}
$$




$$
Q=e^{T}=X\left(I-P_{e} P_{e}^{T}\right) X^{T} \leq \delta_{Q}^{2}
$$

This results in one of four possible outcomes, as shown in Table 1, where within limits is designated as WL and outside the limits is designated as OL.

Table 1: Possible Scenarios

\begin{tabular}{|c|c|c|c|}
\hline Scenarios & Q statistic & $\mathbf{T}^{2}$ statistic & Conclusion \\
\hline 1 & WL & WL & Normal Condition \\
\hline 2 & WL & OL & Expanded Condition \\
\hline 3 & OL & WL & Fault \\
\hline 4 & OL & OL & Fault \\
\hline
\end{tabular}

If the new observation is found to be within the PC model of normal operation or a symptom of expanded normal operation, then the ANPM adapts to that condition by including it in the AAKR memory matrix, as shown in Figure 8. However, if the observation is determined to be due to a fault, then that observation is not added to the memory matrix and the health monitoring system moves to fault diagnosis and prognosis.

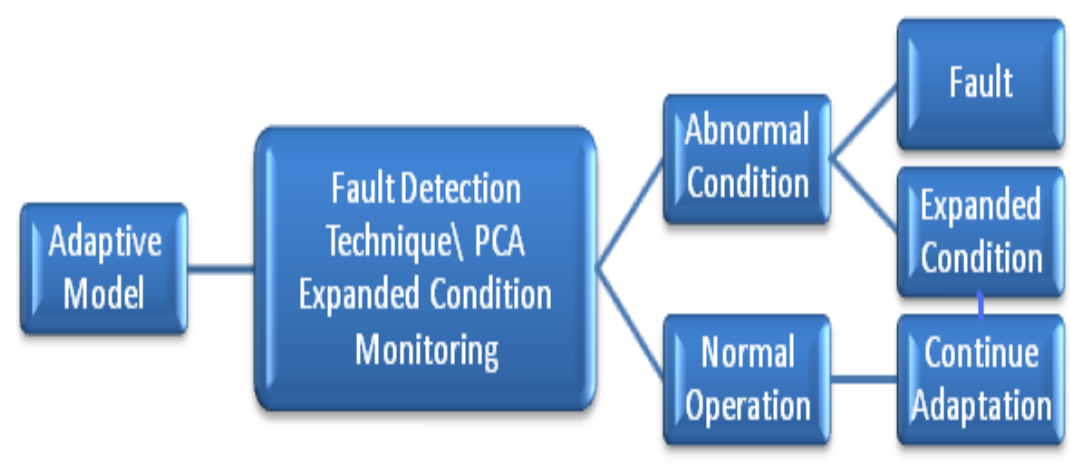

Figure 8: Adaptive Modeling Fault Procedure

PCA-ECM is coded in the ANPM functions; it is not independently available. The results of this analysis are shown below, as extracted from the code, but there is no need to run this algorithm independently of the model development discussed above.

\section{Application - Expanded Operation}

The results of the PCA ECM analysis on the expanded condition data are shown below, in Figure 9. These results were extracted from the ANPM_PCA_NT() function discussed previously. The second plot shows the $\mathrm{T}^{2}$ statistic for the training data (black) and the expanded operation data (red). The third plot shows the same results for the $\mathrm{Q}$ statistic. Applying these results to the scenarios in Table 1, we can see that the $\mathrm{T}^{2}$ is outside the limit while the $\mathrm{Q}$ statistic is within limits, indicating that the system is operating in an expanded nominal condition. 


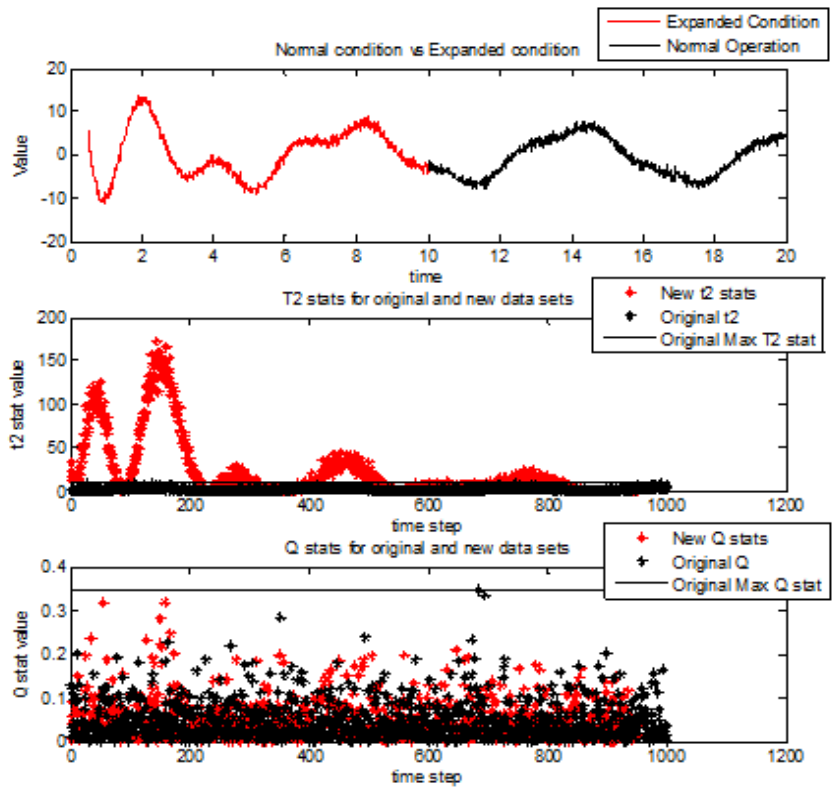

Figure 9: Expanded Nominal Condition PCA-ECM Results

\section{Application - Faulted Operation}

Figure 10 shows the PCA ECM results for the faulted operation. These results were extracted from the ANPM_PCA_NT() function discussed previously. Again, the second plot shows the $\mathrm{T}^{2}$ statistic and the third shows the $\mathrm{Q}$ statistic. The fault occurs at approximately observation 320. The PCA ECM technique is able to detect this fault immediately for all three fault levels, shown by the large jump in Q statistic values at that point. 

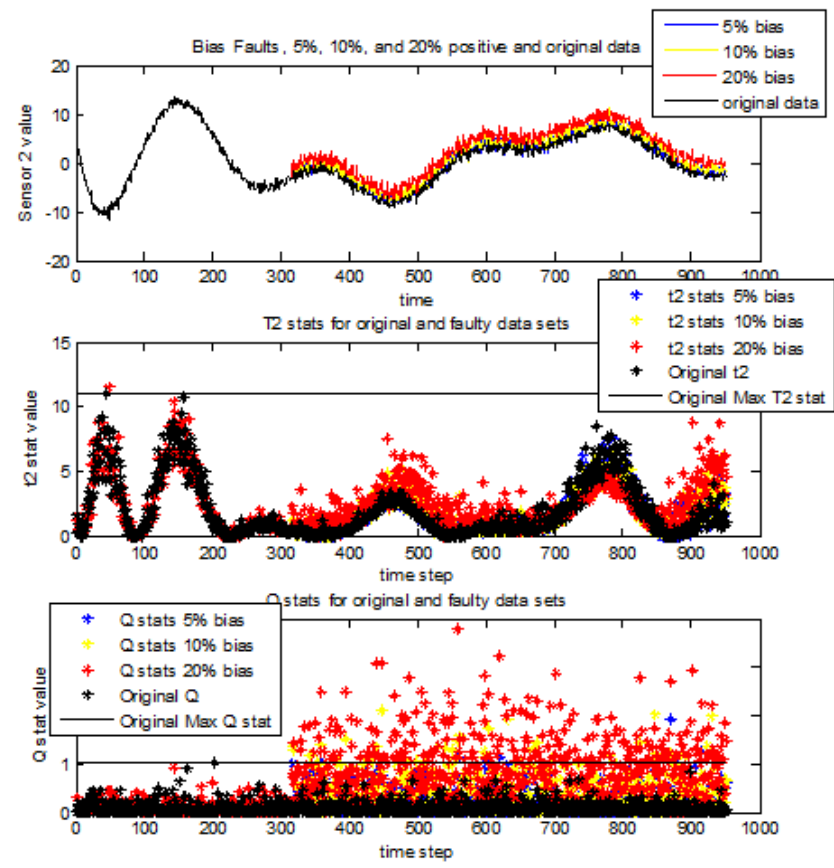

Figure 10: Faulted Condition PCA-ECM Results

\section{Static Fault Detection - SPRT-based Detection}

The PEM toolbox includes automated functions for developing and applying SPRT-based fault detection. While this fault detection method is not suggested for the adaptation phase of monitoring, it may be desirable to fall back to this method after model adaptation is complete. For that reason, the sequential probability ratio test (SPRT) method is discussed here.

The SPRT procedure consists of testing whether a sensor is more likely to be in a normal mode $\mathrm{H}_{0}$ or in a degraded mode $\mathrm{H}_{1}$. The SPRT is optimal in the sense that a minimum number of samples are required to detect a fault (change in mean) existing in the signal.

The general procedure for the SPRT is to first calculate the likelihood ratio, which is given by the following equation where $\left\{x_{n}\right\}$ is a sequence of consecutive $n$ observations of $x$.

$$
L_{n}=\frac{\text { probability of observing } x_{n} \text { given } H_{1} \text { is true }}{\text { probability of observing } x_{n} \text { given } H_{0} \text { is true }}=\frac{p x_{n} \mid H_{1}}{p}
$$

The likelihood ratio is then compared to the a lower $(A)$ and upper $(B)$ bound defined by the false alarm probability $(\alpha)$ and missed alarm probability $(\beta)$ as follows.

$$
A=\frac{\beta}{1-\alpha} \text { and } B=\frac{1-\beta}{\alpha}
$$

If the likelihood ratio is less than $A$ then it is determined to belong to the system's normal mode Ho. Conversely, if the likelihood ratio is greater than $B$ it is determined to belong to the system's degraded mode $\mathrm{H}_{1}$ and a fault is enunciated. 
In the PEM Toolbox, the SPRT is applied to the residuals between a sensor measurement and an empirical model's predictions of the parameter. The residuals are assumed to be normally distributed with a mean of 0 and variance of, which is an estimate of the random variation of the sensor signal. Therefore, the probability distribution function (pdf) for the normal mode of the residuals is given by:

$$
p x \mid H_{0}=\frac{1}{\sqrt{2 \pi \sigma^{2}}} \exp -\frac{x^{2}}{2 \sigma^{2}}
$$

From this description, two degradation modes are readily apparent. These modes are shown in Figure 11. In the first plot there is a mean shift up (+M) and the second plot shows a mean shift down $(-\mathrm{M})$. The random uncertainty is denoted by the spread of the Gaussian function. The SPRT simply determines it the residual sequence is more probably generated from the normal or faulted distributions.

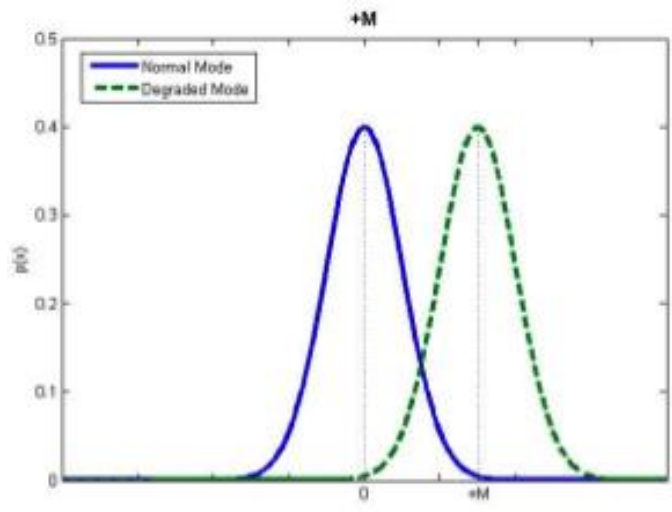

(a)

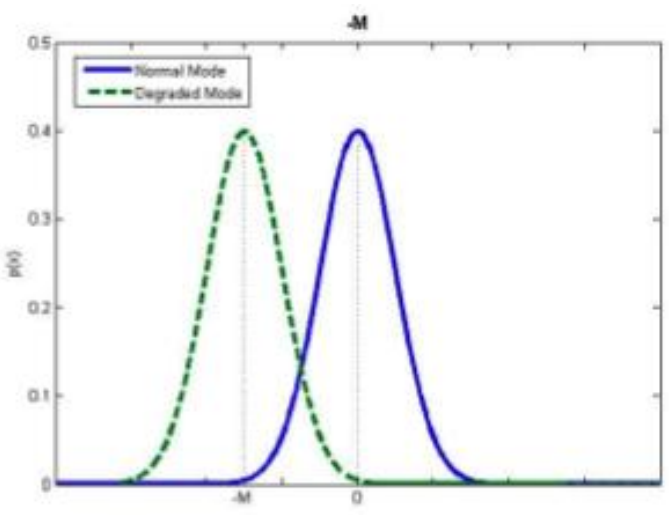

(b)

Figure 11: Illustration of (a) $+M$ and (b) $-M$ degraded modes

\section{Application of SPRT Fault Detection}

SPRT fault detection is implemented in the PEM toolbox for static models. After the model adaptation phase of the ANPM is complete, the system will likely return to a traditional fault detection routine.

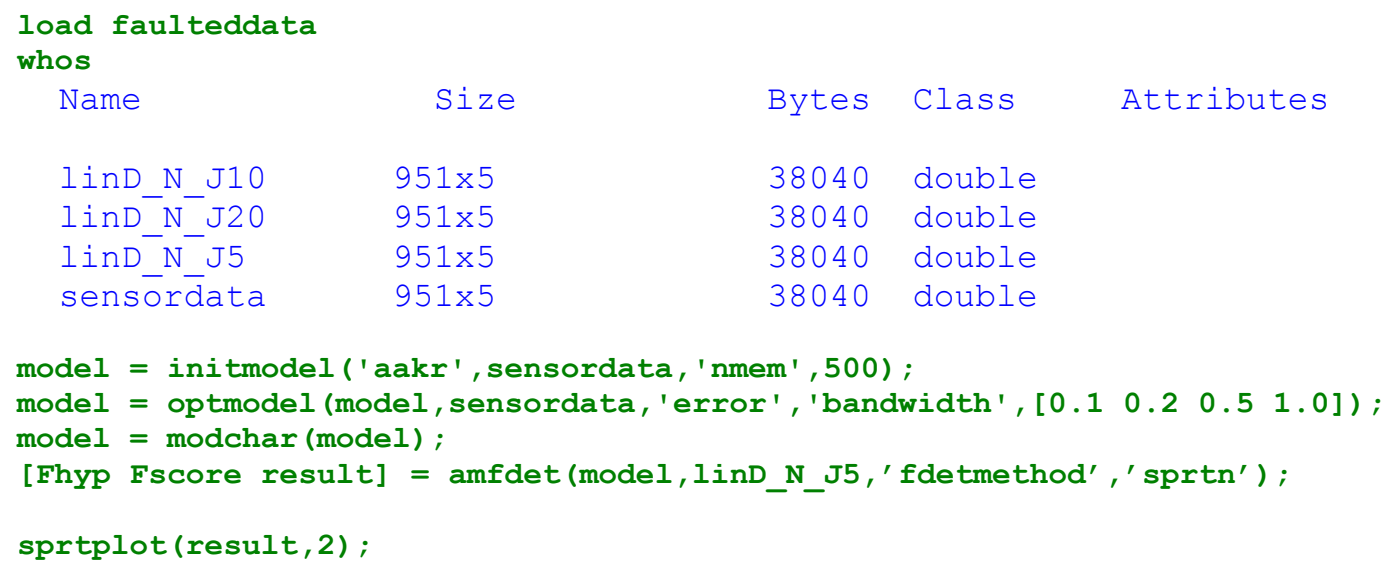



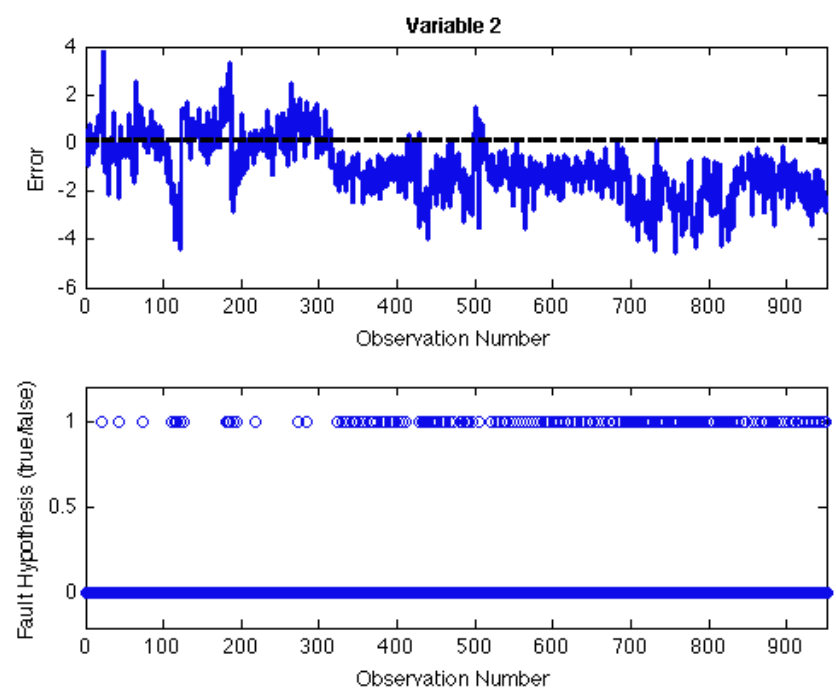

\section{Fault Diagnosis}

Once a fault has been detected, a health monitoring system moves to fault diagnosis. Fault diagnosis is typically accomplished via an expert system, such as a fuzzy inference system, a rule-based system, or a neural network classifier. Often, these systems are trained with all expected faults and remain static after initial training. Elimination Similarity (ES) diagnosis is one method that has been proposed to allow for a more adaptive diagnostic system. This diagnostic method is built on faulted residuals and involves a two-step process, which consists of eliminating fault candidates that do not match the current system configuration and then selecting the most likely candidate of those remaining based on a similarity measure.

After a fault has been detected, the complete fault signature vector is compared to a failure bank of fault signature vectors that correspond to all possible failures. At this point, the fault signatures are compared on qualitative features such as: Residual 1 increases, Residual 2 decreases, ... Residual $N$ remains constant. Fault modes are chosen which match this qualitative description of the fault symptoms. These descriptors can be very simple, as described above, or more specific, such as "increases a lot" or "increases a little." After the dissimilar fault signatures are eliminated on the qualitative basis, multiple possible fault modes may remain which are similar to the current fault signature vector. Then, an historical residual memory matrix of failure symptoms for those fault modes is used to designate which fault is most similar to the current residual signature. The similarity step uses the Euclidean distance between the past failure residuals and the current faulted residuals:

$$
S={ }_{i=1 \quad j=1}^{n} P F R_{i}-C F R_{j}{ }^{2}
$$

where $n$ is the number of faulty residuals; $m$ is the number of observations from the time a fault was initially detected; $P F R$ is the Past Fault Residuals, which constitute the historical data matrix; and CFR is the Current Fault Residual, or the series of residuals of the new system since 
a fault was detected. Figure 12 shows the process of comparing the current failure to the past failures that have the same fault signature vector. This example shows that system failures 1, 4, and 6 have the same fault signature vectors. The Euclidean distances between the actual failure residuals and the historic residuals of the designated failures measure the similarity of the failures. Clearly, smaller Euclidean distances are desirable, meaning that the new residual signature is more similar to an historic fault symptom.

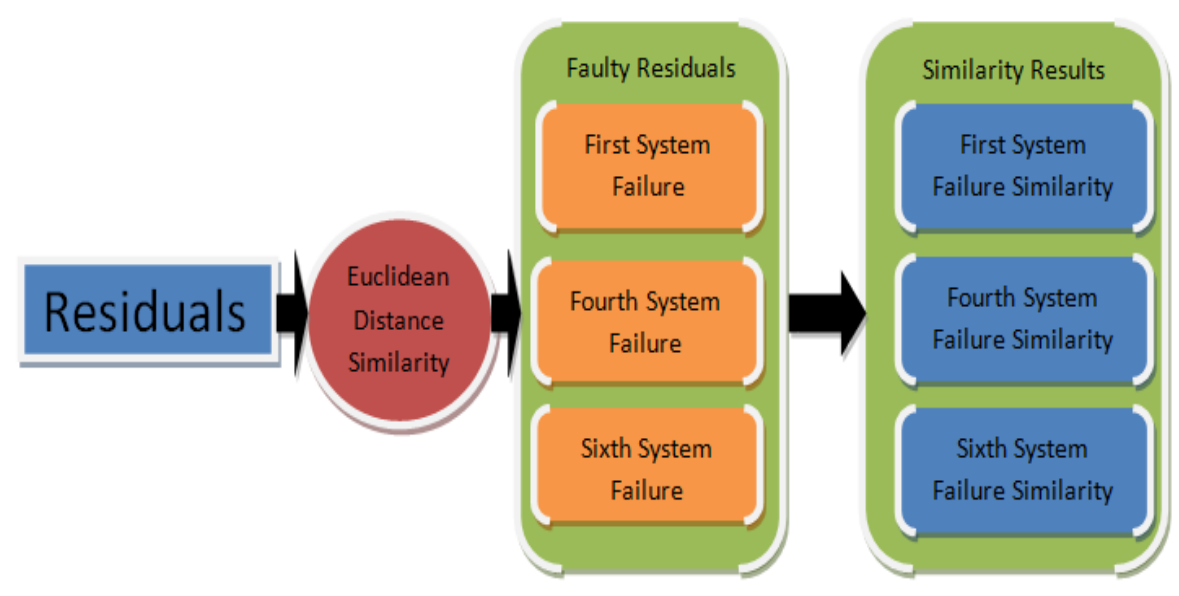

Figure 12: Failure Similarity Process

This fault diagnosis algorithm is attractive for adaptive systems for the same reasons that AAKR is attractive - because it is a nonparametric empirical technique, the diagnoser can easily be updated to include new faults as they appear. If an unanticipated fault is experienced, the new fault symptom is added to the historical matrices, both qualitative and residual signatures, for future fault diagnoses.

\section{Application of ES Diagnosis}

A data set was created to test the ES diagnostic technique. There are 10 residuals with a mean of zero and different variances, which represent a nominal condition. Below in Figure 13 are the non-faulty residuals, which is normally distributed noise with a mean of zero. This is expected when a good monitoring model is used for prediction. The deviation from nominal conditions of the variance or mean of the residual shows some abnormality in the system. 


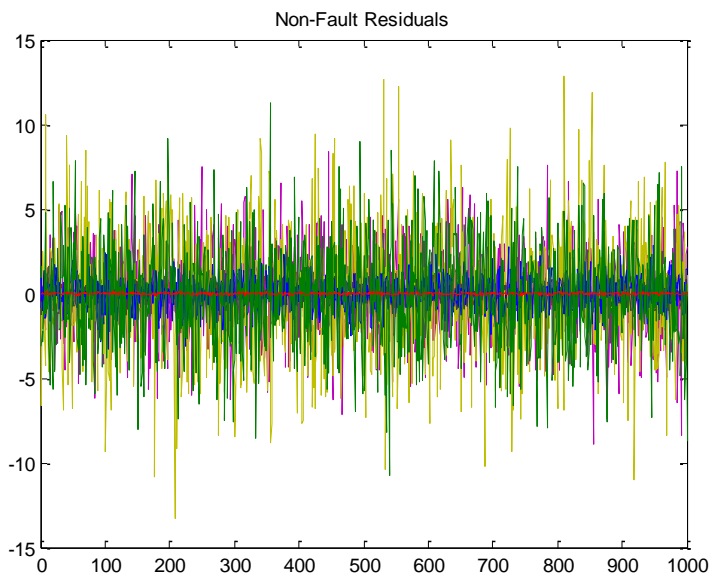

Figure 13: Non-faulty residuals

A historical residual memory matrix of failures was created with fifteen possible failures. These failures map to a corresponding fault signature vector. The historical residual memory matrix consists of ten observations for each failure.

To test the complete capability of the ES diagnostic technique a number of different failures need to have the same fault signature vector. The first two tested failures have the same fault signature vectors which can be seen in Table 2 .

Table 2: Fault Signature Vector for Test 1

\begin{tabular}{|c|c|c|c|c|c|c|c|c|c|}
\hline \multicolumn{1}{|c|}{ Sensors and corresponding fault signatures } \\
\hline 1 & 2 & 3 & 4 & 5 & 6 & 7 & 8 & 9 & 10 \\
\hline 1 & 0 & 0 & 1 & 0 & 0 & 0 & 0 & 0 & 0 \\
\hline
\end{tabular}

This fault signature vector shows that a positive drift or bias was found in sensors 1 and 4. All the other sensors had no detected deviations from nominal conditions. However, for this example, two failures were created that have this fault signature vector, failure a and failure $\mathbf{b}$. These residuals for sensors 1 and 4 can be seen in Figure 14. 

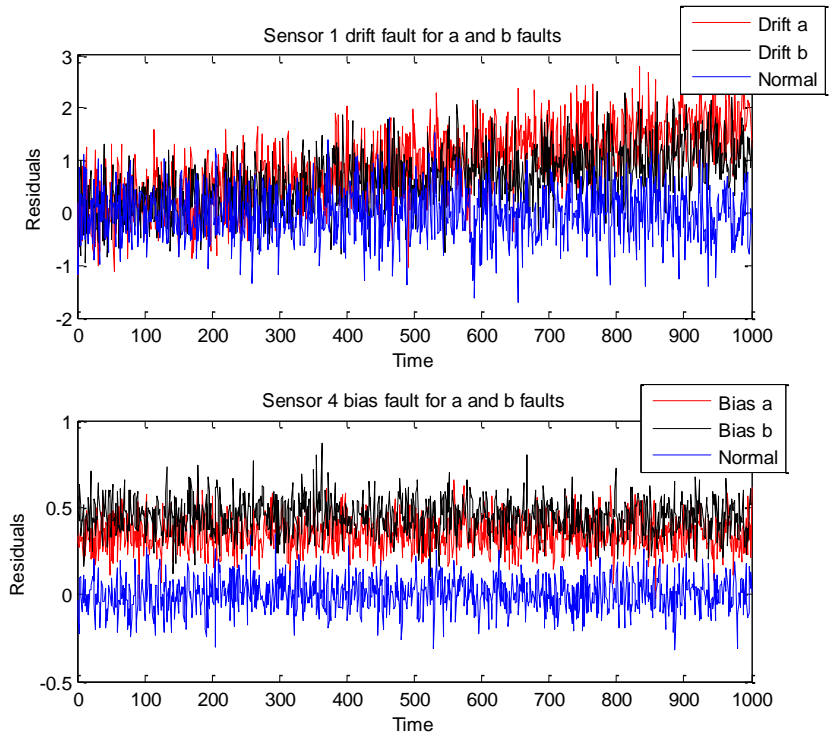

Figure 14: Residuals for Fault A and Fault B

The residuals shown in Figure 15 can be input to the function $\operatorname{ESD}()$ to determine which fault mode the system is running in. Notice that this fault mode is most similar to Fault B above, which is fault mode 5 in the cell array of historical inputs. Fault A is input 4 in the historical inputs.
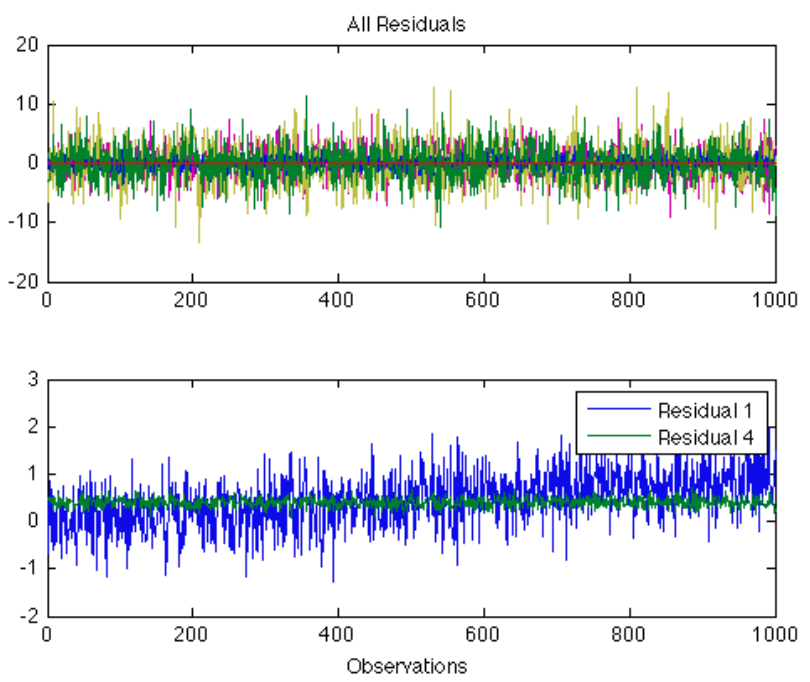

Figure 15: Test Residuals

ESD Elimination/Similarity Diagnosis

Function to diagnose faults using the Elimination Similarity method 


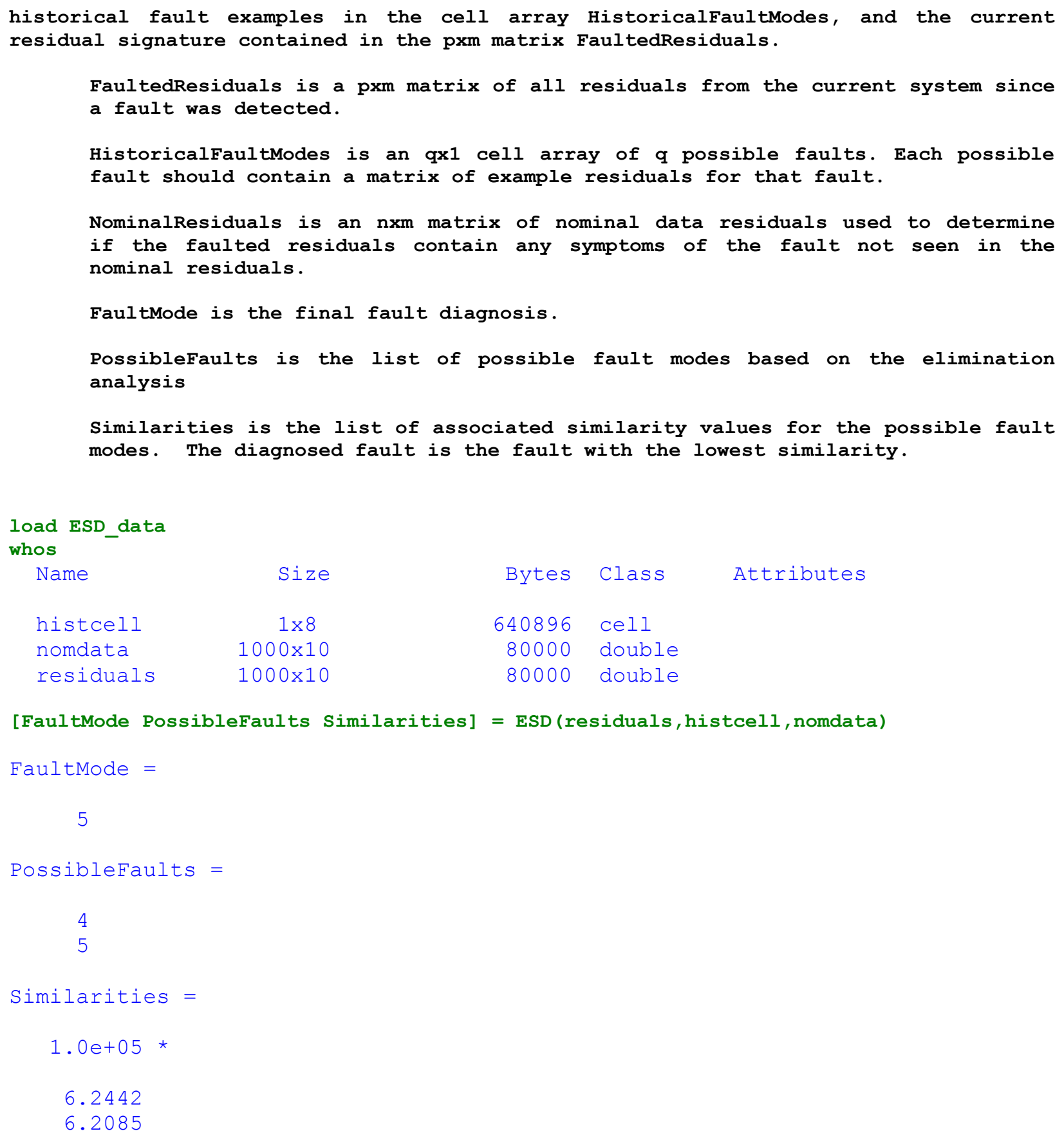

\section{System Prognosis}

The ultimate goal of prognostics for high-value, safety critical equipment and systems is individual-based prognosis. Individual-based prognostics attempt to estimate the remaining useful life of a specific system operating in its specific environment. Several algorithms exist for making individual-based estimates. The PEP toolbox includes two algorithms for individualbased prognosis: the general path model and particle filtering. This section will present the theory and results of a general path model (GPM) analysis, though either is appropriate. For the 
purpose of illustrating the prognosis method, this section will focus on the heat exchanger fouling fault.

\section{General Path Model}

GPM analysis begins with some assumption of an underlying functional form of the degradation path for a specific fault mode. The degradation of the $i^{t h}$ unit at time $t_{j}$ is given by:

$$
y_{i j}=\left(t_{j},,_{i}\right)+{ }_{i j}
$$

where $\varphi$ is a vector of fixed (population) effects, $\theta_{i}$ is a vector of random (individual) effects for the $i^{\text {th }}$ component, and $\varepsilon_{i j} \sim \mathrm{N}\left(0, \sigma_{\varepsilon}^{2}\right)$ is the standard measurement error term. The degradation path model, $y_{i}$, can be extrapolated to the failure threshold, $D$, to estimate the component's time of failure.

First, exemplar degradation paths are used to fit the assumed model. The stage-1 parameter estimates are used to evaluate the random-effects distributions, to determine the mean population random effects, the mean time to failure (MTTF) and their associated standard deviations, and to estimate the noise variance in the degradation paths. The MTTF distribution can be used to estimate the time of failure for any component which has not yet been degraded.

A linear regression model is given by:

$$
Y=b X
$$

The model parameters are estimated as:

$$
b=\left(X^{T}{ }_{y}^{1} X\right){ }^{1} X^{T} \quad{ }_{y}^{1} Y
$$

where $\Sigma_{y}$ is the variance-covariance noise matrix for the response observations. It is important to note that the linear regression model is not necessarily a linear model. The data matrix $X$ can be populated with any function of degradation measures, including higher order terms, interaction terms, and functions such as $\sin (x)$ or $e^{x}$. If prior information is available for a specific model parameter, i.e. $\beta_{j} \sim \mathrm{N}\left(\beta_{j o}, \sigma_{\beta}^{2}\right)$, then the matrix $X$ should be appended with an additional row with value one at the $j^{\text {th }}$ position and zero elsewhere, and the $Y$ matrix should be appended with the a priori value of the $j^{\text {th }}$ parameter.

$$
\begin{aligned}
& X^{*}=\left[\begin{array}{llllllll}
X ; & 0 & \cdots & 0 & 1 & 0 & \cdots & 0
\end{array}\right] \\
& Y^{*}=\left[\begin{array}{ll}
Y ; & j
\end{array}\right]
\end{aligned}
$$

Finally, the variance-covariance matrix is augmented with a final row and column of zeros, with the variance of the a priori information in the diagonal element.

$$
{ }_{y}^{*}=\begin{array}{cccc}
2 & 0 & \ldots & 0 \\
0 & \ddots & 0 & \vdots \\
0 & \cdots & 2 & 0 \\
0 & \cdots & 0 & 2
\end{array}
$$


If knowledge is available about multiple regression parameters, the matrices should be appended multiple times with one additional row for each parameter.

It is convenient to assume that the noise in the degradation measurements is constant and uncorrelated. Some a priori knowledge of the noise variance is available from the exemplar degradation paths. If this assumption is not valid for a particular problem, then other methods of estimating the noise variance must be used. The assumption of uncorrelated noise allows the variance-covariance matrix to be a diagonal matrix consisting of noise variance estimates and a priori knowledge variance estimates. If this assumption is not valid, including covariance terms is trivial; again these terms can be estimated from historical degradation paths.

After a priori knowledge is used to obtain a posterior estimate of degradation parameters, this estimate becomes the new prior distribution for the next estimation of degradation parameters. The variance of this new knowledge is estimated as:

$$
\frac{1}{{ }_{\text {post }}^{2}}=\frac{n}{2}+\frac{1}{{ }_{\text {prior }}^{2}}
$$

where $n$ is the number of observations used to fit the current model.

Each fault mode should have its own prognostic model; lumping multiple fault modes into one model can reduce model accuracy and precision. In this way, if a new fault mode is experienced, it is straightforward to develop a prognostic model for that fault and add it to the bank of prognostic models. As any fault mode is experienced, the information gleaned there can be included in the prior information matrix for future faults. This allows the prognostic model to develop and adapt to the specific system by updating prior information as any new information becomes available.

The PEP Toolbox employs GPM models. The application of such a model to a simulated set of failure data is shown here.

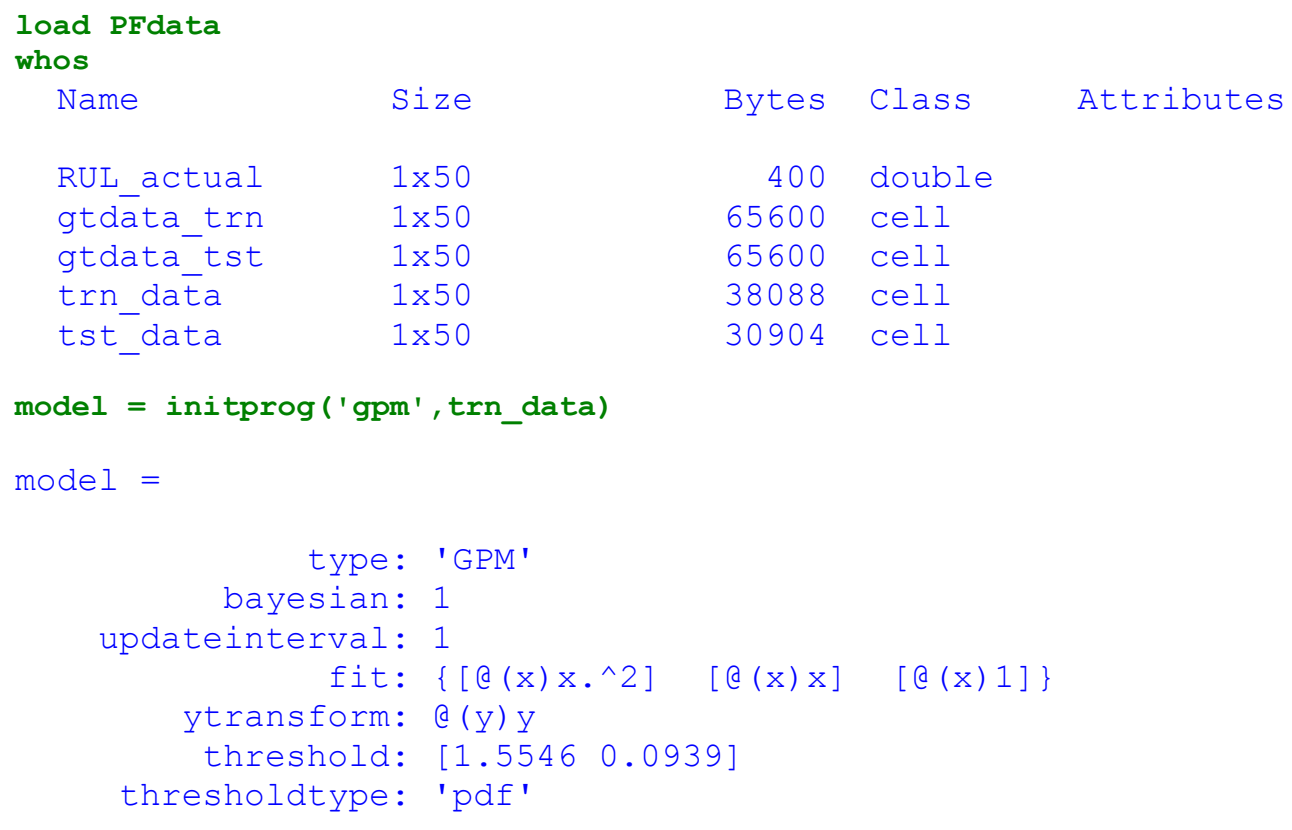



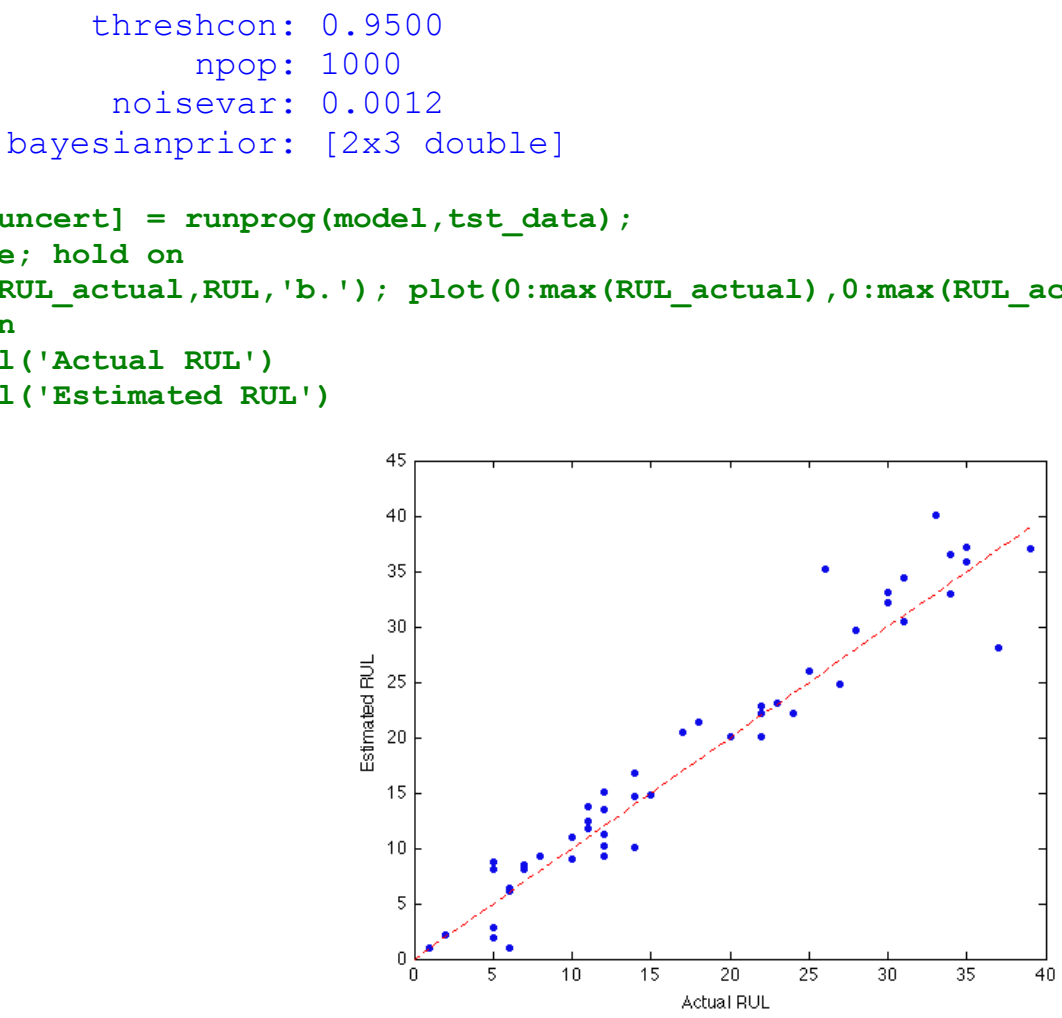

\section{Module Integration}

The integration of the adaptive modules described above is straightforward, as shown in Figure 16. Data from the system is fed into the monitoring module. The residuals from the monitoring module are used to determine if a fault is present. If no fault is present, the system continues to run only the monitoring module; there is no need to look at fault diagnosis or prognosis if a fault has not been detected. If a fault is detected, this should activate the fault diagnosis module. After a diagnosis is made, the appropriate prognostic model for that fault type can be employed to estimate the remaining useful life of the system. As discussed previously, each of these modules can be adapted as new information about the system comes available to continuously improve the health monitoring system.

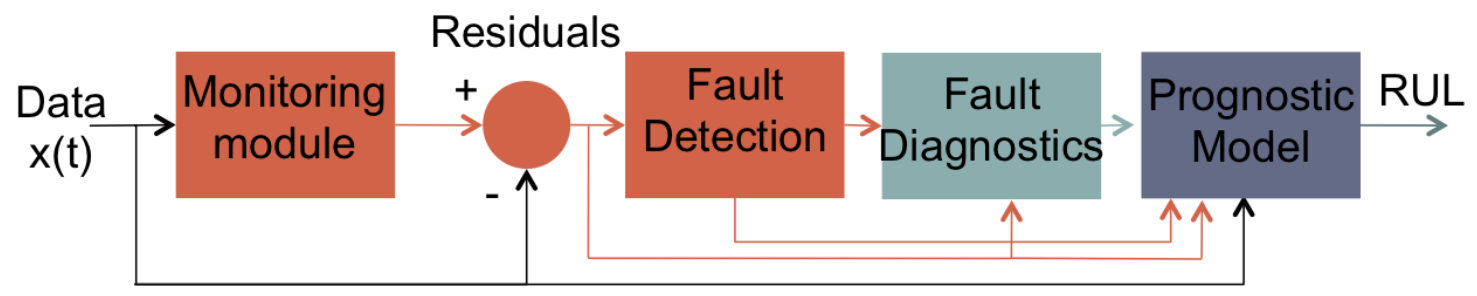

Figure 16: Integration of Health Monitoring Modules 


\title{
Advanced Instrumentation and Control Methods for Small and Medium Reactors with IRIS Demonstration
}

\section{Final Report \\ Volume 7}

On-Line Prognostics Methods with Applications to the IRIS System

Report No. DE-FG07-07ID14895/UTNE/2011-9

NERI-C Grant Number: DE-FG07-07ID14895

\author{
J. Wesley Hines \\ (Principal Investigator) \\ Jamie Coble \\ The University of Tennessee \\ Nuclear Engineering Department \\ Knoxville, TN 37996-2300 \\ E-mail: jhines2@utk.edu
}

May 2011

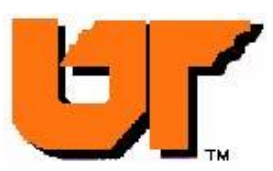




\begin{abstract}
Unforeseen equipment failure is cost-prohibitive, both in terms of equipment repair costs and lost revenue. Discovery of unanticipated pressure vessel head degradation at the Davis-Besse nuclear plant led to a 25-month outage and estimated repair costs exceeding $\$ 600$ million. In September, 2008, a turbine generator malfunction at the D.C. Cook nuclear plant resulted in a fire which led to eventual manual plant shutdown. Turbine repairs totaled $\$ 332$ million in addition to lost revenue during the one-year outage. Enterprise server downtime can be even more costly, resulting in a possible loss of \$6.4 million per hour for brokerage operations or \$2.6 million per hour for credit card authorization services. Small- and medium-sized reactors (SMRs) for export will have additional prognostic needs compared to traditional reactor designs, which include increased expectations of availability, safety, proliferation resistance, extended maintenance intervals, reduced staff, and extended operation intervals, all of which are a result of remote operation in countries that may have limited infrastructure and skill sets. Obviously, it is of paramount importance to be aware of impending equipment failures in SMRs so that operations can be adjusted or auxiliary equipment can be employed to avoid unexpected downtime or failure when possible.

The ultimate goal of most prognostic systems is accurate prediction of the remaining useful life (RUL) of individual systems or components based on their specific use and performance. As equipment degrades, measured parameters of the system tend to change; these sensed measurements, or appropriate transformations thereof, may be used to characterize the system degradation. An appropriate degradation measure, also called a prognostic parameter, may be used with a General Path Model (GPM) to make RUL estimates for specific systems or components. A dynamic Bayesian updating methodology is introduced to incorporate prior information in the GPM regression parameters, thereby capitalizing on all available information. Incorporating prior knowledge into the regression is particularly useful when only a few observations of the degradation parameter are available or the available observations are contaminated with high noise levels. Degradation measures may include sensed measurements, such as temperature or vibration level, or inferred measurements, such as model residuals or physics-based model predictions. Often, it is beneficial to combine several measures of degradation into a single parameter to provide a more robust prognostic model. Selection of an
\end{abstract}


appropriate parameter is a key for making useful individual-based RUL estimates; typically, identification of a prognostic parameter is left to expert analysis, visual inspection of available data, and knowledge of the degradation mechanisms. This approach is tedious and costly, and scales with the number of available data sources and possible fault modes.

This research introduces a set of metrics which characterize the suitability of a prognostic parameter. Parameter features such as trendability, monotonicity, and prognosability can be used to compare candidate prognostic parameters to determine which is most useful for individual-based prognosis. Trendability indicates the degree to which the parameters of a population of systems have the same underlying shape. Monotonicity characterizes the underlying positive or negative trend of the parameter. Finally, prognosability gives a measure of the variance in the critical failure value of a population of systems. By quantifying these features for a given parameter, the metrics can be used with any traditional optimization technique, such as Genetic Algorithms, to identify the optimal parameter for a given system.

The proposed parameter identification method and prognostic algorithms are illustrated with three applications: first, to simulated failure data of an International Reactor Innovative and Secure (IRIS) system; then, to the simulated turbofan engine data proved in the 2008 Prognostics and Health Management Conference Prognostics Challenge; and, finally, to data collected in a laboratory milling equipment wear experiment. The automated system was shown to identify appropriate parameters in both situations and facilitate Type III prognostic model development.

\section{DISCLAIMER}

Any opinions, findings, and conclusions or recommendations expressed in this report are those of the authors, and do not necessarily reflect the views of the U.S. Department of Energy or Westinghouse Electric Company, LLC. 


\section{TABLE OF CONTENTS}

1 INTRODUCTION 1

1.1 Objective 3 Overview …………………………............................... 2

1.2 Significant Contributions of this Research Task ........................................ 3

1.3 Organization of the Report .................................................................. 4

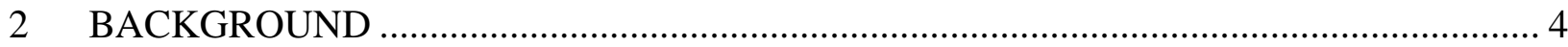

2.1 Prognosis in Health Monitoring.............................................................. 8

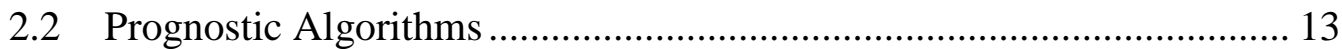

2.2.1 Type I: Traditional Time-to-Failure Analysis................................................. 14

2.2.2 Type II: Stressor-based Prediction ............................................................. 17

2.2.3 Type III: Degradation-based Prediction......................................................... 23

2.3 Data Fusion for Prognostics.................................................................. 27

2.4 Metrics for Evaluating Algorithm Performance ……………………..... 28

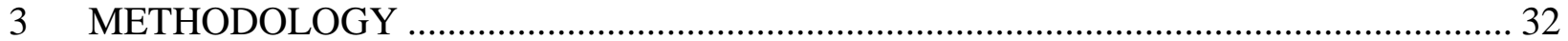

3.1 The General Path Model ........................................................................... 32

3.2 GPM for Prognostics ..................................................................... 35

3.3 Incorporating Prior Information with Bayesian Methods .......................... 36

3.4 Choosing a Prognostic Parameter ............................................................... 37

3.5 Prognostic Parameter Optimization .......................................................... 43

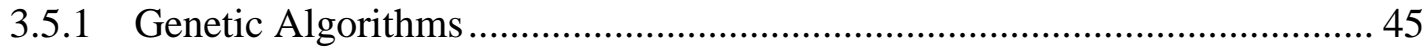

3.5.2 Prognostic Parameter Input Selection ......................................................... 48

3.6 Combined Monitoring and Prognostic Systems ………………………... 49

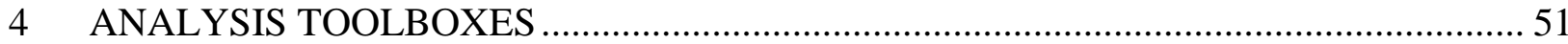

4.1 Process and Equipment Monitoring Toolbox …………………………... 52

4.2 Process and Equipment Prognostics Toolbox............................................ 53

4.2.1 Type I: Reliability Data-Based ............................................................... 53

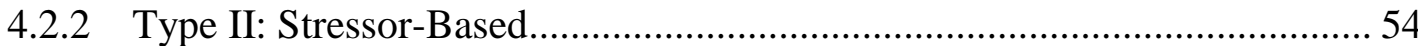

4.2.3 Type III: Degradation-Based ..................................................................... 54

4.2.4 Life Cycle Prognostics ................................................................................ 55

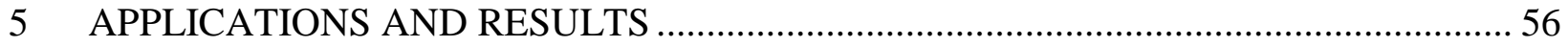

5.1 The Effect of Noise on Model Performance.............................................. 57 
5.2 Prognostic Models for the IRIS System

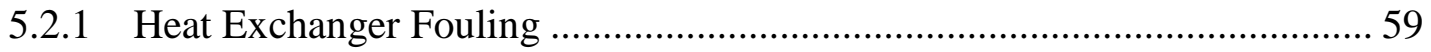

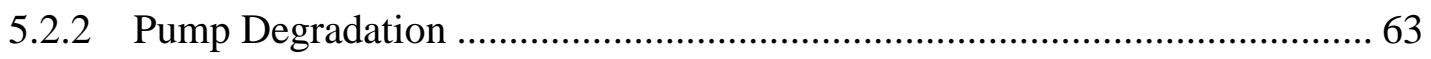

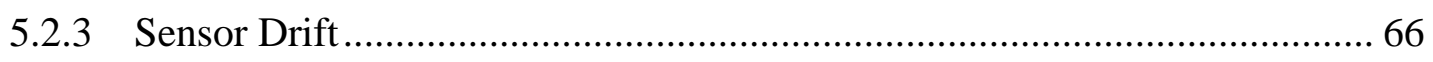

5.3 PHM Challenge Data Description ................................................. 70

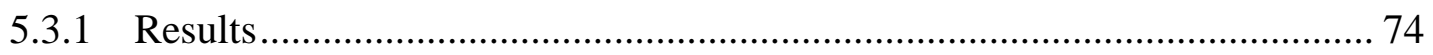

5.3.2 Performance Comparison of the Three Prognostic Types ........................... 84

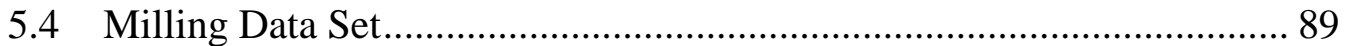

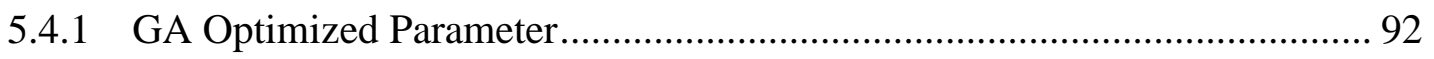

5.4.2 Material Specific Parameters ............................................................ 94

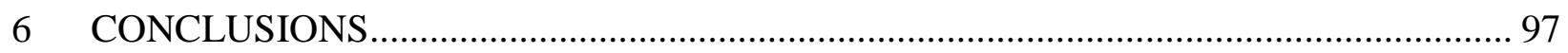

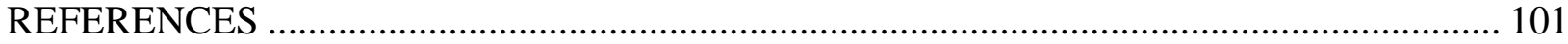

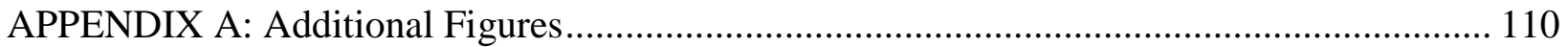

A.1 Noisy Prognostic Parameters ...................................................... 111

A.2 The Effect of Noise on Model Performance...................................... 114

A.3 PHM Challenge Data Monitoring System Residuals ......................... 124

A.4 Milling Data Mean and Standard Deviation Features ........................ 130

APPENDIX B: Process and Equipment Prognostics Toolbox User Guide ............................. 138 


\section{LIST OF FIGURES}

Figure 1-1: Operating and Maintenance Cost Chart [Toms, 1994] ......................................... 2

Figure 2-1: RUL vs Time for time-autonomous systems .................................................. 7

Figure 2-2: RUL vs. Time for a non-time-autonomous system [Mishra et al., 2004] .................. 7

Figure 2-3: Suite of Modules in a Health Monitoring System.............................................. 10

Figure 2-4: Prognostic Algorithm Categorization ............................................................. 14

Figure 2-5: Weibull Failure Distributions with Different Shape Parameters ........................... 16

Figure 2-6: Use of Prognostic Monitor Failure Times to Estimate RUL of Actual Circuit [Mishra

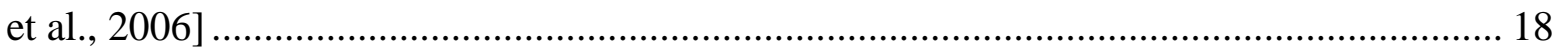

Figure 2-7: A Typical Progression of the Environmental Conditions ...................................... 20

Figure 2-8: A Collection of Possible Degradation Measures ................................................ 20

Figure 2-9: LEAP Short Term Regression [Greitzer, 2001] .................................................. 26

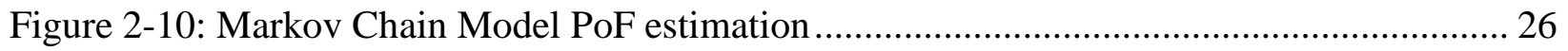

Figure 2-11: $\alpha-\lambda$ Performance for Accuracy [Saxena et al., 2008] ........................................ 31

Figure 3-1: Population of "Good" Prognostic Parameters ................................................... 38

Figure 3-2: Population of "Bad" Prognostic Parameters .......................................................... 39

Figure 3-3: Prognostic Parameter vs (a) Time in Cycles and (b) Time in \% of Full Lifetime ..... 41

Figure 3-4: Flowchart of Genetic Algorithm Optimization ............................................... 45

Figure 3-5: Combined Monitoring and Prognostic System ................................................ 49

Figure 4-1: Integration of the PEM and PEP Toolboxes ................................................. 52

Figure 4-2: PEM Toolbox Architecture .......................................................................... 53

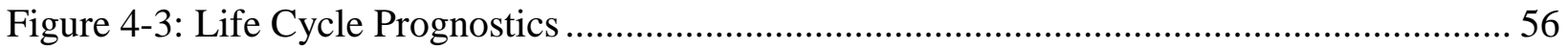

Figure 5-1: RUL Estimates Using Noisy Parameters ...................................................... 58

Figure 5-2: Change in Thermal Resistivity due to Fouling [Upadhyaya et al., NEER] ............. 61

Figure 5-3: Steam Generator Exit Temperature High Power Residuals .................................. 62 
Figure 5-4: Heat Exchanger Fouling RUL Estimation 63

Figure 5-5: Identification of the actual duty point (recreated from [Grist 1999]) ......................... 64

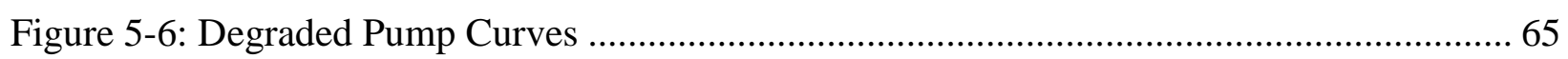

Figure 5-7: (a) Hot and (b) Cold Leg Temperature Residuals for Pump Degradation .................. 66

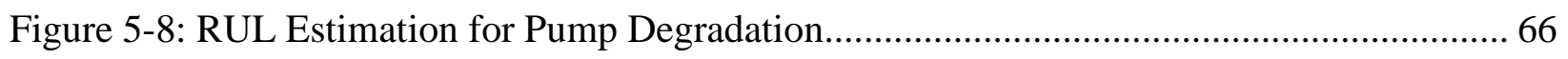

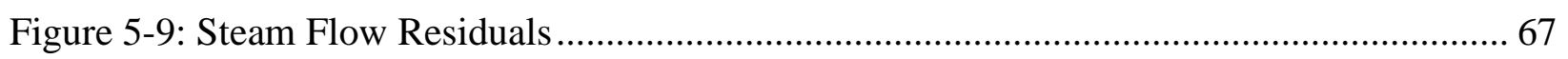

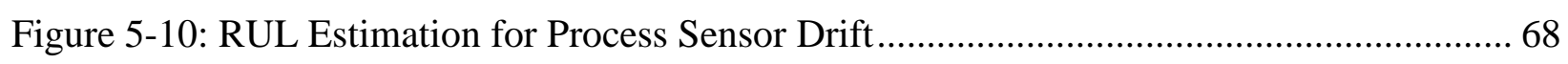

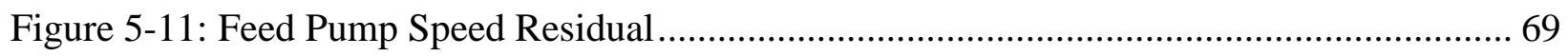

Figure 5-12: RUL Estimation for Control Sensor Drift.................................................................. 69

Figure 5-13: Correlation Coefficient Matrix for Eleven Monitored Variables............................... 71

Figure 5-14: Eleven PHM Data Set Variables ......................................................................... 72

Figure 5-15: Residuals which are expected to be (a) useful and (b) un-useful for prognostics ... 73

Figure 5-16: Prognostic Parameter Identified by Visual Inspection.............................................. 75

Figure 5-17: Prognostic parameter trending and RUL estimation.............................................. 77

Figure 5-18: Visual Inspection RUL Estimate Results ............................................................ 78

Figure 5-19: GPM Results With and Without Bayesian Updating ................................................ 79

Figure 5-20: Prognostic Parameter Identified by GA Optimization ............................................... 81

Figure 5-21: Prognostic Parameter Identified by Reduced GA Optimization................................. 81

Figure 5-22: RUL Estimates for First GA-optimized Parameter.................................................. 83

Figure 5-23: RUL Estimates for Second GA-optimized Parameter ............................................. 83

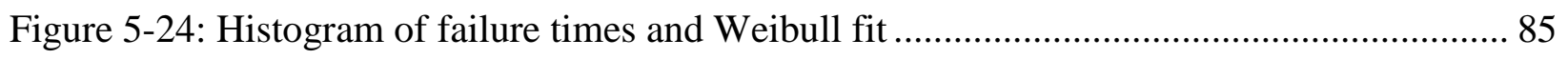

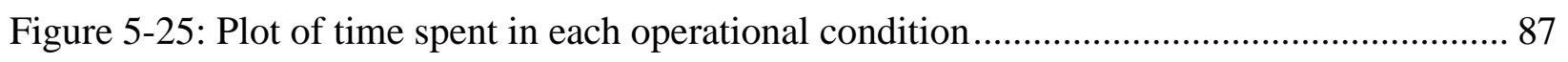

Figure 5-26: Flank Wear Measurements................................................................................... 90

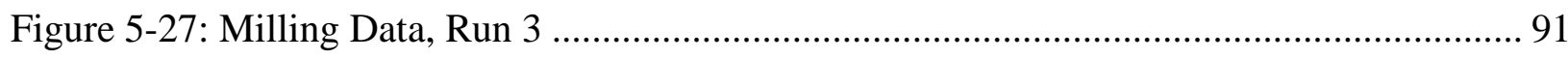

Figure 5-28: Mean and Standard Deviation of Milling Data, Run 3 .......................................... 92 
Figure 5-29: GA-Optimized Prognostic Parameter for Milling Data

Figure 5-30: RUL estimates for Milling Data Run 3 .......................................................... 93

Figure 5-31: GA-Optimized Prognostic Parameter for Runs Boring Iron................................ 95

Figure 5-32: GA-Optimized Prognostic Parameter for Runs Boring Steel .............................. 95

Figure 5-33: RUL Estimates for Lumped and Material-Specific Models ................................ 96 


\section{LIST OF TABLES}

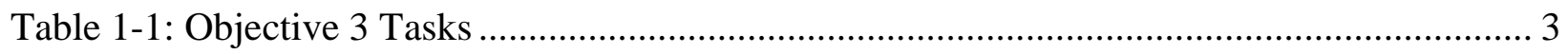

Table 3-1: Example Prognostic Parameter Suitability Metrics.............................................. 41

Table 3-2: Effect of Noise on Parameter Suitability Metrics .................................................. 42

Table 5-1: Prognostic Parameter Suitability Metrics .......................................................... 75

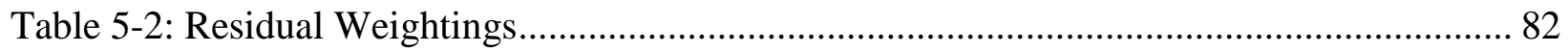

Table 5-3: PHM Challenge Data Parameter Suitability Metrics ........................................... 82

Table 5-4: Correlation of Operating Condition to Lifetime................................................. 87

Table 5-5: Markov Chain Transition Probability Matrix ..................................................... 88

Table 5-6: Prior Distribution for Quadratic Parameters ......................................................... 89

Table 5-7: Milling Data Experimental Conditions ................................................................. 90

Table 5-8: Parameter Suitability Metrics for Milling Data.................................................... 95 


\section{ABBREVIATIONS}

\begin{tabular}{|c|c|}
\hline AAKR & Auto-Associative Kernel Regression \\
\hline AANN & Auto-Associative Neural Network \\
\hline BFGS & Broyden-Fletcher-Goldfarb-Shanno \\
\hline BIST & Built-In Self Test \\
\hline $\mathrm{CBM}$ & Condition Based Maintenance \\
\hline $\mathrm{CBM}+$ & Condition Based Maintenance Plus \\
\hline C-MAPSS & Commercial Modular Aero-Propulsion System Simulation \\
\hline CPU & Central Processing Unit \\
\hline FMEA & Failure Modes and Effects Analysis \\
\hline GA & Genetic Algorithm \\
\hline GPM & General Path Model \\
\hline IRIS & International Reactor Innovative and Secure \\
\hline JSF & Joint Strike Fighter \\
\hline LCM & Life Consumption Model \\
\hline LEAP & Life Extension Analysis and Prognostics \\
\hline MAPE & Mean Absolute Percent Error \\
\hline MOO & Multi-Objective Optimization \\
\hline MRL & Mean Residual Life \\
\hline MTTF & Mean Time to Failure \\
\hline OLM & On-Line Monitoring \\
\hline PACE & Path Classification and Estimation \\
\hline PCA & Principle Component Analysis \\
\hline $\mathrm{PDF}$ & Probability Density Function \\
\hline PEM & Process and Equipment Monitoring Toolbox \\
\hline PEP & Process and Equipment Prognostics Toolbox \\
\hline PHM & Prognostics and Health Management \\
\hline
\end{tabular}




$\begin{array}{ll}\text { PH } & \text { Proportional Hazards } \\ \text { PI } & \text { Prediction Interval } \\ \text { POF } & \text { Probability of Failure } \\ \text { PROaCT } & \text { Prognostics, Reliability Optimization and Control Technologies } \\ \text { PWR } & \text { Pressurized Water Reactor } \\ \text { RCP } & \text { Reactor Coolant Pump } \\ \text { ROI } & \text { Return on Investment } \\ \text { RUL } & \text { Remaining Useful Life } \\ \text { SG } & \text { Steam Generator } \\ \text { SMR } & \text { Small and Medium Reactor } \\ \text { SPRT } & \text { Sequential Probability Ratio Test } \\ \text { SWO } & \text { Sum of Weighted Objectives } \\ \text { ToF } & \text { Time of Failure } \\ \text { WPP } & \text { Weighted Path Prognostics }\end{array}$




\section{INTRODUCTION}

Unexpected equipment failure can lead to large costs, both for maintenance activities and loss of revenue. Discovery of unanticipated pressure vessel head degradation at the Davis-Besse nuclear plant led to a 25 -month outage and estimated repair costs exceeding $\$ 600$ million [Union of Concerned Scientists, accessed Jan, 2010]. In September, 2008, a turbine generator malfunction at the D.C. Cook nuclear plant resulted in a fire which led to eventual manual plant shutdown. Turbine repairs totaled $\$ 332$ million in addition to lost revenue during the one-year outage. Enterprise server downtime can be even more costly, resulting in a possible loss of $\$ 6.4$ million per hour for brokerage operations or $\$ 2.6$ million per hour for credit card authorization services [Feng, 2003]. Small- and medium-sized reactors (SMRs) for export will have additional prognostic needs compared to traditional reactor designs, which include increased expectations of availability, safety, proliferation resistance, extended maintenance intervals, reduced staff, and extended operation intervals, all of which are a result of remote operation in countries that may have limited infrastructure and skill sets. Obviously, it is of paramount importance to be aware of impending equipment failures in SMRs so that operations can be adjusted or auxiliary equipment can be employed to avoid unexpected downtime or failure when possible.

Traditionally, maintenance activities have taken one of two approaches: preventive and corrective. Preventive maintenance includes routine maintenance activities scheduled on a time basis designed to prevent failure from occurring. While these activities may minimize operating cost, they typically involve the highest maintenance costs. Conversely, corrective maintenance involves performing maintenance only when a failure occurs. While this eliminates unnecessary maintenance, it ensures that every piece of equipment in the system will fail. Between these two extremes lies condition-based maintenance, wherein maintenance actions are performed as needed based on the condition of the equipment. Ideally in this strategy, maintenance is performed after a fault occurs but before it leads to failure to reduce any unnecessary maintenance or unplanned downtime. The chart in Figure 1-1, adapted from [Toms, 1994], shows the relative costs associated with each maintenance strategy. The lowest total costs tend to occur with a condition-based maintenance strategy. However, in order to fully realize the 
benefit of such a strategy, it is important to accurately trend the effect of a fault on system performance through prognostics.

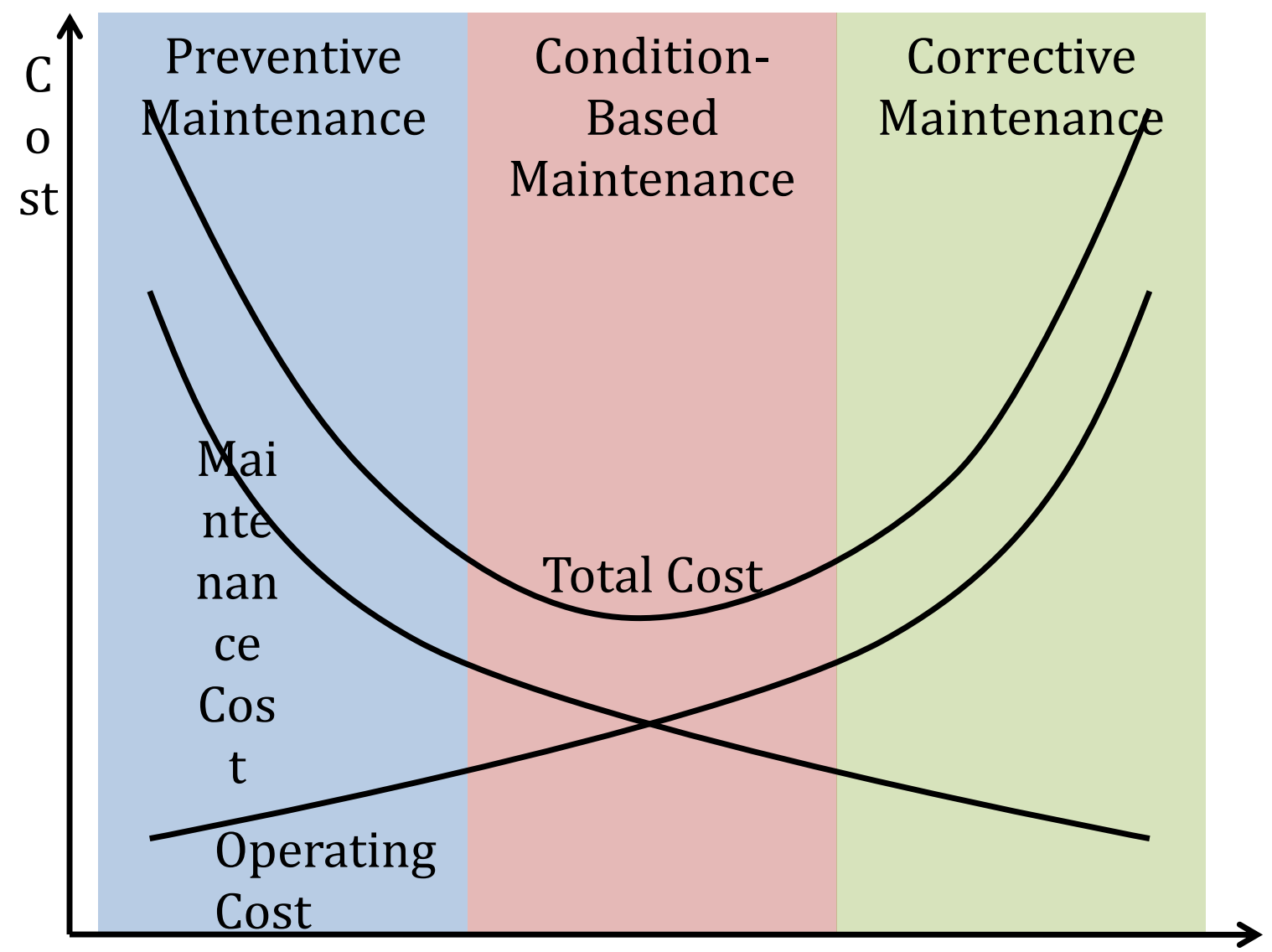

\section{Number of Failure Events}

Figure 1-1: Operating and Maintenance Cost Chart [Toms, 1994]

Objective 3 of this research states: Develop and test an integrated monitoring, diagnostic, and prognostic system for SMRs using an experimental flow control loop and the International Reactor Innovative and Secure (IRIS) design as test platforms. This volume will focus on the development of individual-based prognostic methods. Adaptive monitoring, fault detection, and fault diagnostic methods are discussed in Volume 6 of this report.

\subsection{Objective 3 Overview}

Objective 3 includes twelve tasks, spanning three years, as summarized in Table 1-1. These twelve tasks can be separated into four main research topics: monitoring and fault 
detection, fault diagnostics, system prognostics, and the integration of these three functions into one system. This report focuses on the work performed over the last three years in completion of the prognostics-related research tasks, specifically tasks 3.7 and 3.9, as well as several enabling research topics not specified in the original task list. The remaining tasks, related to system monitoring, fault detection, and fault diagnostics, are discussed in Volume 6 of this report, as well as system integration.

Table 1-1: Objective 3 Tasks

\begin{tabular}{|c|c|c|}
\hline Year 1 & Year 2 & Year 3 \\
\hline $\begin{array}{c}\text { 3.1 Develop IRIS system } \\
\text { hybrid models }\end{array}$ & $\begin{array}{l}\text { 3.5 Develop and test the } \\
\text { case-based fault } \\
\text { identification module }\end{array}$ & $\begin{array}{l}\text { 3.8 Integrate the } \\
\text { degradation models from } \\
\text { Task } 3.4 \text { into the simulation } \\
\text { developed in Topic } 1 \text { and } \\
\text { generate failure data }\end{array}$ \\
\hline $\begin{array}{l}\text { 3.2 Develop an automated } \\
\text { method for hybrid model } \\
\text { adaptation }\end{array}$ & $\begin{array}{l}\text { 3.6 Apply the modeling and } \\
\text { adaptation methods to a } \\
\text { heat exchanger test system } \\
\text { and validate in real time }\end{array}$ & $\begin{array}{l}\text { 3.9 Validate the prognostic } \\
\text { algorithm development } \\
\text { methodologies using } \\
\text { simulated data. }\end{array}$ \\
\hline $\begin{array}{c}\text { 3.3 Integrate the adaptive } \\
\text { system into a model based } \\
\text { FDI system }\end{array}$ & \multirow{3}{*}{$\begin{array}{l}\text { 3.7 Develop a hybrid } \\
\text { prognostic framework that } \\
\text { combines Type I, II, and III } \\
\text { prognostic models }\end{array}$} & $\begin{array}{l}\text { 3.10 Integrate monitoring, } \\
\text { detection, identification, and } \\
\text { prognostic algorithms }\end{array}$ \\
\hline \multirow{2}{*}{$\begin{array}{l}\text { 3.4 Develop degradation } \\
\text { models for example key } \\
\text { systems and components }\end{array}$} & & $\begin{array}{l}\text { 3.11 Test the system using } \\
\text { simulated and laboratory } \\
\text { data }\end{array}$ \\
\hline & & $\begin{array}{c}\text { 3.12 Develop a Matlab- } \\
\text { based system development } \\
\text { toolbox manual }\end{array}$ \\
\hline
\end{tabular}

\subsection{Significant Contributions of this Research Task}

The research work presented here in completion of Objective 3 of this project has resulted in several significant contributions to the field of health monitoring.

1. Development of individual-based prognostic models for simulated common fault types in nuclear power plants. The techniques used to develop these models are straightforward, such that additional models can be developed as new failure modes are identified. 
2. Development of a set of metrics to characterize the suitability of a population of parameters for application to the GPM prognostic method, namely monotonicity, prognosability, and trendability.

3. Development of an automated routine to use the proposed suitability metrics to identify an optimal or near-optimal prognostic parameter, including an input selection method to remove un-useful data sources in order to alleviate the computational burden of the optimization routine.

4. Development of the MATLAB-based Process and Equipment Prognostics (PEP) toolbox to facilitate application of the three types of prognostics to general prognostic model development. Methods to aid in, and to some extent automate, prognostic model development are also included in the PEP toolbox, such as the prognostic parameter identification routine.

\subsection{Organization of the Report}

This report summarizes the work performed in completion of the prognostic-related tasks of Objective 3 over approximately three years. The following chapter discusses pertinent background information about health monitoring and system prognostics. Next, the specific methodologies developed for this research are described. The Process and Equipment Monitoring (PEM) and Process and Equipment Prognostics (PEP) toolboxes are discussed, as they pertain to the current research. Then, results of the application of developed techniques to appropriate test bed data are summarized. Finally, conclusions of this research and areas of future work are outlined.

\section{BACKGROUND}

Compared to the more established areas of condition monitoring, fault detection, and diagnostics, prognostics is a fairly immature field. As such, scholarly publications concerning prognostics have focused on the need for prognostics [Hess et al., 2005; Condition Based Maintenance Plus, 2005; Heo, 2008; Greitzer et al., 1999], the challenges in prognostic model 
development [Engel et al., 2000], the many and varied applications of prognostics [Vichare and Pecht, 2006; Ridgetop Group, 2004; Mishra and Pecht, 2002; Kalgren et al., 2007; Heng et al., 2009; Carden and Fanning, 2004; Doebling et al.; Catbas and Atkan, 2002; Vachtsevanos et al., 1997; Wang and Vachtsevanos, 2001; Orchard and Vachtsevanos, 2007; Keller et al., 2006; Orsagh et al., 2005; Roemer et al., 2005; Ferrell, 1999; Smith et al., 1997; Hess and Fila, 2002; Line and Clements, 2005; Kacprzynski et al., 2004], etc. In fact, in many cases, papers purported to study prognostics consider only monitoring, fault detection, and diagnostics while pointing to prognostics as an area of future work or simply overlooking the estimation of RUL as a key function of prognostics [Heo, 2008; Keller et al., 2006; Liao et al., 2006; Goodman et al., 2005; Urmanov and Hines, 2007; Han et al., 2007; Orsagh et al., 2006]. Only in recent years have specific prognostic methodologies and applications been presented. These papers will be discussed in more detail in subsequent sections.

One cause for the confusion in developing and applying prognostic methodologies is the lack of a unified definition of Prognostics. For instance, the Joint Strike Fighter (JSF) research group includes fault detection and isolation, enhanced diagnostics, material condition assessment, performance monitoring, and estimation of remaining useful life under the umbrella of prognostics [Hess et al., 2005]. However, this collection of activities seems better suited to the common moniker of Prognostics and Health Management (PHM) or the program suggested by the U.S. Deputy Under Secretary of Defense for Logistics and Material Readiness, Condition Based Maintenance plus (CBM+) [CBM+, 2010]. Sheppard, Kaufman, and Wilmering [2008] review the relevant standards in IEEE and other organizations related to prognostics and highlight the importance of predicting RUL as a key feature of prognostics; however, they propose no single, unifying standard for prognostics. For this research, a more specific definition of prognostics will be used, namely estimation of RUL and associated uncertainty. Because fault progression is not deterministic, it is impossible to estimate the RUL exactly. Any RUL estimate given by a prognostic algorithm should be accompanied by an estimate of the uncertainty, giving a confidence interval during which failure is expected to occur or a POF distribution. Prognostics applications in the literature are often remiss in this aspect of the 
analysis; however, the importance of uncertainty estimation is widely discussed [Hess et al., 2005].

Because prognostics is defined as an estimate of the RUL of a system, the time of failure must also be explicitly defined. Two types of failure are commonly considered: hard failure and soft failure [Meeker and Hamada, 1995]. Hard failure is generally considered to have occurred when a product stops working, e.g. an incandescent light bulb burns out or a car tire pops. In hard failure, failure time does not usually occur at a particular degradation level. Instead, loss of functionality occurs at widely varying levels across units. These failures are generally modeled as random failure. Conversely, soft failures are said to have occurred when the degradation level of a system reaches some predefined critical failure threshold, e.g. light output from fluorescent light bulbs decreases below a certain level or car tire tread is below some specified depth. These failures generally do not concur with complete loss of functionality, as in hard failure; however, they correspond with the time when an operator is no longer confident that equipment will continue to work to its specifications. Soft failures, or non-catastrophic failures, are the failures of interest in this research. System degradation can be monitored and trended to make estimates of RUL due to soft failures, whereas hard failures are random in nature and usually can only be predicted if some failure precursor can be monitored.

Finally, the exact nature of RUL should be considered. For a failed unit, it is easy to determine the failure time. It is traditional to consider the RUL at any time before failure to be simply the time between the current time and the failure time (Figure 2-1). Some argue that this simplistic approach is inappropriate. However, Klinger [1992] shows that the assumption that failure time is inversely proportional to the degradation rate is valid for systems which are autonomous in the variable representing time. That is, the differential equations describing failure for these systems are independent of any explicit time dependence. The author further argues that this assumption is generally valid. The inverse relationship should be accepted for systems running under a constant load and environment. In some systems, the operating conditions can be altered to mitigate degradation; in this case the system is not autonomous in time and the inverse relationship is not valid. This more complicated RUL relationship can be seen in Figure 2-2 [Mishra et al., 2004] where a large shock reduces the RUL significantly. Had 
this shock not occurred, the circuit board under test could be expected to continue to perform for the originally estimated lifetime.

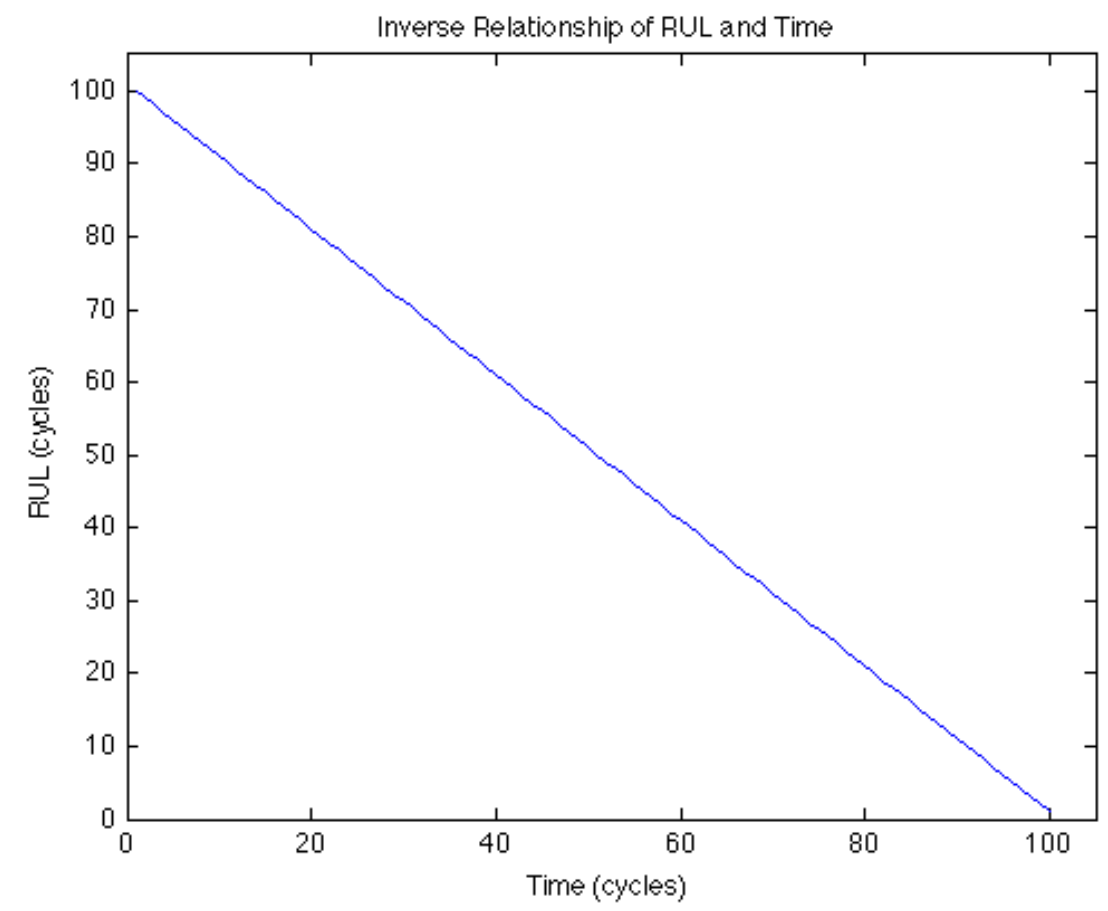

Figure 2-1: RUL vs Time for time-autonomous systems

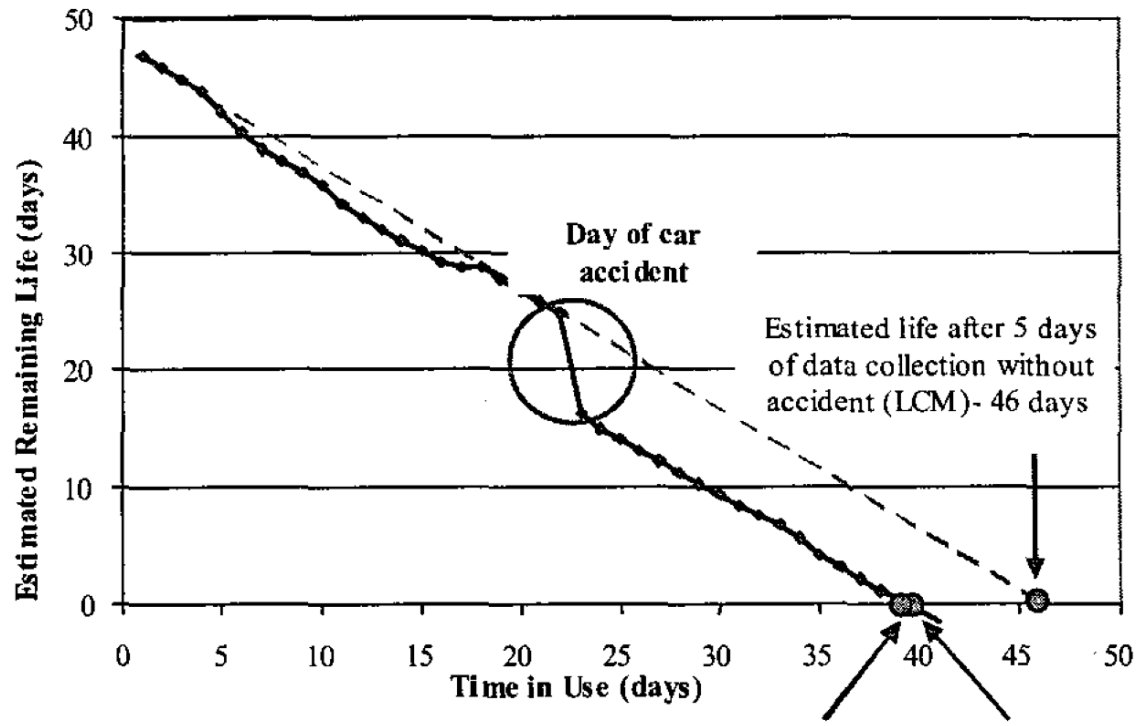

Actual life from resistance Estimated life immediately monitoring - 39 days after accident (LCM) 40 days

Figure 2-2: RUL vs. Time for a non-time-autonomous system [Mishra et al., 2004] 
A significant portion of the published literature in prognostics research focuses on solutions to specific problems, such as electronic prognostics [Vichare and Pecht, 2006; Ridgetop Group, 2004; Mishra and Pecht, 2002; Kalgren et al., 2007], vibration analysis [Carden and Fanning, 2004; Doebling et al.; Catbas and Atkan, 2002], helicopter gearbox monitoring [Engel et al., 2000; Heng et al., 2009; Vachtsevanos et al., 1997; Wang and Vachtsevanos, 2001; Orchard and Vatchsevanos, 2007; Kacprzynski et al., 2004], JSF applications [Keller et al., 2006; Orsagh et al., 2005; Roemer et al., 2005; Ferrell, 1999; Ferrell, 2000; Smith et al., 1997; Hess and Fila, 2002; Line and Clements, 2005], etc. While this approach may result in very good point solutions for these specific problems, generic prognostic algorithms which may be more broadly applicable are of more interest. The goal in developing generic prognostic algorithms is to develop methods which may be rapidly configured for a new system to allow for effective and efficient deployment of Condition Based Maintenance (CBM) technology on large, complex systems [Baruah et al., 2006].

The following sections discuss specific areas of the prognostics work reported in the literature. First, prognostics as a component of a larger health monitoring system is discussed, such as that suggested by the JSF research group. The next section discusses prognostic algorithms, classifying them into three types based on the information used to make prognostic estimates. Then, metrics for evaluating prognostic model performance are outlined. Finally, data fusion methods for combining information for making prognostic estimates are covered.

\subsection{Prognosis in Health Monitoring}

Prognosis is one component in a larger health monitoring system which also includes system monitoring, fault detection, and diagnostic modules. Full health monitoring systems, also called Condition Based Maintenance (CBM) systems, are the focus of much research. Kothamasu, et al. [2006] describe prognostics as part of a full CBM system. The authors describe using prognostic estimates to aid maintenance scheduling and planning; they also suggest prognostics for optimal control algorithms. Pipe [2008] and Hess, et al. [2005] suggest the use of RUL estimates for maintenance planning and logistics systems. Callan, et al. [2006] outline a five-step CBM system which includes: Data Acquisition, Data Manipulation, Condition Monitoring, Health Assessment, and Prognostics. By applying the entire suite of modules, one 
can accomplish the goals of most prognostic systems: increased productivity; reduced downtime; reduced number and severity of failures, particularly unanticipated failures; optimized operating performance; extended operating periods between maintenance; reduced unnecessary planned maintenance; and reduced life-cycle cost. Figure 2-3 gives a diagram of a typical health monitoring system. Data collected from a system of interest is monitored for deviations from normal behavior. Monitoring can be accomplished through a variety of methods, including first principle models, empirical models, and statistical analysis [Hines et al., 2006]. The monitoring module can be considered an error correction routine; the model gives its best estimate of the true value of the system variables. These estimates are compared to the data collected from the system to generate a time-series of residuals. Residuals characterize system deviations from normal behavior and can be used to determine if the system is operating in an abnormal state. A common test for anomalous behavior is the Sequential Probability Ratio Test (SPRT) [Wald, 1945]. This statistical test considers a sequence of residuals and determines if they are more likely from the distribution that represents normal behavior or a faulted distribution, which may have a shifted mean value or altered standard deviation from the nominal distribution. If a fault is detected, it is often important to identify the type of fault; systems will likely degrade in different ways depending on the type of fault and so different prognostic models will be applicable. Expert systems, such as fuzzy rule-based systems, are common fault diagnosers. Finally, a prognostic model is employed to estimate the Remaining Useful Life (RUL) of the system. This model may include information from the original data, the monitoring system residuals, and the results of the fault detection and isolation routines. 


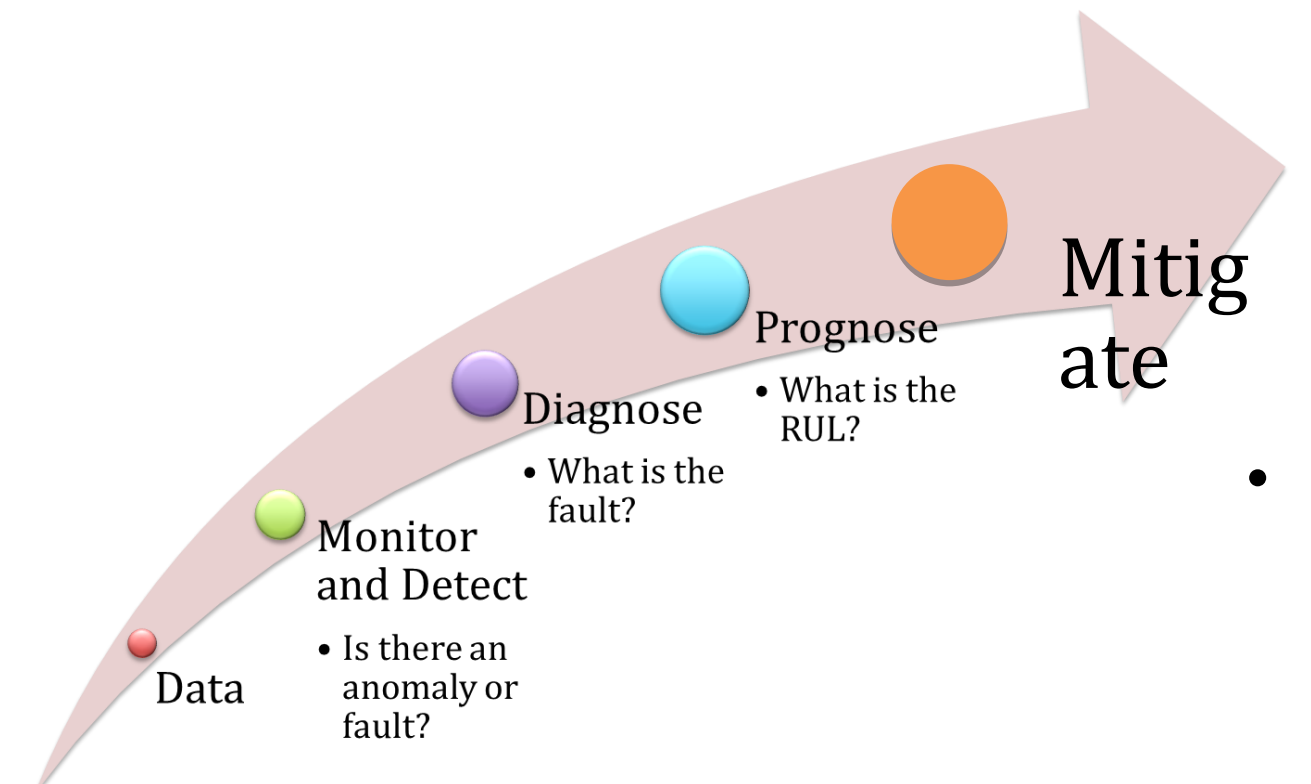

Figure 2-3: Suite of Modules in a Health Monitoring System

Many prognostic algorithms have been proposed; these will be discussed in more detail in following sections. Despite the many differences between these algorithms, they all have several common features [Upadhyaya et al., 1994; Hess et al., 2005; Line and Clements, 2006]. Prognostic systems must naturally build on monitoring and diagnostic systems. It is near impossible to determine the failure time of a device without first detecting and identifying a failure-causing fault.

This leads to the first requirement of a prognostic system: notice period [Line and Clements, 2006]. Also called lead time or fault-to-failure time, the notice period is the amount of time between fault detection and predicted failure. It is easy to agree that in most cases a prognostic system which gives only a few seconds of notice before an impending failure is virtually worthless. The notice period must be sufficiently long to allow for operator action, maintenance, automatic reconfiguration, etc.

Beyond this sensitivity, this also means that fault progression must be sufficiently slow for detection, diagnosis, and prognosis to occur with an acceptable notice period. That is, a precipitating fault must occur and become detectable a reasonable time before the resulting failure. This implies an assumption of most prognostic systems: monotonic fault progression. 
Most prognostics systems assume that once a fault occurs, a failure will also predictably occur in the absence of human intervention; that is, prognostic systems assume that faults cannot selfheal.

A final requirement specific to data-driven prognostics systems is the availability of failure data, specifically several instances of the same type and trend for each failure mode of interest. Failure modes must be carefully identified to differentiate between different fault types and fault progression rates. Similar failure modes which progress at different rates should often be considered different failures. Because of individual, random differences and noisy measurements, it is important to collect many historical fault progressions for each failure mode of interest in order to build a robust and complete prognostic system.

Several procedures have been outlined for developing prognostic systems [Gu et al., 2007; Janasak and Beshears, 2007; Kelkar et al., 1996; Greitzer and Ferryman, 2001]. The specific methodologies vary in the breakdown of steps necessary, but they all generally include the same 3 steps, often subdivided into a total of five to seven steps:

1. Identify important failure modes. This may be accomplished through a failure modes and effects analysis (FMEA) which identifies possible failure modes for a system and ranks them according to severity of failure and risk of occurrence.

2. Identify key parameters that need to be monitored in order to detect and identify these failure modes. This step is sometimes governed by the funds available for the monitoring sensor suite, the effects sensors may have on performance, and the availability of accurate sensors.

3. Identify and develop appropriate methods to monitor the key failure modes. This may include a variety of approaches, including canary devices or a prognostic algorithm. Typically, one prognostic model should be developed for each anticipated failure mode; significant accuracy may be lost by lumping multiple fault modes into one model.

Prognostic algorithms can be classified in many ways. The following section suggests a classification based on the type of information used to make prognostic estimates: reliabilitybased, stressor-based, or condition-based. Prognostic algorithms, like all modeling methods, can 
also be classified as physics-based or empirical. The following sections touch on both types of models; however, the PEP toolbox and the current research focus on empirical prognostics methods. Physics-based models, also called physics of failure models or first principle models, can be expensive and tedious to develop. Often times, the underlying physical processes leading to failure are not completely understood, and simplifying assumptions must be made to facilitate model development. Assumptions made in model development may not be fully applicable to real world systems, which limits the applicability of physics of failure models. In addition, these models are often very computationally expensive, particularly if Monte Carlo simulations are used to estimate confidence intervals about model predictions. Empirical models, on the other hand, use data to fit a model to the relationships seen in real world operation. These models typically provide no additional information about the physical mechanisms leading to failure. Empirical models are preferable to physics of failure models because they are simple to develop, they capture real world relationships, and they require no expertise in the underlying physical phenomena leading to failure. Empirical models have several drawbacks, though. These models rely on operational data for model development. As such, the models are generally only applicable to systems operating within the range of the training data used in model development. This poses additional problems for prognostics models, which rely on run-to-failure data for model training. Very few expensive or safety critical systems are allowed to run to failure; in this case, physics of failure models may be used to simulate failure data for model development. Often, failure data which is available may be the result of accelerated life testing performed during the product design phase. Several methods are available for extracting useful data which describes actual operation from accelerated testing data [Elsayed and Chen, 1998; Carey and Koenig, 1991]. Lemoine and Wenocur [1985] give a comprehensive overview of failure modeling. Tang and Chang [1995] use the Eyring model to explain degradation measure dependence on accelerated factors. Park and Padgett [2006] describe a method for extracting information from accelerated degradation testing with multiple degradation factors. By applying these methods, it is possible to utilize accelerated life test data collected during product design and development phases for prognostics applications. However, care must be given to ensure that the failures seen during accelerated testing are analogous to real-world failures. Accelerated 
testing conditions can result in fault modes which only occur under the accelerated conditions. The following sections discuss a variety of prognostic models, both physics-based and empirical, classified according to the type of data used to make prognostic estimates.

\subsection{Prognostic Algorithms}

As suggested by the "No Free Lunch" Theorem, no one prognostic algorithm is ideal for every situation [Ho and Pepyne, 2002; Koppen, 2004]. A variety of models have been developed for application to specific situations or specific classes of systems. The efficacy of these algorithms for a new process depends on the type and quality of data available, the assumptions inherent in the algorithm, and the assumptions which can validly be made about the system. As such, these prognostic algorithms can be categorized according to many criteria. One proposed categorization focuses on the type of information used to make prognostic estimates; this results in three classes of prognostic algorithms (Figure 2-4) [Hines and Usynin, 2008]. Type I prognostics is traditional time to failure analysis; this type of prognostic algorithm characterizes the expected lifetime of an average system operating in an historically average environment. These methods may be applied if no data specific to the current system is available. Examples of Type I prognostics include Weibull analysis, exponential or normal distribution analysis, and nonparametric distribution analysis. A readily apparent shortcoming of this group of methods is the absence of consideration for operating conditions and environment in making RUL estimates. Typically, systems operating in harsher conditions will fail more quickly while those in milder environments more slowly. Type II, or stressor-based, prognostics use operational and environmental condition data to estimate RUL. This type of prognostics characterizes the lifetime of an average system or component operating in the specific environment. Type II methods can be used if operating conditions, such as load, input current and voltage, ambient temperature, vibration, etc., are measurable and correlated to system degradation. Algorithms in this class include specific formulations of the Markov Chain model, shock model, and proportional hazards model, and the path classification and estimation (PACE) [Garvey and Hines, 2007; Garvey, 2007] or weighted path prognostics (WPP) method. Although more specific than Type I models, Type II models are deficient because they neglect unit-to-unit variance which may be due to manufacturing-, installation-, and maintenance action-variability. 
The final class of algorithms, Type III or condition-based, prognostics, characterize the lifetime of a specific unit or system operating in its specific environment. Extrapolation of a general path model (GPM) is the most common Type III method. GPM extrapolation involves trending a prognostic parameter and extrapolating it to some predefined failure threshold. A prognostic parameter is a measure, either directly sensed from the system or inferred from a set of sensor readings, which characterizes system degradation or health. System failure is commonly indicated by a soft failure threshold at which the system no longer performs to its specifications or cannot be expected to perform for an appreciable amount of time; this is generally some point before a catastrophic failure occurs. A Bayesian updating technique has been developed to incorporate prior knowledge in the model. Type III prognostics are the only truly individualbased prognostic type. This is generally considered the ultimate goal of prognostics for safetycritical components and systems. Each of these classes of prognostic algorithms, as well as appropriate model architectures, is discussed in the following sections.

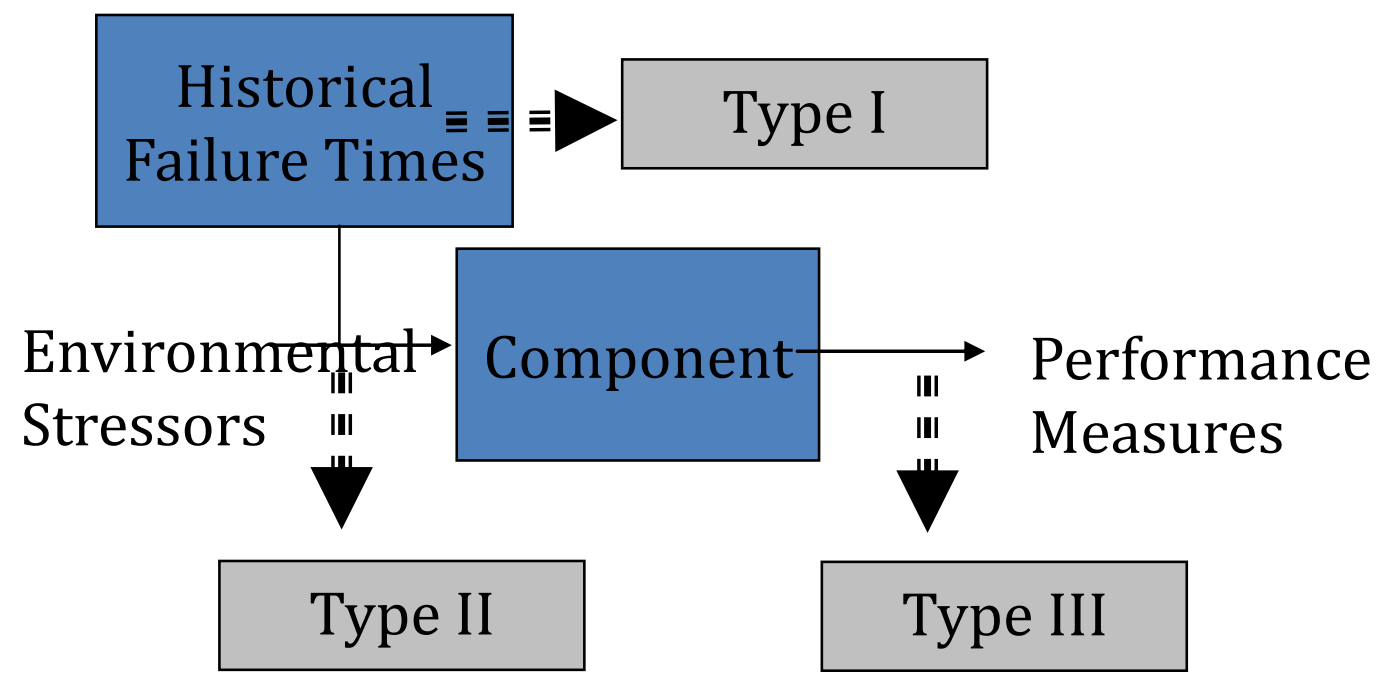

Figure 2-4: Prognostic Algorithm Categorization

\subsubsection{Type I: Traditional Time-to-Failure Analysis}

Type I methods are a simple extension of traditional reliability analysis, based entirely on an a priori distribution of failure times for similar systems in the past. Prognostic algorithms in this class characterize the average lifetime of an average system operating in historically average conditions; they do not utilize any information specific to the system at hand. The main 
assumption made when applying Type I methods is that future systems will operate under similar conditions to those seen in the past and will fail in similar ways.

Typically, Type I prognostic models track a population of systems over their lifetime and record only the failure time of each system. In addition, the total runtime of each system which hasn't failed at the end of the observation is recorded; this is called censored data and is also included in the analysis. A probability distribution is fit to these runtimes to give an estimate of the time of failure (ToF) distribution of the population. The most common parametric model used in reliability analysis is the Weibull distribution. This model is used because it is flexible enough to model a variety of failure rates. The formula for the failure rate (eqn. 1) is a two parameter model with a shape parameter $(\beta)$ and a characteristic life $(\theta)$ :

$$
(t)=-\frac{t}{-} \div
$$

These two parameters provide the modeling flexibility for components exhibiting an increasing failure rate $(\beta>1)$, a constant failure rate $(\beta=1)$, and a decreasing failure rate $(\beta<1)$. With the correct choice of shape parameter, the Weibull distribution adequately models the exponential, normal, or Rayleigh distributions. Examples of different shape parameters are given in Figure 2-5. Additional information on Weibull modeling is available in [Abernethy, 1996].

Traditional reliability methods consider only the total runtime of a system and the historic total lifetimes of similar systems. However, several methods are available to include additional information in reliability analysis, which may make it more useful for prognostics. Yang and Xue [1996] suggest a method for analyzing both catastrophic and soft failures simultaneously using random process simulation and state tree analysis. 


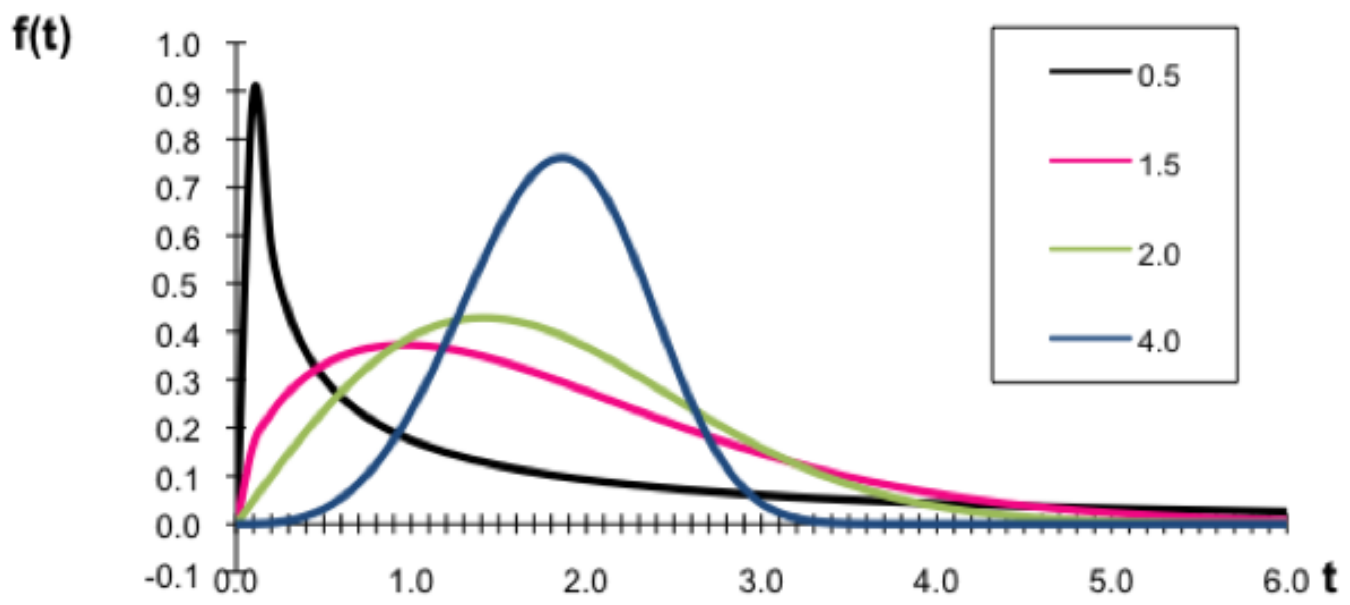

Figure 2-5: Weibull Failure Distributions with Different Shape Parameters

Several studies suggest the use of degradation data in estimating reliability distributions. Lu and Meeker first proposed the General Path Model (GPM) in [1993], which shifts reliability analysis from failure time to failure mode analysis. Improvements to their seminal work are proposed by Girish, Lam, and Jayaram who used neural networks to estimate the failure times for censored systems [2003]; Kharoufeh and Cox apply Markovian degradation models to estimate the failure time for censored systems [2005]; Chen and Zhang attempt to infer the lifetime distribution itself instead of the distribution parameters from the available data [2005]; and $\mathrm{Xu}$ and Zhao extend the approach to use multivariate degradation measures [2005]. These algorithms are the basis for the Type III prognostic GPM algorithm and are discussed in more detail in that section.

Most commonly, the Mean Residual Life (MRL) is used to estimate the RUL of a system. For an unit of age $t$, the MRL method assumes that the remaining life is a random variable, and the MRL is given by the expected value of this random variable [Guess and Proschan, 1985]:

$$
\operatorname{MRL}(t)=\frac{1}{S(t)} \quad S(u) d u
$$

where $S(\cdot)$ is the survival function and $t$ is the current time. The MRL at time $t$ can be calculated from either parametric or nonparametric distributions, which makes it particularly flexible for application to real world data. 
Studies applying Type I prognostics to estimation of RUL have shown the method to be unsatisfactory [Pecht et al., 2002; Lall et al., 1997]. This is to be expected since the MRL method assumes that the remaining life is a random variable; clearly it is not. In fact, because mean residual life is an "average" measure, it is expected to underestimate RUL about half the time and overestimate RUL about half the time. Vichare, et al. [2004] show that Type I methods are insufficient for electronic prognostics; the authors go on to suggest that in situ monitoring of operating conditions, such as temperature, humidity, vibration, and shock, may improve prognostic model performance. This leads to Type II prognostic methods.

\subsubsection{Type II: Stressor-based Prediction}

As suggested by [Vichare et al., 2004; Azzam, 1997; Baybutt et al., 2009], it is intuitive to consider usage conditions, both past and future, when estimating the RUL of a system. Type II methods attempt to do this by characterizing the lifetime of an average component operating in the specific environment. Here, it is assumed that systems operating in the same conditions will fail in similar ways; there is little unit-to-unit variance. Methods which commonly fall into this category include regression analysis with prognostic monitors [Goodman, 2000; Hofmeister et al., 2006; Hofmeister, et al., 2006; Goodman et al., 2006; Mishra et al., 2006], a specific formulation of the Markov Chain model [Kharoufeh and Cox, 2005; Bogdanoff and Kozin, 1985; Kwan et al., 2005], Proportional Hazards Models [Liao et al., 2006; Dale, 1985; Kumar and Klefjo, 1994], physics-of-failure models [Kacprzynski et al., 2004; Oja et al., 2007; Valentin et al., 2003; Pecht and Dasgupta, 1995; Kacprzynski et al., 2002], and Life Consumption models [Mishra et al., 2004; Ramakrishnan and Pecht, 2003]. Studies utilizing each of these architectures are described below.

\subsubsection{Regression Analysis}

Electronic system prognostics on the board or circuit level commonly utilize a built-in self test (BIST) prognostic monitor or canary [Goodman, 2000; Hofmeister et al., 2006; Hofmeister et al., 2006; Mishra et al., 2006]. A prognostic monitor is a "pre-calibrated semiconductor cell that is co-located with the actual circuit on a semi-conductor device" [Mishra et al., 2006]. The prognostic cell is designed to experience a higher current level than the actual circuit by decreasing the cross sectional area of the current-carrying path in the canary. Because the canary 
cell undergoes a higher current density, it is expected to fail in a predictably faster way than the actual circuit. By locating several prognostic monitors on a circuit with different known accelerating factors, the failure times of each of the cells can be trended to predict failure in the actual circuit (Figure 2-6). While this method is convenient and uncomplicated, Pecht, et al. [2001] argue that BIST monitors are not always sufficient for detecting and identifying failures. The authors found BIST results to suffer from a high false alarm rate and a low correlation between the fault indicated by the BIST and the actual fault. These shortcomings should be considered before applying this type of prognostic monitoring module.

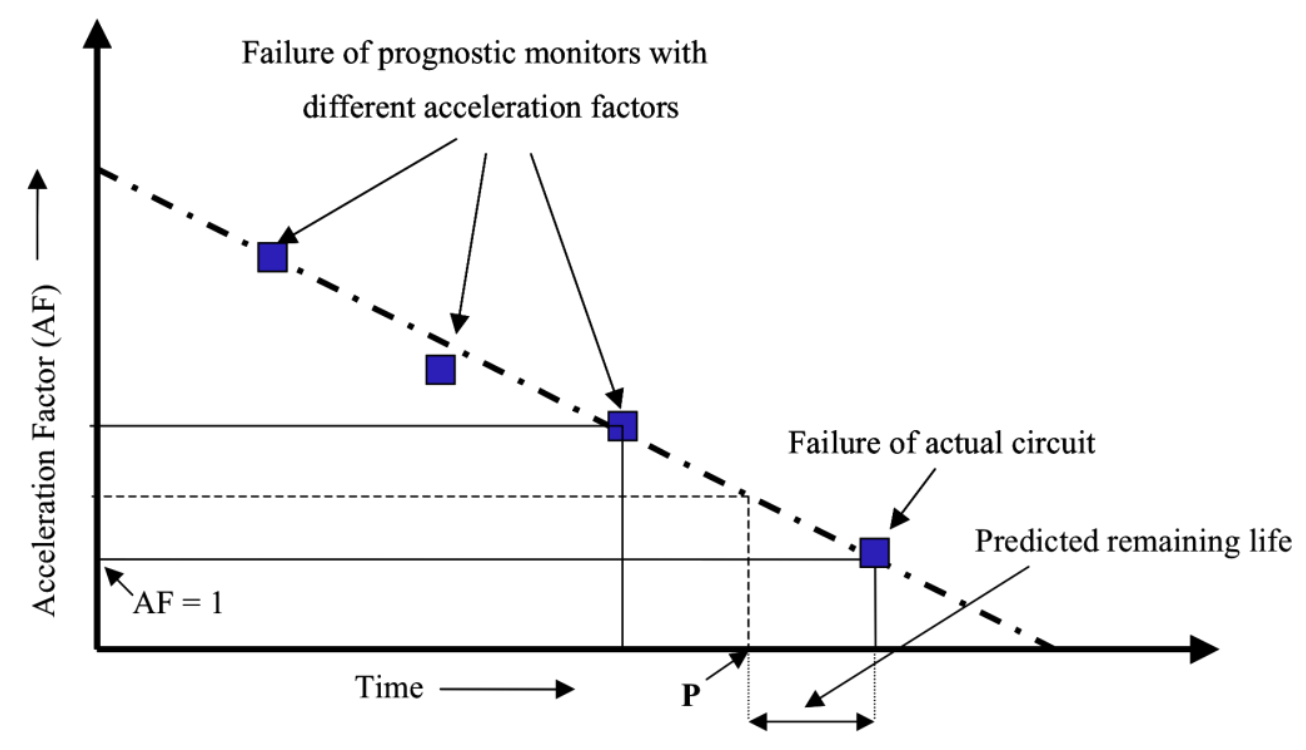

Figure 2-6: Use of Prognostic Monitor Failure Times to Estimate RUL of Actual Circuit [Mishra et al., 2006]

\subsubsection{Markov Chain Models}

Markov Chains and Hidden Markov Chains are common in many simulation exercises [Bogdanoff and Kozin, 1985]. The Markov Chain model is based on the assumption that the next state which a system will occupy depends only on the current state; past states do not affect the probability of transitioning to a new state. There are two types of Markov Chain prognostic models, which vary only in the information they use to simulate possible future states. Type II Markov Chain models, which will be discussed here, really include two models. 
The first model, called the environmental model, is a Markov Chain simulation which produces possible future operating state progressions based on transition probabilities seen in the past and the current operating state. The environment model is needed for making a prediction as to how the environment and operating conditions evolve in the future. The environmental model is defined by the transition probability matrix:

$$
Q=\begin{array}{ccc}
p_{11} & \cdots & p_{1 n} \\
\vdots & \ddots & \vdots \\
p_{n 1} & \cdots & p_{n n}
\end{array}
$$

where $\mathrm{p}_{\mathrm{ij}}$ is the probability of transitioning from state $i$ to state $j$. Often this probability matrix is assumed to be static, but it is straightforward to extend the method to time-dependent or degradation level-dependent transition probabilities. This model is used to simulate many possible future state progressions beginning at the current state.

These state progressions are then mapped to a degradation measure, which is the second model necessary in the Type II Markov Chain algorithm. The degradation measure is represented as a function of observable environmental conditions. To be useful for making a reliability prediction, the function should reflect the manner in which the environmental conditions affect the component reliability. Usually, environmental stressors tend to deteriorate the component reliability in a cumulative manner. Hence, the function to relate the environment conditions to the prognostic parameter is commonly of a cumulative form:

$$
Y\left(t_{k}\right)=\sum_{i=1}^{k} g\left(E\left(t_{i}, t_{i}+\Delta t_{i}\right)\right) \Delta t_{i}
$$

where $Y\left(t_{k}\right)$ is the degradation measure value at time $t_{k}, E\left(t_{i}, t_{i}+\Delta t\right)$ is the environmental condition observed at the time interval $\left[t_{i}, t_{i}+\Delta t\right]$, and $g($.$) is an appropriate function of environmental$ conditions.

When the estimated degradation measure is found to cross some pre-defined threshold, failure is said to have occurred. At each time of interest, many possible state progressions are simulated and mapped to degradation measures. These measures are then used to define a ToF distribution for the system. Figure 2-7 shows a typical progression of the environmental conditions in time. An example of trajectories is given in Figure 2-8 in which the function $\mathrm{g}($.$) is$ assumed to be an identity function. 


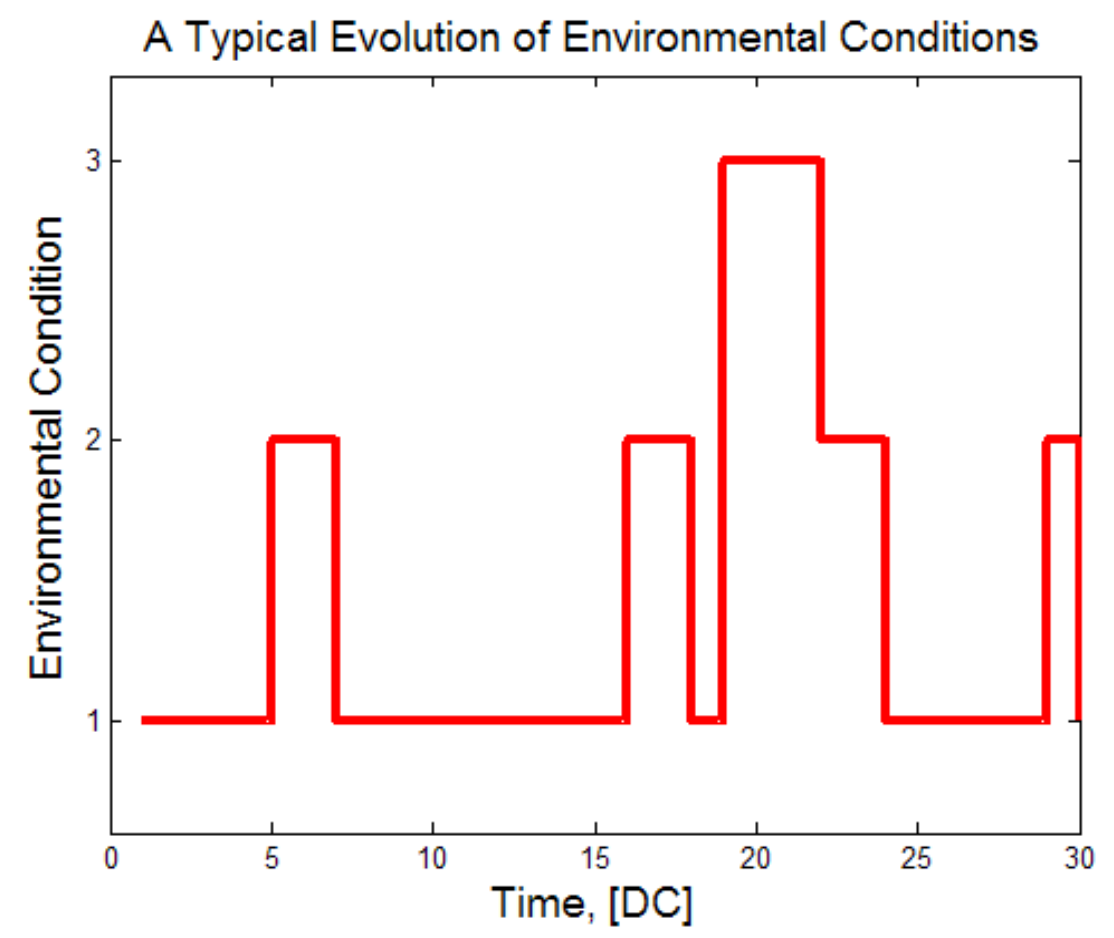

Figure 2-7: A Typical Progression of the Environmental Conditions

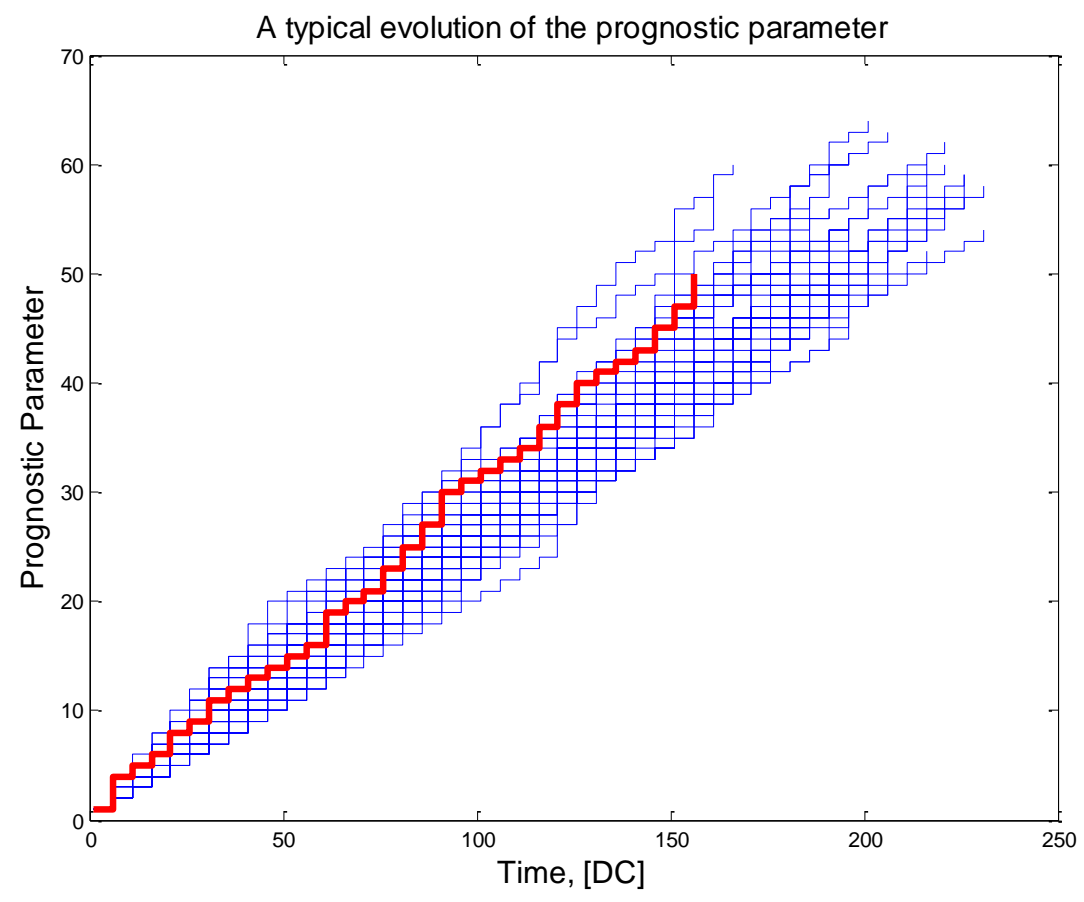

Figure 2-8: A Collection of Possible Degradation Measures 


\subsubsection{Proportional Hazards Model}

The Proportional Hazards (PH) Model developed by Cox and Oakes [1984] merges failure time data and stress data to make RUL estimates. The model uses operating conditions, called covariates, to modify the baseline hazard rate to give a new hazard rate for the system's specific usage conditions:

$$
(t ; z)={ }_{0}(t) \exp { }_{j=1}^{q} z_{j} \dot{\doteqdot}
$$

Failure data collected at covariate operating conditions are used to solve for the parameters $\left(\beta_{\mathrm{j}}\right)$ using an ordinary least squares algorithm. The baseline hazard is the hazard rate when covariates have little or no influence on the failure rate. A basic assumption of the proportional hazards model is that the effects of these covariates are multiplicative; this means that when the ratio of two covariates is evaluated, their hazard rates are proportional. A full discussion of developing a proportional hazards model can be found in [Kumar and Klefjo, 1994]. Dale [1985] applied the proportional hazard model to estimation of product reliability,

applying it to heterogeneous data from non-repairable systems. Liao, et al. [2006] suggest the use of proportional hazards models for estimating RUL, though the authors give no specific results of such an application.

\subsubsection{Physics of Failure Models}

Physics of failure models, or first principles models, are often desirable in engineering applications because they provide a greater understanding of the mechanisms by which systems and components may fail. Physics of failure models are also desirable for high-cost, high-risk systems that cannot be run to failure many times to collect the data needed for development of empirical models [Pecht and Dasgupta, 1995]. The use of physics of failure models for estimating RUL has focused mainly on electronic system prognostics [Oja et al., 2007; Valentin et al., 2003]. These models may be readily available for single components or single fault modes; however, developing accurate physics of failure models for large, complex systems has always been a daunting task. Physics of failure models often suffer from inaccuracies due to the assumptions made in developing the model, the inclusion of physical interactions which 
themselves are not completely understood, and long runtimes. Kacprzynski, et al. [2002] attempt to alleviate the inaccuracies of physics of failure models by fusing the results with other data sources such as diagnostic results, prognostic architectures, inspection information, and real-time system level features. The authors applied their system to a gear with a seeded fault and found very promising results for the data fusion technique. In [Kacprzynski et al., 2004], the same methodology was applied to helicopter gearboxes with similar success. Physics of failure models offer a better understanding of the mechanisms of failure for a component or system, but they are costly and tedious to develop for large, complex systems which experience many fault modes. In addition, the run time needed for many damage propagation models may make them impractical for real-time analysis. For systems with accurate physics of failure models available, it may be prudent to use these models to simulate system failure data. That simulated data may then be used to develop empirical models which may be run very quickly. This alleviates the burden of collecting failure data for expensive or safety critical systems. Physics of failure models are a key component of Life Consumption Models.

\subsubsection{Life Consumption Models}

Life Consumption models (LCM) were first proposed by Ramakrishnan and Pecht [2003] for monitoring RUL in electronic systems. The LCM methodology monitors the environment of a component or system during its entire lifecycle to determine the amount of damage incurred by the various loads and conditions experienced. This damage is translated to lost "life" which is subtracted from the expected life of an average system or component. The incurred damage is estimated through physics of failure models; this damage amount is related to a fraction of life lost by considering the total amount of operation under the same conditions which would result in failure of an undamaged part. LCM is illustrated in [Ramakrishnan and Pecht, 2003] and [Mishra et al., 2004] by application to a mounted printed circuit board operated under the hood of a moving vehicle. Both temperature and vibration levels were monitored on the board during use. The methodology was shown to effectively estimate RUL of the circuit board, even in the event of unexpected damage accumulation caused by a large, random shock. The major drawback of the LCM methodology is the need for accurate physics of failure models to estimate the accumulated damage. As mentioned above, physics of failure models are often not available, 
not accurate, or not time-effective for large, complex systems. Development of a more general LCM methodology which utilizes empirical models, such as neural networks, kernel regression models, or simple regression, for damage estimation would increase the applicability of this algorithm.

\subsubsection{Type III: Degradation-based Prediction}

Finally, the last category of prognostic algorithms attempts to characterize the lifetime of the specific system operating in its specific environment. These methods are termed degradation-based (or condition-based). The most common method in this class is the General Path model (GPM), which is described in great detail later. Basically, the GPM attempts to track some measure of degradation called a prognostic parameter and extrapolate it to failure [Lu and Meeker, 1993; Updhyaya et al., 1994; Chinnam, 1999; Brown et al., 2007; Engel et al, 2000; Heo, 2008; Keller et al., 2006; Hines et al., 2006; Byington et al., 2004; Luo et al., 2003]. This measure may be something measured directly from the system that gives information about system condition and fault severity, or it may be inferred from measurements made on the system. The specific details of this method are left to later sections because it is the focus of this work; however, fundamentals and results of this algorithm given in the literature are summarized here. Additional algorithms included in Type III prognostics are a different formulation of Markov Chain models [Hines and Usynin, 2007; Hines et al., 2007; He et al., 2006] and Shock models [Esary and Marshall, 1973; Mallor and Santos, 2003; Gut, 1990].

\subsubsection{Parametric Methods}

The General Path Model (GPM) was first proposed by Lu and Meeker [1993] to move reliability analysis from failure time to failure mode analysis. Extension of their original methodology to prognostics is discussed in detail in later sections. The first work to make this extension was by Upadhyaya, et al. [1994]. The authors in that work apply traditional regression models and neural networks to trend system degradation. In later years, the extrapolation methodology of traditional regression models was applied to helicopter gearboxes [Engel et al., 2000], flight control actuators [Byington et al., 2004], aircraft power systems [Keller et al., 2006], computer power supplies [Hines et al., 2006], and global positioning systems [Brown et al., 2007]. In addition, work by Chinnam [1999] applied the GPM methodology to feed forward 
neural networks for estimating degradation levels. Each of these studies attempt to model a degradation measure and extrapolate it to some pre-defined threshold to estimate the system RUL. Only a few of the studies consider the problem of uncertainty measurements [Engel et al., 2000; Hines et al., 2006; Byington et al., 2004]. The studies tend to take two approaches to uncertainty estimation. Uncertainty measurements in [Hines et al., 2006] and [Engel et al., 2000] are estimated based on the model architecture used to make the prediction, while Byington, et al. [2004] utilize Bayesian belief models to estimate the uncertainty. In addition, bootstrap methods can also be used to make uncertainty estimates. If accurate physics of failure models are available, these may be used to trend the system degradation. In these models, the physics of failure model utilizes measurements directly from the system of interest to estimate the hidden damage [Luo et al., 2003]. Here, the authors utilize a residual monitoring system to estimate the hidden damage in a system; this basic methodology can be applied to physics of failure models or empirical models. As in the previous discussion, Type III physics of failure models suffer from difficulty in development, inaccuracies due to the assumptions made in model development, and long runtimes. However, if accurate physics of failure models are available, they can be applied to prognostic algorithms and used with the GPM methodology.

Greitzer, et al. [1999; 2001; 2001] propose a slightly different formulation of the GPM, which he coined the Life Extension Analysis and Prognostics (LEAP) method. LEAP differs from GPM primarily in that it is a short-term regression model. Instead of regressing a model onto the entire operating history of a system or component, LEAP utilizes some recent window of data for the regression. Although using more data tends to lead to more stable predictions, using the entire operating history may mask recent changes in behavior which are of critical importance. While these predictions have greater variability, they tend to be more sensitive to recent and abrupt changes in condition, as seen in Figure 2-9. The LEAP methodology is proposed as a Type II method using operating conditions to estimate some figure of merit and then trending that figure of merit to failure. However, the extension to Type III prognostics is clear, where the figure of merit is directly measured or inferred from direct measurements of the system. This methodology is an improvement on the traditional GPM in that it is more sensitive 
to abrupt changes, but it is also less robust to noisy measurements. Identification of an optimal window size for regression is a critical task in applying this technique.

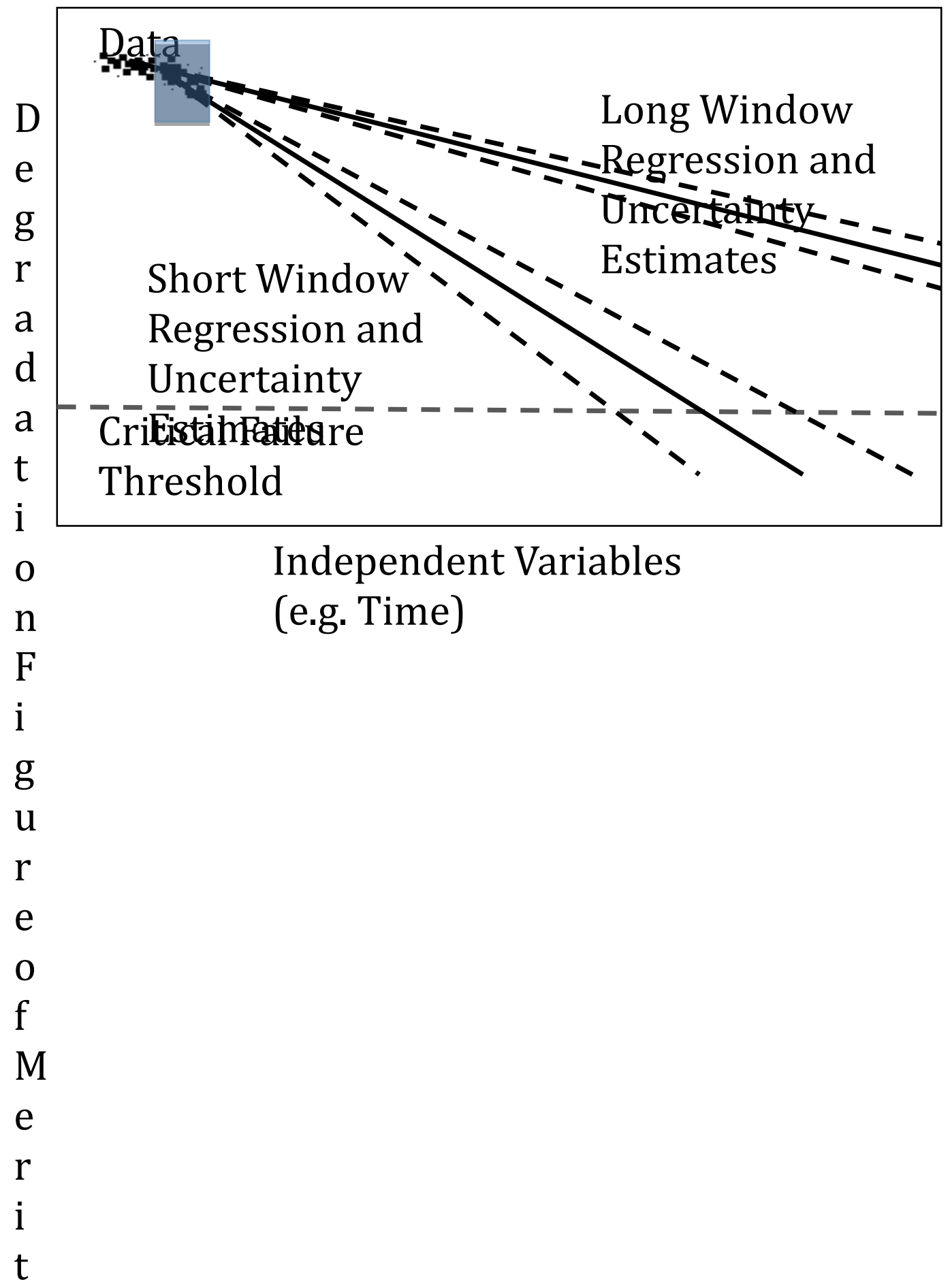


As mentioned previously, Markov Chain models can fall into the Type II or Type III category. While Type II Markov Chain models use the Markov assumption to generate possible future operating condition progressions, Type III Markov Chain models use the Markov assumption to generate random shock arrival times based on the current degradation level [Hines and Usynin, 2008; Hines et al., 2007; He et al., 2006]. These random shocks contribute some deterministic amount of degradation, usually one unit, to the degradation measure. The time of failure is calculated as the time when this simulated degradation measure crosses the failure threshold. At each time of interest, many degradation paths are simulated, and a probability of failure distribution is estimated from the collection as shown in Figure 2-10. The Markov Chain model is continuous in the time domain, but discrete in the degradation measure.

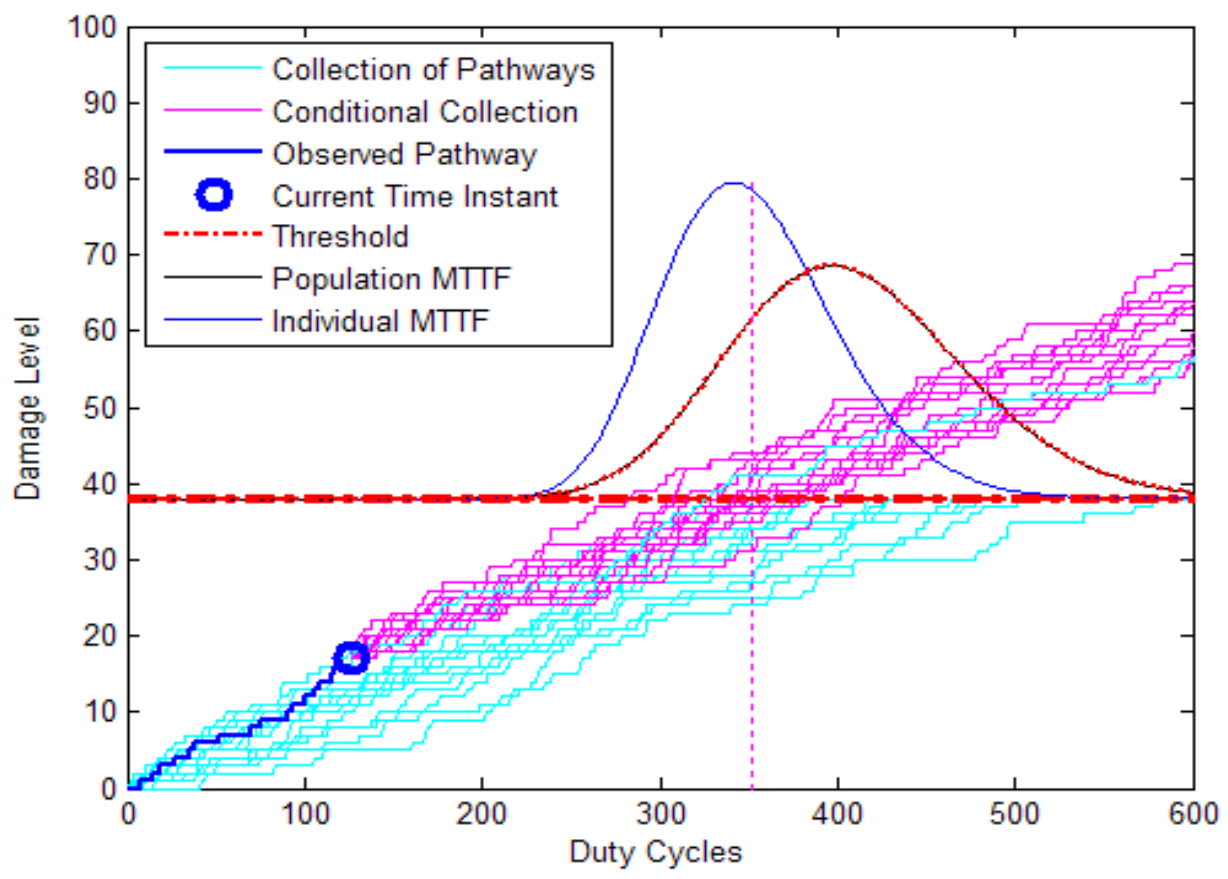

Figure 2-10: Markov Chain Model PoF estimation

A more general formulation is the Shock model [Esary and Marshall, 1973; Mallor and Santos, 2003; Gut, 1990]. Instead of experiencing some known amount of shock at each random 
shock occurrence, the shock model allows for a shock of random size. In this model, the time between random shocks is a continuous variable, with the probability of shock often determined by the current degradation state, the operating conditions, or some combination thereof. The size of the shock may be based on a single shock size distribution, or other features such as the current degradation measure, the operating condition, or other measures available from the system may define it. Again, when the cumulative degradation measure crosses some predetermined threshold, failure is said to have occurred; a probability of failure distribution is estimated from multiple simulated degradation measures.

\subsection{Data Fusion for Prognostics}

In any CBM system, many data sources are available for making prognostic estimates. These data sources may be redundant, such as measurements from a group of redundant sensors; as measurements from different subsystems within the same system. The main concern in any attempt at combining these data sources is to avoid producing a fused result which performs worse than the best performing constituent result [Roemer et al., 2001]. This can happen if data sources with little or no prognostic capability are included in the data fusion set. Several data fusion techniques have been proposed specifically for application in prognostic algorithms. These will be briefly described here.

Jardine, et al. [2006] explain the need for appropriate data fusion methods, stating "When multiple sensors are used, data collected from different sensors may contain different partial information about the same machine condition." The authors describe two classes of fusion methods: instantaneous and convolutive. Instantaneous methods involve time-independent, or memoryless, mixing functions; convolutive methods include some time dependence. Choi and $\mathrm{Li}$ [2006] apply instantaneous mixing functions to fuse time and frequency data to estimate crack size in gear tooth traverse cracks. Goebel and Bonissone [2004] use PCA-based data fusion methods to give a strong prognostic indicator when none is available in the data. Their method includes data analysis, PCA, adaptive network based fuzzy inference, and trending analysis. They apply the method to constant load, wet-end papermill breaks. The resulting model minimizes false alarms and provides adequate coverage of multiple types of breaks resulting from unknown causes. 
Roemer, et al. [2001] attempt to optimize data fusion results by reducing false alarm rates, increasing confidence levels in fault detection, and optimizing time to failure predictions. The authors specify three types of data fusion: feature creation, feature combination, and knowledge fusion. They specify knowledge fusion as a method to incorporate legacy information, physical model predictions, and signal-based information to improve prognostic estimates. The authors give an overview of common data fusion methods, including Bayesian Inference, Dempster-Shafer Method, Weighting/Voting Fusion, Fuzzy Logic Inference, and Neural Network Fusion. The authors illustrate the application of these methods to engine test cell sensor validation. They do not present results specific to prognostic applications.

Hughes, et al. [2002] combine multiple fault mode indicators using traditional logical tests. They compare the performance of logical 'OR' tests and rank sum tests. These methods are used to reduce false alarms and improve fault isolation result. They apply their approach to disk drive failures. The two logical tests gave similar performance for each of the cases studied.

Wang and Coit [2004] use joint PDFs to determine when any one of a collection of degradation measures crosses its threshold. They apply their methodology to reliability prediction based on degradation modeling of multiple degradation parameters. This allows for the combination of the information from multiple degradation models. The authors apply their methodology to simulated degradation data. Though the method is proposed for reliability monitoring, extension to prognostics is straightforward.

Several methods for prognostic estimation have been described in the previous sections, including algorithms in each of the three types: reliability-based, stressor-based, and degradation-based. Data fusion methods which have been applied to prognostics have also been described. Because a plethora of diverse algorithms and methodologies is available for prognostic estimation, it is necessary to develop a set of performance metrics by which the models can be compared.

\subsection{Metrics for Evaluating Algorithm Performance}

A major area of ongoing research in prognostics is model performance characterization. Performance metrics are necessary to allow model developers to compare two competing models, to understand the validity of a prognostic estimate, and to characterize model 
performance over different operating regimes, fault modes, or systems. Performance metrics for monitoring, fault detection, and diagnostic systems are well established [Hines et al., 2008], including accuracy, robustness or auto-sensitivity, spill-over or cross-sensitivity, fault detectability, uncertainty measures, fault detection time, and false alarm/missed alarm rates. Appropriate prognostic performance metrics, however, are less understood. Research in prognostic model performance metrics has focused on three areas: algorithm performance, computational speed, and economic incentive metrics. Obviously, it is desirable for prognostic algorithms to make accurate and precise RUL estimates. However, by the very nature of prognostics, these estimates ideally are made in an online fashion, which means the algorithm must have an acceptably short computation time. This is of particular import for systems which intend to have real-time data collection and RUL estimation running during system use. Metrics to characterize the computational cost of prognostic algorithms include complexity [Byington et al., 2005], specificity [Vachtsevanos, 2003], CPU time [Saxena et al., 2008], and "Big O" runtime notation [Aho et al., 1983]. It seems reasonable to assume that, given ample funds, the rate of increasing computer speed, and the use of more sophisticated computational methods [Volkov and Demmel, 2008], a system will be available to make an RUL prediction in any prescribed time frame. Economic metrics, such as Return on Investment (ROI), may consider model performance in making cost benefit analyses [He et al., 2006; Saxena et al., 2008; Wood and Goodman, 2006; Banks and Merenich, 2007]. Generally, these analyses show that PHM systems decrease maintenance costs, while increasing availability and improving safety. However, these metrics are primarily intended for a management group and are not considered model performance metrics for this research.

Measures of prognostic algorithm performance are of more interest to the current research. Current research largely focuses on method development; however, algorithm performance metrics have been the focus of several studies in recent years [Line and Clements, 2006; Vachtsevanos, 2003; Saxena et al., 2008; Banks and Merenich, 2007; Kacprzynski et al., 2004; Saxena et al., 2009]. Each of these studies resulted in different, and sometimes competing, performance metric definitions. However, each study highlights key shortcomings in traditional performance metrics. Prognostic algorithm performance metrics tend to fall into one of two 
categories: accuracy metrics or precision metrics. The field, however, is plagued by a problem common to many areas of prediction: "The more precise the remaining life estimate, the less probability that this estimate will be correct" [Hess et al., 2005]. Bearing this in mind, prognostic algorithm performance measures must be developed which will allow researchers and developers to accurately choose between competing prognostic models.

Unlike monitoring system models which produce one estimate of the system value at each point in time, prognostic models result in a time-series of RUL estimates. That is, the same value is being estimated at each prediction. In general, we are willing to suffer large errors and uncertainties early in life if a model will provide better performance as the system approaches failure [Line and Clements, 2006; Saxena et al., 2008; Saxena et al., 2009]. Traditional error measures do not account for these progressive acceptable accuracy and precision levels. Saxena, et al. [2008] suggest several metrics to account for this, the most interesting of which are the $\alpha-\lambda$ performance metrics for accuracy and precision. The $\alpha-\lambda$ performance dictates that the accuracy (or uncertainty) should be within some specified $\alpha^{*} 100 \%$ of the actual value within a relative distance, $\lambda$, to the actual failure, as shown in Figure 2-11. The formulation proposed by the authors gives a true or false value for the $\alpha-\lambda$ performance; namely, are the algorithm predictions correct to within $\alpha^{*} 100 \%$ at the $\lambda$-relative distance to failure. It may be more useful to consider when the algorithm is within the $\alpha^{*} 100 \%$ precision level or the fraction of time that the algorithm is within this requirement. 


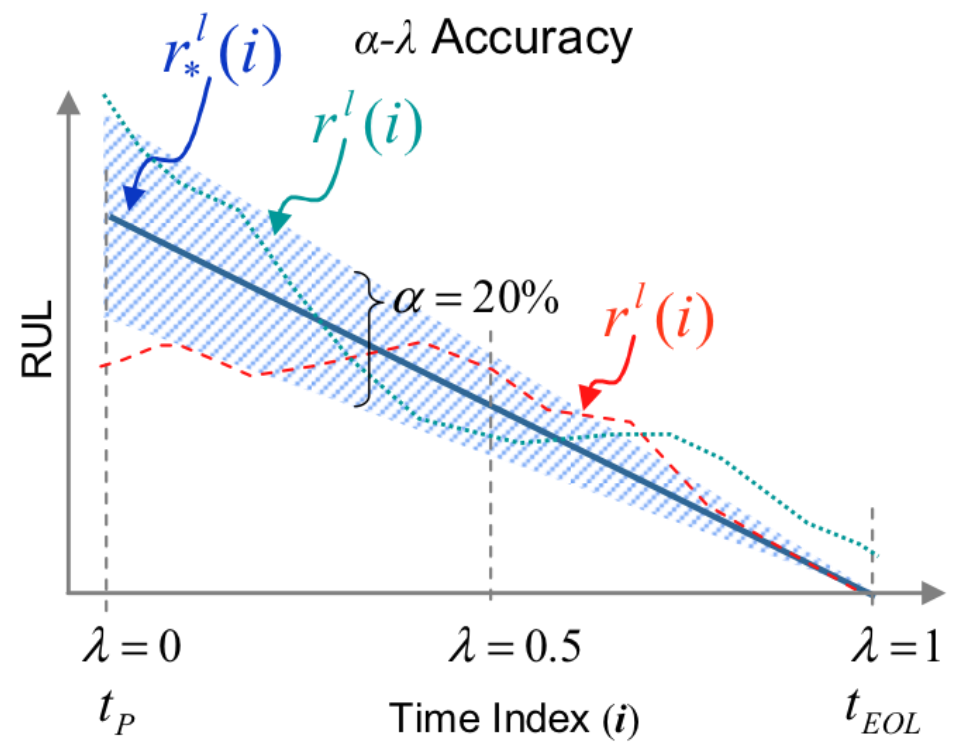

Figure 2-11: $\alpha-\lambda$ Performance for Accuracy [Saxena et al., 2008]

In addition to concerns about the importance of correctly accounting for temporal needs, prognostic models that predict that failure will occur before actual failure are generally considered better than those that predict failure will occur after the actual failure. RUL estimates greater than the actual remaining life leave room for unexpected failures and unplanned maintenance. Saxena, et al. [2008], suggest an exponentially weighted accuracy metric to account for this, which gives more weight to late predictions and less weight to early predictions. This metric considers the RUL predictions made at one point in time, instead of the entire time series of predictions. A similar error metric was used in the 2008 PHM data challenge [Saxena et al., 2008].

Although it is not generally applicable, for systems with known RUL, the model accuracy for a specific system at any given prediction time can easily be measured by the absolute percent error:

$$
A=\frac{\left|R U L_{\text {actual }} R U L_{\text {estimated }}\right|}{R U L_{\text {actual }}}
$$

The accuracy of the model can be calculated across a population of systems as the mean absolute percent error (MAPE) at a given prediction time (or fraction of total life). This gives a time series of expected accuracy for the algorithm. Along with the measure of accuracy, a measure of 
RUL uncertainty should be included. This uncertainty estimate may be derived from the specific model architecture or estimated through a Monte Carlo bootstrap method; these methods of uncertainty estimation are described in great detail in [Hines et al., 2008; Tamhane and Dunlop, 2000; Kutner et al., 2004].

This chapter has given an overview of the related prognostics research available in the open literature. The following chapter presents the prognostic methodology used in this research.

\section{METHODOLOGY}

The problem of accurately and precisely predicting remaining useful life is very complicated; as such many methodologies and algorithms have been proposed to address this problem, which were discussed previously. This work focuses on Type III, or condition-based prognostics. Extrapolation of a general path model (GPM) to some pre-defined failure threshold is the most common Type III method. The GPM algorithm employed is a formulation of the model originally proposed by Lu and Meeker [1993]. This method is described in detail in the following sections, including a Bayesian updating methodology for incorporating prior information into the GPM fit. This is followed by a discussion of the features necessary in an appropriate prognostic parameter for trending. Finally some discussion of automated methods for identifying the optimal parameter is given.

\subsection{The General Path Model}

The General Path Model (GPM), also called degradation modeling, was first proposed by Lu and Meeker [1993] to move reliability analysis methods from failure-time analysis to failureprocess analysis. Traditional methods of reliability estimation use failure times recorded during normal use or accelerated testing to estimate a ToF distribution for a population of identical components. In contrast, GPM uses degradation measures to estimate the ToF distribution. The use of historical degradation measures allows for the direct inclusion of censored data, which gives additional information on unit-wise variations in a population. 
GPM analysis begins with some assumption of an underlying functional form of the degradation path for a specific fault mode. The degradation of the $i^{\text {th }}$ unit at time $t_{j}$ is given by:

$$
y_{i j}=\left(t_{j},,_{i}\right)+{ }_{i j}
$$

where $\varphi$ is a vector of fixed (population) effects, $\theta_{i}$ is a vector of random (individual) effects for the $i^{\text {th }}$ component, and $\varepsilon_{i j} \sim N\left(0, \sigma_{\varepsilon}^{2}\right)$ is the standard measurement error term. Application of the GPM methodology involves several assumptions. First, the degradation data must be describable by a function, $\eta$; this function may be derived from physics-of-failure models or from the degradation data itself. In order to fit this model, the second assumption is that historical degradation data from a population of identical components or systems is available. This data should be collected under similar use (or accelerated test) conditions and should reasonably span the range of individual variations between components. Because GPM uses degradation measures instead of failure times, it is also not necessary that all historical units are run to failure; censored data contains information useful to GPM forecasting. The final assumption of the GPM model is that there exists some defined critical level of degradation, $D$, beyond which a component no longer meets its design specifications, i.e. the component has failed. Therefore, some components should be run to failure in order to quantify this degradation level. Alternatively, engineering judgment may be used if the nature of the degradation parameter is explicitly known.

Several methods are available to estimate the degradation model parameters, $\varphi$ and $\theta$. In some cases, the population parameters may be known in advance, such as the initial level of degradation. If the population parameters are unknown, estimation of the vector of population characteristics, $\varphi$, is trivial; by fitting the model to each exemplar degradation path, the fixed effects parameters can be taken as the mean of the fitted values for each unit. The variance of these estimates should be examined to ensure that the parameters are in fact population-wide parameters. If significant variability is present, the parameters should be considered random and moved to the $\theta$ vector. A two-stage method of parameter estimation was proposed by $\mathrm{Lu}$ and Meeker [1993] to estimate distribution parameters for the random effects.

In the first stage, the degradation model is fit to each degradation path to obtain an estimate of $\theta$ for that unit; these $\theta$ 's are referred to as stage- 1 estimates. It is convenient to 
assume that the stage-1 estimates, or an appropriate transformation, $\Theta=H(\theta$ ), is normally (or multivariate normally) distributed so that the random effects can be fully described using only a mean vector and variance-covariance matrix without significant loss of information. If this assumption is not justifiable, the GPM methodology can be extended in a natural way to allow for other random effects distributions.

In the second stage, the stage-1 estimates (or an appropriate transformation thereof) are combined to estimate $\varphi, \mu \theta$, and $\Sigma \theta$. At this stage, if the variance of some previously assumed random parameter is effectively zero, this parameter should be considered a fixed effects parameter and should be removed from the random parameter distribution.

In their seminal paper, Lu and Meeker [1993] describe Monte Carlo methods for using the GPM parameter estimates to estimate a time to failure distribution and corresponding confidence intervals. Because the focus of this paper is estimating time to failure of an individual component, these methods will not be described here.

Several limitations and areas of future work of the GPM have been identified by Meeker, et al. [1998]. Some of these areas have been addressed in work by other authors. First, Meeker, et al. cite the need for more accurate physics of failure models. While such models are helpful for understanding degradation models, they may not be necessary for RUL estimation. In fact, if exemplar data sets cover the range of likely degradation paths, it may be adequate to fit a function which does not explain failure modes but accurately models the underlying relationships. With this idea, neural networks have been applied to GPM reliability analysis [Upadhyaya et al., 1994; Chinnam, 1999; Girish et al., 2003].

In addition, the GPM was originally developed for reliability analysis of only one fault mode. In practical applications, the system of interest may consist of several components each with different fault modes, or of one component with several possible, even simultaneous fault modes. These multiple degradation paths may be uncorrelated, in which case extension of the GPM is trivial: reliability of a component for all degradation modes is simply the product of the individual reliabilities, and RUL can be considered some function of the RULs for each fault mode, such as the minimum. If, however, the degradation measures are correlated, extension of the GPM is more complicated. For example, in the case of tire monitoring, several degradation 
measures may contain information about tire reliability, including wall thickness, tire pressure, and tire temperature. However, it is easy to see that these measures may be correlated; a higher temperature would cause a higher pressure, etc. The case of multiple, competing degradation modes is beyond the scope of the current work. A discussion of the problem can be found in Wang and Coit [2004].

\subsection{GPM for Prognostics}

The GPM reliability methodology has a natural extension to estimation of remaining useful life of an individual component or system; the degradation path model, yi, can be extrapolated to the failure threshold, D, to estimate the component's time of failure. This type of degradation extrapolation was proposed early on by Upadhyaya, et al. [1994]. In that work, the authors used both neural networks and nonlinear regression models to predict the RUL of a small induction motor. The prognostic methodology used for the current research is described below.

First, exemplar degradation paths are used to fit the assumed model. The stage-1 parameter estimates are used to evaluate the random-effects distributions, to determine the mean population random effects, the mean time to failure (MTTF) and their associated standard deviations, and to estimate the noise variance in the degradation paths. The MTTF distribution can be used to estimate the time of failure for any component which has not yet been degraded.

As data is collected during use, the degradation model can be fit for the individual component. This specific model can be used to project a time of failure for the component. Because of noise in the degradation signal, the projected time of failure is not perfect. A prediction interval (PI) about the estimated parameters can be evaluated as:

$$
\left[\wedge t_{n 1, / 2} s \sqrt{1+1 / n}, \hat{+}+t_{n 1, / 2} s \sqrt{1+1 / n}\right]
$$

where $t_{n-1, \alpha / 2}$ is the student's t-distribution, $n$ is the number of observations used to fit the model, and $s$ is the standard deviation of the degradation model parameters. The standard deviation of the parameters can be estimated through traditional linear regression techniques. The range of model parameters can be used to project an PI about the estimated time of failure.

The methodology described considers only the data collected on the current unit to fit the degradation model. However, prior information is available from the historic degradation paths 
used for initial model fitting, including the mean degradation path and associated distributions. This data can provide valuable knowledge for fitting the degradation model of an individual component, particularly when only a few data points have been collected or the collected data suffers from excessive noise. Bayesian updating methods have been developed to incorporate this additional information in the fitted model.

\subsection{Incorporating Prior Information with Bayesian Methods}

The current research investigates using Bayesian methods to include prior information for linear regression problems. However, as discussed above, the GPM methodology can be applied to nonlinear regression problems as well as other parametric modeling techniques such as Neural Networks. Other Bayesian methods could be applied to these types of models, but such application is beyond the scope of the current research. For a complete discussion of Bayesian statistics including other Bayesian update methods, the interested reader is referred to [Lindely and Smith, 1972; Gelman et al., 2004; Carlin and Louis, 2000]. In addition, work by Robinson and Crowder [2000] focuses on Bayesian methods for nonlinear regression GPM.

A linear regression model is given by:

$$
Y=b X
$$

The model parameters are estimated as:

$$
b=\left(X^{T}{ }_{y}^{1} X\right){ }^{1} X^{T}{ }_{y}^{1} Y
$$

where $\Sigma_{y}$ is the variance-covariance noise matrix for the response observations. It is important to note that the linear regression model is not necessarily a linear model. The data matrix $X$ can be populated with any function of degradation measures, including higher order terms, interaction terms, and functions such as $\sin (x)$ or $e^{x}$. If prior information is available for a specific model parameter, i.e. $\beta_{j} \sim \mathrm{N}\left(\beta_{j o}, \sigma_{\beta}^{2}\right)$, then the matrix $X$ should be appended with an additional row with value one at the $j^{\text {th }}$ position and zero elsewhere, and the $Y$ matrix should be appended with the a priori value of the $j^{\text {th }}$ parameter.

$$
\begin{aligned}
& X^{*}=\left[\begin{array}{llllllll}
X ; & 0 & \cdots & 0 & 1 & 0 & \cdots & 0
\end{array}\right] \\
& Y^{*}=\left[\begin{array}{ll}
Y ; & j
\end{array}\right]
\end{aligned}
$$


Finally, the variance-covariance matrix is augmented with a final row and column of zeros, with the variance of the a priori information in the diagonal element.

$$
{ }_{y}^{*}=\begin{array}{cccc}
2 & 0 & \cdots & 0 \\
y & \ddots & 0 & \vdots \\
0 & \cdots & 2 & 0 \\
0 & \cdots & 0 & 2
\end{array}
$$

If knowledge is available about multiple regression parameters, the matrices should be appended multiple times with one additional row for each parameter.

It is convenient to assume that the noise in the degradation measurements is constant and uncorrelated. Some a priori knowledge of the noise variance is available from the exemplar degradation paths. If this assumption is not valid for a particular problem, then other methods of estimating the noise variance must be used. The assumption of uncorrelated noise allows the variance-covariance matrix to be a diagonal matrix consisting of noise variance estimates and a priori knowledge variance estimates. If this assumption is not valid, including covariance terms is trivial; again these terms can be estimated from historical degradation paths.

After a priori knowledge is used to obtain a posterior estimate of degradation parameters, this estimate becomes the new prior distribution for the next estimation of degradation parameters. The variance of this new knowledge is estimated as:

$$
\frac{1}{{\underset{p o s t}{j}}_{j}}=\frac{n}{2}+\frac{1}{\text { prior }_{j}}
$$

where $n$ is the number of observations used to fit the current model.

\subsection{Choosing a Prognostic Parameter}

Identification of an appropriate prognostic parameter is key for applying a GPM prognostic model to a system. An ideal prognostic parameter has three key qualities: monotonicity, prognosability, and trendability [Coble and Hines, 2009]. Monotonicity characterizes the underlying positive or negative trend of the parameter. This is an important feature of a prognostic parameter because it is generally assumed that systems do not undergo self-healing, which would be indicated by a non-monotonic parameter. However, this assumption is not valid for some components such as batteries, which may experience some 
degree of self-repair during short periods of nonuse. The monotonic trend is considered valid when considering an entire system, even if individual components or sub-systems may experience some self-repair. Prognosability gives a measure of the variance in the critical failure value of a population of systems. Ideally, failure should occur at a crisp, well-defined degradation level. A wide spread in critical failure values can make it difficult to accurately extrapolate a prognostic parameter to failure. Finally, trendability indicates the degree to which the parameters of a population of systems have the same underlying shape and can be described by the same functional form. These three intuitive metrics can be formalized to give a quantitative measure of prognostic parameter suitableness. Ideally, these metrics would each range from zero to one, one indicating a very high score on that metric and zero indicating that the parameter is not suitable according to the particular metric. Figures 15 and 16 give two populations of prognostic parameters; the parameter shown in Figure 3-1 is considered useful for prognostics, while the parameter in Figure 3-2 is not. These two populations will be used to illustrate the three suitability metrics as they are discussed.

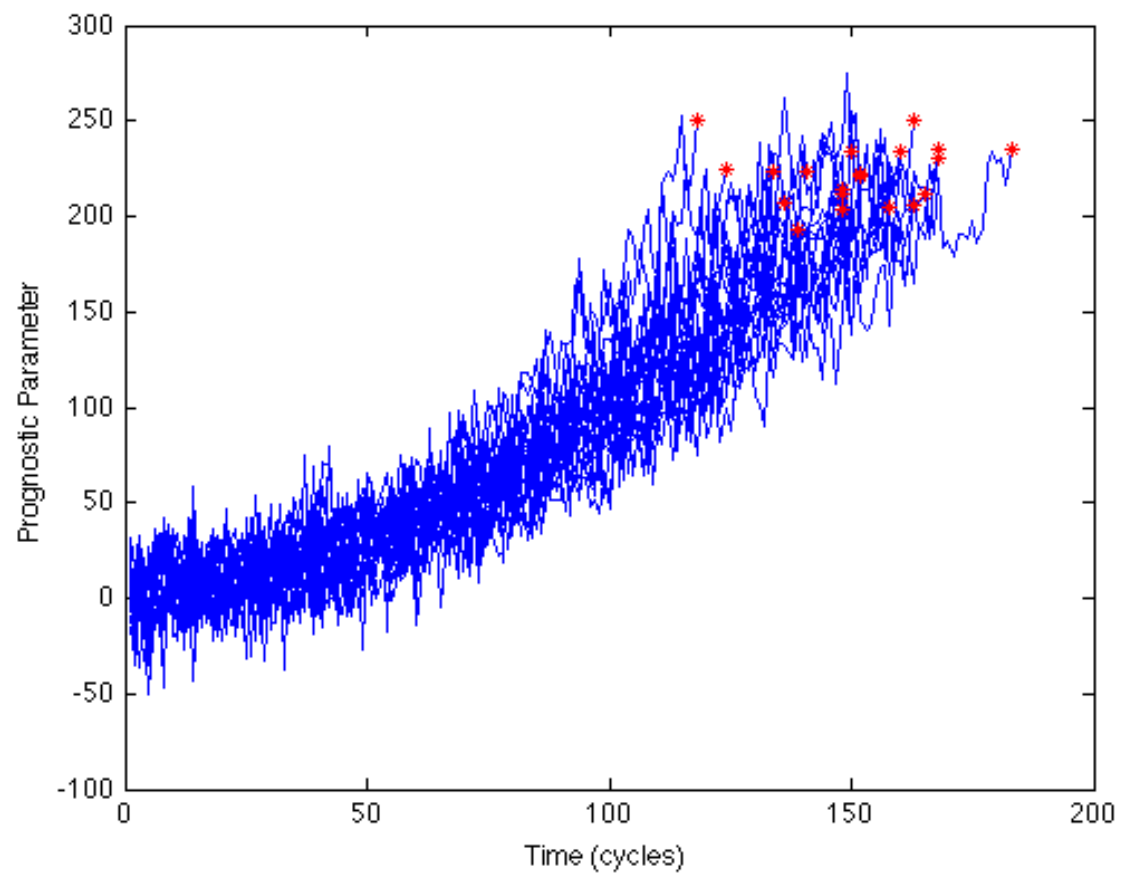

Figure 3-1: Population of "Good" Prognostic Parameters 


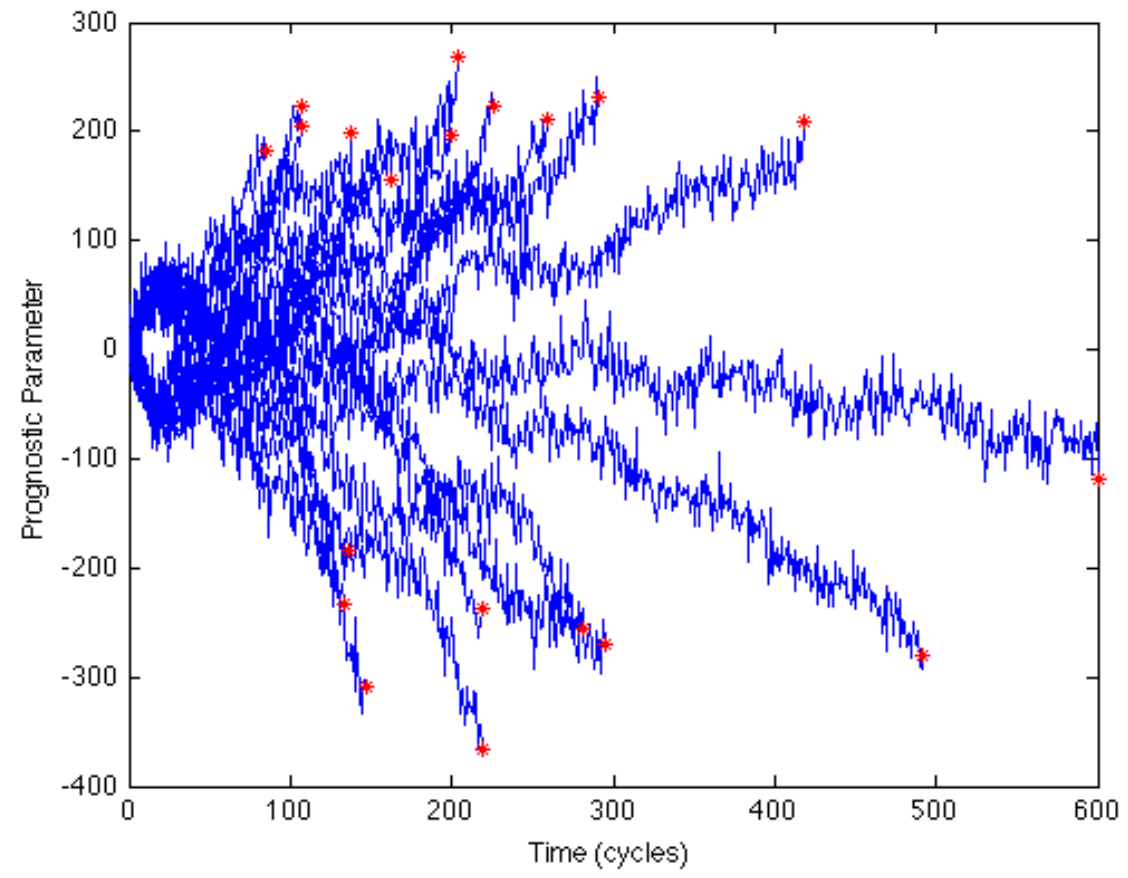

Figure 3-2: Population of "Bad" Prognostic Parameters

Monotonicity is a straightforward measure given by:

$$
\text { Monotonicity = mean }\left|\frac{\# \operatorname{pos} d / d x}{n} 1 \frac{\# n e g d / d x}{n 1}\right| \div
$$

where $n$ is the number of observations in a particular history. The monotonicity of a population of parameters is given by the average difference of the fraction of positive and negative derivatives for each path. When using data collected or inferred from actual systems, it is important to adequately smooth the data to give more accurate estimates of the derivatives. Numerical calculation of a function derivative should rarely be left to a simple difference function; the addition of noise makes this method inaccurate and impractical. In practice, fitting a line to a small portion of the data, perhaps five or ten observations, and taking the derivative to be the slope of that line will give a more realistic measure of the slope. When this method is employed, the above equation for monotonicity need only be adjusted for the number of calculated derivatives. Instead of $n-1$ derivatives, $n-m$ derivatives may be calculated, where $m$ is the number of data points used to calculate one derivative. The population of good parameters 
shown in Figure 3-1 is clearly monotonically increasing, in the absence of noise; the monotonicity metric for this parameter is 0.940. Conversely, the bad parameter shown in Figure 3-2 has monotonicity of 0.501 . It is important to note that the current formulation of monotonicity does not consider if the entire population is monotonic in the same direction, only that each individual exhibits an either generally increasing or decreasing trend. This is an undesirable feature in the prognostic parameter; however, it is considered in characterizing the prognosability which looks at how well clustered failure values are. If failure values for the entire population are well clustered and the individual parameters are monotonic, then the population must have either an increasing or decreasing monotonicity.

Prognosability is calculated as the deviation of the final failure values for each path divided by the mean range of the path. This is exponentially weighted to give the desired zero to one scale:

$$
\text { Prognosability }=\exp \frac{\text { std }(\text { failurevalues })}{\text { mean }(\mid \text { failurevalue startingvalue })} \dot{\doteqdot}
$$

This measure encourages well-clustered failure values, i.e. small standard deviation of failure values, and large parameter ranges. This gives the model a long range to predict a very precise value, which can be related to the notice period discussed previously. The failure values for the good prognostic parameter are very well clustered, following a wide range; the prognosability is 0.930. The failure values of the population of bad prognostic parameters cover a wide range of values; this parameter has prognosability of only 0.346 .

Characterizing the trendability of a population of parameters poses significant difficulty compared to the other two metrics. A candidate parameter is trendable if the same underlying functional form can model each parameter in the population. Initially, trendability was characterized by comparing the fraction of positive first and second derivatives in each parameter. However, this naïve approach was highly susceptible to noise and did not provide a clear distinction between trendable and not-trendable parameters. An improved method for characterizing trendability is used in which prognostic parameters are re-sampled with respect to the fraction of total lifetime. This results in each prognostic parameter containing exactly 100 observations, with each observation corresponding to $1 \%$ of lifetime. The linear correlation is 
calculated across the population of prognostic parameters, and the trendability is given by the smallest absolute correlation:

$$
\text { Trendability }=\min \left(\left|\operatorname{corrcoef}_{i j}\right|\right)
$$

Figure 3-3 gives a comparison of a population of prognostic parameters as a function of cycles and as a function of the percent of life. It is visually obvious in Figure 3-3(a) that the parameters of this population can be described by the same underlying function. By transforming to the percent lifetime space in Figure 3-3(b), it is straightforward for a computer to recognize this relationship as well. The "good" population shown in Figure 3-1 has trendability of 0.984, while the population of "bad" parameters shown in Figure 3-2 has trendability of 0.288 . The three suitability metrics for both populations are summarized in Table 3-1.

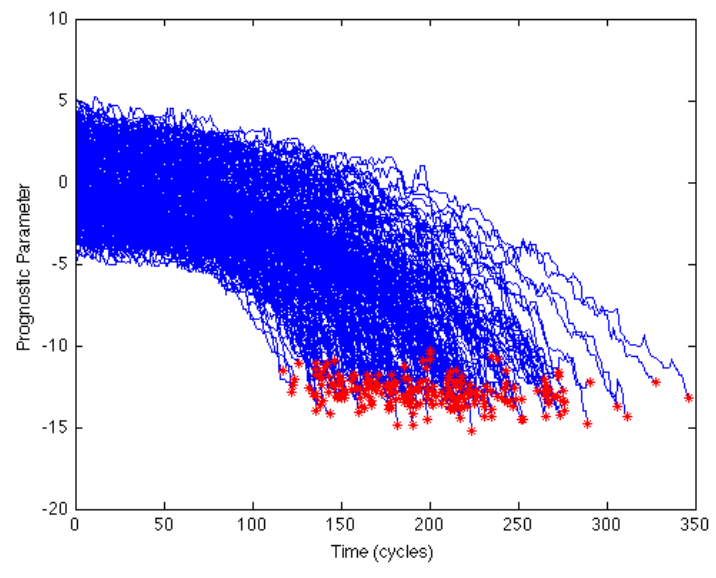

(a)

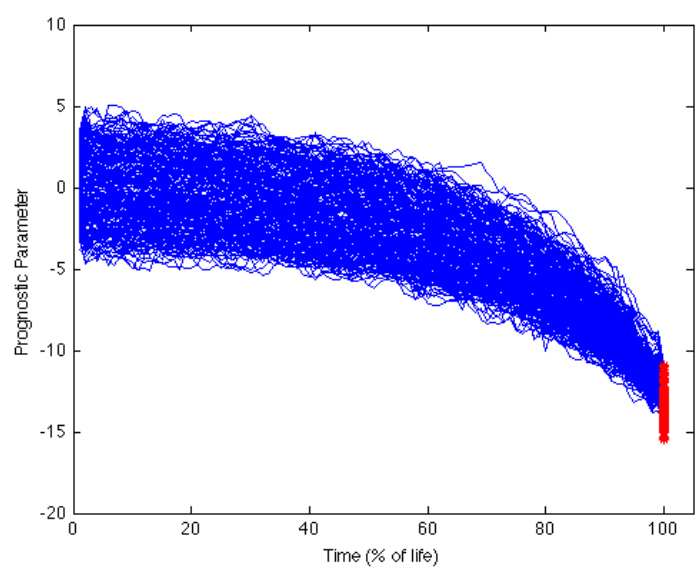

(b)

Figure 3-3: Prognostic Parameter vs (a) Time in Cycles and (b) Time in \% of Full Lifetime

Table 3-1: Example Prognostic Parameter Suitability Metrics

\begin{tabular}{|l|l|l|l|}
\hline & Monotonicity & Prognosability & Trendability \\
\hline Good Parameter (Figure 3-1) & 0.940 & 0.930 & 0.984 \\
\hline Bad Parameter (Figure 3-2) & 0.510 & 0.346 & 0.288 \\
\hline
\end{tabular}

The effect of noise on the parameter suitability metrics is of particular import. Specifically, bad parameters which are noisy should still be identified as bad. It is slightly trickier for good parameters. Some noise contamination can be tolerated by the GPM 
methodology, but significant levels of noise will appreciably reduce model performance. Three sets of good and bad parameters are tested; each of the six parameters is plotted in Appendix A. The first set is completely noise free; the second set is contaminated with Gaussian noise at a level of $20 \%$ of the mean parameter value; the third set is contaminated with Gaussian noise at $80 \%$ of the mean parameter value. The suitability metrics for each of these parameters are given in Table 3-2. As the table shows, the parameter suitability metrics for a "good" prognostic parameter are only slightly degraded at a noise level of $20 \%$. The suitability metrics for the very noisy parameter, however, are significantly degraded, indicating that this parameter would result in degraded model performance. The three "good" parameters are used in the following chapter to investigate the effect of noise on model performance. The suitability metrics for the "bad" parameter are not significantly changed by the addition of noise. This is a useful result indicating that even the noise-free bad parameter is poorly suited to prognostics.

Table 3-2: Effect of Noise on Parameter Suitability Metrics

\begin{tabular}{|l|c|c|c|}
\hline & Monotonicity & Prognosability & Trendability \\
\hline \multicolumn{4}{|c|}{ Good Parameter } \\
\hline Noise Free & 1.000 & 0.937 & 1.000 \\
\hline $20 \%$ Noise & 0.955 & 0.913 & 0.986 \\
\hline 80\% Noise & 0.715 & 0.761 & 0.713 \\
\hline \multicolumn{4}{|c|}{ Bad Parameter } \\
\hline Noise Free & 0.439 & 0.344 & 0.228 \\
\hline 20\% Noise & 0.409 & 0.340 & 0.192 \\
\hline 80\% Noise & 0.441 & 0.343 & 0.153 \\
\hline
\end{tabular}

Several methods are available for identifying candidate prognostic parameters, including visual inspection of sensed data and model residuals, Principal Component Analysis, traditional optimization method, and Genetic Algorithms approaches. Traditionally, parameter identification is done through visual inspection and engineering judgment. While visual inspection can lead to the identification of useful prognostic parameters, it can be tedious and time consuming when parameters are needed for several components or fault modes, and the optimal parameter may be overlooked in favor of a suitable one. Automated methods for identifying prognostic parameters are possible with a formalized set of metrics to characterize their suitability. By defining a fitness function as a weighted sum of the three metrics: 


\section{fitness $=w_{m}$ monotonicity $+w_{p}$ prognosability $+w_{t}$ trendability}

a set of prognostic parameters can be compared to determine the most suitable one. Here, the constants $w_{m}, w_{p}$, and $w_{t}$ control how important each metric is in the optimization. For most applications, these constants can each be identically one to give equal weight to each parameter feature. However, as discussed previously, monotonicity may not be an appropriate prognostic parameter feature for some applications, such as for battery health monitoring. In that case, monotonicity may be excluded from the parameter fitness calculation by giving it a weight of zero. In addition to optimizing for the best combination of parameter suitability metrics, the prognostic parameter can also be optimized for other features perhaps not directly related to parameter performance. For instance, minimizing the weights of each of the inputs in a linear combination may be of value because it reduces the complexity of the solution and forces unimportant weights to go to zero; this can be achieved by adding an additional term to the

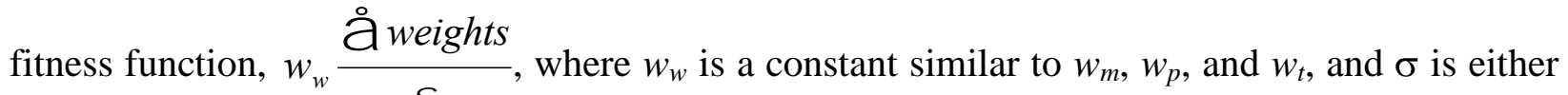
the standard deviation or the range of the input. Additionally, in order to reduce the uncertainty in the RUL prediction, it is beneficial for the first and second derivatives to have the same sign, i.e. increasing functions are concave up and decreasing functions are concave down. This can be included in the fitness function by simply adding a large penalty for mismatch of first and second derivatives. Other such features may also be included as they apply to the desired parameter optimization. The fitness function is used with optimization techniques such as gradient descent, genetic algorithms, and machine learning methods to identify useful prognostic parameters.

\subsection{Prognostic Parameter Optimization}

After developing an appropriate fitness function, defined by the suitability metrics given above and other desirable features, any number of well-established optimization methods may be applied to identify the optimal prognostic parameter. Optimization methods can be broken into two major groups: deterministic methods and stochastic methods. Deterministic methods include gradient descent, Newton method, quasi-Newton method, Nelder-Mead, and brute-force search [Horst and Hoang, 1996]. These methods suffer from high computational cost and sensitivity to 
local optima. Gradient descent methods attempt to traverse the fitness landscape to identify the optimal solution, but are easily fooled by local optima. Newton's method uses the second order Taylor expansion to find the roots of the derivative of the fitness function, although this method breaks down for derivatives which are not locally well approximated by a quadratic function. The Quasi-Newton method uses a mixed quadratic and cubic line search procedure and the Broyden-Fletcher-Goldfarb-Shanno (BFGS) formula for updating the approximation of the Hessian matrix. Nelder-Mead is a direct-search method that uses only function values, that is, it does not require computation of derivatives; this method works well for non-smooth objective functions. These methods are further complicated by multiple optimization parameters. For complicated fitness functions, these methods are likely to settle in a local optimal solution instead of the global optimum. Brute force methods attempt to identify every possible solution to a given optimization problem and compare the performance of all solutions to identify the optimal one. Brute force methods are obviously computationally intensive, and are well suited to optimizing parameters with discrete values. They are not readily applicable to large, continuous optimization spaces.

Stochastic methods include genetic algorithms, particle swarm, stochastic gradient descent, and simulated annealing [Schneider, 2006]. These methods are more robust to local optima because they search the entire solution space stochastically instead of moving along a given path. Given enough time, stochastic methods are guaranteed to find the global optimal solution; given a set amount of time, they are able to find near-optimal solutions [Horst and Hoang, 1996]. Stochastic methods are often coupled with a traditional gradient descent to refine the results of the optimization. Because stochastic methods are more robust to local optima and handle multiple optimization parameters well, this research will focus on these methods for parameter selection. The most common stochastic optimization method is genetic algorithms (GA), which is described in some detail in the next section. Additional multi-objective stochastic optimization methods include normal boundary intersection method, normal constraint method, and successive pareto optimization method, but GA optimization is the method of choice for the current research. A brief overview of GA optimization is given next. 


\subsubsection{Genetic Algorithms}

Classical optimization attempts to minimize the cost function by starting at an initial set of parameter values and utilizing function and derivative information to hone in on a minimum value. This type of optimization can quickly breakdown if the initial values are close to a local minimum; classical optimization will assume the first minimum it finds is the global minimum which is not necessarily true. Genetic algorithm (GA) optimization works differently by testing a random population of initial parameter values and mimicking the processes of evolution to optimize these values, as outlined in Figure 3-4. Because of the pseudo-random nature of the parameter populations evaluated, GA optimization is sometimes able to ignore local minima in search of a global minimum. Each of the steps in genetic algorithm optimization is quickly discussed below. A full discussion of continuous genetic algorithms is available in Haupt and Haupt [2004].

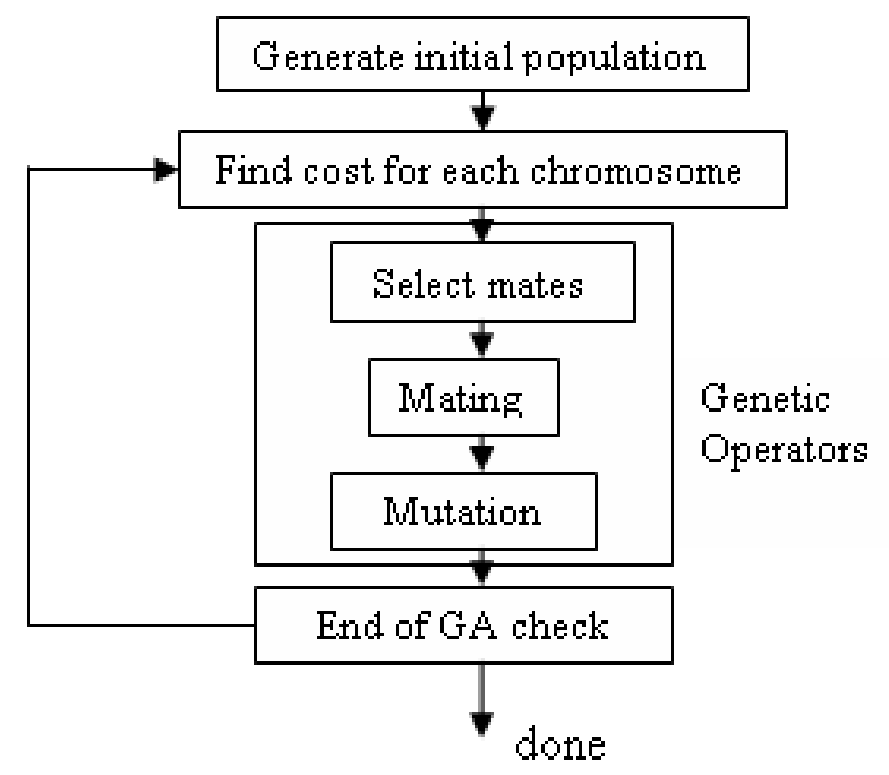

Figure 3-4: Flowchart of Genetic Algorithm Optimization

\subsubsection{Initial Population and Cost}

The goal of genetic algorithms is to search for an optimal solution of a given problem. This process begins by defining a "chromosome" to be a vector of variable values, a possible 
solution to the optimization. If a particular optimization problem has $N_{v a r}$ variables, then a possible chromosome for that optimization is

$$
\text { chromosome }=\left[p_{1}, p_{2}, p_{3}, \ldots, p_{N_{\mathrm{var}}}\right]
$$

where $\mathrm{p}_{\mathrm{i}}$ is a continuous-valued variable. These $p_{i}$ variables are called "genes." A "population" is the set of all chromosomes in one generation. Each chromosome has an associated fitness value found by evaluating the fitness function for the gene values in that chromosome.

Fitness functions are mathematical functions used to assign a value to a set of variables (chromosome). Fitness functions characterize the values and parameters to be optimized. Development of appropriate fitness functions is critical for genetic algorithm optimization: "The success of the GA on a particular function is certainly related to how the function is 'encoded"' [Mitchell et al., 1992]. Overly complex fitness functions can slow GA performance; overly simplified functions may not adequately describe the fitness surface.

After the fitness function has been evaluated for each parameter (or set of parameters), the initial population is ordered in terms of its performance. The genetic algorithm then uses three genetic operators to generate a new, hopefully fitter generation of solution chromosomes.

\subsubsection{Genetic Operators}

Three evolutionary modes exist for generating a subsequent generation: reproduction, crossover, and mutation.

Reproduction, or selection, results in an exact copy of the best performing chromosomes into the new population. This echoes Darwin's theory of Survival of the Fittest. Because of this mode, it is always guaranteed that the best fitness function cost will either improve or at least remain constant between successive generations; the best cost across generations will never worsen. In creating a new generation, a set number of chromosomes are produced via reproduction: the number of elite individuals.

Crossover, or mating, is the creation of offspring chromosomes from two parent chromosomes retained from the previous generation. This method is used to generate a specified fraction of the chromosomes in the new generation, the crossover fraction. Several methods have been developed to perform this crossover. The GA crossover of this research utilizes a "scattered" crossover function. Scattered crossover creates a random binary vector of length 
Nvar, called the mask. A child is generated by accepting the genes of the first parent when the mask vector is valued 1 . Genes of the second parent are selected when the mask vector is values 0 :

$$
\begin{array}{cc}
\text { parent }_{1} & {\left[p_{1}, p_{2}, p_{3}, \ldots, p_{N_{\text {var }}}\right]} \\
\text { parent }_{2} & {\left[q_{1}, q_{2}, q_{3}, \ldots, q_{N_{\text {var }}}\right]} \\
\text { mask } & {[0,1,1, \ldots, 0]} \\
\text { offspring } & {\left[q_{1}, p_{2}, p_{3}, \ldots, q_{N_{\text {var }}}\right]}
\end{array}
$$

The final model of new chromosome generation is mutation, which has obvious connections to evolutionary biology. In each generation, random mutations alter a set percent of the variables in the list of chromosomes, the mutation rate. Mutations prevent overly fast convergence and facilitate exploration of other areas of the fitness surface. In general, a gene is randomly to be mutated. This gene can either be replaced by a new, random value or a random value can be added to the gene. For the research presented here, mutation is based on a Gaussian distribution. This distribution uses random numbers taken from a Gaussian distribution centered on zero. These random numbers are added to the genes chosen for mutation.

Through these three methods, a new generation is created which is then evaluated for fitness. This cycle is repeated until one of several stopping criteria is met: a set number of generations is reached, a minimum average change between generations is not met, or the optimal value of the fitness function is obtained.

Like other optimization methods, GA has several advantages and disadvantages. One obvious advantage is the use of a fitness function to determine which parameter values to keep; classical optimization follows gradient curves and can get stuck in a local minimum. Because of the threat of local minimum, the initial parameter values in classical optimization must be close to the optimal values. GA optimization does not have this problem of initial values because of the inherent randomness in generation construction. As mentioned above, this randomness may allow GA to ignore local minima in search of the global minimum. If given enough time, GA will find the optimal, or near optimal solution to most problems. However, the disadvantage is that this can be computationally more intensive than a classical approach. Another disadvantage lies in identifying an appropriate fitness function for the problem. For model optimization, the 
mean squared error of the model is an apparent and appropriate fitness function. In some situations, however, additional parameters are needed to produce an optimal and appropriate solution. In these cases, multi-objective optimization (MOO) is used to produce a solution which is acceptable for several criteria.

\subsubsection{Multi-Objective Optimization}

In some applications, model optimization has several, sometimes competing objectives. In this case, a solution must be identified which satisfies some or all of these objectives to a greater or lesser degree. Bentley and Wakefield [1996] identify six methods for combining multiple objectives in one fitness function. This research will implement the sum of weighted objectives (SWO) method. This is the simplest and, therefore, most common method. To create a SWO fitness function, each objective is given a weight to specify its relative importance; the multiple objectives are then summed to produce a fitness function:

$$
\text { fitness }=w_{i} f_{i}
$$

where $f_{i}$ is the fitness function for the $i^{\text {th }}$ objective. The SWO method forces the GA to converge to a compromise solution and results in a best compromise solution based on the weights of each objective. It should be noted that in practice determining appropriate weight values for each objective can be very difficult.

\subsubsection{Prognostic Parameter Input Selection}

The computation time necessary to execute a GA optimization increases exponentially with the number of parameters being optimized [Ding and $\mathrm{Yu}, 2005$ ]. Many data sources are available as inputs to a prognostic parameter, including sensor readings, monitoring system residuals, fault detection and diagnostic results, and operating condition indicators. In order to alleviate the computational burden, possible prognostic parameter inputs can be pre-screened to determine if they are expected to contribute to the overall prognostic parameter suitability. In fact, removing unnecessary inputs may improve the optimization results. While the optimization should give these inputs weights of zero, this rarely happens before the optimization is terminated. When optimizing a weighted sum of possible inputs, it is straightforward to determine which inputs will improve the prognostic parameter and which will be detrimental. 
The proposed input selection method involves simply evaluating the fitness function for each candidate input and rejecting any inputs with fitness below a specified threshold. When using the simple sum of the three suitability metrics as a fitness function, a threshold of $1.0-1.5$ is generally appropriate for identifying useful inputs.

\subsection{Combined Monitoring and Prognostic Systems}

Figure 3-5 gives a combined monitoring, fault detection, and prognostics system similar to the one used in this research. The main difference between the system shown here and that shown in Figure 2-3 is the absence of the fault diagnostic module. The research presented in this dissertation does not consider different fault modes when making prognostic estimations; however, it would be trivial to use diagnostic information to inform the prognostic model. If fault diagnostics are available, then multiple prognostic models should be developed, one for each fault mode of interest. The monitoring system employs an Auto-Associative Kernel Regression (AAKR) model for monitoring and the Sequential Probability Ratio Test (SPRT) for fault detection. Both of these methods are described in broad detail below. The interested reader is referred to [Hines et al., 2008] for a more complete discussion of AAKR and [Wald, 1945] for SPRT.

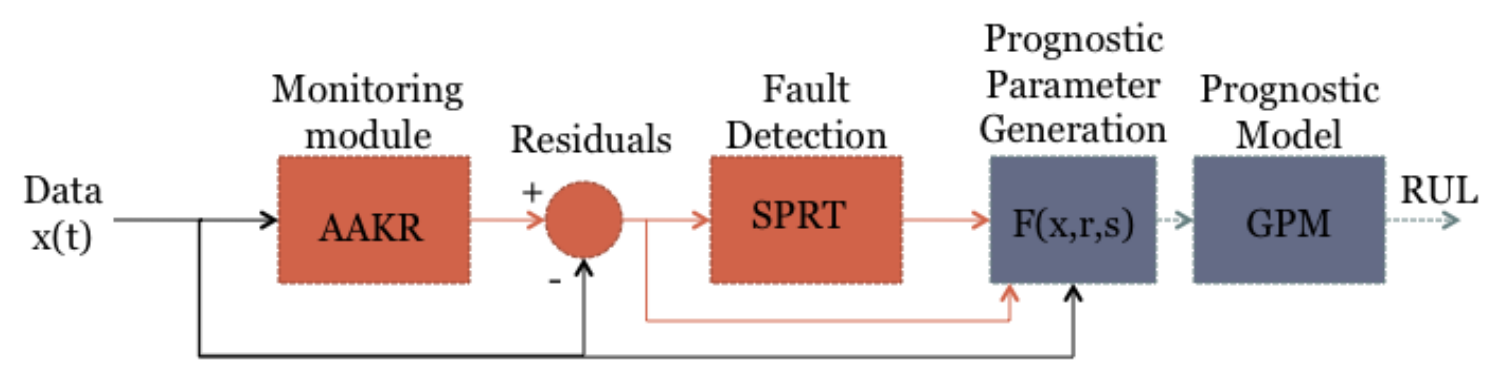

Figure 3-5: Combined Monitoring and Prognostic System

Auto-Associative models can generally be considered an error correction technique. These models compare a new observation to those seen in the past to estimate how the system "should" be running. These corrected predictions can be compared to the measured data to identify faulted operation. Several auto-associative architectures are available, including autoassociative neural networks, auto-associative kernel regression (AAKR), and multivariate state 
estimation technique [Hines et al. 2008]. This research employs the AAKR algorithm for system monitoring.

Auto-associative kernel regression (AAKR) is a non-parametric, empirical technique. Exemplar historical observations of system operation are stored in a data matrix. As a new observation is collected, it is compared to each of the exemplar observations to determine how similar the new observation is to each of the exemplars. This similarity is quantified by evaluating the distance between the new observation and the exemplar. Most commonly, the Euclidean distance is used:

$$
d_{i}=\sqrt{m=1}\left(\begin{array}{ll}
X_{j} & \left.x_{i j}\right)^{2}
\end{array}\right.
$$

where $d_{i}$ is the distance between the new observation, $\boldsymbol{X}$, and the $i^{\text {th }}$ exemplar, $x_{i}$. The distance is converted to a similarity measure through the use of a kernel. Many kernels are available; this research uses the Gaussian kernel:

$$
s_{i}=\exp \frac{d_{i}^{2}}{h^{2}} \div
$$

where $s_{\mathrm{i}}$ is the similarity of the new observation to the $i^{\text {th }}$ exemplar. Finally, the "corrected" observation value is calculated as a weighted average of the exemplar observations:

$$
\hat{X}=\frac{s_{i} x_{i}}{s_{i}}
$$

Monitoring system residuals are then generated as the difference between the actual observation and the estimated, corrected observation. These residuals are used with a SPRT to determine if the system is operating in a faulted or nominal condition. As the name suggests, the SPRT looks at a sequence of residuals to determine if the time series of data is more likely from a nominal distribution or a pre-specified faulted distribution. As new observations are made, the SPRT compares the cumulative sum of the log-likelihood ratio:

$$
s_{i}=s_{i 1}+\log i_{i}
$$

to two thresholds, which depend on the acceptable Type I and Type II fault rates: 


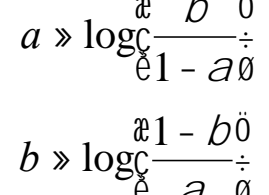

where $\alpha$ is the acceptable false alarm (false positive) rate and $\beta$ is the acceptable missed alarm (false negative) rate. For this research, false alarm and missed alarm rates of $1 \%$ and $10 \%$ respectively are used. If $s_{i}<\mathrm{a}$, then the null hypothesis is accepted; that is, the system is operating in a nominal condition. If $s_{i}>\mathrm{b}$, then the alternative hypothesis is accepted; that is, the system is operating in a faulted condition. When a determination is made, the sum, $s_{i}$, is reset to zero and the test is restarted.

After a fault is detected in the system, the prognostic system can be engaged to determine the RUL for the system. As discussed, GPM prognostic methods use a measure of system degradation to estimate RUL. Monitoring system residuals, or combinations of residuals, are natural candidates for these prognostic parameters because they inherently give a measure of the deviation of a system from normal operation. The following chapter investigates the application of this combined monitoring/prognostic system and the previously developed prognostic parameter identification method to example data sets.

\section{ANALYSIS TOOLBOXES}

Development of an integrated health management system can be daunting because of the high level of complexity involved in identifying appropriate algorithms at each stage. To support this, a suite of MATLAB-based toolboxes have been developed at the University of Tennessee Prognostics, Reliability Optimization and Control Technologies (PROaCT) lab with a range of monitoring, fault detection and prognostic capabilities. The Process and Equipment Monitoring (PEM) toolbox was developed to facilitate auto-associative modeling of process and system data, and fault detection [Hines and Garvey, 2005]. The PEM toolbox includes autoassociative kernel regression models, auto-associative neural networks, and linear regression models for system monitoring; and sequential probability ratio test and error uncertainty limit monitoring fault detection methods. The results of both of these modules, as well as an 
independently developed diagnostic module if available, can be used to facilitate prognostic analysis. The Process and Equipment Prognostics (PEP) toolbox is a MATLAB-based toolbox developed to aid in development of empirical prognostic models of each of the three types [Coble and Hines, 2009]. The PEP toolbox is designed to integrate with the previously developed PEM Toolbox. The results of process monitoring and fault detection produced by the PEM toolbox as well as the original system data are utilized by the PEP toolbox to make RUL predictions for the system, as shown in Figure 4-1.

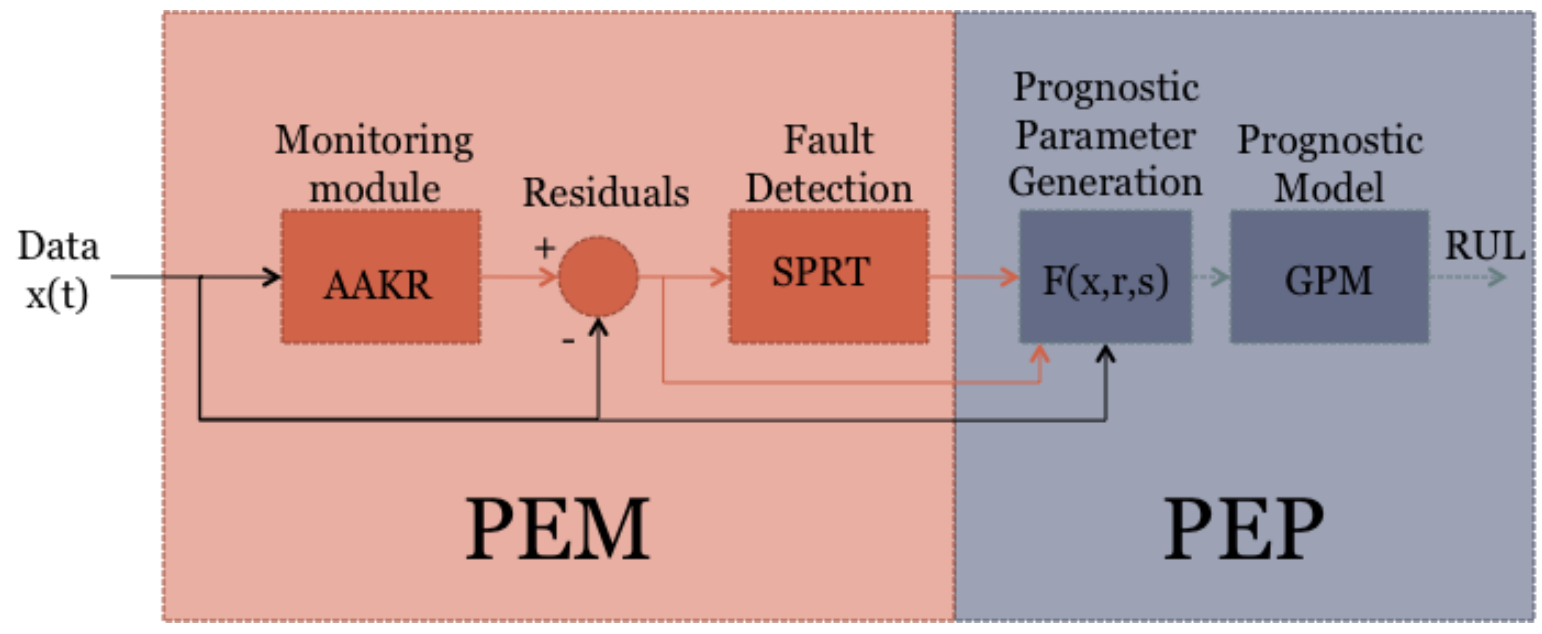

Figure 4-1: Integration of the PEM and PEP Toolboxes

\subsection{Process and Equipment Monitoring Toolbox}

The previously developed PEM Toolbox is a MATLAB based set of tools that currently provide a generalized set of functions for use in process and equipment monitoring applications, specifically online monitoring systems (OLM). The current architecture of the PEM Toolbox is organized into six function categories as shown in Figure 4-2. The first category allows for data to be acquired from multiple sources and conditioned to assure data quality. The next category includes tools to aid in model development including variable grouping and multivariate model optimization. Several model types are supported by PEM, including AAKR, autoassociative neural networks (AANN), and linear regression models. Once a model is developed, functions for parameter prediction and performance analysis are available to compare competing models. The final two function categories provide methods for uncertainty estimation and fault detection. 


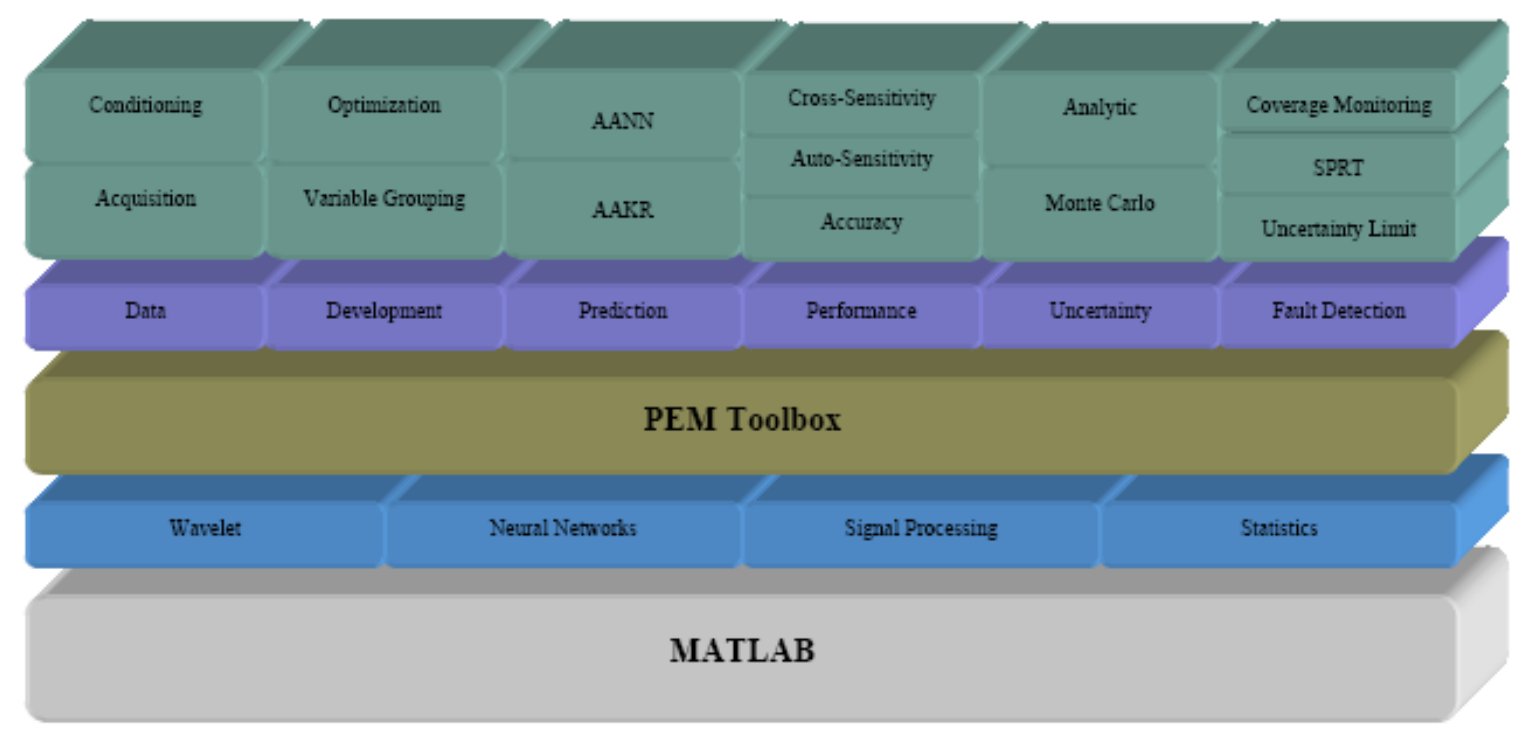

Figure 4-2: PEM Toolbox Architecture

\subsection{Process and Equipment Prognostics Toolbox}

The purpose of the PEP toolbox is to provide a complete set of tools to facilitate prognostic model development. A myriad of prognostic algorithms have been developed which use a variety of information sources, models, data processing algorithms, etc. Typically, prognostic model development depends highly on the expertise of the developer. The PEP toolbox reduces the development burden on the system designer and facilitates the rapid development of competing models. As described in the previous chapter, prognostic algorithms can be classified by the type of information used to make RUL estimates. Algorithms in each of the three classes are included in the PEP toolbox. Appendix B includes the User's Guide for the first release version of the PEP Toolbox, version 1.0.

\subsubsection{Type I: Reliability Data-Based}

These methods consider historical time to failure data which are used to model the failure time distribution. They estimate the life of an average component operating under historically average usage conditions. The PEP toolbox includes several statistical analysis techniques for Type I prognostics: Weibull, exponential, and Gaussian distribution models. Because of its flexibility in modeling infant mortality, random failure, and wear-out failure, the Weibull distribution is used most commonly for reliability analysis. These methods do not consider any 
information specific to the current system, except the total runtime without failure. Type I algorithms result in a distribution of possible failure times; the RUL for a system is determined as the MRL at the current lifetime.

\subsubsection{Type II: Stressor-Based}

A readily apparent disadvantage of reliability-based prognostics is that it does not consider the operating condition of the component. However, components operating under harsh conditions would be expected to fail sooner and components operating under mild conditions to last longer. Type II methods account for environmental stresses and usage conditions (e.g. temperature, load, vibration, etc.) when estimating the system RUL. These models estimate the lifetime of an average component operating under the given usage conditions. The PEP toolbox includes three common Type II algorithms: Markov Chain models, Shock models, and Proportional Hazards models. Due to the stochastic nature of these algorithms, each results in a distribution of failure times. The RUL estimate here is estimated for a given reliability confidence level. A confidence level of 0.5 may be chosen to obtain the average RUL for nonsafety critical components. For safety-critical or high value systems, a conservative reliability confidence level of 0.95 may be more appropriate.

\subsubsection{Type III: Degradation-Based}

The final class of algorithms considers the way in which a specific component responds to its specific usage. It is most commonly constructed through the identification or development of a degradation parameter, which is trended towards failure. The PEP toolbox includes two algorithms for Type III estimates: the general path model and particle filtering. It also includes functionality to include prior information in the regression parameters of a new component or system via Bayesian updating techniques. If a Type III model is applicable to a given system, identification of an appropriate prognostic parameter is paramount to model performance. An automated methodology for identifying prognostic parameters from multiple data sources is described in Coble [2010], which uses measures of parameter suitability to identify a parameter. By formalizing the suitability of a prognostic parameter, identification of an appropriate parameter becomes a straightforward optimization problem; the methodology included in the 
PEP utilizes Genetic Algorithm optimization to find an optimal or near-optimal parameter. The parameter suitability metrics and identification methodology is fully included in the PEP toolbox and can make use of many available data sources, including the sensed measurements from the system, monitoring system residuals and fault detection results obtained from the PEM toolbox.

\subsubsection{Life Cycle Prognostics}

As a system moves through life, different prognostic model types may be appropriate, as shown in Figure 4-3. The PEP toolbox allows a prognostic system to transition between the three prognostics types as more information about the system and its use becomes available. For a newly fabricated component or system, the only possible estimate of RUL is reliability based, because no information is available about how the unit will be used and no data is available from its use. For instance, a new car tire is said to last 40,000 miles based on data collected from similar tires operating in "normal" conditions. However, during operation planning and early life, specific information about the future use of the unit should be available. At this point, Type II methods can be used to make RUL estimates. Because these estimates consider the specific use of the unit, they should provide more accurate and precise RUL predictions. If it is known that the tire will operating on primarily unpaved roads, the failure time distribution will shift down to account for the harsher usage condition. Finally, as the unit is used and data becomes available from its use and degradation, Type III prognostics may be applicable to obtain an even more accurate estimate of RUL. If performance characteristics such as tread-depth or pressure can be measured on the tire, an individual-based estimate of the RUL can be made for that specific tire. Because the PEP toolbox includes functionality to develop all three types of prognostic models, transitioning between the RUL estimation methods is more readily performed as the operator sees fit. A key area of future work is developing a method to seamlessly shift between the three prognostic types as is appropriate, considering the RUL information of two types of models during the transition period. 


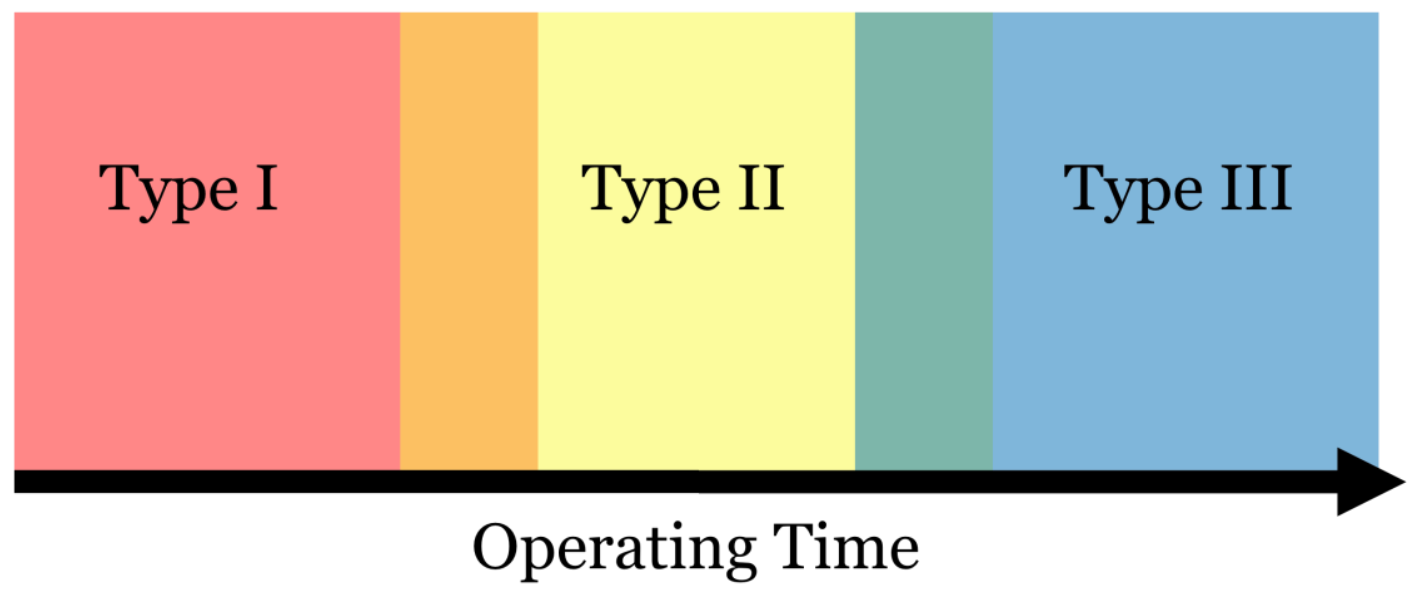

Figure 4-3: Life Cycle Prognostics

The PEP toolbox is a set of MATLAB-based tools designed as a unified platform for developing data-based prognostic models. It is designed to couple with the PEM toolbox to allow for a full monitoring, fault detection, and prognostic system. The toolbox facilitates and automates prognostic model development by implementing the three prognostic types and additional methods to ease the developmental burden on the prognostic system developer.

\section{APPLICATIONS AND RESULTS}

This section presents the results of applying the GPM/Bayes prognostic model to four data sets. First, the effect of noise in the prognostic parameter on GPM/Bayes model performance is investigated with simulated prognostic parameters. The second example application applies the GPM/Bayes methodology to the simulated faulty IRIS data described in Volume 6. This application investigates applying a prognostic model to each of the four simulated fault modes: heat exchanger fouling, pump degradation, process sensor drift, and control sensor drift. The data set given in the 2008 PHM Challenge Problem, which resulted from a turbofan engine simulator, is the basis of the third example application. Additional data from the same simulator, which is available at the NASA Prognostics Data Repository [Saxena and Goebel, 2008], was also used in this application. The final example investigates a milling equipment degradation data set, also obtained from the NASA repository [Agogino and Goebel, 2007]. 


\subsection{The Effect of Noise on Model Performance}

Prognostic models using the "good" prognostic parameters discussed in section 3.4 and plotted in Appendix A were developed to investigate the effect of noise on prognostic model performance. As shown in Table 3-2, the introduction of noise in the prognostic parameter can have a significant effect on parameter suitability metrics. It is shown here that the noisier parameters are useful for prognostics, but show degraded results over the less noisy parameters.

This application of the GPM/Bayes methodology investigated the three populations of "good" parameters given in Appendix A. The first population is noise free; the second is contaminated with Gaussian noise with zero mean and standard deviation equal to $20 \%$ of the mean parameter value; and the third set is contaminated with $80 \%$ mean value noise. Twenty example parameters are available for each population. For each parameter, a GPM/Bayes model is developed using the remaining 19 paths. This model is used to determine the RUL at 100 equally spaced intervals along the exemplar path; each observation is $1 \%$ of the total lifetime. Figure 5-1 gives the resulting RUL estimates for run 6. The results for each run are plotted in Appendix A. RUL estimates for each case begin at roughly 150 cycles. This is the average lifetime which is predicted by the Bayesian prior distributions. As more observations are collected, the regression coefficients shift from being highly dependent on the prior distributions to depending on the collected data from the actual system. As this happens, the RUL estimates become more appropriate for the specific system being modeled. As indicated in Figure 5-1 and the figures in Appendix A, the number of observations needed to shift the model from the population-based prior information to the system-specific observations depends on the noise in the prognostic parameter. The noise-free parameter responds quickly to the collected data while the noisy parameters take longer to reach the correct solution. In addition, the three parameters tend to converge near the end of life. At this point, the regression model has learned the underlying functional form of the prognostic parameter and noise is less of an issue. 


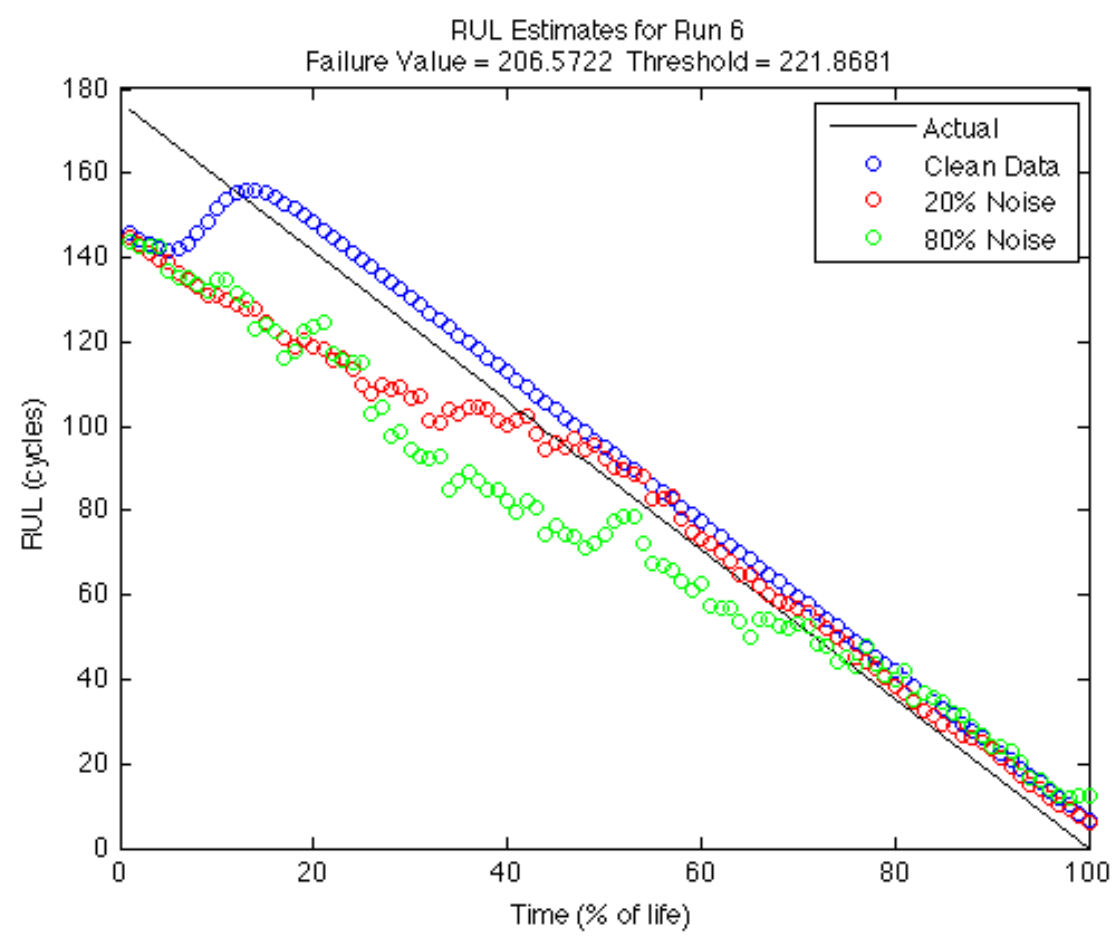

Figure 5-1: RUL Estimates Using Noisy Parameters

These results also highlight the effect of threshold values on RUL bias. For cases with a failure value that is less than the model threshold, the RUL is consistently overestimated, as in run 6. Cases with failure values greater than the model threshold, such as run 2 , result in underestimated RUL. This highlights the need for a more sophisticated thresholding technique. The application of a single, static threshold to all runs will often result in a biased RUL estimation. Several solutions to this problem have been proposed, including the use of probabilistic thresholds as opposed to deterministic thresholds [Wang and Coit, 2007; Usynin et al., 2008]. However, a more accurate method may be to account for system-specific features when determining the failure threshold. A system-specific failure threshold may be informed by usage conditions or features of the data. Methods which do not apply the same threshold, deterministic or stochastic, have been suggested [Garvey and Hines, 2007; Sharp and Hines, 2009]. Integration of a system-specific threshold with the GPM/Bayes model described here will afford more accurate RUL prediction for systems without a clearly defined failure threshold. 
The next example application looks at the full monitoring/prognostic system to make RUL estimates. By monitoring the system measurements, degradation is characterized by monitoring system residuals. The proposed parameter identification method is applied to these residuals to identify an appropriate prognostic parameter and the GPM/Bayes model is applied to several identified parameters for prognostics.

\subsection{Prognostic Models for the IRIS System}

For each fault type under consideration, only one exemplar run to failure was simulated due to time constraints and the computational expense of running the NCSU simulator. For each fault, twelve one-day "snapshots" of data were simulated to mimic a year-long degradation process. Simulated nominal operation, wherein no fault is present, was used to develop two models for system monitoring. The first model contains 13 well-correlated measurements from the simulation. The second model contains only the generated redundant signals for flow rates and steam pressure. These two models, along with appropriate fault detection routines were used to monitor the system and detect faults. After a fault was detected, the remaining data was analyzed to identify an appropriate prognostic parameter through visual inspection. One hundred additional examples of each parameter were generated in order to build a GPM model for each fault type. The following sections describe the developed prognostic models for each of the four faults described in Volume 6. The fault modes are also described here for completion.

\subsubsection{Heat Exchanger Fouling}

In general, fouling is the accumulation of unwanted material on the surface of process equipment. Heat exchanger fouling is of particular import for two reasons: first, fouling causes an increase in thermal resistivity which reduces heat exchanger effectiveness; second, it reduces the cross sectional area for flow which increases the pressure drop across the flow path. This work will focus on the effect of fouling on the thermal resistivity of the steam generator (SG) heat exchanger. The thermal resistance due to fouling, $\mathrm{R}_{\mathrm{f}}$, is included in the equation for the overall heat transfer coefficient as follows [Piezo Systems]:

$$
\frac{1}{U_{o} A_{o}}=\frac{1}{{ }_{0} A_{o}}+\frac{\mathrm{R}_{\mathrm{f}, \mathrm{i}}}{\mathrm{A}_{\mathrm{i}}}+\frac{\mathrm{R}_{\mathrm{w}}}{\mathrm{A}_{\mathrm{w}}}+\frac{\mathrm{R}_{\mathrm{f}, \mathrm{o}}}{\mathrm{A}_{\mathrm{o}}}+\frac{1}{{ }_{\mathrm{i}} A_{i}}
$$

$\mathrm{U}_{\mathrm{o}}=$ overall heat transfer coefficient based on outside area of tube wall 
$\mathrm{A}=$ tube wall area

$\mathrm{A}_{\mathrm{w}}=$ mean wall area

$\mathrm{R}_{\mathrm{f}}=$ thermal resistance due to fouling

$\mathrm{R}_{\mathrm{w}}=$ thermal resistance of wall

$\alpha=$ convective heat transfer coefficient.

$\mathrm{U}_{\mathrm{o}}$ decreases as the deposit thickness or $\mathrm{R}_{\mathrm{f}}$ increases with time. The thermal fouling resistance, $R_{f}$, is defined as:

$$
R_{f}=\frac{1}{\mathrm{U}_{\mathrm{o} \text {,dirty }}}-\frac{1}{\mathrm{U}_{\mathrm{o}, \text { clean }}}
$$

The overall heat transfer coefficient determines how much heat can be transferred between the hot and the cold fluid in a given heat exchanger. When it falls below a tolerable value, the heat exchanger has to be serviced.

To simulate SG heat exchanger fouling, we need only to define the overall heat transfer coefficient of the dirty tubes. Experimental results obtained in [Upadhyaya et al., NEER] are shown below. Figure 1 gives the percent change in overall thermal resistance as a function of runtime. This curve can be used to generate fouled data for the SG heat exchanger with some modifications. Though the results shown below are given for a matter of approximately 170 hours, we would like to extend this degradation mode to occur over the course of approximately one year. In addition, the total change in thermal resistance should be limited to approximately $30 \%$ of the nominal value; this degradation may be unusually high for legacy systems, but it is accepted here because the system is new and untested. In addition to nominal operation data, we would like to have 12 ten-minute "snap-shots" of simulated operation at 12 equally spaced intervals along the curve, to simulate the year long degradation process. 


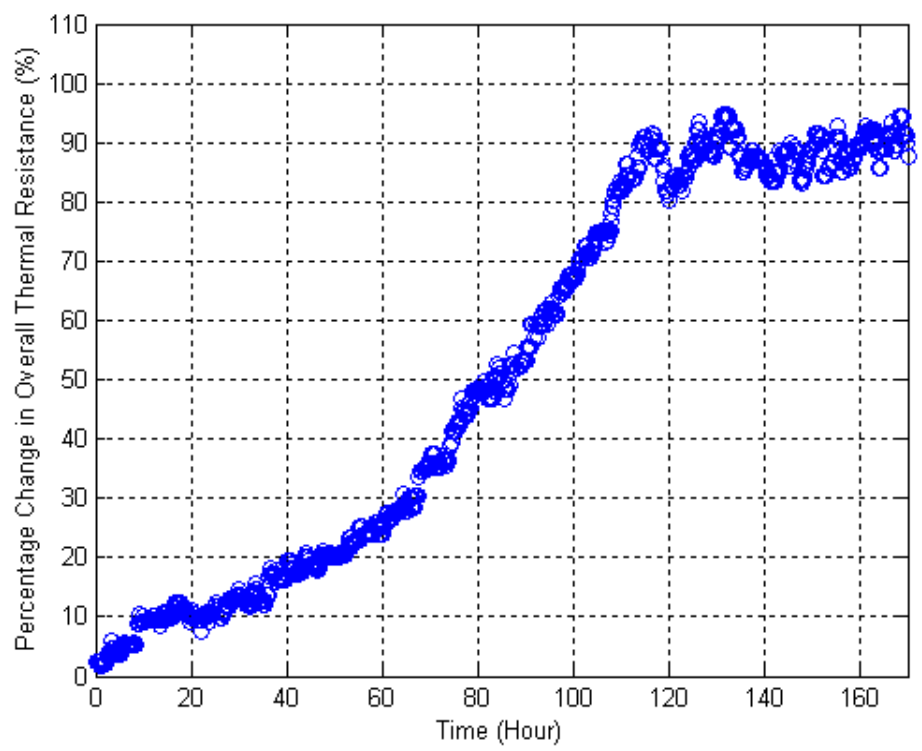

Figure 5-2: Change in Thermal Resistivity due to Fouling [Upadhyaya et al., NEER]

A GPM prognostic model was used to estimate RUL for heat exchanger fouling faults. This failure mode presents an interesting case. When the plant is operating in a lower power demand, the heat exchanger is not stressed as highly, and the effects of the fault are somewhat muted. However, when only the periods of high power operation and the resulting residuals are considered, the fault has a very noticeable trend in the Steam Generator Exit Temperature, as shown in Figure 5-3. This example was used to generate additional degradation paths to develop a GPM model. 


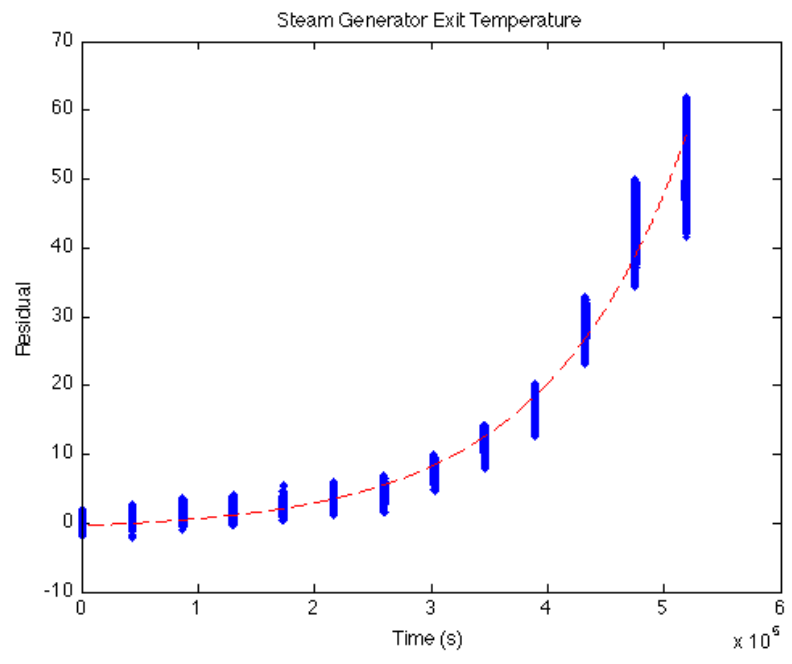

Figure 5-3: Steam Generator Exit Temperature High Power Residuals

One hundred generated degradation paths were used to develop a GPM model, which the simulated data shown above was applied to for testing. The RUL estimation results are shown in Figure 5-4. The blue line shows the actual system RUL over thirteen months of operation. During the first month, operation is nominal and no prognostic estimate is made. As discussed previously, during the first month of faulted operation, the fault detection routine does not detect the small degradation of the heat exchanger; again, no prognostic model is activated. Finally, at the second month of faulted operation, the fault is detected and the prognostic algorithm is activated to make RUL estimates. The prognostic model performs well, with very accurate estimates by the 7 th month of faulted operation, five months before failure. 


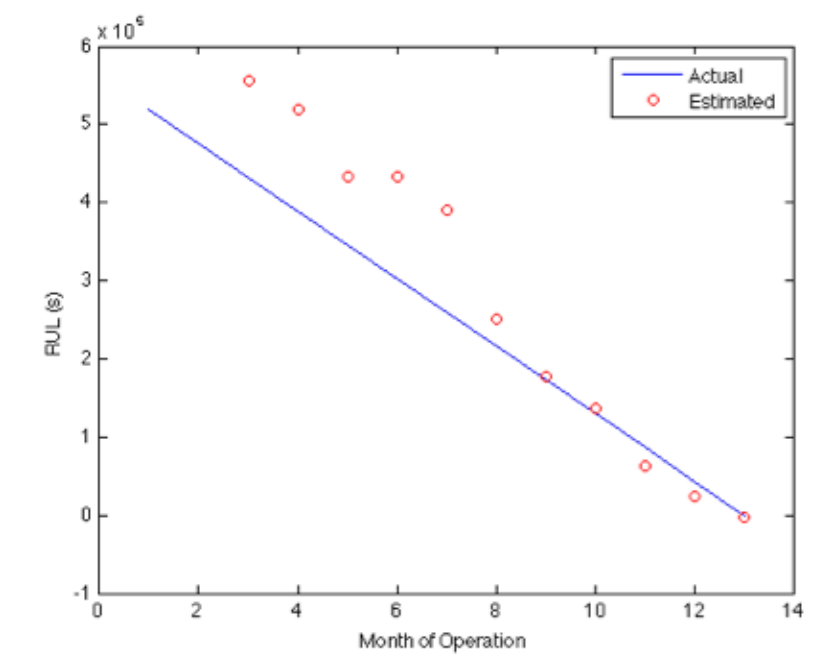

Figure 5-4: Heat Exchanger Fouling RUL Estimation

\subsubsection{Pump Degradation}

Cavitation of centrifugal pumps can limit performance and compromise safe operation of the plant. Grist [1999] identifies four categories of cavitation-induced pump failure: hydraulic performance loss, hydrodynamically induced cavitation surging, thermodynamically induced cavitation surging, and cavitation erosion. This research will focus on hydraulic performance loss due to cavitation in the reactor coolant pump (RCP). This mode of pump failure leads to a continuing failure to provide the required coolant flowrate, which reduces volumetric performance. Such a failure mode appears as a head-drop below the non-cavitating performance characteristics as shown in Figure 5-5. In order to model this type of failure, pump curves corresponding to the cavitated pump performance should be generated and used to give the appropriate flowrate/head-loss relationship. It may be possible to modify the existing pump curves by a simple transformation, which accounts for the loss in flow rate at a given time. 


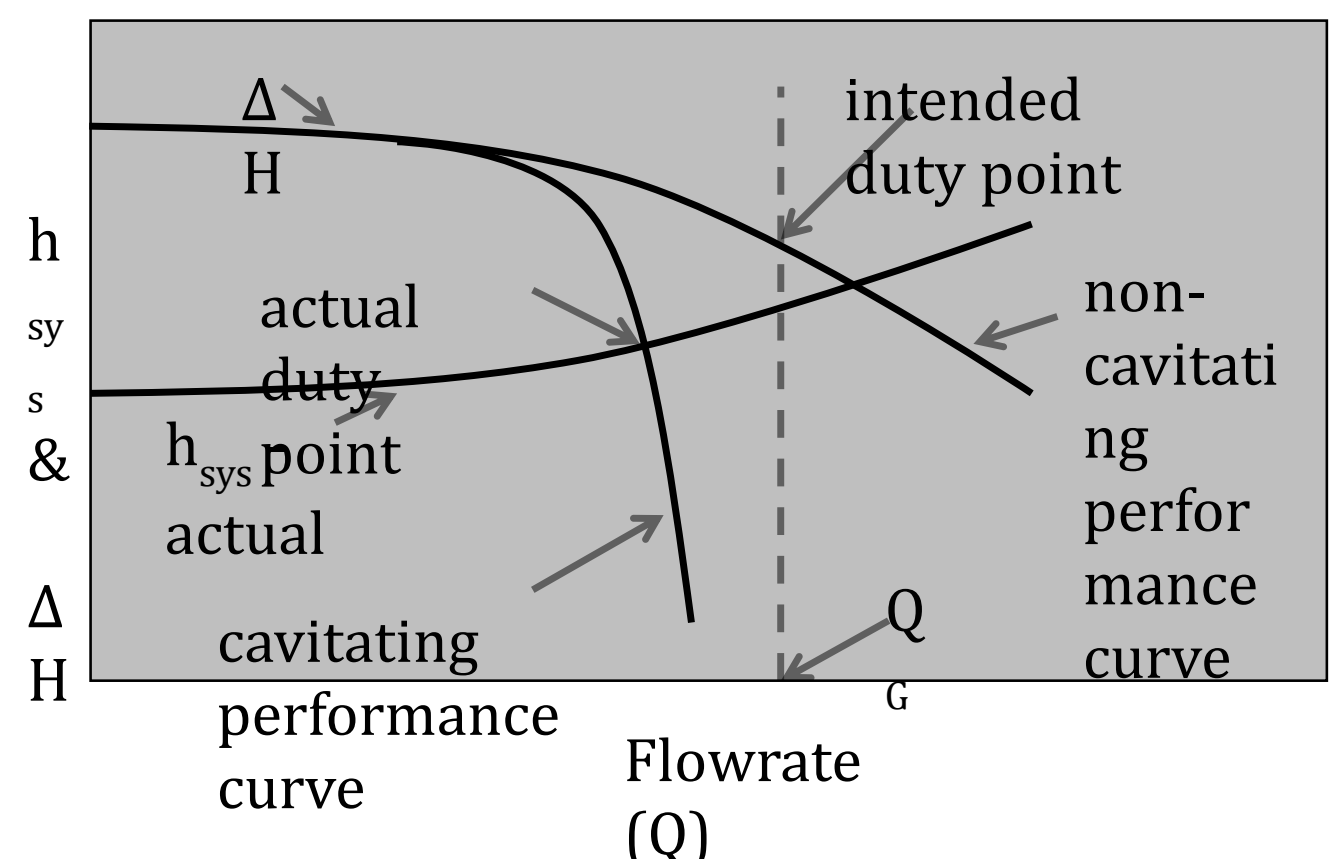

Figure 5-5: Identification of the actual duty point (recreated from [Grist 1999])

Once an appropriate transform to move between nominal and degraded pump performance curves has been identified, then data can be simulated to give "snapshots" of the degraded pump performance over the course of a year. Ideally, we would like to have ten minutes of un-degraded data representing nominal operation. In addition, ten-minute blocks of degraded operation giving a flowrate degradation of $1 \%$ with each block should be simulated resulting in a total degradation of $10 \%$ of flow rate. This can be achieved by regenerating the pump curves as shown in Figure 5-6, where the pump curve is regenerated for each degradation level by making the following transform of the flow rate:

$$
Q_{p}^{*}=\frac{Q}{p}
$$

where $p$ is the fraction of flowrate remaining, i.e. for the first degraded condition, where $99 \%$ of the flow is still available, $p=0.99$. This effectively shifts the pump curves in along the flow variable, adjusting for the lost flow rate. We will consider flow loss of $10 \%$ to be failure. Two types of degradation simulation are desired: first a degradation in only one of the RCPs, and then a degradation in all the parallel RCPs. As in the other fault modes, several histories of these 
progressions are needed, wherein the fraction of flowrate available may vary randomly by $\sim 0.1 \%$ about the expected loss value.

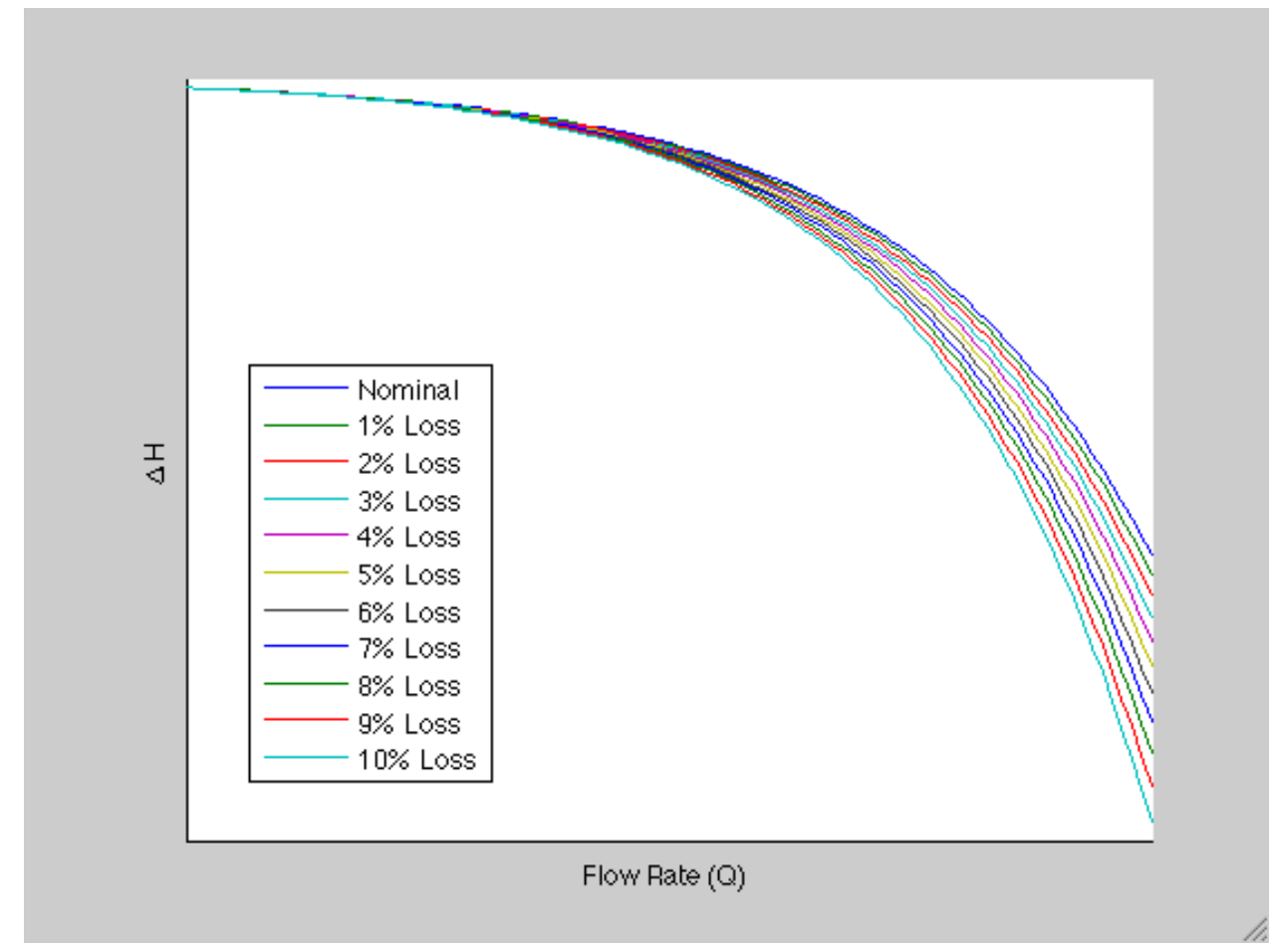

Figure 5-6: Degraded Pump Curves

As the pump degrades, the feedwater flow rate will decrease, which leads to decreased Hot Leg Temperature and increased Cold Leg temperature. Both of these effects are seen in the simulated data, as shown in Figure 5-7. The differences are quite small, less than one degree Fahrenheit at $10 \%$ pump degradation. However, there is a very clear linear trend in both that can be exploited for building a GPM model. Additional degradation paths were generated to simulate the Hot Leg Temperature degradation to build a prognostic model. The model was then applied to the data shown in Figure 5-7a. The result of this RUL estimation is shown in Figure 5-8. The RUL estimations have a slight bias toward longer lifetimes, but the average percent error is less than $10 \%$. 


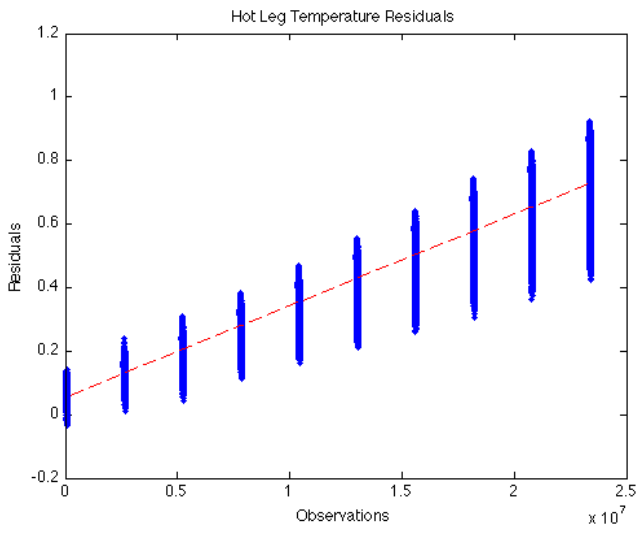

(a)

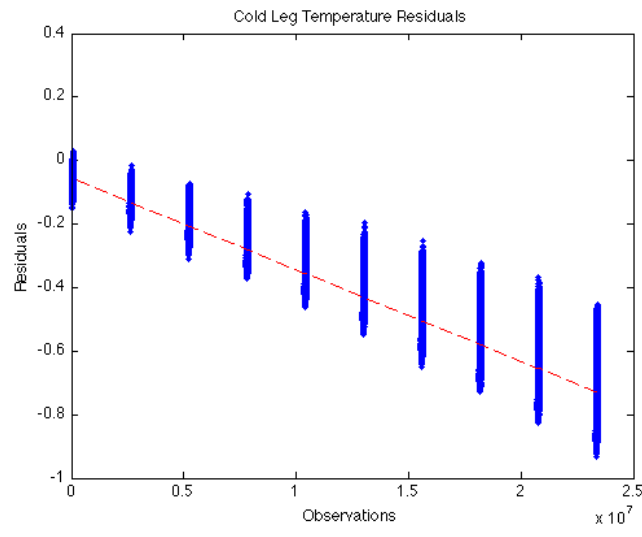

(b)

Figure 5-7: (a) Hot and (b) Cold Leg Temperature Residuals for Pump Degradation

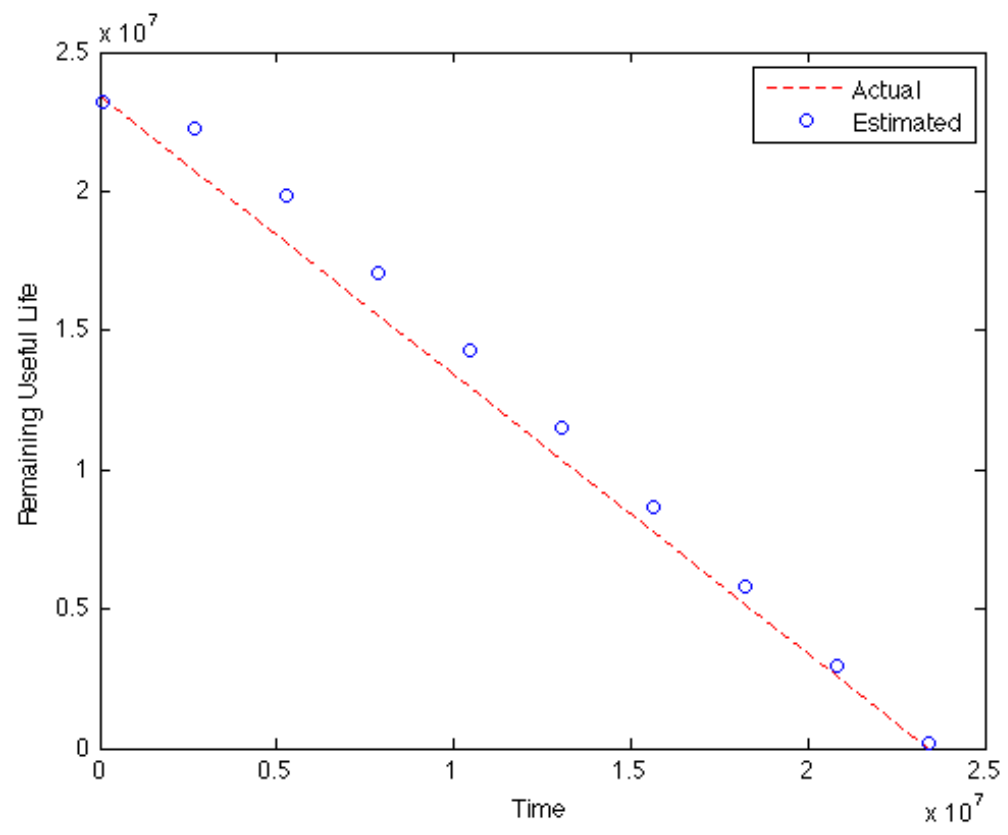

Figure 5-8: RUL Estimation for Pump Degradation

\subsubsection{Sensor Drift}

Sensor drift is possibly the most straightforward fault. This involves a slow, linear drift in one sensor. This particular fault should be simulated for two different sensors: one process sensor and one control sensor. A process sensor refers to any sensor which measures a variable of the process but is not used by the control algorithms, whereas a control sensor is used for 
system control. Feedwater flow is a process sensor of particular interest because it is known to have problems in current pressurized water reactor (PWR) plant designs. The drift in the process sensor should not affect the values of any other sensors and will show up simply as a linear drift. The drift in the control sensor, however, will affect the other variables in the control scheme, leading to sensor fault masking. Sensor drift should occur linearly, eventually adding up to a drift of $1 \%$ of the actual sensor value.

\subsubsection{Process Sensor Drift}

This drift was simulated as a slow drift which grew linearly over time to a final size of $1 \%$ of the sensor span, which is considered failed by current guidelines. Because process sensors don't play into any control algorithms, detecting and identifying the drifted sensor is straightforward. In this research, one steam flow sensor is drifting. This is easy to detect with the use of redundant sensors; the flow/pressure model described earlier was used to monitor for this sensor drift. Figure 5-9 clearly shows the residuals of the drifting sensor departing from those of the nominal sensor. The results of the RUL estimation for this fault are shown in Figure 5-10; because the sensor drift is known to be linear, fitting a GPM model is rudimentary. The RUL predictions for this fault are very accurate, with errors less than $1 \%$.

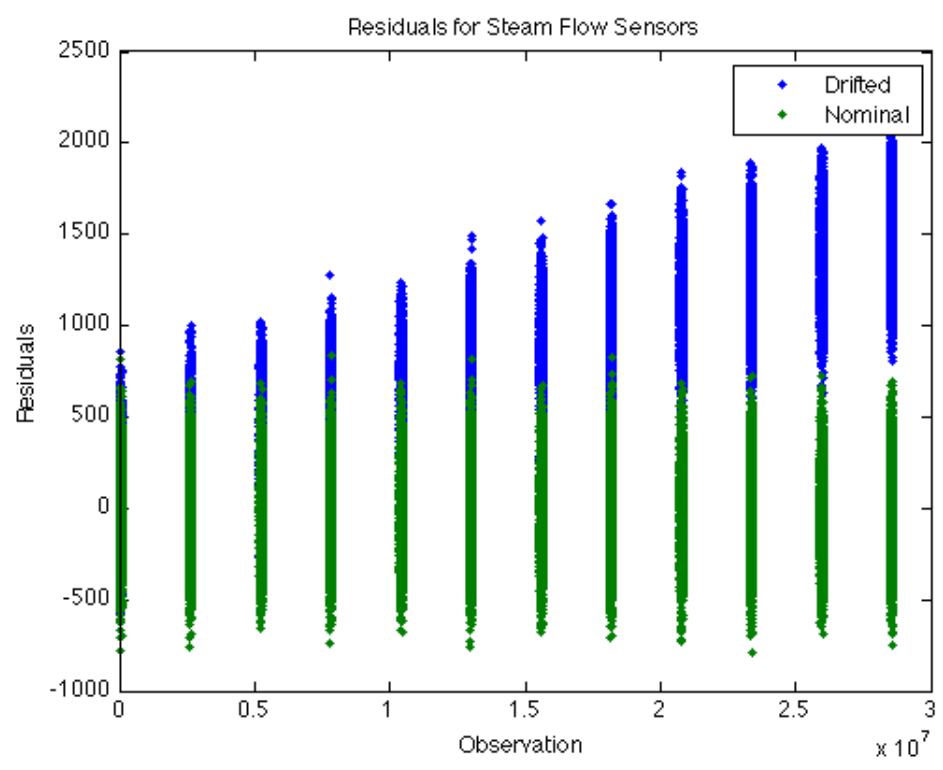

Figure 5-9: Steam Flow Residuals 


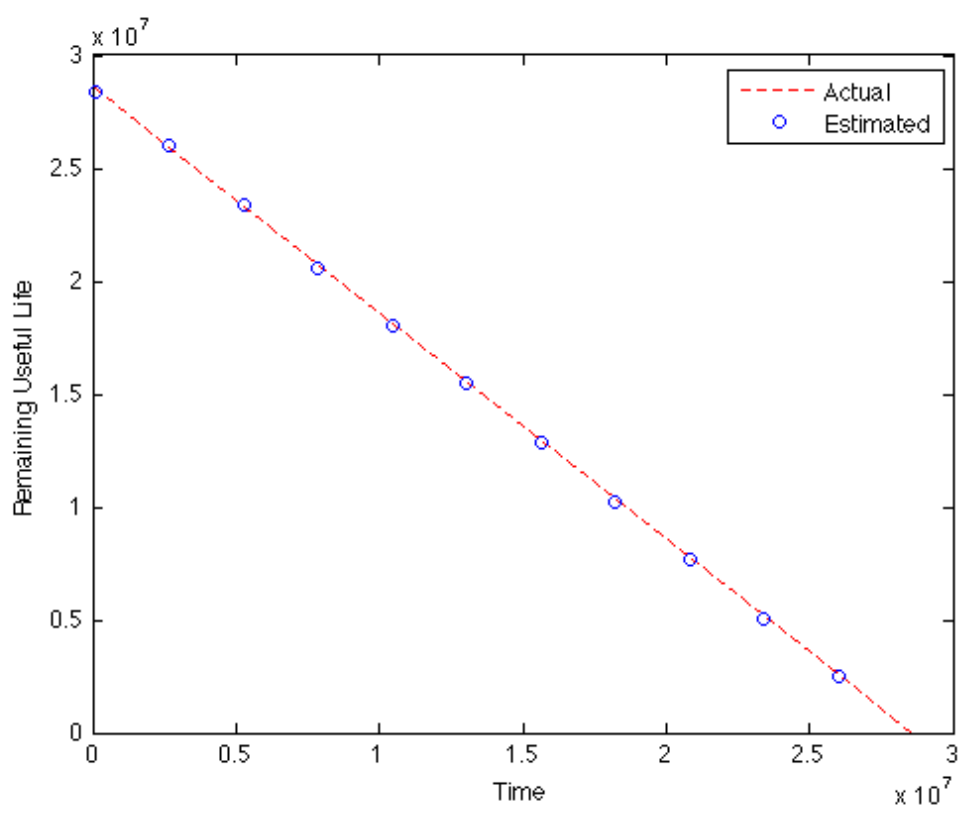

Figure 5-10: RUL Estimation for Process Sensor Drift

\subsubsection{Control Sensor Drift}

The final fault considered in this research is control sensor drift. This is similar to process sensor drift in that the sensor reading is offset by an amount which increases with time. However, it is not as simple to detect because the drifting sensor is used in the control system. This leads to sensor masking, where the control system maintains the sensor reading at a set level while the actual value being measured is changing. Feeding a faulty value into the control system will cause other measurements to change. In this case, one of the most noticeable and trendable changes is in the Feed Pump Speed, which is controlled based on the Steam Pressure (Figure 5-11). Because the drift introduced in the control sensor is linear, the resulting effect on pump speed is also linear. This is used to build a linear GPM model similar to those used for pump degradation and process sensor drift. The results of applying this prognostic model to the data below are shown in Figure 5-12. This model slightly underestimates the lifetime of the sensor, but it is accurate to within $1 \%$. 


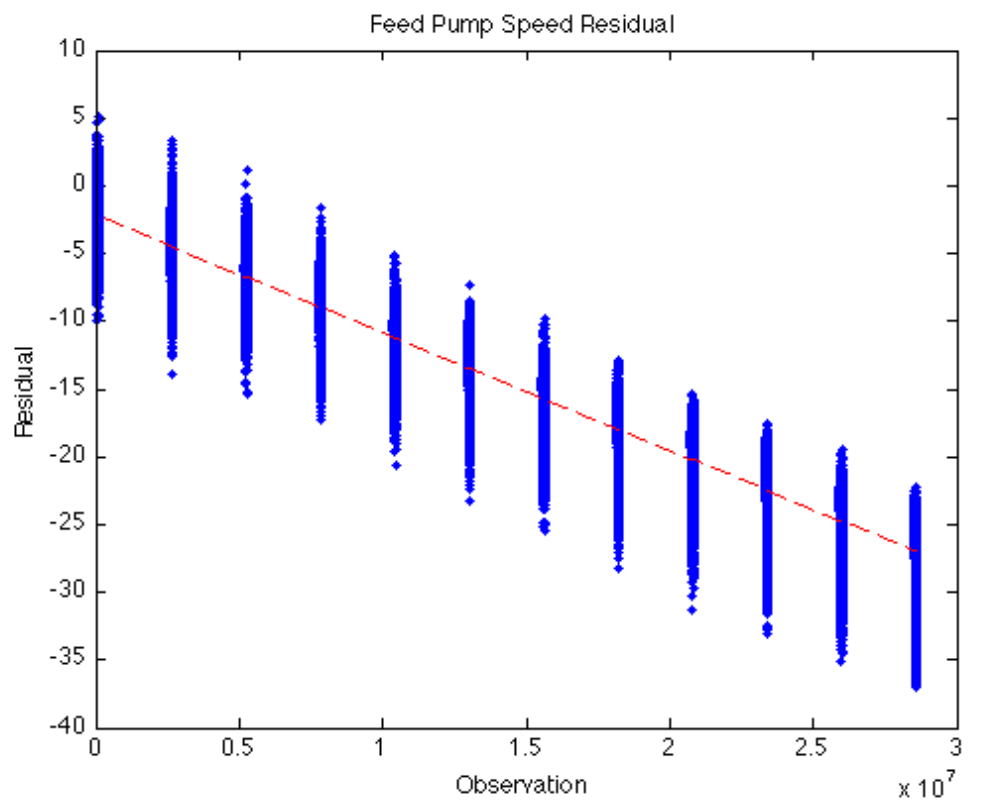

Figure 5-11: Feed Pump Speed Residual

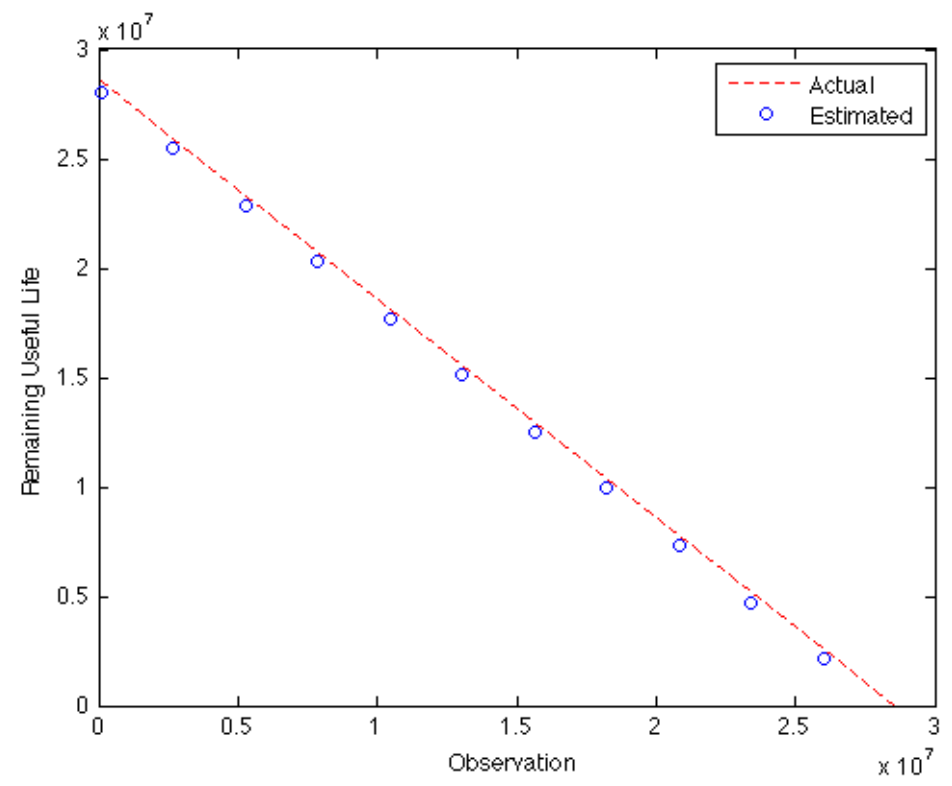

Figure 5-12: RUL Estimation for Control Sensor Drift 


\subsection{PHM Challenge Data Description}

The PHM Challenge data set consists of 218 cases of multivariate data that track from nominal operation through fault onset to system failure. Data were provided which modeled the damage propagation of aircraft gas turbine engines using the Commercial Modular AeroPropulsion System Simulation (C-MAPSS). This engine simulator allows faults to be injected in any of the five rotating components and gives output responses for 58 sensed engine variables. The PHM Challenge data set included 21 of these 58 output variables as well as three operating condition indicators. Each simulated engine was given some initial level of wear which would be considered within normal limits, and faults were initiated at some random time during the simulation. Fault propagation was assumed to evolve in an exponential way based on common fault propagation models and the results seen in practice. Engine health was determined as the minimum health margin of the rotating equipment, where the health margin was a function of efficiency and flow for that particular component; when this health indicator reached zero, the simulated engine was considered failed. The interested reader is referred to [Saxena et al., 2008] for a more complete description of the data simulation.

The data have three operational variables - altitude, Mach number, and TRA - and 21 sensor measurements. Initial data analysis resulted in the identification of six distinct operational settings; based on this result, the operating condition indicators were collapsed into one indicator which fully defined in which of the six modes the engine was operating. In addition, ten sensed variables were identified whose residuals changed in a meaningful way through time and were well correlated to each other. In this way, the 24 sensor data set was reduced to 11 variables, with original variable numbers: 1 (the operating condition indicator), 5, 6, 7, 12, 14, 17, 18, 20, 23, and 24. Because the nature of the prognostics challenge was to develop a prognostic algorithm with no knowledge of the system under test or the variables available, no effort is made to physically relate these eleven sensors. However, they are considered suitable for autoassociative modeling due to the strong inter-correlations within the group. The correlations between these eleven variables are shown in Figure 5-13. 


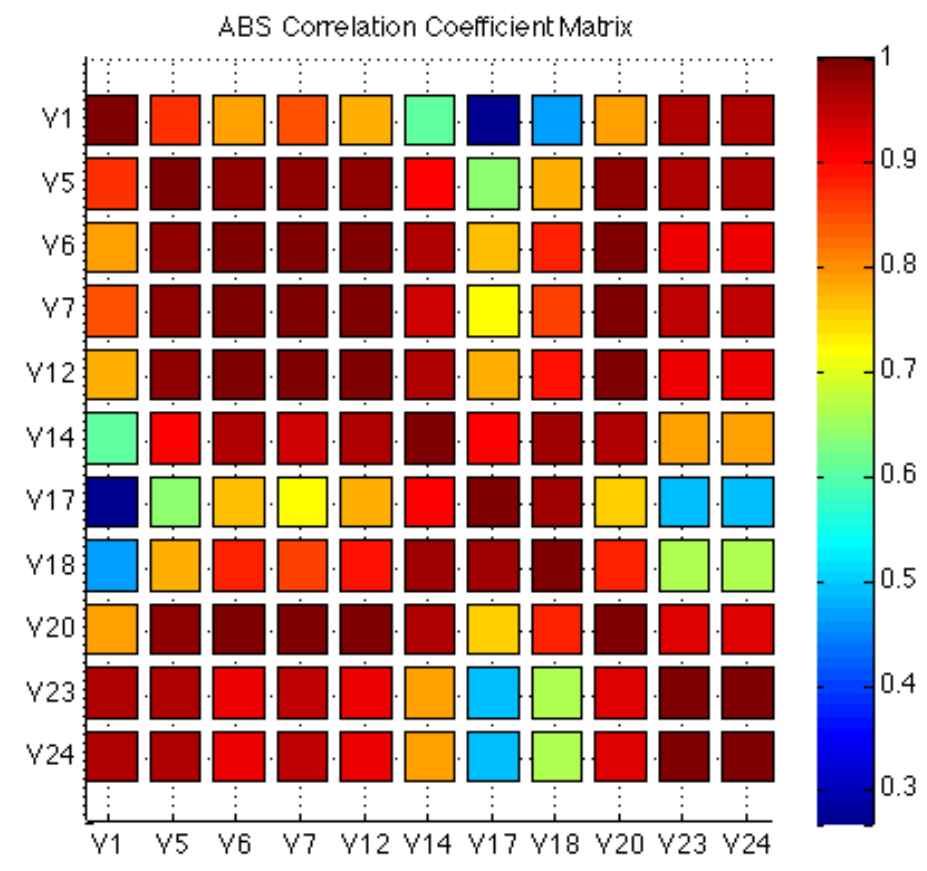

Figure 5-13: Correlation Coefficient Matrix for Eleven Monitored Variables

The GPM method uses degradation information, either directly measured or inferred, to estimate the system RUL. Initial analysis of the raw data does not reveal any trendable degradation parameter. That is, no sensed measurement has an identifiable trend toward failure. Figure 5-14 is a plot of the eleven variables that were determined to statistically change with time. These variables were used to develop a monitoring and prognostics system. 


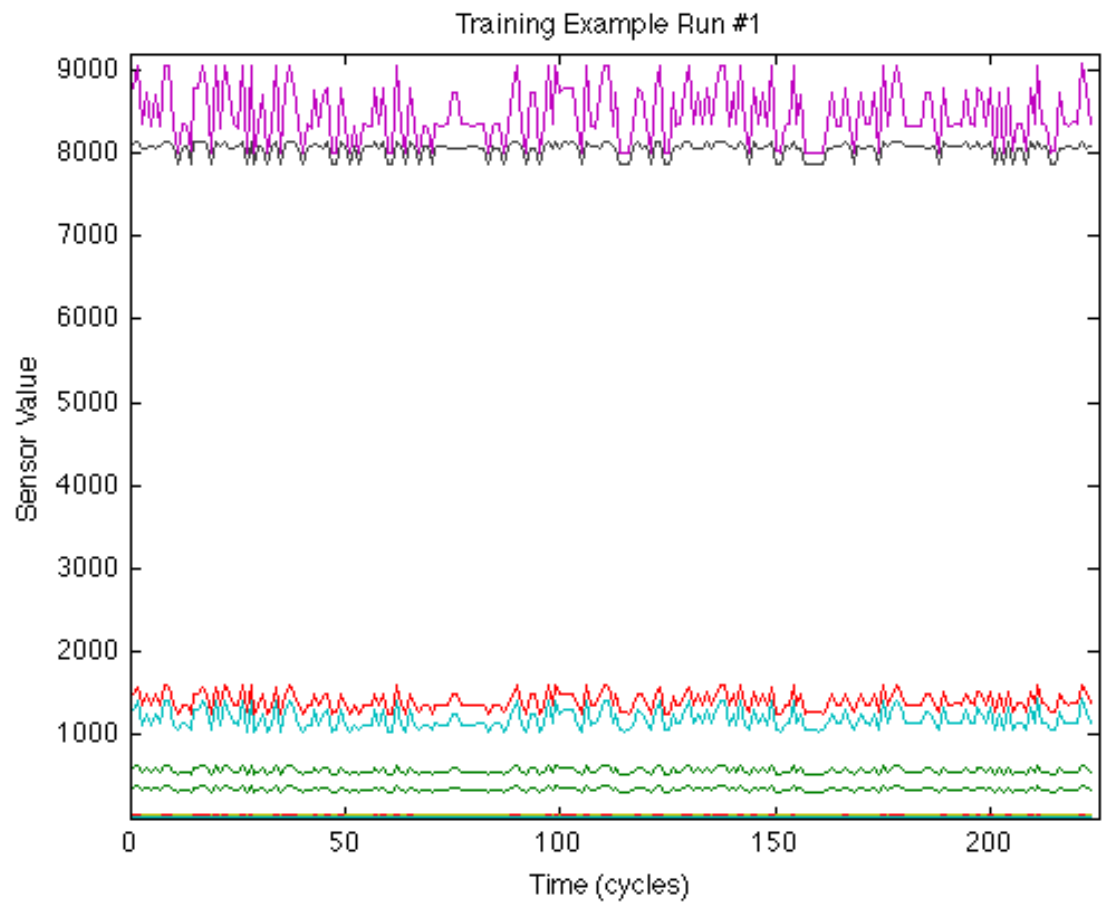

Figure 5-14: Eleven PHM Data Set Variables

The eleven variables are monitored with an auto associative kernel regression (AAKR) model. The residuals between the measured values and the AAKR "corrected" values are candidates for inclusion in the prognostic parameter. Two of these residuals are shown below in Figure 5-15. The residual shown on the left is expected to be useful for prognostic predictions, while the residual shown on the right is not expected to be useful because the population of residuals do not all have similar shapes or equal failure values. It may be possible to classify these residuals into several groups of similar residuals; this may be indicative of different failure modes, but this idea was not pursued for this work. All eleven residuals are plotted in Appendix A, for reference. For this simple application, only linear combinations of the residuals are considered for possible prognostic parameters. However, it is a straightforward extension of the method to include other features, such as the measured data or fault detection results, or to allow for higher order terms such as nonlinear combinations of several inputs, exponential terms, etc. 


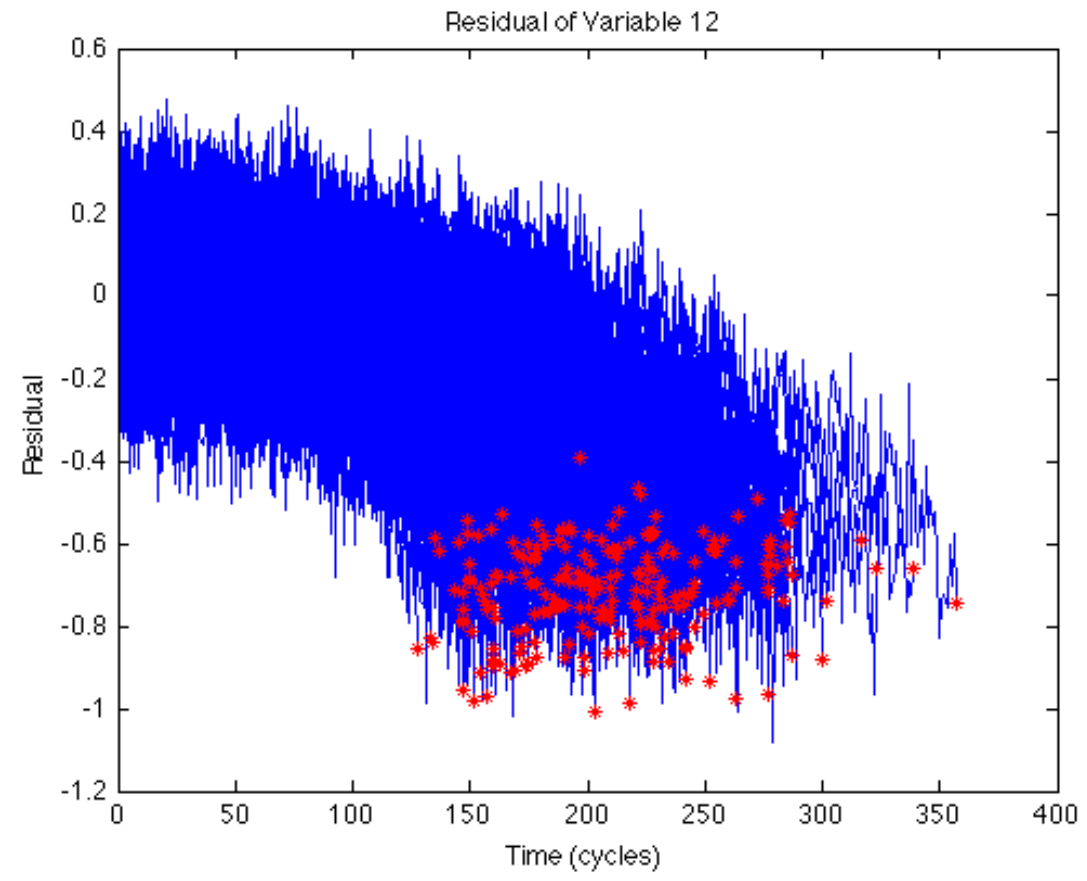

(a)

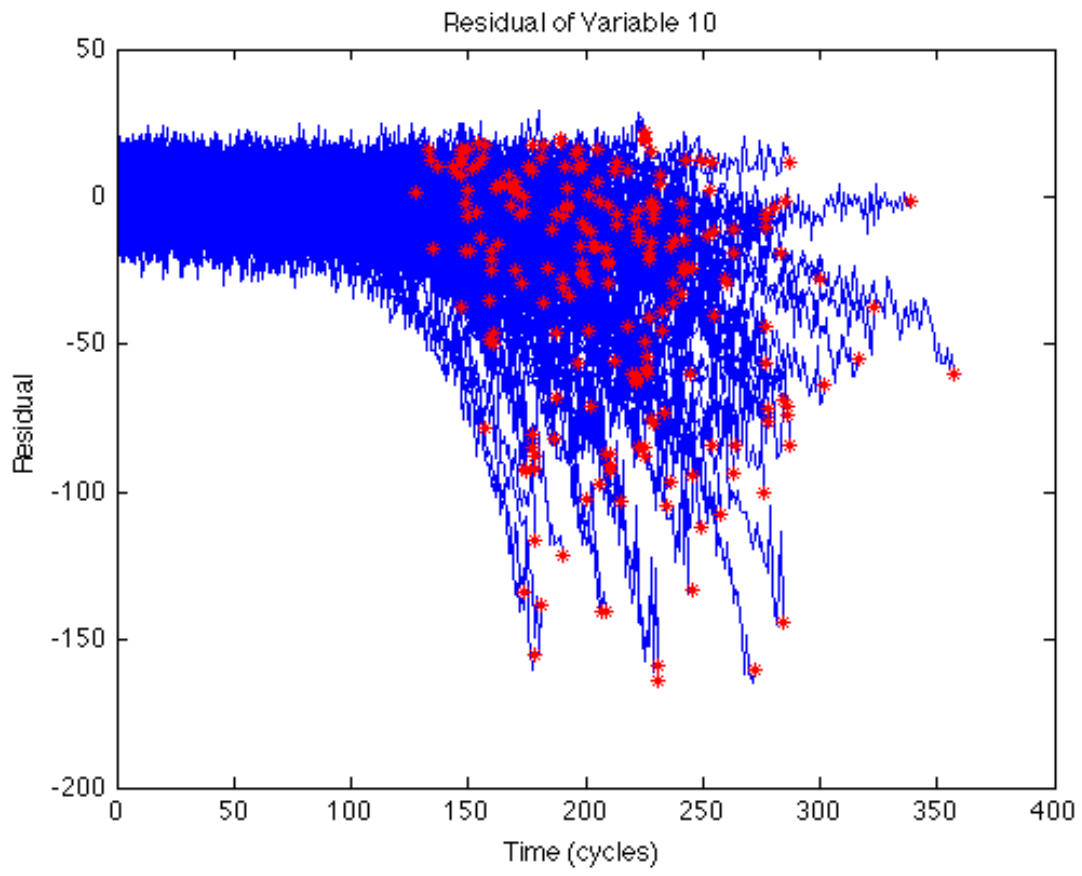

(b)

Figure 5-15: Residuals which are expected to be (a) useful and (b) un-useful for prognostics 
Three competing prognostic parameters are identified from the monitoring system residuals, one identified through visual inspection and two identified using the proposed automated method. Each of these parameters is used to develop a basic prognostic model and make RUL estimations for the test cases. The following section presents the results of prognostic parameter identification and RUL estimation for both of the methods.

\subsubsection{Results}

Three competing prognostic parameters are identified. The first parameter is based on visual inspection and expert analysis. The second parameter is identified using the proposed automated identification method. The third parameter is also identified via the automated method, but makes use of the input selection method described to remove un-useful inputs and relieve the computational burden. The three resulting prognostic parameters and their respective prognostic models are given below. A GPM model with dynamic Bayesian updating is developed using each candidate parameter. The prognostic models are then tested on the 218 test cases given in the PHM '08 challenge. Model performance is characterized using the MAPE of the known RULs for the 218 test cases.

\subsubsection{Visual Inspection}

Visual inspection of the residuals suggests that an appropriate parameter might be a weighted average of the residuals for variables 6,7,14,18, and 20. All eleven model residuals are plotted in Appendix A. The five residuals are weighted by the inverse of their average range and summed to give the prognostic parameter identified through visual inspection. By scaling the residuals, the relative importance of each contributor to the prognostic parameter is equal. Because no engineering judgment can be made concerning the physical characteristics of the system, weighting each input equally is a reasonable approach. The inputs can be scaled with respect to several measures, including the standard deviation, the input range, the correlation to RUL, etc. By combining several similar residuals the spread in the failure value is reduced, as shown in Figure 5-16. This is sometimes referred to as parameter bagging and is a common variance reduction technique. Table 5-1 gives the parameter suitability metrics for each of the residuals. The five residuals with total suitability (given by the sum of the three suitability 
metrics) greater than 1.5 were combined to create the final parameter, which has greater suitability metrics than any one constituent residual. Identification of this parameter involved several weeks of expert analysis of the available data.

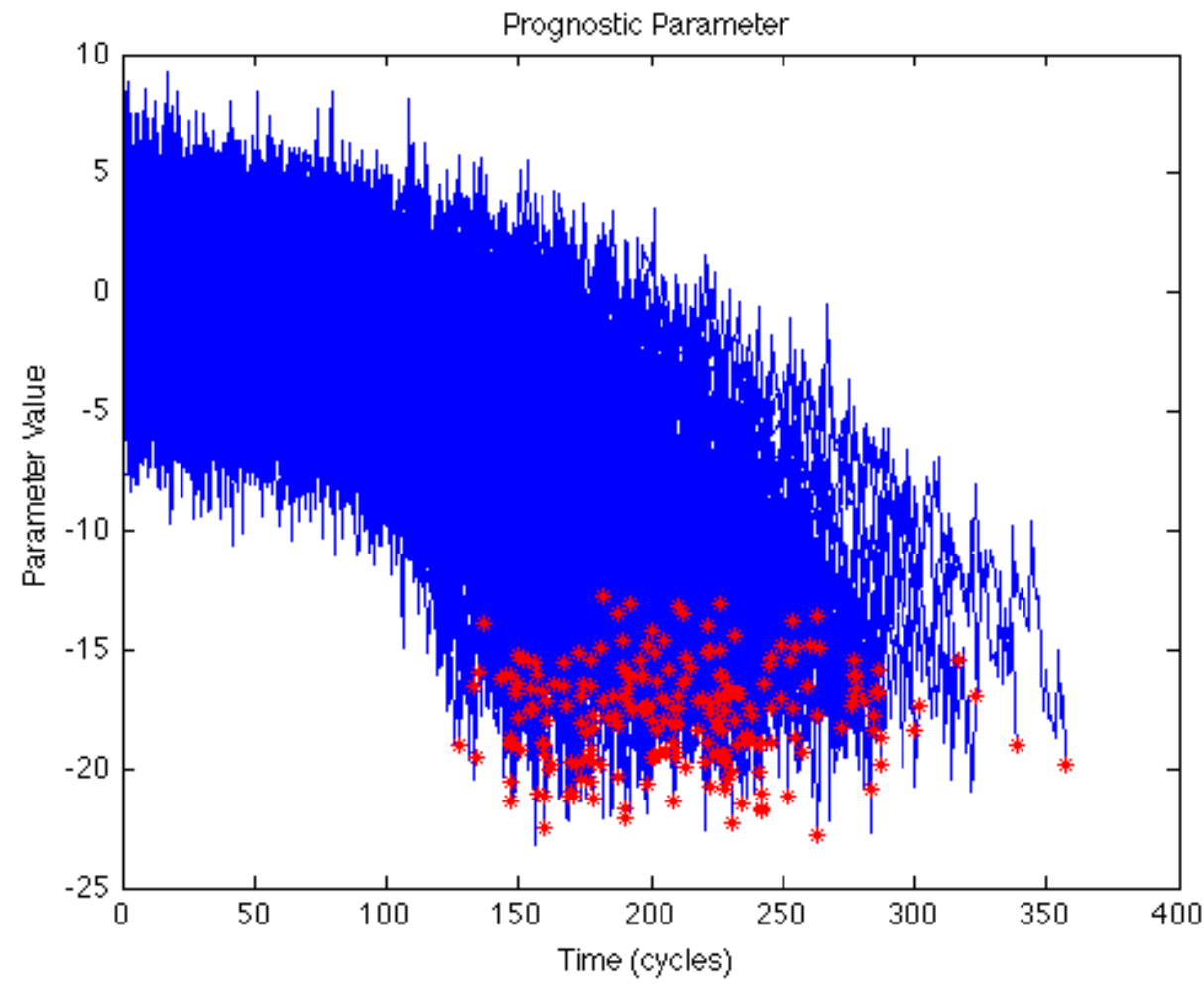

Figure 5-16: Prognostic Parameter Identified by Visual Inspection

Table 5-1: Prognostic Parameter Suitability Metrics

\begin{tabular}{|c|c|c|c|c|}
\hline Variable & Monotonicity & Prognosability & Trendability & Suitability \\
\hline 3 & 0.435 & 0.370 & 0.000 & 0.805 \\
\hline 5 & 0.537 & 0.613 & 0.018 & 1.168 \\
\hline 6 & 0.604 & 0.727 & 0.291 & 1.622 \\
\hline 7 & 0.749 & 0.818 & 0.726 & 2.293 \\
\hline 12 & 0.654 & 0.314 & 0.001 & 0.968 \\
\hline 14 & 0.782 & 0.851 & 0.814 & 2.447 \\
\hline 17 & 0.639 & 0.282 & 0.000 & 0.921 \\
\hline 18 & 0.703 & 0.731 & 0.649 & 2.083 \\
\hline 20 & 0.625 & 0.737 & 0.476 & 1.838 \\
\hline 23 & 0.447 & 0.497 & 0.000 & 0.945 \\
\hline 24 & 0.457 & 0.510 & 0.000 & 0.967 \\
\hline
\end{tabular}




\begin{tabular}{|l|l|l|l|l|}
\hline VI Parameter & 0.846 & 0.891 & 0.903 & 2.640 \\
\hline
\end{tabular}

A second order polynomial model can been used to model the degradation parameter. While an exponential model may be more physically appropriate, and was certainly found to be after the data simulation method was made public, the quadratic model is more robust to noise and better describes the data fit for the chosen prognostic parameter. For the methodology proposed, the model must be linear in parameters; however, simple exponential models, such as $y=\exp (a x+b)$ parameterized as $\ln (y)=a x+b$, cannot be used with negative $y$-values, because the natural logarithm of a negative number is undefined in the real number system. This adds unnecessary complexity to the modeling method. Quadratic equations, on the other hand, are naturally linear in parameters and can be used without significant concern for the effects of noise on the model fit. Shifting the prognostic parameter to the positive quadrant eliminates the problem of taking the logarithm of negative values; however, the quadratic fit results in a lower fitting error than the exponential fit, with mean squared errors of 1.53 and 2.33 respectively. Because of its robustness to noise and reduced modeling error, the quadratic fit is chosen for this research.

Figure 5-17 gives an example of a polynomial fit of the prognostic parameter with the time the model crosses the critical failure threshold indicated. The threshold of -13.9 was chosen as the upper $95 \%$ level of the distribution of failure values for the known failed cases. This gives an estimated system reliability of $95 \%$ which is a conservative estimate of failure time and reduces the possibility of overestimating RUL and having a failure. The time between the last sample and that star is the estimate of RUL, as indicated by the blue area. For this case, the estimated RUL is exactly correct, with an estimated remaining life of 36 cycles. 


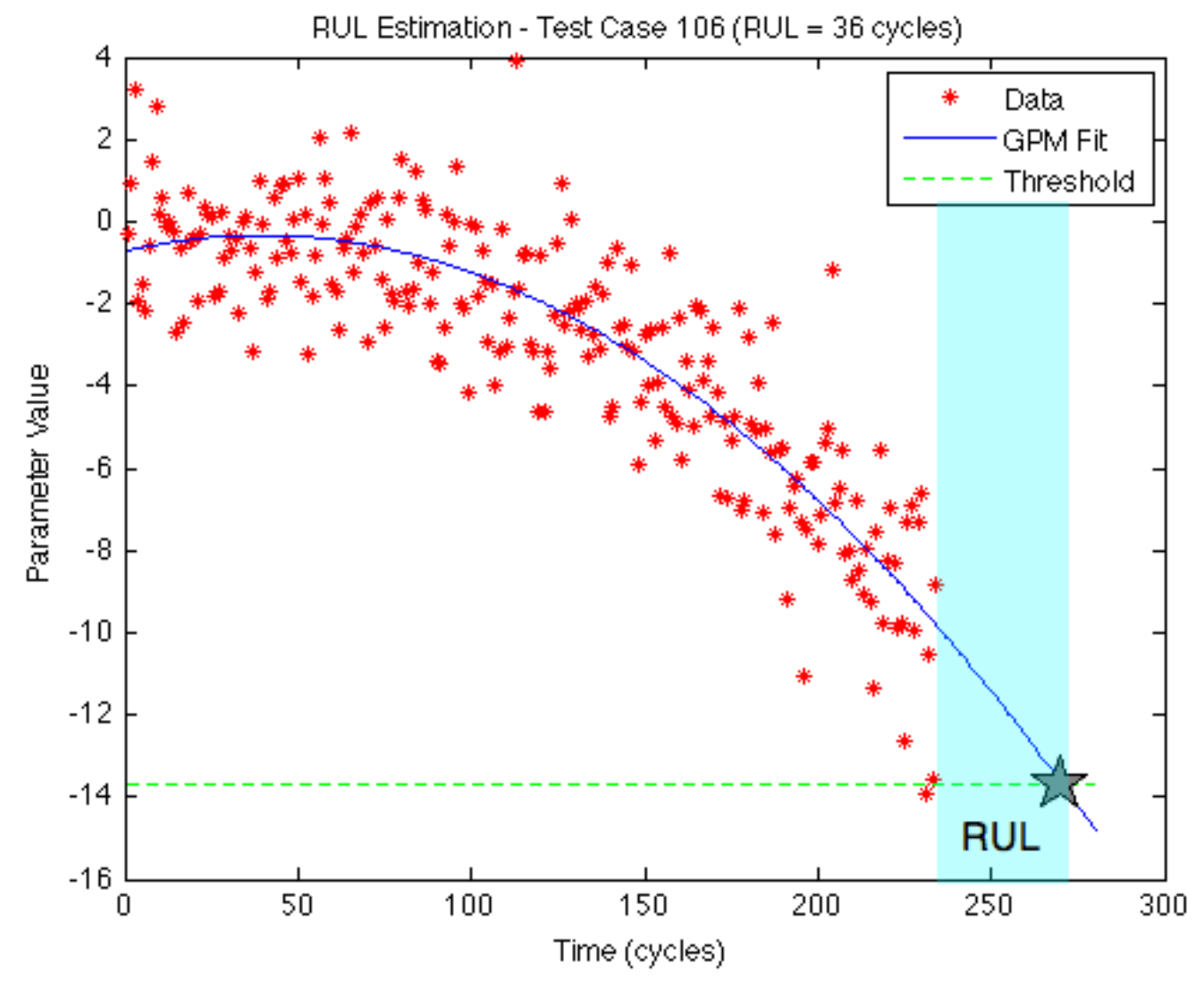

Figure 5-17: Prognostic parameter trending and RUL estimation

This prognostic parameter was used to develop a GPM prognostic model with Bayesian updating. The model was developed using the prognostic parameter resulting from 218 training cases which ran from beginning of life to failure, and was tested with 218 test cases which ran from beginning of life to some point after a failure-inducing fault occurred but before actual failure. The result of each of these cases is shown below, in Figure 5-18. The GPM model developed with this prognostic parameter resulted in a MAPE of $22.8 \%$. While this result is larger than would be accepted in practice, it is important to note that in approximately half of the 218 test cases the RUL is predicted to within $10 \%$ accuracy. The large MAPE is due to poor performance on a few cases, which skews the results. 


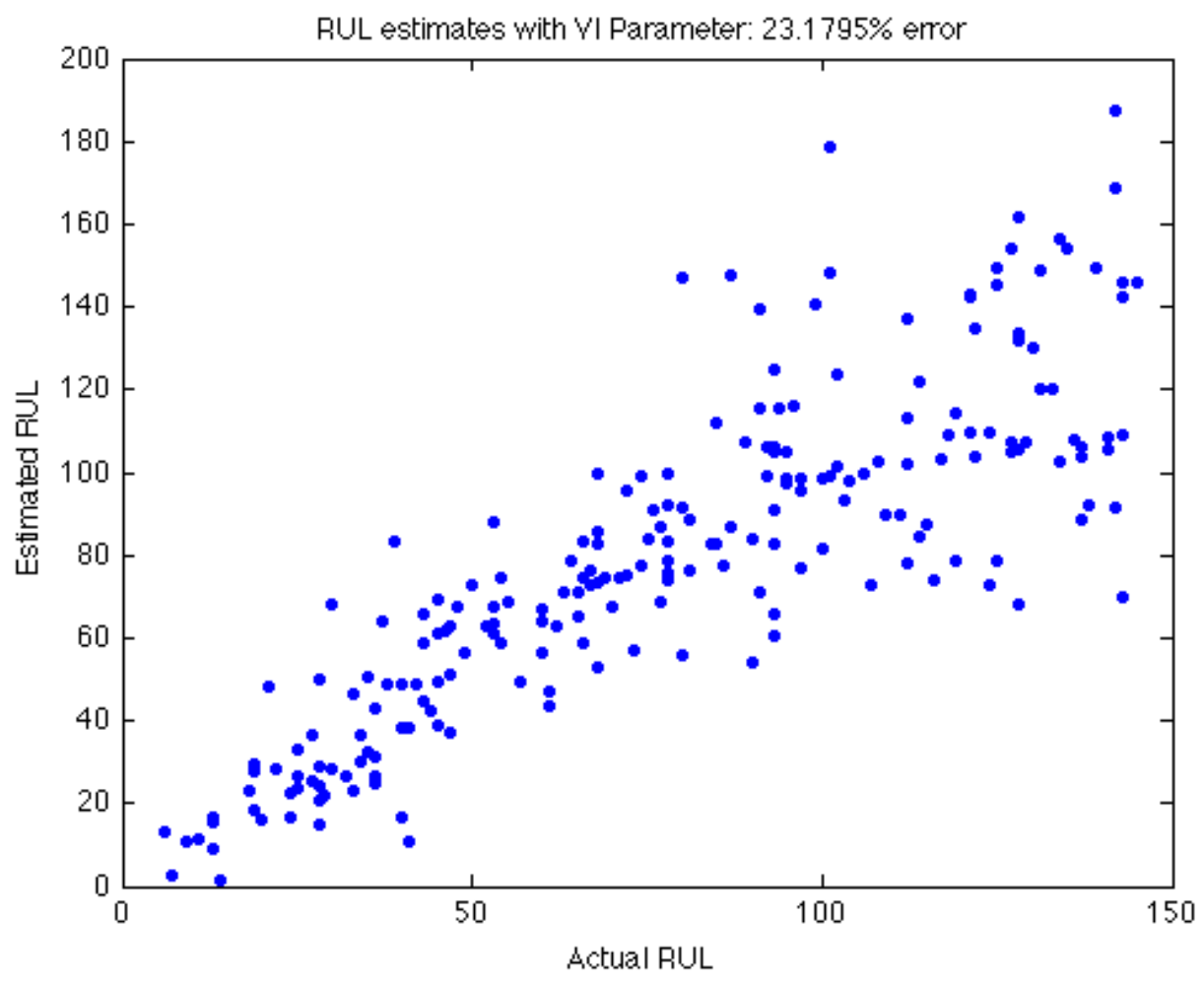

Figure 5-18: Visual Inspection RUL Estimate Results

To illustrate the utility of the Bayesian updating method, a GPM model with no updating was also employed. The advantage of including prior information via dynamic Bayesian updating is to improve RUL estimates when very few observations are available, the data are very noisy, or both. A comparison of the performance through time of the straight GPM and the GPM with Bayesian updating is given in Figure 5-19. In this experiment, the two methodologies were applied to each of the training cases using only a fraction of the full lifetime. The models were applied to subsets of each lifetime in 5\% increments, i.e. the models were run using $5 \%$ of the full lifetime, $10 \%, 15 \%$, etc. The RUL error at each percentage was calculated across the 218 full training cases to determine how the error decreases as more data become available. As was seen in the example case, the non-Bayesian method may result in an undeterminable RUL. In fact, for the data used here, nearly half the runs resulted in an indeterminate RUL estimate using the GPM methodology without Bayesian updating for runs using less than half the total lifetime. For these cases, the RUL is estimated using a Type I, or traditional reliability-based, 
method in order to give an estimate of RUL prediction error. The mean residual life is found at each time using a Weibull fit of the failure times and the current lifetime. Mean Residual Life (MRL) is found by:

$$
\operatorname{MRL}(t)=\frac{1}{R(t)} \quad R(s) d s
$$

where $\mathrm{R}(\mathrm{t})$ is the reliability function at time $\mathrm{t}$. In practice, the prognostic method would likely fall back to a more rudimentary method such as this if the Type III model did not produce a reasonable answer.

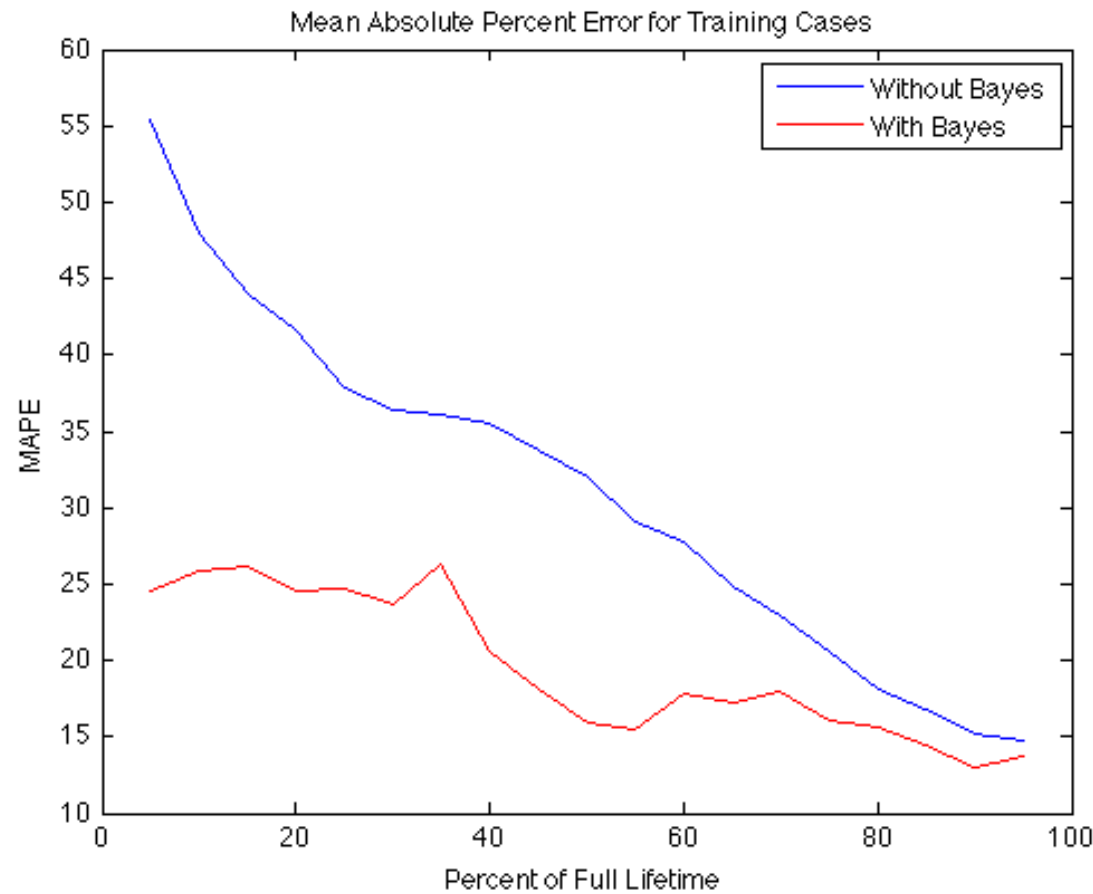

Figure 5-19: GPM Results With and Without Bayesian Updating

The GPM/Type I model which does not include prior information gives an average error of approximately $55 \%$ when only $5 \%$ of the full lifetime is available and relies on the Type I method for approximately half of the cases. Conversely, the GPM/Bayes method gives approximately 25\% error and is able to predict an RUL for every case. As Figure 5-19 shows, the average error of both methods decreases as more data becomes available and eventually 
converges to approximately equal error values when the available data overpowers the prior information.

\subsubsection{GA Optimized Parameters}

Two prognostic parameters were identified using the automated method described with a GA optimization. The GA was used to optimize the weighting coefficients in a weighted sum of the eleven monitoring system residuals. For this application, the fitness function was given by a straight sum of the three suitability metrics:

$$
\text { fitness = monotonicity }+ \text { prognosability }+ \text { trendability }
$$

This gives equal weight to each of the three parameter suitability measures, but does not consider anything else. While the visual inspection parameter involved several weeks of expert analysis, the GA optimization involved only a few minutes of worker time to set up and approximately 1.68 hours of unsupervised computer runtime. While the time needed for the GA optimization to run will scale with the number of possible inputs, it involves mainly computer runtime and is only a fraction of the time needed for parameter identification through expert opinion. A second GA optimization was run using the input selection method described previously with a suitability cutoff of 1.5 for inclusion in the genetic algorithm. This optimization considered only five inputs and ran in 0.93 hours, nearly half the time the full optimization needed. The parameter identified by the first GA optimization is given in Figure 5-20, and the second in Figure 5-21. 


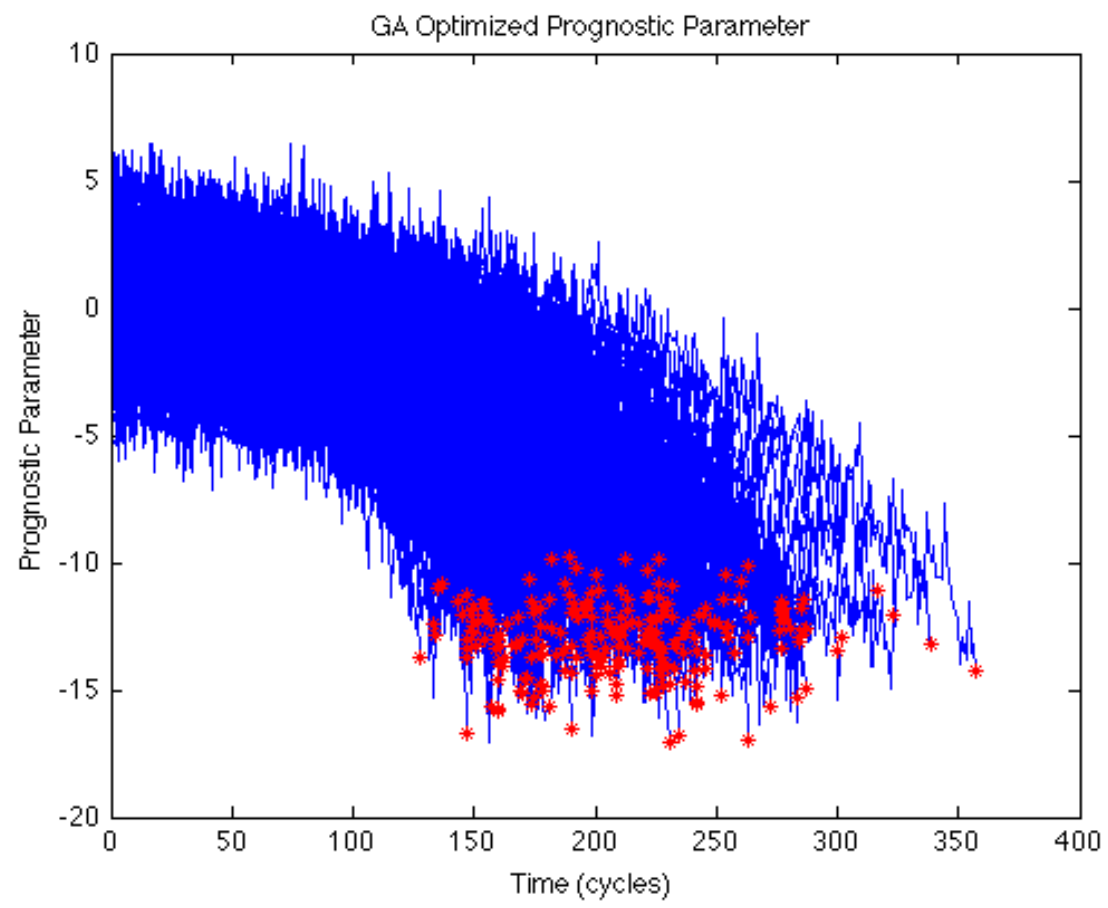

Figure 5-20: Prognostic Parameter Identified by GA Optimization

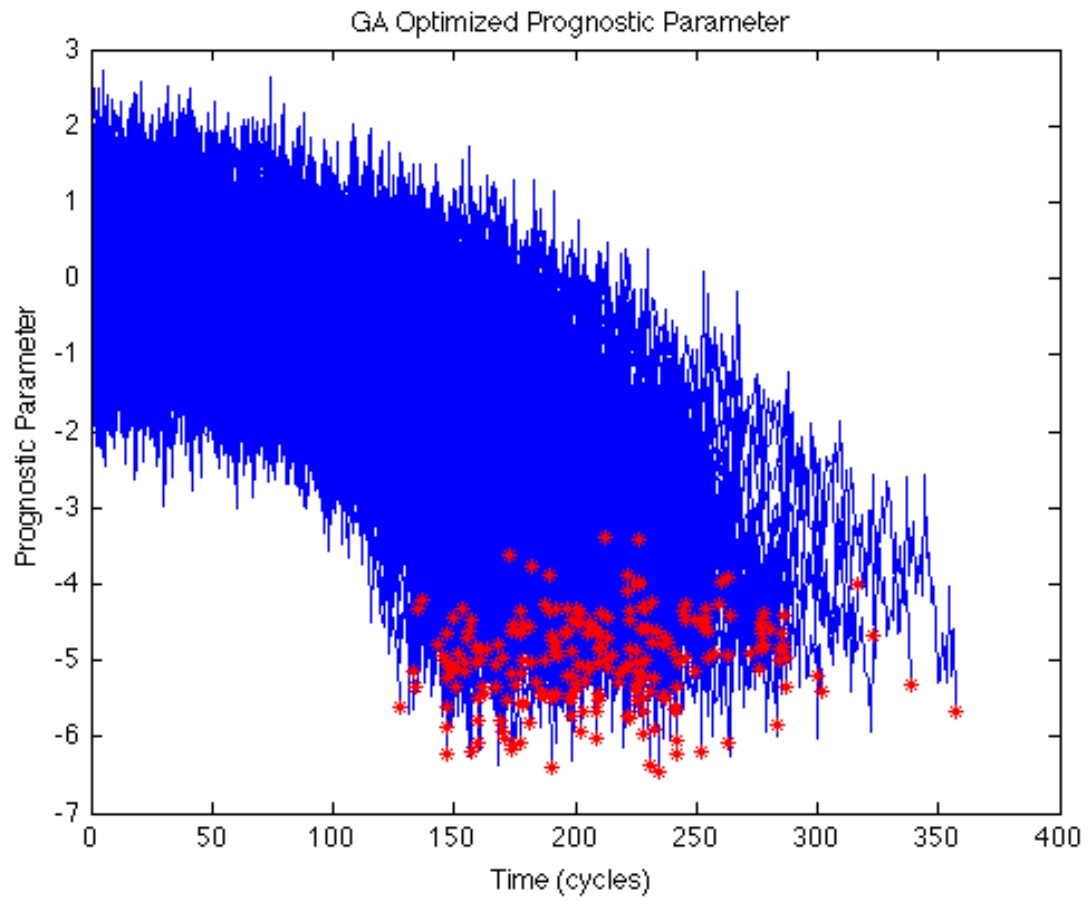

Figure 5-21: Prognostic Parameter Identified by Reduced GA Optimization 
Table 5-2 gives a comparison of the weights for the parameter identified by visual inspection and the two selected by the GA. The parameter suitability metrics for each parameter are given in Table 5-3; the suitability of each parameter is calculated as the sum of the three suitability metrics, as it was determined in the fitness function. The fitness of both of the GAoptimized parameters is basically equivalent to that of the parameter identified via visual inspection. This may be further improved by standard GA improvement techniques, such as coupling the result with a gradient descent optimization or running the GA several times to find the best result.

Table 5-2: Residual Weightings

\begin{tabular}{|c|c|c|c|c|c|c|c|c|c|c|c|}
\cline { 2 - 11 } \multicolumn{1}{c|}{} & \multicolumn{10}{c|}{ Residual } \\
\hline Parameter & 1 & 3 & 4 & 5 & 10 & 12 & 15 & 16 & 18 & 21 & 22 \\
\hline $\begin{array}{c}\text { VI } \\
\text { Parameter }\end{array}$ & 0.00 & 0.00 & 0.19 & 0.14 & 0.00 & 4.76 & 0.00 & 28.5 & 0.74 & 0.00 & 0.00 \\
\hline $\begin{array}{c}\text { GA } \\
\text { Parameter 1 }\end{array}$ & -0.28 & 0.81 & 0.07 & 0.14 & 0.00 & 4.93 & 0.02 & 29.1 & 0.35 & 0.32 & 0.10 \\
\hline $\begin{array}{c}\text { GA } \\
\text { Parameter 2 }\end{array}$ & 0.00 & 0.00 & 0.02 & 0.07 & 0.00 & 2.38 & 0.00 & 5.92 & 0.25 & 0.00 & 0.00 \\
\hline
\end{tabular}

Table 5-3: PHM Challenge Data Parameter Suitability Metrics

\begin{tabular}{|c|c|c|c|c|}
\hline Parameter & Monotonicity & Prognosability & Trendability & Suitability \\
\hline VI Parameter & 0.846 & 0.891 & 0.903 & 2.640 \\
\hline GA Parameter \#1 & 0.941 & 0.895 & 0.931 & 2.766 \\
\hline GA Parameter \#2 & 0.901 & 0.889 & 0.909 & 2.698 \\
\hline
\end{tabular}

These GA-optimized prognostic parameters were also used to develop GPM prognostic models. Figure 5-22 gives the results for the prognostic model developed with the first parameter population. Figure 5-23 gives the results for the second parameter. Overall model performance with all three parameters is comparable, and it is nonsensical to claim one parameter as best based on the model performance. However, this application has shown that the proposed automated parameter identification method can identify prognostic parameters comparable to those identified by visual inspection and expert analysis in a fraction of the time. 


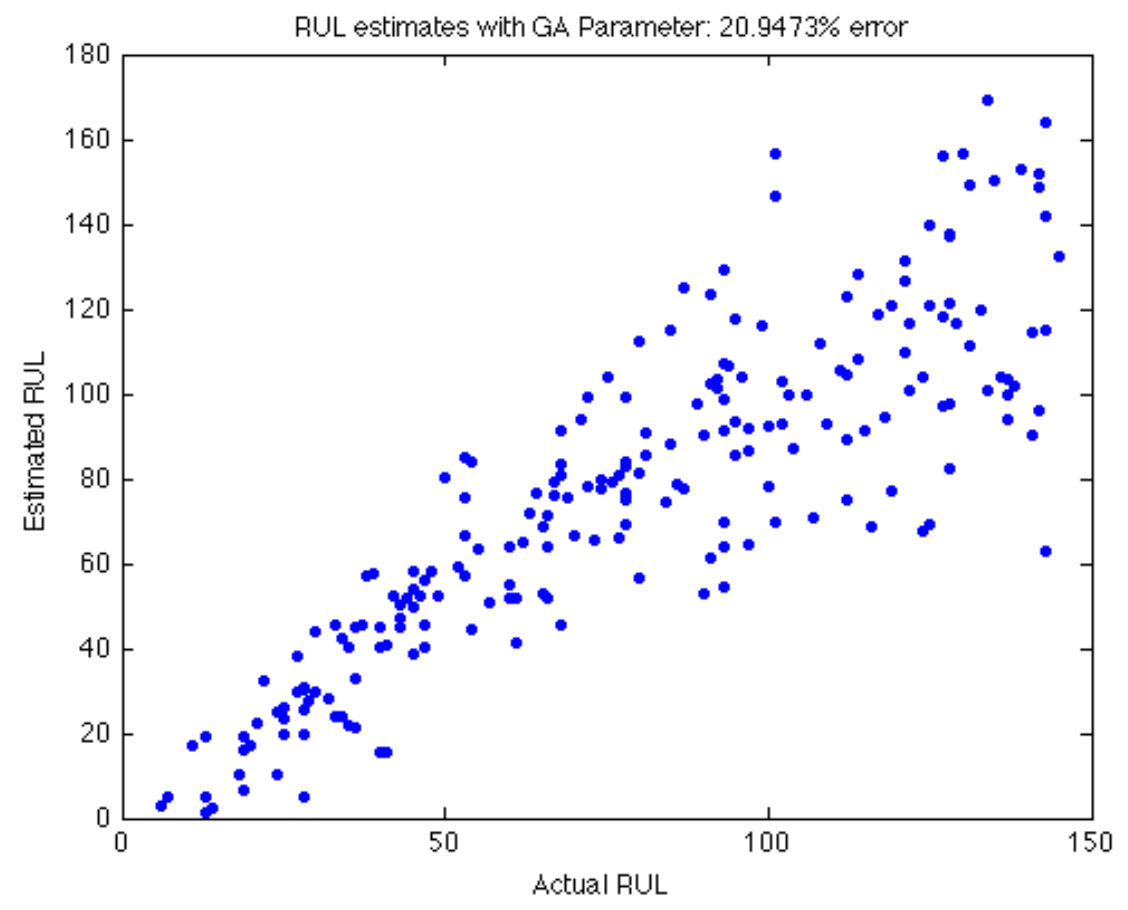

Figure 5-22: RUL Estimates for First GA-optimized Parameter

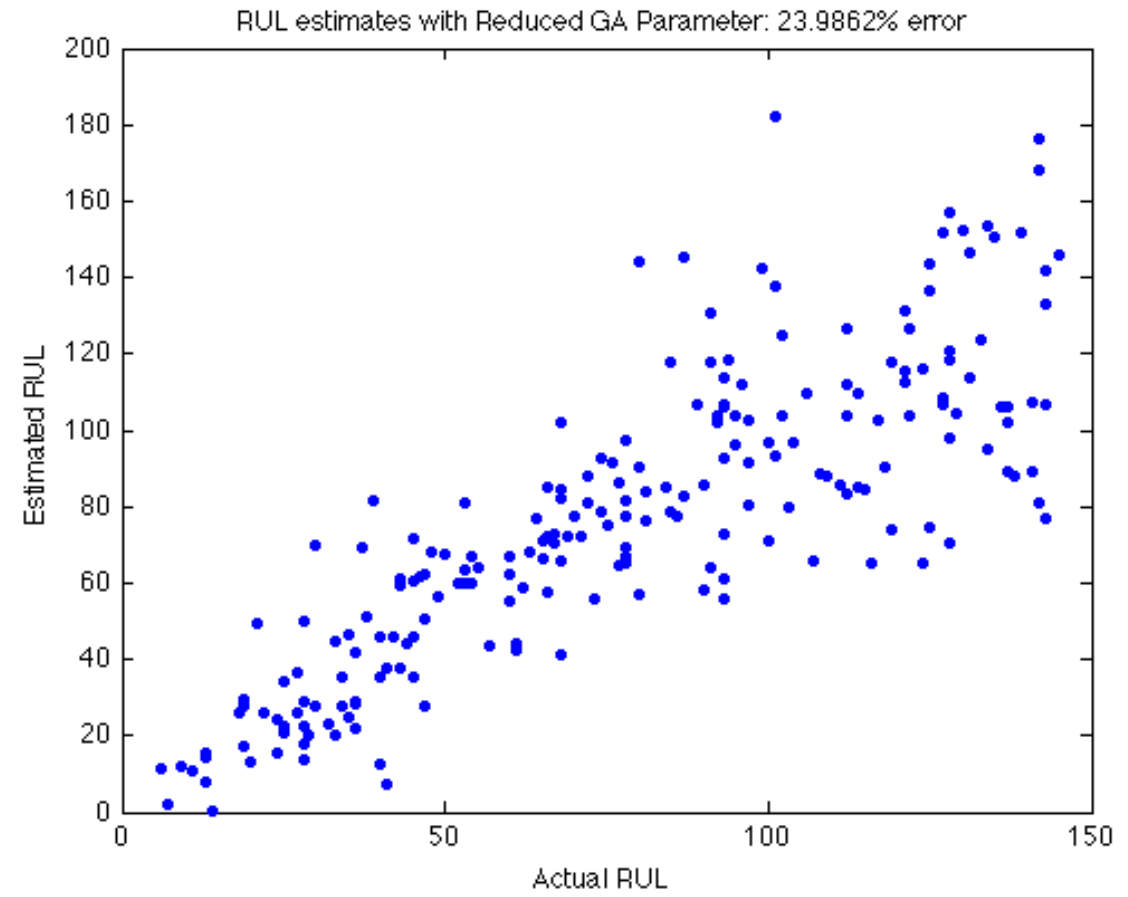

Figure 5-23: RUL Estimates for Second GA-optimized Parameter 
The sample application presented here benefited from the extensive expert analysis needed to identify a parameter through visual inspection, in that a subset of possible parameter inputs had already been identified and parameter optimization was restricted to only consider the monitoring system residuals. In applications with a larger domain of inputs, which could include the actual signals, the monitoring system predictions, information on usage, environment, and load, and fault alarm and diagnostic results, as well as higher order terms of any of these inputs, the input selection technique applied in identifying the second GA parameter will be paramount to timely and accurate parameter identification. It will greatly reduce GA runtime and help ensure that a near optimal parameter is identified.

The prognostic parameters identified here were used to develop a Type III model for prognostic estimation. However, this data set includes both sensed measurements and operating condition measurements. Type I and II models may also be applicable to this type of data. The following section looks at several competing prognostic models of each type and compares their performance on this data set to illustrate the efficacy of the individual-based algorithm.

\subsubsection{Performance Comparison of the Three Prognostic Types}

Three competing prognostic models were developed, one of each prognostic model class, to illustrate the performance improvements offered by including additional information sources. The three models are compared based on the "score" given by the challenge scoring function:

$$
\begin{aligned}
& d=R U L_{\text {estimated }} \quad R U L_{\text {actual }} \\
& \operatorname{score}(d<0)=\exp (d / 13) \quad 1 \\
& \operatorname{score}(d>0)=\exp (d / 10) \quad 1
\end{aligned}
$$

The Type I prognostic model utilizes a Weibull distribution-based reliability analysis. Two Type II models were developed to estimate RUL based on the operating history. The first uses a simple regression model considering the time spent in each operating condition; the second, a Markov Chain model as described earlier. Finally, a GPM model using the GAoptimized prognostic parameter given in Figure 5-20 gives the Type III estimates. 


\subsubsection{Type I Prognostics}

Type I prognostics are traditional time-to-failure analysis methods, such as Weibull analysis. A histogram of the failure times for the 218 training cases is given in Figure 5-24 along with the Weibull probability density function. The maximum likelihood Weibull fit of this data gives parameter estimates of 228.5 and 5.1 for the scale parameter and shape parameter, respectively. The scale parameter indicates that $63.2 \%$ of the observed systems can be expected to fail by cycle 228.5. Since the shape parameter is much greater than 1 , failure is occurring due to wear-out degradation. For each new system, the RUL is estimated using the MRL calculation previously discussed. Because the failure data has a large variance, accurate RUL prediction is not possible. From the figure, one can see that the most probably value is around 210 cycles; however, the values have a range that covers approximately two hundred cycles. Using this Weibull fit and the MRL calculation to estimate the RUL for each of the 218 test cases resulted in a score of approximately 22,500 .

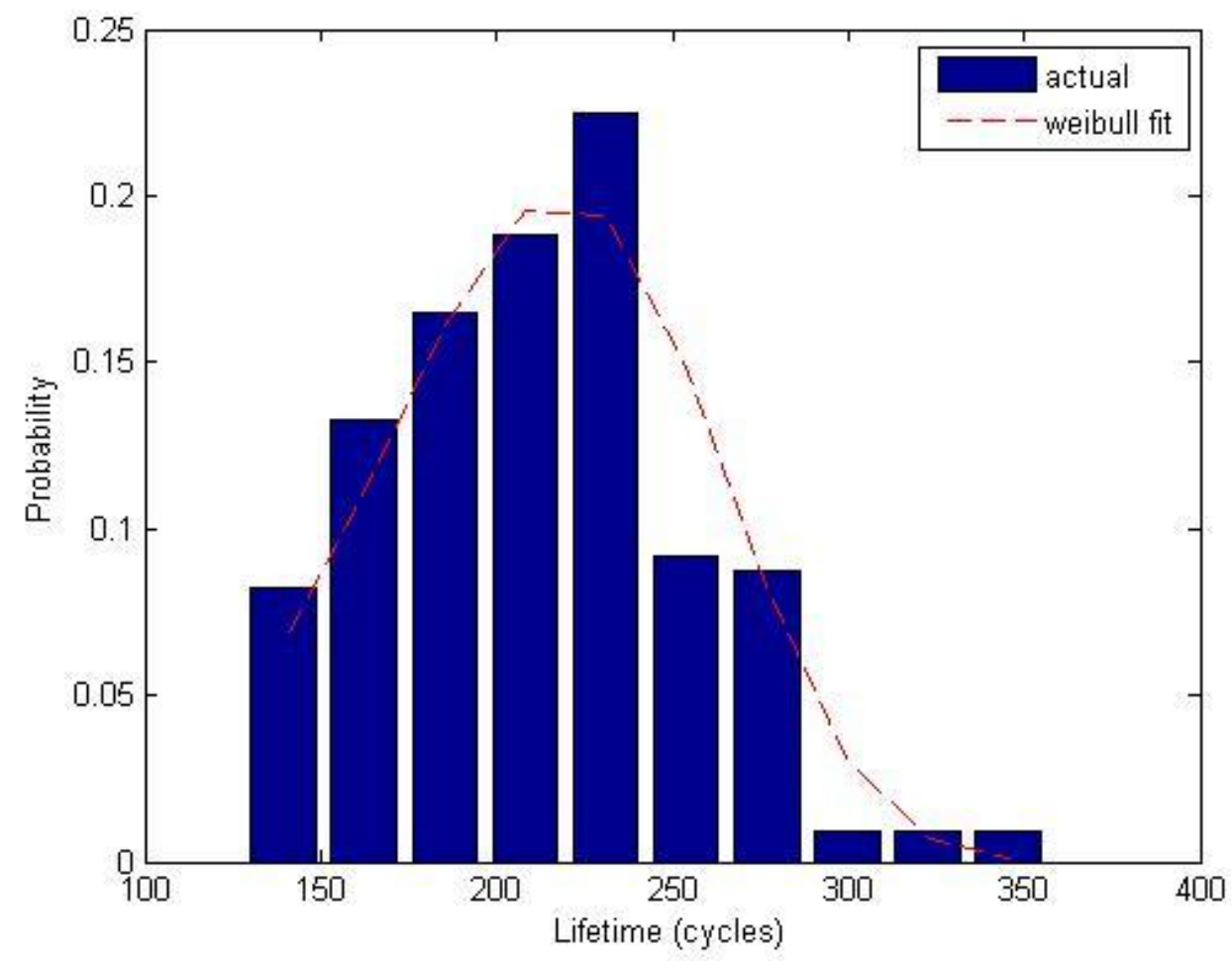

Figure 5-24: Histogram of failure times and Weibull fit 


\subsubsection{Type II Prognostics}

Type II methods use operational data, such as load, environment, input current, etc, to make predictions of RUL. As discussed earlier, the data can easily be divided into six operational conditions; the percentage of full lifetime spent in each condition is shown in Figure 5-25. This figure shows that the variation in the percent of time spent in each condition is small $(\sim 10 \%)$, but the variation in failure times is much larger $(\sim 20 \%)$. Therefore, it is improbable that the variation in time spent in each operational condition is the source for the variation of failure time. 


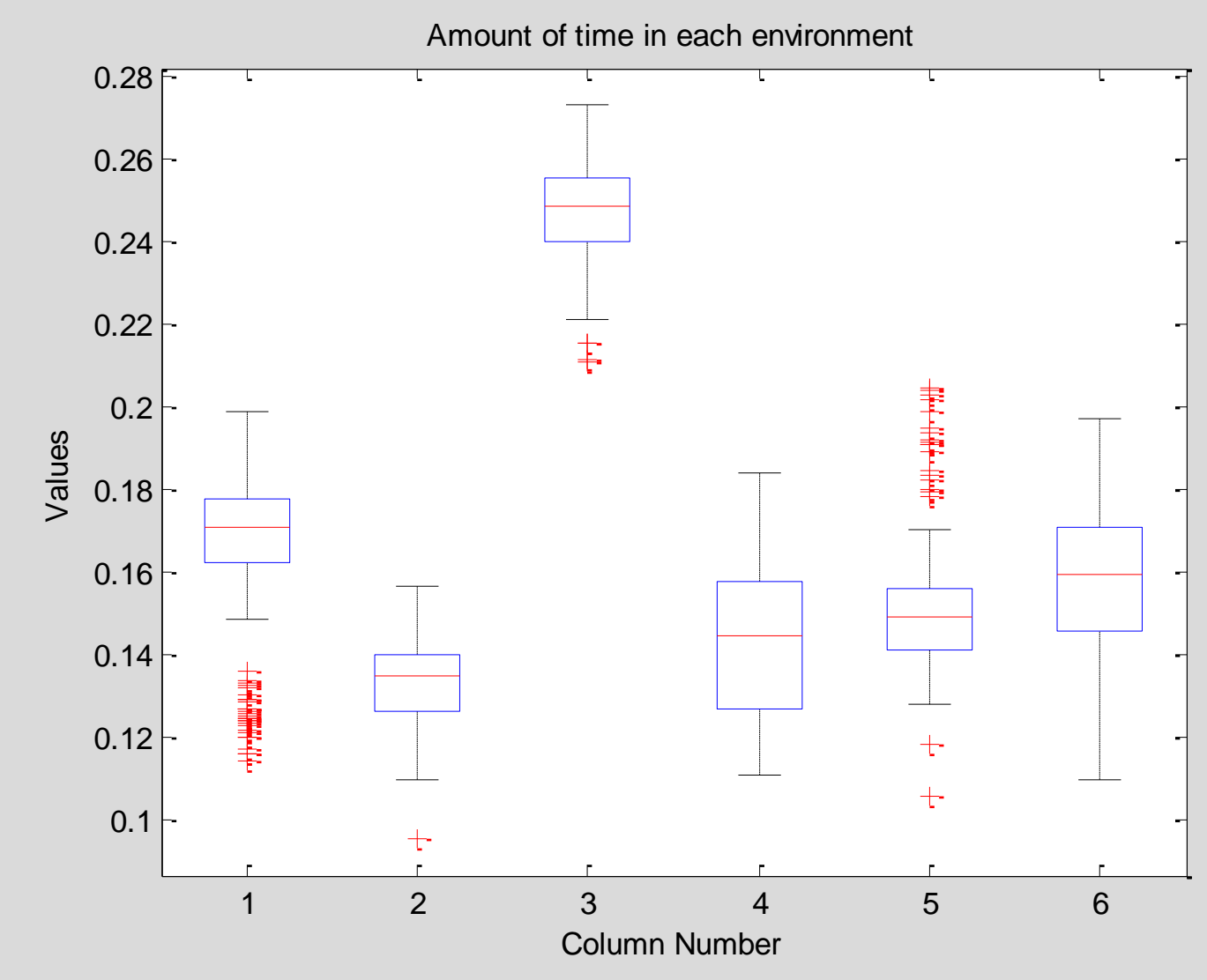

Figure 5-25: Plot of time spent in each operational condition

Several methods can be used to estimate the RUL from this usage data. Simplest, perhaps, is a regression using the total amount of time spent in each condition to predict RUL (the percent of time spent in each condition may also be used). Regressions of this type have been performed using a simple linear regression, inferential kernel regression, and neural networks. The results for all regressions have been poor, with little improvement over Type I estimates. This is most likely because the amount of time spent in any one operating condition is not well correlated to the total lifetime, as shown in Table 5-4. The cost function for an inferential kernel regression was approximately 20,600 .

Table 5-4: Correlation of Operating Condition to Lifetime

\begin{tabular}{|c|c|}
\hline \multicolumn{2}{|c|}{ Correlation to Lifetime } \\
\hline 1 & 0.12 \\
\hline 2 & -0.037 \\
\hline
\end{tabular}




\begin{tabular}{|c|c|}
\hline 3 & 0.041 \\
\hline 4 & 0.021 \\
\hline 5 & -0.064 \\
\hline 6 & -0.046 \\
\hline
\end{tabular}

In addition to simple regression approaches, a Markov Chain (MC) model was developed to simulate possible future operating condition progressions. The transition probably matrix calculated from the training cases is given in

Table 5-5. These operating condition progressions are used to determine a distribution of failure times for the system. However, as with the inferential regression, it was difficult to map the simulated operating condition information to an RUL estimate or degradation parameter to determine end of life for each simulated path. The results of the MC study were also poor, with a score of approximately 19,200.

Table 5-5: Markov Chain Transition Probability Matrix

\begin{tabular}{|c|c|c|c|c|c|c|}
\hline \multicolumn{1}{r|}{ To } & & & & & & \\
\cline { 2 - 7 } & 1 & 2 & 3 & 4 & 5 & 6 \\
\hline 1 & 0.14 & 0.16 & 0.15 & 0.16 & 0.15 & 0.24 \\
\hline 2 & 0.16 & 0.15 & 0.14 & 0.14 & 0.15 & 0.25 \\
\hline 3 & 0.15 & 0.14 & 0.15 & 0.15 & 0.15 & 0.26 \\
\hline 4 & 0.16 & 0.15 & 0.15 & 0.15 & 0.15 & 0.24 \\
\hline 5 & 0.15 & 0.15 & 0.15 & 0.15 & 0.15 & 0.26 \\
\hline 6 & 0.15 & 0.14 & 0.15 & 0.15 & 0.15 & 0.26 \\
\hline
\end{tabular}

\subsubsection{Type III Prognostics}

A GPM/Bayes model as described in Chapter 4 was developed using the GAoptimized prognostic parameter given in Figure 5-20. For the current problem, quadratic regression models were fit to the full degradation parameter for each of the 218 training cases. The resulting prior distributions for the parameter fits are given in Table 5-6. Very precise estimates of $\mathrm{p} 1$ and $\mathrm{p} 2$ are available. More variance is seen in $\mathrm{p} 3$, which is assumed to correspond to the random level of initial degradation and is retained for that reason. The variance of the degradation parameter can be estimated from the training 
examples by smoothing each example path and subtracting the smoothed path from the actual path. This gives an estimate of the noise; the noise variance can be estimated directly as the variance of this data set. For this problem, the noise variance in the degradation parameter is estimated to be 0.0588 . The cost for the test set is much improved over the previous two methods at approximately 2,500.

Table 5-6: Prior Distribution for Quadratic Parameters

\begin{tabular}{|c|c|c|}
\hline & Mean & Std Dev \\
\hline$p_{1}$ & -0.0001 & $4.30 E-05$ \\
\hline$P_{2}$ & 0.0075 & 0.0028 \\
\hline$p_{3}$ & -0.2057 & 0.370 \\
\hline
\end{tabular}

The next example application utilizes a data set collected in a laboratory testing experiment. This example will further investigate the efficacy of the parameter identification method and input selection technique.

\subsection{Milling Data Set}

The second data set used for this method is the Milling Data set collected by Agogino and Goebel, also available at the NASA Prognostic Data Repository [2007]. This data set presents milling machine wear measurements made in a laboratory experiment. Figure 5-26 shows the measured flank wear for each of 15 runs; the original data set includes 16 runs, but Run 6 includes only one set of measurements which do not indicate failure so it is excluded from this analysis. The experimental conditions for each run are given in Table 5-7. For this application, failure was assumed to occur at flank wear of $0.45 \mathrm{~mm}$, as indicated. Failure times given in Table 5-7 are interpolated from the available flank wear measurements, assuming that the degradation is linear between measurements. In real-world applications, flank wear measurements would not be available. A prognostic parameter for this system should be able to characterize the milling machine degradation from the available information sources: operating condition indicators, including depth of cut, feed, and material; and available sensor measurements, including AC spindle motor current, DC spindle motor current, table vibration, spindle vibration, table acoustic emissions, and spindle acoustic emissions, or features of these measures. 


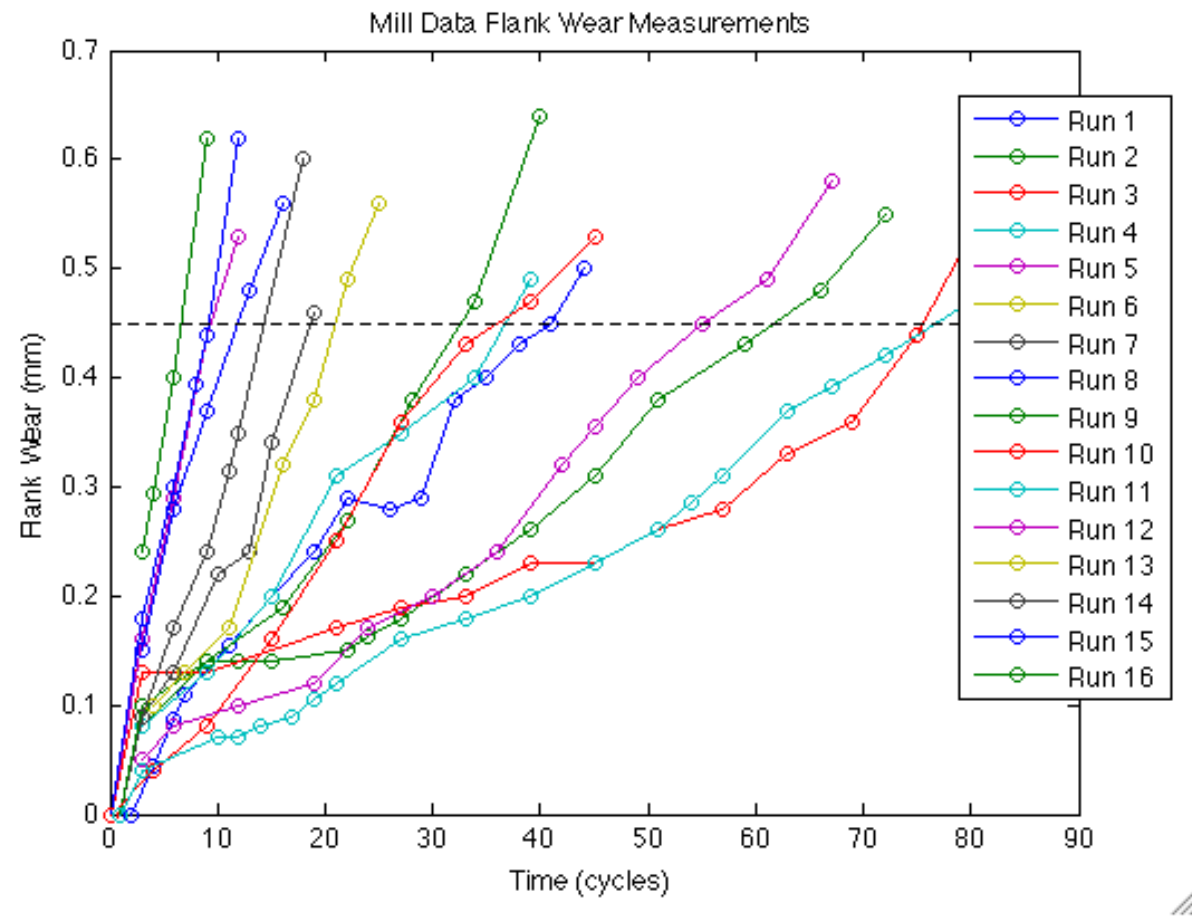

Figure 5-26: Flank Wear Measurements

Table 5-7: Milling Data Experimental Conditions

\begin{tabular}{|c|c|c|c|c|}
\hline Run & Dept & Feed & Mate & Failur \\
\hline 1 & 1.5 & 0.5 & cast & 41.0 \\
\hline 2 & 0.75 & 0.5 & cast & 61.8 \\
\hline 3 & 0.75 & 0.25 & cast & 75.5 \\
\hline 4 & 1.5 & 0.25 & cast & 36.7 \\
\hline 5 & 1.5 & 0.5 & steel & 9.33 \\
\hline 6 & 1.5 & 0.25 & steel & $\mathrm{N} / \mathrm{A}$ \\
\hline 7 & 0.75 & 0.25 & steel & 18.6 \\
\hline 8 & 0.75 & 0.5 & steel & 9.17 \\
\hline 9 & 1.5 & 0.5 & cast & 32.6 \\
\hline 10 & 1.5 & 0.25 & cast & 36.0 \\
\hline 11 & 0.75 & 0.25 & cast & 76.8 \\
\hline 12 & 0.75 & 0.5 & cast & 55.0 \\
\hline 13 & 0.75 & 0.25 & steel & 20.9 \\
\hline 14 & 0.75 & 0.5 & steel & 14.4 \\
\hline 15 & 1.5 & 0.25 & steel & 11.9 \\
\hline 16 & 1.5 & 0.5 & steel & 6.68 \\
\hline & & & & \\
\hline
\end{tabular}

Figure 5-27 shows the raw data for run 3. Because each of the sensors measure highly oscillatory variables, this data is not well suited to the AAKR monitoring system 
described previously. However, there are underlying trends in the data which can be used to monitor the system. Of particular interest here are the mean and standard deviation of the signals. By monitoring these features of the measured variables, changes in the system can be characterized and trended to failure. The means and standard deviations of the six signals for run 3 are shown in Figure 5-28. Signal features are calculated for each timestep reported. These steps are not equally spaced in time; however, no information about sampling rate is given to allow for different time averaging. Each time step includes 9000 observations, but time steps span different durations. Because it is not precisely known how these measurements are made, this is considered the best option for this data set. Plots of the mean and standard deviation for all fifteen runs are given in Appendix A.

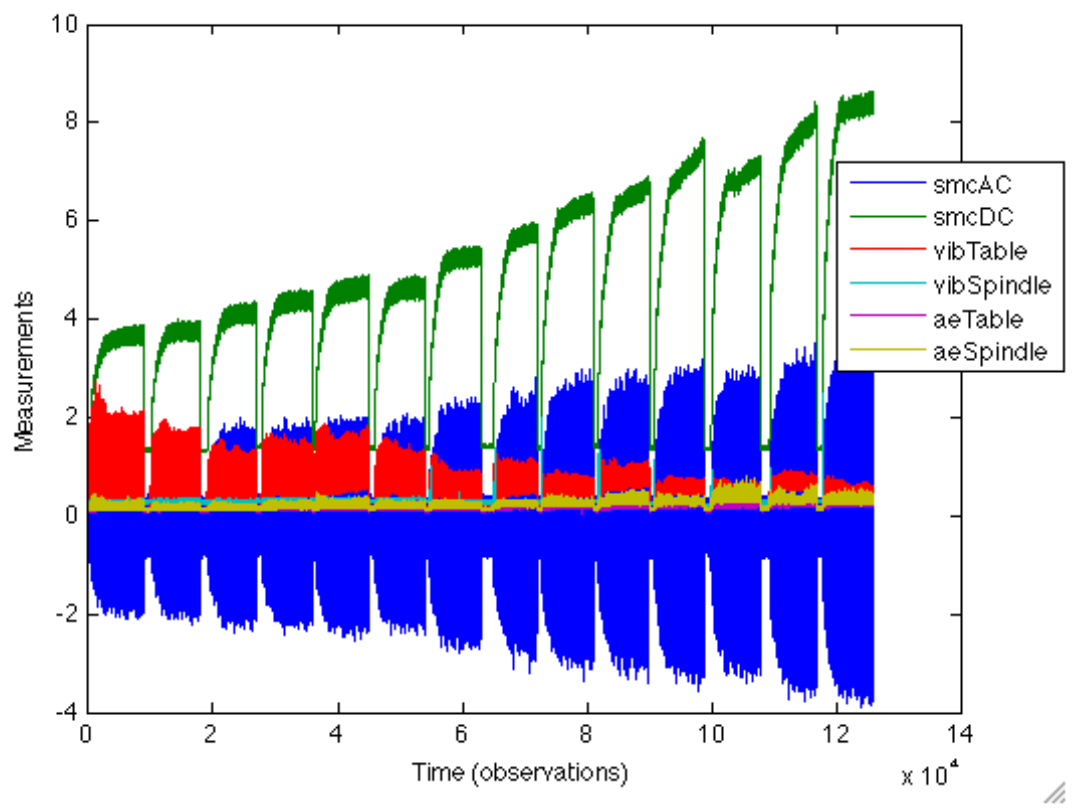

Figure 5-27: Milling Data, Run 3 

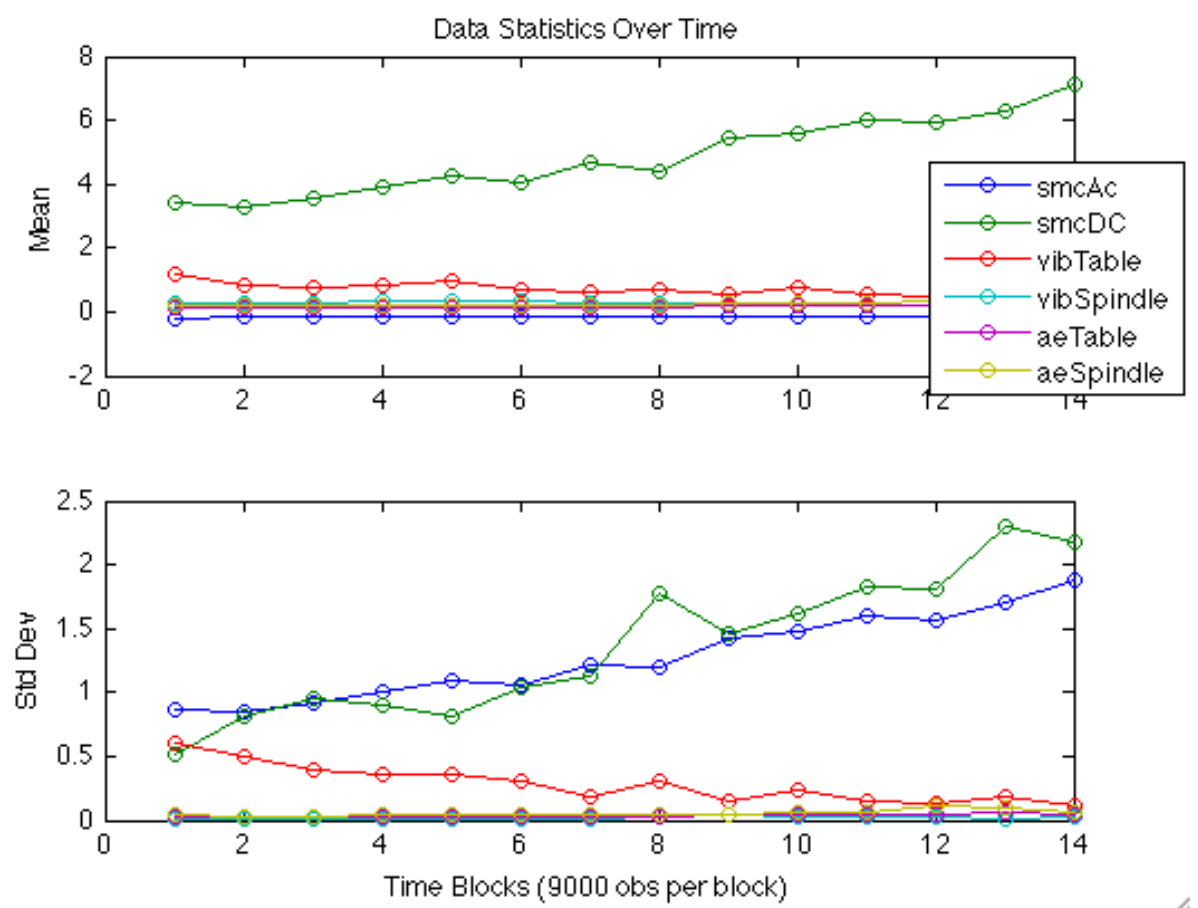

Figure 5-28: Mean and Standard Deviation of Milling Data, Run 3

\subsubsection{GA Optimized Parameter}

The parameter identification method was applied to these twelve features to identify an optimal prognostic parameter for the system. The parameter identified for the fifteen runs is shown in Figure 5-29. This parameter has monotonicity, prognosability, and trendability metrics of $0.889,0.807$, and 0.829 , respectively. The parameter is used to develop a GPM/Bayes model for the entire population of milling experiments. Because only fifteen example runs are available, the performance of the model is measured through a leave-one-out method. That is, for each of the fifteen cases, the remaining fourteen cases are used with the prognostic parameter to develop a GPM/Bayes model. RUL estimates are made for the left out case using this model. In each case, the RUL is estimated at each observation. Figure 5-30 gives the results of the prognostic model for Run 3 using the GA optimized parameter. The model on a whole performed well, with an MAPE of $21.5 \%$ over the total lifetime. As a component approaches failure, the error decreases to an average of $10.2 \%$ or better within the last three cycles before failure. 


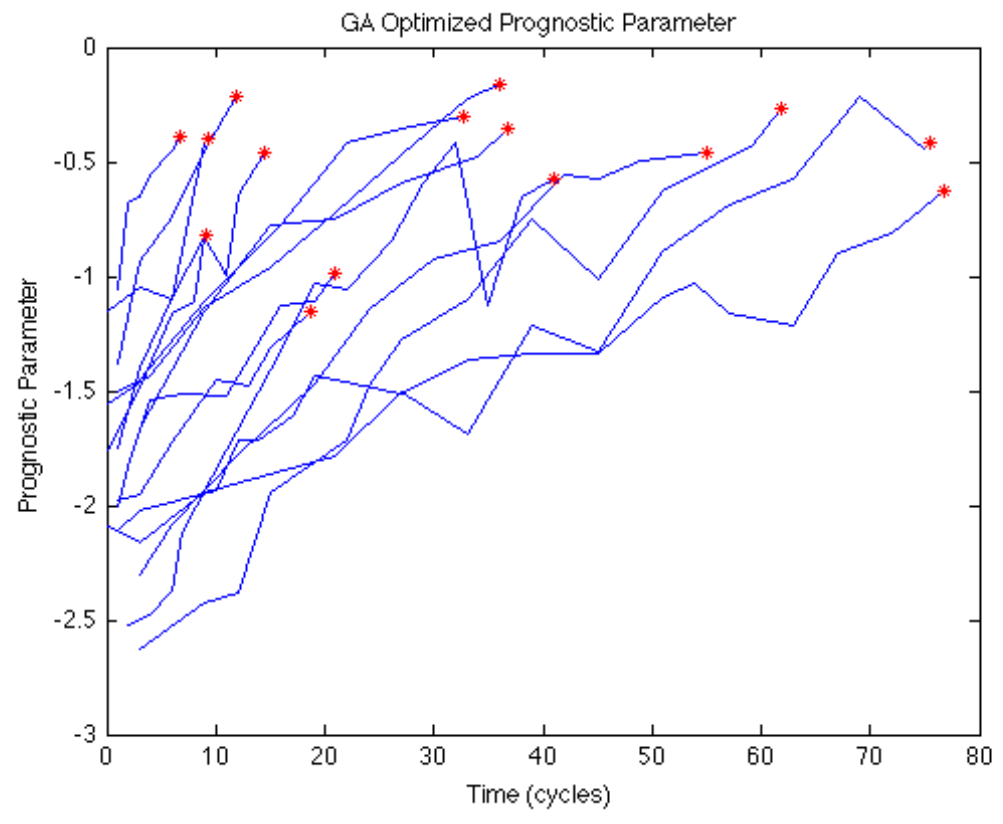

Figure 5-29: GA-Optimized Prognostic Parameter for Milling Data

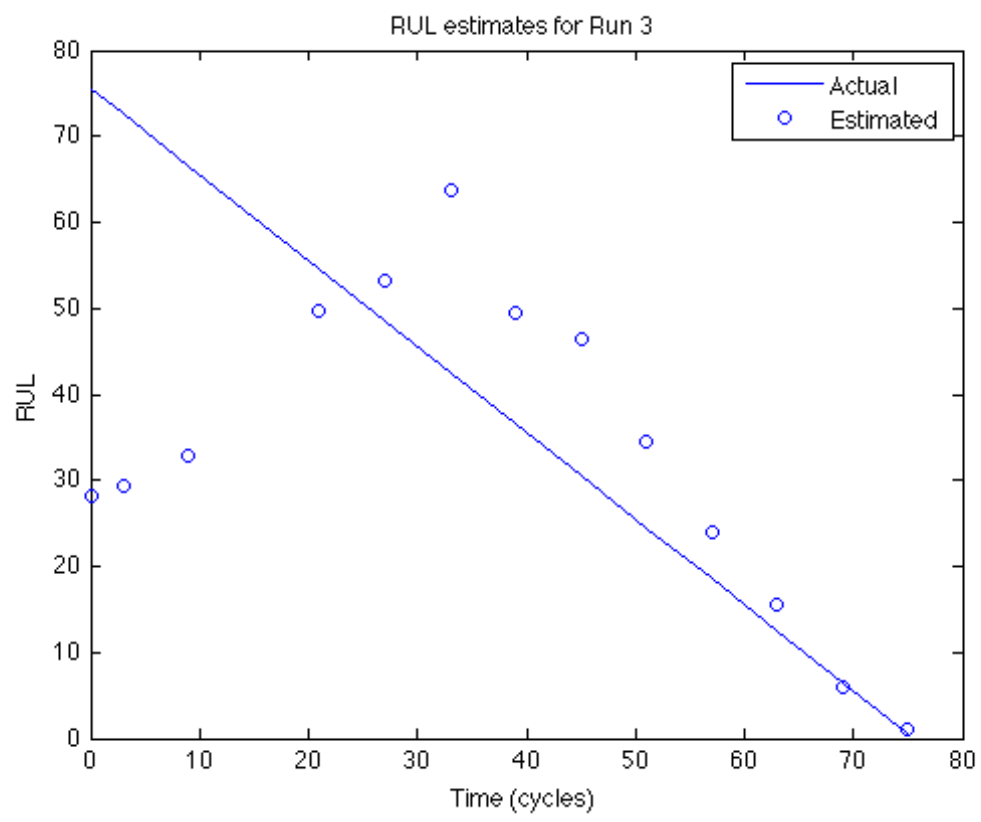

Figure 5-30: RUL estimates for Milling Data Run 3

Because the identification of appropriate prognostic parameters is automated, it is straightforward to develop additional prognostic parameters beyond the single parameter for each system. This data includes three operating conditions: depth of cut, feed, and material. A single mill run involves only one set of operating conditions for each cut. 
Instead of applying one prognostic model to the entire population, it may be more effective to classify runs according to one or more operating condition and develop multiple prognostic models. The functions available in the PEP toolbox make this extra step trivial for prognostic parameter optimization. The next section presents the results of developing separate prognostic models for each of the two materials being milled: cast iron and steel.

\subsubsection{Material Specific Parameters}

Two additional prognostic parameters are identified for the milling data based on the type of material being milled. As indicated in Table 5-7, the milling experiments using steel have a much shorter mean lifetime, only 13.0 cycles, compared to those using cast iron, which have a mean life of 51.9 cycles. This indicates that it should be beneficial to separate these cases into two separate models. Three operating conditions total are available; prognostic model performance may be further improved by dividing the data according to two or all of these parameters. However, because only fifteen runs are available, it is not possible with the available data set. Should more data become available, this is an interesting course to pursue.

The optimized parameter for cast iron is shown in Figure 5-31, and that for steel in Figure 5-32. The prognostic parameter suitability metrics and total suitability, given by the sum of the three metrics, for these two parameters and the single parameter given earlier are presented in Table 5-8. By separating the runs according to material, the prognostic parameter suitability increased slightly for both cases. The resulting RUL estimations using these improved parameters showed improvement over the single population parameter. Using the same leave-one-out methodology, each of the fifteen

runs were prognosed using the appropriate model. Separating the data according to material improved prognostic performance by nearly 50\%, giving an average RUL error of $12.3 \%$, with a reduced error of $8.2 \%$ or less within three cycles of failure. Figure 5-33 compares the performance of the two models. This figure shows that the materialspecific models have better performance than the lumped model, which should be expected from the improved parameter suitability metrics. 


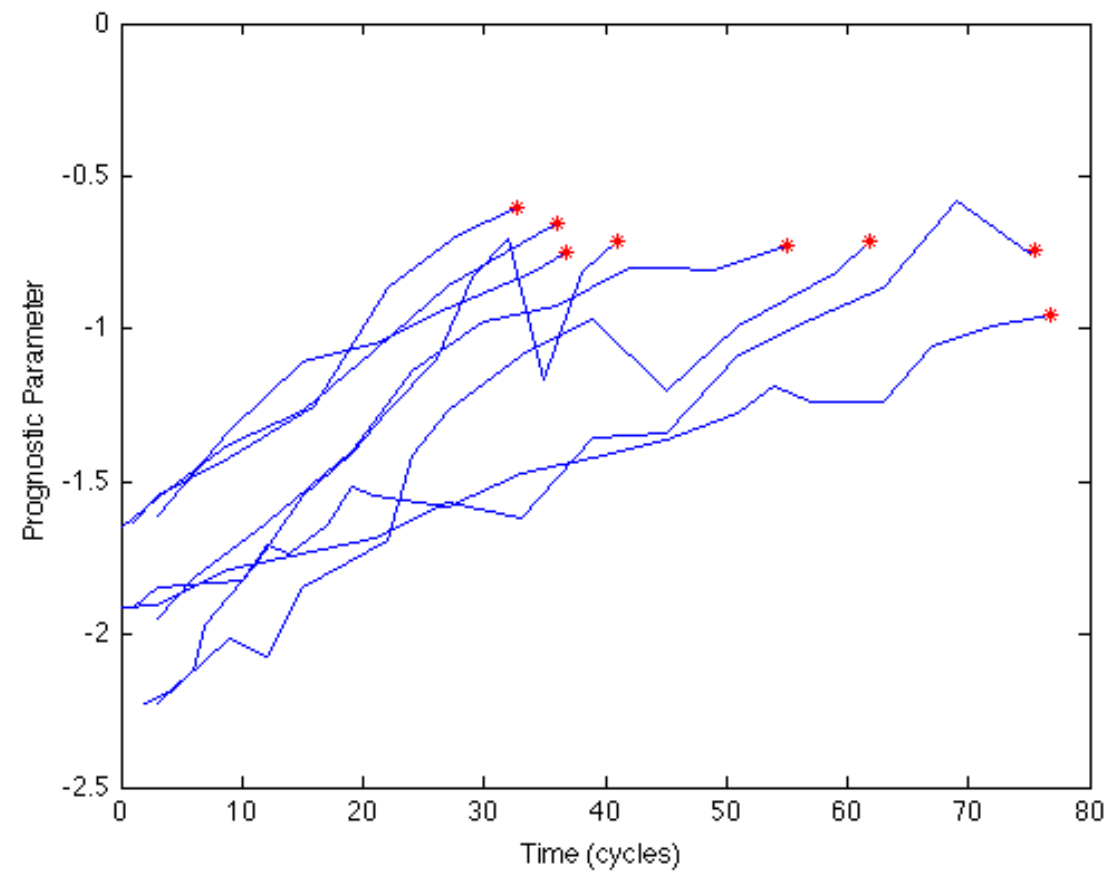

Figure 5-31: GA-Optimized Prognostic Parameter for Runs Boring Iron

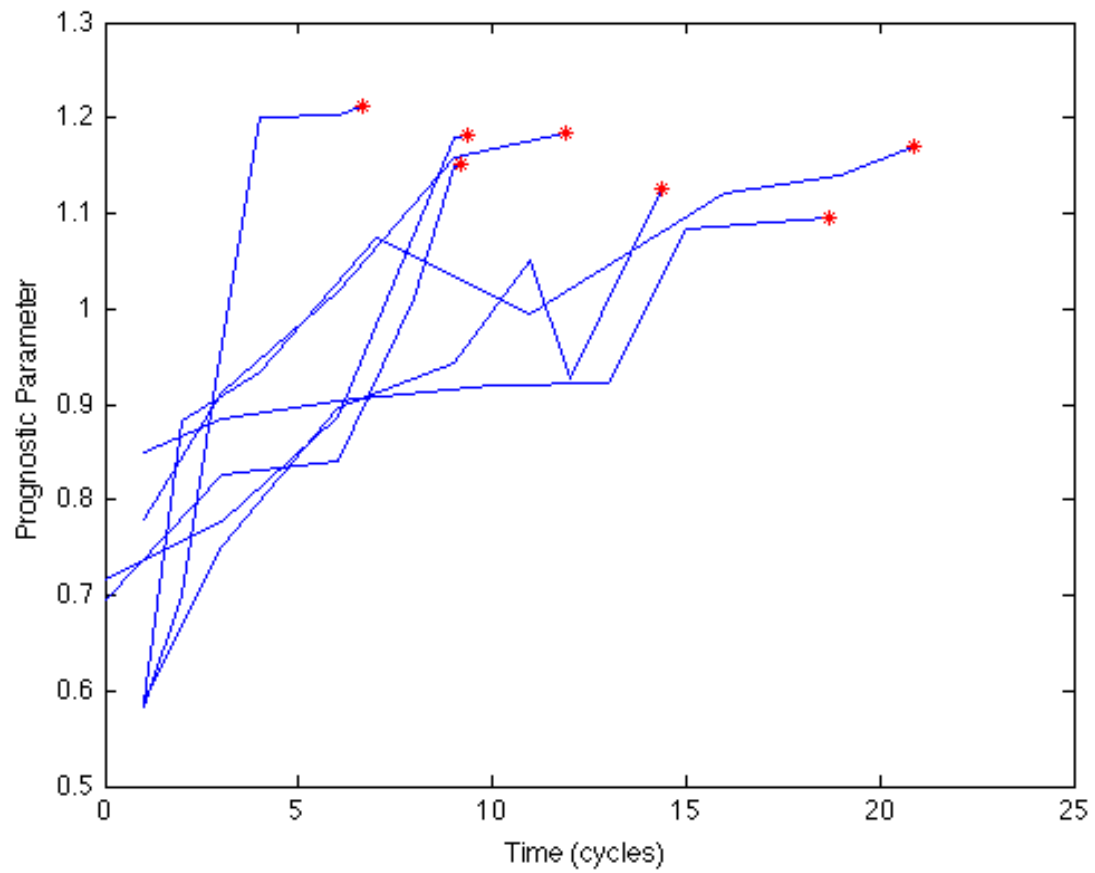

Figure 5-32: GA-Optimized Prognostic Parameter for Runs Boring Steel

Table 5-8: Parameter Suitability Metrics for Milling Data 


\begin{tabular}{|c|c|c|c|c|}
\hline & Monotonicity & Prognosability & Trendability & Suitability \\
\hline Full Parameter & 0.889 & 0.807 & 0.829 & 2.526 \\
\hline Iron Parameter & 0.969 & 0.917 & 0.898 & 2.783 \\
\hline Steel Parameter & 0.929 & 0.947 & 0.768 & 2.643 \\
\hline
\end{tabular}

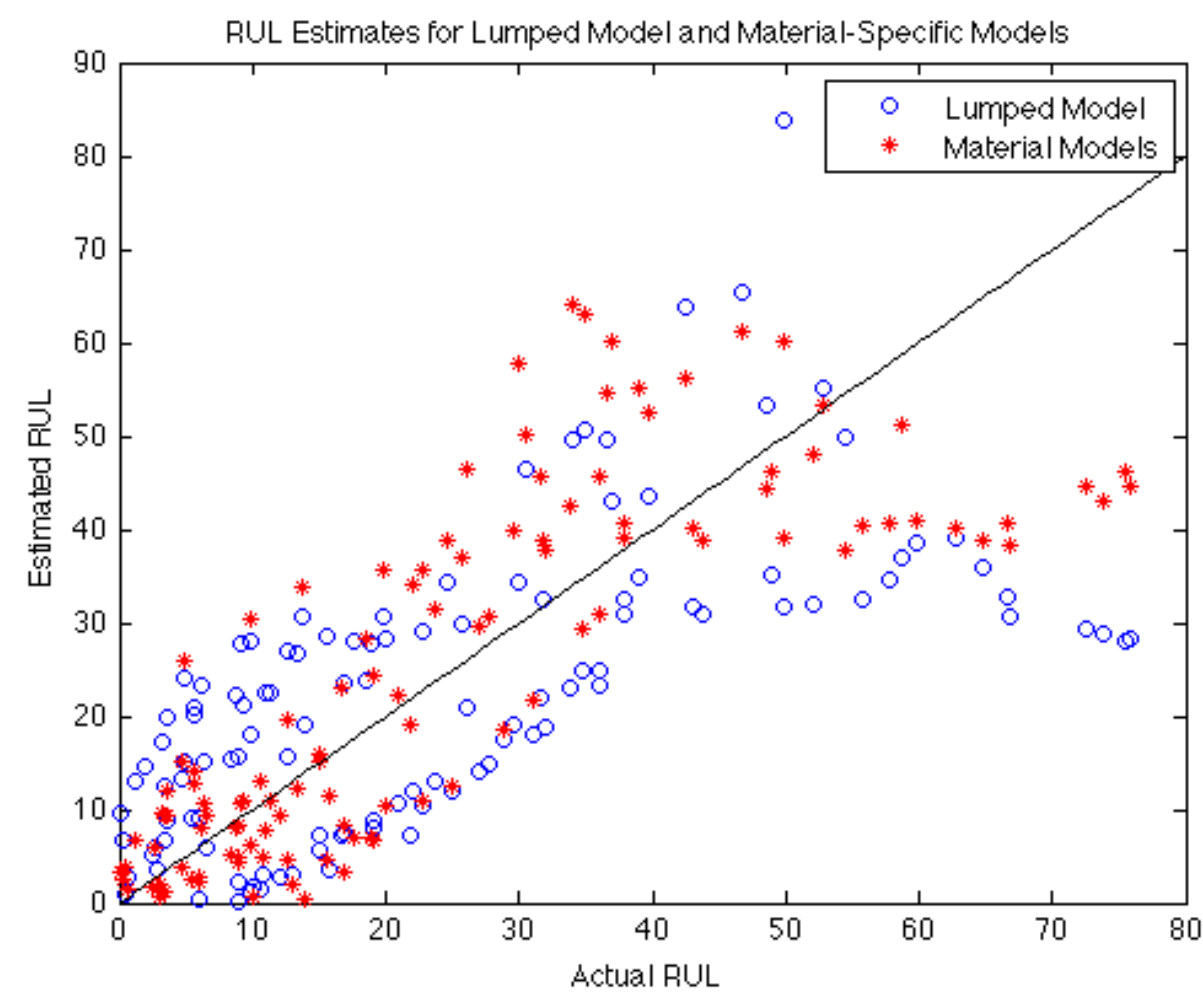

Figure 5-33: RUL Estimates for Lumped and Material-Specific Models

Using the automated parameter identification method for this data allowed for easily identifying separate prognostic parameters for data in two groups: steel and cast iron. Allowing the computer to do all the computationally heavy work allows a prognostic system designer to investigate many possible competing parameters and choose among them. The PEP toolbox has the ability to identify prognostic parameters for any number of data groups for easy comparison. For this example application, dividing the cases by the material being bored afforded some improvement in the RUL estimation, particularly at end of life when such estimation is most important. 


\section{CONCLUSIONS}

This report has presented the work pursued in completion of the prognostic system tasks of Objective 3 of the NERI-C project. Volume 6 of this report series discussed system monitoring, fault detection, and fault diagnostics. Completing the health monitoring system, after a fault has been detected and identified, the appropriate prognostic model may be used to estimate the remaining lifetime of the system. Individual-based, or Type III, prognostics are most desirable for safety-critical or highvalue equipment and systems such as power plants. A Type III prognostic model, the General Path Model (GPM), was described. This prognostic algorithm trends a measure related to system health called a prognostic parameter to estimate RUL. The prognostic parameter may be a direct measurement of system health, such as tire tread depth, or it may be inferred from other measurements from the system, such as tire pressure or operating temperature. A parametric model is fit to the prognostic parameter and extrapolated to a pre-defined critical failure level. The RUL is estimated as the difference between the current time and the time at which the extrapolated model crosses the failure threshold. This research proposed a modification to the traditional GPM method which uses Bayesian updating methods to incorporate prior information about the expected prognostic parameter path. Incorporating prior information allows the GPM to be applied to systems with very few observations or whose observations are contaminated by noise. A comparison of the application of the traditional GPM method and the proposed GPM/Bayes method showed that the latter was able to make prognostic estimates in every case, whereas the former method was often unable to make RUL estimates very early in life. In addition, the GPM/Bayes model had smaller Mean Absolute Percent Error (MAPE) for predictions made early in system life; as more information became available, the error of the two methods converged because in both cases prognostic estimates were based primarily on the available prognostic parameter values with little emphasis on the prior information.

Additionally, the effect of noisy prognostic parameters on model performance was investigated. It was shown that the GPM/Bayes methodology can perform in the face of significant noise contamination. Parameter noise slows the response time of the regression model to transition from the population-based prior information to the 
information contained in the system-specific observations. However, as more observations become available, the model is able to determine the correct underlying regression function despite high levels of noise. The RUL estimates made with clean data, data contaminated with $20 \%$ noise, and data contaminated with $80 \%$ noise converged as the system aged and approached end of life.

Additionally, GPM models for each of four faults common in nuclear power plants were developed and applied to test cases of the faults generated from a first principle simulation of the IRIS plant.

When Type III algorithms are applicable, their performance is highly dependent on identification of an appropriate prognostic parameter. Ideally, a prognostic parameter should have three key qualities: monotonicity, prognosability, and trendability. Monotonicity is a measure of the general positive or negative trend of the prognostic parameter. Damage is generally assumed to be cumulative and irreversible. Because the prognostic parameter can be considered an indicator of system health, if the parameter were not monotonic, it would indicate some measure of self-healing is taking place. This assumption is not valid for some specific components, such as batteries which do experience some increased capacity after a period of rest. However, it is usually considered valid when dealing with an entire system. Prognosability characterizes how well clustered the failure values are for a population of systems. Because a critical failure threshold must be identified for the GPM model, failure values should be well clustered in order to give a crisp failure value and reduce RUL uncertainty. Finally, trendability measures how well each parameter for a population of systems can be described by the same underlying function. The GPM depends on fitting a parametric model to the prognostic parameter and extrapolating it to failure, so it is key that the same parametric function be applicable to the entire population of systems with the same type of failure mechanism.

By formalizing these metrics so that the suitability of a candidate prognostic parameter can be quantified, it is straightforward to apply conventional optimization methods to identify an optimal, or near-optimal, prognostic parameter from many possible data sources including sensed data, monitoring system residuals, environmental and operational conditions, fault detection and diagnostic results, etc. This research 
applied Genetic Algorithm (GA) optimization to identify appropriate prognostic parameters for two very different data sets: the turbofan engine simulation data given in the PHM '08 prognostic challenge and milling data, both available at the NASA Prognostic Data Repository. GA runtime scales exponentially with the number of parameters to optimize; an input selection method was presented to alleviate this computational burden. Inputs which have total suitability less than some threshold, generally 1.5, are removed from the candidate parameter inputs. Application to the PHM challenge data set showed that the GA of the full set of possible inputs and the reduced set resulted in prognostic parameters of comparable performance, but with significantly reduced optimization runtime. In addition, the examples investigating the effect of noise on parameter suitability and performance and the milling data set indicate that parameter suitability is positively correlated with model performance; that is, parameters with higher suitability metrics result in greater model performance on the measure of mean absolute percent error of the failure time.

The PHM challenge data application utilized the results of a condition monitoring and fault detection system to characterize the degradation in a given system. Prognostic parameters were generated from a subset of the monitoring system residuals; monitoring system residuals are natural components of a prognostic parameter because they inherently measure the deviation of a system from normal operation. Three prognostic parameters were identified for the PHM '08 challenge data set: one through visual inspection and two using the proposed optimization method. The three parameters had comparable suitability metrics and resulted in comparable model performance on the test runs. The parameter identified through visual inspection involved several weeks of expert analysis to form the final parameter. Conversely, the first GA-optimized parameter, which included 11 monitoring system residuals as possible inputs, took a fraction of a man-hour to set up and 1.68 hours of unsupervised computer runtime. The second GA-optimized parameter considered a subset of the eleven residuals, using only five of the possible inputs. This optimization ran in 0.93 hours, nearly half the time of the full optimization. This example application suggests that the proposed prognostic parameter selection method performs comparably to expert analysis in a fraction of the 
time. This application, however, benefited from extensive analysis given to the system before the optimization method was developed.

The final example application utilized milling data available at the NASA repository. Several prognostic parameters were quickly identified for this data set. The first parameter optimization lumped all fifteen available runs together to develop one parameter for the entire population. In addition, two separate parameters were developed for the two materials being bored: cast iron and steel. By applying the automated method, it is simple to develop many competing prognostic models to determine if additional factors, such as operating conditions or system set-up, should be considered in model development.

This research presented the development of the GPM/Bayes prognostic model and an automated method for identifying appropriate prognostic parameters from many available data sources. The efficacy of both methodologies was illustrated. Additionally, the development of a MATLAB-based Process and Equipment Prognostics (PEP) toolbox was discussed. The original methods developed for this dissertation, as well as other prognostic algorithms, are available in the PEP toolbox to aid in rapid model prototyping and full health monitoring in conjunction with the Process and Equipment Monitoring (PEM) toolbox. 


\section{REFERENCES}

Abernethy, R. B., The New Weibull Handbook, 2nd edn. ISBN 096530620 8. Abernethy, North Palm Beach, 1996.

Agogino, A. and K. Goebel (2007). "Mill Data Set," BEST lab, UC Berkeley. NASA Ames Prognostics Data Repository, [http://ti.arc.nasa.gov/project/prognostic-data-repository], NASA Ames, Moffett Field, CA.

Aho, A.V., J.D. Ullman, and J.E. Hopcroft, Data Structures and Algorithms. Addison Wesley, 1983.

Azzam, H., "A Practical Approach for the Indirect Prediction of Structural Fatigue from Measured Flight Parameters," Journal of Aerospace Engineering 211 (G) 1997: 29 - 38.

Banks, J. and J. Merenich, "Cost Benefit Analysis for Asset Health Management Technology," Proceedings of the IEEE Reliability and Maintainability Symposium (RAMS), 2007: 95 100.

Baruah, P., R.B. Chinnam, and D. Filev, "An Autonomous Diagnostics and Prognostics Framework for Condition-Based Maintenance," 2006 International Joint Conference on Neural Networks, Vancouver, BC, Canada: July 16 - 21, 2006.

Baybutt, M., C. Minnella, A. Ginart, P.W. Kalgren, and M.J. Roemer, "Improving Digital System Diagnostics Through Prognostic and Health Management (PHM) Technology," IEEE Transactions on Instrumentation and Measurement 58 (2), 2009: 255 - 262.

Bogdanoff, J.L., and F. Kozin, Probabilistic Models of Cumulative Damage, John Wiley and Sons, New York: 1985.

Byington, C.S., M. Watson, D. Edwards, P. Stoelting, “A Model-Based Approach to Prognostics and Health Management for Flight Control Actuators," Proceedings of the IEEE Aerospace Conference, 2004: 3551 - 3562.

Byington, C.S., M.J. Roemer, P.W. Kalgren, "Verification and Validation of Diagnostic/Prognostic Algorithms," Machinery Failure Prevention Technology (MFPT) Conference, 2005: Virginia Beach, VA.

Callan, R., B. Larder, and J. Sandiford, "An Integrated Approach to the Development of an Intelligent Prognostic Health Management System," Proceedings of the IEEE Aerospace Conference, 2006: 12.

Carden, E.P. and P. Fanning, "Vibration Based Condition Monitoring: A Review," Structural Health Monitoring 3 (4) 2004: 355 - 377.

Carey, M.B., and R.H. Koenig, "Reliability Assessment Based on Accelerated Degradation: A Case Study," IEEE Transactions on Reliability 40 (5) 1991: 499 - 506. 
Carlin, B.P and T.A. Louis, Bayes and Empirical Bayes Methods for Data Analysis, 2nd ed. Boca Raton: Chapman and Hall/CRC: 2000.

Catbas, F.N. and A.E. Atkan, "Condition and Damage Assessment: Issues and Some Promising Indices,” Journal of Structural Engineering 128 (8) 2002: 1026 - 1036.

Chen, Z. and S. Zheng, "Lifetime Distribution Based Degradation Analysis," IEEE Transactions on Reliability, 54 (1), March 2005, 3 - 10.

Choi, S. and C.J. Li, "Estimation of Gear Tooth Transverse Crack Size from Vibration by Fusing Selected Gear Condition Indices," Measurement Science and Technology 17 2006: 2395 2400.

Coble, J. and J.W. Hines, "Fusing Data Sources for Optimal Prognostic Parameter Selection." Sixth American Nuclear Society International Topical Meeting on Nuclear Plant Instrumentation, Control, and Human-Machine Interface Technologies NPIC\&HMIT 2009, Knoxville, Tennessee, April 5-9, 2009.

Condition Based Maintenance Plus (2005). Online: http://www.acq.osd.mil/log/mppr/CBM\%2B.htm

Cox, D.R. and D. Oakes, Analysis of Survival Data, Chapman and Hall: 1984.

Dale, C.J., "Application of the Proportional Hazards Model in the Reliability Field," Reliability Engineering 10, 1985:1 - 14.

Doebling, S.W., C.R. Farrar, M.B. Prime, and D.W. Shevitz, "Damage Identification and Health Monitoring of Structural and Mechanical Systems from Changes in their Vibration Characterisstics: A Literature Review," Technical Report LA-13070-MS, Los Alamos National Lab.

Elsayed, E.A., and A.C-K. Chen, "Recent Research and Current Issues in Accelerated Testing," Proceedings of the IEEE Conference on Systems, Man, and Cybernetics, 1998: 4704 - 4709.

Esary, J.D. and A.W. Marshall, "Shock Models and Wear Processes," The Annals of Probability 1 (4) 1973: $627-649$.

Feng, W.C., "Making a Case for Efficient Super Computing," Queue Oct 2003: pp 54 - 64.

Ferrell, B.L., "JSF Prognostics and Health Management," Proceedings of the IEEE Aerospace Conference, 1999: 471.

Ferrell, B.L., "Air Vehicle Prognostics and Health Management," Proceedings of the IEEE Aerospace Conference, 2000: 145 - 146.

Engel, S., B. Gilmartin, K. Bongort, and A. Hess, "Prognostics, the Real Issues Involved with Predicting Life Remaining," Proceedings of the IEEE Aerospace Conference, pp. 457-469, 2000.

Garvey, D. and J.W. Hines, "Dynamic Prognoser Architecture via the Path Classification and Estimation (PACE) Model," AAAI Fall Symposium on Artificial Intelligence for Prognostics, Nov 9-11, 2007, Arlington, VA. 
Garvey, Dustin R. (2007), “An Integrated Fuzzy Inference Based Monitoring, Diagnostic, and Prognostic System," Ph.D. Dissertation, Nuclear Engineering Department, University of Tennessee, Knoxville: 2007.

Gelman, A., J. Carlin, H. Stern, and D. Rubin, Bayesian Data Analysis 2nd ed. Boca Raton: Chapman and Hall/CRC: 2004.

Girish, T., S.W. Lam, and S.R. Jayaram, "Reliability Prediction Using Degradation Data - A Preliminary Study Using Neural Network-based Approach," European Safety and Reliability Conference, Jun 15-18, 2003: Maastricht, The Netherlands.

Goebel, K. and P. Bonissonne, "Prognostic Information Fusion for Constant Load Systems," 7th International Conference on Information Fusion, 2004: Stockholm, Sweden.

Goodman, D.L, S. Wood, and A. Turner, "Return-on-Investment (ROI) for Electronic Prognostics in MIL/AERO Systems," Proceedings of the IEEE Systems Readiness Technology Conference (AUTOTESTCON), 2005: 73 - 75.

Goodman, D., "Prognostic Techniques for Semiconductor Failure Modes," Ridgetop Group, Inc. white paper, 2000: available at www.Ridgetop-Group.com.

Goodman, D., B. Vermeire, P. Spuhler, and H. Venkatramani, "Practical Application of PHM/Prognostics to COTS Power Converters," Proceedings of the IEEE Aerospace Conference, 2005: 3573 - 3578 .

Goodman, D., B. Vermeire, J. Ralston-Good, and R. Graves, "A Board-Level Prognostic Monitor for MOSFET TDDB," IEEE Aerospace Conference, 2006: Big Sky, MT.

Greitzer, F.L., E.J. Stahlman, T.A. Ferryman, B.W. Wilson, L.J. Kangas, and D.R. Sisk, "Development of a Framework for Predicting Life of Mechanical Systems: Life Extension Analysis and Prognostics (LEAP)," International Society of Logistics (SOLE) Symposium, Las Vegas, Aug 30 - Sept 2, 1999.

Greitzer, F.L., and T.A. Ferryman, "Predicting Remaining Life of Mechanical Systems," Intelligent Ship Symposium IV, April 2 - 3, 2001.

Greitzer, F.L., "Life Extension Analysis and Prognostics (LEAP) Architectures," FY 2001 Laboratory Directed Research and Development Annual Report PNNL: 312 - 316.

Grist, Edward, Cavitation and the Centrifugal Pump: A Guide for Pump Users, Taylor \& Francis: Philadelphia, PA. (1999).

Gu, J., N. Vichare, T. Tracy, and M. Pecht, "Prognostics Implementation Method for Electronics," Proceedings of the Reliability and Maintainability Symposium (RAMS), 2007: $101-106$.

Guess, F. and F. Proschan, "Mean Residual Life: Theory and Applications," FSU Statistics Report M702. AFOSR Technical Report No. 85-178, June 1985.

Gut, A., "Cumulative Shock Models," Advances in Applied Probability 22 (2) 1990: 504 - 507. 
Han, D.K., M. G. Pecht, D.K. Anand, and R. Kavetsky, "Energetic Material/Systems Prognostics," $53^{\text {rd }}$ Annual Reliability and Maintainability Symposium (RAMS), Orlando, FL, Jan 22-25, 2007.

He, D., S. Wu, P. Banerjee, and E. Bechhoefer, "Probabilistic Model Based Algorithms for Prognostics,” IEEE Aerospace Conference, 2006: Big Sky, MT.

Heng, A., S. Zhang, A. Tan, and J. Mathew, "Rotating Machinery Prognostics: State of the Art, Challenges, and Opportunities," Mechanical Systems and Signal Processing 23 2009: 724 739.

Heo, G.Y., "Condition Monitoring Using Empirical Models: Technical Review and Prospects for Nuclear Applications," Nuclear Engineering and Technology 40 (1) 2008: 49 - 68.

Hess, A. and L. Fila, "The Joint Strike Fighter (JSF) PHM Concept: Potential Impact on Aging Aircraft Problems," Proceedings of the IEEE Aerospace Conference, 2002: 3021 - 3026.

Hess, A., G. Calvello, and P. Frith, "Challenges, Issues, and Lessons Learned Chasing the 'Big P': Real Predictive Prognostics Part 1," Proceedings of the IEEE Aerospace Conference, pp. 3610 - 3619, March 2005.

Hines, J.W., R. Seibert, S.A. Arndt, "Technical Review of On-Line Monitoring Techniques for Performance Assessment (NUREG/CR-6895) Vol. 1, State-of-the-Art." Published January, 2006.

Hines, J.W., A. Usynin, and A. Urmanov, "Prognosis of Remaining Useful Life for Complex Engineering Systems," American Nuclear Society International Topical Meeting on Nuclear Plant Instrumentation, Control, and Human-Machine Interface Technologies (NPIC\&HMIT), 2006: Albuquerue, NM.

Hines, J.W., J. Garvey, J. Preston, and A. Usynin, "Empirical Methods for Process and Equipment Prognostics," Tutorial presented at the IEEE Reliability and Maintainability Symposium (RAMS), 2007.

Hines, J.W. and A. Usynin, "Current Computational Trends in Equipment Prognostics," International Journal of Computational Intelligence Systems 1 (1) 2008: 1 - 9.

Hines, J.W., D. Garvey, R. Seibert, A. Usynin, and S.A. Arndt, "Technical Review of On-Line Monitoring Techniques for Performance Assessment (NUREG/CR-6895) Vol. 2, Theoretical Issues," Published May, 2008.

Ho, Y.-C., and D. L. Pepyne, "Simple Explanation of the No Free Lunch Theorem of Optimization”, Cybernetics and Systems Analysis, 38 (2), 2002, pp 292 - 298.

Hofmeister, J.P., P. Lall, R. Graves, "In-Situ, Real-Time Detector for Faults in Solder Joint Networks Belonging to Operational, Fully Programmed Field Programmable Gate Arrays (FPGAs)," Proceedings of the IEEE AUTOTESTCON, 2006: 237 - 243.

Hofmeister, J.P., J. Judkins, E. Oritz, D. Goodman, and P. Lall, "Real-time BIST Detector for BGA Faults in Field Programmable Gate Arrays (FPGAs)," Ridgetop Group, Inc. white paper, 2006: available at www.Ridgetop-Group.com. 
Horst, R. and T. Hoang, Global Optimization: Deterministic Approaches Springer: New York: 1996.

"Hot Carrier (HC) Prognostic Cell," Ridgetop Semiconductor-Sentinel Silicon Library, Aug 2004.

Hughes, G.F., J.F. Murray, K. Kreutz-Delgado, and C. Elkan, "Improved Disk Drive Failure Warnings," IEEE Transactions on Reliablity 51 (3) 2002: 350 - 357.

Janasak, K.M., and R.R. Beshears, "Diagnostics to Prognostics - A Product Availability Technology Evolution," Proceedings of the Reliability and Maintainability Symposium (RAMS), 2007: 113 - 118.

Jardine, A.K.S., D. Lin, and D. Banjevic, "A Review on Machinery Diagnostics and Prognostics Implementing Condition-Based Maintenance," Mechanical Systems and Signal Processing 20 2006: $1483-1510$.

Kacprzynski, G.J., M.J. Roemer, G. Modgil, A. Palladino, K. Maynard, "Enhancement of Physics-of-Failure Prognostic Models with System Level Features," IEEE Aerospace Conference, 2002: Big Sky, MT.

Kacprzynski, G.J., A. Liberson, A. Palladino, M.J. Roemer, A.J. Hess, and M. Begin, "Metrics and Development Tools for Prognostic Algorithms," Proceedings of the IEEE Aerospace Conference, 2004: 3809 - 3815.

Kacprzynski, G.J., A. Sarlashkar, M.J. Roemer, A. Hess, and W. Hardman, "Predicting Remaining Life by Fusing the Physics of Failure Modeling with Diagnostis," Journal of the Mineral, Metals, and Materials Society 56 (3) 2004: 29 - 35.

Kalgren, P.W., M. Baybutt, A. Ginart, C. Minnella, M.J. Roemer, and T. Dabney, "Application of Prognostic Health Management in Digital Electronic Systems," Proceedings of the 2007 IEEE Aerospace Conference, March, 2007: 1 - 9.

Kelkar, N., A. Dasgupta, M. Pecht, I.Knowles, M. Hawley, and D. Jennings, "SMART" Electronic Systems for Condition-Based Health Management," Proceedings of 9th International Congress and Exhibition on Condition Monitoring and Diagnostic Engineering Management (COMADEM), 1996: 591 - 602.

Keller, K., K. Swearingen, J. Sheahan, M. Baily, J. Dunsdon, B. Cleeve, K.W. Przytula, and B. Jordan, "Aircraft Electrical Power Systems Prognostics and Health Management," IEEE Aerospace Conference, 2006: Big Sky, MT.

Kharoufeh, J.P. and S.M. Cox, "Stochastic Models for Degradation-based Reliability," IIE Transactions (2005) 37, 533 - 542.

Klinger, D.J., "Failure Time and Rate Constant of Degradation: An Argument for the Inverse Relationship," Microelectronic Reliability 32 (7) 1992: 987 - 994.

Koppen, M., "No-Free-Lunch Theorems and the Diversity of Algorithms", Congress on Evolutionary Computation, 1, 2004, pp 235 - 241. 
Kothamasu, R., S.H. Huang, and W.H. VerDuin, "System Health Monitoring and Prognostics A Review of Current Paradigms and Practices," International Journal of Advanced Manufacturing Technology 28 2006: 1012 - 1024.

Kutner, M.H., C.J. Nachtsheim, and J. Neter, Applied Linear Regression Models, McGrawHill/Irwin, New York, New York: 2004 (4 ed).

Kumar, D. and B. Klefjo, "Proportional Hazards Model: A Review," Reliability Engineering and System Safety 44, 1994: 177 - 188.

Kwan, C., X. Zhang, R. Xu, and L. Haynes, "A Novel Approach to Fault Diagnostics and Prognostics," Proceedings of the IEEE International Conference on Robotics \& Automation, 2005: $604-609$.

Lall, P., M. Pecht, and E. Harkim, Influence of Temperature on Microelectronics and System Reliability, New York: CRC Press, 1997.

Leao, B. P., T. Yoneyama, G. C. Rocha, and K.T. Fitzgibbon, "Prognostic Performance Metrics and their Relation to Requirements, Design, Verification, and Cost-Benefit." International Conference on Prognostics and Health Management, 2008: Denver, CO.

Lemoine, A.J., and M.L. Wenocor, “On Failure Modeling,” Navel Research Logistics Quarterly 32 1985: $497-508$.

Liao, H., W. Zhao, and H. Guo, "Predicting Remaining Useful Life of an Individual Unit Using Proportional Hazards Model and Logistic Regression Model." Proceedings of the Reliability and Maintainability Symposium (RAMS), 2006: 127 - 132.

Lindely, D.V. and A.F. Smith, "Bayes Estimates for Linear Models," Journal of the Royal Statistical Society (B) 34 (1) 1972: 1 - 41.

Line, J.K. and N.S. Clements, "A Systematic Approach for Developing Prognostic Algorithms on Large Complex Systems," Proceedings of the IEEE Aerospace Conference, 2005: 1 - 7.

Line, J.K., and N.S. Clements, "Prognostics Usefulness Criteria," Proceedings of the IEEE Aerospace Conference, 2006: 7.

Luo, J., M. Namburu, K. Pattipati, L. Qiao, M. Kawamoto, S. Chigusa, "Model-based Prognostic Techniques," IEEE Systems Readiness Technology Conference (AUTOTESTCON), Sep 2225 2003: Anaheim, CA

Mallor, F. and J. Santos, "Classification of Shock Models in System Reliability," Monograf' 1as del Semin. Matem. Garc'1a de Galdeano. 27 2003: 405-412.

Meeker, W.Q., L.A. Escobar, and C.J. Lu, "Accelerated degradation tests: modeling and analysis," Technometrics, vol. 40, no. 2, pp. 89-99, 1998.

Meeker, W.Q., and M. Hamada, "Statistical Tools for the Rapid Development and Evaluation of High-Reliability Products," IEEE Transactions on Reliability 44 (2) 1995: 187 - 198.

Mishra, S. and M. Pecht, "In-situ Sensors for Product Reliability Monitoring," Proceedings of SPIE, vol 4755 (2002) pp 10-19. 
Mishra, S., S. Ganesan, M. Pecht, and J. Xie, "Life Consumption Monitoring for Electronics Prognostics," Proceedings of the IEEE Aerospace Conference, 2004: 3455 - 3467.

Oja, M., J.K. Line, G. Krishnan, R.G. Tryon, "Electronic Prognostics with Analytical Models using Existing Measurands," $61^{\text {st }}$ Conference of the Society for Machinery Failure Prevention Technology (MFPT), April 17 - 19, 2007: Virginia Beach, VA.

Orchard, M., and Vachtsevanos, G., "A particle filtering approach for on-line failure prognosis in a planetary carrier plate," International Journal of Fuzzy Logic and Intelligent Systems, vol. 7, no. 4, pp. 221-227, 2007.

Orsagh, R., D. Brown, M. Roemer, T. Dabnev, and A. Hess, "Prognostic Health Management for Avionics System Power Supplies," Proceedings of the IEEE Aerospace Conference, pp.3585-3591, 5-12 March 2005: Big Sky, MT.

Orsagh, R.F., D.W. Brown, P.W. Kalgren, C.S. Byington, A.J. Hess, and T. Dabney, "Prognostic Health Management for Avionic Systems," IEEE Aerospace Conference, 2006: Big Sky, MT.

Park, C. and W.J. Padgett, "Stochastic Degradation Models with Several Accelerating Variables," IEEE Transactions on Reliability 55 (2) 2006: 379 - 390.

Pecht, M. and A. Dasgupta, "Physics of Failure: An Approach to Reliable Product Development," Journal of the Institute of Environmental Sciences 38 (5) 1995: 30 - 34.

Pecht, M., M. Dube, M. Natishan, R. Williams, J. Banner, and I. Knowles, "Evaluation of Builtin Test," IEEE Transactions on Aerospace and Electronic Systems 37 (1) 2001: 266 - 271.

Pecht, M., D. Das, and A. Ramakrishnan, "The IEEE Standards on Reliability Program and Reliability Prediction Methods for Electronic Equipment," Microelectronic Reliability, 42 $1259-1266,2002$.

Piezo Systems, Inc., Cambridge, Massachusetts, Introduction to Piezo Transducers, (2001). www.piezo.com

Pipe, K., "Practical Prognostics for Condition Based Maintenance," 2008 International Conference on Prognostics and Health Management, Oct 6 - 9, 2008: Denver, CO.

Ramakrishnan, A. and M.G. Pecht, "A Life Consumption Monitoring Methodology for Electronic Systems," IEEE Transactions on Components and Packaging Technologies 26 (3) 2003: $625-634$.

Robinson, M.E. and M.T. Crowder, "Bayesian Methods for a Growth-Curve Degradation Model with Repeated Measures," Lifetime Data Analysis 6, 2000: 357 - 374.

Roemer, M.J., G.J. Kacprzyniski, and M.H. Schoeller, "Improved Diagnostic and Prognostic Assessments using Health Management Information Fusion," IEEE Systems Readiness Technology Conference (AUTOTESTCON), 2001: 365 - 377.

Roemer, M.J., J. Dzakowic, R.F. Orsagh, C.S. Byington, G. Vachtsevanos, "Validation and Verification of Prognostic and Health Management Technologies," Proceedings of the IEEE Aerospace Conference, 2005: 3941 - 3947. 
Saxena, A., K. Goebel, D. Simon, N. Eklund, "Prognostics Challenge Competition Summary: Damage Propagation Modeling for Aircraft Engine Run-to-Failure Simulation," International Conference on Prognostics and Health Management, 2008: Denver, CO.

Saxena, A. and K. Goebel (2008). "C-MAPSS Data Set," NASA Ames Prognostics Data Repository, [http://ti.arc.nasa.gov/project/prognostic-data-repository], NASA Ames, Moffett Field, CA.

Saxena, A., J. Selaya, E. Balaban, K. Goebel, B. Saha, S. Saha, and M. Schwabacher, "Metrics for Evaluating Performance of Prognositc Techniques." International Conference on Prognostics and Health Management, 2008: Denver, CO.

Saxena, A., J. Celaya, B. Saha, S. Saha, and K. Goebel, "Evaluating Algorithm Performance Metrics Tailored for Prognostics," Proceedings of the IEEE Aerospace Conference, 2009: 1 13.

Sharp, M. and J.W. Hines, "Analysis of Prognostic Opportunities in Power Industry with Demonstration" Sixth American Nuclear Society International Topical Meeting on Nuclear Plant Instrumentation, Control, and Human-Machine Interface Technologies NPIC\&HMIT 2009, Knoxville, Tennessee, April 5-9, 2009.

Sheppard, J.W., M.A. Kaufman, and T.J. Wilmering, "IEEE Standards for Prognostics and Health Management," 2008 IEEE Autotestcon, Salt Lake City, UT: Sept 8- 11, 2008.

Smith, G., J.B. Schroeder, S. Navarro, and D. Haldeman, "Development of a Prognostics and Health Management Capability for the Joint Strike Fighter," Proceedings of AUTOTESTCON, 1997: 676 - 682.

Tamhane, A.C. and D.D. Dunlop, Statistics and Data Analysis from Elementary to Intermediate, Prentice-Hall, Inc, Upper Saddle River, New Jersey: 2000.

Tang, L.C., and D.S. Chang, "Reliability Prediction Using Nondestructive AcceleratedDegradation Data: Case Study on Power Supplies," IEEE Transactions on Reliability 44 (4) 1995: $562-566$.

Toms, L.A., Machinery Oil Analysis - Methods, Automation \& Benefits, 1st Edition, Larry A Toms Technical Services, Pensacola, Florida, 1994.

Union of Concerned Scientists, Davis-Besse Outage Report: <http://www.ucsusa.org/assets/documents/nuclear_power/davis-besse-ii.pdf>, accessed Jan 2010.

Upadhyaya, B, J.W. Hines, B. Lu, and X. Huang, "On-Line Monitoring and Diagnostics of the Integrity of Nuclear Plant Steam Generators and Heat Exchangers," Phase III NEER Annual Report, Report No. DE-FG07-01ID14114/UTNE-06, NEER Grant Number: DE-FG0701ID14114.

Urmanov, A. and J.W. Hines, "Electronic Prognostics Through Continuous System Telemetry and Empirical Models," $53^{\text {rd }}$ Annual Reliability and Maintainability Symposium (RAMS), Orlando, FL, Jan 22-25, 2007. 
Usynin, A., J.W. Hines, and A. Urmanov, "Uncertain Failure Thresholds in Cumulative Damage Models, "Proceedings of the IEEE Reliability and Maintainability Symposium (RAMS), 2008: 334 - 340.

Vachtsevanos, G., Kim, W., Al-Hasan, S., Rufus, F., Simon, M., Schrage, D. and Prasad, J.V.R., "Mission Planning and Flight Control: Meeting the Challenge with Intelligent

Techniques," Journal of Advanced Computational Intelligence, Vol. 1, No. 1, pp. 62-70, October 1997.

Vachtsevanos, G. "Performance Metrics for Fault Prognosis of Complex Systems." Proceedings of the IEEE Systems Readiness Technology Conference (AUTOTESTCON), 2003: 341 - 345.

Valentin, R., M. Osterman, B. Newman, "Remaining Life Assessment of Aging Electronics in Avionic Applications," Proceedings of the Annual Reliability and Maintainability Symposium (RAMS), 2003: $313-318$.

Vichare, N., P. Rodgers, V. Eveloy, and M. Pecht, "In Situ Temperature Measurement of a Notebook Computer - A Case Study of Health and Usage Monitoring of Electronics," IEEE Transactions on Device and Materials Reliability 4 (4), December 2004: 658 - 663.

Vichare, N., and M. Pecht, "Prognostics and Health Management of Electronics," IEEE Transactions on Components and Packaging Technologies, 29 (1), 2006, pp 222 - 229.

Volkov, V. and J.W. Demmel, "Benchmarking GPUs to Tune Dense Linear Algebra," Proceedings of the ACM/IEEE Conference on Supercomputing, 2008: 1-11.

Wald, A. "Sequential Tests of Statistical Hypotheses." Annals of Mathematical Statistics 16 (2): 117-186.

Wang, P. and Vachtsevanos, G. "Fault Prognostics Using Dynamic Wavelet Neural Networks", Journal of Artificial Intelligence for Engineering Design, Analysis and Manufacturing, vol.15, pp. 349-365, 2001.

Wang, P. and D.W. Coit, "Reliability Prediction based on Degradation Modeling for Systems with Multiple Degradation Measures," Proceedings of the Reliability and Maintainability Symposium (RAMS), 2004: $302-307$.

Wang, P. and D. Coit, "Reliability and Degradation Modeling with Random or Uncertain Failure Threshold," Proceedings of the IEEE Reliability and Maintainability Symposium (RAMS), 2007: 392 - 397.

Wood, S. and D. L. Goodman, "Return-on-Investment (ROI) for Electronic Prognostics in High Reliability Telecom Applications." Proceedings of the $28^{\text {th }}$ Annual International Telecommunications Energy Conference (INTELEC), 2006: 1 - 3 .

Xu, D. and W. Zhao, "Reliability Prediction using Multivariate Degradation Data," Proceedings of the Annual Reliability and Maintainability Symposium (RAMS), 2005: 337 - 341.

Yang, K. and J. Xue, "Continuous State Reliability Analysis," Proceedings of the Annual Reliability and Maintainability Symposium (RAMS), 1996: 251 - 257. 
APPENDIX A: Additional Figures 


\section{A.1 Noisy Prognostic Parameters}
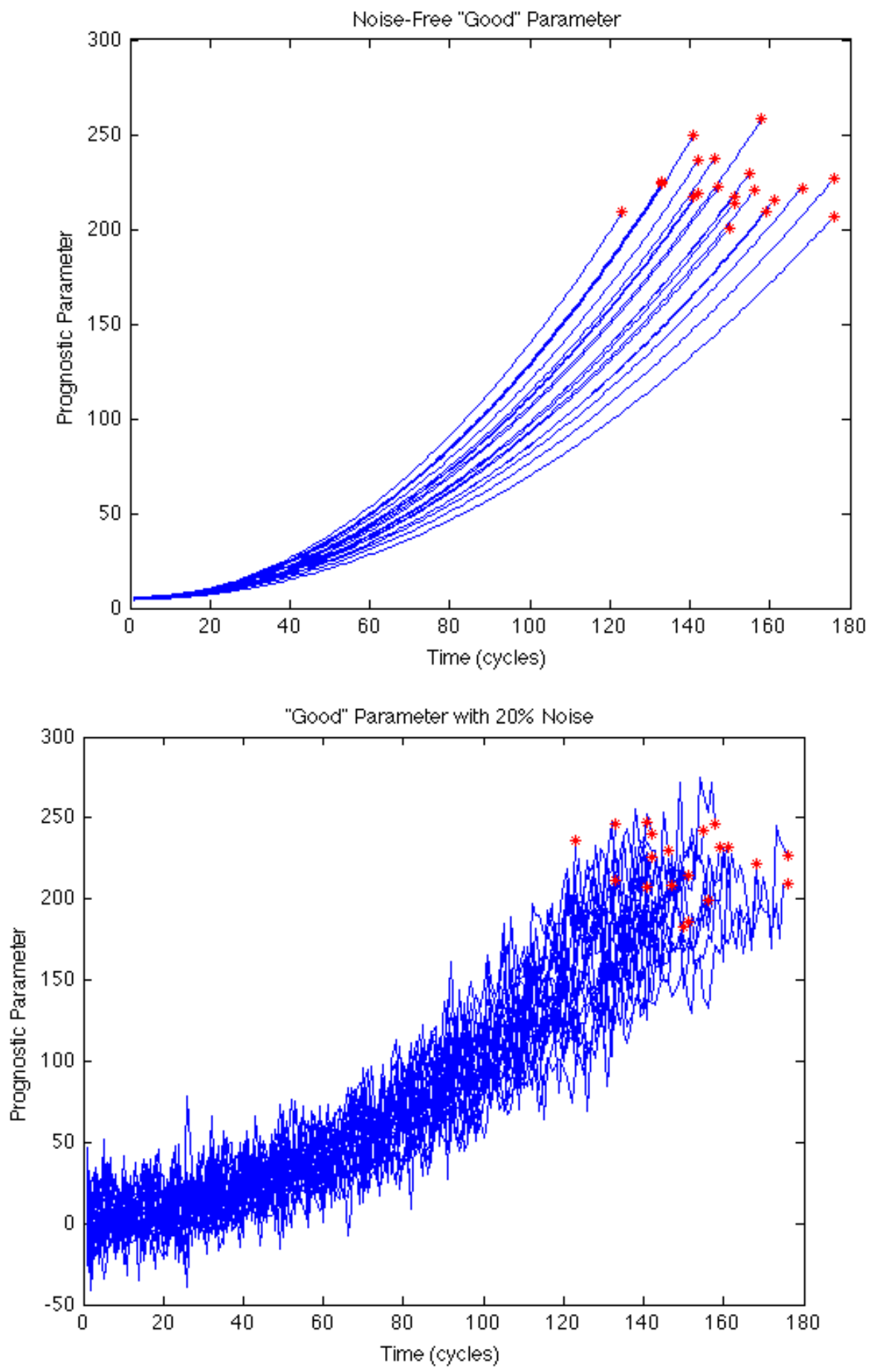

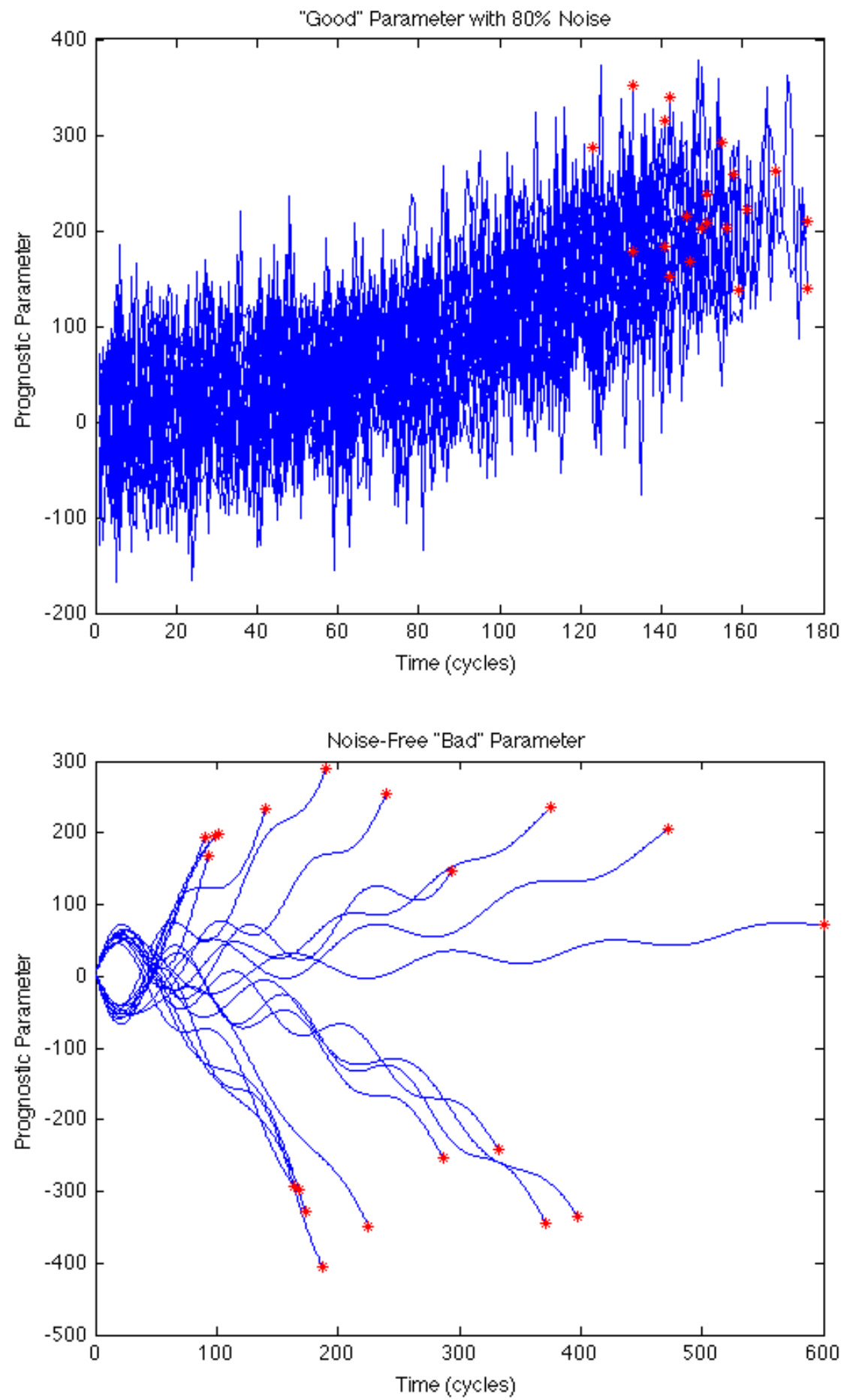

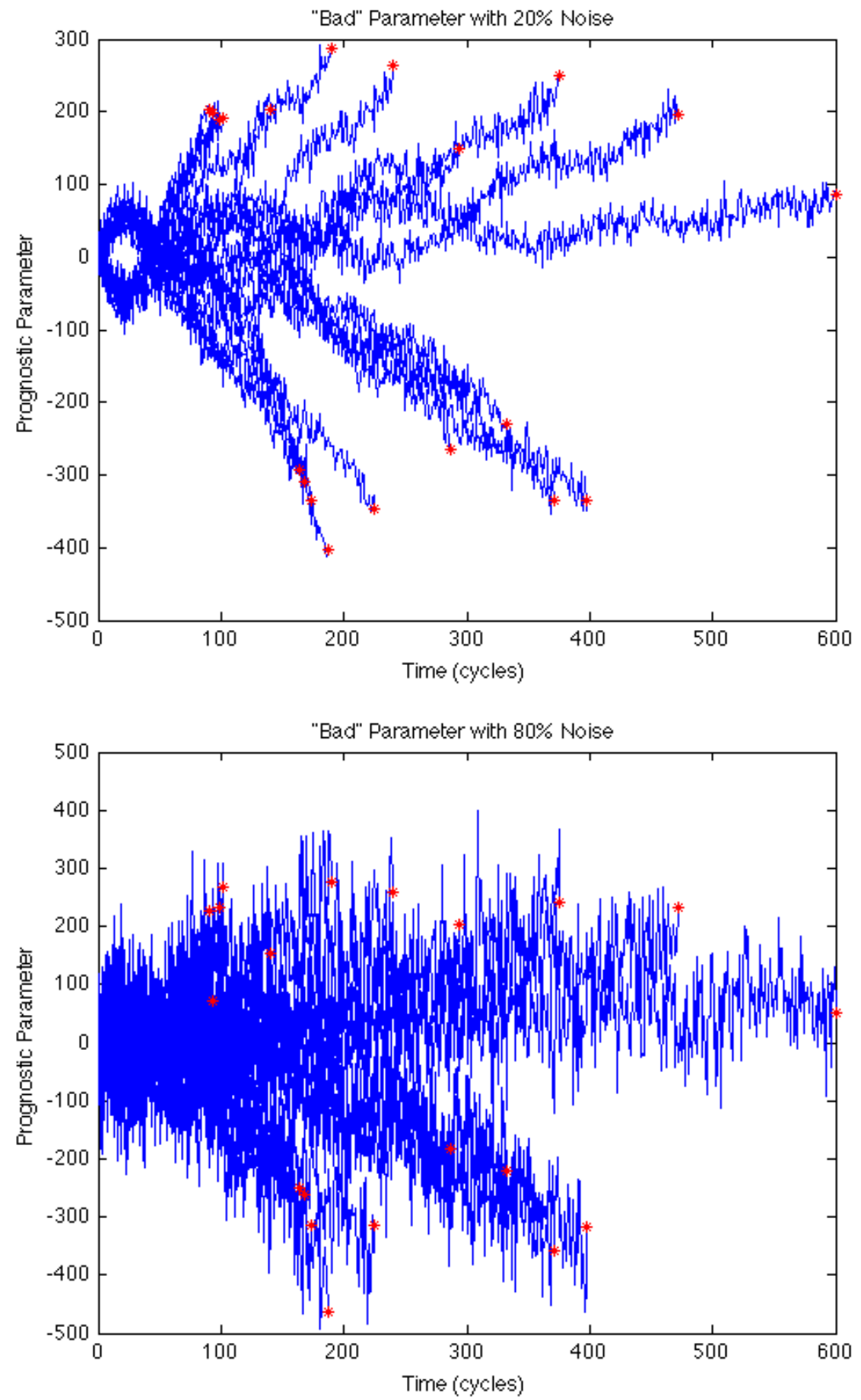


\section{A.2 The Effect of Noise on Model Performance}

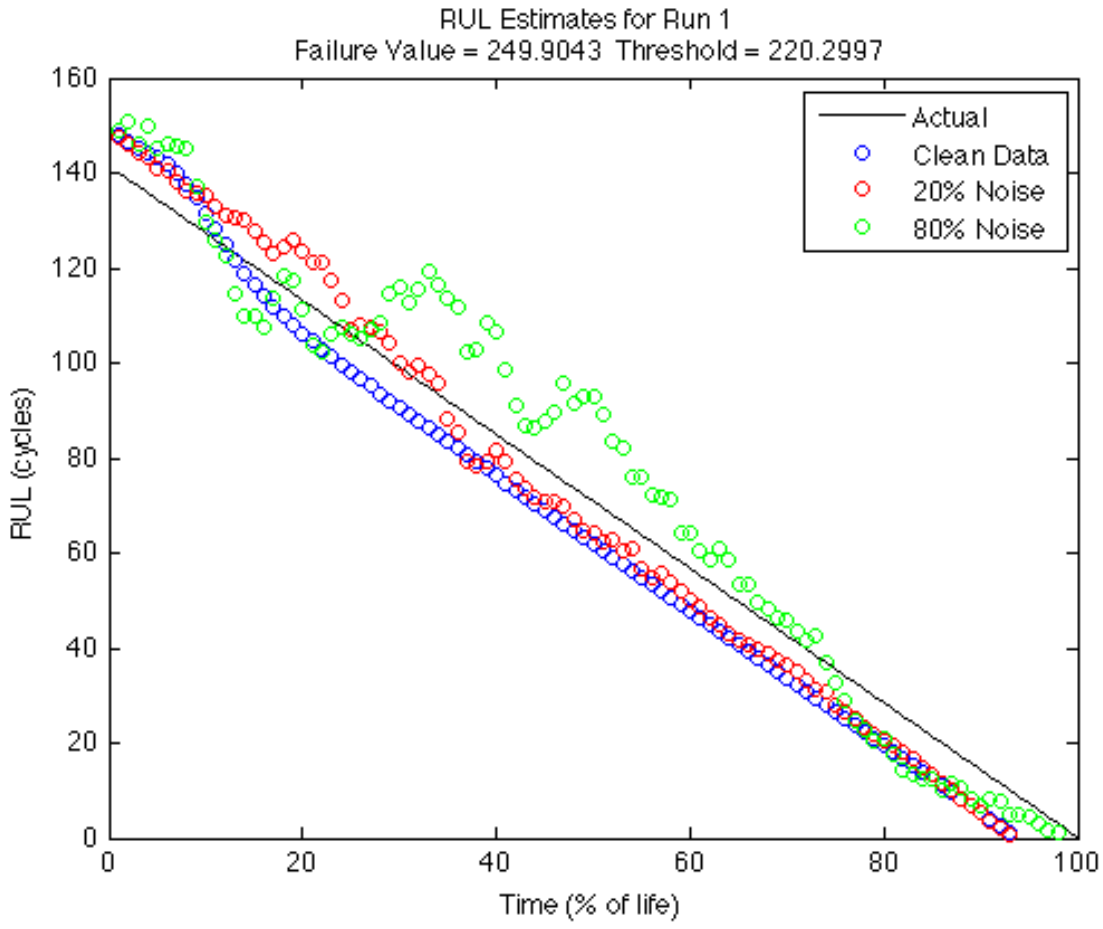

RUL Estimates for Run 2

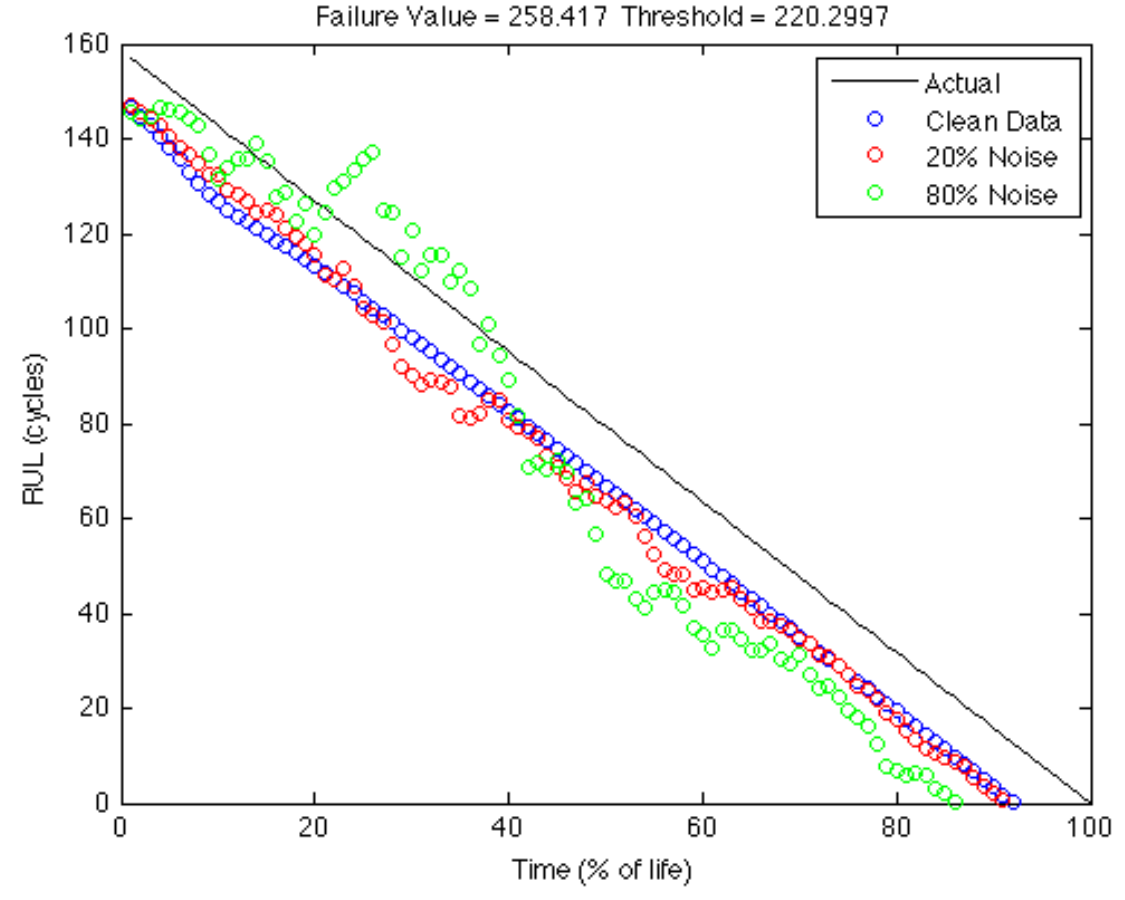


RUL Estimates for Run 3

Failure Value $=220.2997$ Threshold $=221.8681$

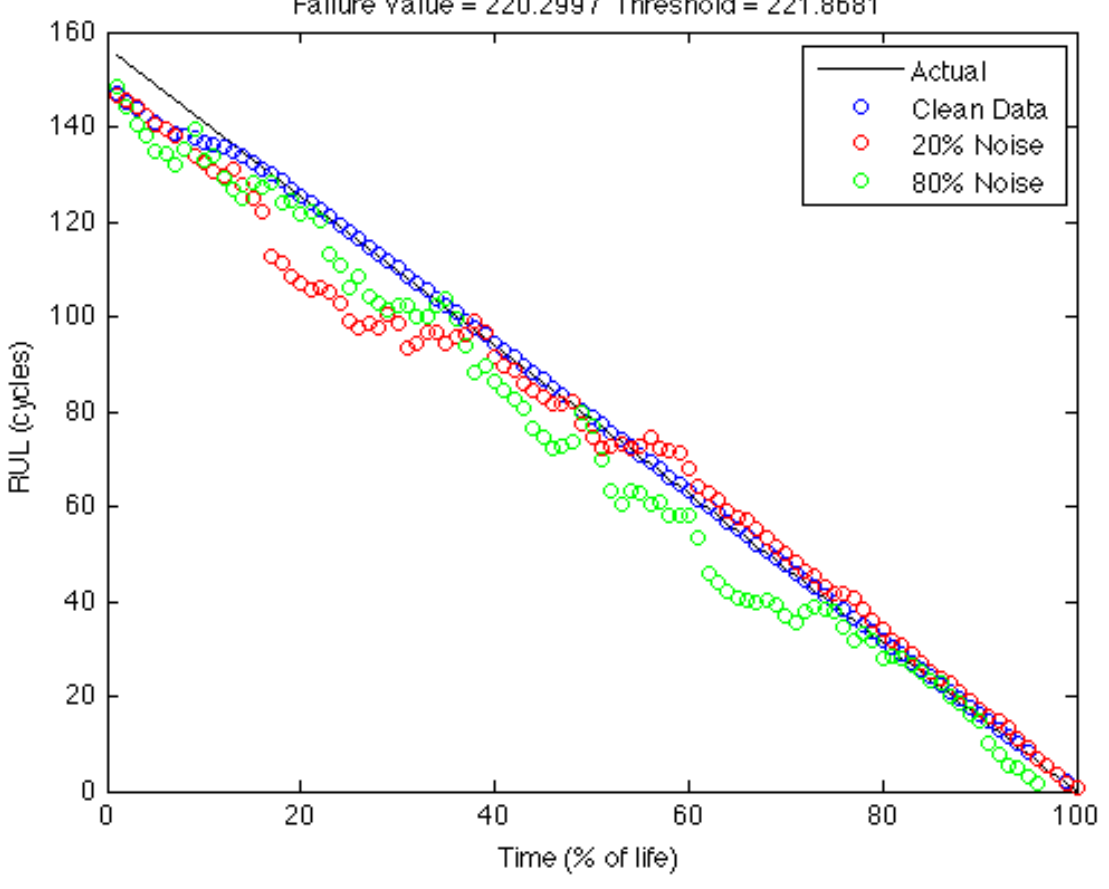

RUL Estimates for Run 4

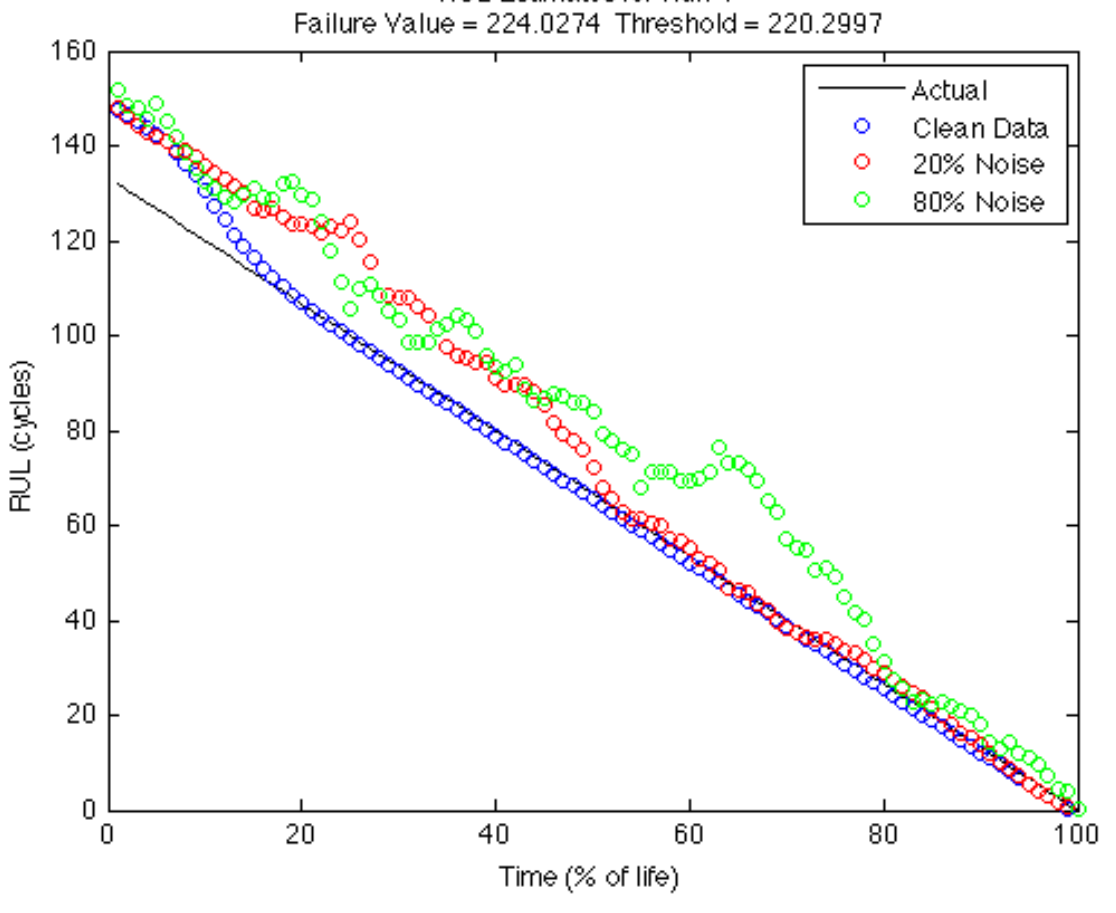


RUL Estimates for Run 5

Failure Value $=236.3859$ Threshold $=220.2997$

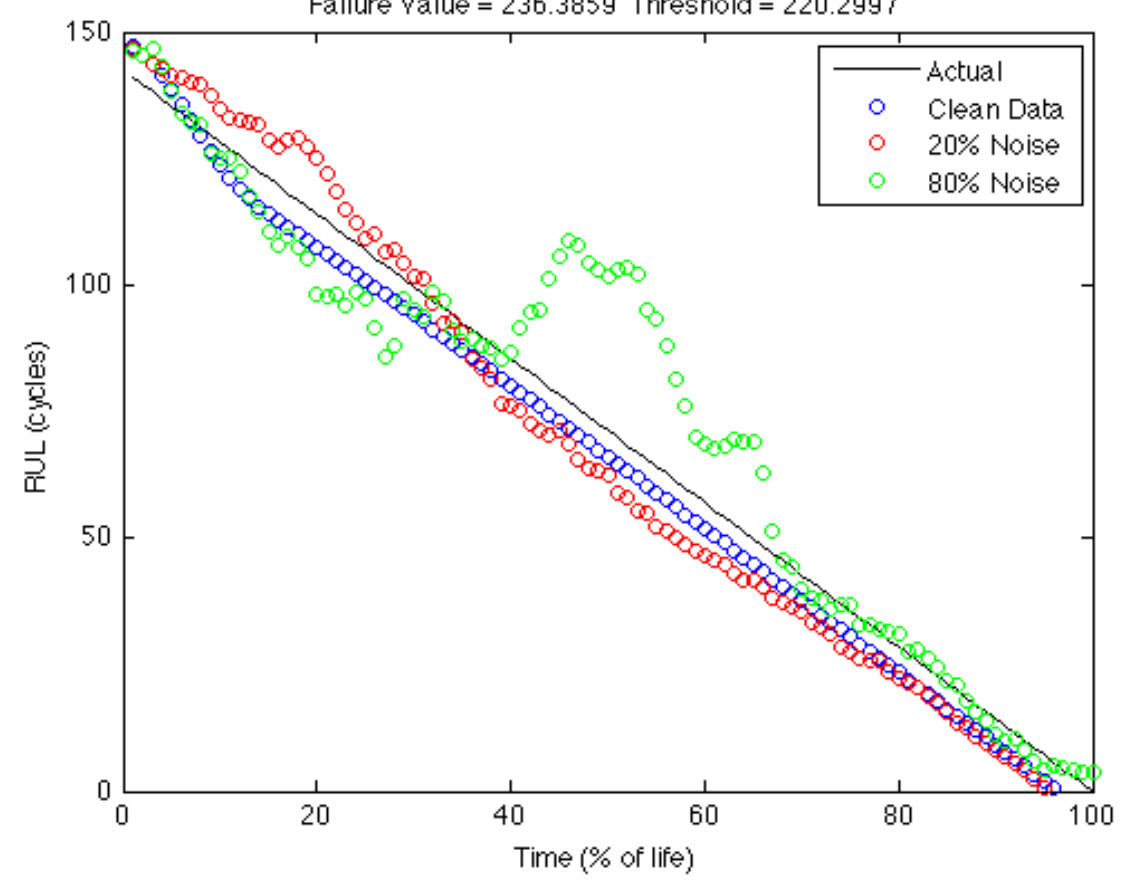

RUL Estimates for Run 7

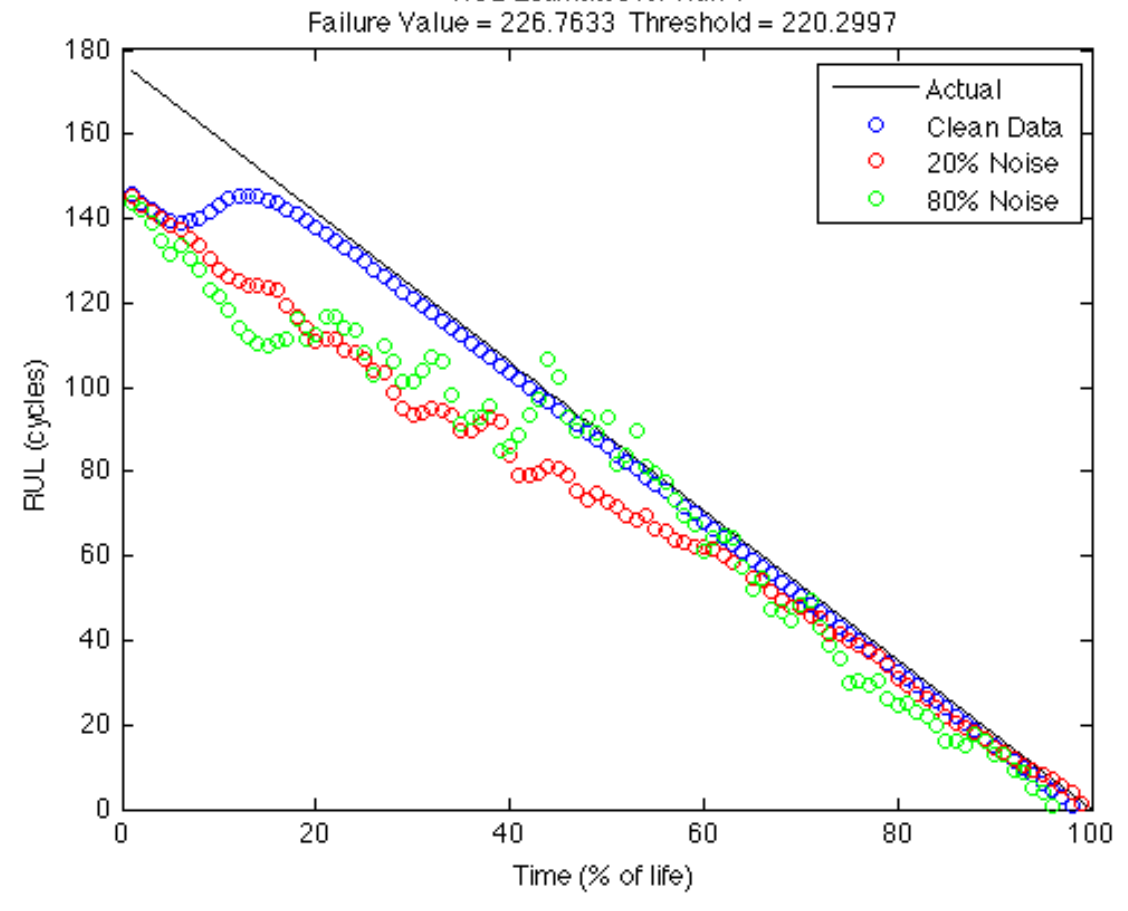


RUL Estimates for Run 8

Failure Value $=215.0172$ Threshold $=221.8681$

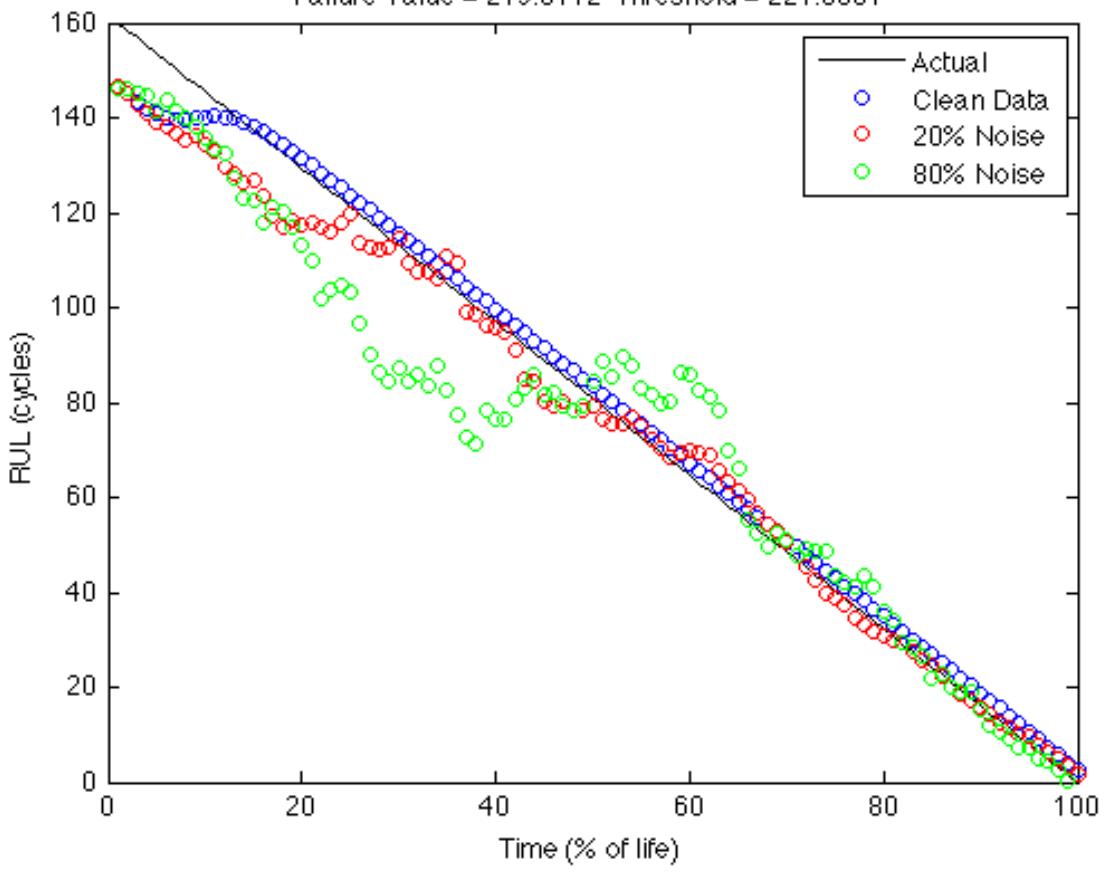

RUL Estimates for Run 9

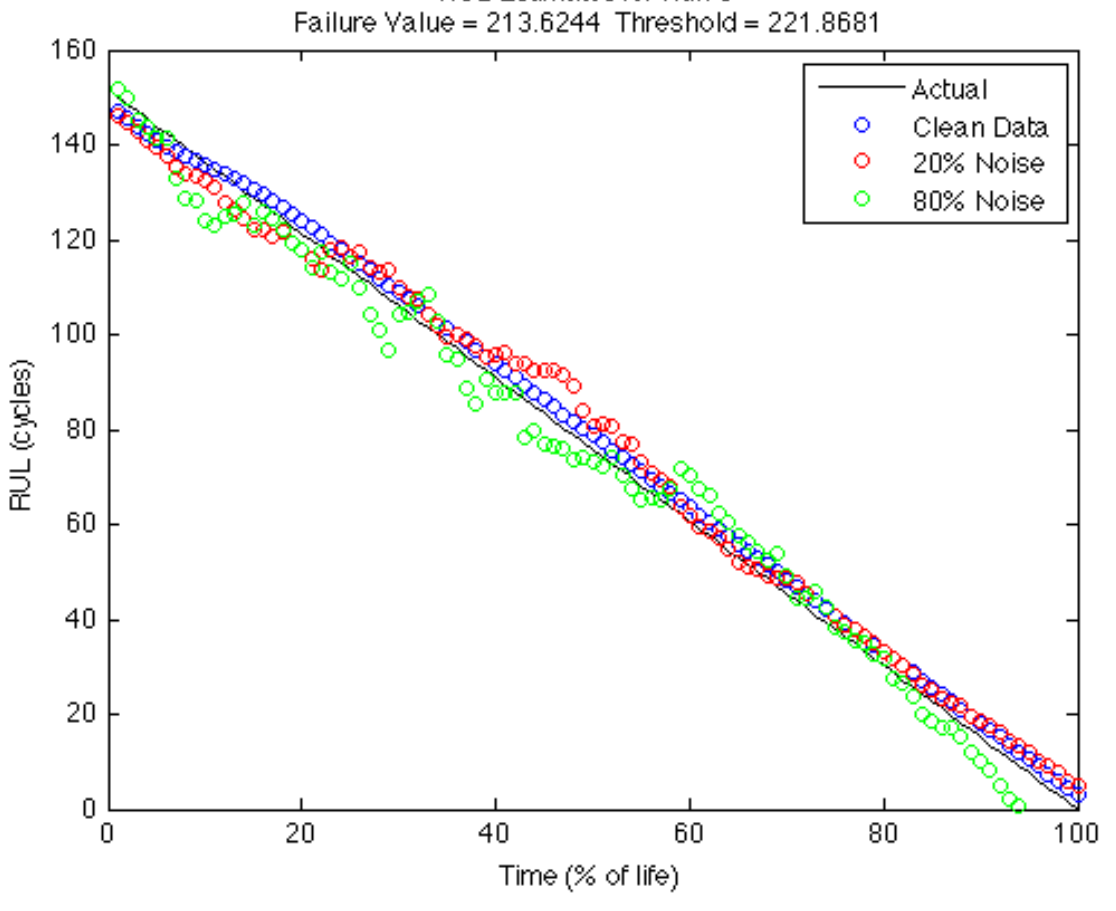


RUL Estimates for Run 10

Failure Value $=218.5916$ Threshold $=221.8681$

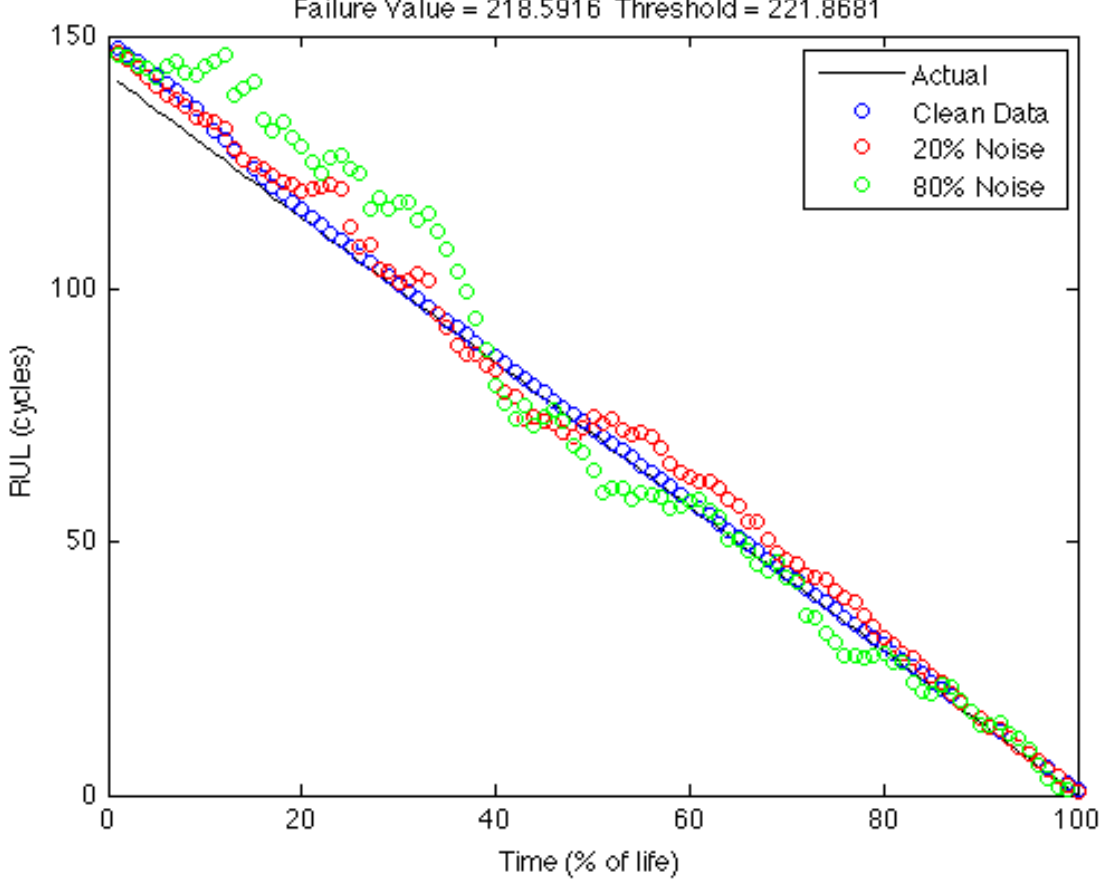

RUL Estimates for Run 11

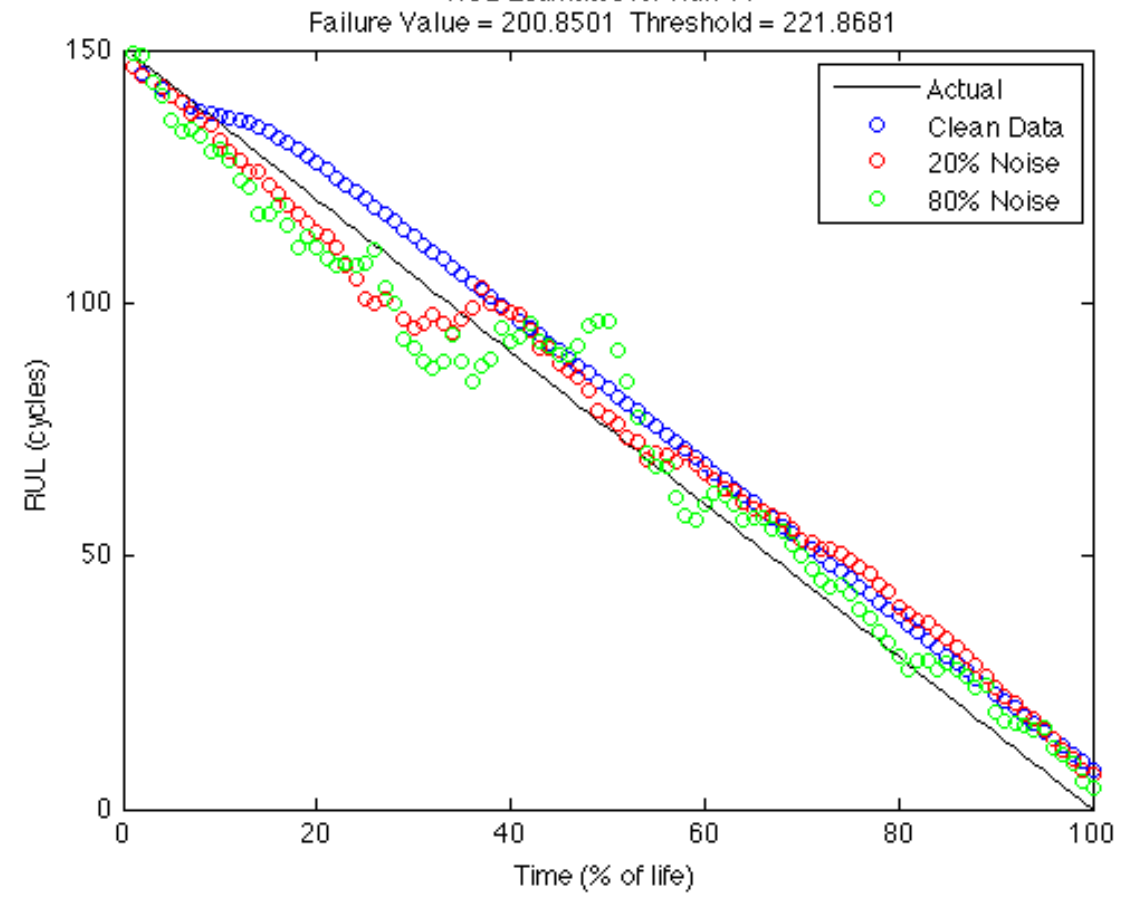


RUL Estimates for Run 12

Failure Value $=224.8747$ Threshold $=220.2997$

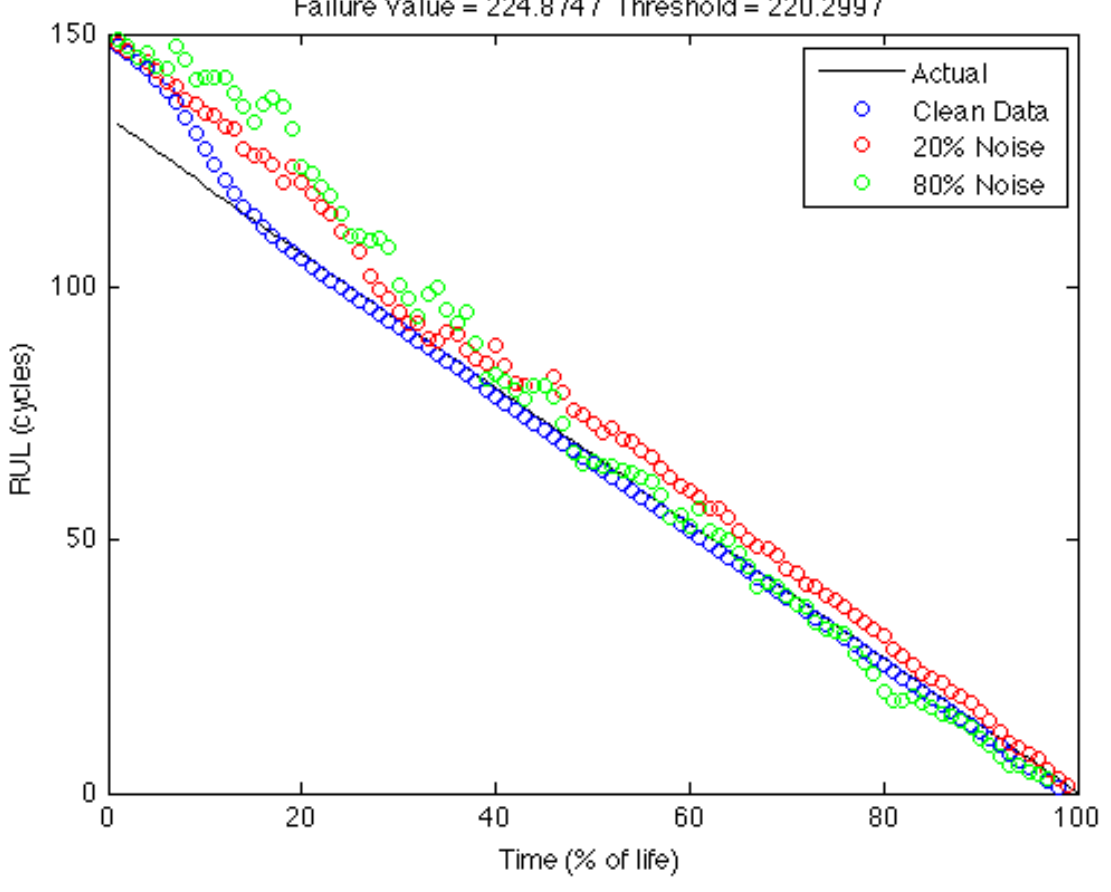

RUL Estimates for Run 13

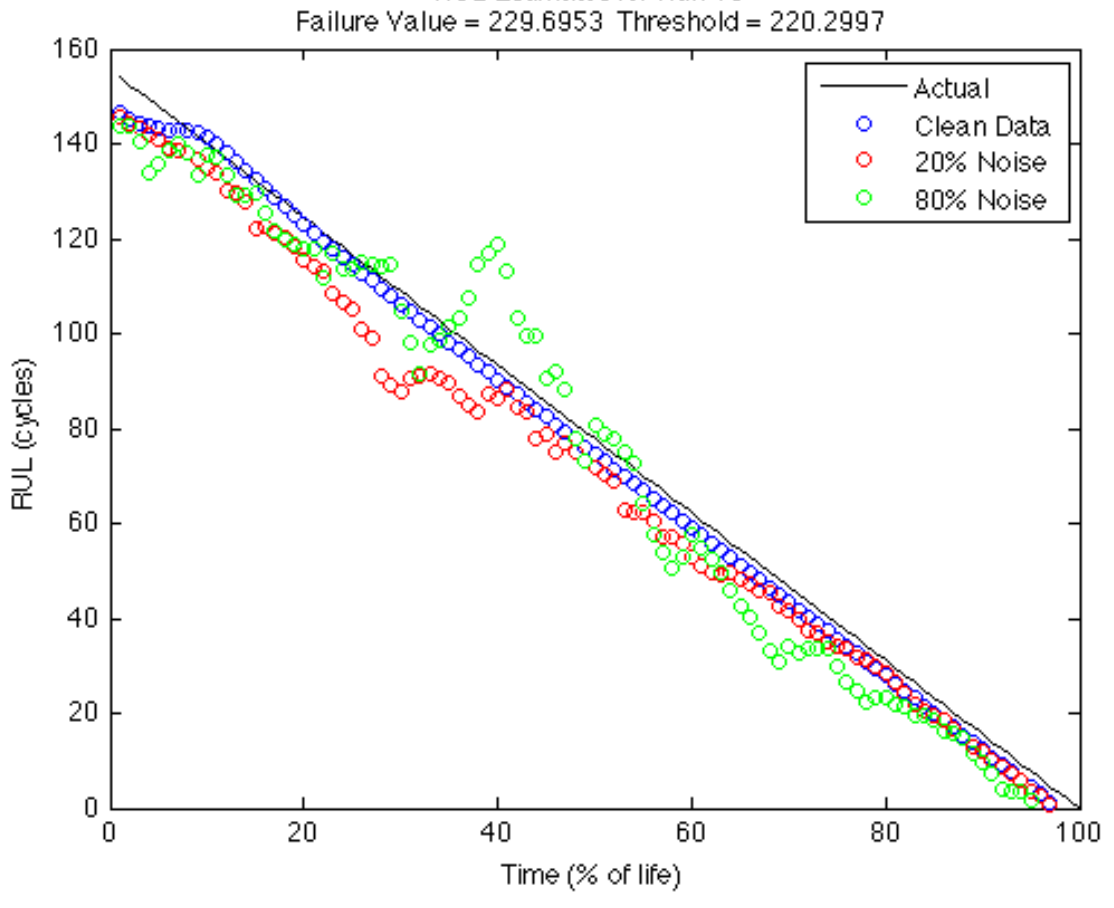


RUL Estimates for Run 14

Failure Value $=216.9345$ Threshold $=221.8681$

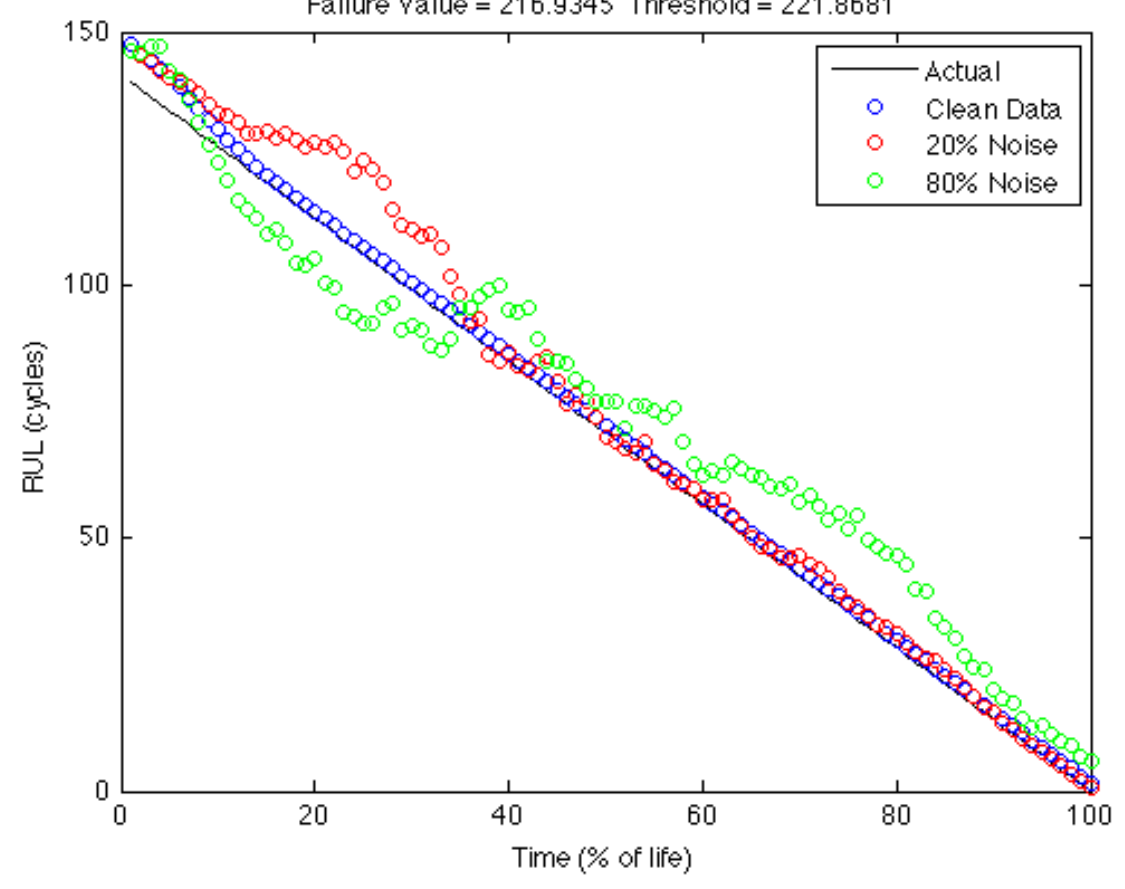

RUL Estimates for Run 15

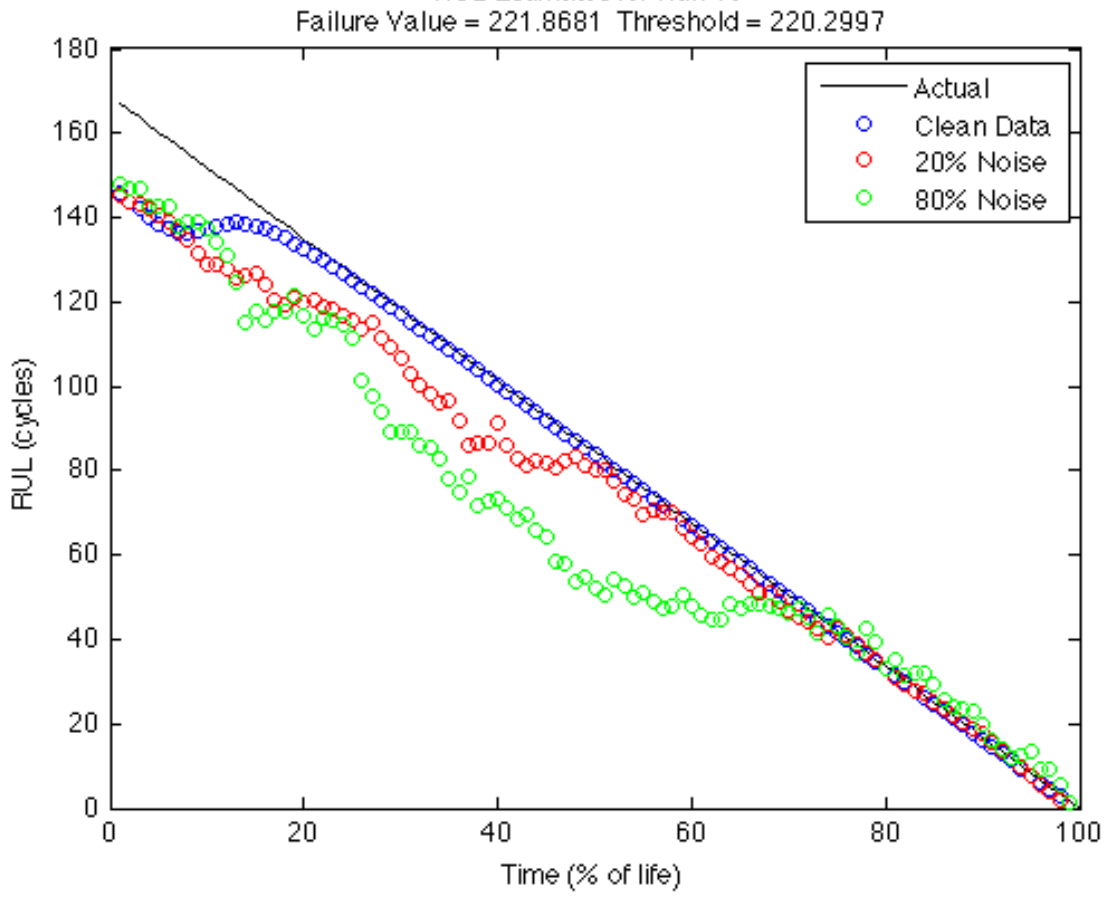


RUL Estimates for Run 16

Failure Value $=208.9028$ Threshold $=221.8681$

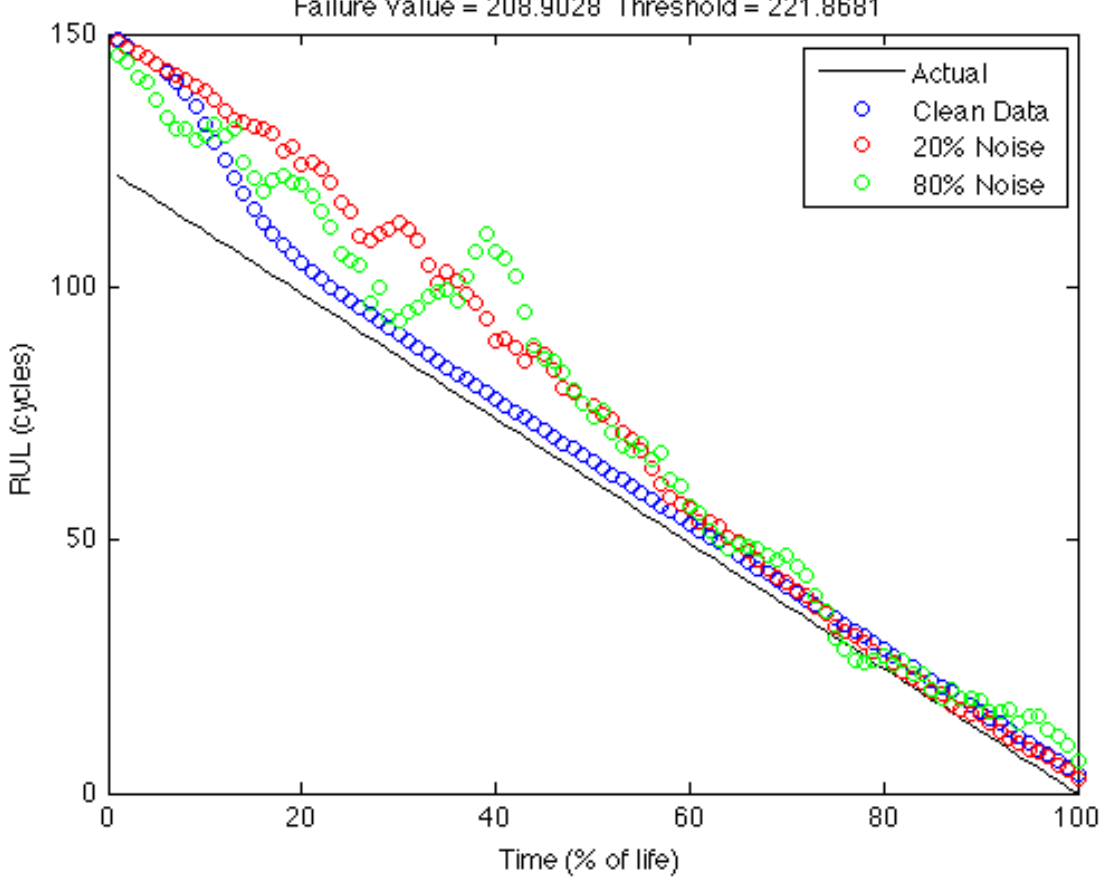

RUL Estimates for Run 17

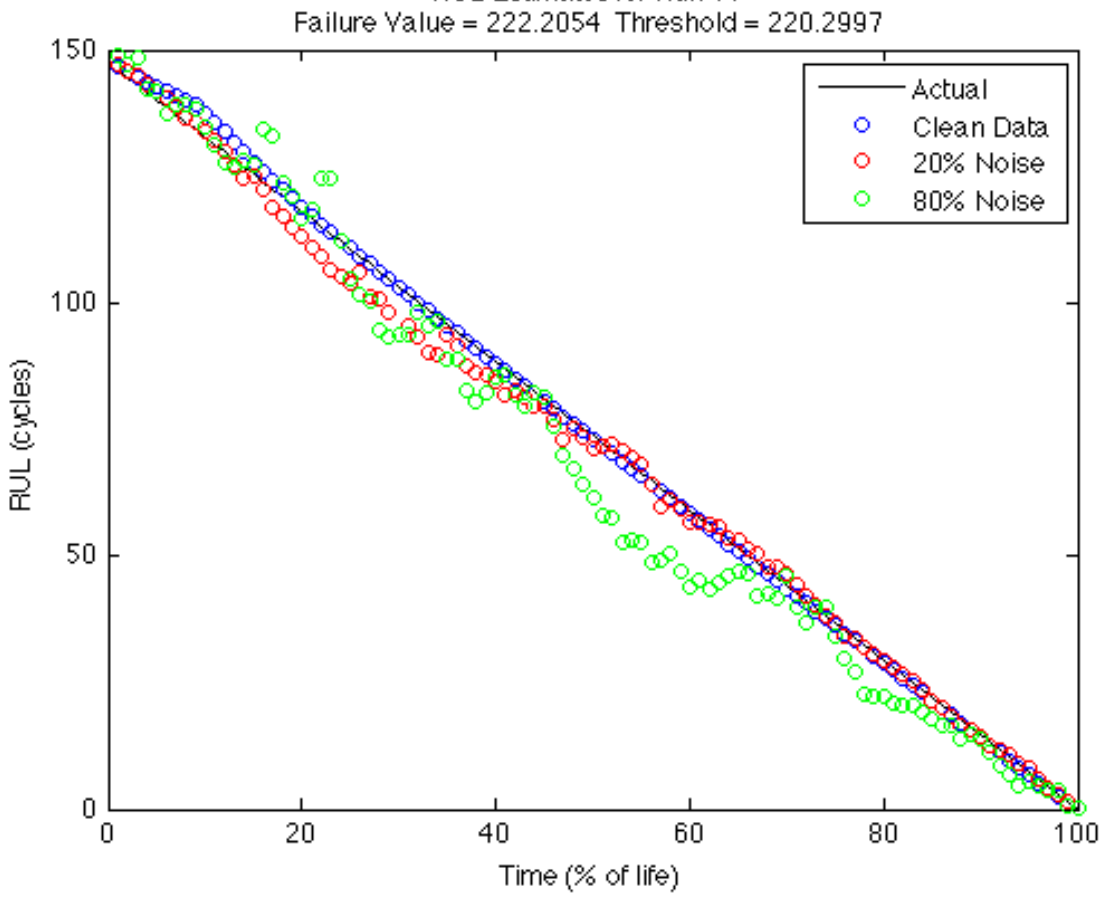


RUL Estimates for Run 18

Failure Value $=217.5092$ Threshold $=221.8681$

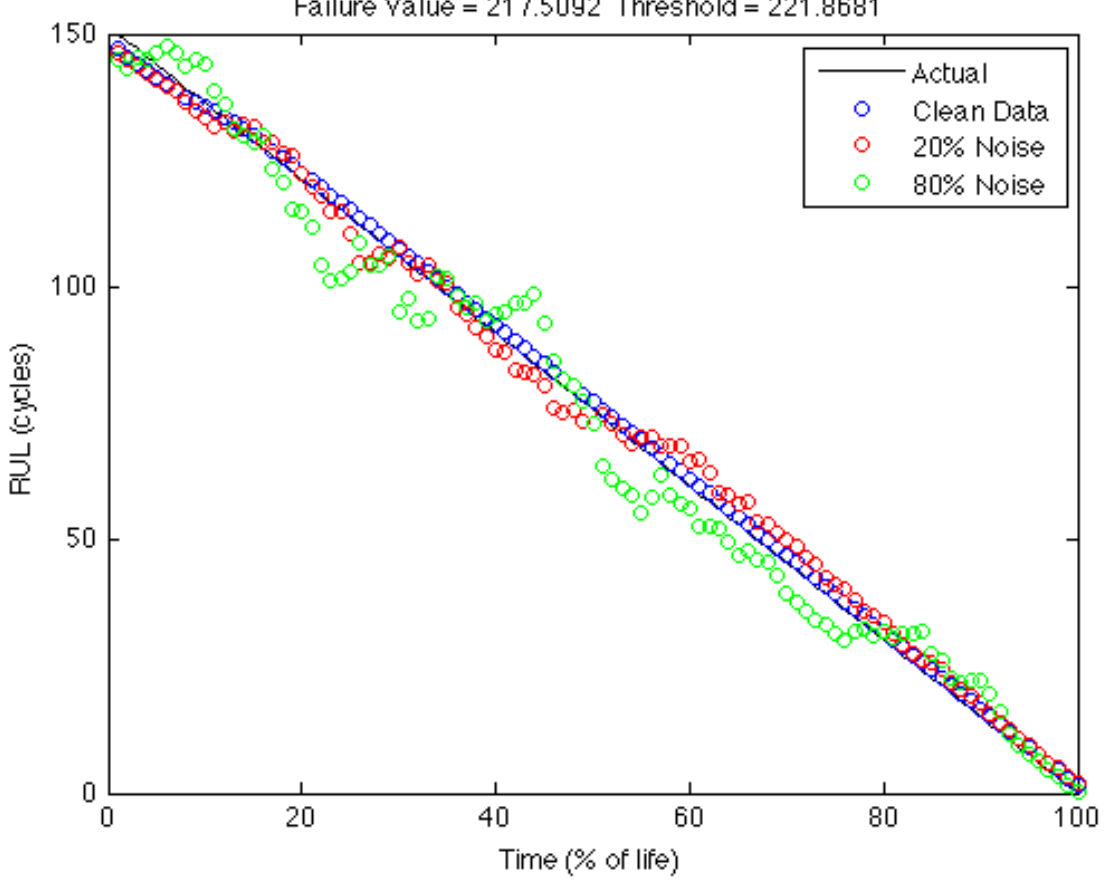

RUL Estimates for Run 19

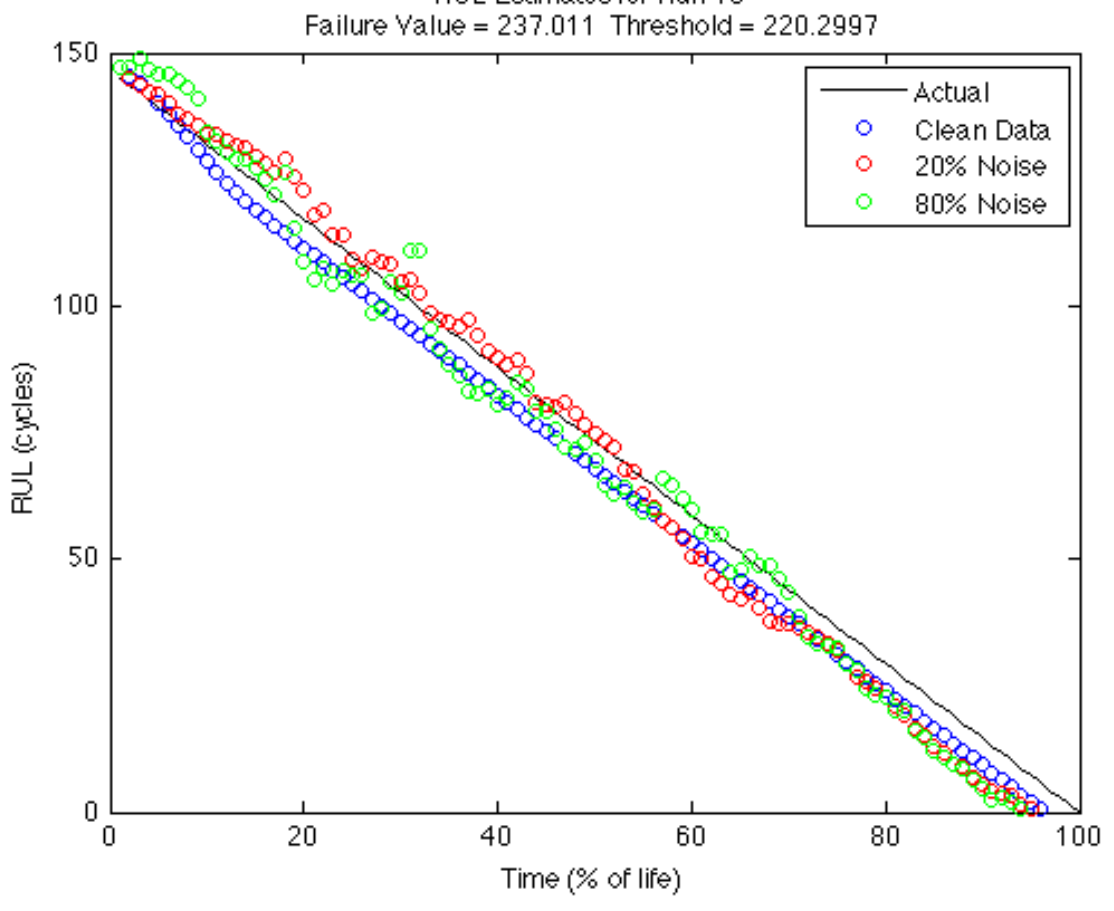


RUL Estimates for Run 20

Failure Value $=208.8713$ Threshold $=221.8681$

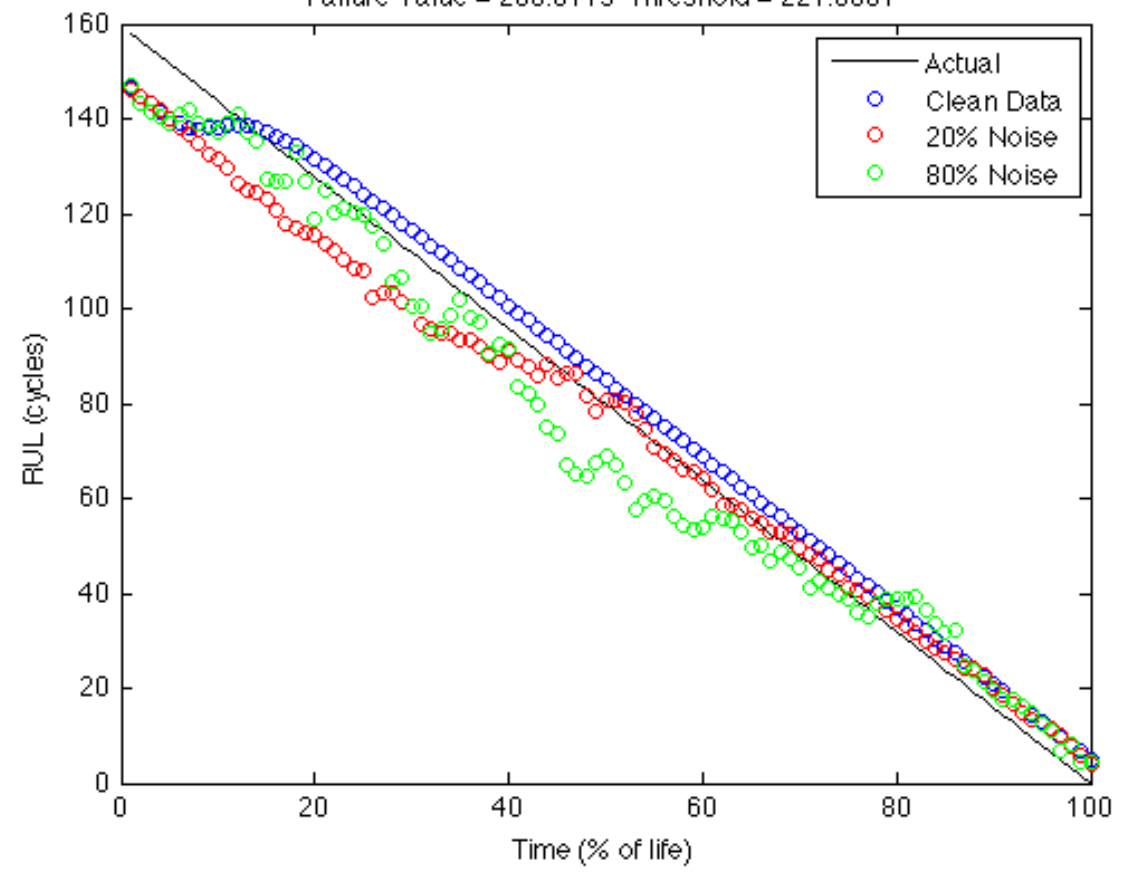




\section{A.3 PHM Challenge Data Monitoring System Residuals}
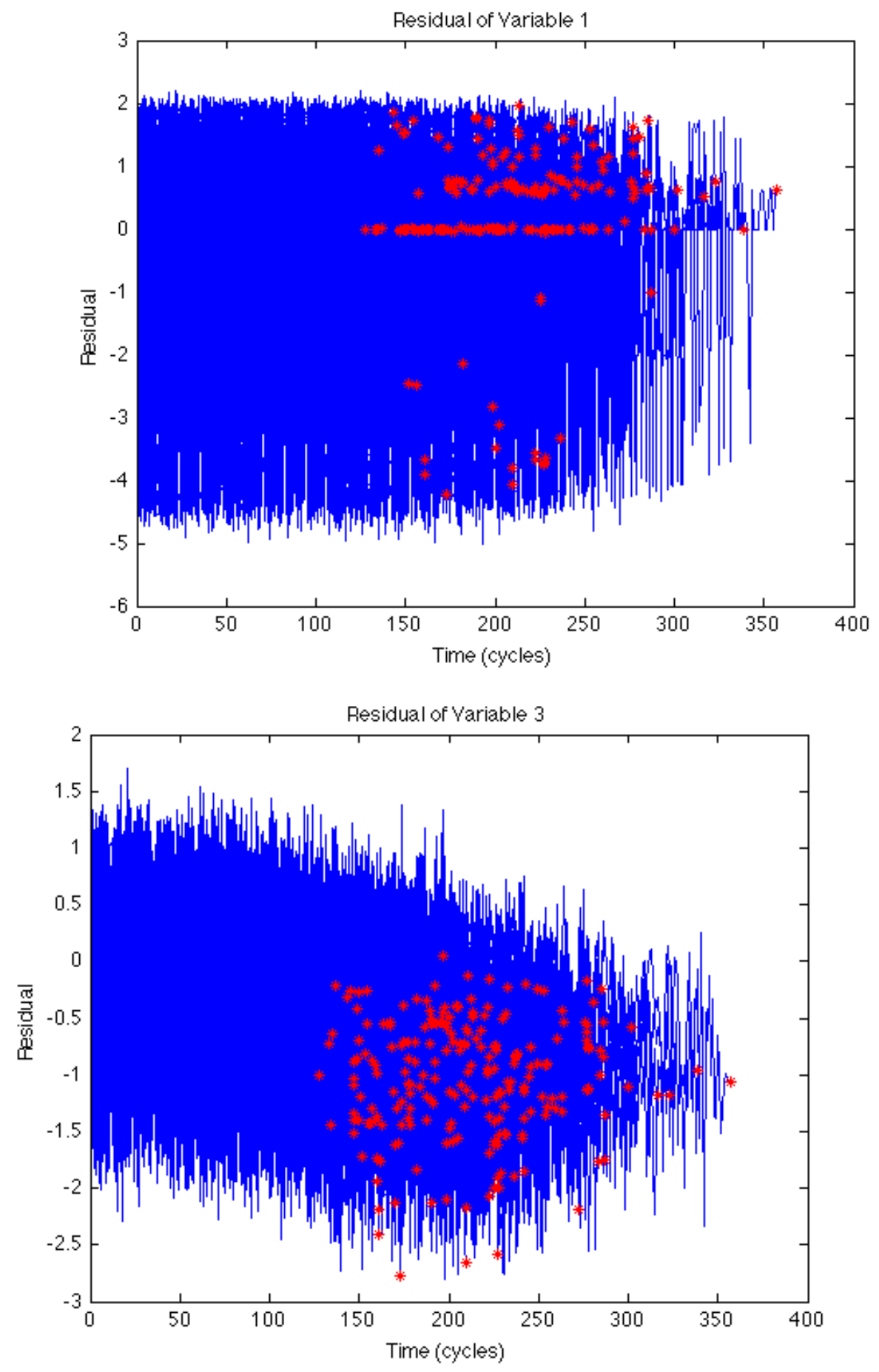

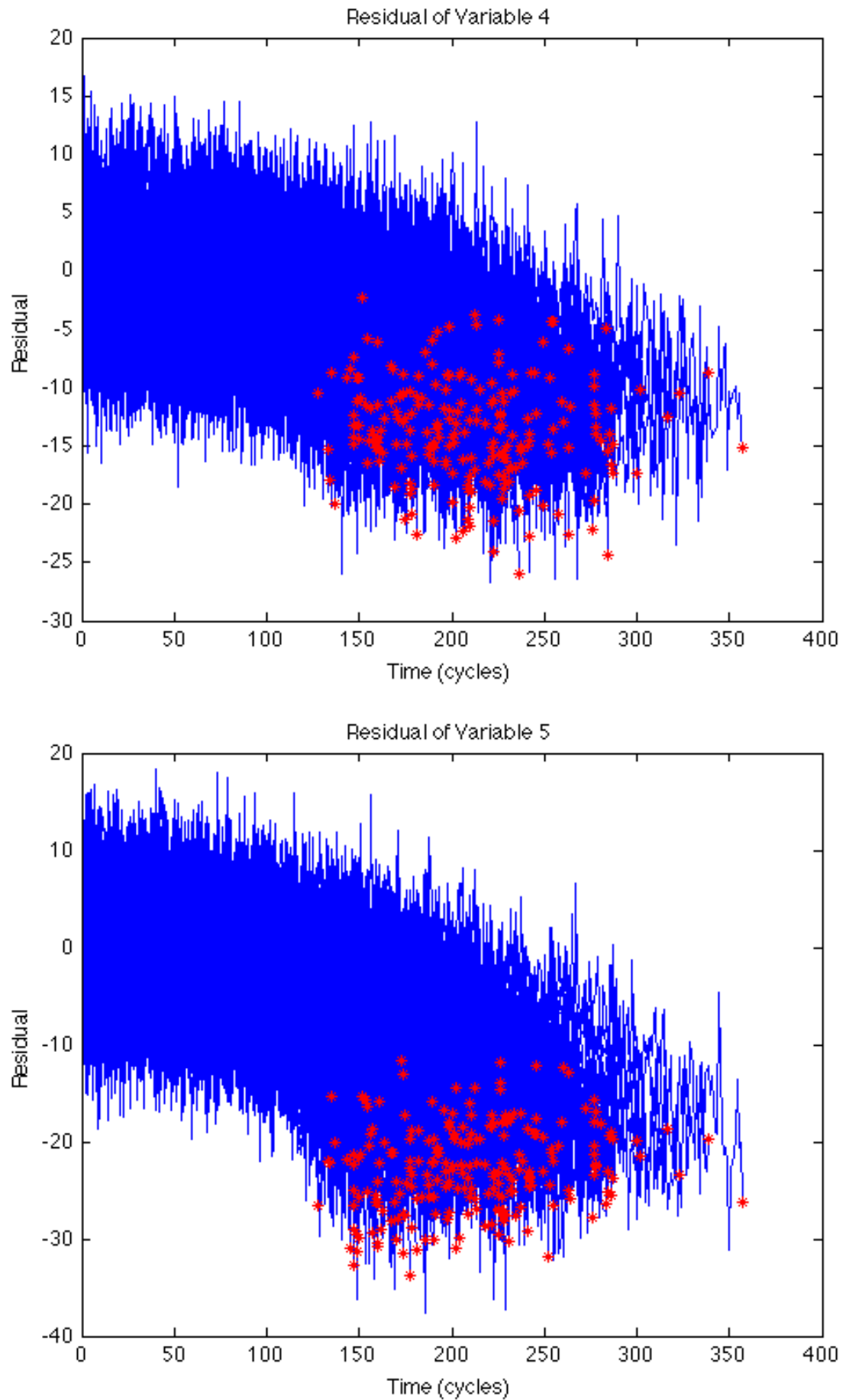

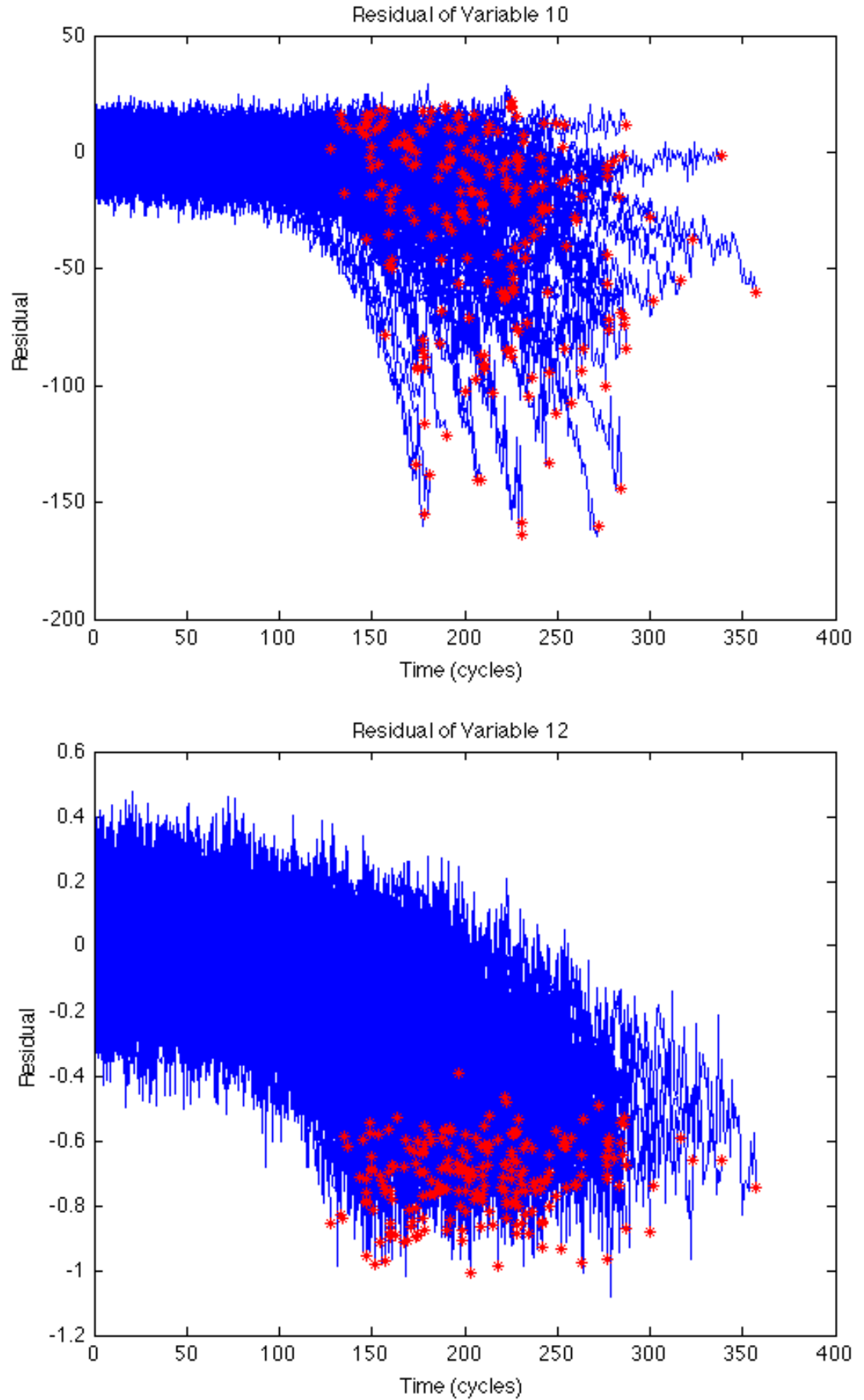

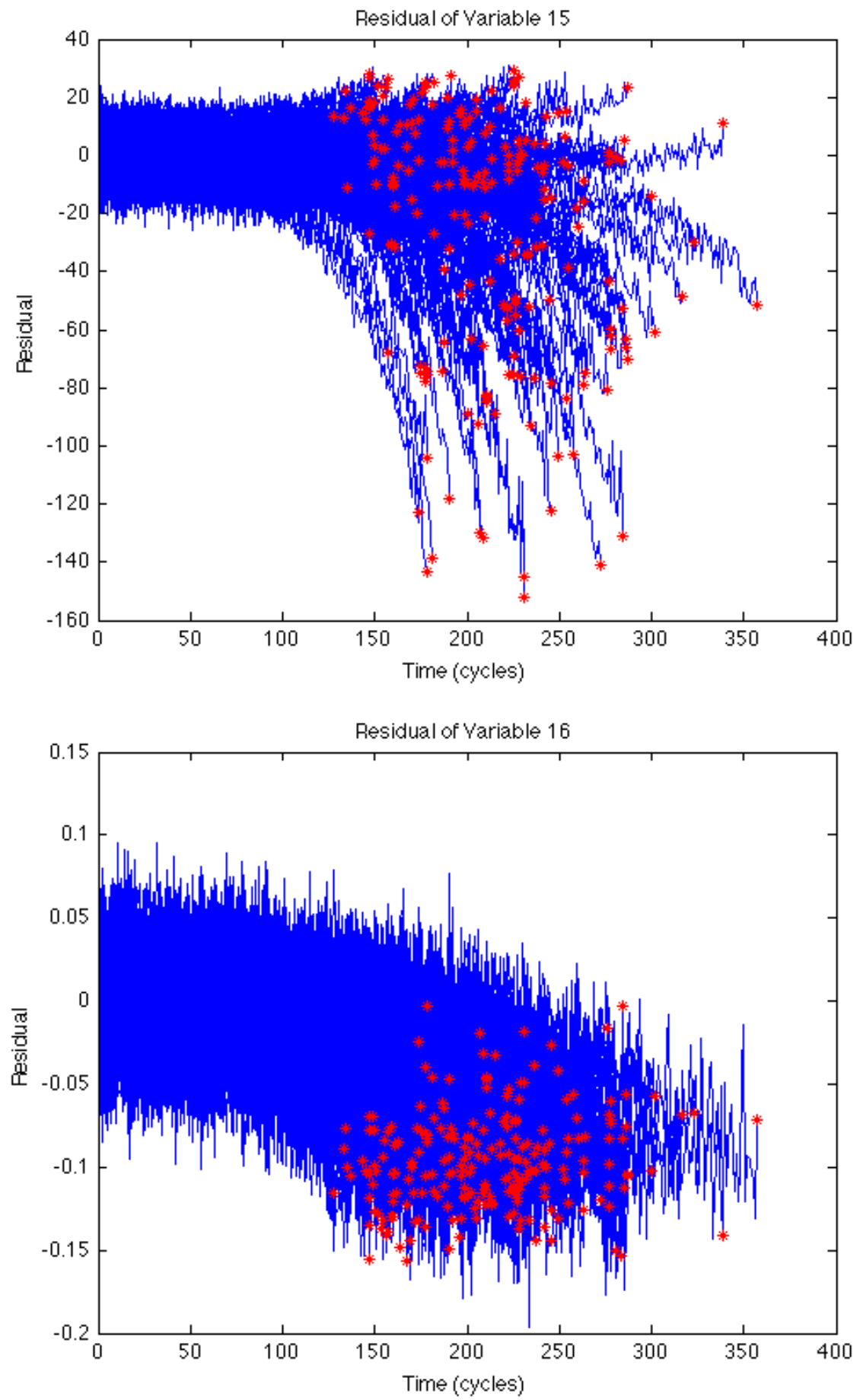

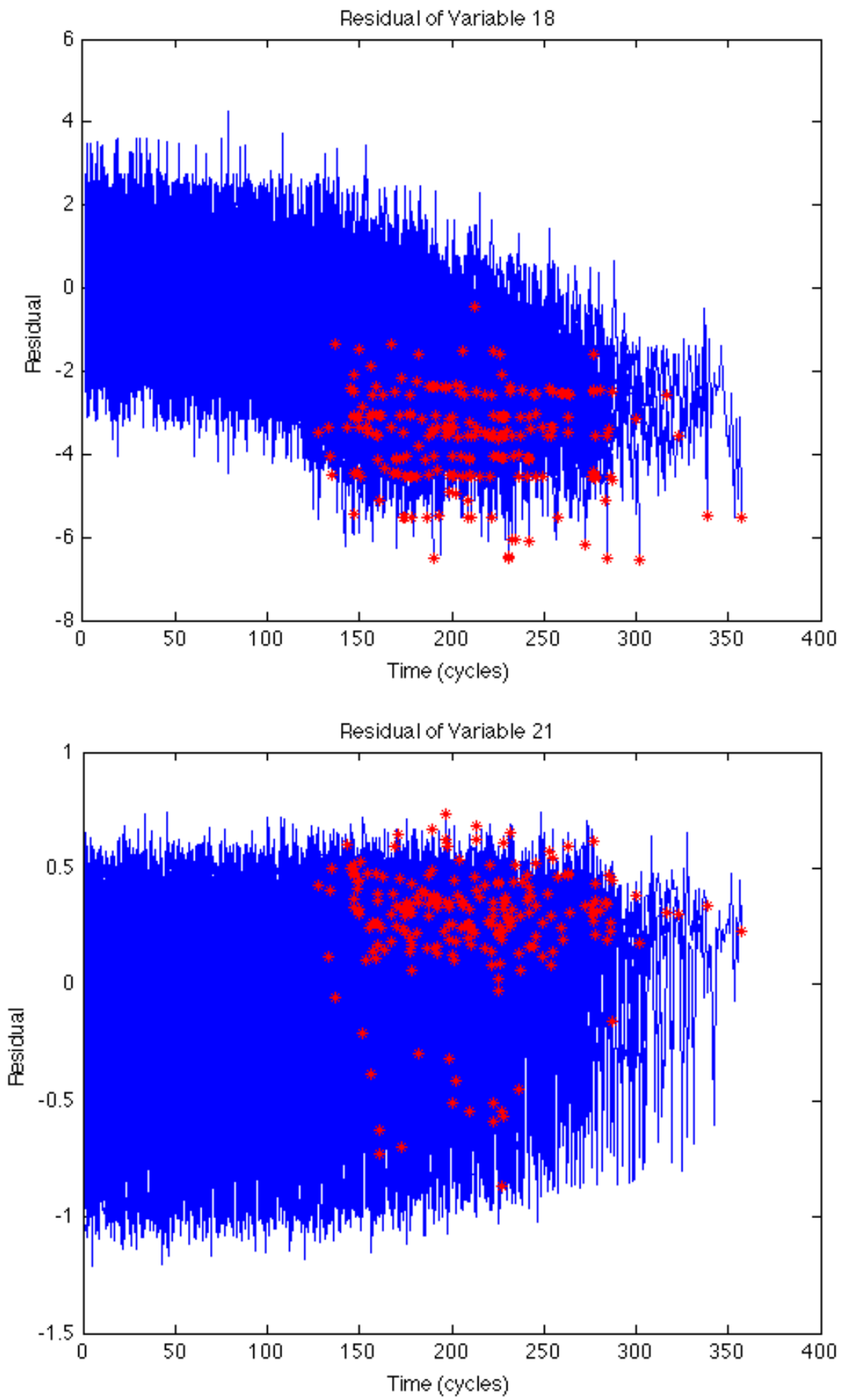


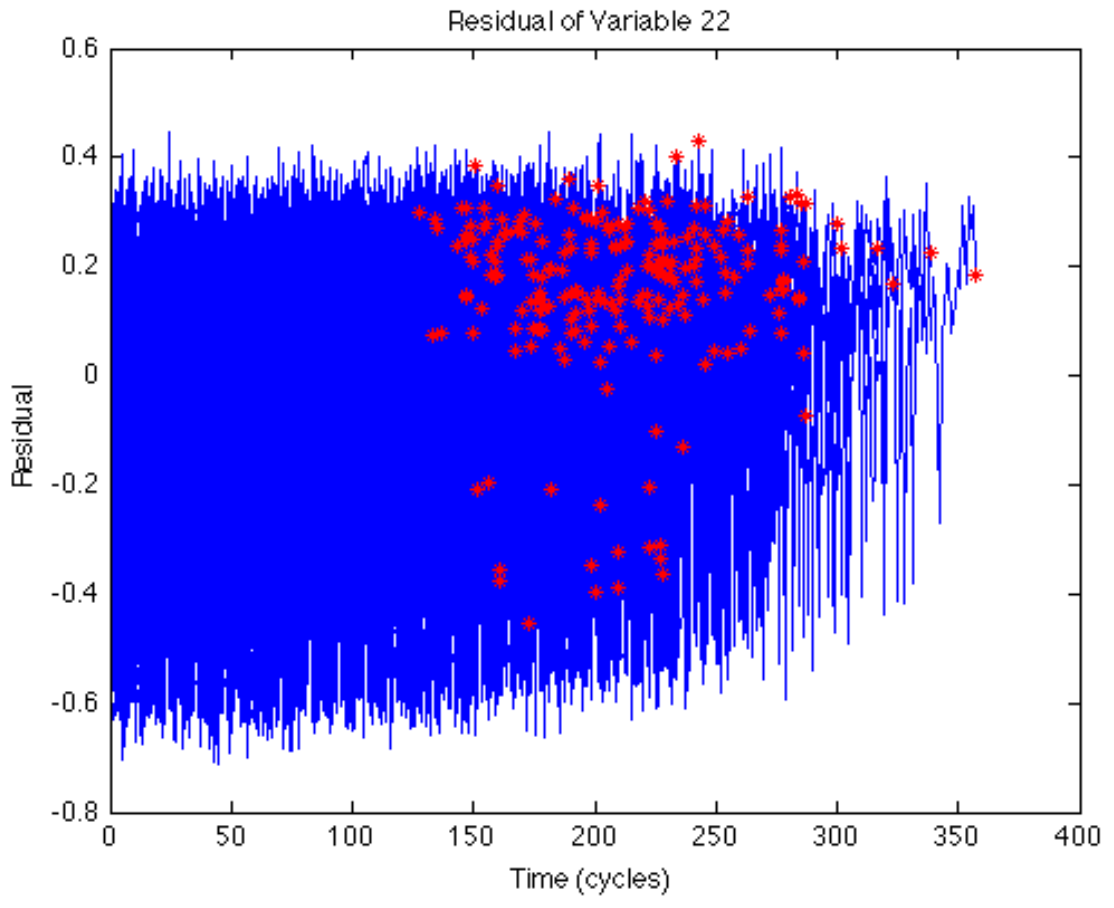




\section{A.4 Milling Data Mean and Standard Deviation Features}
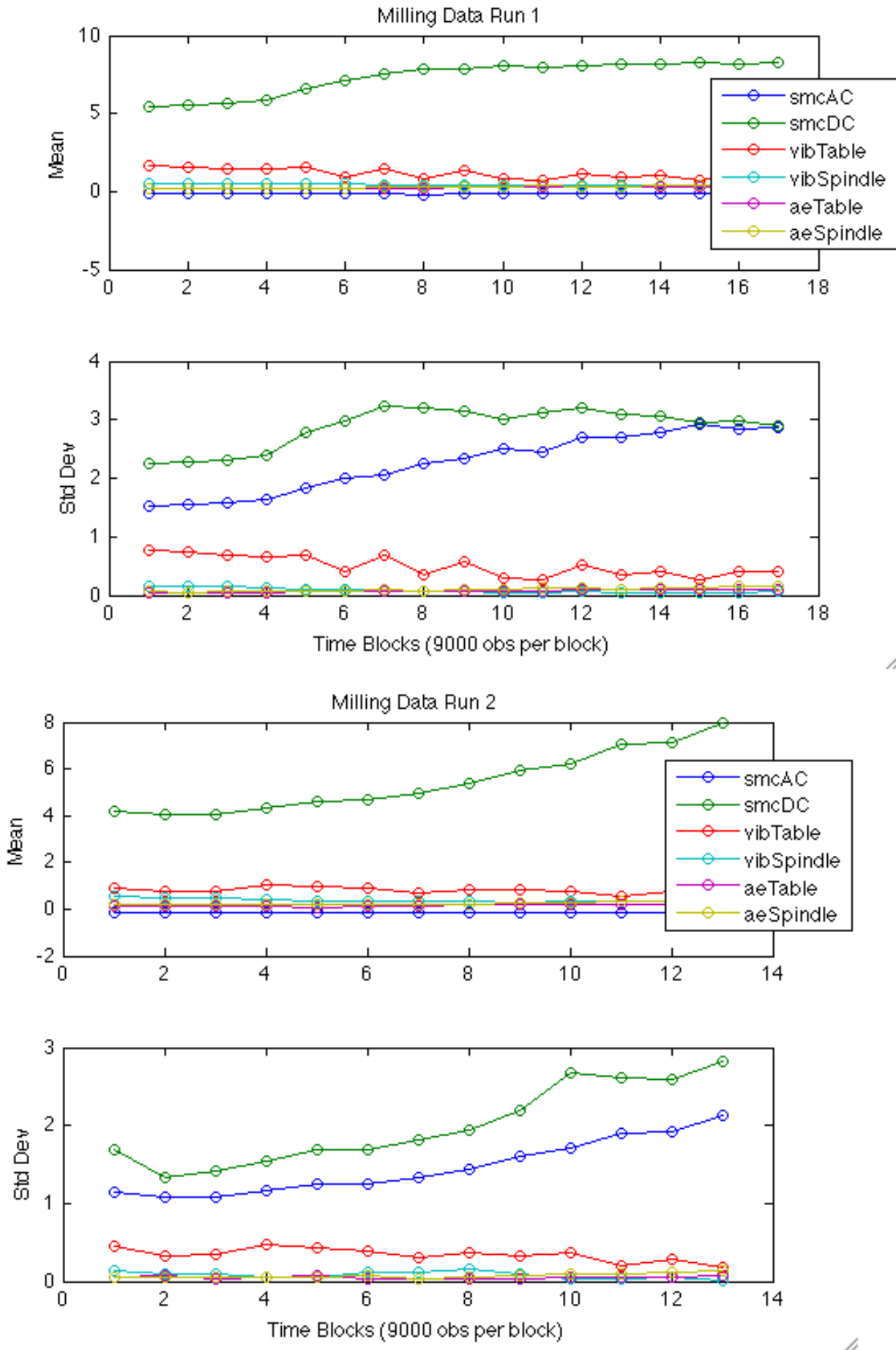

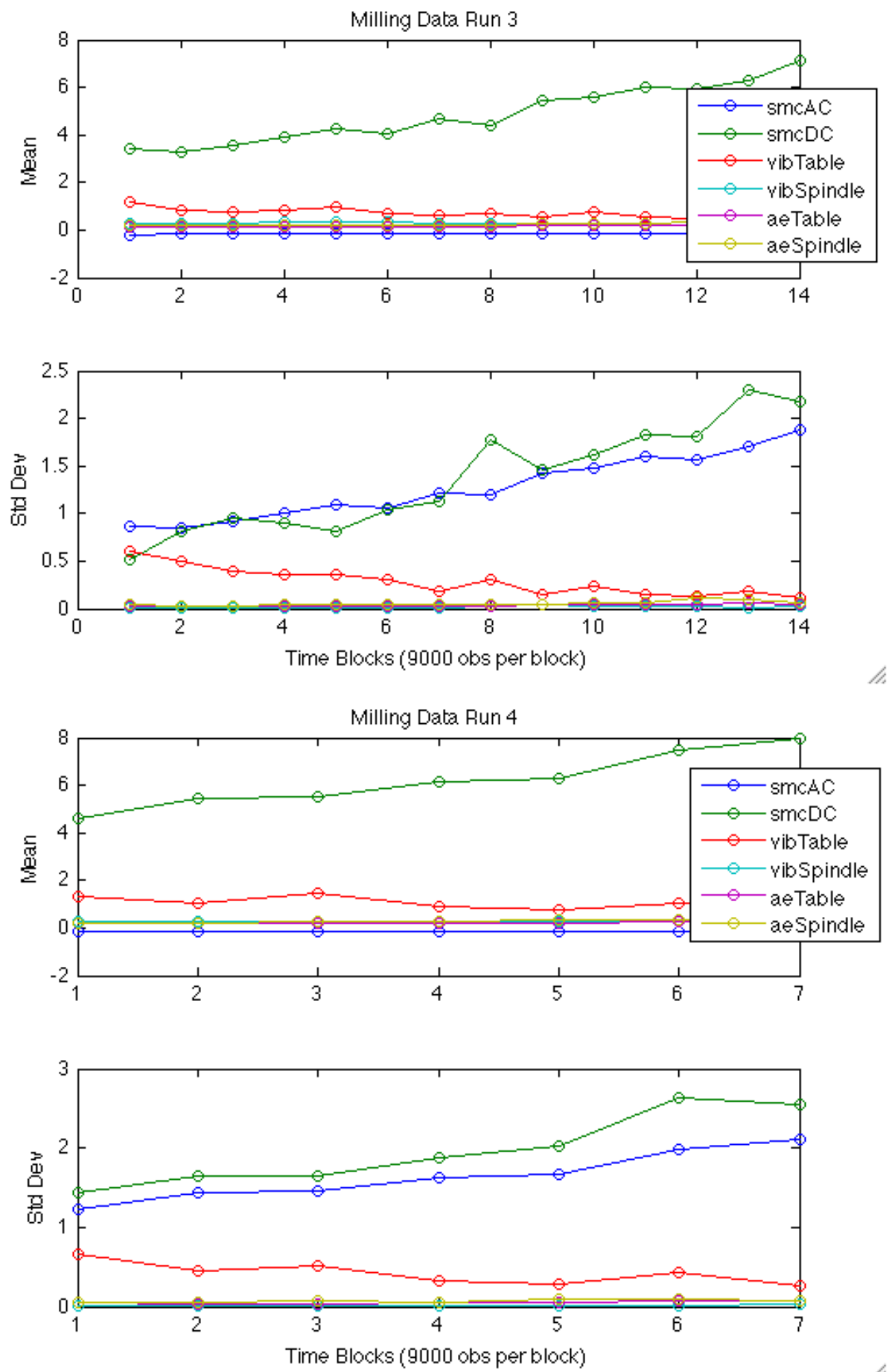

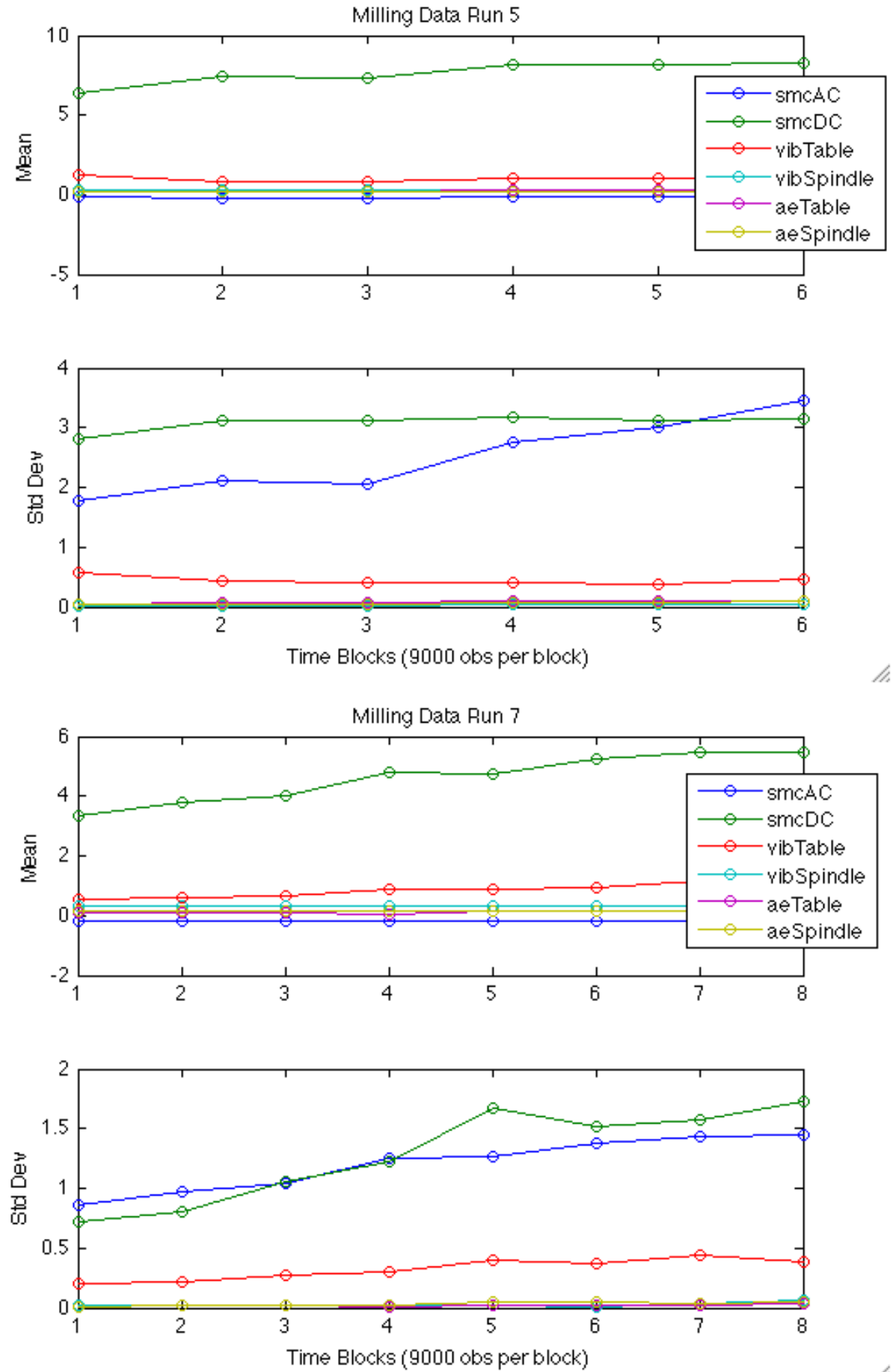

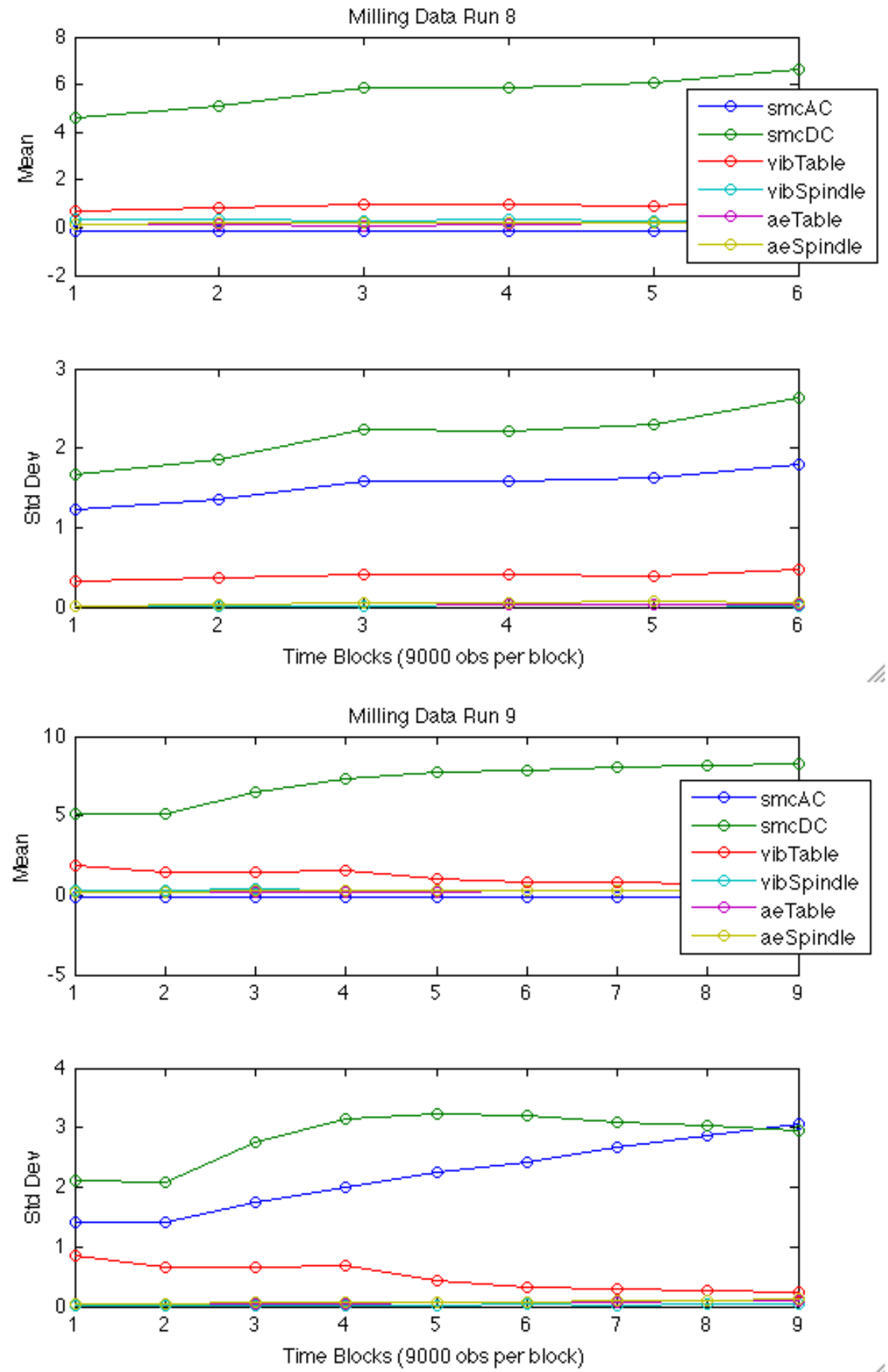

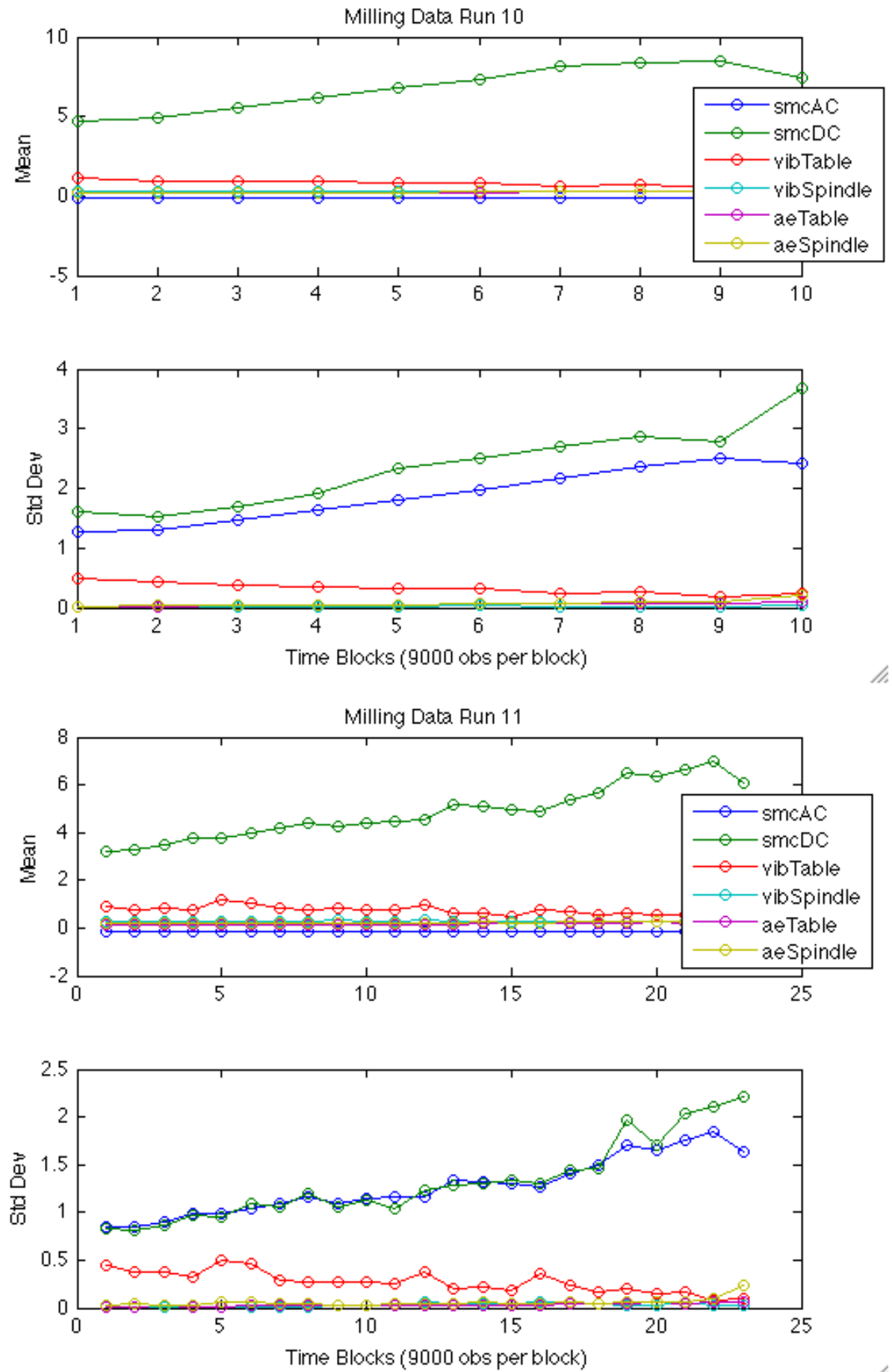

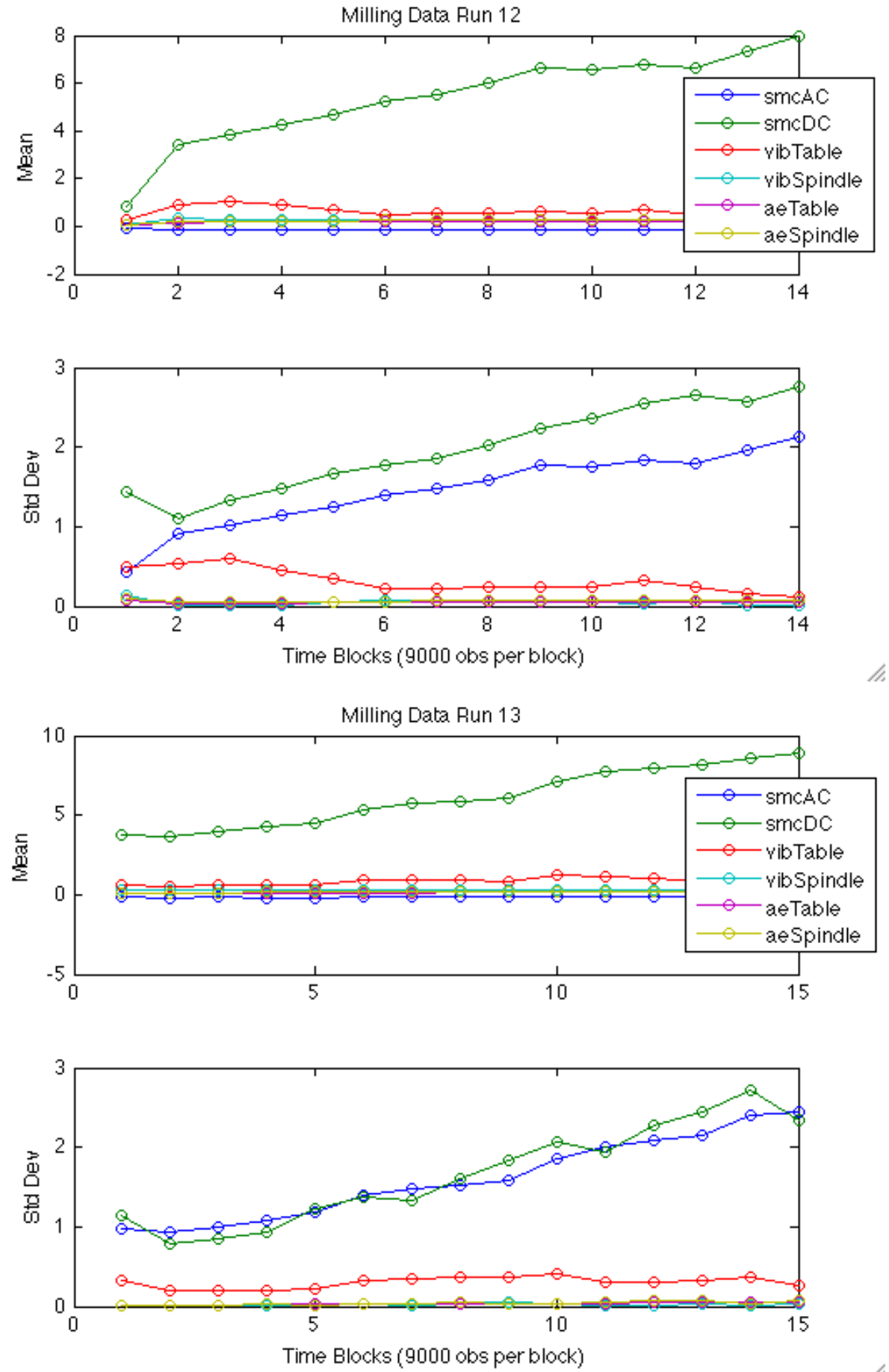

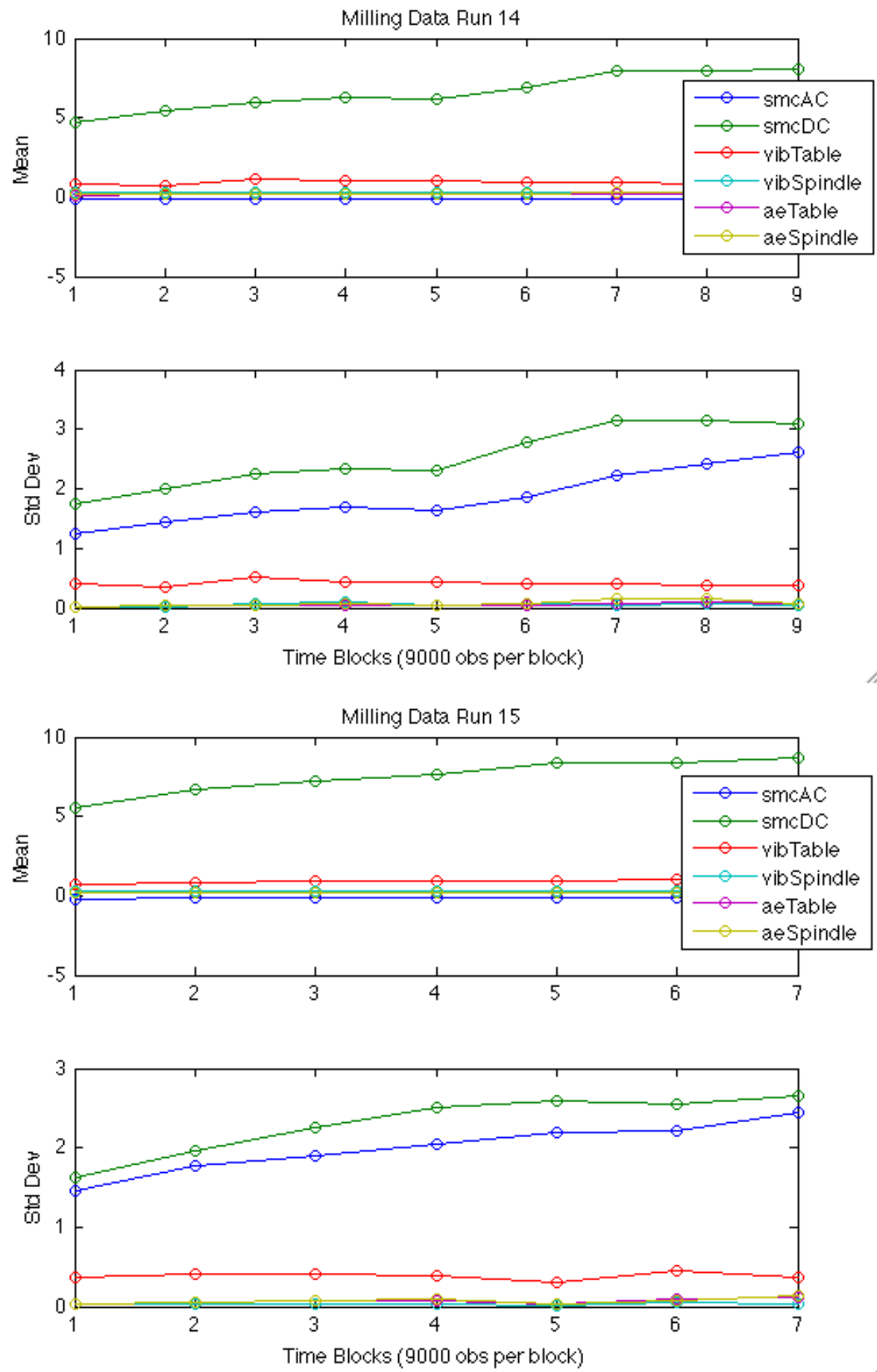

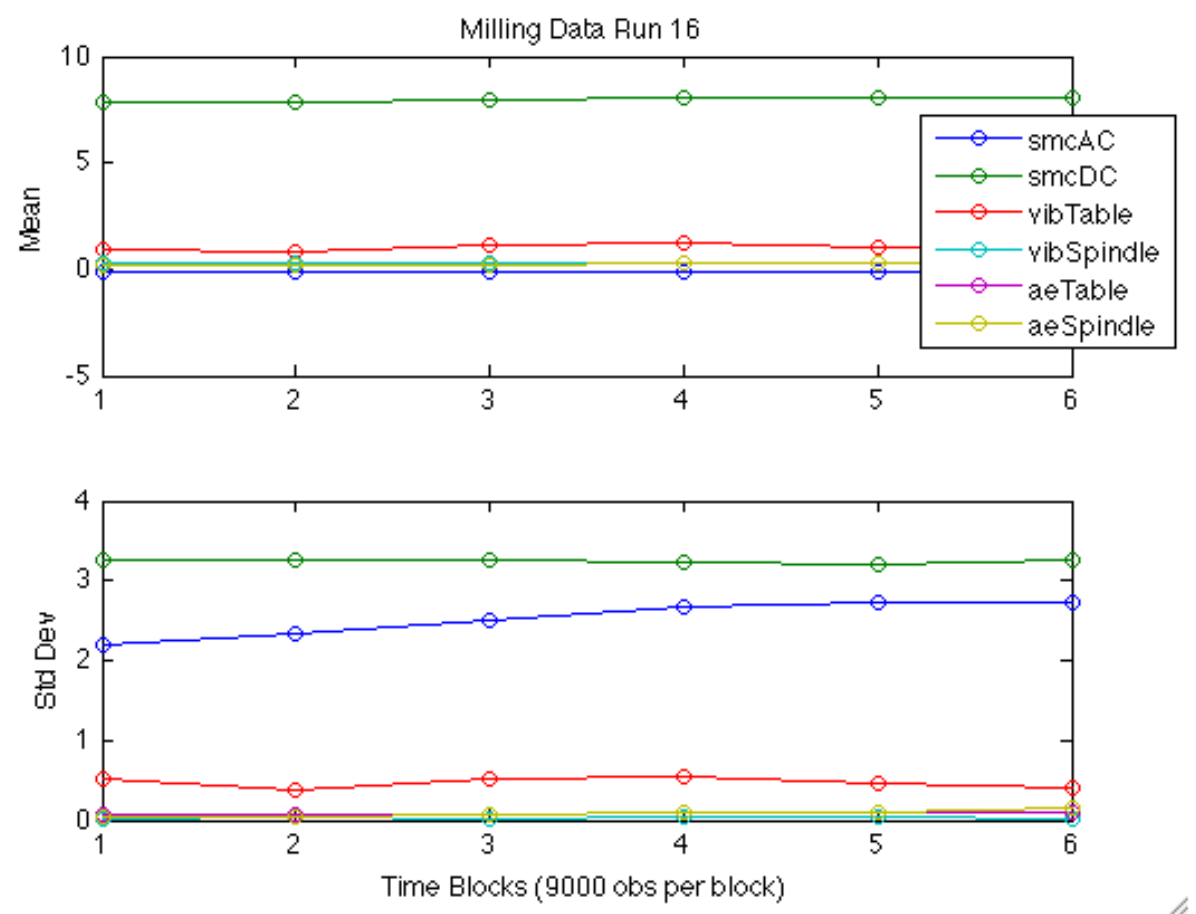
APPENDIX B: Process and Equipment Prognostics Toolbox User Guide 


\section{Process \& Equipment Prognostics Toolbox}

Dr.Jamie Coble, jcoble1@utk.edu

Dr.J.Wesley Hines, jhines2@utk.edu

Nuclear Engineering Department

The University of Tennessee

Knoxville, TN 37996-2300

\section{User's Guide}

Version 1.0 
Copyright (C) 2011 by Jamie B. Coble All rights reserved. 


\section{Acknowledgements}

The authors would like to thank our co-researchers for their help in developing the PEP toolbox and this User's Guide. Much gratitude goes to Brian Wood and Siobhan O'Reilly for their help in drafting this document. We would also like to acknowledge David Hooper, Dr. Alexander Usynin, and James Henkel for their research contributions, which led respectively to the development of generic frameworks of Particle Filtering, Markov Chain and Shock, and Proportional Hazards models in the PEP toolbox. 


\section{Table of Contents}

1 INTRODUCTION

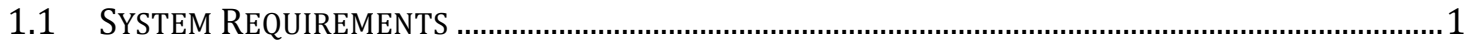

1.2 TOOLBOX ARCHITECTURE

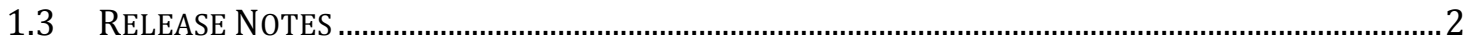

2 BACKGROUND

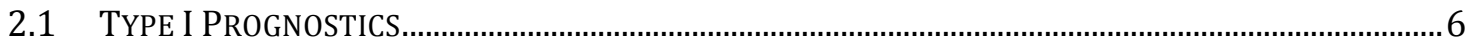

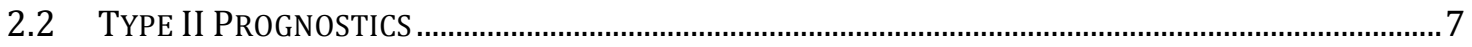

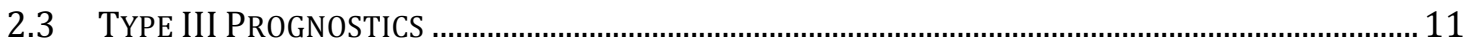

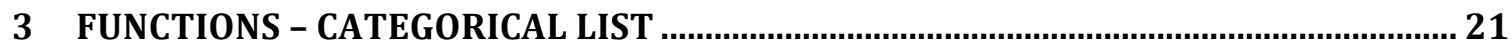

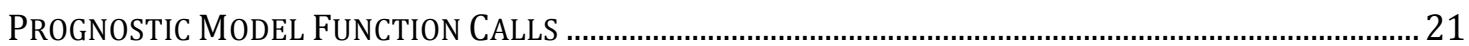

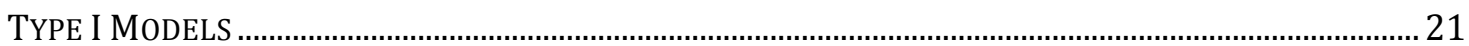

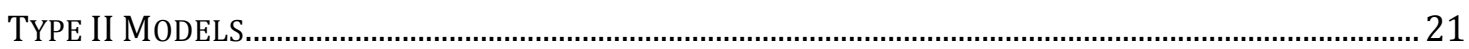

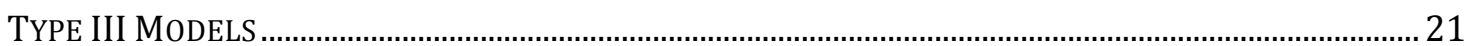

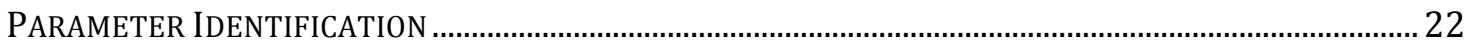

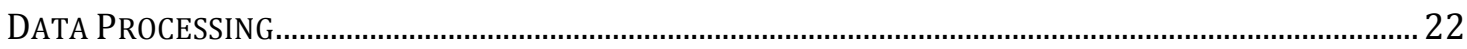

4 FUNCTIONS - ALPHABETICAL LIST

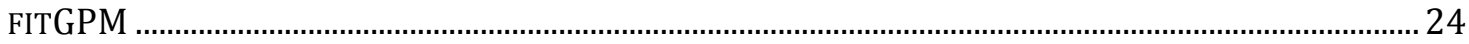

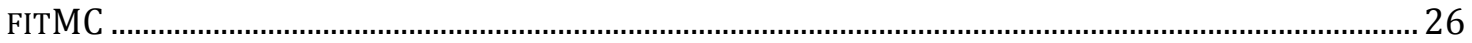

FITPF

INITBAYES

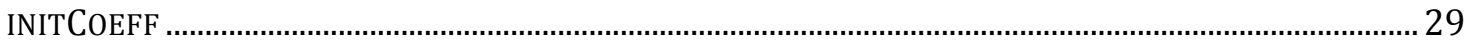

INITGPM

INITMC

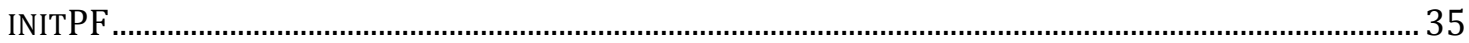

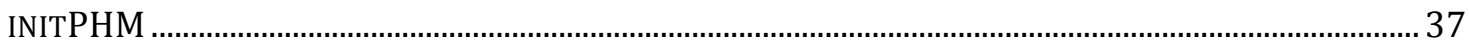

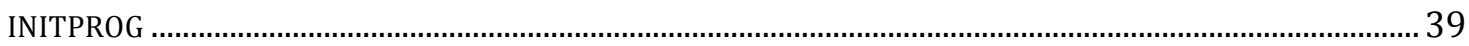

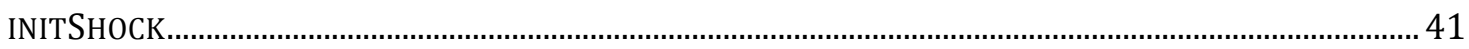

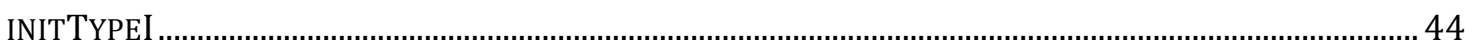

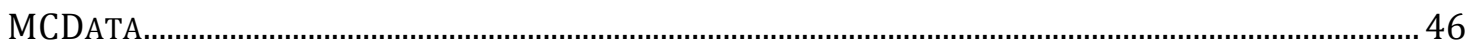

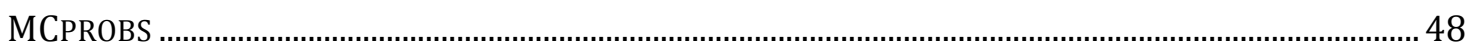

OPTPARAM .

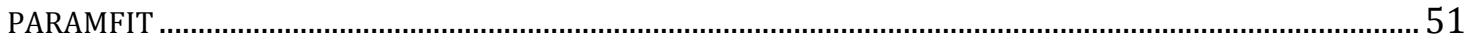

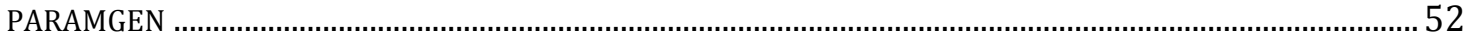

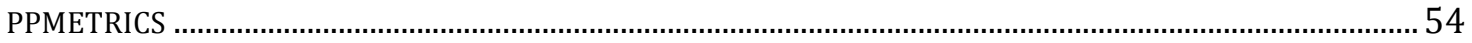

RESIDGEN

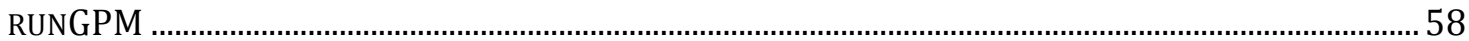

RUNMC

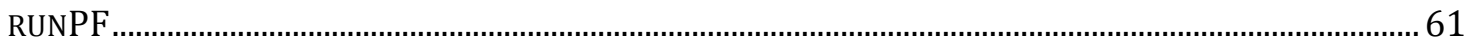

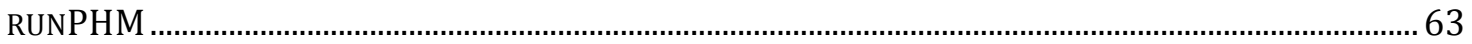

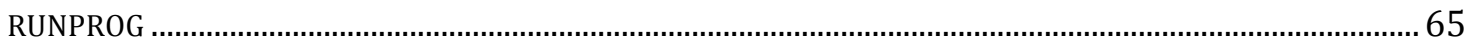

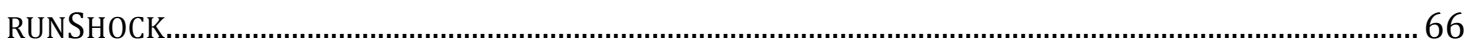

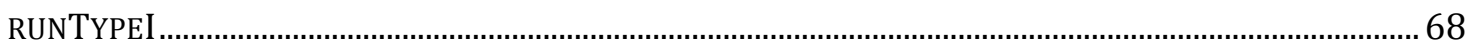

THRESHGPM

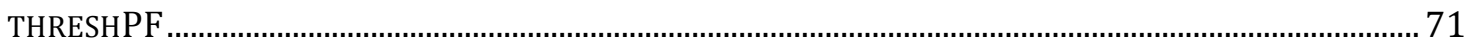

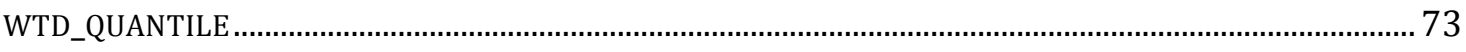

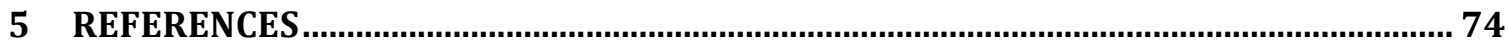




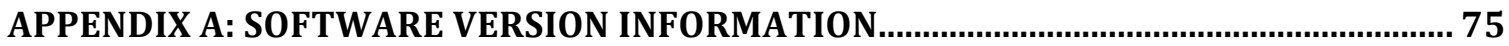

V1.0.

APPENDIX B: HEALTH MONITORING EXAMPLE USING THE PEM AND PEP TOOLBOXES76 


\section{List of Figures}

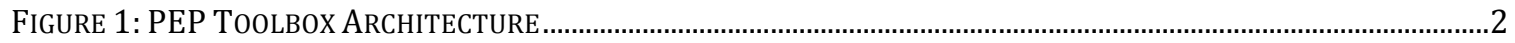

Figure 2: Complete HEalth ManAgement SyStEm (Coble \& Hines 2008) ..........................................................

FIGURE 2: EXAMPLE OF RESIDUALS USED AS A PROGNOSTIC PARAMETER

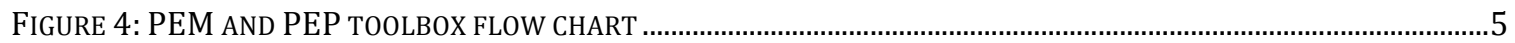

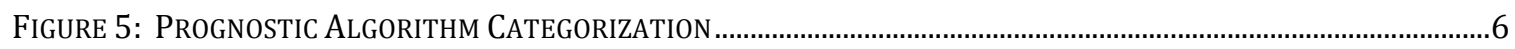

FiguRE 6: WeIBULl FAILURE DiSTRIBUTIONS WiTh DifFERENT SHAPE PARAMETERS ...................................................

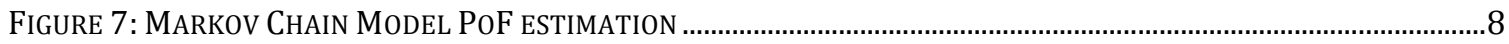

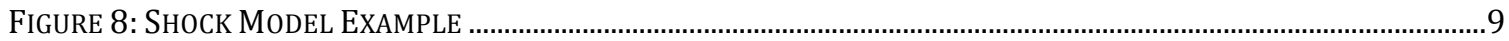

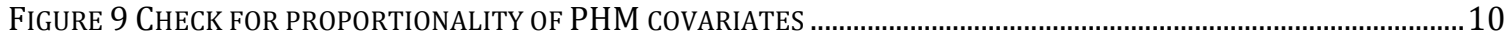

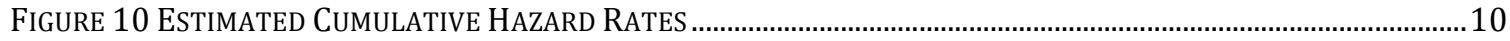

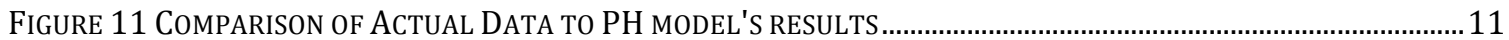

FIGURE 12: BAYESIAN UPDATING METHODOLOGY ……........................................................................................... 13

FIGURE 13: PROGNOSTIC PARAMETER FOR A POPULATION OF FAILED SYSTEMS ..........................................................15

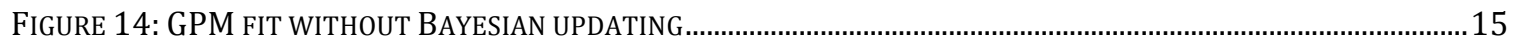

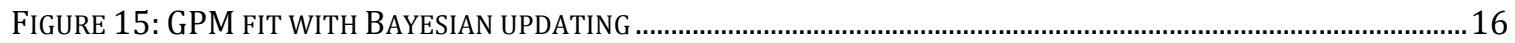

FIGURE 16: SAMPLE ESTIMATES OF THE SYSTEM STATE PRIOR TO WEIGHTING...........................................................17

FIGURE 17: SAMPLE ESTIMATES OF THE SYSTEM STATE AFTER WEIGHTING .............................................................. 18 


\section{Introduction}

The Process and Equipment Prognostics (PEP) Toolbox is a set of MATLAB-based tools that facilitate fast prototyping of empirical-based prognostic models developed at the University of Tennessee Nuclear Engineering Department. The goal of this User's Guide is to present the pertinent background information and basic syntactic information needed to employ the PEP Toolbox.

This guide begins by providing an introduction to the PEP Toolbox, including an overview of the toolbox architecture and requirements for employing the available functions. The relevant background of prognostics and the empirical techniques available in the PEP is summarized with appropriate references for the interested reader to find additional information. The algorithms and techniques employed in this toolbox are all openly available in literature. The PEP does not employ any protected or patented material, but instead provides a foundation for building empirical prognostic models for fast prototyping and model comparison. Appendix A includes an example application of the Process Equipment Monitoring (PEM) toolbox, discussed later, and PEP toolboxes for health monitoring of the turbofan engine data presented in the 2008 PHM Challenge.

\subsection{System Requirrements}

The system requirements for the PEP Toolbox are based on the requirements of MATLAB 7.11, which was used for development. Although some functions will execute properly on previous releases of MATLAB, it is suggested that that the PEP Toolbox be run on MATLAB 7 to ensure all functions execute as intended.

\subsubsection{Toolbox Requirements}

The PEP Toolbox employs functions from several existing MATLAB toolboxes. In order to use all functions in the PEP Toolbox, the following toolboxes must be available:

1) Process and Equipment Monitoring (PEM) Toolbox, release 1.5 or later[Hines, 2005]

2) Statistics Toolbox, release 7.4 or later

3) Signal Processing Toolbox, release 6.14 or later

4) Global Optimization Toolbox, release 3.1 or later

\subsection{Toolbox Architecture}

The current architecture of the PEP Toolbox, shown in Figure 1, includes functions supporting each of the three types of prognostics, which are discussed in the next chapter. Each of these modeling algorithms includes methods for estimating the 95\% uncertainty interval of the remaining useful life (RUL) estimates. Additional functionality is available to support model development. Each of the available functions and their use is discussed in later chapters. 


\section{Uncertainty Estimation}

\section{Reliability Models \\ Markov \\ Chain \\ Shock \\ Proportional
Hazards \\ General \\ Particle \\ Path Model \\ Filters}

\section{Type I}

Type II

Type III

Process and Equipment Prognostics (PEP) Toolbox

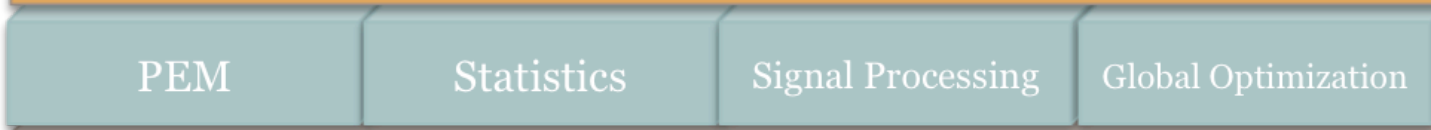

\section{MATLAB}

Figure 1: PEP Toolbox Architecture

\subsection{Release Notes}

This documentation has been prepared for the first formal release (version 1.0) of the PEP Toolbox. This release includes core functions of the PEP Toolbox to facilitate conventional reliability models, Markov chain, shock, proportional hazards, general path, and particle filtering prognostic models. It also includes methods for estimating the RUL uncertainty for each model type, commonly based on Monte Carlo procedures. Finally, algorithms are included to identify prognostic parameters from multiple data sources for Type III models. All methods and algorithms employed in the PEP Toolbox are widely available in the open literature; as such, there is no alternate research version of the PEP Toolbox as there is in the PEM toolbox. 


\section{Background}

Prognostics is one component of a complete health monitoring system which also includes system monitoring, fault detection, diagnostic modules, and operation and maintenance planning as shown in Figure 2. Full health monitoring systems, also called Condition Based Maintenance (CBM) systems, are the focus of much research. Data collected from a system of interest is monitored for deviations from normal behavior. Monitoring can be accomplished through a variety of methods, including first principle models, empirical models, and statistical analysis [Hines et al., 2006]. The monitoring module can be considered an error correction routine; the model gives its best estimate of the true value of the system variables. These estimates are compared to the data collected from the system to generate a time-series of residuals. Residuals characterize system deviations from normal behavior and can be used to determine if the system is operating in an abnormal state. In Figure 3 below, the residuals have the same shape and fail at similar values thus they can be used as a prognostic parameter.

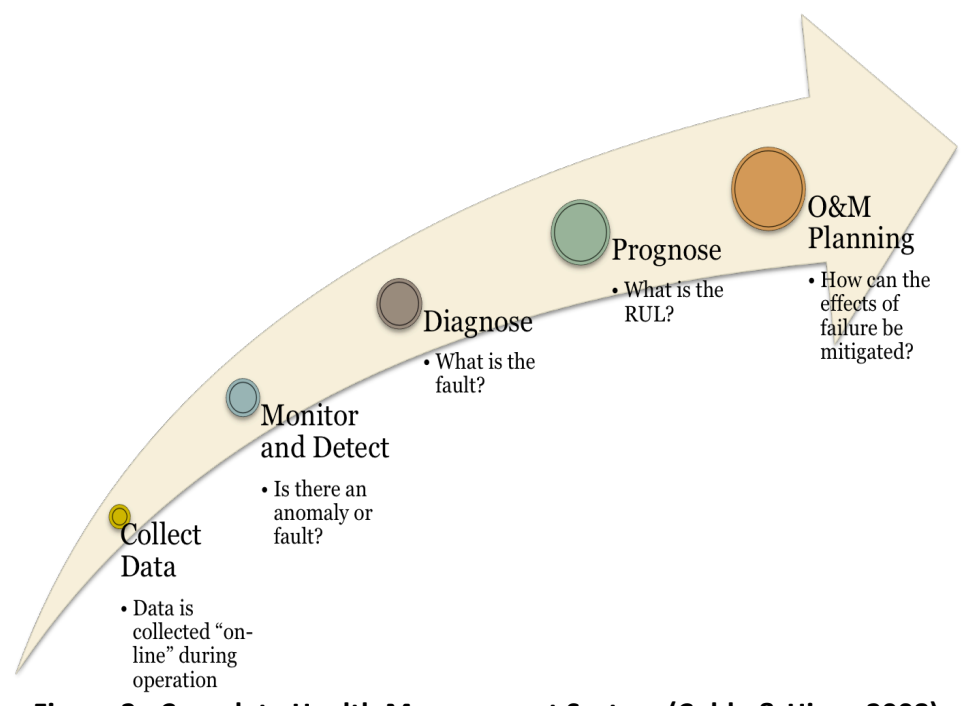

Figure 2: Complete Health Management System (Coble \& Hines 2008) 

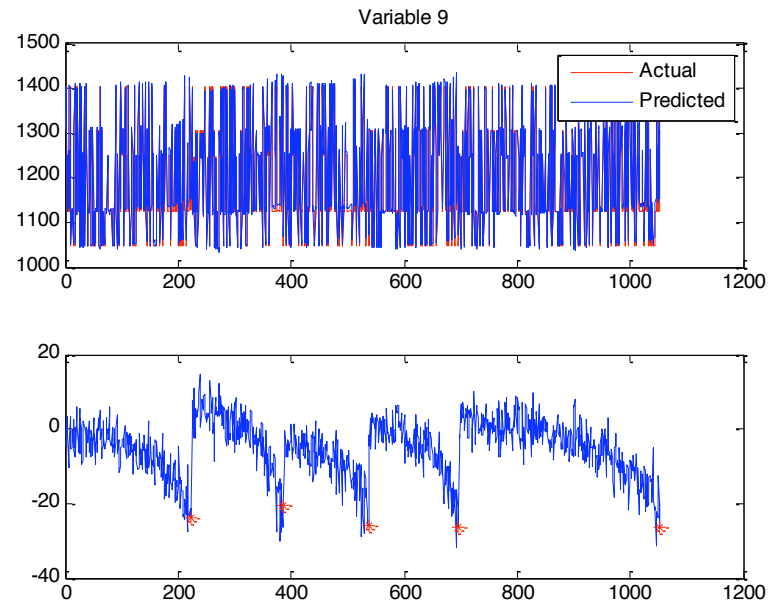

Figure 3: Example of Residuals used as a Prognostic Parameter

A common residual test for anomalous behavior is the Sequential Probability Ratio Test (SPRT) [Wald, 1945]. This statistical test considers a sequence of residuals and determines if they are more likely from the distribution that represents normal behavior or a faulted distribution, which may have a shifted mean value or altered standard deviation from the nominal distribution. If a fault is detected, it is often important to identify the type of fault; systems will likely degrade in different ways depending on the type of fault and so different prognostic models will be applicable. Expert systems, such as fuzzy rulebased systems, are common fault diagnosers. Finally, a prognostic model is employed to estimate the Remaining Useful Life (RUL) of the system or component. This model may include information from the original data, the monitoring system residuals, and the results of the fault detection and isolation routines. By applying the entire suite of modules, one can accomplish the goals of most prognostic systems: increased productivity; reduced downtime; reduced number and severity of failures, particularly unanticipated failures; optimized operating performance; extended operating periods between maintenance; reduced unnecessary planned maintenance; and reduced life-cycle cost.

Development of an integrated health management system can be daunting because of the high level of complexity involved in identifying appropriate algorithms at each stage. To support this, a suite of MATLAB-based toolboxes have been developed at the University of Tennessee PROaCT lab with a range of monitoring, fault detection and prognostic capabilities. The Process and Equipment Monitoring (PEM) toolbox was developed to facilitate auto-associative modeling of process and system data, and fault detection [2]. The PEM toolbox includes auto-associative kernel regression models, auto-associative neural networks, and linear regression models for system monitoring and sequential probability ratio test and error uncertainty limit monitoring fault detection methods. The results of both of these modules, as well as an independently developed diagnostic module, if available, can be used to facilitate prognostic analysis. The Process and Equipment Prognostics (PEP) toolbox is a MATLAB-based toolbox developed to aid in development of empirical prognostic models of each of the three types. The PEP toolbox is designed to integrate with the previously developed PEM Toolbox. The results of process monitoring and fault detection produced by the PEM toolbox as well as the original system data are utilized by the PEP toolbox to make RUL predictions for the system, as shown in Figure 4. 


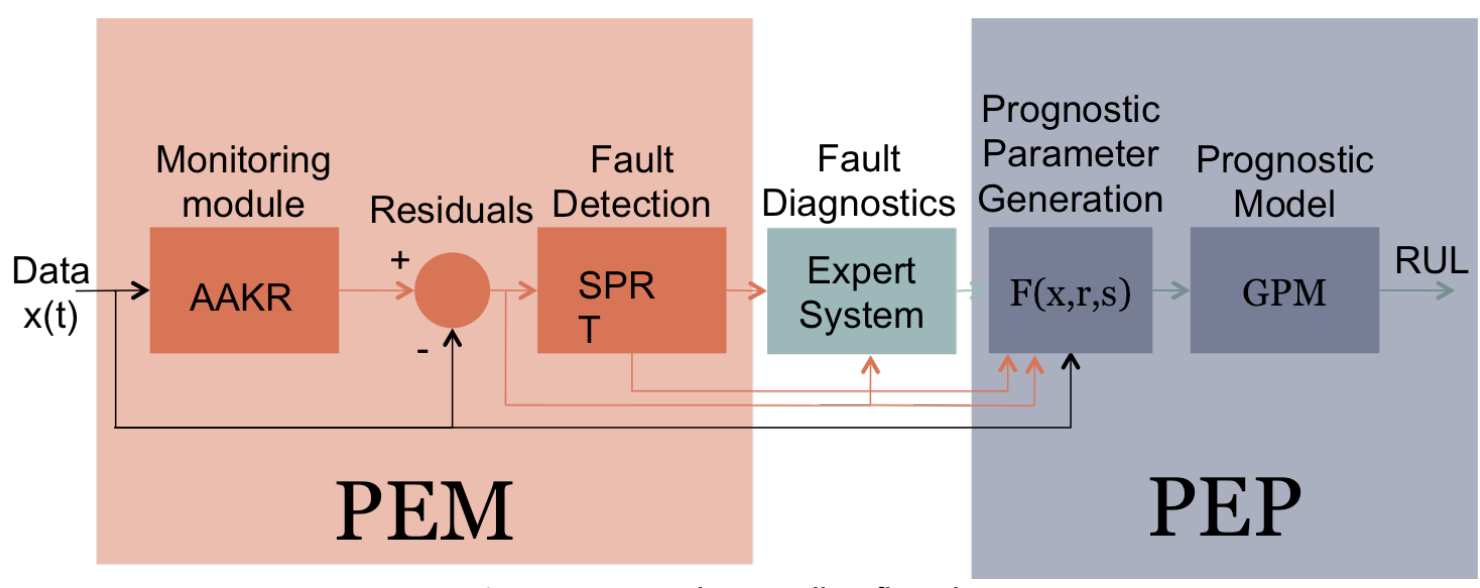

Figure 4: PEM and PEP toolbox flow chart

The purpose of the PEP toolbox is to provide a base set of tools to facilitate prognostic model development. A myriad of prognostic algorithms have been developed which use a variety of information sources, models, data processing algorithms, etc. Typically, prognostic model development depends highly on the expertise of the developer. The PEP toolbox reduces the development burden on the system designer and facilitates the rapid development and quantitative performance characterization of competing models.

A variety of algorithms have been developed for application to specific systems or classes of systems. The efficacy of these algorithms for a new process depends on the type and quality of data available, the assumptions inherent in the algorithm, and the assumptions that can validly be made about the system. As such, these prognostic algorithms can be categorized according to many criteria. One proposed categorization is based on the type of information used to make prognostic estimates; this results in three classes of prognostic algorithms, as shown in Figure 5. Type I prognostics is traditional time to failure reliability analysis; this type of prognostic algorithm characterizes the expected lifetime of an average system operating in a historically average environment. Type II methods characterize the average life under specific usage conditions. They can be used if operating condition stressors, such as load, input current and voltage, ambient temperature, vibration, etc., are measurable and correlated to system degradation. Algorithms in this class include specific formulations of the Markov Chain model, shock model, and proportional hazards model.[Hines, 2007] The final class of algorithms, Type III, or condition-based prognostics, characterizes the lifetime of a specific unit or system operating in its specific environment; these are the only truly individual-based prognostic models. These methods attempt to trend some measure of degradation, either directly measured from the system or inferred from other measurements, to a pre-defined failure threshold. The PEP toolbox includes algorithms representing each of the three categories. These algorithms are described in broad detail in the following sections. References to more detailed discussions are given for the interested reader. 


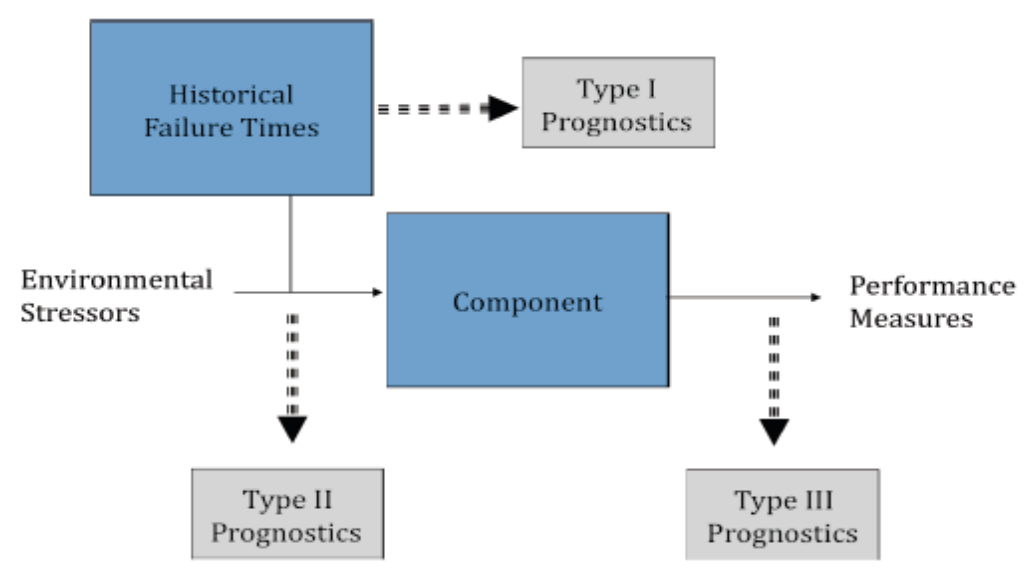

Figure 5: Prognostic Algorithm Categorization

\subsection{Type I Prognostics}

Type I methods are a simple extension of traditional reliability analysis, based entirely on an a priori distribution of failure times for similar systems in the past. Prognostic algorithms in this class characterize the average lifetime of an average system operating in historically average conditions; they do not utilize any information specific to the system at hand. The main assumption made when applying Type I methods is that future systems will operate under similar conditions to those seen in the past and will fail in similar ways.

Typically, Type I prognostic models track a population of systems over their lifetime and record only the failure time of each system. In addition, the total runtime of each system which hasn't failed at the end of the observation is recorded; this is called censored data and is also included in the analysis. A probability distribution is fit to these runtimes to give an estimate of the time of failure (ToF) distribution of the population. The most common parametric model used in reliability analysis is the Weibull distribution. This model is used because it is flexible enough to model a variety of failure rates. The formula for the failure rate, $\lambda(t)$, is a two parameter model with a shape parameter $(\beta)$ and a characteristic life $(\theta)$ :

$$
\lambda(t)=\frac{\beta}{\theta}\left(\frac{t}{\theta}\right)^{\beta-1}
$$

and the failure probability density is given by:

$$
f(t)=\left(\frac{\beta}{\theta}\right)\left(\frac{t}{\theta}\right)^{\beta-1} e^{-\left(\frac{t}{\theta}\right)^{\beta}}
$$

The two parameters in the Weibull model provide the modeling flexibility for components exhibiting an increasing failure rate $(\beta>1)$, a constant failure rate $(\beta=1)$, and a decreasing failure rate $(\beta<1)$. With the correct choice of shape parameter, the Weibull distribution adequately models the exponential, normal, or Rayleigh distributions. Examples of different shape parameters are given in Figure 6 , where $f(t)$ is the probability density of the Weibull distribution. Additional information on Weibull modeling is available in Abernethy [1996]. 


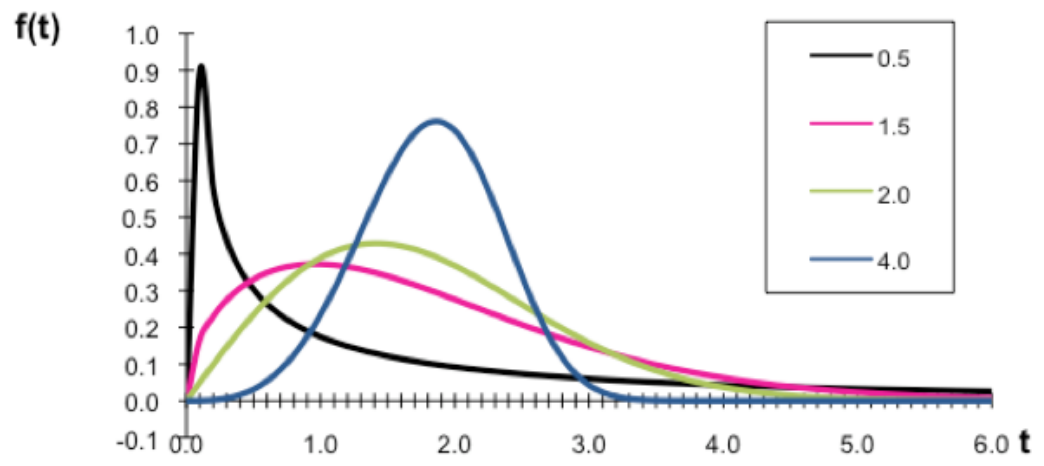

Figure 6: Weibull Failure Distributions with Different Shape Parameters

Most commonly, the Mean Residual Life (MRL) is used to estimate the RUL of a system using Type I prognostics. For a unit of age $t$, the MRL method assumes that the remaining life is a random variable, and the MRL is given by the expected value of this random variable [Guess and Proschan, 1985]:

$$
\operatorname{MRL}(t)=\frac{1}{S(t)} \int_{t}^{\infty} S(u) d u
$$

where $S(\cdot)$ is the survival function and $t$ is the current time. The survival function is the complemet of the CDF and gives the probability of surviving beyond time t. The MRL at time $t$ can be calculated from either parametric or nonparametric distributions, which makes it particularly flexible for application to real world data.

\subsection{Type II Prognostics}

Type II prognostic algorithms incorporate information about the operating conditions of the system into estimates of RUL. The PEP toolbox supports three Type II algorithms: Markov Chain models, shock models, and proportional hazards models.

\subsubsection{Markov Chain Models}

The Markov Chain model is based on the assumption that the next state which a system will occupy depends only on the current state; past states do not affect the probability of transitioning to a new state. There are two types of Markov Chain prognostic models, which vary only in the information they use to simulate possible future states. Type II Markov Chain models are composed of two models.

The first model, called the environmental model, is a Markov Chain simulation which produces possible future operating state progressions based on transition probabilities seen in the past and the current operating state. The environment model is needed for making a prediction as to how the environment and operating conditions evolve in the future. The environmental model is defined by the transition probability matrix, Q:

$$
Q=\left[\begin{array}{ccc}
p_{11} & \cdots & p_{1 n} \\
\vdots & \ddots & \vdots \\
p_{n 1} & \cdots & p_{n n}
\end{array}\right]
$$


where $\mathrm{p}_{\mathrm{ij}}$ is the probability of transitioning from state $i$ to state $j$. Often this probability matrix is assumed to be static, but it is straightforward to extend the method to time-dependent or degradation level-dependent transition probabilities. This model is used to simulate many possible future state progressions beginning at the current state.

These state progressions are then mapped to a degradation measure, which is the second model necessary in the Type II Markov Chain algorithm. The degradation measure is represented as a function of observable environmental conditions. To be useful for making a reliability prediction, the function should reflect the manner in which the environmental conditions affect the component reliability. Usually, environmental stressors tend to deteriorate the component reliability in a cumulative manner. Hence, the function to relate the environment conditions to the prognostic parameter is commonly of a cumulative form:

$$
Y\left(t_{k}\right)=\sum_{i=1}^{k} g\left(E\left(t_{i}, t_{i}+\Delta t_{i}\right)\right) \Delta t_{i}
$$

where $Y\left(t_{k}\right)$ is the degradation measure value at time $t_{k}, E\left(t_{i}, t_{i}+\Delta t\right)$ is the environmental condition observed at the time interval $\left[t_{i}, t_{i}+\Delta t\right]$, and $g($.$) is an appropriate function of environmental conditions.$

When the estimated degradation measure is found to cross some pre-defined threshold, failure is said to have occurred. At each time of interest, many possible state progressions are simulated and mapped to degradation measures. These measures are then used to define a time of failure (ToF) distribution for the system. At each time of interest, many degradation paths are simulated, and a probability of failure distribution is estimated from the collection as shown in Figure 7.

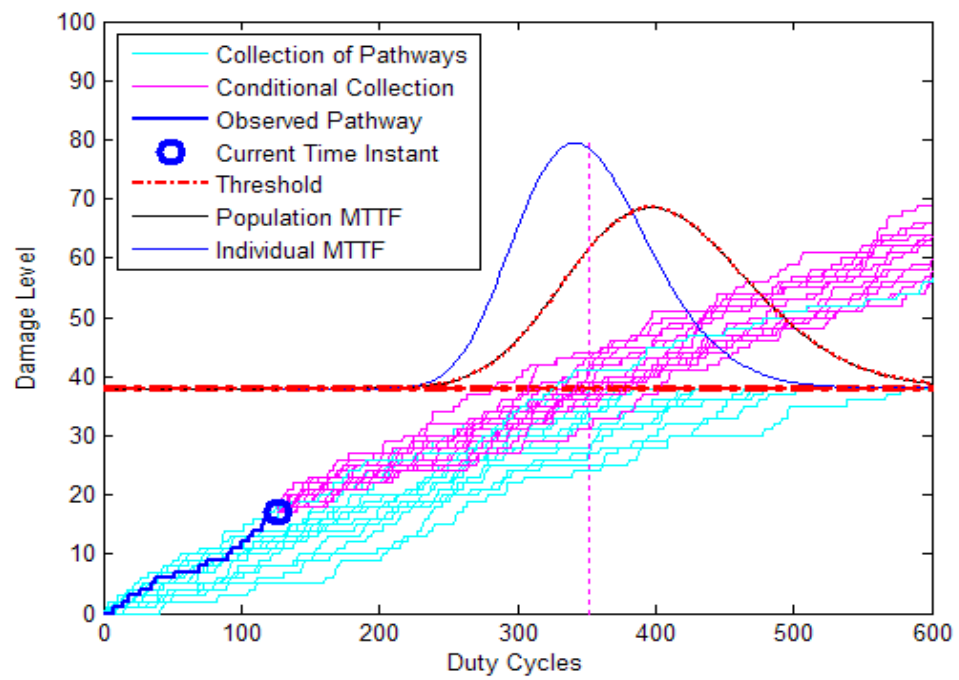

Figure 7: Markov Chain Model PoF estimation

In Figure 6, the blue lines are a collection of degradation pathways that grow towards the failure threshold over time. If the actual degradation is measureable, indicated by a dark blue path, then the model can be used to simulate future pathways from the current state. These are represented by the purple paths. The collection of degradation paths can be used to predict the failure distribution. The red distribution represents the population failure distribution while the blue distribution is the predicted distribution for the individual. [Hines, 2007] 


\subsubsection{Shock Models}

The Markov Chain model is continuous in the time domain, but discrete in the degradation measure. A more general formulation is the Shock model [Esary and Marshall, 1973; Gut, 1990; Mallor and Santos, 2003]. Instead of experiencing some known amount of shock at each random shock occurrence, the shock model allows for a shock of random size. Shock models have three parameters that are estimated from historical data: time between successive shocks, $t \sim \operatorname{Exp}(\lambda)$, magnitude of the shocks, $x \sim F(x)$, and the critical failure threshold.

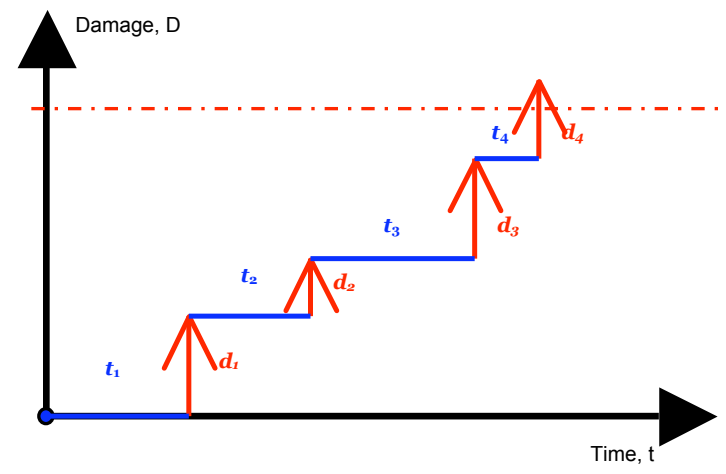

Figure 8: Shock Model Example

In this model, the time between random shocks is a continuous random variable, with the probability of shock often determined by the current degradation state, the operating conditions, or some combination thereof. The size of the shock may be based on a single shock size distribution, or other features such as the current degradation measure, the operating condition, or other measures available from the system may define it. Again, when the cumulative degradation measure crosses some predetermined threshold, failure is said to have occurred; a probability of failure distribution is estimated from multiple simulated degradation measures.

\subsubsection{Proportional Hazards Models}

The Proportional Hazards (PH) Model developed by Cox [1984] merges failure time data and stress data to make RUL estimates. The basic proportional hazard model assumes that the observed hazard rate is separable into a baseline hazard rate dependent only on time, $\lambda_{o}(t)$, and a second function which is independent of time but dependent on operating conditions, called covariates, $\psi(z ; \beta)$, where $z$ is a numerical quantification of the covariates and $\beta$ is regression parameters. The model uses the covariates to modify the baseline hazard rate to give a new hazard rate for the system's specific usage conditions:

$$
\lambda(t ; z)=\lambda_{0}(t) \exp \left(\sum_{j=1}^{q} \beta_{j} z_{j}\right)
$$

Therefore the observed hazard rate at any given covariate condition is always a multiple of the baseline hazard rate, $\lambda_{o}(t)$, and a proportionality constant determined by the covariate function, $\psi(z ; \beta)$, hence the name proportional hazards model. Generally $\psi(z ; \beta)$ is assumed to have some type of known functional form such as logistic, linear, inverse linear, or exponential. Typically, and in the PEP Toolbox, this function is assumed to be exponential. Generally $\lambda_{o}(t)$ does not have any assumed functional form, and the PEP Toolbox utilizes a non-parametric, empirical-based baseline hazard rate. 
Failure data collected at a variety of covariate operating conditions are used to solve for the parameters $\left(\beta_{j}\right)$ using an ordinary least squares algorithm. The baseline hazard is the hazard rate when covariates have little or no influence on the failure rate. A basic assumption of the proportional hazards model is that the effects of these covariates are multiplicative; this means that when the ratio of two covariates is evaluated, their hazard rates are proportional.

In the following example, a tire is operated in one of three operating conditions: normal, off-road, and high slip. To check for proportionality of the covariates of all three operating conditions, the log of the negative log of the reliability function is plotted as seen in Figure 9 below.

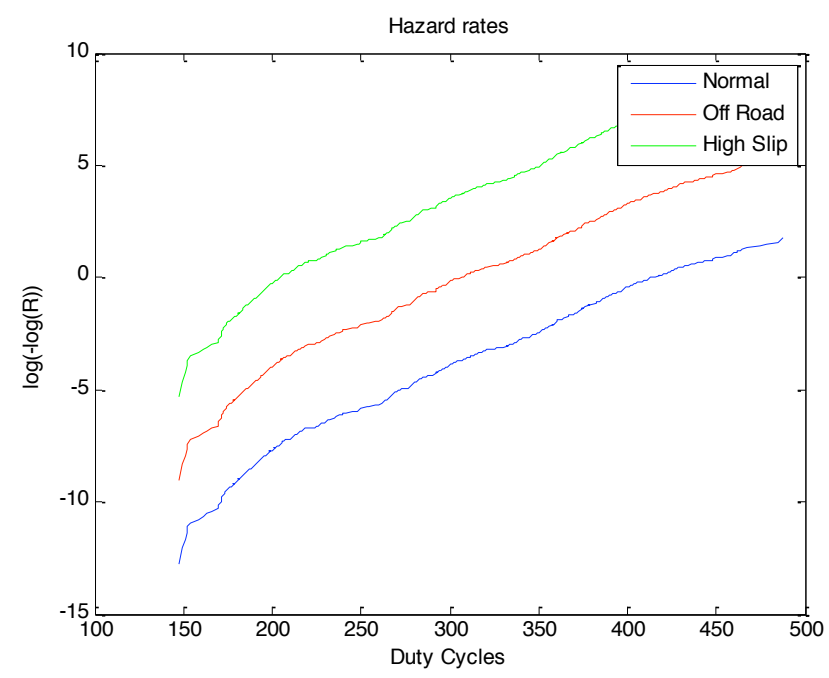

Figure 9 Check for proportionality of PHM covariates

The baseline hazard rate is then estimated with the covariate value of 0 for normal conditions. The predicted hazard rate can be seen in Figure 10 below.

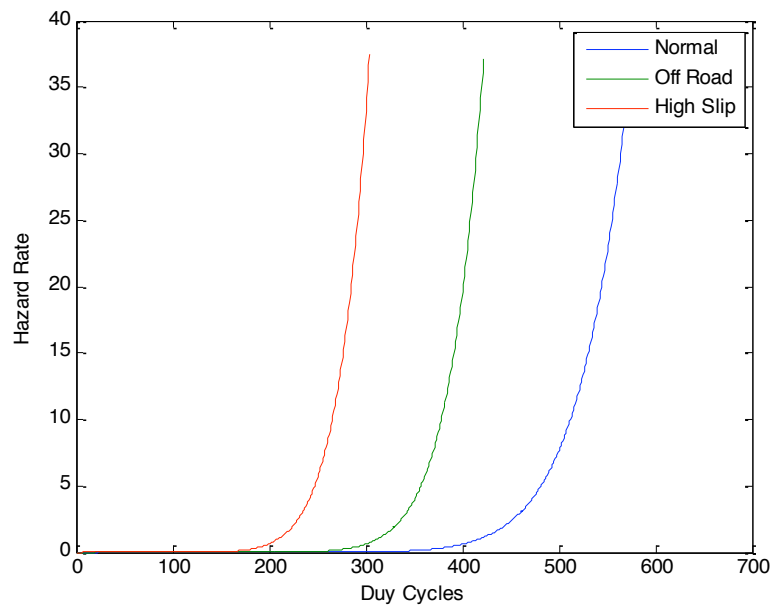

Figure 10 Estimated Cumulative Hazard Rates 
Finally, the samples are used to collect the PH model's coefficients with the covariates of 1 for off road and 2 for high slip. The model is then run and results in a regression coefficient that can then be used to model the hazard rates. Figure 11 below shows the comparison of the actual data to the PH model's results. A full discussion of developing a proportional hazards model can be found in [Kumar and Kelfjo, 1994].

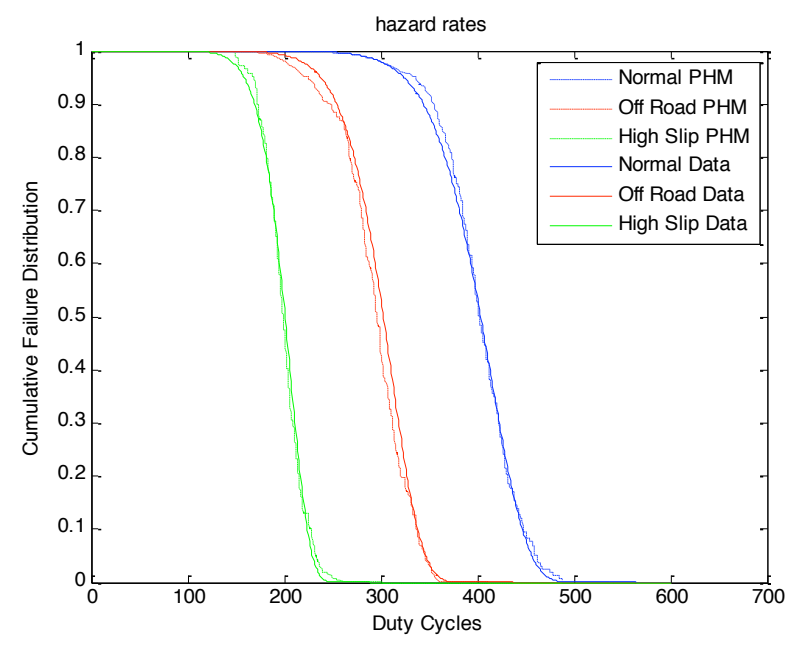

Figure 11 Comparison of Actual Data to PH model's results

\subsection{Type III Prognostics}

Type III models consider the actual condition of the system, either directly measured from the system or inferred from other measurements. These condition measurements, often called degradation measures or prognostic parameters, are used to extrapolate from the current condition to a pre-defined critical failure threshold. The following section introduces the idea of the prognostic parameter. This is followed by descriptions the two Type III models available in the PEP Toolbox: general path model and particle filtering model. Finally, a short discussion of selecting an appropriate prognostic parameter is given, which necessary for either algorithm.

\subsubsection{Prognostic Parameter}

Effects-based prognostics uses degradation measures to form a prognostic prediction. A prognostic parameter, also called a degradation measure, is a scalar or vector quantity that numerically reflects the current ability of the system to perform its designated functions properly. It is a quantity that is correlated with the probability of failure at a given moment. A degradation path is a trajectory along which the degradation measure is evolving in time towards the critical level corresponding to a failure event. Type III prognostics attempt to extrapolate along this degradation path to determine the RUL of a component or system.

The degradation measure does not have to be a directly measured parameter. It could be a function of several measured variables that provide a quantitative measure of degradation. It could also be an empirical model prediction of the degradation that cannot be measured. For example, pipe wall thickness may be an appropriate degradation parameter but there may not be an unobtrusive method to directly measure it. However, there may be related measurable variables that can be used to predict 
the wall thickness. In this case the degradation parameter is not a directly measurable parameter but a function of several measurable parameters. Monitoring system residuals are intuitive candidates for prognostic parameters because they naturally characterize how "far" a system is from normal operation.

When the degradation level of a system reaches some predefined critical failure threshold, the system is said to have experienced a soft failure; for example, car tire tread is below some specified depth. These failures generally do not concur with complete loss of functionality, as in a hard failure; however, they correspond with the time when an operator is no longer confident that equipment will continue to work to its specifications. Both general path models and particle filters attempt to extrapolate the prognostic parameter to a critical failure threshold to estimate the RUL; these algorithms are described in the following sections.

\subsubsection{Generall Path Model}

The General Path Model (GPM) was first proposed by Lu and Meeker [1993] to move reliability analysis from failure time to failure mode analysis. The GPM reliability methodology has a natural extension to estimation of RUL of an individual component or system. GPM analysis begins with some assumption of an underlying functional form of the degradation path for a specific fault mode. The degradation of the $i^{\text {th }}$ unit at time $t_{j}$ is given by:

$$
y_{i j}=\eta\left(t_{j}, \phi, \theta_{i}\right)+\varepsilon_{i j}
$$

where $\phi$ is a vector of fixed (population) effects, $\theta_{i}$ is a vector of random (individual) effects for the $i^{\text {th }}$ component, and $\varepsilon_{i j} \sim N\left(0, \sigma_{\varepsilon}^{2}\right)$ is the standard measurement error term. The model parameters are estimated from the available data. This degradation path model, $y_{i}$, can be extrapolated to the failure threshold, $D$, to estimate the component's time of failure.

As data is collected during use, the degradation model is fit for the individual component. This specific model can be used to project a time of failure for the component. Because of noise in the degradation signal and uncertainty in the failure threshold, the projected time of failure is not exact. Monte Carlo techniques are used with estimates of the uncertainty sources to project a $95 \%$ uncertainty interval around the RUL estimate.

The traditional GPM methodology considers only the data collected on the current unit to fit the degradation model. However, prior information is available from the historic degradation paths used for initial model fitting, including the mean degradation path and associated distributions. This data can provide valuable knowledge for fitting the degradation model of an individual component, particularly when only a few data points have been collected or the collected data suffers from excessive noise. Bayesian updating methods are used to incorporate this additional information in the fitted model.

Bayesian updating is a method for combining prior information about the set of model parameters with new data observations to give a posterior distribution of the model parameters (Figure 12). This allows both current observation and past knowledge to be considered in model fitting. 


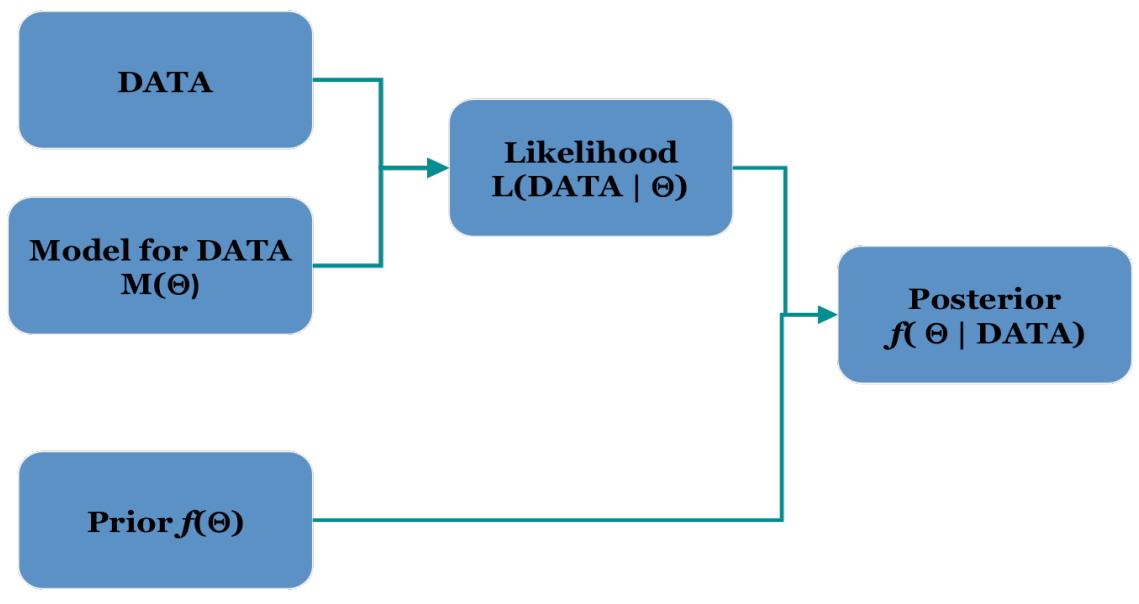

Figure 12: Bayesian Updating Methodology

The PEP Toolbox allows for Bayesian updating of linear regression models. These models are not necessarily linear models, but are linear-in-parameters. In this vein, a linear regression model is given by:

$$
Y=b X
$$

The model parameters are estimated as:

$$
b=\left(X^{T} \Sigma_{y}^{-1} X\right)^{-1} X^{T} \Sigma_{y}^{-1} Y
$$

where $\Sigma_{y}$ is the variance-covariance noise matrix for the response observations. It is important to note that the linear regression model is not necessarily a linear model. The data matrix $X$ can be populated with any function of degradation measures, including higher order terms, interaction terms, and functions such as $\sin (x)$ or $e^{x}$. If prior information is available for a specific model parameter, i.e. $B_{j} \sim N\left(B_{j o}, \sigma^{2}{ }_{B}\right)$, then the matrix $X$ should be appended with an additional row with value one at the $j^{\text {th }}$ position and zero elsewhere, and the $Y$ matrix should be appended with the a priori value of the $j^{\text {th }}$ parameter.

$$
\begin{aligned}
X^{*} & =\left[\begin{array}{llllllll}
X ; & 0 & \cdots & 0 & 1 & 0 & \cdots & 0
\end{array}\right] \\
Y^{*} & =\left[\begin{array}{lll}
Y ; & \beta_{j}
\end{array}\right]
\end{aligned}
$$

Finally, the variance-covariance matrix is augmented with a final row and column of zeros, with the variance of the a priori information in the diagonal element.

$$
\Sigma_{y}^{*}=\left[\begin{array}{cccc}
\sigma_{y}^{2} & 0 & \cdots & 0 \\
0 & \ddots & 0 & \vdots \\
0 & \cdots & \sigma_{y}^{2} & 0 \\
0 & \cdots & 0 & \sigma_{\beta_{j}}^{2}
\end{array}\right]
$$

If knowledge is available about multiple regression parameters, the matrices should be appended multiple times with one additional row for each parameter. 
It is convenient to assume that the noise in the degradation measurements is constant and uncorrelated. Some a priori knowledge of the noise variance is available from the exemplar degradation paths. If this assumption is not valid for a particular problem, then other methods of estimating the noise variance must be used. The assumption of uncorrelated noise allows the variance-covariance matrix to be a diagonal matrix consisting of noise variance estimates and a priori knowledge variance estimates. If this assumption is not valid, including covariance terms is trivial; again these terms can be estimated from historical degradation paths.

After a priori knowledge is used to obtain a posterior estimate of degradation parameters, this estimate becomes the new prior distribution for the next estimation of degradation parameters. The variance of this new knowledge is estimated as:

$$
\frac{1}{\sigma_{\text {post }, \beta_{j}}^{2}}=\frac{n}{\sigma_{y}^{2}}+\frac{1}{\sigma_{\text {prior }, \beta_{j}}^{2}}
$$

where $n$ is the number of observations used to fit the current model.

Bayesian updating is particularly effective when few data points are available or the data is contaminated with a high level of noise. An example application is given here to illustrate the efficacy of Bayesian techniques. Figure 13 shows the prognostic parameter for a population of failed systems. These parameters have a clear, negative trend toward failure which is roughly quadratic in nature. For this GPM system, a quadratic function is fit to data from a new system and extrapolated to a critical failure threshold of approximately -20 . Figure 14 shows the results of applying the GPM to approximately 90 observations of noisy data from a new system. As the figure shows, the small amount of data available includes noise levels which preclude appropriate model fitting. Figure 15 shows the prognostic result for the same system when Bayesian updating is employed. In this model, the inadequate data available from the system is augmented with prior information to "force" the model fit to take the downward shape which is historically expected. The RUL estimate obtained with the Bayesian approach is 135 cycles, versus an undeterminable estimate obtained from the non-Bayesian approach. The actual RUL after the first 84 observations is 170 cycles, resulting in an RUL error of approximately $20 \%$. While this error is still high, it is within a reasonable accuracy considering the amount of data available and will improve further as more data is collected. 


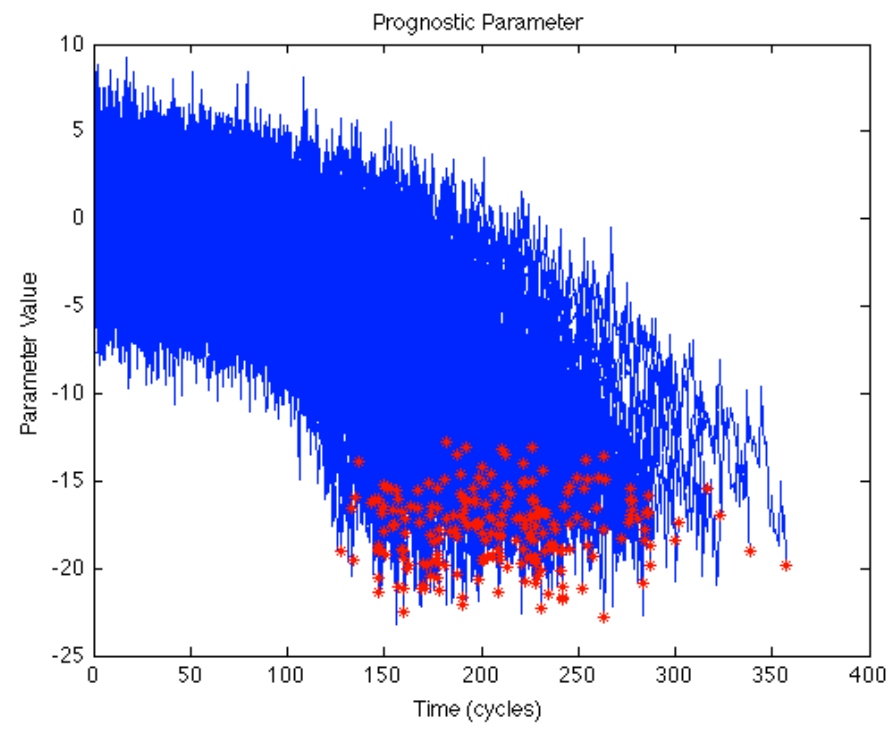

Figure 13: Prognostic Parameter for a Population of Failed Systems

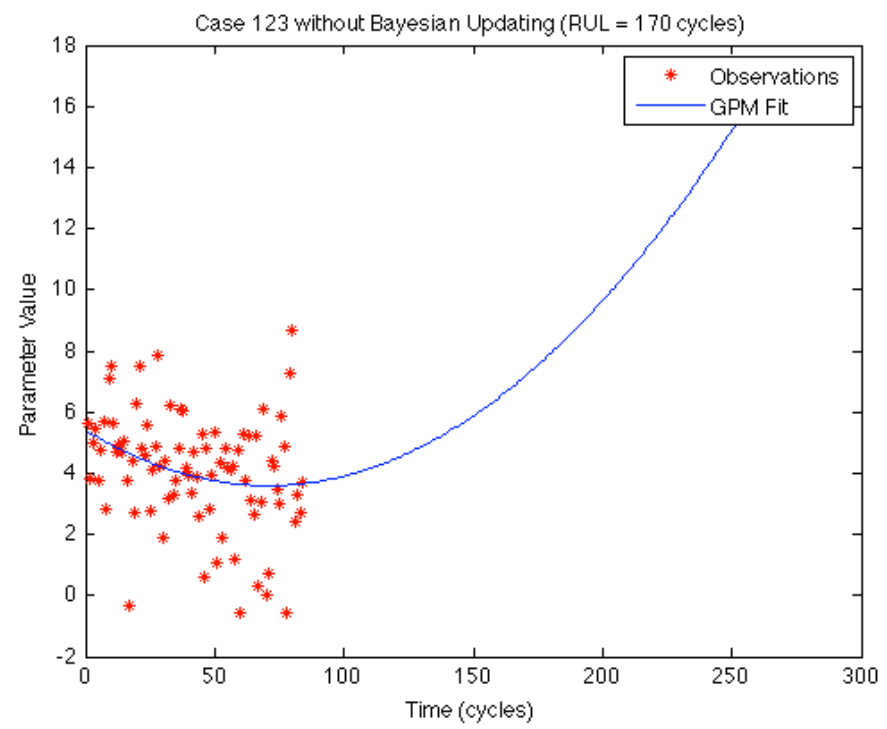

Figure 14: GPM fit without Bayesian updating 


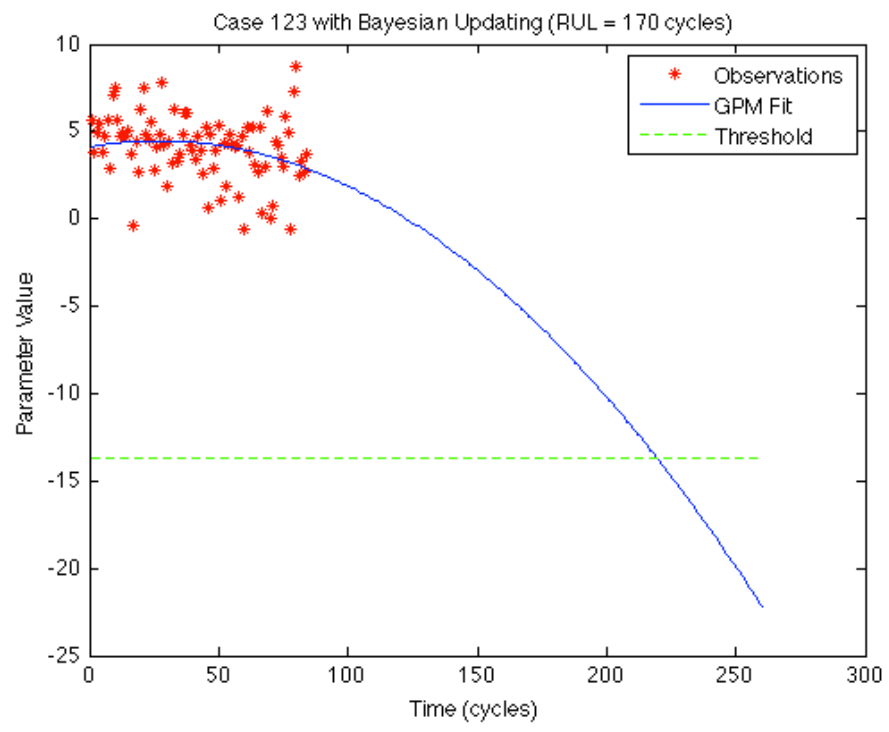

Figure 15: GPM fit with Bayesian updating

\subsubsection{Particle Filtering Model}

Particle filtering was originally developed to provide an estimation of the marginal probability in Bayes' Theorem that would allow for modeling of nonlinear systems and potentially non-Gaussian noise [Cadini, 2009]. The particle filter method utilizes Monte Carlo simulation to provide an approximate solution to the marginal distribution by generating artificial random samples and comparing their distribution to that of the measurements. The particle filter method first starts with Bayes' Theorem:

$$
p\left(\boldsymbol{x}_{k} \mid \mathbf{z}_{0: k}\right)=\frac{p\left(\mathbf{z}_{k} \mid \boldsymbol{x}_{k}\right) * p\left(\boldsymbol{x}_{k} \mid \mathbf{z}_{0: k-1}\right)}{p\left(\mathbf{z}_{k} \mid \mathbf{z}_{0: k-1}\right)}
$$

Here, $\mathbf{x}$ represents the state-space vector of the system, which is not directly measured. In condition monitoring, this is typically the prognostic parameter or other measure of system health. The term $\mathbf{z}$ represents the vector of measurements. Measurements are first taken at time $=0$; the "current" time, or the time of interest is represented by the subscript $k$, and the previous time step is $k-1$. The term $p\left(\boldsymbol{x}_{k} \mid \boldsymbol{z}_{0: k}\right)$ is defined as the posterior distribution and represents the distribution of the likelihood of a system state $\mathbf{x}_{\mathrm{k}}$ existing given the measurements $\mathbf{z}_{0: k}$ (i.e. all measurements, including the current measurement). The term $p\left(\mathbf{z}_{k} \mid \boldsymbol{x}_{k}\right)$ is the conditional probability and represents the likelihood that a given state would yield the current measurements. The term $p\left(\boldsymbol{x}_{k} \mid \mathbf{z}_{0: k-1}\right)$ is the prior distribution and is the likelihood that a given state would exist based on all measurements prior to the current measurement. Finally, $p\left(\boldsymbol{z}_{k} \mid \boldsymbol{z}_{0: k-1}\right)$ is the marginal probability and represents the likelihood that the current measurements would occur given all previous measurements.

The long-standing difficulty with Bayes' Theorem is determining the marginal probability, and this is the purpose of particle filtering. The first step in particle filtering is Sequential Importance Sampling (SIS), where a known distribution is used to generate random samples for $\mathbf{x}_{0: k}$. (In contrast to GPM, SIS updates all particles simultaneously at a given time step rather than updating a single particle all the way to failure.) The distribution only needs to ensure that the range of possibilities is covered, though a distribution that closely resembles the true distribution of probabilities should provide faster convergence and more reliable results. This distribution is defined as an importance function: 


$$
q\left(\boldsymbol{x}_{k} \mid \boldsymbol{z}_{0: k}\right)
$$

Next, weights for the sample particles are defined by relating the importance function to the posterior distribution:

$$
w_{k}^{i} \propto \frac{p\left(x_{k}^{i} \mid z_{0: k}\right)}{q\left(x_{k}^{i} \mid z_{0: k}\right)}
$$

In the above equation, both terms in the ratio are unknown. However, the weights from the previous time step are known and can be used to approximate the weights according to (1). The approximation may require normalization to form a true pdf; this can be readily performed after all weights are estimated.

$$
w_{k}^{i} \propto w_{k-1}^{i} * \frac{p\left(\mathbf{z}_{k} \mid x_{k}^{i}\right) p\left(x_{k}^{i} \mid x_{k-1}^{i}\right)}{q\left(x_{k}^{i} \mid x_{0: k-1}^{i}, \mathbf{z}_{0: k}\right)}
$$

A visual example of the weighting process may be seen in Figure 16 and Figure 17. Prior to weighting, all samples have equal weight. At time step 20, those particles whose states have a higher likelihood of representing the true state of the system (as estimated through measurements) receive greater weight; the particles with less likelihood of representing the system receive less weight. Having re-weighted the particles, they now represent an updated posterior distribution of the marginal probability and the new posterior distribution may be estimated.

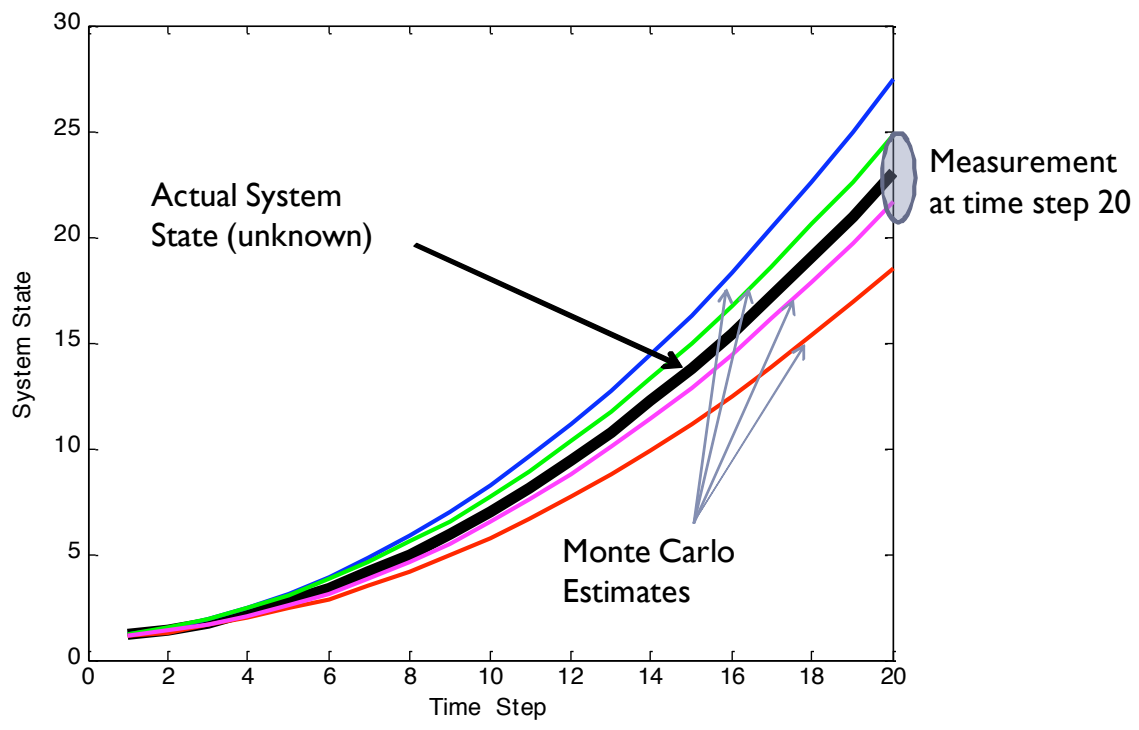

Figure 16: Sample Estimates of the System State Prior to Weighting 


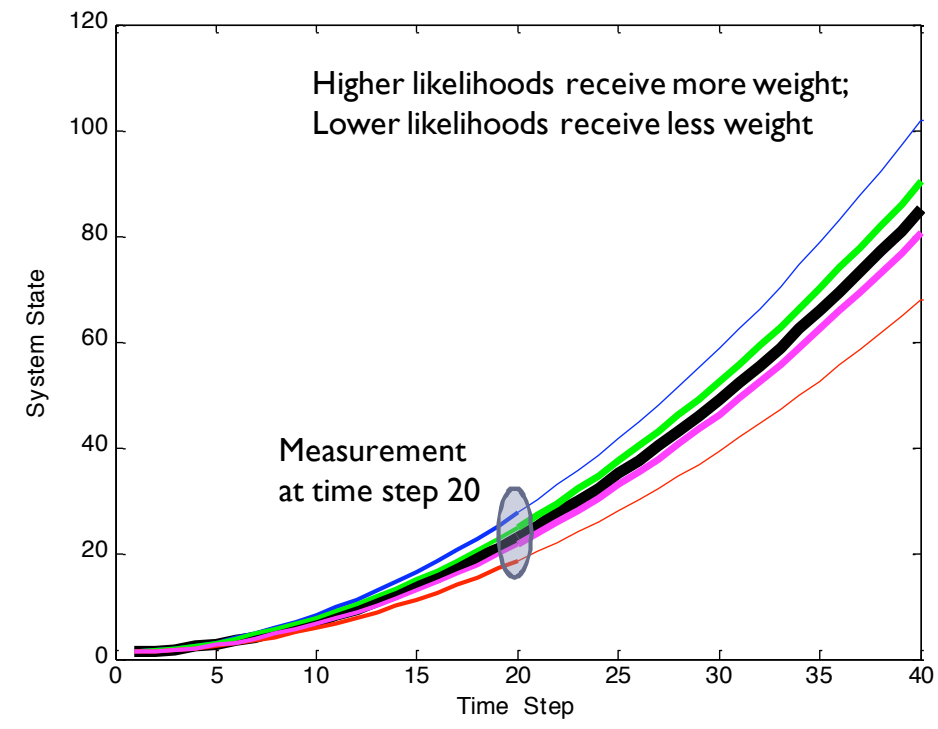

Figure 17: Sample Estimates of the System State after Weighting

As this process is repeated, the particle weights are continually updated every time Bayes' Rule is applied. Since SIS will add weight to particles with the highest likelihood at the expense of lowerlikelihood particles, the process will eventually drive the weights of all particles to zero except for the highest-likelihood particle, a problem known as degeneracy. To avoid degeneracy, the particles are occasionally redistributed by Sequential Importance Resampling (SIR).

SIR may be conducted by a variety of methods, but the general approach is to replace the existing weighted particles with new unweighted particles chosen by the posterior distribution provided by SIS. In review, SIS may be seen as a filter of particle weights where the weights are updated to fit the estimated posterior distribution. SIR may therefore be seen as a filter of the particles themselves, where new particles are chosen based on the weighted particles after SIS. This two-stage process is the heart of particle filtering.

Because particle filtering employs a Monte Carlo process, uncertainty estimates may be readily provided by the existing particle distributions. The state estimate at the present time is given by (2). The failure probability estimate at a future time $k+i$ is given by (3). Finally, the failure time distribution at a future time $k+i$ is given by (4).

$$
\begin{gathered}
p\left(\boldsymbol{x}_{k} \mid \mathbf{z}_{0: k}\right) \approx \sum_{i=1}^{N} w_{k}^{i} \delta\left(\boldsymbol{x}_{0: k}-\boldsymbol{x}_{0: k}^{i}\right) \\
\hat{p}(k+i) \approx \frac{\sum_{m, x_{k}^{m}>d^{*}} w_{k}^{m}}{\sum_{n, x_{k}^{n}>d^{*}} w_{k}^{n}} \\
p\left(\tau_{k} \mid \mathbf{z}_{0: k}\right) \approx \sum_{i=1}^{N} w_{k}^{i} \delta\left(\tau_{k}-t^{i}\right)
\end{gathered}
$$

Additional statistical inferences may be made via the particle distributions, such as $95 \%$ confidence intervals, hypothesis tests, etc.

Both Type III prognostic algorithms described rely on an appropriate prognostic parameter for modeling and extrapolating to failure. The PEP toolbox includes functionality to automatically select an 
appropriate, near-optimal prognostic parameter from available data sources. The following section briefly describes that process.

\subsubsection{Selecting a Prognostic Parameter}

An ideal prognostic parameter has three key qualities: monotonicity, prognosability, and trendability [Coble and Hines, 2009]. Monotonicity characterizes the underlying positive or negative trend of the parameter. This is an important feature of a prognostic parameter because it is generally assumed that systems do not undergo self-healing, which would be indicated by a non-monotonic parameter. However, this assumption is not valid for some components such as batteries, which may experience some degree of self-repair during short periods of nonuse. The monotonic trend is considered valid when considering an entire system, even if individual components or sub-systems may experience some self-repair. Prognosability gives a measure of the variance in the critical failure value of a population of systems. Ideally, failure should occur at a crisp, well-defined degradation level. A wide spread in critical failure values can make it difficult to accurately extrapolate a prognostic parameter to failure. Finally, trendability indicates the degree to which the parameters of a population of systems have the same underlying shape and can be described by the same functional form. These three intuitive metrics are formalized in [Coble, 2010] to give a quantitative measure of prognostic parameter suitability.

Monotonicity is a straightforward measure given by:

$$
\text { Monotonicity }=\text { mean }\left(\left|\frac{\# \operatorname{pos} d / d x}{n-1}-\frac{\# n e g d / d x}{n-1}\right|\right)
$$

where $\mathrm{n}$ is the number of observations in a particular history. The monotonicity of a population of parameters is given by the average difference of the fraction of positive and negative derivatives for each path. When using data collected or inferred from actual systems, it is important to adequately smooth the data to give more accurate estimates of the derivatives. Numerical calculation of a function derivative should rarely be left to a simple difference function; the addition of noise makes this method inaccurate and impractical. In practice, fitting a line to a small portion of the data, perhaps five or ten observations, and taking the derivative to be the slope of that line will give a more realistic measure of the slope. When this method is employed, the above equation for monotonicity need only be adjusted for the number of calculated derivatives. Instead of $n-1$ derivatives, $n-m$ derivatives may be calculated, where $\mathrm{m}$ is the number of data points used to calculate one derivative. The population of good parameters shown in Error! Reference source not found. is clearly monotonically increasing, in the absence of noise; the monotonicity metric for this parameter is 0.940 . Conversely, the bad parameter shown in Error! Reference source not found. has monotonicity of 0.501 . It is important to note that the current formulation of monotonicity does not consider if the entire population is monotonic in the same direction, only that each individual exhibits an either generally increasing or decreasing trend. This is an undesirable feature in the prognostic parameter; however, it is considered in characterizing the prognosability which looks at how well clustered failure values are. If failure values for the entire population are well clustered and the individual parameters are monotonic, then the population must have either an increasing or decreasing monotonicity.

Prognosability is calculated as the deviation of the final failure values for each path divided by the mean range of the path. This is exponentially weighted to give the desired zero to one scale: 


$$
\operatorname{Pr} \text { ognosability }=\exp \left(-\frac{\text { std }(\text { failurevalues })}{\text { mean }(\mid \text { failurevalue }- \text { startingvalue } \mid)}\right)
$$

This measure encourages well-clustered failure values, i.e. small standard deviation of failure values, and large parameter ranges. This gives the model a long range to predict a very precise value, which can be related to the notice period discussed previously. The failure values for the good prognostic parameter are very well clustered, following a wide range; the prognosability is 0.930 . The failure values of the population of bad prognostic parameters cover a wide range of values; this parameter has prognosability of only 0.346 .

Characterizing the trendability of a population of parameters poses significant difficulty compared to the other two metrics. A candidate parameter is trendable if the same underlying functional form can model each parameter in the population. Initially, trendability was characterized by comparing the fraction of positive first and second derivatives in each parameter. However, this naïve approach was highly susceptible to noise and did not provide a clear distinction between trendable and not-trendable parameters. An improved method for characterizing trendability is used in which prognostic parameters are re-sampled with respect to the fraction of total lifetime. This results in each prognostic parameter containing exactly 100 observations, with each observation corresponding to $1 \%$ of lifetime. The linear correlation is calculated across the population of prognostic parameters, and the trendability is given by the smallest absolute correlation:

$$
\text { Trendability }=\min \left(\left|\operatorname{corrcoef}_{i j}\right|\right)
$$

The PEP toolbox utilizes these suitability metric with genetic algorithm optimization to identify a nearoptimal prognostic parameter from the available data sources. The prognostic parameter is optimized according to the following fitness function:

$$
\text { fitness }=w_{m} \text { monotonicity }+w_{p} \text { prognosability }+w_{t} \text { trendability }
$$

The previous sections briefly described the algorithms available in the PEP toolbox. The following chapters list the functions included in the PEP toolbox and introduces their use. 


\section{Functions - Categorical List}

The Process and Equipment Prognostics (PEP) Toolbox is designed to work with the previously developed Process and Equipment Monitoring (PEM) Toolbox to develop a suite of health management tools. The PEP includes functionality to perform the three types of prognostic models described previously in this guide. Each of the functions currently integrated in the PEP Toolbox are described below, categorized by function purpose. This list is continuously being updated as the PEP toolbox is developed and improved.

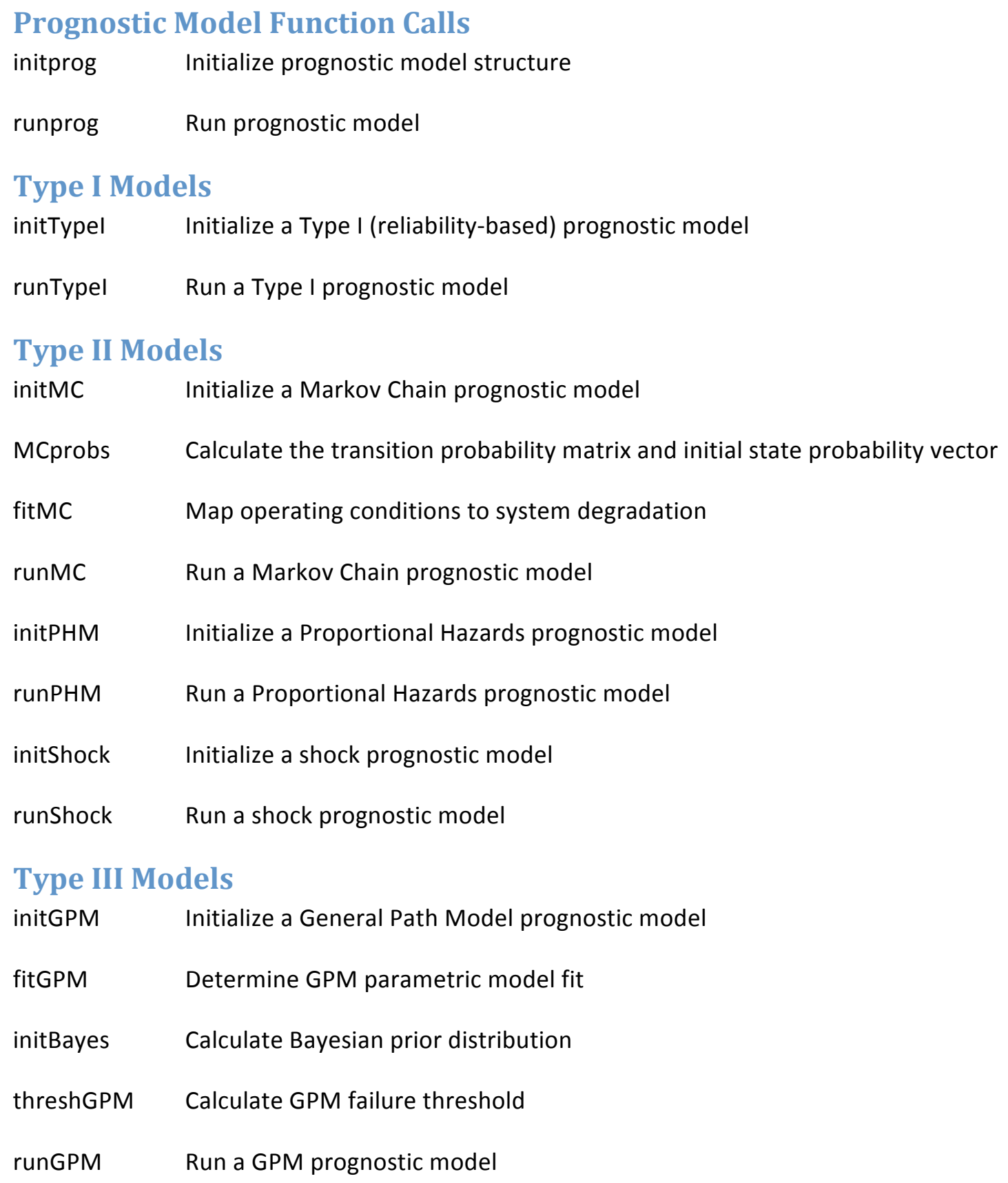




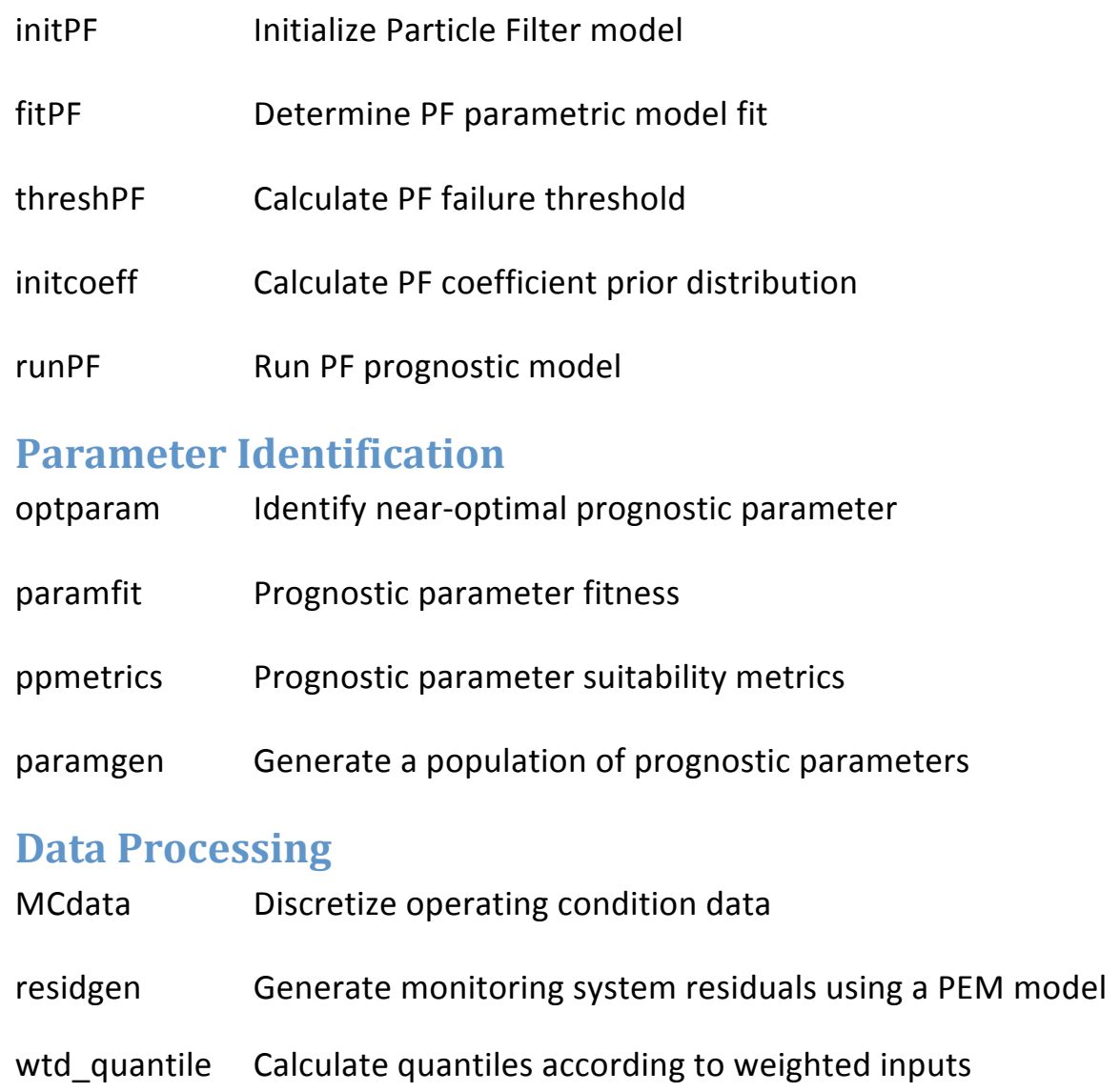




\section{Functions - Alphabetical List}

This section presents the help information for each of the PEP Toolbox functions. This help information includes syntax, descriptions, flag/value pairs if applicable, and example usages. For a categorical list of functions, refer to the previous chapter. 


\section{fitGPM}

Determine GPM parametric model fit

Syntax:

[Fit Ytransform] $=$ FITGPM(prognosticparameters)

Description:

This function determines the best fit for a GPM prognostic model between linear, quadratic, and exponential fits.

[Function Ytransform] = FITGPM(prognosticparameters) determines the best fit for the historic paths contained in the cell array prognosticparameters. The function considers linear, quadratic, and exponential fits. Ytransform gives the transformation of y needed to make the fit linear in parameters. This has value @(y)log(y) for exponential functions and @(y)y (indicating no transformation is needed) for linear and quadratic functions.

Example:

load degradation

whos

$\begin{array}{lcrlr}\text { Name } & \text { Size } & \text { Bytes Class } & \text { Attributes } \\ & & 1 \times 376 & \text { cell } \\ \text { failed } & 1 \times 25 & 40 & \text { double } & \\ \text { failtimes } & 1 \times 5 & 1536 & \text { cell }\end{array}$

failval $=\operatorname{NaN}(1,25)$;

failtime $=\operatorname{NaN}(1,25)$;

figure

hold on

for $i=1: 25$

plot (failed $\{i\}(:, 1)$, failed $\{i\}(:, 2), b^{\prime}$ )

failval $(i)=$ failed $\{i\}($ end, 2$)$;

failtime $(i)=$ failed $\{i\}($ end, 1$)$;

end

plot (failtime, failval, 'r*')

hold off

xlabel ('Time (cycles)')

ylabel('Prognostic Parameter')

title('Population of Prognostic Parameters') 


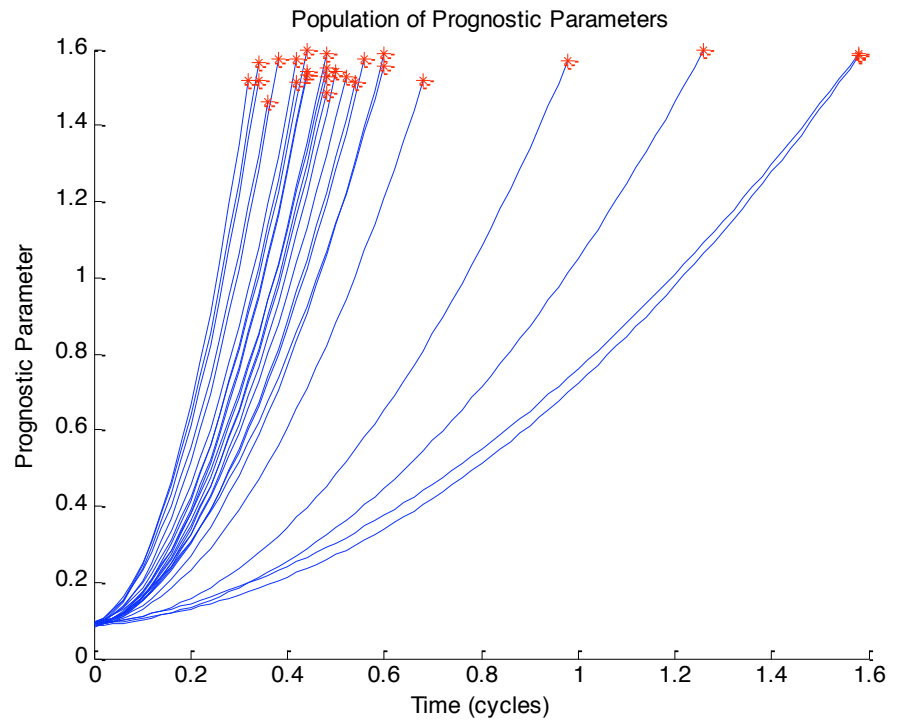

[fit ytrans]=fitGPM (failed)

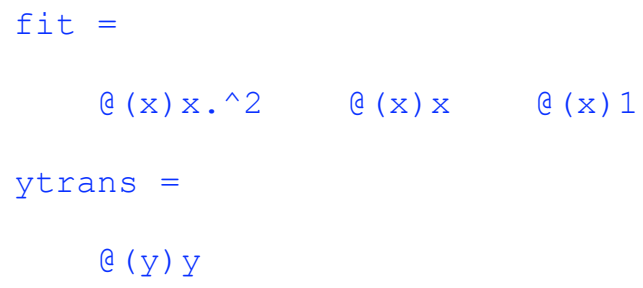




\section{fitMC}

Map operating conditions to system degradation

Syntax:

b = FITMC(operatingconditions, model,threshold)

Description:

This function determines the coefficients to map time spent in each operating condition to system degradation.

$b=$ MCFIT(operatingconditions, model,threshold) calculates the appropriate coefficients to fit the time spent in each operating condition, calculated from the operating conditions progressions to failure contained in the cell array operatingconditions, to the model (given by an anonymous function of the form @ $(b, t) f(b, t))$ with ending value equal to threshold

Example:

load tire

whos

\begin{tabular}{|c|c|c|c|}
\hline Name & ize & Bytes & Class \\
\hline fail & $1 \times 100$ & 297936 & cell \\
\hline unfail & $1 \times 3$ & 3436 & cell \\
\hline
\end{tabular}

fit $=a(b, t) t * b$;

fitMC (fail, fit, 100)

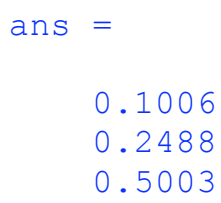

0.2488

0.5003 


\section{fitPF}

Determine PF parametric model fit

Syntax:

[Function xtransform] $=$ FITPF $($ prognosticparameters $)$

Description:

This function determines the best fit for a PF prognostic model between linear, quadratic, and exponential recursive fits.

[Function xtransform] $=$ FITPF (prognosticparameters) determines the best fit for the historic paths contained in the cell array prognosticparameters. The function considers linear, quadratic, and exponential recursive fits. These "fits" are the additive portion of $x t[x(n)]=x t[x(n-1)]+f(x(0: n-1), t(0: n))$, where $x t[]$ is the appropriate xtransform. This has value @ $(x) \log (x)$ for exponential functions and @ $(x) x$ (indicating no transformation is needed) for linear and quadratic functions.

Example:

load PFdata

whos

\begin{tabular}{|c|c|c|c|}
\hline Name & Size & Bytes & Class \\
\hline RUL_actual & $1 \times 50$ & 400 & double \\
\hline gtdāta_trn & $1 \times 50$ & 65600 & cell \\
\hline gtdata_tst & $1 \times 50$ & 65600 & cell \\
\hline trn_data & $1 \times 50$ & 38088 & cell \\
\hline tst_data & $1 \times 50$ & 30904 & cell \\
\hline
\end{tabular}

[fit xtransform] = fitPF(trn_data)

fit $=$

a $(t, t 1) t \cdot \wedge 2-t 1 \cdot \wedge 2 \quad a(t, t 1) t-t 1$

xtransform =

a $(x) x$ 


\section{initBayes}

Calculate Bayesian prior distribution

Syntax:

[prior nvar] = initBayes $($ progparam, $\mathrm{f}, \mathrm{yt})$

Description:

This function calculates the initial Bayesian prior distribution for a GPM model.

Prior = INITBAYES(prognosticparameters, fit,ytransform) calculates the Bayesian prior distribution for the coefficients, $b$, of the functional fit $f(b, t)$ where fit is a cell array of the form $f(x)=\{@(x) f 1(x) @(x) f 2(x) \ldots$ $@(x) f n(x)\}$ and ytransform is a function handle $P(y)=@(y) f y(y)$, where $P(y)=f(x)^{*} b$ for the historic prognostic parameter paths contained in the cell array prognosticparameters. It is assumed that the coefficients are normally distributed with mean and variance calculated from the population of fits. Prior is a matrix which contains the mean value for each parameter in the first row and the standard deviation in the second row:

$$
\text { prior }=\left[\begin{array}{ccc}
m_{1} & \ldots & m_{n} \\
s_{1} & \ldots & s_{n}
\end{array}\right]
$$

Example:

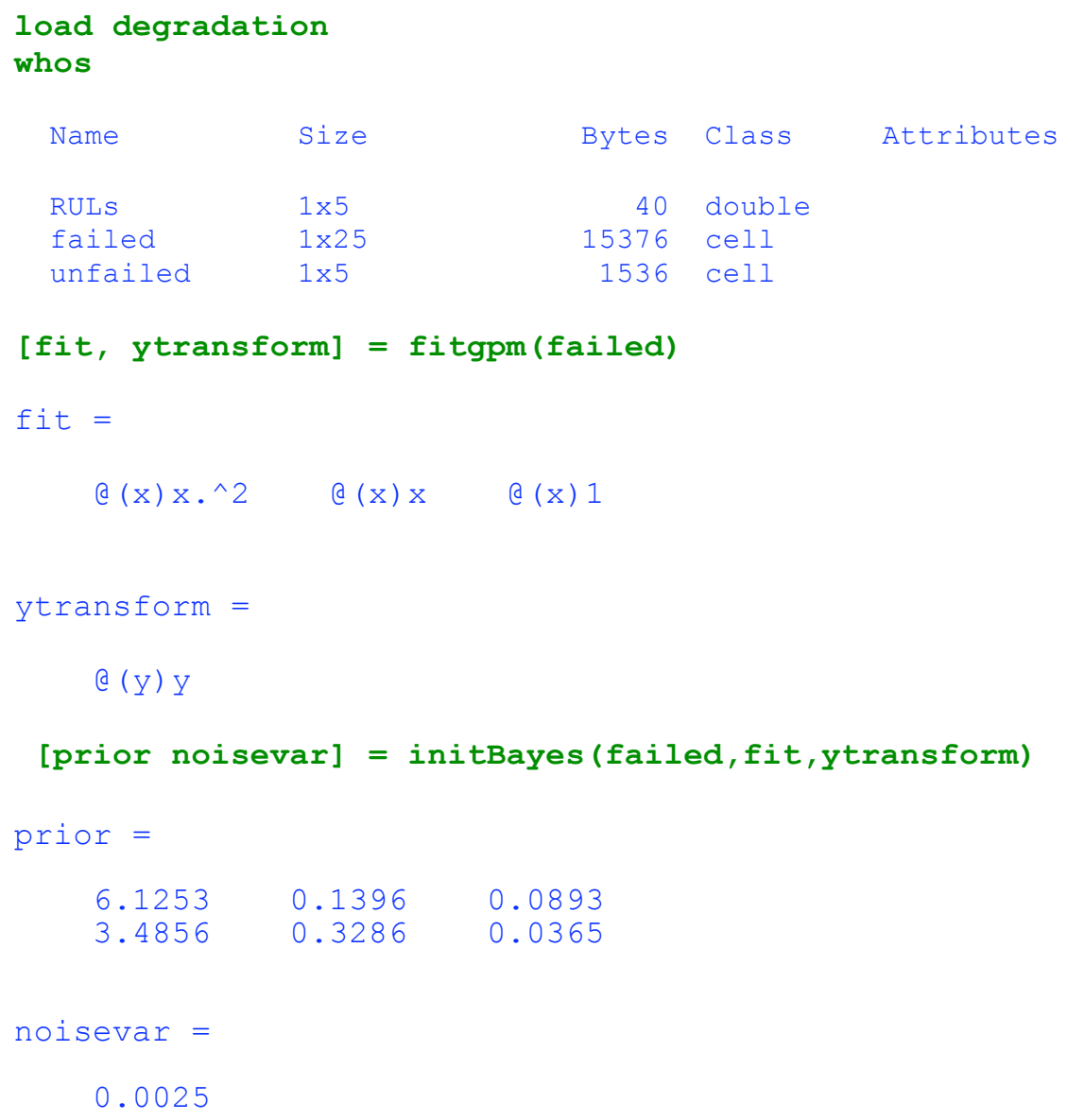




\section{initCoeff}

Calculate PF coefficient prior distribution

Syntax:

[Prior noisevar] = INITCOEFF(prognosticparameters, fit,xtransform)

Description:

This function calculates the initial Bayesian prior distribution for a PF model.

[Prior noisevar] = INITCOEFF(prognosticparameters, fit, xtransform) calculates the Bayesian prior distribution for the coefficients, $b$, of the functional fit $f(b, t)$ where fit is a cell array of the form $f(t, t 1)=$ $\{@(t, t 1) f 1(t, t 1) @(t, t 1) f 2(t, t 1) \ldots @(t, t 1) f n(t, t 1)\}$ and $x$ transform is a function handle $P(x)=@(x) f x(x) . x 1$ and $t 1$ indicate the respective values at the previous observation, where $P(x)=P(x 1)+f(t, t 1) * b$ for the historic prognostic parameter paths contained in the cell array prognosticparameters. It is assumed that the coefficients are normally distributed with mean and variance calculated from the population of fits. Prior is a matrix that contains the mean value for each parameter in the first row and the standard deviation in the second row:

$$
\text { prior }=\left[\begin{array}{ccc}
m_{1} & \ldots & m_{n} \\
s_{1} & \ldots & s_{n}
\end{array}\right]
$$

noisevar is a scalar which gives an estimate of the variance of the measurement noise.

Example:

load PFdata

whos

\begin{tabular}{|c|c|c|c|}
\hline Name & Size & Bytes & Class \\
\hline RUL_actual & $1 \times 50$ & 400 & double \\
\hline gtdata_trn & $1 \times 50$ & 65600 & cell \\
\hline gtdata_tst & $1 \times 50$ & 65600 & cell \\
\hline trn_data & $1 \times 50$ & 38088 & cell \\
\hline tst data & $1 \times 50$ & 30904 & $\mathrm{cell}$ \\
\hline
\end{tabular}

[fit xtransform] $=$ fitPF(trn_data);

[prior noisevar] = initCoeff(trn_data,fit,xtransform)

prior $=$

$0.0002 \quad 0.0002$

$0.0000 \quad 0.0041$

noisevar $=$

0.0012 


\section{initGPM}

Initialize a General Path Model prognostic model

\section{Syntax:}

model $=$ initGPM(prognosticparameters)

model $=$ initGPM(prognosticparameters,'flag',value,,..$)$

\section{Description:}

This function initializes an GPM prognostic toolbox structure

Model = INITGPM(prognosticparameters) initializes a GPM model using the historic paths contained in the cell array prognosticparameters. The most appropriate fit is chosen from quadratic, linear, and exponential fits.

Model = INITGPM(prognosticparameters,'flag',value,...) initializes a GPM model using the historical prognostic parameters and the values indicated by the 'flag' - value pairs. 'flag' may be set to any of the following:

'bayesian' (true) Use Bayesian updating to include prior information in function fitting (true or false)

'noise' (calculated using enovar() in PEM) An estimate of the noise variance

'updateinterval' (1) Number of time steps between fitting updates ( $n$, an integer) 'once' indicates the prior will be used once after all data is collected (in a nondynamic way)

'fit' (optimization) Functional fit to use for GPM fitting (entered as a cell array of function handles, i.e. $f=\left\{@(x) f 1(x) @ x(f 2(x) . . . @(x) f n(x)\}\right.$ where $\left.P(x)=f(x)^{*} B\right)$. Function must be linear in parameters for this type of entry. If a non-zero intercept is required, an "@(x)1" entry must be included. If no fit is given, an optimization is performed for the best fit among linear, quadratic, and exponential models.

'ytransform' (@(y)y) Functional transformation of $y$ to make the prognostic parameter linear in parameters, i.e. if $y=a^{*} \exp (b x)$ then $\log (y)=\log (a)+b^{*} x$, entered as 'ytransform' $=$ $@(y) \log (y)$ and 'fit' = \{@(x)1 @(x)x $\}$

'threshold' (calculated) Specify the critical threshold value (for hard thresholds) or the mean and standard deviation in the form [m s] (for threshold distributions)

'thresholdtype' ('pdf') Hard threshold or distribution ('hard'or 'pdf')

'threshcon' (0.95) The confidence level at which the threshold is calculated. Only applicable for "hard" type thresholds.

'npop' (1000) Number of Monte Carlo simulations used to make uncertainty estimates.

\section{Example:}


load degradation

whos

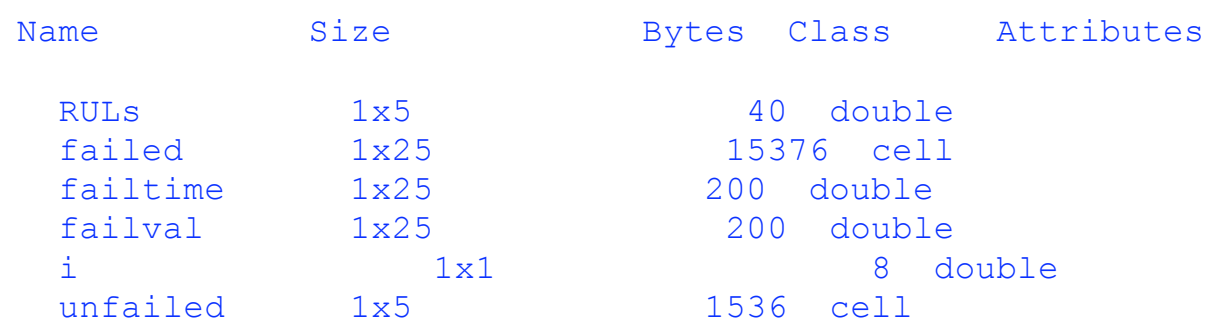

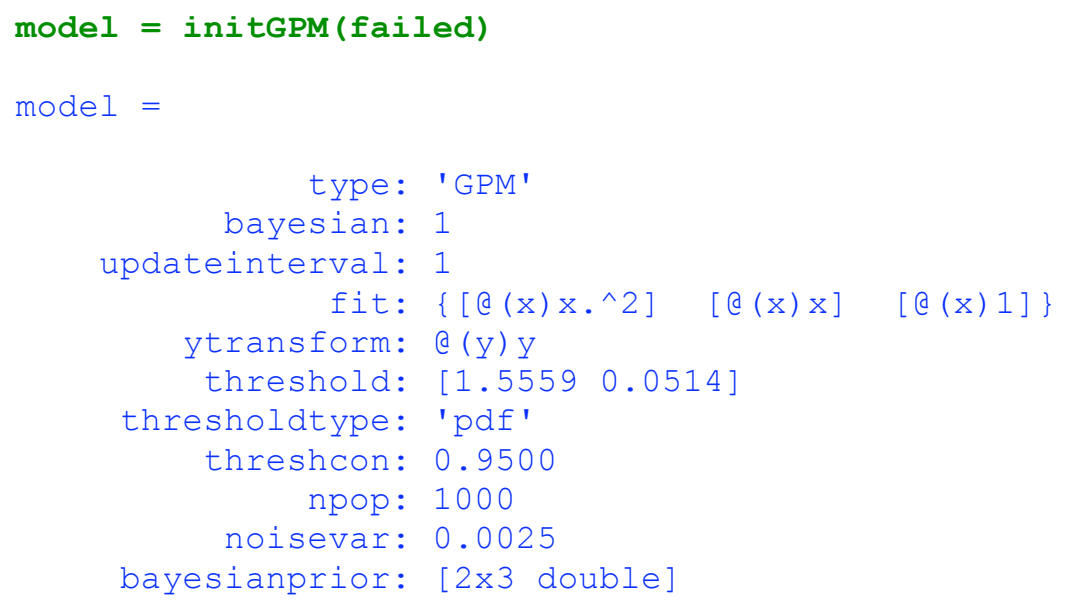




\section{initMC}

Initialize a Markov Chain prognostic model

\section{Syntax:}

Model = INITMC(operatingconditions)

Model = INITMC(operatingconditions,'flag', value,...)

\section{Description:}

This function initializes an MC model prognostics toolbox structure.

Model = INITMC(operatingconditions) initializes an MC model using the historic operating parameter progressions to failure contained in the cell array operatingconditions. These conditions should be discrete and numbered 1-n (no ordinal relation is implied by the numbering). The degradation parameter is assumed to be a weighted linear combination of the time spent in each operating condition. Initial degradation is assumed to be identically zero. The critical failure threshold for this parameter is assumed to be 100 (effectively measuring 100\% degradation). If the data contained in operatingconditions is not numbered from 1 to $n$, the data may be reformatted in MCdata() prior to initializing the model.

Model = INITMC (operatingconditions, 'flag', value,...) initializes an MC model using the historic operating condition progressions to failure and the user-supplied inputs indicated by the 'flag'-value pairs. 'flag' may be set to any of the following, with the default values given:

'Q' (calculated) The transition probability matrix for moving between operating conditions

'u' (calculated) The initial state probability vector

' $f$ ' (weighted sum of time spent in each condition) A function for mapping operating conditions to a degradation parameter. Should be input as an anonymous function @ $(b, t) f(b, t)$ where $b$ is a column vector of coefficients and $t$ is a row vector of time spent in each condition, 1-n

' $b$ ' (calculated from data) The vector of coefficients which is used with $f$ to map the operating conditions to a degradation parameter

'threshold' (100) The critical failure threshold applied to the degradation parameter to indicate failure

'npop' (1000) The number of Monte Carlo simulations generated for each RUL estimation

'RULcon' (0.95) The point in the resulting RUL distribution which defines the RUL. 0.95 indicates that RUL estimate is the point where the reliability of the system is 0.95 (Failure probability is 0.05 )

Example:

load tire

whos 


\begin{tabular}{|c|c|c|c|c|}
\hline Name & Size & Bytes & Class & Attributes \\
\hline fail & $1 \times 100$ & 303136 & cell & \\
\hline unfail & $1 \times 3$ & 3592 & cell & \\
\hline
\end{tabular}

figure

plot (fail $\{1\}, '$.')

axis ([-inf inf 0 4])

xlabel('Time (cycles)')

ylabel ('Operating Condition')

title('Example Operating History')

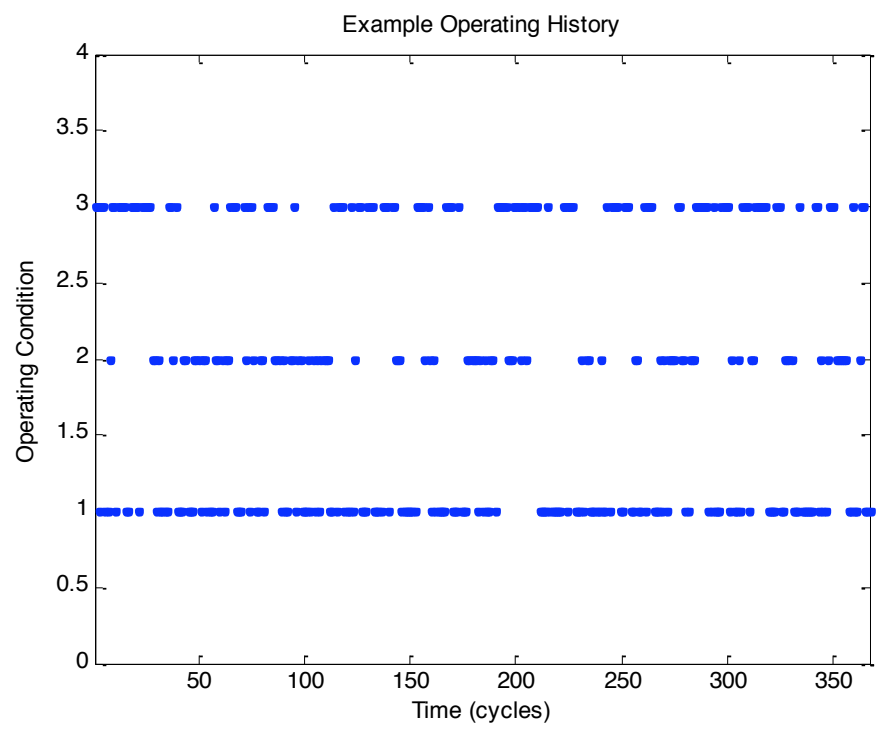

model = initMC (fail)

model $=$

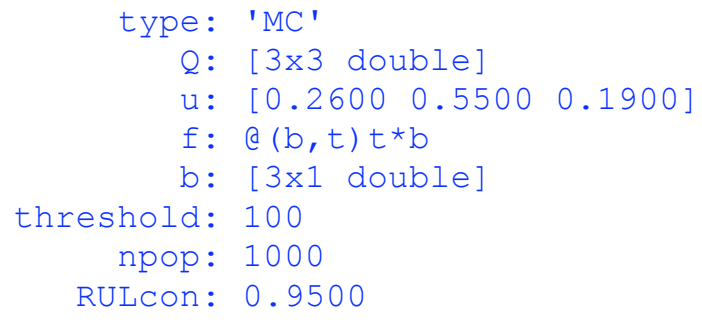

model.Q

ans $=$

$\begin{array}{lll}0.4963 & 0.3092 & 0.1945 \\ 0.4047 & 0.3935 & 0.2018 \\ 0.2473 & 0.1540 & 0.5988\end{array}$


model.b

$\mathrm{ans}=$

0.1006

0.2488

0.5003 


\section{initPF}

Initialize Particle Filter model

\section{Syntax:}

Model $=$ INITPF(prognosticparameters)

Model = INITPF (prognosticparameters,'flag',value,,..$)$

\section{Description:}

This funciton initializes a particle filter prognostic toolbox structure.

Model = INITPF(prognosticparameters) initializes a PF model using the historic paths contained in the cell array prognosticparameters. The most appropriate recursive fit is chosen from quadratic, linear, and exponential fits.

Model = INITPF(prognosticparameters,'flag',value,...) initializes a PF model using the historical prognostic parameters and the values indicated by the 'flag' - value pairs. 'flag' may be set to any of the following:

'bayesian' (true) Use Bayesian updating to adjust model parameter distributions when re-sampling

'noisevar' (calculated using enovar() in PEM) An estimate of the noise variance

'fit' (optimized) Functional fit to use for PF recursive fitting (entered as a cellarray of recursive function handles, i.e. $f=\{@(x, x 1) f 1(x, x 1) @(x, x 1) f 2(x, x 1) \ldots @(x, x 1) f n(x, x 1)\}$ where $x$ is the current observation and $x 1$ is the previous observation and $P(x)=f(x) * B)$ Function must be linear in parameters for this type of entry. If a non-zero intercept is required, an "@(x)1" entry must be included. If no fit is given, an optimization is performed for the best fit among recursive linear, quadratic, and exponential models

'transform'(@(y)y) Functional transformation of $\mathrm{y}$ to make the prognostic parameter linear in parameters, i.e. if the function is logarithmic, it can be considered as $\log (y)=$ $\log (y 1)+b(x-x 1)$, then ytransform = @(y) $\log (y)$ and fit $=@(x, x 1) x-x 1$

'modcoeff' (calculated) Distribution of model coefficients for sampling for particle forward prediction

'threshold' (calculated) Specify the critical threshold value (for hard thresholds) or the mean and standard deviation in the form [m s] (for threshold distributions)

'thresholdtype' ('pdf') Hard threshold or distribution ('hard' or 'pdf')

'threshcon' (0.95) The confidence level at which the threshold is calculated. Only applicable for "hard" type thresholds

'npart' (1000) Number of particles to simulate for each population

'particlethresh' (0.5) Fraction of effective particles which causes particle/coefficient resampling 
'RULcon' (0.95) The point in the resulting RULdistribution which defines the RUL. 0.95 indicates that RUL estimate is the point where the reliability of the system is 0.95 (Failure probability is 0.05 )

Example:

load PFdata

whos

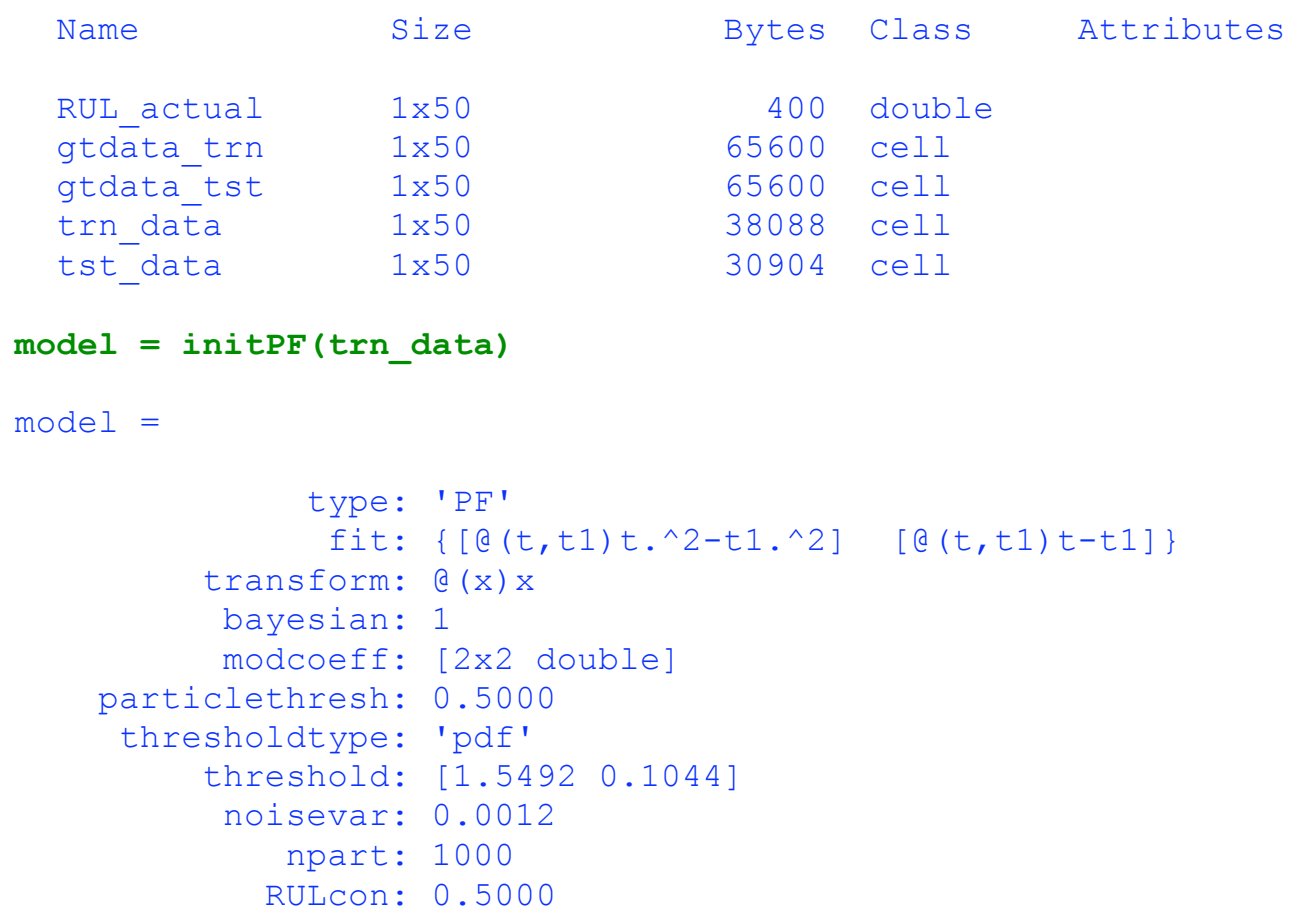




\section{initPHM}

Initialize a Proportional Hazards prognostic model

Syntax:

Model = INITPHM(cov, times)

Model = INITPHM(cov,times,'flag', value...)

\section{Description:}

This function initializes a PHM prognostics toolbox structure.

Model = INITPHM(cov,times) initializes a proportional hazards model with the covariates defined in the nxp matrix cov and the failure times in the $n \times 1$ column matrix times. The baseline value is taken to be zero.

Model = INITPHM(cov,times,'flag', value...) initializes a proportional hazards model using the covariates and failures times in the matrics cov and times, and the 'flag'-value pairs given. 'flag' may be set to any of the following, with the default values given:

'frequency' (one for each observation) The jth element of this $\mathrm{nx} 1$ column vector indicates the number of times that the combination of the jth set of covariates and the jth failure time are observed together.

'censoring' (all zeros) The jth element of this $\mathrm{nx} 1$ column vector indicates whether that observation is a failure time (0) or a censored time (1).

'baseline' (zero) This 1xp row vector indicates the baseline values for each of the covariates.

'beta' (calculated) The coefficients of the Cox proportional hazards regression.

'hazard' (calculated) The baseline cumulative hazard function given as a 2-column matrix with failure times in the first column and the corresponding estimated cumulative hazard rate in the second column.

'RULcon' (0.95) The point in the resulting RUL distribution which defines the RUL. 0.95 indicates that RUL estimate is the point where the reliability of the system is 0.95 (Failure probability is 0.05 ).

Example:

load PHMchalldata

whos

$\begin{array}{lcrlr}\text { Name } & \text { Size } & \text { Bytes } & \text { Class } & \text { Attributes } \\ & & & \\ \text { RUL } & 259 \times 1 & 2072 & \text { double } & \\ \text { trn } & 1 \times 260 & 10350848 & \text { cell } & \\ \text { tst } & 1 \times 259 & 6555280 & \text { cell }\end{array}$

trn_cov $=\operatorname{cell}(\operatorname{size}(\operatorname{trn}))$;

failtimes $=\mathrm{NaN}($ numel $(\operatorname{trn}), 1)$; 


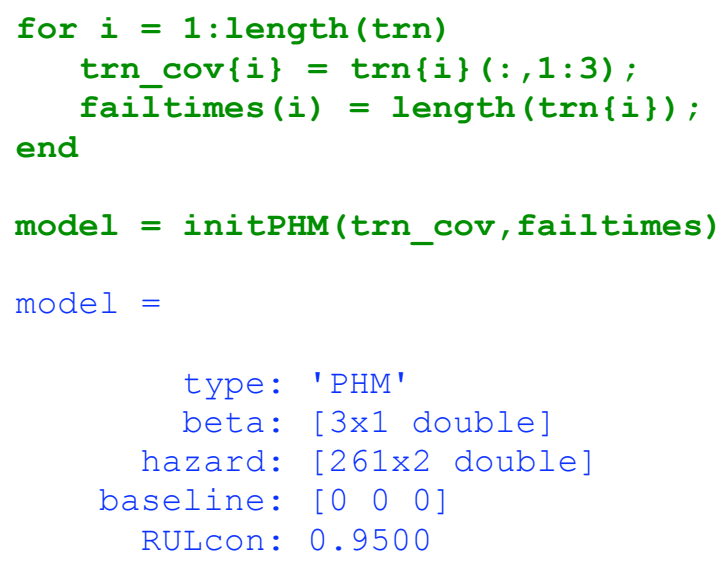




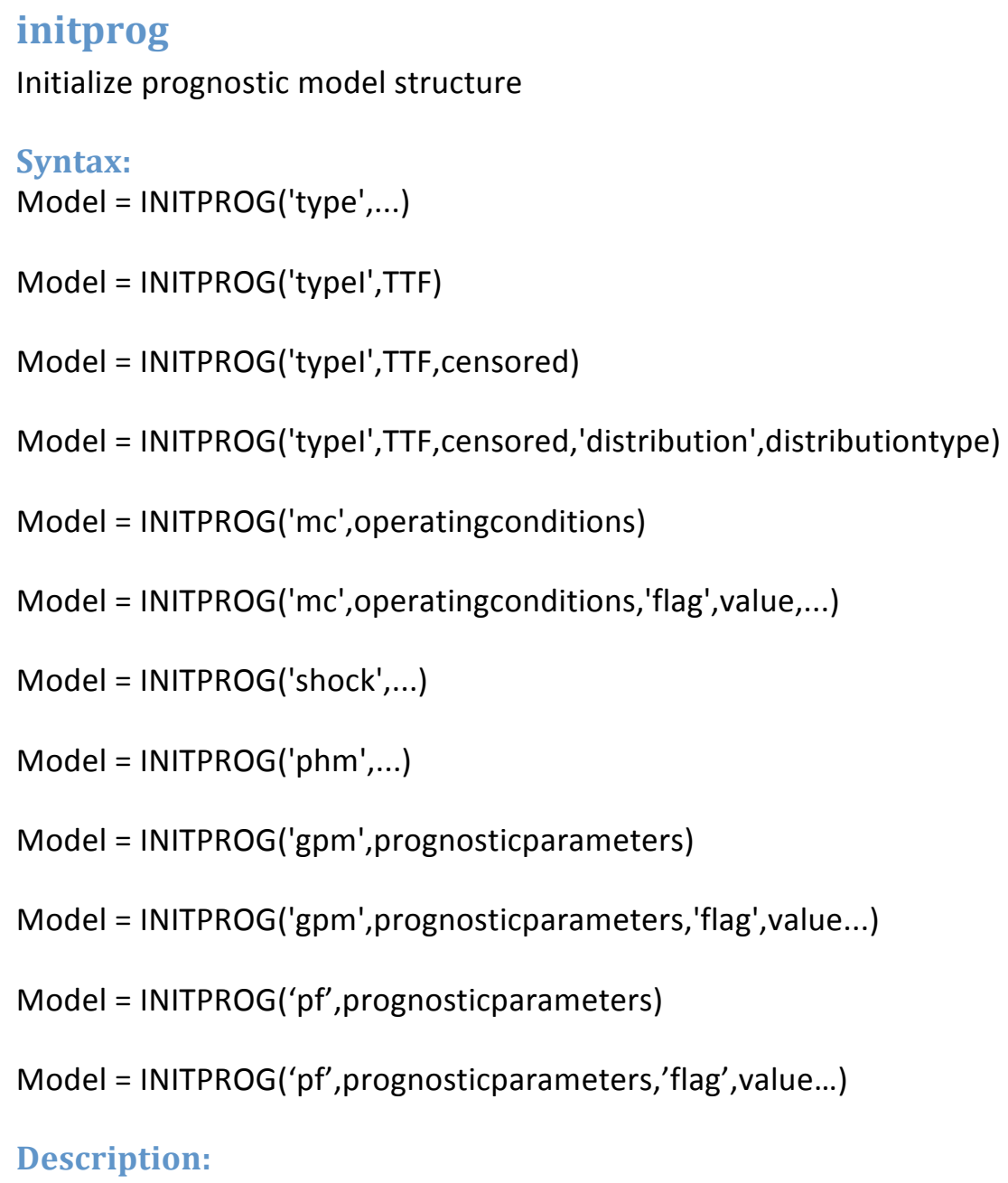
set to any of the following strings:

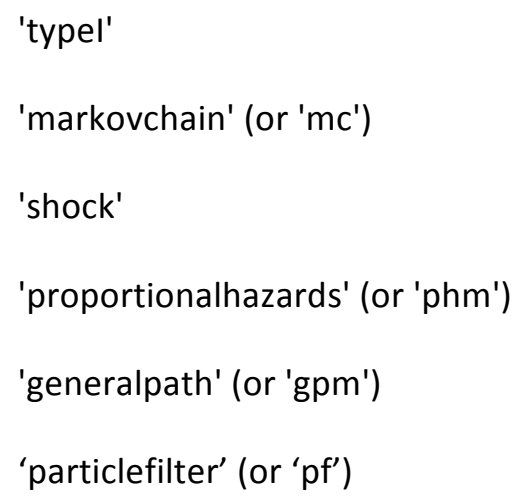

For necessary inputs and available flag/value options, please see the appropriate individual "init" functions: initTypel, initMC, initShock, initPHM, initGPM, initPF. 
Example:

For examples of each model type, please see the appropriate init functions, as listed above. To utilize the initprog wrapper function, simply indicate the appropriate type as the first input. 


\section{initShock}

Initialize a shock prognostic model

Syntax:

Model = INITSHOCK(shocks)

Model = INITSHOCK(shocks,'flag', value, ,...)

\section{Description:}

This function initializes a shock model prognostics toolbox structure

Model = INITSHOCK(shocks) initializes a shock model using the readings (such as vibration or current) which contain a progression of random shocks contained in the cell array shocks. Each cell may contain a vector of the measurement of interest or an $\mathrm{nX2}$ matrix where the first column is time and the second column is the sensed value. Here, the indication of failure is the sum of the shock sizes.

Model $=$ INITSHOCK(shocks,'flag',value,...) initializes a shock model using the progression of random shocks contained in the cell array shocks and the values indicated by the 'flag' - value pairs. 'flag' may be set to any of the following:

'operatingcond' (none) A cell array of column vectors that indicate the operating conditions for each shock progression. The operating condition at each time step should be an integer from 1 to $n$, which indicates which of $n$ discrete operating conditions the system is in. This can account for the difference in probability of a shock occurring for each operating condition. This results in a Type II model. Note that this option CANNOT be used with a degradation dependant distribution. The function MCdata() can be used to put operating conditions into discrete groups numbered 1 to $\mathrm{n}$. Indicated in model structure as model. . Typell $=$ true.

'degdepend' (false) Parameter of the time distribution can be dependent on the degradation level, as might happen in a vibrating bearing or failing power supply. This effectively makes the shock model a Type III model. Lambda is assumed to depend on the degradation according to a polynomial of degree 2 unless otherwise specified by 'timeorder'. A non-polynomial function can be specified with 'timefon'. Note that this option CANNOT be used with operating condition information. Indicated in model structure as model. TypellI = true.

'degcondition' Measurements of equipment degradation can be used to develop a degradation dependent shock model. Input should be a cell array of degradation measures of the same size as shocks.

'timeorder' (2) The order of polynomial used to describe the dependence on degradation.

'timefcn' (polynomial) An anonymous function of the form @(d)f(d) for degradation-dependent distributions. For instance, if the distribution is dependent on the exponential function, then the timefon would be input as: @(b,d)exp(b(1)*d+b(2)) The parameters in $b$ will be fit to the available data. Notice that your function 
parameters must be a vector called ' $b$ ' to be fit. If they are known a priori, they can be specified with 'timecoef'.

'timecoef' (fit) A vector which contains the coefficients of the degradation dependent function used to define the time distribution.

'timepar' (distribution fit) Parameter of a static (not degradation dependent) time distribution, which is assumed to be exponential. This is the lambda parameter of the exponential distribution.

'magdist' (normal) Distribution of the magnitude of shocks ('constant', 'normal', 'nonparametric' or 'np')

'magpar' (distribution fit) Parameters of magnitude distribution

'minshock' (calculated) The minimum size of the input that is considered a shock. It is calculated as the mean non-shock input value plus three standard deviations of the noise.

'npop' (1000) The number of Monte Carlo simulations generated for each RUL estimation

'RULcon' (0.50) The point in the resulting RULdistribution which defines the RUL. 0.5 indicates that RUL estimate is the point where the reliability of the system is 0.5 (Failure probability is 0.5 )

'thresholdtype' ('pdf') Hard threshold or distribution ('hard' or 'pdf')

'threshold' (calculation) Specify the critical threshold value (for hard thresholds) or the mean and standard deviation in the form [m s] (for threshold distributions)

'threshcon' (0.50) Confidence level for determining threshold.

\section{Example:}

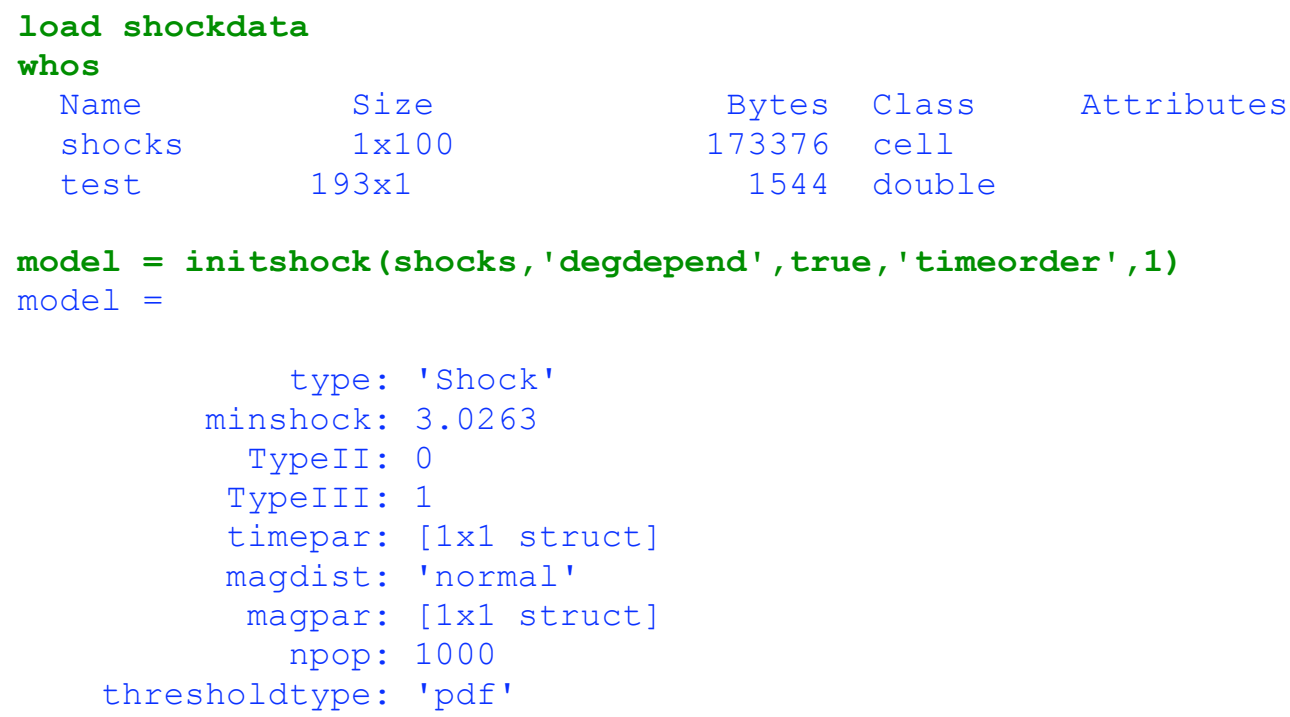


threshold: [1x1 struct]

threshcon: 0.5000

RULcon: 0.5000 


\section{initTypeI}

Initialize a Type I (reliability-based) prognostic model

Syntax:

Model $=$ INITTYPEI(TTF)

Model = INITTYPEI(TTF, censored $)$

Model = INITTYPEI(TTF,censored,'distribution', distributiontype)

Description:

This function initializes a reliability-based prognostic model structure.

Model = INITTYPEI(TTF) initializes a reliability-based prognostic model using the failure times in the vector TTF. This model uses a Weibull distribution to model failure times.

Model = INITTYPEI(TTF, censored) initializes a model using the failure and censoring times in the vector TTF. The variable censored indicates whether each observed time is a failure (0) or censored (1) time.

Model = INITTYPEI(TTF, censored,'distribution',distributiontype) initializes a Type I model using the failure time distribution indicated by distributiontype. distributiontype can be set to any of the following strings: 'weibull', 'exponential' (or 'exp'), 'normal'. The default distribution type is Weibull. Note that the variable censored may be omitted if all data points are actual failure times.

\section{Example:}

load failuretimes

whos

\begin{tabular}{|c|c|c|c|c|}
\hline Name & Size & Bytes & Class & Attributes \\
\hline failuretimes & $135 \times 1$ & 1080 & double & \\
\hline
\end{tabular}

typeI = inittypeI (failuretimes)

typeI.parameters

histfit (failuretimes, 10, 'wbl')

type $I=$

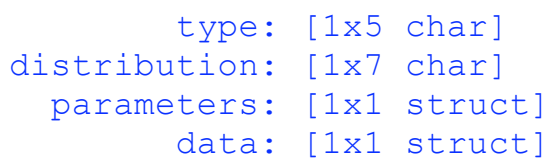

ans $=$

beta: 13.0851

theta: 127.5712 


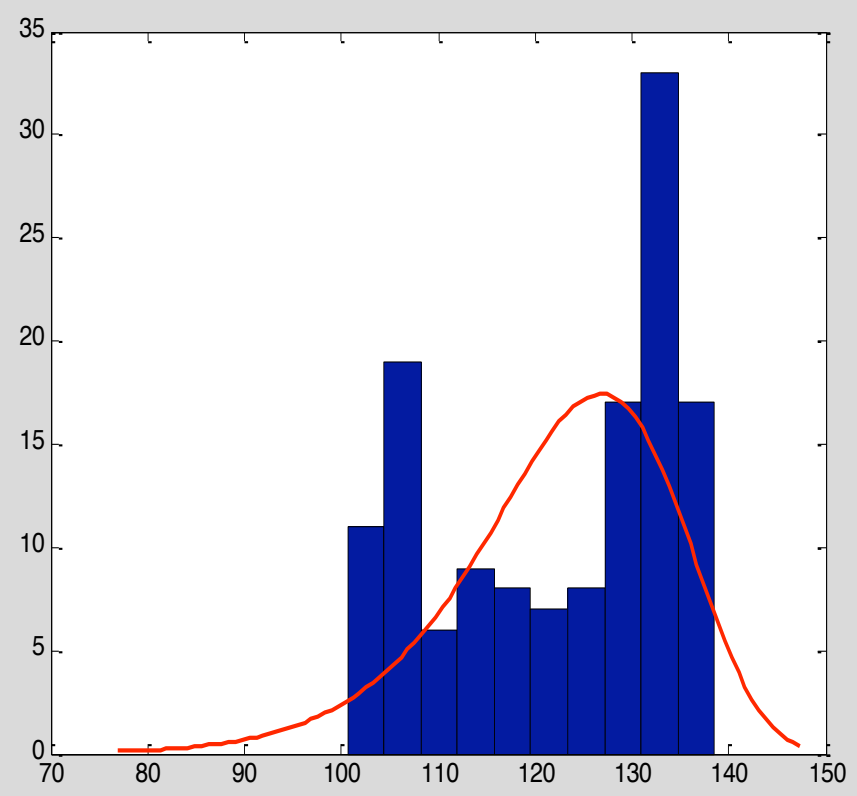




\section{MCData}

Discretize operating condition data

Syntax:

[newoperatingconditions map] $=$ MCDATA(operatingconditions)

[newoperatingconditions map] = MCDATA(operatingconditions, 'flag', value)

\section{Description:}

This function converts operating condition data into data needed for Markov Chain Models

[newoperatingconditions map] $=$ MCDATA(operatingconditions) converts the operating condition progressions to failure contained in the cell array operatingconditions into conditions numbered 1-n (no ordinal relation is implied by the new numbers). The progressions in the cell array operatingconditions may be of size $n x m$ where $n$ is the number of observations in one history and $m$ is the number of variables which fully define the operating condition. These values need to be discrete (or discretized by some outside method) to apply this function correctly. The output map is a matrix which indicates the relationship between the original operating conditions (of size $1 \mathrm{xn}$ ) to an operating condition class. The first row in map defines the first operating condition, the second row, the second operating condition, and so on.

[newoperatingconditions map] $=$ MCDATA(operatingconditions, 'flag', value) separates the operating conditions progressions contained in the cell array operatingconditions into MC appropriate conditions. The 'flag'/value pairs may be set to any of the following (defaults):

'tol' (0.10) The noise tolerance for separating operating conditions.

'map' (determined) The map for moving from measured operating conditions to the MC numbered conditions where the first row in map defines the first operating condition, the second row in map defines the second operating condition, and so on.

\section{Example:}

load PHMchalldata

whos

$\begin{array}{lrrlr}\text { Name } & \text { Size } & \text { Bytes } & \text { Class } & \text { Attributes } \\ & & & \\ \text { RUL } & 259 \times 1 & 2072 & \text { double } \\ \text { trn } & 1 \times 260 & 10337328 & \text { cell } \\ \text { tst } & 1 \times 259 & 6541812 & \text { cell }\end{array}$

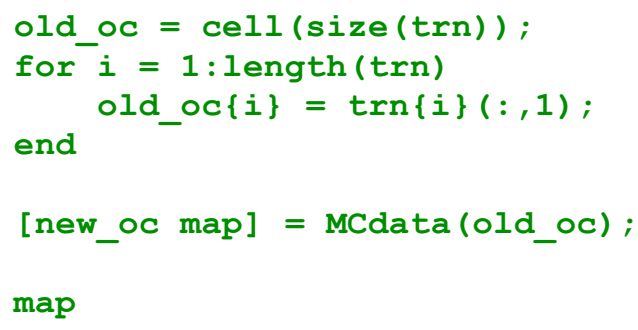



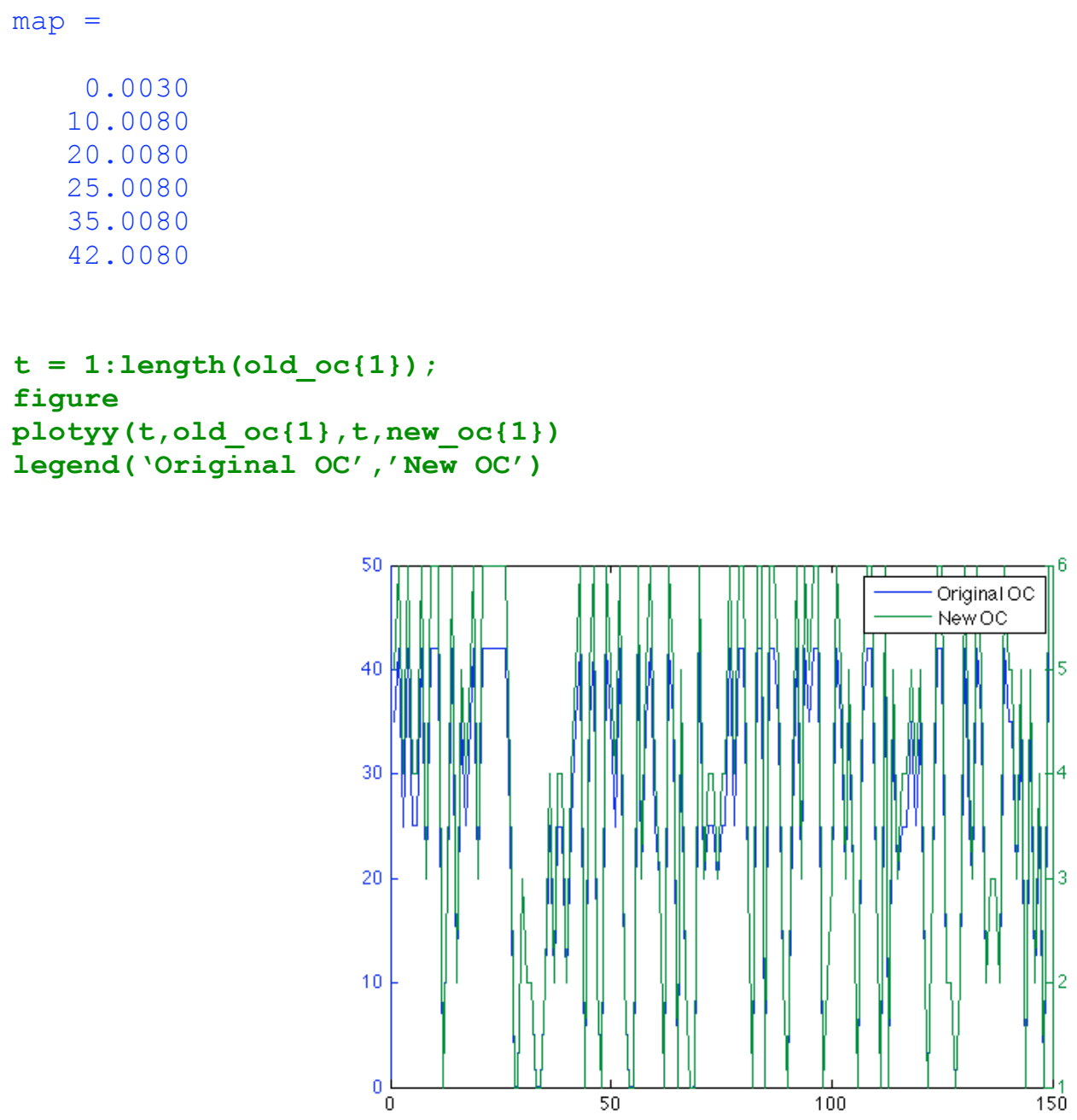


\section{MCprobs}

Calculate the transition probability matrix and initial state probability vector

Syntax:

$[\mathrm{Q} u]=\mathrm{MCPROBS}$ (operatingconditions)

Description:

MCPROBS calculates the transition and initial condition probabilities. This function calculates the transition probability matrix and the initial condition probability vector for a Type II Markov Chain model

$[Q \mathrm{u}]=\mathrm{MCPROBS}$ (operatingconditions) calculates the transition probability matrix $(\mathrm{Q})$ and the initial condition vector $(u)$ for a Type II Markov Chain model using the operating condition progressions to failure contained in the cell array operatingconditions.

Example:

load PHMchalldata

whos

$\begin{array}{lrrlr}\text { Name } & \text { Size } & \text { Bytes } & \text { Class } & \text { Attributes } \\ \text { RUL } & 259 \times 1 & 2072 & \text { double } \\ \text { trn } & 1 \times 260 & 10337328 & \text { cell } \\ \text { tst } & 1 \times 259 & 6541812 & \text { cell }\end{array}$

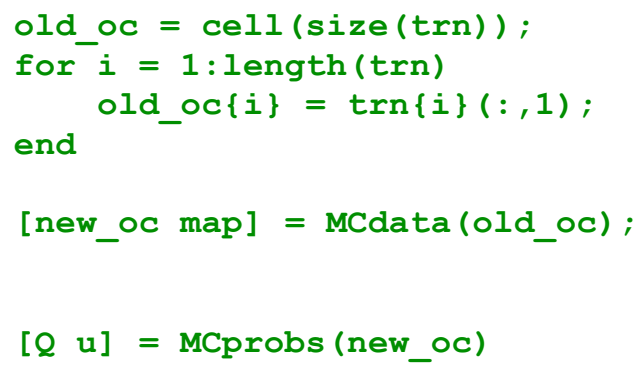
0.1474
0.1520
0.1512
0.1521
0.1430
0.2544
0.1440
0.1508
0.1502
0.1472
0.1524
0.2554
0.1455
0.1466
0.1517
0.1468
0.1588
0.2506
0.1543
0.1568
0.1479
0.1507
0.1415
0.2488
0.1502
0.1551
0.1542
0.1465
0.1536
0.1460
0.1518
0.1488
0.1511
0.2431
$0.1500 \quad 0.2498$

$\mathrm{u}=$
0.1577
0.1346
0.1231
0.1885
0.1385
0.2577 


\section{optparam}

Identify near-optimal prognostic parameter

Syntax:

param = OPTPARAM(inputs, 'flag', value,...$)$

\section{Description:}

This function uses Genetic Algorithms and parameter suitability metrics to identify a near-optimal prognostic parameter from several data sources.

param = OPTPARAM(inputs, 'flag', value, ...) identifies a near-optimal prognostic parameter from the available data in the cell array inputs. The function uses genetic algorithms to optimize the weights in a linear combination of the available signal inputs. Function returns a structure, param, which includes the resulting weights and all information needed to obtain the parameter from a new data run using function PARAMGEN. Flag/value pairs may be set to any of the following:

'inputs' ('all'): $\quad$ Determines how many of the inputs should be considered by the GA. Can be set to 'all', indicating that all should be used, or 'subset', indicating that a subset of useful parameters should be used.

'cutoff' (1.5): Cutoff value for determining which input parameters are useful when choosing a subset of inputs for optimization.

'fitness' (sum of $M, P, T)$ : Identifies the fitness function to be used, input as @fitness. fitness(w,inputs) must be a matlab m-file which takes only the candidate solution from the GA and the cell array of possible inputs to determine the fitness of a candidate solution.

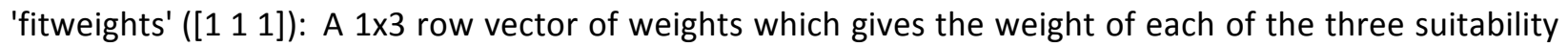
metrics in determining the fitness. The first entry corresponds to monotonicity, the second to prognosability, and the third to trendability.

'initpop' ([]): $\quad$ Any candidate weightings that the GA should consider. If visual inspection or expert analysis has lead to any suitable parameters, these can be included in the GA to allow it to explicitly consider them in the optimization. They should be included as an $\mathrm{nXm}$ matrix where $\mathrm{n}$ is the number of possible prognostic parameters to be included and $m$ is the number of candidate inputs to the parameter. Each row should contain the weights associated with one possible parameter.

'group' (one group): Multiple prognostic parameters may be optimized to compare groups. Associated value must be a cell array of matrices where each matrix indicates the runs included in a specific group. Groups may be overlapping. Output in this case will be a structure array where the ith entry corresponds to the optimum parameter for the ith group.

'smoothing' (false): May be set to 'true' or 'false', indicates whether the resulting parameter should be smoothed prior to final model development. 


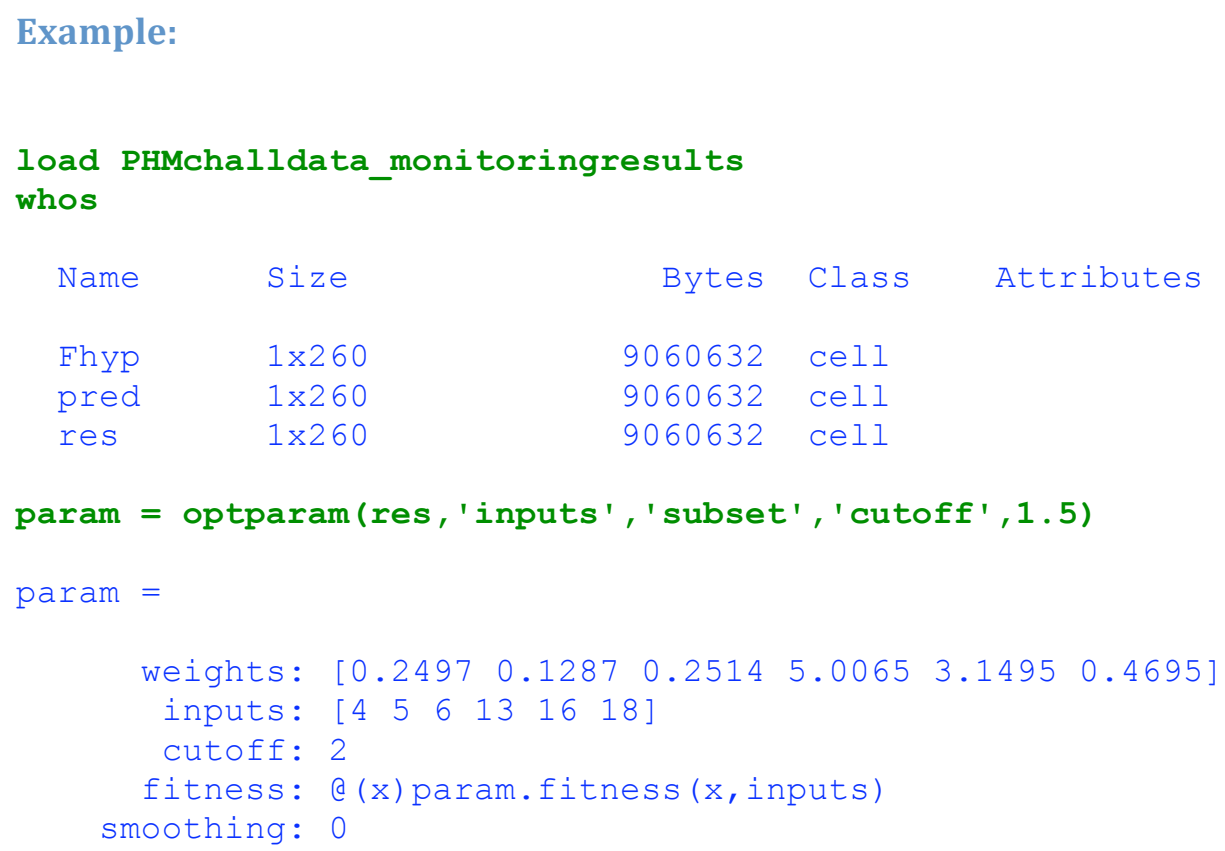




\section{paramfit}

Prognostic parameter fitness

Syntax:

fitness = PARAMFIT(weights, inputs)

fitness = PARAMFIT(weights, inputs, suitabilityweights)

Description:

PARAMFIT determines the fitness of a candidate prognostic parameter. This function determines the fitness of a candidate prognostic parameter by calculating the sum of monotonicity, prognosability, and trendability. These three prognostics metrics are computed using the function PPMETRICS.

fitness = PARAMFIT(weights,inputs) calculates the fitness of a linear combination of inputs, weighted according to the row vector weight, as the sum of the parameter suitability metrics monotonicity, prognosability, and trendability.

fitness = PARAMFIT(weights, inputs, suitabilityweights) calculates the fitness of a linear combination of inputs, weighted according to the row vector weight, as the weighted sum of the parameter suitability metrics monotonicity, prognosability, and trendability, where suitabilityweights is a 1x3 row vector with the weight of monotonicity in the first entry, prognosability in the second, and trendability in the third.

fitness = PARAMFIT(weights, inputs, suitabilityweights, filter) filters the resulting prognostic parameter before calculating the suitability. An exponential filter is used via expfilt().

\section{Example:}

load PHMchalldata_monitoringresults

whos

\begin{tabular}{|c|c|c|c|}
\hline Name & Size & Bytes & Class \\
\hline Fhyp & $1 \times 260$ & 9060632 & cell \\
\hline pred & $1 \times 260$ & 9060632 & cell \\
\hline res & $1 \times 260$ & 9060632 & cell \\
\hline
\end{tabular}

fitness $=$ paramfit $($ ones $(1,21)$, res $)$

fitness $=$

$-1.0485$ 


\section{paramgen}

Generate a population of prognostic parameters

Syntax:

progparam = PARAMGEN(param_struct,inputs)

progparam = PARAMGEN(param_struct,inputs,time $)$

\section{Description:}

This function generates a population of prognostic parameters according to the options saved in the structure param_struct.

progparam = PARAMGEN (param_struct,inputs) creates a population of prognostic parameters using the options saved in param_struct and the candidate inputs in the cell array inputs. OPTPARAM() can be used to generate the parameter structure.

progparam = PARAMGEN(param_struct,inputs,time) creates a population of prognostic parameters which includes the time variable for each case in the first column and the prognostic parameter in the second. Both inputs and time should be cell arrays where the number of observations (rows) in each cell of inputs should be equal to the corresponding cell of time. PARAMGEN calls also produce a plot of the population of prognostic parameters.

progparam $=$ PARAMGEN (...,plot) plots resulting prognostic parameters if set to true. Default is false.

\section{Example:}

load PHMchalldata ga

load PHMchalldata monitoringresults

whos

\begin{tabular}{|c|c|c|c|}
\hline Name & Size & Bytes & Class \\
\hline Fhyp & $1 \times 260$ & 9060632 & cell \\
\hline $\mathrm{m}$ & $1 \times 1$ & 8 & double \\
\hline $\mathrm{p}$ & $1 \times 1$ & 8 & double \\
\hline par & $1 \times 260$ & 459192 & cell \\
\hline param & $1 \times 1$ & 1017 & struct \\
\hline pred & $1 \times 260$ & 9060632 & cell \\
\hline res & $1 \times 260$ & 9060632 & cell \\
\hline t & $1 \times 1$ & 8 & double \\
\hline
\end{tabular}

prognosticparameter = paramgen (param, res, true) ; 


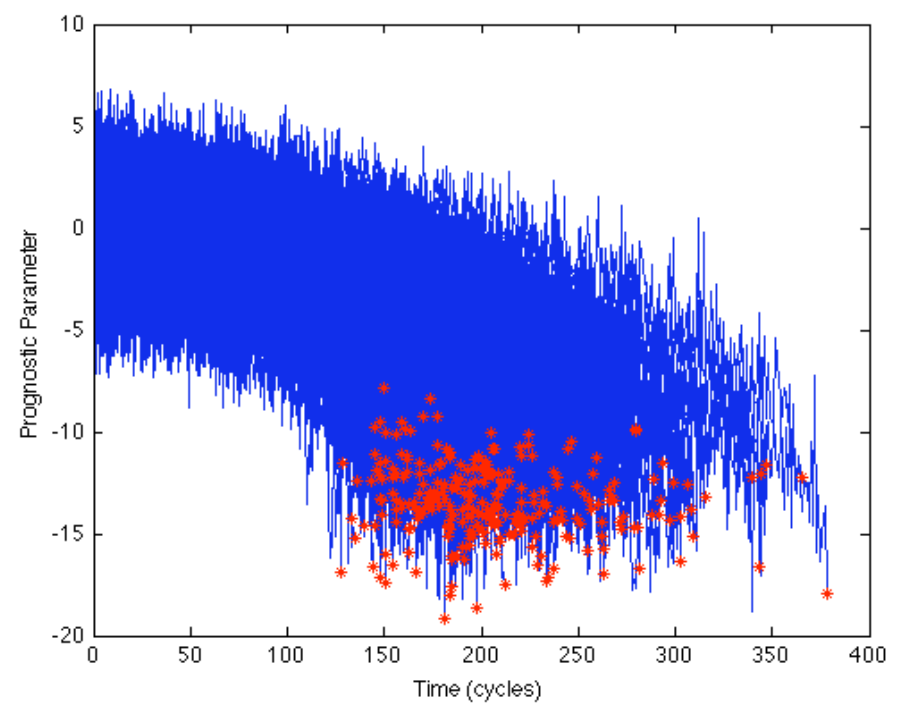




\section{ppmetrics}

Prognostic parameter suitability metrics

\section{Syntax:}

[monotonicity prognosability trendability] = PPMETRICS(params)

\section{Description:}

This function characterizes the appropriateness of a prognostic parameter based on three metrics.

[monotonicity prognosability trendability] = PPMETRICS(params) evaluates the population of prognostic parameters contained in the cell array params for three metrics of adequacy.

Montonicity measures the general increasing or decreasing trend of the parameter. Because the assumption is made that systems do not self heal and no corrective action is taken, prognostic parameters are assumed to be monotonic. This assumption may not be valid for some systems such as batteries that do exhibit some self healing during periods of rest, or systems which experience some outside intervention to improve the condition.

Prognosability is a measure of the variance of the failure values for a population of parameters.

Trendability characterizes how well a population of parameters can be fit by the same functional form. It measures the similarity of the general trend of the parameter for a population of systems.

\section{Example:}

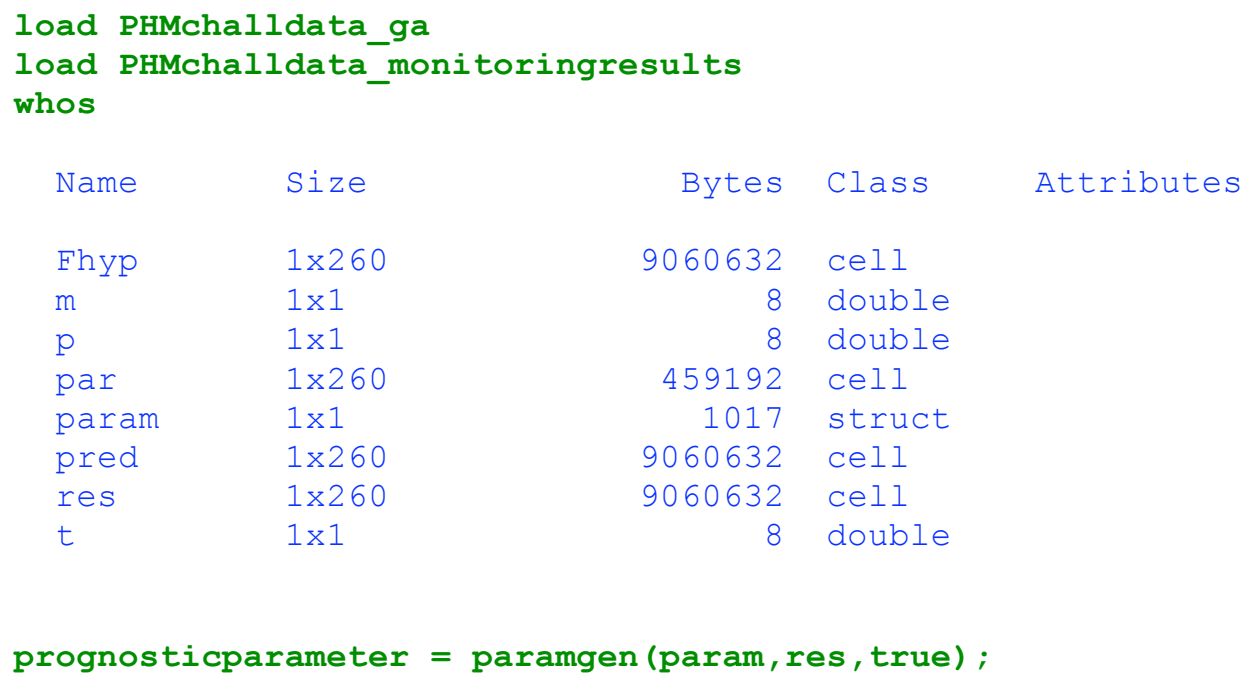




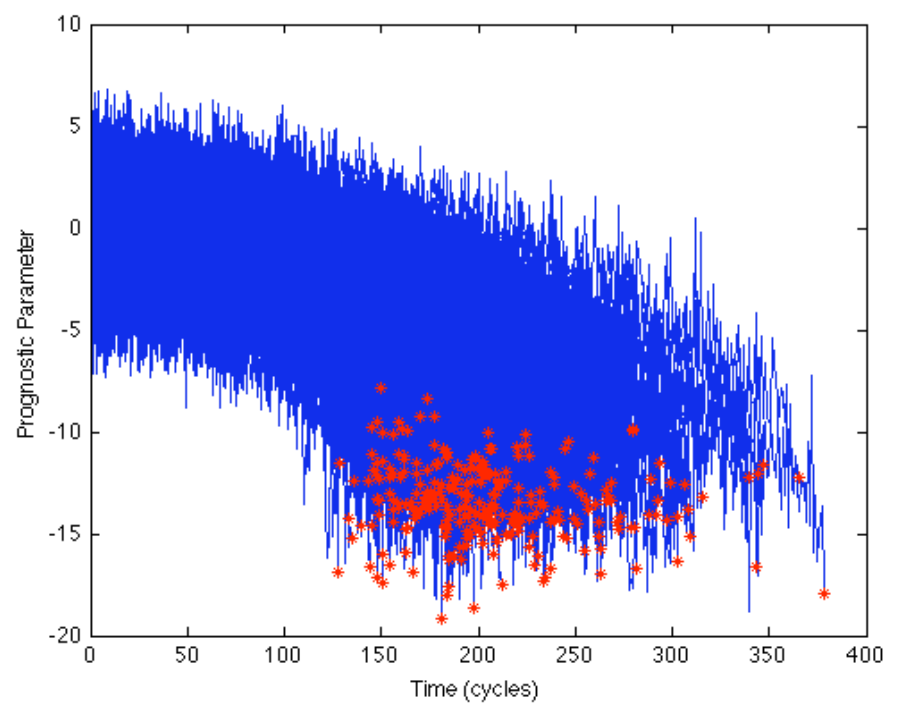

$\left[\begin{array}{lll}m & p & t\end{array}\right]=\operatorname{ppmetrics}($ prognosticparameter)
$\mathrm{m}=$
0.6916
$\mathrm{p}=$
0.8674
$t=$
0.8684 


\section{residgen}

Generate monitoring system residuals using a PEM model

Syntax:

RESID = RESIDGEN(model, data)

Description:

This function uses a PEM model to calculate the residuals of a population of systems.

RESID = RESIDGEN (model,data) generates residuals using the previously developed PEM toolbox model and a cell array of data for a population of systems. Note that the generated residuals are predictions actual.

Example:

load PHMchalldata

load PHMchalldata_models

whos

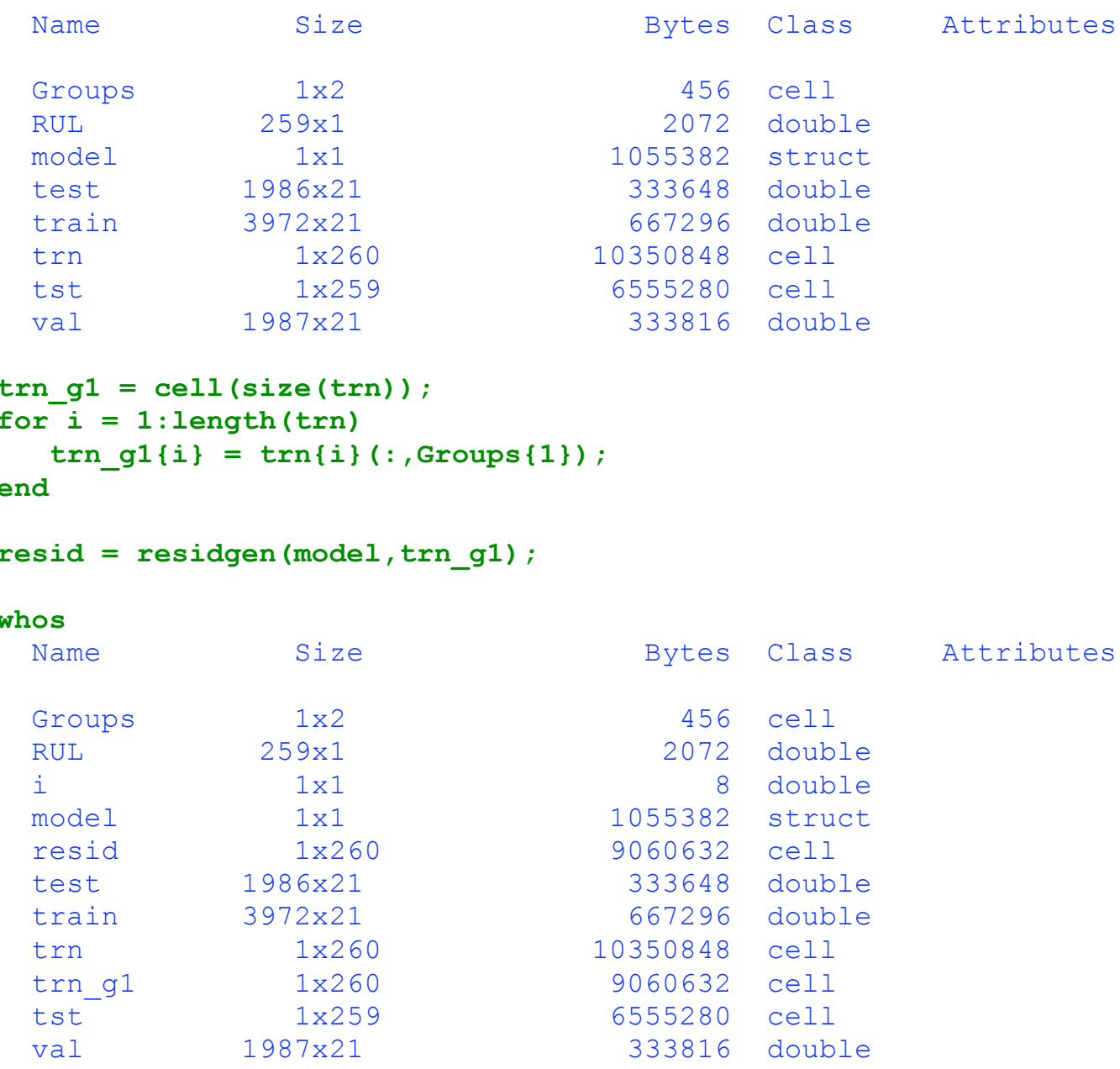

figure

plot (resid $\{1\}$ ) 
xlabel ('Observation')

ylabel ('Residual')

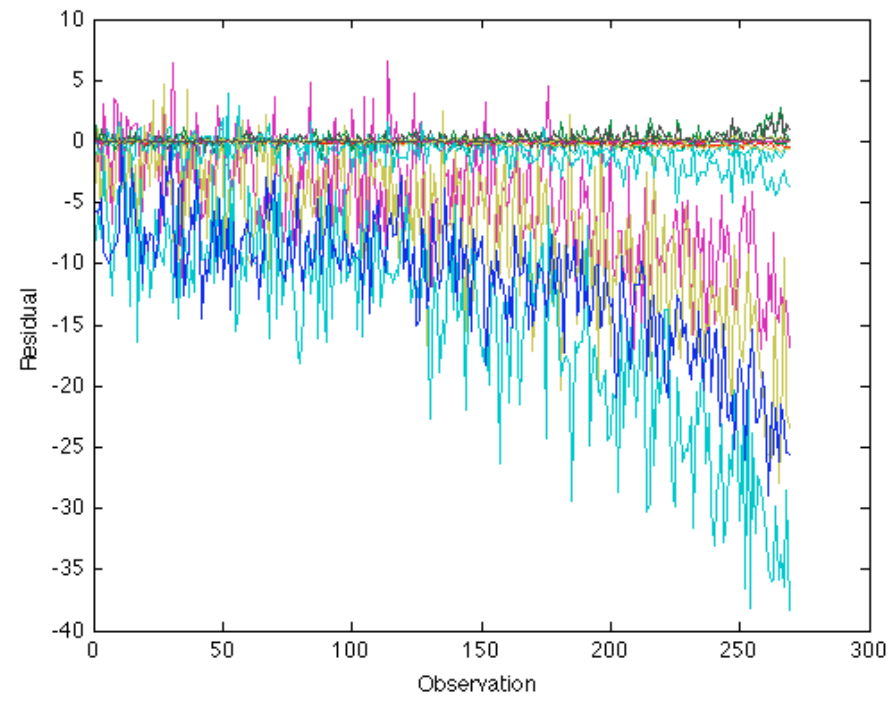




\section{runGPM}

Run a GPM prognostic model

Syntax:

RUL = RUNGPM(model,currentparam)

RUL = RUNGPM(model,timestamp,currentparam)

$[$ RUL uncert model $]=$ RUNGPM $(\ldots)$

\section{Description:}

This function makes RUL estimates using a general path model.

RUL = RUNGPM(model,currentparam) makes prognostic estimates using a Type III model. 'model' should be of type 'gpm'. 'currentparam' may be a column vector, matrix, or cell array. If only one system is under surveillance, currentparam should be a column vector containing observations of that system up to the current time. If multiple systems are under surveillance, and parameter observations are available for the same time steps for each system, currentparam may be a matrix whose columns contain the parameter observations for a single system. If multiple systems are under surveillance which have been running for different amounts of time, currentparam may be a cell array containing the parameter observations for each system in a column vector contained in separate cells. Here, it is assumed that observations are made every time unit, with an equal sampling interval.

RUL = RUNGPM(model,currentparam) may also be used for multiple systems under surveillance where the time stamp for each system is not the same. In this case, currentparam should be a cell array whose cells contain an $n \times 2$ matrix where the first column is the time stamp for that particular unit and the second column is the parameter values at each time.

RUL = RUNGPM(model,timestamp,currentparam) can be used when the sampling interval is not equal across observations. If currentparam is a column vector, then timestamp should also be a column vector. If currentparam is a matrix, then timestamp may be a column vector of times (if each unit is surveyed at the same time) or it may be a matrix of times (if surveillance times for each unit are different).

$[R U L$ uncert model $]=$ RUNGPM $(. .$.$) returns the estimated RUL, the associated 95 \%$ confidence intervals, and the model structure with an updated Bayesian prior for future estimates. No other fields are changed.

\section{Example:}

load degradation

whos

\begin{tabular}{|c|c|c|c|}
\hline Name & Size & Bytes & Class \\
\hline RULs & $1 \times 5$ & 40 & double \\
\hline failed & $1 \times 25$ & 15376 & $\mathrm{cell}$ \\
\hline unfailed & $1 \times 5$ & 1536 & $\mathrm{cell}$ \\
\hline
\end{tabular}

model $=$ initprog ('gpm',failed)

model $=$ 


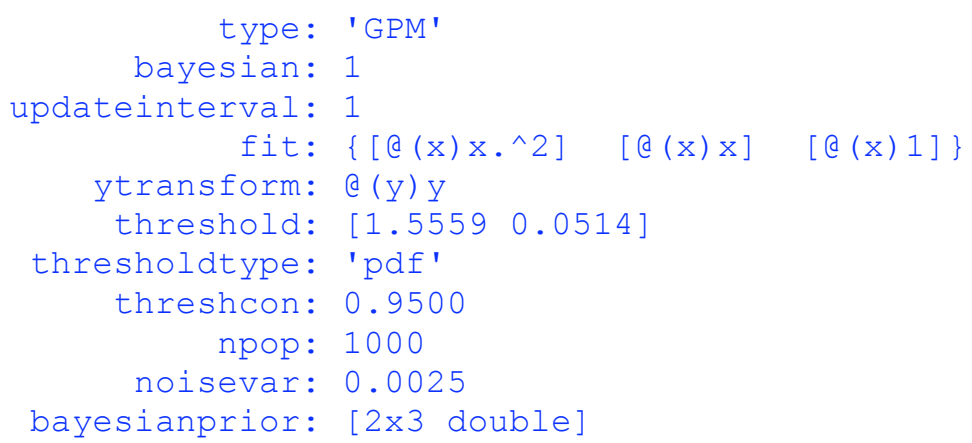

[RUL uncert] = runGPM (model, unfailed)

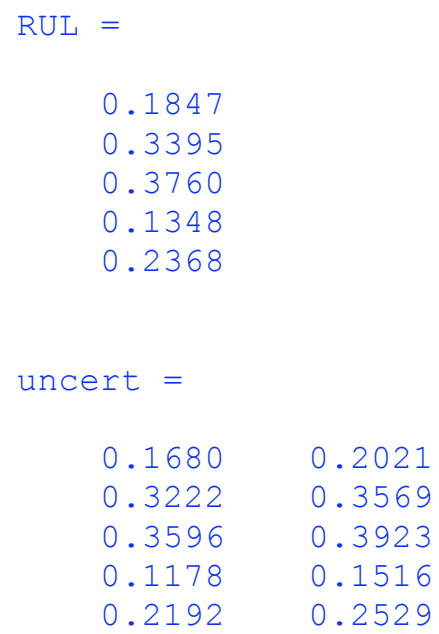




\section{runMC}

Run a Markov Chain prognostic model

Syntax:

RUL = RUNMC(model,operatingconditions)

RUL = RUNMC(model,'new')

$[R U L$ uncert $]=\operatorname{RUNMC}(\ldots)$

\section{Description:}

This function makes RUL estimates using a Type II MC model

$\mathrm{RUL}=\mathrm{RUNMC}$ (model,operatingconditions) makes prognostic estimates using a Type II Markov Chain model. model should be of type ' $M C^{\prime}$ '. operating conditions is a column vector of the operating conditions seen by the unit under test up to the current time. If operatingconditions is a cell array of column vectors, one vector for the operation of a single unit to the current time, then RUL is a row vector of RUL times.

RUL = RUNMC(model,'new') calculates the expected RUL of a system starting from new, with no information about its operating states.

$[R U L$ uncert $]=R U N M C(\ldots)$ returns the RUL prediction and the associated $95 \%$ uncertainty interval.

\section{Example:}

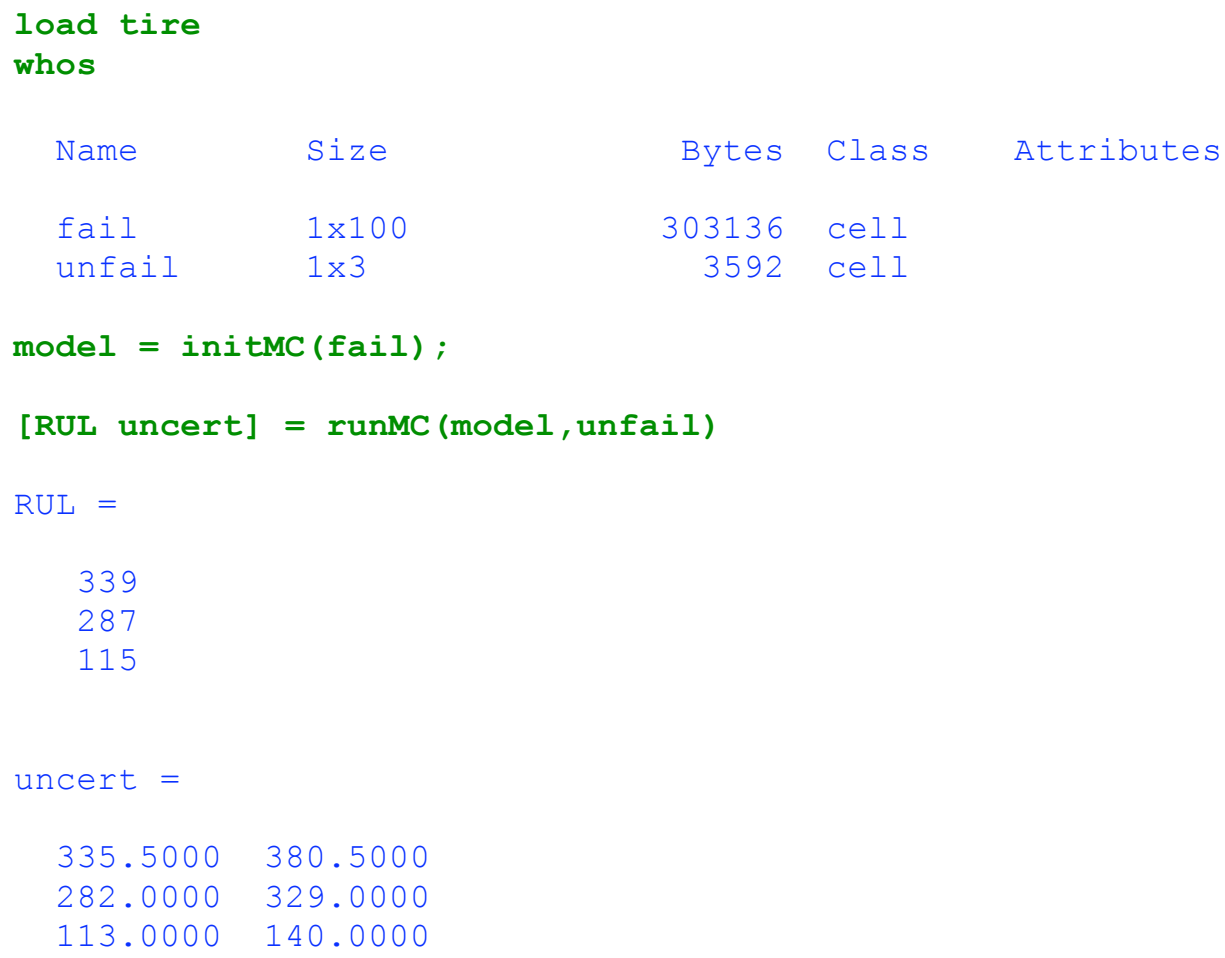




\section{runPF}

Run PF prognostic model

Syntax:

RUL $=$ RUNPF(model, currentparam)

RUL $=$ RUNPF(model,timestamp,currentparam)

$[R U L$ uncert $]=\operatorname{RUNPF}(\ldots)$

\section{Description}

This function makes RUL estimates using a particle filtering model.

RUL $=$ RUNPF(model,currentparam) makes prognostic estimates using a particle filtering model. The model should be of type 'PF'. currentparam may be a column vector, matrix, or cell array. If only one system is under surveillance, currentparam should be a column vector containing observations of that system up to the current time. If multiple systems are under surveillance, and parameter observations are available for the same time steps for each system, currentparam may be a matrix whose columns contain the parameter observations for a single system. If multiple systems are under surveillance which have been running for different amounts of time, may be a cell array containing the parameter observations for each system in a column vector contained in separate cells. Here, it is assumed that observations are made every time unit, with an equal sampling interval.

RUL = RUNPF(model,currentparam) may also be used for multiple systems under surveillance where the time stamp for each system is not the same. In this case, currentparam should be a cell array whose cells contain an $n \times 2$ matrix where the first column is the time stamp for that particular unit and the second column is the parameter values at each time.

RUL $=$ RUNPF(model,timestamp,currentparam) can be used when the sampling interval is not equal across observations. If currentparam is a column vector, then timestamp should also be a column vector. If currentparam is a matrix, then timestamp may be a column vector of times (if each unit is surveyed at the same time) or it may be a matrix of times (if surveillance times for each unit are different).

$[R U L$ uncert $]=\operatorname{RUNPF}(\ldots)$ returns the estimated $R U L(s)$ and the associated $95 \%$ uncertainty interval(s).

\section{Example:}

load PFdata

whos

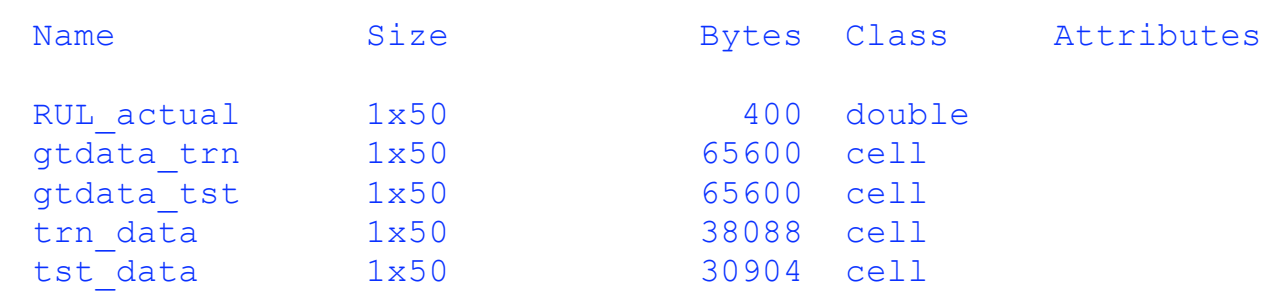

model = initPF(trn data);

[RUL uncert $]=\operatorname{run} \bar{P} F($ model, tst_data $)$; 
figure; hold on

plot (RUL_actual, RUL, ' O')

plot $(1: 4 \overline{0}, 1: 40$, 'r--')

xlabel ('Actual RUL (cycles)')

ylabel ('Estimated RUL (cycles)')

title(['Particle Filter RUL Estimates: MAPE = ' num2str(mean((RULRUL_actual'). /RUL_actual'*100)) ])

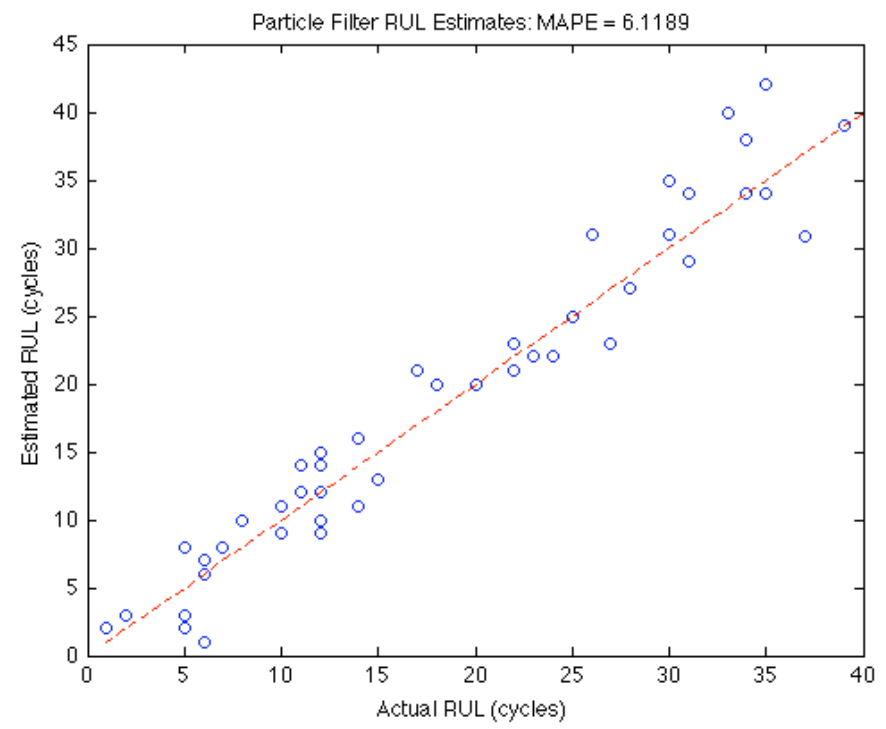




\section{runPHM}

Run a Proportional Hazards prognostic model

Syntax:

$[$ RUL uncert $]=$ RUNPHM(model,covariates)

Description:

This function makes RUL estimates using a Proportional Hazards Model.

[RUL uncert $]=$ RUNPHM(model,currentsystem) makes prognostic estimates using a Proportional Hazards model. currentsystem is an $n \times 2$ matrix where the first column is the current time for a particular system and the second column is the covariate value for that system. The output RUL is an $n \times 1$ matrix of RUL estimates based on the reliability confidence level and uncert is an $n \times 2$ matrix of $95 \%$ uncertainty intervals.

Example:

load PHdata

whos

\begin{tabular}{|c|c|c|c|c|}
\hline Name & Size & Bytes & Class & Attributes \\
\hline baseline & $1 \times 1$ & 8 & double & \\
\hline censoring & $16 \times 1$ & 128 & double & \\
\hline covariates & $16 \times 1$ & 128 & double & \\
\hline failuretimes & $16 \times 1$ & 128 & double & \\
\hline frequency & $16 \times 1$ & 128 & double & \\
\hline
\end{tabular}

model = initPHM (covariates, failuretimes, 'baseline', baseline,...

'frequency' , frequency, 'censoring', censoring) ;

covariate $=1 /(170+273)$;

times $=0: 500: 2000$;

currentsystem $=[$ times' covariate*ones $(5,1)]$;

[RUL uncert] = runPHM (model, currentsystem)

RUL $=$

$1.0 e+03 \star$

1. 4104

0.9470

0.5603

0.1300

0.3046

uncert $=$

$1.0 e+03 *$

0.99296 .6823

$1.2072 \quad 6.6831$

$1.4634 \quad 6.6862$ 
1.5350

6.6881

2.0343

6.6965 


\section{runprog}

Run prognostic model

Syntax:

$[$ RUL uncert $]=$ RUNPROG $($ model,$\ldots)$

[RUL uncert $]=$ RUNPROG (model,currenttime)

$[$ RUL uncert $]=$ RUNPROG (model)

$[R U L$ uncert $]=$ RUNPROG (model,currentenvir)

$[$ RUL uncert $]=$ RUNPHM(model,covariates)

$[$ RUL uncert $]=$ RUNPROG (model,currentparam)

Description:

This function estimates the Remaining Useful Life (RUL) using the model previously developed.

For necessary inputs, please see the appropriate individual "run" functions: runTypel, runMC, runShock, runPHM, runGPM, runPF.

Example:

For examples of each model type, please see the appropriate run functions, as listed above. To utilize the runprog wrapper function, provide an initialized model of the appropriate model type. 


\section{runShock}

Run a shock prognostic model

Syntax:

RUL = RUNSHOCK(model, shocks)

RUL = RUNSHOCK(model,shocks,operatingconditions)

RUL = RUNSHOCK(model, shocks,condition)

$[R U L$ uncert $]=$ RUNSHOCK $(\ldots)$

\section{Description:}

This function makes RUL estimates using a Shock Model.

RUL = RUNSHOCK(model,shocks) makes prognostic estimates using a Type II shock model. shocks is a vector of measurements, such as vibration or voltage, which indicate the measure of interest. shocks may be an $n \times 2$ matrix where the first column is the time stamp and the second column is the sensor measurement.

RUL = RUNSHOCK(model,shocks,operatingconditions) considers the planned future operating condition in generating random shocks. operatingconditions may be a column vector of discrete condition indicators for possible future time, an $\mathrm{n} \times 2$ matrix where the first column is the planned time steps and the second is the operating condition, or a single scalar indicating the operating condition for all future use.

RUL = RUNSHOCK(model,shocks) makes prognostic estimates using a Type III shock model. shocks is a vector of measurements, such as vibration or voltage, which indicate the measure of interest. In this case, model includes shock probabilities dependent on the current estimated equipment condition.

RUL = RUNSHOCK(model,shocks,condition) makes prognostic estimates using a Type III shock model and the current measured equipment condition.

$[R U L$ uncert $]=$ RUNSHOCK $(\ldots)$ returns RUL predictions and the associated $95 \%$ uncertainty interval.

\section{Example:}

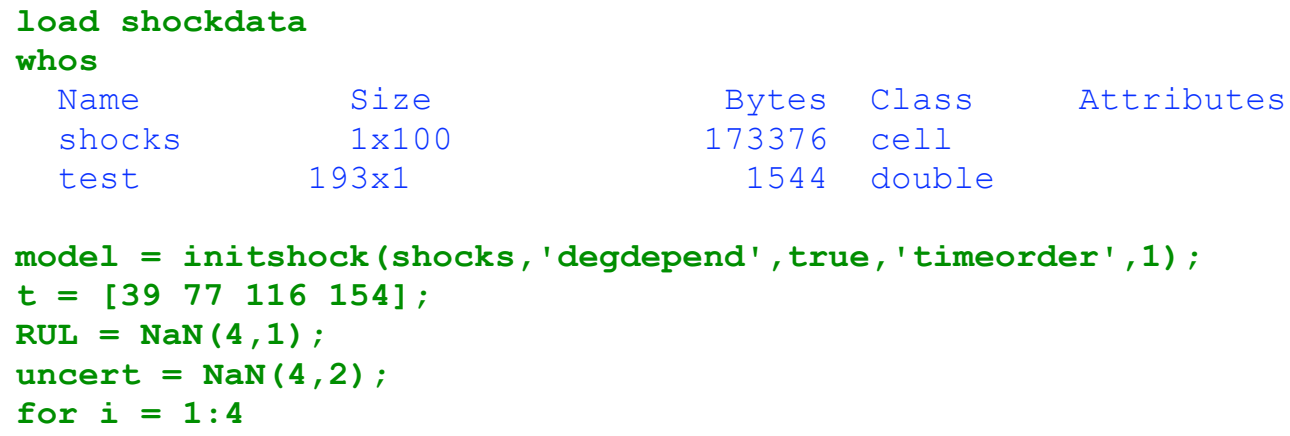


[RUL(i) uncert(i,:)] = runShock(model, test $(1: t(i)))$; end

RUL =

78.0986

61.5199

38.5305

36.3906

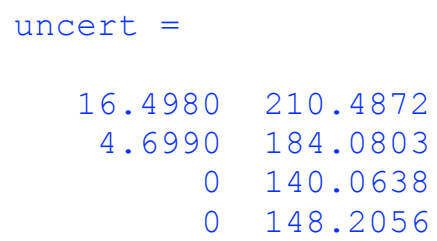




\section{runTypeI}

Run a Type I prognostic model

Syntax:

RUL = RUNTYPEI(model,currenttime)

Description:

This function makes RUL estimats using a Type I (reliability based) prognostics model.

RUL = RUNTYPEI(model,currenttime) makes prognostic estimates for a component or system that has lasted to time currenttime using a Type I model. currenttime can be a scalar indicating the current time for a single component or system, or it may be a vector indicating the current time for a population of components or systems. The RUL estimate for each individual in the population is made independently. currenttime may be input as a column or row vector; the RUL predictions will be returned in the same format.

RUL is calculated as the 0.5 -percentile of the conditional probability $R(t \mid t>T)=\frac{R(t)}{R(T)}$

[RUL uncert $]=$ RUNTYPEI (...) returns the RUL and the associated 95\% uncertainty interval for each time in currenttime. No other fields are changed.

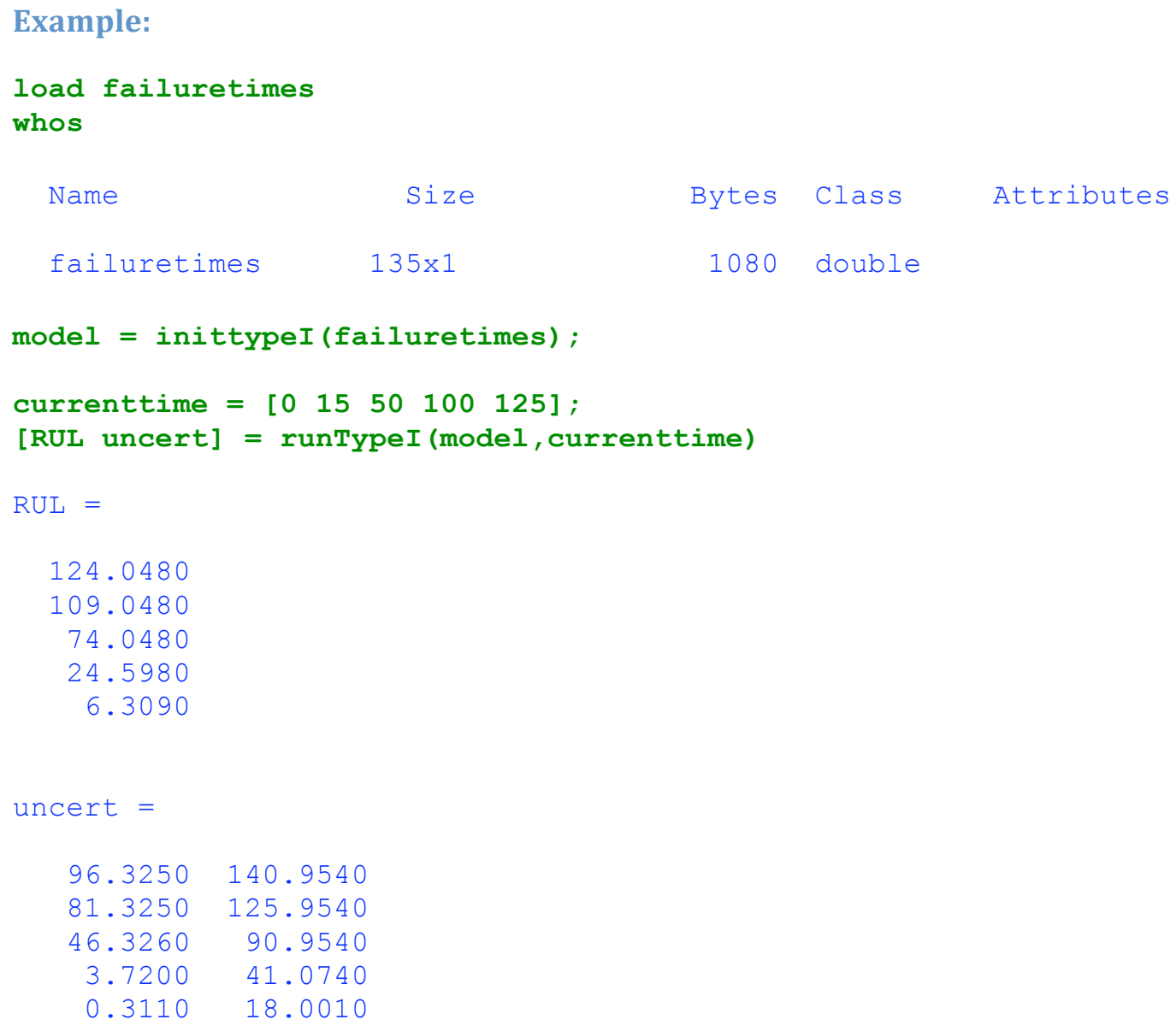




\section{thresh GPM}

Calculate GPM failure threshold

Syntax:

Threshold $=$ THRESHGPM(prognosticparameters, fit,yt $)$

Threshold = THRESHGPM(prognosticparameters, fit,yt,thresholdtype,threshcon)

\section{Description:}

This function calculates the critical failure threshold for a GPM model based on the full historic failure paths contained in 'progparam'.

Threshold $=$ THRESHGPM(prognosticparameters,fit,yt) determines the critical failure threshold for the paths contained in the cell array 'prognosticparameters'. The function calculates a hard threshold as the conservative 95th percentile of the failure values for the historic paths.

Threshold $=$ THRESHGPM(prognosticparameters,fit,yt,thresholdtype,threshcon) determines the critical failure threshold using the type specified by 'thresholdtype'. 'thresholdtype' may be set to either:

'hard' A constant value is used for the critical failure threshold. This value is the conservative $95^{\text {th }}$ quantile of the failure values for the historic paths.

'pdf' A distribution of critical threshold values is determined. The failure values are assumed to be normally distributed with mean and variance as determined by the historic paths. The threshold distribution is returned as a $1 \times 2$ vector of the form [mean standarddeviation].

'threshcon' indicates the confidence level at which the threshold is calculated from the failure values.

\section{Example:}

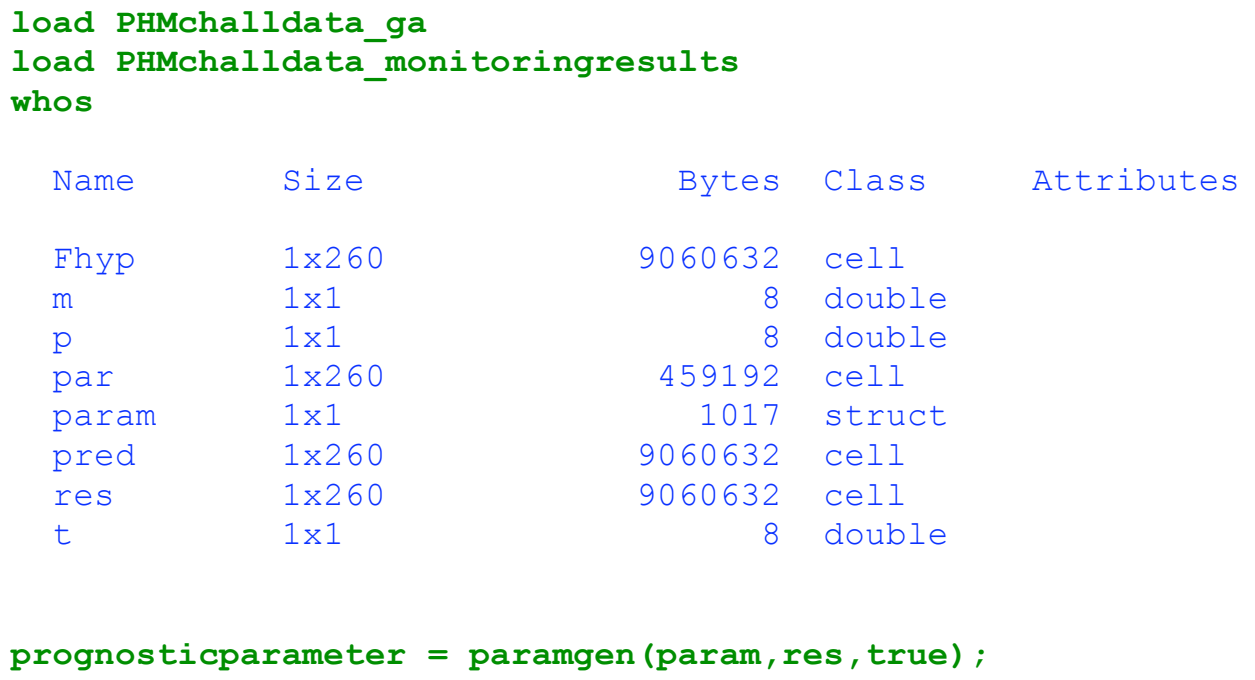




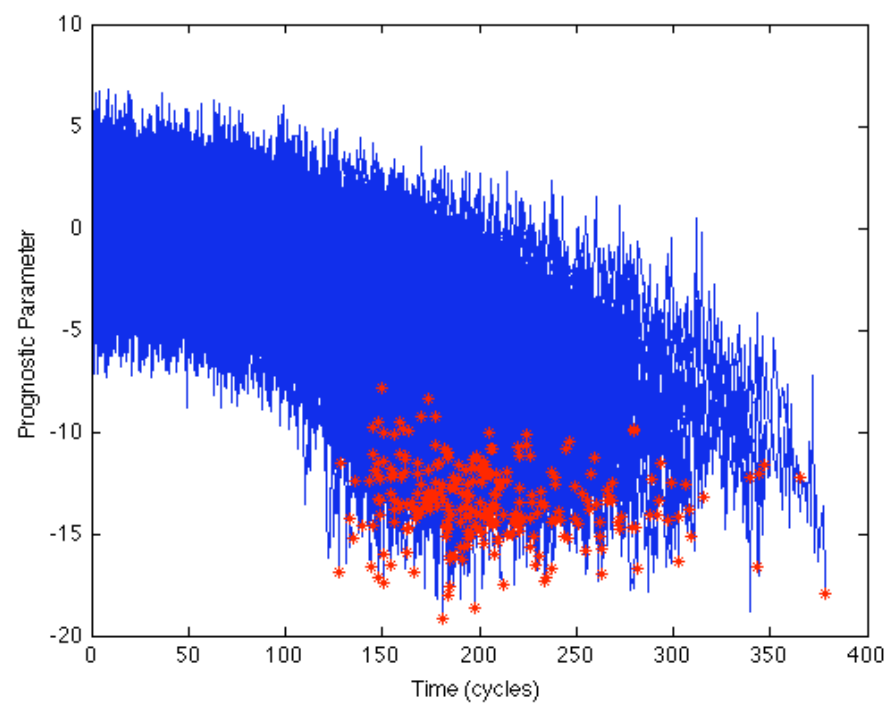

[fit ytrans]=fitGPM (prognosticparameter)

fit $=$

a (x)x.^2 $\quad$ a ( $x) x \quad$ a (x) 1

ytrans $=$

a (y) $y$

threshold = threshGPM (prognosticparameter, fit,ytrans)

threshold =

$-9.9524$

threshold = threshGPM (prognosticparameter, fit,ytrans, 'pdf' )

threshold =

$-10.9743 \quad 0.5743$ 


\section{threshPF}

Calculate PF failure threshold

Syntax:

Threshold $=$ THRESHPF $($ prognosticparameters, fit,yt $)$

Threshold $=$ THRESHPF(prognosticparameters, fit,yt,thresholdtype,threshcon)

\section{Description:}

This function calculates the critical failure threshold for a PF model based on the full historic failure paths contained in 'progparam'.

Threshold $=$ THRESHPF(prognosticparameters,fit,yt) determines the critical failure threshold for the paths contained in the cell array 'prognosticparameters'. The function calculates a hard threshold as the conservative 95th percentile of the failure values for the historic paths.

Threshold $=$ THRESHPF(prognosticparameters, fit,yt,thresholdtype,threshcon) determines the critical failure threshold using the type specified by 'thresholdtype'. 'thresholdtype' may be set to either:

'hard'

A constant value is used for the critical failure threshold. This value is the threshcon quantile of the failure values for the historic paths.

'pdf'

A distribution of critical threshold values is determined. The failure values are assumed to be normally distributed with mean and variance as determined by the historic paths. The threshold distribution is returned as a $1 \times 2$ vector of the form [mean standarddeviation].

'threshcon' indicates the confidence level at which the threshold is calculated from the failure values.

\section{Example:}

load PFdata

whos

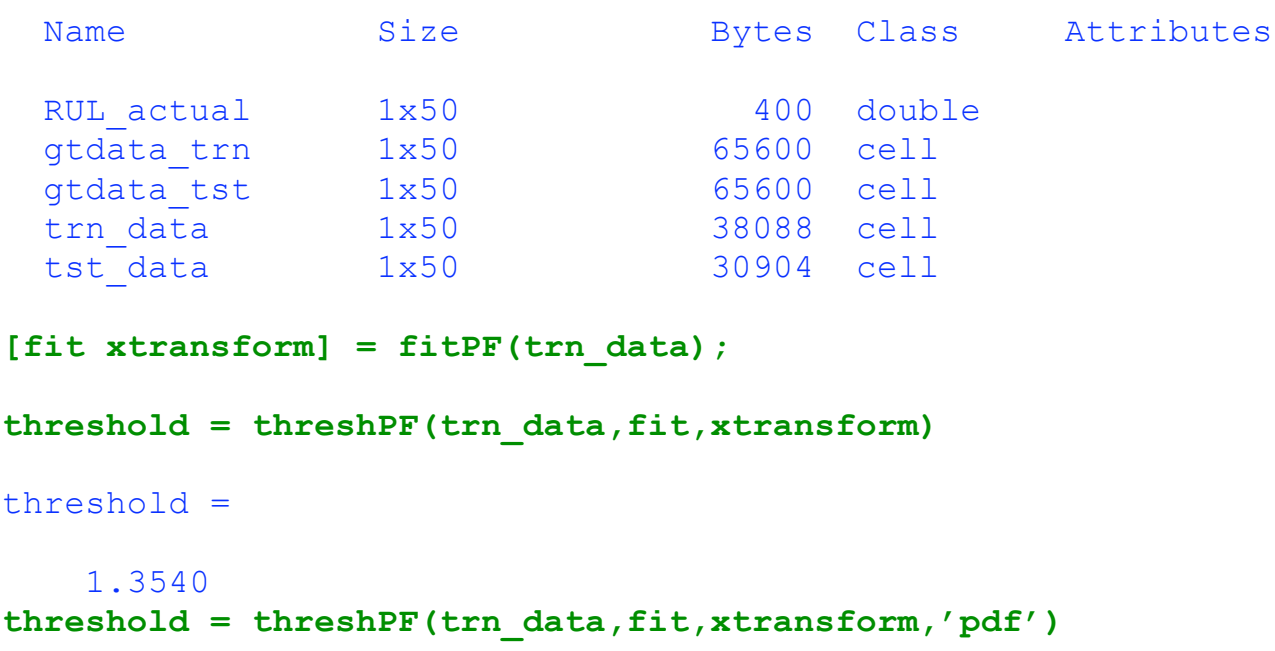


threshold =

$1.5492 \quad 0.1044$ 


\section{wtd_quantile}

Calculate quantiles according to weighted inputs

Syntax:

$Y=$ WTD_QUANTILE $(X, W, Q)$

\section{Description:}

This function estimates quantiles of a sample while considering weighted importance of each sample value

$\mathrm{Y}=$ WTD_QUANTILE $(\mathrm{X}, \mathrm{W}, \mathrm{Q})$ returns the quantiles of the values in $\mathrm{X}$ while considering weights of values given in $W . Q$ is a scalar or a vector of cumulative probability values. Both $X$ and $W$ are vectors of the same size. $Y$ is the same size as $Q$, and $Y(i)$ contains the $P(i)$-th quantile of $X$. Linear interpolation is used to estimate the quantile based on the weights in $W$. If the weights in $W$ are equal, wtd_quatile() returns the same result as quantile()

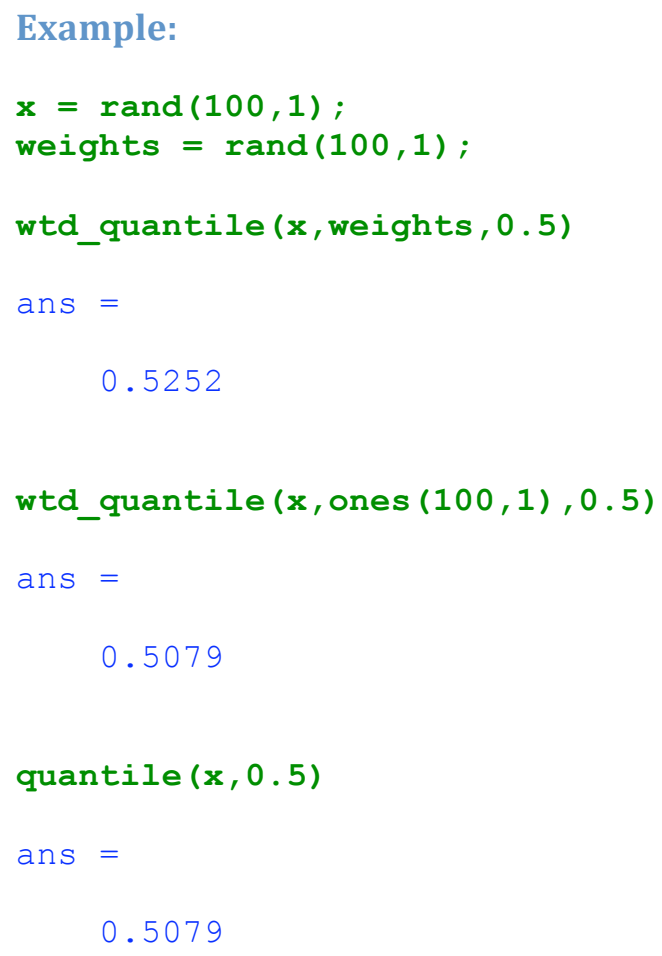




\section{References}

Abernethy, R. B., The New Weibull Handbook, 2nd edn. ISBN 096530620 8. Abernethy, North Palm Beach, 1996.

Coble, J. and J.W. Hines, "Fusing Data Sources for Optimal Prognostic Parameter Selection." Sixth American Nuclear Society International Topical Meeting on Nuclear Plant Instrumentation, Control, and Human-Machine Interface Technologies NPIC\&HMIT 2009, Knoxville, Tennessee, April 5-9, 2009.

Coble, J.B., "Merging Data Sources to Predict Remaining Useful Life - An Automated Method to Identify Prognostic Parameters." PhD dissertation, University of Tennessee, 2010. <http://trace.tennessee.edu/utk_graddiss/683>.

Cox, D.R. and D. Oakes, Analysis of Survival Data, Chapman and Hall: 1984.

Esary, J.D. and A.W. Marshall, "Shock Models and Wear Processes," The Annals of Probability 1 (4) 1973: $627-649$.

Guess, F. and F. Proschan, "Mean Residual Life: Theory and Applications," FSU Statistics Report M702. AFOSR Technical Report No. 85-178, June 1985.

Gut, A., "Cumulative Shock Models," Advances in Applied Probability 22 (2) 1990: 504 - 507.

Hines, J.W. and D. Garvey (2005). "The Development of a Process and Equipment Monitoring (PEM) Toolbox and its Application to Sensor Calibration Monitoring." Fourth International Conference on Quality and Reliability (ICQR4), August 9 - 11, 2005: Beijing, China.

Hines, J.W., J. Garvey, J. Preston, and A. Usynin, "Empirical Methods for Process and Equipment Prognostics," Reliability and Maintainability Symposium RAMS, 2007.

Kumar, D. and B. Klefjo, "Proportional Hazards Model: A Review," Reliability Engineering and System Safety 44, 1994: $177-188$.

Lu, C.J., and W. Meeker, "Using Degradation Measures to Estimate a Time-to-Failure Distribution," Technometrics 35 (2) 1993: 161 - 174.

Mallor, F. and J. Santos, "Classification of Shock Models in System Reliability," Monograf'ıas del Semin. Matem. Garc'ia de Galdeano. 27 2003: 405-412.

Upadhyaya, B.R., M. Naghedolfeizi, and B. Raychaudhuri, "Residual Life Estimation of Plant Components," P/PM Technology June, 1994: 22 - 29. 


\section{Appendix A: Software Version Information}

This Appendix contains information regarding the versions of the PEP Toolbox. The main purpose of this section is to provide a documented history of the PEP Toolbox development, beginning with version 1.0.

\section{v1.0}

This version is the first release of the PEP Toolbox. It includes functionality to facilitate development and execution Type I, Markov Chain, Shock, Proportional Hazards, General Path, and Particle Filtering prognostic models. Uncertainty estimation techniques are included with each RUL estimation method, commonly based on Monte Carlo analysis. It also includes algorithms to identify an optimal or nearoptimal prognostic parameter for Type III prognostic models. This version is the foundation for future PEP Toolbox releases. 


\section{Appendix B: Health Monitoring Example using the PEM and PEP Toolboxes}

The following example employs the PEM and PEP Toolboxes to develop monitoring, fault detection, and prognostic models for a simulated turbofan engine. The data and a description of its simulation are available at http://ti.arc.nasa.gov/project/prognostic-data-repository. The necessary data file is included in the PEP Toolbox. The code below can be pasted into MATLAB; results are shown after the generating code.

The PHM Challenge data set consists of 218 cases of multivariate data that track from nominal operation through fault onset to system failure. Data were provided which modeled the damage propagation of aircraft gas turbine engines using the Commercial Modular Aero-Propulsion System Simulation (CMAPSS). This engine simulator allows faults to be injected in any of the five rotating components and gives output responses for 58 sensed engine variables. The PHM Challenge data set included 21 of these 58 output variables as well as three operating condition indicators, for a total of 24 measured variables. Each simulated engine was given some initial level of wear, which would be considered within normal limits, and faults were initiated at some random time during the simulation. Fault propagation was assumed to evolve in an exponential way based on common fault propagation models and the results seen in practice. Engine health was determined as the minimum health margin of the rotating equipment, where the health margin was a function of efficiency and flow for that particular component; when this health indicator reached zero, the simulated engine was considered failed.

This example application of health monitoring will look at developing a system without the benefit of the domain knowledge described above. Here, the available data is used to develop each module from sensor selection through modeling, fault detection, and prognosis.

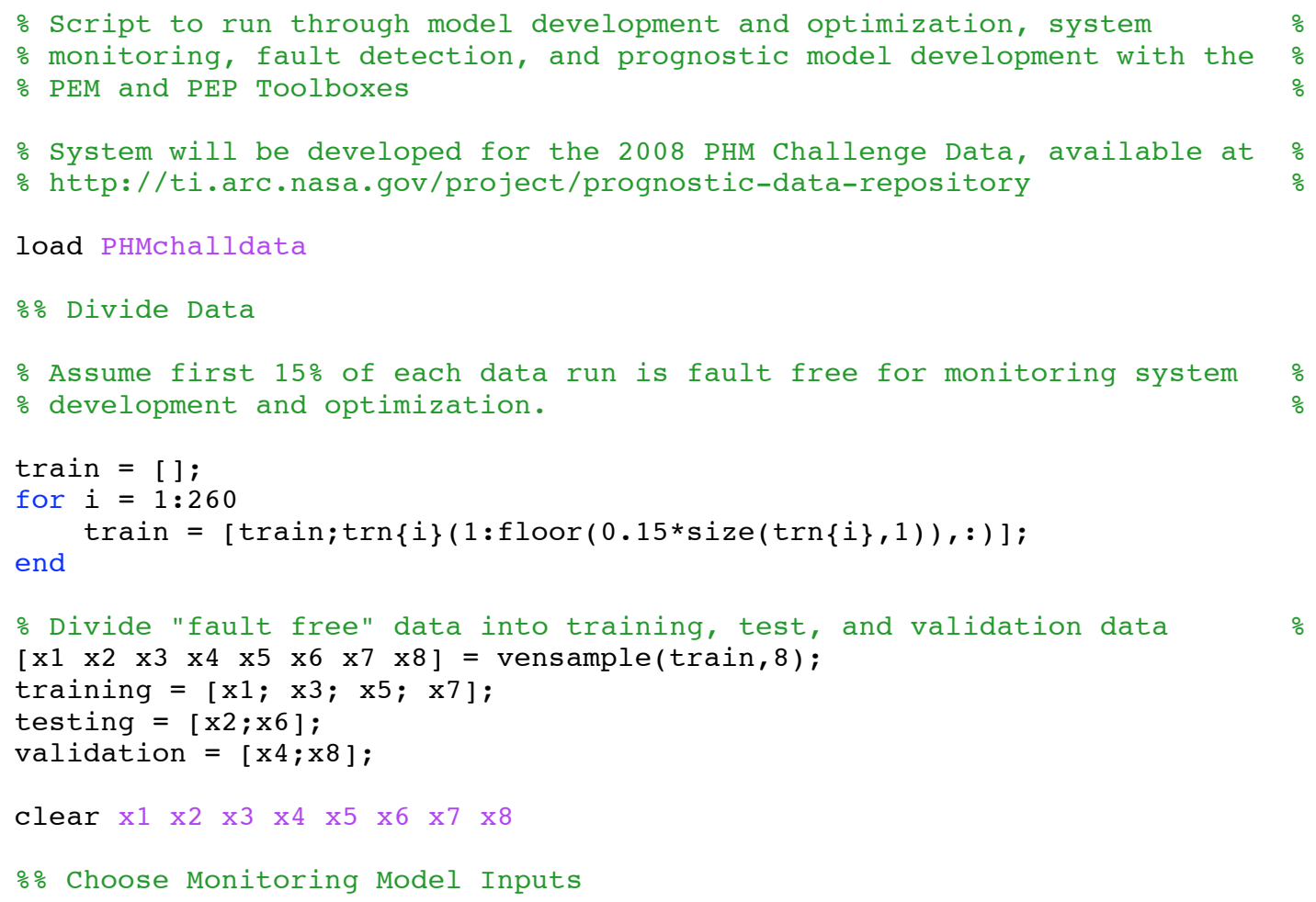


\% Model inputs are chosen based on linear correlations between available $\%$

\% variables. Correlations with magnitude less than 0.3 are considered

\% unuseful; those with magnitude between 0.3 and 0.7 may have some

$\%$ predictive power, and those with magnitude greater than 0.7 are

\% considered good predictors.

\% The PEM function AAGROUP() divides data into groups for auto-associative\%

\% model development. It uses a cut-off of 0.7 to identify appropriate

\% groups from the data. This function gives us two groups, as shown.

\% Notice that some variables are members of both groups.

\section{Groups = aagroup(training);}

$\operatorname{disp}([$ 'Group $1:$ ', 10])

$\operatorname{disp}$ (Groups $\{1\}$ )

$\operatorname{disp}([$ 'Group $2:$ ', 10$]$ )

disp (Groups $\{2\}$ )

gcplot(training, Groups)

Variables: 24

Observations: 3972

Removed Sensors:

The number of the removed sensors:0

Group 1 :

Columns 1 through 20

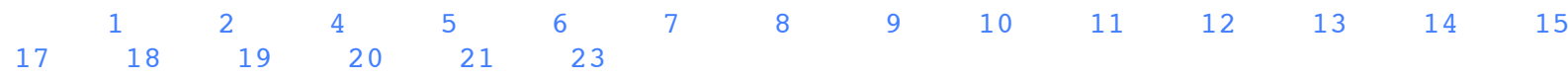

Column 21

24

Group 2 :

$\begin{array}{llllllll}3 & 11 & 14 & 16 & 17 & 18 & 21 & 22\end{array}$

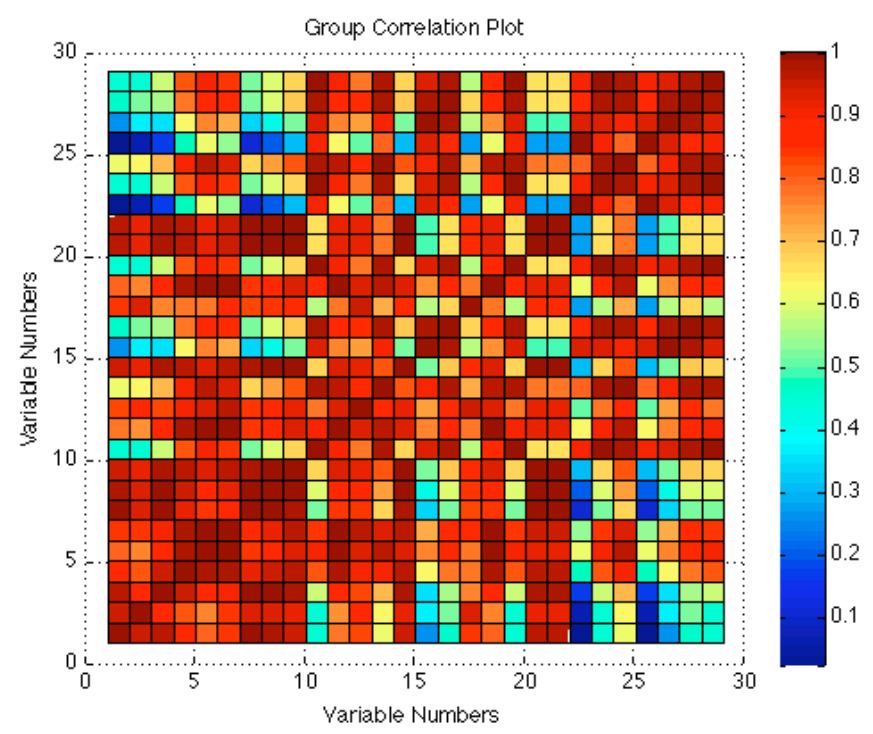

$\because$ We can use the variables in Group 1 to make a monitoring system. This $\%$ \% model will have 21 variables. 


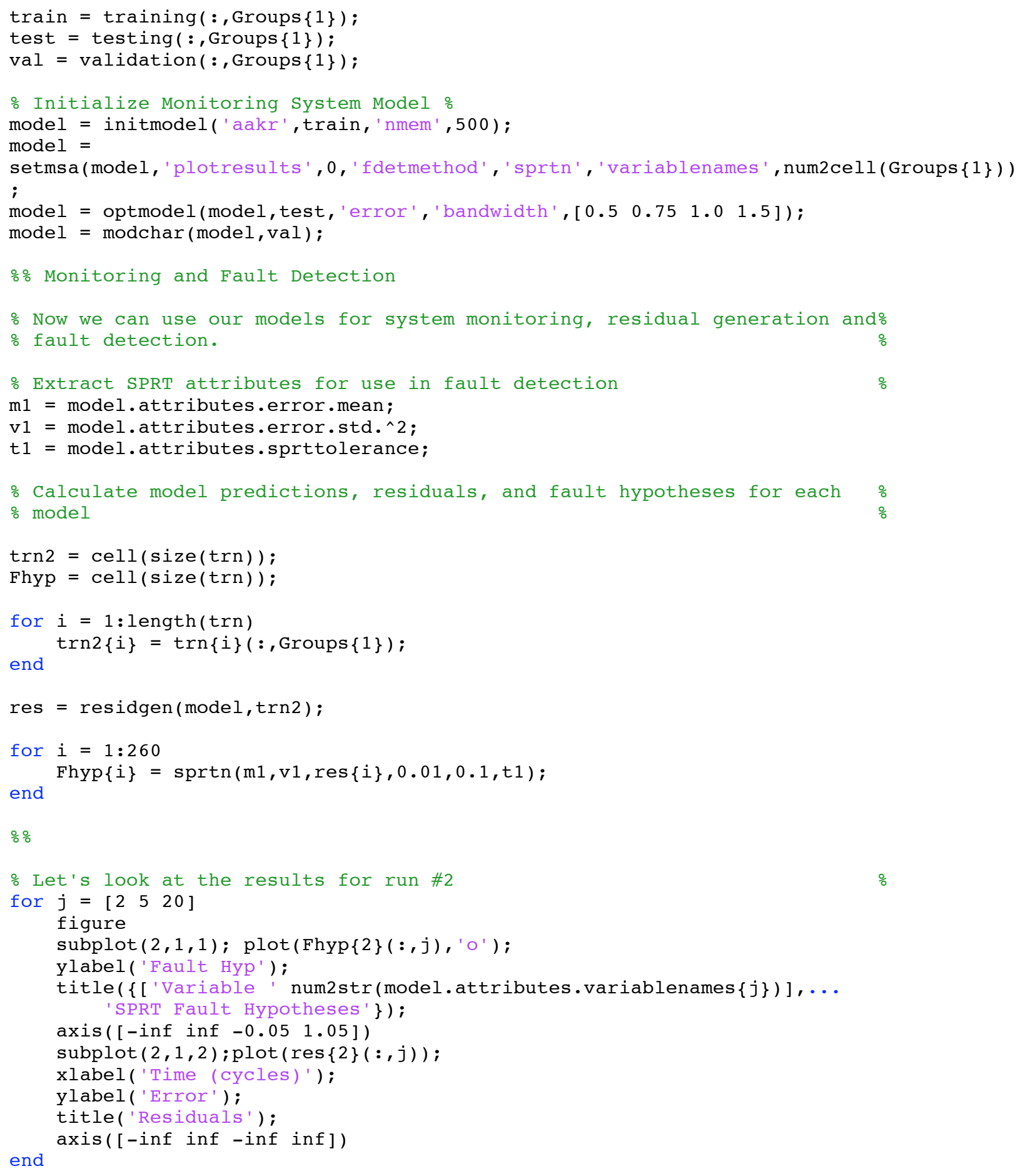



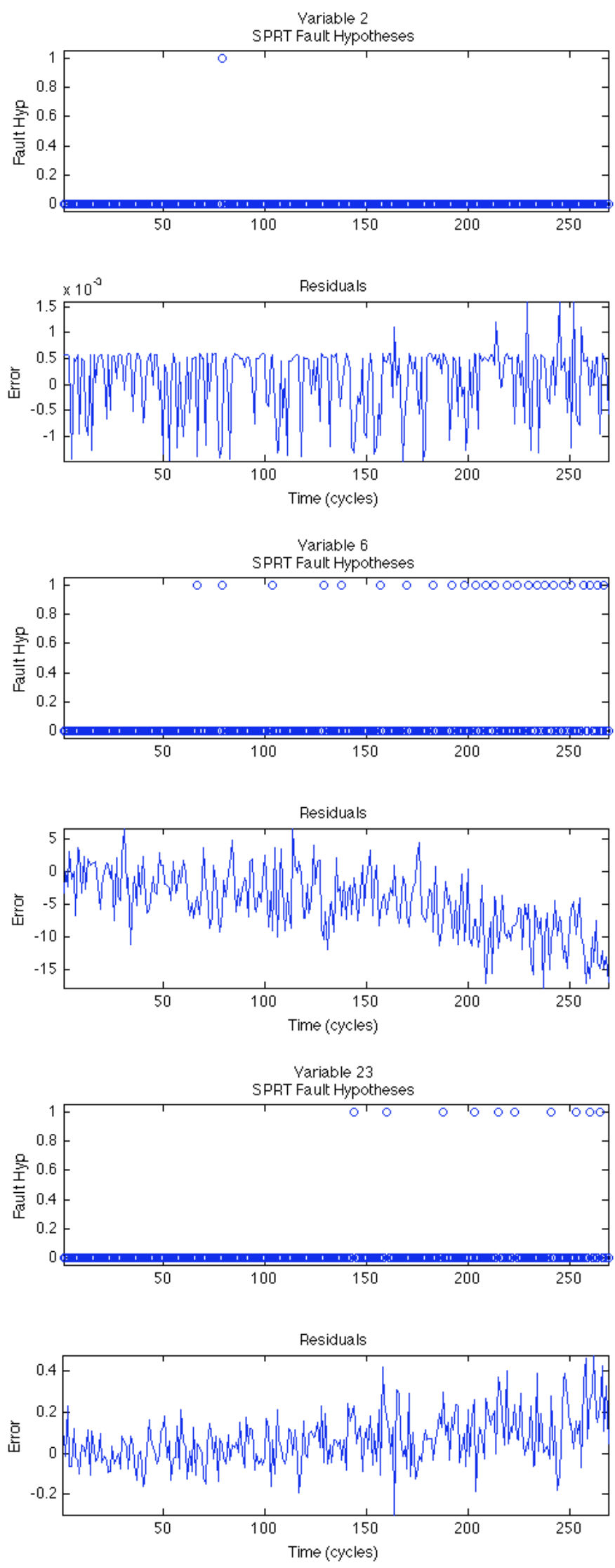


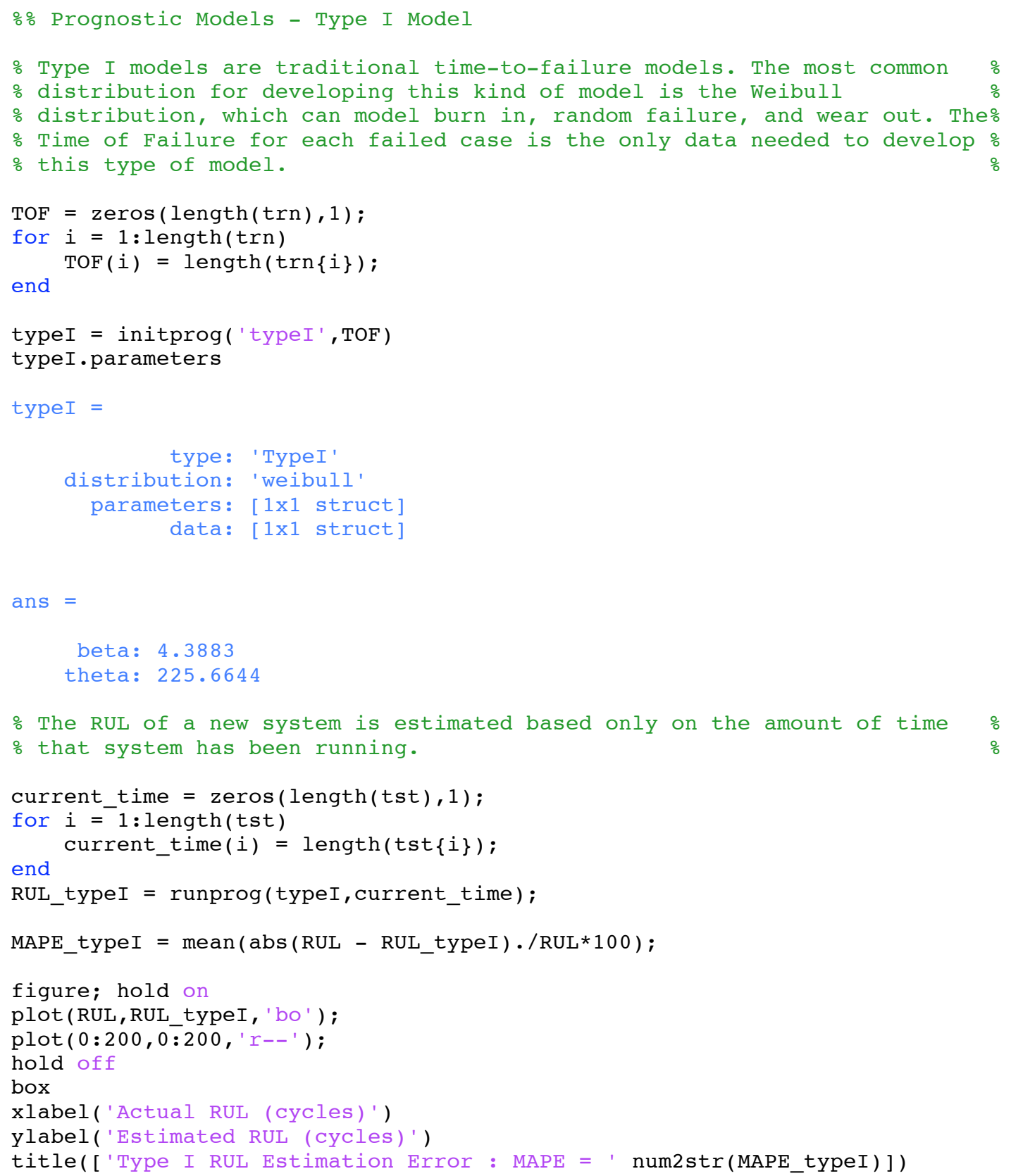




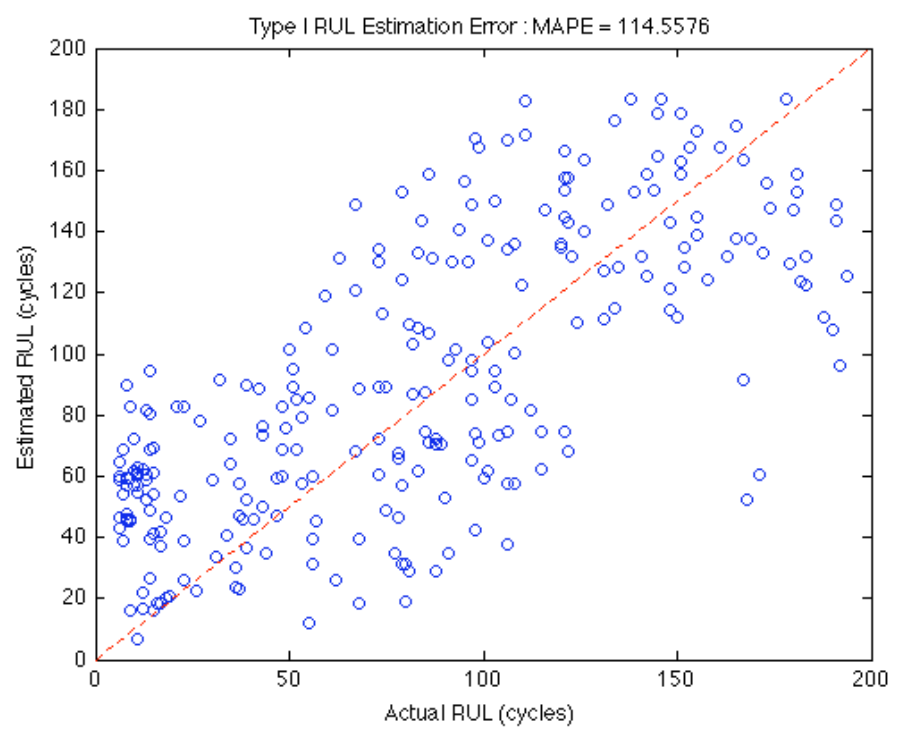

\% Prognostic Models - Type II Model

$\%$ Type II models consider the past and expected future operating $\%$

$\%$ conditions of a system when making prognostic estimates. One example of\%

$\%$ a Type II algorithm is the Markov Chain model. This model uses a

\% transition probability matrix to simulate possible future operating \%

$\%$ conditions and relates these conditions to a degradation measure. The \%

\% PHM Challenge Data has six distinct operating condtions, so it may be $\%$

\% well suited to this type of model.

\% Format historic operation condition progressions to be numbered $1-6$.

\% These numbers have no ordinal relationship.

old_oc $=\operatorname{cell}(\operatorname{size}(\operatorname{trn}))$

for $i=1:$ length (trn)

old_oc $\{i\}=\operatorname{trn}\{i\}(:, 1)$;

end

[new_oc map $]=$ MCdata (old_oc) $;$

typeII = initprog( 'MC', new_oc, 'RULcon', 0.5$)$

typeII.Q

typeII.b

typeII =

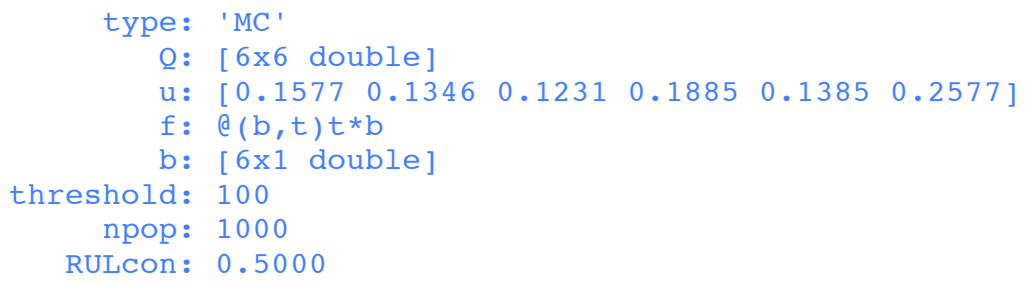

typeII.Q $=$

$\begin{array}{llllll}0.1474 & 0.1520 & 0.1512 & 0.1521 & 0.1430 & 0.2544 \\ 0.1440 & 0.1508 & 0.1502 & 0.1472 & 0.1524 & 0.2554 \\ 0.1455 & 0.1466 & 0.1517 & 0.1468 & 0.1588 & 0.2506 \\ 0.1543 & 0.1568 & 0.1479 & 0.1507 & 0.1415 & 0.2488\end{array}$



0.1502
0.1551
0.1542
0.1465
0.1511
0.2431
0.1536
0.1460
0.1518
0.1488
0.1500
0.2498
typeII.b $=$
0.6506
0.3333
0.4624
0.5269
0.7096
0.2334

$\%$ Format test path operating conditions to the MC classes 1 - 6 using the $\%$ \% map identified previously.
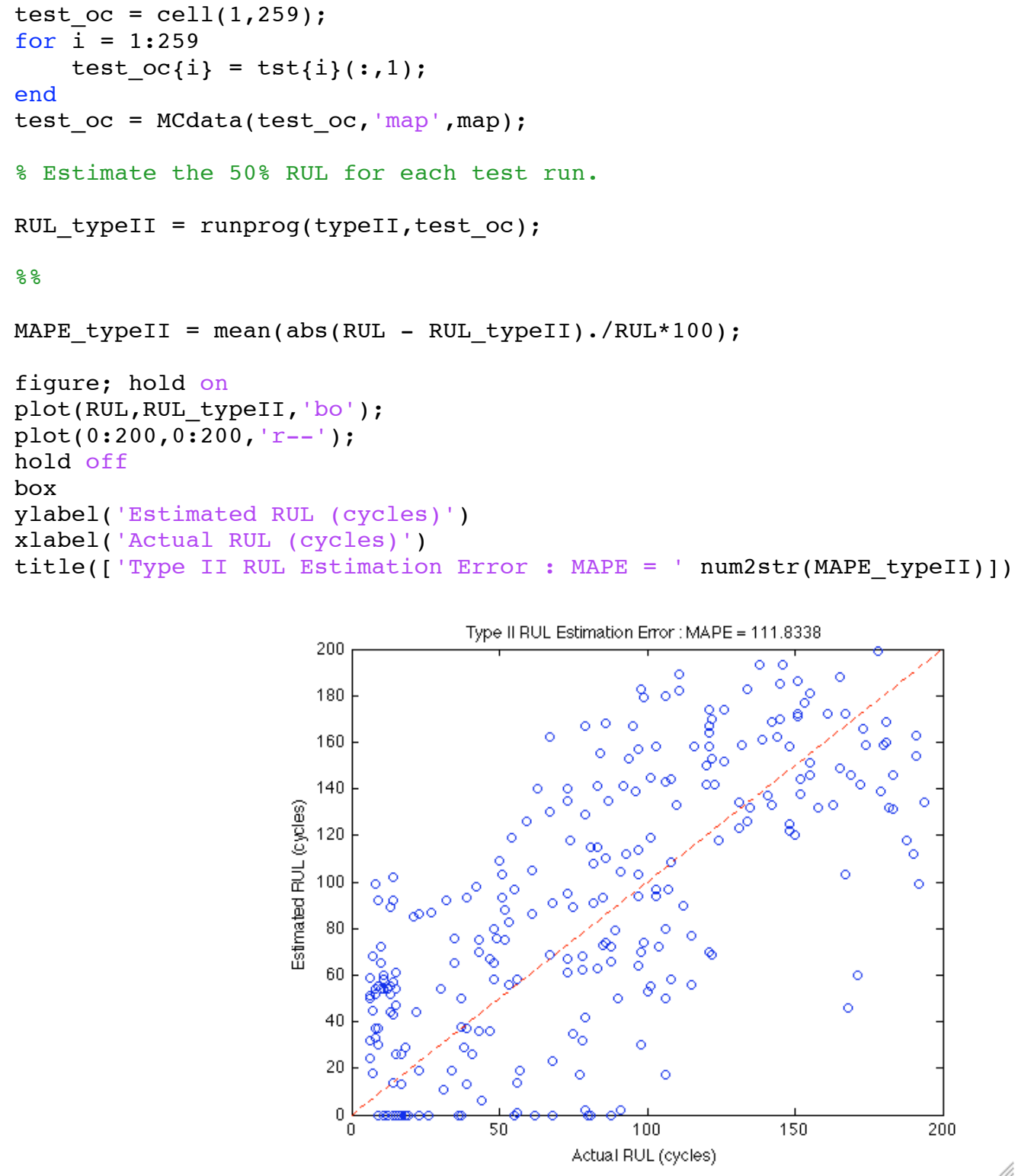

$\%$ We see that the results of this model are not very good. If we look back\% \% at the transition probability matrix and linear fit, we see that we do \%

\% not get much extra information from the markov chain formalism. The 
$\%$ probability of transitioning between any two states is nearly equivalent\%

$\%$ and the total time spent in any one operating condition does not seem to\%

\% give us much information about the degradation level.

\% Prognostic Models - Type III Model

\% Type III models consider the actual condition of the system, either

$\%$ measured or inferred from other measurements. These condition

\% measurements are fit to a parametric model which is then extrapolated to\%

\% a pre-defined critical failure threshold. \%

\% The first step is to identify an appropriate prognostic parameter.

\% Monitoring model residuals are a natural choice for prognostic

\% parameters because they naturally characterize how "far" the system is \%

\% operating from normal behavior. For this example, we are looking for the\%

\% optimal linear combination of the 21 residuals based on a sum of the

\% three suitability metrics: monotonicity, prognosability, and

o trendability.

\% The optimized parameter includes a subset of the monitoring system

\% residuals. Residuals are chosen for inclusion in the GA by calculating \%

\% the fitness of each individual residual and including only those with $\%$

\% total suitability over 2.0

param = optparam (res, 'inputs', 'subset', 'cutoff',1.0)

par = paramgen (param, res, true);

title('GA Parameter')

$\left[\begin{array}{lll}\mathrm{m} & \mathrm{p} & \mathrm{t}\end{array}\right]=\operatorname{ppmetrics}(\operatorname{par})$

$\mathrm{m}=$

0.6916

$\mathrm{p}=$

0.8674

$t=$

0.8684

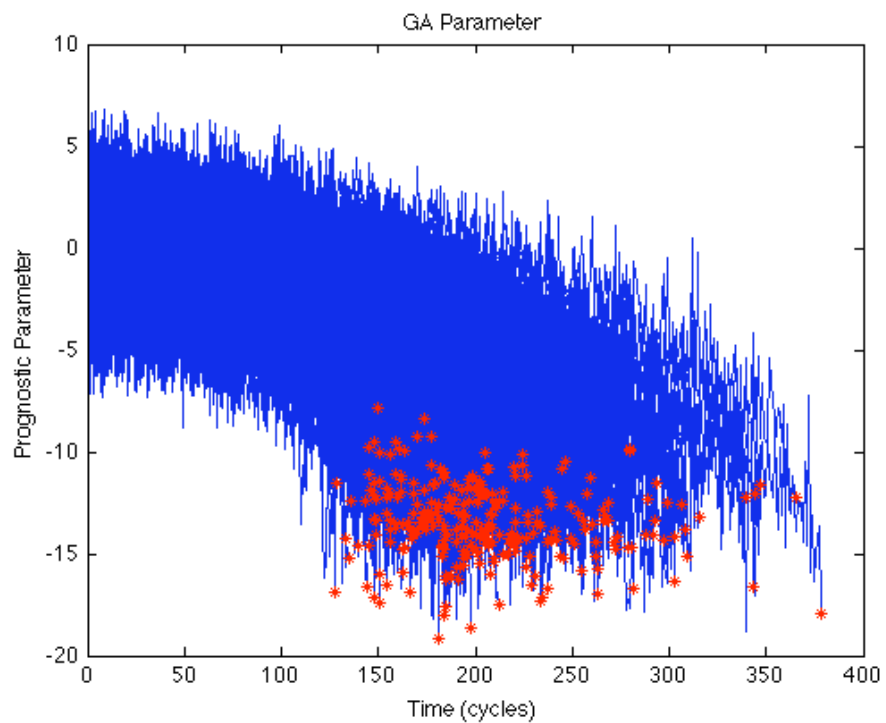

\% A prognostic model is developed for the GA optimized parameter 


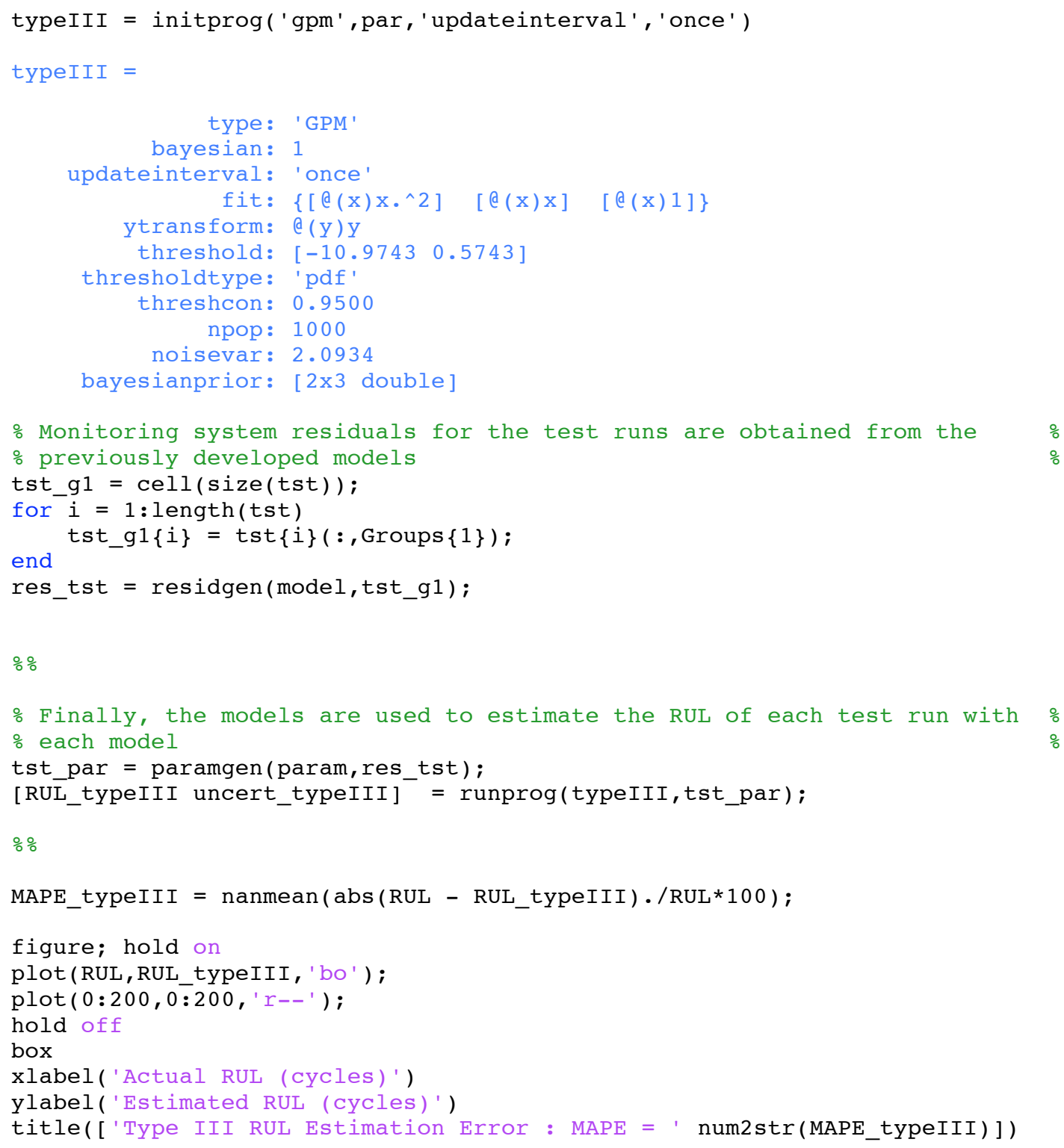




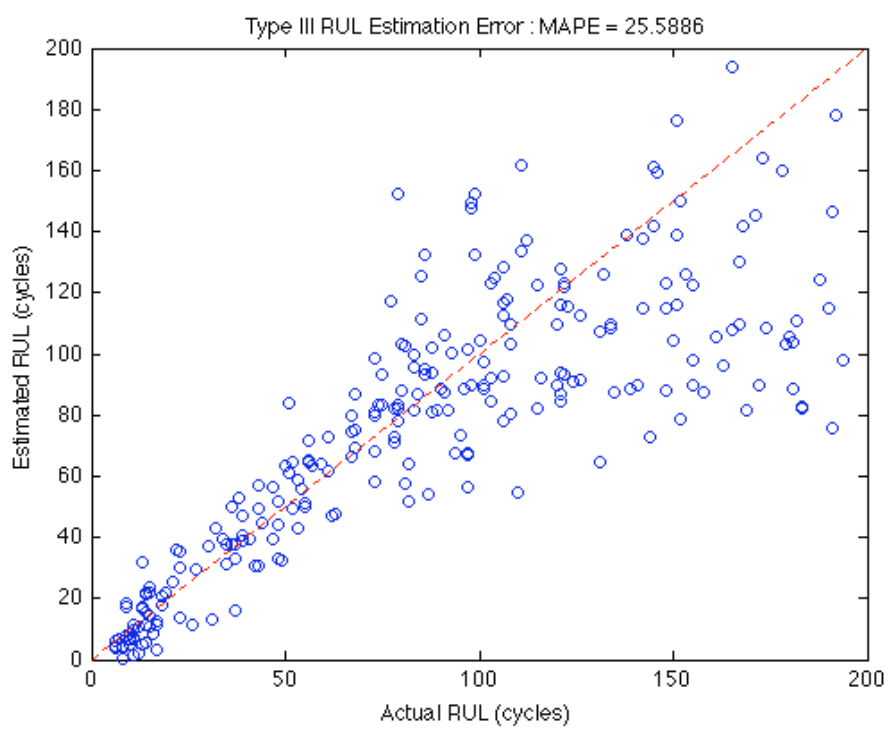

